\title{
A RE-EXAMINATION OF CODEX EPHRAEMI RESCRIPTUS
}

\author{
Robert William Lyon
}

A Thesis Submitted for the Degree of PhD at the University of St Andrews

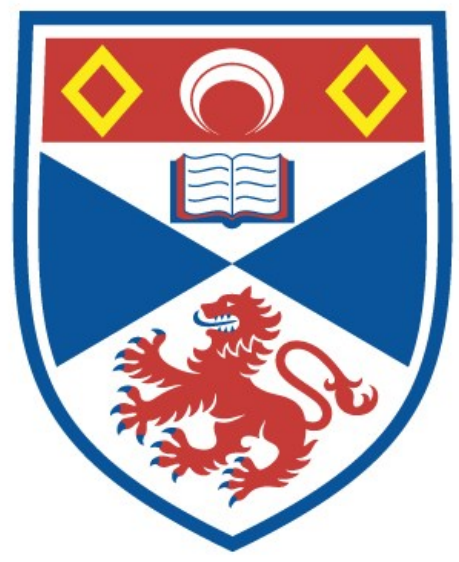

1959

Full metadata for this item is available in

St Andrews Research Repository

at:

http://research-repository.st-andrews.ac.uk/

Please use this identifier to cite or link to this item: http://hdl.handle.net/10023/11010

This item is protected by original copyright 
A RE-MRAUTNATIOH

OF

CODEX EPHRAEII RESCIIPTUS

being a Thesis presented by Robert W. Ivon, B.S., B.D., Th.I.

The University of St. Andrews in application for the degree of Ph.D.

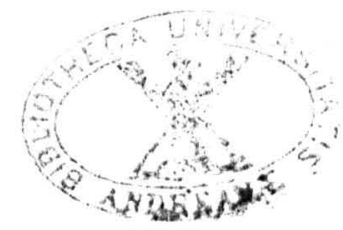




\section{IMAGING SERVICES NORTH}

Boston Spa, Wetherby

West Yorkshire, LS23 7BQ

www.bl.uk

\section{BEST COPY AVAILABLE.}

VARIABLE PRINT QUALITY 


\section{IMAGING SERVICES NORTH}

Boston Spa, Wetherby

West Yorkshire, LS23 7BQ

www.bl.uk

PAGE NUMBERING AS

ORIGINAL 
Boston Spa, Wetherby

West Yorkshire, LS23 7BQ

www.bl.uk

\section{BEST COPY AVAILABLE.}

\section{TEXT IN ORIGINAL IS}

CLOSE TO THE EDGE OF

$$
\text { THE PAGE }
$$


I hereby declare that the following phesis is based on tho results of invostigations carried out by me, that the Theols is xy ow corposition, and that it has not prevlously been presented for a higher degree.

The Research was carried out in St. Lary's College of the University of St. Axdixews. 
Acadente Careor

I matriculated in Ohio thiversity, Athens, Ohto in September, 1947 and followed a course tealing to the B. D. degree in June, 1951 .

I matriculated in Astury Theological Sentrary, Filnore, Kentucky in Septenber, 195'I, being avarded the B. D. degree in June, 1954.

I matriculated in Princeton theological Sominary in September, 195\%, being avarded the Th. $\mathrm{x}$. degree in June, 1956.

In October, 1956 I comenced research at St. Yary's College of the Umivergtity of st. Andrews, the results of which are being submitter as a Ph. D. Thesis. 
I certify that Robert $W_{\text {. }}$ Lyon has spent 8 torras in Iresearch on the subject, the Rewexantination of Codex Bphraens Rescriptus, that he has furfilled the conditions of Ordinance No. 16 (St. Andrews), and that he is qualified to submit the accompanying thesis in application for the degree of $\mathrm{Ph}$. D.

The Very Reverend Natthew Black

N.A., B.D., Phe D., D. Li.tt., D. D., F.B.A. Professor of Divintty and Bibitical Crettelsm Principal of St. Iary's College

St. Andrews University 
The Iight of the World 


\section{PREFACE}

Codex Ephraemi Rescriptus has been the neglectod menber of the family of great uncials. Photographic facsimilos have been produced of S., $A, B, D$, and the others, but only a sample page or two of the valuabl.e palimpsest is available in textbooks. All the other important codices have been studied nore than onee. But as regards Codex $\mathrm{C}$ only Mischendorf has labored seriously over it. In textbooks on textual. criticisn, Codex $C$ has been given - almost without exception - less than half the space of any of the other main uncials. Fo be sure, It is a difficult manuscript to read, and neny lacunae exist. Yot because of Its ase and the quality of its text, every possible pice of information should be accurately extracted from this once boautiful codex. Due to the unwarranted neglect of Codex $\mathrm{C}$, this study was undertaken. The suggestion for a re-examination of the manuscript originated with Professor D. Kurt Aland of Halle in conjunction with the intemational comittee sponsored by the Anerican Bible Society, Ihe National Bible Society of Scotland and the Whrttemberg Bable Society in Gormany.

During the study of the mamuscript the work by R. B. Haselden was consulted in the hope that some scientific means might be used to recover more of the lost text of $\mathrm{G}$. Several publications by the Palimpsestmor schung Institut of the Beuron Honastery were also consulted. Inttlo benefit was realized from these reperezes. Some of the possible scientific aids were not available at the Bibliotheque Hationale in Paris. Others which were used proved to be of negligible value. If ght filters and ul traviolet photographs revealed nothing that could not be seen by the unatded eye. Inframed photographs were able to pierce the "gioberti" tincture, but they did not recover what has been lost to time and the eraser. This was rather disconcerting, because at several points the codex conzd give valuable assis ance on troublesone readings. On the other hand $99 \%$ of the miting on the extant folios has been transcribed: we shall be satisfied that more has not been taken from us.

1. R. B. Haselden, Gctentific Alds for the Study of Mamuscripts, Irensactions of the Btbliographical Society, Supp Loment 10. T0 Toxford: The University Press, 1935), $108 \mathrm{pp}$. 
This odtion varios in several ways from that of tychendore. The seriptio continua text with uncial letters (used by rischendorf to imitate the codex, not to provide a facsimile) has been abandoned in favor of the more readable form used in modern editions of manseripts. Word divisions, when more than one possibility exists, are purely arbitrary and not the witness of the codex. I have not written the text of the correctors in the body of the text as mischendors did, but rather have relegatod them entirely in the Appendices. Furthemore, Tischendor's edition contained only one Appendix with all necessary notes. I have seen fit to separate the work of the different scribes wo have worked on the codex. As a rosult separate Appendices are found containing notes on the text of the original scribe, the two correctors, and finally an Appendix with notes on the edition of mischendorf to point out where I have corrected his text. In this way the rork of each man has been separated and can be referred to more eastly. Wherever necessary, cross references are noted. Appropriate romarks are always found when and why I have disagreed with tischondoxt.

I have now the pleasure of cxpressing my gratitude to those who have helped ne in he process of edfting this manuscript: to Principal Mathew Black of St. Mary's College, St. Andrews University, for gutdance and helpful advice throughout the course of this study. His enthusiam for pure research is contagious. To Professor Bruce 15. Hetzger of Princeton, New Je: ry for his general. interest in the project and for several suggestions in the line of bibliography. To Dr. R. WeI. Wilson of St. Mary's College for reading the introductory chapter and nalling valuable criticisms and to Robert Way, student of United College, for proolreading part of the text of the codex. A special vord of appreciation is extendad to Professor Lerrinl I. Parvis of Enory University, Bnory Georgla, for the use of the micro-filin of Codex $c$, and to the American Bible Society for undermiting the cost of photo raphs and for providing me with a Greek typewiter. A word of appreciation is also due to the stafe of the Hanuscript Departaent of the Bibliotheque Nationale for their cooperation in more ways than one can mention. I aust also mention the 
quiet assistance and encouragenent eivon by ny wife; to her also I an gratoful for her part in typing the thesis. Finally, I mat ac nowledge the Grace of the Sovereign God mo intervened when eye disease threatened to halt this project. Phrough this goodness and merey alone is this thosis presented as a finished work. 


\section{CABSE OF CONIENTS}

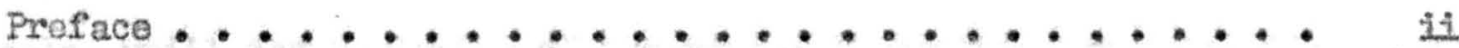

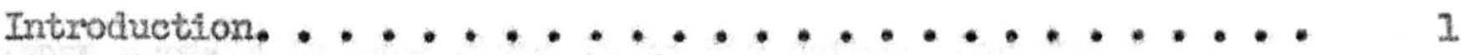

A. History of Previous Stuntes of Codex C ........ I

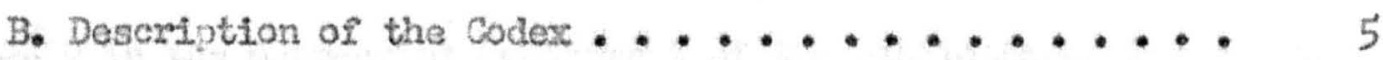

1. Condition and appearance............ 5

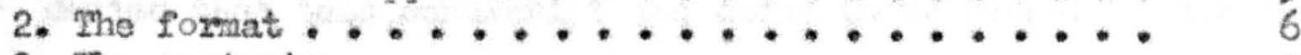

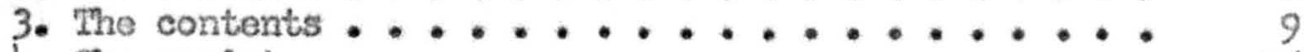

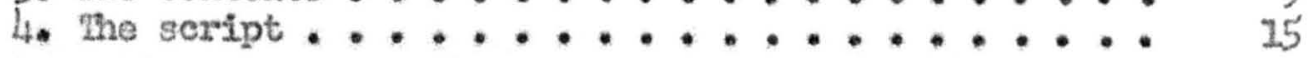

C. The Provenance of the Hamiscript......... 18

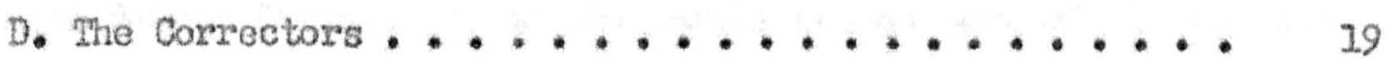

1. Comections by the original scribe ....... 20

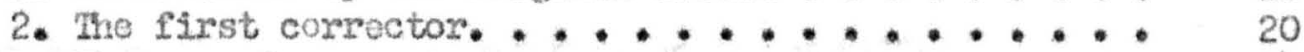

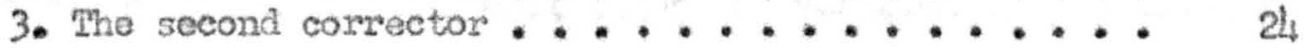

E. The Results of the Present study ......... 26

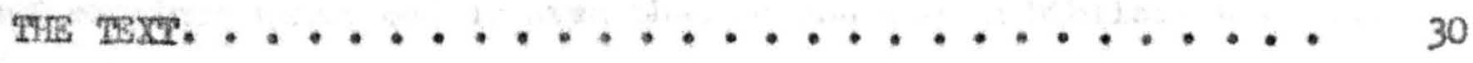

Appendices: Explanations and Sigla. . . . . . . . . 320

A. Notes on the Text of the riginal sartbe . . . . 322

B. Alterations Made by the First Corrector. . . . . 328

c. Alterations llade by the Second Corrector...... 380

D. Corrections to the Edition of Mischendorf. ...... 404

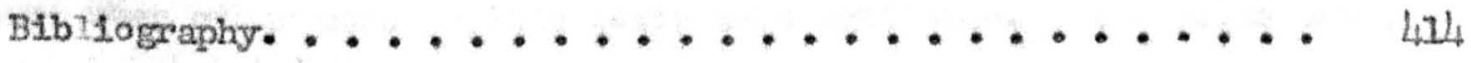




\section{IIITRODUCTIOH}

Codex Ephraemi Rescriptus (Gregory: OL; von Soden: 0 3) has been in its present home in Paris since 1602 when it was brought from Italy by Catherine de' Hedici. The manuscript bolonged to the de' Nedici fanily less than one hundred years. It was in the east, perhaps at Constantinople, until the beginning of the sixteenth century, at which time Andrew Lascar appears to have given it to his patron, Iorenzo de' Medici. In Paris the Codex originally was Given the number 1905, but now is listed as Gree 9 in the manuscript deparinent of the Bibliotheque Nationale. Scrivener denfed that this ranuscript was anons those bought by larshall Strozai.

Until Hetstein studied the manuscript it had been assuned that the Ilbrarian Boivin first detected the partly erased text of the Old and Now pestamonts. Wetstein, however, credited this duscovery to the French protestant Peter Allix, and his contention was verffied by 2 ischendorf. To this writer it secms difficult to believe that the earlier writing had remained umoticed or even that no one knev a biblical text once existed under the thirty-eight semons of sphraen of syria. On many pages from one to six lines stand out clearly at eithor the top or bottom of the page where the twelftin century text was not witten. It is more likely that, because of the conditton of the codex, very little significance was attached in pre-textreritical days to the partially hidden text whon other more legible texts were available. It remains true, nevertheless, that Peter Allix was the first to make lmown the earlier contents of the manuscript and thereby establish its value.

Altix made no use of his discovery. The first to cite any readings

1. F.H.A. Scrivener, A Plain Introduction to the Criticism of the New Testanent, 4 th ed. edited by Edward ifliler, vol. I (Iondon: Coorge Bo17 and Sons, 1894), p. 127 .

2. J.J. Hetsteln, Novur Tostamentum Graecun, Tornus I (Anstelaedani, 1751), p. 27. In his prolegomona twonty-one years earlier tetstein assumed, as did everyone else at that time, that Boivin was the discoverer of the lower text. 
from Codex $\mathrm{O}$ was Kuster, who in 1710 and again In 1723 published 1411's Greok New Testanent and Ancluded such readings as were forwaxded to him by Boivin. These eltations of Codex C (1isted in Kugtor's edition as Paris 9) were very sporadic and in no way a complete or systematic presentation of the text of the manuseript. Furtier interest in the codex was aroused by the brief description given by lontfaucon and nore especially by his facsimile gpecimen of the script. The first serious attempt to study Codex $C$ and its text mas made in 1726 by J.J. Fotstein, then in his early twenties. He first rade sanple extracts of Its text and shoved these to Rzchard Bentley, the Carbridge philow logian, who encouraged hin to complete his collation. This he did while on lasve fros his chaolatincy duties. For his work Bentley paid hin fifty pounds sterling. Although it was deposited with Bontley in Cambridge, this collation, of course, was never incorporated into Bentley's projected edition of the Greek New Testanent. The first publication of the exact contents of the codex was made in Wetstein's Prolegomena, issued anonymously from Ansterdam in $17300^{3}$ This was followed in 2751 and 1752 by his creek New Testament, throughout which the readings of the codex are first cited by its present symbol. Wrotstoin's collation was made against the 1654 Amsterdan edition of the Fextus Receptus. Itis citations of Codex 0 represented a highly significant contribution to textrual studies although it was not complately satisfactory when judged by later critical standards. Less gfmificant variations, such as addition or omisstion of the article and differences in word order, were not noted. Wotstein made no indication of places where he was unable to read the text. Furthemore, his atterrots to distinguish between the original scribe and the two correctors were quite unsatisfactory. Fimally, a

1. Iudolph Kuster, Novan Iestanentum Craecus, atc. 2nd ed. (Lipsiae: Filit J. Friderici Gleditschis, 1723). This second edition was meroly the unsold stocks of the first edition with a new titla page. On page lib of his. Praefatio Kuster described bxlef'ly the manuscript and states that the cltations in the apparatus were leceived from Botvin. In all. likelihood Kuster had not even see the codex.

2. Bemardi de Vontfaucon, Palaeoraphia Greeca sive de Ortu et Pro gressu Literarun Graccarum, (Paris, 1708), p. 2131 .

3. J.J. Hetsteln, Prolegomena ad Novi Testamenti Grsee Editionem dami: i. \& Jo Wetstenios and G. Smith, $\overline{1730}, \mathrm{p}, 12$. 
not insigniflcant number of exrors had been found in his collation.

After Fetstein numberous scholars examined the codex and testified to the competeney of his work. Hore than one, however, voiced the sentinent that nore could yet be gamered by another carepul study of the text. Griesbach desired that as much as was still legible be printed letter for letter. Ints concern on the part of Griesbach was probably hoightenod by wat ho thought was a marked deterioration in the condition of the nanuseript. He was unable to detect maxy places which Wotstein had read. Although ho studied the manuscript to sone extent, he added only two roadings (fiarle $6.2,4$ ) to those cited by Wetstein. A generation Later Iachmann indicatod the farne that was waiting for the seholar who would undertake to publish a conplete edi3 tion of the Codex. He was the first, it appears, to suggest the application of a chenteal remagent to bring out the latent text.

The next significant move was made by F.F. Fleck of Ieipzig who 4 studied the manuscript in 1834-35\%. At his instance the "gioberti" tineture was applied by the library authorities to the vast majority of folios. This application undoubtedy factiltated the deciphering of the codex, and has restored previously illegible sections of the text. In numerous places, howover, the workers have betrayed their lack of experience in this type of sanuscript work by leaving large black spots where the tincture was almost splashed on the folios. His study indicated certatn facts relative to the history of the codex and will be discussed later. He added nothing to the list of variant readings. Ho left that task to another greater than hinself who was to come after him.

1. cf. John David Wichelis, Introductionto the Nev Testament, trans, from the 4 th edftion by Herbert larsh, Vol. II, Part I (Cambidge: J. Archdeacon, 1793), p. 260. p. vi:

2. J.J. Criesbach, Symbolae Criticae, Tomus Prior (Ho7ae, 1785),

3. Caxl Lachmann, Weachenschaft ther seine Ausgabe des neuen Testaments," Theologische Studien und Kritiken, III (1830), p. 831f.

4. F.F. Fleck, HUber die Handschrift des neuen Testamentes gewohnlich Codex Ephraemi Syri Rescriptus genannt in der königlichen Bibliothek zu Paris, Theologische Studien und Kritiken, XIV (184i), pp. $126 \mathrm{ff}$. 
In $1840 \mathrm{C}$. Mschendore at the age of 25 and fresh from his appointw ment to the faculty at Ieipzig came to Paris to produce the first conplete tert of the codex with a thorough Introduction and coptous notes In an Appendix. Alnost without exception tho scholasily world accorded Its unreserved praise and many assuned that the codex need not be further examined. Yet others did look at the manuscript, If for no reason other than to gain an adequate appreciation of Tischendori"s accomplishont. Tragelles looked at the codex several. tines to satisty his own mind as regards the variant reading in I 14mothy $3.16,2$ but he made no serious atterapt to test the accuracy of THschendorf. The loamed Italian, A. 3 Ceriant, however, did question the accuracy of Tischendorf's work, but his call for a new examination of the codex fell on deafe ears. It is probable that no one felt the Iinted laprovement on the text of Iischendoref would be worth the immense amount of painstalcing labour Lnvolved in reading so difficult a manscript. Hemann von soden shows his acquiescence in Itischendorf's edition and maires no mention that either he or his assistants looked at the codox. On the other hand ho lists as Sehreibfehler readings in misehentorf's text which are cleerily wrong and which could have been easily corrected had he felt the need to verify those readings. 4

Nove than one hundred years have passed sirce Ischondore published his edtion of codex 0 . This witer has not been able to note a single instance of a critic finding any errors in this standerd edition. With

1. Constantinus "tischendorf, Codex Johraemi Syzi. Rescriptus sive Framonta veteris Restamenti e Codice Graeco Parisiensi Coleberrimo Quinti ut Videtur post Christur Soculi, (Isipsiae: Bernh. Tauchntz, 1845 ). The low Testament was printed and published separately in 1843. In the 1845 edition various printer's errors found in the former adition have been corrected. Throughout this edition the 1845 edition of msehendore is used.

2. Saranel P. Tragelles, An Account of the Printed Text of the Groek

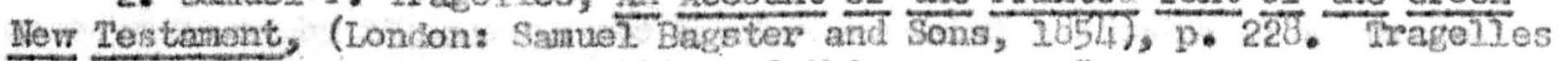
Fefors to his "repeatod inspection of this passage."

3. A. Ceriani, "Letture della Classe di lettere e Scienze Storiche - Morali, Critica Biblica," Reale Instituto Lombardo di Scienze e Lettore Rendiconti, Seri.es II, Vol. XXI (1838), p. 5T7.

4. Hearamn von Soden, Die schriften des neuon Testanents, L. Teil (Berlin: Verlag von Arthur Claue, 1902-1910), p./935I. 
the availability of new photographic techniques and the possibility that another century might have favorably affected the low Testement text, the time seened right for a now and thorough excanination of this vory valuable and significant witness to the ancient toxt of the New Testament. II Description of the Godex

A. Condition and Appearance.

The present condition of our codex is far fron its original glory. "It is," as Gregory has satd, "Iike a man who hus been mained in the wars. Its besuty and fulness are depurted." Yot vogtiges of its forver glory aro seen clearly in 1ts square, smooth script as the lines of its solid singlo column stretch across the page. Its early color was light tan vellum with a dark brom ink. On a very fer pages unaffected by the tineture, these colors are seen to be only slichtly modified by time. The first three lines of each book of the llow lestanent were written in a vory non-durablo vernillion ink, of which no traces can be seen. Iost of the pages, however, are a greenish blue with every shade from light green to dark blue. The original letterg are now dark blue or blue green and, at times, alnost black. The upper trelf th century script is black and often gives a blotted appearance due to the tincture. At several points where the tincture appears to have been spilled, black or blue-black smudges have obscured the text.

By those who have boen able to matco the comparison, the vellun is not considered to have as high a quality as the other prinary New Testament uncials, especially Codex A. The present condition of the individual folios varies considerably. Iany are quite firm and do not show any appreaiable amount of wear and tear. These leaves havo no holos and their narging are still close to their original size. Others have not fared so well. Holes have been caused either by the erasures of the early correctors or by the person who first dimantied the codex to write the semong of Ephraem. In many places these exasures have made the follos

15. C.R. Gregory, Canon and Text of the Now Testament (Edinburgh: T \& T ciexk, 1907), p. 346. 
transparently thin. A very Iinited nuber are quito vitiated throughout with holes and creases or folds, and are held intact by tape. The twolfth century seribe who wrote the upper text was not unduly concemed to exase the early toxt, but rather he depended on his hoavier pen and darker Ank to obscure the bibjical text. For this we car be thanifful. Wost of the text can be copied with absolute certainty. On the other hand numerous significant readings are quite indistinet or even peramnenty lost.

At tho present time the codex is nade up of two hunired-eight leaves, of which one hundred-forty-five are of the New testanent. When Ilschendorf studied the ranuscript there was one nore folfo, but for some unexplained reason folio 138 of the present binding - the one used for a 1. facsinatle by mischendorf - has disappeared. The present binding is according to the upper text; the lover text has been thoroughly mixed. More than a fer folios were reversed when the later toxt was written so that the top of a page of the sermons is the bot on of the page of the biblical text. The folios are preceded by four taodern leaves. The first, dated 23 Hovenber 1883, contains the following note: Volune de 209 feuiltets Ie fol. 138 manque/ C. Tisohendorf a dome dans son Codex Sphraemi syrt rescriptus (Lipsiae, 1845, he) un fac-sintile d'une page de ce Reuizlot, qui contonait un fragnent de 1.Ecclestaste, $V, 5-V I, 10$. Two others are Indices for locating the biblical text. One has the follos numbered consecutively and notes the portion of the biblical text contained on dich follo. The other is a revorse indate which lists the chapters of the Now Testament and then gives the foltio or folios wisch contain the separate chaptors. The fourth leaf contains notes on the text and specimens of the script.

B. The Eormat.

It has already been mentioned that Codex $C$ has only one column per page. The full lines contain approximately 40 letters, although on some

1. That this pige 1 misging was, apparently, Ixist noted in 1883 at which time a note to this effoct was placed in the beginning of the codex. As far as I know no one has, directly or fndirectly, laid the blame for its absence on ilschendorf. 
pages the number of letters per 3 ine is onty 35 or 36 . There are generally 41 Innes per page; but on three pages 42 Innes have been written, while the two extant folios of I Peter have 46 Iines on all sour pages. On six occasions the scribe added wo to five letters below the last letters of the last line. The lines aro evenly spaced, having been marked out by a sharp stylus whose imprint can usually be seen quito clearly. The scribe has freely used the paragraph systent. From four to twelvo Innes per page have not been filled. Thjs characteristic is seen much less in Pevelation and somemat less in some of the Pauline epistios than elsewhere. Iarge marginal letters have been used regularly, but apm parently not according to any pattem. They are used at the beginnings of paragraphs and Anwonian sections, but thoy have also been used in the middlo of words. They probably provided a jurited artistic touch in an otherwise straightformard format.

Two punctuation marks are seen throughout the manuscript. The high poriod is placed approxinately at the top of the letters and usually ocoupies a little less space than one letter. The other sign is a small cross. From these punctuation marks two questions arise: (1) Does the period represent one mark throughout the codex, or wore than one depending on its height above the line? The latter proposition was defended by Hug who stated that a stop was placed at the foot of a letter for a comm ma; for a colon the stop was placed in the middle of the letter. This contention was accepted by others, but was rightly rejected by rischendort as an invention of the critics. Ho such technical system was used by the original scribe. (2) The second question is whether the originat. scribe ever used the small cross, especially at the end of a paragraph, or whether in every instance this crosa is to be credicted to a corrector.

1. Wone of the pages of the manuscript has only 40 Iines, despite what Pischendorf has sald to tho contrary. On the page he mentions as having only 40 lines he has skipped one line complotely. The page containing Phileran, however, does have 40 75nes, but this is because the text of that letter ends on Iine 40 . Fsischondorf also notes that the first page of I Corinthians has 42 ilnes. But on this see Appendix A.

2. J. Ho Hug, An Introduction to the Whitings of the Nev Testament,

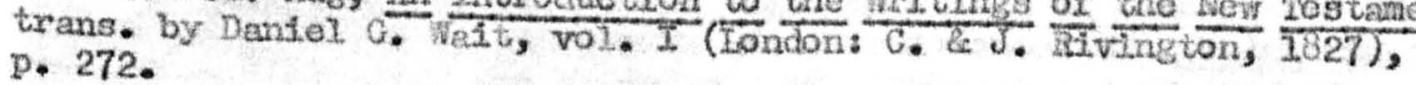


This problea cannot be set led so peremptorily. In most places the cross is easily assigned to the corrector because it is squeezod between letters or added above the line. Only at the end of a major passage is there any question. Hug elaimed the orighal scribe diet use the cross in these in1.

stances. Tischendore was not propared to bo so dogratic as in the previous question regarding the high period: yet ho did not hesitite to differ again with Hug. Very often this cross is quito indistinct, but when it is seen clearly, it does not have the same solid stroke characteristic of the first scribe. Fven more daraging to Hug's position is the fact that at the end of a paragraph both the high period and the eross are often seon. It is highly untikely that one scribe would have placed two different punetuation maries together. In the light of these observations we can definitely reject the statements of ling and accopt the conclusions of Mischendorf that the first scribe used only one pumetuation nark, nanely, the high pertod.

The uncial writing is contimuous except for the linited use of the high period. No accents or breathing marks were included by the original scribe, but were added by a later hand. For the diaeresis over the 1 and $\underline{U}$ at the beginning of a gyllable a straight line is used xather than one or two dots. In the margin the Amonian sections are found in alf Lowr gospels, but at present the Eusebian canoni are not seen. It is quite Iikely, as othors have nentioned, that these were written with the same verminjion ink that was used ot the beginning of each book and have Iikewise disappoared. There are no $\underline{x \varphi p a \lambda a \text { la }}$ on the pages of the text, but Iists of $\tau_{1} \tau \lambda_{0}$, preceded the four gospels. Of these $\tau_{1} \tau \lambda_{0}$, , how ever, only those for Iuke and John are now extant. The titles at the begiming of each book and subscriptions at the ond are extrenely sinple with no added artistic touch sucin as is found, for exarple, in the sinat t-

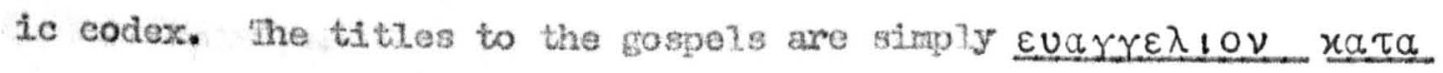

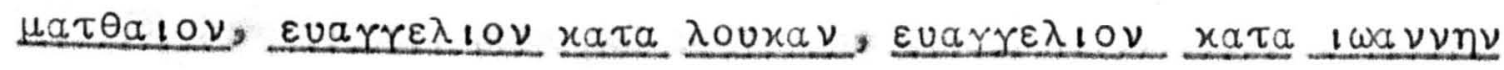

3. Loe. clt., On netther of these questions of punctuation did Ilug alter his position in the fourth Cerran edition of his Binleitung published in 1847 after the appearance of Tischendor't's Prologomena.

2. S.g., Sarivener, op. cit., p. 123. 
(the first page of Hark is miseing) and are witten with the more permw anent brom ink in letters slightly larger than those of the text. The subseriptions at the end of the gospels are identical to the tfties. No trace 18 loft of the title of Acts. For the Pautine epistles the subscrlptions are simply $\pi \rho 0 s, \rho \omega \mu \alpha$ ovs, and so forth. Those for the

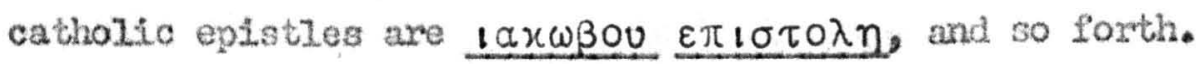

The nomina sacra written by the seribe are those generally use at that time in Hew Testanent manuscipts:

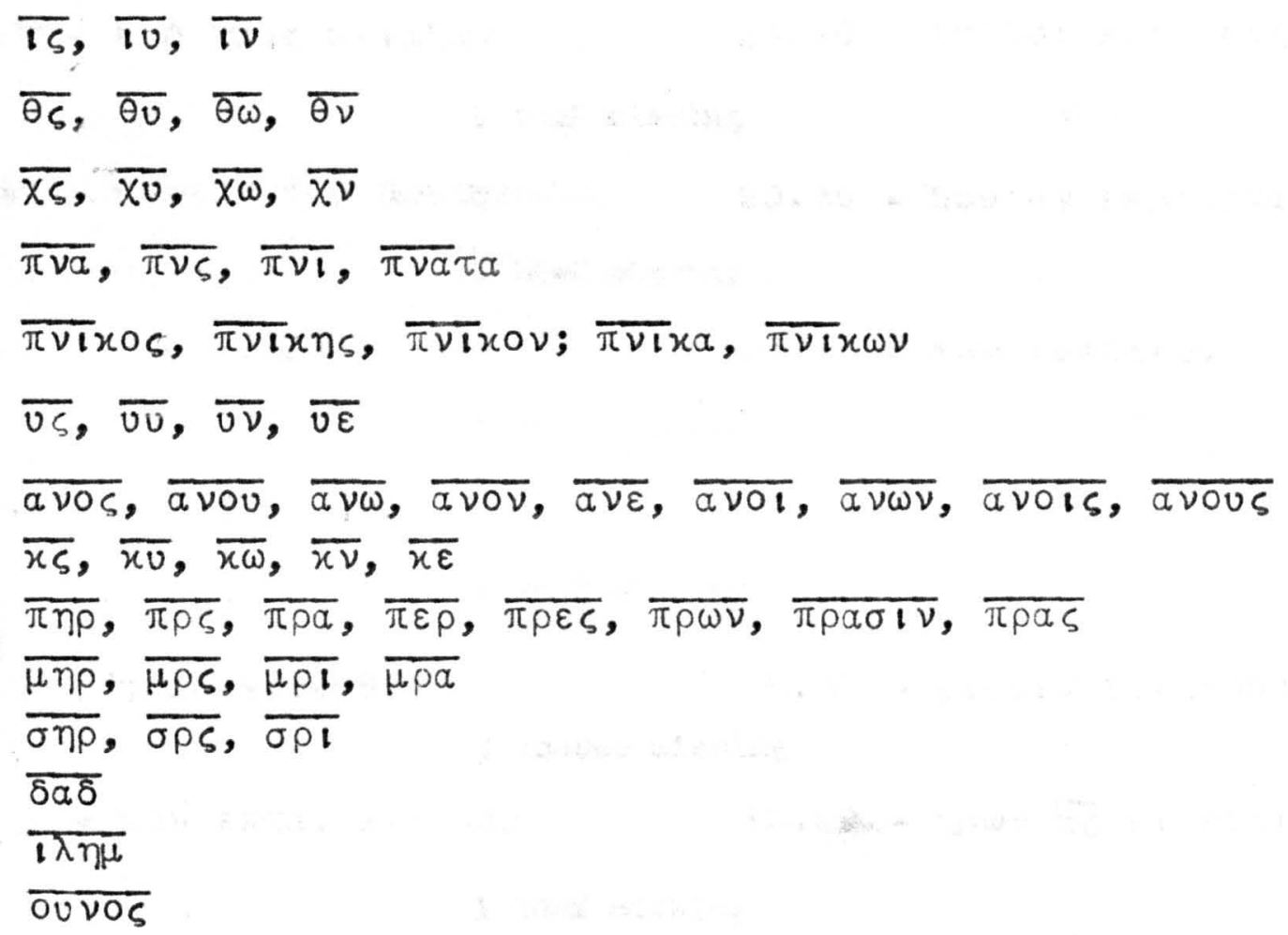

$\overline{\beta \lambda \varepsilon \varepsilon \mu}$ for $\beta \eta \theta \lambda \varepsilon \varepsilon \mu$ is used once, in Natther 2.16. The word oupavos

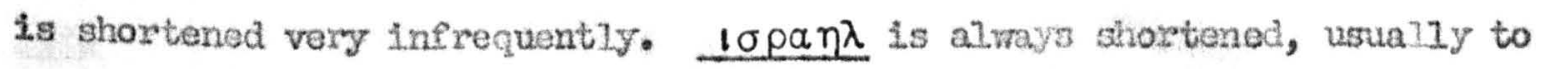
$\overline{I n \lambda}$, but once in Inke to $\overline{1 \varepsilon \lambda}$, and in Acts everytime but once to $\overline{1 \sigma \lambda}$. The $x \alpha_{1}$ compendium is never used and the same may be seid for all othor ligatures. Once (in Romans 26.21) at the ond of the line the orifinal scribe wrote $\mu$ ou in the following fashion: the end of a line for 2 is used regularly, but not always. Wo other abbreviations or variations are saade from the stratght lettered text. c. The Contents.

All twenty-seven canonteal books were contalined in Codex C. There is no evicience that any extramcenonical books were included. The following are the exact contents of the codex in its present forma 
Beginning with:

Ending with:

Matthow

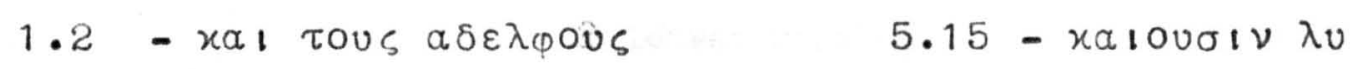

2 leaves missing

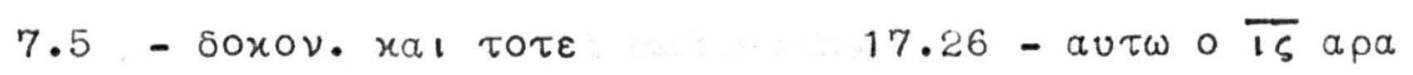

1 leaf missing

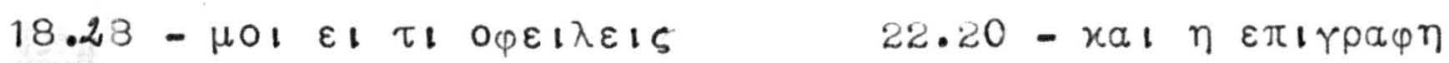

1. Jeas missing

$23.17-\eta$ - vaos arlabwv

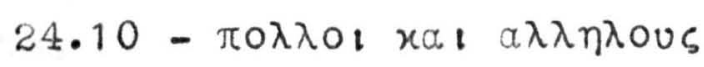

1 leaf missing

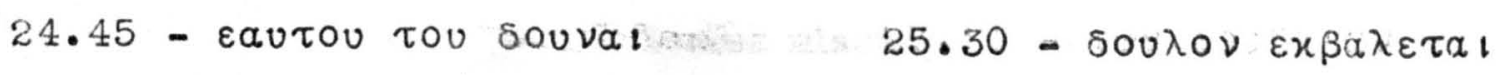

1. Leaf misuing

$26.22-\varepsilon \imath \varsigma \varepsilon x a \sigma \tau 0 \zeta \mu \eta \tau \imath$

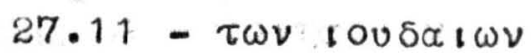

1. loaf mising

$27.47-\tau \imath \nu \varepsilon \varsigma \delta \varepsilon \tau \omega \nu$

$28.14-\pi 01$ เ

\section{Mark}

1. Leaf missing

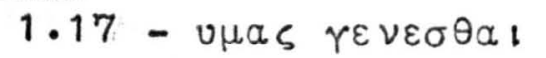

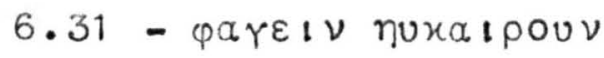

2 leaves missing

$$
8.5-\pi 0 \nu \varepsilon \pi \tau a \cdot x a \downarrow \pi a \rho \quad 12.29-\eta \mu \omega \nu \overline{x \zeta} \varepsilon \downarrow \varepsilon \sigma \tau \iota \nu
$$

1 leaf missing

$13.19-\tau \alpha_{1} \operatorname{rap} a_{\imath} \eta \eta \varepsilon \rho a !$ to the end. $(16.20)$

\section{Luke}

$$
1.2-x a_{1} u \pi \eta \rho \varepsilon \tau a_{1} \quad 2.5-\varepsilon \mu \nu \eta \sigma \tau \varepsilon u \mu \varepsilon \nu \eta a u \tau \omega
$$

2 leaf missing

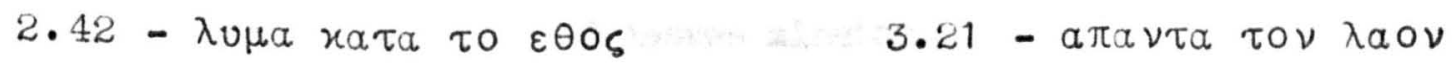

1 leas missing

$$
4.25-\varepsilon \pi \imath \varepsilon \tau \eta \rho_{\imath} \alpha \quad 6.4-\varepsilon \varphi \alpha_{\gamma} \varepsilon \nu \text { x } 1 \varepsilon \delta \omega x \varepsilon^{-}
$$

1 leaf missing

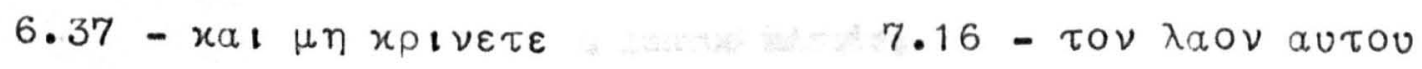

2 Leaves missing

$$
8.28-\nu \eta \mu \varepsilon \gamma a \lambda \eta \varepsilon เ \pi \varepsilon \nu \quad 12.3-\varepsilon \pi \iota \tau \omega \nu \delta \omega \mu \alpha \tau \omega \nu
$$

9 Leaves missing $v$

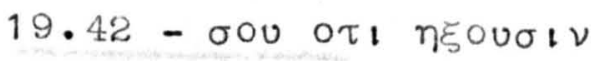


I leaf missing
$21.21-\tau 0 \tau \varepsilon$ ०1 $\varepsilon \nu \tau \eta$
$22.19-\pi 0 เ \varepsilon \downarrow \tau \varepsilon \varepsilon \downarrow \varsigma \tau \eta \nu$

2. leaves missing

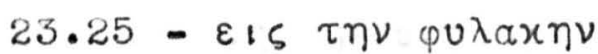

$24.7-\tau o \nu \overline{v \nu} \tau o v \overline{a \nu O v}$ o $\tau_{1} \delta \varepsilon_{1}$

1 leaf missing

$24.46-x a ! \varepsilon ı \pi \varepsilon \nu$ autols to the end.

John

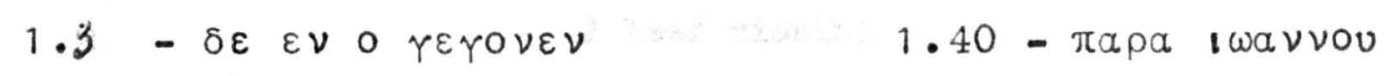

2. Leaves missing

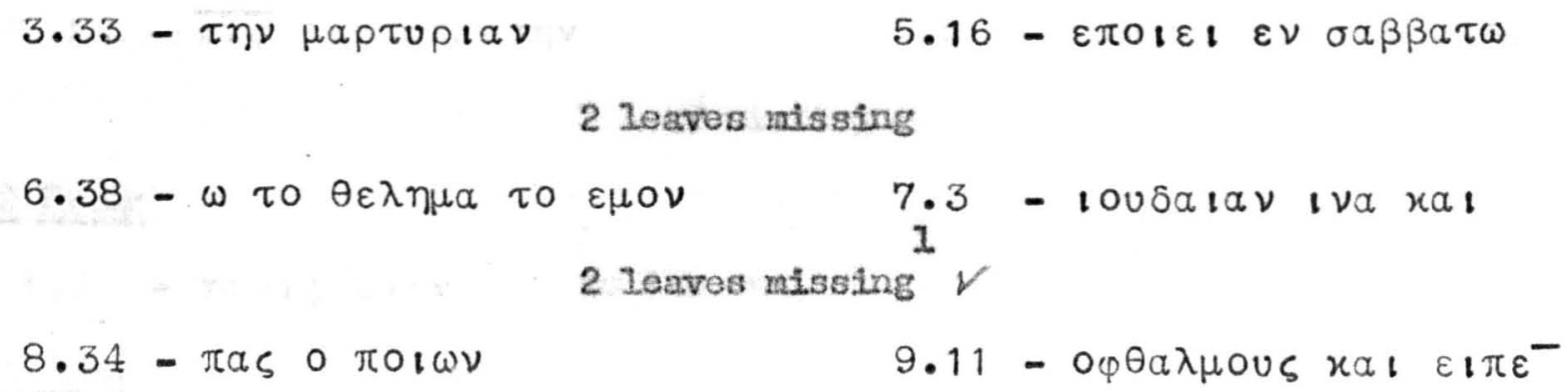

\section{Ieaves missing}

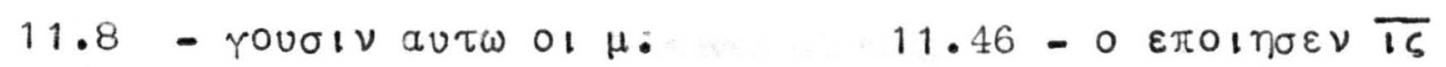

2 Ieaves missing

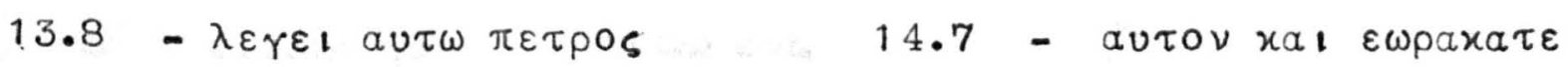

2 leaves missing

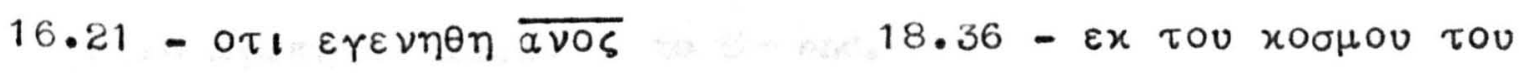

2 leaves missing

$20.26-x a \downarrow \mu \varepsilon \theta$ $\eta \mu \varepsilon \rho a s$ to the end.

Acts

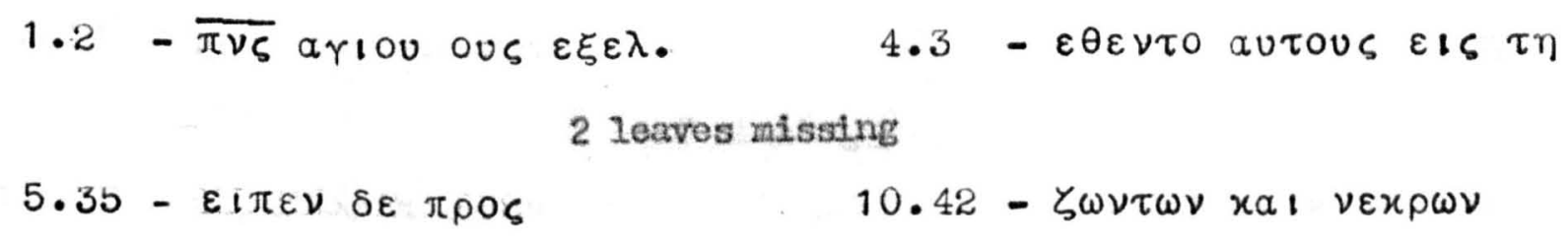

2 leaves missing

$13.1-0 \varsigma \mu \alpha \nu a \eta \nu \tau \varepsilon$

$16.36-\pi \circ \rho \varepsilon v \varepsilon \sigma \theta \varepsilon \varepsilon \nu \varepsilon ı \rho \eta \nu \eta$

4. Ieaves missing $v$

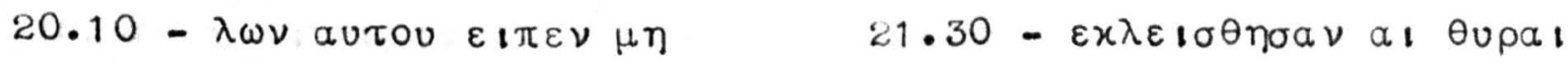

1 leaf missing

$22.21-x a 1 \varepsilon เ \pi \varepsilon \nu \pi \rho{ }_{1} \quad 23.18-\pi \rho{ }_{5} \tau o \nu x_{1} \lambda_{1} \rho \rho$

1. Because of the amount of space available it is not possibjo for the missing leaves to have contained the pertcope de adultery. 
1. leaf missing

$24.15-\pi 1 \delta a \cdot \varepsilon x \omega \nu \pi \rho \circ \varsigma \quad 26.19-a \pi \varepsilon ı \theta_{\zeta} \tau \eta$

1 leaf missing

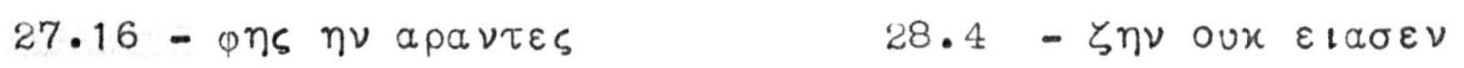

1. Ieaf missing

James

$1.2-\pi \varepsilon \rho ! \pi \varepsilon \sigma \eta \tau \varepsilon \pi 0 เ x . \quad 4.2-x \alpha \downarrow \pi 0 \lambda \varepsilon \mu \varepsilon \downarrow \tau \varepsilon$

1 leas missing

I Peter:

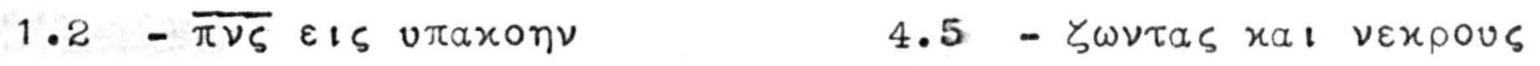

1 leaf missing

\section{Peter}

$1.2-\chi \alpha \rho i s$ viv to the end.

1 John

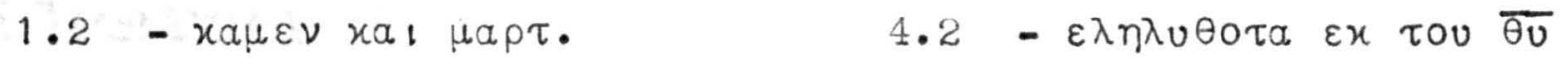

2 leaves missing

3 John

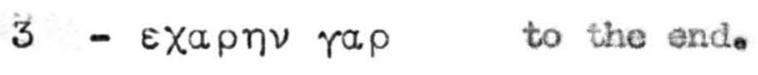

Jude

$3-\alpha \gamma \alpha \pi \eta \tau \circ$ l $\pi \alpha \sigma \alpha \nu$ to the end.

\section{Romans}

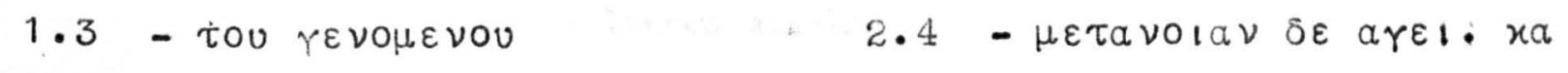

1. leaf missing

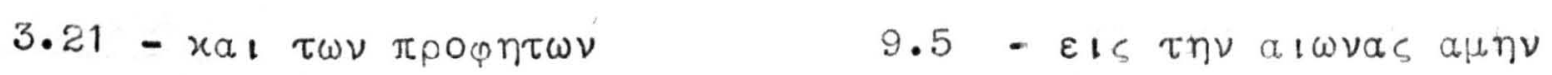

I. leaf rissing

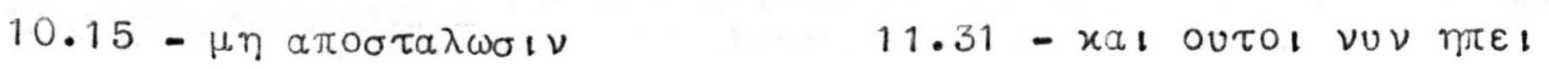

1. leaf missting

13.10 - ouv vouou $\eta$ a yajn to the end.

\section{Gorinthians}

$$
1.3-x a \rho ı \omega_{\mu} \downarrow \nu \quad 7.18-\mu \eta \varepsilon \pi 1 \sigma \pi a \sigma \theta \omega
$$

1. Ieaf mitssing

$$
\begin{aligned}
& 9.7-\gamma a \zeta \varepsilon \sigma \theta \alpha \iota \tau 1 \varsigma \sigma \tau \rho \alpha \tau . \quad 13.8-\varepsilon เ \tau \varepsilon \gamma \lambda \omega \sigma \sigma \alpha \iota \\
& 2 \text { leaves missing } \\
& 15.40-\mu \varepsilon \nu \eta \tau \omega \nu \varepsilon \pi \text {. to the ent. }
\end{aligned}
$$


2 Corinthians

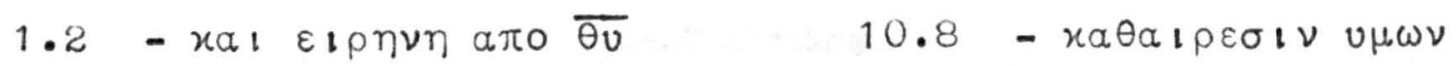

3 leaves missing

Galatians

$1.21-\varepsilon \pi \varepsilon เ \tau a$ $\eta \lambda \theta 0 \nu$ to the end.

Ephesians

1 lear missing

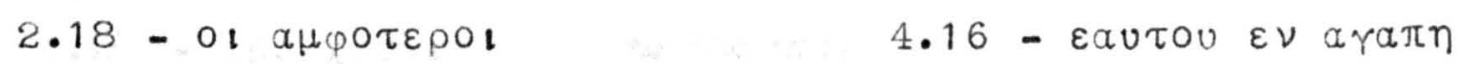

2 leaves missing

\section{Philippians}

1.22 - $\rho \eta \sigma o \mu \varepsilon$ ov $\gamma \nu \omega \rho \iota \zeta \omega \quad 3.5$ - $\operatorname{i\eta \lambda } \varphi v \lambda \eta \varsigma \beta \varepsilon \nu \imath a$

\section{I leaf nissing}

\section{Colossings}

$1.2-x a \rho ı \varsigma_{1} u \mu 1 \nu$ to the end.

I Thessatonians

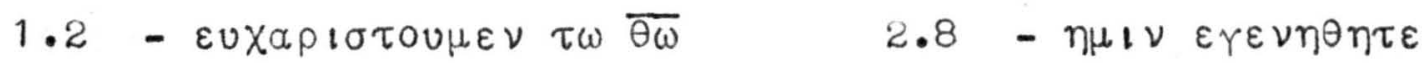

$$
\begin{aligned}
& 4 \text { leaves missing }
\end{aligned}
$$

Hebrevs

$$
2.4-\mu \varepsilon \rho \text { เ }
$$

1 leaf inssing

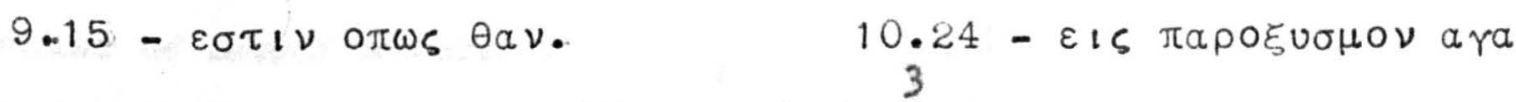

2 leaves missing

$12.16-\mu \eta \tau i s$ Topvos to the end.

1. Eberhard Nestle, in his Introduction to the Textual Criticism of the Greek New Testament (Iondon: Williams and Norgate, 1901), p. 63, erroneously stated that the whole of I Thessalonians had been lost.

2. In his Prolegomena (p.15) Tischendorf explains sufficiently the reasons for stating that Hebrews originally followed 2 Thessalonians.

3. It 1s possible that Wetstein saw the follo that contajned Hebrows 11.15 - 12.16? In his Prolegonena Wetstein stated thit Codex $\mathrm{C}$ wes desunt from 10.24-11.15 instead of Prom $70.21-12.16$ - that is, only one foltio was lost instead of two. His statement could be dismissed lichtly as an inaccuracy except for the fact that 11.15 is precisely where the second of the two missing pages would have begun. He must hitve had a reason for pieteing out verse 11.15. Against the possibility that he sav this follo is the port that in his New Testament he stops cjting codex C at 10.24 and doeshot wosure again unti1 12.16. This does not prove, however, that he nevor san wis foltio. 
I thimothy

1. 1eaf missing

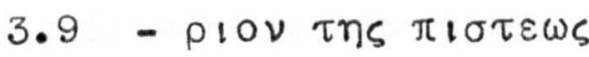
$5.19-\eta \tau \rho \imath \omega \nu \mu \alpha \rho \tau u \rho \nu$

1 lear missing

2 21mothy

$1.3-\chi \alpha \rho ı \nu \varepsilon \chi \omega \tau \omega \overline{\theta \omega}$ to the end.

Iytus

$1.2-\pi \rho \circ \chi \rho \circ \nu \omega \nu$ to the end.

Philienon

$3-x a \rho t s u \mu t \nu$ to the end

Revelation

$$
\begin{aligned}
& 1.2 \text { - os } \varepsilon \mu a \rho \tau u \rho \eta \sigma \varepsilon \nu \\
& 3.19-\zeta \eta \lambda \varepsilon v \varepsilon \text { ouv } x a_{1} \mu \varepsilon \tau a
\end{aligned}
$$

Ieaf missing

$5.14-x u \nu \eta \sigma a \nu x a ! \varepsilon เ \delta \circ \nu$

$7.14-\mu 01$ outol $\varepsilon l \sigma 1 \nu$

1 leaf nissing

$9.17-x a 1$ outws $180 \mathrm{v}$

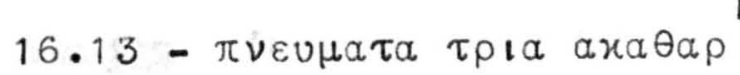

1. leaf uissing

$18.2-\pi a \nu \tau o s$ opveov

$19.5-$ - autov oเ $\mu \imath x \rho \circ$

3 Ieaves missing

From these contents we may present the followtng table:

Number of leaves preserved:

145

Number of leares missing:

\begin{tabular}{lc} 
Matthew & $7 \frac{2}{2}$ \\
Uark & $3 \frac{7}{2}$ \\
Zuke & 18 \\
John & 14 \\
Acts & 12 \\
Catholic Episties & 4 \\
Pauline Episties & 22 \\
Revelation & 6 \\
\hline
\end{tabular}

Potal

$87^{2}$

Total Folios originally in Now Tostament:

1. Because of a fortuitous textual transposition on the leaf containing Rev. 10.9 - 11.12, the contents of that pago should be given in 
It is easily seen that all the New Testanent books are represented except

2 Thessalonians and 2 John. Several of the smaller epistles are extant

In thoir entirety except for the first three colored lines. On the other hand Irake and, more especialy, John are sonewhat fragmentary. Luke 12.4 -19.42 is the largest lost section.

D. The Script.

The letters in this coder are larger than those of the other abig three" Nev Testanent uncials. In excecution the letters are more like the Alexcancian Codex in the British Museva than either tho Vatican or Sinaitic manuscripts. The letters most similar to codex A are $\underline{a}, \underline{b}$ (although a little nore erect in Codex $\mathrm{C}$ ), $\eta$ (the hoxizontal stroke is high), $\underline{\theta}, \underline{x}, \underline{\lambda}, \underline{\mu}, \underline{\underline{e}}$ (at times the body is almost dianond-shaped: $\Delta)$, and $\underline{\omega}$. The hooks on the ends of $\underline{\varepsilon}, \underline{\sigma}$, and $\underline{\tau}$ are quite pronounced. Gama is only two-thirds as vide as other square letters. The base of deIta extends beyond its two legs and sometines to the next letter. This base has a smaller hook than, for example, the tau. The diagonal stroke of mu jolns the vertical strokes just short of the ends of those strokes: $N$. The tail of the pho curls at the end: $\rho$. The two arms of ypsizon

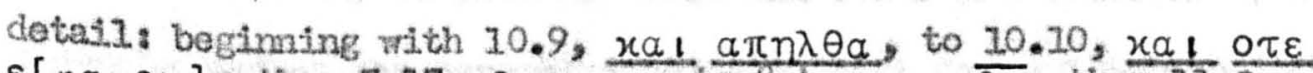

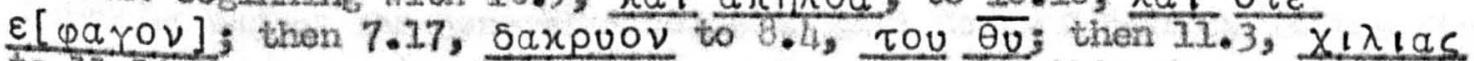
to 11.12 גeyouons An adequate explanation for this phenomenon is given by Harold Oliver, "A Textual Transposition in Codox $C_{\text {, }}$ "JBL IXXVI (1957), pp. 233-236. A further note right well be added on the toxt of Revelation. R. H. Charles in his comentary on the Apocalypse has stated that the text of 0 is "most carefulyy mitten." (The Revelation of St. John, ICC Series,

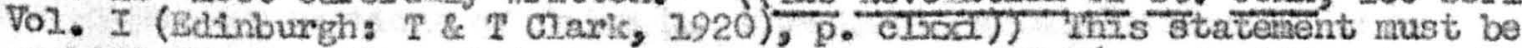
qualified. One iten will show whys In chapters 10-16 a significant number of omisstions - often by homoiotolouton - is noted. Because they may have a relationship to the conclusions reached by 0liver, those orissions are

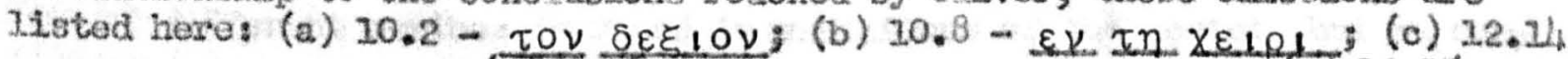

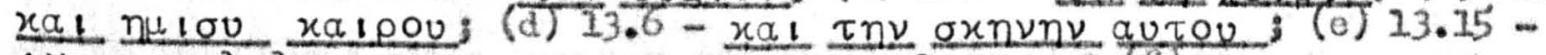

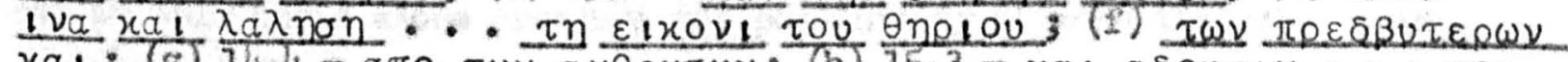

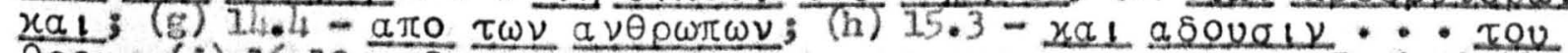

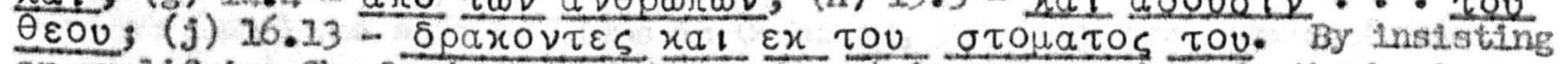
on qualifyine Charles! statonent, we do not in any way degrade the basic quality of the text of $\mathrm{C}$ in the Apocalypse. But the scribe'a peculiarities are stillz frequent and apparent.

2. If, as was undoubtedy so, the $\tau 1 \tau \lambda 0_{1}$ for lathew and llark wero included in the codex the nubber or missing folios is 89 and the total. number origtnaly in the coder was 234 .

3. In his Handbook to the Dextual Griticism of the New Testament p. 71. F.G. Kenyon stated, "When complete, the New Testanent would have occupsod 238 leaves." Did he include pages for $\tau$ I $\tau \lambda O_{1}$ of Acts, the catholic epistles, the Paurine epistles and Revelation? No evidence excists for such an assumption. 
meet at or very near the base line. One of the strolkes of chi curls slightly: $x$. The vertical stroke of psi. is not so long as in Codex A. The impression of this script is one of najesty and indicates that the nanuscript was almost certainly produced in a scriptorium or, at least, by a professional scribe.

It is scarcely worthwhile to note the coment rade by Wetstein as regards the date of Codex $\mathrm{C}$. He dren attention to a note added to the text of Hebrews and concluded the manuscript was written before A.D. 5 h2. Aside from the validity of the argunont, the conclusion is insignificant since no one is inclined to date the manuscript so late. The question of the date of Codex $C$ is usually decided lareoly on paleographic grounds. Our codex is Iater than both Codex Vaticanus and Codex Sinaiticus, but not later than Codex Alexandrinus. In addition to the writing Tischendorf cited the limited punctuation, the very simple subscriptions, and its textual afinitios os evidence of the manuscript's antiquitit. The fact that its single column represents, to a certain extent, a clevelopment from the three and four colurns of $B$ and $S$ must also be considered. The absence of any Euthaltan apparstus in the pauline epistles indicates a limit on how late the codex may be placed. When all itens are considered, we see no wasson to reject the general conclusion that Codex $C$ belongs to the first hatf or the filthe century.

Hitschendorf correctly noted that different sartbes wote the old and New Nostanent sections of codex $C$. Wuch less certainty renains as to whether more than one scribe had a hand in copying the Now Testament. Io hint of different scribes is found in variations of the script, but we cannot on this basis alone preclude the possibility of more than one scribe. Scholars faced the sarne problens in Codex $L$, yet they have successfurly "located" the different writers. Severa? notes on the orthography of the manuscript shoutd be instructive. The itacism 1 for $\underline{\varepsilon}$ is secn throughout the manuscript. The verbal suffixes, $\underline{\theta \alpha_{1}}, \underline{\theta \varepsilon}$ and $\underline{\tau} a_{1}, \underline{\tau \varepsilon}$ are interchenged regularly wh the exception: they are not confused in the fourth gospel. This indication of a change in John can be followed up 
by other observations. Aside frotn one Instance in luke, the codex always has the ppelling $\pi i \lambda \alpha \tau o s . ~ B u t$ in John $\pi \varepsilon t \lambda \alpha \tau o s$ is found ovexy time. A peculiarity found only, but regulariy, in John is papeloatol. In none of the other gospels is the numoveable omitted so frequenty as in John. Furthermore, John alone of the gospels frequently has $\lambda$ nџoua I rather than

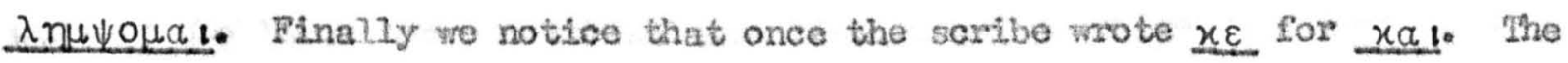
combined weight of these differences in John, if they camot be explajned by

a differance in the history of its text, suggest that another hand nitght have vritten the fourth pospel.

4

It has been suggested by athers that a different scribe wrote the book of Acts. The use of $\overline{1 \sigma \lambda}$ Instead of $\overline{1 \eta \lambda}$ for $10 p a \eta \lambda$ is taken as a clear Indication of a different scxibe. Taken by itself this is probably not sufficient to substantiate the theory of another hand. But the more than usual carelessness of the scmibe is also impressive. One gets the idea that the whiter of Acts ras not thoroughly at hora with the Greek language - or at least with Iukan symtax and vocabulary. This fact of the carelessness of the scribe, however, ean be stressed too much. Any number of factors - such as hoalth, emotional problens, lighting - could explain a tempoxary lapse from the scribe's customary accuracy.

Only one other possibility is worth mentioning as regards the different scribes. In the text of Revelation the seribe has written $\eta$ for $\varepsilon$ ? several cimes. This orthographic exror is not found elsewhere in the manuscript. As in Acts the scribe has been more caroless than usual, but this

1. A number of times the name is not found because the pages aro lost, but in each gospej. the name is found more than once.

2. This omission of the nu-noveable is found even more often in the Pauline episties, but this part of the codex shows none of the other characteristies of the fourth gospel so as to suggest that the scribe of John was also the scribe of the Pauline corpus.

3. Of even greater signtficance is the slight difference in the subscription at the end of John. Here the subseription is vaitten on iwo Innes: EYAPTEAION and KATAISANNHN. In Mark and Iuke (the end of Hat thew is missing) the subscxiption is watten on one Iine:

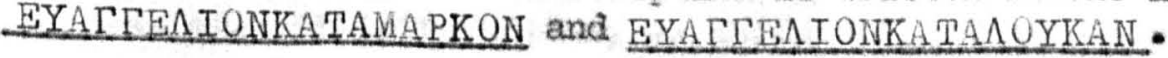

4. Iudidig Traube, Nomina Sacra, Quellen und Untorguchungen zur lat-

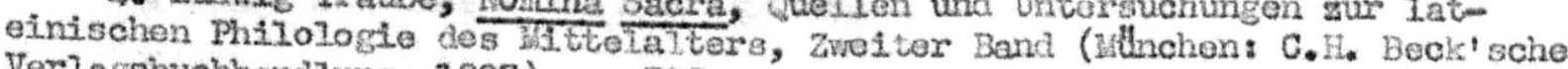
Verlagsbuch

5. On this basis wo could allege that for Mischendorf's edition of Codex $\mathrm{C}$ different persons copied the text of Iatthow (vinere I have found 1. 3 errors per folio) and tho catholic epistlos (whero I have found 3.4
exrorg per folio). 
Is of cloubtiful value in assessing the possibilities of different scribes. The general problem of the text in Revelation is so corplex in itself.

No other variationg have been found to suggest different seribes. The horizontal Iine for $\underline{\nu}$ at the end of a line is used with equal frequency and is raxked identically throughout the codex. The crowded letters at the ends of the lines show no variation. Punctuation does not vary except in the fourth gospel where the author's style probably explains the increased use of the high period. We conclude by emphasizing that cases for different scribes are best supported in John and Acts, especially the former, and that slight evidence is also found in Revelation for another witer.

\section{Provenance of the lanuscript.}

Our discussion of the provenance of the Codex 0 might most profitabiy begin with a stmuxy of Iischendorf's comnents. Tischendorf traces the travels of the codex backwards, noting first that it was probably brought to Italy either from the ares of Constantinople or Coristantinople itself. or this we nay be fairly certedn. To go beyond this Tischendorf rolied more on conjecture and a personal interpretation of the very linited evidence in themanuscript itself. He assumed that the difforence between the first and third hands could not be axplained entirely by the lapse of time. A diference in location was also necessary. Ho tumed, therefore, to Mlexandria. This was probably tnevitable sineo noro is know of the text and hanuseripts of this area than any other corner of the ancient world. Hits bases for olatining Legpt or Alexundria as the honeland of Codex 0 are its similarity to Codex Alexandrinus and, to a lettex extent, other codices Generally assumed to have cone from lecypt. He then notes various spellings and exrors in the text which sugsest an veyptian origin. He falled to note, however, one fairly solid plece of evidence mentioned by tiline and skeat in support of an Eeyptian origin. These scholars noted that Codex $c$ has

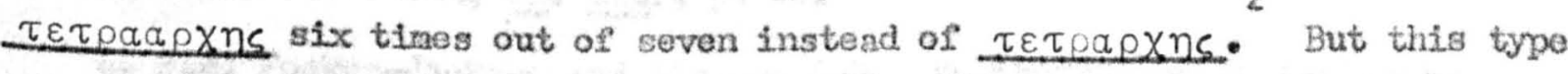

1. ef. his Introduction, p. $25 \mathrm{fe}$.

2. Liline and skeat, op. cit., p. 67 . 
of evidence must be used cantiously. Attention should be draw to words of pregelles mritten more than a century ago". . the occurance of Alexandrian forma in a MS of the llew restament does not prove (itrities his) Egrpt to be the country of such a lis as to Lts origin." Still unsolved is the problen of how widely diffused Alexandatian Greek was in New Iestament tines and during the early history of the transwisgion of the text. Until we know this we carnot assign a nanuseript to Egypt simply because it contains some Gerptian forms. If Mlexarditan critics set the pace as regards the New rostanent text, as Zuntz asserts, then the stream of influence worked out from Alexandria and oux limited evidence romatns anbiguous. Hilno and Skeat should again be quoted. "Throrotunately we have no evidence to show hor unform the scribal tradition of the Graeco-Roman worlds was at this pertod, and until that is decided no amount of similarity can be used to decide origin." Thero are no fortuitous spellings or confusion of proper names as have been found in Coder Sinaiticus to suggest a possible origin. Critics have generally voiced theis hositancy to locate Codex C. Our escanination adcts nothing to a possible solution. Fe nust be concent to pose the problen and give a Iikely solution.

\section{The Correctors}

Wo have already noted that two different correctors have alterod or added to the text of Codex $G$. Before their work is discussed a few comments will be made concerning corrections made to his own text by the original scribe.

\section{Tragalies, go. cit., p. 125.}

2. Cunther Zuntz, The Text of the Boisties, A Disquisition toon The Corgus Paulinum, The Schweich Lectures of 1976 (Ioncion: Geoferey Cuuberlege, oxford thiversity Press, 1953) p. 263 fe.

3. Mijne and Skeat, op. cit., p. 66.

4. F.J. Foakes-Jackson and Kirsopp Lake, Boginings of Chrigtiantiy, Part I The Acts of the Aposties, Vol. III the Foxt of Acts, by Jares Ropes (Iondon: WaelfT, an and Co., Ltd., 1226) p.iv. "There soens to be no sufficient reason for any confident assertion that it is of Bgrotian origin." 
A. Corrections by the Original scribe.

The first saribe has rarely corrected his om text. These instances are all noted in the appendices and need not be emunemated here. What Is somewhat surprising in a defluse manuscript such as Codex $C$ is for the scribe to show so little concern with his corrections. Ihese corrections may be divided into two classes according to the way they have been made. (1) Nost of the tine the scribe has erased some lotters and written over the erasures. This erasing has not been done so thoroughly as the erasIng done by the first corrector. The original. lotters can usually be seen fairly easily under the present text. The erasures are not vory extensive. He never exased a full Inne to add letters. The most he has erased is about twolve letters; usually the erasure is limited to three or four letters. (2) A limited number of corrections have been made, not by erasing, but by adding a single smaller lecter either between letters or above a letter. In Revelation 13.15 , for exanple, the scribe wrote $\varepsilon \delta 0 \theta$ and then added $\eta$ above the theta: $\varepsilon \delta 0 \theta$. Very seldom are any of the corrections by the original scribe significant for textual. criticism. Usually they correct obvious seribal blunders. B. The First Corrector.

Because of the style of his script the first corrector is usually dated a century after the first scribe, or about the middle of the sixth century. Inis need not be questionod, but it might be safor to be more general and date him anytime in the sixth century. The stroke of his pen is not so heavy as that of the original scribe; neither is his script too elegant, though it is far from the slovenliness of the second corrector. The letters aro slifhtly smaller and normaliy lean slightly to the right. Sven if he has arplo space, his letters are still smaller.

This corrector has made an attempt to preserve the good appearance of the manuscript. He never crosses out words or letters to write between the lines. Erasing was his coman (though not sole) rethod of emending the text. These erasures vary in thoroughness: in some places the original letters are clearly seen; in others even inframred photographs have not brought out the erased text. The erasures also vary fron a singlo 
letter to four or fitve complete lines. In this way the comector has tried to avold miting in the nargins, although from tisse to tine he does violate the right nargin. Many times the comrector erased letters or words without adjing anything to the text. In these instances of onissions he has left the place blank and has not atteripted to space the remalning lotters eveniy. It may woll be asked how we knos that such enfifendations were made by the eirst corrector since there is no seript by which to Identify him. To this we can only say that such erasures are not known to have been made by the second corrector who either crossed out or enefreled with dots whet he wished to ond from the text. These erasures are not by the second corrector. They are, therofore, elthor by the first correotor or by another corrector who erased the text before the codex eane into the hands of the second corrector. Some indeed have questionod if there might not be more than two conroctors. But if sone of the ontssions are to be assigned to a third hypothetical corrector, then this corrector's ropk was strictly Innted to erasing. Only two hands other than the original scribe are seen to have rorlsed on the manuscript. We cannot belleve a correotor did nothing but erase. We endorse without reservation, therefore, the bwo correctors as described by Tischendorf. The practice of erasing by the first scribe has been dise cussed at length. At tijnes, however, he has emended the text by adding sxall letters above the line ruch the sane way as the omiginal scribo. These small. letters are sweficiently different from those of the first seribe so that they are not confused.

The Iirst corrector has worked throughout the manuscript, but not always with the same frequency. He has made alnost bwice as many changes In esch of Mark, Luke, and John as in Matthew. Acts and the Pauline epistles are between these extromes. He has scarcely bothered to alter the text of Ravelation. The per-folio corrections of the different books or groups of books is as follows: 


\begin{tabular}{lcccc} 
Book & Folios & Corrections & Average per Sollo \\
\cline { 2 - 3 } Hatthew & 24 & 91 & 3.75 \\
Hark & 16 & 121 & 7.5 \\
Iulke & $15 t$ & 100 & 6.7 \\
John & 10 & 70 & 7.0 \\
Acts & 20 & 96 & 4.8 \\
Cath. epistles & 97 & 66 & 7.0 \\
Pavi. episties & 39 & 177 & 4.5 \\
Revelation & 8 & 10 & 1.25
\end{tabular}

on the following page is given a more complete and detailed breakioum of the distribution of empandations nade by both comectors. No tondency is seen for the correctors to maice fewer changos as they get farther Into the manuscript. Although nore scribal blunders are found in Acts than elsewhere, the corrector has not been more active here. He has matle no effort to correct all such emors.

For cxitical purposes lort congidered the text of the first correctm or as almost equally valuable with the original text. I Tis must have been because a few of the corrections agreed with codex B. But many more of the erefendations agree with Codex $A$ or other Byzantine uncials in the gospels. Its nost frequent ally is probably Codex II, especially in Maxk mere the corrector agrees with Codex II on thirty-seven readings in which the testirnony is divided. Then one considers the nuraber of obvious errors which have been corrected and the number of singular or sub-gingular readings of codex $C$ which have been uade to arree with the mass of witnesses, this total represents a high degreo of vinilarity. It is doubtrul if the readings of this corrector can be considered as significant for an early text, although its textual affinities are worth noting for the history of the text and the study of relationsizips betwen various witnesses.

Where wes the home of the first corroctor? mischendorf conjecturod that it was probably somewhere in Palestine, Syria or Asia Minor, because 
Toxt

Leaves $\frac{\text { First }}{\text { totals }} \frac{\text { Corrector }}{\text { per page }} \frac{\text { Second }}{\text { totals }} \frac{\text { Corrector }}{\text { per page }}$

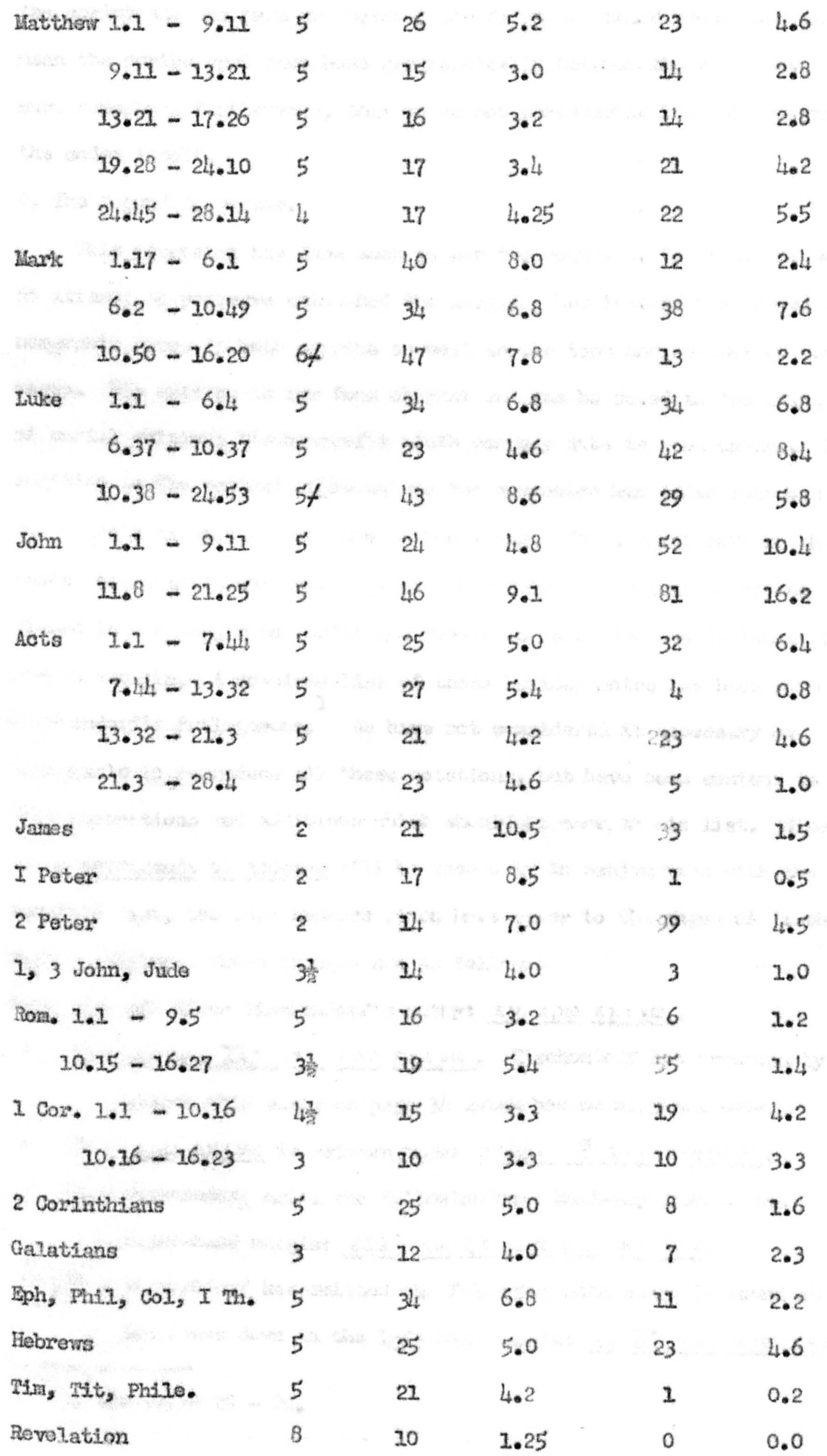


he secmod to be between the first scribe in Beypt ard the second forrector in Constantinople. But this is without foundation. The fact that both the script and the text are between the first and third hands doos not mean the scribe must have been geographically between the other two. We must reneaber, furthermore, that wo do not necessamily lanow tic origin of the codex itself.

\section{c. The second Gormector.}

This corrector has done much to max the beauty of Colox $\mathrm{C}$. Wh made no attengt to preserve untouched the nargins, but instead has placed inm numerable notes in both margins as woll as the tops and boitoms of the pages. Hits writing is far from elegant and can be dated in the dying days of uncial whting: Thschendore's ninth century date is good enough. In addition to the textual onfendations the corrector has added spirants and accents as volJ as the punctuation marks. The lareest part of the second carrector's work conststs of the regular litwagical notations placed in the margin to assist the reader in using the Now festament in church warkhip. A complote list of these lexical notes has been given in Tischendorf's Prolegomena. We have not considered it necessary or desixablo to reproduce all these notations, but have been entent to Ijst corrections and additions which should be made to his 1ist. Since these corrigenda et addenda will be used only in conjunction with the complete 14st, the page numbers given here refer to the pages of is schendorf's edition. These changes are as follows:

Page 30 - add after Mschendore's entry: $\underline{\underline{\nu}} \underline{x \omega} \underline{\varepsilon x \perp \nu \omega}$.

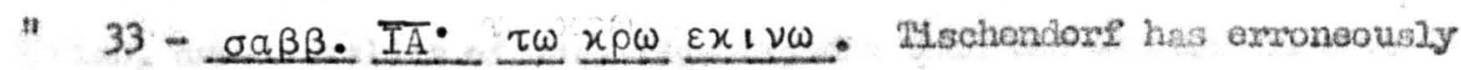
placed this entary on page 34 which has no maxginal note.

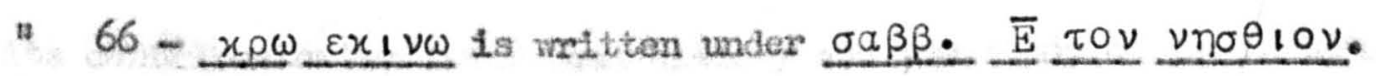

" 83 - llsehendore omits the following note half-way down in the

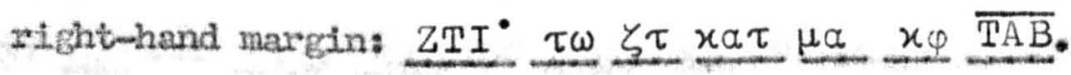

" 110 - Thschendore has ontthed the following note wition is found about

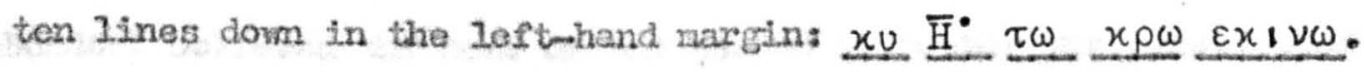


Page 172 - Instead of what Tischendore has, read the following gaß3 6 .

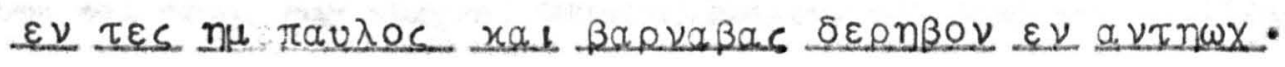

" 173 - A 11tile over halfway down the page th the righthand aargin,

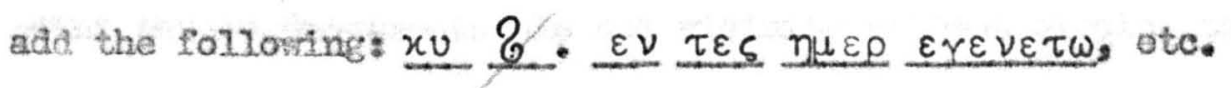

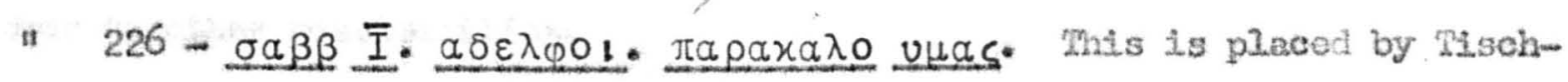
endorf or both paces 225 and 226. Page 225 has nothing.

The second corrector has atso made fundations in 2.17 parts of the text except Revelation; he has made no changes thore. HIfs corrections tend to be moro sporadic than those of the first corrector. In the cath ollo opisties his empribations are linited to $1.7 \mathrm{per}$ folio, wile in the fourth gospel there are 13.3 carrections per folfo - much higher than any average by the first corrector. We note the following results:

\begin{tabular}{lcccc} 
Book & Ieaves & Corrections & Per Folio \\
\cline { 2 - 4 } Hathew & 24 & 94 & 4.0 \\
Hark & 16 & 63 & 4.0 \\
Jukke & $15 t$ & 105 & 7.0 \\
John & 10 & 133 & 13.3 \\
Acts & 20 & 64 & 3.2 \\
Cath. episties & 97 & 16 & 1.7 \\
Paul. epistles & 39 & 90 & 2.35 \\
Revelation & 8 & 0 & 0.0
\end{tabular}

A further brealkdom in the distribution of corrections may be seen in the table on page 23.

The toxt of the corrector is pure Byzantine, or Nostcott and Hort's Syrtan, and is seldon of nore significance than the average cursive text of the ninth or tenth century. Fis work is nore likely to be studied for its contribution to Ifturetal. history and the study of lectionaries. The orthography is more Byzantine. The following spellings are found

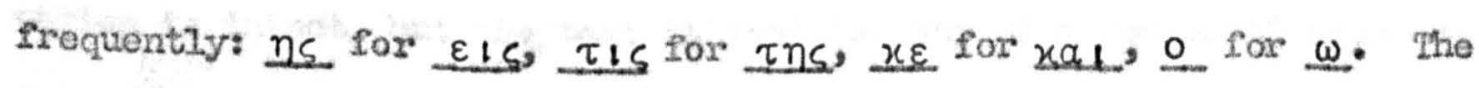
xay compendium is also used as well as other ligatures.

Tischendorf belfeved the hone of the second corrector was Constantinople. But again the evidence is less than conclusive. We are fairly certain the manuscript was in the Constantinople aroa at the beginning 
of the sixteonth century, but this ts a long time after the ninth contury. Certain notes and names may suggest Constantinople, but they are surficiently anabierus as to be open to nore than one interpretation. Te should be sefe to point torard Constartinople and vicinity without closing the door to other possibilities.

\section{$\checkmark$ Results of the Present Study}

We mat imediately mention that the work of rischendore, by and large, has been verified by the present study. This is not to say that errors have not been found. Wven the nost caroful coliator wi11 make errors. The errors we have found are listed in Appondix A. Wary are insigntficant; but a significant number are important for the textual criticism of the Ner Testament. For example, arrangenonts will be made to correct nore than seventy citations of Codex $c$ in Nestle's odition of the Greek text.

This odition differs in a number of wys from that of tischendor. He reproduced the large marginal letters as they are found in the Codex. His text was printed in scriptio continya with the uneial letters. We have been content to reproduce the text without any effort toward initation. Then the text had been erased and re-mitten by the first corrector, he printed the corrector's text in allihtly smaller letters even though he was able to decipher some or all of he original lettore. When the vellum ms tom or when nothing could be seon, he left his toxt blank and indicated by comas the number of letters probably lost. This edition adopts the nore modern mothod of using brackets to print what camnot be seen. We have made one modification: to differentiate between the two reasons for not being able to recover the origlnat text we have used different signs. The square brackets [] indicate the follo is torn so that thore is no vellum to hold the letters. Parentheses () are used when the vellum is intact, but the text is lost for any of a muber of reasons. This distinction, it is hoped, will avoid unnecessary work for anyone who may want to consult the codex for a specific reading. If he notes that our text has the reading in square brackets, he will know that nothing will bo gained from again consulting the codex Atself. A.7. notes on the two 
correctors will be found in Appendices $B$ and $C$, and reference is always made when, and why, we have disagreed with lischendorf.

Only with great hesitation is ons oncouraged to disagree with wischondorf in obscure natters. He was a grast paleographer. Anyone who is acquainted with the story of how he disagreed with 271 othors in declaring that the Iast verse of John's gospel in Codor Sinaiticus was added later and how his contention was vertfied by an inframed photograph will know the hesftancy we felt whenever we had to depart from his conclusions. Yet Thschendore was young when he tackled Codex C. It reprosents his first work on an iraportant manuscript. Even when possible detorioration in the manuscript is considered, the jupression is gained that on a few occasions Hischendors wrote more than he saw. One significant incident is the text

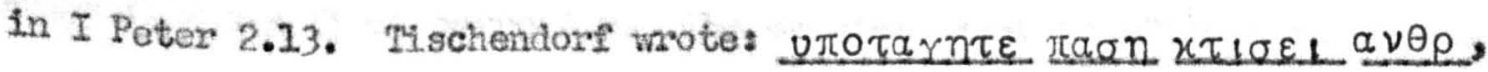

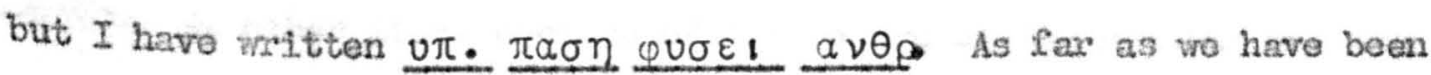
able to find, $\varphi v \sigma \varepsilon_{1}$ represents a sincular roadine of Codex $C$, so that Is schendorf could not have expected it. We did not expect it either. But wo studiod the reading in the manuscript itsete, in a natural photoEraph, and in an infra-red photograph. They all tended to confirm our first inprossion. The lowrer hate of both phi and upstion are seen. Thy

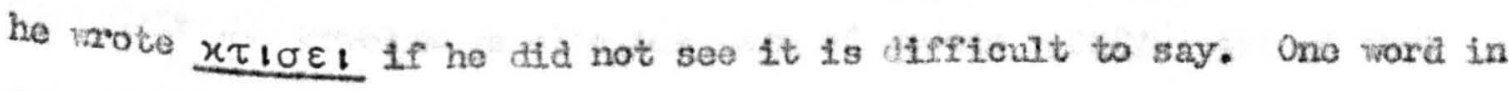
his defense, hovever, ought to be said. In a manuscript of this type when so many words and lettors are indistinet, and when the reador knows what ho expects to see, it is often difficult to draw the line between when he thinks he see and when he acturaly sees. Theibest illustration of this dilenme is when one is driving into paxis in tho exry morning fog. Il looks for the tifiel Tower and knows what he expects to see. Ho nay be deceived by vague tracos in the fog and probably is not able to say exactly when he clefinitely sam the tower. whis will be the experionce of anjone who reads codex C. For this reason we have tried to stay on the safe gide and wrte only what we definitely saw, including in parentheses what could not be seen or what we were not sure we saw. This one criticism of Tischendorf must not detract from his work. His edition of Codex $C$ was a nagnificent achievenent. 
Throughout the text and notes of this edition wo have triod to keep In mind the needs and problens of the editor of a Greek text and the eitations in his critical apparatus. Ho faces difricult problens in cling a manseript which has so many lacumae as does Coder $C_{\text {. }}$ Is it satisfactory, for example, to Include our nanusertpt awong others unter a comon sign? In Nestle's apromatus the Hesychian symbol includes Codox $C$ unless stated othemise. In reality, however, who knows all the places where Coders $\mathrm{C}$ is missing? The Hosychian syubol is still used. It Is true that Nestle's text only attarpts to Indicate generally the witm nesses for and against dffferent readings. Tot it carnot afford to be ambiguous.

A more serious and difficult problens arises when the text has been changed by the first corrector. The original text is often losi perrian nontly. At other times because of the nature of the reading and the anount of space available we can be quite ceriain what the corrector added, but we cannot know if he rewrote the original toxt after adding sonething to the text. At vartous places the compotor has arased beyond recovery severa? lines of the text to add a word or phrase. It carnot be assuned that the corrector rewnote the ortinal text. To fllustrate the problem we cite the text of iomans 7.2heff. All of the text in Ronans 7.24-8.2 is remintiten. Nothing in the rewwitten text can be said to represent the text of the original scribe. In his eighth edition Tischendorf has made this mistake. Another excamle where Mischendoref cites Codex $c^{*}$ for what wes written by $\mathrm{c}^{2}$ is Romans 14.5 . Wwo full lines have been erased and rempitien by the corrector. Wischendorf cites Codex $\mathrm{G}$ as a witness for os $\mu \varepsilon \nu$ yap rather than os $\mu \varepsilon \nu$. But this is the corrected text. We do not know wat the ritst scribe wrote. Anothor problem related to this is when to cite 02 for a variant reading. When several words have been erased to add some letters, should all the rords then rewritten - even though they ropresent the text of the original scribe - be cited as the text of the corrector? In lark

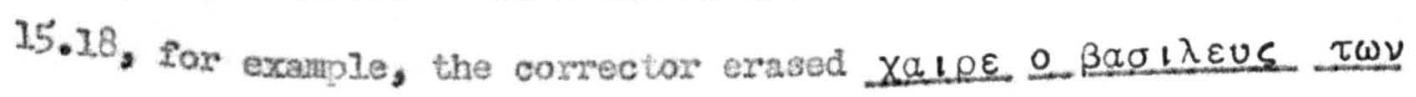

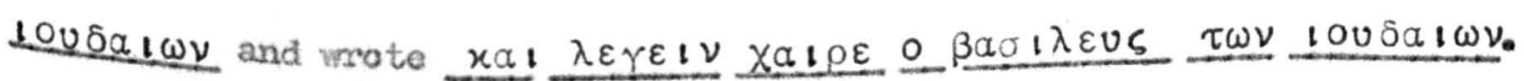




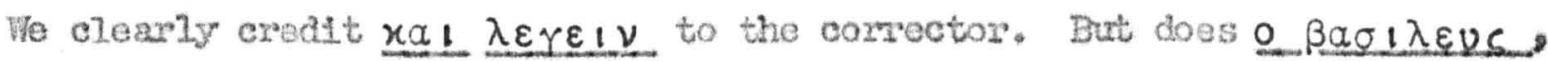
(instead of $\beta a \sigma \mid \lambda \varepsilon v)$ which the ortginal. scribe aloo wrote, represent a critical judgment on the part of the corrector? or after adding na $\lambda \varepsilon \gamma \varepsilon 1 \nu$ did he simply roproduce the eisst teact?

Wo are content to stress these problems in olting Codex $\mathrm{C}$. In the notes in the appendices we have tried to indicate clearly the condition of the text and conclusions we have reached so as to cutde the eritics and editors who alone nust malce the decisions for thest own works. 
THE TEXT 


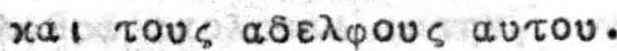

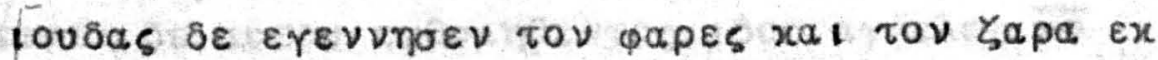

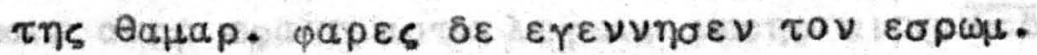

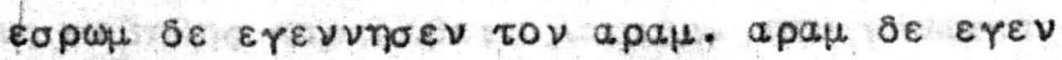

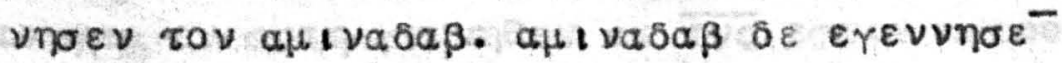

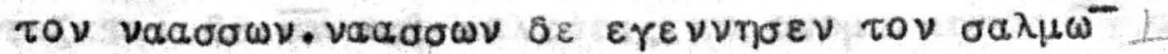

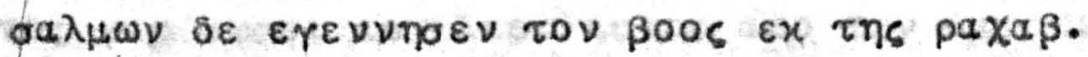

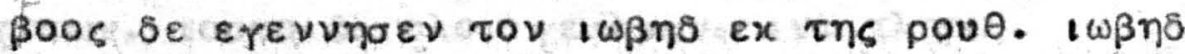

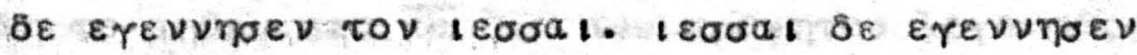

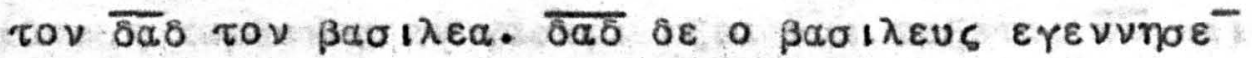

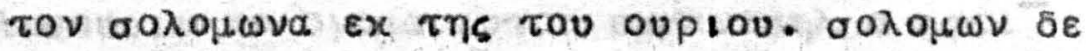

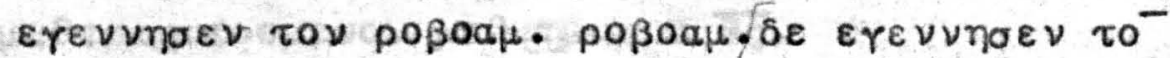

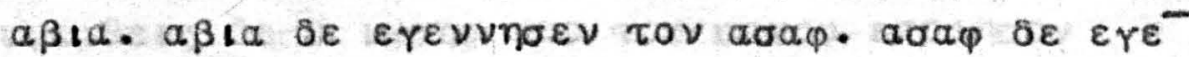

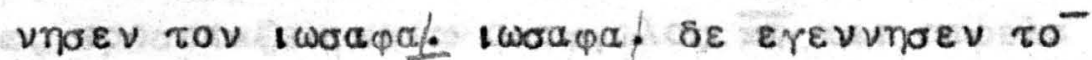
twpaj.

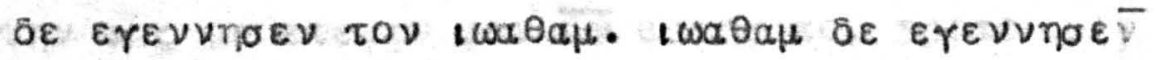

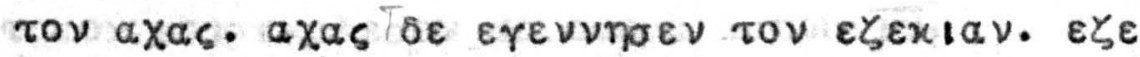

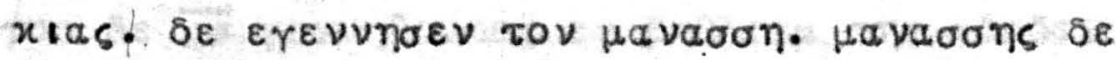

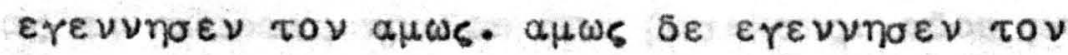

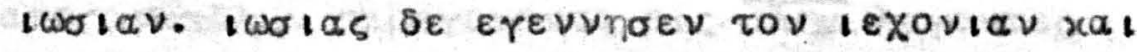

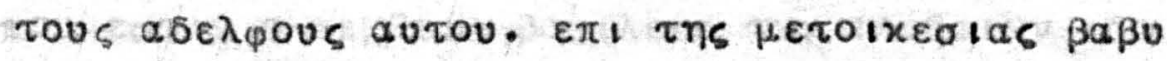

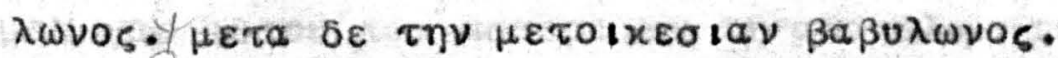

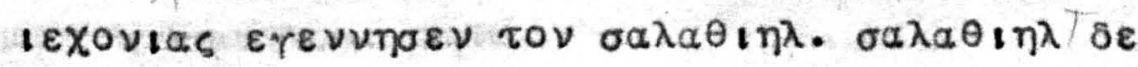

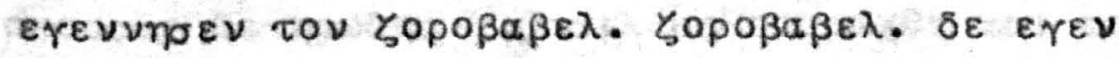

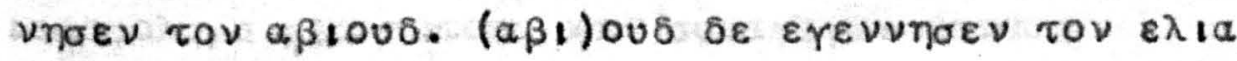

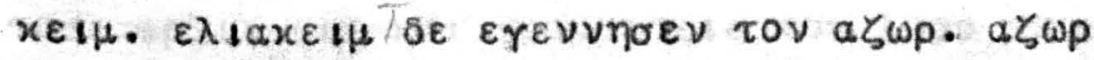

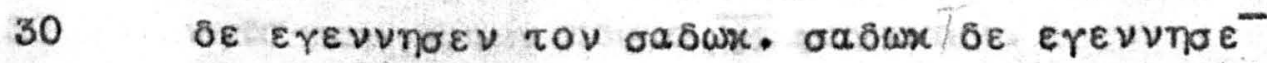

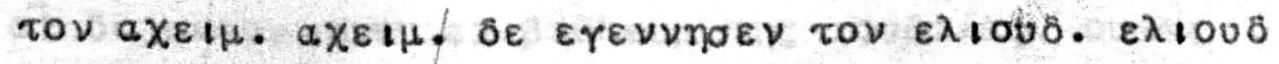

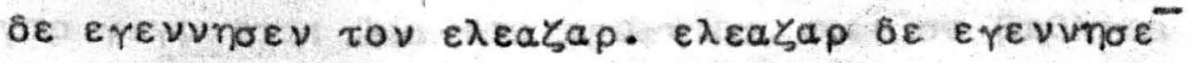

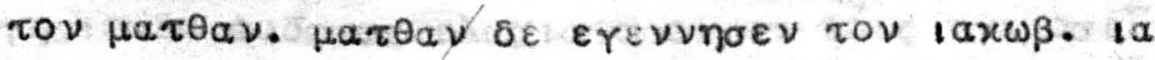

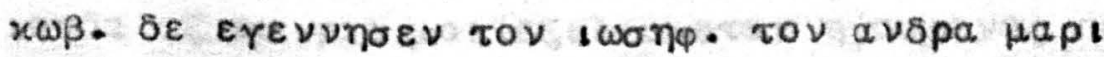

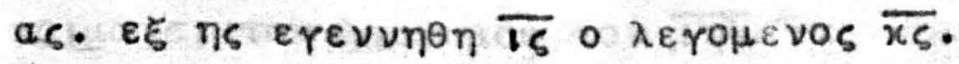

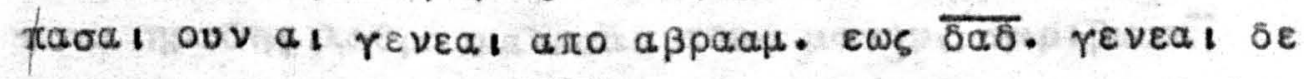

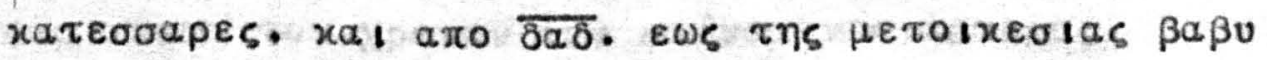

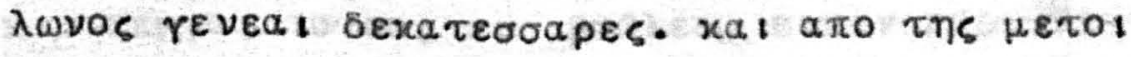

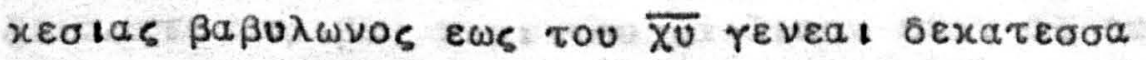

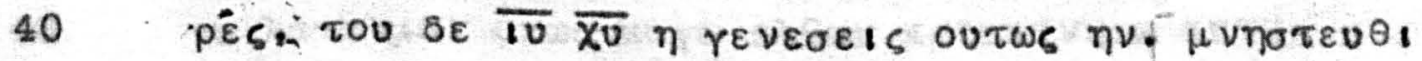

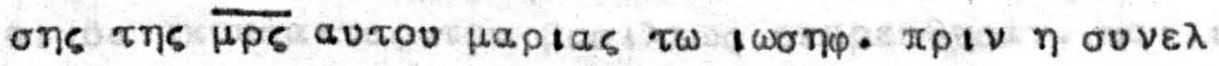




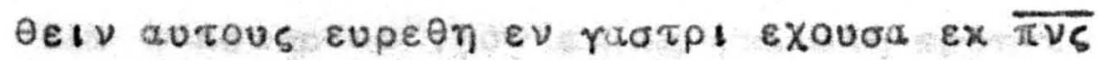
ariov. twanp of o avnp autns oixalog we

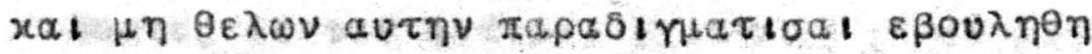

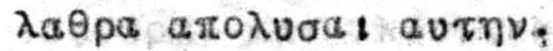

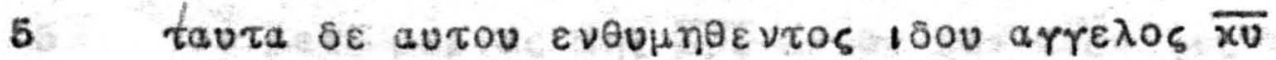

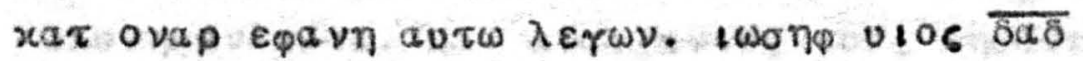

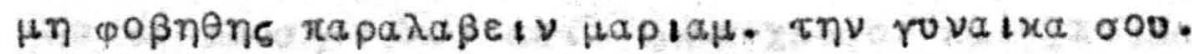

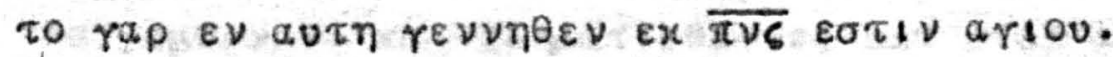

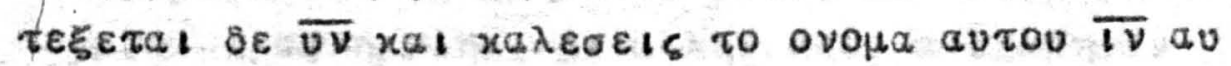

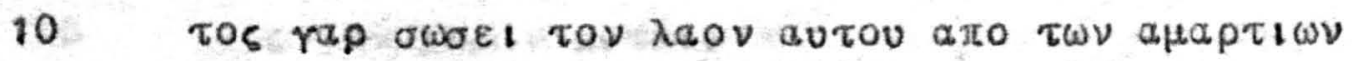

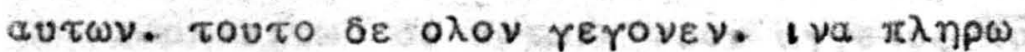

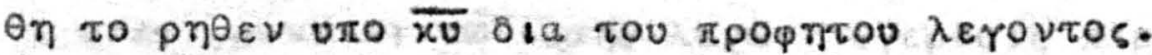

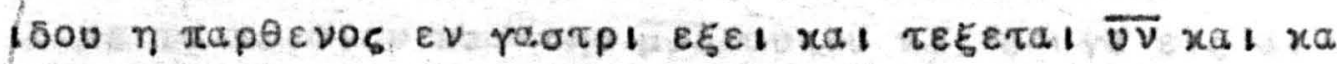

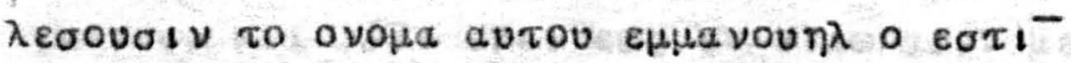

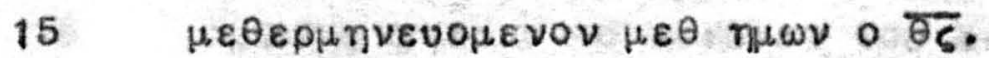

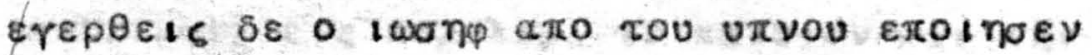

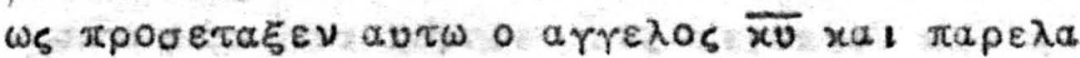

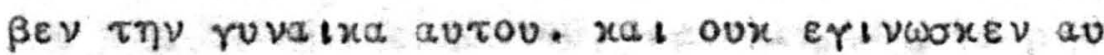

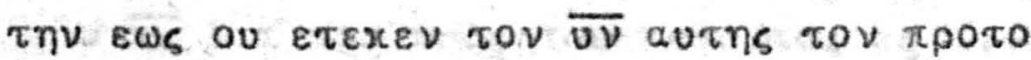

20 toxov xal $\varepsilon x a \lambda \varepsilon \sigma \varepsilon \nu$ to ovoua autou TV.

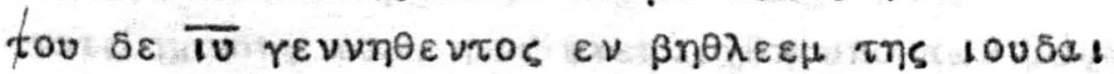

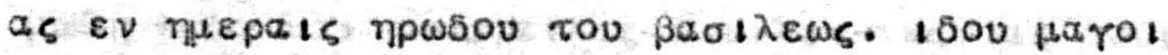

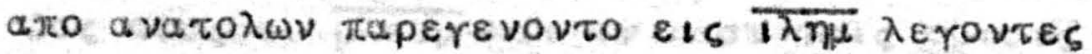

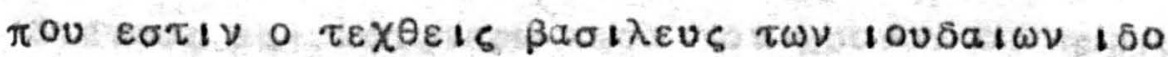

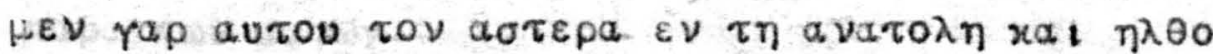

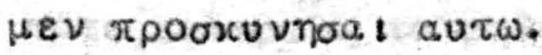

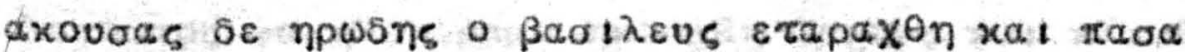

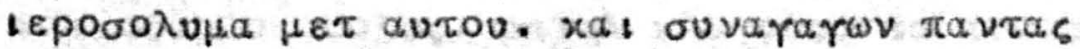

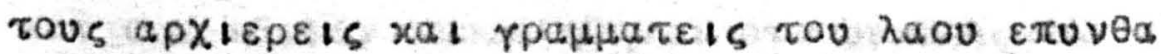

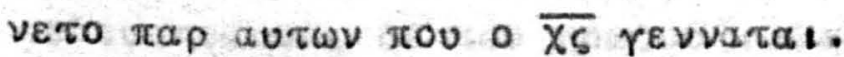

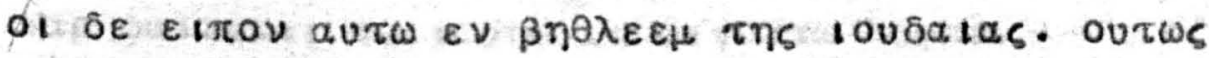

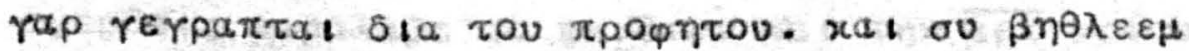

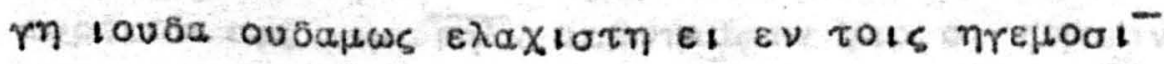

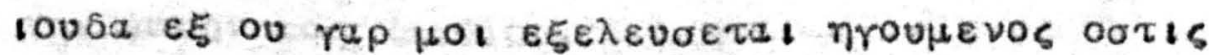

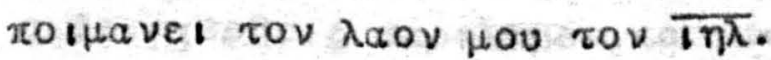

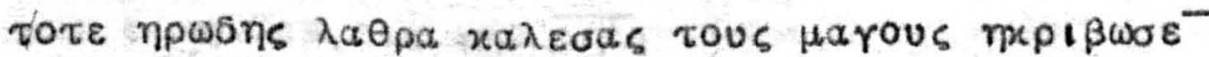

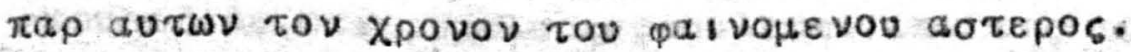

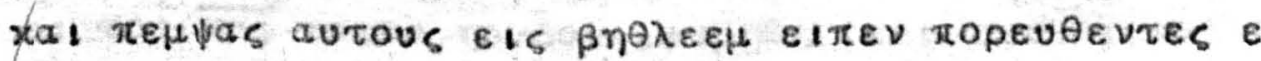

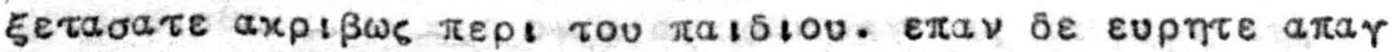

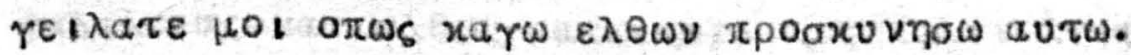

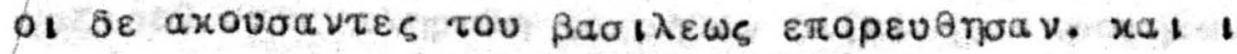




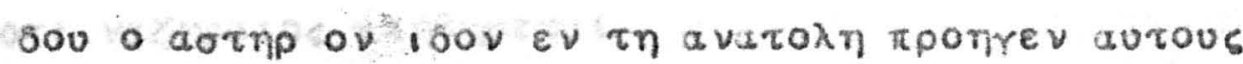

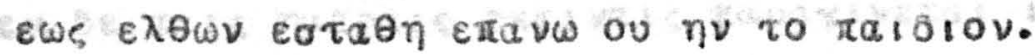
1

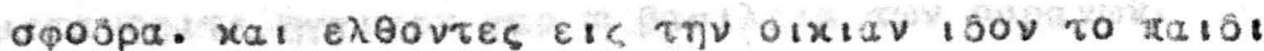
ov Heta Haptas ths $\overline{\mu \rho \zeta}$ autou xal tedovtes tpoo

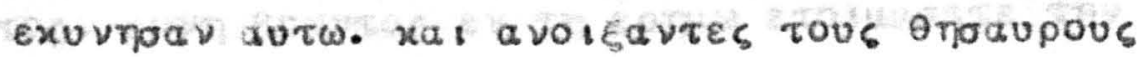

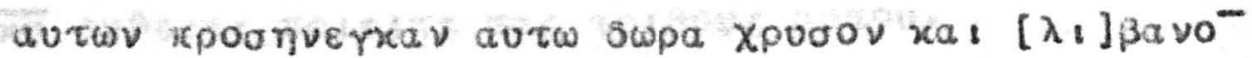

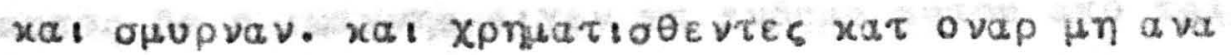

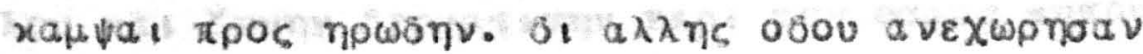

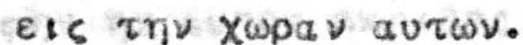

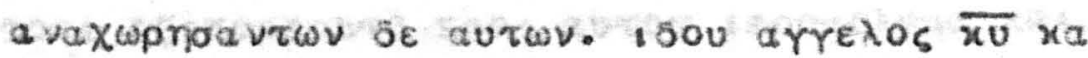

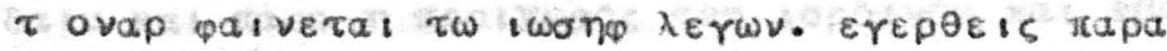

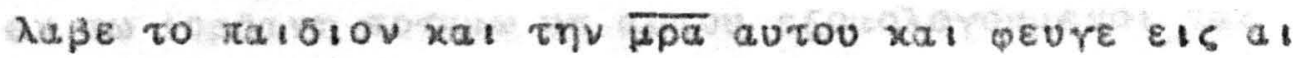

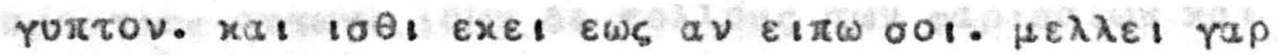

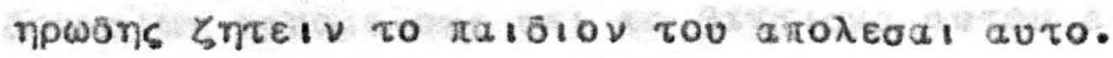

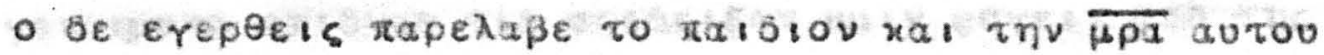

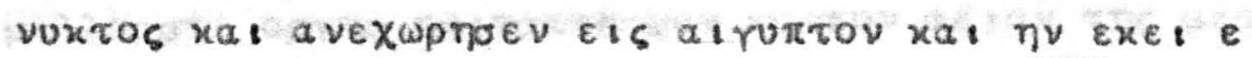

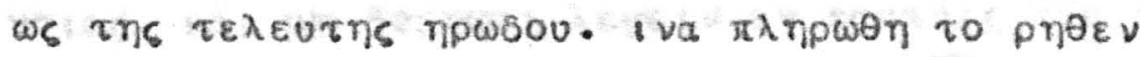

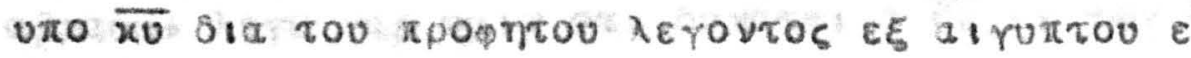

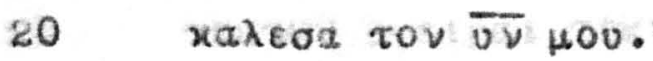

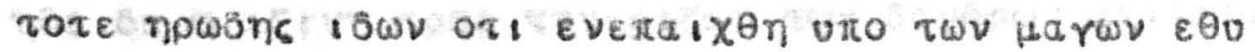

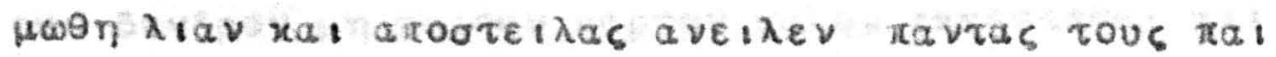
Sas tous $\varepsilon \nu \overline{\beta \lambda \varepsilon \varepsilon \mu}$ xal $\varepsilon v$ rad tois optois autns a

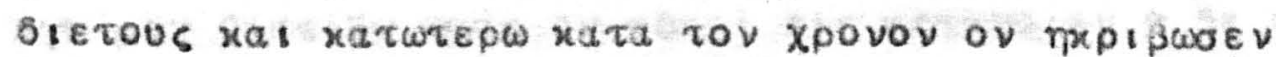

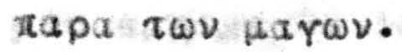

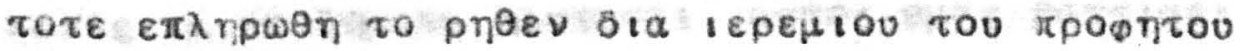

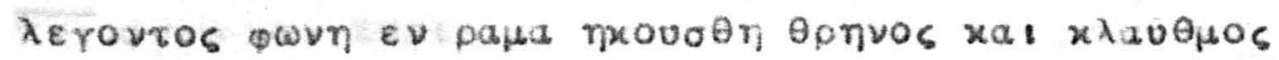

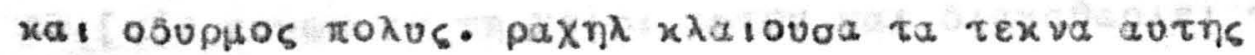

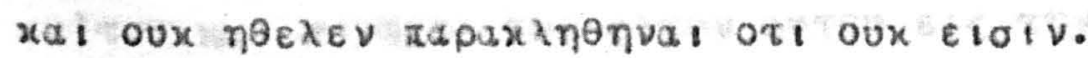

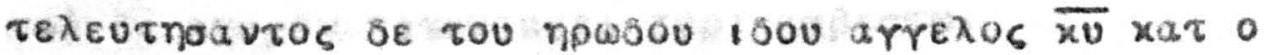

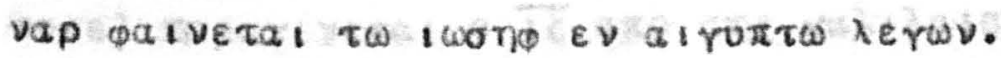

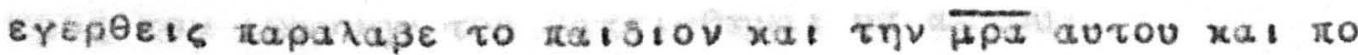

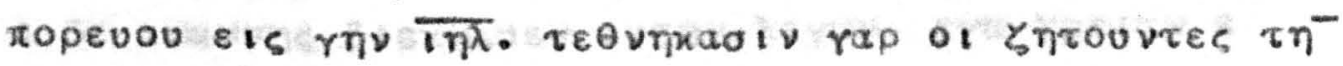

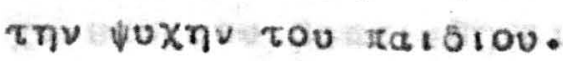

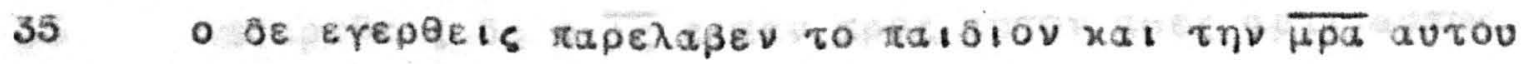

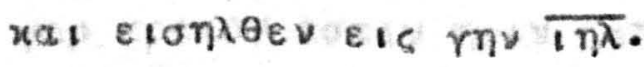

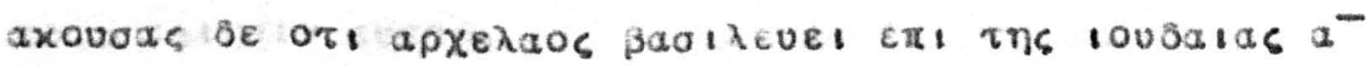

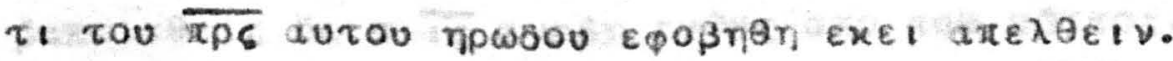

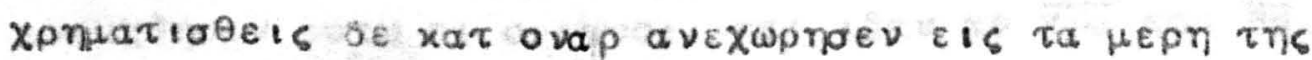

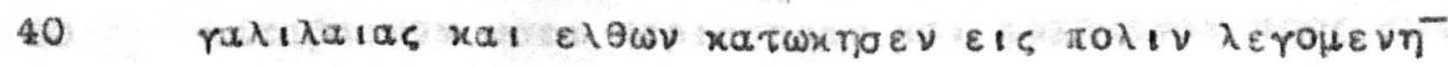

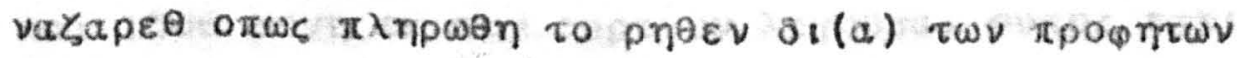




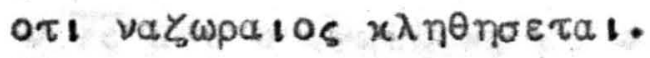

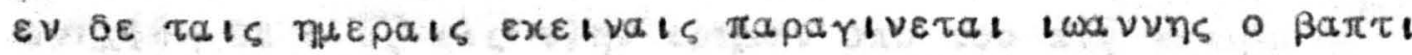

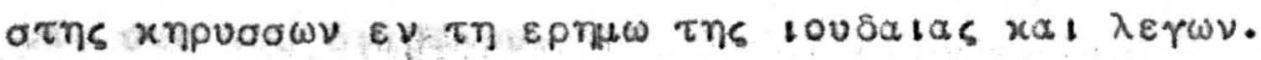

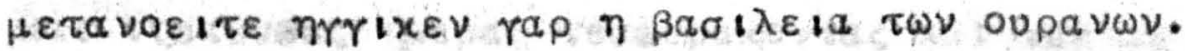

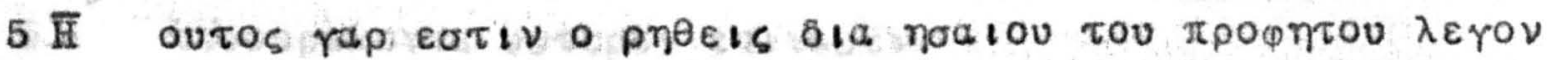

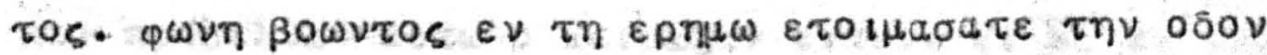

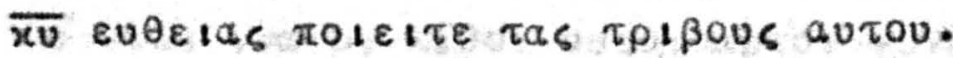

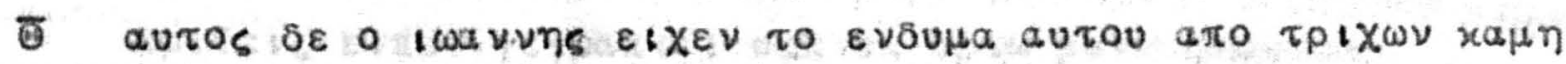

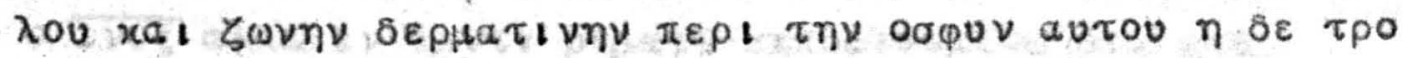

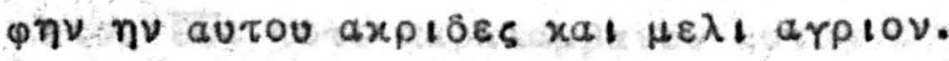

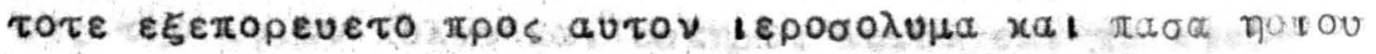

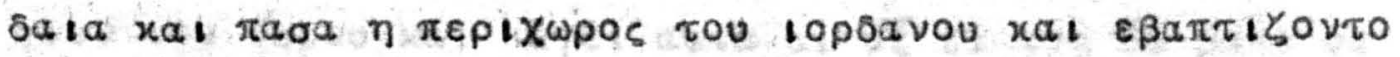

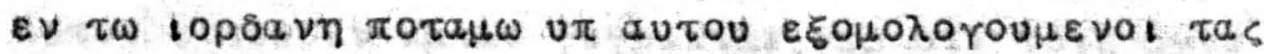

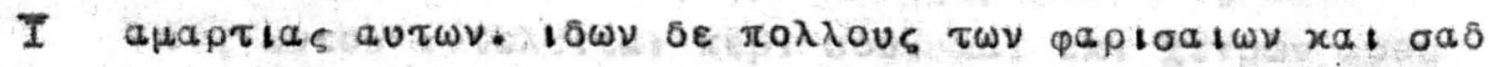

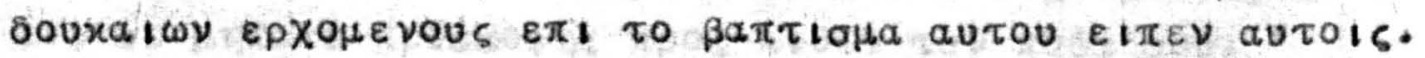

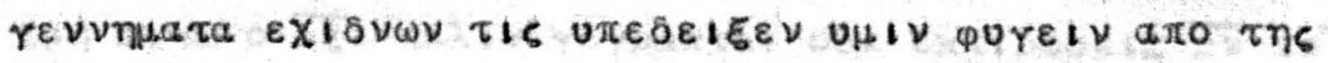

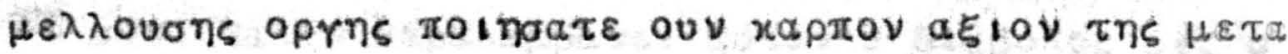

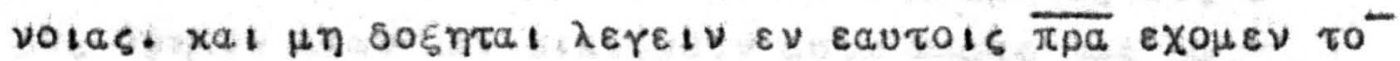

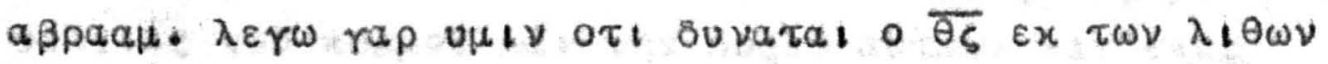

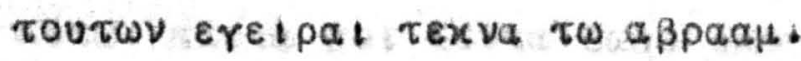

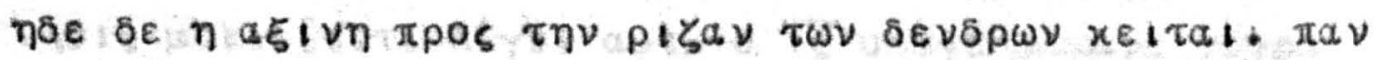

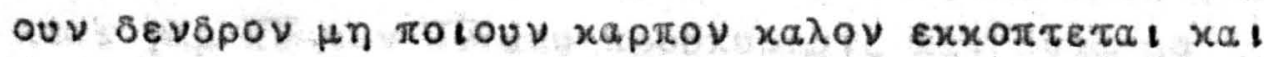
\&is $\pi u p \beta a \lambda \lambda \varepsilon \tau a$ l.

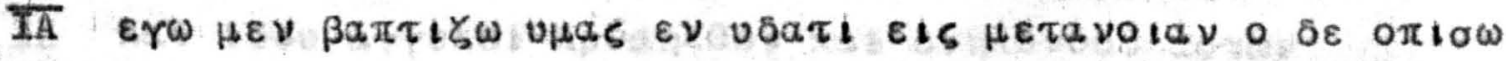

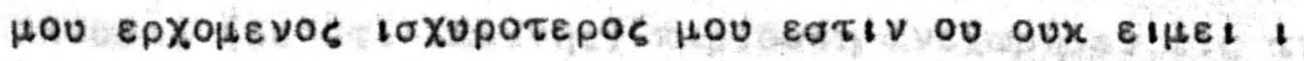

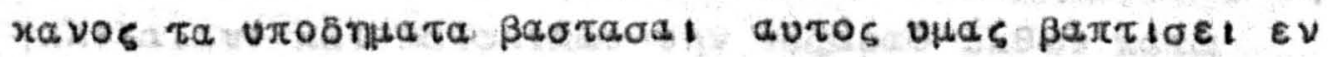
$\pi \nu$ ariw $x a i$ tupl.

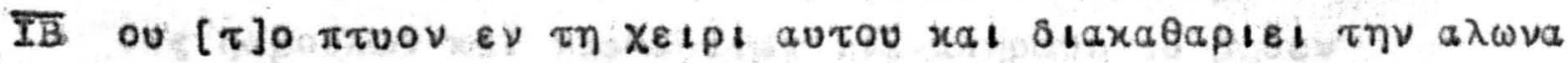

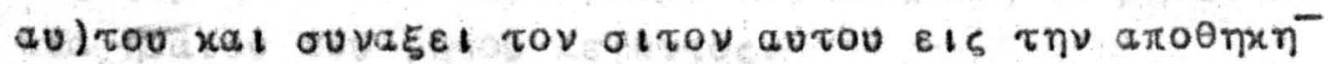

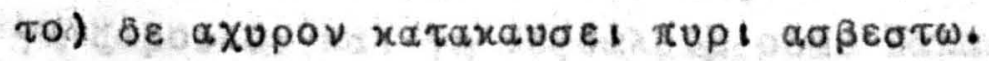

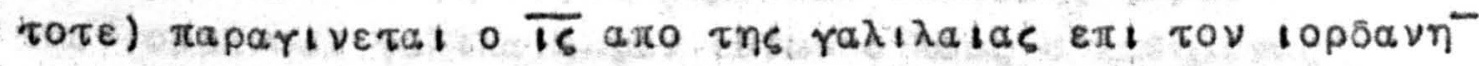

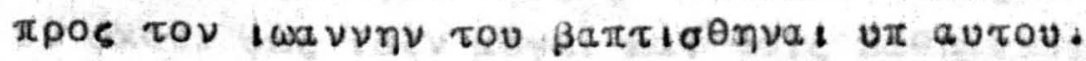

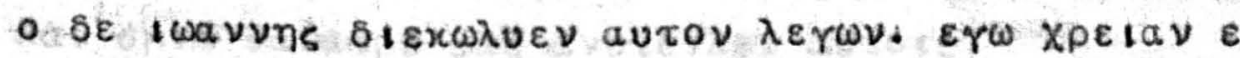
$\chi \omega$ v

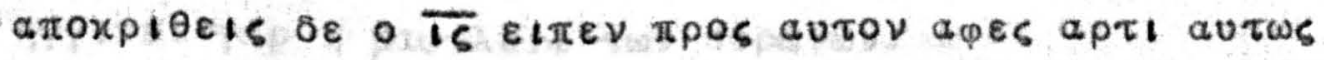

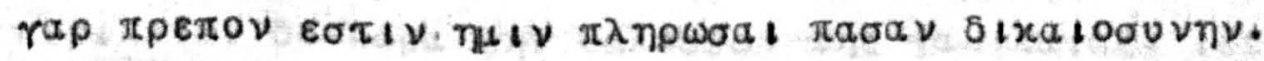
Tote apinotv autov.

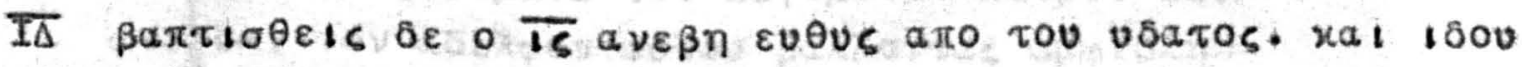

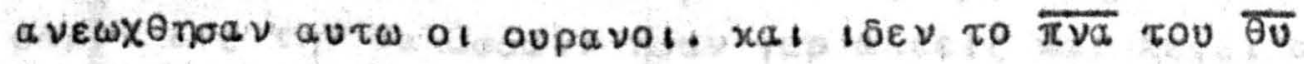

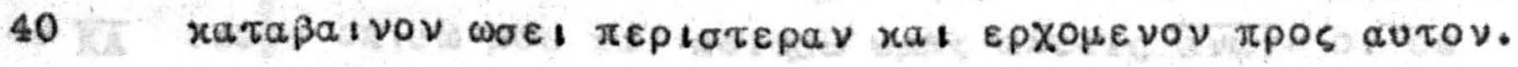

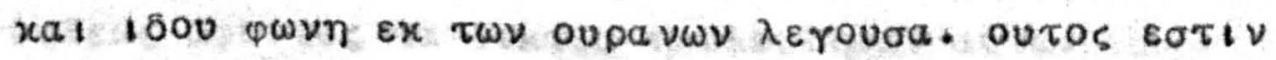




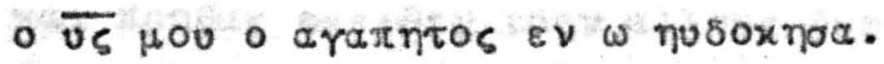

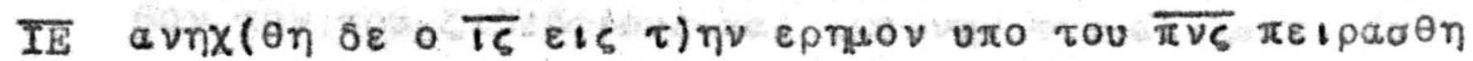
val v

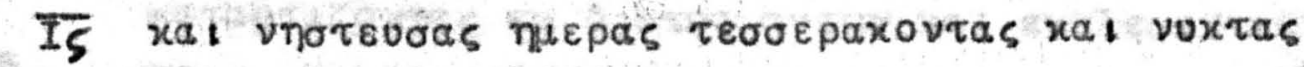

5

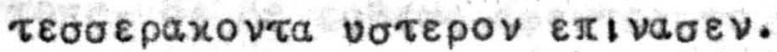

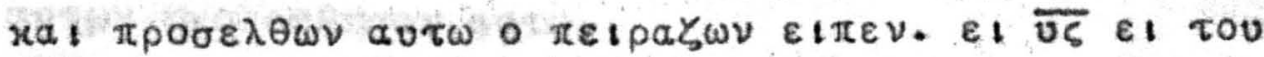

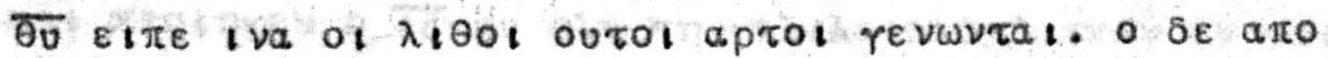

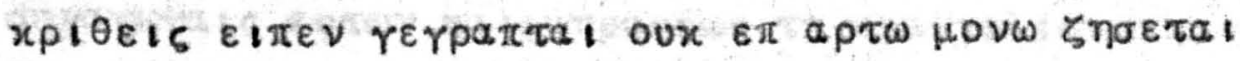

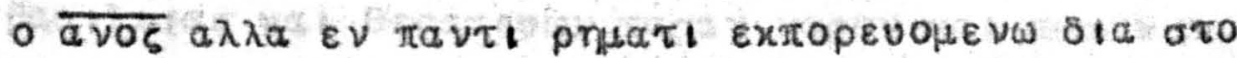

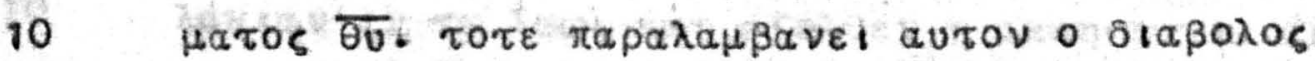

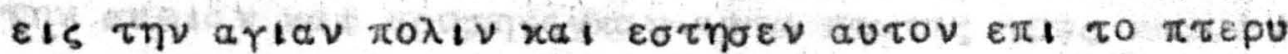

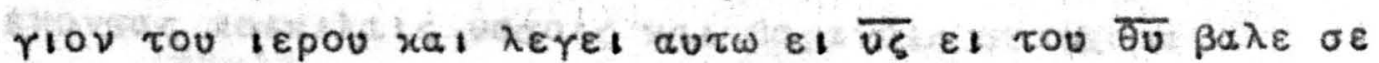

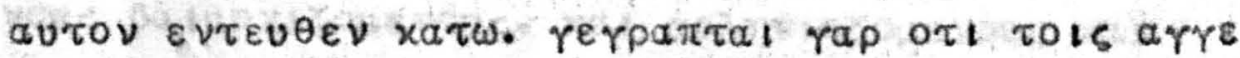

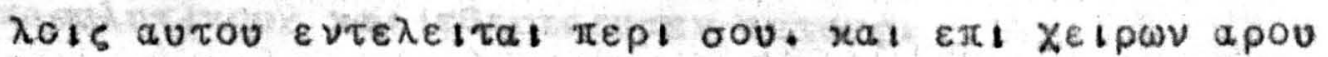

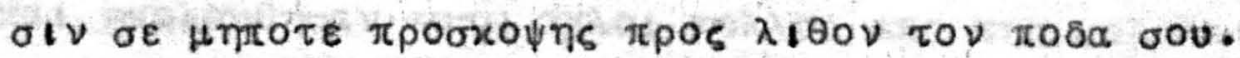

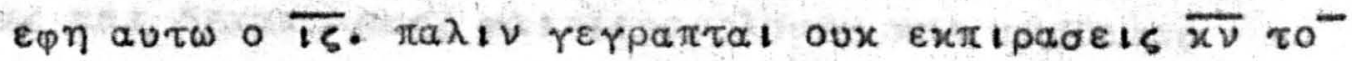

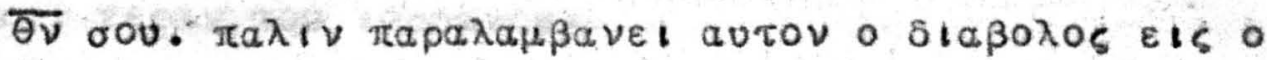

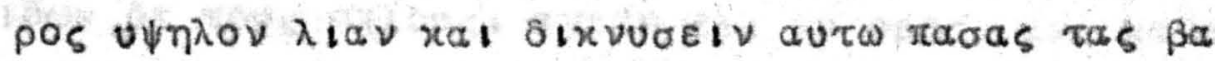

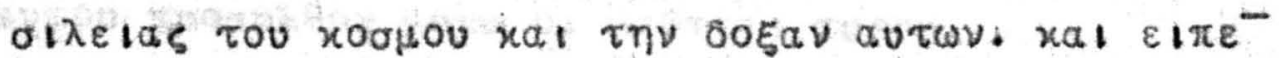

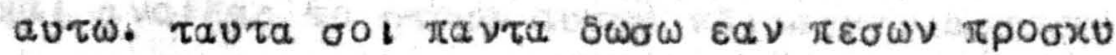

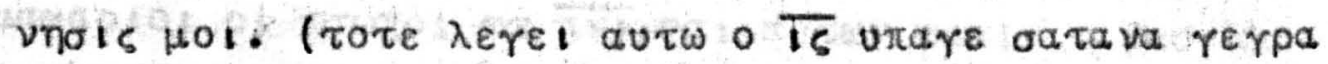

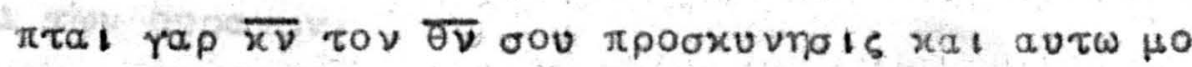

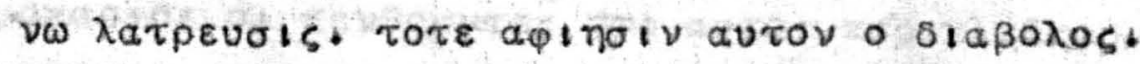

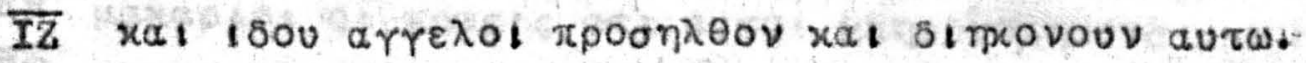

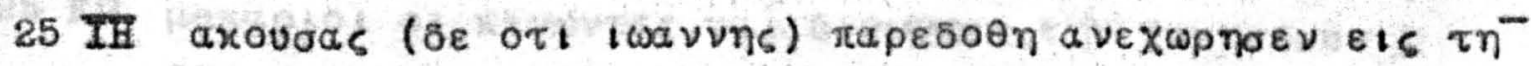

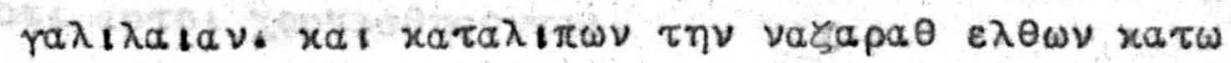

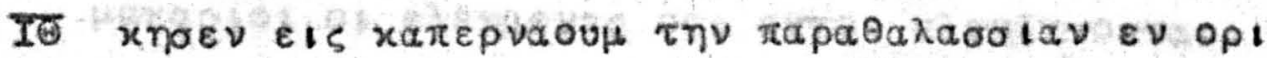

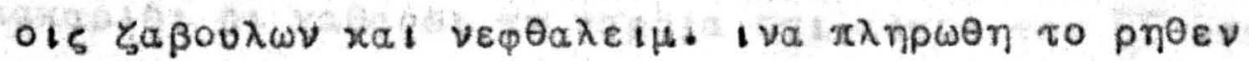

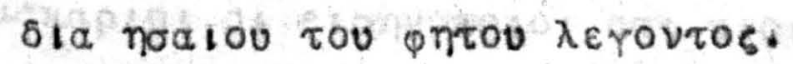

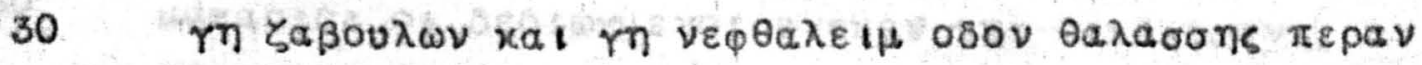

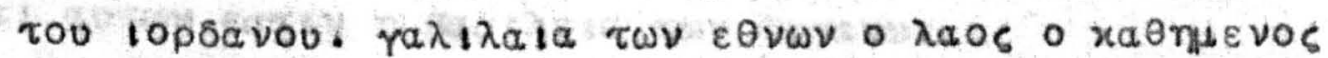

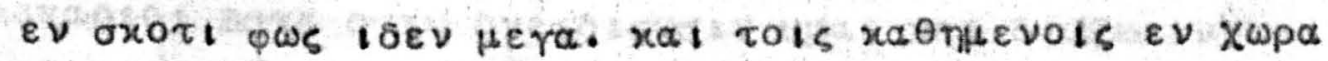

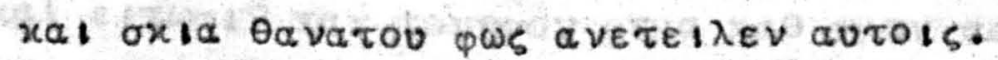

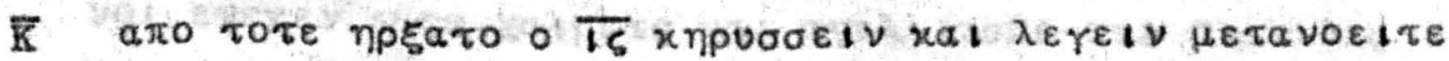
35

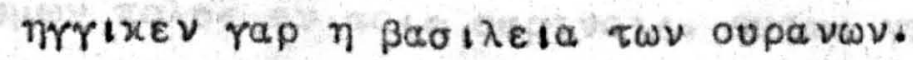

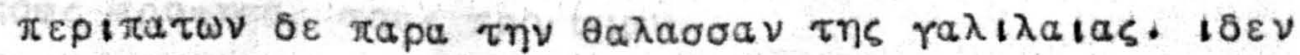

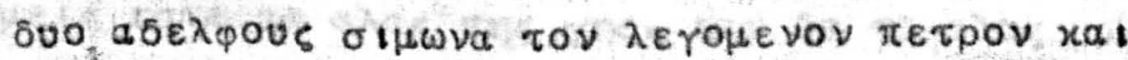

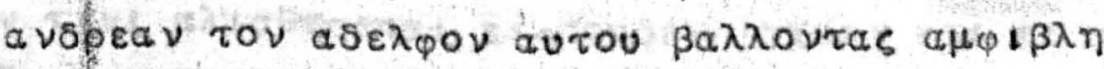

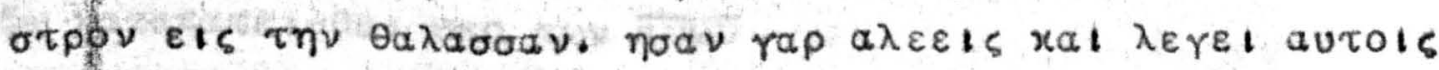

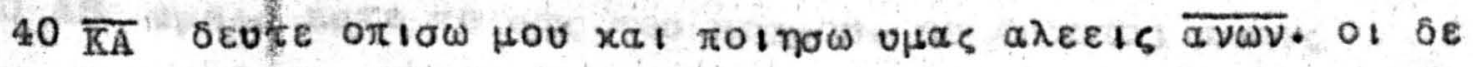

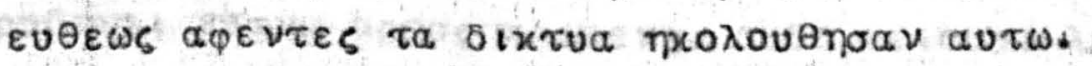




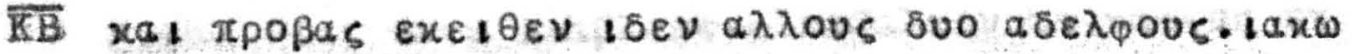

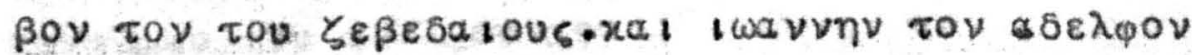

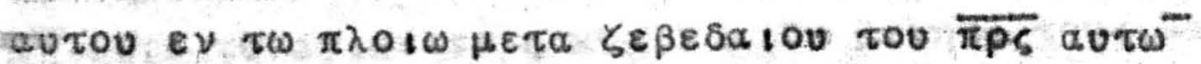

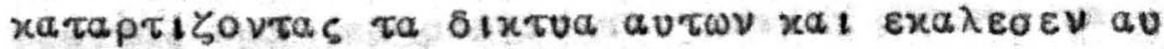

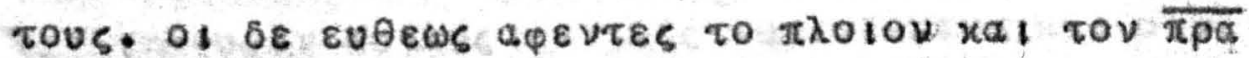

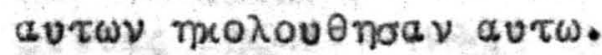

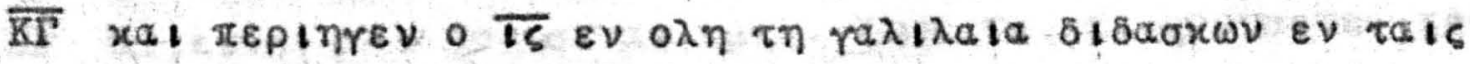

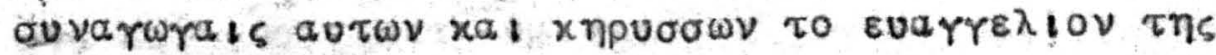

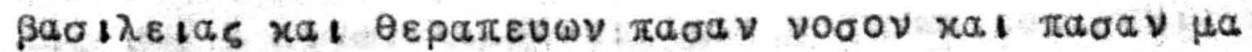

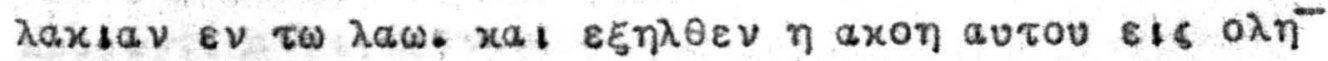

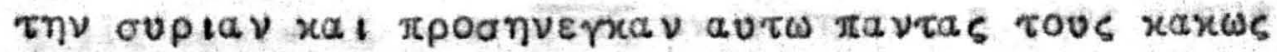

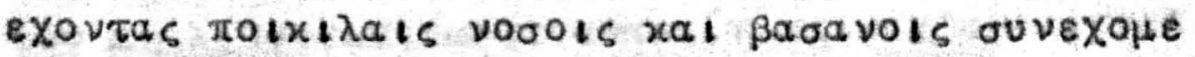

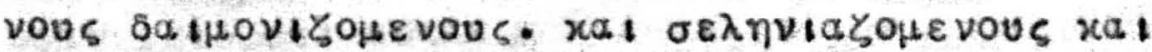

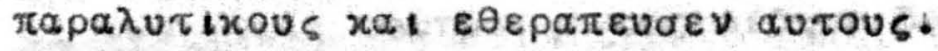

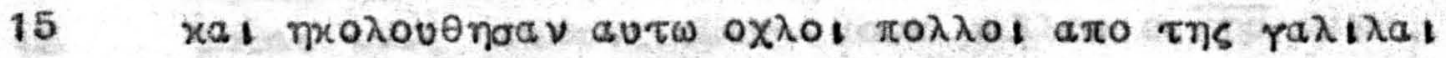

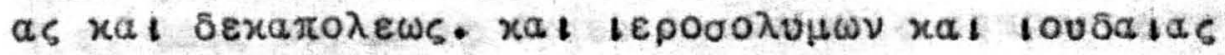
xat $x \varepsilon p a \nu$ tou topos vou.

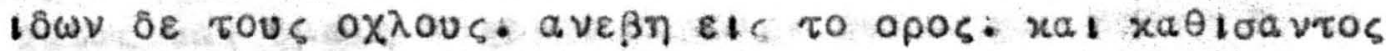

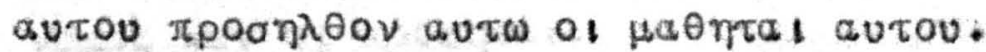

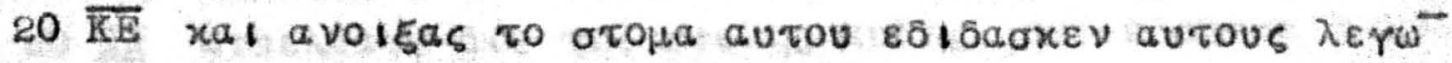

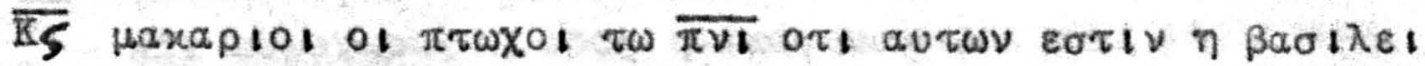
a $\tau \omega v$ oupavwv.

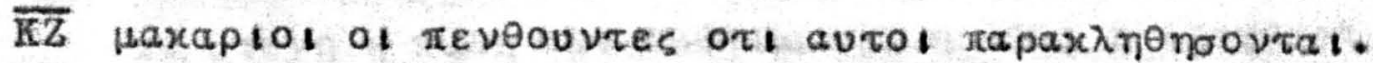

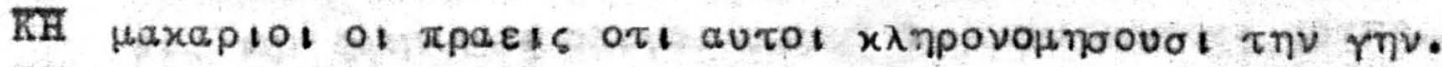

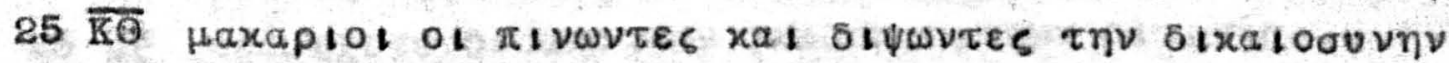

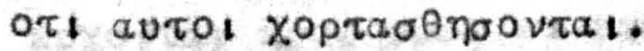

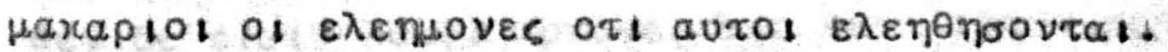

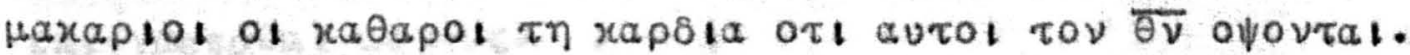

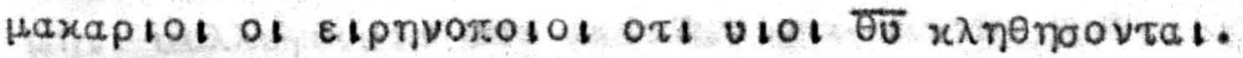

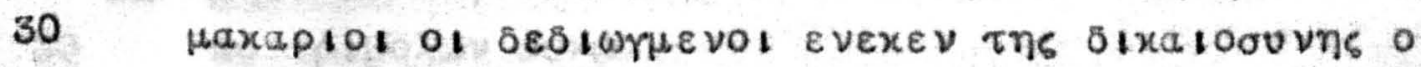

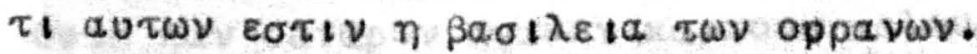

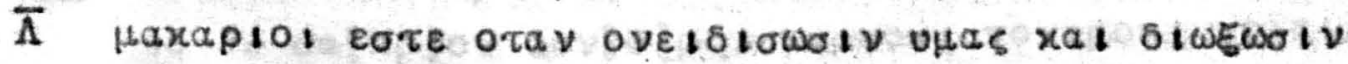

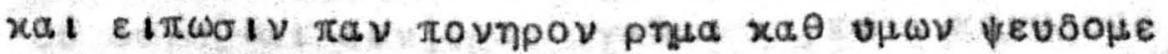

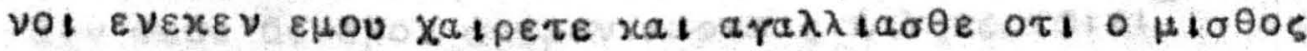

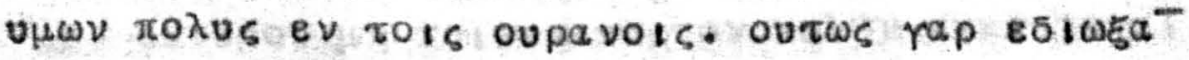

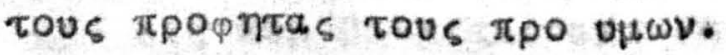

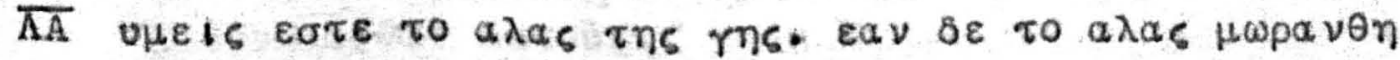

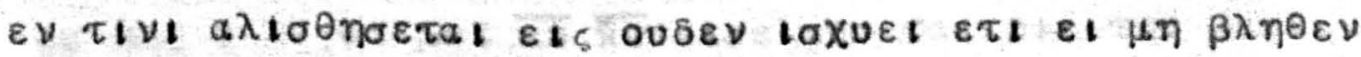

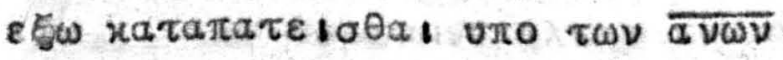

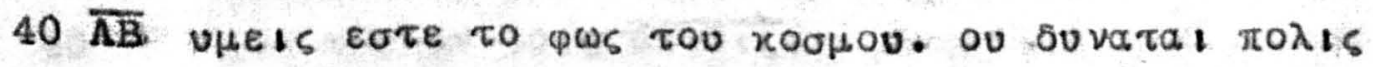

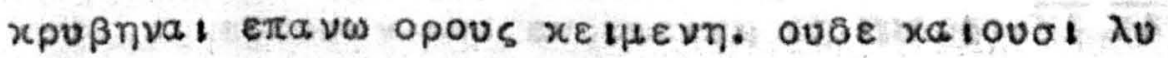




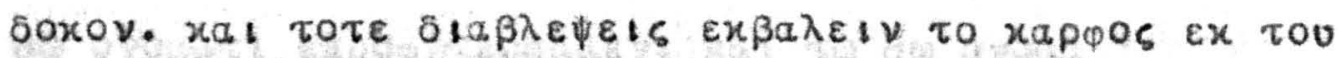

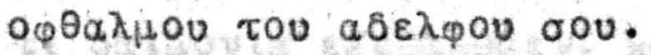

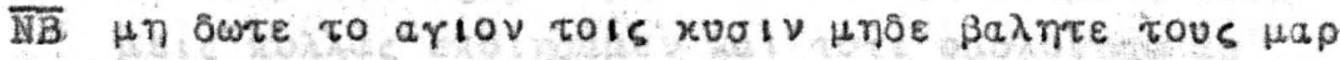

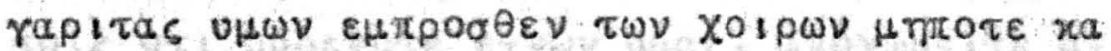

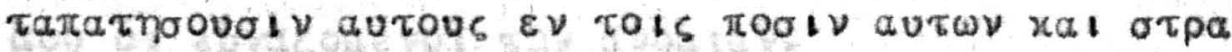

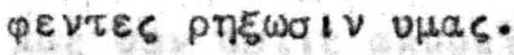

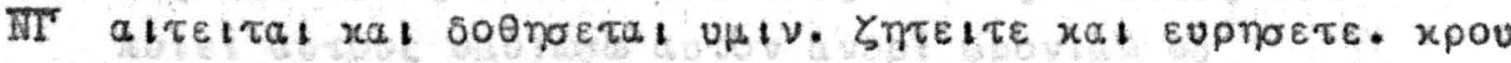

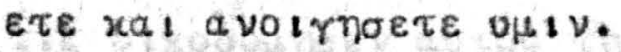

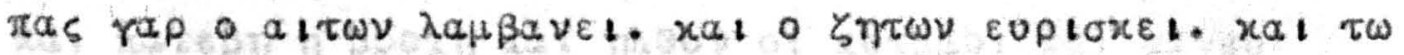
10 xpovovi avolrnoetal.

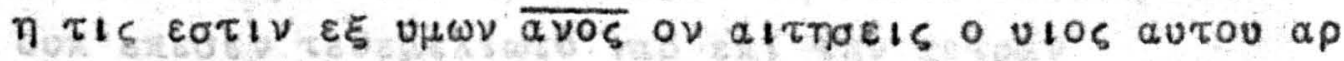

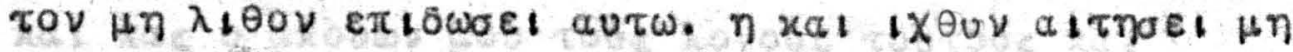

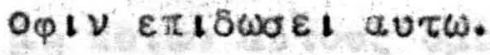

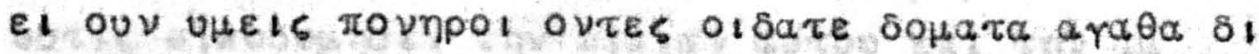

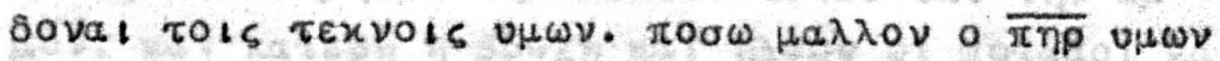

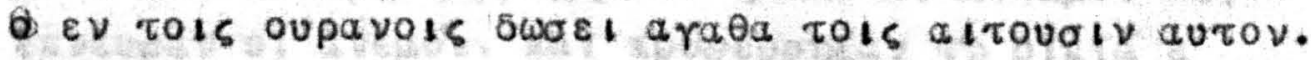

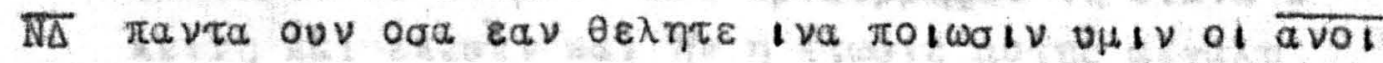

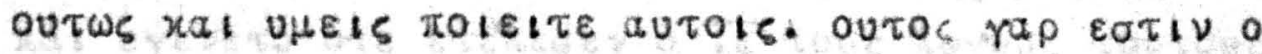
VOHOS xal of $\pi p \circ p \eta \tau \alpha_{1}$.

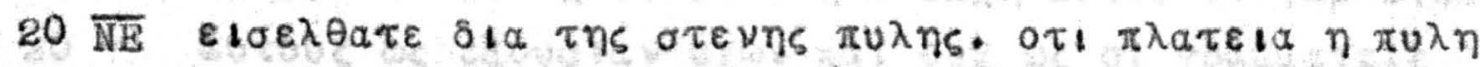

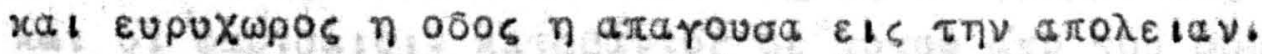

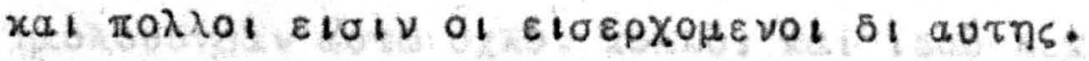

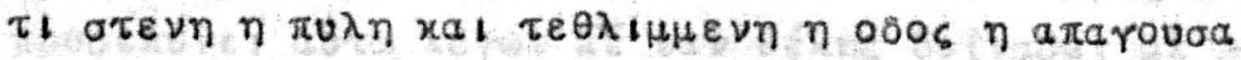

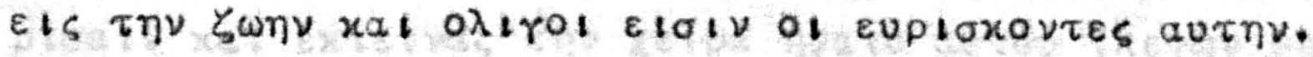

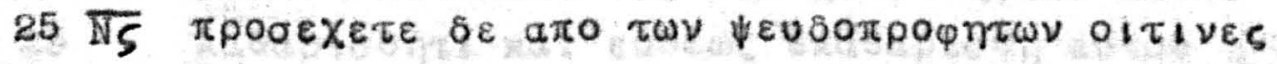

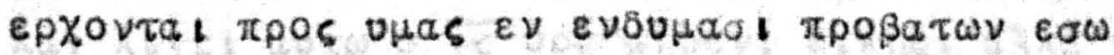

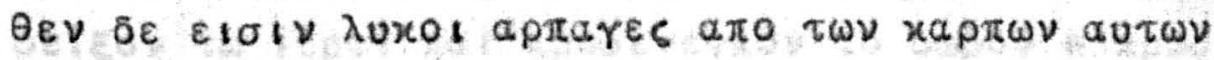

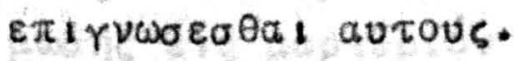

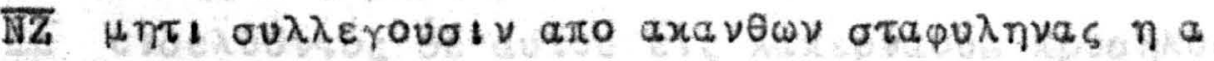
To $\tau p \perp \beta O \lambda \omega v$ ovxa.

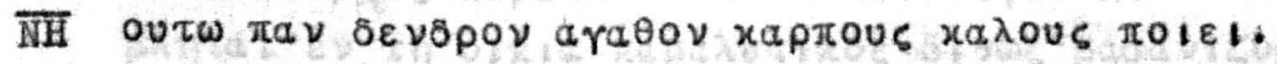

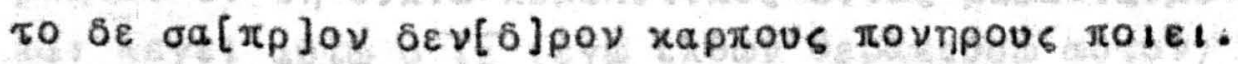

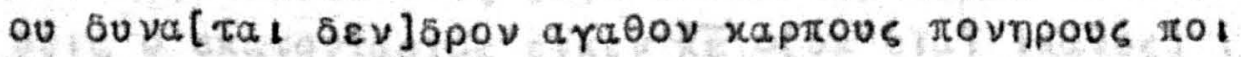

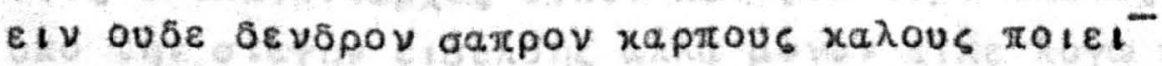

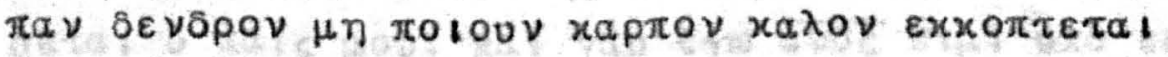

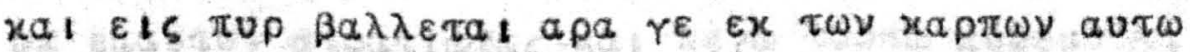
$\varepsilon \pi \imath \gamma \nu \omega \sigma \varepsilon \sigma \theta a$ autous.

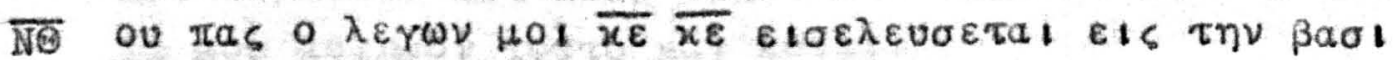

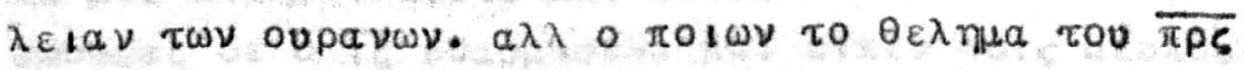
40 HOV TOU $\varepsilon V$ TOLS OUVOIS.

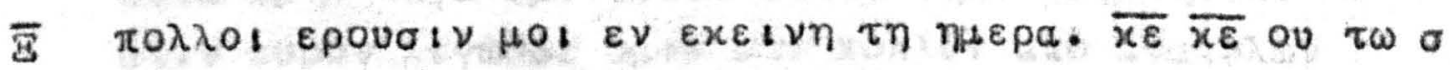




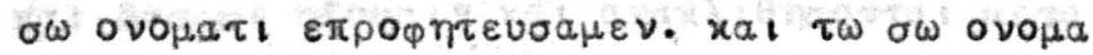

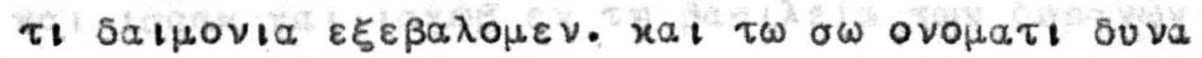

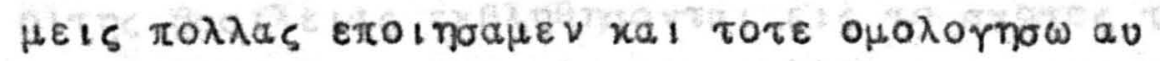

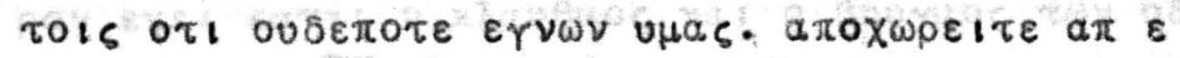

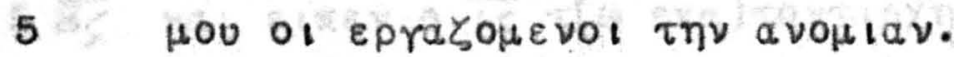

इA sas ouv ootis axovel pov tous dorous toutous $x a l$

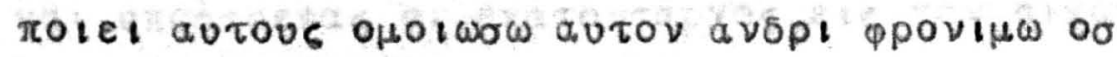

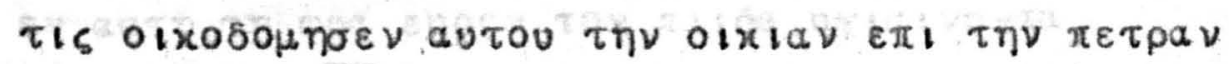

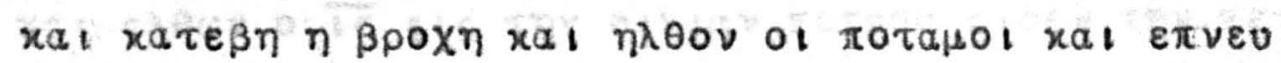

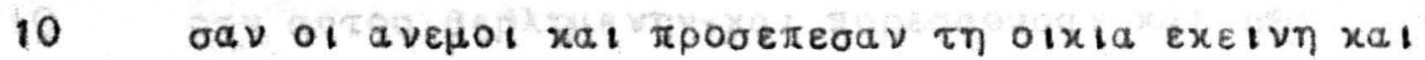
oux $\varepsilon \pi \varepsilon \sigma \varepsilon \nu \tau \varepsilon \theta \varepsilon \mu \varepsilon \lambda l \omega \tau \sigma$ rap $\varepsilon \pi \imath \tau \eta \nu \pi \varepsilon \tau \rho \alpha \nu$ xal $\pi a_{5}$ o axouw Hou tous doyous toutous $x_{1}$

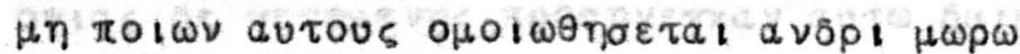

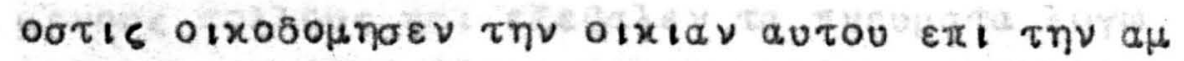

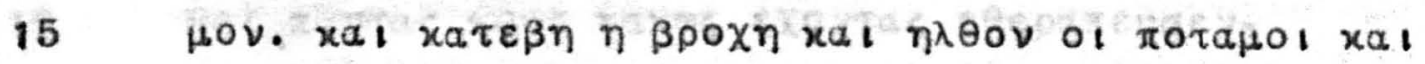

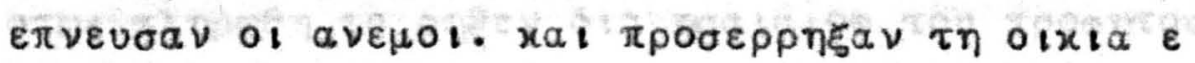

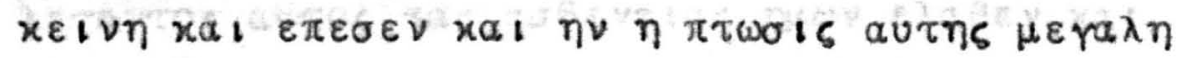

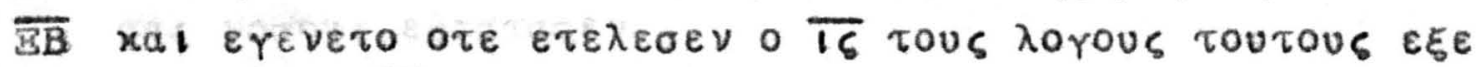

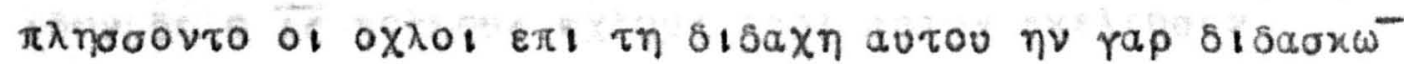

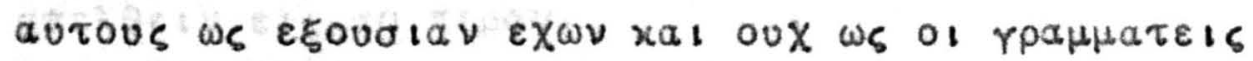

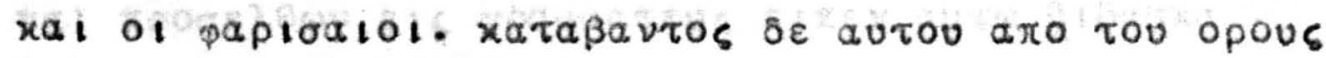

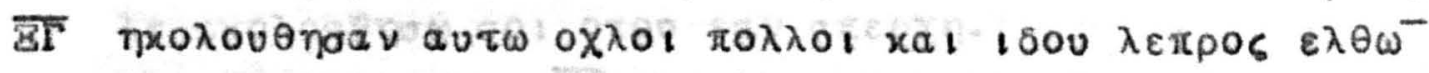

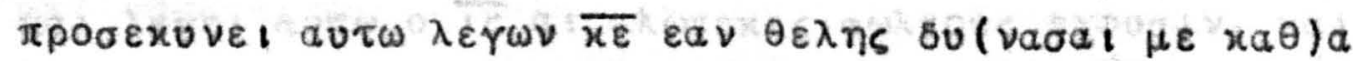

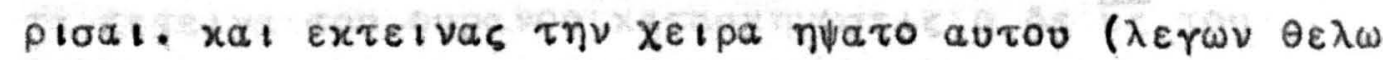

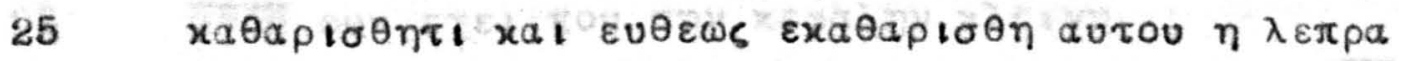

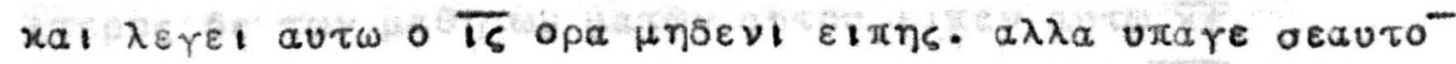

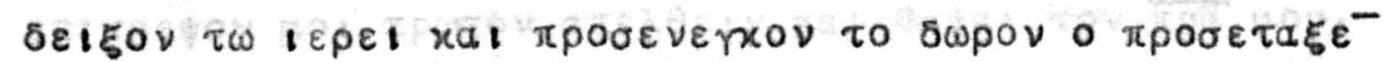
Hwans E Is Haptupiov autois.

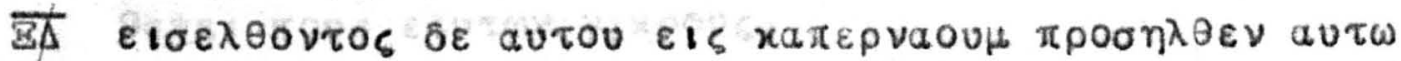

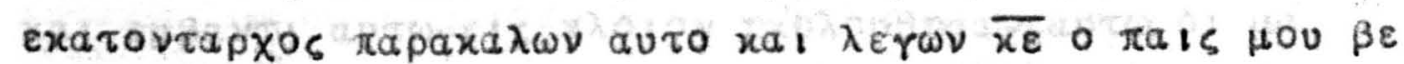

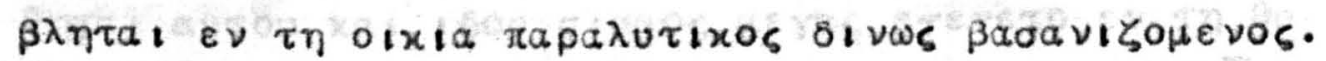

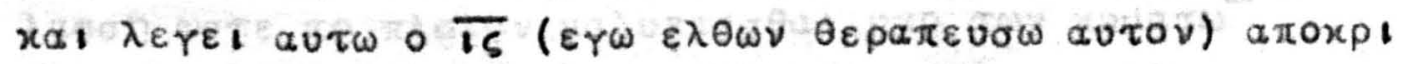

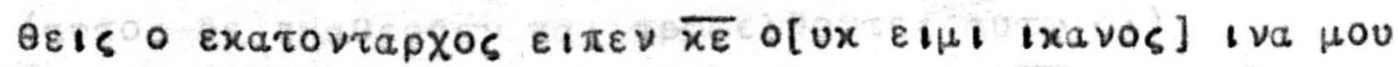

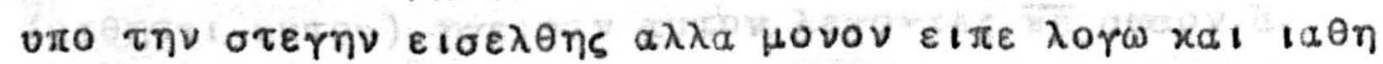

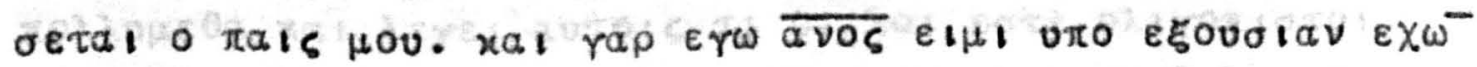

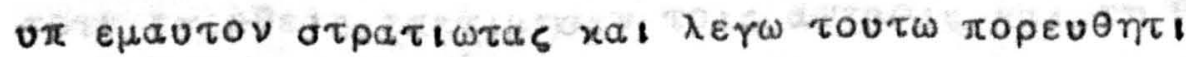

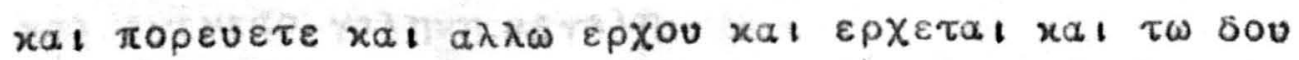

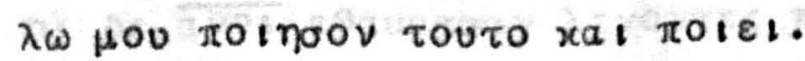

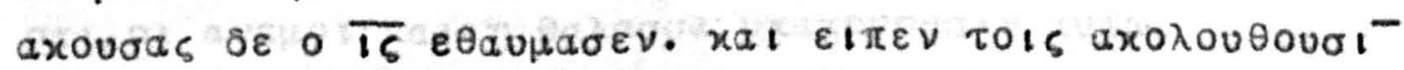

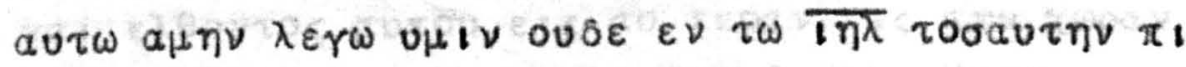

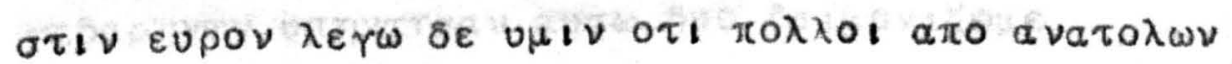




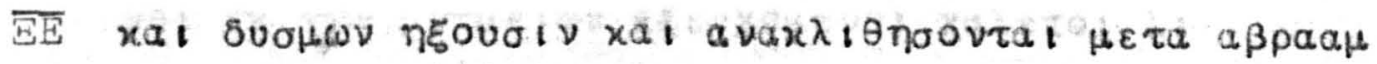

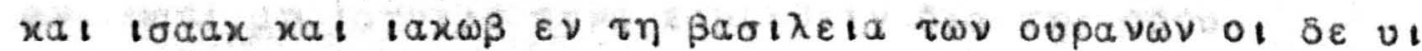

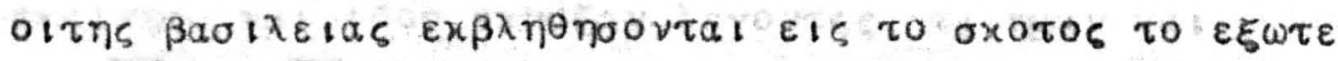

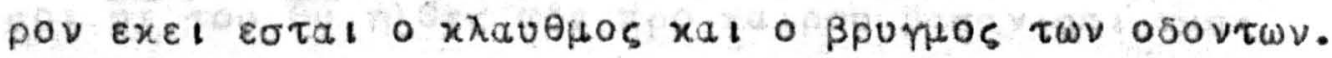

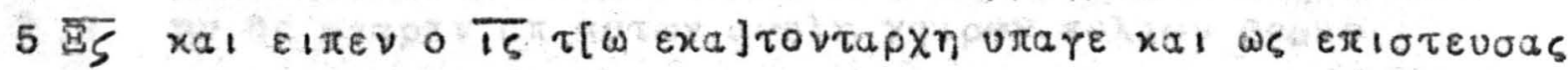

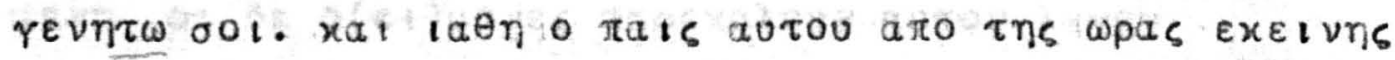

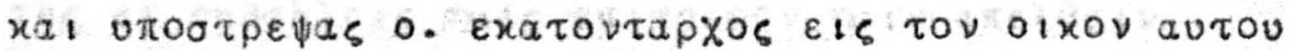

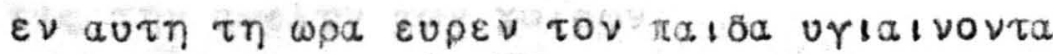

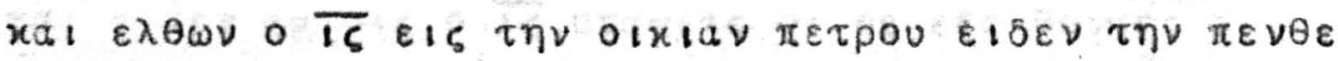

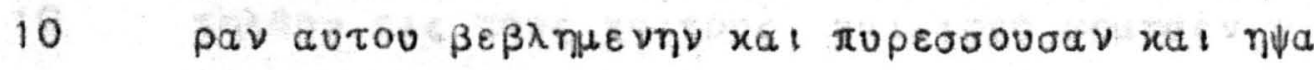

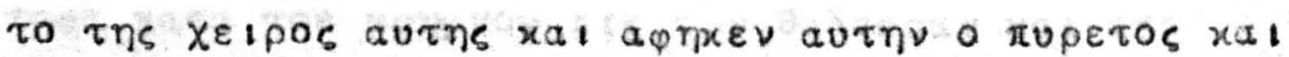

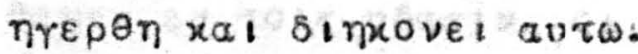

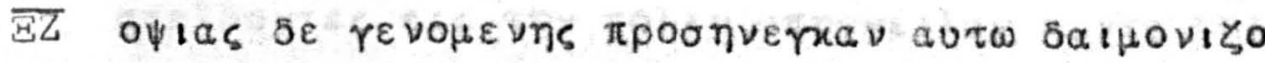

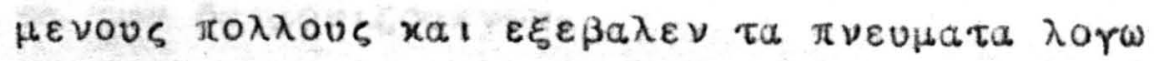

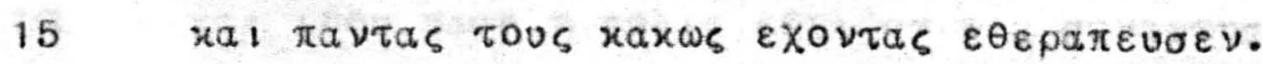

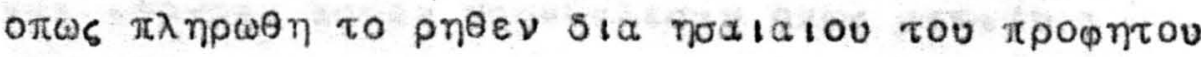

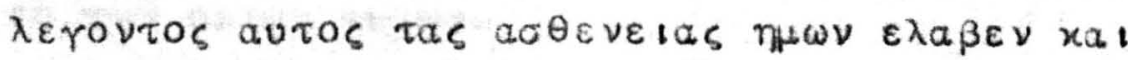

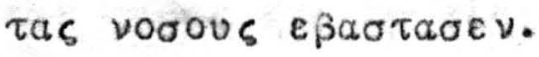
$1 \delta \omega \nu$ $5 \varepsilon \circ \overline{L \zeta} \pi 0 \lambda \lambda$ OU $20 \quad a \pi \varepsilon \lambda \theta \varepsilon \downarrow \nu \varepsilon i s$ to $\pi \varepsilon p a \nu$.

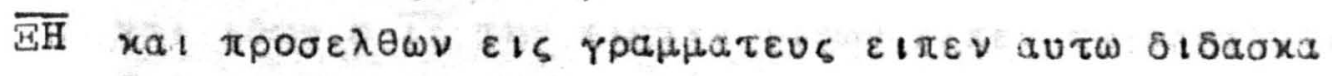

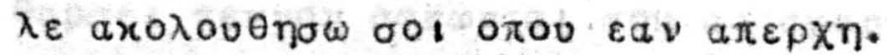

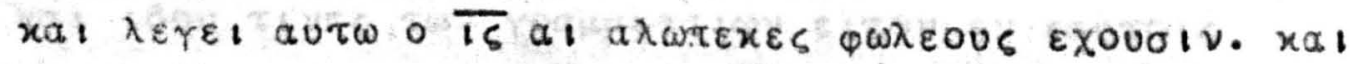

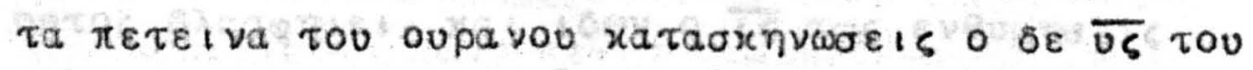

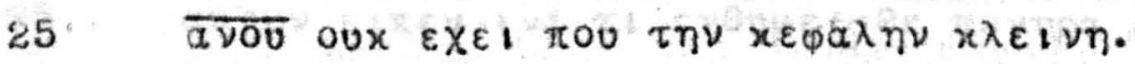

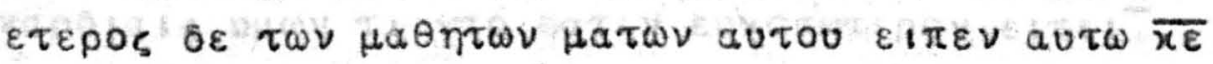

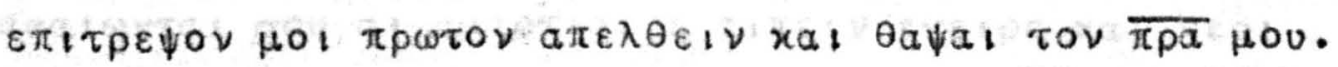

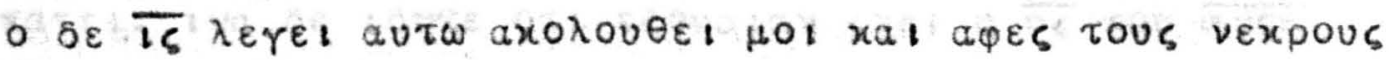

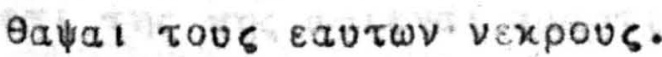

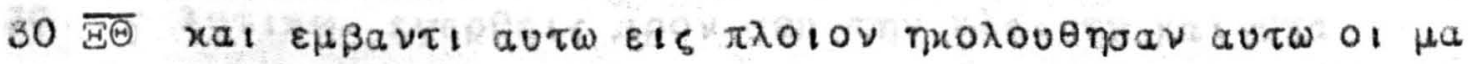

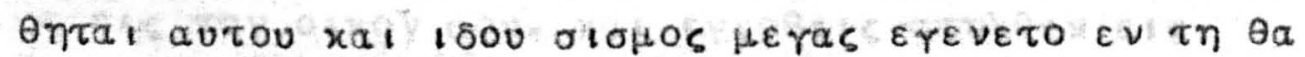

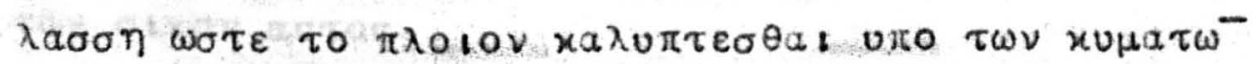
(

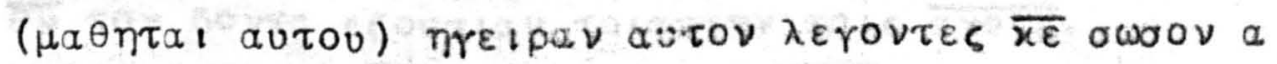

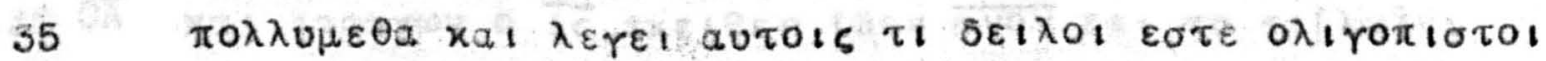

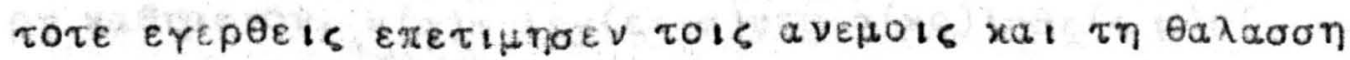
xal $\varepsilon \gamma \varepsilon \nu \varepsilon \tau o$ $\gamma a \lambda \eta \eta \eta \eta$

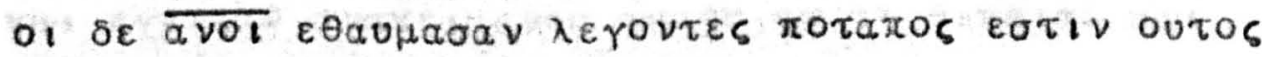

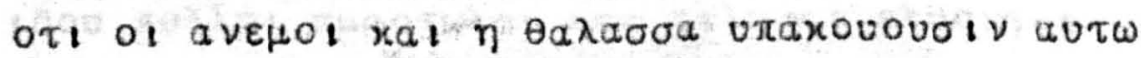

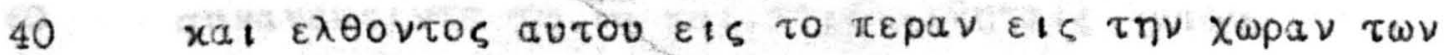

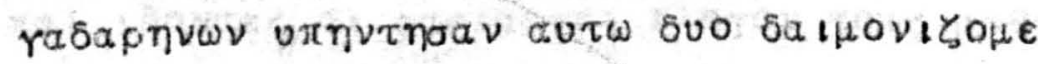




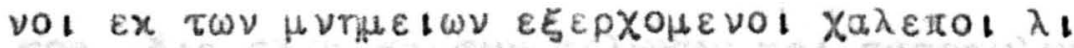

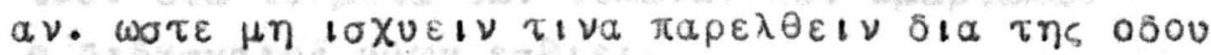

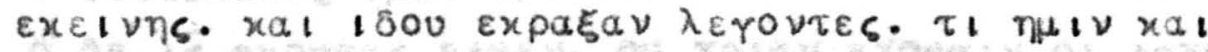

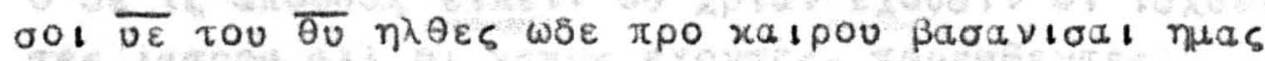

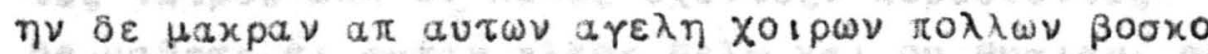

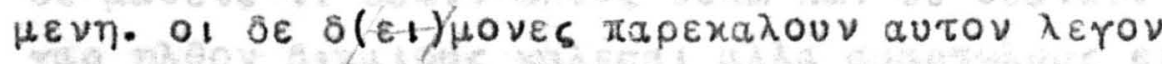

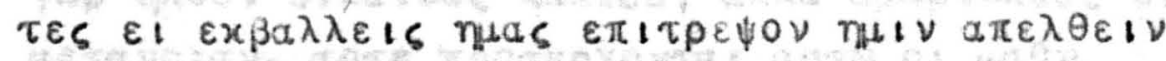

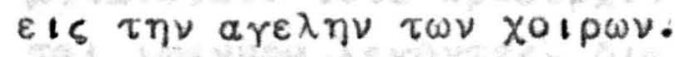

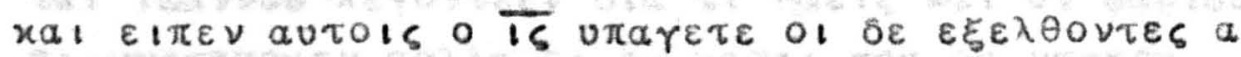
$10 \pi \eta \lambda \theta 0 \nu$ \& 15 tous xoltous. xal 1500 wpH

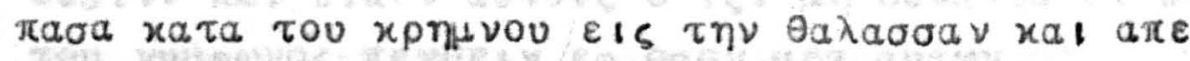

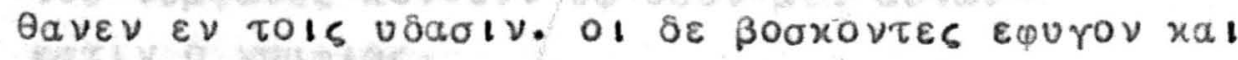

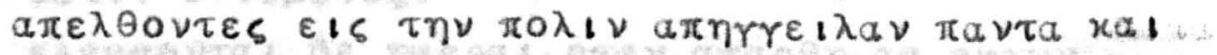

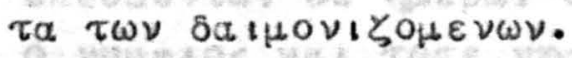

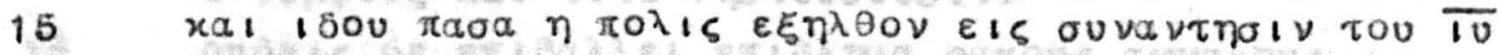

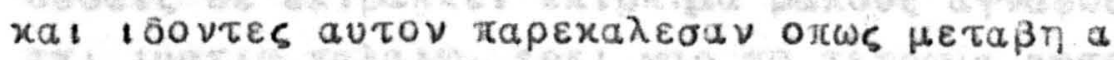
$\pi 0 \tau \omega \nu$ opl $\omega \nu$ a $\tau \tau \omega \nu$.

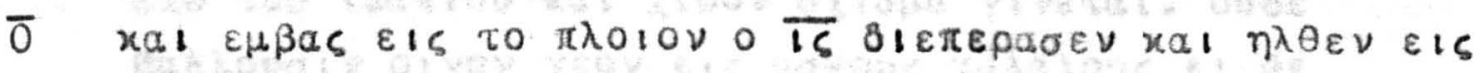

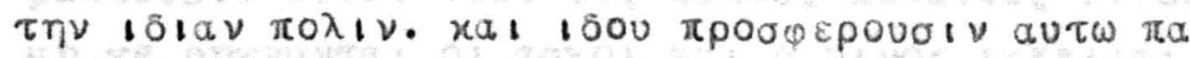

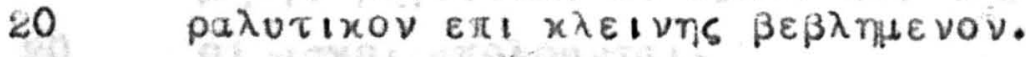

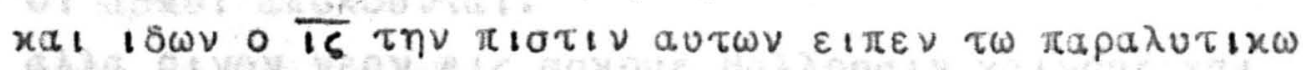

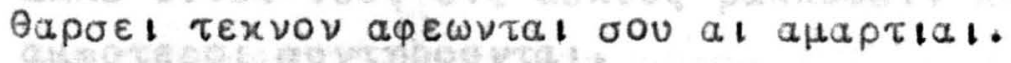

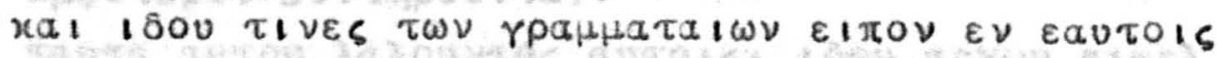

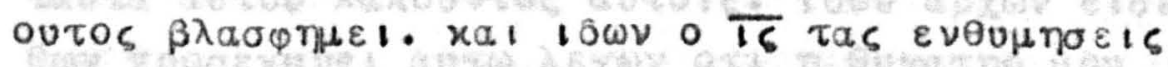

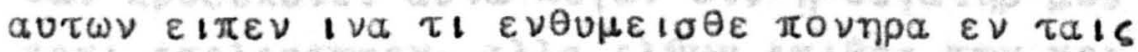

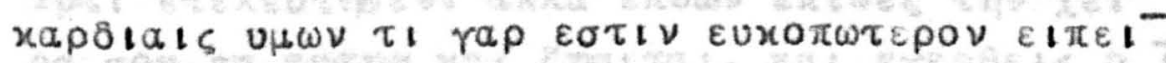

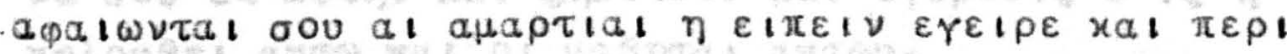

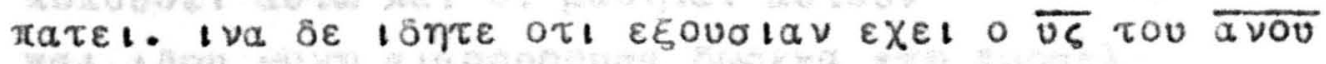

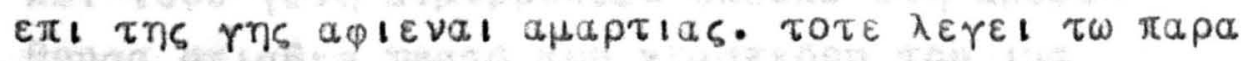

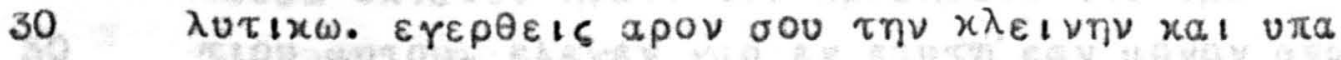

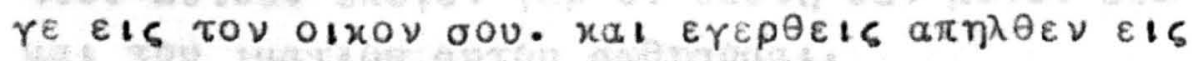
TOV olxov autov.

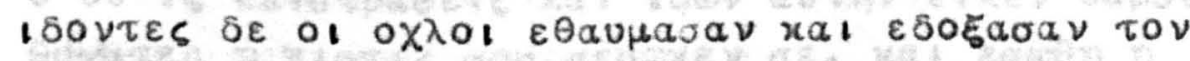

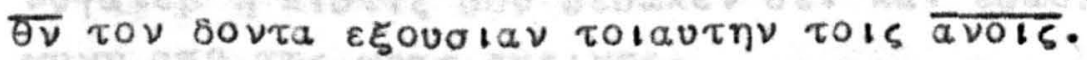

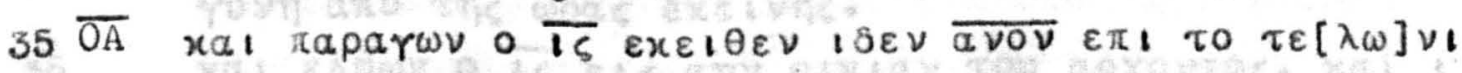
ov $x a \theta \eta \mu \varepsilon v o v . x(\alpha), \lambda \varepsilon \gamma(\varepsilon, \alpha) \cup \tau \omega$ a $(x \circ) \lambda v \theta \varepsilon(1 \mu) \circ(1 \quad x a l$

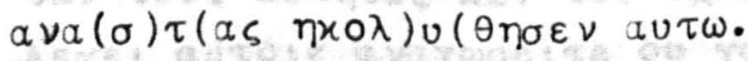

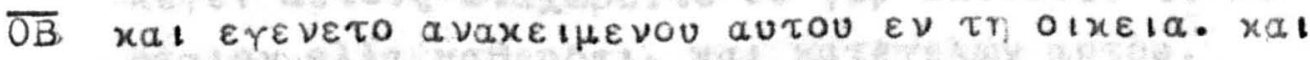

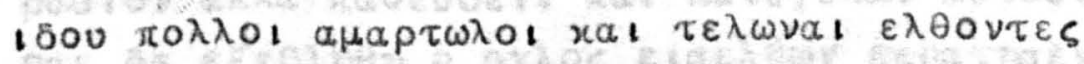

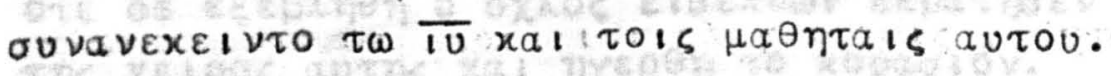

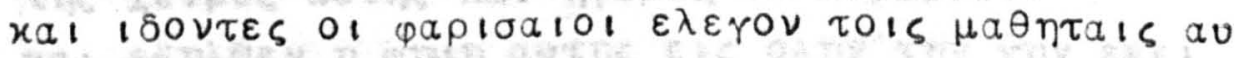




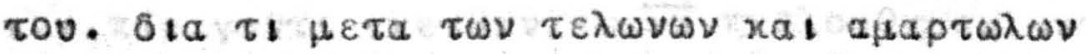
-

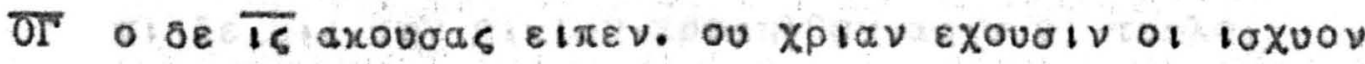

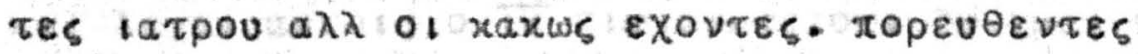

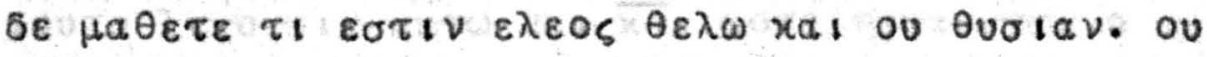

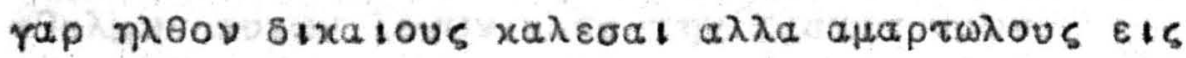

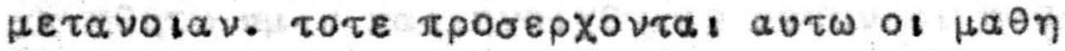

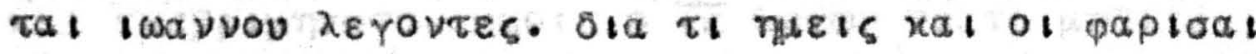

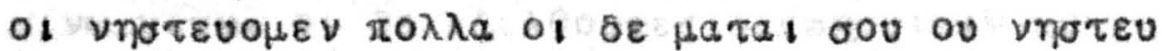

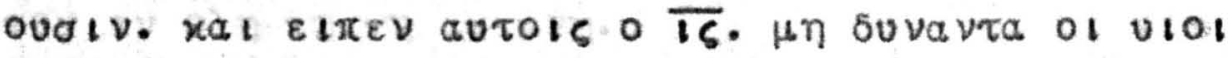

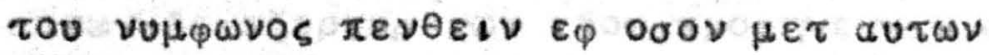

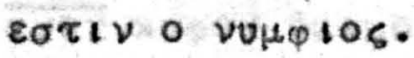

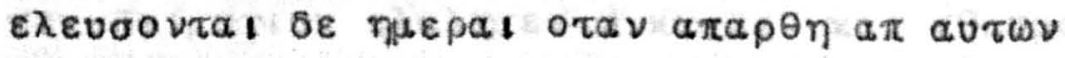

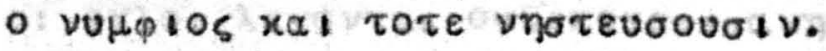

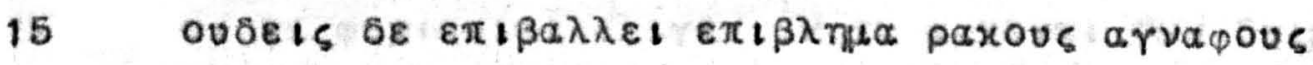

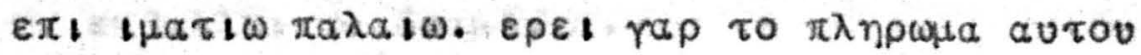

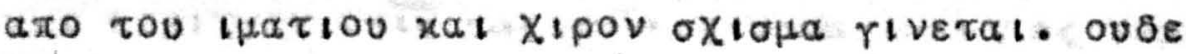

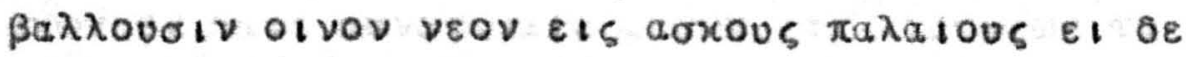

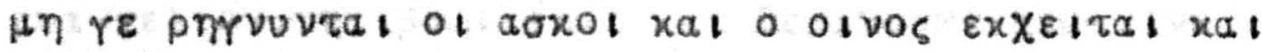
20 ol aбxol aroxouvtal.

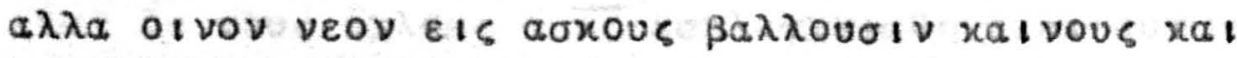

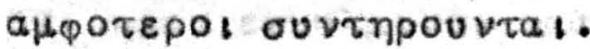

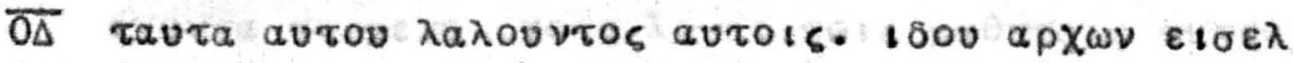

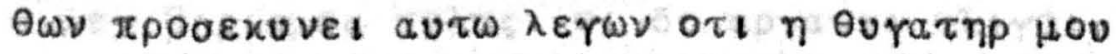

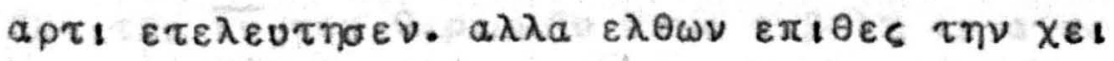

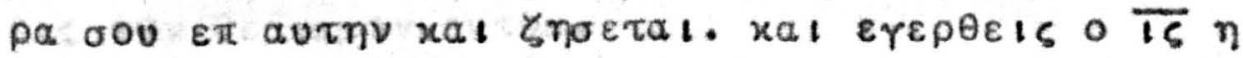

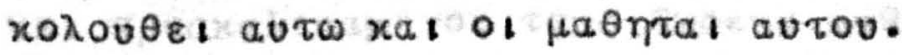

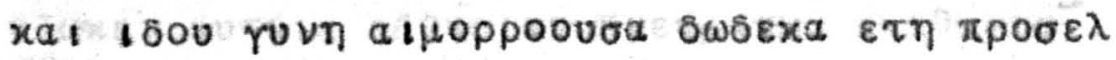

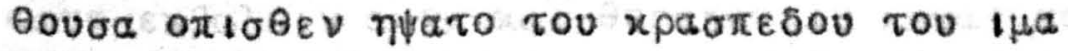

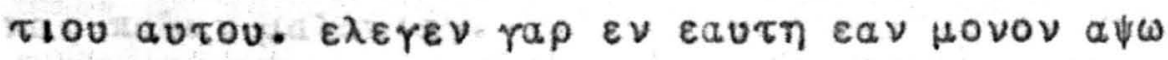

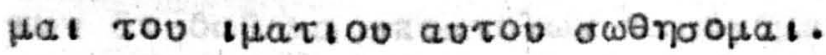

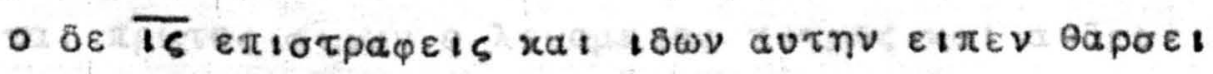

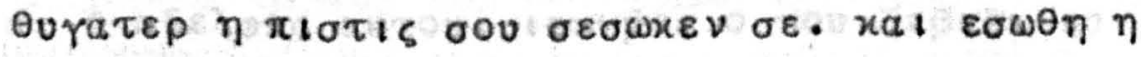

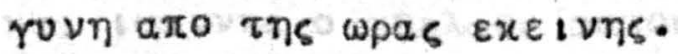

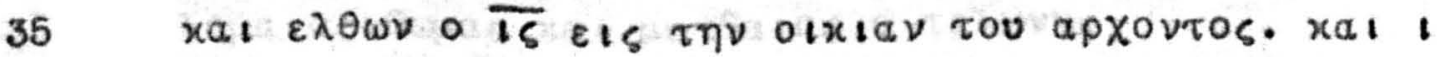

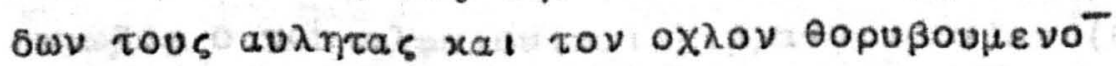

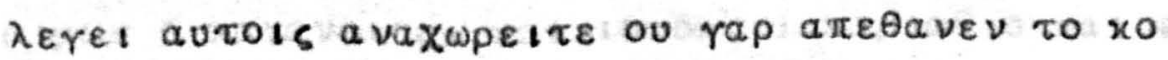

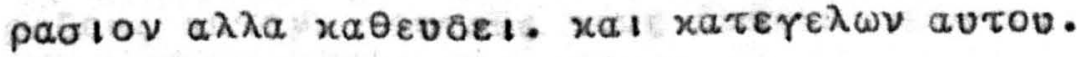

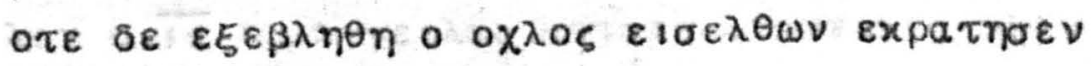

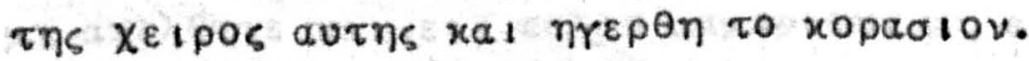

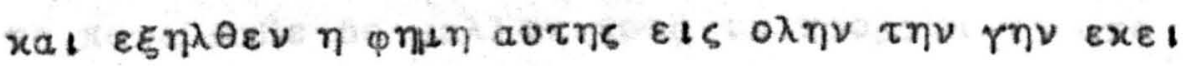




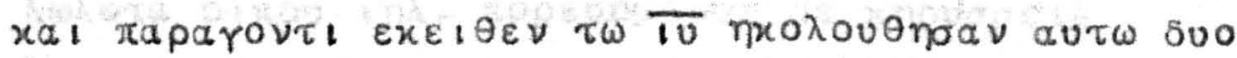

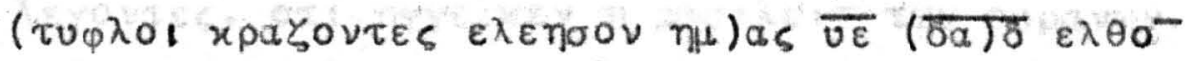

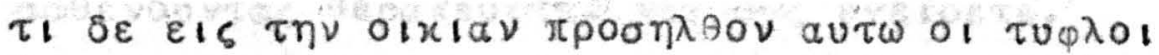

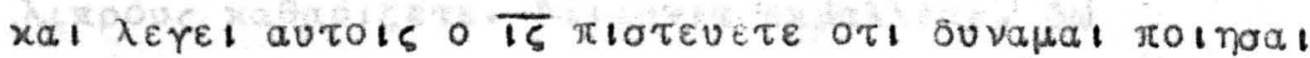

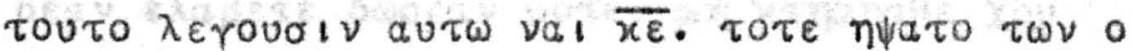

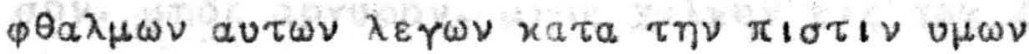

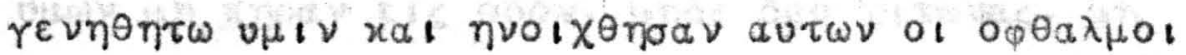

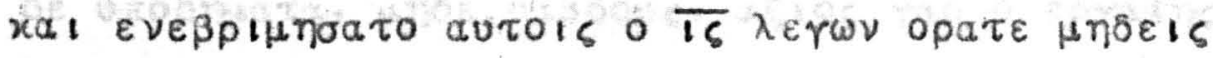

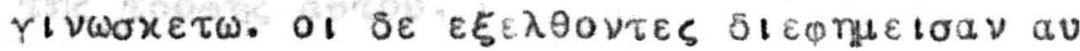

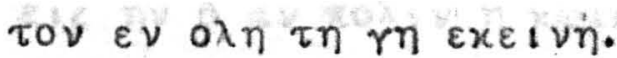

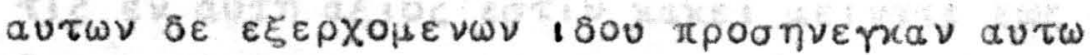

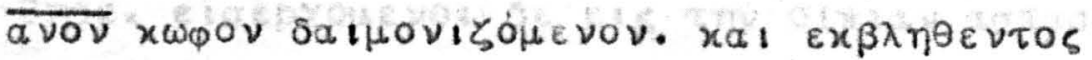

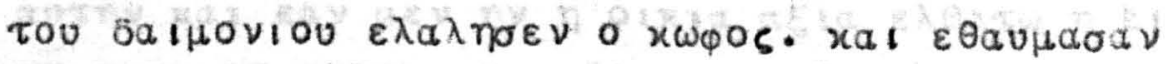

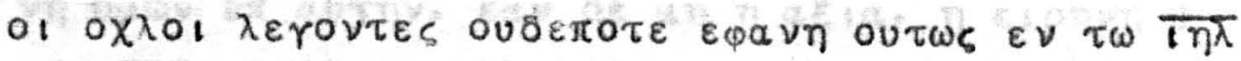

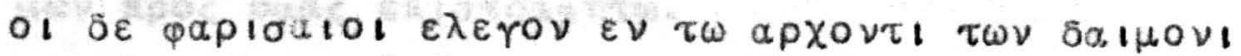

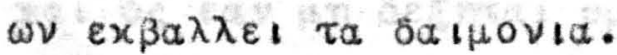

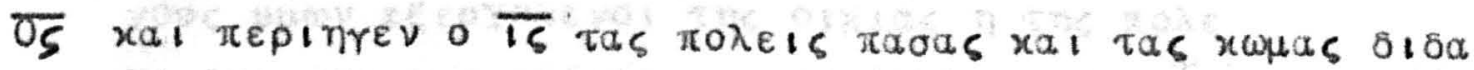

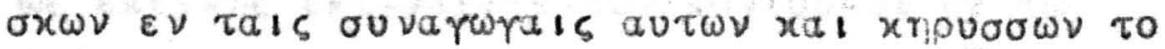

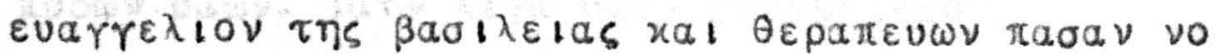

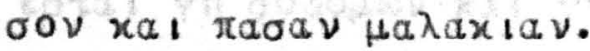

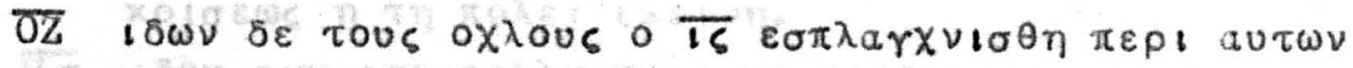

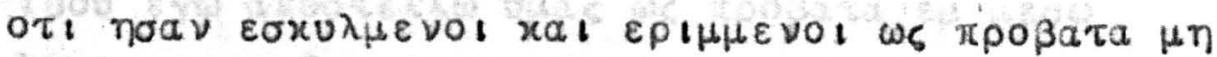

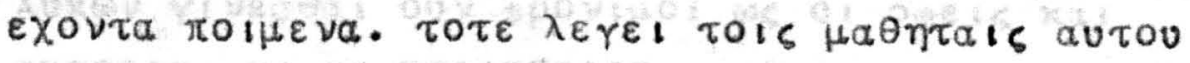

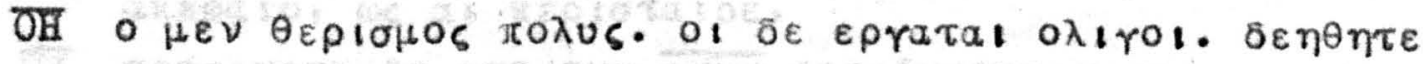

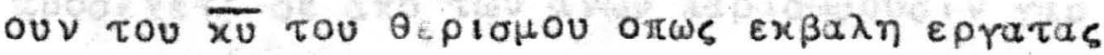

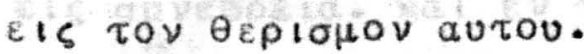

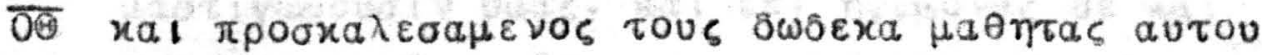

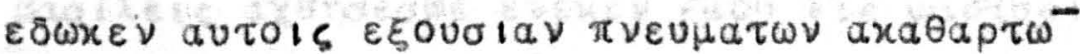

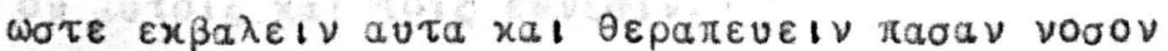

30 II $x a \uparrow \pi a \sigma a \nu \mu a \lambda a x\llcorner a \nu$.

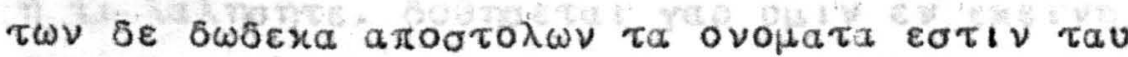

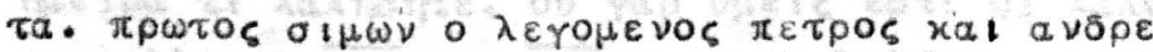

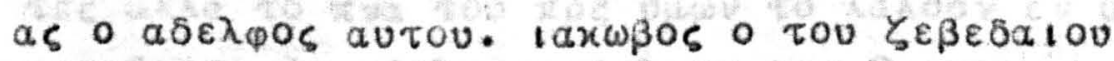

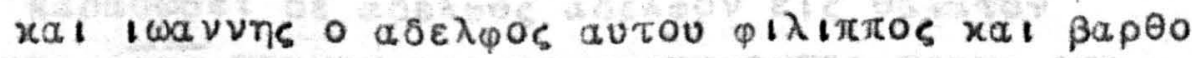

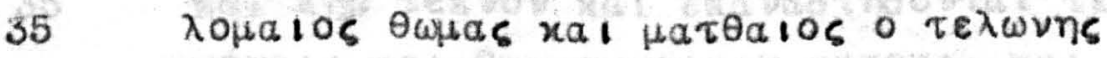

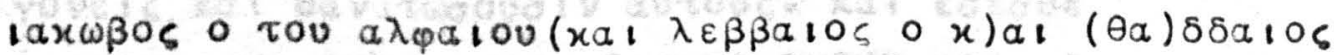
$\sigma l \mu \omega \nu$ o xavavalos. $x a l$ lovbas $l \sigma x a p l \omega \theta$ o xal $\pi a$

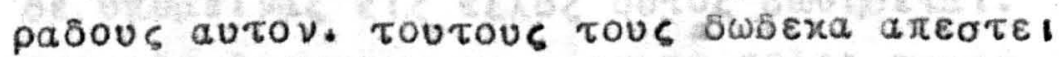

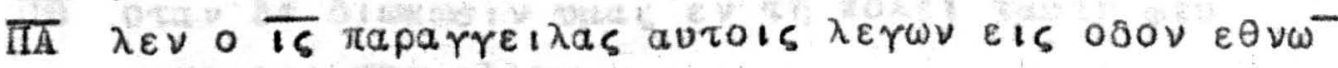
40

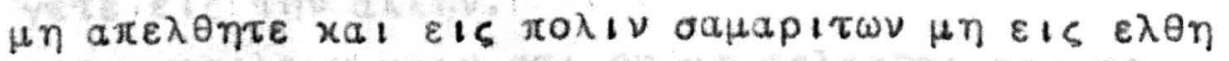

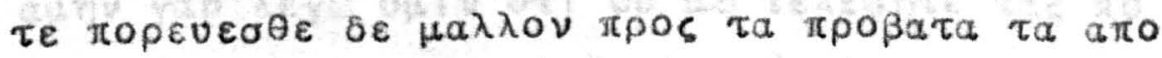




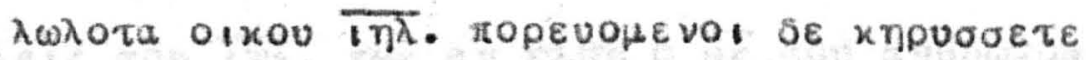

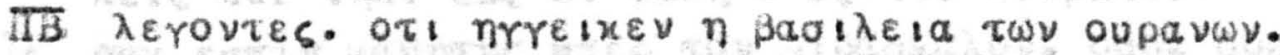
a

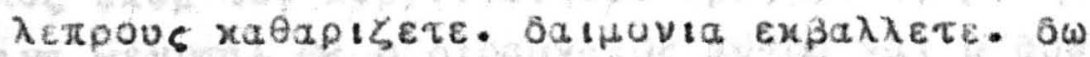

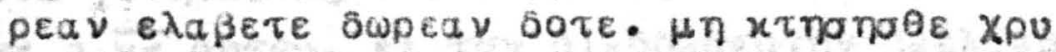

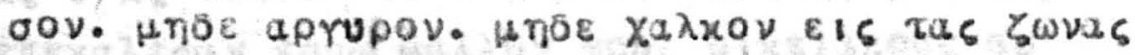

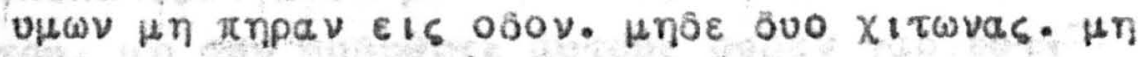

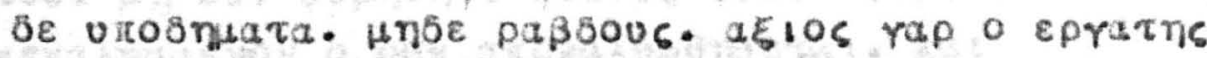

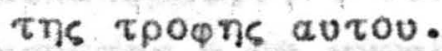

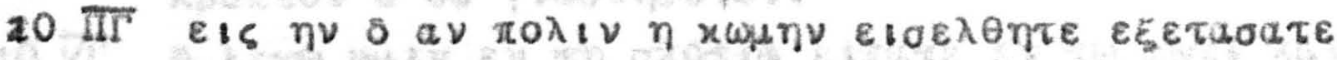

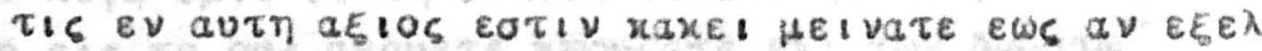

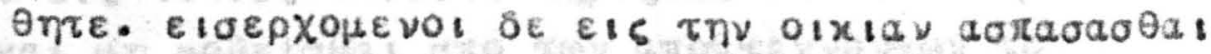

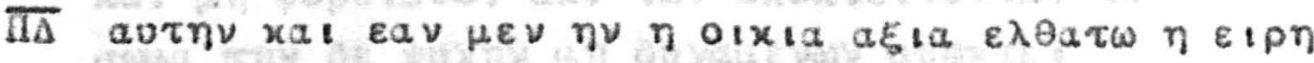
v

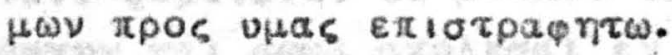

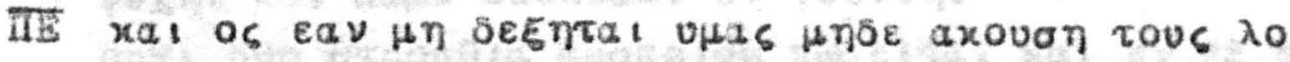

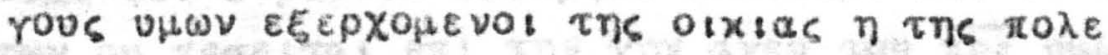

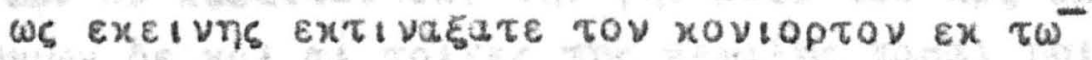

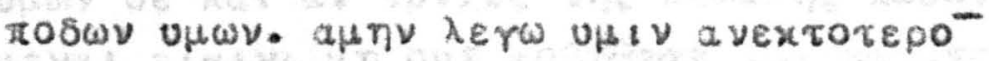

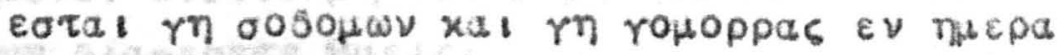

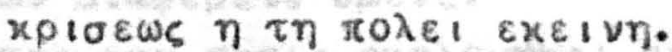

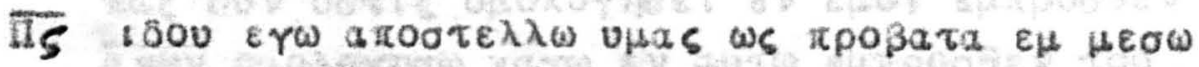

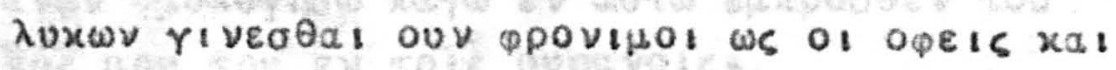

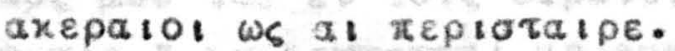

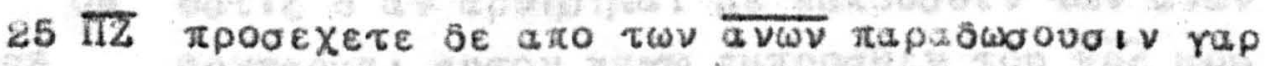
Eis ouve $3 \rho e_{1 a}$. xal ev tais ouvarwrais autw

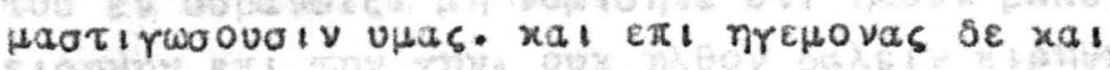

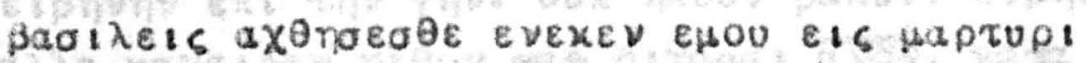

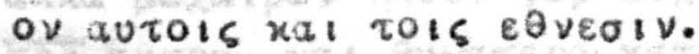

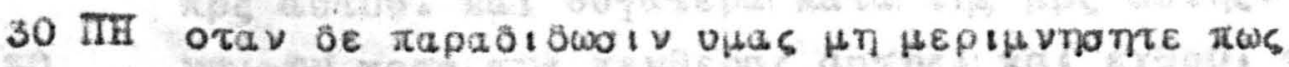

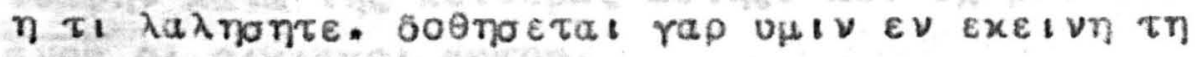

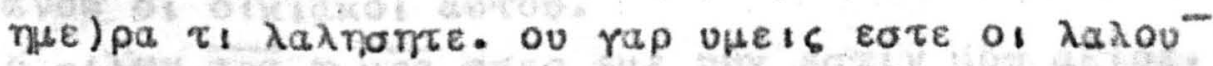

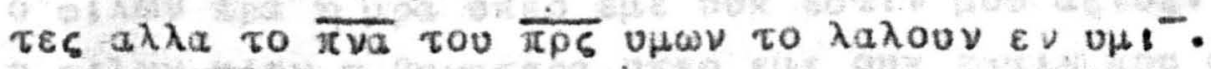

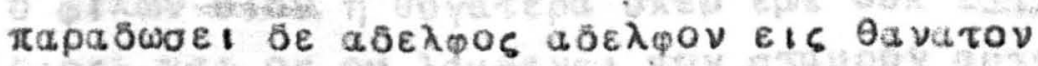

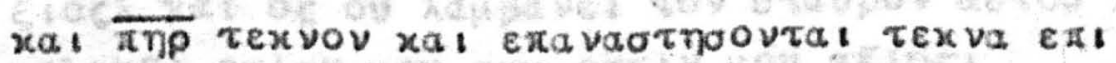

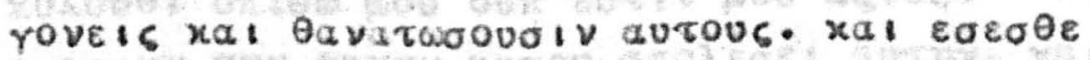

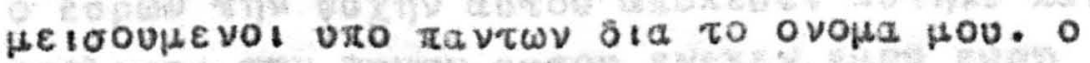

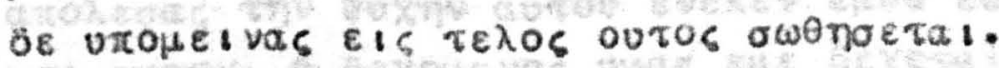

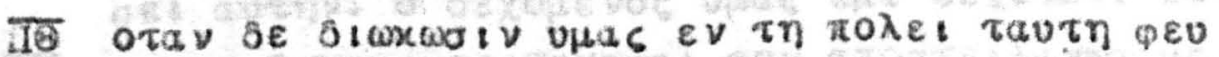

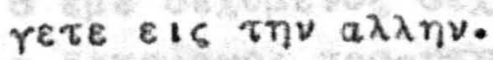

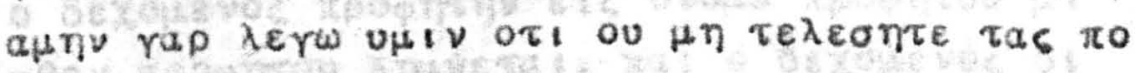




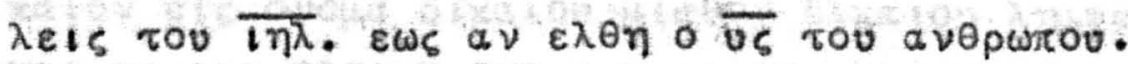

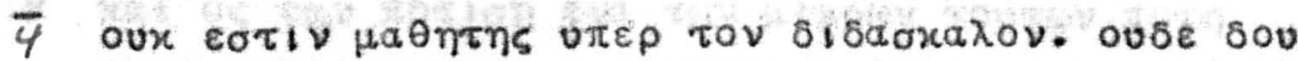

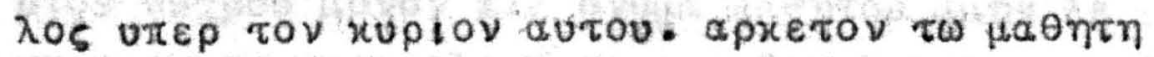

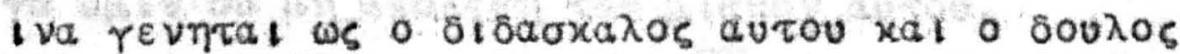

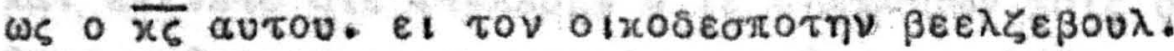

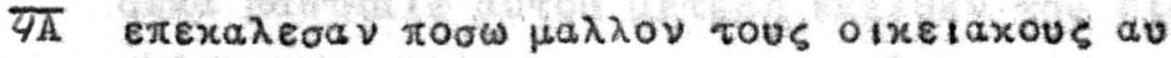

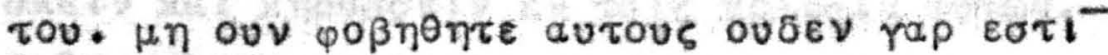

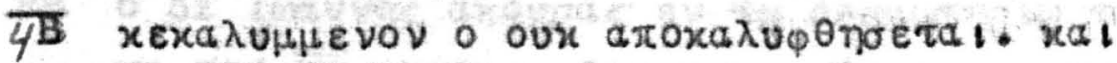

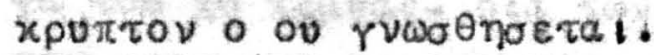

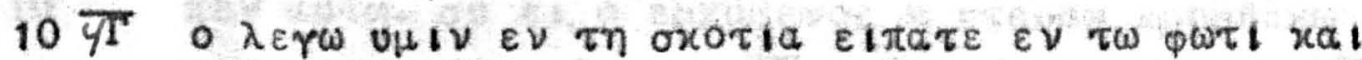

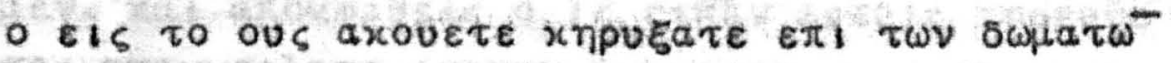

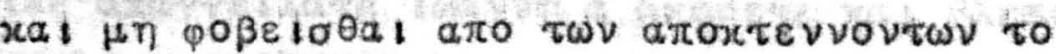

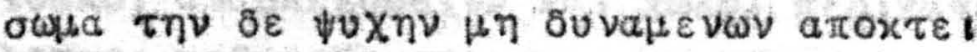

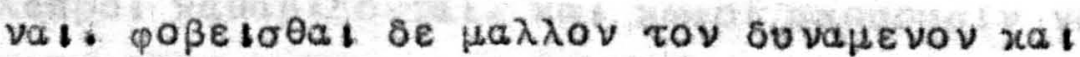

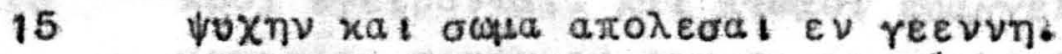

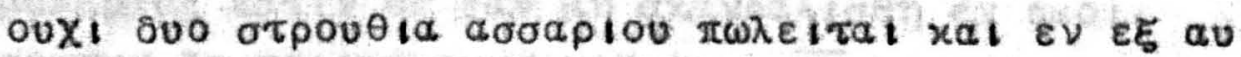

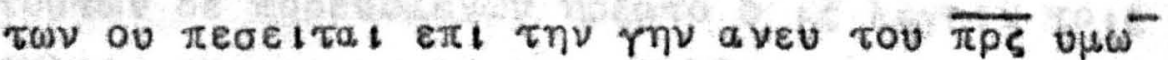

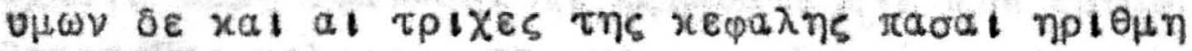

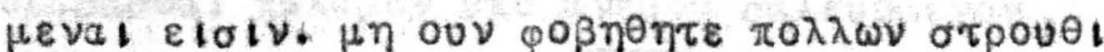

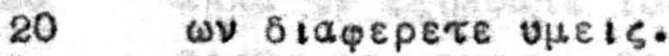

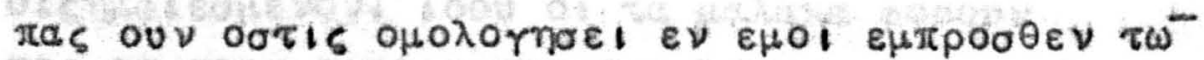

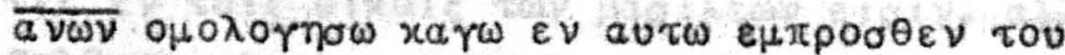
$\bar{\pi} \rho \zeta$ Hov tov $\varepsilon \nu$ tols oupavols.

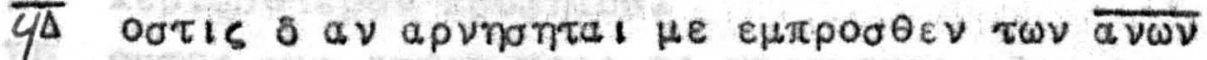

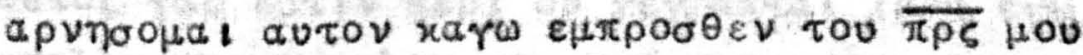

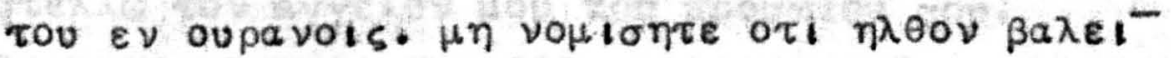

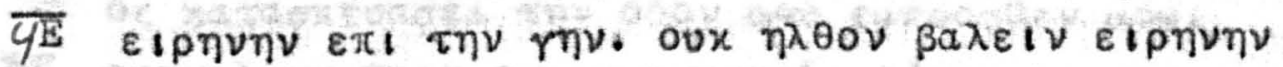

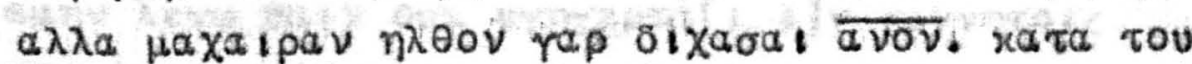

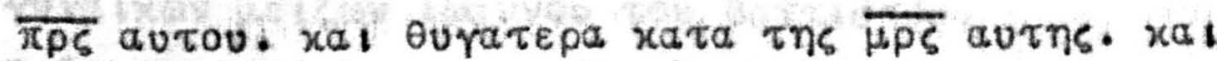

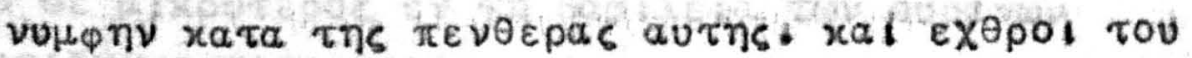
avov ot otxtaxol autov.

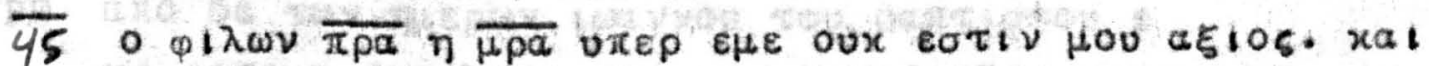

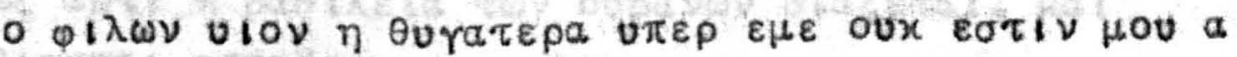

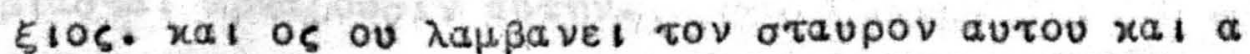

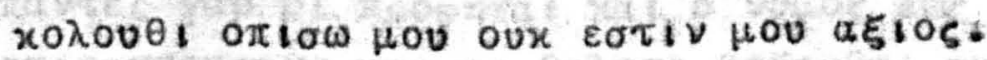

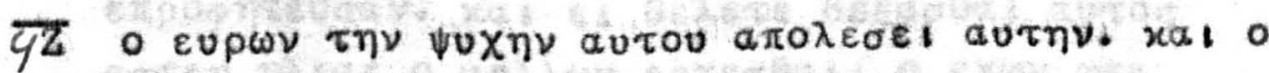

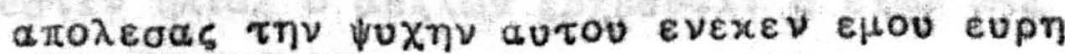

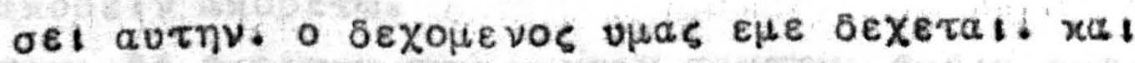

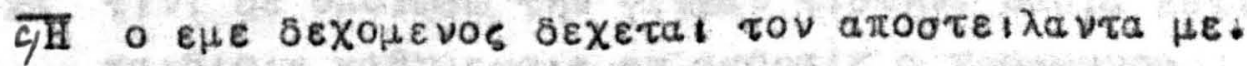

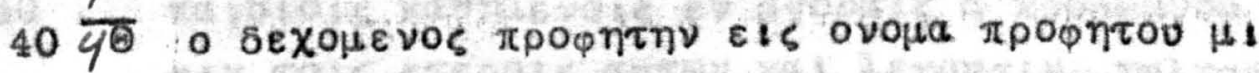

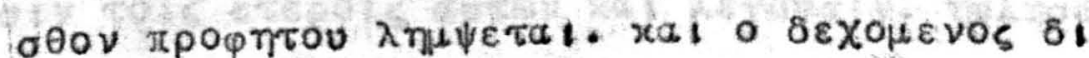




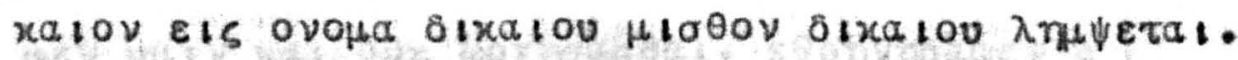

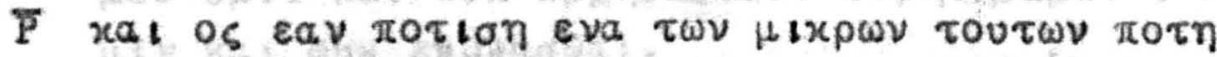

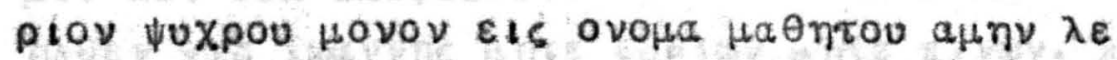

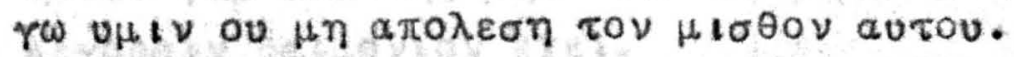

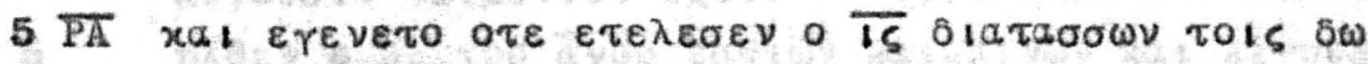

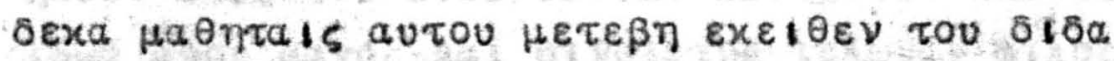

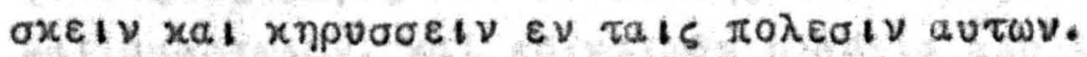

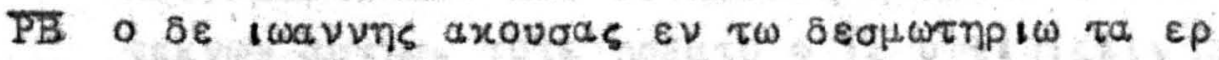

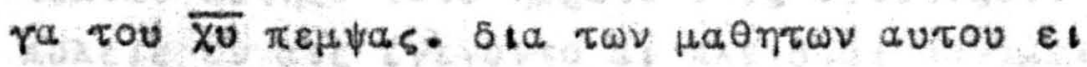

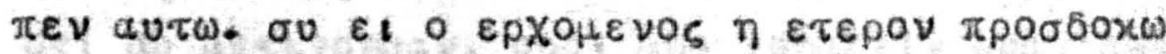

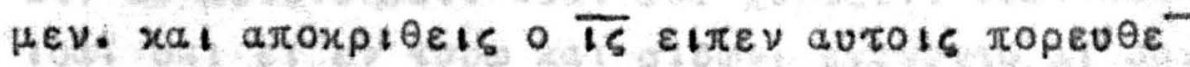

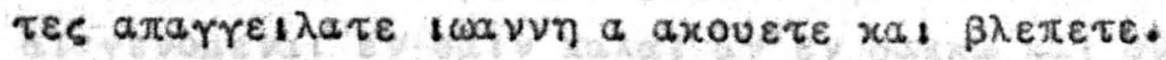

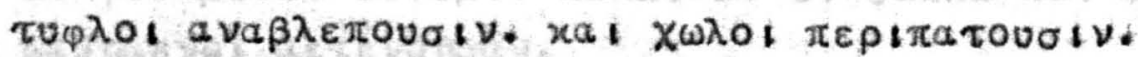

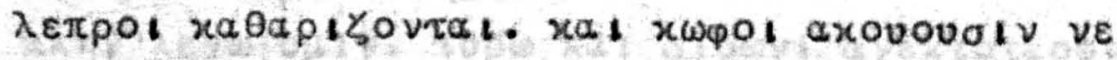

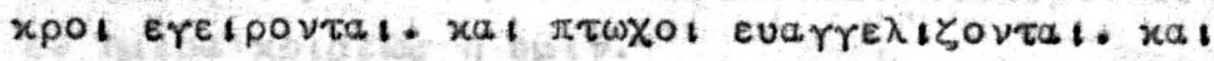

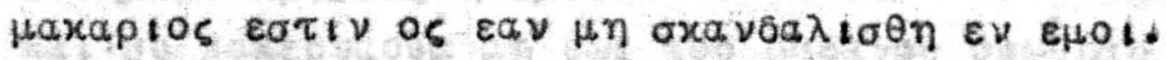

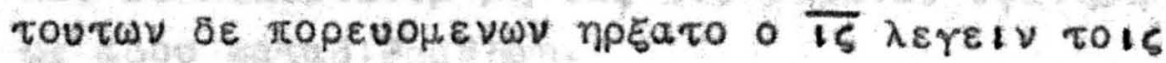

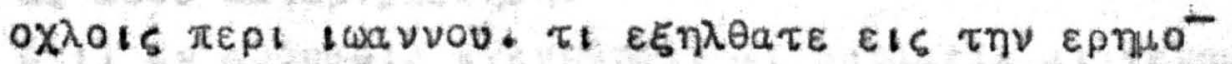

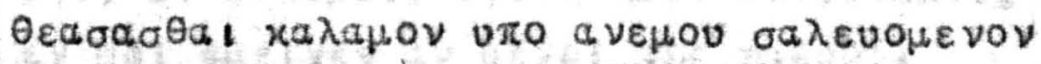

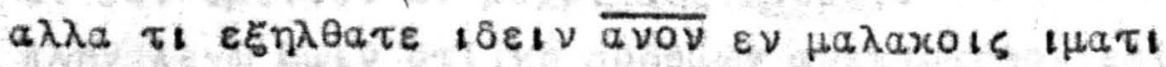

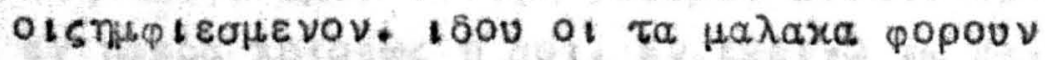

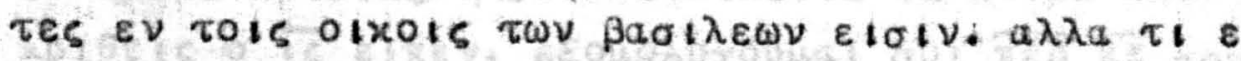

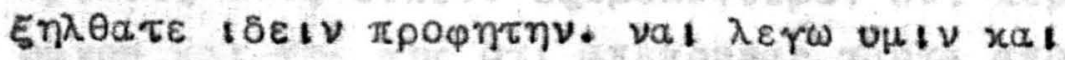
$\pi \varepsilon \rho เ \sigma \sigma o \tau \varepsilon \rho \circ \nu \pi \rho \circ \eta \eta \tau 0$.

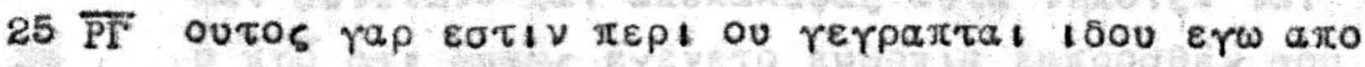

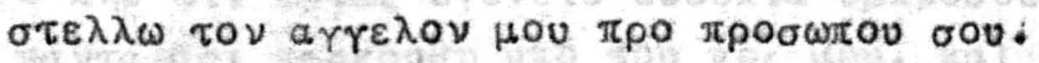

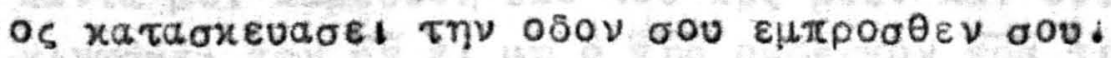

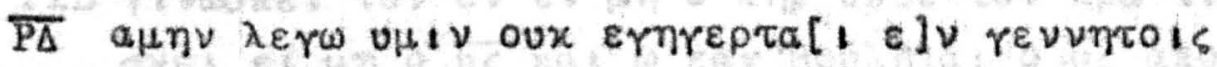

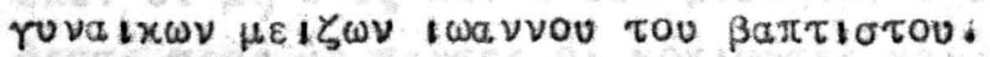

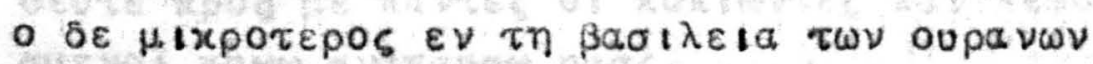

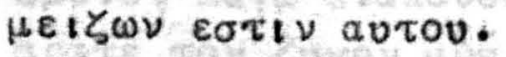

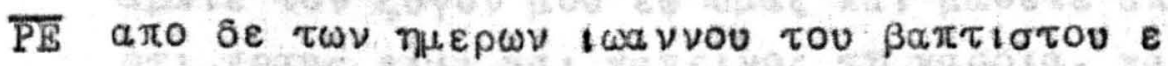

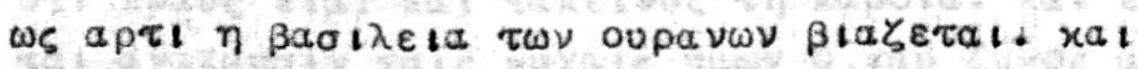

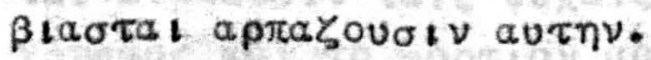

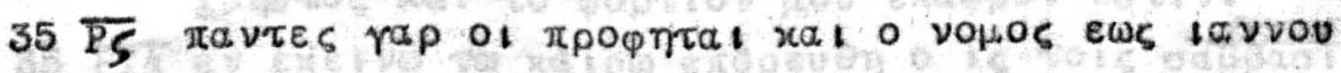

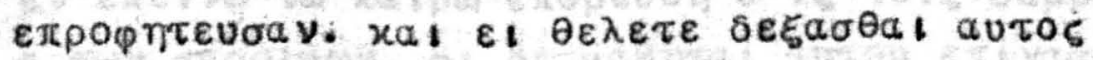

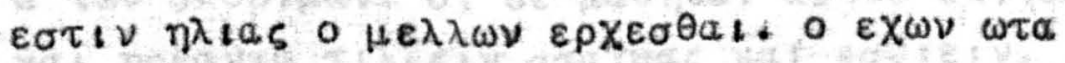
axOVEIV axoverw.

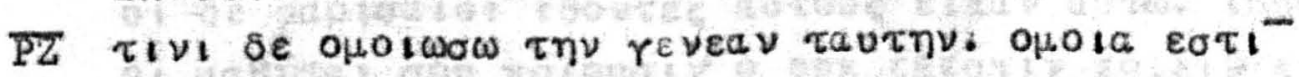

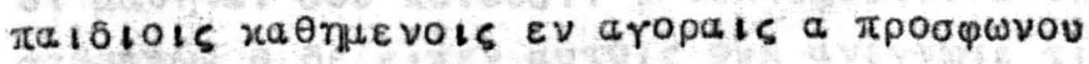

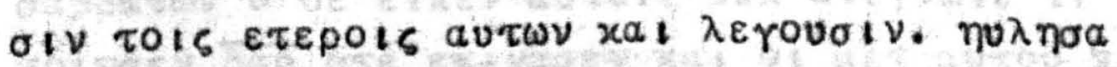




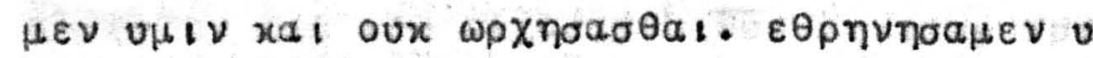
सIV xal ovx $\varepsilon x \circ \downarrow a \sigma \theta a l$.

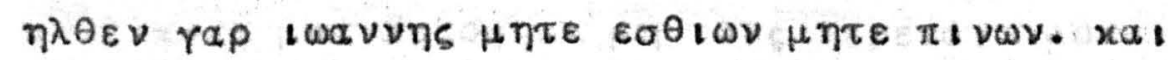

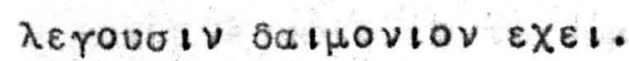

$\eta \lambda \theta \varepsilon \nu$ o $\overline{v \zeta}$ tov $\overline{a \nu 0 u} \varepsilon \sigma \theta i \omega \nu$ xal $\pi l v \omega \nu$ xal $\lambda \varepsilon$ roval-

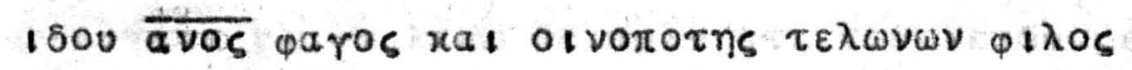

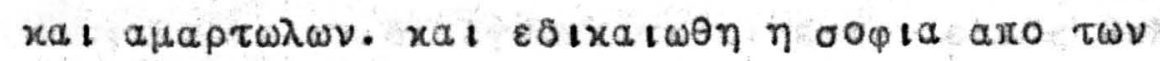

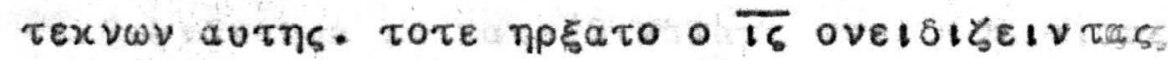

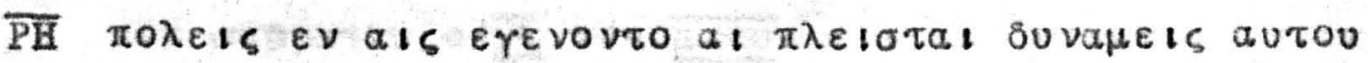

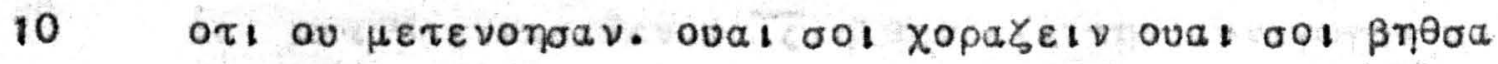

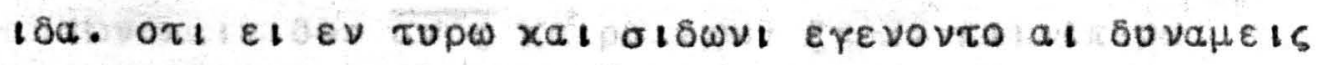

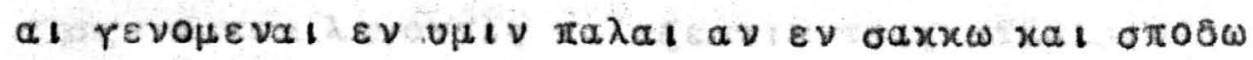

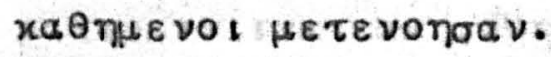

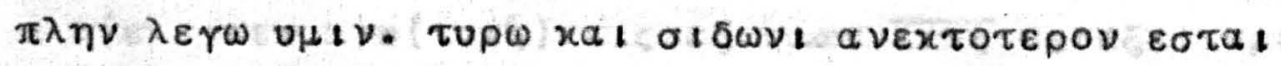

$15 \varepsilon v$ пнера xp

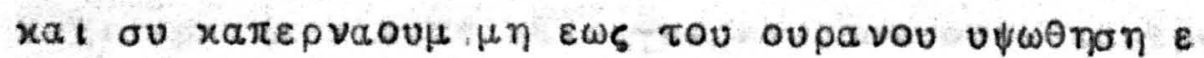

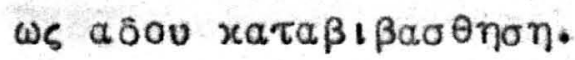

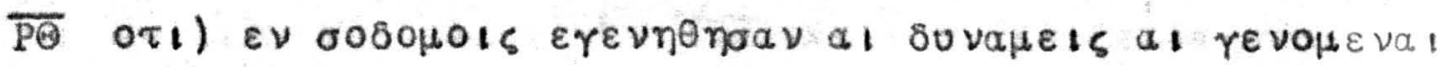

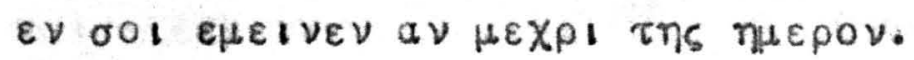

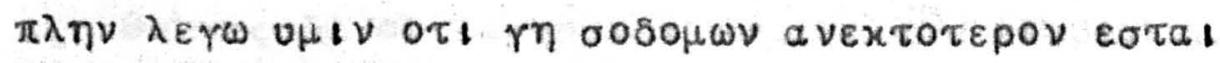

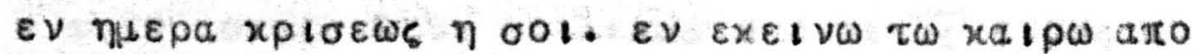

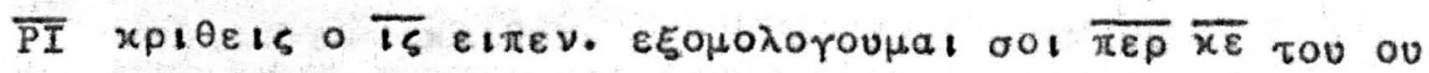

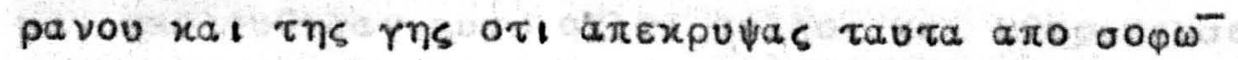

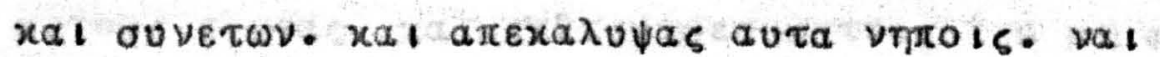

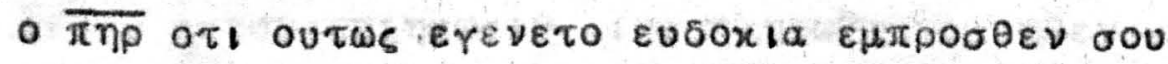

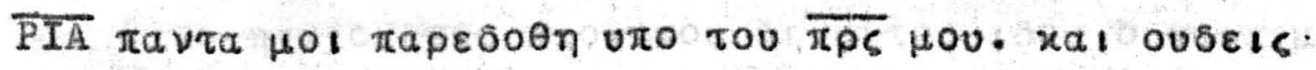

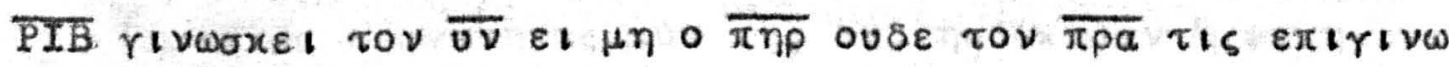

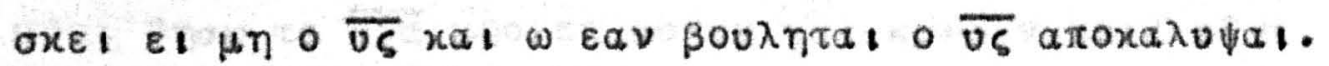

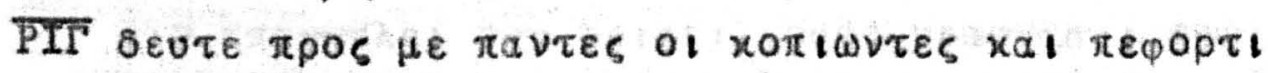

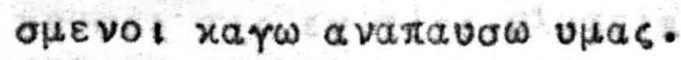

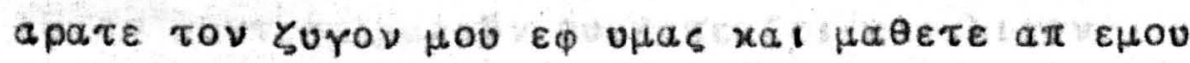

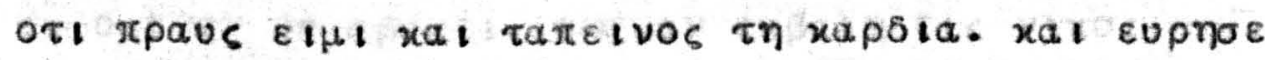

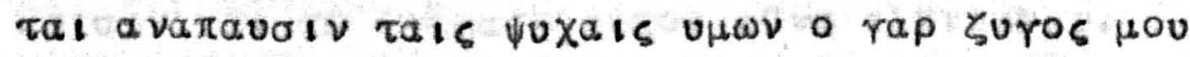

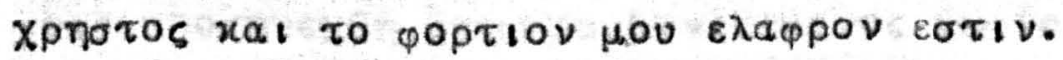

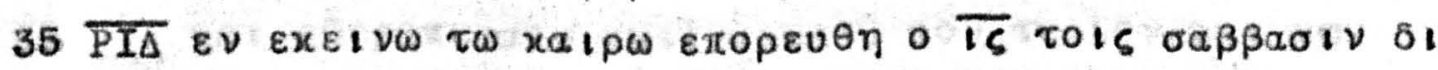

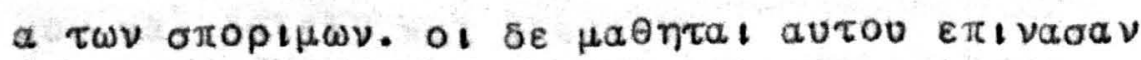

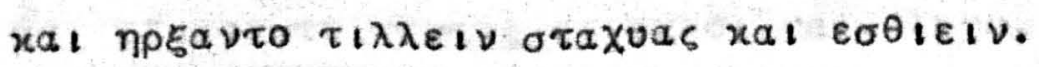

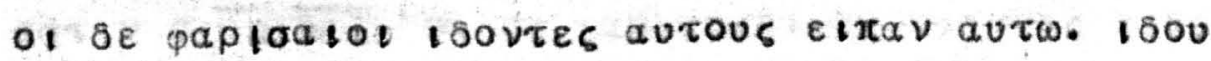

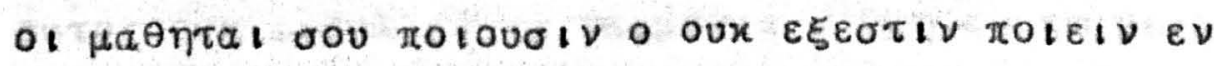

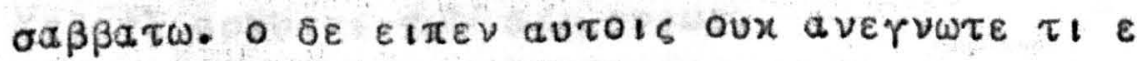

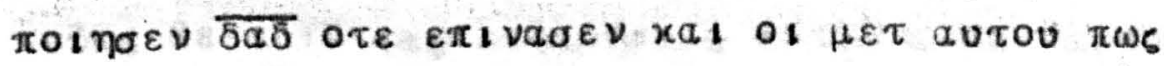




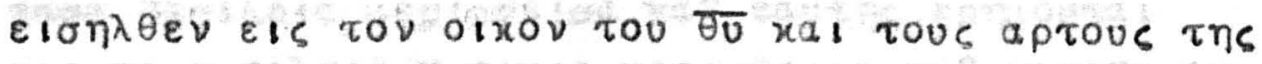

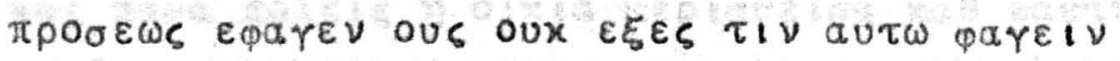

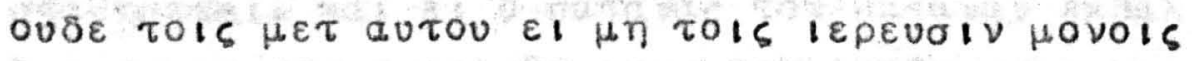

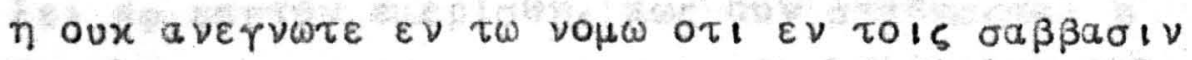

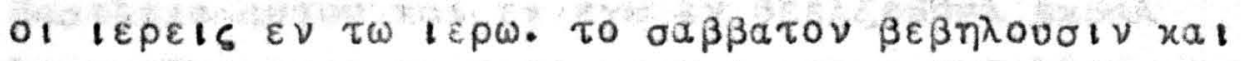

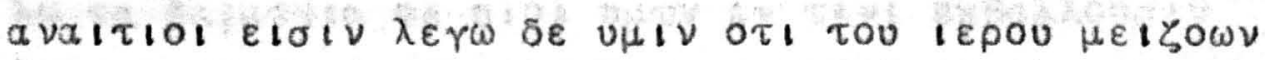

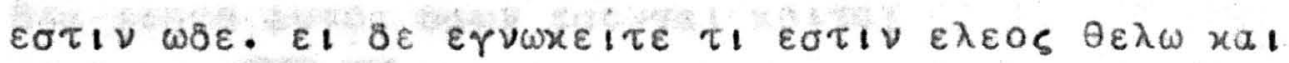

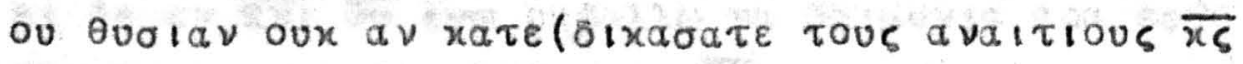

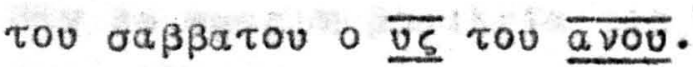

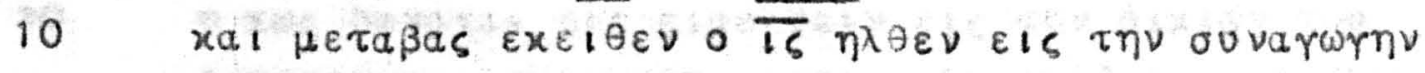

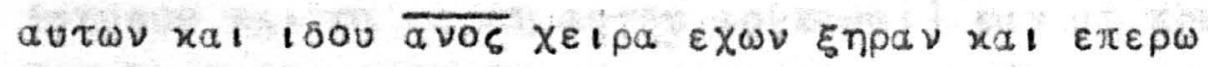

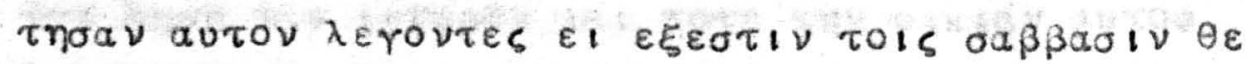

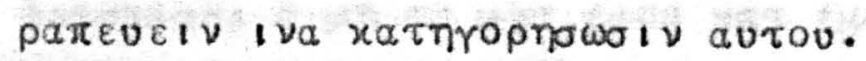

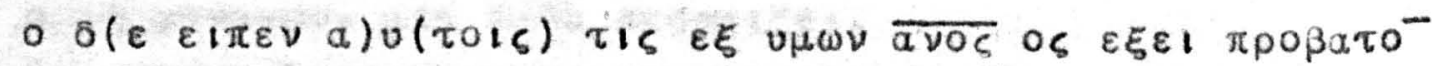

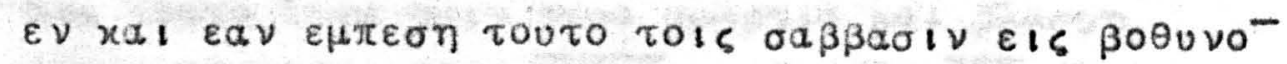

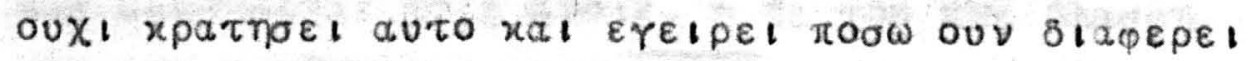

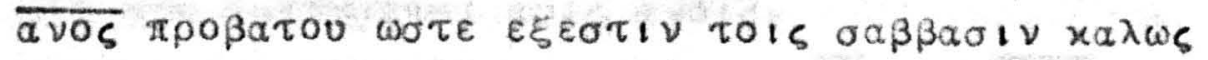

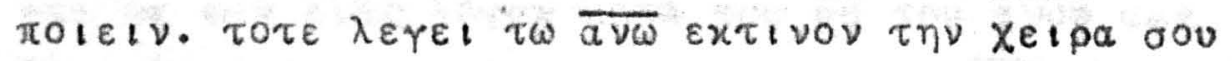
xa! $\varepsilon \xi \varepsilon \tau \varepsilon เ v \varepsilon$

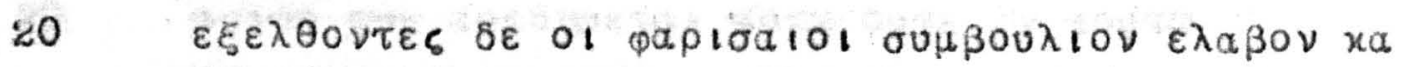

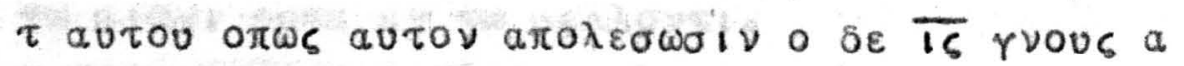
$\nu \varepsilon x \omega p \eta n \sigma \varepsilon \nu \varepsilon x\llcorner\theta \varepsilon \nu$.

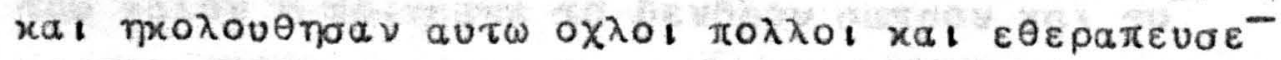

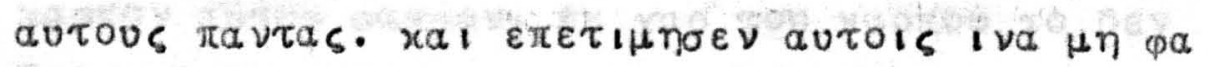

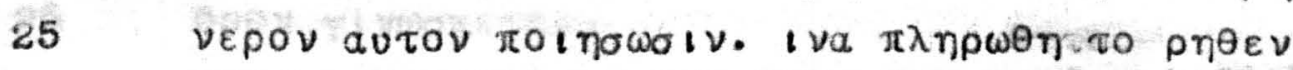

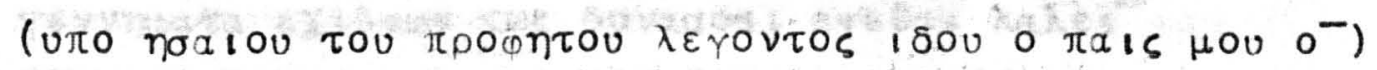
(

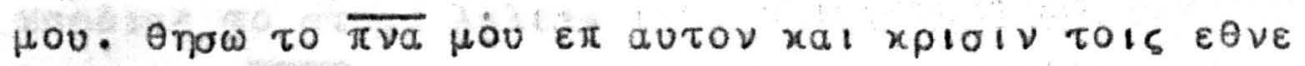

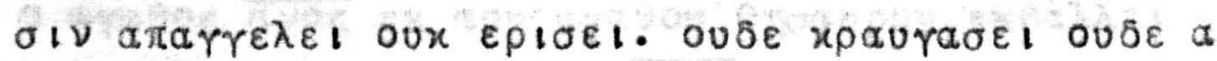
xovael tis $\varepsilon \nu$ tals $\pi \lambda a \tau \varepsilon l a$ is $\tau \eta \nu$ Q

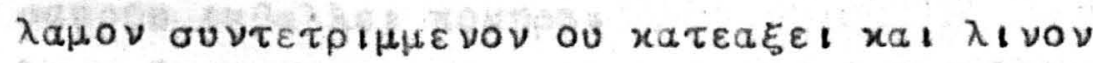

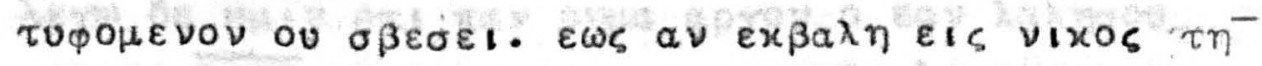

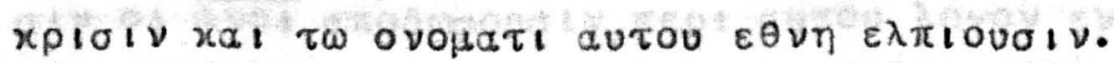

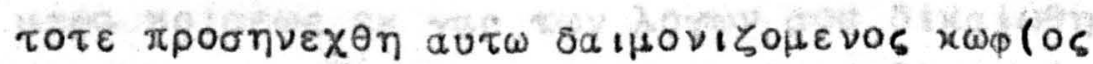

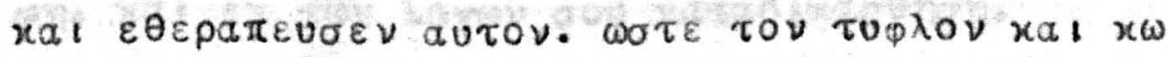

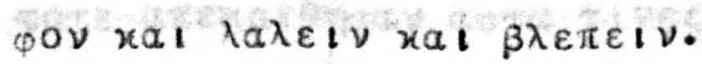

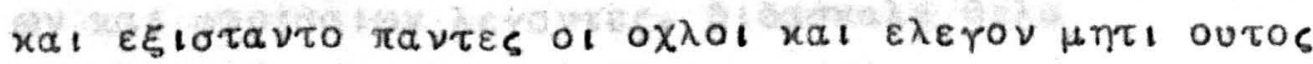

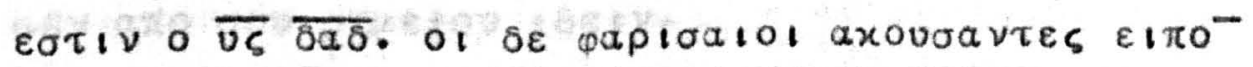

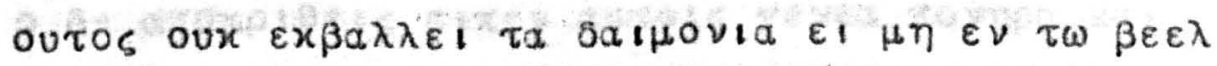

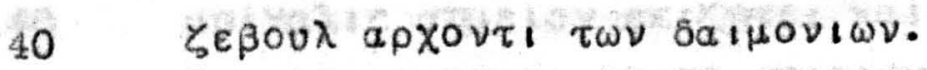

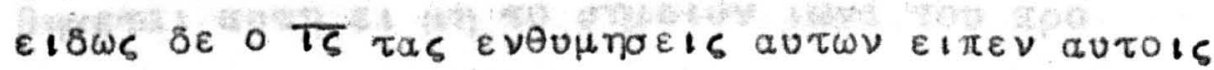




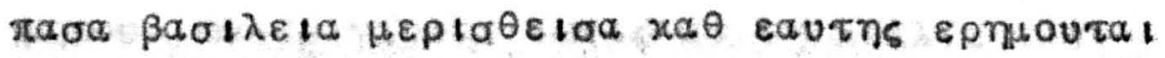

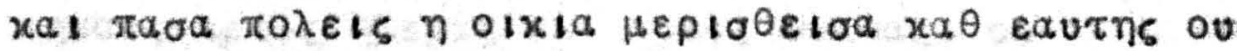

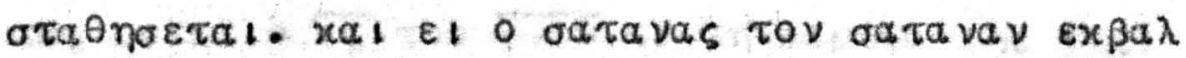

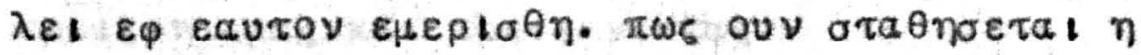

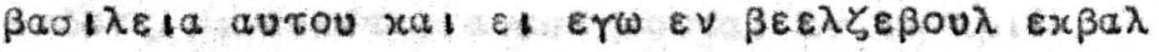

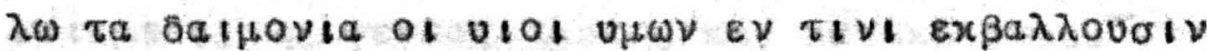

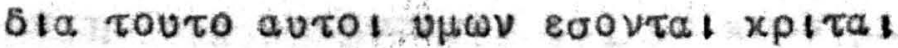

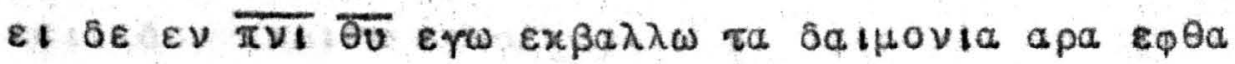

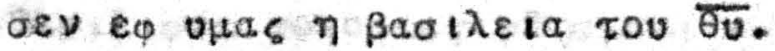

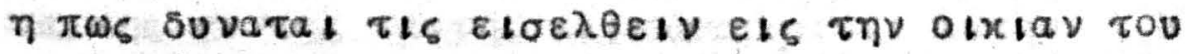

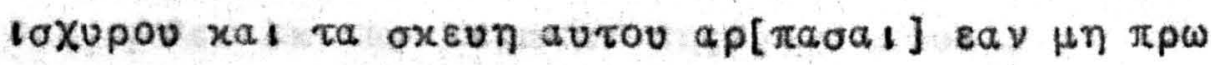

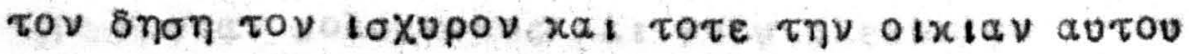

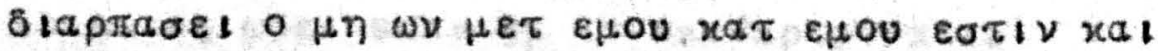

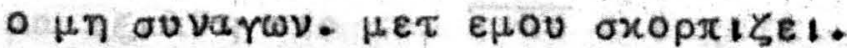

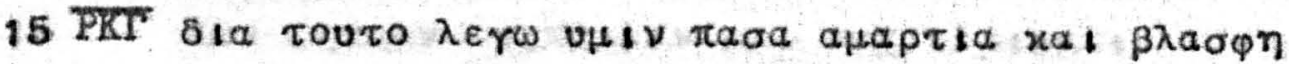

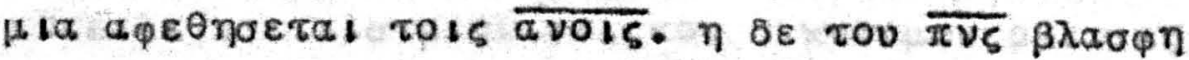

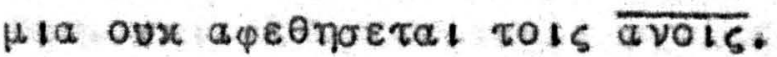

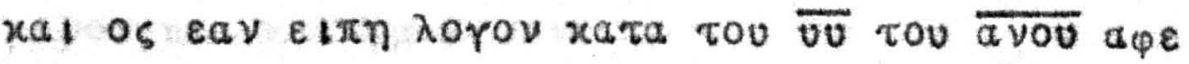

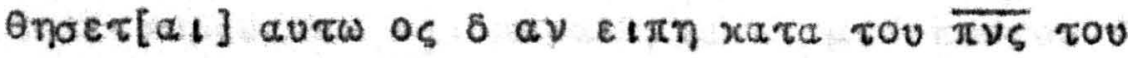

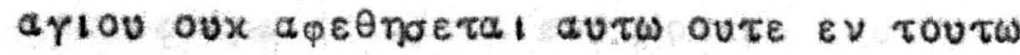

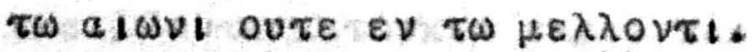

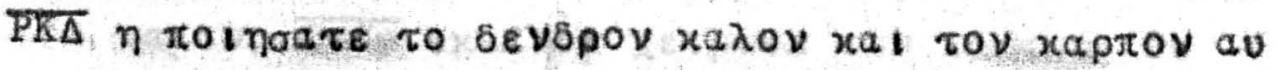

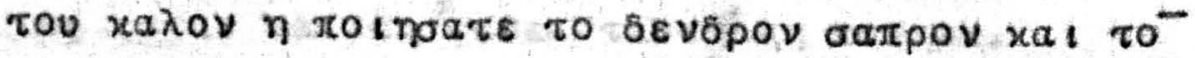
xaptov autou aatpov, ex rap tov xaptov to $\delta 8 V$ spov rivwoxetal.

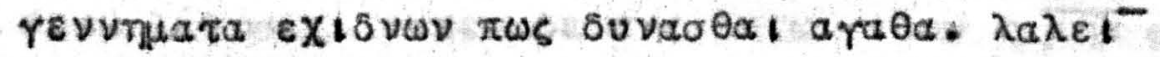

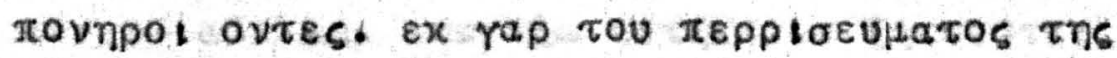
xapôtas to atoua $\lambda a \lambda \varepsilon t$.

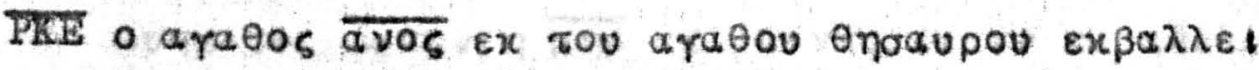

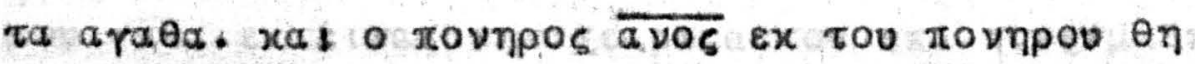

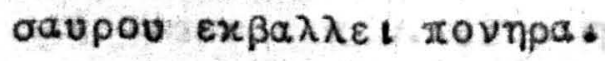

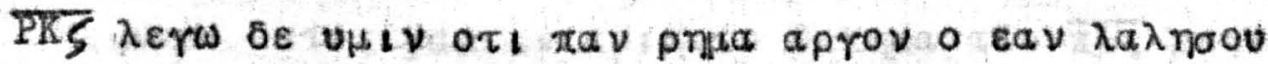

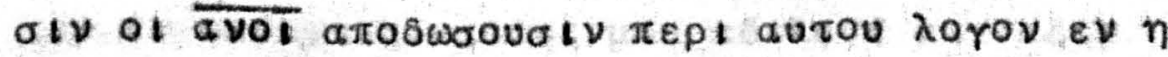

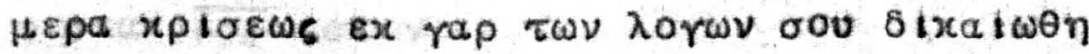

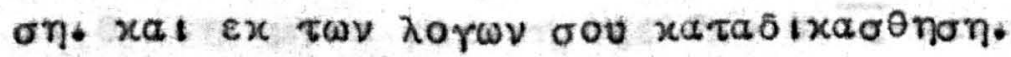

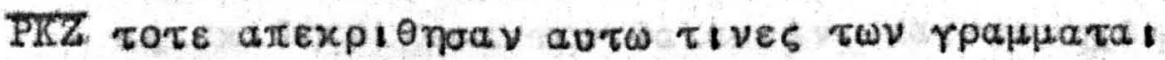

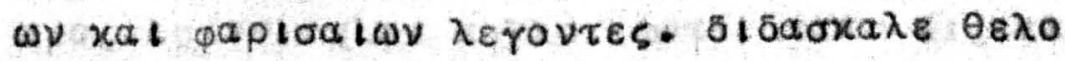
$\mu \varepsilon \nu$ arto $\sigma 0 v$ onuetov $t \sigma \varepsilon t \nu$.

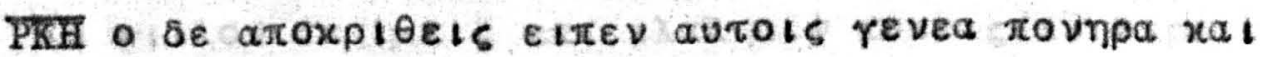

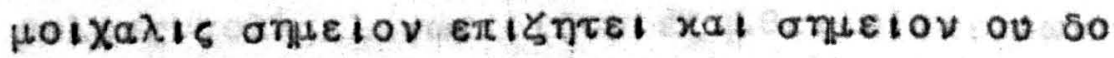

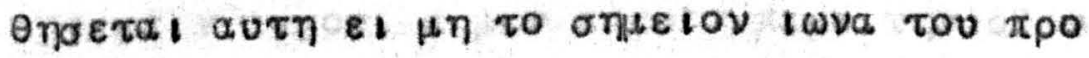




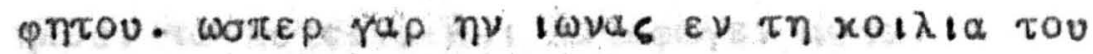

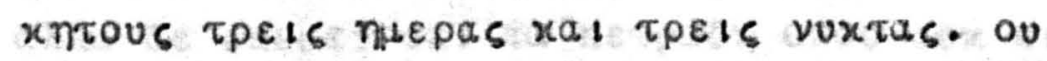

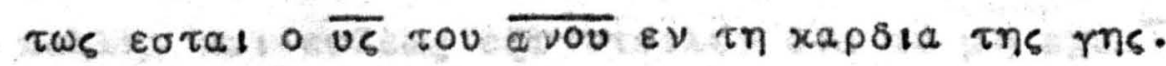

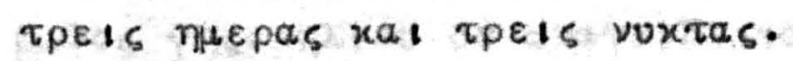

a

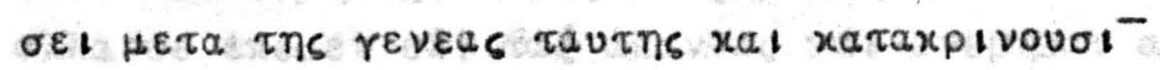

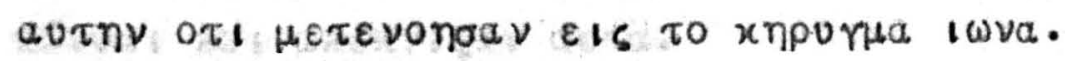

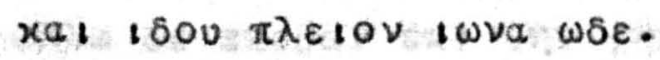

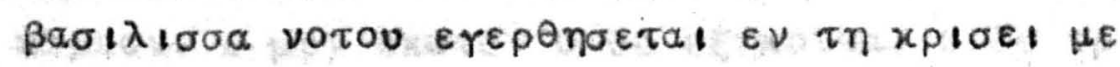

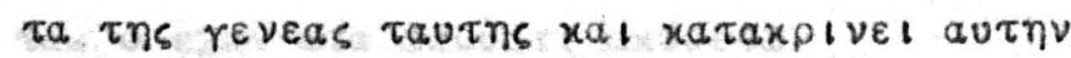

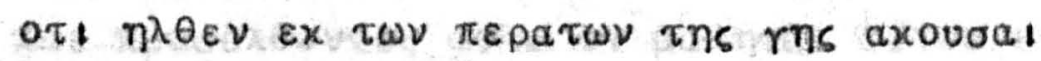

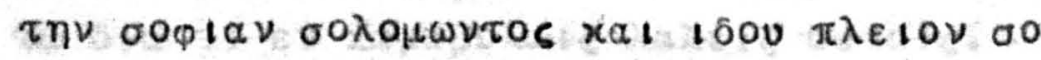

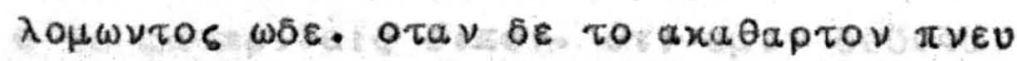

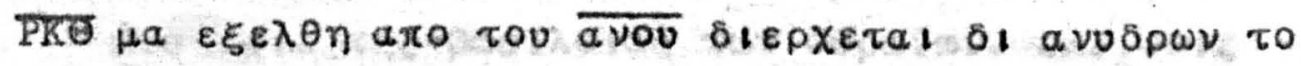

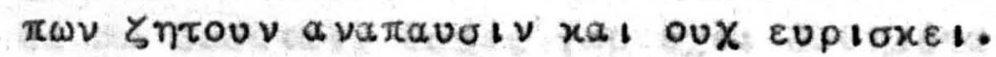

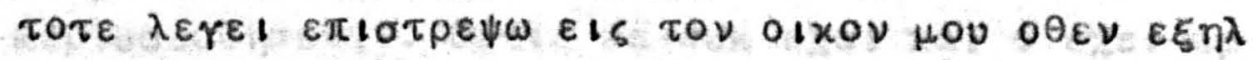

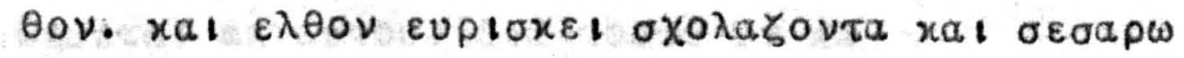
$\mu \varepsilon \nu O \nu$ xal $x \in x o \sigma \mu \eta \mu \varepsilon \nu o \nu$.

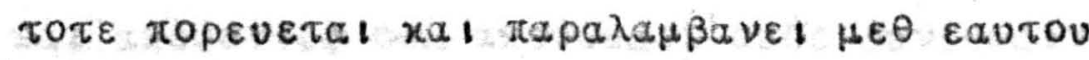

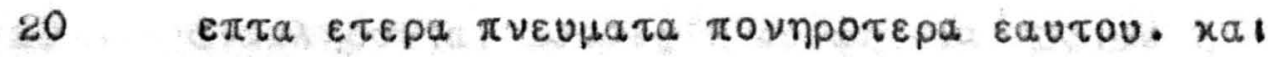

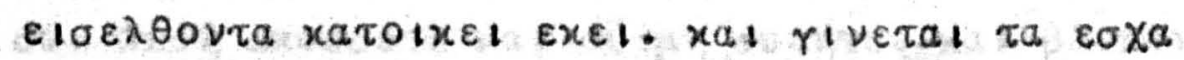

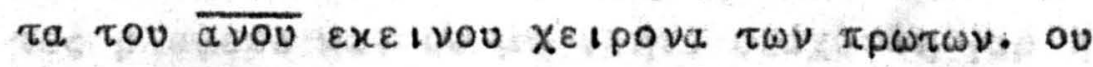

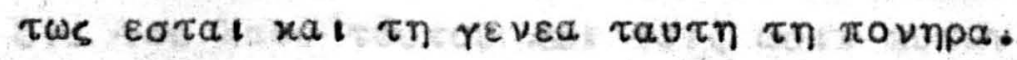

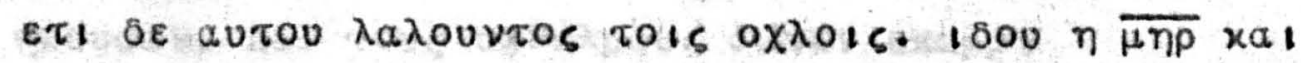

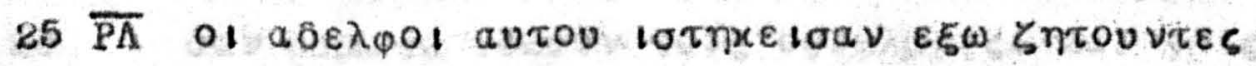

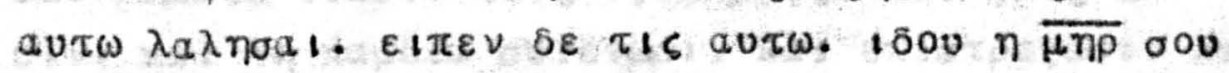

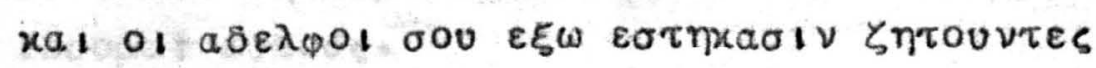

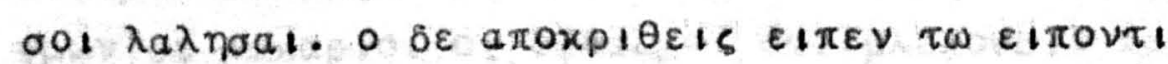

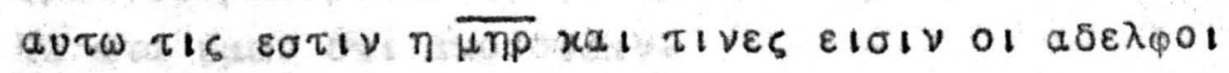

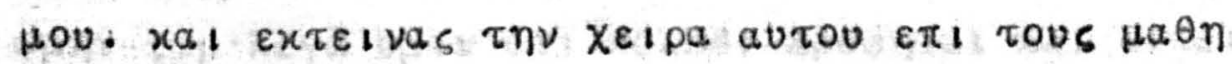

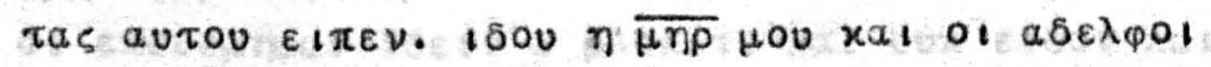

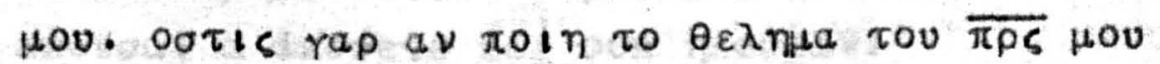

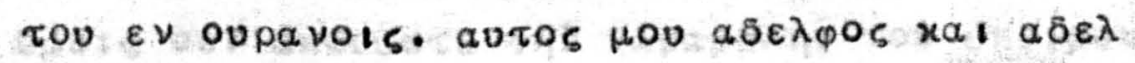

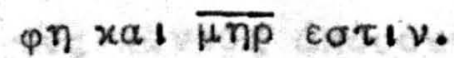

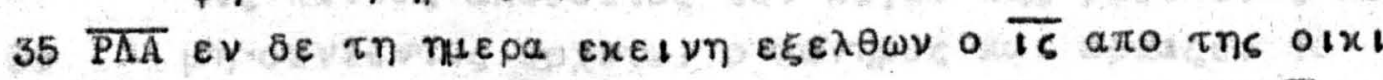

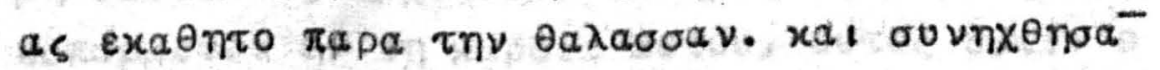

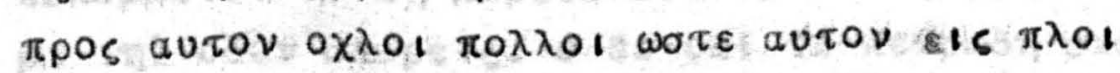

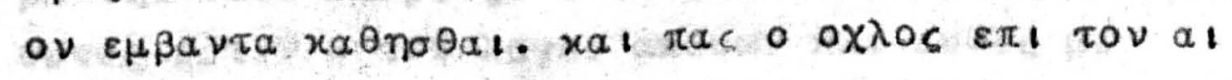

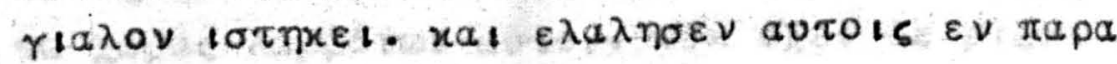

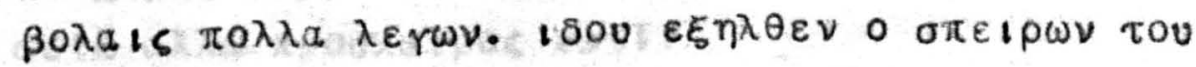

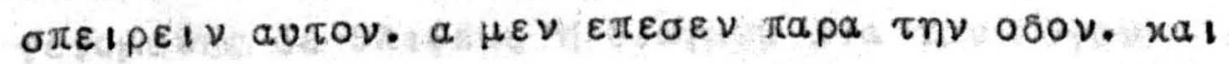




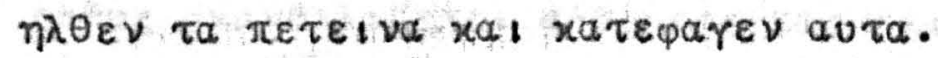

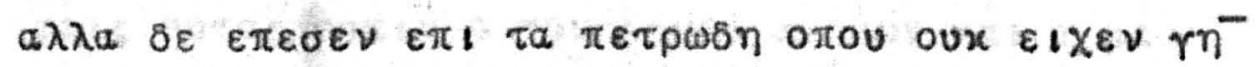

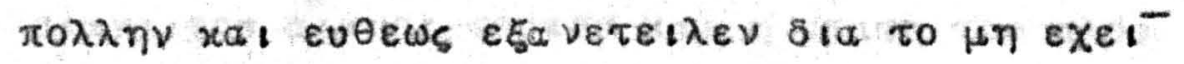

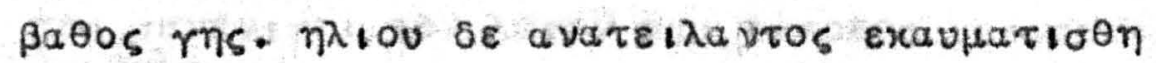

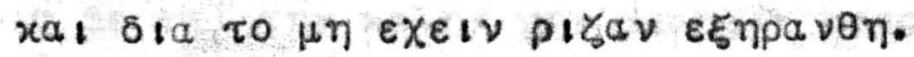

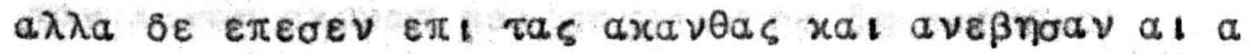

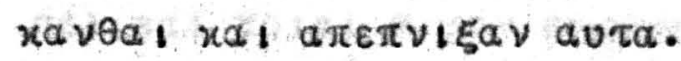

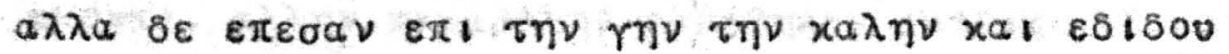

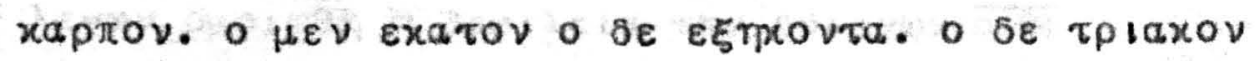

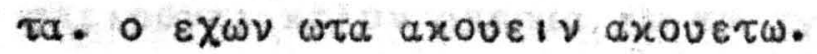

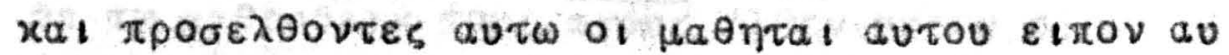

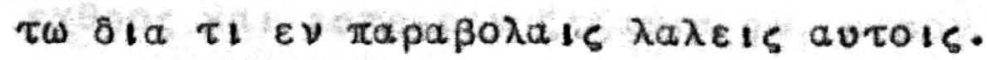

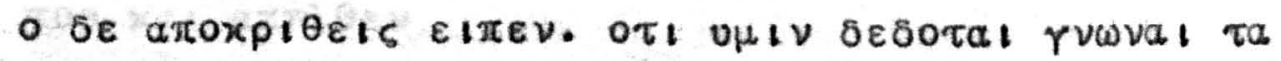

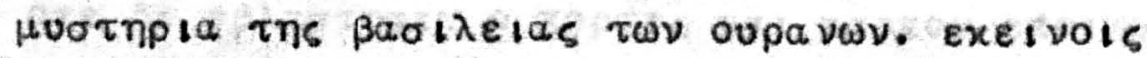

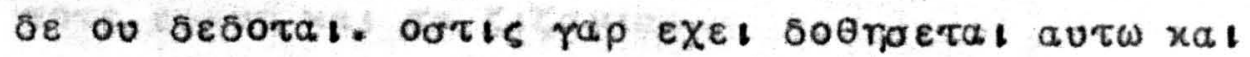

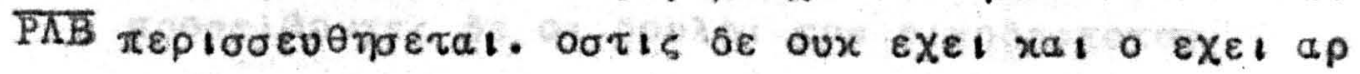

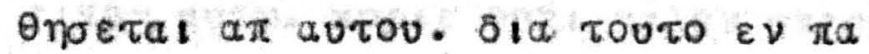

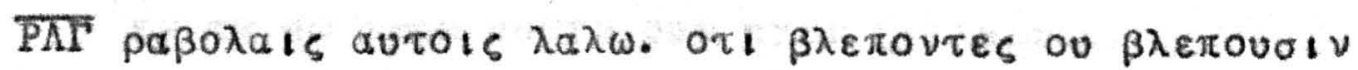
xal axovovtec oux axovovotv ouse auviovolv. $x a l$

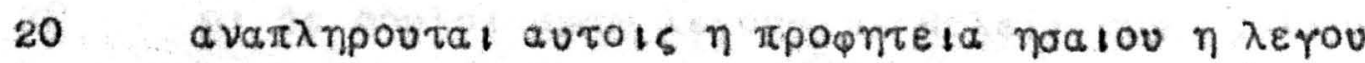

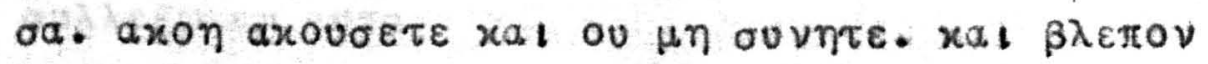

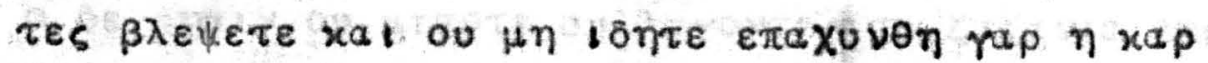

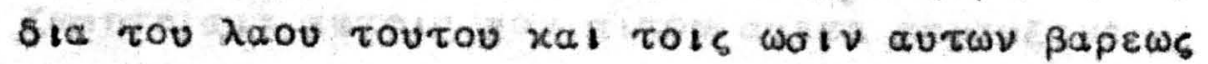

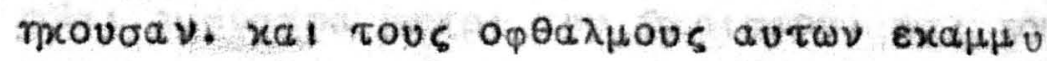

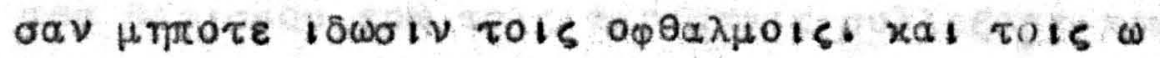

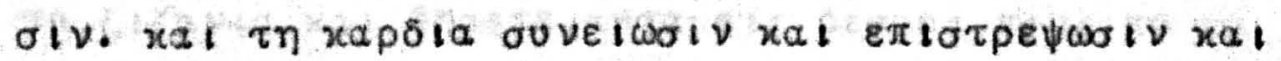
laophat aurous.

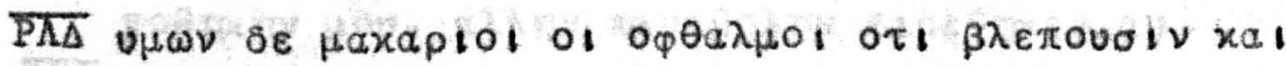

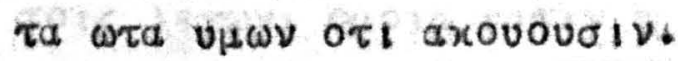

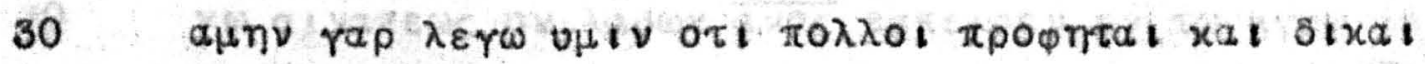

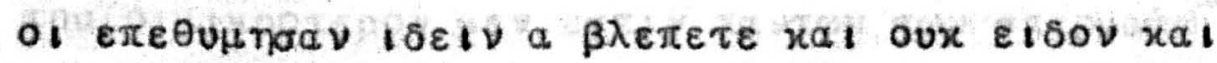
axovoal a axovere xal oux pxovaav.

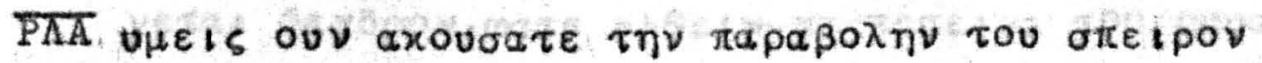

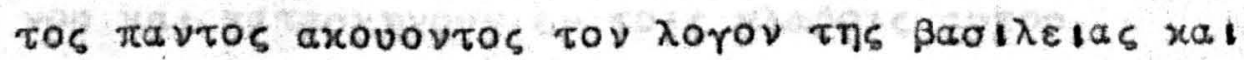

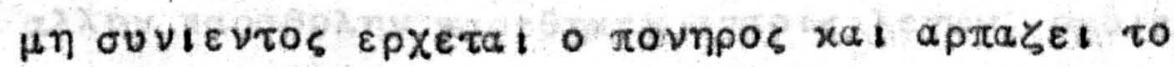

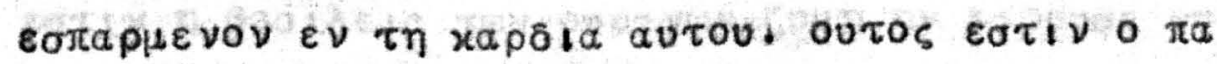

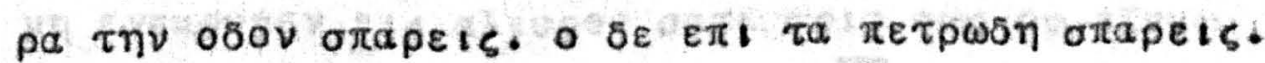

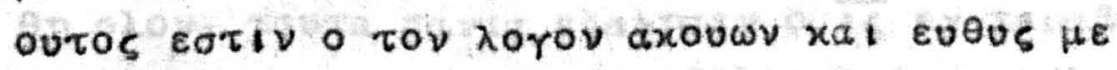

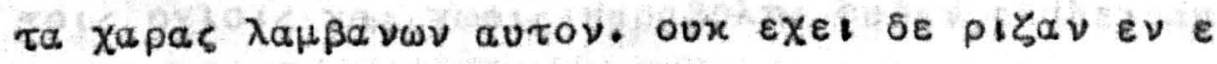

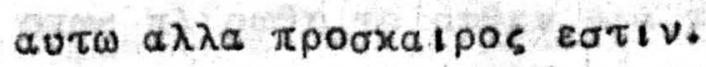

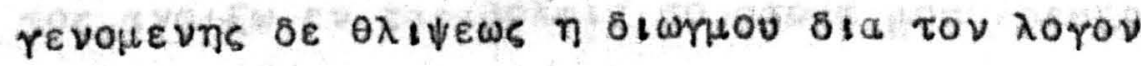




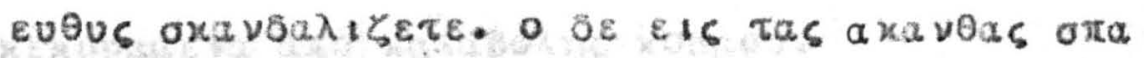
peis outos eativ o tov hayov arouw hat $\eta \mu \varepsilon$

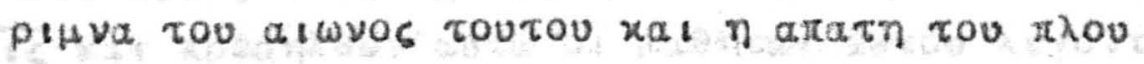
tov ouvivirel tov hapoy xal axapros re!vetal.

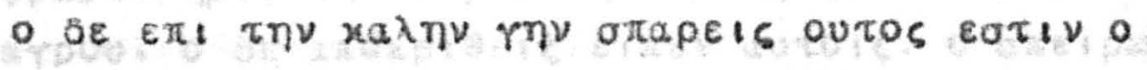
Tov dorov axovwy hat ouvelwy of on xapropo

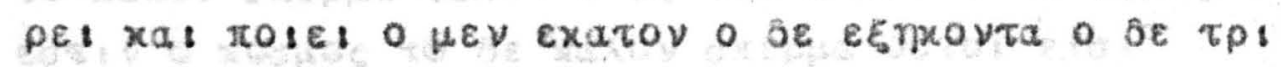

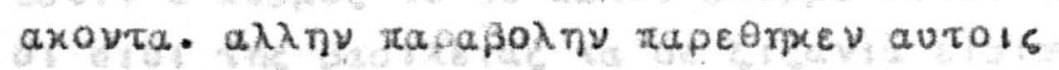

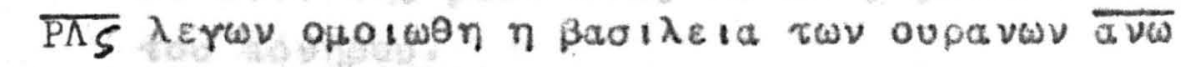

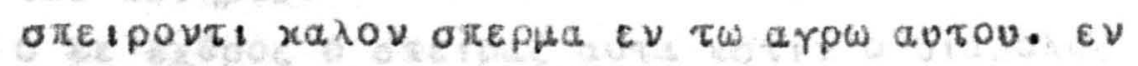

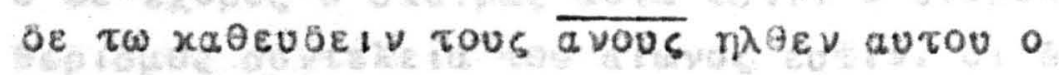

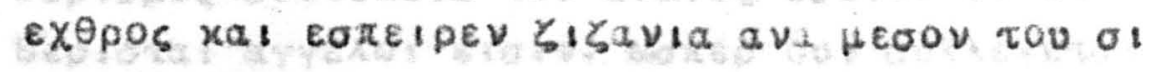
Tou xat $a \pi \eta \lambda \lambda \theta \varepsilon v$

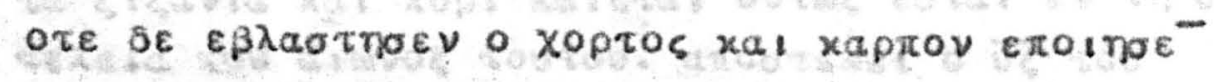

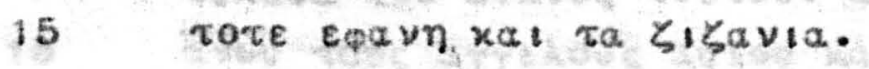

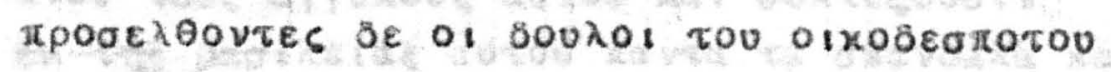

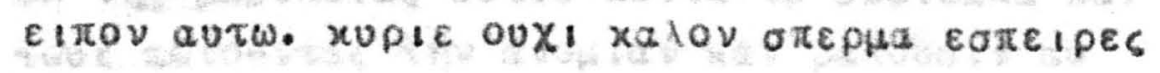

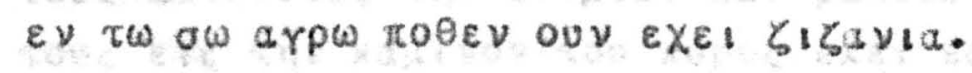

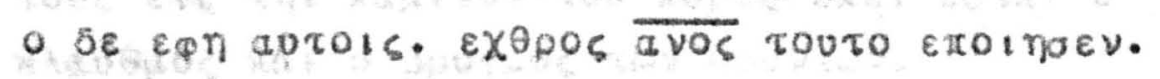

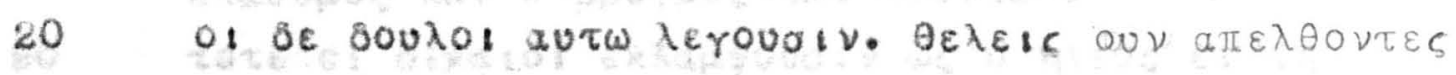

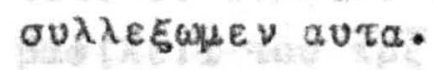

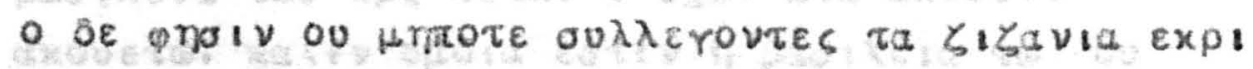

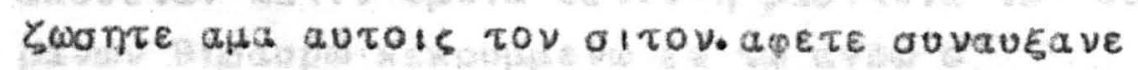

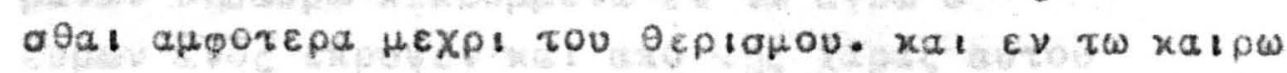

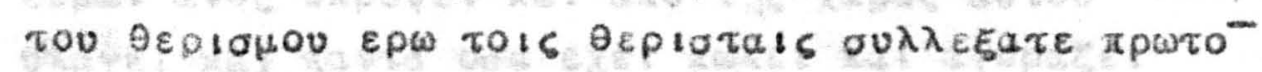

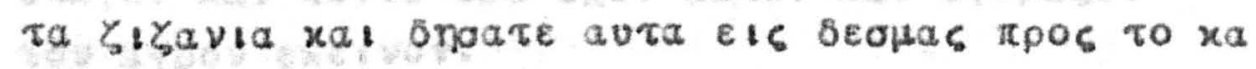

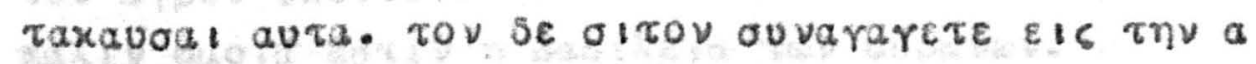

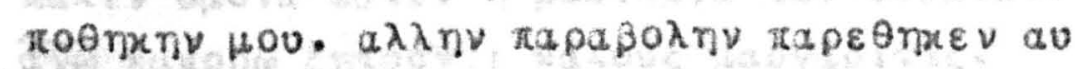

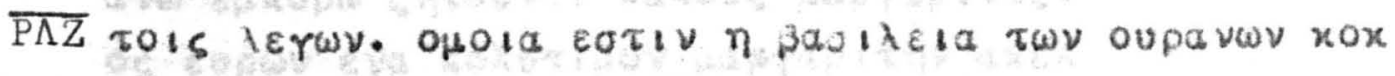
so

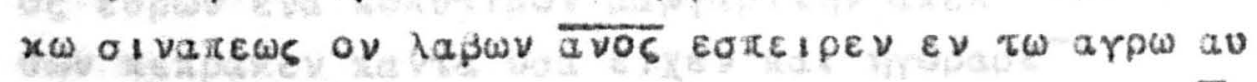

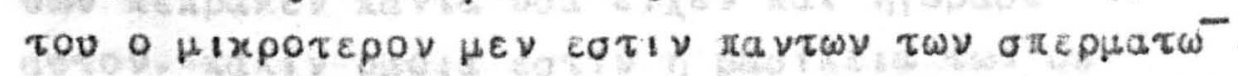

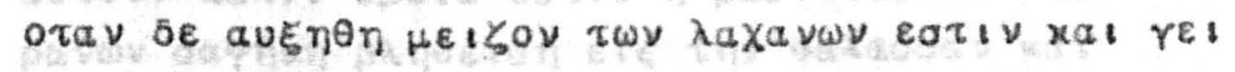

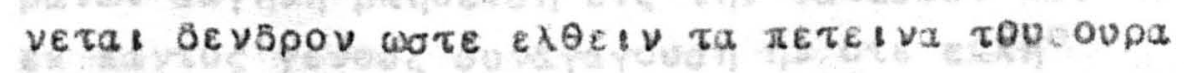
vou xal xaraaxnuouv $\varepsilon v$ tols xhabois autou.

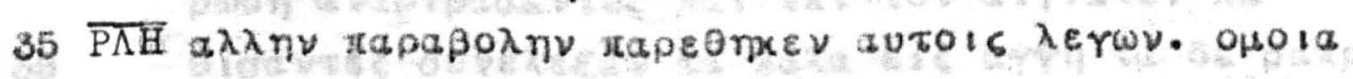

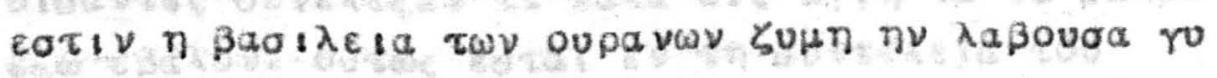

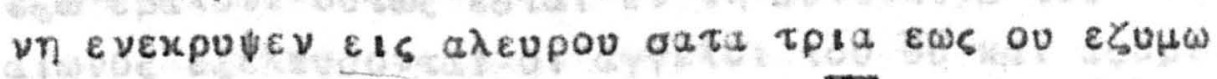

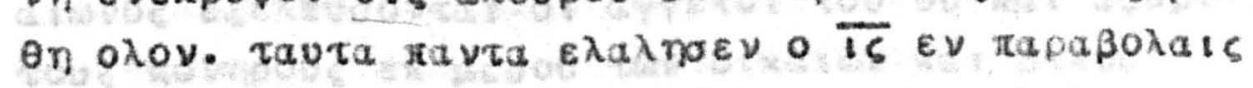

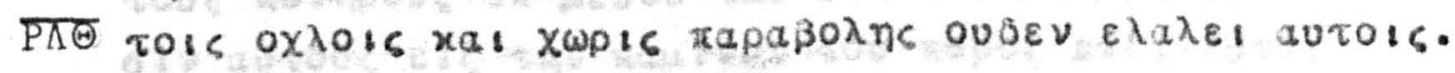

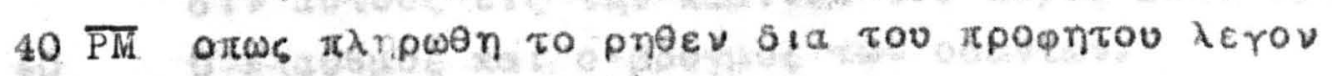

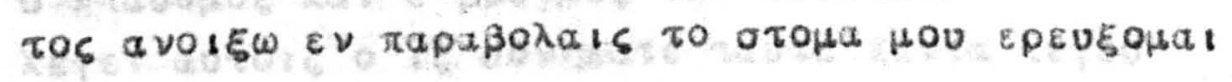

$$
\text { xata } \mu a \theta \theta a 10 v 13.21-\$ 5
$$




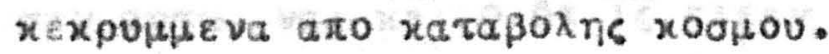

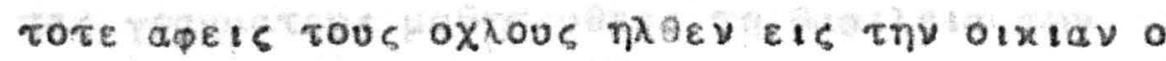

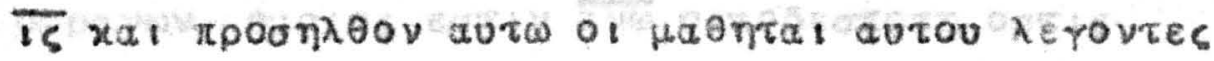

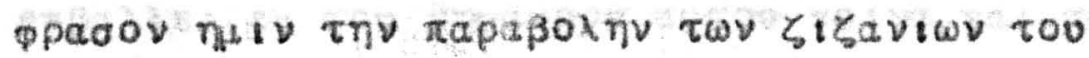

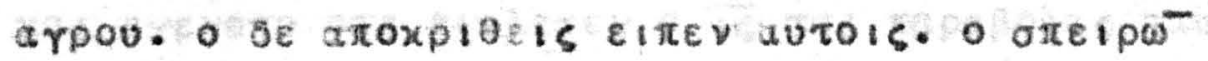

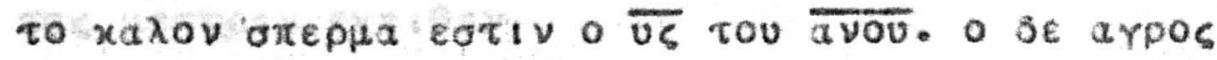

Eativ o xoajos to $6 \varepsilon$ xalov orepha outot $\varepsilon t^{-}$

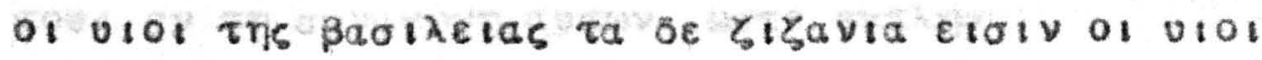
rou rovmpou.

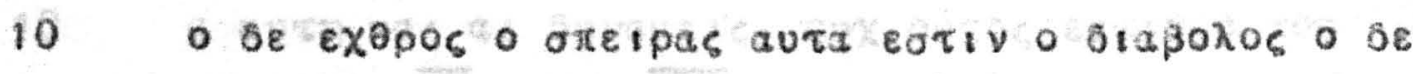

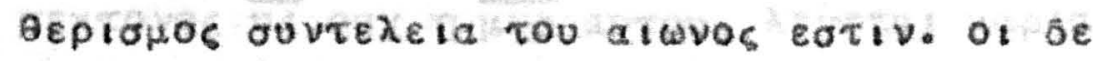

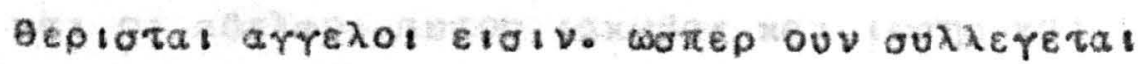

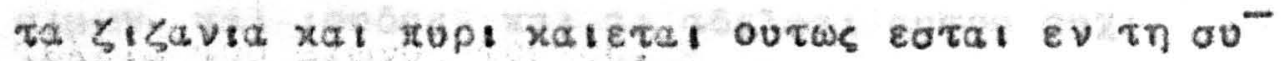

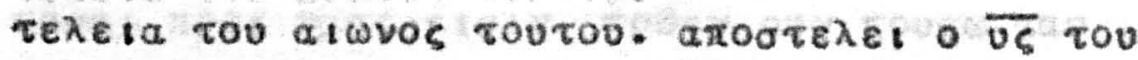

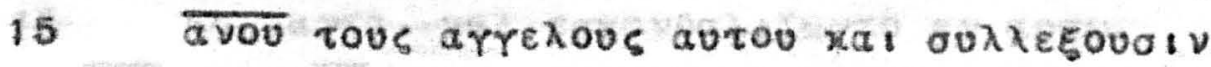

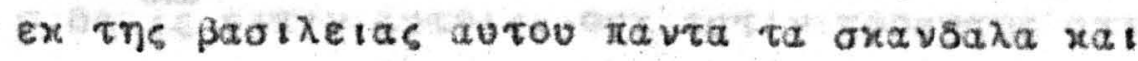

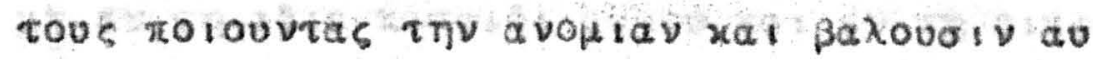

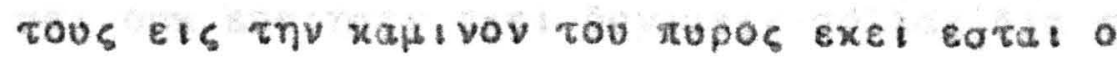

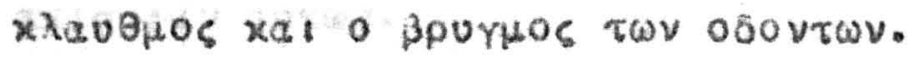

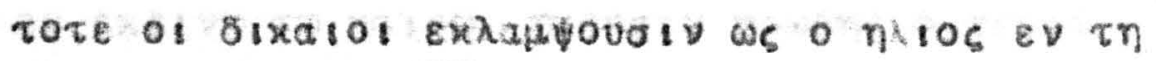

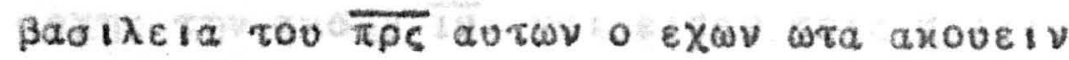

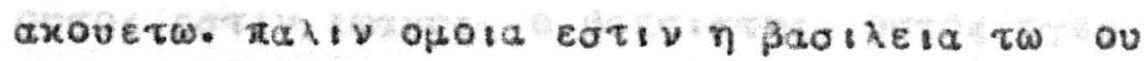

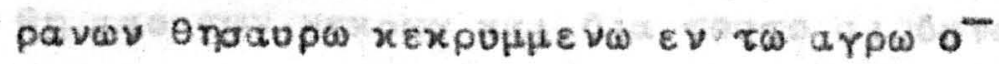

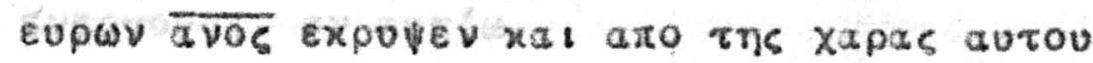

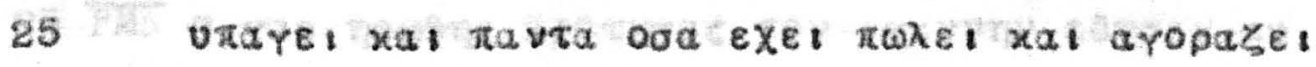
tov appov $\varepsilon x t_{\text {vov. }}$

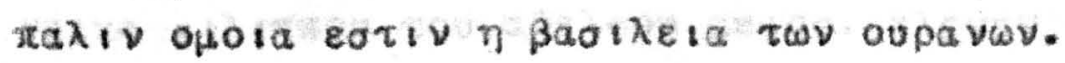

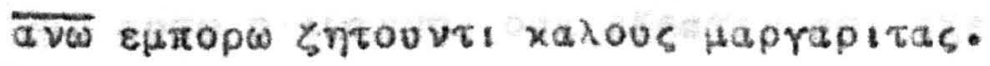

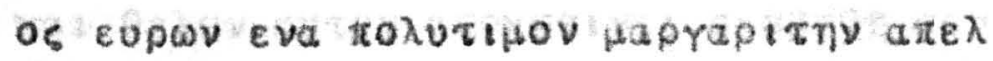

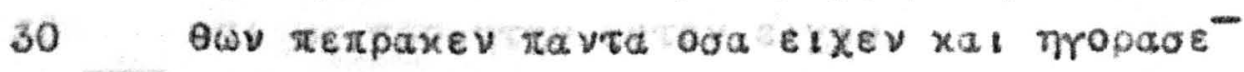

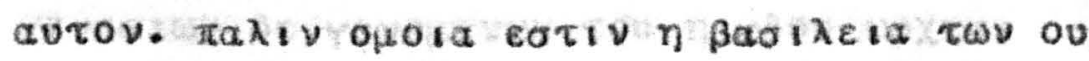

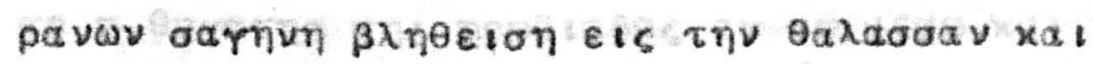

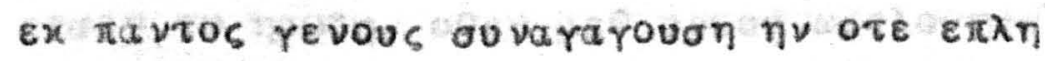

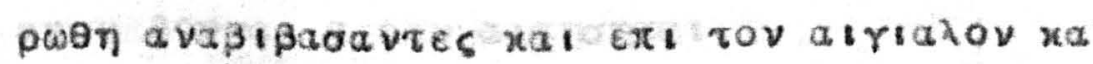

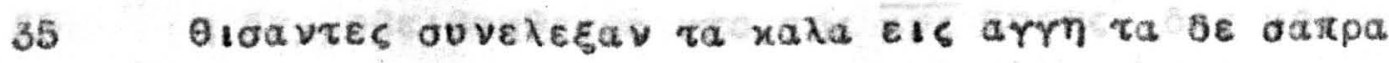

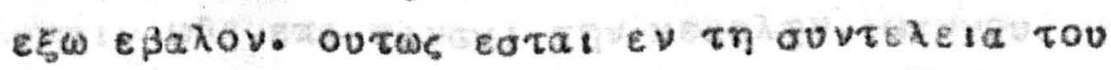

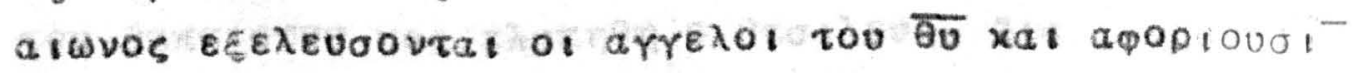

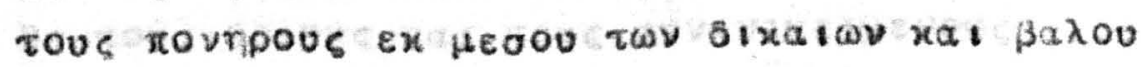

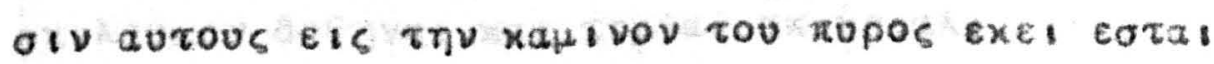

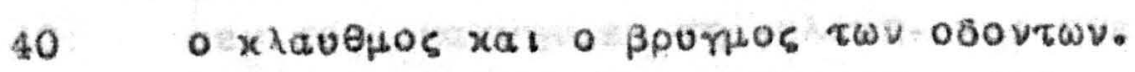

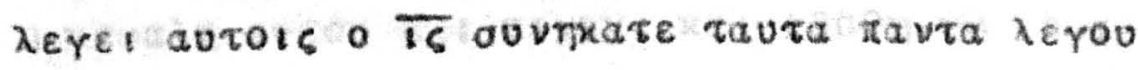




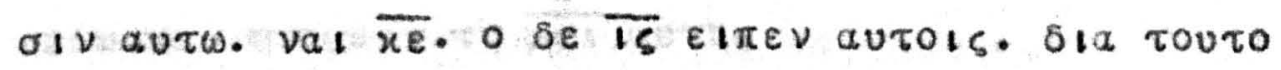

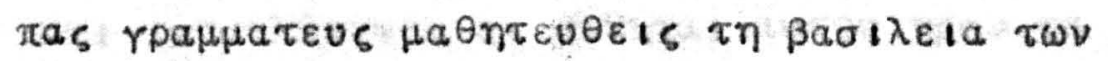

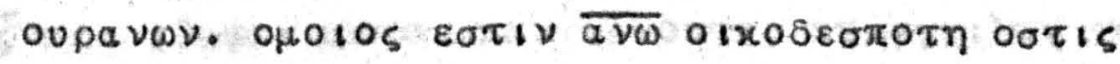

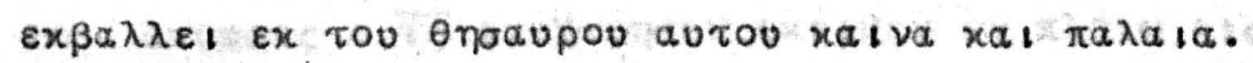

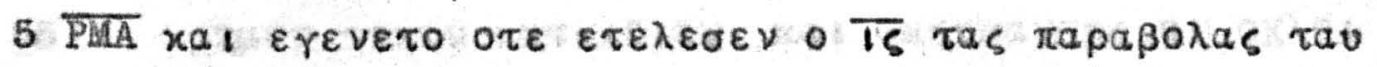
$\tau a_{5} \mu \varepsilon \tau \eta \rho \varepsilon \nu$ ex $1 \theta \varepsilon \nu$.

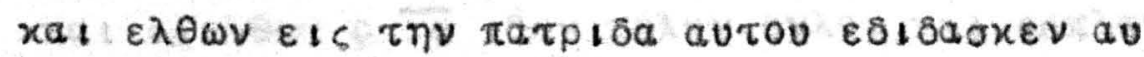

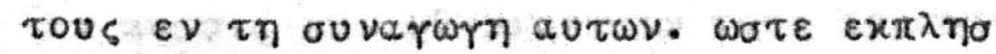

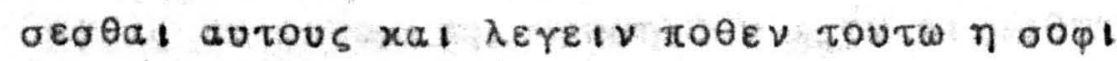

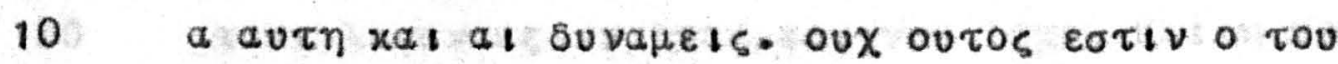

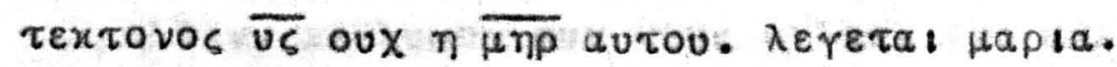

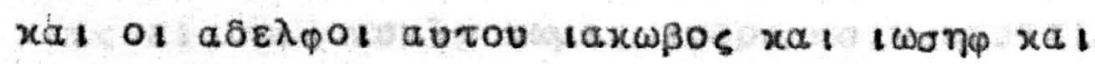

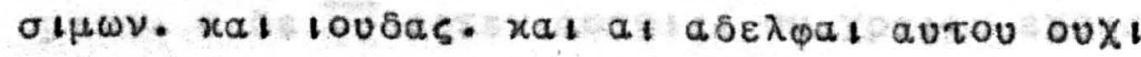

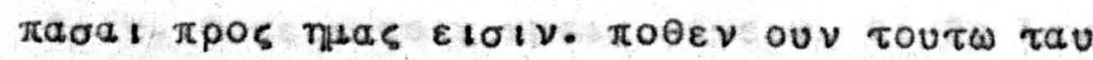

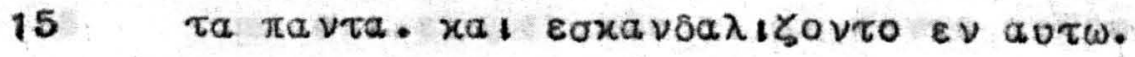

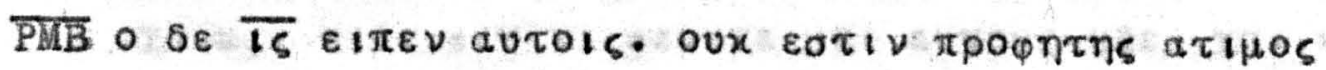

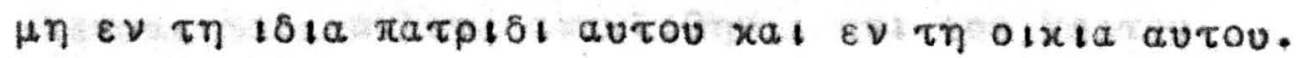

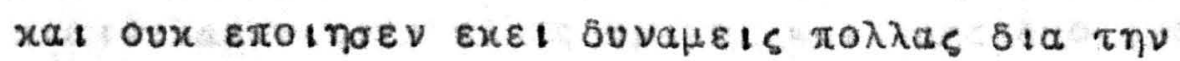

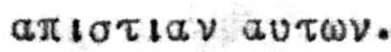

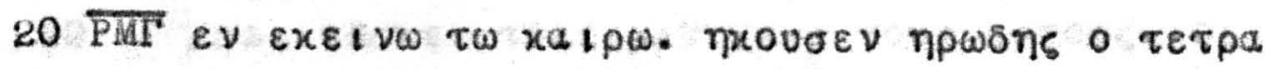

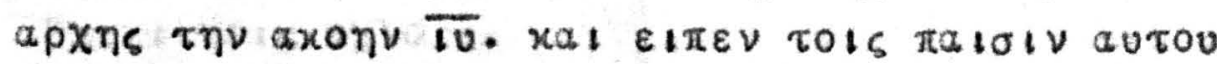

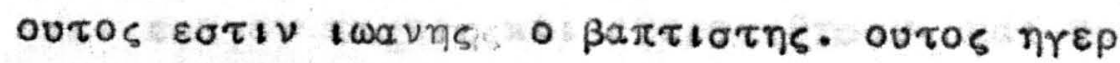

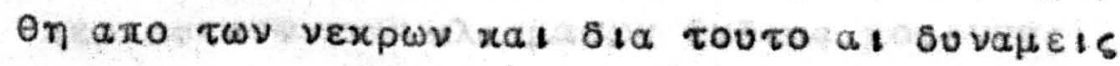

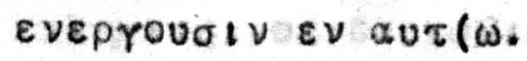

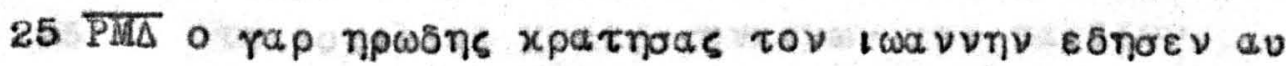

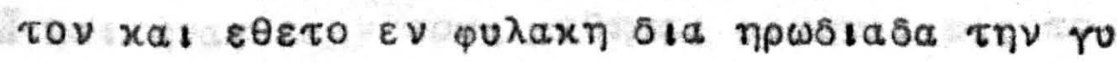

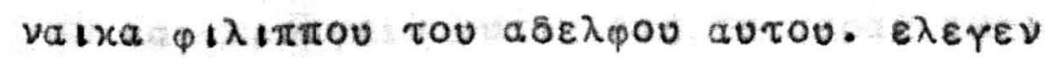

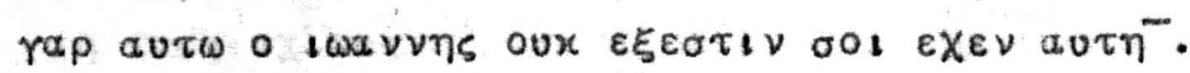

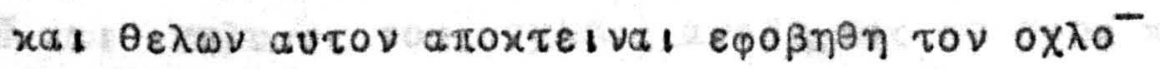

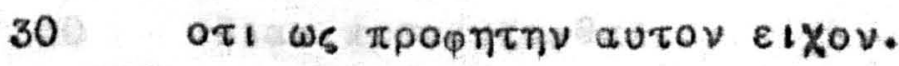

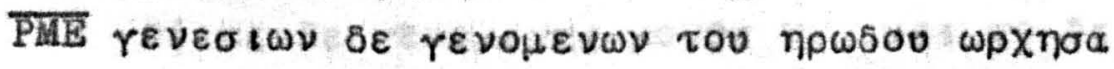

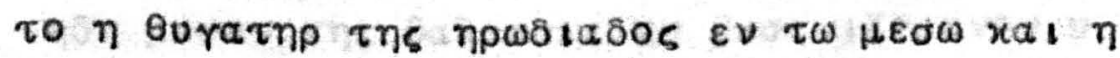

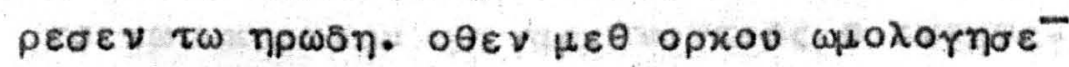

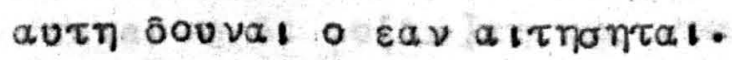

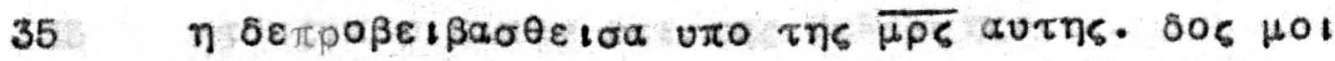

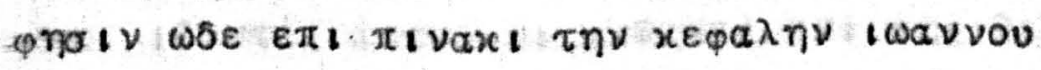

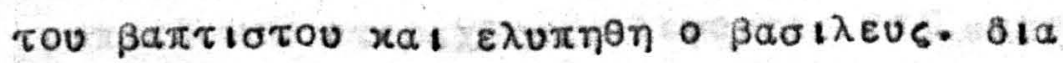

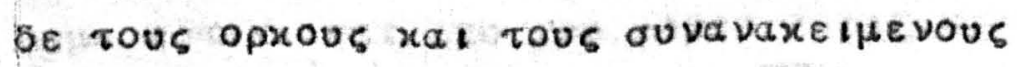
$\varepsilon x \varepsilon \lambda \varepsilon v \sigma \varepsilon \nu \delta 0 \theta \eta \nu \alpha, x \alpha$ । $\pi \varepsilon \mu \psi \alpha s$ a $\delta \varepsilon x \varepsilon \varphi \alpha \lambda_{l}$

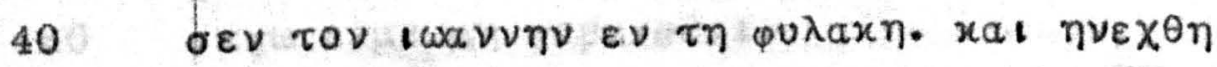

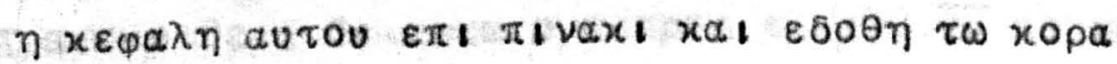




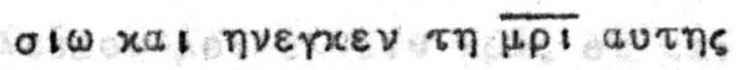

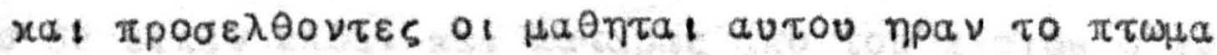

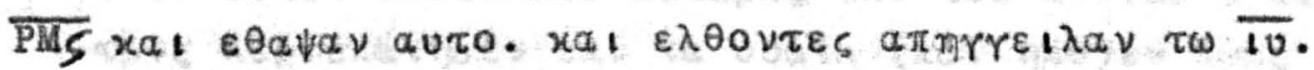

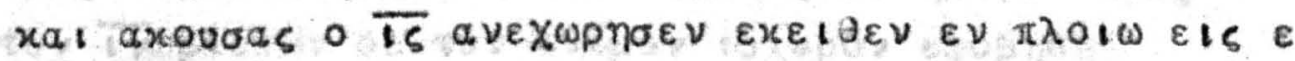

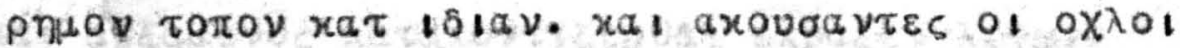

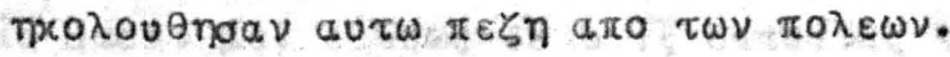

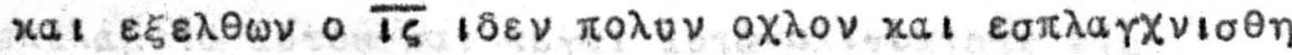

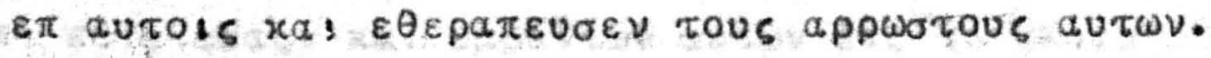

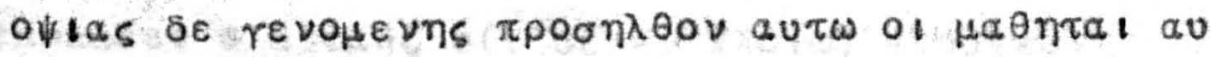

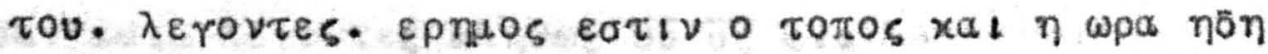

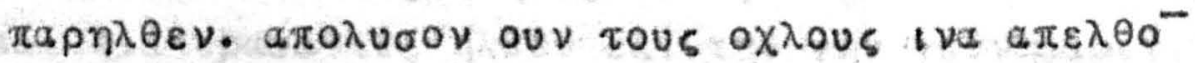

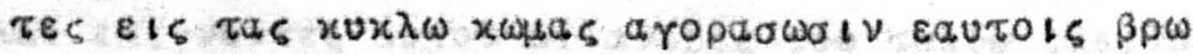

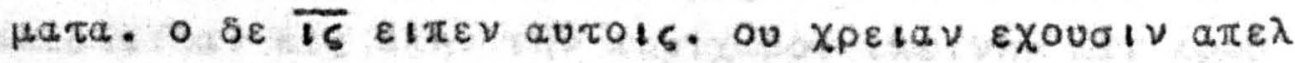

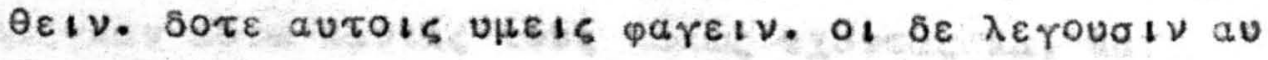

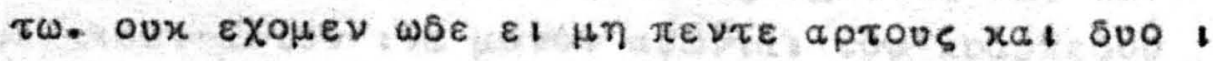

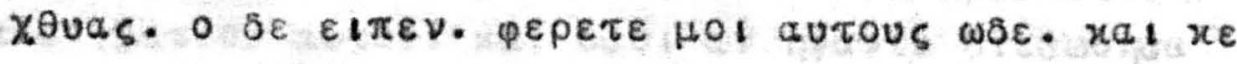

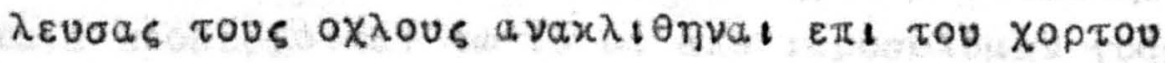

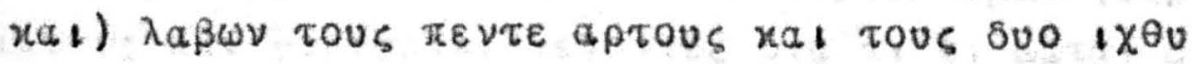

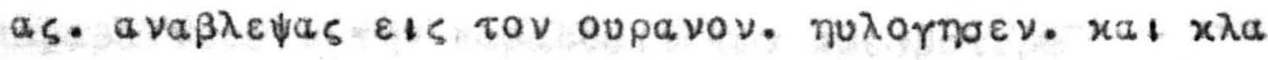

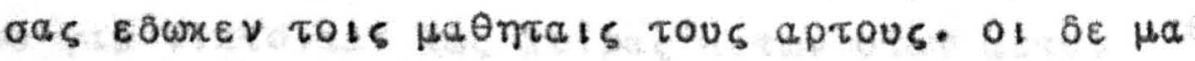

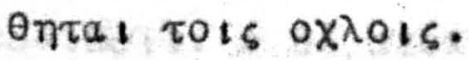

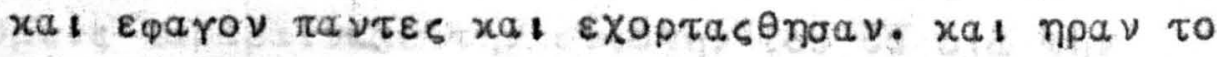

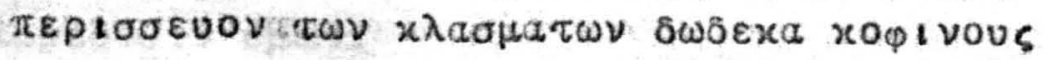

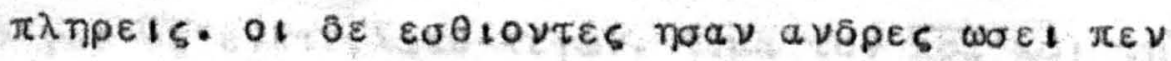

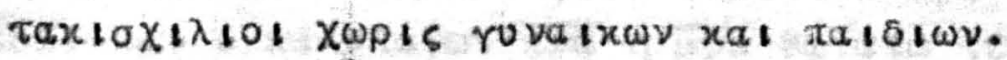

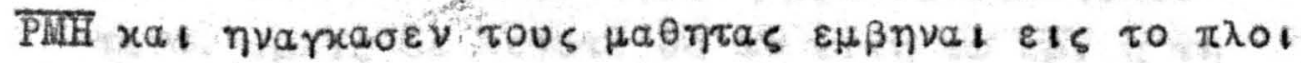

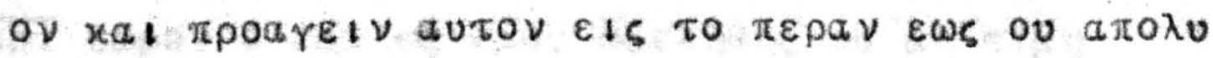
on rovs oxious.

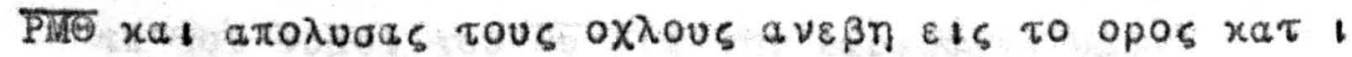
30

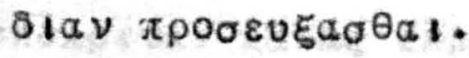

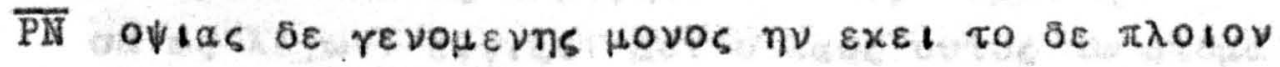

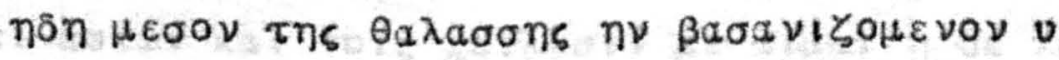

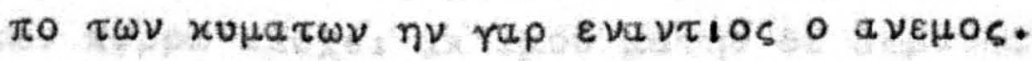

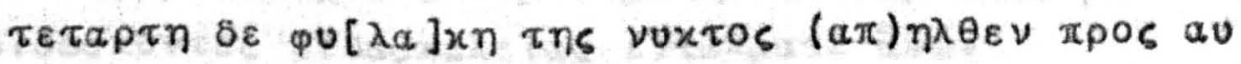

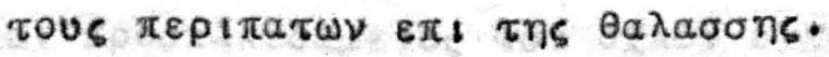

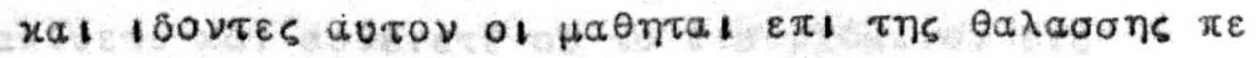

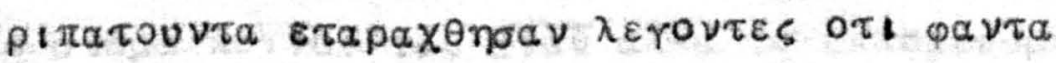

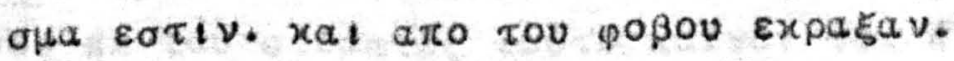

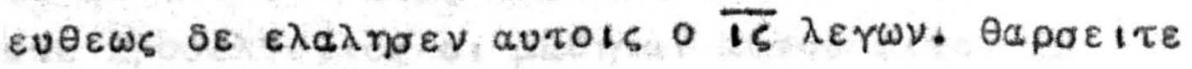
$\varepsilon \gamma \omega \varepsilon t \mu \iota \mu \eta \varphi \beta \varepsilon \iota \sigma \theta a \downarrow$.

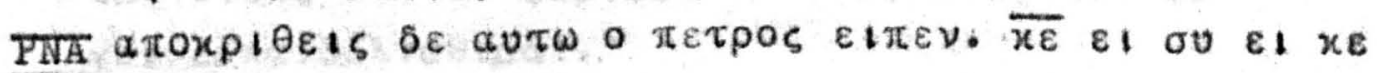




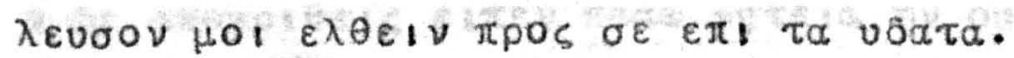

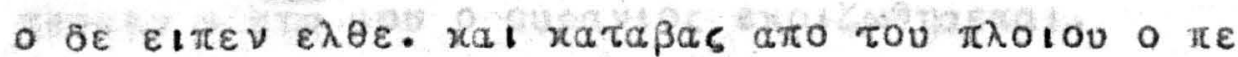

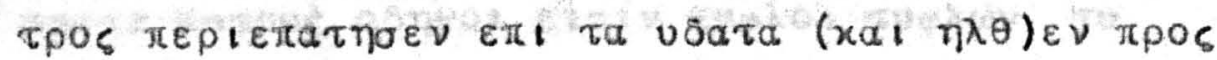

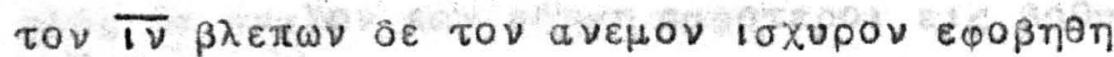

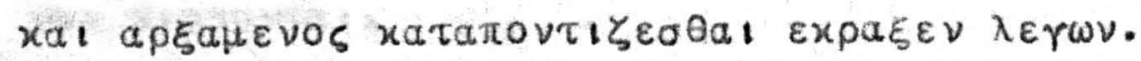

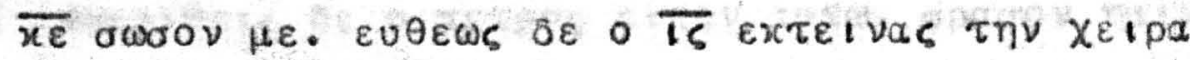

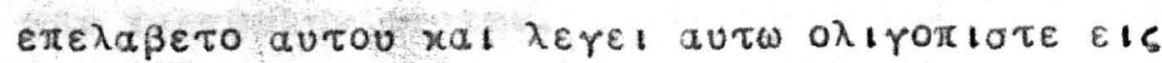

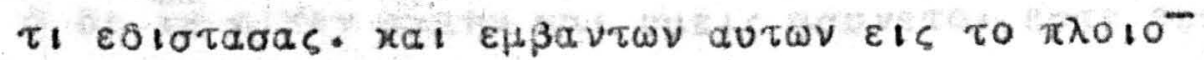

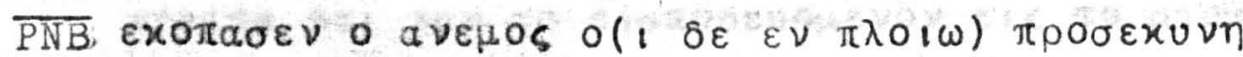

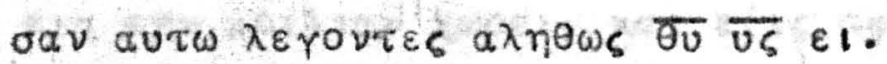

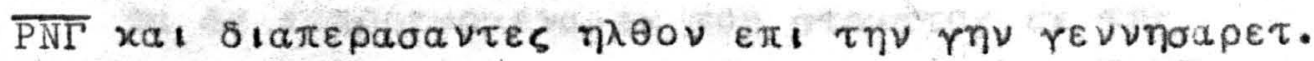

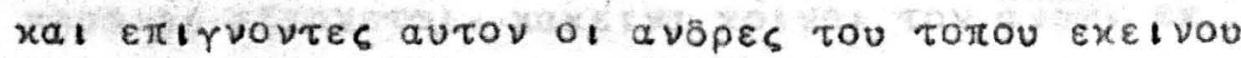

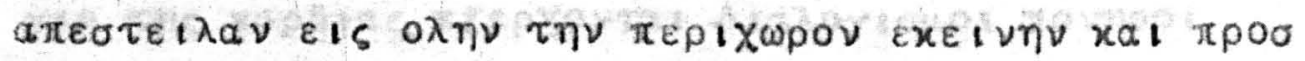

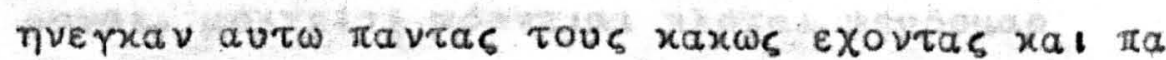

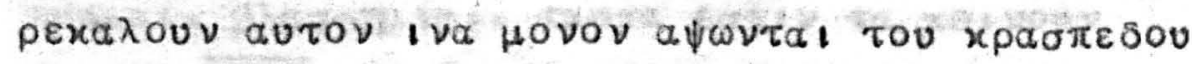

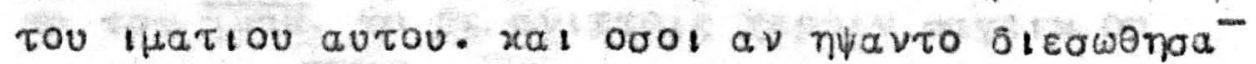

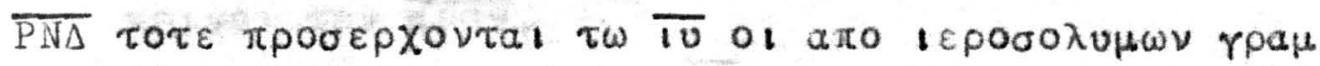

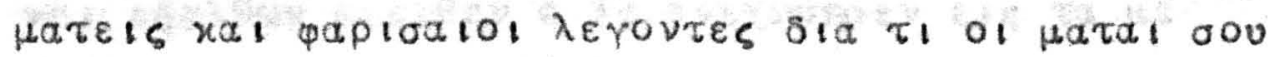

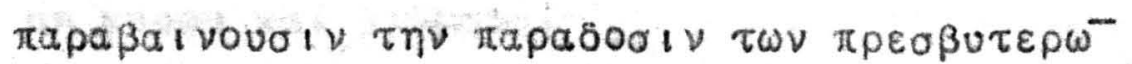

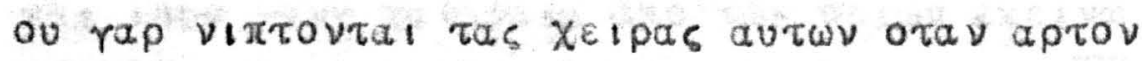

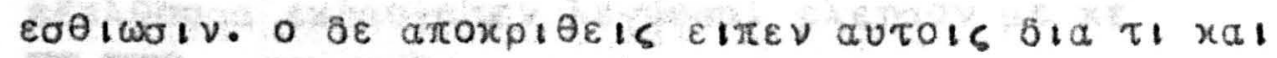

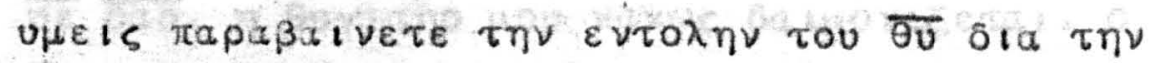
Tapasooiv visw.

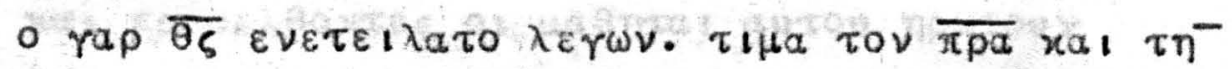

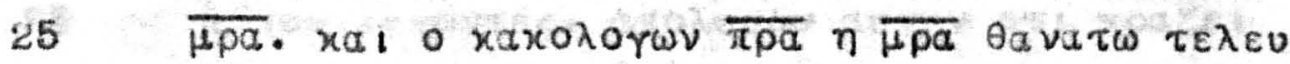

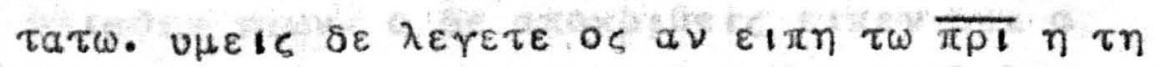

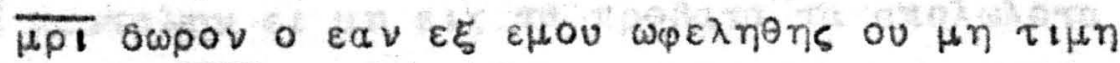

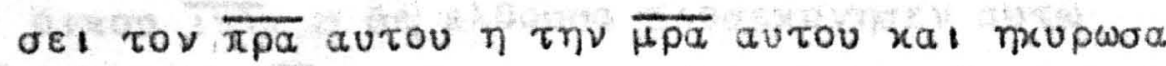

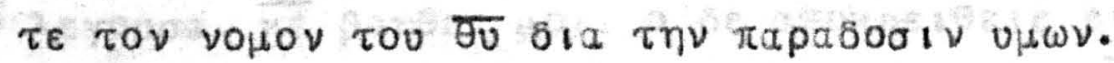
ง

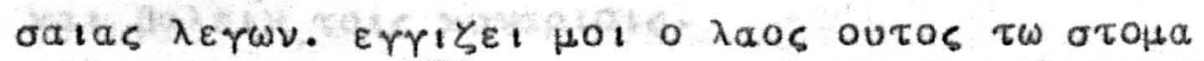

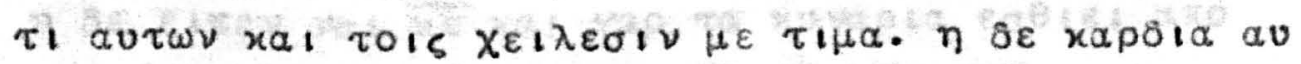

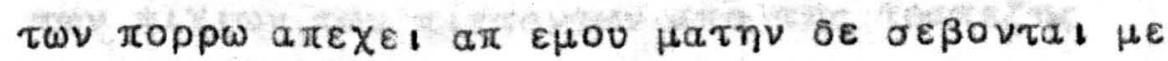

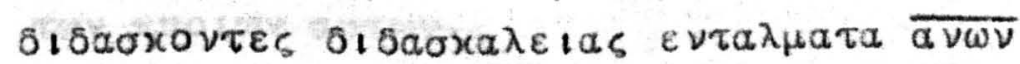

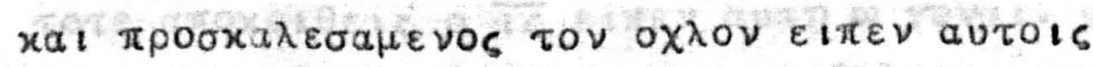

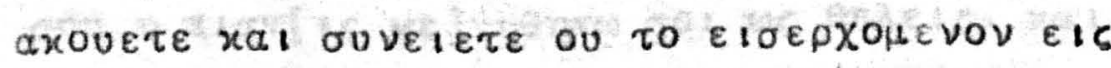

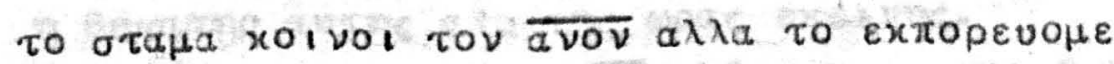

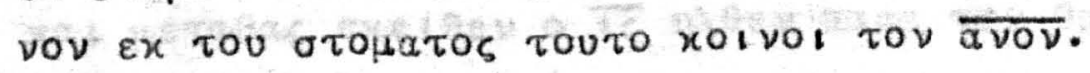

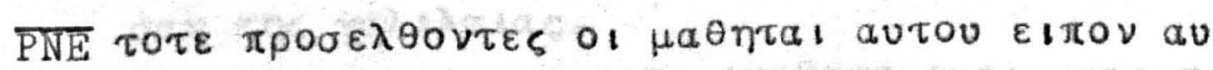

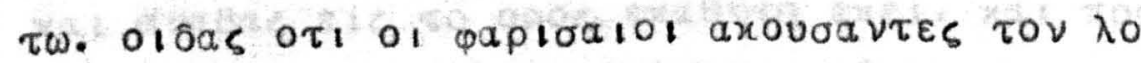
rov $\varepsilon \sigma x a \nu \delta a \lambda เ \sigma \theta$ noav. 


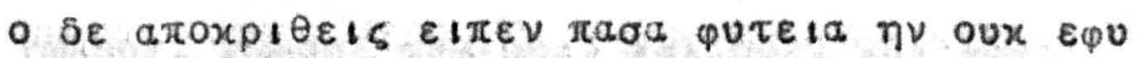

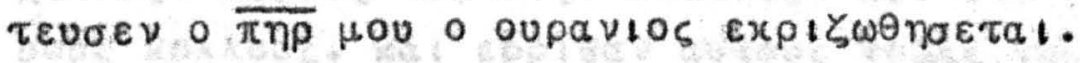

PNS a

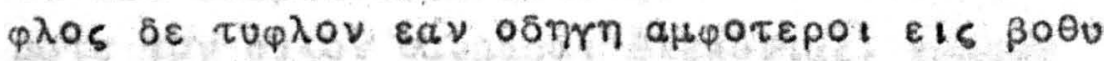
vov $\pi \varepsilon$ वOU vंal.

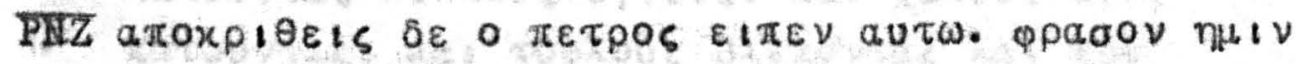

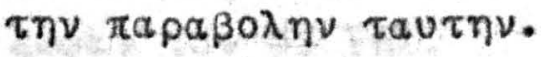

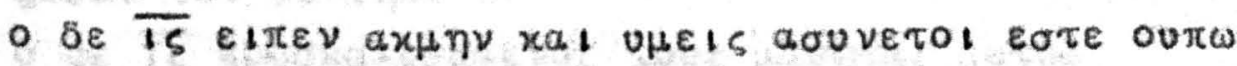

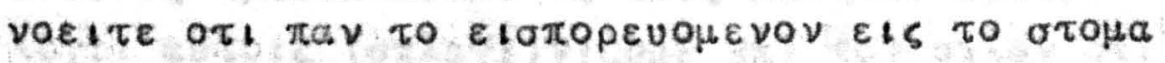

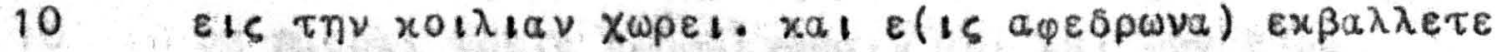

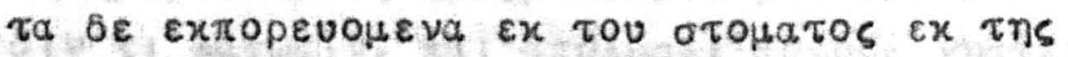

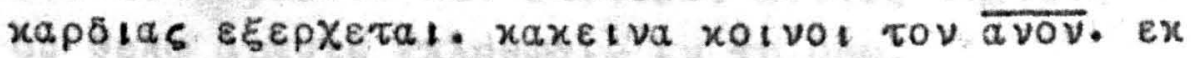

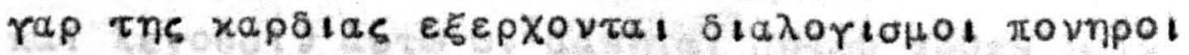

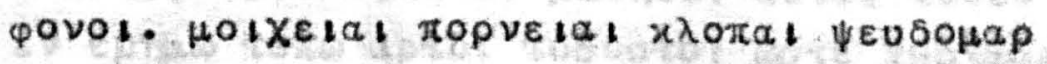

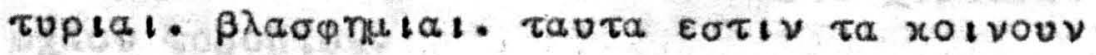

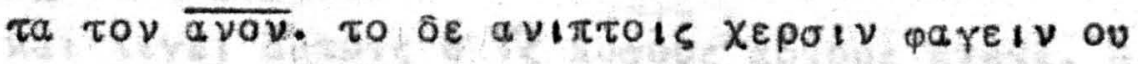
xolvol Tov $\overline{a v O V}$.

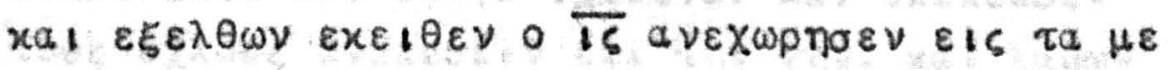

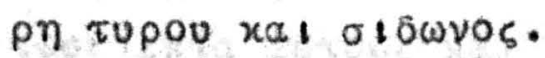

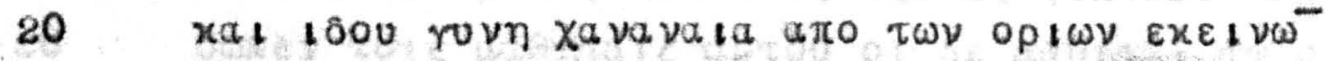

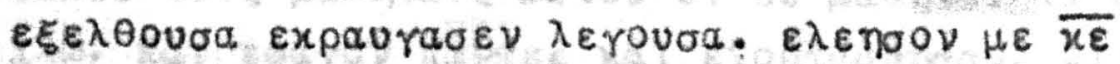

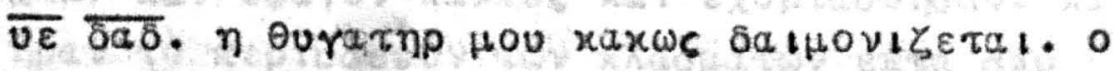

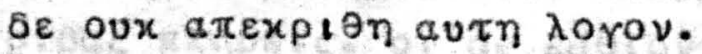

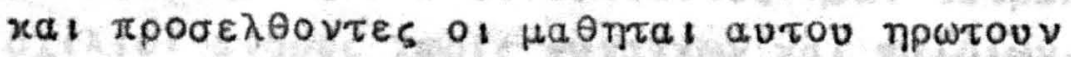

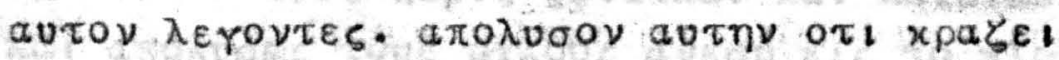

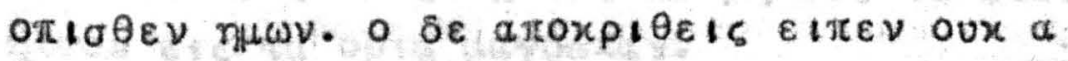

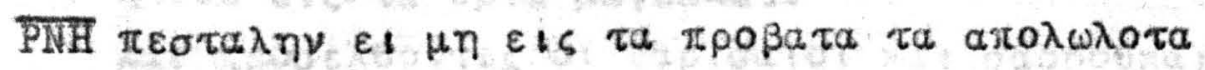

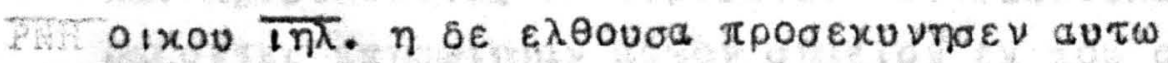

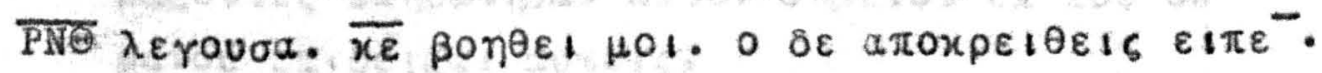

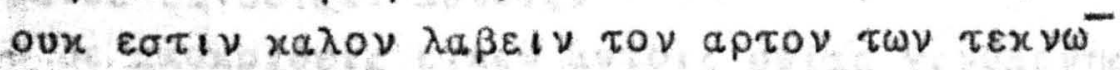
xa! $\beta a \lambda \varepsilon เ V$ Tols xuvaplo!s. $\eta \delta \varepsilon \varepsilon ! \pi \varepsilon v$ vat $\overline{x \varepsilon}$ xal rap $\tau a$ xuvapıa $\varepsilon \sigma \theta i \varepsilon !$ a

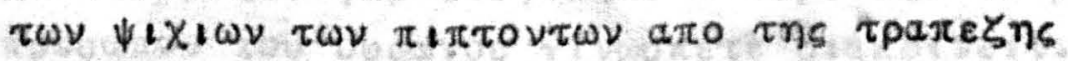
$\tau \omega v$ xup $\omega$ w $\alpha u \tau \omega v$.

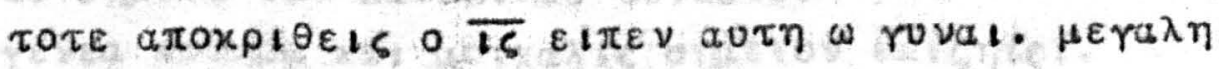

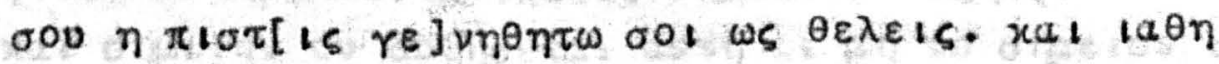

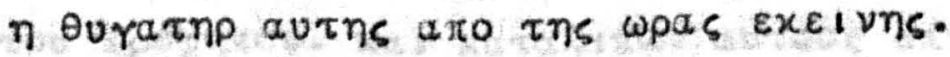

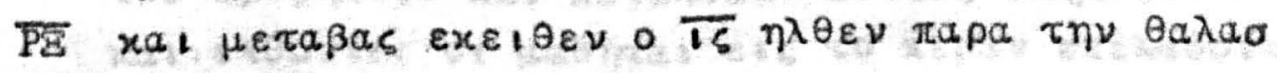

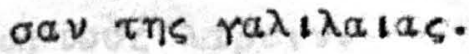

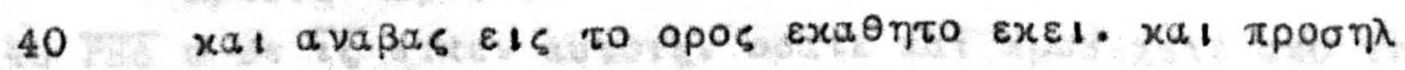

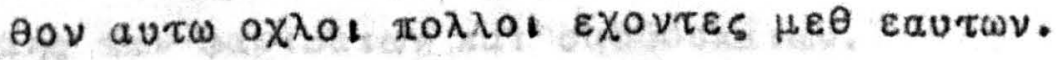




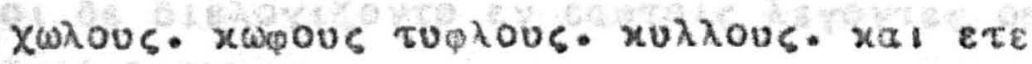

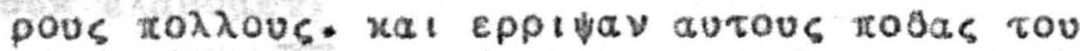

TU xa, $\varepsilon \theta \varepsilon p a \pi \varepsilon v \sigma \varepsilon \nu$ aviols.

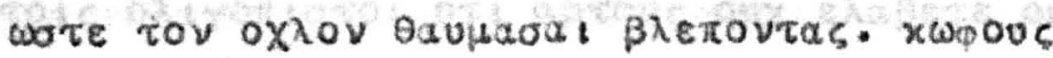

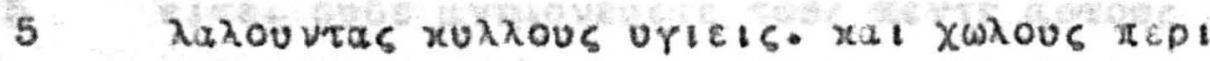
rarouvtas. $x a !$ to gaoav tov $\bar{\theta}$ int.

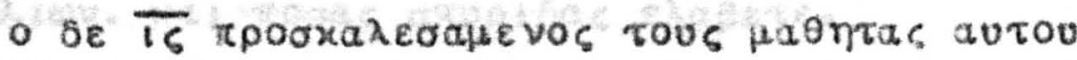

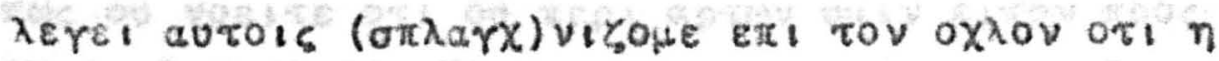

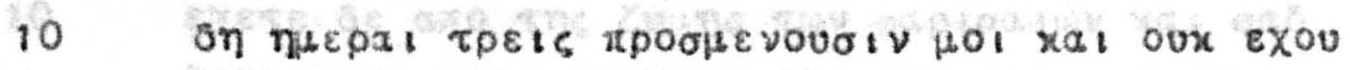

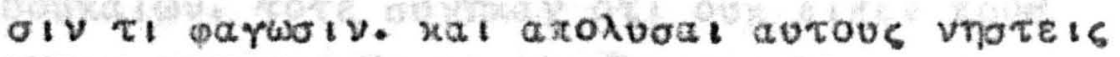

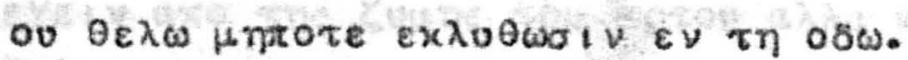
xal Aerovoiv autw of HaOntal autou rooev nulv

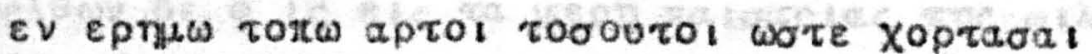
oxiov toooutov. xat leyel autols o Ts rooove aptous exete. ol be

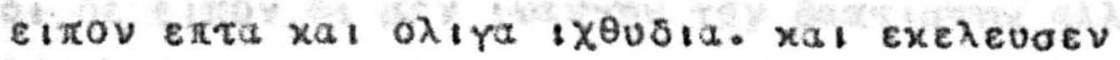

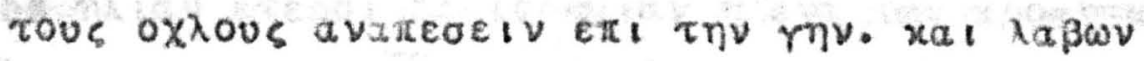
toue erta aptous hat rous 1 xouas $(\varepsilon x \lambda a g e v$ xal) $\varepsilon$

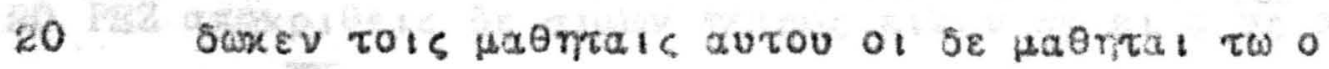

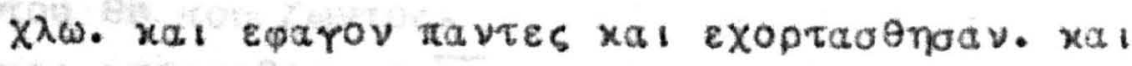

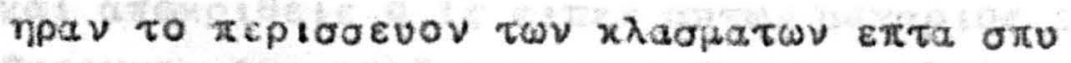

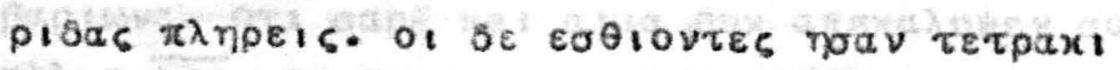

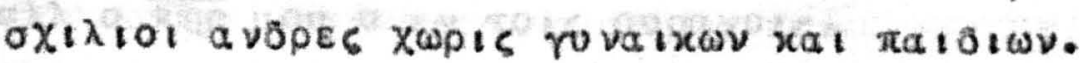

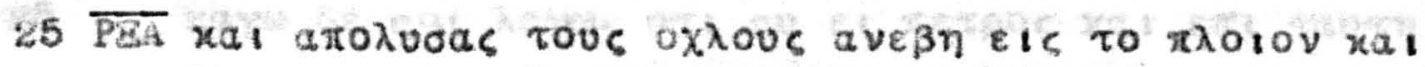

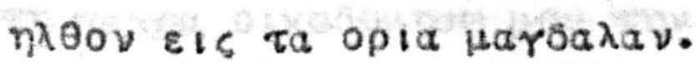

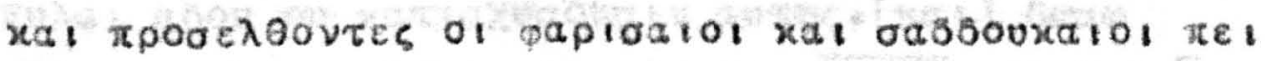

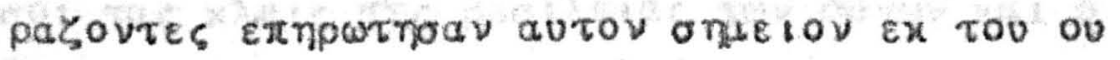

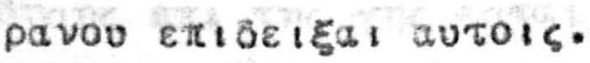

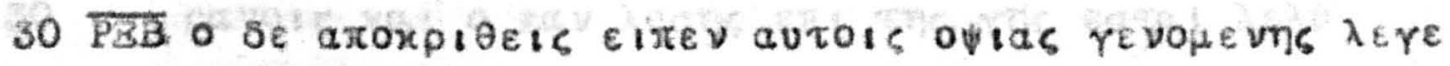

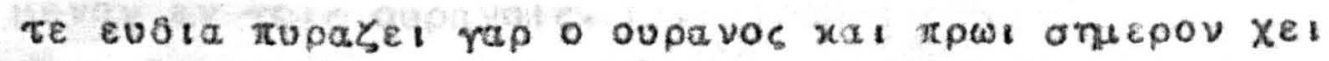

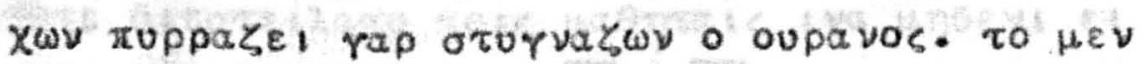

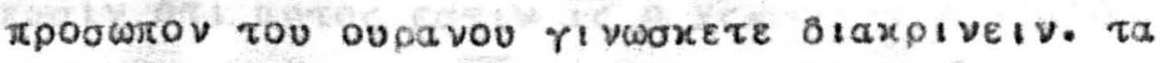

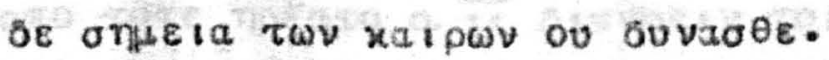

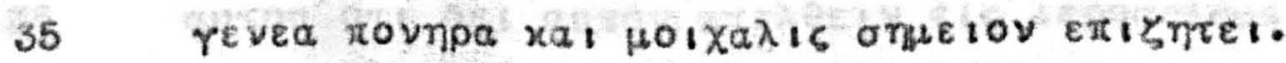

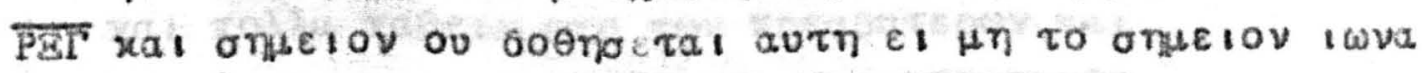

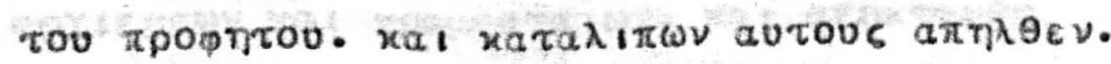

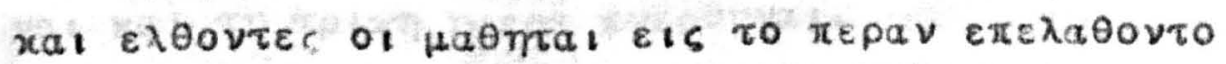
aptous $\lambda \alpha \beta \varepsilon i v$.

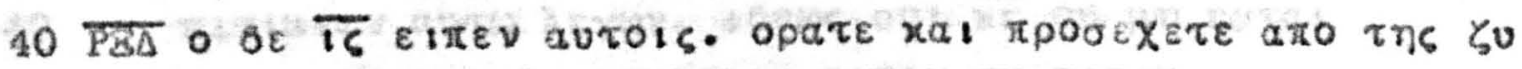

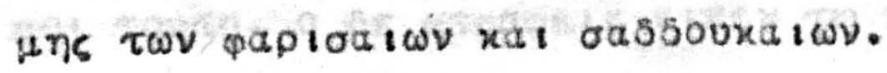




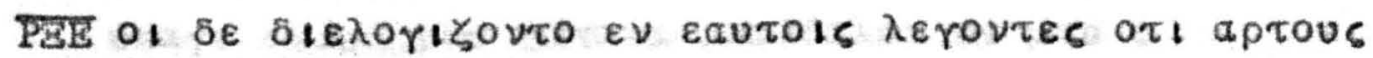
oux $\varepsilon \lambda \alpha \beta о \mu \varepsilon \nu$.

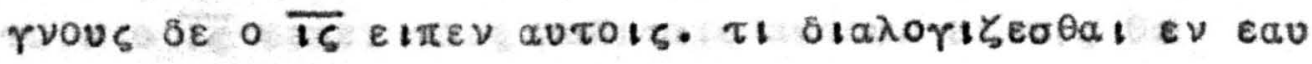

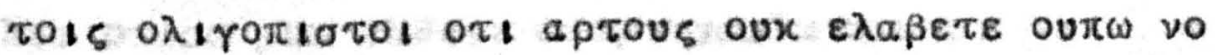

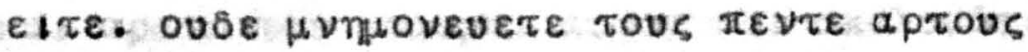

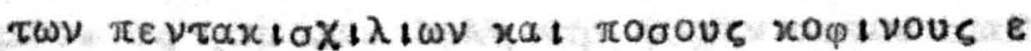

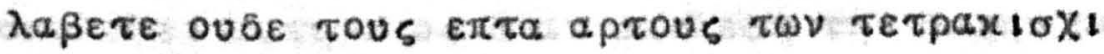

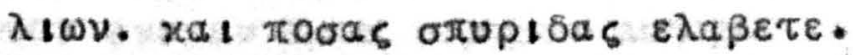

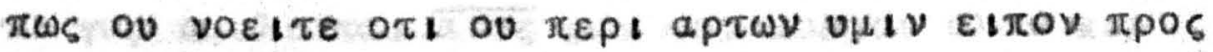

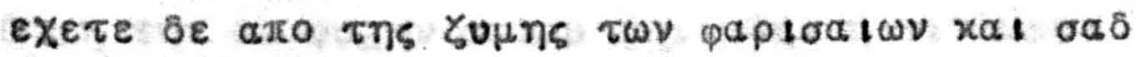

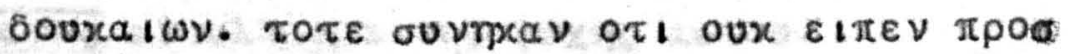

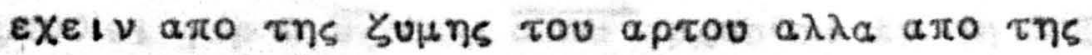

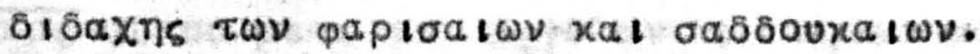

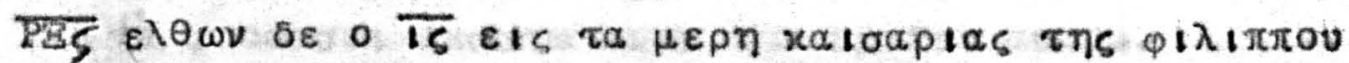
15

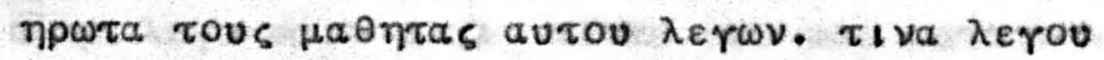

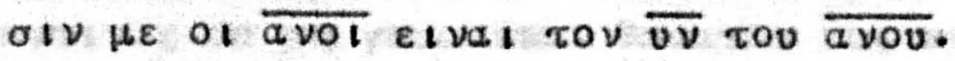

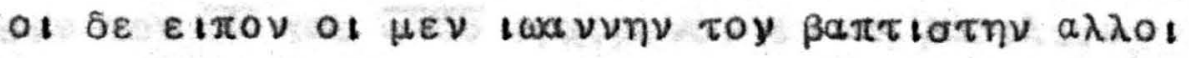

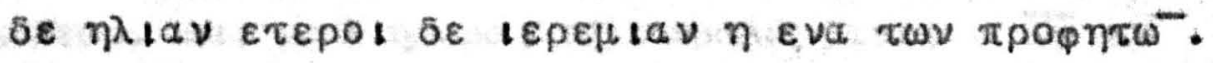

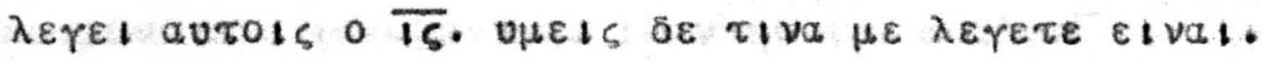

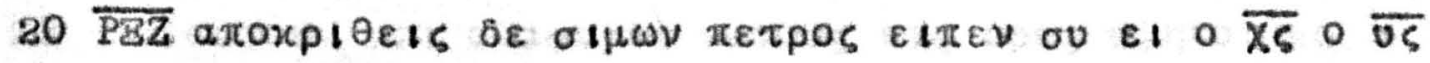

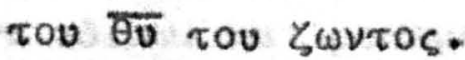

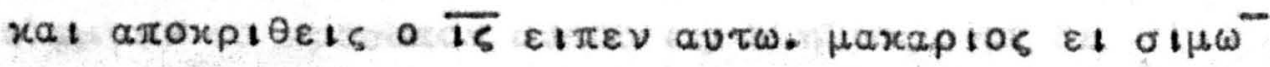

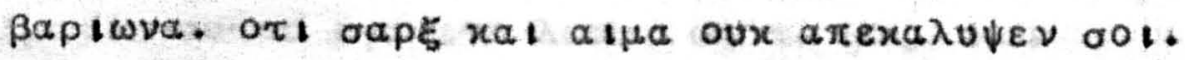
$\alpha \lambda \lambda$ ० $\overline{\pi \eta p} \mu$ ov $0 \varepsilon \nu$ TOLs oupavois.

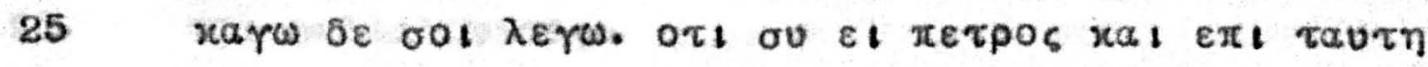

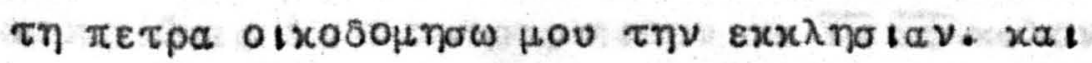

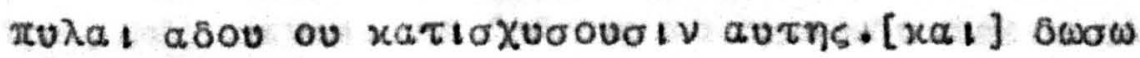

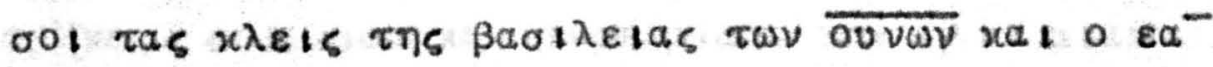

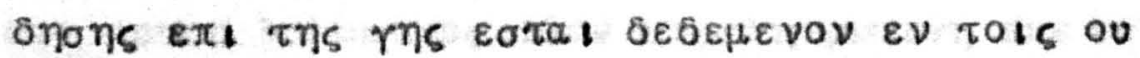

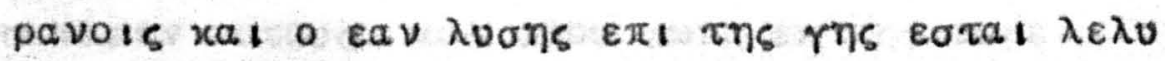
Hevov $\varepsilon \nu$ Tols oupavois.

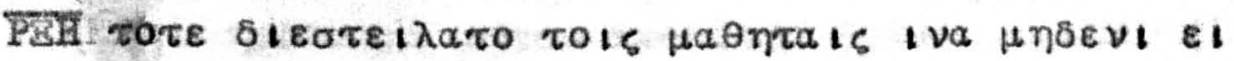

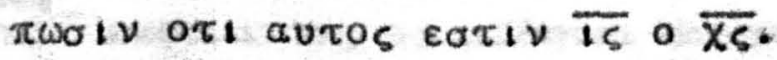

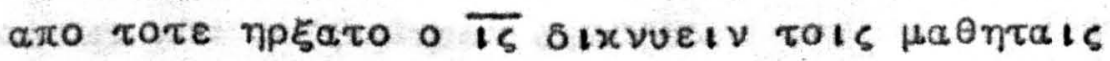

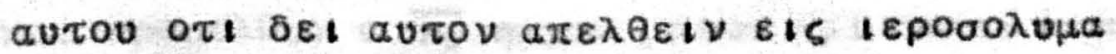

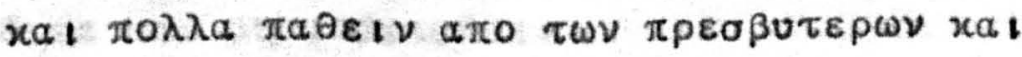

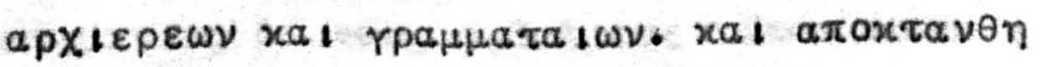

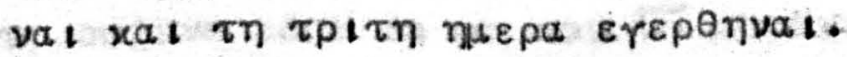

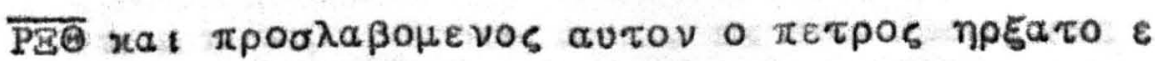

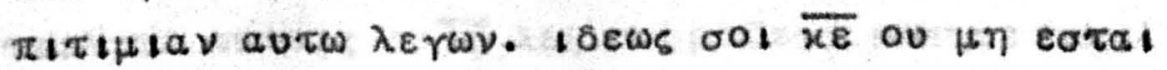

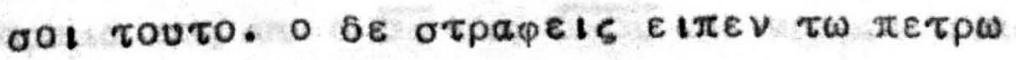




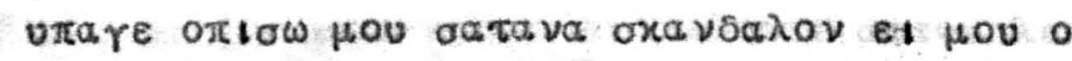

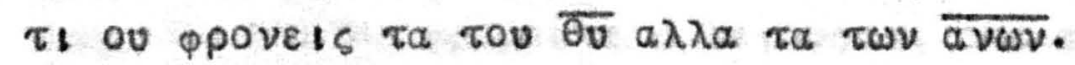

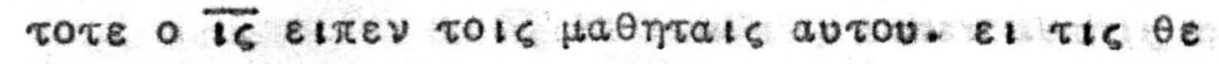

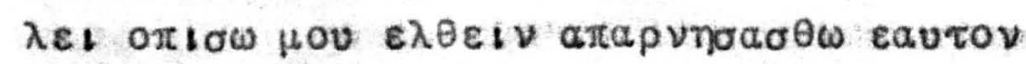

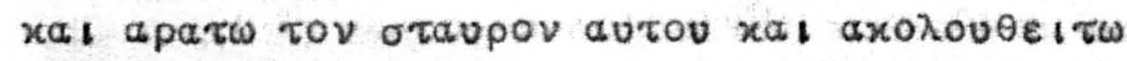

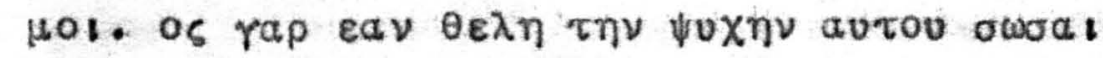

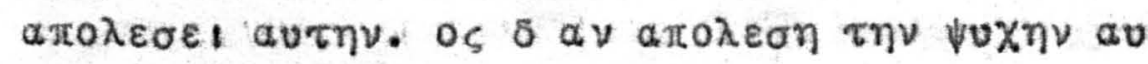

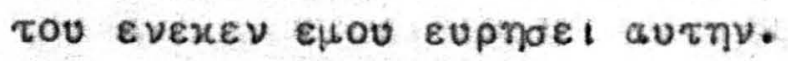

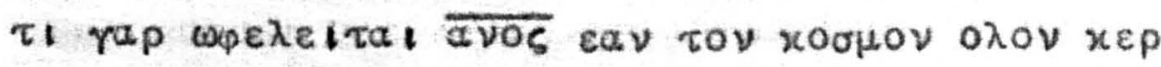

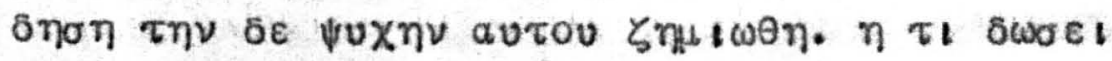

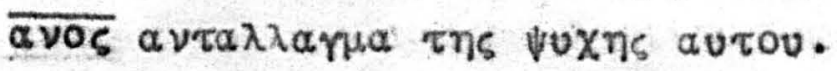

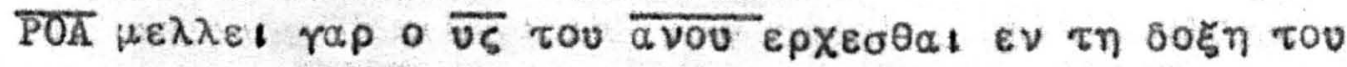

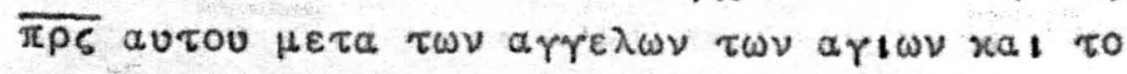
$\tau \varepsilon$ a

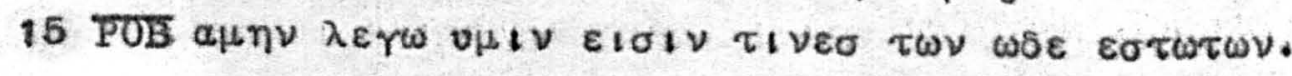

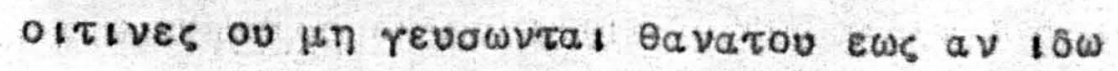

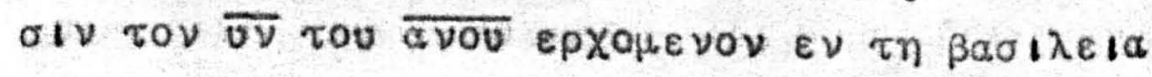

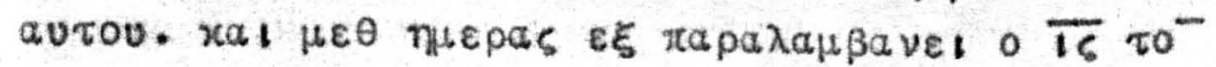

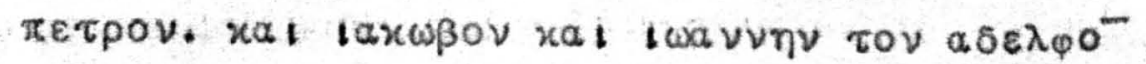

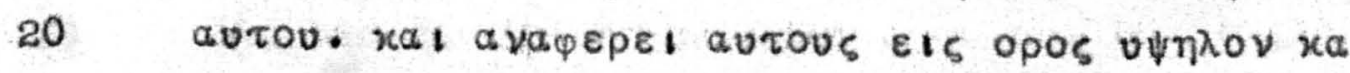

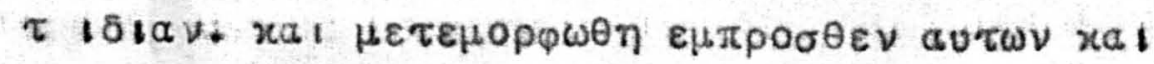

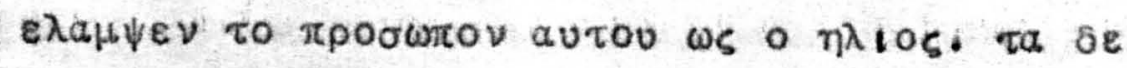

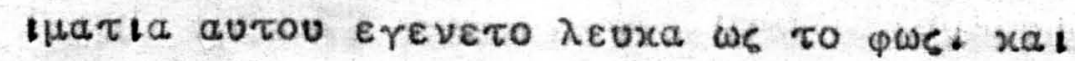

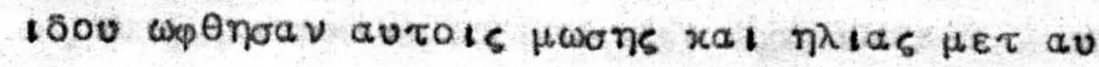

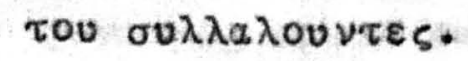

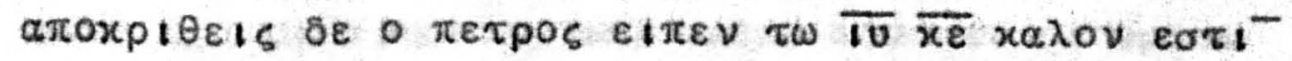

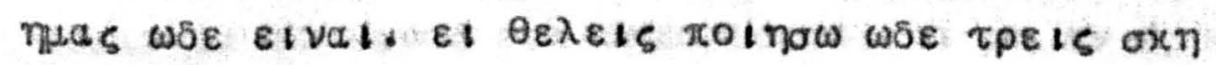

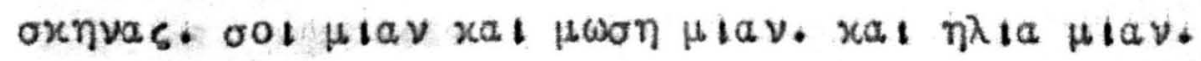

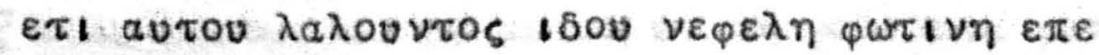

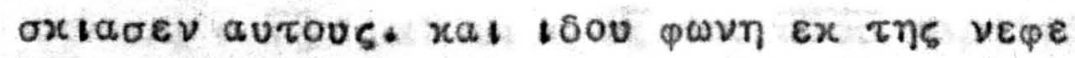

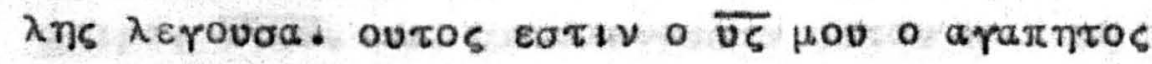

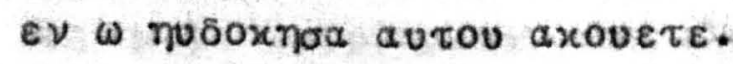

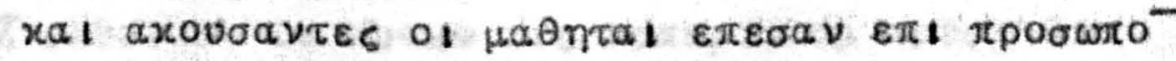

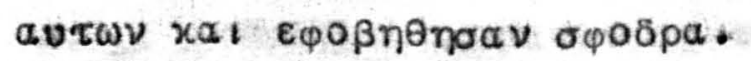

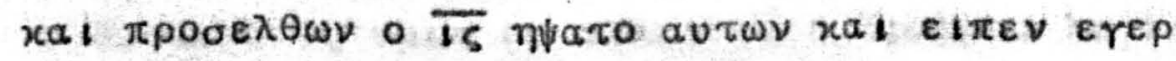

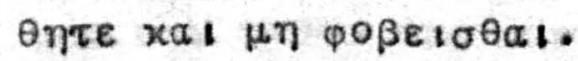

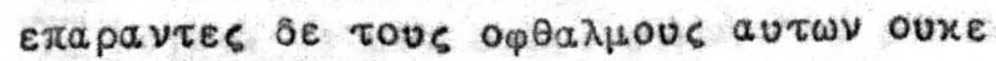

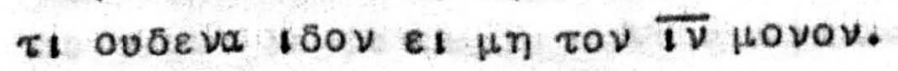

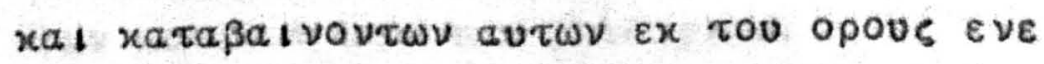

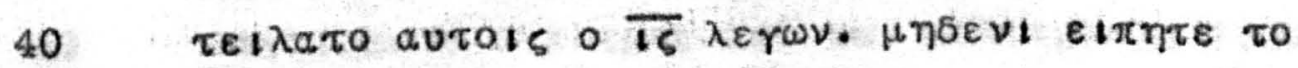

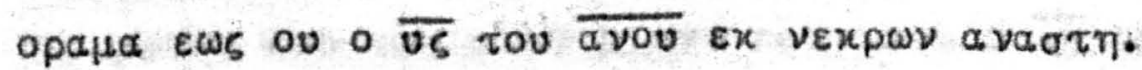


POF xal $\varepsilon \pi \varepsilon \rho \omega \tau \eta$ a

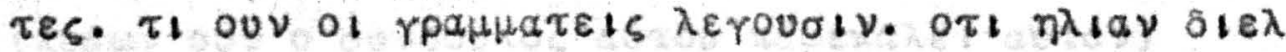

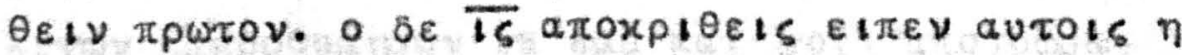

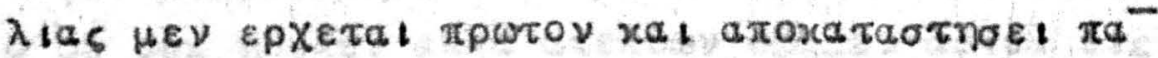

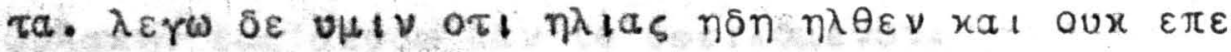

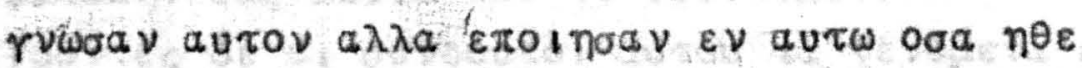

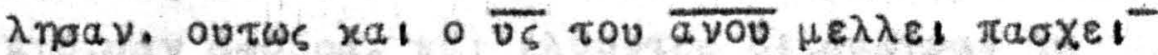

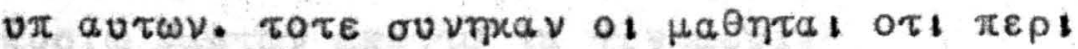

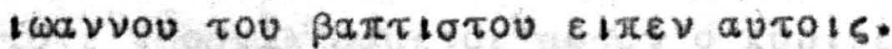

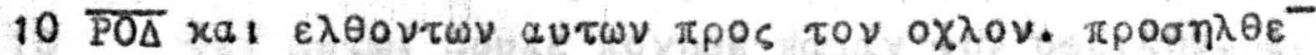

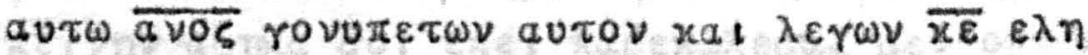

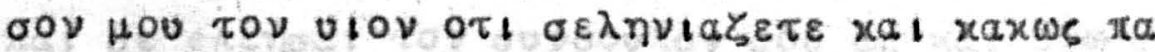

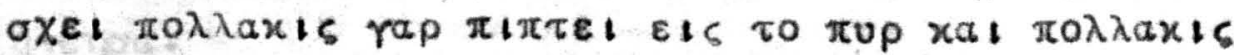

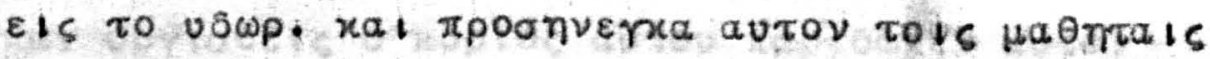

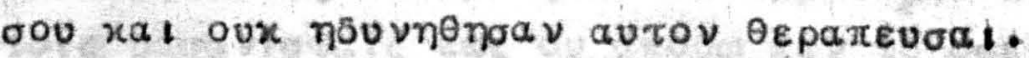

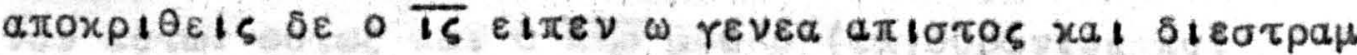

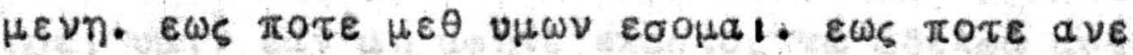

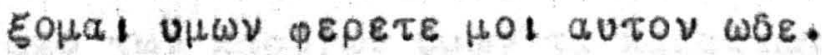

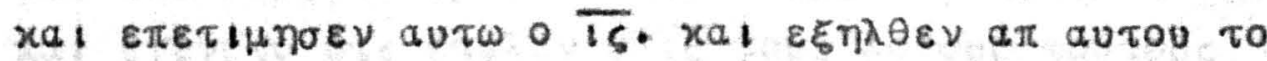

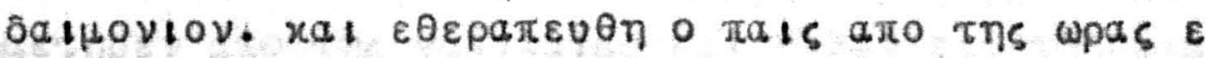

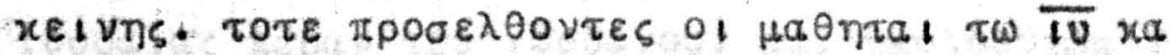

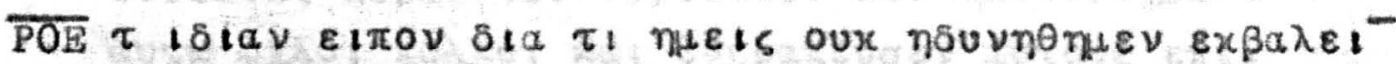

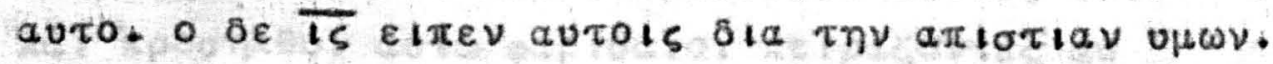

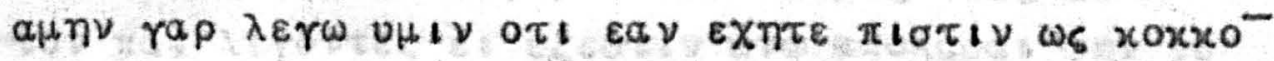

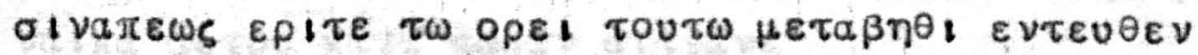

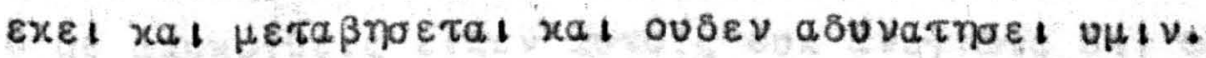

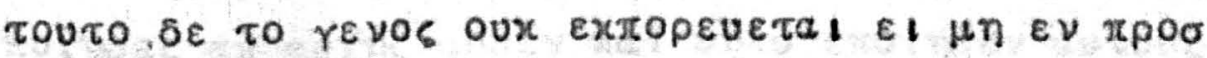
$\varepsilon u \times \eta$ xa! vnore!a.

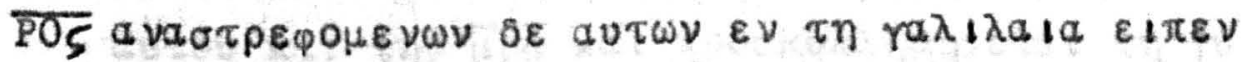

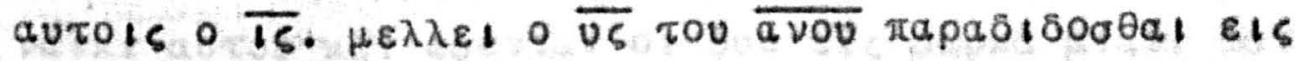

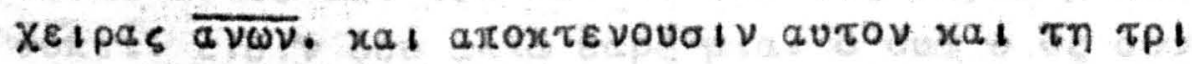

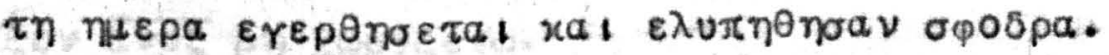

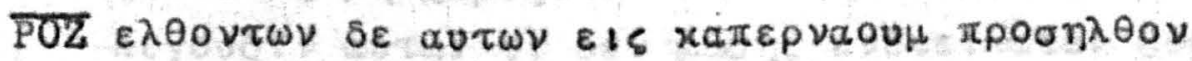

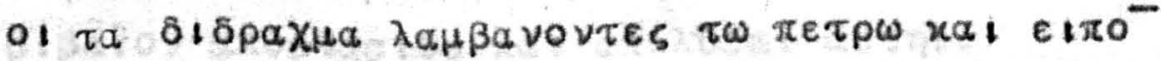

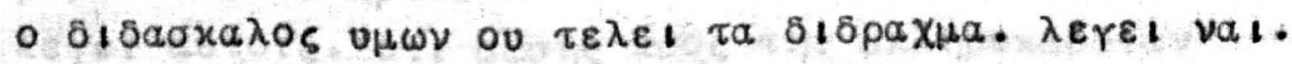

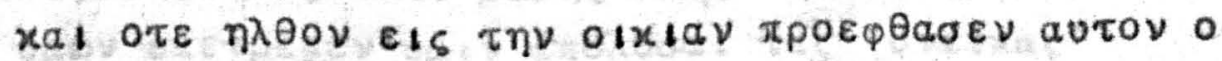

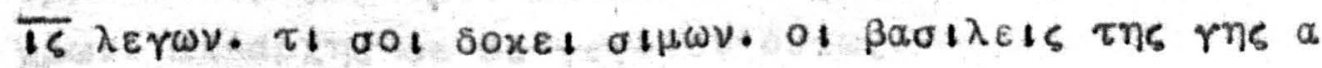

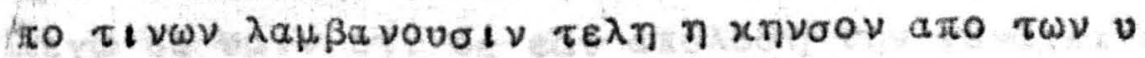

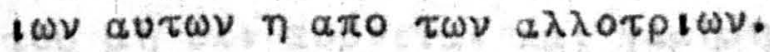

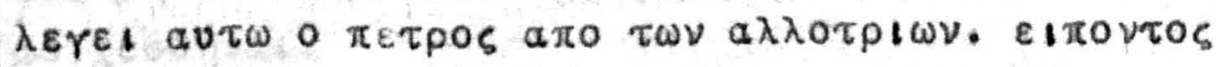

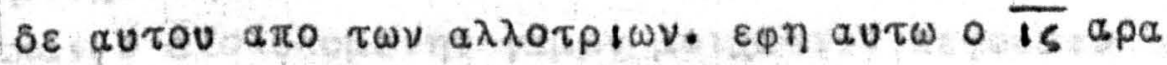


Hol $\varepsilon$ i $\tau$ Opeineis.

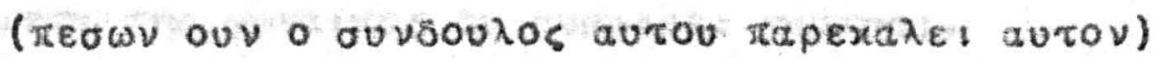
$\lambda \varepsilon \gamma \omega \nu \mu a x p o \theta u \mu \eta \sigma o v e \pi \varepsilon \mu \varepsilon$ xa $\sigma 0(1$ a $\pi 0) \delta \omega \sigma \omega$

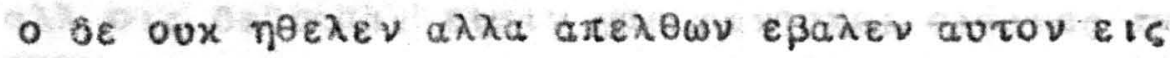

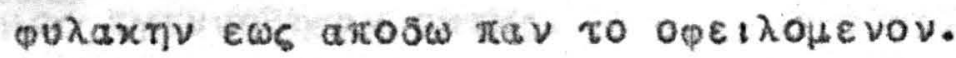
sovies oe ol ouvsounor autou ta revopeva enu

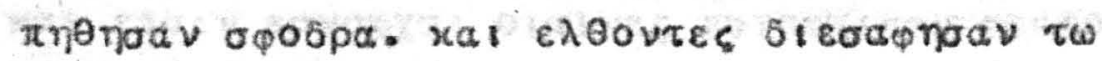
xup

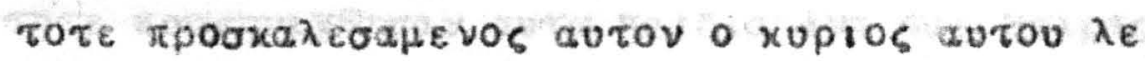

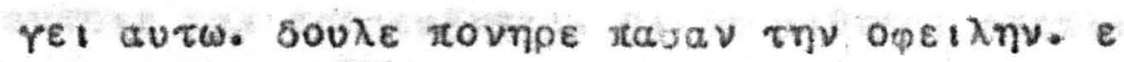

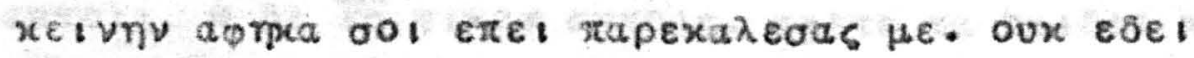

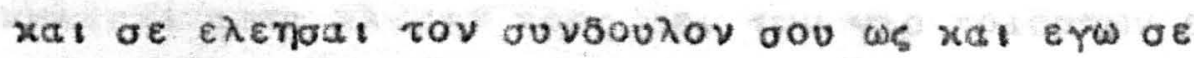

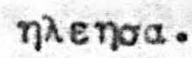

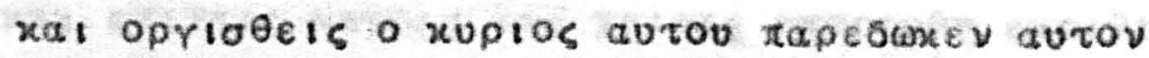

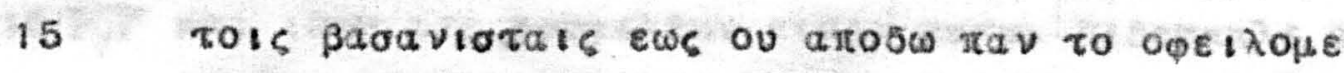

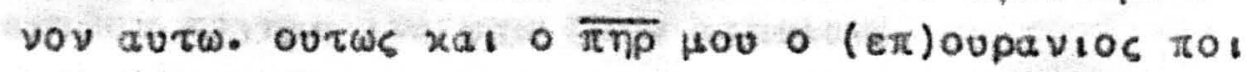

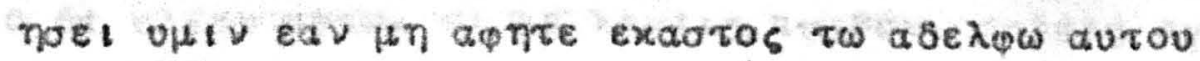

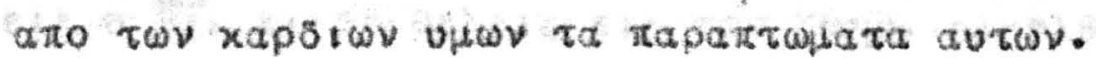

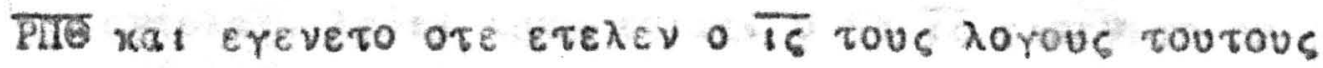

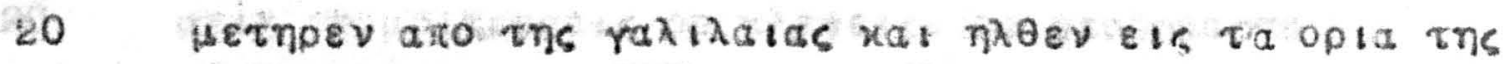
lousalas tepav tou lopsavov. xal mionoverjay

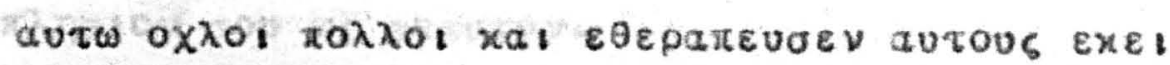

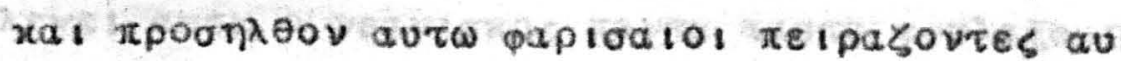

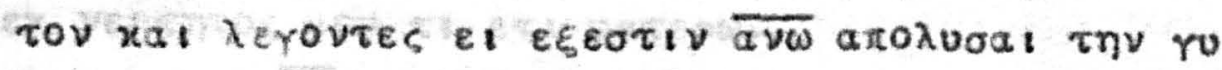

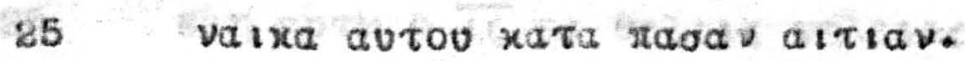

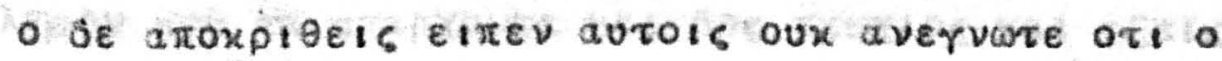

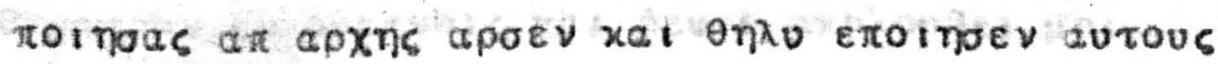

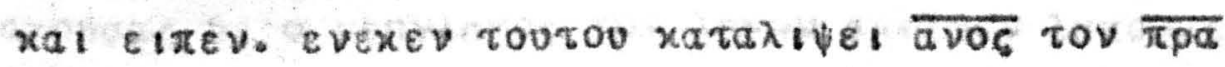

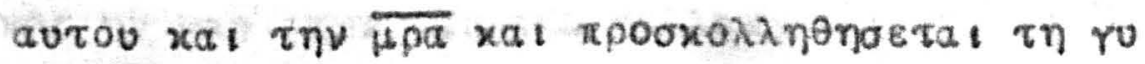

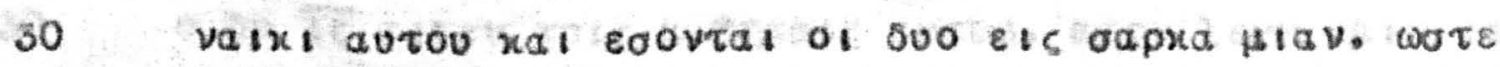

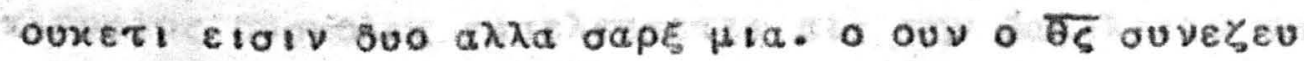
$\xi \varepsilon \nu \overline{\alpha v 0 \zeta} \mu \eta x \omega \rho ! \zeta \varepsilon \tau \omega$.

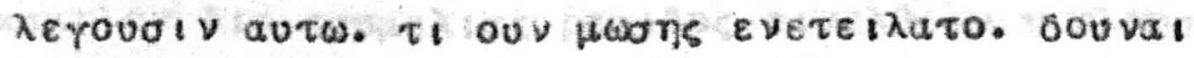

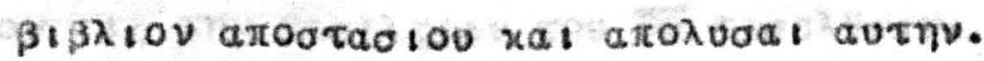

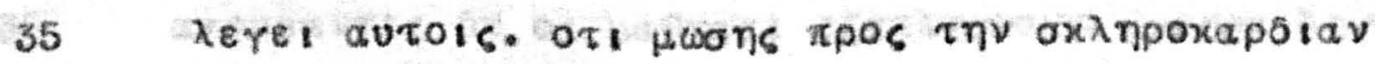

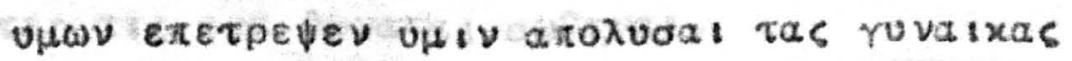

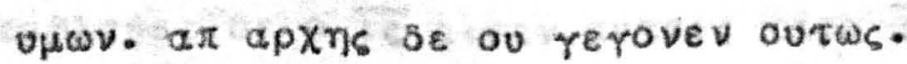

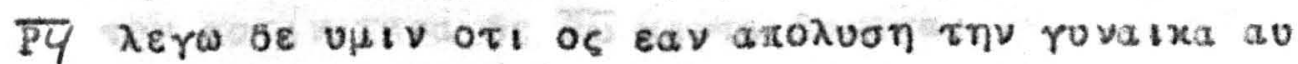

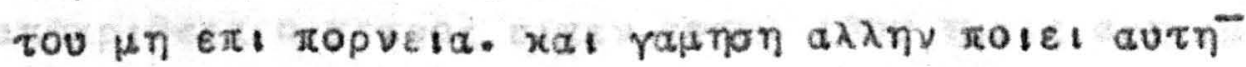

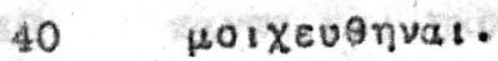

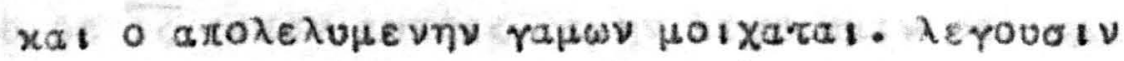




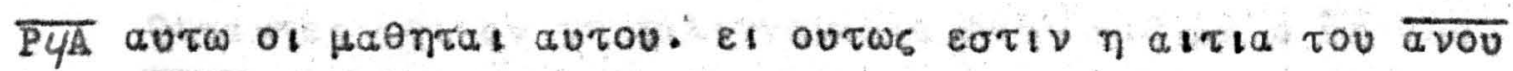
$\mu \varepsilon \tau a$ ths rovalixos ov ouнpepe! rap noal.

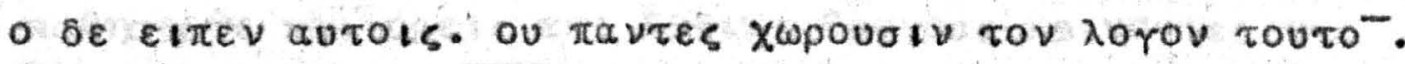

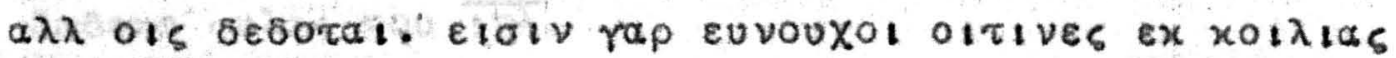

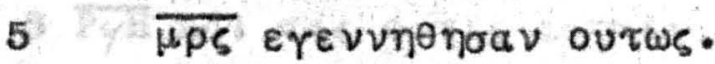

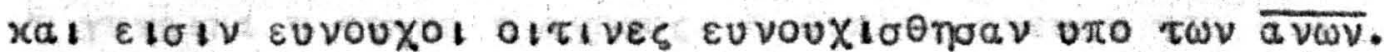

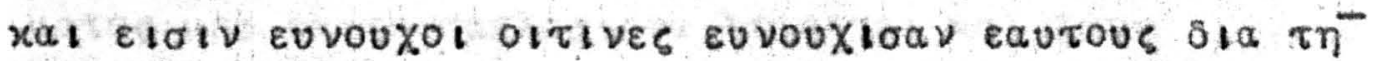

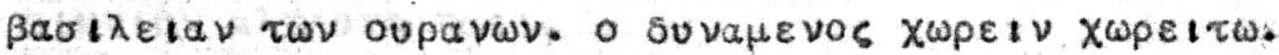

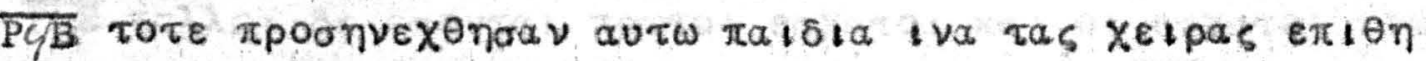

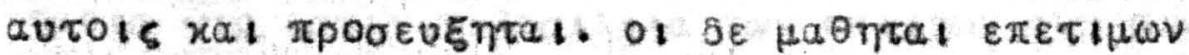

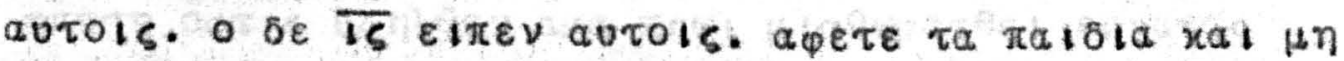

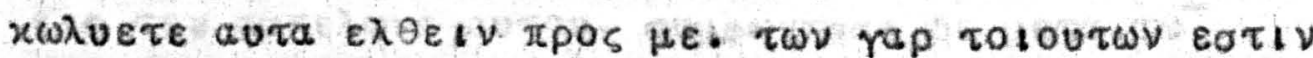

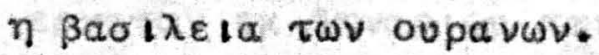

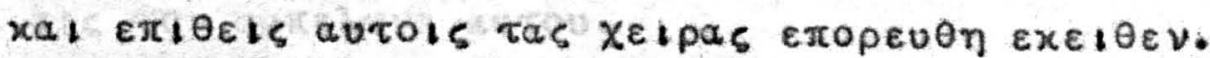

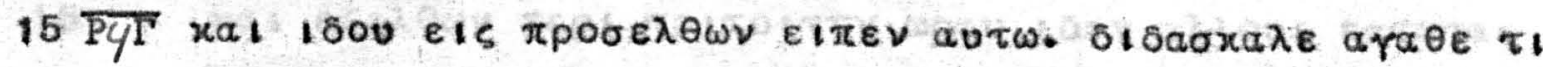

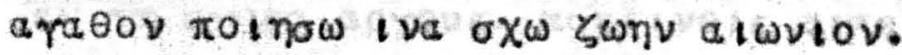

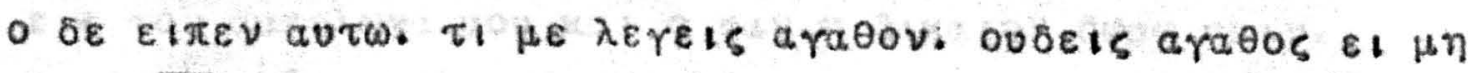

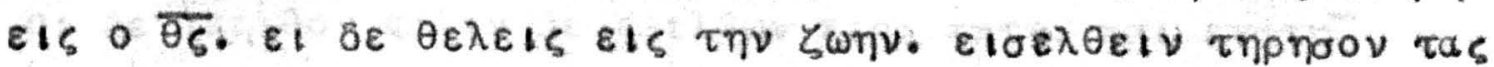

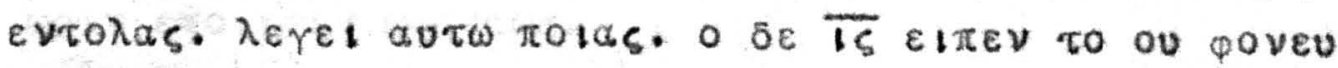

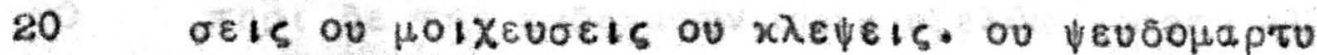

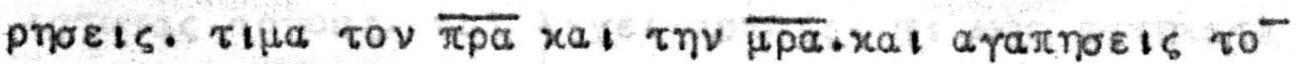

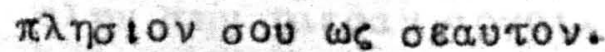

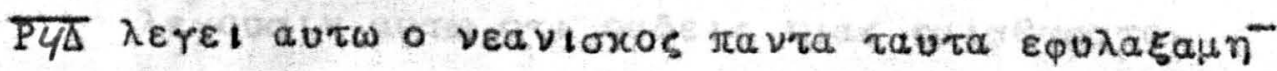

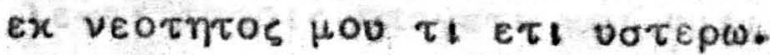

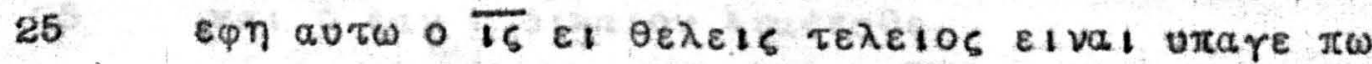

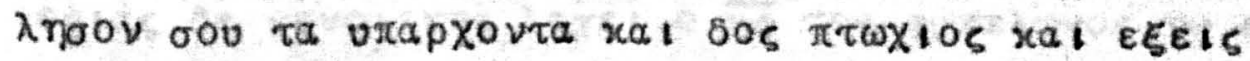

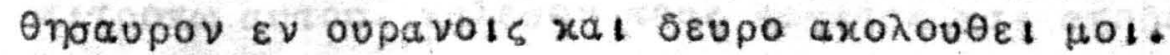

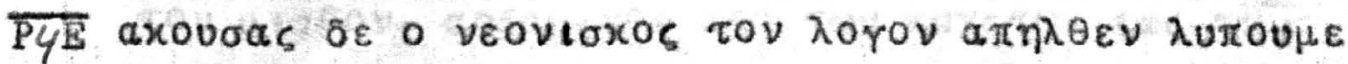

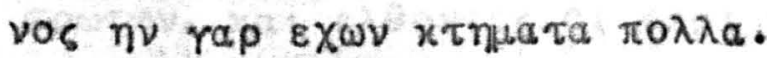

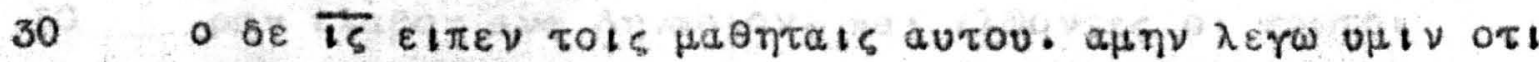

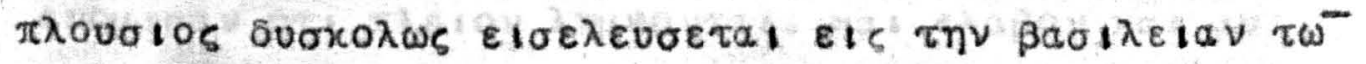

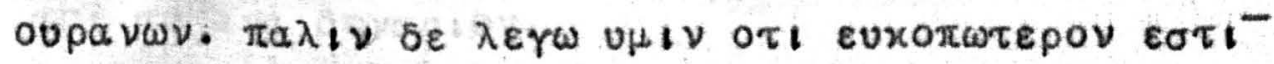

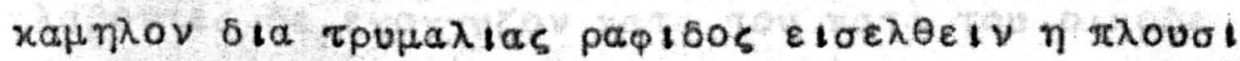

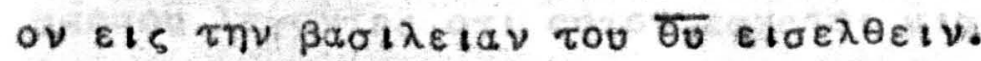

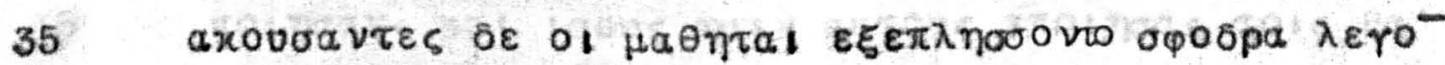

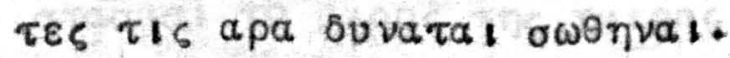

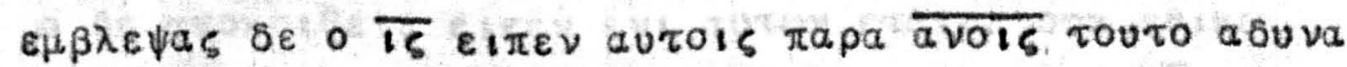

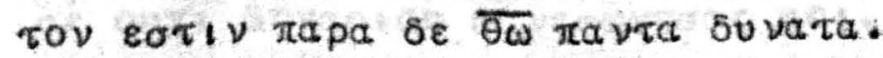

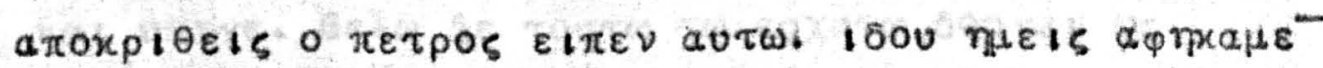

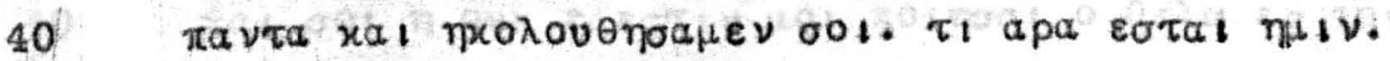

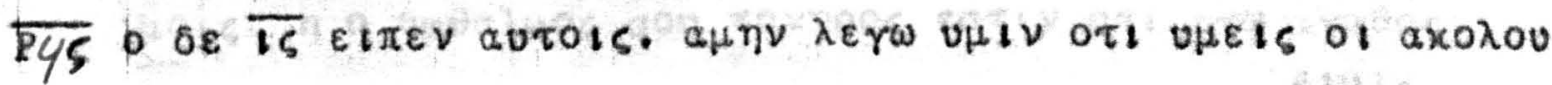




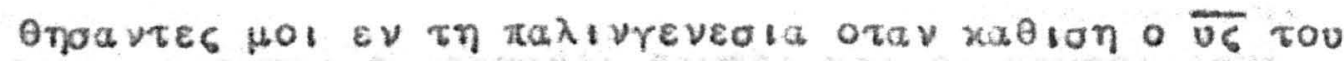

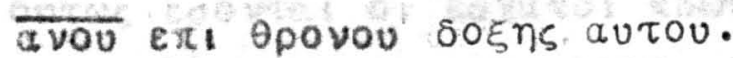

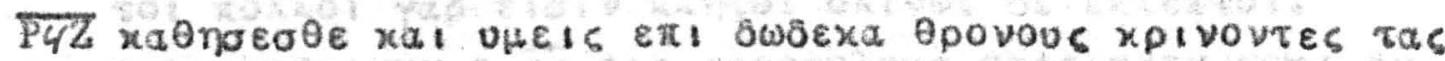
swbexa pulac rov iñ.

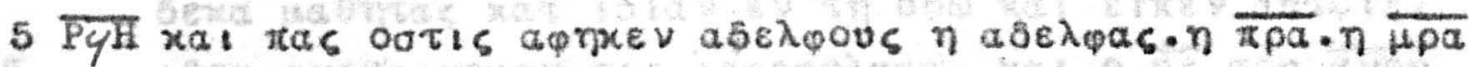
$\eta$ rovaixa $\eta$ texva $\eta$ arpous. $\eta$ oixtas evexev tou ovo

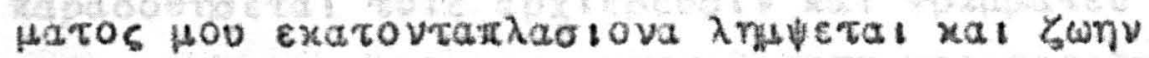
alwviov x npovounoel.

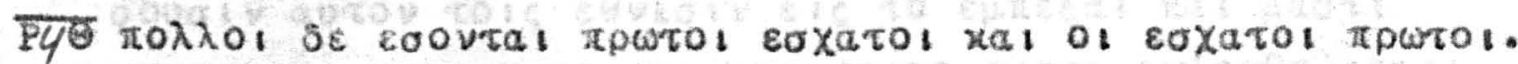

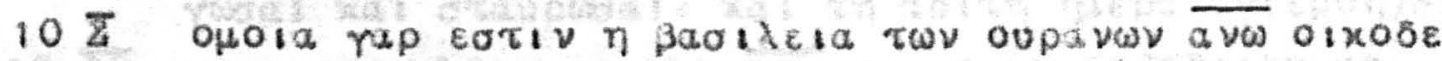

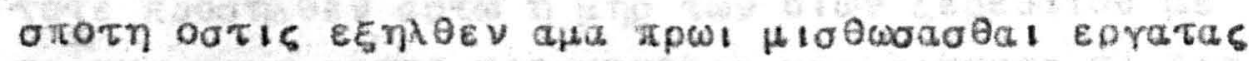

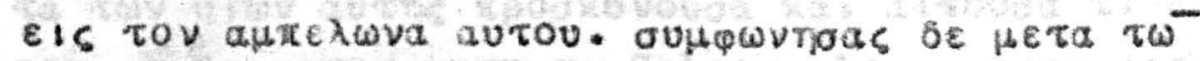

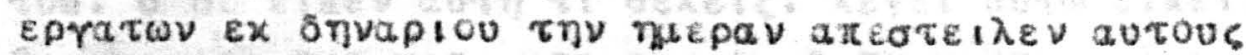

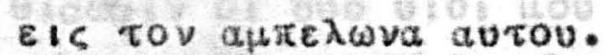

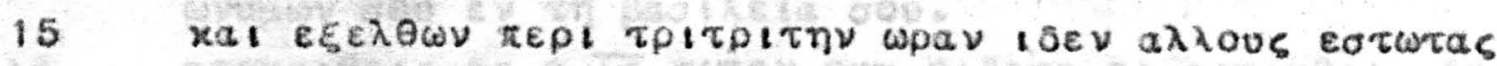

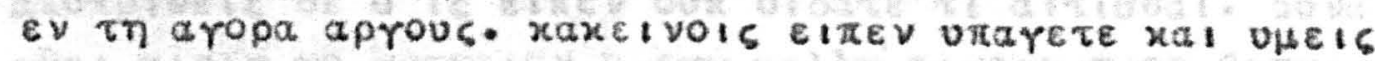

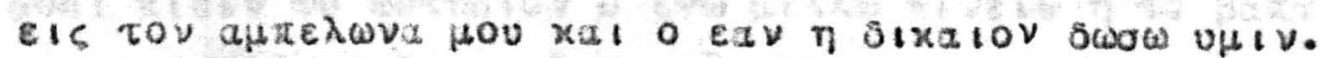

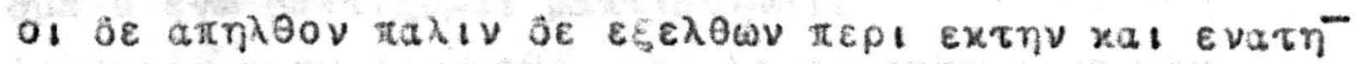

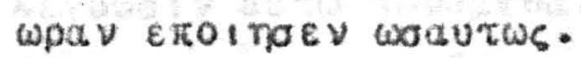

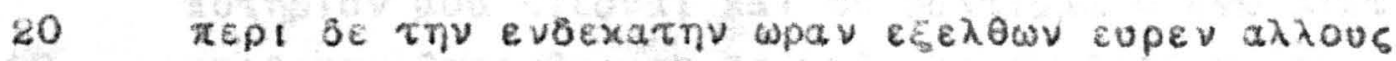

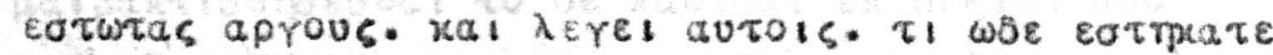

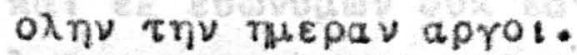

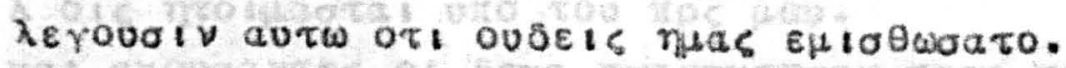

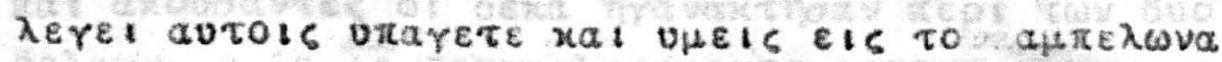
25 xal 0 eav $\eta$ otxatov $\lambda \eta_{\mu} \downarrow \varepsilon a \theta \varepsilon$.

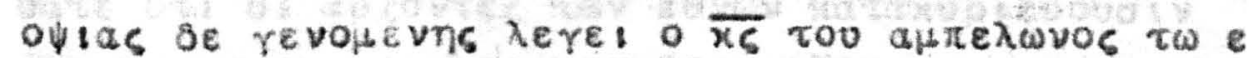

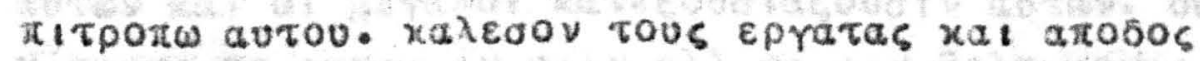

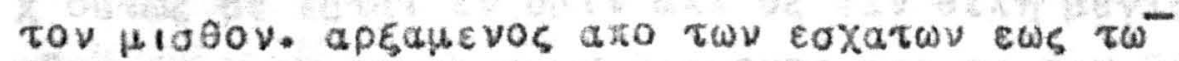

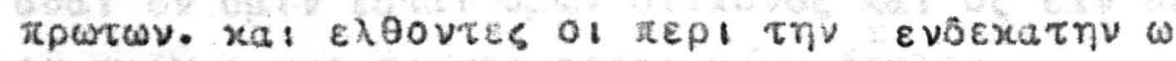

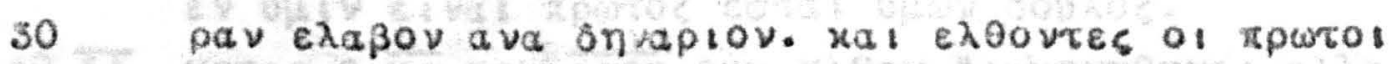

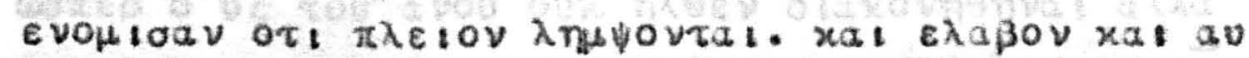
Tot to a va ônvapıov.

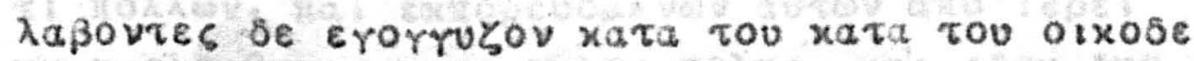

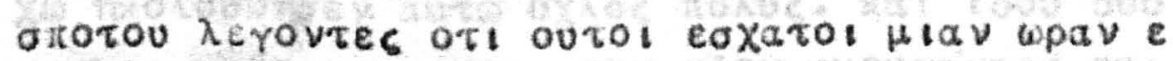

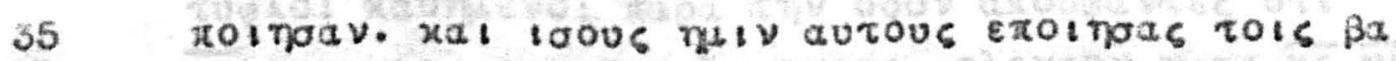
ataoad to Bapos the nuepas kat tov kavowva.

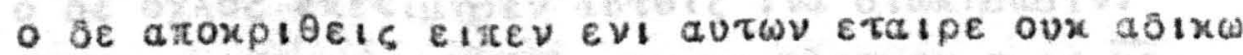
aE ouxl ónvapiou auvequvnaas Hol. apov to $00^{-}$

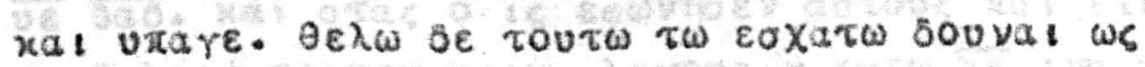

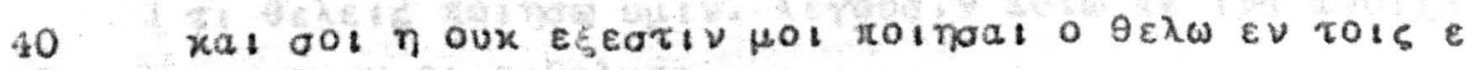

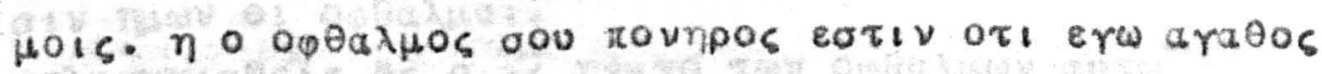




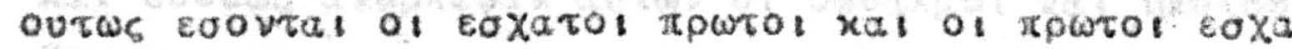

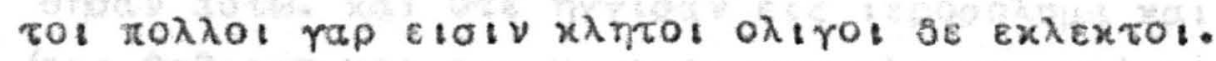

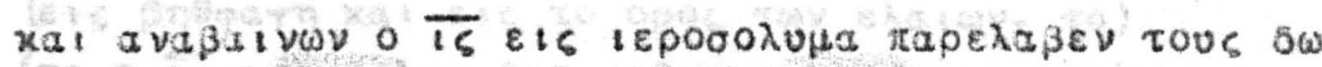

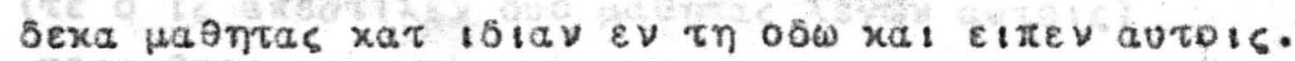

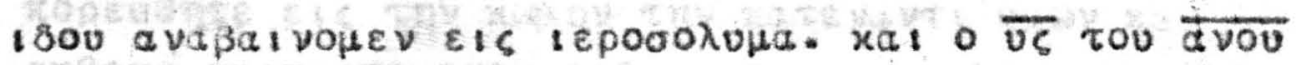

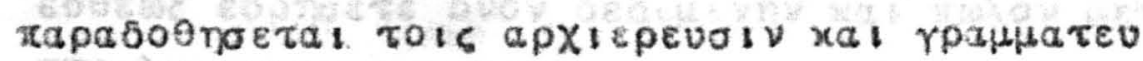

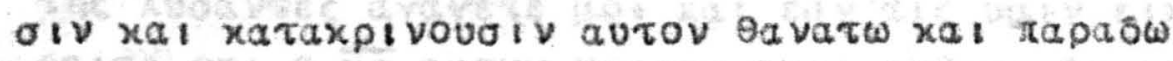

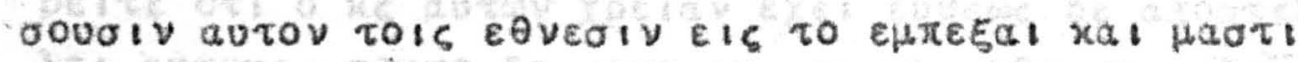

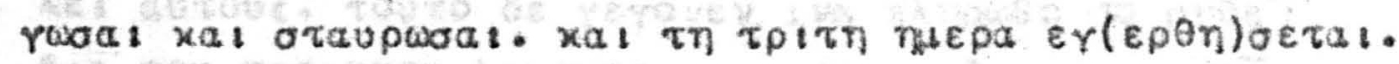

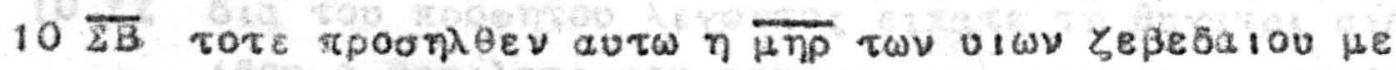

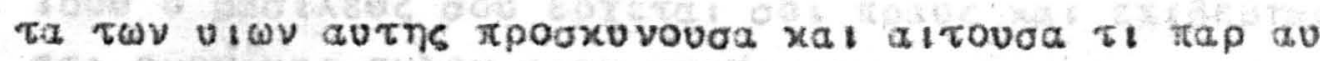

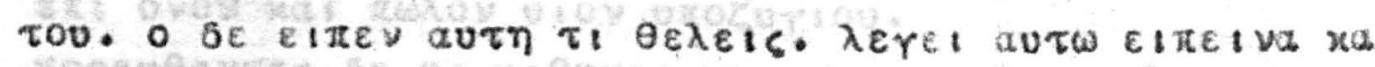

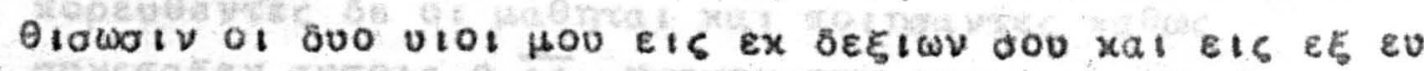

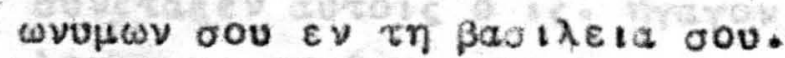

15 a

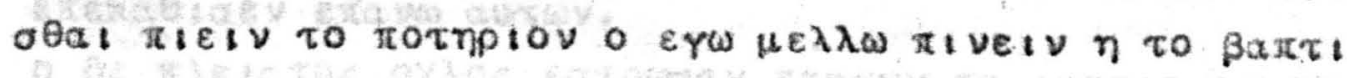

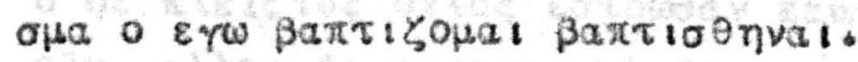

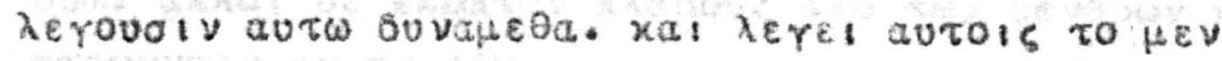

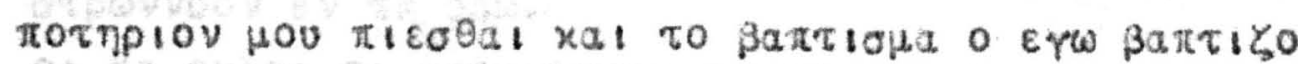

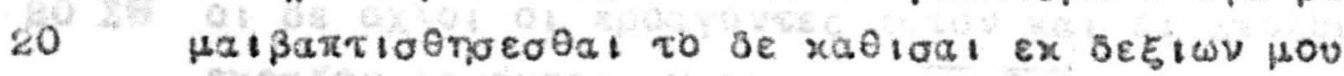

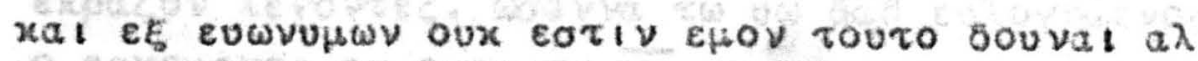

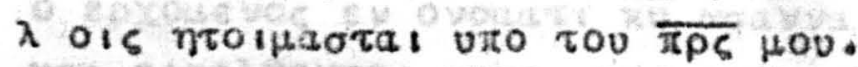

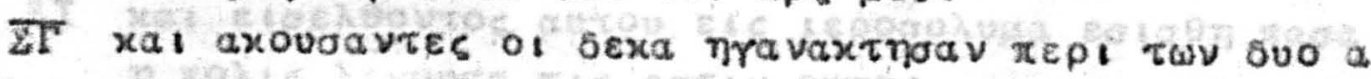

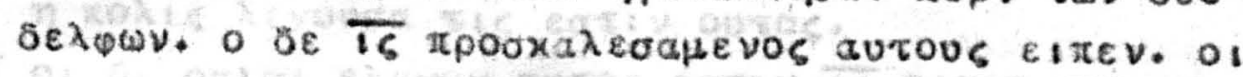

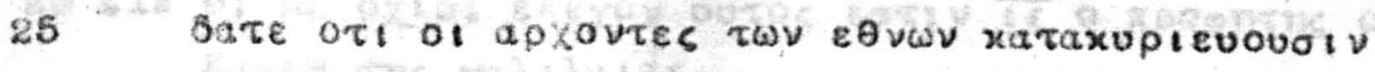

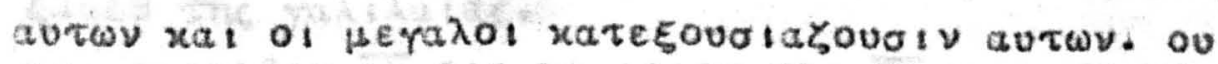

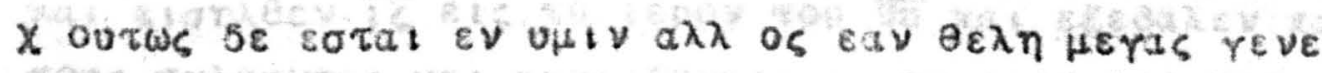

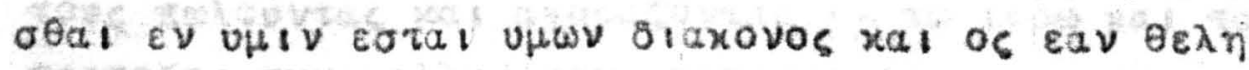

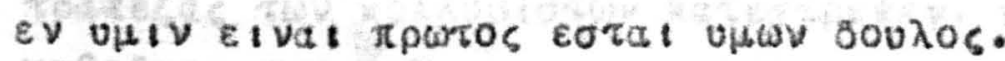

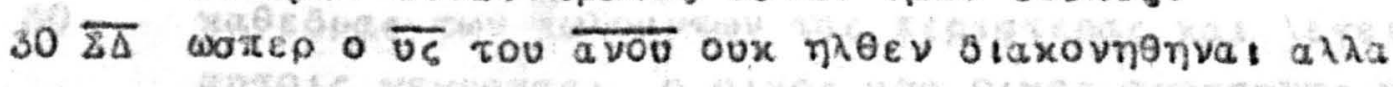

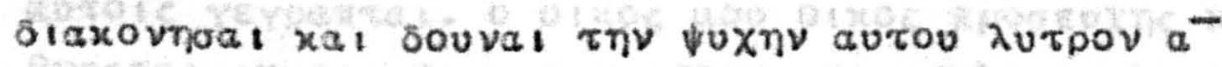

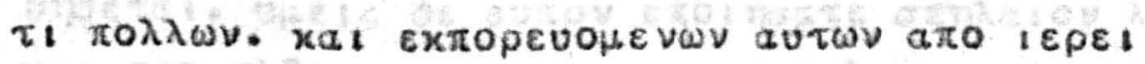

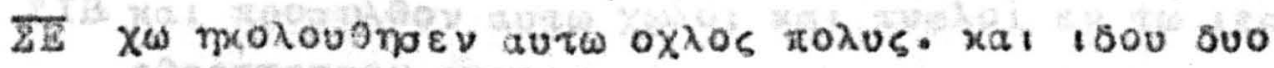

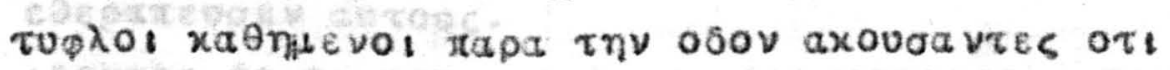

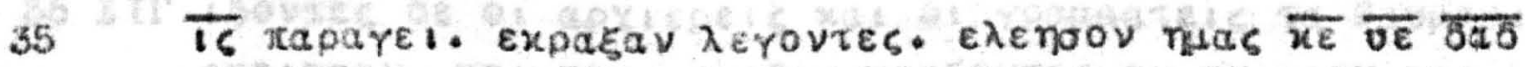

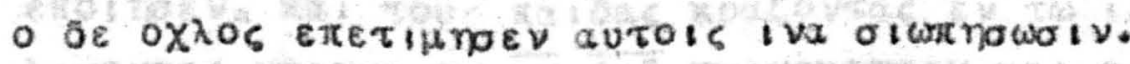

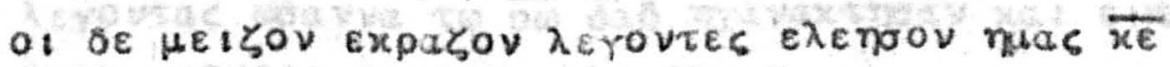

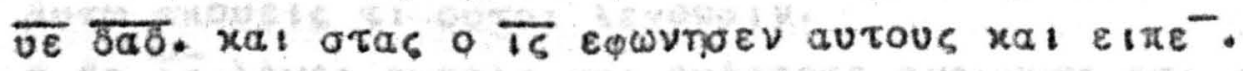

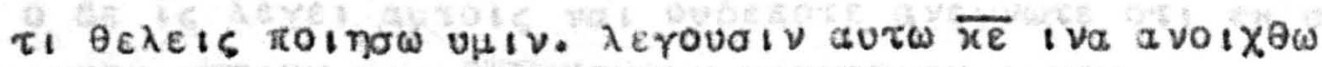
oiv nuwv oi $0_{\varphi} \ominus \alpha \lambda \mu 01$.

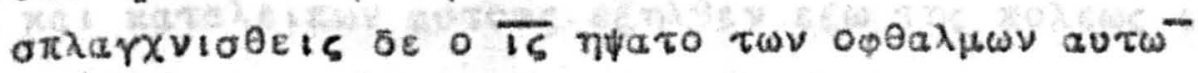




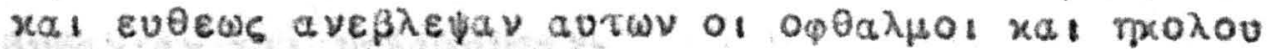

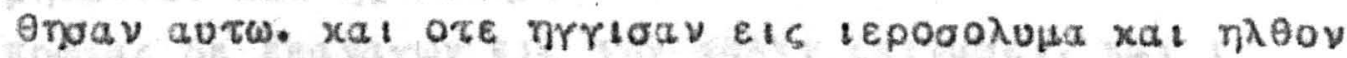

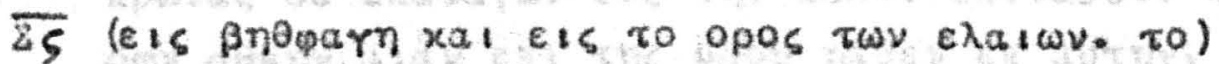

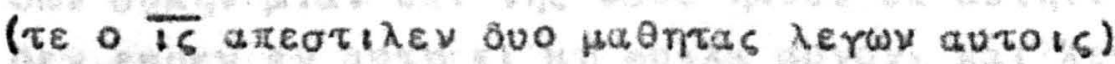

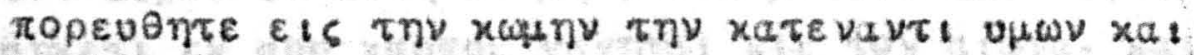

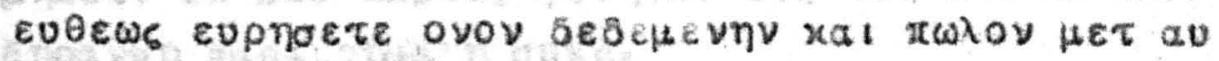

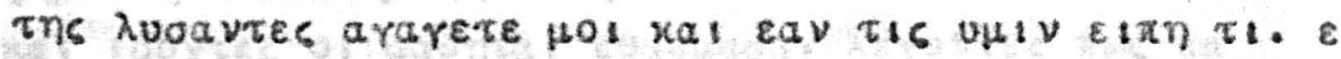

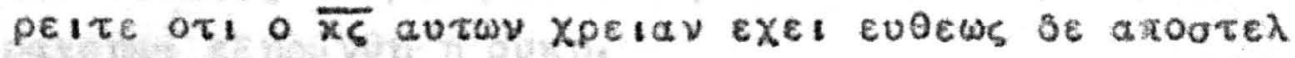

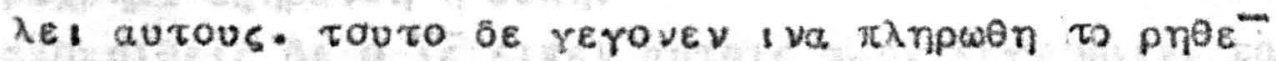

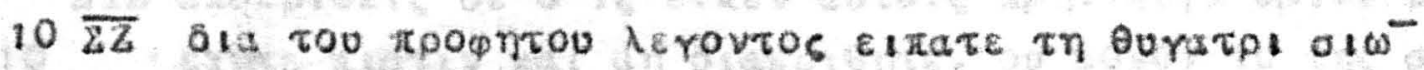

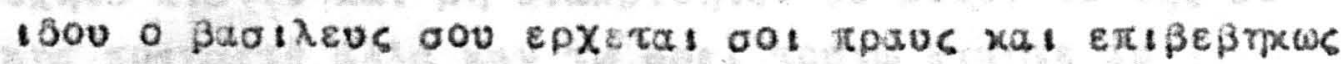
\&ut ovov xat twhov utov viroburiou.

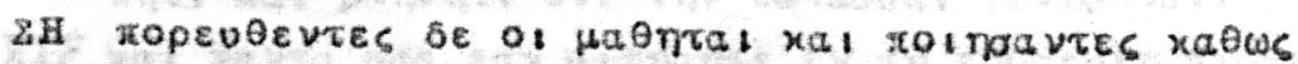

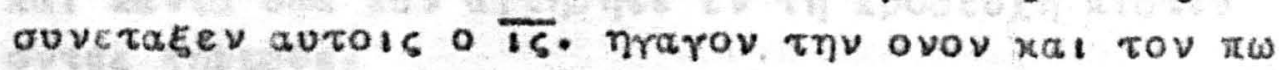

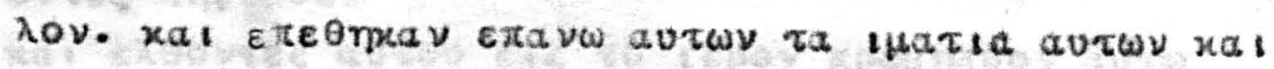

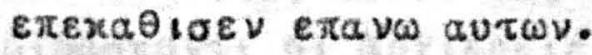

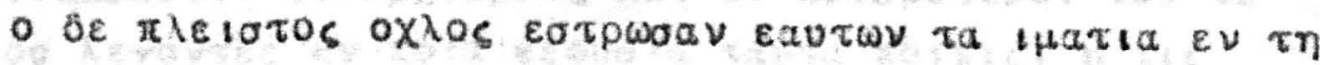

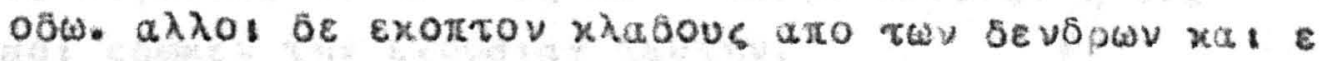

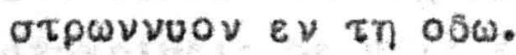

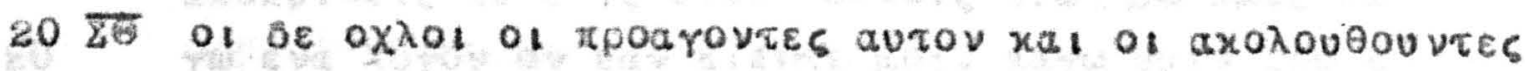

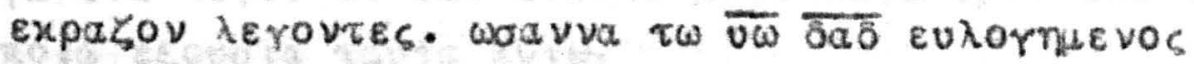

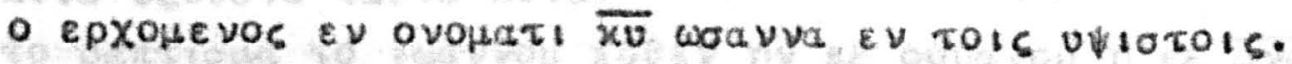

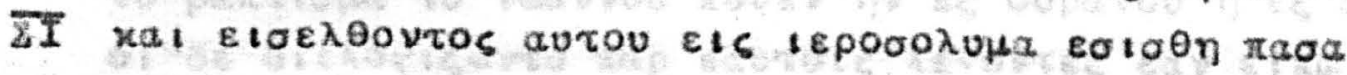

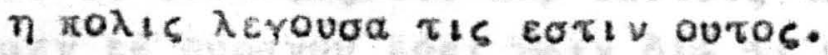

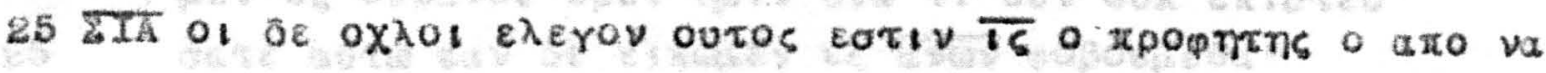

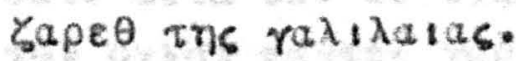

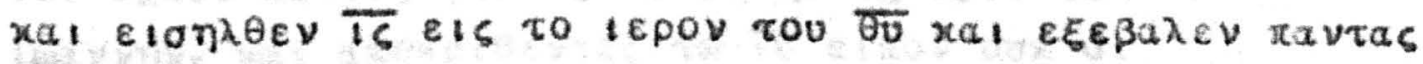

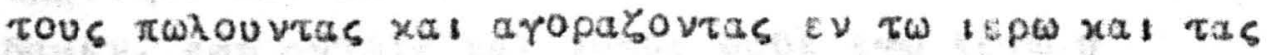

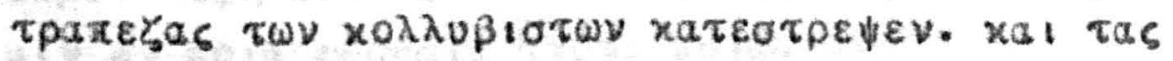

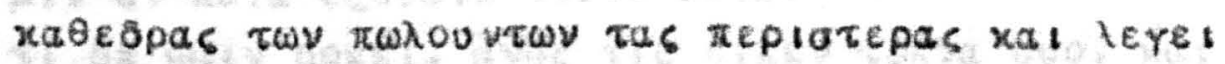
autols revpartal. o otxos hou oixos kpooevxns $x \lambda \eta$

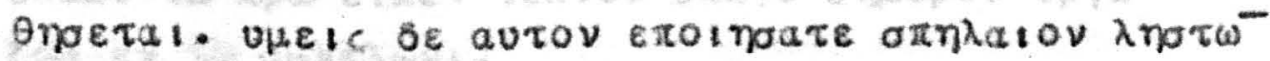

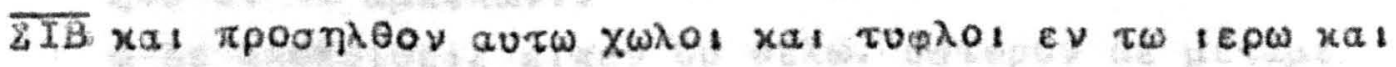
$\varepsilon \theta \varepsilon p a \pi \varepsilon v \sigma \varepsilon v$ autovs.

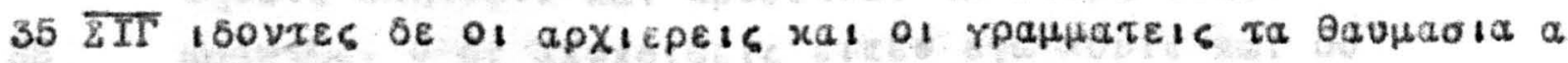

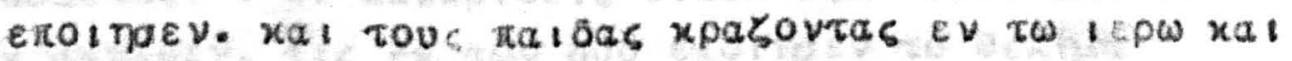

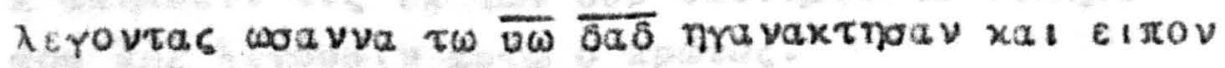
autw axoueis TI outal Aeyoudiv.

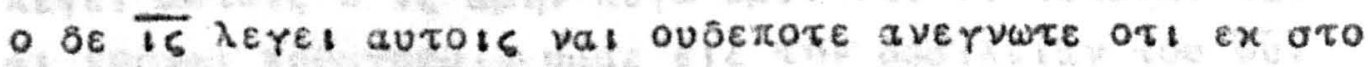

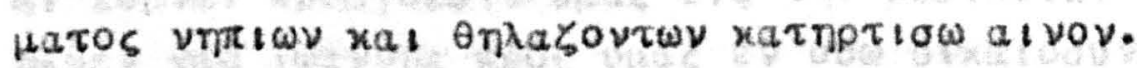

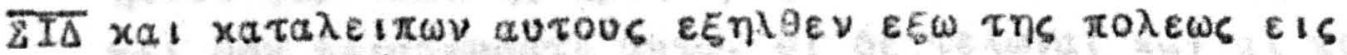




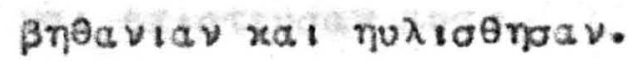

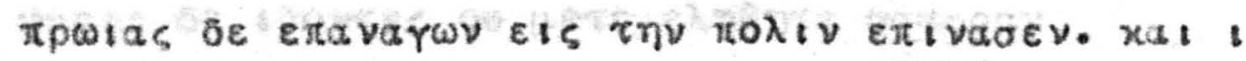

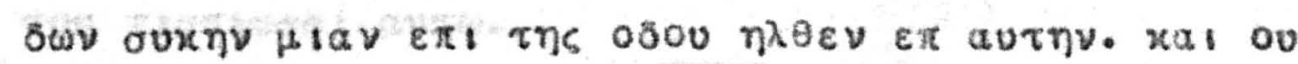

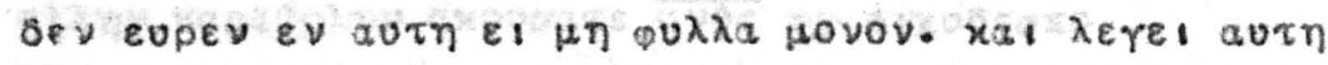

нтрет, $\varepsilon x$ aov xaptos reyntal ets tov atwva. xal $\varepsilon$

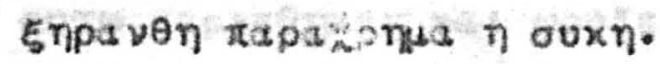

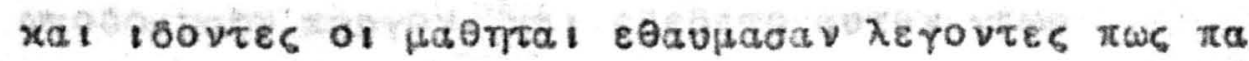

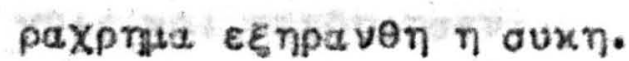

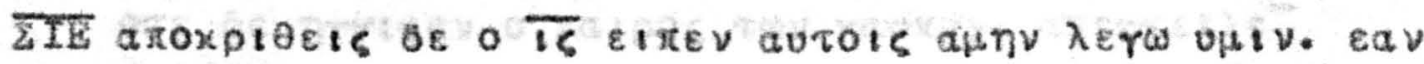

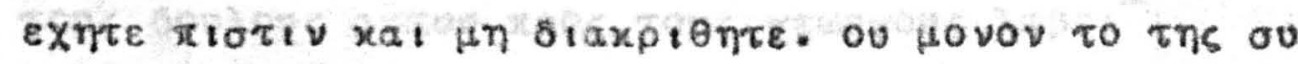

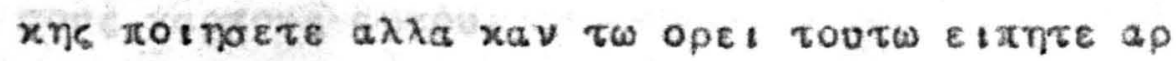

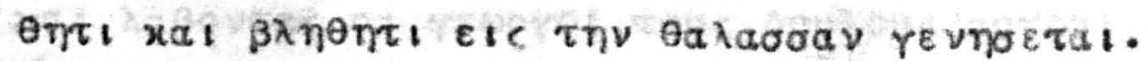

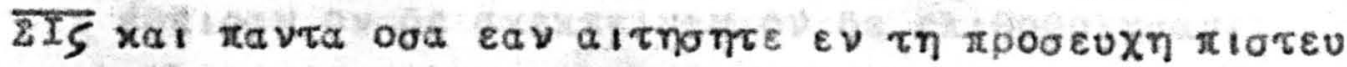
o vтes $\lambda \eta \Downarrow \varepsilon \sigma \theta \varepsilon$.

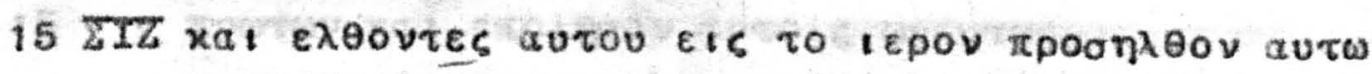

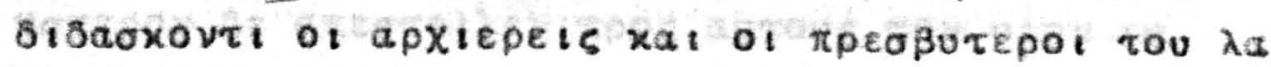

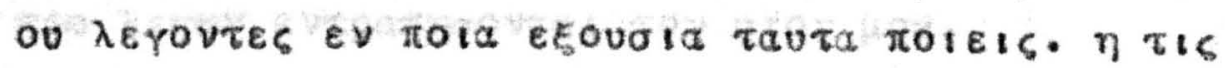

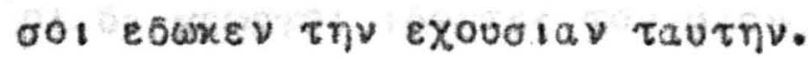

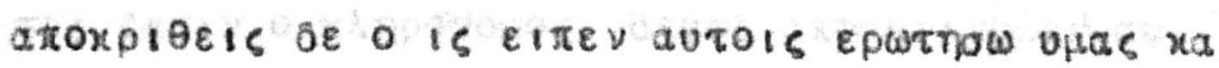

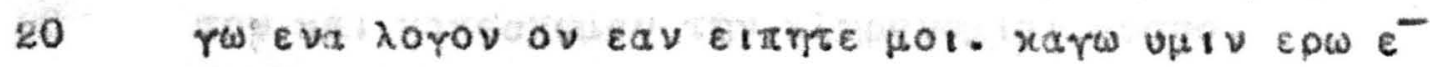
Tola ez̧ovota taura rotw.

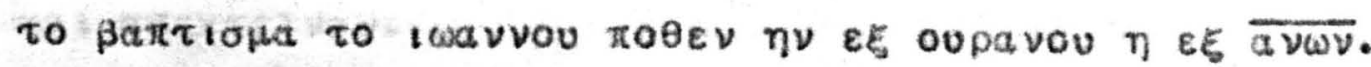

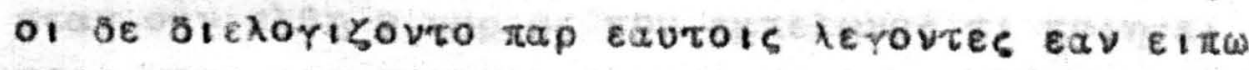

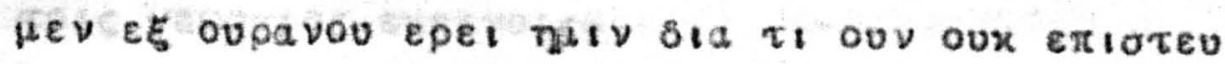

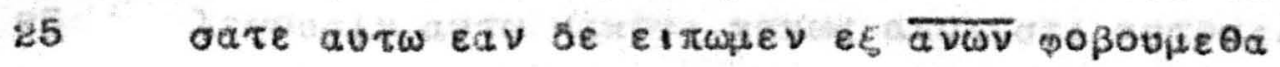

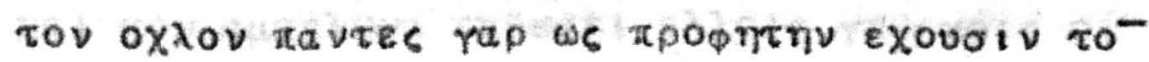

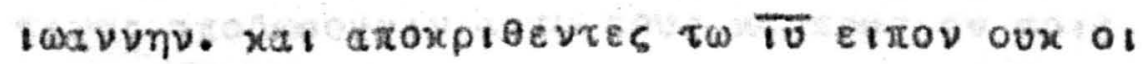

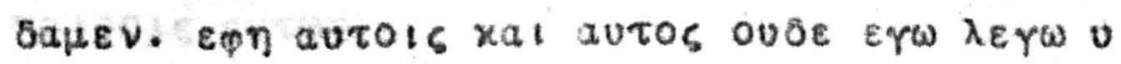

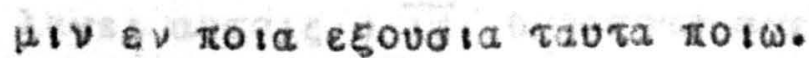

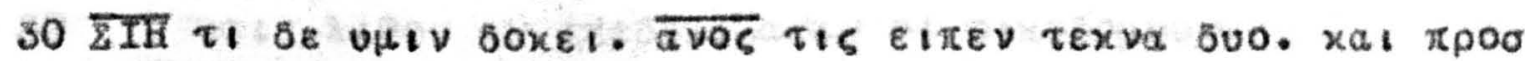

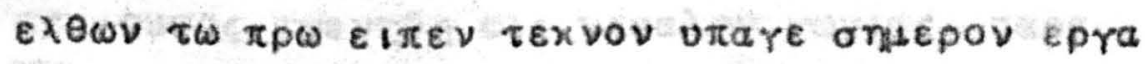

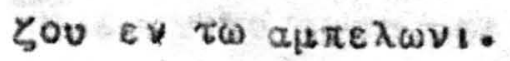

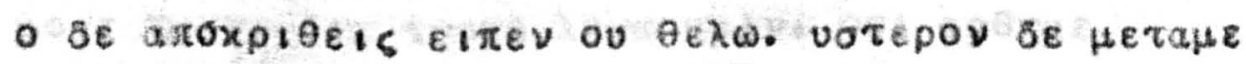

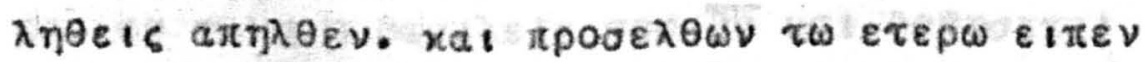

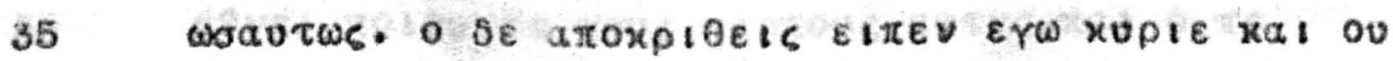

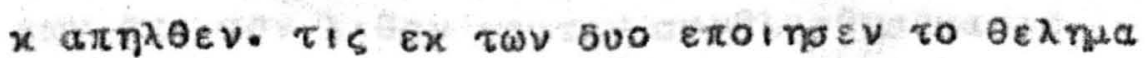

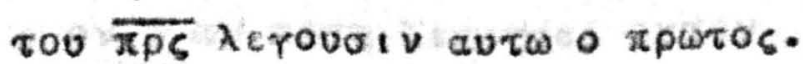

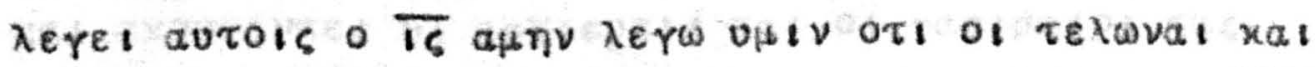

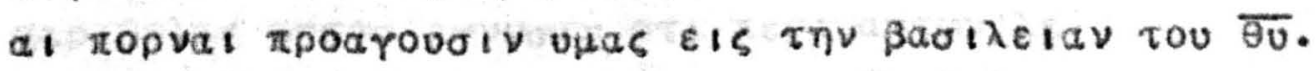

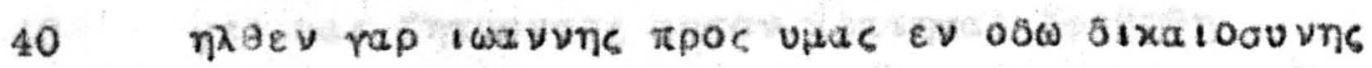

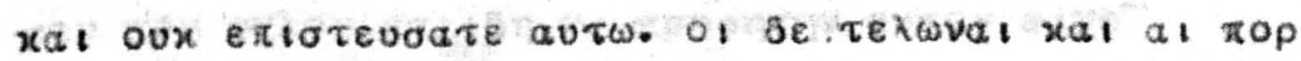


val $\varepsilon \pi เ \sigma \tau \varepsilon v \sigma a \nu$ av $\tau$.

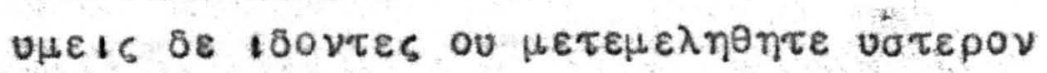

$\tau$

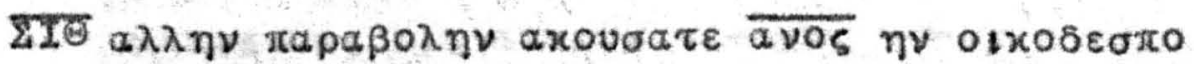

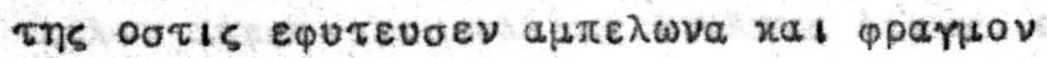

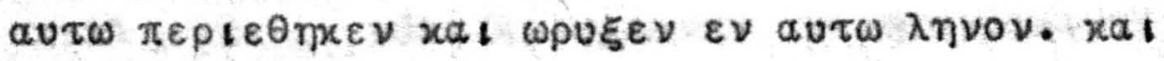

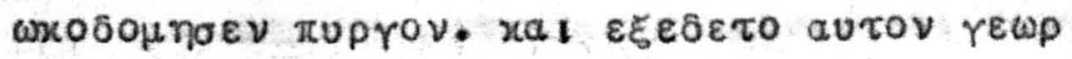

rois xal arebnunoev.

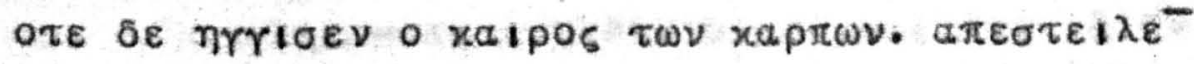

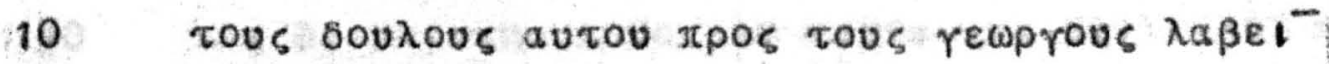

Tous xaprous aurou.

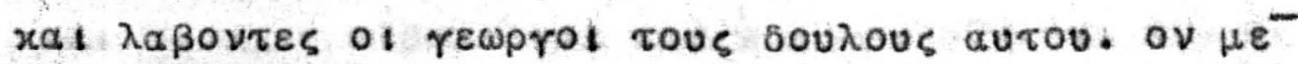

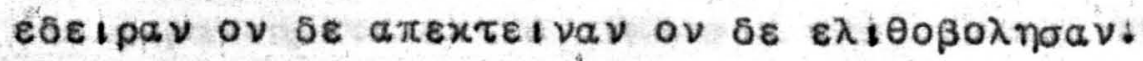

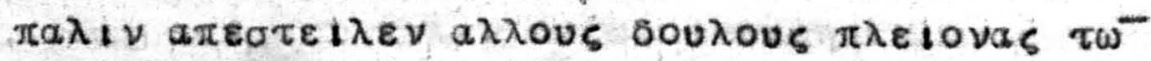

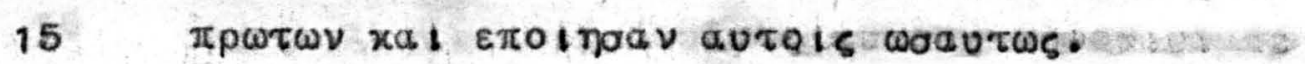

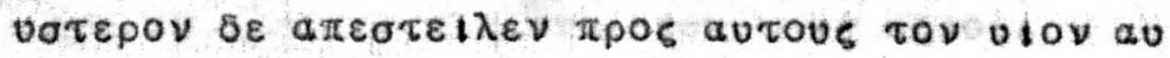

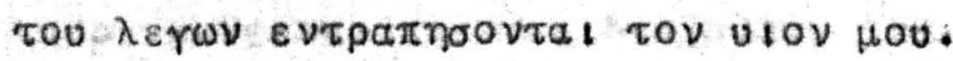

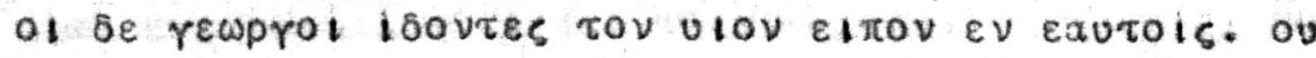

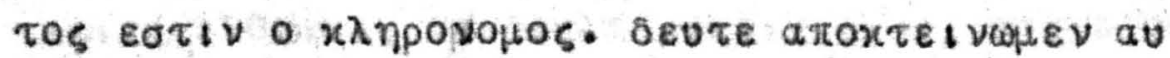

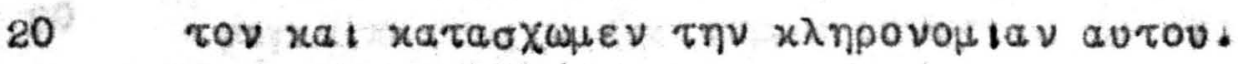

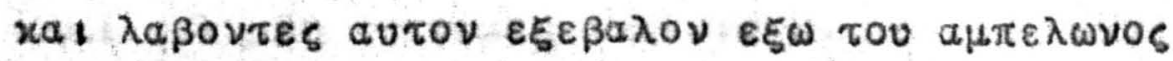

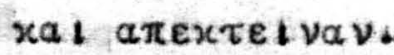

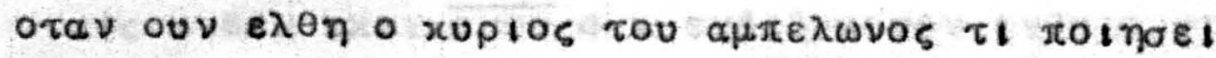

Tots rewprtos exetvols.

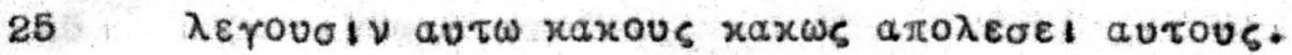

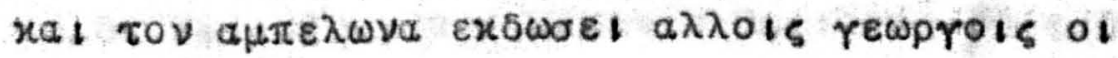

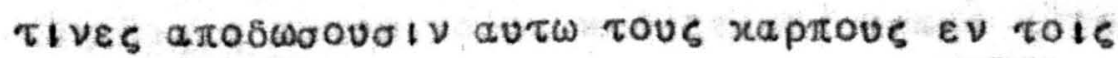
xalpois autw.

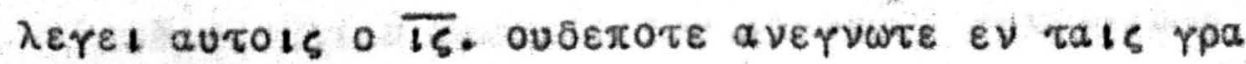

$30 \varphi$ Q $15 . \lambda t \theta 0 \nu$ ov a

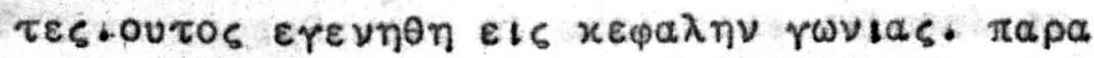

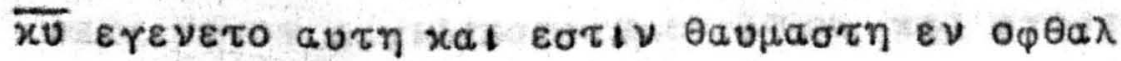

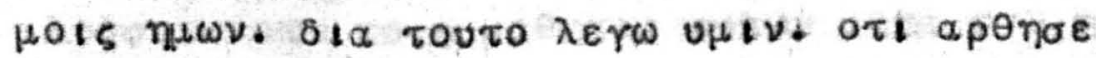

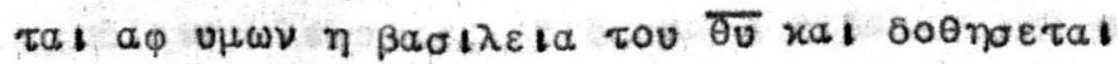

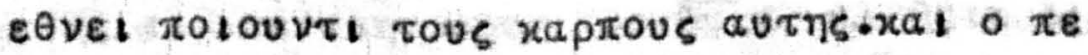

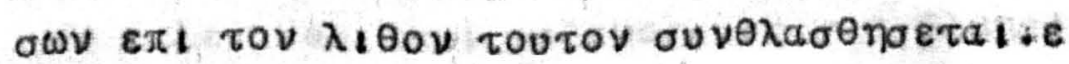

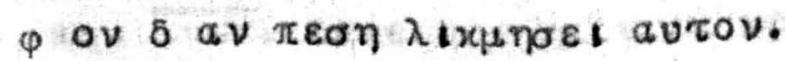

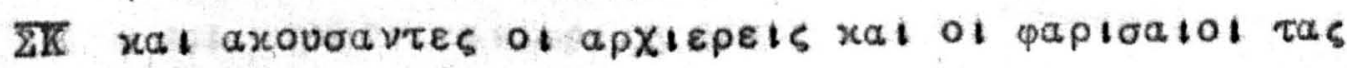

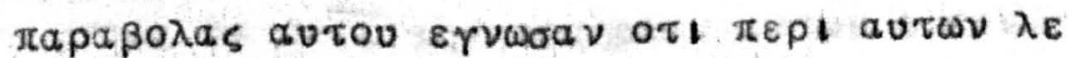

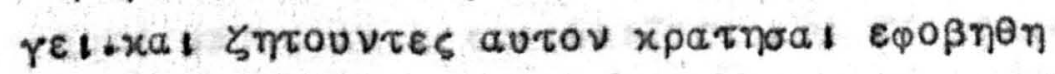

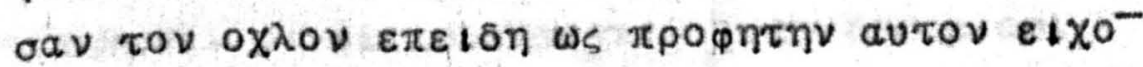

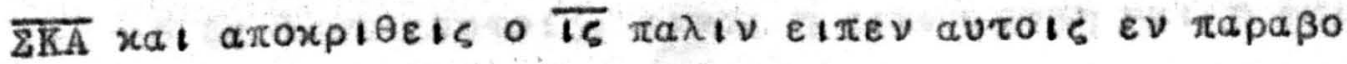




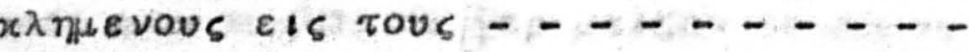

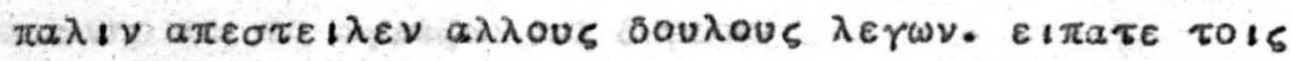

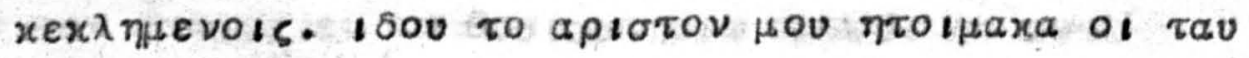

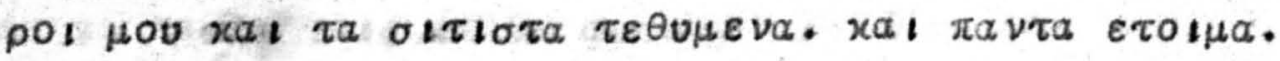
seute els rous rapovs.

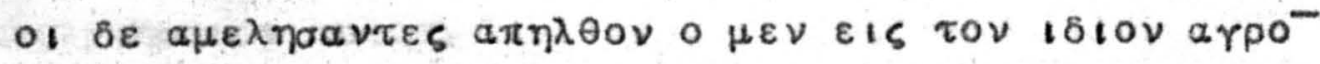

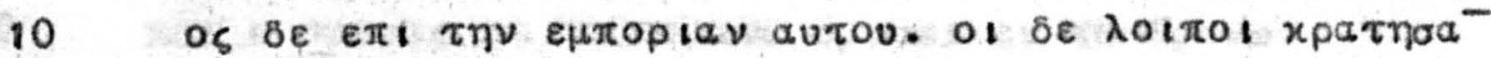

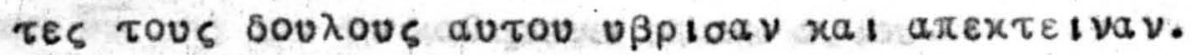

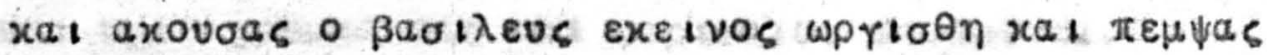

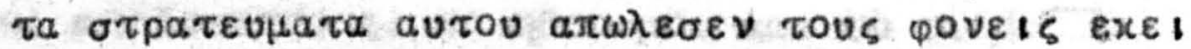

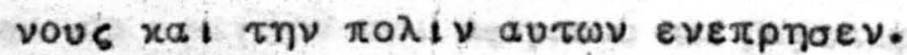

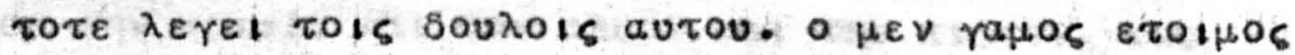

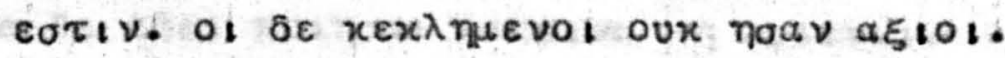

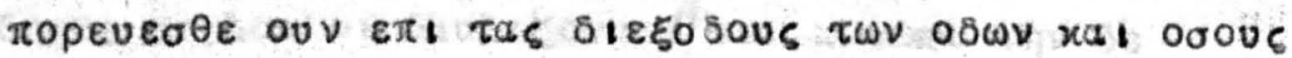

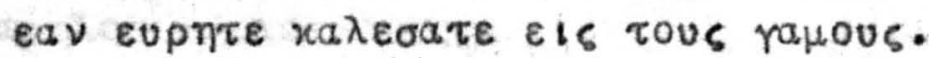

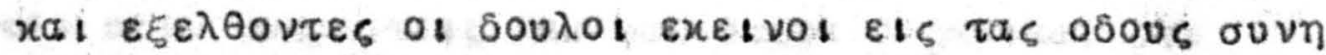

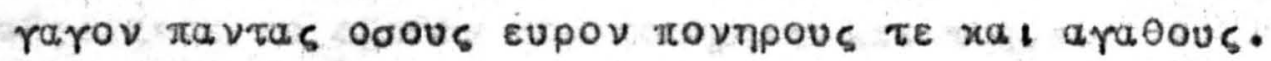
$x a ! e \pi \lambda \eta \sigma \theta \eta$ a arajoc a vax

$\overline{\mathrm{KKB}} \varepsilon$

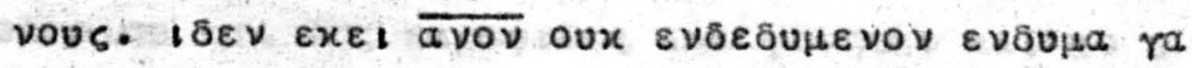

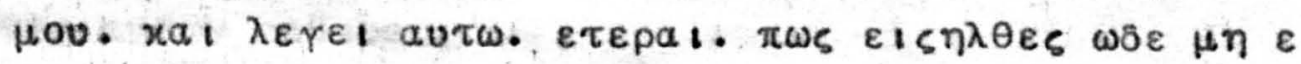

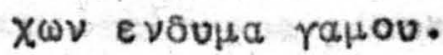

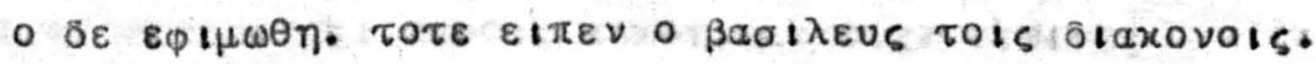

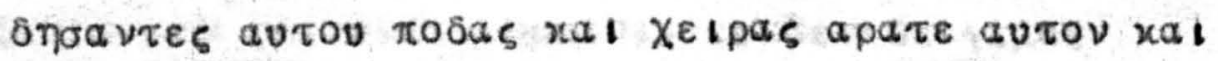

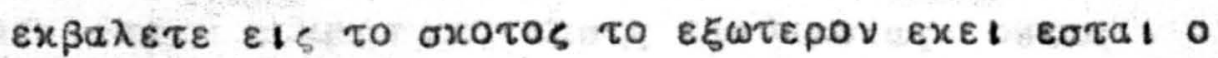

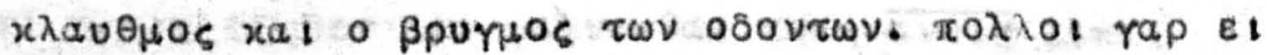

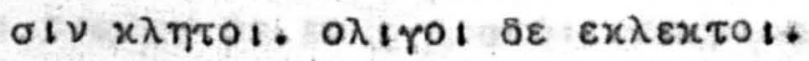

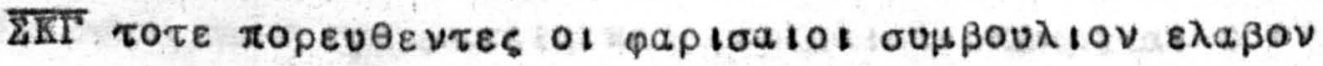

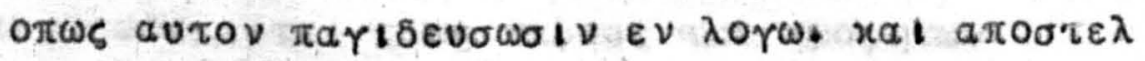

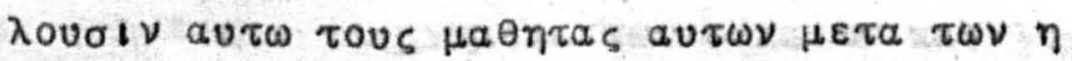

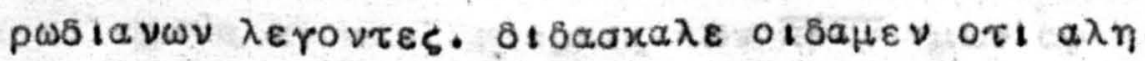

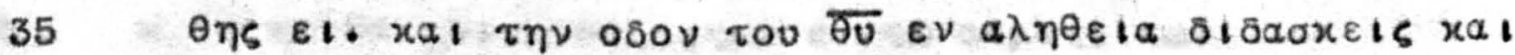

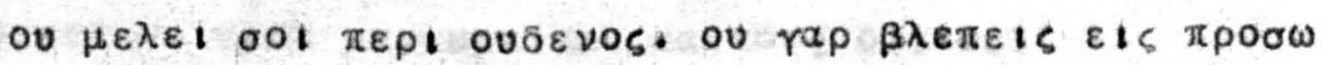

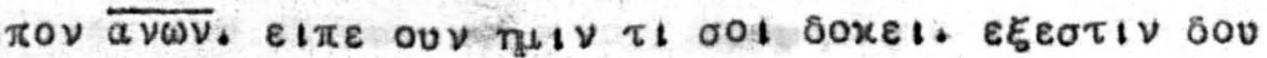

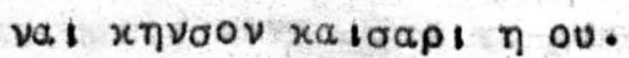

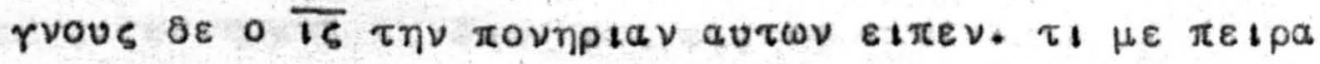

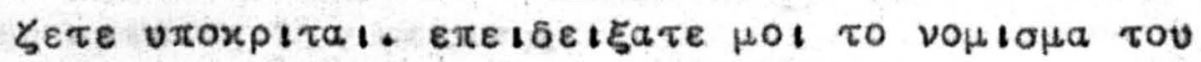

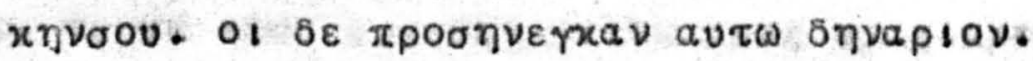

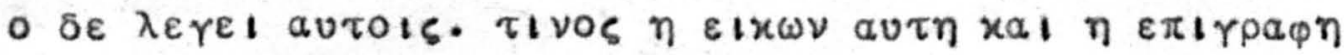


$\eta$ a vaos a arlacas tov xpuaov.

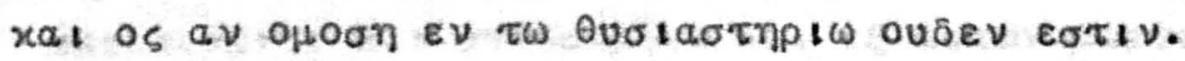

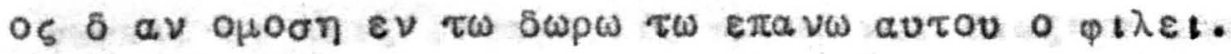

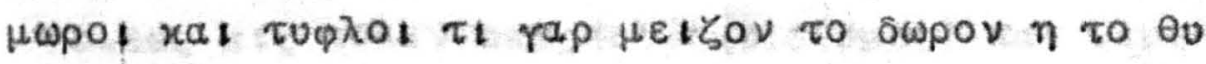

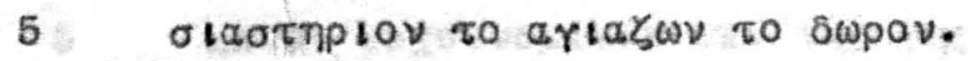

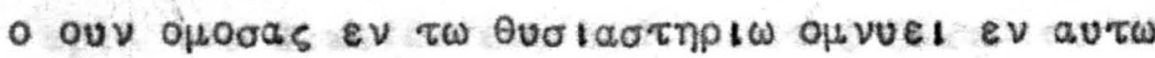

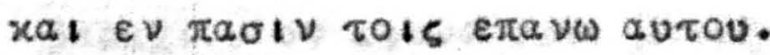

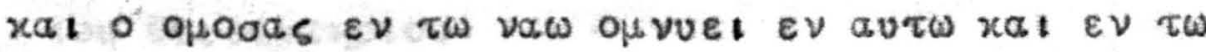

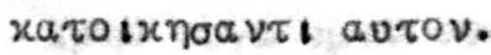

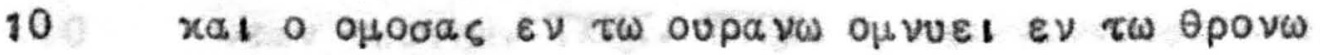

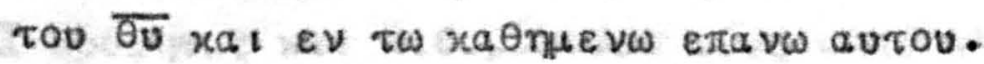

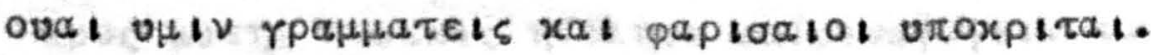

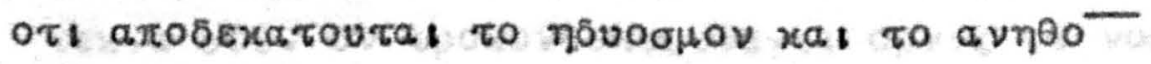

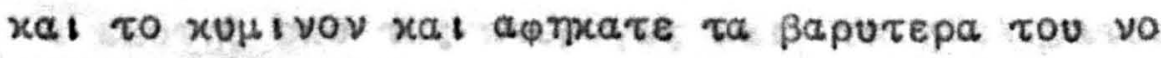

15

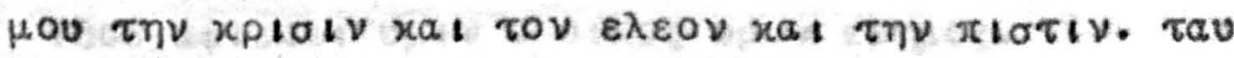

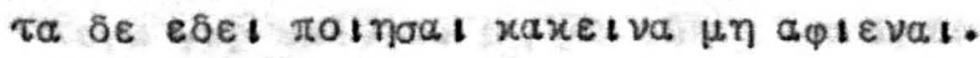

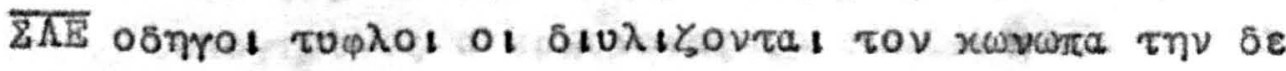

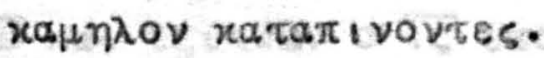

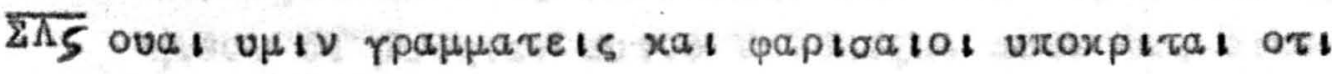

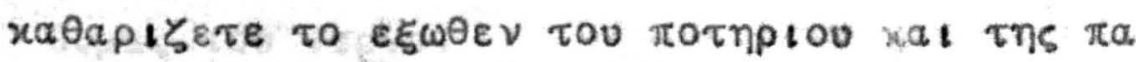

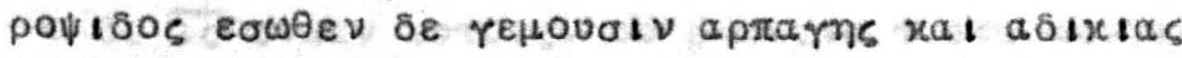

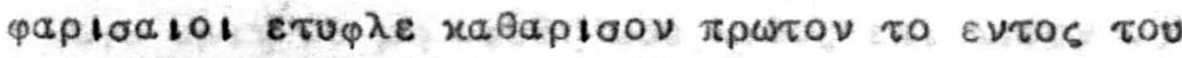

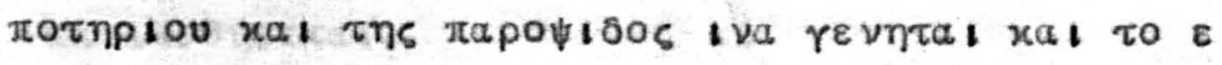
xTos autwr xatapov.

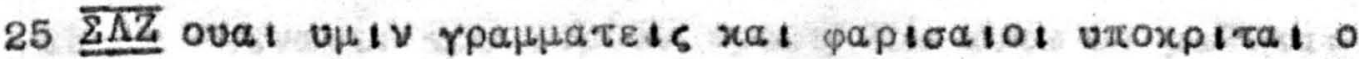

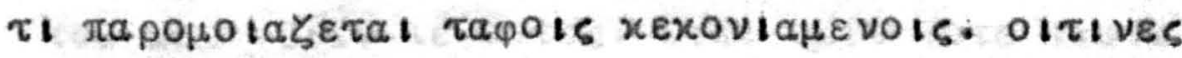

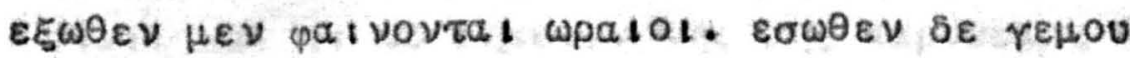

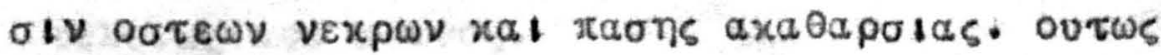

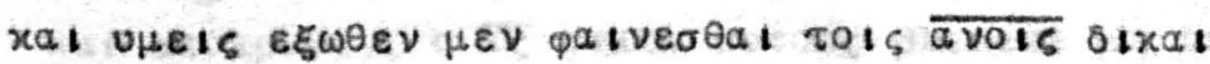

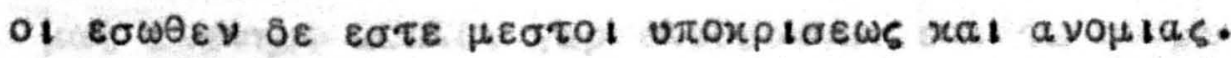

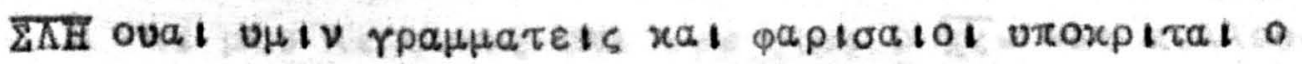

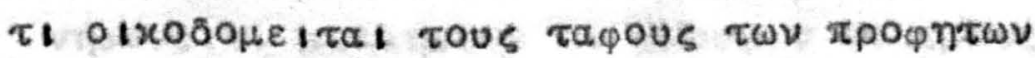

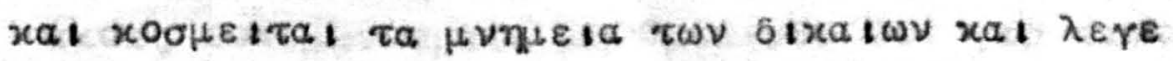

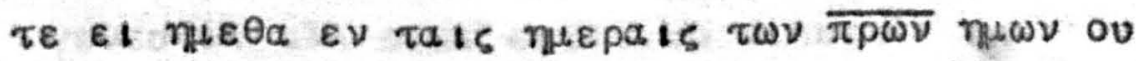

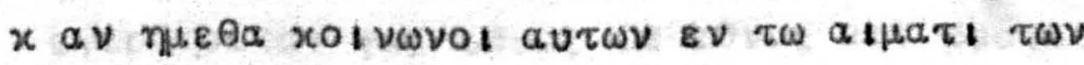

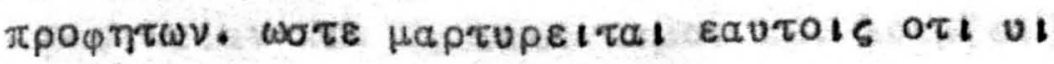
ol $\varepsilon \sigma \tau \varepsilon \tau \omega \nu$ фо

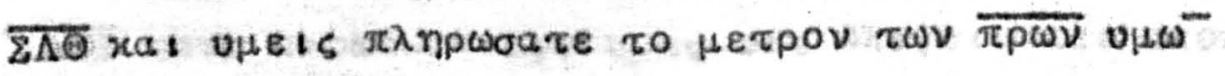
opels revvnuata exı tns xplaews tns reevuns.

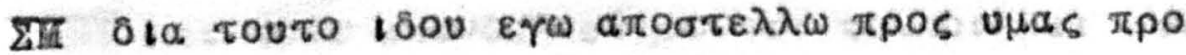




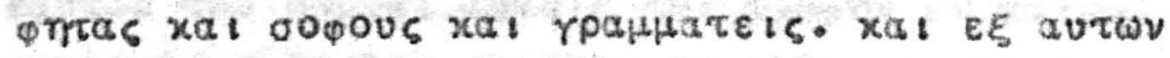

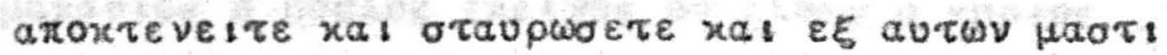

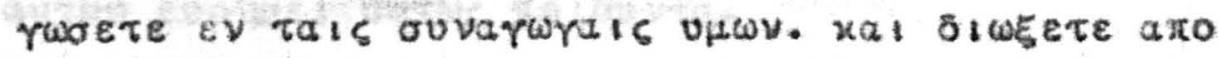

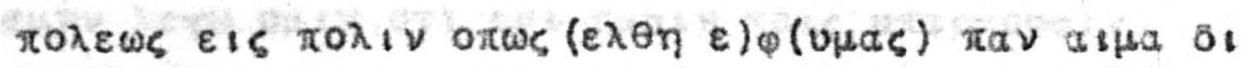

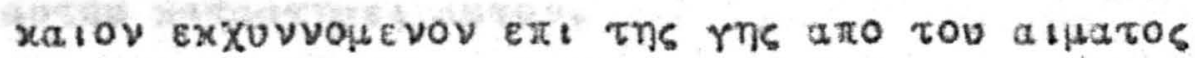

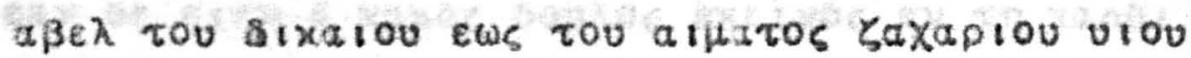
Bapaxiou ov eqovevaate hetagu tou vaou xal Tov Ovorartmptou.

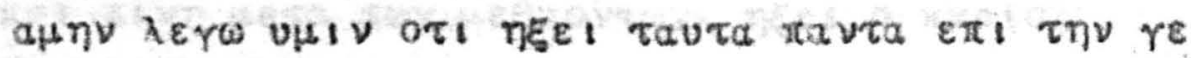

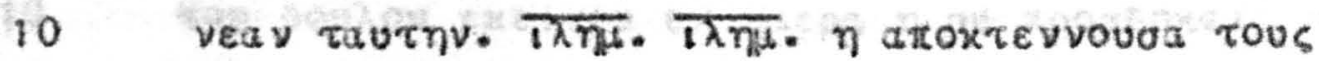

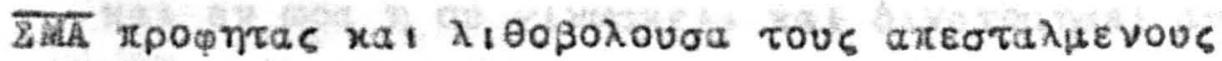

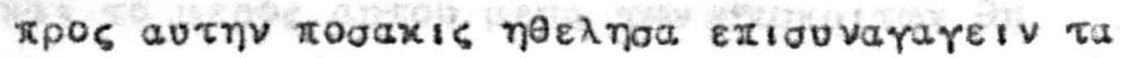

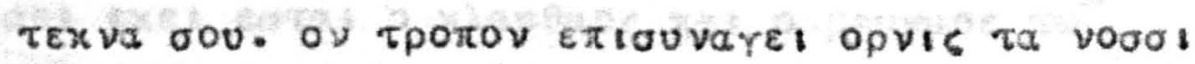

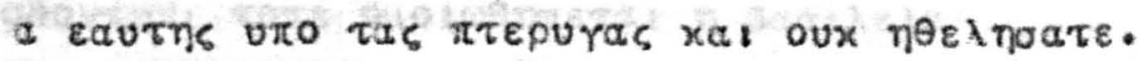

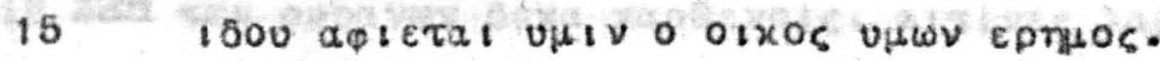

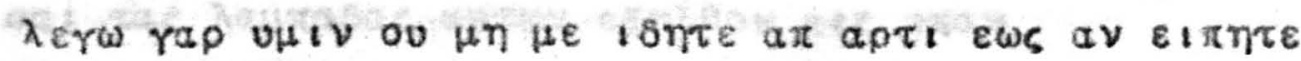

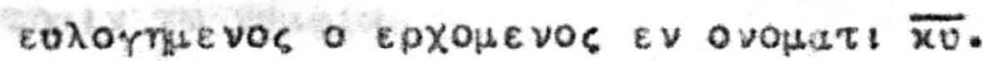

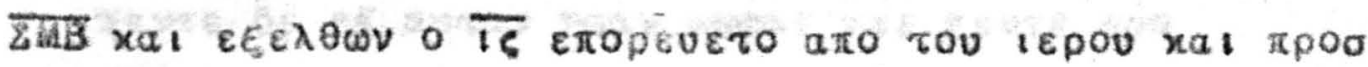

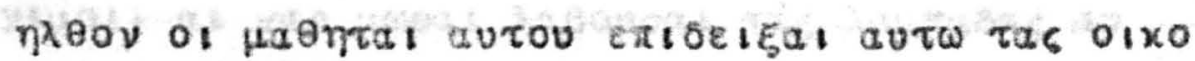
ôpus tou $1 \varepsilon p 00$.

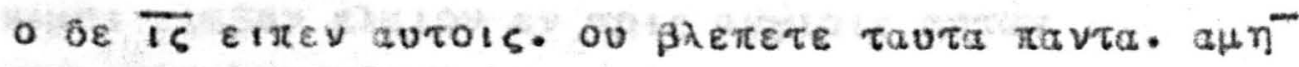

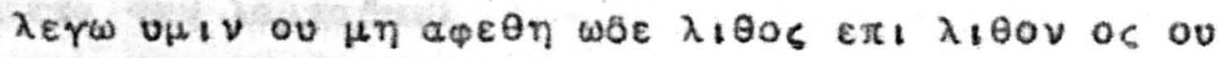

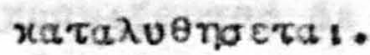

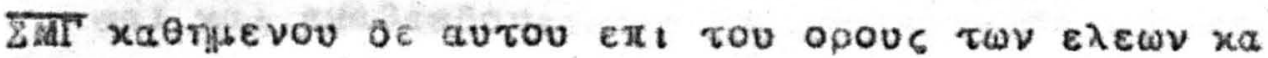

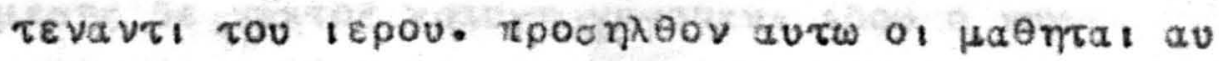

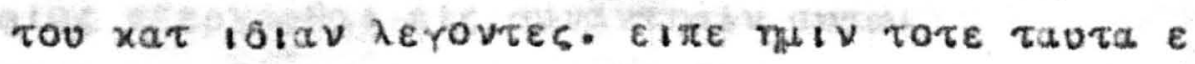

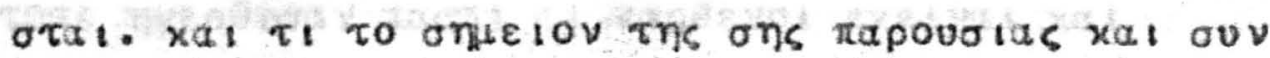
$\tau e \lambda \varepsilon$ las $\tau$ Tov a twVos.

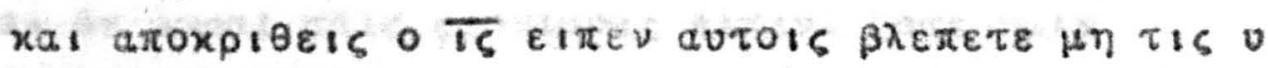

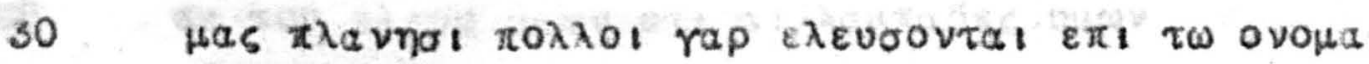

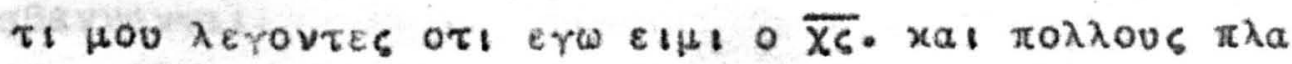

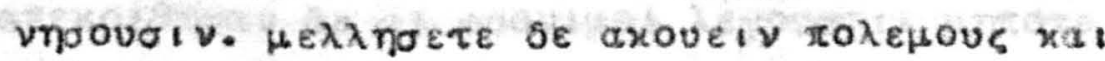

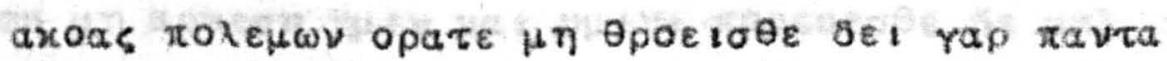

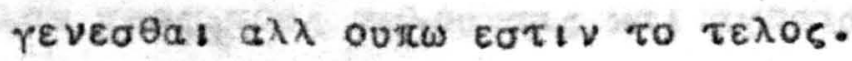

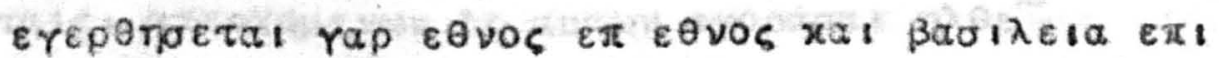

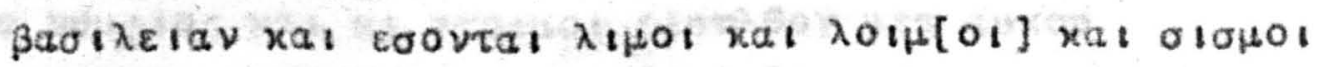

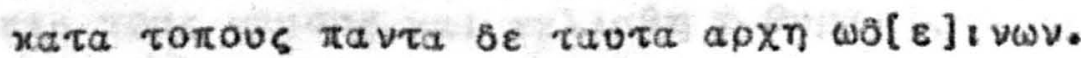

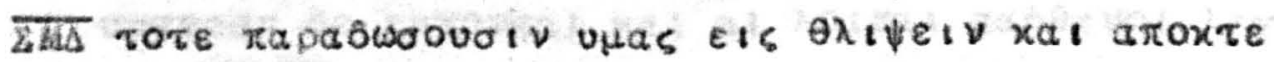

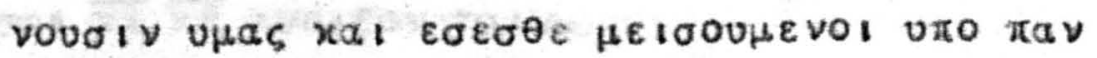
$\tau \omega \nu$ sia to ovoua $\mu$ ou.

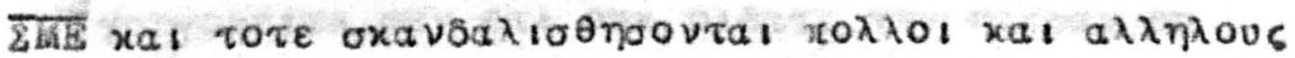




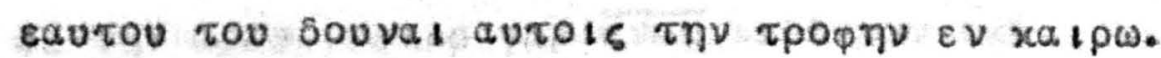

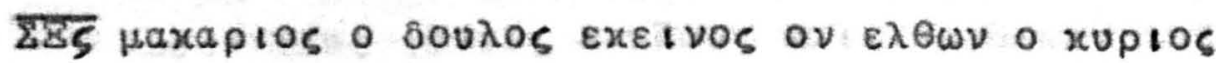

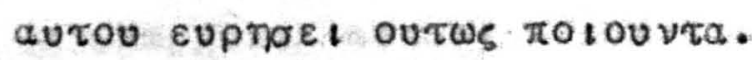

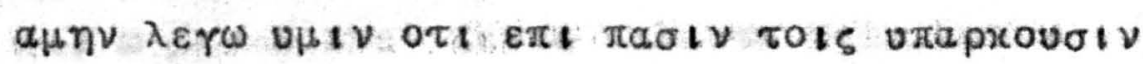

5

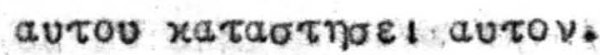

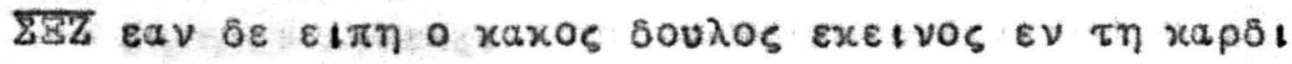

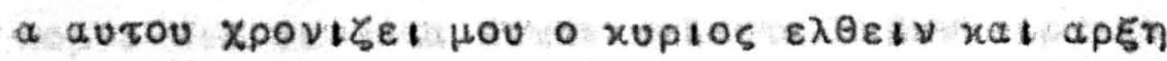

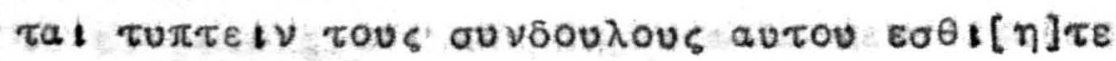

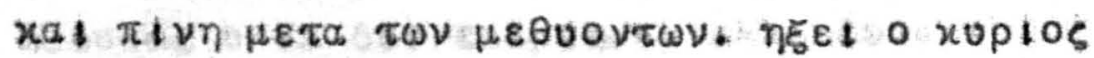
Tou sou

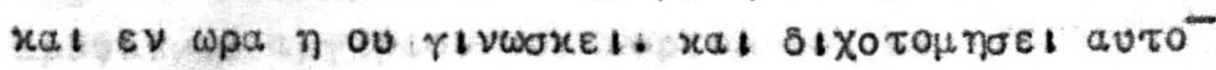

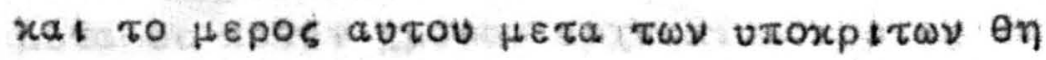

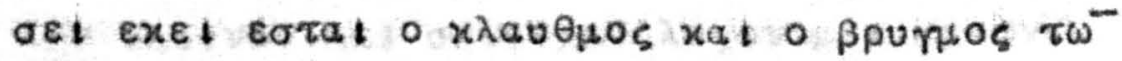

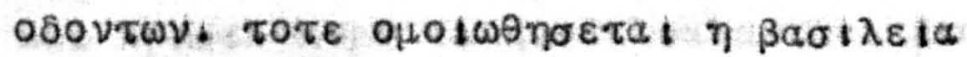

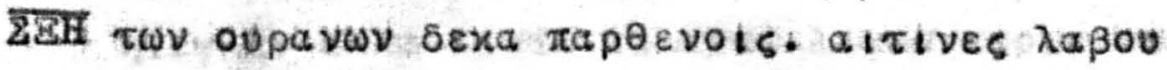

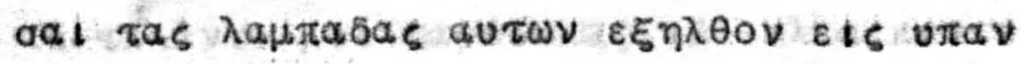

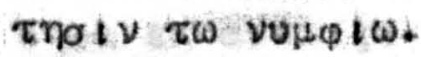

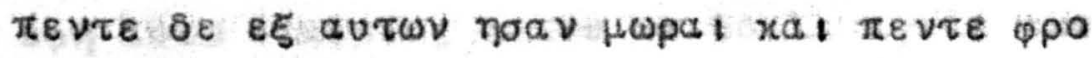

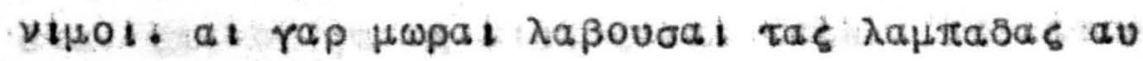

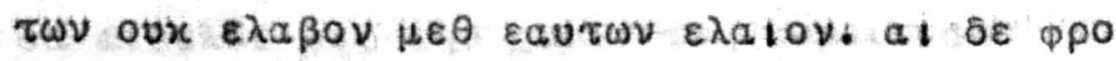

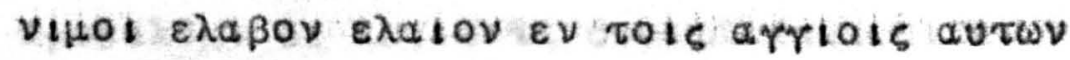

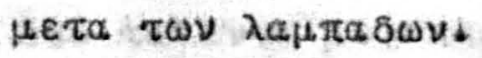

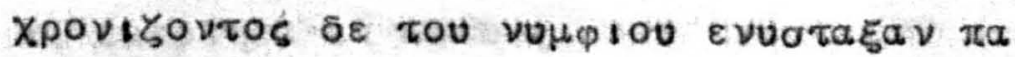

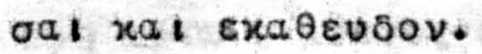

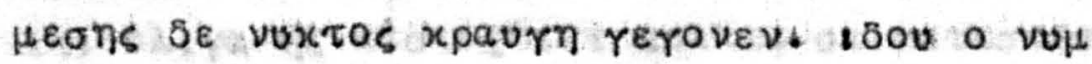

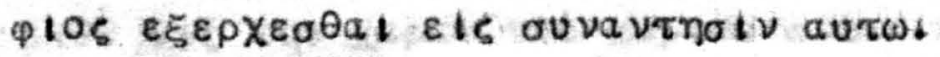

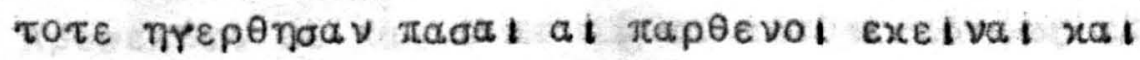

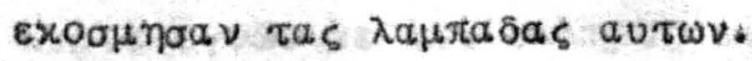

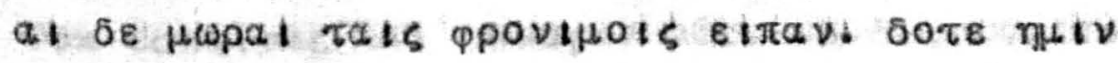

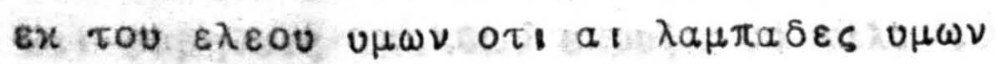

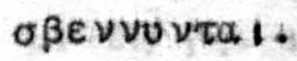

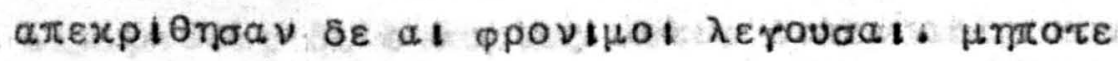

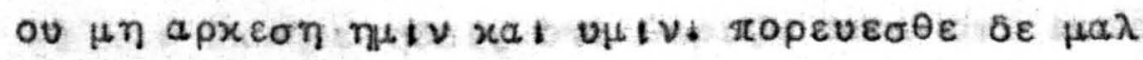

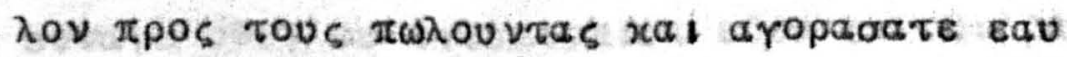

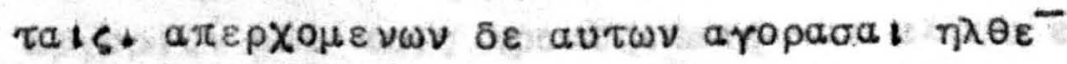

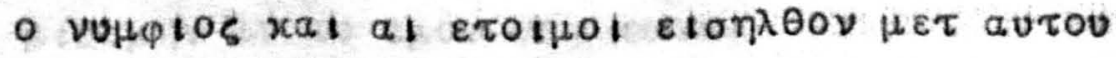

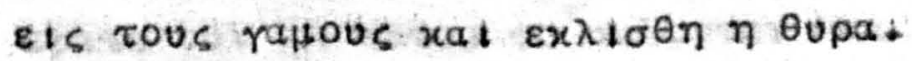

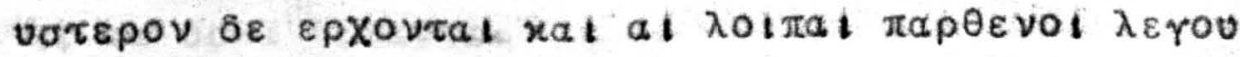
oat $\overline{x \varepsilon} \overline{x \varepsilon}$ avotzov nutv.

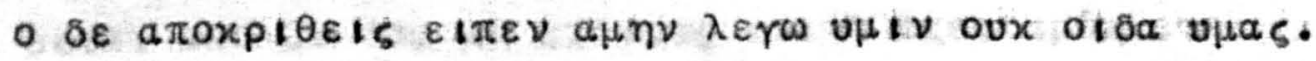

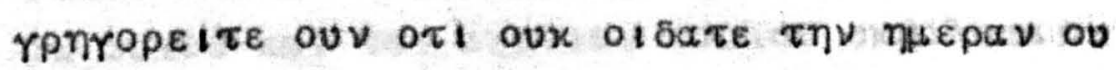




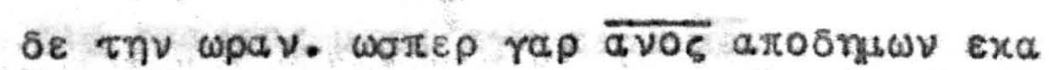

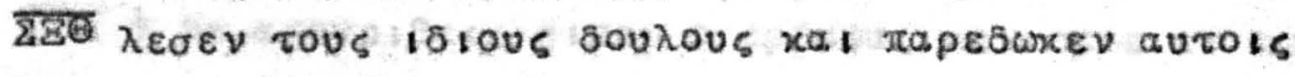
ra. viapxovia aurou.

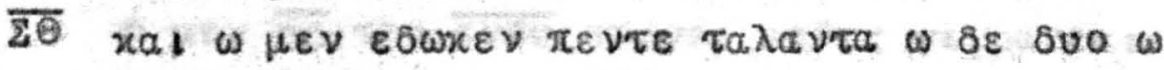

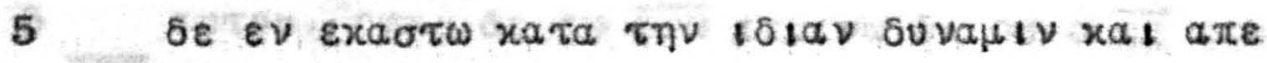

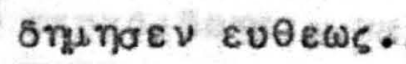

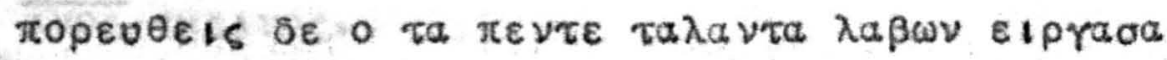

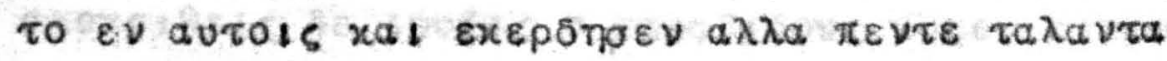

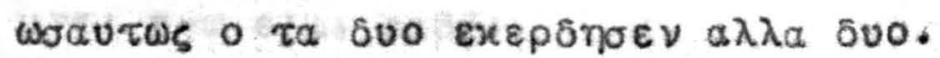

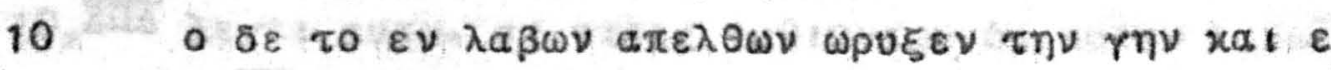

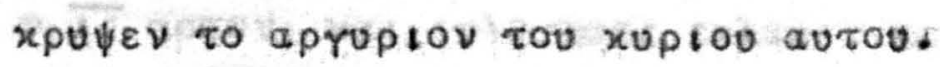

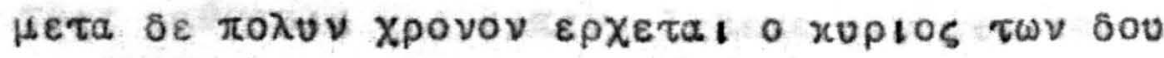

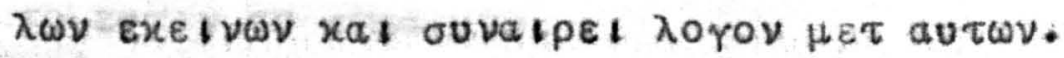

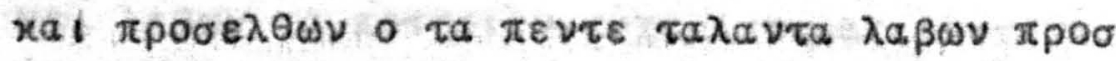

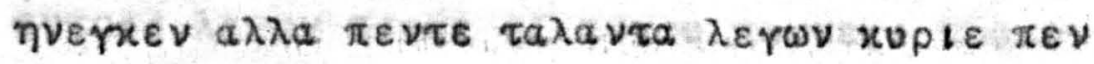

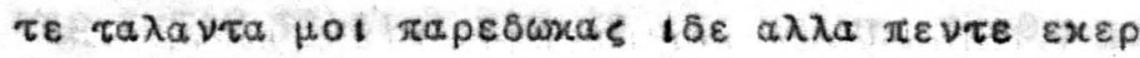
snoa $\varepsilon \pi$ autots.

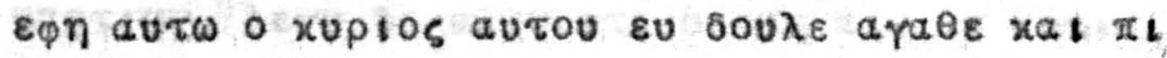

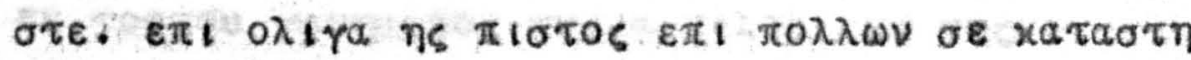

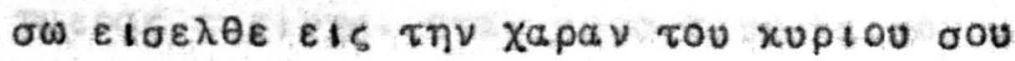
$\pi p \circ \sigma \varepsilon \lambda \theta \omega \nu$ $\delta \varepsilon$ x

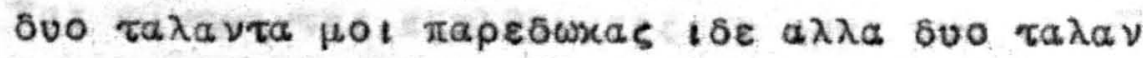

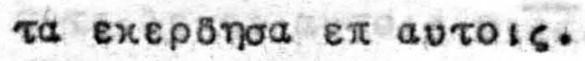

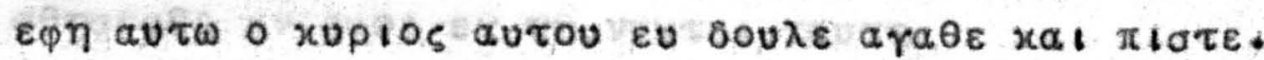

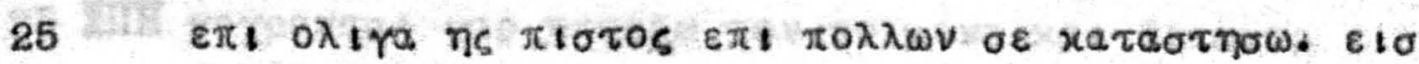

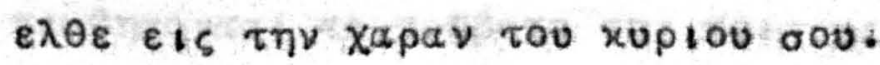

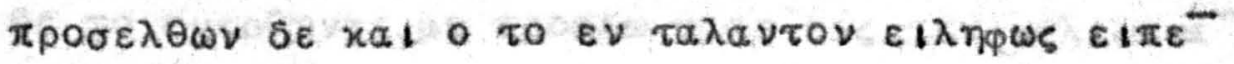

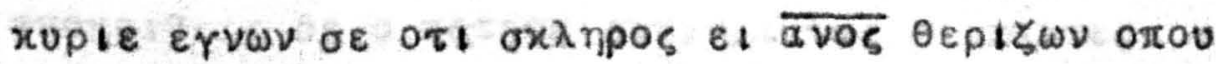

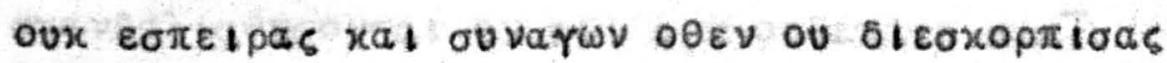

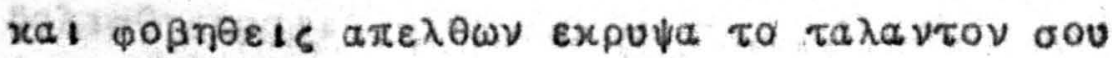

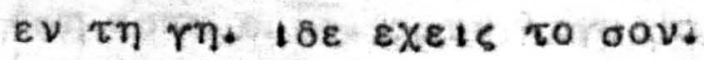
a

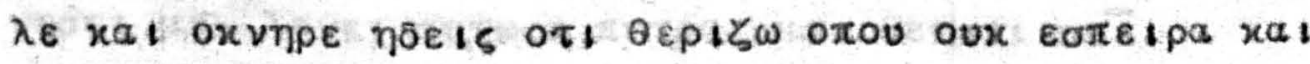

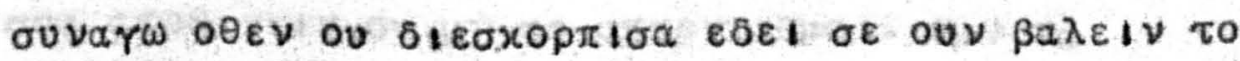

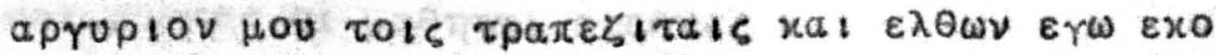

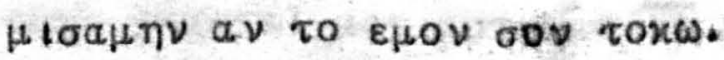

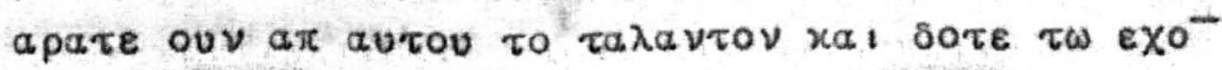

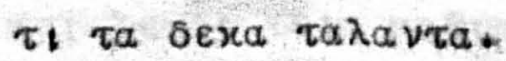

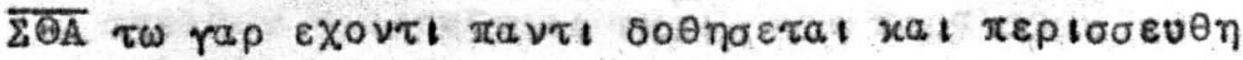

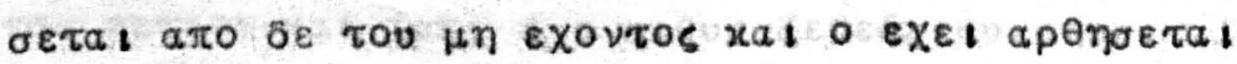

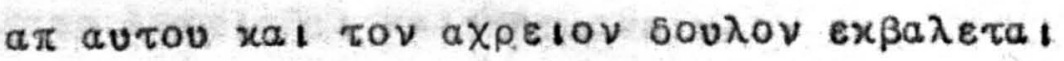




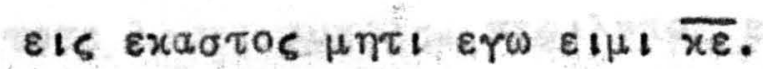

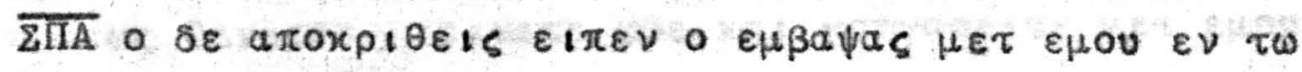

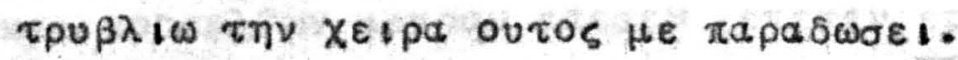

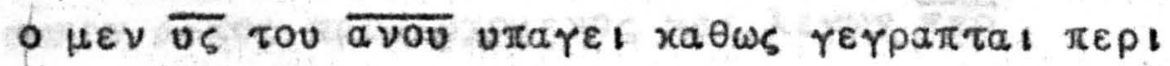

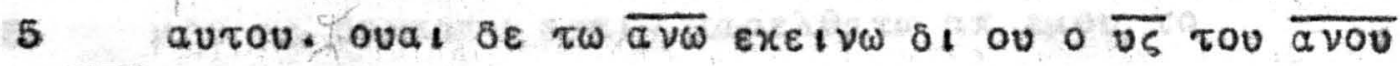

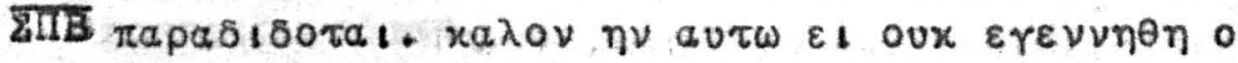
बरणु ExeIvos.

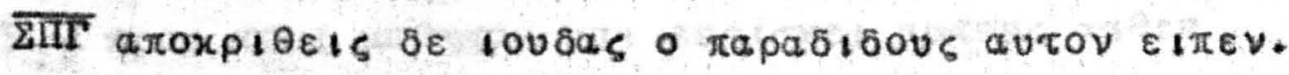
$\mu \eta \tau t \varepsilon \gamma \omega \varepsilon t \mu l \rho \beta \beta \imath$.

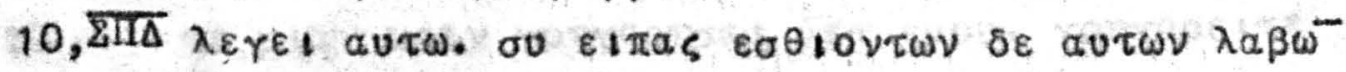

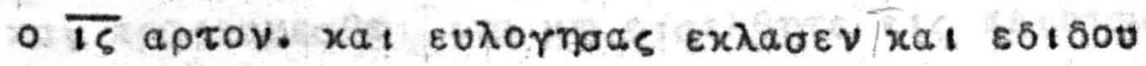

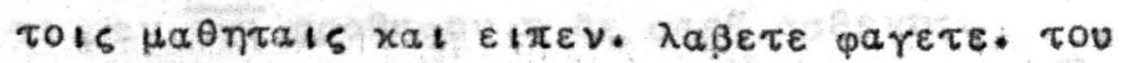

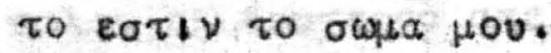

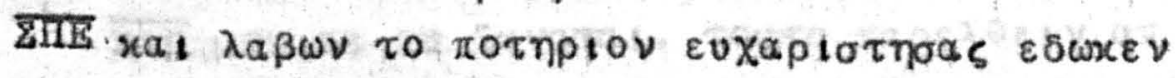

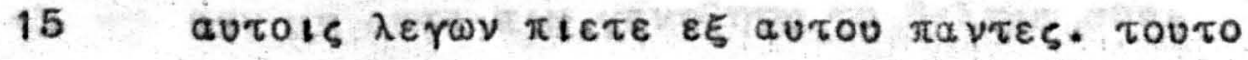

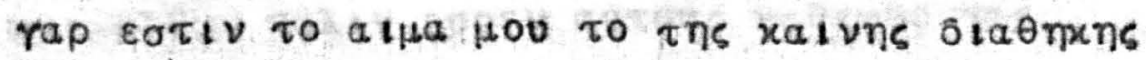

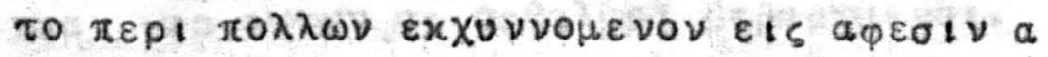

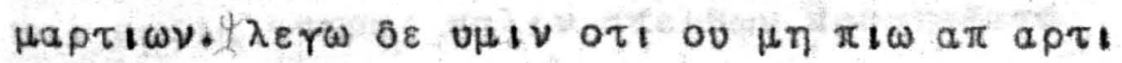

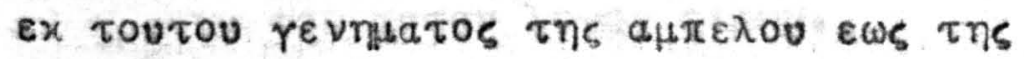

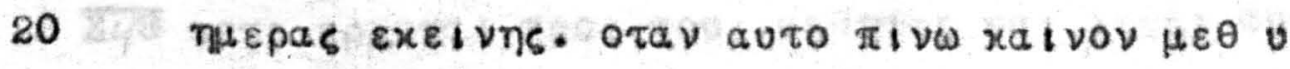

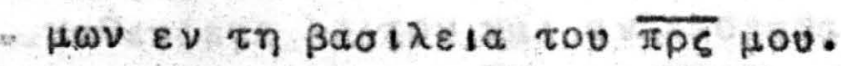

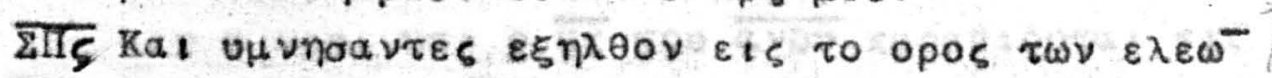

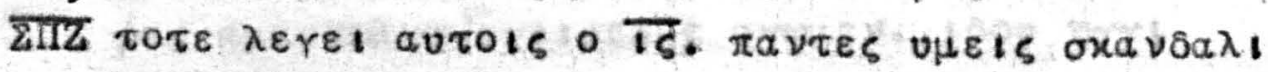

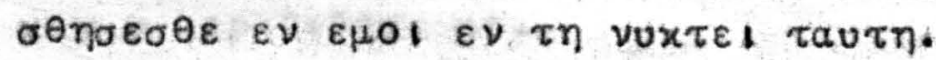

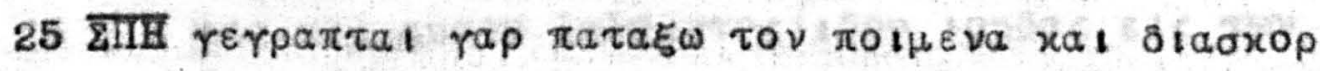

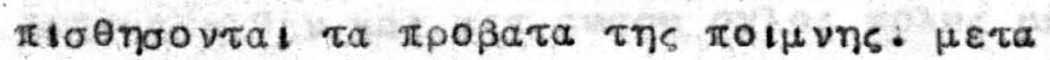

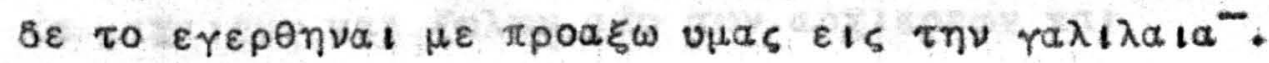

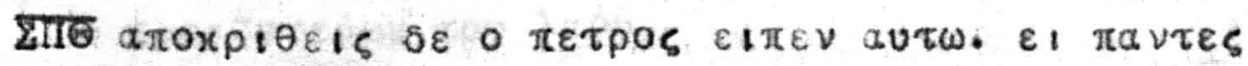

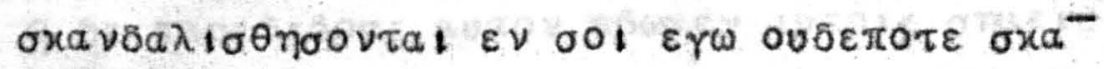

30 saxtoonoopal.

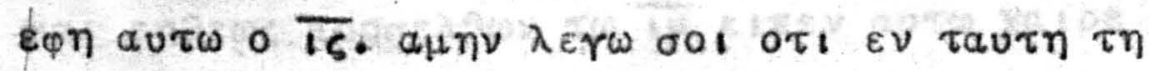

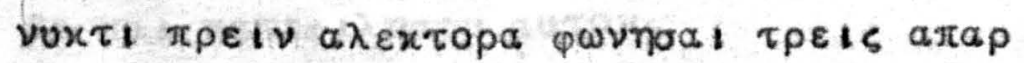

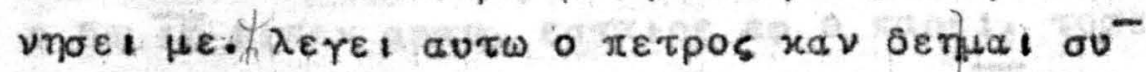

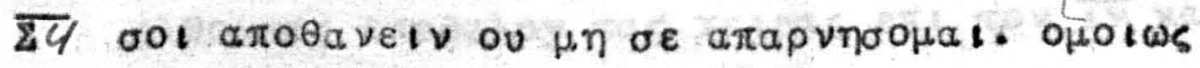
35

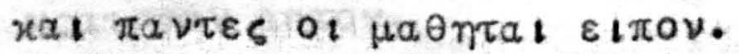

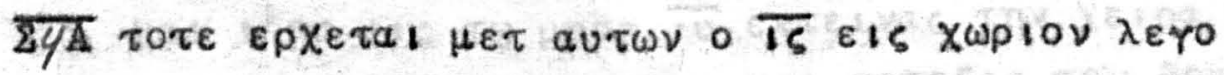
$\mu \varepsilon$ vov retonuavel.

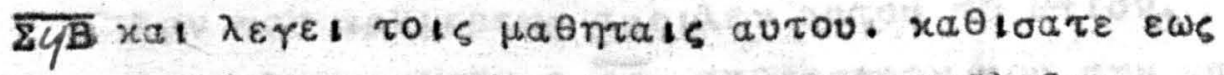

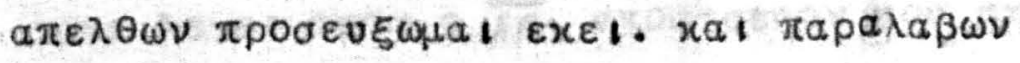

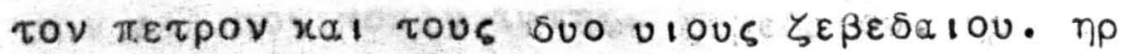

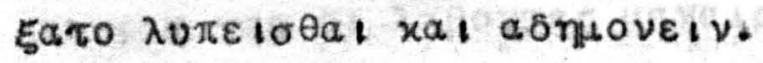




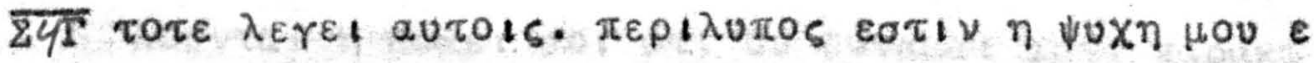

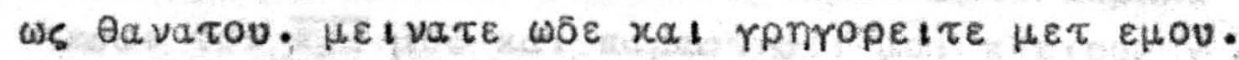

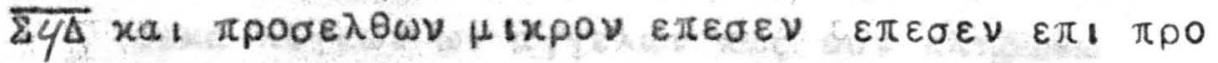

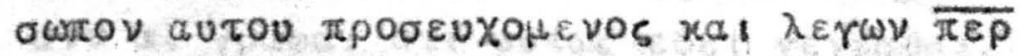

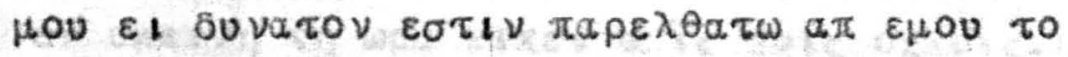

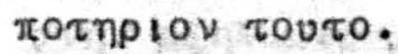

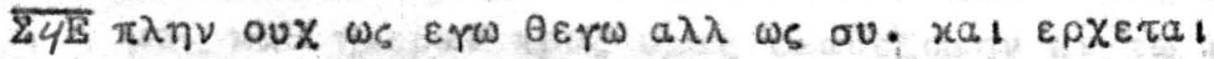

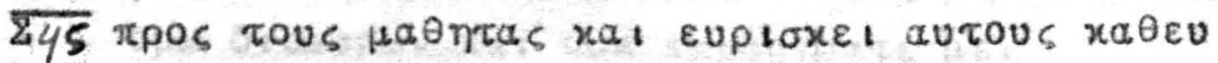

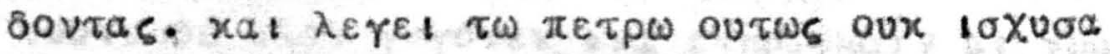

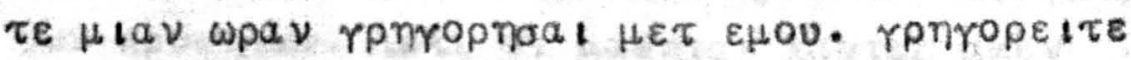

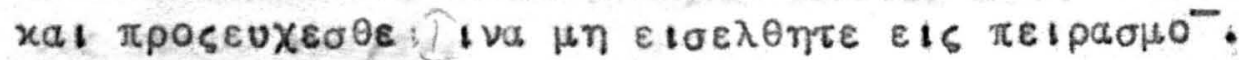

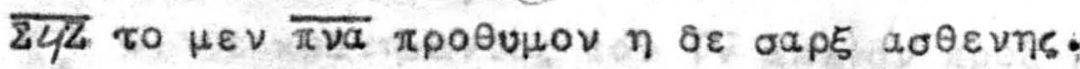

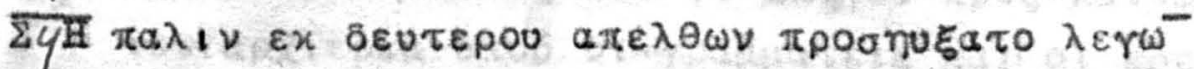

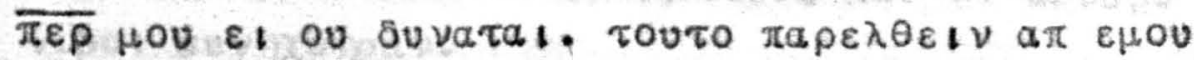

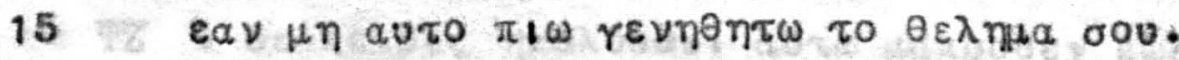

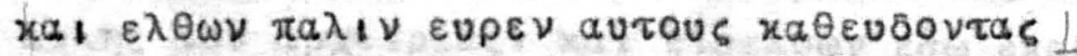

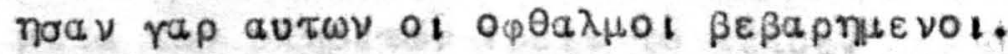

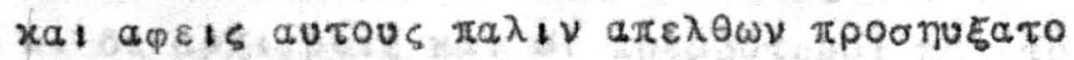

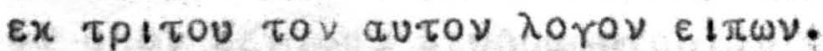

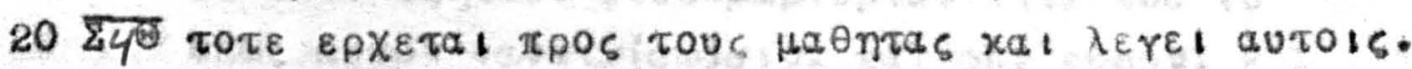

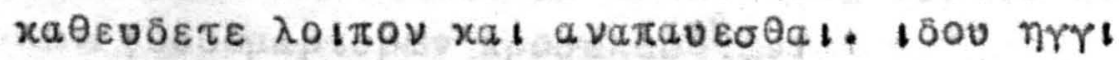

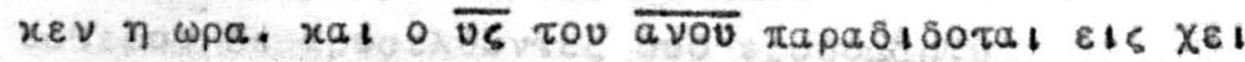

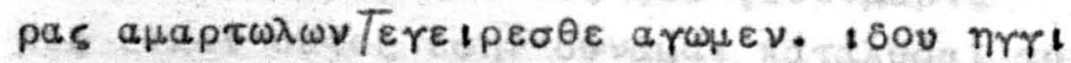

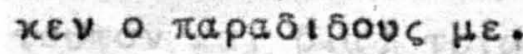

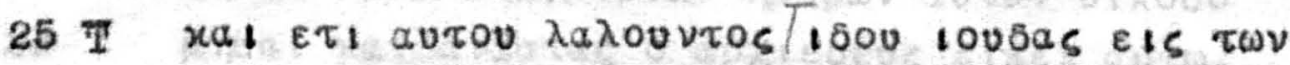

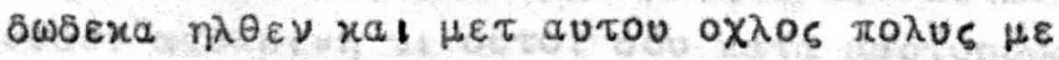

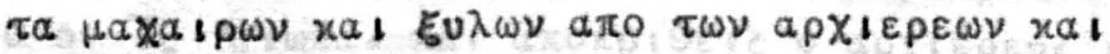

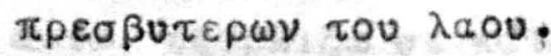

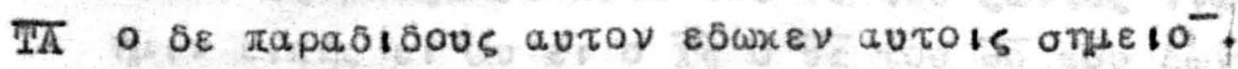

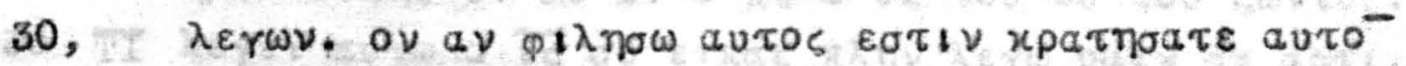

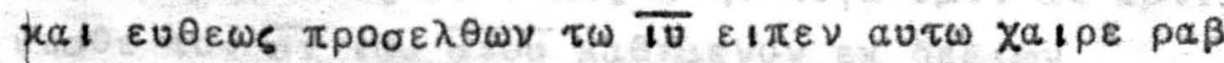

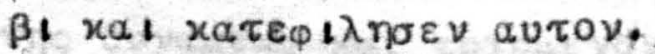

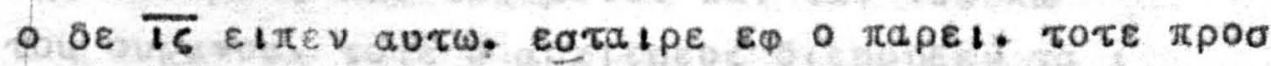

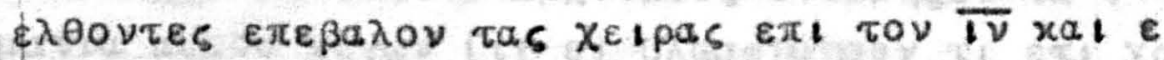

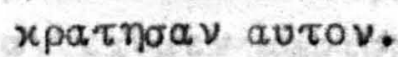

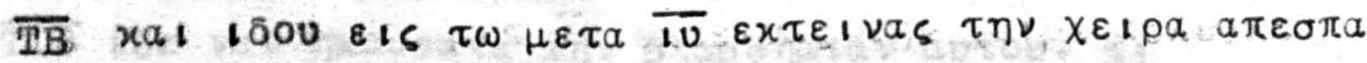

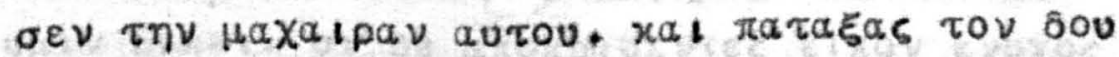

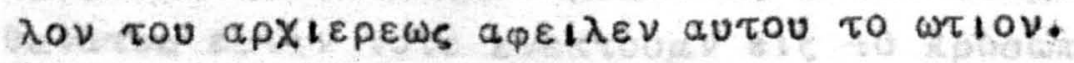

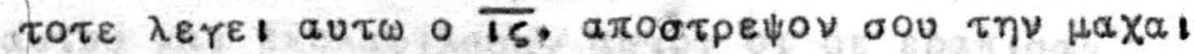
40 pav Eis tov torov autns.

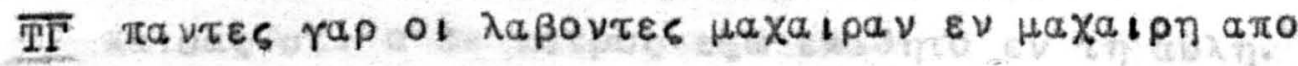




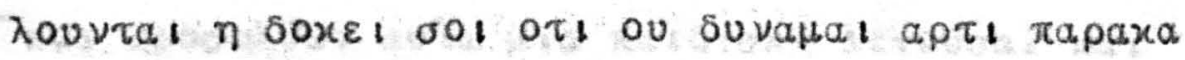

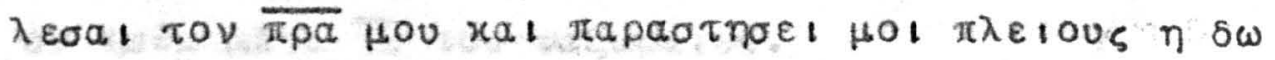

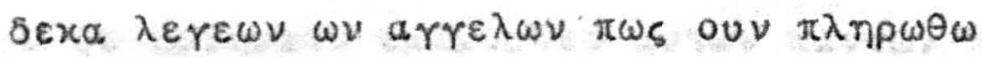

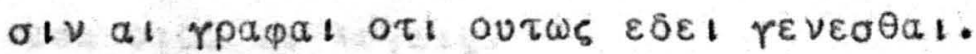

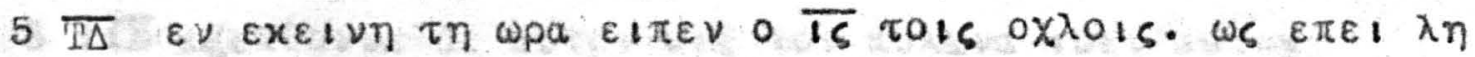

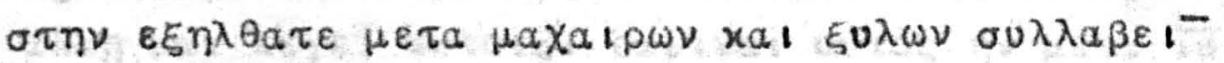

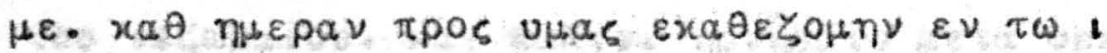

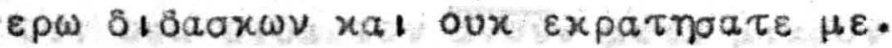

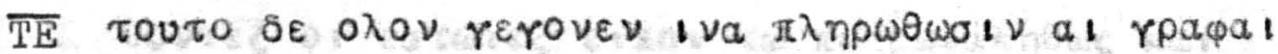

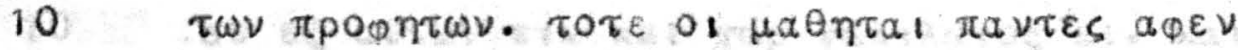
Tes autov $\varepsilon$ urov.

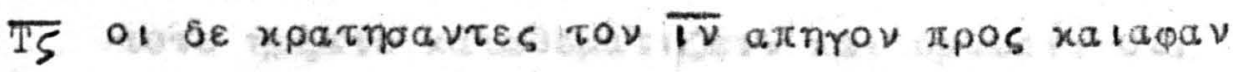

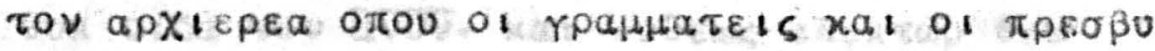

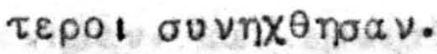

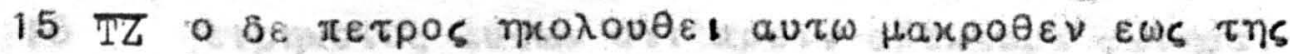

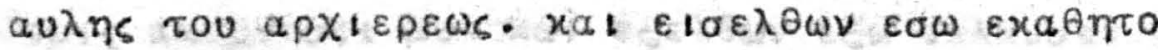

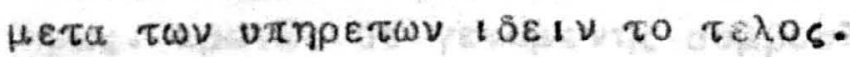

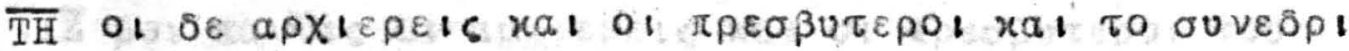

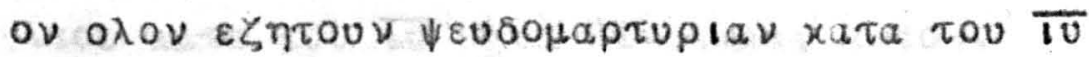

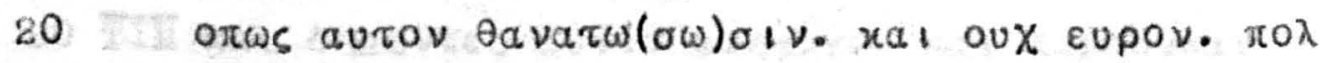

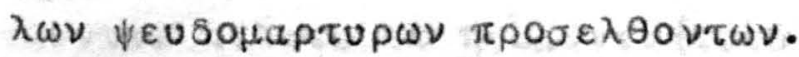

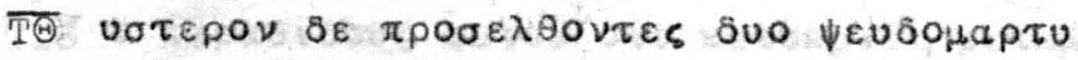

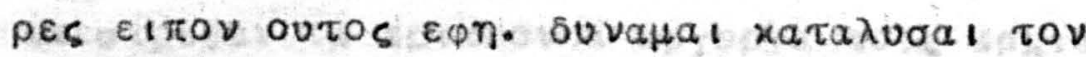

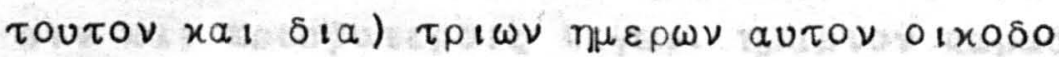

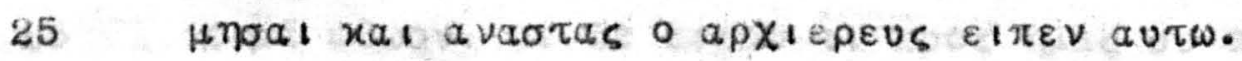

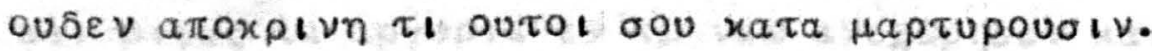

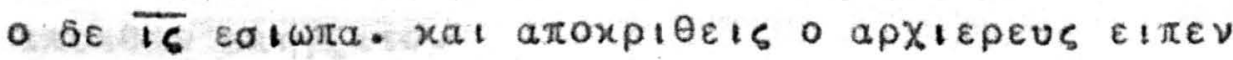

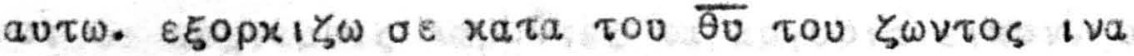

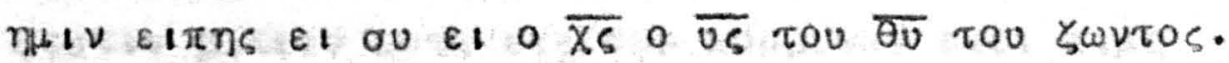

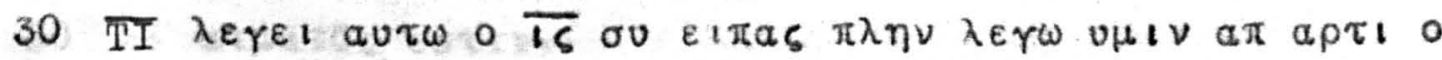

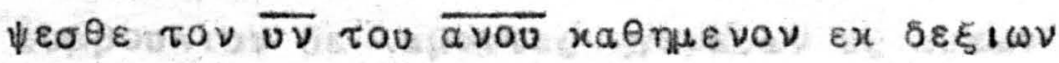

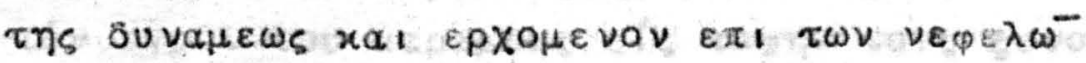

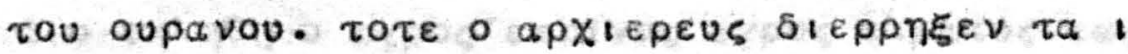

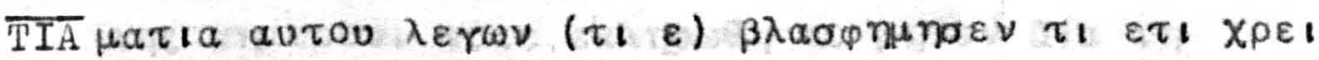
$a \nu \varepsilon \times 0 \mu \varepsilon \nu \mu a \rho \tau u p \omega v$.

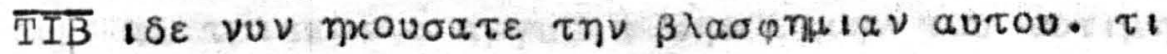
UH

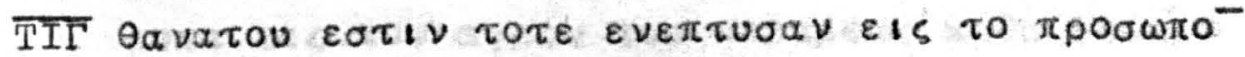

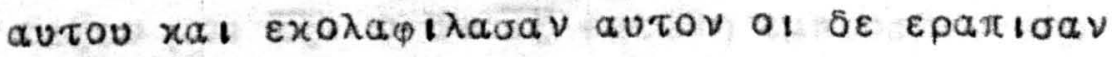

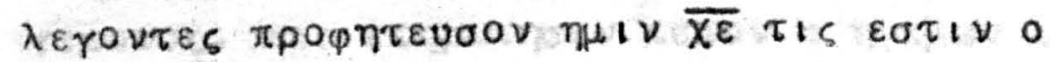

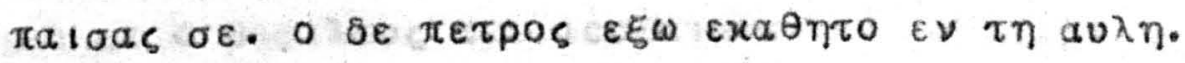




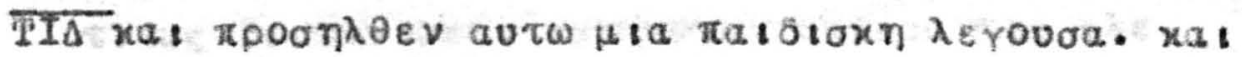

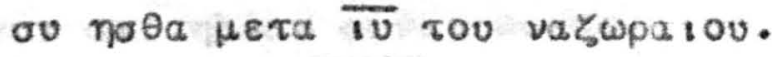

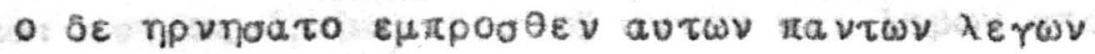

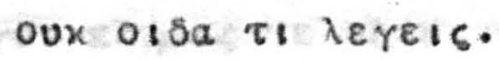

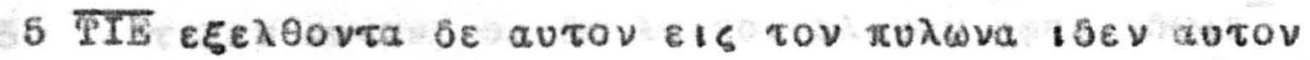

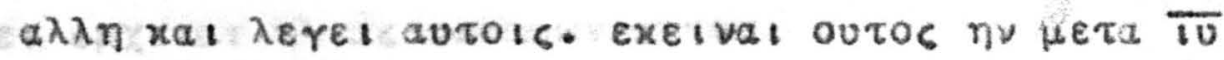

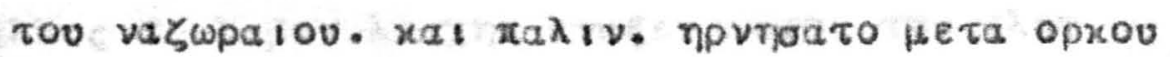

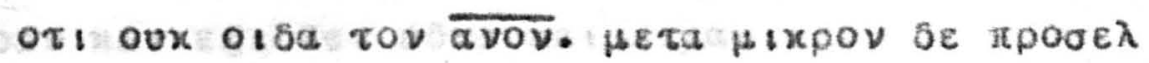

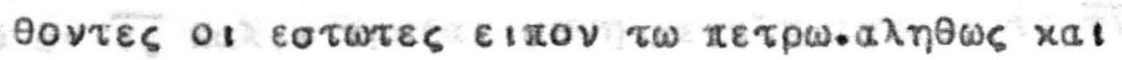

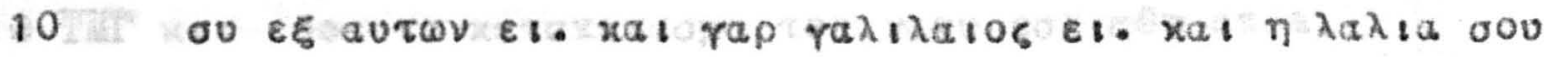

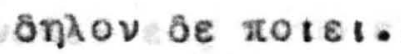

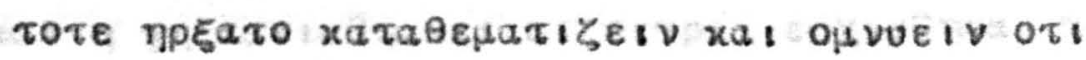

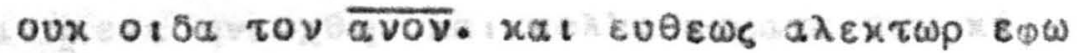

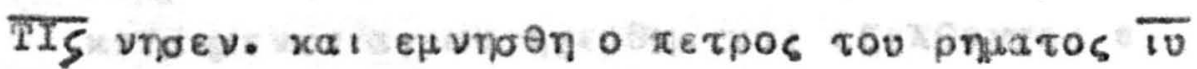

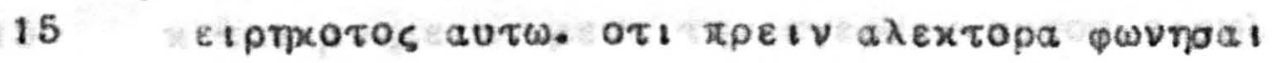

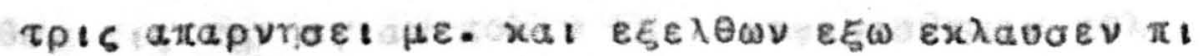
upws. apwias ge

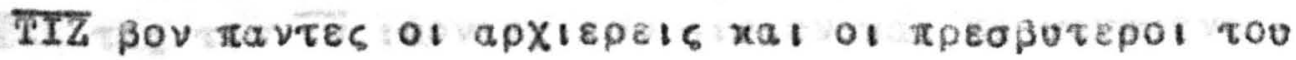

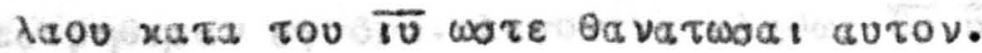

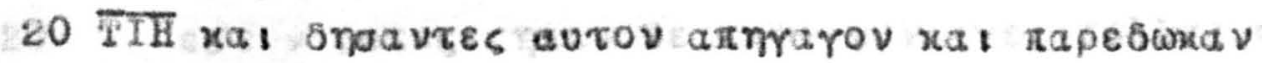

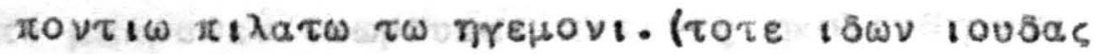

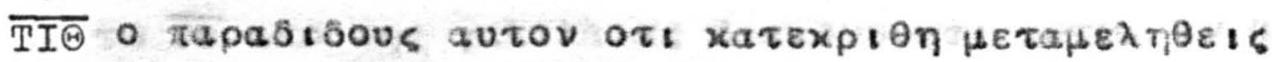

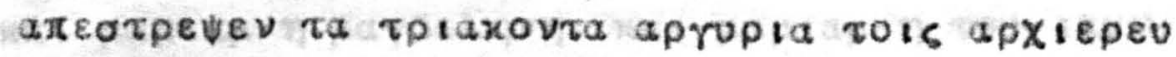

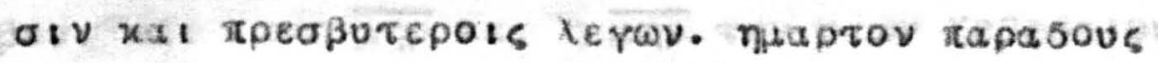

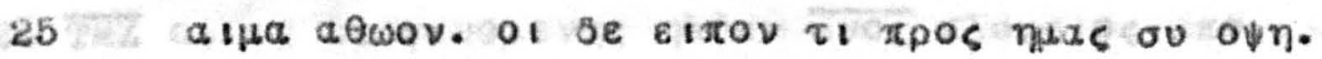
$x a l$ pl

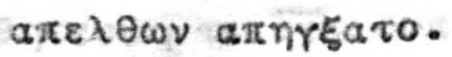

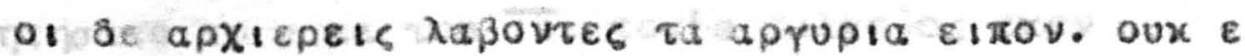

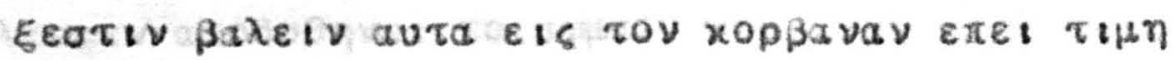

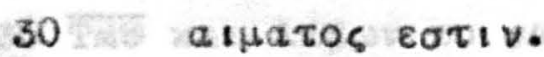

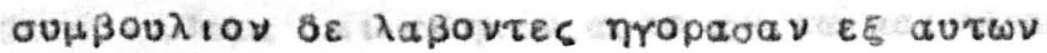

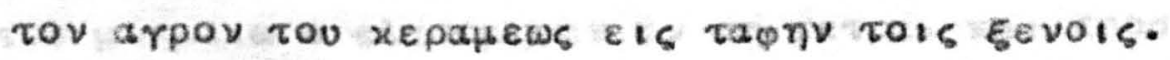

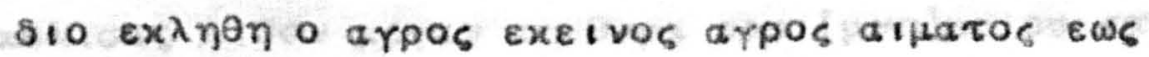

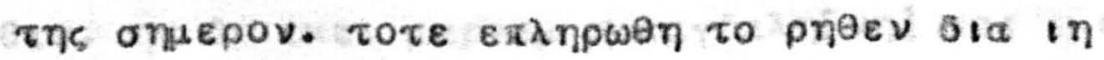

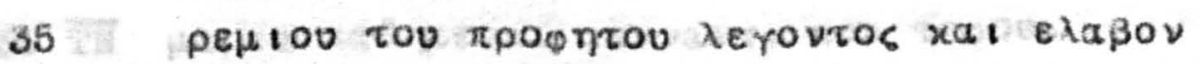

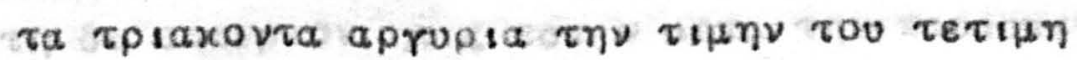

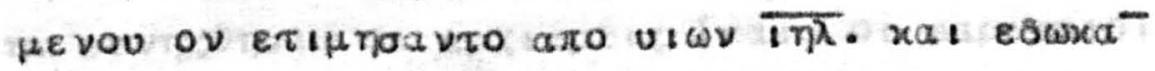

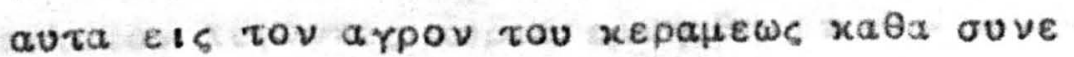

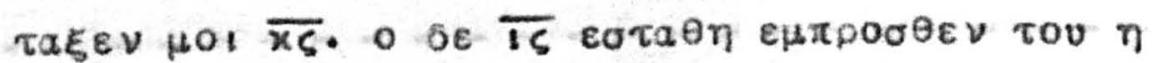

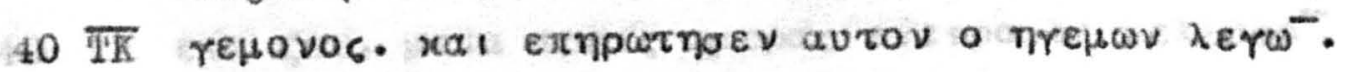

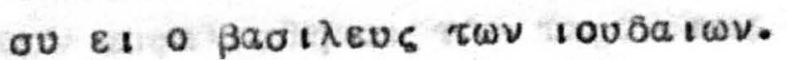




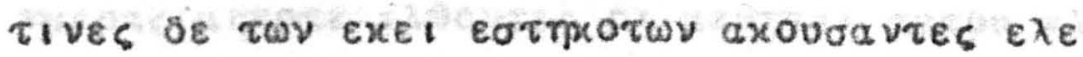
rov ori $\eta \lambda_{1} \alpha \nu$ pwe: outos.

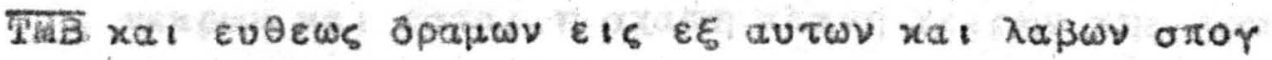

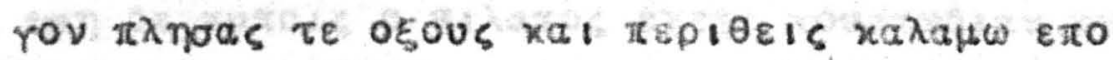

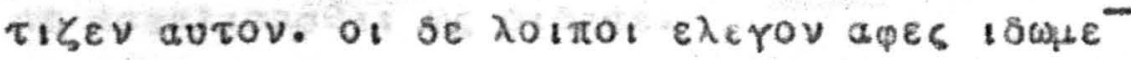

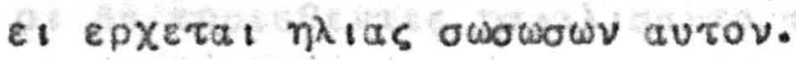

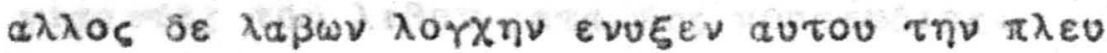

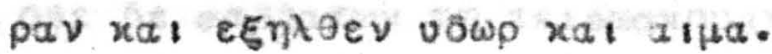

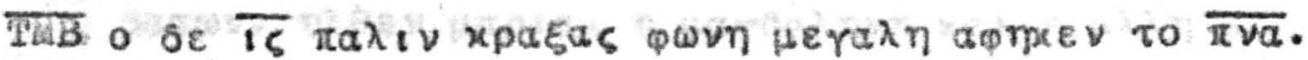

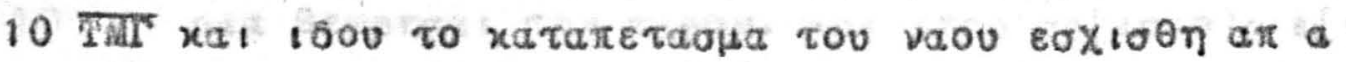

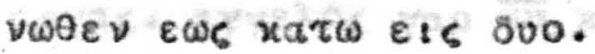

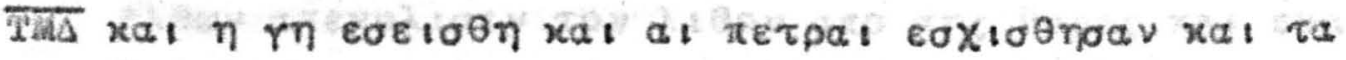

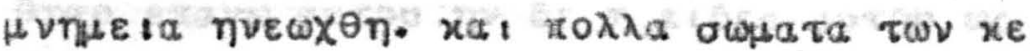

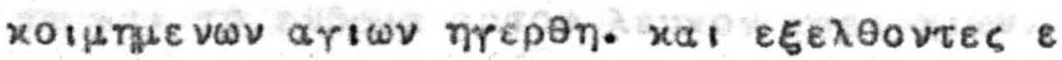

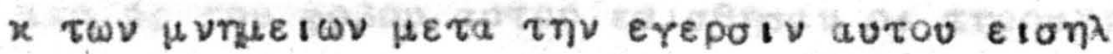

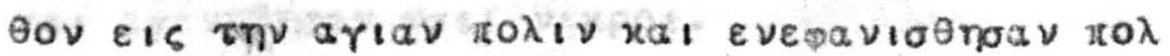

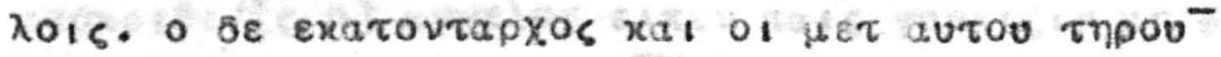

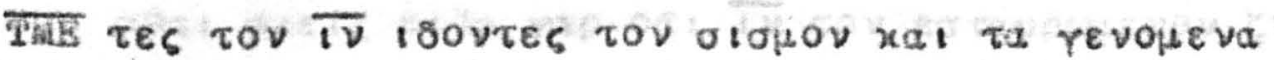

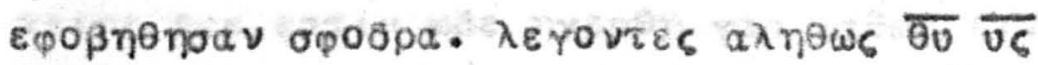

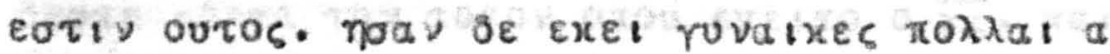

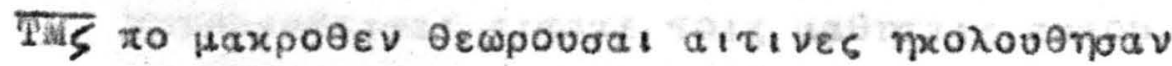

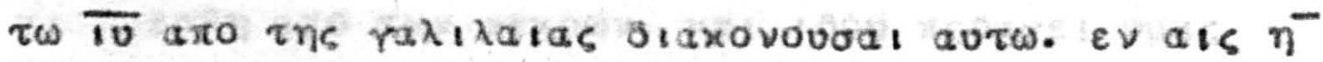

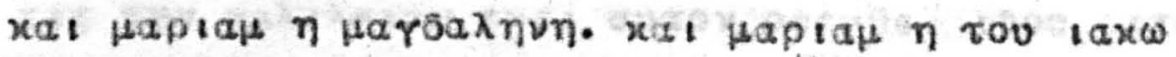

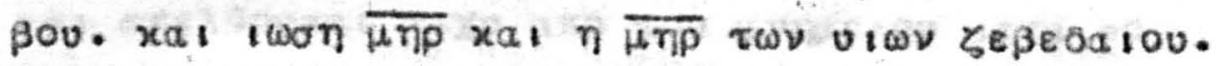

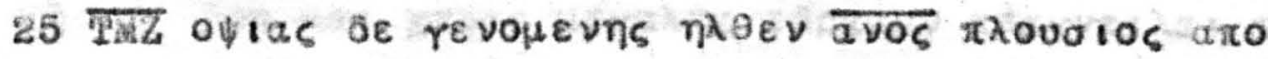

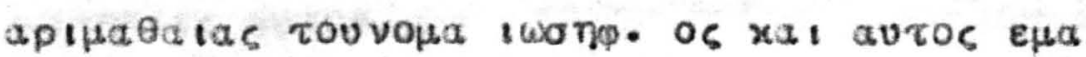

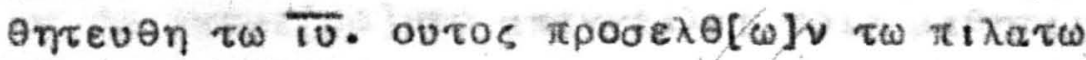

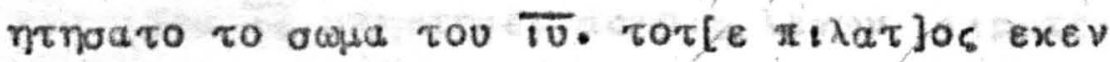

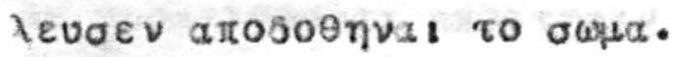

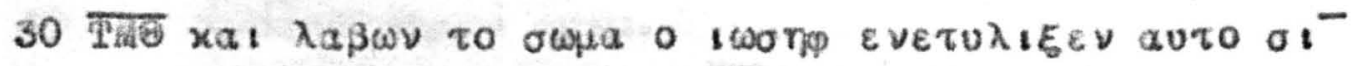

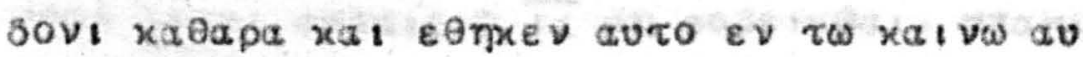

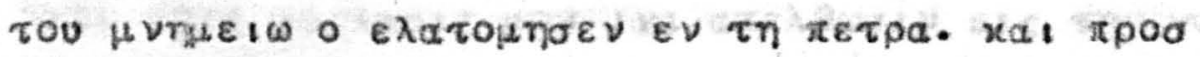

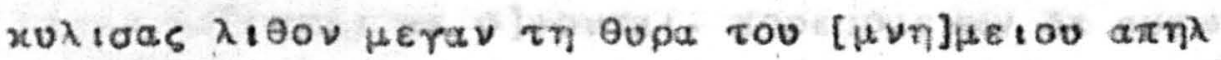

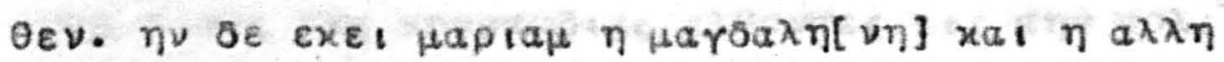

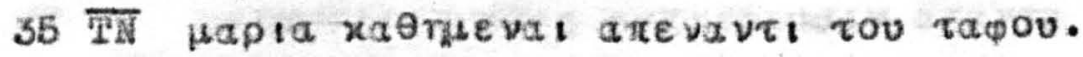

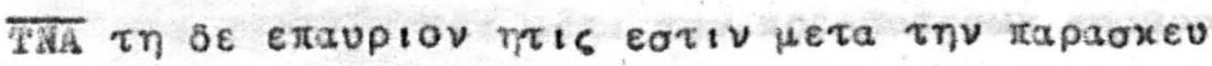

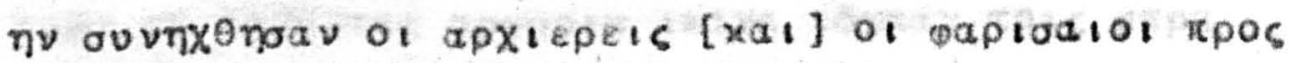

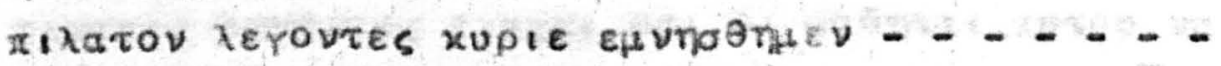

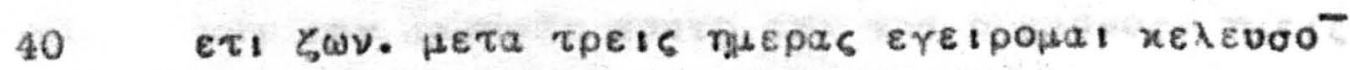

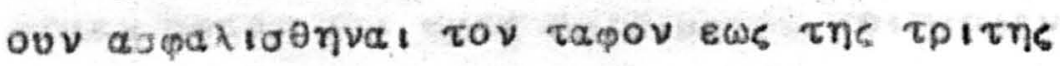




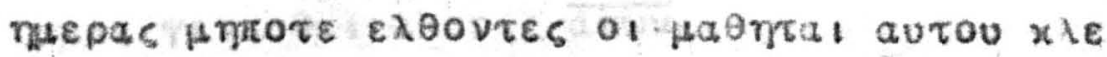

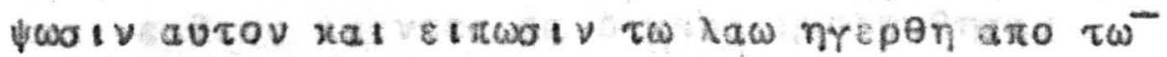

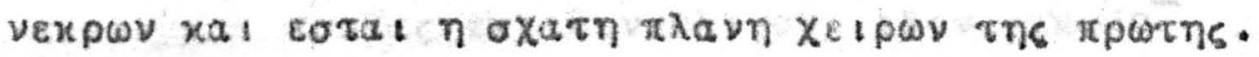

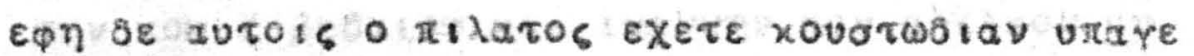

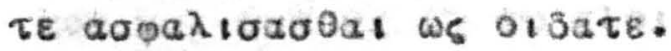

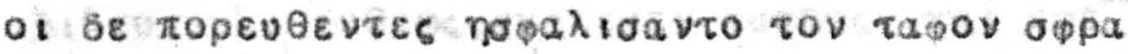

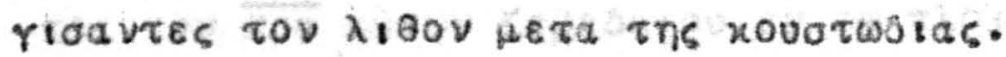

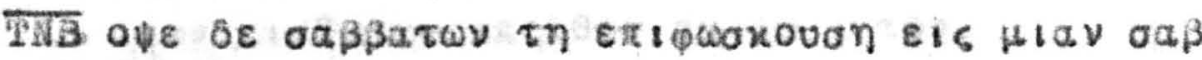

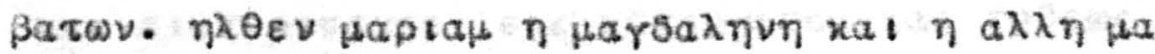

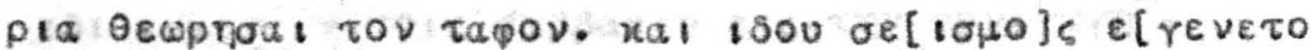

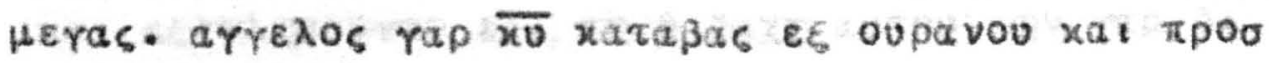

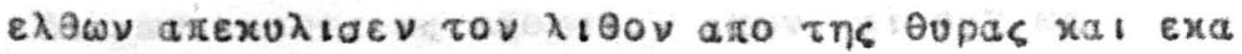

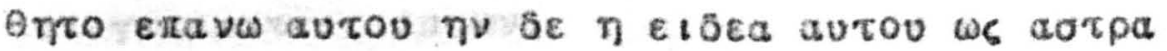

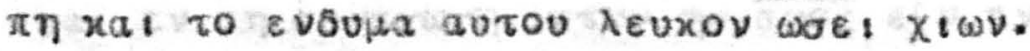

15 TII a

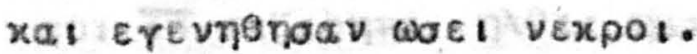

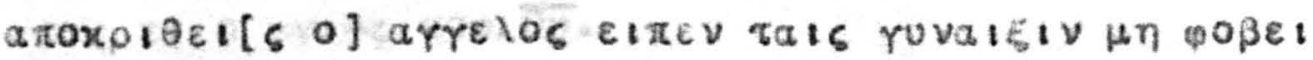

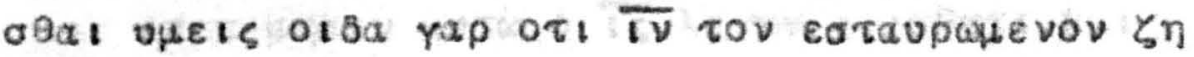

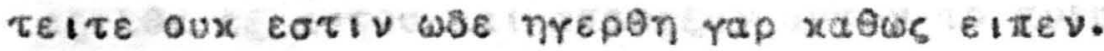

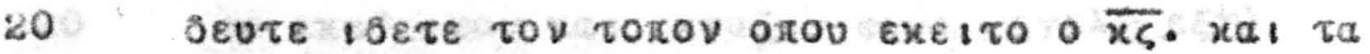

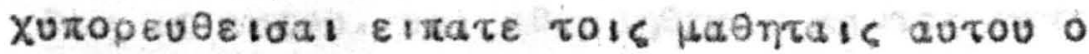

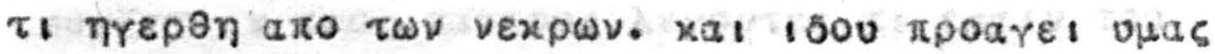

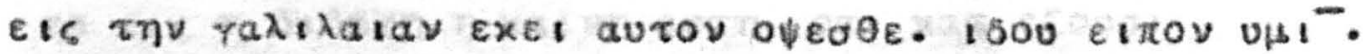

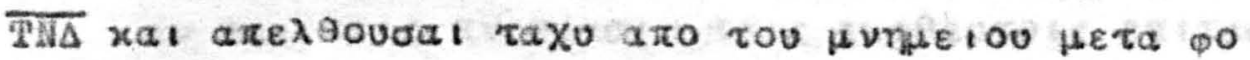

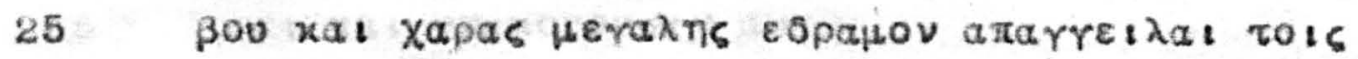

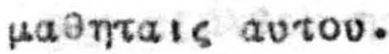

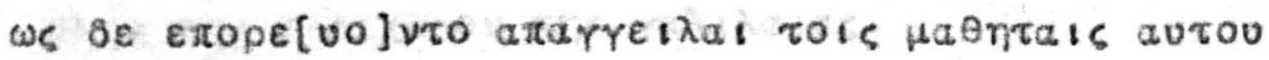

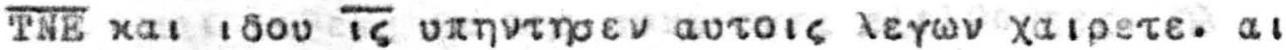

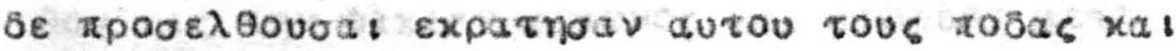

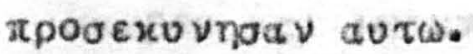

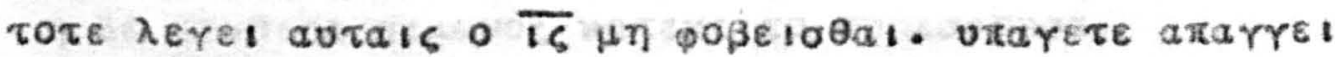

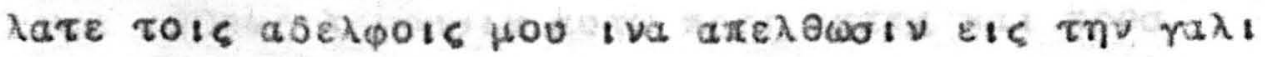

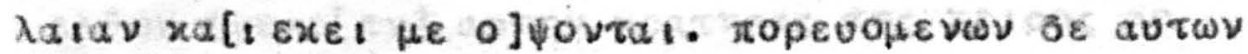

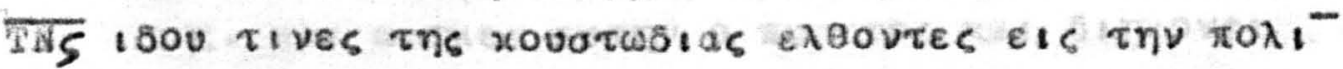

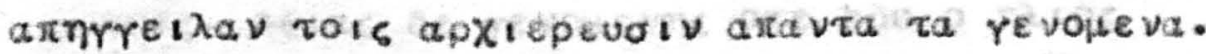

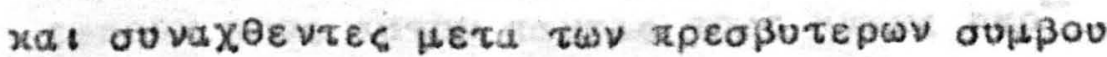

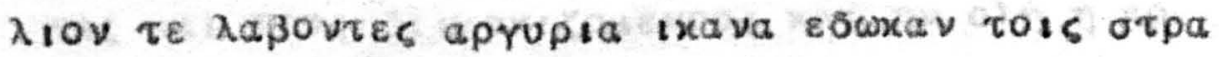

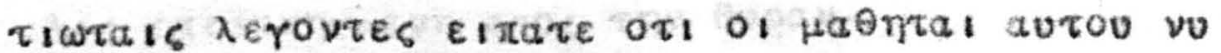

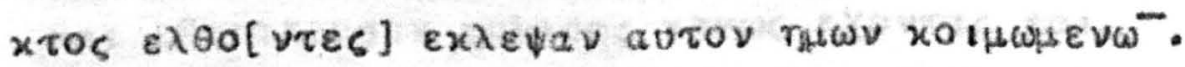

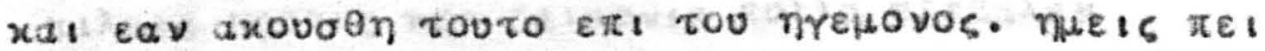

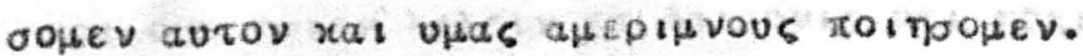




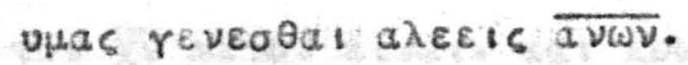

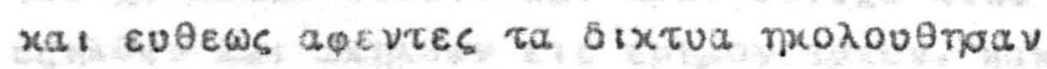

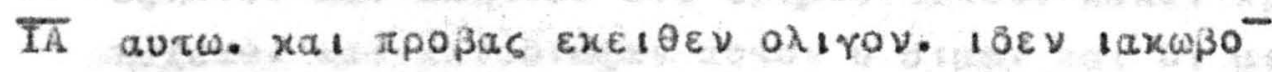

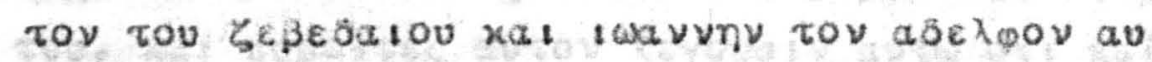

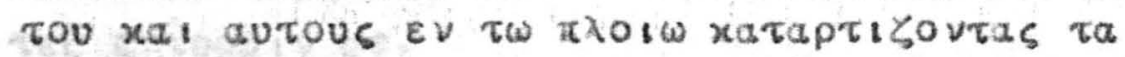
Sixtua xat cuoews exadeorv autous. xal ap $\varepsilon^{-}$

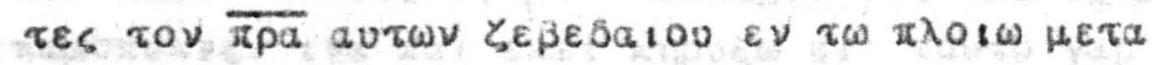

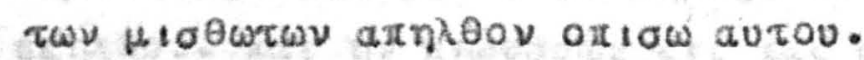

Is xal elaxopevovial ets xaxepvaoup xal eveews

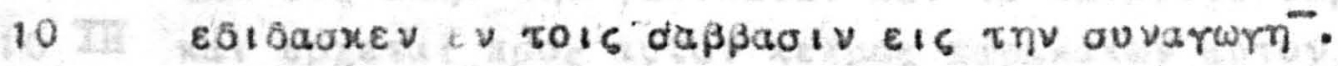

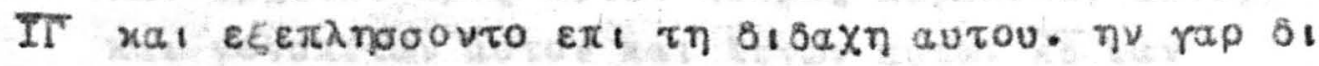

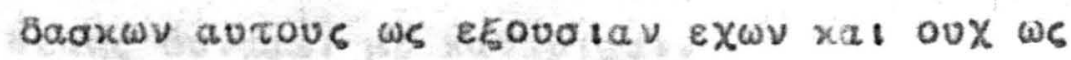
ot rpapiateis autwv.

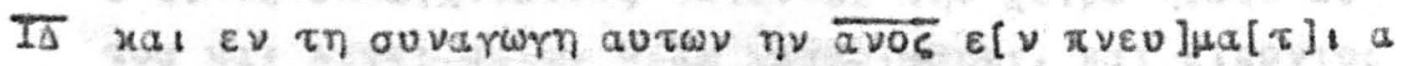

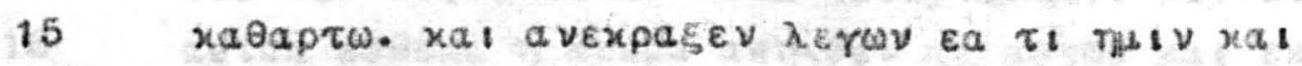

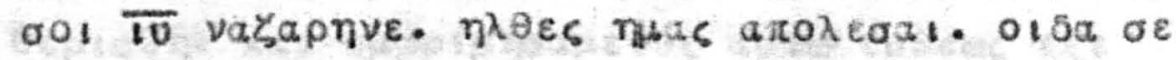
Tis El o arsos tov बु.

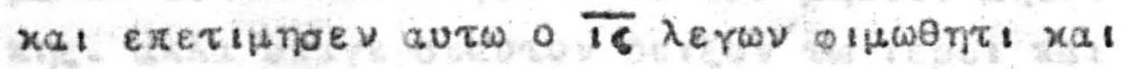
$\varepsilon \xi \varepsilon \lambda \theta \varepsilon \varepsilon \xi$ av

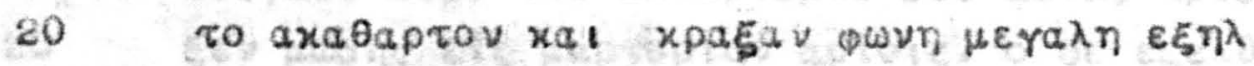

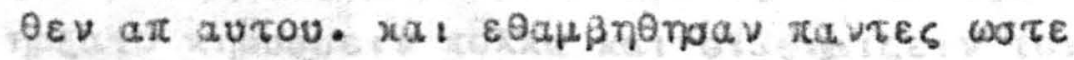

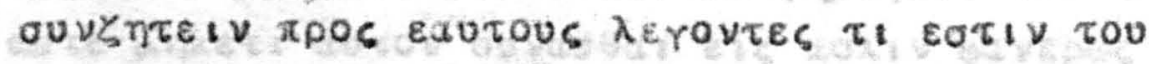

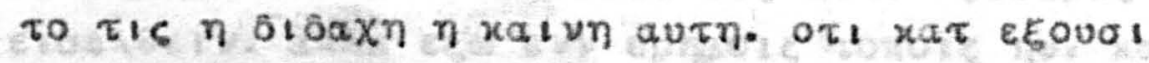

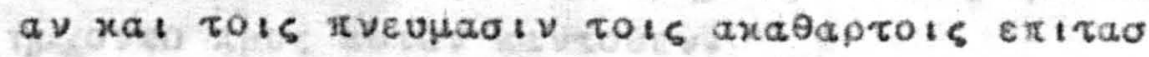
25 oet xat uxaxovovatv avtw.

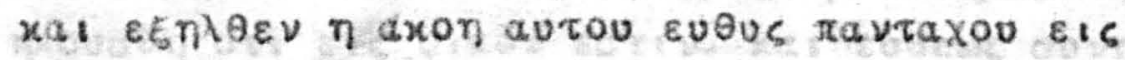

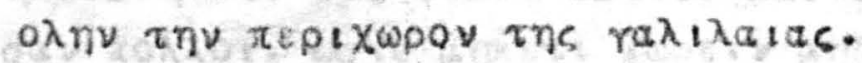

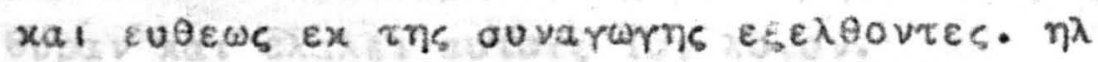

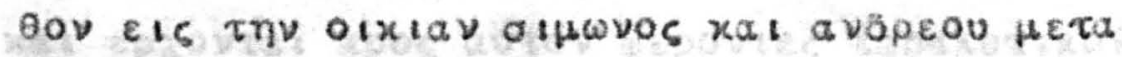
30 laxcopou xal lwavvou.

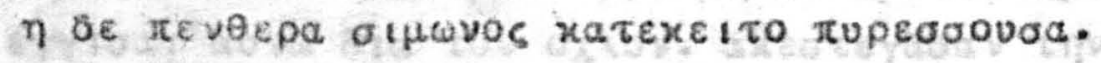

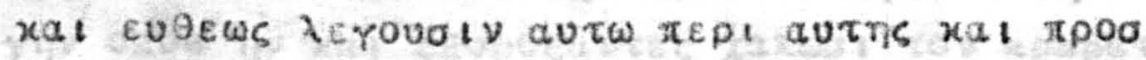

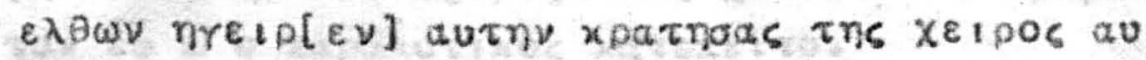

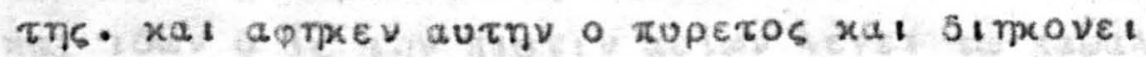

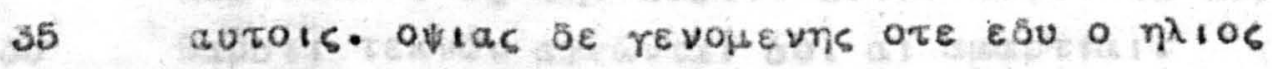

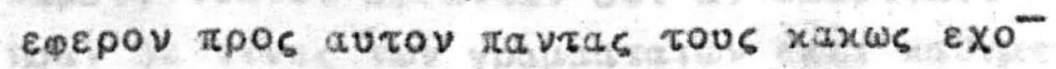

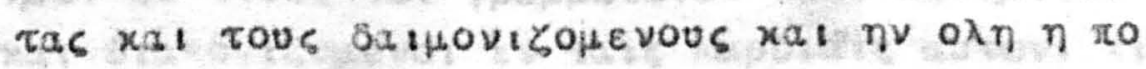

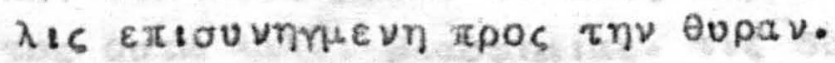

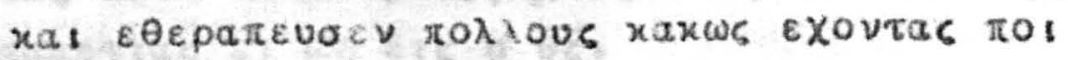

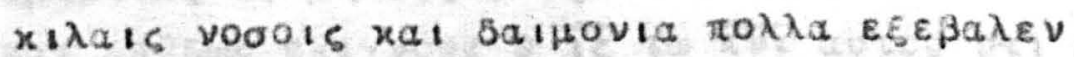

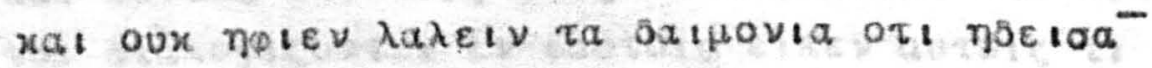




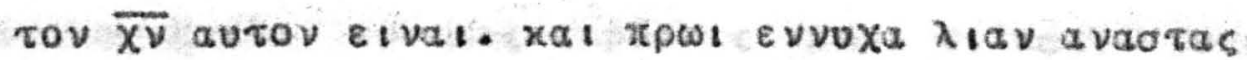

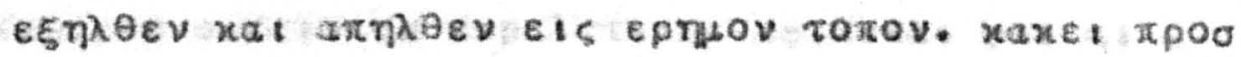

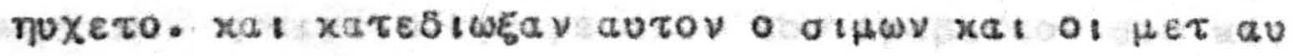

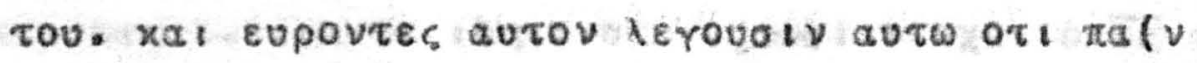

xal $\lambda \varepsilon \gamma \varepsilon$ a

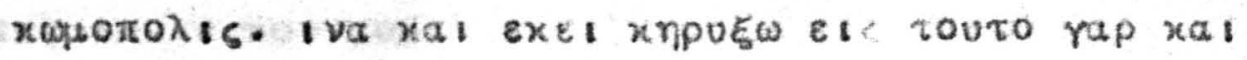

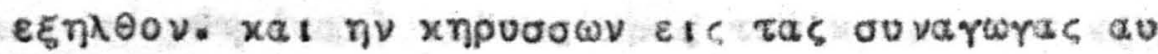

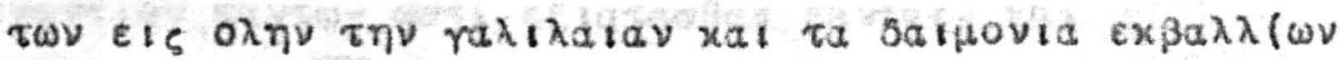

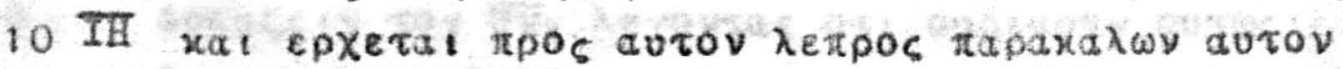

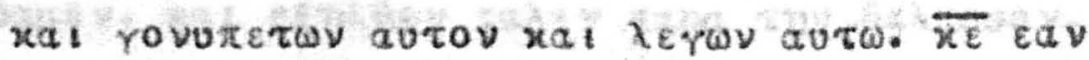

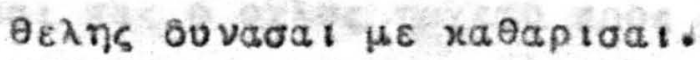

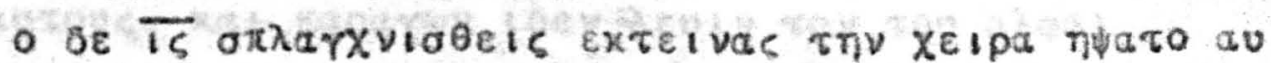

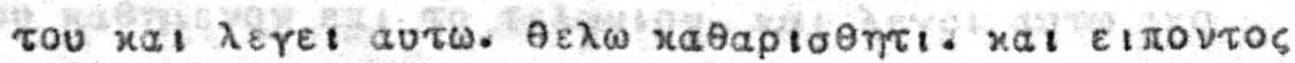

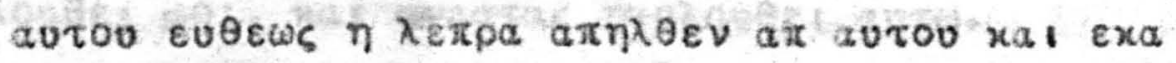

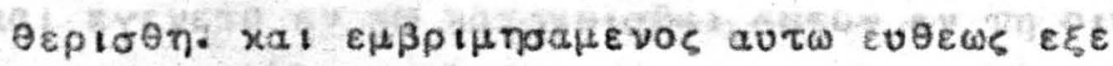

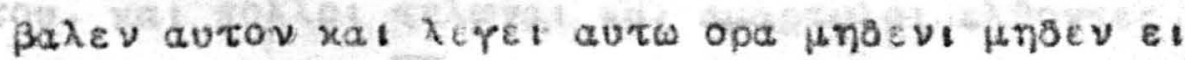

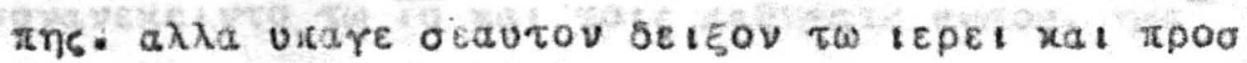
evema । $\pi \varepsilon \rho$ i tou $x a \theta \alpha \rho$ op

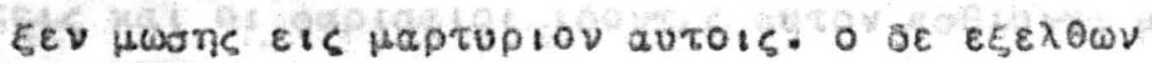

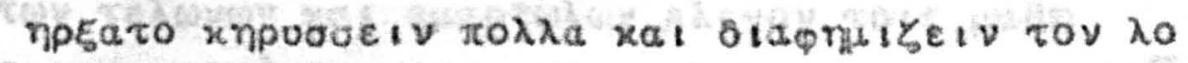

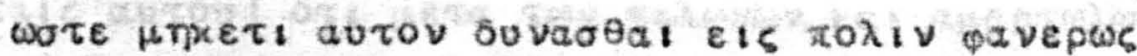

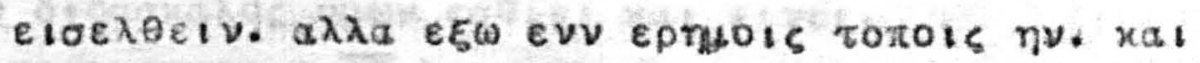

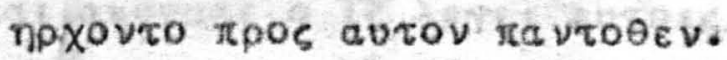

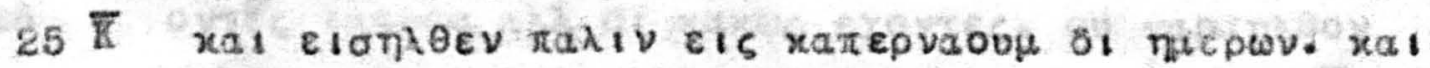

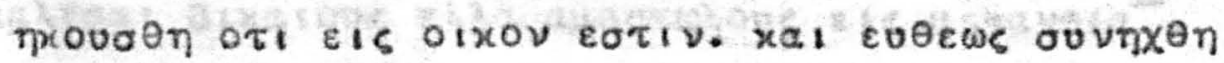

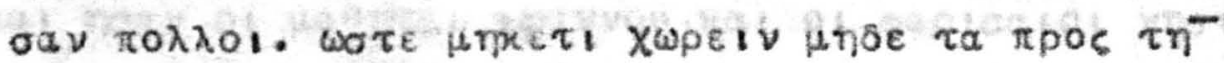

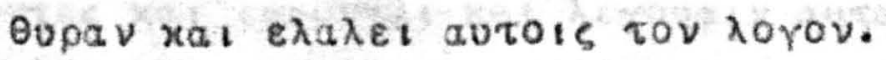

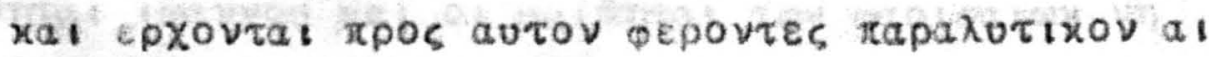

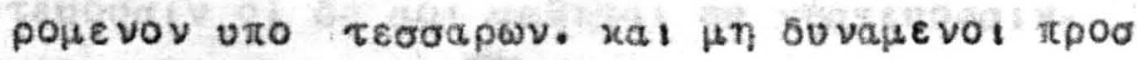

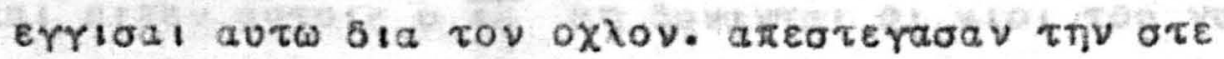

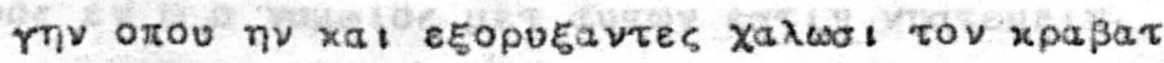

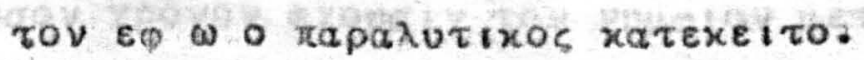

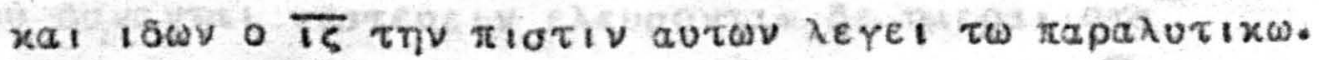

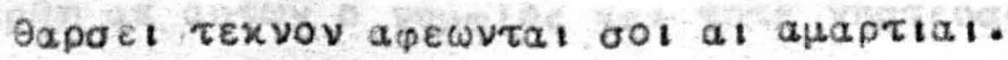

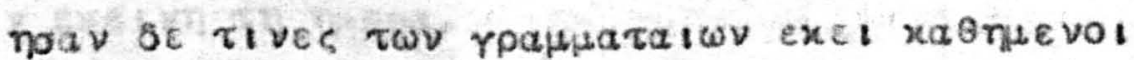

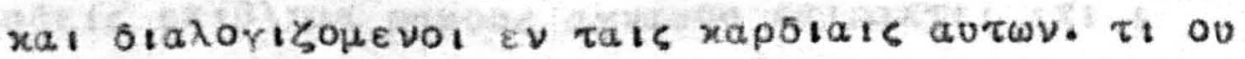

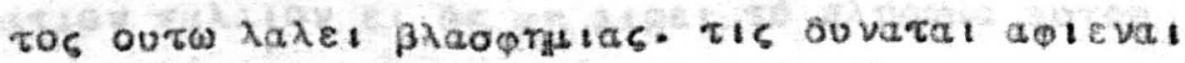
apaptias $\varepsilon / \mu \eta$ Els 0 बढ़.

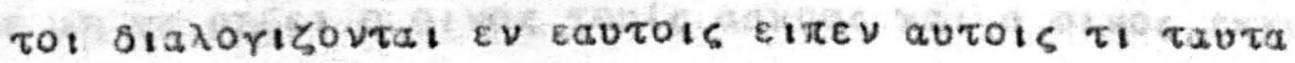


oิ

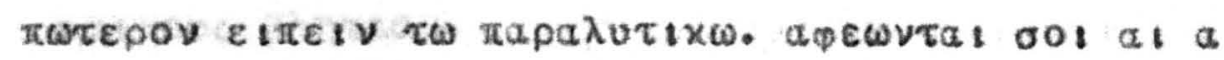

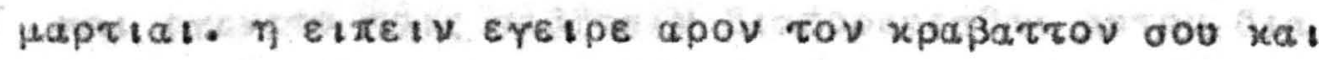

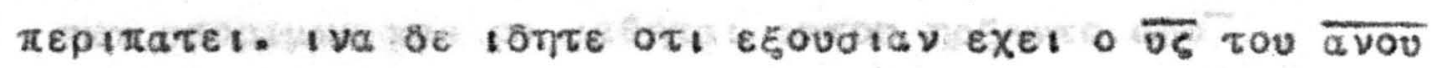

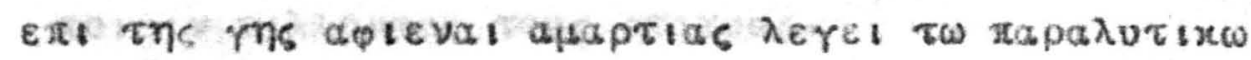

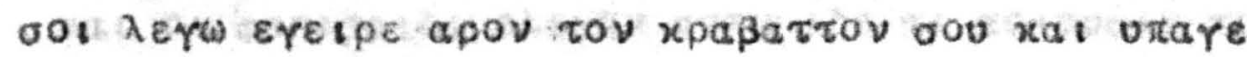
Eis rov oixov oou.

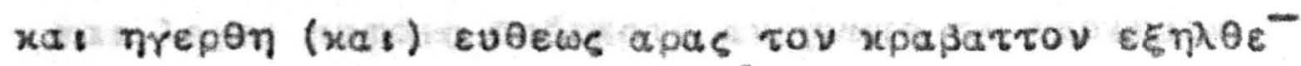

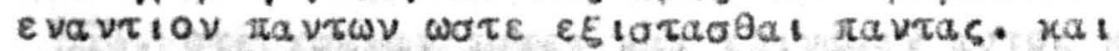

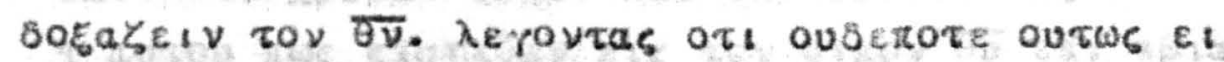

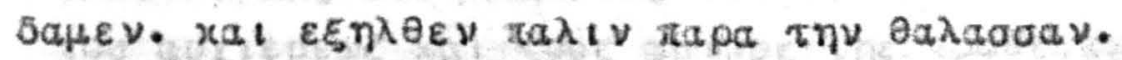

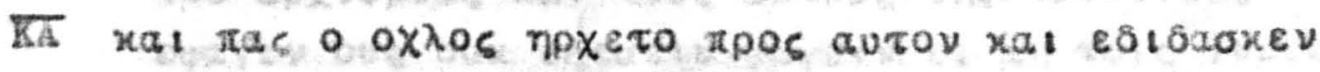

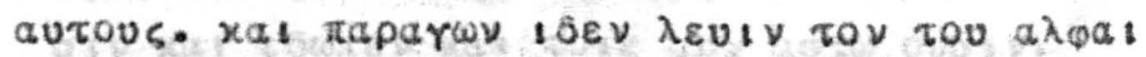

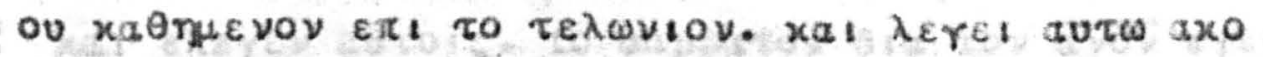
$\lambda 00 \theta \varepsilon 1$ Hol. nal avaotas ppodover, autw.

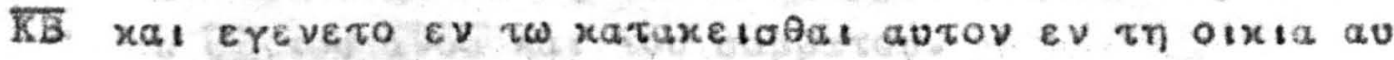

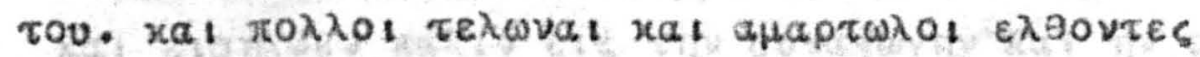

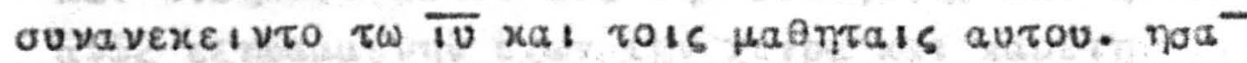

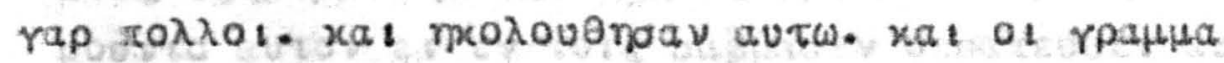

20 Tels xal of paploalot lovers avtov eaglovta Heta

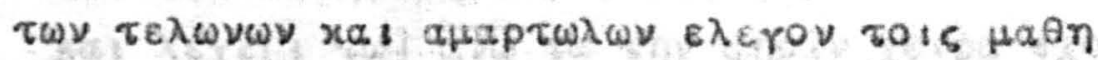

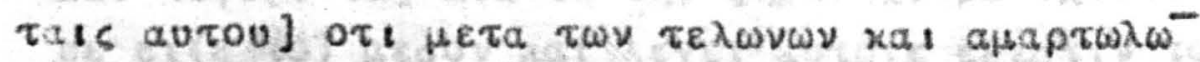

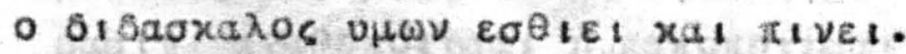

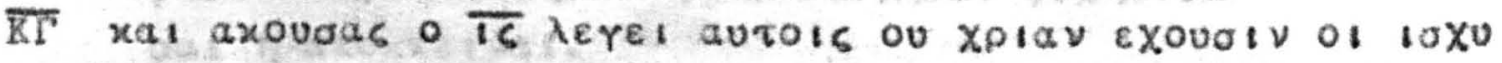

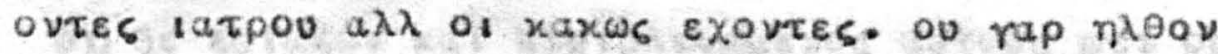

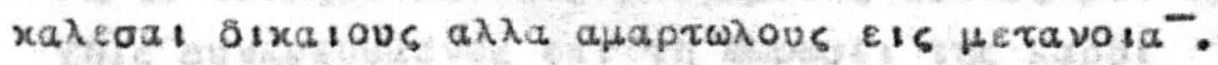

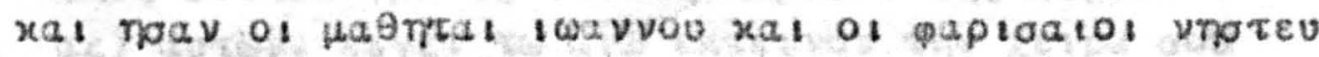

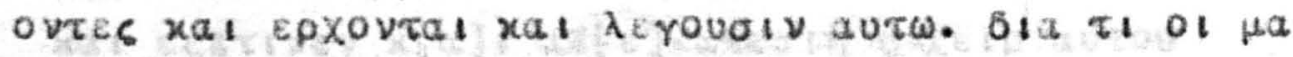

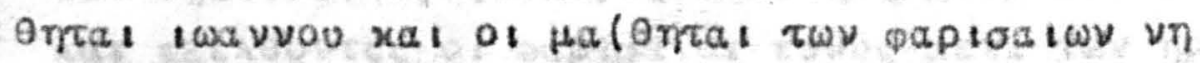

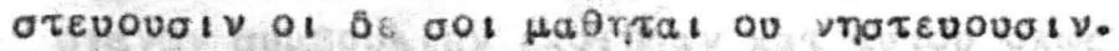

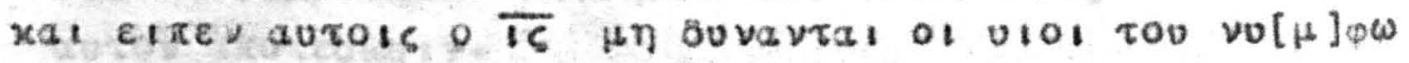

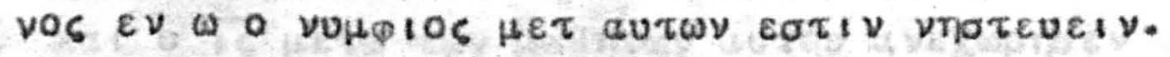

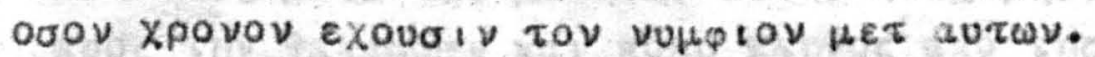

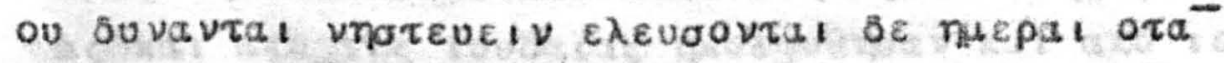

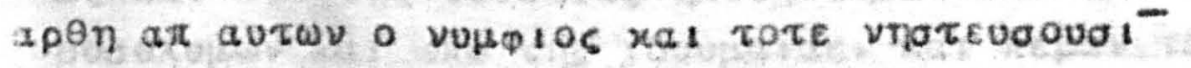

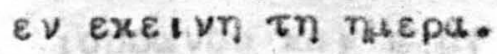

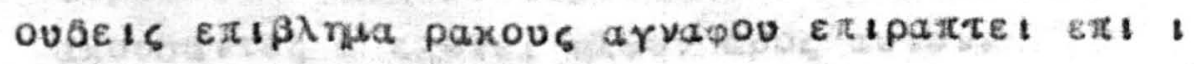

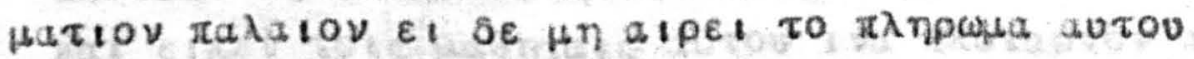

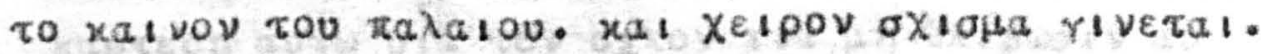

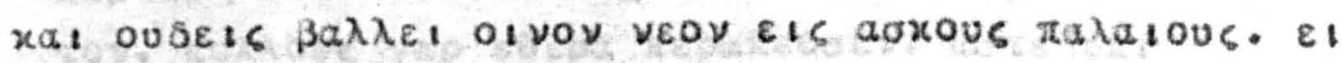

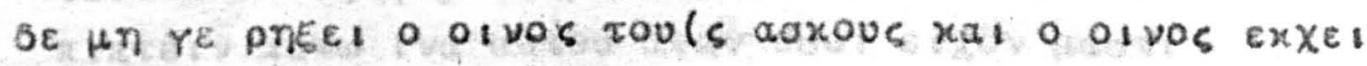




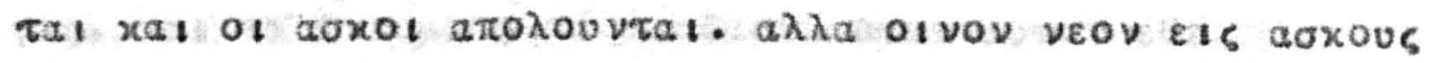
xaเvovs $\beta \lambda \eta \eta \varepsilon \circ$.

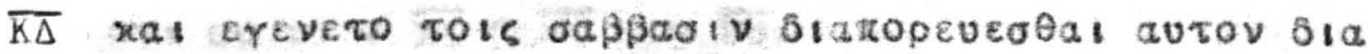

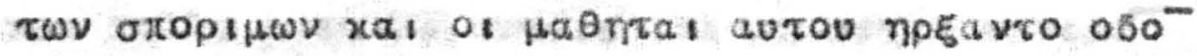

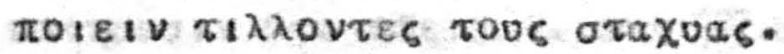

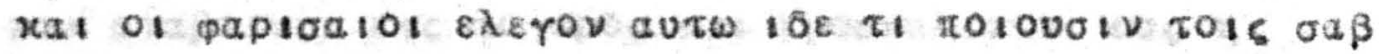

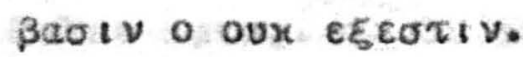

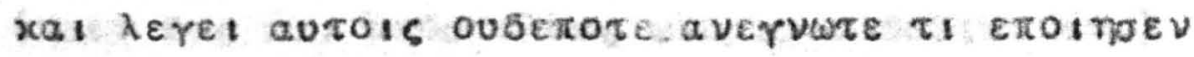

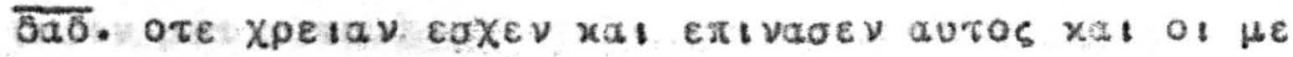

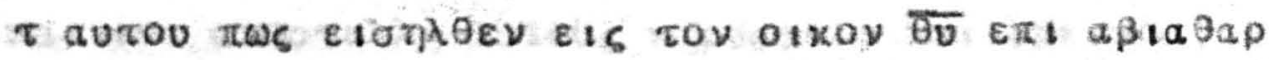

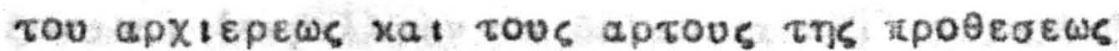

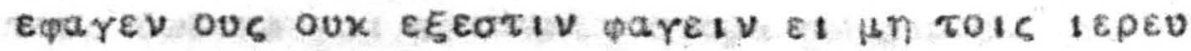

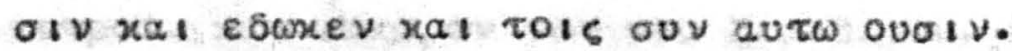

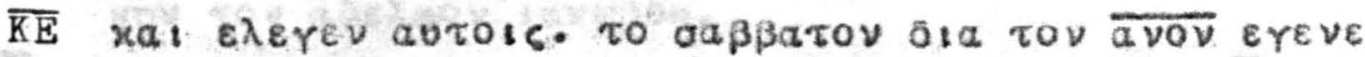

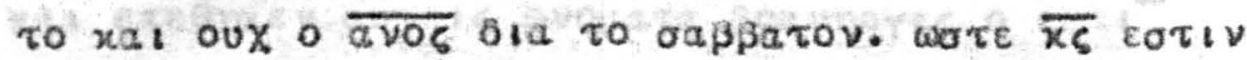

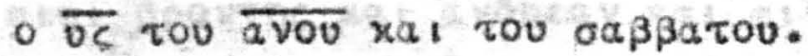

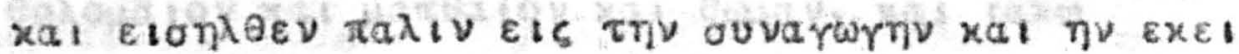

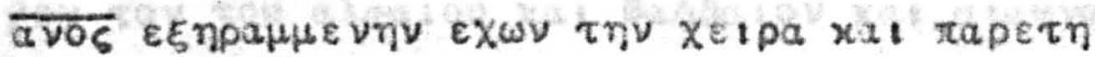

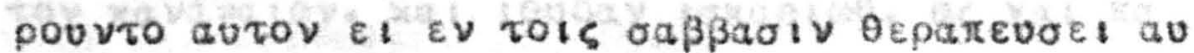

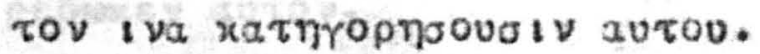

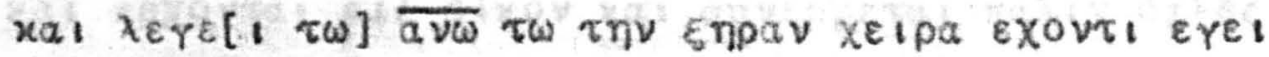

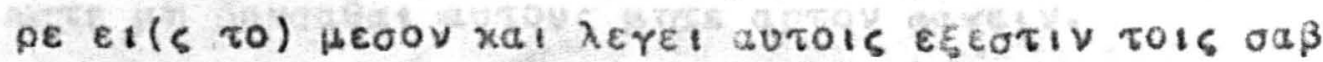

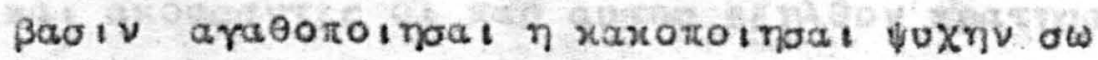
oat $\eta$ atoxe เ val.

01) $8 \varepsilon^{3}$ ea

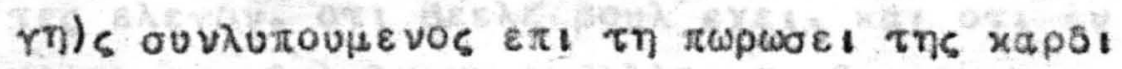

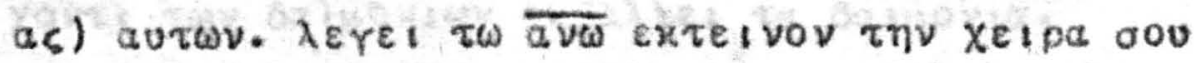

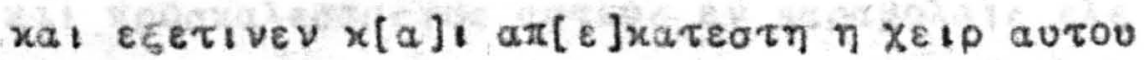

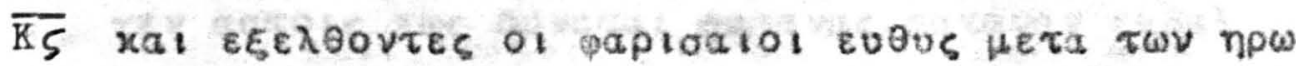

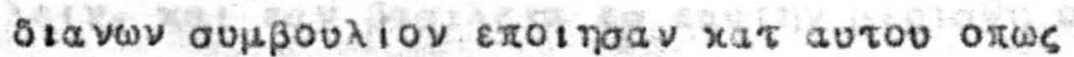
aurov aroleowotv.

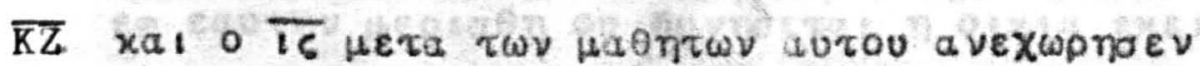

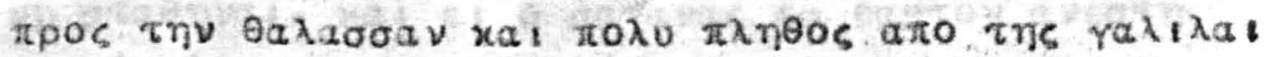
as $x a l$ aro ths lovalas mxoxovenoav xat aro is

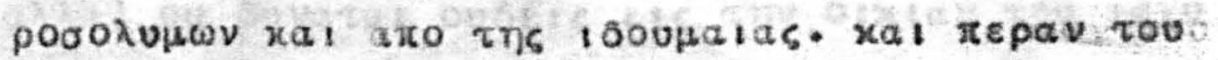

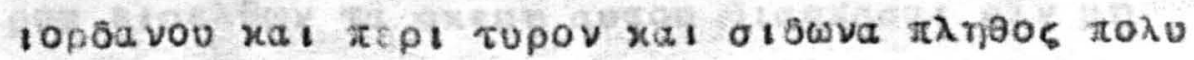

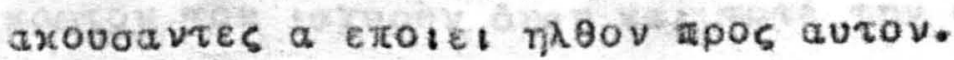

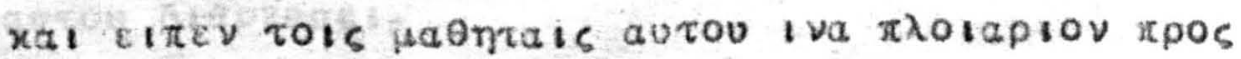

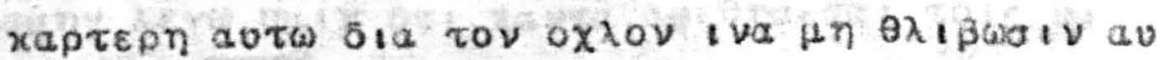

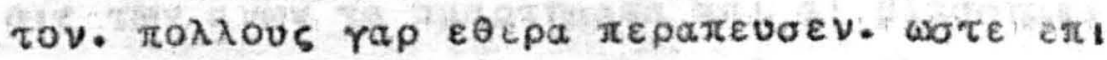

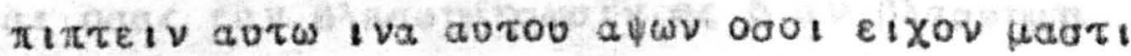




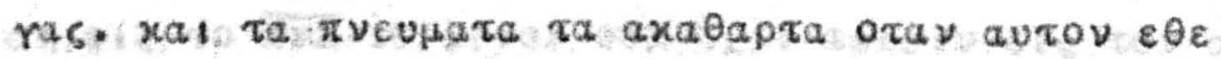

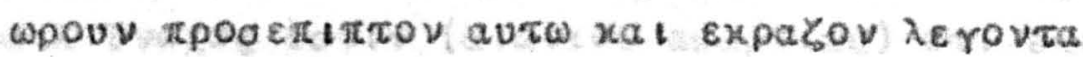

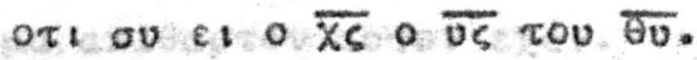

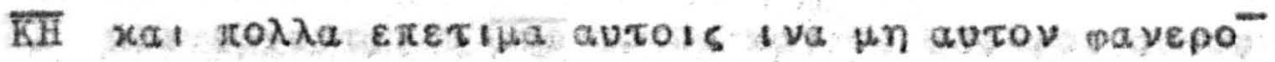

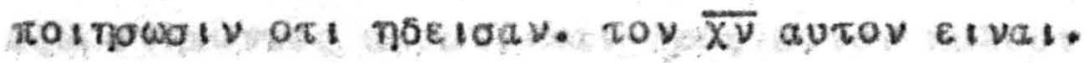

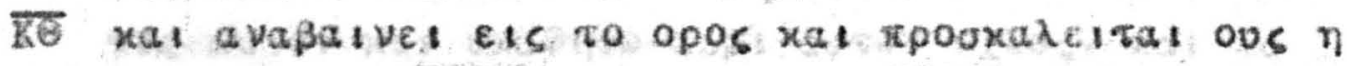

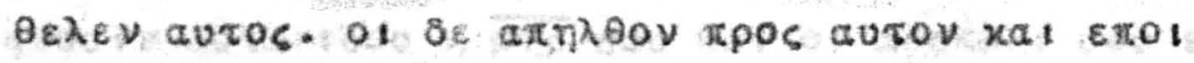
(noev ous xal aroorodous wvopaoev owsera iva) (woiv $\mu \varepsilon \tau$ aurou)

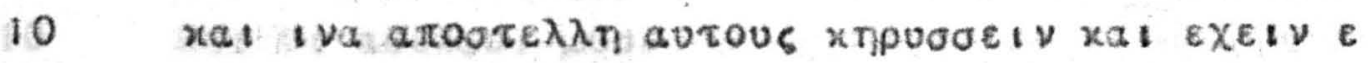

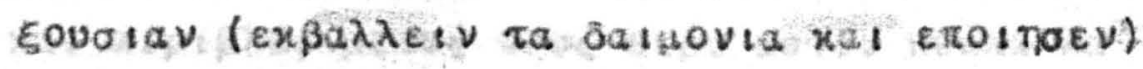

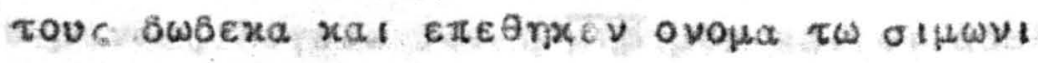

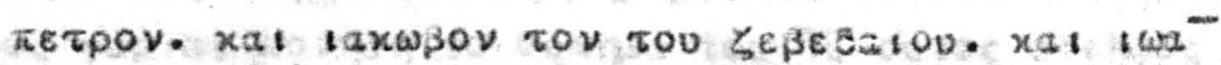

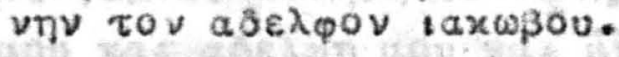

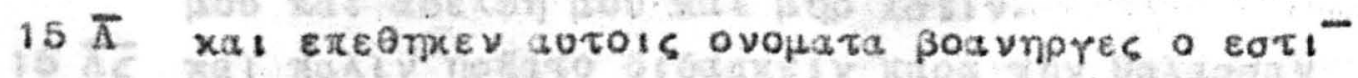

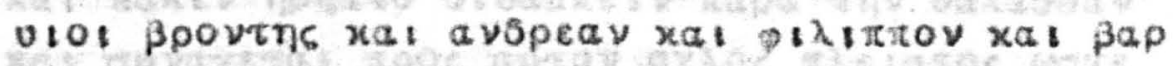

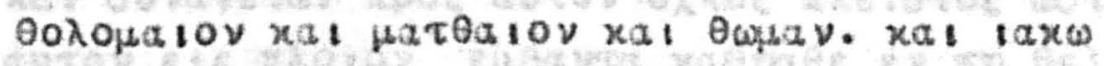
Bov tov tou a teas 100 xal Oasbatov xal othava Tov xavavalov. xal $1008 a \mathrm{~V}$ laxaptwo. of xal $\pi$ a

20 pesaxev autov.

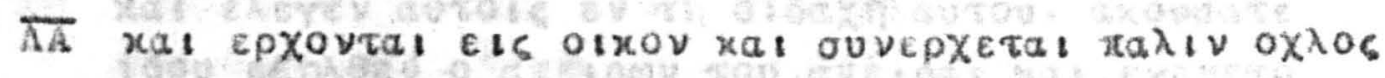

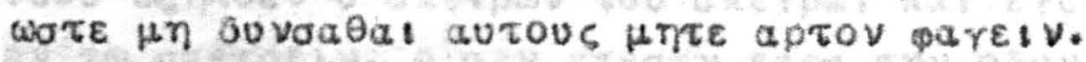

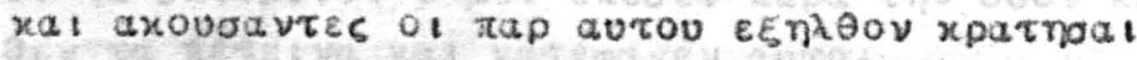
autov eterov rap ort eEcotr.

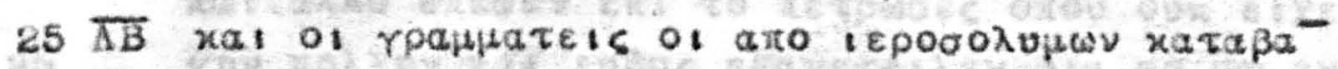

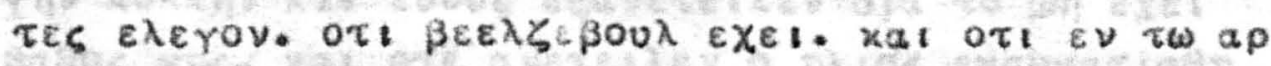

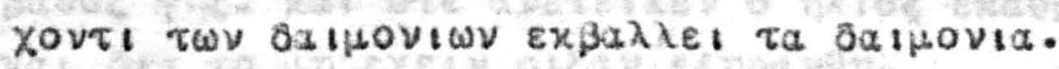

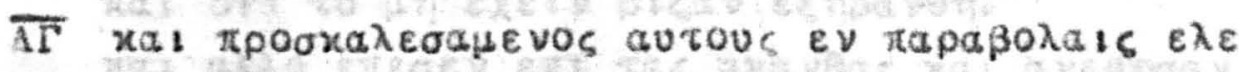

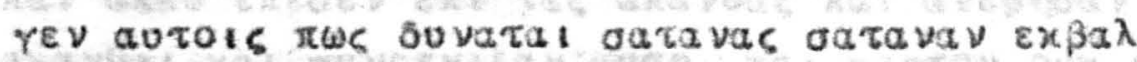

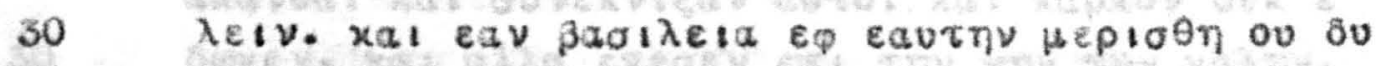

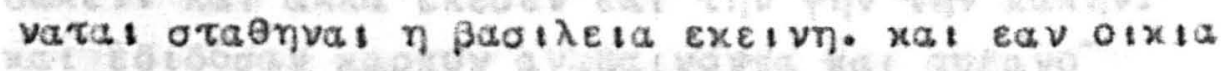

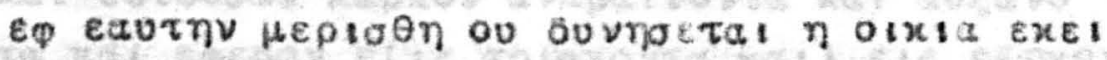

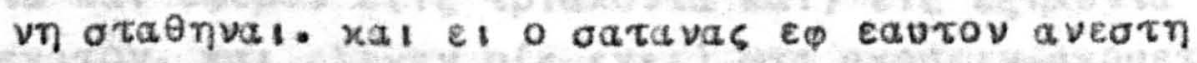

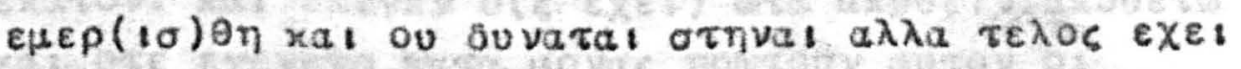

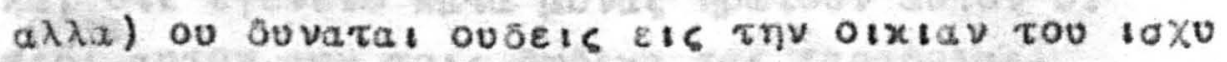

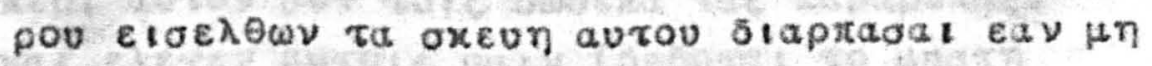

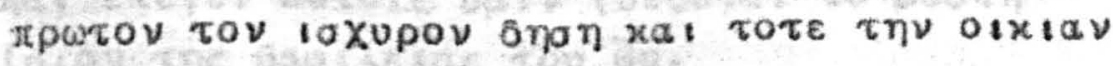
aurou Blapraot.

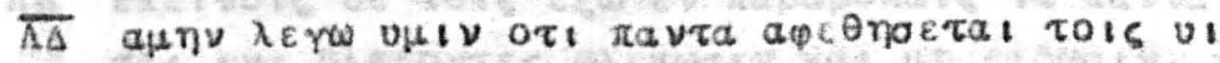

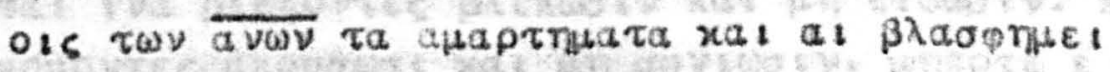

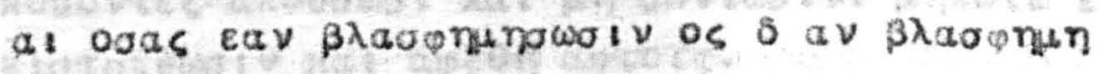




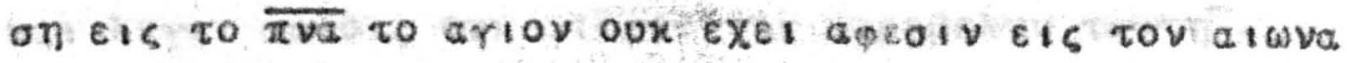

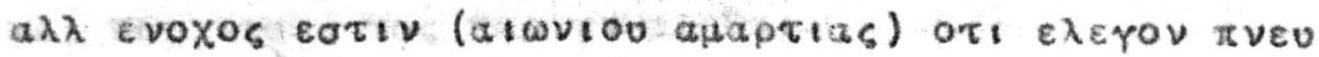

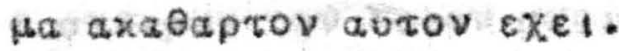

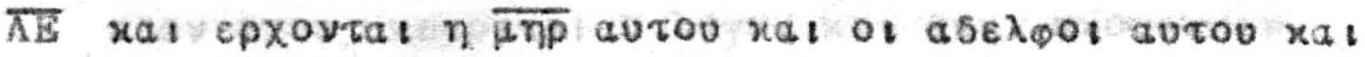
$\varepsilon \xi \omega \sigma \tau$ or

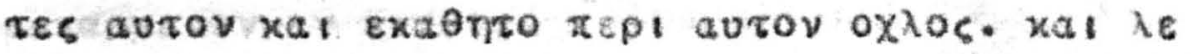

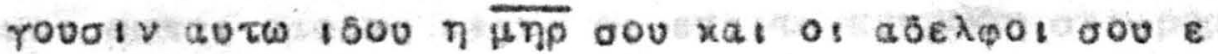

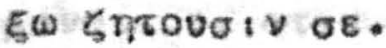

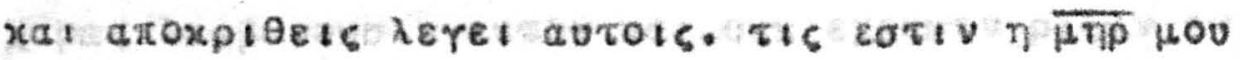

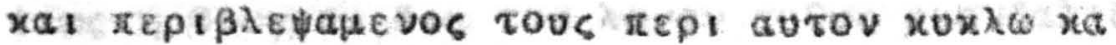

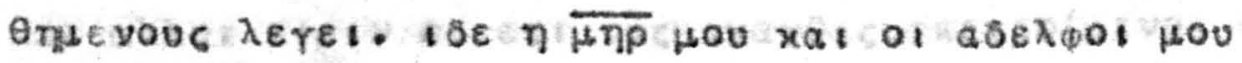

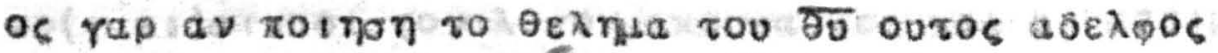

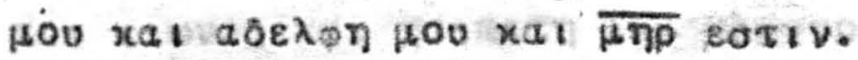

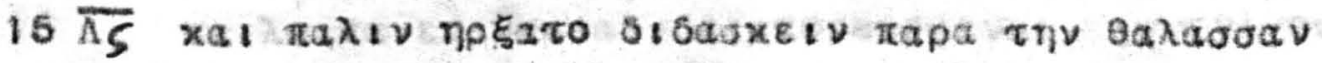

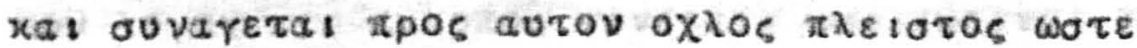

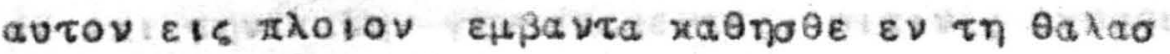

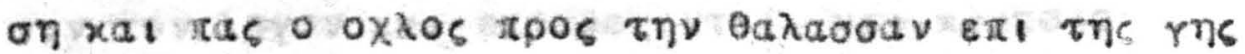

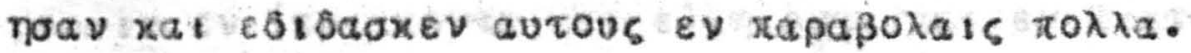

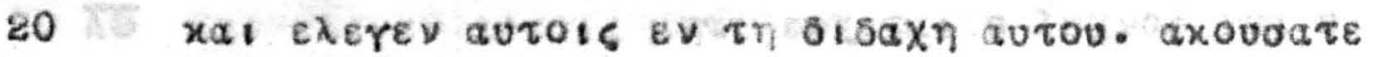

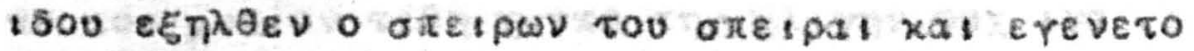

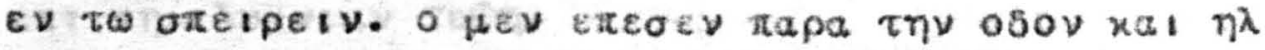

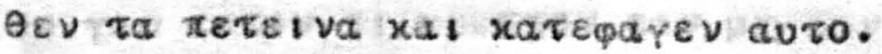

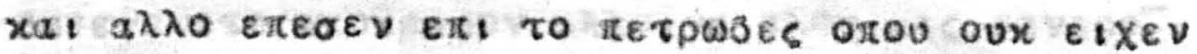

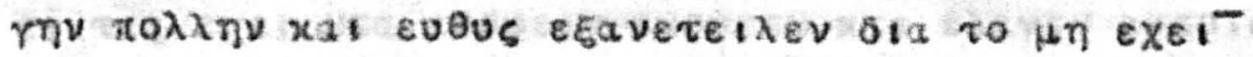

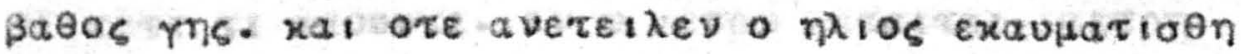

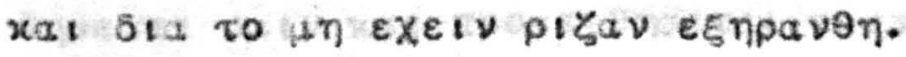

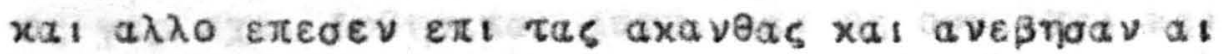

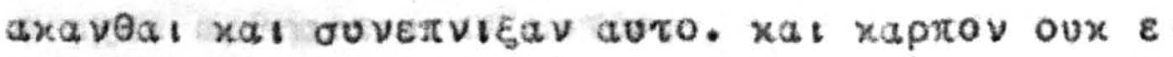

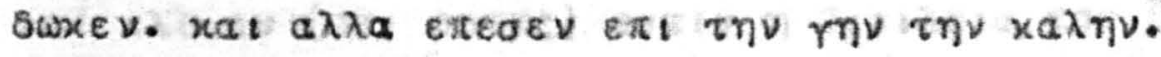

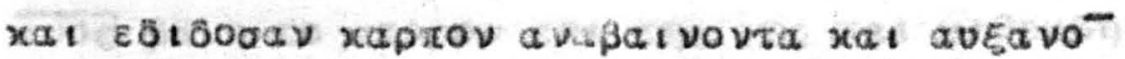

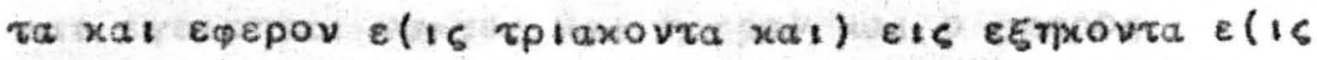

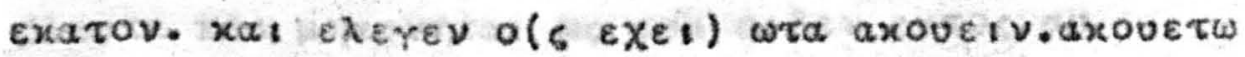

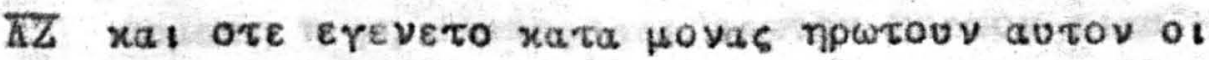

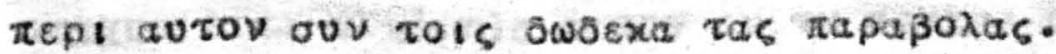

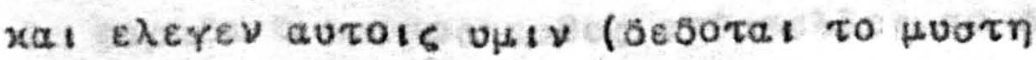
lpiov ths paoidelas tou $\overline{\theta 0}$.

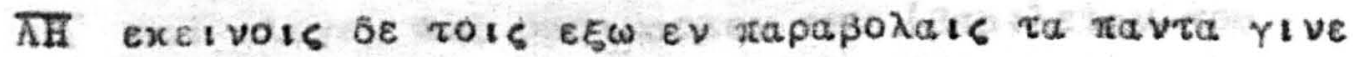

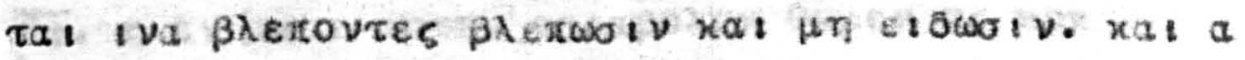

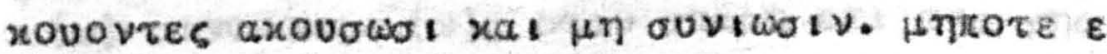

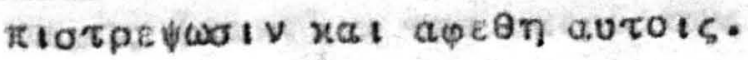




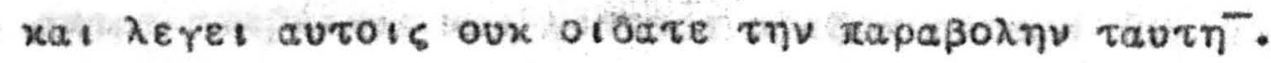

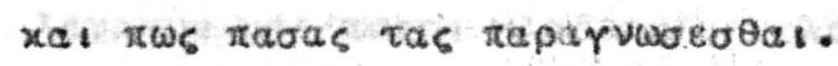

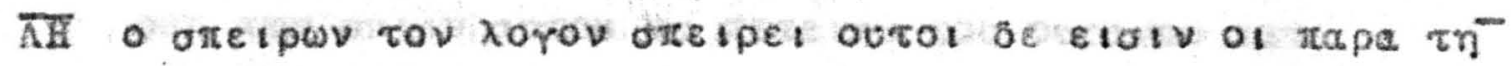

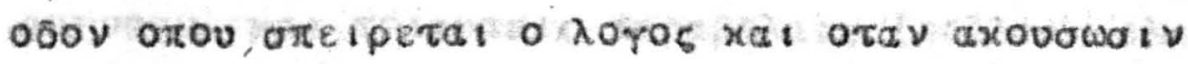

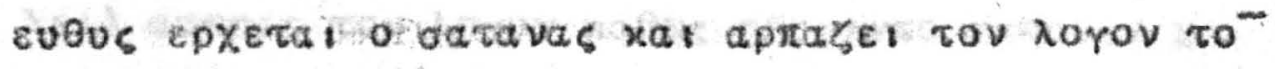
eataphevov ev autois:

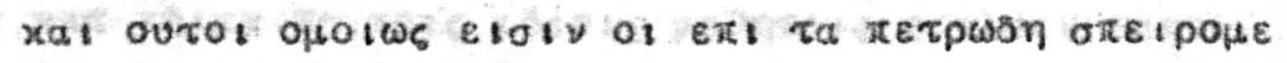
Hevol. ot otav axouowat rov hoyov eveus $\mu \varepsilon \tau a$

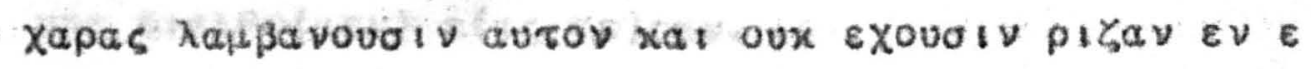

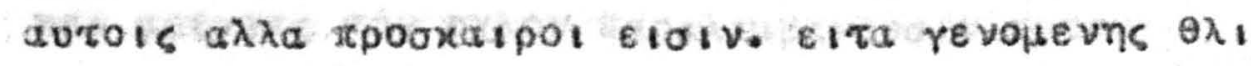

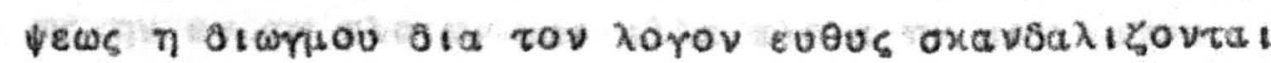

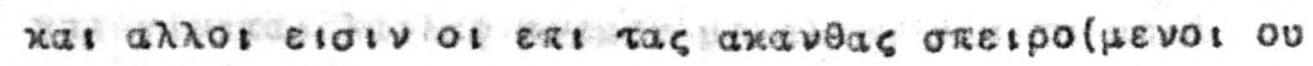

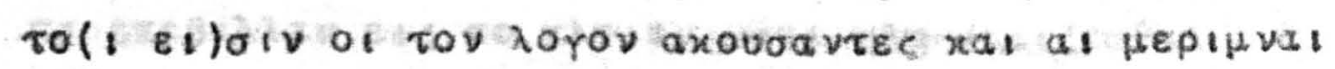

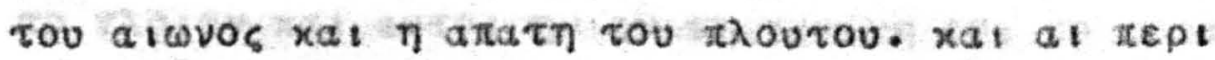

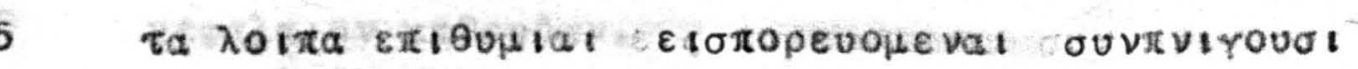

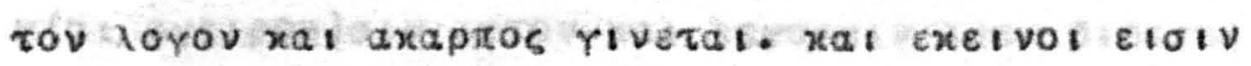

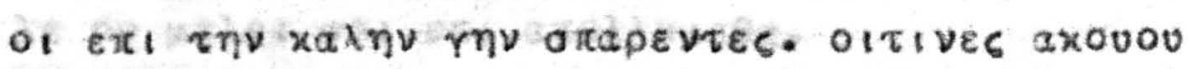
oiv rov torov xai rapabexovtat. xai xapropo

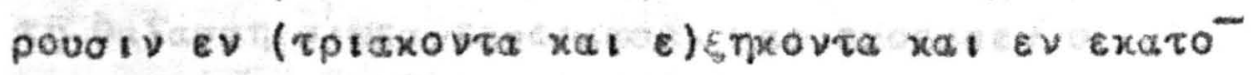

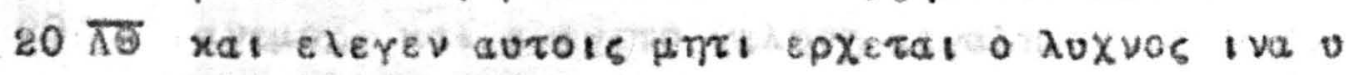

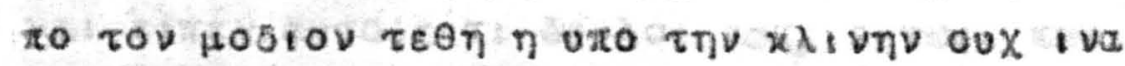

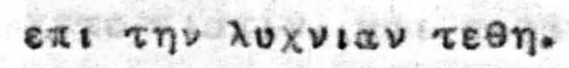

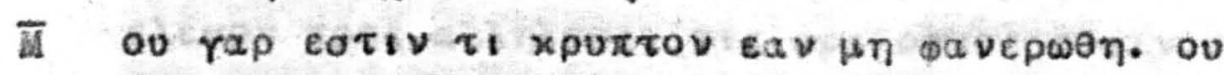

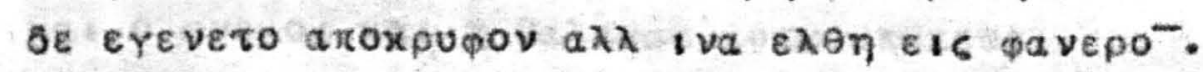

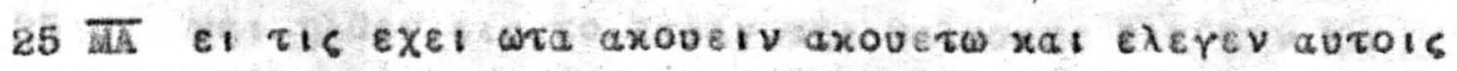

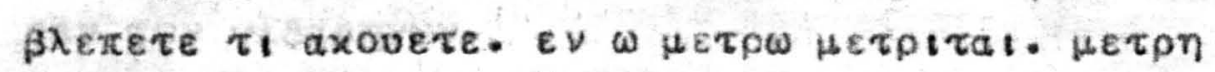

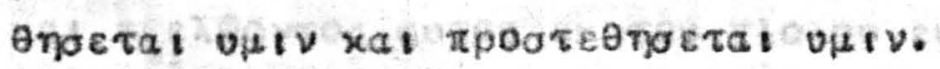

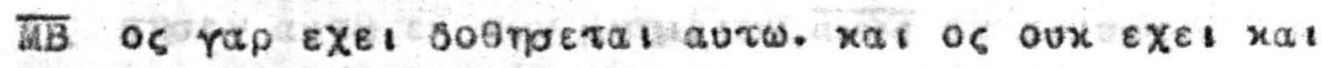
- Exet aponoeral an aurou.

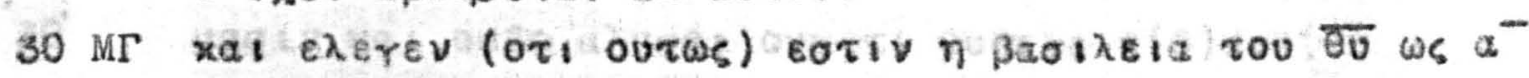

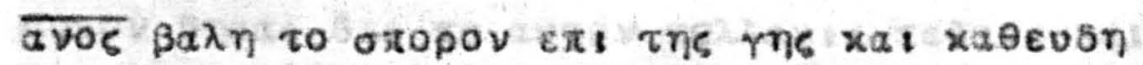
xat eyeiptra, voxtos $x a l$ nuepav xal o atopos

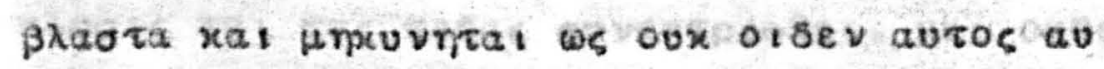

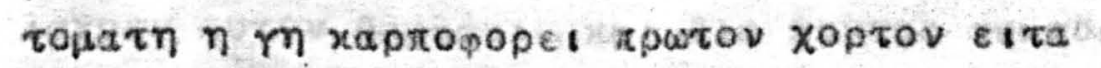

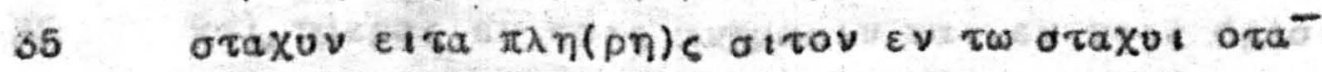

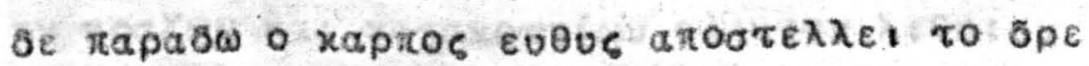

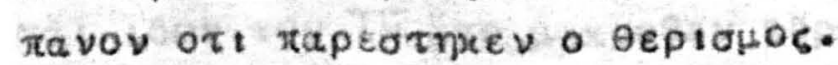

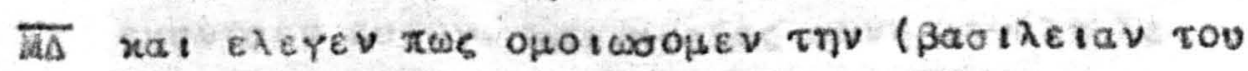

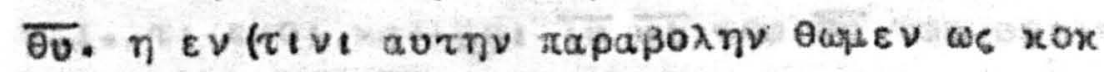

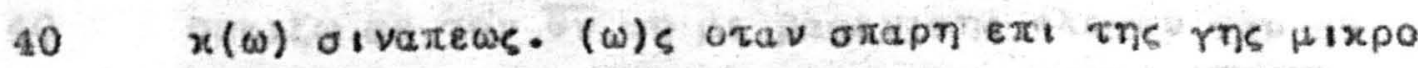

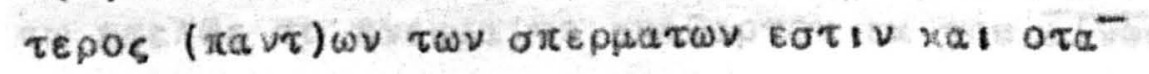




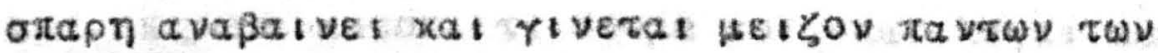

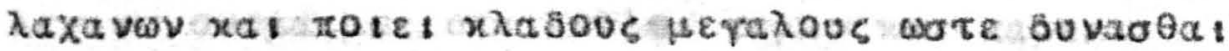

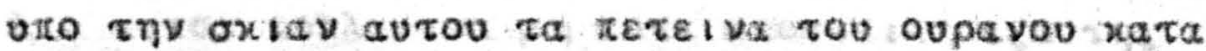

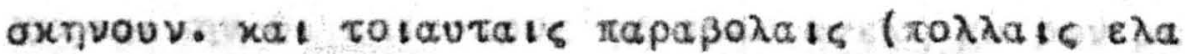

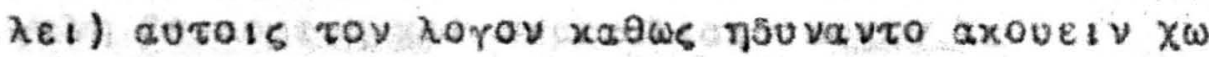

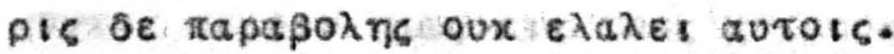

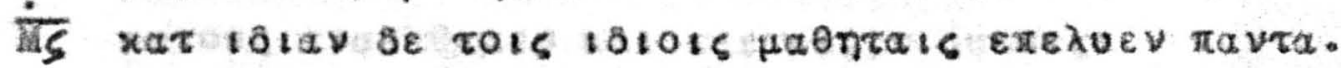

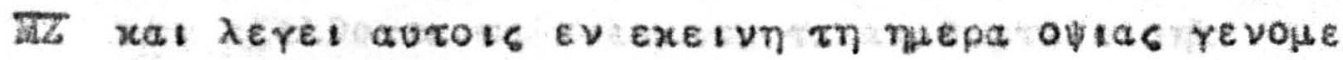
vis $51 \varepsilon \lambda \theta \omega(\mu \varepsilon \nu) \varepsilon(16 . \pi \varepsilon)$ pav.

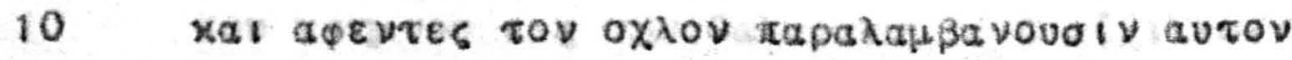

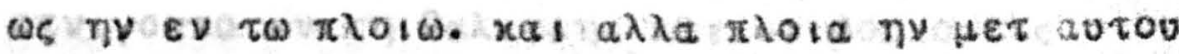

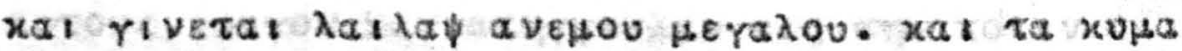

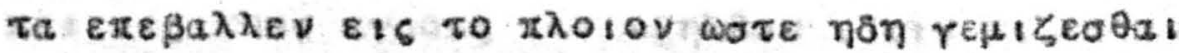

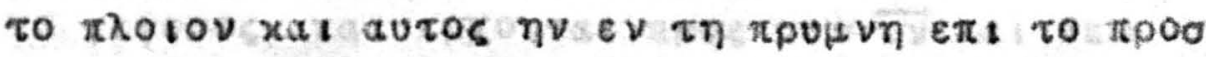

$15 x \varepsilon \varphi a \lambda a t \sigma v i x a \theta \varepsilon v \delta \omega \nu$.

$x$ (a) eyeipoulaty autov xas ie rovoty autw oisaoxa

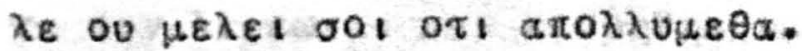
xaı ठิ

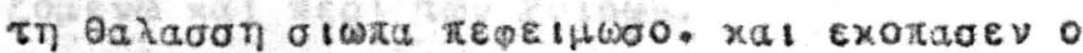

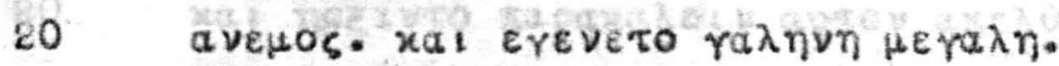

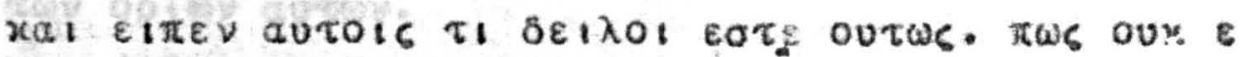

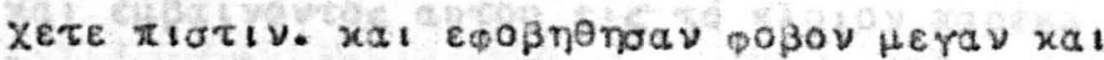

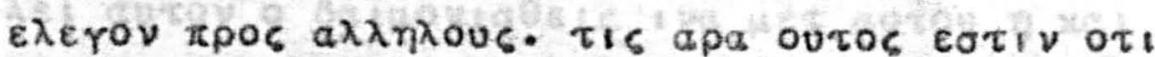

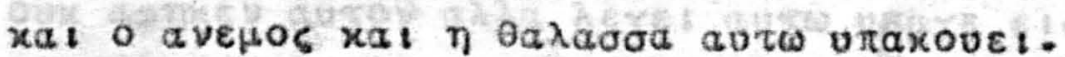

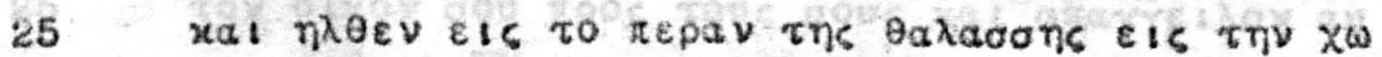

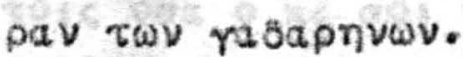

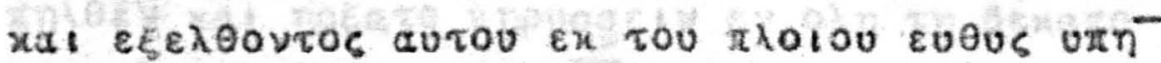

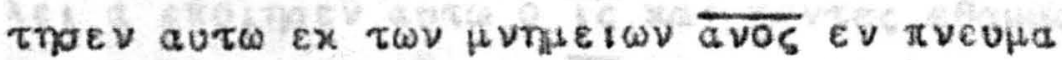

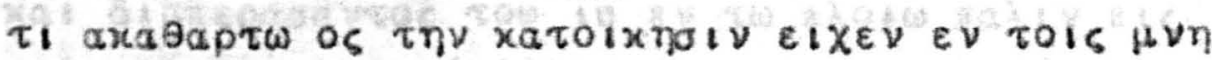
Haaiv xal oube a

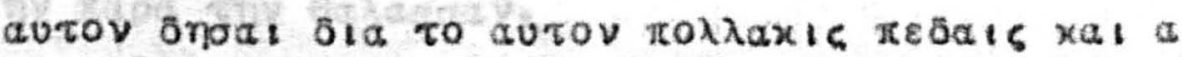

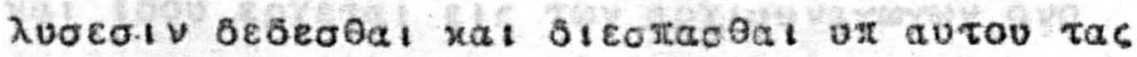

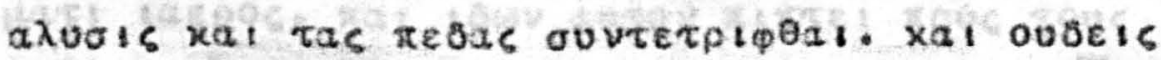

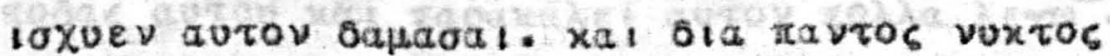

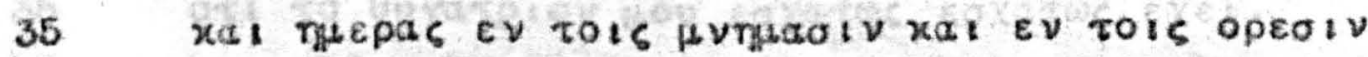

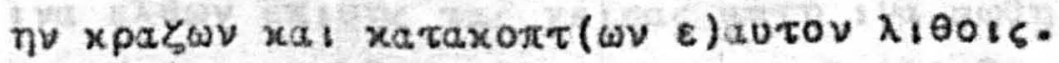

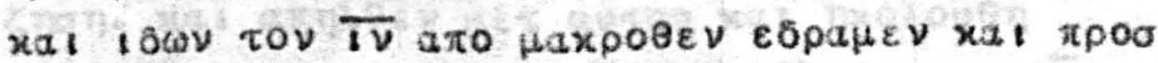

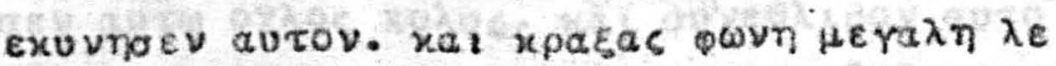

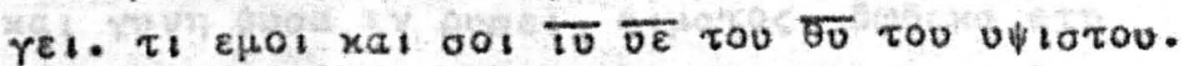

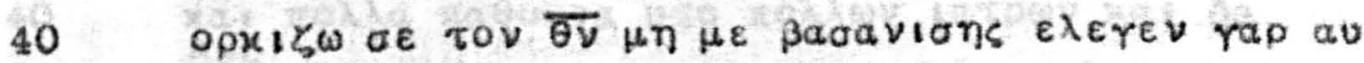

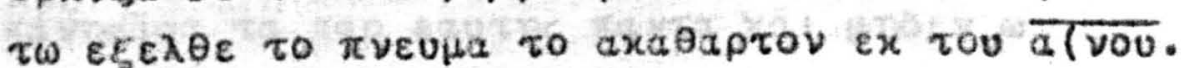




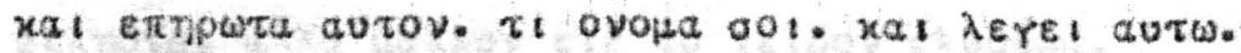

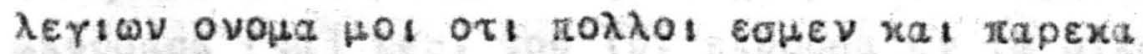

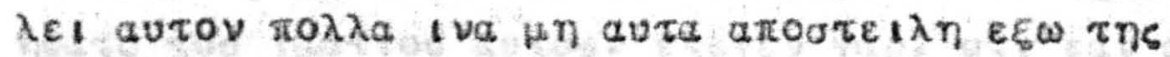

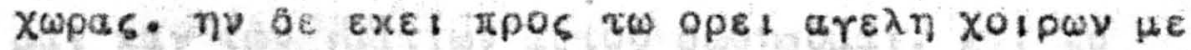

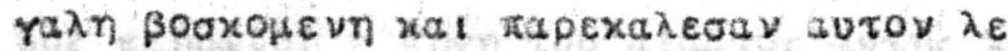

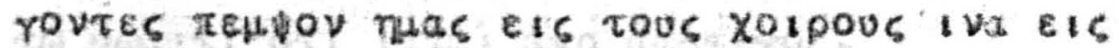
autov \& \&

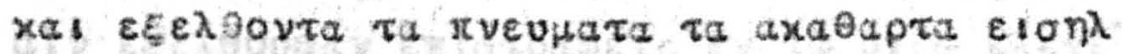

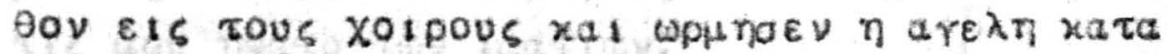

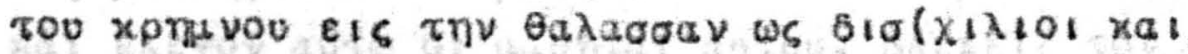

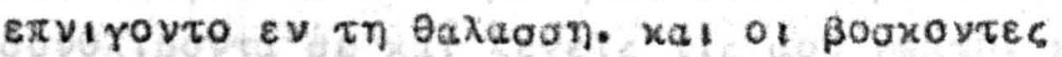

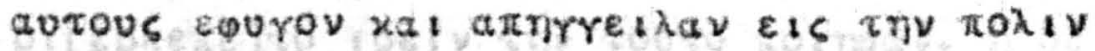

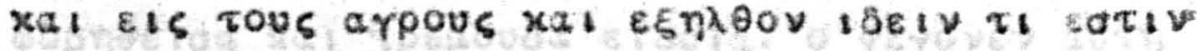

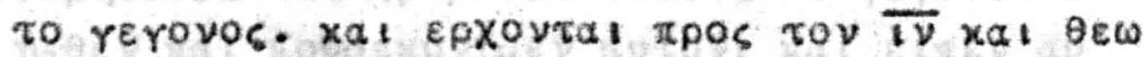

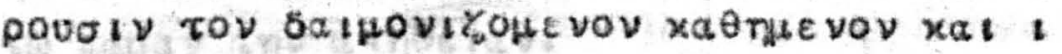

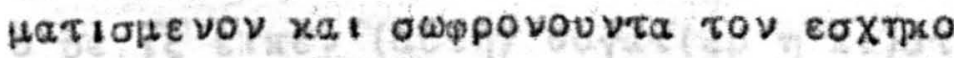

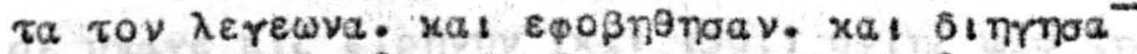

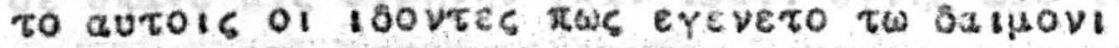

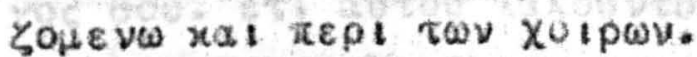

0

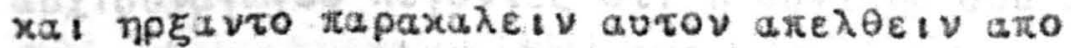
$\tau \omega v$ oplwv autw.

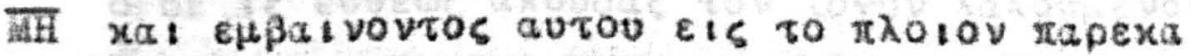

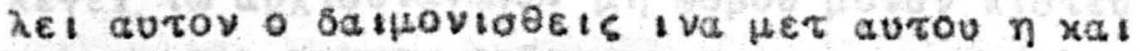

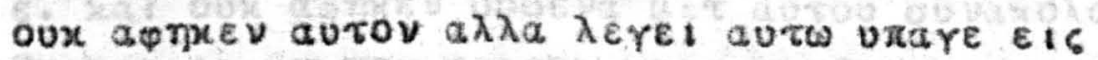
tov oixov aOv xpos tous oous xat ataryeitov av

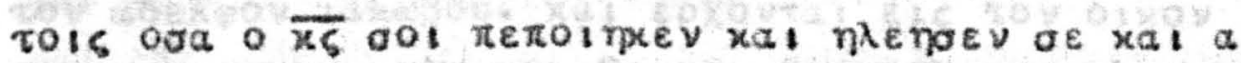

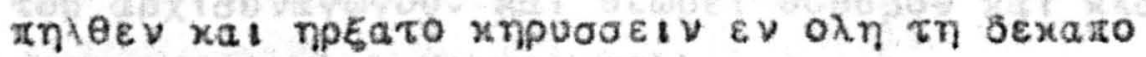

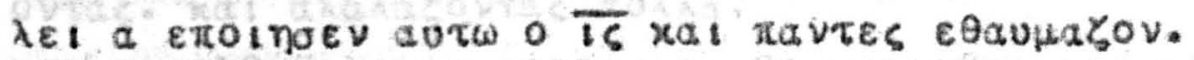

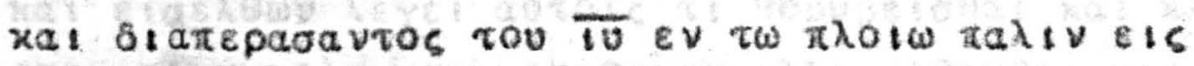

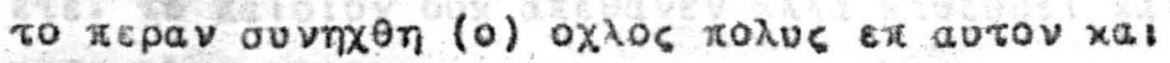

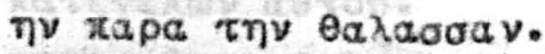

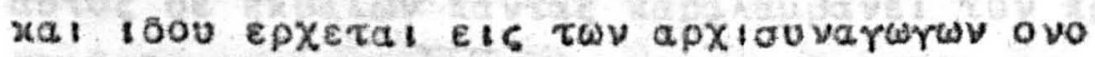

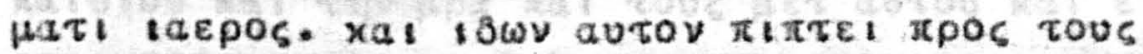

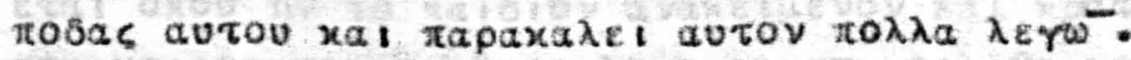

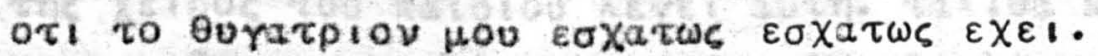

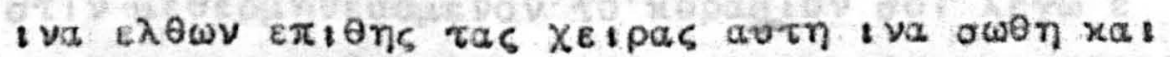

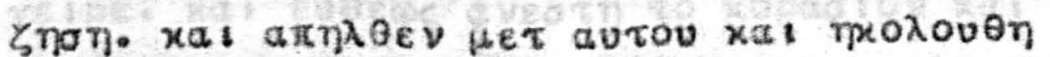

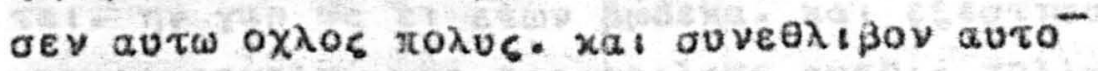

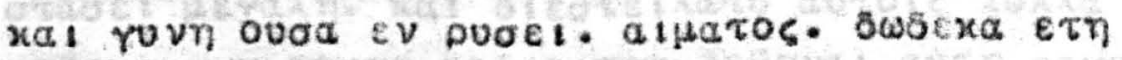

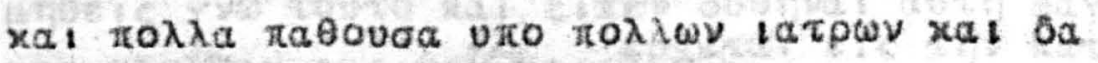

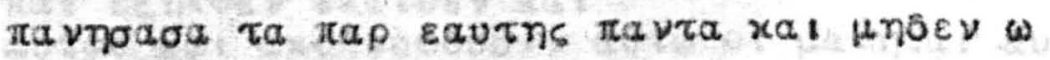




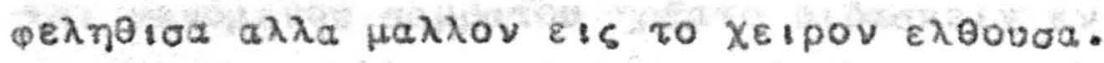

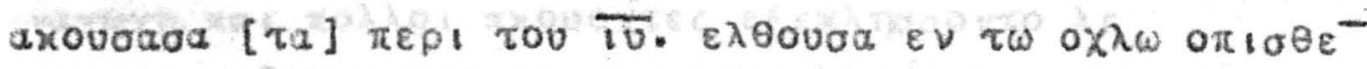

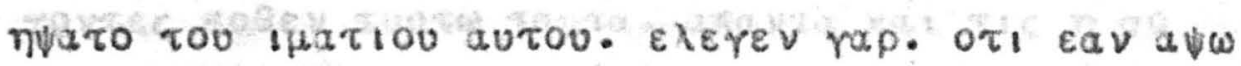

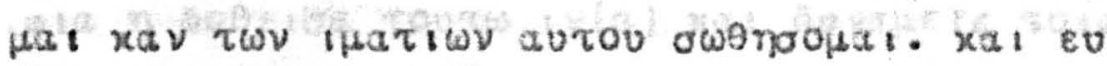

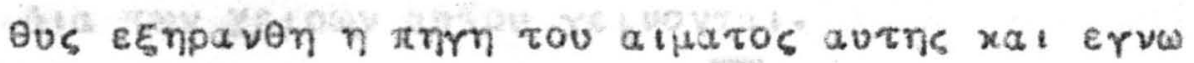

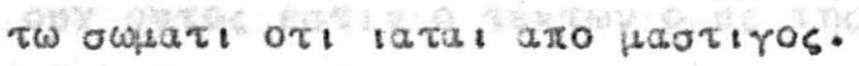

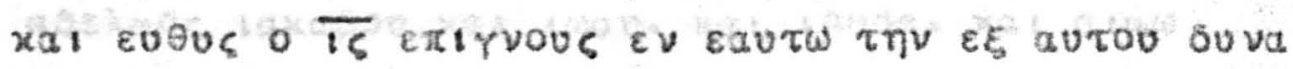

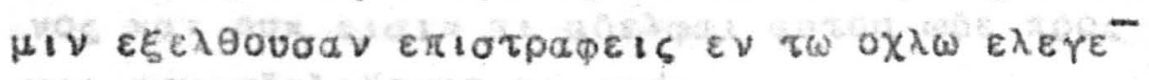

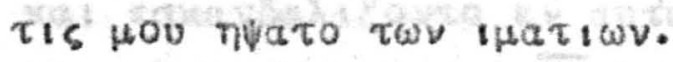

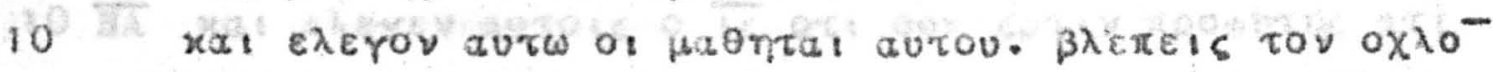

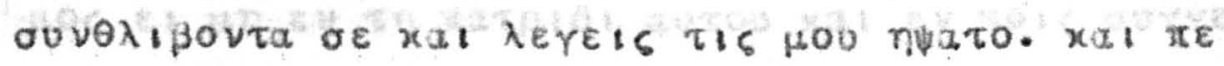

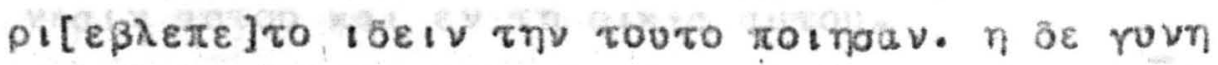

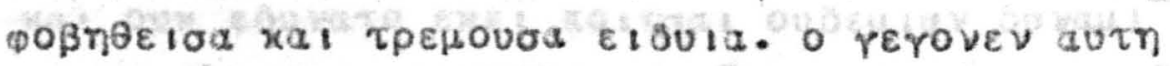

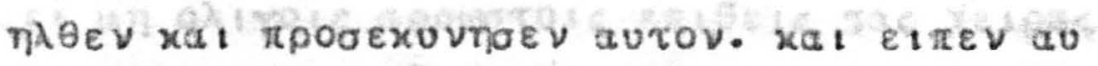

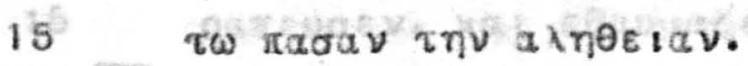

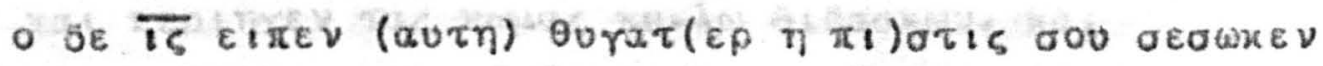

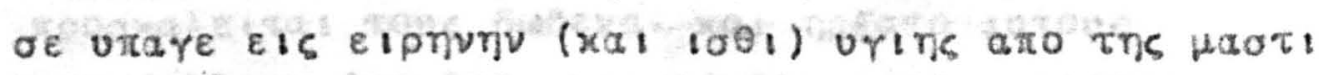

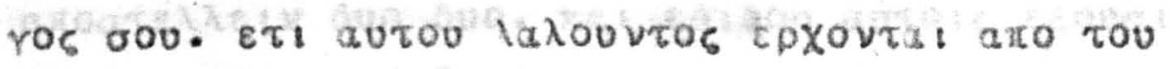

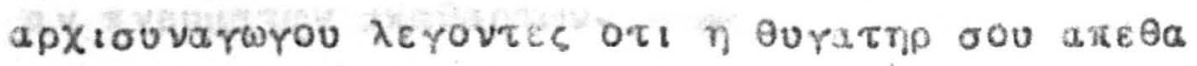

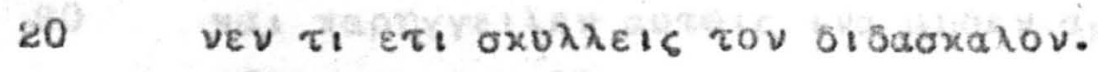

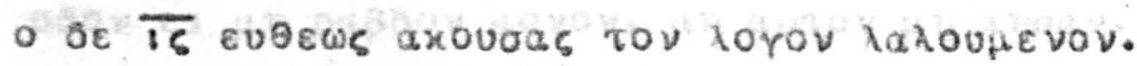

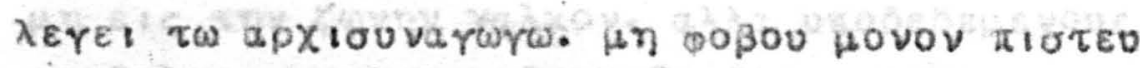

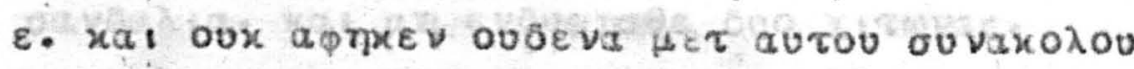

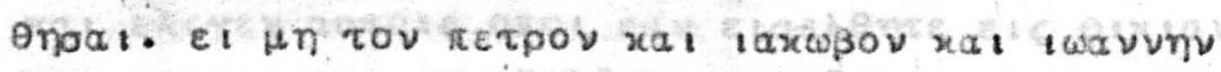

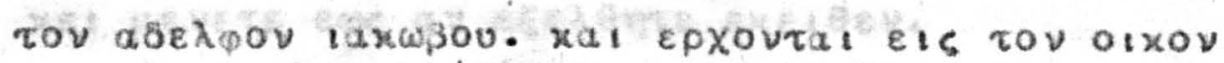

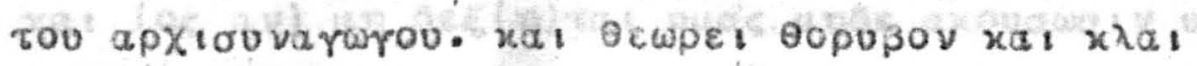

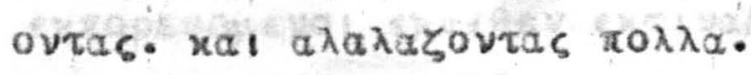

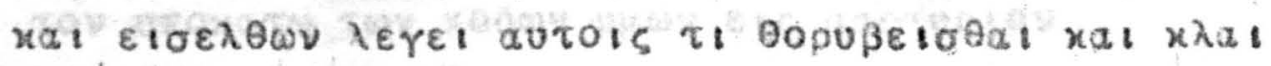

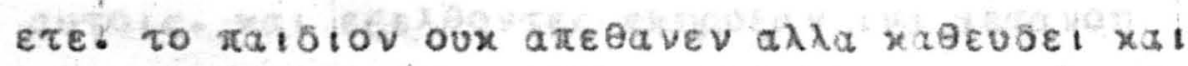

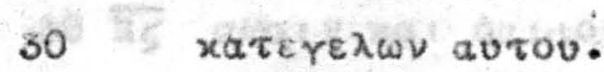

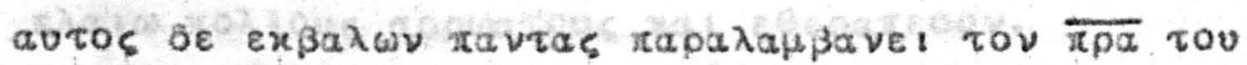

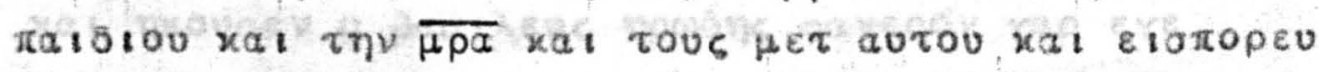

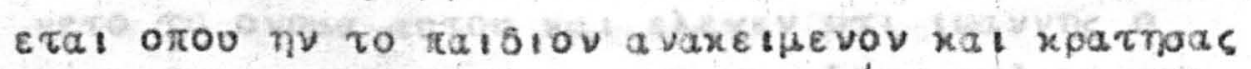

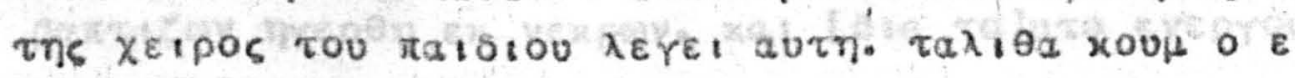

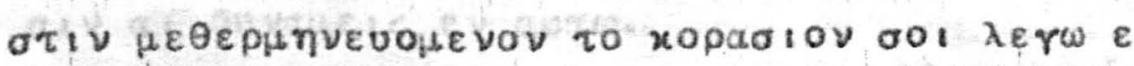

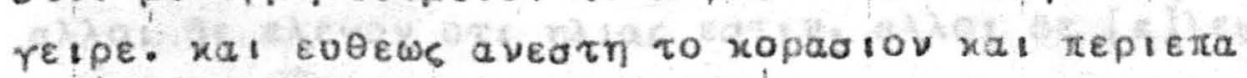

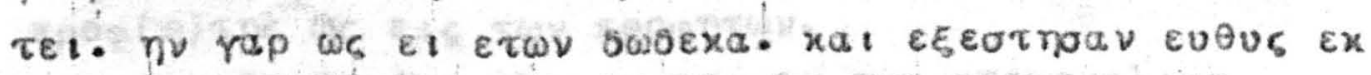

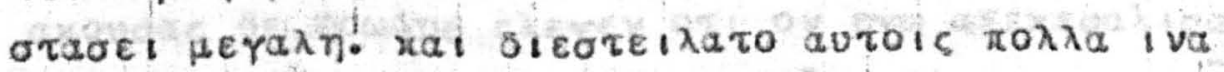

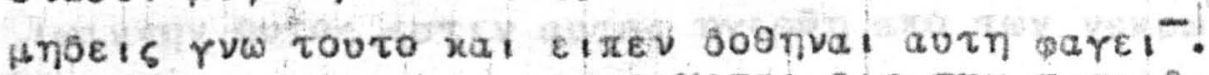

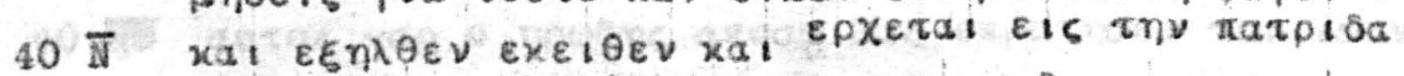

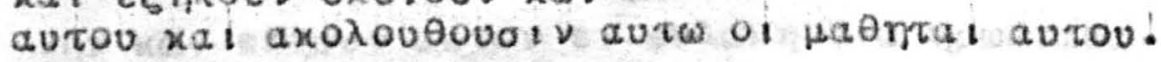




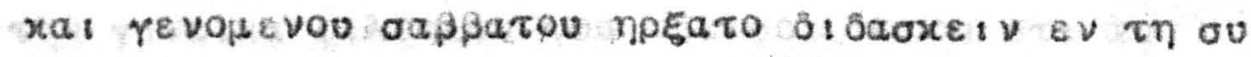

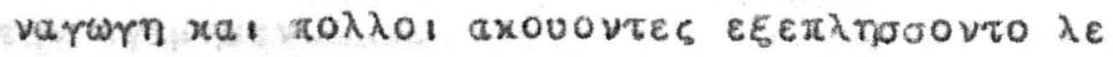

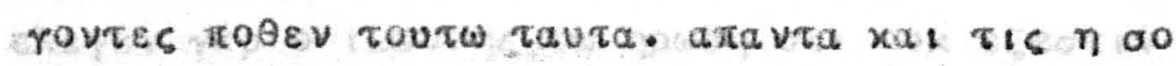

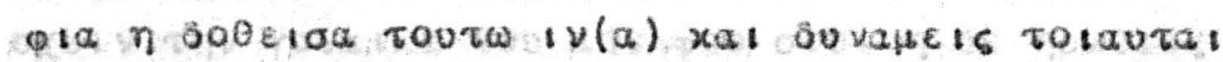

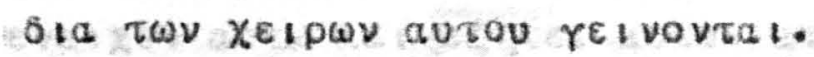

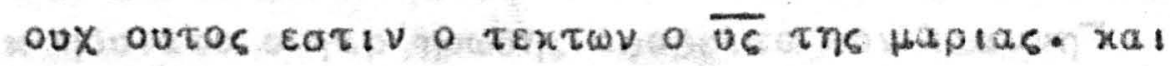

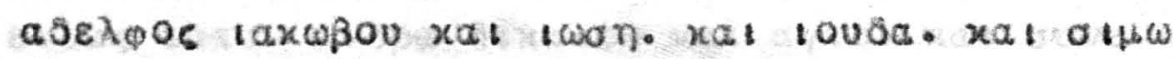

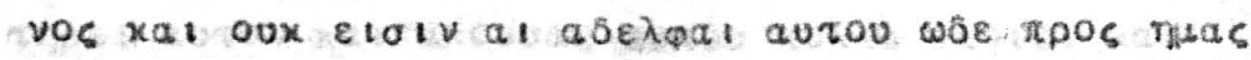

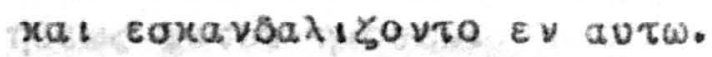

10 मA xal exerevautois a $\overline{16}$ ort oux eotiv xpontins at

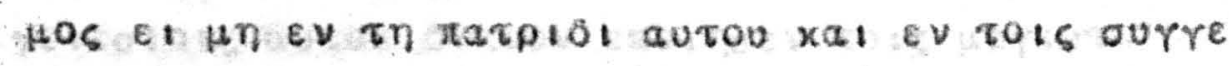
Veaiv autoo xal eV Th oixta autou.

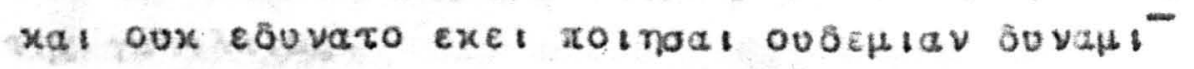

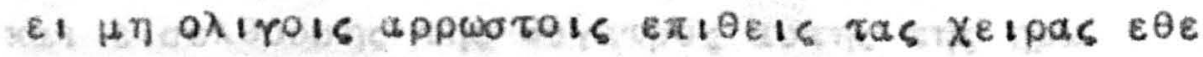

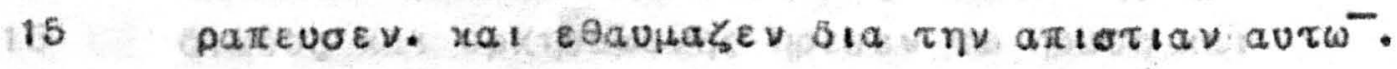

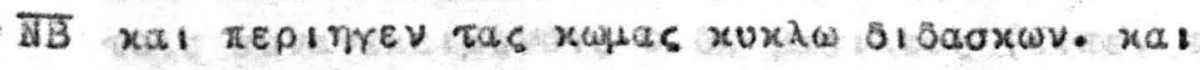

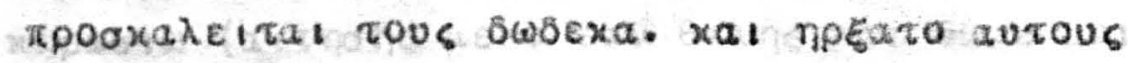

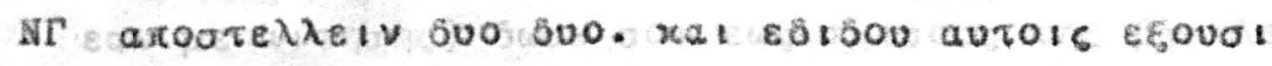

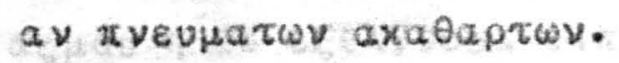

20 xal tapnrretiev autols iva Hnôev apwotv $\varepsilon$ is

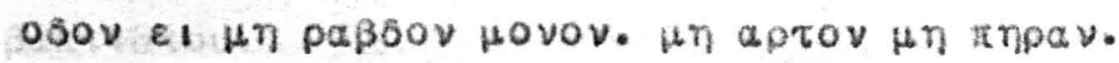

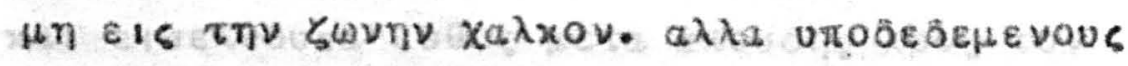
oavola

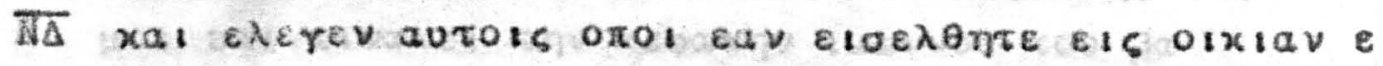
25

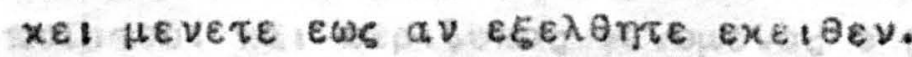

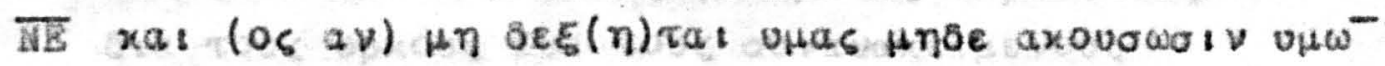

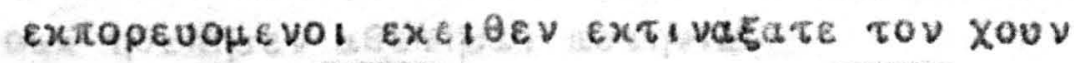

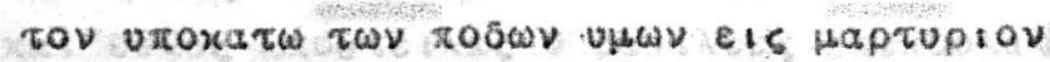

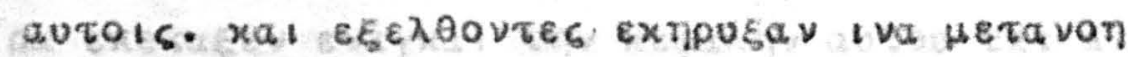

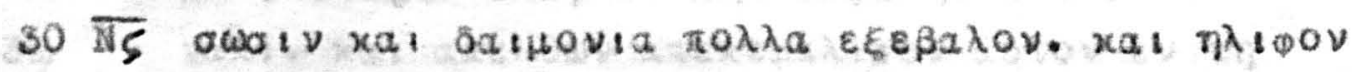

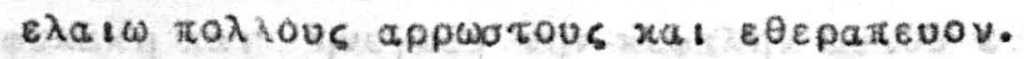

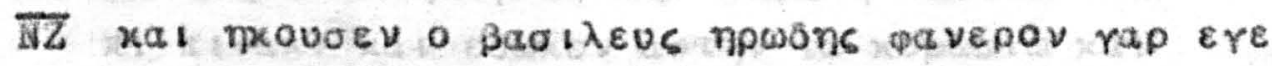

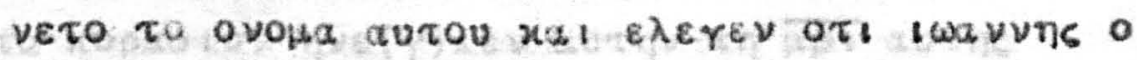

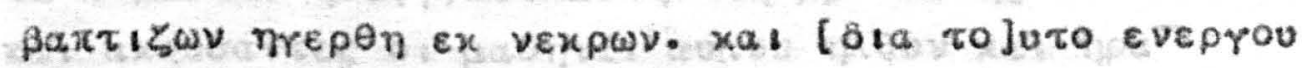
otv at suvajeis $\varepsilon \nu$ autw.

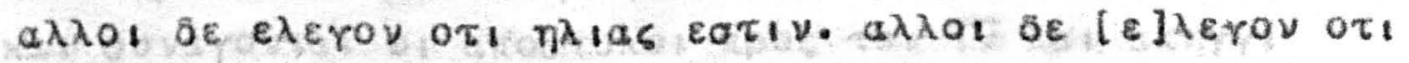

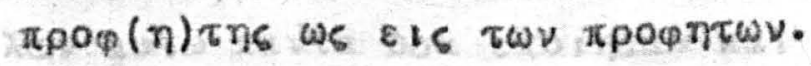

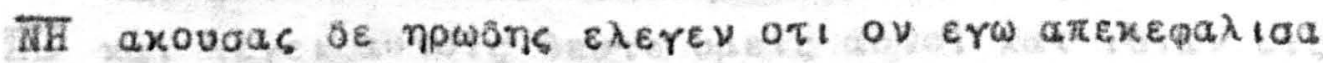

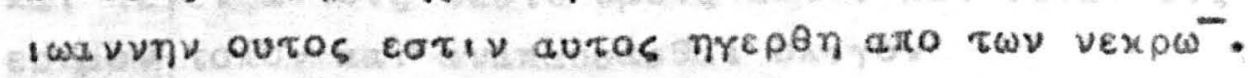

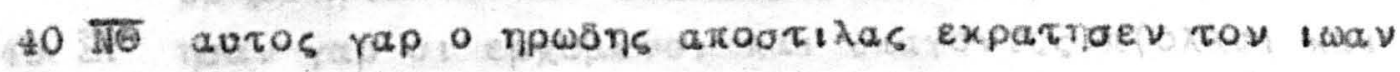

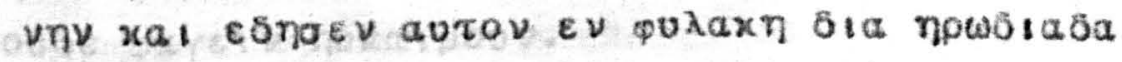




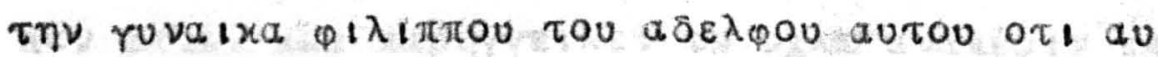

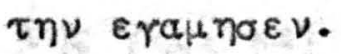

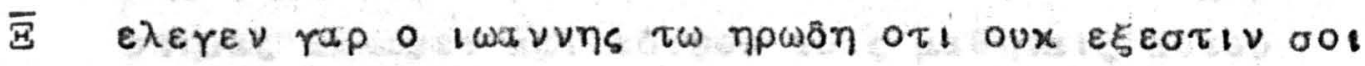

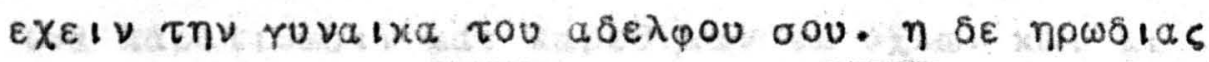

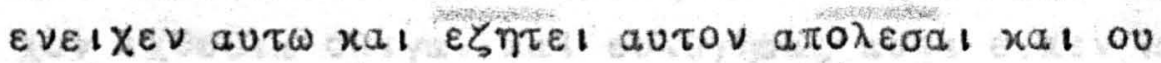

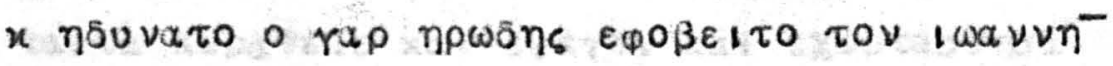
\&!

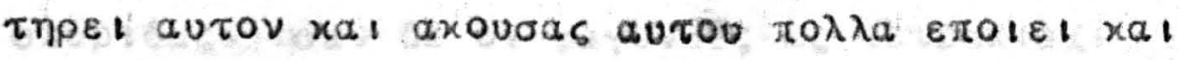

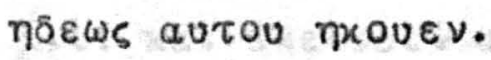

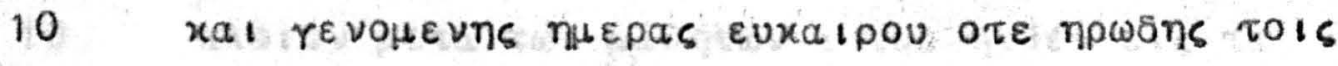

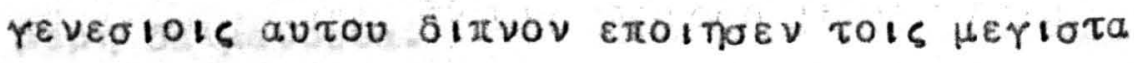
oiv autou xal rois Xidtapxois xal tols mpwtols

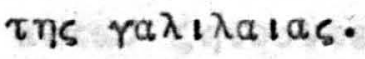

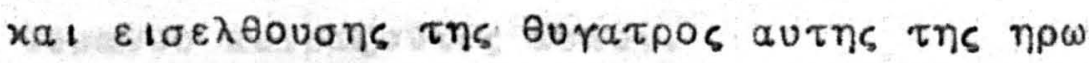

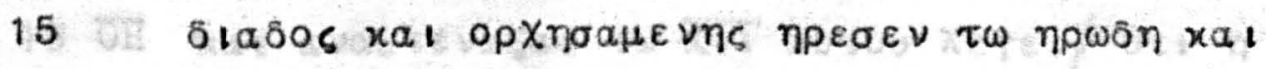

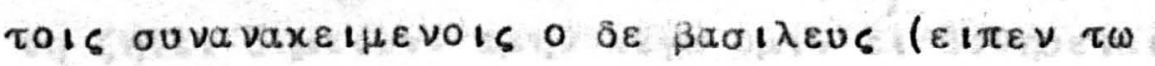

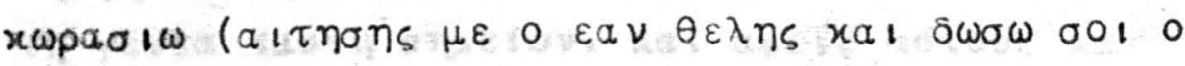

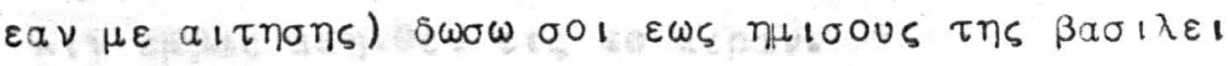

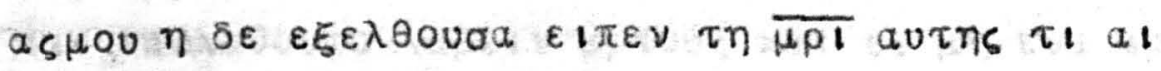

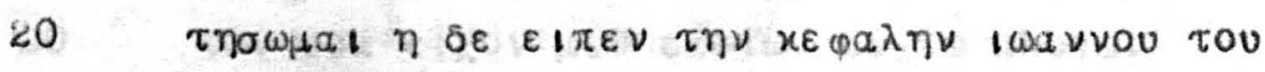

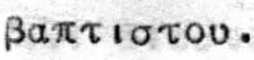

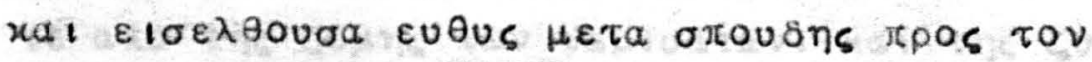

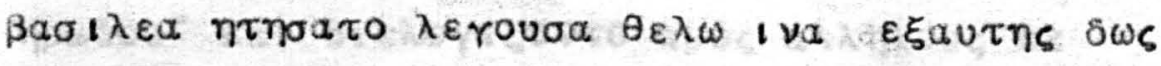

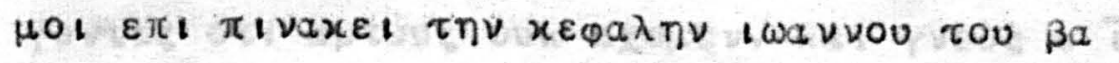

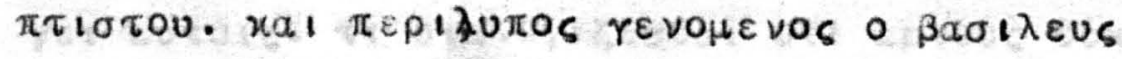

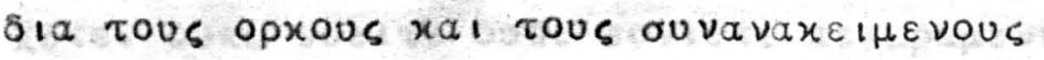

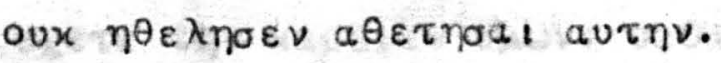

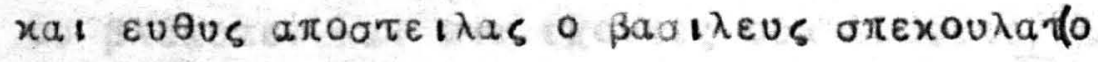

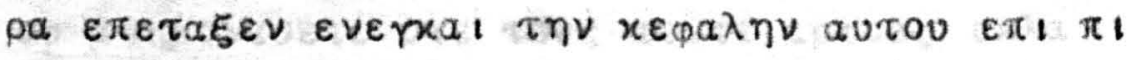
$\nu a x 1 . x a 1$ a $\pi \varepsilon \lambda \theta \omega \nu$ a $\pi \varepsilon x \varepsilon \varphi \alpha \lambda I \sigma \varepsilon \nu$ autov $\varepsilon \nu \tau \eta$

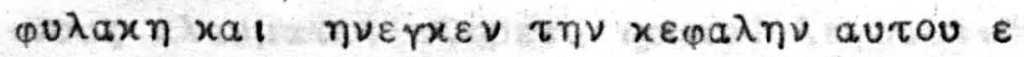

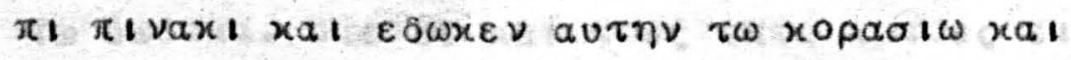

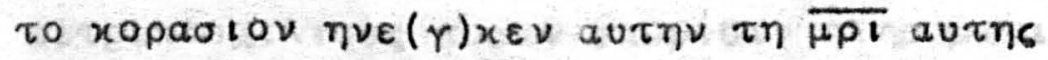

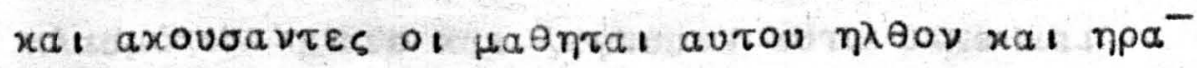

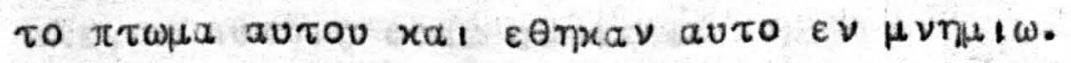

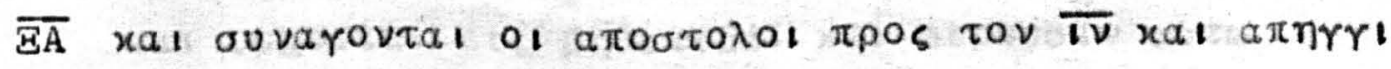

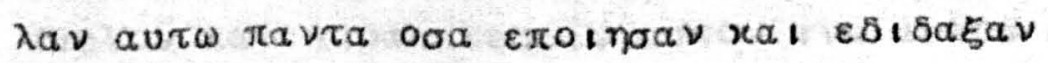

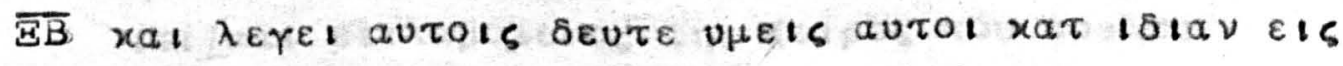

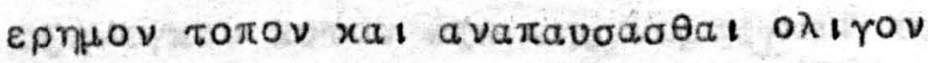

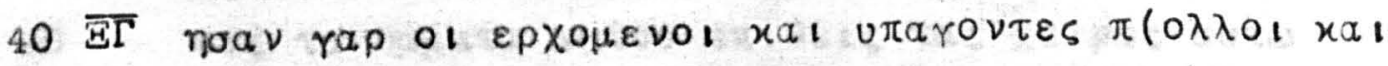
ouธ์ pareı nuxalpouv. 


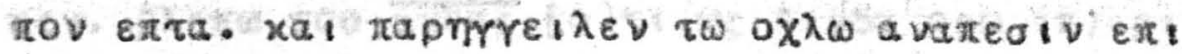
tris rns. xat $\lambda a \beta \omega v$ tous etra aptous xal euxapt

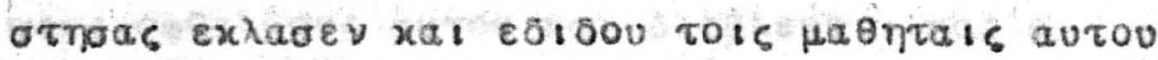

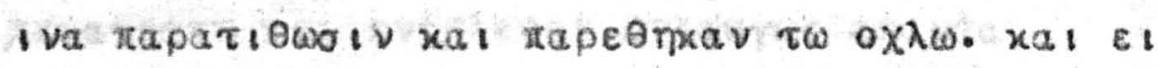

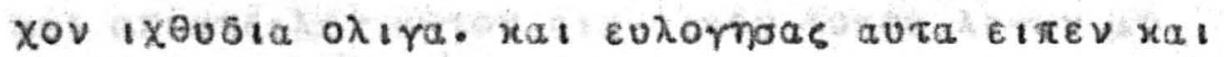

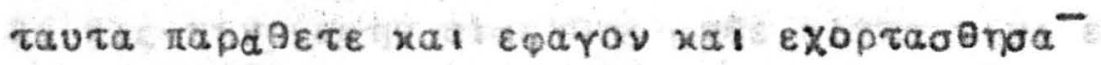

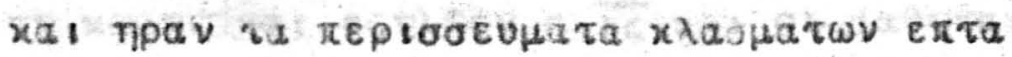

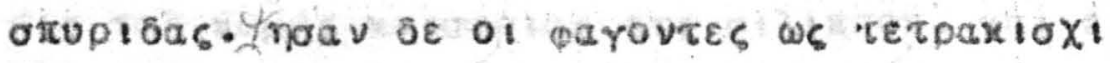

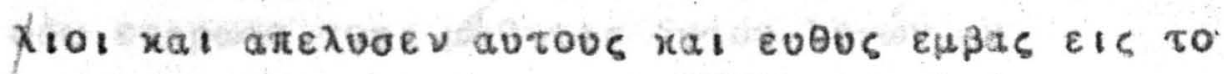

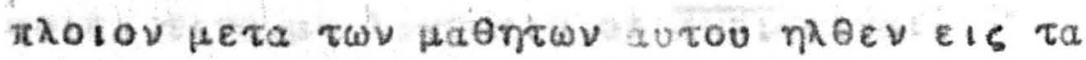
$\mu \varepsilon \rho \eta \delta a \lambda \mu a v 0 v \theta a$.

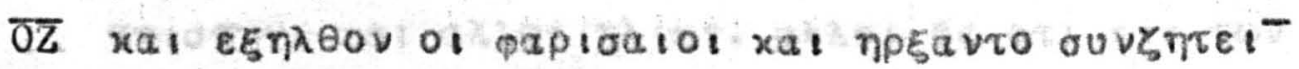

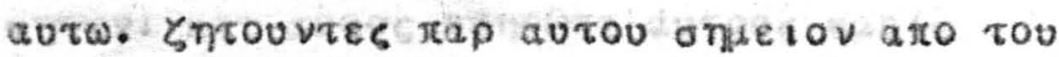

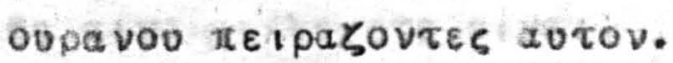

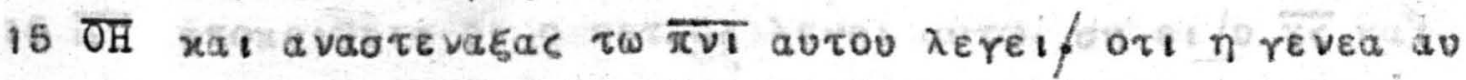

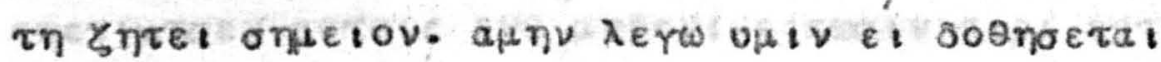

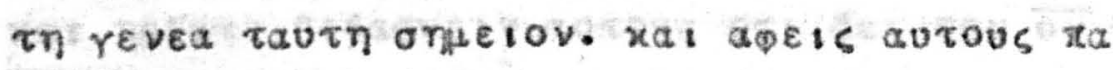

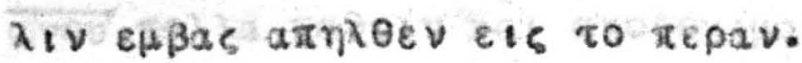

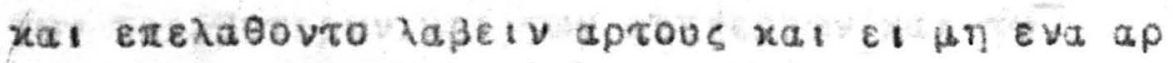

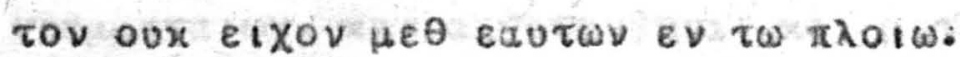

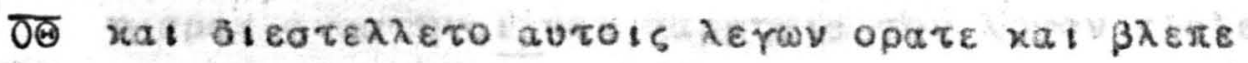

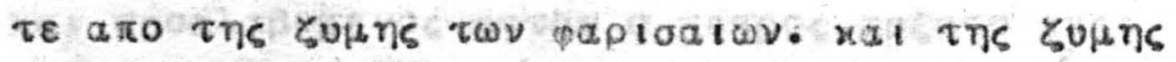

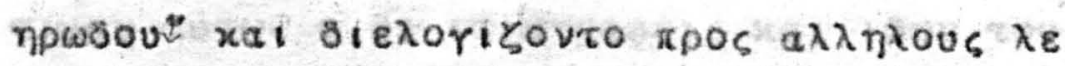

II fovtes oti aptous oux exopev. xai yuovs o $T$ is $\lambda e$

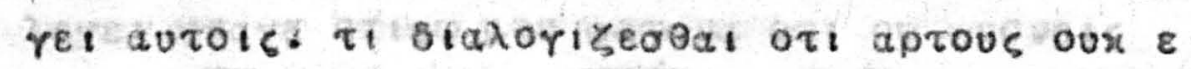

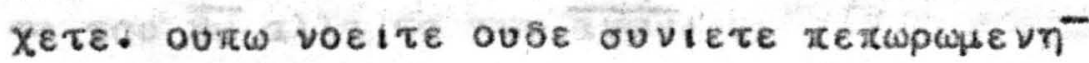

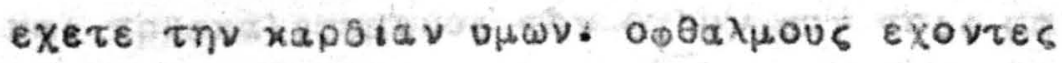

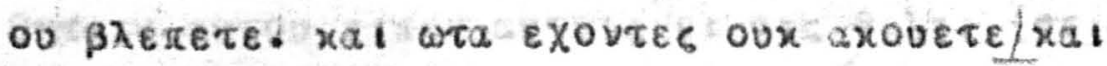

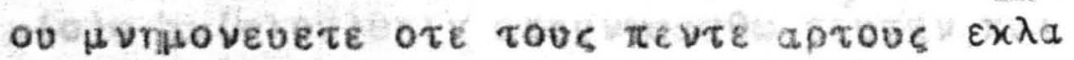

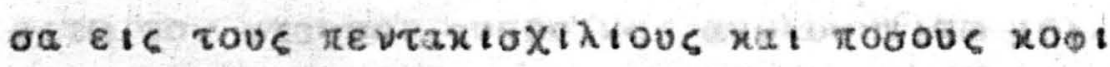

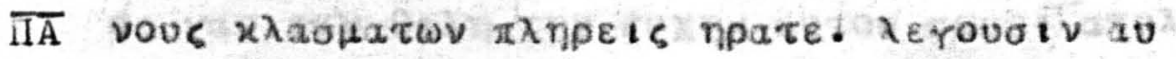

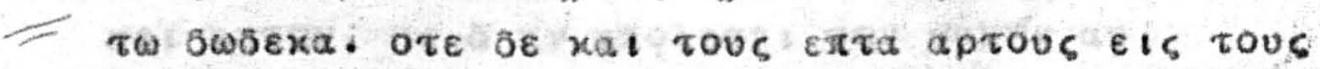

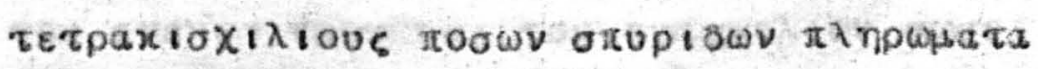

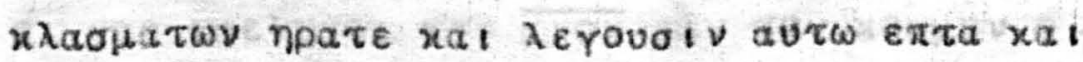
exerev autois outw ouviete.

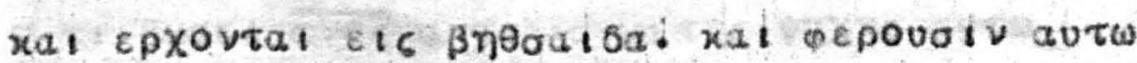
rugtov xal rapaxisovaiv aurov tva aurou atn

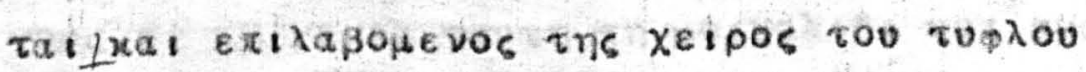

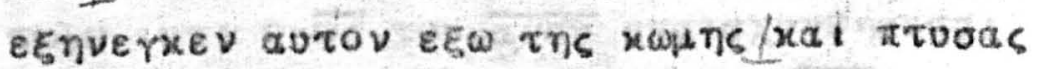

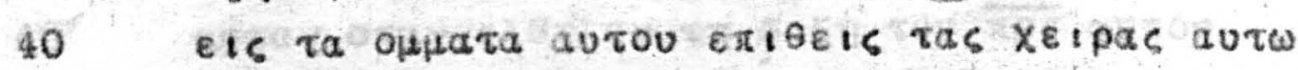

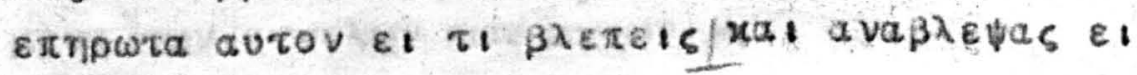
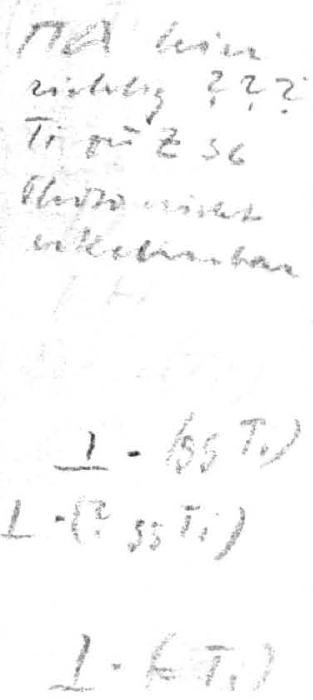


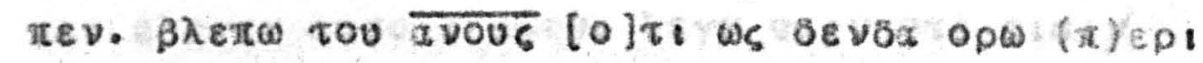

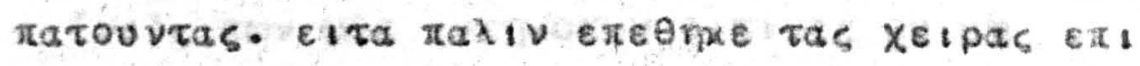

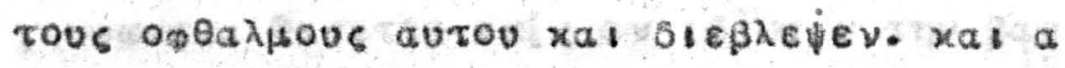

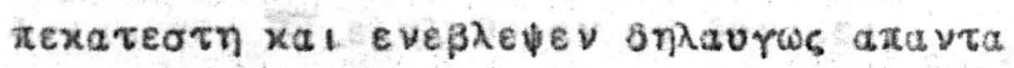

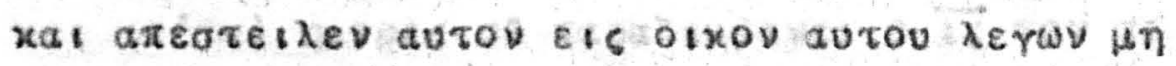

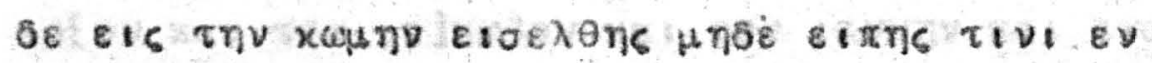

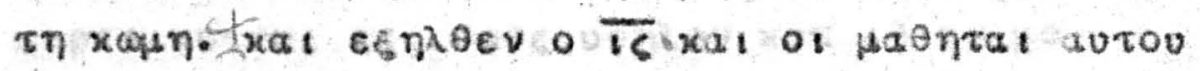

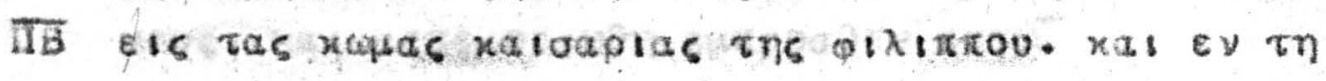

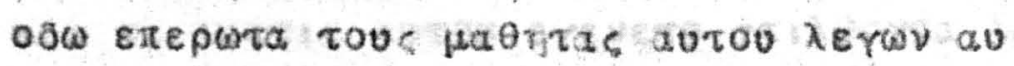

10 Tols. Tiva he Aerovotv ot avol eival.

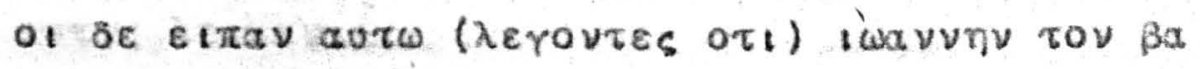

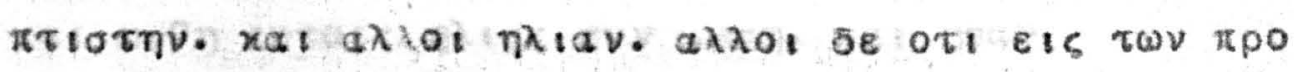

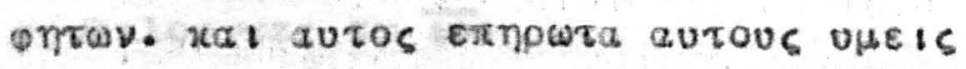
se $\tau$ เ

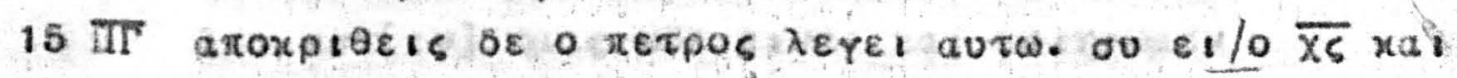

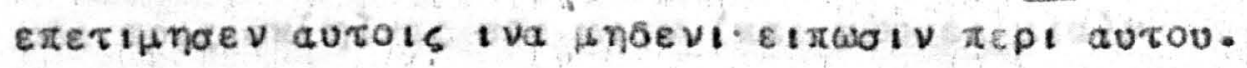

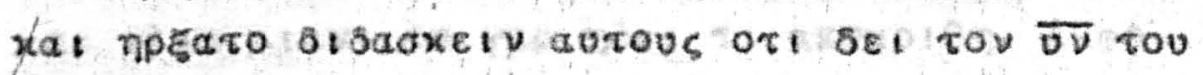

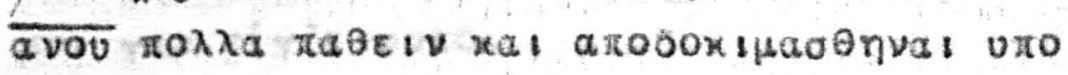

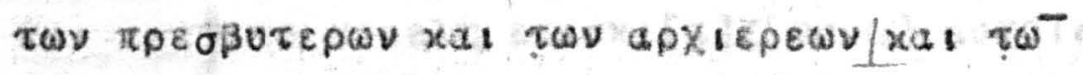

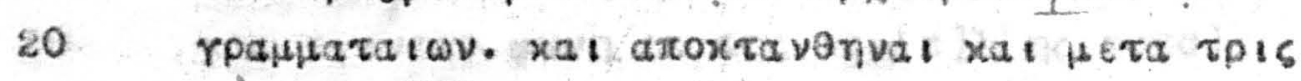

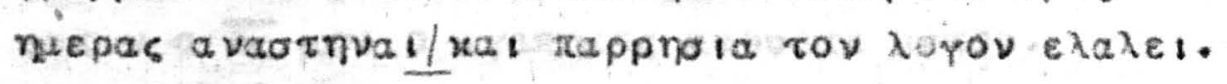

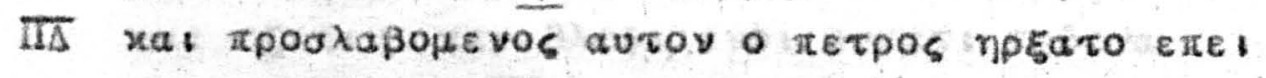

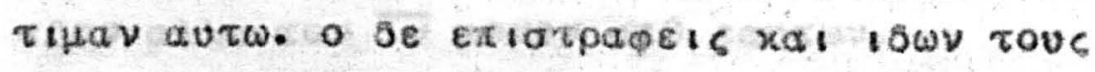

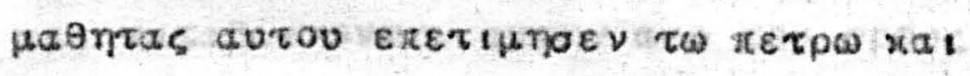

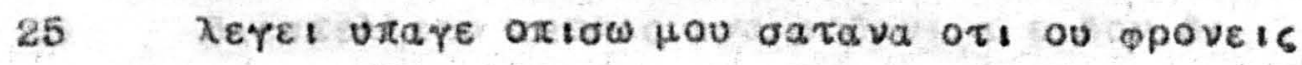

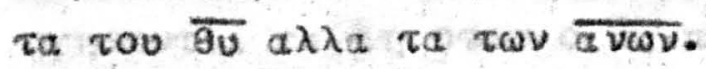

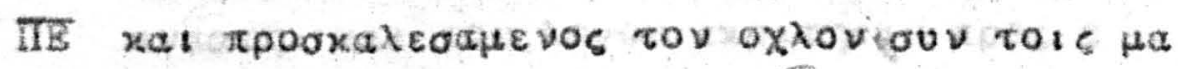

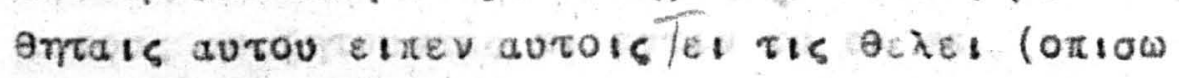

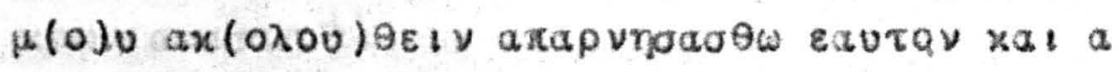

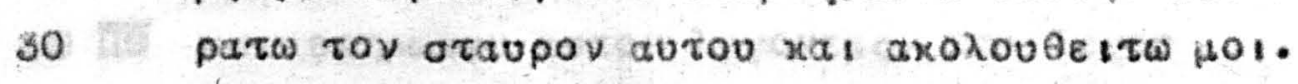

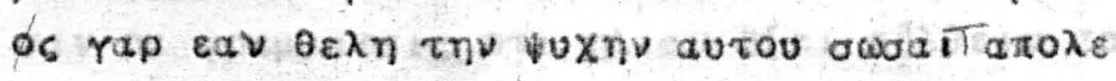

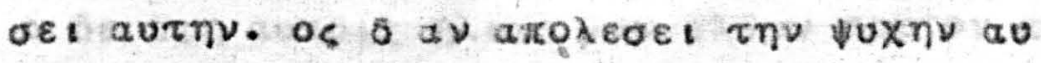

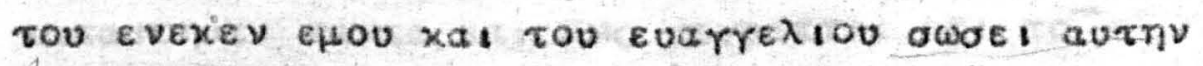
ti rap woelnoet tov avov exv tov xodiov o

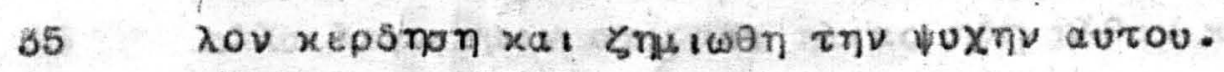

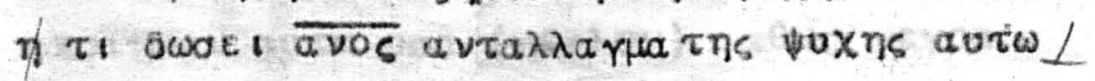

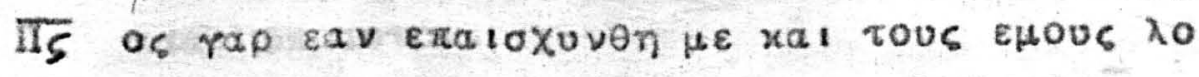
rous $\varepsilon 2$ ข

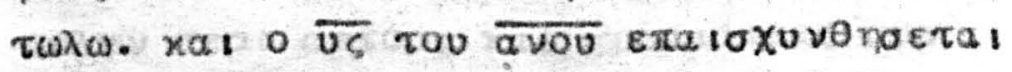

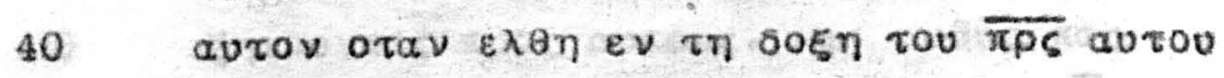

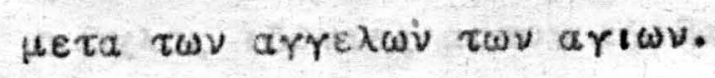




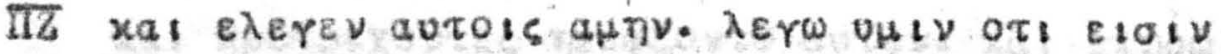

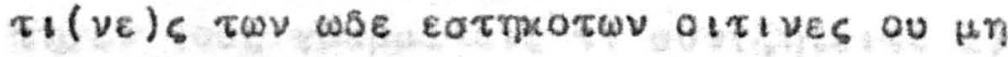

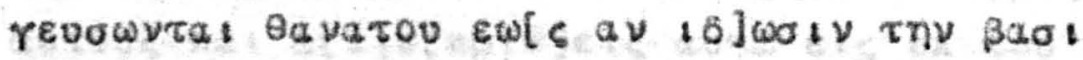

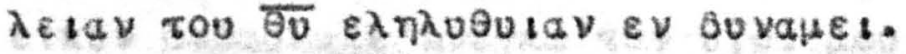

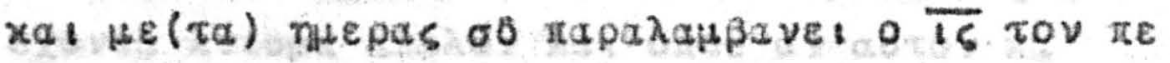
tplov xat tov la]xwpov xa! roy tavyny xas a va

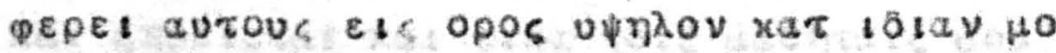

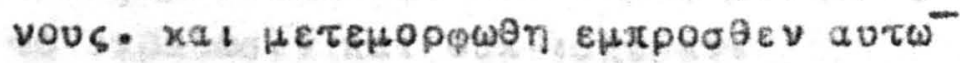

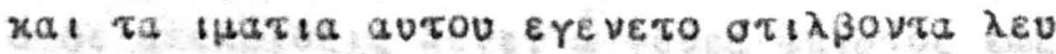

10 xa diav ola rvageus ext ths ms ou juvatat

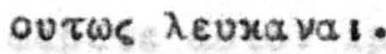

xal $\omega p \theta \eta$ aurois ndias ouv Hwan. xal noav

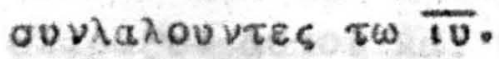

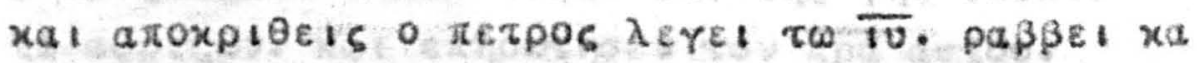

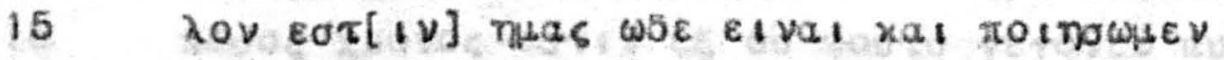

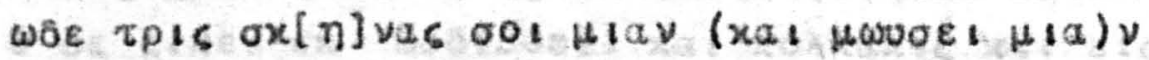

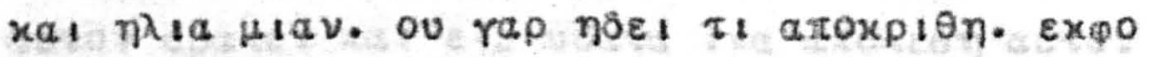
Bot $r a \rho$ e[r]evovto.

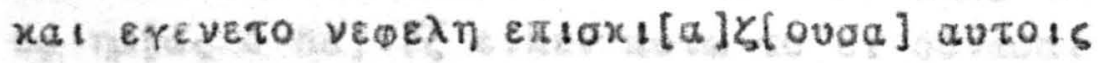

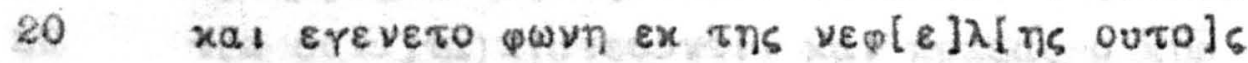
eativ O प्S HOU o ararntos axouere autou.

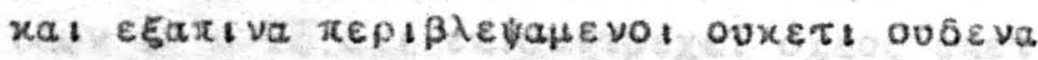

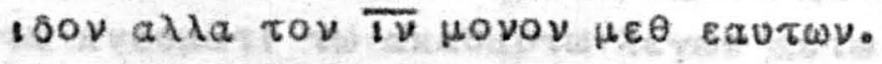

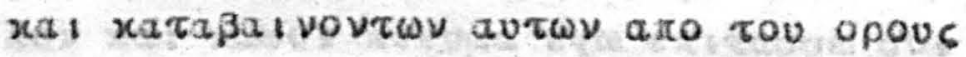

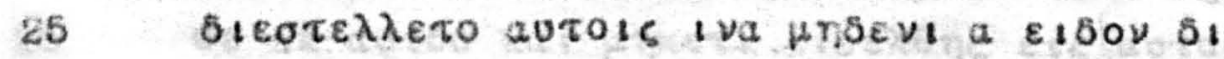

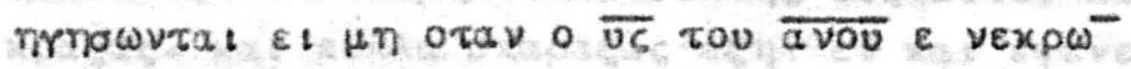
avarin. xal tov hayov exparnoav $\pi \rho \sigma_{6} \mathrm{E}$

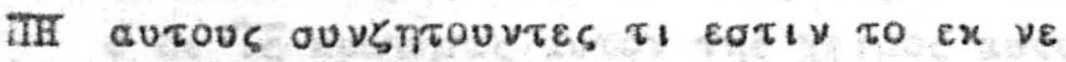

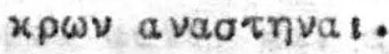

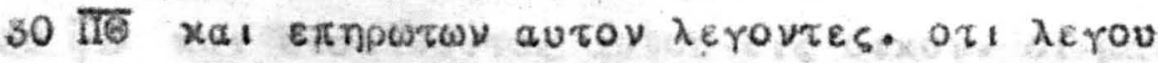

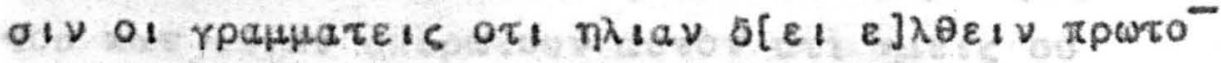

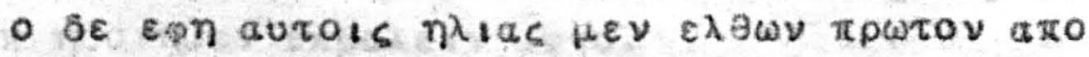

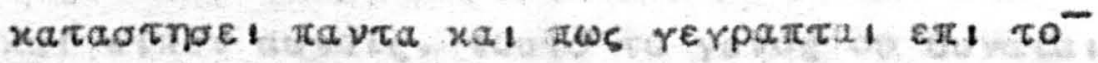

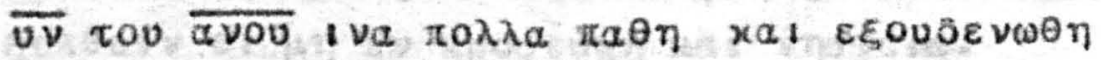

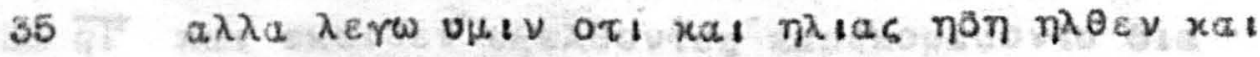

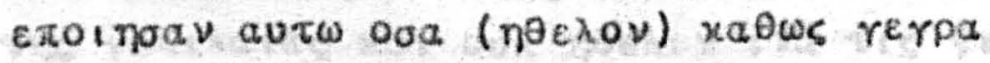

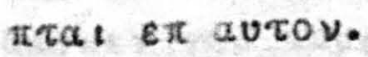

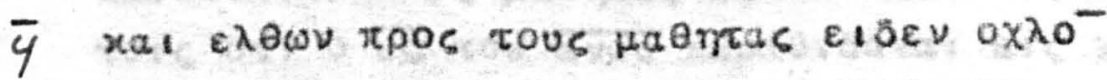

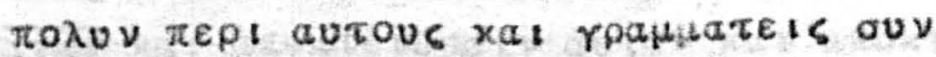

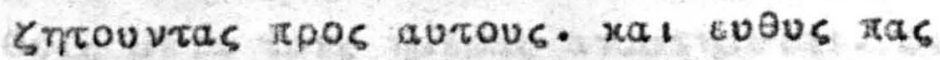

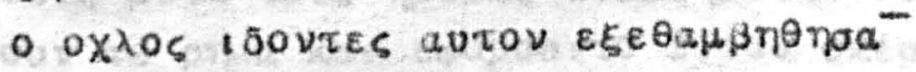




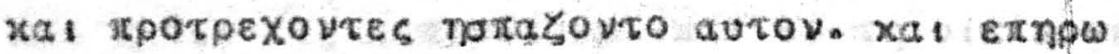

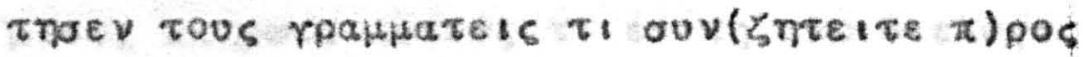

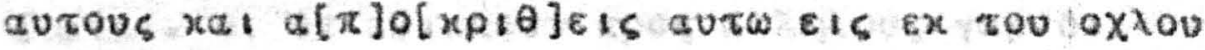

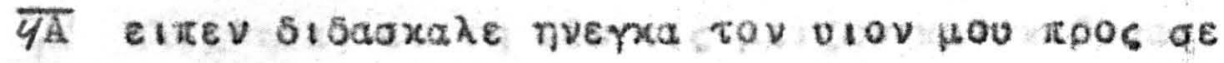

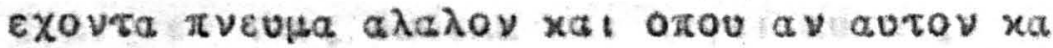

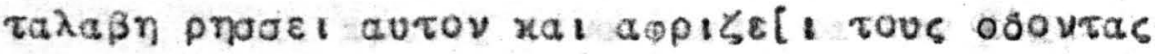

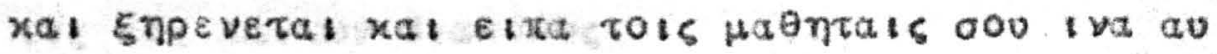

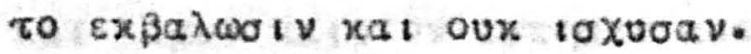

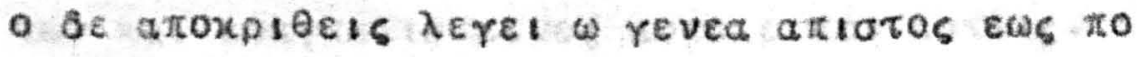

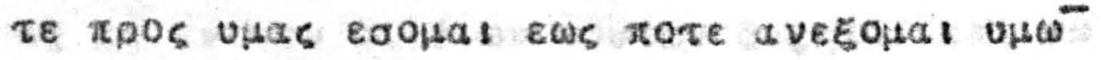

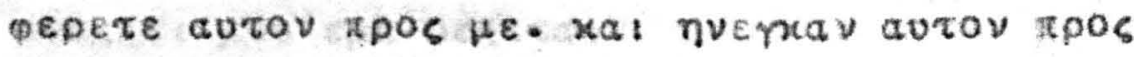
autov xal $\$$ sov autov to aveupa cuous auve

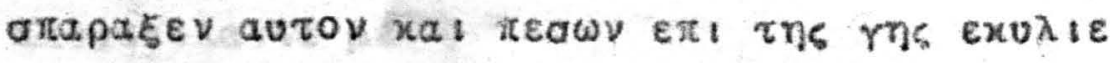

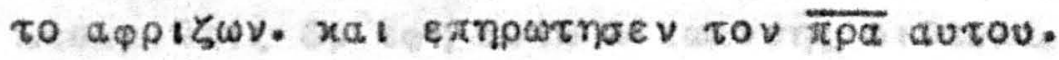
Tooos xpovos eativ $\varepsilon \xi$ ou routo reroveV autw.

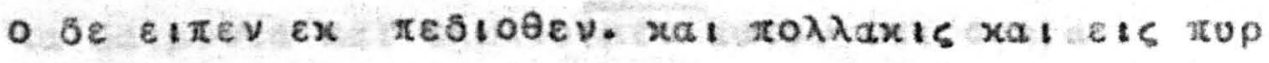

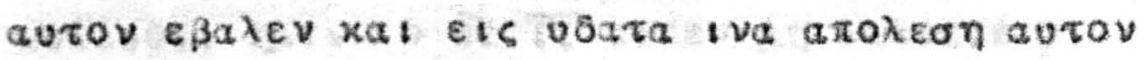

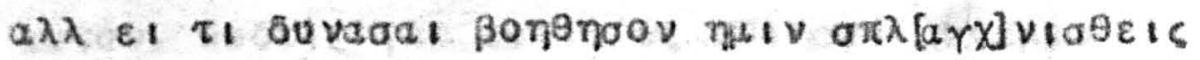

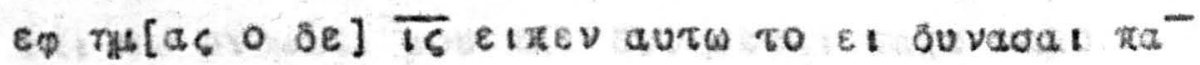

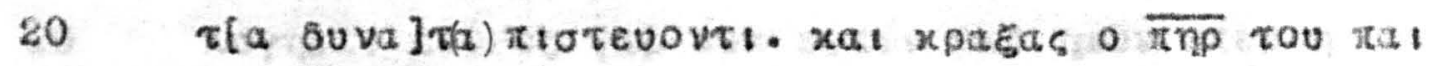

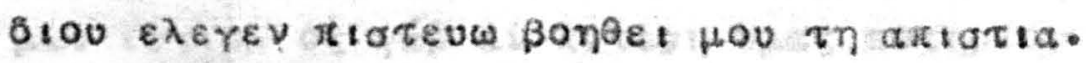
is

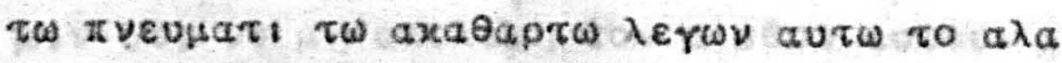

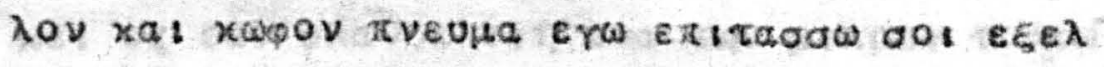

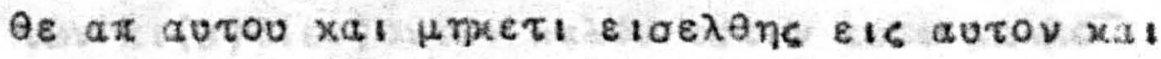

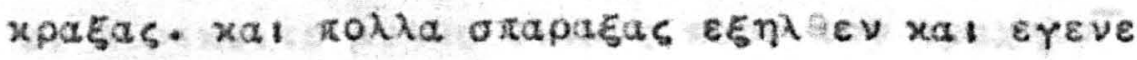

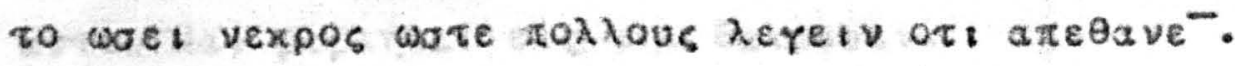
- $\delta \varepsilon$ is xpatnoas auroy tns $x e i p o s$ autou nret pev autov xal aveart.

$30 \overline{4 B}$ xal etoexoovtos aurou ets otxov. or Haentat av

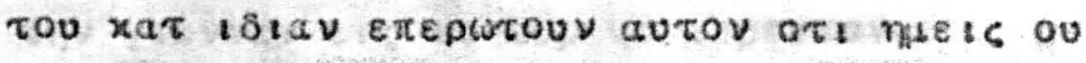

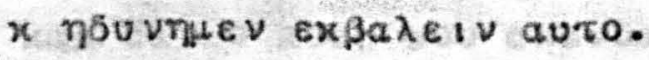
xat etrev autots. Touto to revos ou buvatal $\varepsilon$

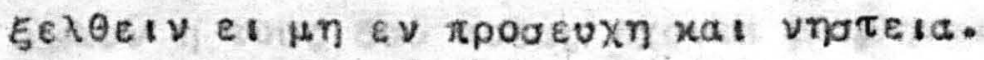

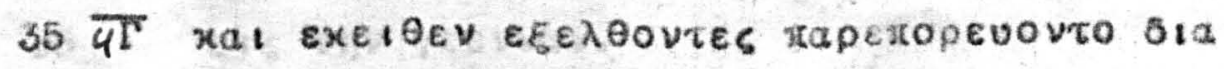

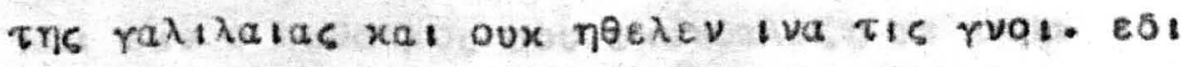

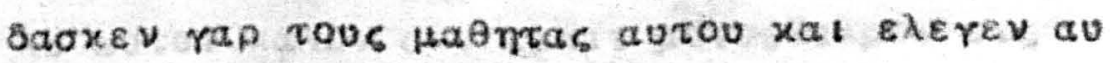

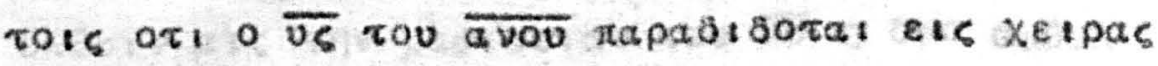

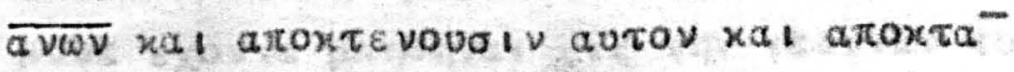

40 Oels Heta tpls nuepas a vaatnoetat. Ol $5 \varepsilon$ nrvo

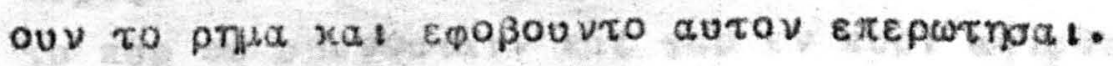




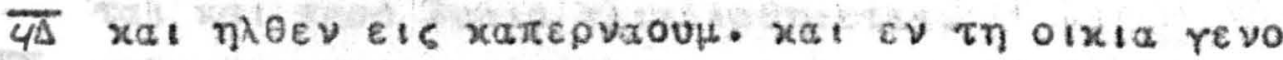

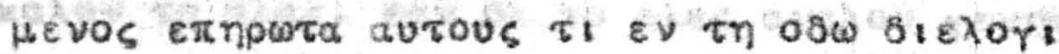

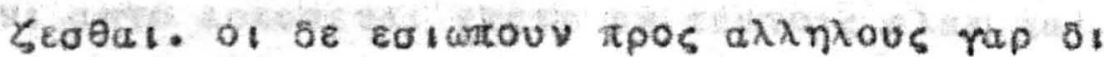

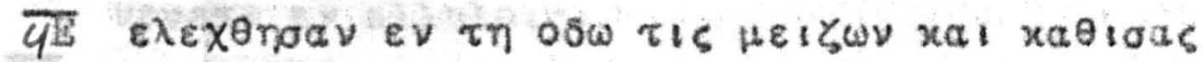

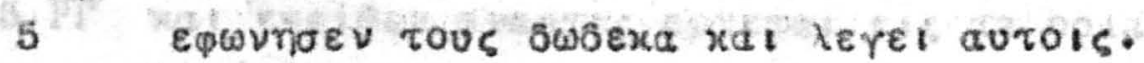

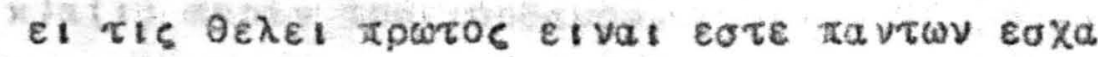
Tos $x a 1 \pi a v t \omega y$ siaxovos.

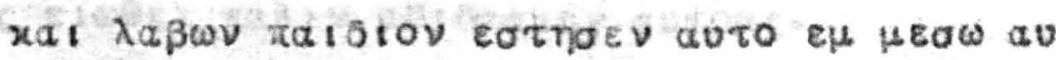

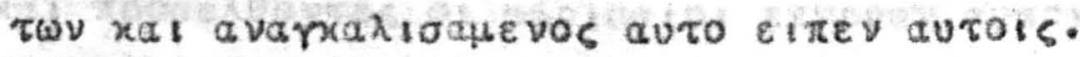

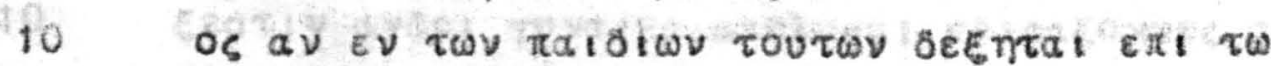

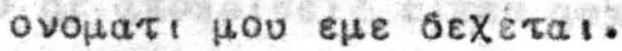

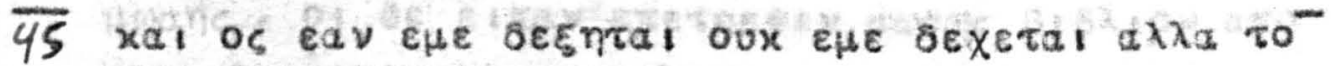

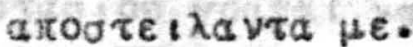

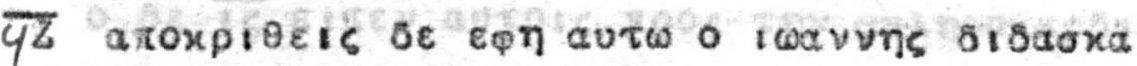

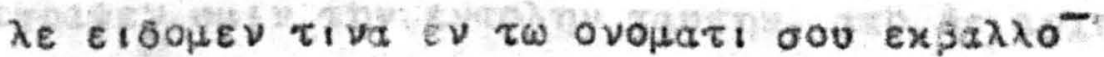

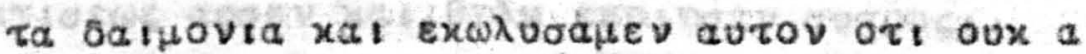

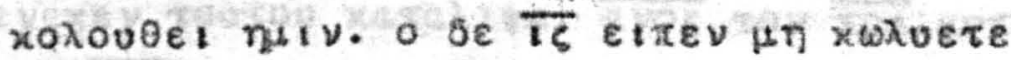

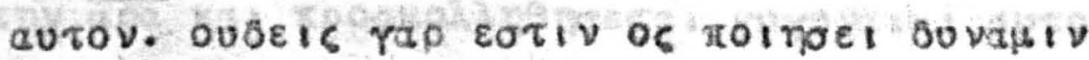
$\varepsilon \pi, \tau \omega$ ovouat, $\mu 00$ xal ovyroetai $\tau \alpha x u$ xaxo

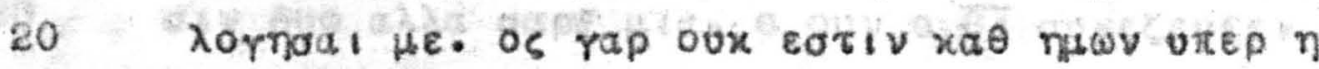

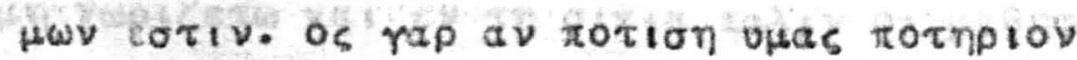

पस

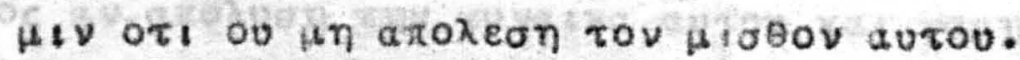

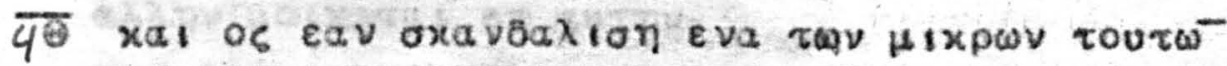

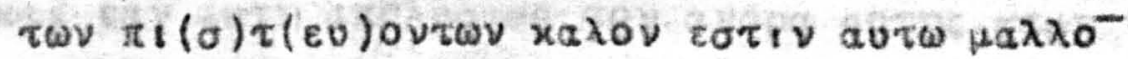

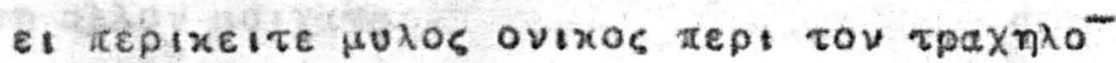

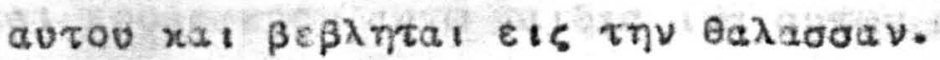

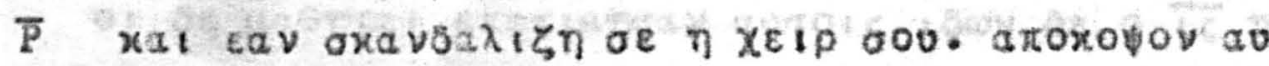

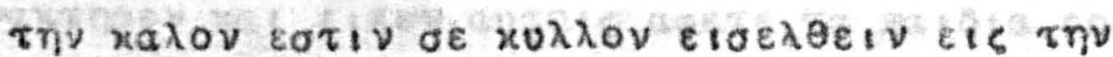

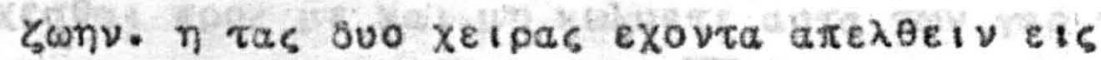

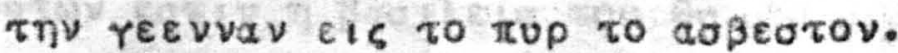

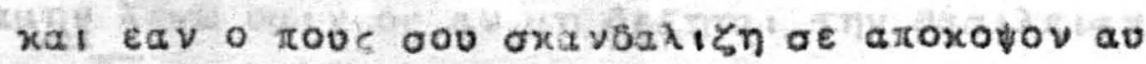

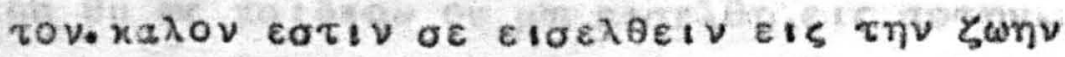

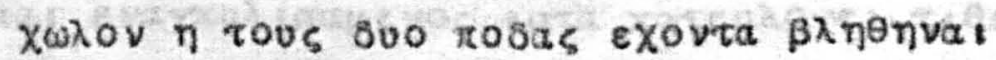

35 is tกv reevvav.

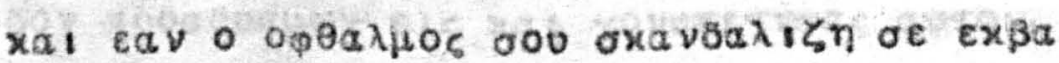

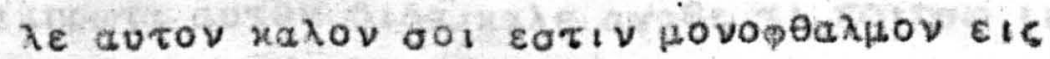

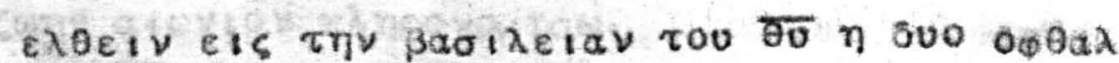

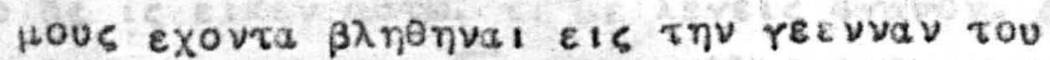

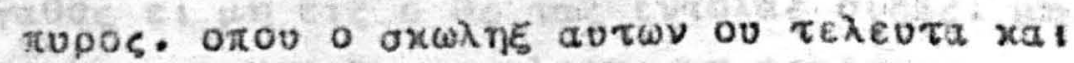

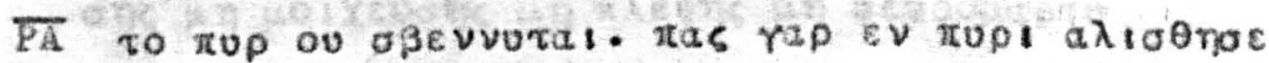




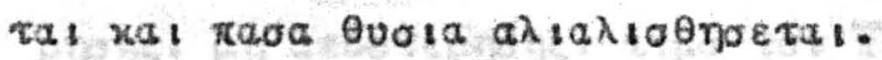

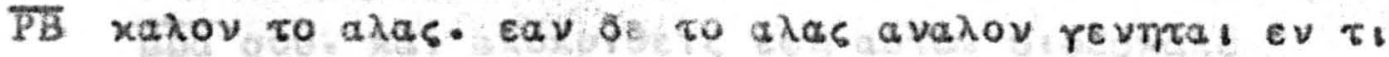

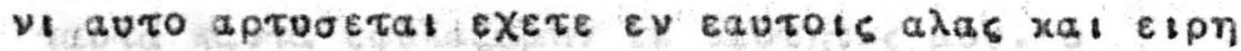

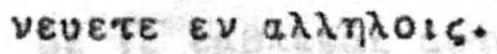

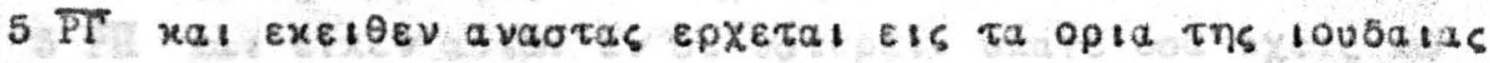
$x)$ (1) repav tou 1006 avou.

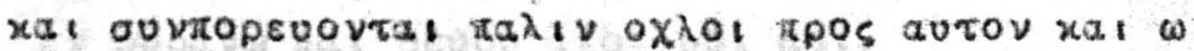

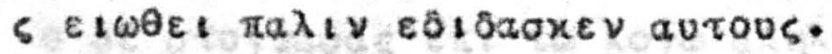

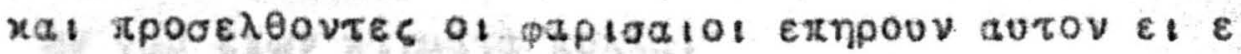

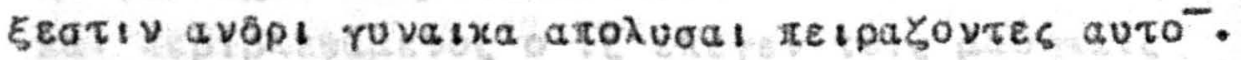

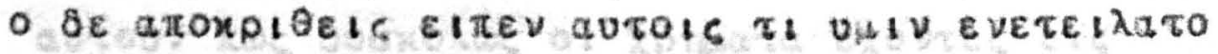

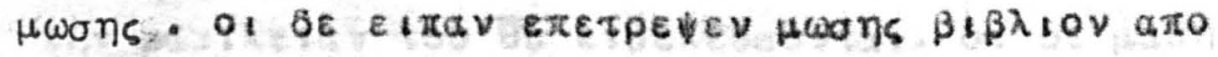

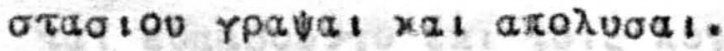

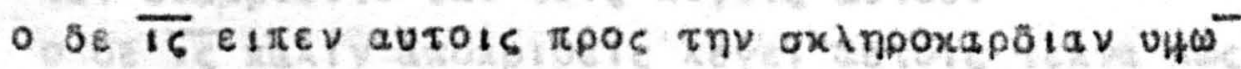

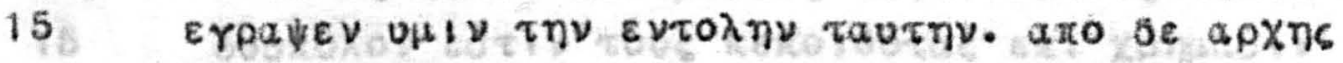

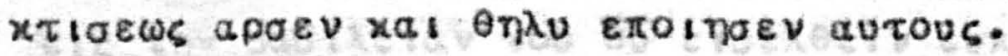

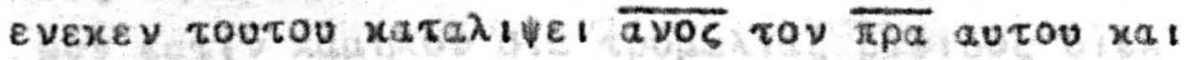

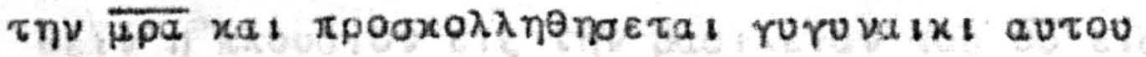

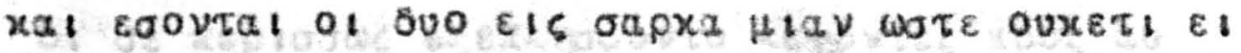

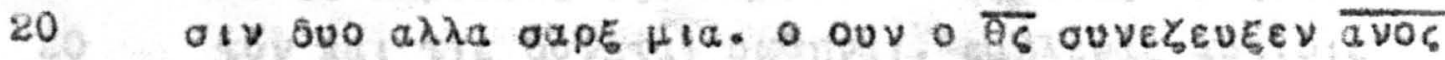

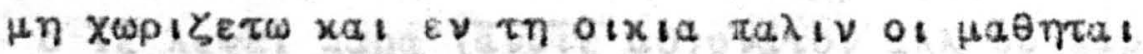

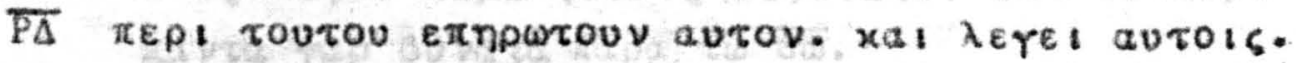

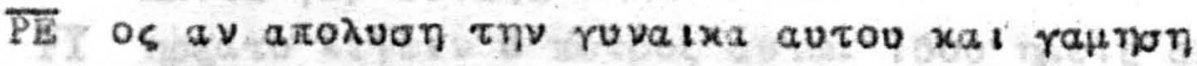

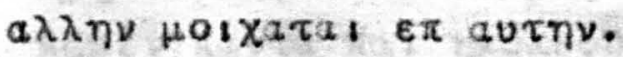

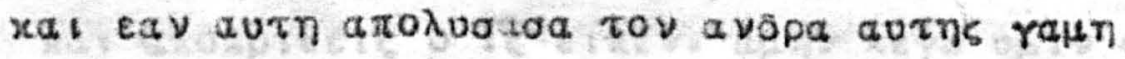

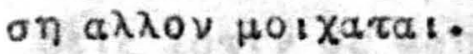

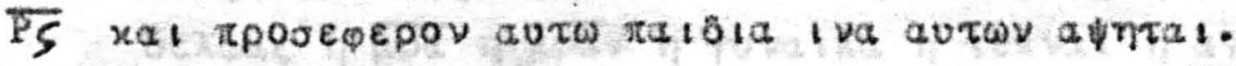

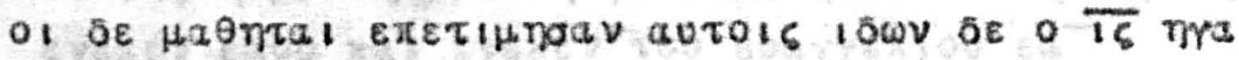

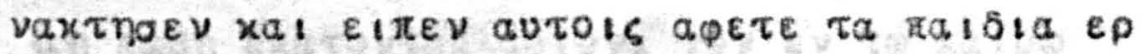

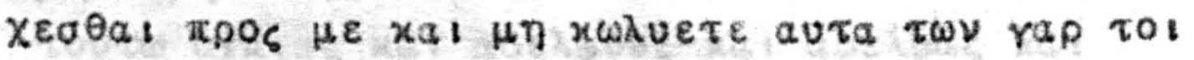

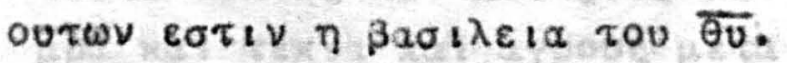

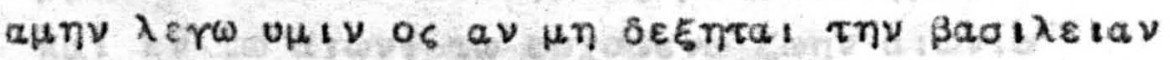

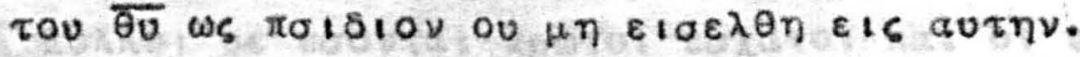

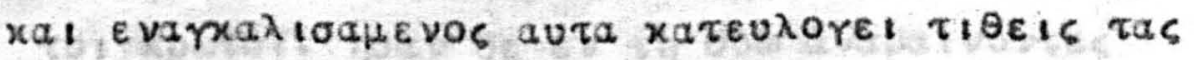

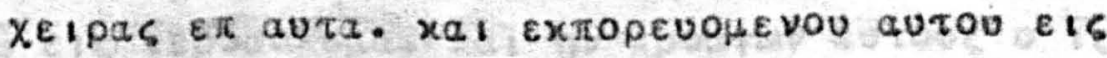

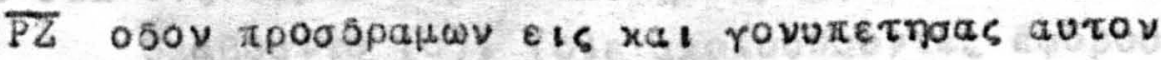

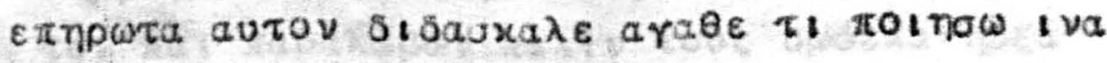

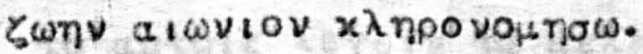

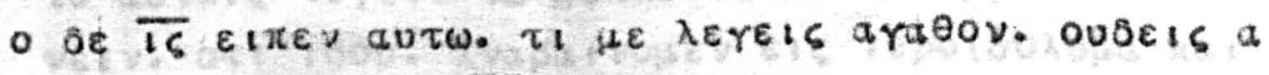

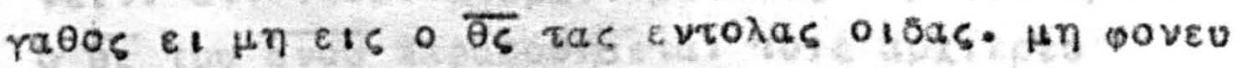

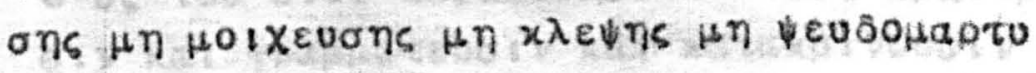




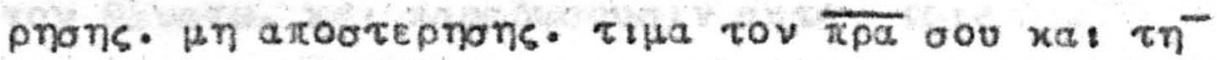

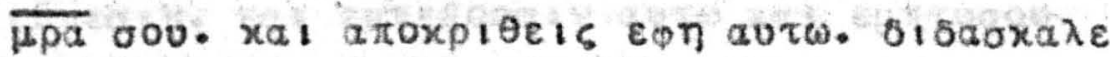

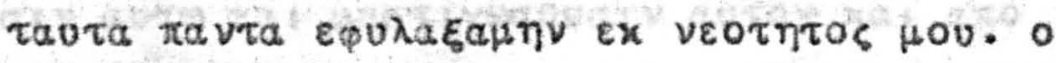

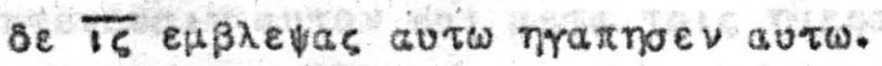

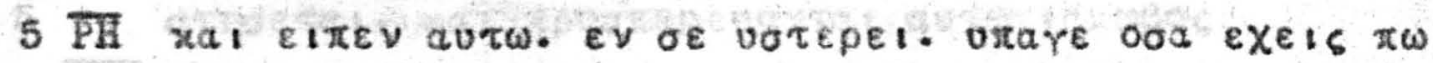

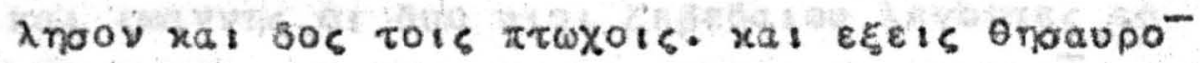

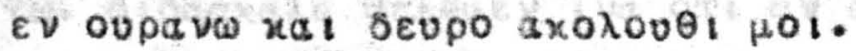

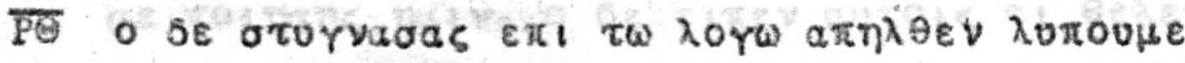

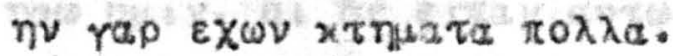

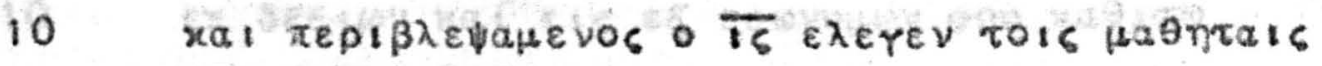

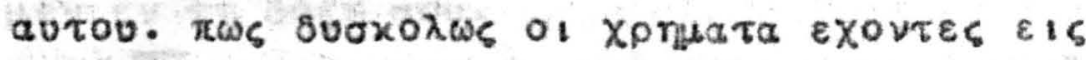

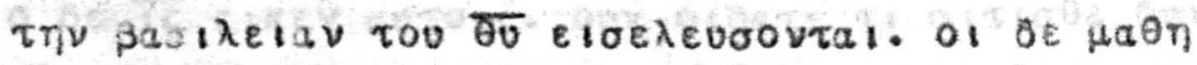

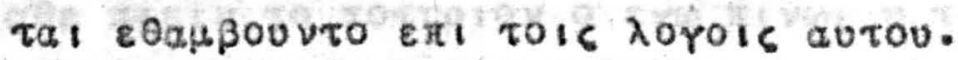

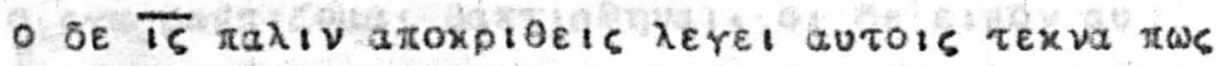

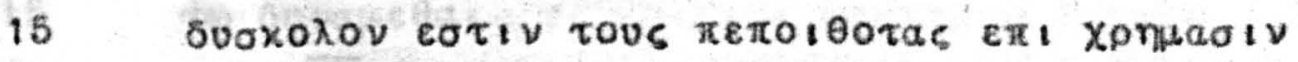

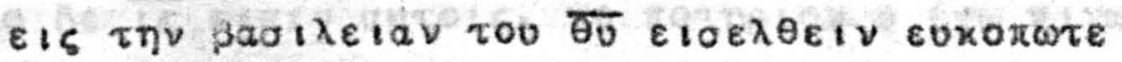

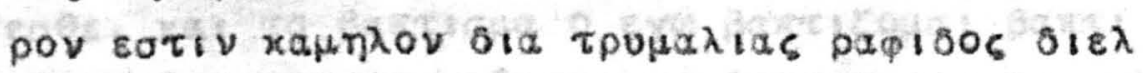

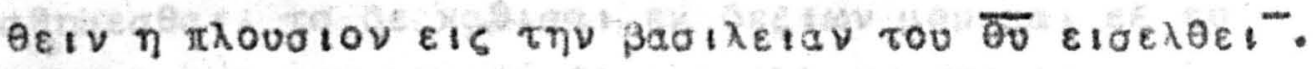

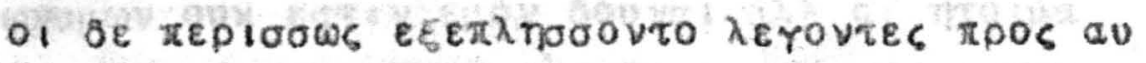

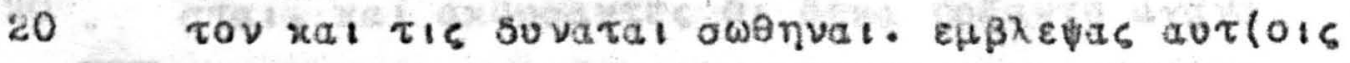

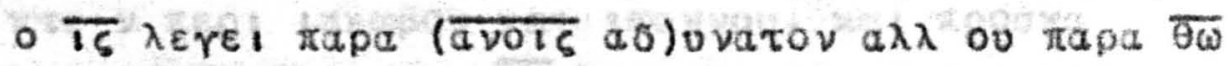

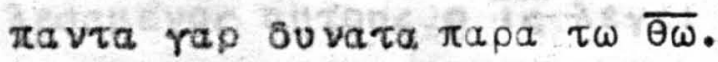

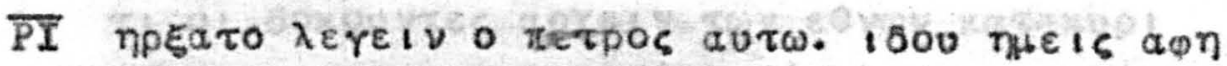

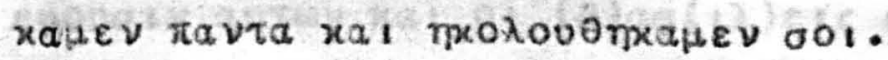

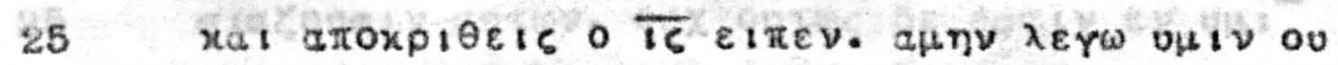

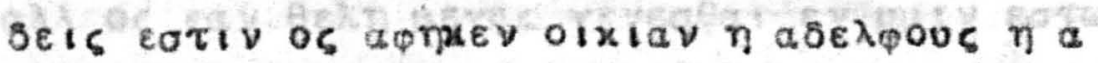

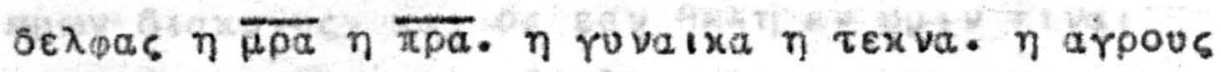

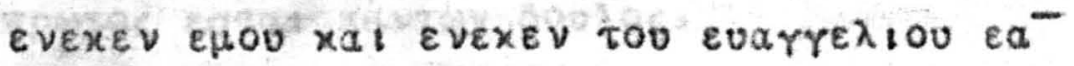

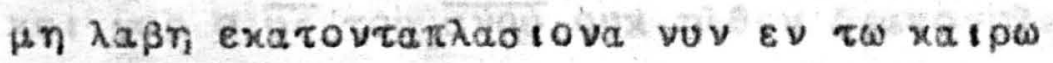

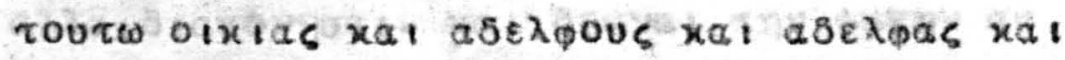

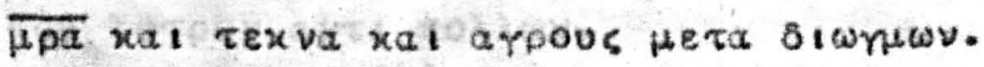

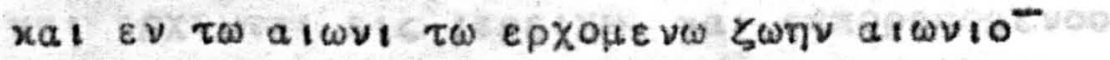

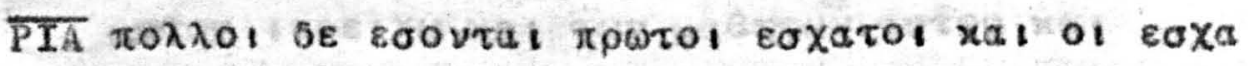

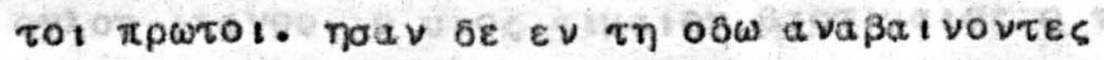

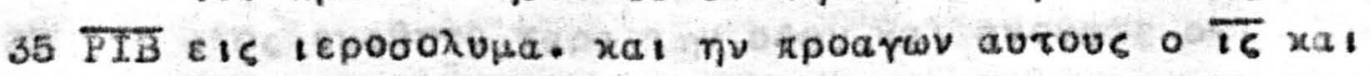

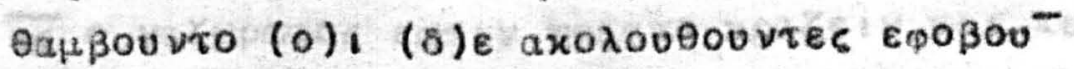

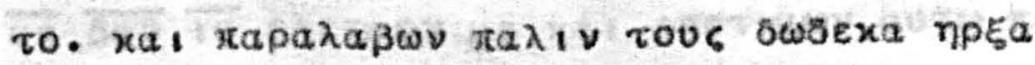

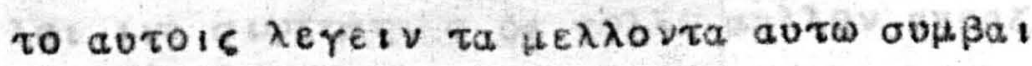

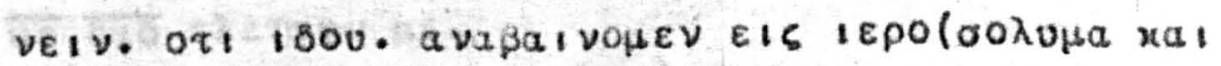

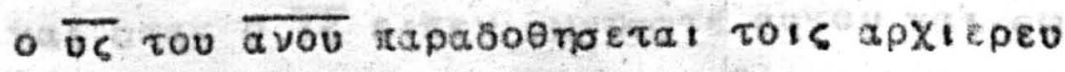

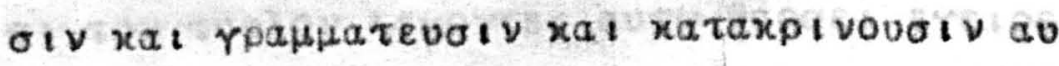




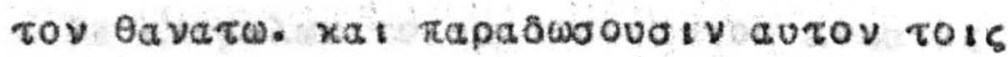

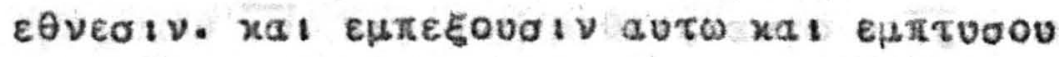
oiv autw xai Haotirwovoiv avTov xal aro

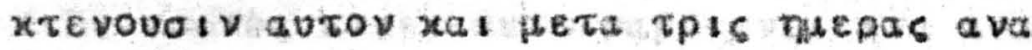

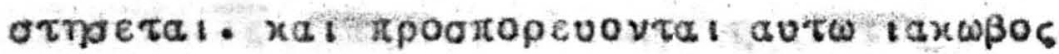

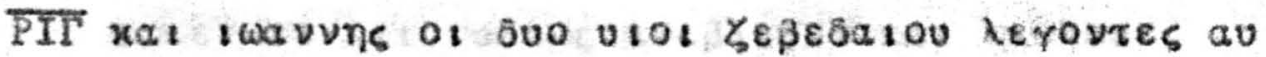

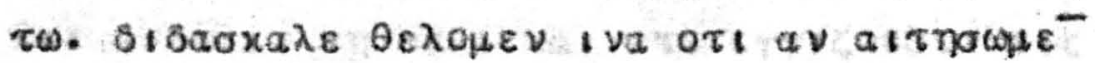

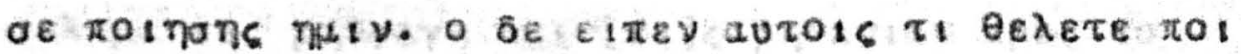

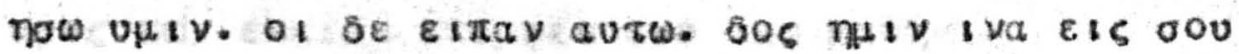

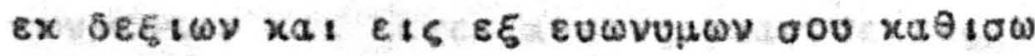

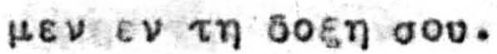

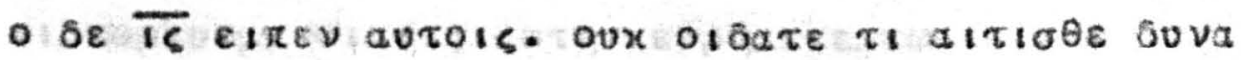

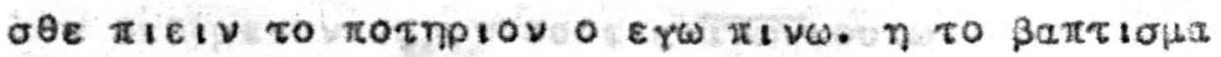

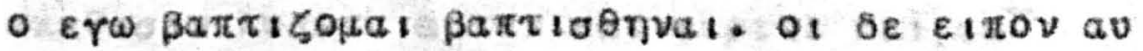
$\tau \omega$ ou $\vee \alpha \varepsilon \varepsilon \theta a$.

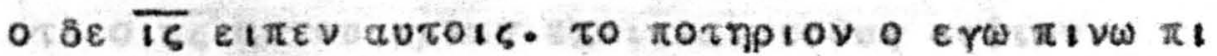

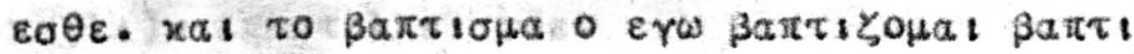

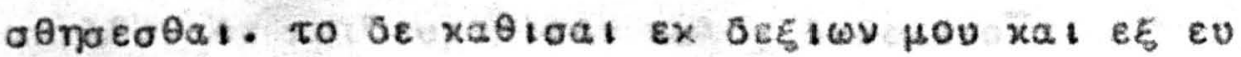

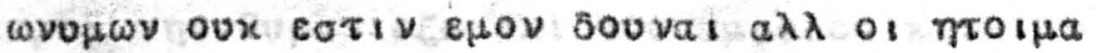

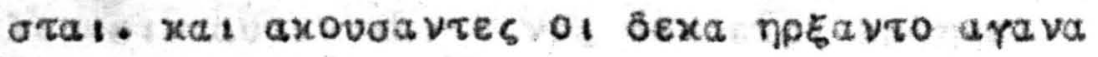

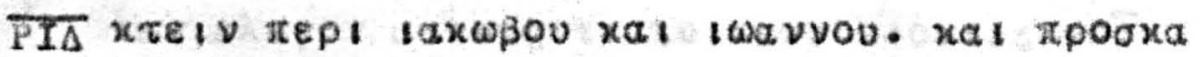

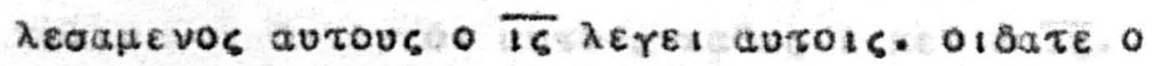

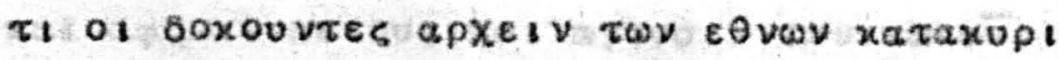

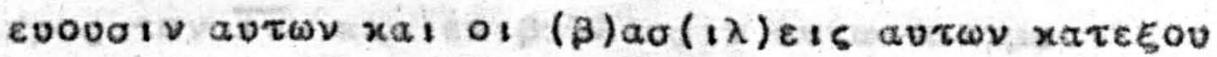

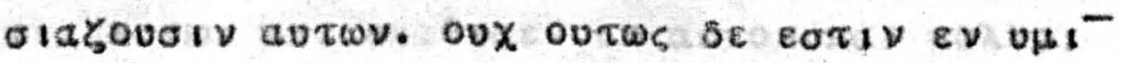

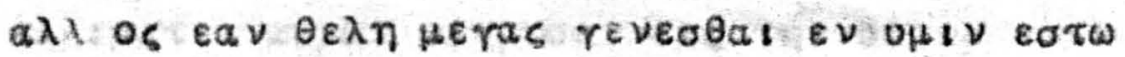

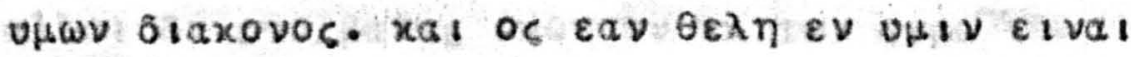

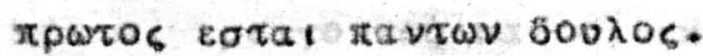

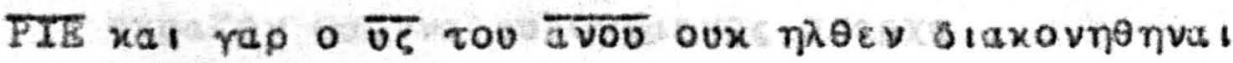

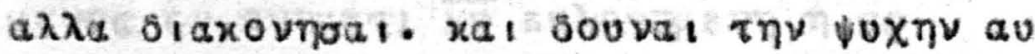

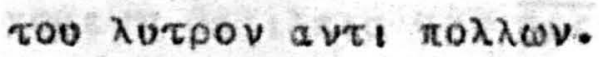

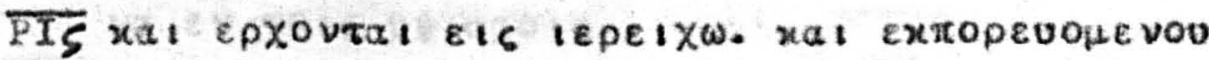
aurov $\mid \varepsilon p \varepsilon i \chi \omega$ xa! $\tau \omega \nu \mu \alpha \theta \eta \tau \omega \nu$ aurov $x \alpha !$

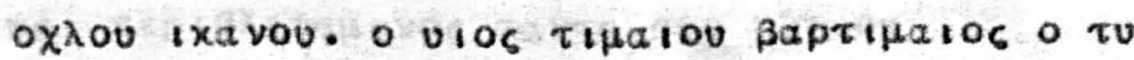

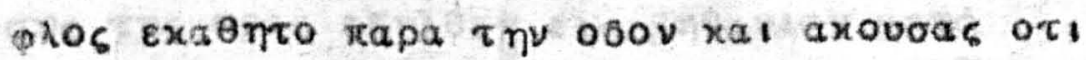

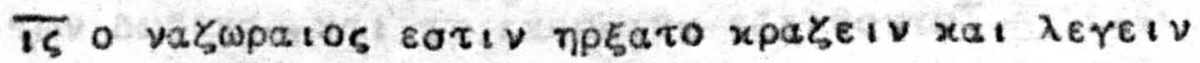

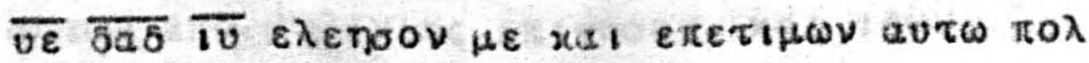

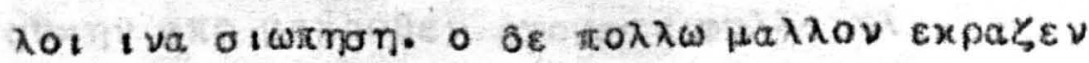

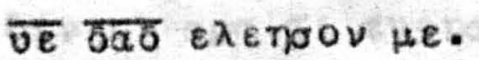

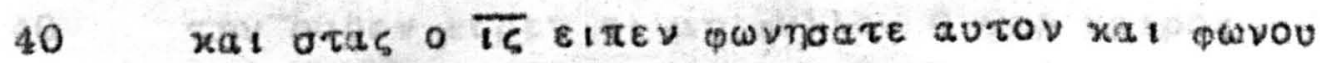

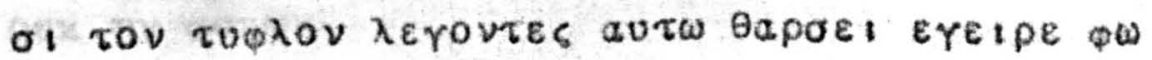
VE เoe. 


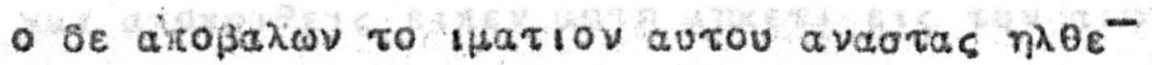

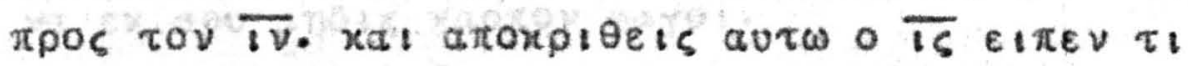

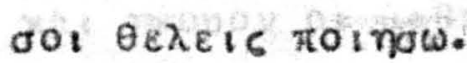

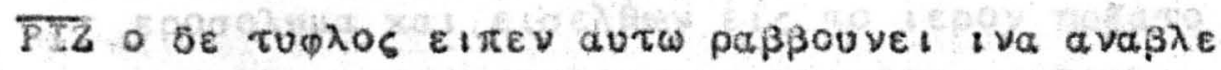

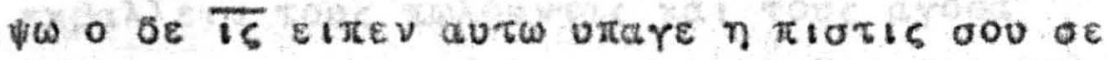

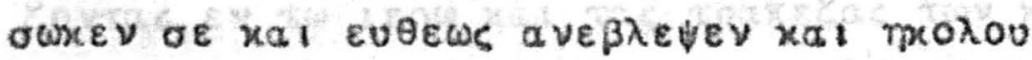

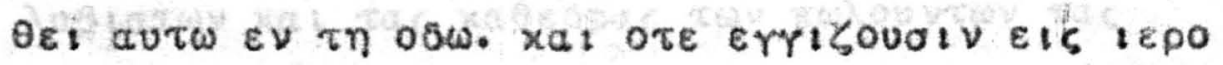

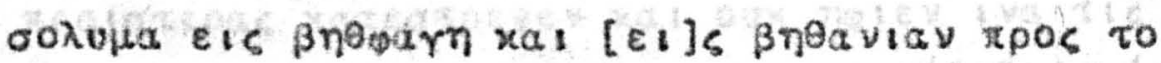

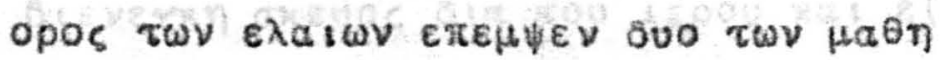

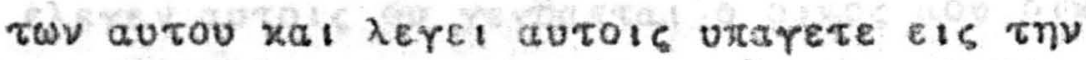

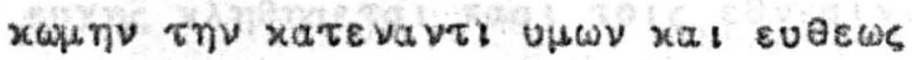

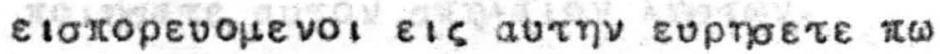

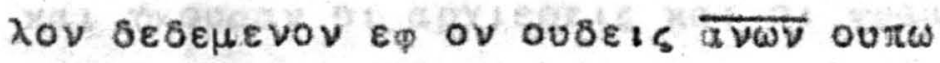

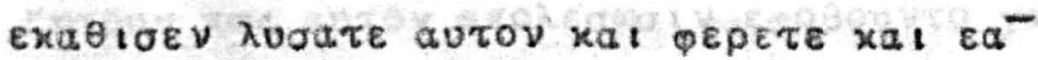

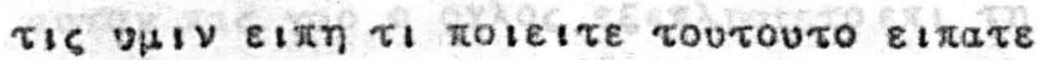

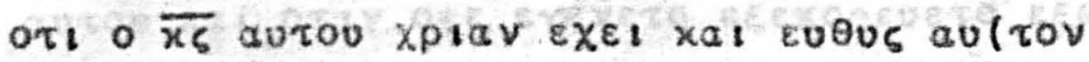

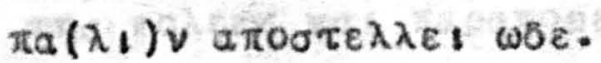

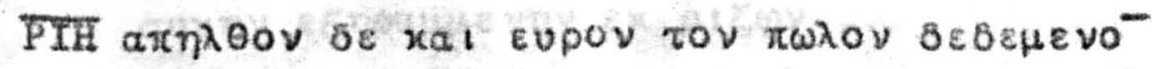

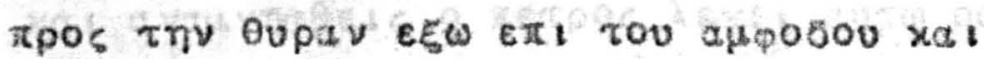

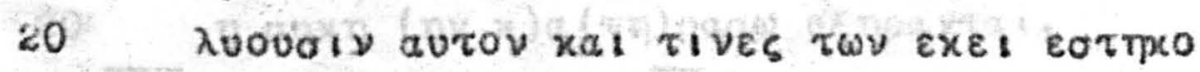

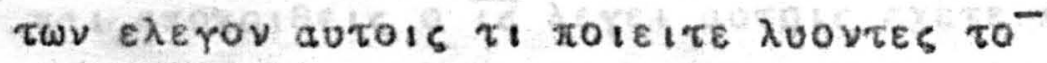

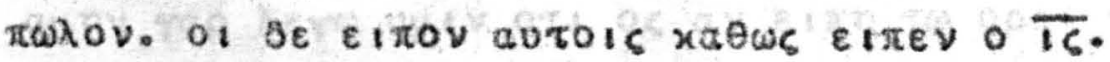

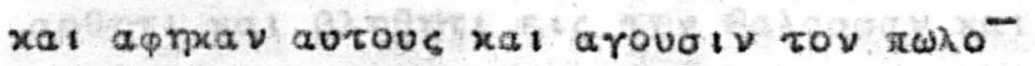

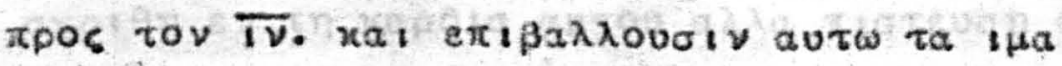

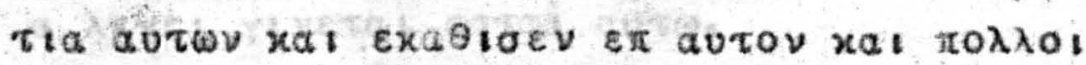

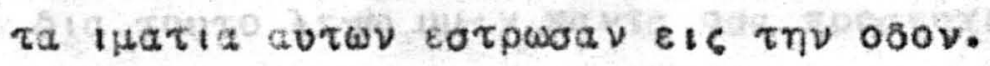

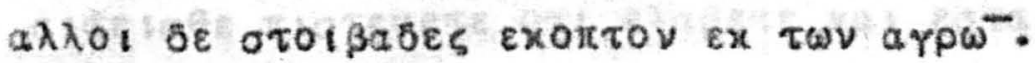

PIE xal of rpoarovtes xat of axoloveouvtes expacov wauva euxorna vos 0 epxo

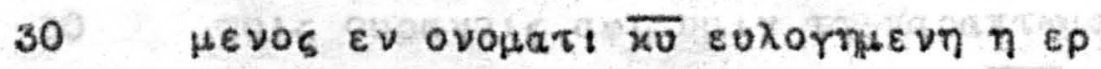

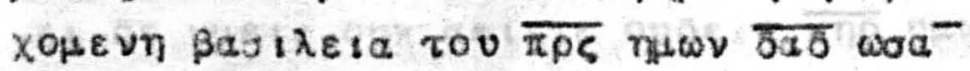
va $\varepsilon \nu$ Tols vilotois.

PI xat $\varepsilon t a \eta \lambda \theta \varepsilon v$ \&is $i \varepsilon p 000 \lambda u \mu a$ eis to tepov

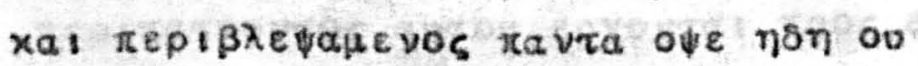

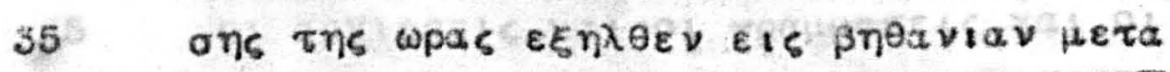

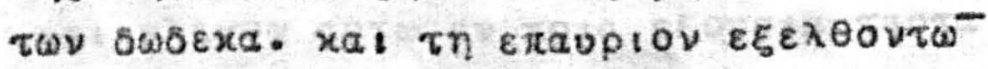

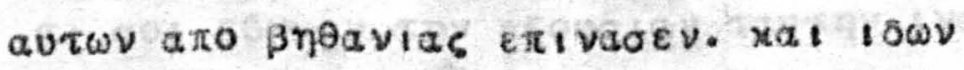

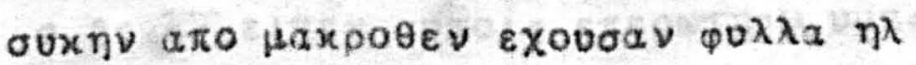

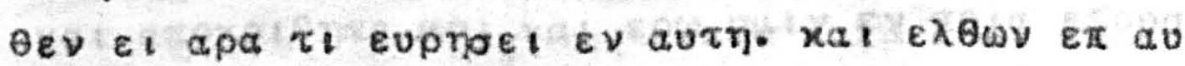

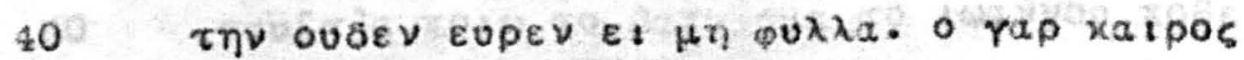
oux nv ouxwv. 


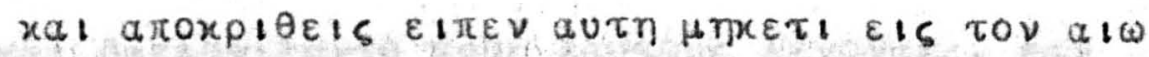

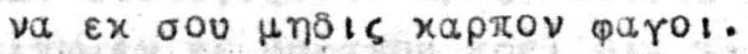

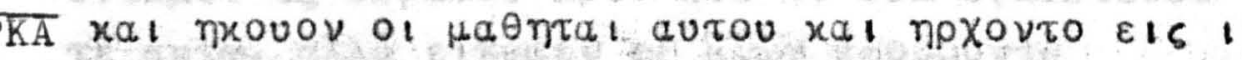

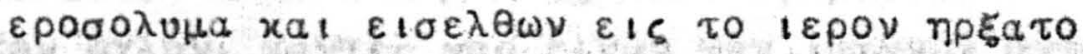

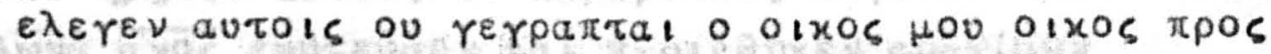

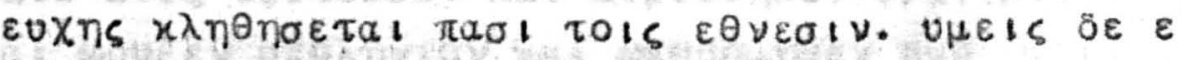

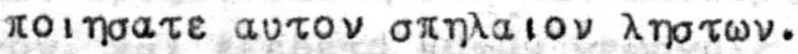

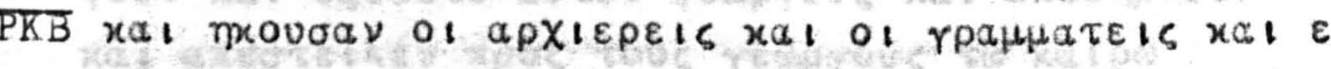

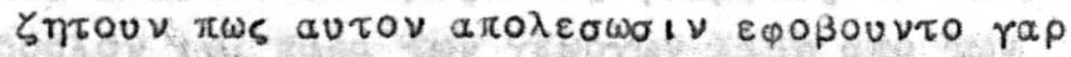

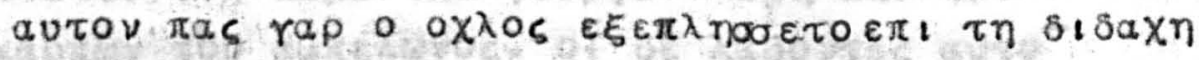

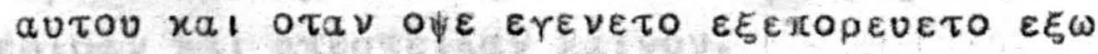

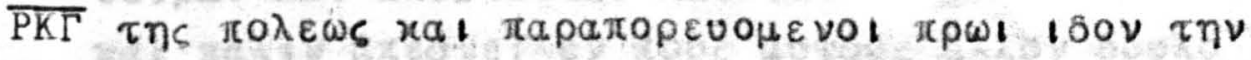

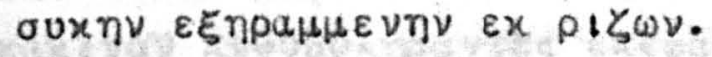

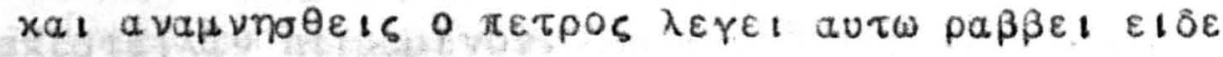

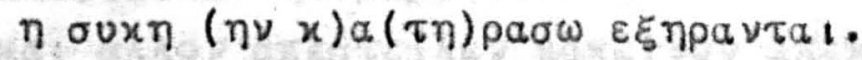

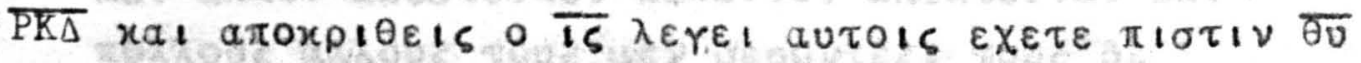

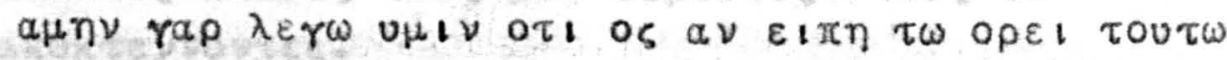

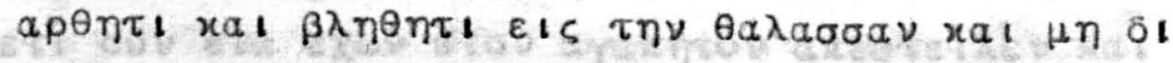

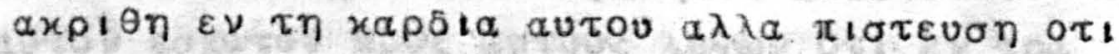

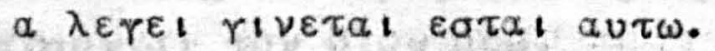

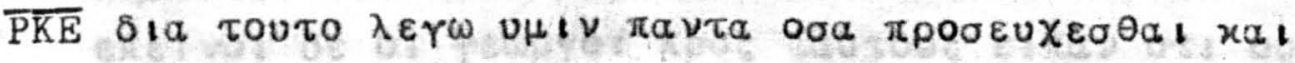

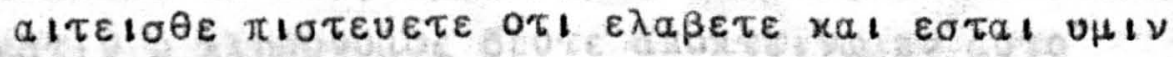

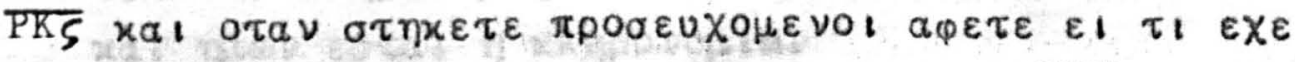

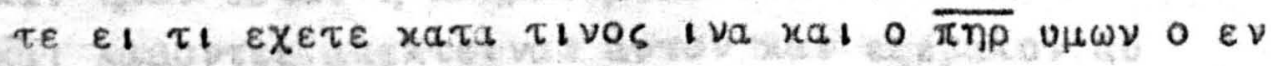

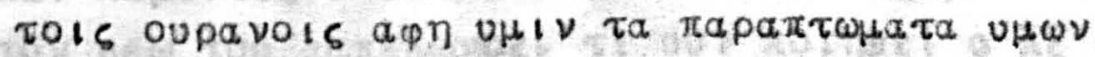

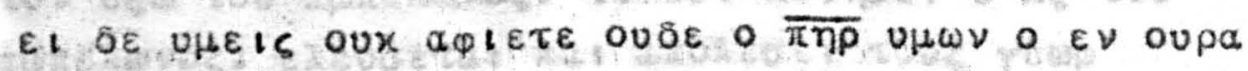

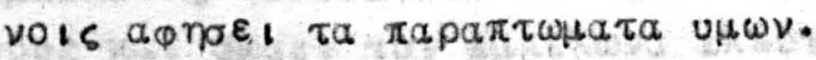

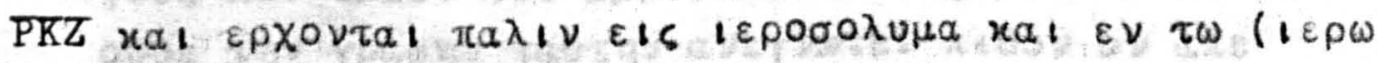

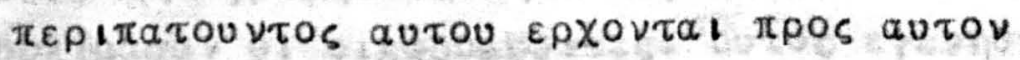

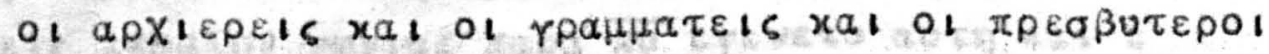

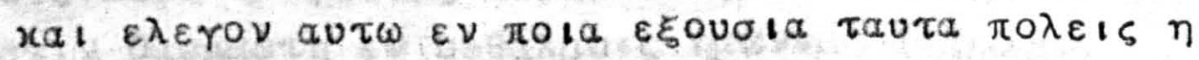

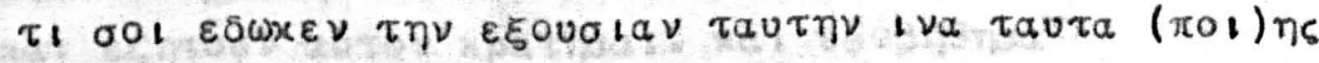

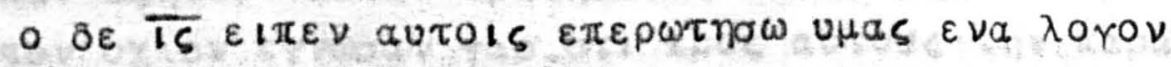

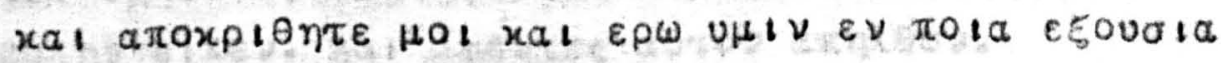

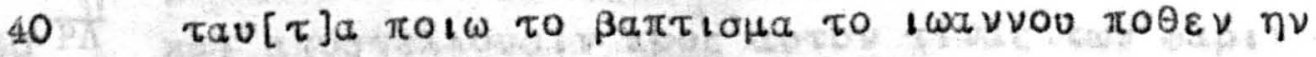

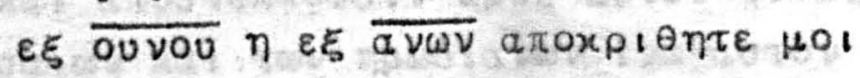




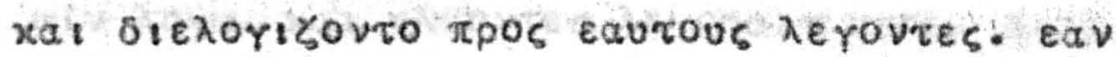

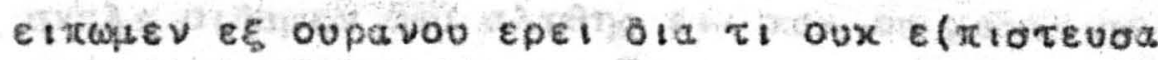

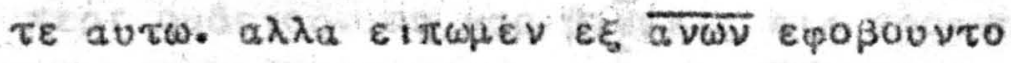

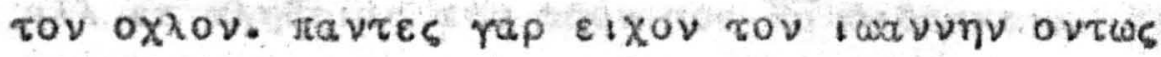

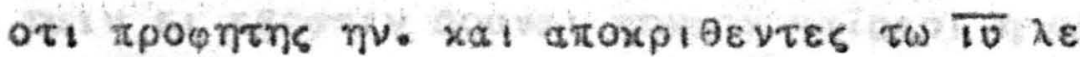
rovoiv oux oibatev.

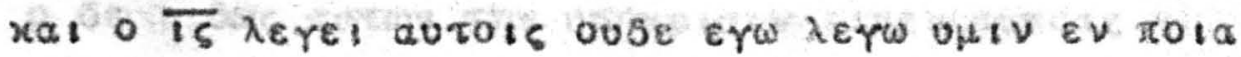

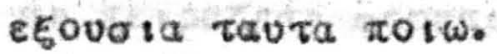

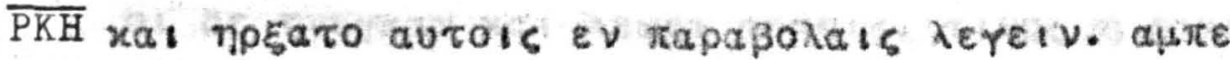

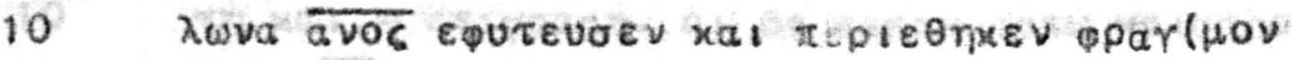

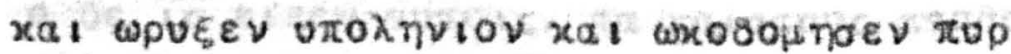

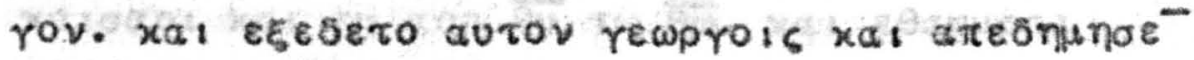
$x a$ a a

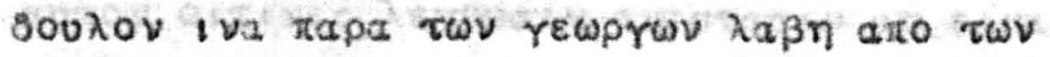

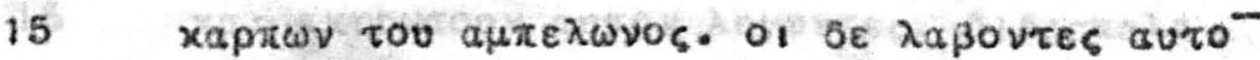

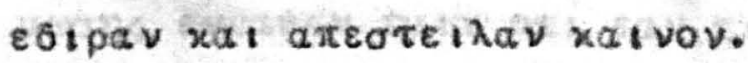
$x a$ I $\pi$ a IV areorei

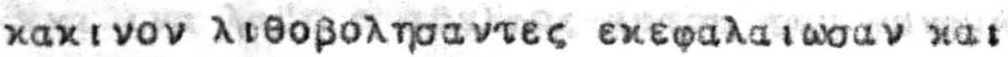

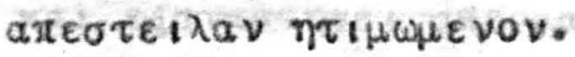

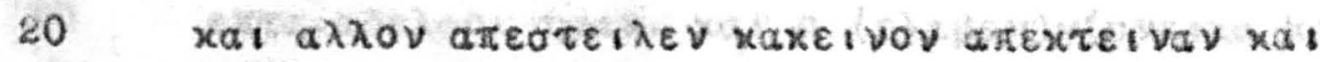

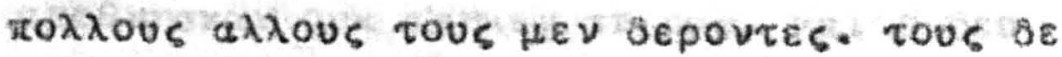
aroxtevvovies.

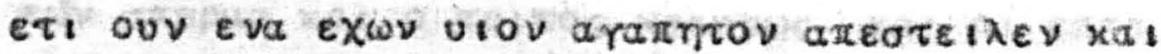

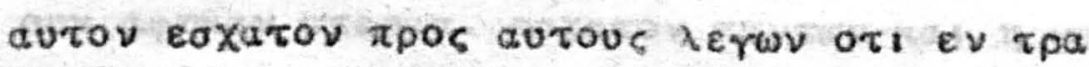

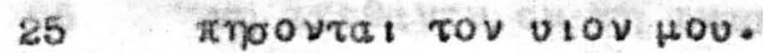

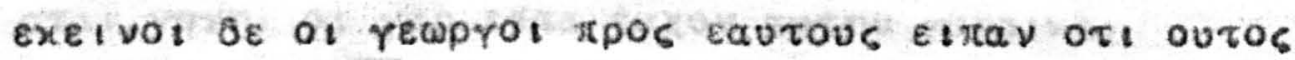

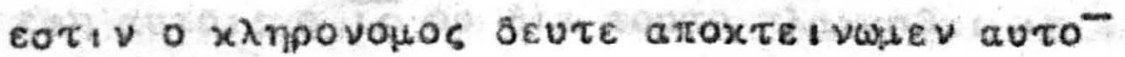

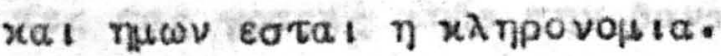

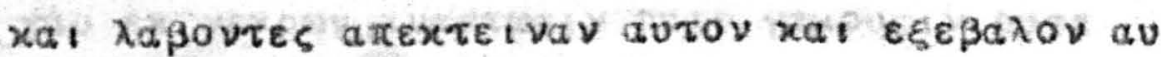

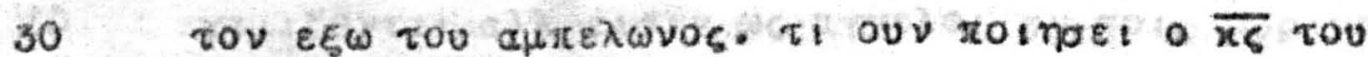

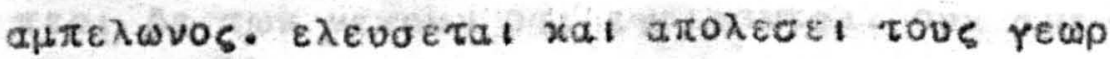

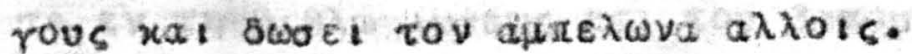

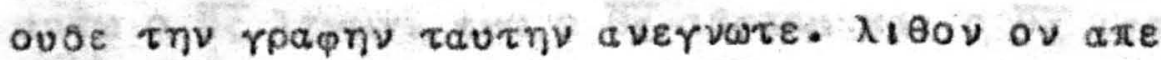

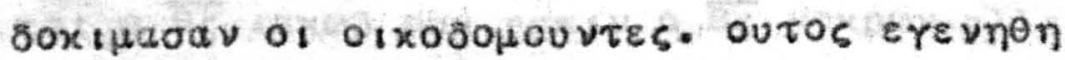

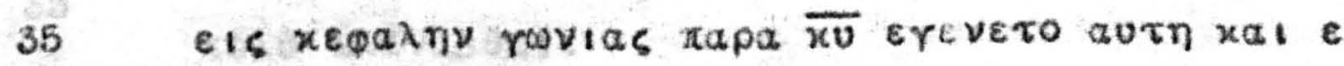

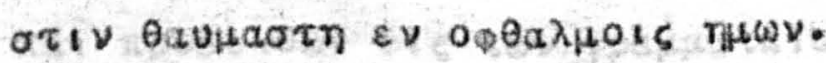

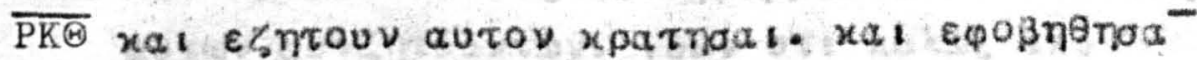

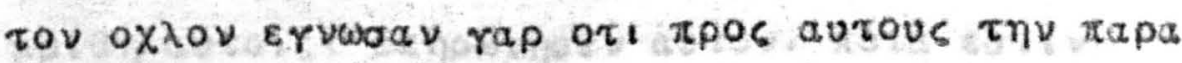

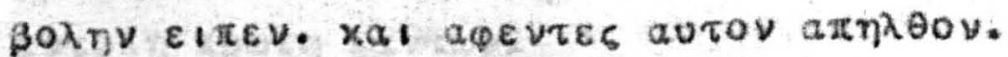

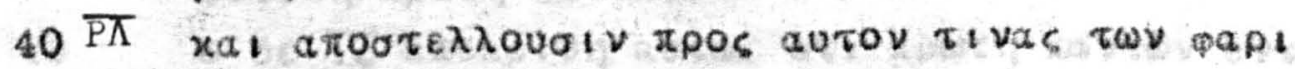

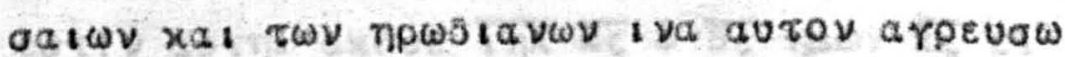




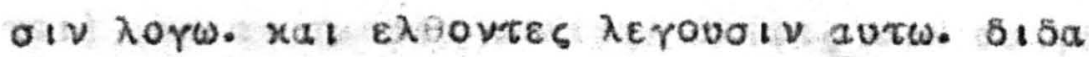

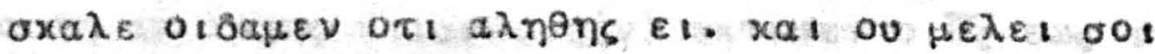

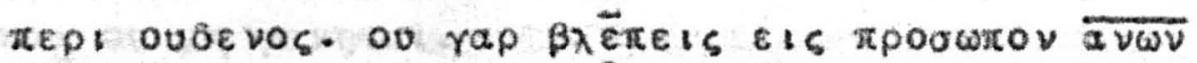

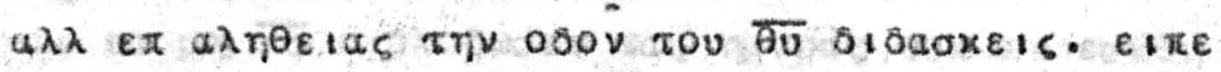

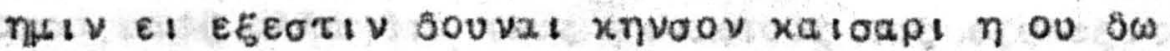
$\mu \varepsilon \nu \eta \mu \eta \delta \omega \beta \varepsilon \nu$.

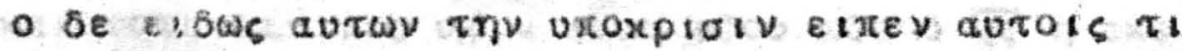

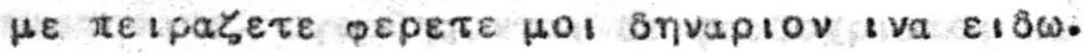

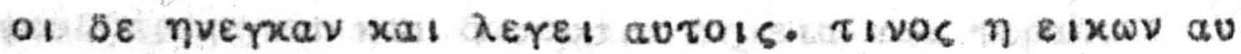

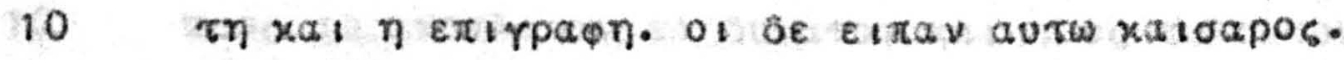

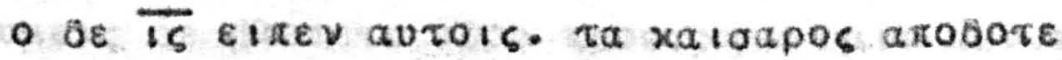

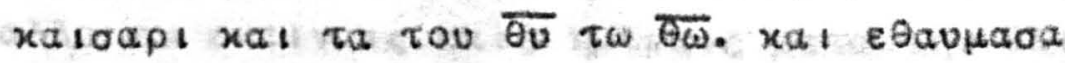

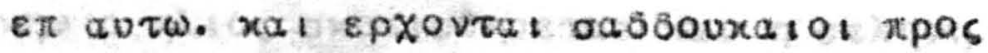

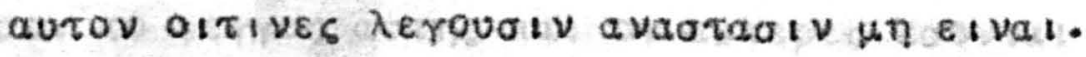

15 xat expowtouv autov $\lambda e$ roves. of

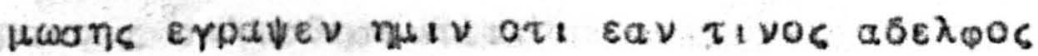

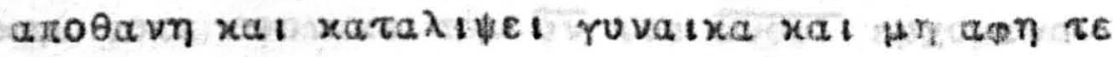

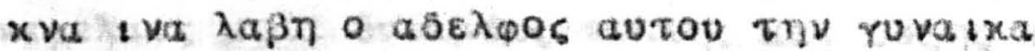

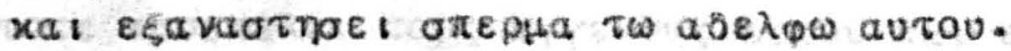

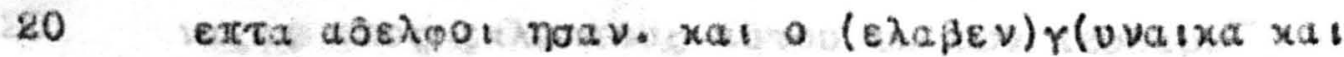

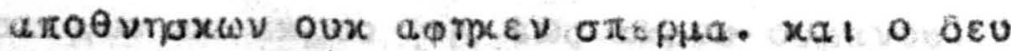

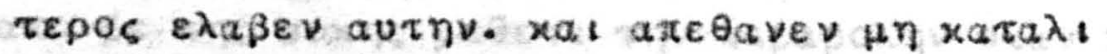

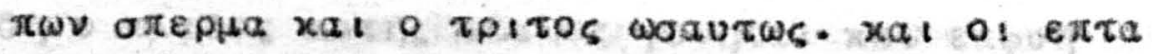

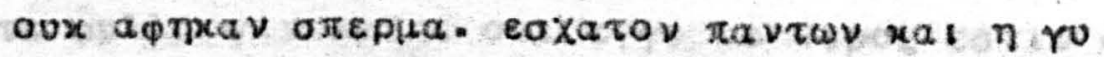

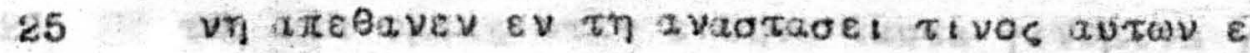

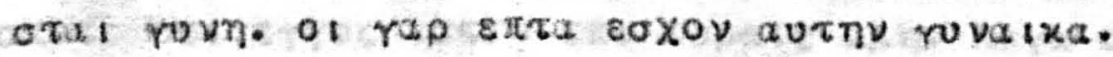

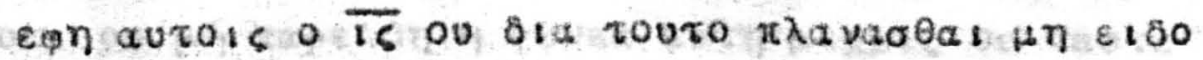

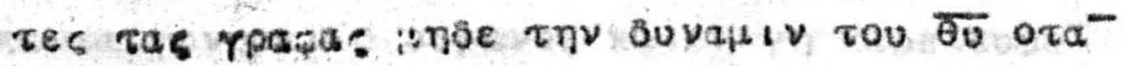

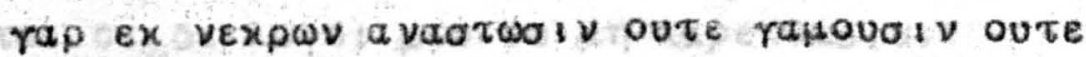

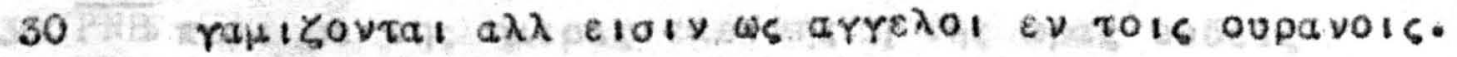

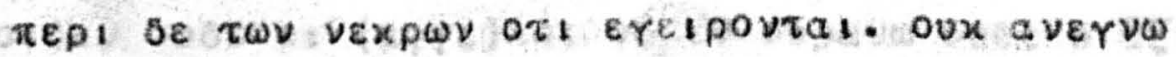

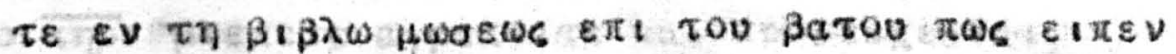

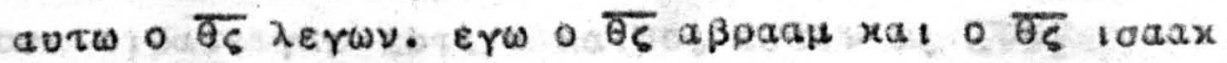

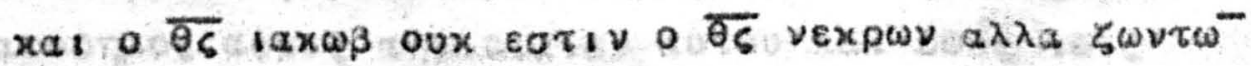

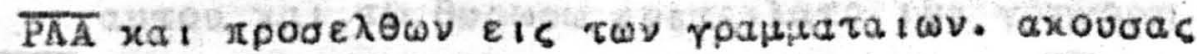

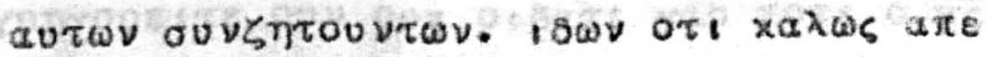

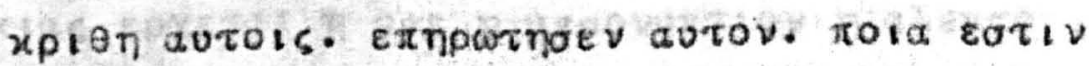

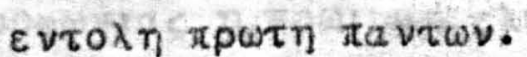

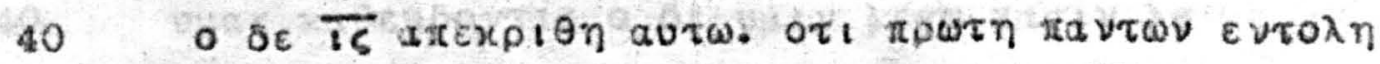

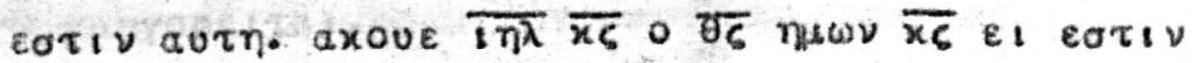




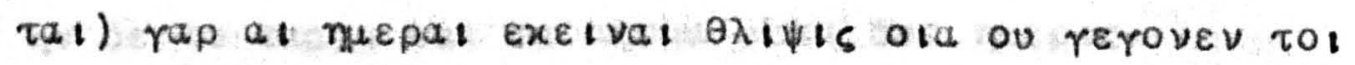

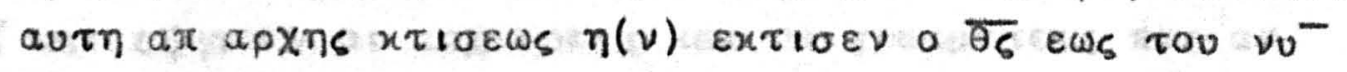
xal ou $\mu \eta$ $\gamma \varepsilon v \eta \tau a l$.

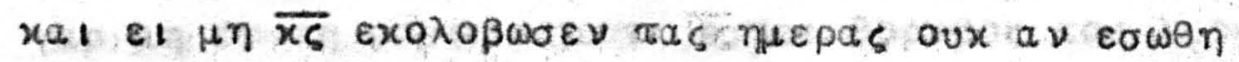

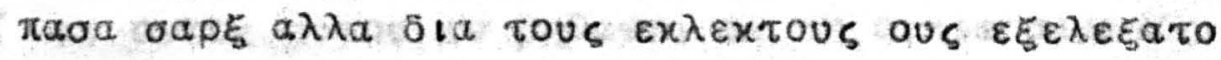

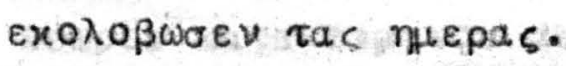

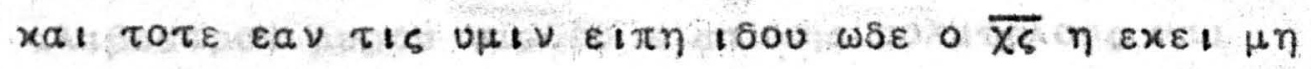

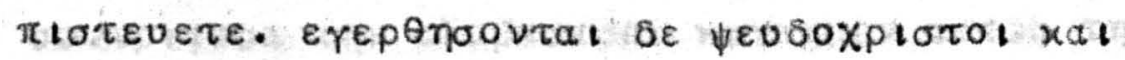

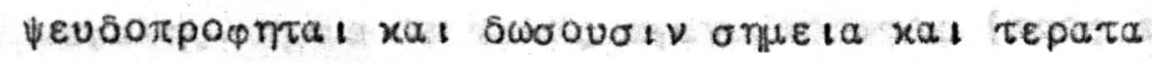

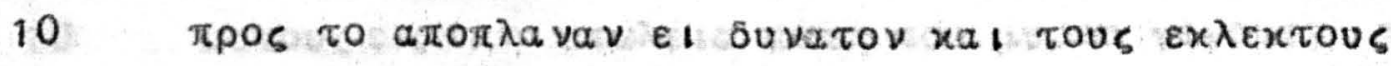

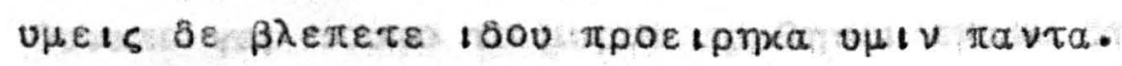

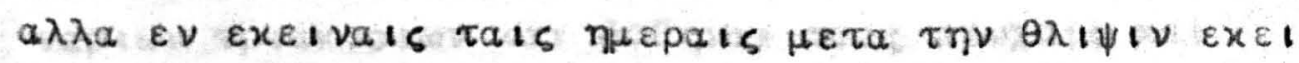

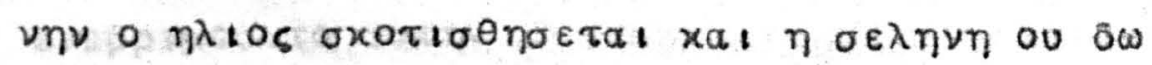

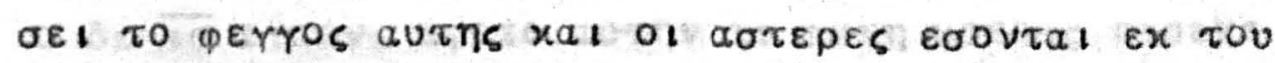

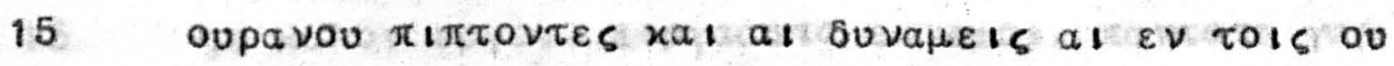
pavols aadevonoovial.

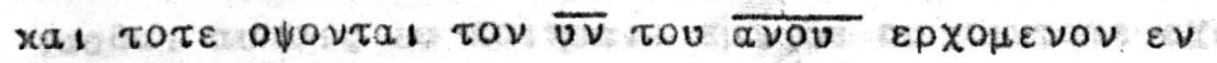

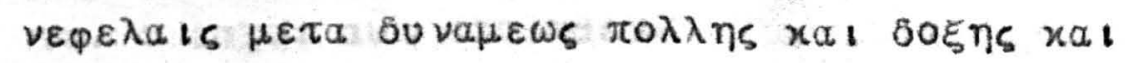

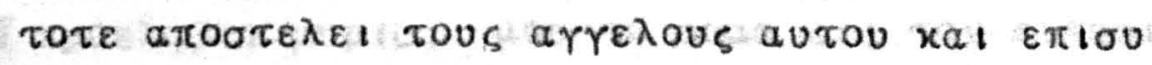

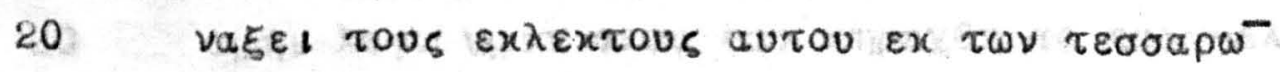

a $\Sigma \varepsilon \mu \omega \nu$ ar axpou rns $\varepsilon \omega \zeta$ axpov oupavou.

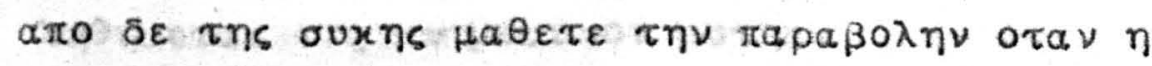

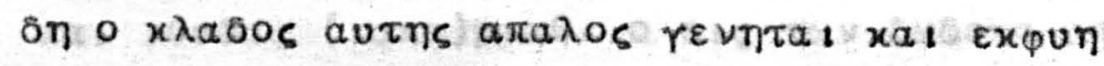

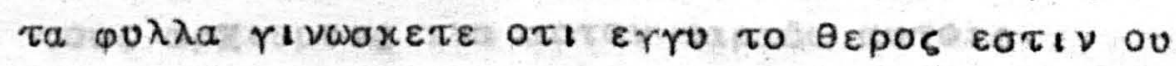

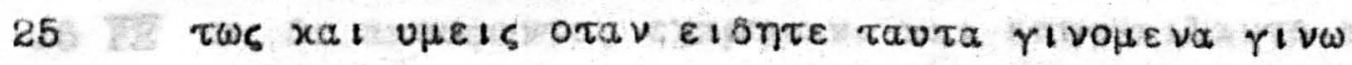

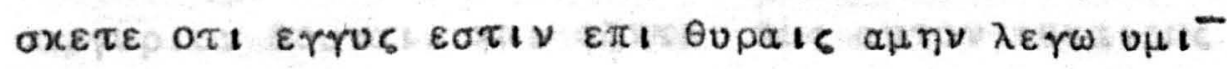

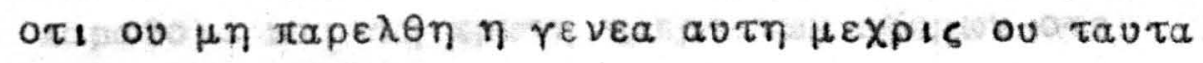

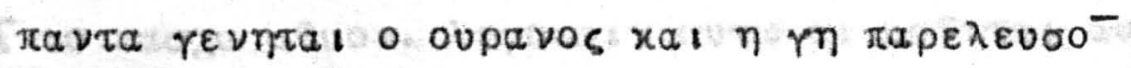

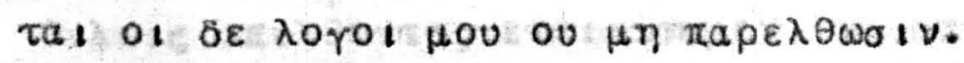

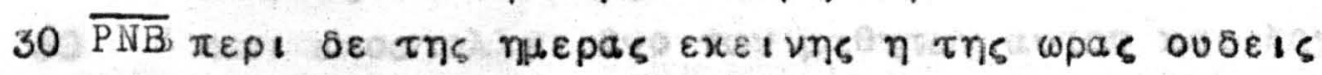

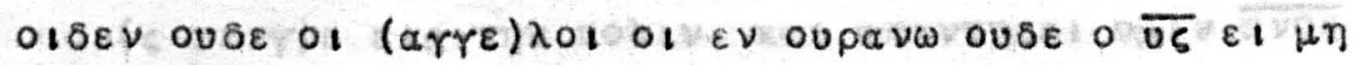

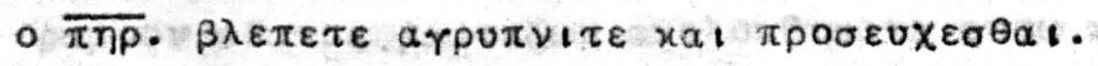

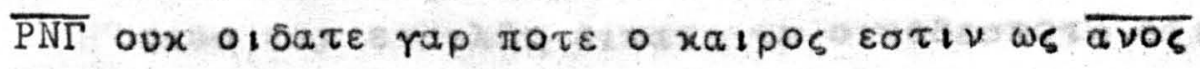

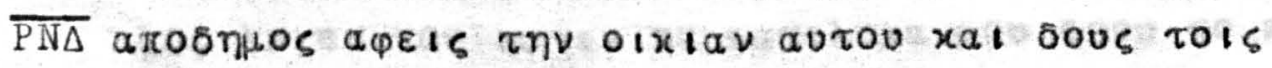

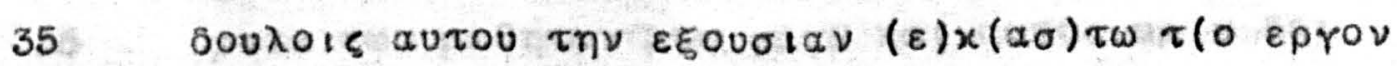

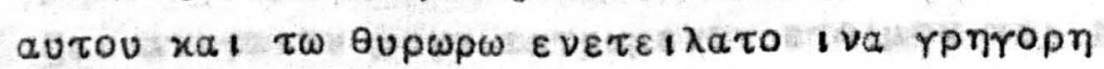

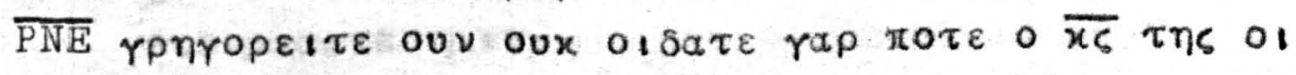

xıas $\varepsilon \rho X \varepsilon \tau a ! ~ \eta$

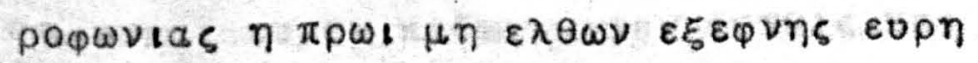

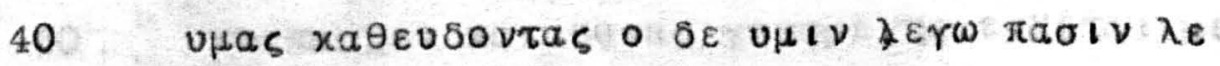

ro ronropeıtนl. 


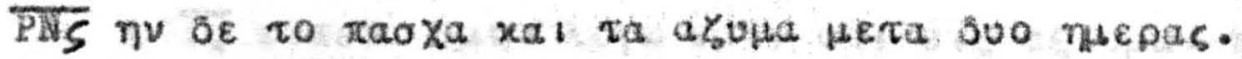

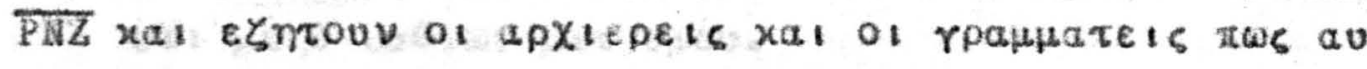

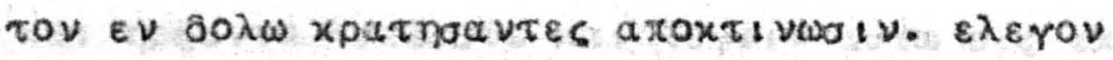

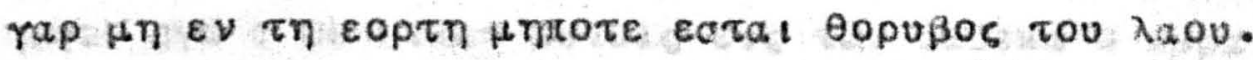

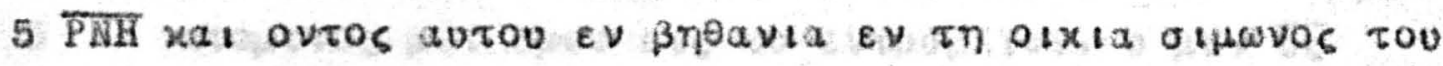
גexpou katake

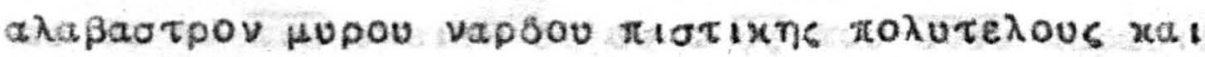

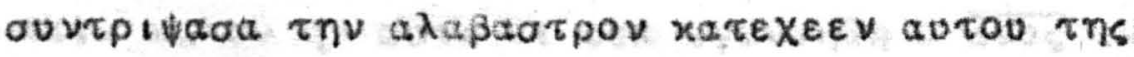

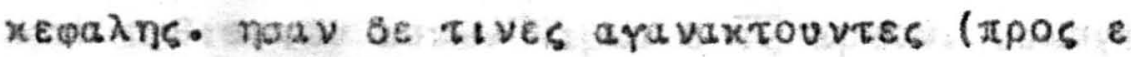

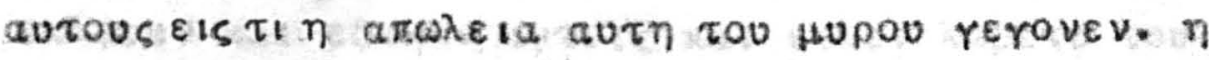

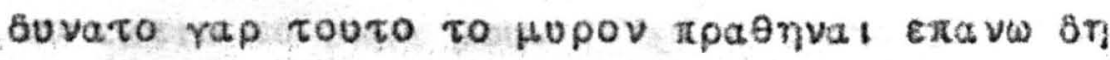

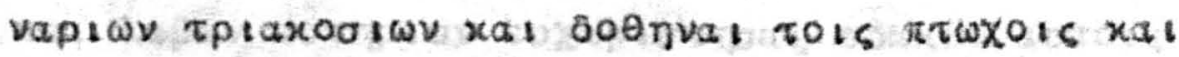

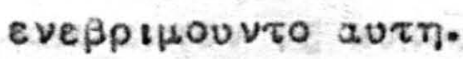

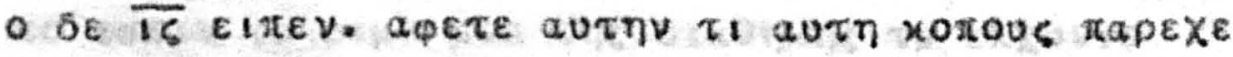

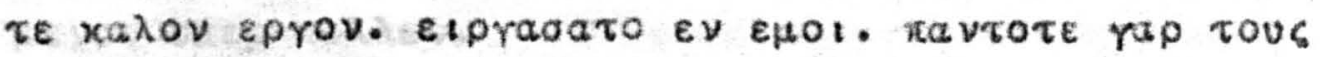

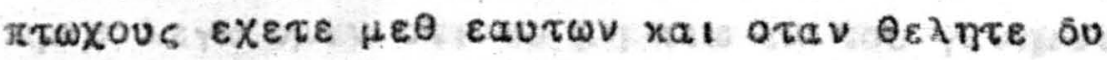

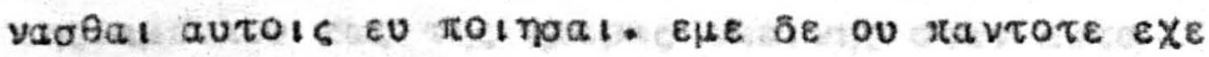

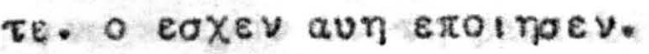

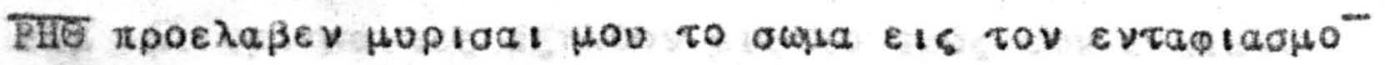

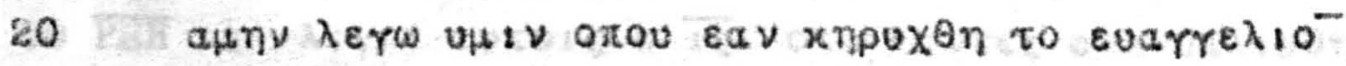

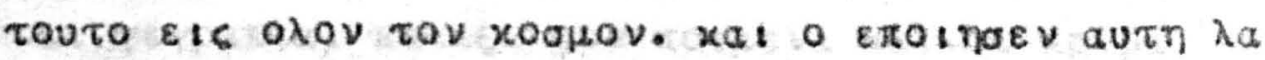

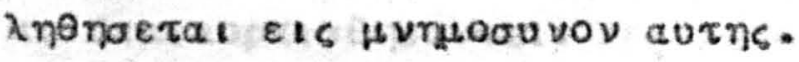

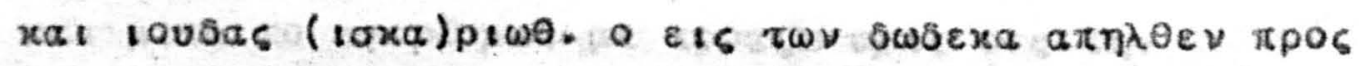
Tous apXiepeis tva autov tapasw autois.

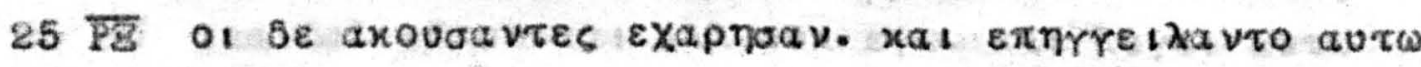

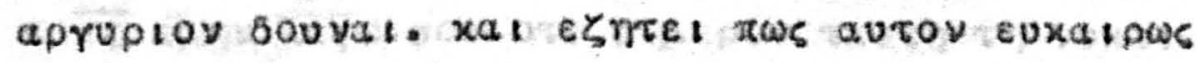

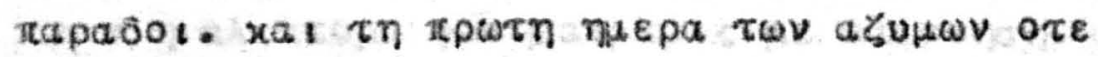

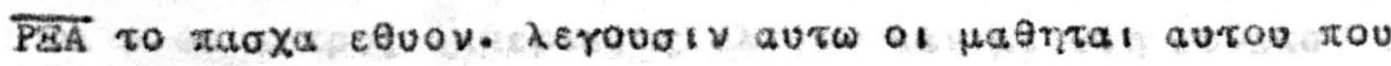

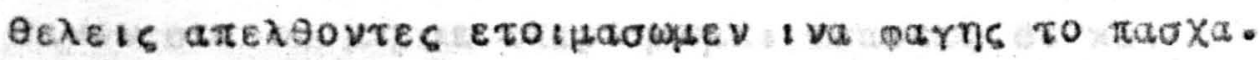

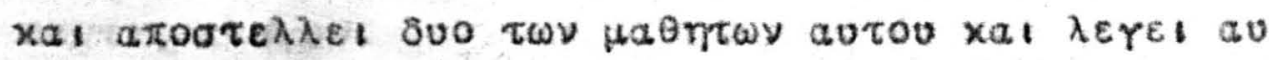

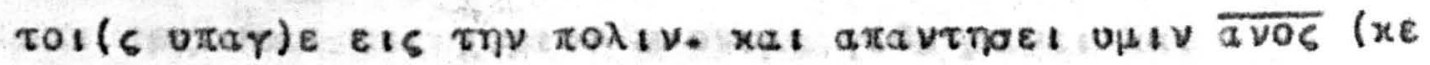

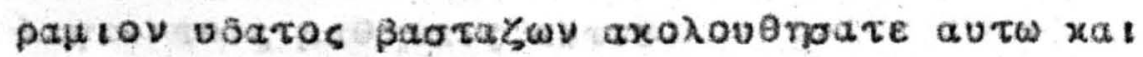

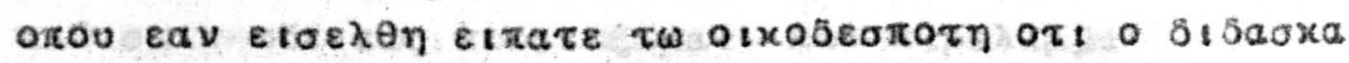

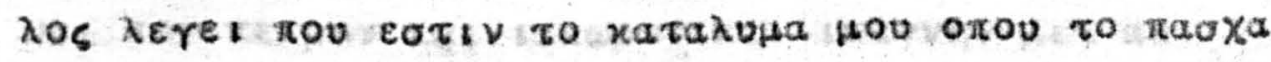

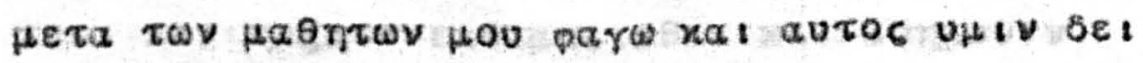
Set avaratov hera eatpusevov erothov xal exe! ecoupaaare nutv.

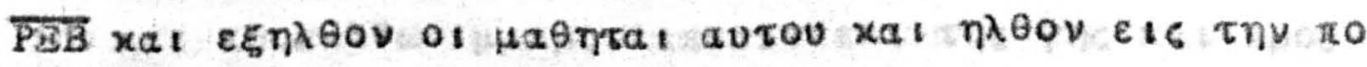

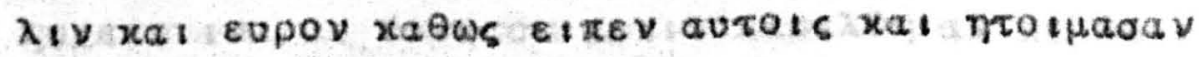

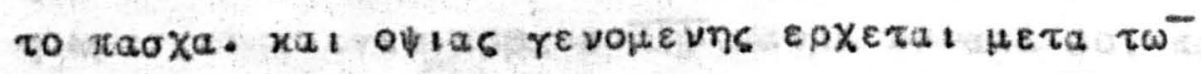

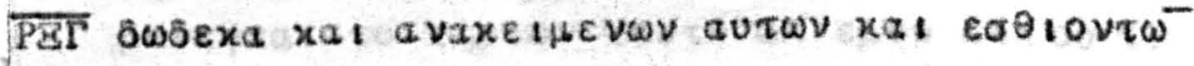




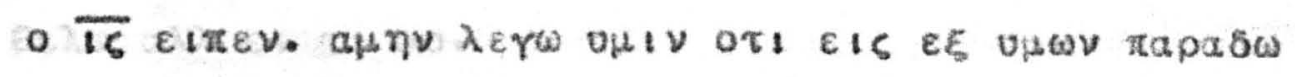
oथ $\mu \varepsilon$ o $\varepsilon \sigma \theta \imath \omega \nu \mu \varepsilon \tau \varepsilon \mu o v$.

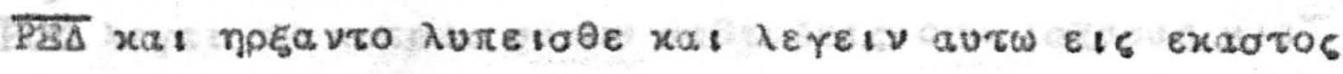

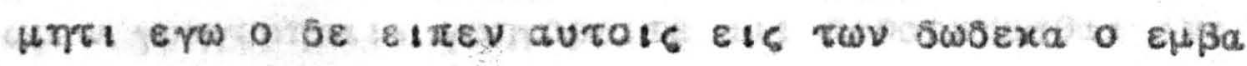

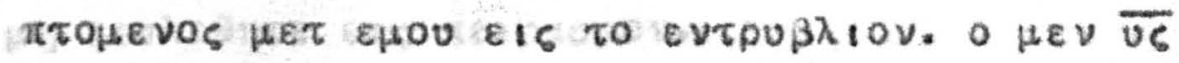

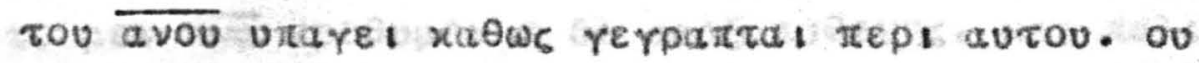

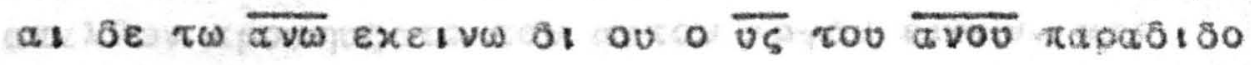

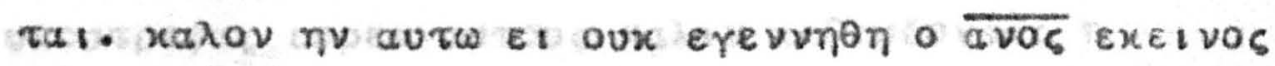

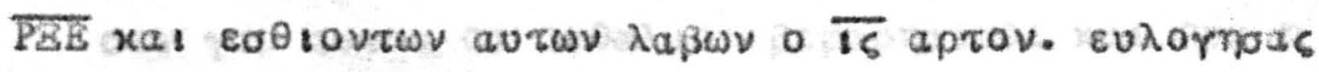

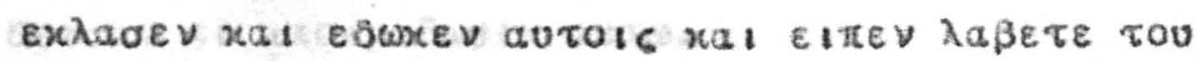

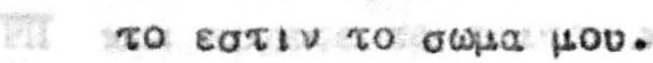

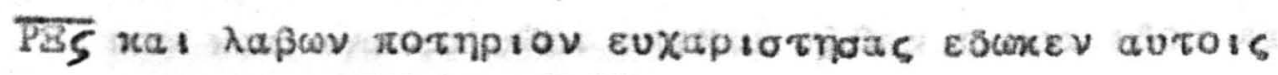

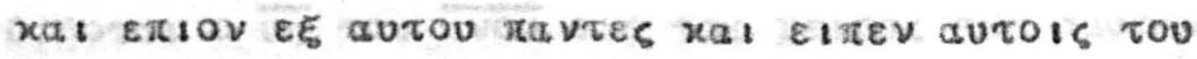

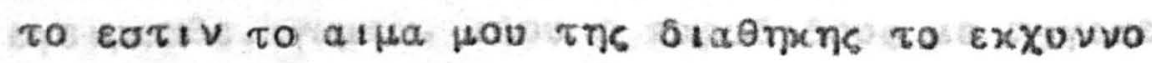

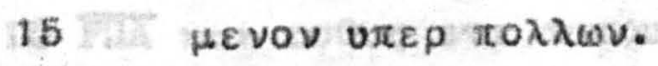

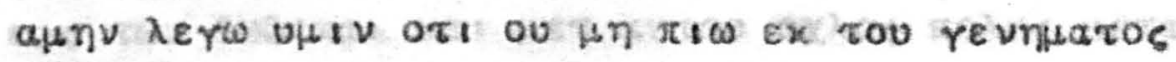

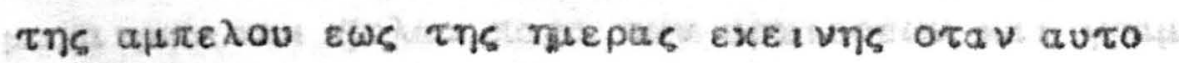

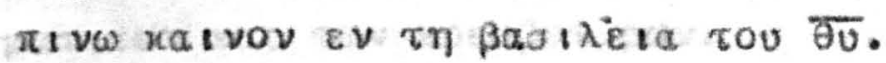

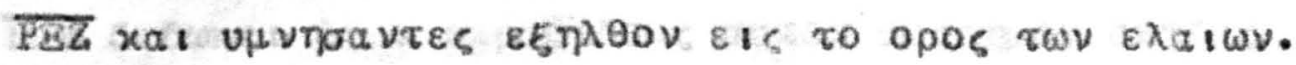

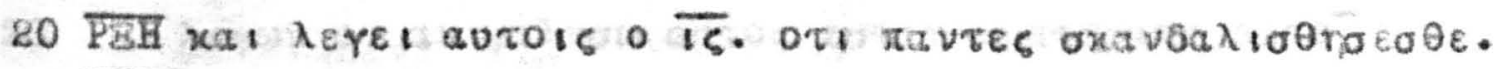

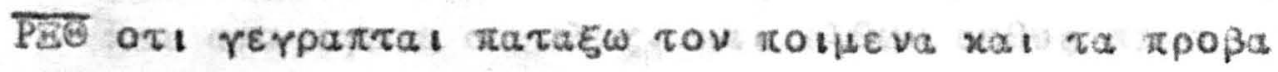

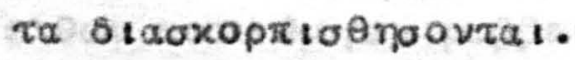

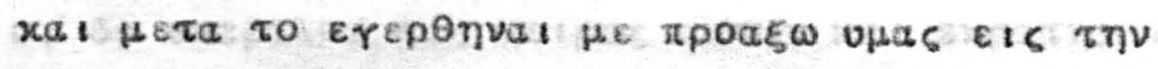

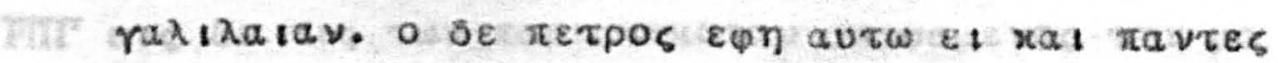

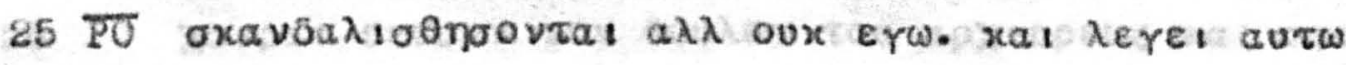

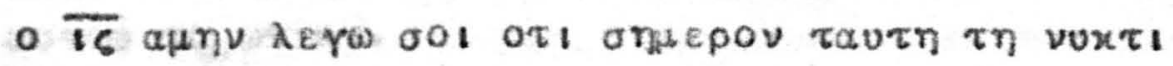

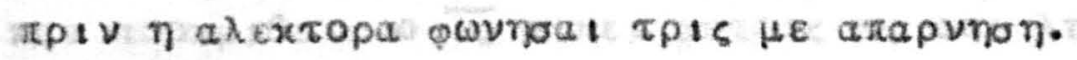

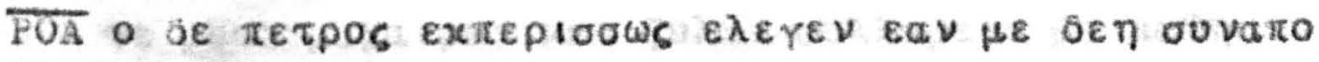

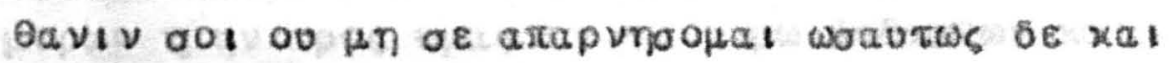

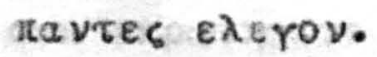

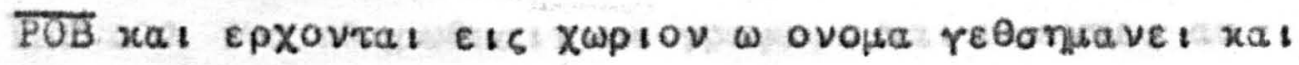

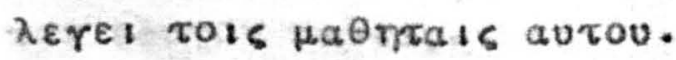

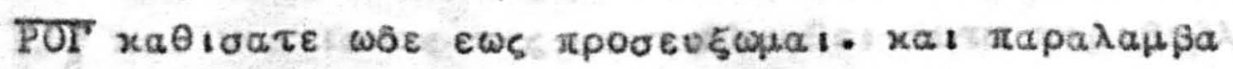

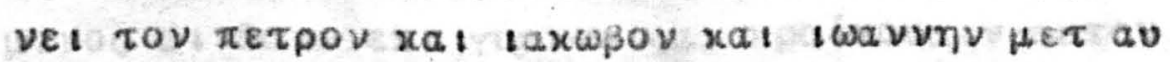

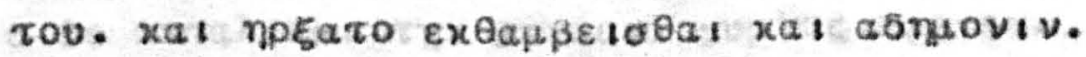

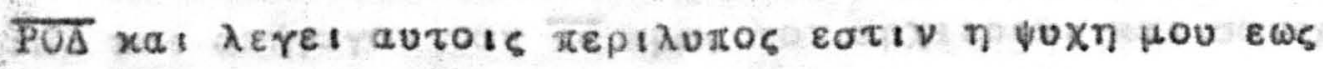

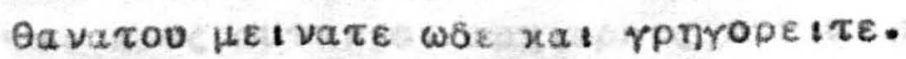

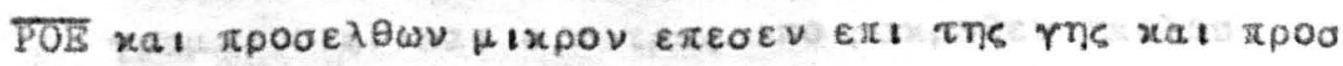

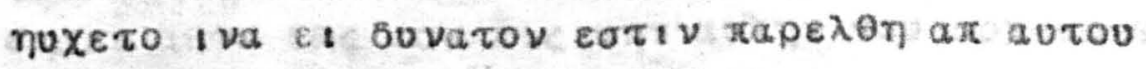

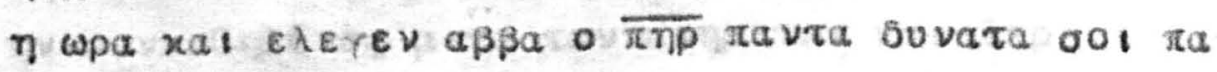

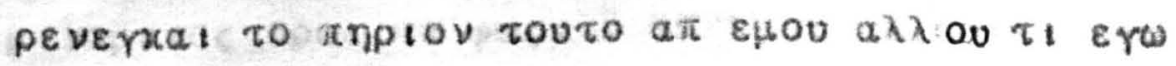


$\theta \varepsilon \lambda \omega$ a $a \lambda \quad \varepsilon \downarrow \tau_{l} \quad \sigma u$.

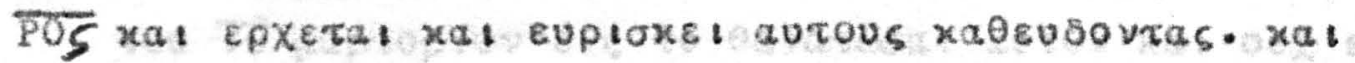

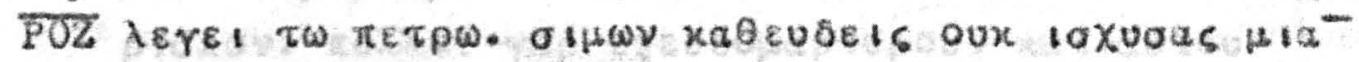

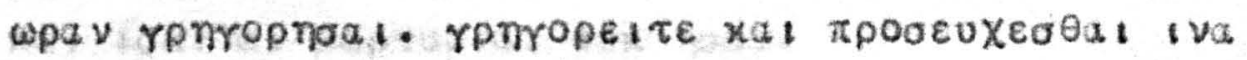

5

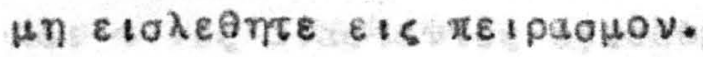

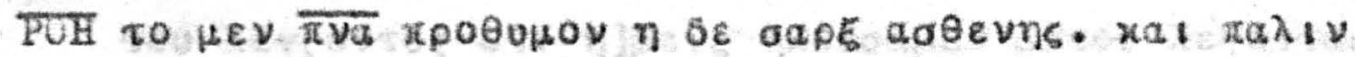

POE aic

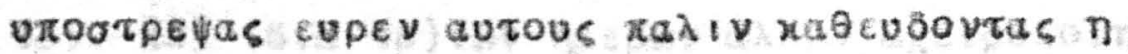

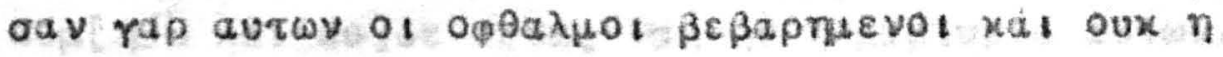

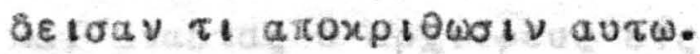

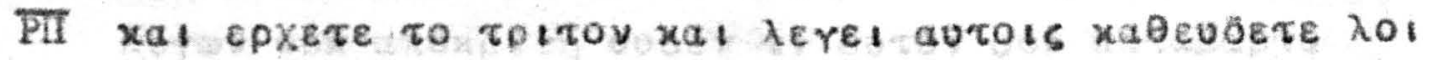

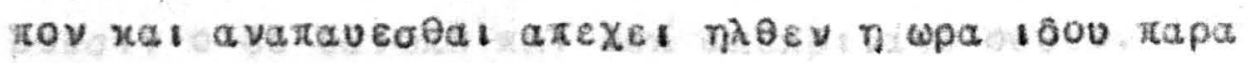

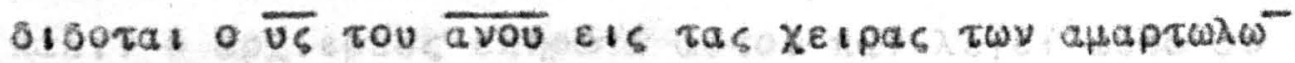

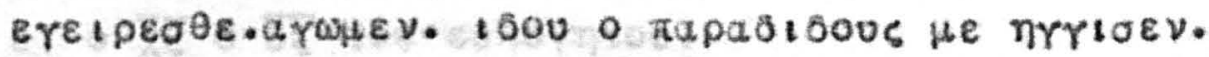

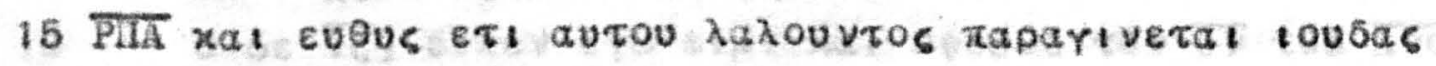

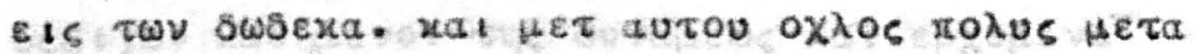

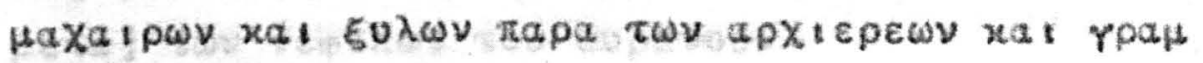

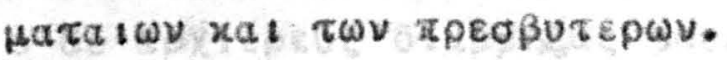

PIB sebwne 1 of o rapastoovs autov avoaruov autots

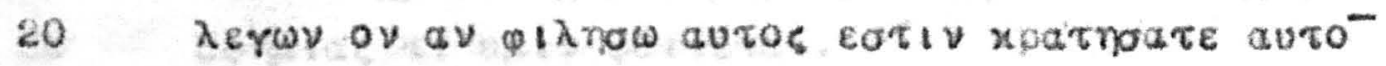

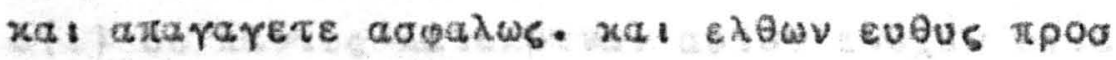

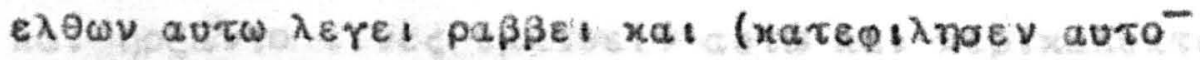

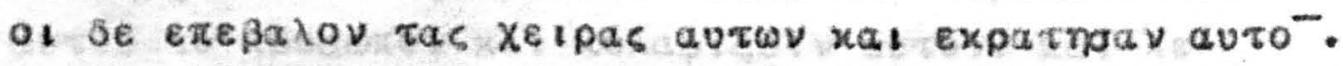

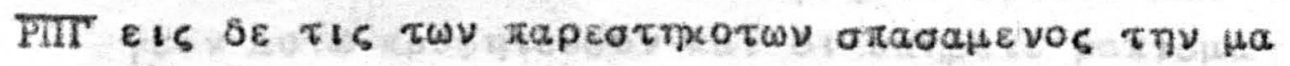

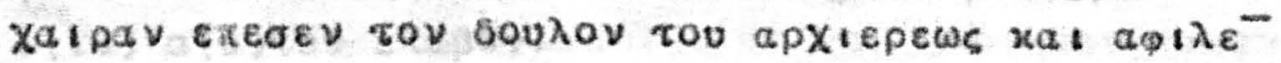
autou to cotiov.

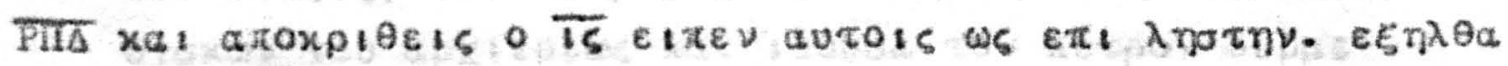

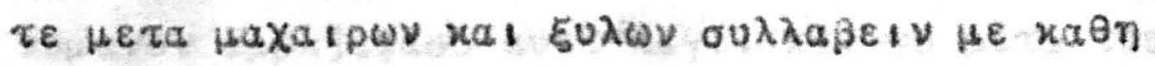

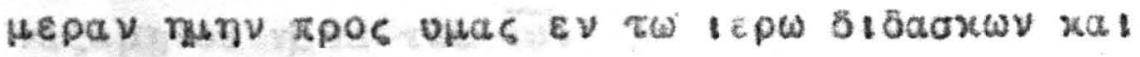

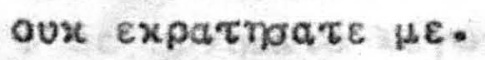

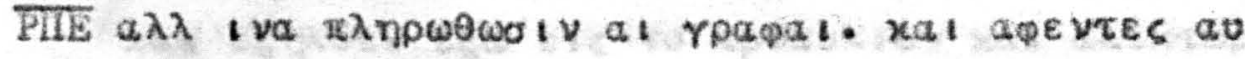

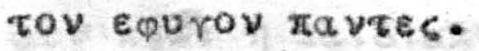

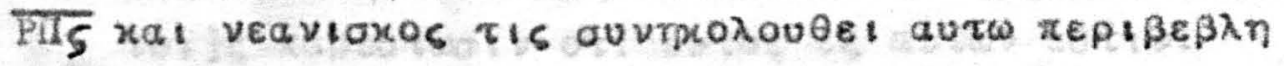

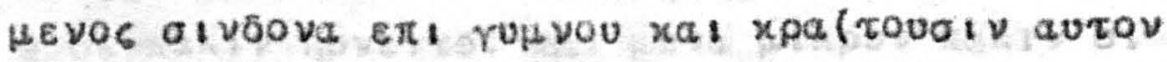

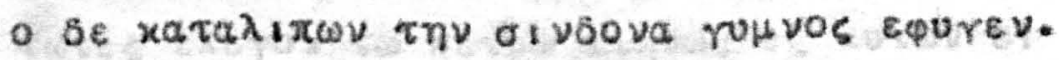

FIz xal arnrarov tov TV Ipos tov apXiepea. xal ouvepxo-

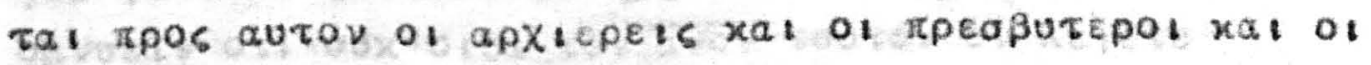

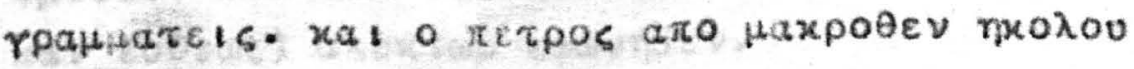

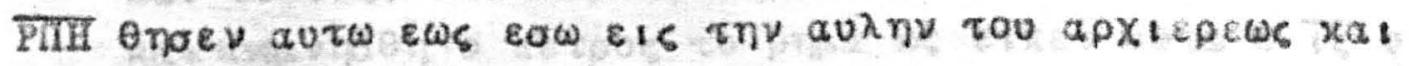

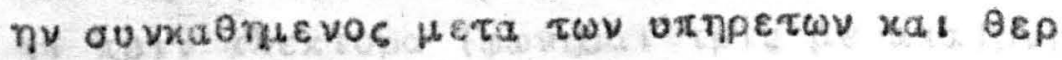

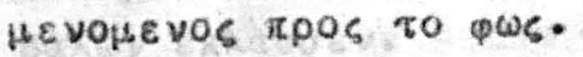




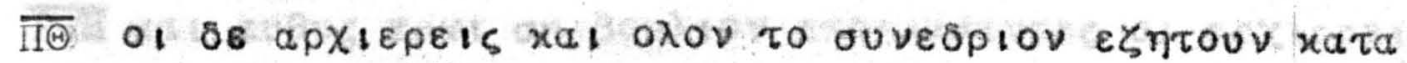

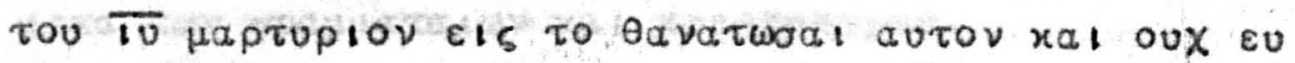

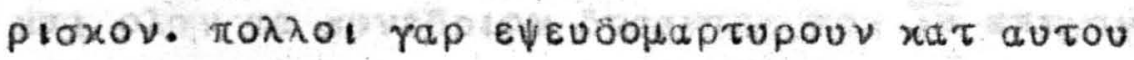
xat toal al japtuptat oux noav.

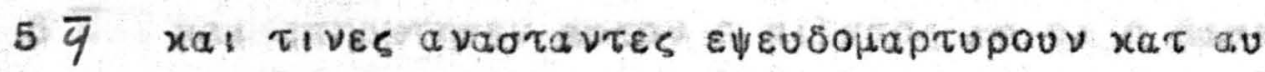

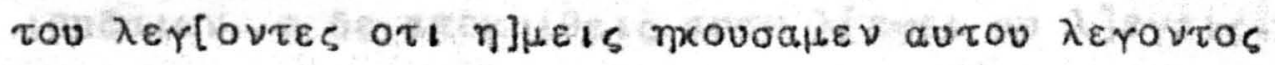

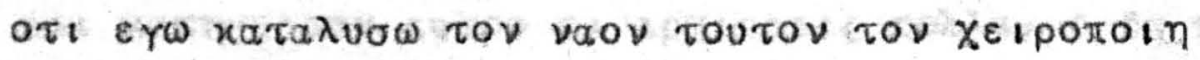

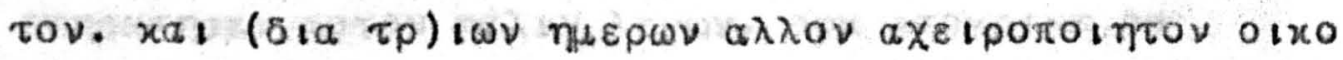

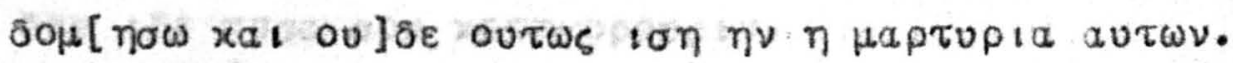

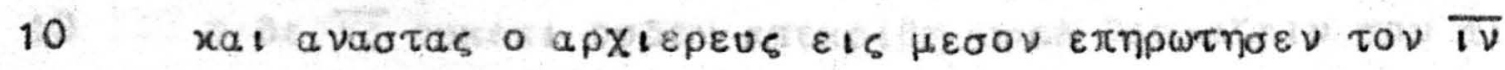

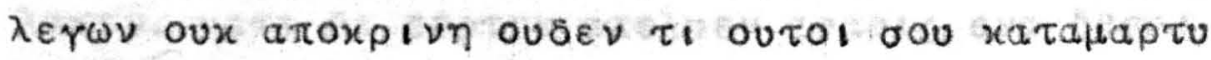

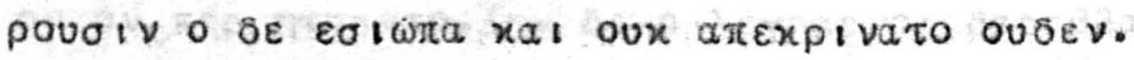

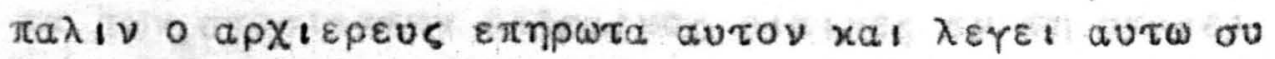

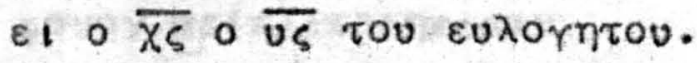

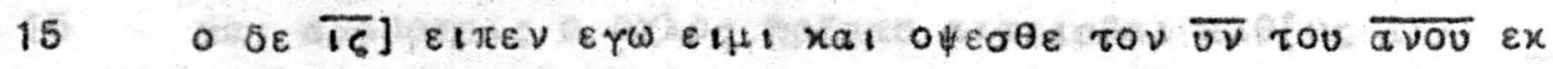

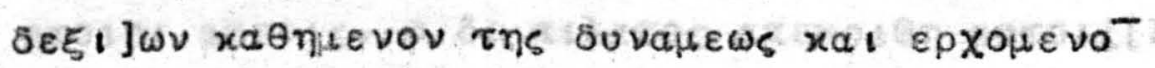

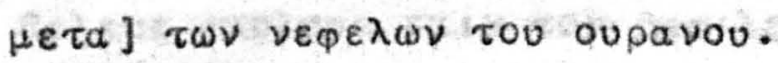

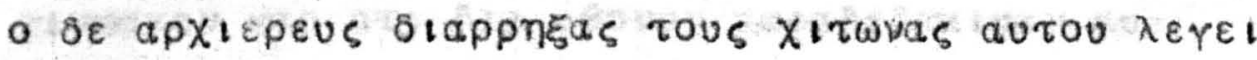

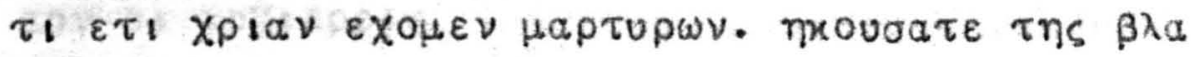

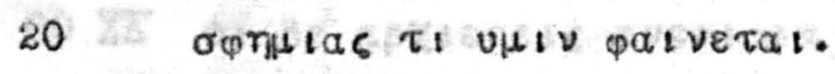

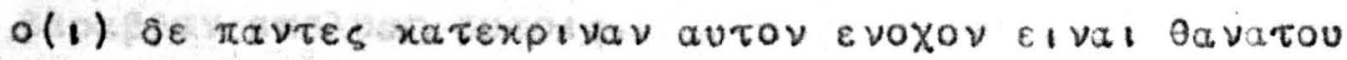

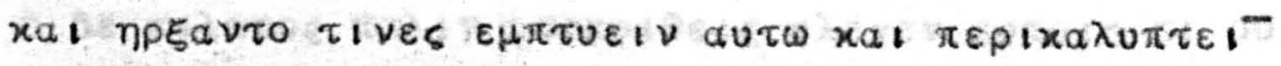

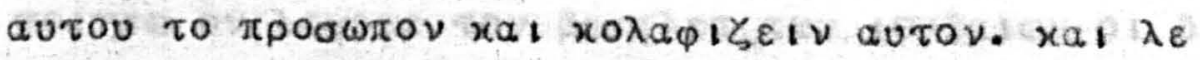

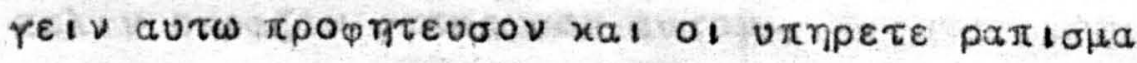

$25 \sigma(t v) a(u) \tau o v$ exaßov.

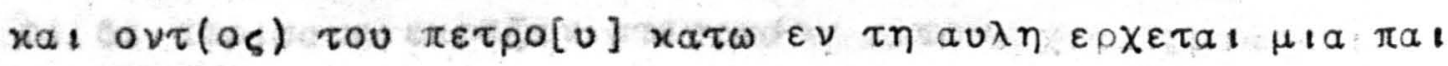

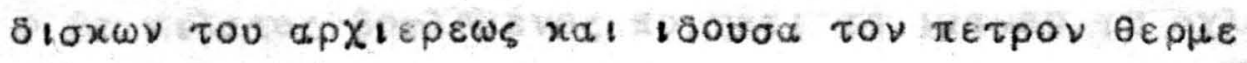

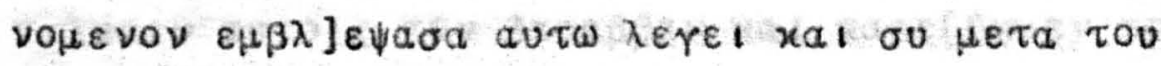

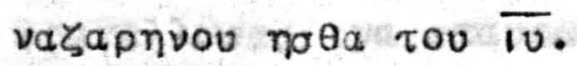

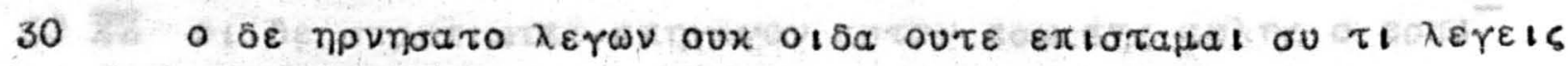

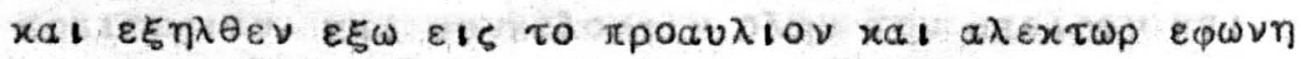

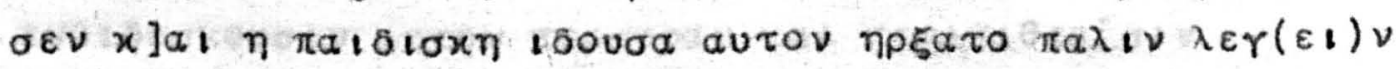

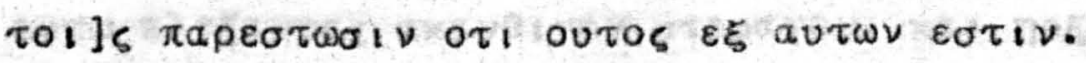

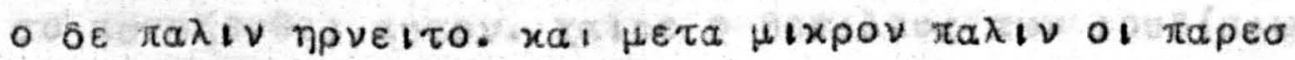

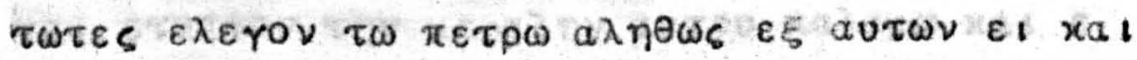

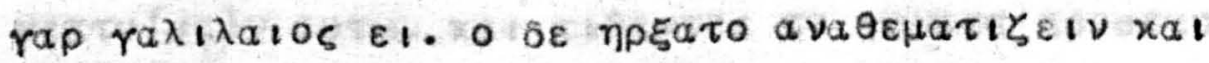

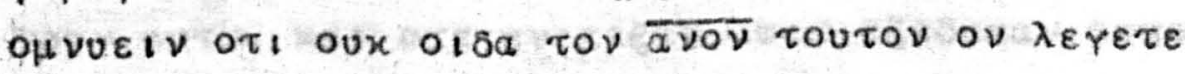
xal $\varepsilon v \theta \varepsilon \omega \varsigma) ~ a \lambda \varepsilon x \tau \omega \rho \varepsilon \varphi \omega \nu \eta \sigma \varepsilon \nu$.

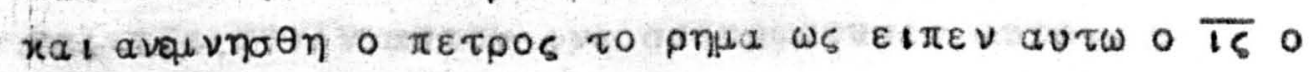

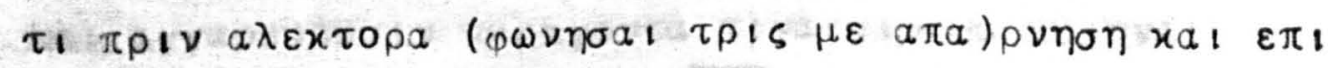

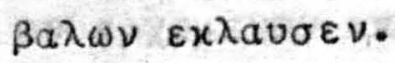




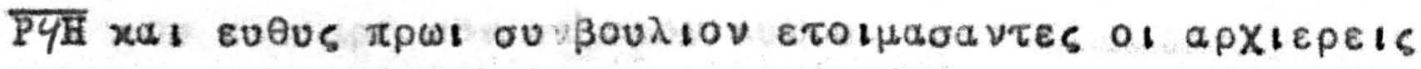

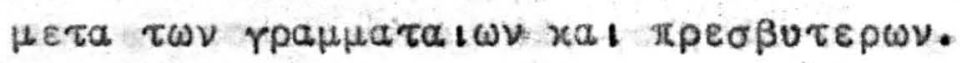

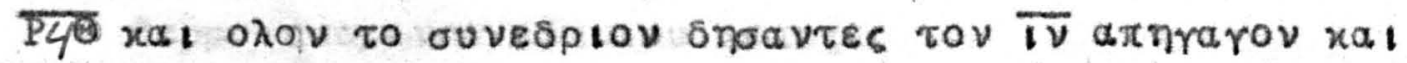
$\pi \alpha \rho \varepsilon \delta \omega x a v \pi i \lambda a \tau \omega$.

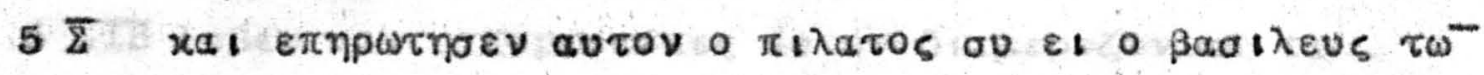

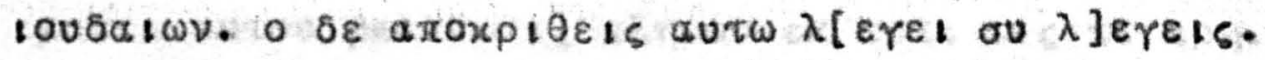

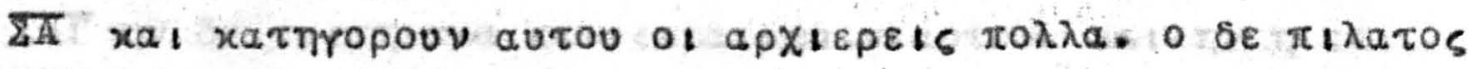

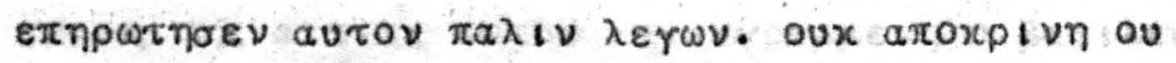

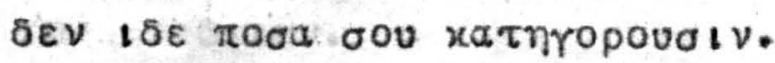

10

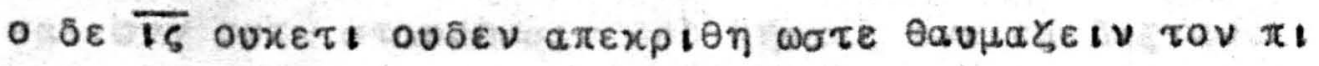

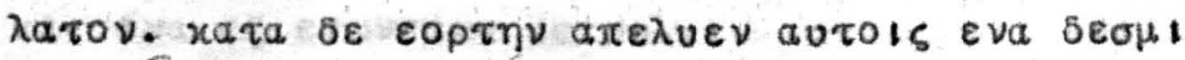

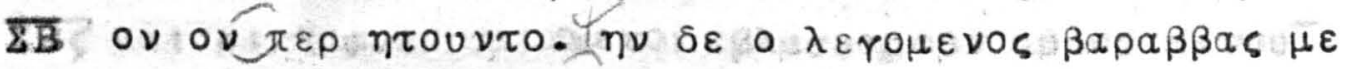

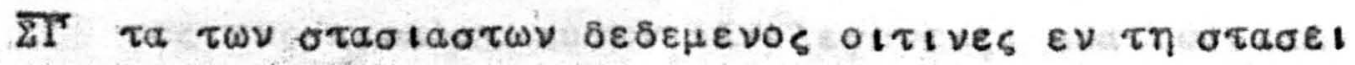

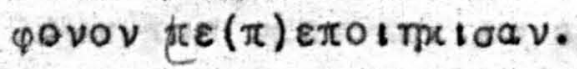

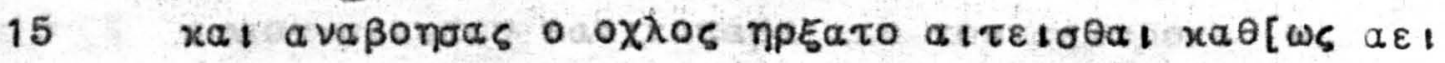

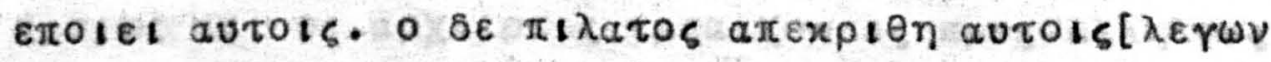

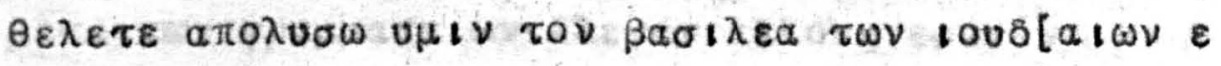

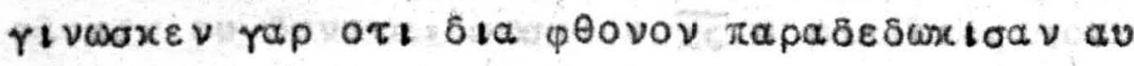

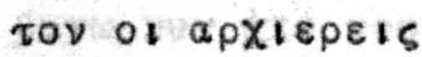

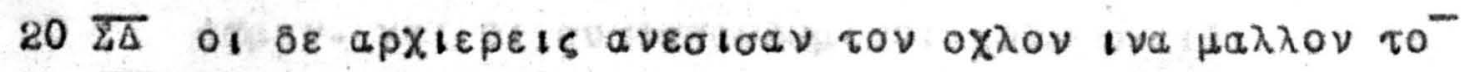

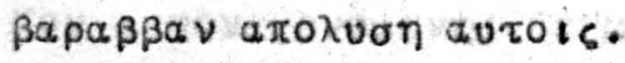

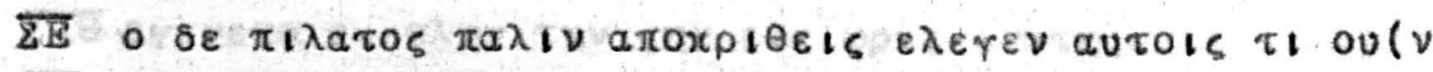

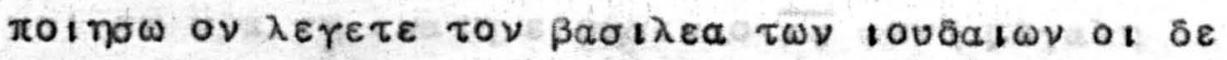

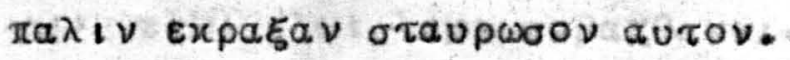

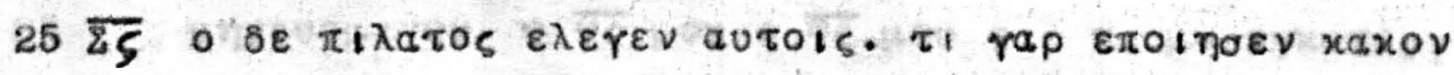

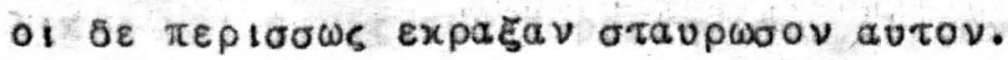

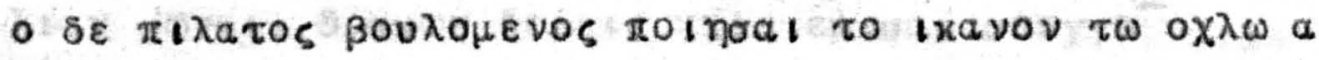

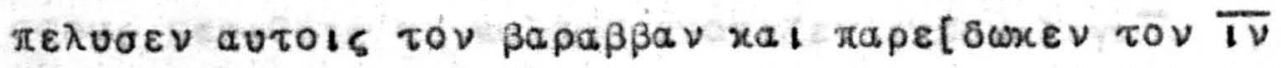

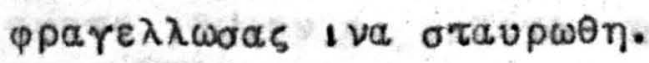

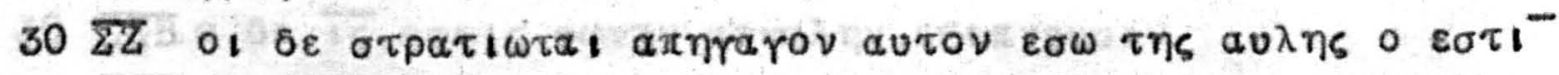

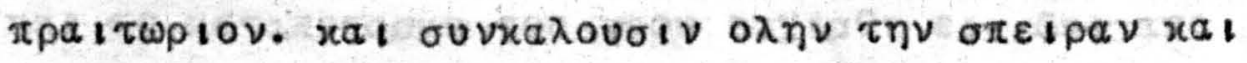

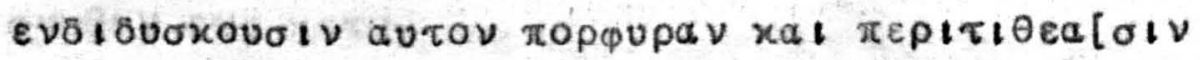

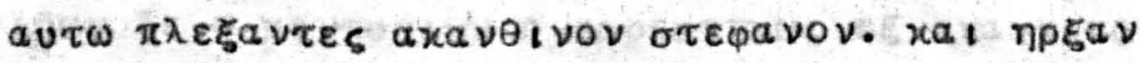

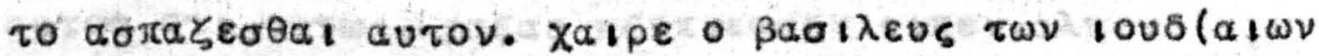

$35 x a$ «

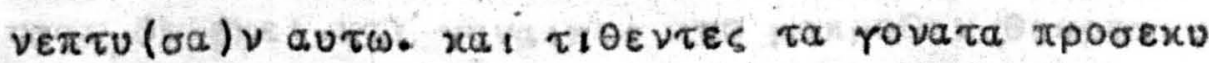

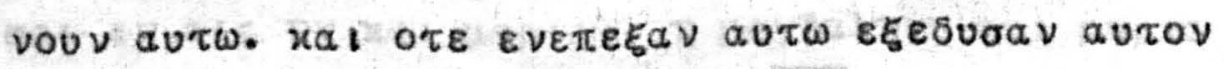

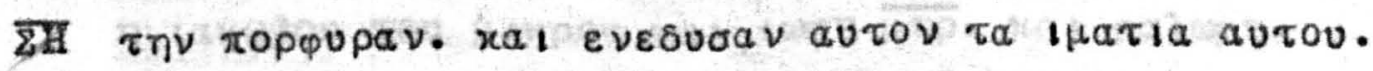

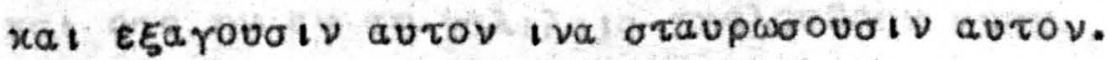

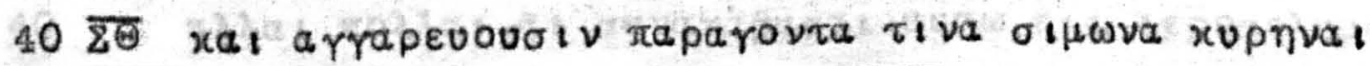

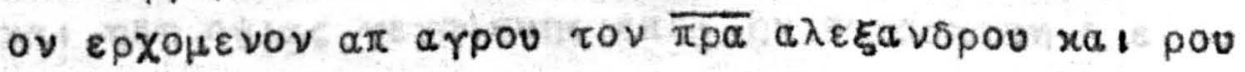




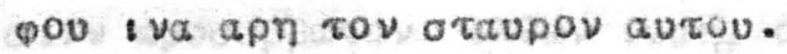

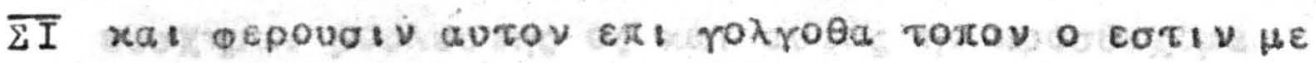

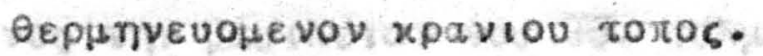

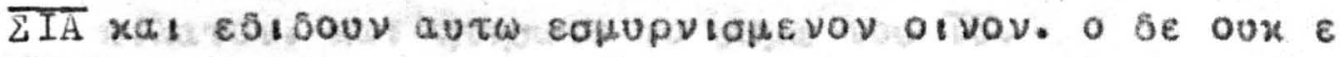

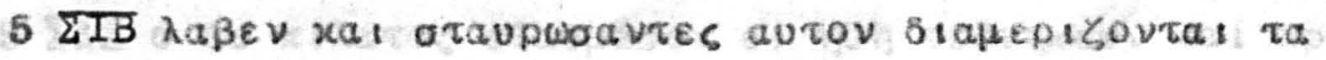

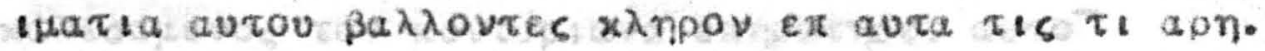

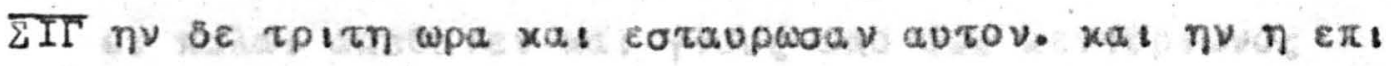

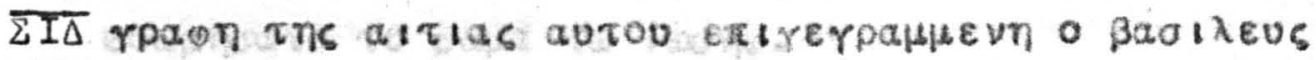
$\tau \omega \nu$ sovo̊ $1 \omega \nu$.

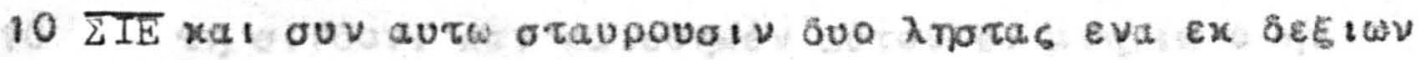

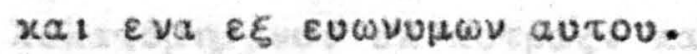

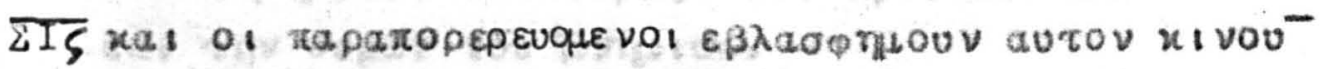

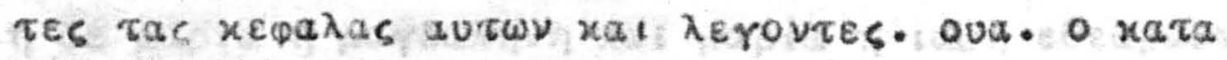

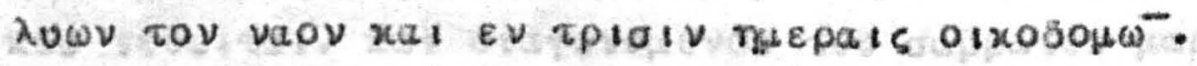

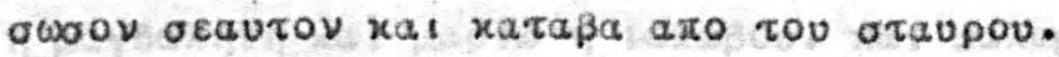

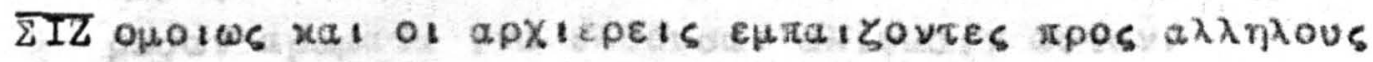

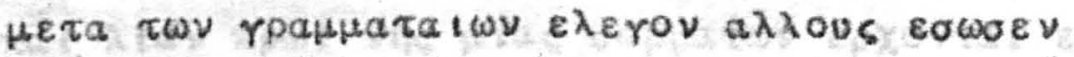

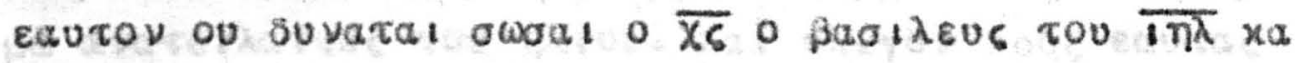

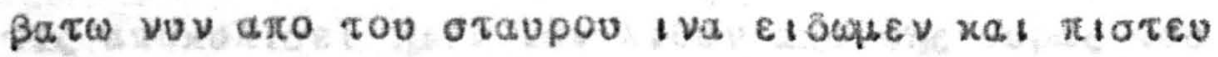

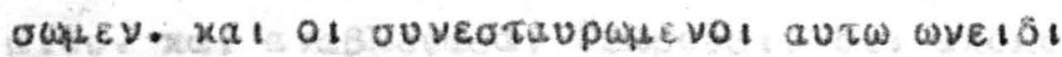

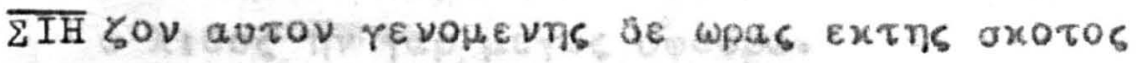

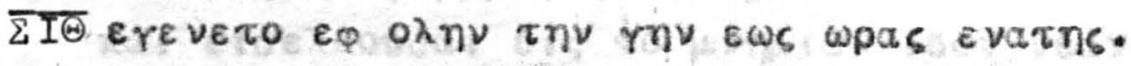

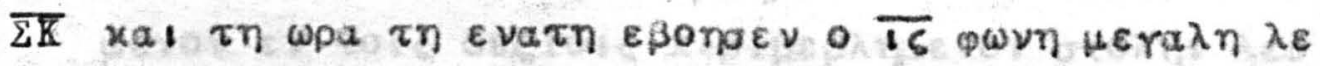

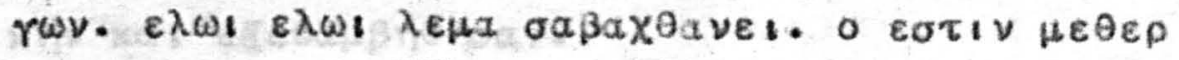

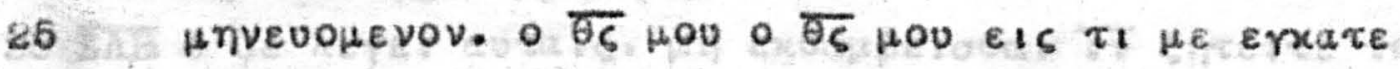

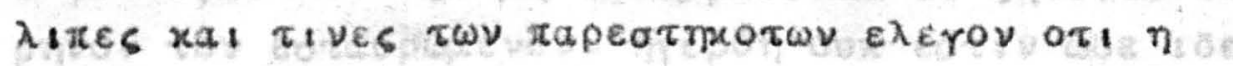

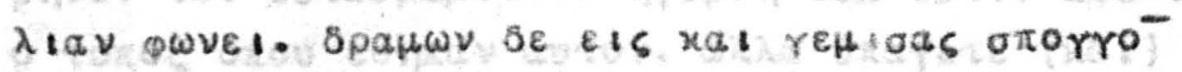

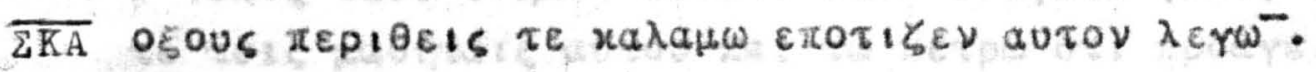

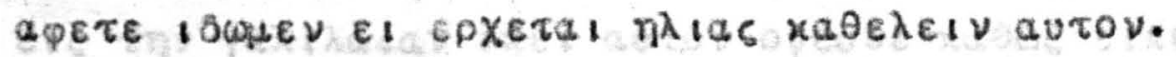

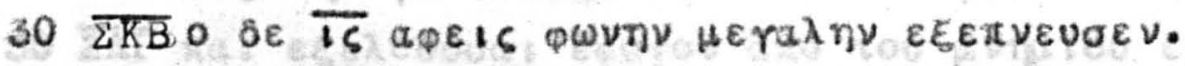

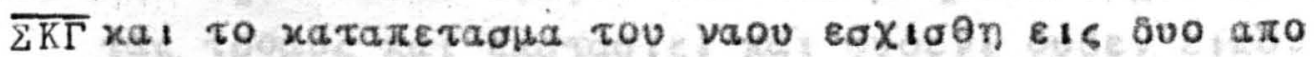
a $v \omega \theta \varepsilon \vee \varepsilon \omega \varsigma$ $x a \tau \omega$.

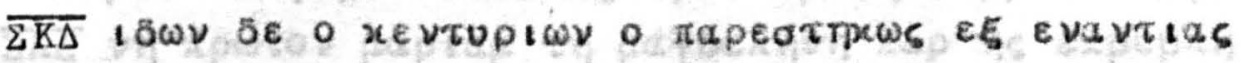

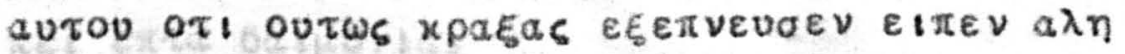

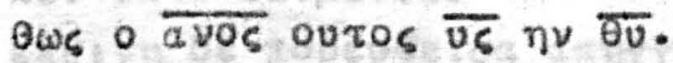

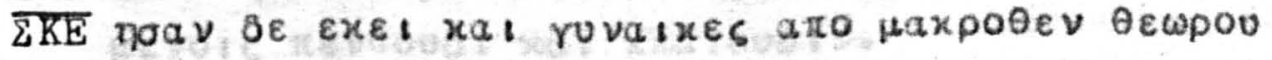

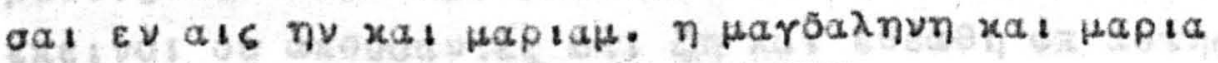

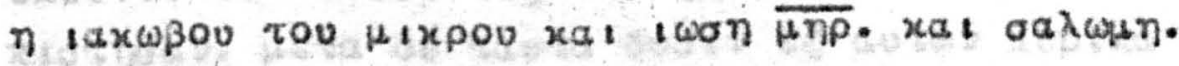

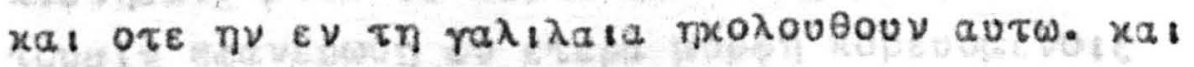

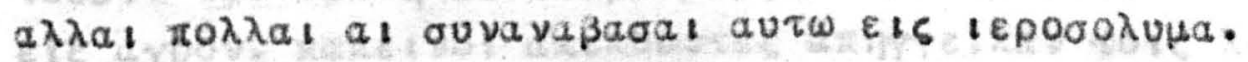

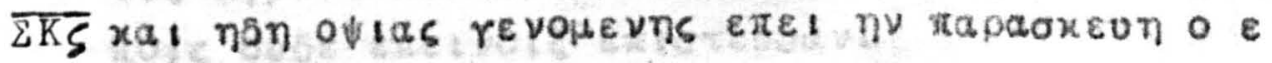




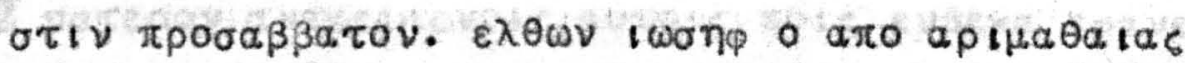

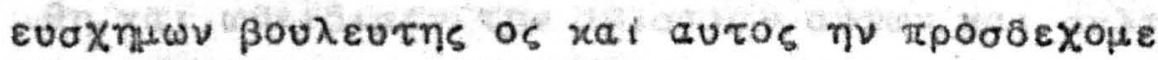

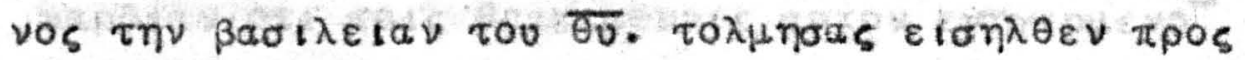

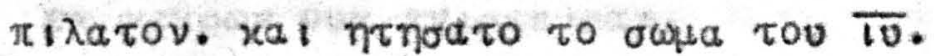

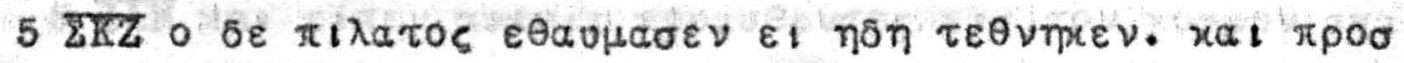

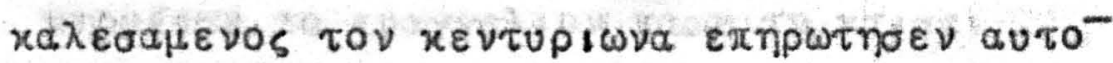
et $\pi a \lambda a$ I $a \pi \varepsilon \theta a v \varepsilon v$ xa! fvous axo tou xevtoplwos

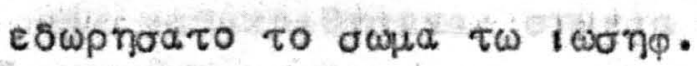

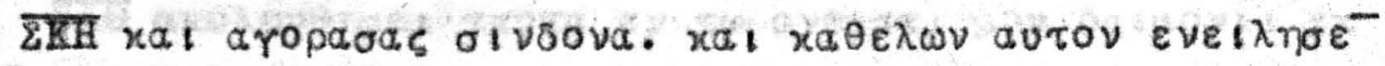
10

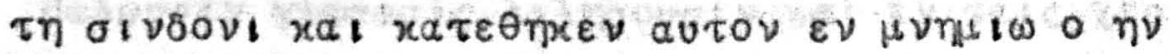

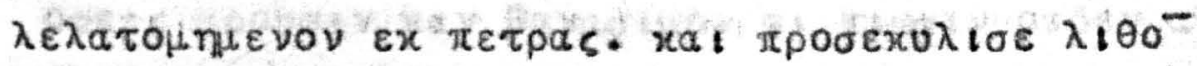

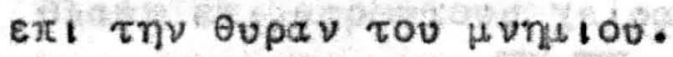

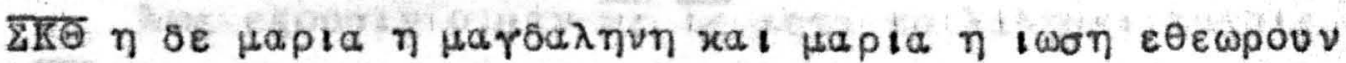

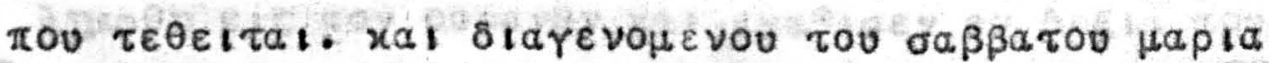

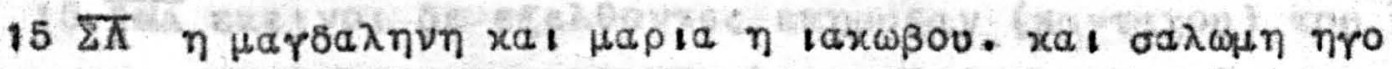

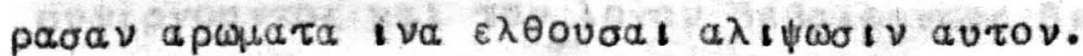

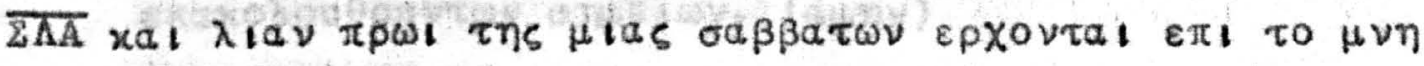

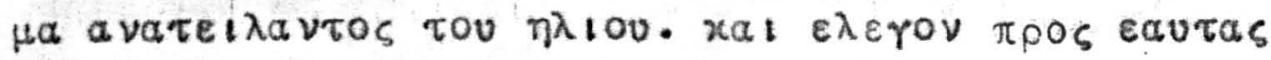

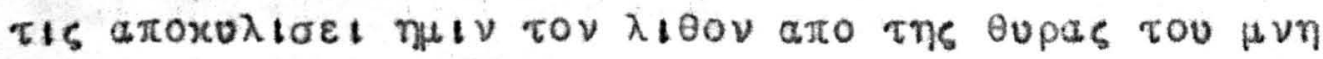

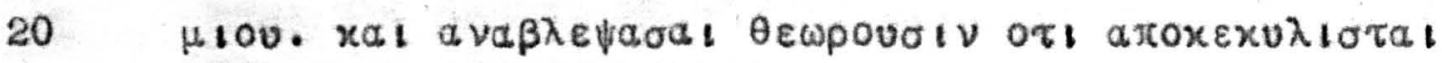

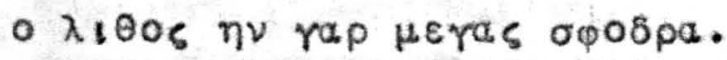

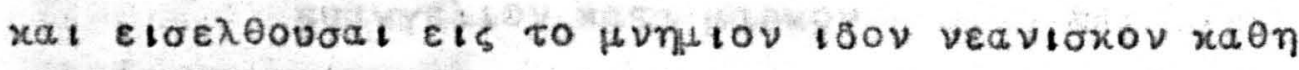

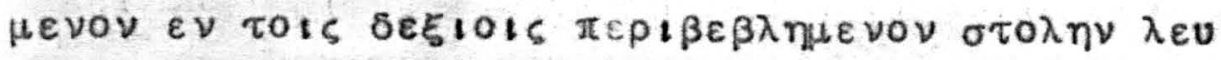

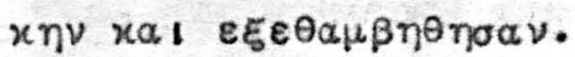

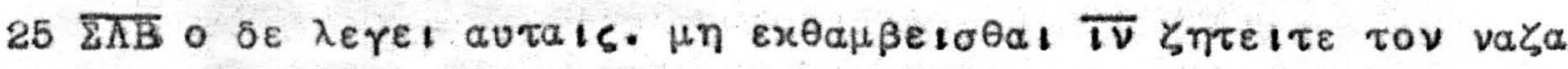

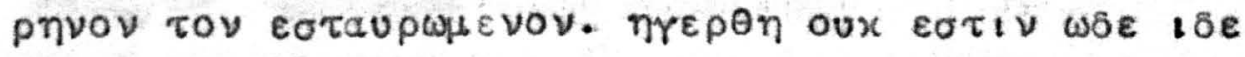

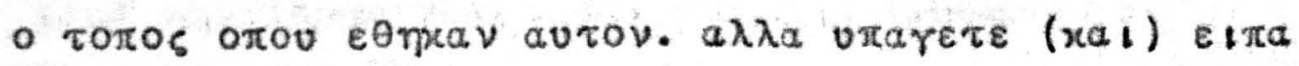

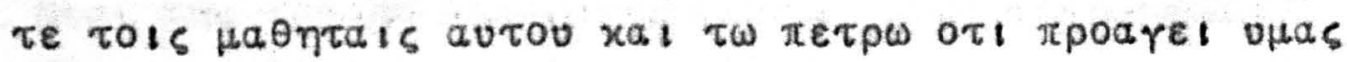

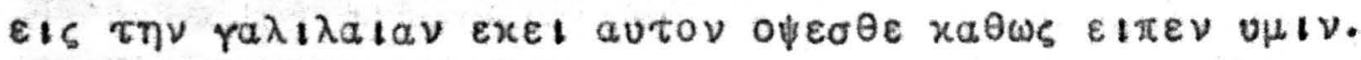

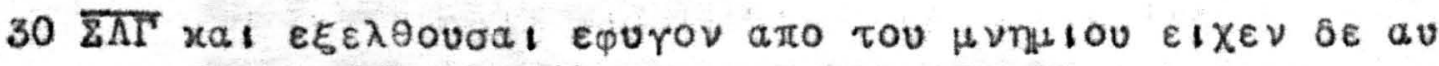

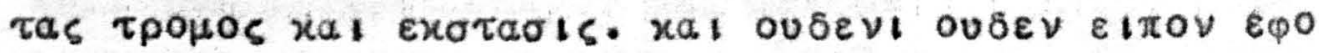

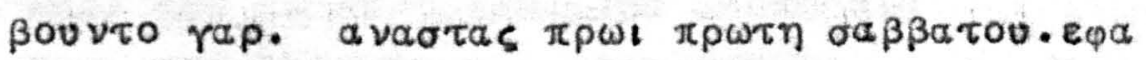

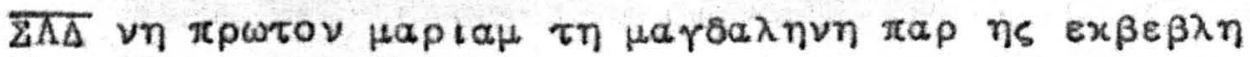

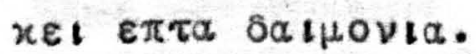

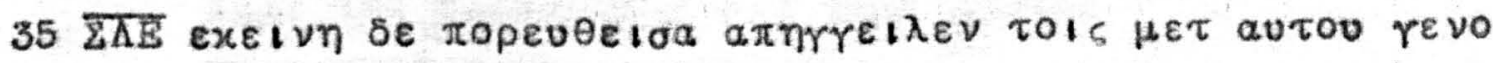

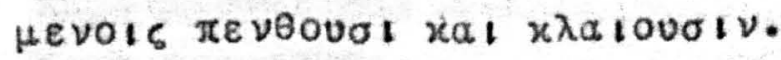

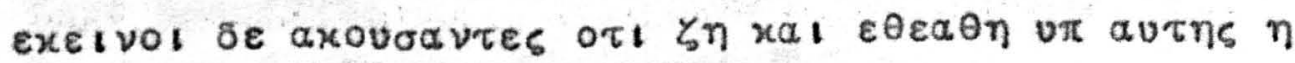

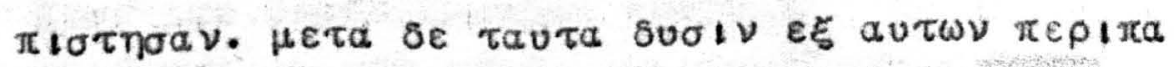

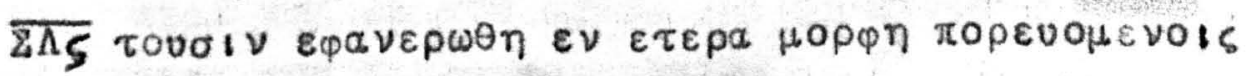

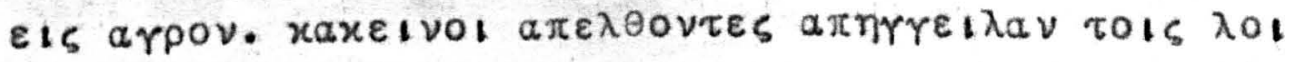

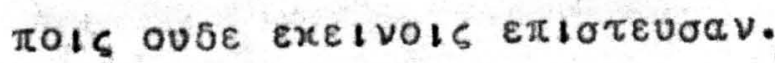




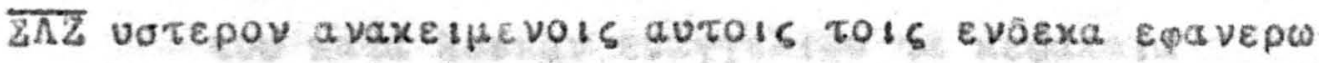

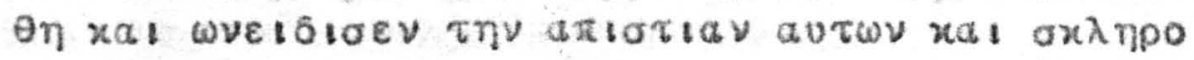

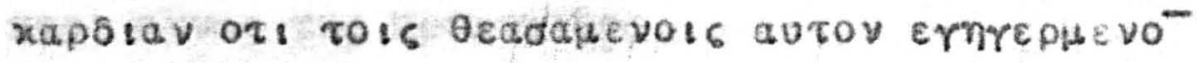

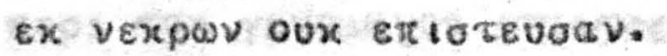

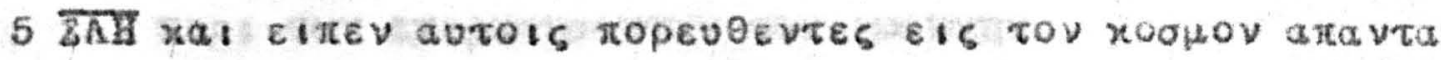

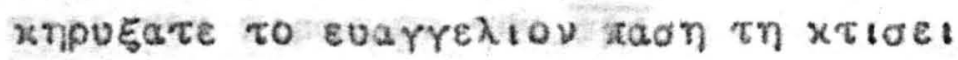

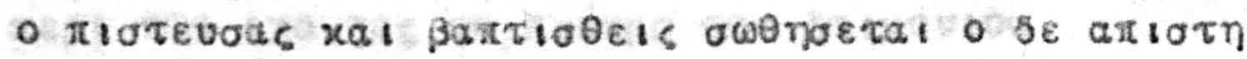

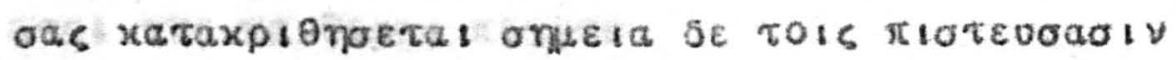

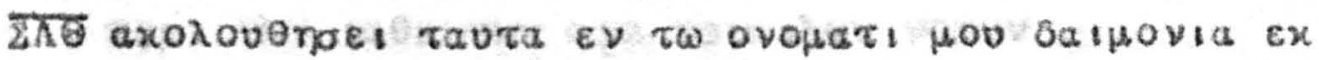

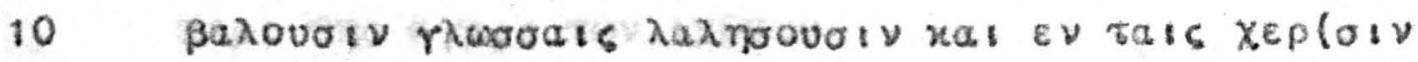

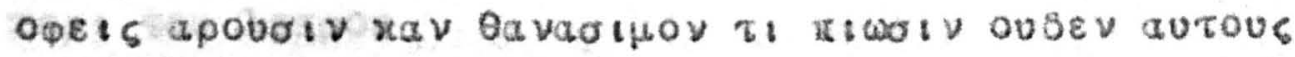

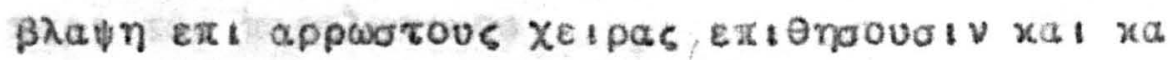

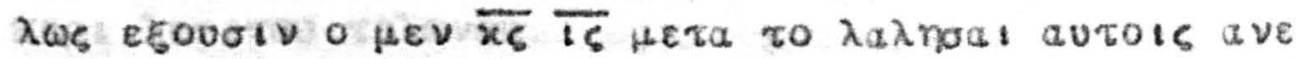
2. $\lambda$ In

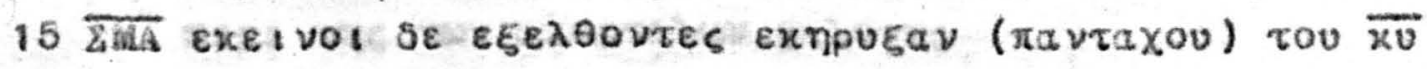

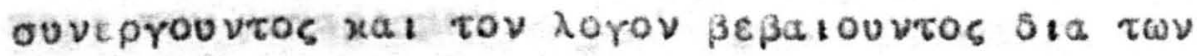

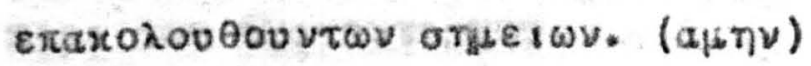

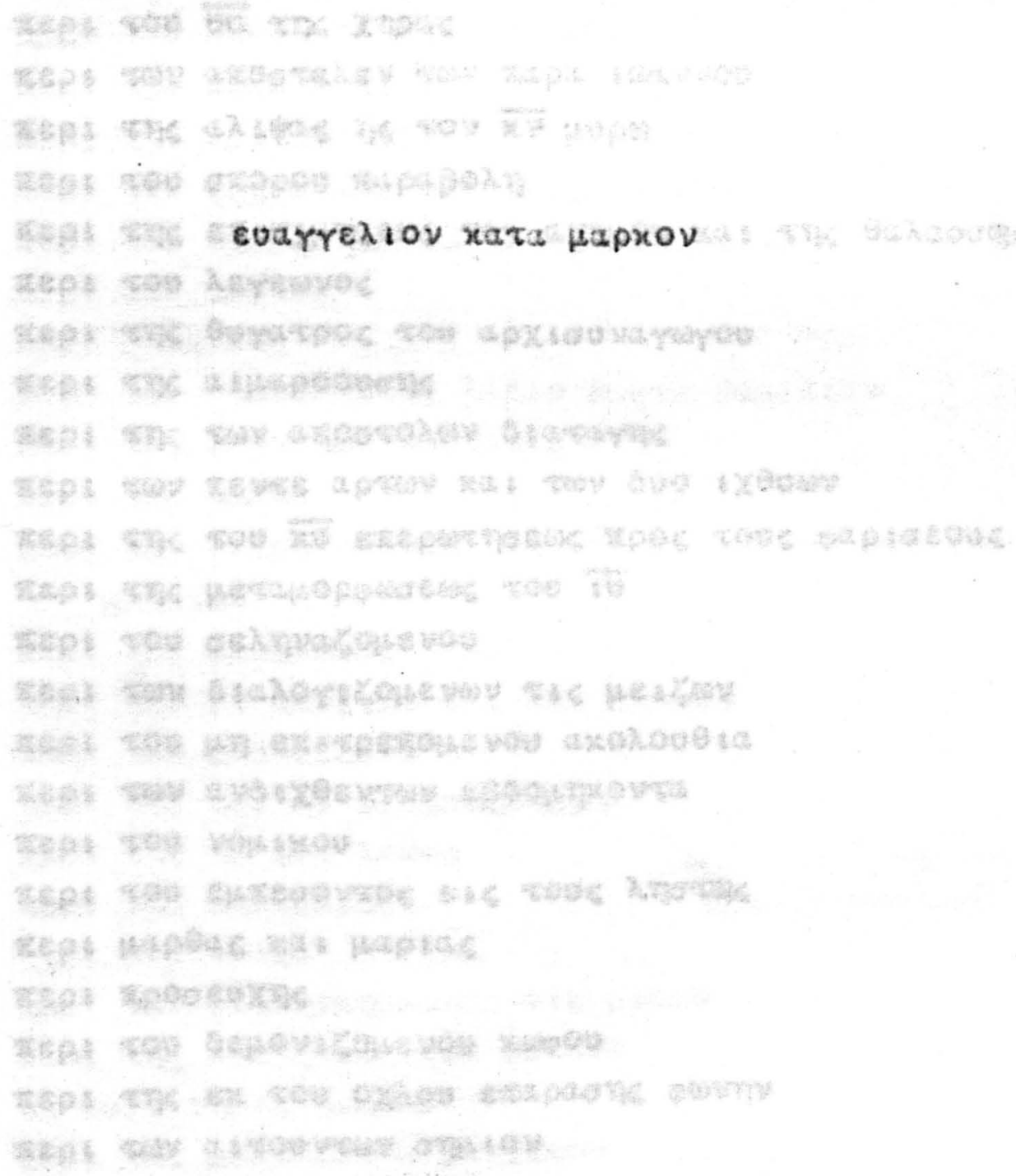




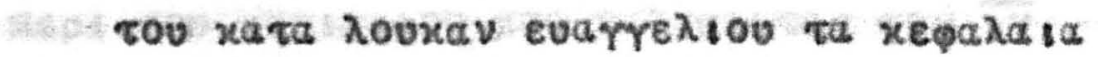

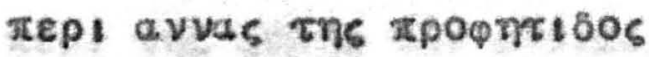

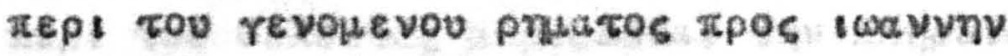

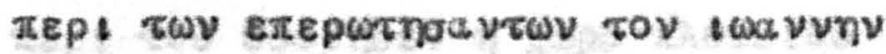

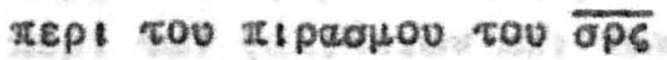

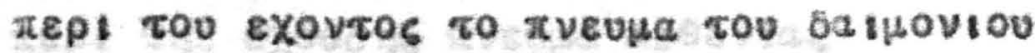

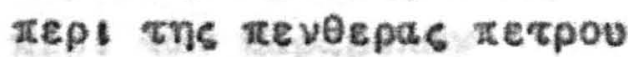

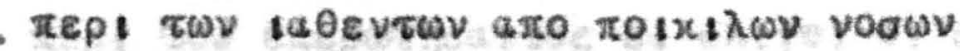

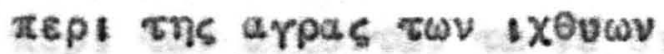

тері тоu $\lambda$ expov

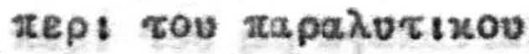

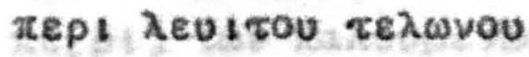

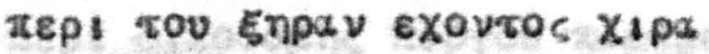

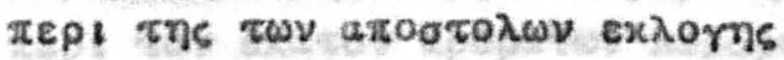

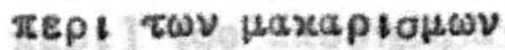

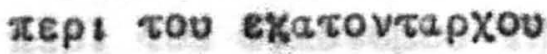

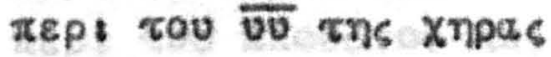

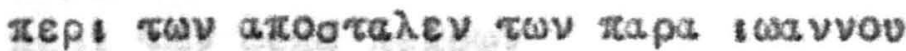

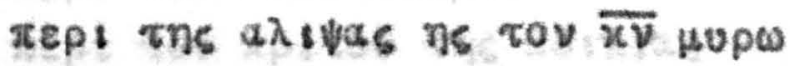

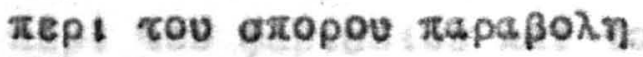

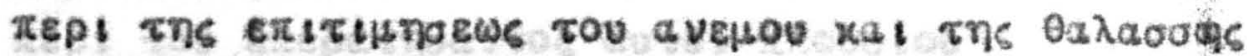

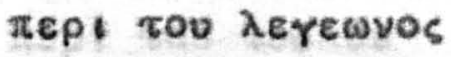

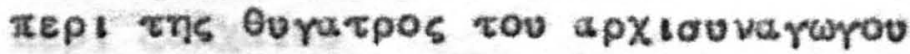

тep। тทs a нароovons

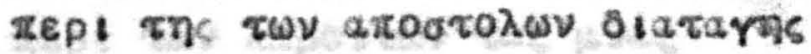

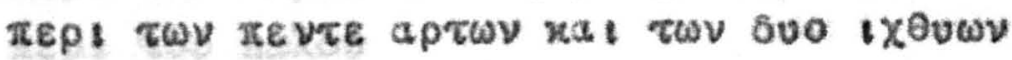

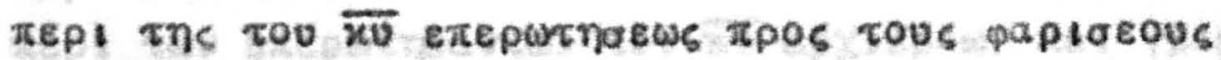

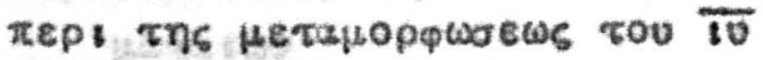

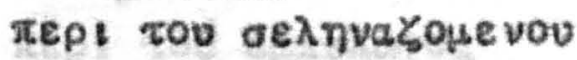

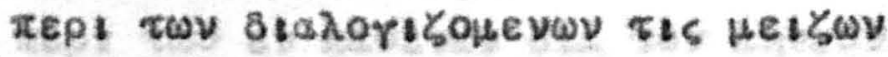

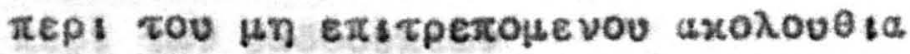

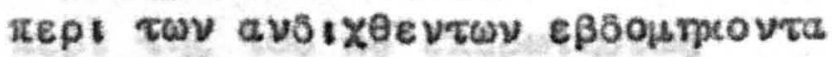

xep! то0 vouixov

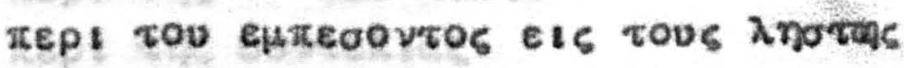

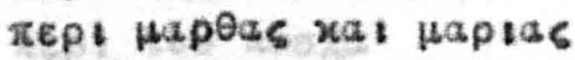

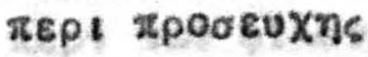

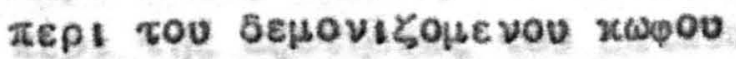

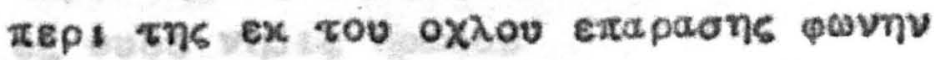

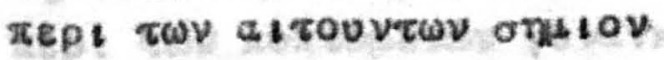




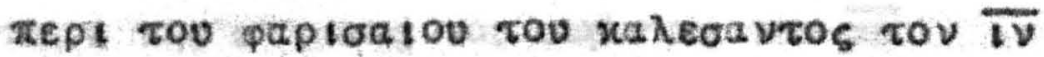

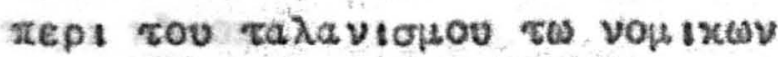

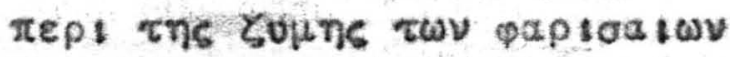

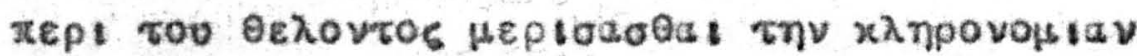

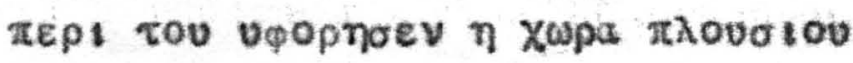

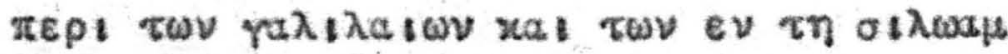

zept the exooons tveupa aooeviac

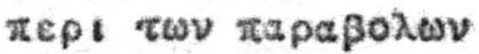

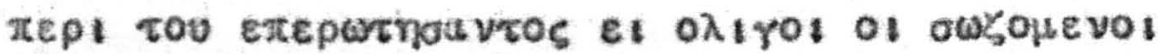

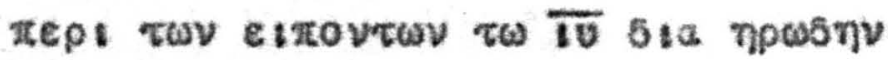

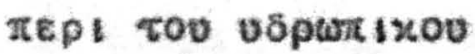

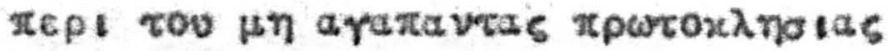

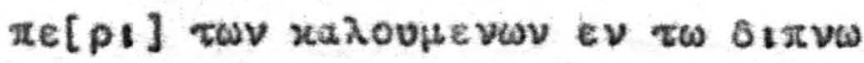

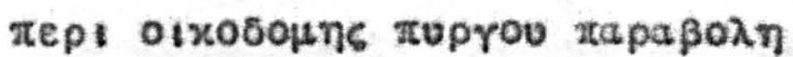

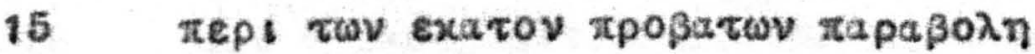

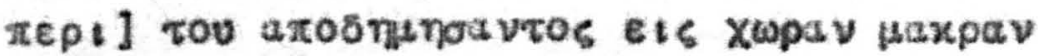

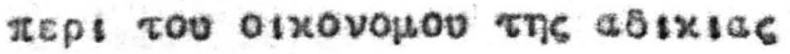

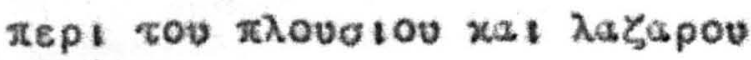

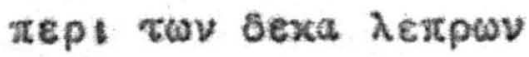

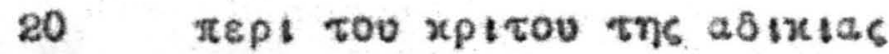

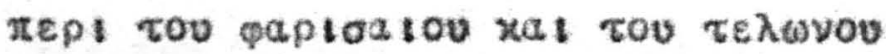

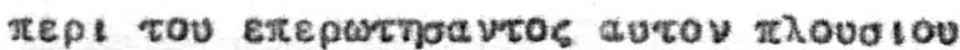

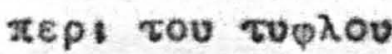

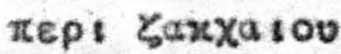

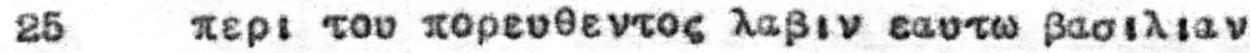

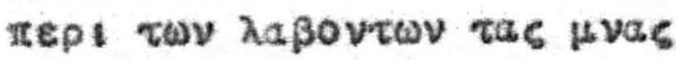

тepi rov twiou

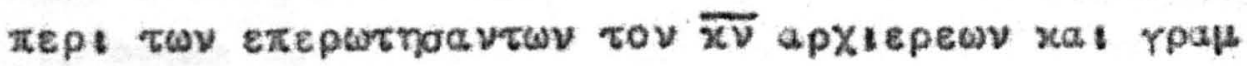
HaTa $1 \omega \mathrm{N}$

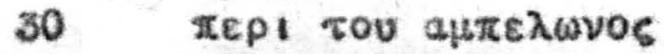

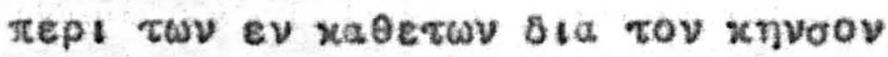

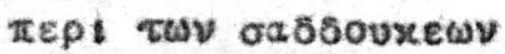

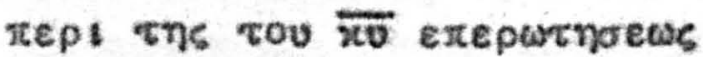

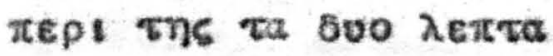

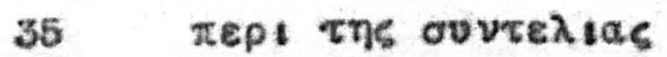

$\pi e \rho i$ rov raoxa

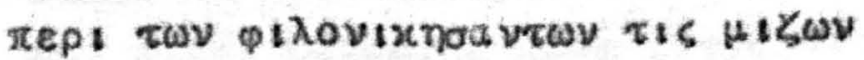

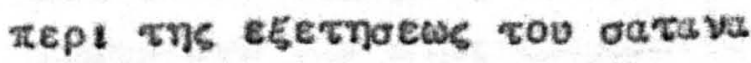

eE ovoe vinats npassou

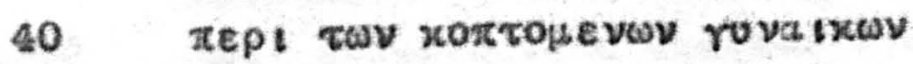

Tepl tov Heravangaveros Angrou 


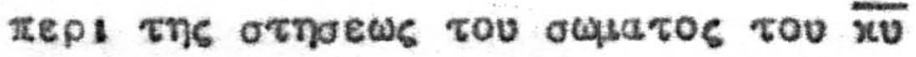

тер! xieorta

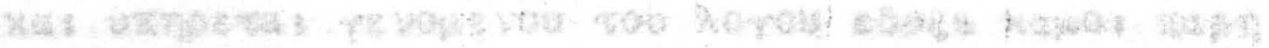

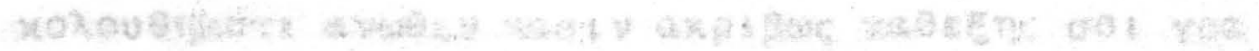

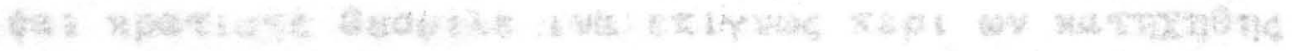

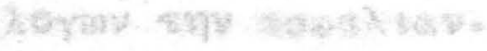

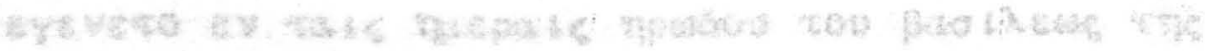

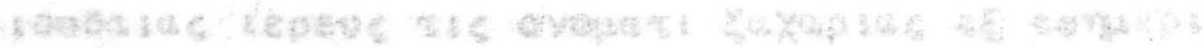

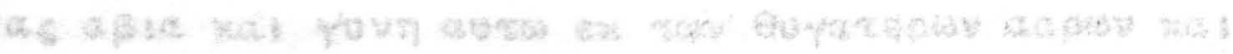

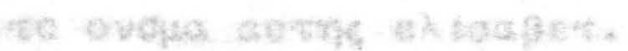

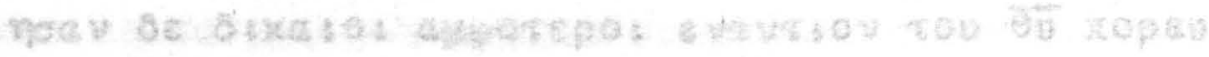

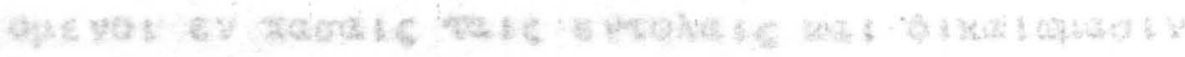

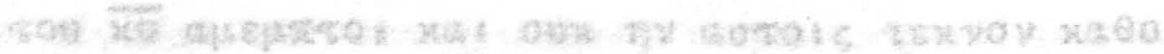

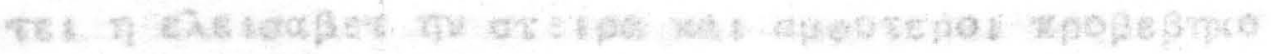

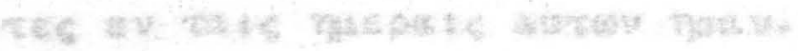

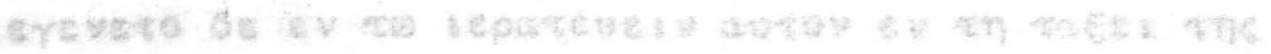

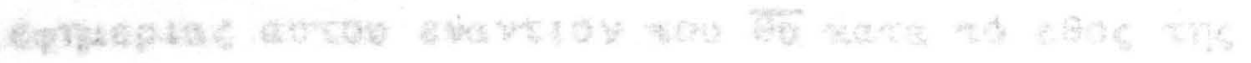

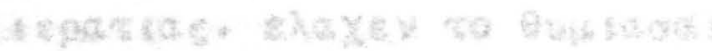

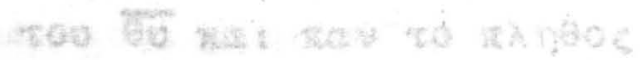

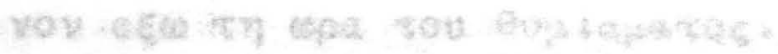

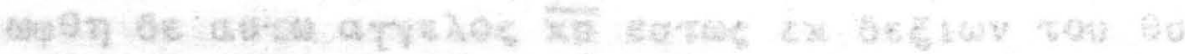

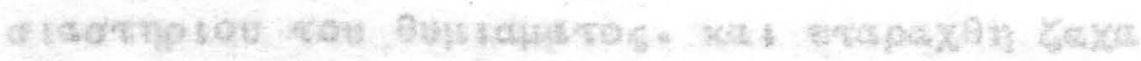

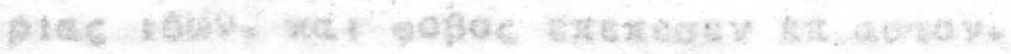

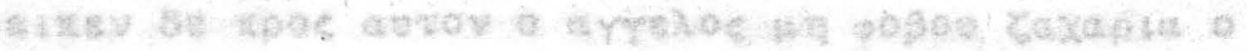

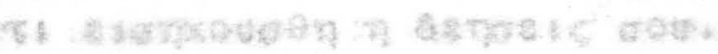

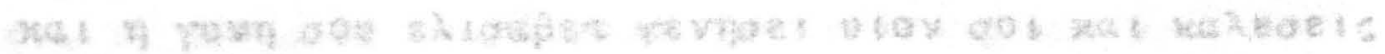

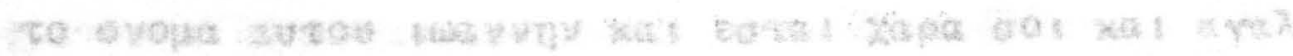

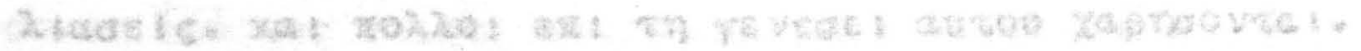

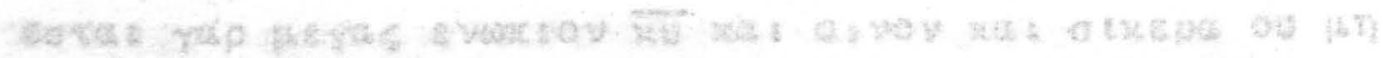

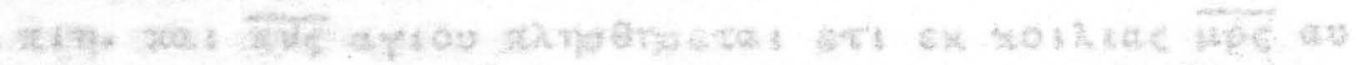

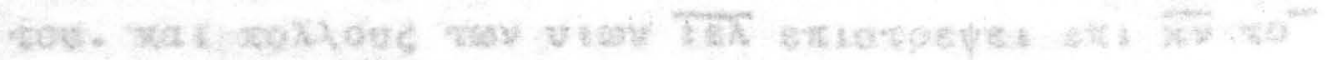

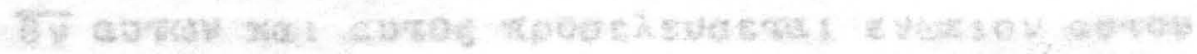

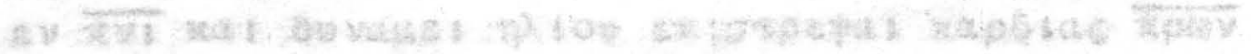

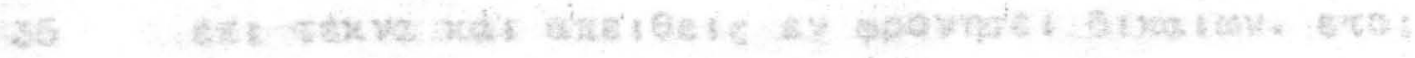

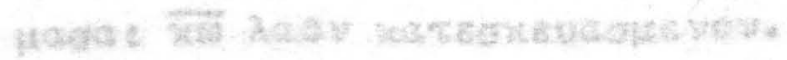

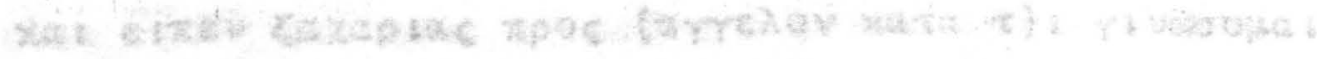

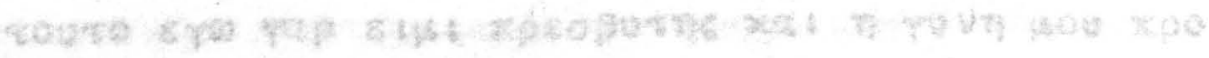

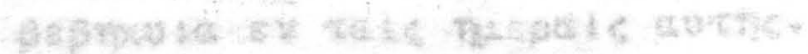




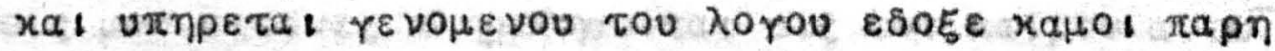

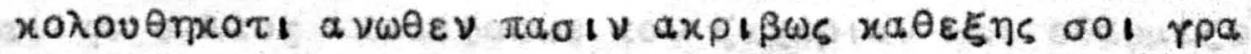

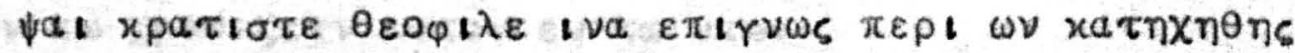

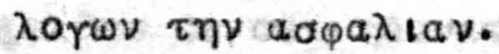

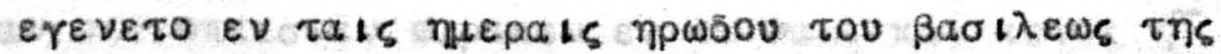

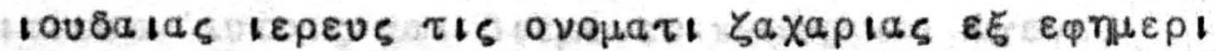

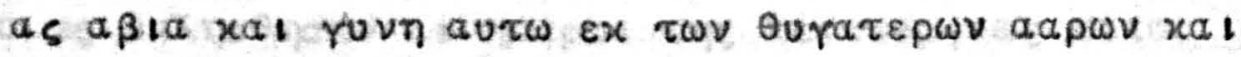

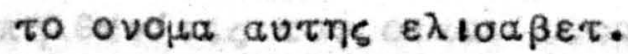

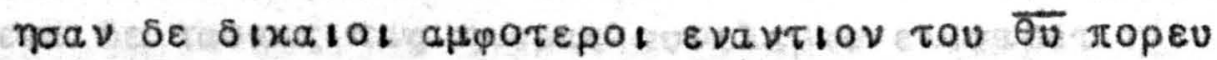

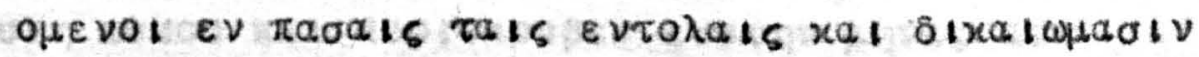

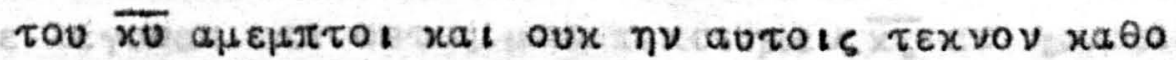

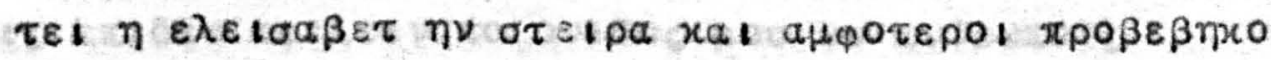

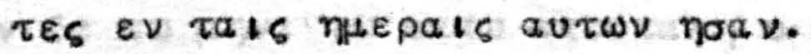

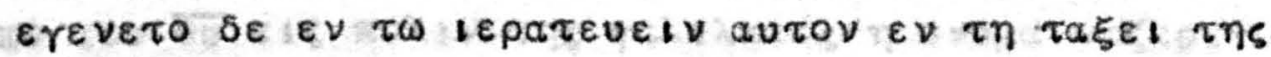

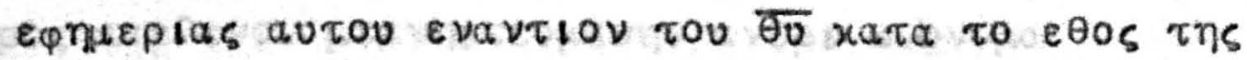

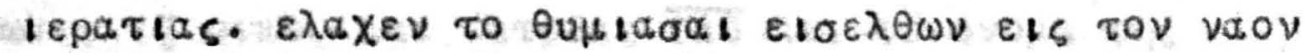

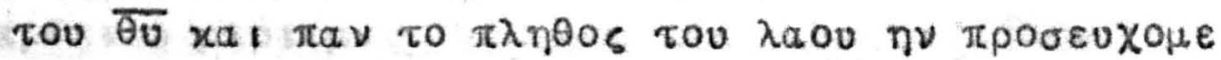

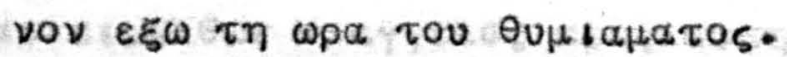

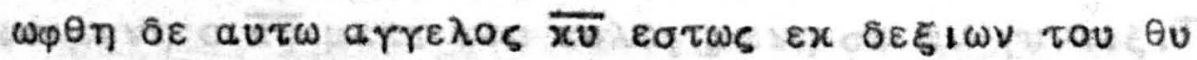

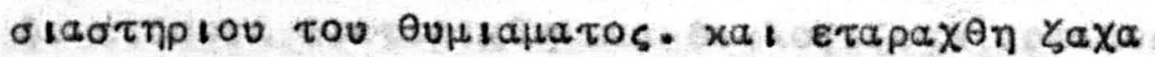
plas tôw. xal

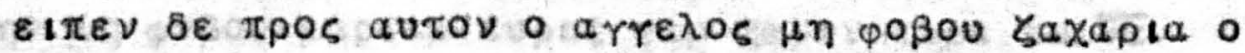

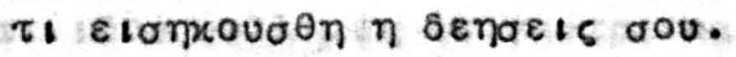

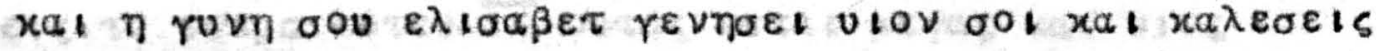

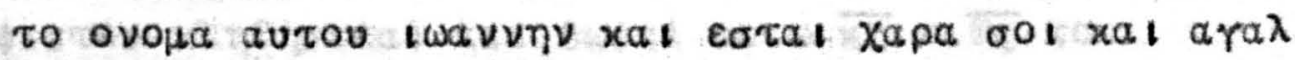

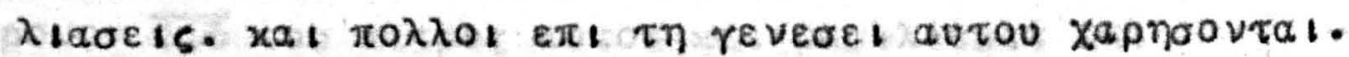

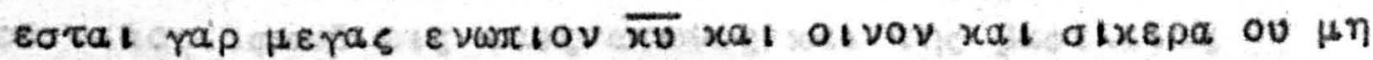

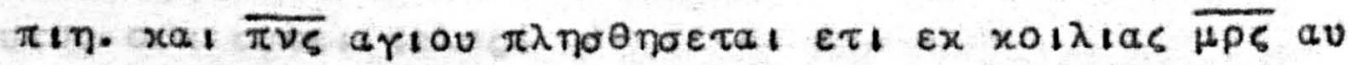

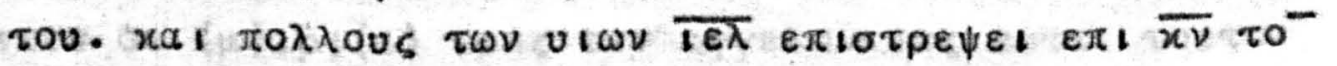

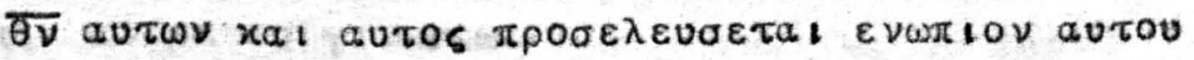

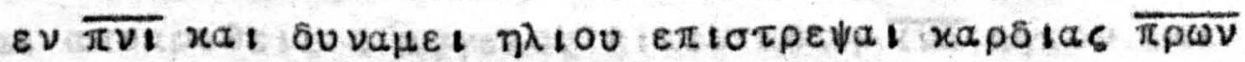

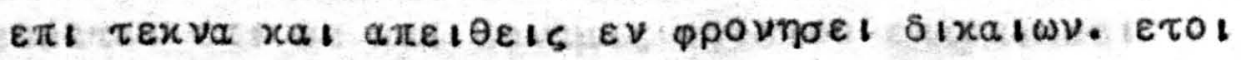

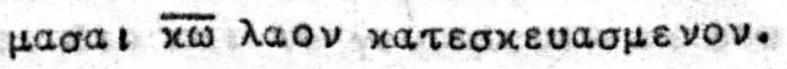

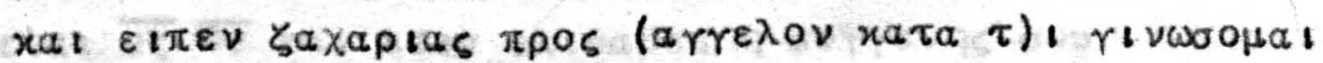

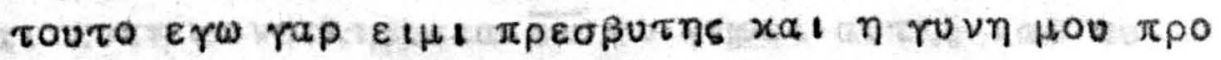
$\beta \varepsilon \beta \eta u t a \varepsilon v$ tals nuepals autns.

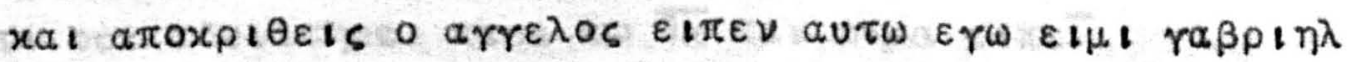

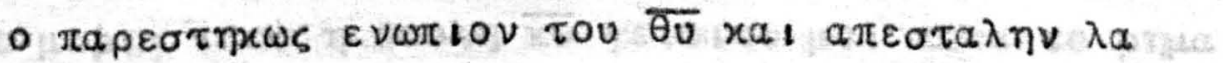




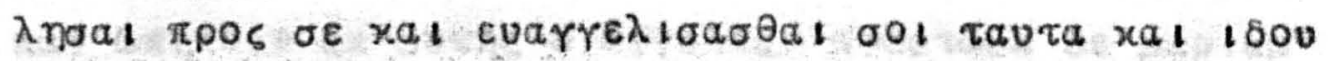

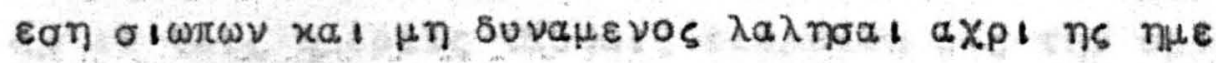

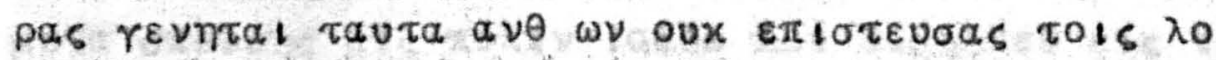

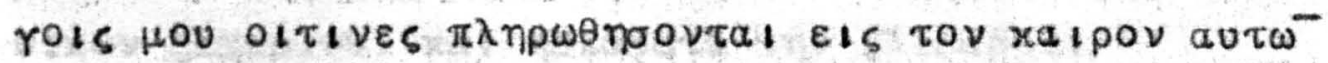

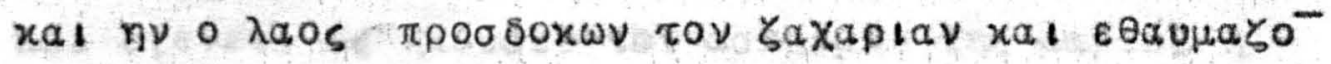

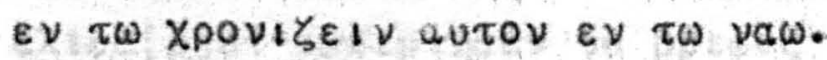

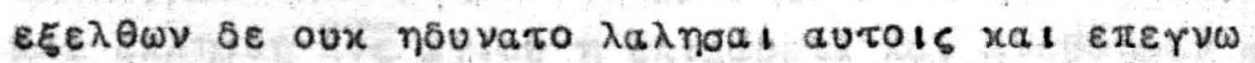

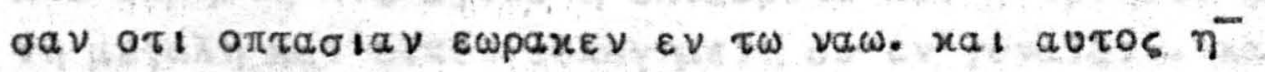

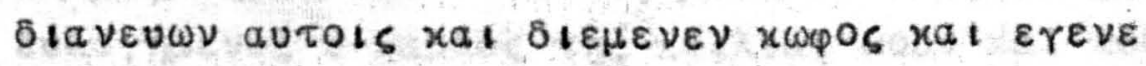

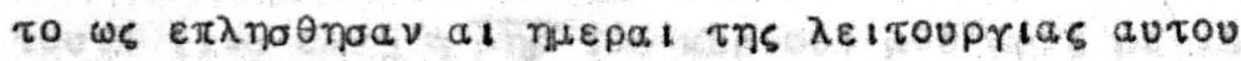

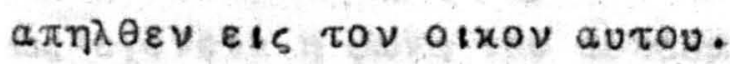

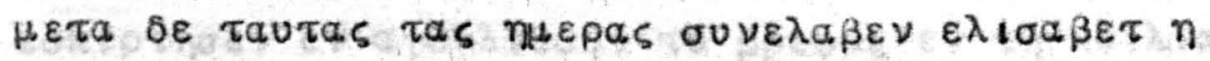

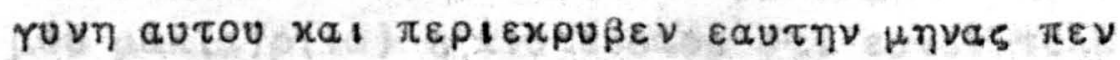

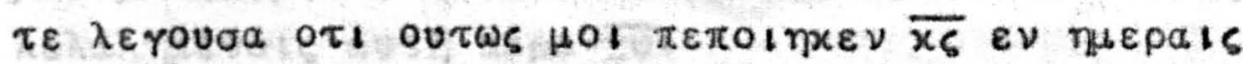

15 ats $\varepsilon \pi \delta \delta \varepsilon \nu$ a

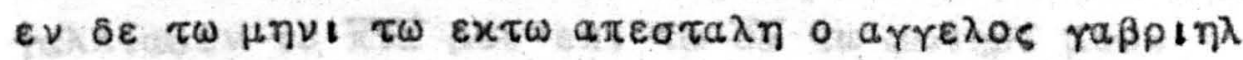

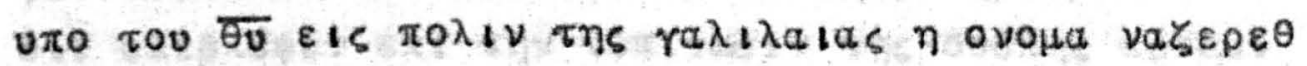

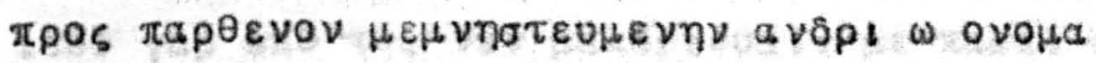

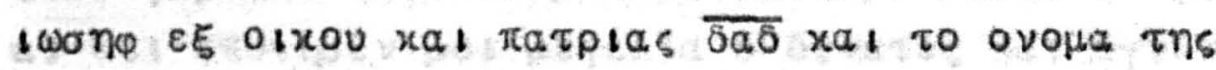

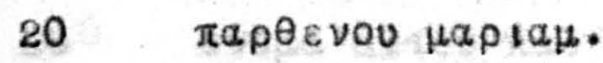

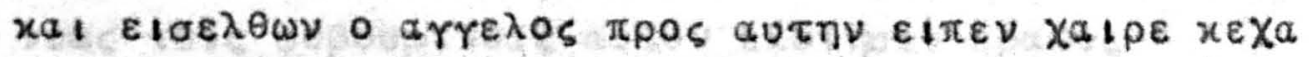

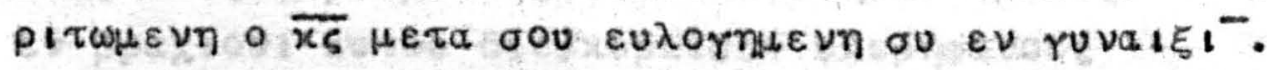

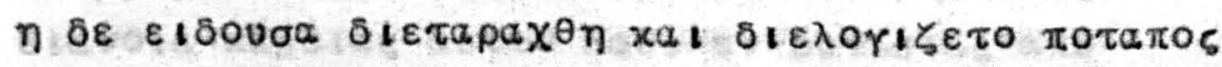

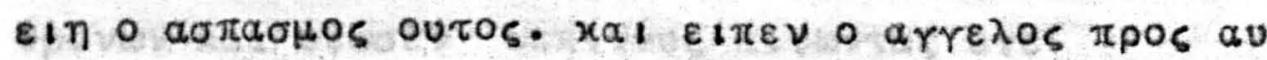

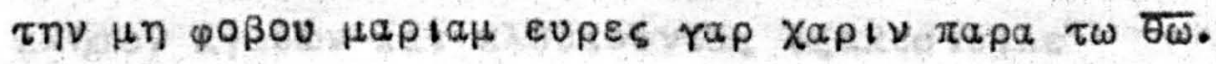

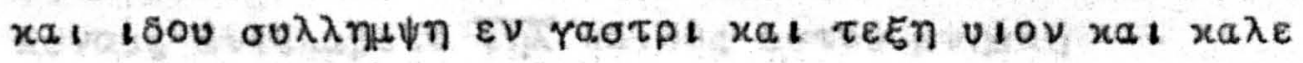
aris to ovoua autou TV outos earal Heras $x a l$ vios

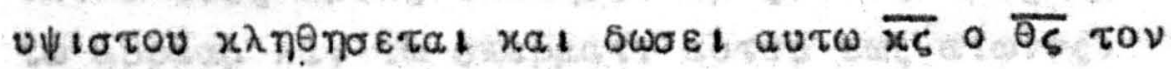

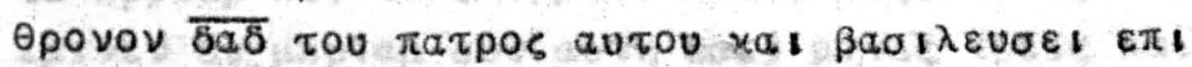

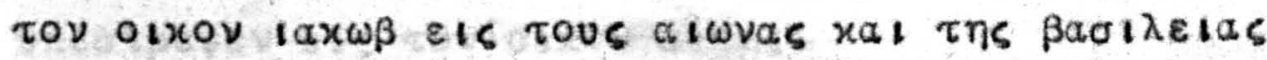

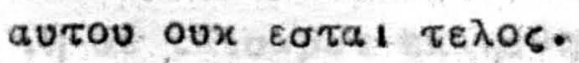

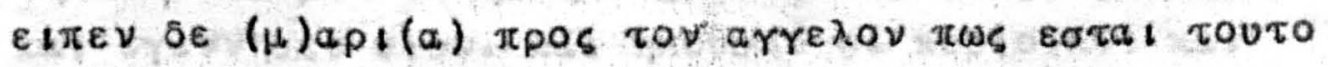

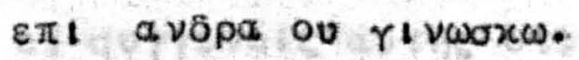

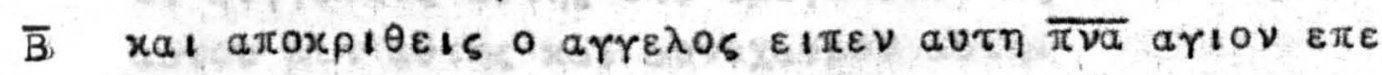

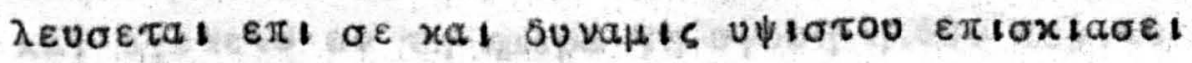
oot $810 x a 1$ to revvauevov $\varepsilon x$ oov ariov $x \lambda \eta \theta \eta$

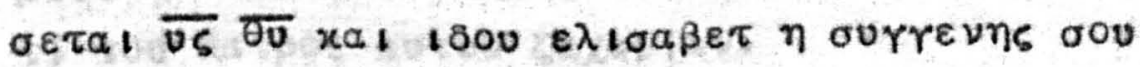

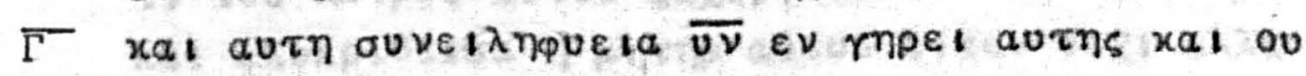

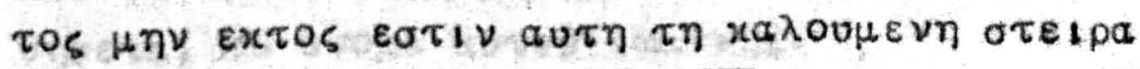

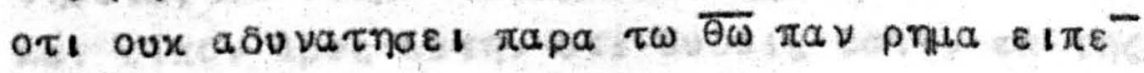

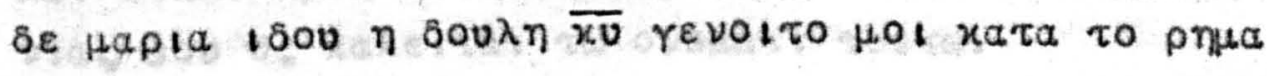




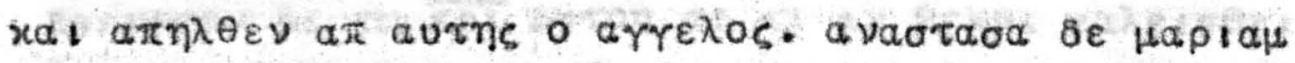

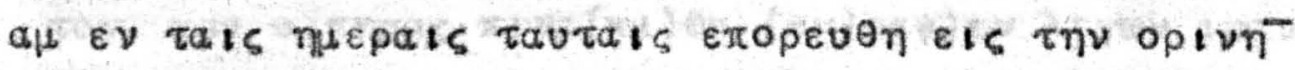

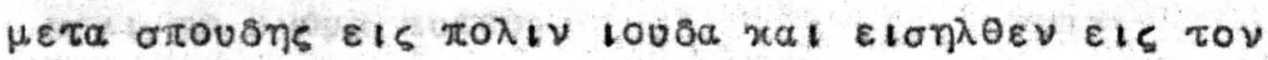

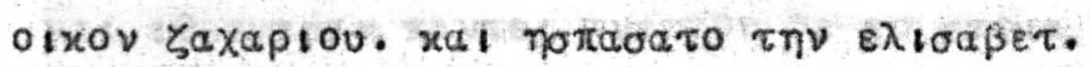

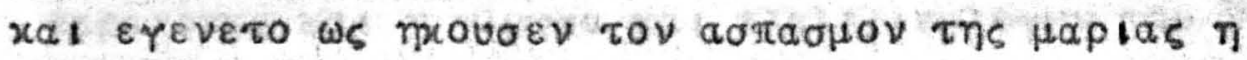

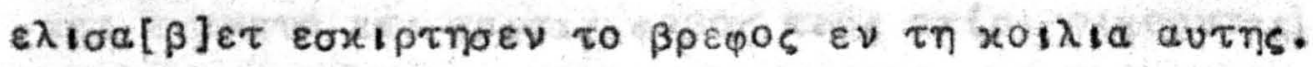

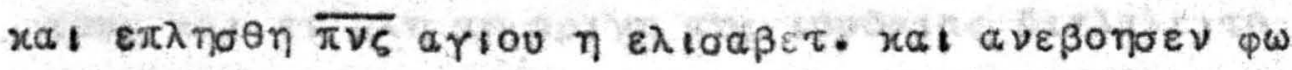

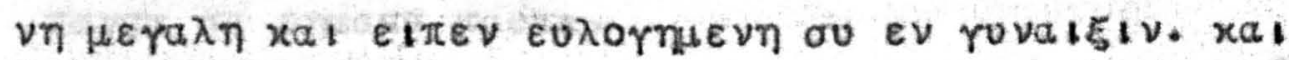

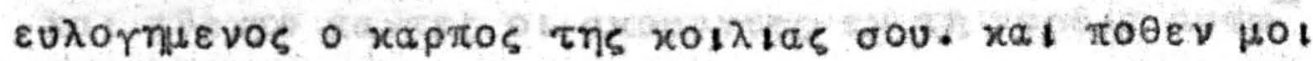

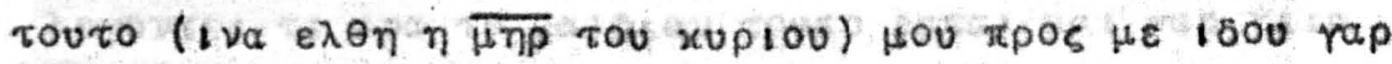

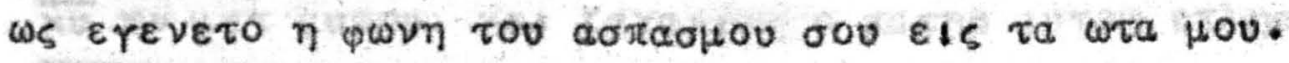

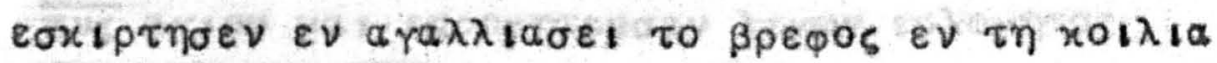

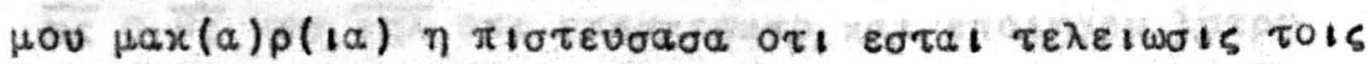

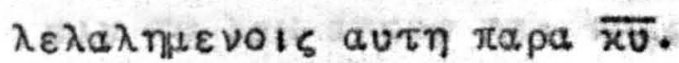

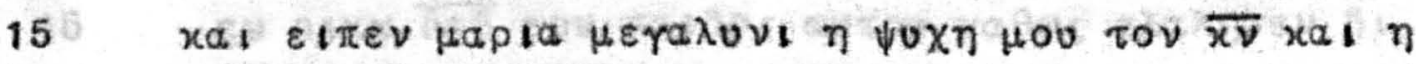

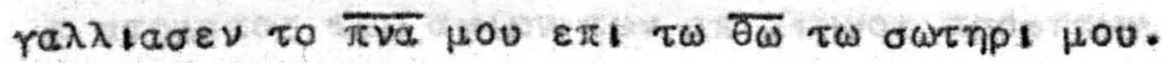

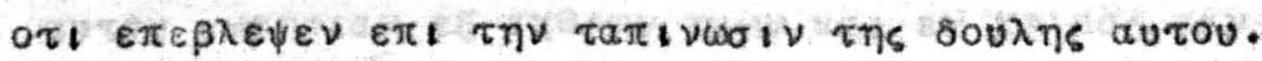

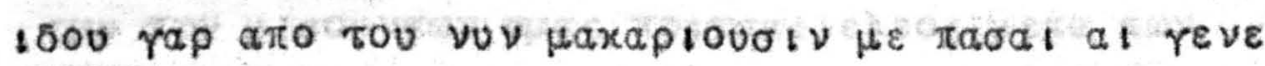

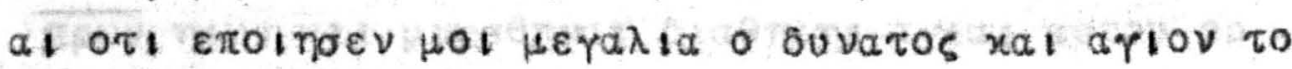

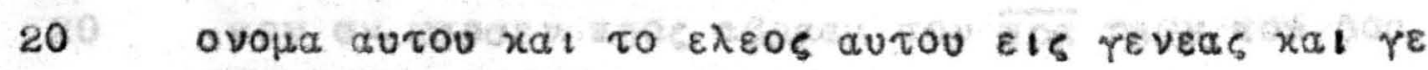
veas tois poßoufevols autov eto Inoev xpatos

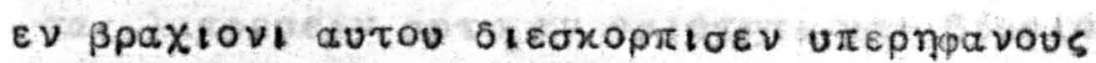

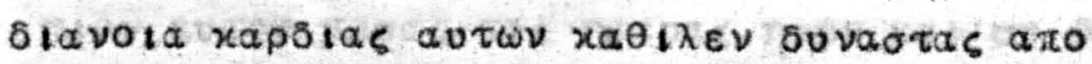

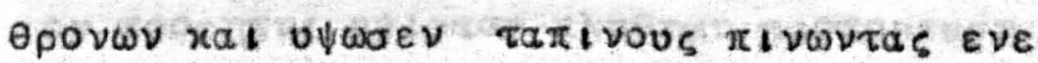

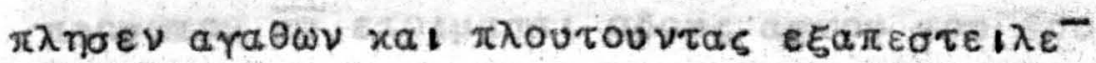

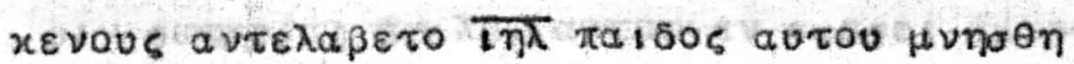

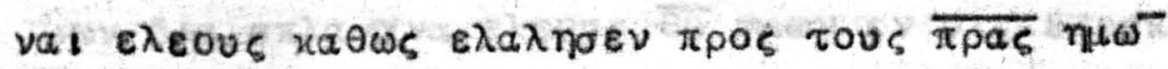

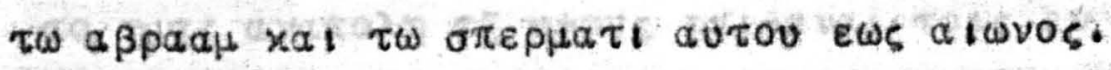

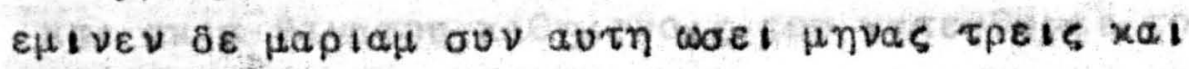

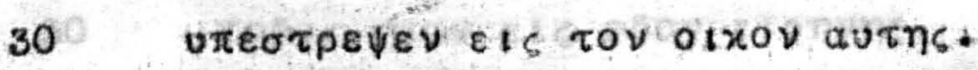

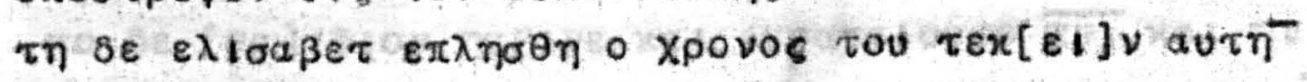

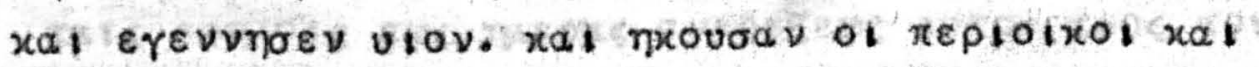

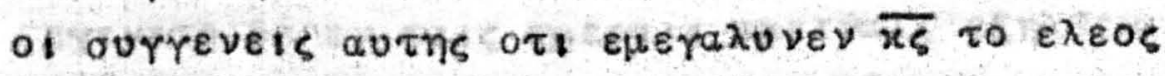

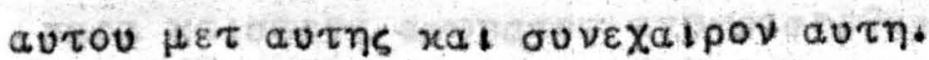

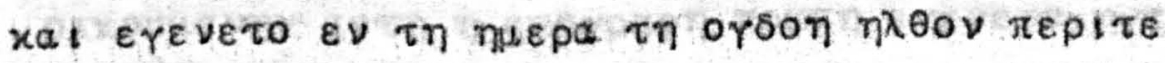

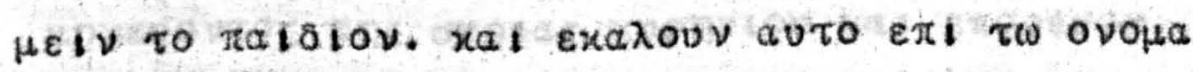

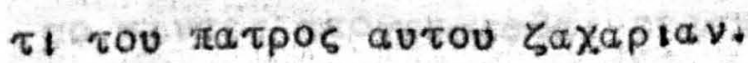

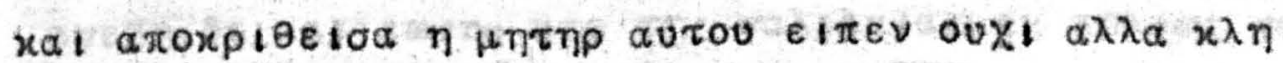

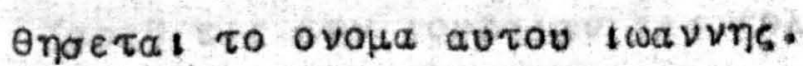

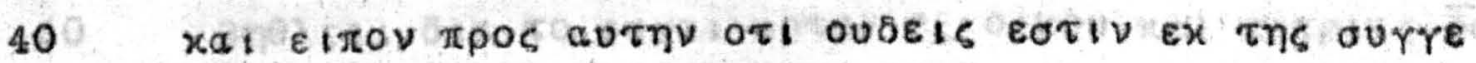

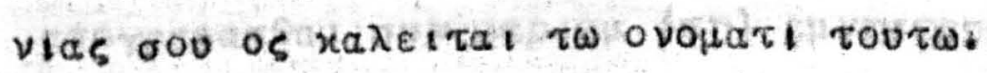




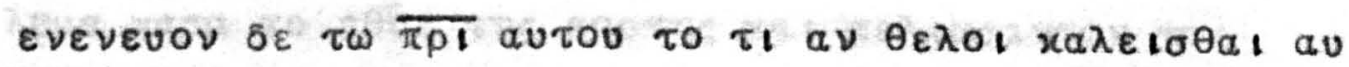

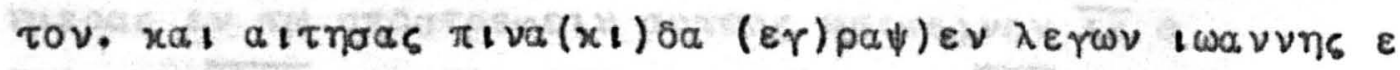

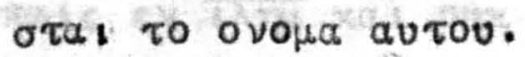

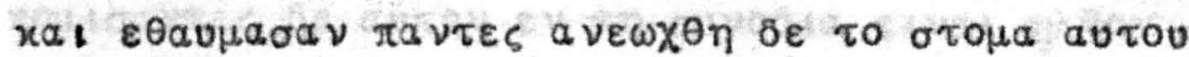

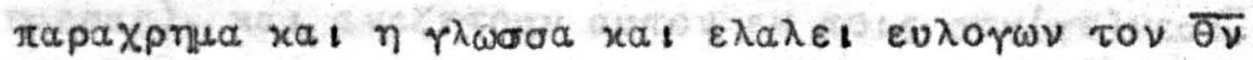

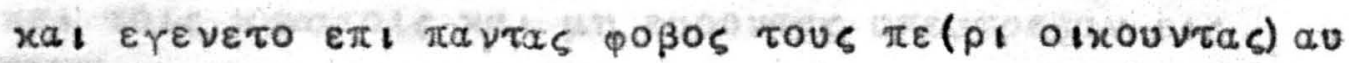

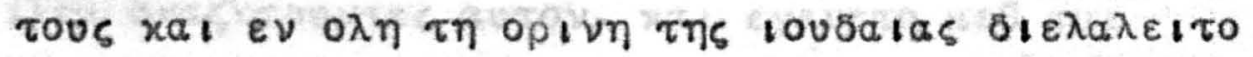

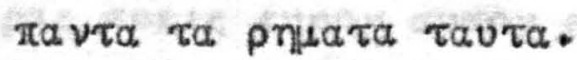

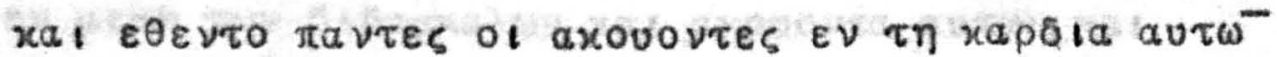

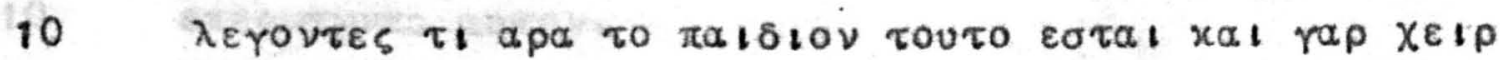

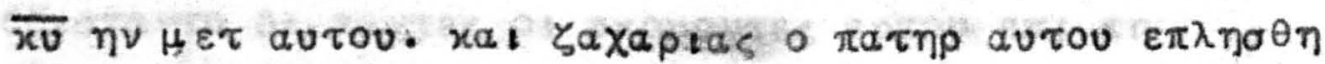

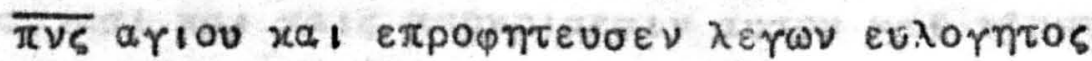
$\overline{x_{\zeta}} \circ \overline{\theta_{\zeta}} \tau$

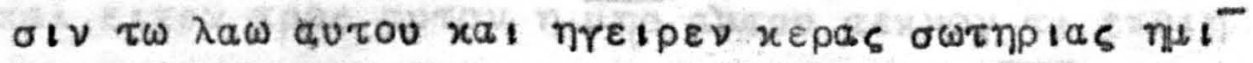

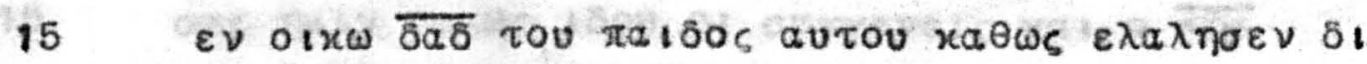

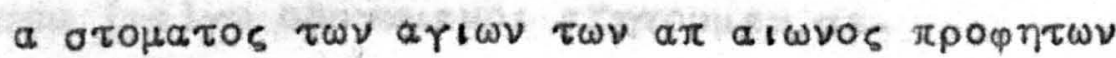

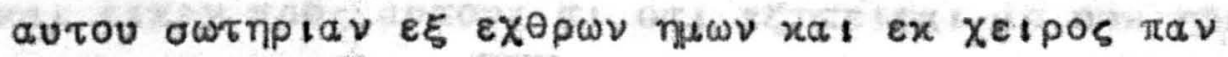

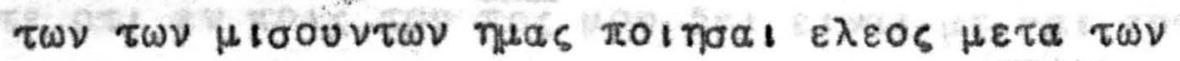

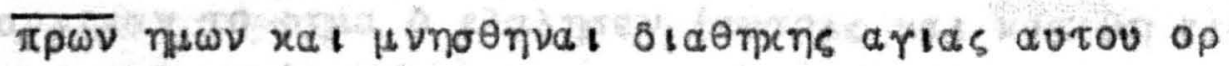

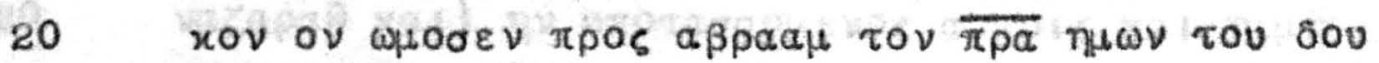

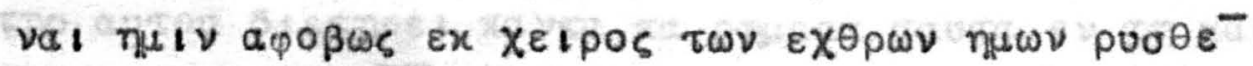

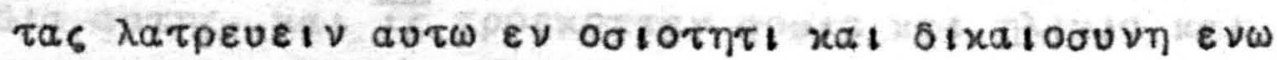

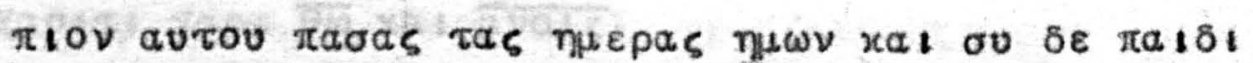

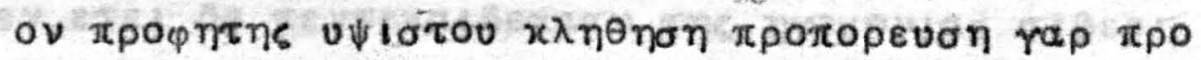

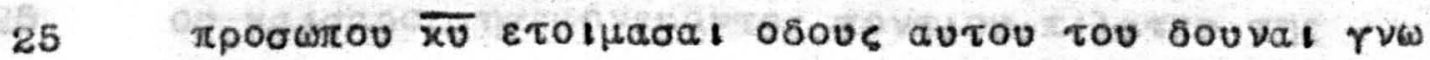

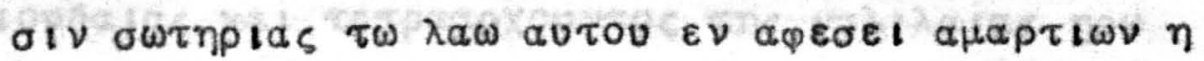

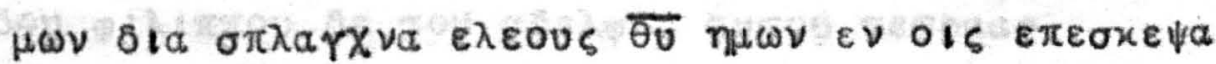

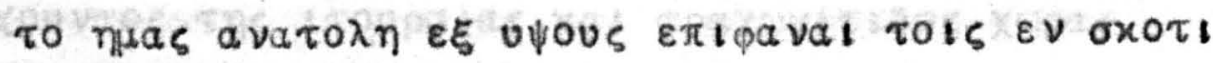

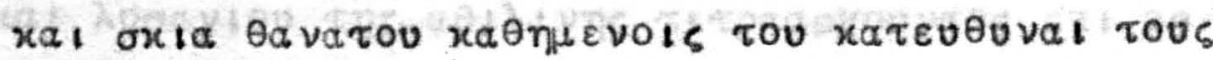

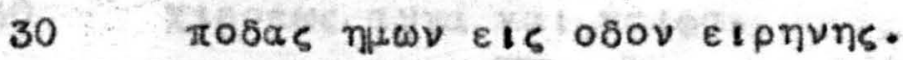

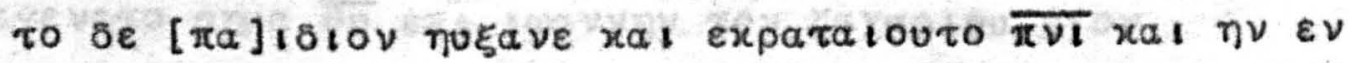

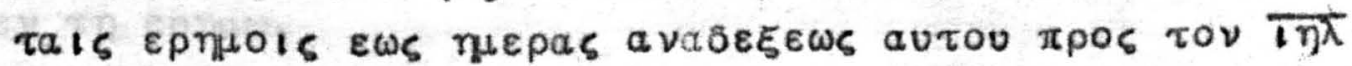

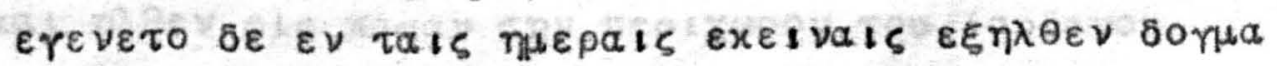

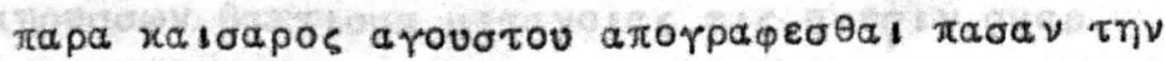

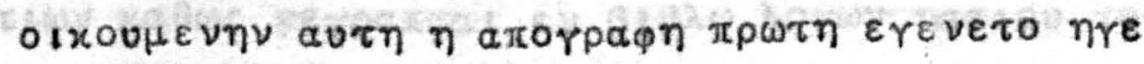

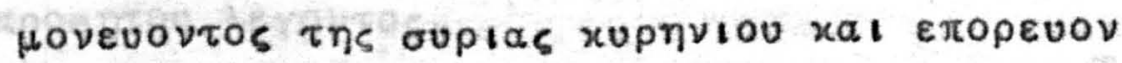

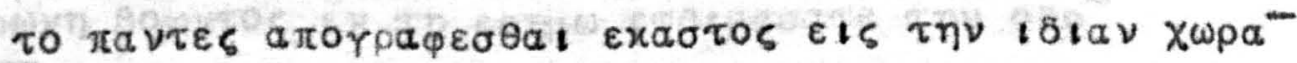

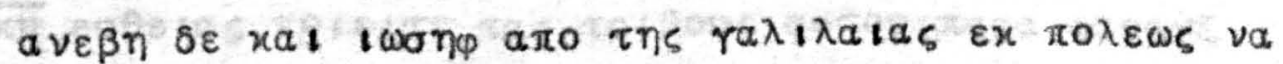

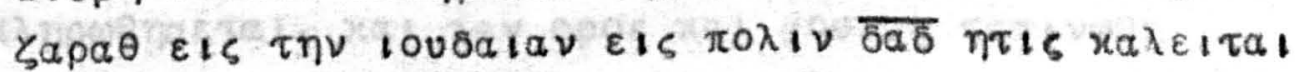

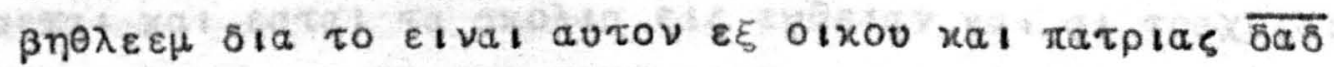

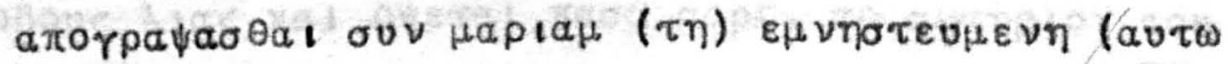




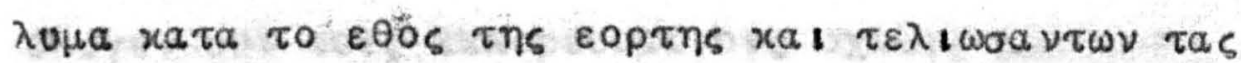

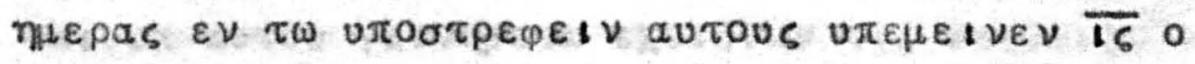

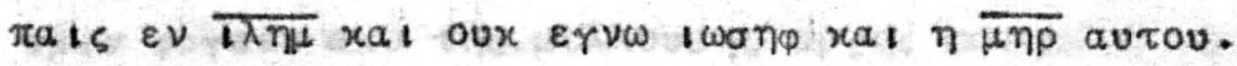

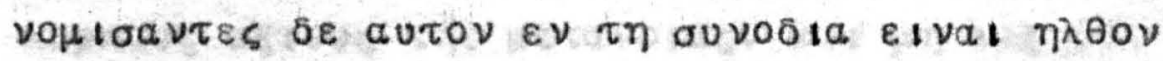

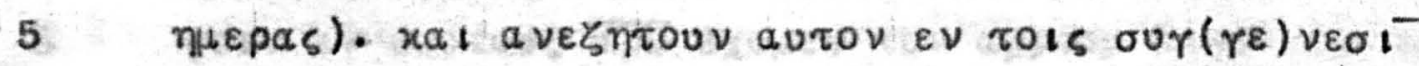

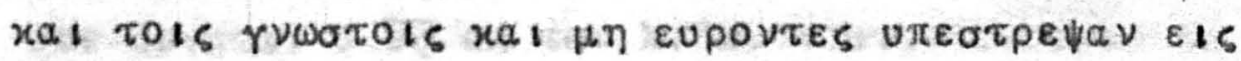

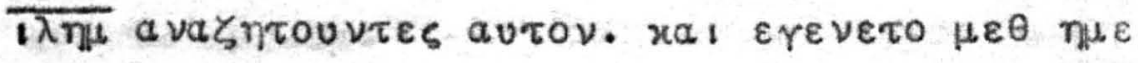

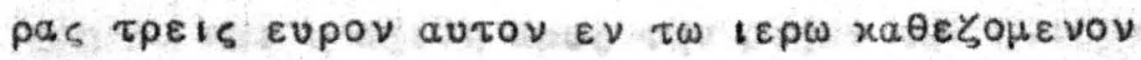

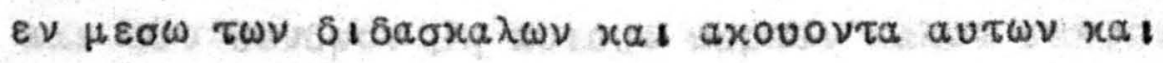

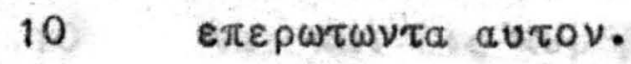

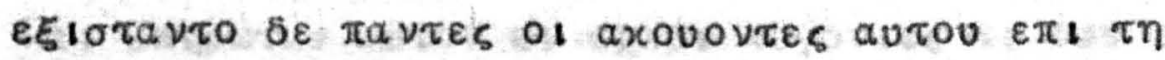

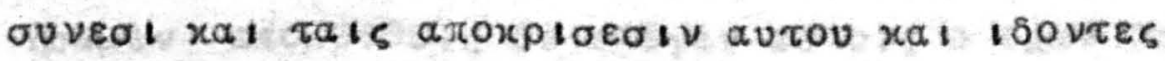

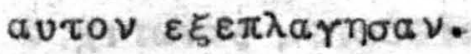

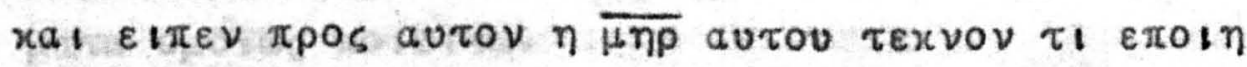

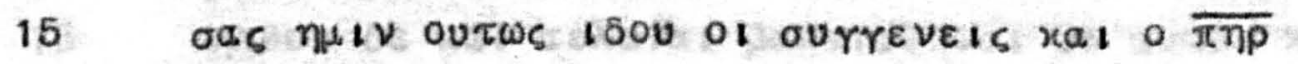

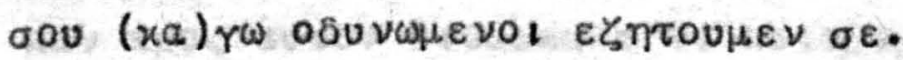

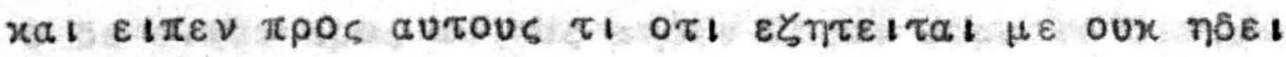

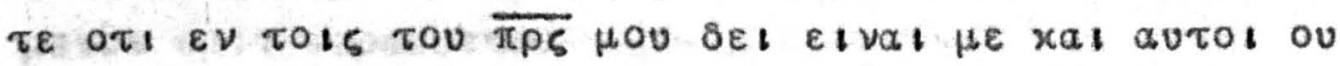

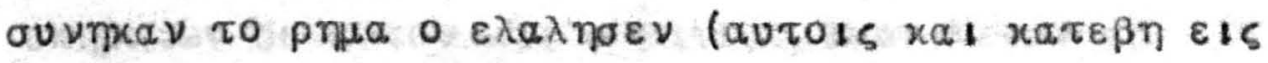

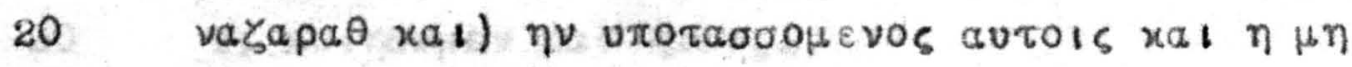

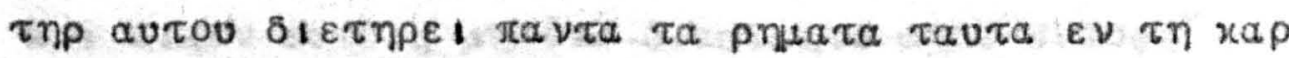

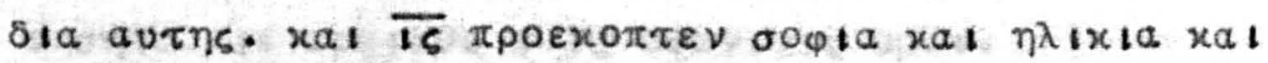

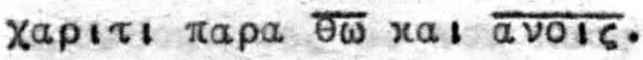

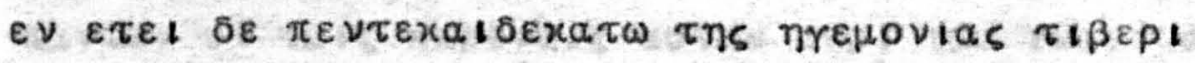

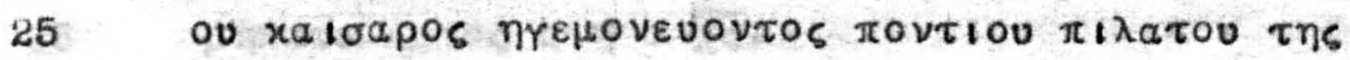

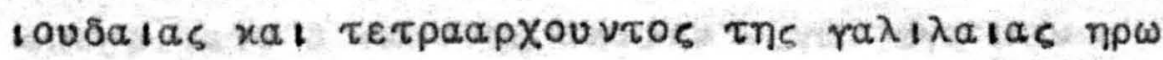
$\delta 00$

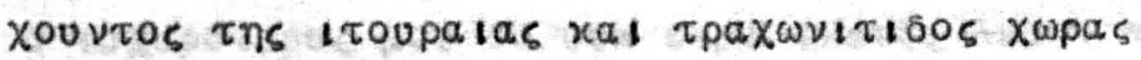

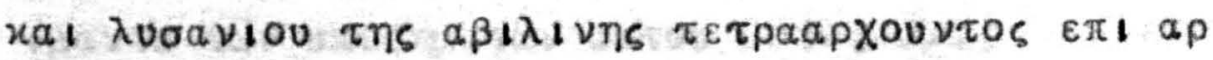

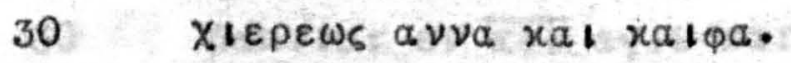

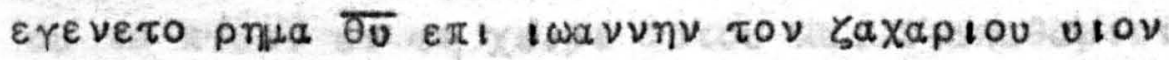
$\varepsilon \nu \tau \eta \varepsilon \rho \eta \mu \omega$.

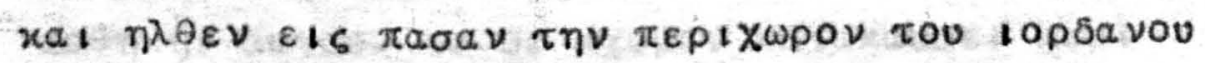

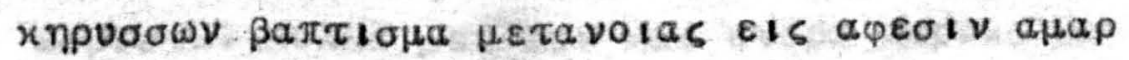

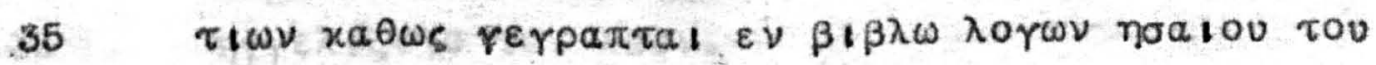
троф

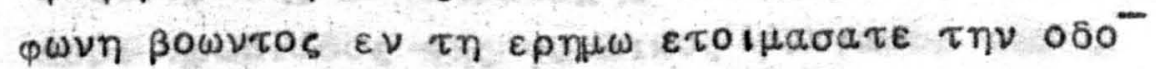

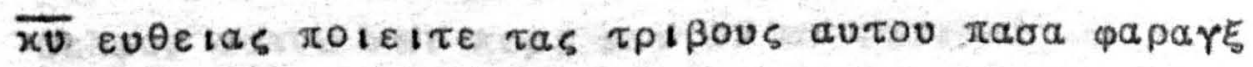

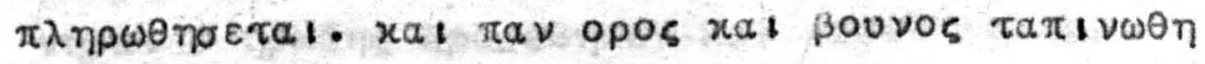

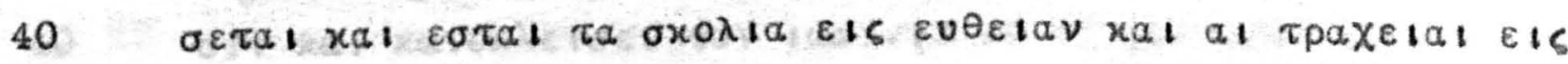

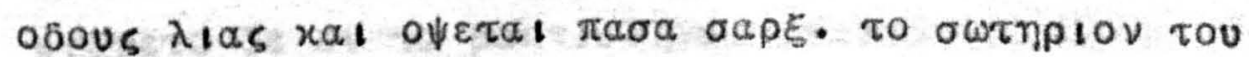




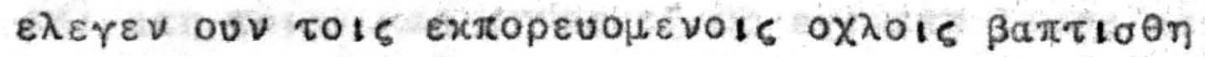

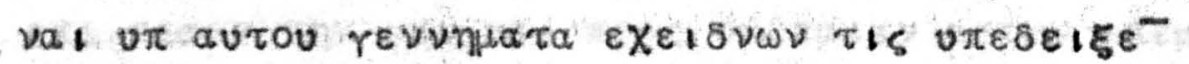

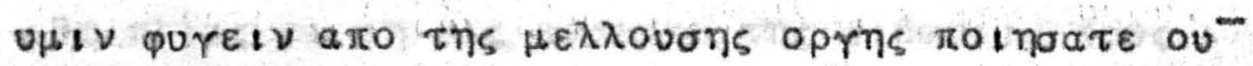

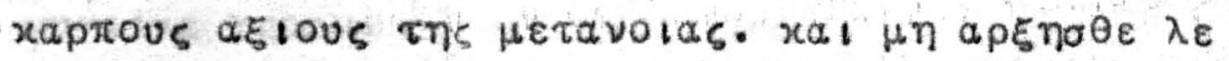

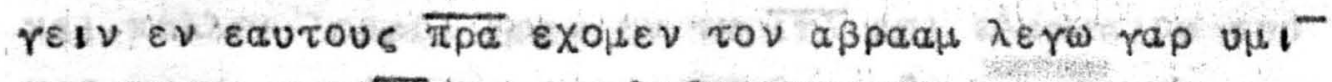

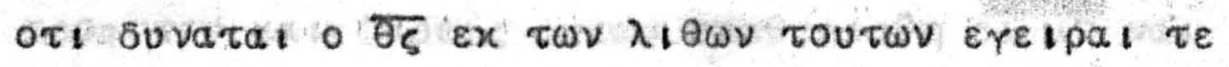

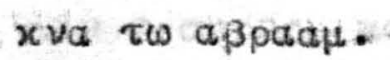

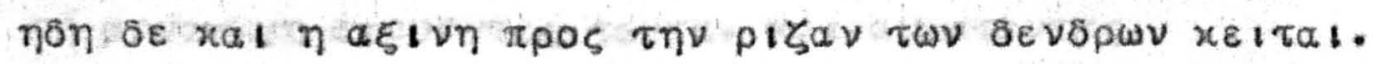

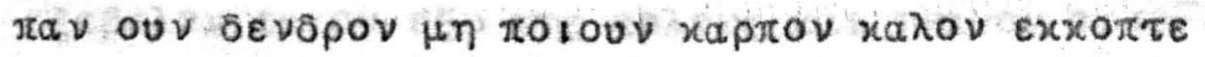

$10 \tau a l$ xal $\varepsilon l s \pi \rho \rho \beta \lambda \lambda \varepsilon \tau a \imath$.

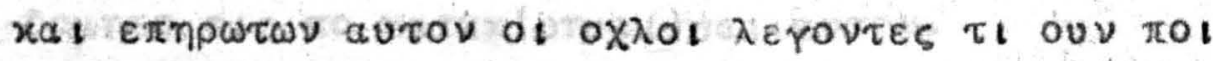

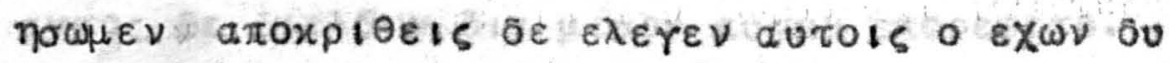

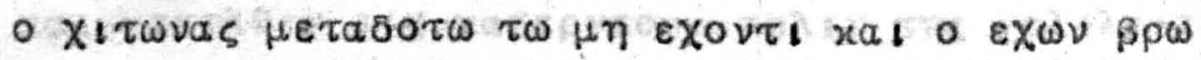
Hata quolws tole $1 \tau \omega$.

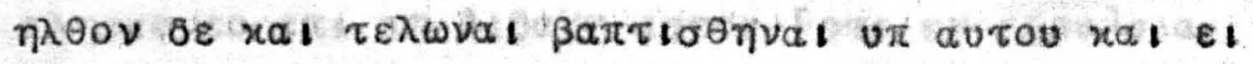

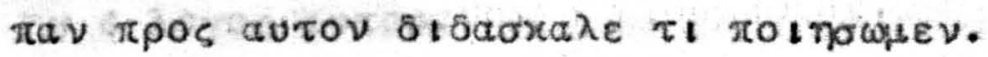

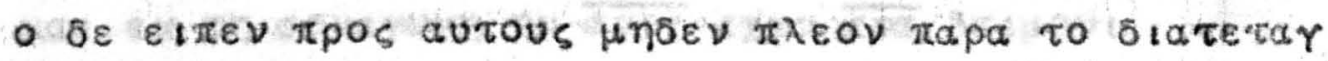

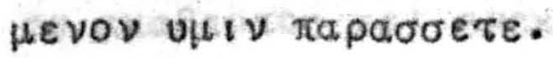

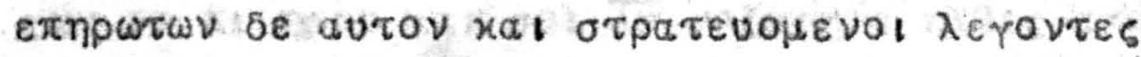
$\tau t$ to Inowiev xal nue is.

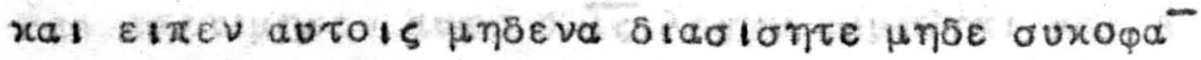

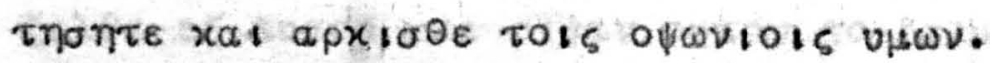

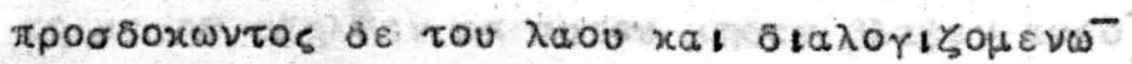

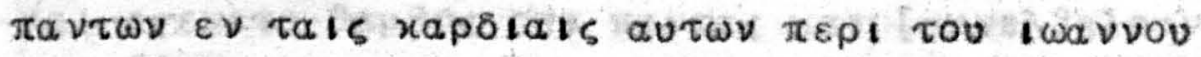
$\mu \eta \eta \tau$ वutos $\varepsilon$ in $\circ \overline{X \zeta}$.

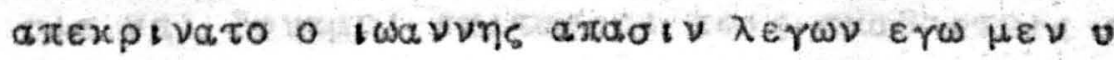

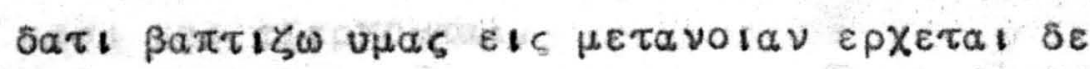

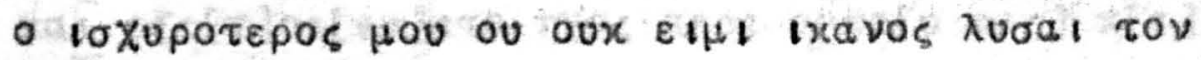

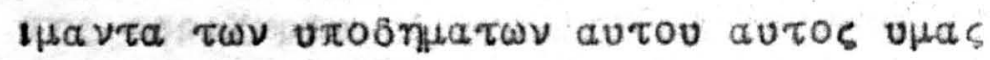

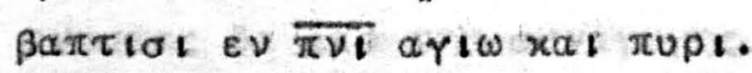

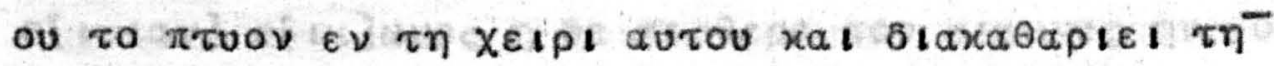

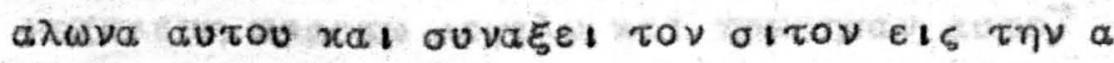

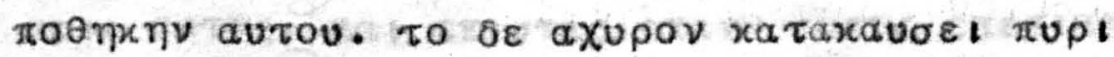

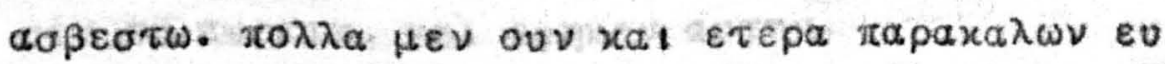

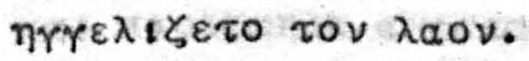

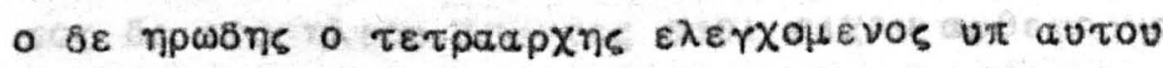

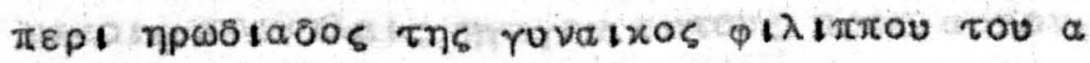

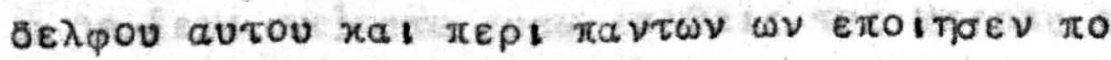

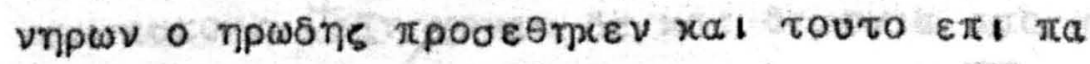

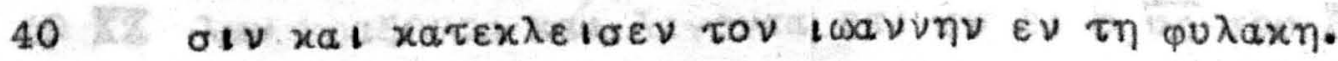

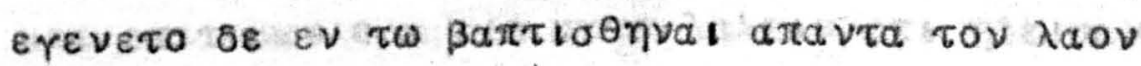




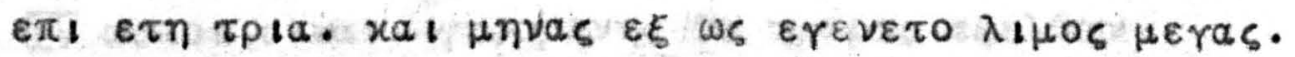

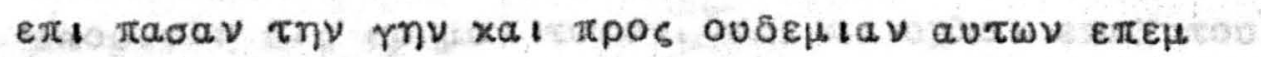

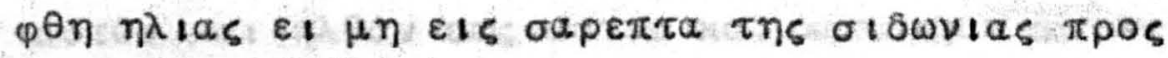
ruvalxa xnpav.

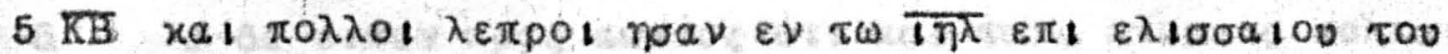

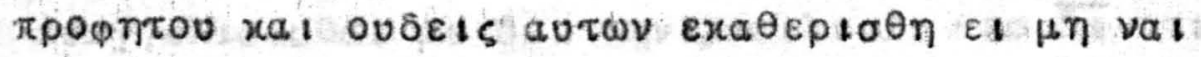

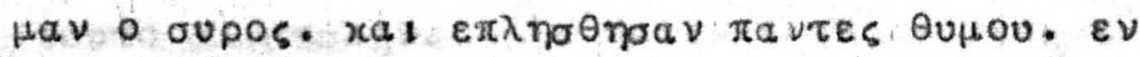

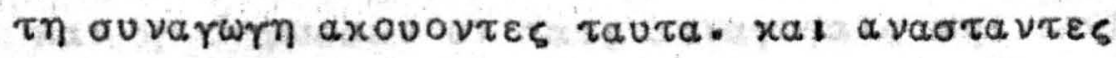

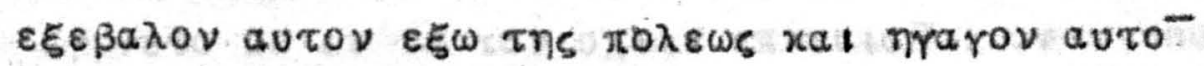

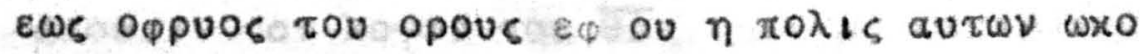

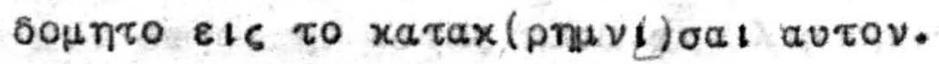

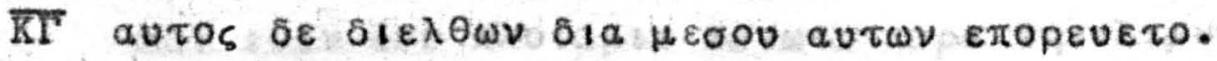

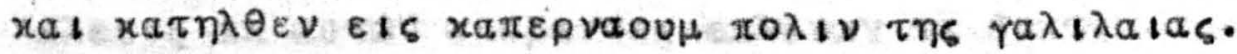

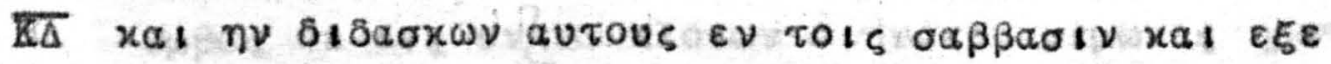
$\pi \lambda \eta \sigma_{0}$

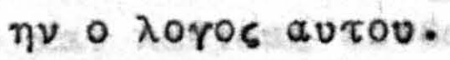

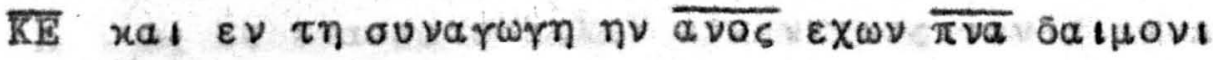

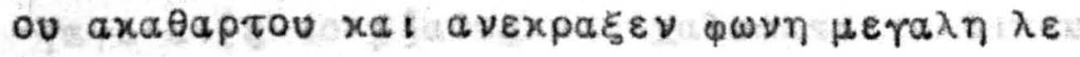

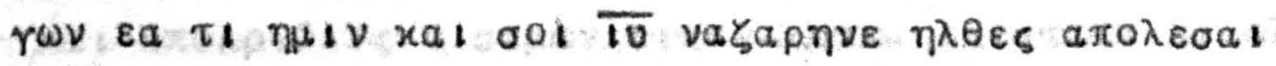

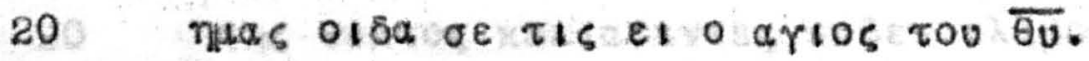

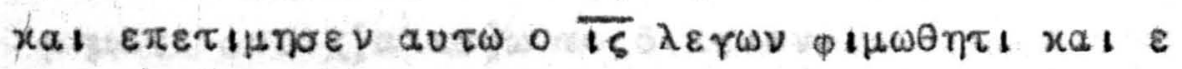

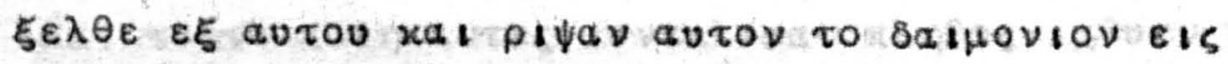

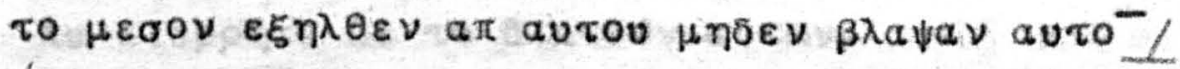

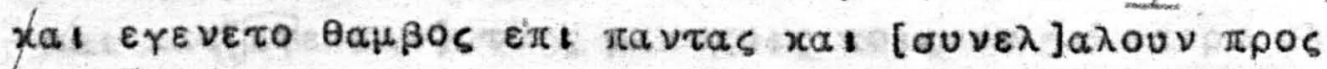

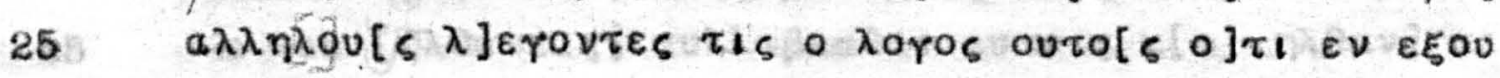

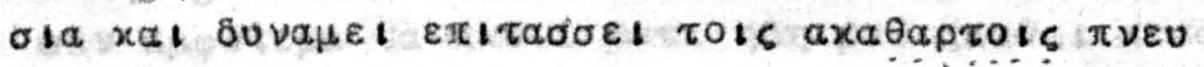

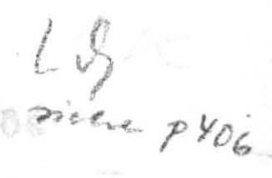

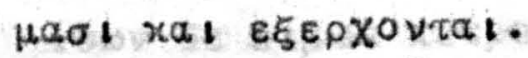

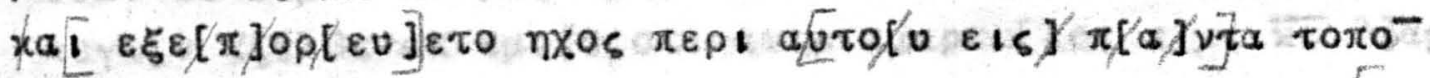

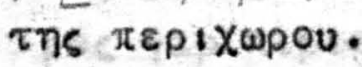

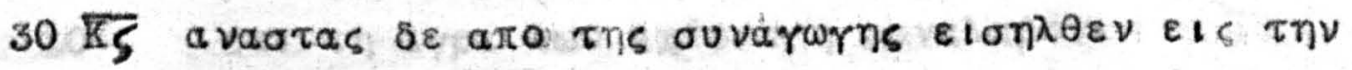

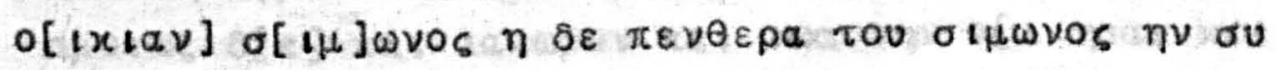

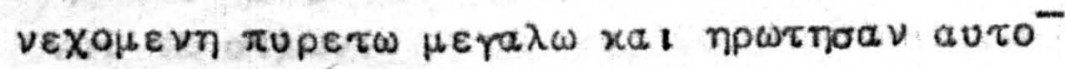

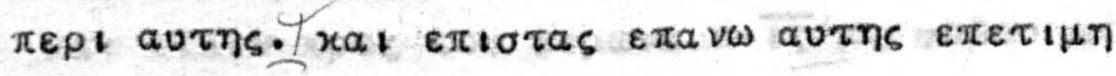

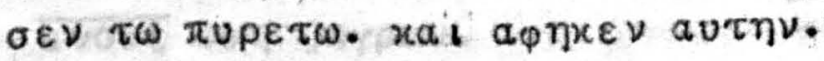

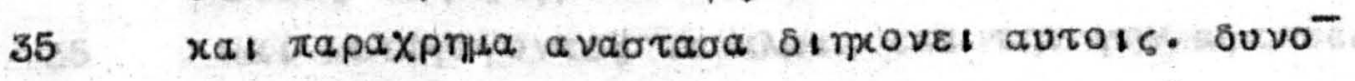
tos $\delta \varepsilon$ rou $\eta \lambda$ lou araves $\frac{1}{2}=$

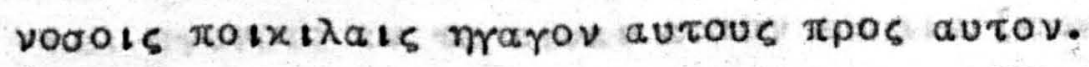

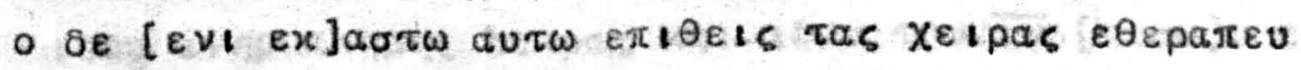

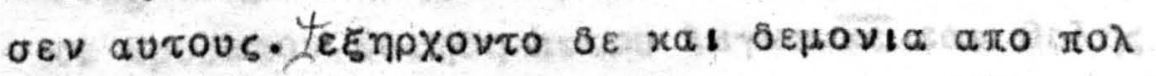

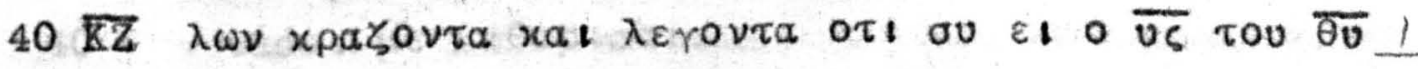

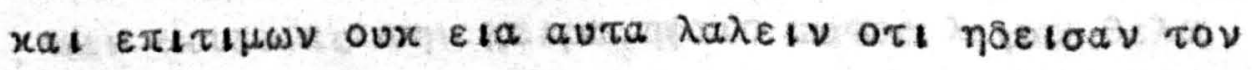




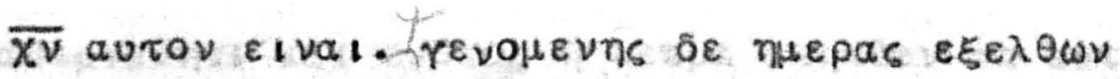

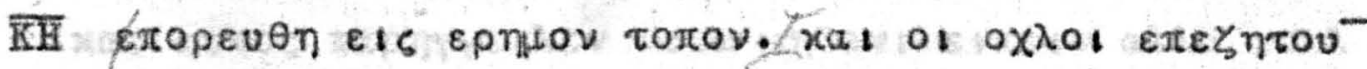

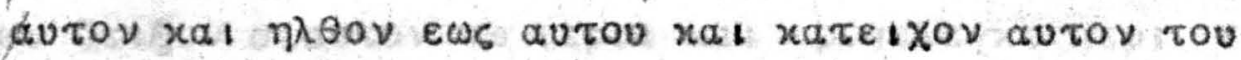

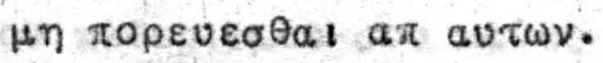

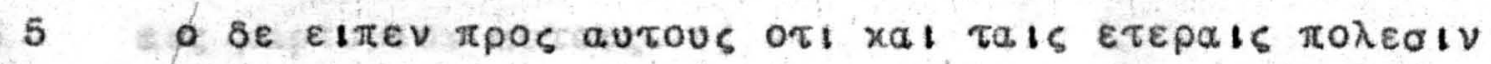

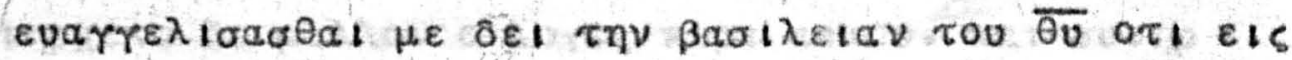

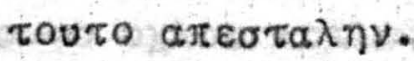

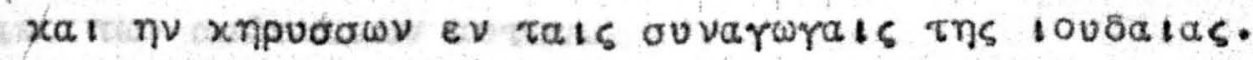

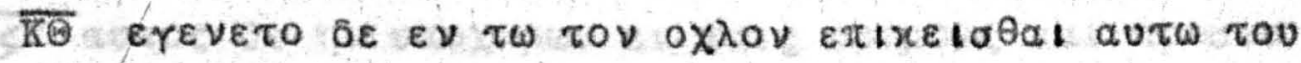

10 axovelv tov horov tou $\overline{00}$.

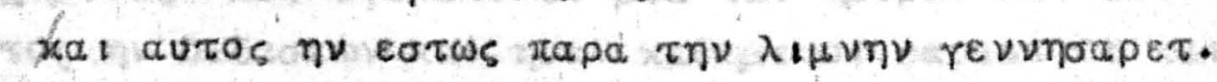

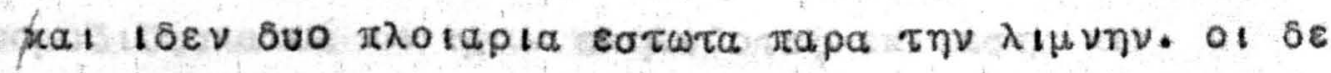

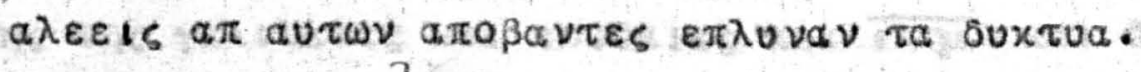

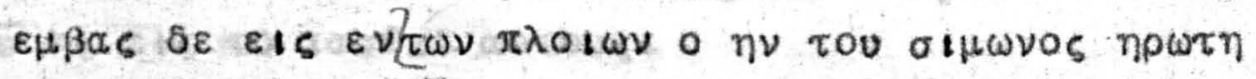
oev autov lato] the ms exavarareiv odirov.

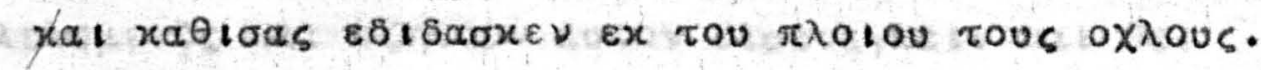

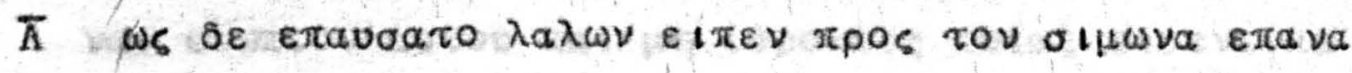

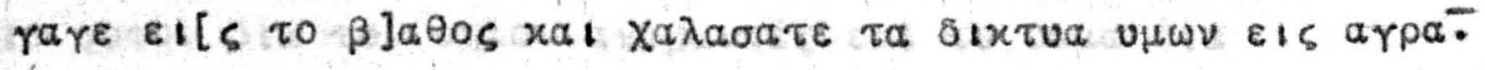

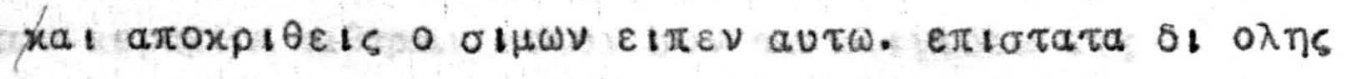

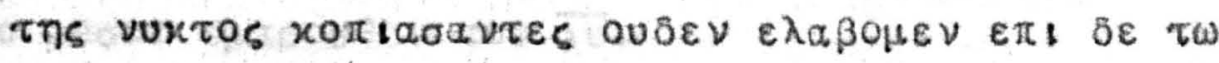

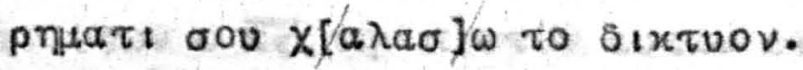

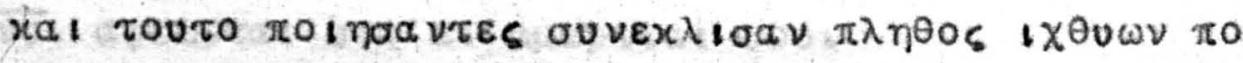

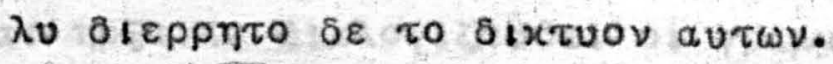

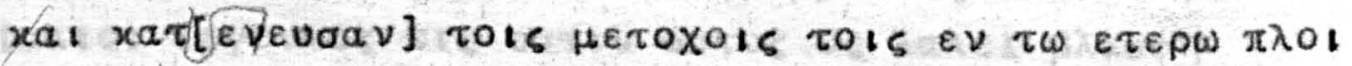
$\omega \tau$ $\sigma a \nu$ a

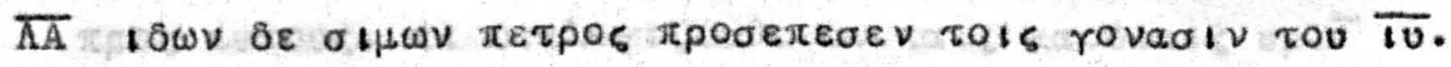

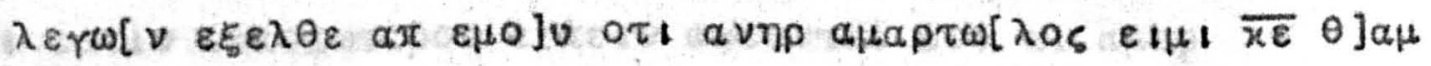

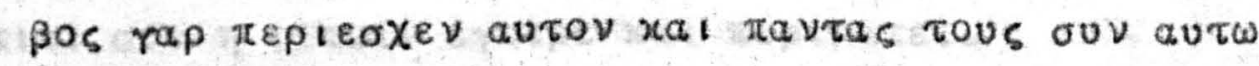

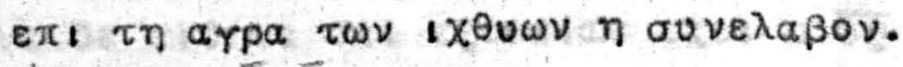

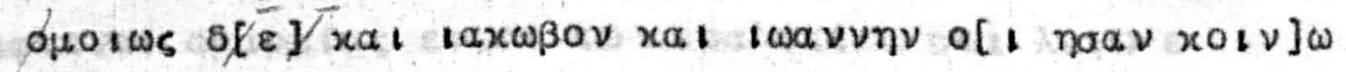

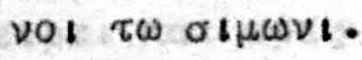

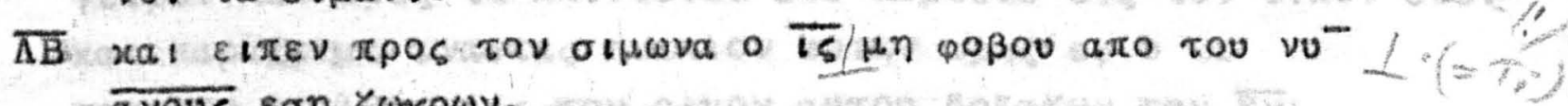

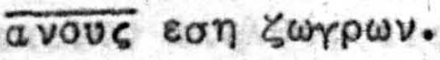

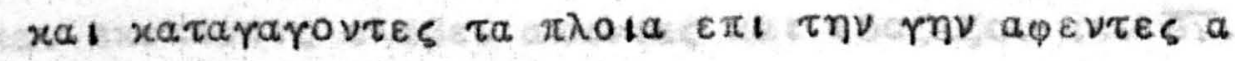

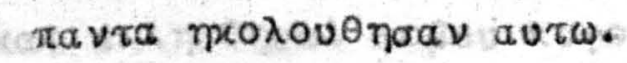

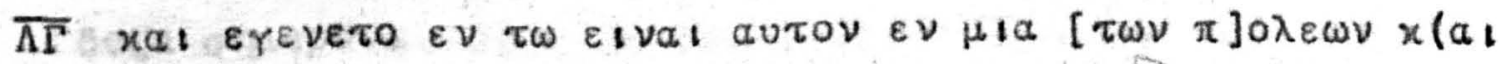

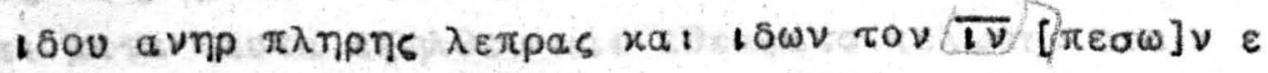

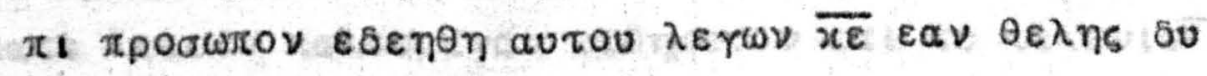

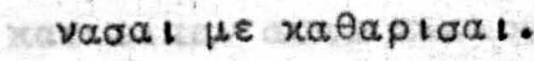

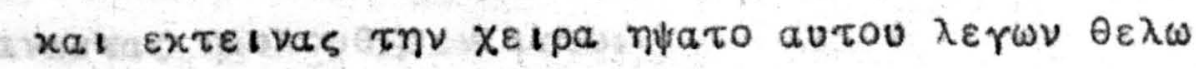




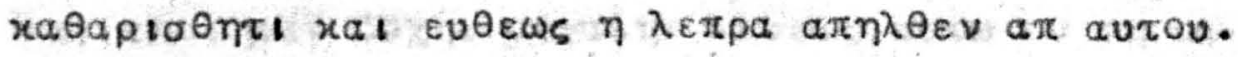

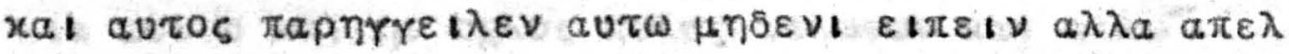

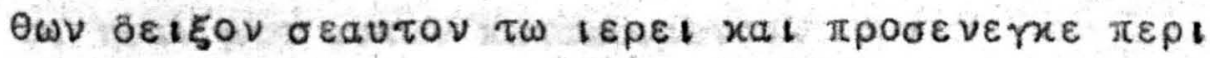

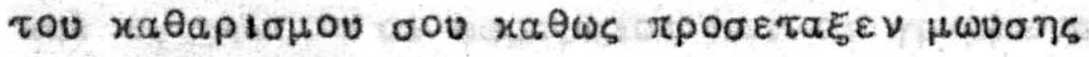
Eis Haptupiov autois.

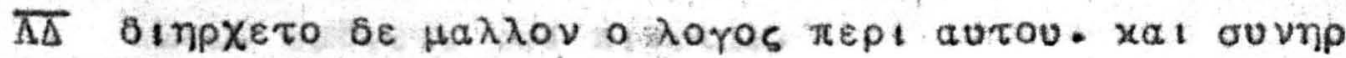

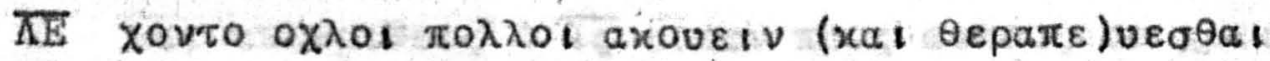

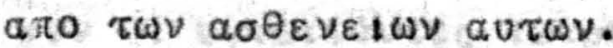

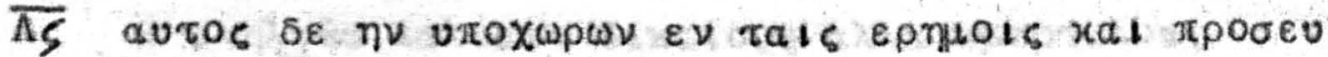

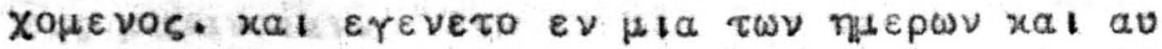

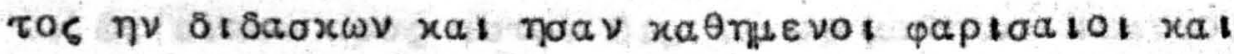

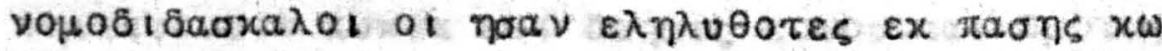

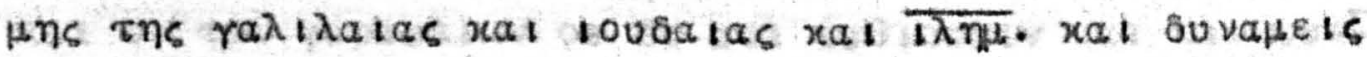

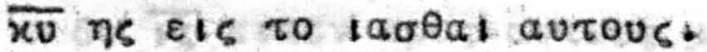

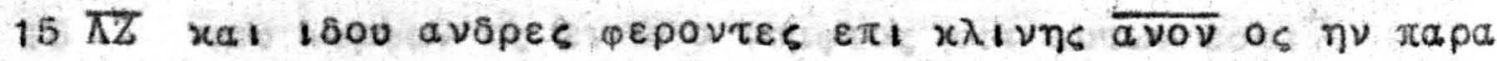

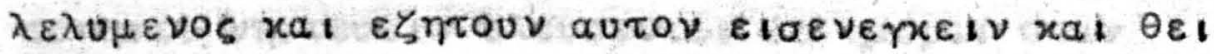

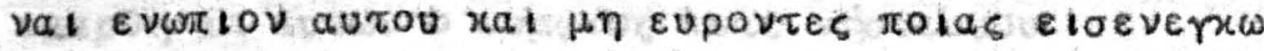

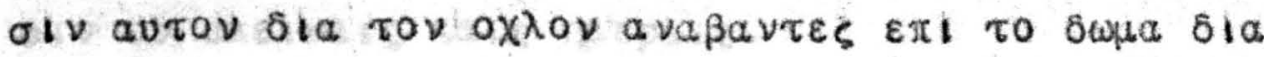

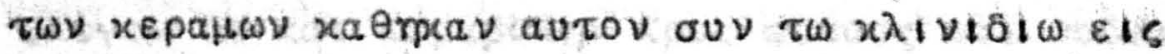

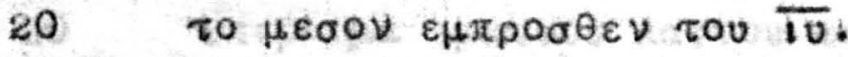

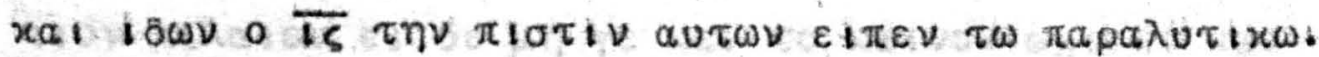

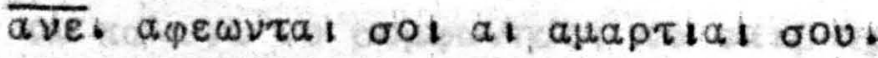

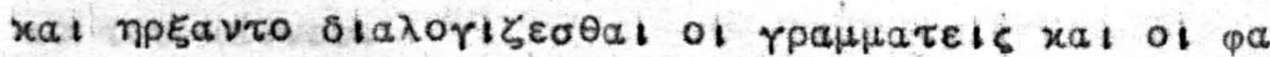

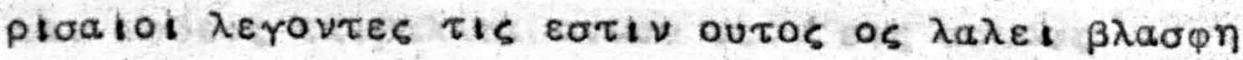

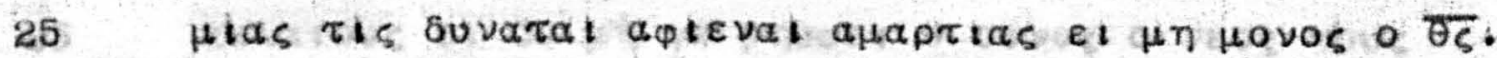

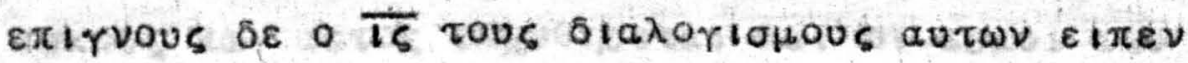

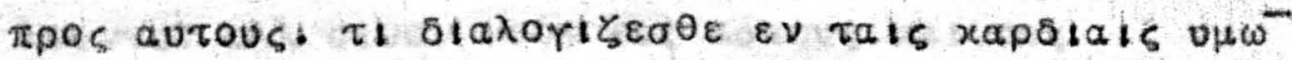

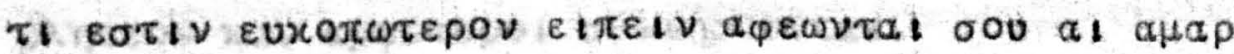

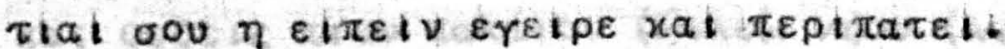

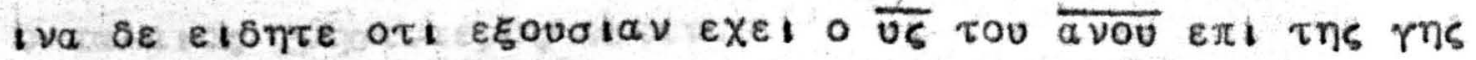

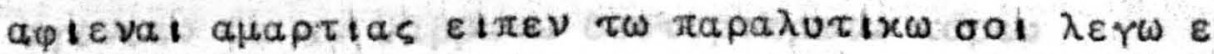

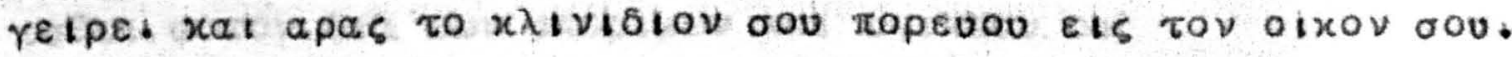

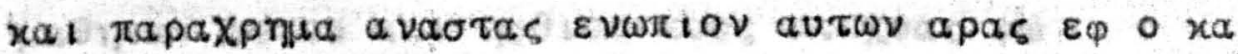

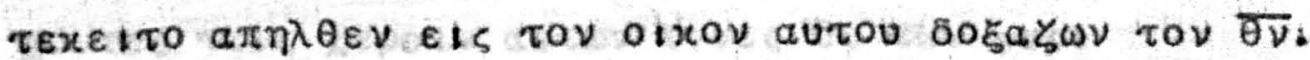

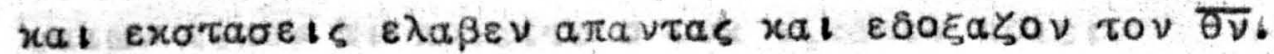

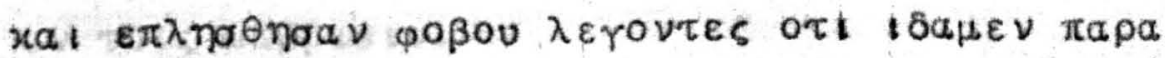
ธоĔ $\alpha$ an

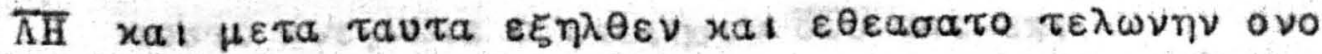

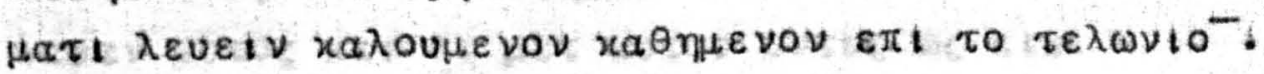

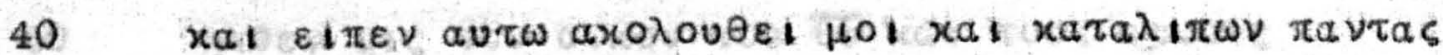

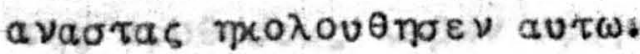




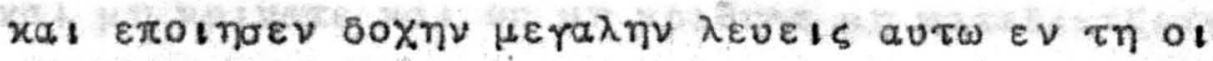

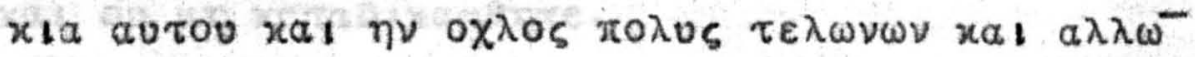

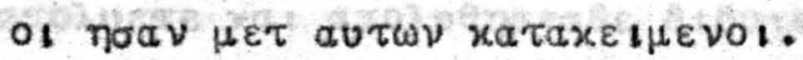

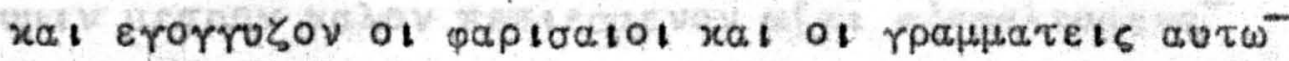

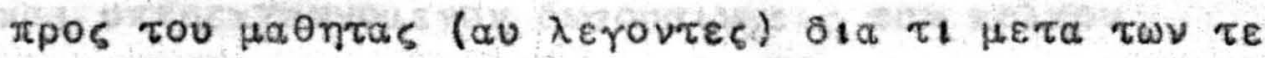
$\lambda \omega v \omega v \varepsilon \sigma \theta i \varepsilon \tau \varepsilon$ xal $\pi$ เ $\varepsilon \tau a l$.

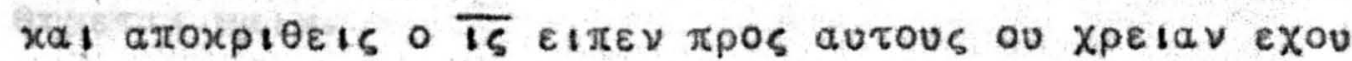

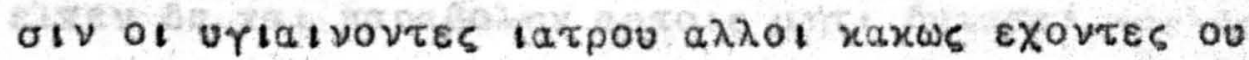

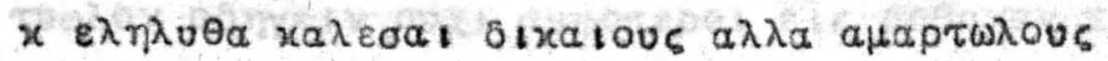

10 Eis Hetavoiav.

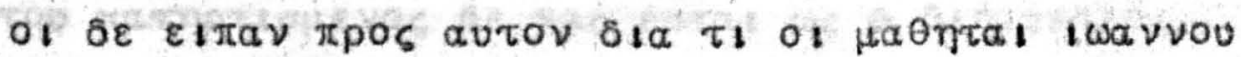

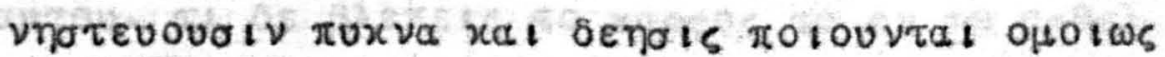

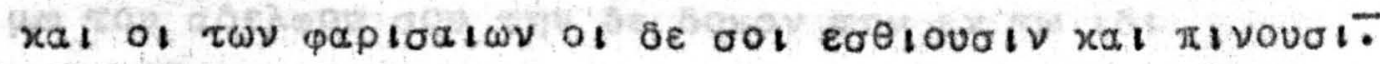

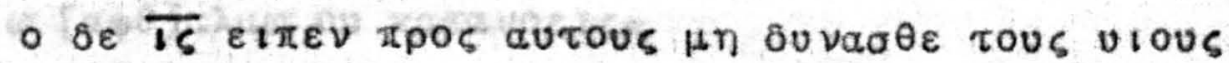

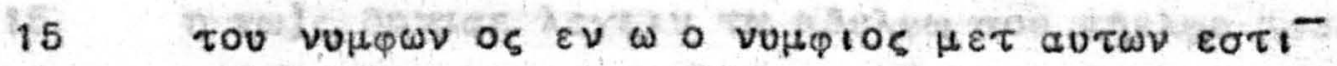

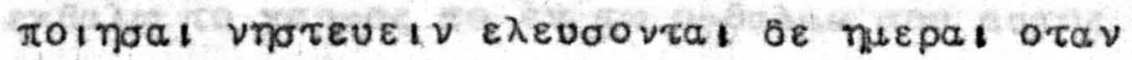

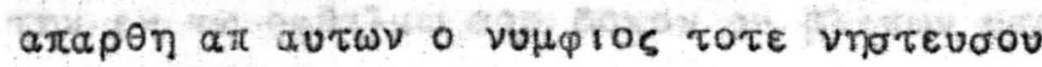

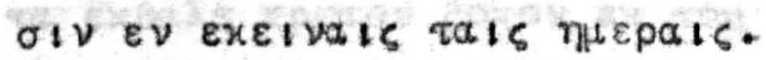

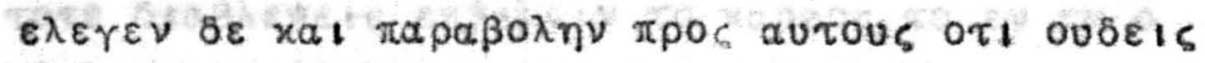

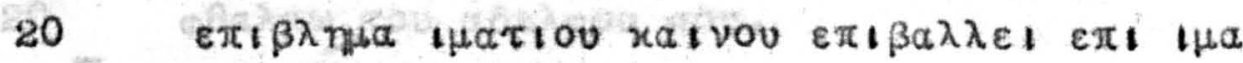

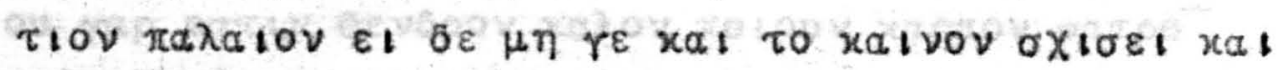

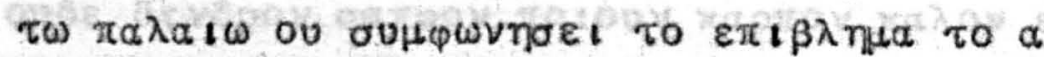
To rov xalvou.

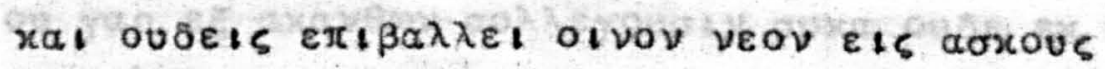

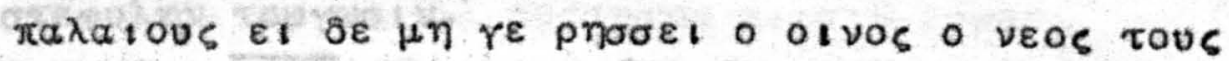

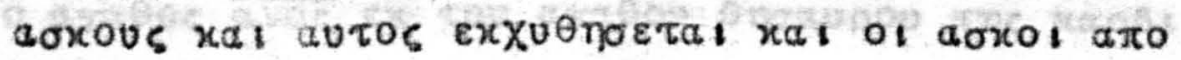

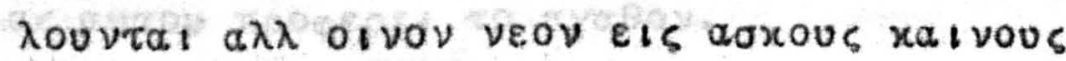

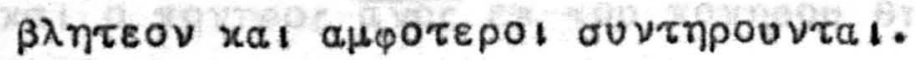

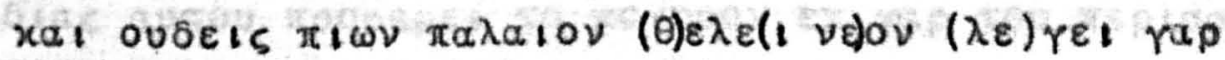

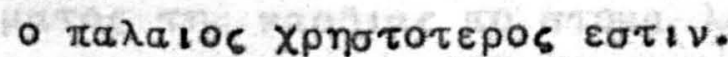

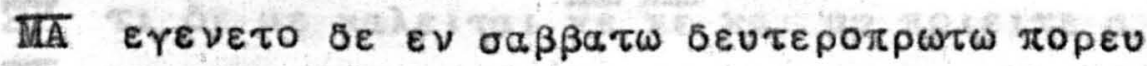

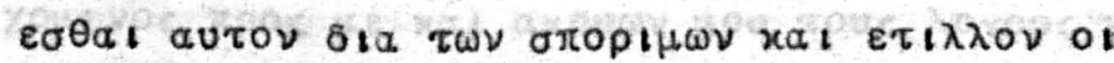

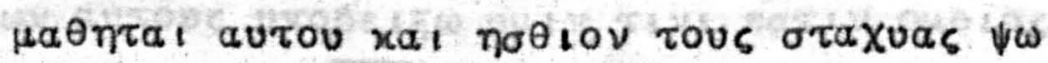

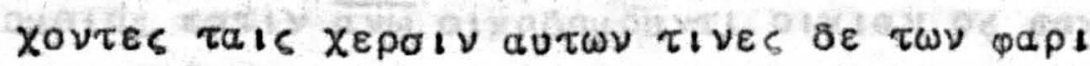

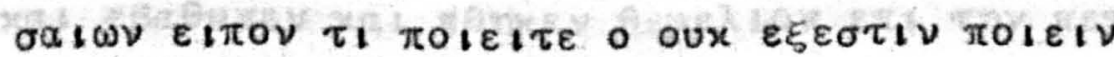

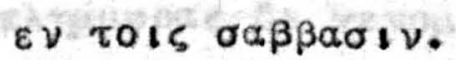

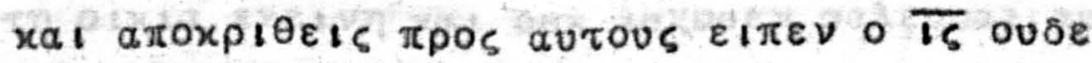

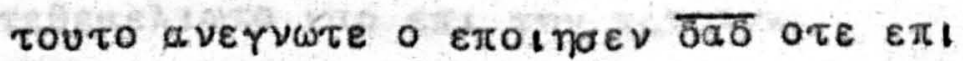

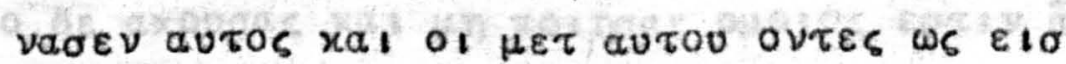

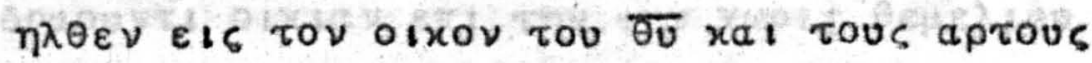

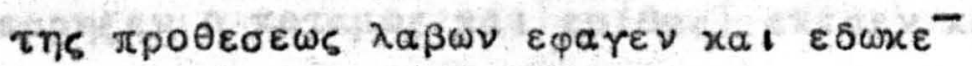




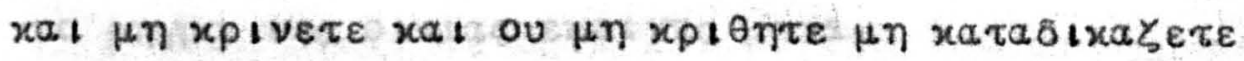

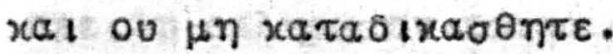

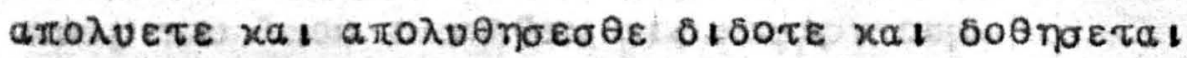

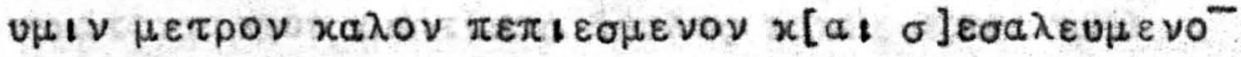

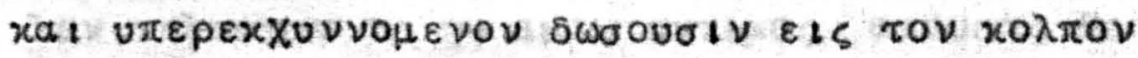

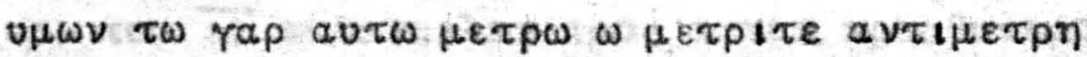

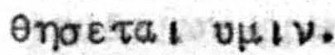

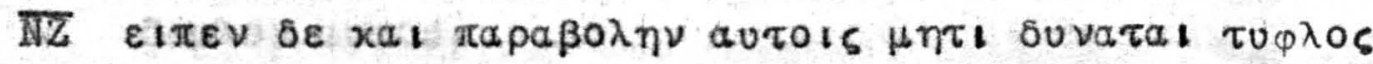

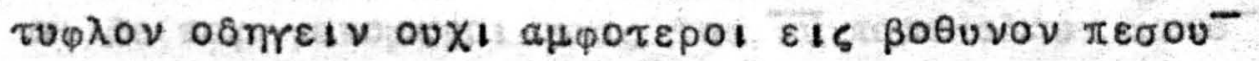

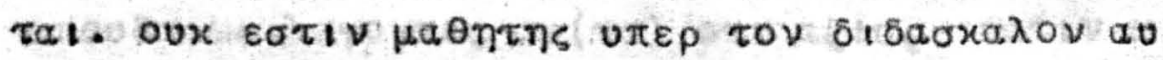

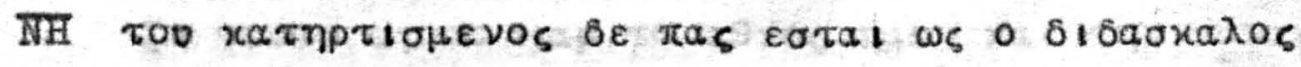

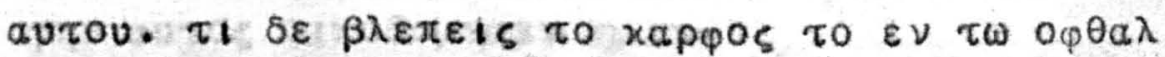

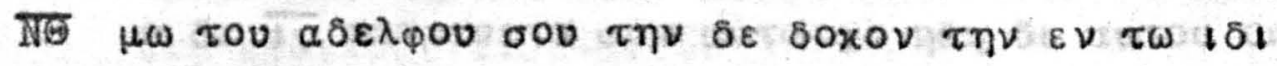

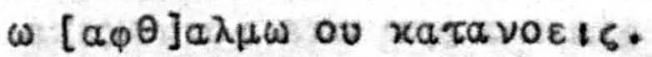

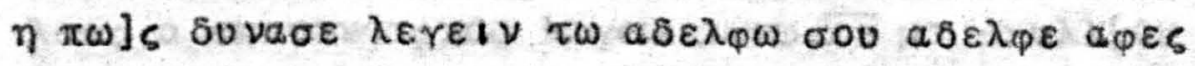

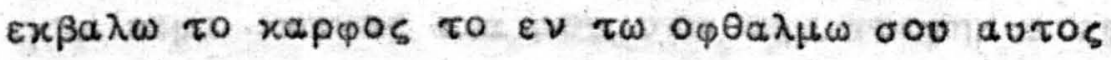

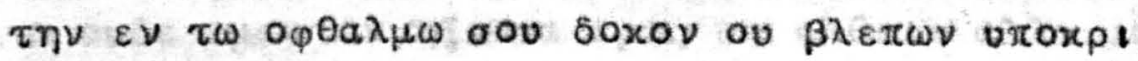

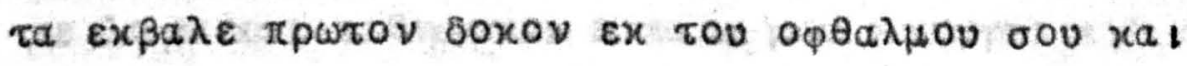

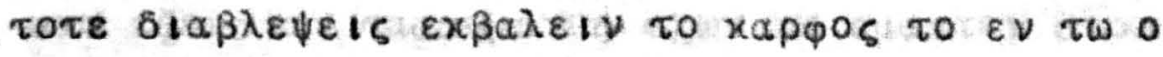

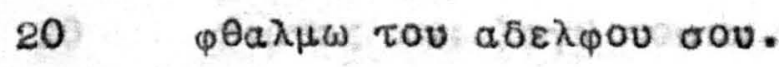

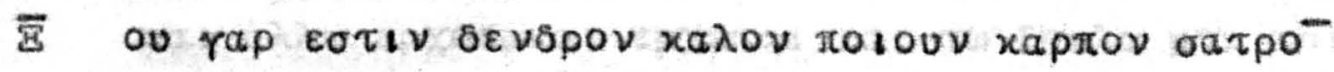

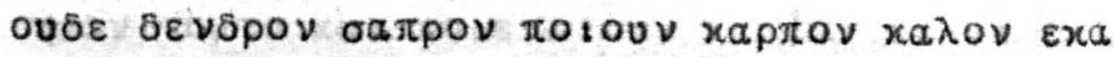

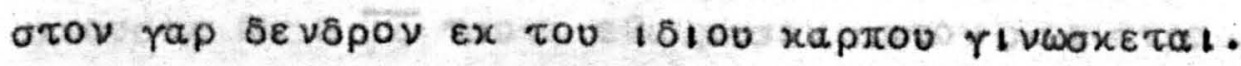

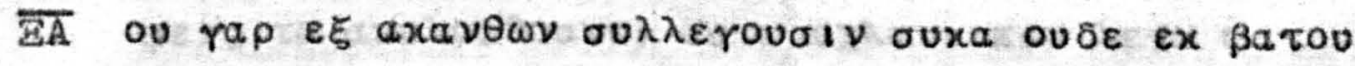
25

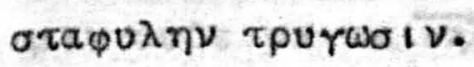

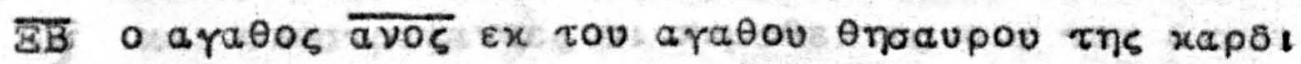

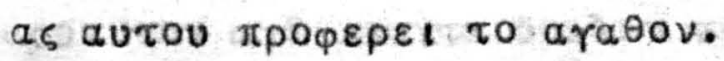

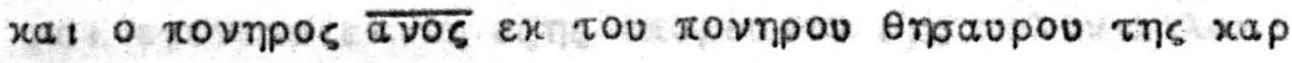

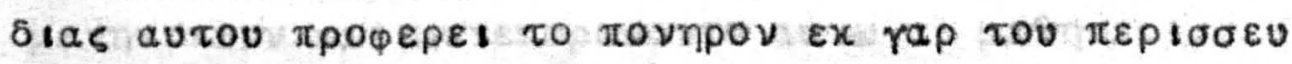

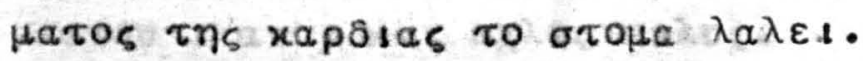

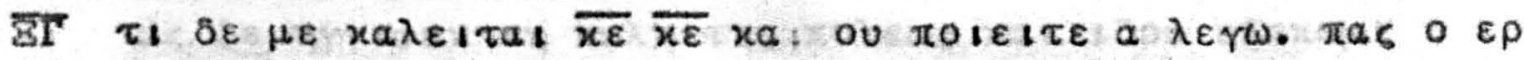

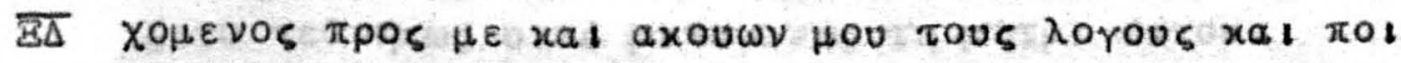

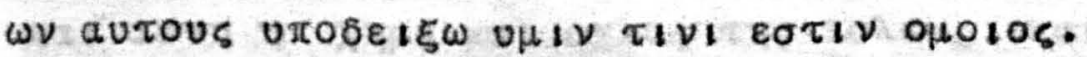

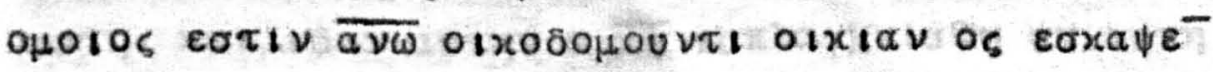

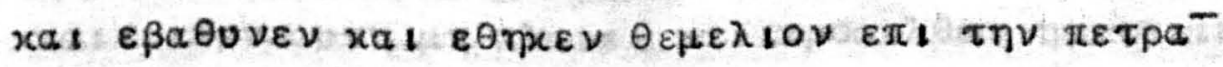

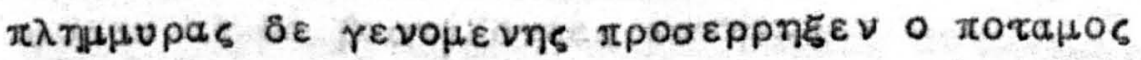

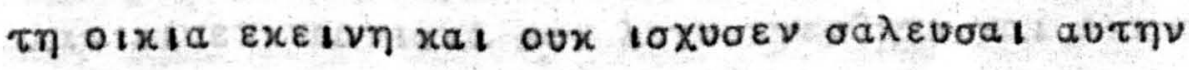
$\tau \varepsilon \theta \varepsilon \beta \varepsilon \lambda \iota \omega \tau o$ rap $\varepsilon \pi \iota \tau \eta \nu \pi \varepsilon \tau \rho \alpha \nu$.

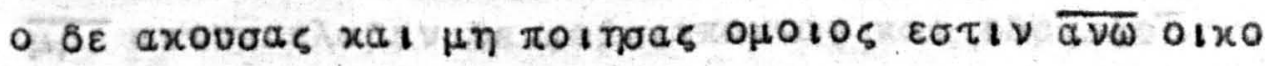

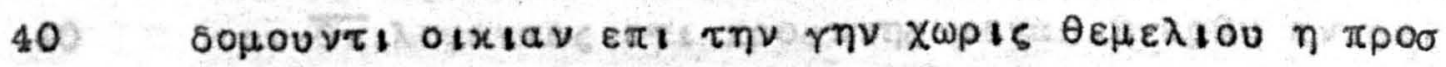

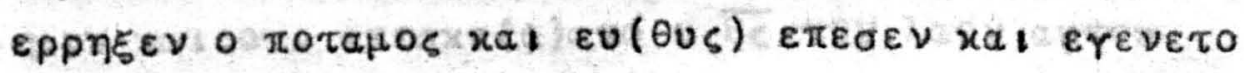




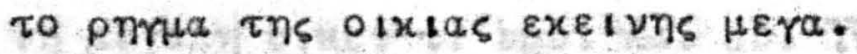

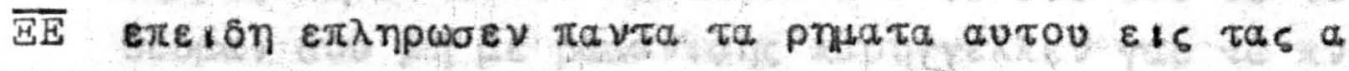

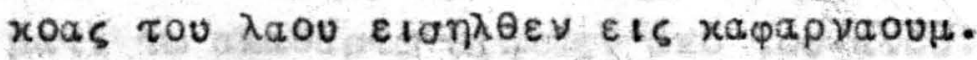

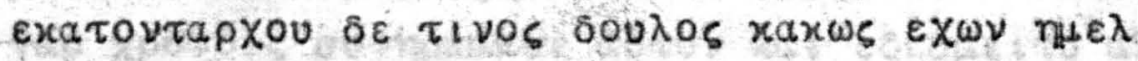

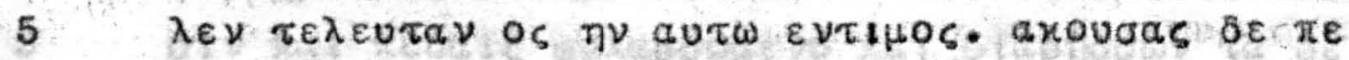

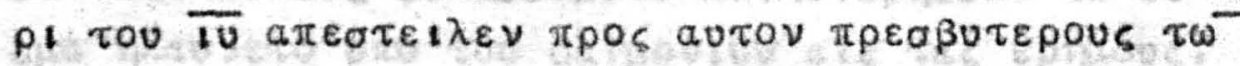

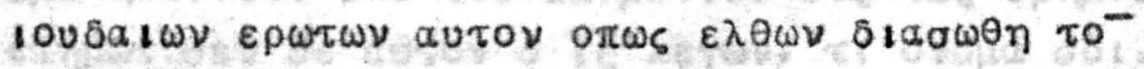
ठouגov autou.

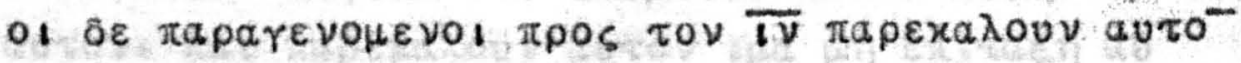

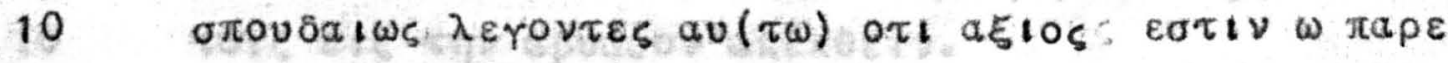

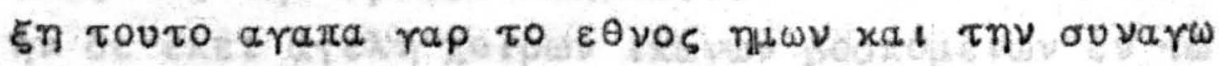
riv autos oixosounoev nueiv.

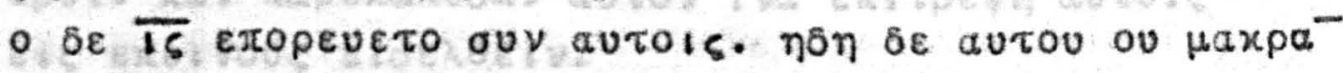

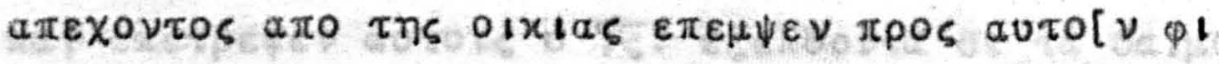

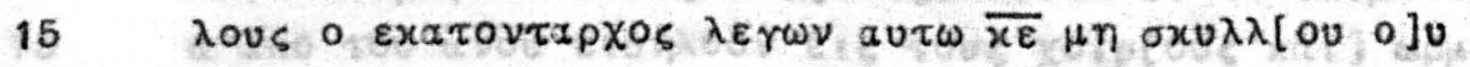

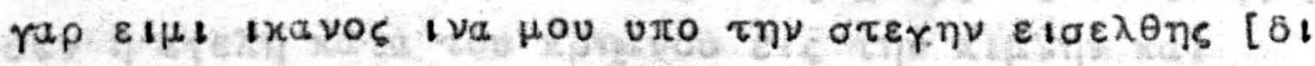

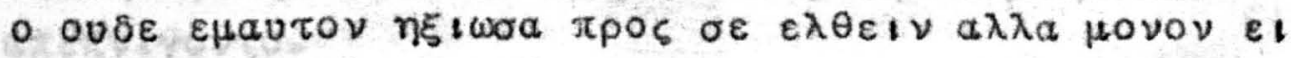

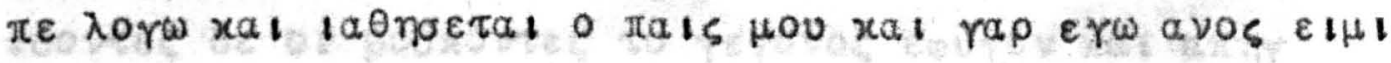

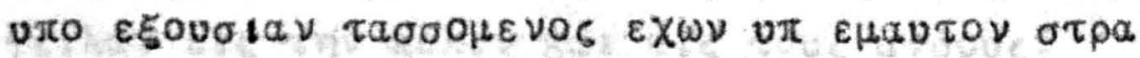

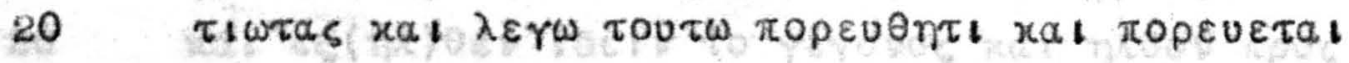

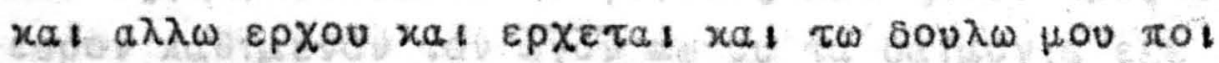

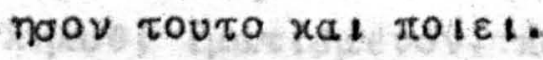

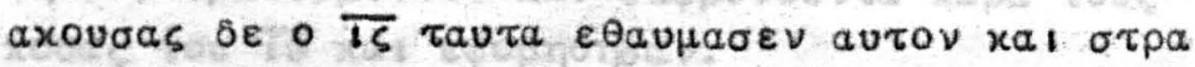

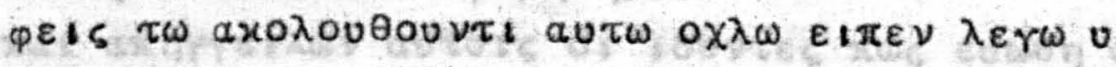

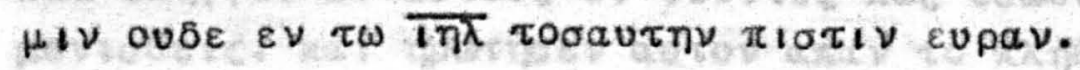

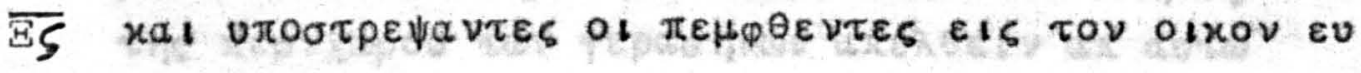

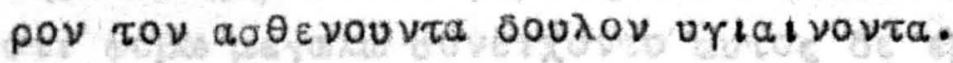

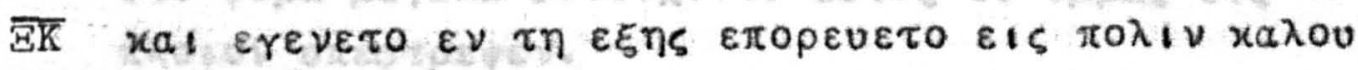

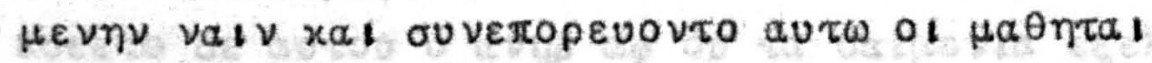

30 autov ixavol $x a l$ oxhos roגus.

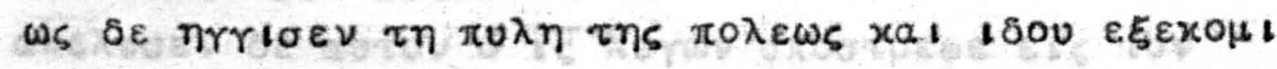

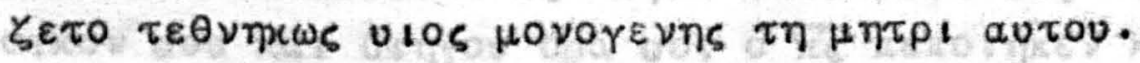

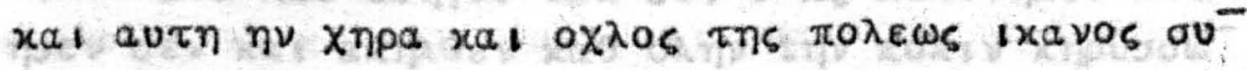

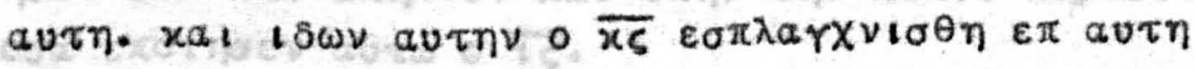

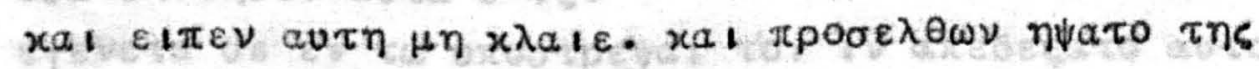

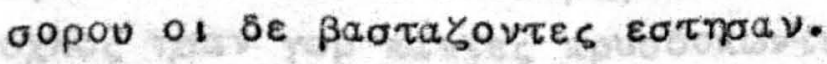

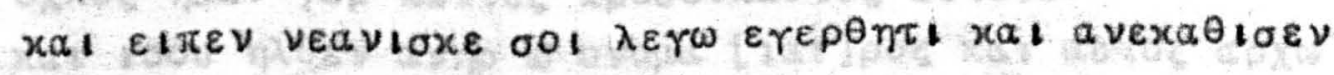

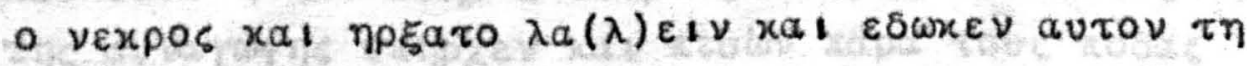

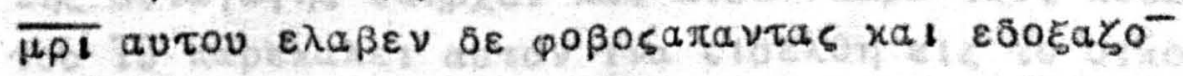

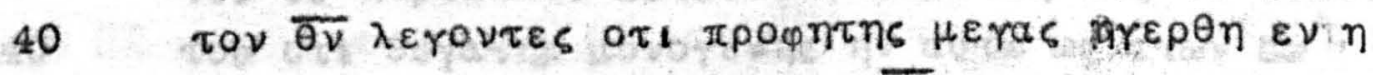

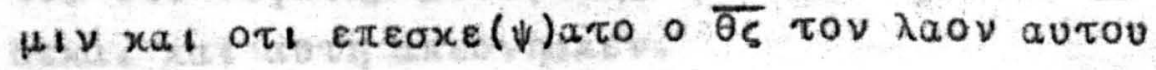




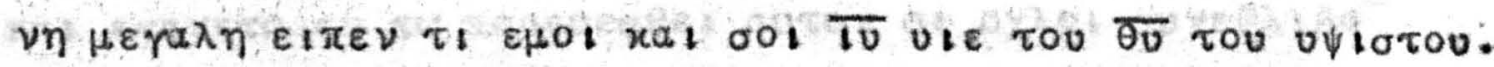

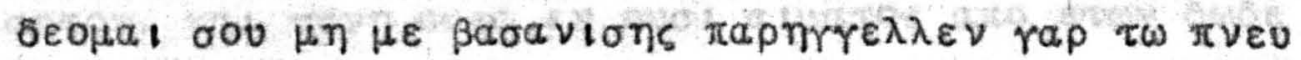

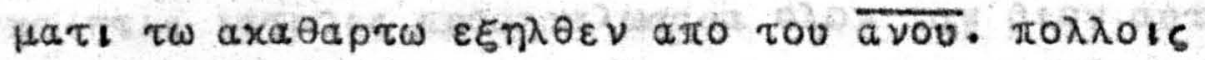

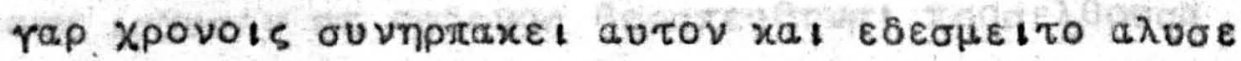

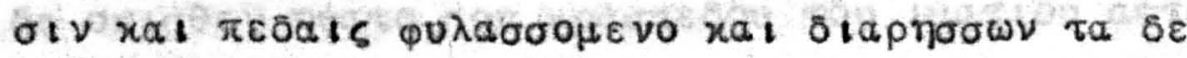

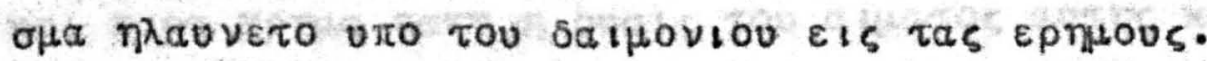

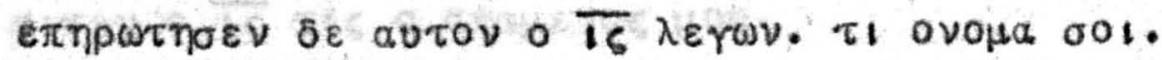

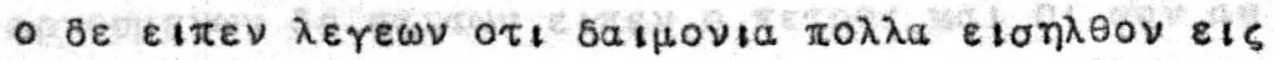

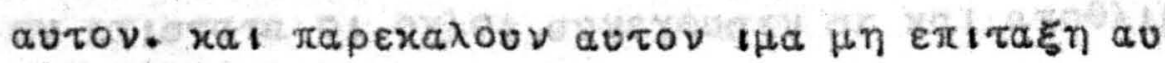

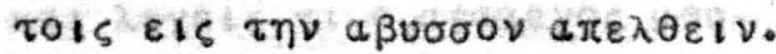

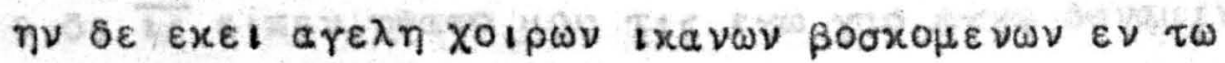

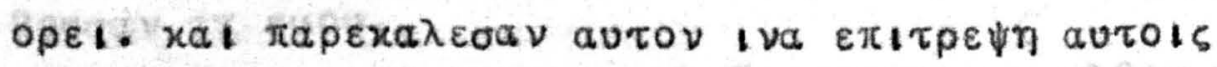

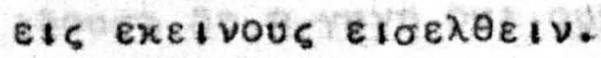

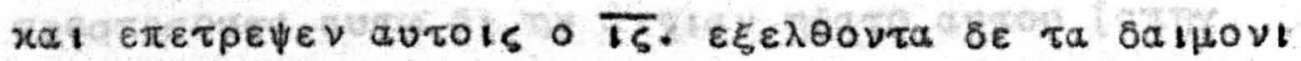

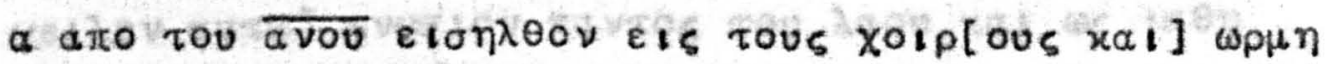

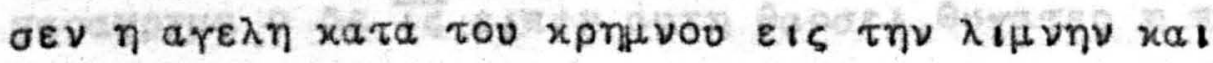

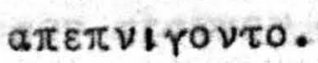

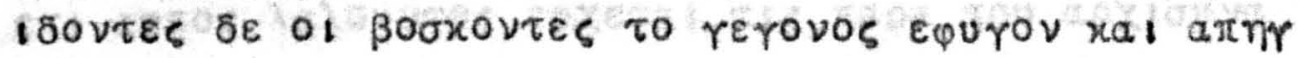

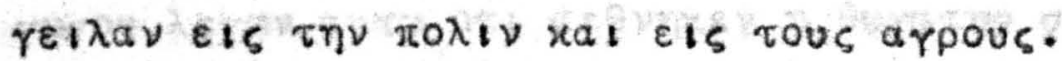

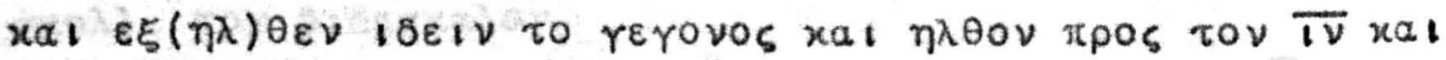

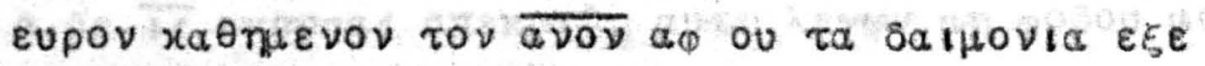

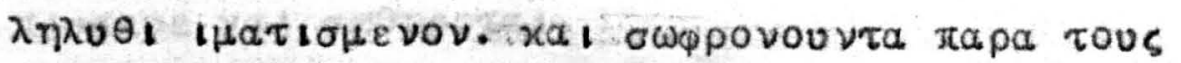

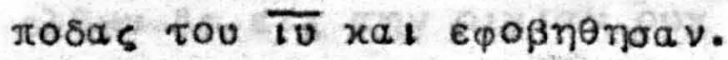

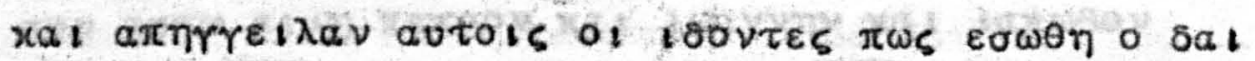

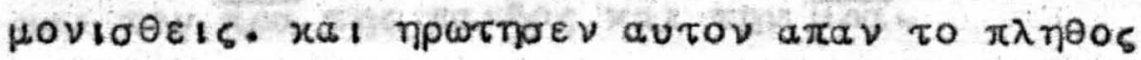

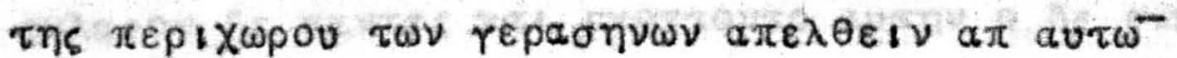

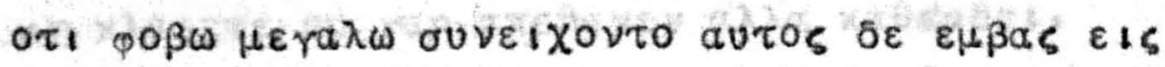

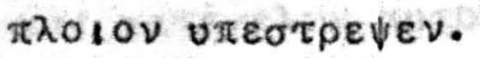

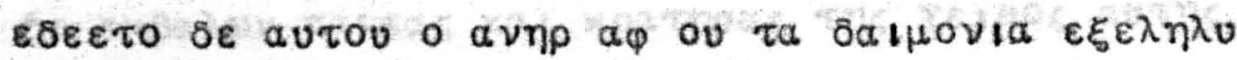

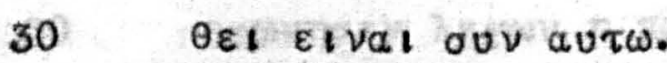

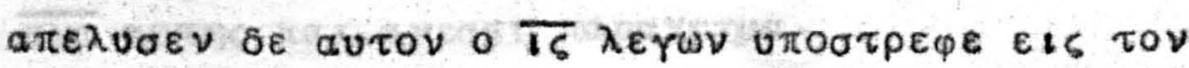

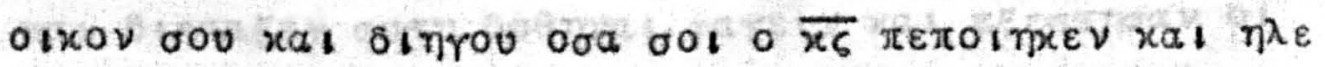

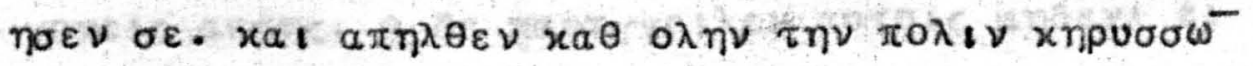

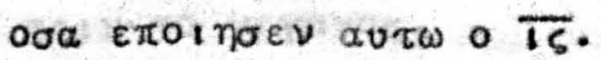

35 EYร

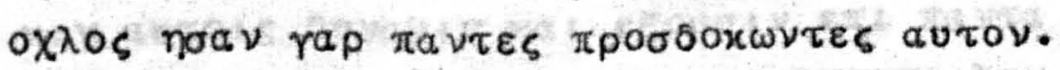

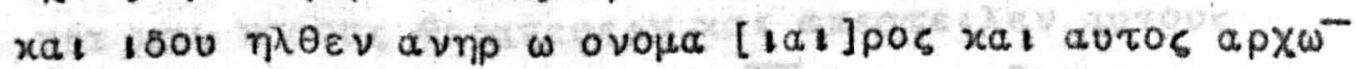

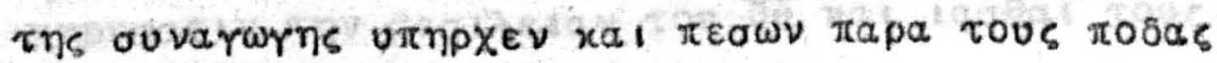

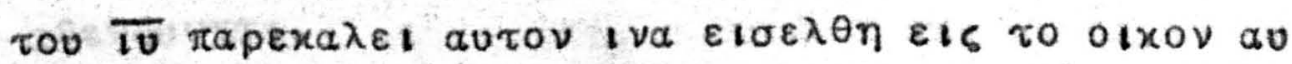

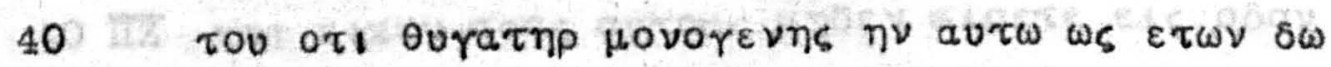

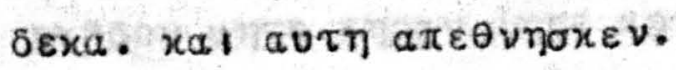




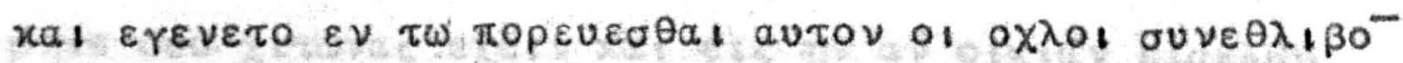

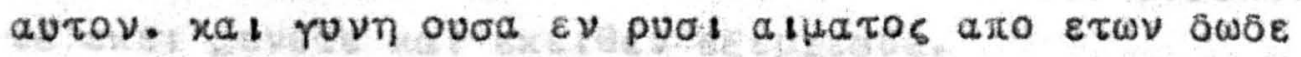

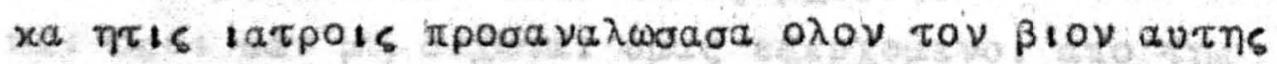

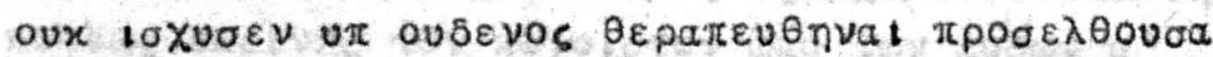

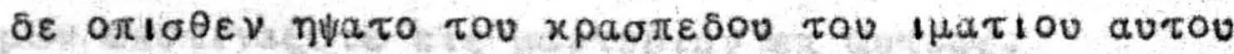

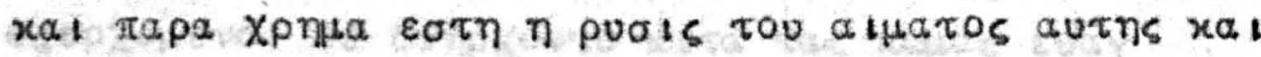

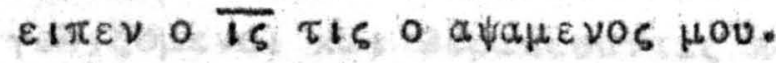

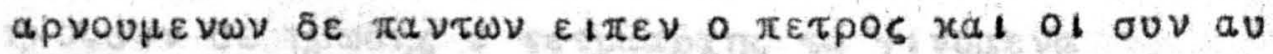

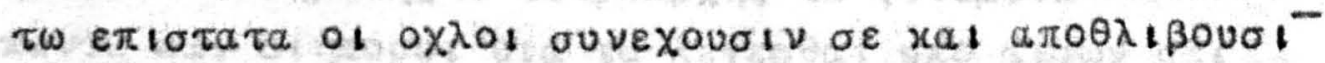

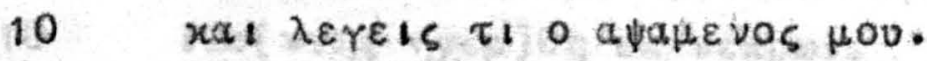

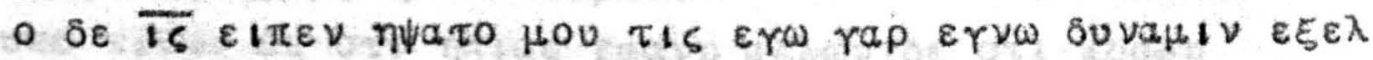
Qovoav a $\varepsilon \mu$ ov.

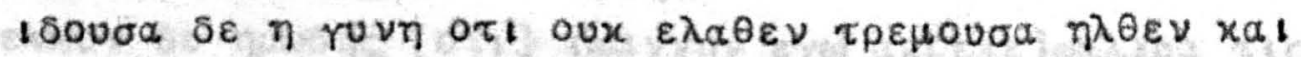

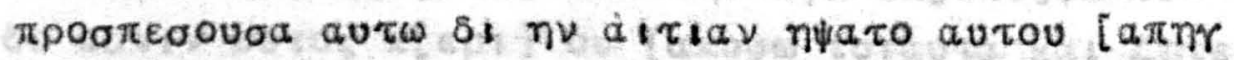

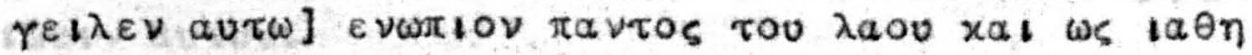

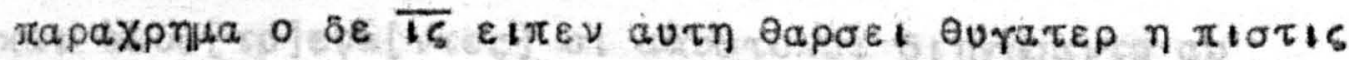

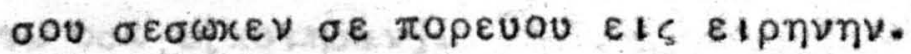

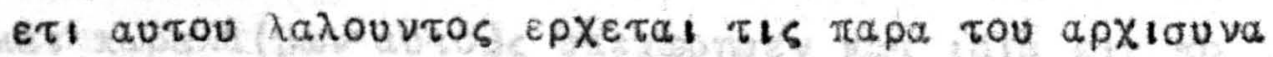

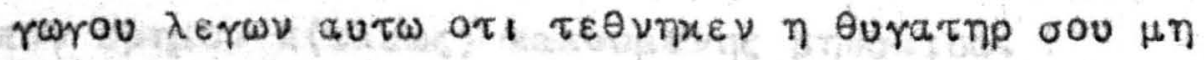
oxv $\lambda \lambda \varepsilon$ Tov $\delta 18 a \sigma x a \lambda o \nu$.

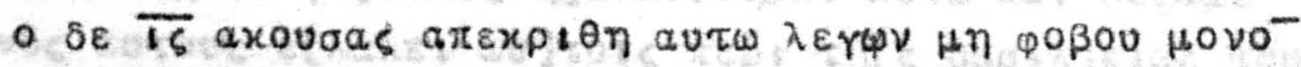

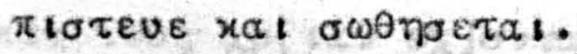

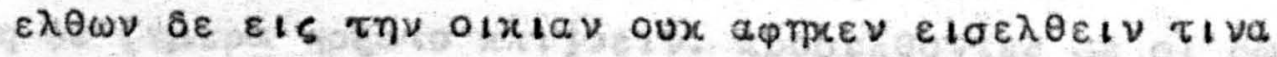

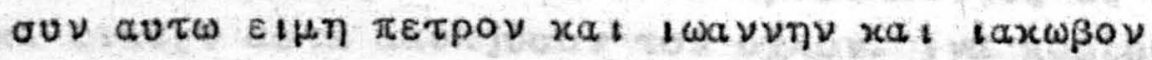

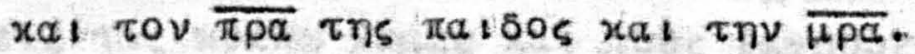

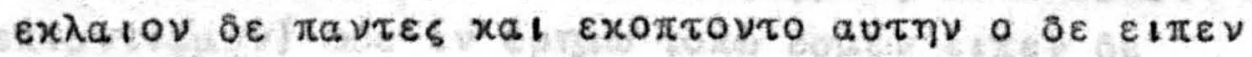

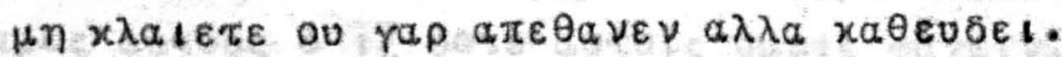

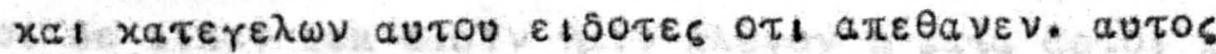

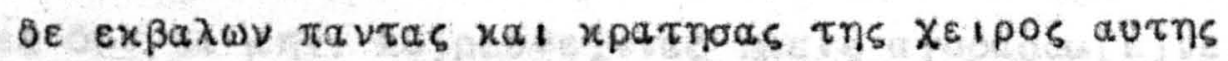

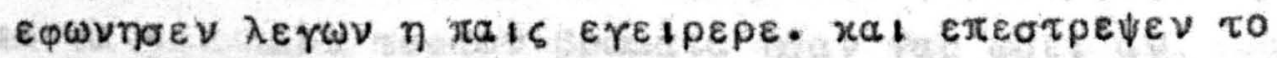

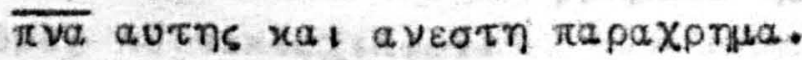

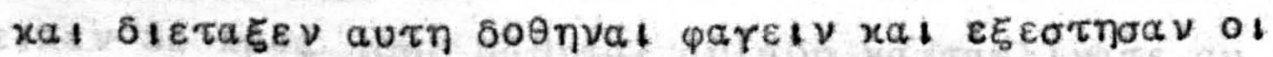

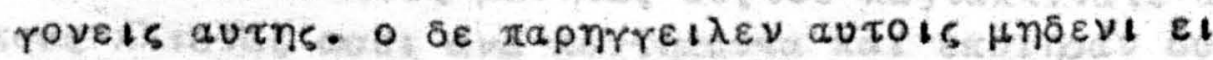
$\pi \varepsilon เ \nu$ to rerovos.

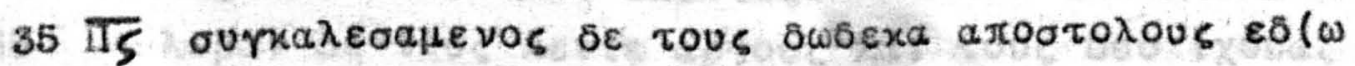

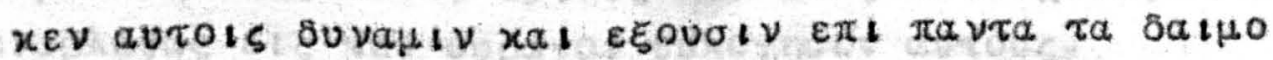

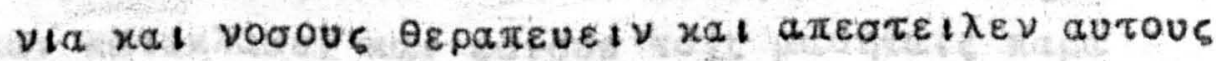

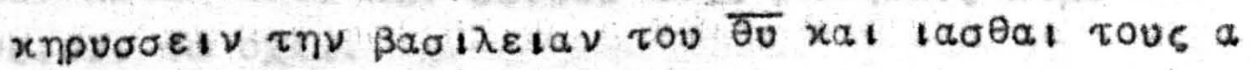

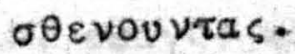

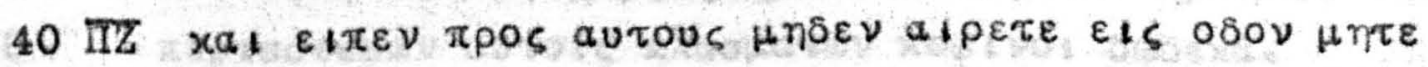

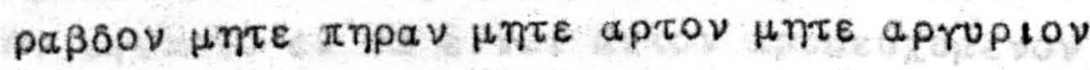




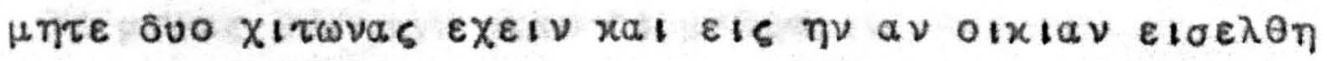
$\tau \varepsilon \varepsilon x \varepsilon \downarrow \mu \varepsilon \nu \varepsilon \tau \varepsilon$ xa! $\varepsilon x \varepsilon \downarrow \theta \varepsilon \nu$ $\varepsilon \xi \varepsilon \rho \chi \varepsilon \sigma \theta \varepsilon$.

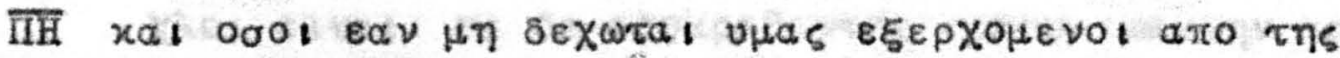

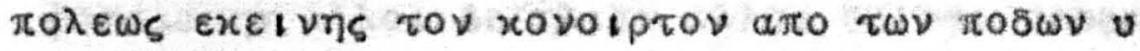

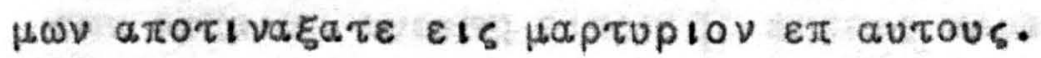

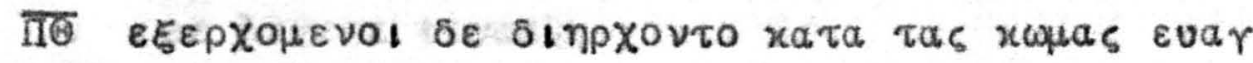

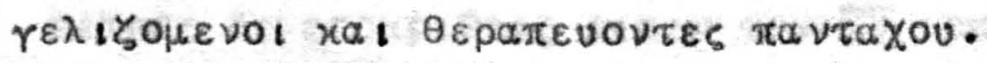

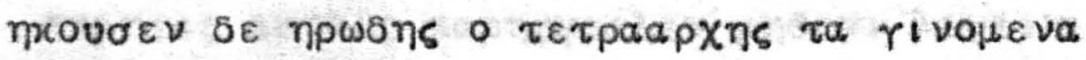

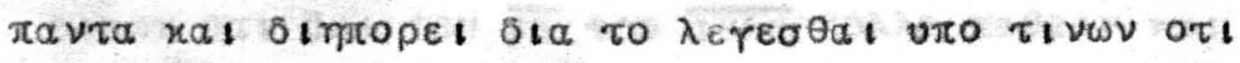

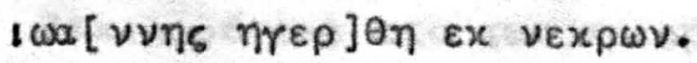

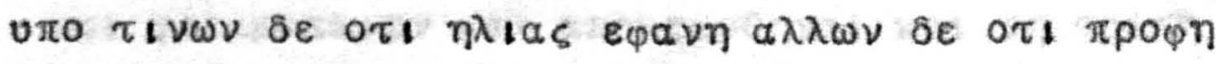

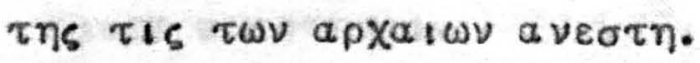

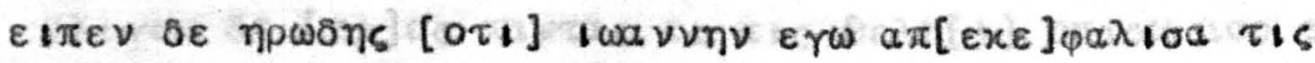

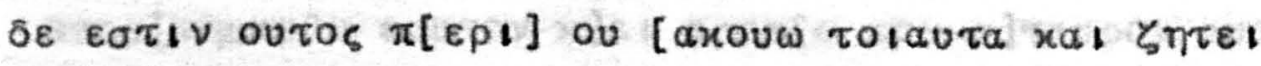
เธeเป aurov.

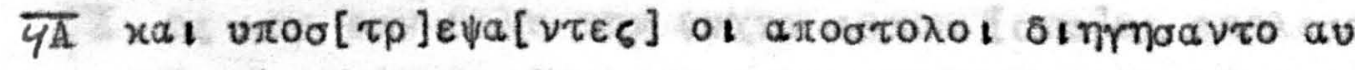
$\tau \omega$ ođa $\varepsilon[\pi \circ$ inoav $]$.

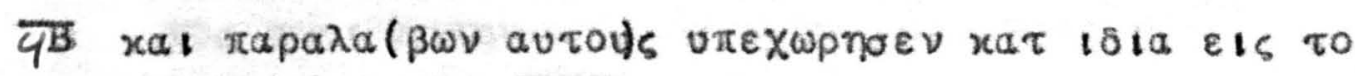

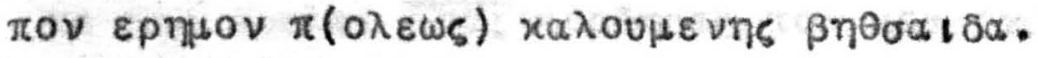

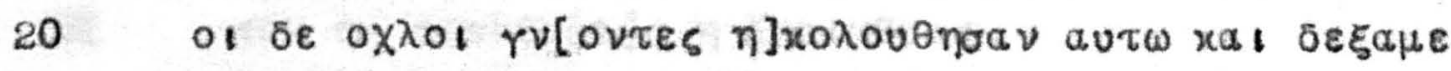

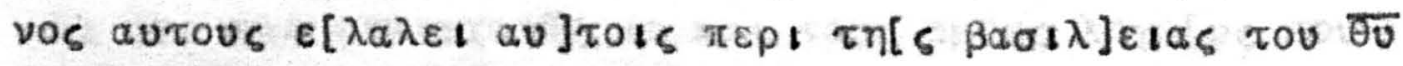

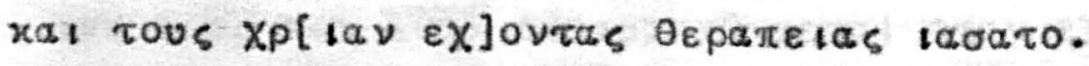

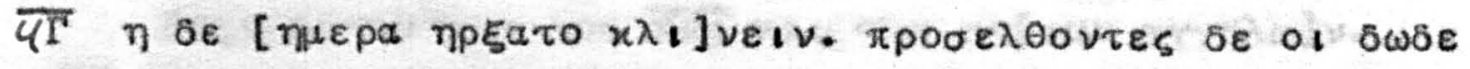

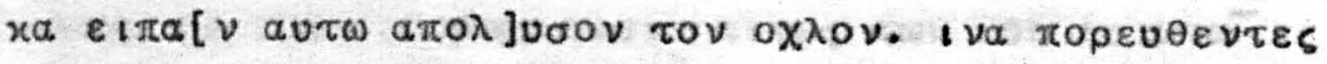

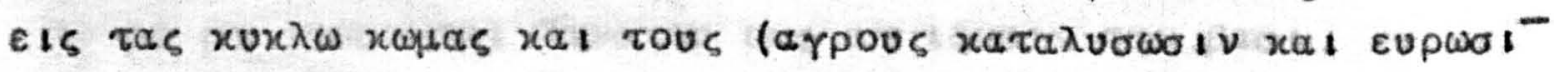

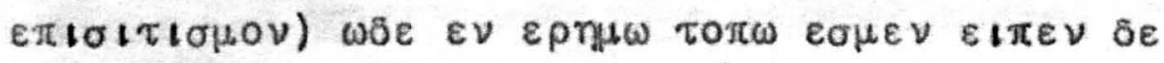

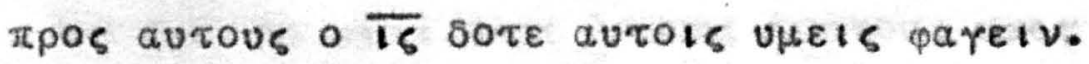

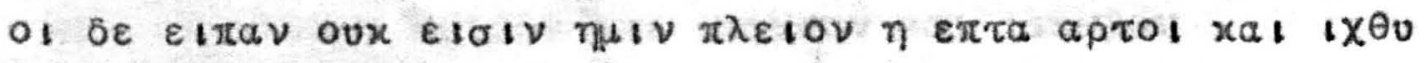

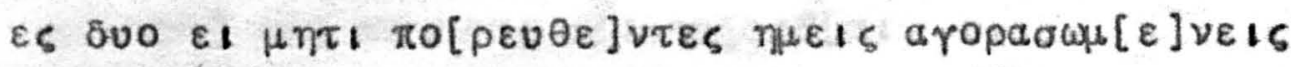

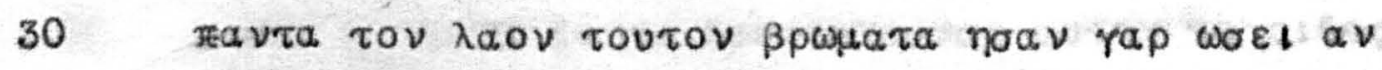

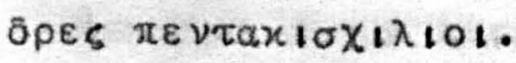

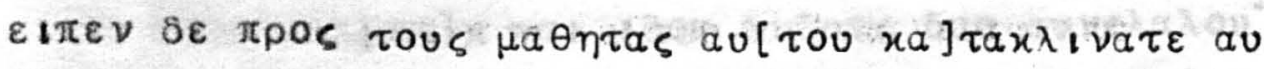

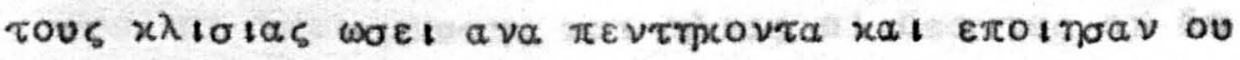

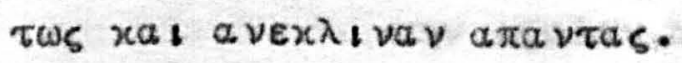

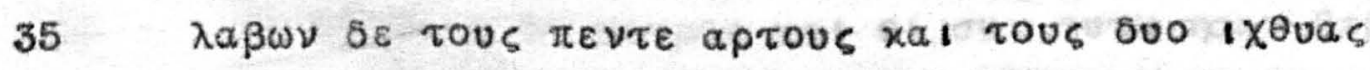

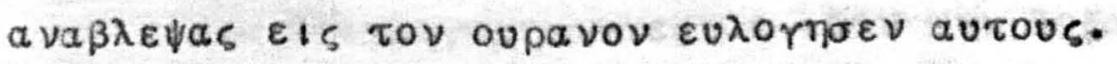

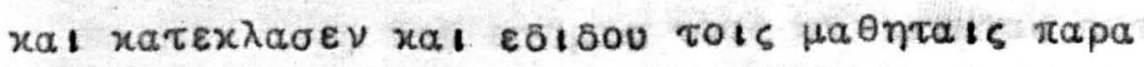

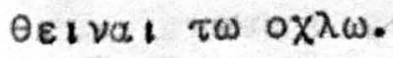

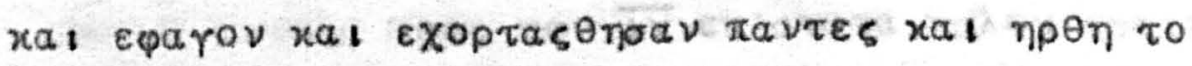

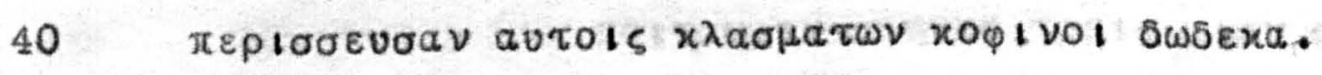

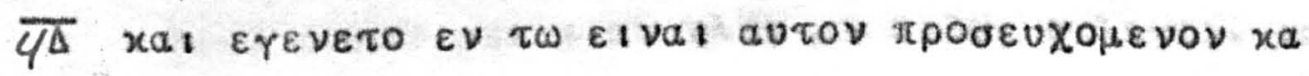




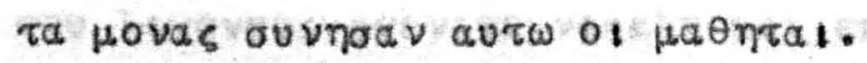

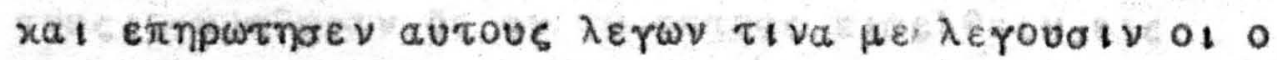

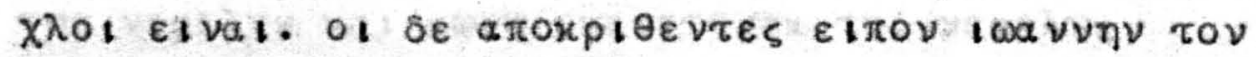

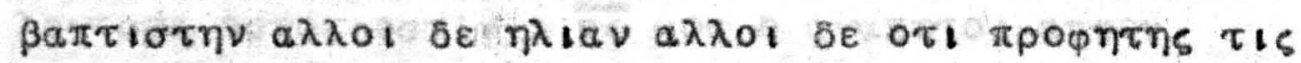

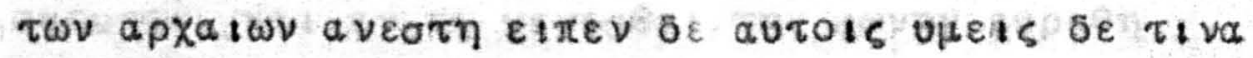
$\mu \varepsilon \lambda \varepsilon \gamma \varepsilon \tau \varepsilon \varepsilon$ เ $\alpha a$ t.

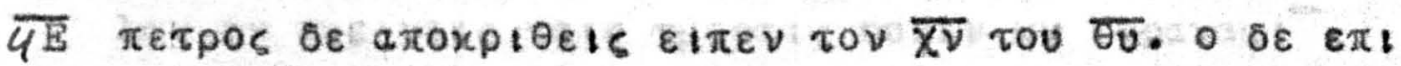

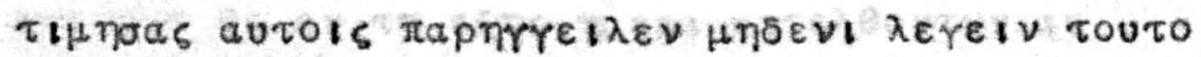

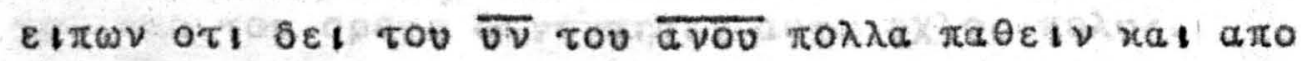

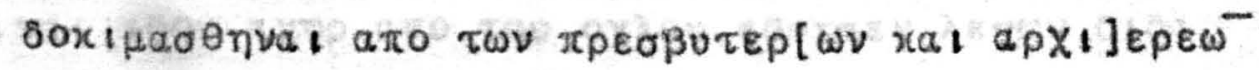

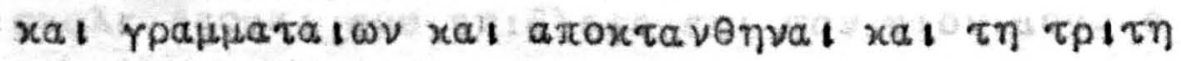

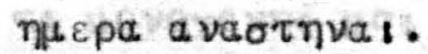

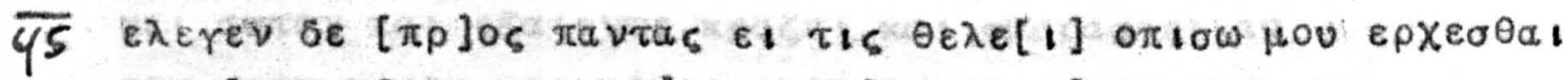

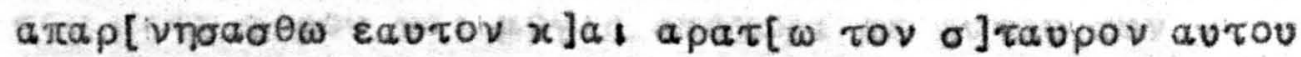

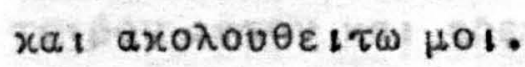

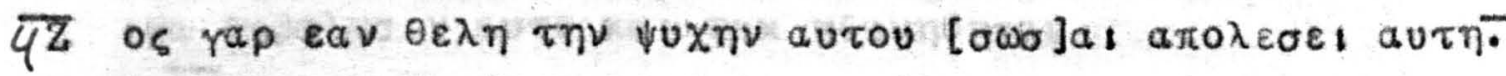

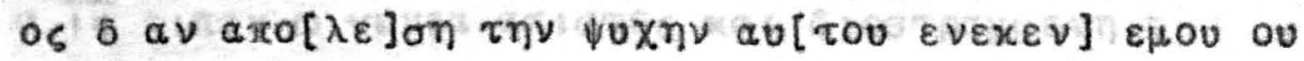

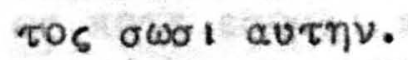

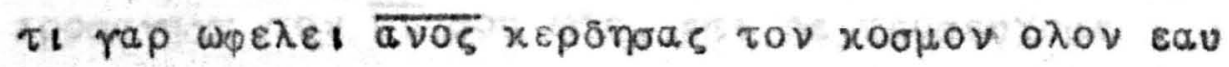

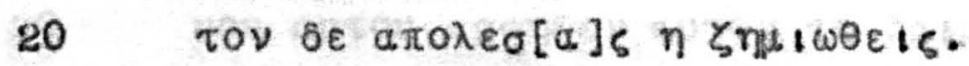

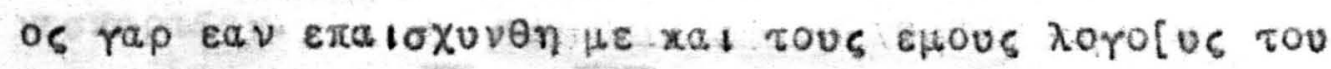

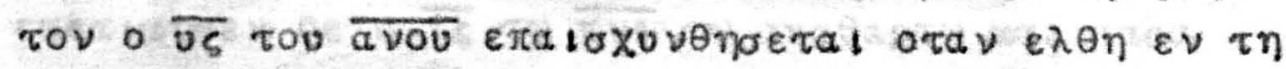

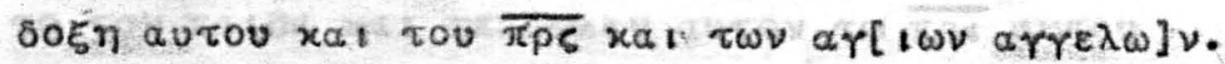

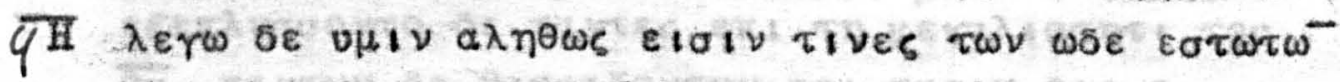

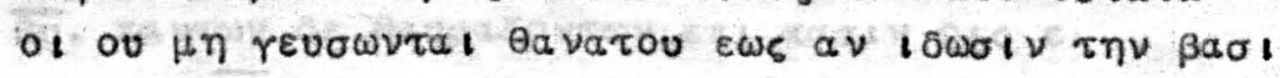
$\lambda \varepsilon i a v$ tou

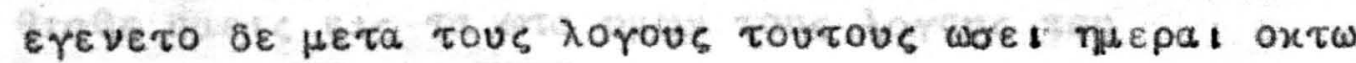

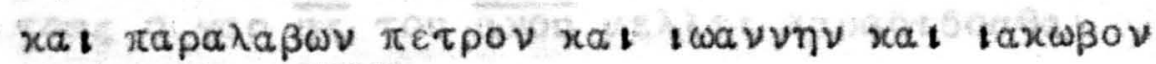

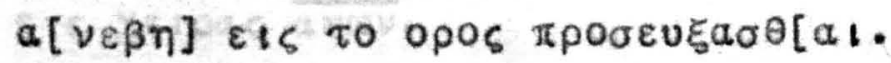

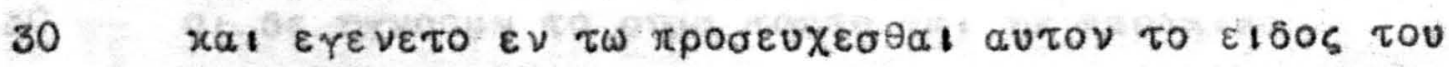

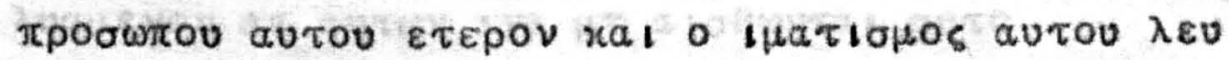

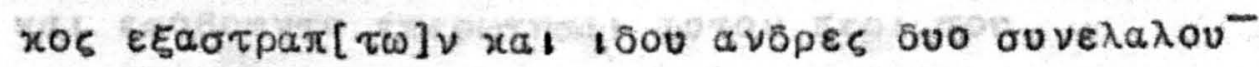

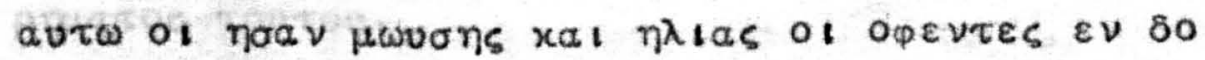

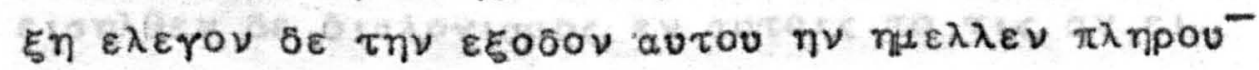

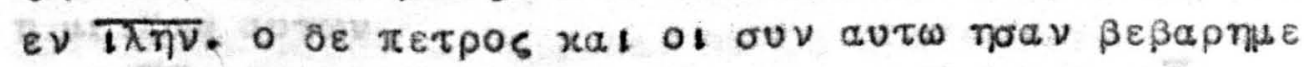

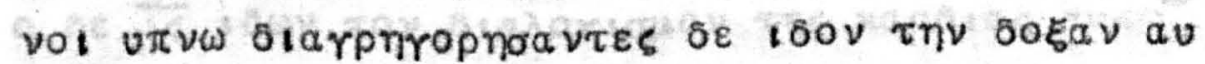

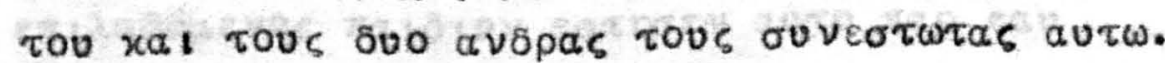

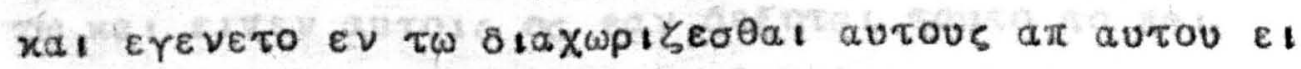

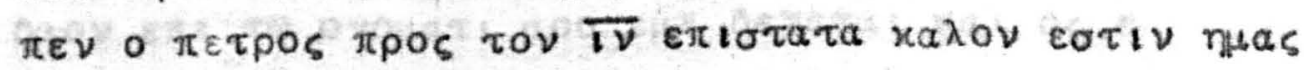

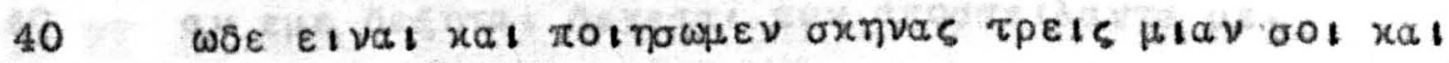

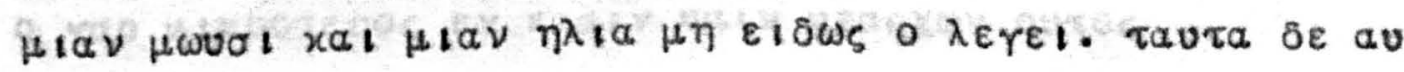




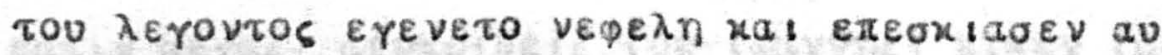

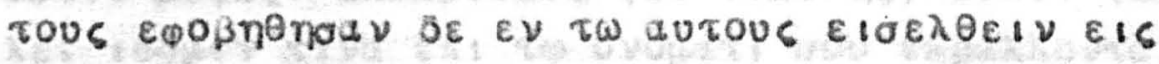

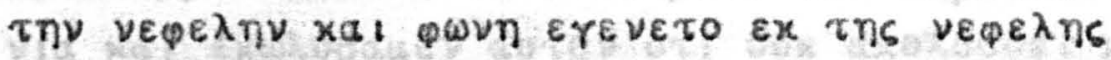

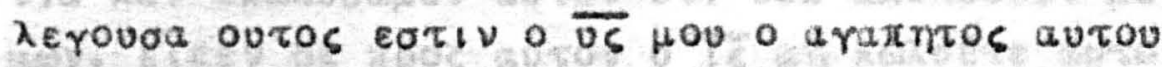

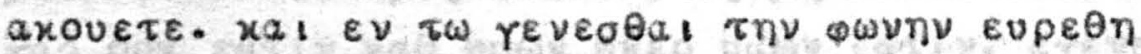
is hovos xat autol eairnoav xal ovoevel axhrre!

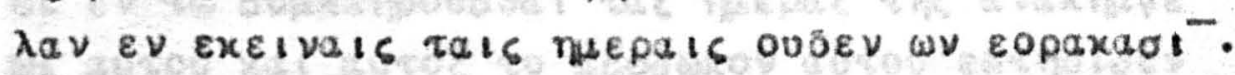

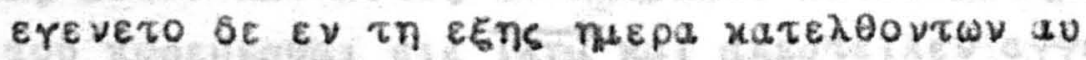

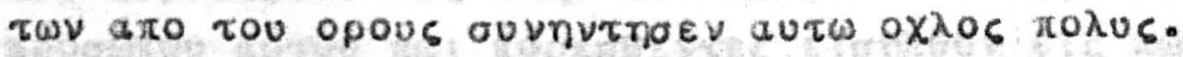

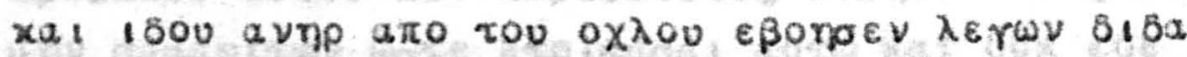

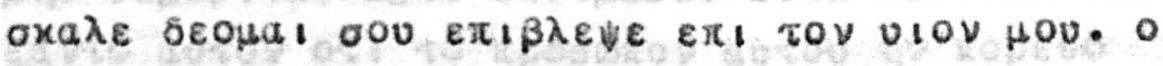

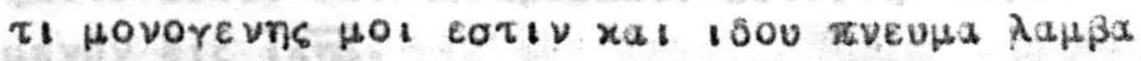

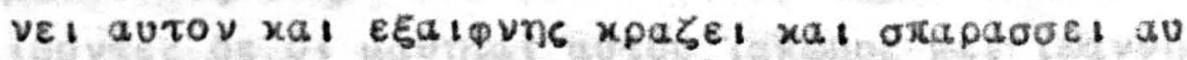

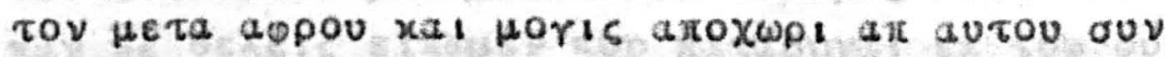

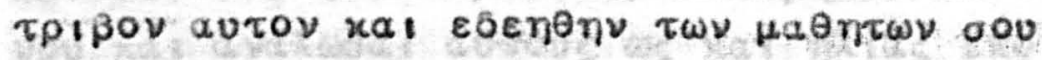

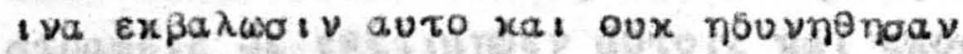

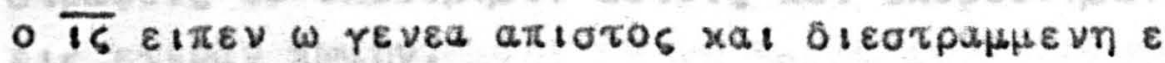

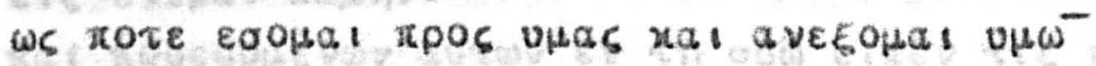

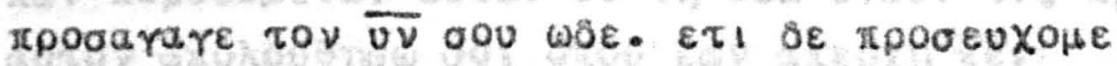
vou autou eppnEev autov to sathoviov xal ou

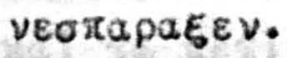

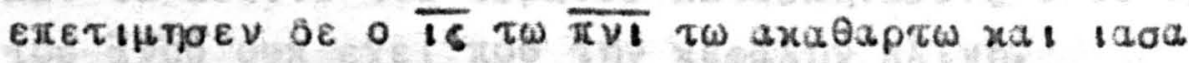

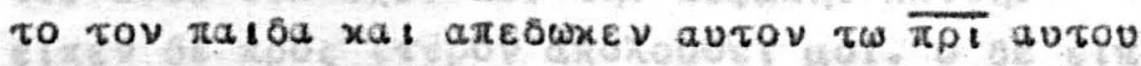

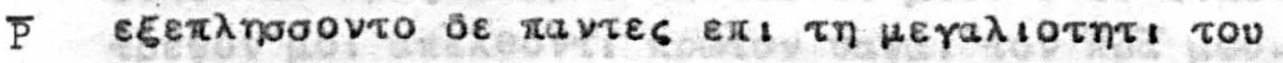

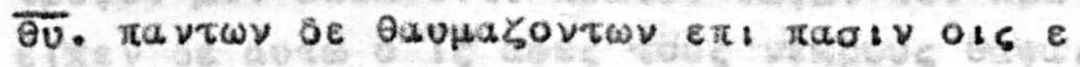

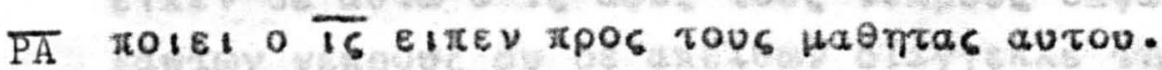

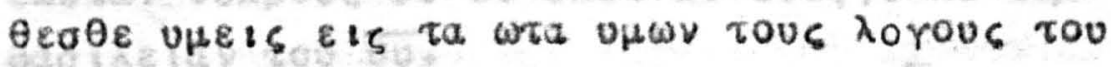

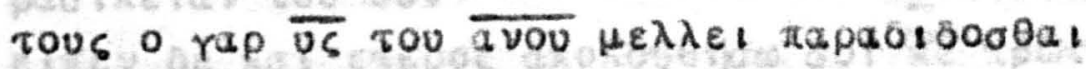
Eis Xeipas a a

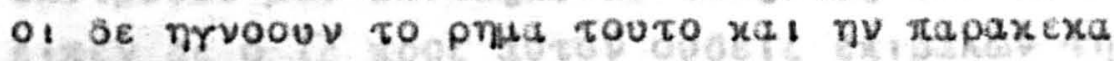

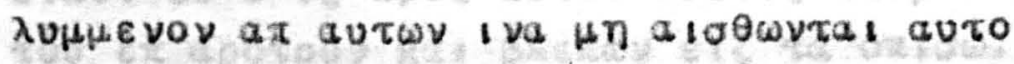

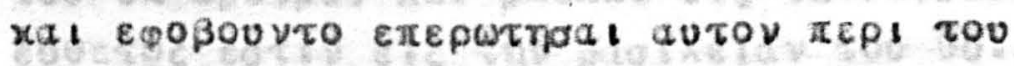
pruatos toutou.

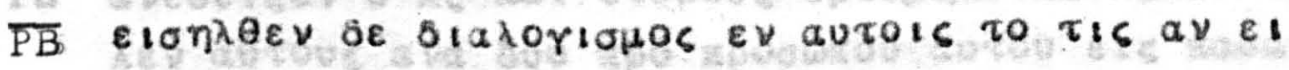
$\eta \mu \varepsilon \downarrow \zeta \omega v$ au $\omega$.

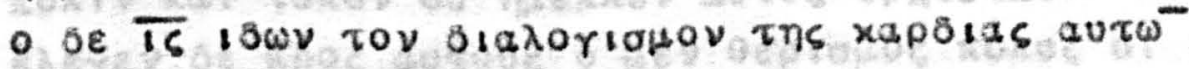

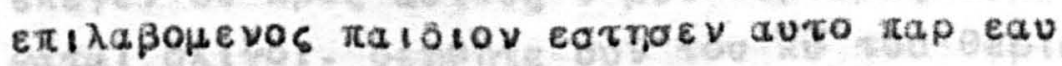

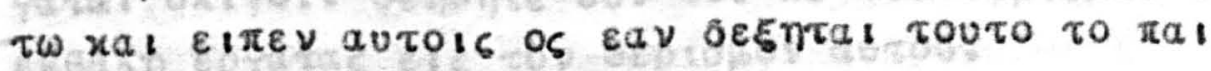

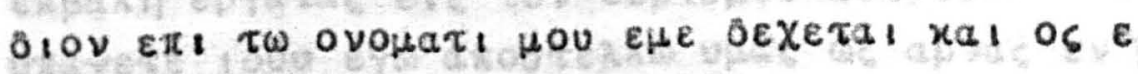

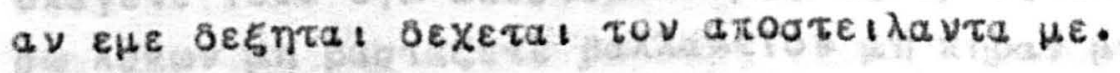

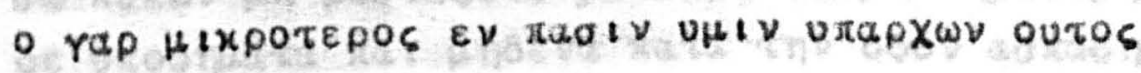




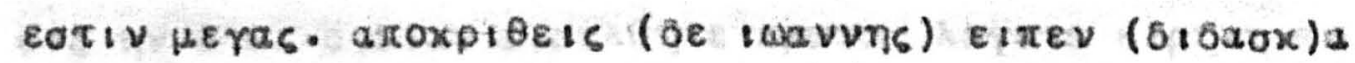

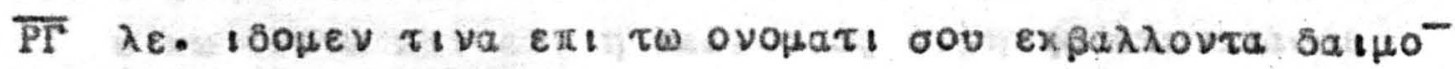

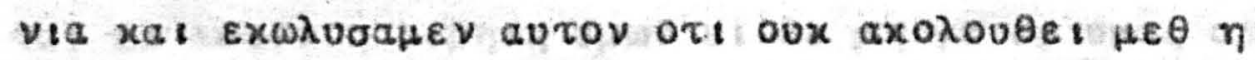

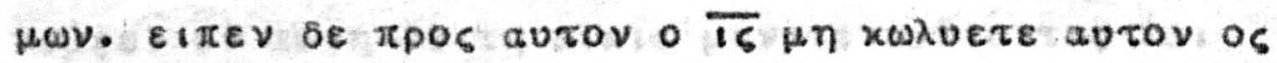

5

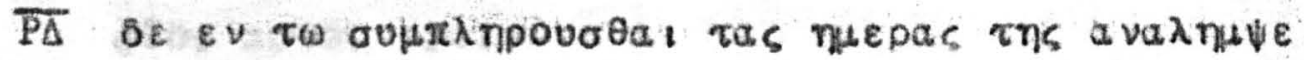

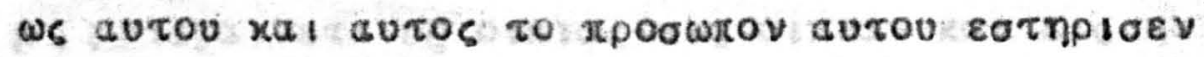

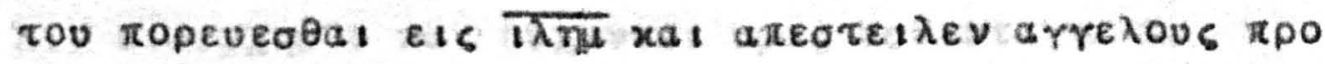

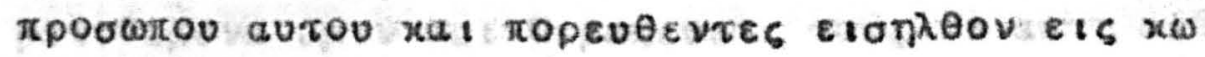

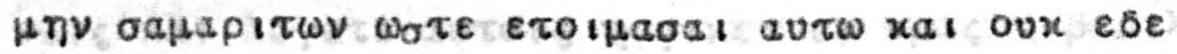

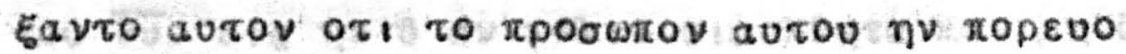
Hevoveis $\overline{\mathrm{t} T \eta \mu}$.

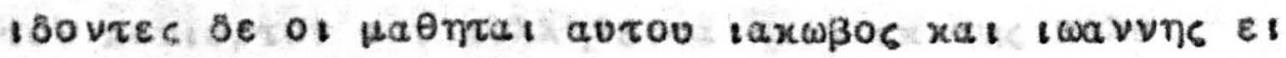

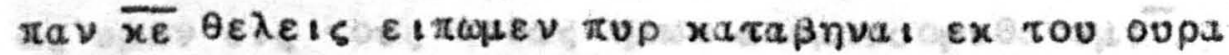

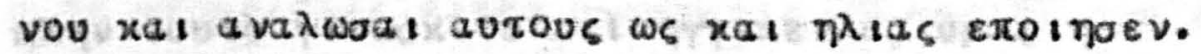

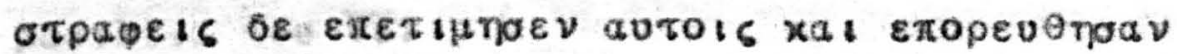
E। $\varepsilon \tau e p a \nu$ x

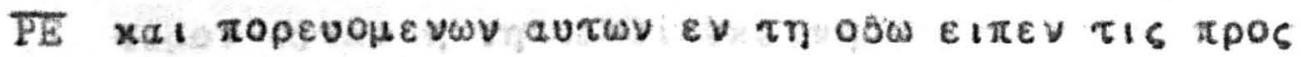

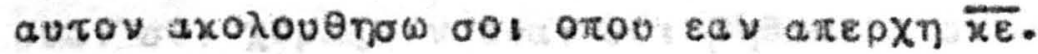
xal eirev autw o $\overline{t s}$ as a

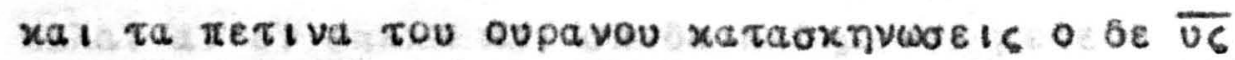

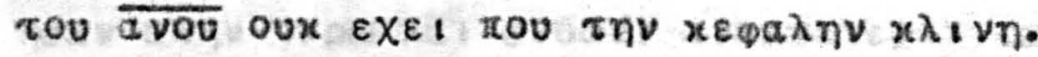

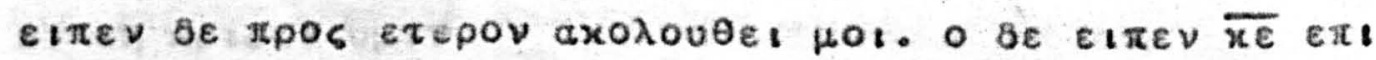

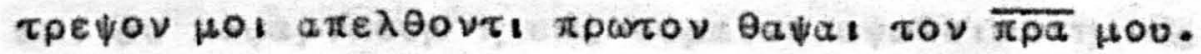

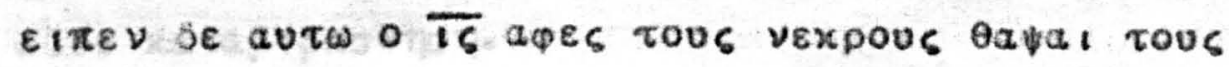

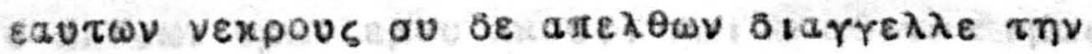
Baoı $\lambda \varepsilon$ เay rov $\overline{\theta 0}$.

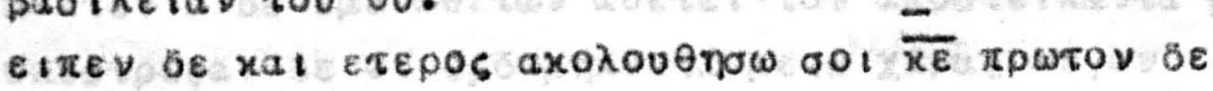

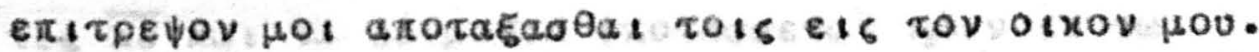

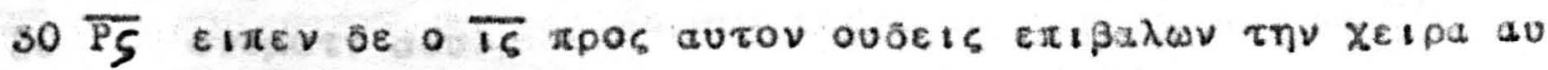

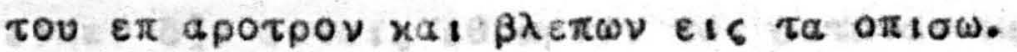

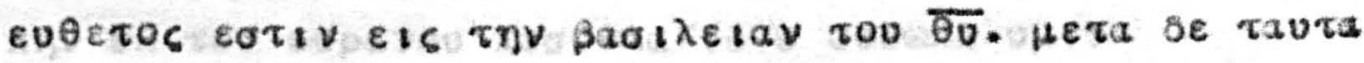

PZ a

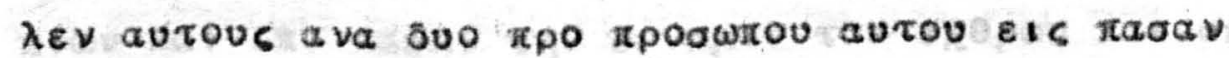

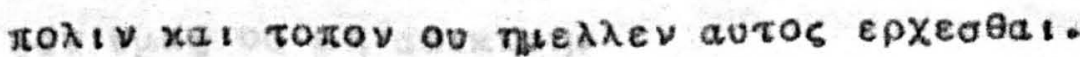

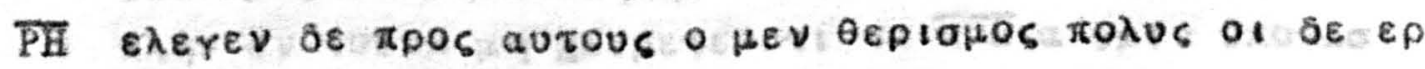

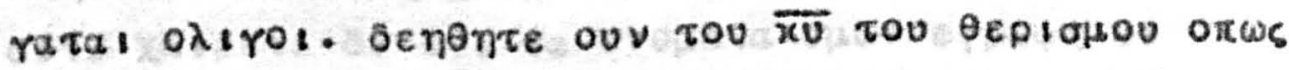

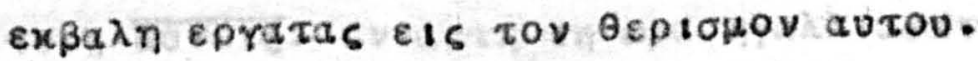

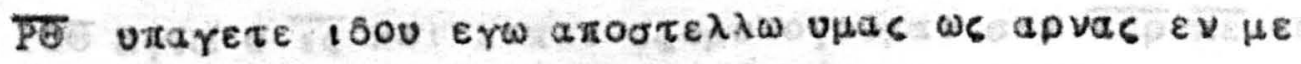

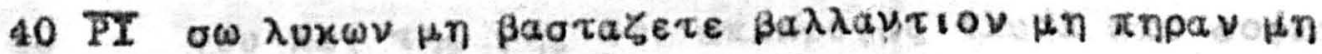

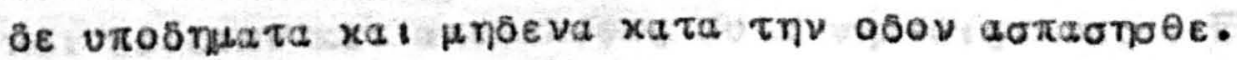




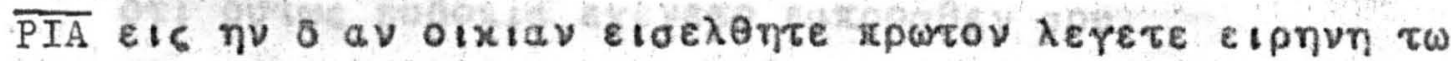

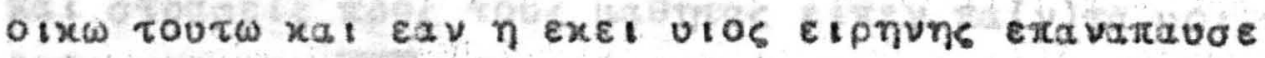

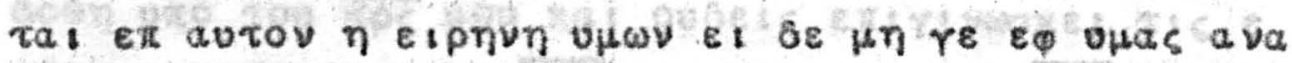

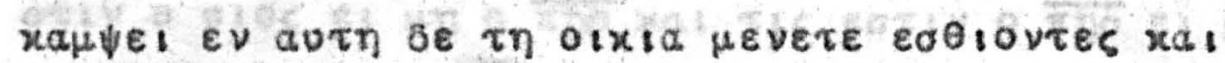

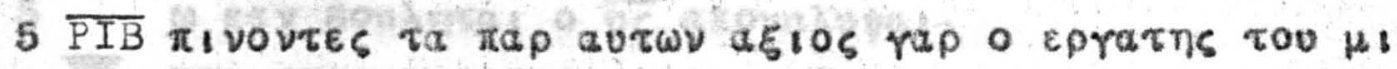

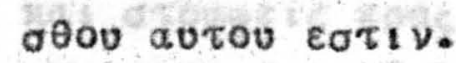

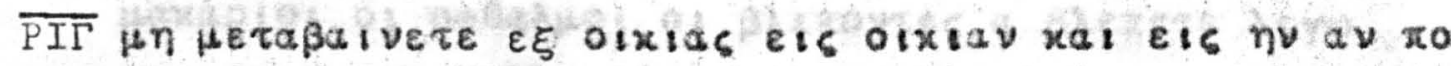

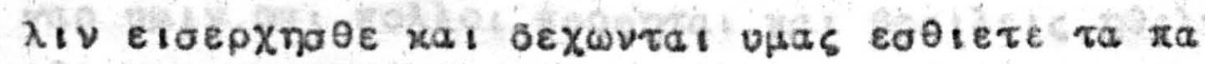

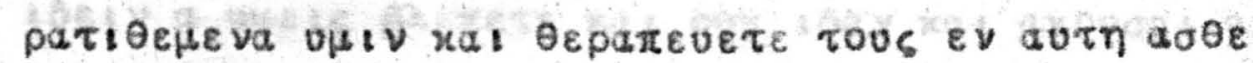

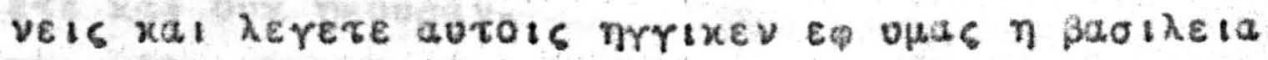

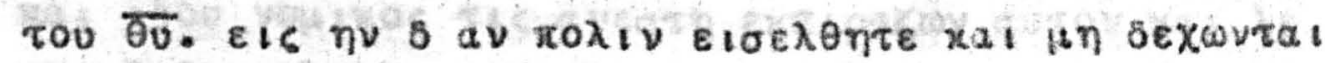

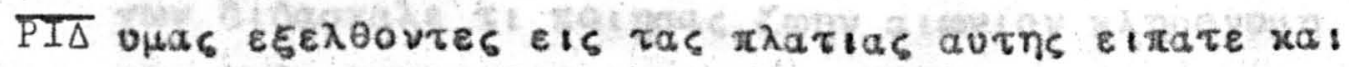

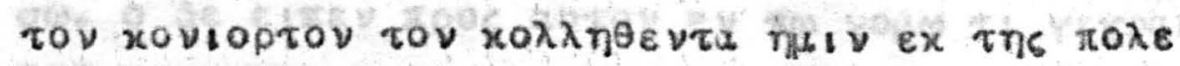

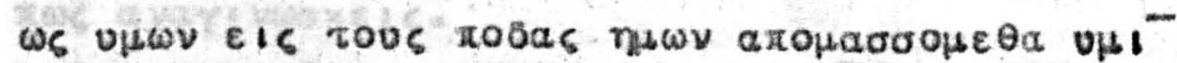

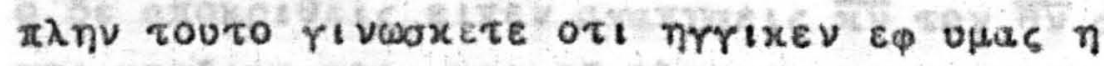

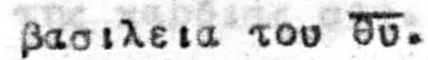

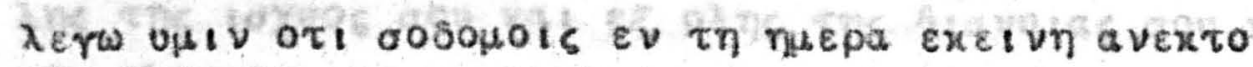

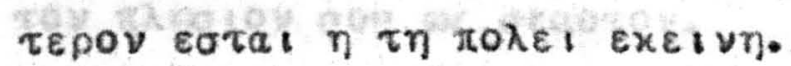

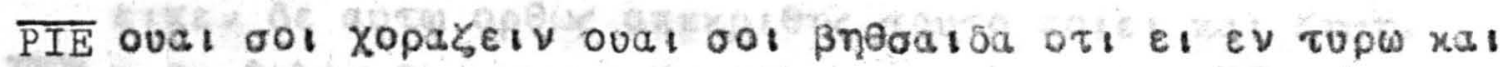

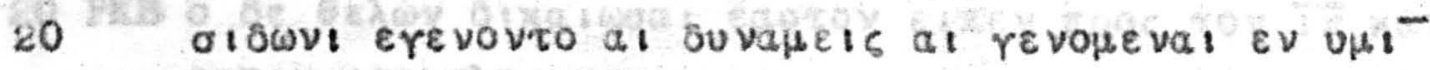

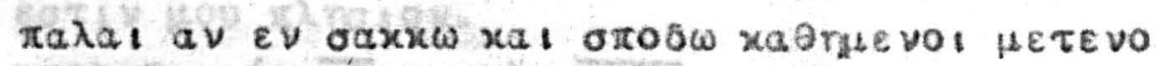

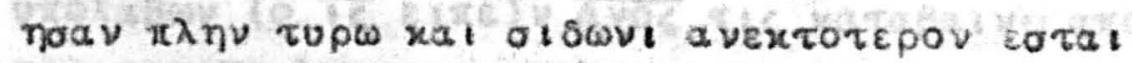

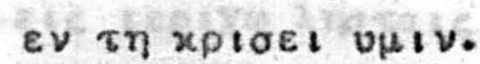

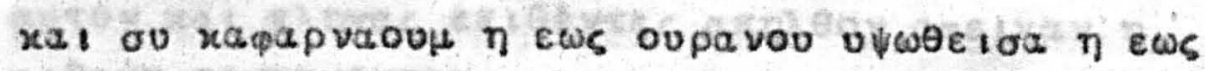

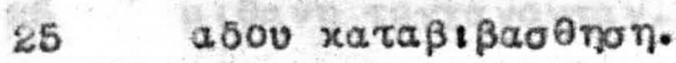

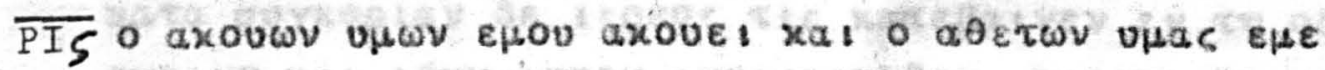

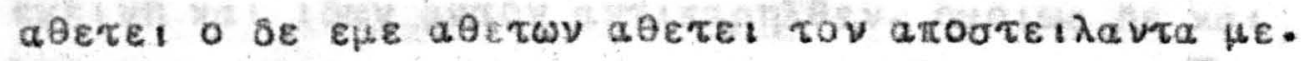

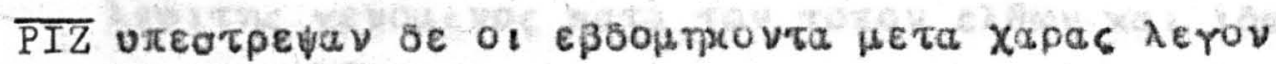

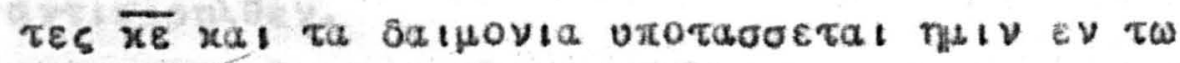
30 ovouat! oov.

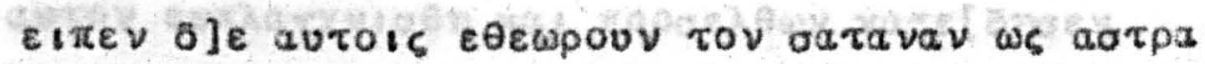

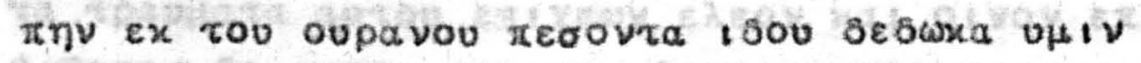

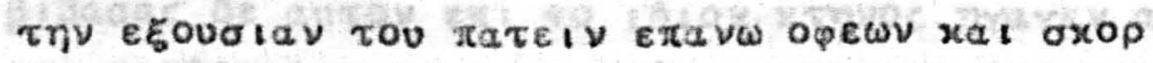

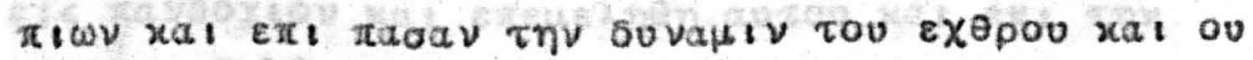

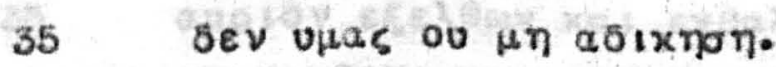

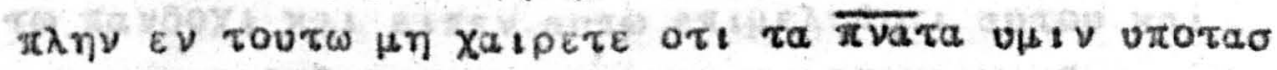

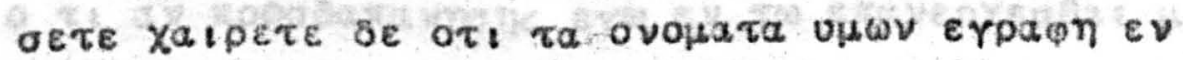

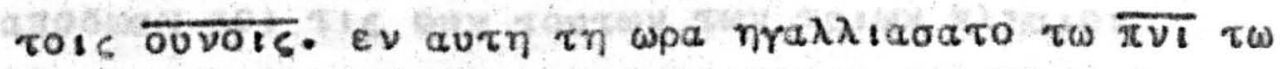

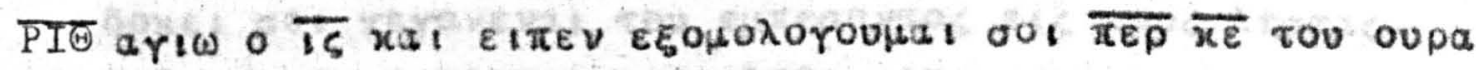

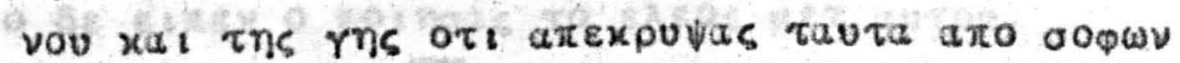

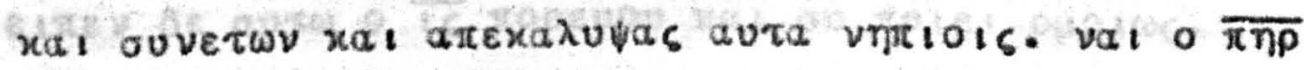




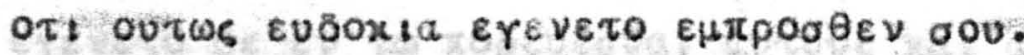

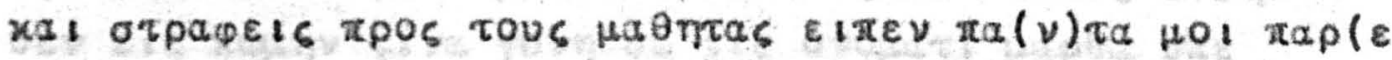

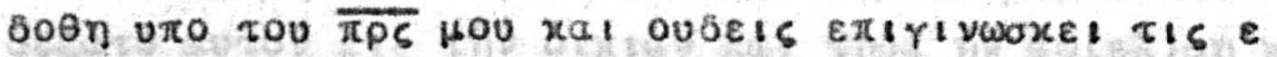

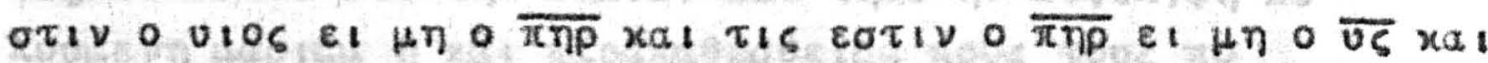

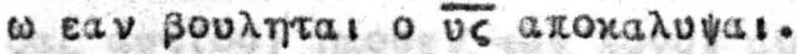

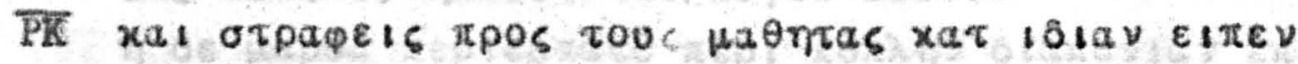

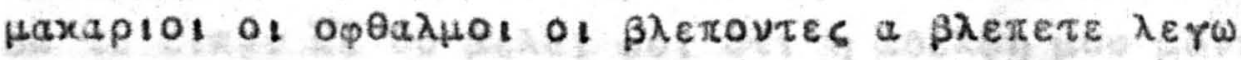

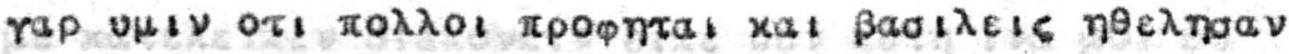

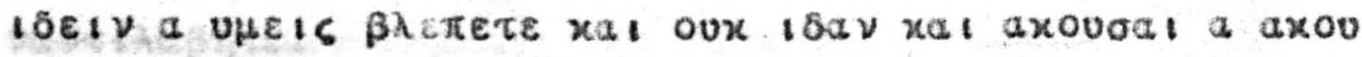

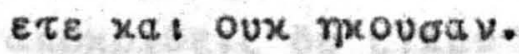

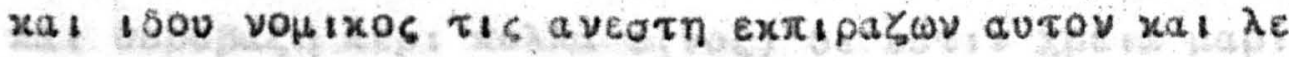

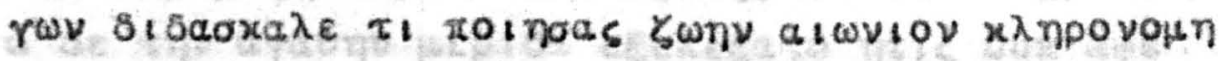

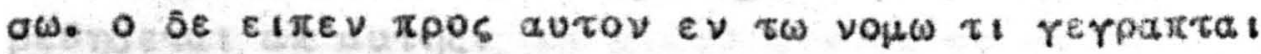
$\pi \omega s$ a varivwaxeis.

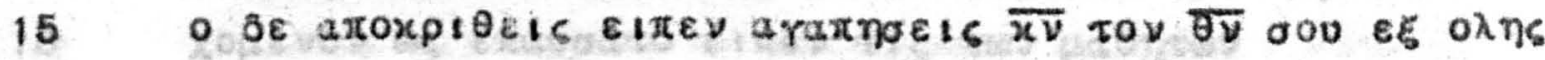

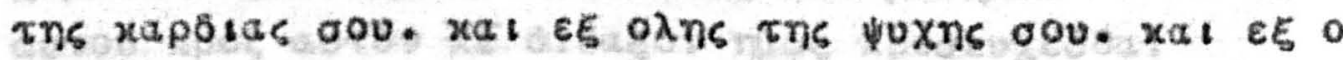

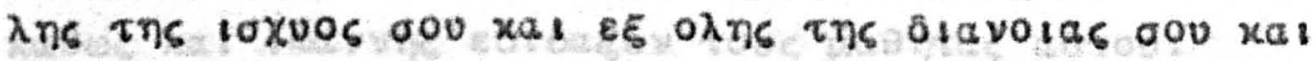
Tov rinaiov oou ws ocautov.

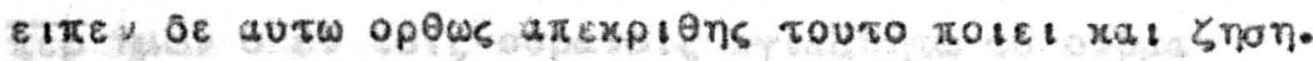

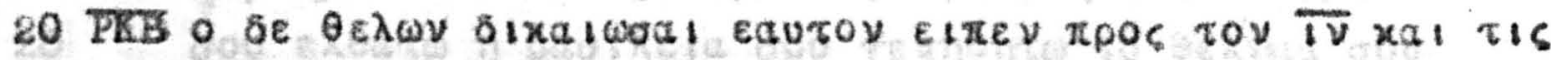
EOTIV HOU II no

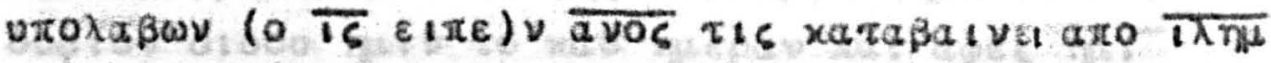

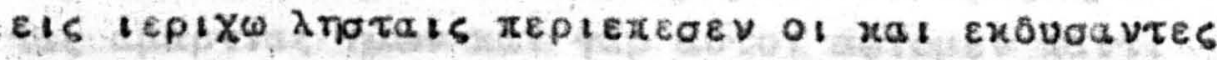

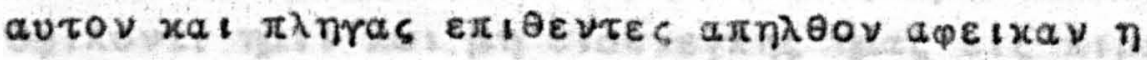

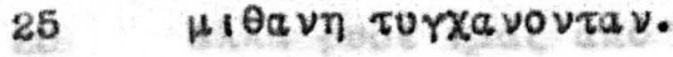

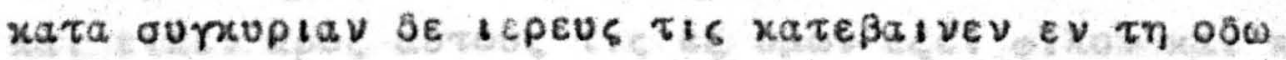

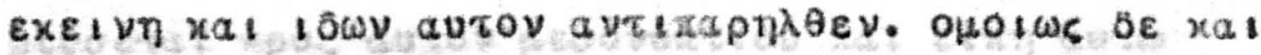

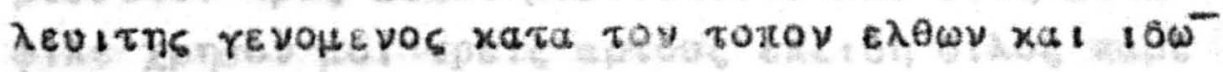
a $v \tau เ \pi 3 \rho \eta \lambda \theta \varepsilon v$.

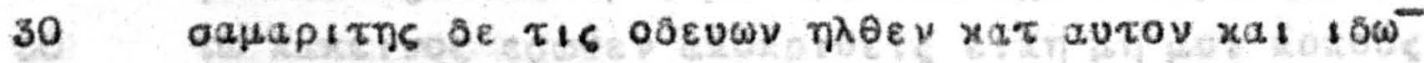

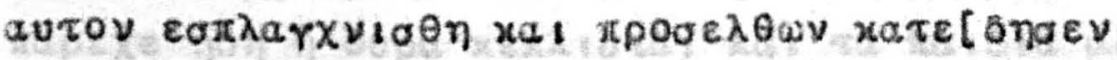

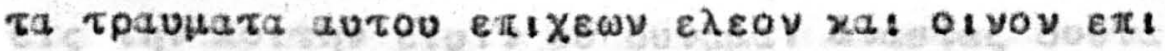

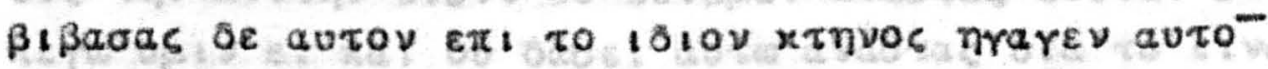

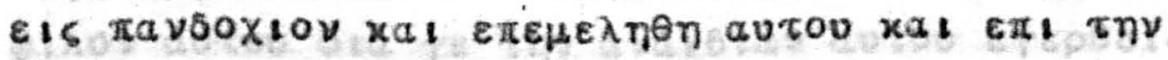
aupiov $\varepsilon \xi \varepsilon \lambda \theta \omega \nu$ xal $\varepsilon x \beta a \lambda \omega \nu$ suo ônvapıa $\varepsilon \delta \omega x \varepsilon \nu$

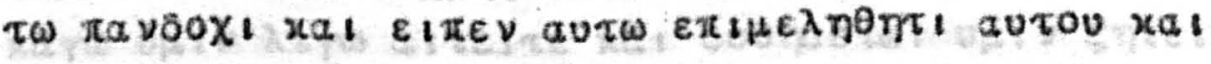

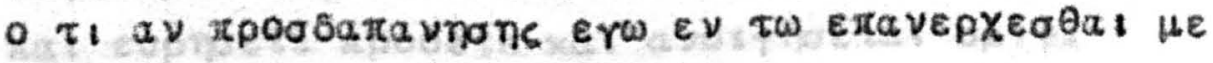

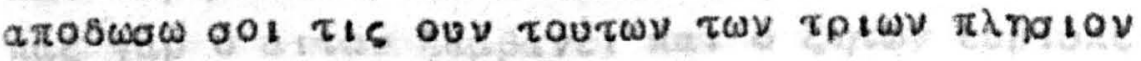

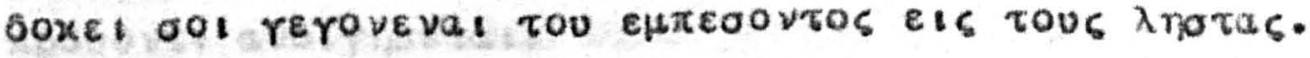

40 o $\delta \varepsilon \varepsilon i \pi \varepsilon \nu$ o $\pi 0$ Inas to $\varepsilon \lambda \varepsilon O \zeta ~ H \varepsilon \tau$ avtov.

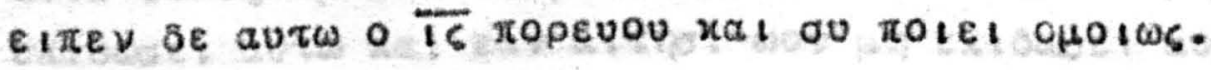




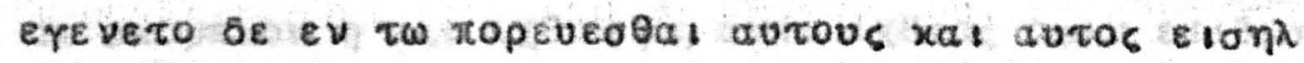

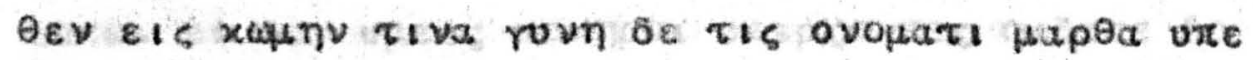

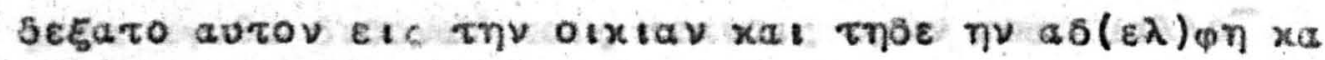

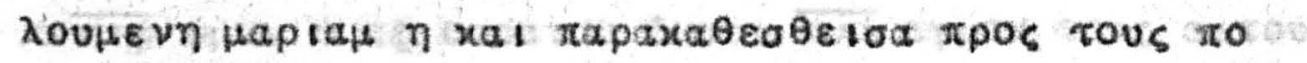
oas aur(ou) mKouev rov torov aurou.

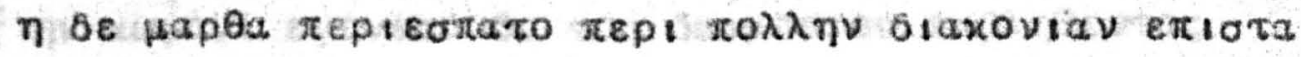

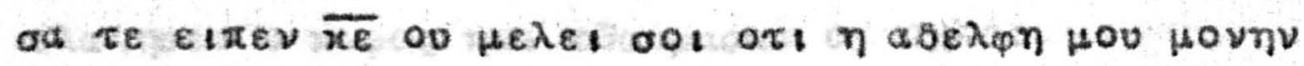

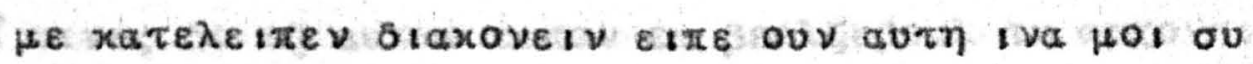

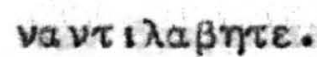

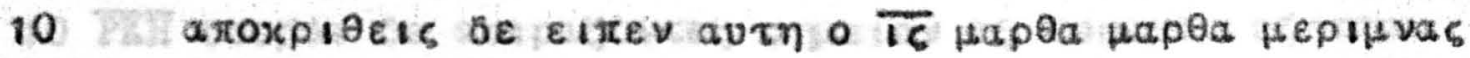

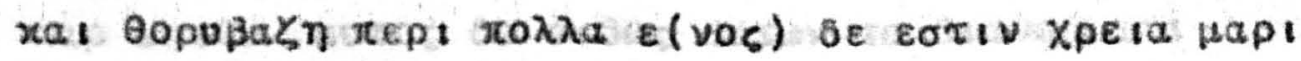

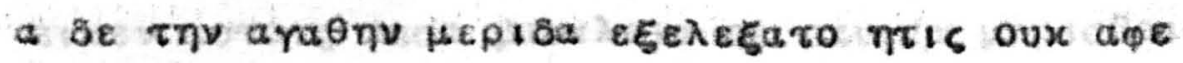

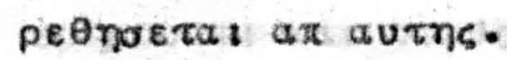

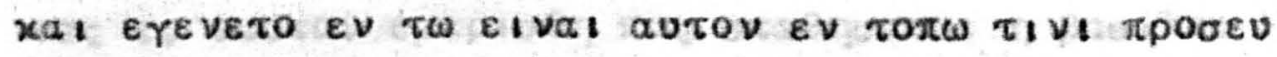

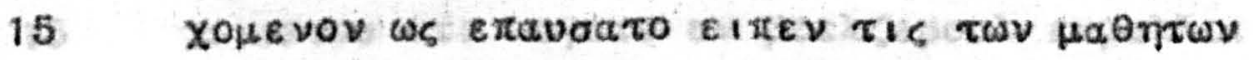

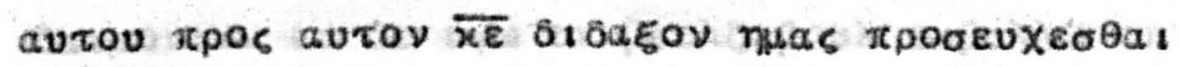

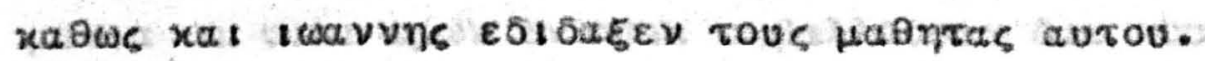

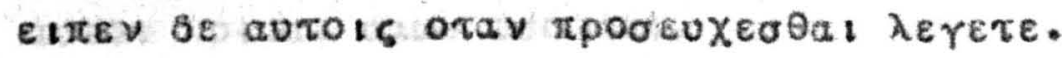

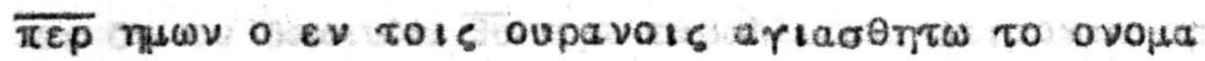

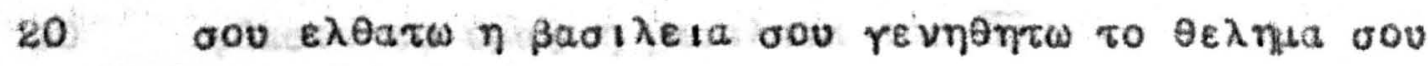

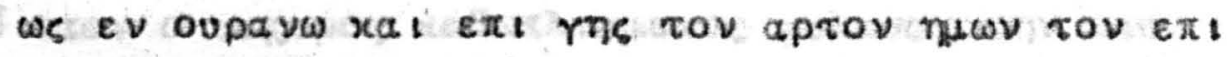

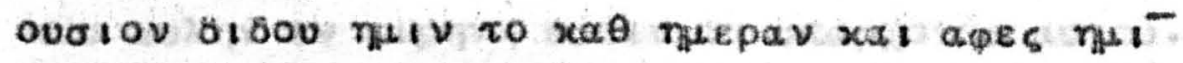

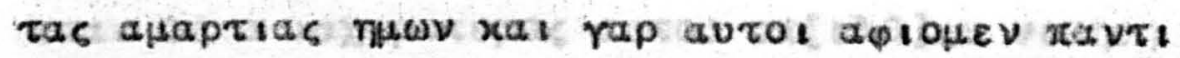

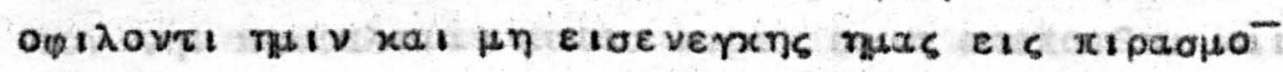

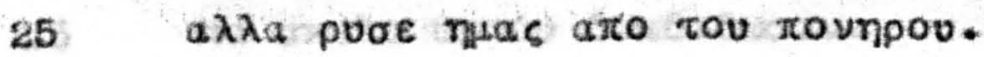

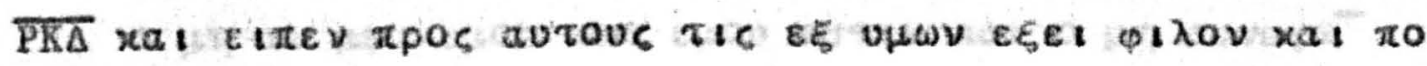

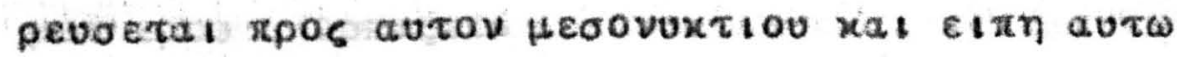

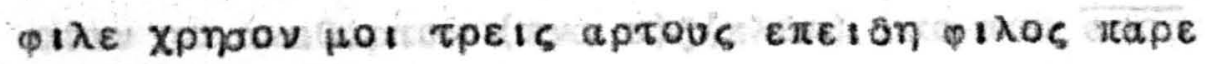

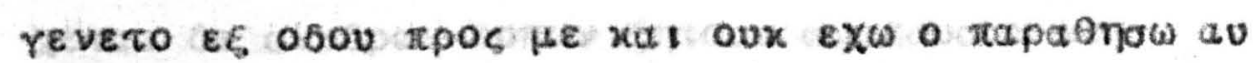

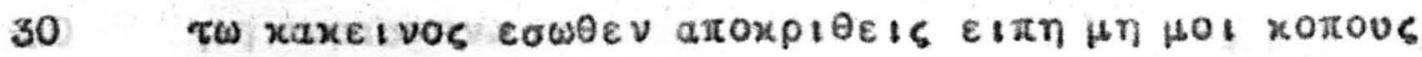

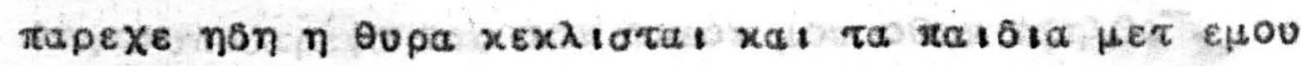

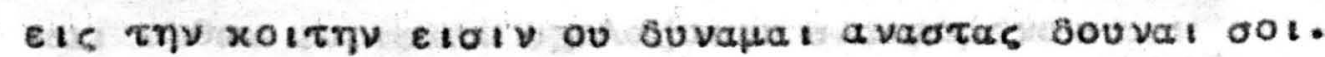

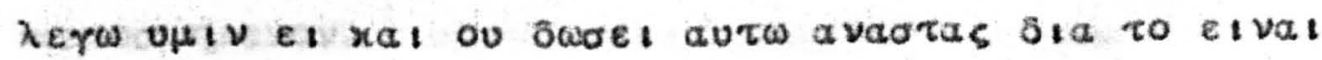
pixov autou sta re tru avatotav autou erepeets

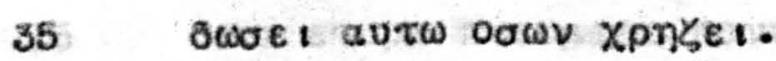

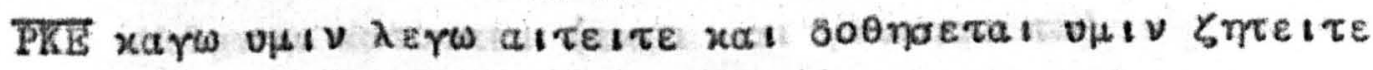

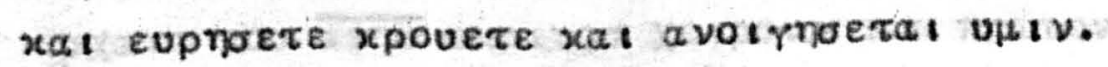

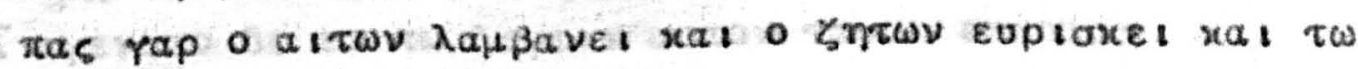
xpovovis avoirnoetal.

40 Tiva $\delta$ e eह upwv tov $\overline{\pi p a}$ aitnoet o vios aptov $\mu \eta \lambda_{1}, 0^{-}$

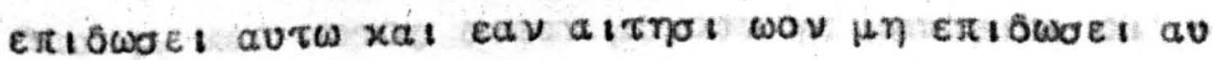




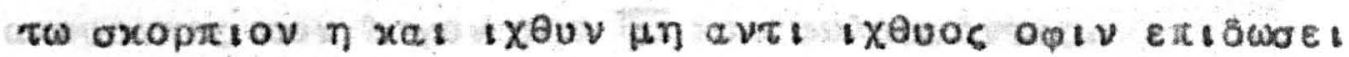

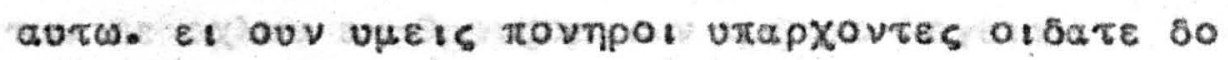

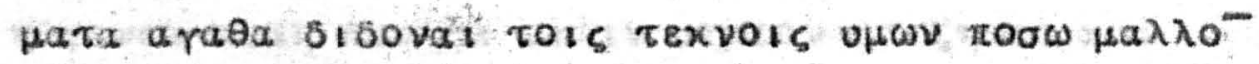

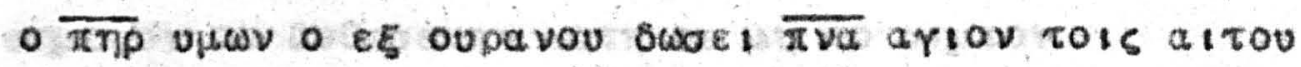

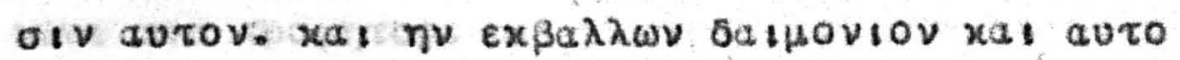

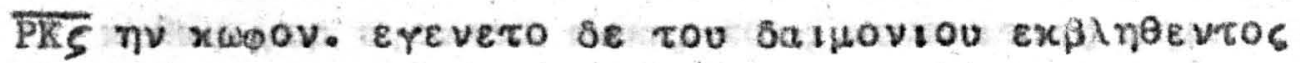

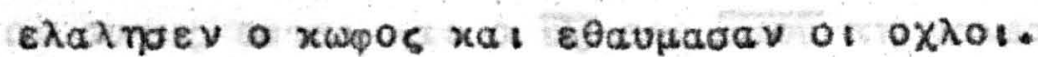

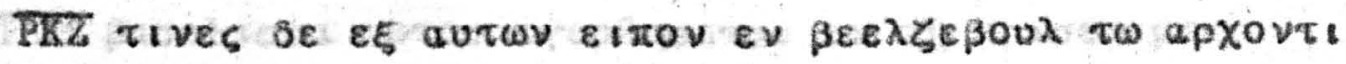

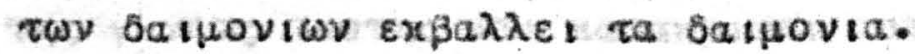

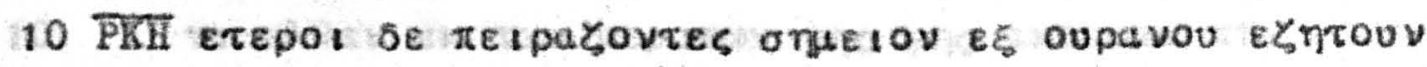

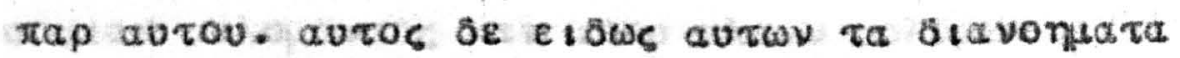

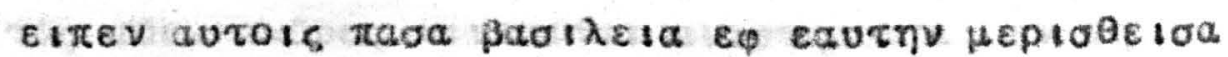

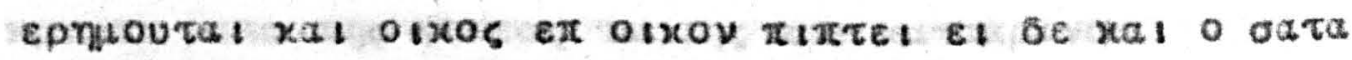

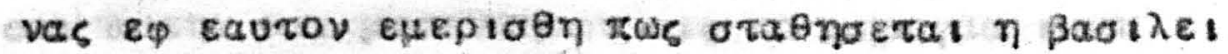

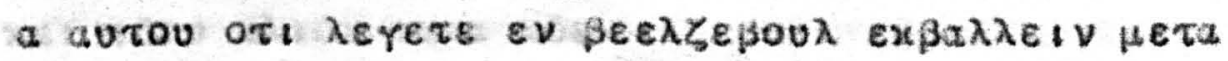

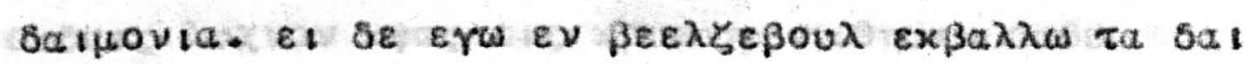

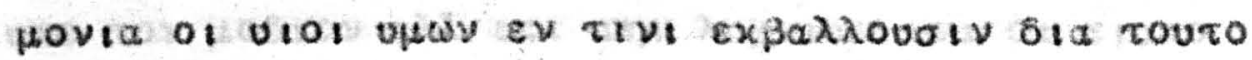

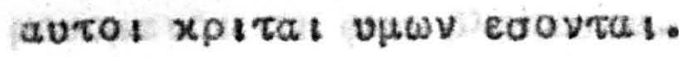

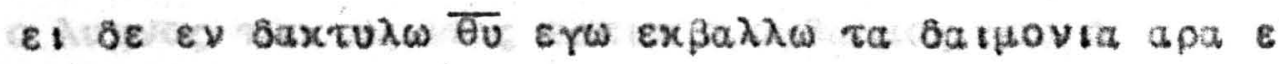

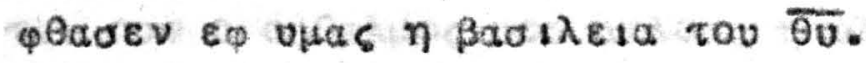

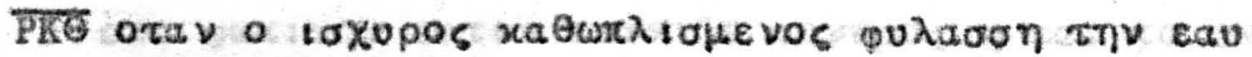

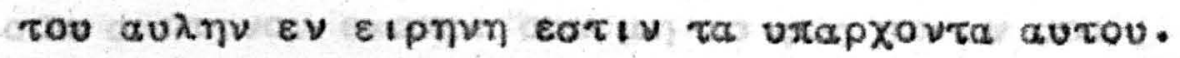

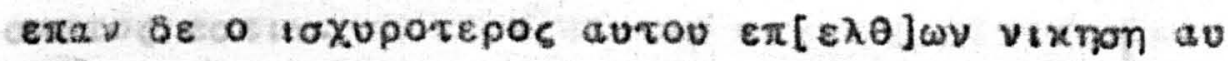

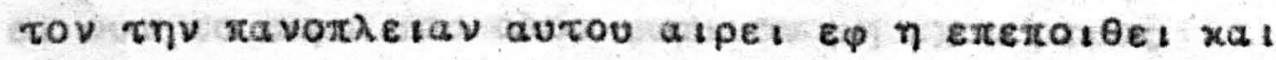

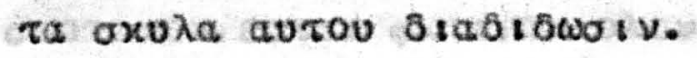

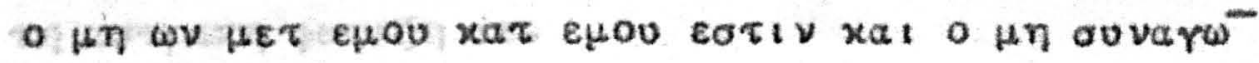

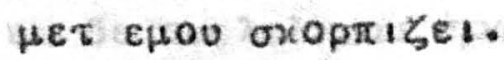

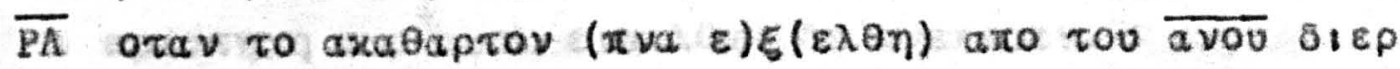

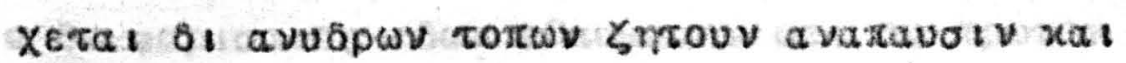

30 Hn Eupiaxov.

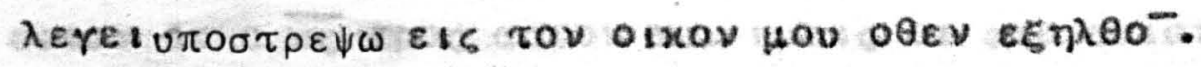

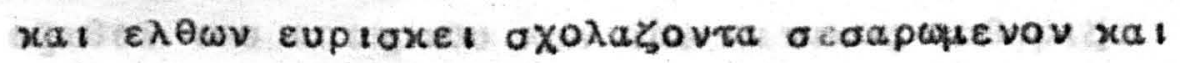
xexoopnievov.

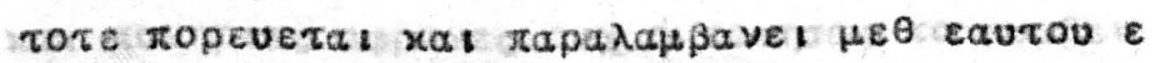

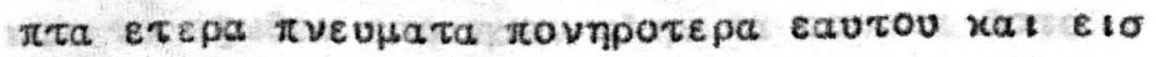

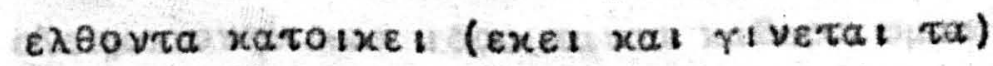

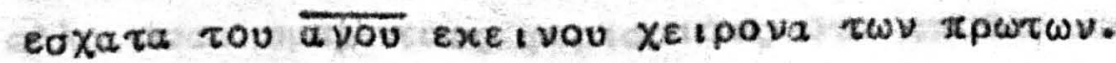

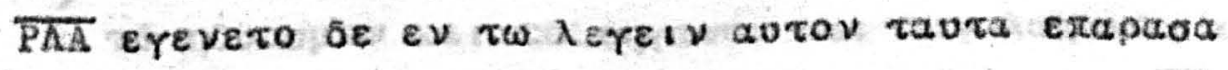

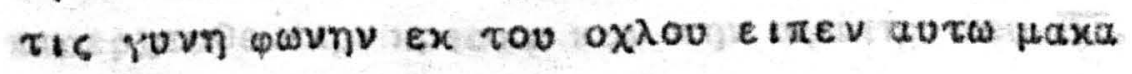

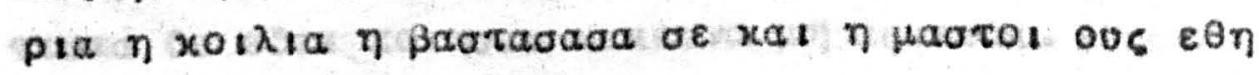

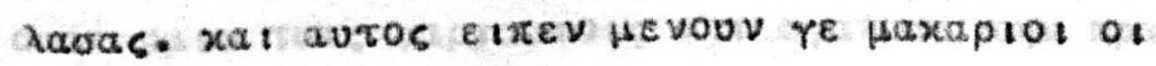




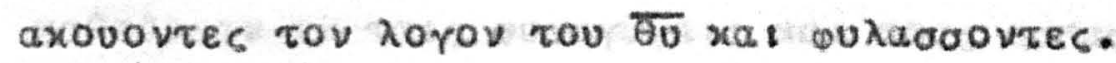

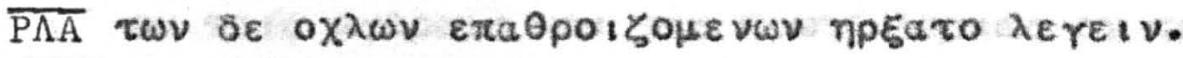

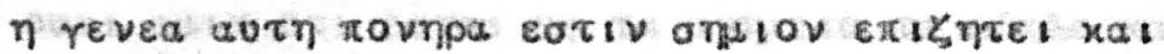

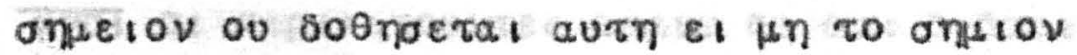
isva rou mpaprou.

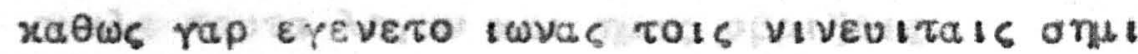

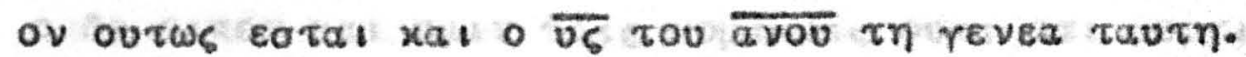

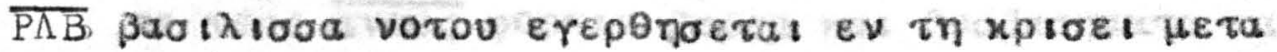

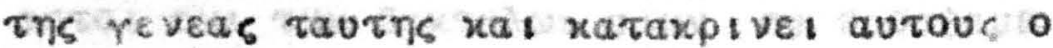

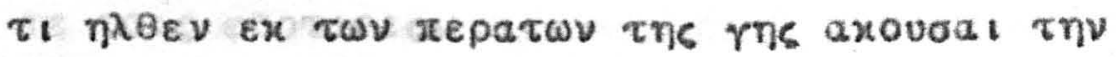

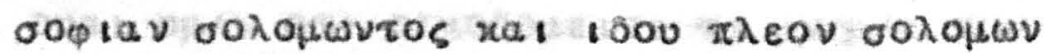

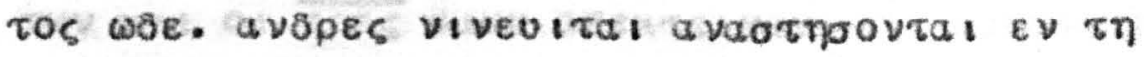
xploe! $\mu \varepsilon \tau a$ ths reveas rautrg xas xataxpivou

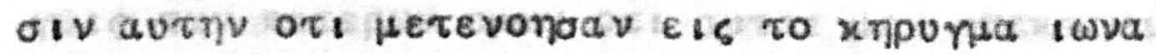

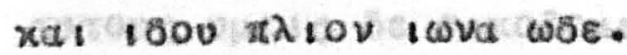

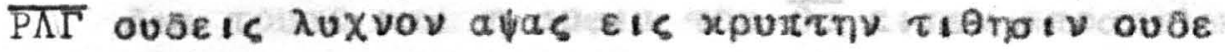

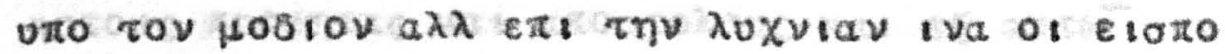

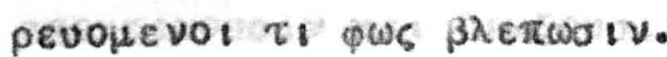

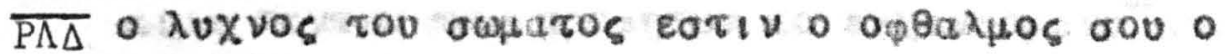
20 Tav ouv o opBajhos oov arious $\eta$ oxov to owha

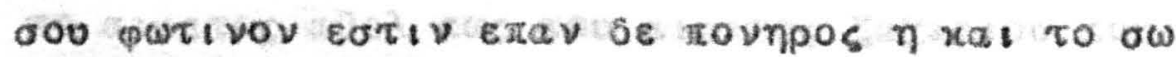

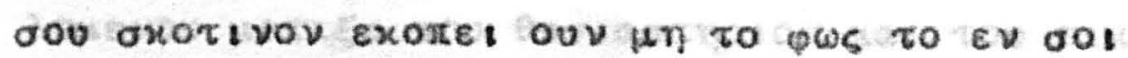

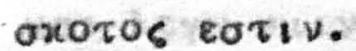

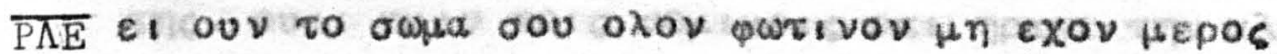

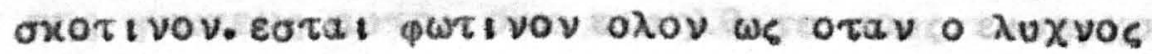

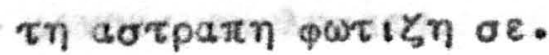

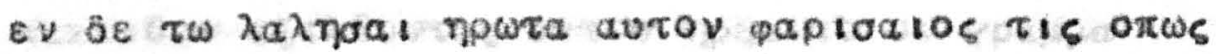

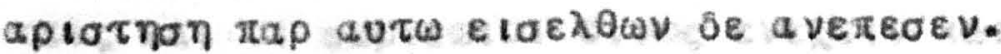

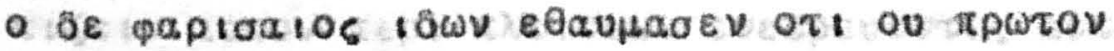

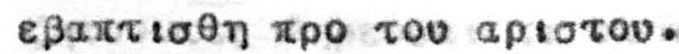

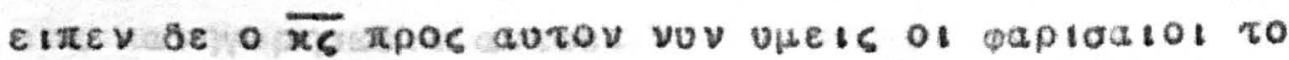

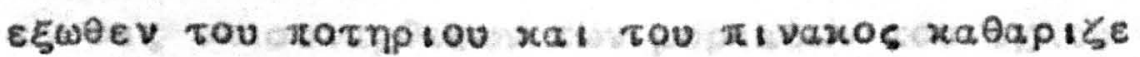

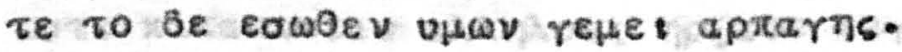

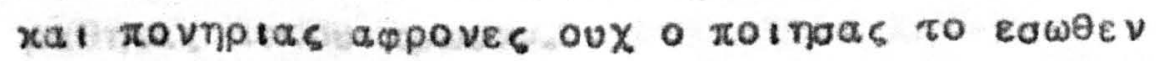

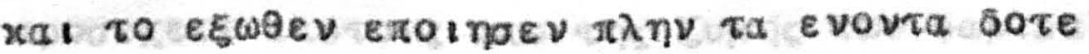

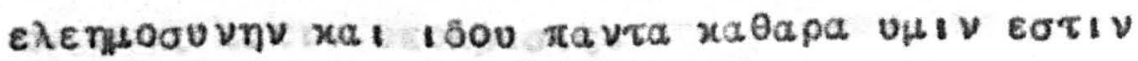

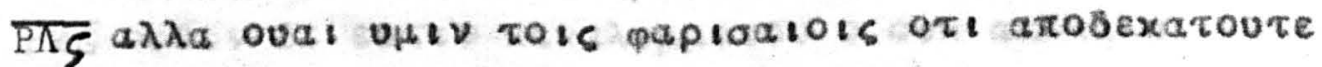

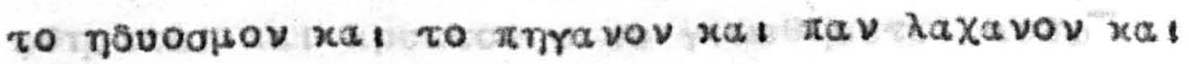

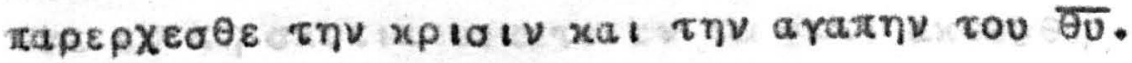

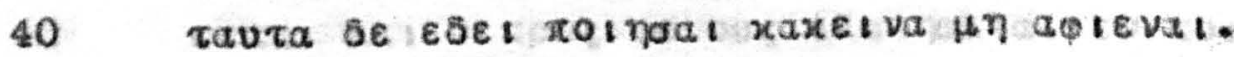

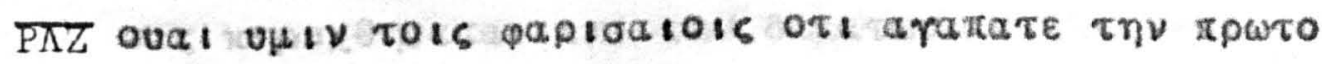




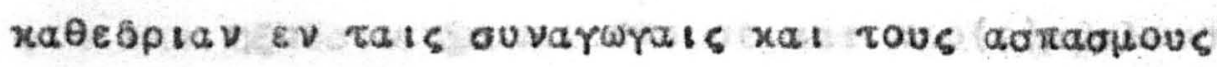

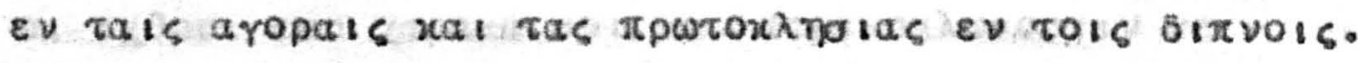

PRI oval vHı

avor oi teptxarouves erava ou oux orsaiv.

5

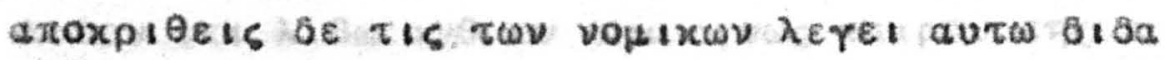

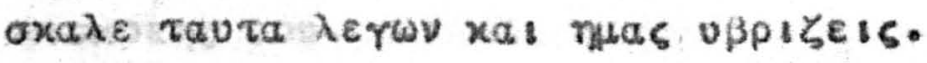

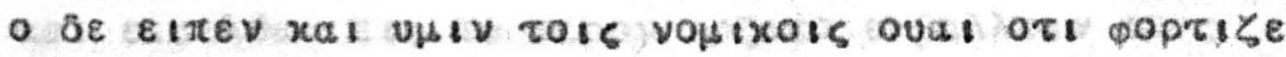

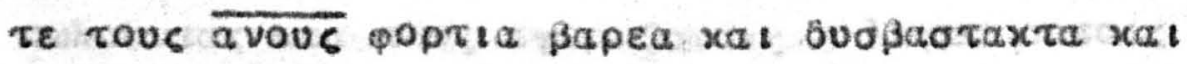

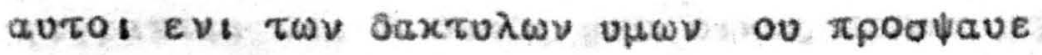

10 Te tois poptiots.

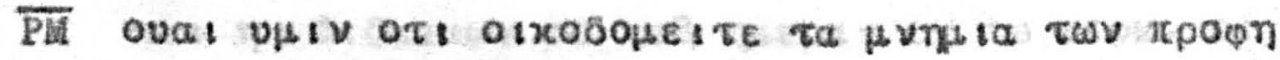

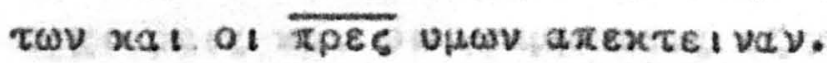

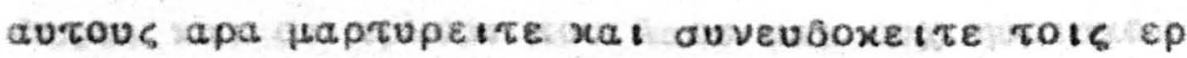

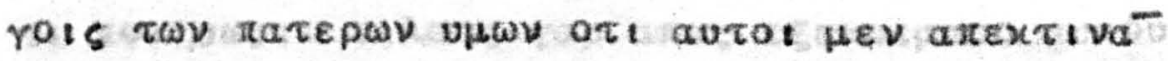

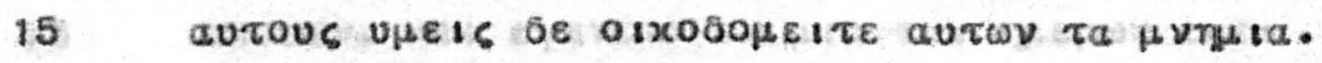

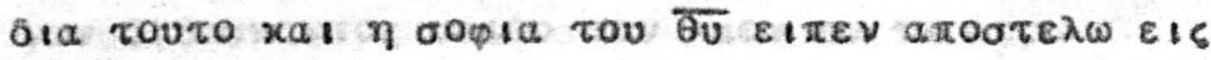

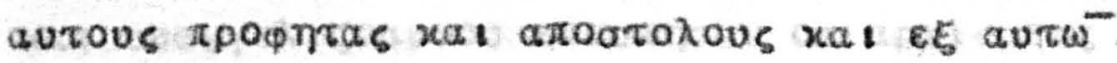

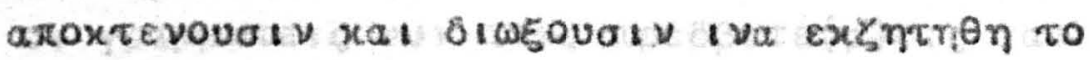

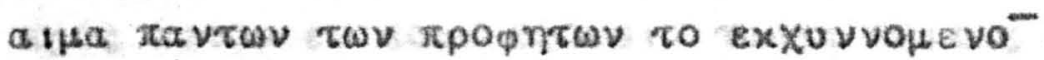

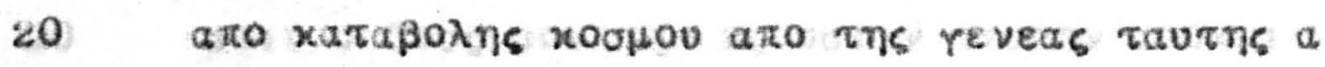

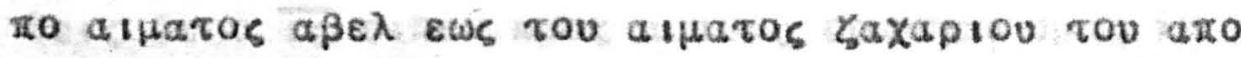

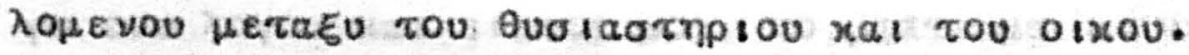

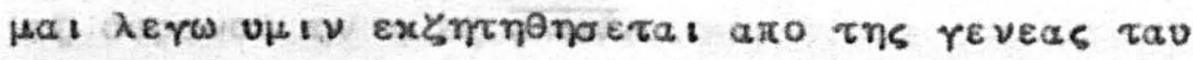

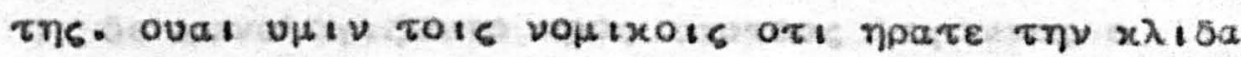

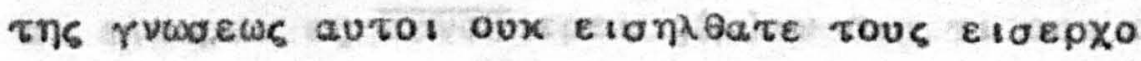
$\mu \varepsilon v o u s$ exwivoare.

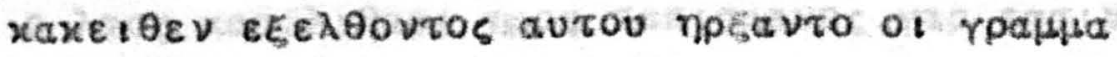

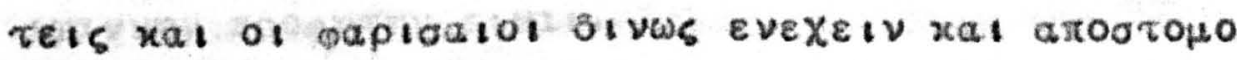

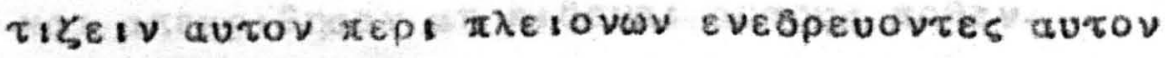

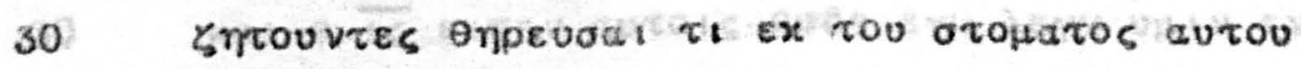
iva xatnropnawaiv autov.

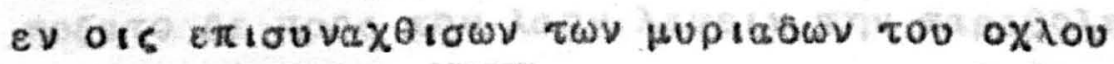

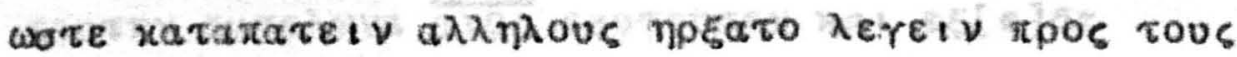
Haerras aviou xpwtov.

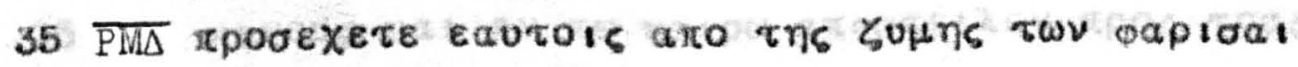

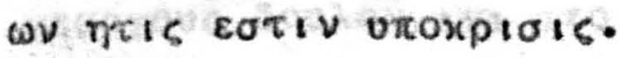

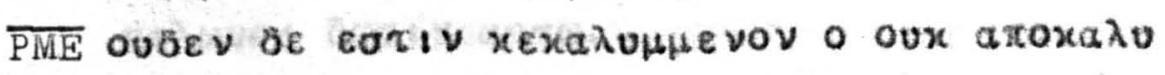

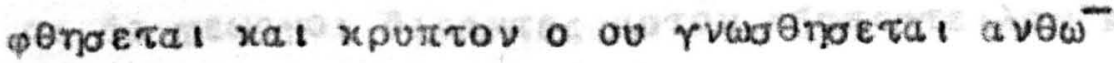

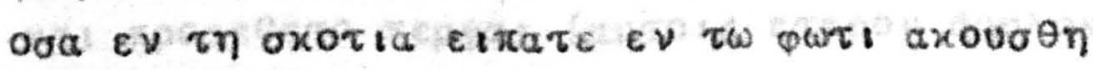

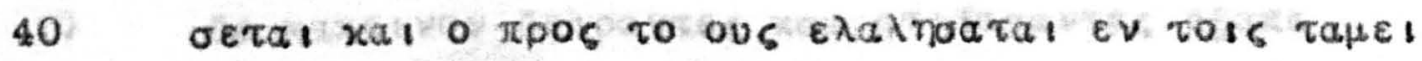

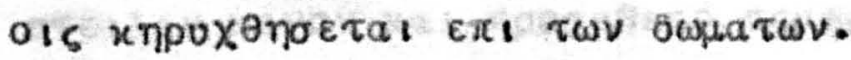




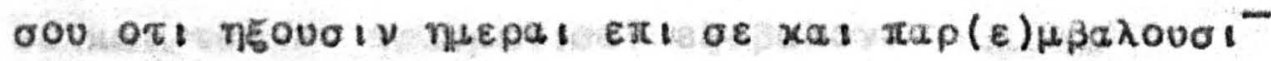

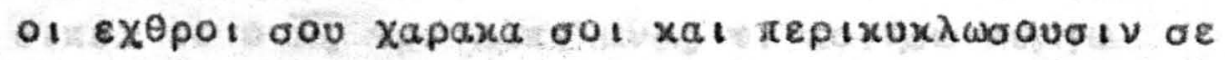

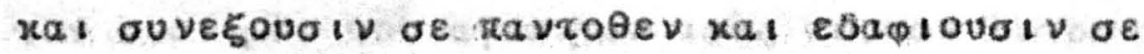

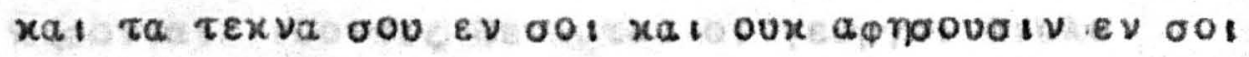

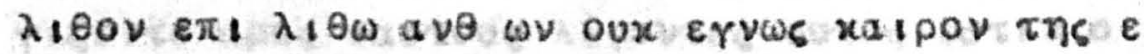
rioxorns gov.

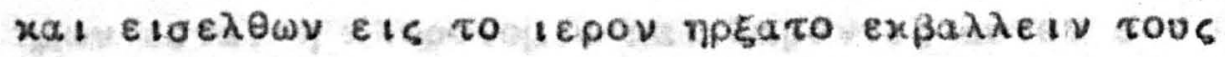

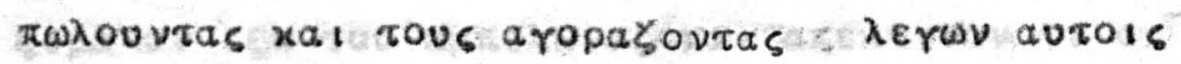

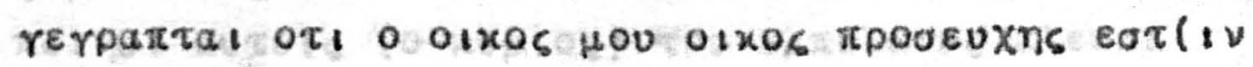

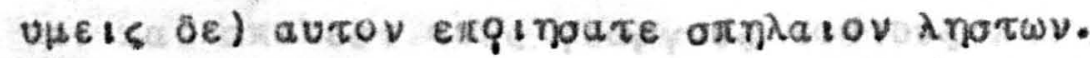

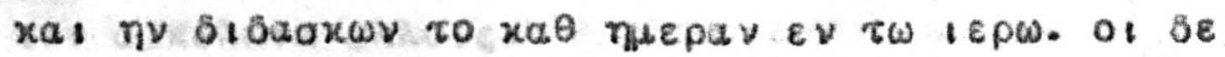

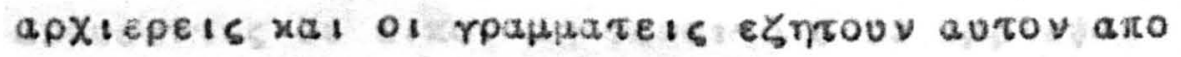

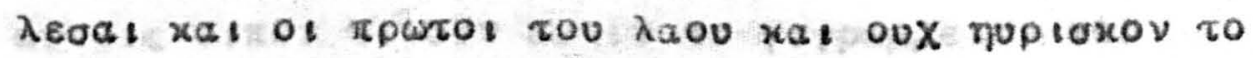

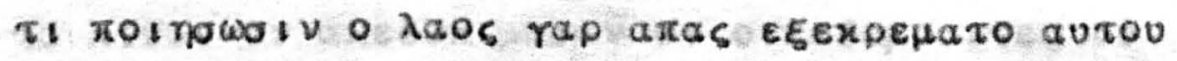

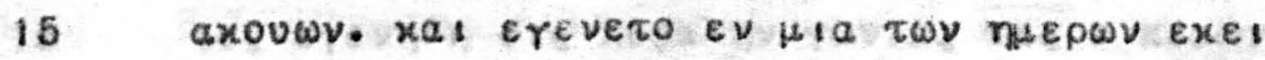

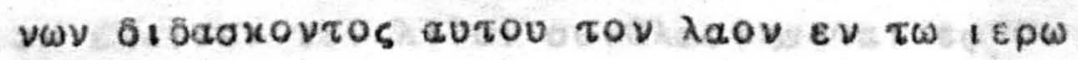

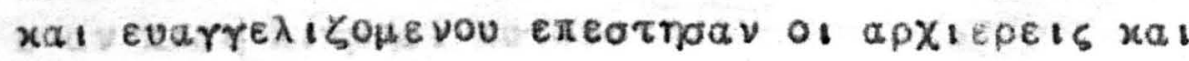

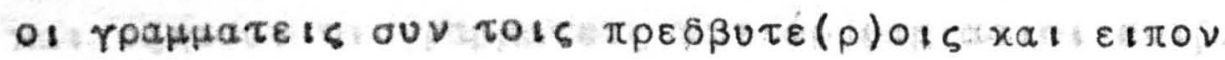

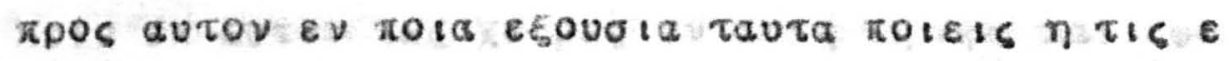

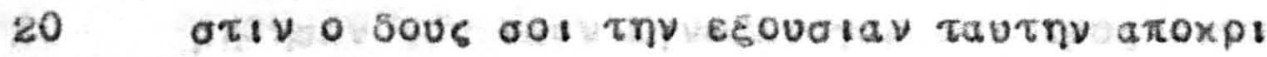

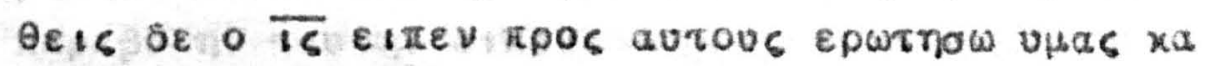

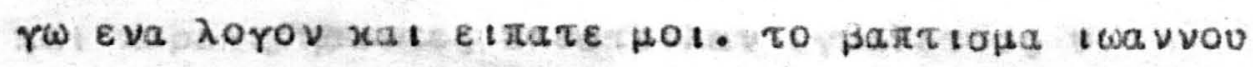
$\varepsilon \xi$ oupavou $\eta \nu \bar{\eta} \varepsilon \bar{\alpha} \overline{\boldsymbol{\nu} \omega \nu}$.

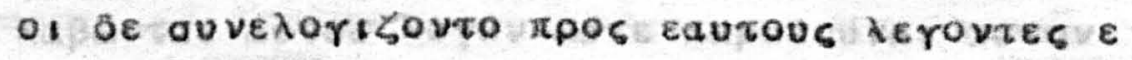
av $\varepsilon$ I

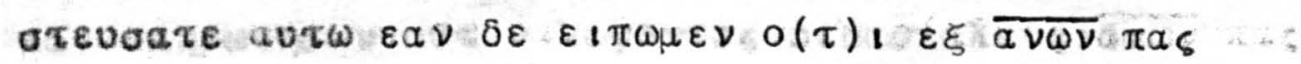

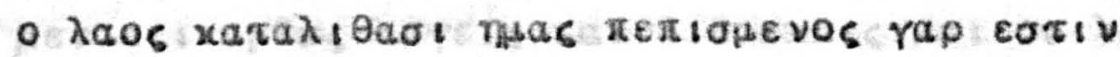

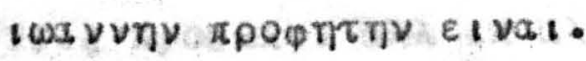

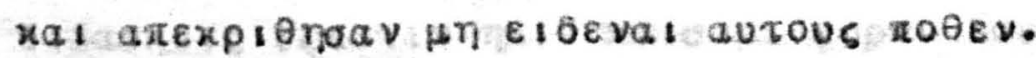

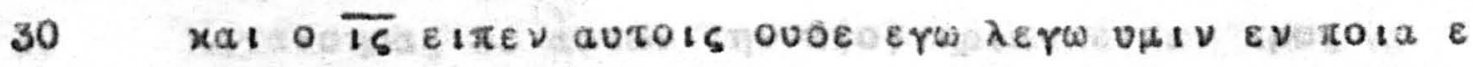
छovata rauta roiw.

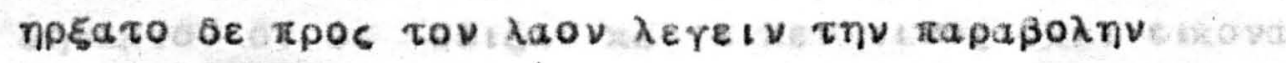

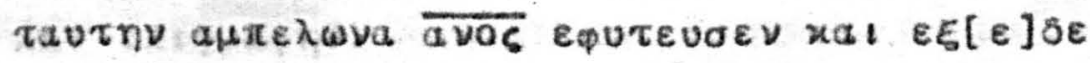
To autov rewprois xal areónumos xpovous.

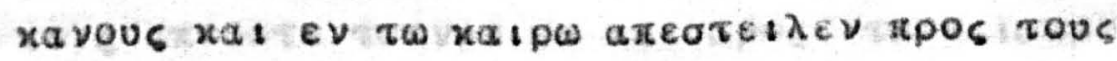
reworous soviov iva ato tou xaprov tou $\alpha \mu$

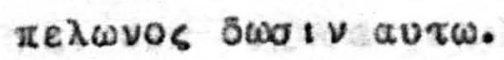

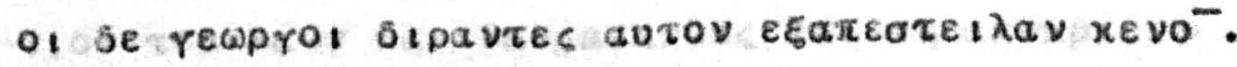

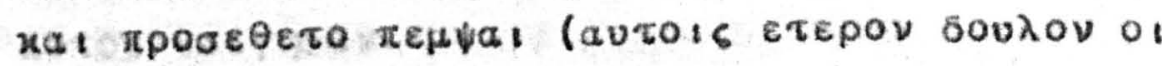

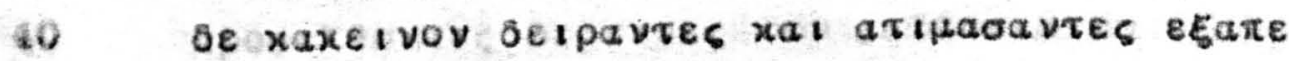

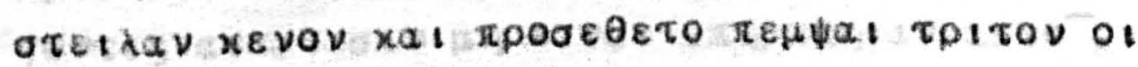




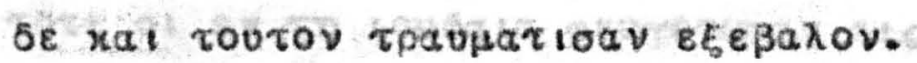

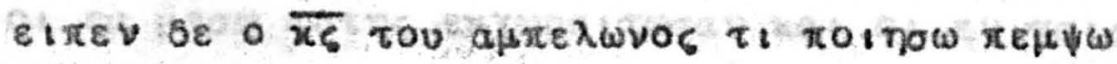

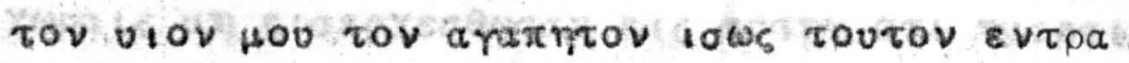

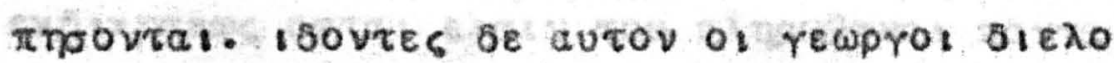

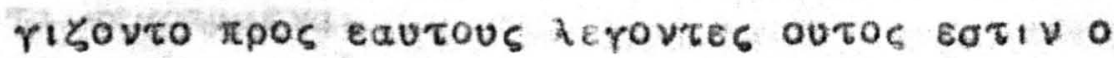

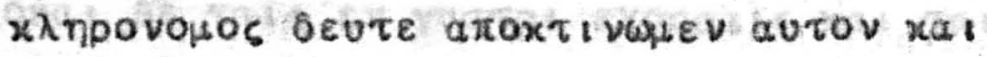

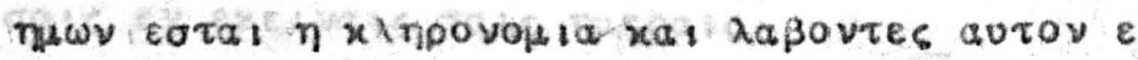

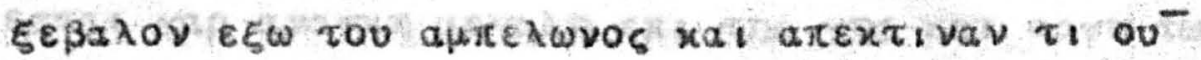

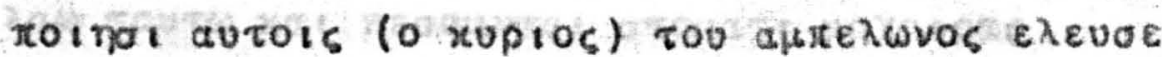

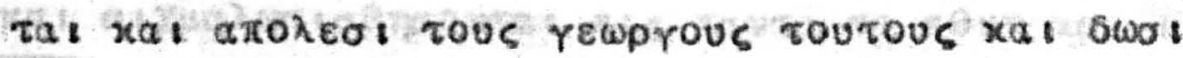

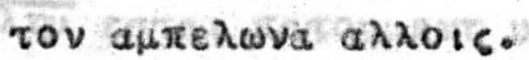

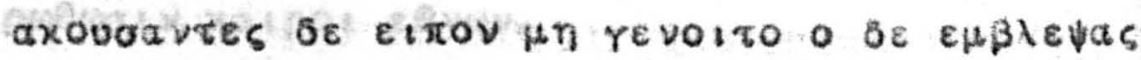

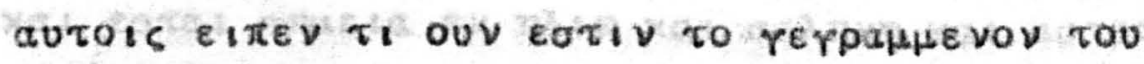
ro $\lambda i \theta 0 y$ ov are

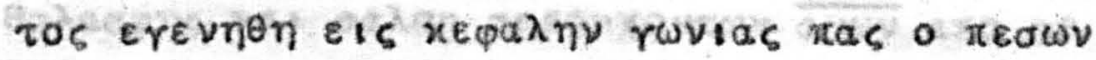

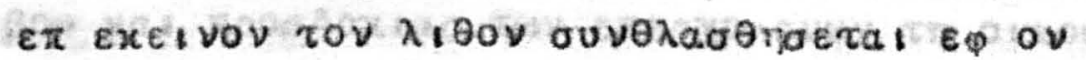
$\delta$ av tron $\lambda$ ixunjo: aurov.

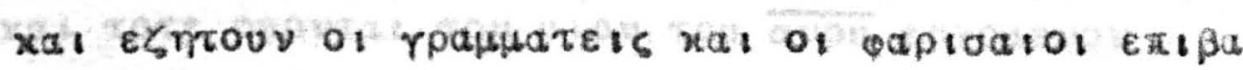

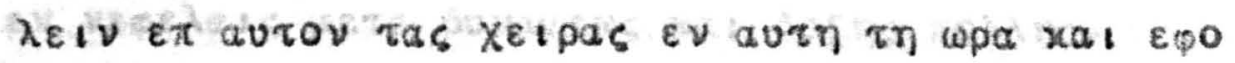

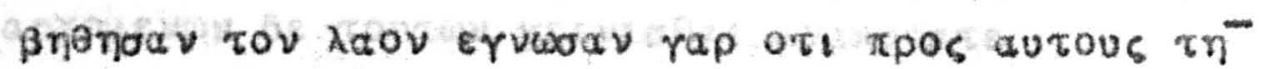

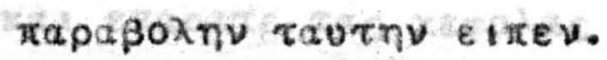

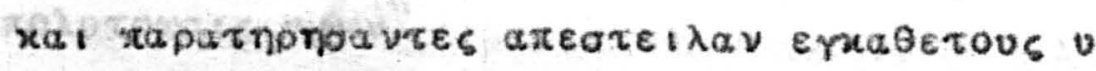
toxp ivouevous eavrou otxal ovs et vat i va ext

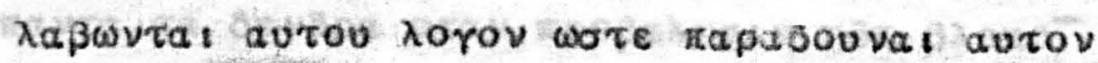

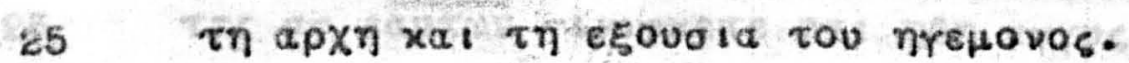

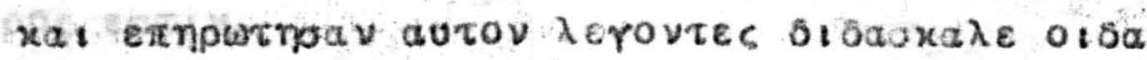

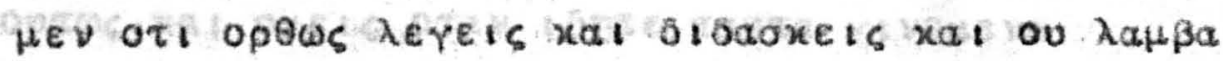

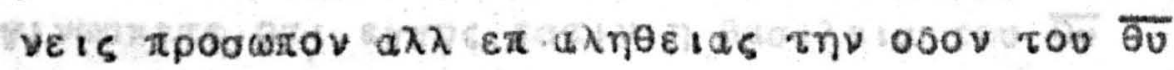

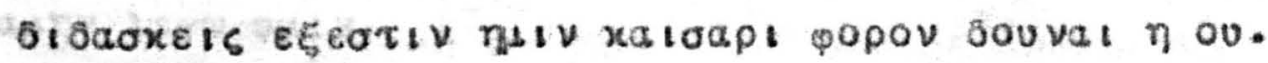

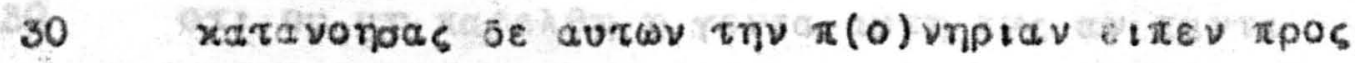

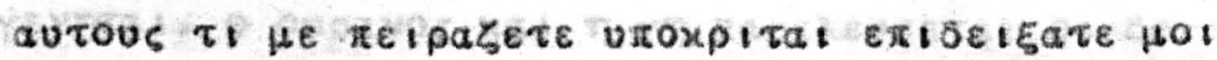

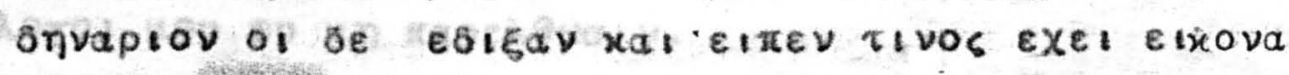

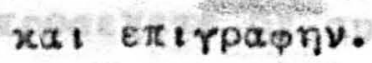

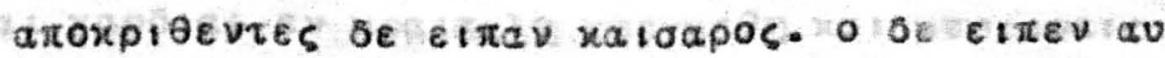

35 tols axobote toivuv ta xaıapos. to xaloap! xa!

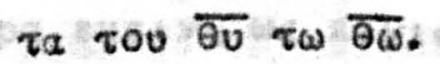

xal oux toxuoav $\varepsilon \pi 1 \lambda a \beta \varepsilon \sigma \theta a 1$ autov pruatos $\varepsilon v a^{-}$

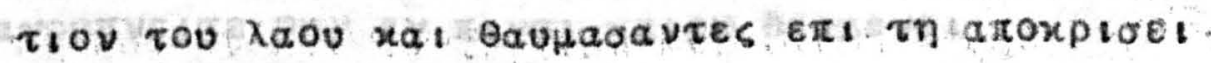
aurov eairmoav.

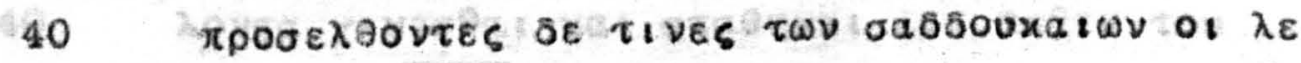
rovies avaorao 


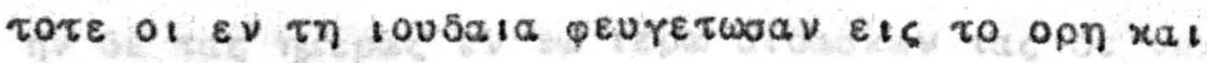

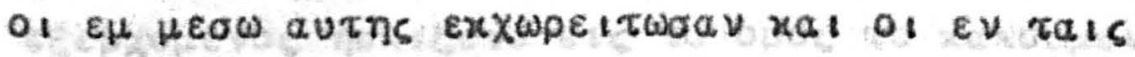

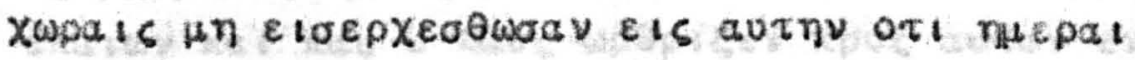

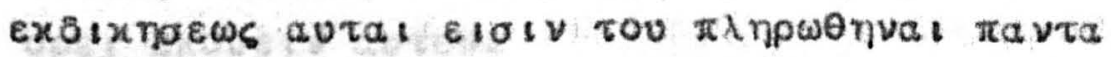

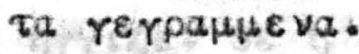

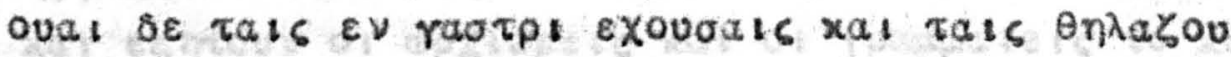
dals ev exeivals tais nuepals.

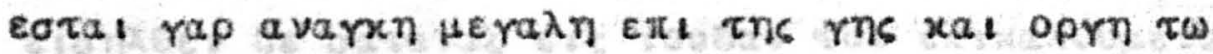

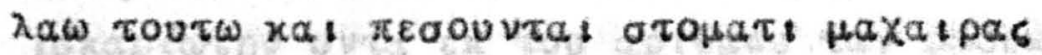

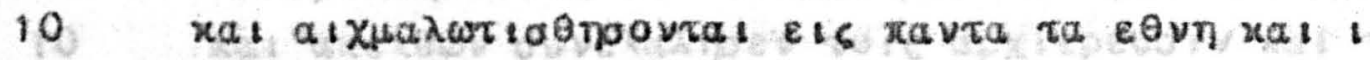

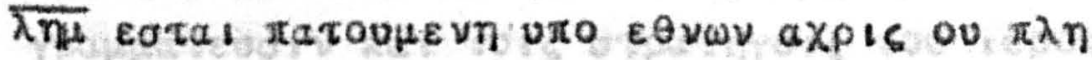
pwowaiv xalpoi zevov.

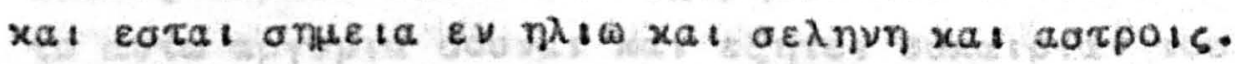
xal ext ths ms ouvoxn $\varepsilon \theta$ vav $\varepsilon \nu$ axopta nxous

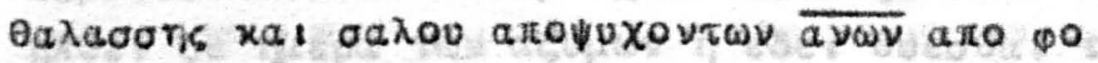

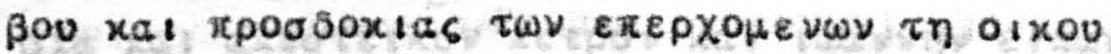

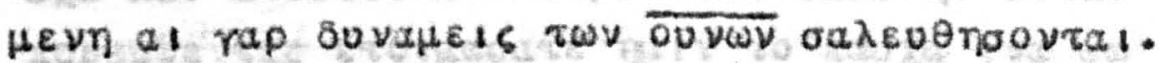

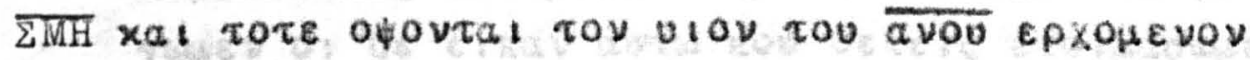

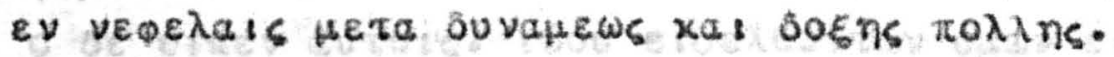

20 apXopevor of toutw resveatas avaxuqate

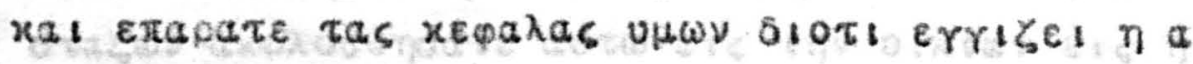

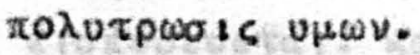

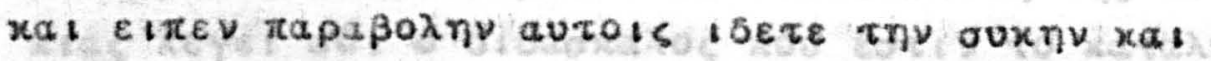

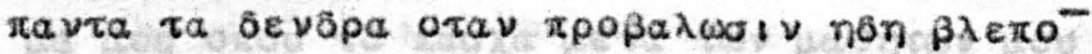

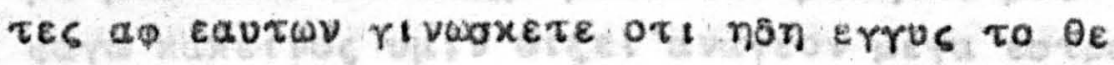
pos eariv.

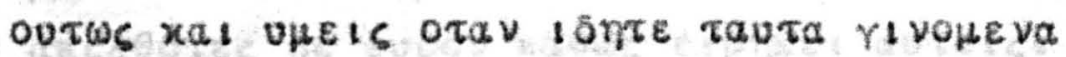

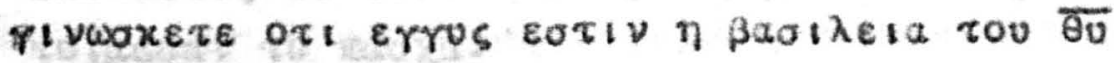
aptiv derw univ.

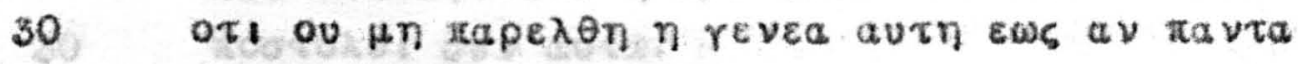

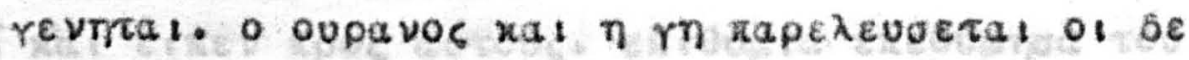

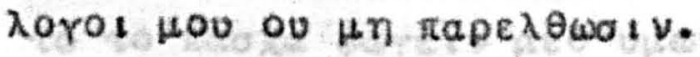

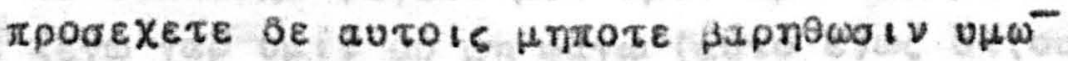
a) xapolat $\varepsilon \nu$ xpa

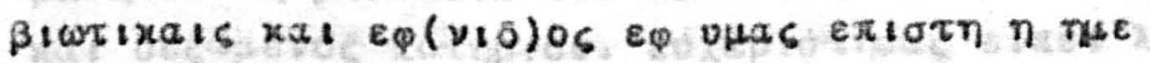

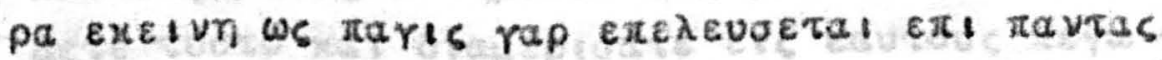

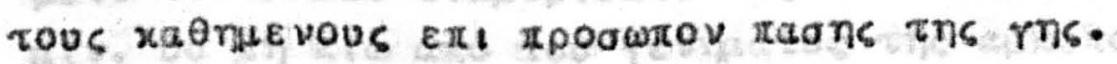

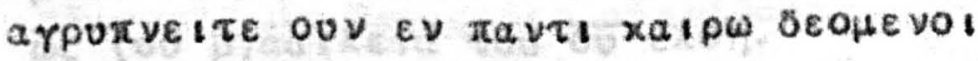

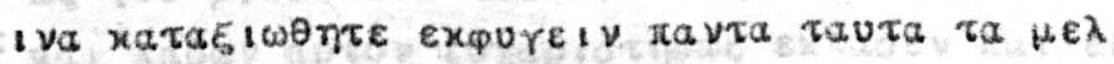

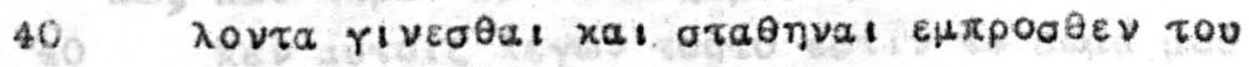
viou tou $\overline{\text { avov. }}$ 


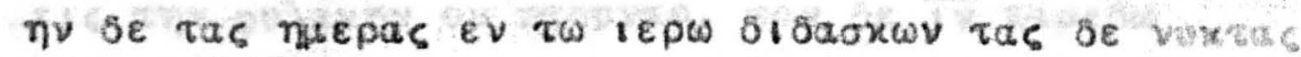

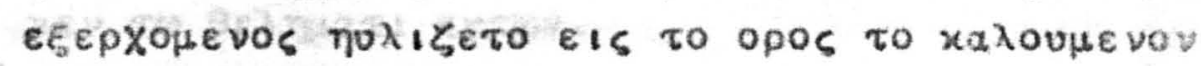

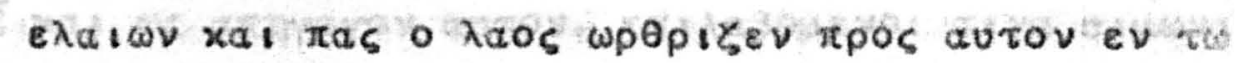
opeı axove tv autou.

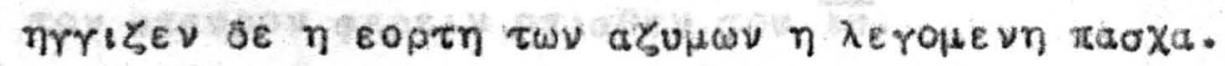

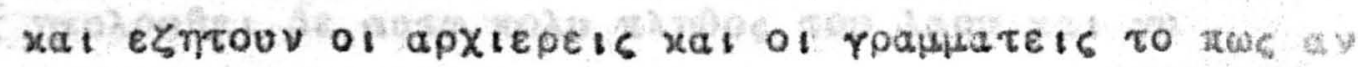

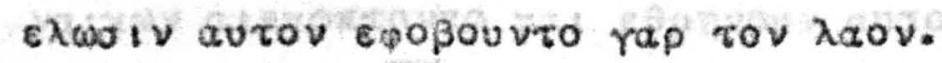

Eı

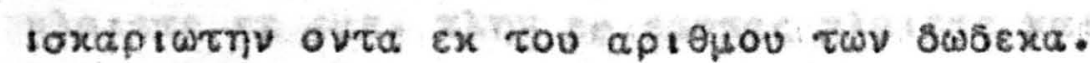

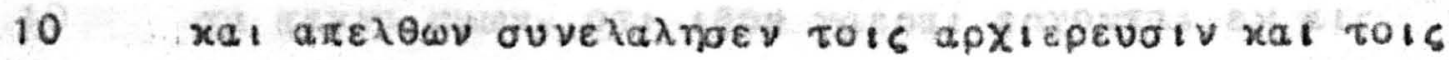

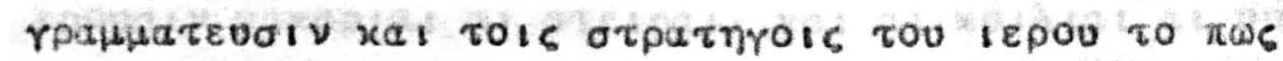

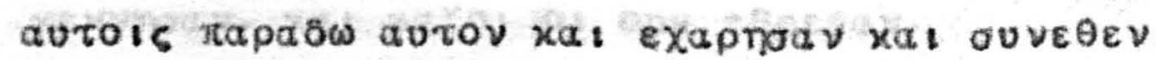

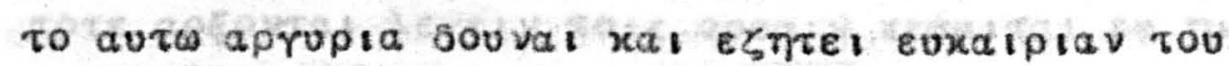
Tapasouvai autov atep oxגou autoic.

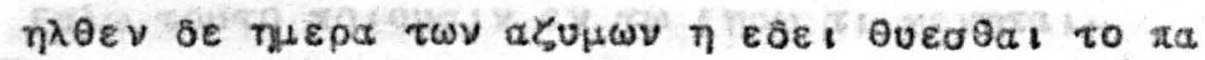

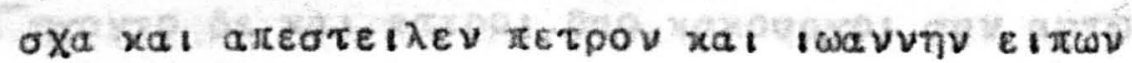

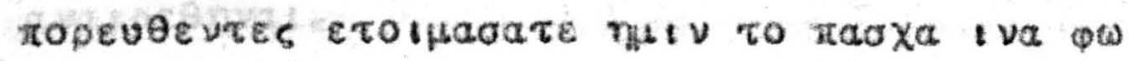

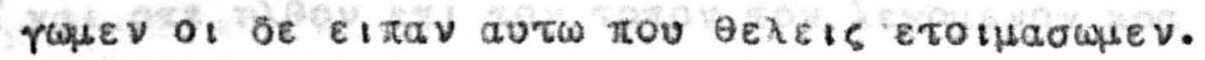

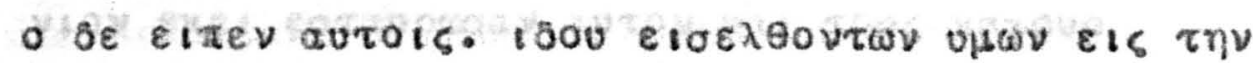

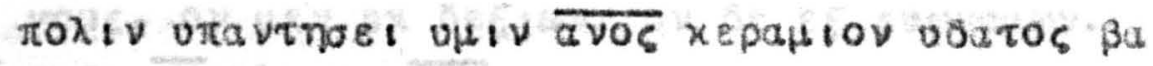

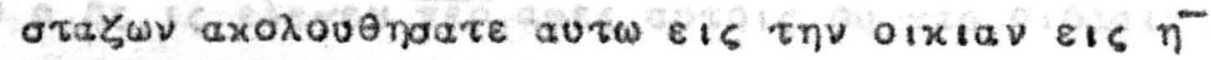

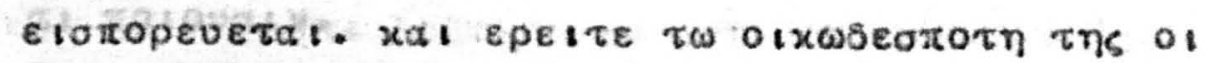

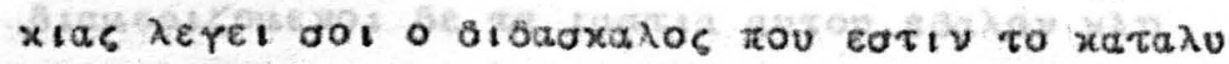

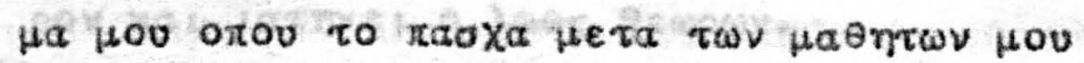

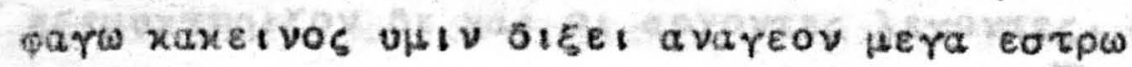

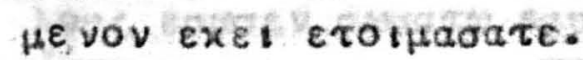

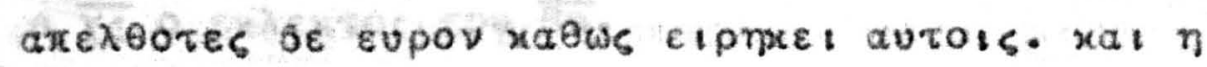
tothaoav to taoxa.

xa 1 ote erevero $\eta$ spa avereosv xal of bubexa a 30 tootonol ouv aurw.

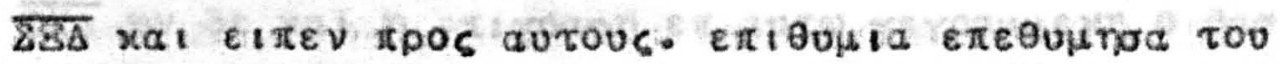

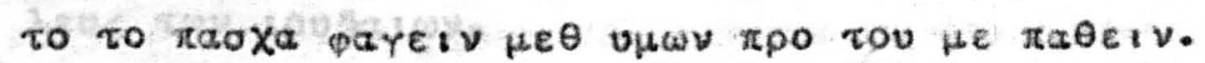

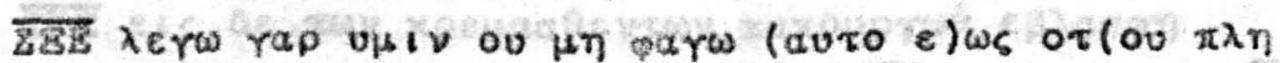

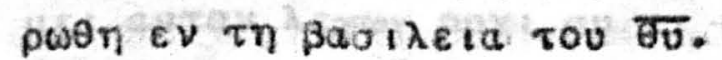

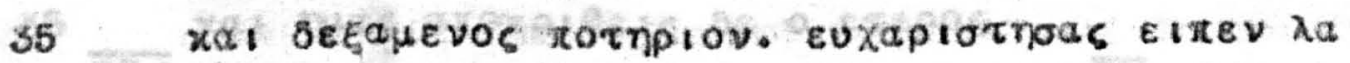

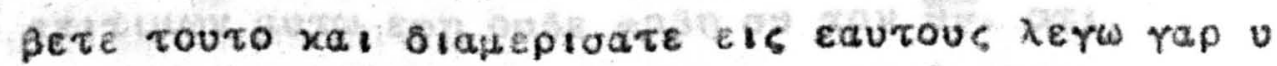

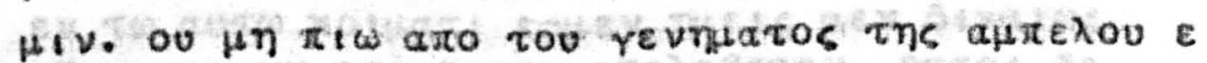

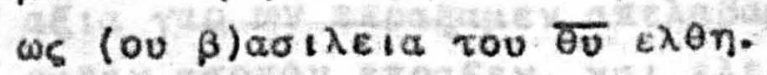

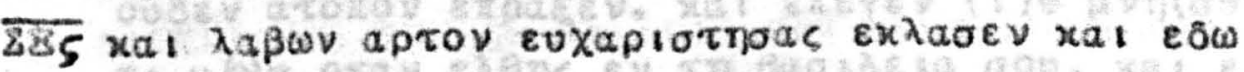
40

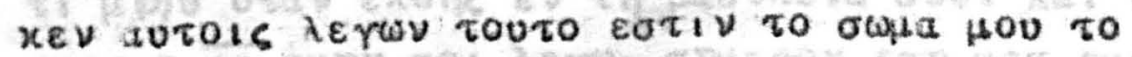

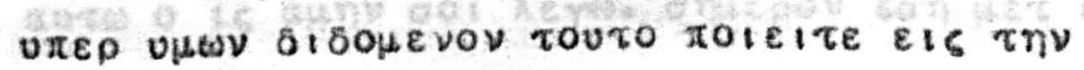




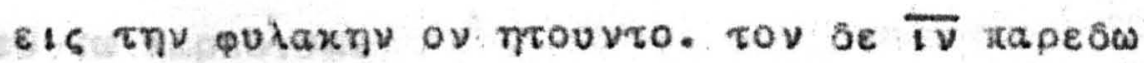

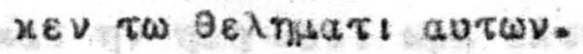

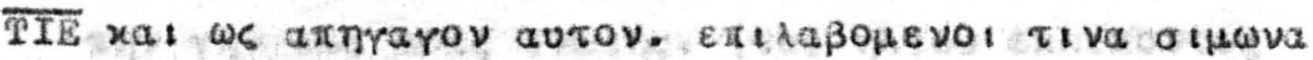

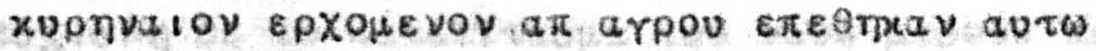

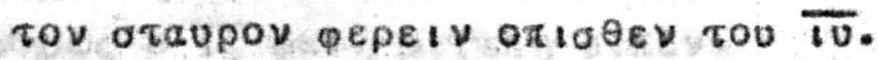

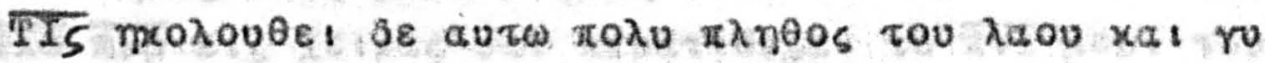

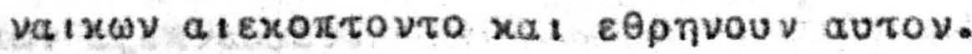

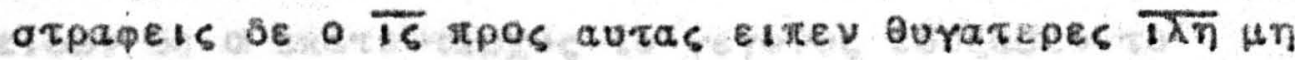

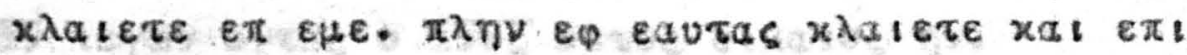

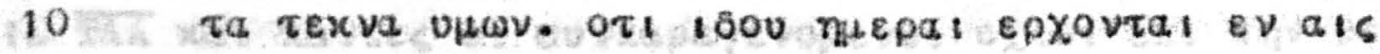

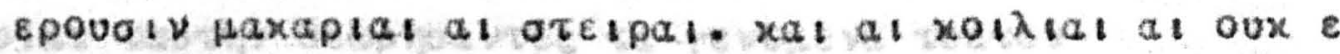

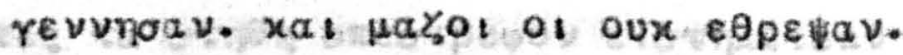

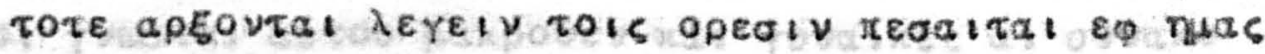

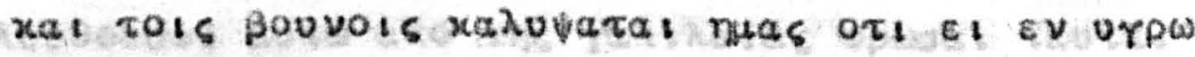

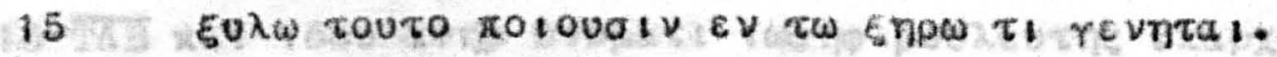

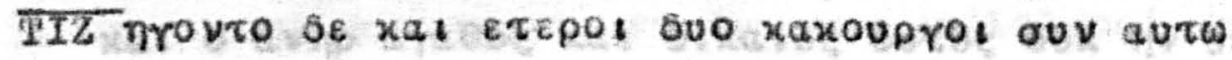
avatpeonvas.

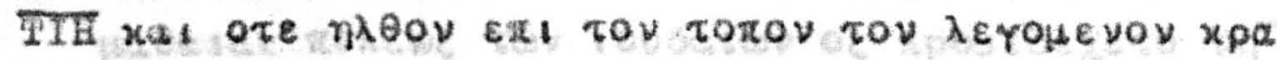
Viov exel eataupway autov xal tous xaxoup

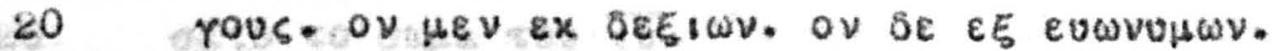

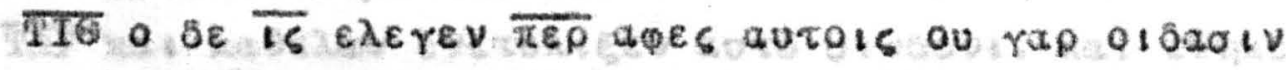
Tl Tolovalv.

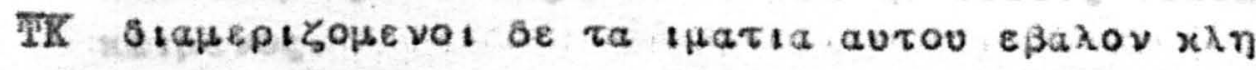
pov xal latmxet o daos $\theta \varepsilon \omega p \omega \nu$.

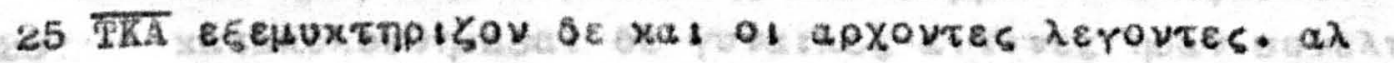

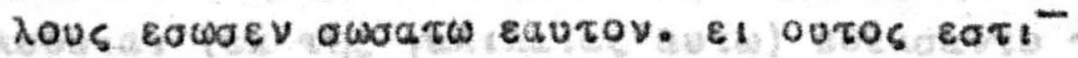

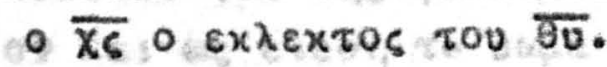

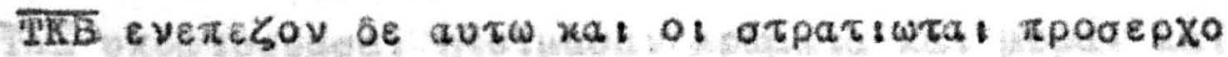

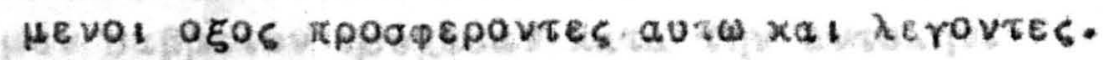

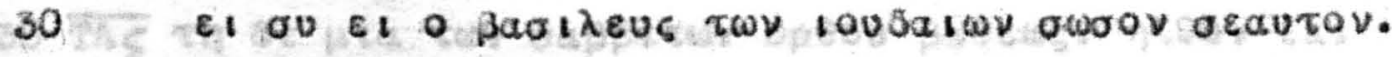

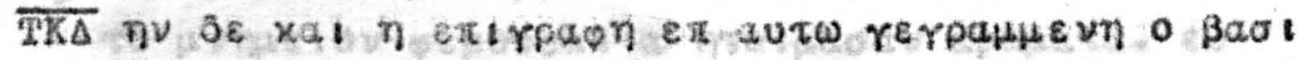

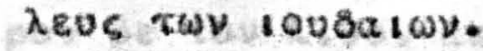

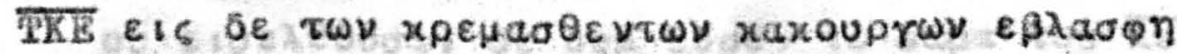

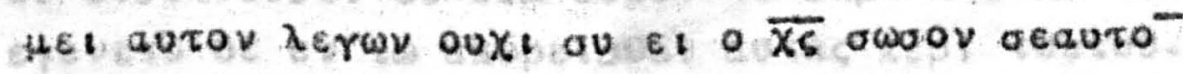
35

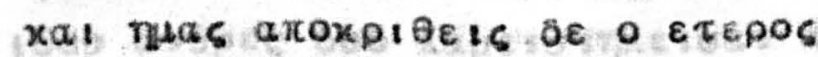

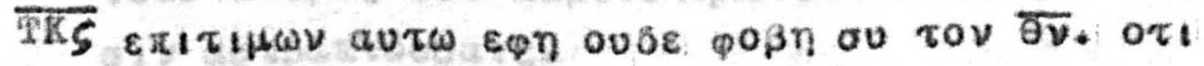

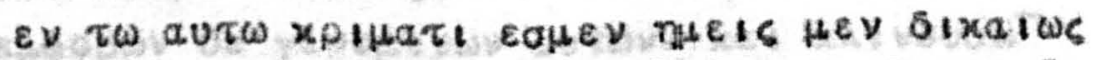

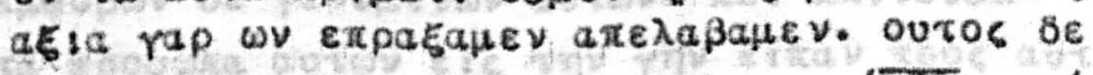

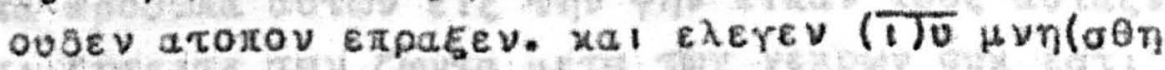

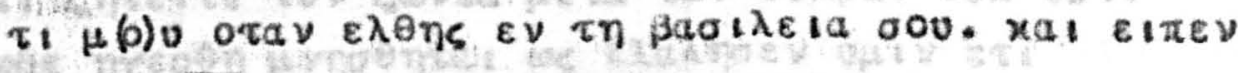

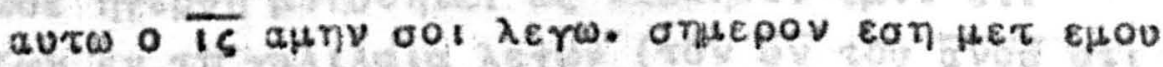


\&v $\tau \omega$ tapası

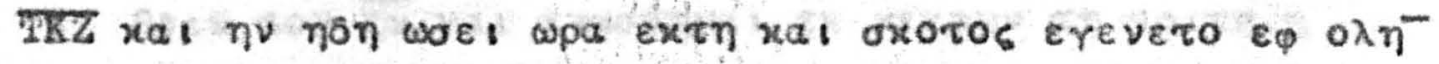

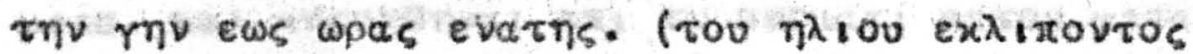

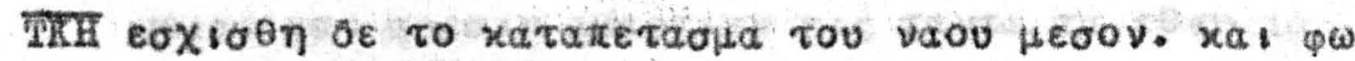

5

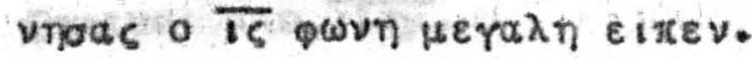

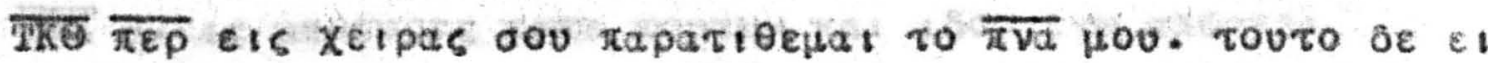
$\pi \omega \nu$ eรg $\pi \nu \varepsilon v \sigma \varepsilon v$.

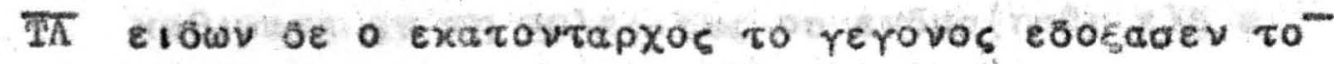

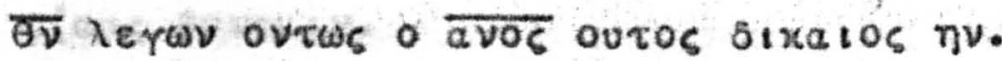

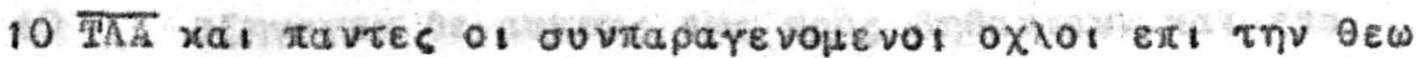

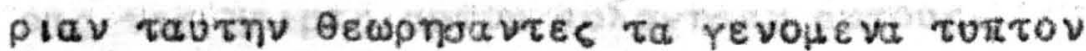

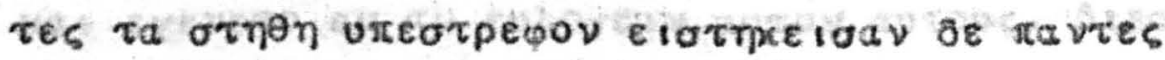

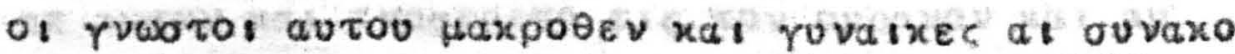

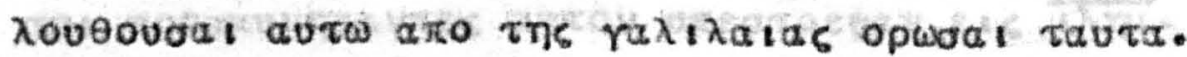

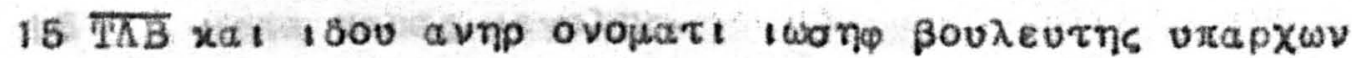
xal o avmp aratos $x a, \delta$ ixaios outos oux nv auna

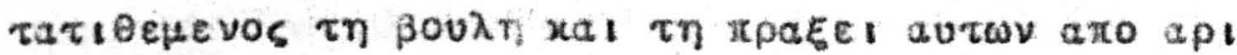

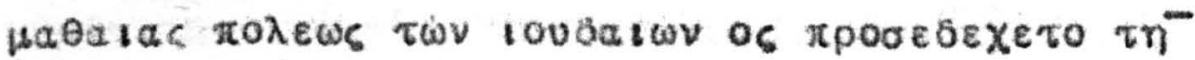

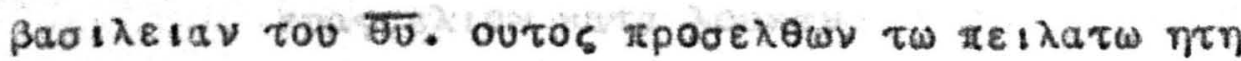
20

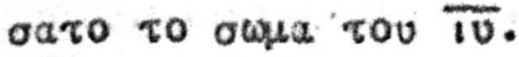

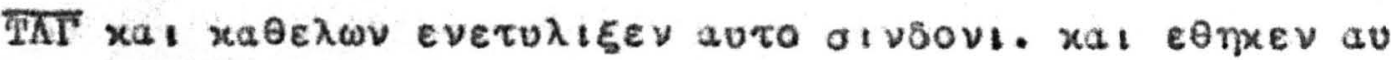

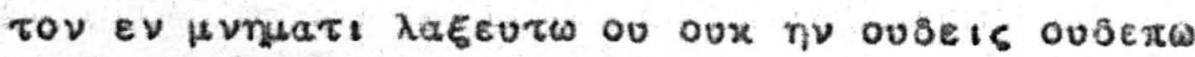

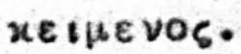

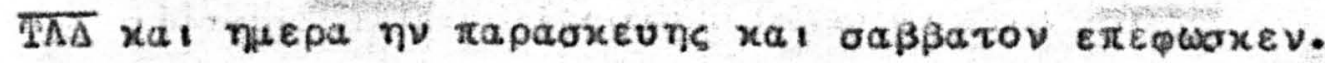
25

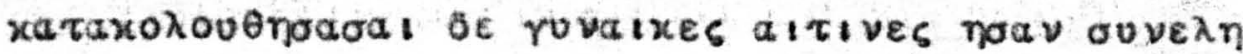

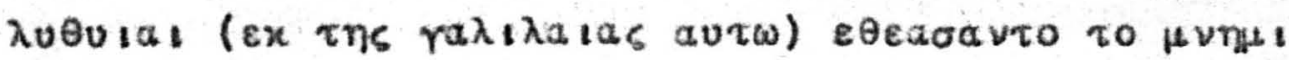
ov $x_{a}$, we $\varepsilon \tau \varepsilon \theta \eta \eta$ to owsa sutou.

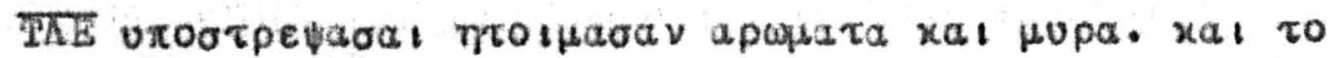

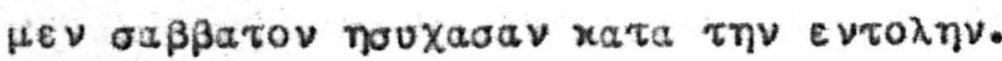

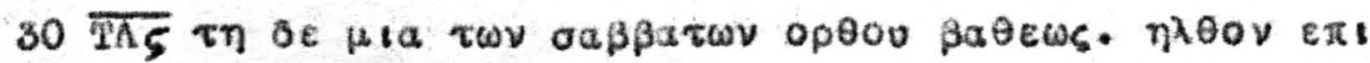

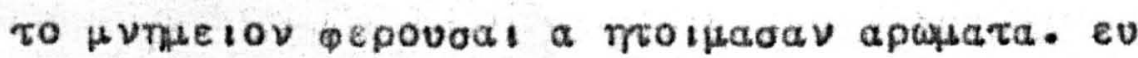

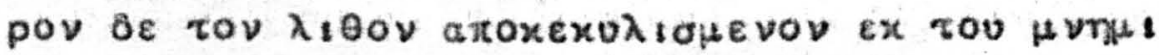

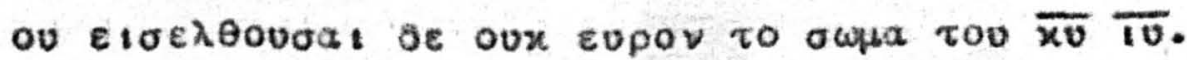

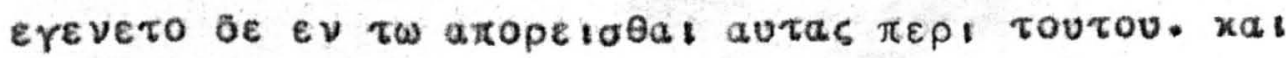

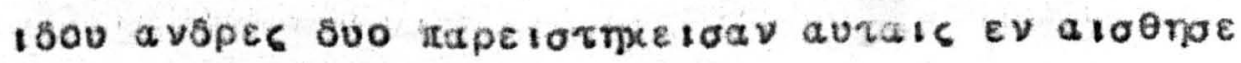

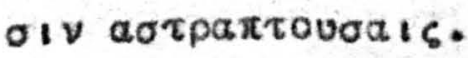

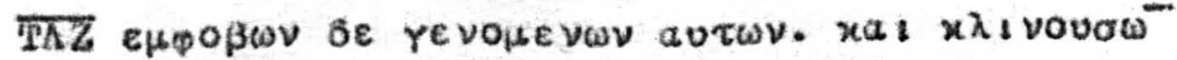

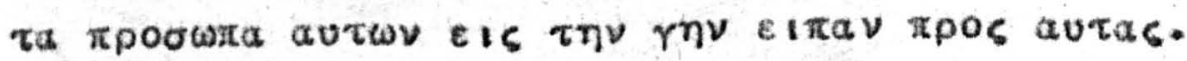

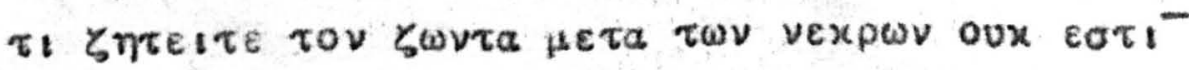

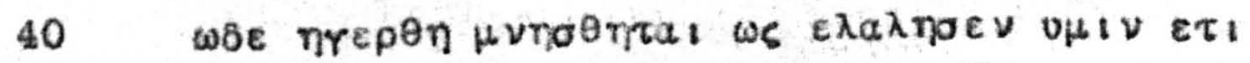

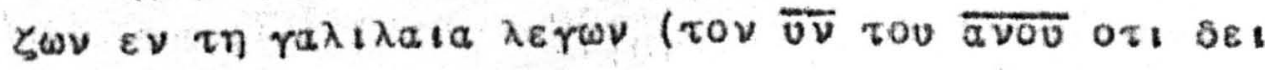




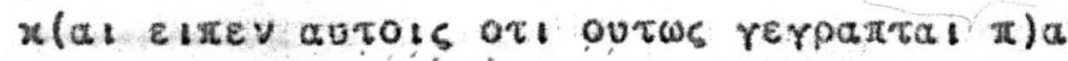

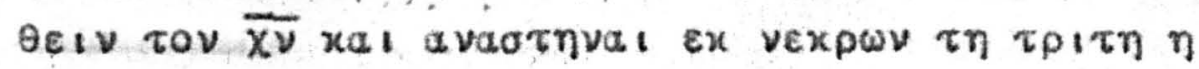

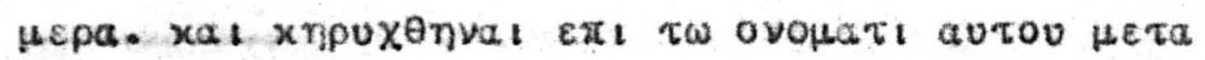

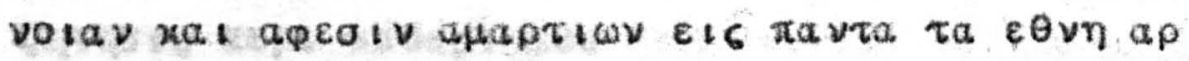

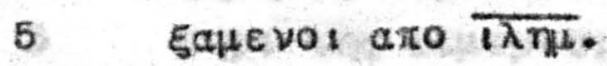

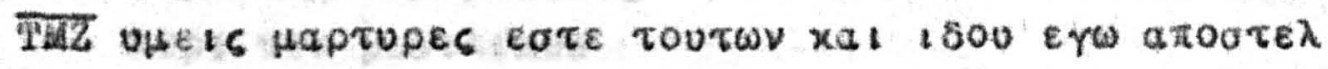

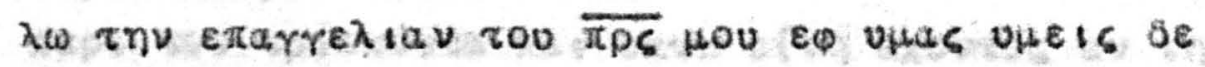

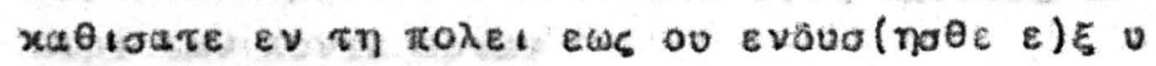
blos suvajtv.

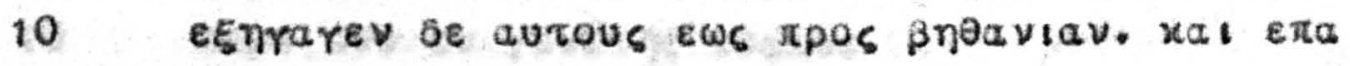

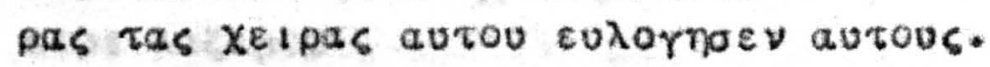

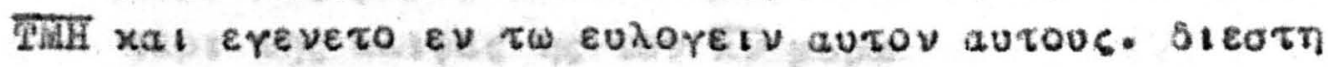

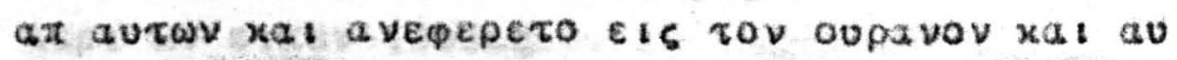

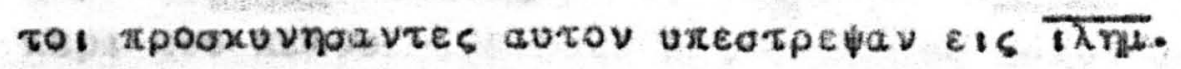

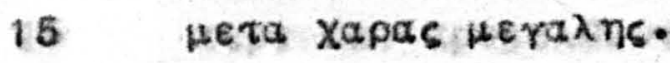

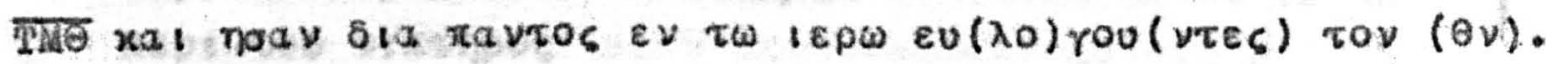

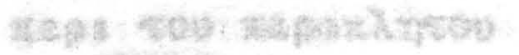
ateps whe as at

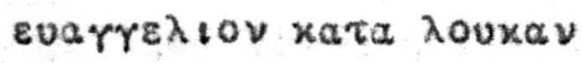




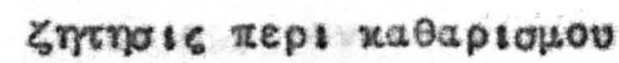

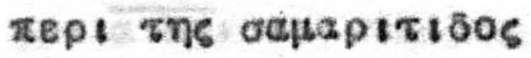

זept tov $\beta$ ao $t \lambda$ trov

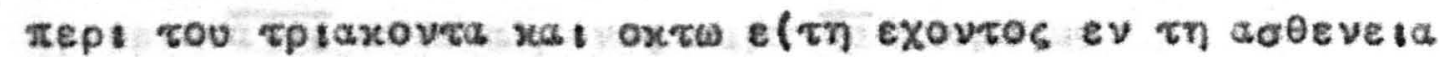

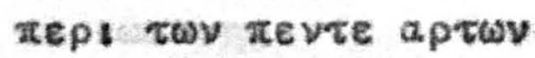

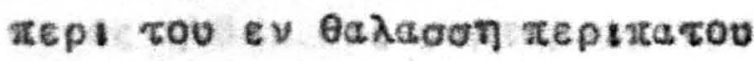

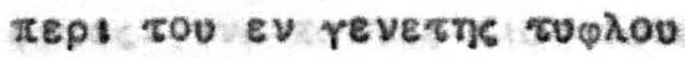

тер גaל̧apou

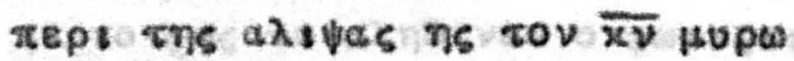

$\pi e p ! \omega \nu$ เ $\pi \varepsilon v$ sovacs

$\pi \varepsilon \rho$ เ $\tau$ เov $\pi \omega \lambda$ ov

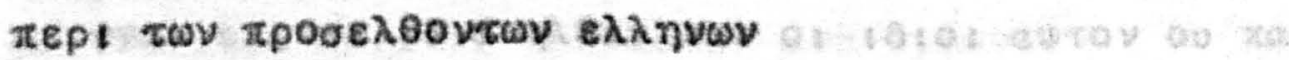

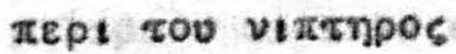

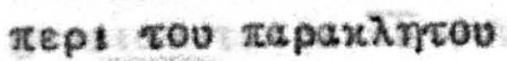

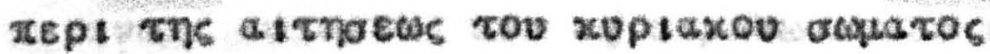

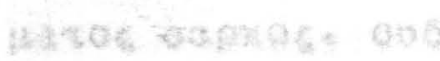

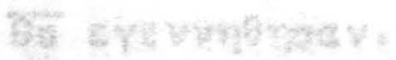

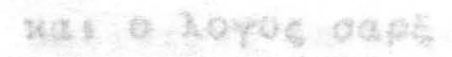

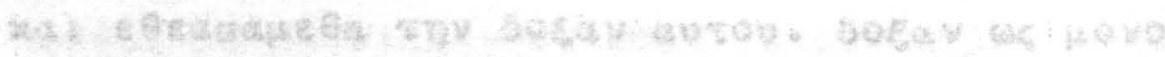

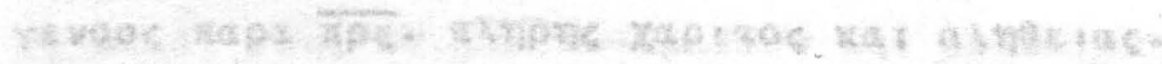

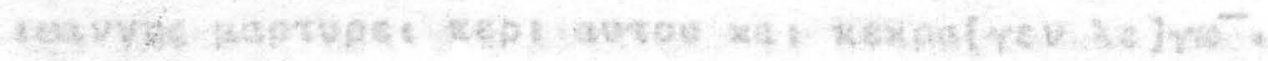

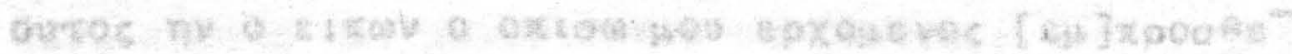

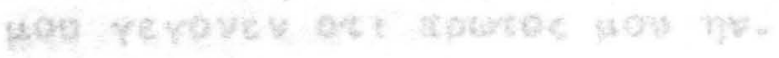

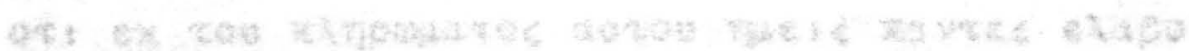

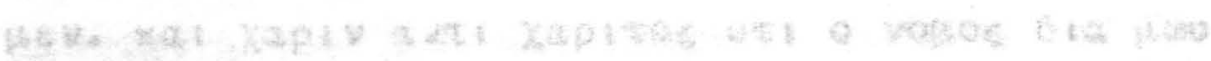

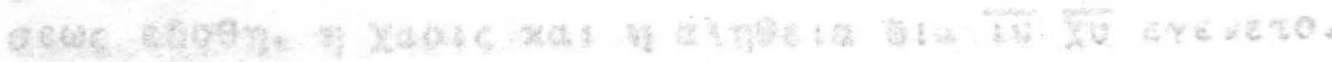

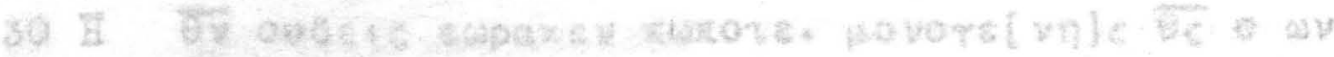

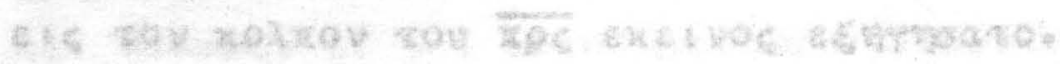

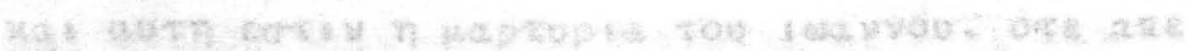

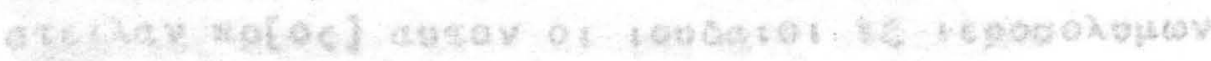

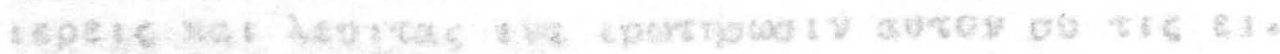

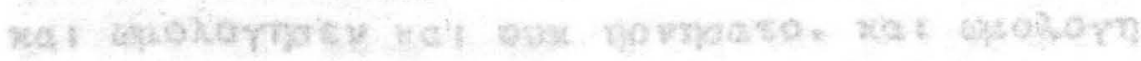

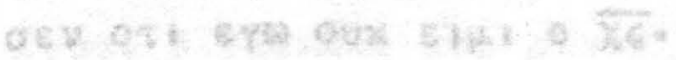

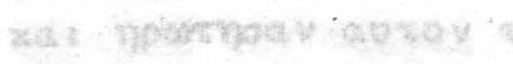

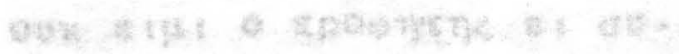

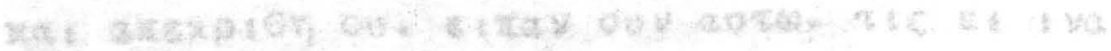

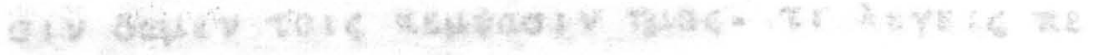

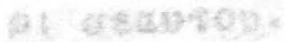

xes. I $100 \mathrm{v}$. 


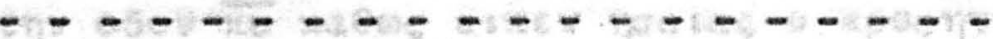

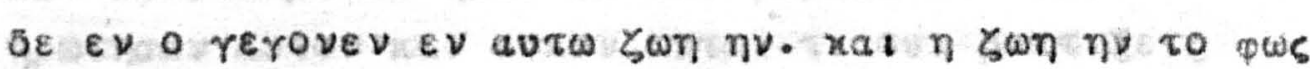

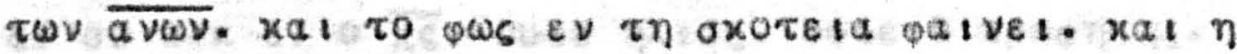

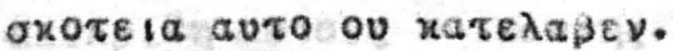

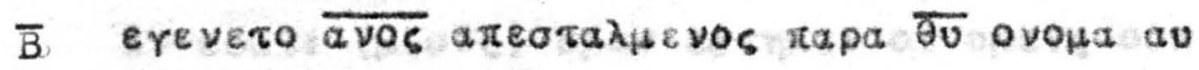
$\tau \omega$ s wavvins.

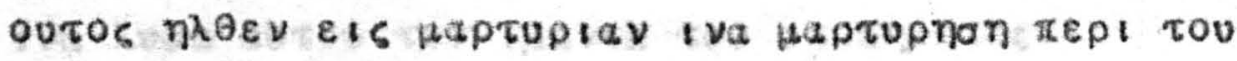

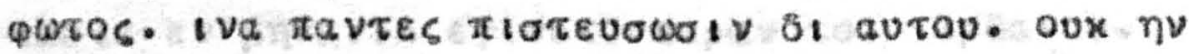

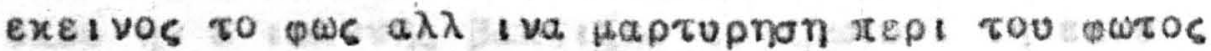

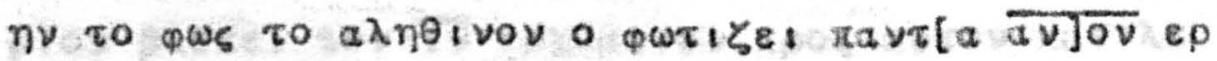

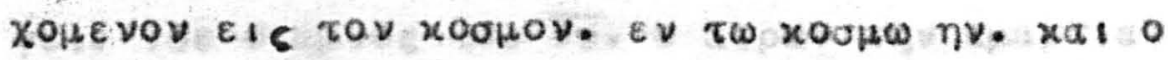

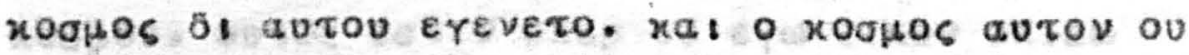

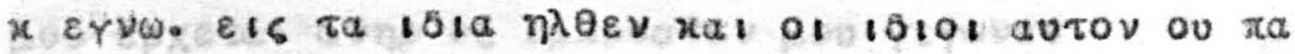

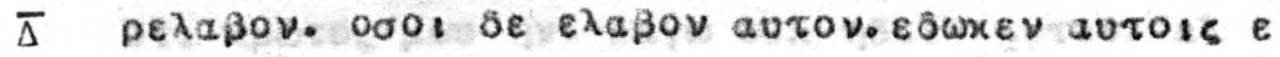

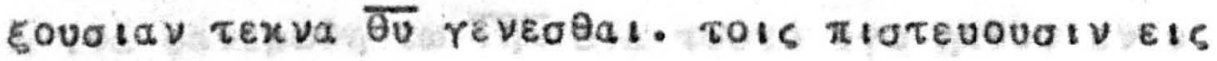

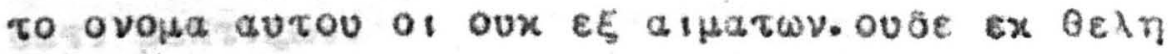

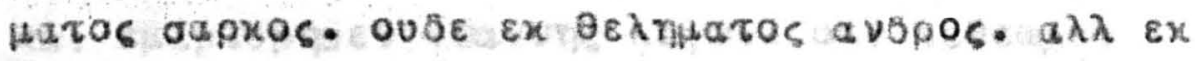
ब̄ erevuningav.

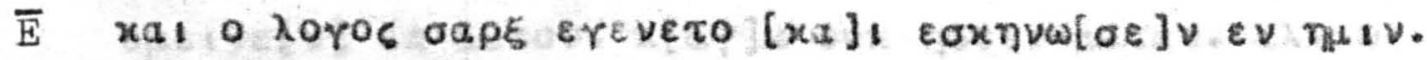

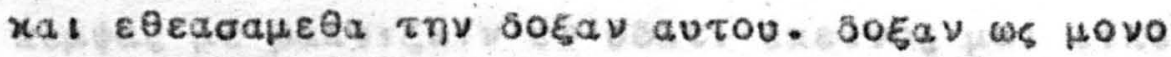

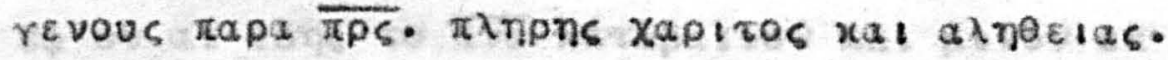

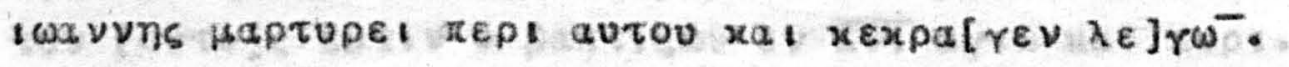

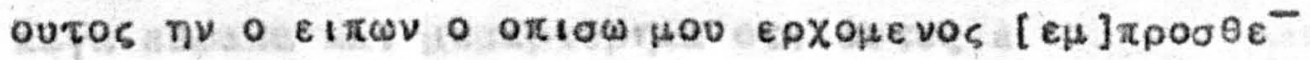
Hov rerovev ot 1 a

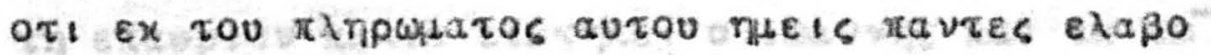

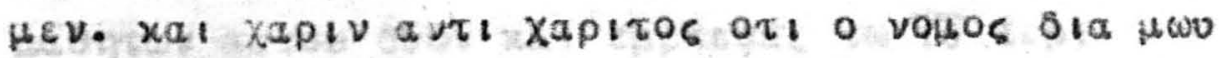

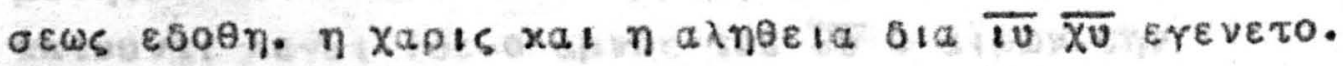

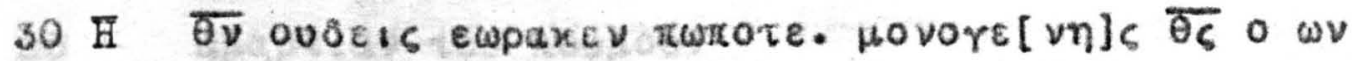

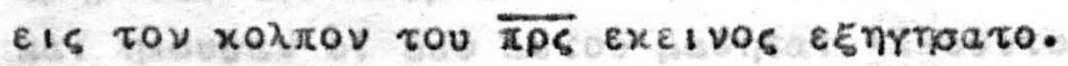

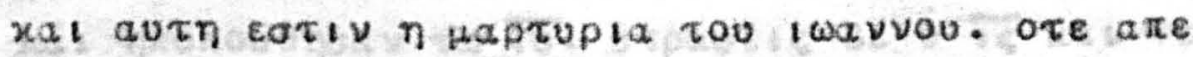

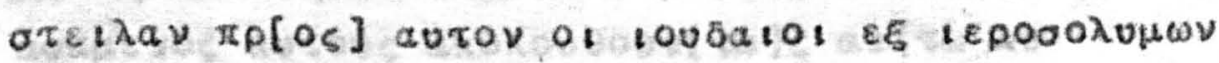

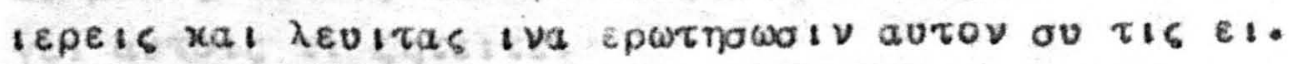
xal wholornoev $x_{1}$ oux npvrjato. xal whoגorn

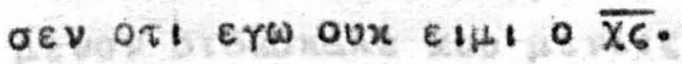

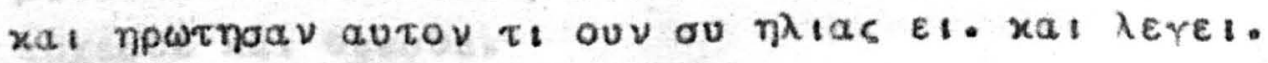

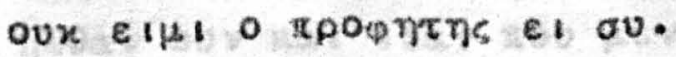

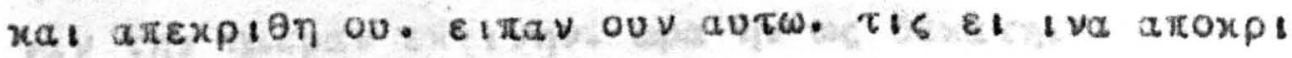

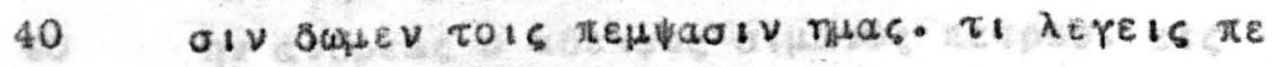
pi oeaurou. 


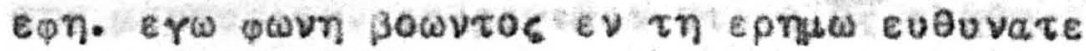

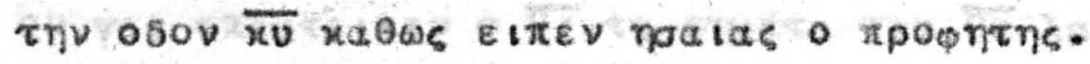

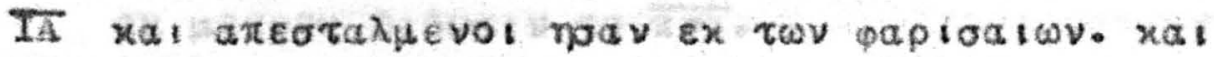

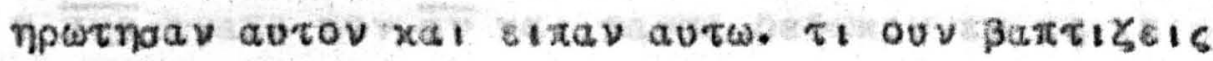

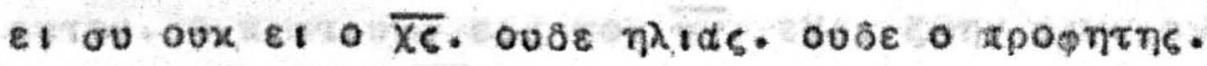

I8 atexpion autoic o twavvnc $\lambda \varepsilon \gamma[\omega] \nu \varepsilon \gamma \omega \beta \pi \tau i \zeta_{\omega} \omega$

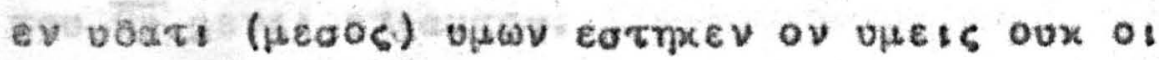

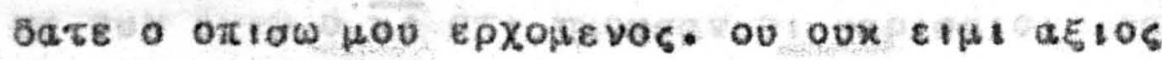

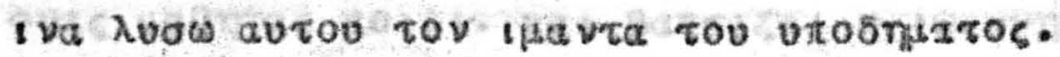

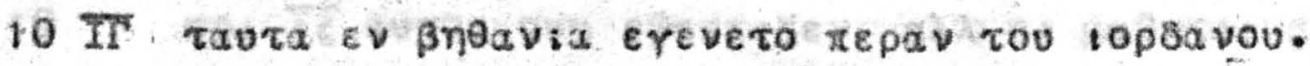

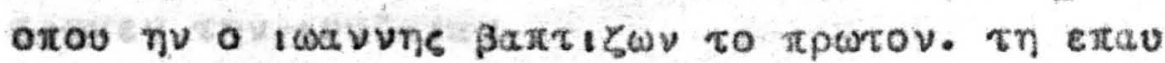
piov $\beta \lambda \varepsilon \pi \varepsilon$ t rov TV epXosevov $\pi \rho \circ$ s avtov xa। $\lambda \varepsilon$

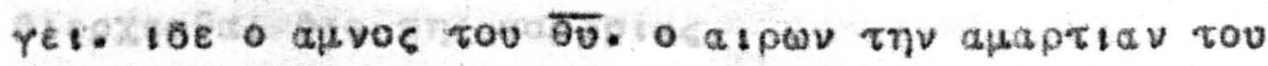
xoopov. outos eativ viep ov erw etsov oxtaw

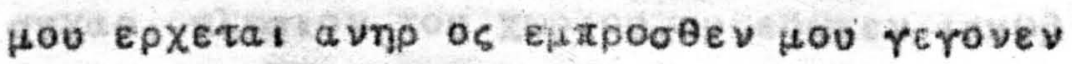

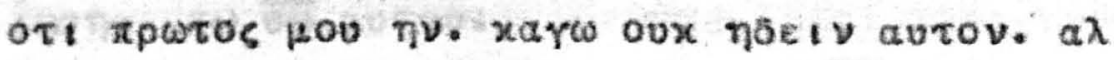

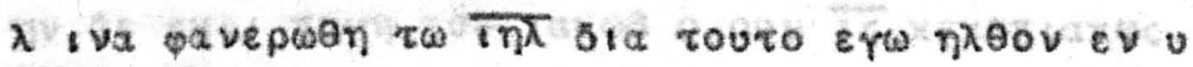

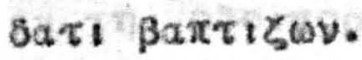

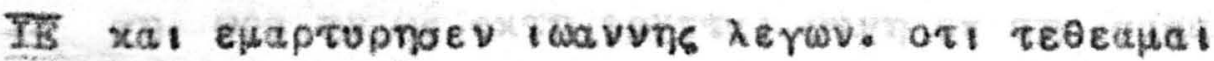

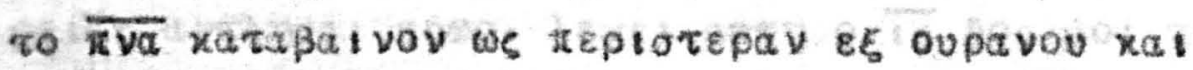

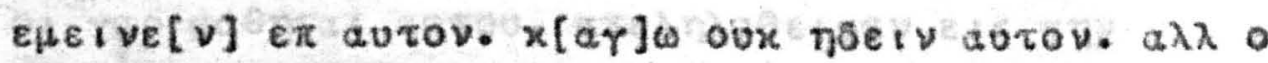

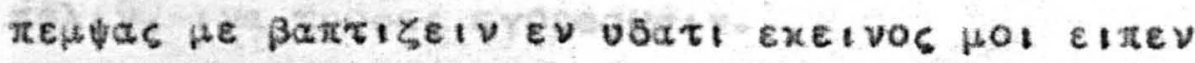

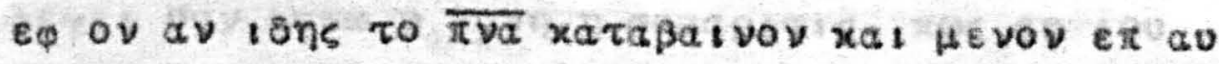

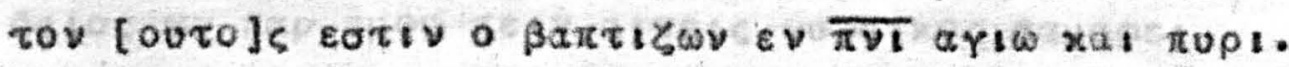
xayd swpaxa xat Heisapropina ot ovtos $\varepsilon$

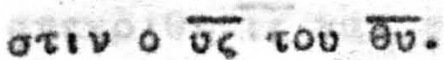

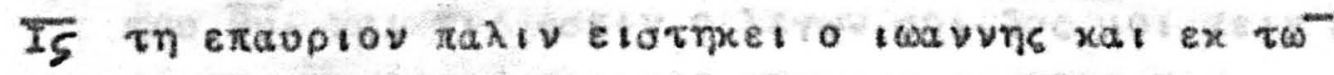

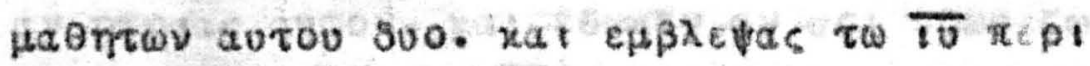

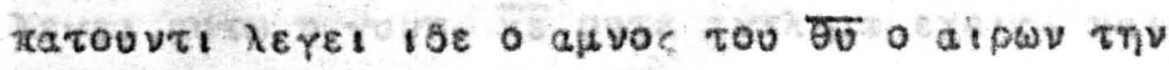
auapriav rou xoonov.

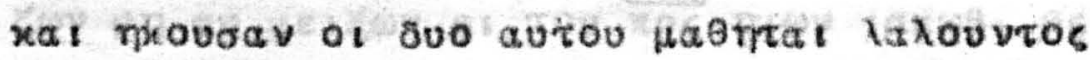
xat nuodovenoav $\tau \omega$ TV. otpapeis se o Ts xal $\theta \varepsilon$

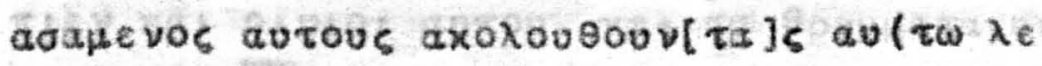

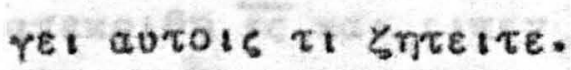

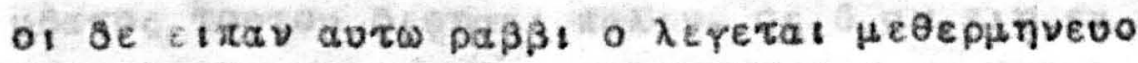

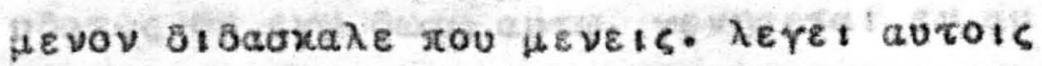
$\varepsilon \rho \times \varepsilon \sigma \theta \varepsilon$ xat $\circ \vee \varepsilon_{\sigma} \theta \varepsilon$.

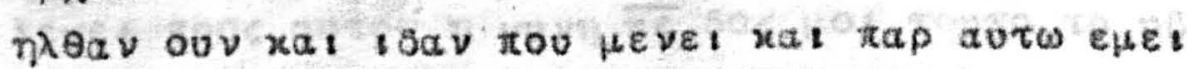

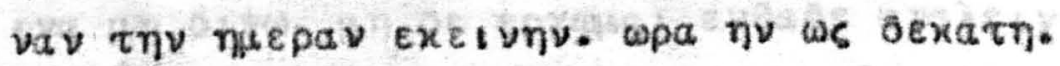

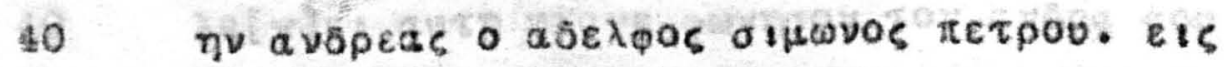

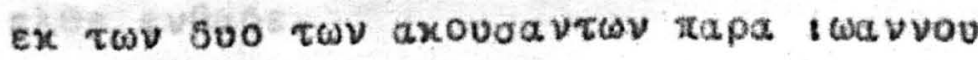




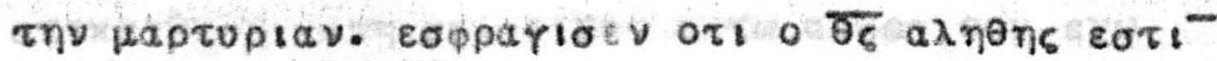

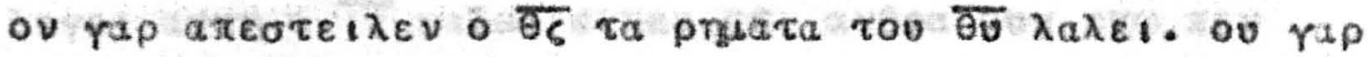

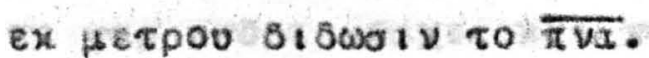

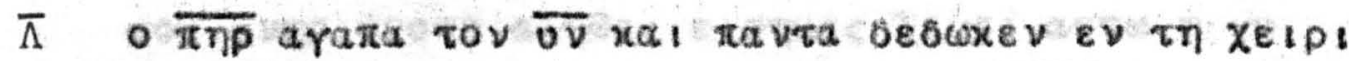

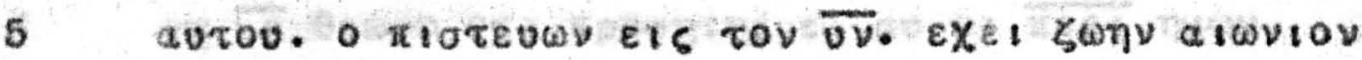

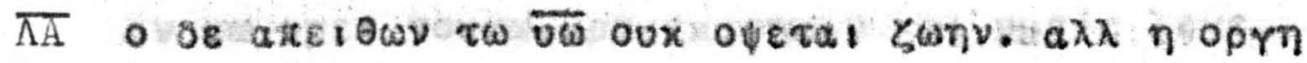

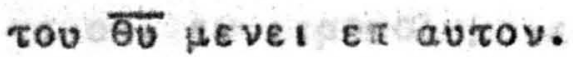

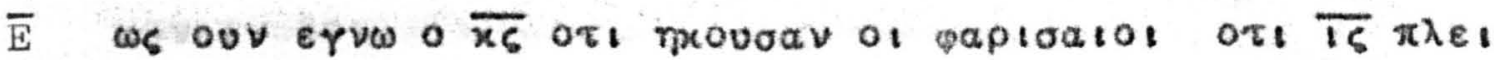

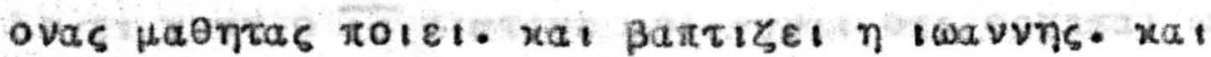

10

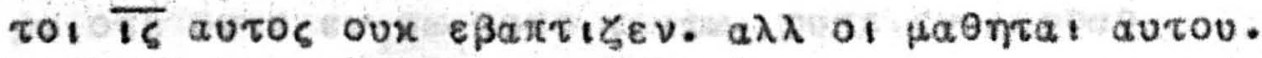

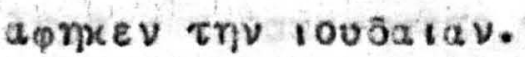

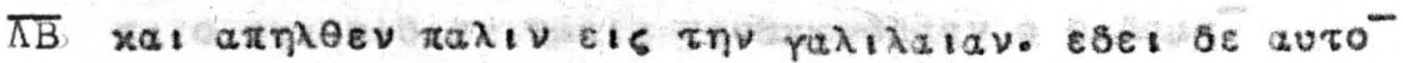

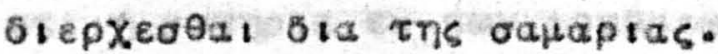

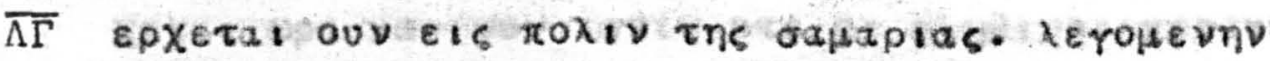

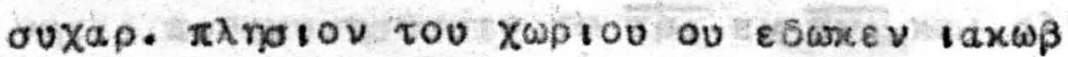

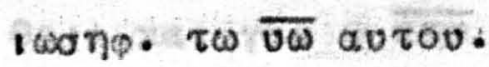

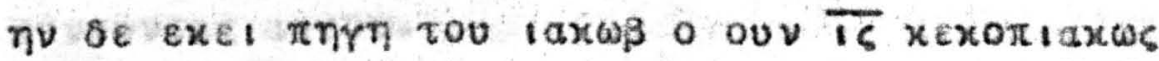

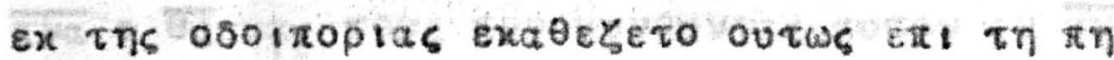

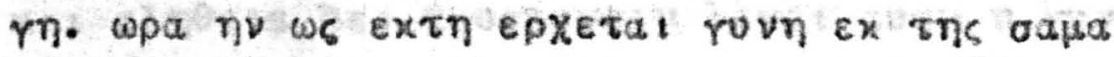

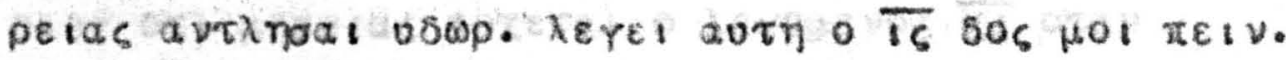

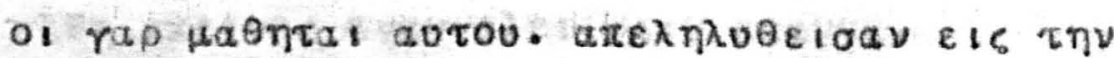
moגiv. Iva tpoeas aropaowatv.

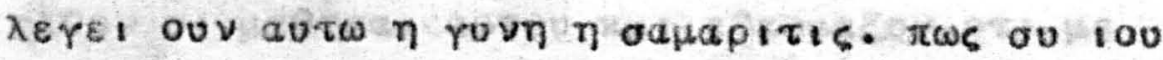

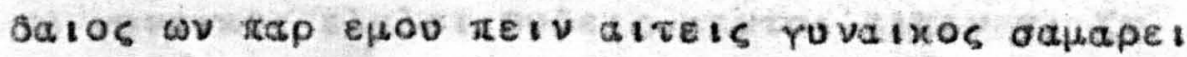

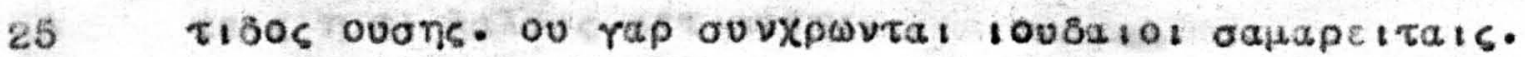

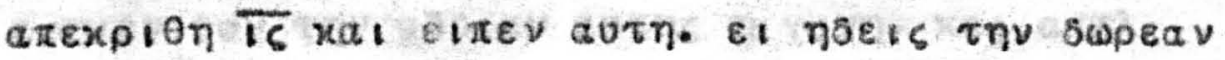

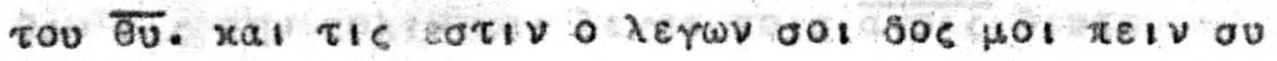

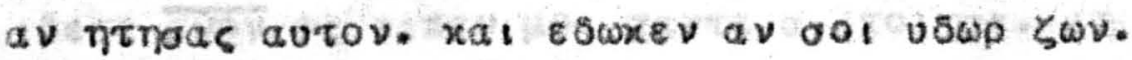

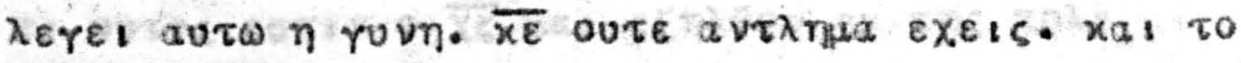

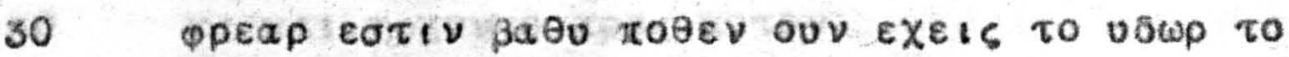

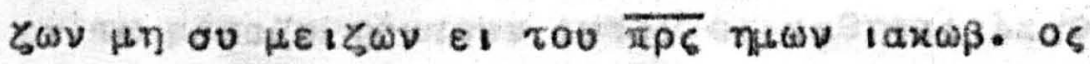
ธะकิ

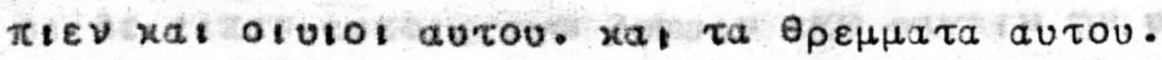

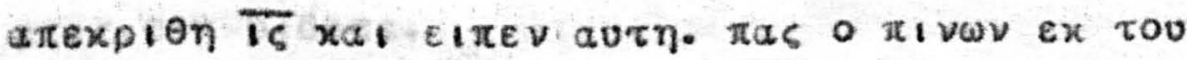

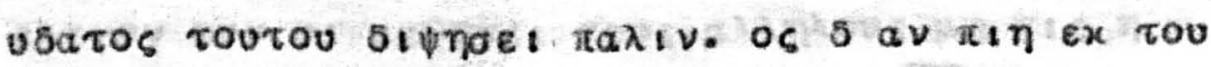

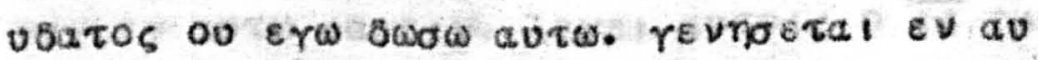

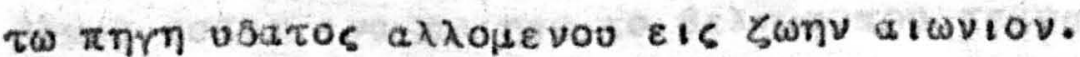

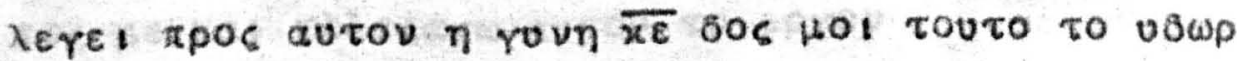

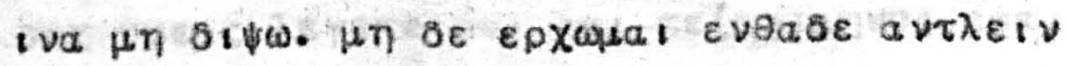

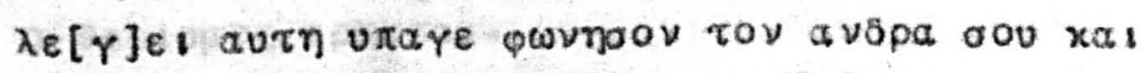

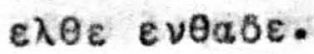




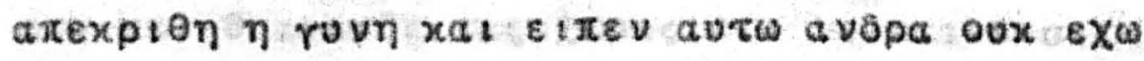

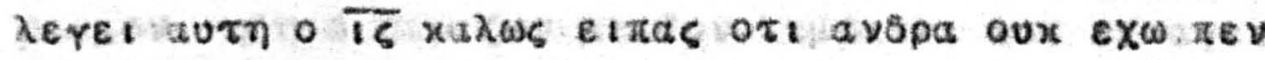

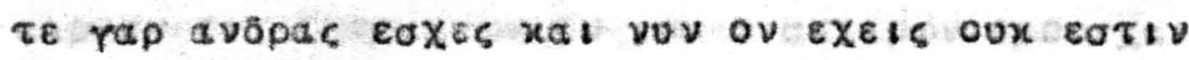

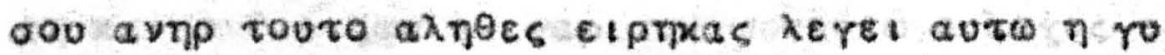

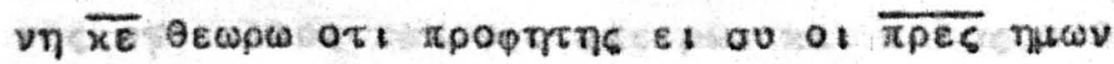

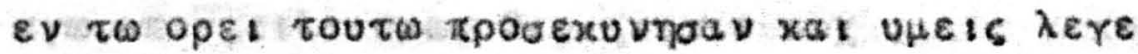

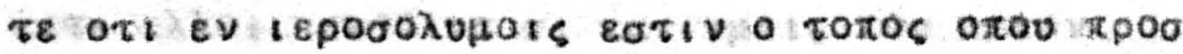
xuveเv set.

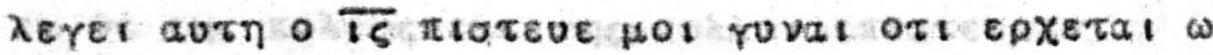

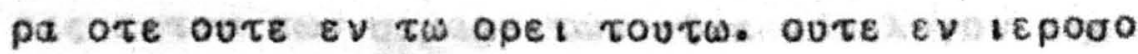

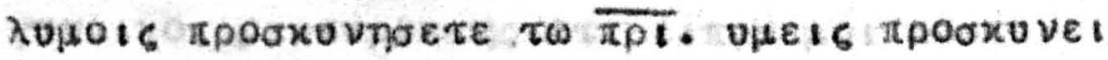
te o oux 0

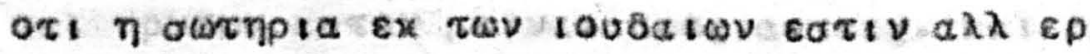

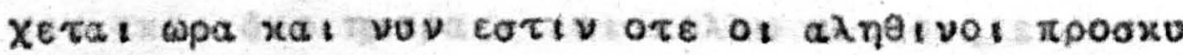

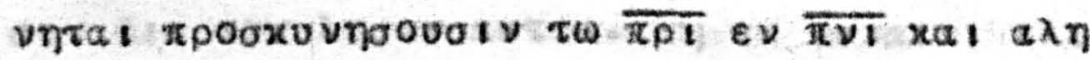

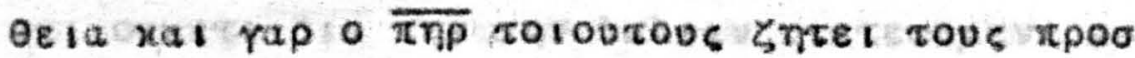
xuvouvtas aurov.

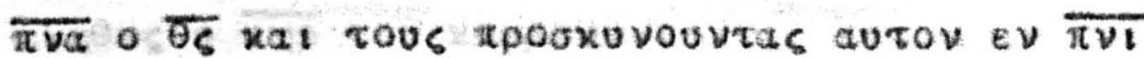

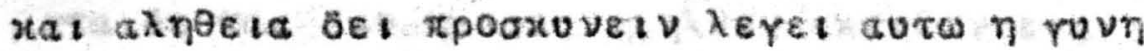

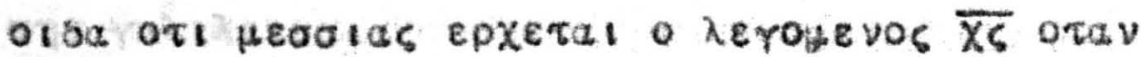

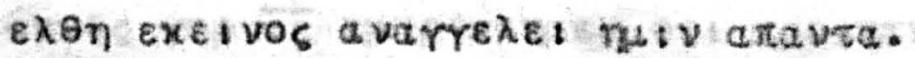

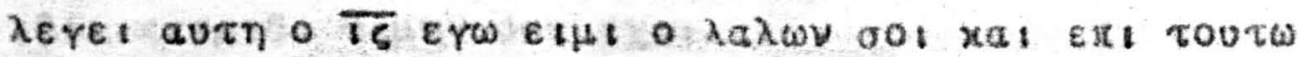

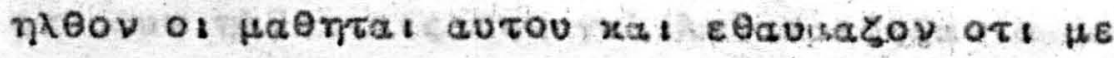

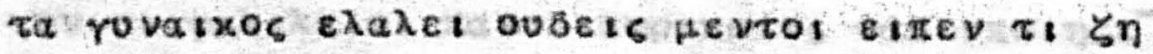

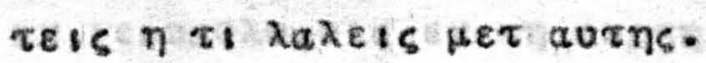

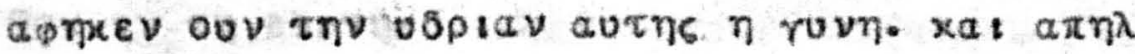

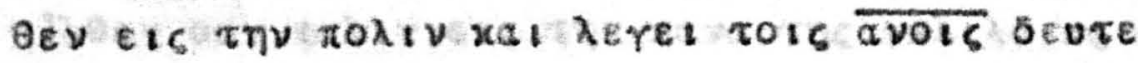

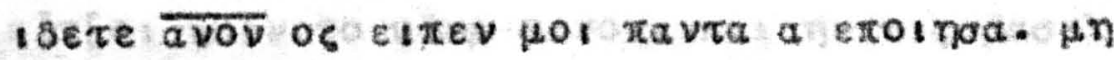

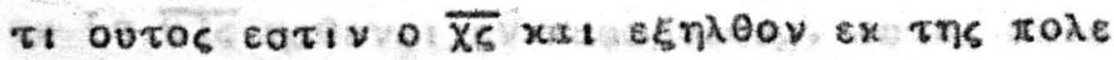

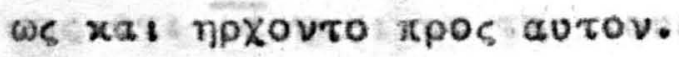

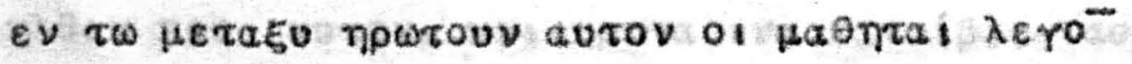

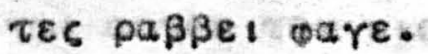

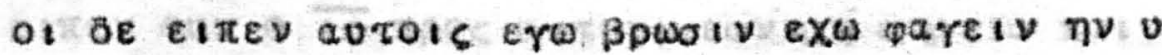
Heis oux 0isate.

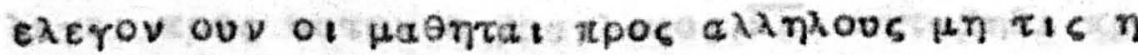
vepev autw pareiv. $\lambda \varepsilon \gamma \varepsilon$ a

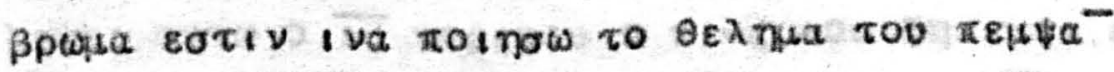

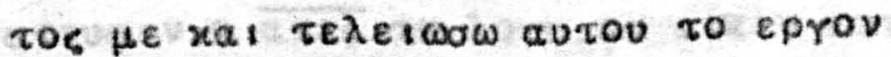

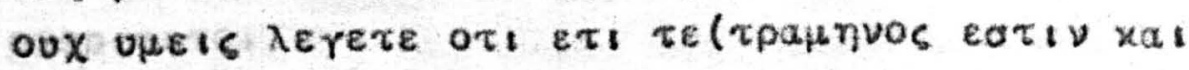

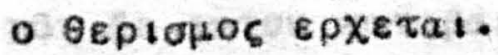

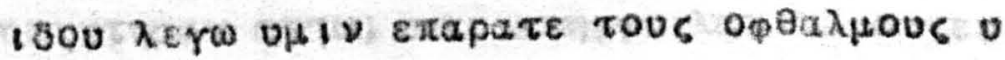




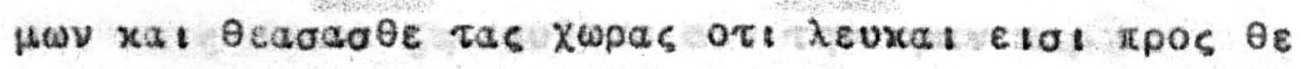

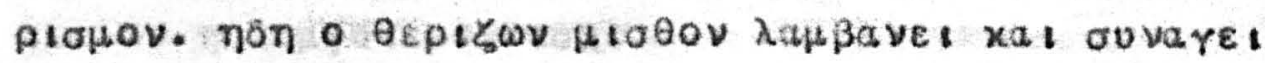

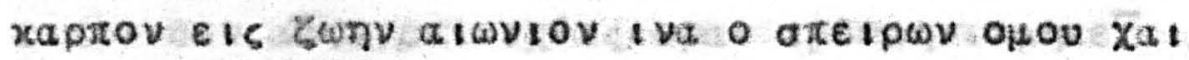

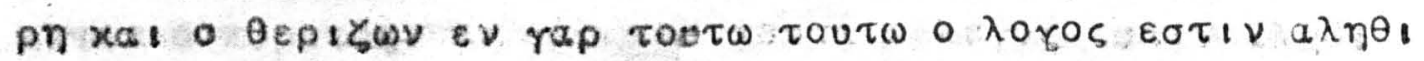

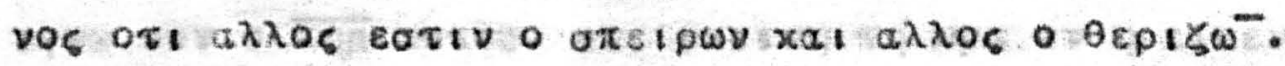

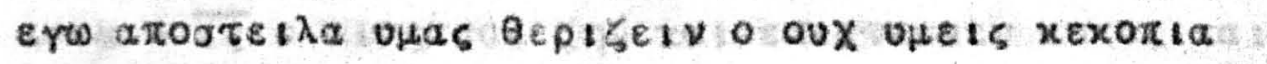

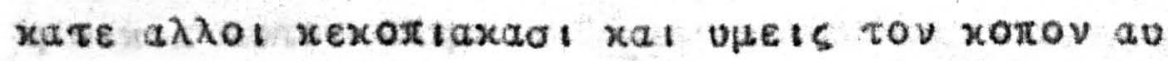

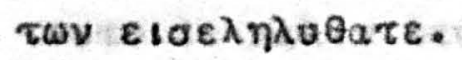

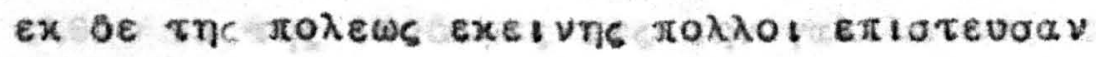

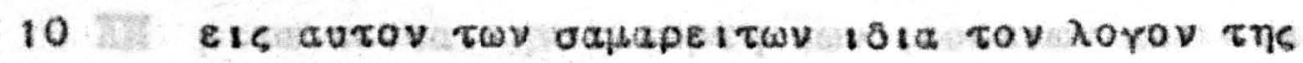

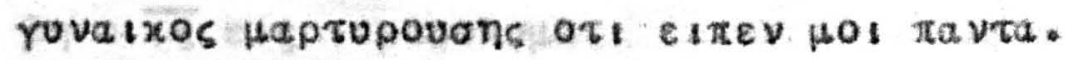

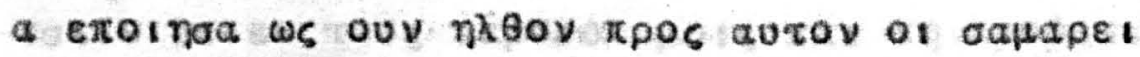

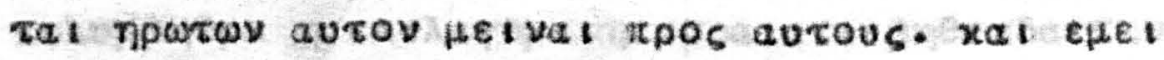

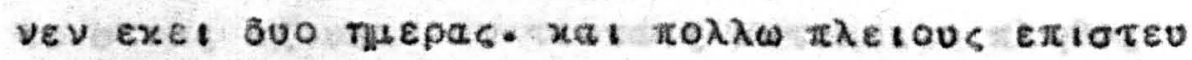

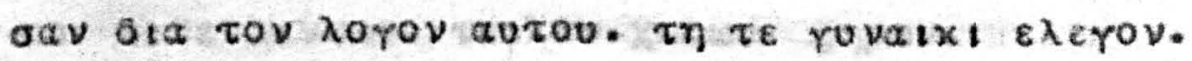

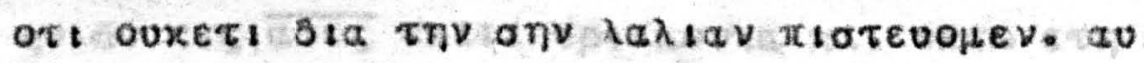

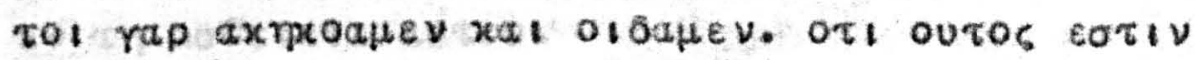
alnows o ODP rou xoOHOU.

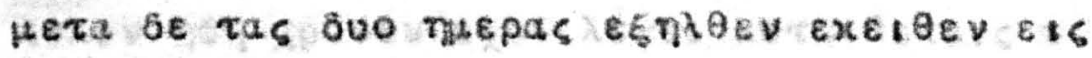
rqv radidatav.

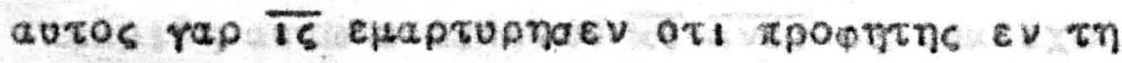

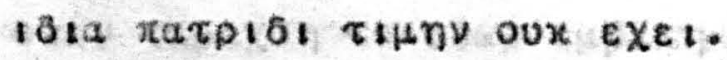

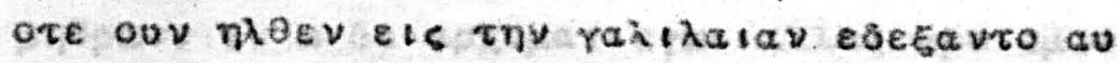

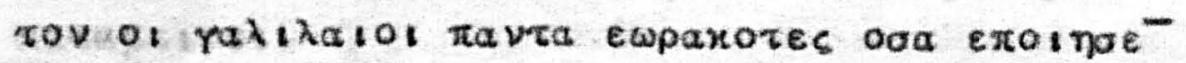

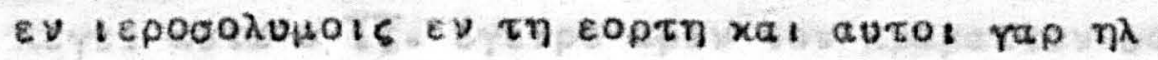
eov ev $\tau \eta$ roptn.

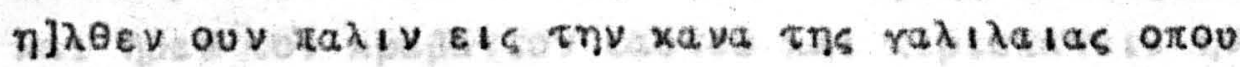

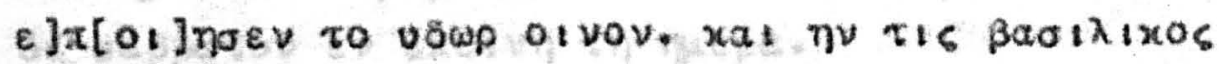

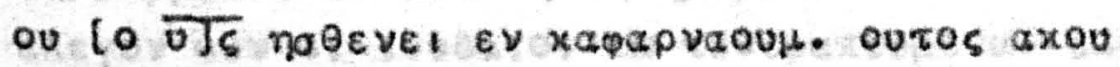

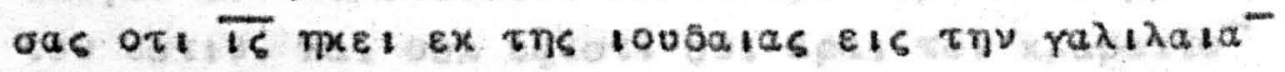

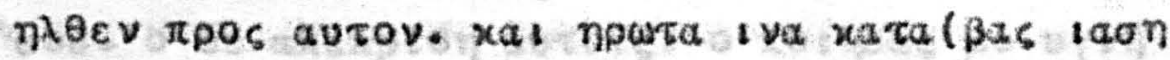

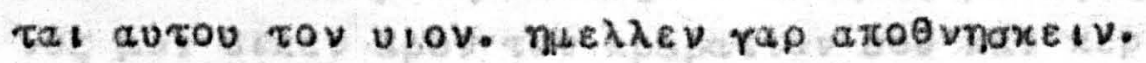

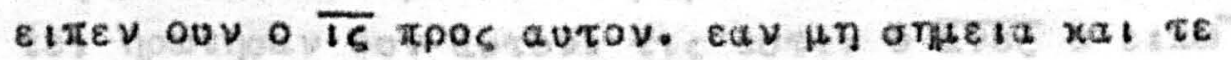

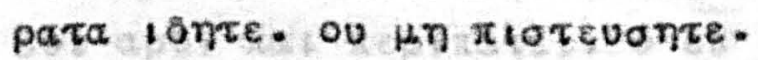

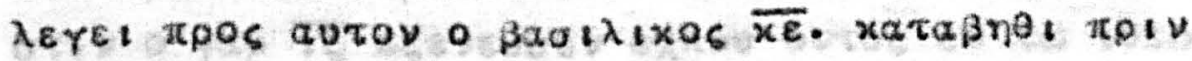
atooaveiv to taisioy Hov.

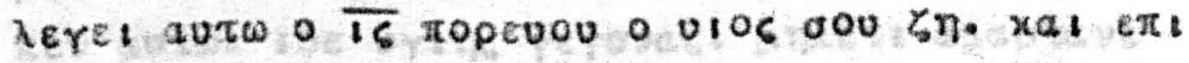

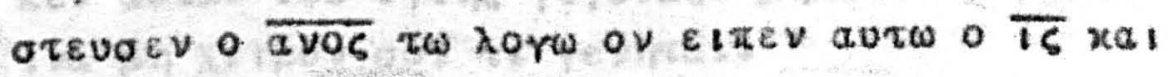
exopevero.

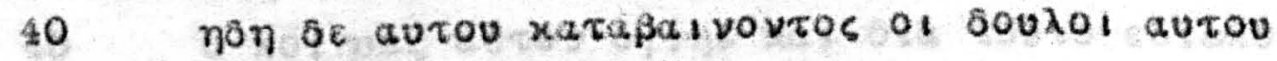

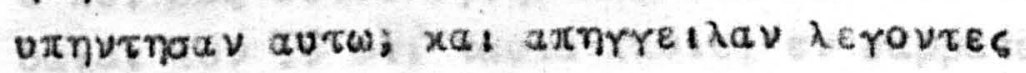




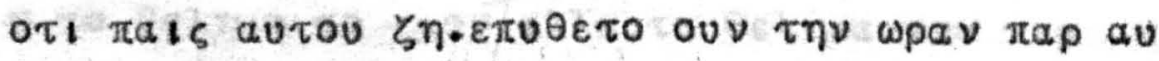

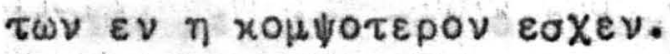

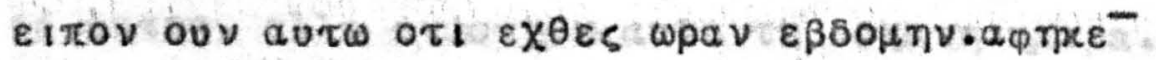

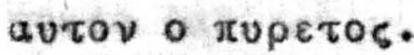

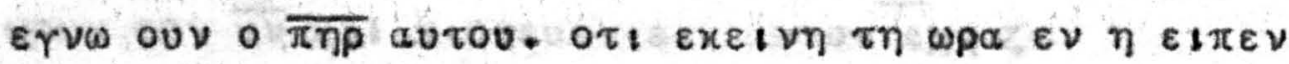

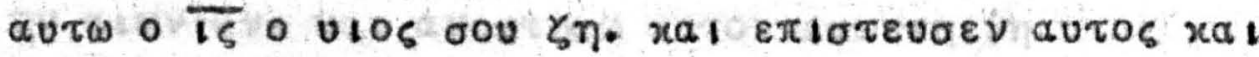

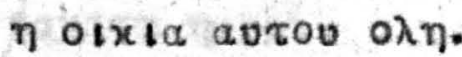

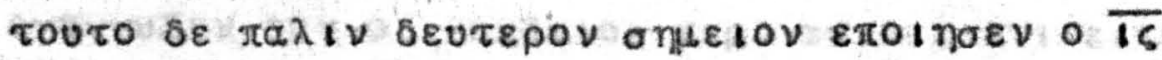

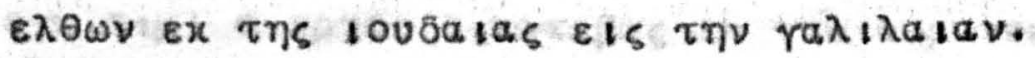

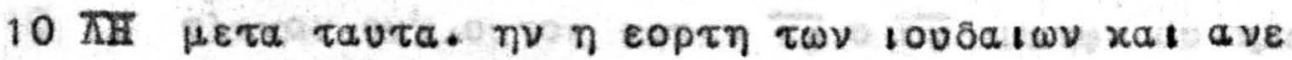

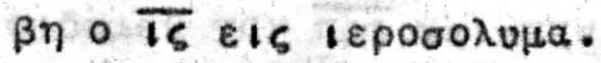

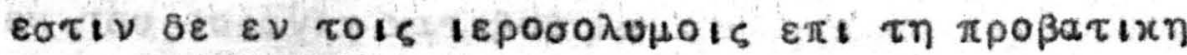

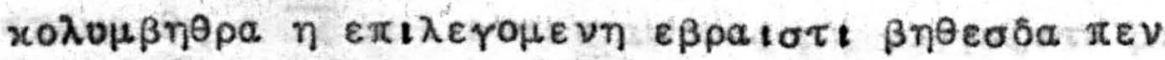

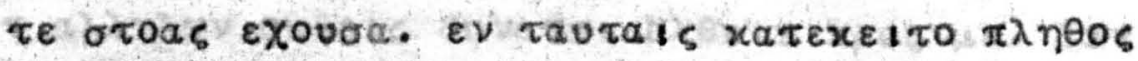

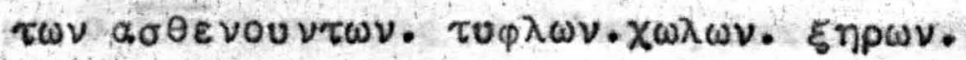

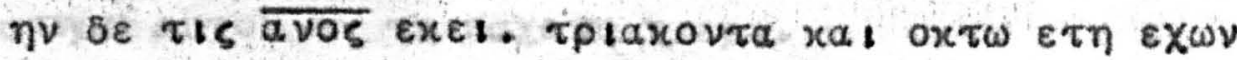
$\varepsilon \nu \tau \eta$ $\alpha \sigma \theta \varepsilon \nu \varepsilon t a$ aúou.

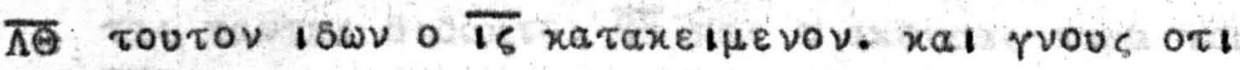

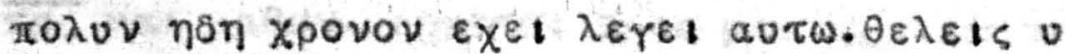
rins reveöal.

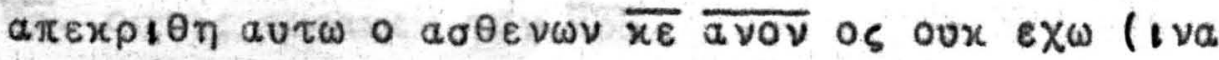

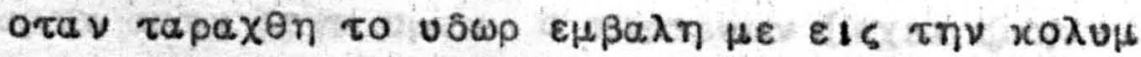

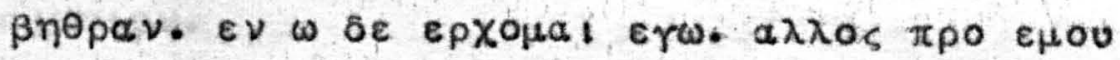

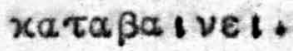

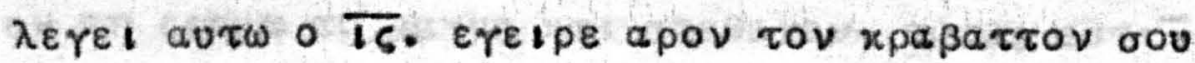

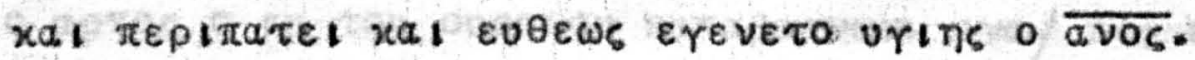

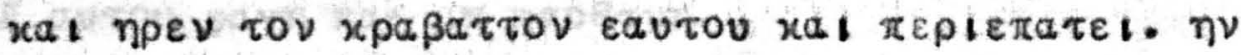

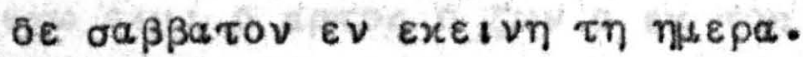
$\varepsilon \lambda \varepsilon$ \%

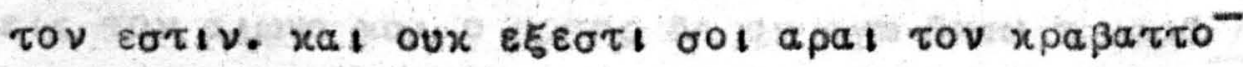

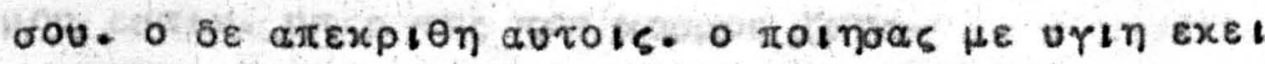

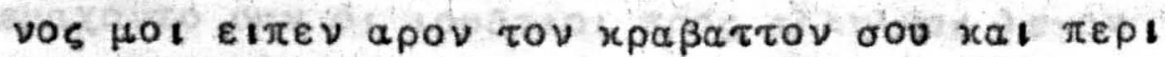

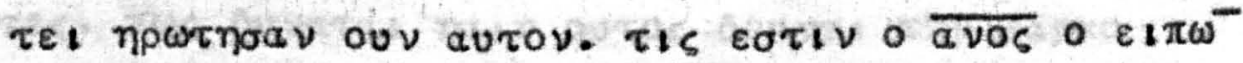

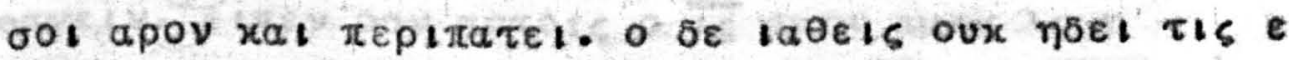

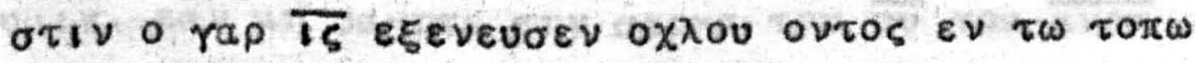

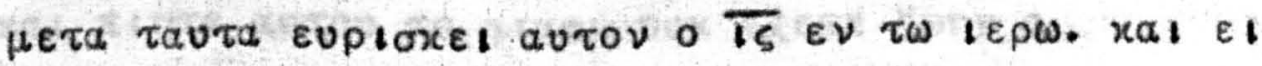

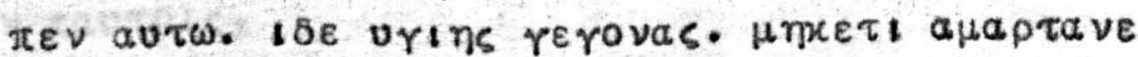

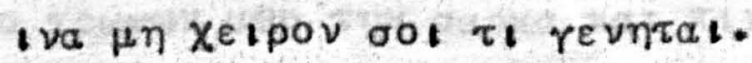

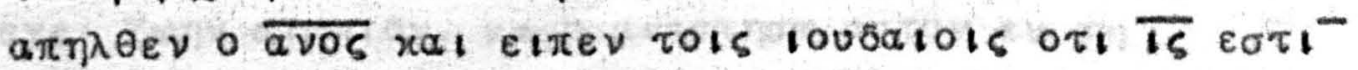

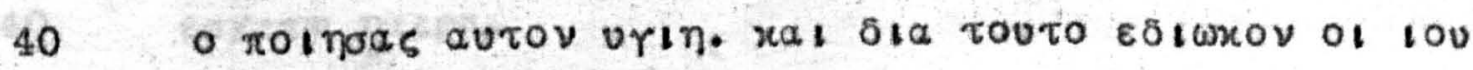

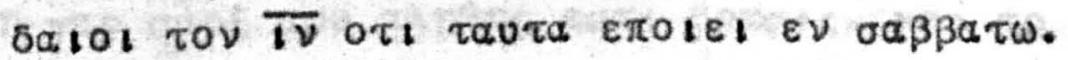


w ro $\theta \varepsilon \lambda r_{h} \alpha$ to $\varepsilon \mu a \nu / a \lambda \lambda \alpha$ to $\theta \varepsilon \lambda \eta \mu a$ tov $\pi \varepsilon \mu$

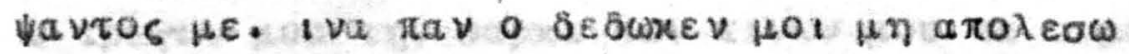

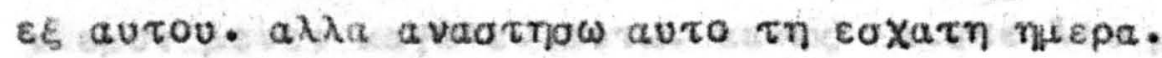

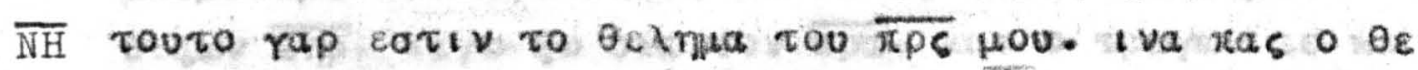

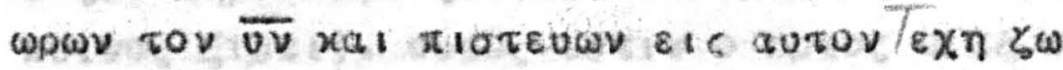

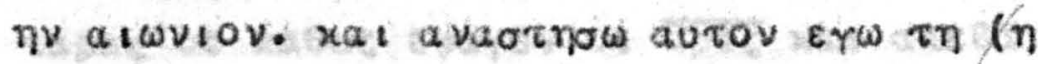
eoxarn nuepa.

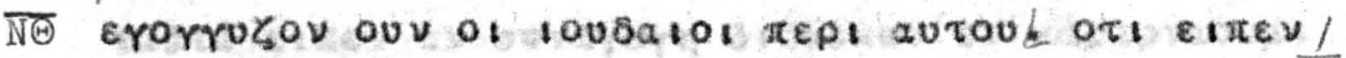
Erw $\varepsilon i \mu l$ o aptos a xatapas $\varepsilon x$ tou oupavou $x a t$

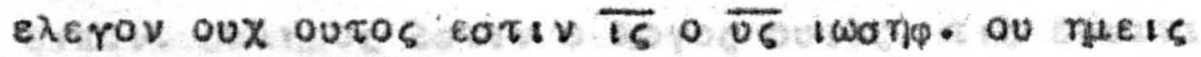

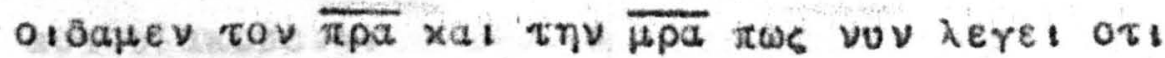
Ex tou oupavou xatußeßrpa.

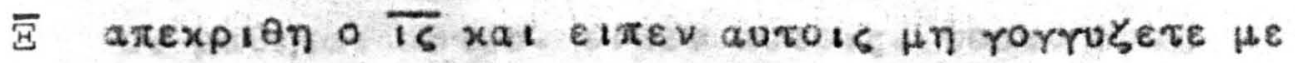

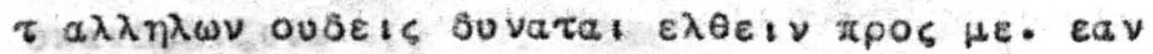

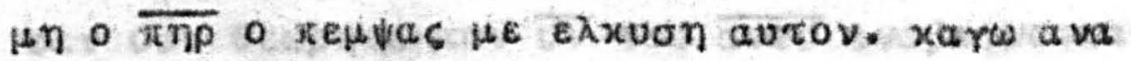

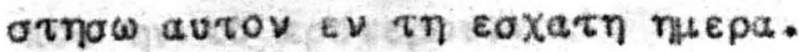

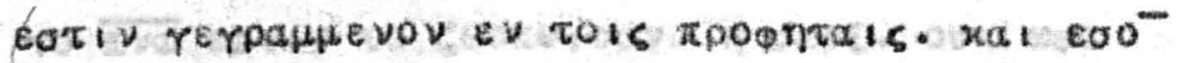

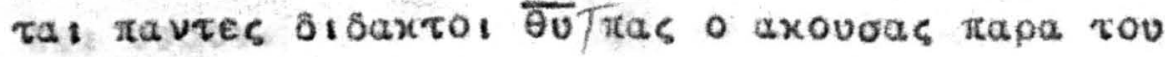

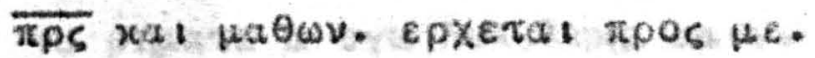

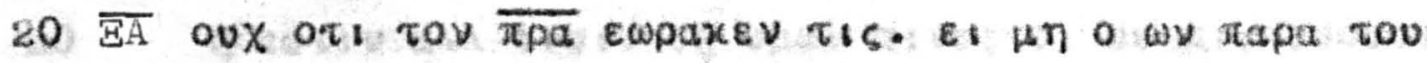

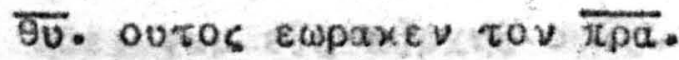

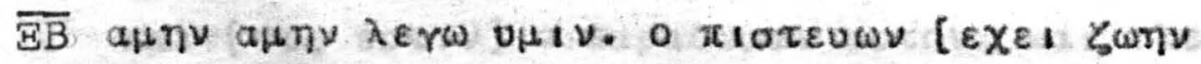
aเwviov.

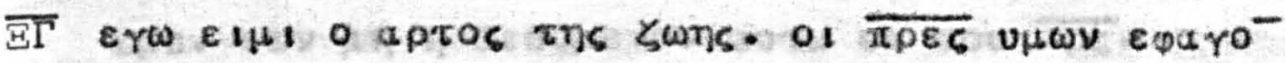

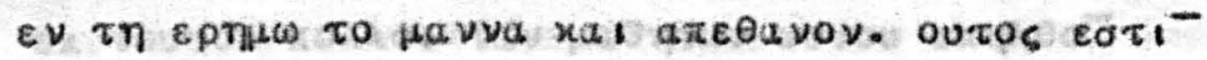

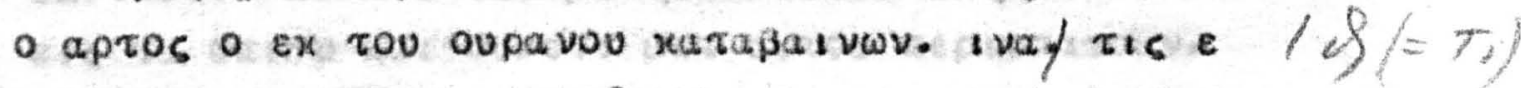
E. aurov warm xal un arooavr.

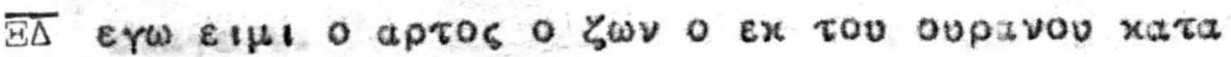

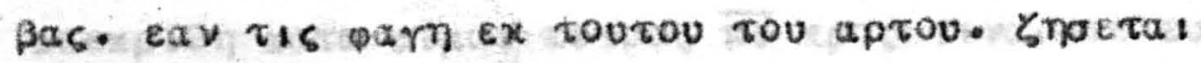

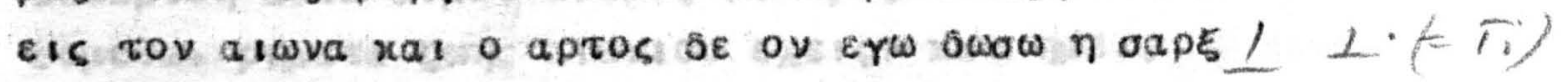

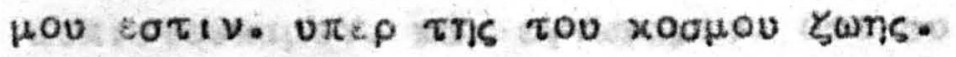

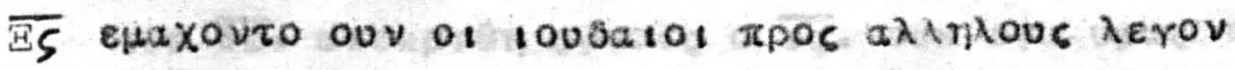

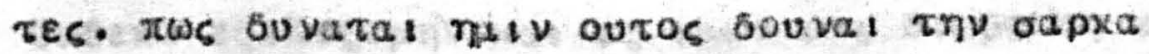

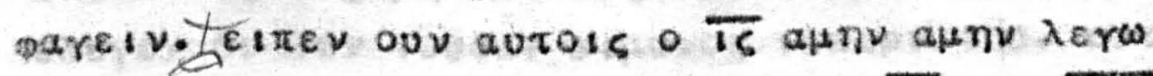

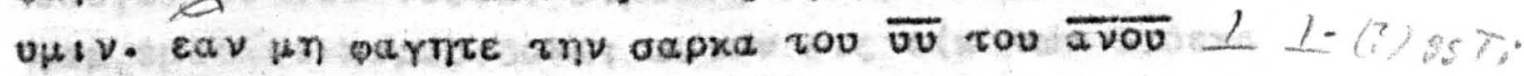

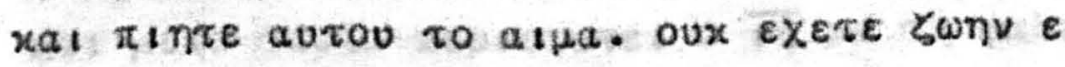

$\checkmark$ eavtors.

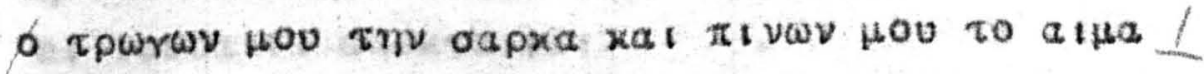

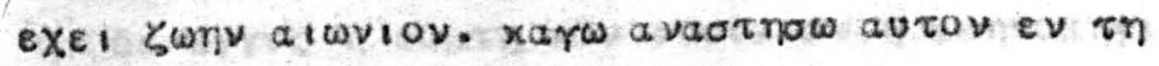

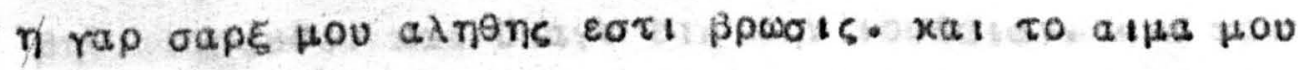




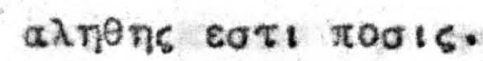

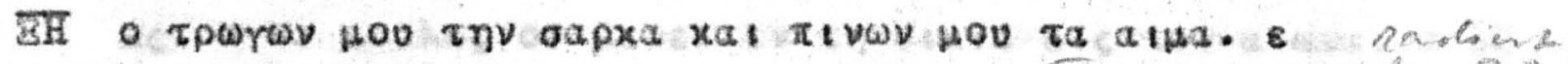

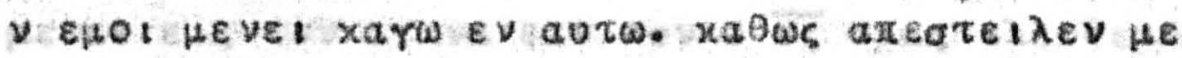

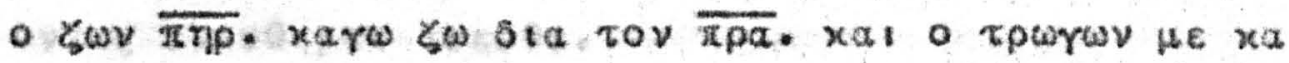

5

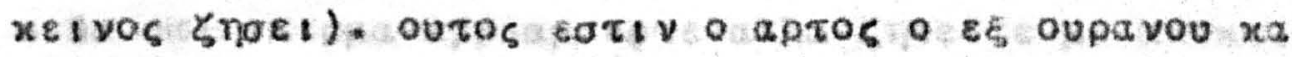

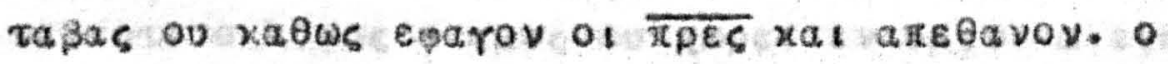

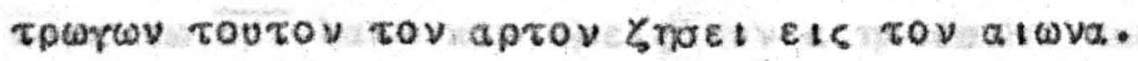

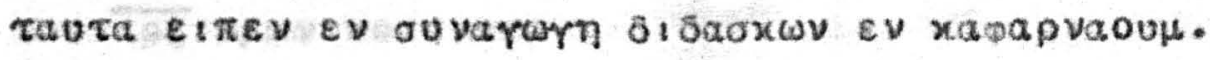

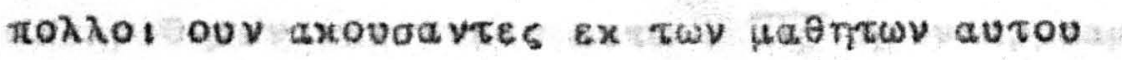

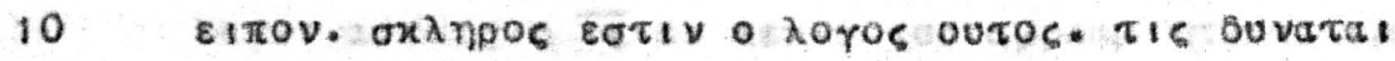
autoo axoue : $v$.

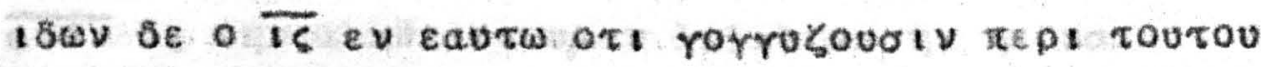

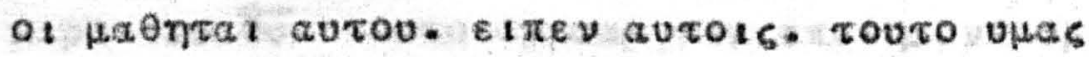
oxavsa

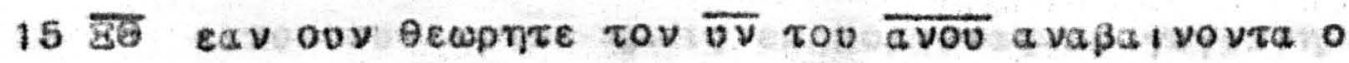

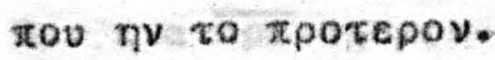

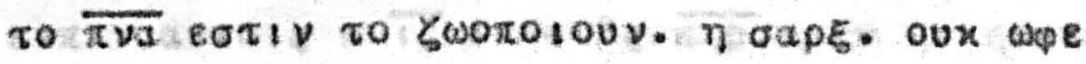

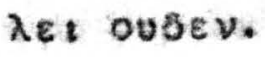

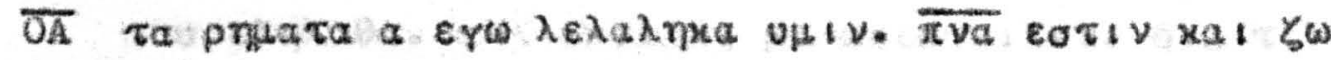

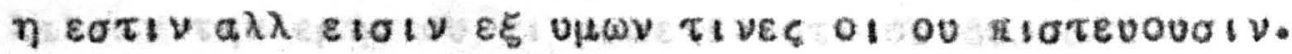

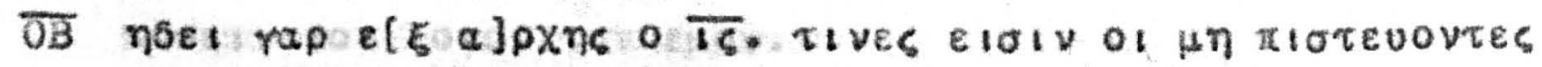

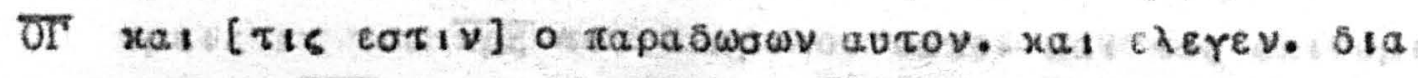

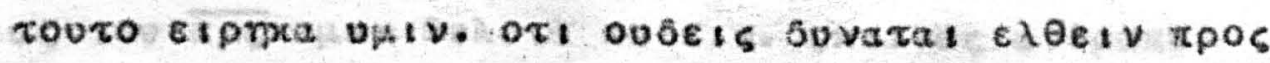

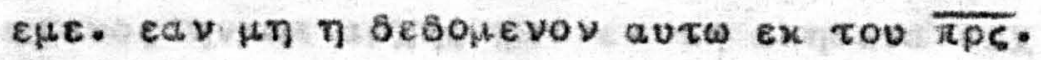

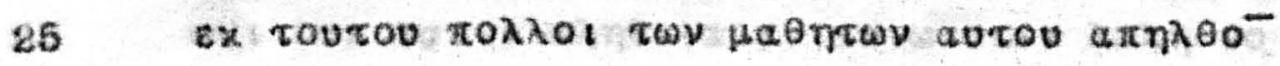

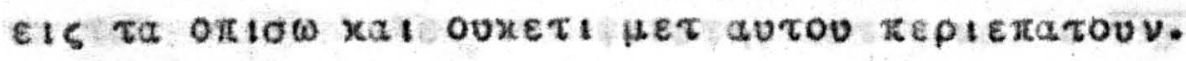
Eเ งสareเv.

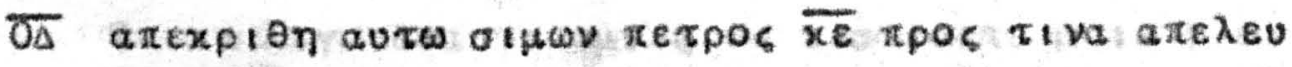

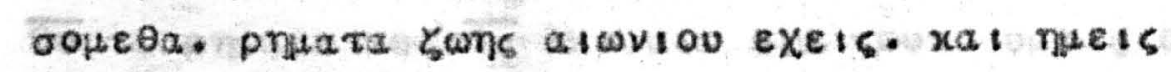

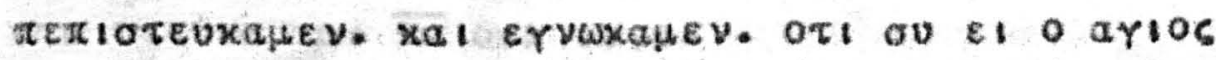

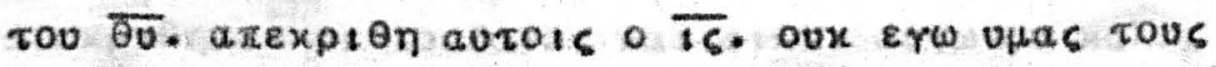

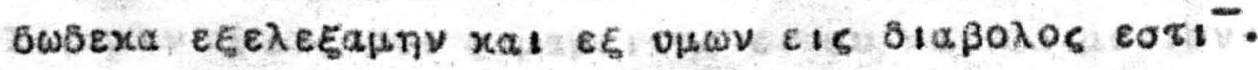

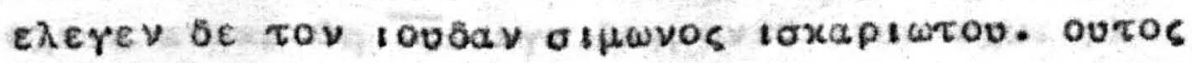

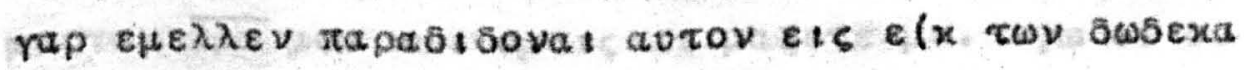

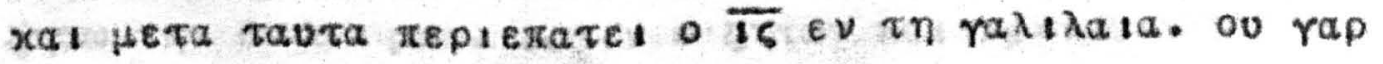

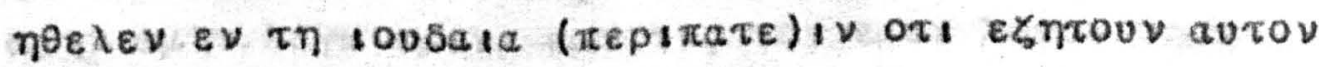
of lovsasot axoxelval.

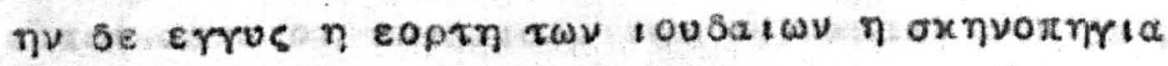

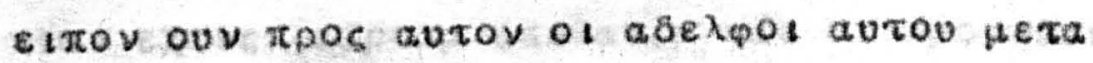

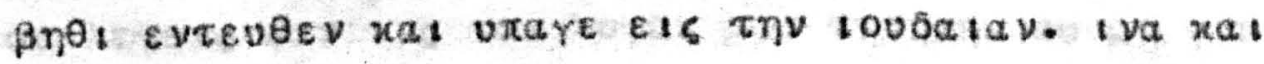




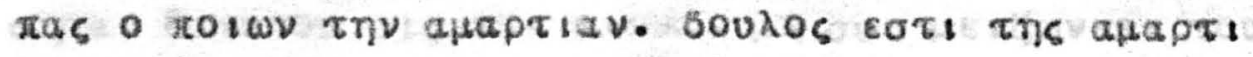
as. 0 b sou

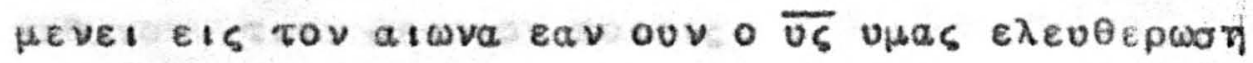

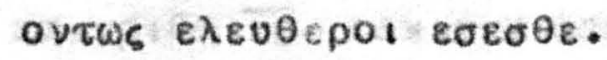

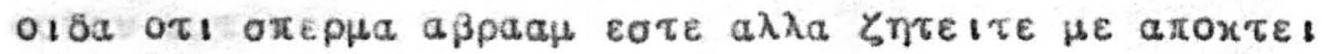

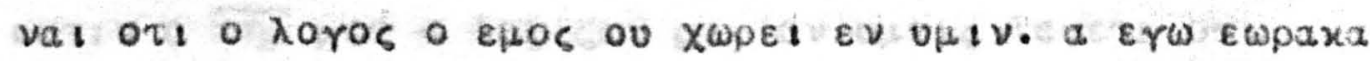

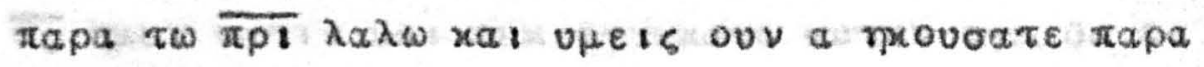

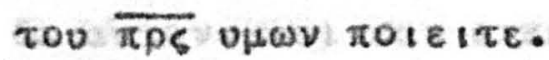

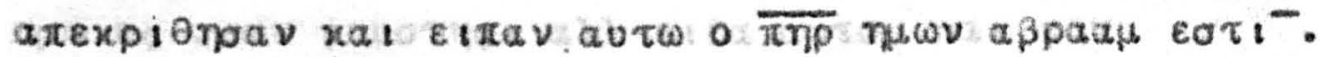

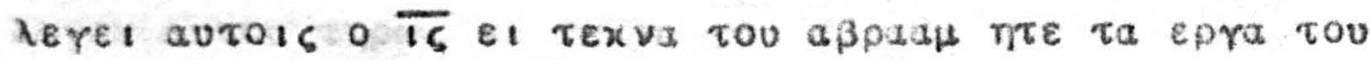

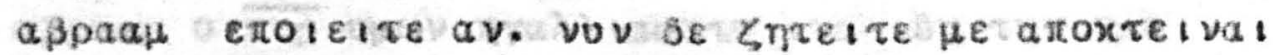

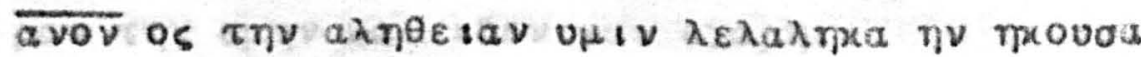

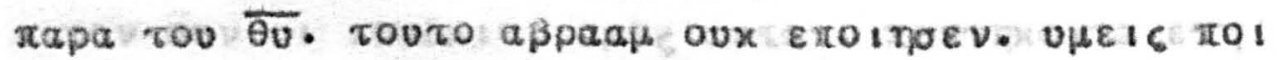

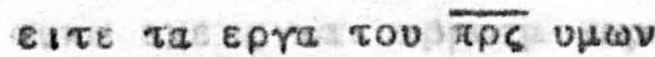
Eirov ouv autw. nueis ex ropveras ov rerevunue

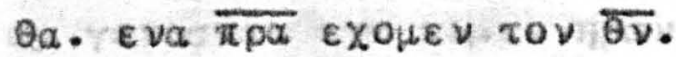

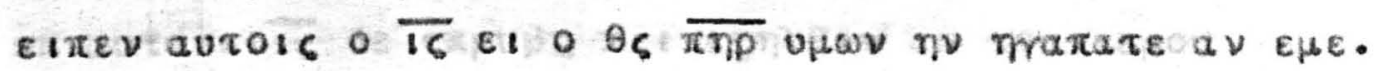

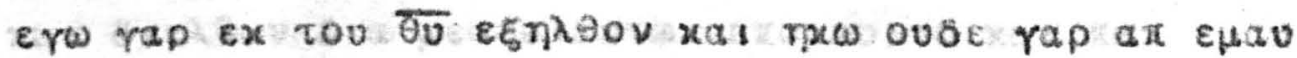

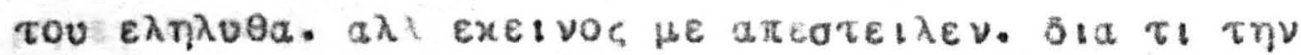

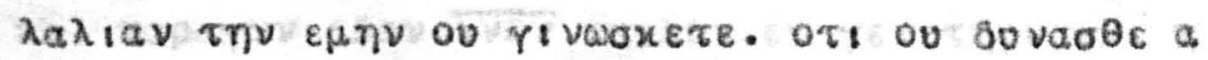

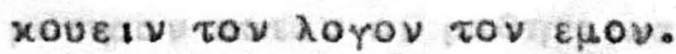

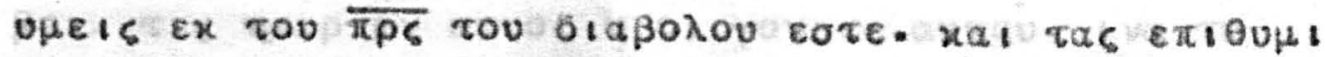

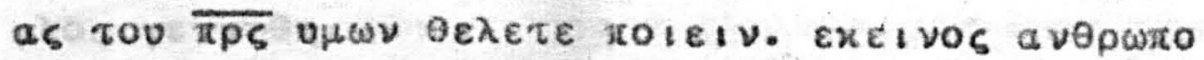

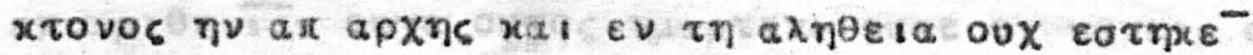
oน

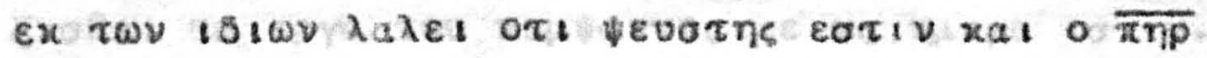

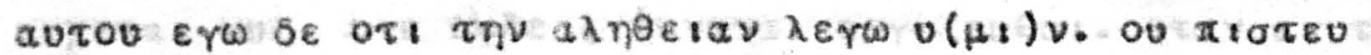

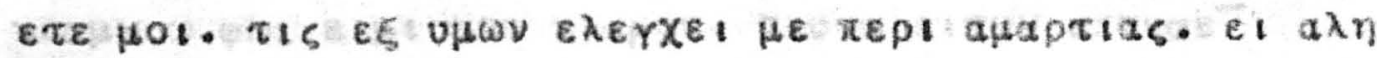

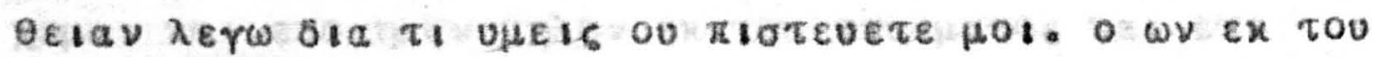
ब0 ta pnuara rou $\overline{\theta 0}$ axovel. ota routo vieis ovx axou

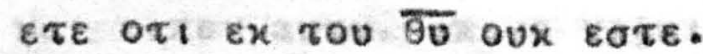

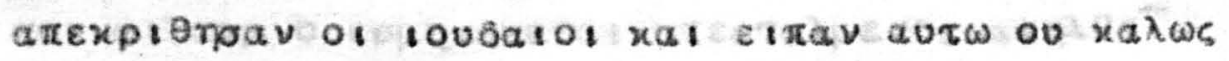

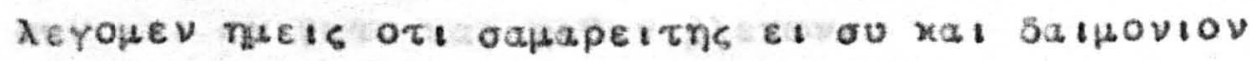

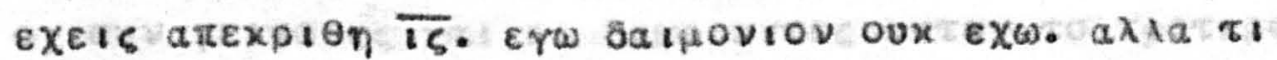

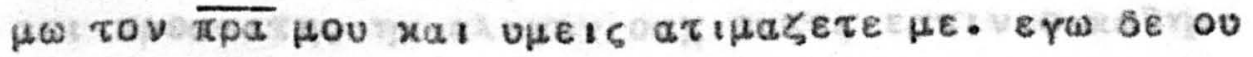

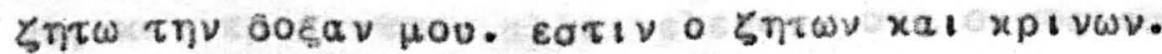

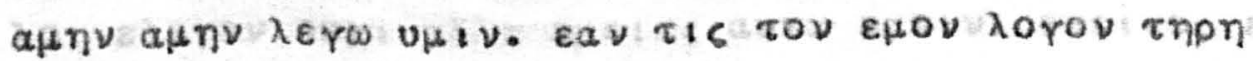

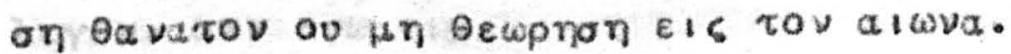

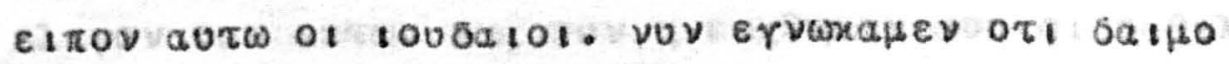

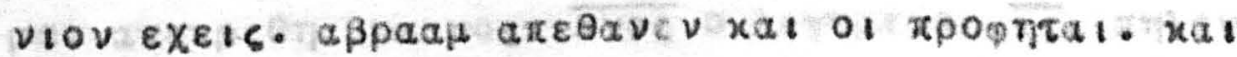

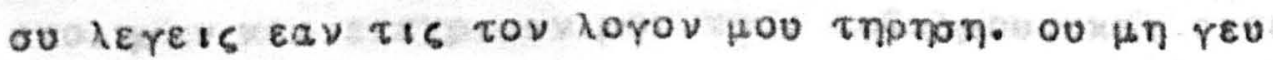




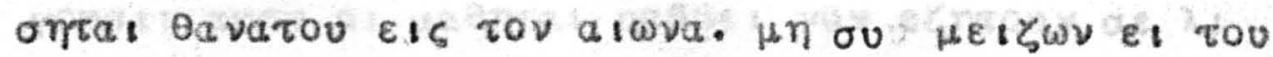

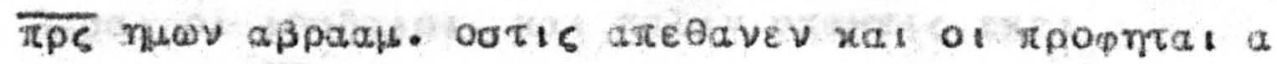

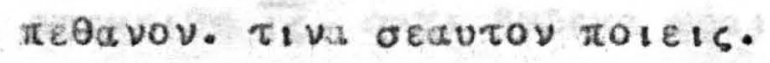

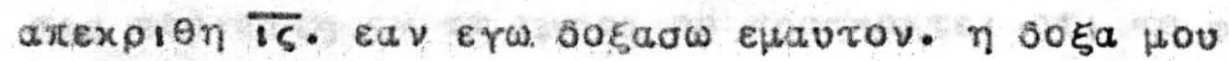

ovor. $\varepsilon \sigma \tau t \nu$.

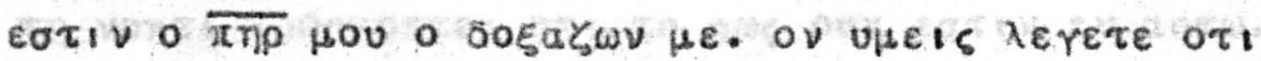

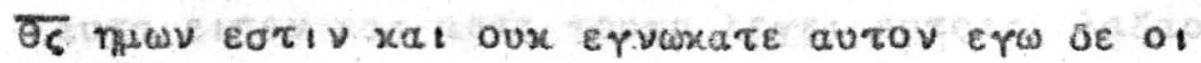

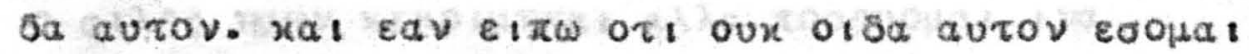

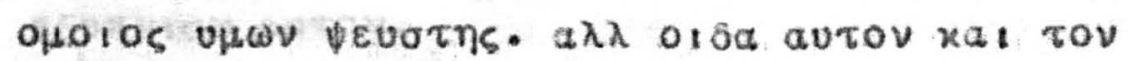

10 norov aurou rnp.

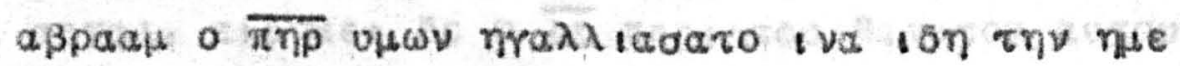

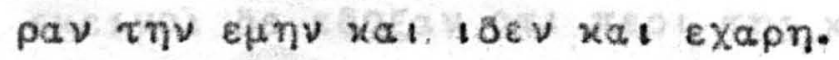

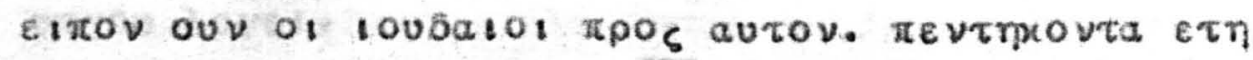
outw exeis xat appaap ewpaxas.

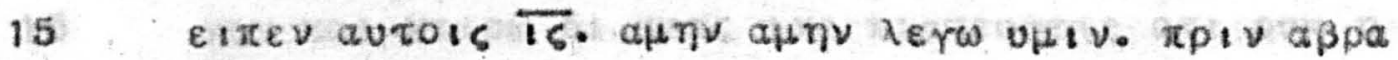

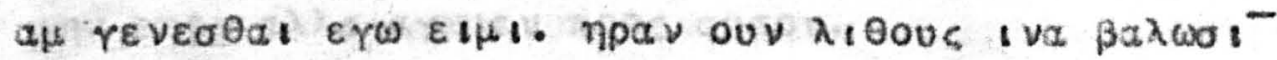

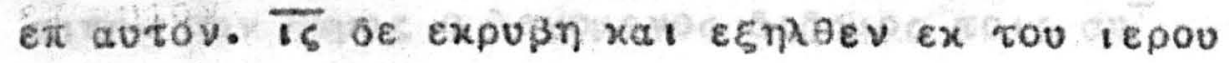

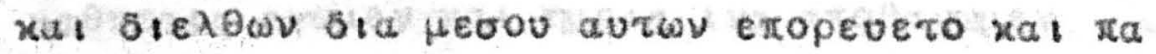
prove orrws.

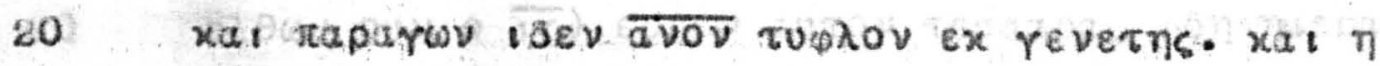

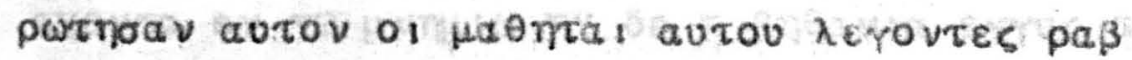
Bei. Tis nastev outos $\eta$ ot roveis autov. ina tu

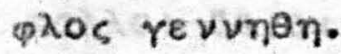
arexpion Ts. oute outos nuaptev. oute oi yoveis autou

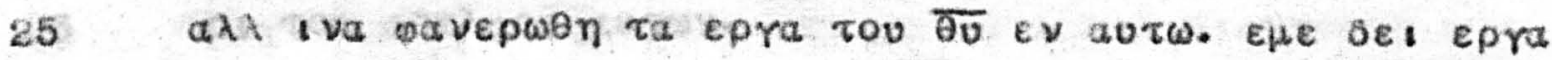

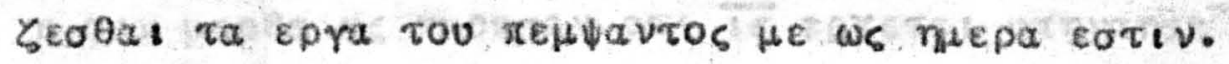

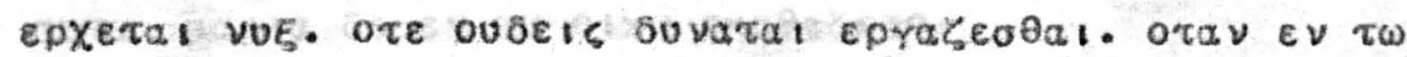

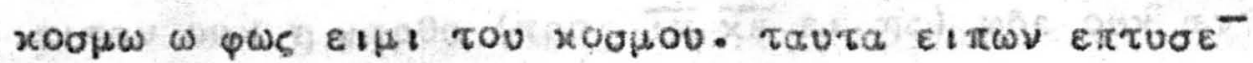

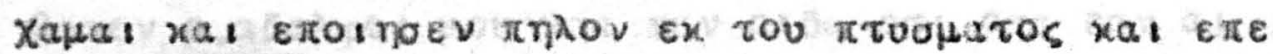

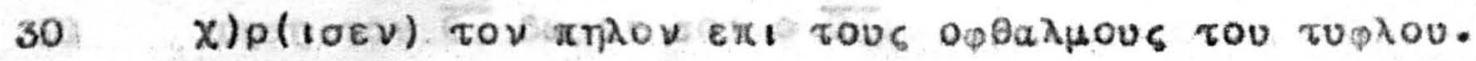

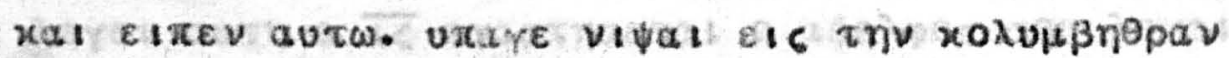
tov oi

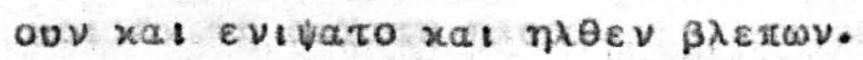

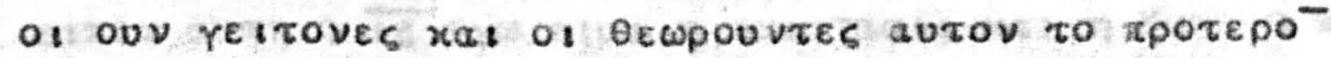

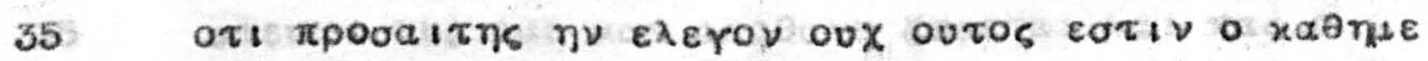

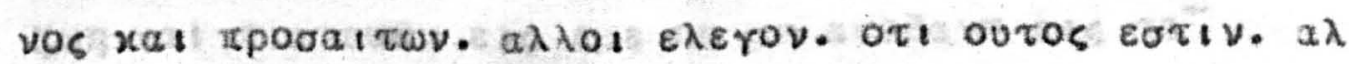

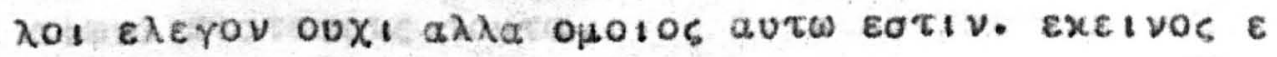

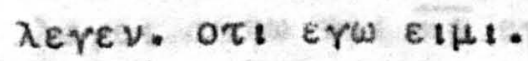

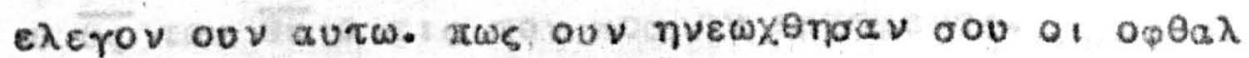

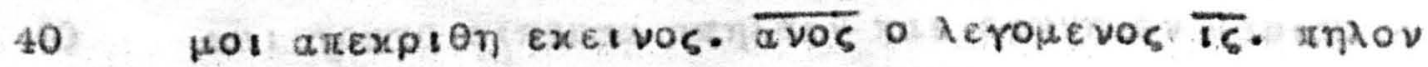

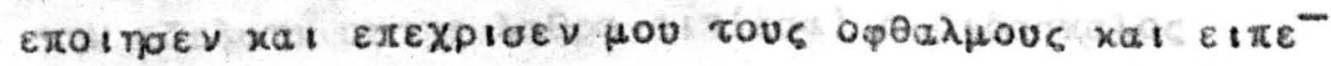

xar twavvทv $8.52-8.11$ 


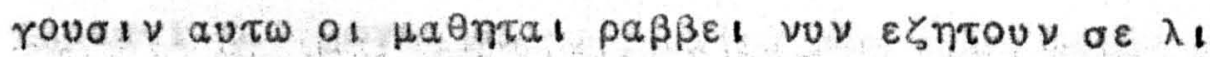

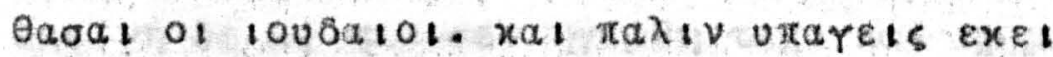

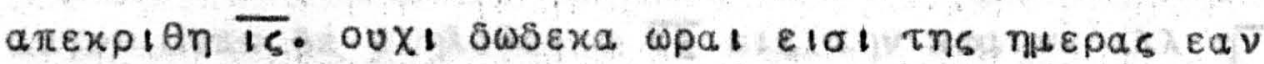

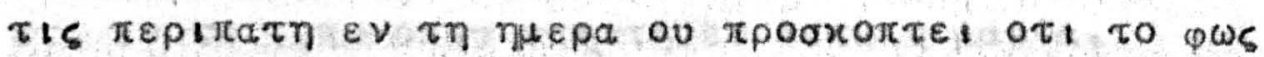

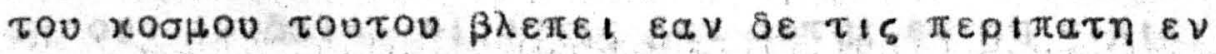

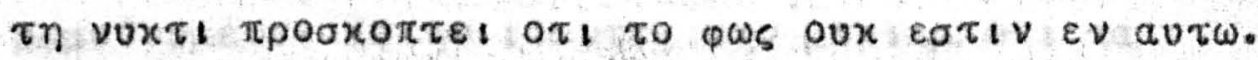

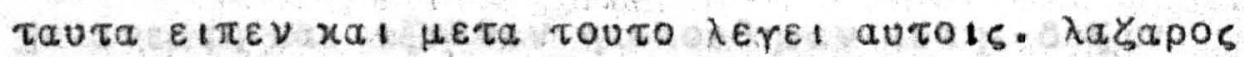

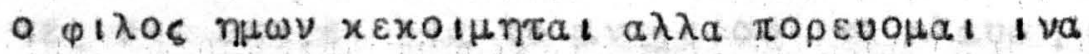
$\varepsilon \xi \cot \sigma \omega$ autov.

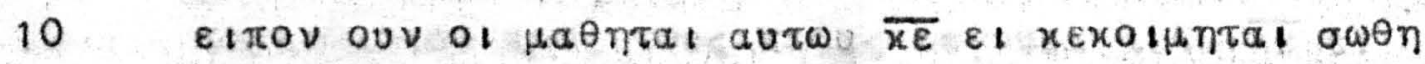

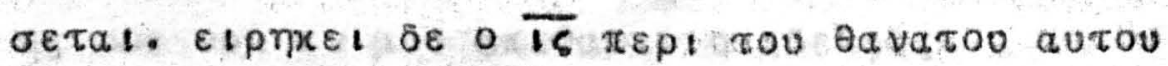

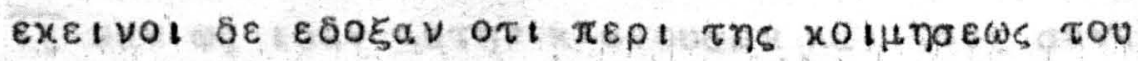
uTvou $\lambda \varepsilon \gamma \varepsilon 1$.

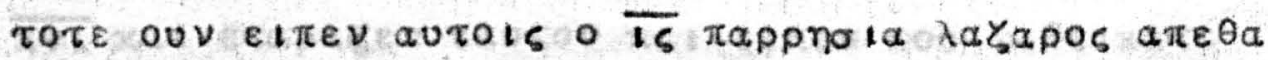

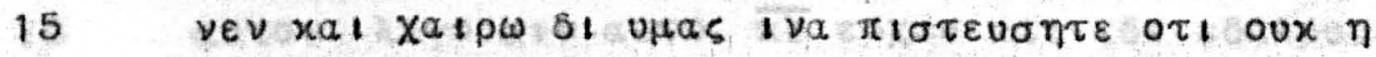

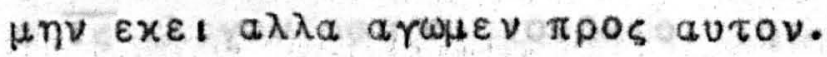

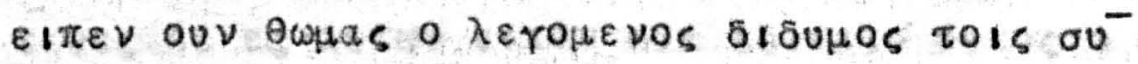

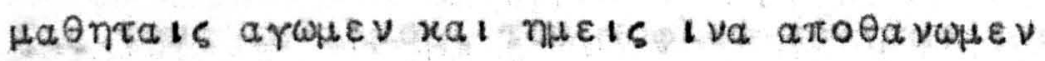

$\mu \varepsilon \tau$ au

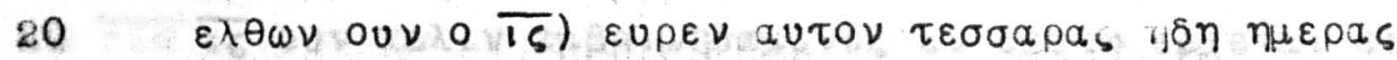

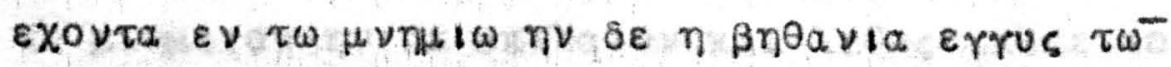

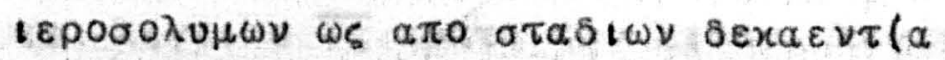

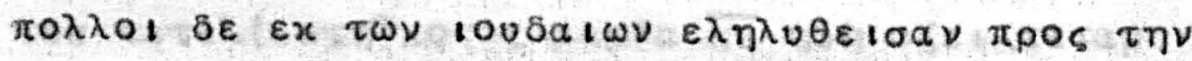

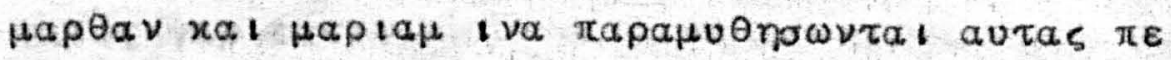

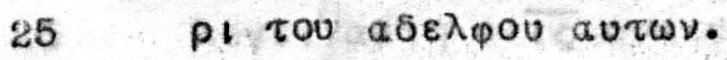

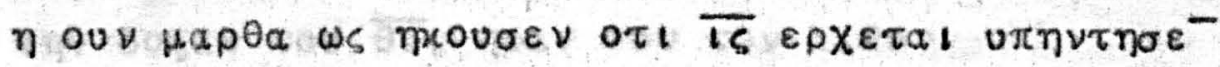

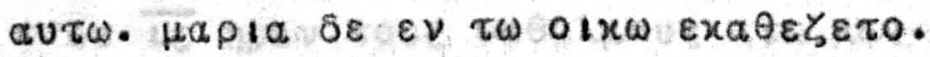

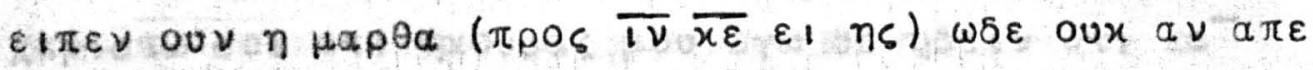

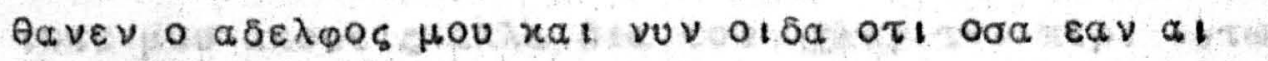

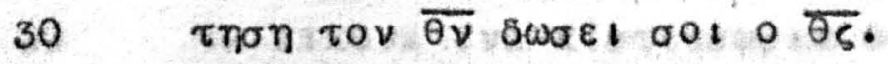

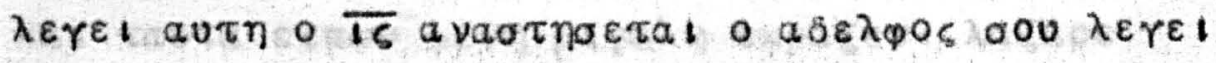

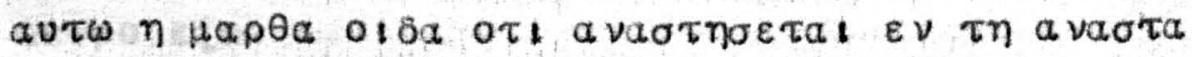

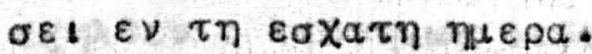

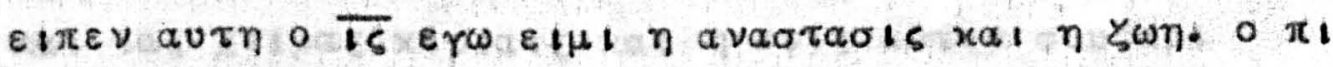

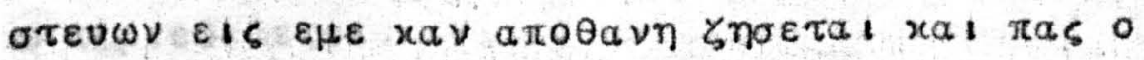

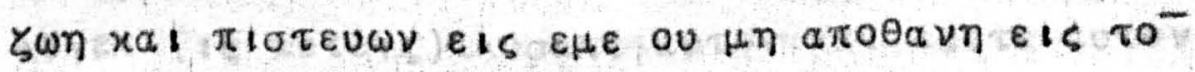

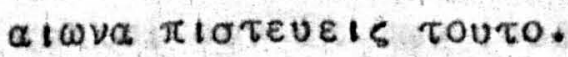

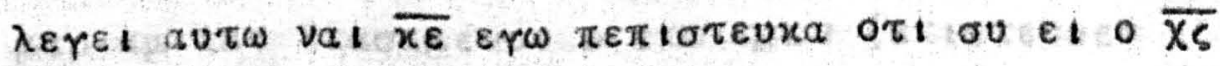

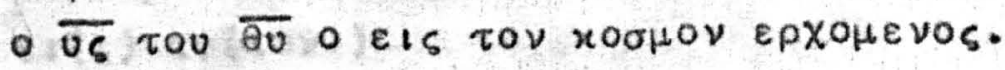

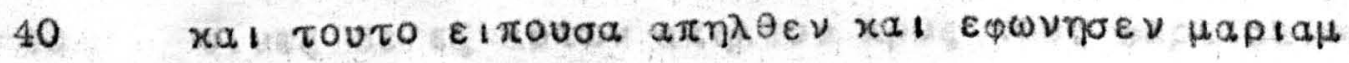

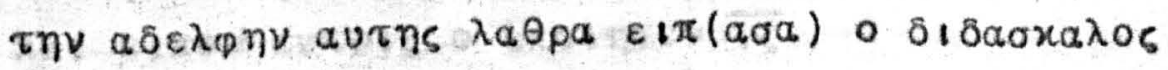


$\pi a \rho \varepsilon \sigma \tau$ เ $x a$ เ $\phi \nu \varepsilon_{\imath}$ o $\varepsilon$

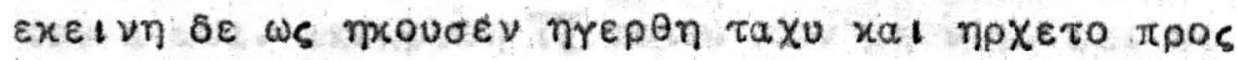

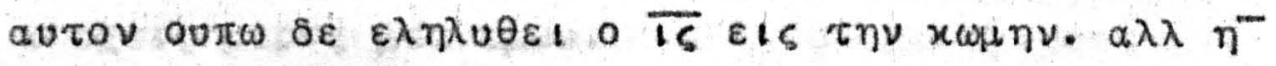

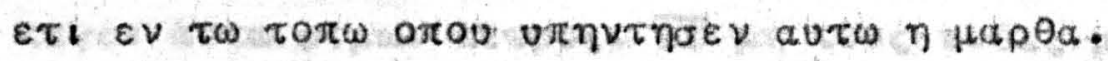

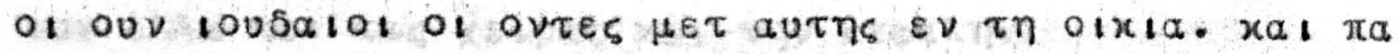

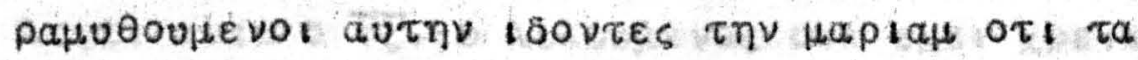

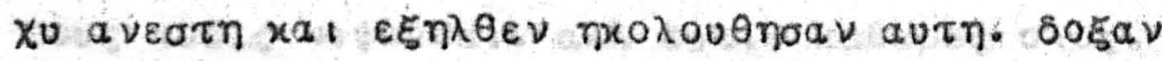

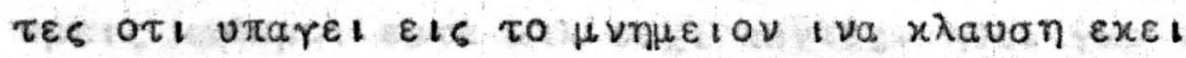

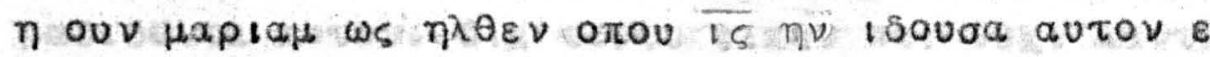

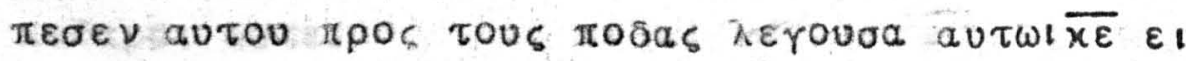

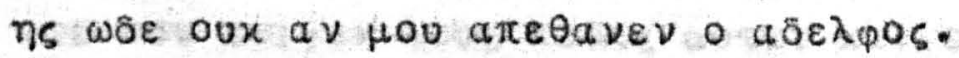

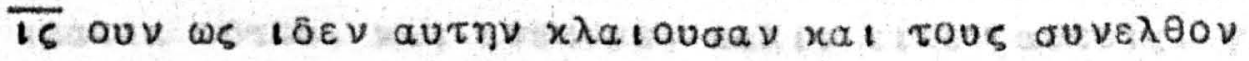

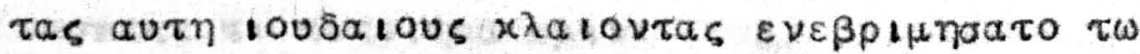

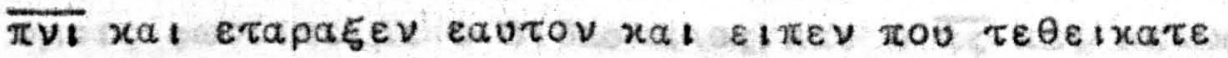

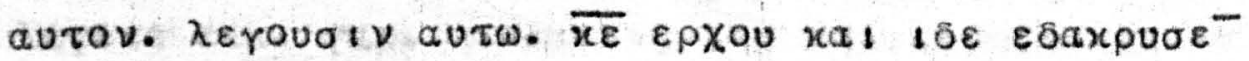

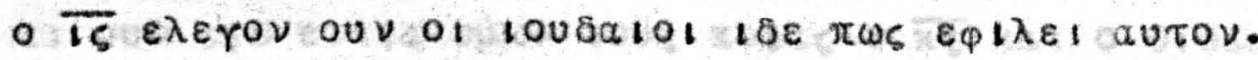

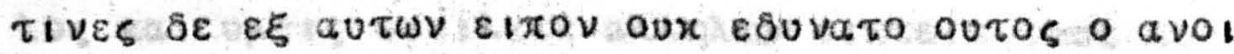

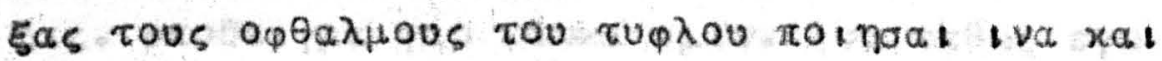

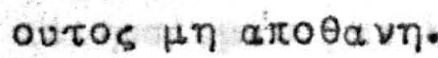

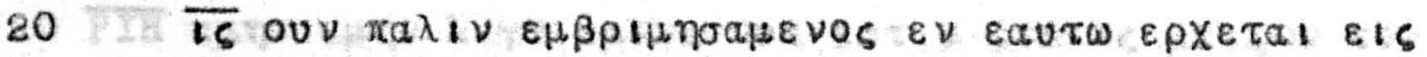

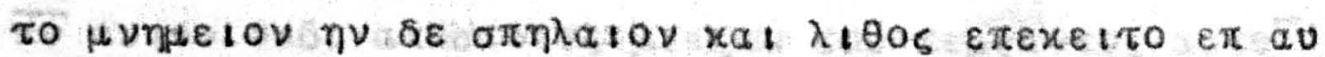

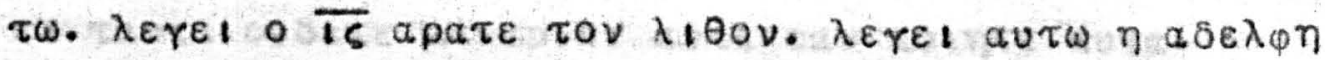

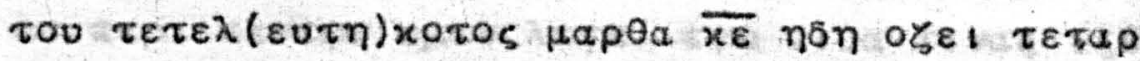
$\tau a$ ios $r a \rho \in \sigma i v$.

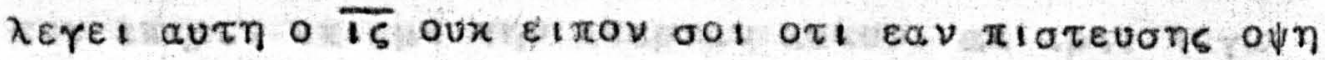

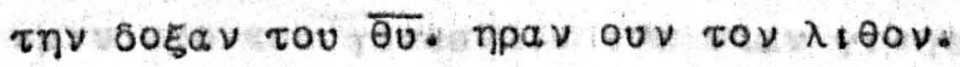

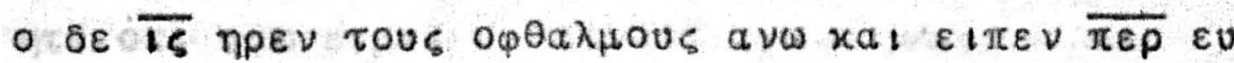

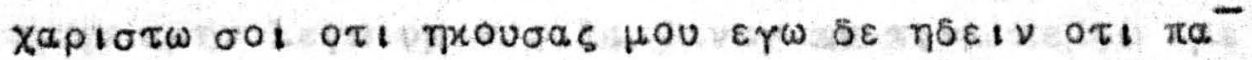

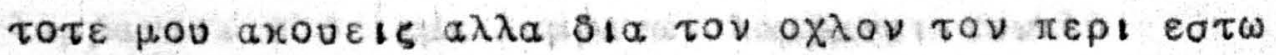

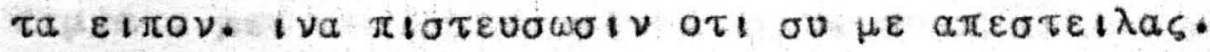

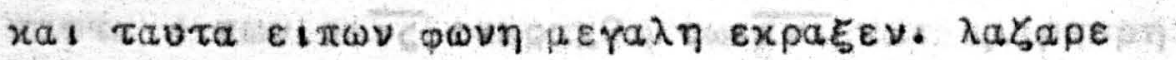

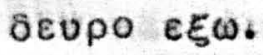

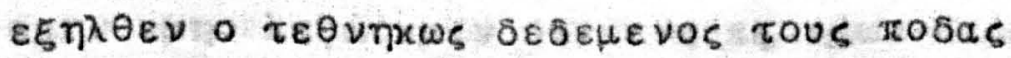

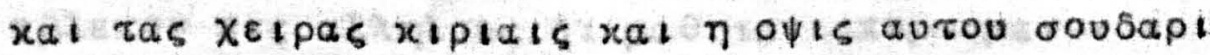
$\omega \pi \varepsilon \rho t \varepsilon \delta \varepsilon \delta \varepsilon \tau 0$.

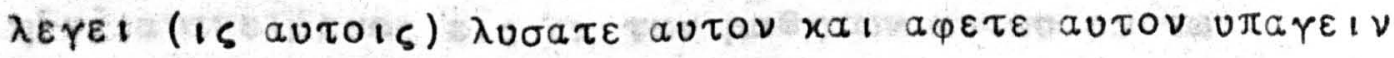

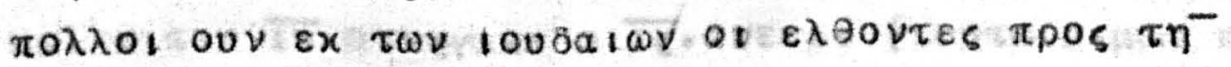

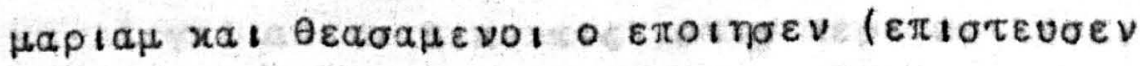
$\varepsilon i(s)$ au (tov.)

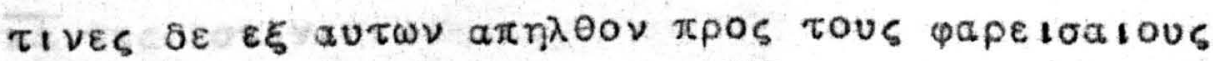

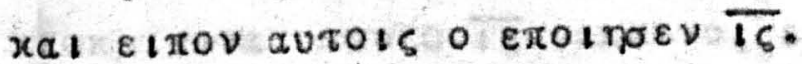




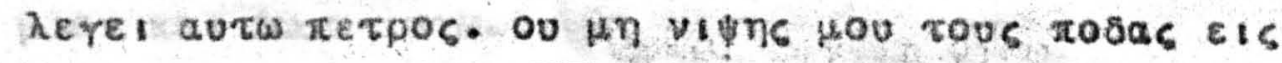

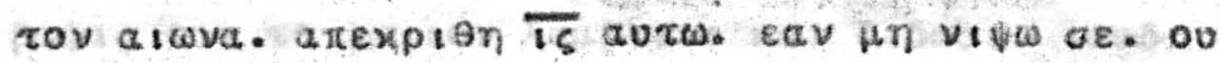

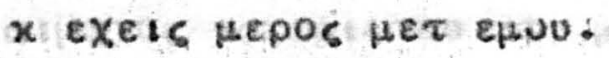

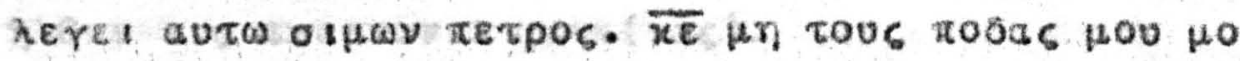

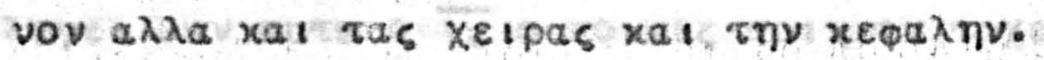

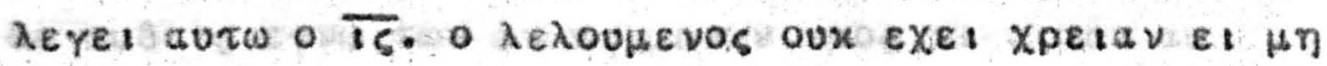

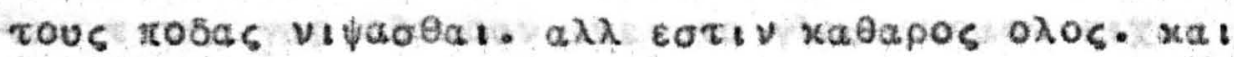

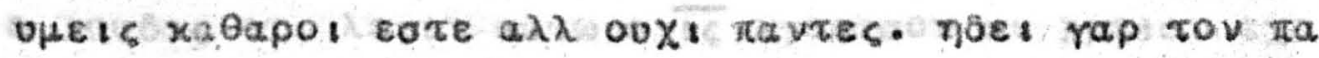

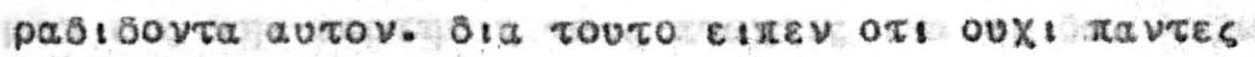
xabapot eare.

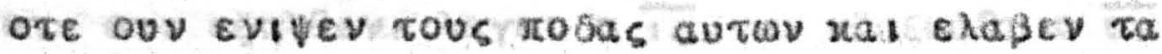

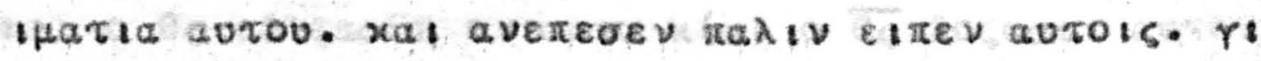

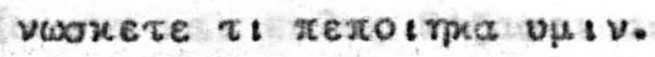

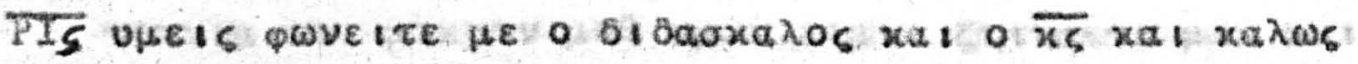
15

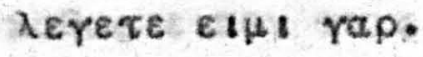

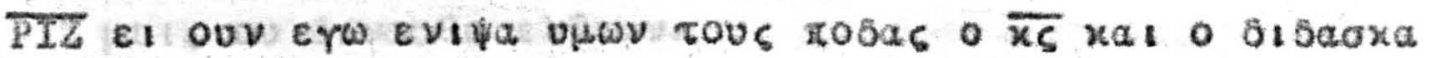

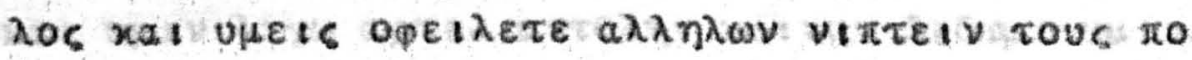

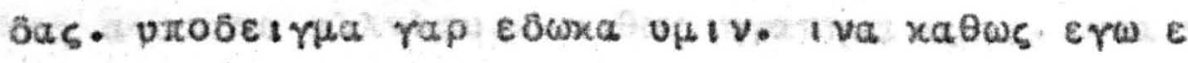

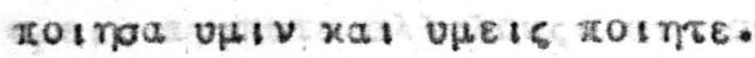

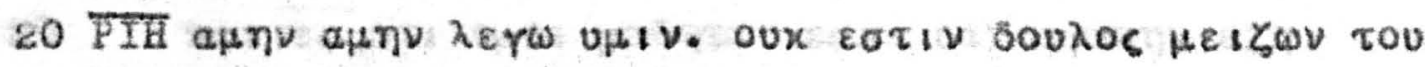

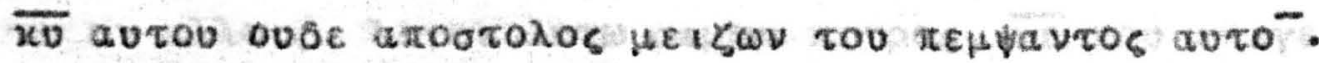

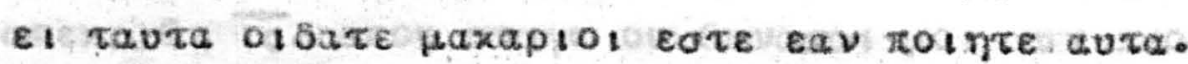

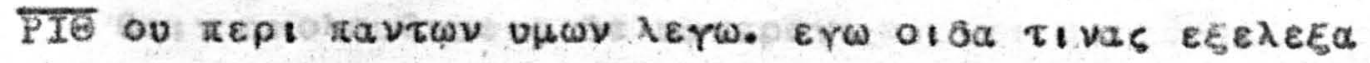

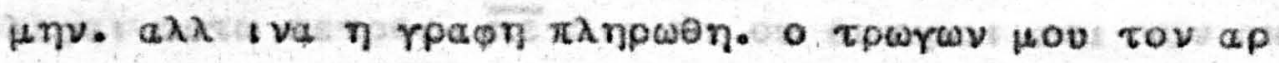

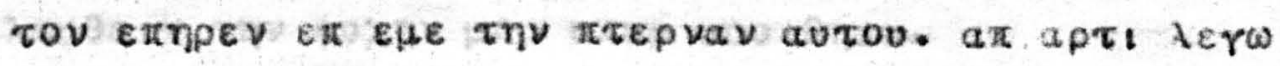

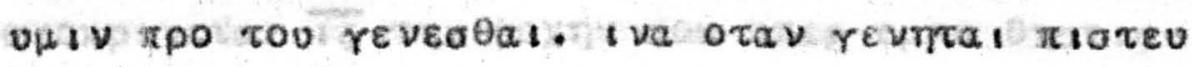

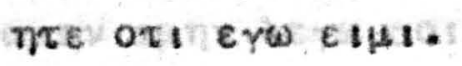

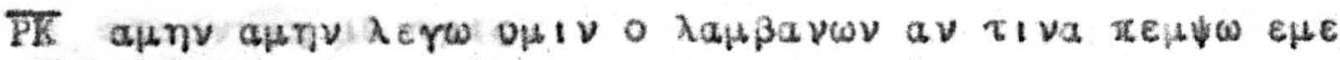

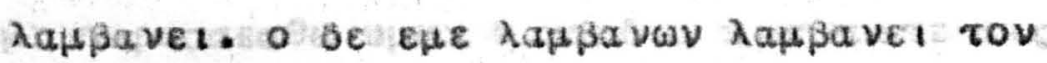
$\pi \varepsilon \mu \psi a \tau a \mu \varepsilon$.

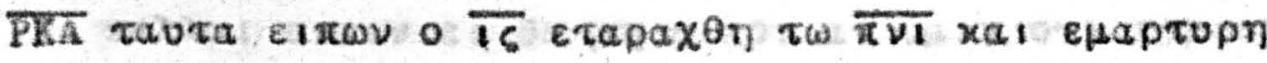
oev xal $\varepsilon เ \pi \varepsilon \nu$.

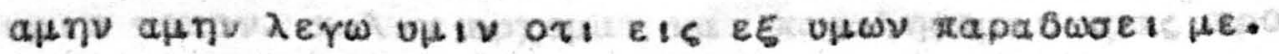

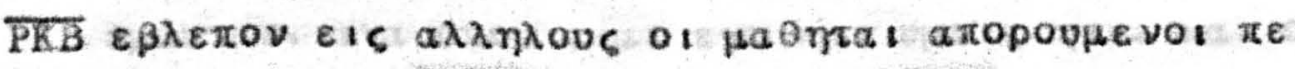
pi Tivog Aereit.

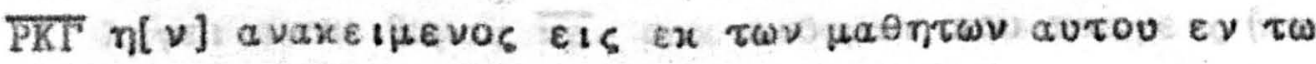

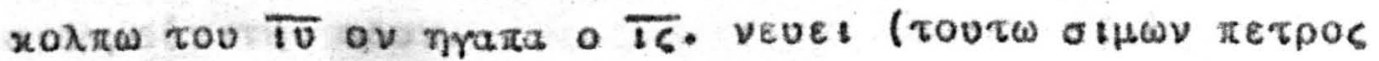

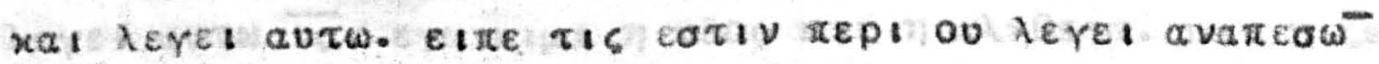

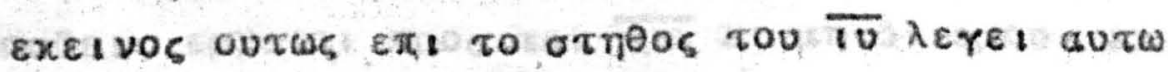


Hiov xat owow avew.

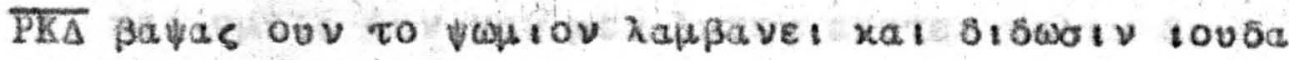

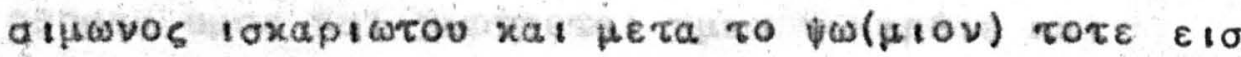

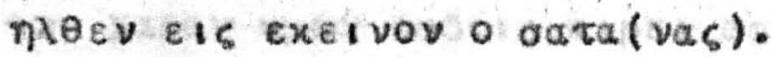

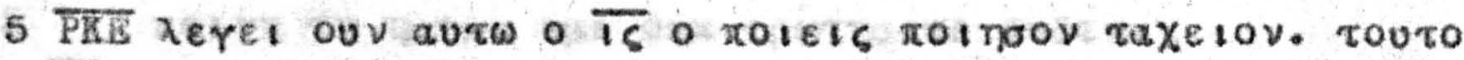

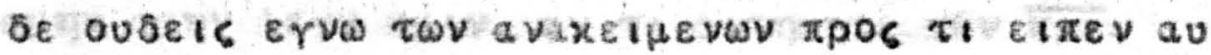

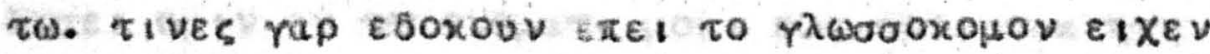

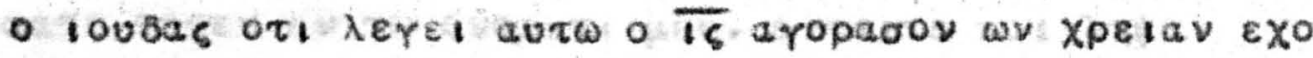

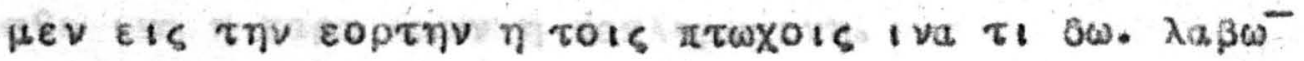

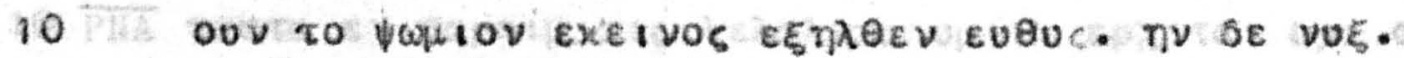

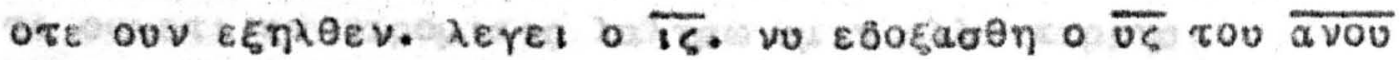

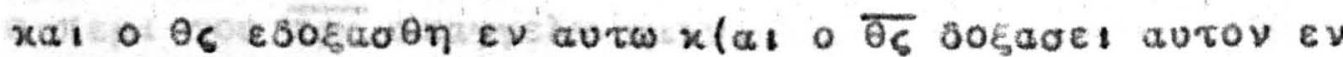

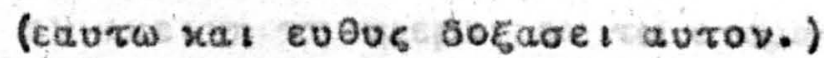

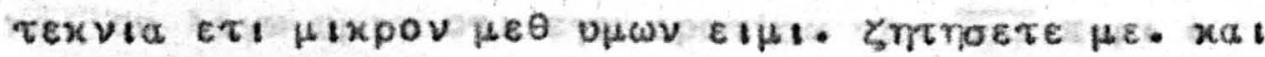

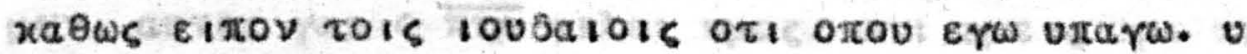
$\mu \varepsilon i s$ ou suvaoge $\varepsilon \lambda \theta \varepsilon i v$.

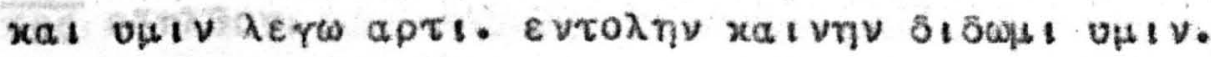

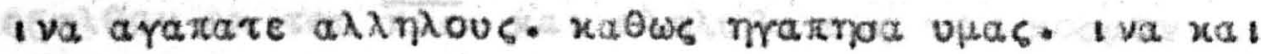

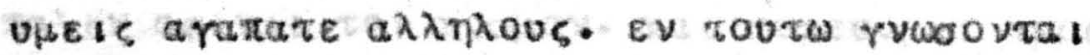

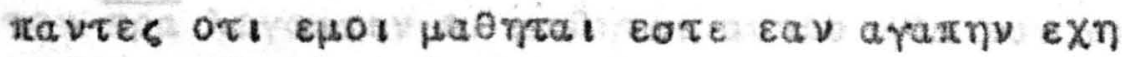

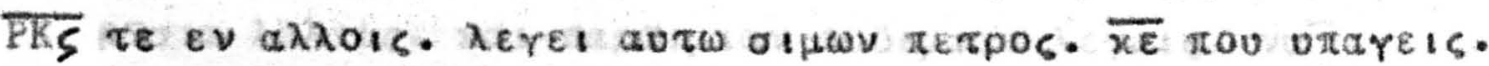

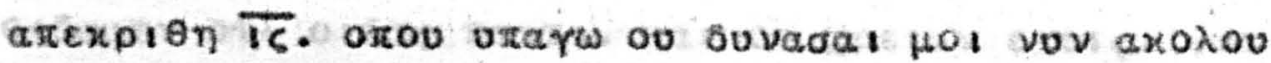
Beiv axoxoutnoels os vorepov.

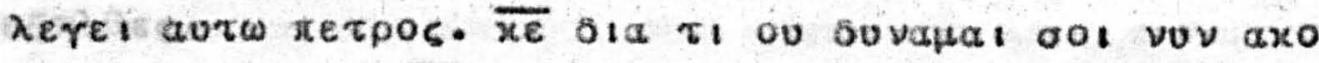

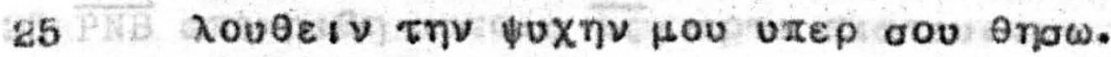

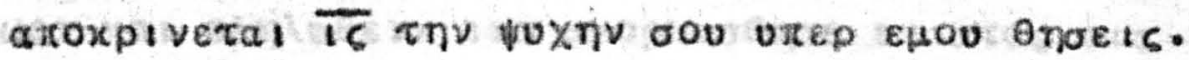

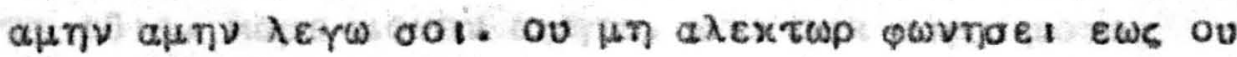

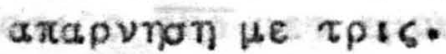

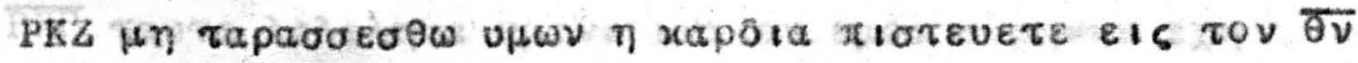

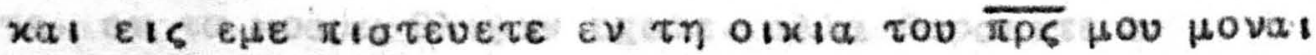

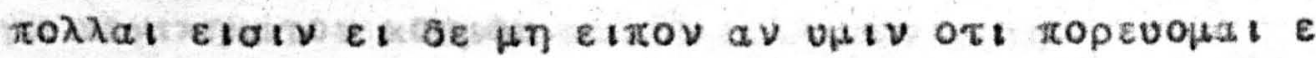

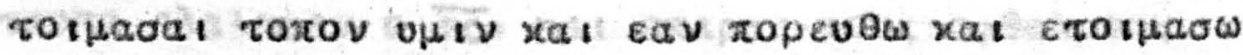

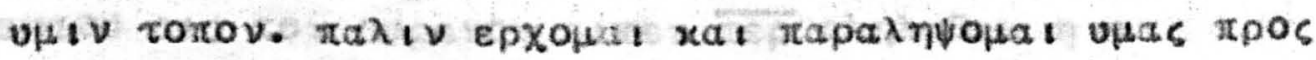

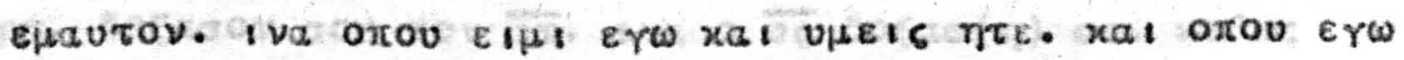

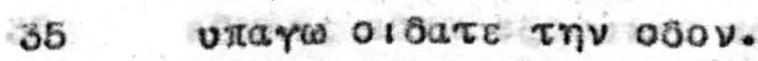

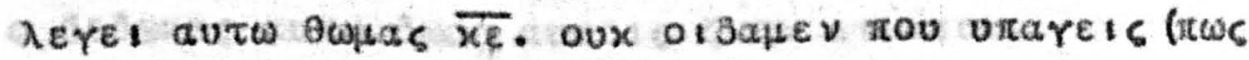

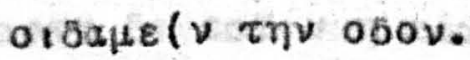

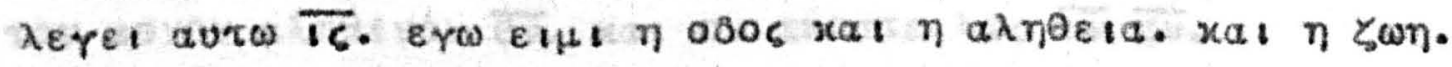

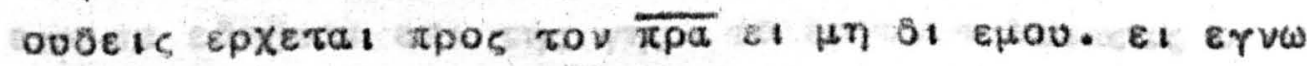

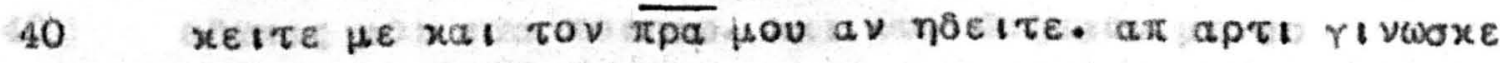
$\tau \varepsilon$ autov xal $\varepsilon \omega p a x a \tau \varepsilon$. 


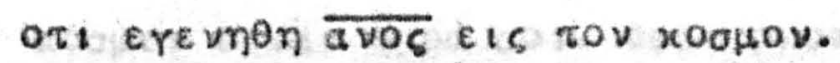

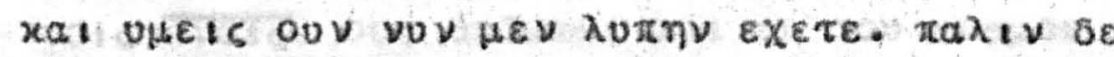

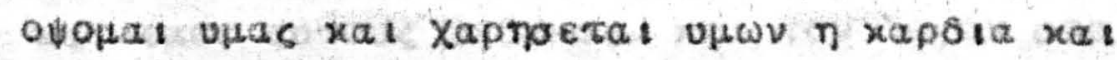

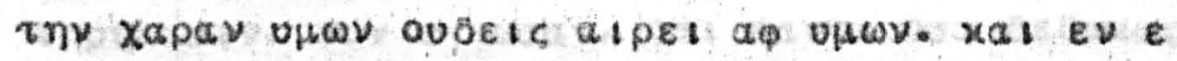

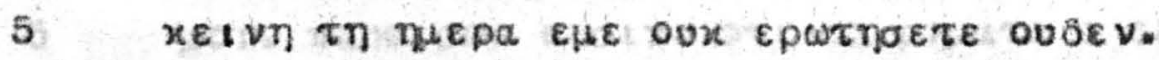

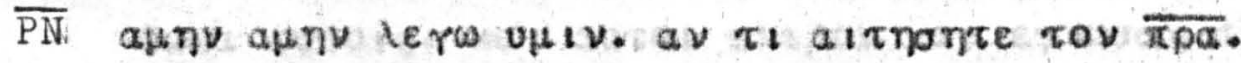

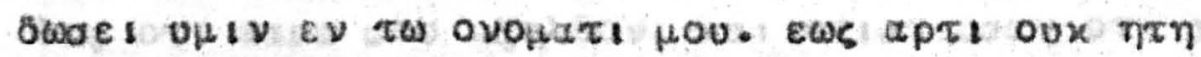

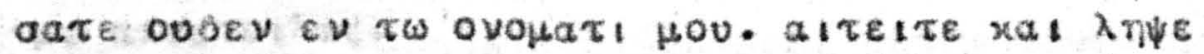

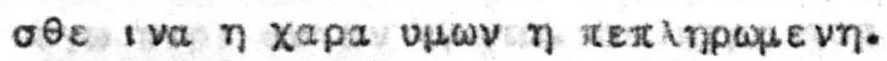

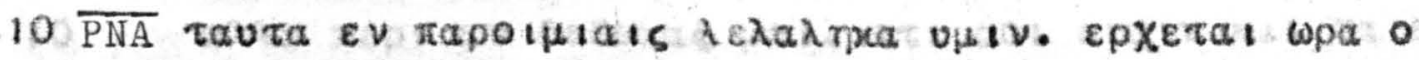

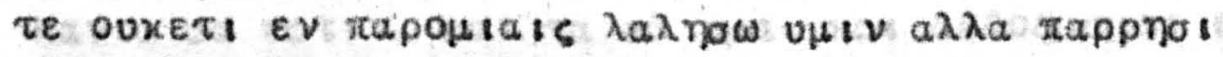

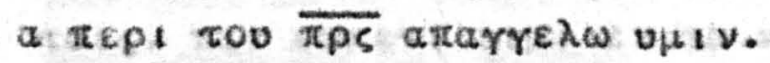

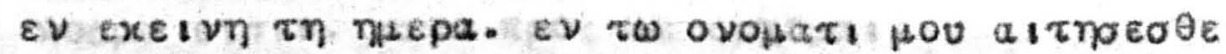

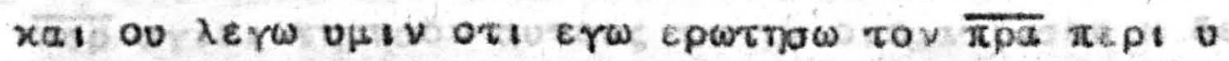

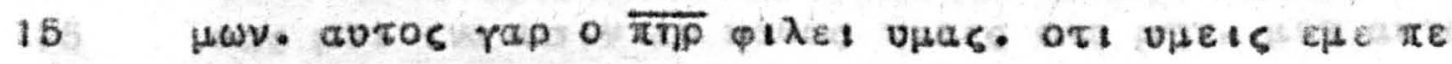

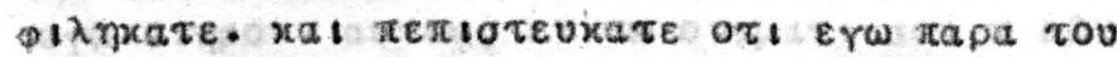

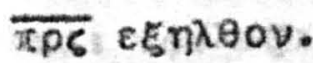

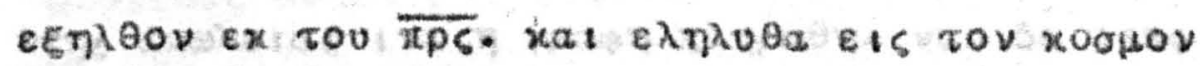

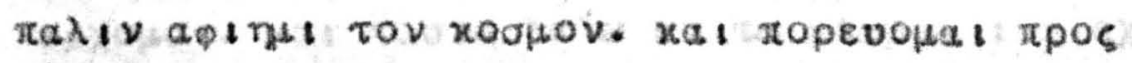

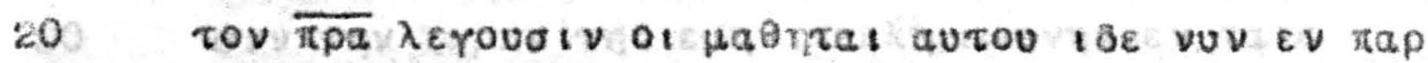

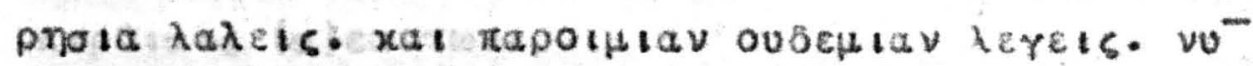

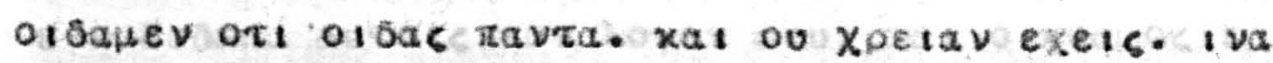

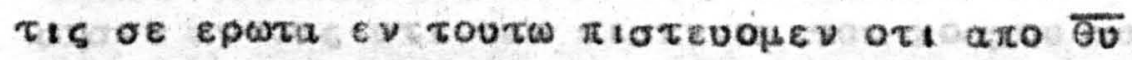
$\varepsilon \varepsilon \eta \lambda \varepsilon_{\varsigma}$.

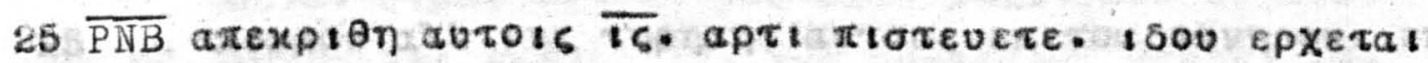

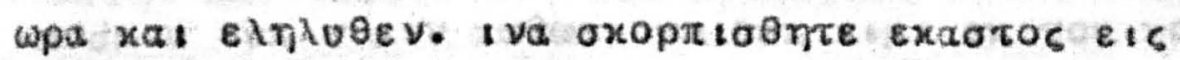

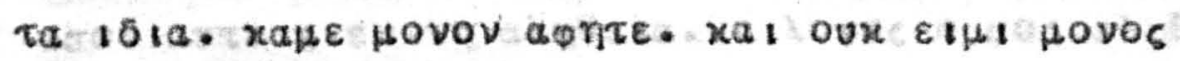

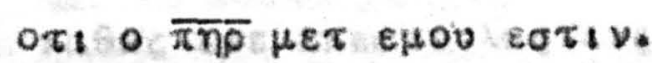

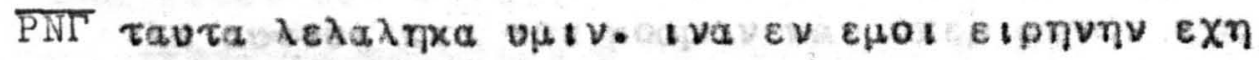
30

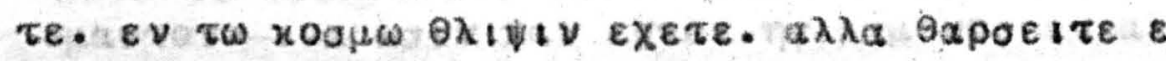

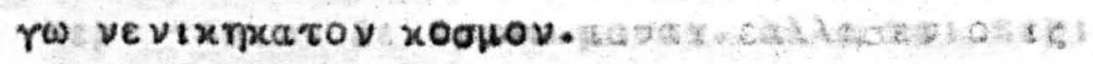

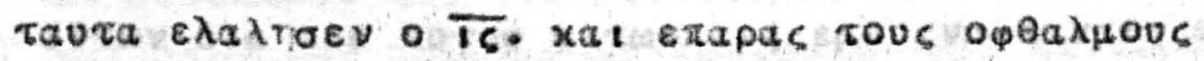
autov ets tov oupavov etrev $\overline{\pi \varepsilon p} \operatorname{e\lambda \eta \lambda v\theta EV~} \omega$

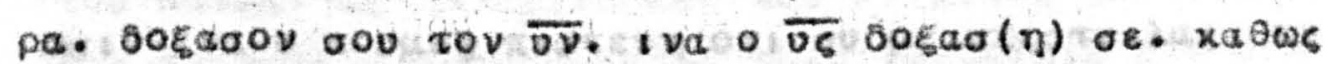

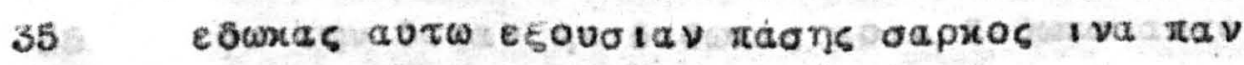

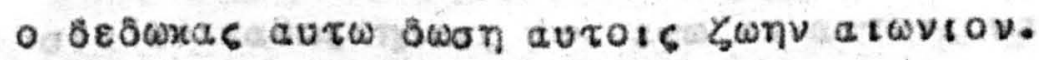

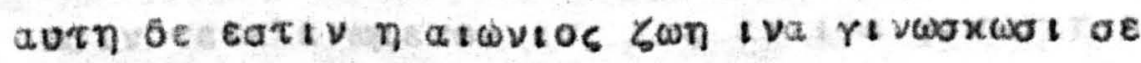

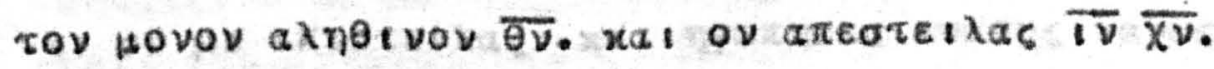

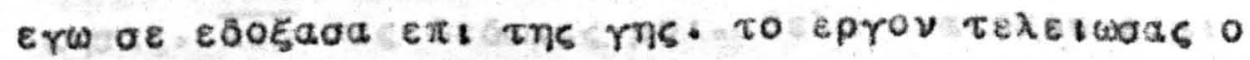

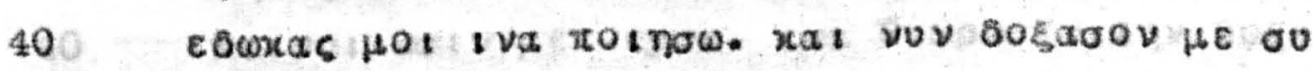

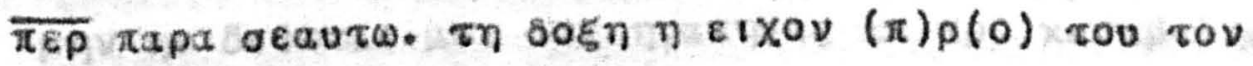


roghov Etvat rapa ool. Eqavepaja jov to ovopa

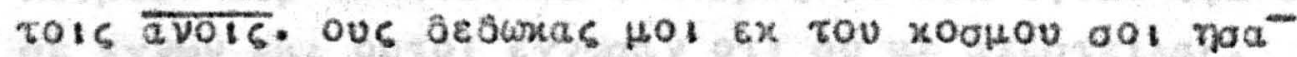
xal efol autove seouxas. xat rov lorov aou te

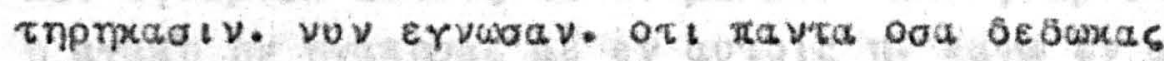

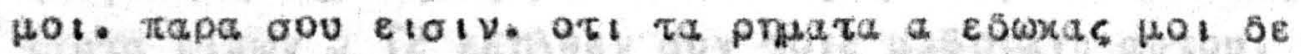

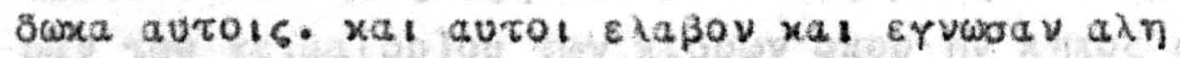
OW or He areoretias.

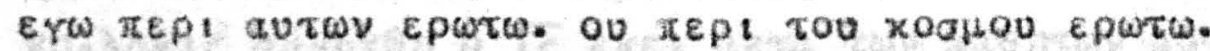

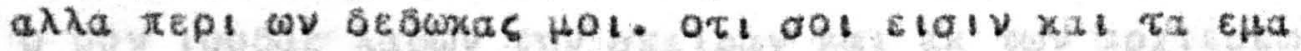
Tavra aa eativ. xat ta a eua. xal jebogaqual ev av

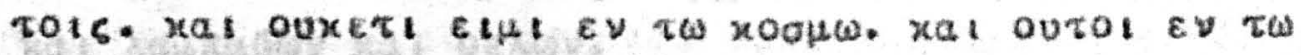
xoaja Etotv. xaras xpor ae epXoual.

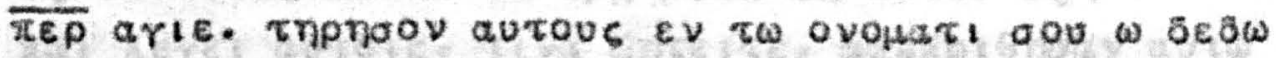

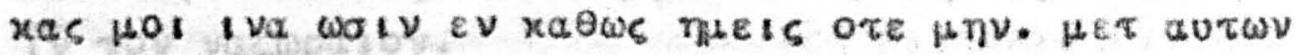

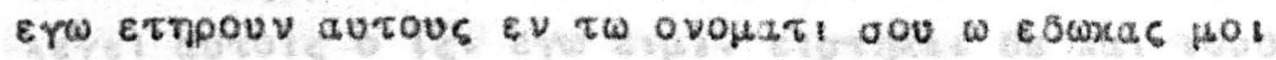

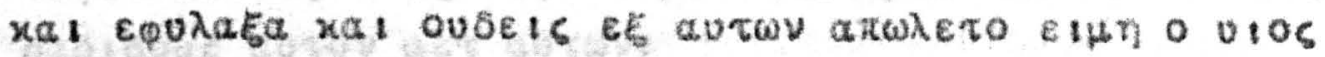

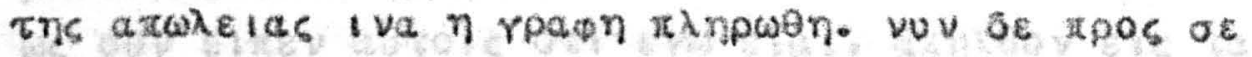

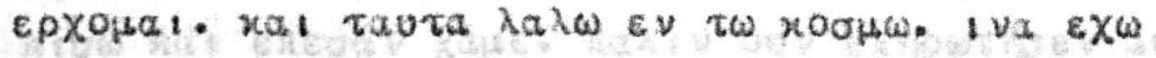

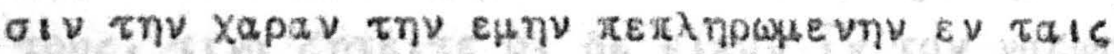
xapbiatc (o) euviw.

erw obowxa aurois tov horov oov. xat o xoouoc esi

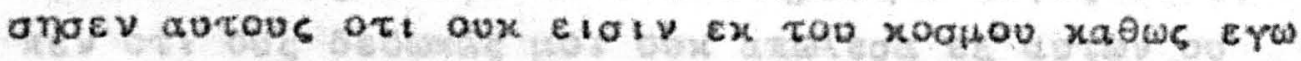

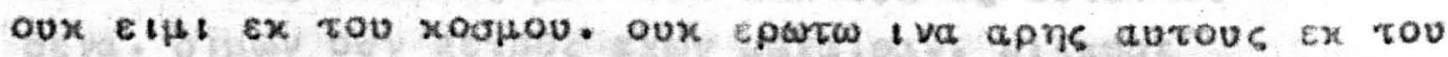
xoamov alt iva mpnons aurous ex rou zovmpou. ex rov xoopov oux elotv xaows erw oux ellu ex rov xoopov art

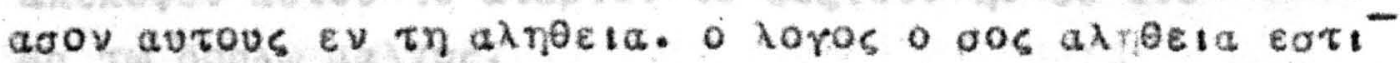

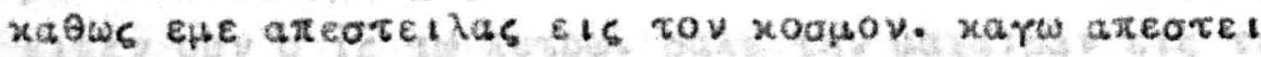

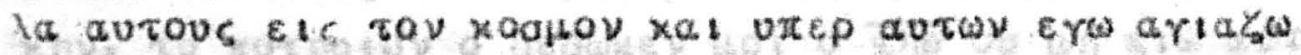

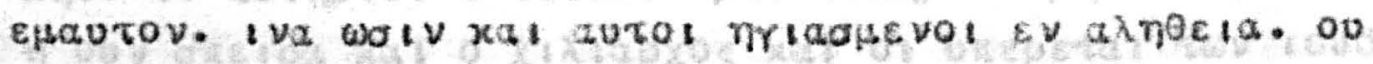

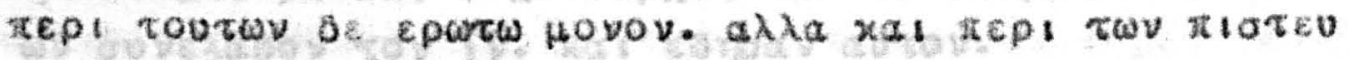

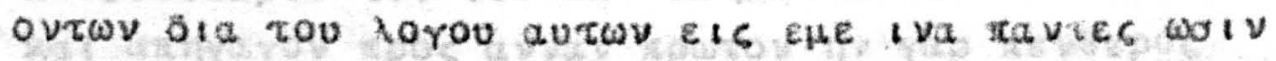

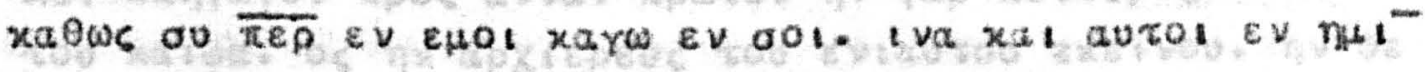

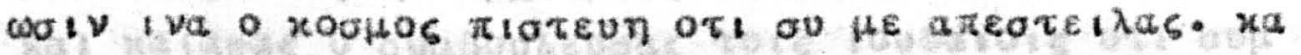

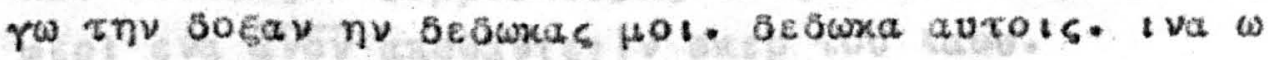

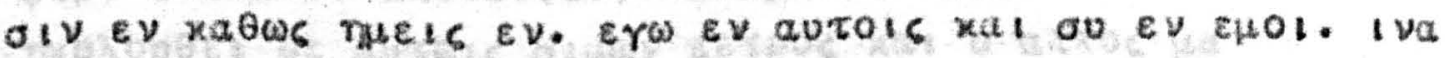

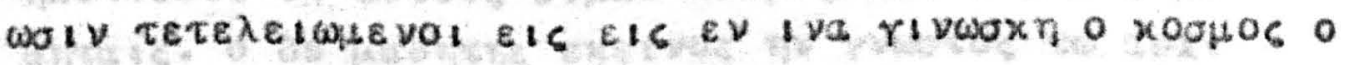

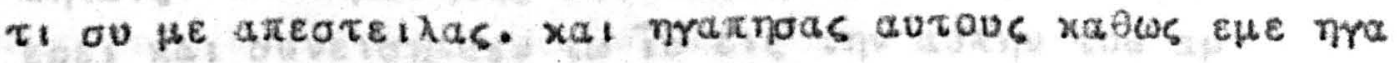

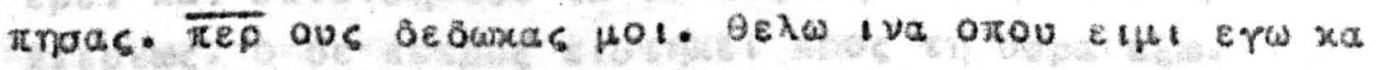

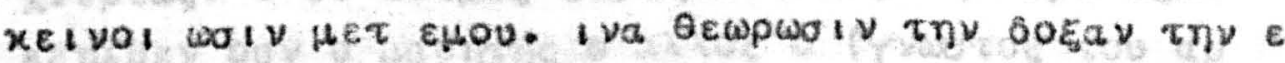

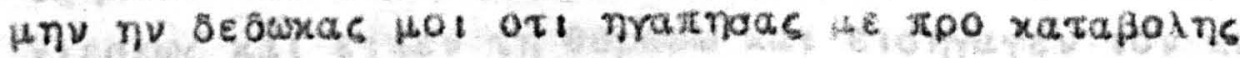




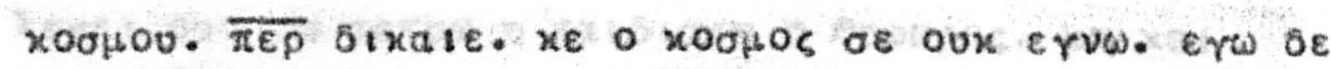

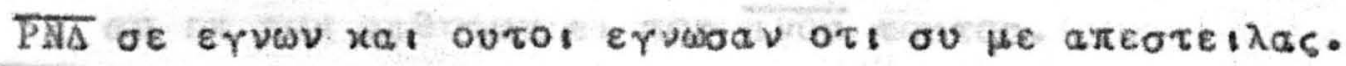

PWE $x a t$ ervaspiga autots to ovoua gov. xat rvwp law. I va

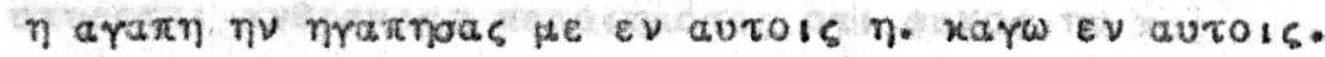

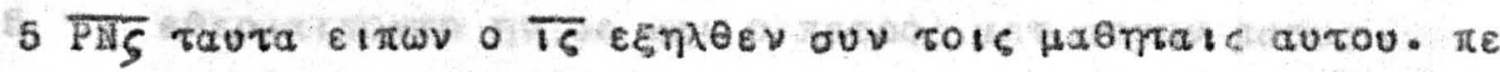

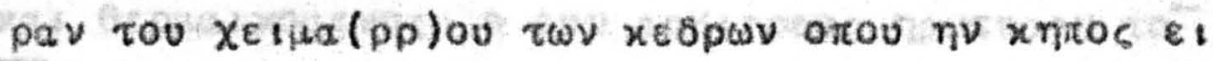

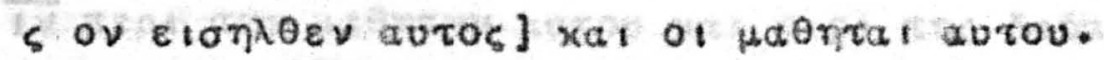

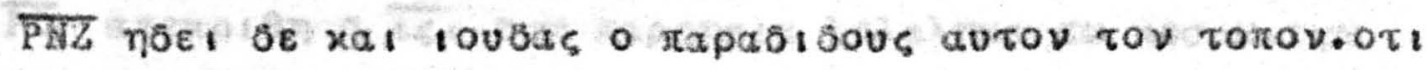

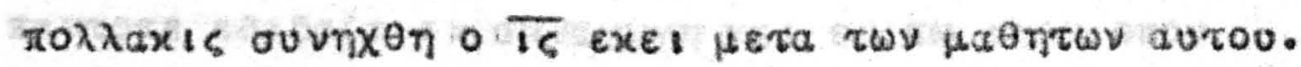

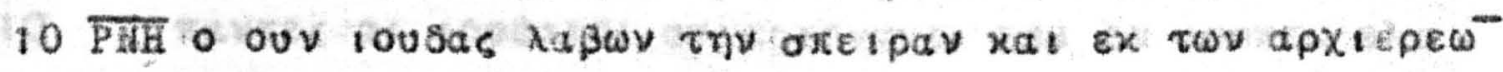

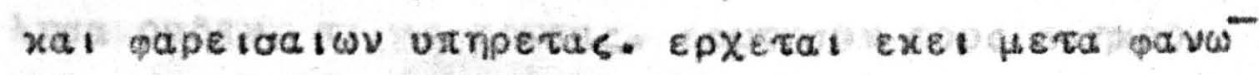

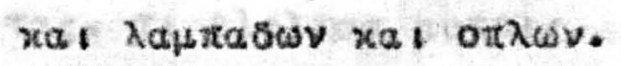

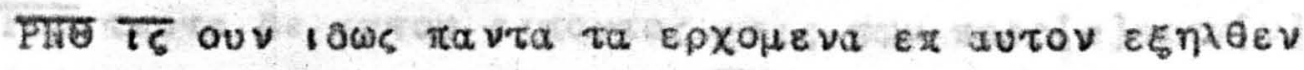

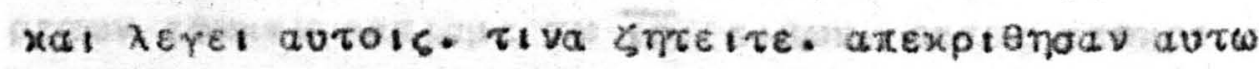

15 TV rov val,opalov.

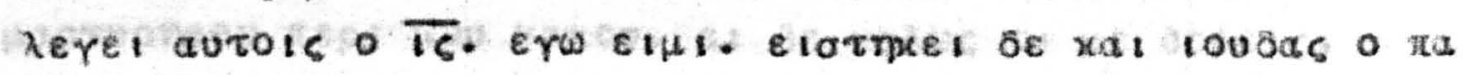
pasısous autov $\mu \varepsilon \tau$ autw.

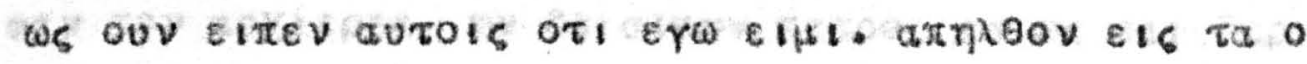

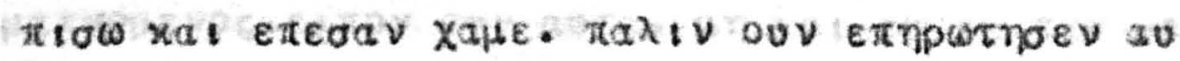

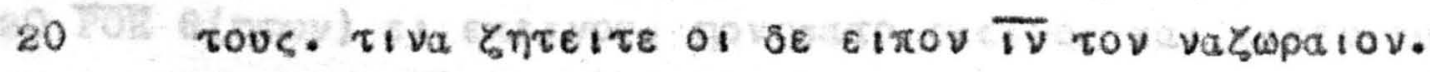

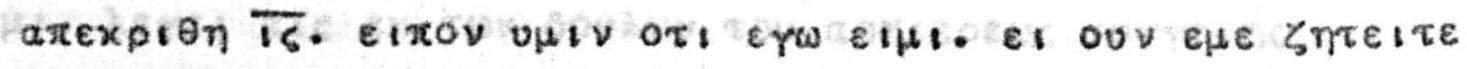

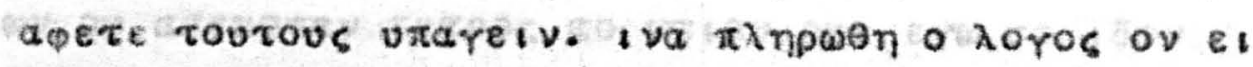

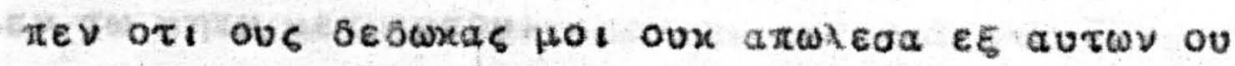

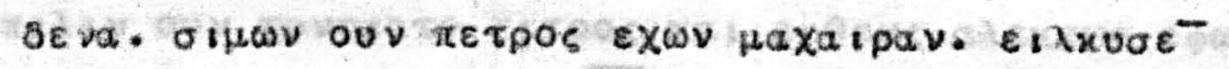

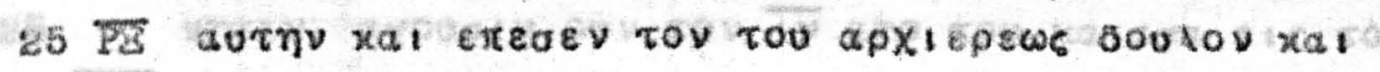

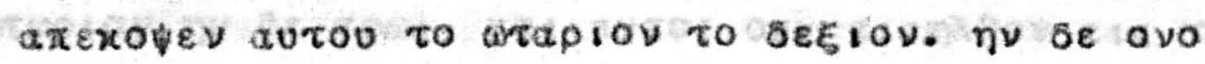
Ha $\tau \omega$ бov $\lambda \omega$ patxos.

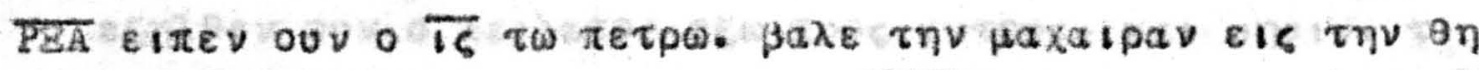

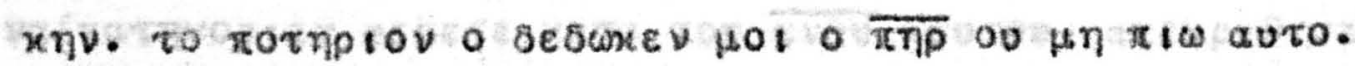

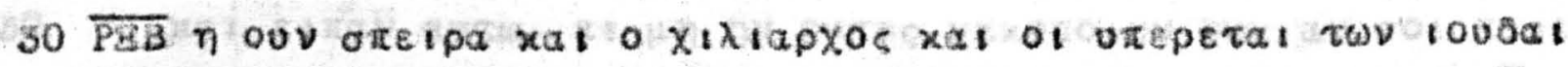

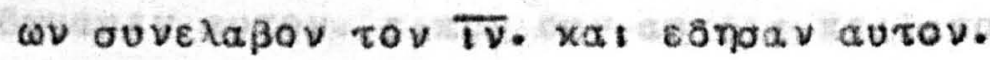

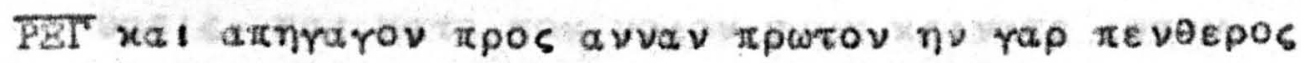

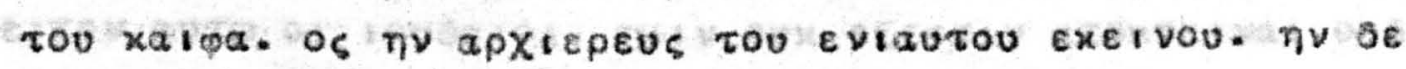

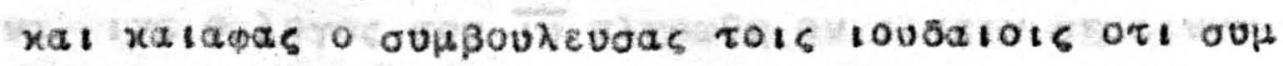

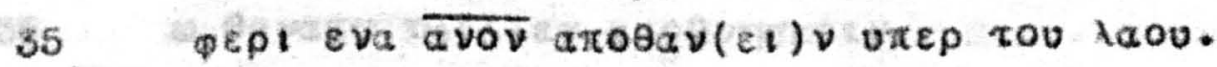

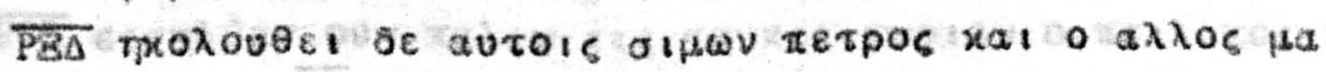

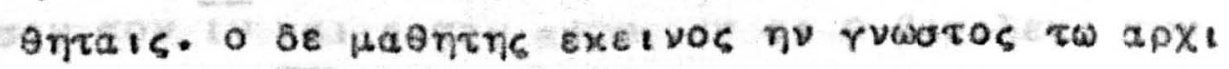

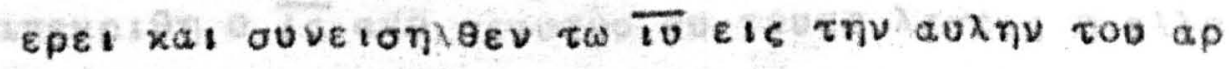

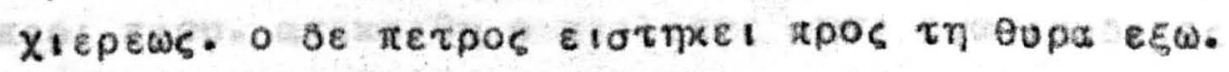

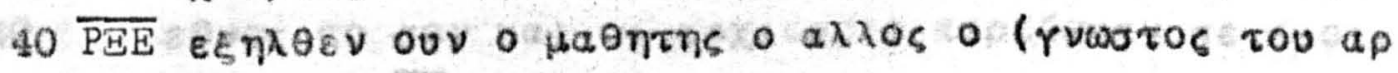

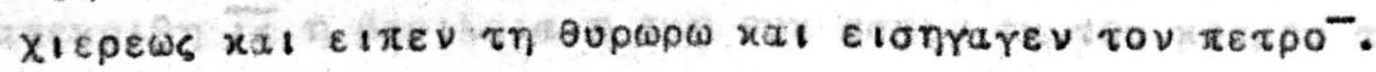




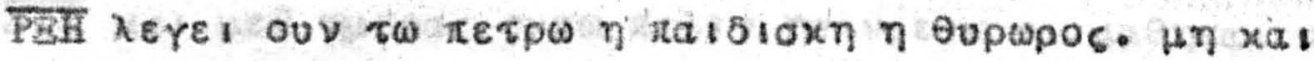

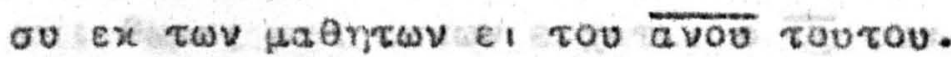

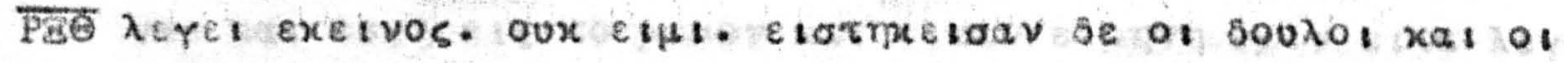

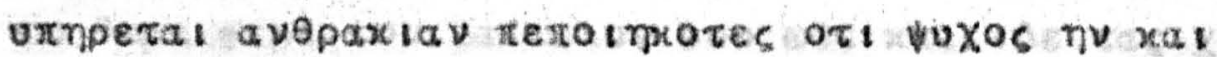

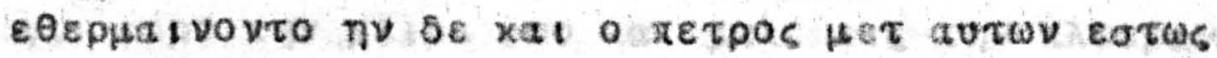
xat $\theta \varepsilon p h a$ ivouevoc. o ouv apXlepeus npwrnoev ro

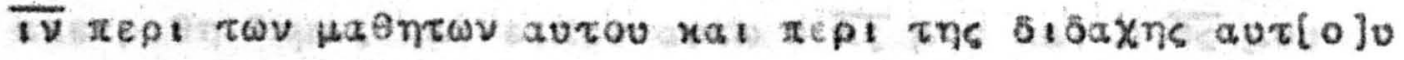

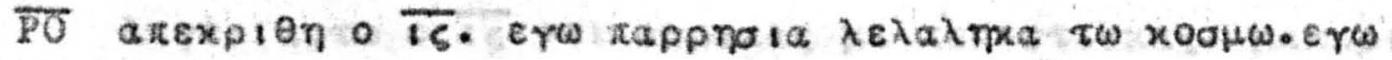

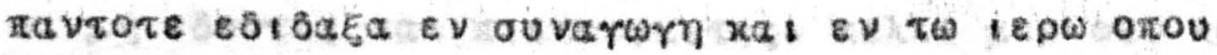

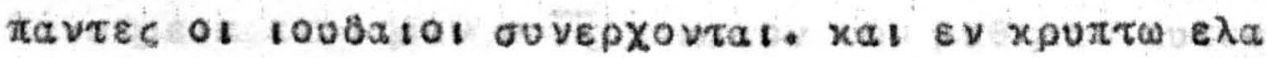
Anoa ousev. Tt $\mu$ e eptrac. eparnoov rous axtpoo

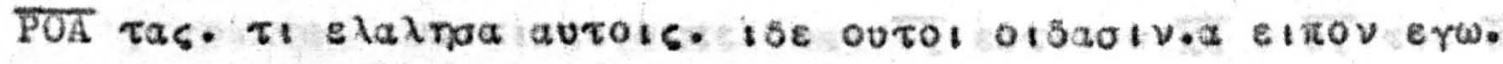

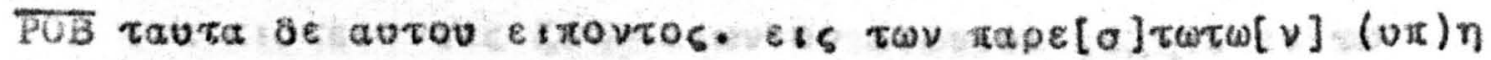

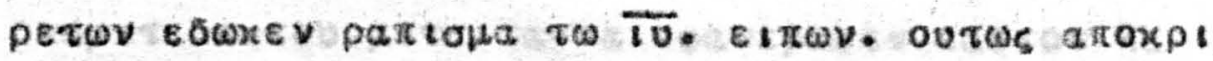

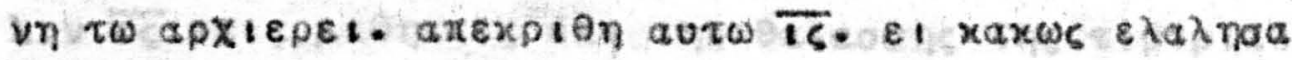

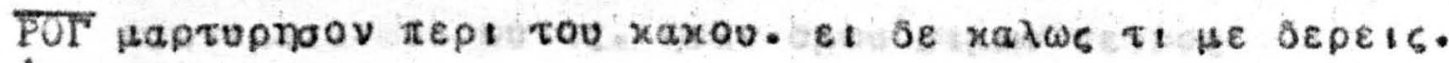
एOS areoteidev auv autov o avvas $8 e \delta e \mu \varepsilon v o v$ rpos xala

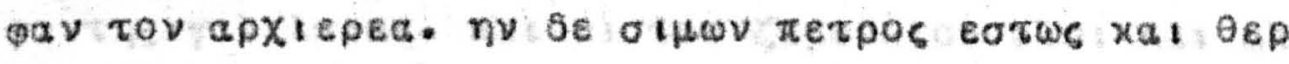

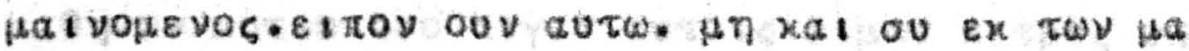

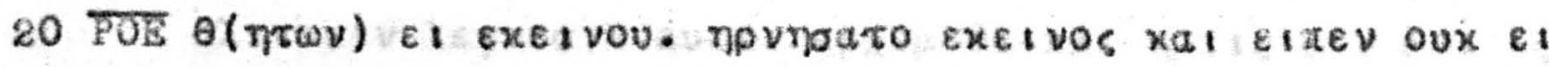

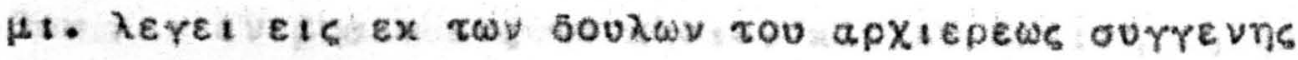

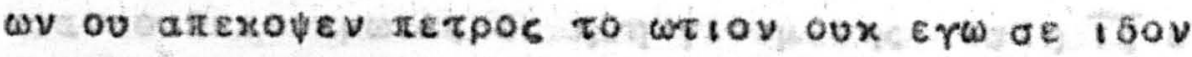

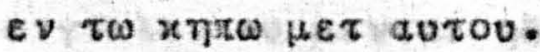

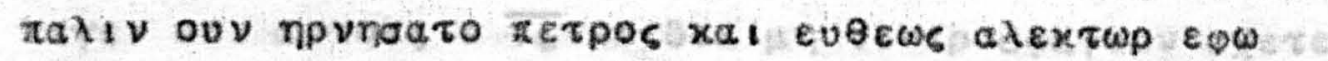

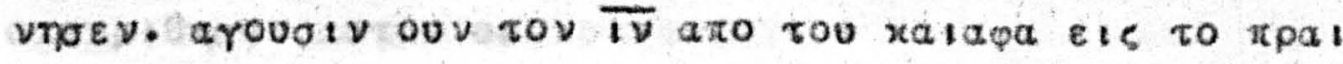

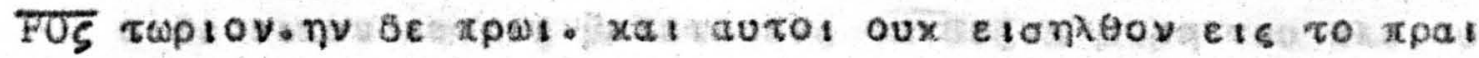

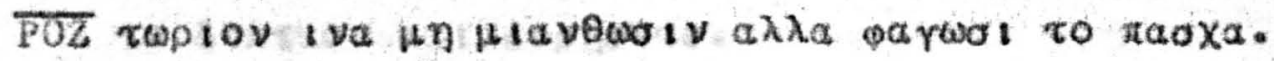

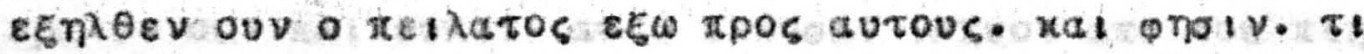

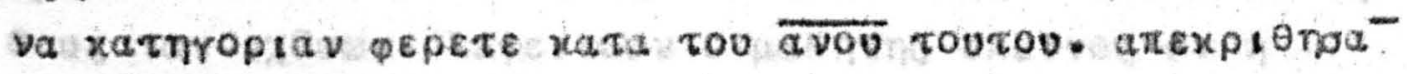

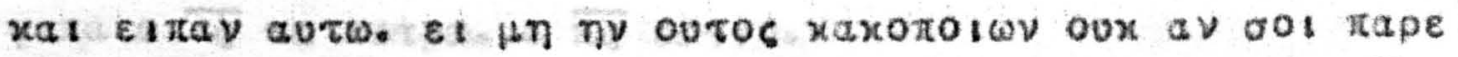

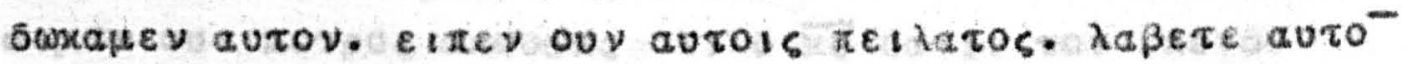
uHeis. hat xata tov volov visw xpivate autov.

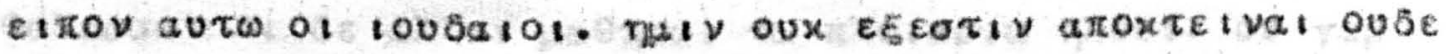

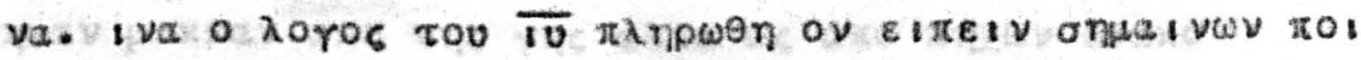

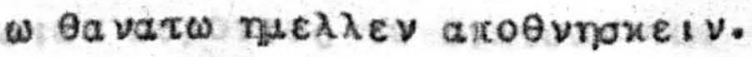

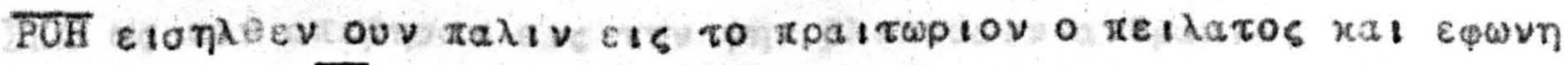

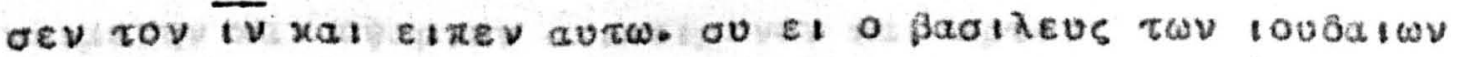

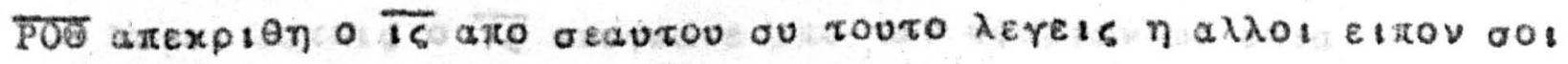

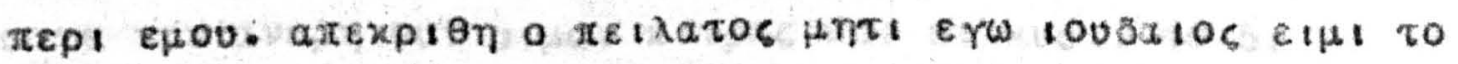

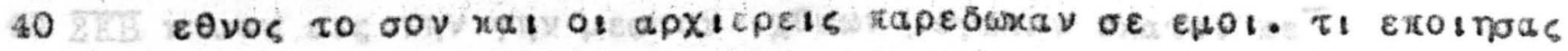

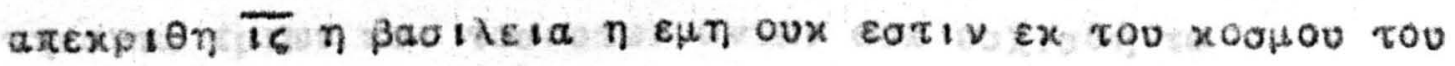




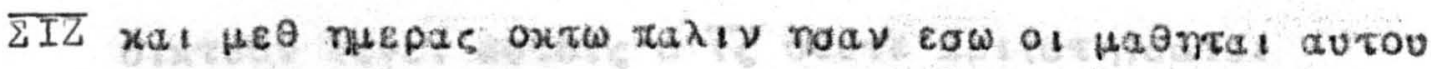

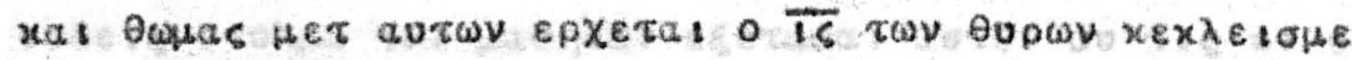

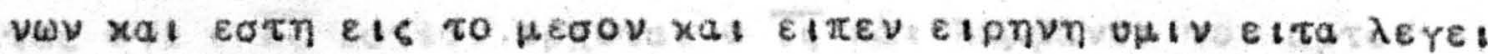

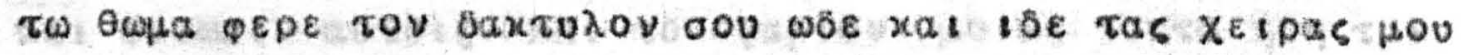

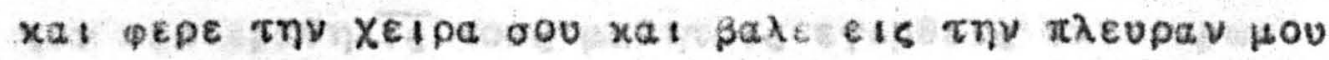

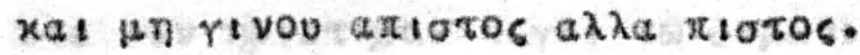

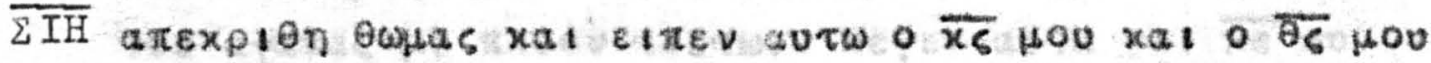

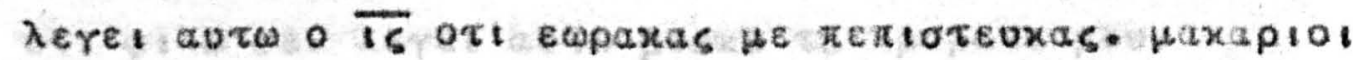

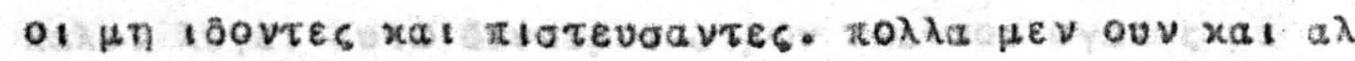

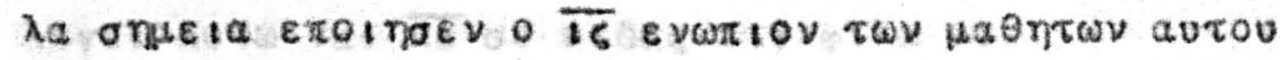

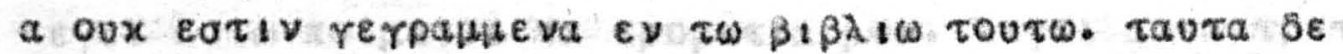

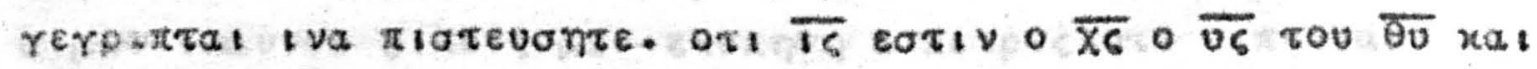

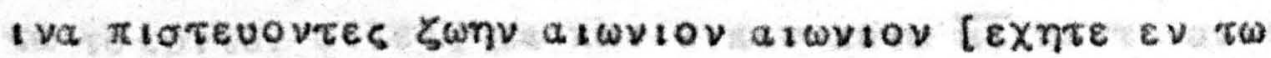

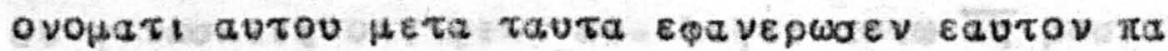

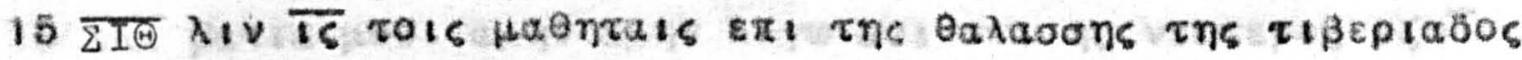

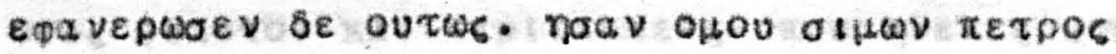

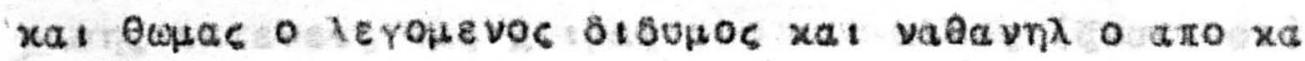

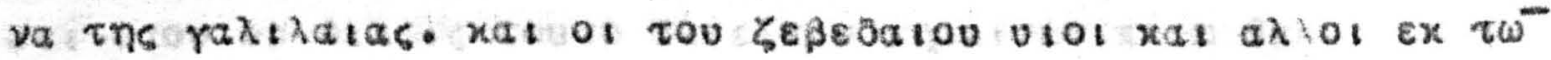
$\mu \alpha \theta \eta \pi \omega v$ auto ô

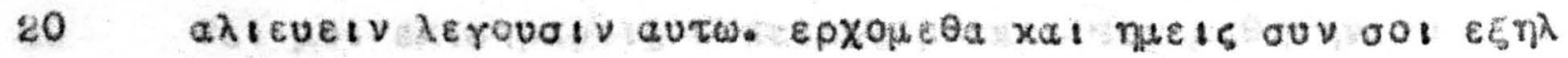

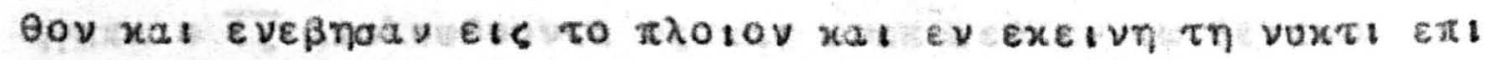

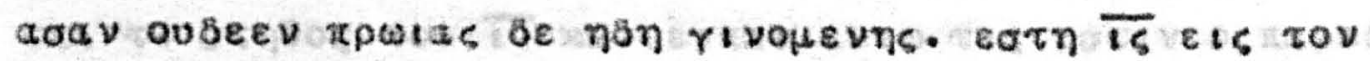

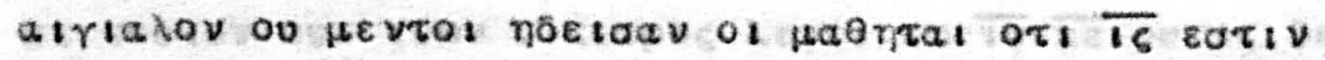

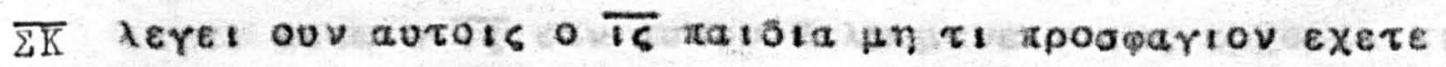

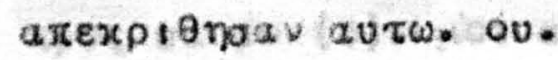

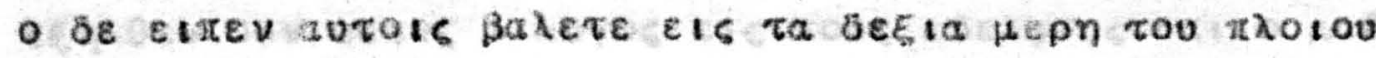

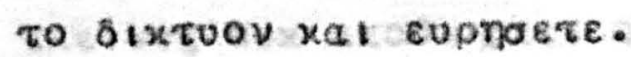

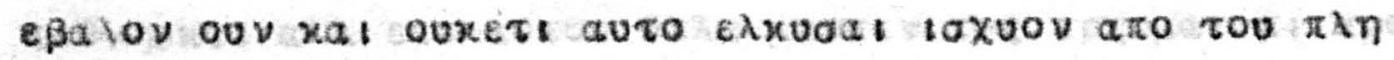

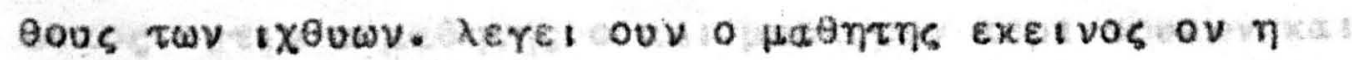

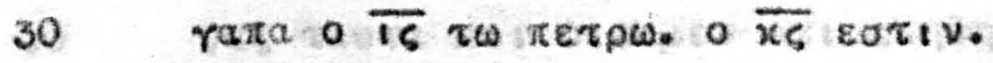

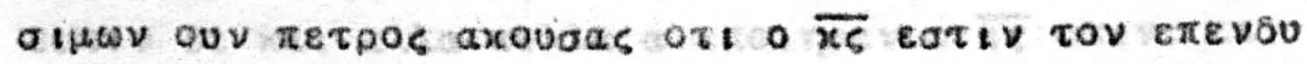

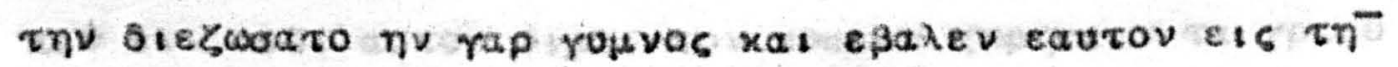

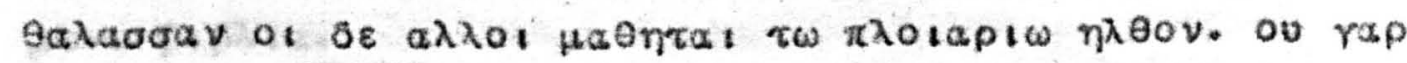

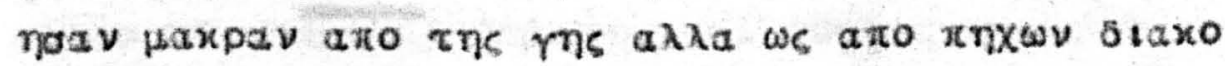

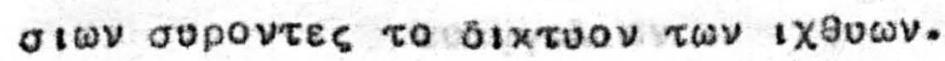

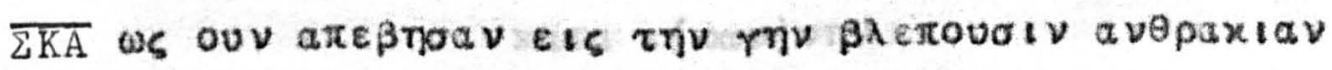

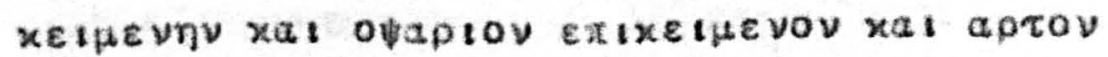

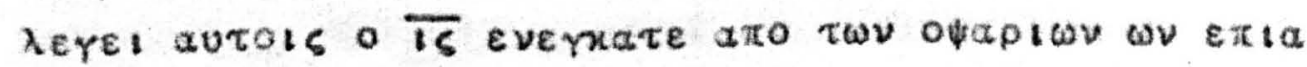

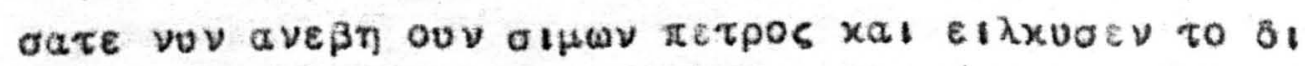

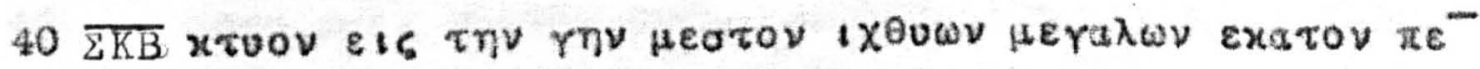

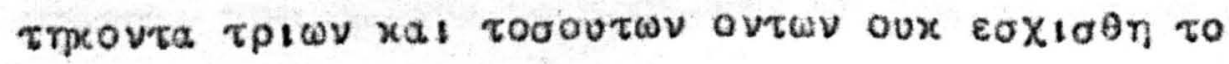




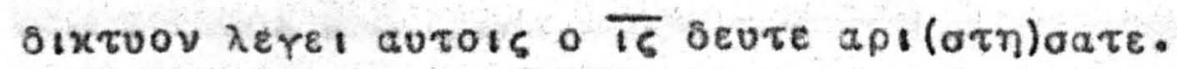

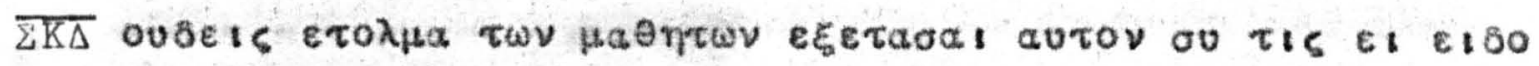

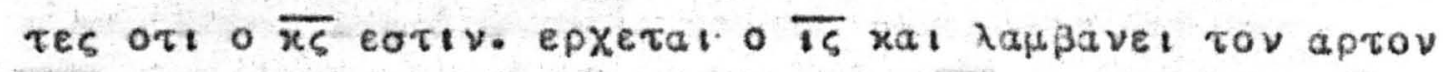

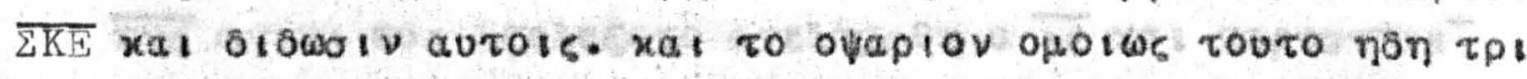

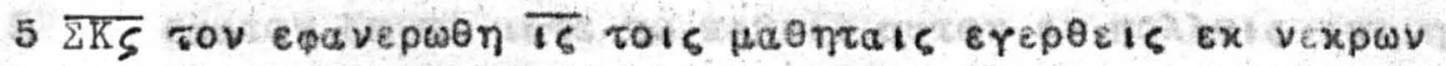

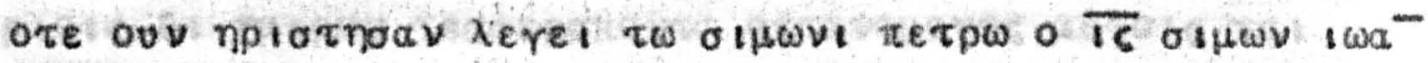

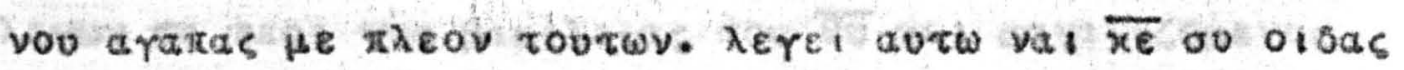

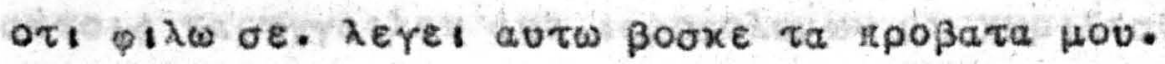

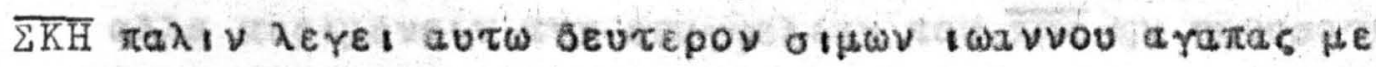

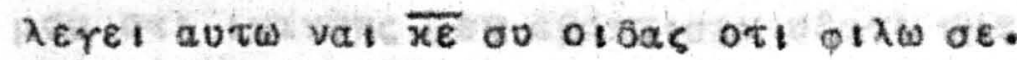

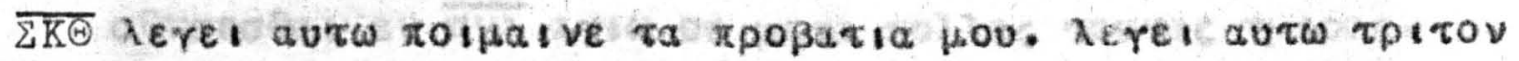

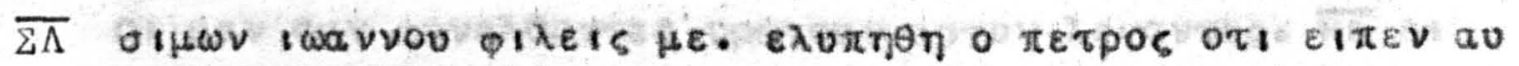

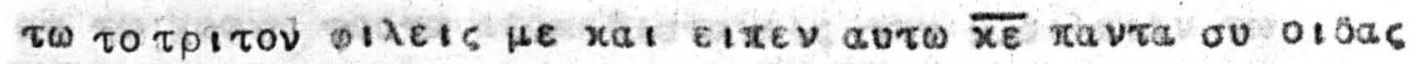

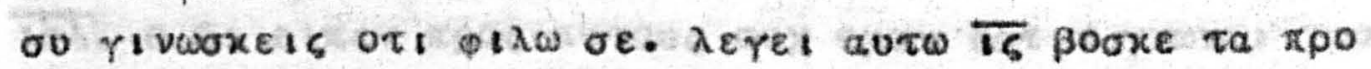

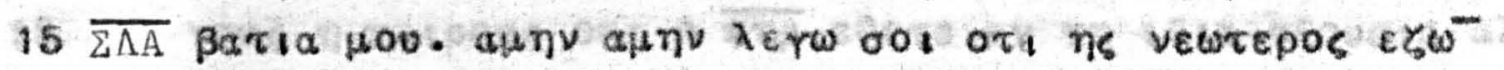

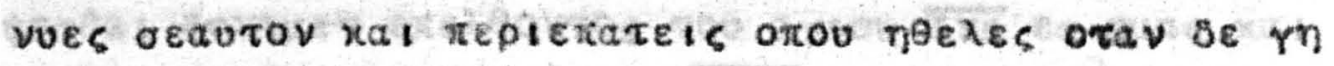

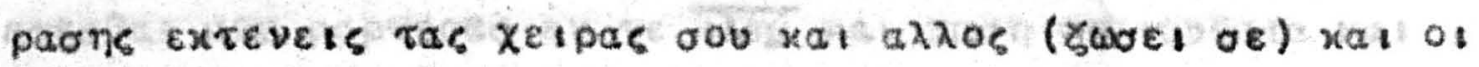

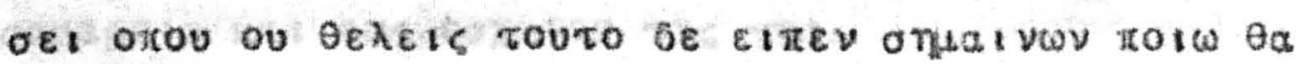

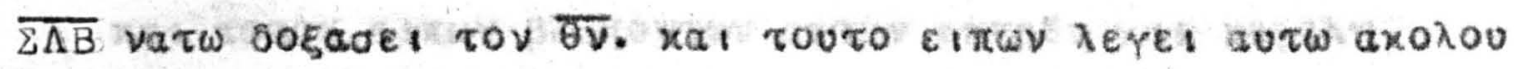

$\theta \varepsilon$ । Hol exı

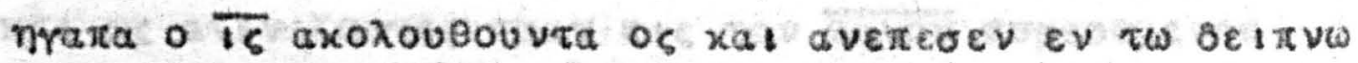

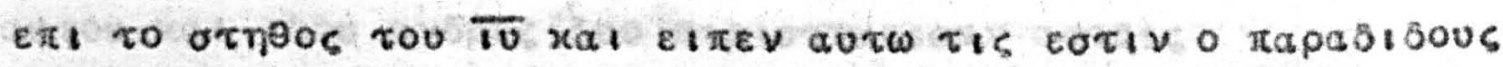

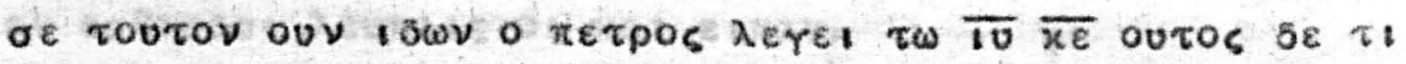

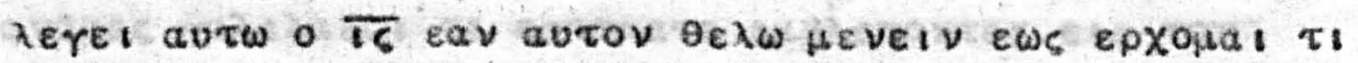

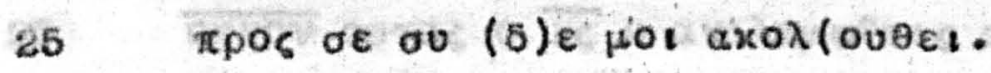

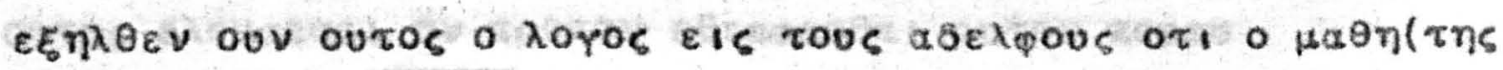
exetvos oux aroovnoxet oux eitev be autw o ts ot oux a

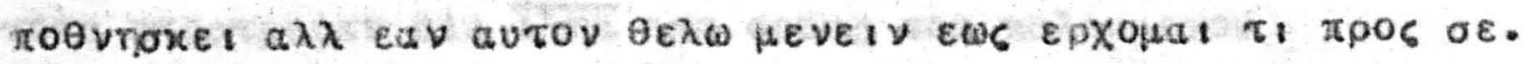

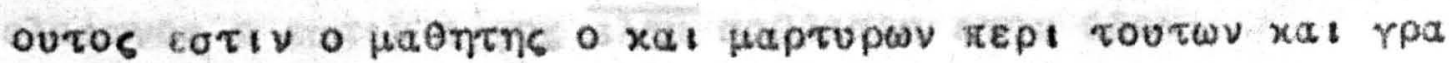

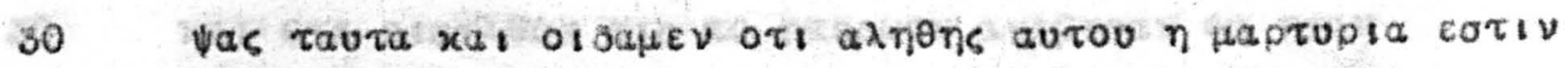

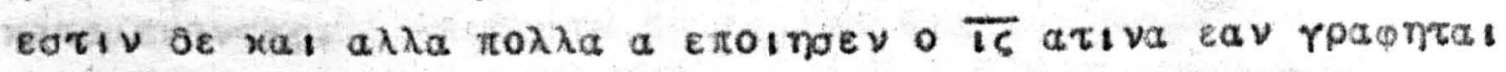

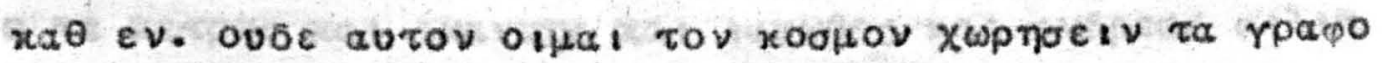
$\mu \varepsilon v a \beta เ \beta \lambda$ ia.

Evaryexiov

xara,$(\omega a v) v \eta \eta v$ 


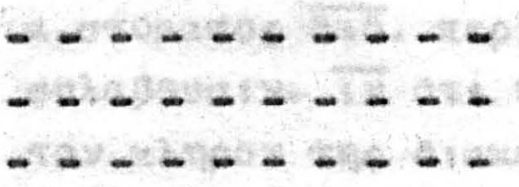

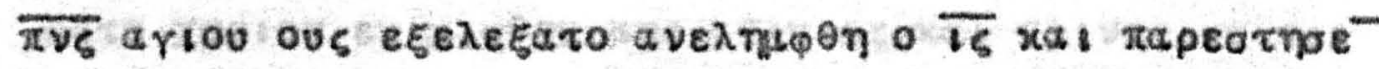

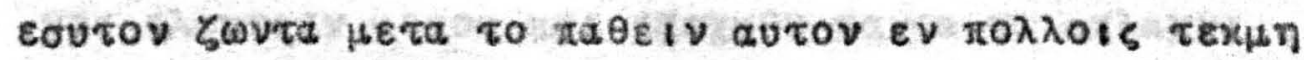

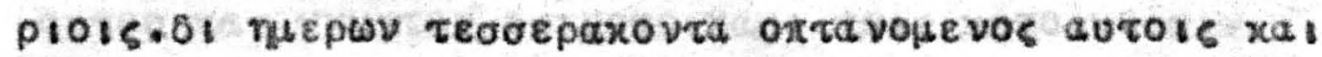

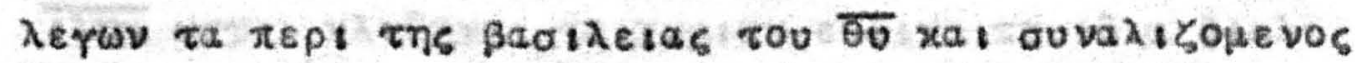

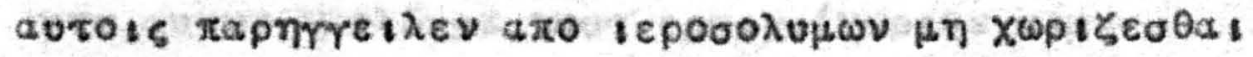

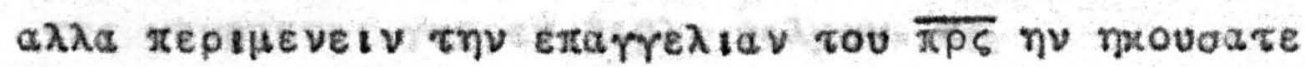

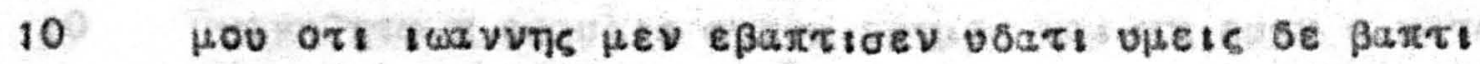

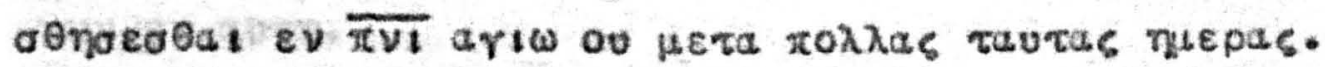

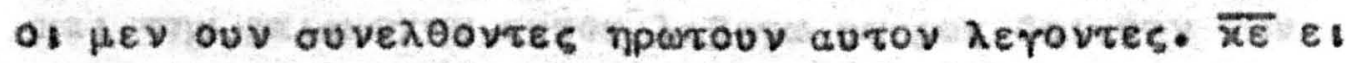

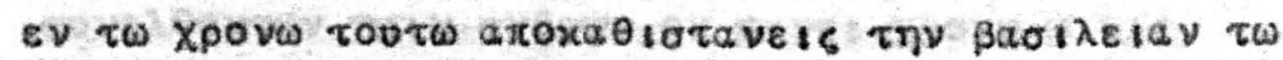

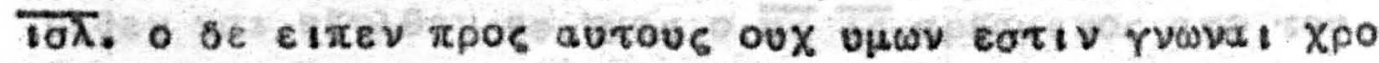

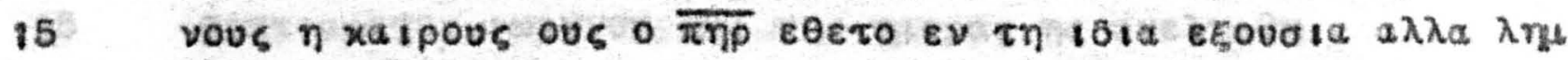

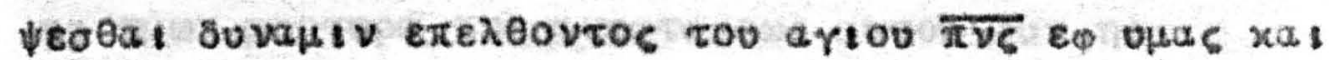

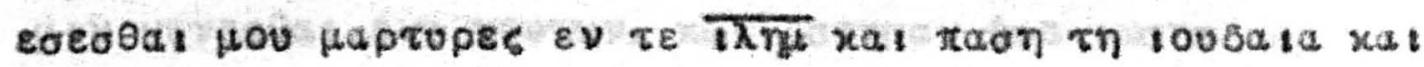

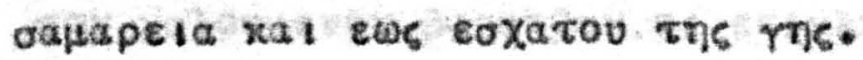

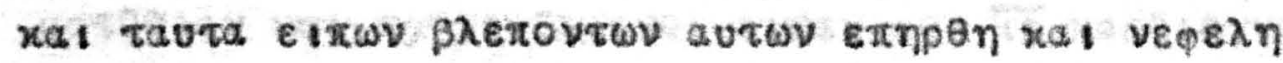

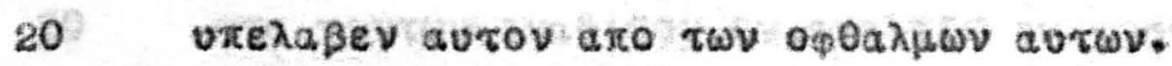

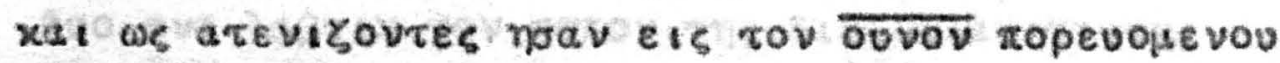
autou.xa, 1800 avopes ovo raptorrpe toav autois

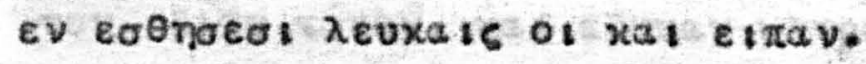

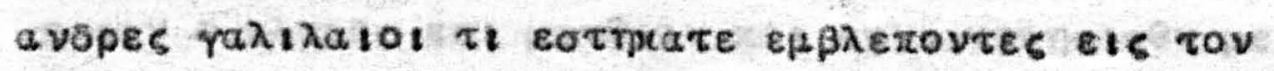

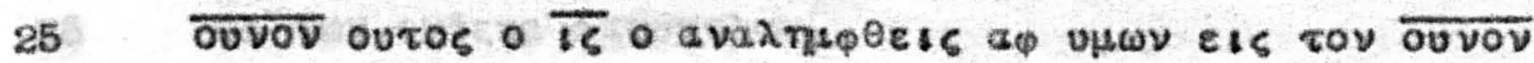

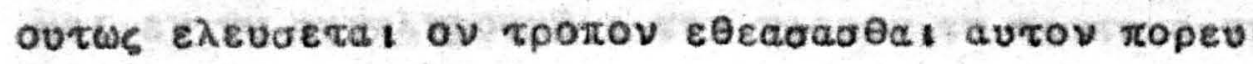
örevov eis rov ouvov.

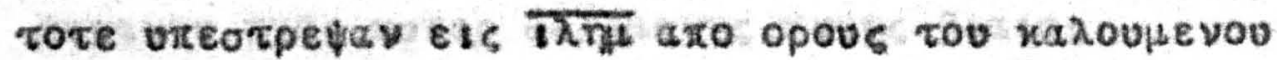

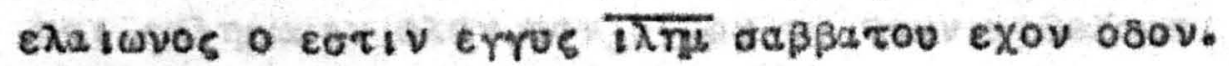

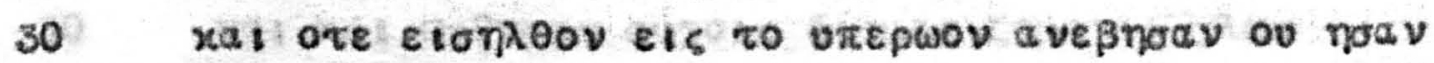
xarauevovies.

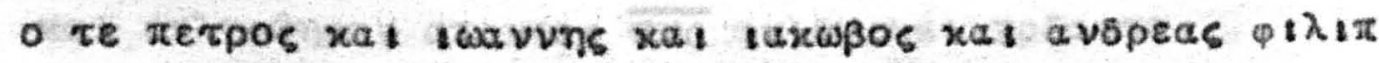

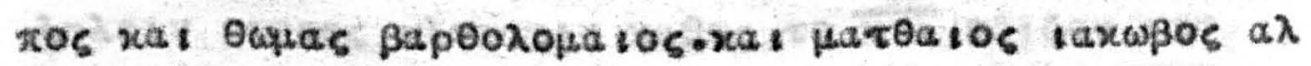

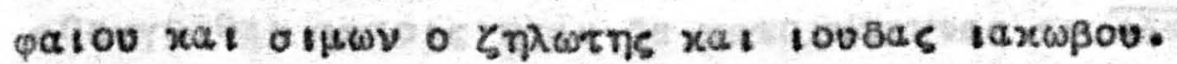

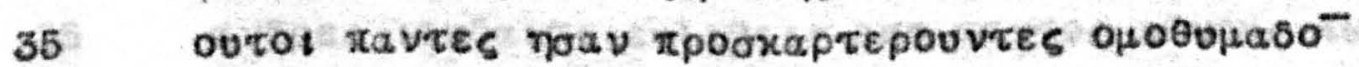

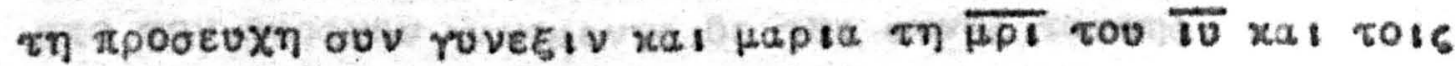
asexpois aurou.

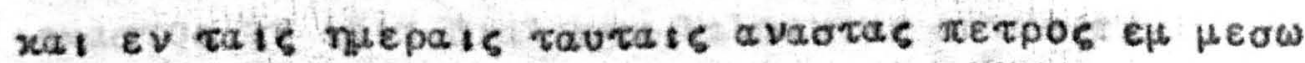

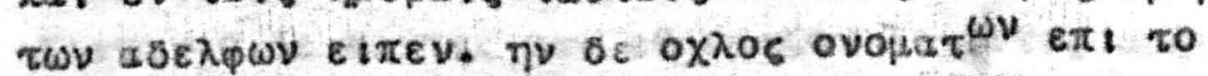

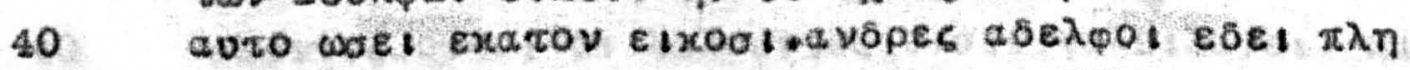

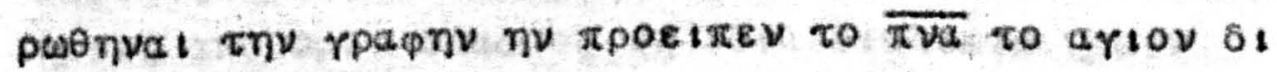




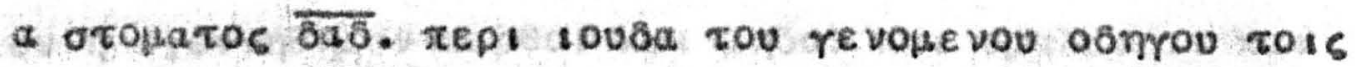

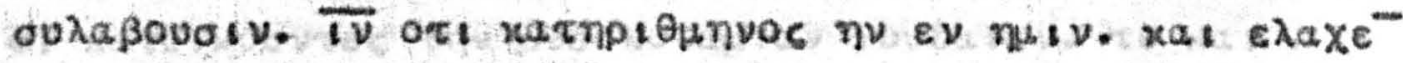

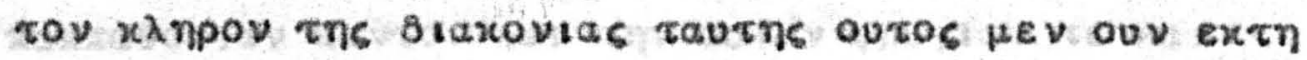

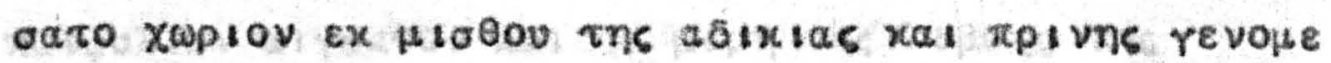

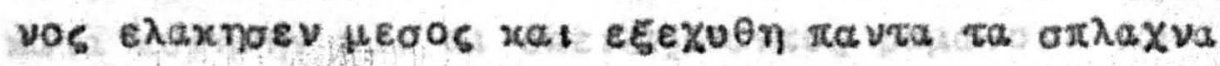
autou nat rutorov erevero nad rois natotxouaty

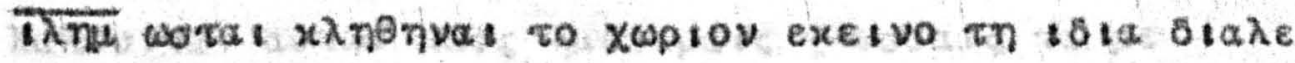

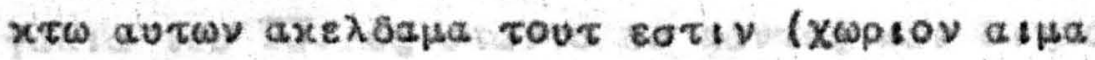

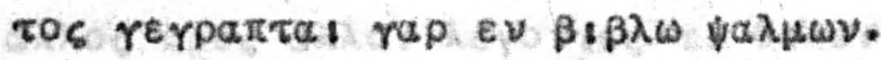

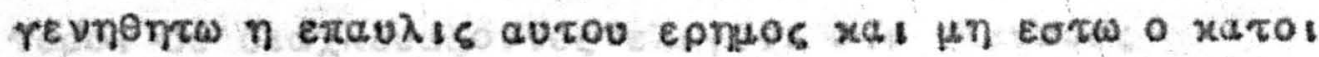
xwV \&V auTn.

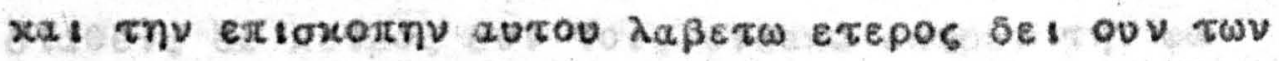

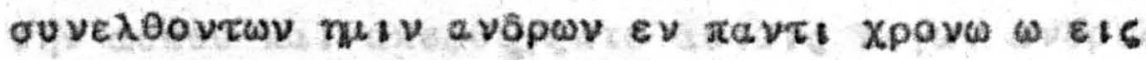

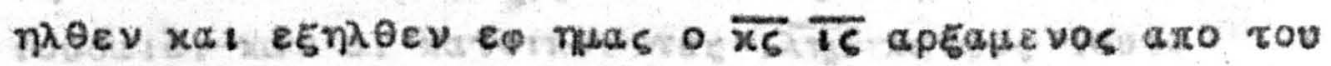

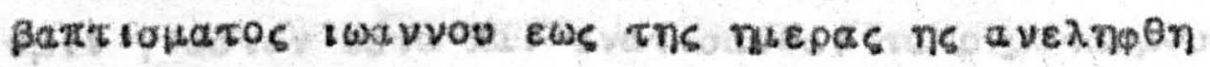

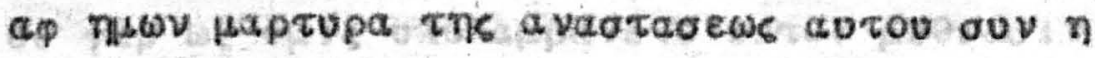

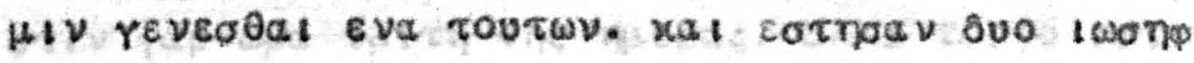

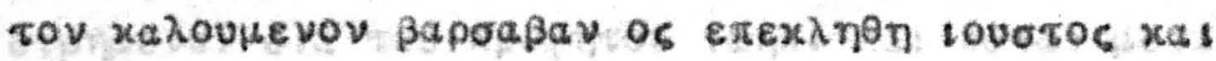

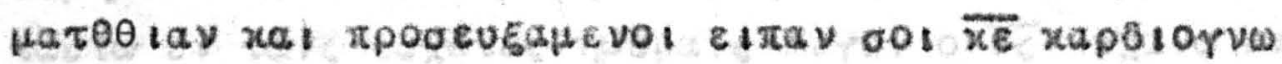

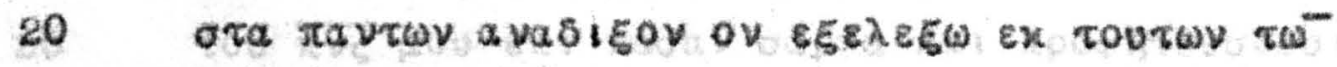

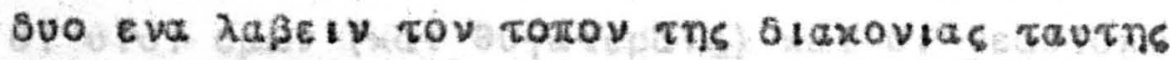

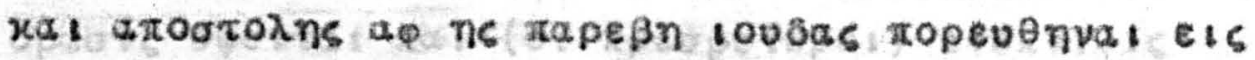
Tov 1810 rotov. nat eönav xampovs avtols xal e

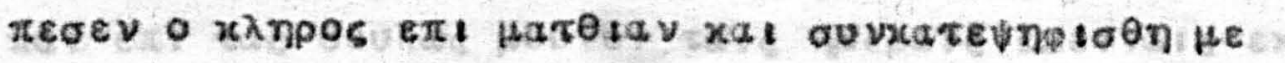

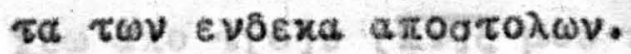

xa। $\varepsilon$ V $\tau$ ou

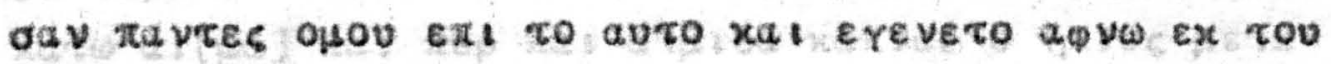

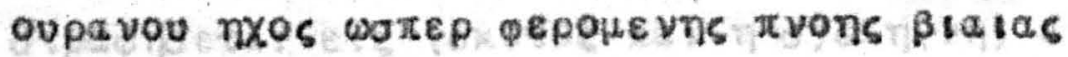

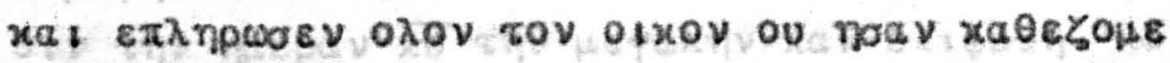

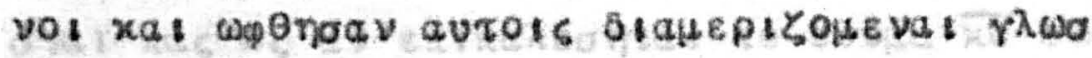

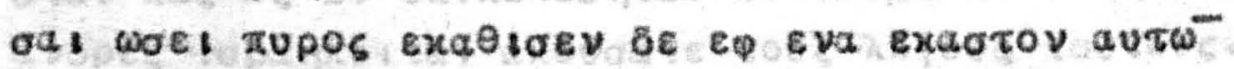

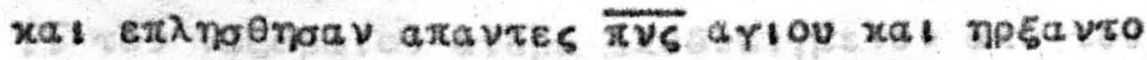

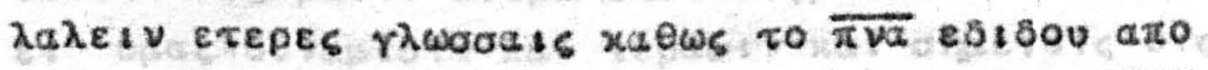

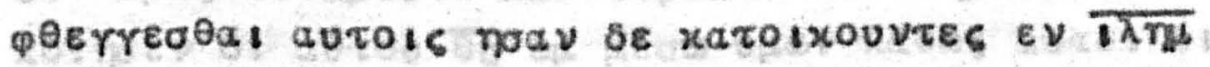

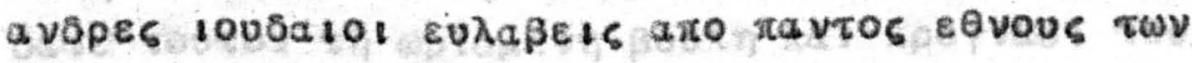

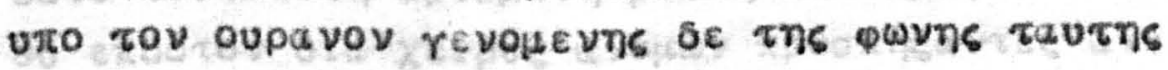

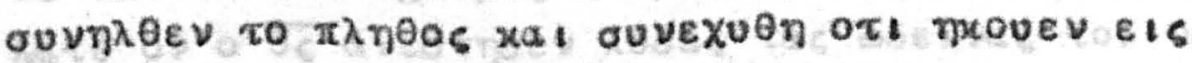

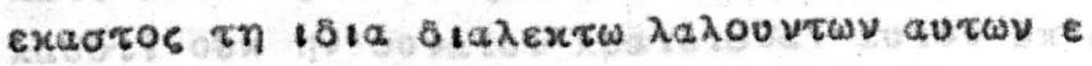

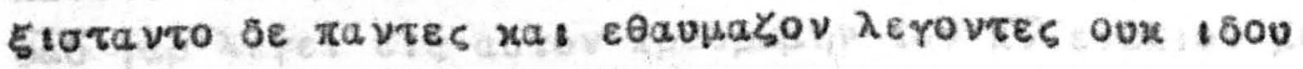

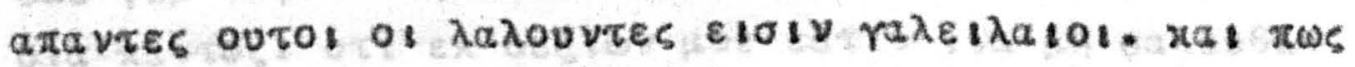

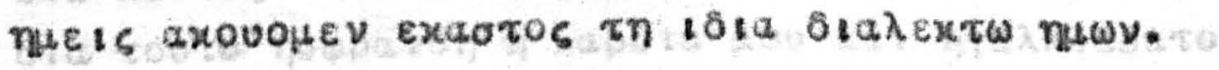




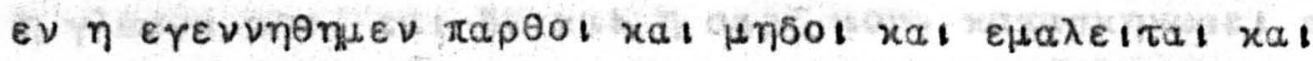

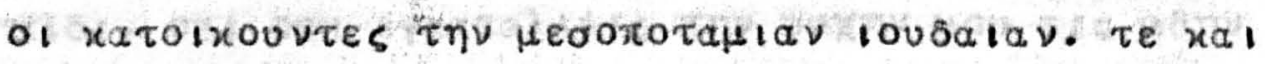

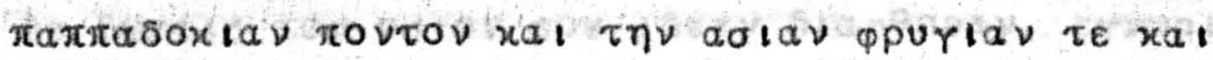

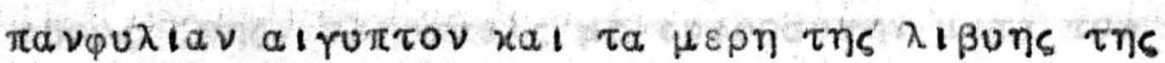

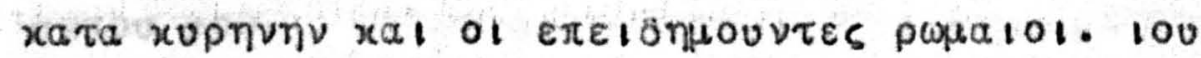

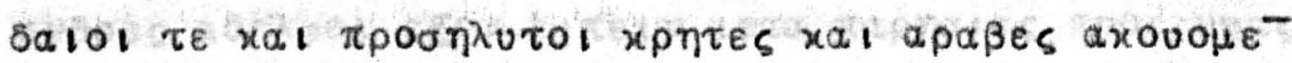

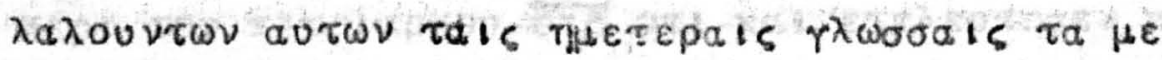

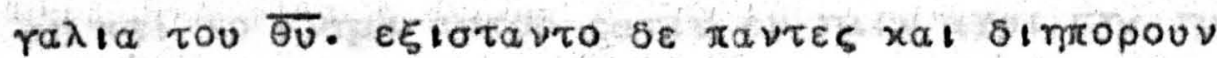

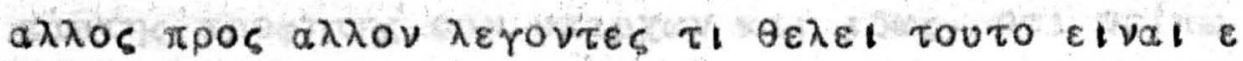

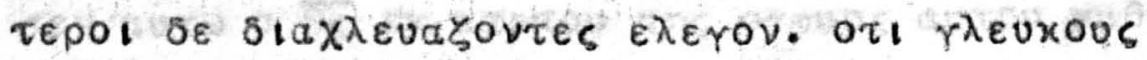
$\mu \varepsilon \mu \varepsilon \sigma \tau \omega \mu \varepsilon$ VO $\varepsilon เ \sigma \downarrow \nu$.

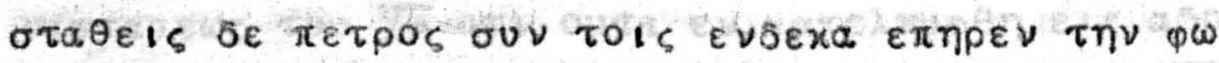

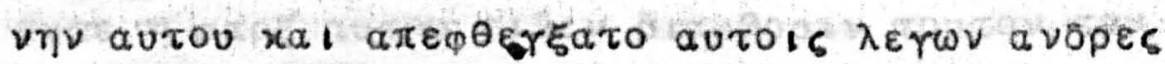

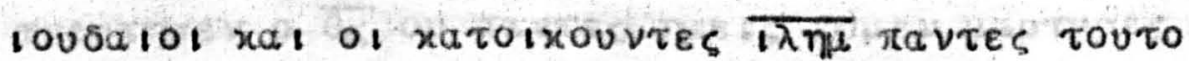

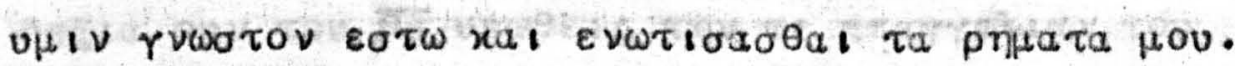

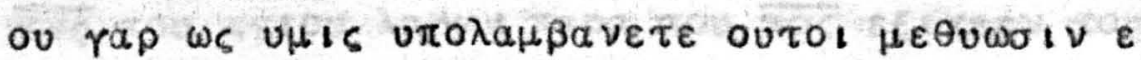

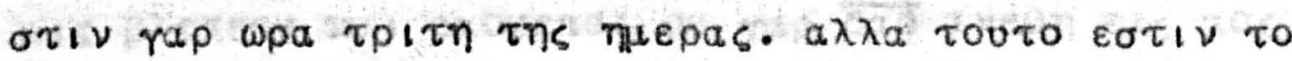

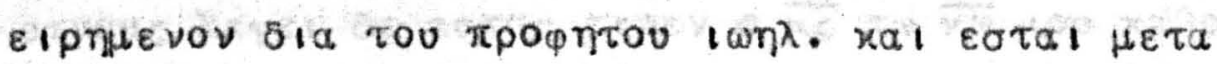

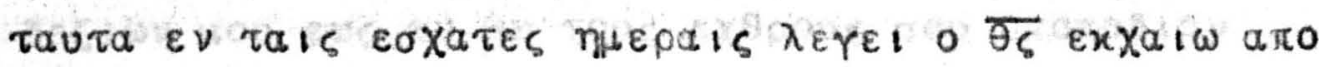

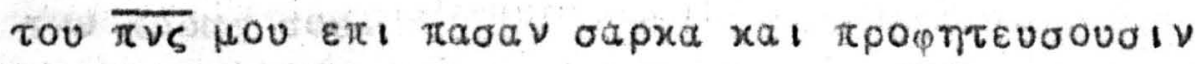

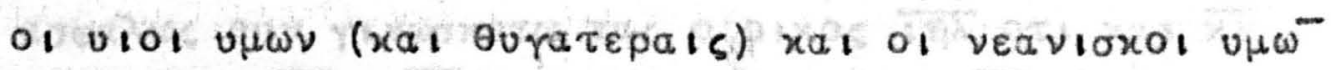

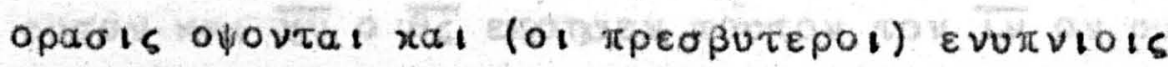

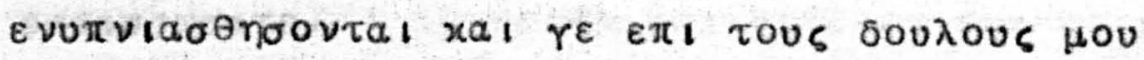

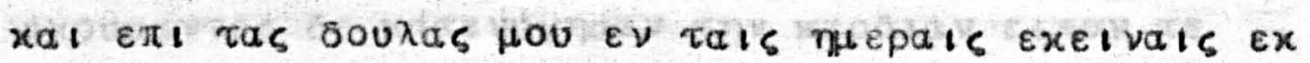

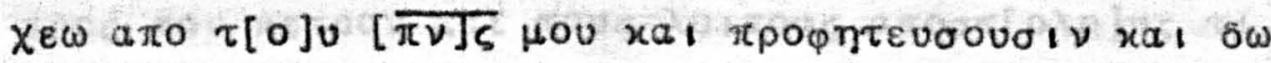

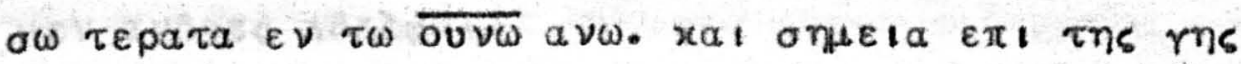

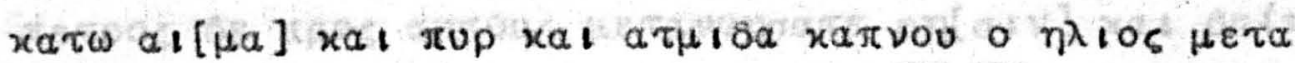

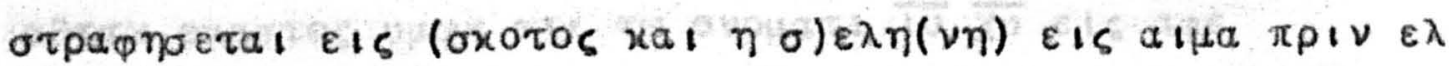

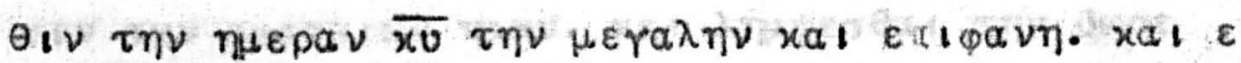

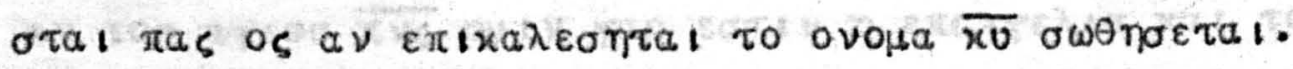

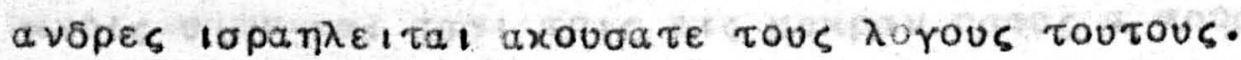

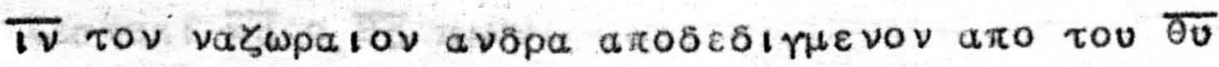

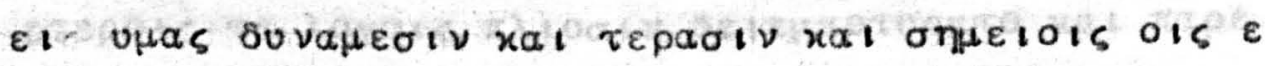

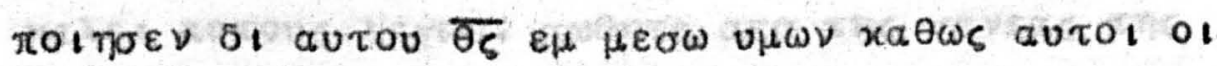

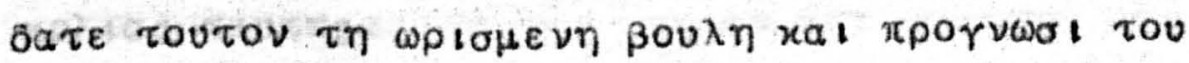

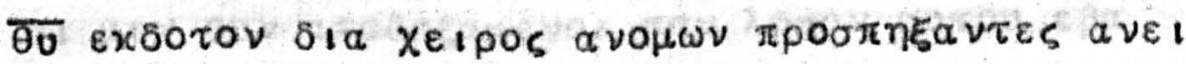

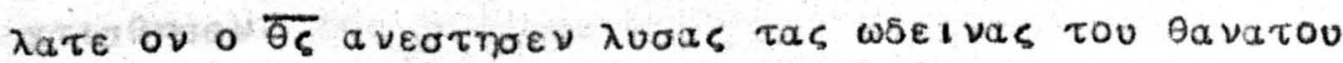

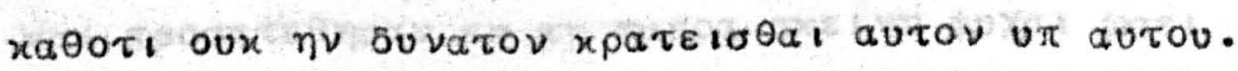

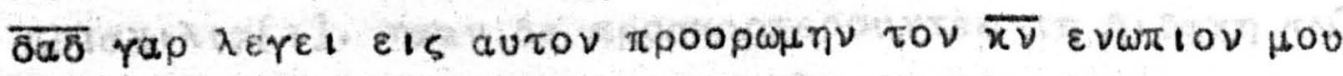

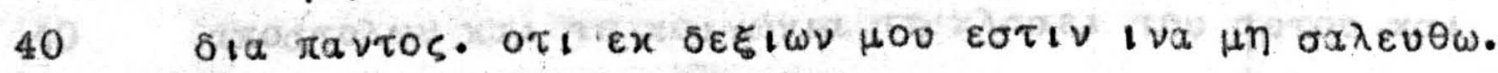

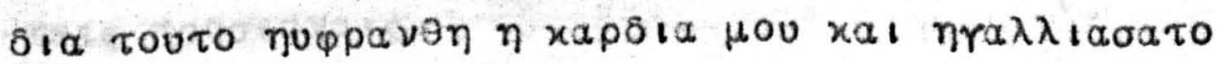




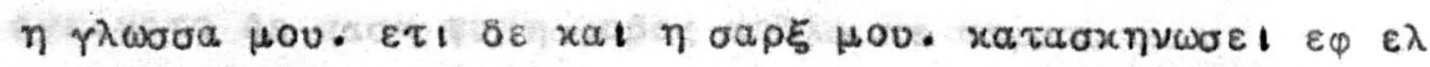

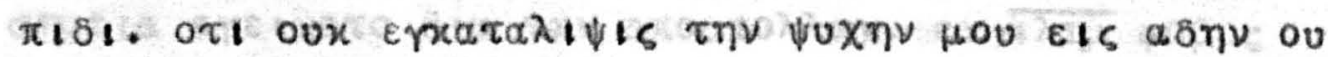

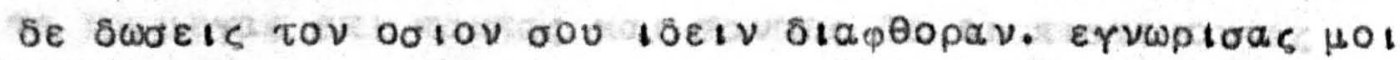

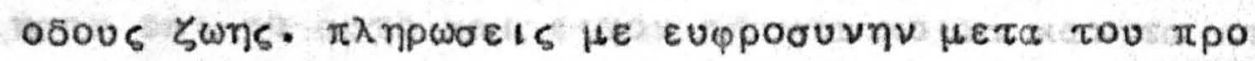
5 owiou oov.

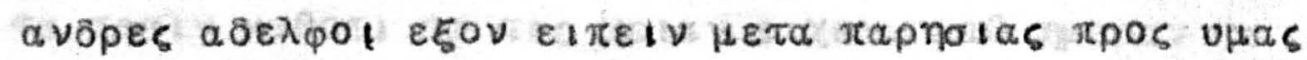

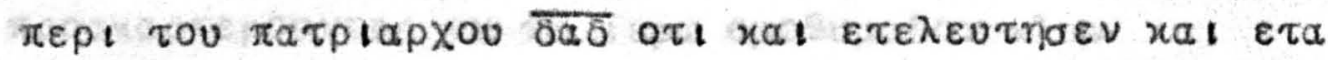

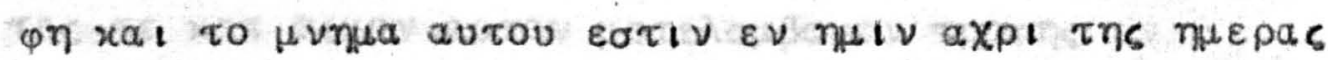

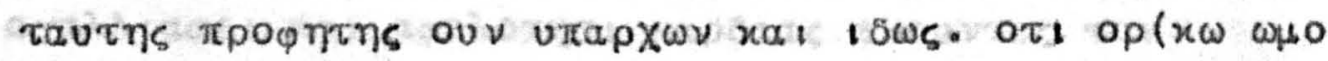

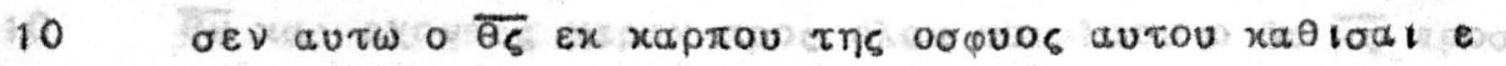

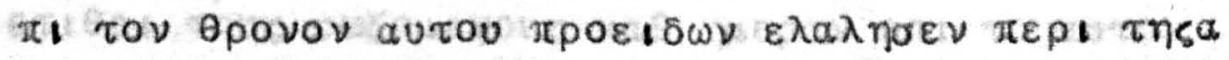

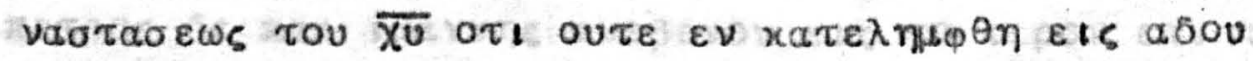

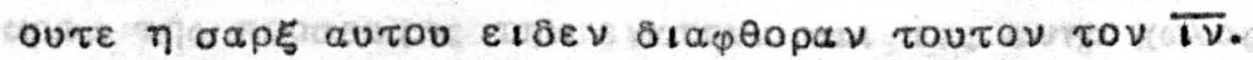

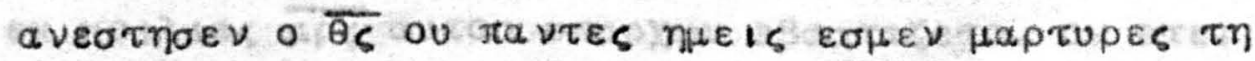

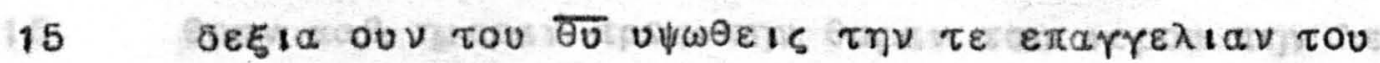

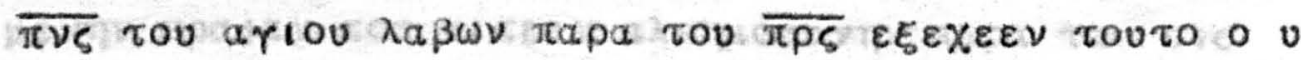

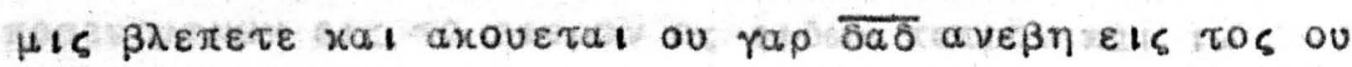
pavous $\lambda \varepsilon \gamma \varepsilon$ i $8 \varepsilon$ autos $\varepsilon ı \pi \varepsilon \nu$ o $\overline{x \zeta} \tau \omega \overline{x \omega} \mu$ Hov $x a \theta 0 u \varepsilon x$

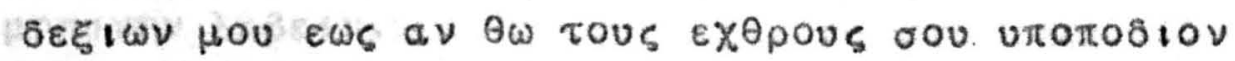

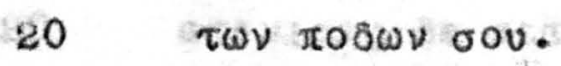

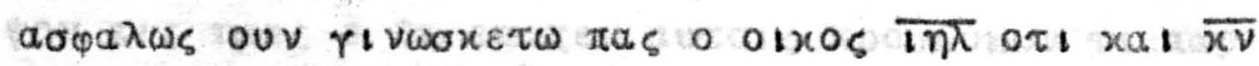

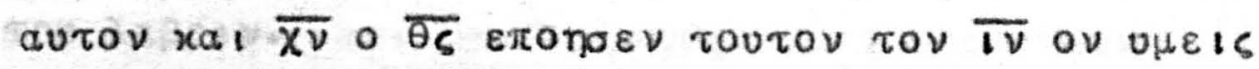
eотаupworte.

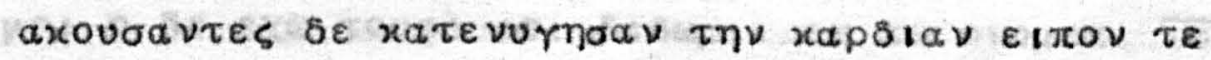

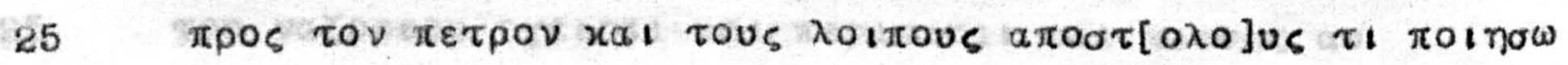
$\mu \varepsilon \nu$ avopes a $\delta \varepsilon \lambda \varphi O r$.

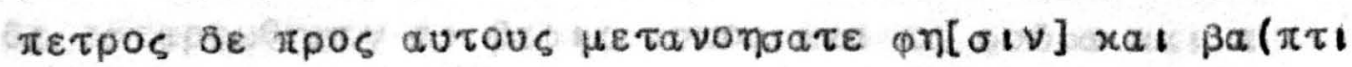

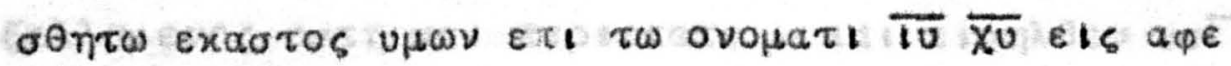

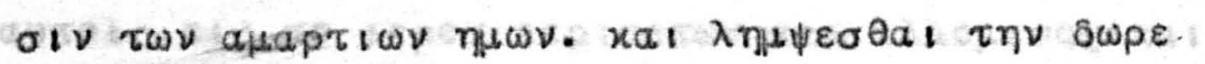

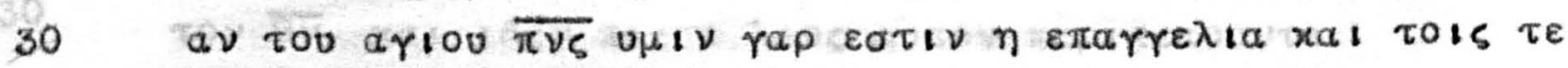

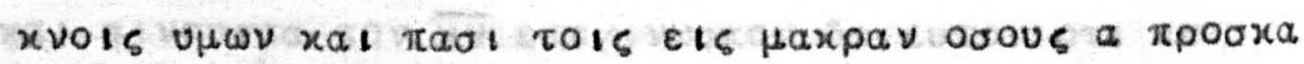

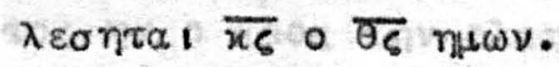

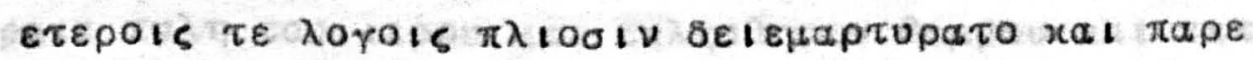

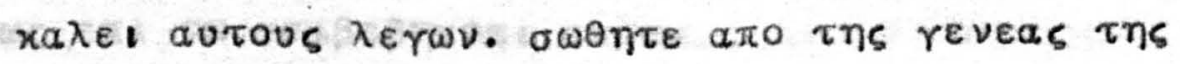

35 xoגias tautns.

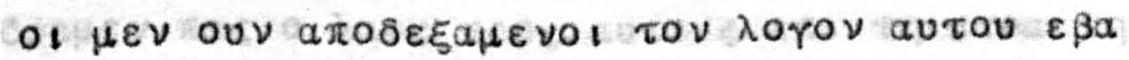

$\pi \tau \operatorname{r} \theta$ ina $\alpha$.

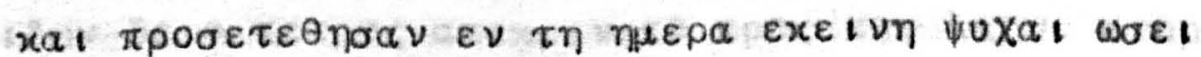

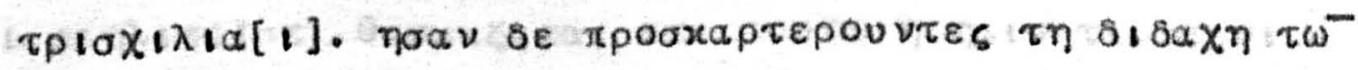

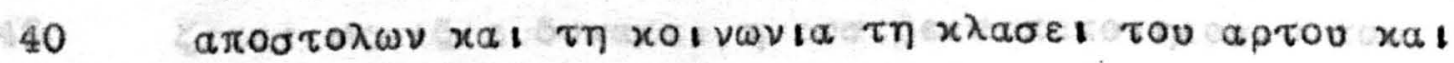

tais $\pi$ poosuxais. 


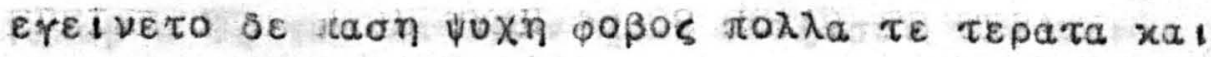

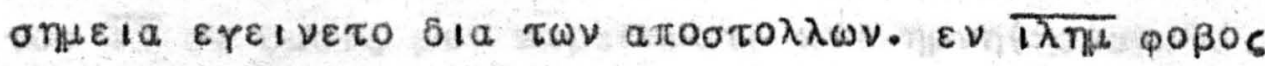

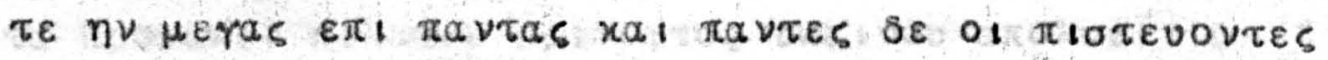

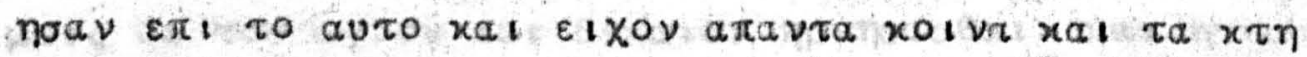

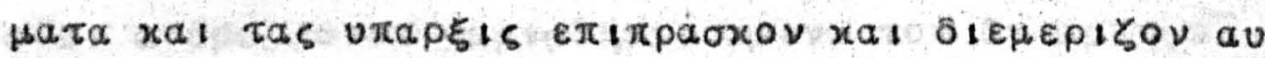

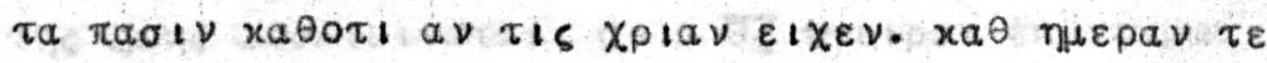

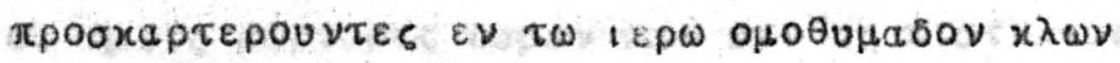

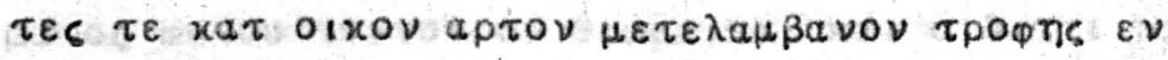

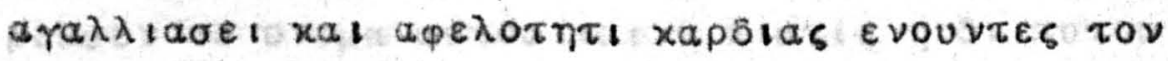

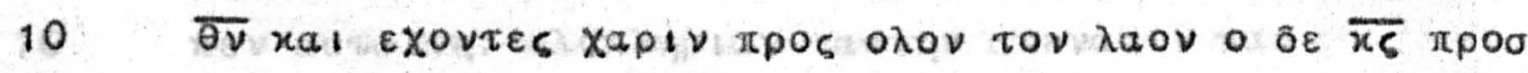

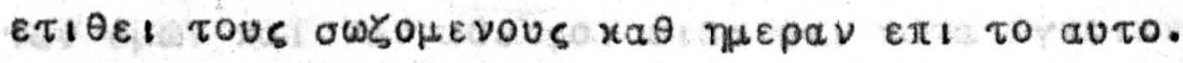

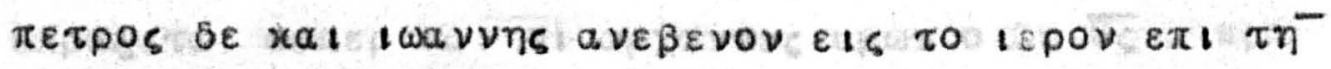

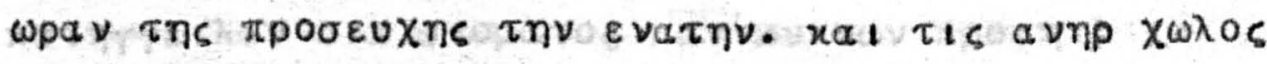

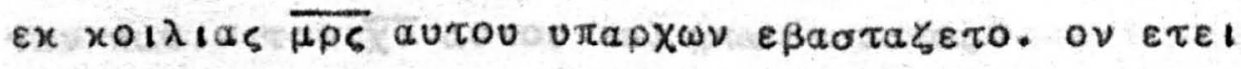

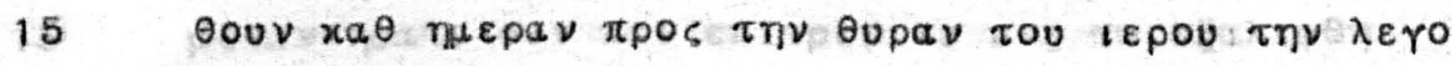

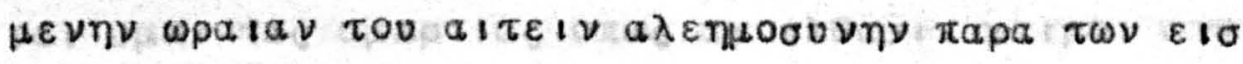

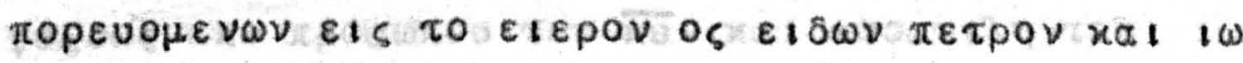

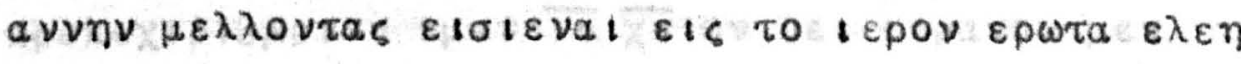

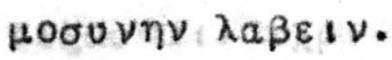

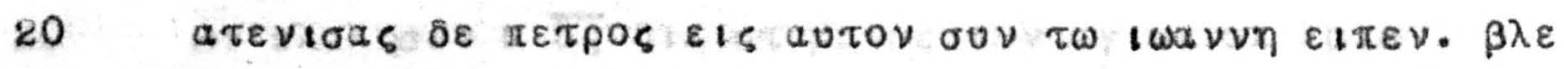

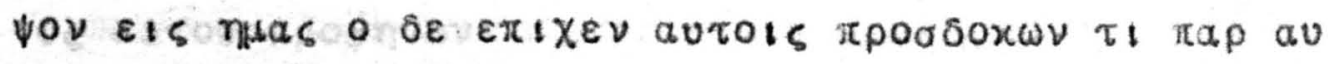
rov $\lambda a \beta \varepsilon เ v$.

$\pi \varepsilon \tau p \circ s$ $\delta \varepsilon \varepsilon t \pi \varepsilon V$ aprupiov xal Xpvotov oux $u \pi a \rho X \varepsilon 1$

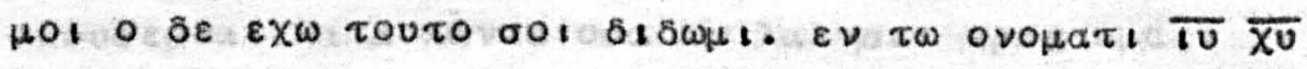

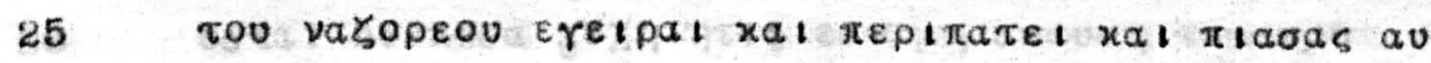

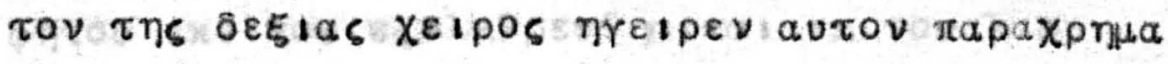

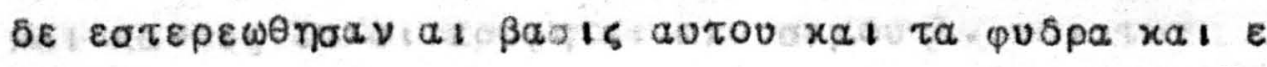

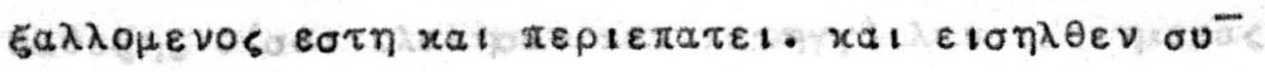

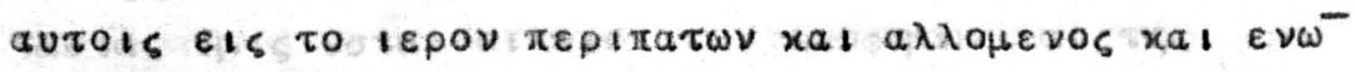

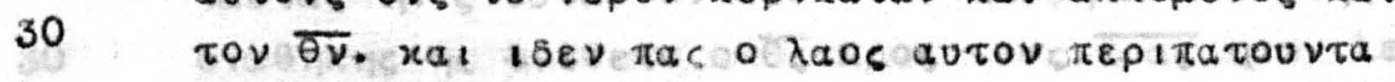

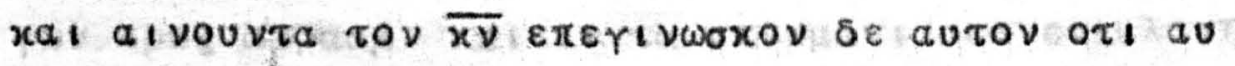

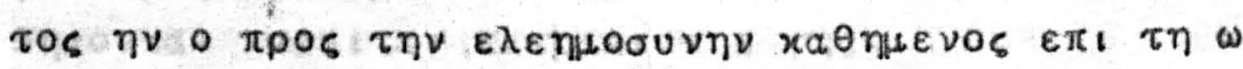

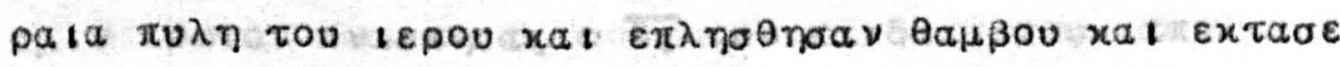

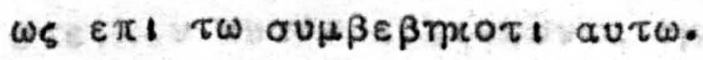

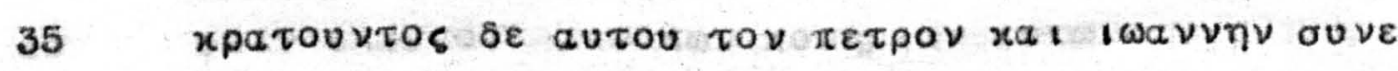

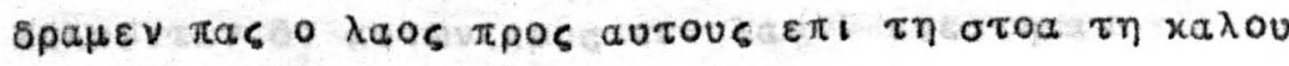

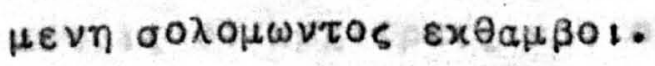

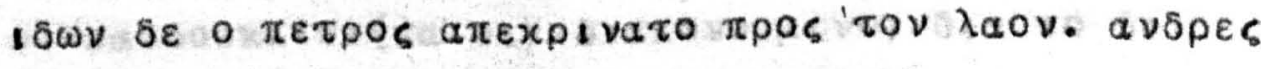

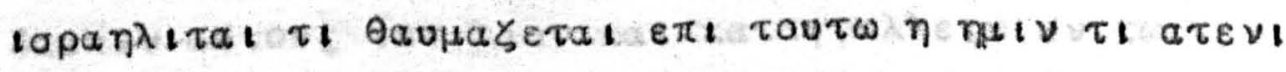

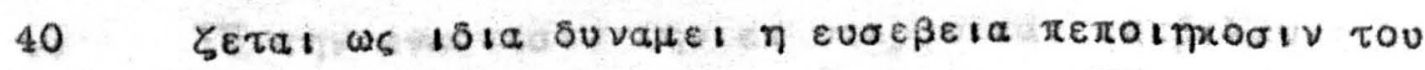

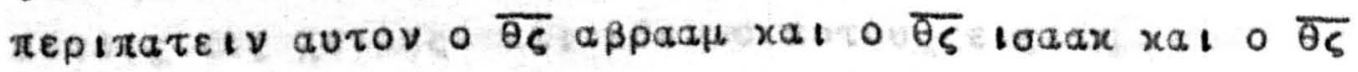




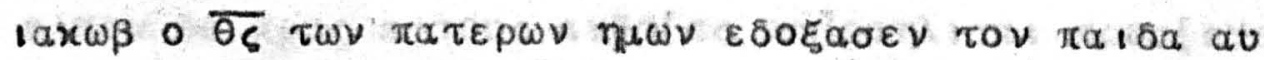

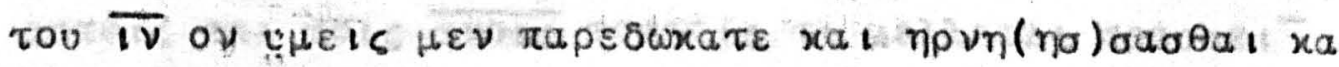

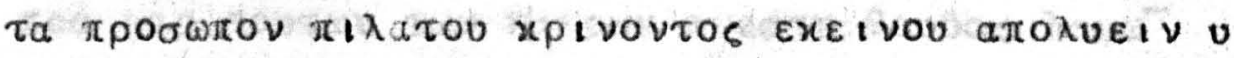

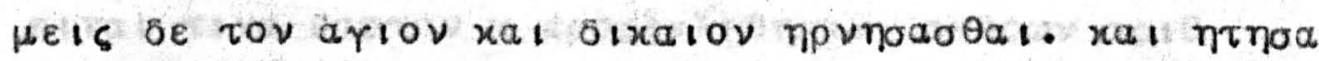

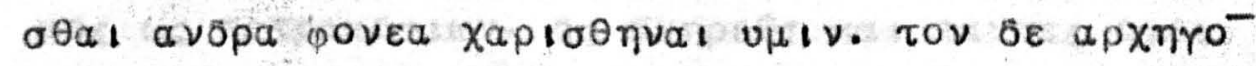

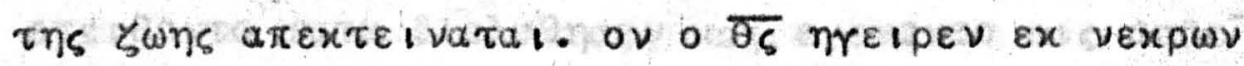

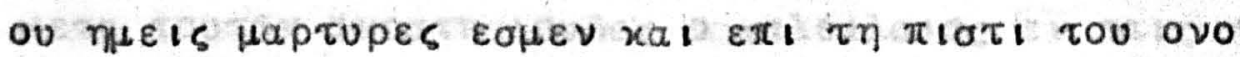

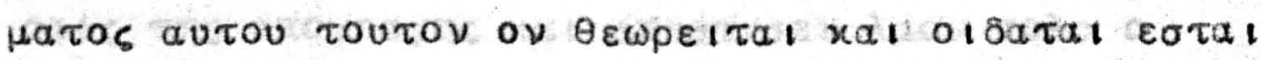

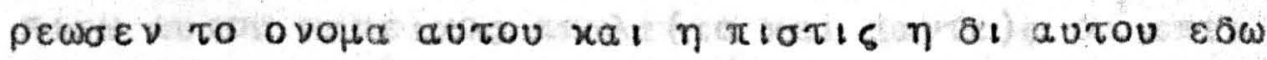

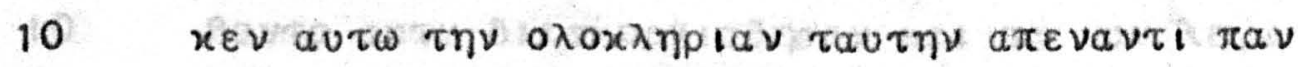

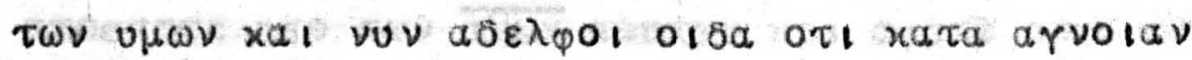

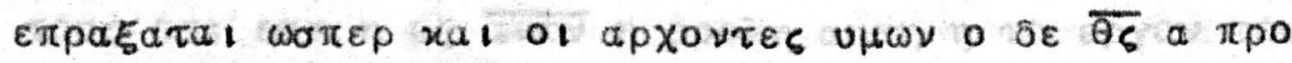

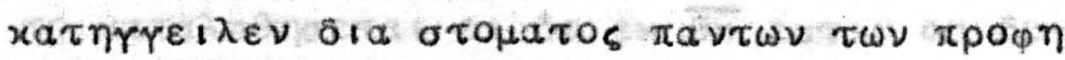

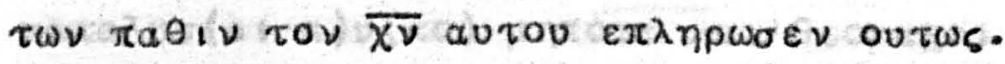

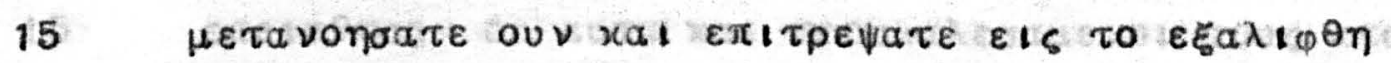

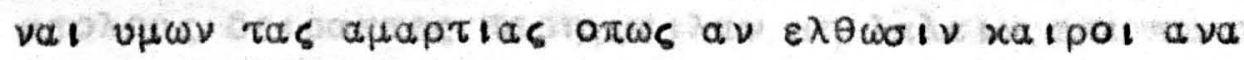

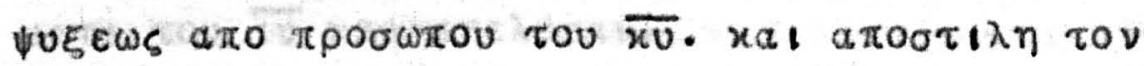

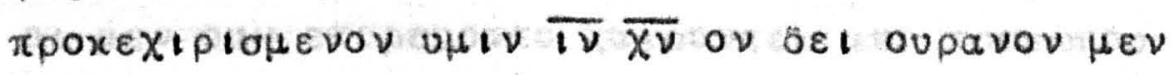

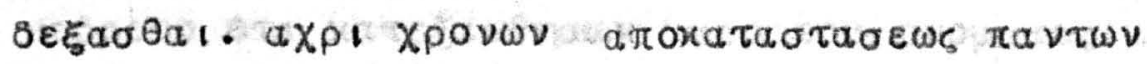

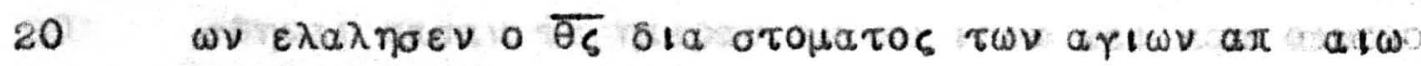

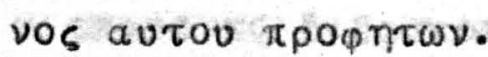

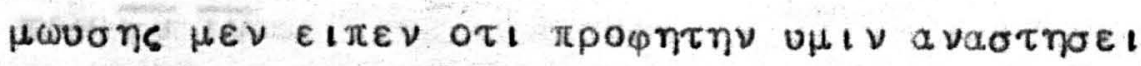

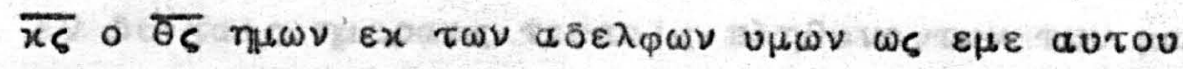

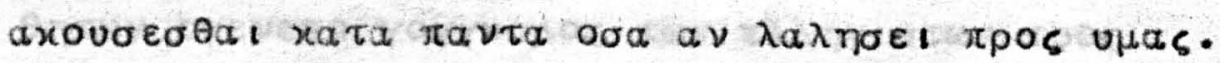

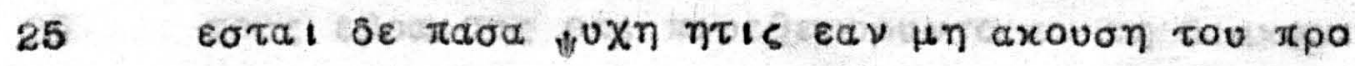

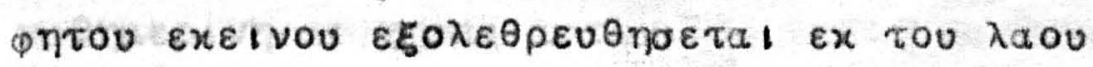

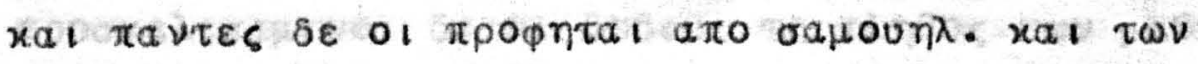

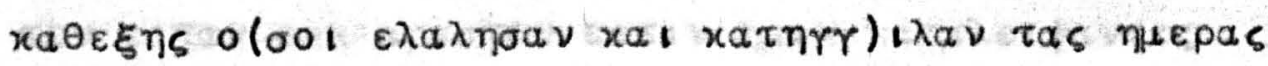

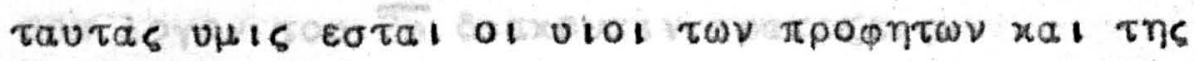

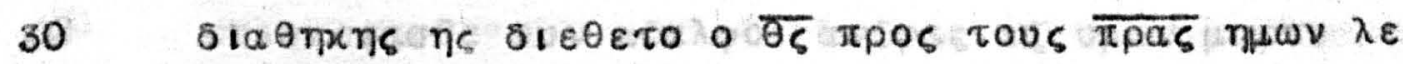

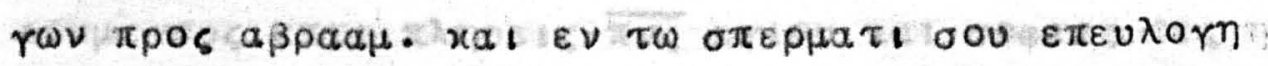

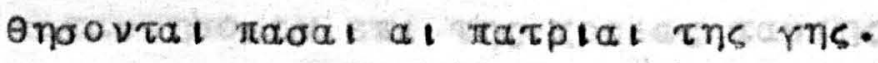

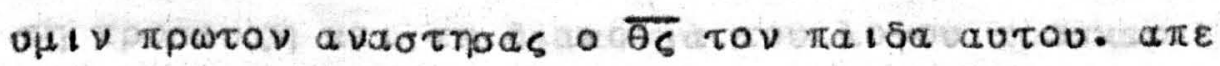

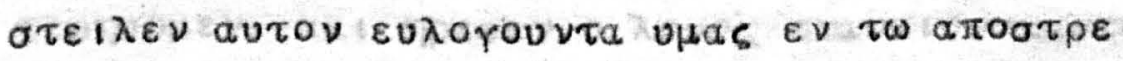

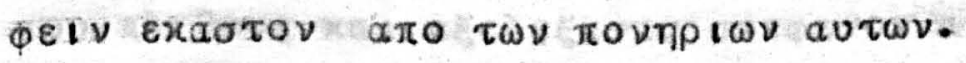

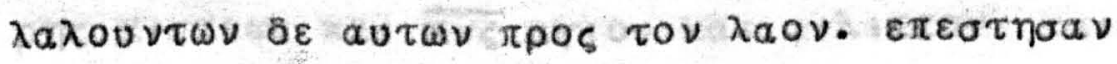

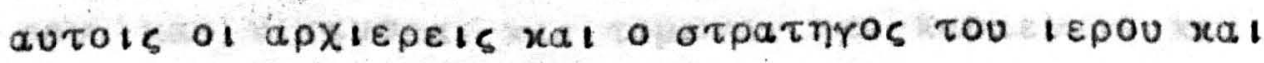
ol $\alpha \alpha \delta \delta \circ x_{\alpha}$

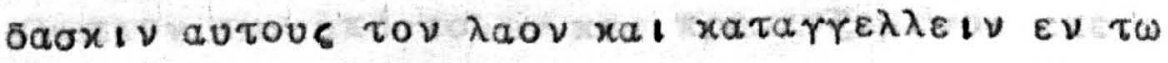

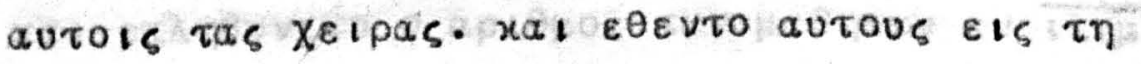




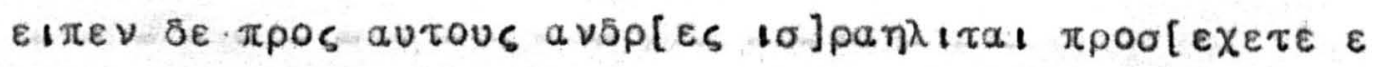

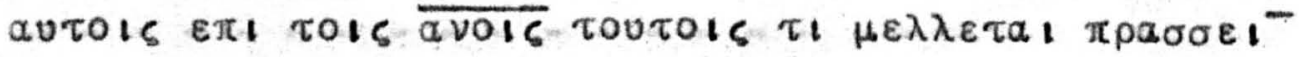

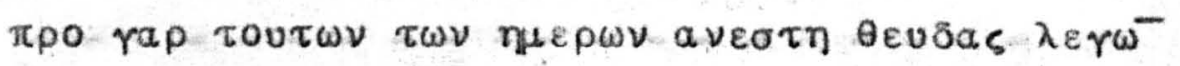

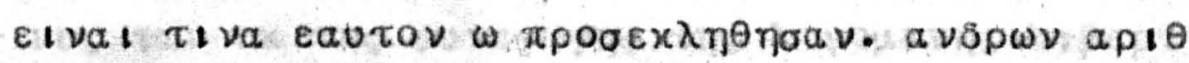

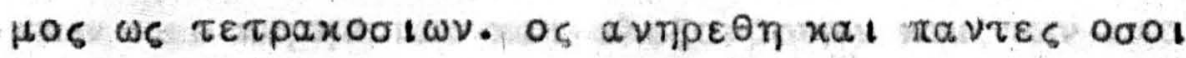

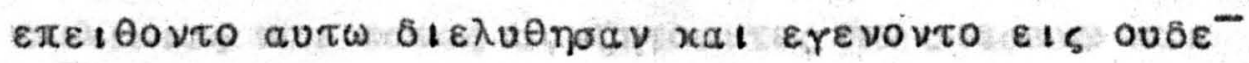

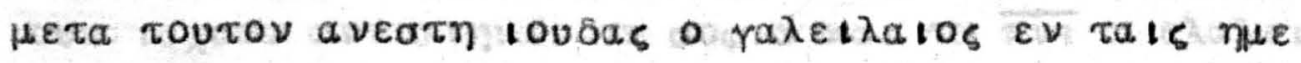

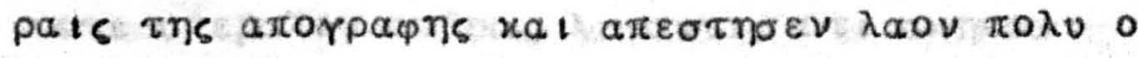

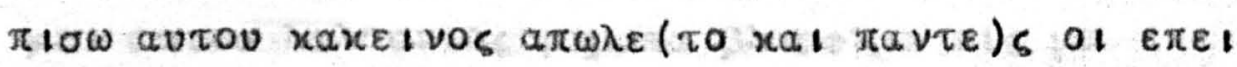

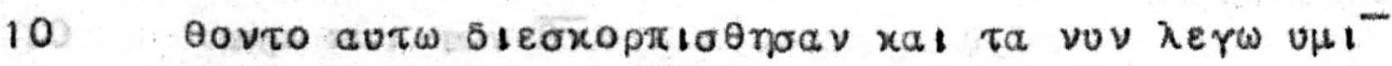

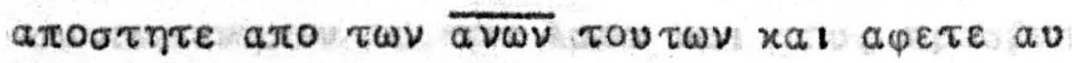

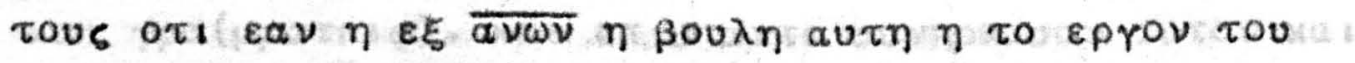

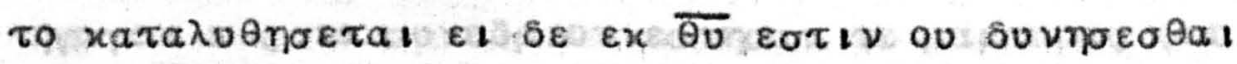

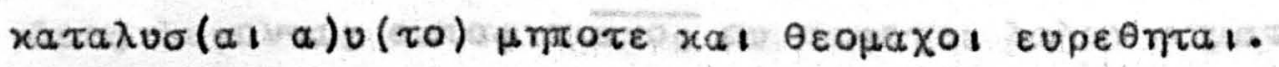

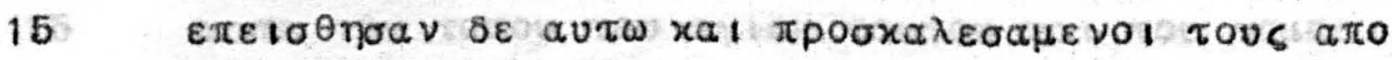

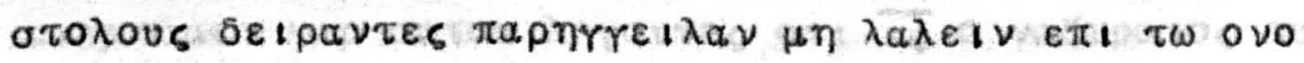

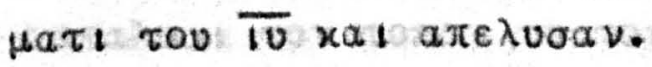

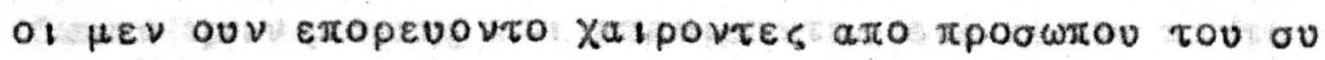

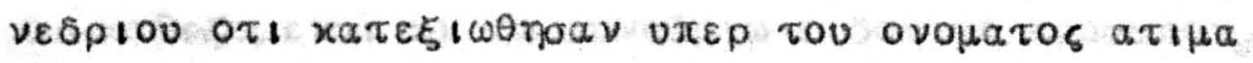

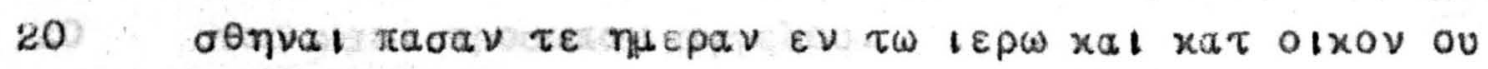

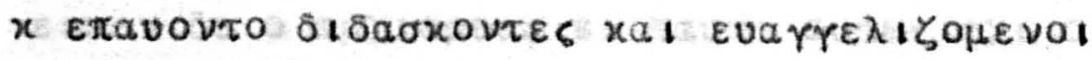
$\operatorname{\tau ov} \overline{\boldsymbol{x} \nu} \overline{\mathrm{TV}}$.

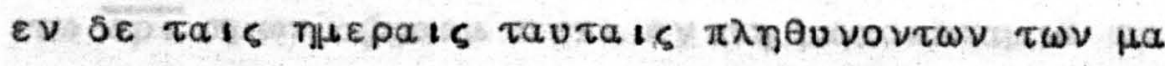

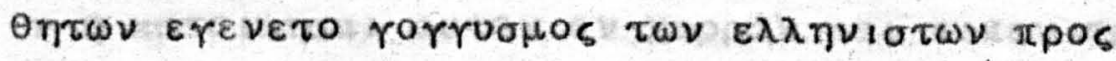

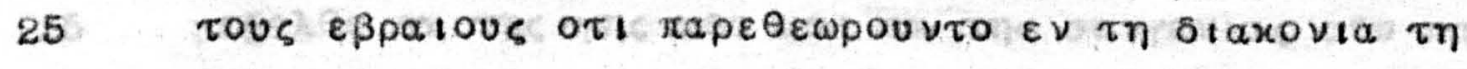

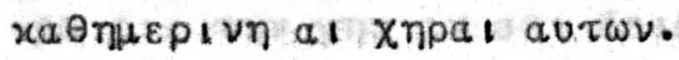

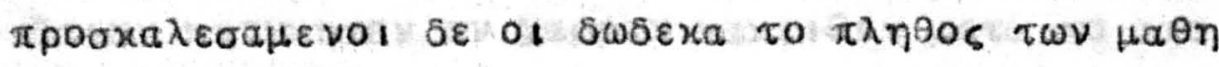

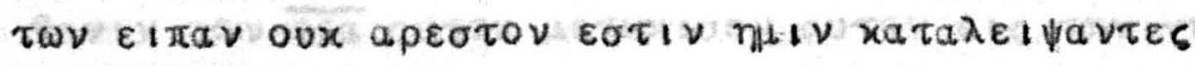

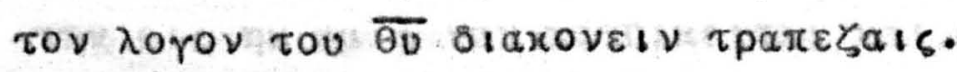

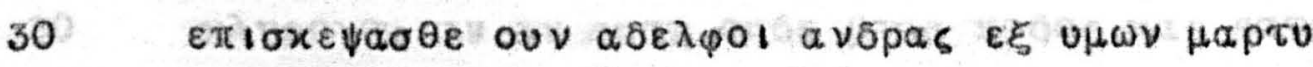

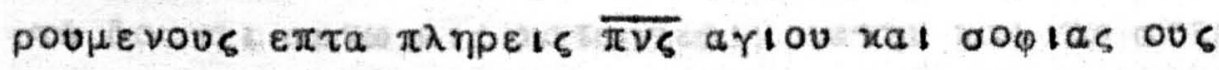

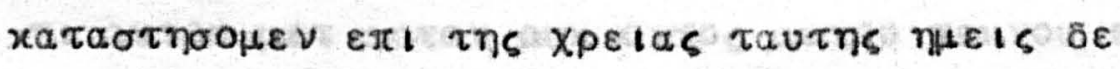

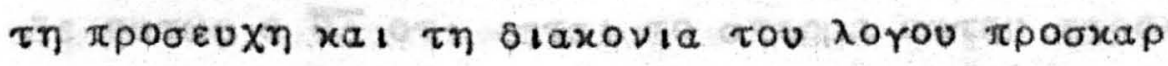

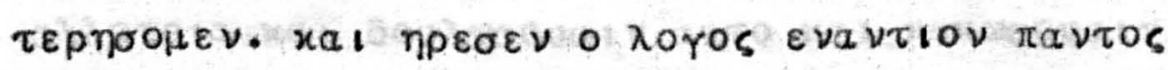

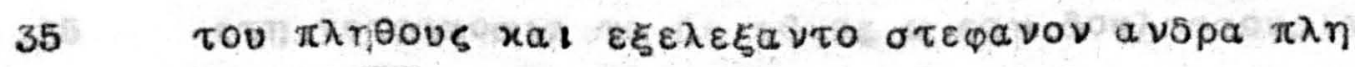

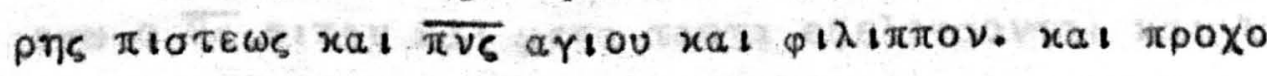
pov. $x a l v i x a v o p a \cdot x a t \tau \imath \mu \omega v \alpha$. xal $\pi \alpha \rho \mu \varepsilon v a v . x a l$

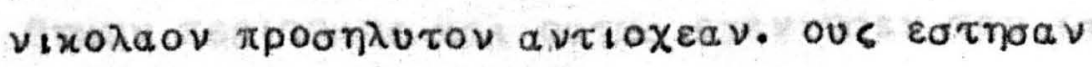

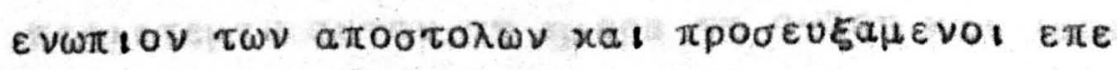

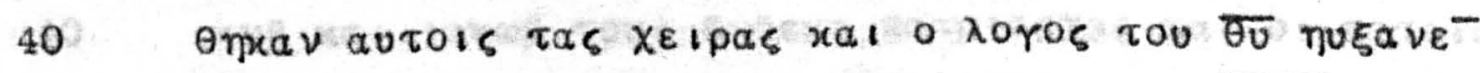

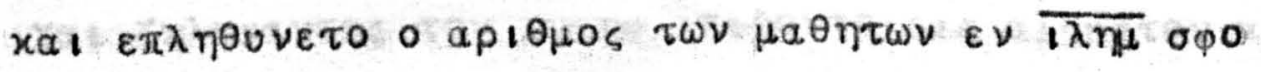




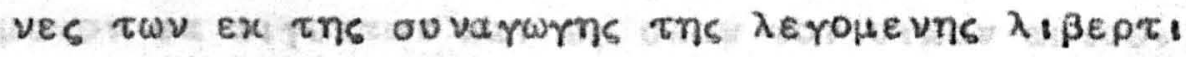

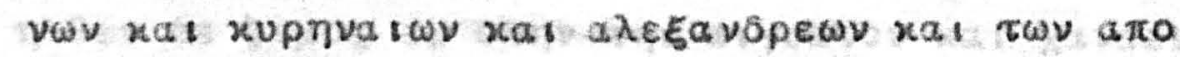

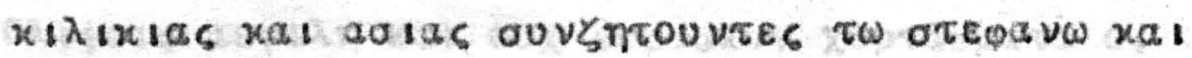

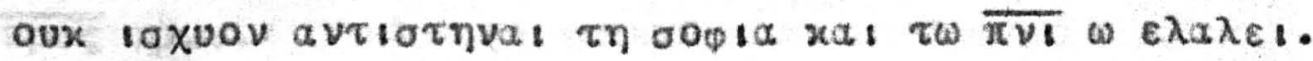

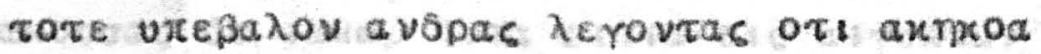

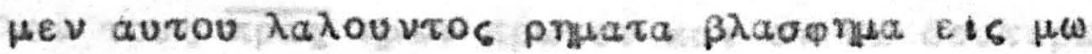

10 vanv xal tov $\overline{\theta \nu}$.

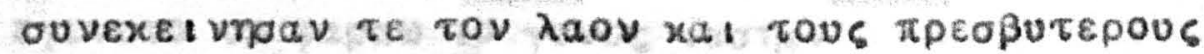

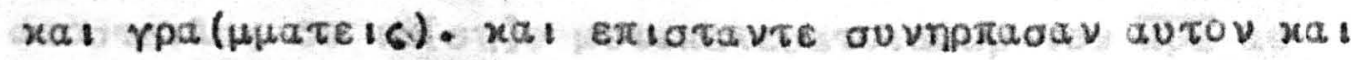

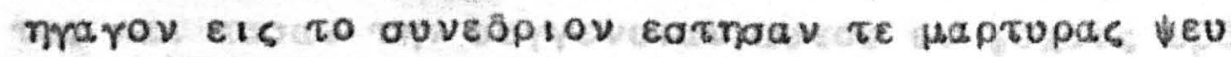

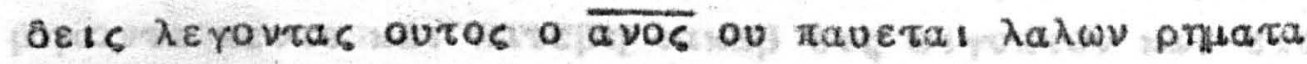
15 nata tou totou tou ariou toutou nat tou vouor.

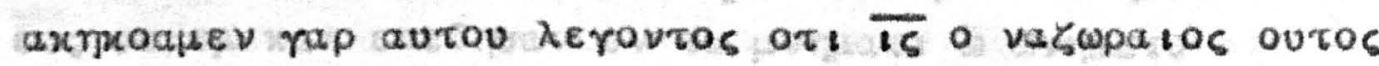

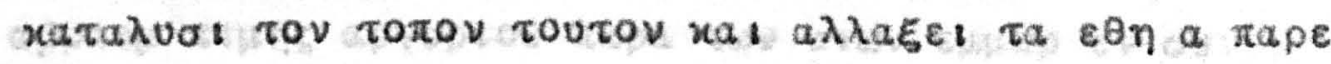

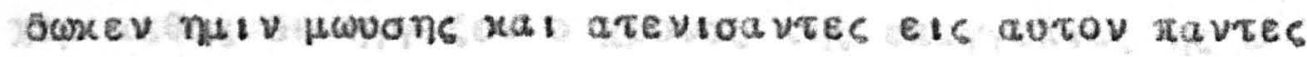

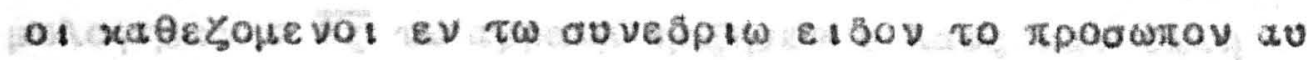
20 Tov was r toowiov arrexov.

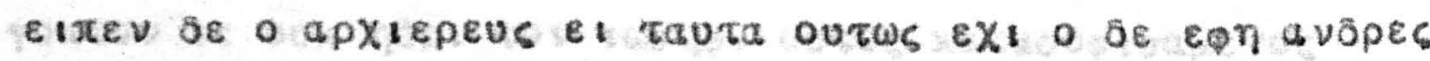

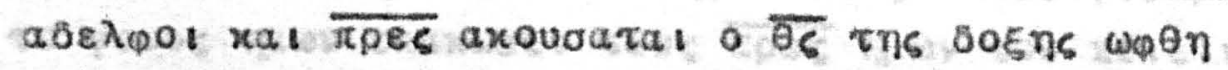

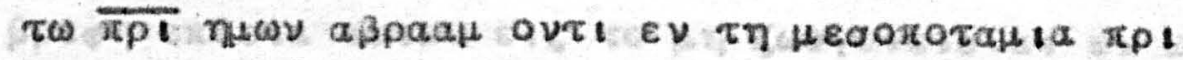

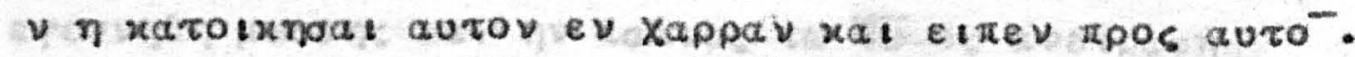

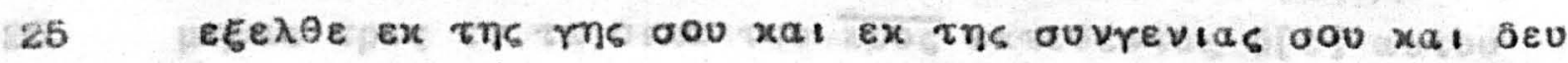

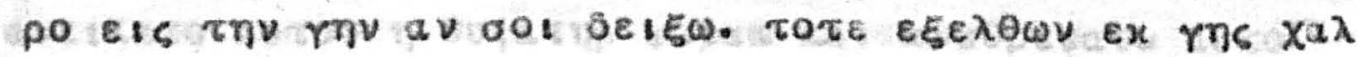

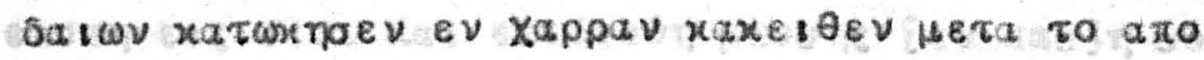

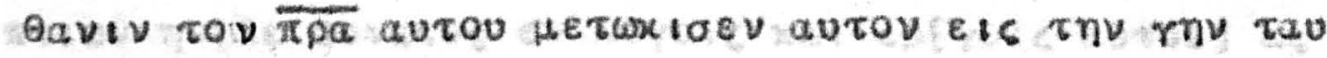

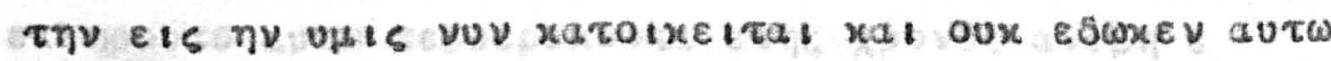

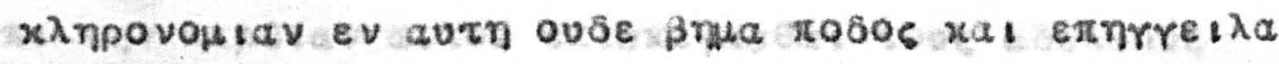

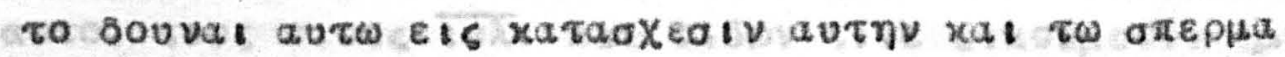

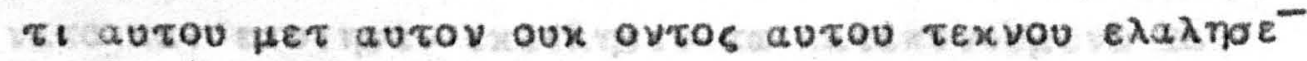

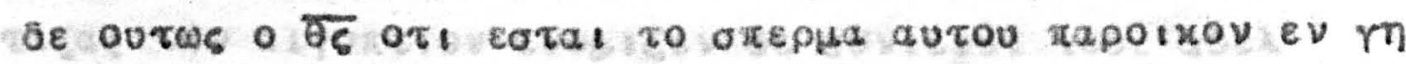

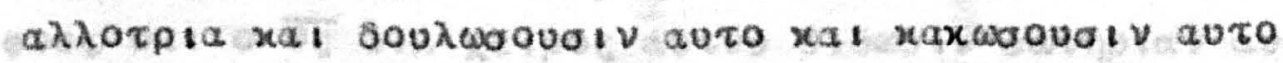

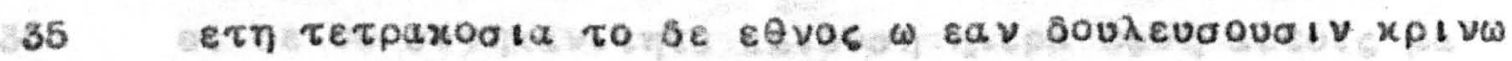

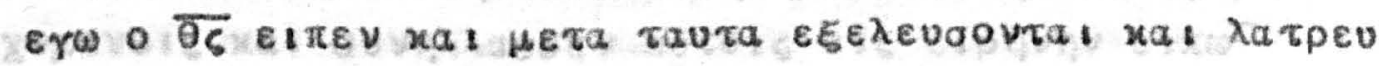

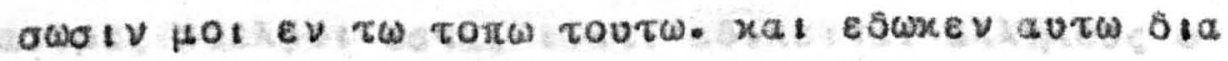

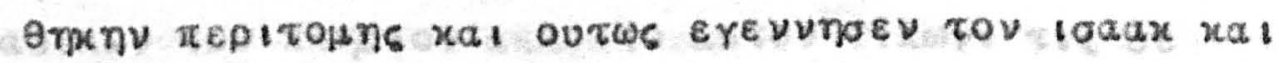

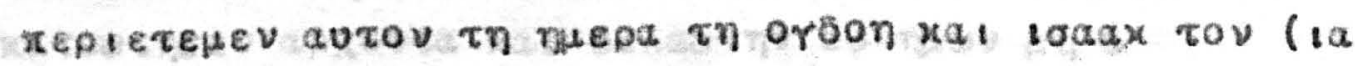

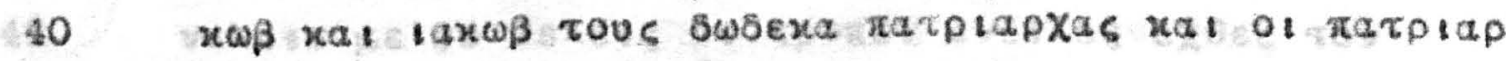

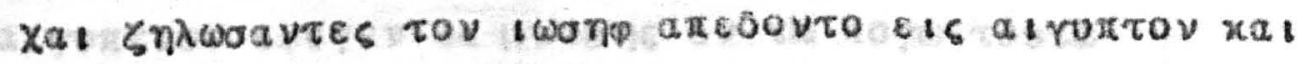




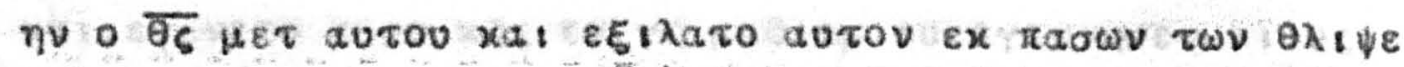

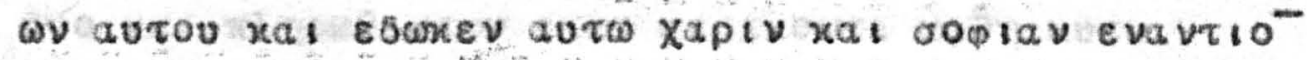

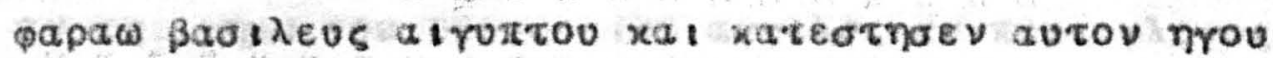
Hevov $\varepsilon \pi$ airutrov xal $\varepsilon$ oxov tov oixov autov.

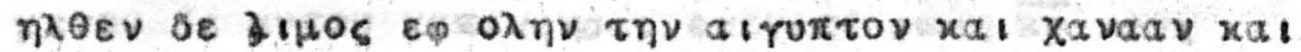

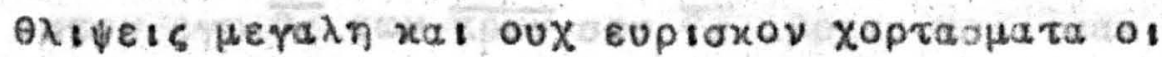
$\overline{\pi p e s}$ nuwv.

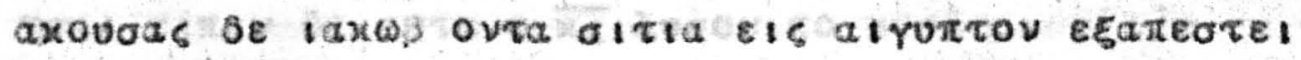

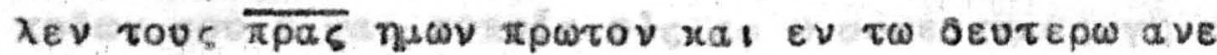

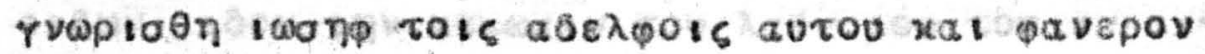

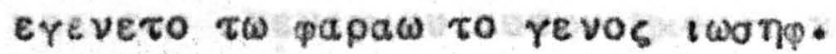

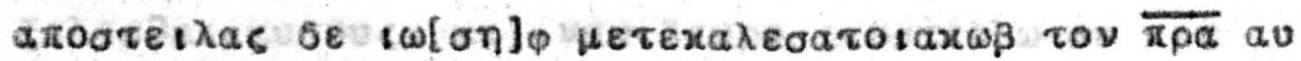

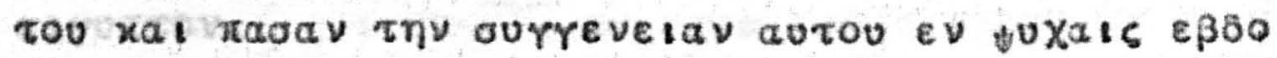

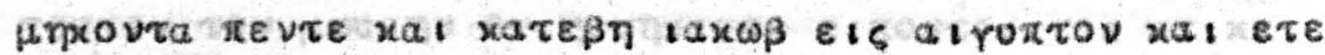

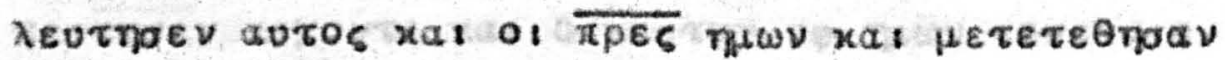

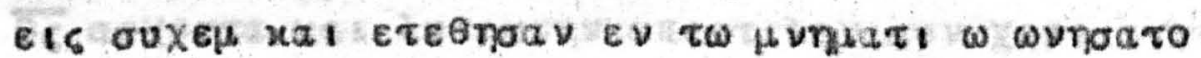

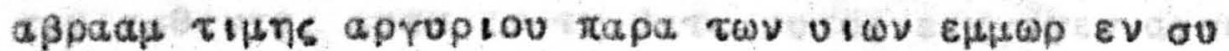

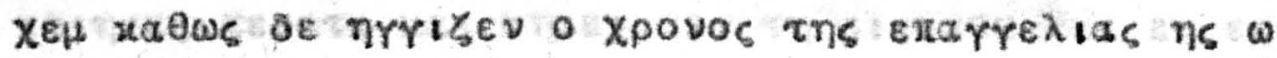

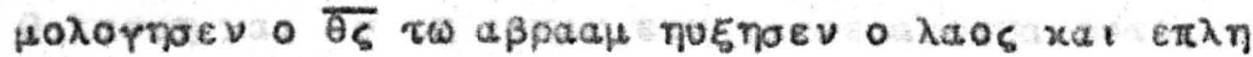

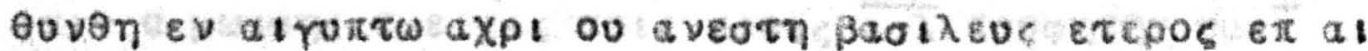

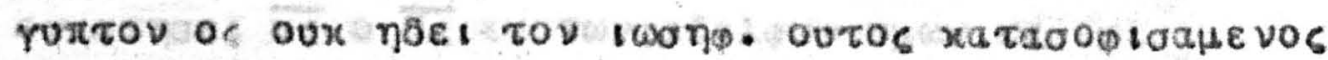

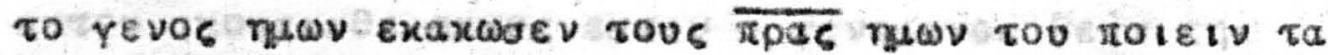

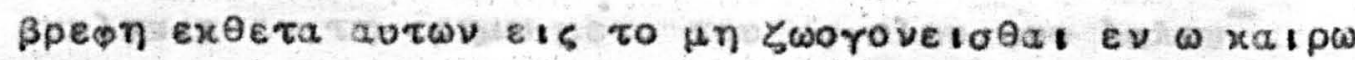

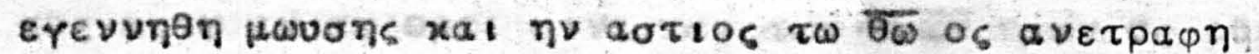

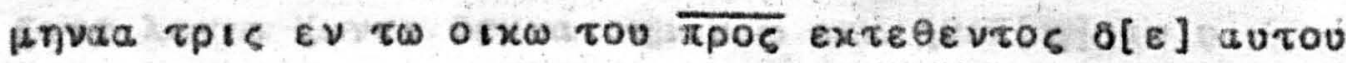

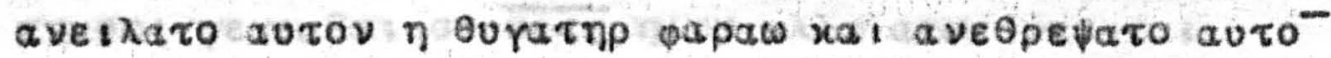

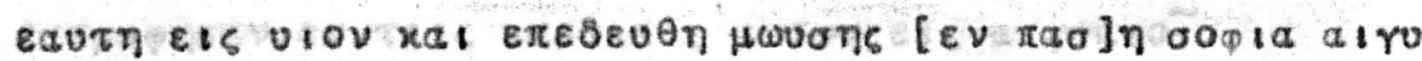

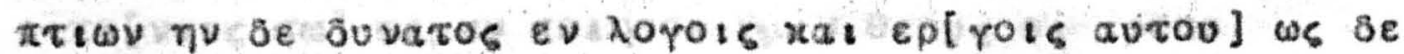

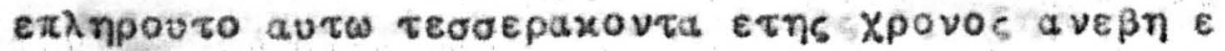

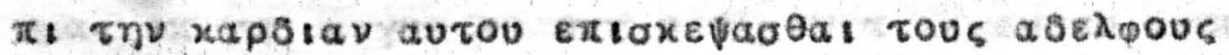

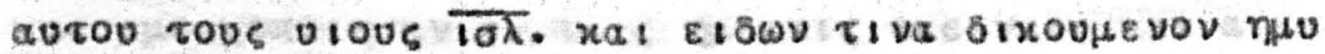

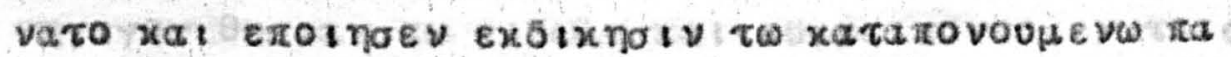

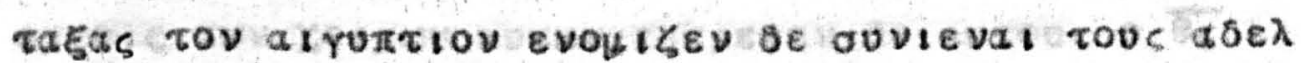

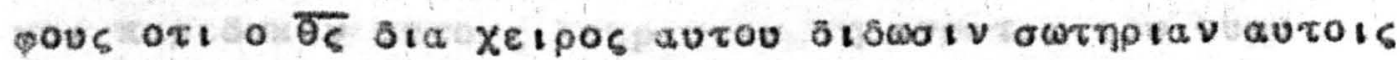

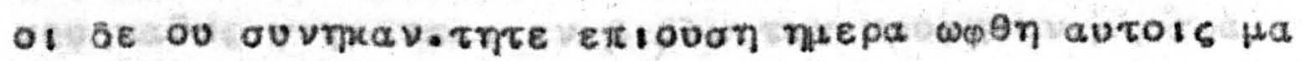

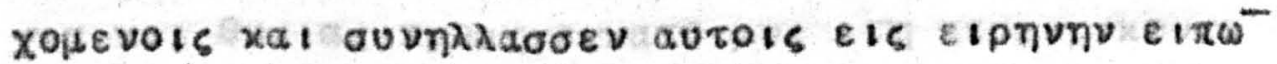

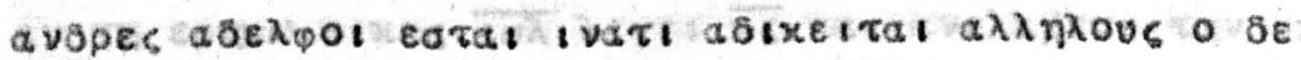

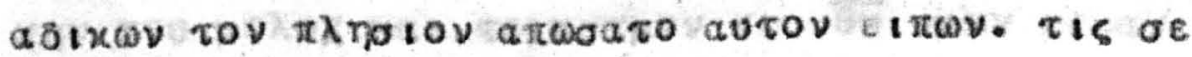

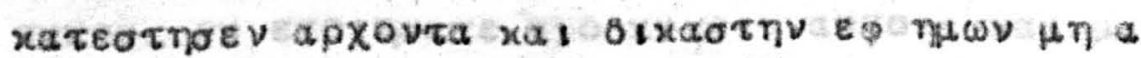

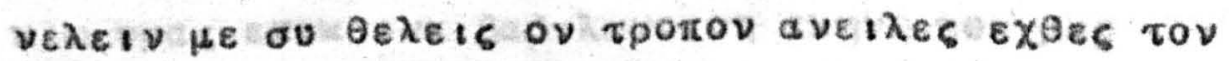
airuteriov $\varepsilon$ puyev $\delta \varepsilon \mu$ wo 


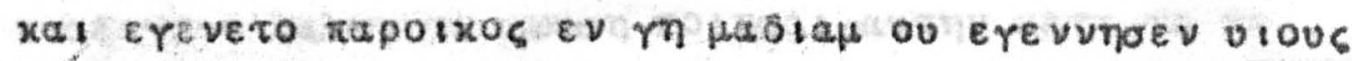

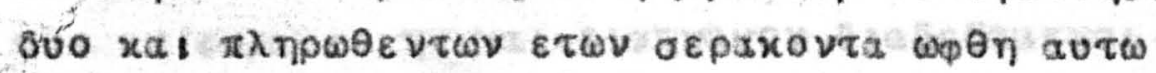

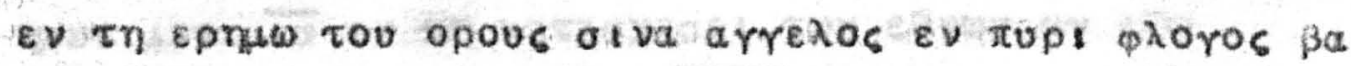

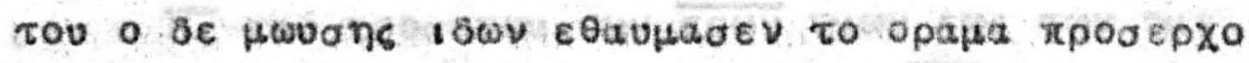

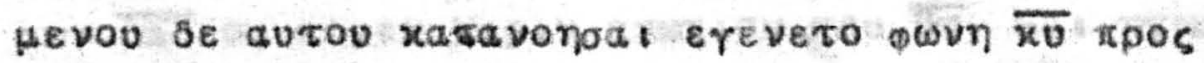

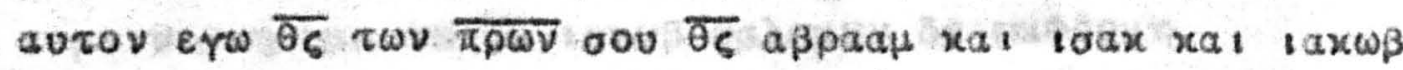

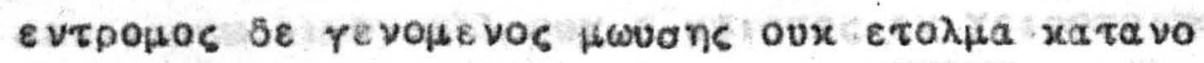

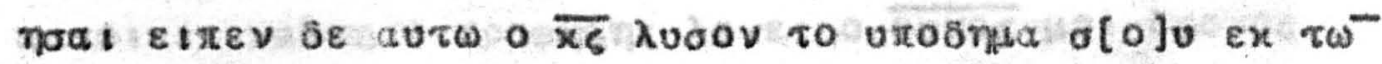

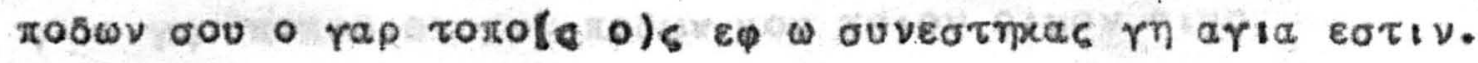

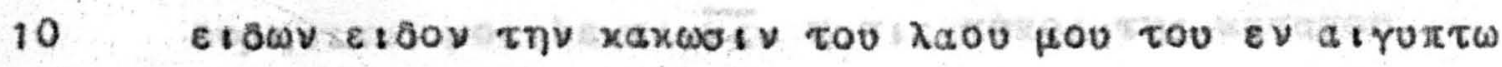

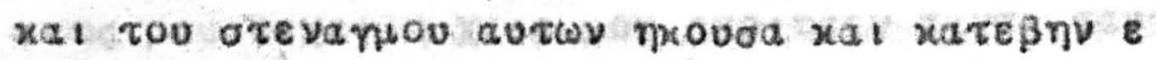

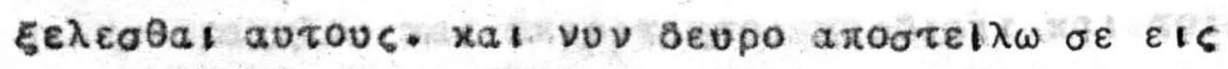
airutiov.

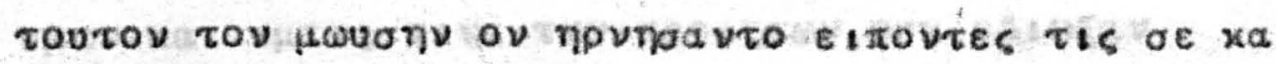

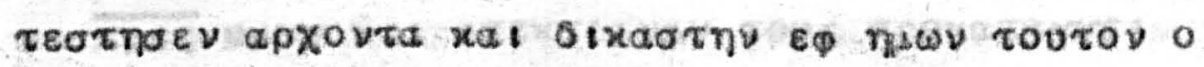

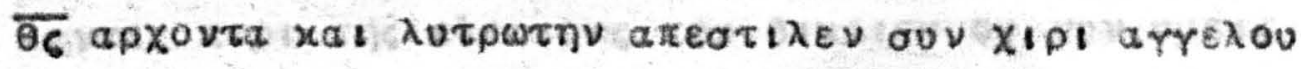

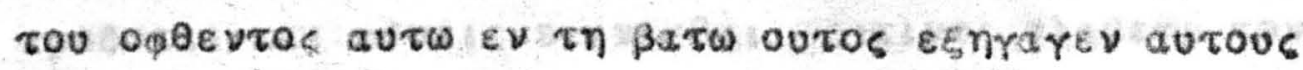

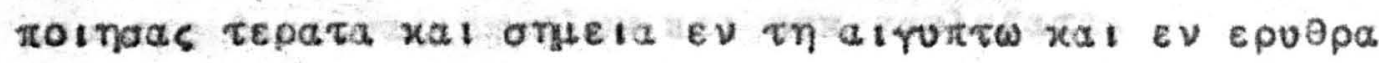

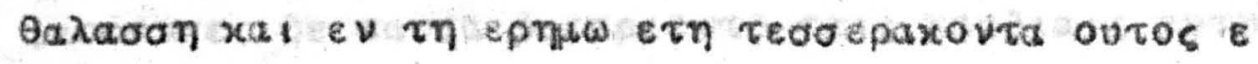

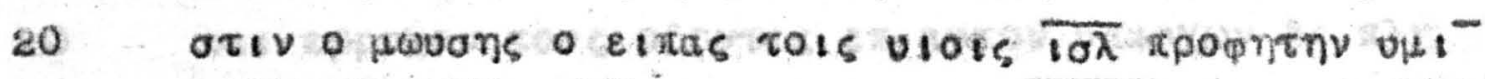

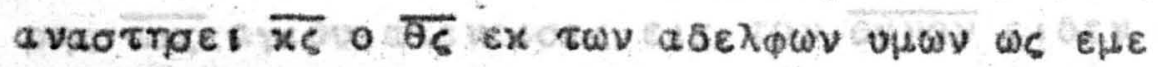

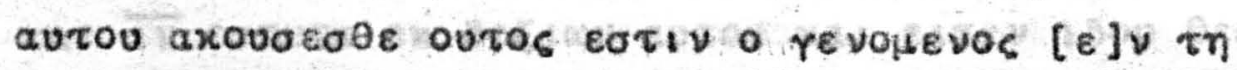

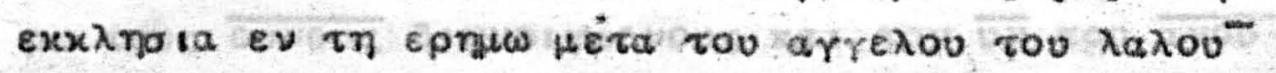

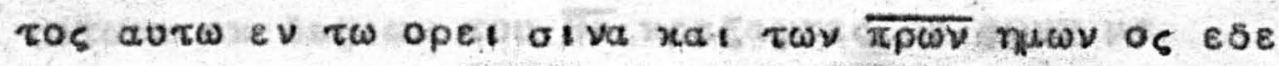

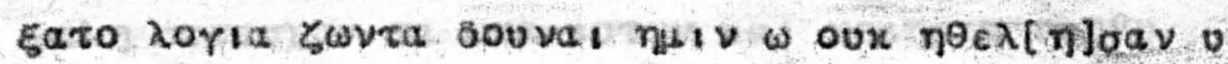

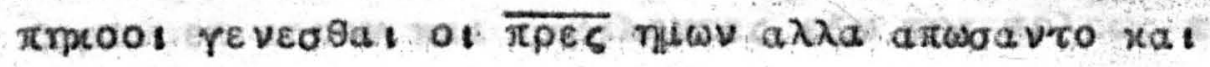

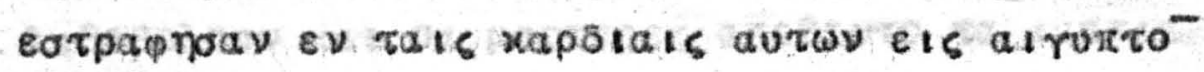

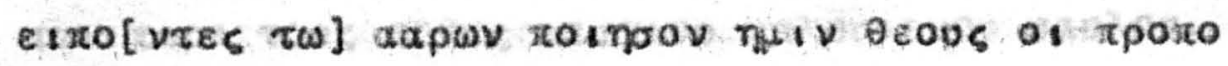

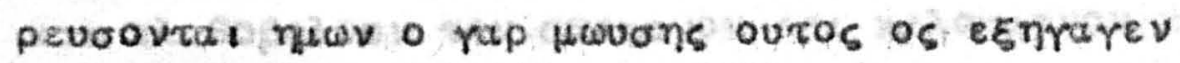

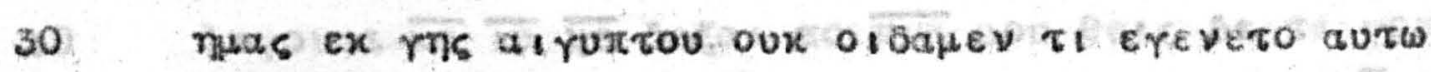

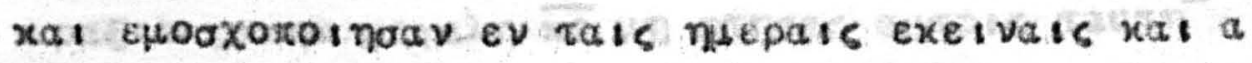

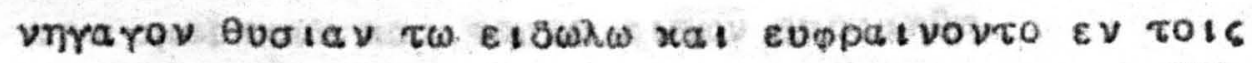

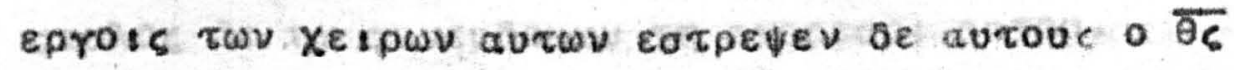

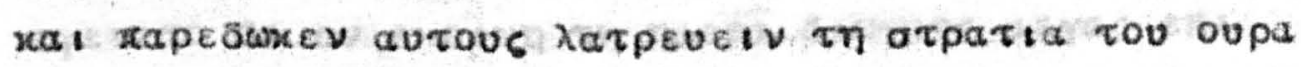

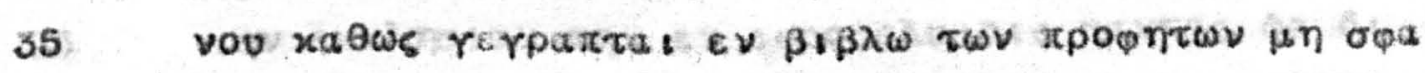

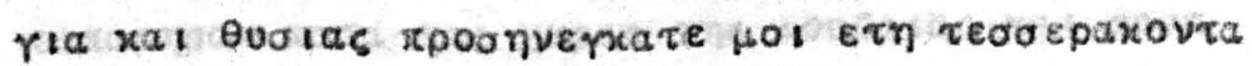

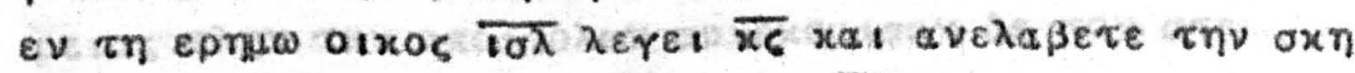

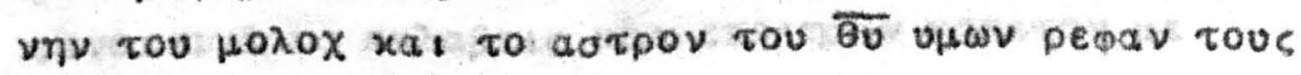

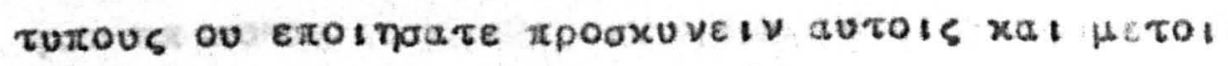

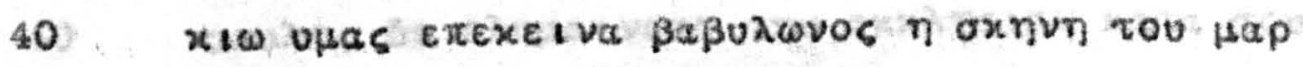

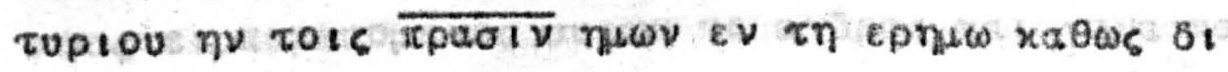




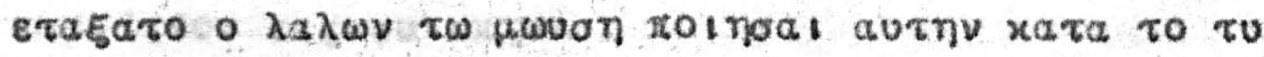

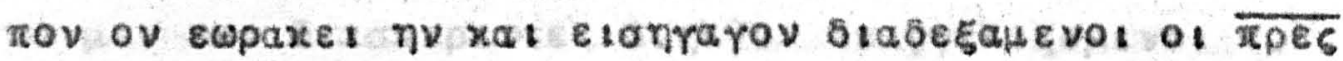

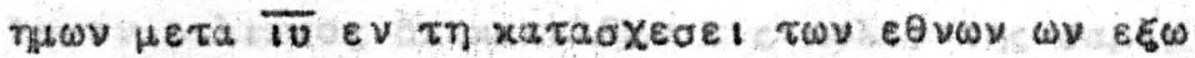

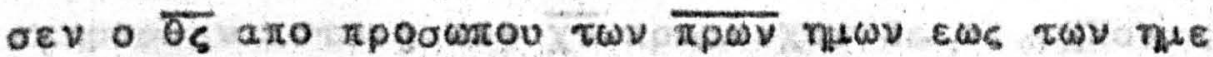

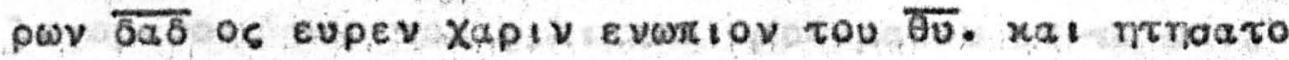

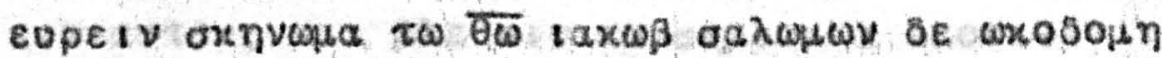

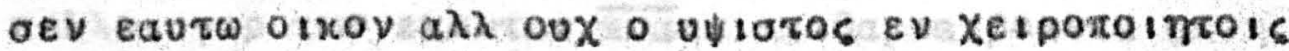

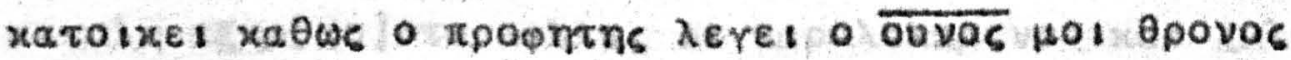

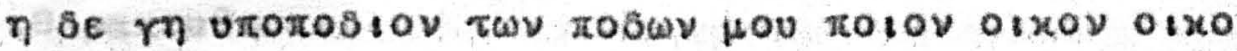

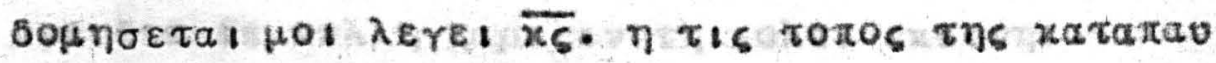

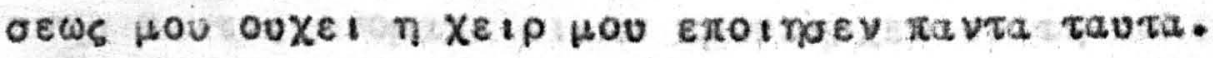

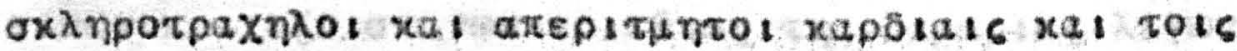

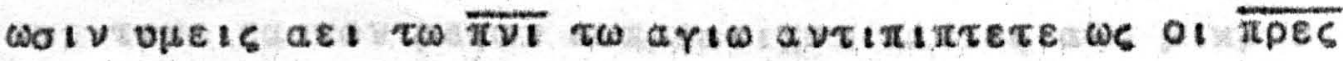

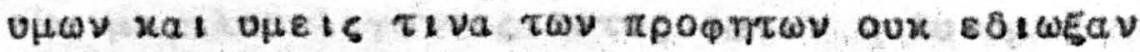

15 of $\overline{\pi p e s}$ unwv xal axexte Ivay rous xpoxararre!

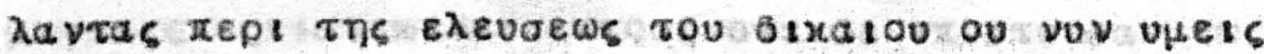

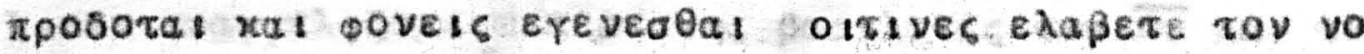

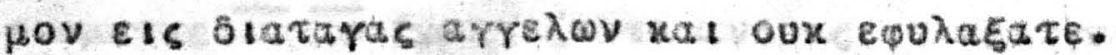

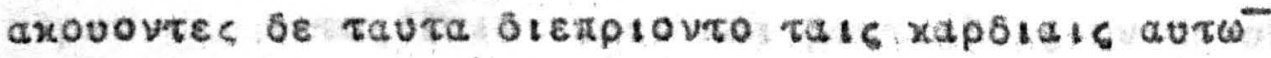

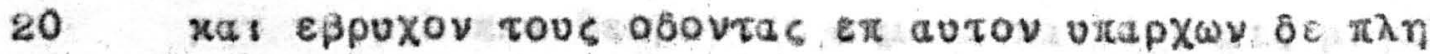

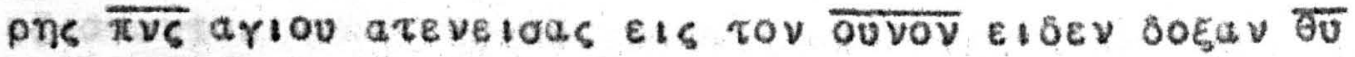

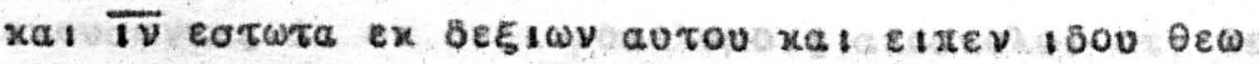

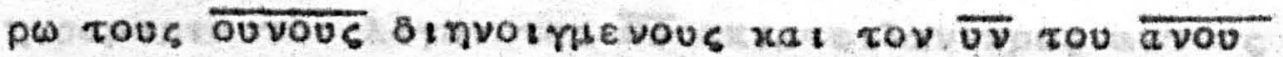

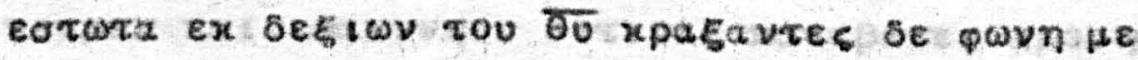

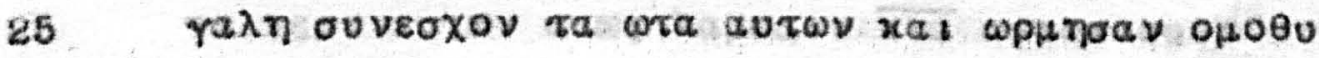

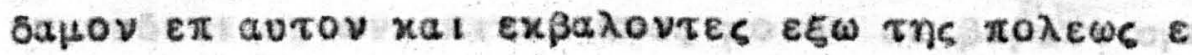

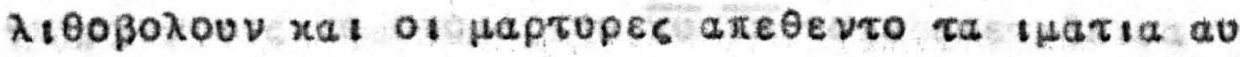

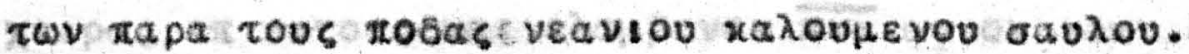

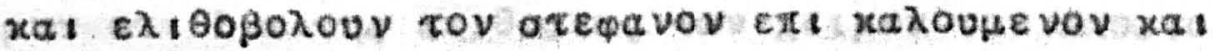

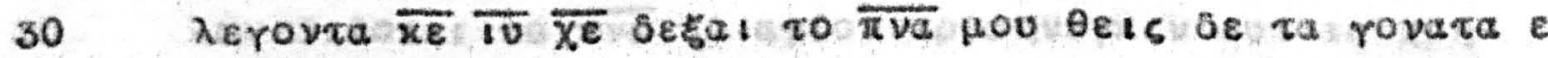

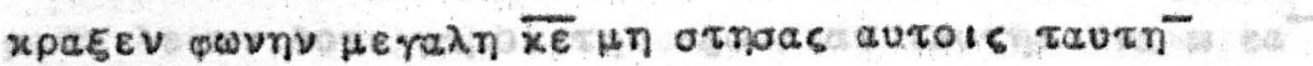

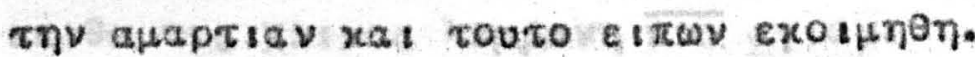

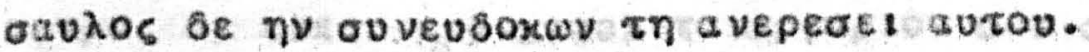

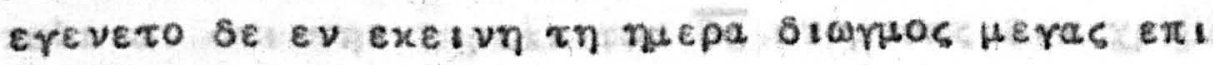

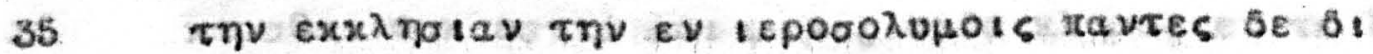

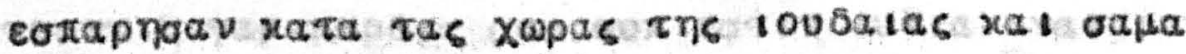

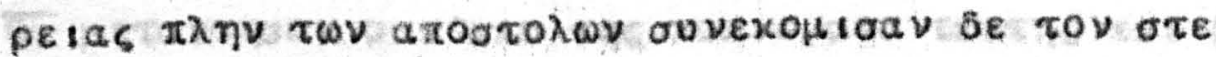

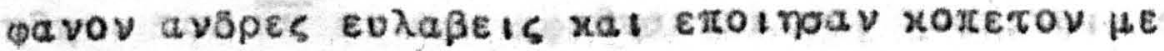
rav $\varepsilon \pi$ avtw.

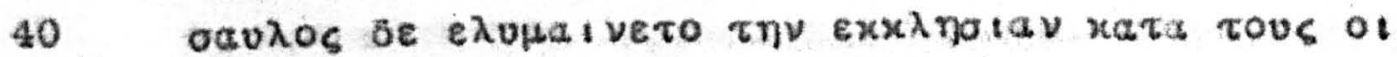

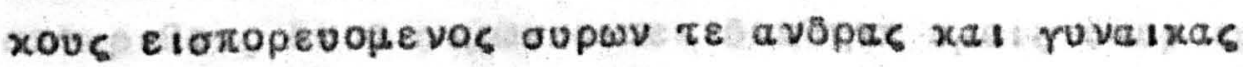




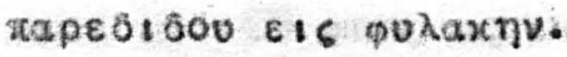

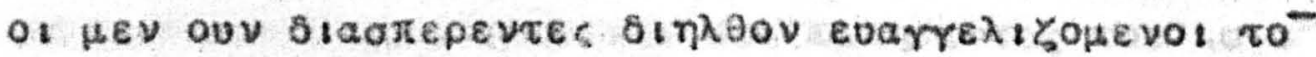

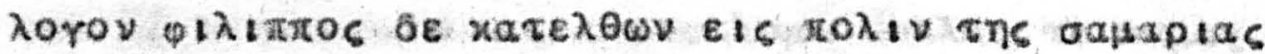

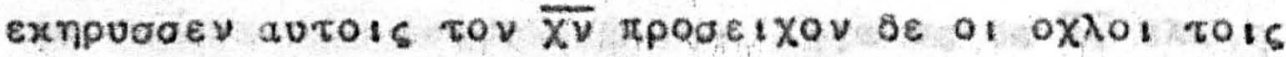
Aeropevols oro tov

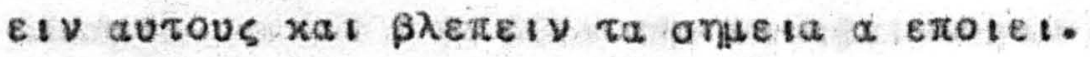

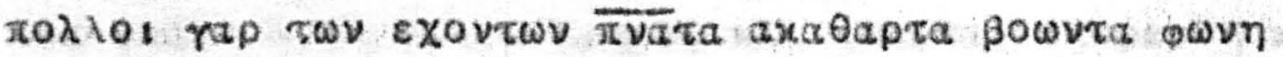

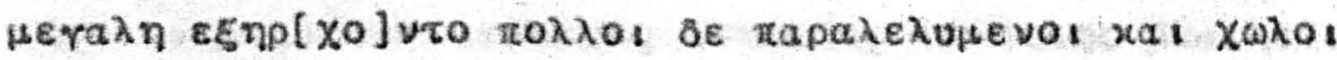
$\varepsilon \theta \varepsilon p a \pi \varepsilon v \theta$ noav.

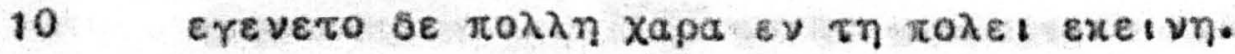

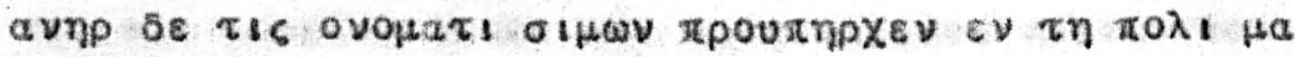

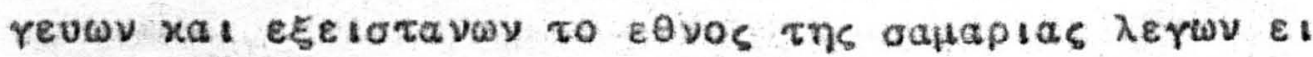

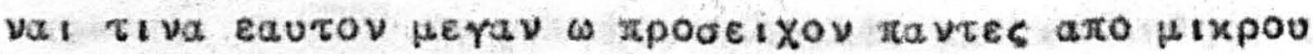

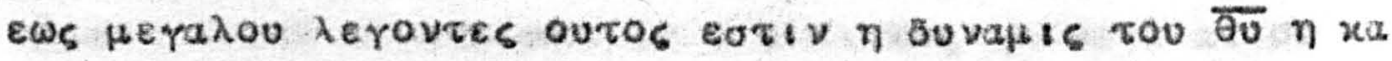

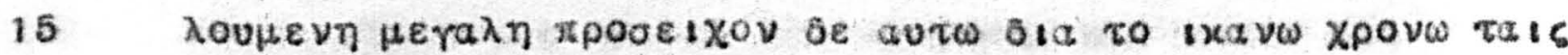

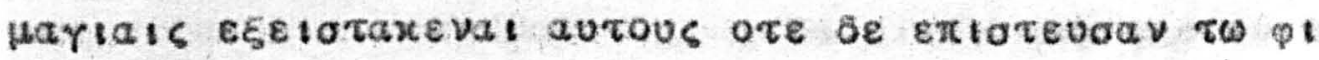

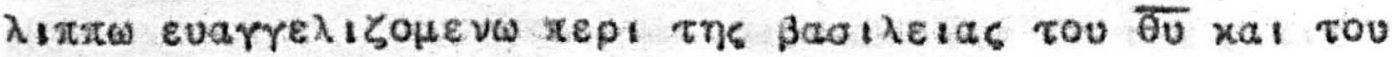

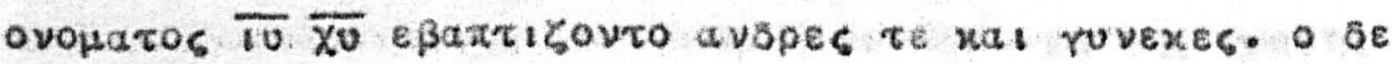

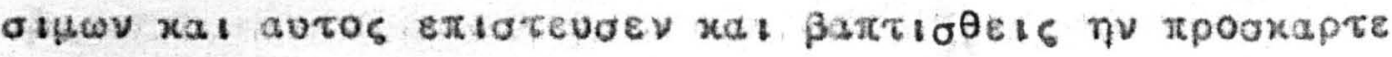

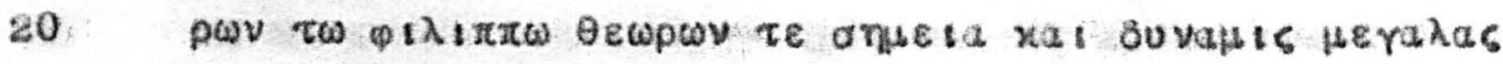

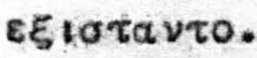

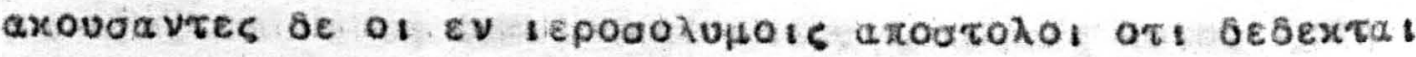

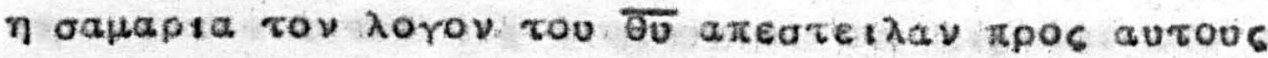

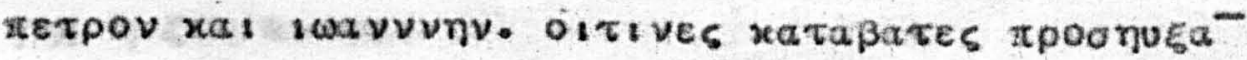

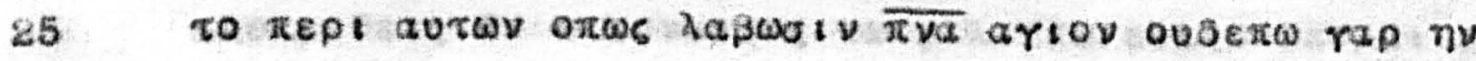

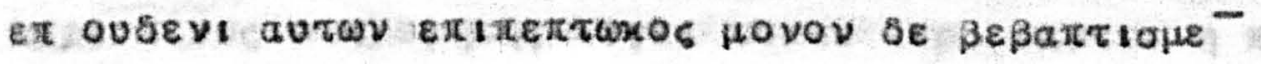

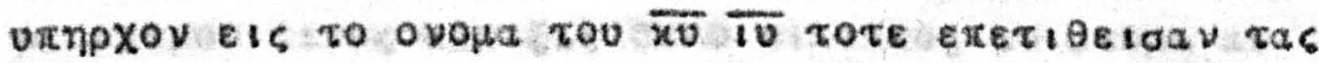

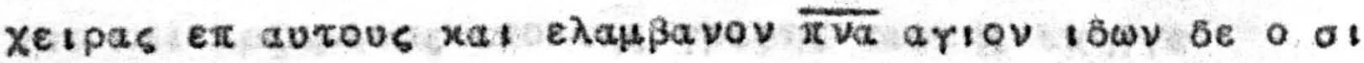

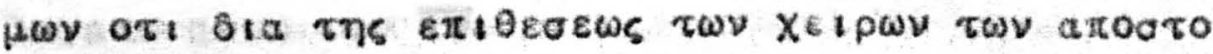

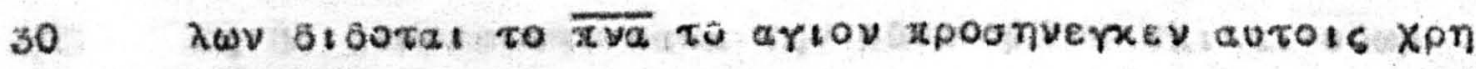

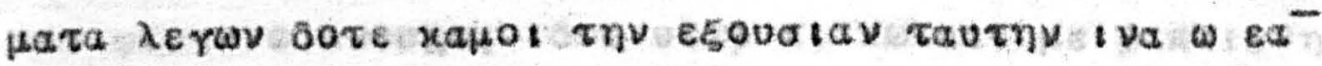

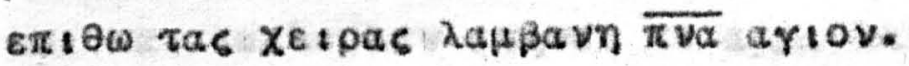

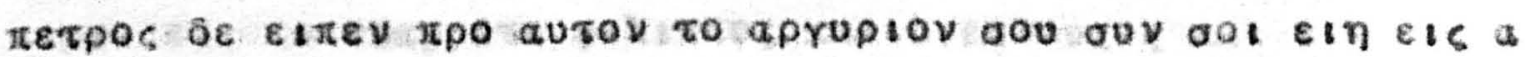

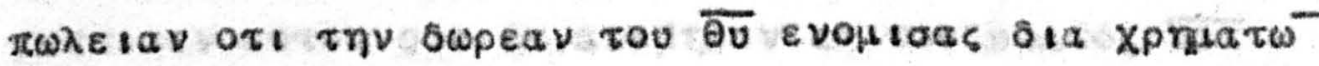

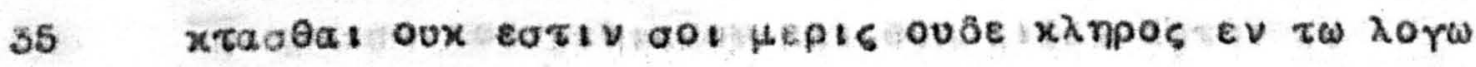

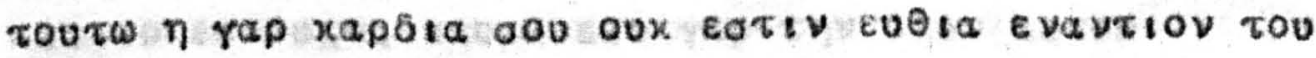

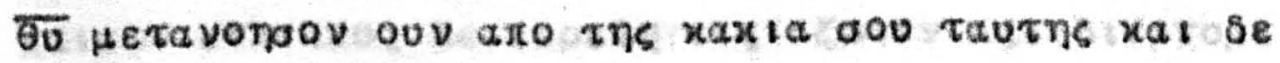

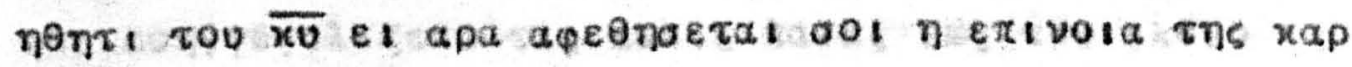

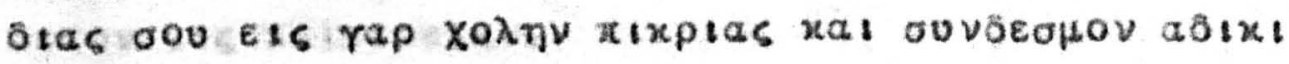

40 as opw oe ovta.

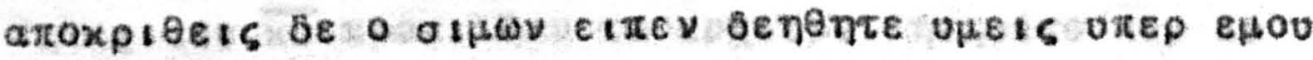




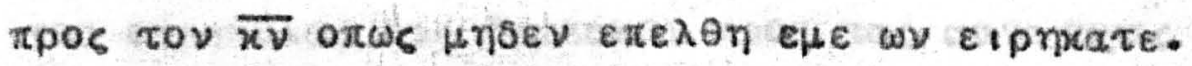

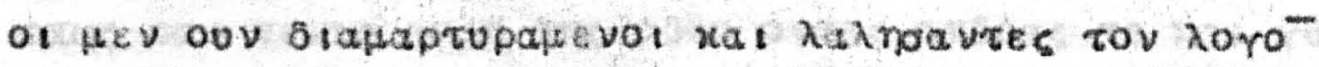

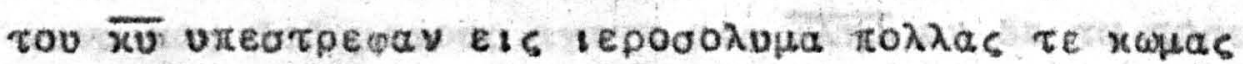

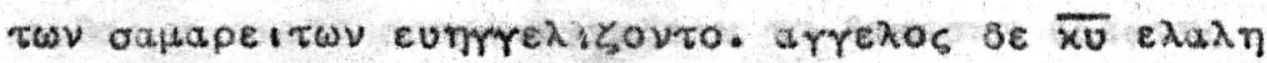

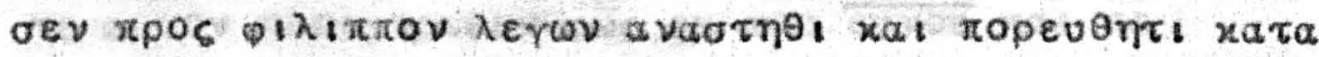

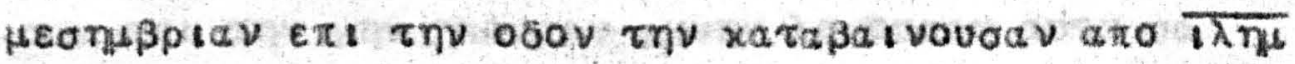

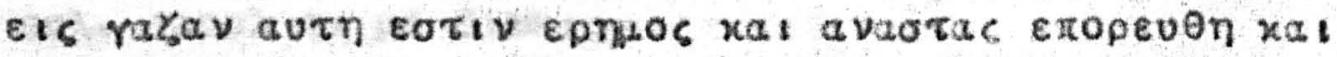

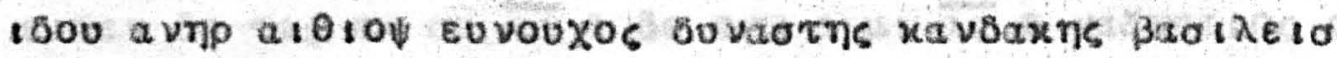

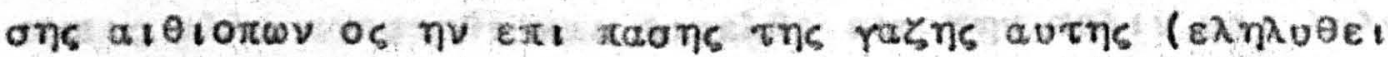

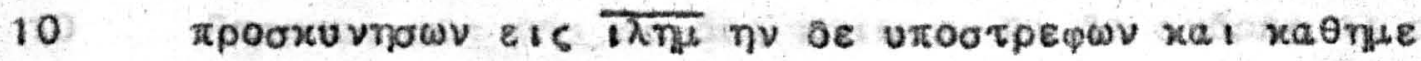
vos ex! aphatog autou $x a t$ a vereivwoxev noalay rov

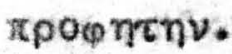

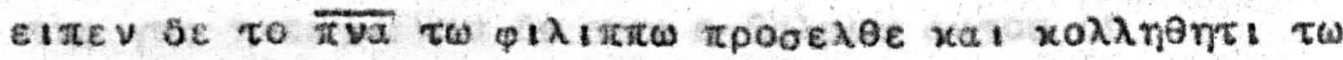

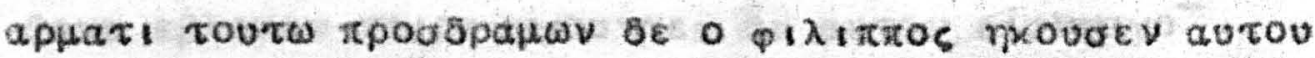

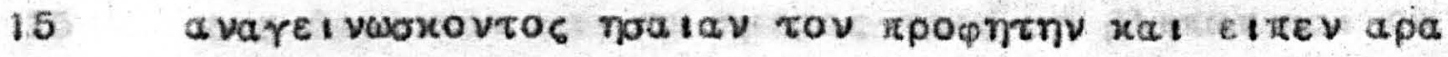

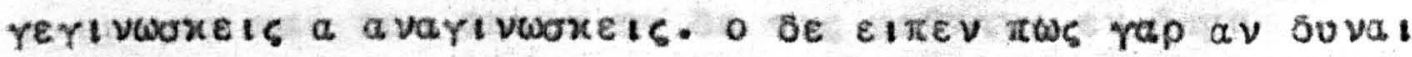

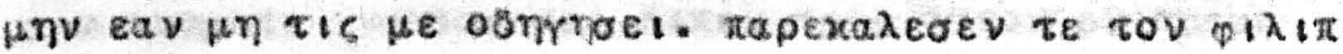

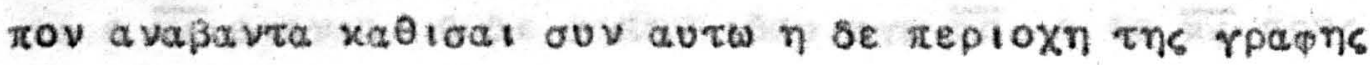

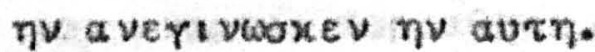

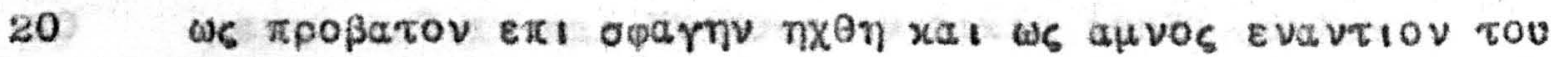
xeipavtos autov apavos outwe oux avotrel to oto

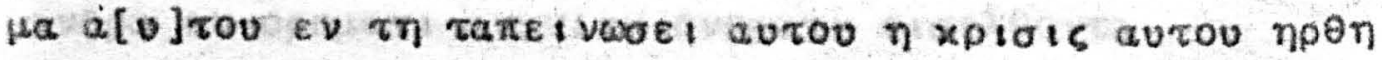

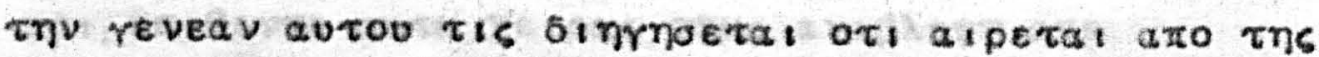

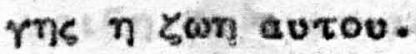

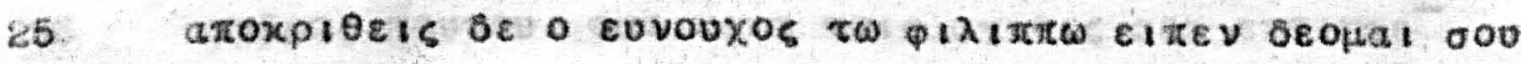

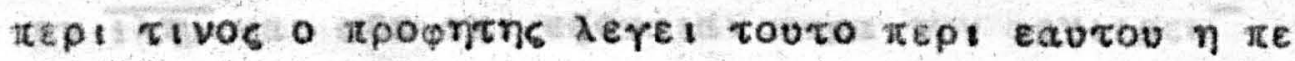

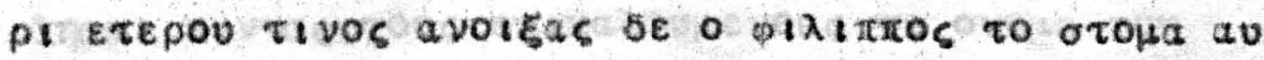

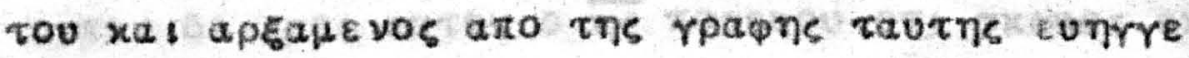

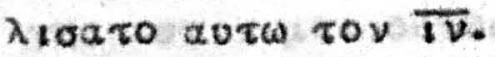

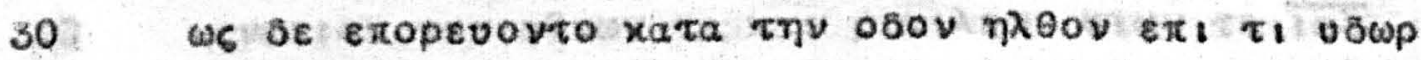

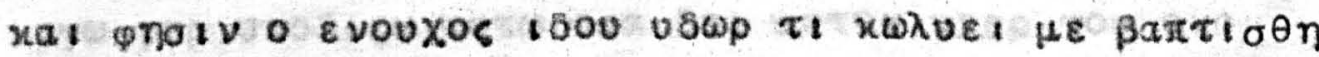

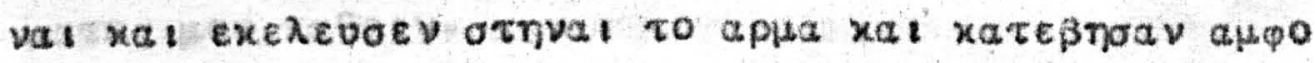

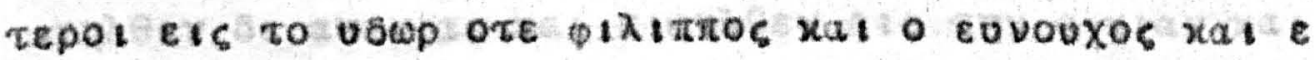

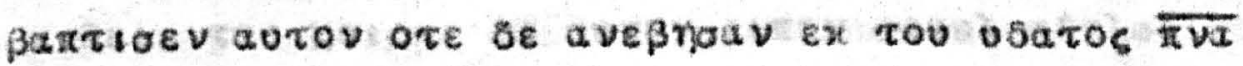

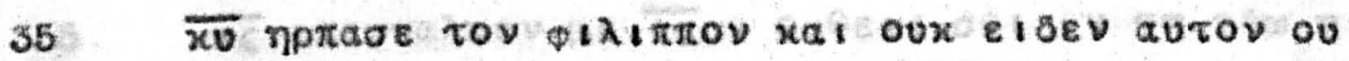

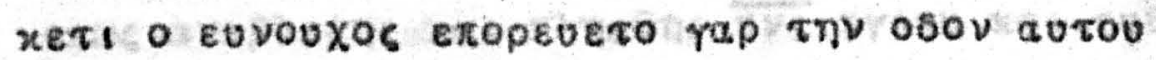
Xaipav

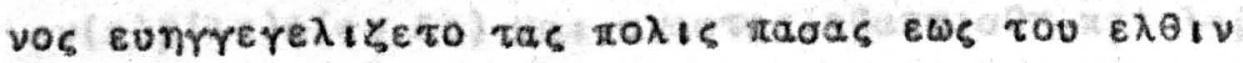
autoveis xatoapelav.

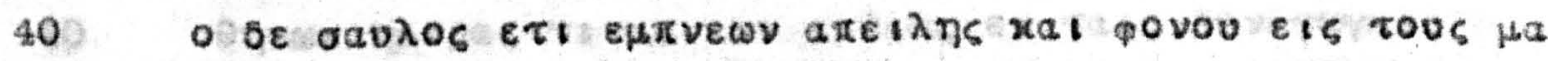

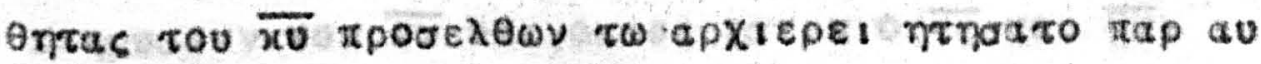


Tov extorodas ets bapaaxov xpos tas avvarwras oxwe eav tivas eupn ths 0000 ovtac avopas te xal ro

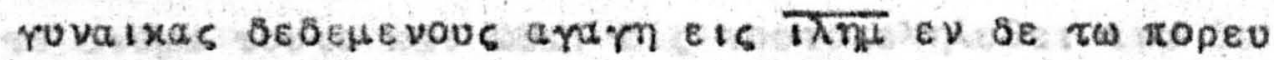

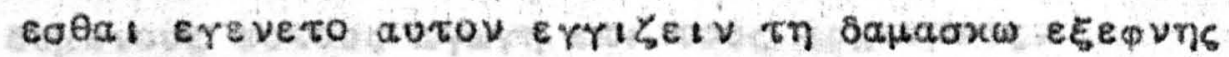

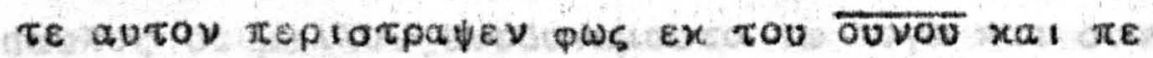

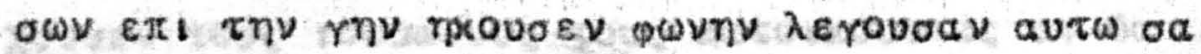

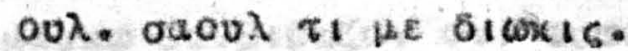

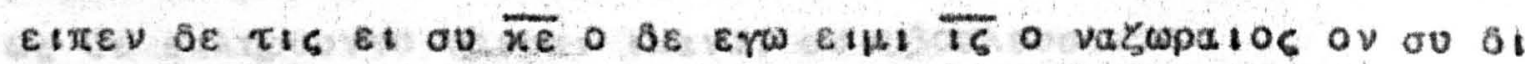

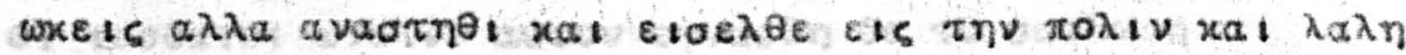

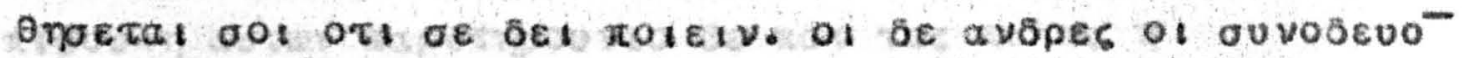

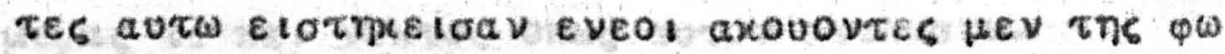

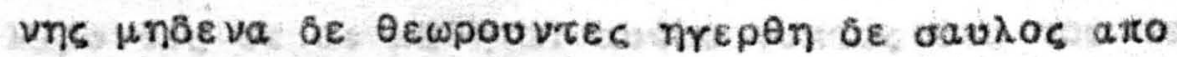

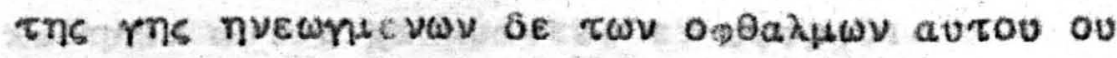

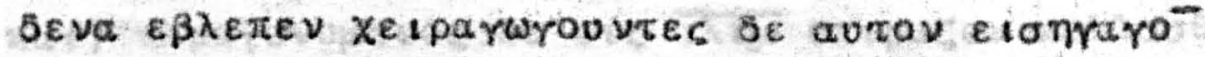

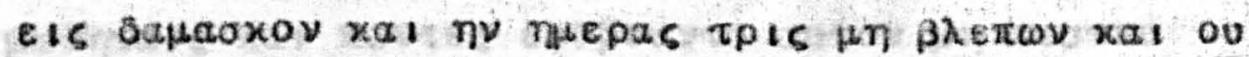

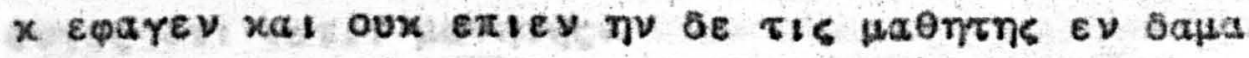
axw ovopart avavias xal eitev ipos avrov ev opasa

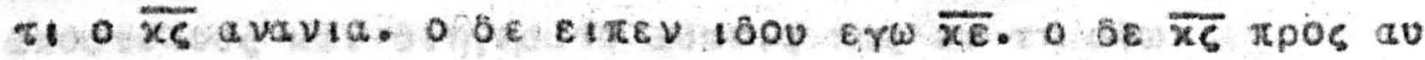

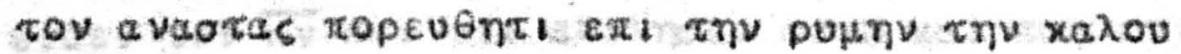

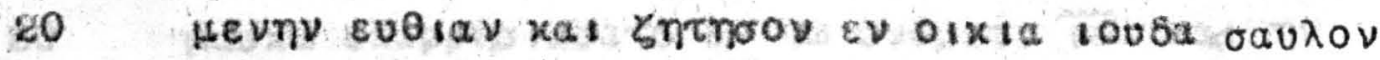

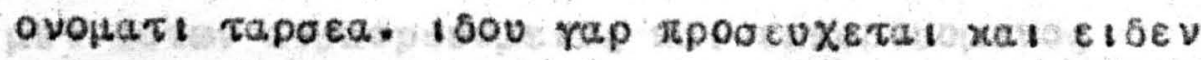

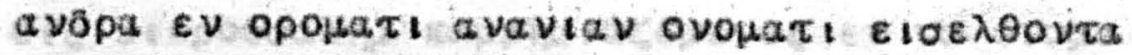

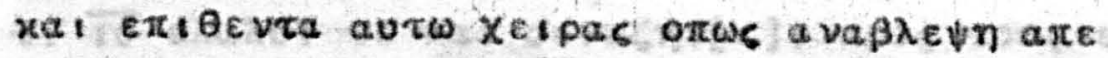

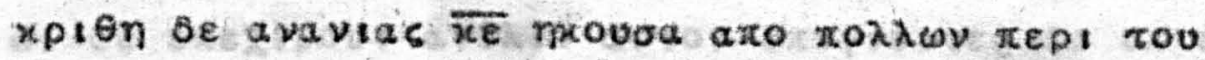
avopos toutou. ooa xaxa rois ariots oov esoinge-

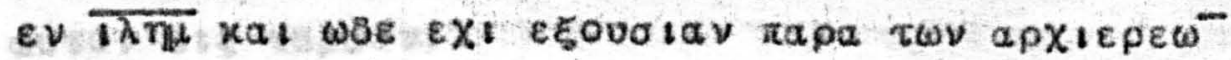

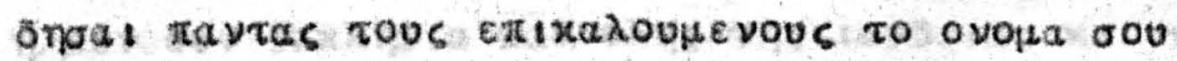

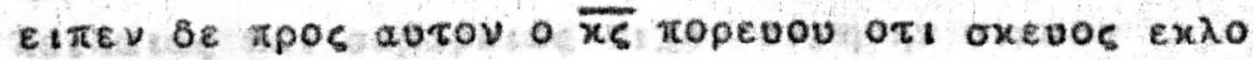

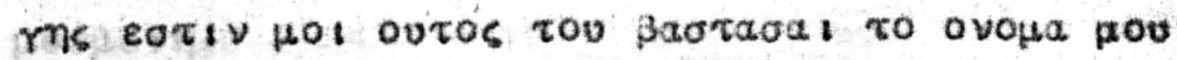

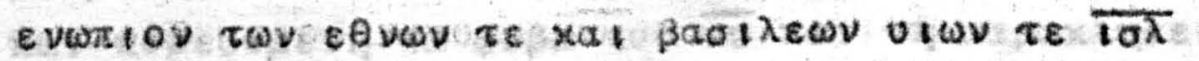
ero rap uxost HaTos Hou $\pi a \theta e t v$.

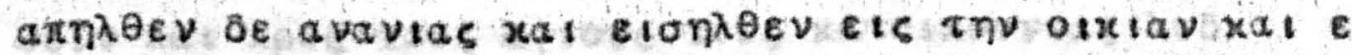

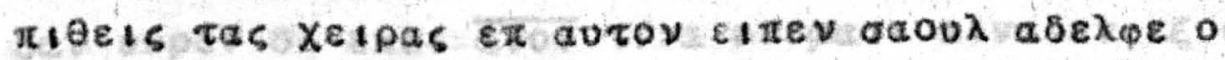

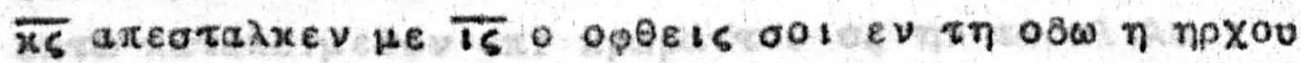

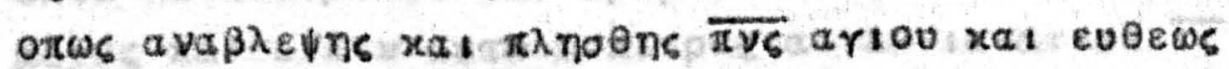

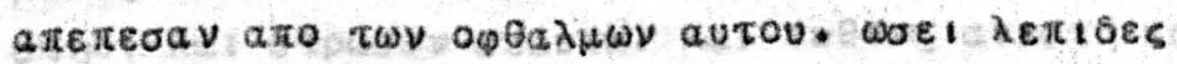

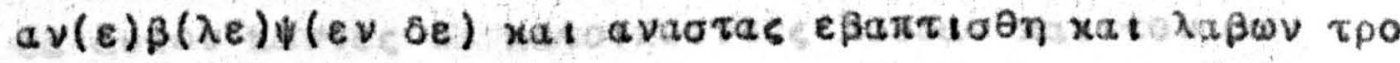

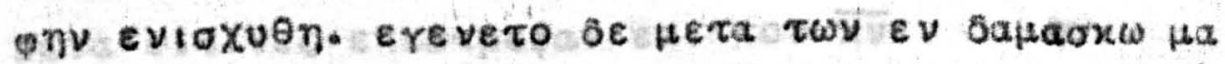

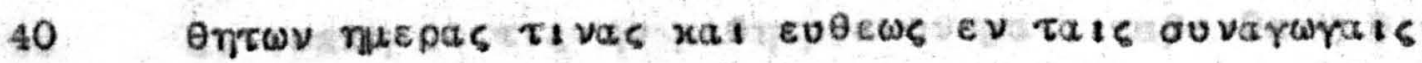

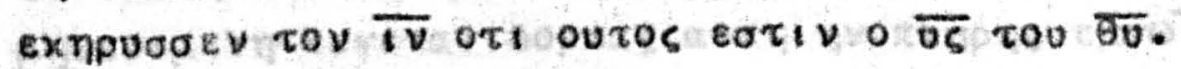




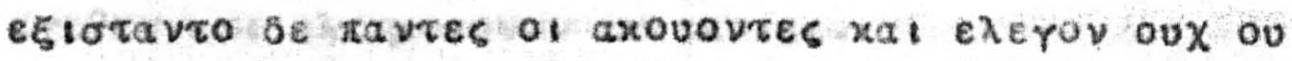

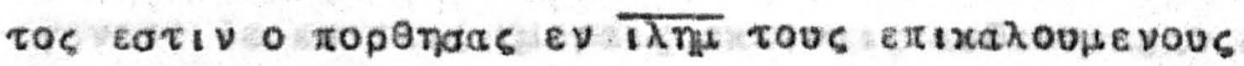

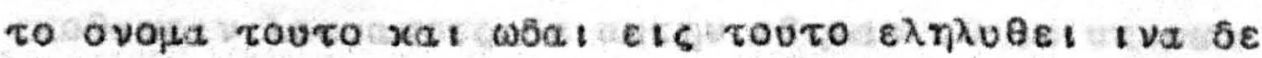
oefevous autous ararn etl tous apXiepeis.

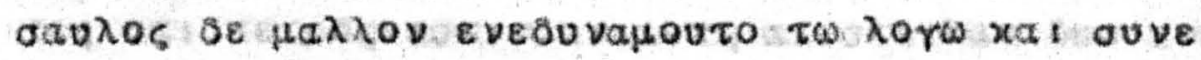
Xuvvey tous iousatous tous xatoixouvtas \&v $6 a$

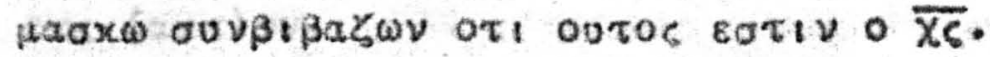

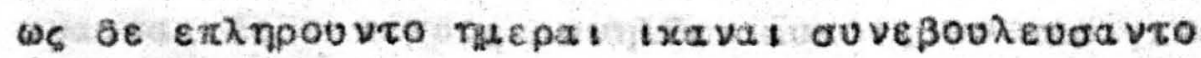

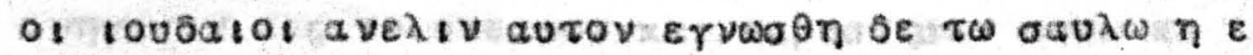

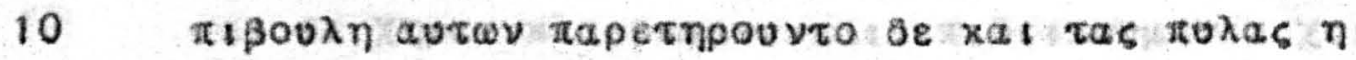
Hepas $\tau e$ xat vuxtos oxtws autov avenwo Iv. $\lambda a \beta o v \tau e s$ of ot paental autou vuxtos óta tou tetxouc

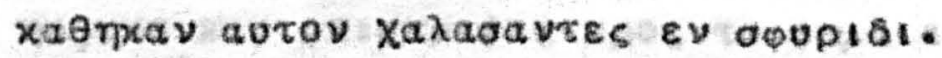

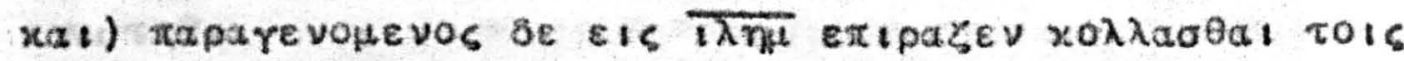

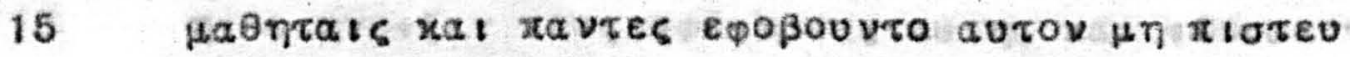

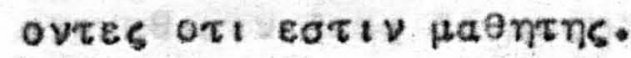

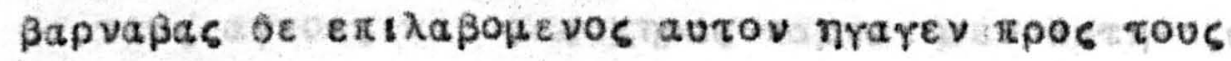

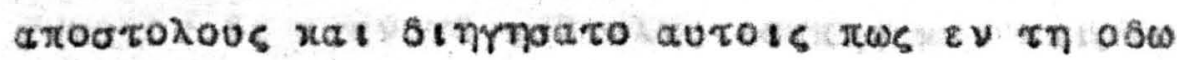

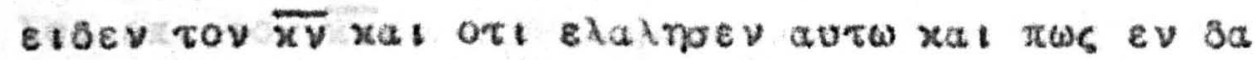

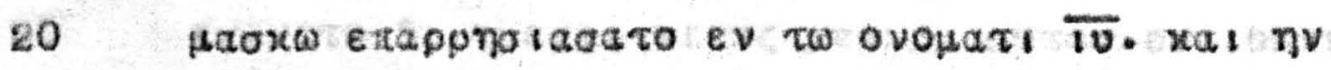

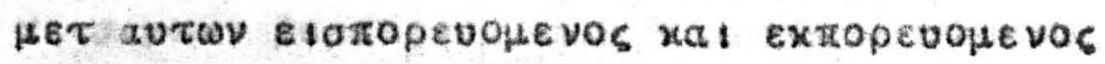

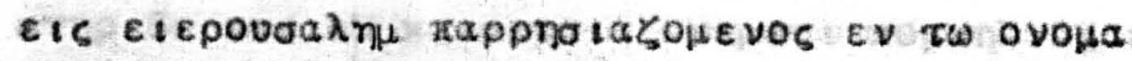

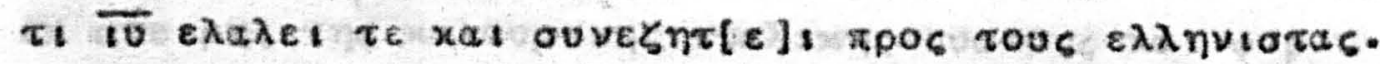

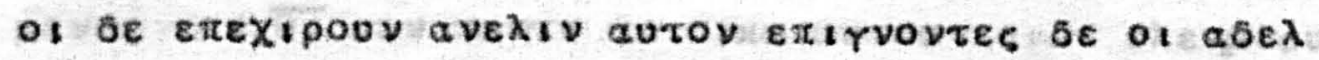
25 poi xatnrapov aurov eis xatoaptav xat egaxeareideautov eis rapoov.

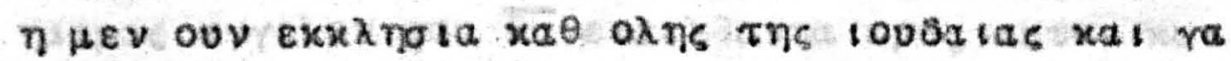

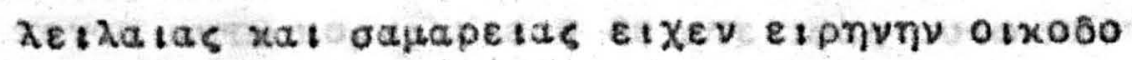
Hovนe vn.

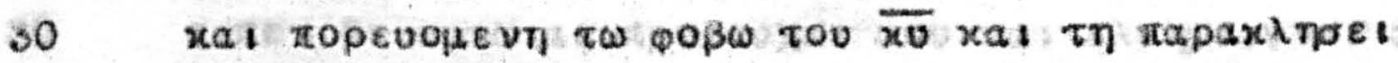

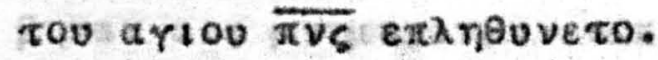

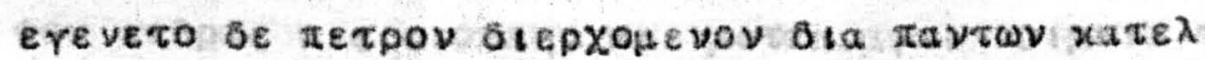
Qeiv xal tpos rous arlove tovs xatorxouvtas $\lambda 08$

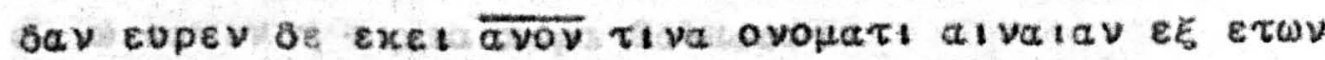

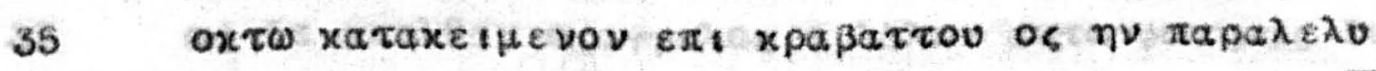

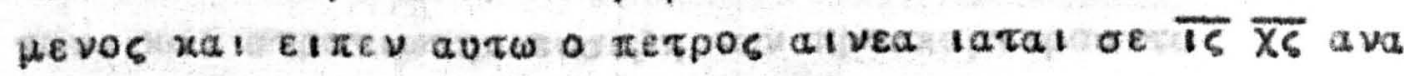

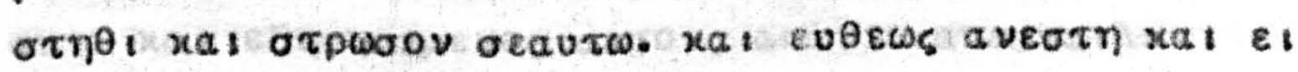

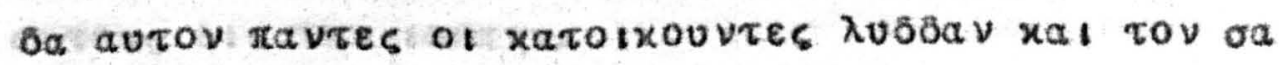

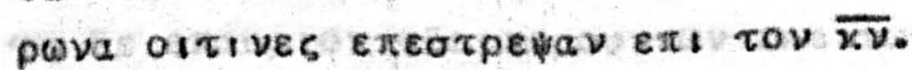

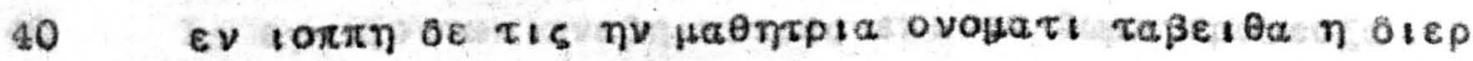

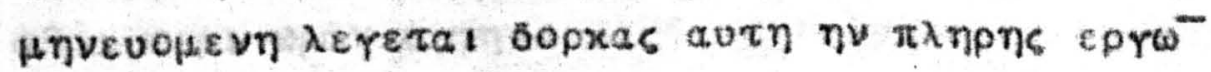


aratov xal exenuoovvts wV exotEt.

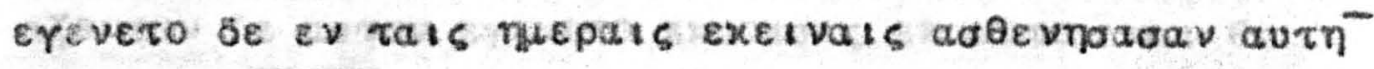

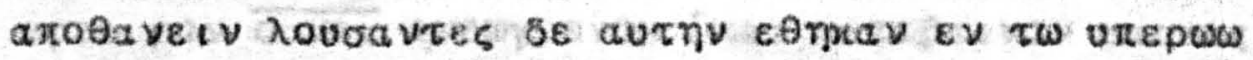

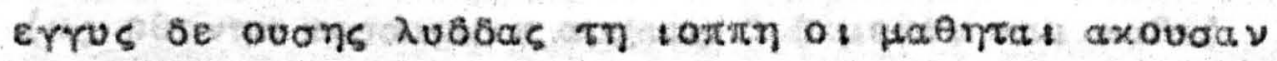

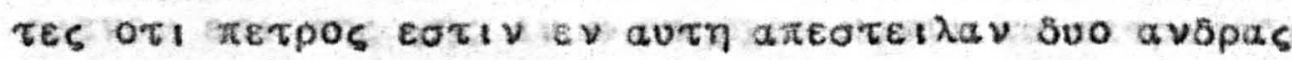

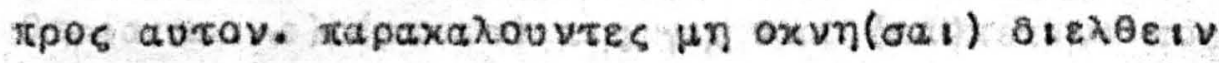
ews $(a v \tau) \omega v$.

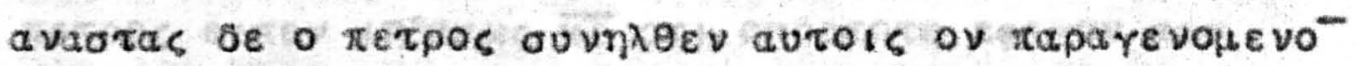

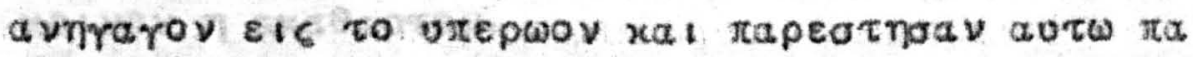

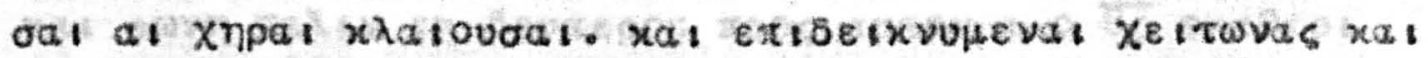

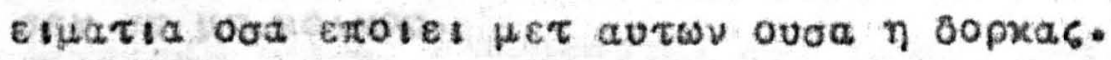

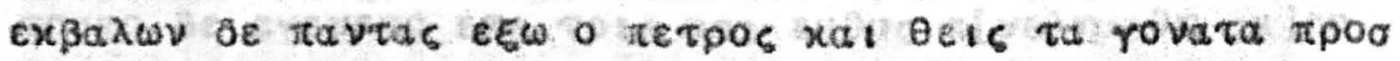

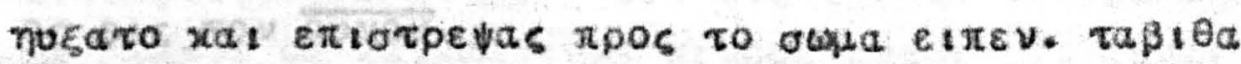
avuarñe.

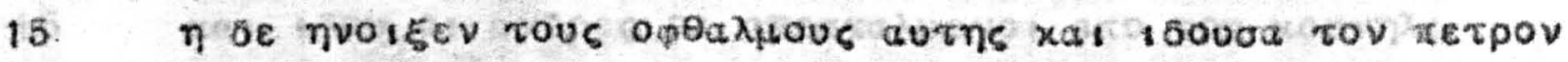

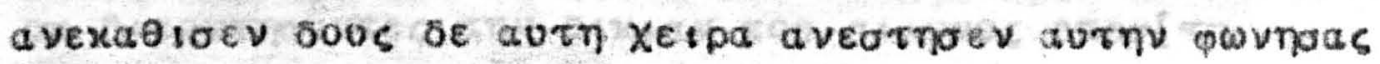

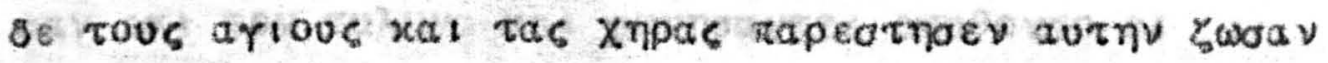

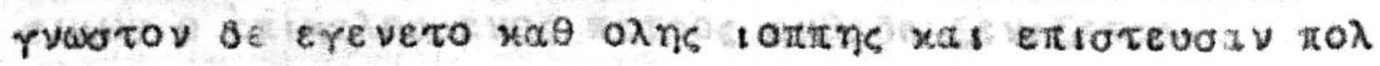

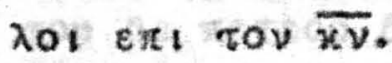

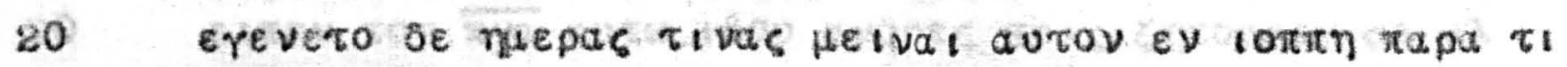

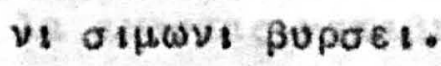

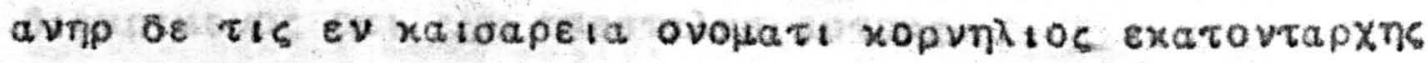

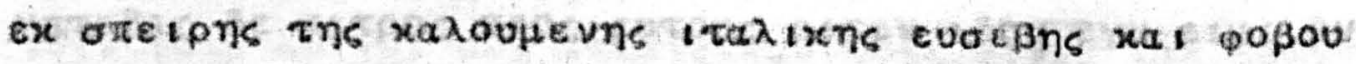

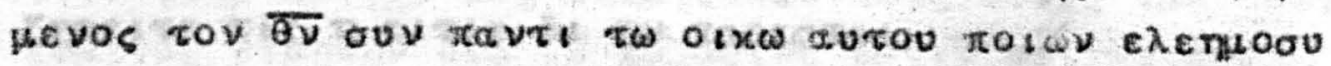

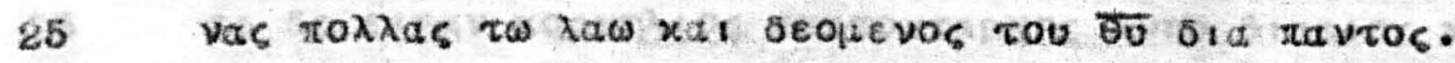

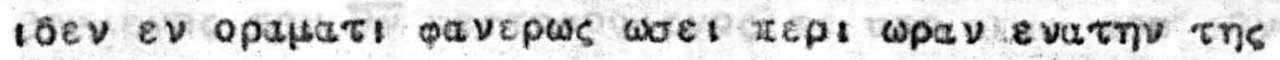

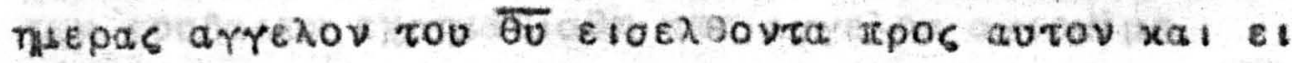

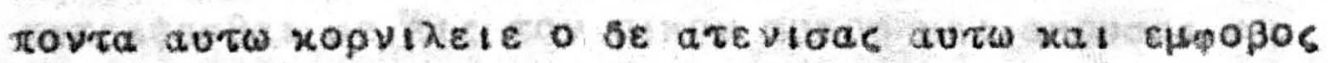

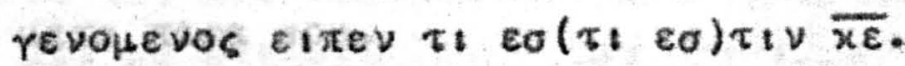

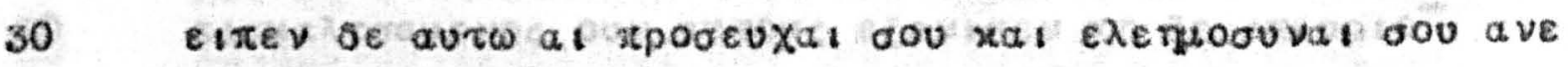

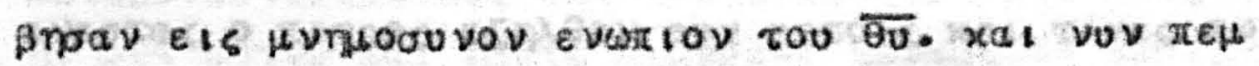

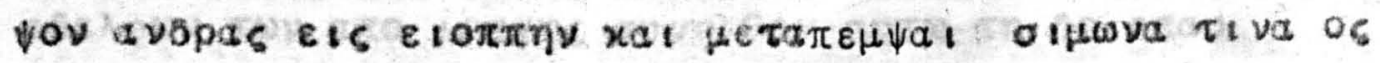
etı ot $\omega$ eativ

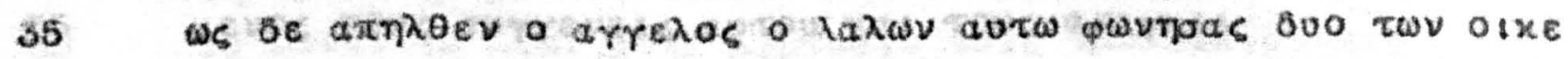

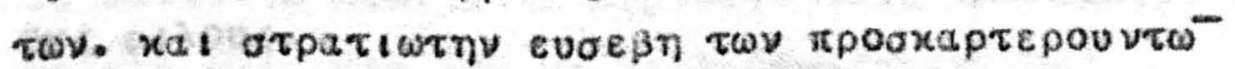

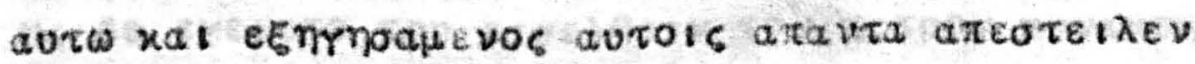
aurovs eis tทे cotinv.

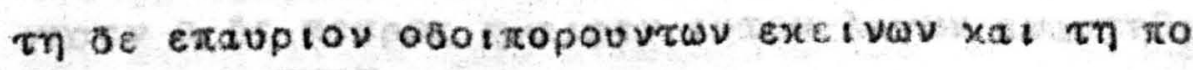

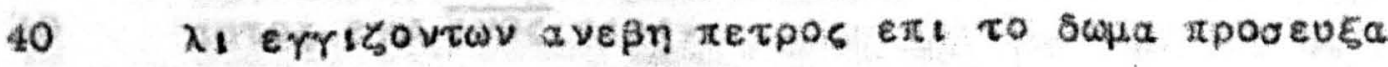

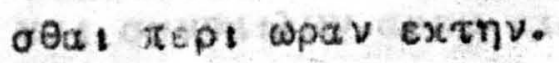




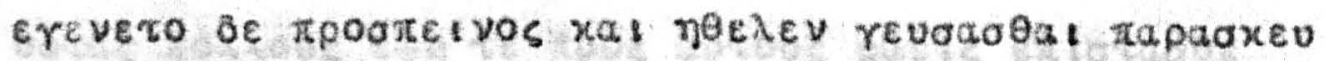

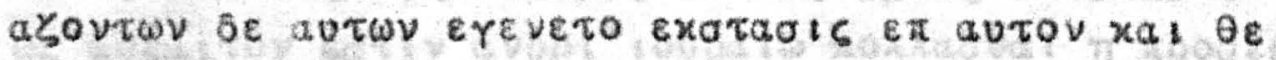

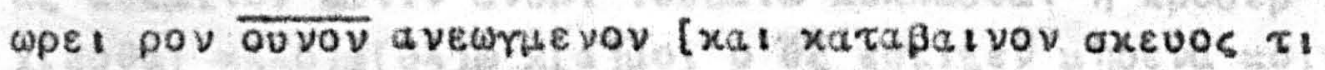

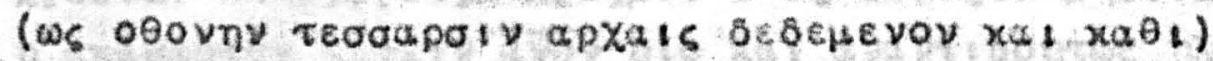

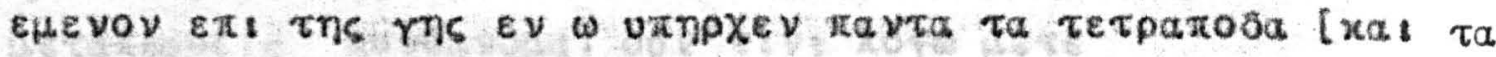

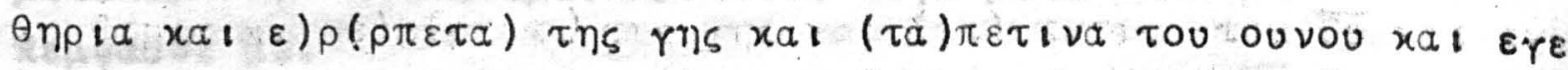

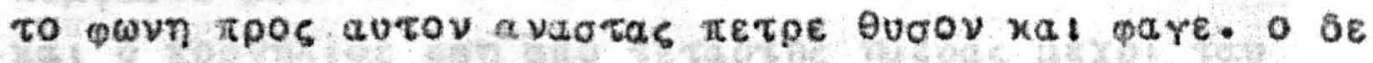

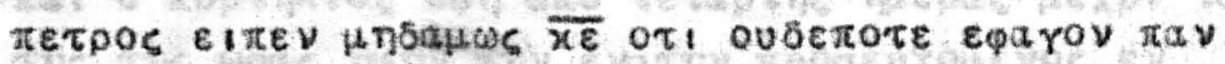
xotvov $\eta$ axataprov.

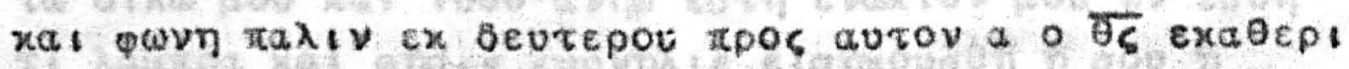

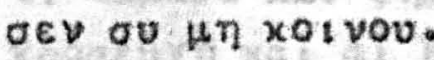

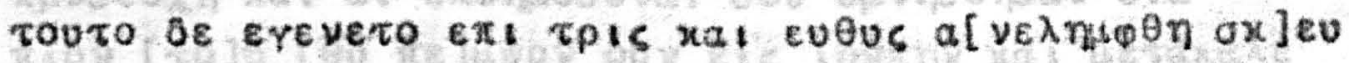
os eis rov OUVOV.

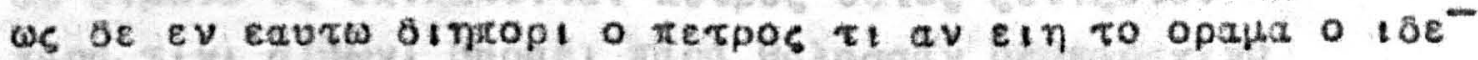

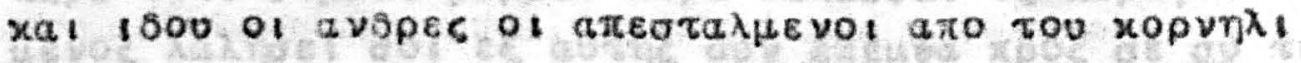

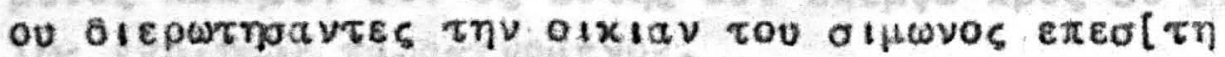

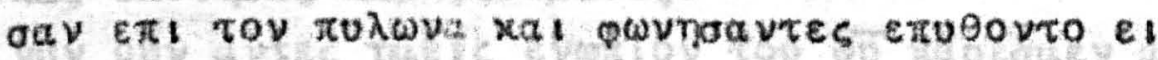

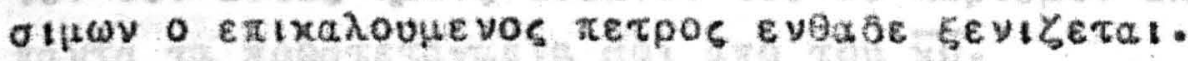

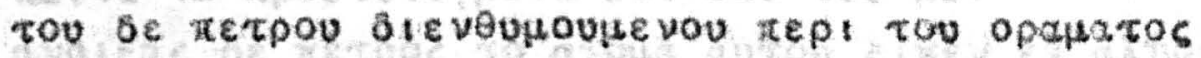

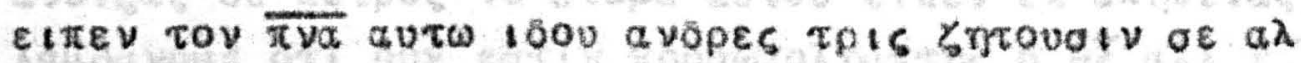
$\lambda a$ a vaotas xaraßnel xa! zopevou ouv aurois Hnōev

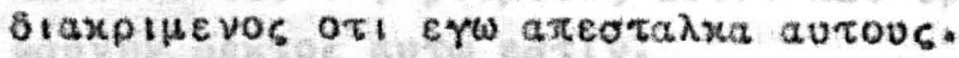

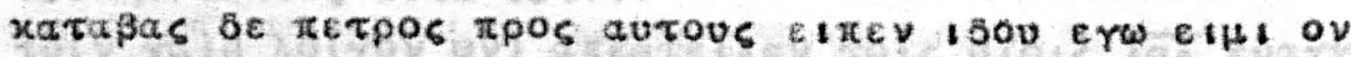

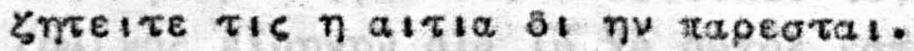

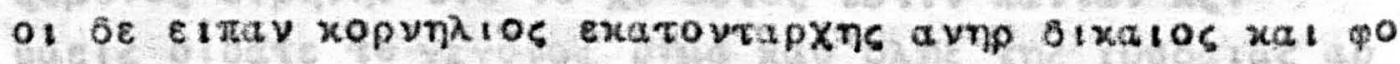

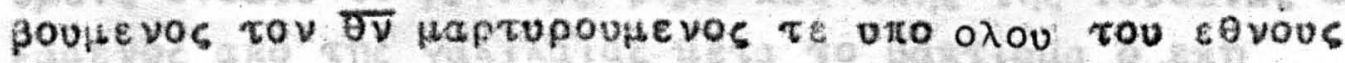

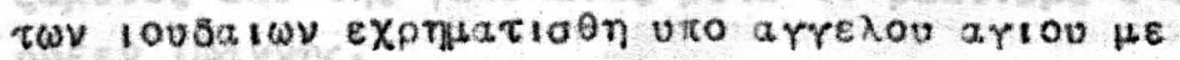

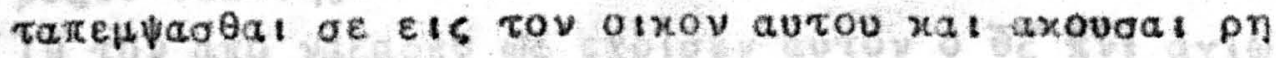

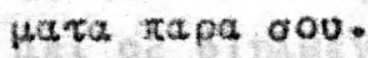

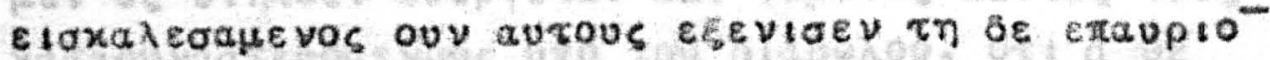

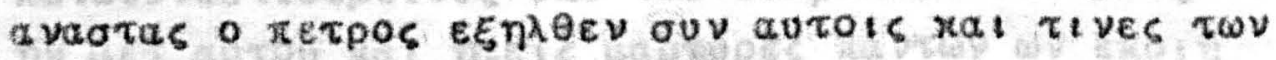

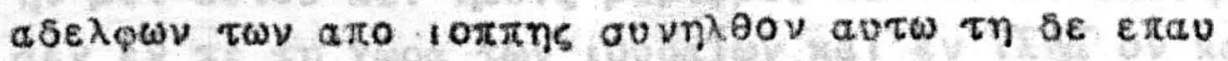

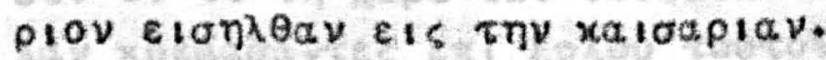

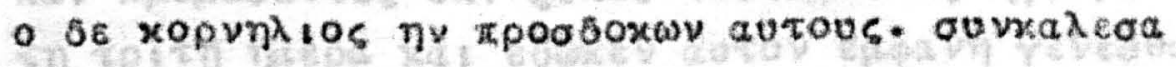
Hevos tous aurrevels autou hat rous avarat OUS PIXOUS.

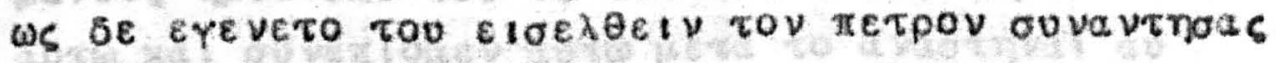

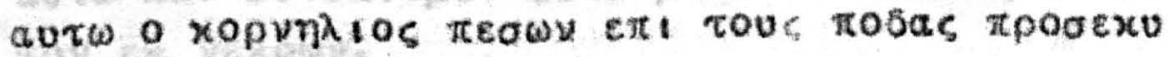

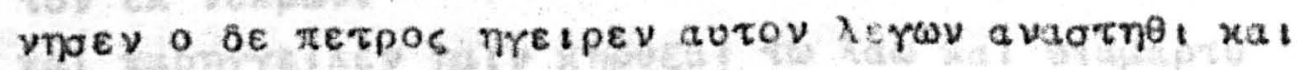

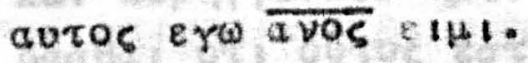

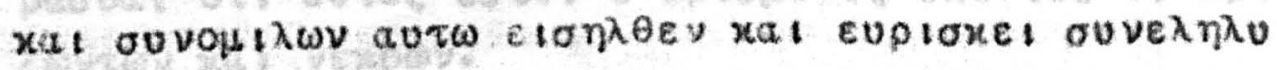




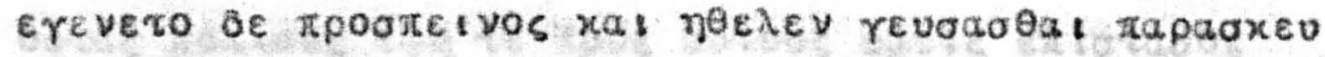

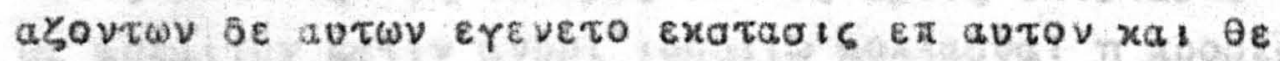

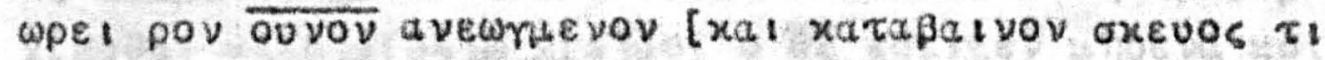

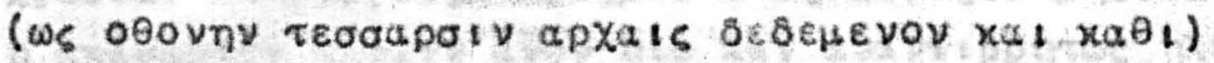

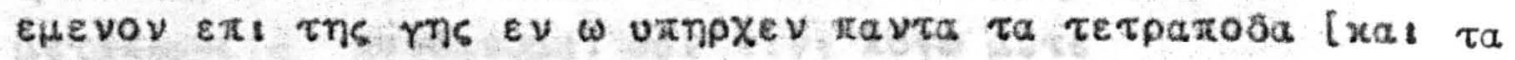

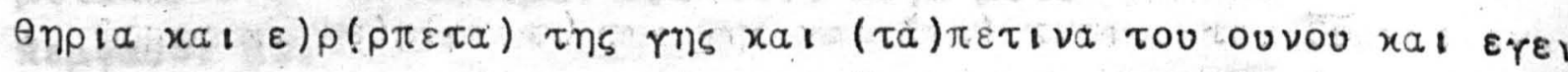

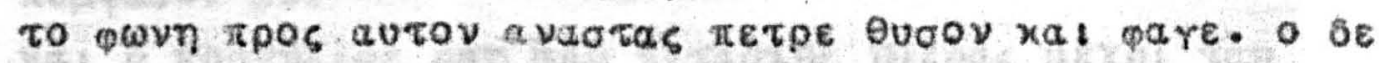

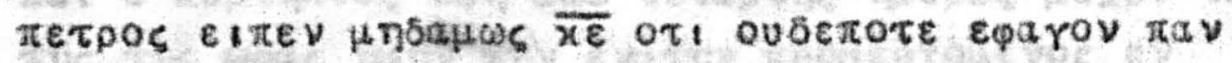
xotvov $\eta$ axaexprov.

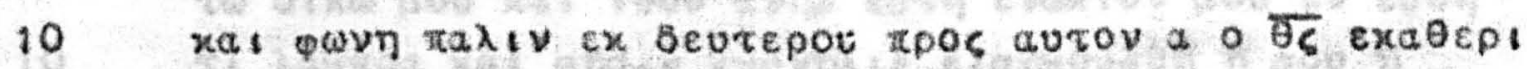

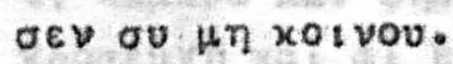

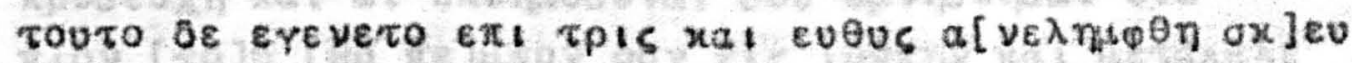
of eis tov ONVOV.

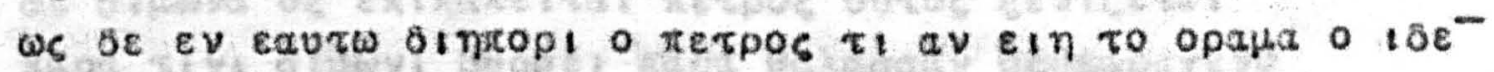
xal 1800 or a ou oi

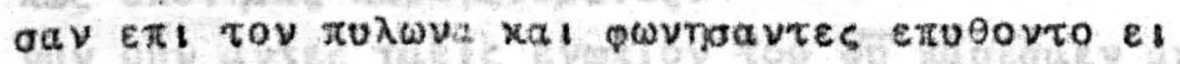

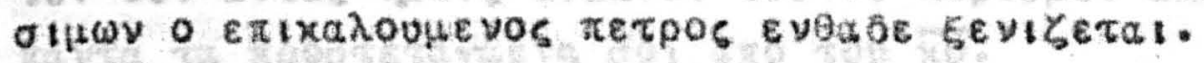
Tou $\delta \varepsilon \pi \varepsilon \tau p \circ$ atevounounevov tep! tou oparatos

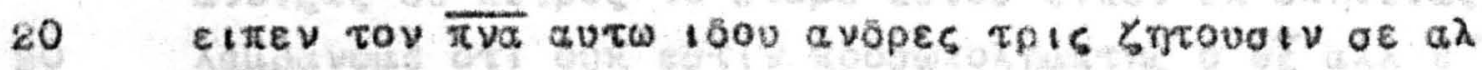

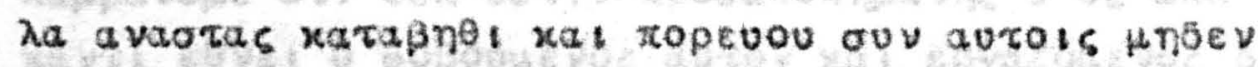

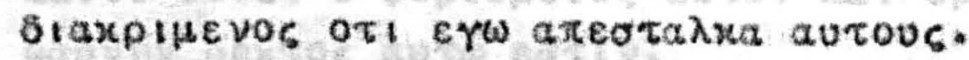

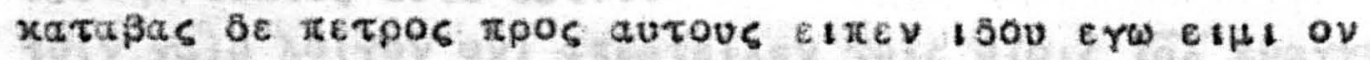

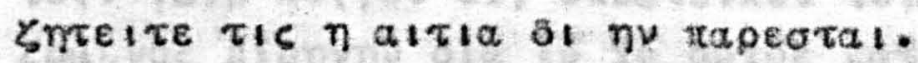

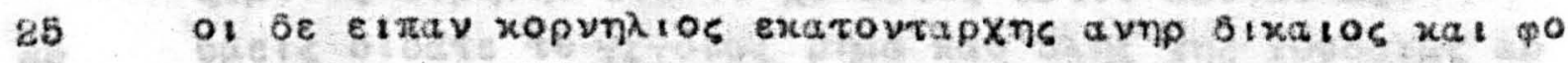

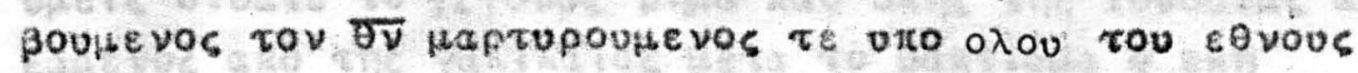

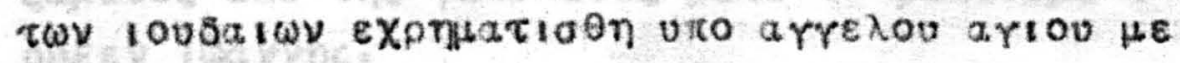

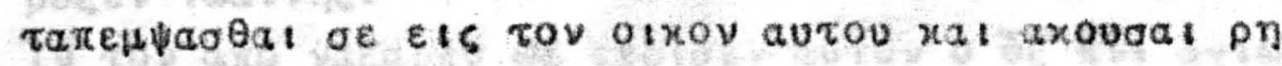
Hara rapa oou.

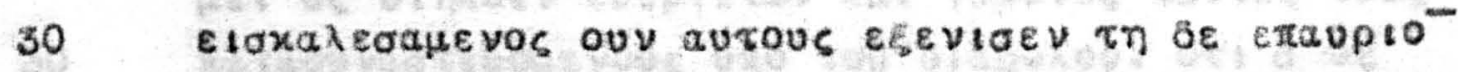

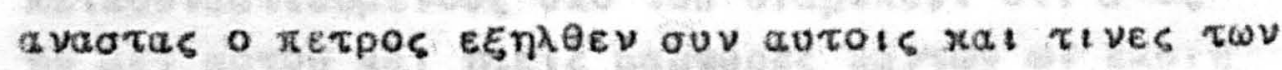

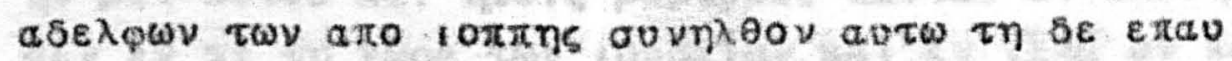

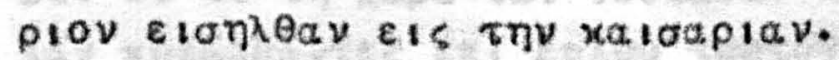

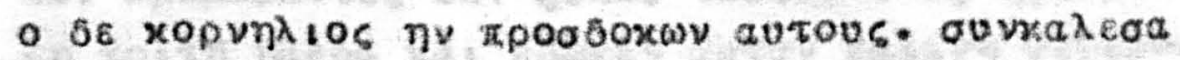
Hevos tous aurreveis autou xal tous avarkal OUS PIXOUS.

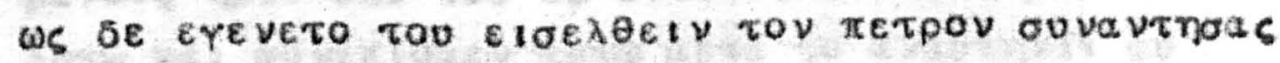

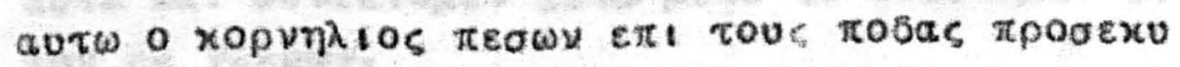

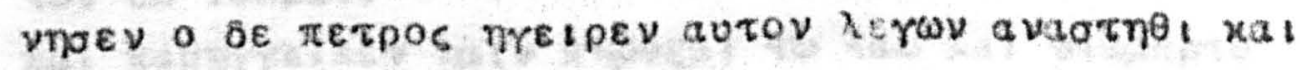

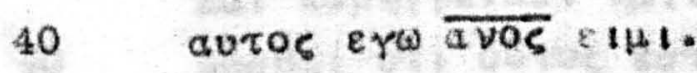

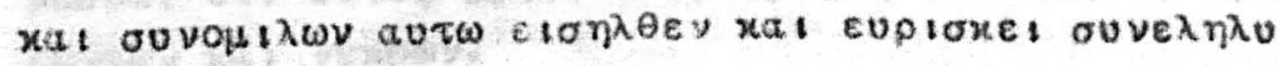




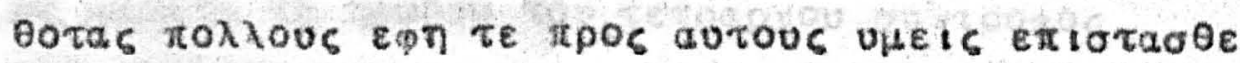

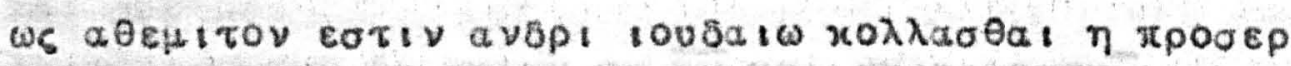

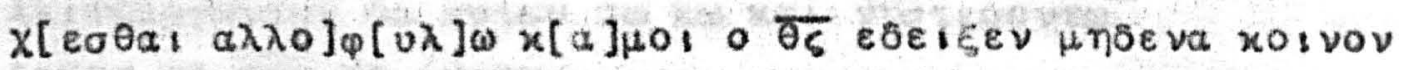

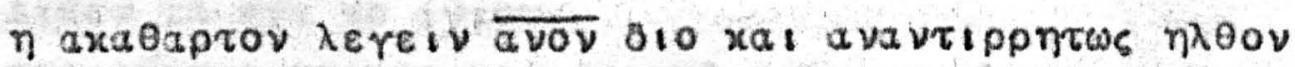

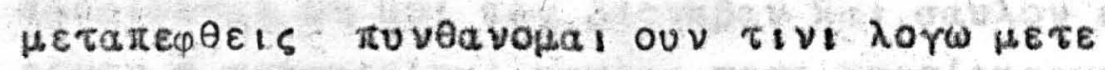

$\pi \varepsilon \mu \downarrow a \sigma \theta \varepsilon \mu \varepsilon$.

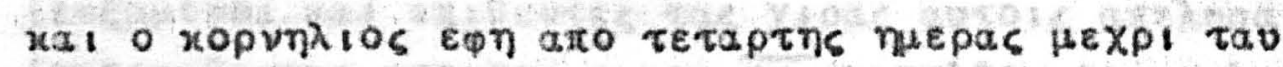

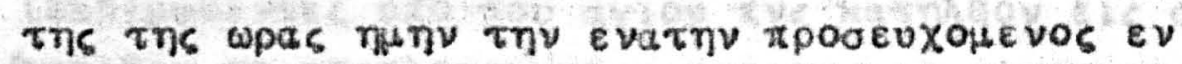

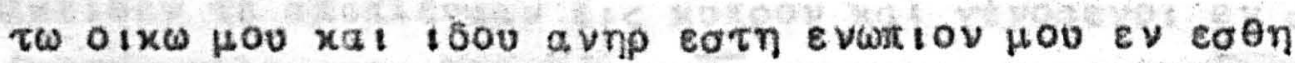

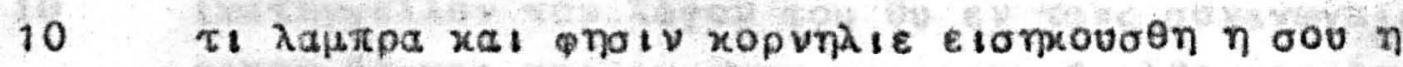

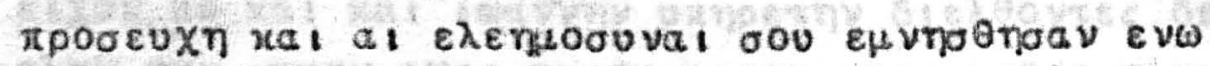

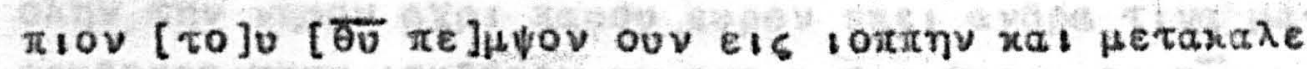

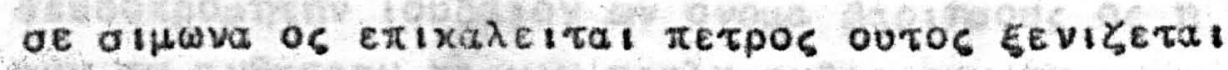

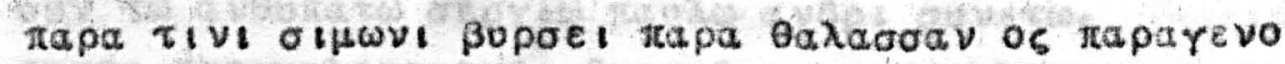

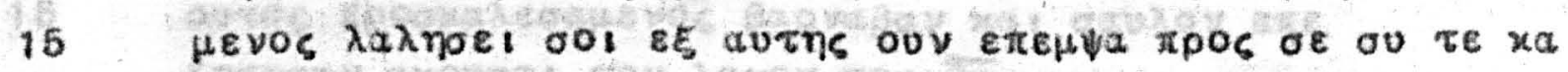

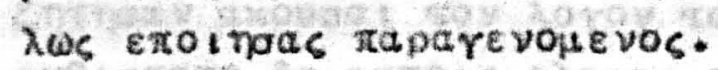

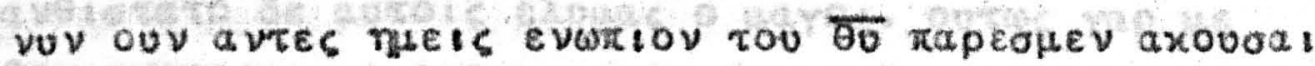

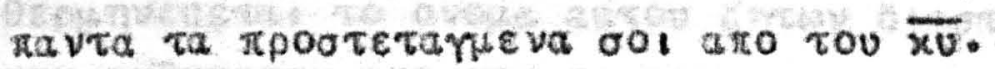

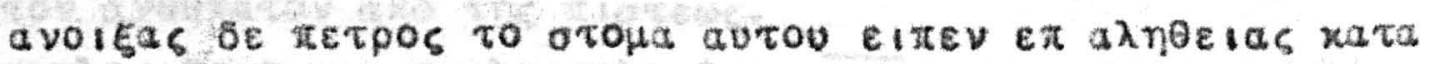

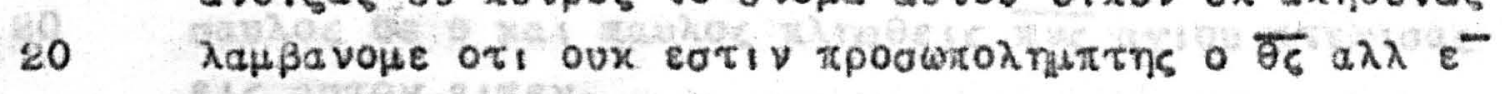

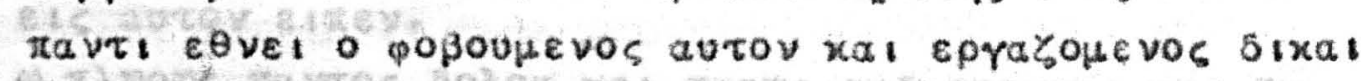

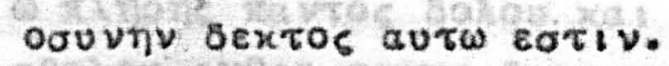

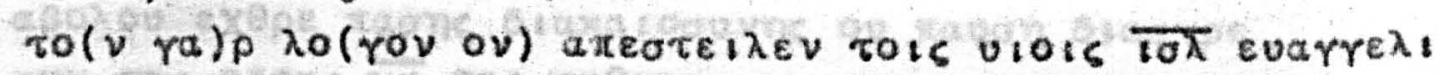

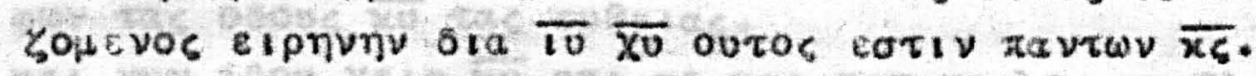

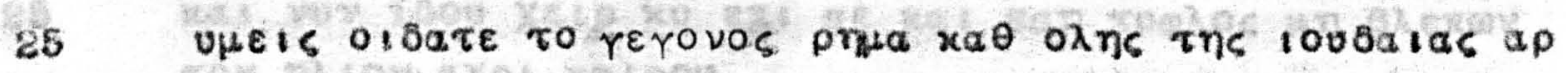

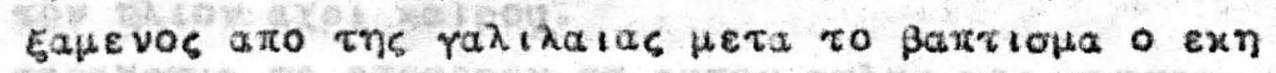
pugev twa vun

TV tov ato vacape日 ws exploev autov o $\overline{\theta_{\zeta}} \overline{\pi v t}$ artw xal suva Hel os sindeev evepretw xal layevos tavtas rous

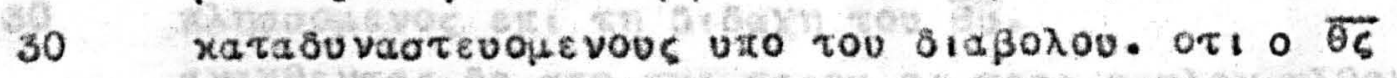

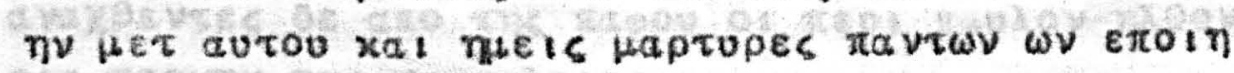

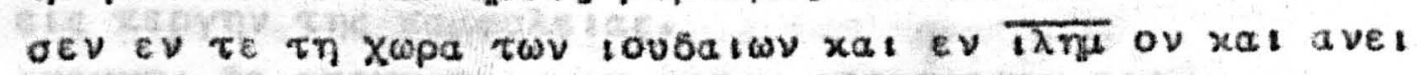

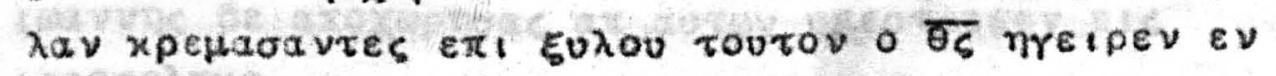

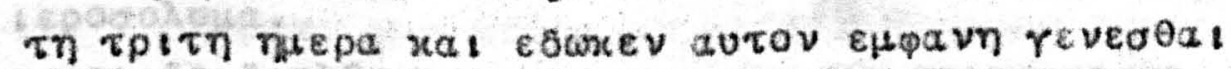

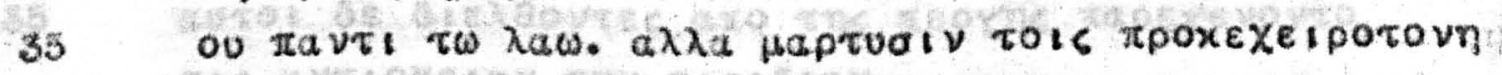
Hevols nuiv vio tou Bo otrives ouveparohev

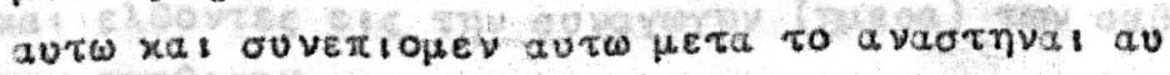
Tov $\varepsilon x$ vexpwv.

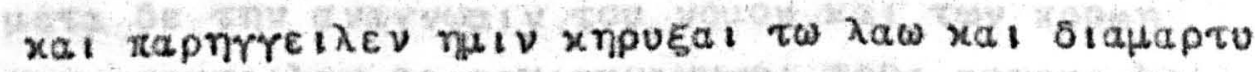

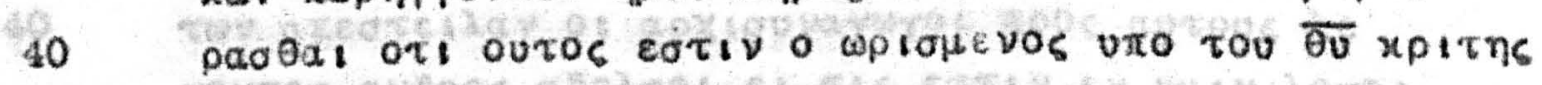

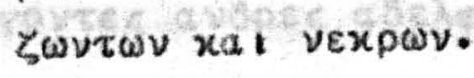




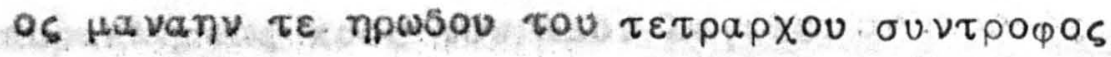

xal oavios.

$\lambda \varepsilon$ เ

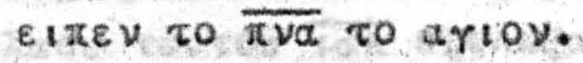

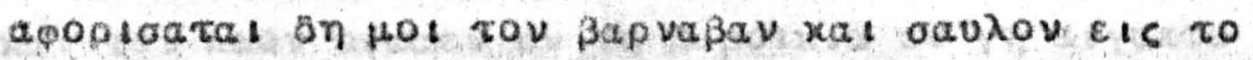

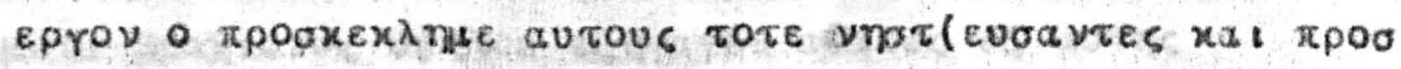

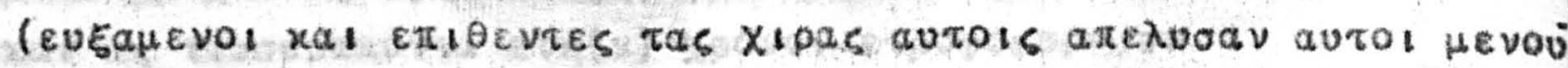

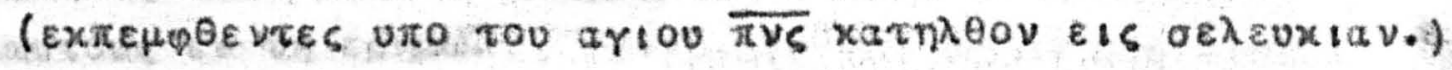

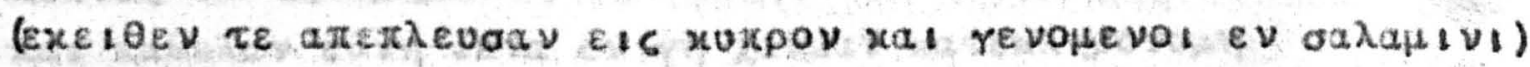

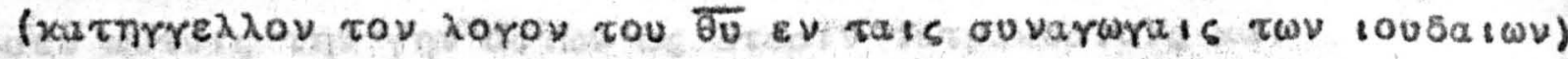

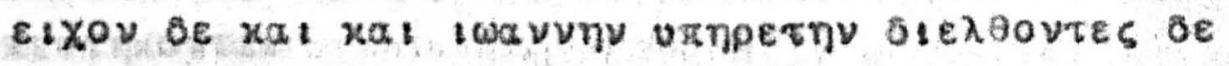

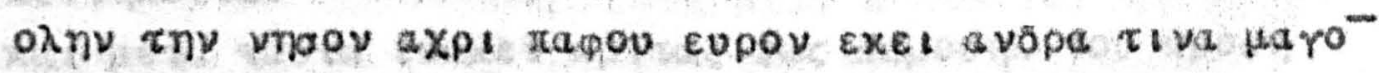

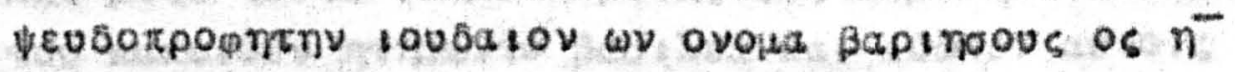

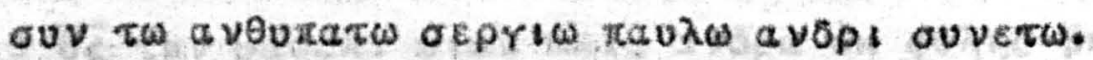

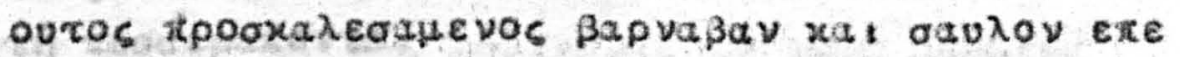

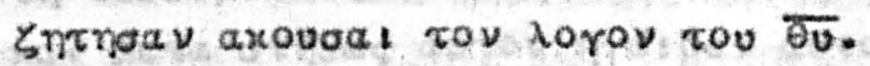

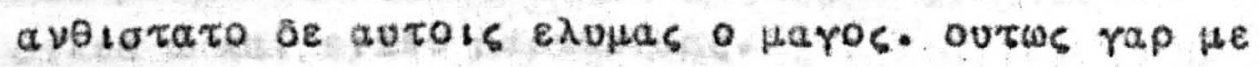

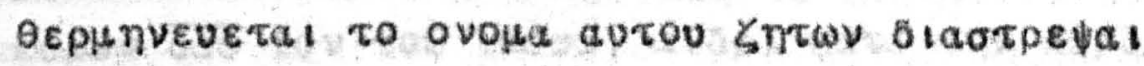
rov avourarov aro tris riarews.

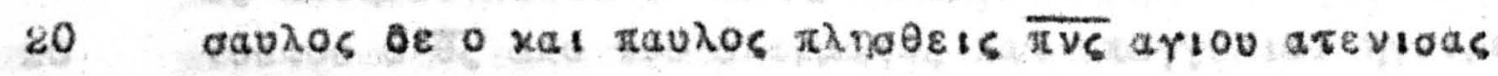
eis autov EtTeV.

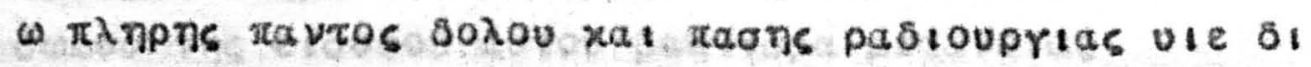

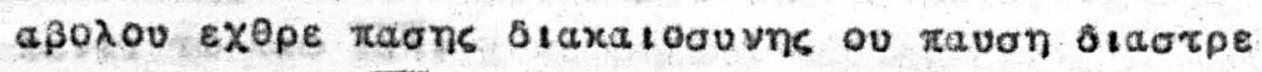

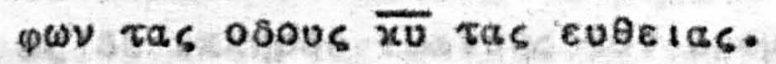

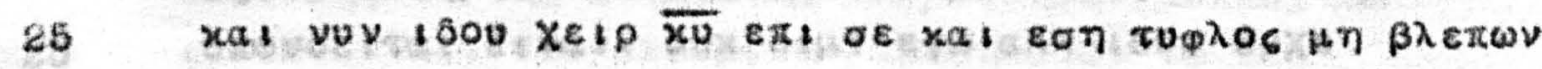
rov nitov axpi xalpou.

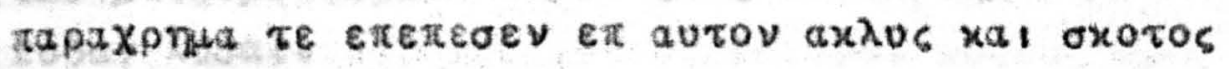

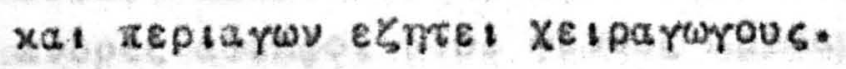

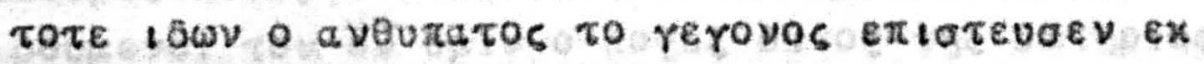

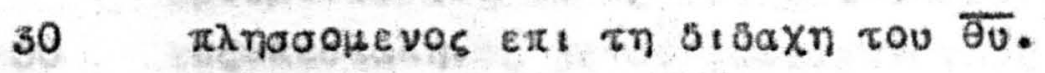

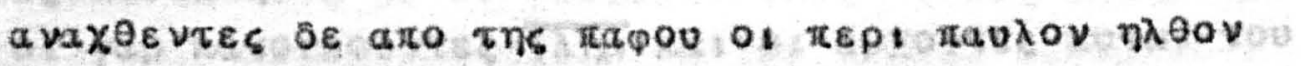

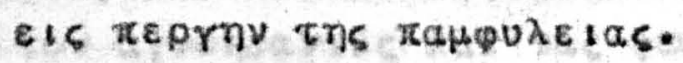

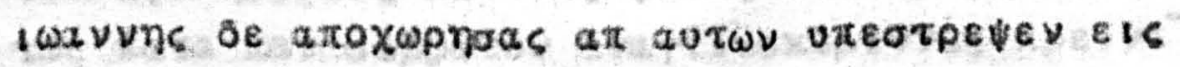
iеролодинa.

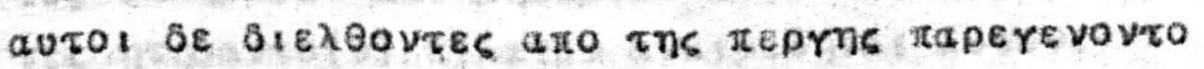

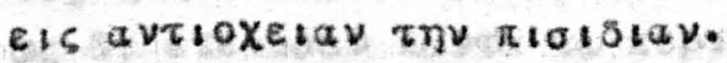

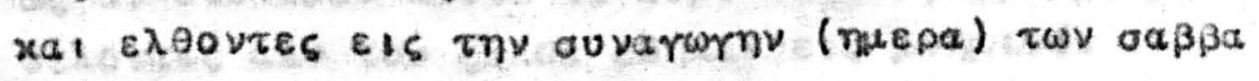

rwo exac laav.

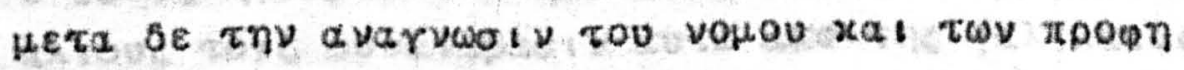

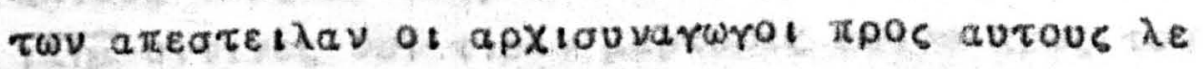

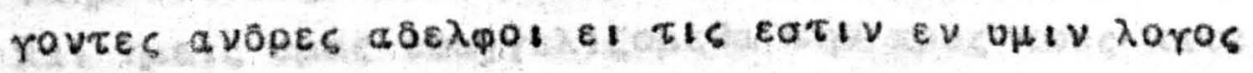




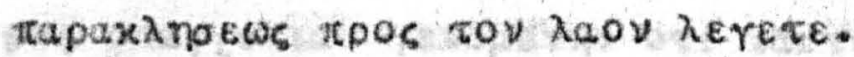

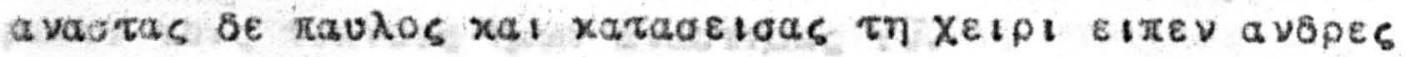

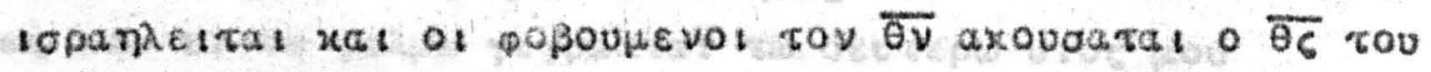

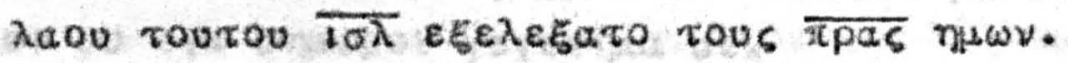

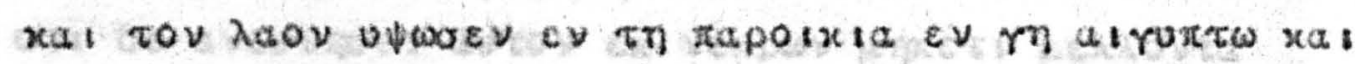

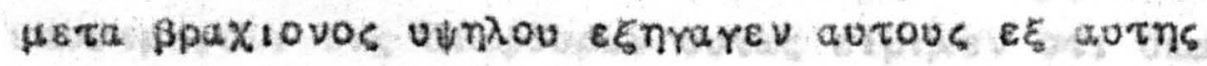

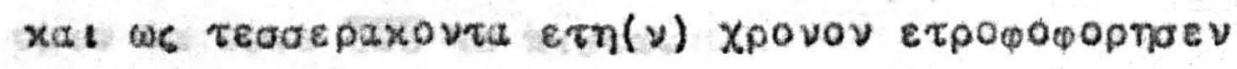
aurous $\varepsilon V$ in $\varepsilon p \pi n i \omega$.

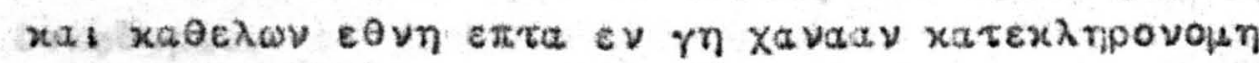

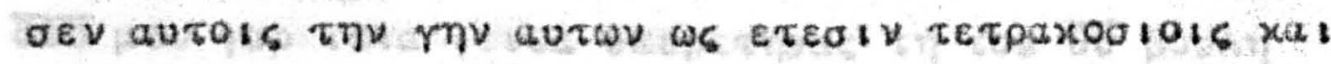
זevтriovra.

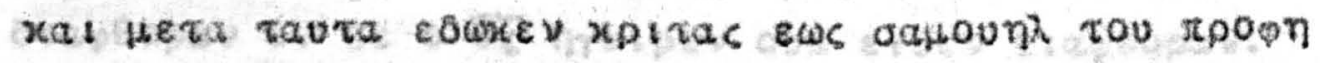

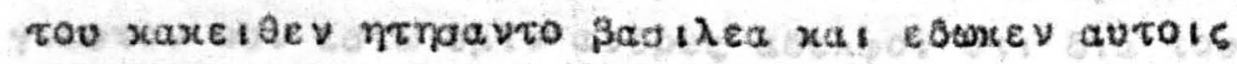

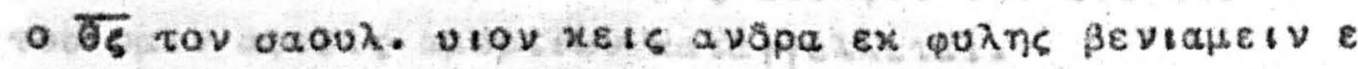

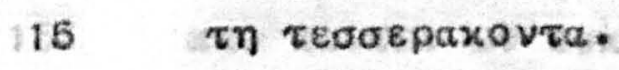

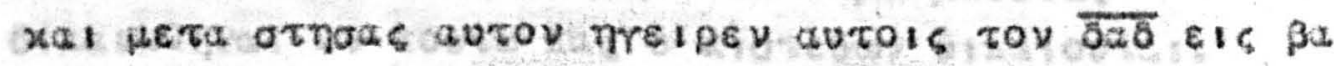

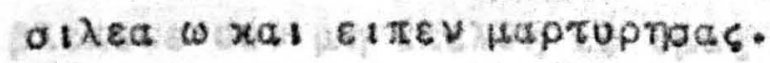

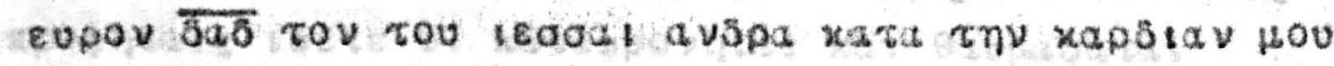

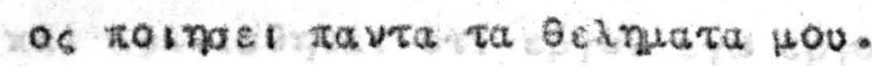

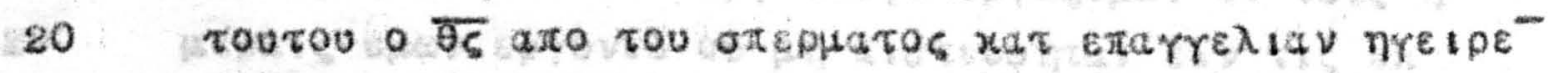

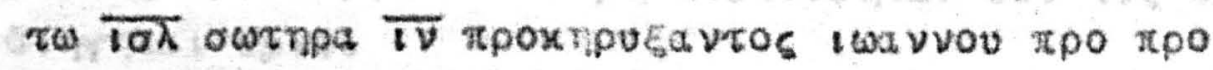

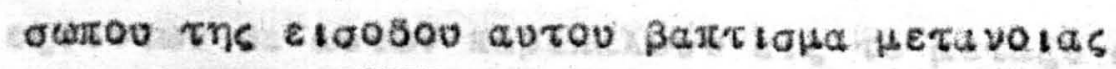

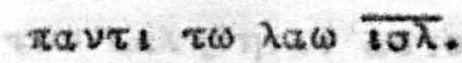

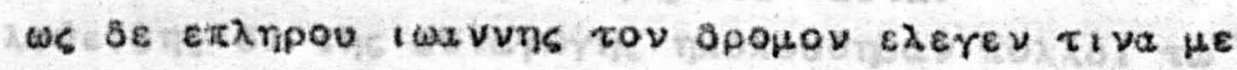

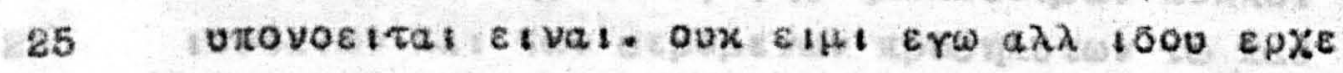

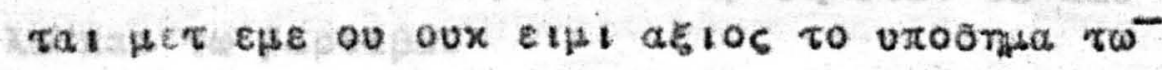
Hostov Avoal. a

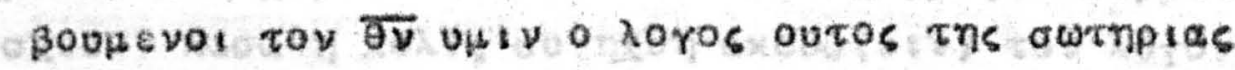

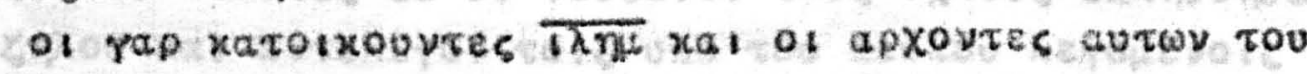

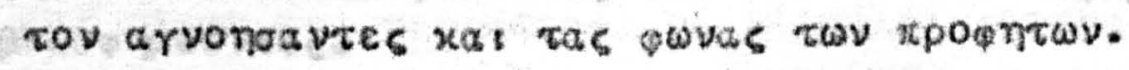

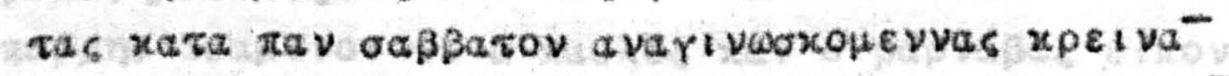

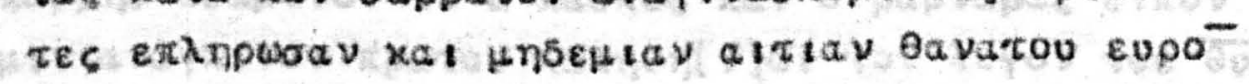
35 Tec nenoavo(v) rilarov avapeanva aurov.

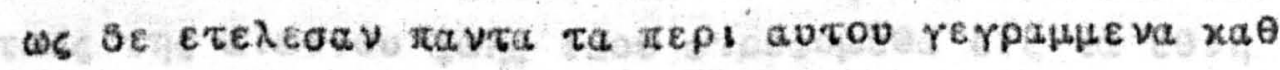

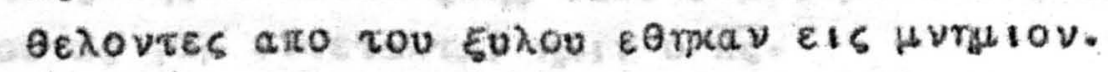

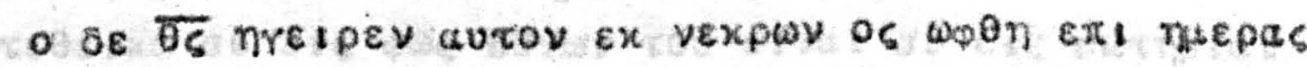

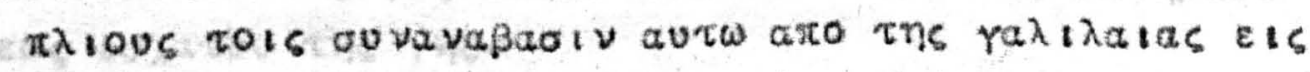

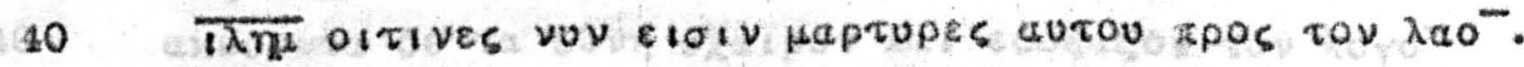

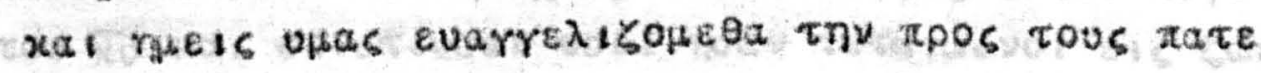




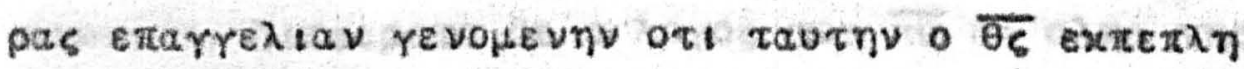

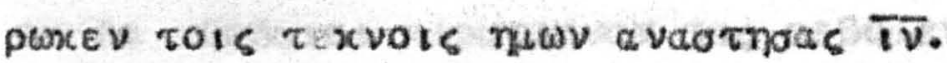

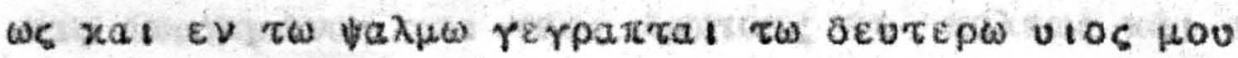

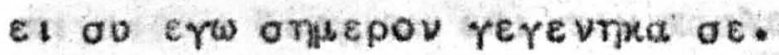

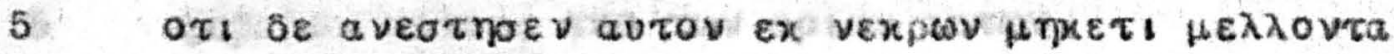

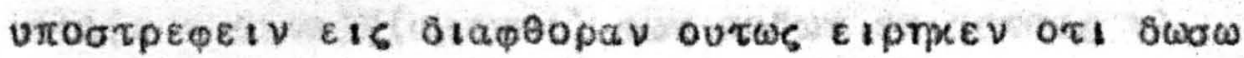

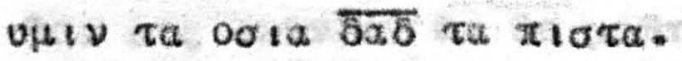

ö

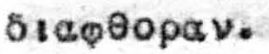

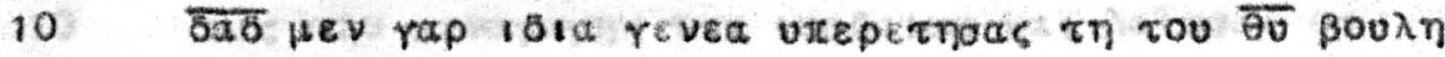

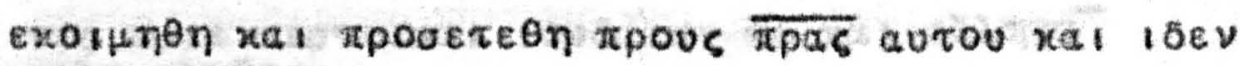

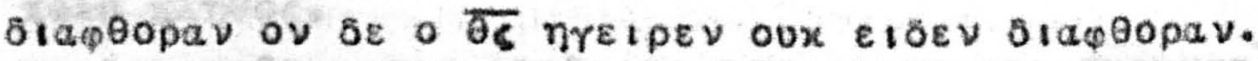

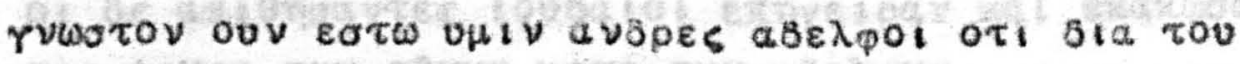

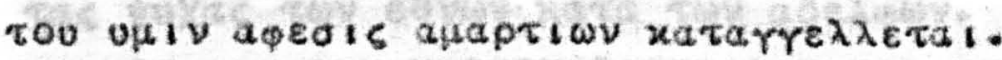

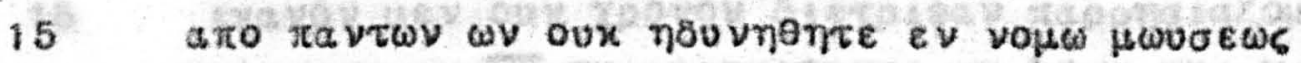

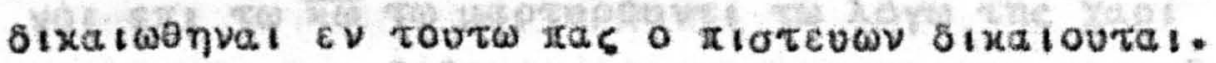

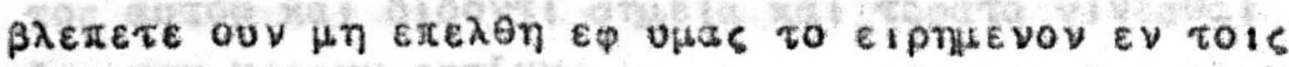

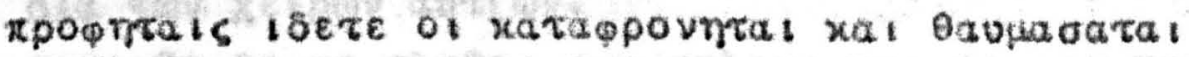

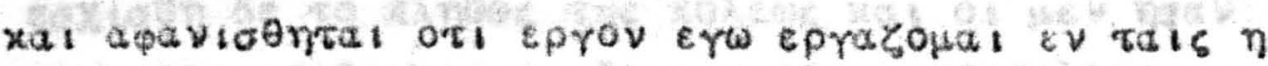

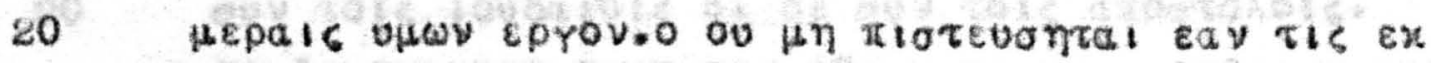
sinrnta! vilv.

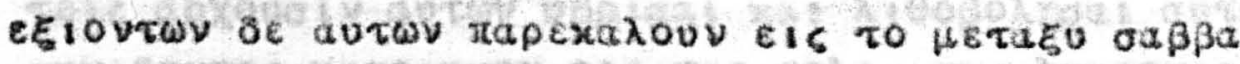

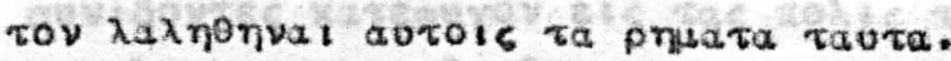

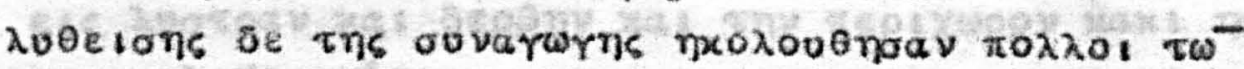

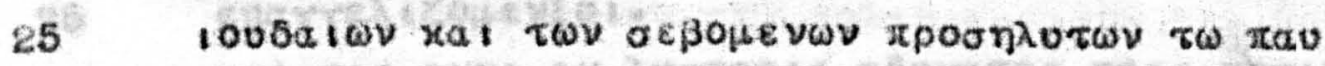

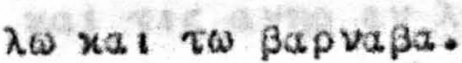

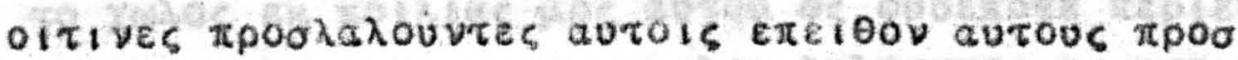

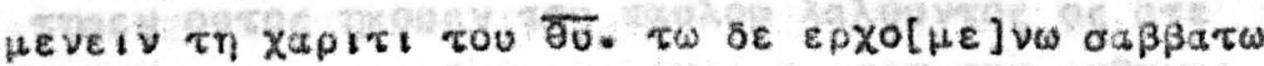

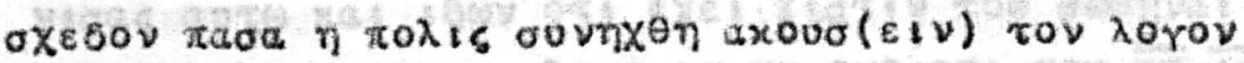

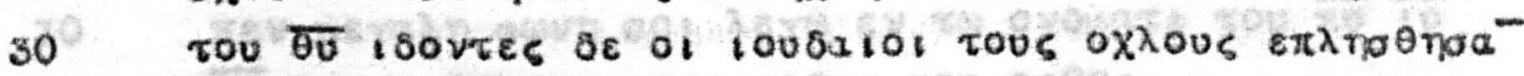

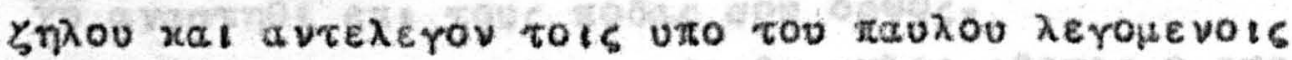

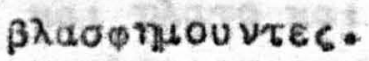

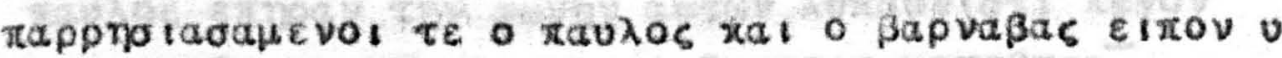

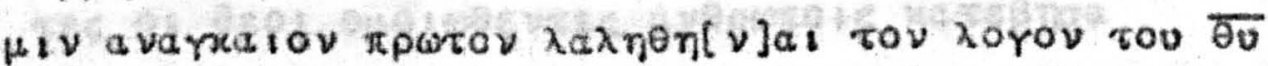

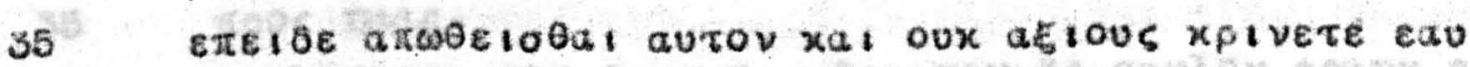

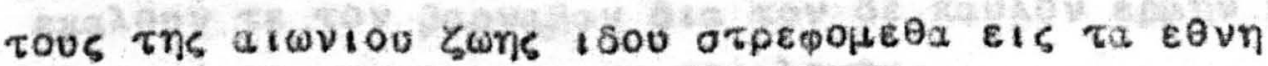

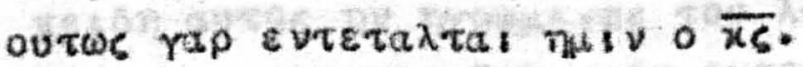

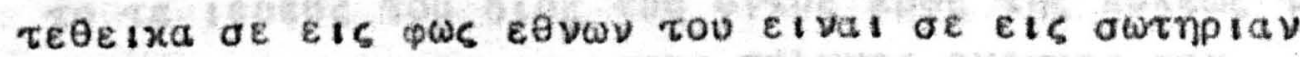

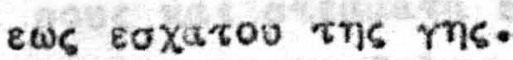

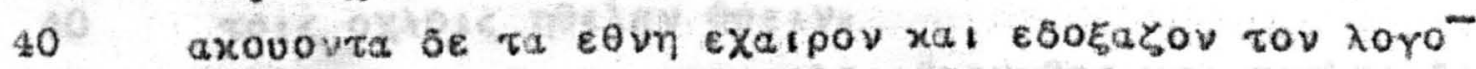

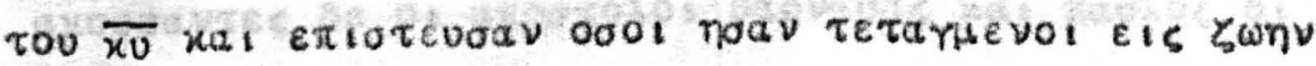




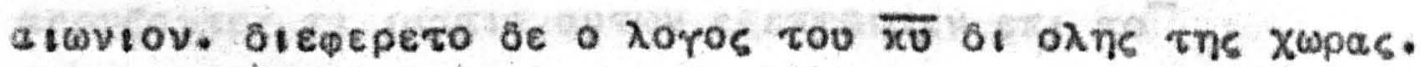

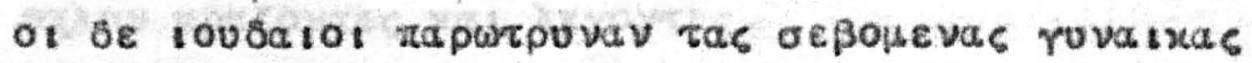

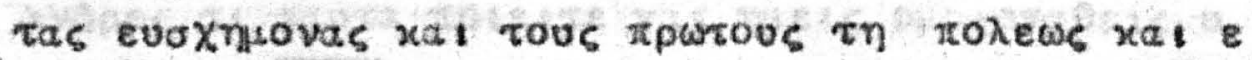

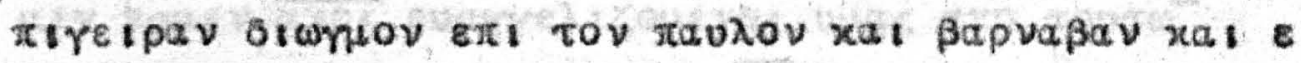

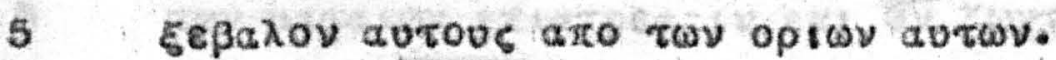

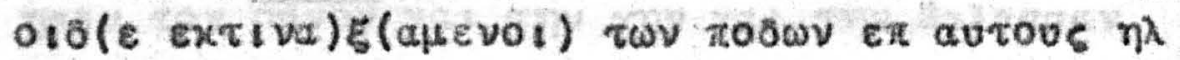

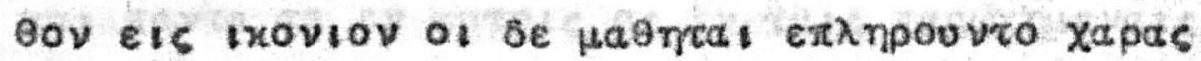
$x_{i}$ तेंद artov.

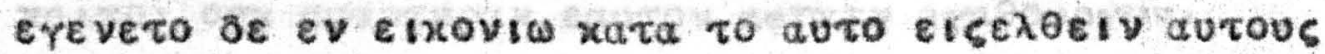

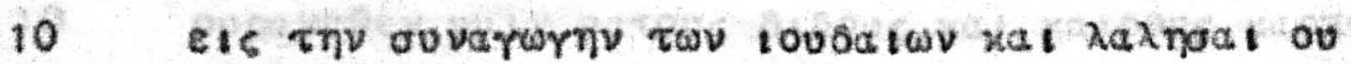

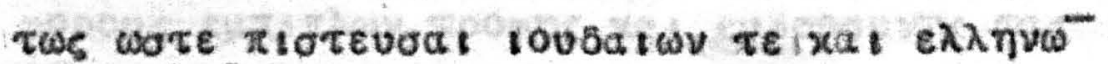

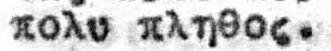

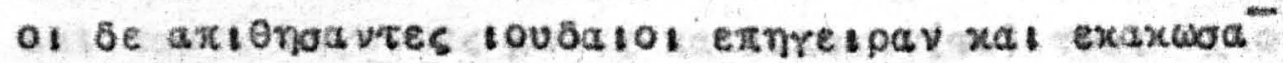

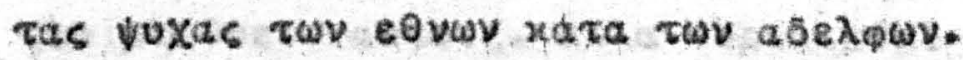

15 ixavov $\mu \varepsilon v$ ouv Xpovov

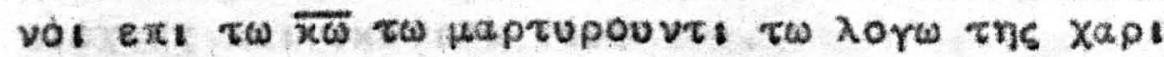
ros aurou xat sิ sta rwv Xetpov avet wv.

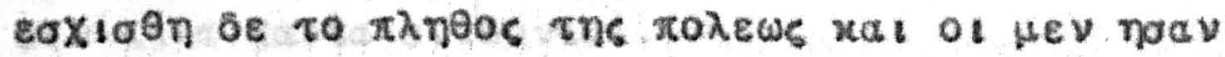
auv Tois loubatats os de av tois arootonots.

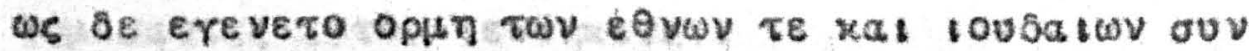

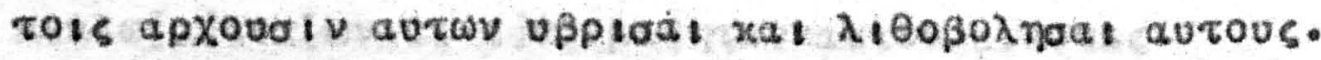

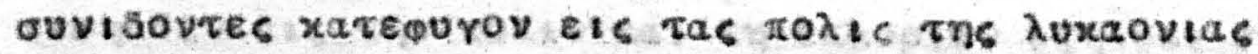

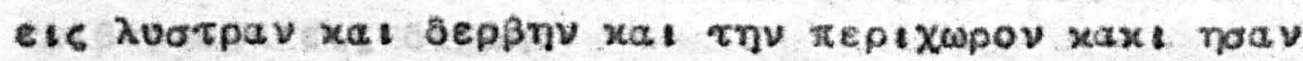

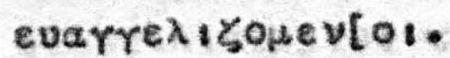

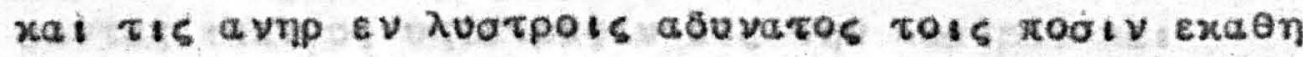

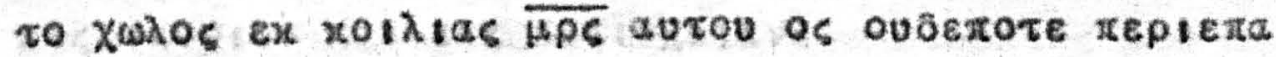

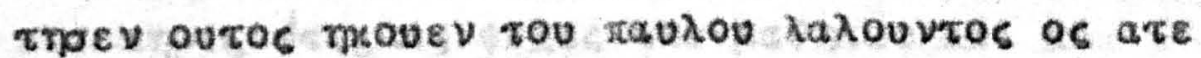

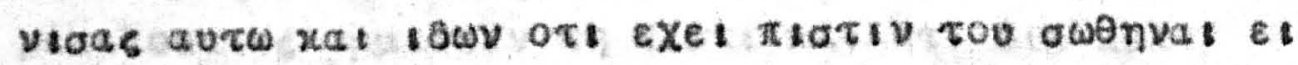

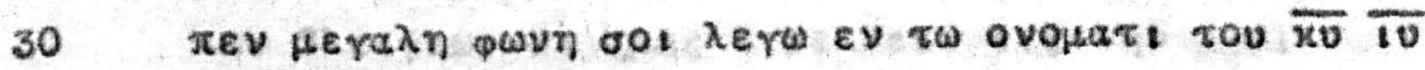

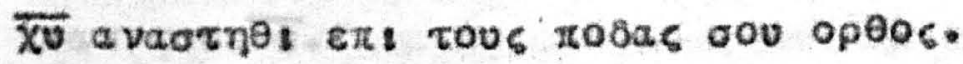

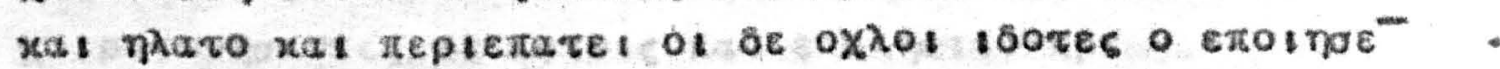

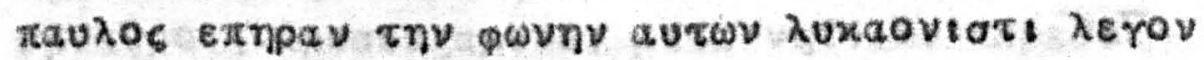

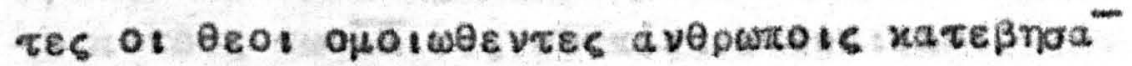

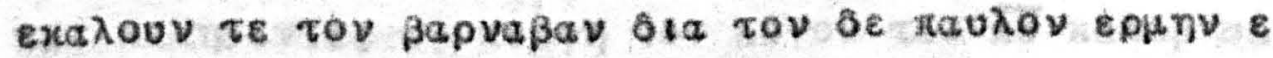

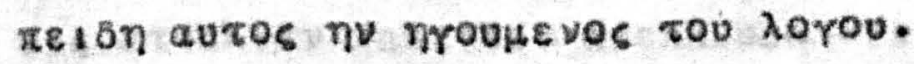
to te lepeus tou 8 tos tou ovros roos the toגews tau

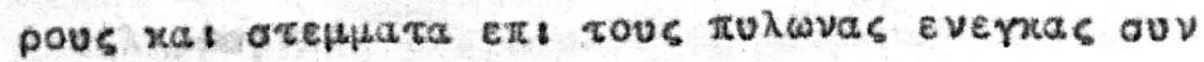

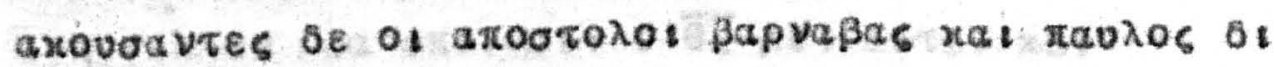




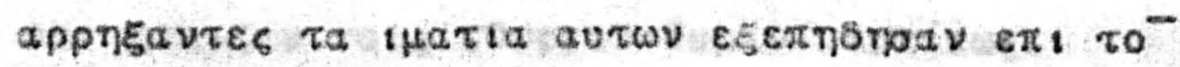

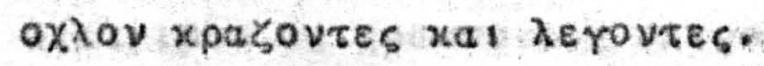

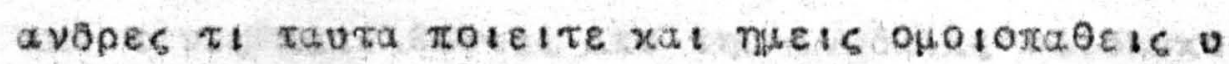

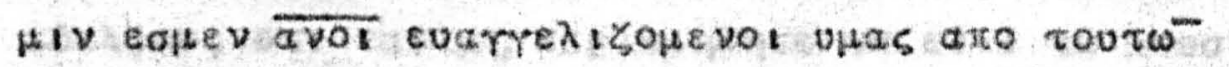

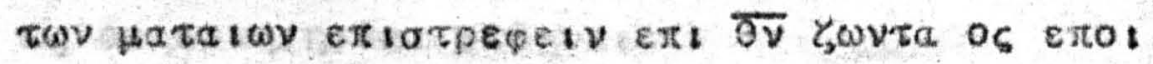

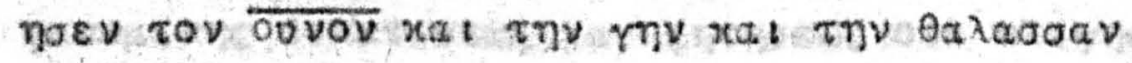

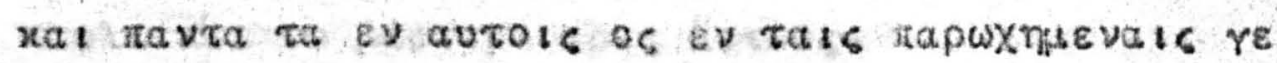

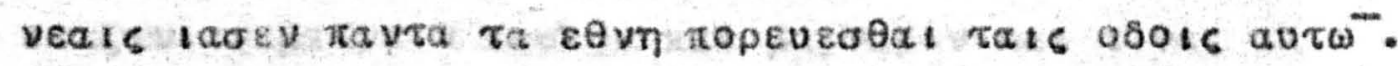

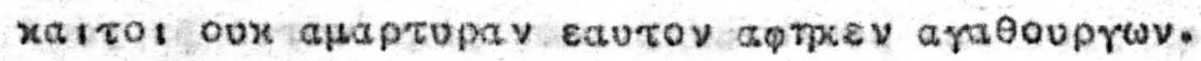

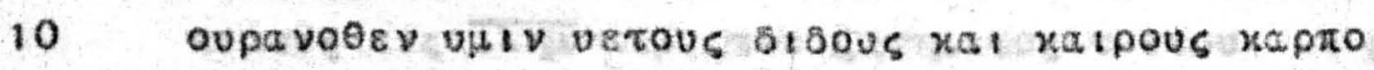
popous eqrithwv tpoens xat evppoouvns tas xaposac viav.

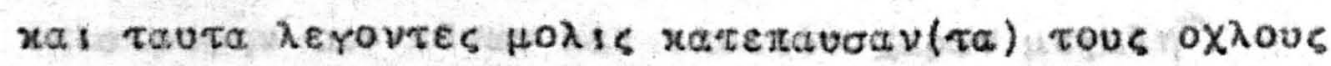

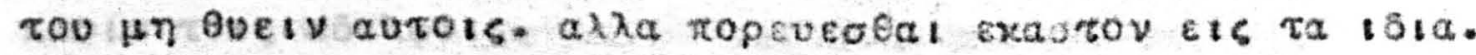

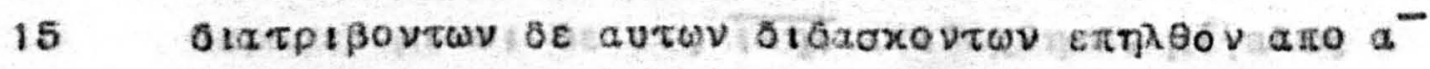
rioxias xas ixoviou tousatol.

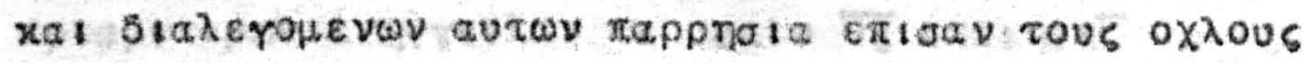

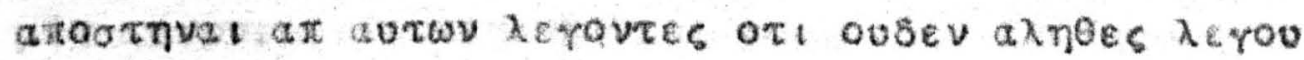

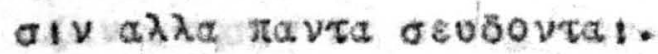

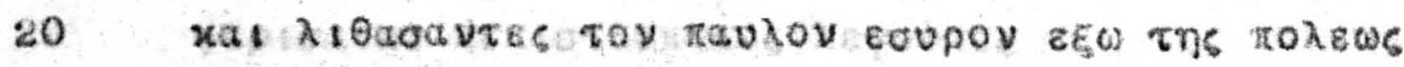

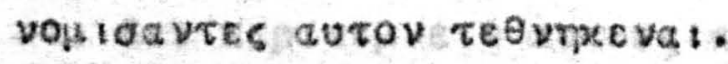

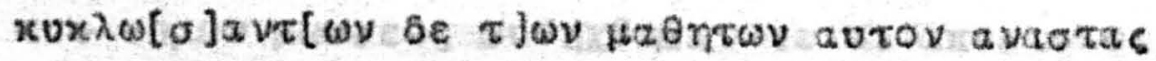

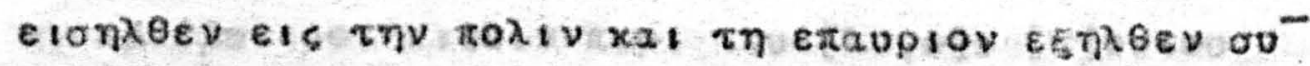

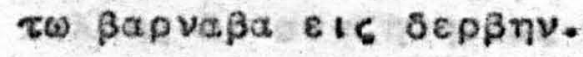

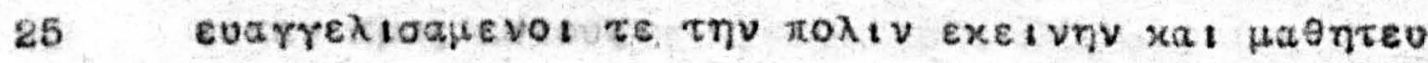

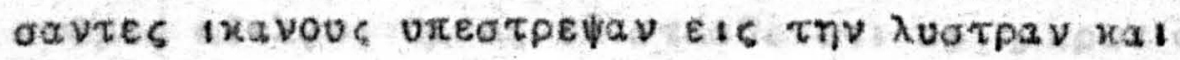
Els hoviov xal ets avtioxetav.

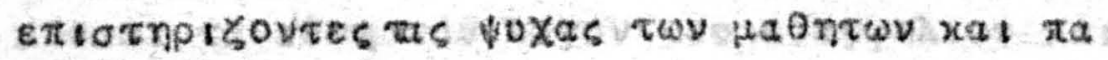

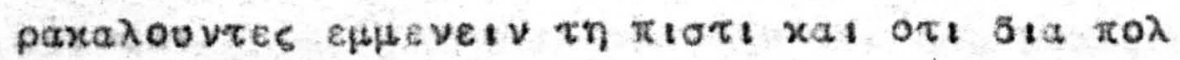

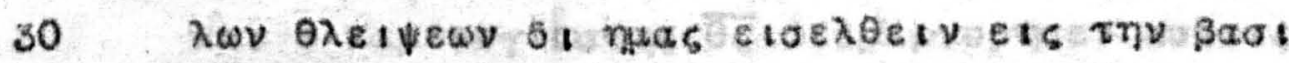

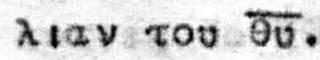

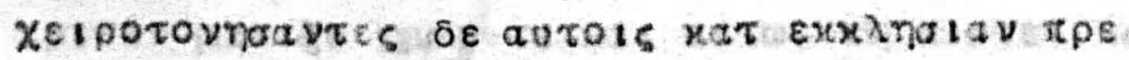

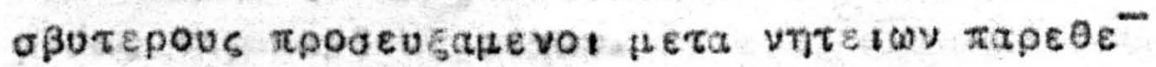
to autous $\tau \omega \overline{x \omega}$ \& 16 oy $\pi$ (eriateuxtady.

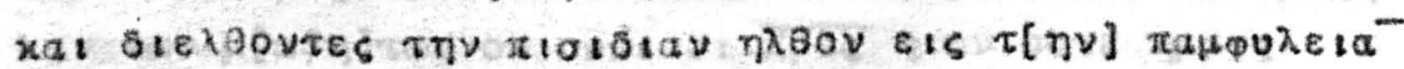

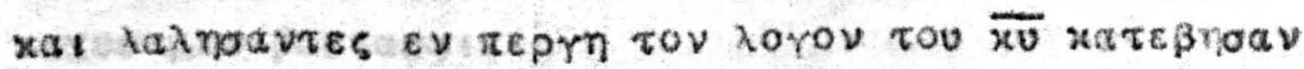

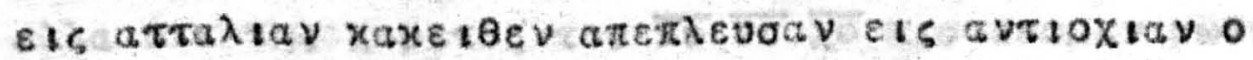

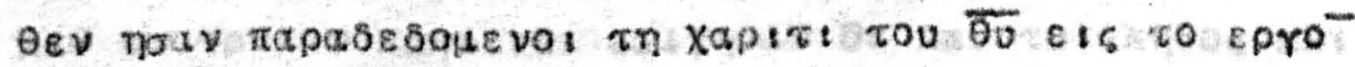
- er $\operatorname{np\omega arv.~}$

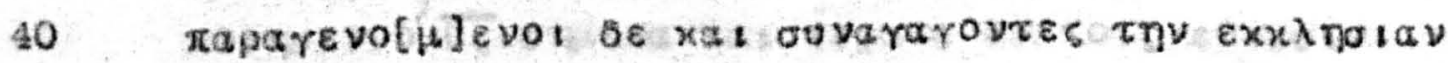

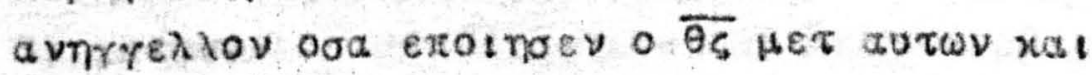




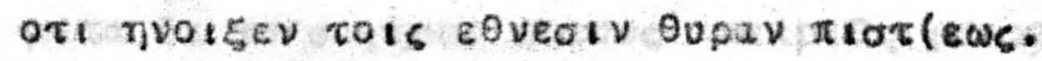

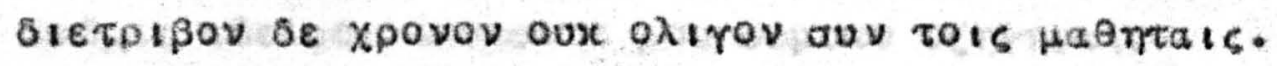

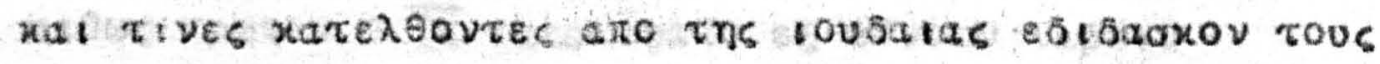

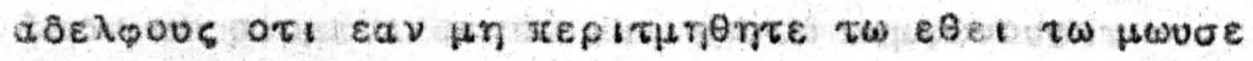
we ov sovnonotas owenval.

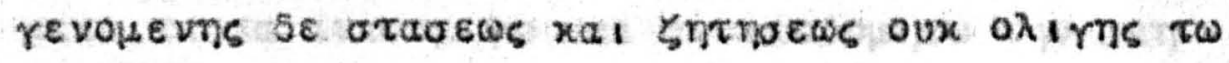

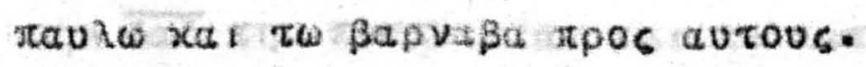

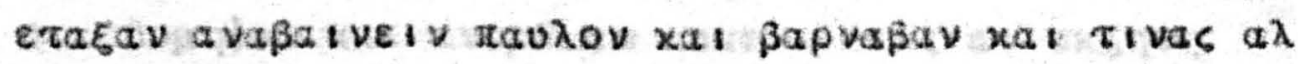

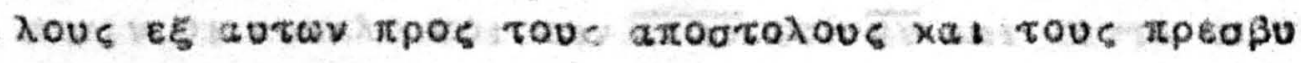

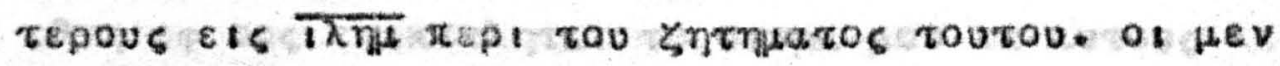

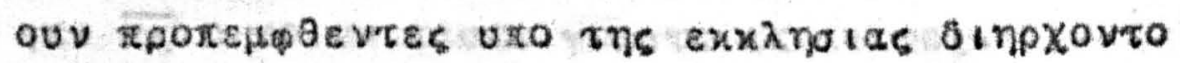

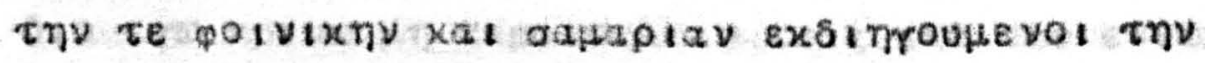

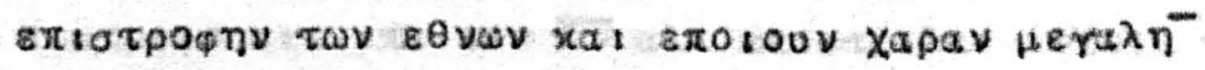
raotv rots ase

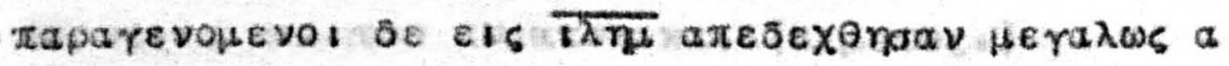

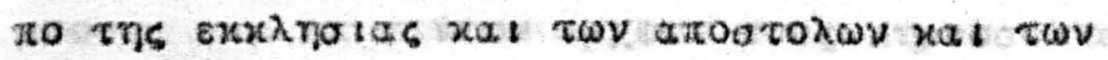

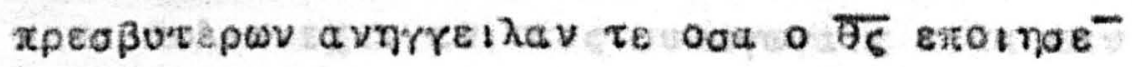
HE $\tau$ aurov.

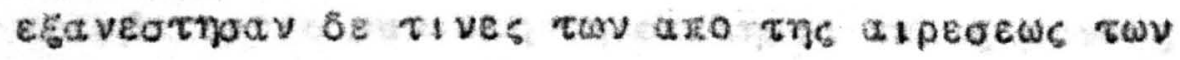

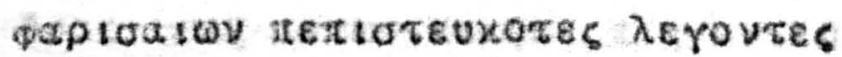

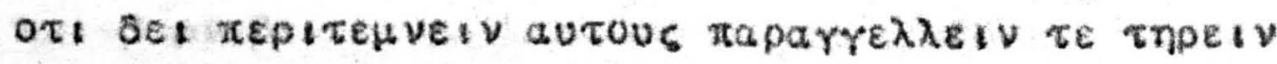
Tov vosav HwV

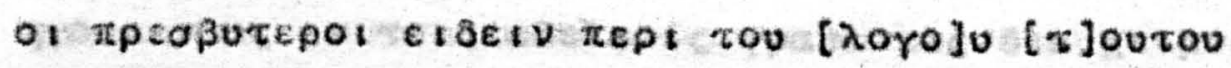

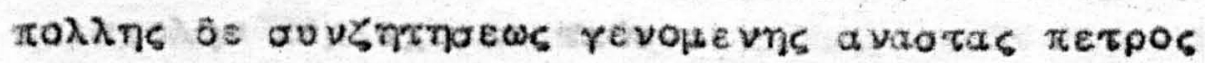

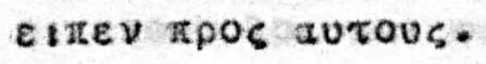

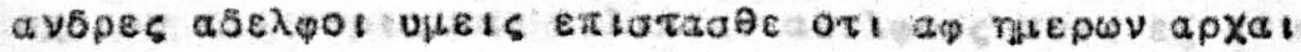

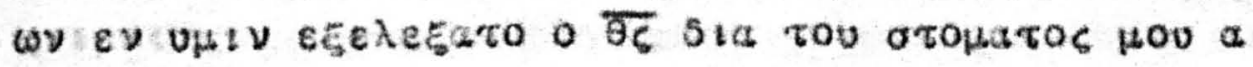

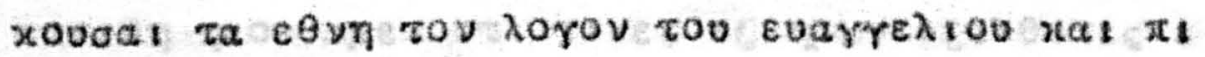
arevort.

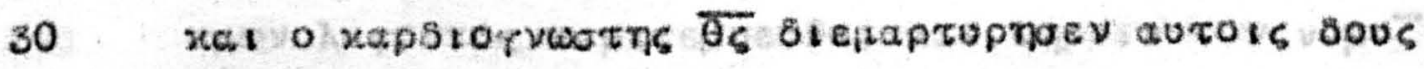

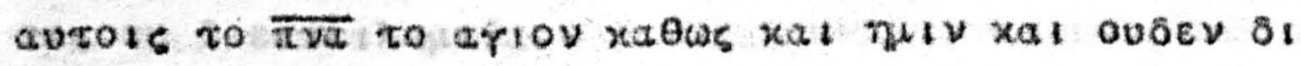

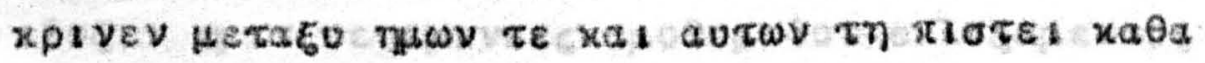
proas tas xaposas avtov.

voy $\tau t)$ )

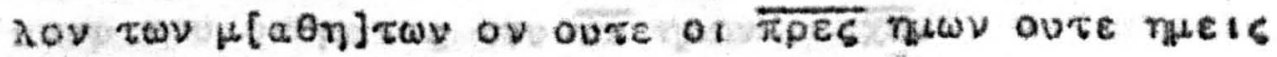
toxvoaper ßaoraoat.

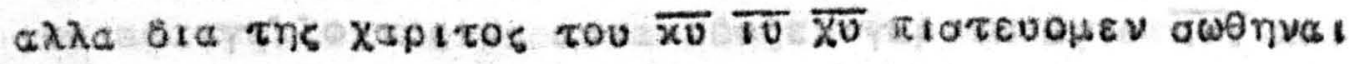
$x a \theta$ ov rporov xax $\varepsilon$ เ

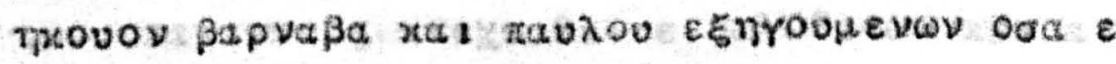

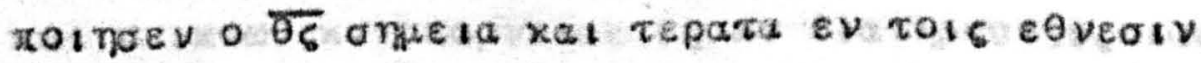
B) aurov. 


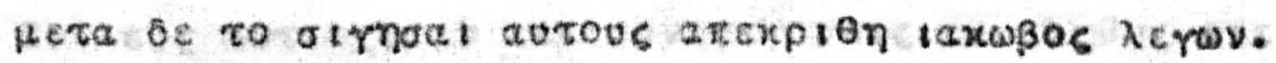

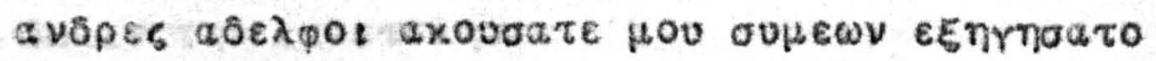

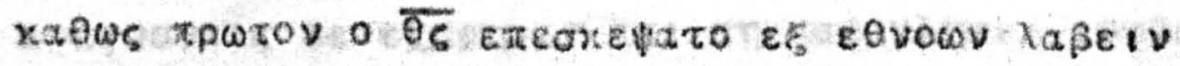

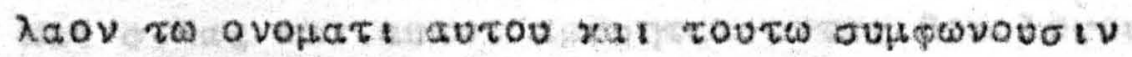

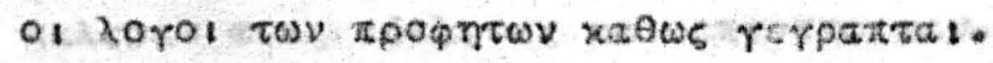

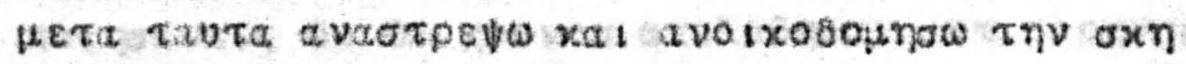

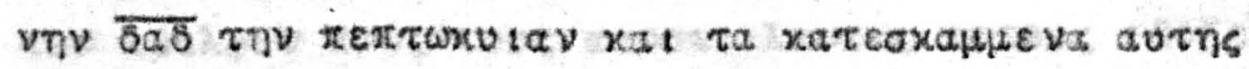

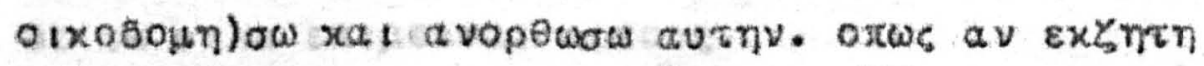

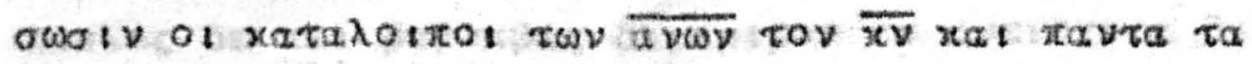

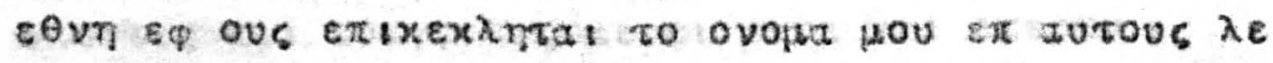

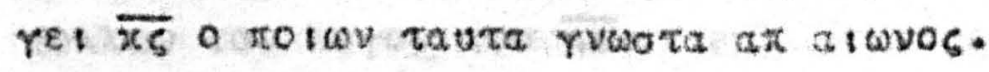

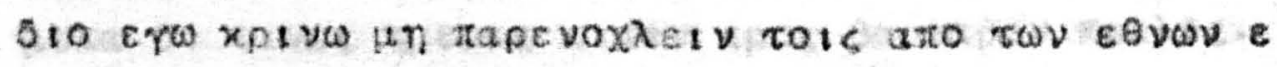

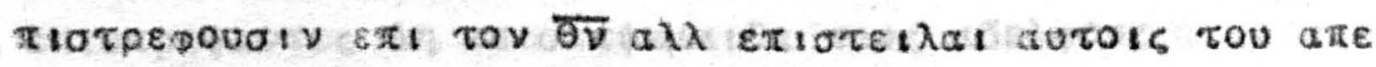

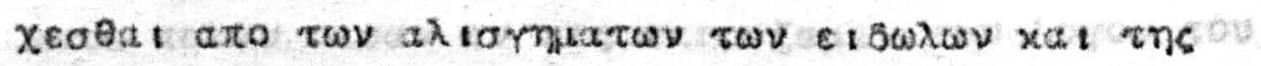

15 Topvetas kat tov gvixrou rai (aliaros.) Hovans rap $\varepsilon x$ revewv apXewv tous unpuosovras auto-

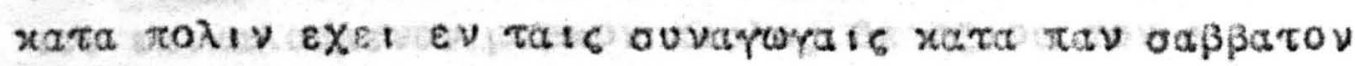

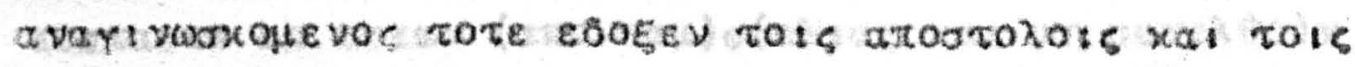

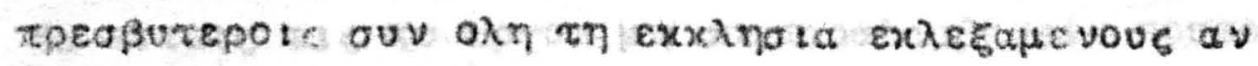

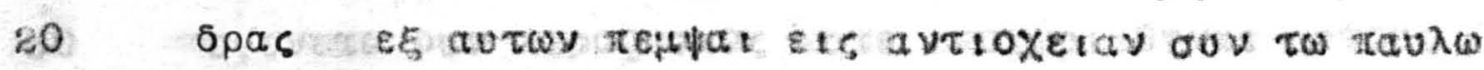

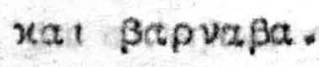

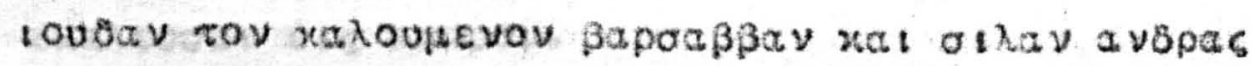

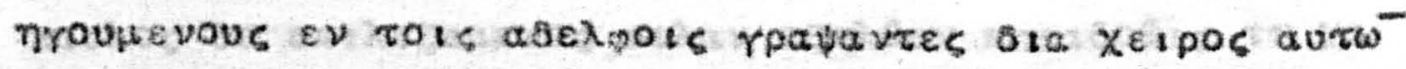

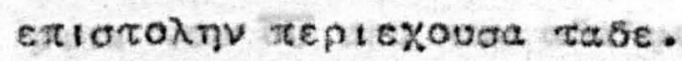

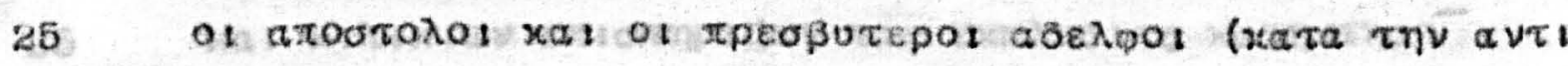

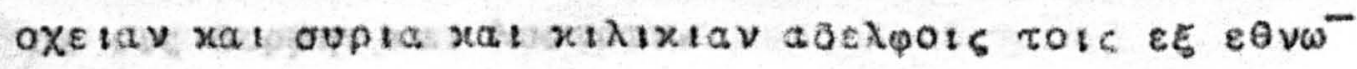
Xatpetv.

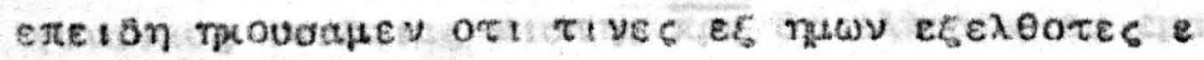

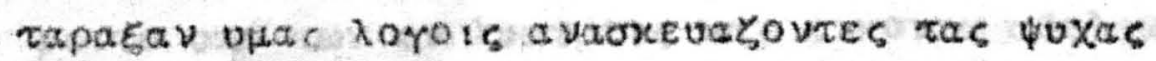

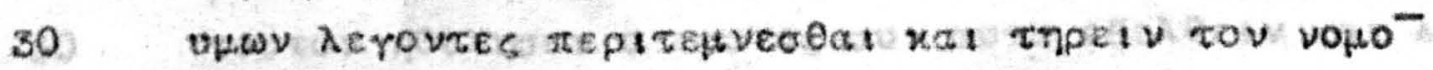
O:5 ov ठิ

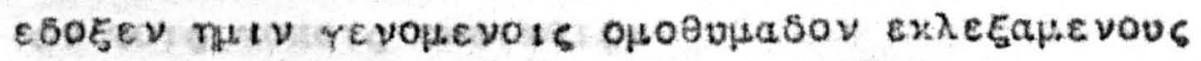

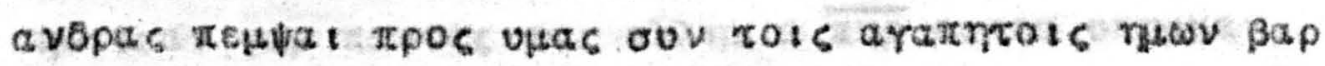

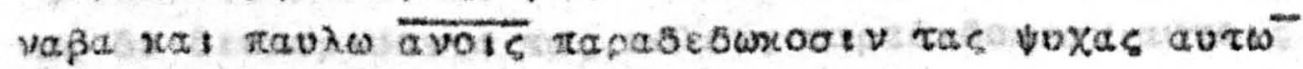
35 vтep tov ovouaros rov $\overline{x v}$ nuesv $\overline{\text { To }} \overline{\mathrm{XV}}$.

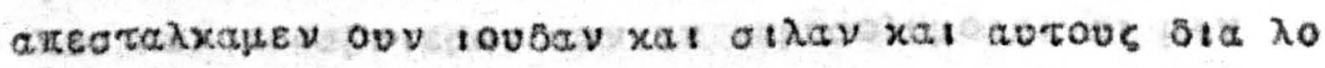

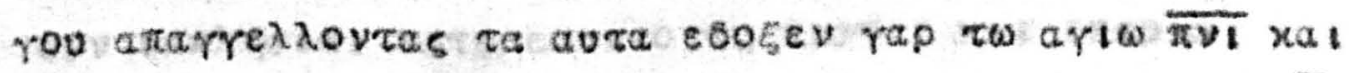

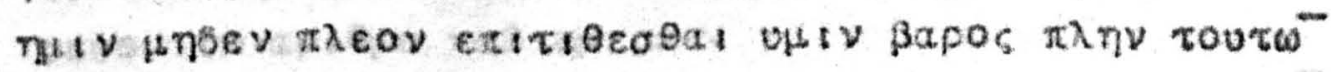

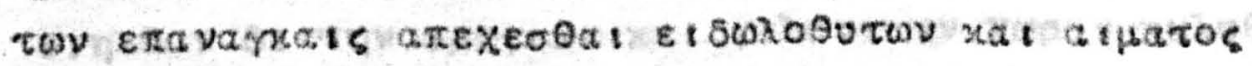

40 xat $\pi v t x \tau \omega \nu$ xat ropyetae $\varepsilon \xi$,

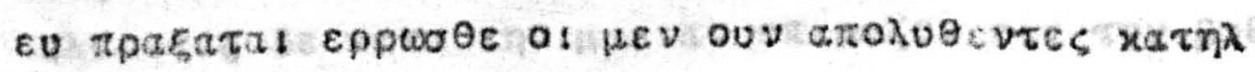




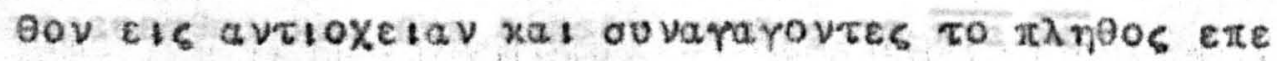

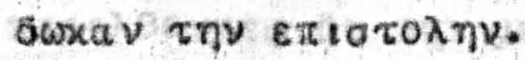

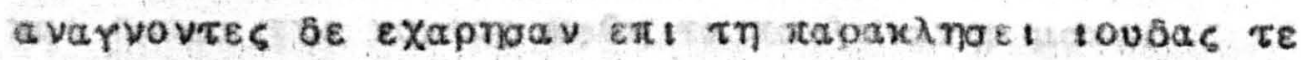

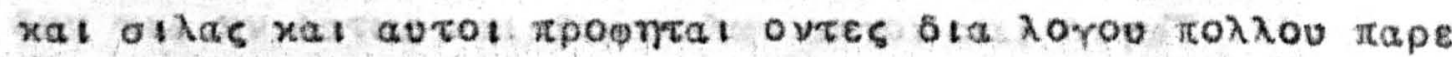

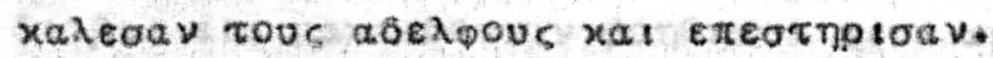

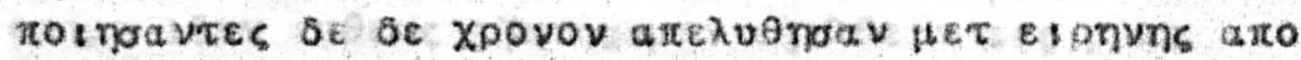

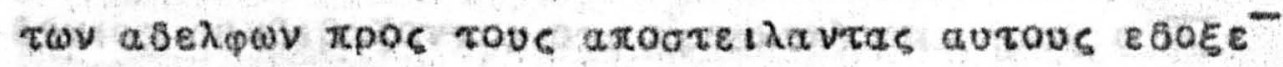

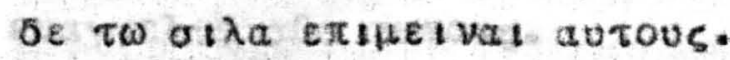

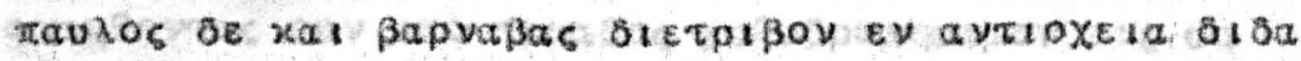

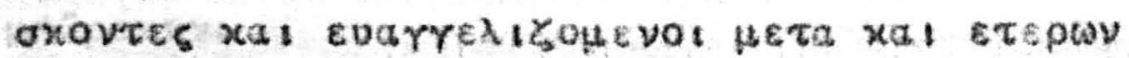

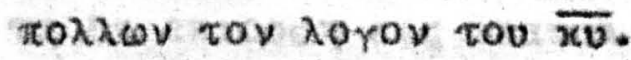

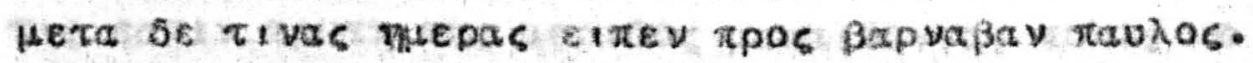

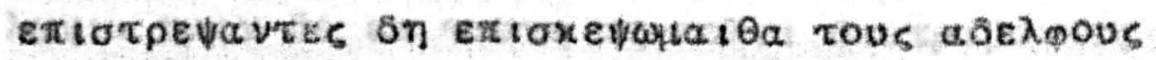

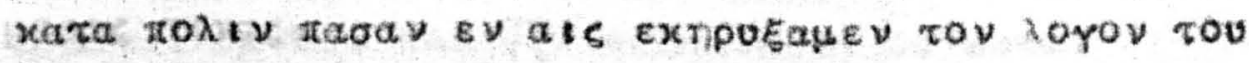
$\overline{x v} \pi \omega s$ exovoiv.

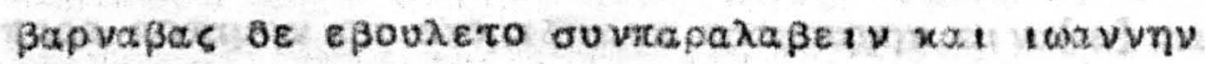

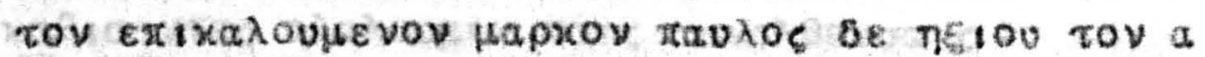

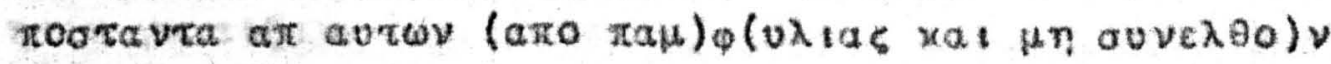

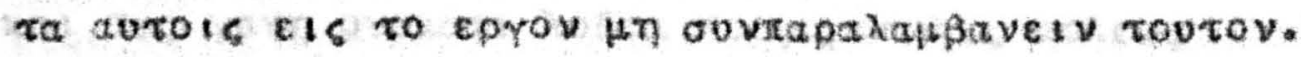

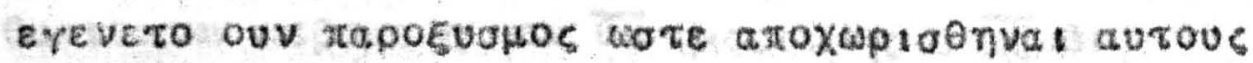

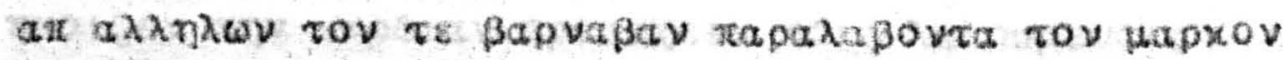
exrievoat $\varepsilon$ is xumpov.

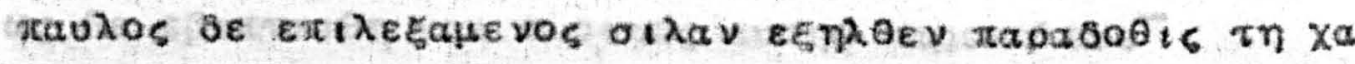

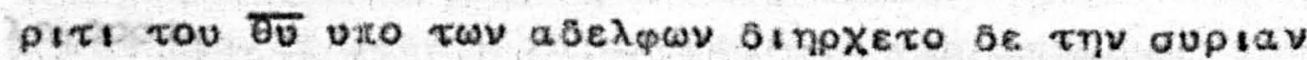

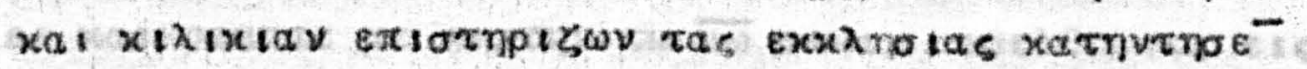

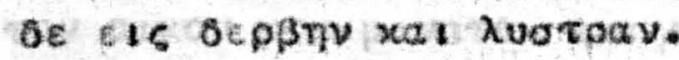

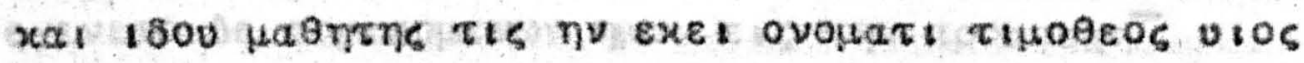

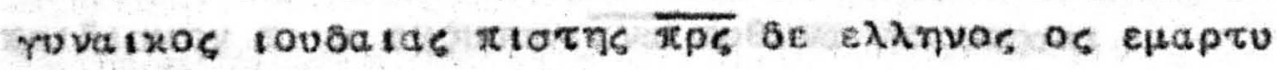

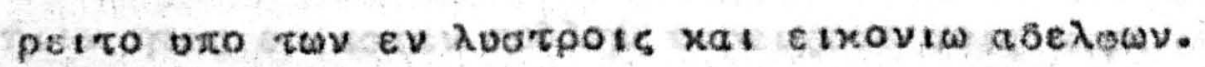

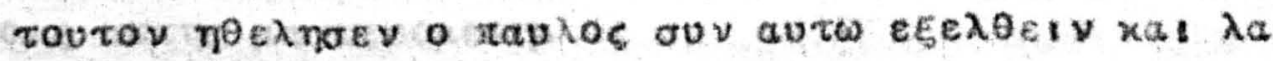

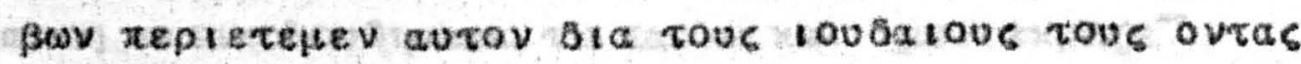
eV rois totols Exetvois.

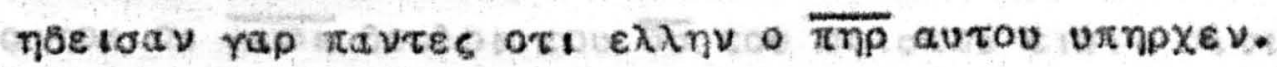
ws $\delta \varepsilon$ ot

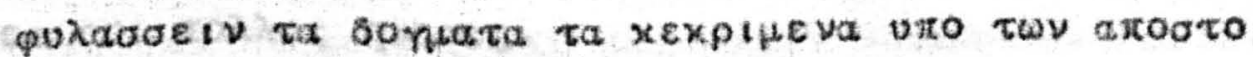

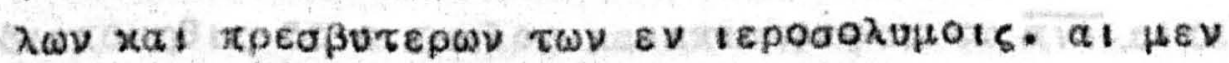

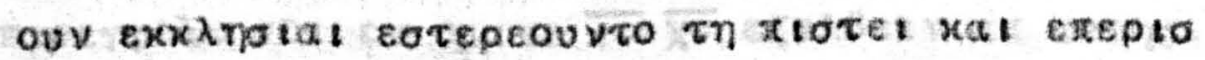
oevov $\tau \omega$ apı

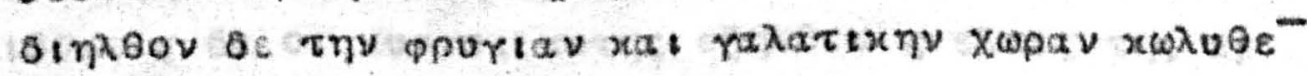

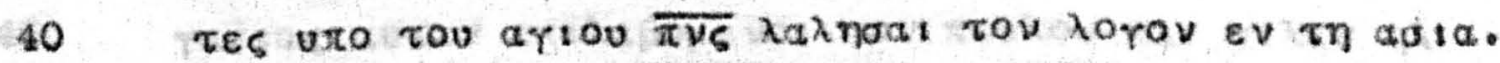

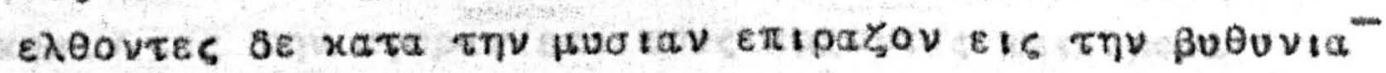




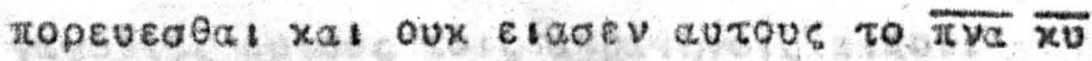

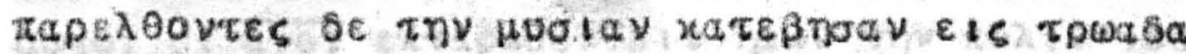

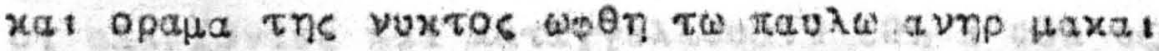

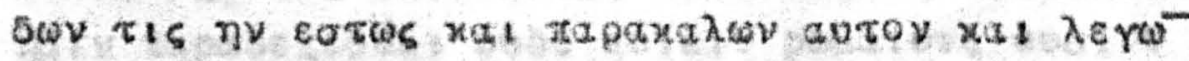

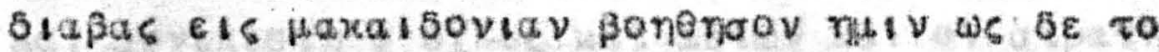

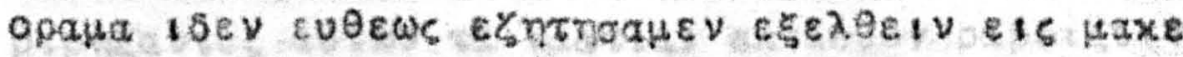

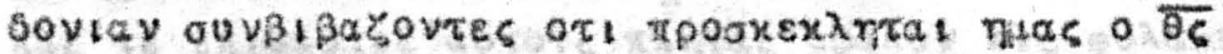
evarredtesobe autous.

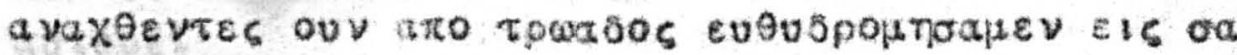

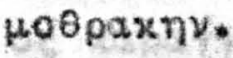

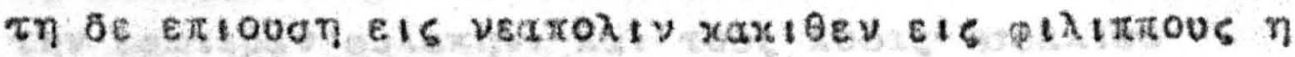

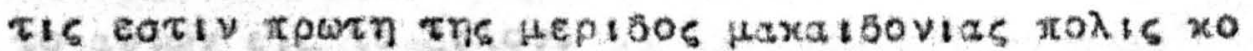

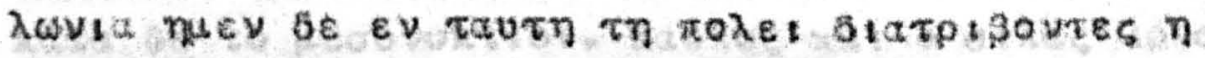
Hepas tivas.

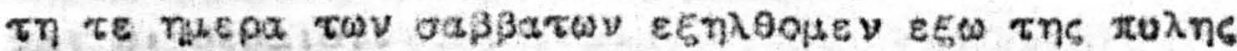

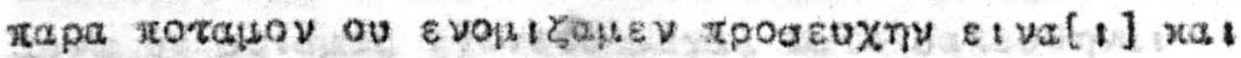

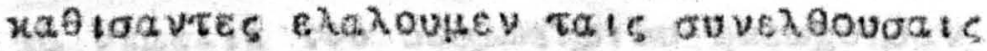

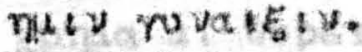

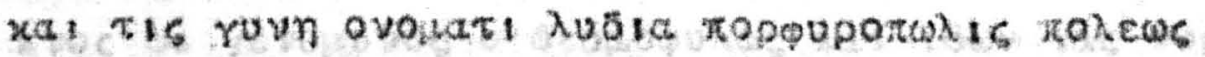

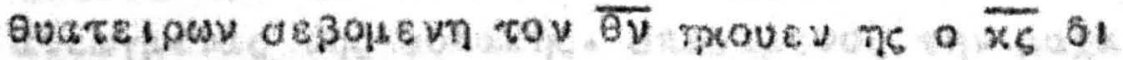

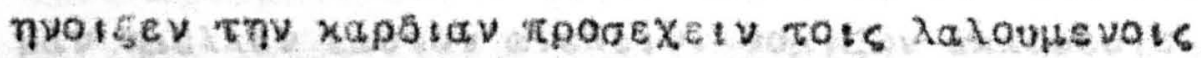
vio rou taviou.

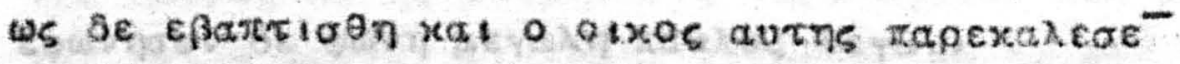
$\lambda \varepsilon$ rovod.

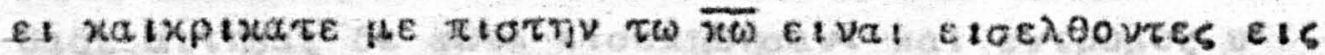

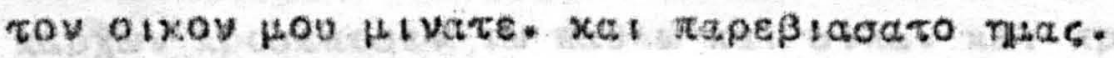

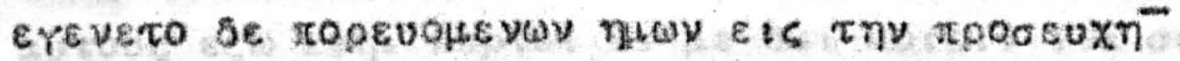

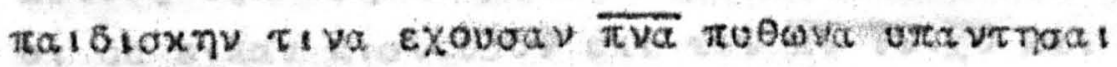

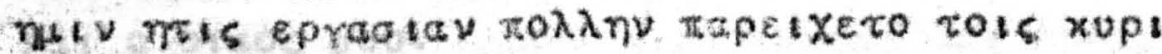
ois autns mavtevopevn.

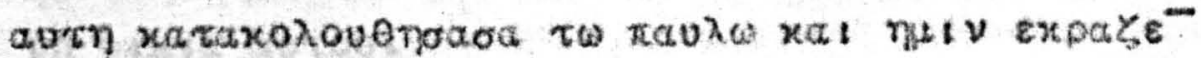
$\lambda$ eyouga.

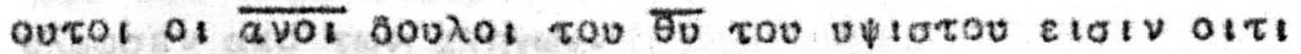

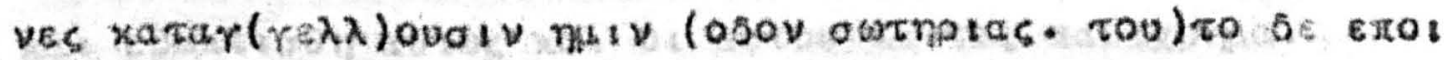

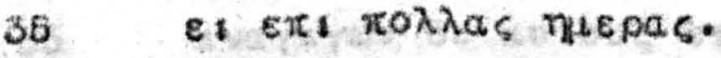

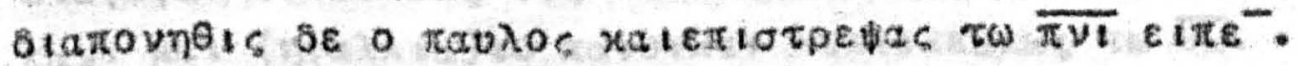

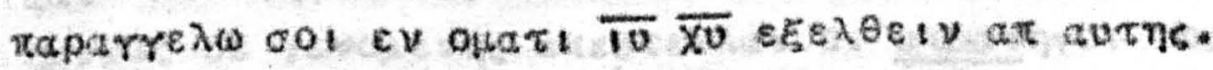

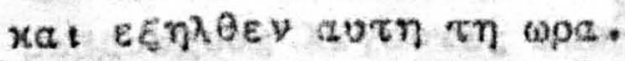

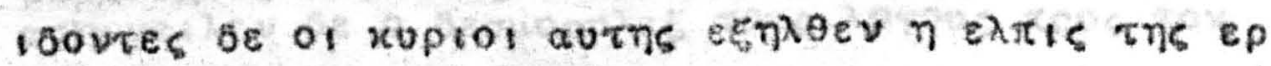

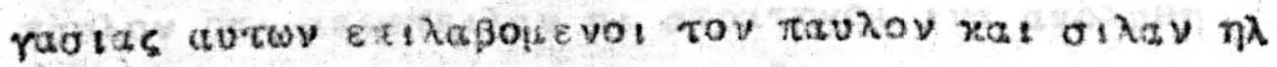

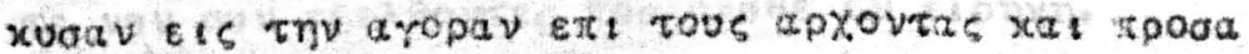


rarovies autous tois arpatnrois eirov.

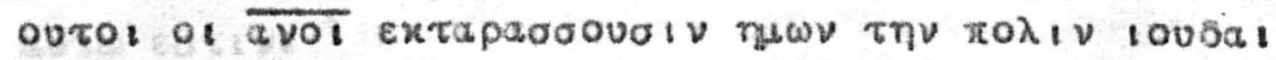

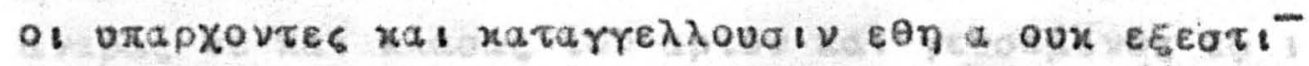

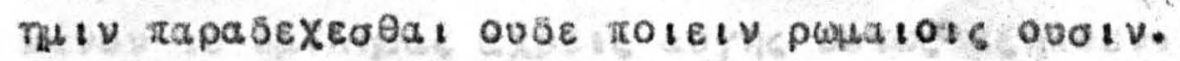

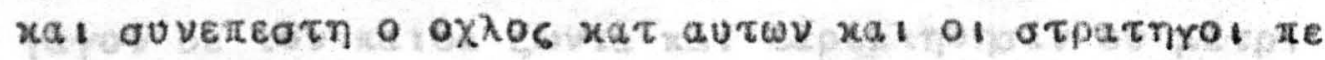

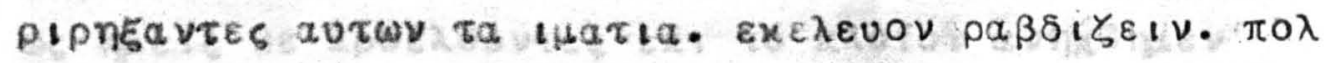

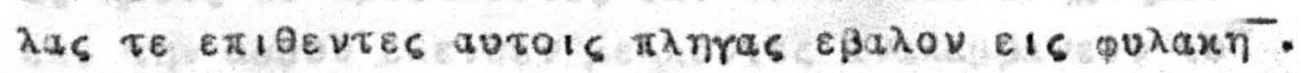
raparreı autous.

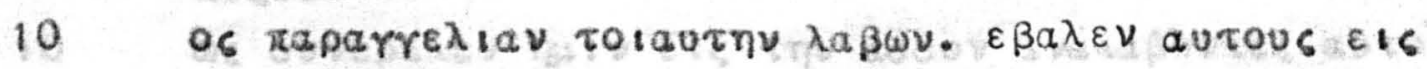

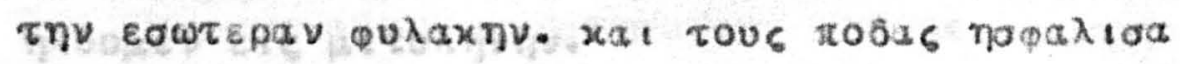

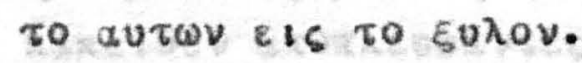

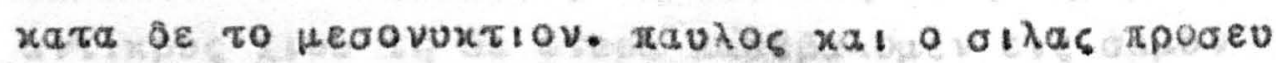

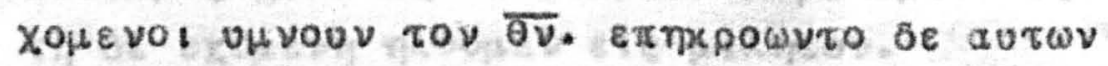

15 xal ol sequloi.

apvw $0 \varepsilon$ a\&lahos erevero Heras wate aAdevenva!

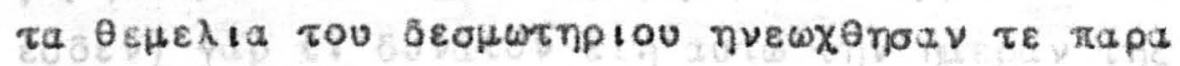

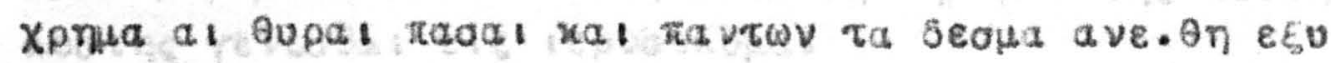

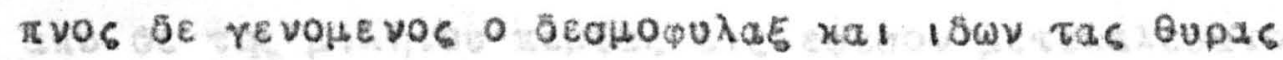

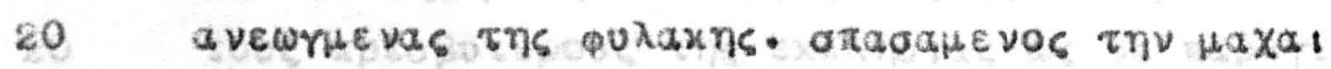

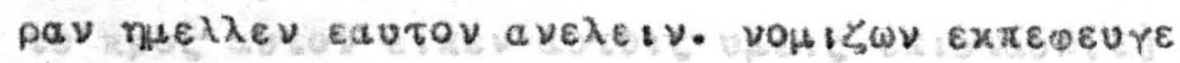
val Tous seantous.

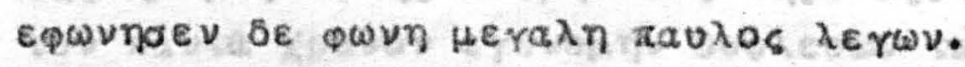

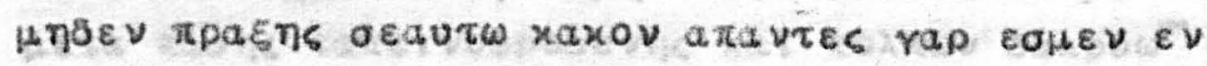

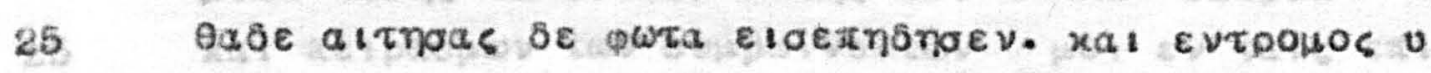

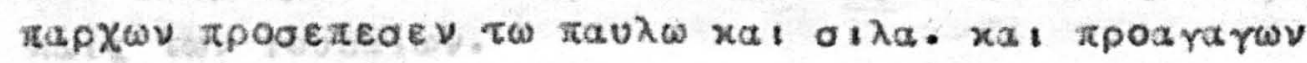

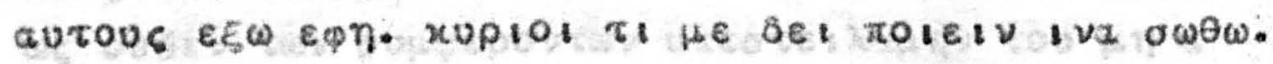

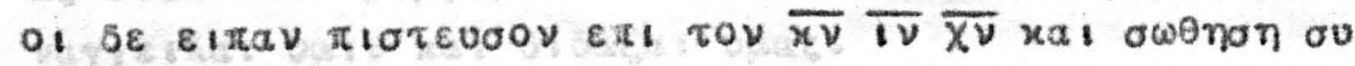
xas o oixos oov.

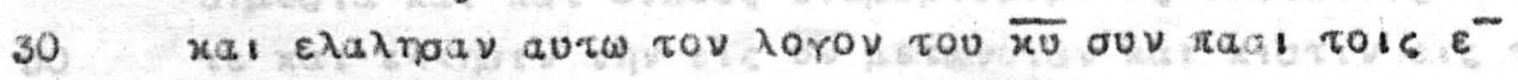

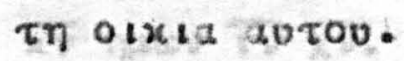

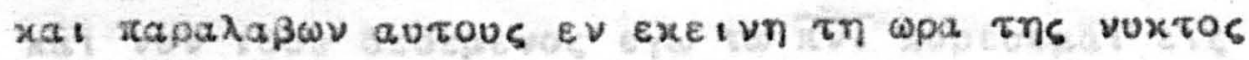

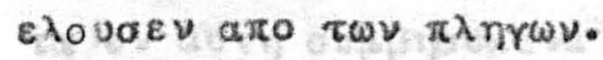

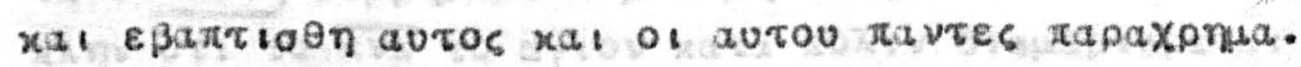

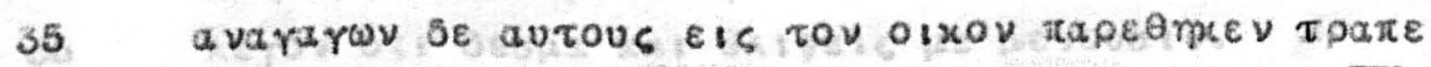

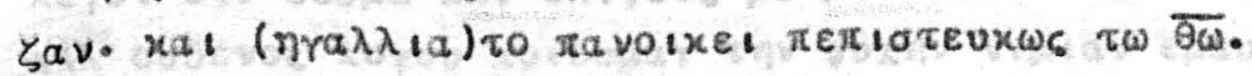

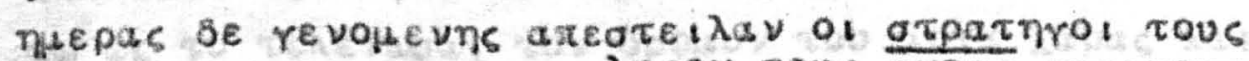

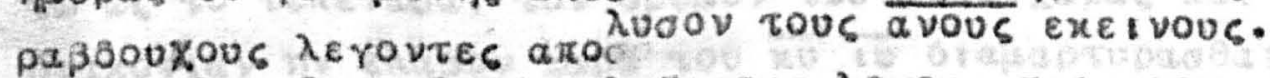

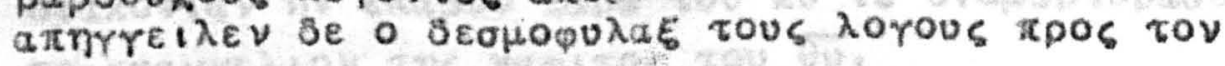

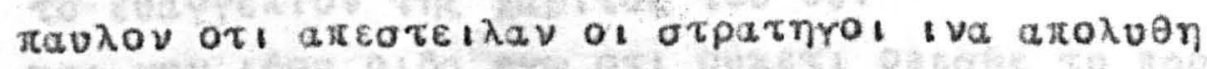
$\tau \varepsilon$ vU 
$\lambda \omega \nu$ a. $\alpha \cup \tau \omega \varepsilon \sigma \tau \iota v$.

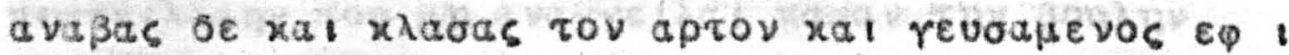

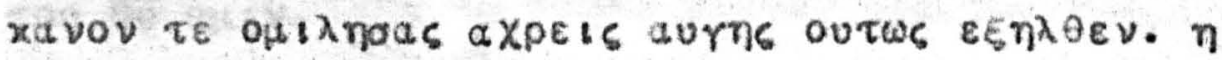

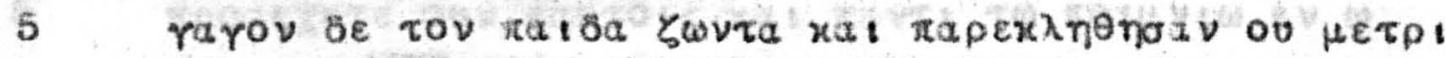

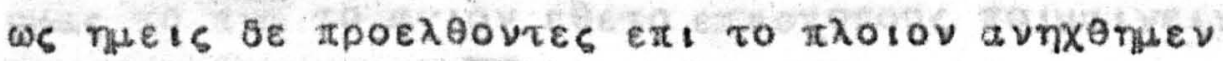

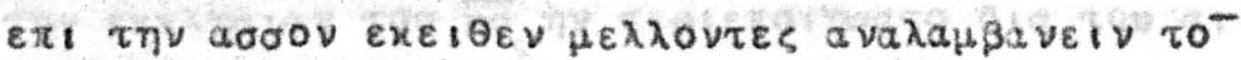

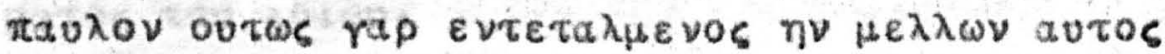
$\pi \varepsilon \zeta \varepsilon \cup \varepsilon เ v$.

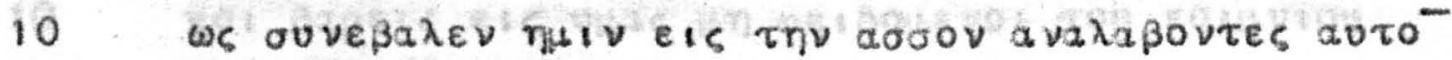

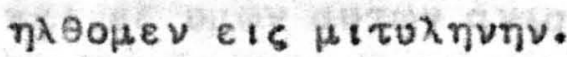

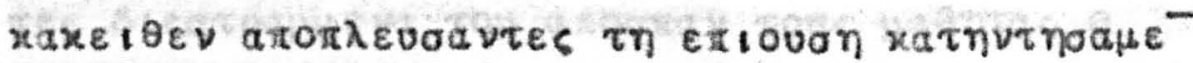

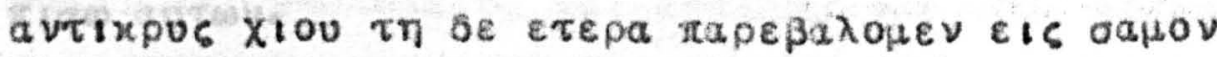

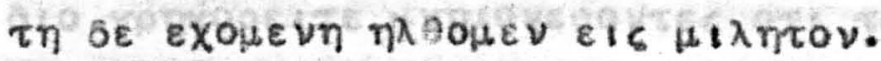

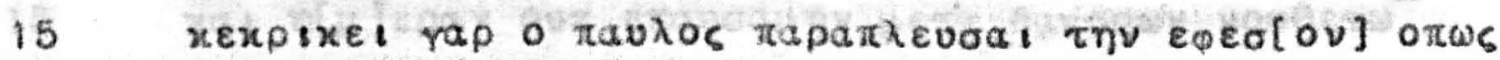

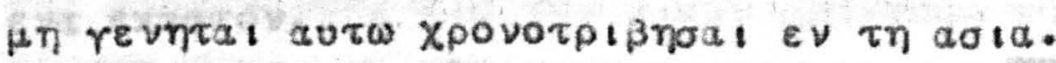

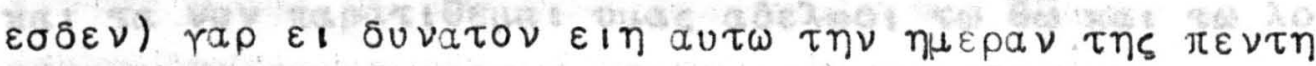

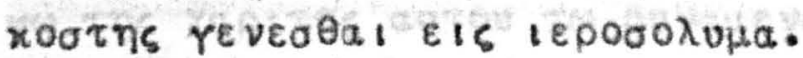

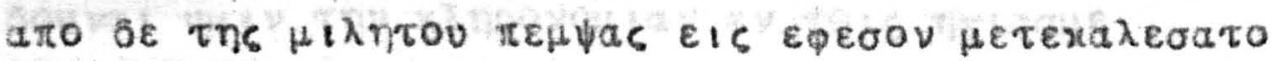

20 tous tpeoputepous ths exxAnotas ws $8 \varepsilon$ staperevov

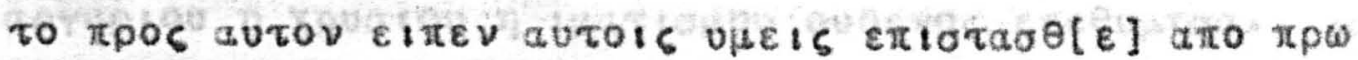

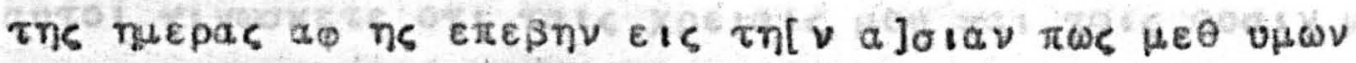

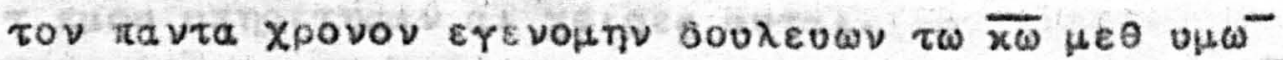

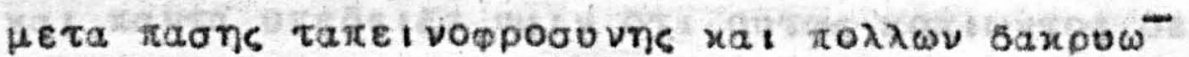

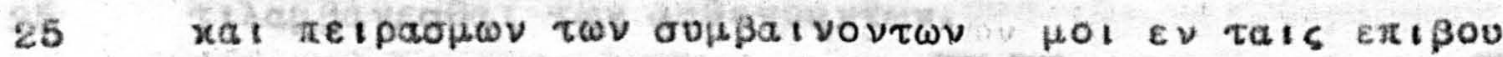

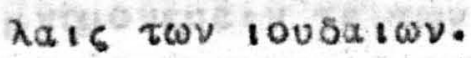

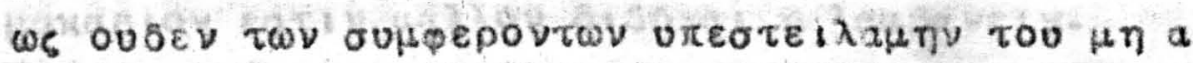

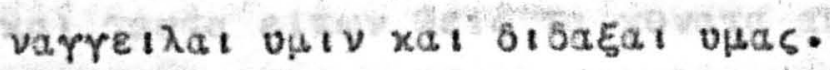

onjoola xat xat olxous olapaptupopevos loubatots

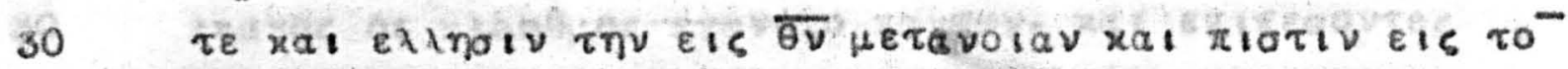

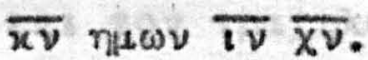

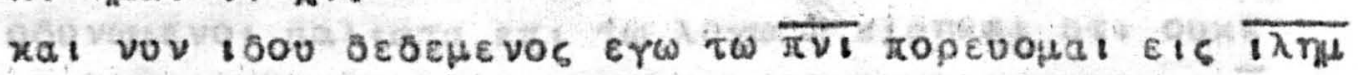

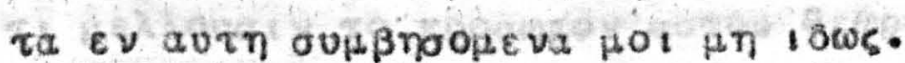

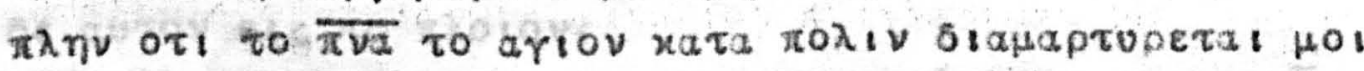

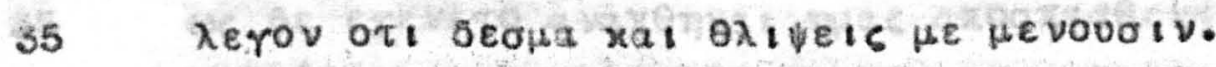

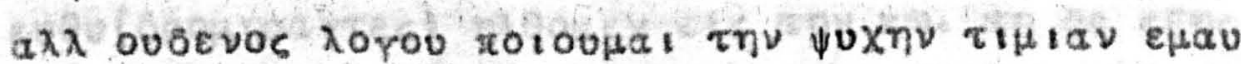

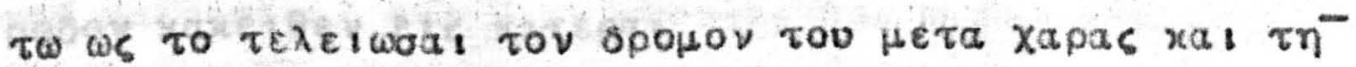

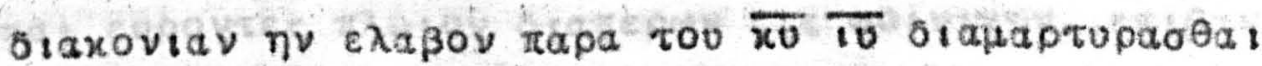

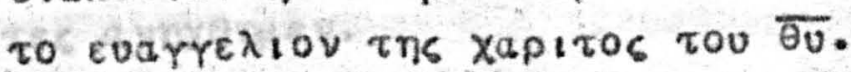

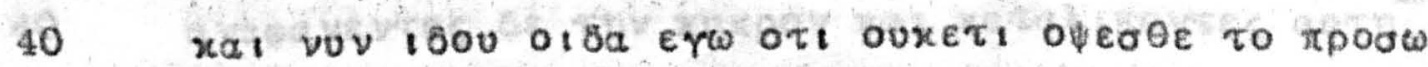

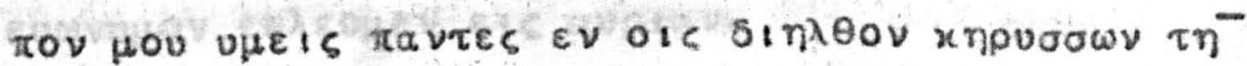




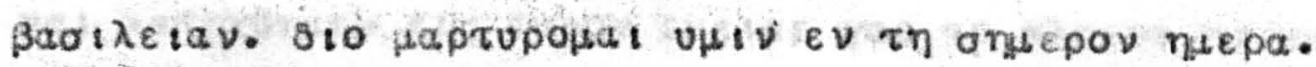

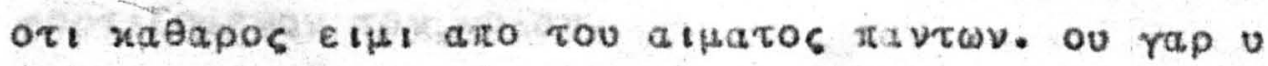

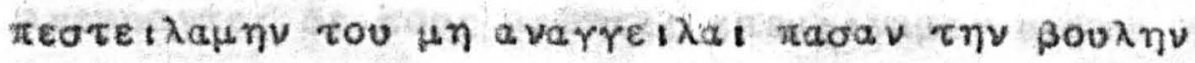
tov बU UนtV.

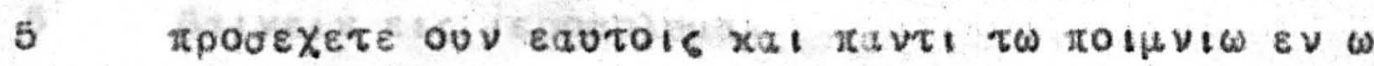

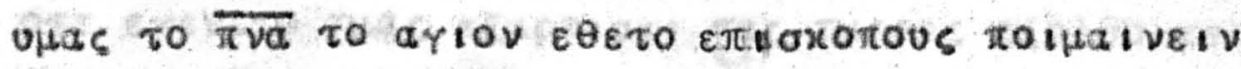

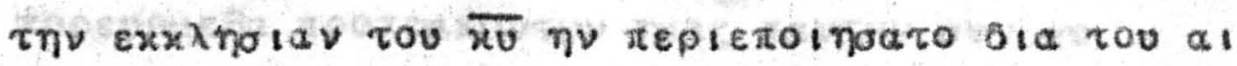
Hatos tov 16100 .

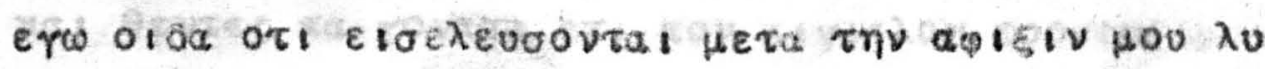

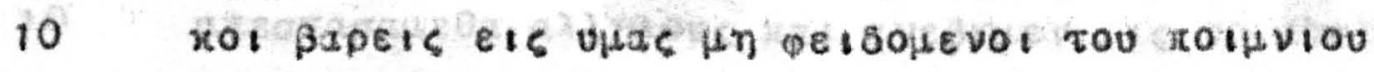

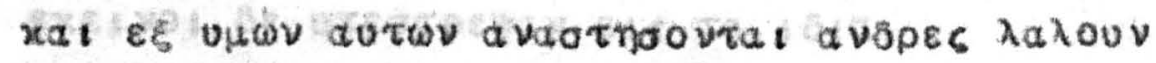

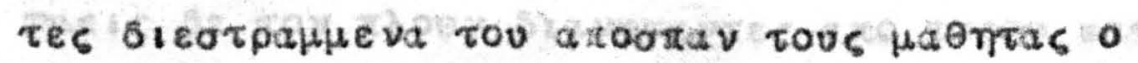
स $10 \omega$ aurw.

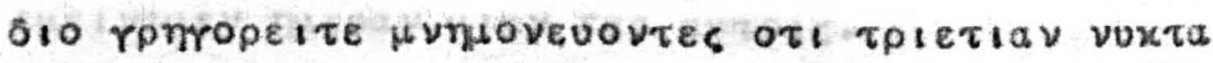

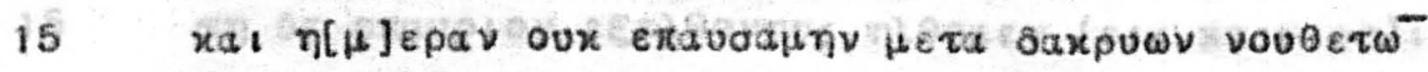
eva exarotov.

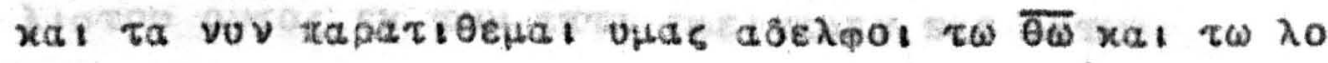

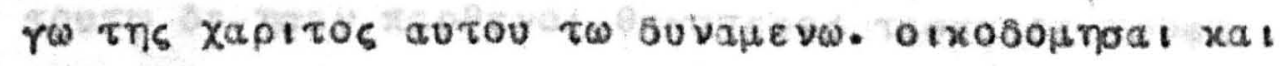

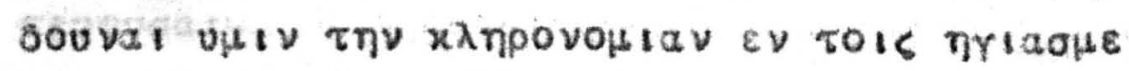

20 vois $\pi a t v$.

apruptou $\eta$ Xpootou

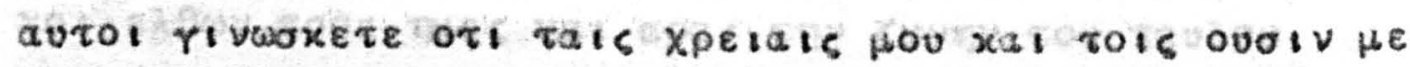

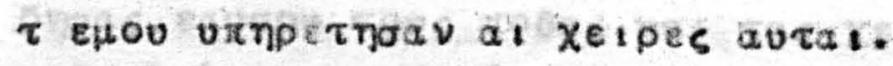

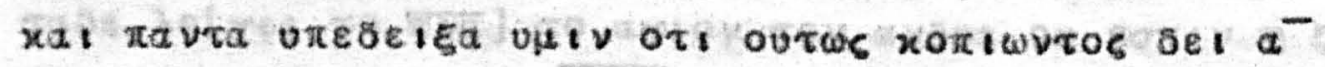

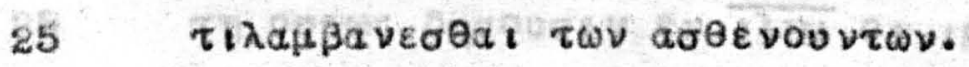

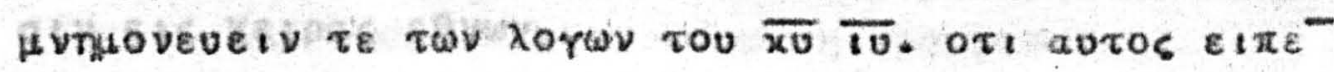

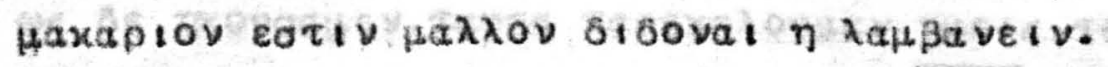

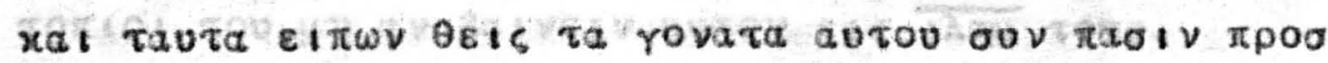

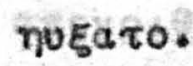

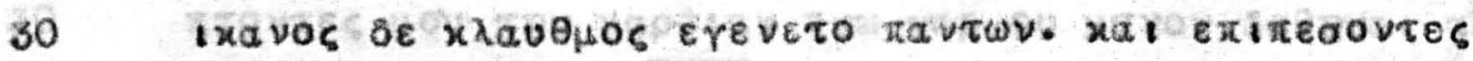

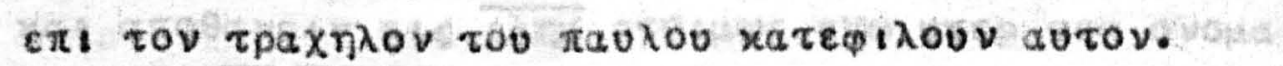

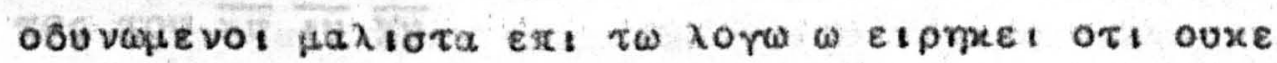

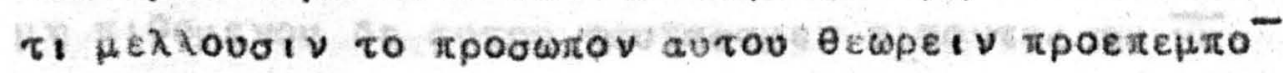
SE autov eis to tinoiov.

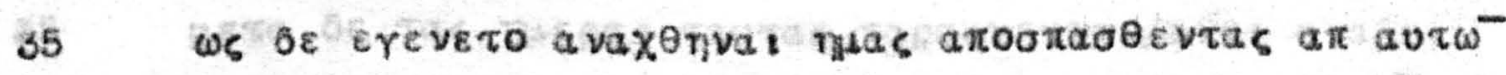

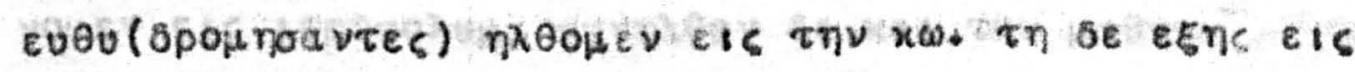

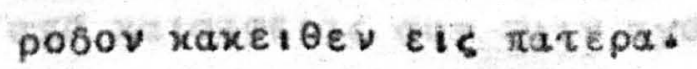

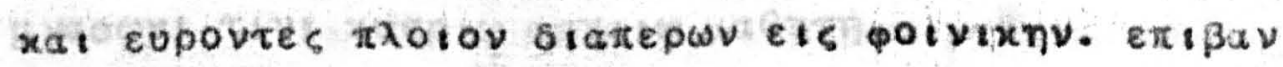
$\tau \varepsilon \zeta$ a vix $\theta$ nuev.

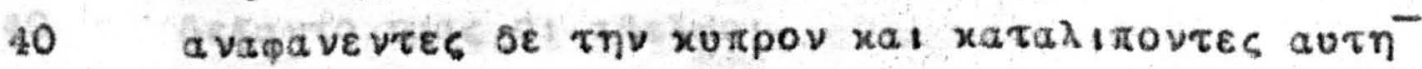

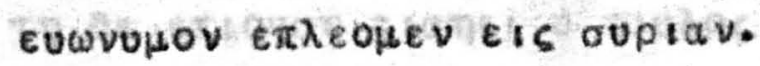




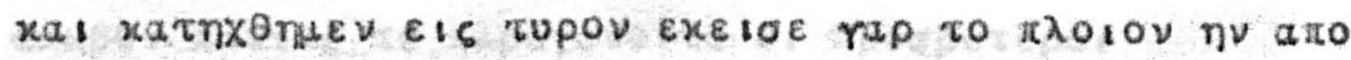

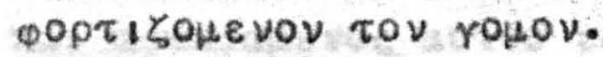

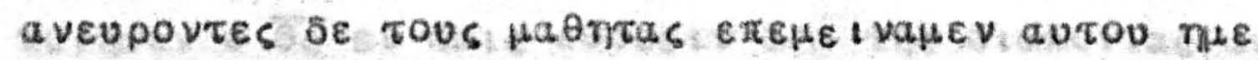

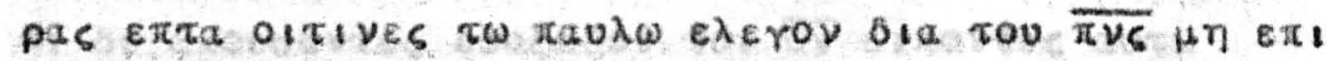

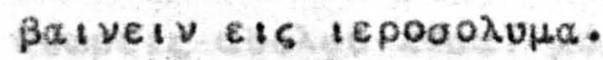

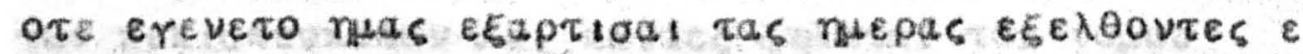

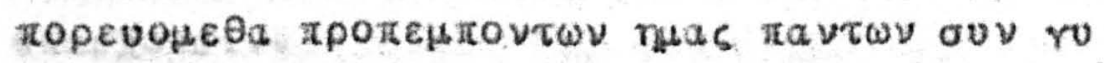

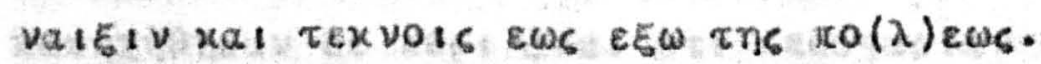

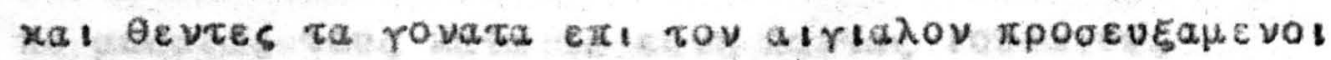

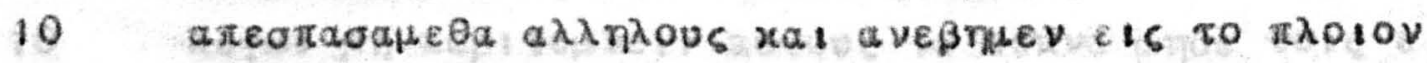
Exe!vol of vieotpetay eis ta $151 a$.

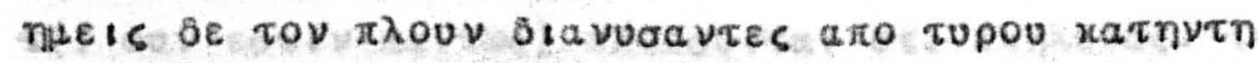

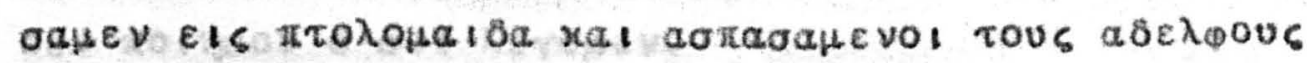

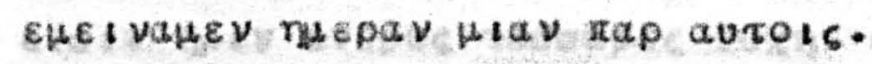

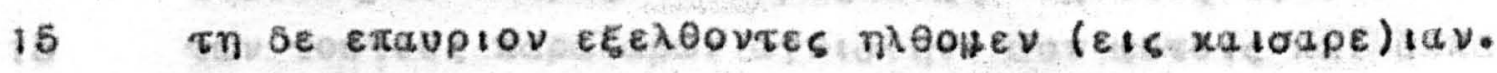

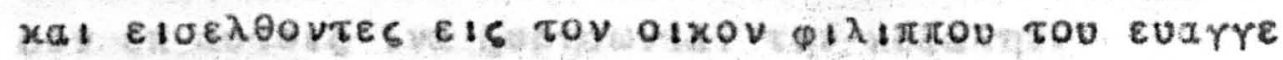

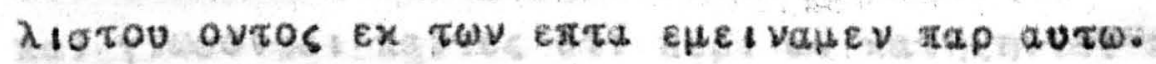

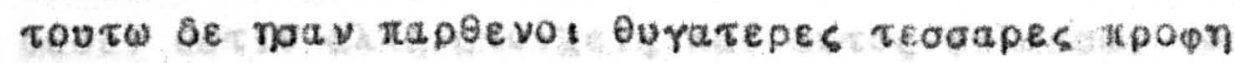
revovoal.

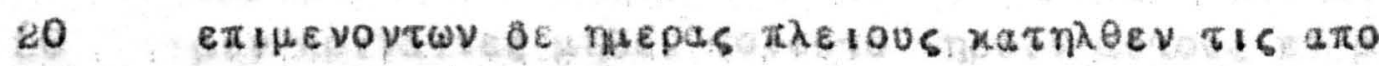
$\tau[\eta] s$ ioubalas xpopnns ovouat araßos.

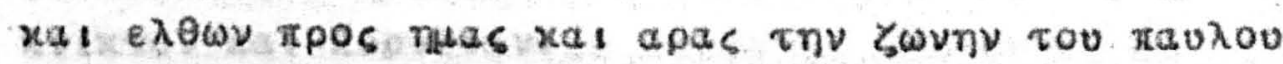
Inoas eautou tous zobas xal tas Xetpas $\varepsilon$ t $\pi \varepsilon v$.

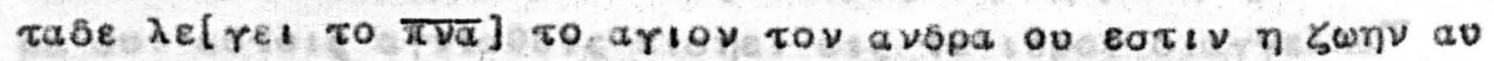

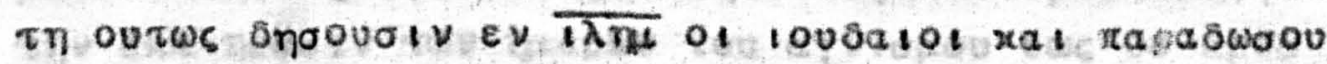
oiv eis Xeipas $\varepsilon \theta v \omega v$.

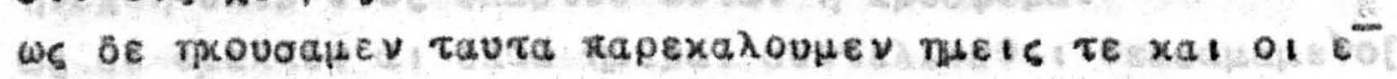

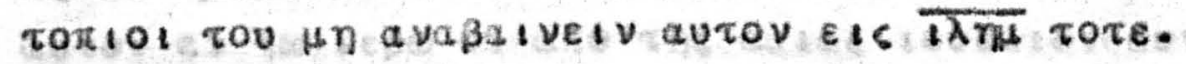

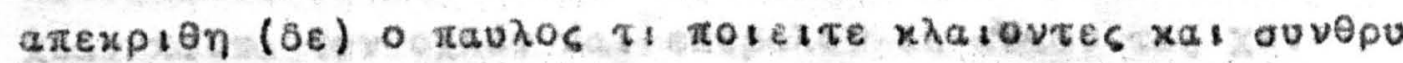

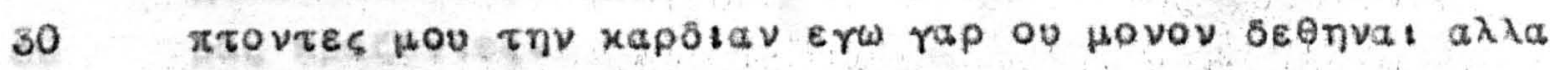

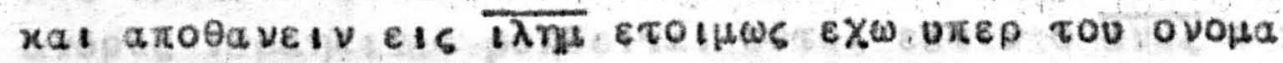
ToG $\tau$ Tov $\overline{x U} \overline{\mathrm{iv}} \overline{\mathrm{XU}}$.

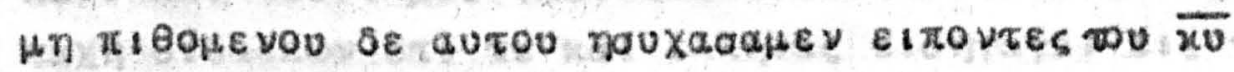

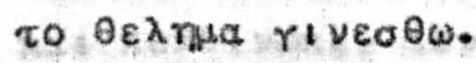

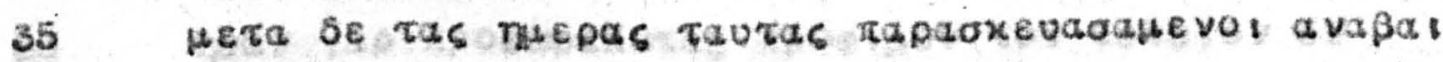

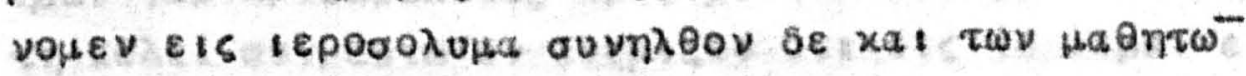

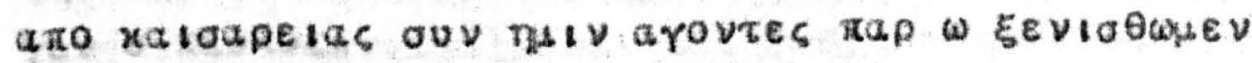

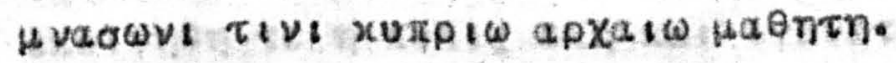

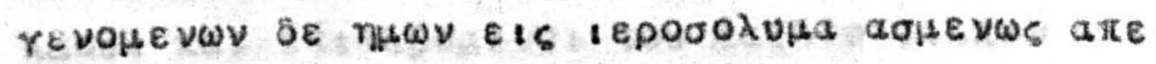

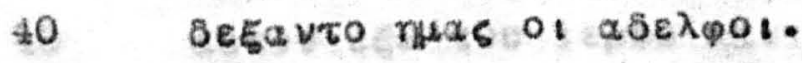

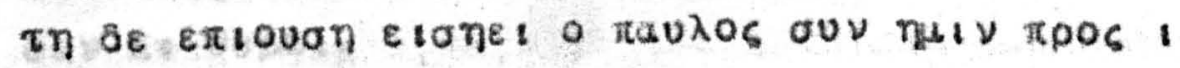




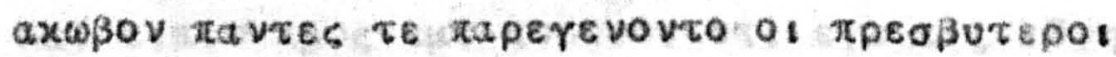

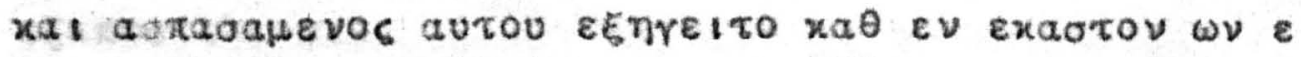

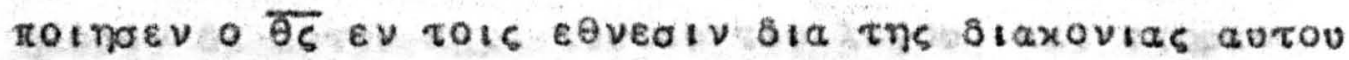

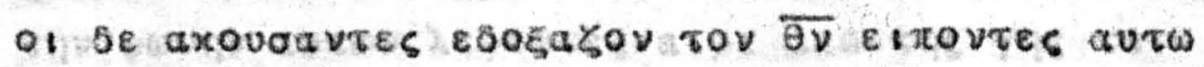

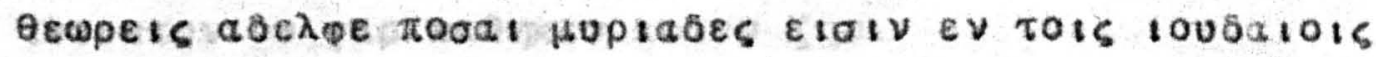

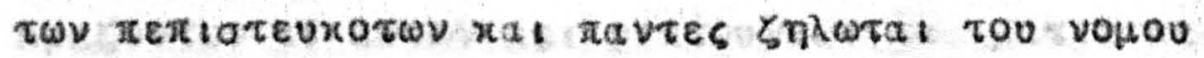

vitapXovaiv.

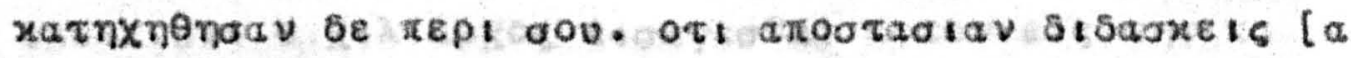

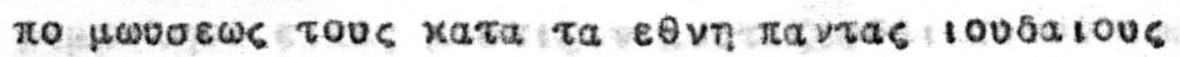

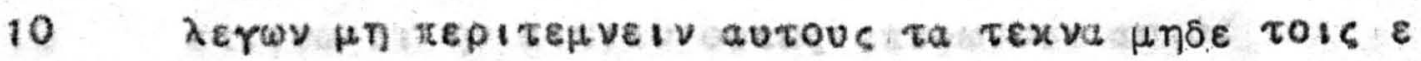
Qeatv xepixateเv.

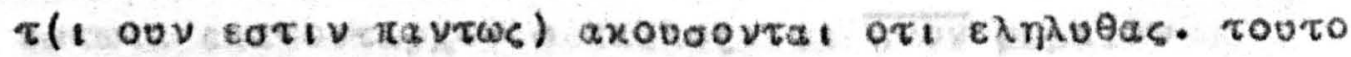
ouv zolnaov a dol Aerojev.

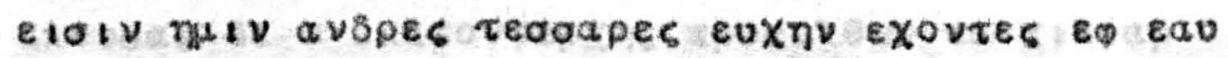

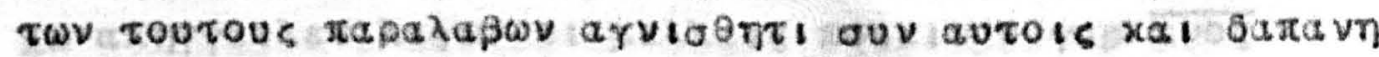

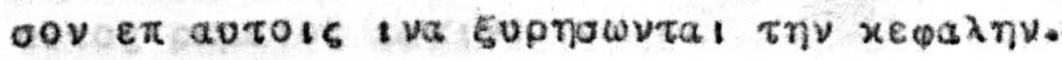

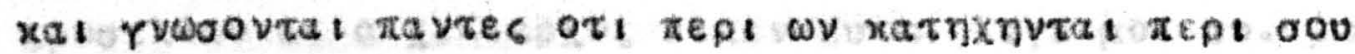

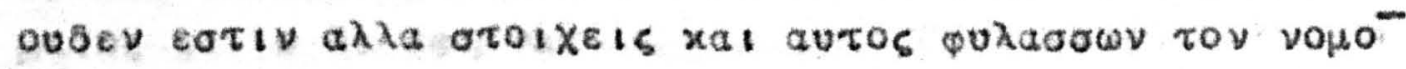

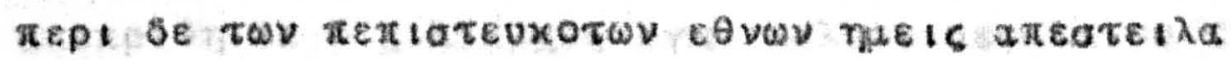

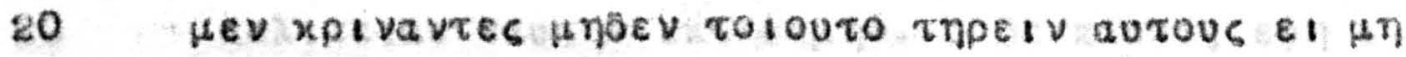

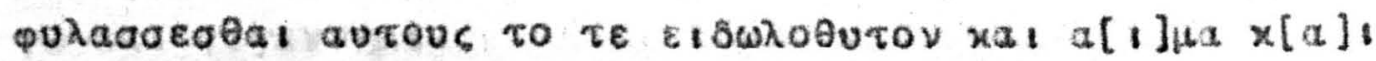
Kvixtov xa! ropvesa.

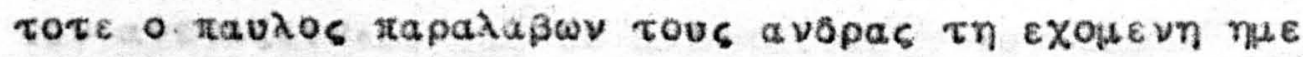

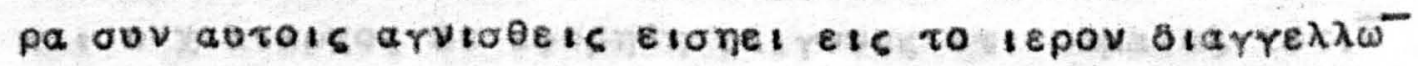

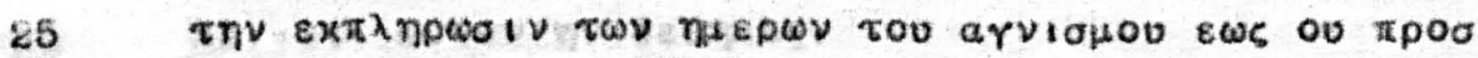

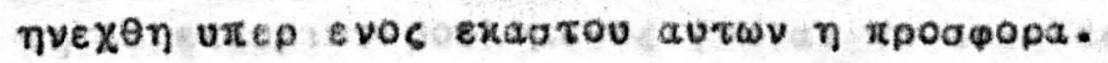

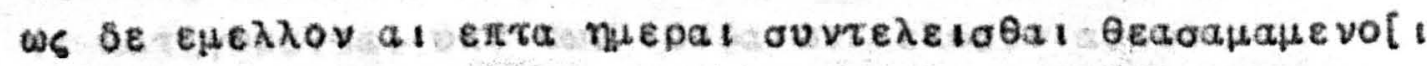

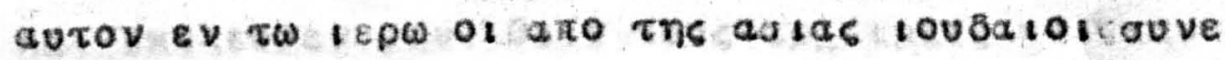

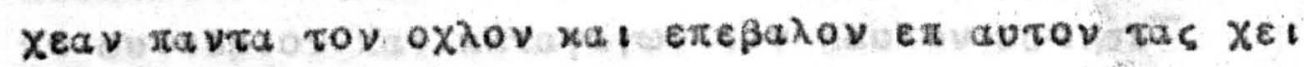

30 pas upazovtec.

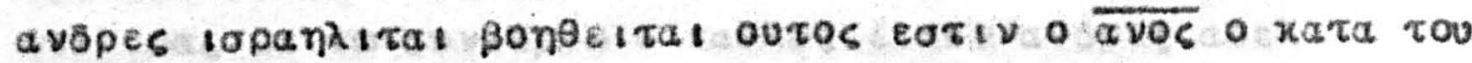

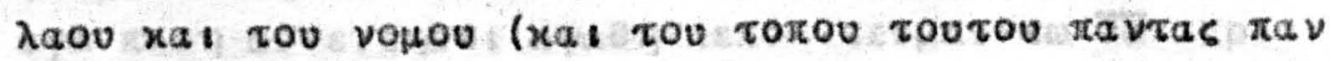

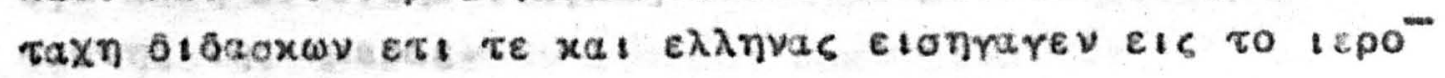
xai xexoivwev tov ariov tomov toutov.

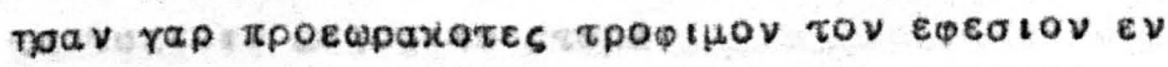

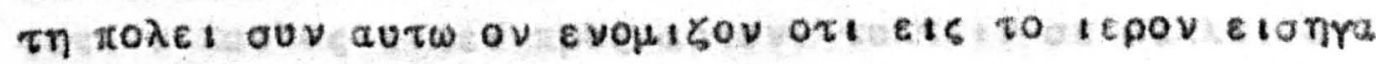

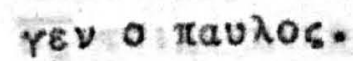

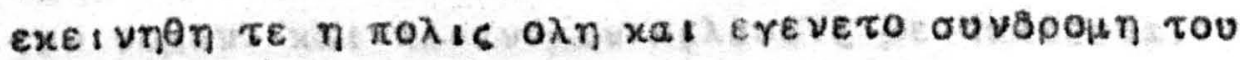

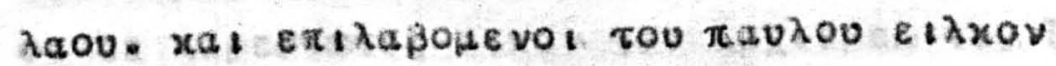

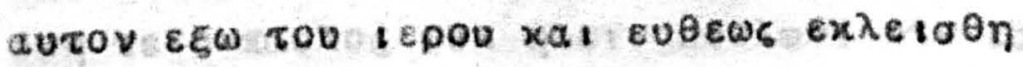
oav at oupal. 


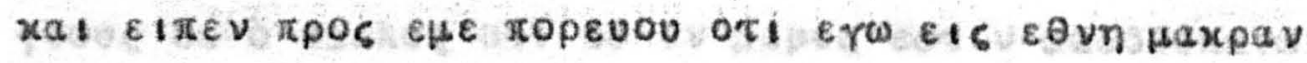
$\varepsilon \xi a \pi \circ \sigma \tau \varepsilon \omega \sigma \varepsilon 6$

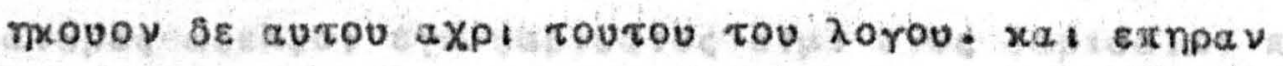

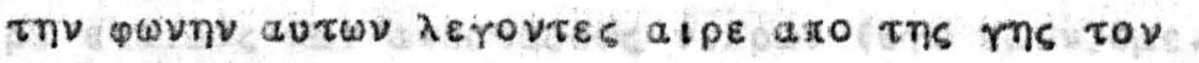
rotoutov ou rap xaerpey autov לnv.

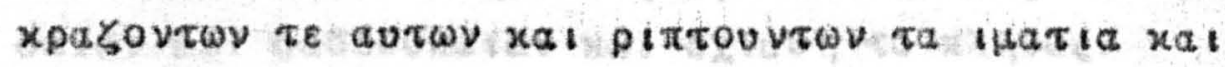

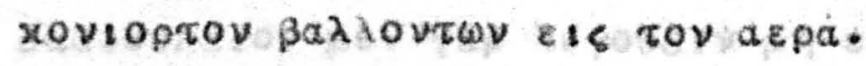

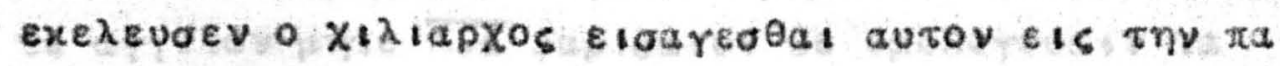

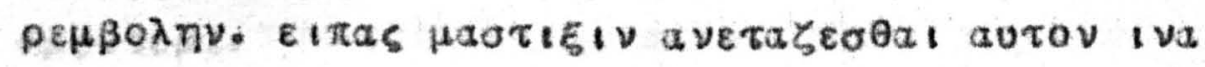

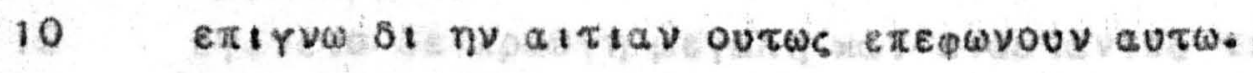

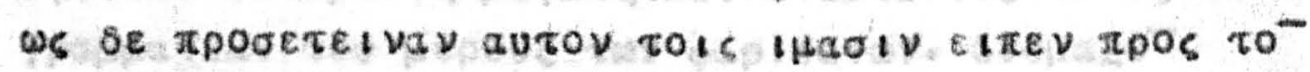

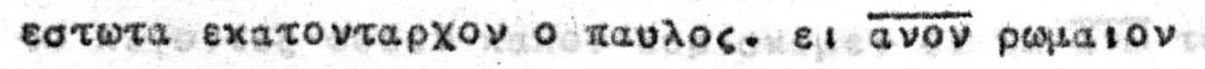

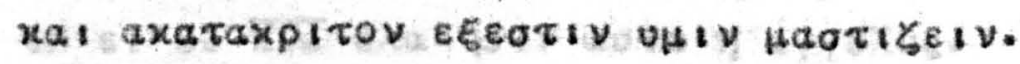

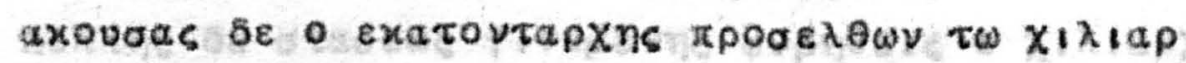

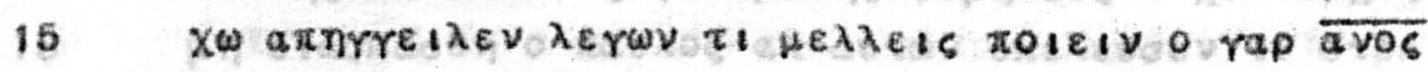
ovTos pwiatos eariv.

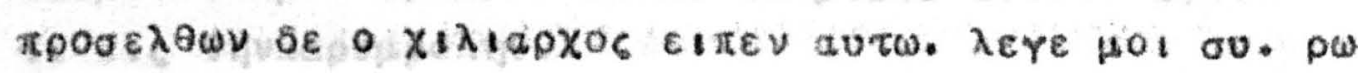
Hatos 81.0 o $\varepsilon$ p val.

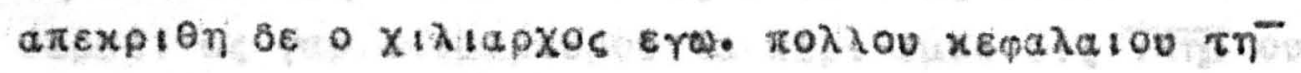

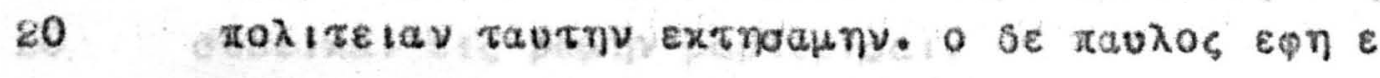
ro xal rerevunue.

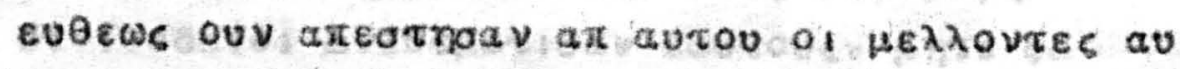
rov averaלs เv.

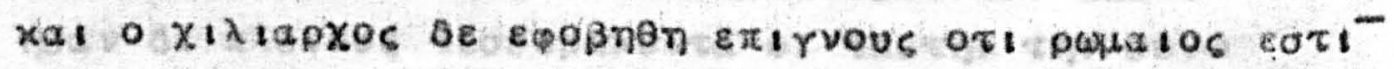
25 xas otr autov nv besnuws.

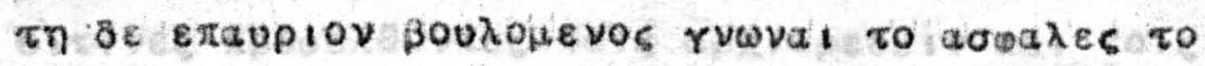

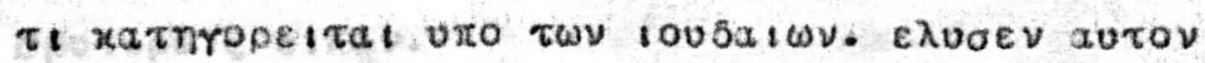

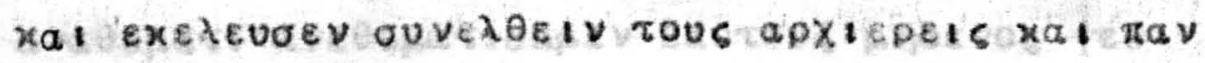

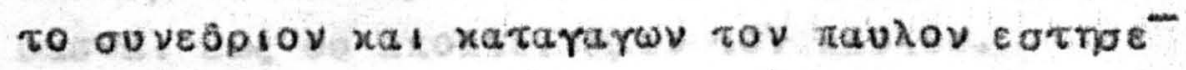

30 e1c aurous.

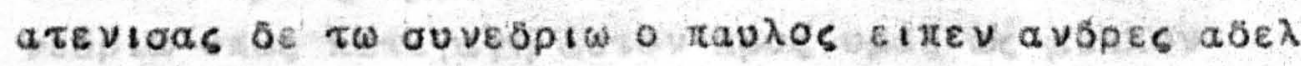

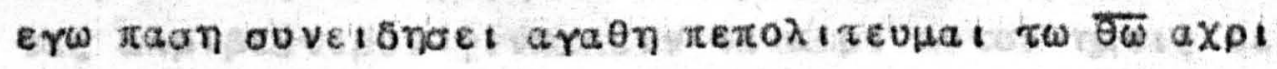
rautns tns nuepas.

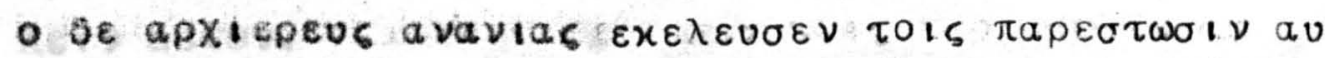

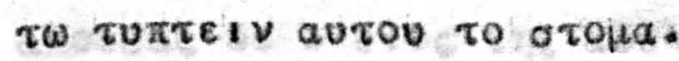

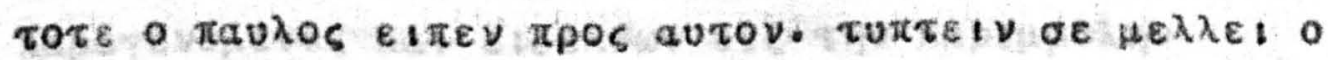

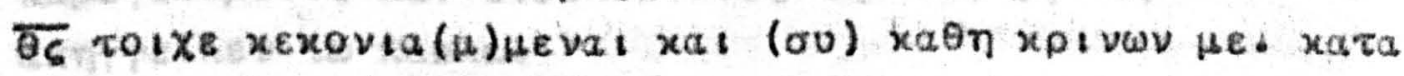

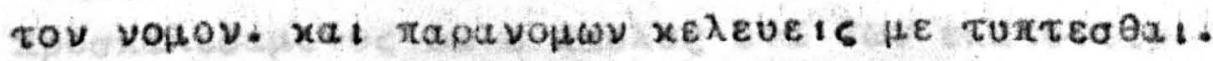

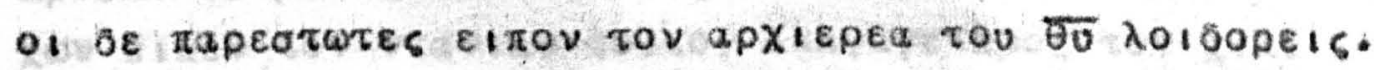

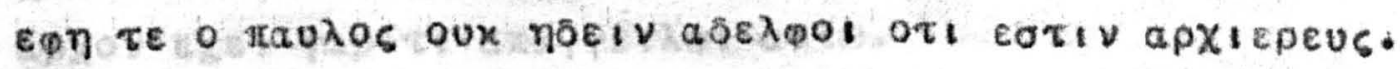
reypartal rap apXo(v)ra tov haou aou oux epe is kaxws 


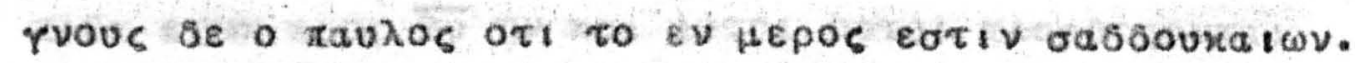

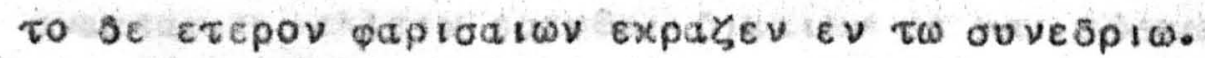

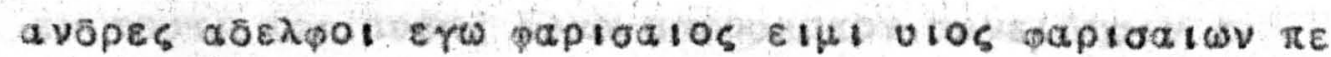

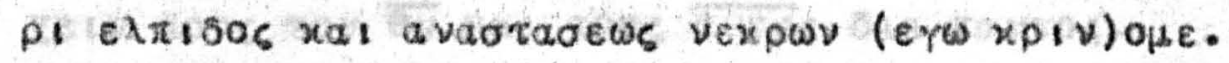

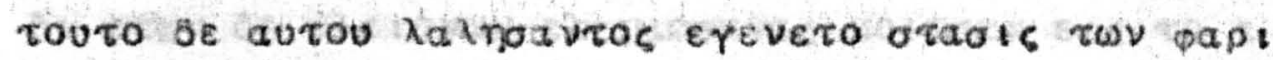

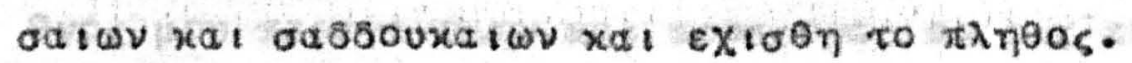

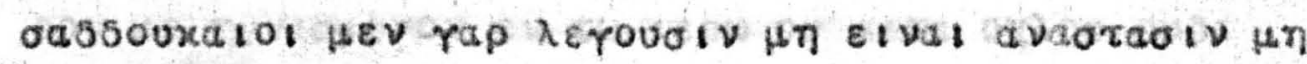

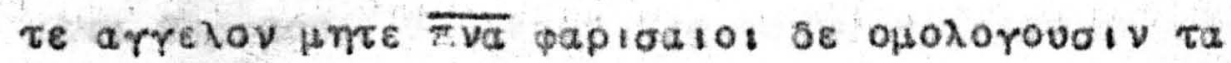
quюогера.

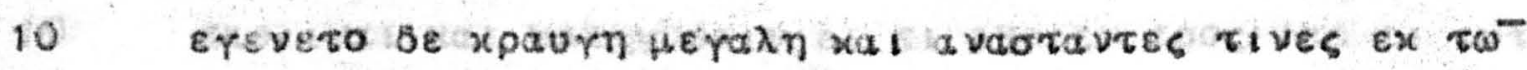

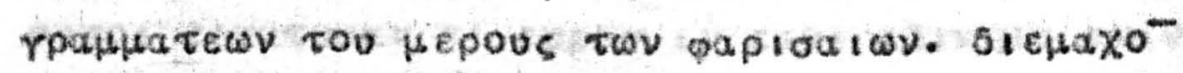

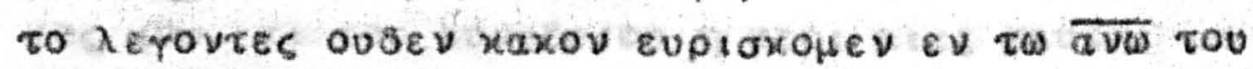

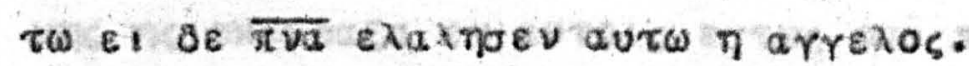

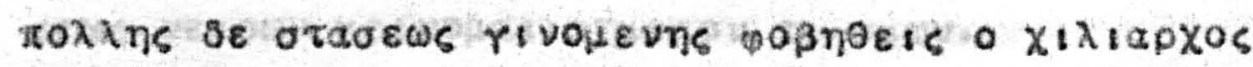

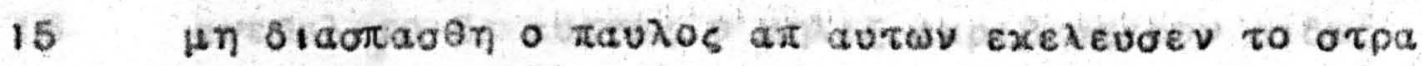

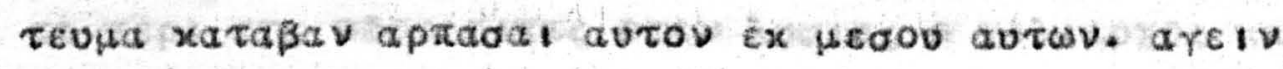

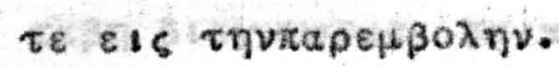

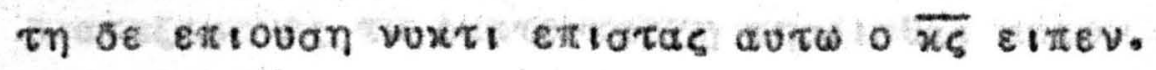

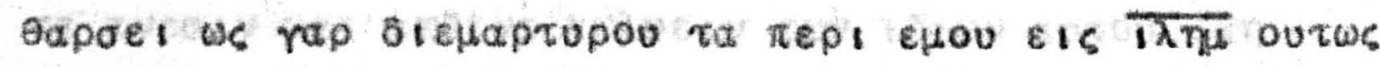

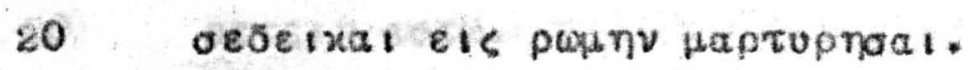

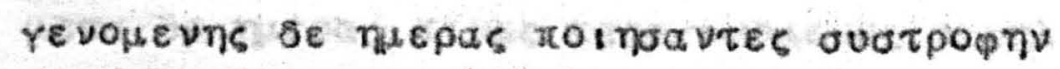

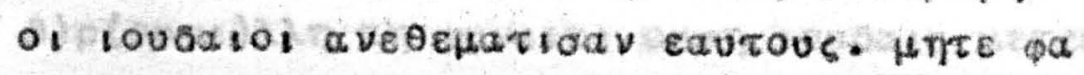

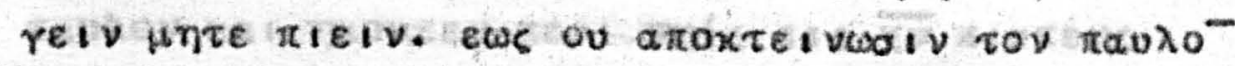

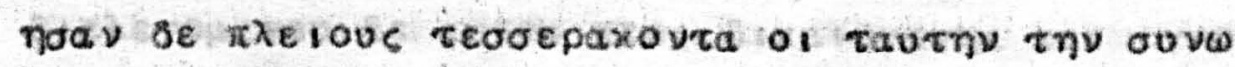
25 Hoolav notnoa

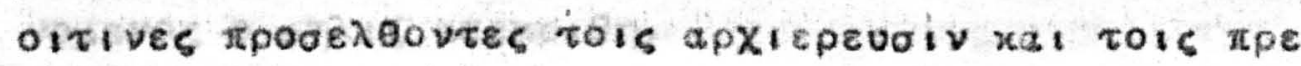
opotepols $\varepsilon$ itav.

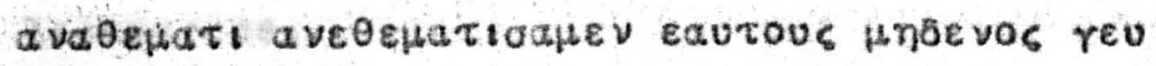
oavea \& eas ov arone ivto, ev rov raviov.

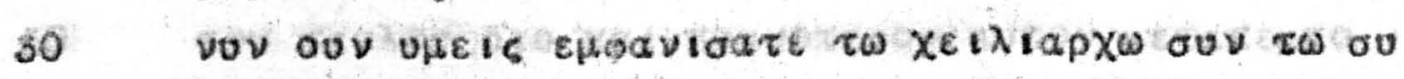

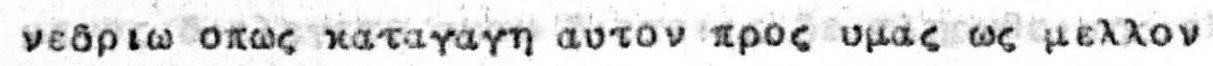
Tas axpipeotepov yivwaxeiv ta $\pi \in \rho t$ dutov Tueis De

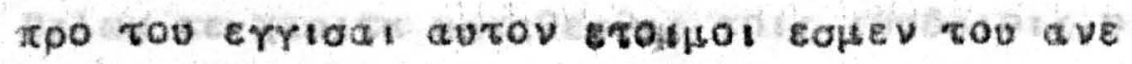
Aeiv autov.

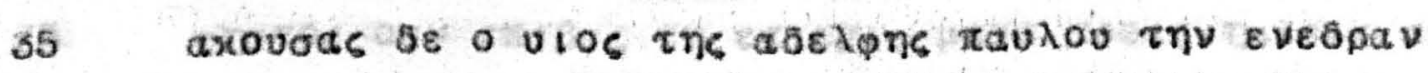

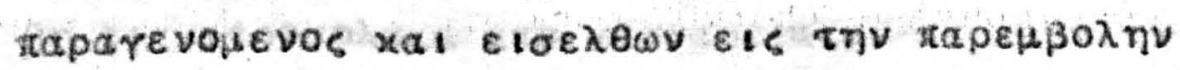

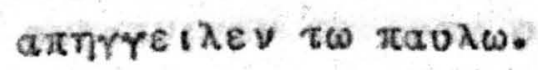

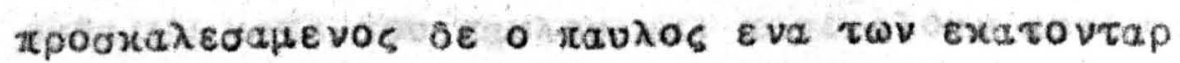

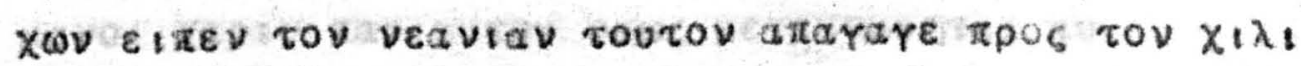

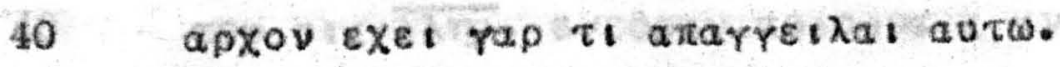

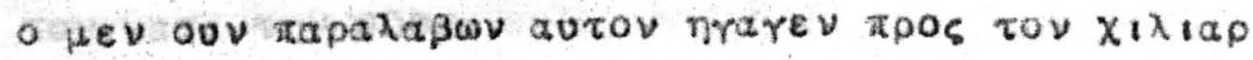




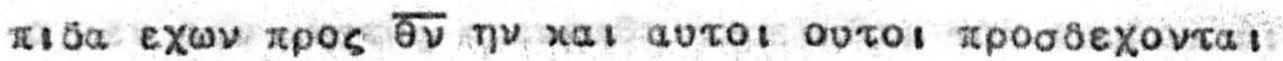

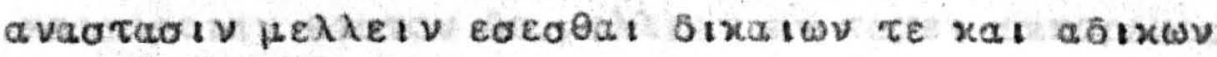

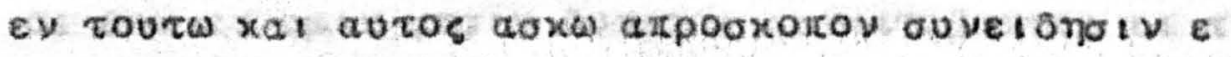
XeIV xpos tov $\overline{\theta \nu}$ xal tovs $\overline{a v o v s}$ ata tavtos.

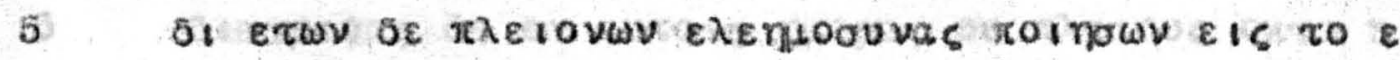

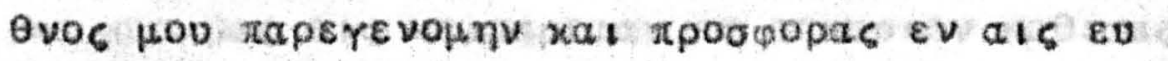

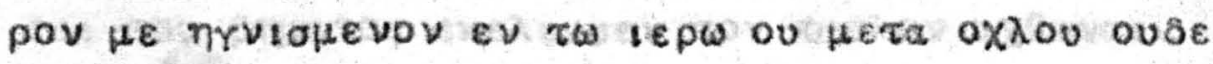

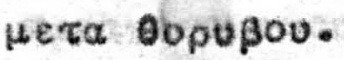

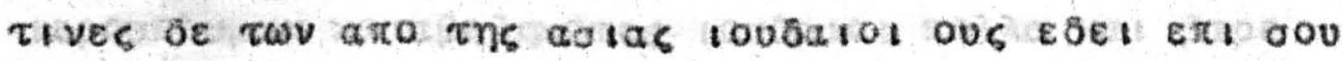

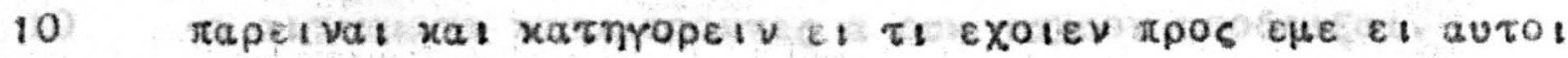

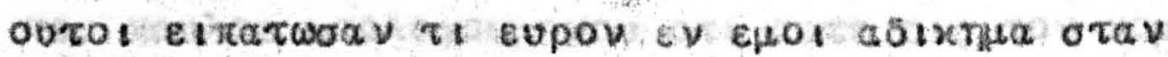

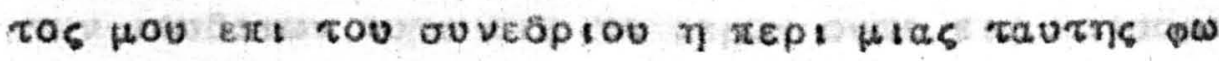

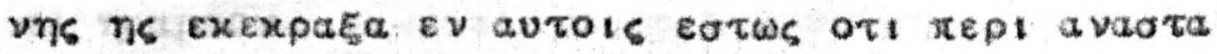

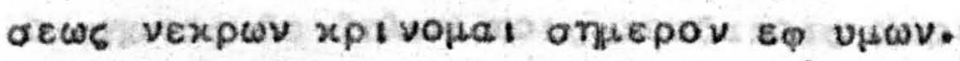

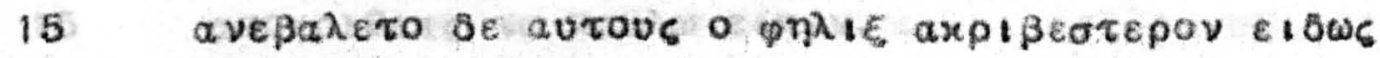

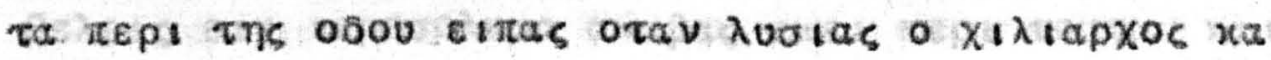

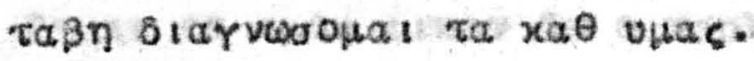

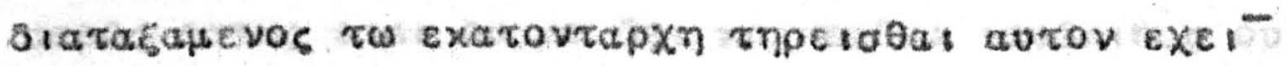

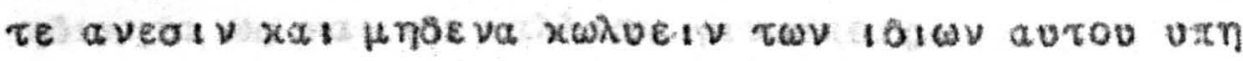

20 peretv aurw.

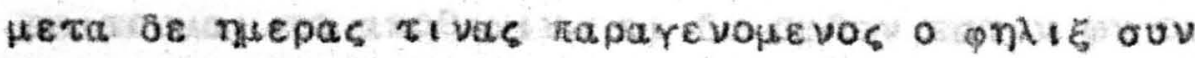

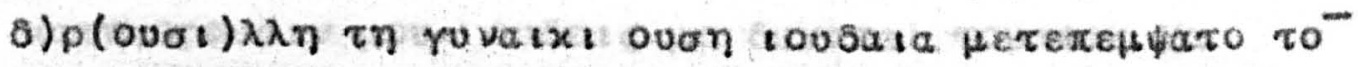

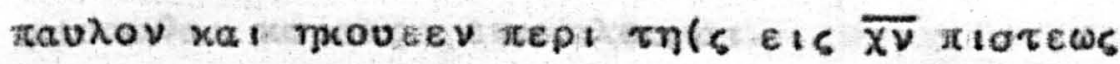

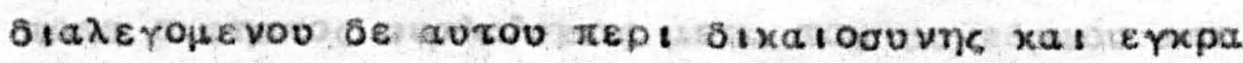

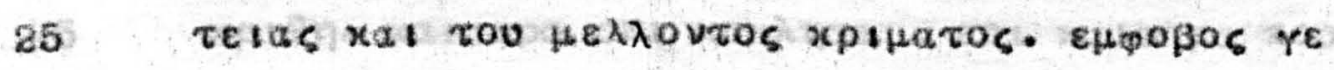

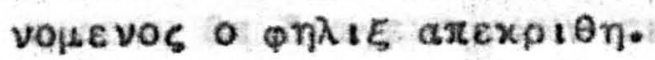

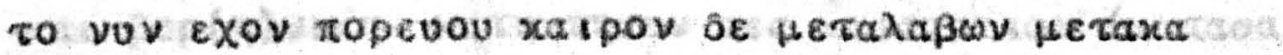

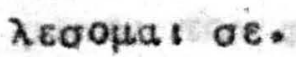

a

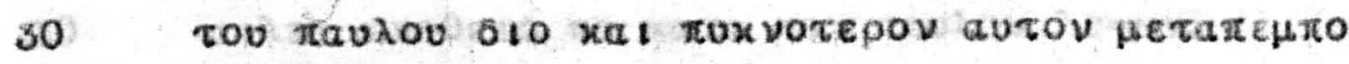

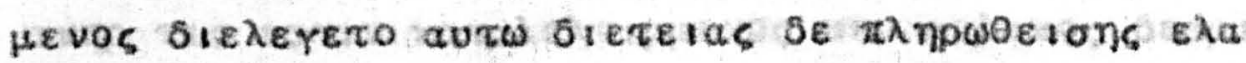

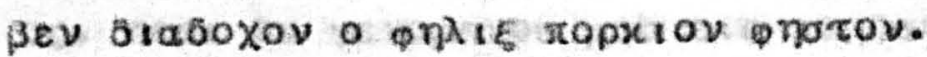

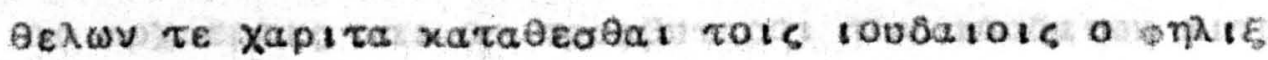

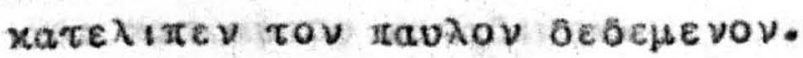

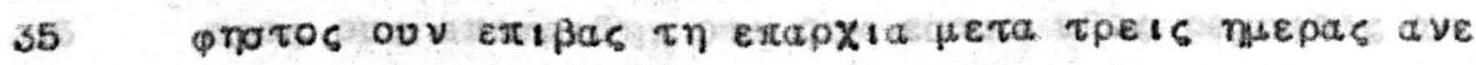

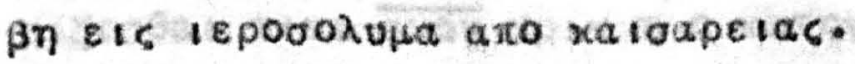

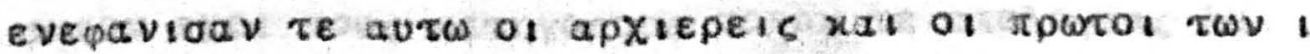

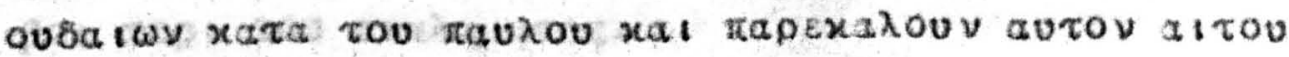

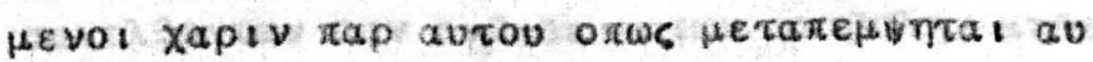

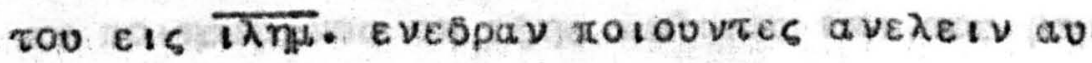

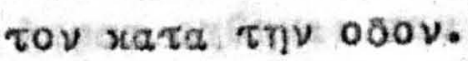




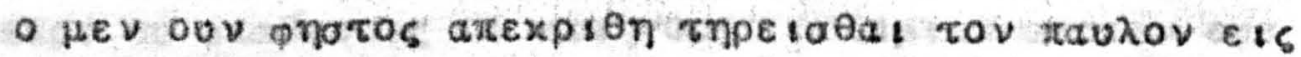

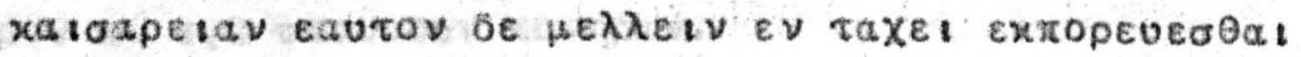
of ouv \& $\nu$ oน

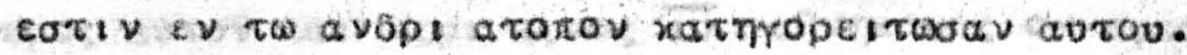

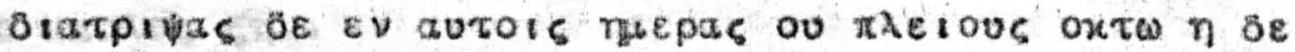

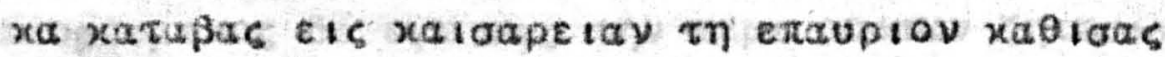

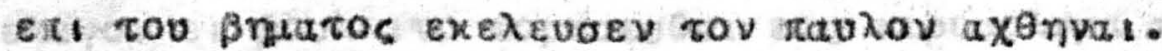

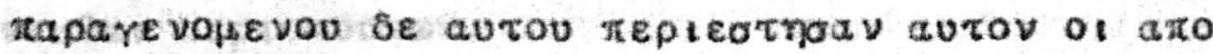

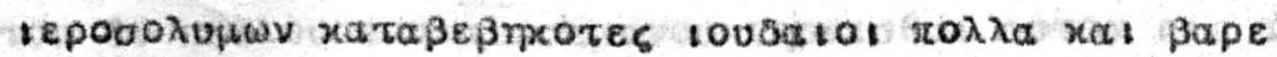
a aitratata xatapepoves a oux raxuov arobetรat.

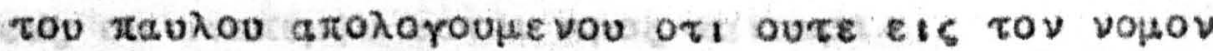
twV loubalwy oute $\varepsilon$ is to tepov oute eis xatoapa $\tau$. nuaptov.

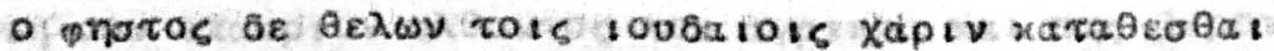

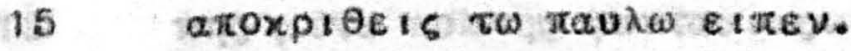

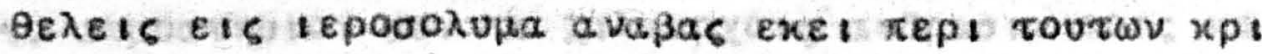

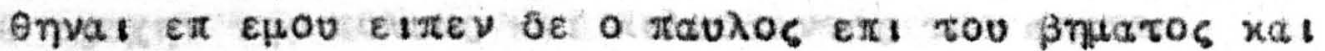

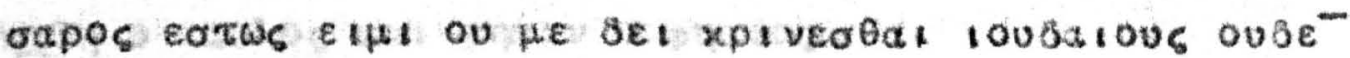
nôtหrya ws xat av xaditov rivwaxels.

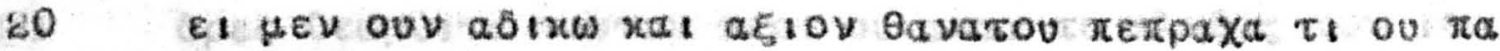

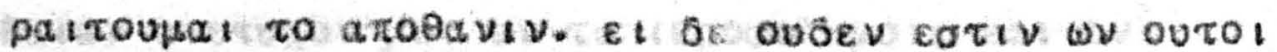

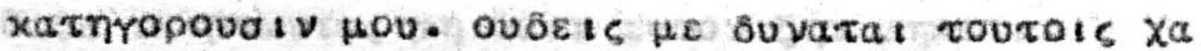

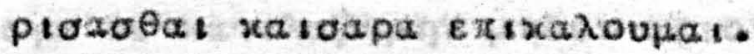

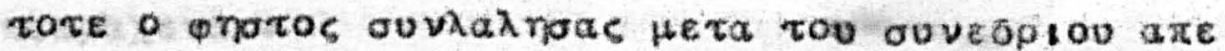

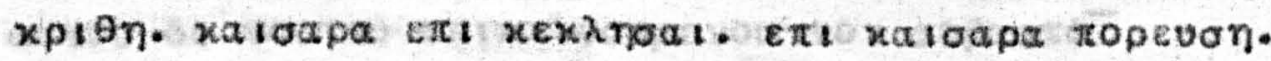

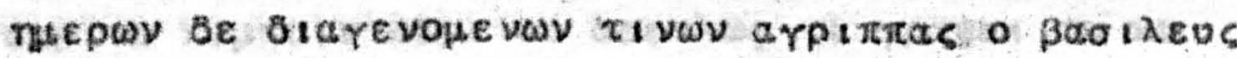

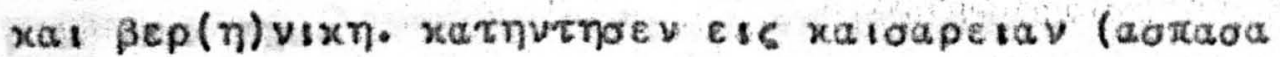
Hevor Tov snotov.

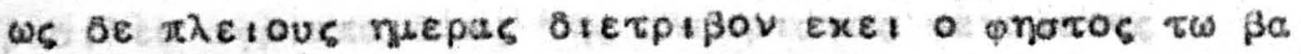

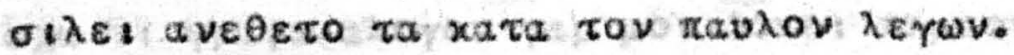

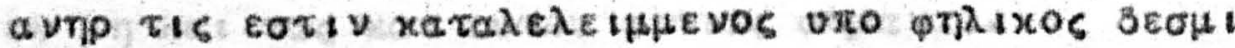

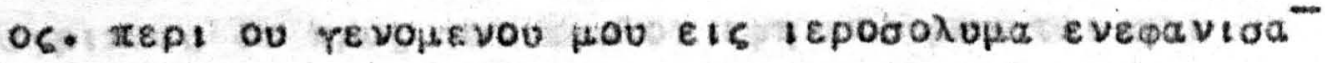

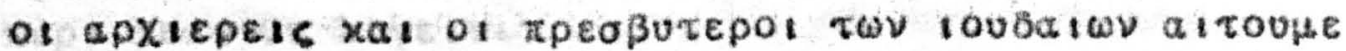
Vot xat autoo xataôtxทv.

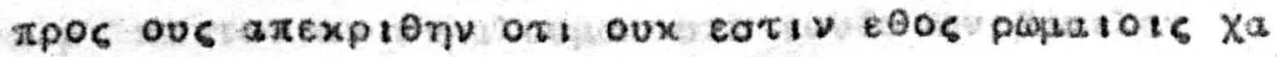

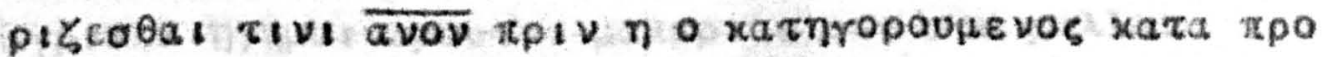
awrov EXor tous xarryopous torov te arodortas la Bos. xept too evxiniatos.

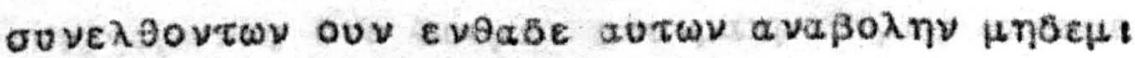

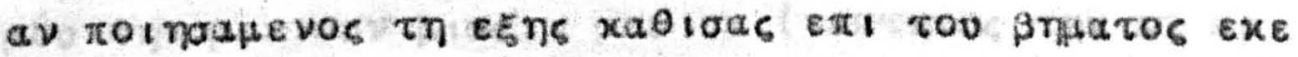

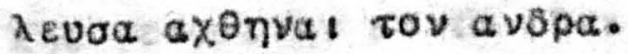




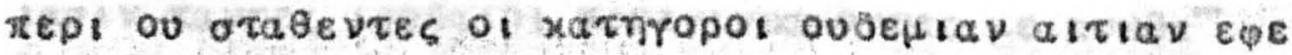

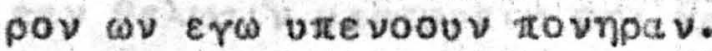

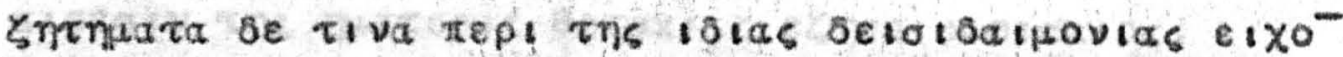

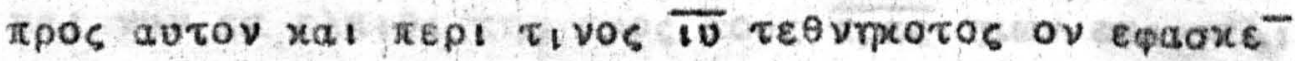

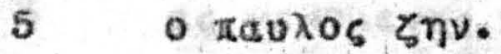

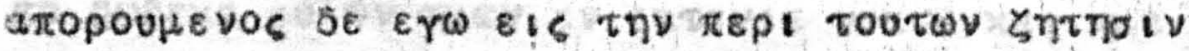

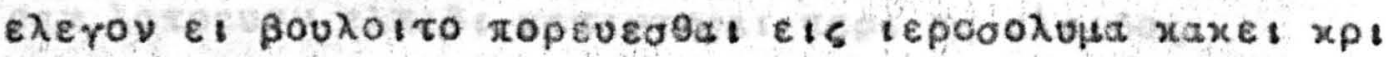

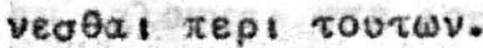

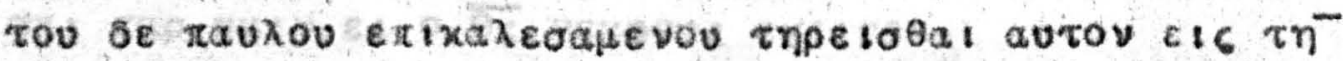

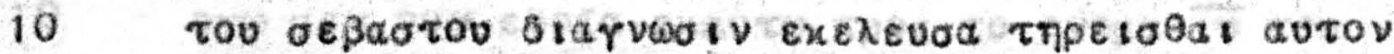
ews ov a varewila autov tpos xaloapa.

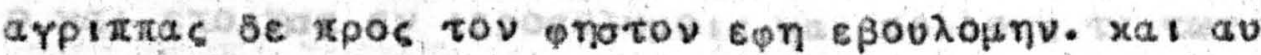
ros tou ayou axovoal. o se duptov pnotv axovan autou.

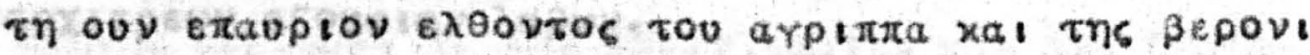

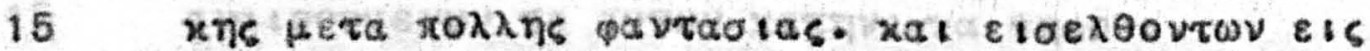

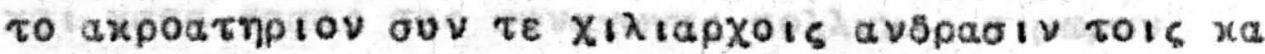

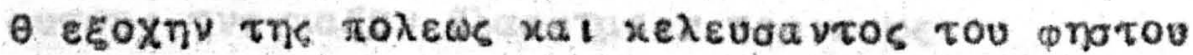

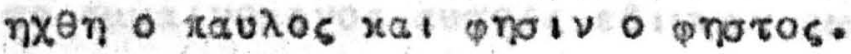

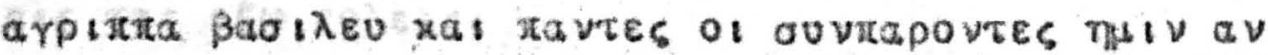

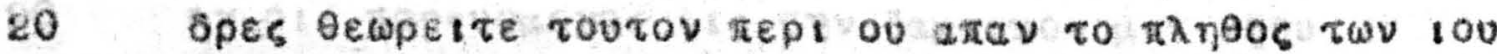

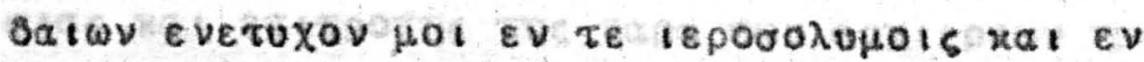

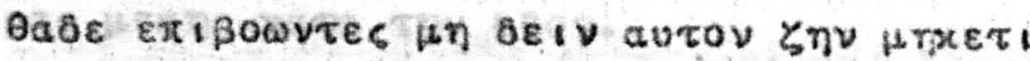

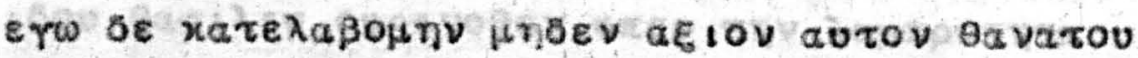

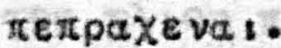

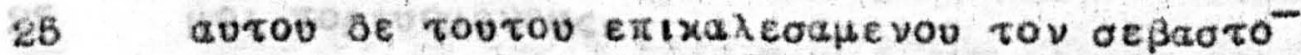

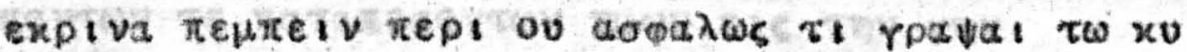

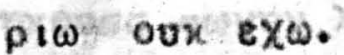

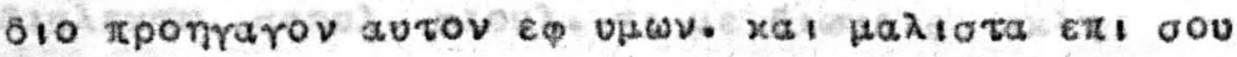
Badinev arpiria otws rns a vaxptoews revouevis

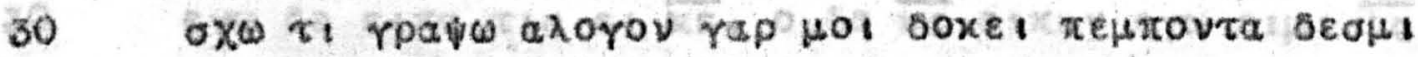

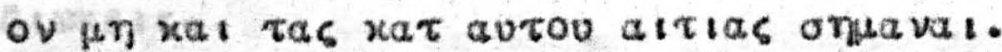

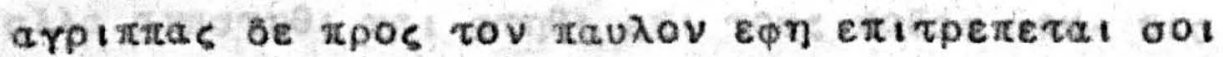

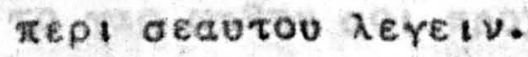

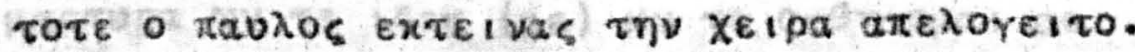

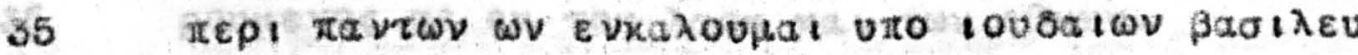

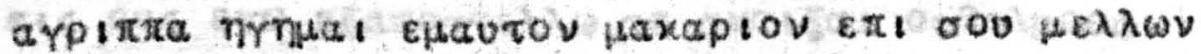

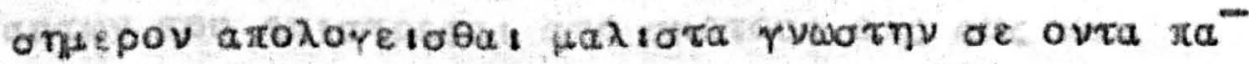

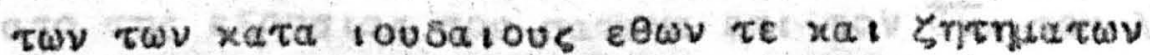

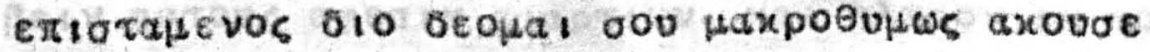

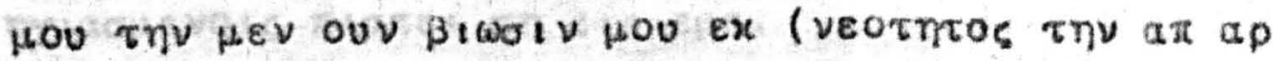

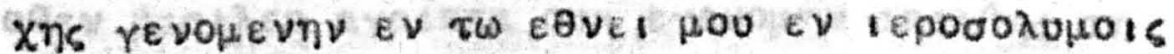




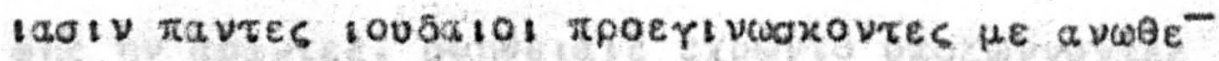

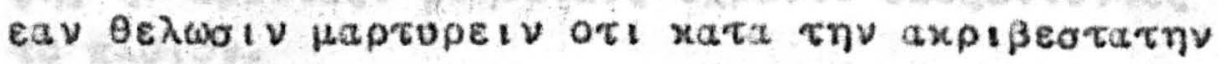

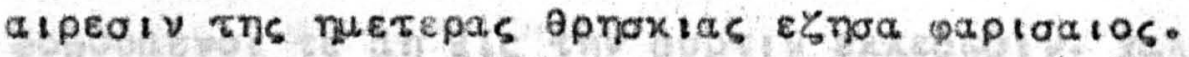

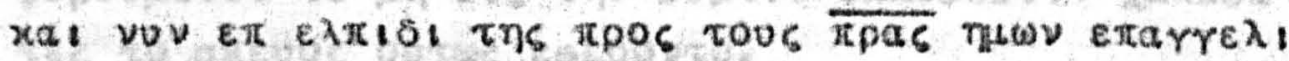

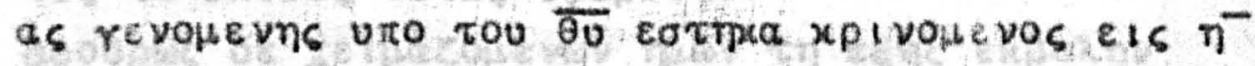

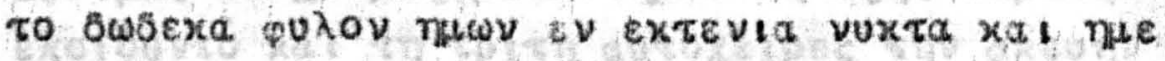

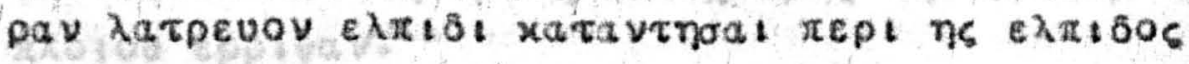

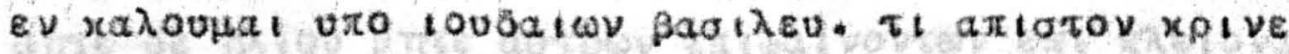

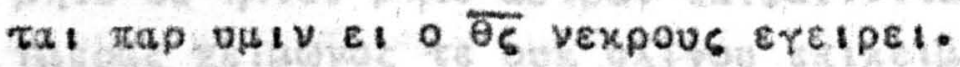

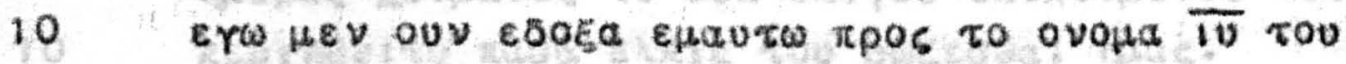
vaלwpa 100 3e

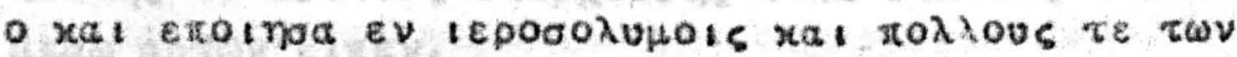

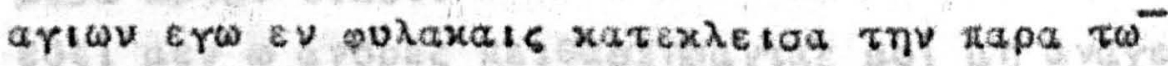

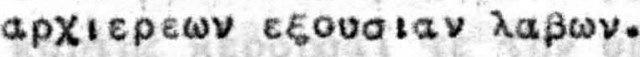

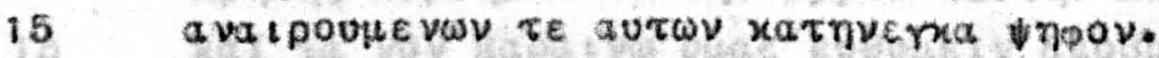

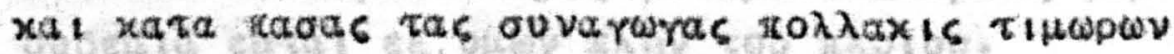

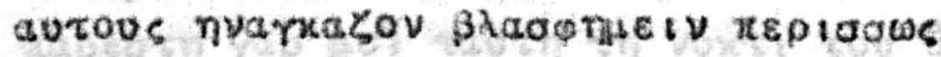

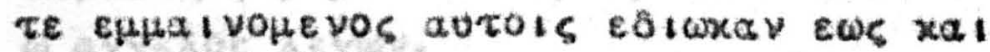

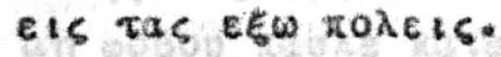

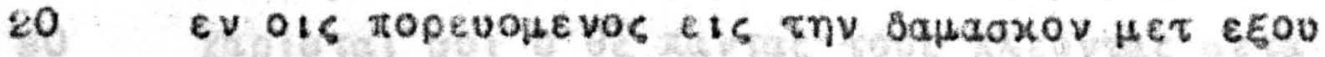

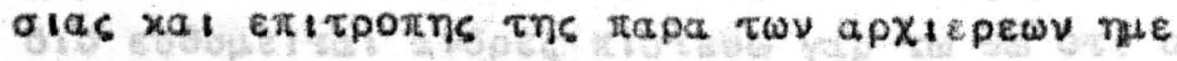

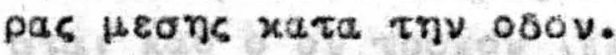

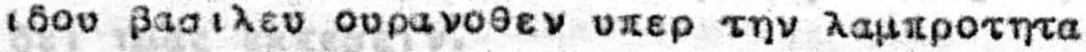

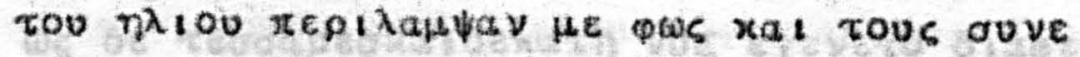

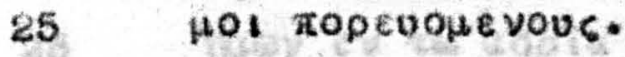

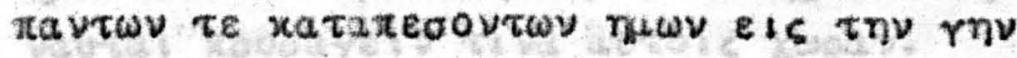

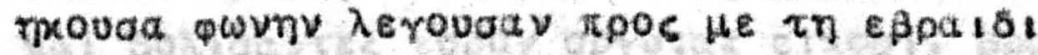

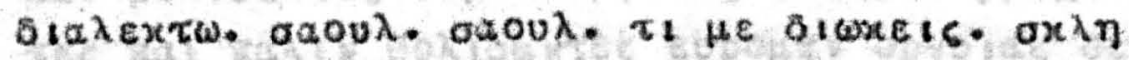

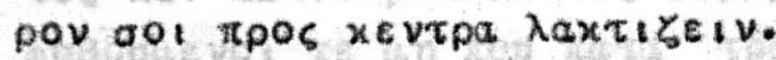

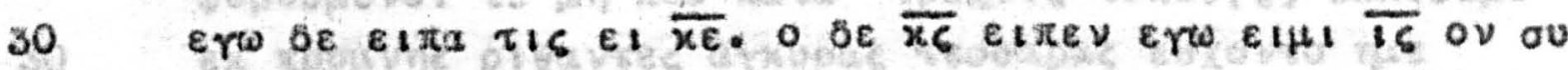
oิ เunets.

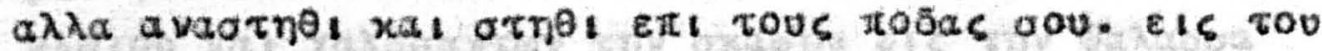

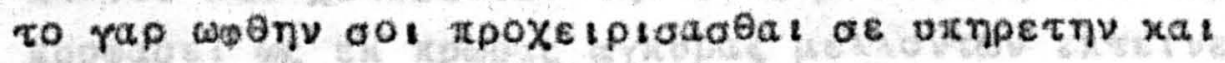

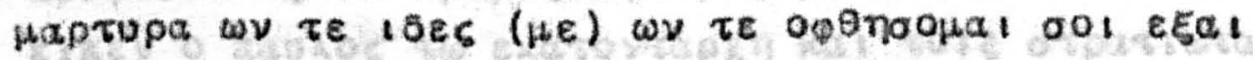

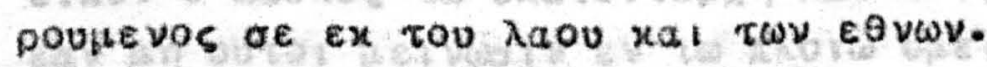

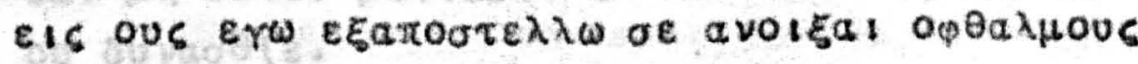

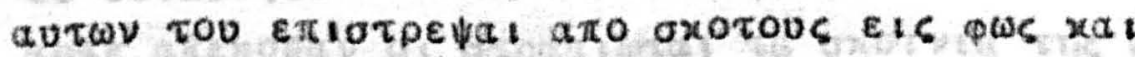

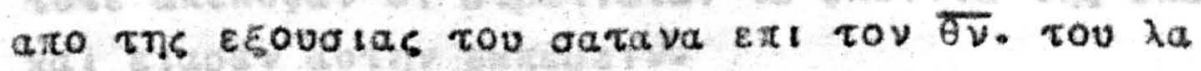

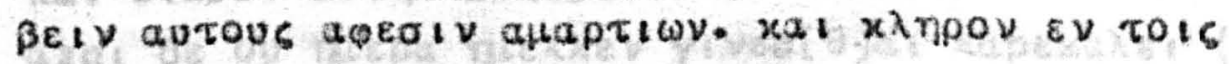




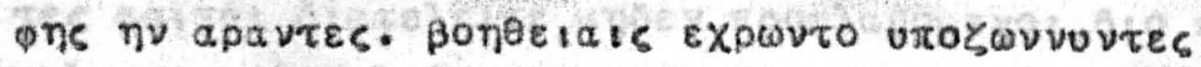

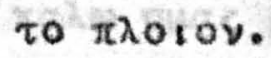

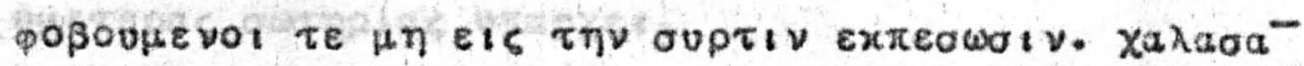
Tes to axevos outws eqepovto.

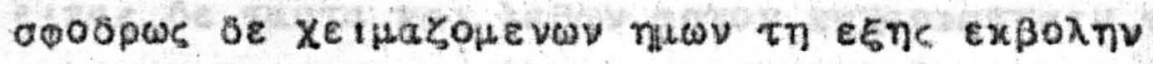

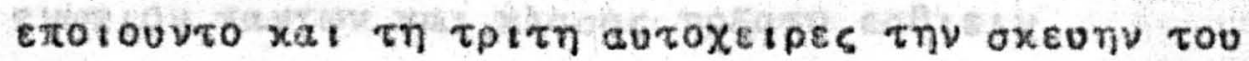

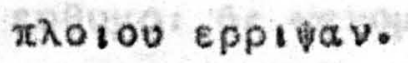

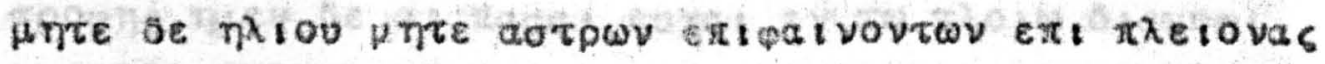

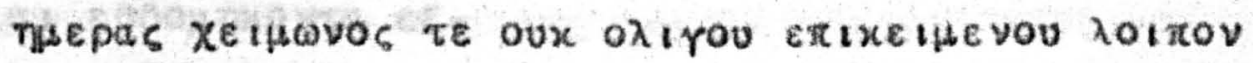

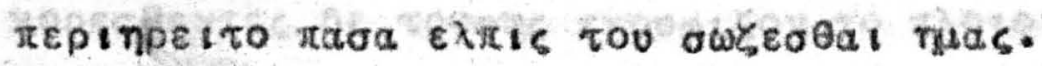

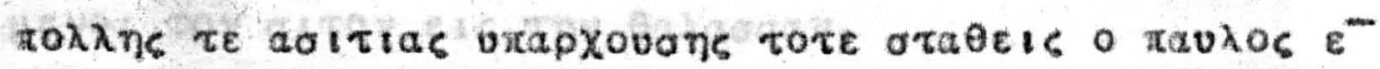
$\mu \varepsilon \sigma \omega$ autwV $\varepsilon เ \pi \varepsilon v$.

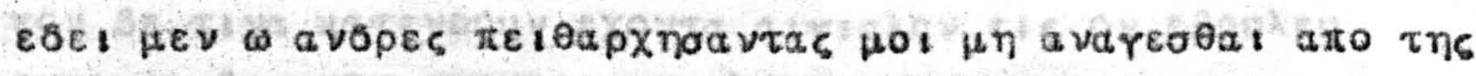

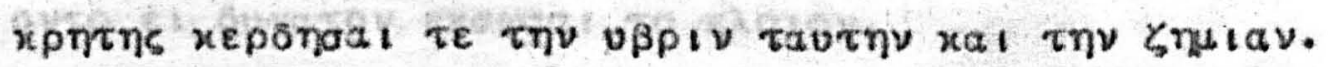

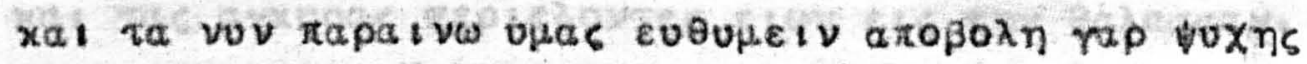

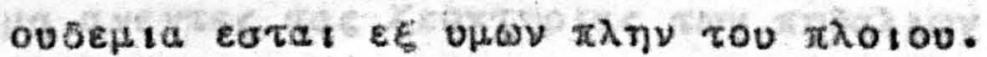

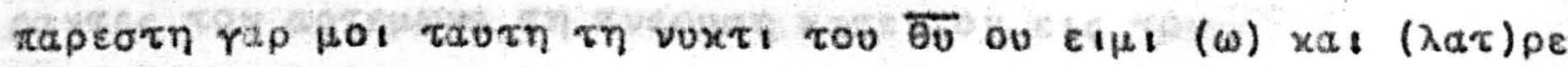
$\omega$ arrexos $\lambda e r s v$.

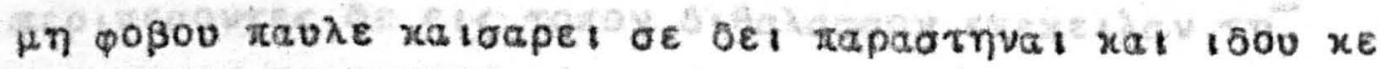

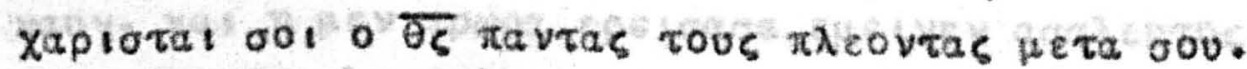

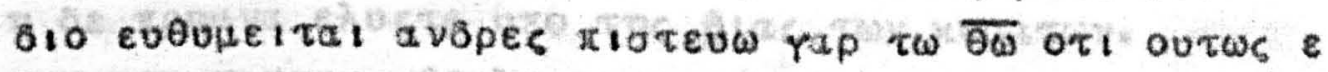

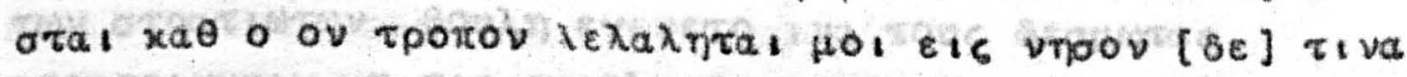

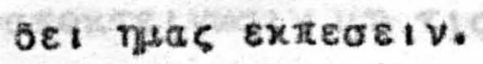

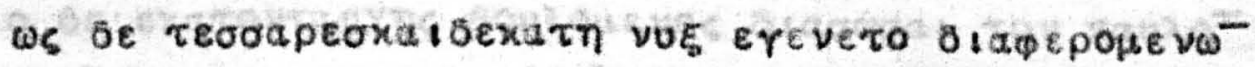

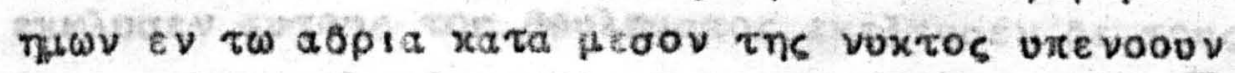

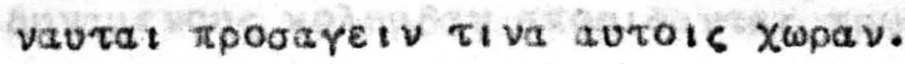

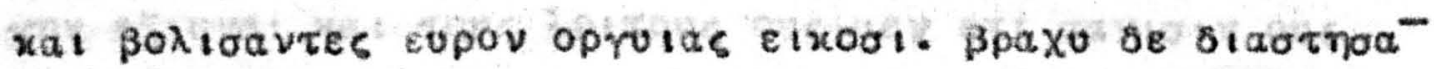

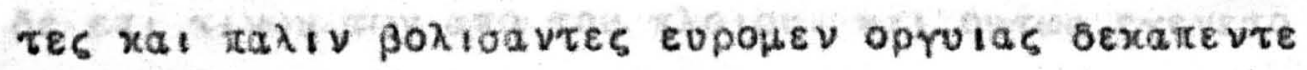

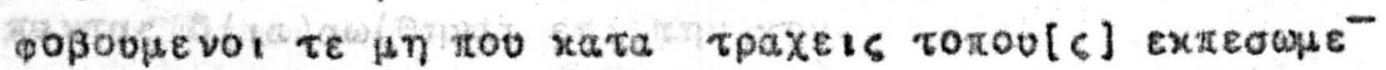

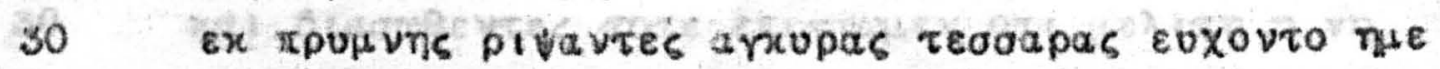

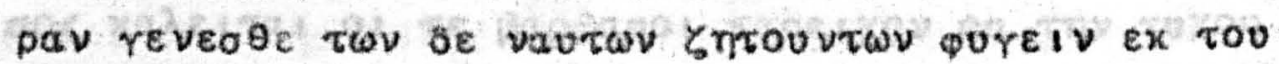

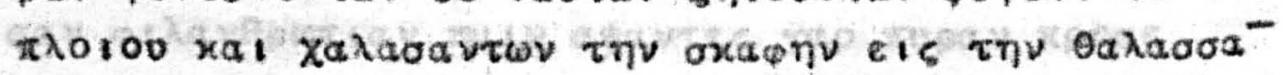

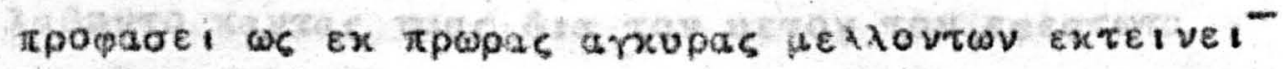

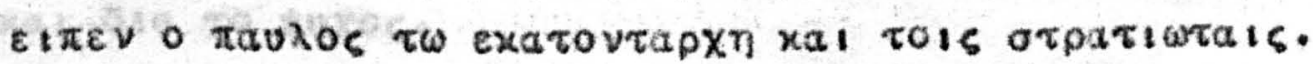

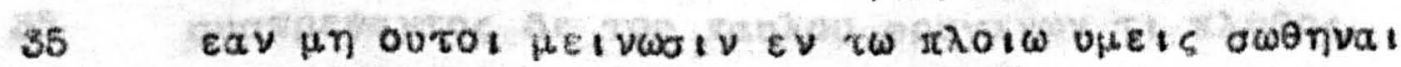
ov $0 v \operatorname{vac} \theta(\varepsilon$.

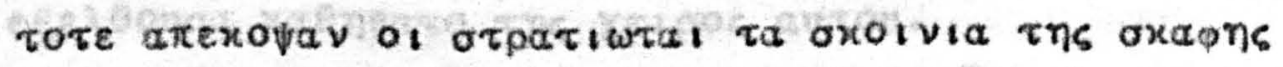
xal Elaaav autnv Exreosiv.

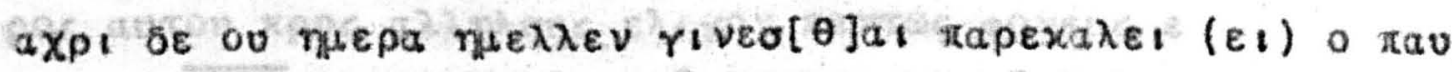

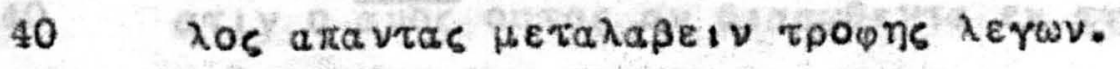

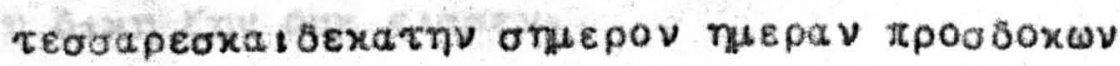




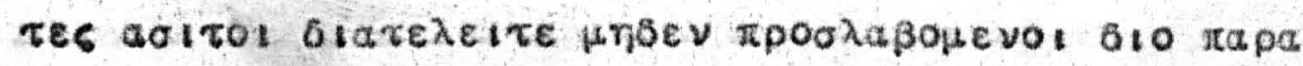

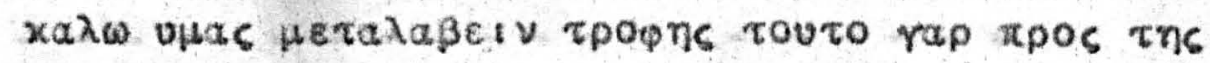

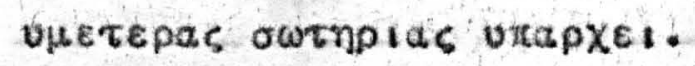

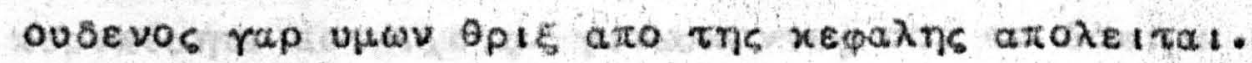

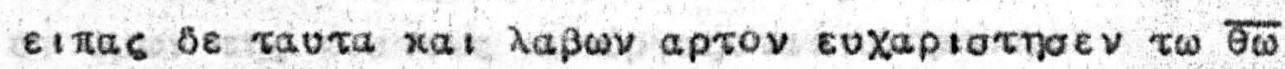

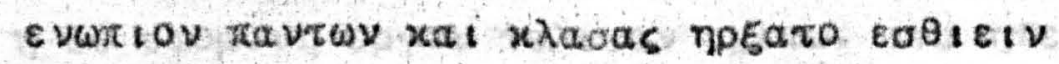

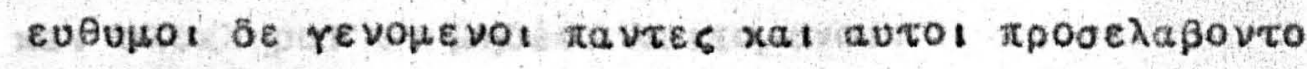

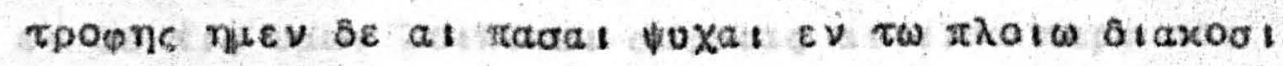

a) $\varepsilon$ psoqniovta $\varepsilon \xi$ *

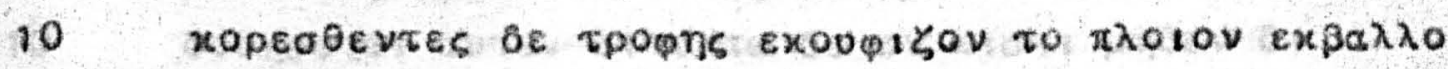

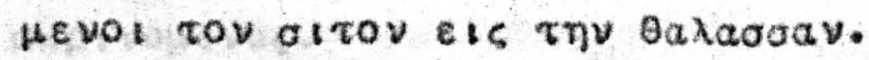

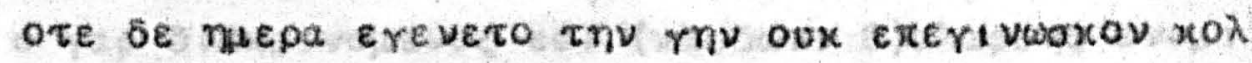

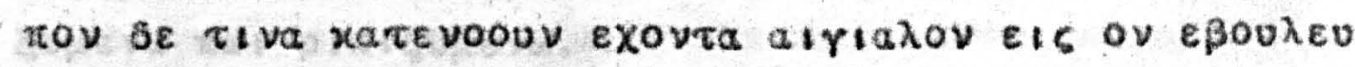

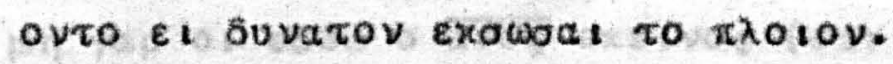

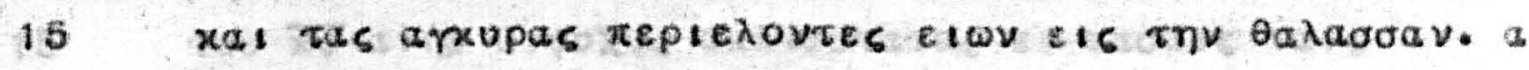

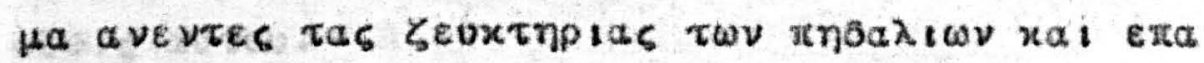

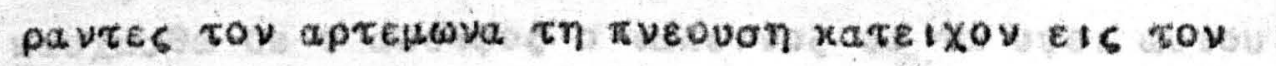
airialov.

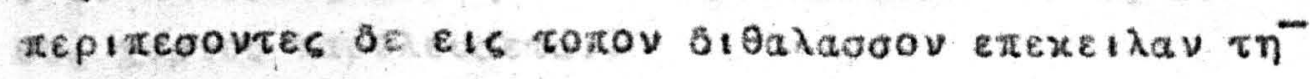

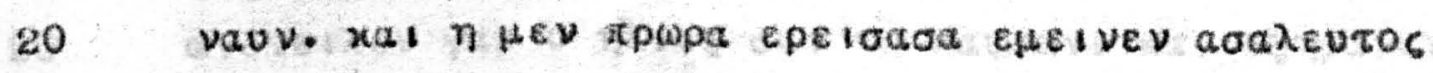

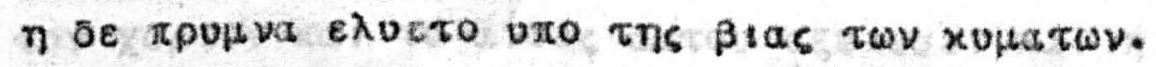

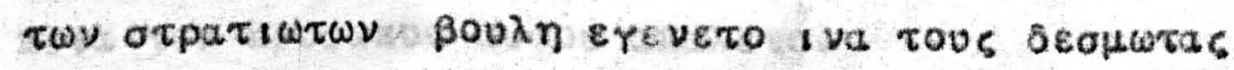

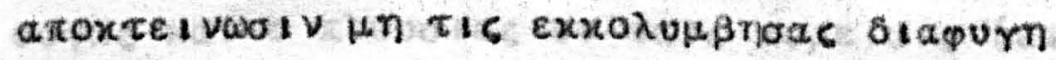

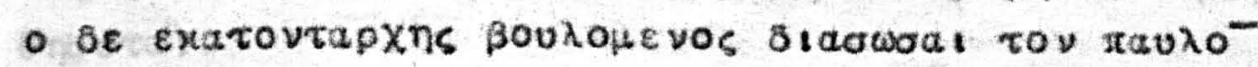

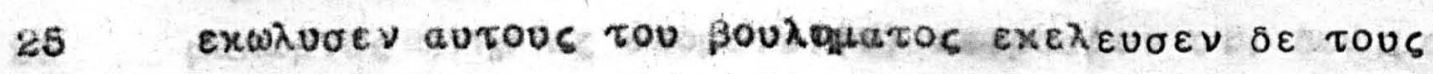

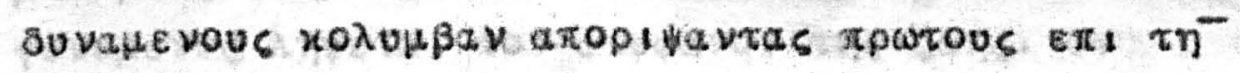

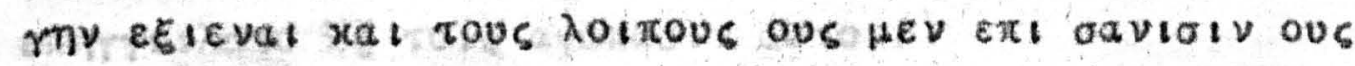

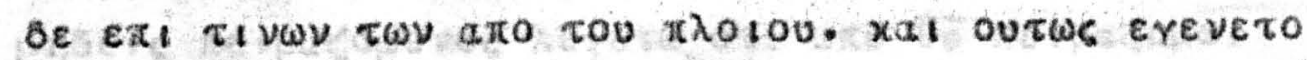

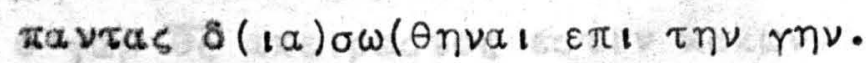

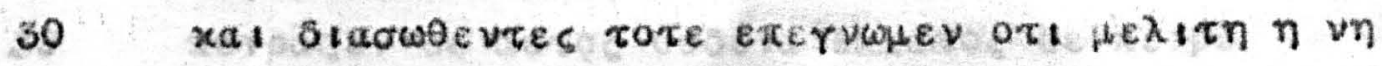

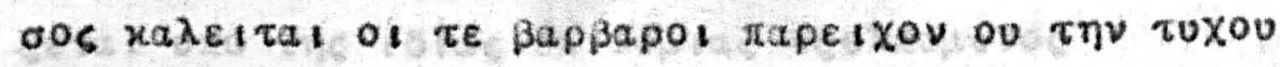

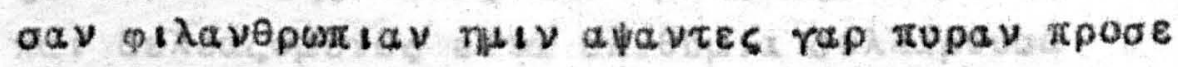

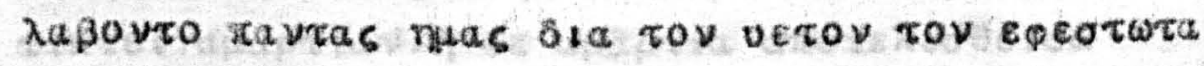
xal ora to

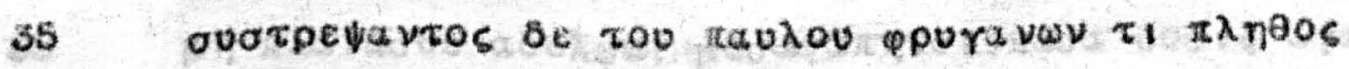

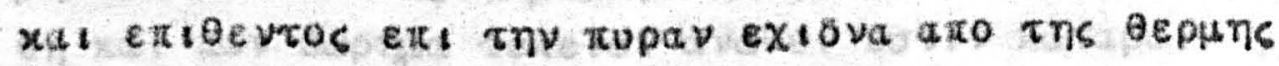
ęe

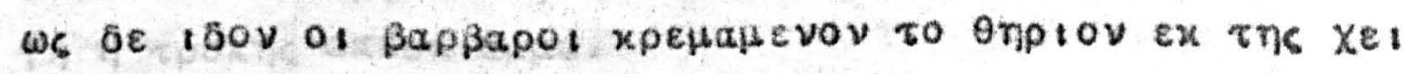

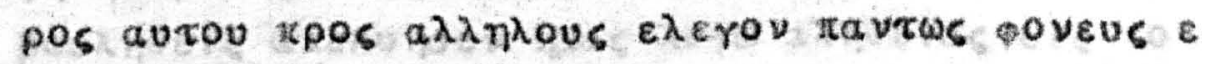

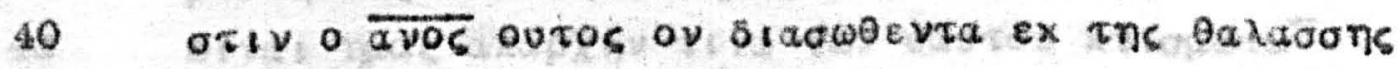
$\eta \operatorname{six\eta } \zeta \eta \nu$ oux e iaaev. 


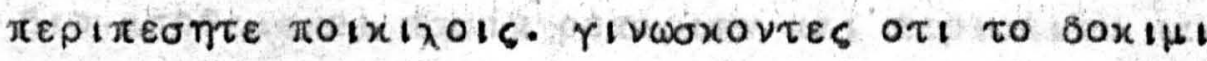

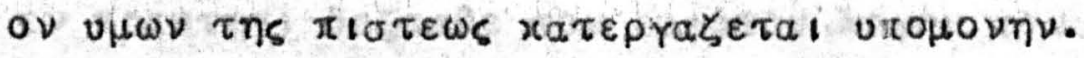

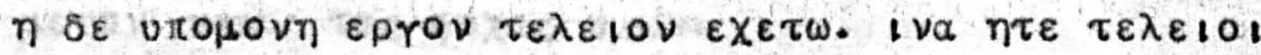

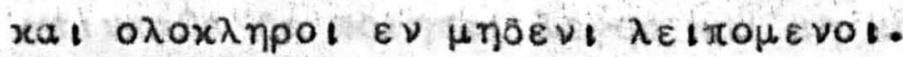

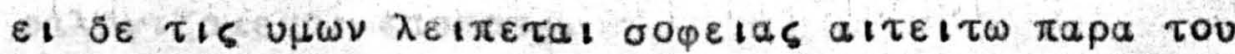

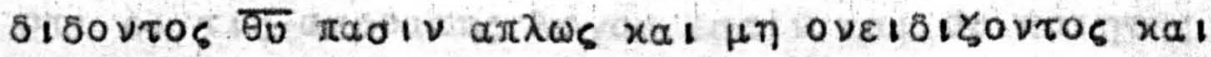

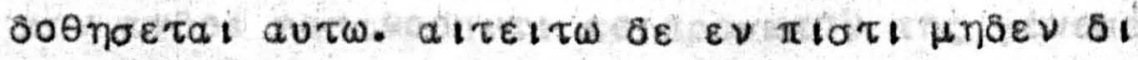
axpivouevos.

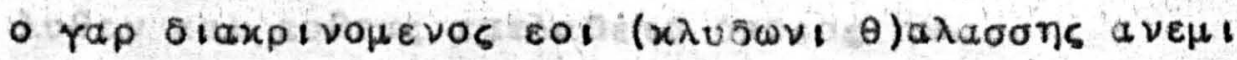

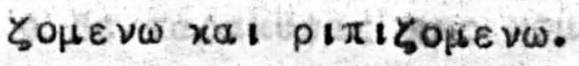

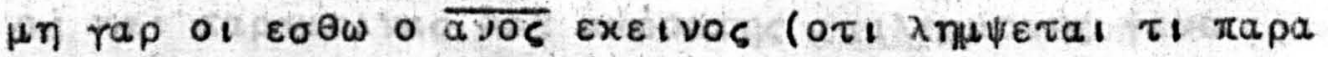

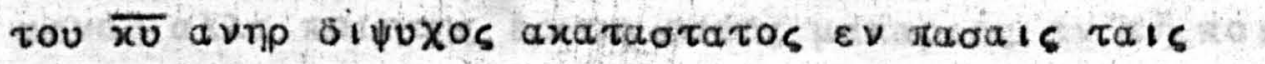
00015 autou.

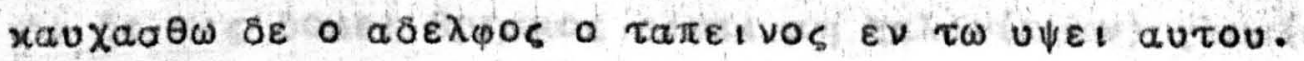

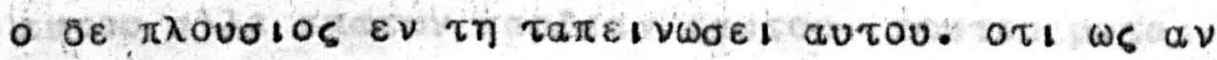

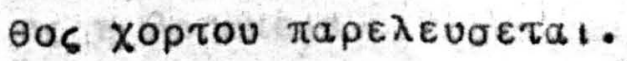

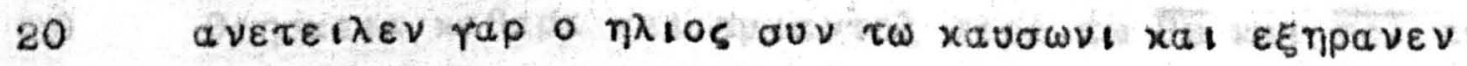

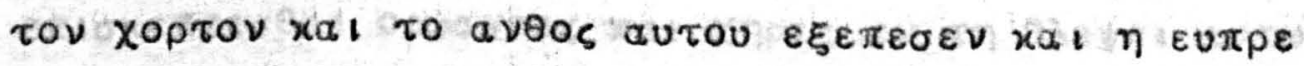

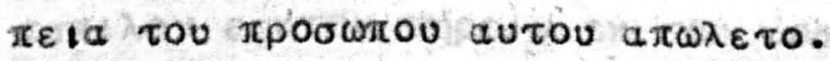

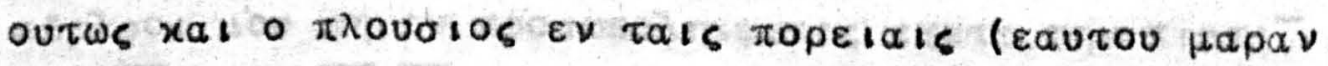

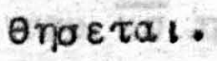

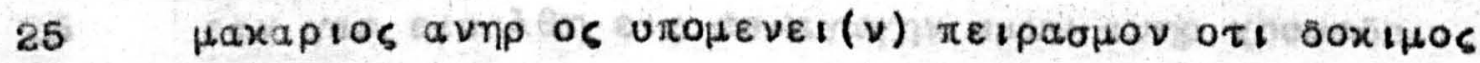

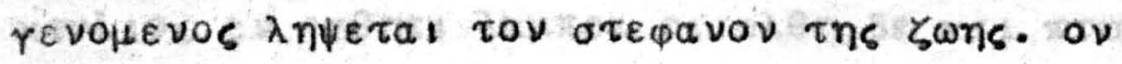

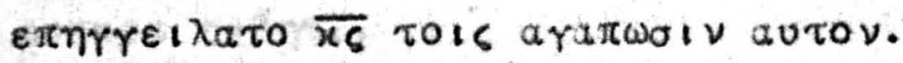

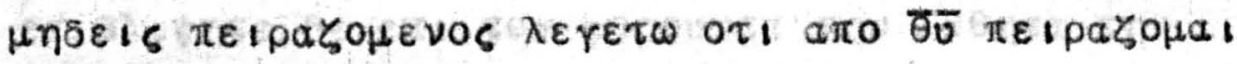

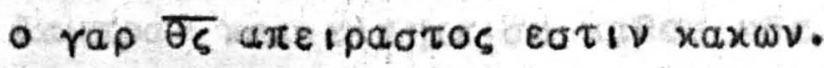

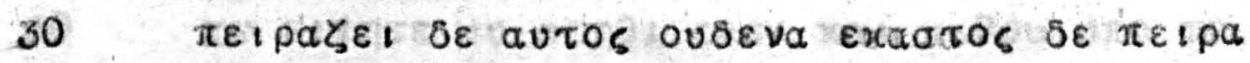

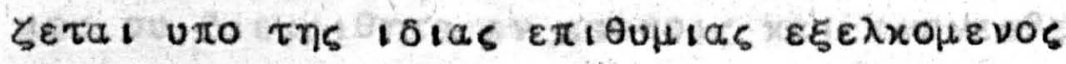

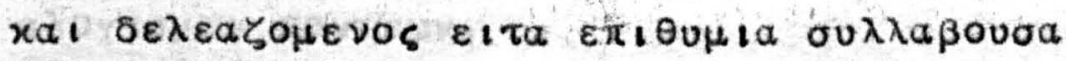

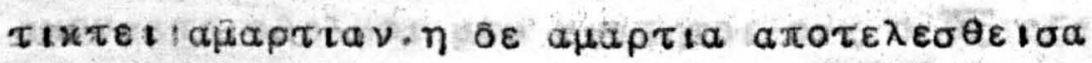

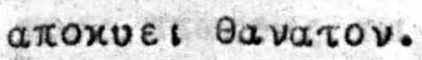

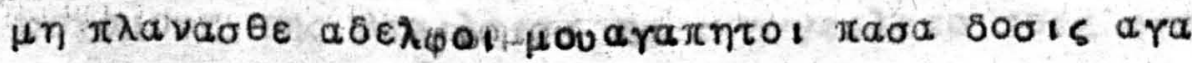

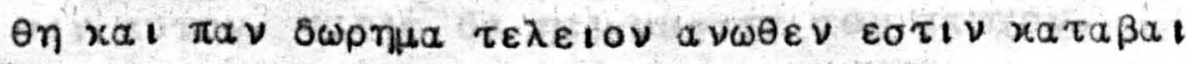

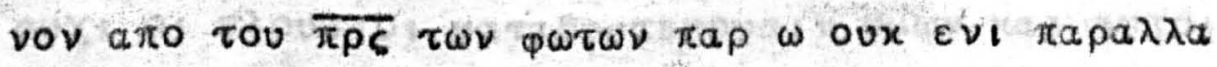
$\eta \eta \tau p o \pi \eta \zeta$ a

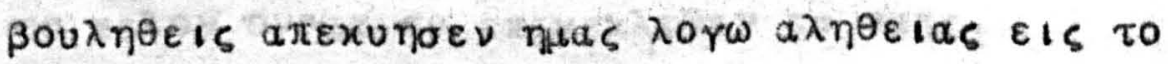

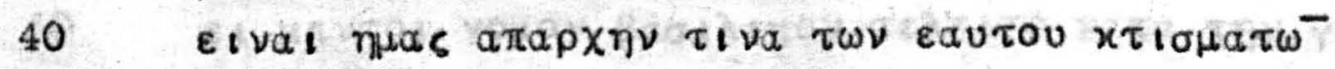

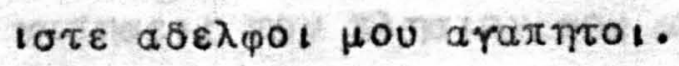




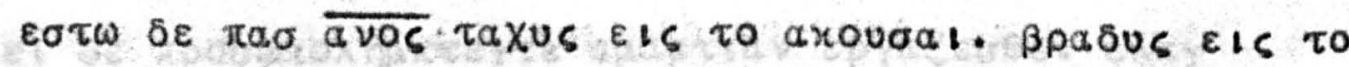

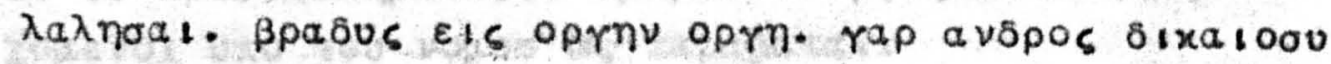

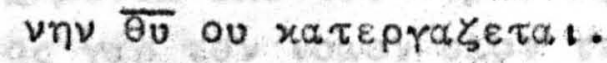

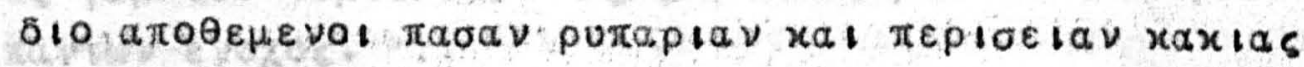

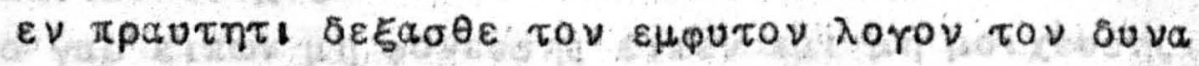

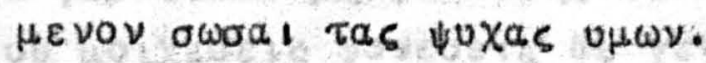

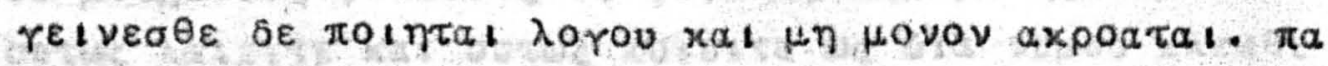

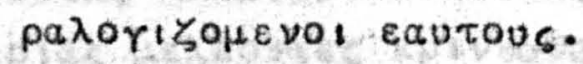

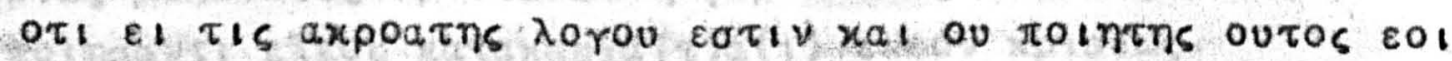

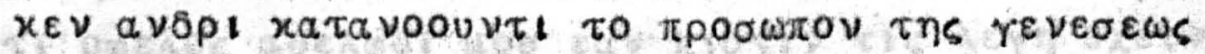

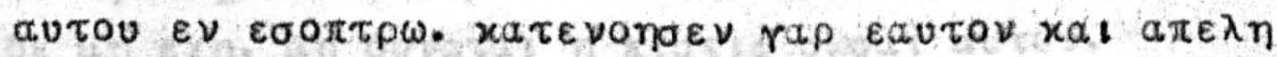

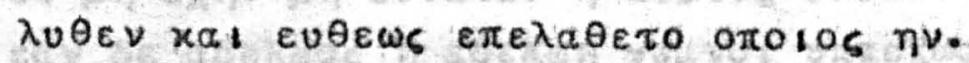

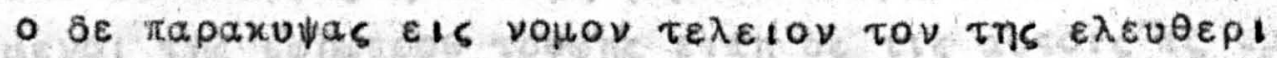

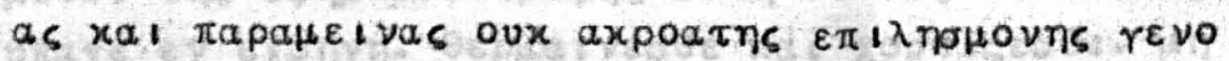

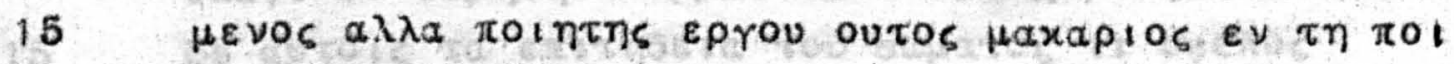
noel autou $\varepsilon \sigma \tau a l$.

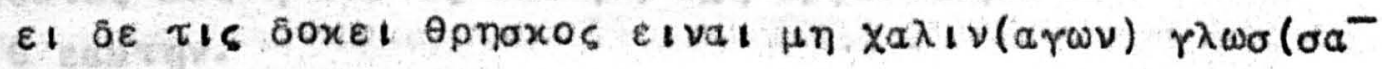

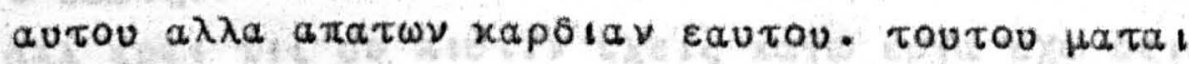

os $\eta$ ep noxela.

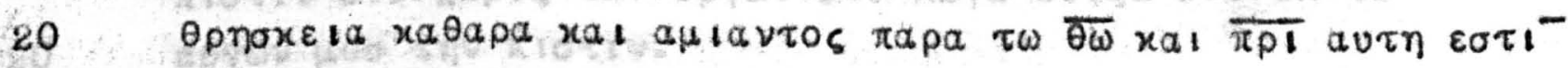

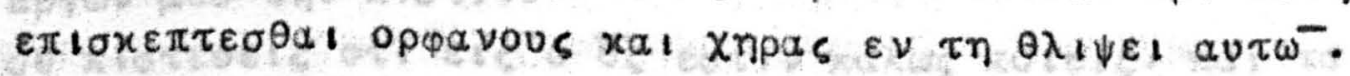

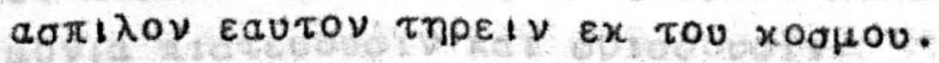

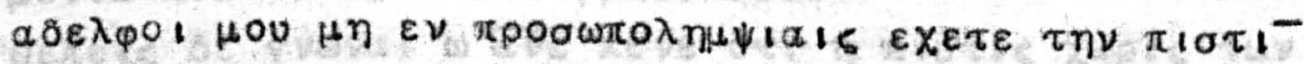

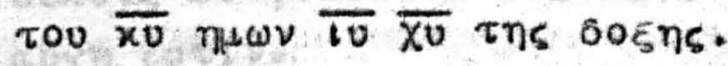

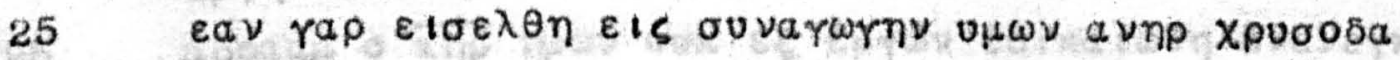

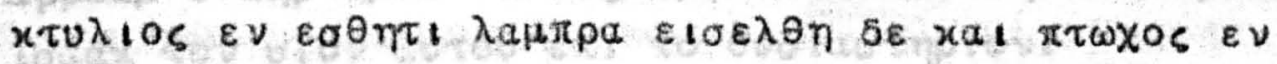
puтapa $\varepsilon \sigma \theta \eta \tau \iota$.

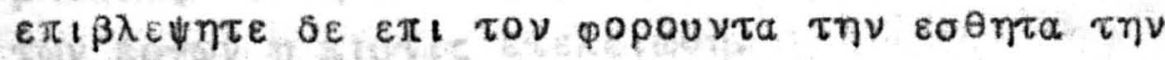

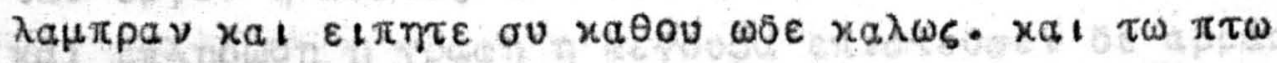

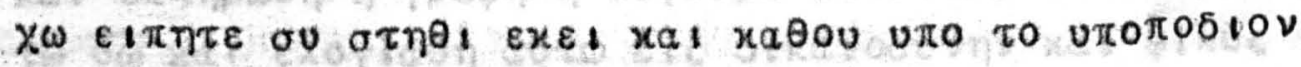

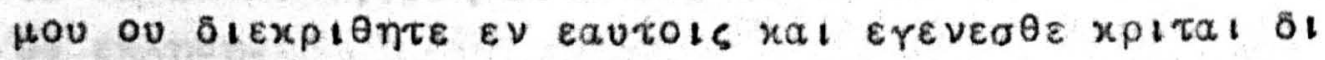

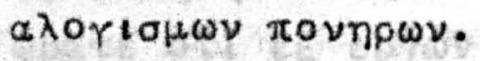

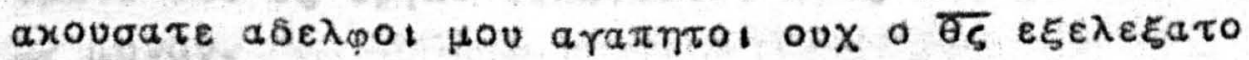

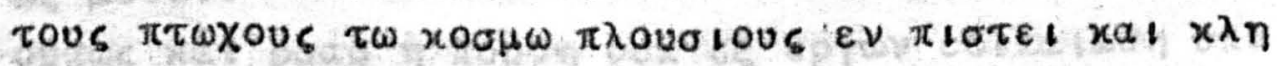

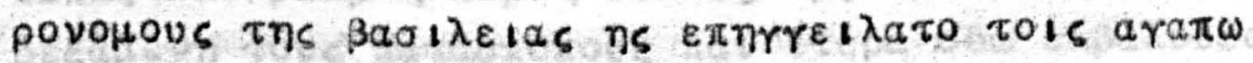

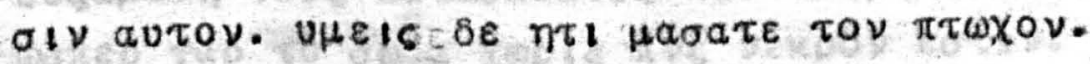

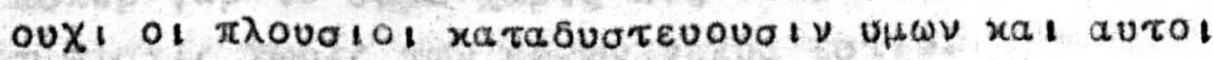

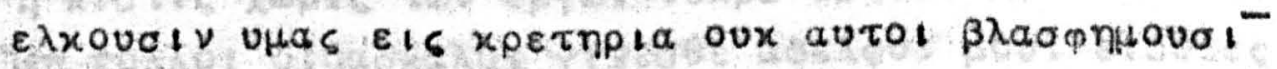

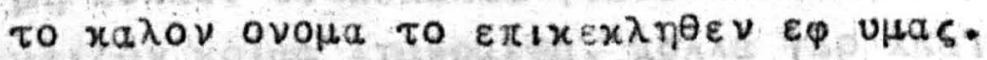

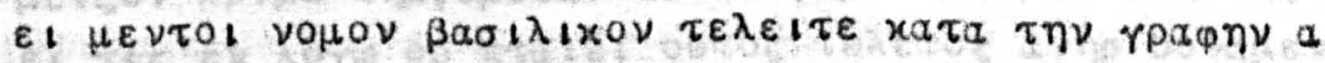

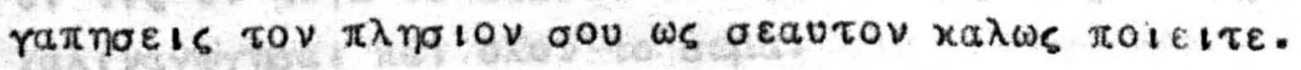




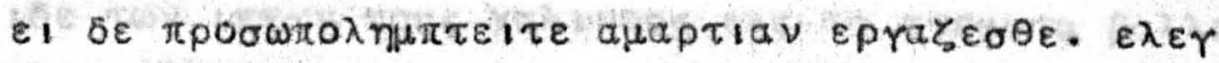

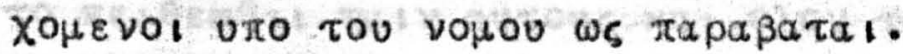

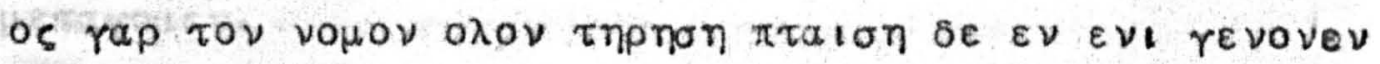
$\pi a \nu \tau \omega \nu$ \& voXOs.

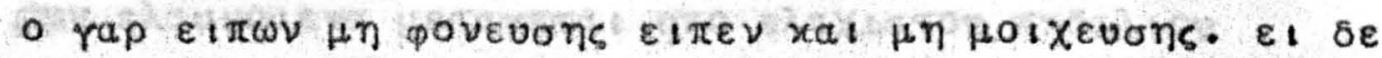

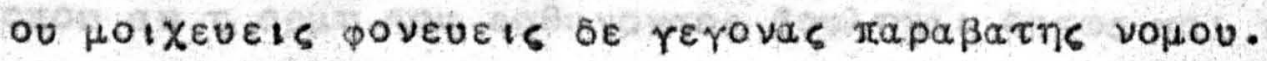

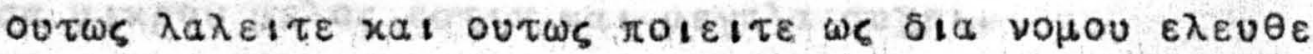

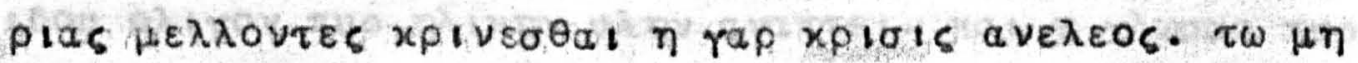

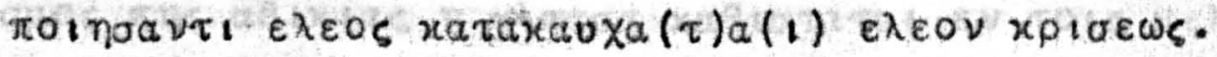

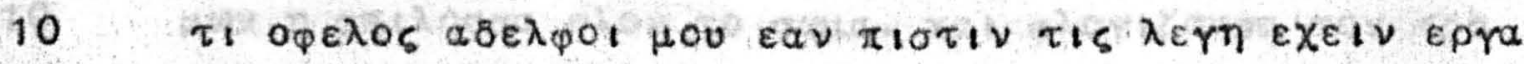

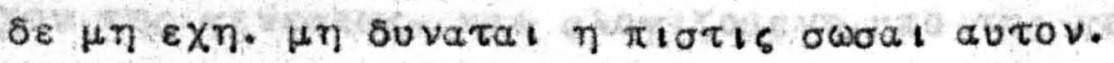

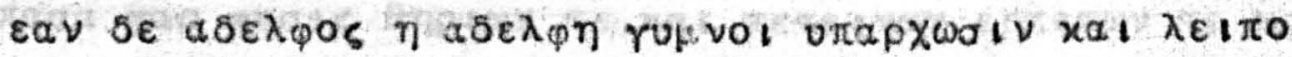

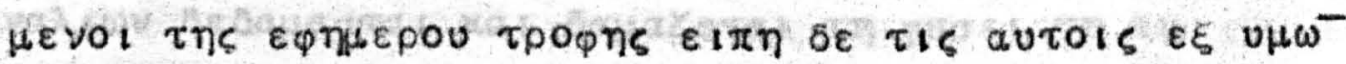

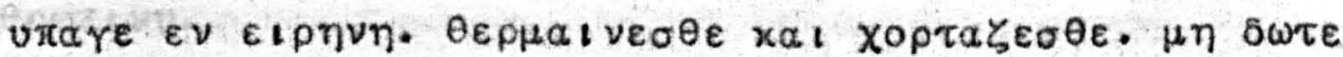

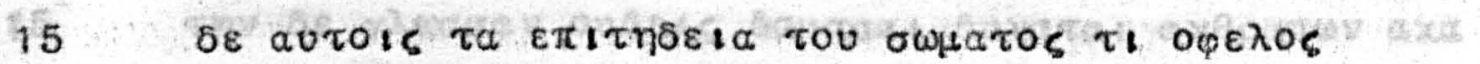

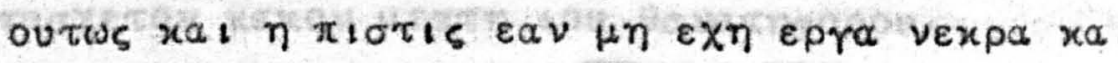

$\theta$ eaurnv.

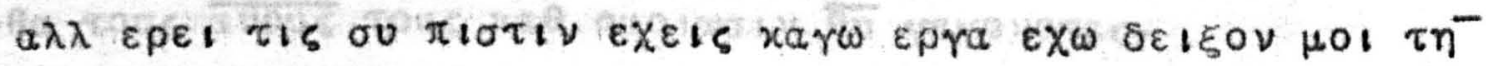

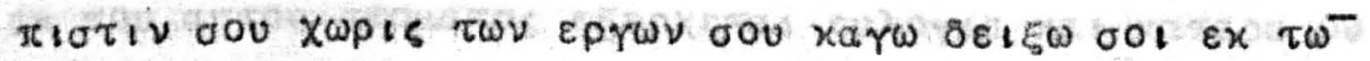

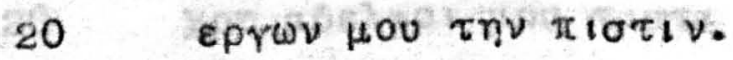

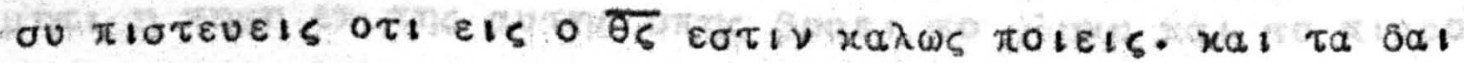

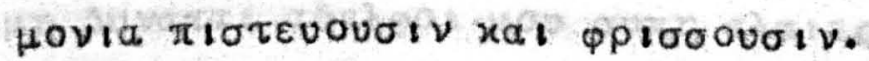

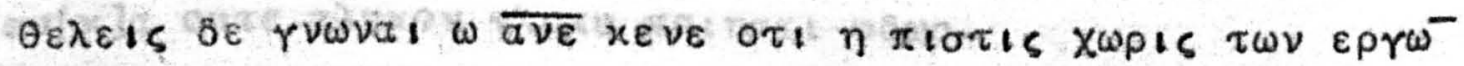

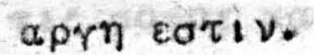

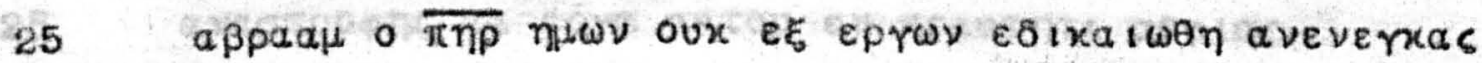

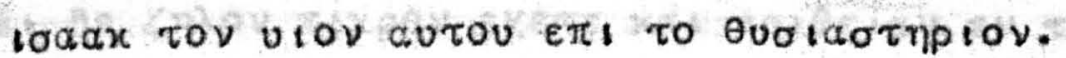

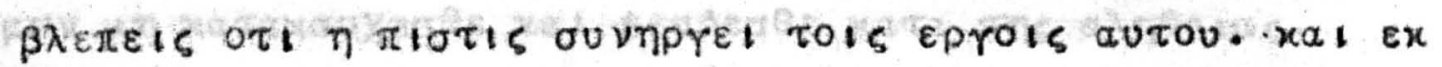

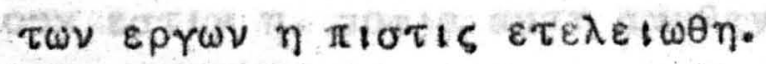

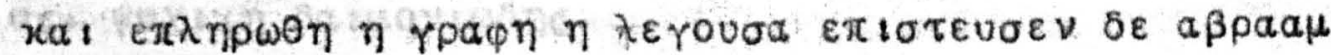

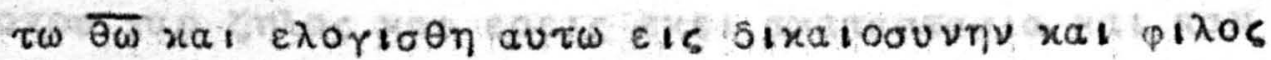

O० $\varepsilon x \lambda \eta \dot{\theta} \eta$.

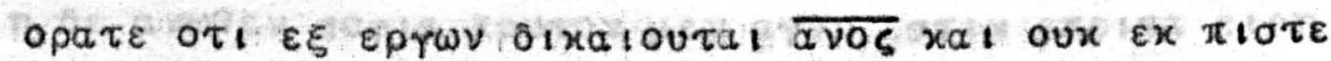
ws Hovov.

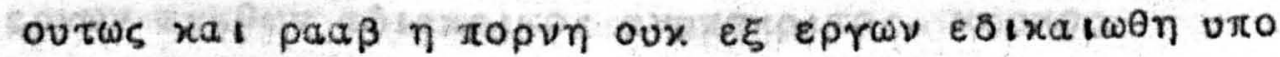

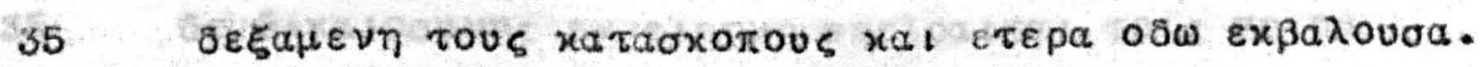

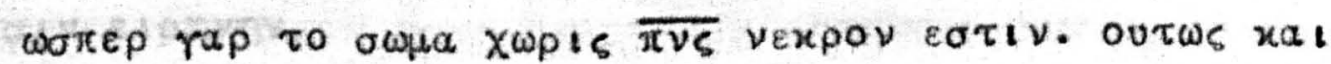

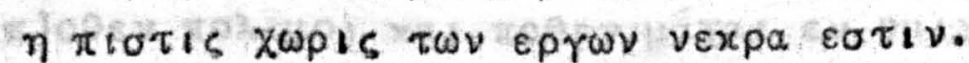

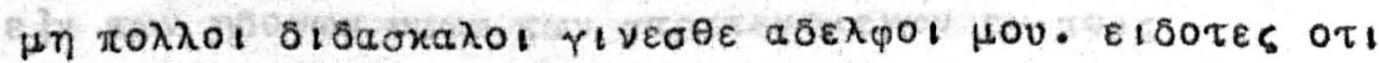

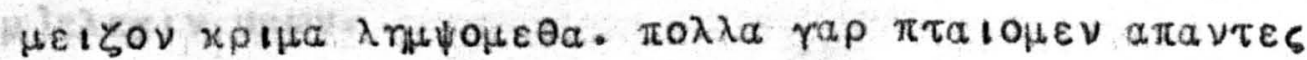

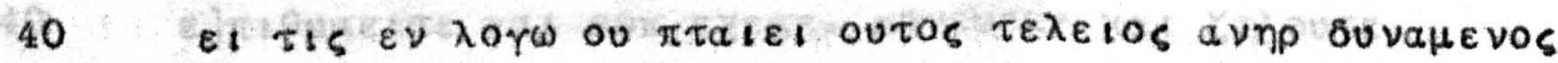

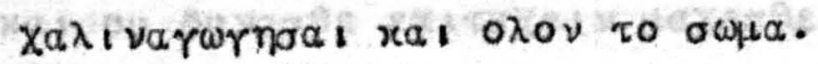




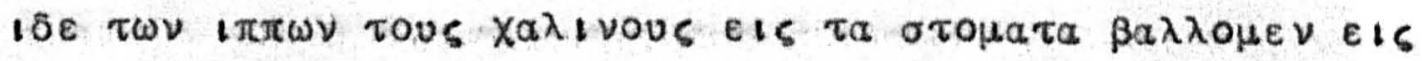

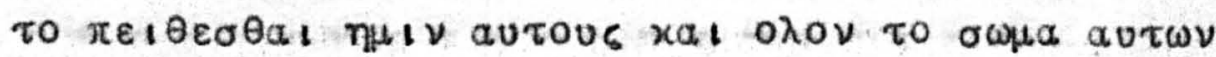

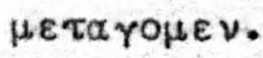

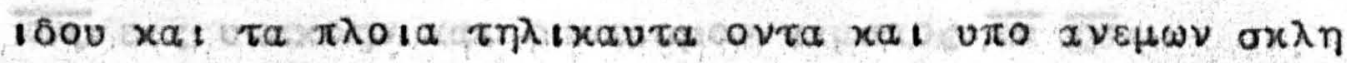

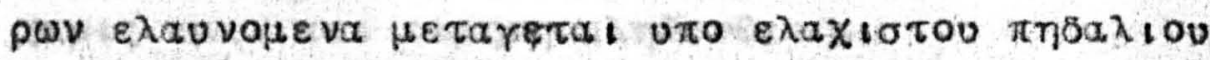

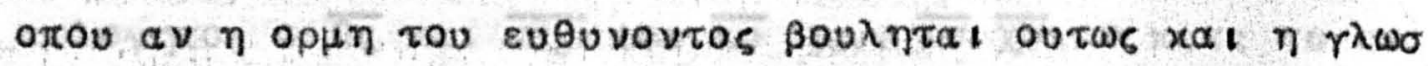

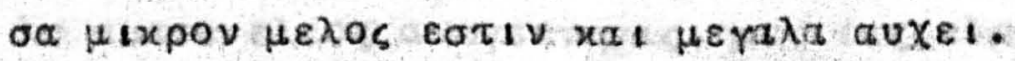

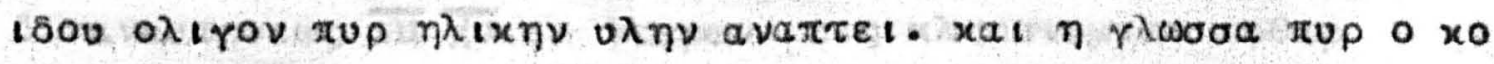

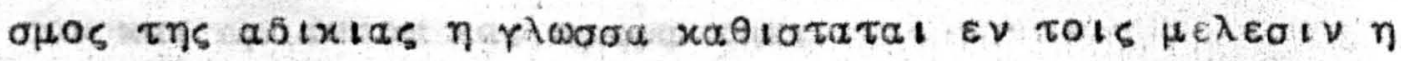

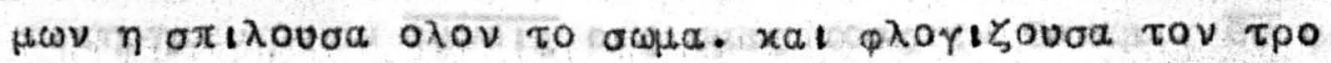

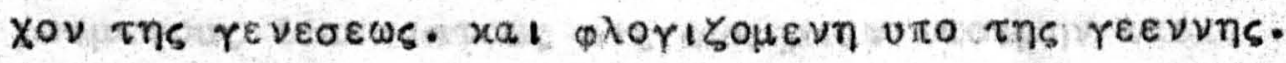

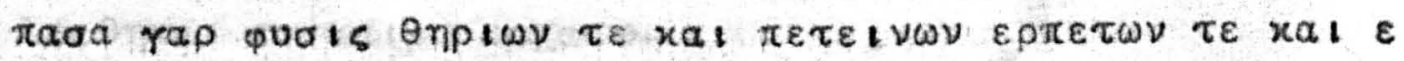

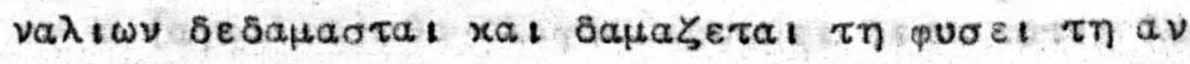
$\theta$ pwrtvi. $\tau \eta \underline{\nu} \delta \varepsilon$ భ

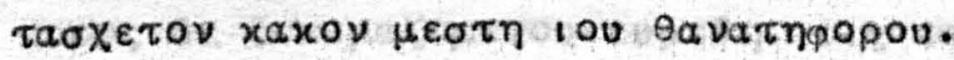

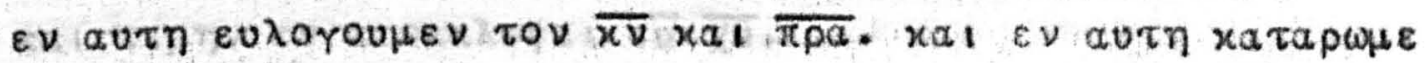
$\theta a$ rovs $\overline{a v o u}$ tovs $x a \theta$ opotwatv $\overline{\theta u}$ repovotas.

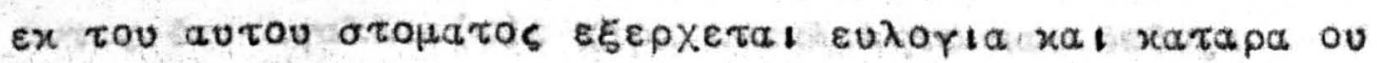

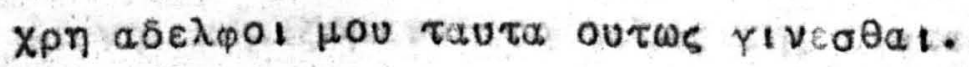

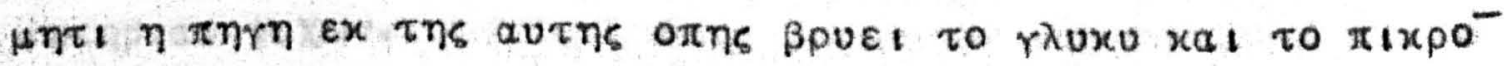

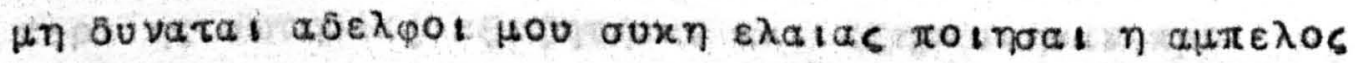

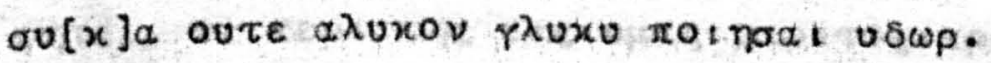

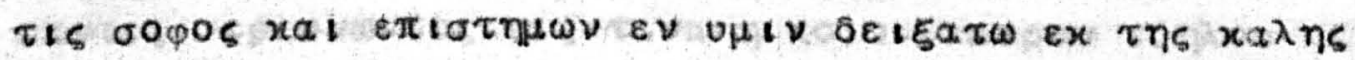

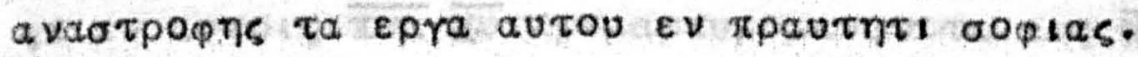

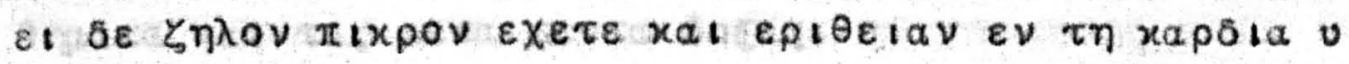

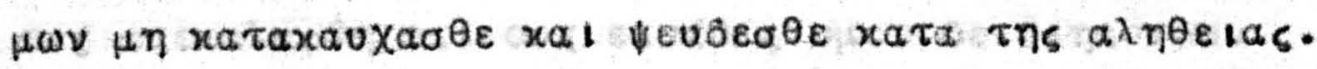

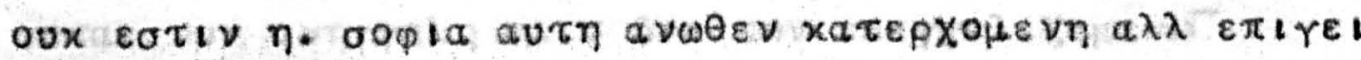

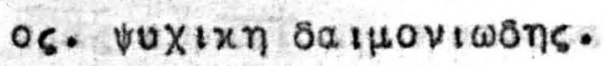

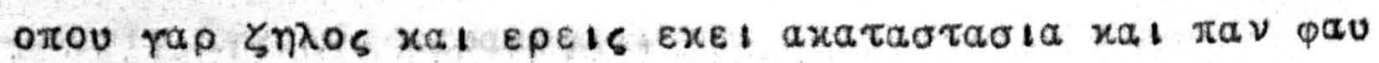

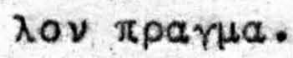

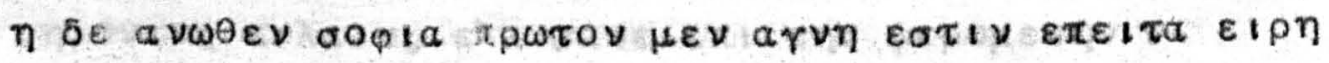

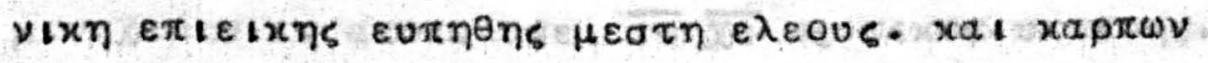

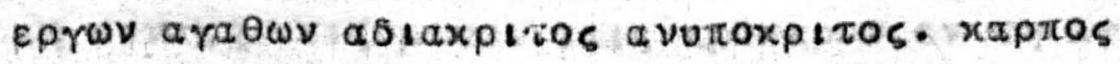

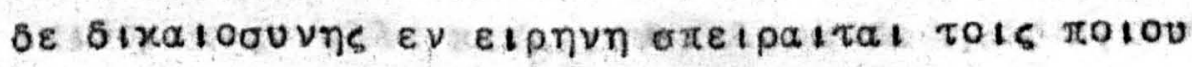

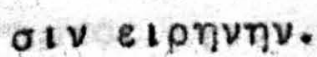

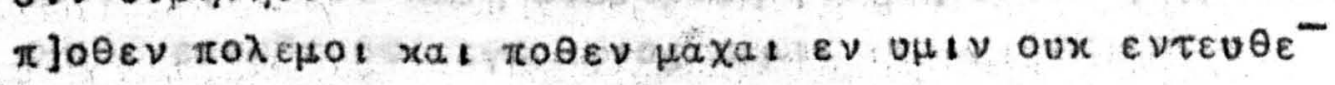

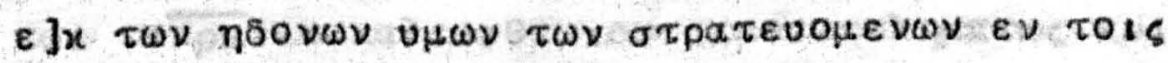
$\mu] \varepsilon \lambda \varepsilon \sigma \iota v$ u $\omega \nu$.

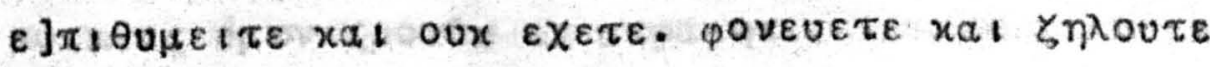

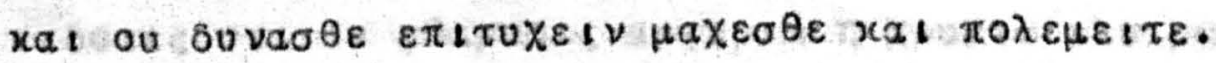




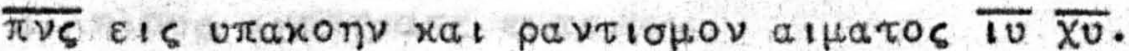

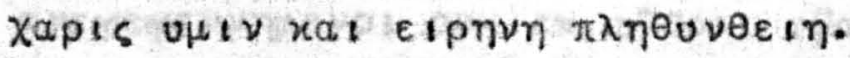

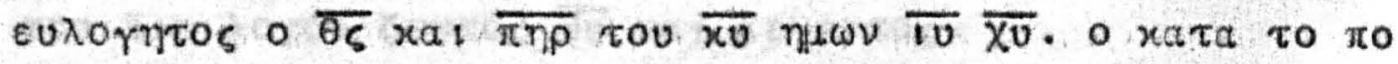

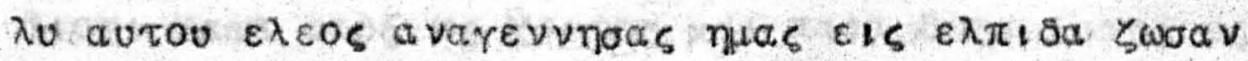

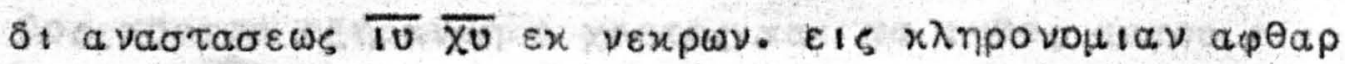

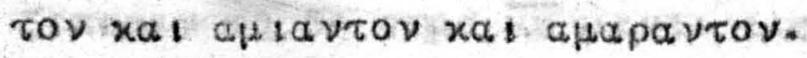

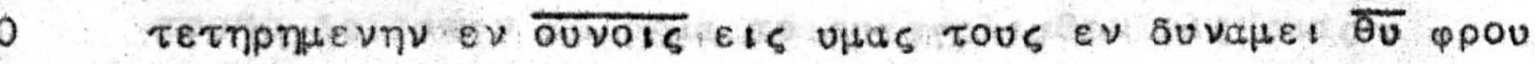

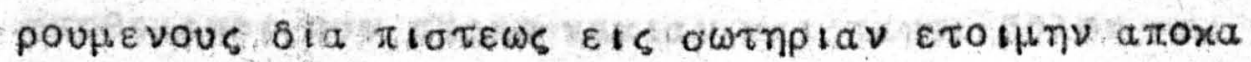

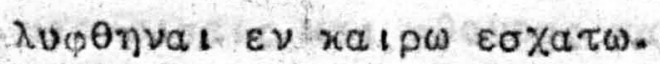

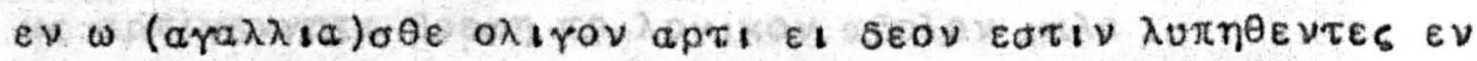

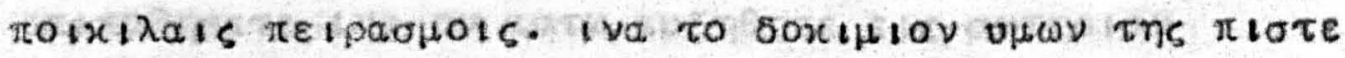

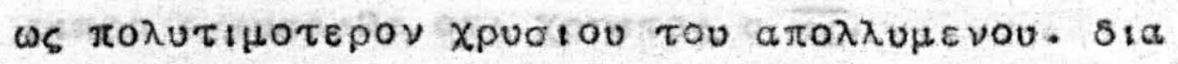

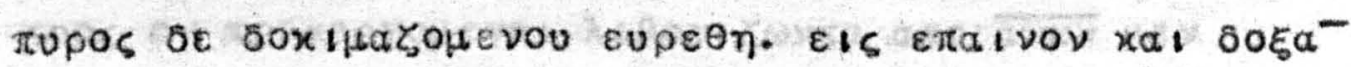

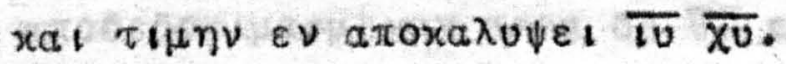

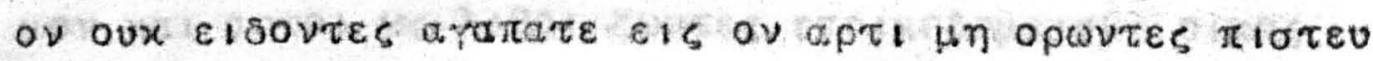

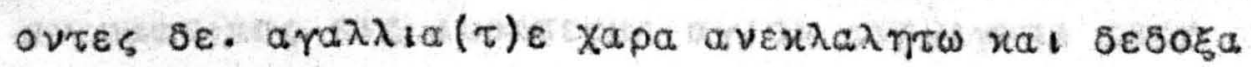

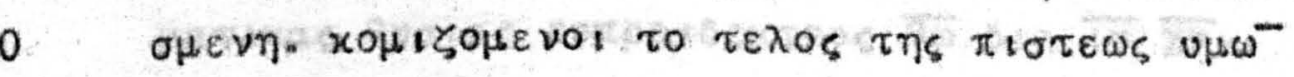

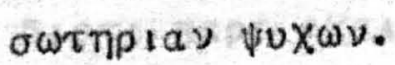

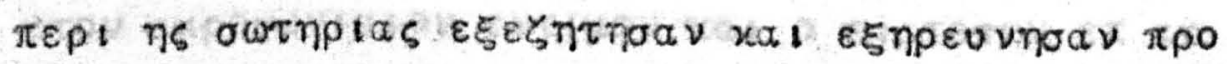

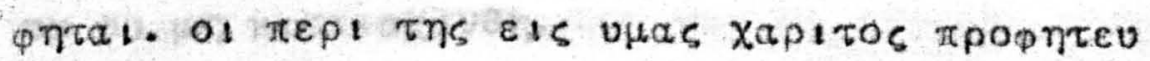

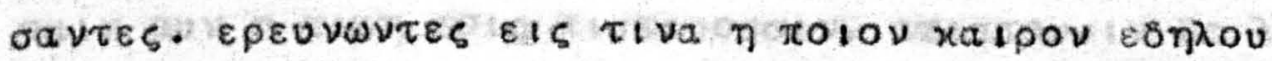

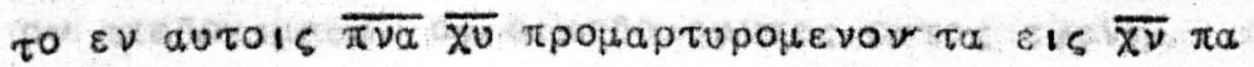

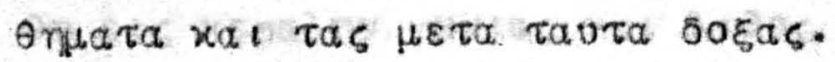

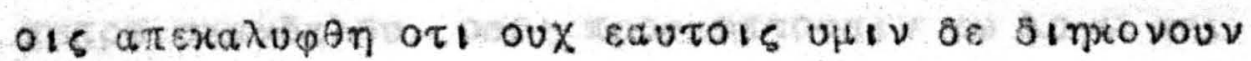

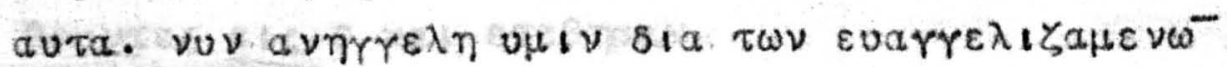

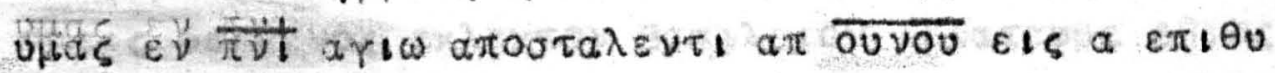

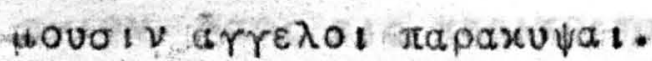

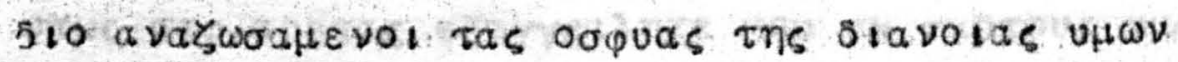

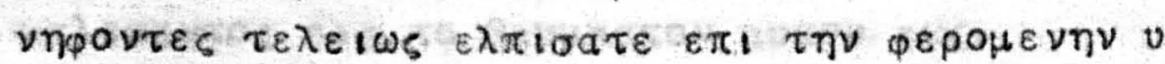

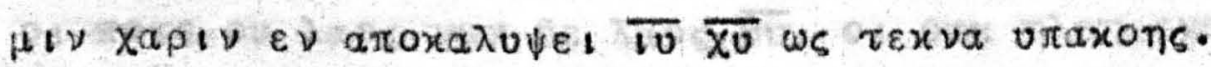

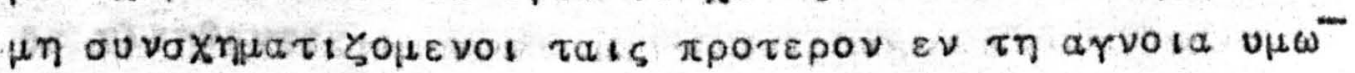

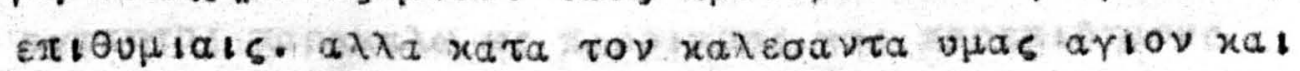

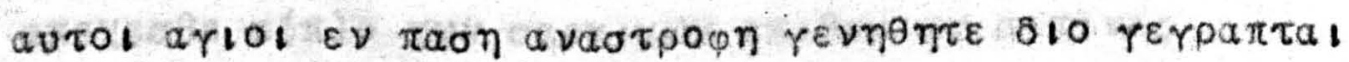

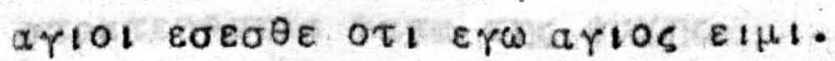

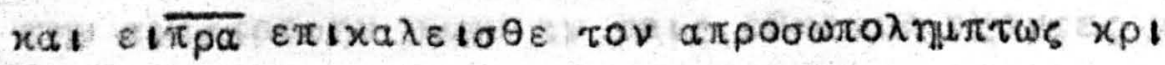

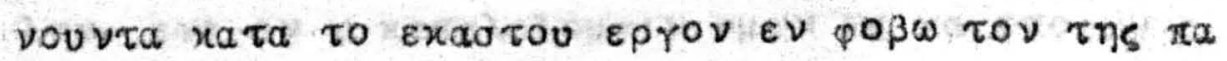

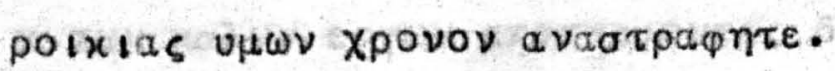

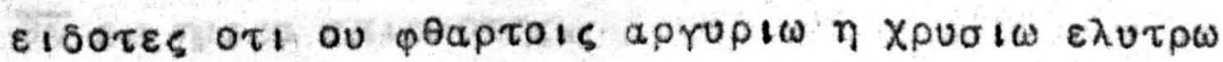

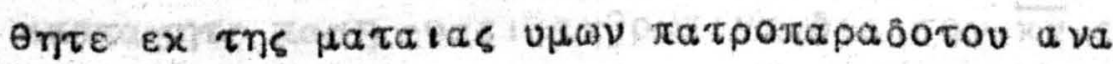

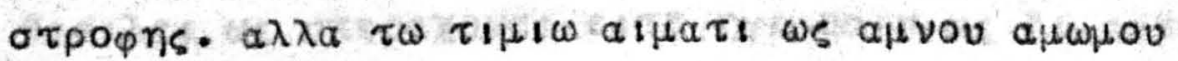
$x a$ i arii

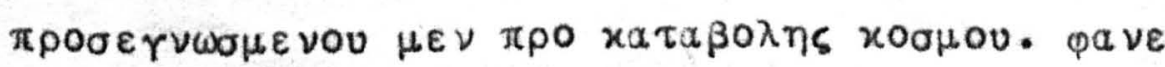

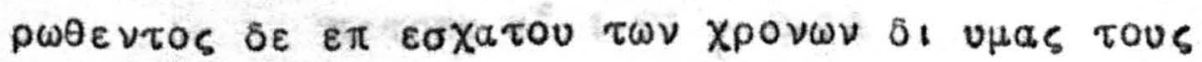
$\pi \varepsilon \tau \rho \circ a^{\prime} 1.1-21$ 


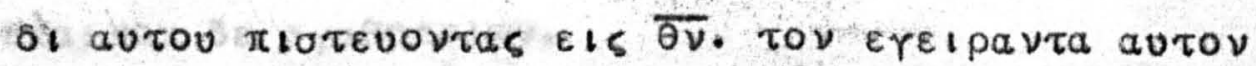

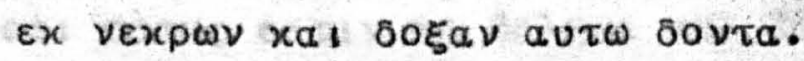

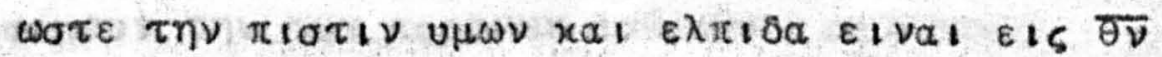

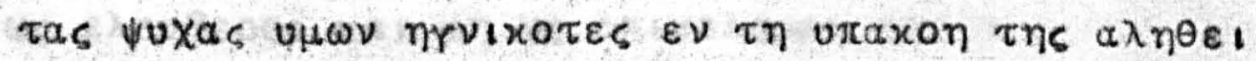

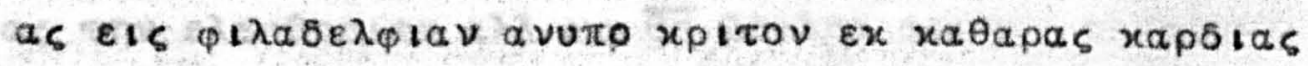

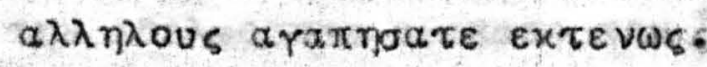

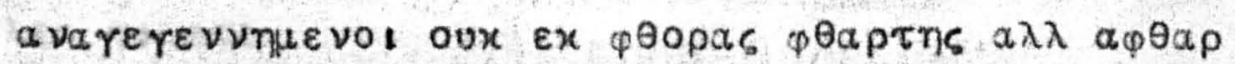

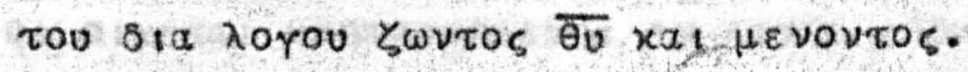

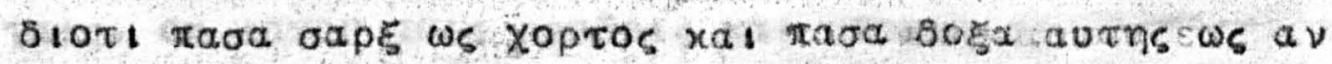

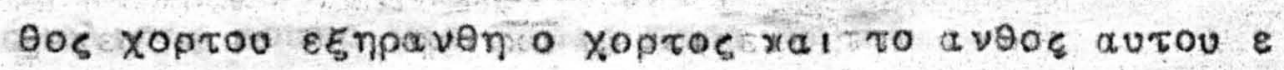

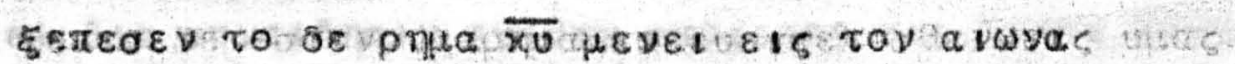

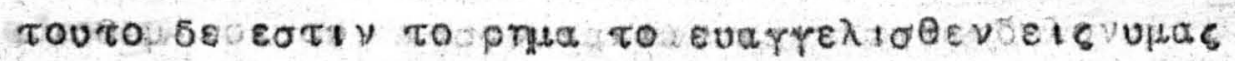

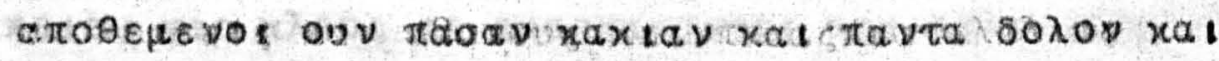
unoxptoels $x a l$ povous $x_{1}$ tacas $x a \tau a \lambda a \lambda$ las

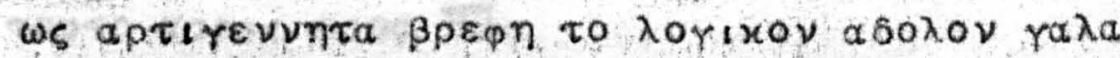

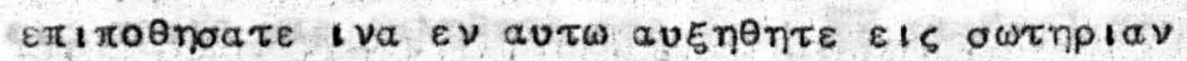

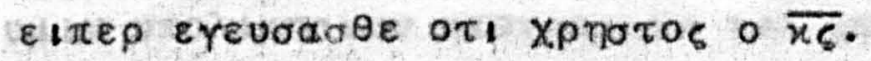

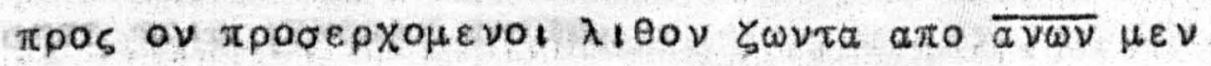

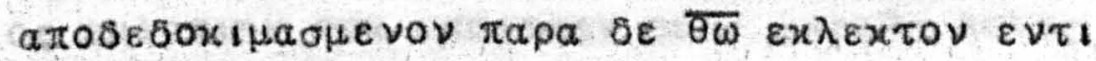

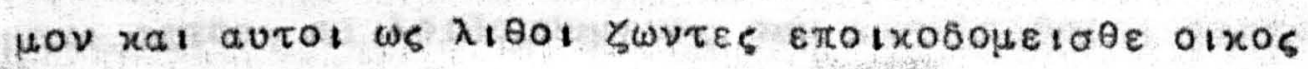

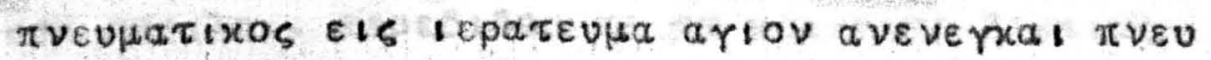

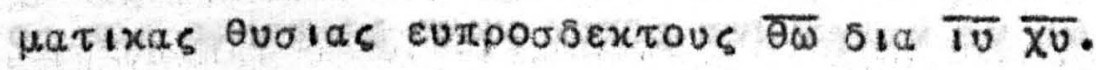
бı

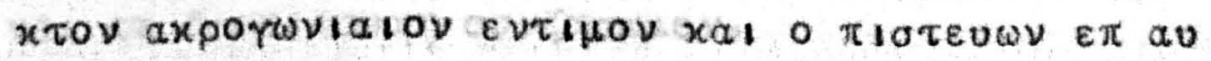

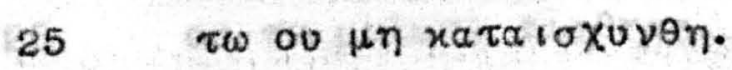

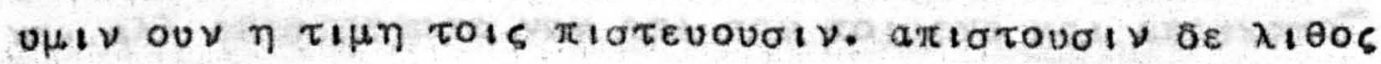

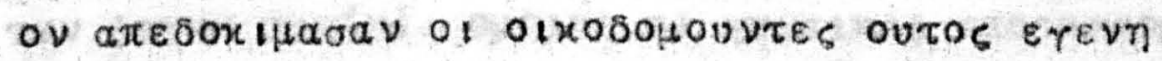

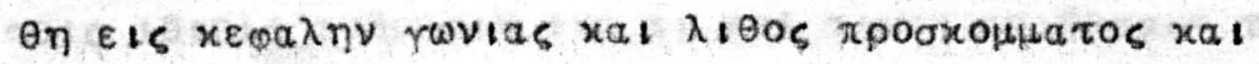

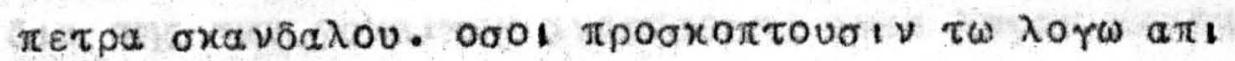

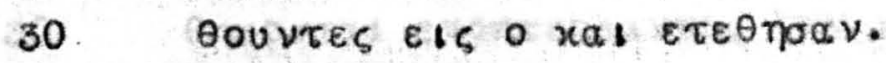

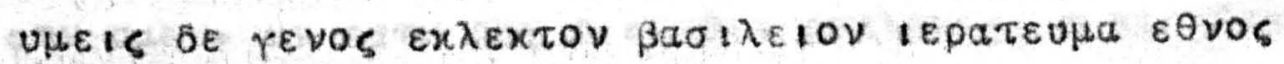

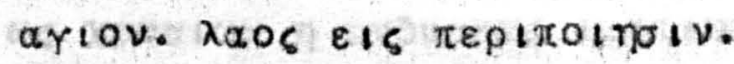

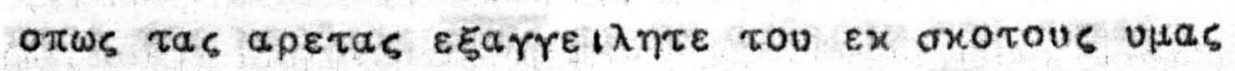

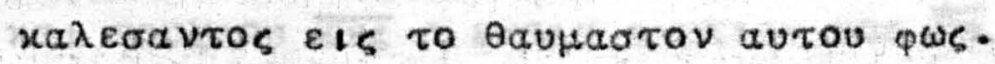

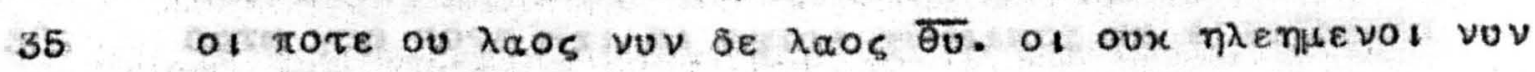

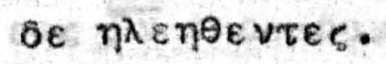

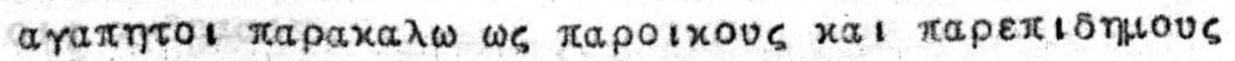

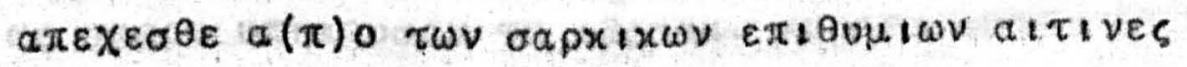

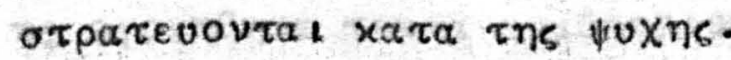

$40 \quad \tau \eta \nu$ ava

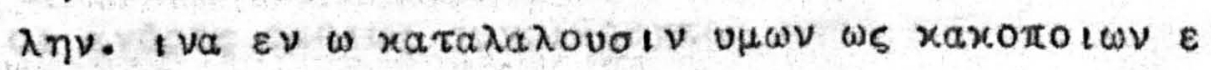

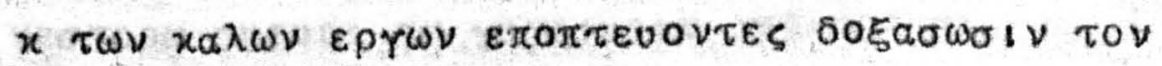

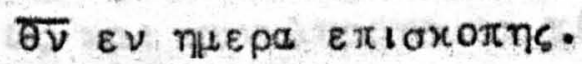

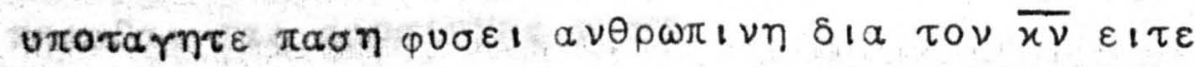

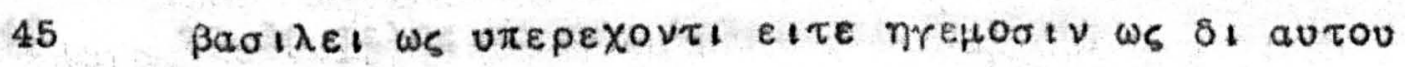

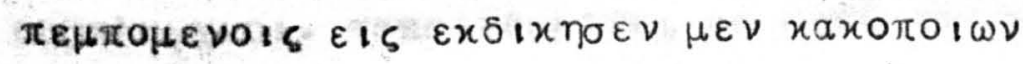




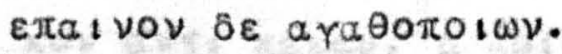

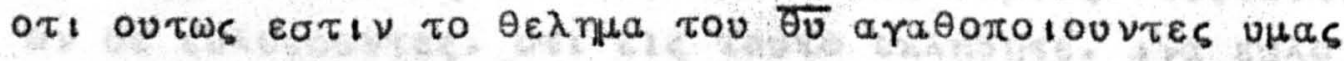

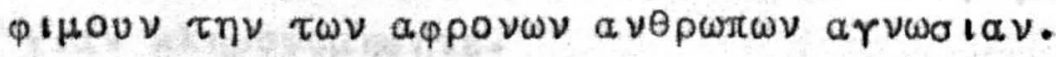

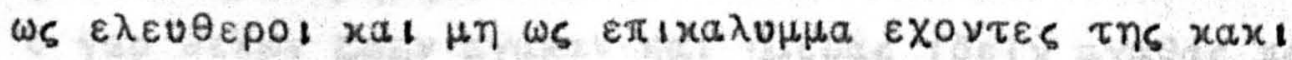

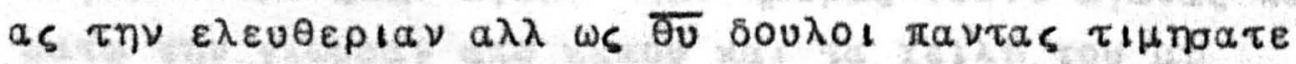

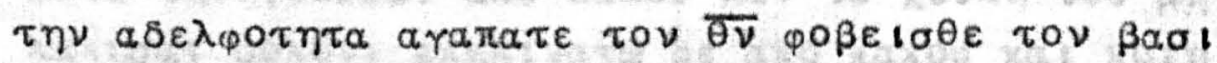

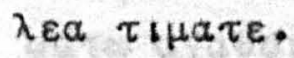

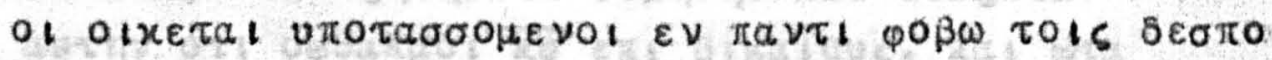

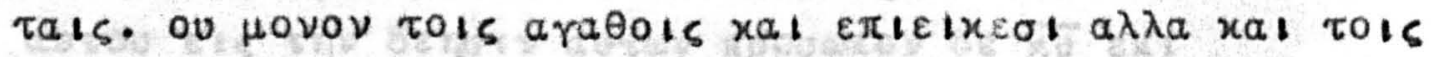

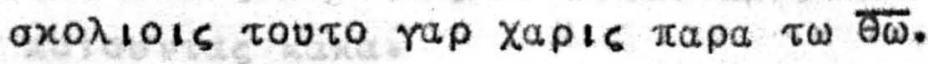

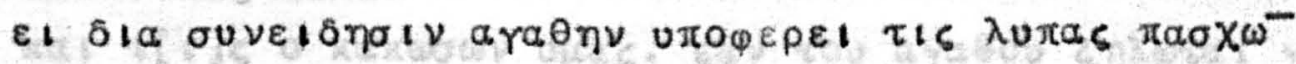
a. $\delta$ ixws.

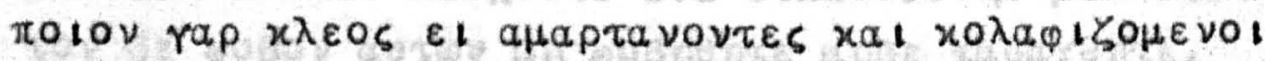

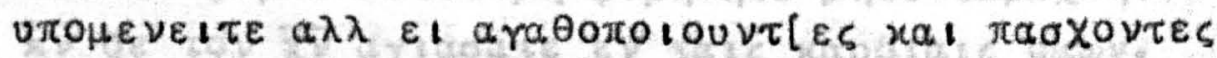

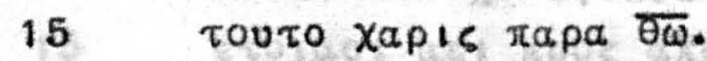

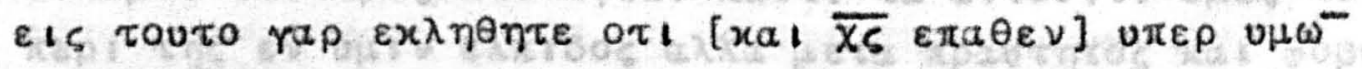

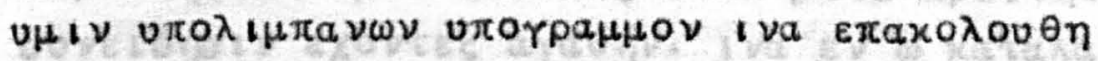

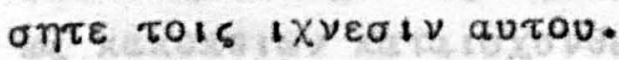

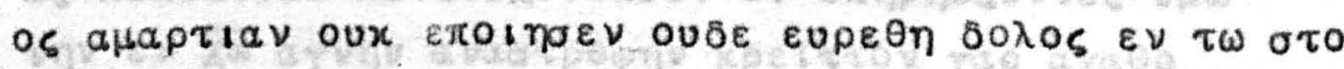

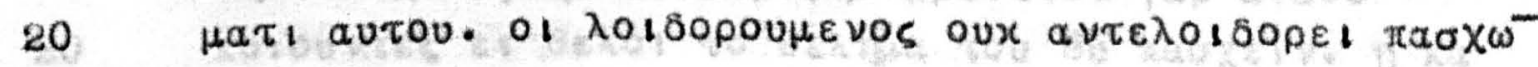

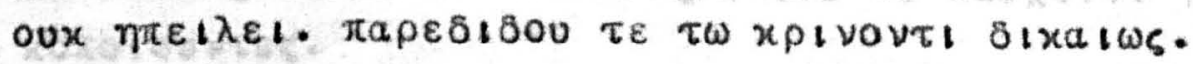

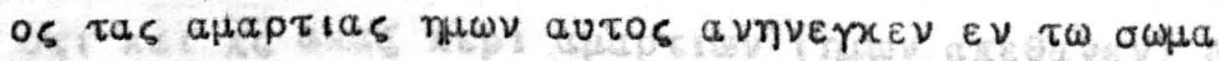

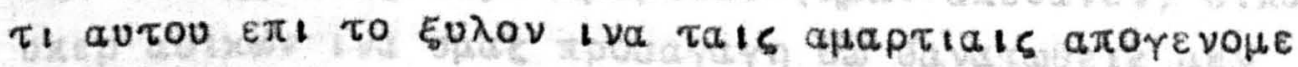

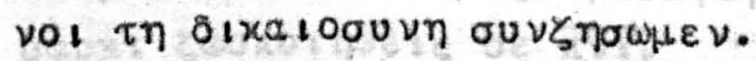

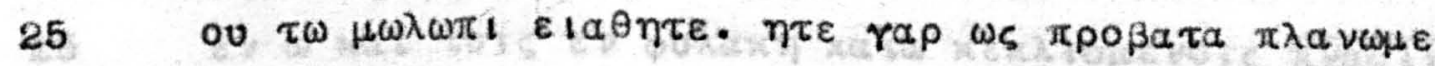

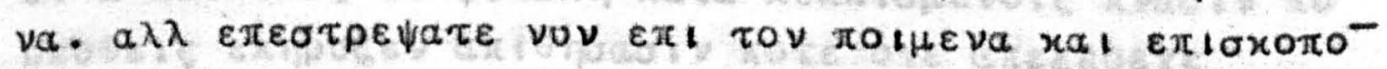

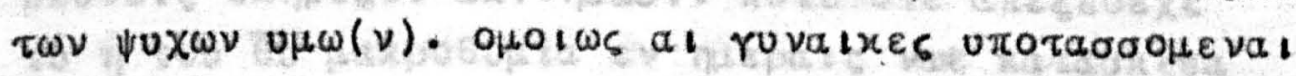
Tols $1 \delta 1015$ a

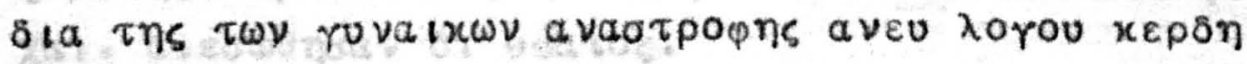

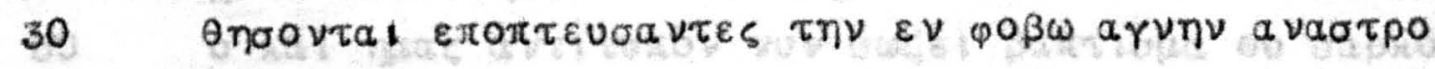

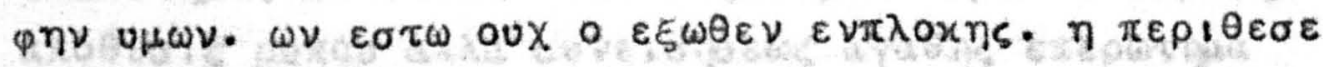

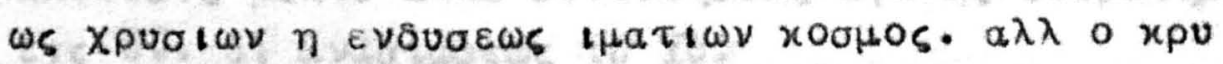

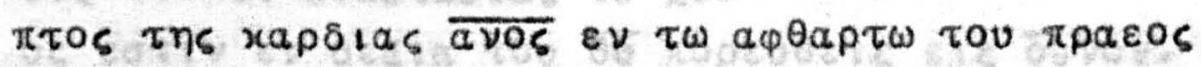

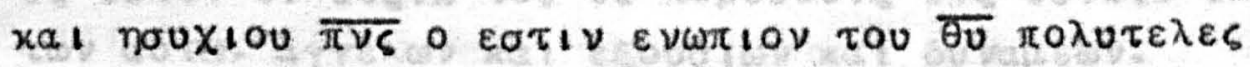

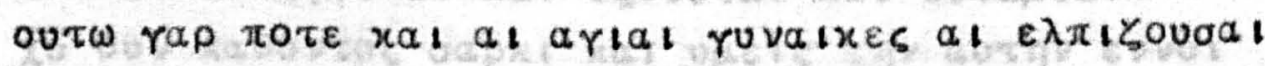

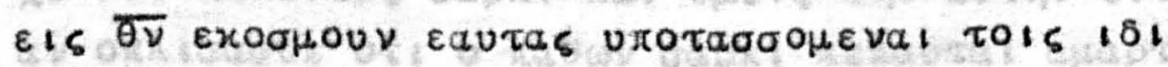

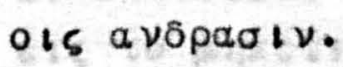

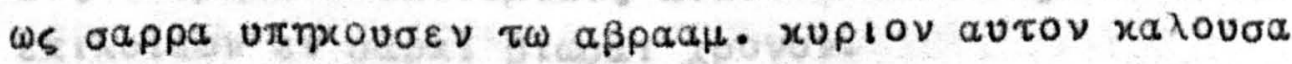

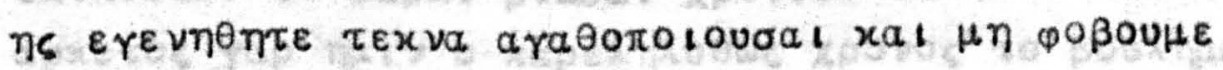

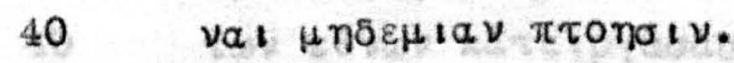
ol a

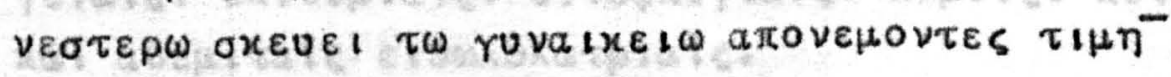

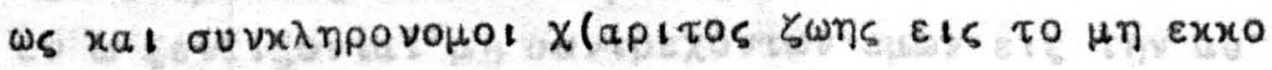

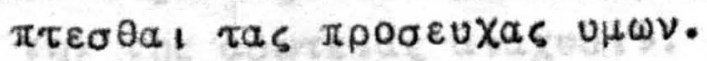

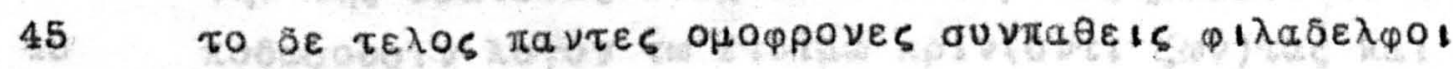

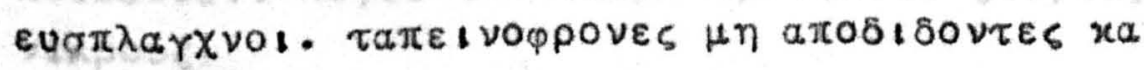




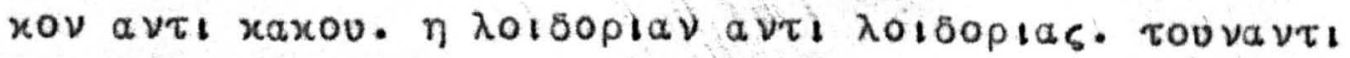

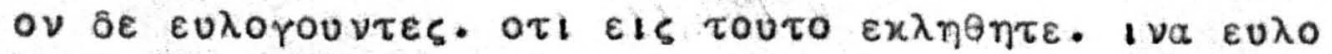

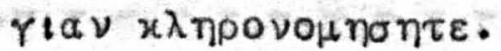

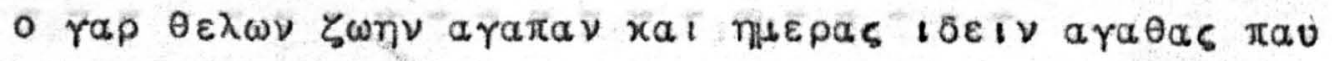

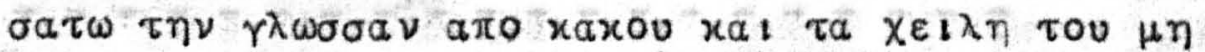

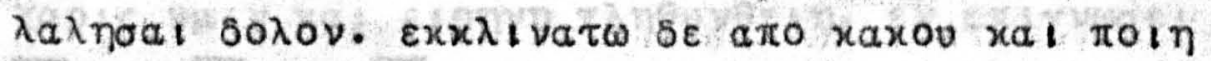

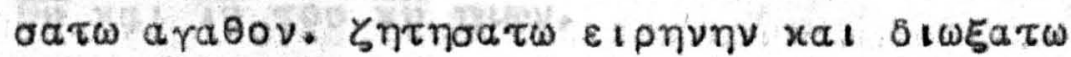

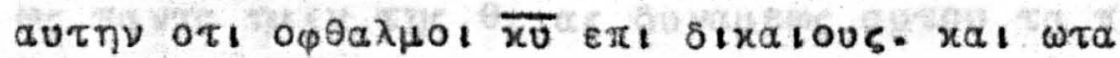

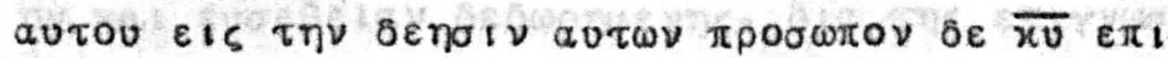
Tolouvras xaxa.

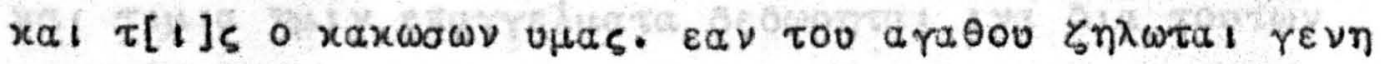

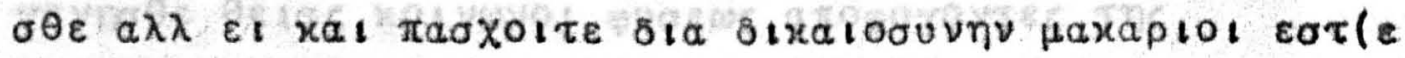

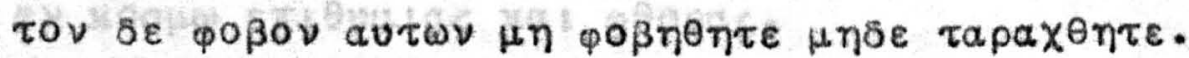

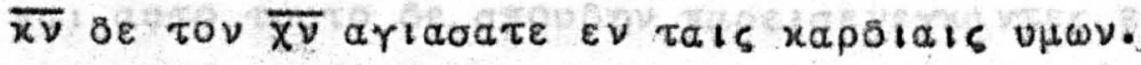

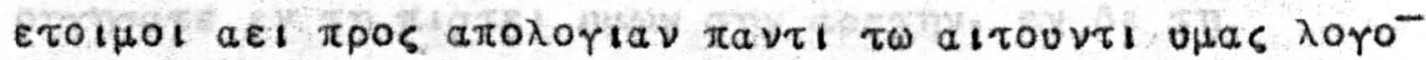

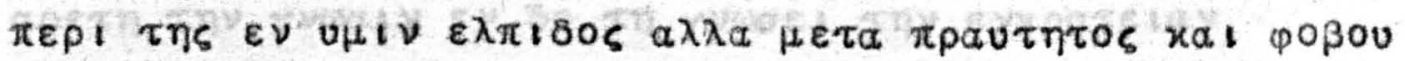

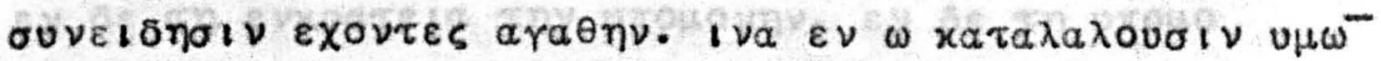

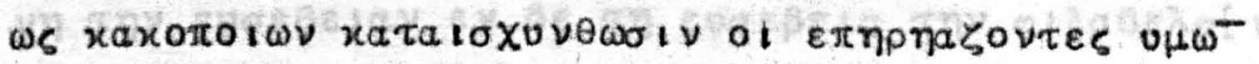

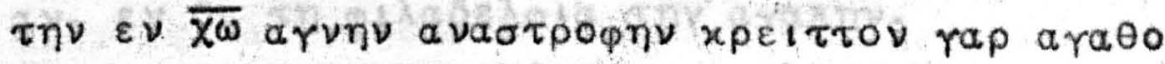

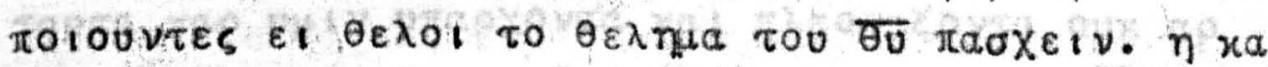
xomolouvies.

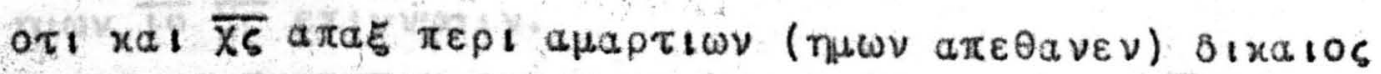

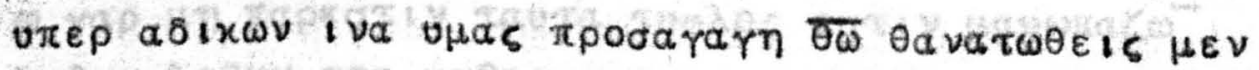

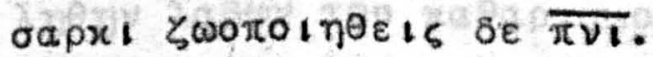

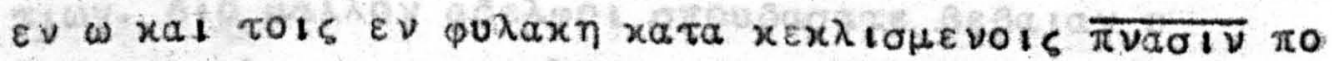

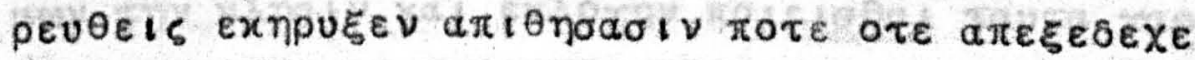

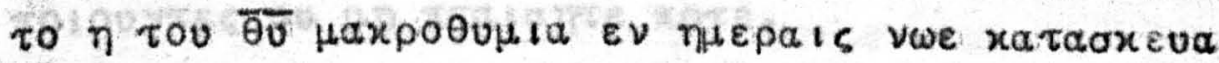

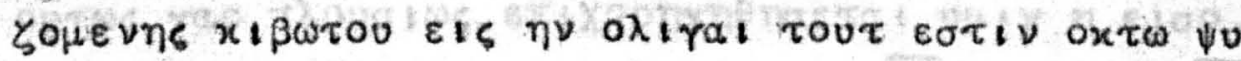

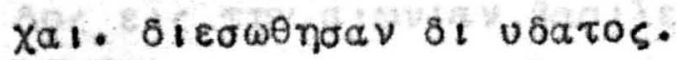

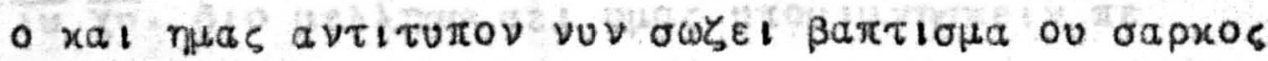

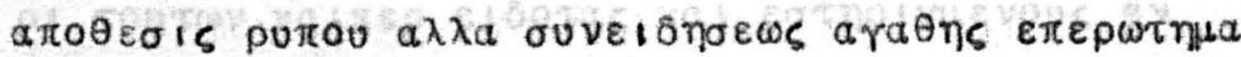

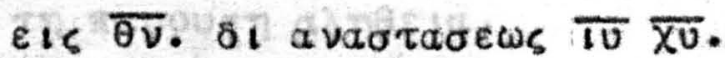

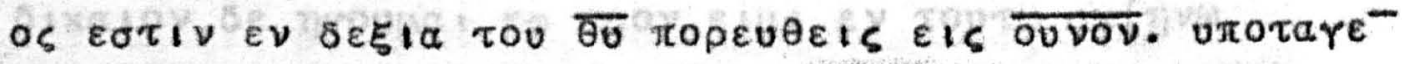

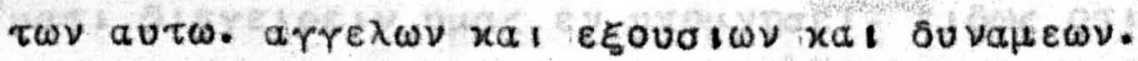

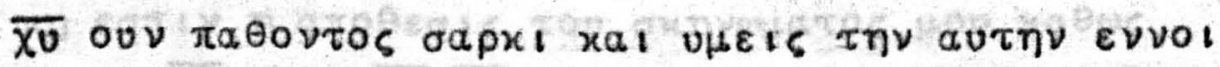

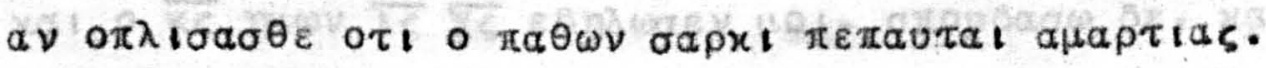

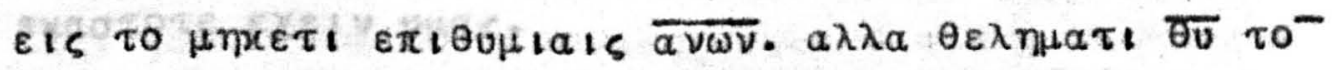

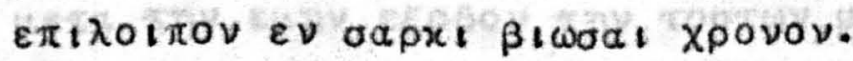

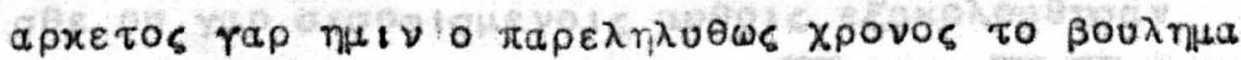

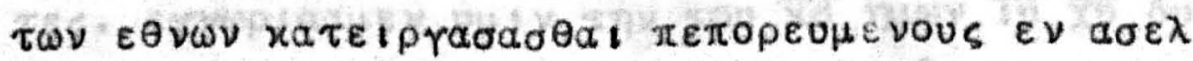

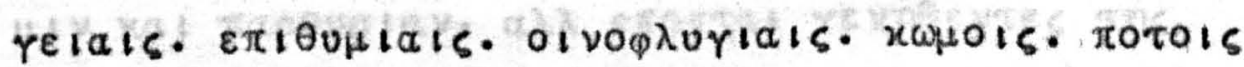

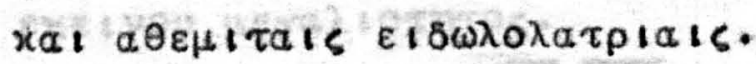

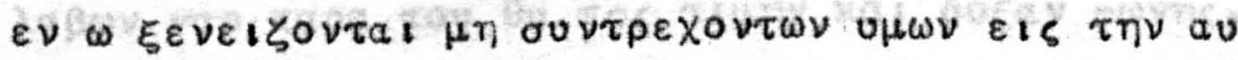

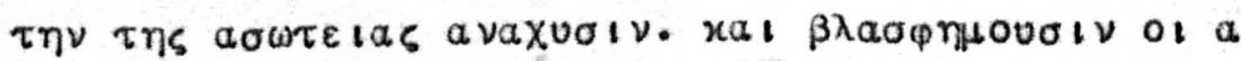

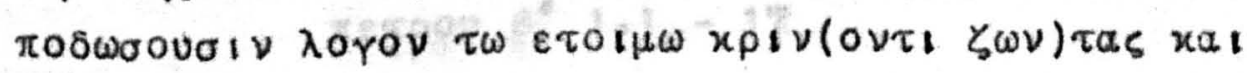
vexpous. 


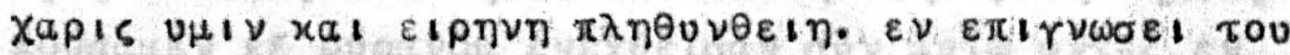

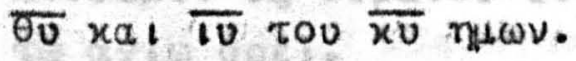

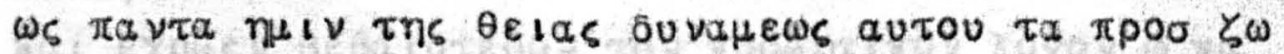

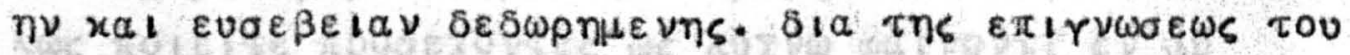

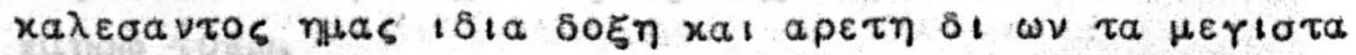

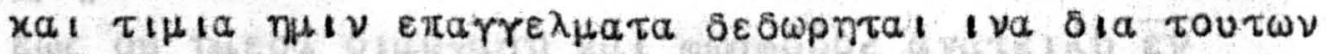

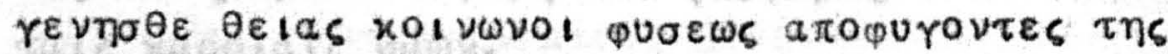

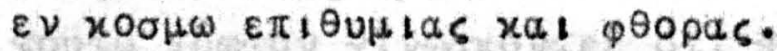

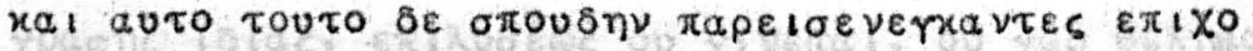

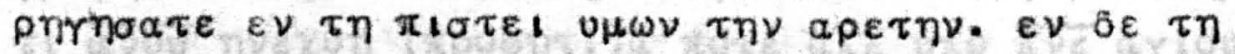

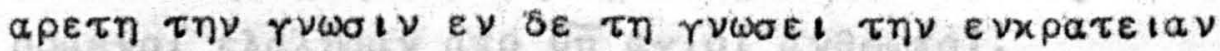

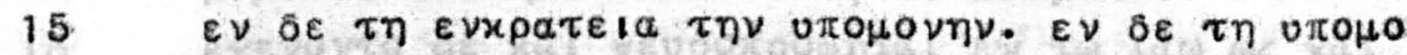

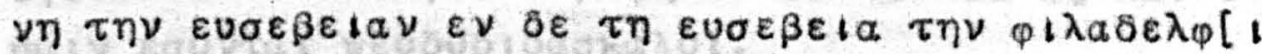

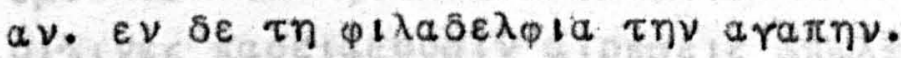

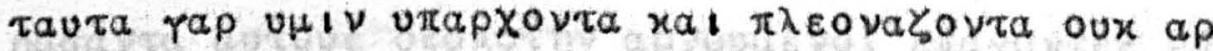

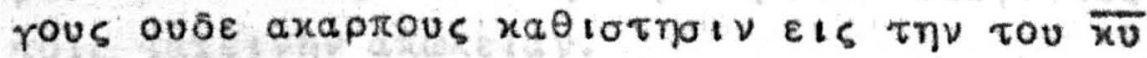

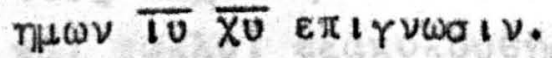

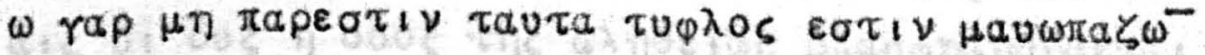

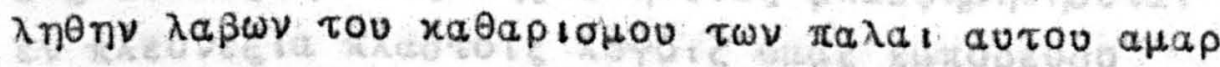

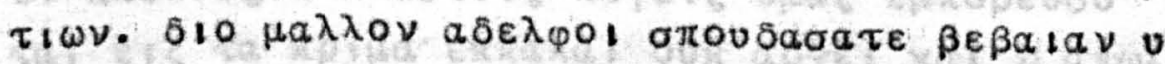

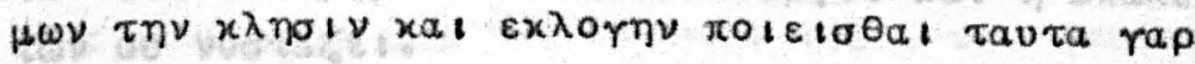

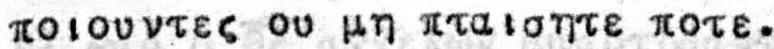

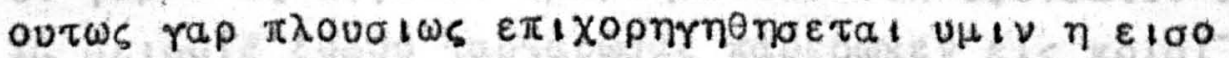

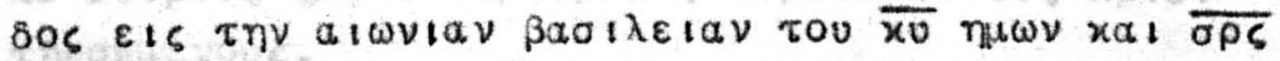
Tv $\overline{\chi v}$. $510 \mu \varepsilon \lambda \lambda \eta \eta \omega$ a

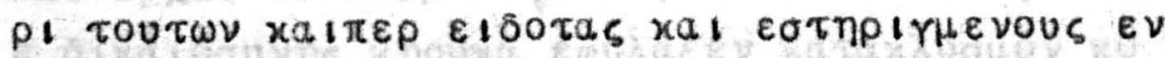

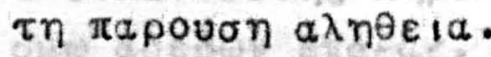

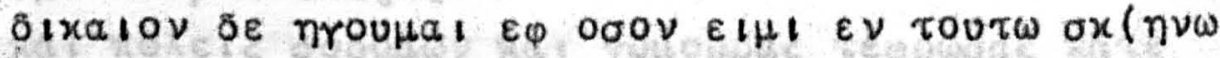

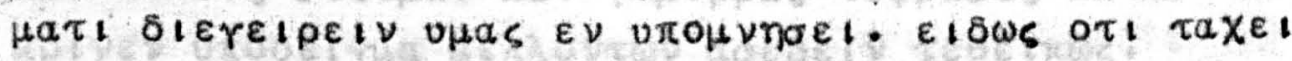

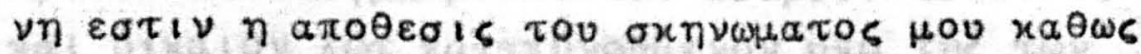

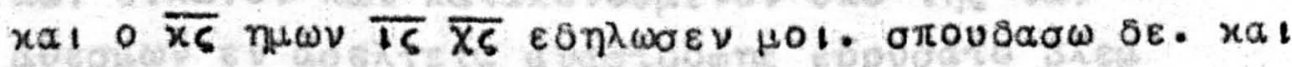

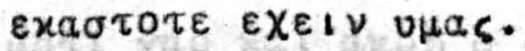

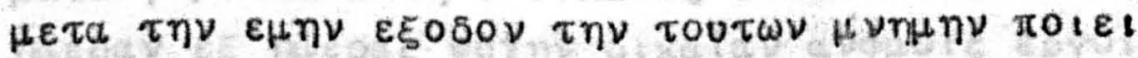

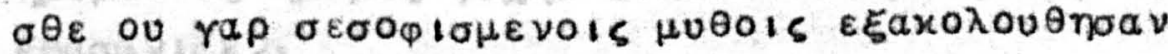

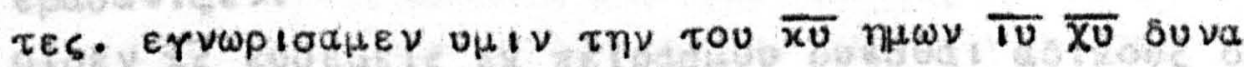

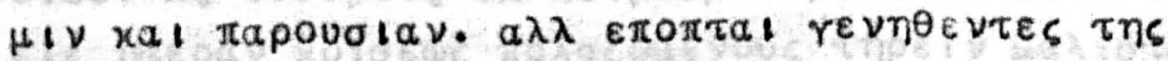

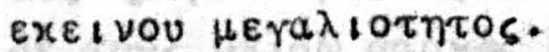

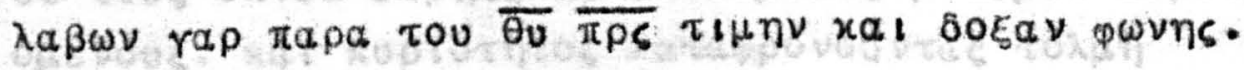




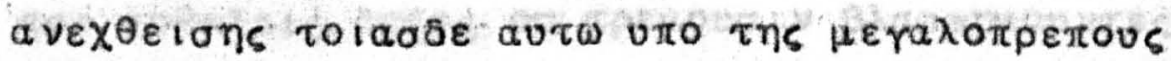

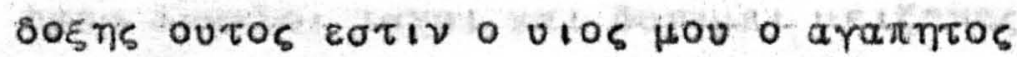

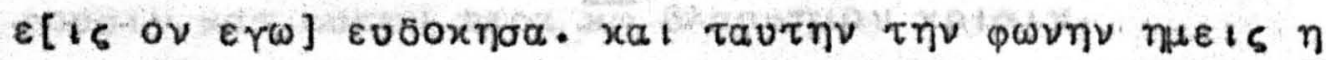

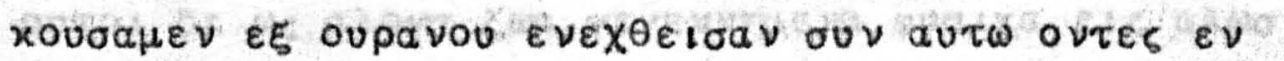
$\tau \omega$ artw ope t.

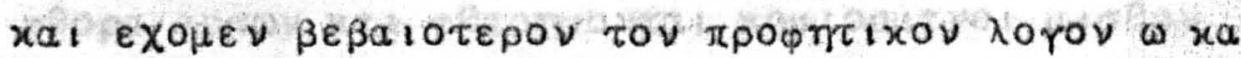

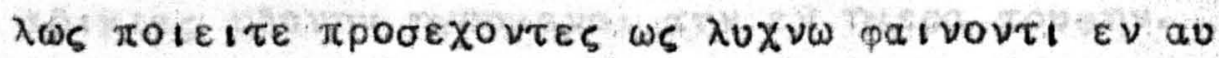

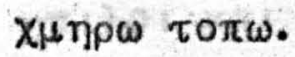

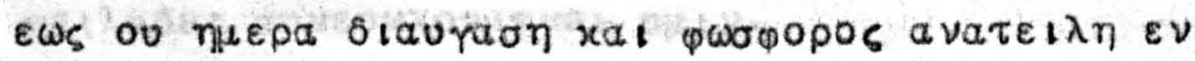
tais xapoitais viw.

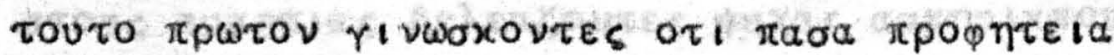

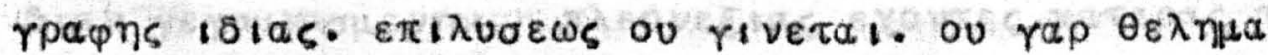

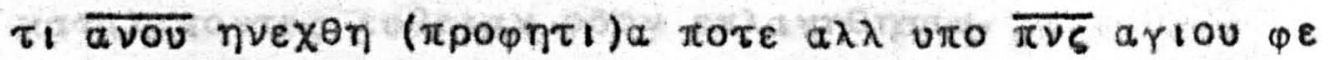

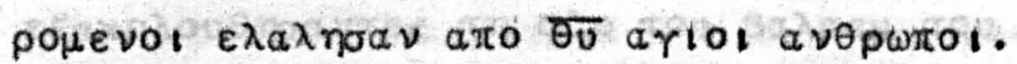

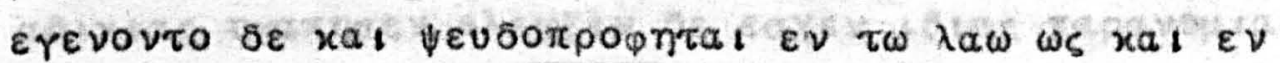

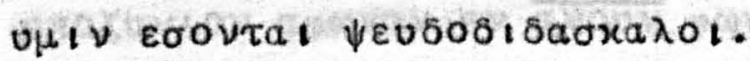

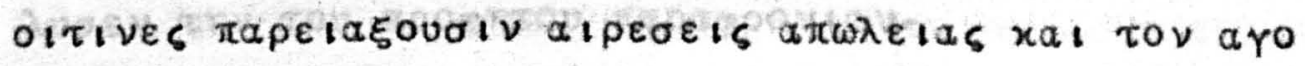

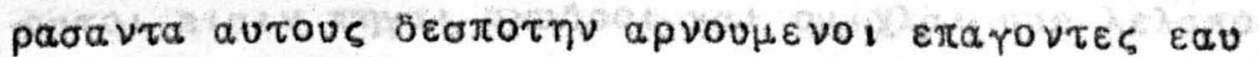

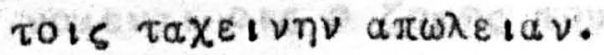

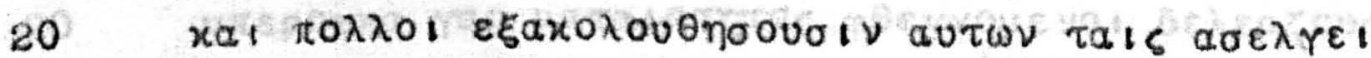

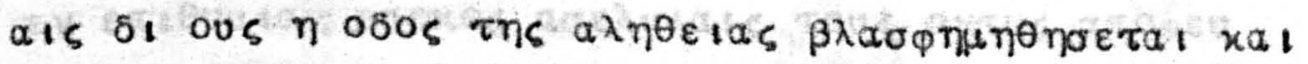

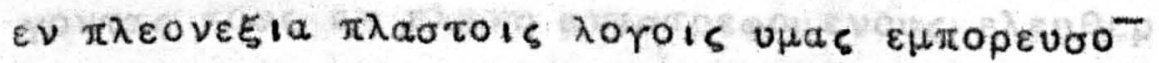

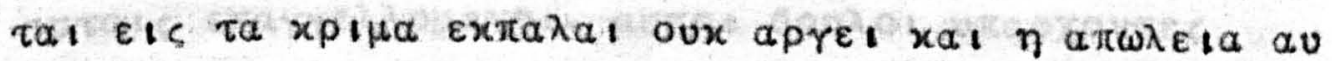

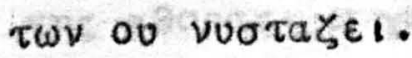

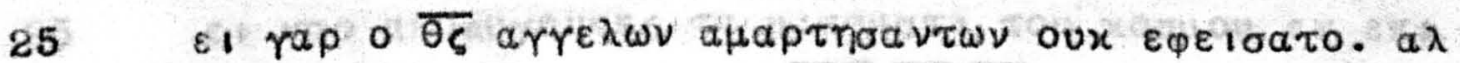

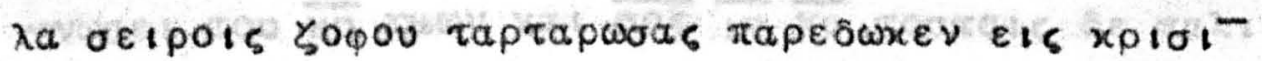

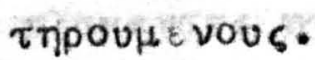

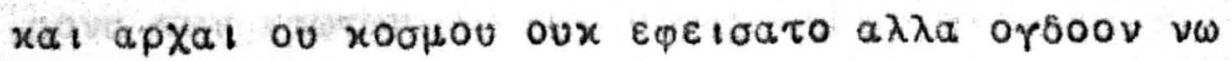

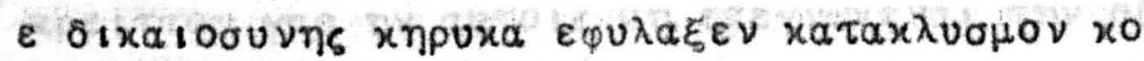

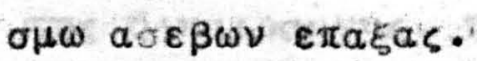

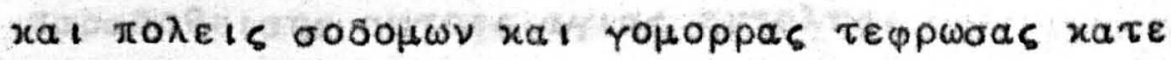

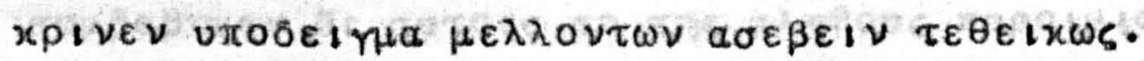

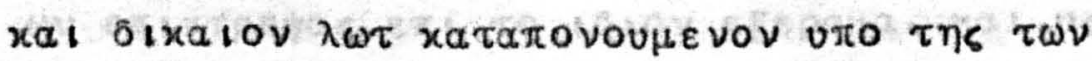

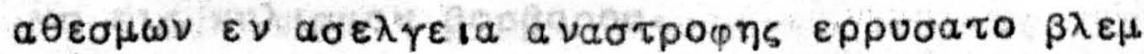

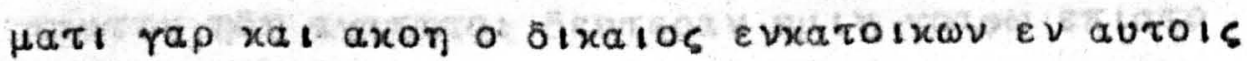

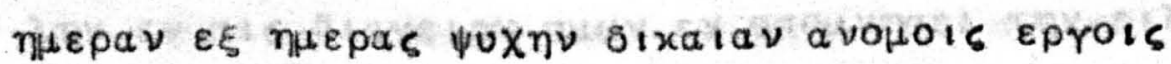
$\varepsilon \beta a \sigma a \nu i \zeta \varepsilon v$.

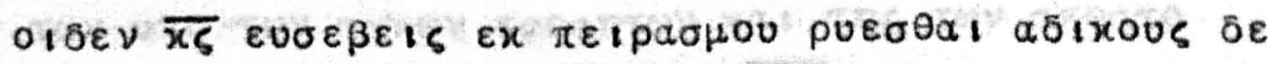

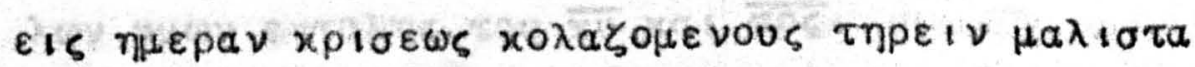

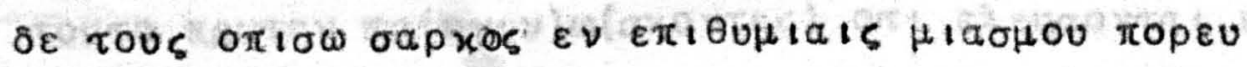

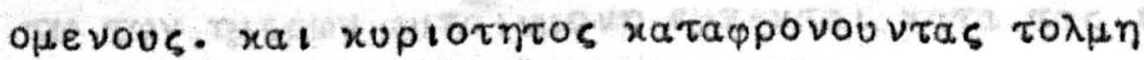




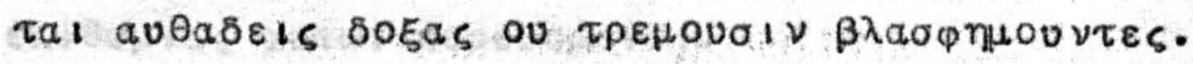

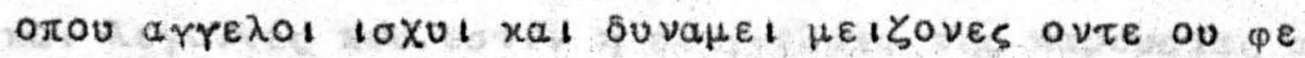

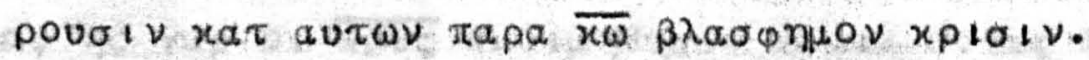

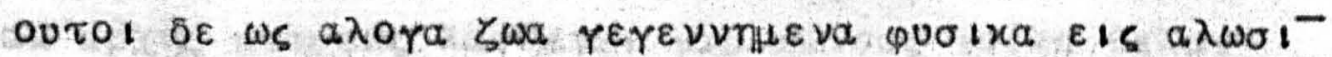

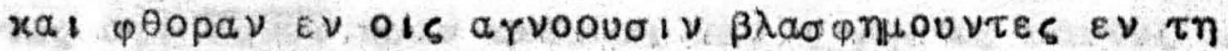

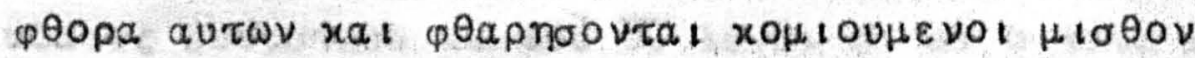

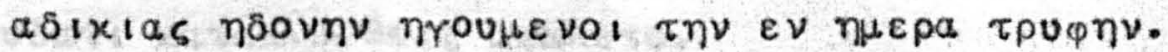

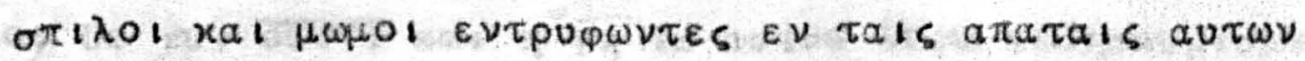

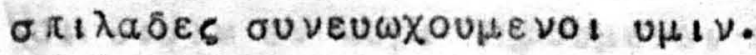

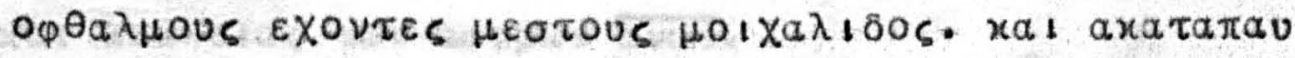

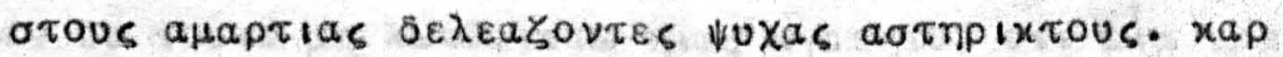

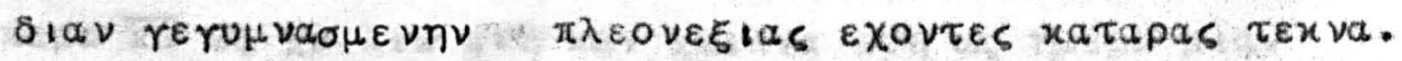

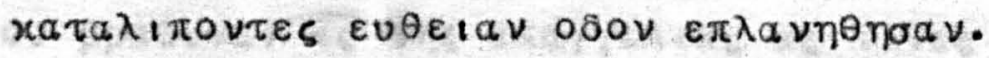

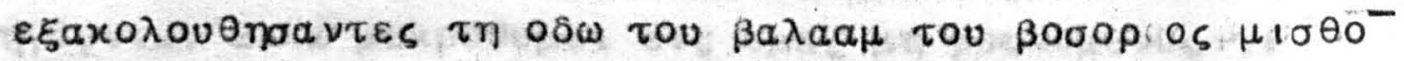

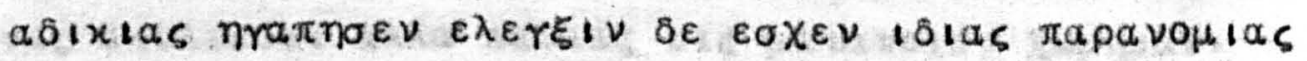

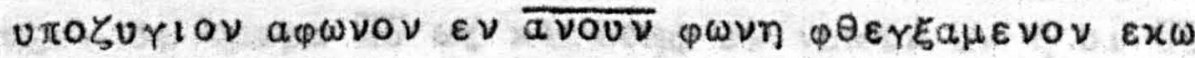

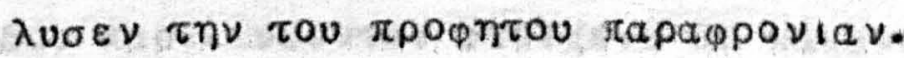

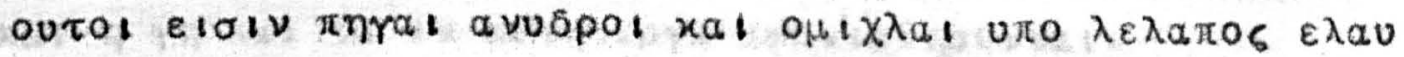

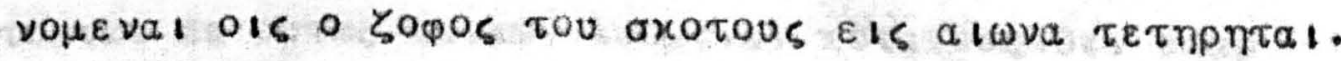

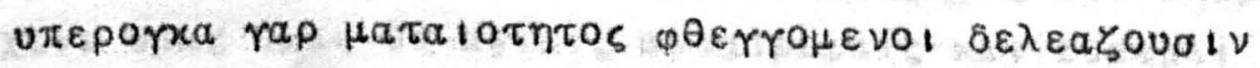

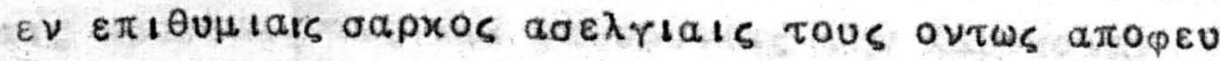

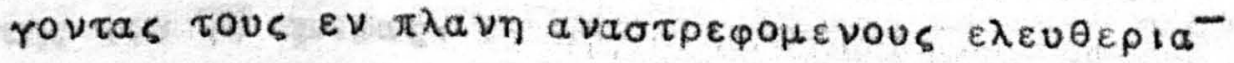

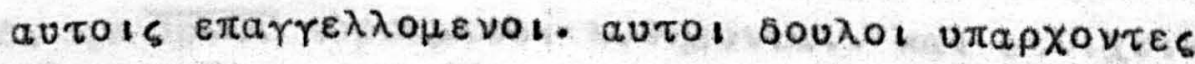

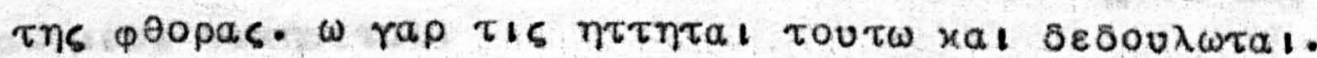

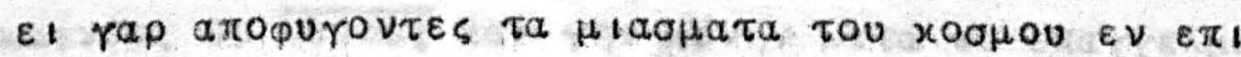

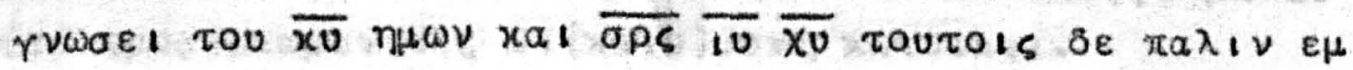

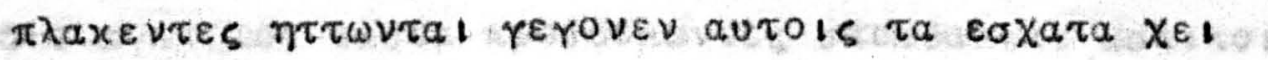
pova $\tau \omega \nu \pi \rho \omega \tau \omega \nu$.

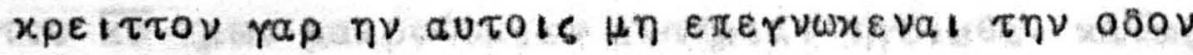

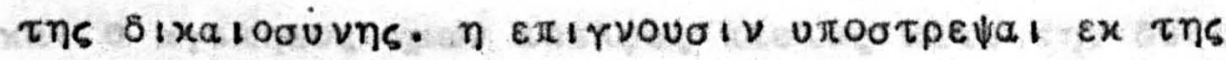

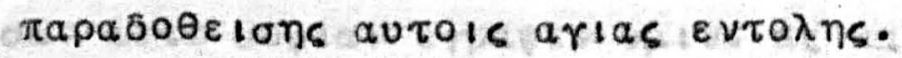

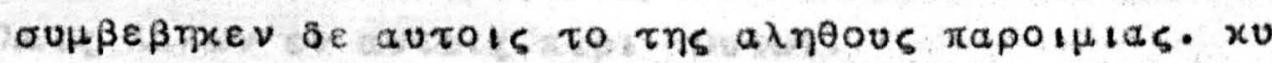

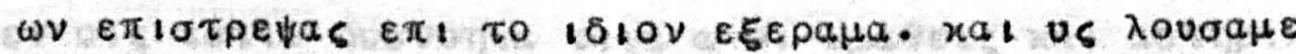

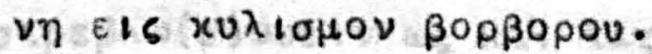

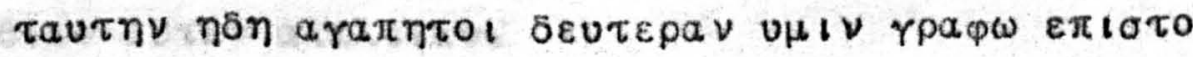

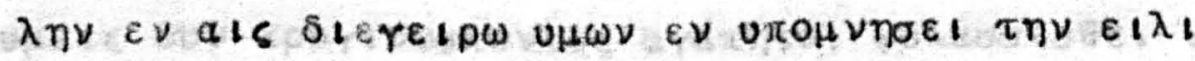

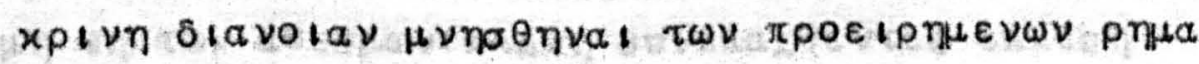

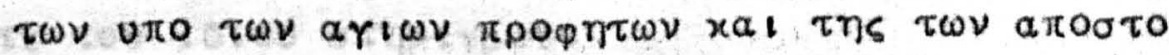

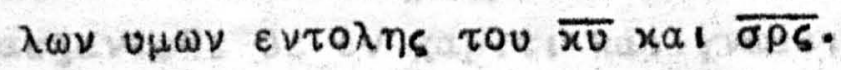

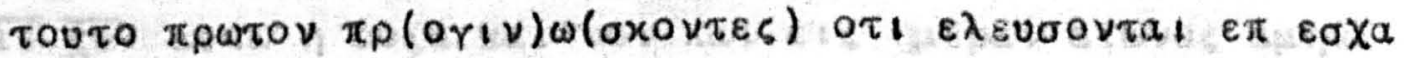

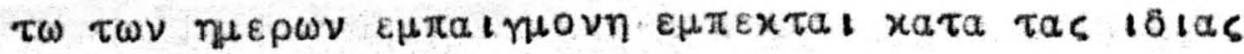




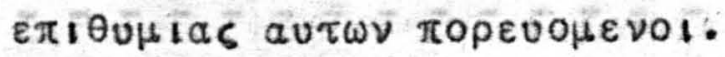

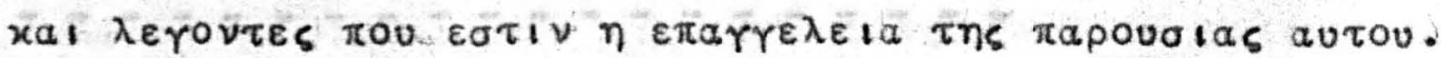

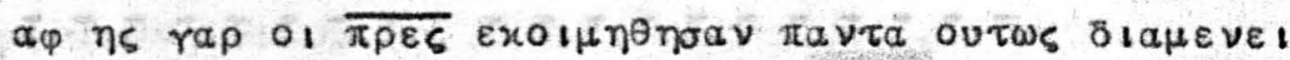

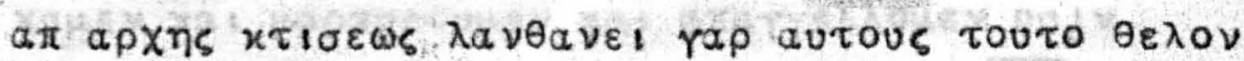

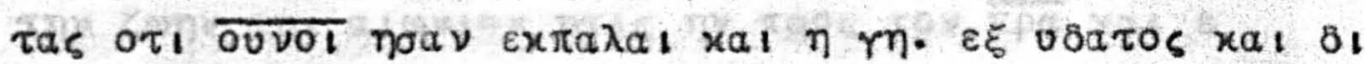

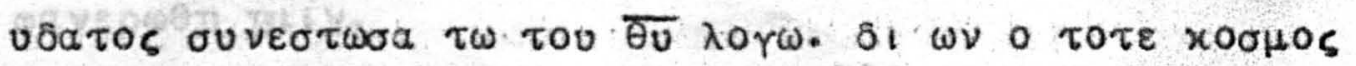

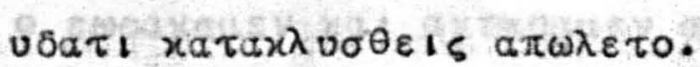

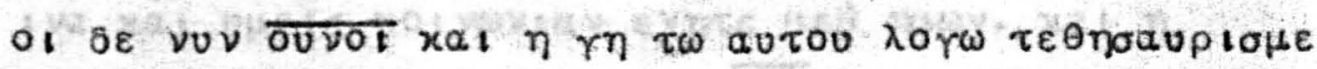

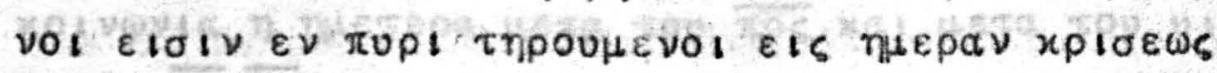
$x a 1 \alpha \pi \omega \lambda \varepsilon i a \zeta \tau \omega \nu$ a $\tau \varepsilon \beta \omega \nu \overline{\alpha \nu \omega \nu}$.

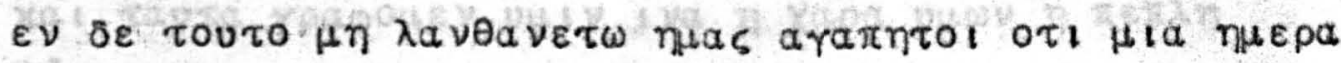

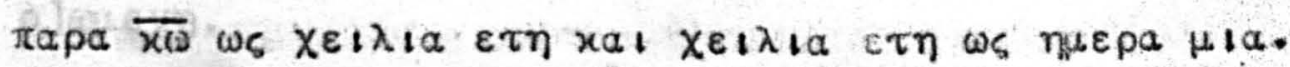

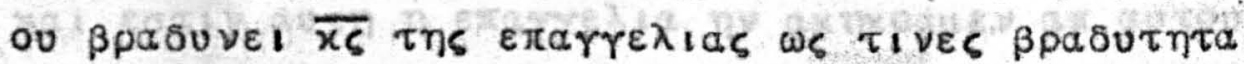

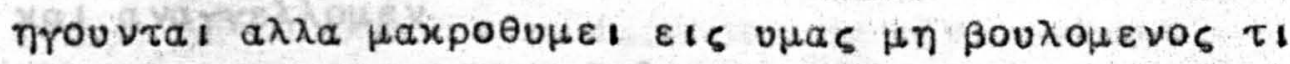

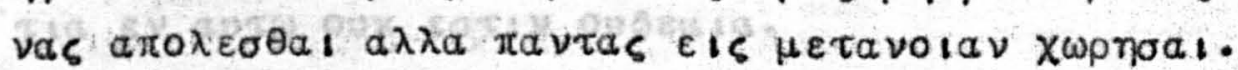

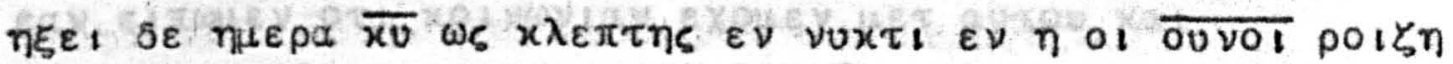

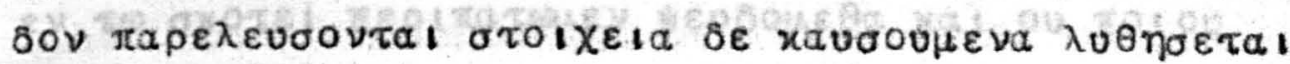

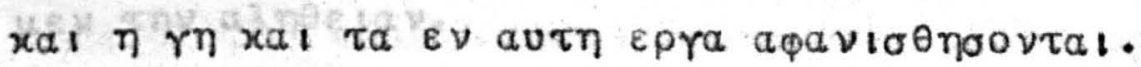

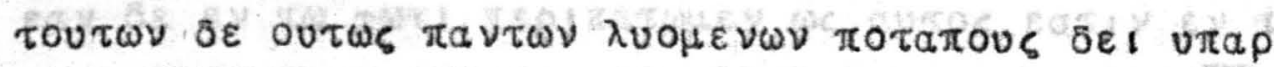

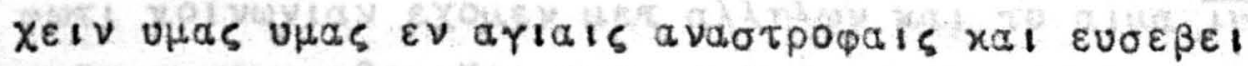

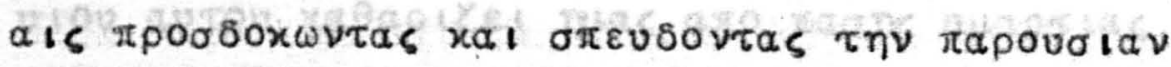

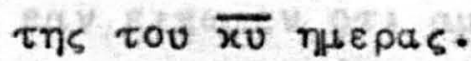

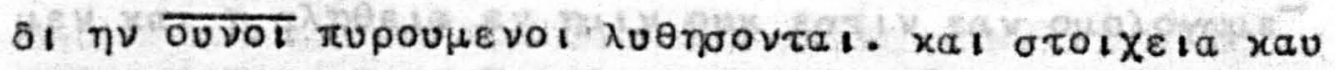

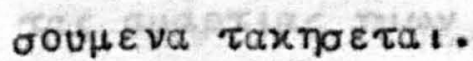

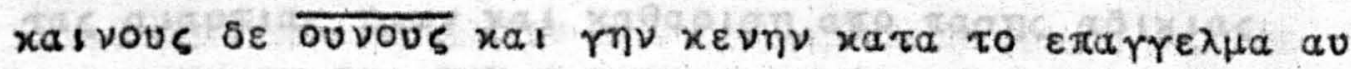

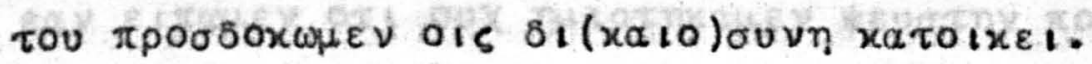

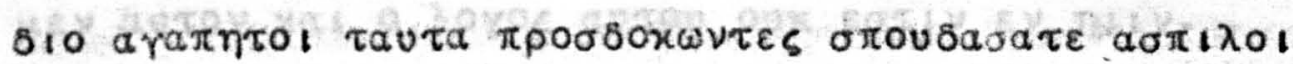

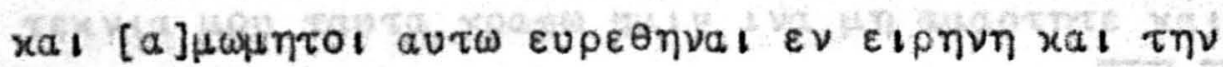

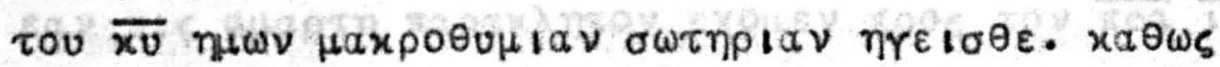

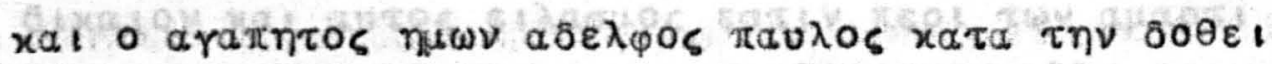

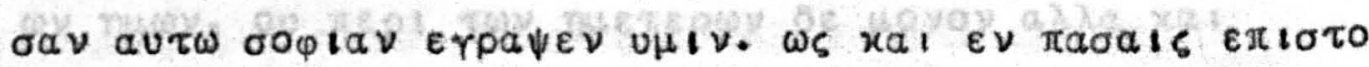

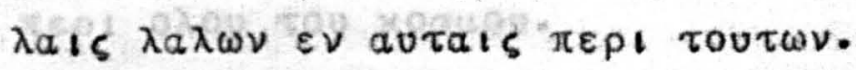

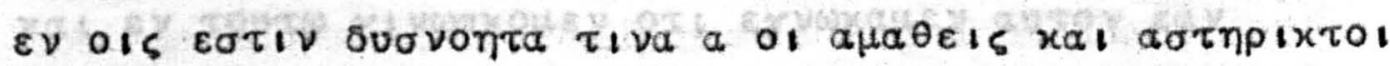

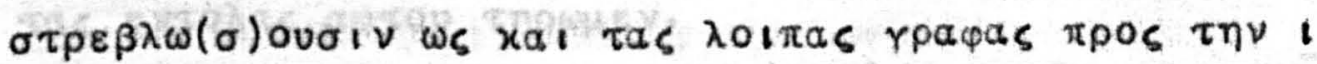

$35 \quad \delta เ a \nu$ aU $\tau \omega \nu$ a

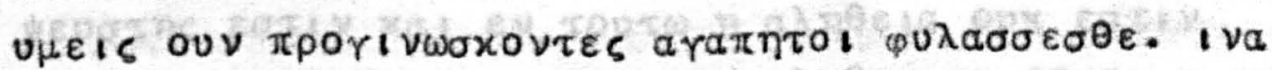

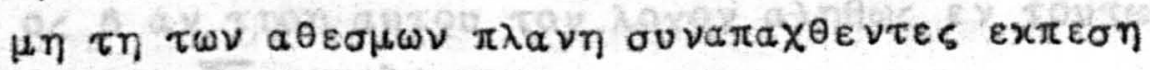

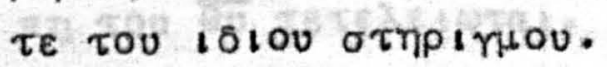

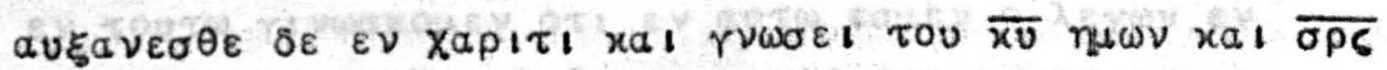

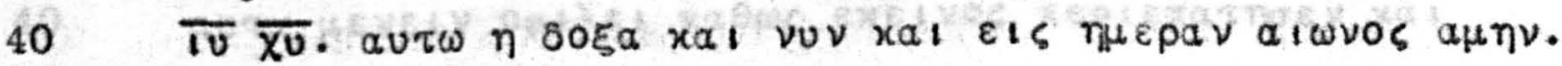




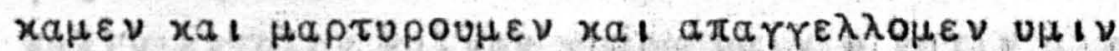

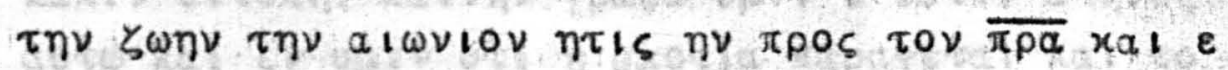

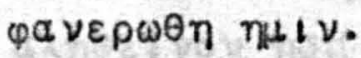

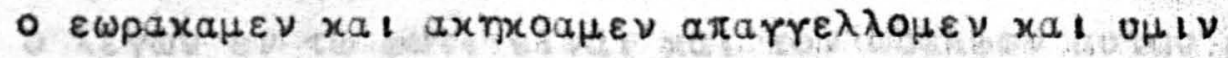

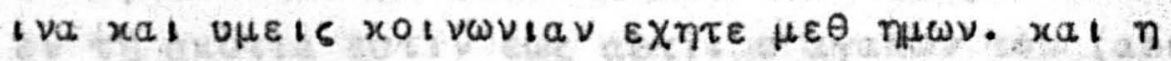

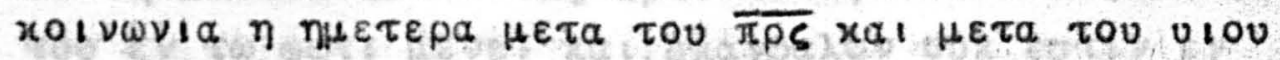

10 autou $\overline{10} \overline{\chi 0}$.

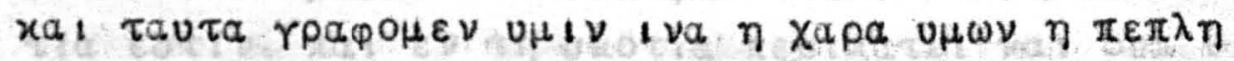
p] $\omega \mu \varepsilon \vee \eta$.

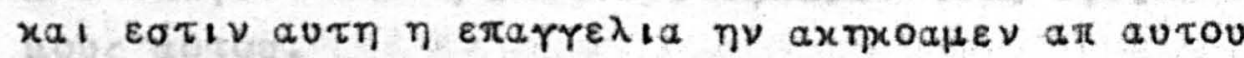

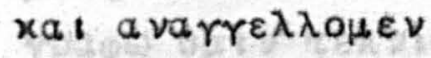

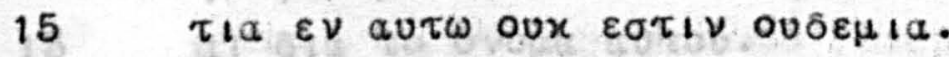

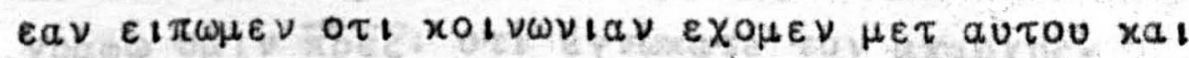

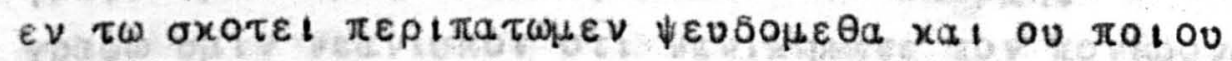
$\mu \varepsilon \nu \tau \eta \nu$ a $\lambda \eta \theta \varepsilon เ a v$.

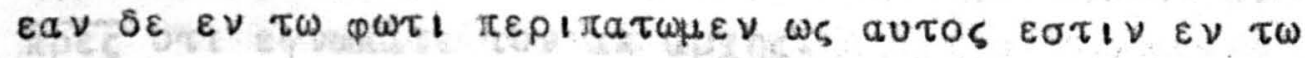

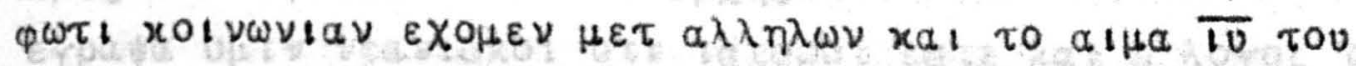

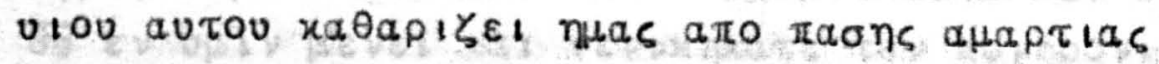

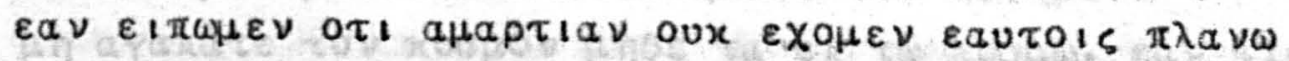

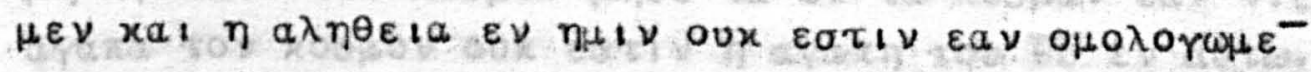

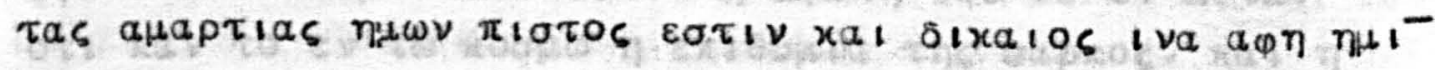

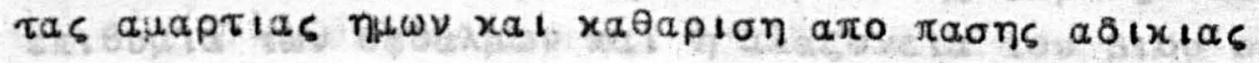

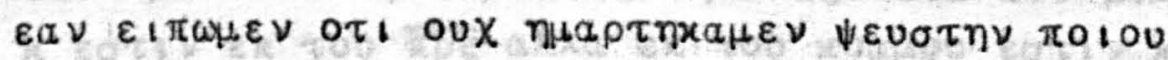

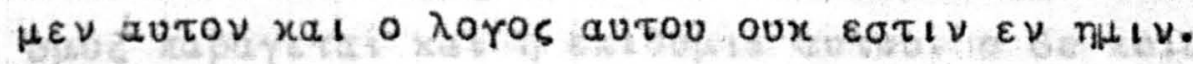

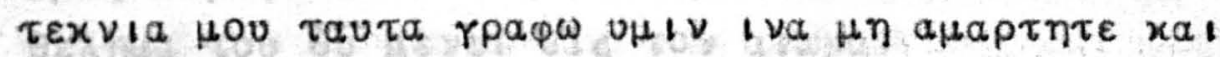

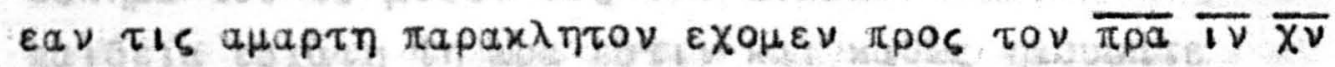

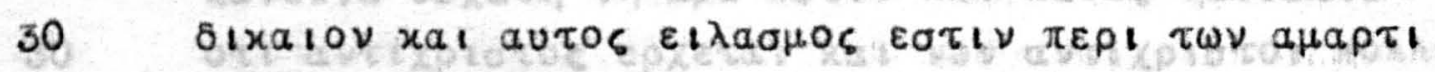

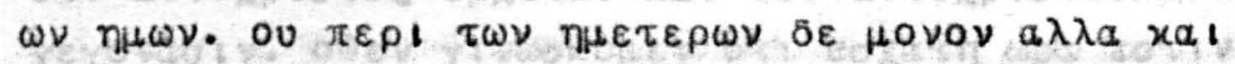

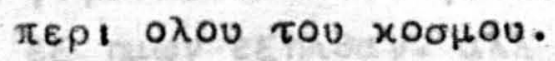

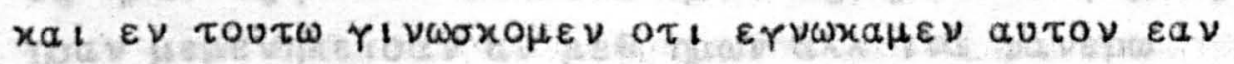

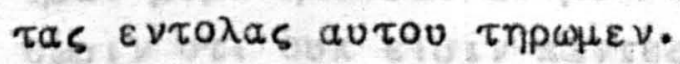

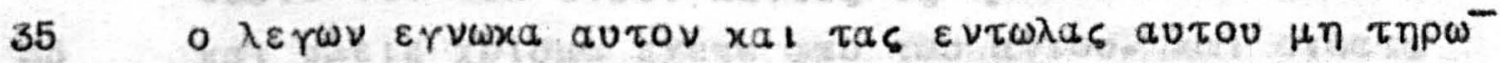

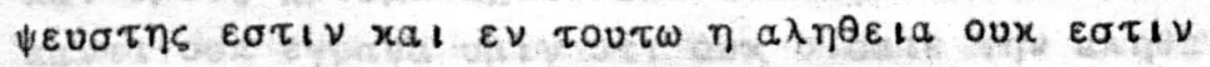

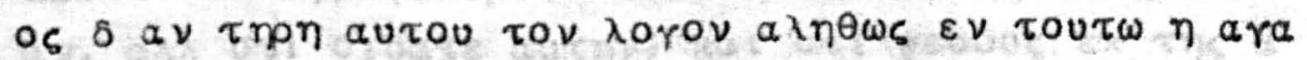
$\pi \eta \tau 0 u \overline{\theta v} \tau \varepsilon \tau \varepsilon \lambda \varepsilon เ \omega \tau \alpha \iota$.

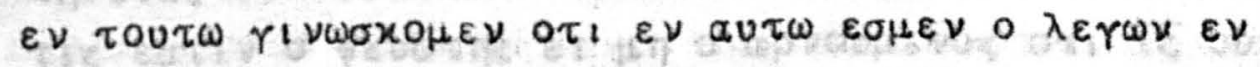
40 aU

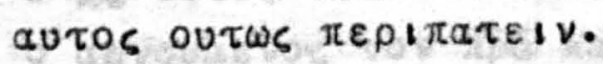

$$
1 \text { wavvou } a^{\prime} 1.1-2.6
$$




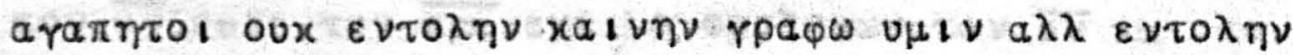

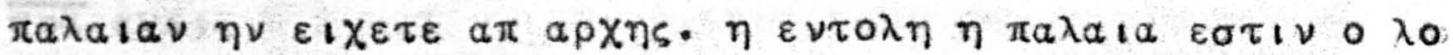

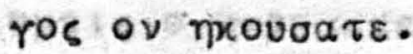

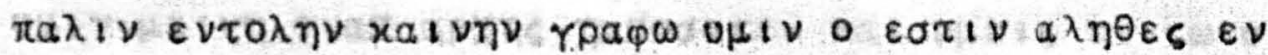

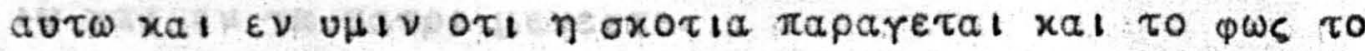

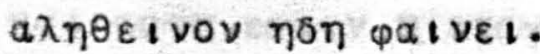

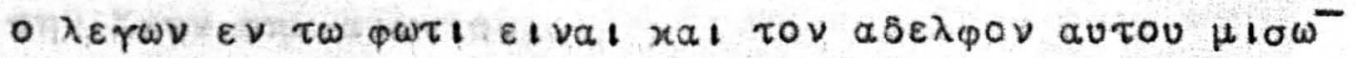

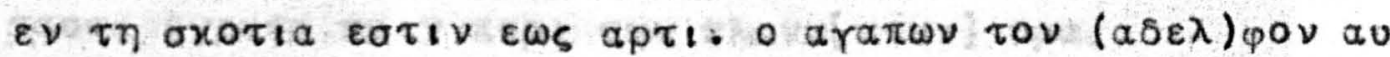
$\tau o v \varepsilon \nu \tau \omega \varphi \omega \tau ! ~ \mu \varepsilon v \varepsilon$, $x \alpha$ เ $\sigma x \alpha \nu \delta a \lambda o \nu$ oux $\varepsilon \sigma \tau ! \nu \varepsilon \nu$

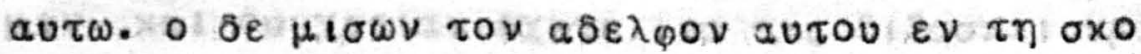

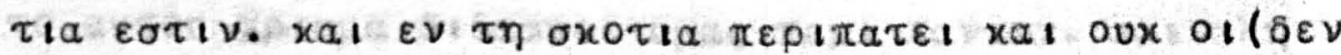

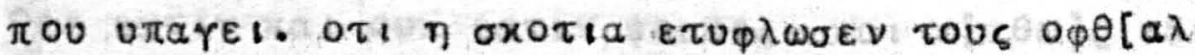
Hov autou.

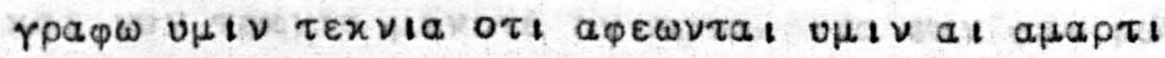

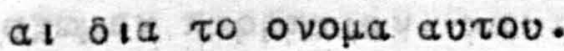

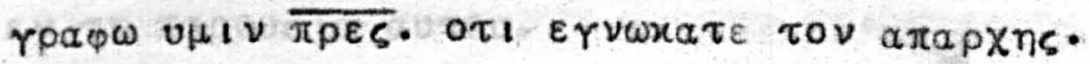

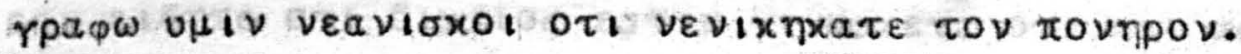

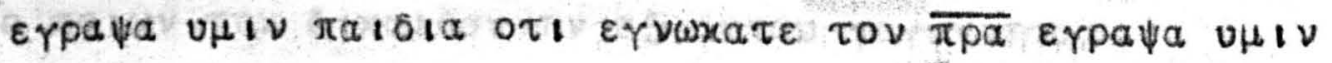

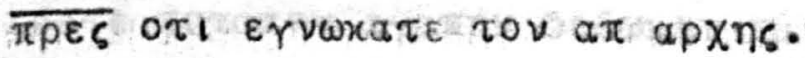

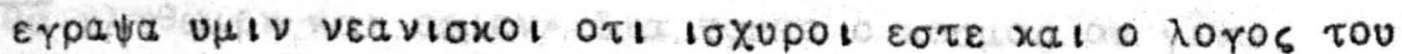

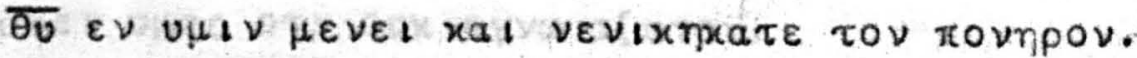

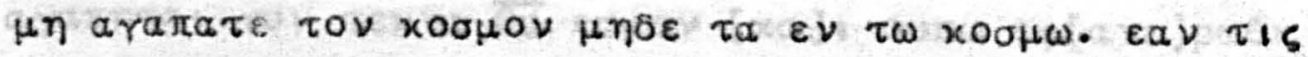

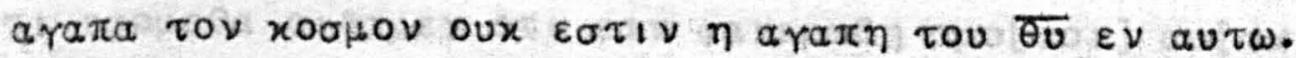

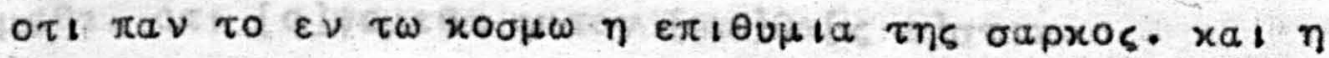

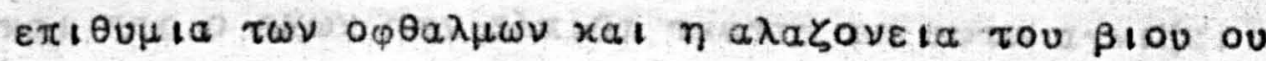

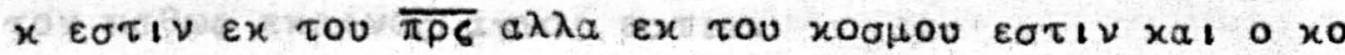

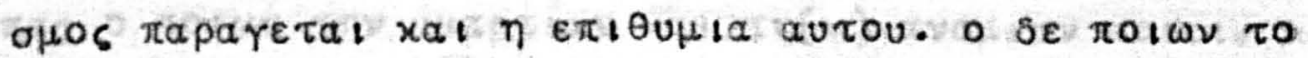

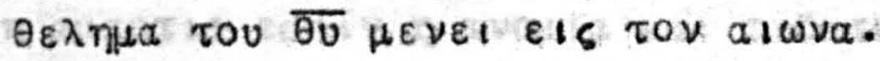
$\pi a 1 \delta \varepsilon เ \alpha \varepsilon \sigma \chi \alpha \tau \eta \tau \eta$

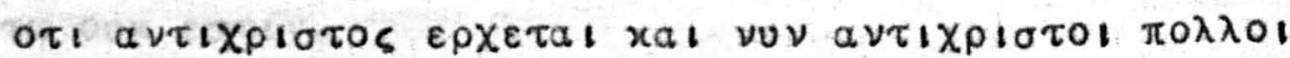

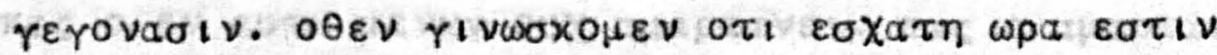

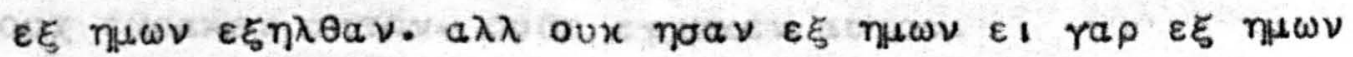

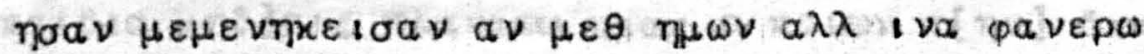
$\theta \omega \sigma i \nu$ ot

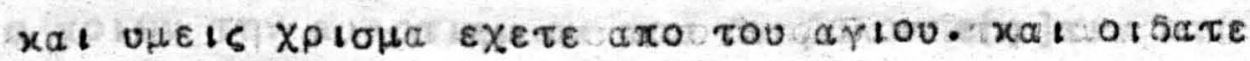

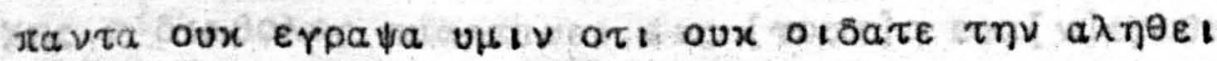

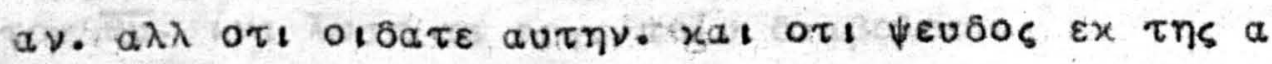
$\lambda \eta \bar{\varepsilon} \iota$ เаs oux $\varepsilon \sigma \tau \iota \nu$.

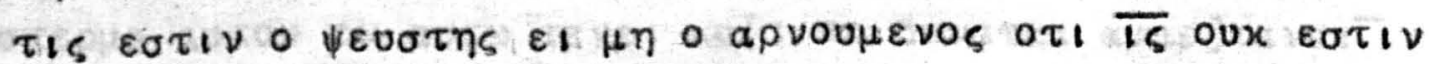

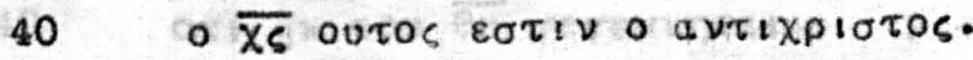

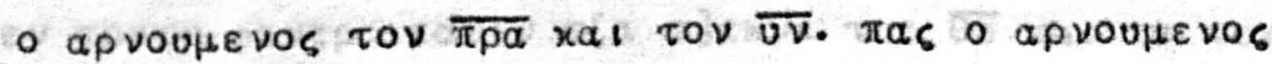




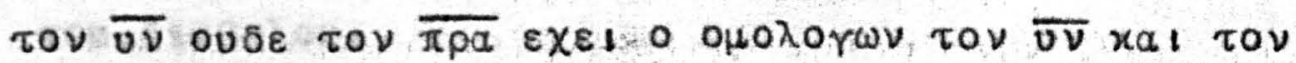
$\overline{\pi p \alpha} \varepsilon \times \varepsilon$.

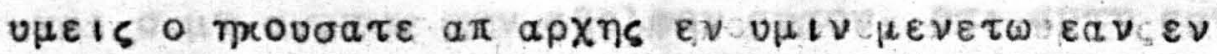

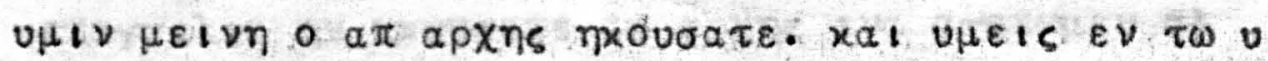

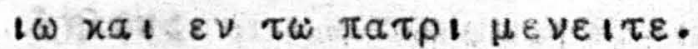

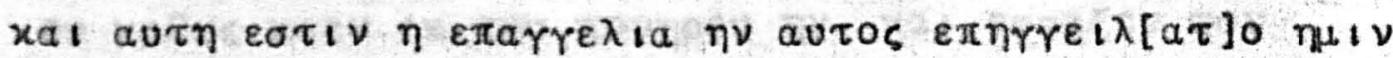

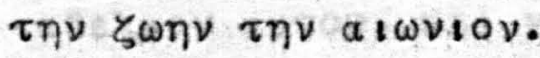

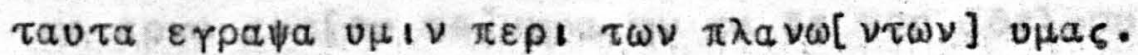

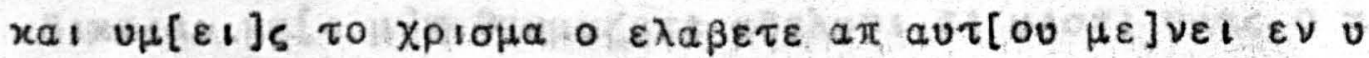

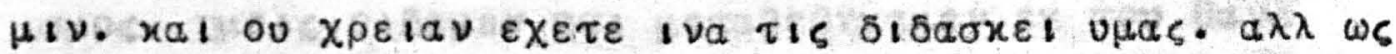

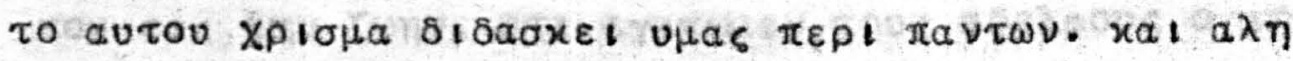

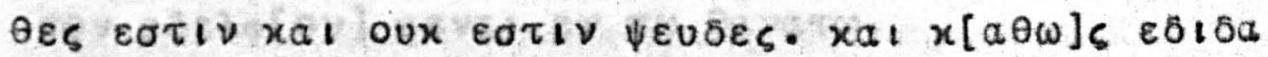
$\xi \varepsilon \nu$ upas $\mu \varepsilon \nu \varepsilon \tau \varepsilon \varepsilon \nu$ au $\tau \omega$.

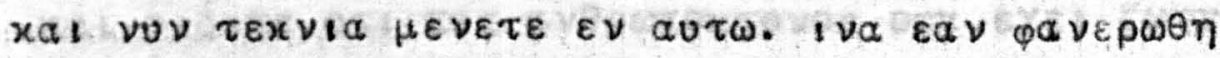

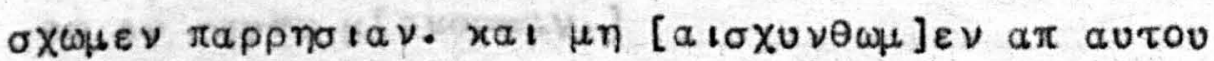
$\varepsilon \vee \tau \eta \pi$ Ta

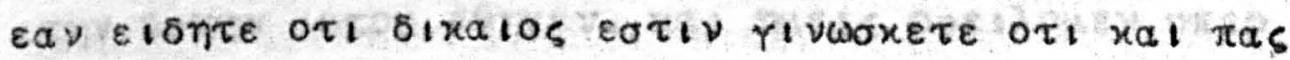

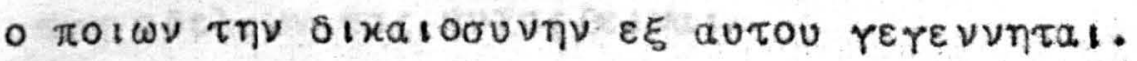

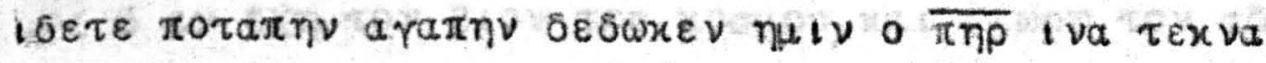

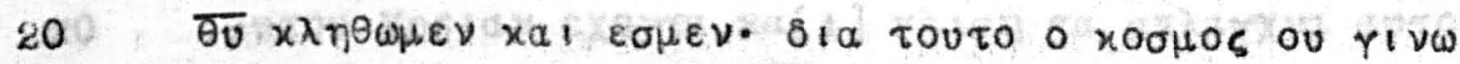

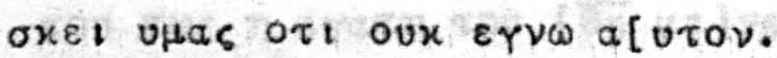

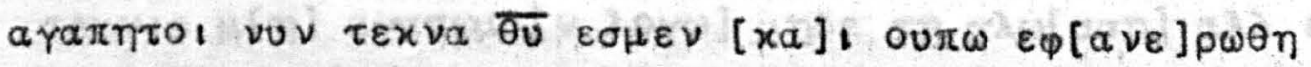

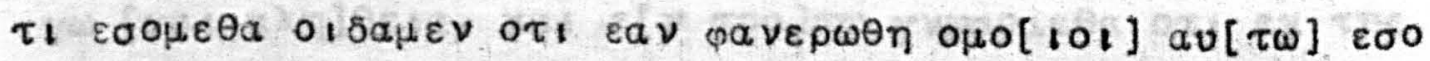

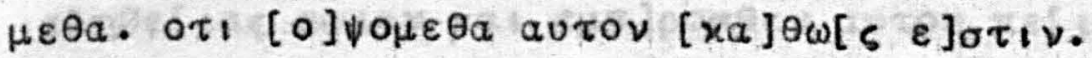

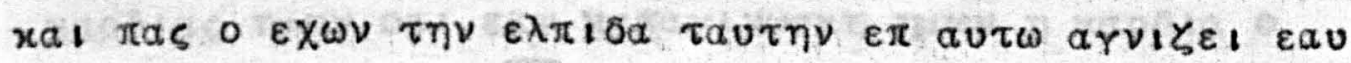

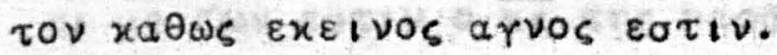

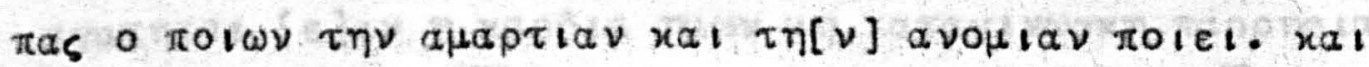

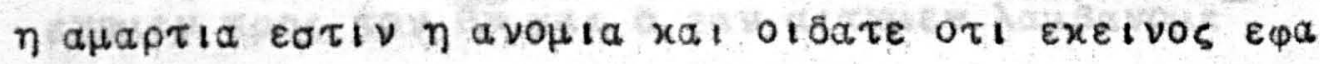

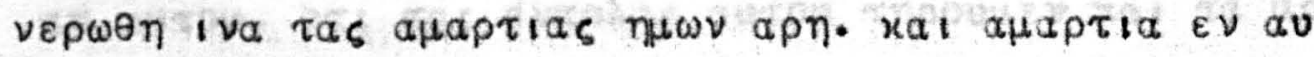
$30 \tau \omega$ oux $\varepsilon \sigma \tau i \nu$.

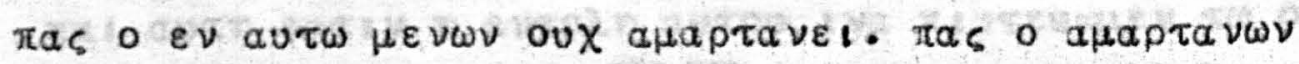

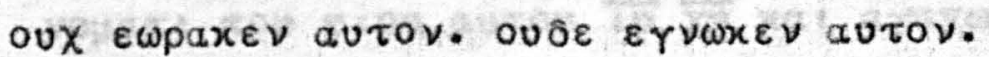

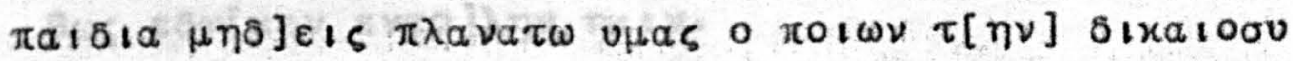

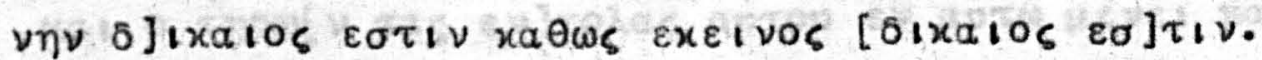

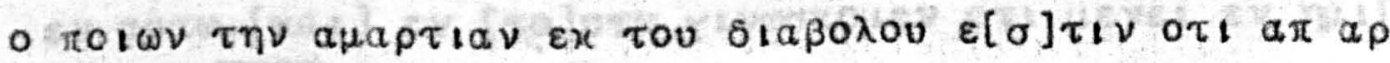

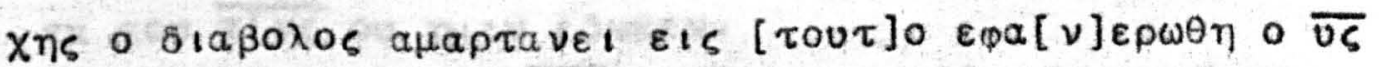

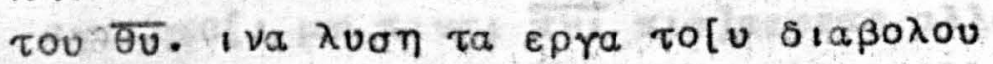

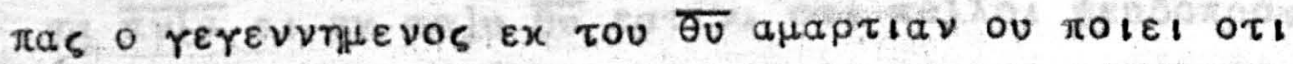

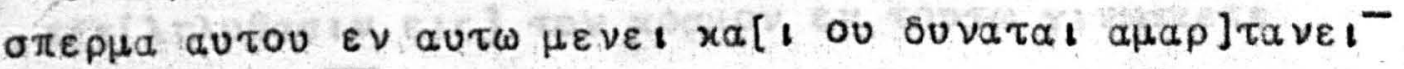

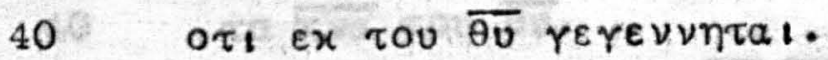

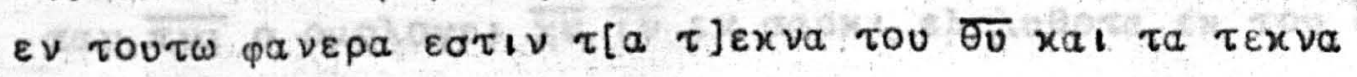




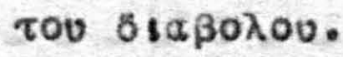

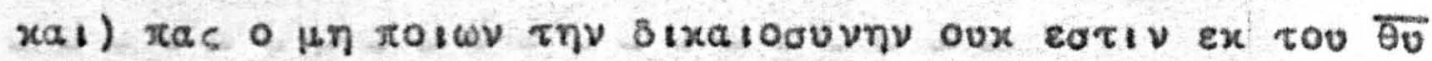

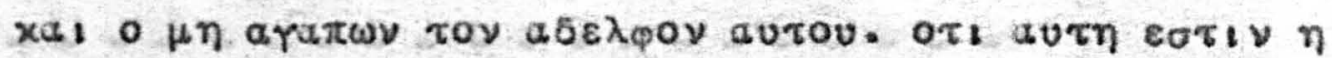

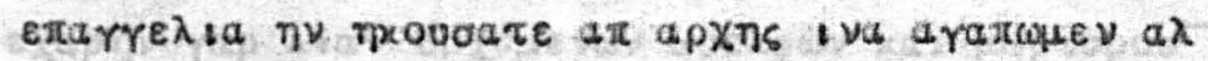
$\lambda \eta \lambda$ ove.

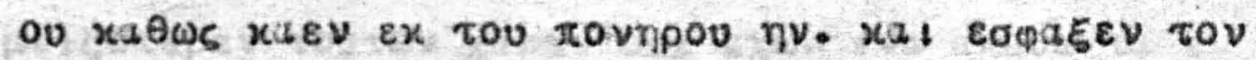

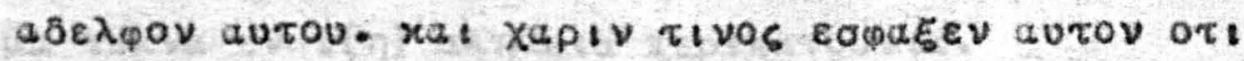

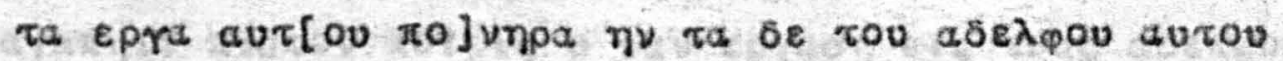

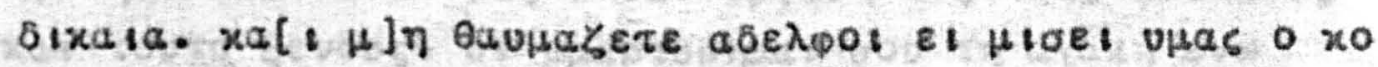

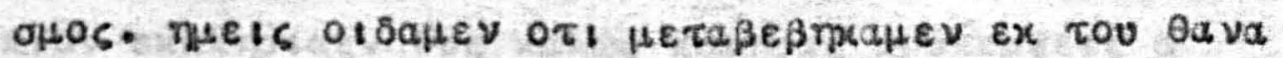

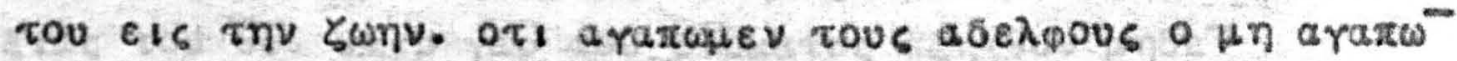

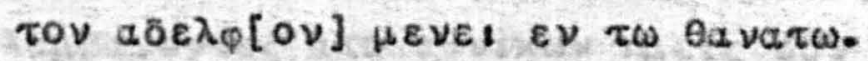

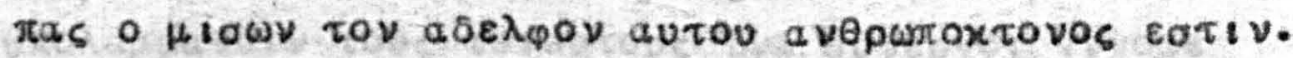

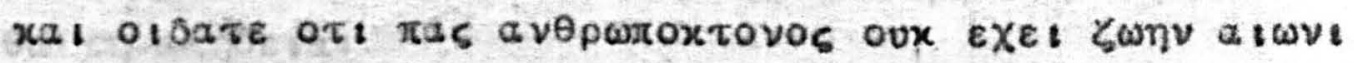

15 ov $\varepsilon \nu$ Eav

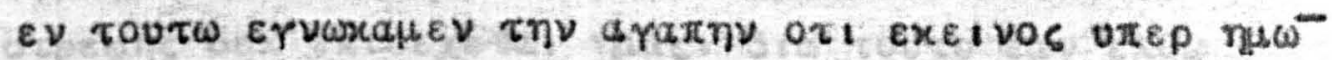

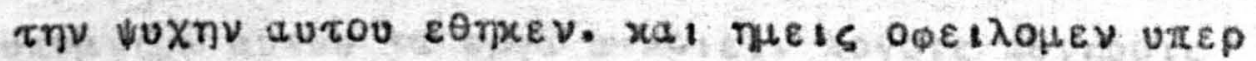

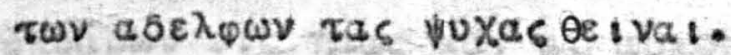

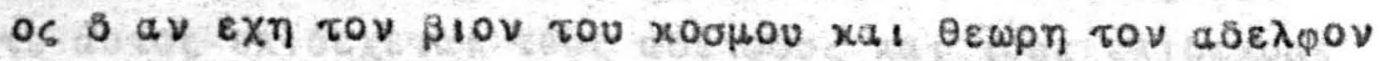

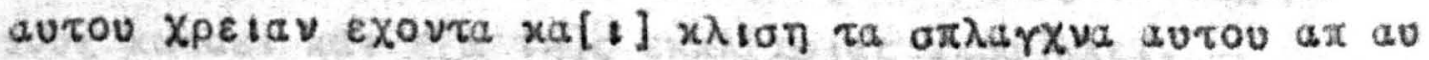

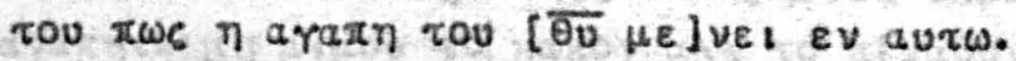

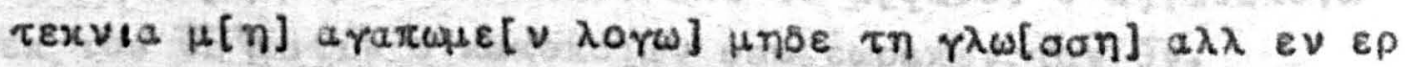

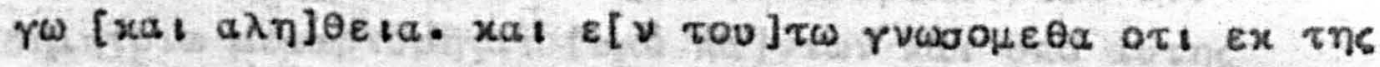

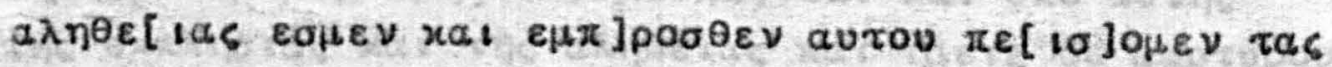

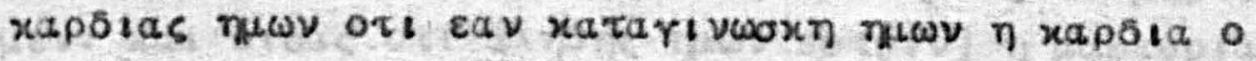

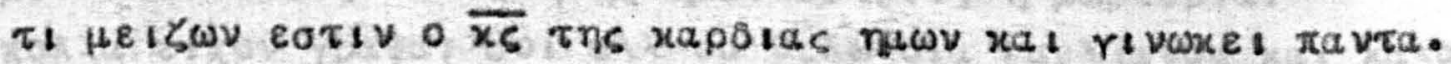

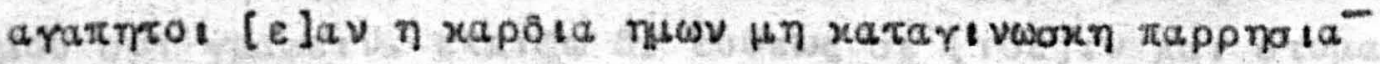

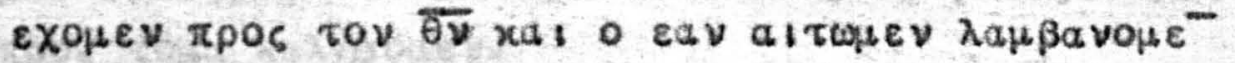

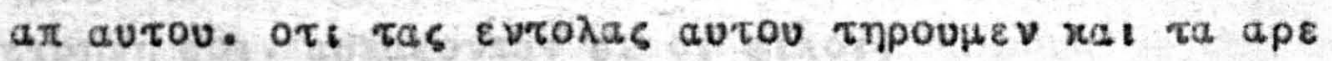

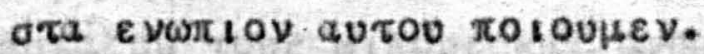

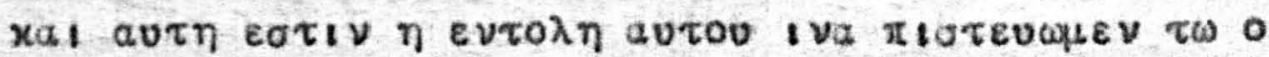

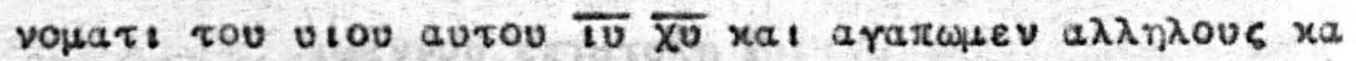

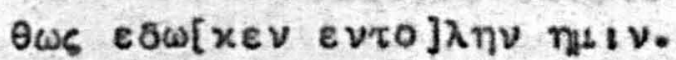

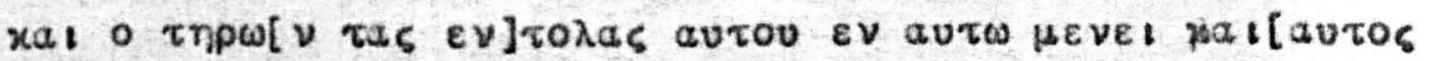

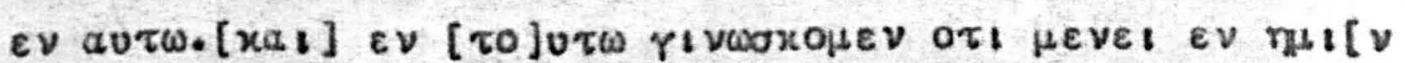
ex tov $\pi[\overline{v s}$ ou nuiv esconev.

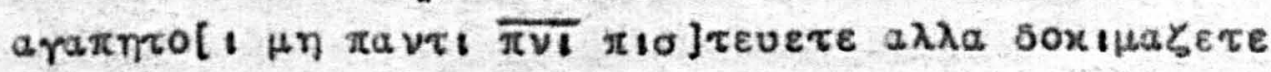

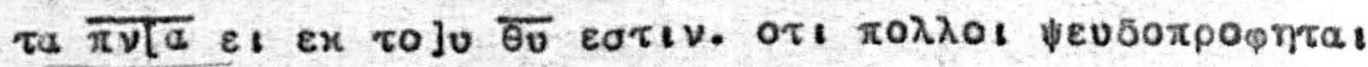

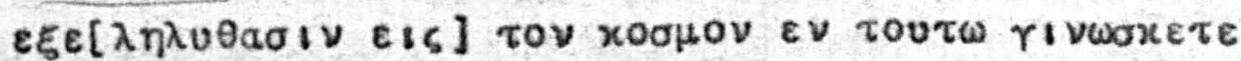

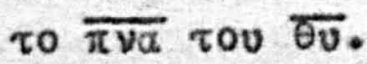

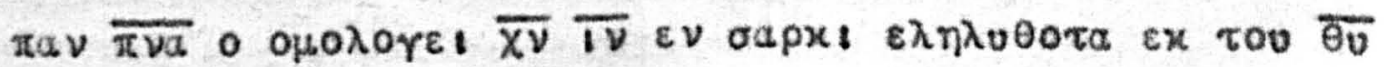




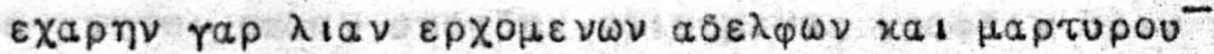

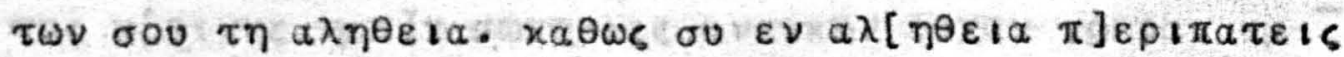

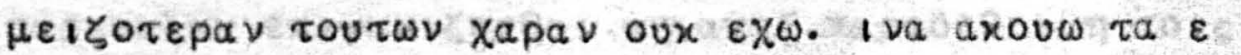

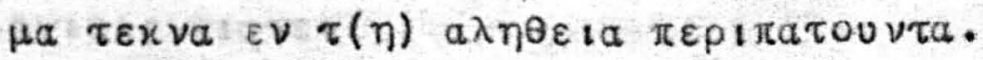

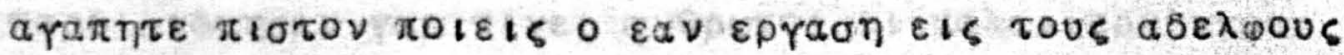

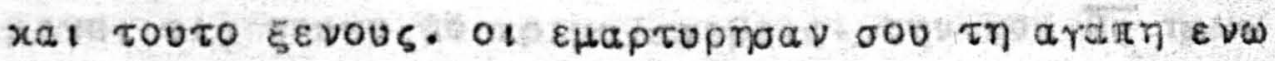

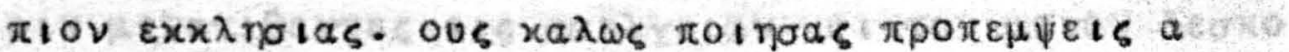

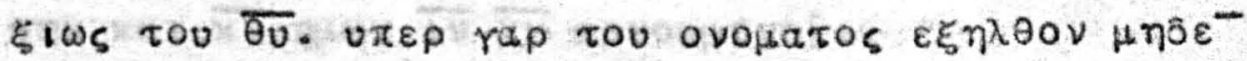

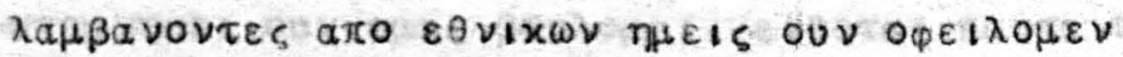

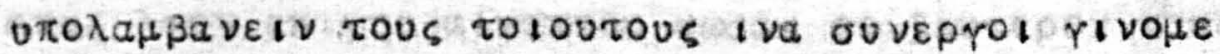

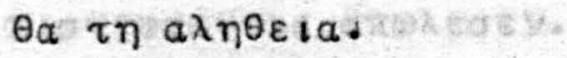

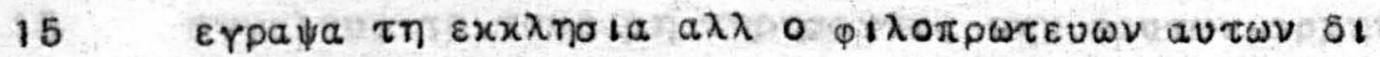

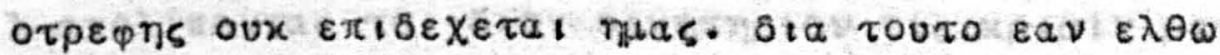

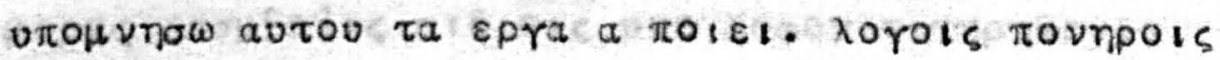

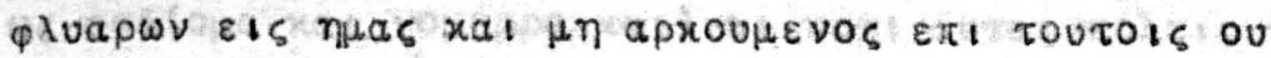

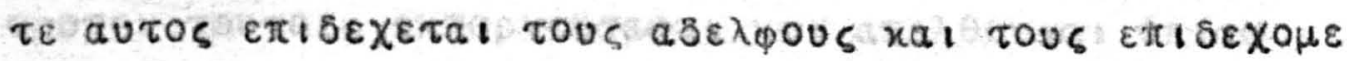

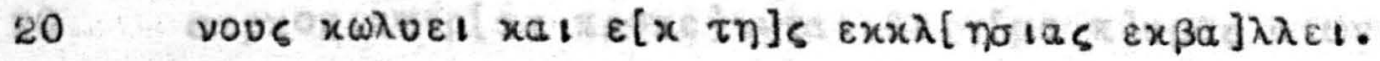

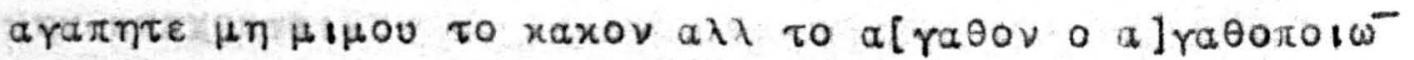

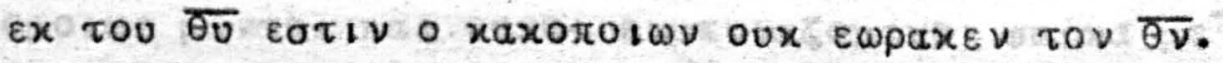

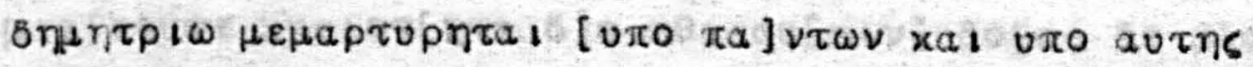

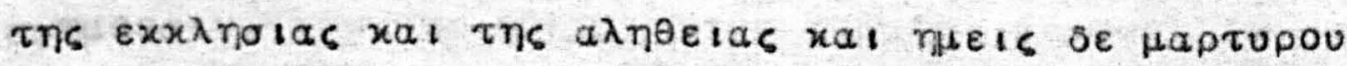

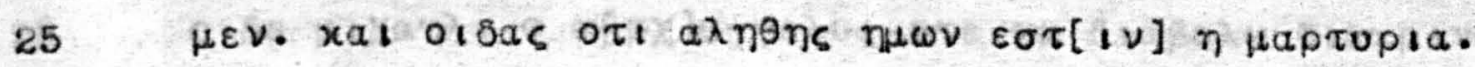

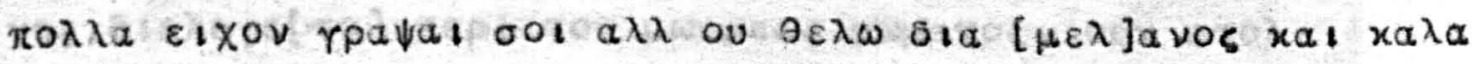

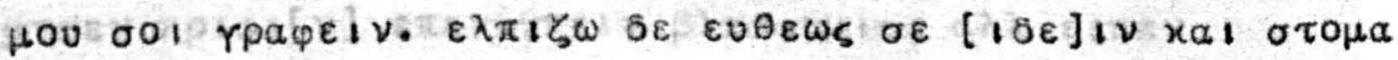

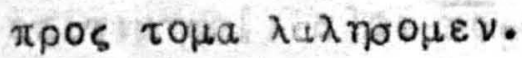

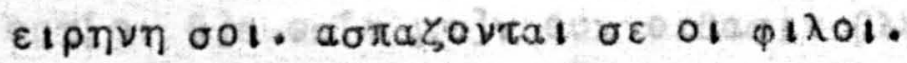

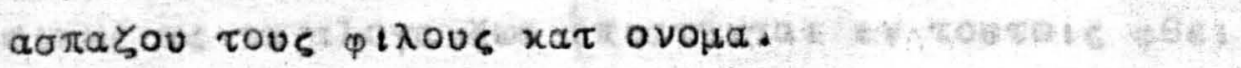

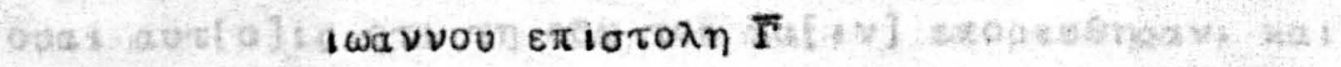

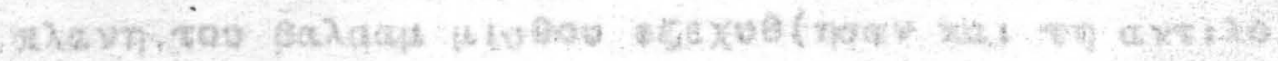

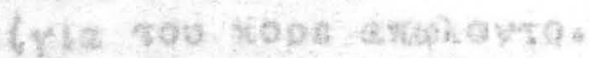

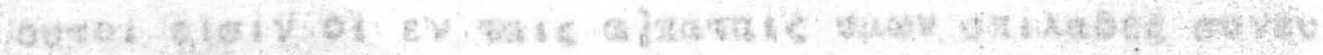

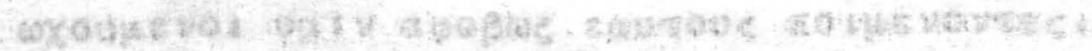




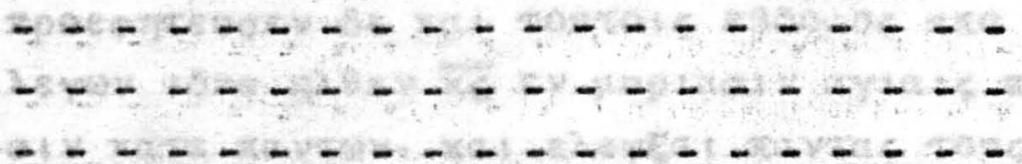

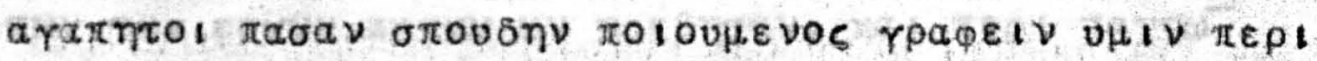

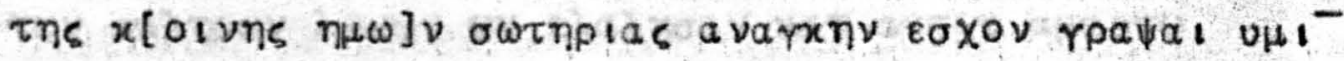

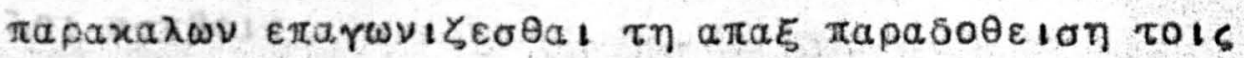

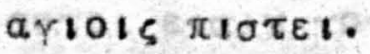

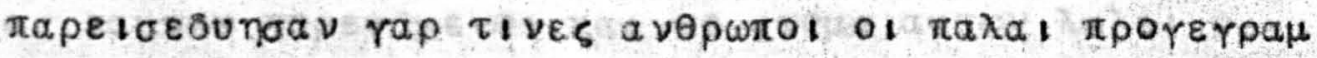

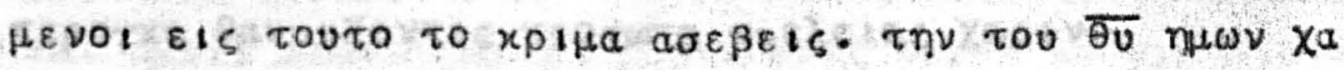

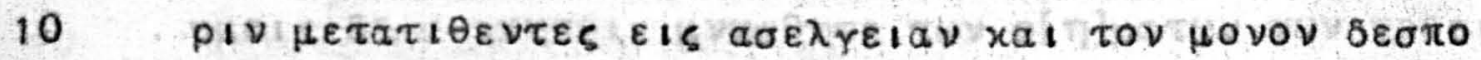

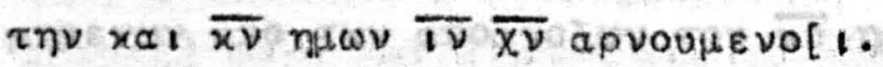

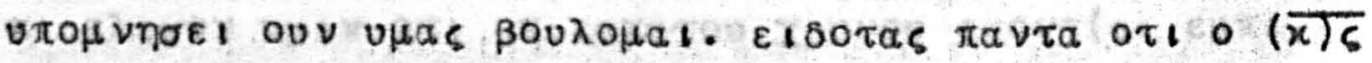

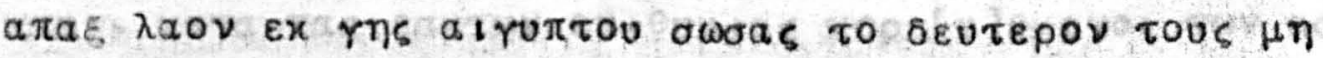
$\pi ı \tau \varepsilon v \sigma a \nu \tau \alpha \zeta$ a $a \omega \lambda \varepsilon \sigma \varepsilon \nu$.

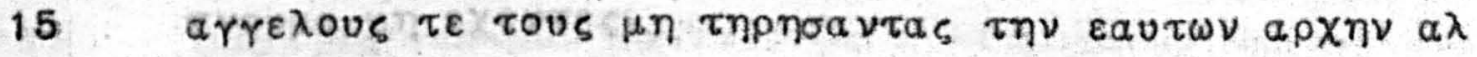

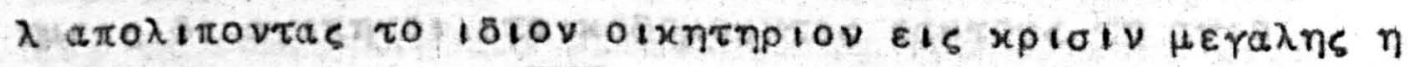

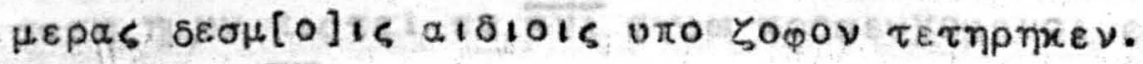

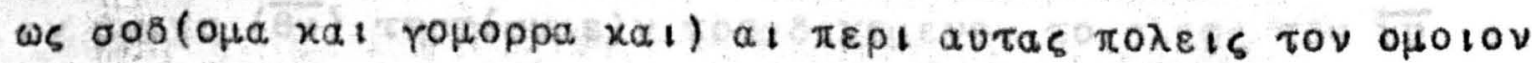

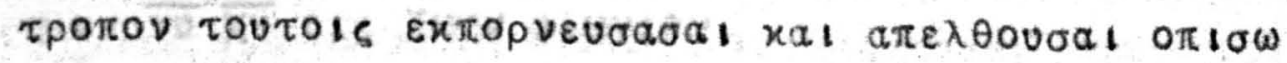

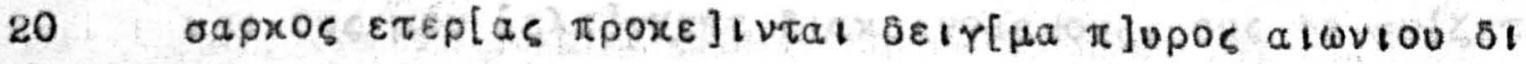

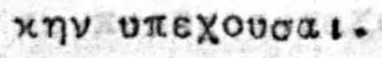

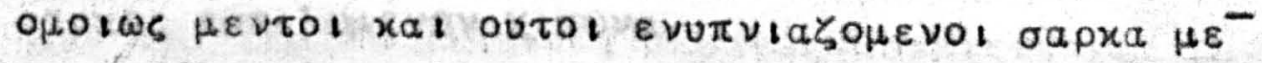

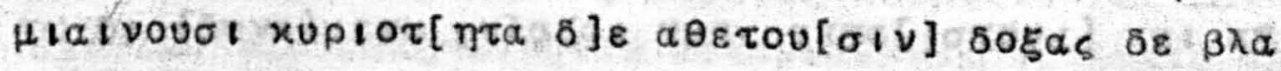
$\sigma \varphi \eta \mu O v \sigma: v$.

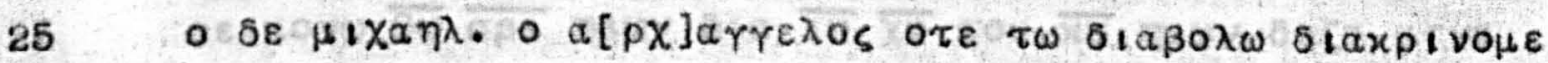

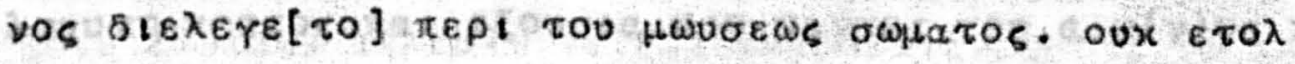

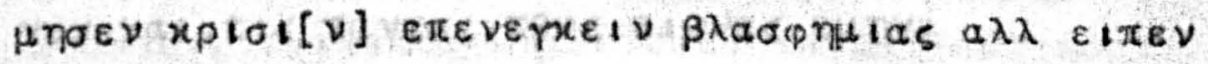
$\varepsilon \pi ! \tau i \mu \eta \eta a[1, \sigma] \circ, \overline{x \zeta}$ :

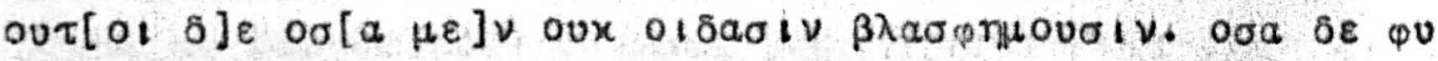

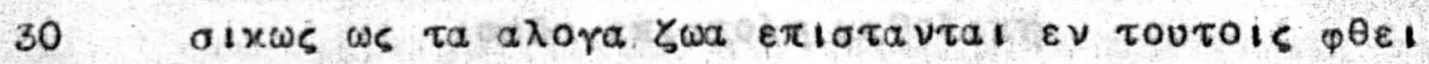
poviai.

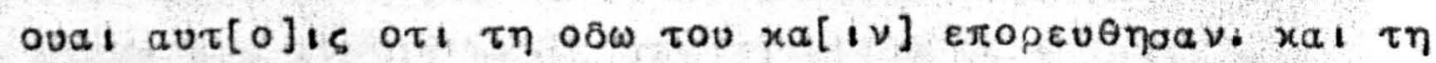

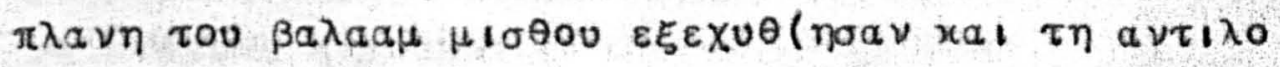

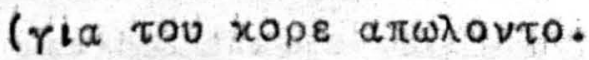

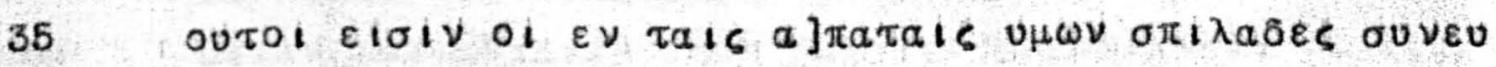

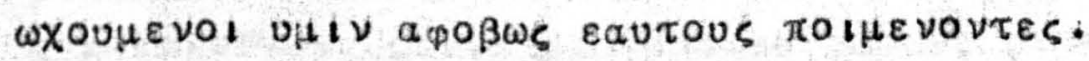
$\nu \varepsilon \varphi \varepsilon \lambda a i$ avobpol

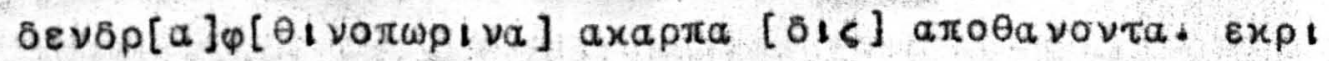

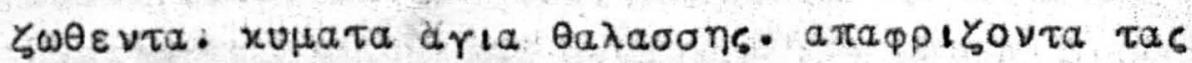

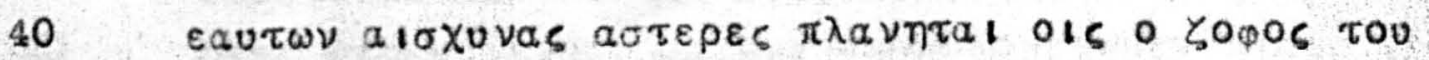

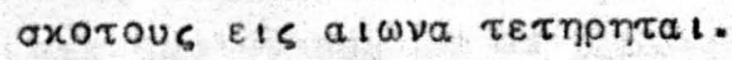




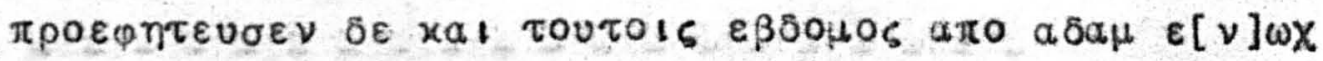

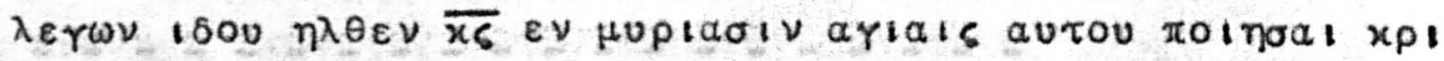

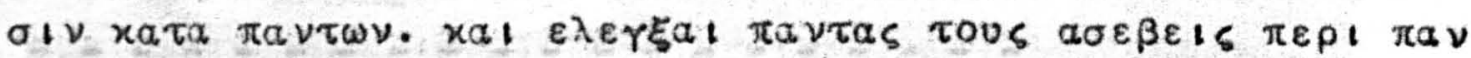

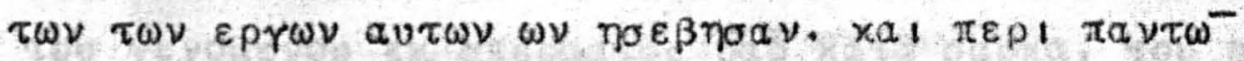

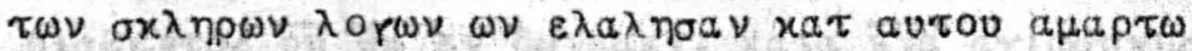

$\lambda \circ \mathrm{i} \alpha \sigma \beta \varepsilon i s$.

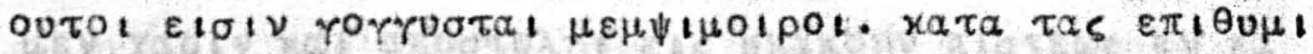

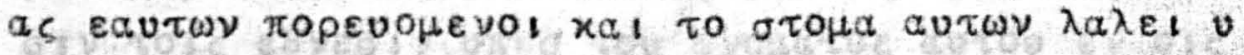

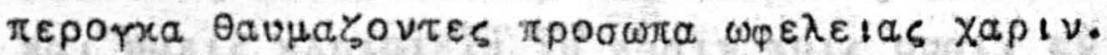

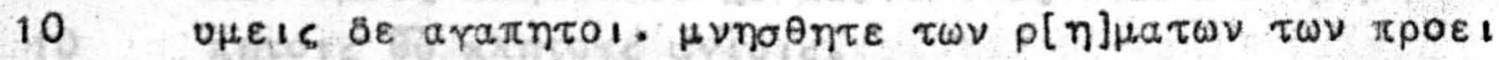

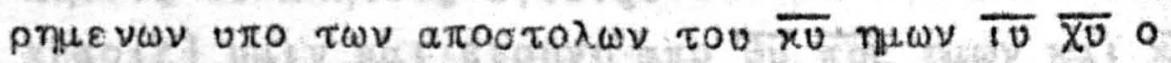

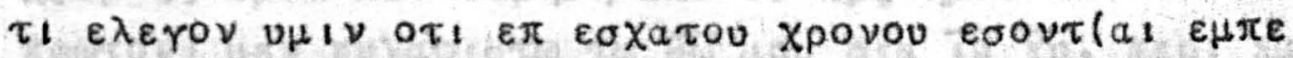

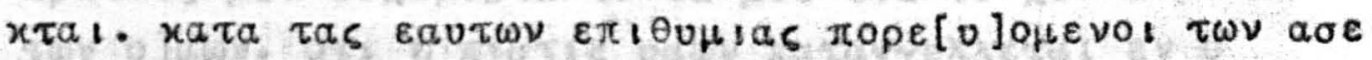

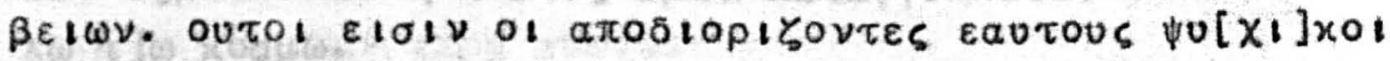

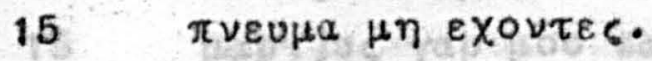

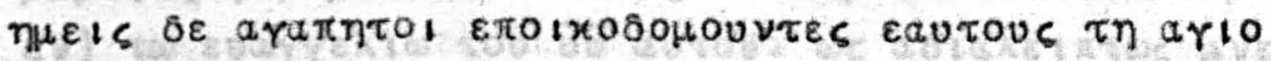

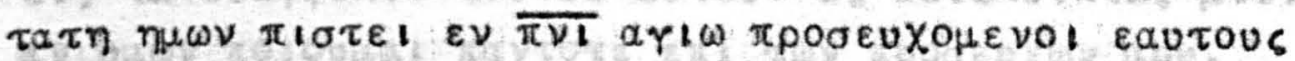

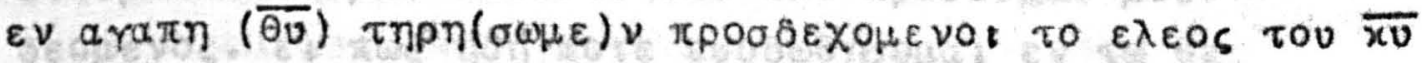

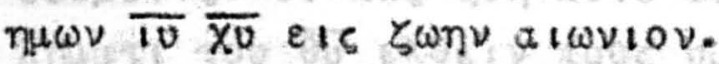

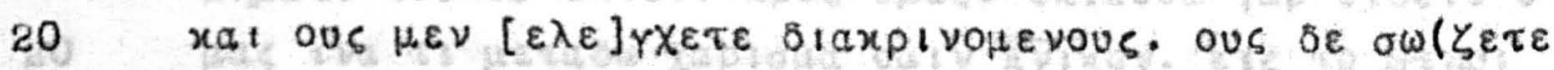

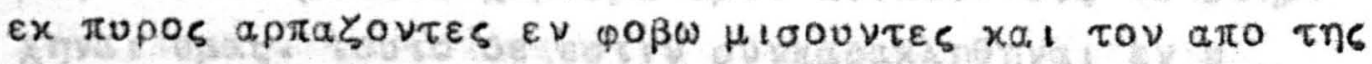

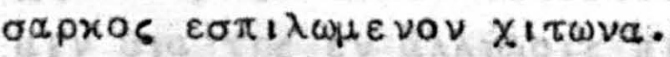

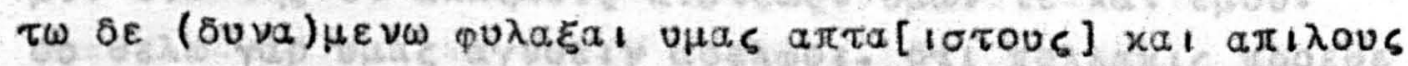

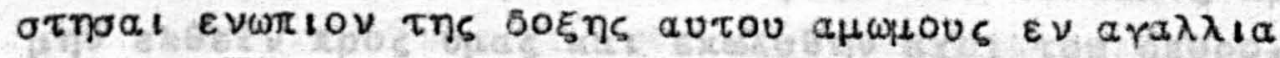

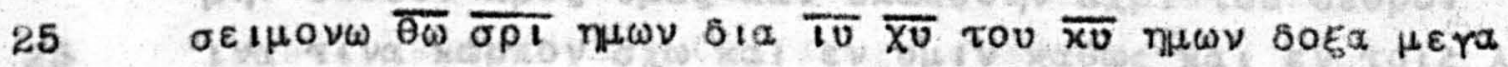

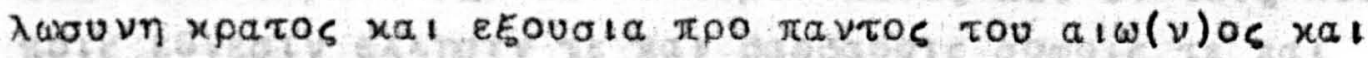

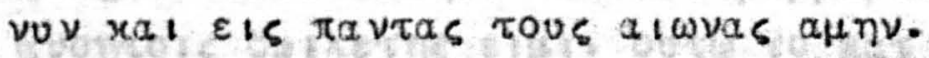

$100 \delta a \varepsilon \pi 1 \sigma \tau \sigma \lambda \eta \quad x a \theta 0 \lambda เ x \eta$

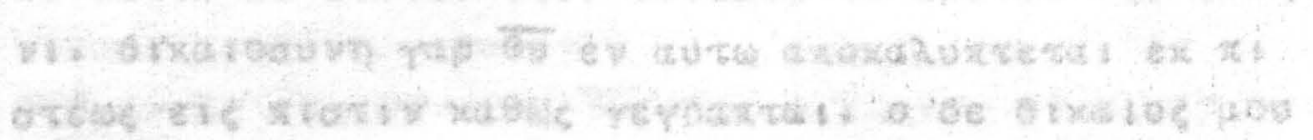

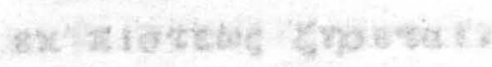

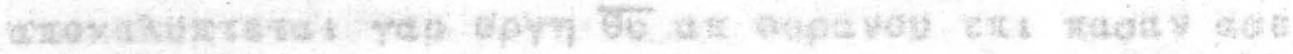

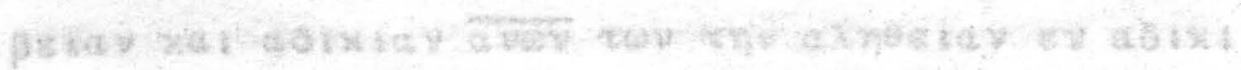

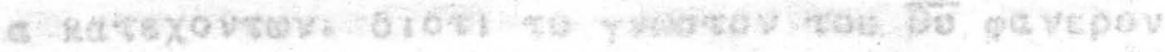

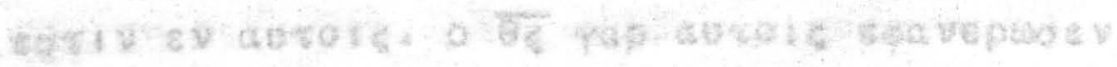

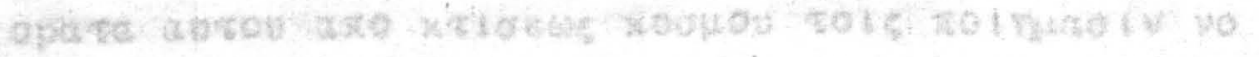

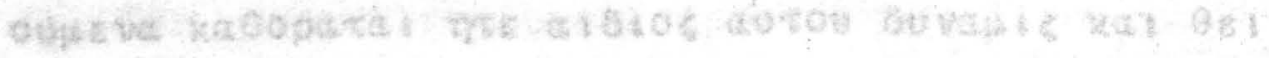




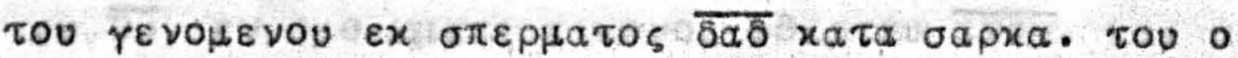

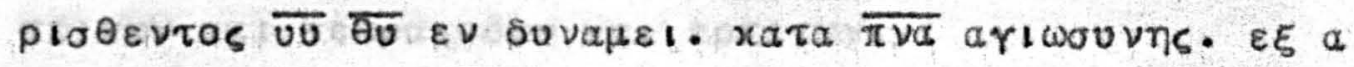

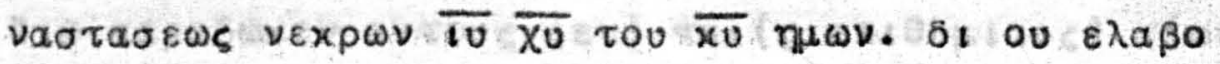

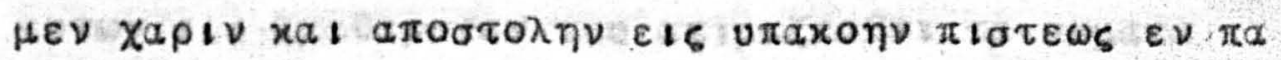

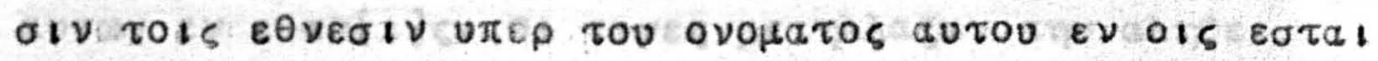

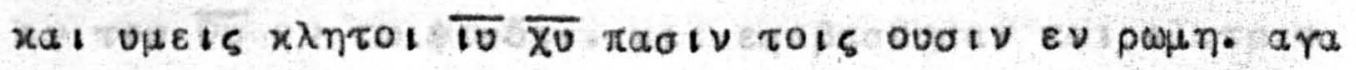
$10 \pi \eta \pi$ ts $\overline{\theta 0} x \lambda \eta \tau$ ts artois.

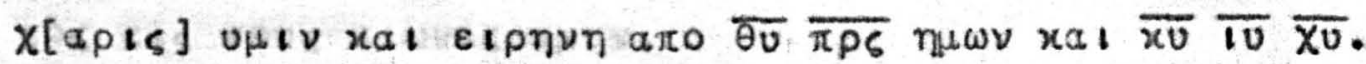

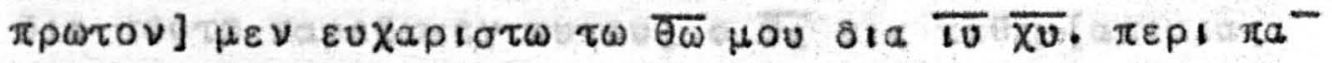

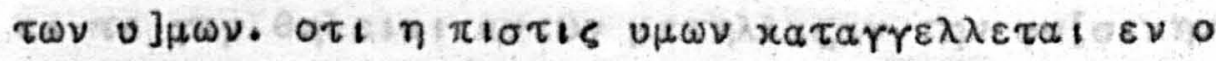

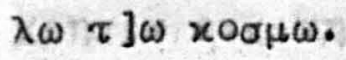

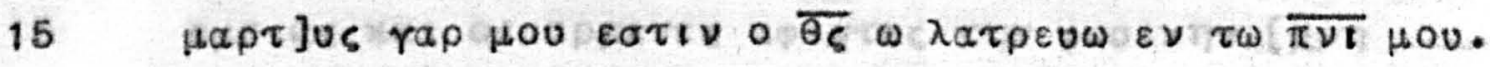

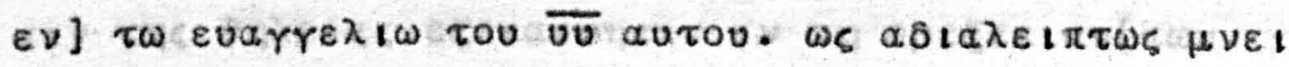

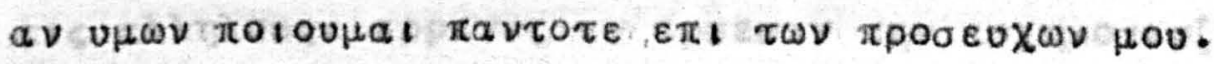

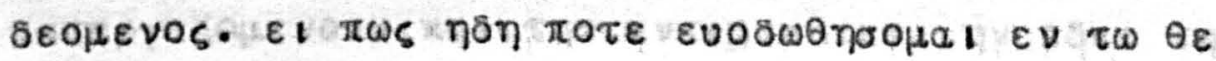

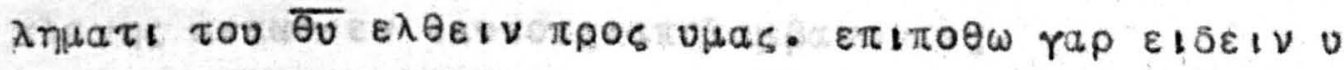

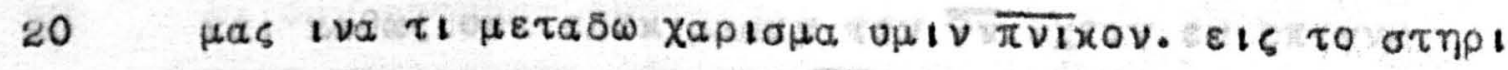

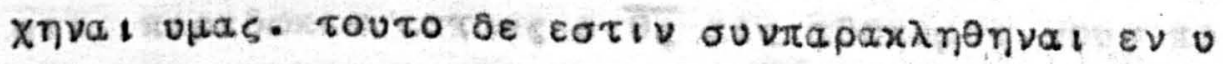
HIV ô

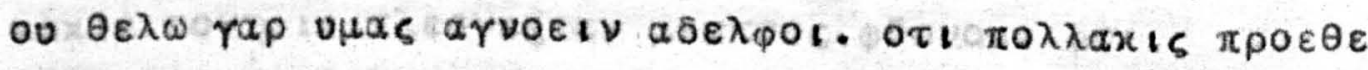

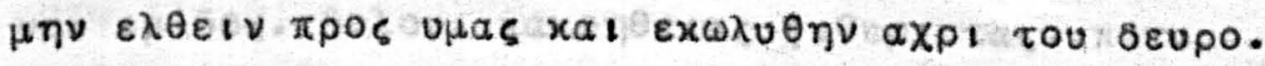

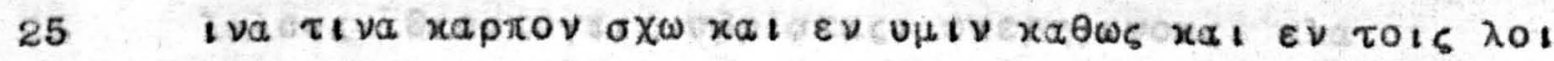

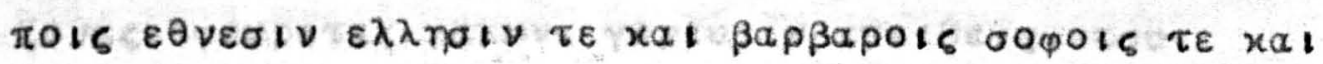

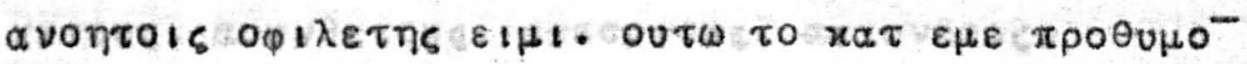

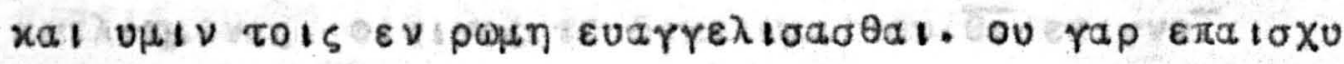

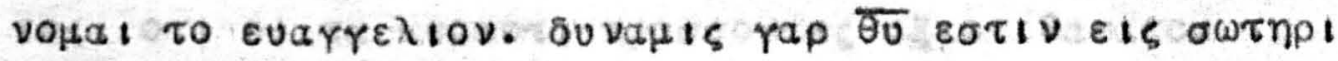

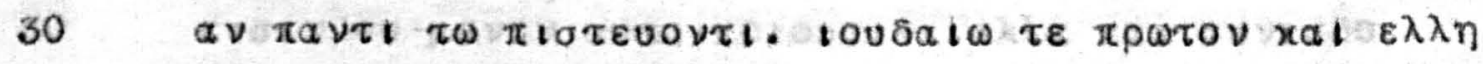

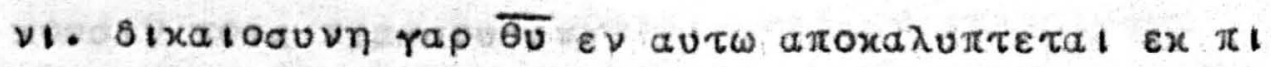

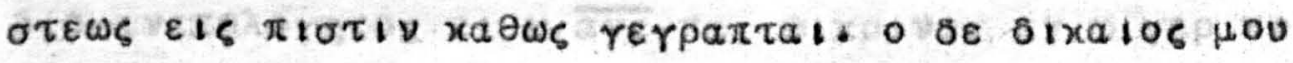

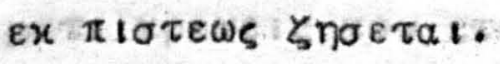
a

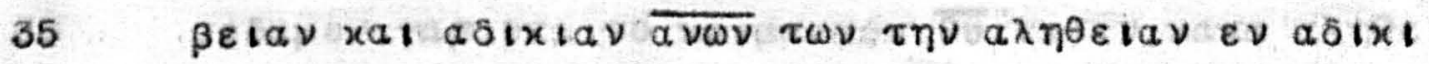

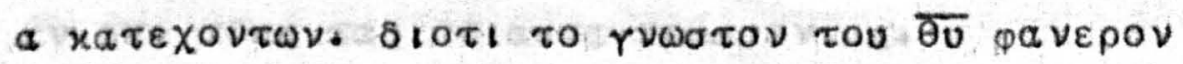

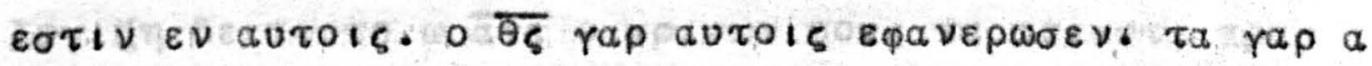

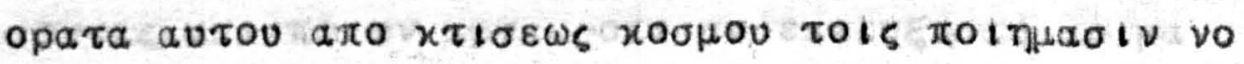

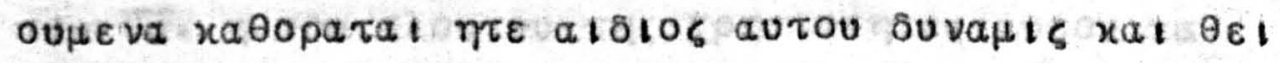

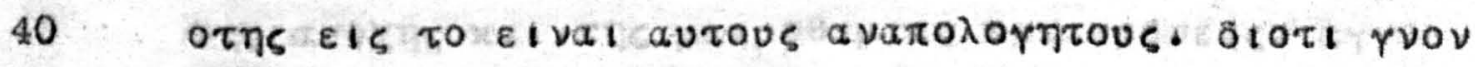

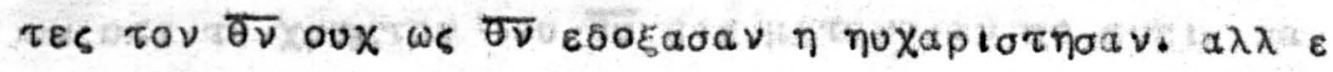




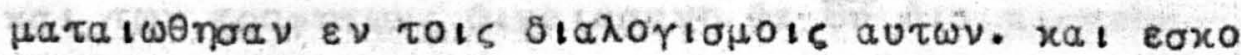

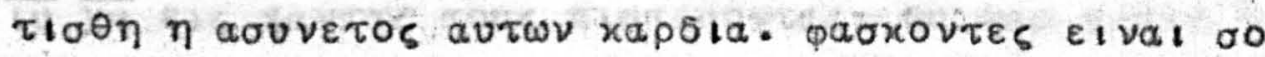

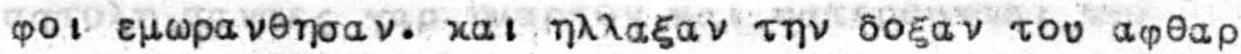

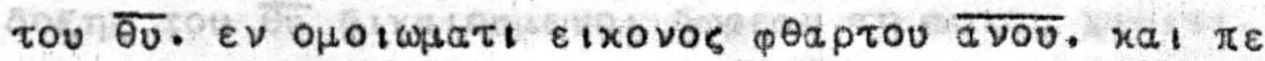
$\tau \varepsilon$ เ

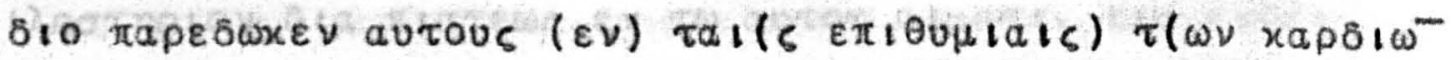

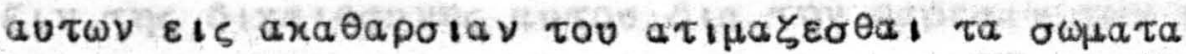

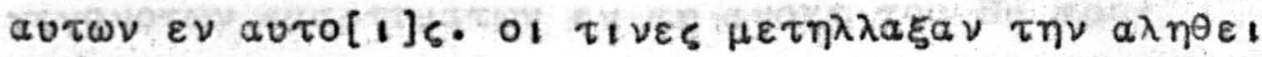

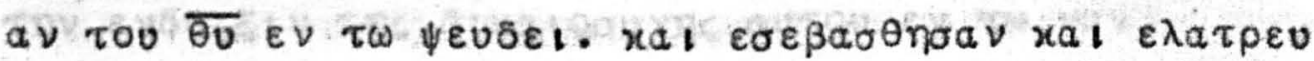

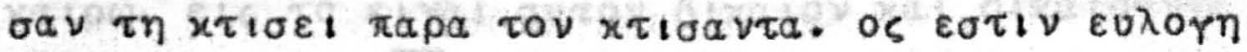
Tos els tous alwvas a $\mu \eta \eta$.

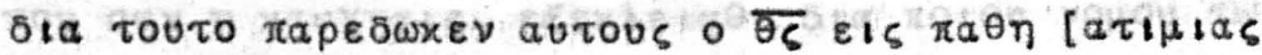

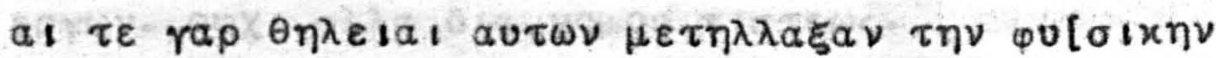

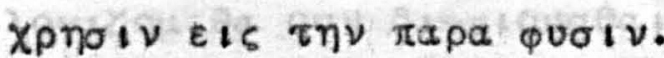

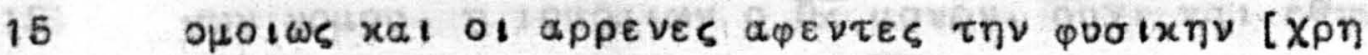

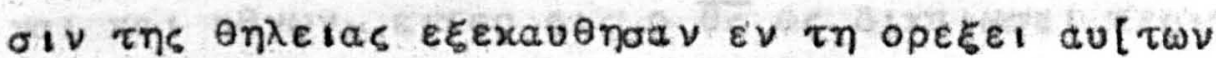

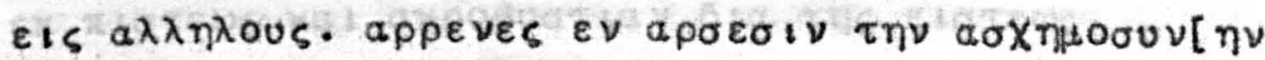

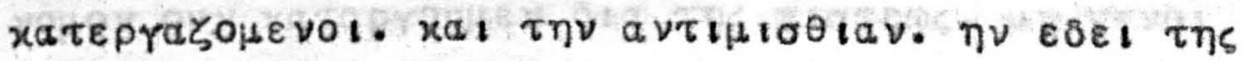

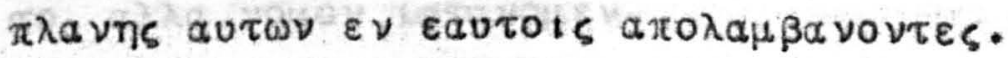

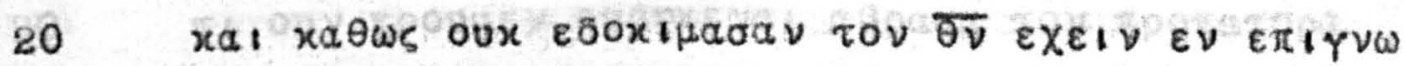

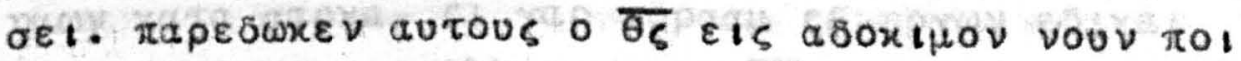

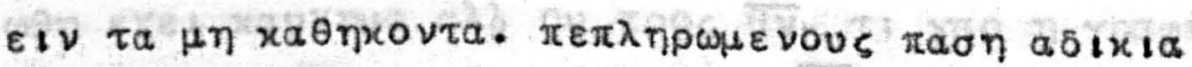

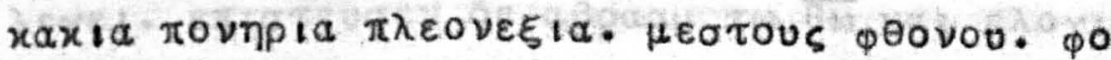

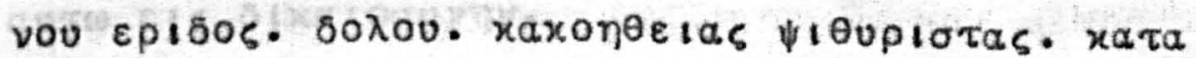

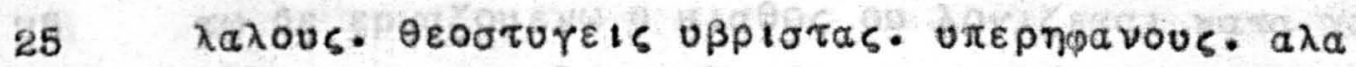

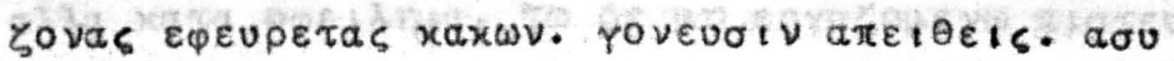

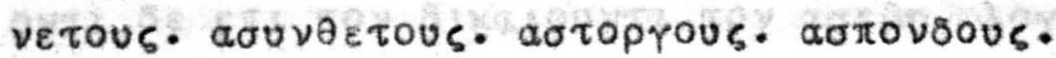

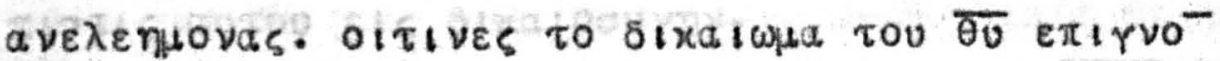

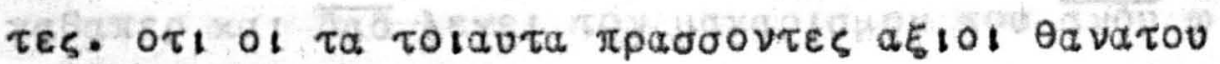

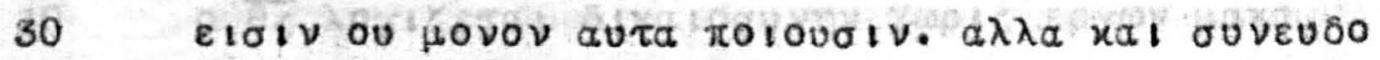
xovoiv tois $\pi \rho \alpha \sigma \sigma o v \sigma t \nu$.

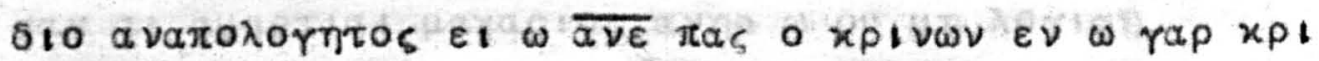

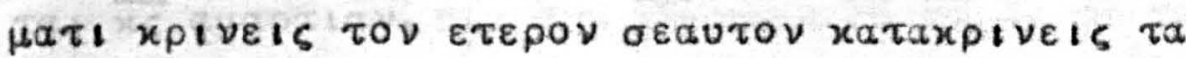

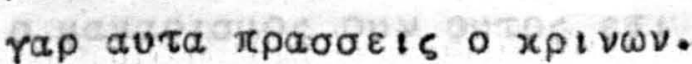

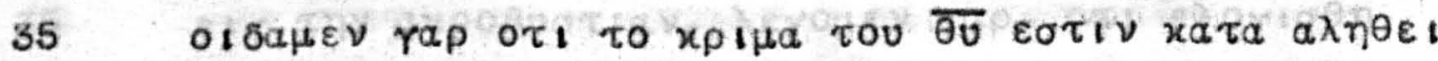

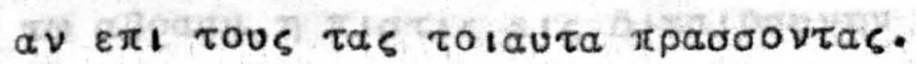

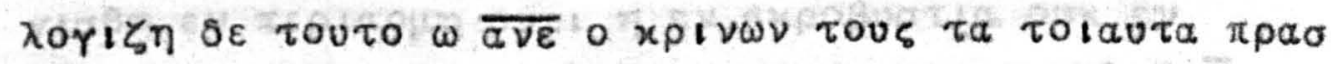

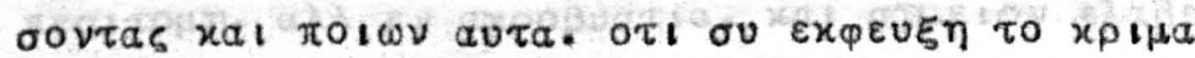

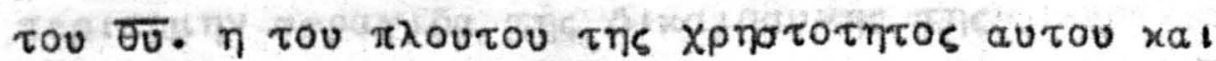

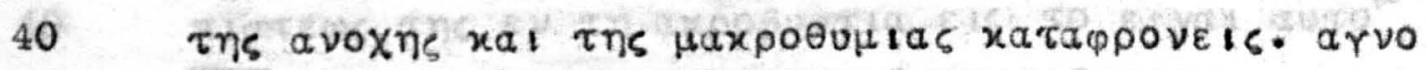

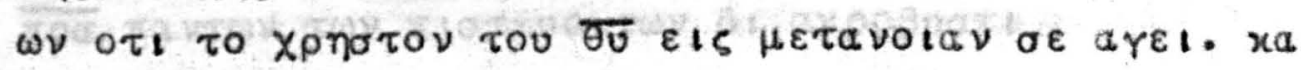




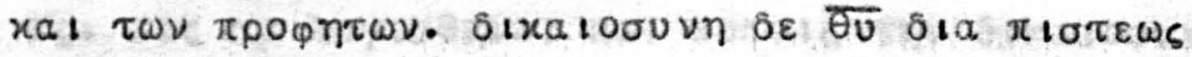

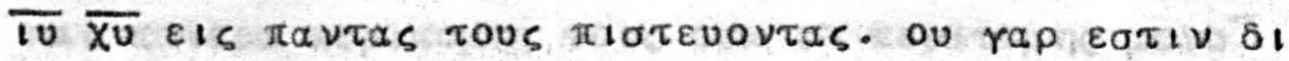

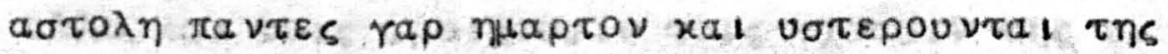

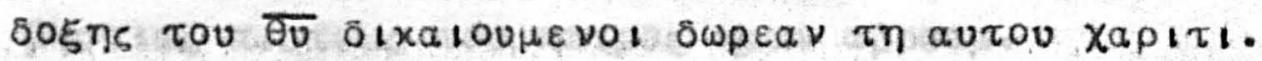

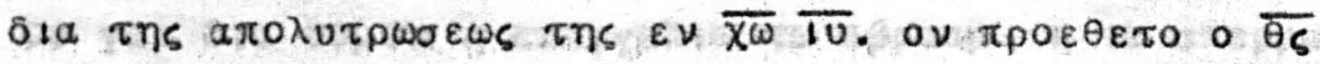

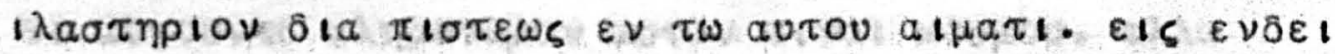

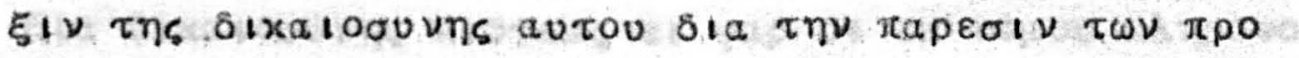

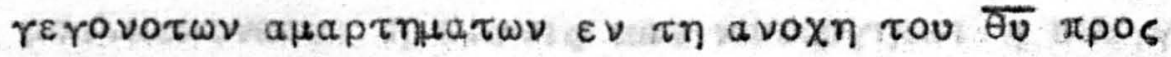

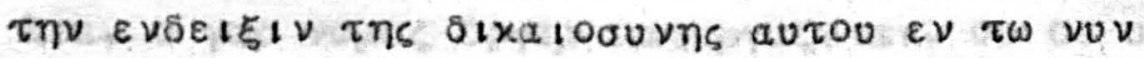
xalpw. Els to EIVal autov sixatov xal olxalouvta Tov $\varepsilon x$ tritews $\overline{10}$.

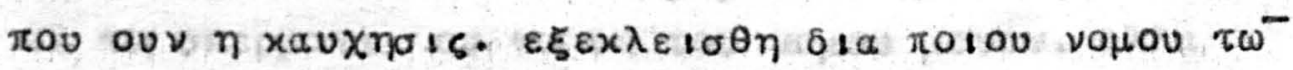

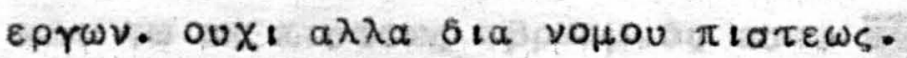

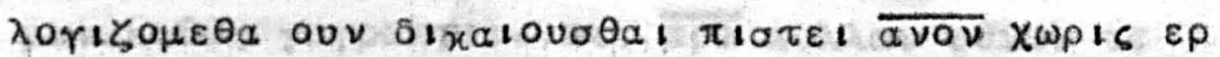

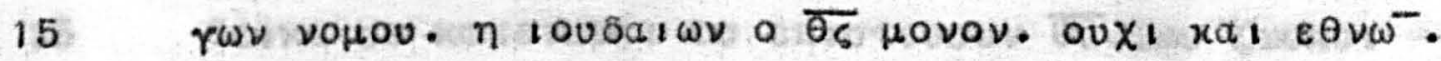

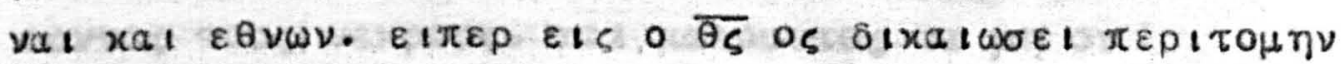

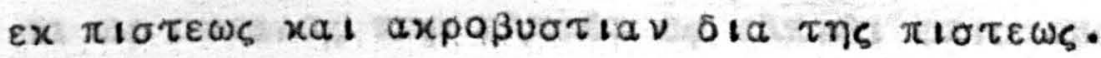

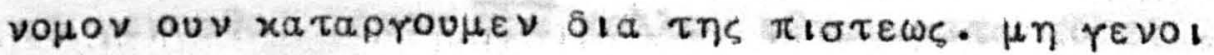

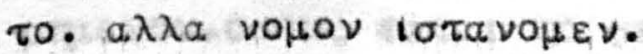

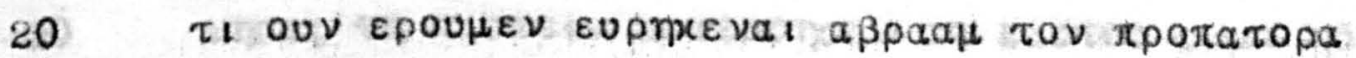

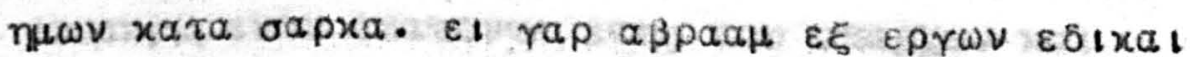

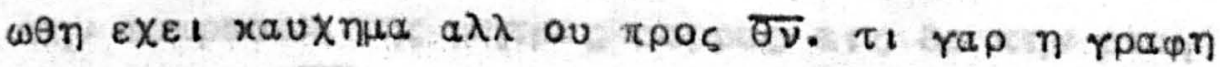

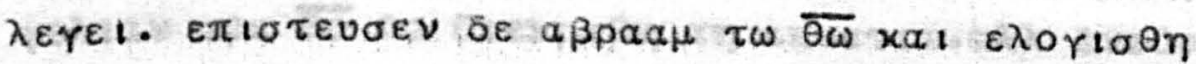

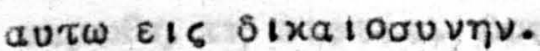

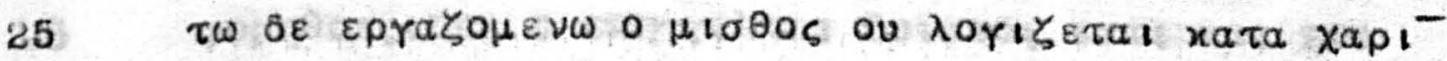

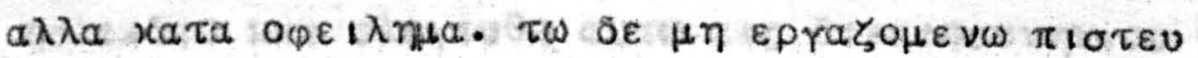

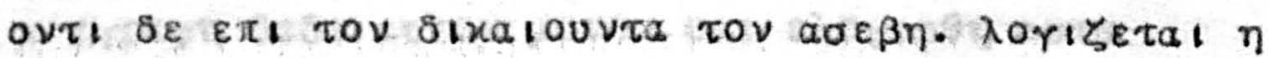

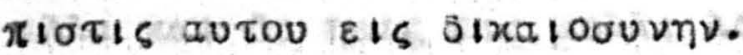

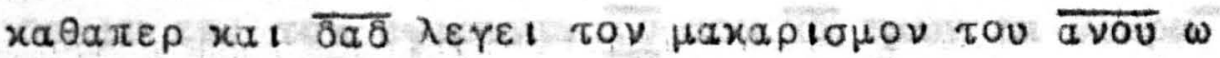

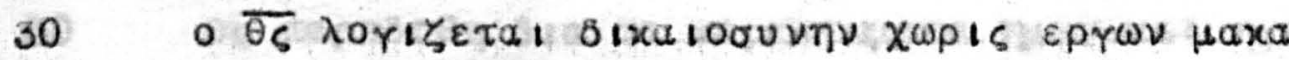

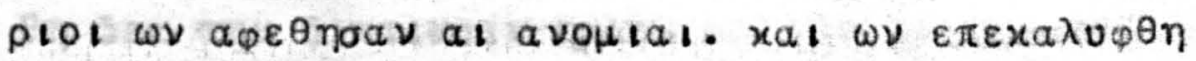

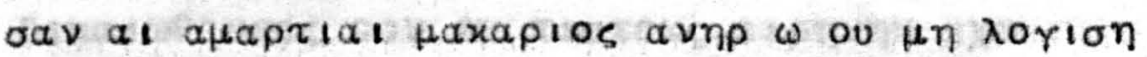

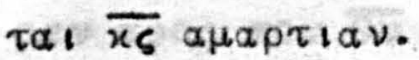

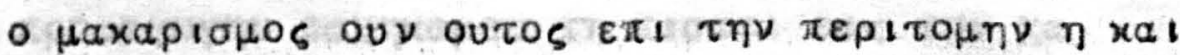

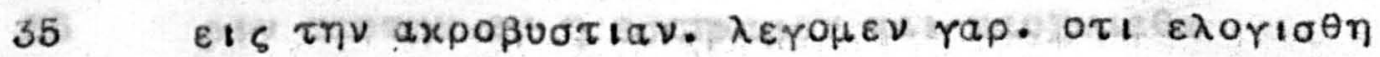

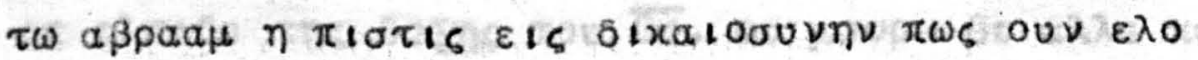

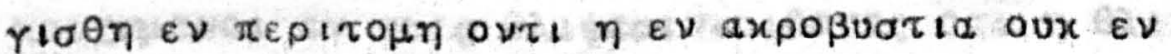

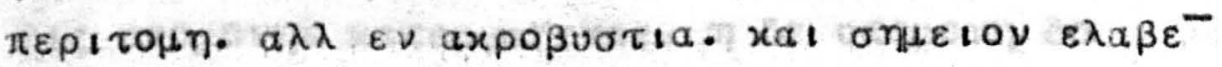

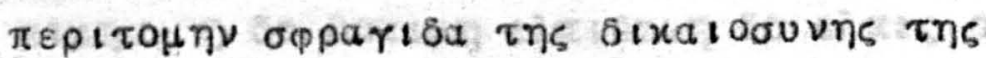

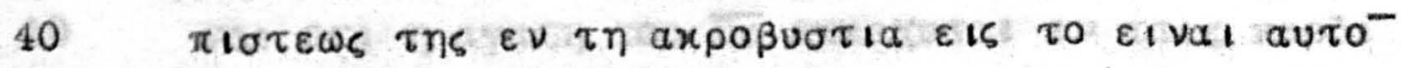

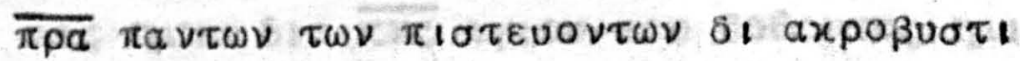




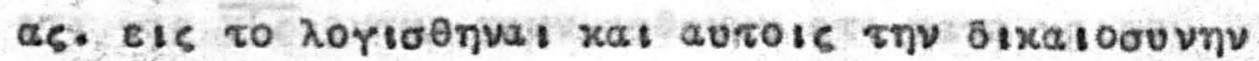

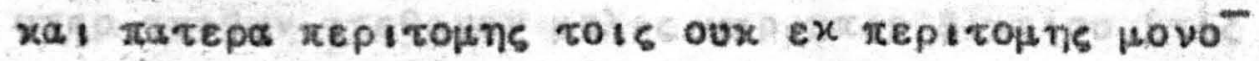

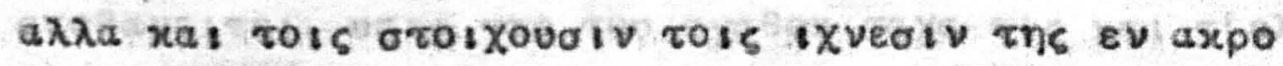

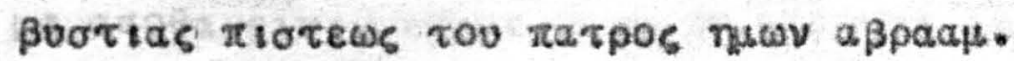

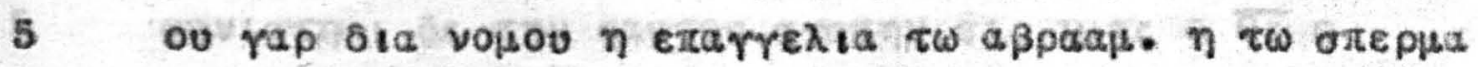

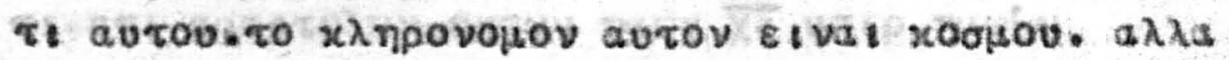
bia oixatoouvn tatorews ei rap of ex vojou $x \lambda$ npovo

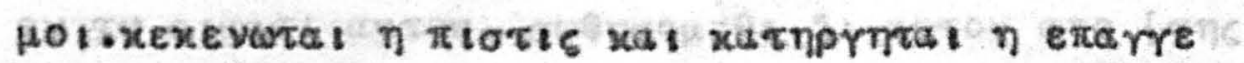

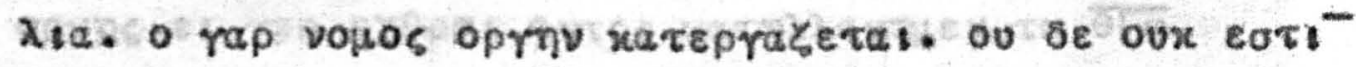

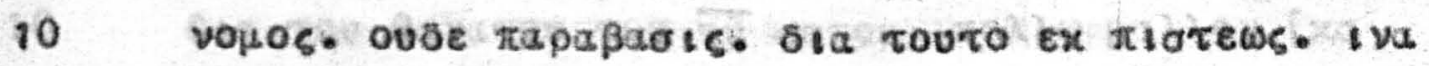

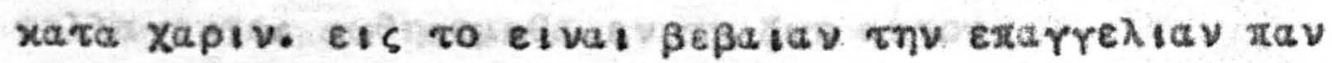

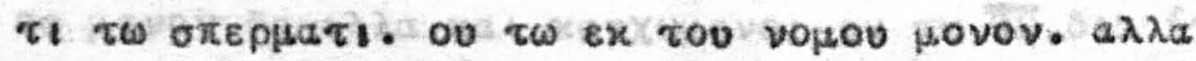

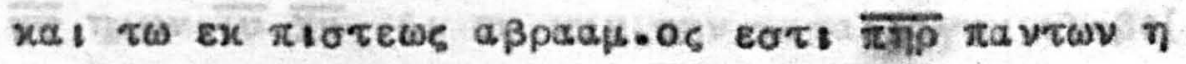

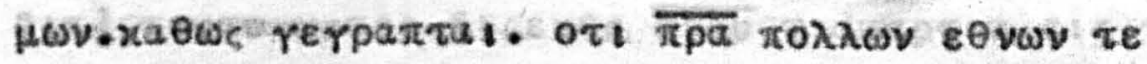

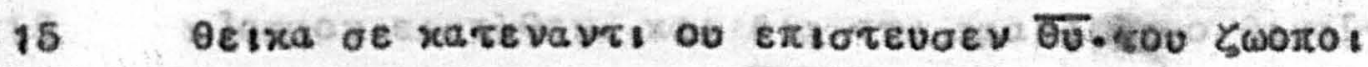

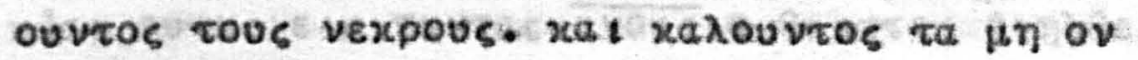

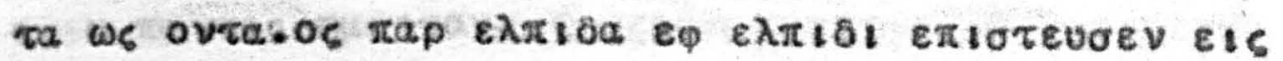

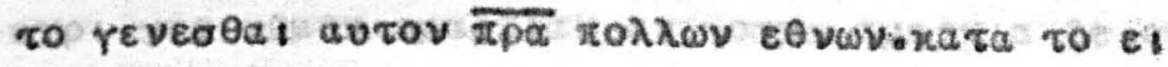

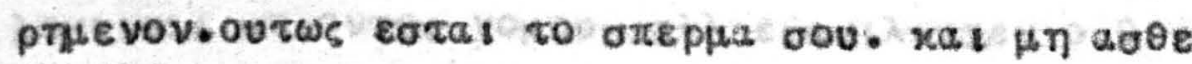

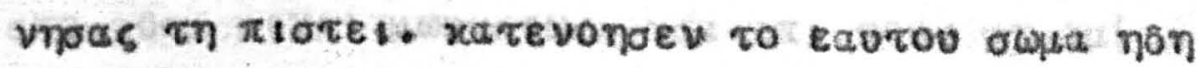

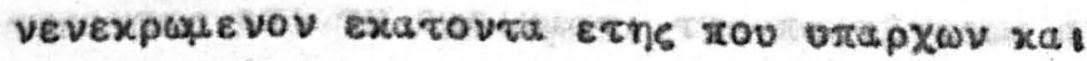

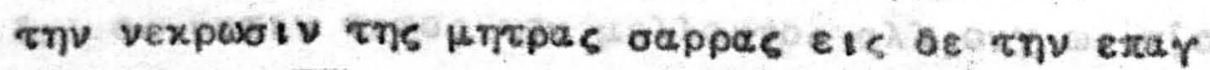

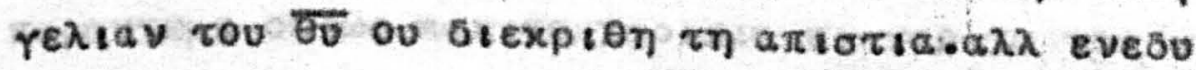
vapw

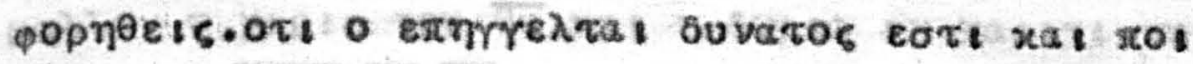

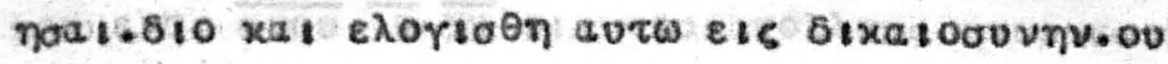

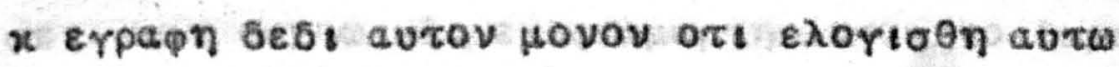

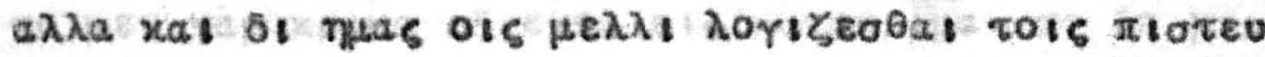

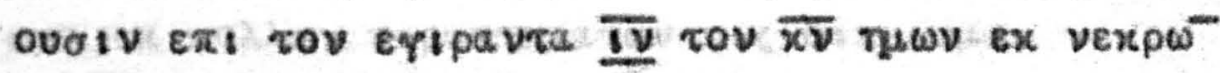

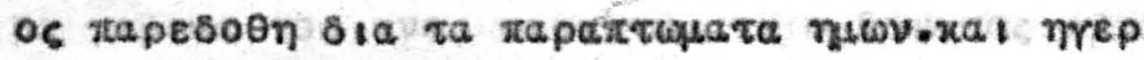

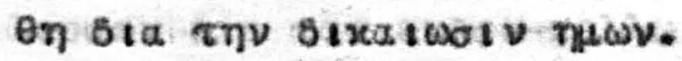

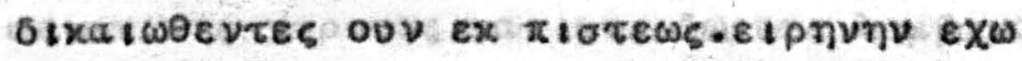

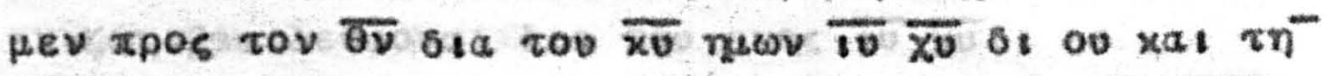

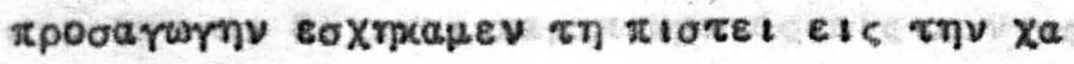

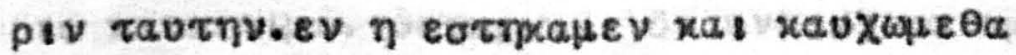

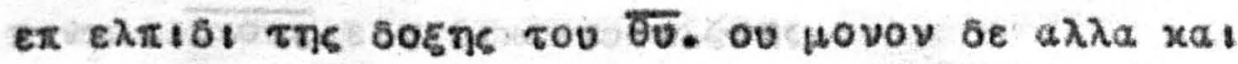
xauxตุน

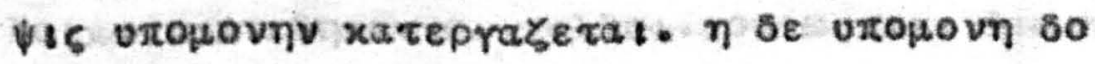

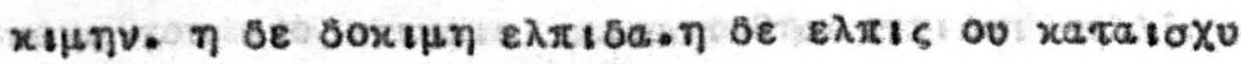

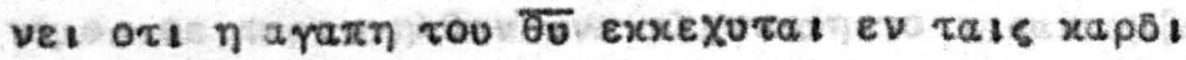

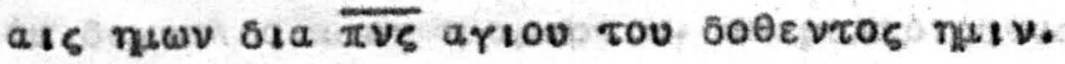




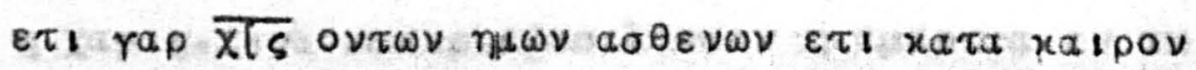

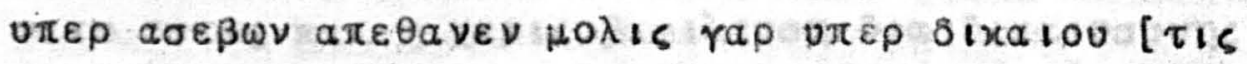
a Ha a $\pi \circ \theta \nu \varepsilon i \nu$.

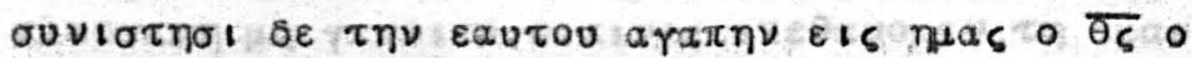

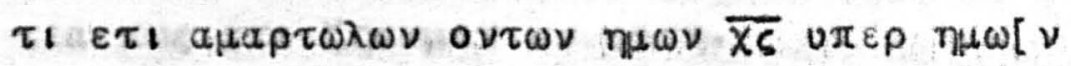

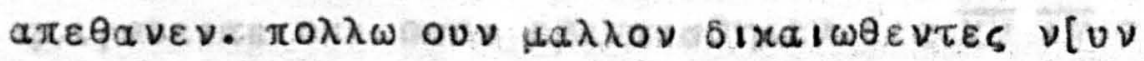

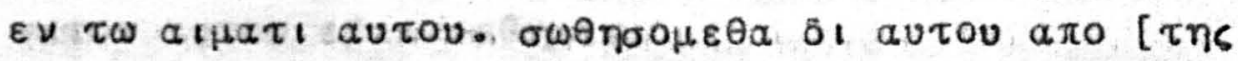

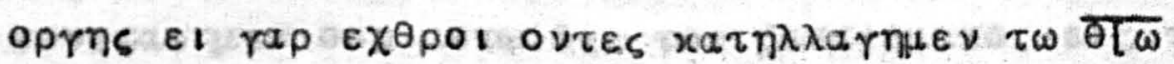

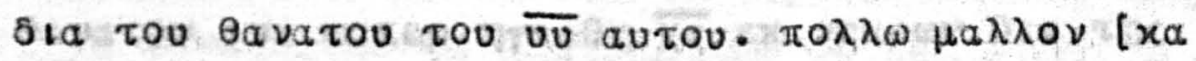

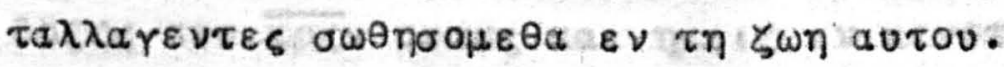

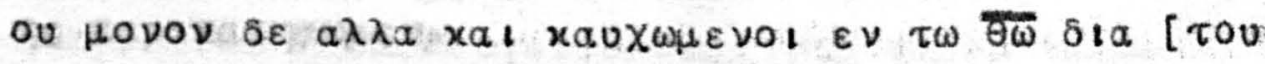

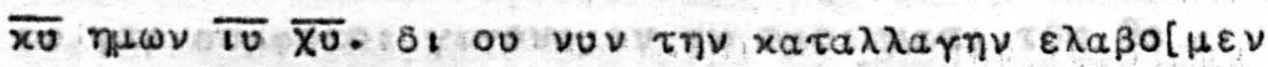

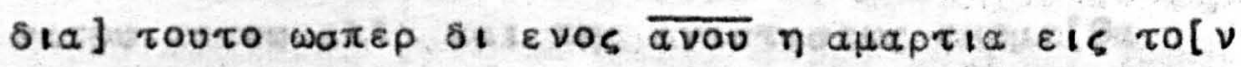

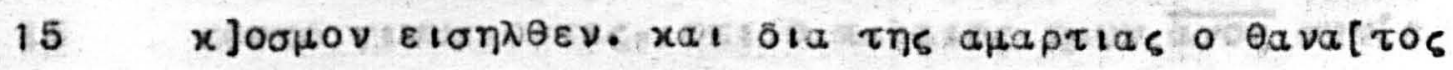

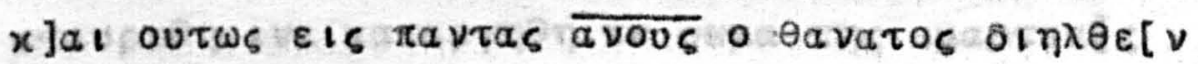

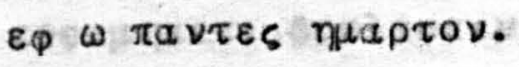

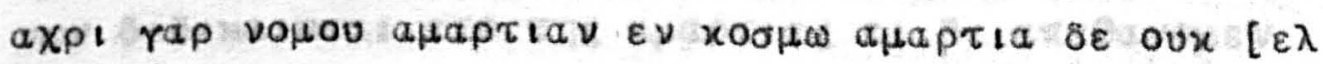

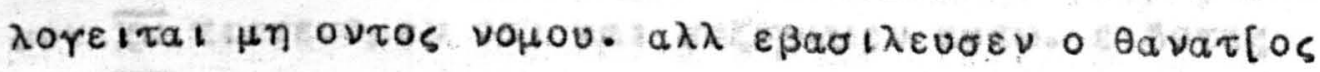

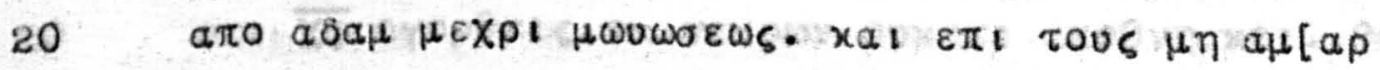

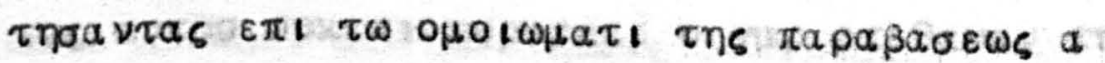

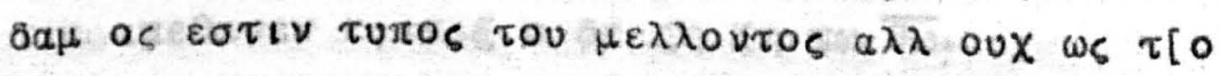

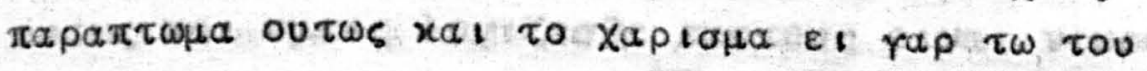

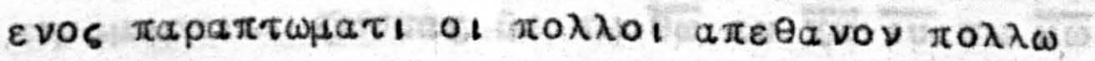

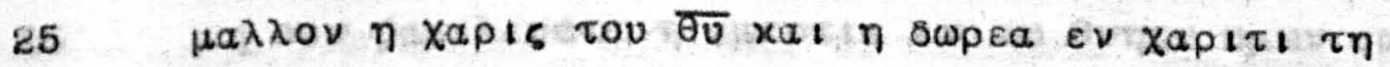

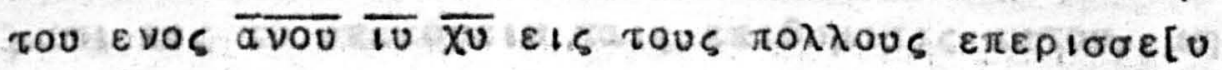

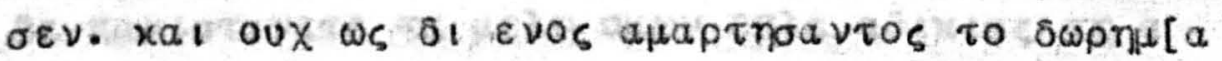

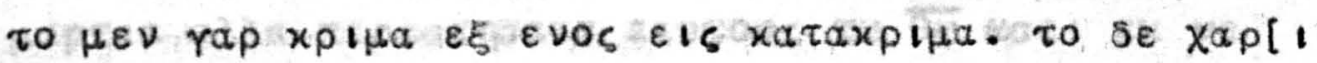
$\sigma \mu \alpha \varepsilon x \pi \alpha \lambda \omega \nu \pi a p a \pi \tau \omega \mu \alpha \tau \omega \nu$ \& is $\delta i x a i \omega \mu \alpha$.

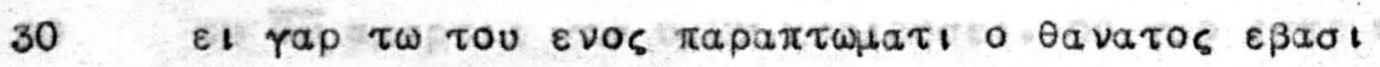
$\lambda \varepsilon v \sigma \varepsilon \nu$ ô

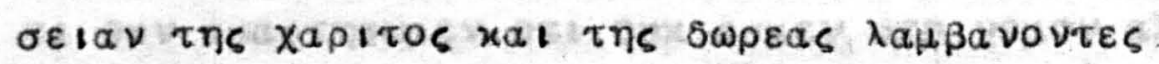

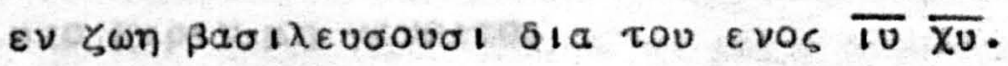

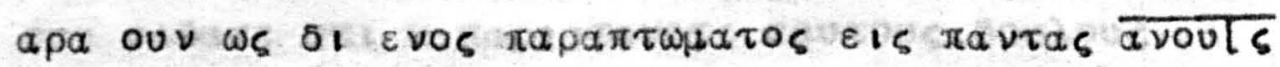

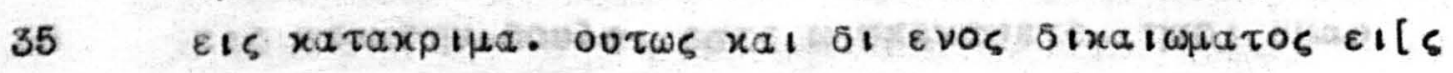

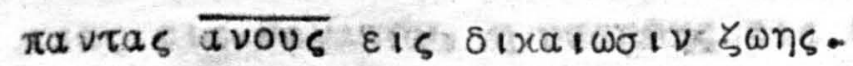

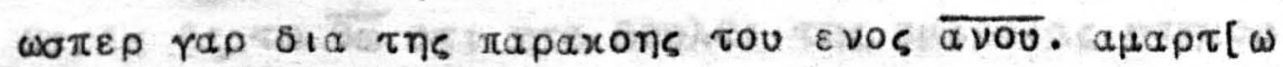

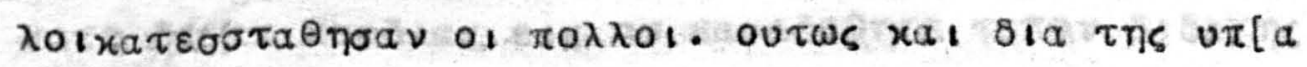

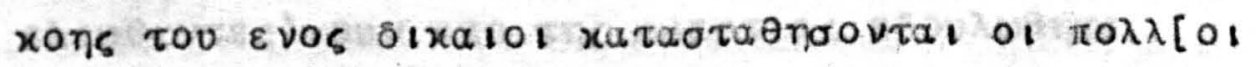

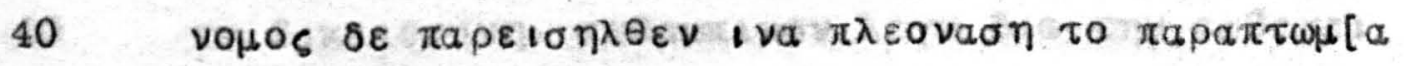
ou $\delta \varepsilon \varepsilon \pi \lambda \varepsilon \sigma \nu a \sigma \varepsilon \nu \eta a \mu a \rho \tau I \alpha$. $v \pi \varepsilon \rho \varepsilon \pi \varepsilon \rho เ \sigma \sigma \varepsilon v \sigma \varepsilon \nu$ 


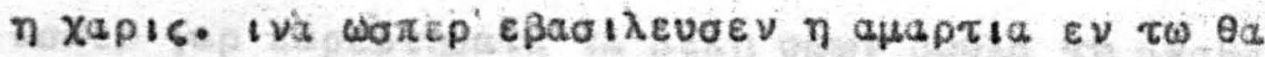

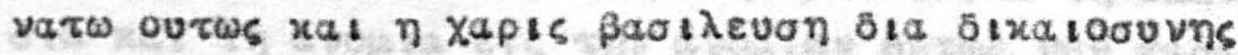

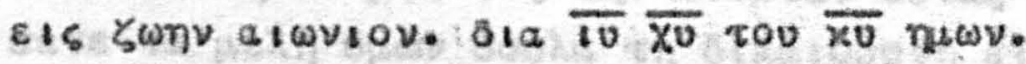

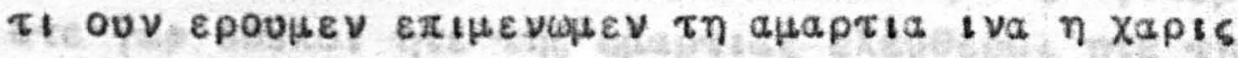

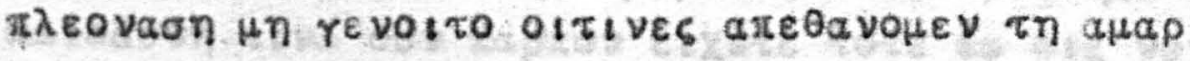

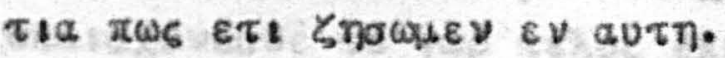

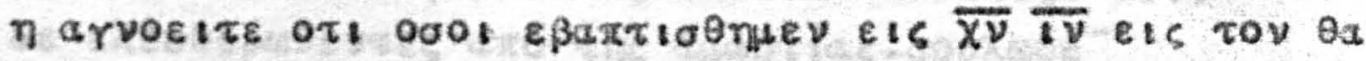

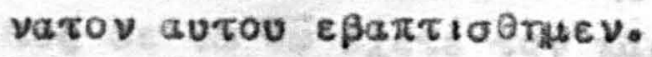

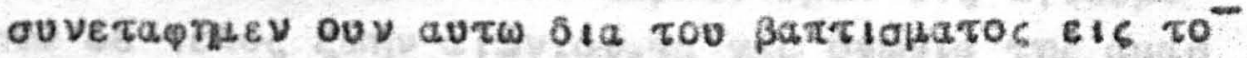

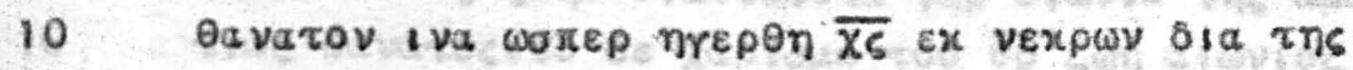

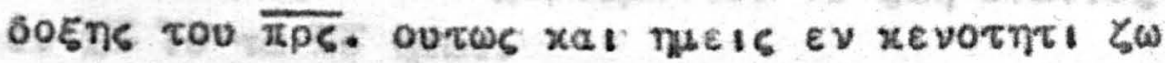

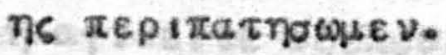

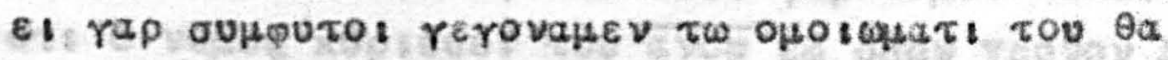

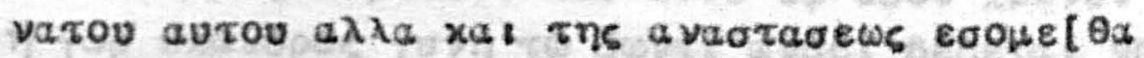

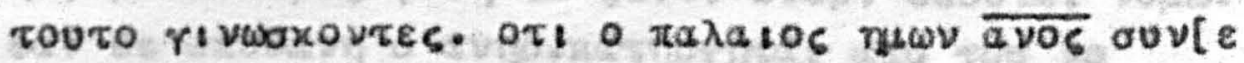

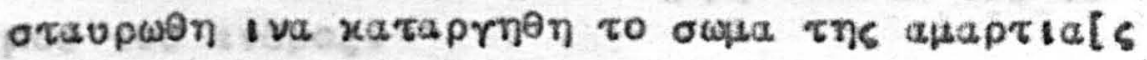

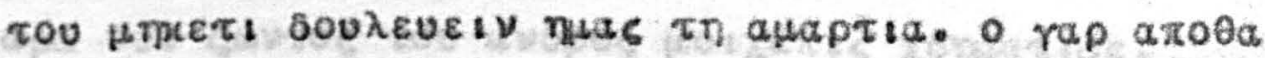

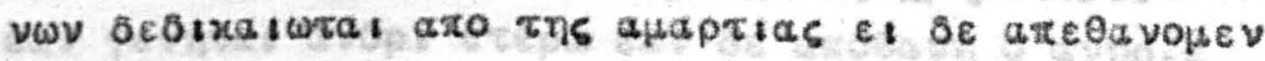

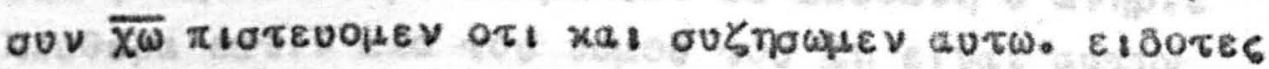

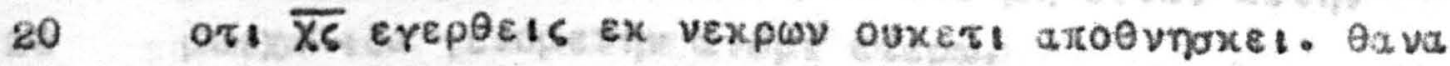

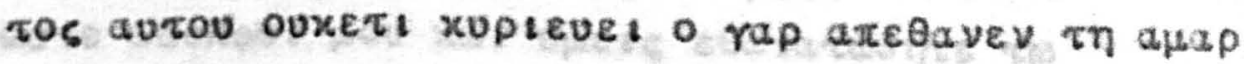

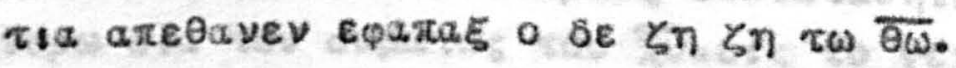

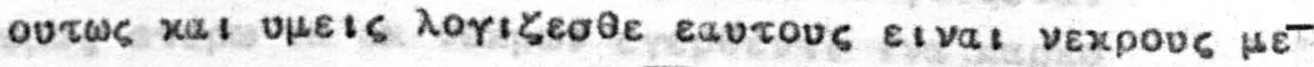

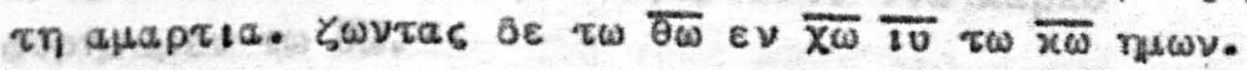

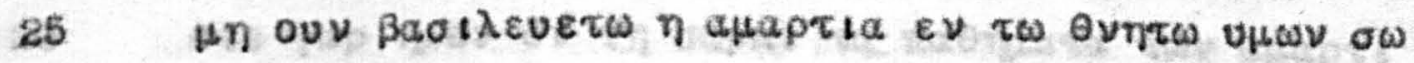

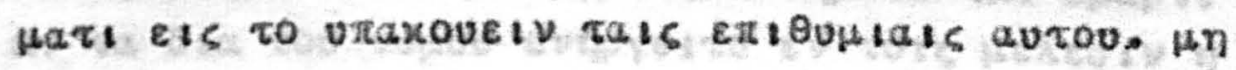

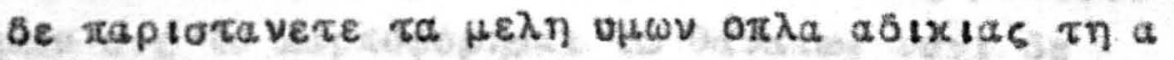

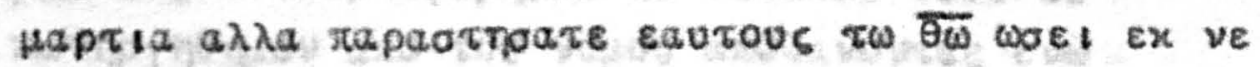

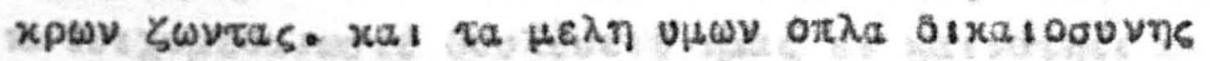

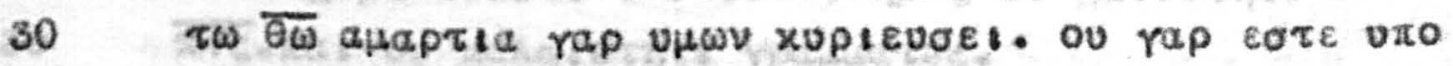

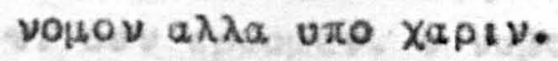

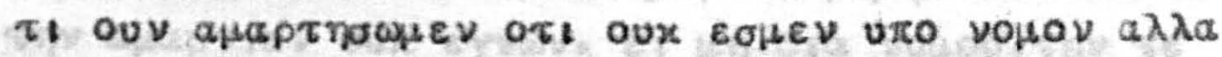
vito Xapiv $\mu \eta$ revotro.

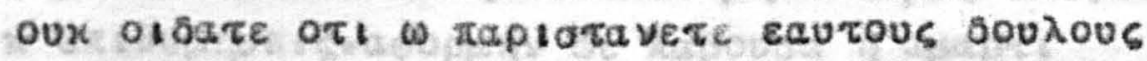

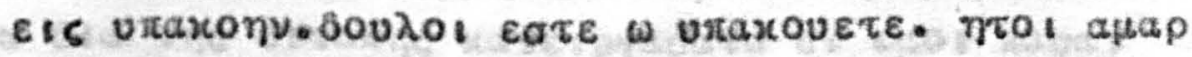

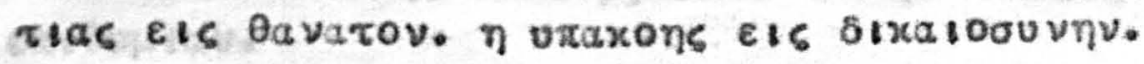

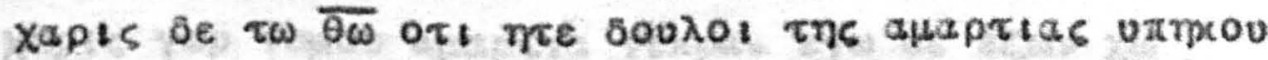

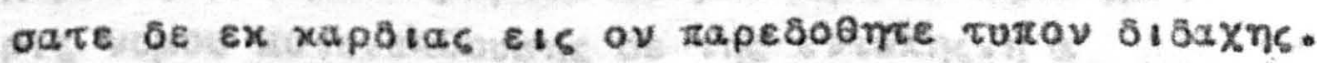

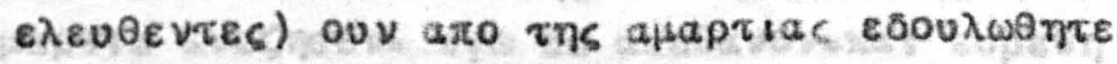

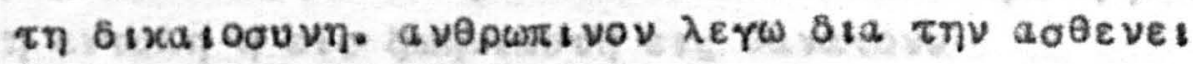

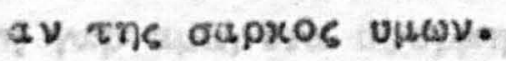




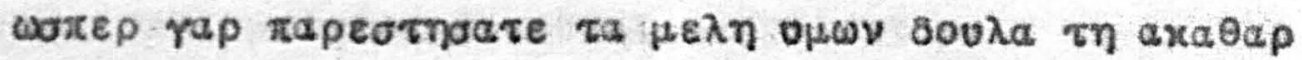

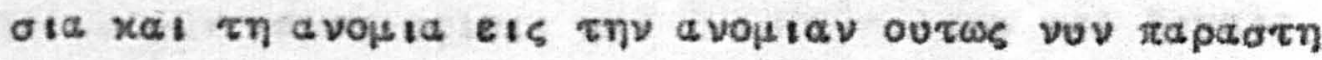

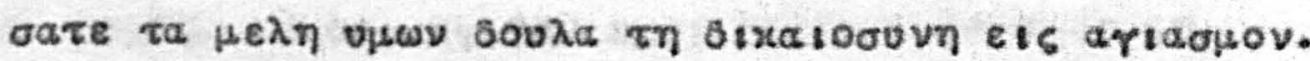

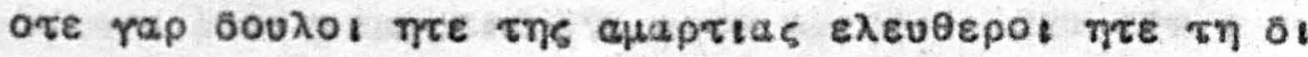

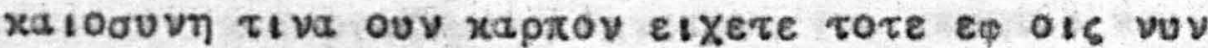

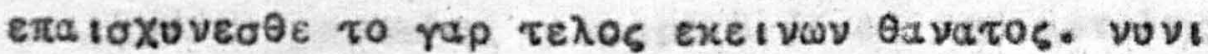

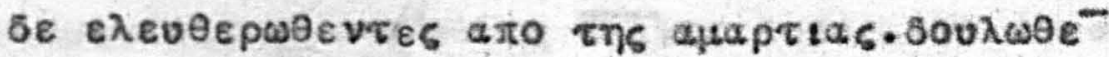

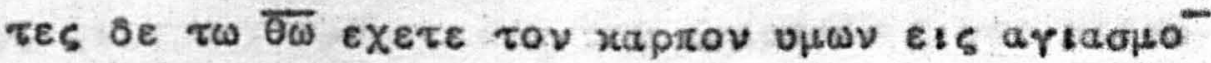

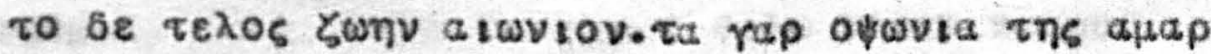

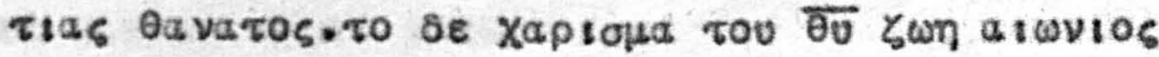
$\varepsilon v \overline{\chi \omega}$ TV $\tau \omega \overline{x \omega}$ nutsv.

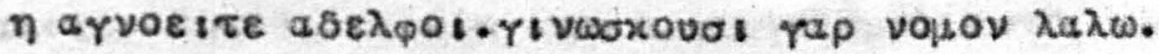

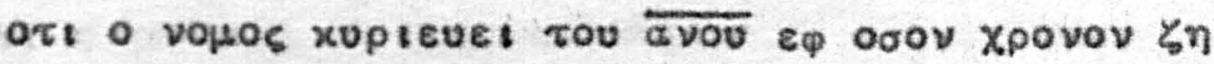

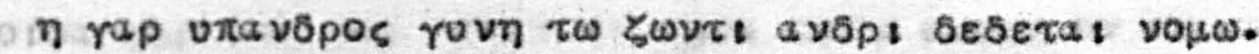

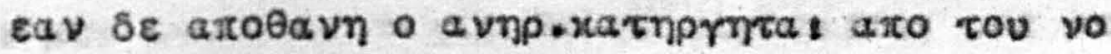
Hov tou avธิpos.

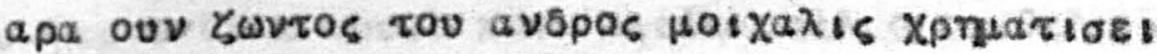

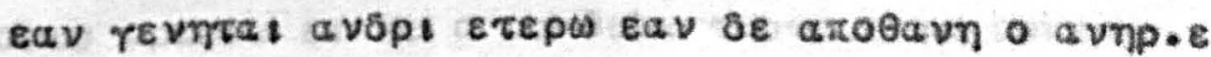

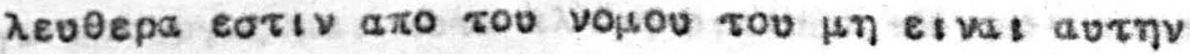

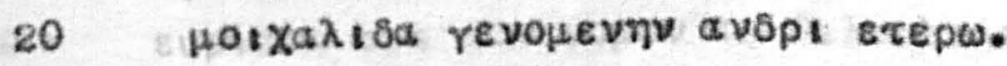

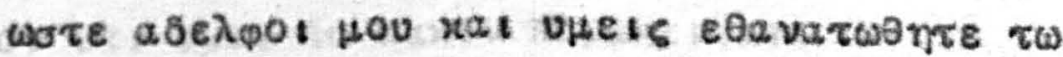

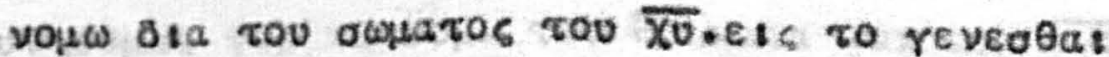

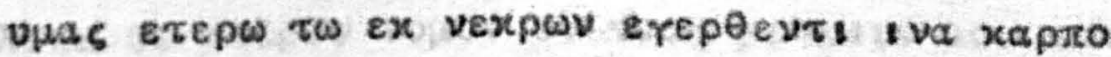

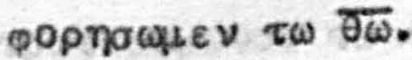

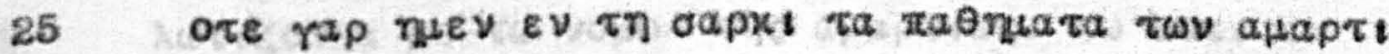

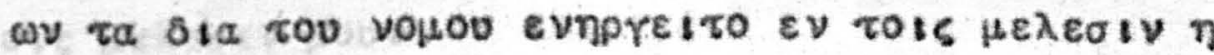

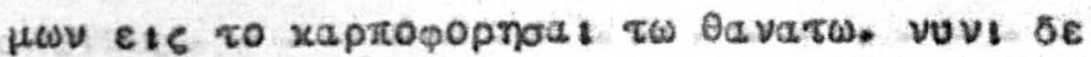

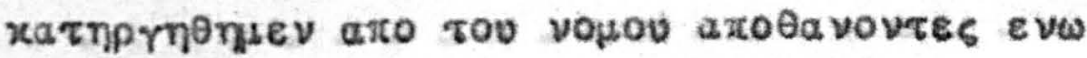

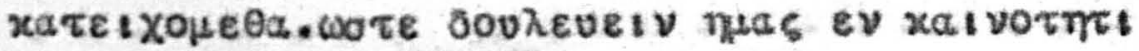

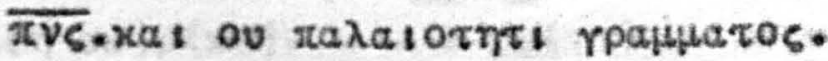

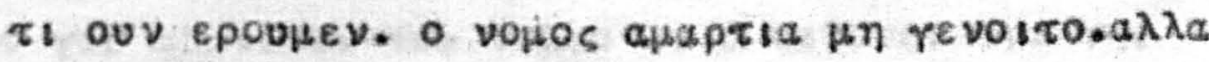

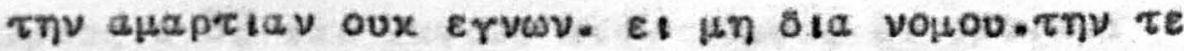

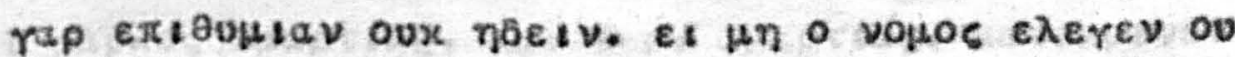

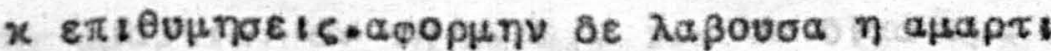

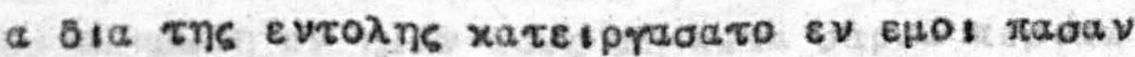
ertoustav.

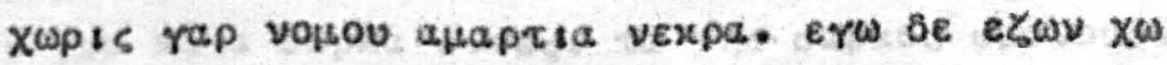

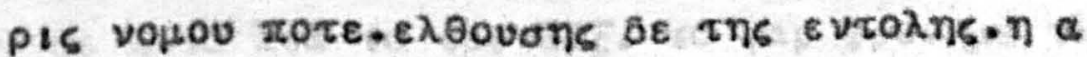

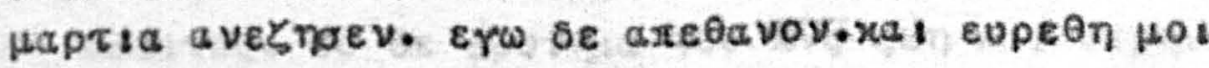

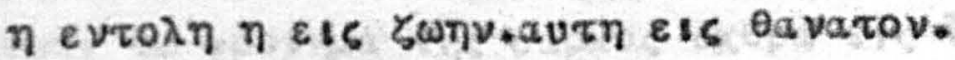

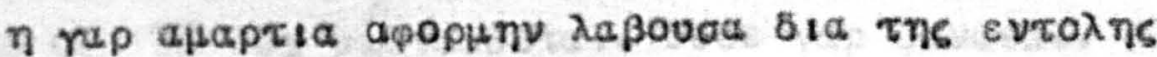




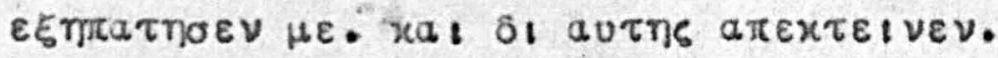

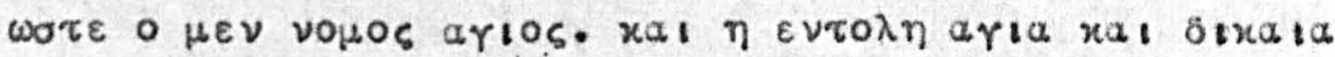

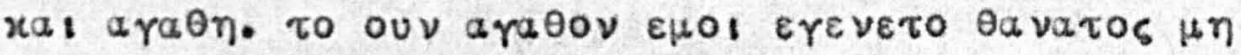

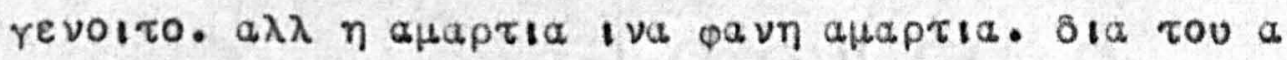

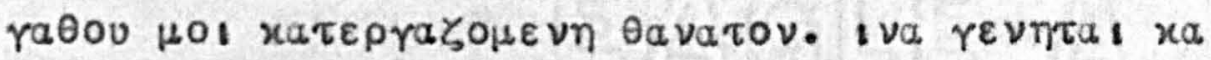

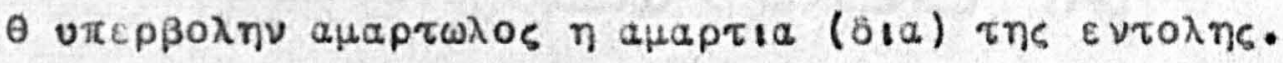

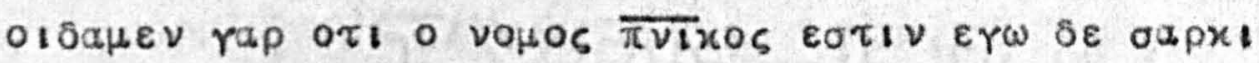

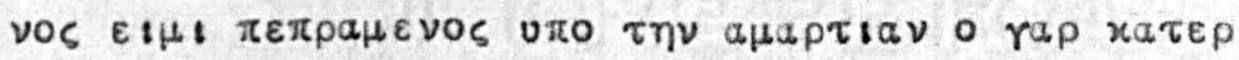

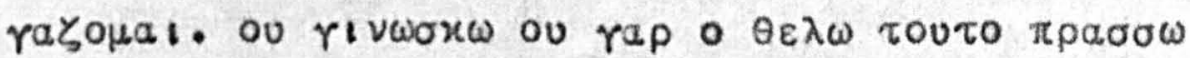

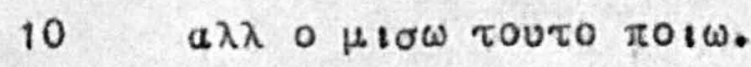

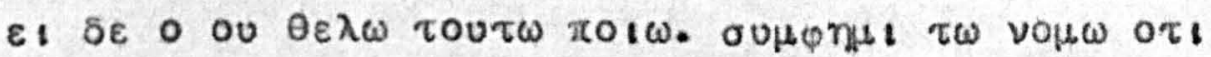

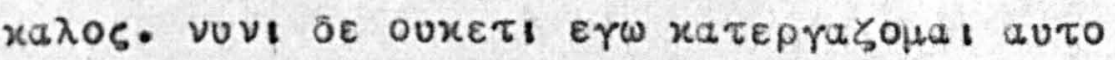

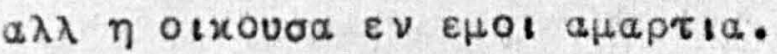

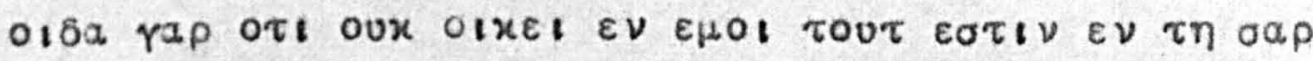

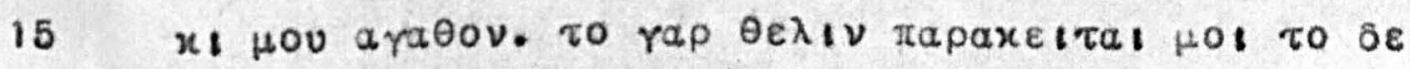

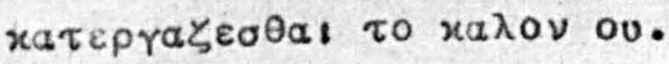

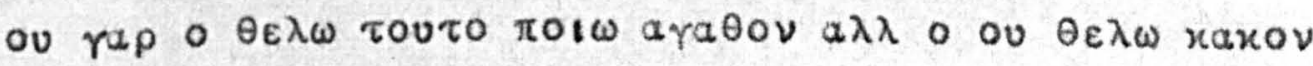

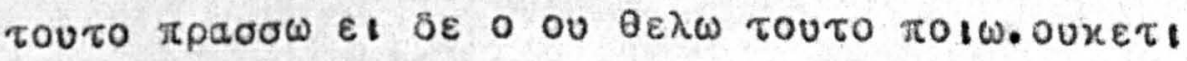

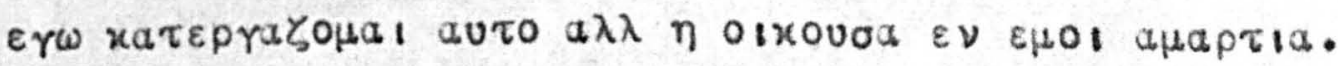

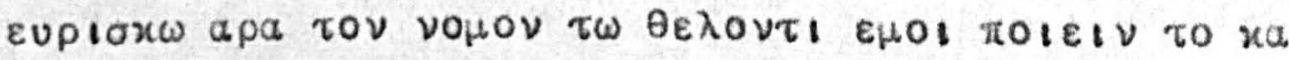

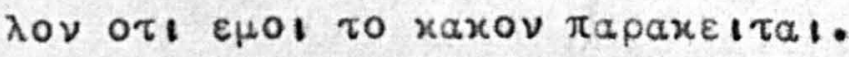

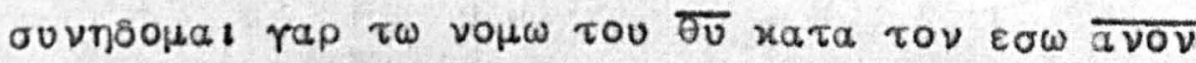

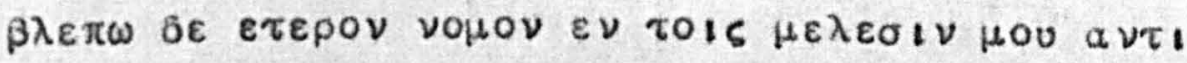

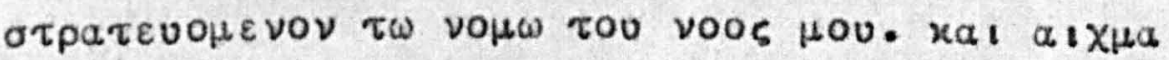

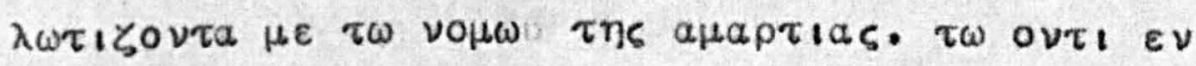
$\tau o$ is $\mu \varepsilon \lambda \varepsilon \sigma i \nu \mu O \nu$.
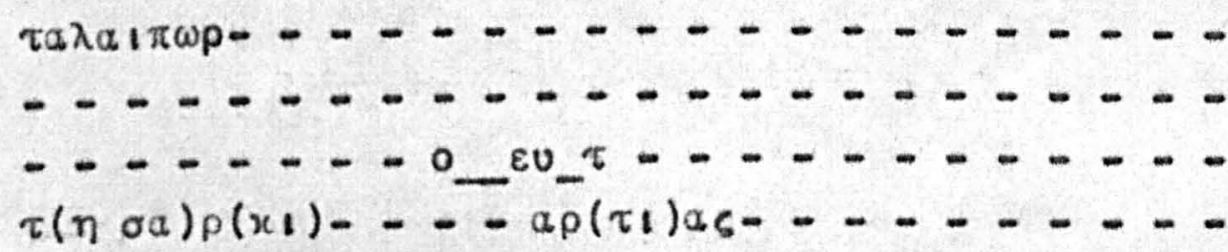

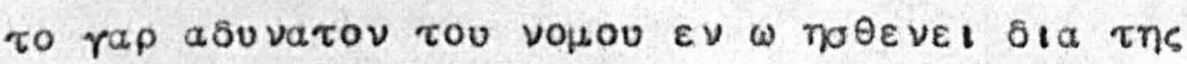

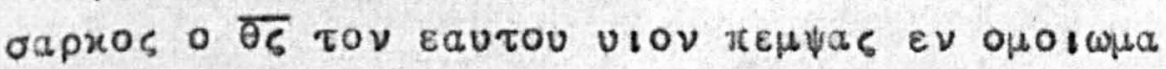

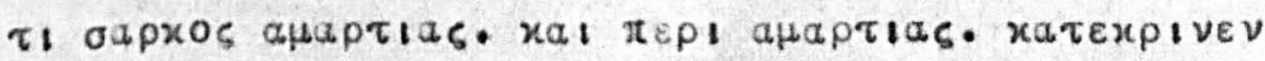

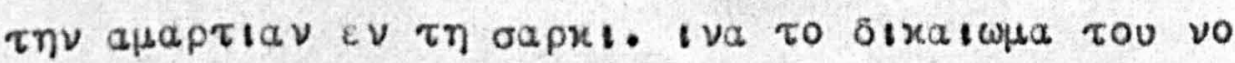

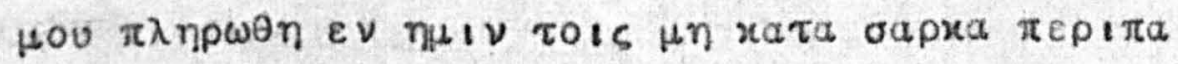

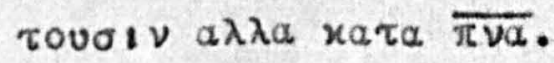

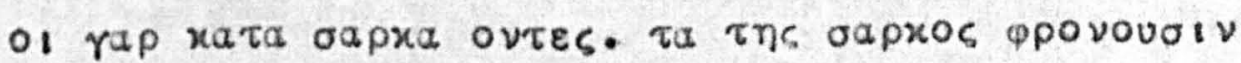

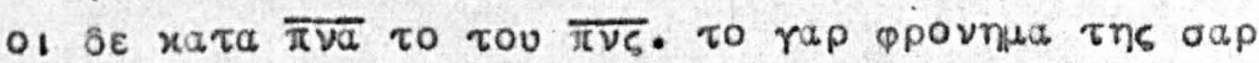

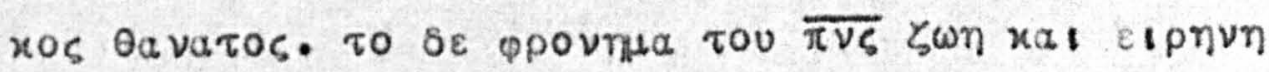

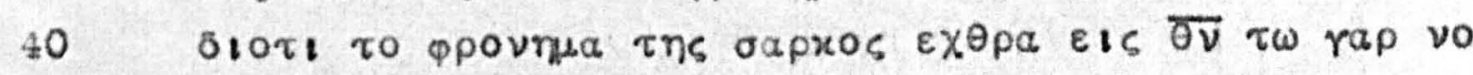

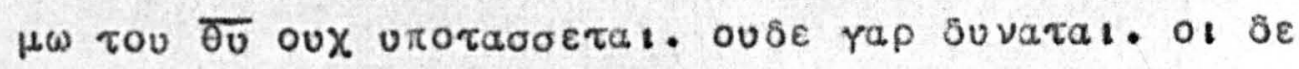




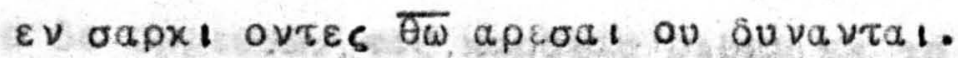

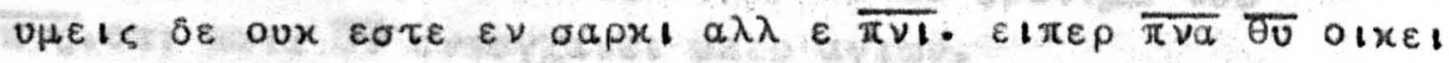

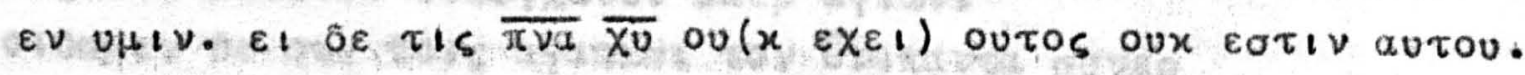

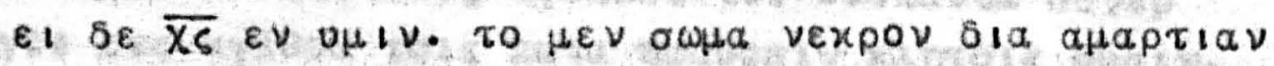

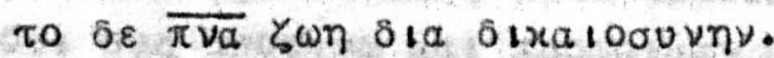

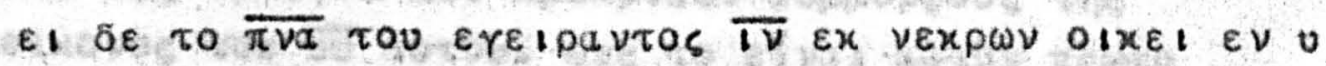

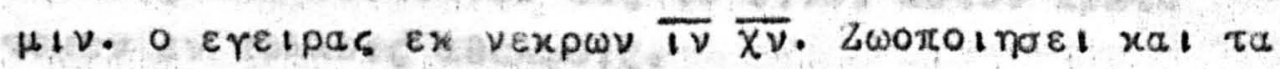

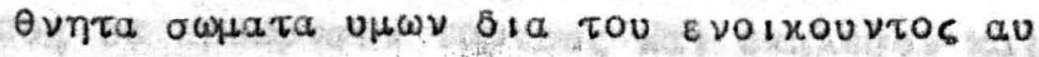

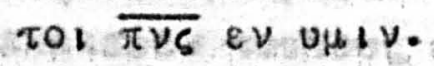

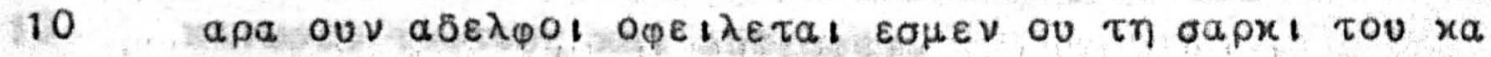

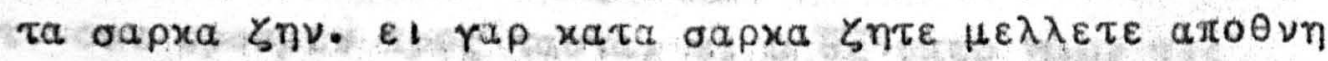

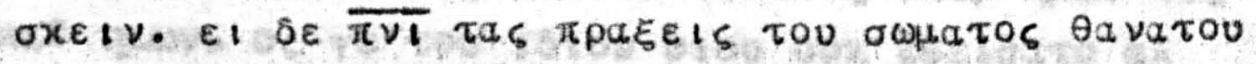

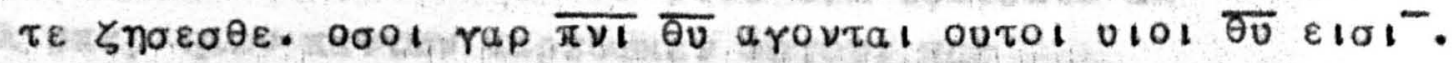

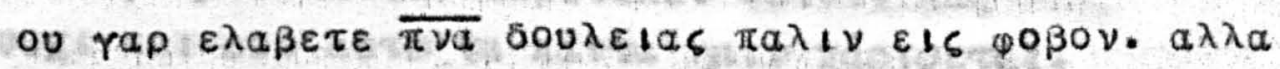

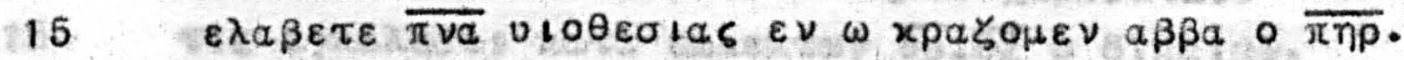

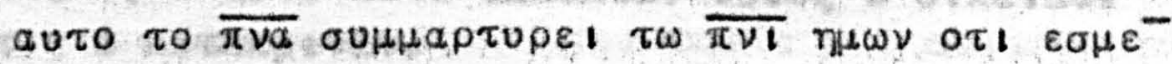

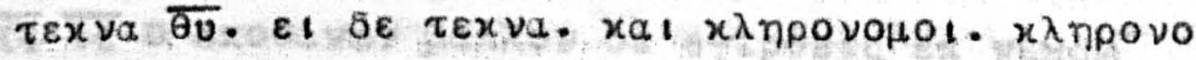

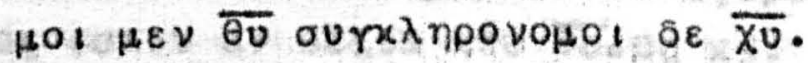

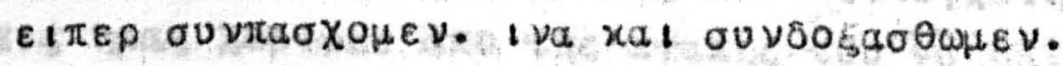

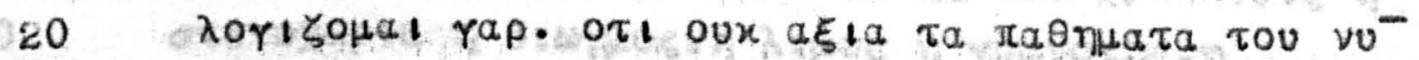

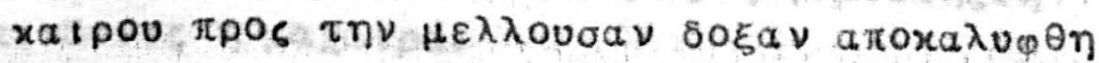

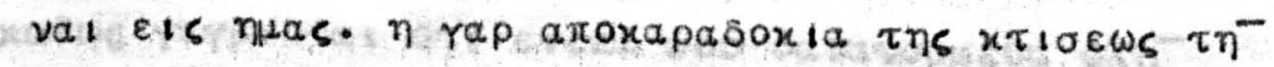

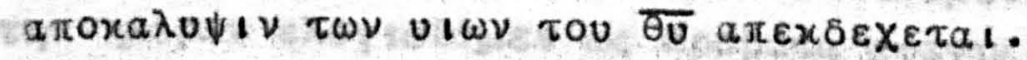

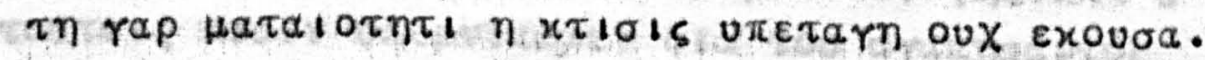
$25 \quad a \lambda \lambda a$ ô

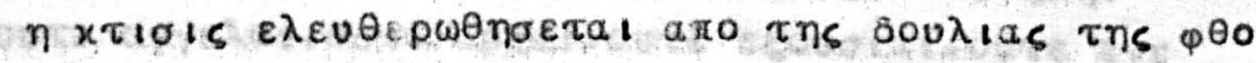

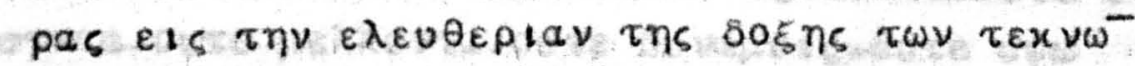

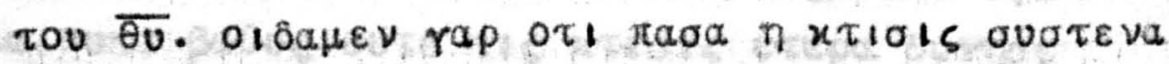

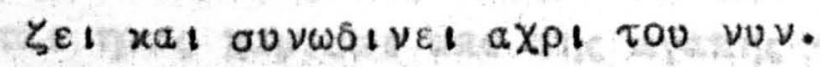

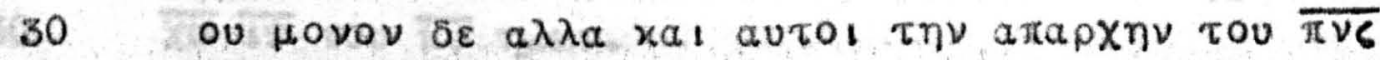

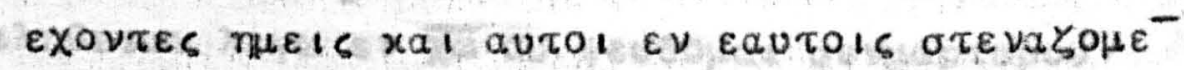

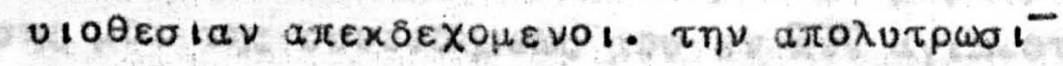

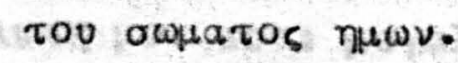

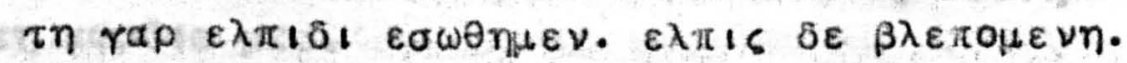

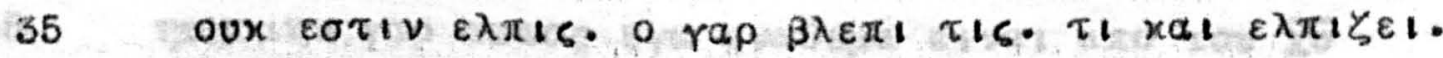

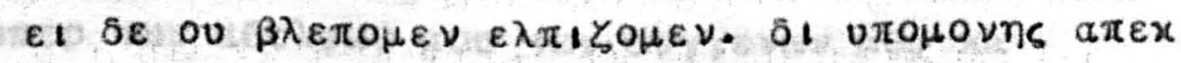

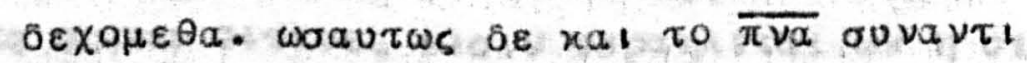

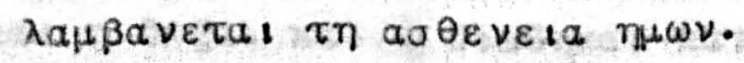

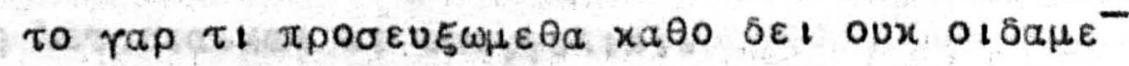
$a \lambda \lambda$ au

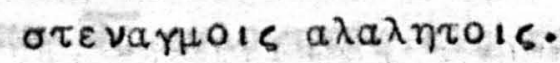




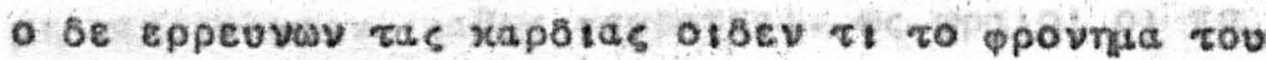

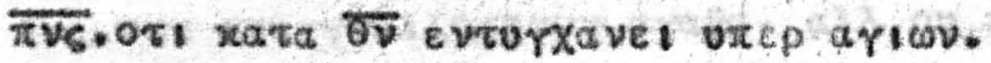

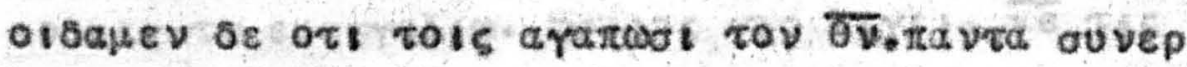

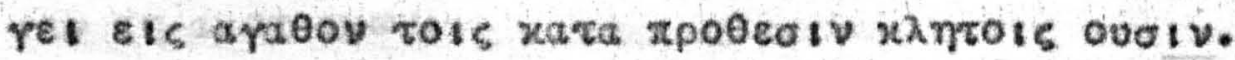

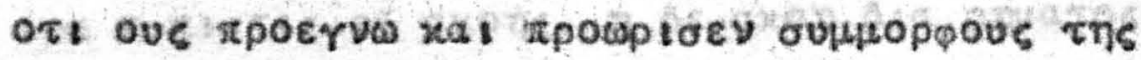
etxovos tou vo autou. els to etvat autov tparo

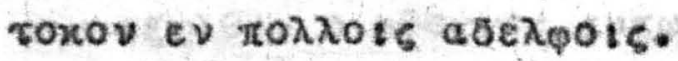

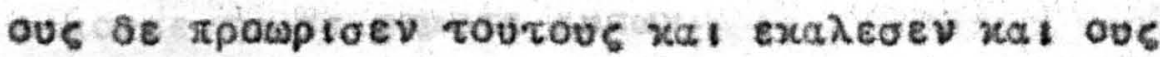

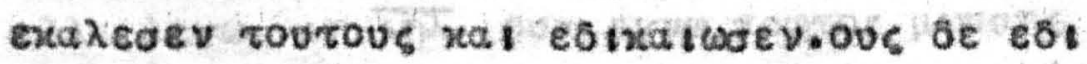
xa lwoev toutous xat esoğacev.

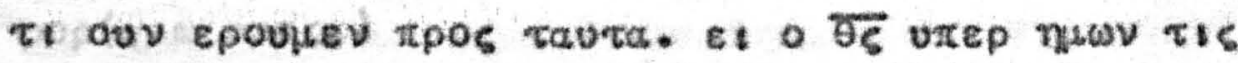
xas nuw. of re rov isiov viov oux eqeioato a $\lambda$

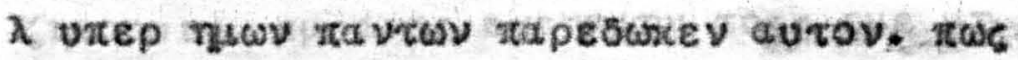
ouxt xat av aurw ta tavea tut $t$ Xaptoetal.

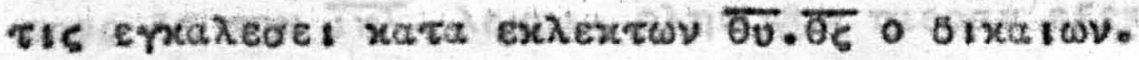
TIC o rartaxpt vesv.

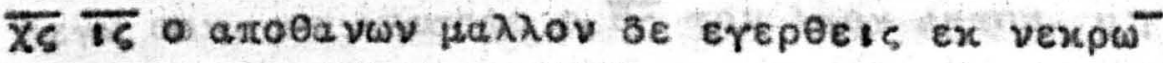

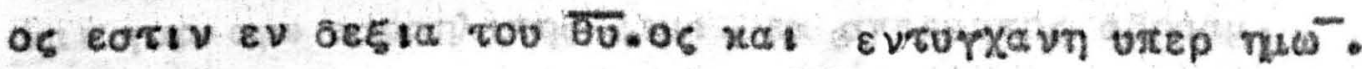

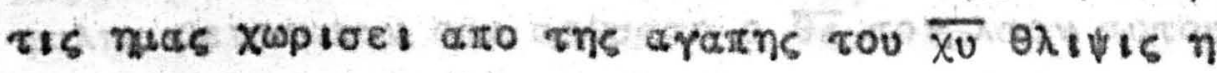

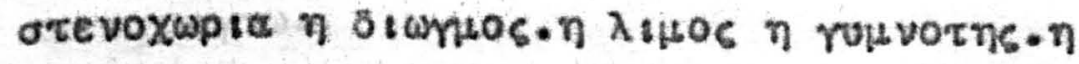

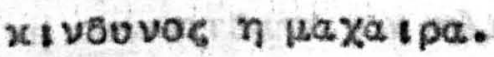

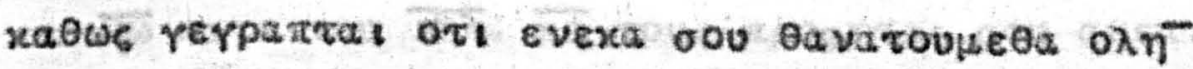

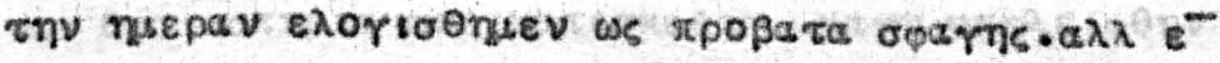

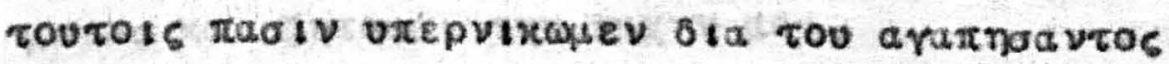

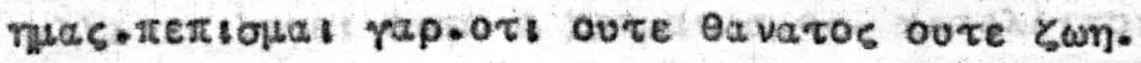
oure arrexol.oure apXa1.0ute egovatal.oure eve

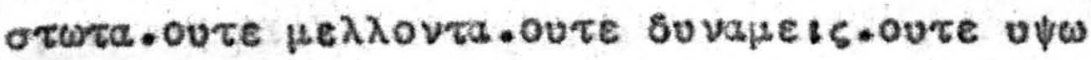

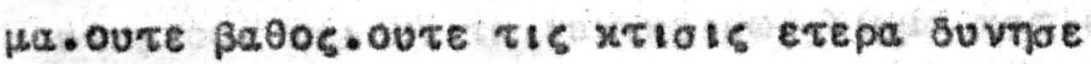

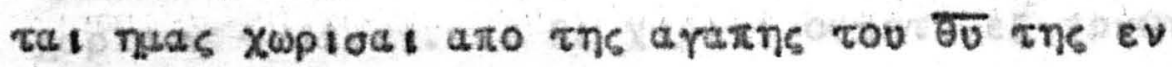

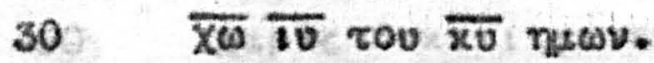

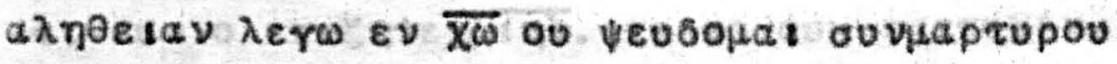

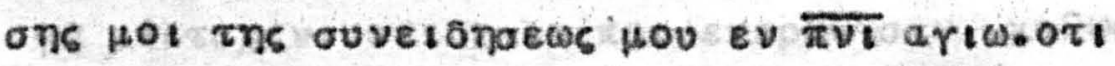

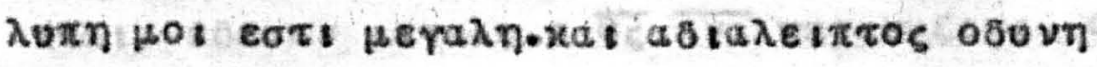

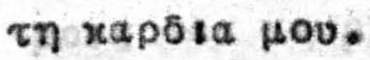

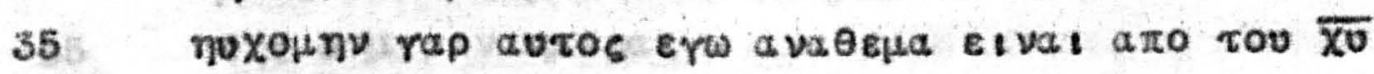

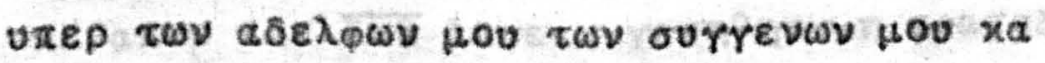

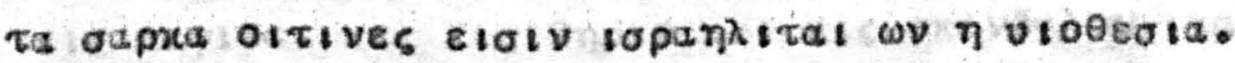

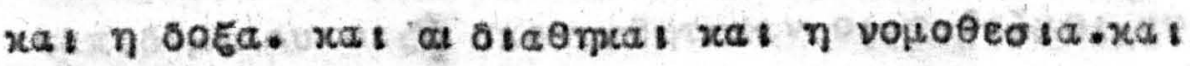

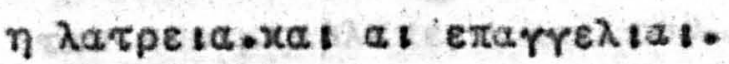

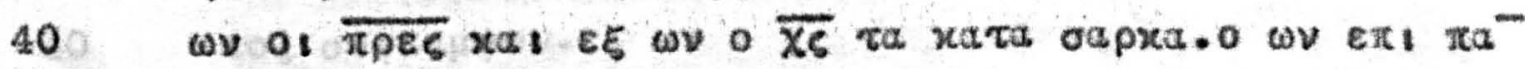

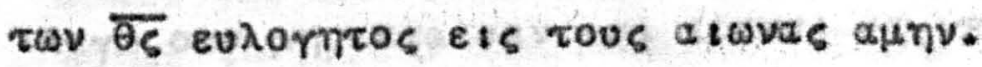




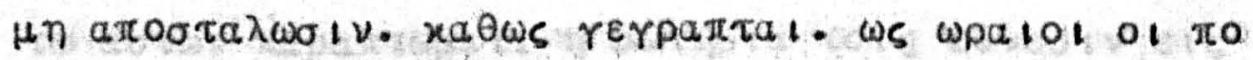

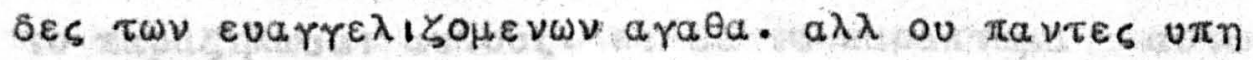

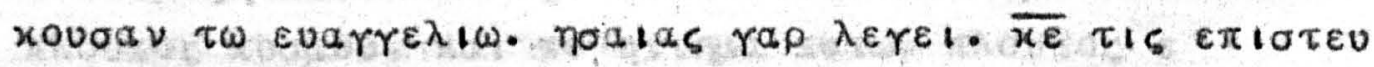

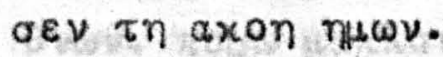

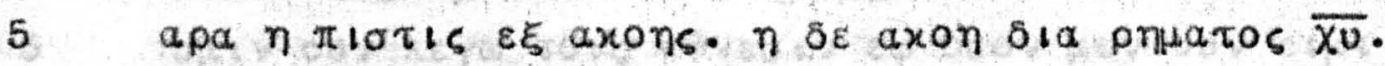

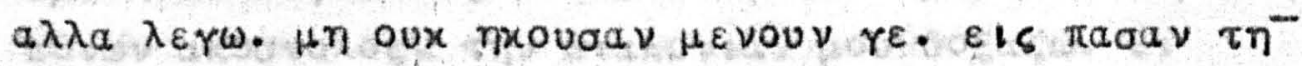

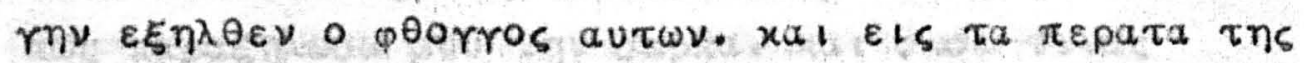

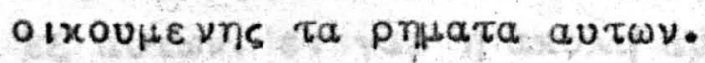

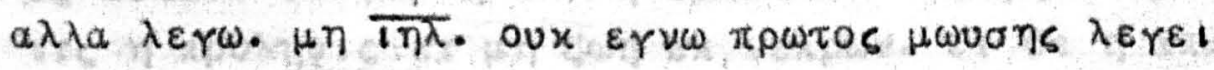

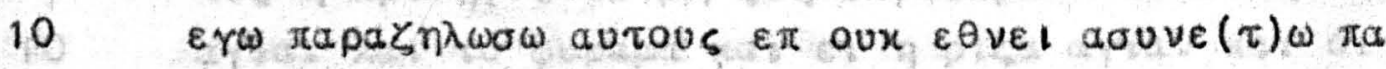
pop (riw uhas.)

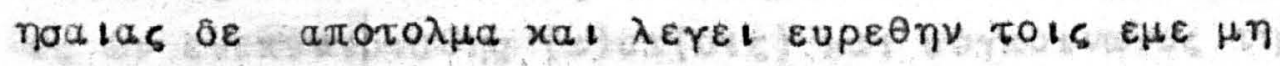

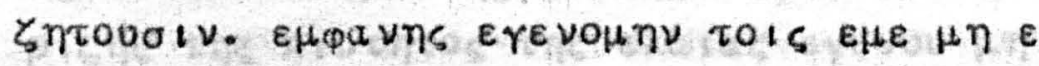
$\pi \varepsilon \rho \omega \tau \omega \sigma$ iv.

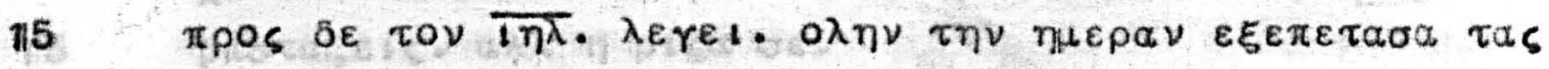

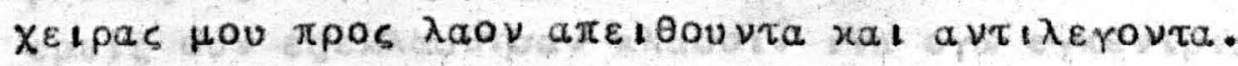

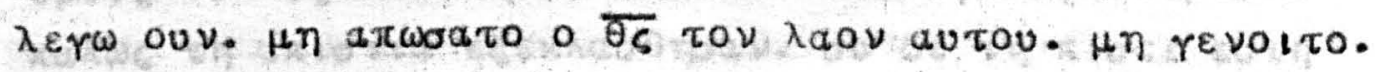

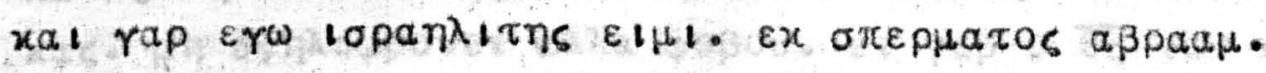

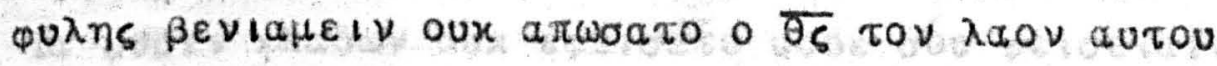

20 ov rporrvw.

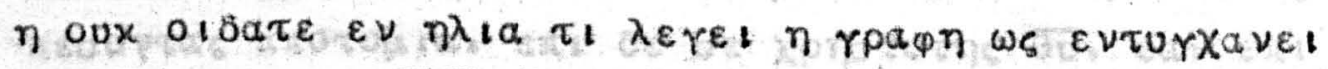

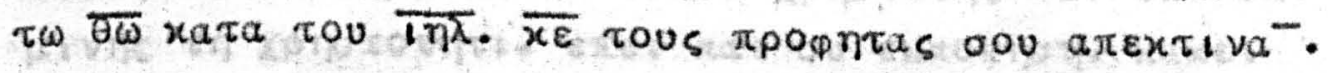

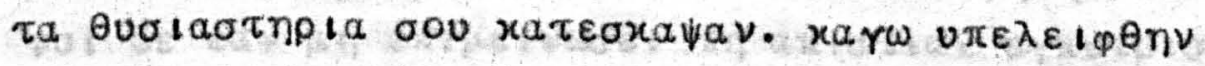

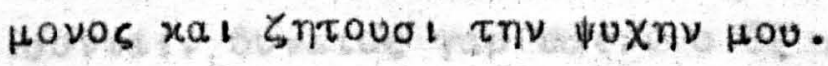

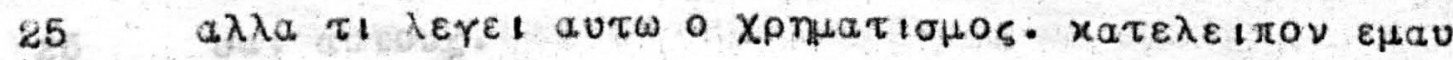

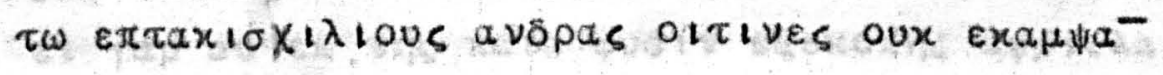
rovo in $\beta$ aad.

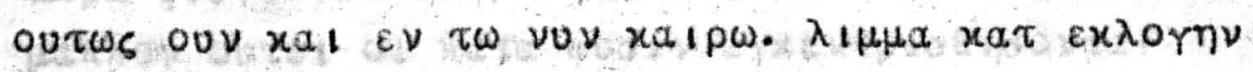

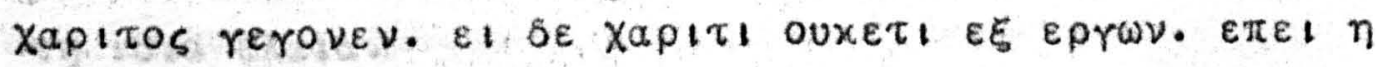
30 Xapls ouxerl rivetal Xapls.

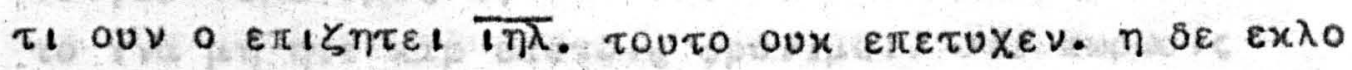

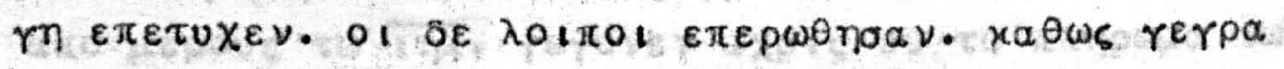

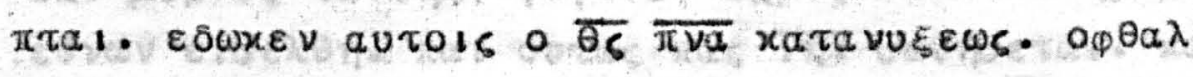
Hous tov $\mu \eta \beta \lambda \varepsilon \pi \varepsilon i v$ xal wra tov $\mu \eta$ axoueiv. ews

35 tns onнepov $\eta \mu \varepsilon p a s$.

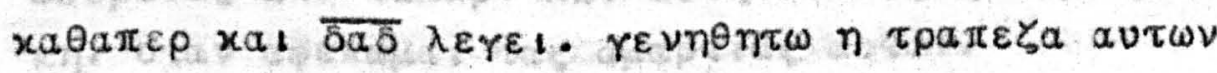

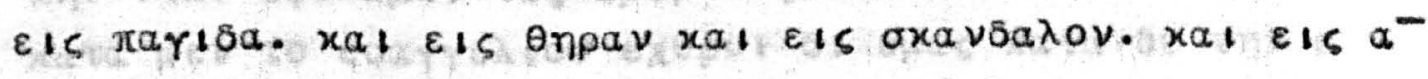

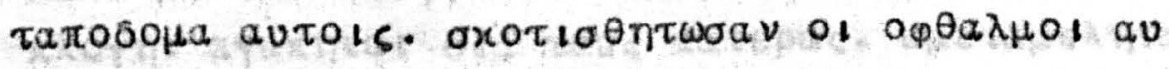

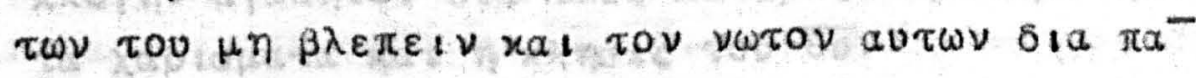
40 tos ouraptov.

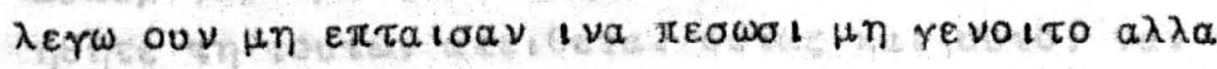




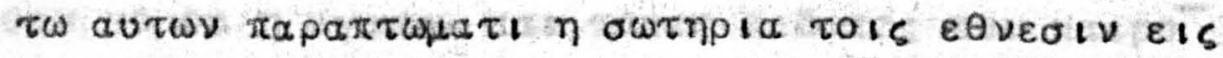

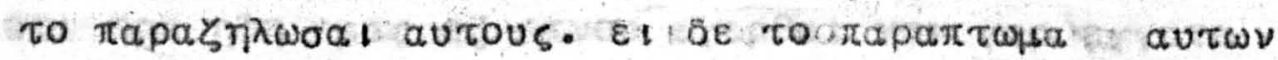

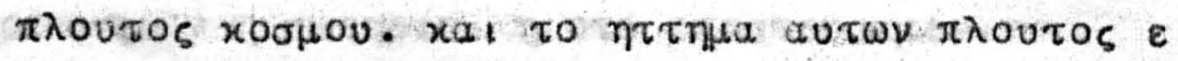

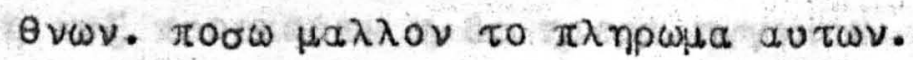

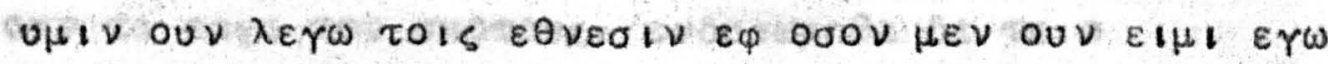

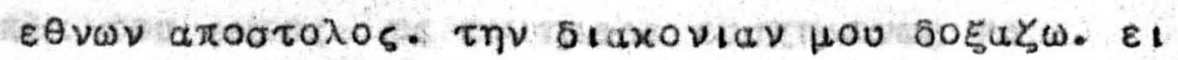

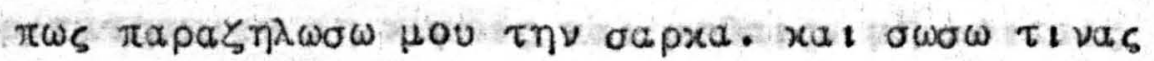

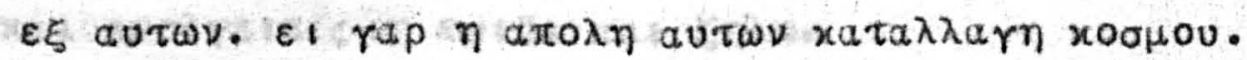

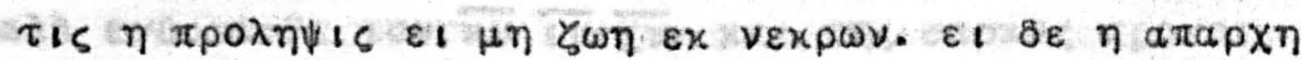

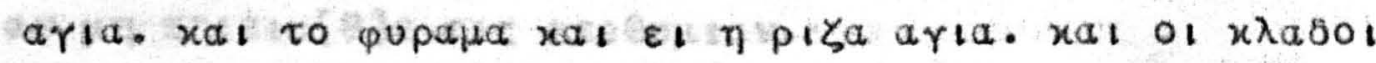

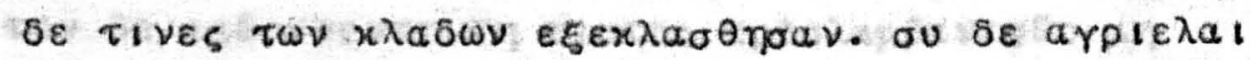

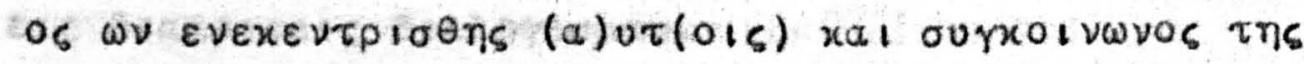

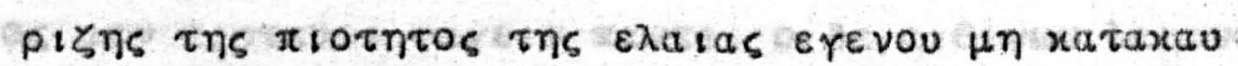

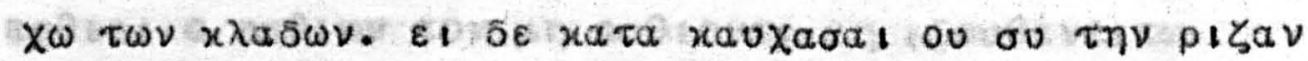

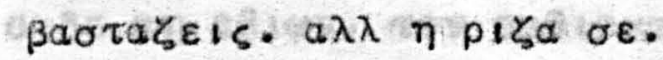

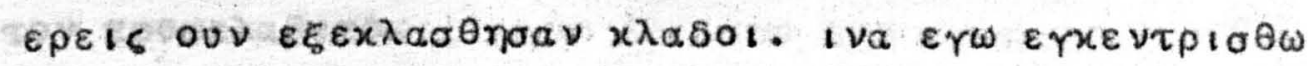

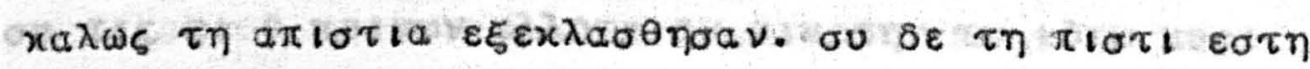

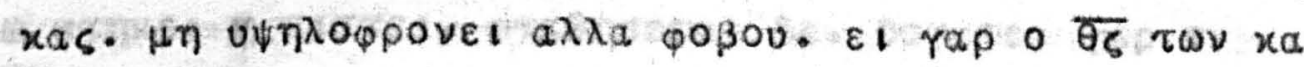

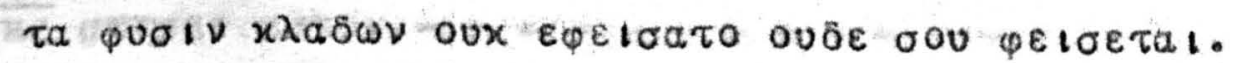

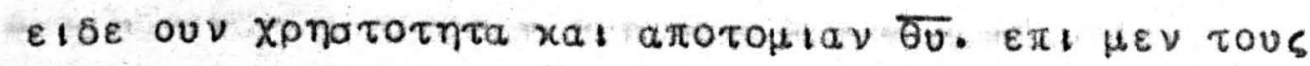

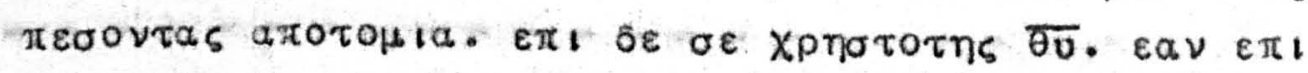

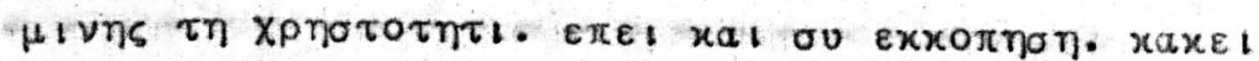

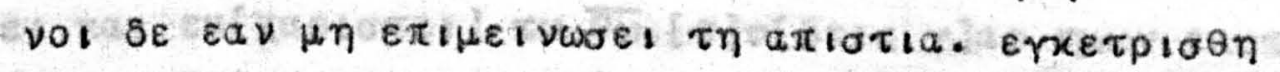

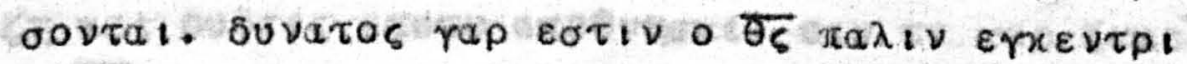
oat avous.

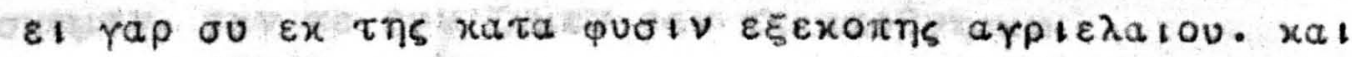

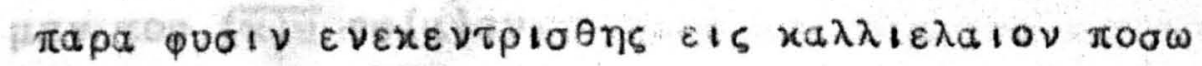

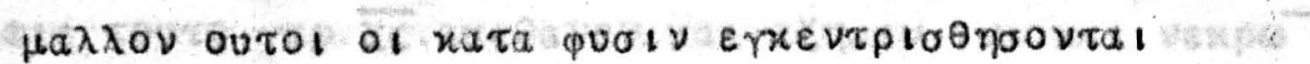

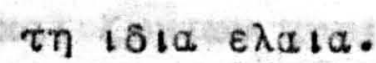

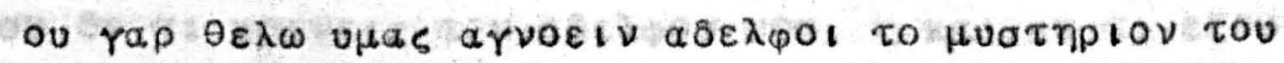

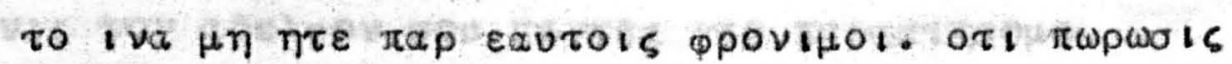

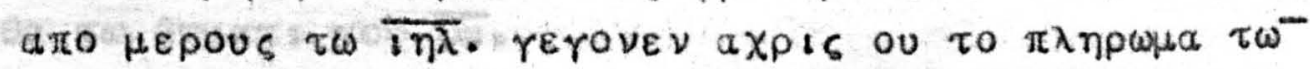

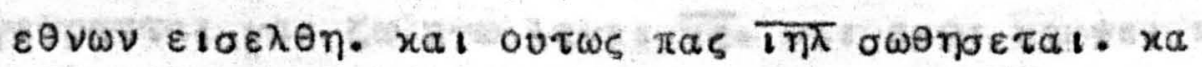

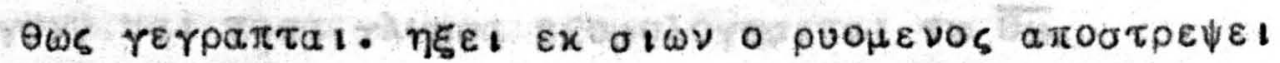

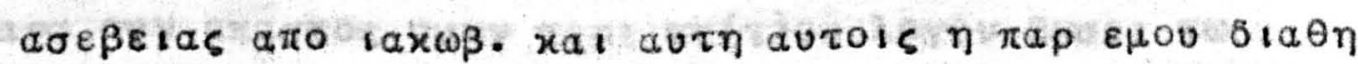

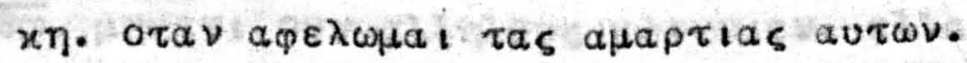

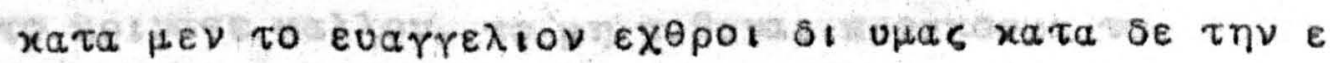

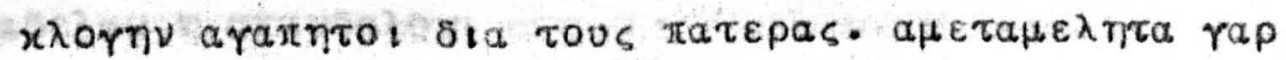

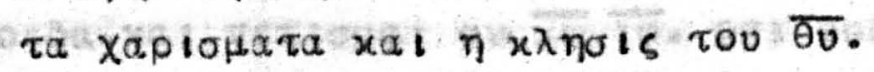

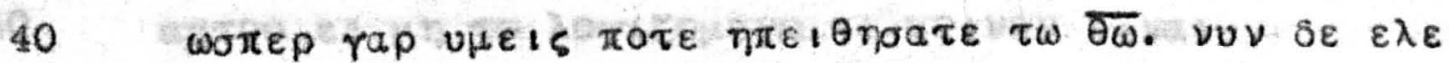

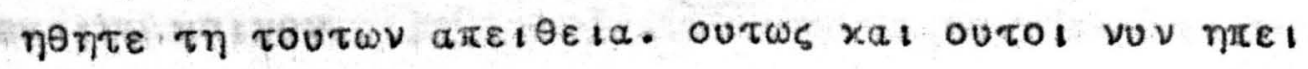




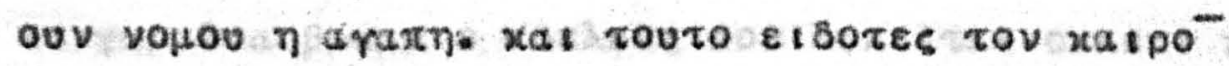

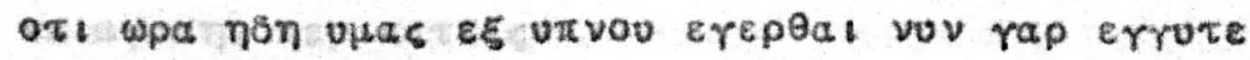

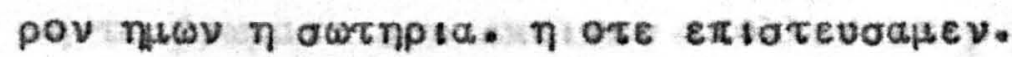

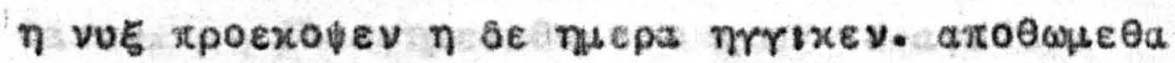

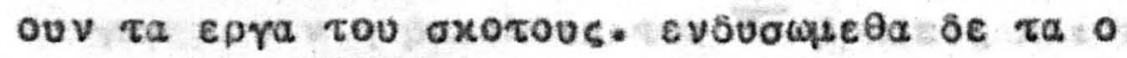

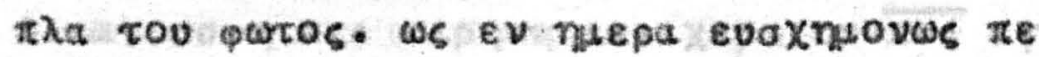

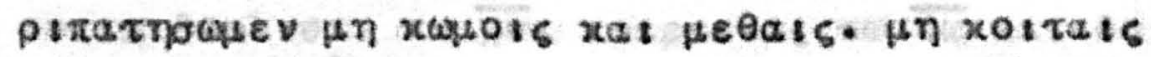

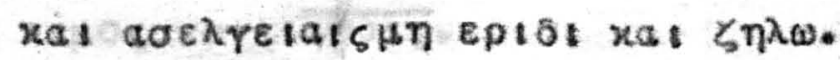

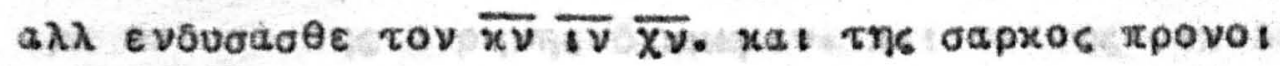

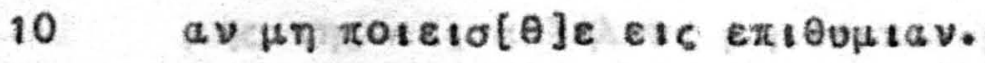

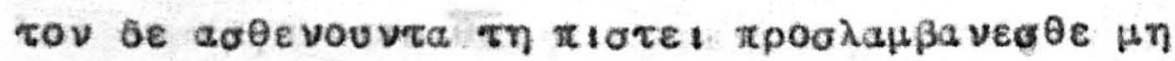
Ets ôtaxptoets otaxoyrohw.

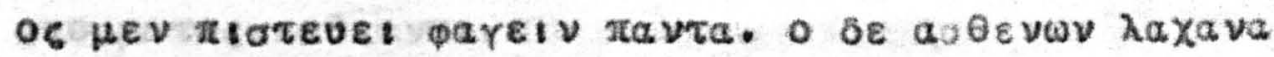

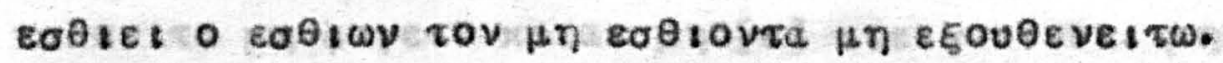

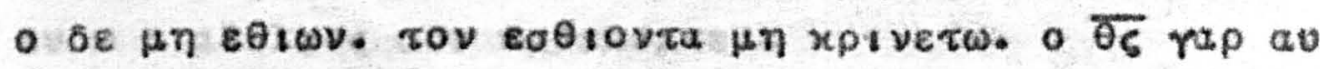

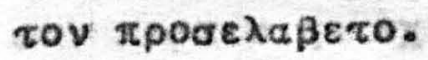

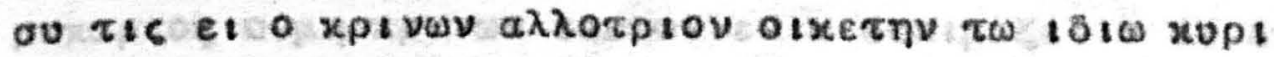

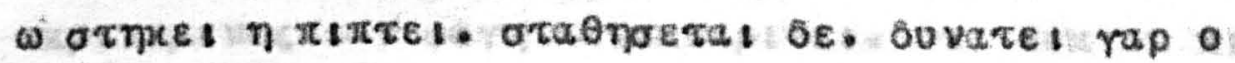

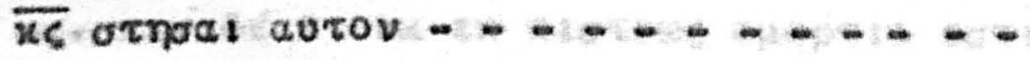

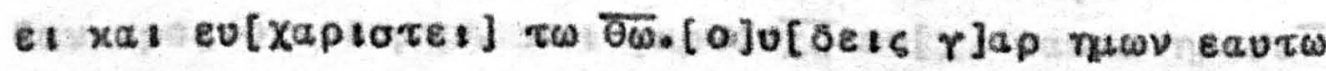

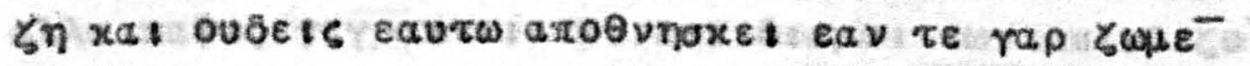

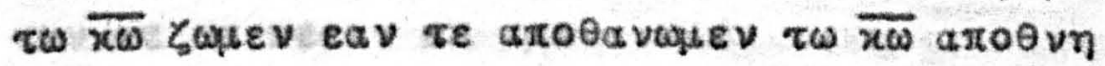

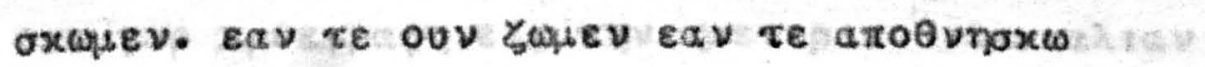
Hev rov $(\bar{x}) 0$ ea $(\mu)$ ev.

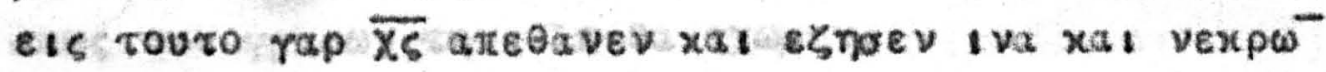
xat לovtuv xeptevorn.

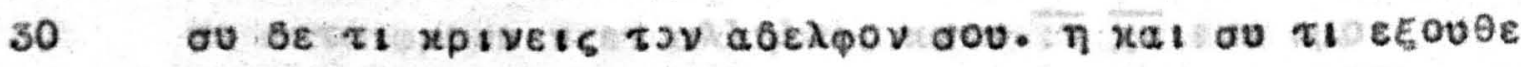

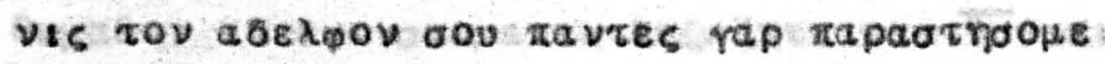

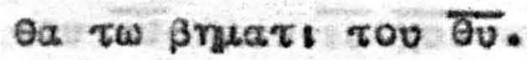

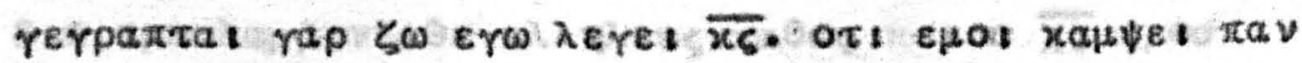

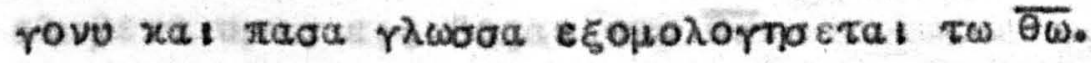

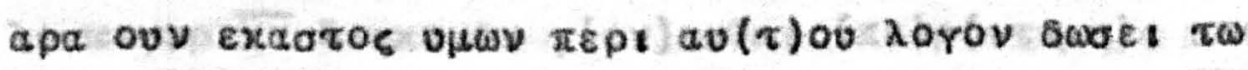

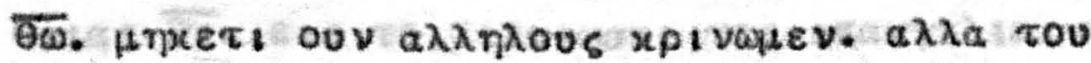

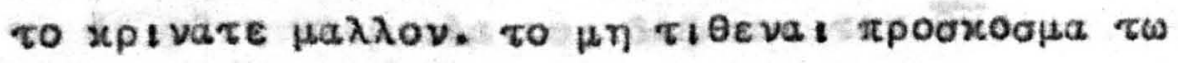

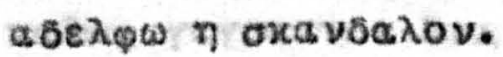

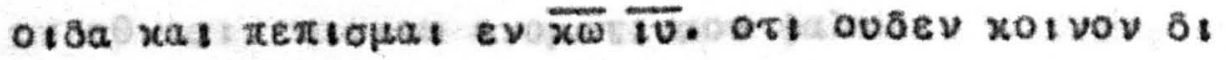

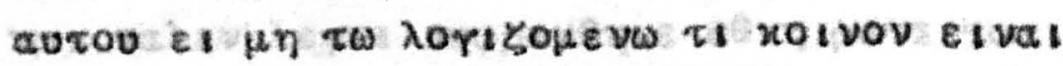
exe IVw xotvov. 


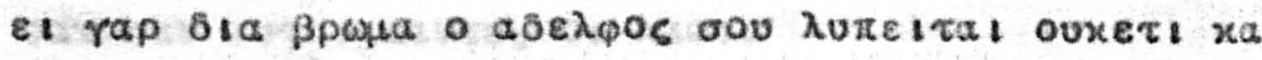

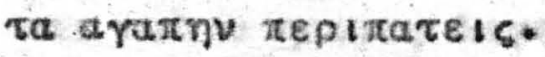

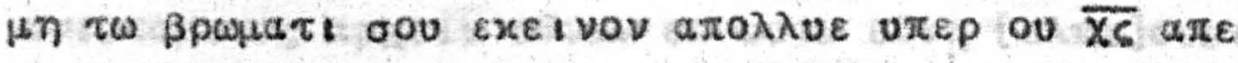

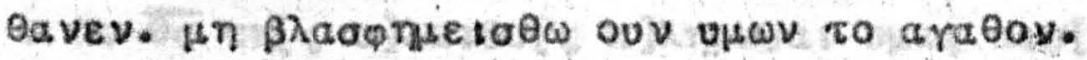

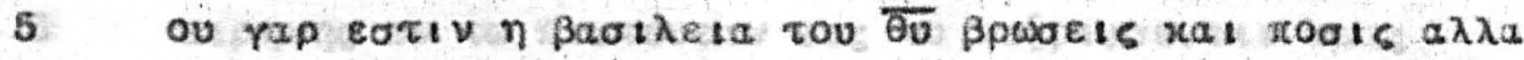

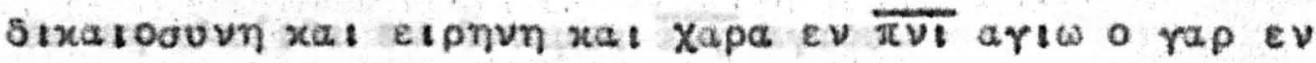

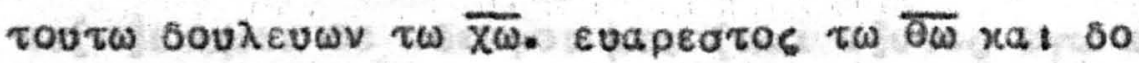

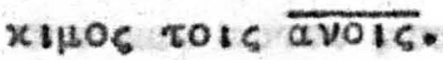

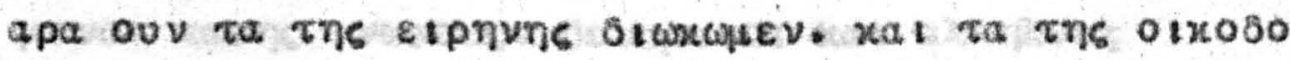

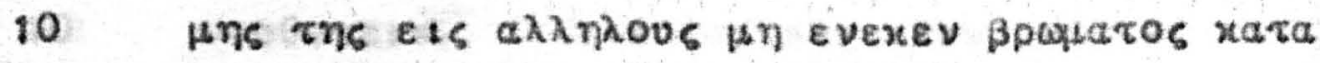
$\lambda v e$ ro eprov rov

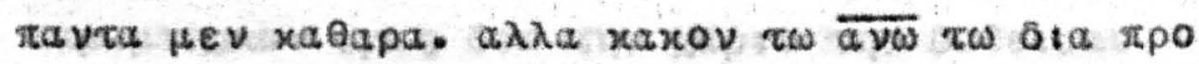
axojuarog $80010 \mathrm{vi}$.

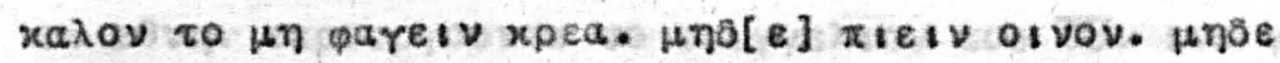

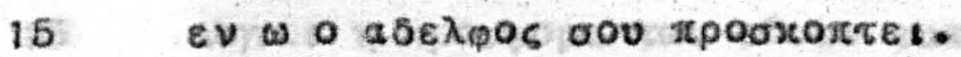

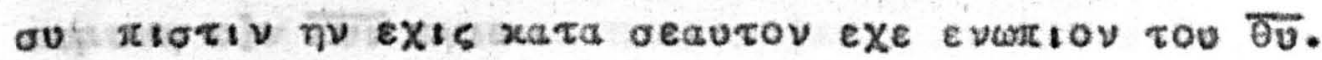

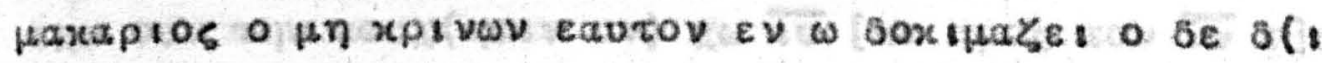

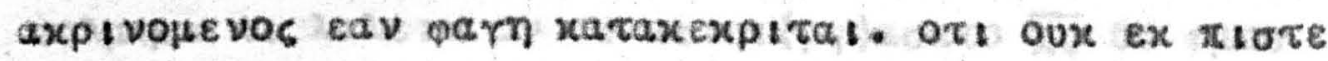

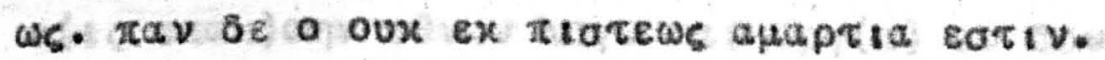

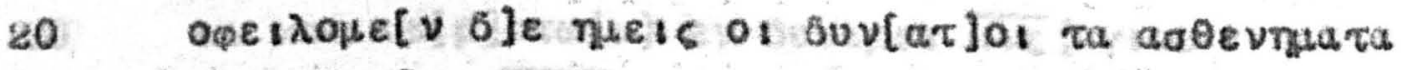

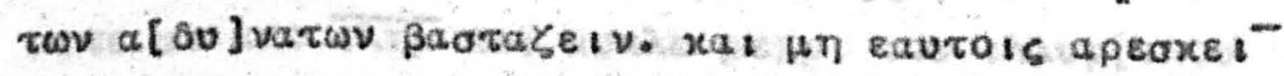

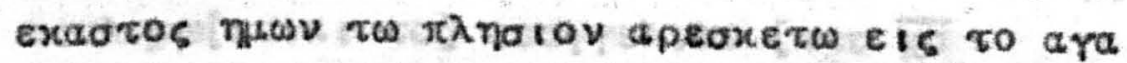

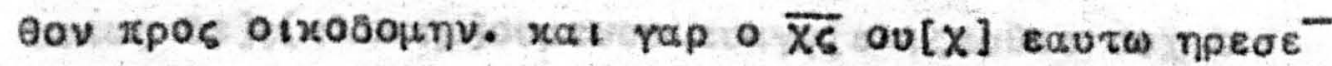

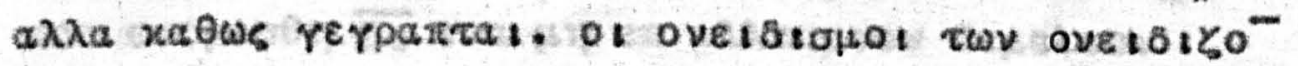

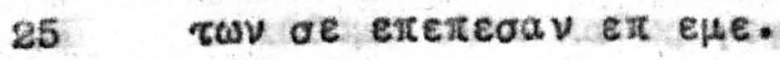

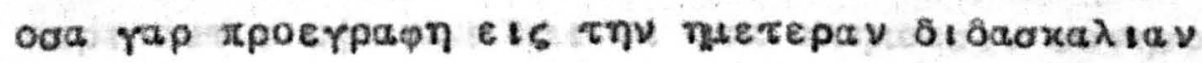

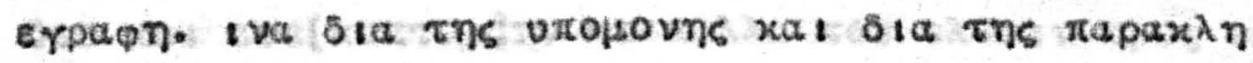

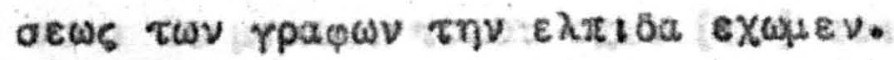

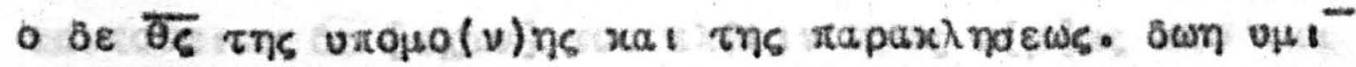

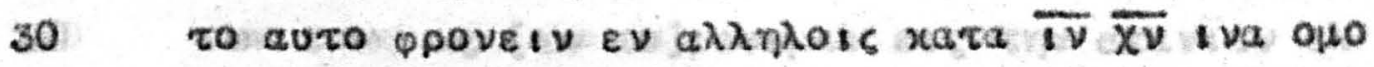

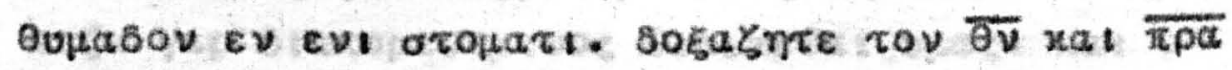

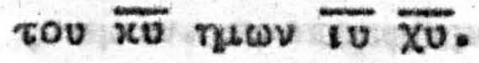

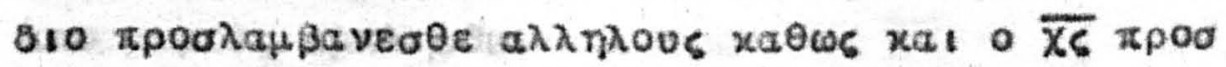

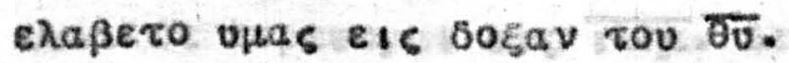

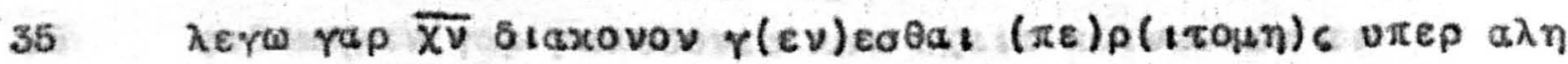

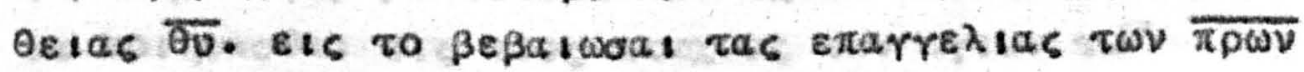

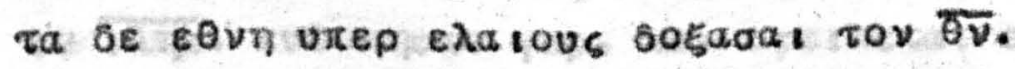

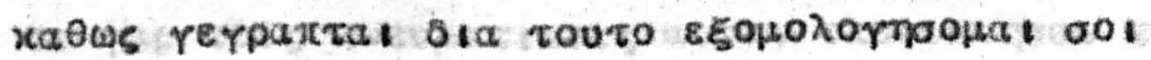

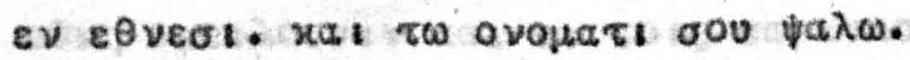

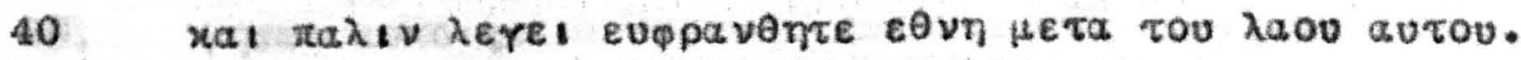

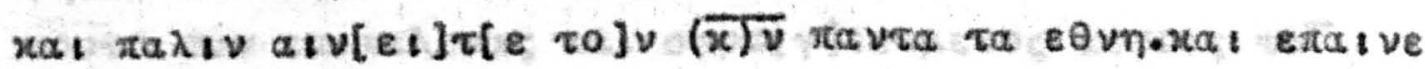




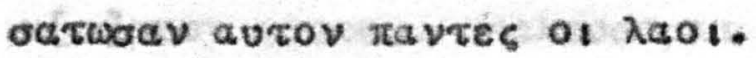

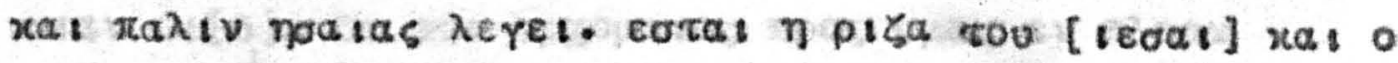

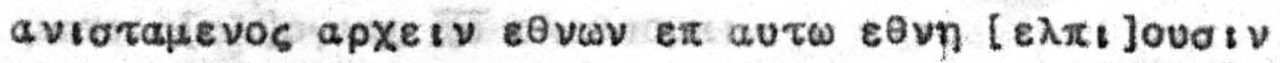

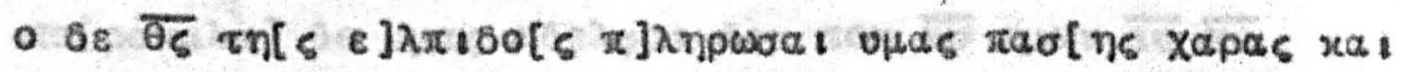

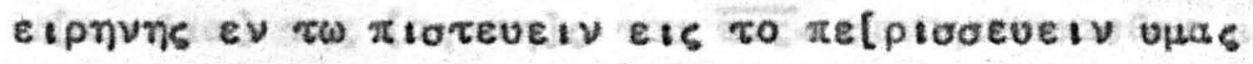

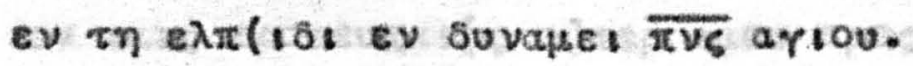

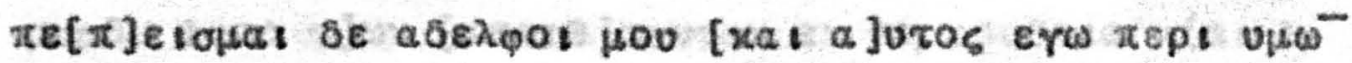

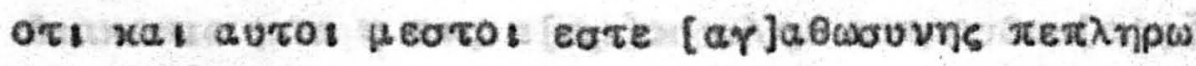

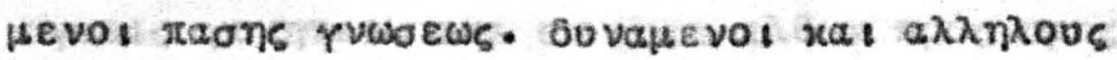
voverceเv.

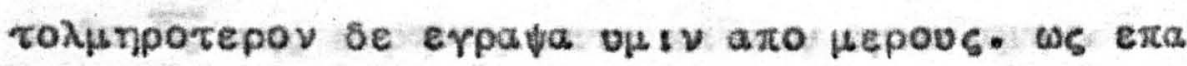
vaน

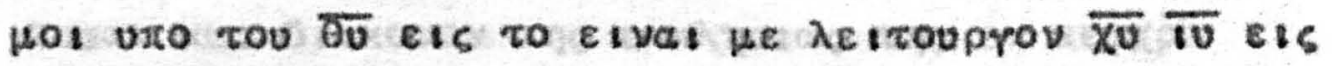

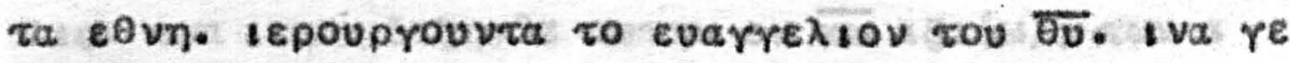

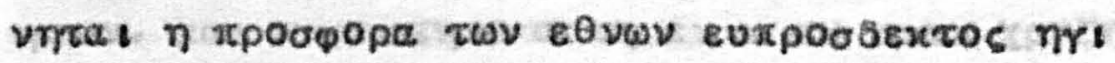

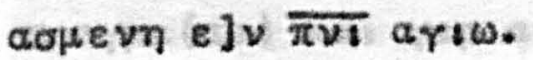

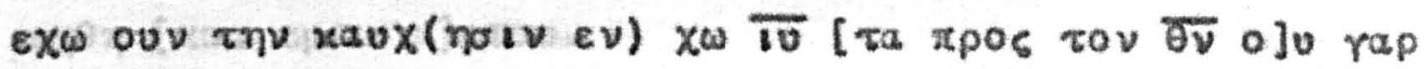

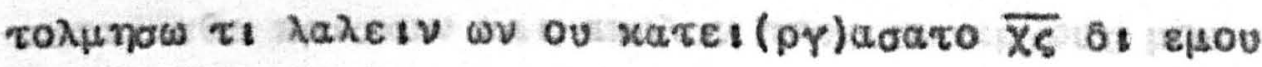

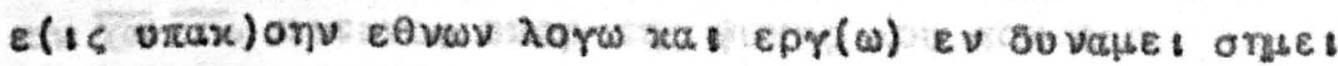

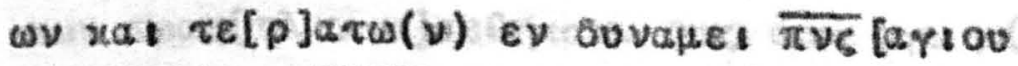

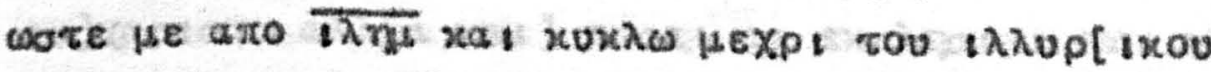

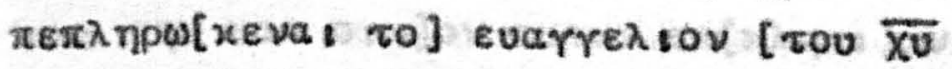

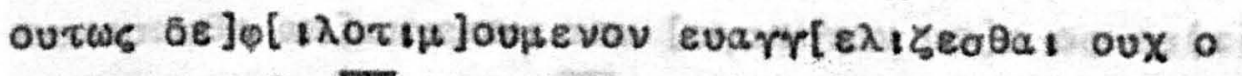

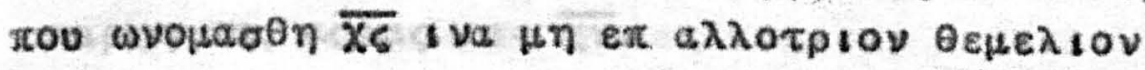

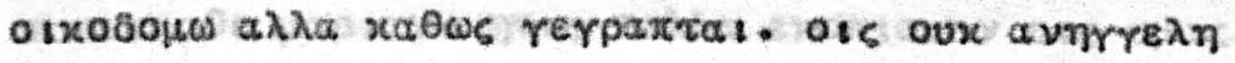

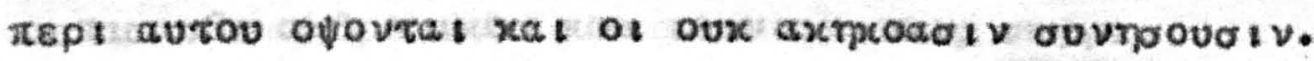

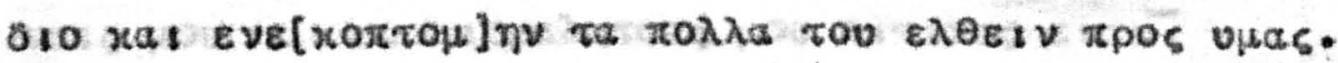

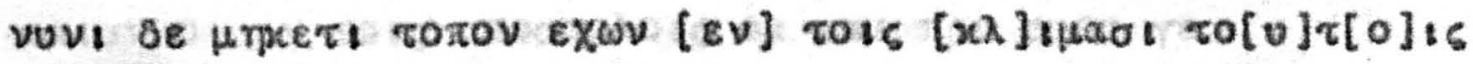

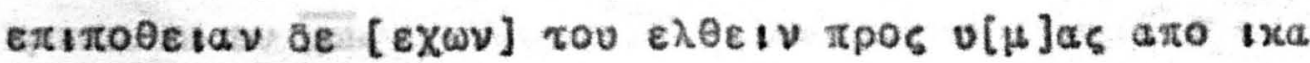

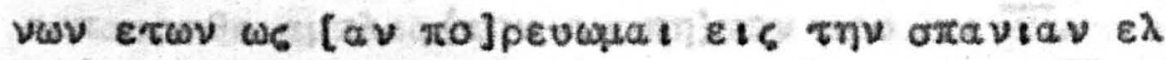

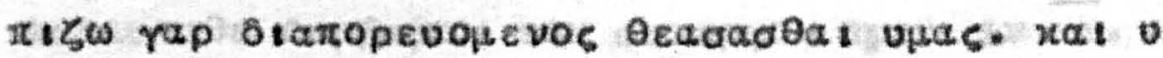

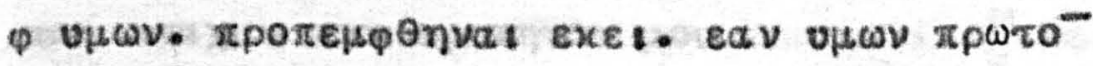

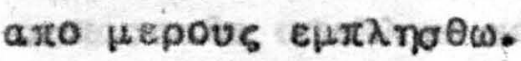

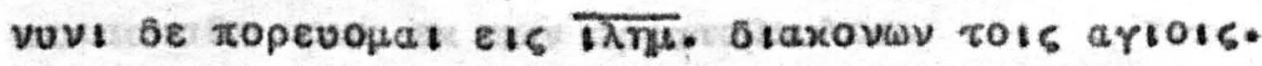

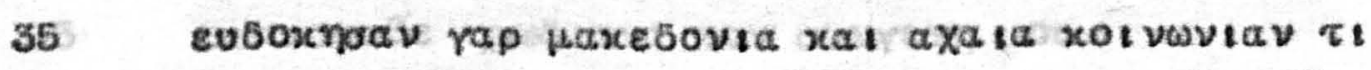

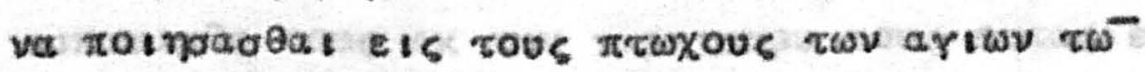

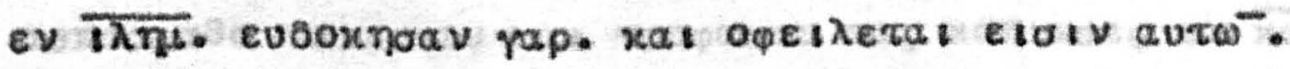

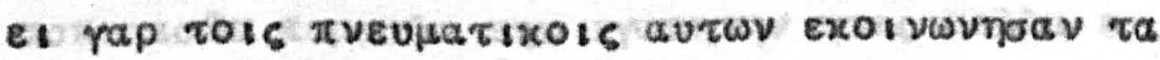

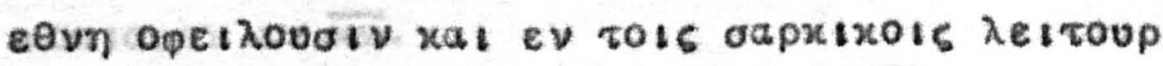

40, mace avrois.

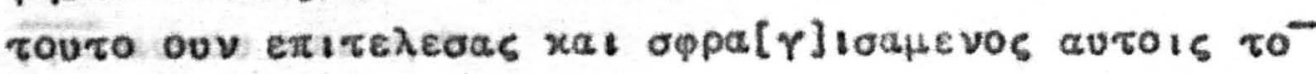




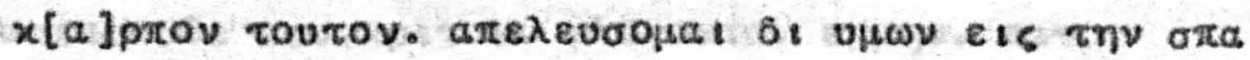

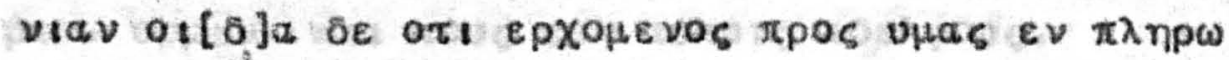

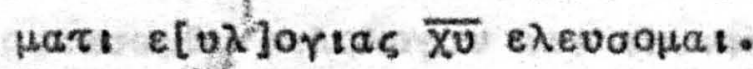

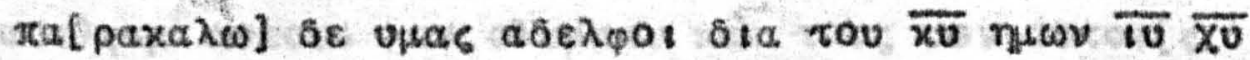

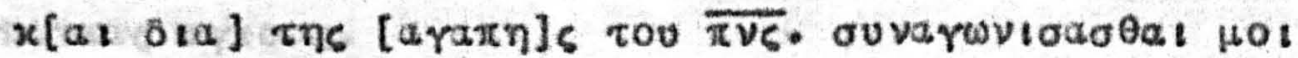

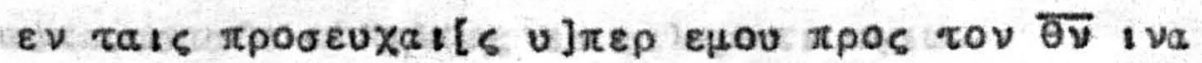

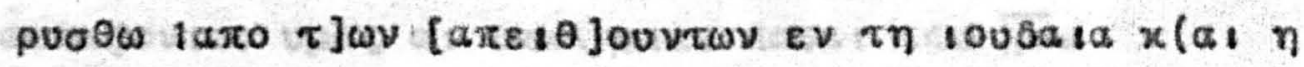

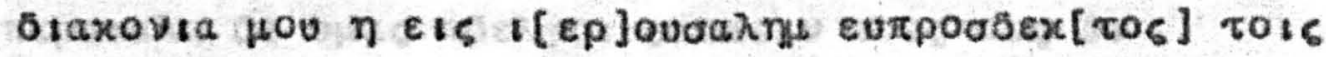

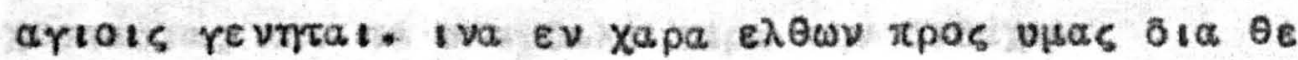

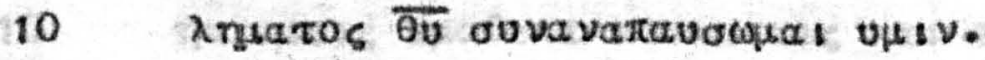

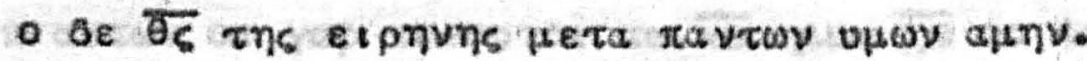

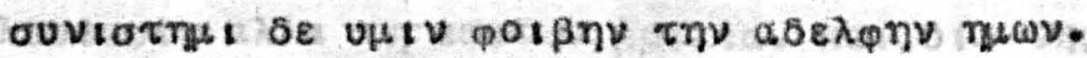

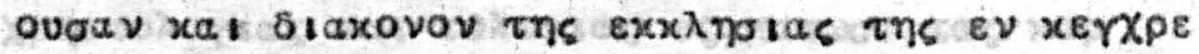

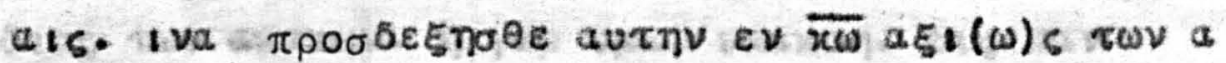

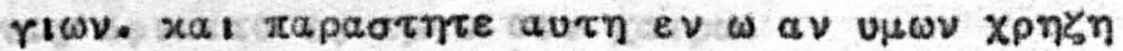

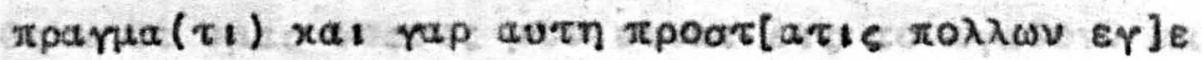

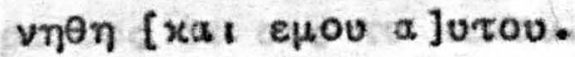

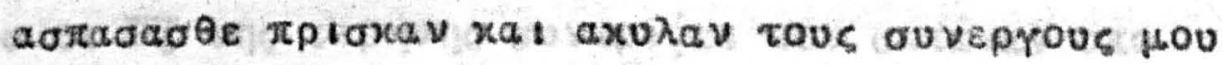

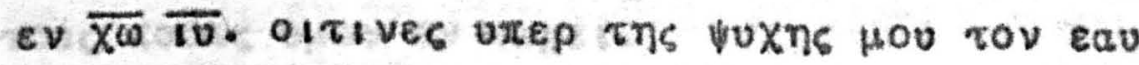

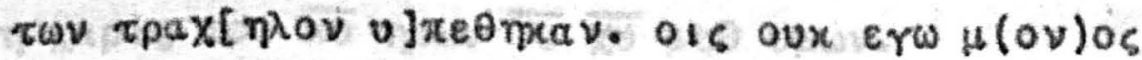

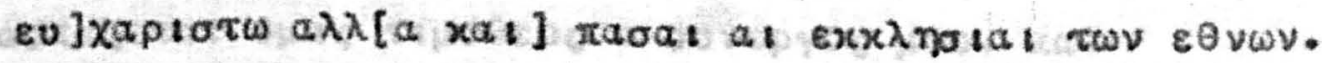

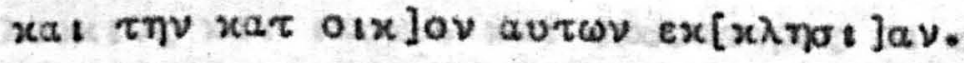

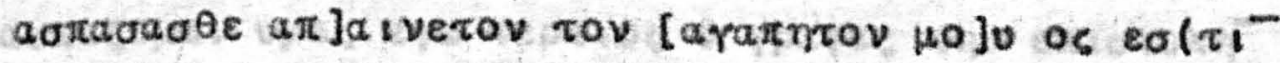
arap $\rho \eta \eta \bar{s}$ arsas $\varepsilon i s \overline{X v}$.

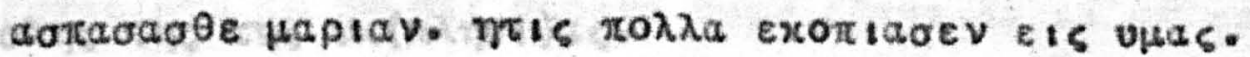

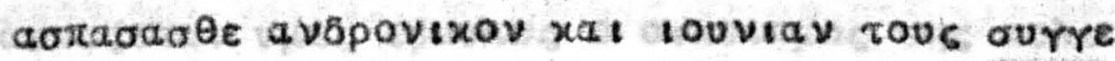

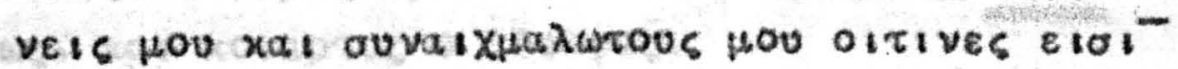

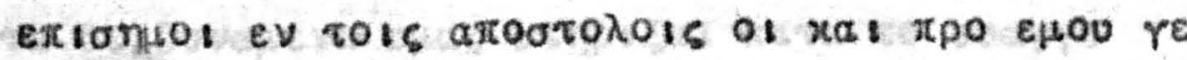
rovact $\varepsilon v \bar{X}$.

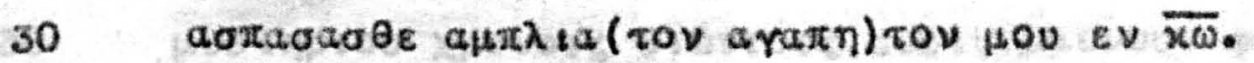

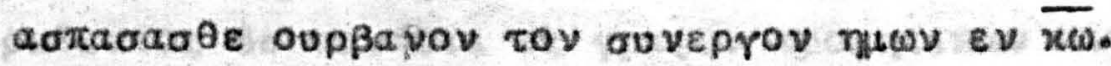

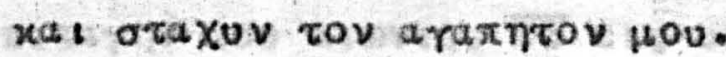

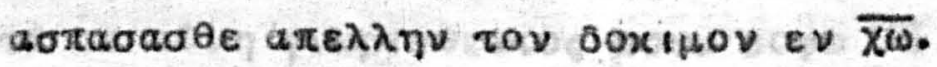

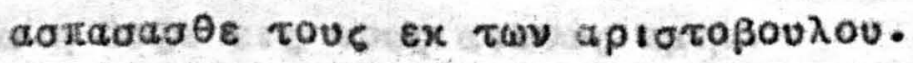

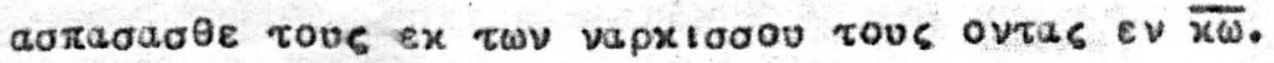

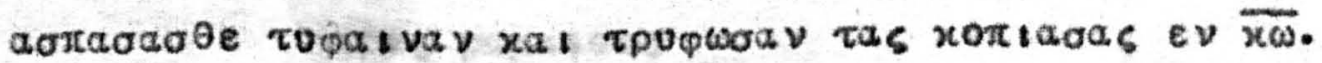

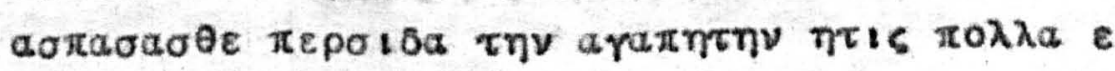
xortaoev $\& \nu \overline{x \omega}$.

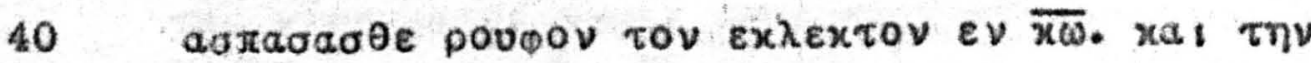

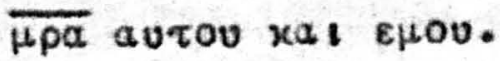




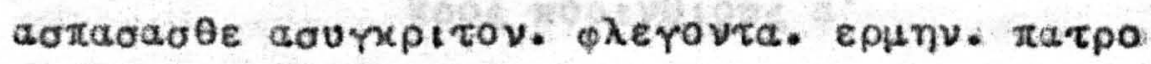

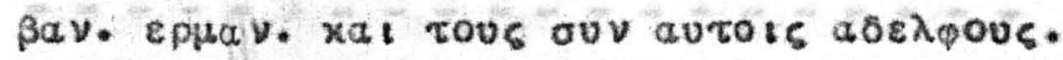

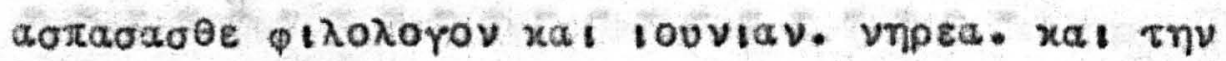

abe

5 ravtas arious.

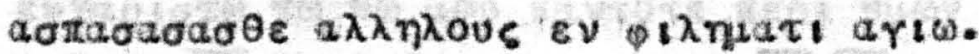

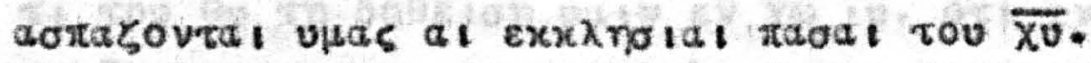

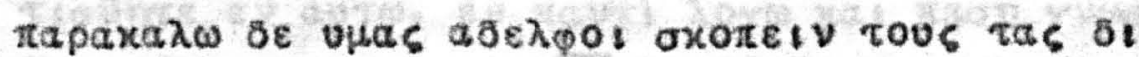

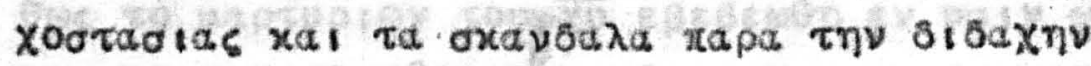

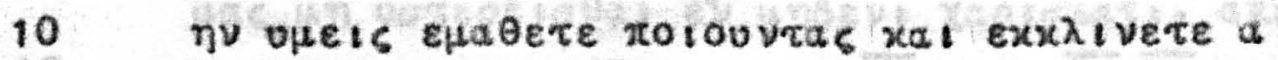

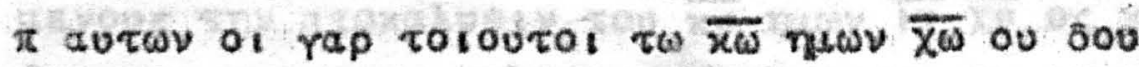

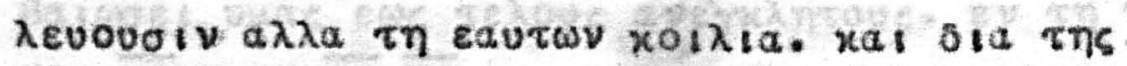

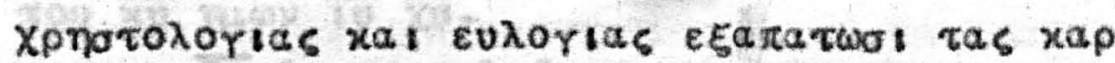

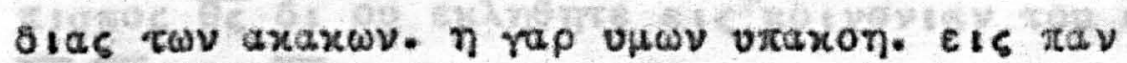

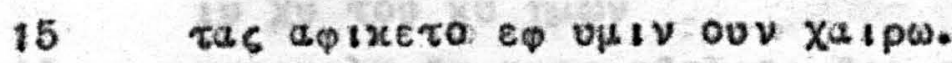

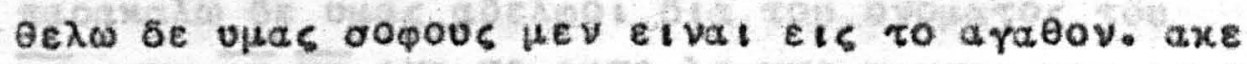
palovs oe els to xaxov.

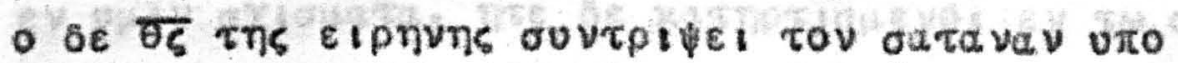

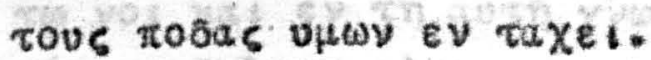

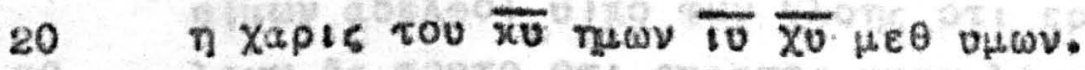

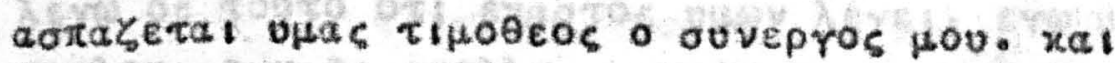

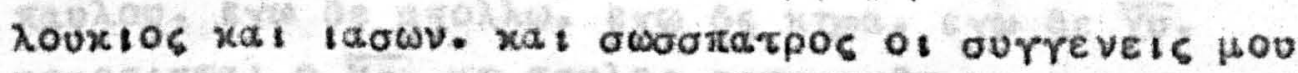

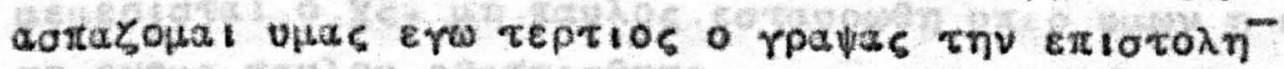

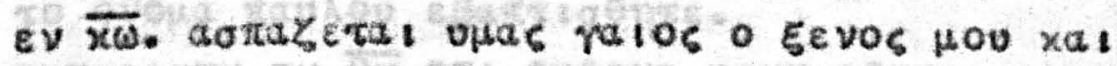

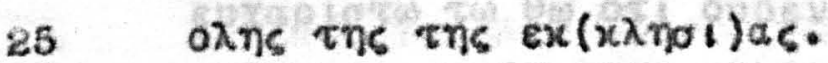

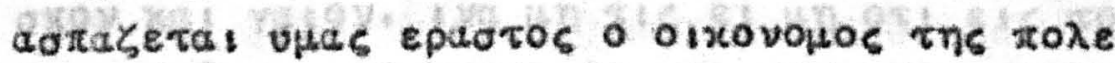

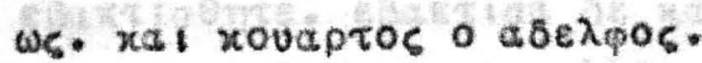

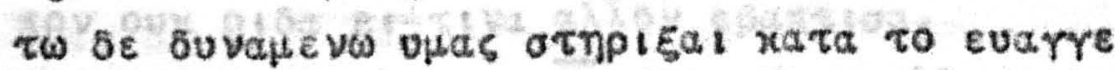

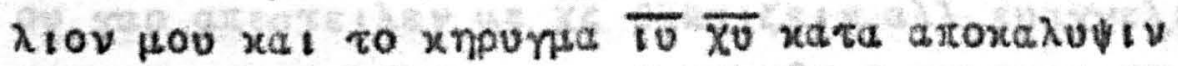

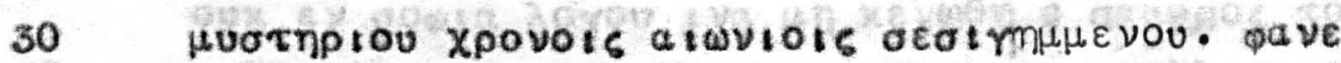

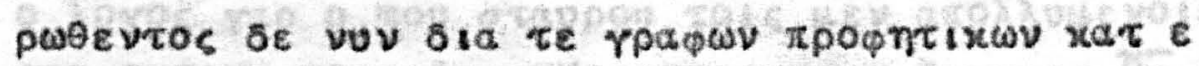

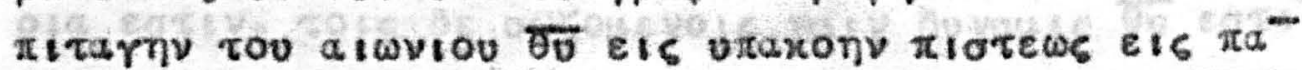

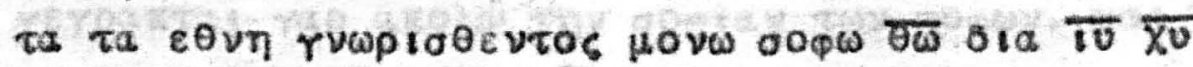

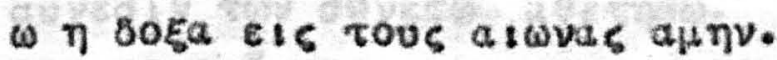

$\pi \rho{ }_{6}$ pwatalove

$\pi$ pos paqa iovs $16.14-27$ 


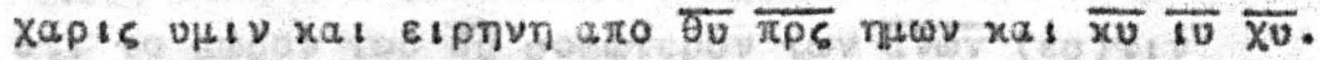

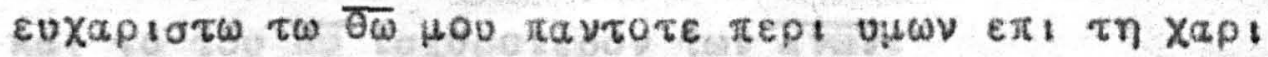

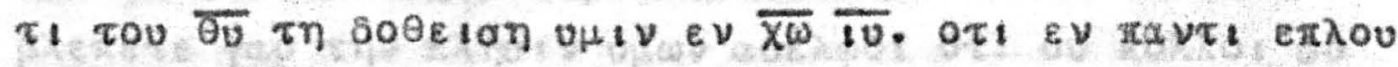

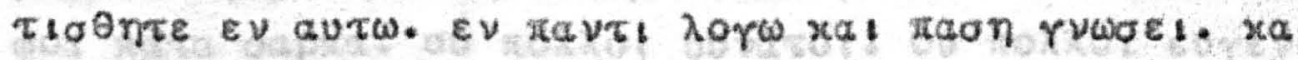

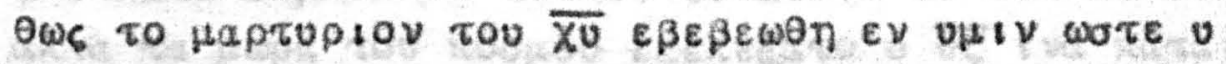

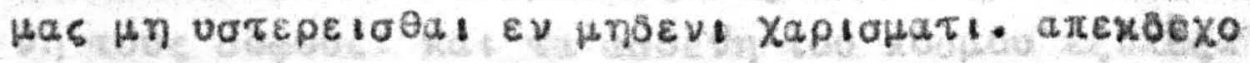

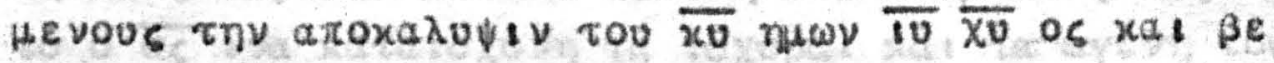

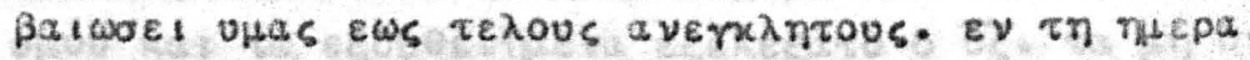

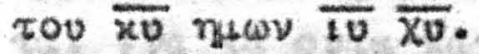

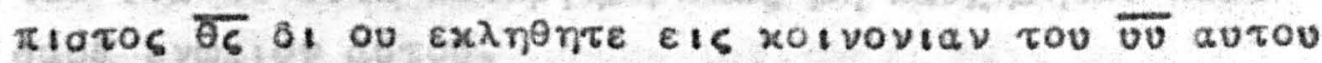
TV $\overline{X 0}$ rov $\overline{x u}$ nuav

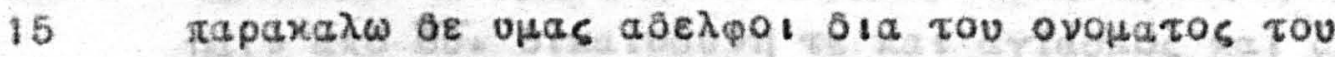

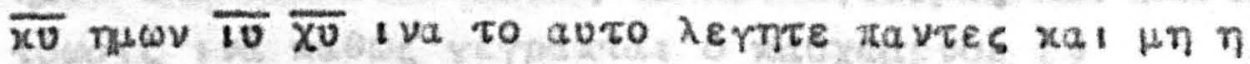

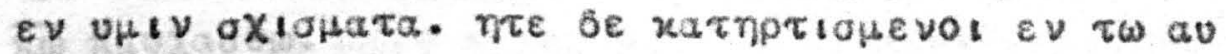

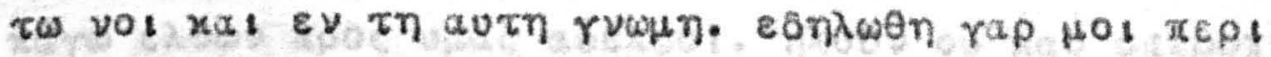

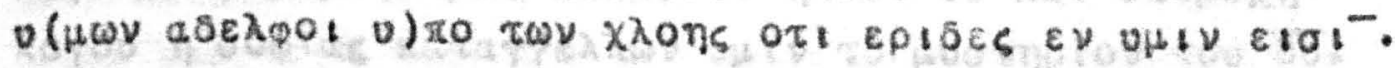

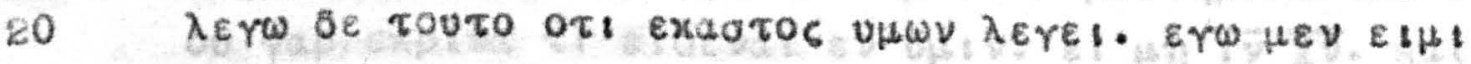

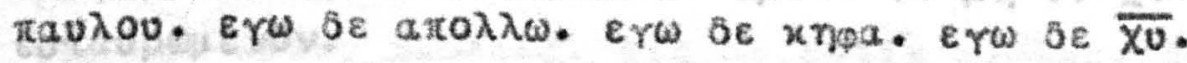

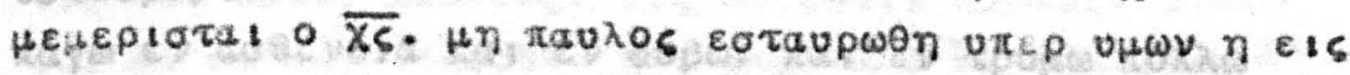

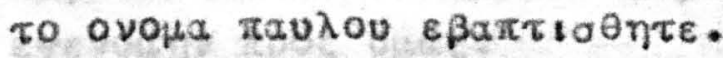

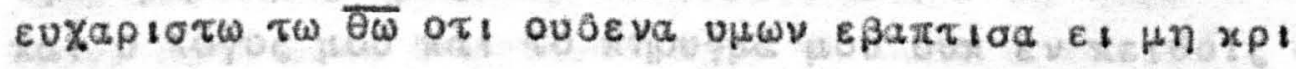

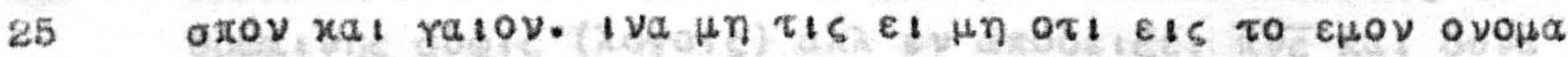

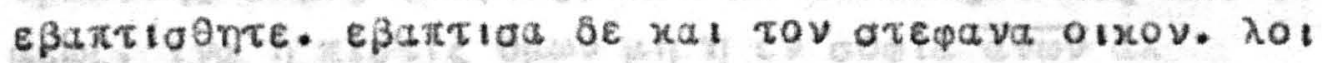

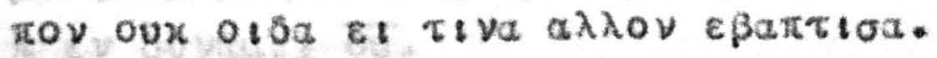

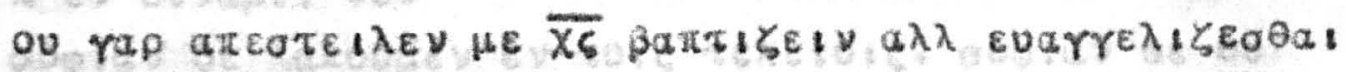

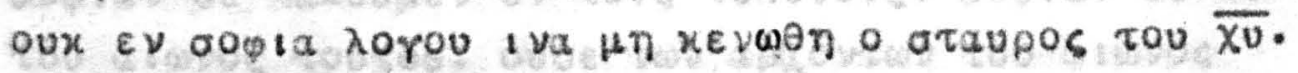

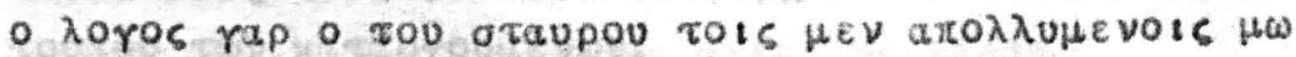

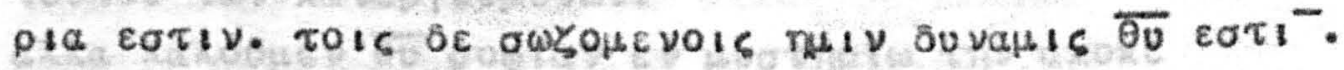

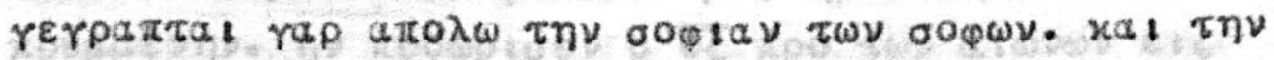

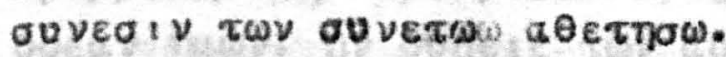

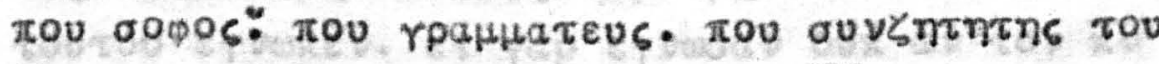

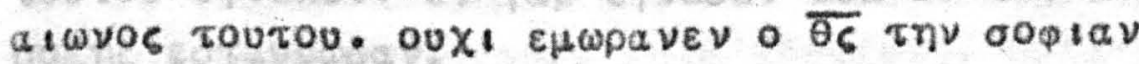

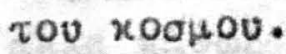

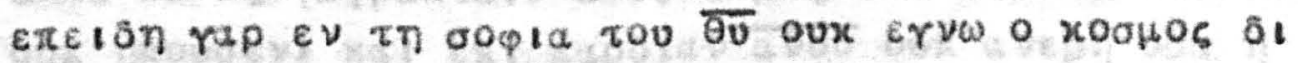

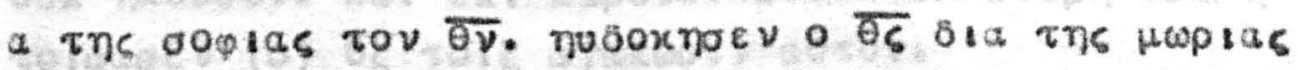
rou xnpurpatos owal tous tiotevortas.

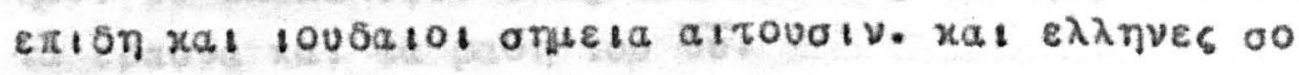

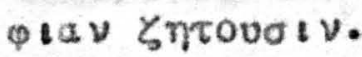




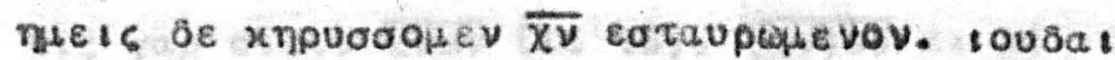

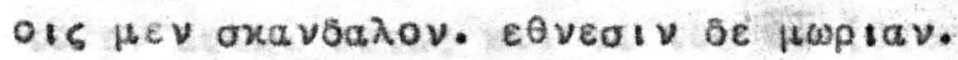

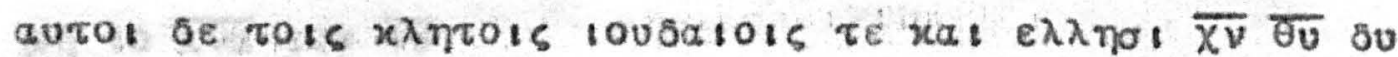
vauiv $x \alpha$ i $\overline{\theta 0}$ oop $1 \alpha \nu$.

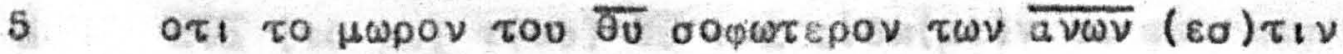

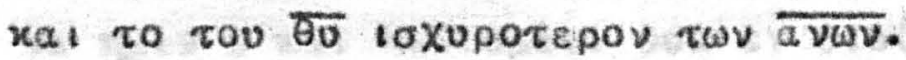

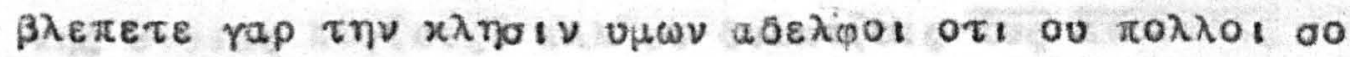

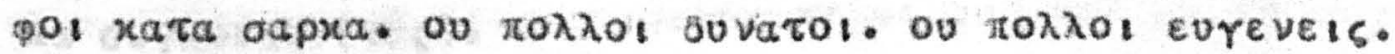

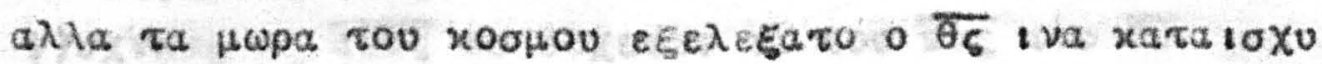

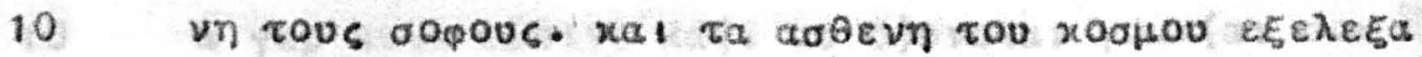

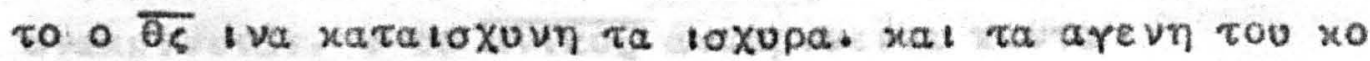

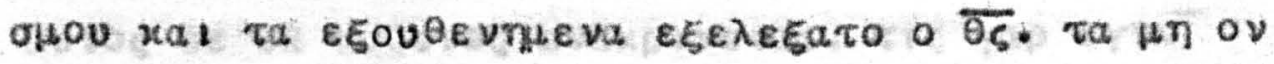

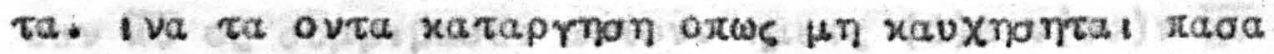
aup $\varepsilon$ vantiov autou.

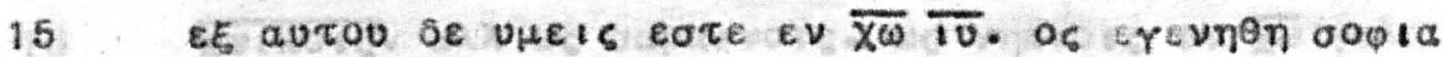

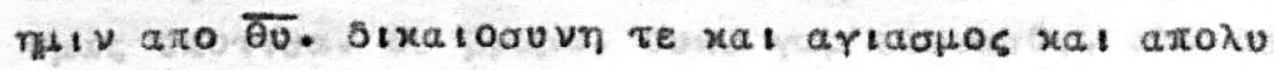
rpwots tva xaOws yerpartal o xauxwievos $\varepsilon v$

$\overline{x \omega}$ xav $\times a \sigma \theta \omega$.

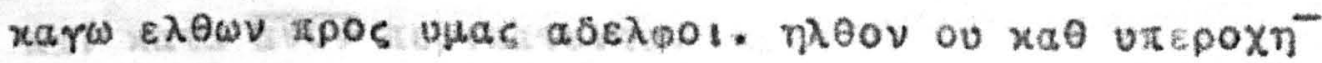

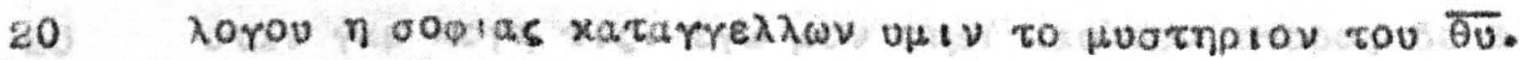

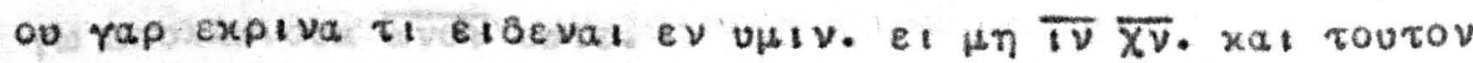
Eoraupwuevov.

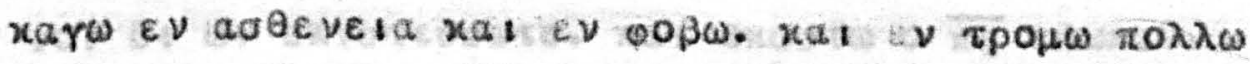

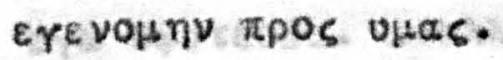

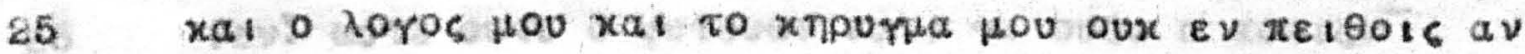

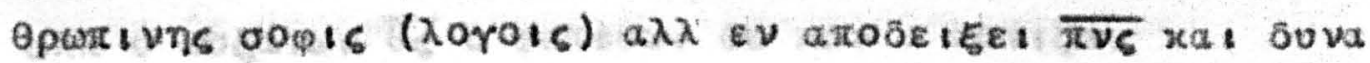

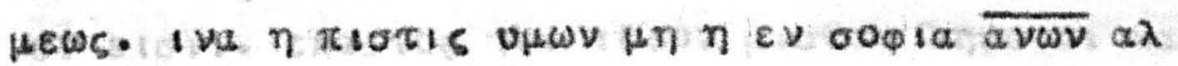
$\lambda \varepsilon v$ ovvauel $\overline{\theta u}$.

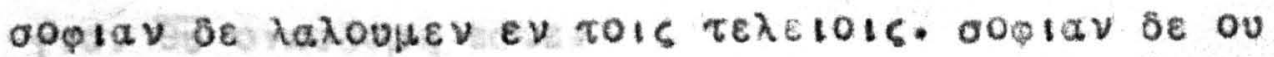

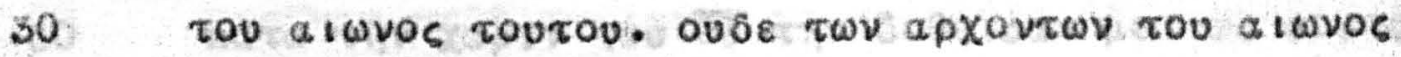

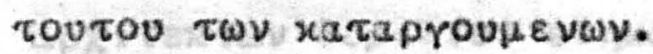

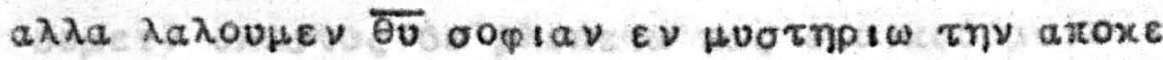

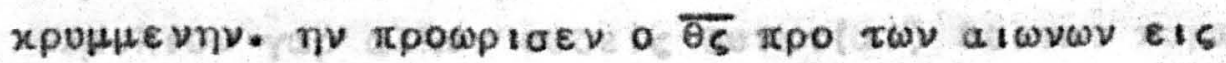

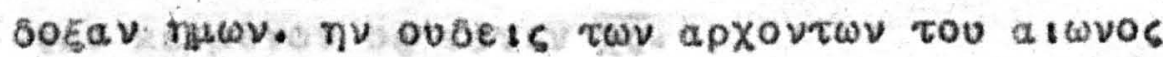

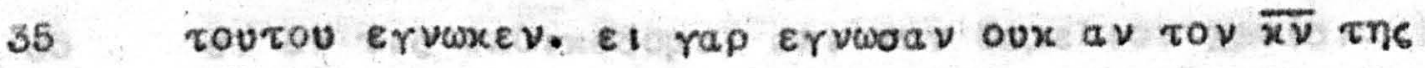
sognc eqtaupwoav.

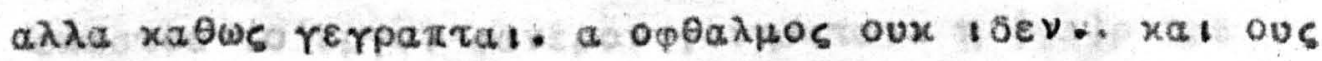

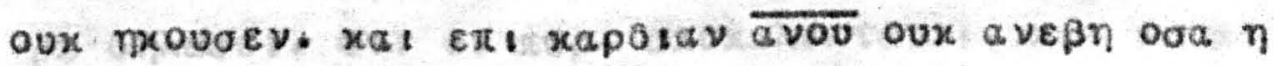

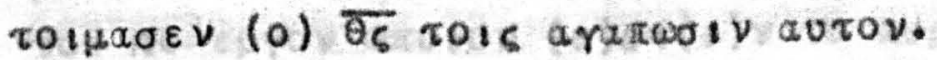

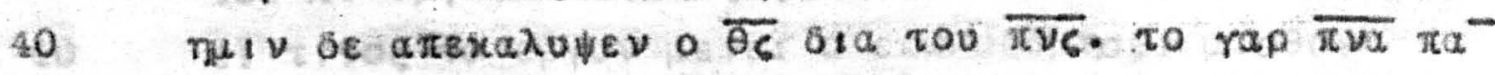

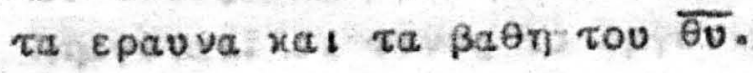

xpos xop:verovs a $1.83-2.10$ 


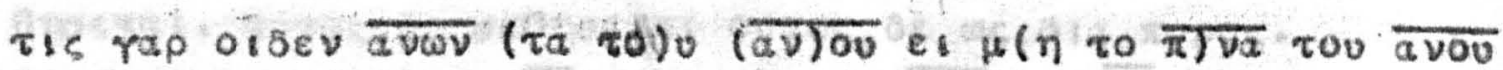

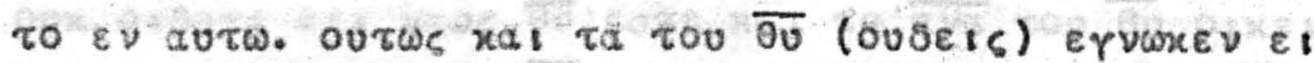

un $\tau_{0} \overline{\pi v a} \tau o v \overline{\theta v}$

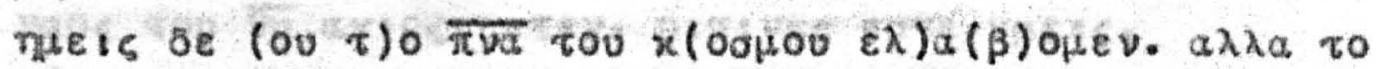

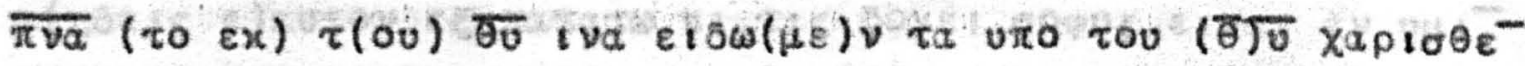

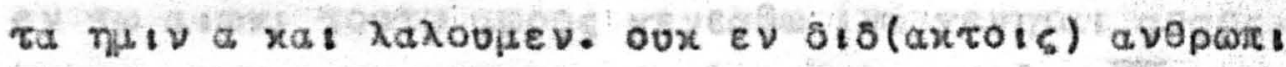

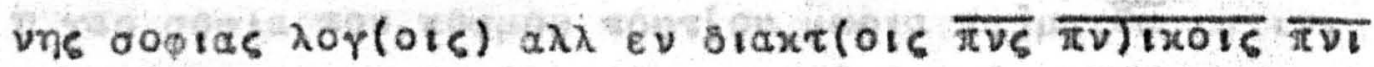

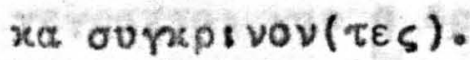

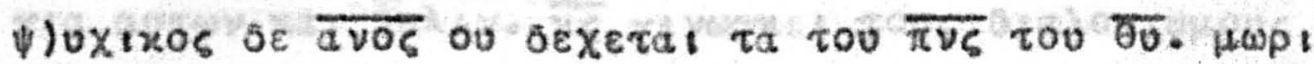

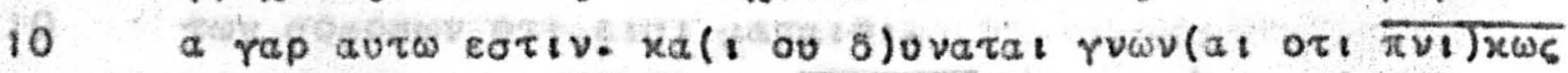

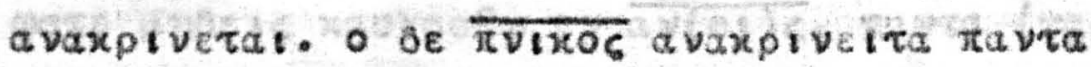

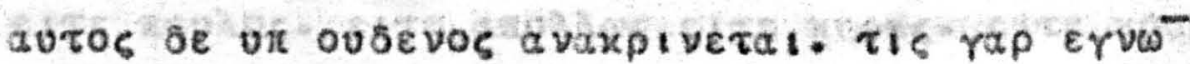

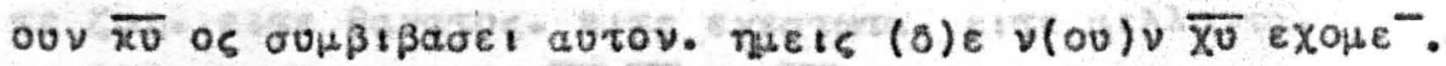

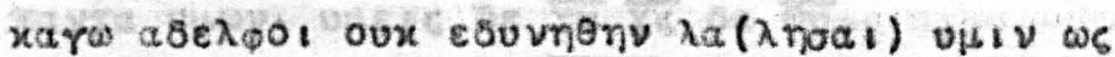

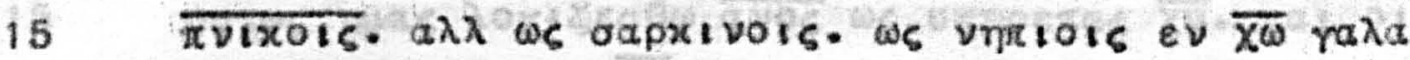

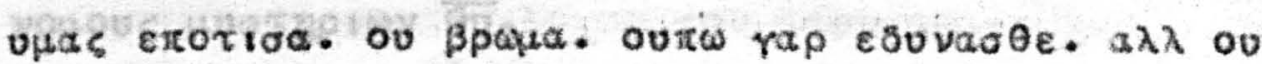

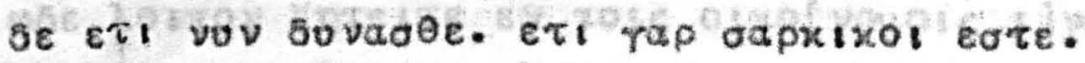

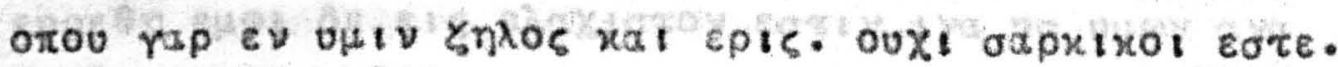
xal xara avov repitareıte.

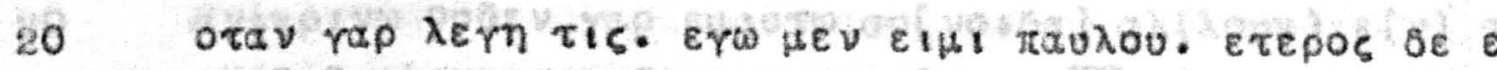

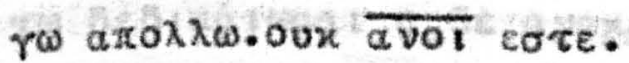

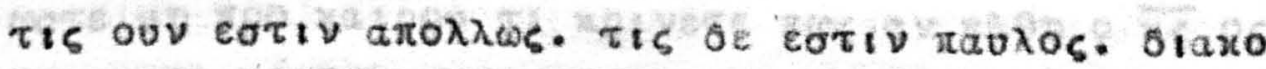

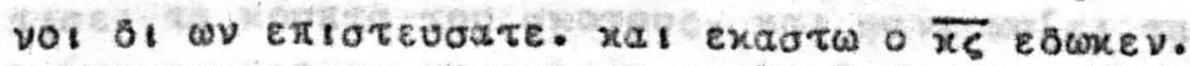

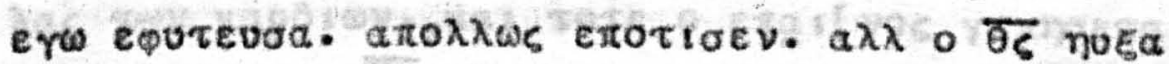

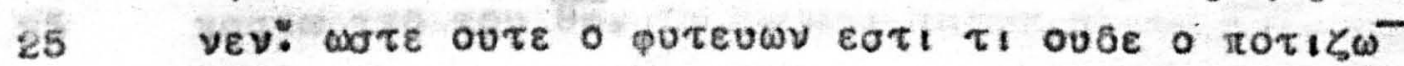

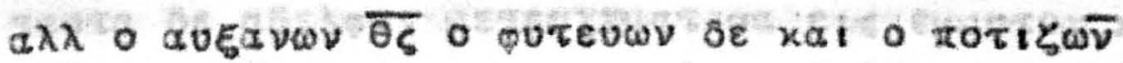

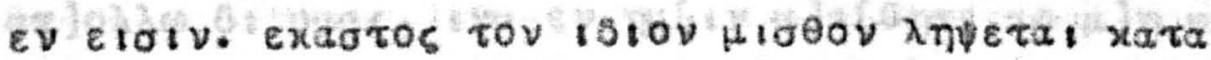

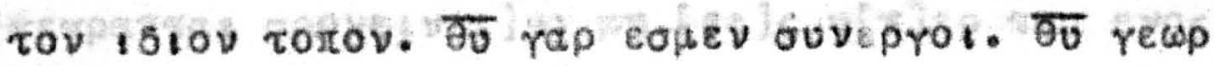

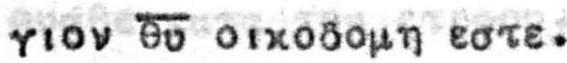

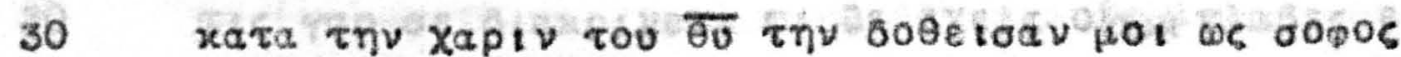

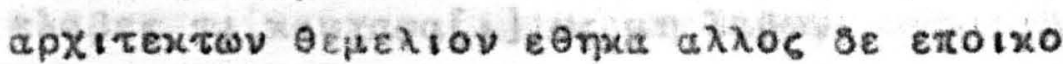

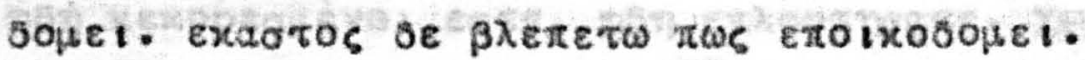

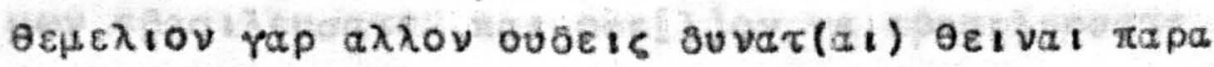
zov xetuevov os $\varepsilon \sigma \tau i v \overline{X \zeta}$.

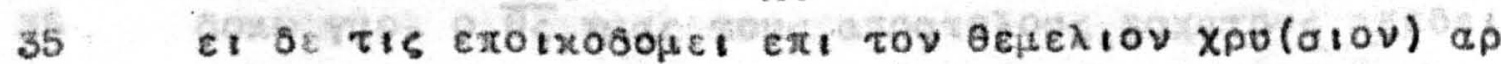

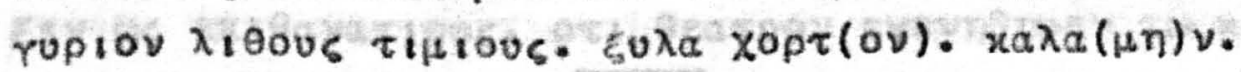

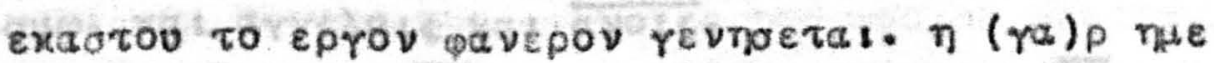
pa on

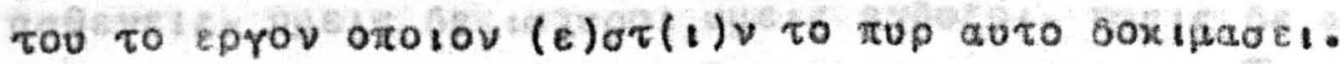

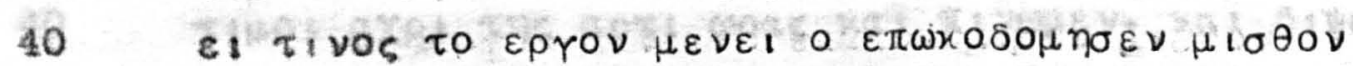

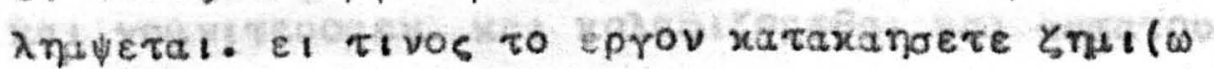




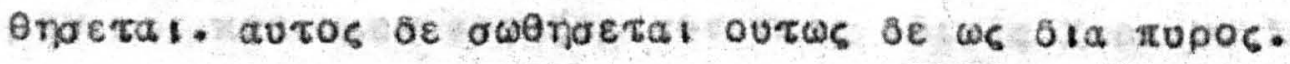

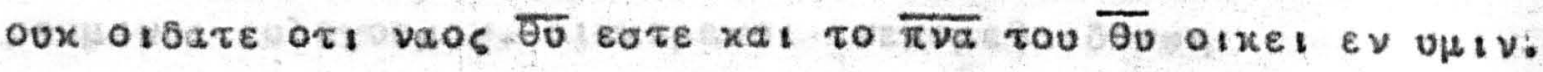

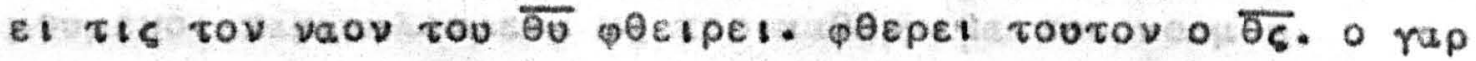

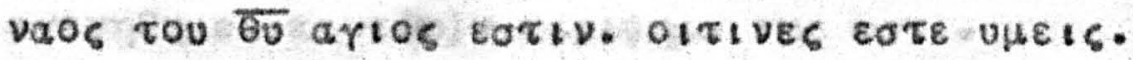

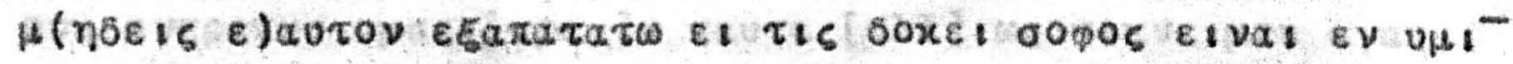

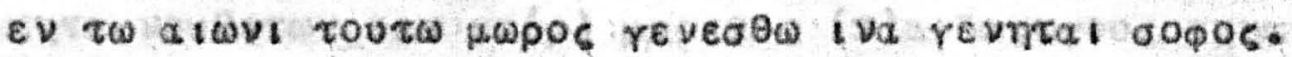

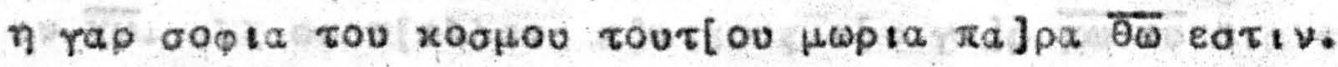

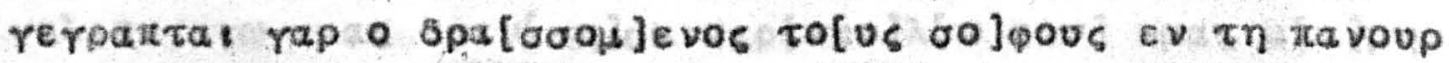

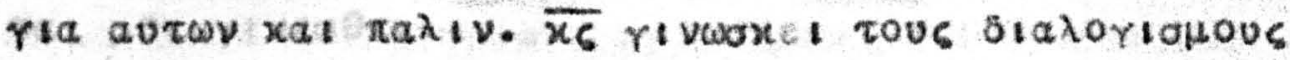

10 tos oopoow ort etol haratol.

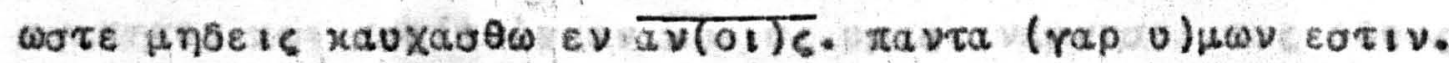

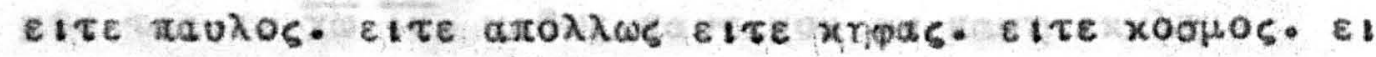

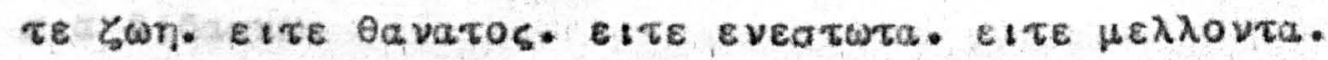

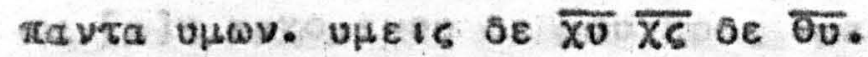

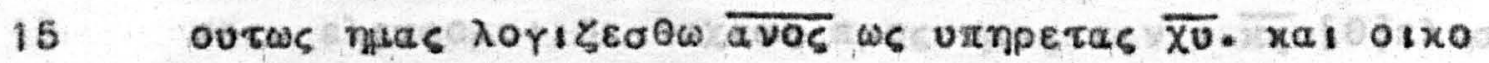

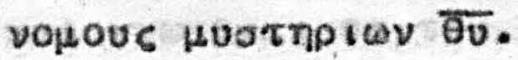

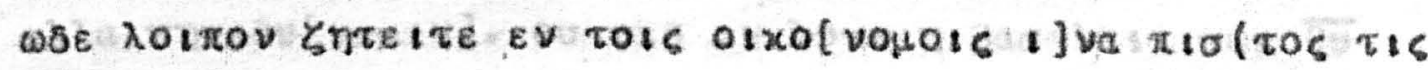

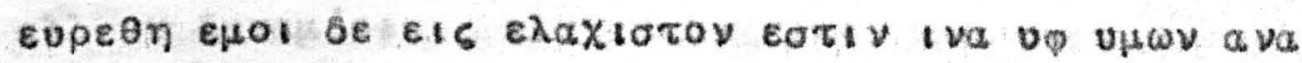

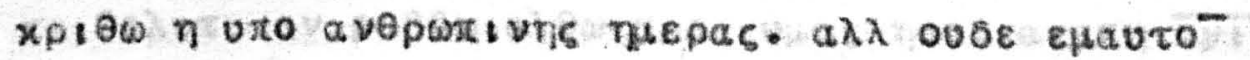

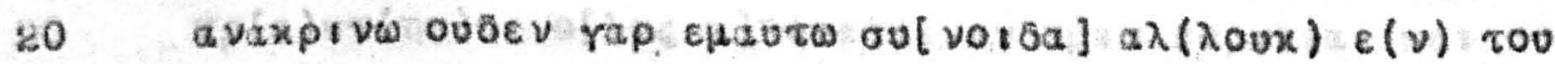

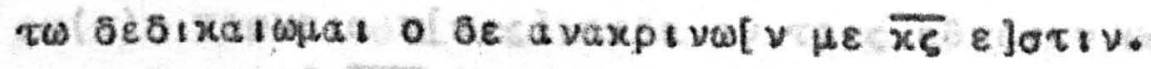

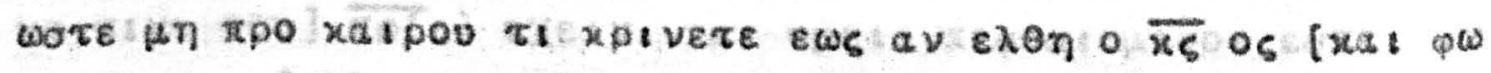

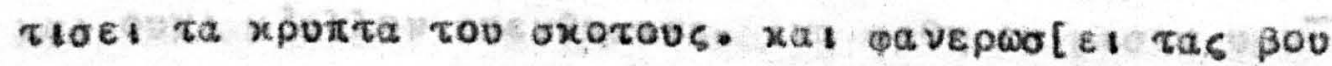

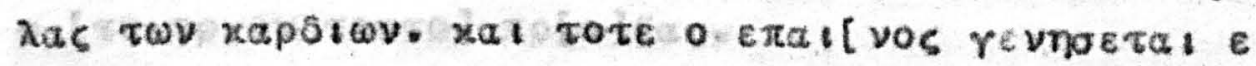

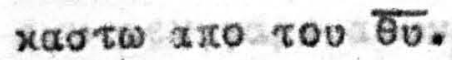

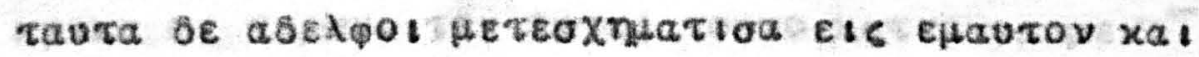

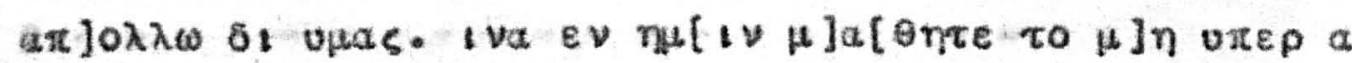

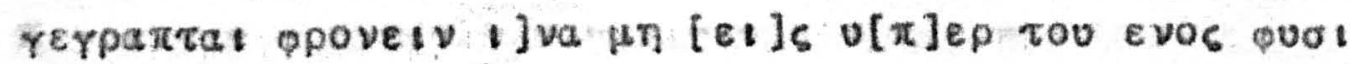

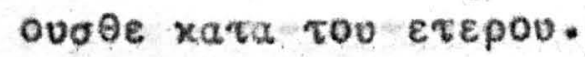

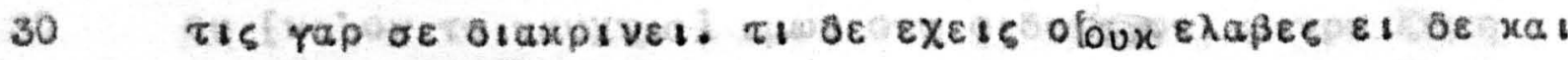
$\varepsilon \lambda a \beta \varepsilon s$,

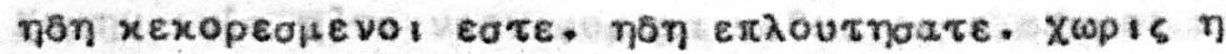

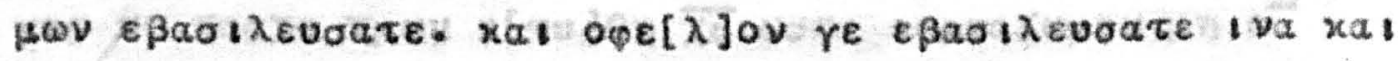

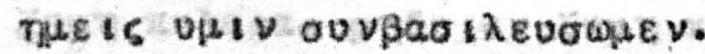

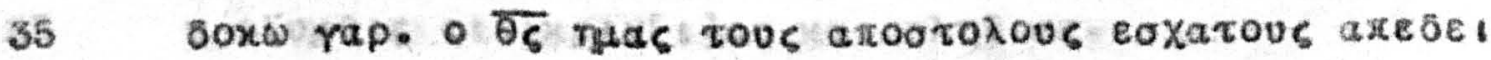

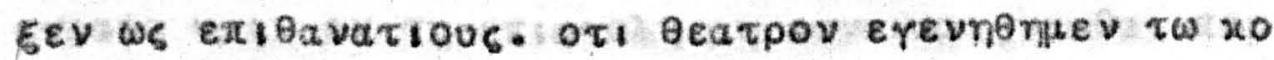

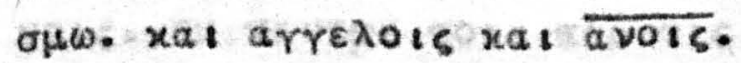

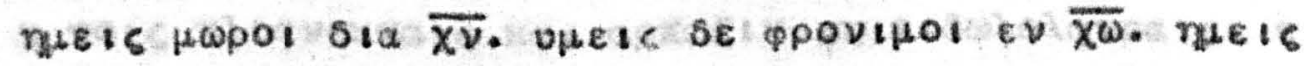

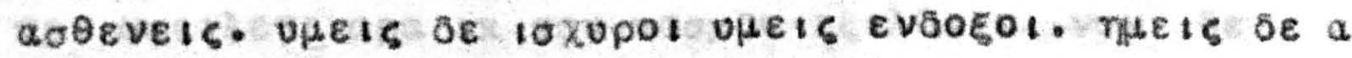

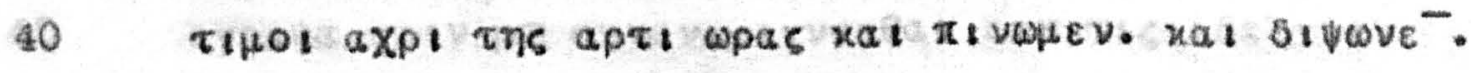

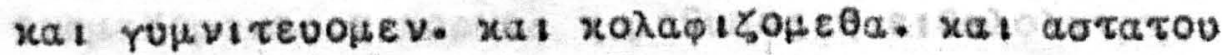




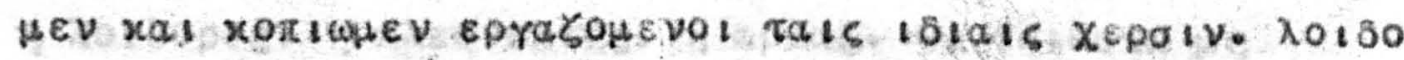

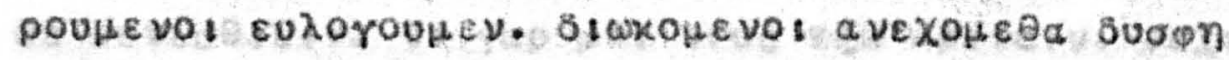

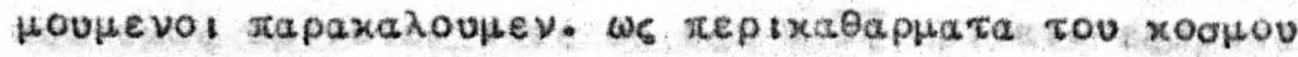

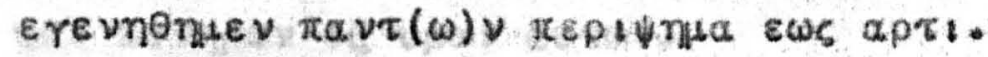

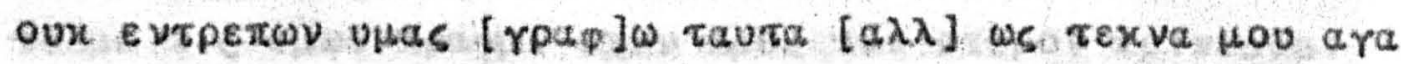

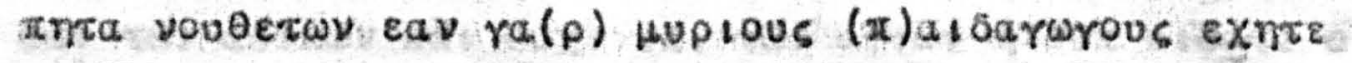

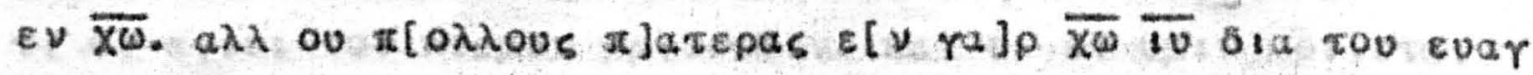

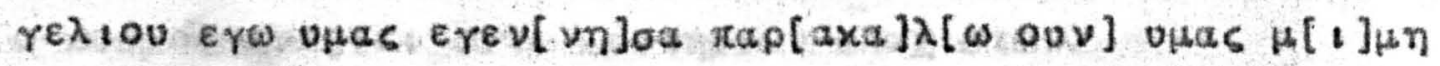
ras 100 riveo $\theta \varepsilon$.

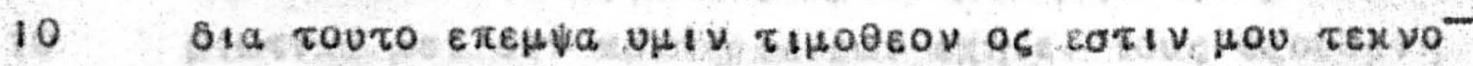

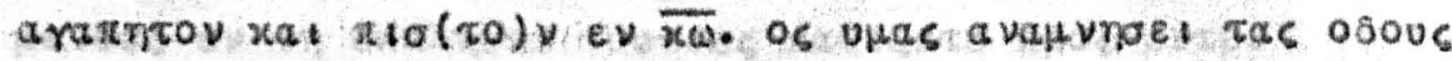

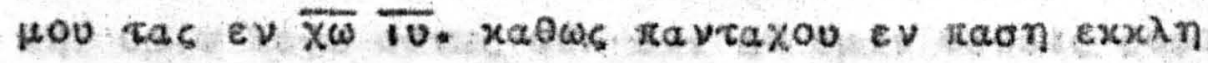
oia $816 a \sigma x \omega s$.

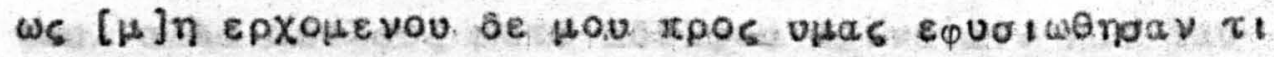

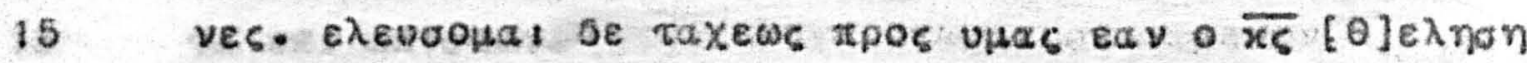

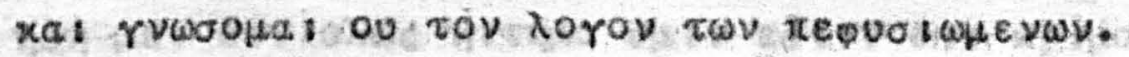

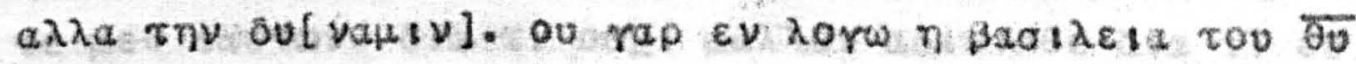

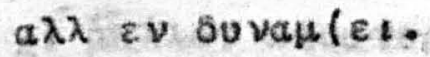

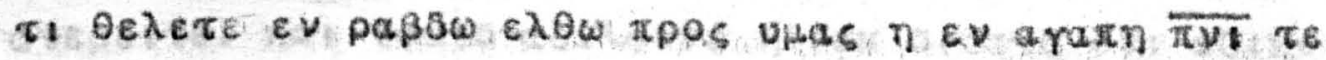

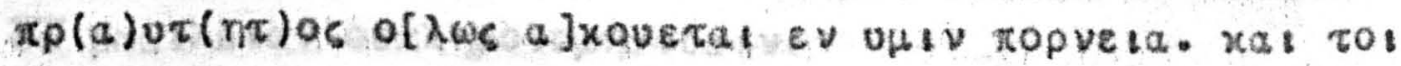

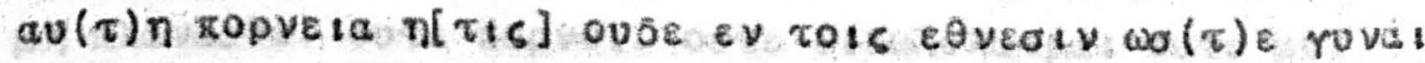

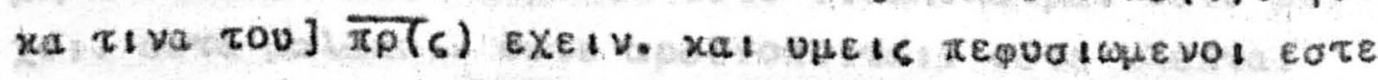

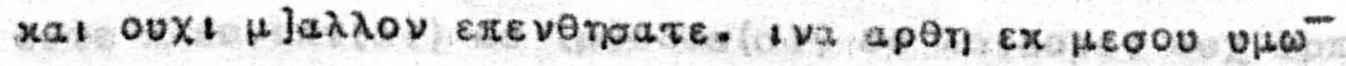
- [ro eprov routo] $\pi \rho[a] \xi a s$.

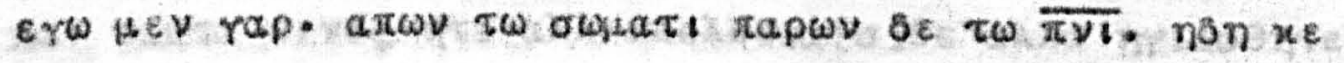

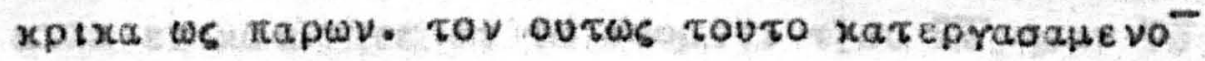

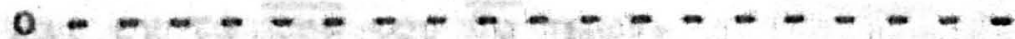

x -------------$\varepsilon---------------$

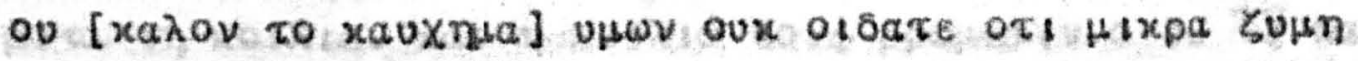

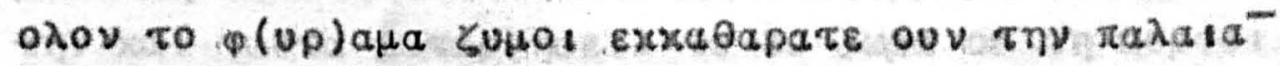

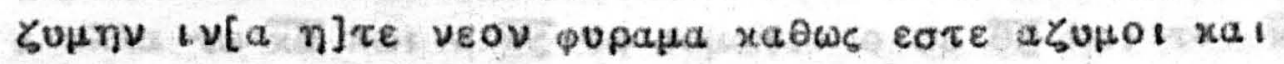

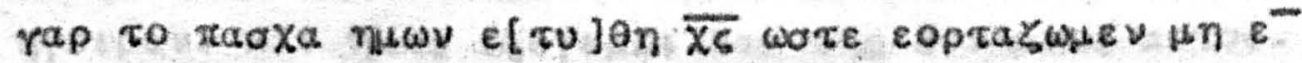

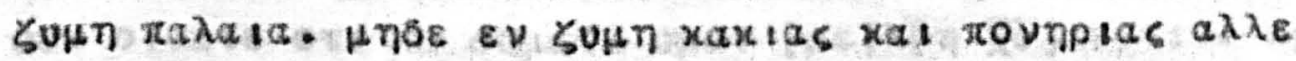

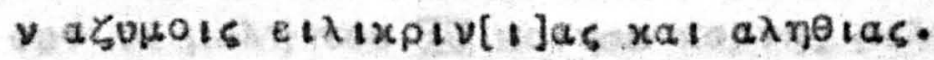

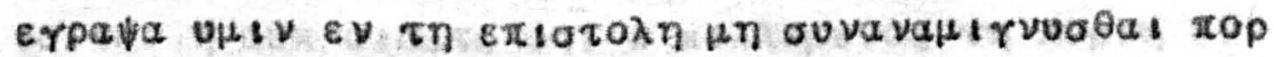

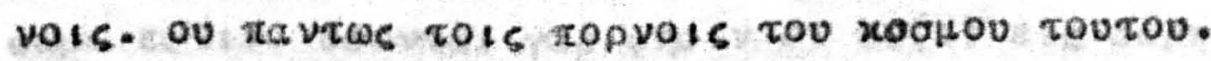

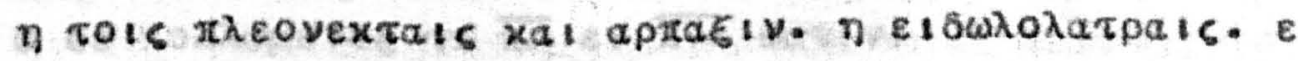

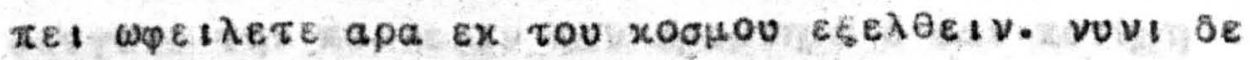

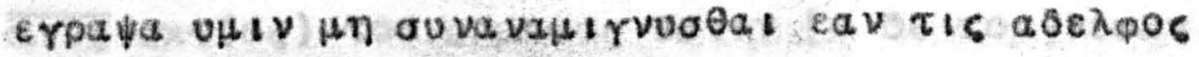

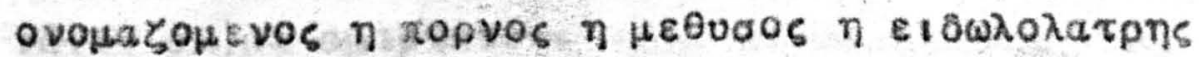




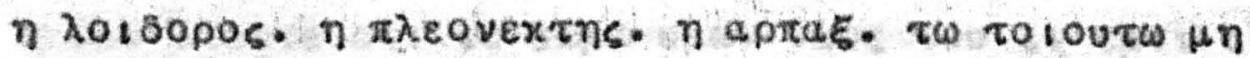

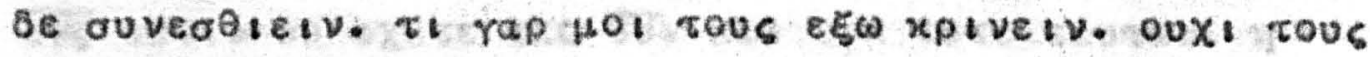

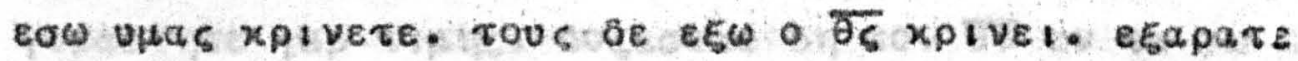

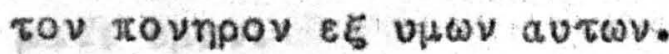

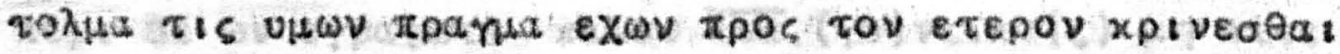

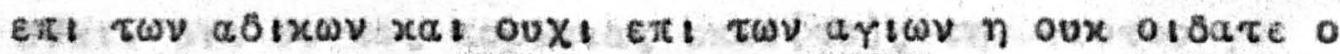

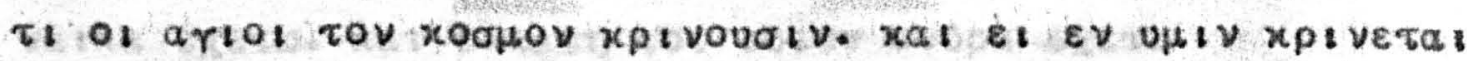

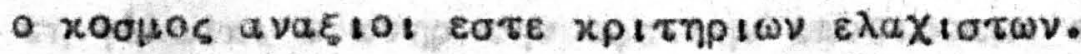

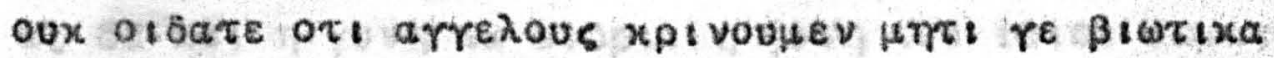

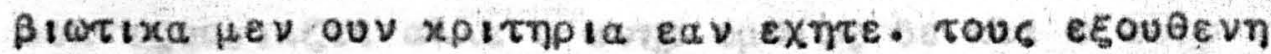

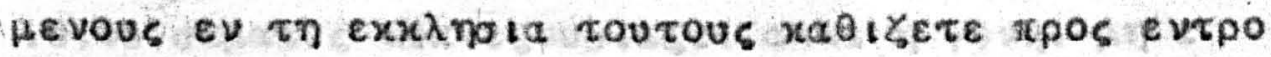

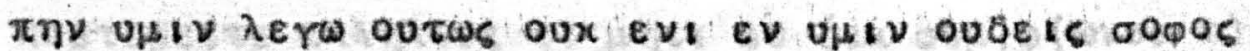

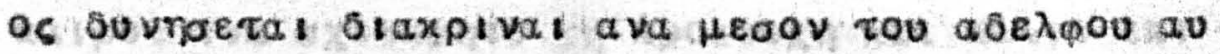

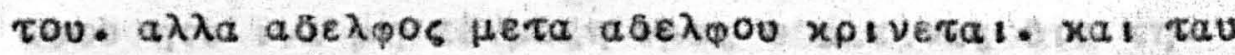

15 Ta $e \pi t$ art $10 \tau \omega \nu$.

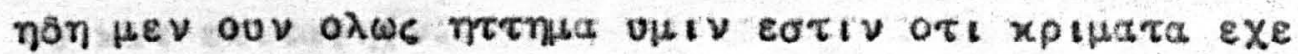

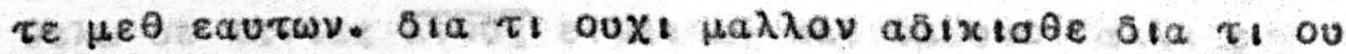

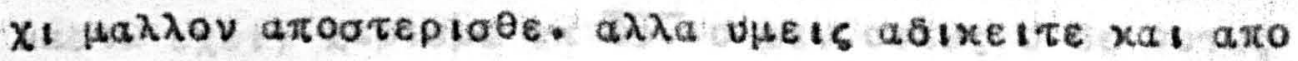

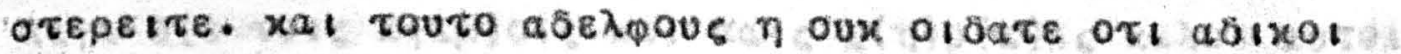

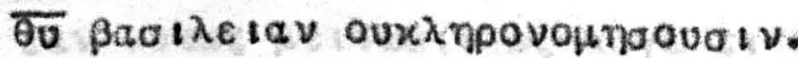

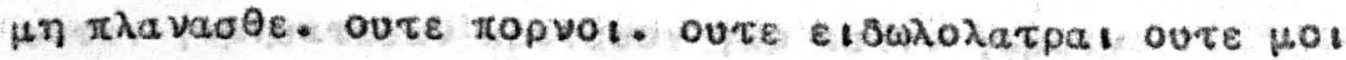

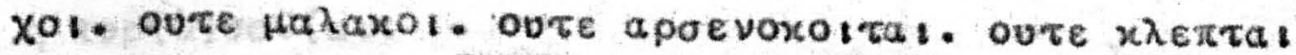

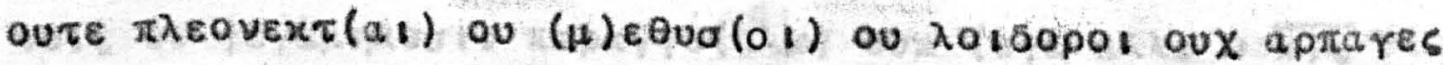

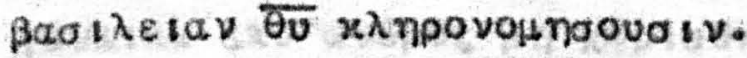

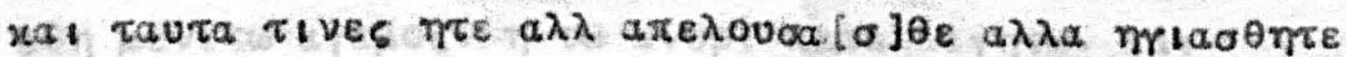

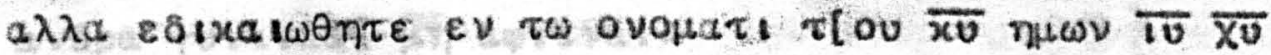

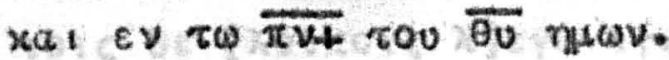

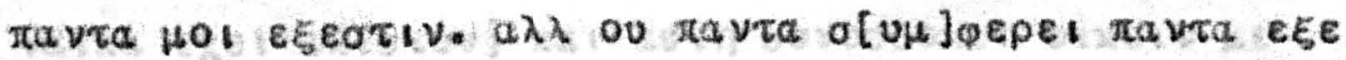

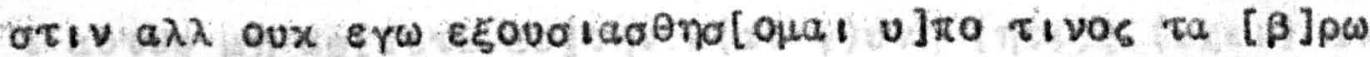

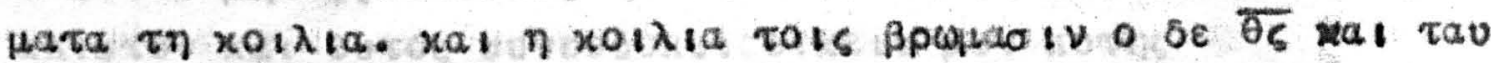

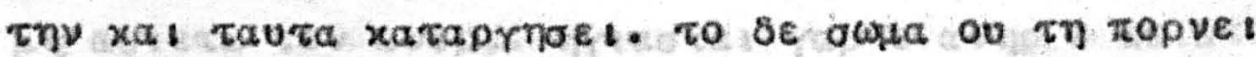

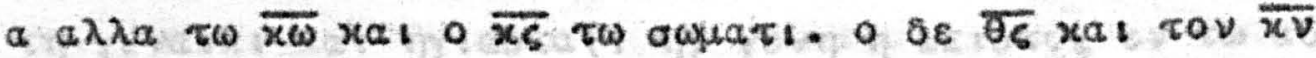

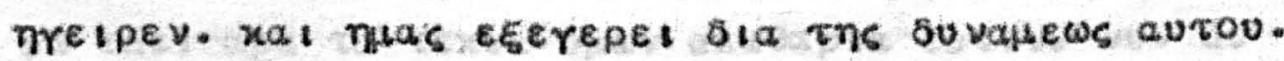

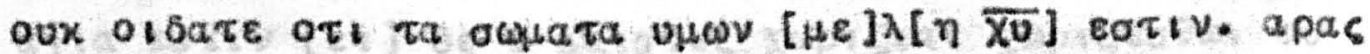

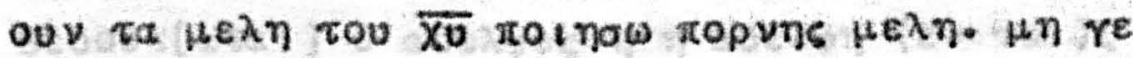

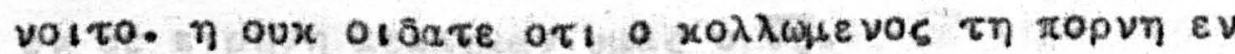

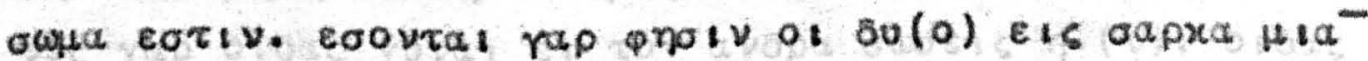

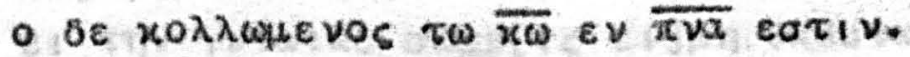

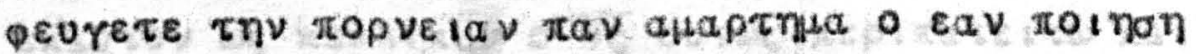

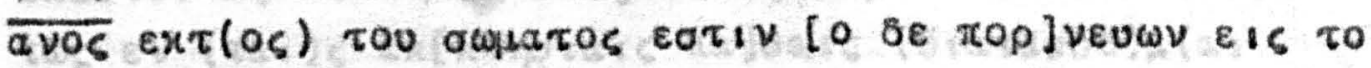

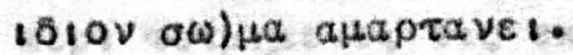




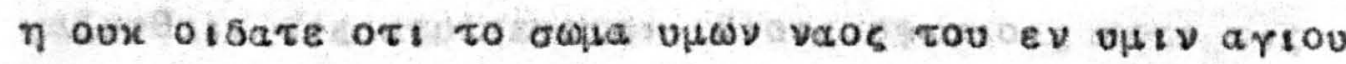

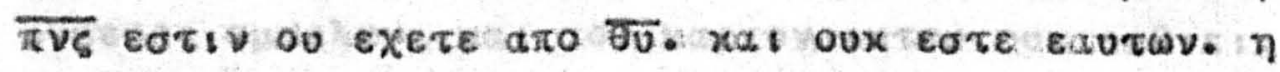

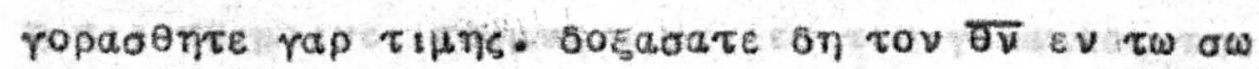

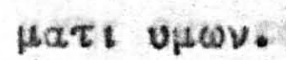

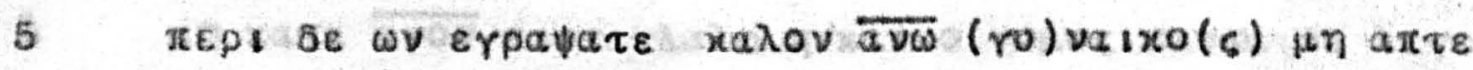

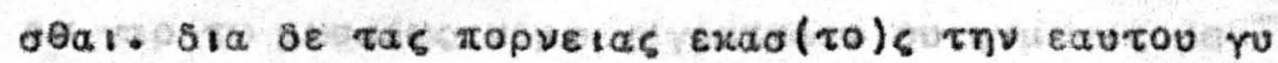

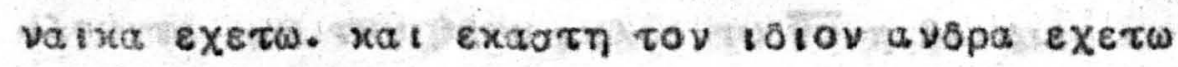

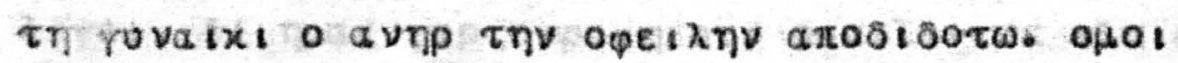

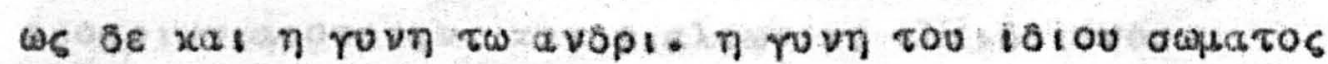

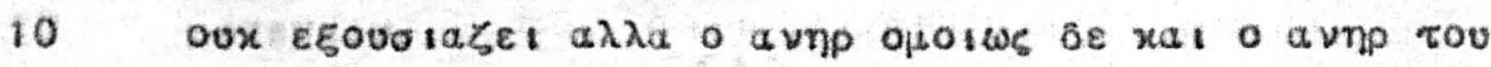
10100 oquaros oux EE

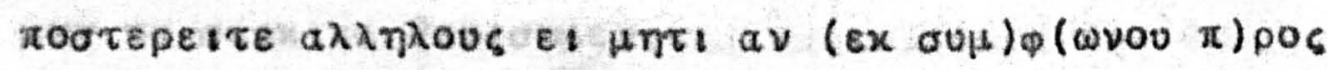

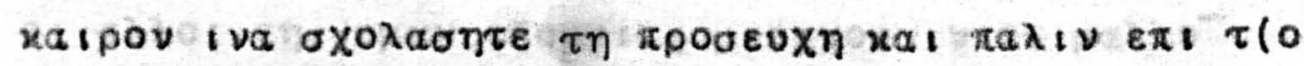

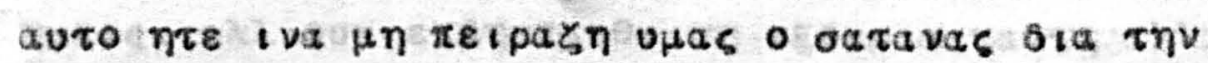

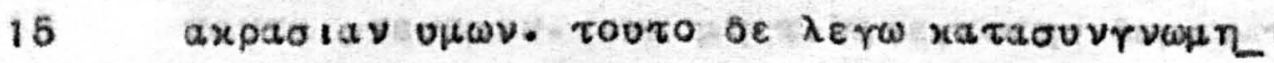
ov xar exirarnilv.

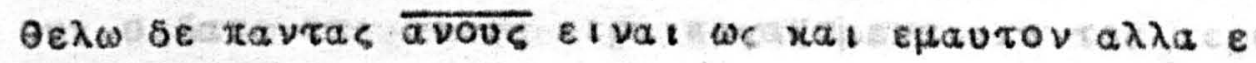

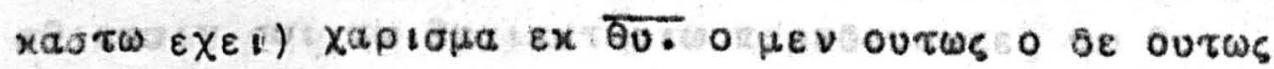
$\lambda \varepsilon$ ro $\delta(\varepsilon$ rois a rap)ois xal tais $X(\eta) \rho(a)$ is xatov avtois

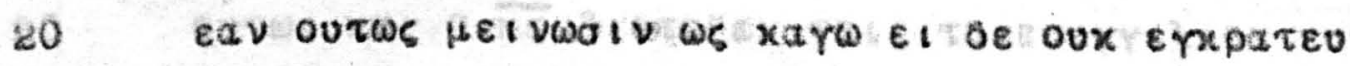

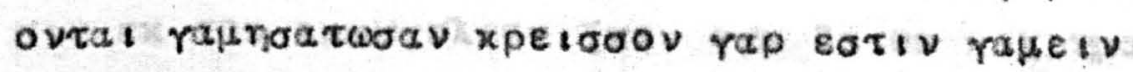
$\eta \pi \operatorname{top}(0) \operatorname{vo\theta }(\alpha$ i.

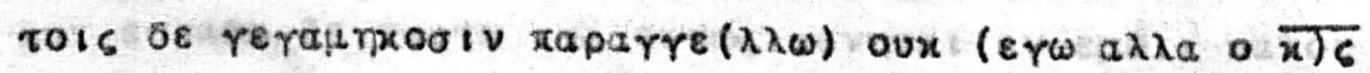

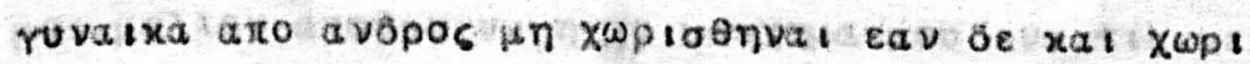

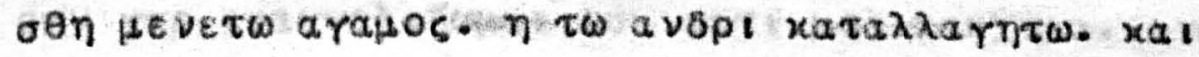
avôpa rovatxa] Hท aptevat.

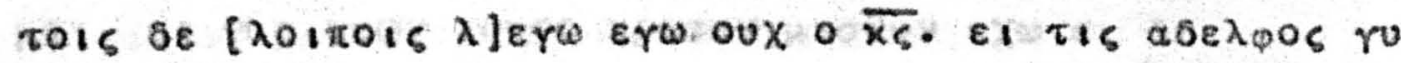

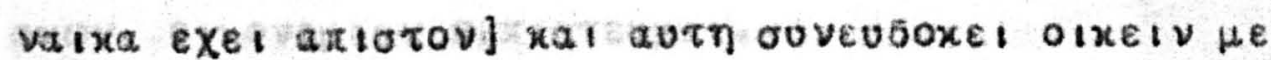

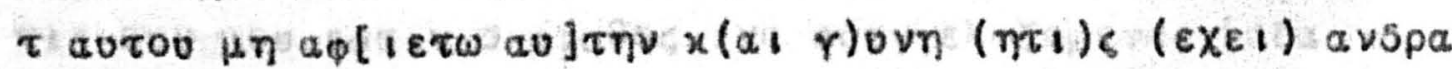

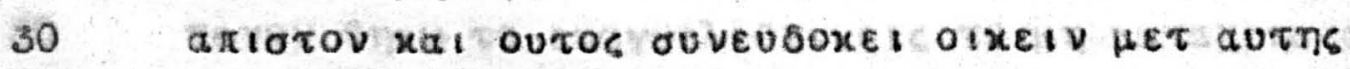

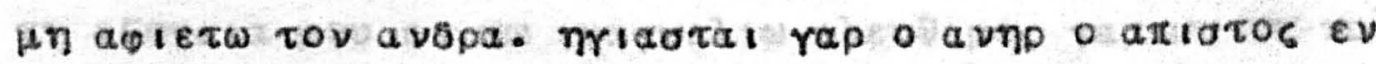

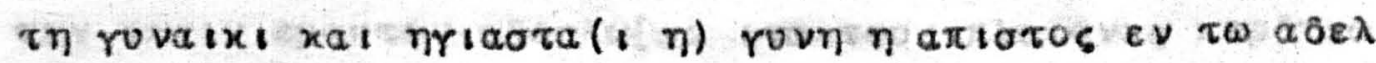

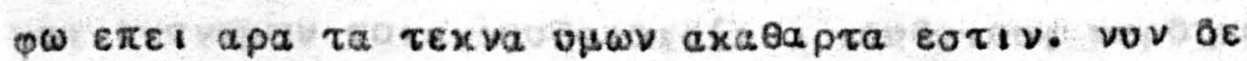

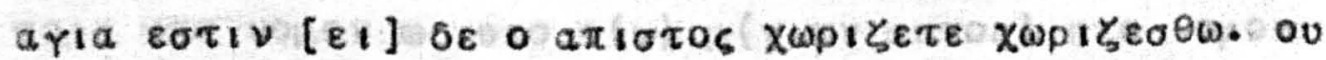

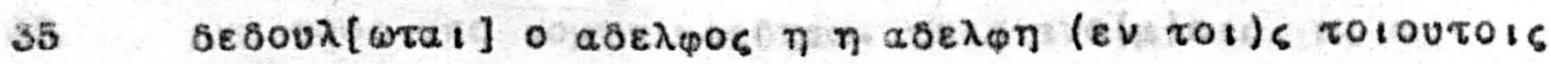

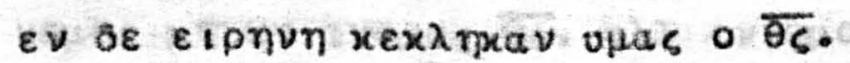

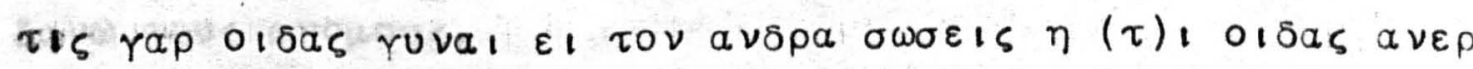

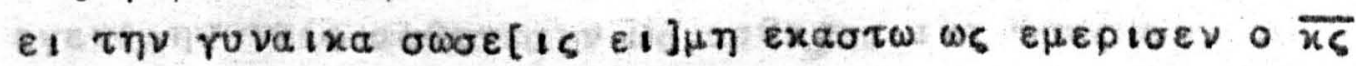

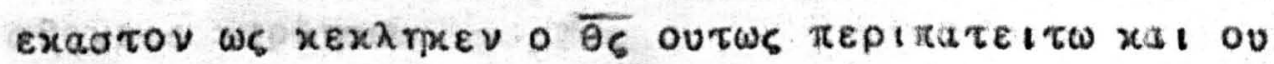

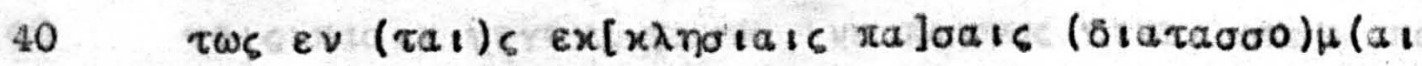

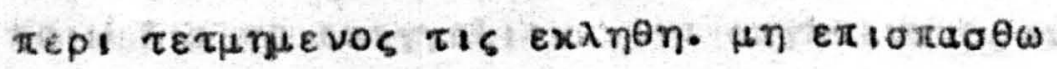




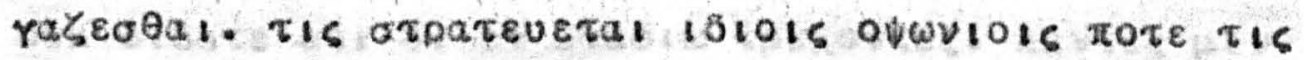

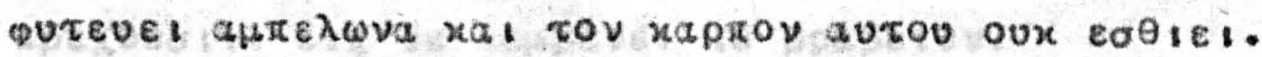

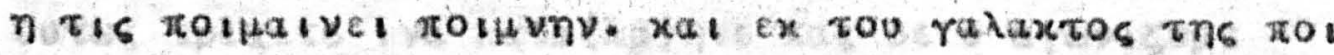

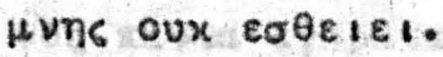

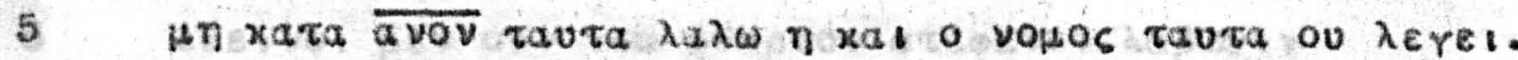

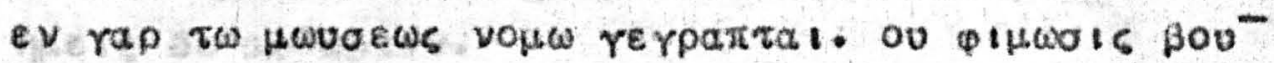

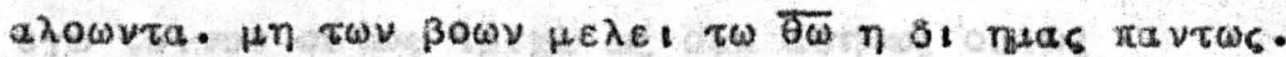

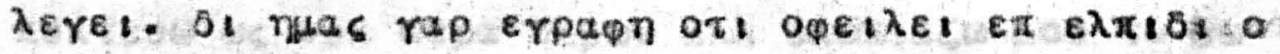

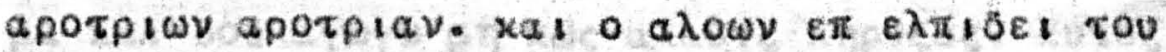

10 घetexeiv.

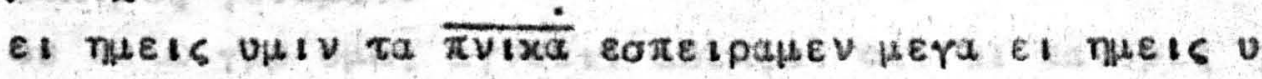

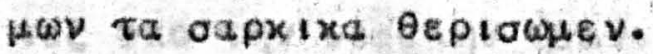

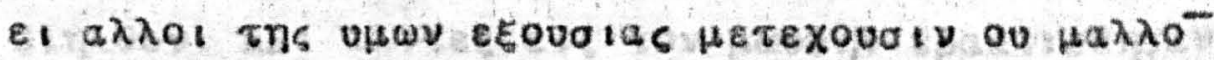

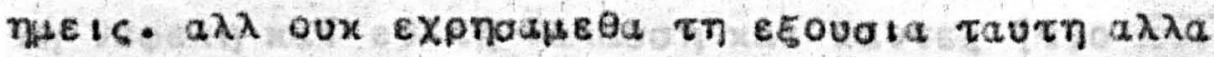

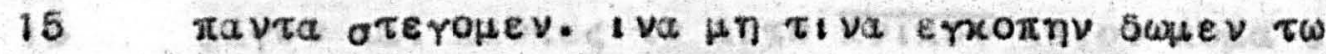

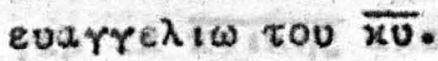

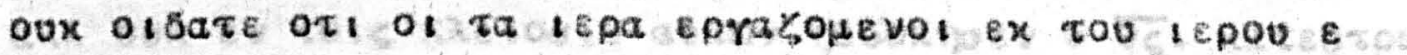

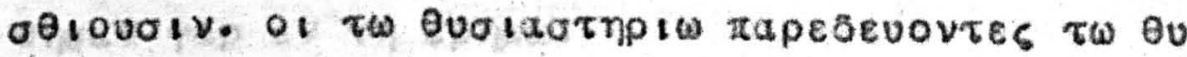

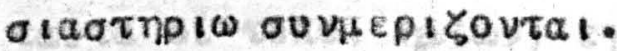

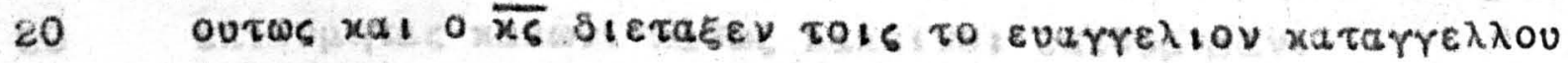

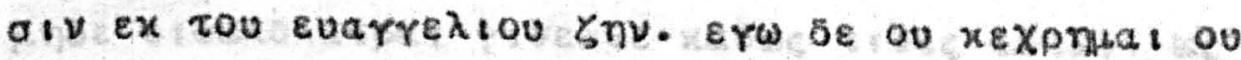

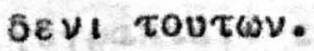

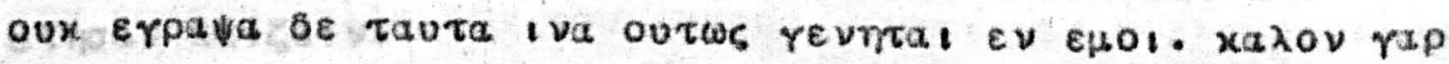

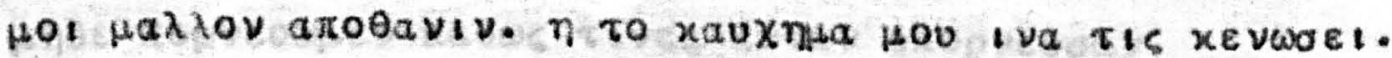

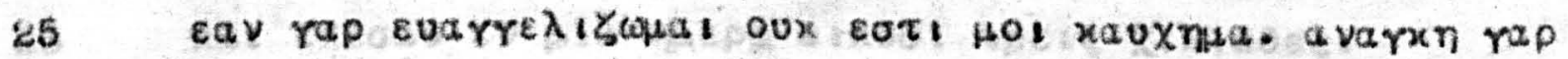

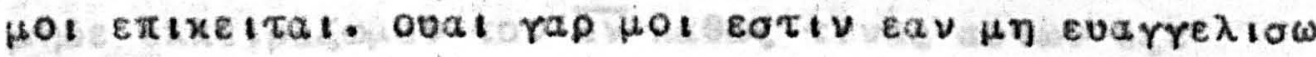

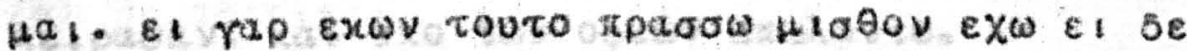

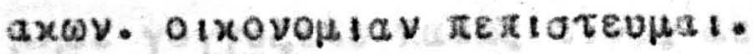

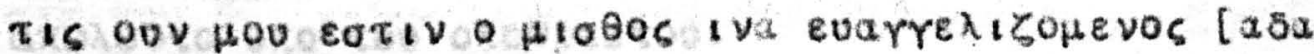

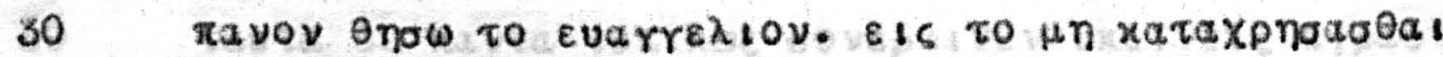

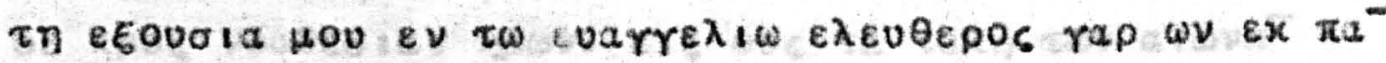

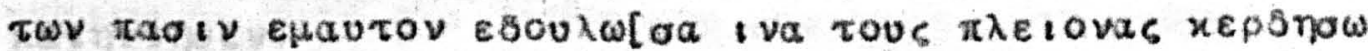

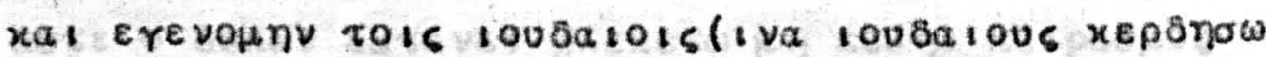

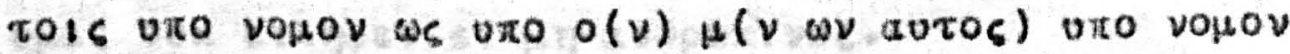

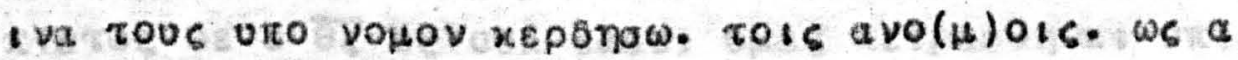

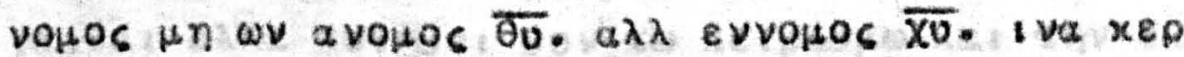
Dave rous a vohous.

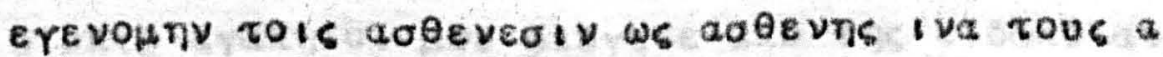

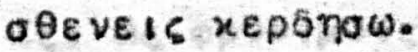

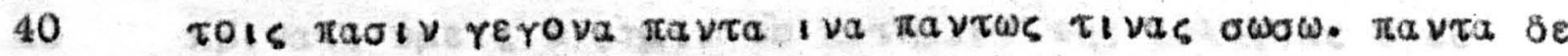

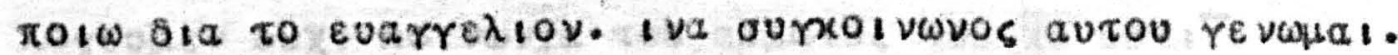




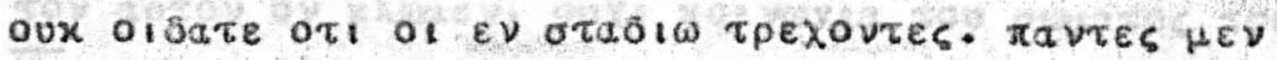

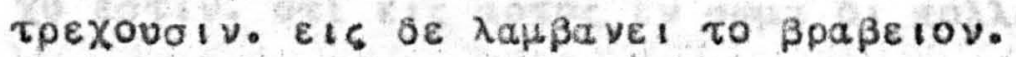

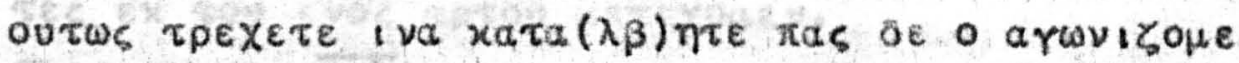

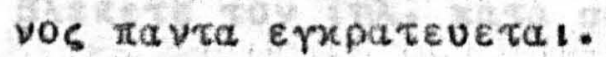

5 exeivoi hev ouv iva papotov arepavov Aaßwatv nue is $8 \varepsilon$ apoaprov.

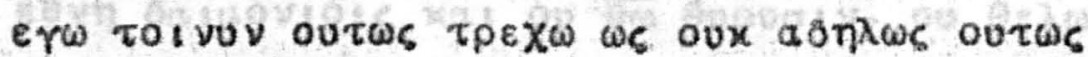

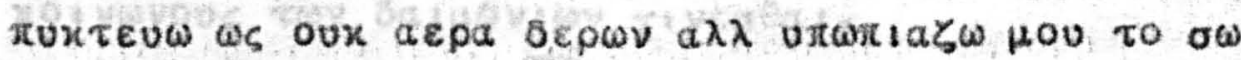

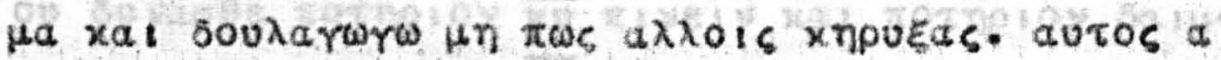

$10 \quad 30 x \mu 0_{6} \gamma \in v \omega \mu a 1$.

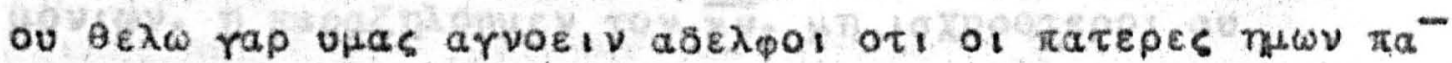

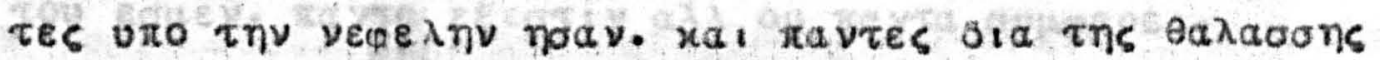

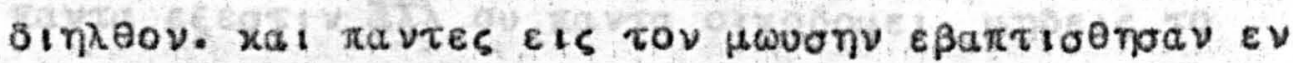

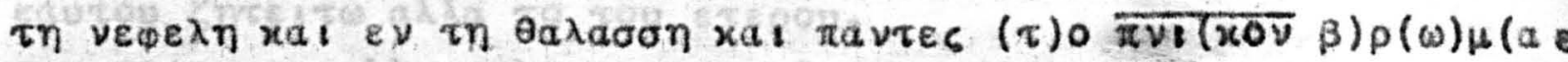

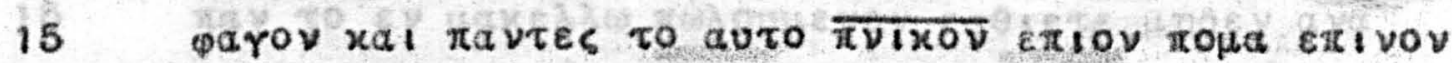

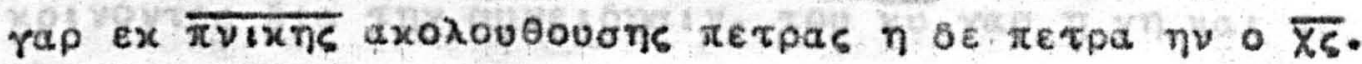

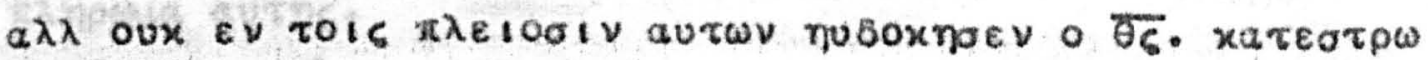
onoav rap $\varepsilon \nu$ in epnuw.

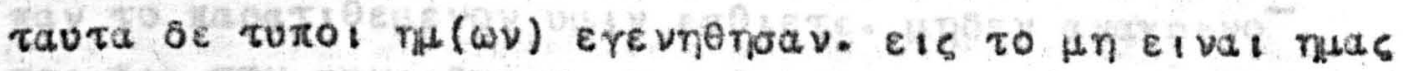

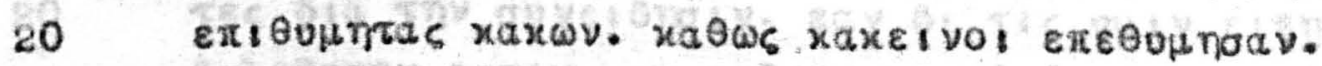

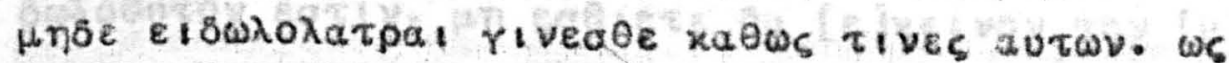
rerpatral. Exabioev o laog pareiv xat $\pi t e t v$ xal ave

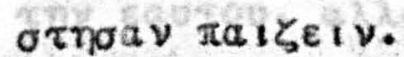

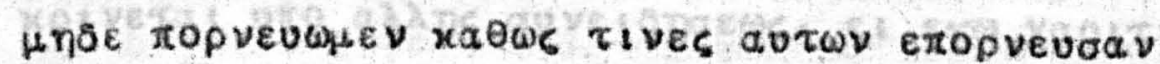

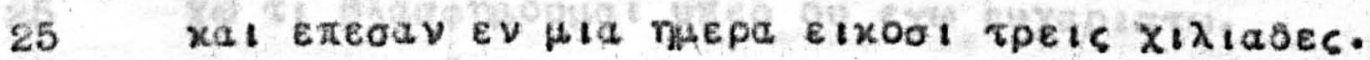

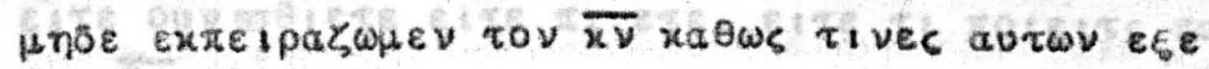

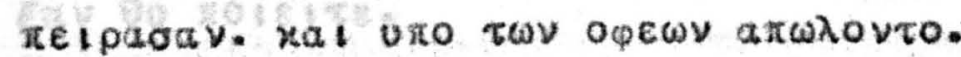

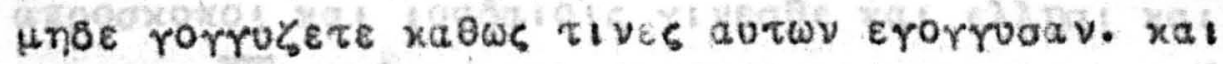
atwiovto vio tov oxoepeutou.

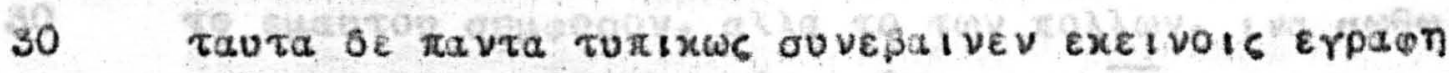

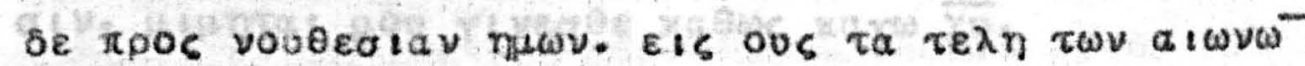

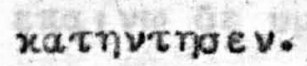

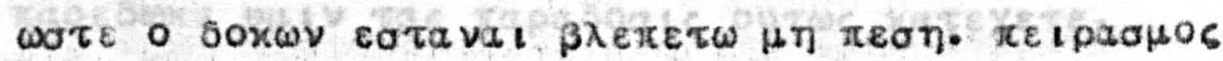

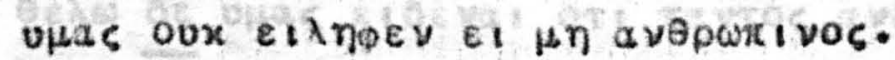

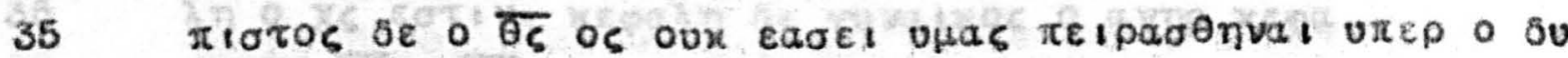

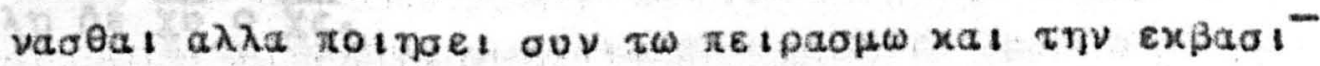

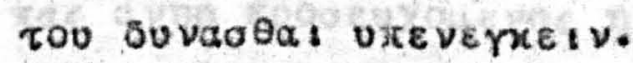

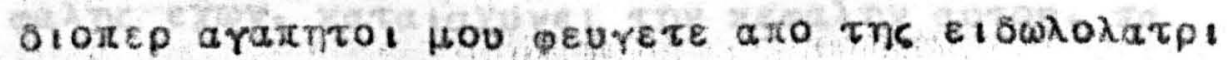
as. Ws ppovifois $\lambda E$ rw.

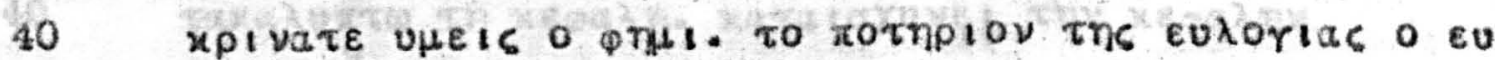

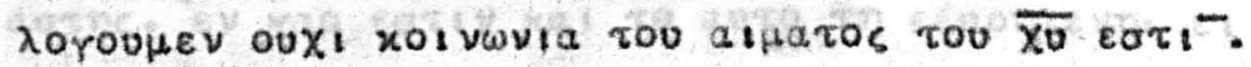




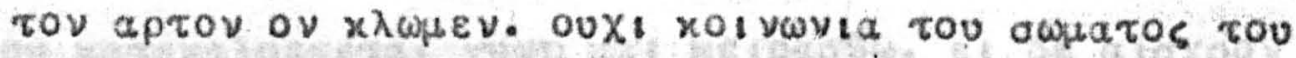

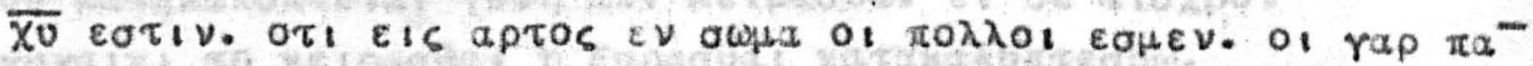

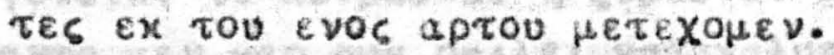

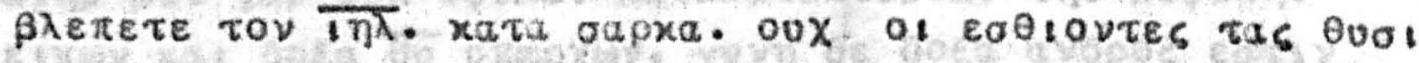

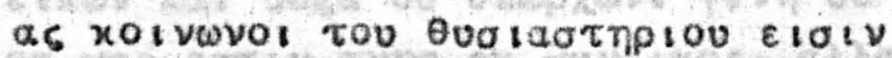

( $\tau$ । oOV QTH

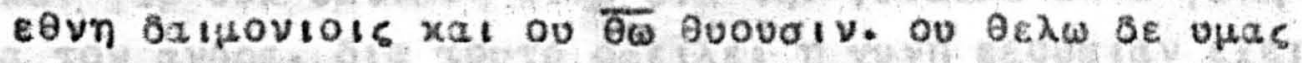
xotvavous rov sathoviov rivealat.

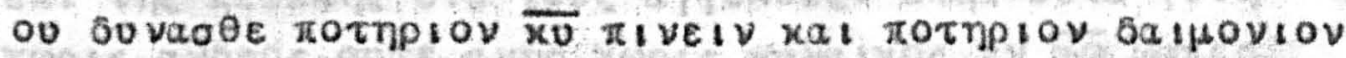

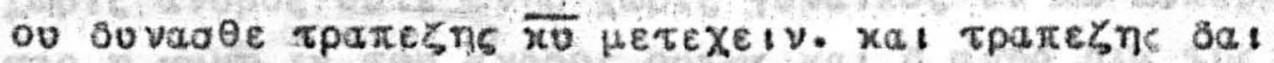

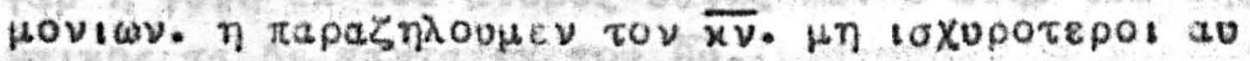

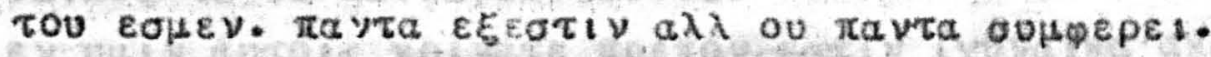

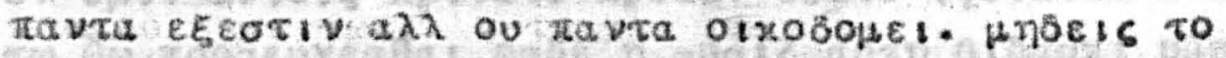

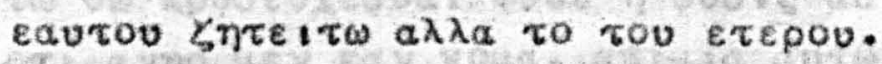

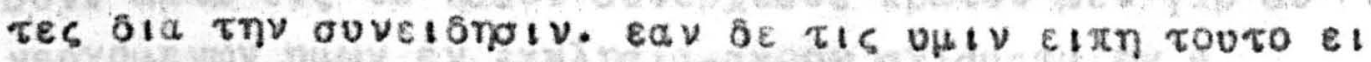

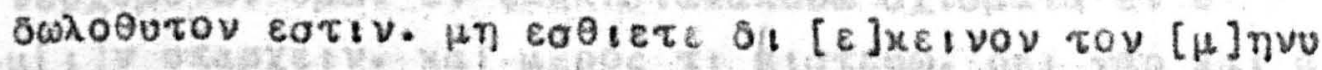

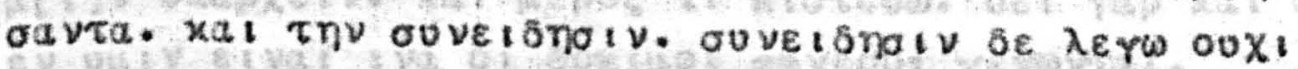

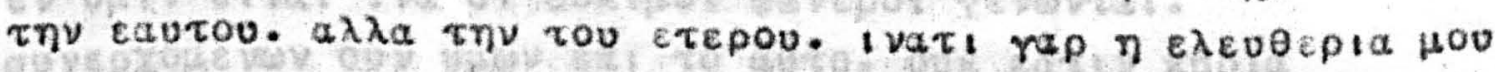

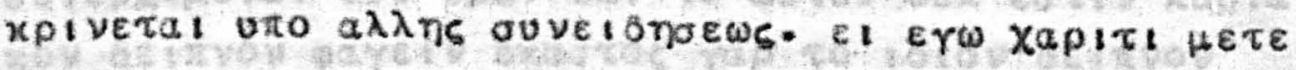

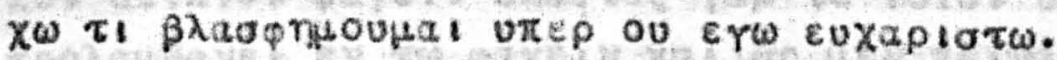

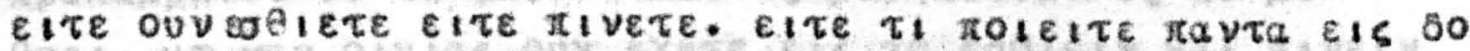

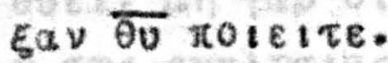

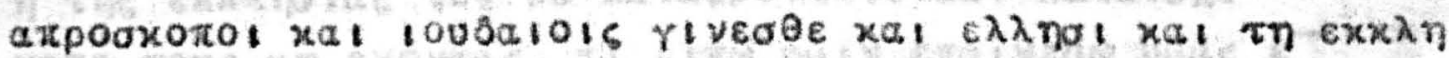

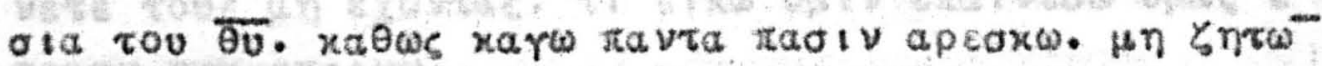

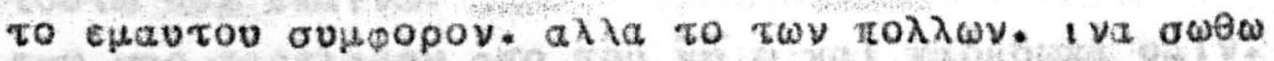

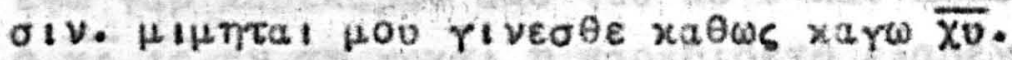

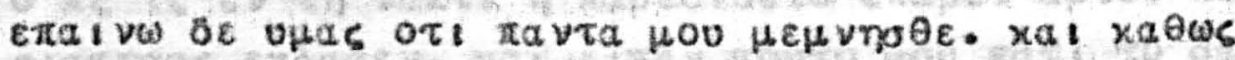

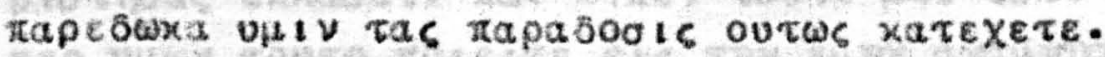

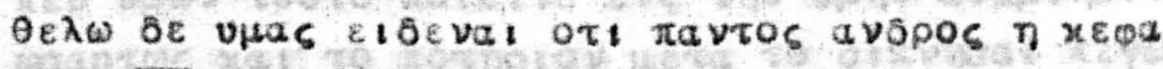
$\lambda \eta \circ \overline{X s} \varepsilon \sigma \tau i v . x \varepsilon p a \lambda \eta$ oe ruvalxos o avmp $x \in \varphi \alpha$ $\lambda \eta \bar{\delta} \overline{\chi 0} \circ \overline{X \zeta}$.

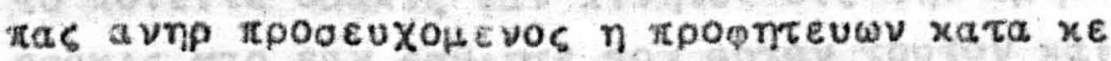

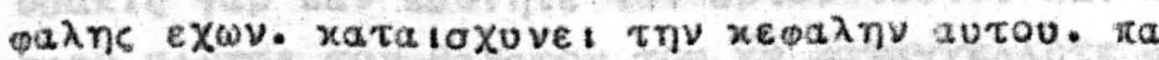

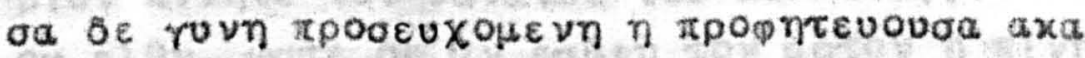

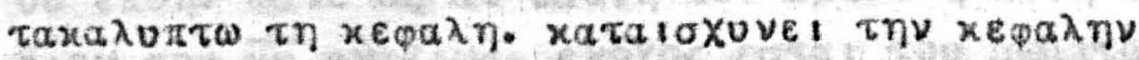

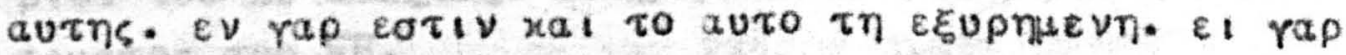




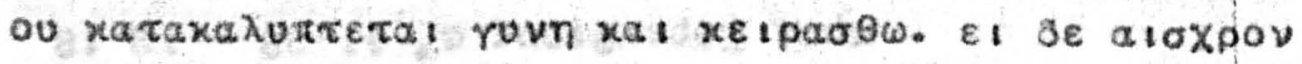

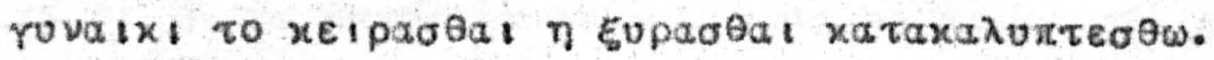

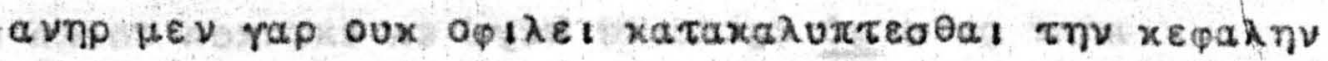

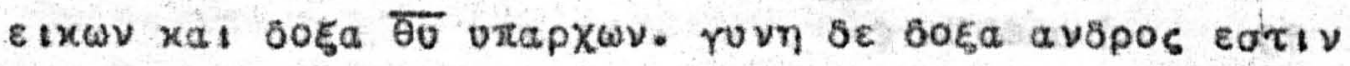

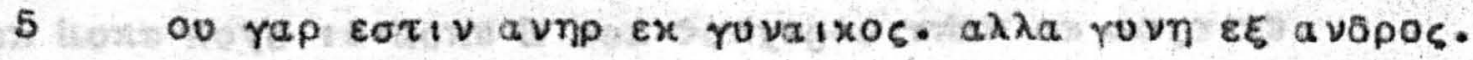

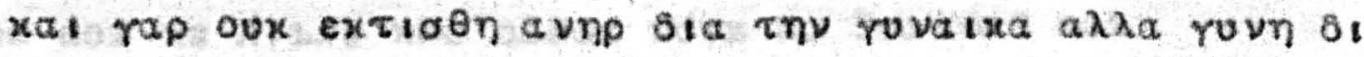

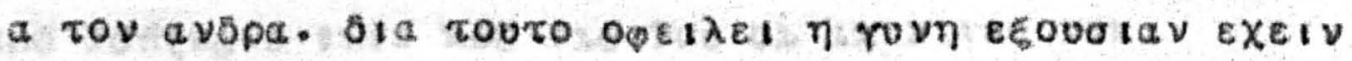

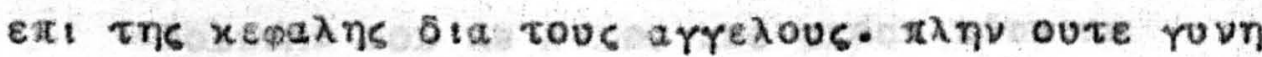

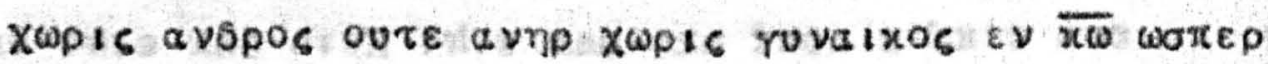

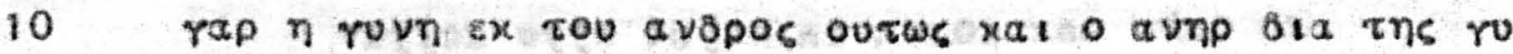
vaixos $\tau a$ be ravra $\varepsilon x$ rou 50 .

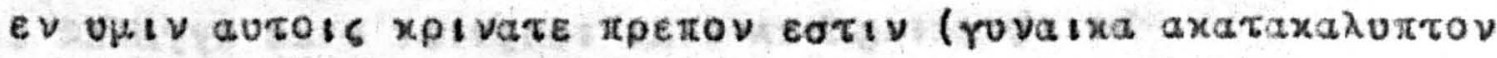

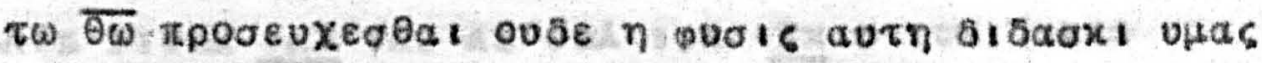

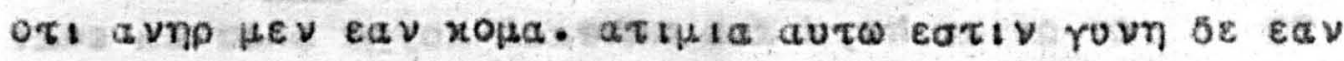

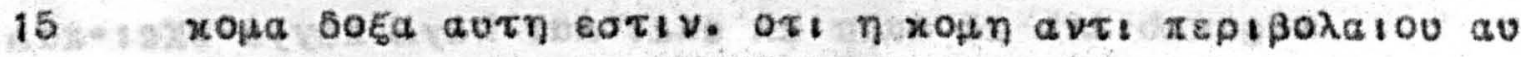

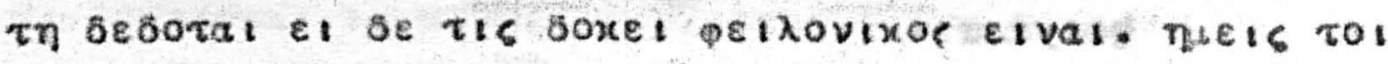

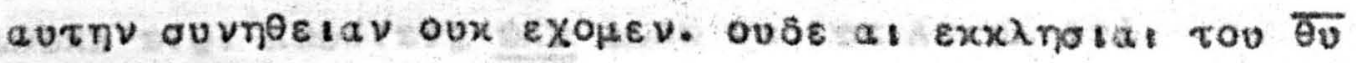

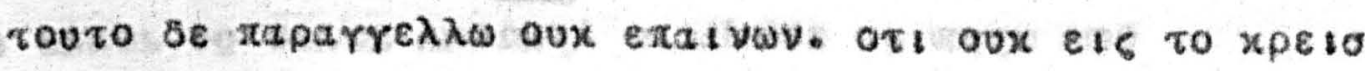

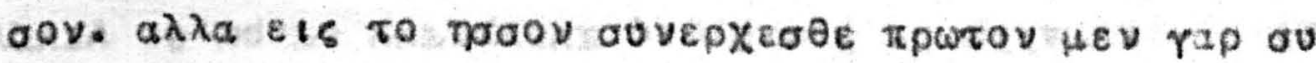

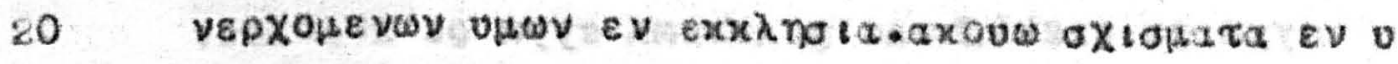
$\mu[1] \nu$ orapxetv. xal hepos $\tau$ t tiareva. bel rap xal atpeoeis

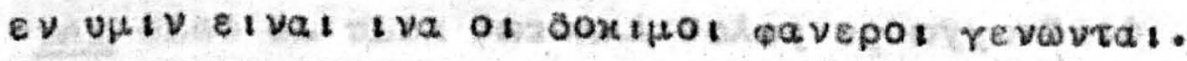

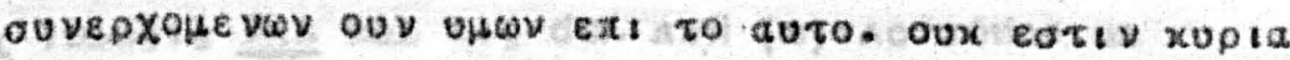

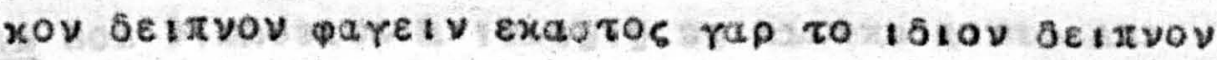

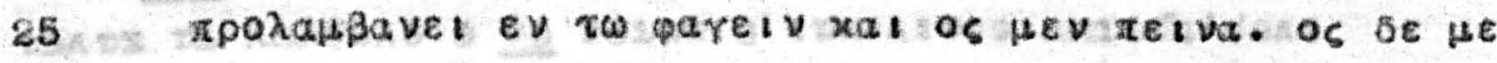

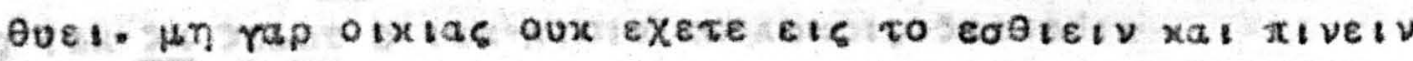

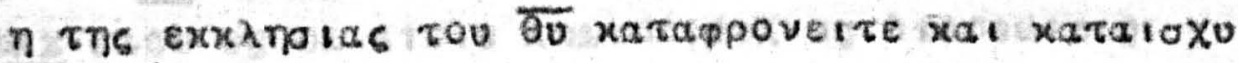

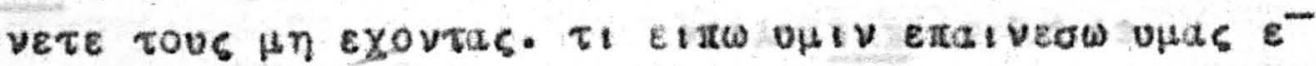

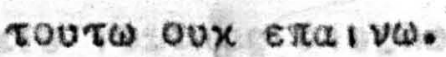

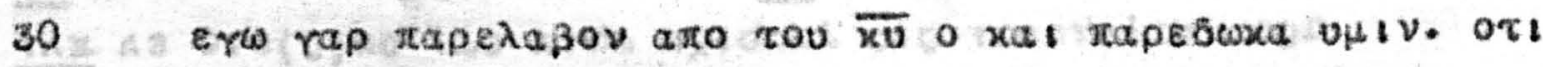

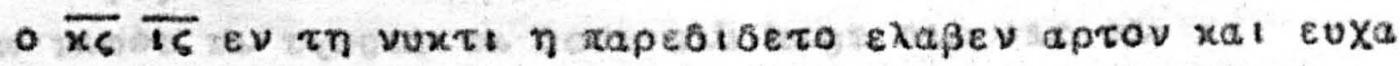

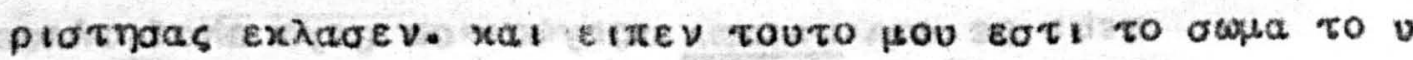

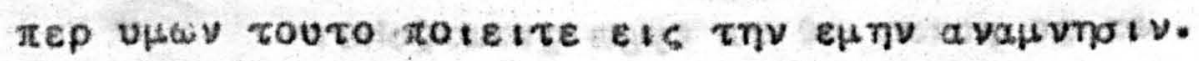

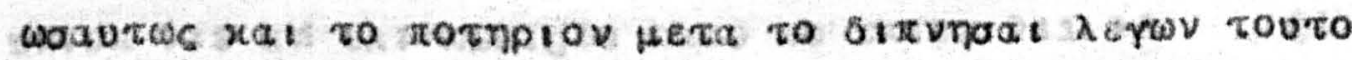

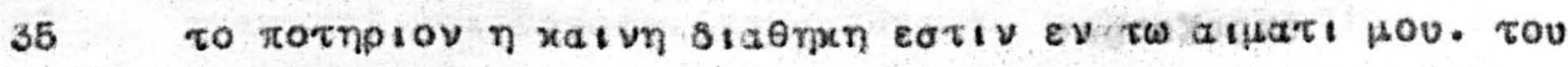

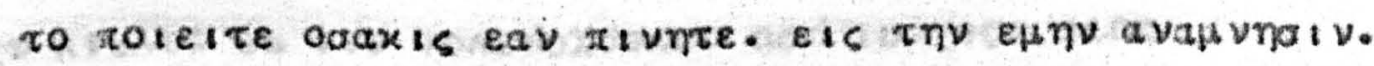

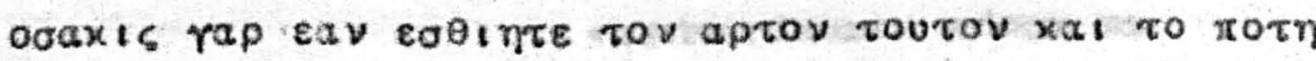

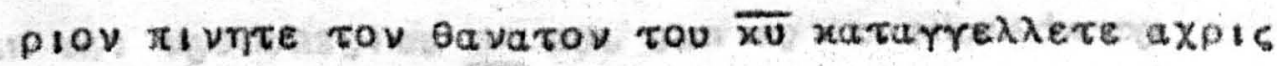

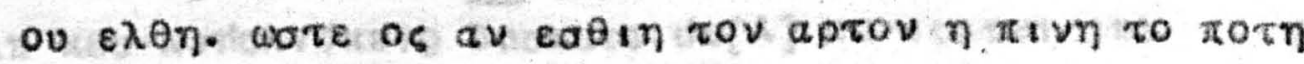

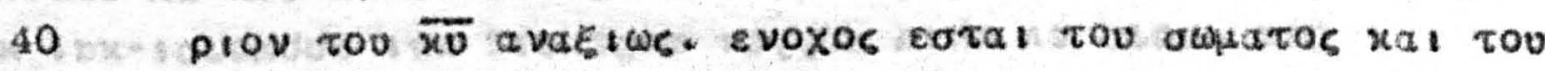
aluaros rov $\overline{x v}$. 


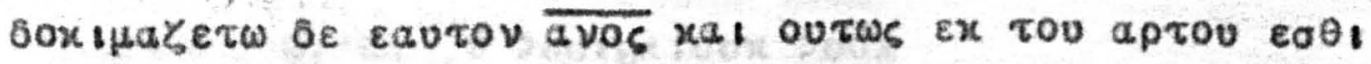

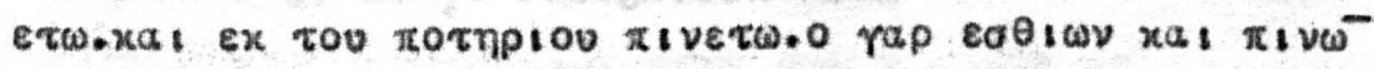

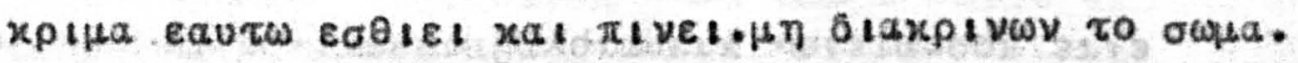

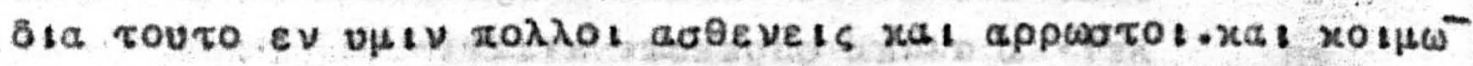

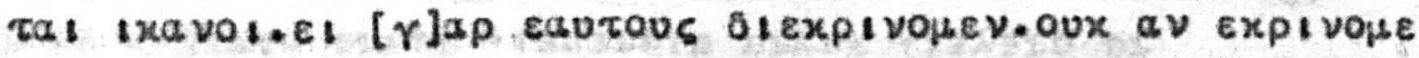

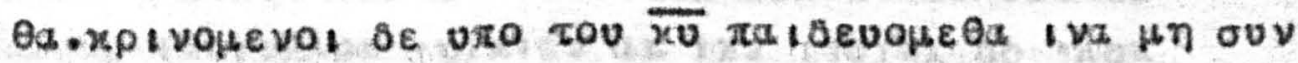

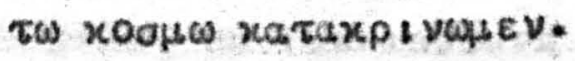

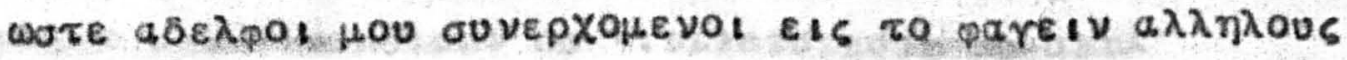
exbexea $\theta \varepsilon$.

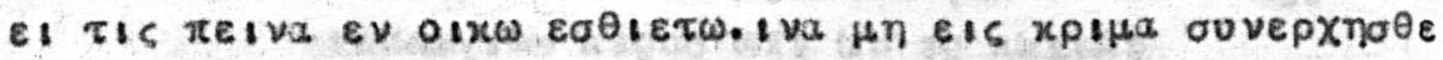

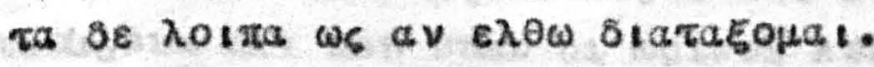

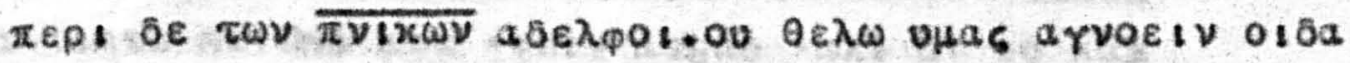

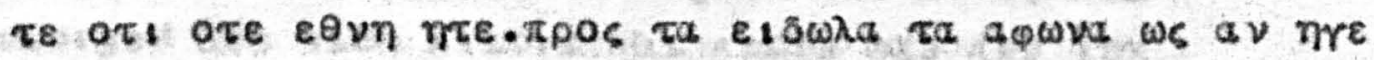

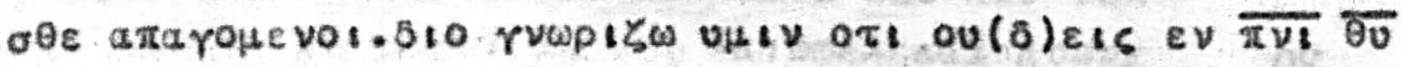

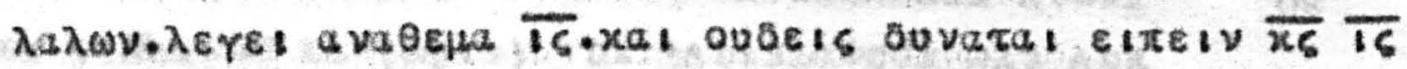
et $\mu \eta \varepsilon \nu \overline{\pi \nu t}$ ariw.

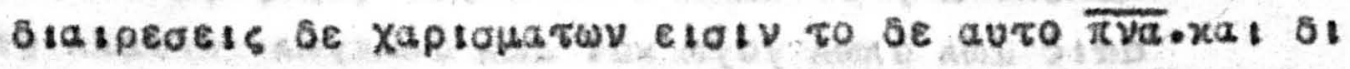
atpearis oftaxoviwy etaiv.xal o autos $\overline{x b}$ xat otaxpiats

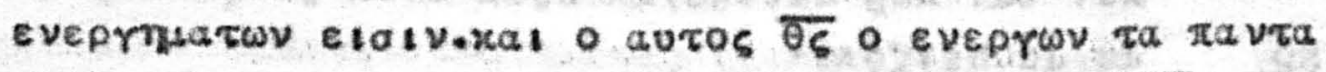

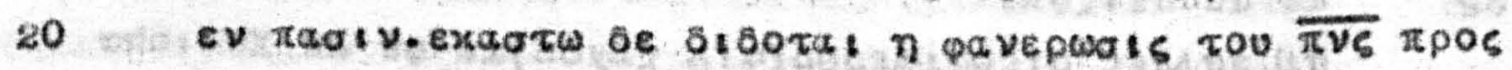

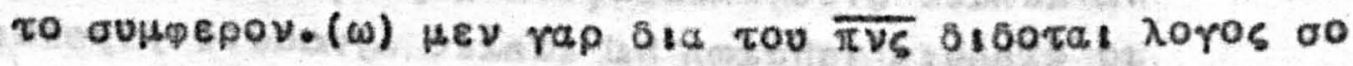

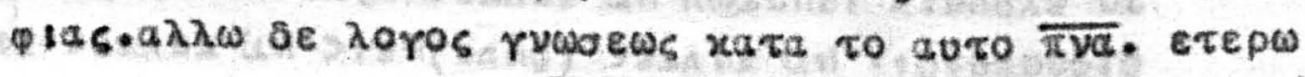

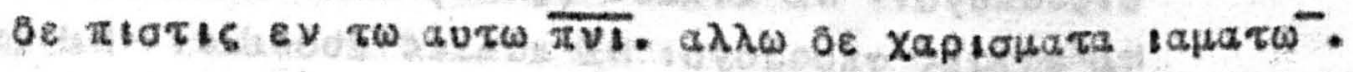

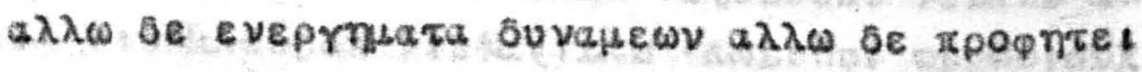

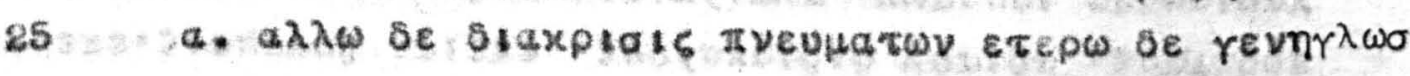

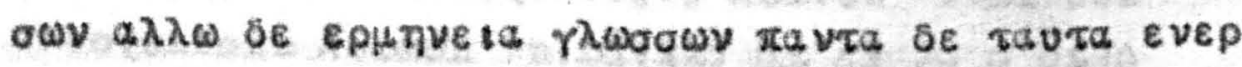

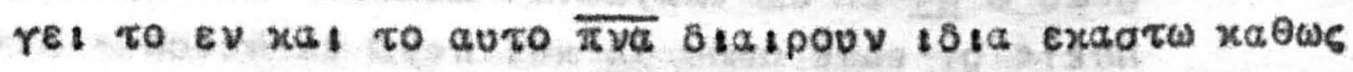
Bouneral.

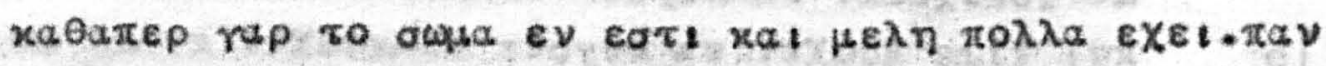

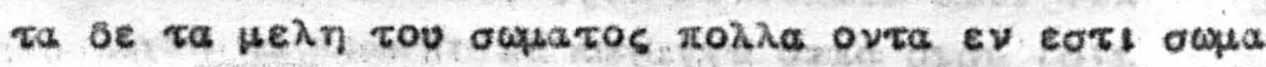

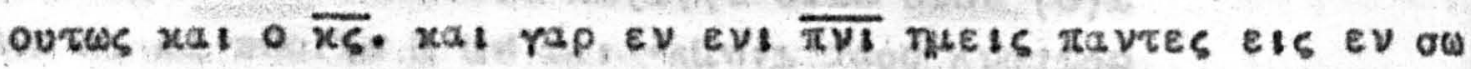

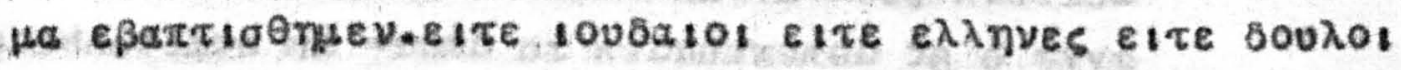

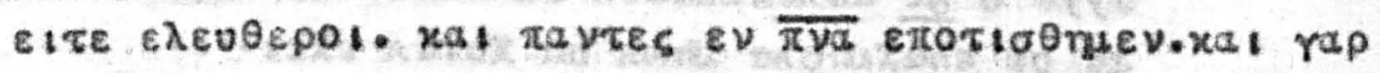

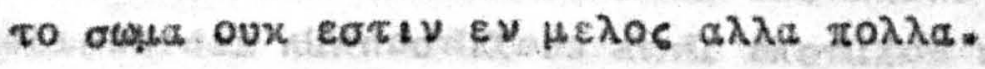

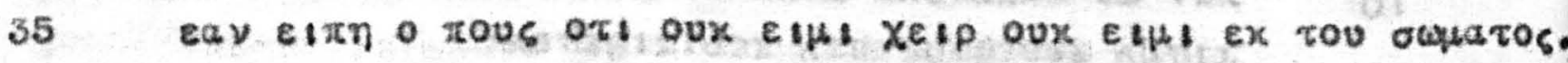

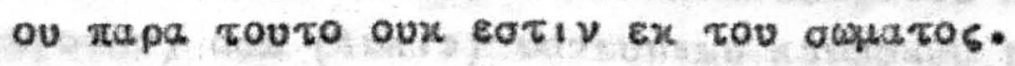

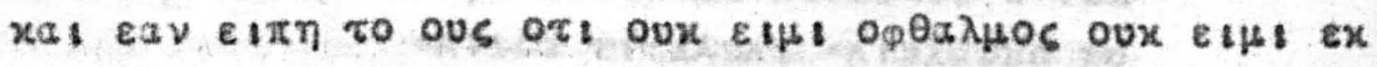

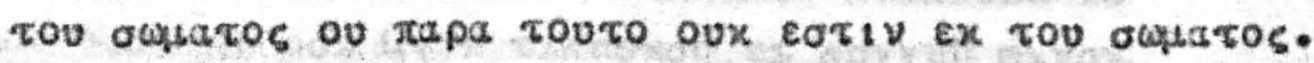

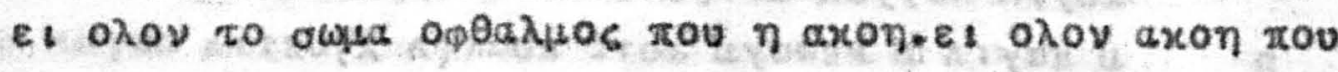

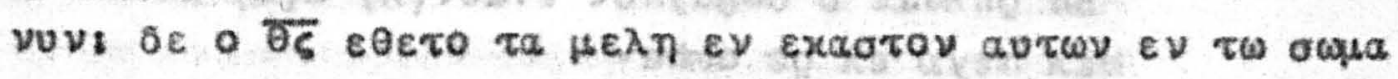




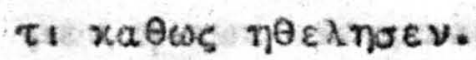

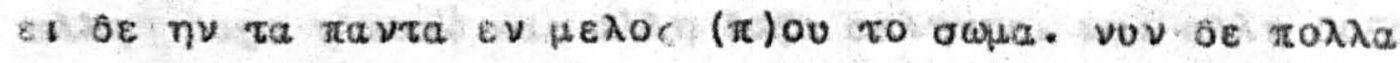

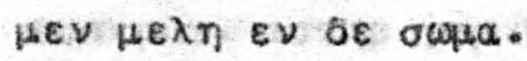

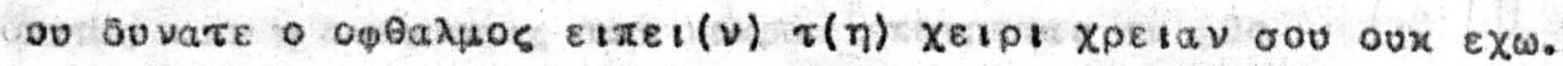

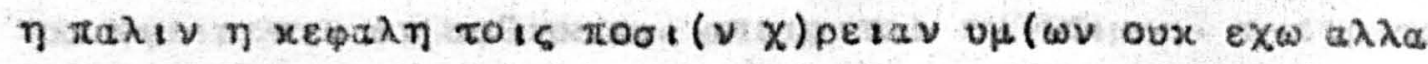

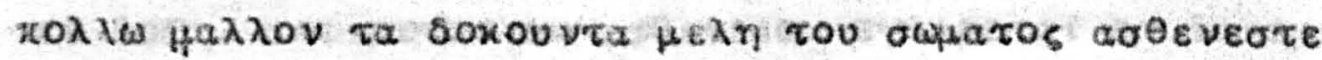
pa vזapXeเv. a varuata eariv.

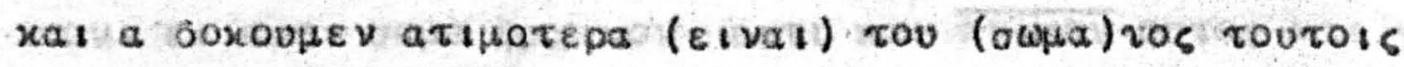

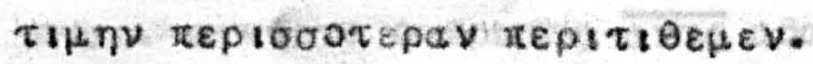

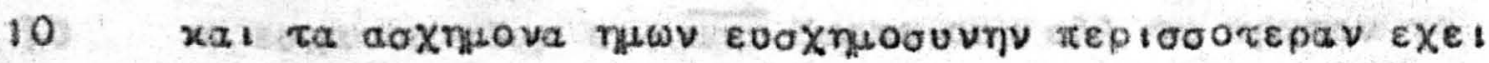

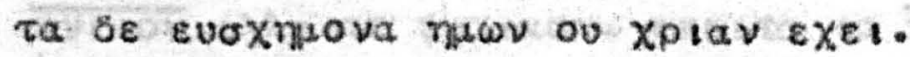

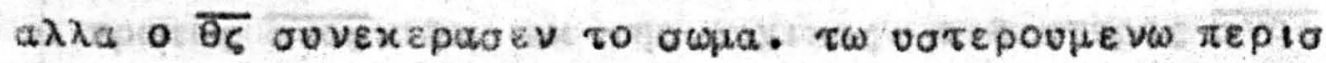

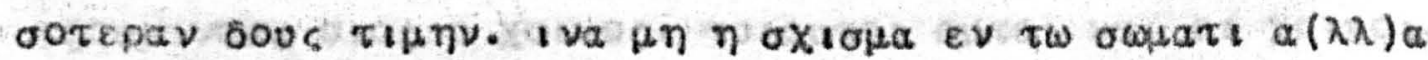

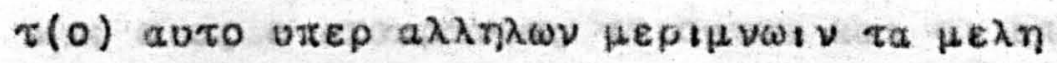

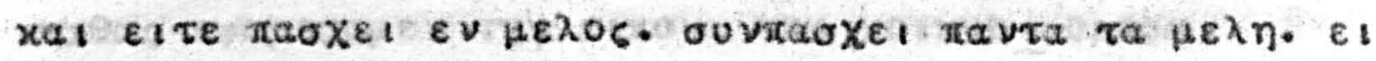

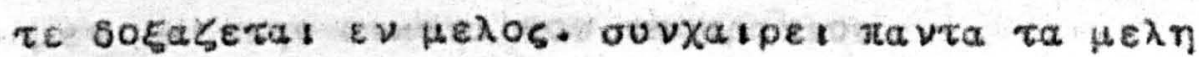

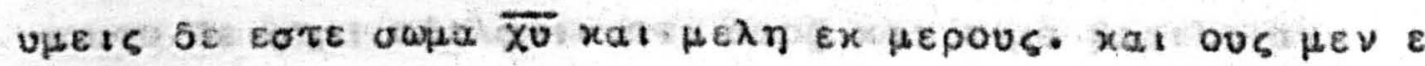

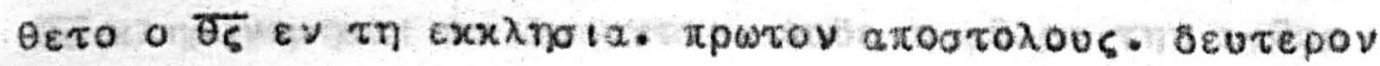

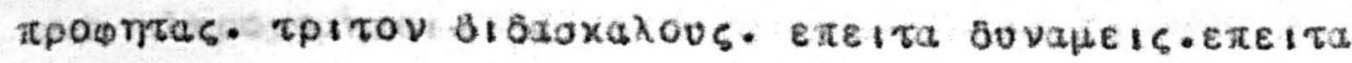

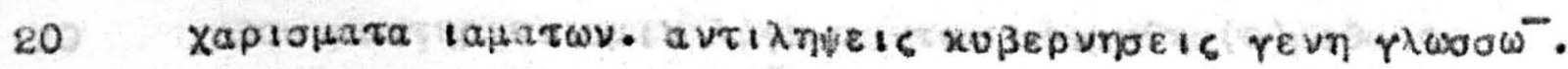

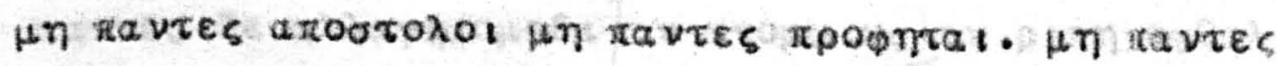

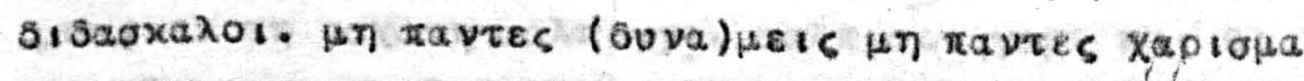

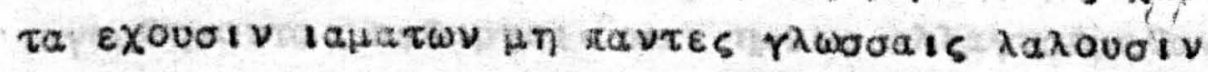

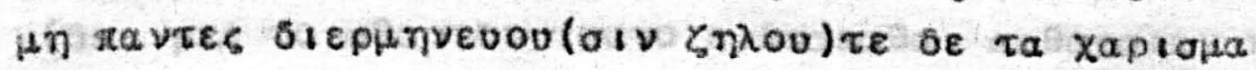

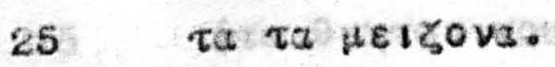

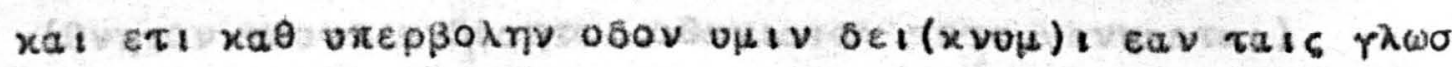

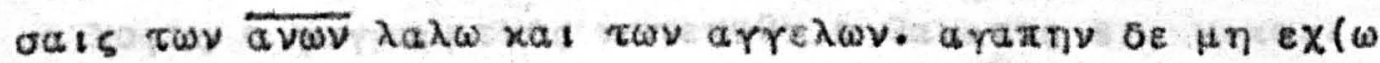

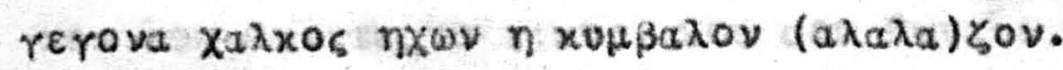

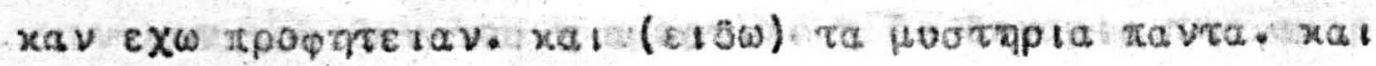

so raoav triv ruwoiv.

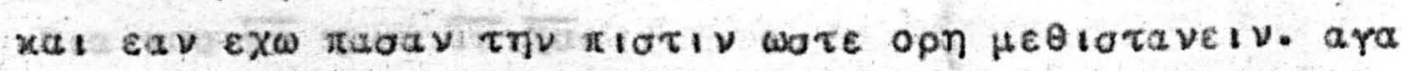

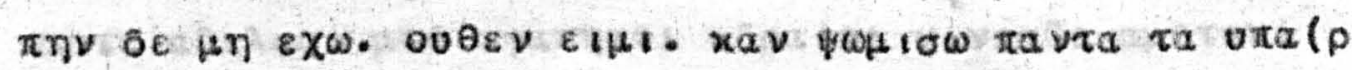

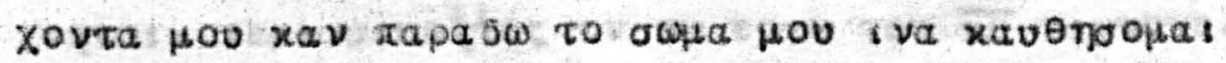

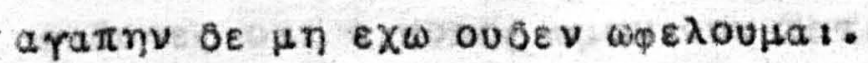

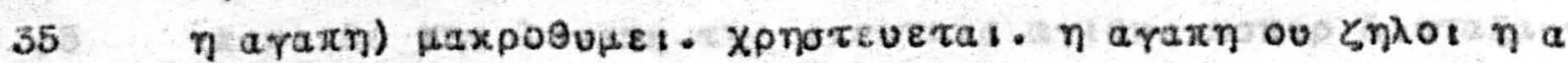

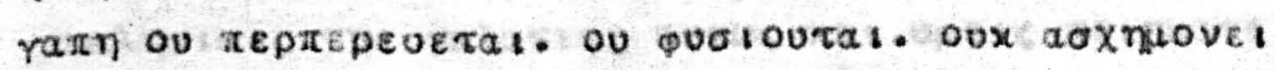

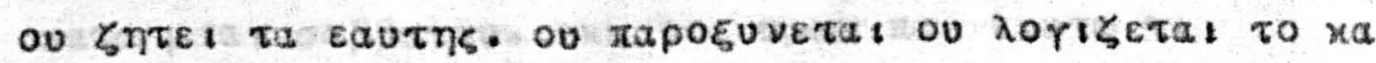

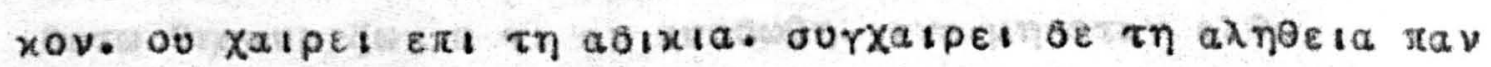

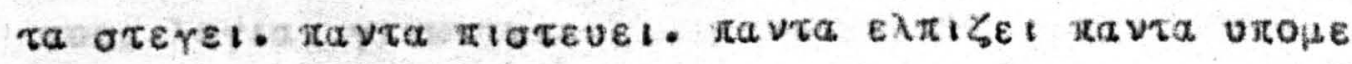

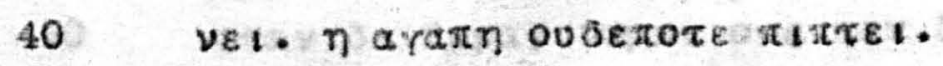

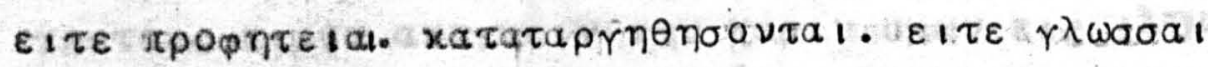

$$
\text { xpos xopivelovs a } 12.18-13.8
$$




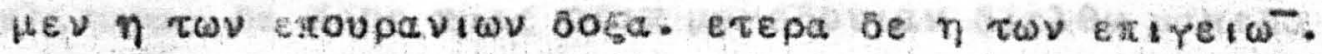

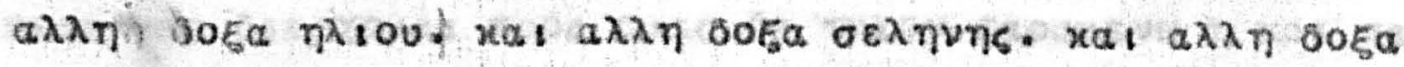

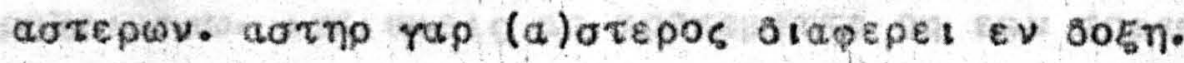

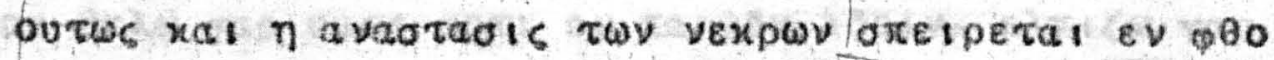

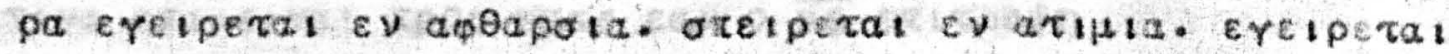

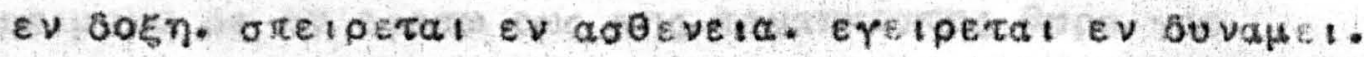

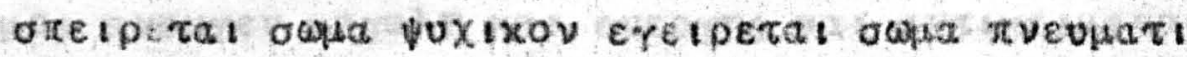

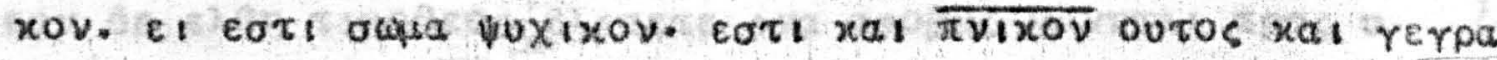

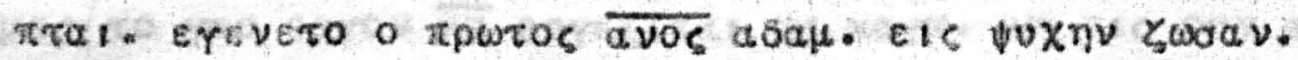

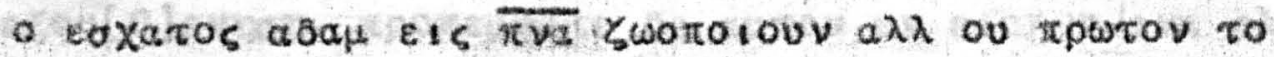

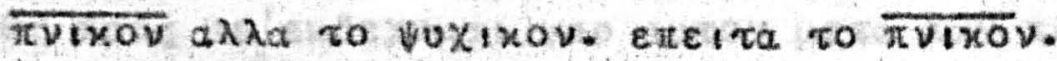

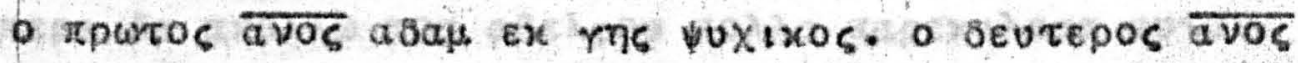
Egouparou. alos a Xolxos. rolourat outol xal ot Xolxas. xal otos o exoupavtos tolourot kat ot exoupaviot. xal

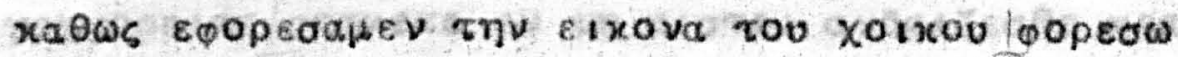

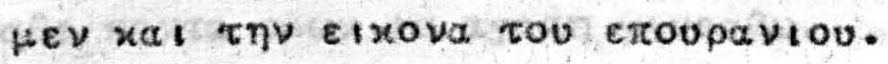

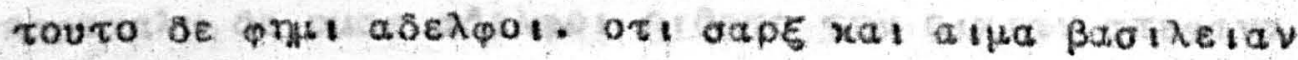

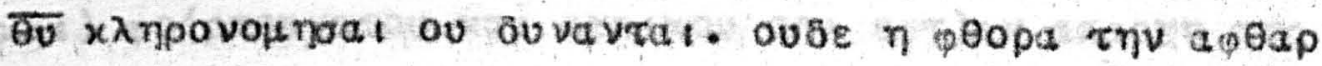
ola xhnpovoptijo 1 .

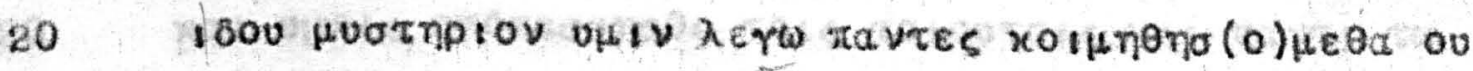

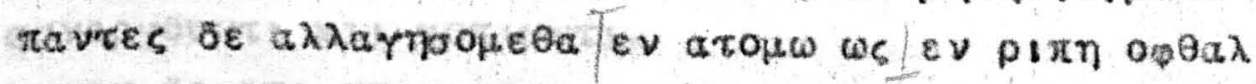

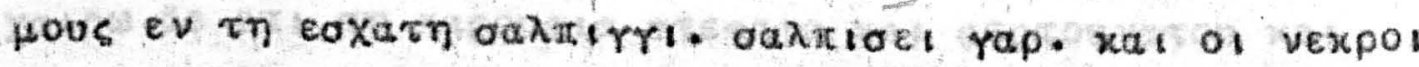

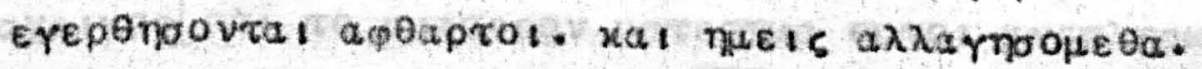

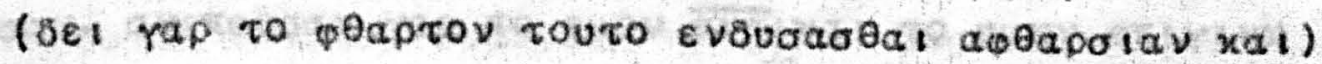

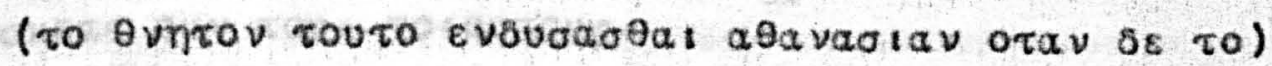

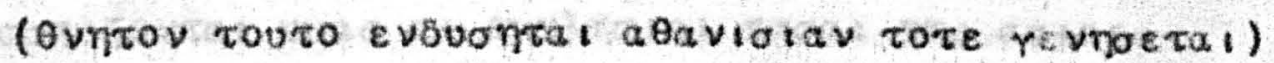

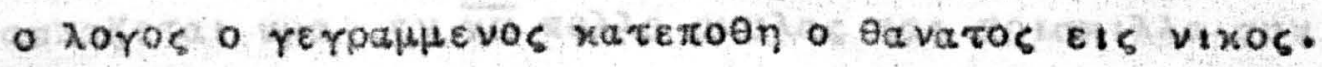

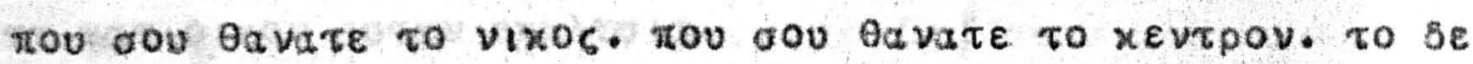

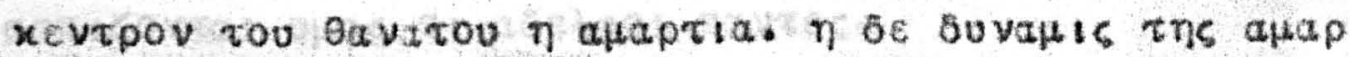

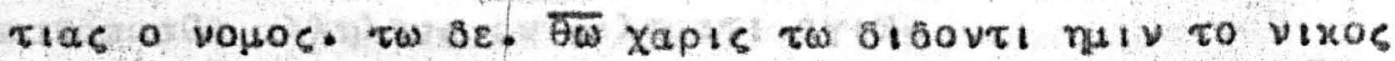
oia rov $\overline{x U}$ nuwV TV $\overline{\mathrm{Xu}}$.

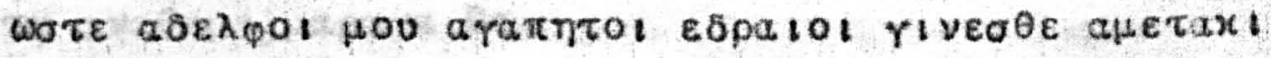

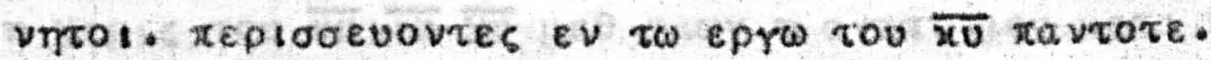

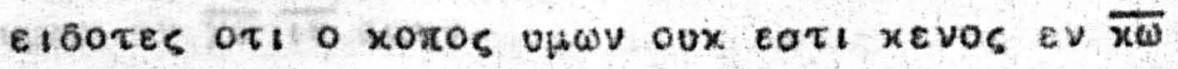

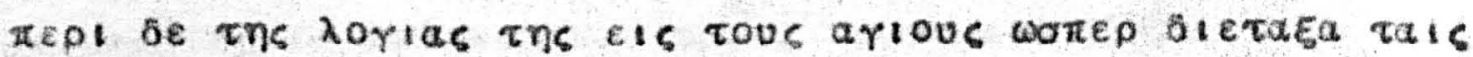
exxinotats tne yolatias outws xat vHets toingare

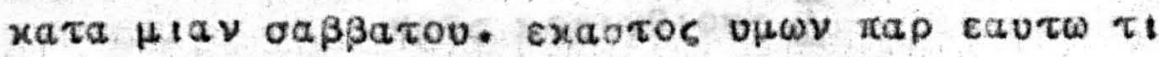

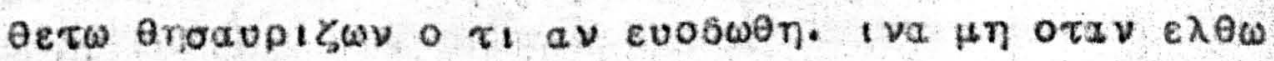

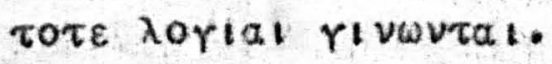

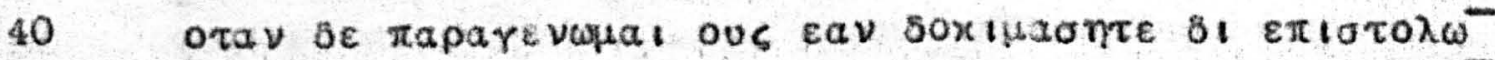

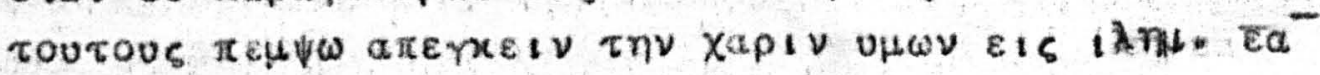

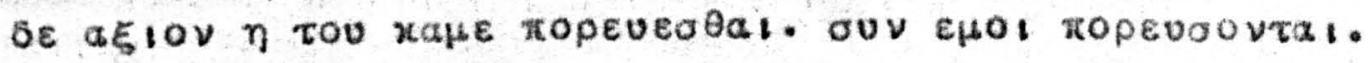




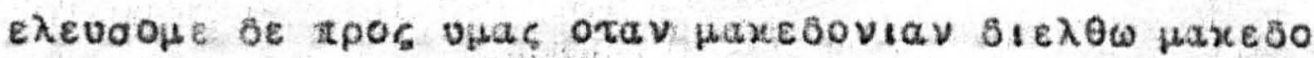

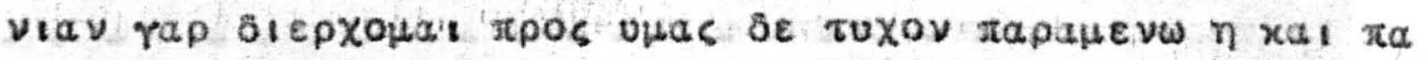

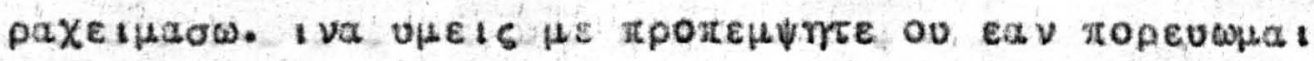

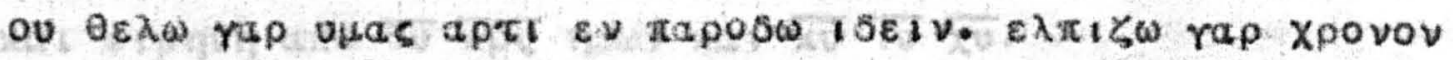

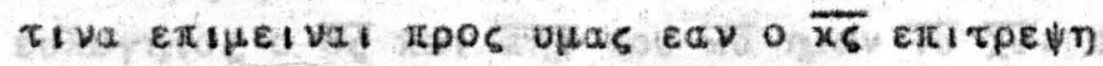

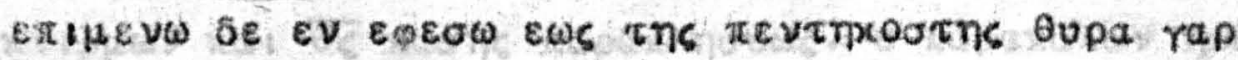

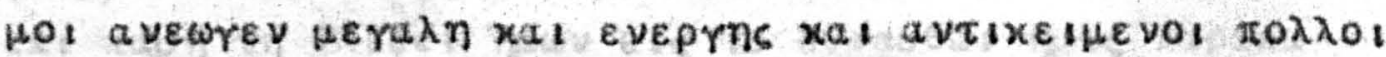

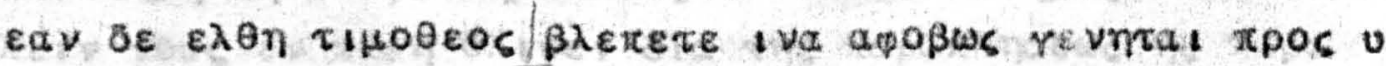

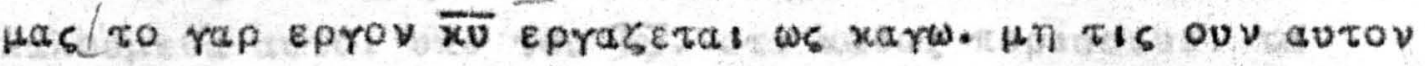

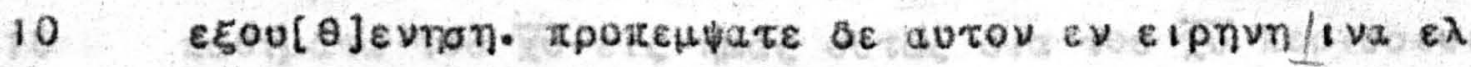

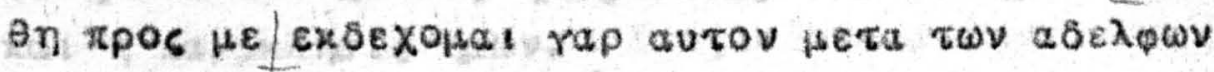

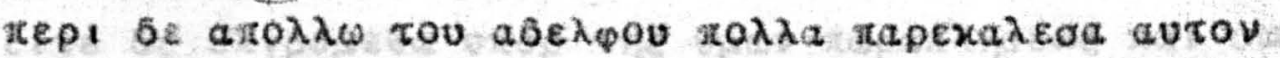

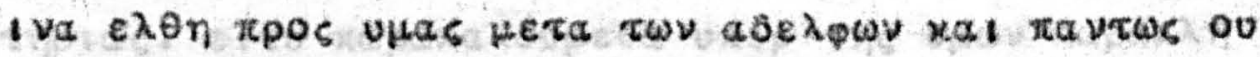

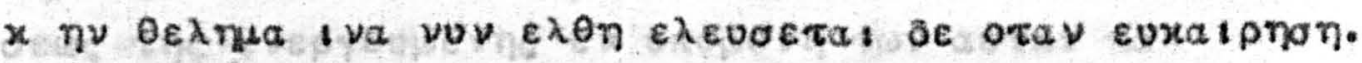

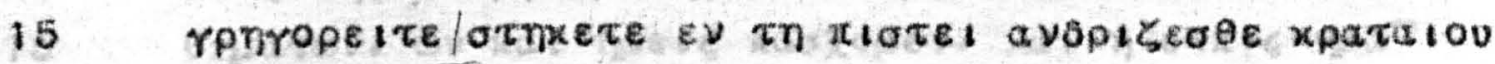

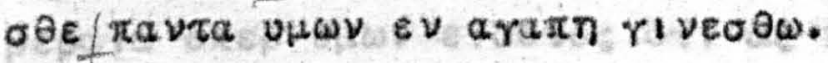

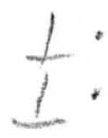

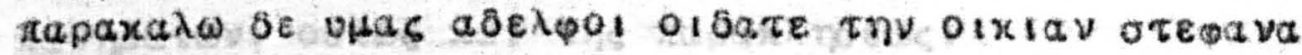

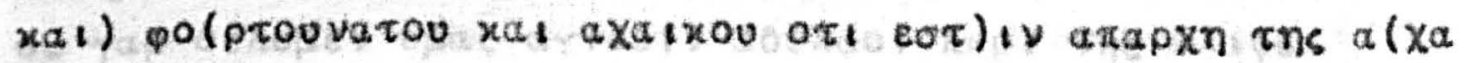
las xat ets staxoviav rols aylots etagay eavtous tva

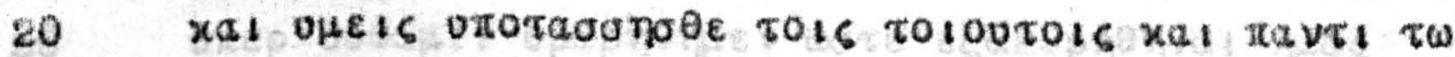
ouveprouvt xal xorlwVt?.

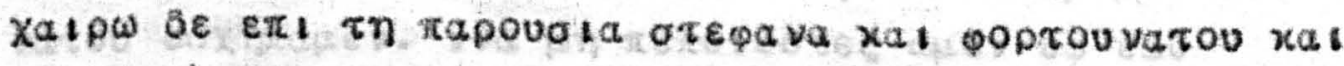

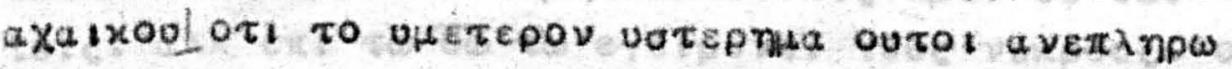

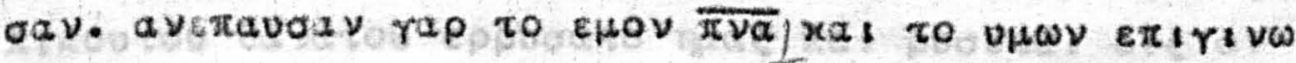
25 axere ouv rous rotourous.

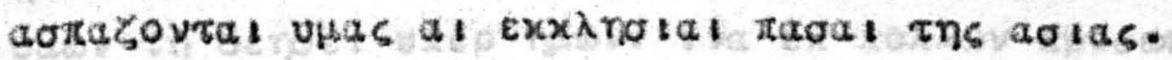

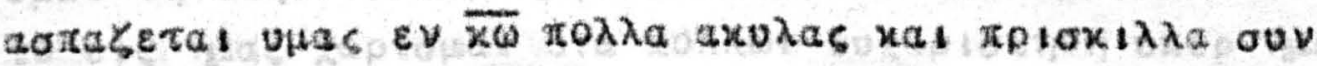
rn $x a \tau$ oixov autwy exxiroia.

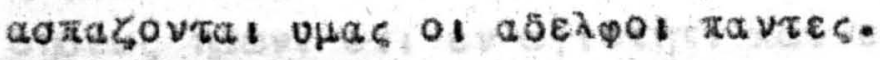

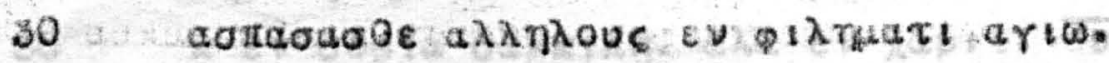

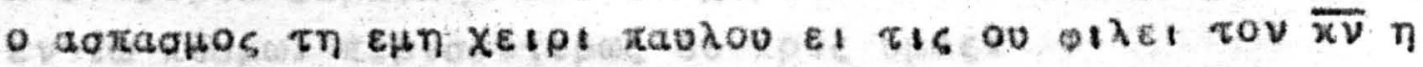
$\tau \omega$ ava $\theta \varepsilon+a$ L papava $\theta \alpha$.

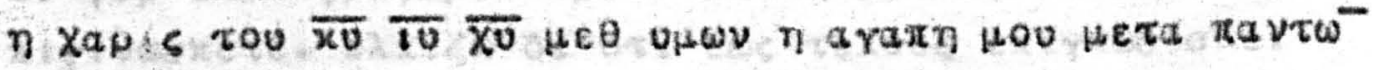

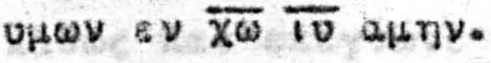

upos xop:ve1006 $\bar{A}$ 


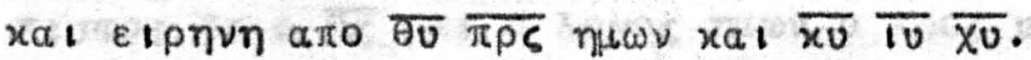

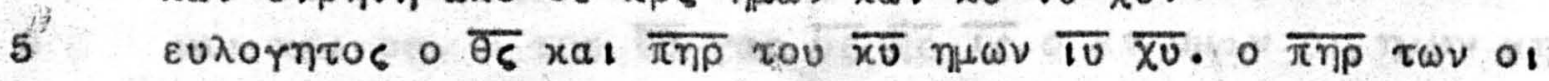

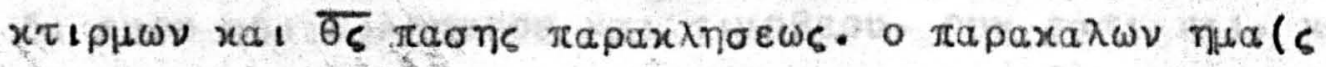
$\varepsilon \pi ! \pi a \sigma \eta \tau \eta$

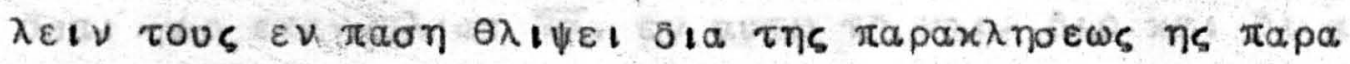

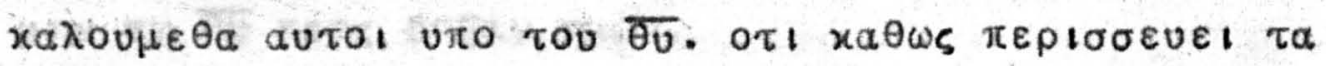

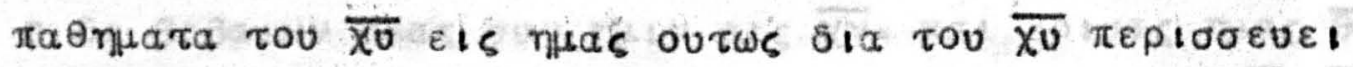

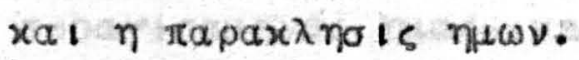

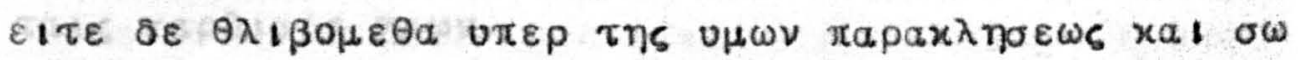

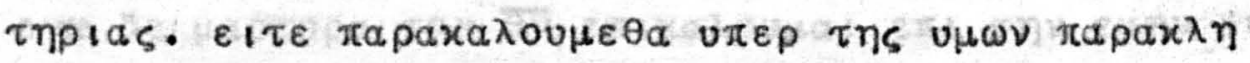

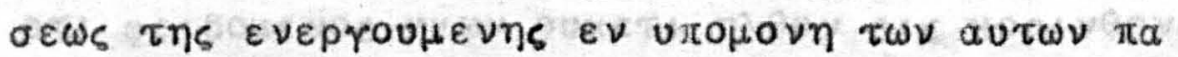

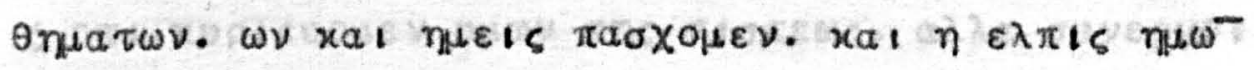

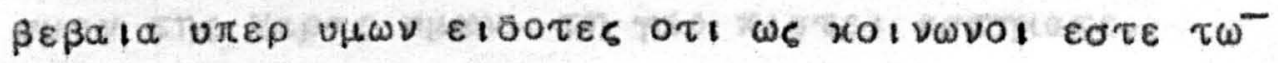
$\pi a \theta \eta \mu a \tau \omega \nu$, ou $\tau \omega s$ xal $\tau \eta_{s} \pi a p a x \lambda \eta_{\sigma} \varepsilon \omega s$.

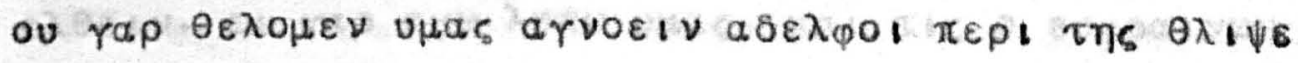

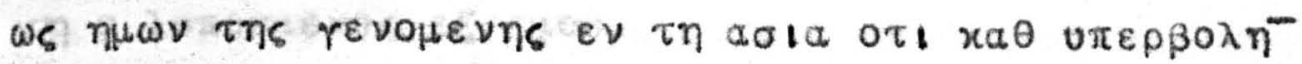

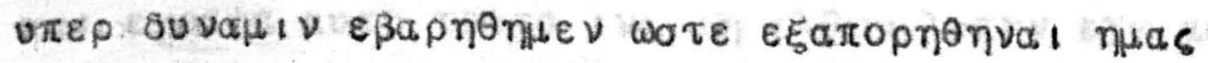

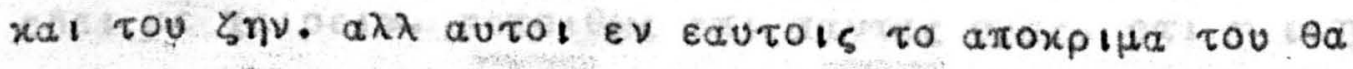

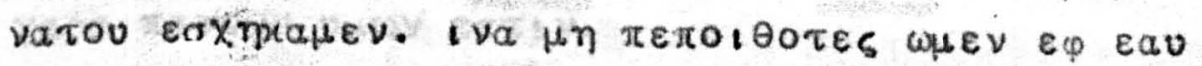

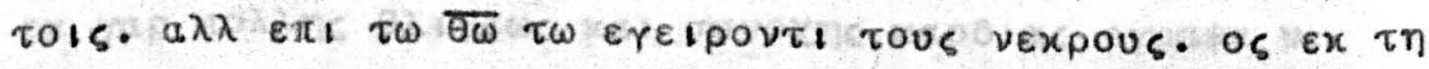

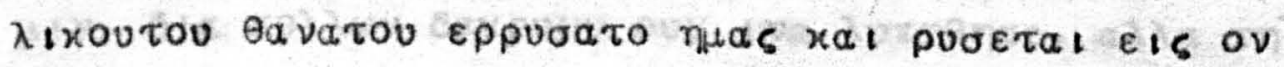

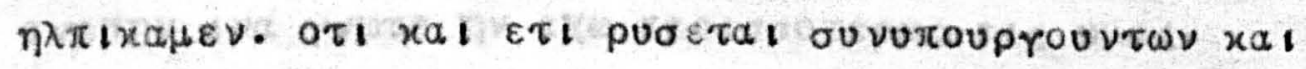

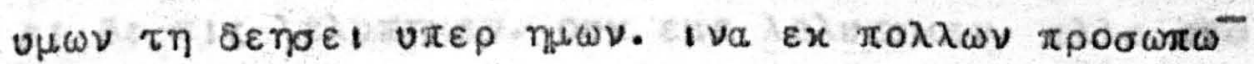

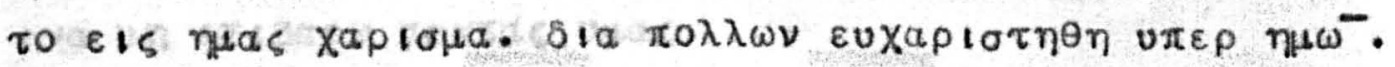

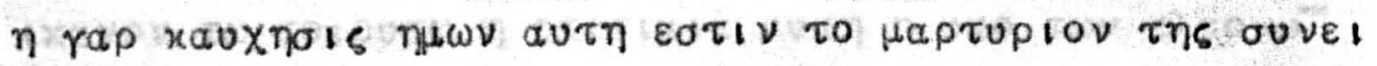

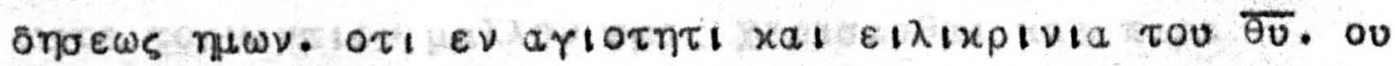

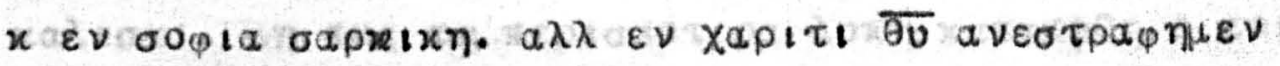

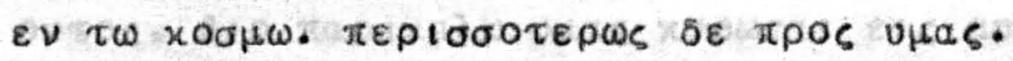

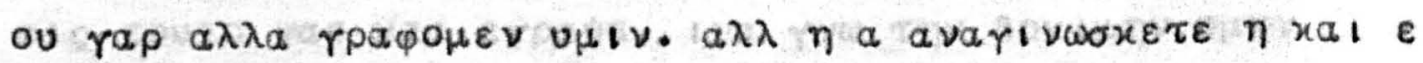

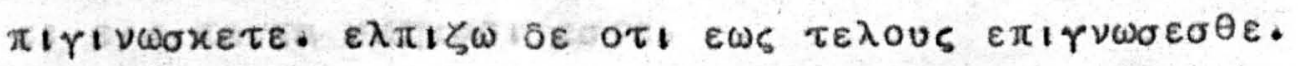

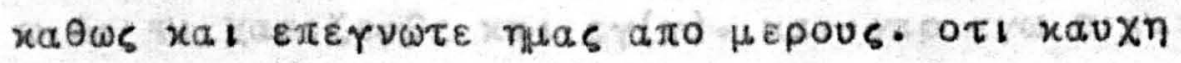

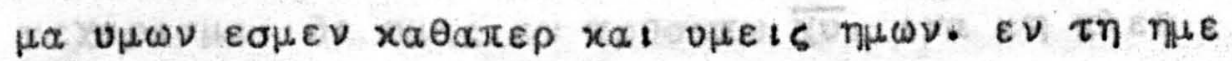

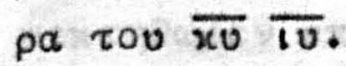

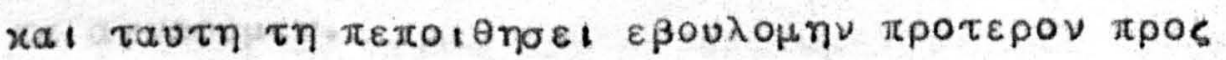

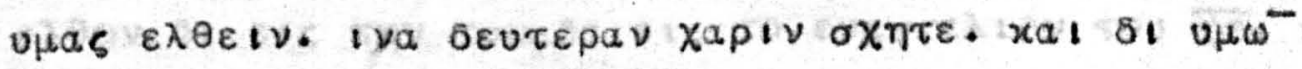

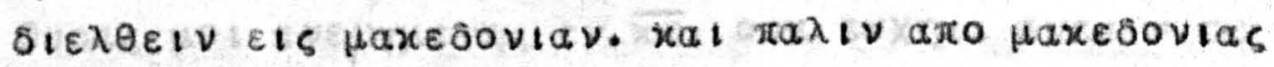

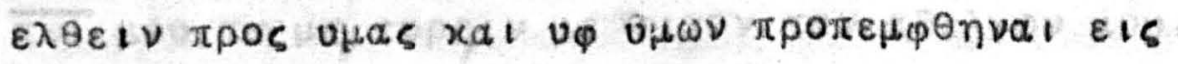

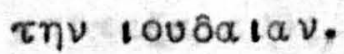




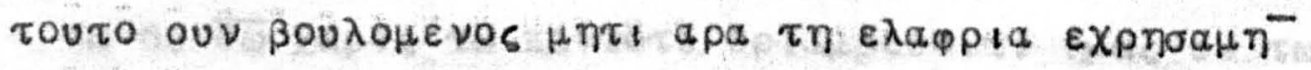

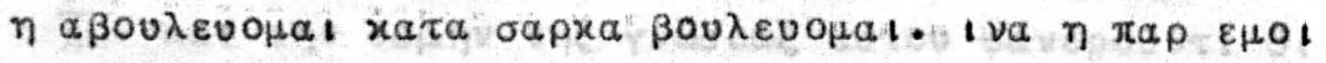
to val. val. xal to ou. ou.

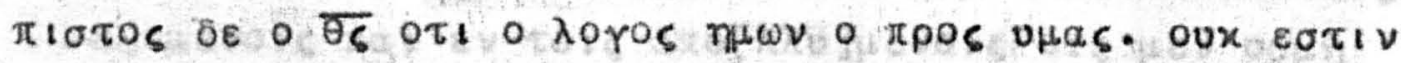

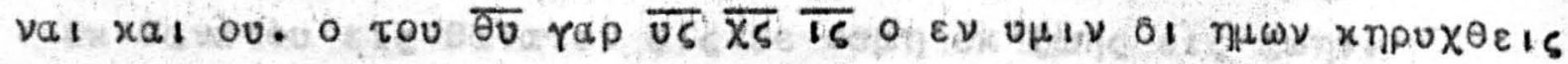

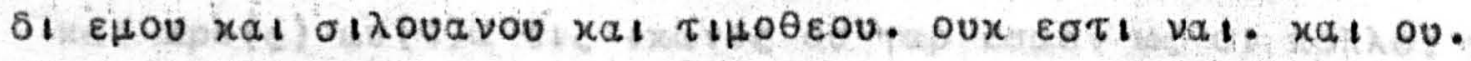

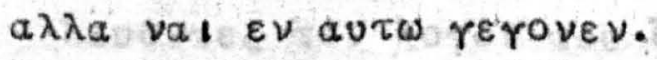

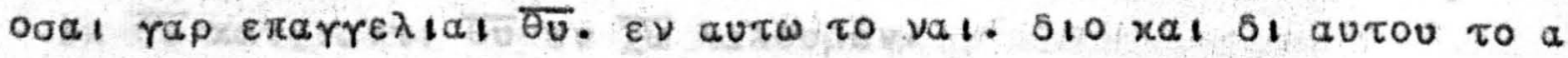

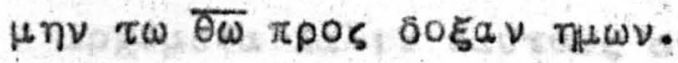

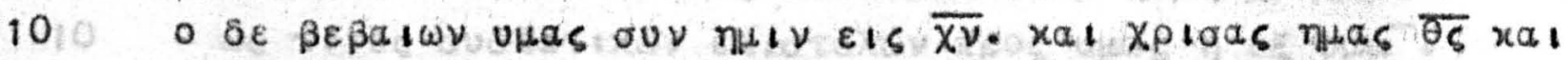

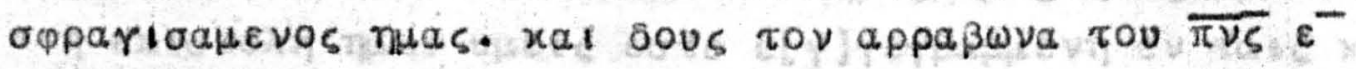
tais xapsiais nuwv.

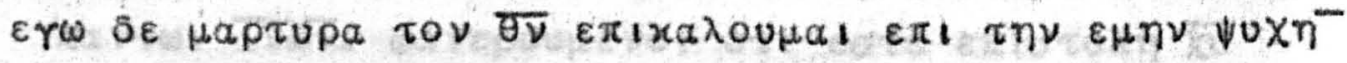

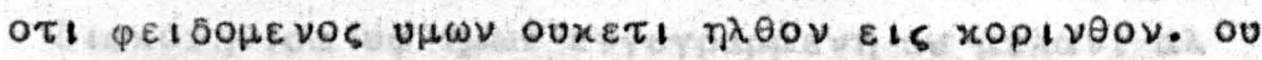

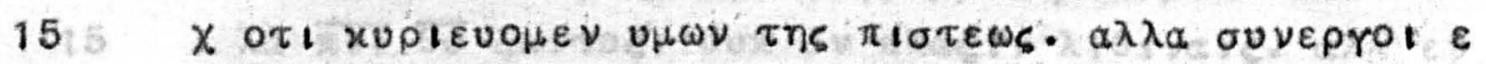

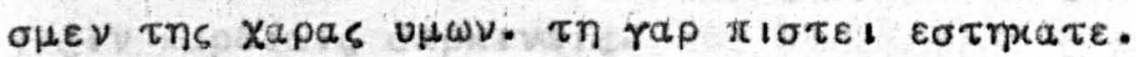

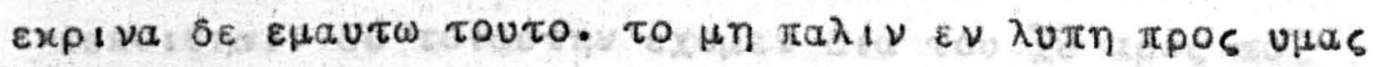

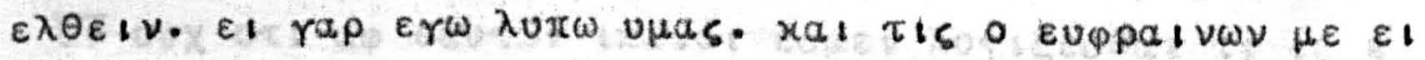

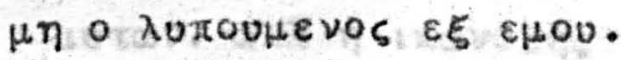

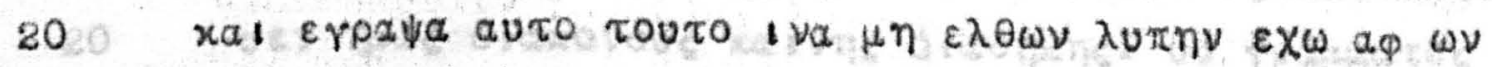

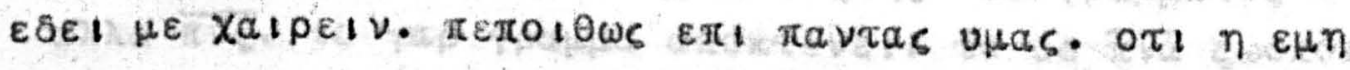

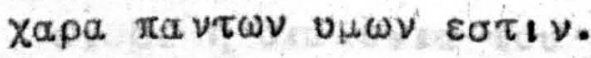

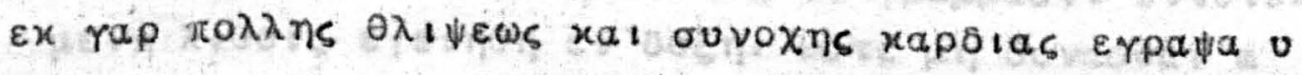

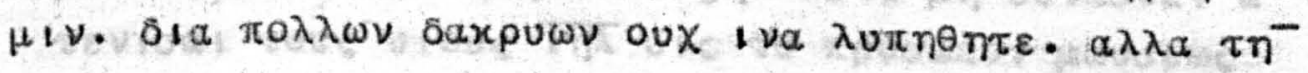

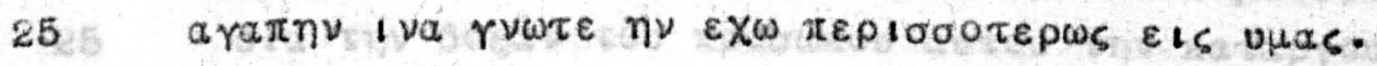

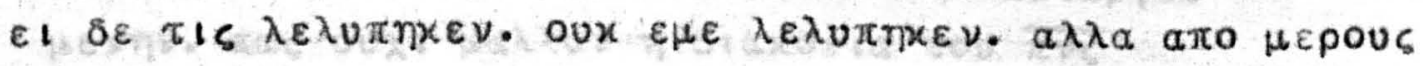

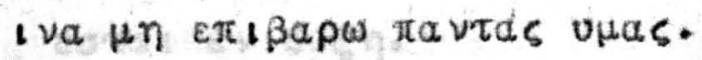

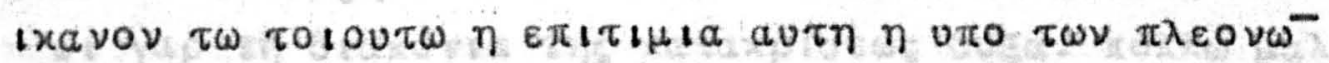

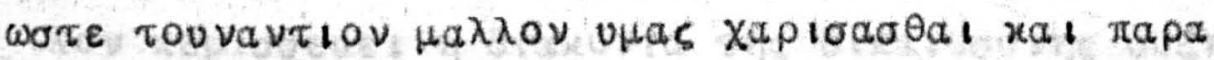

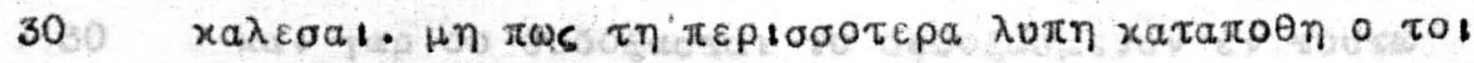

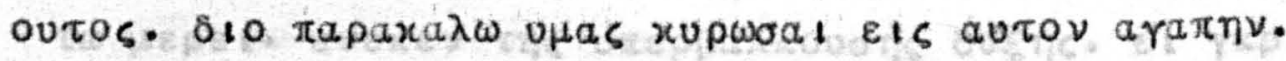

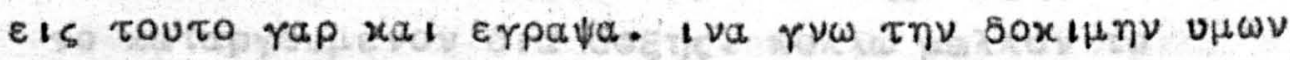

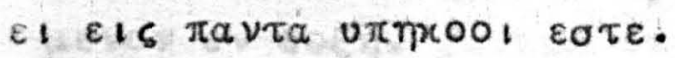

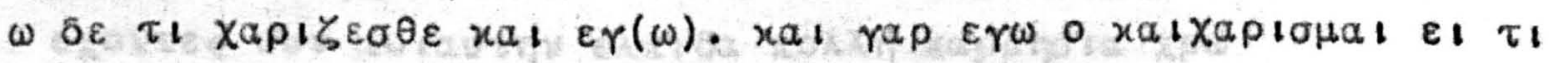

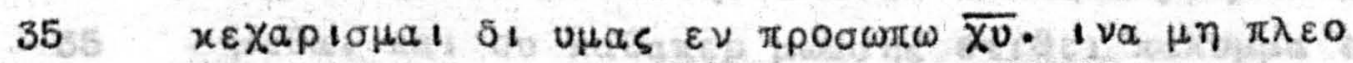

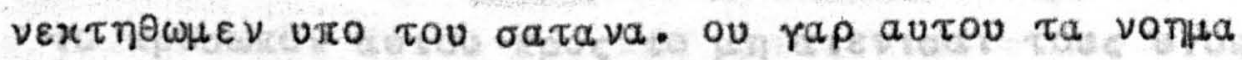
$\tau a$ arvoounev.

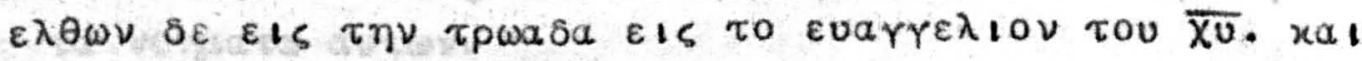

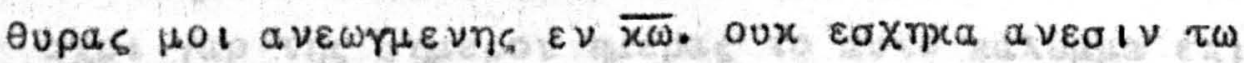

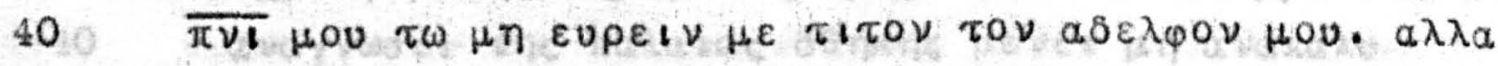

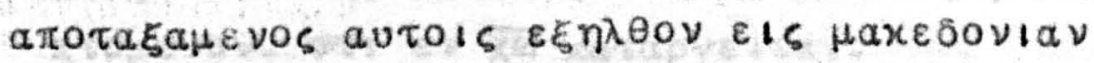




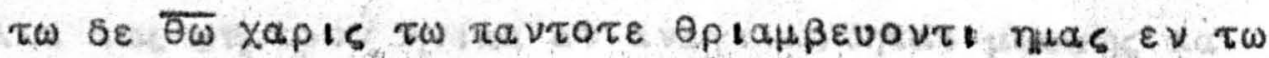

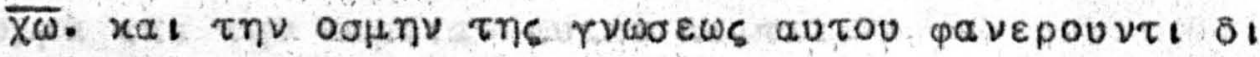

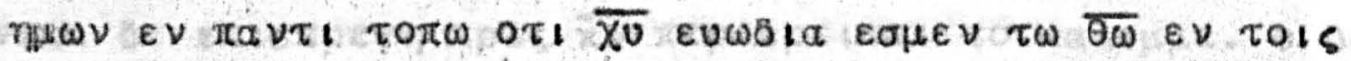

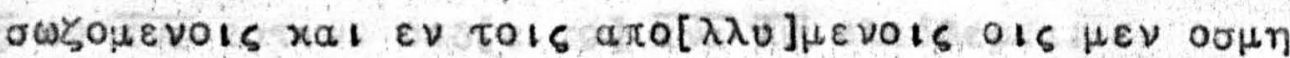

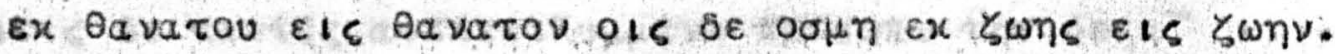

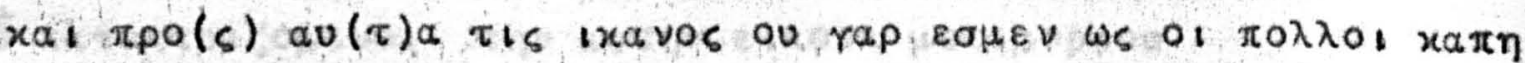

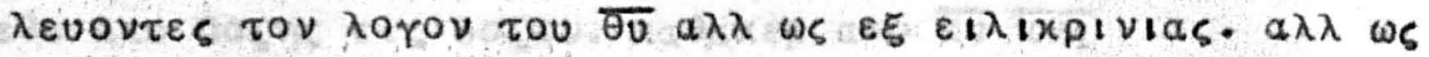

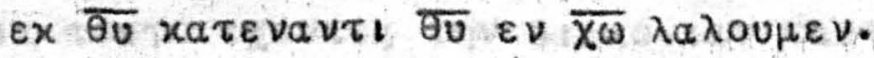

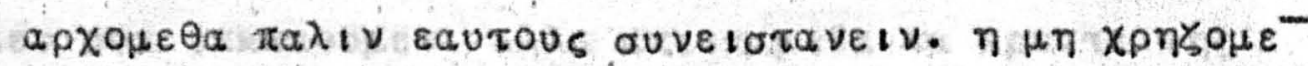

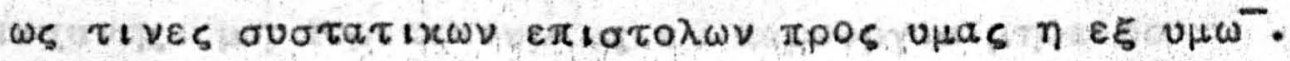

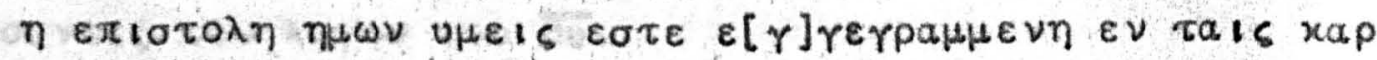

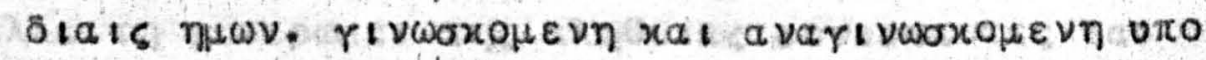

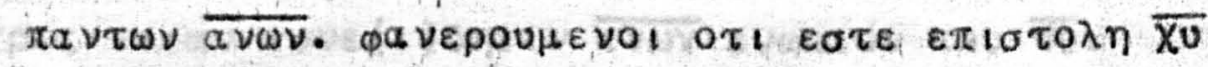

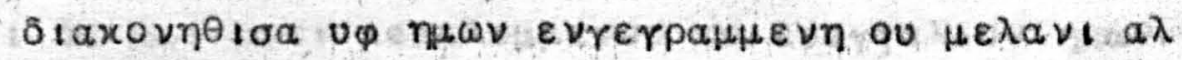

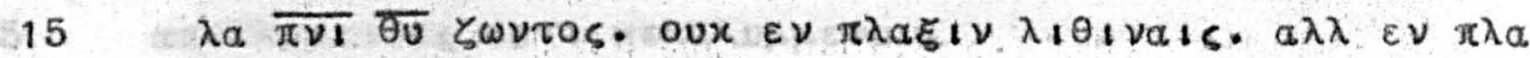
కiv xapótals oapx!vais.

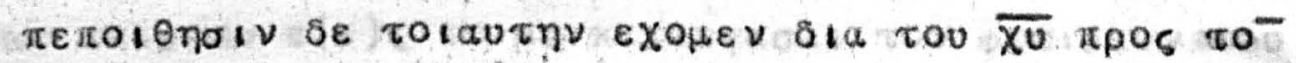

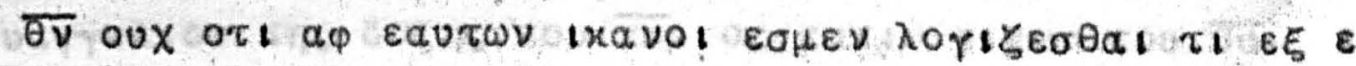

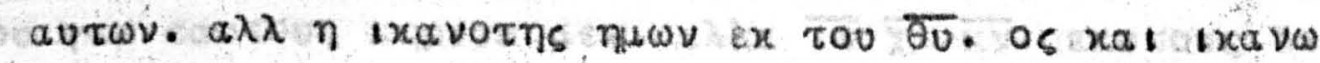

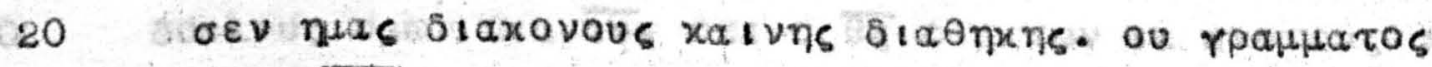

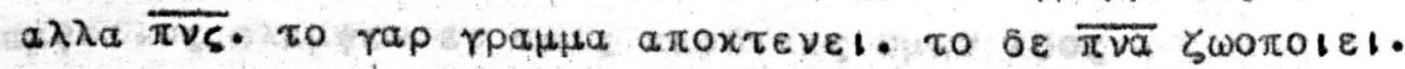

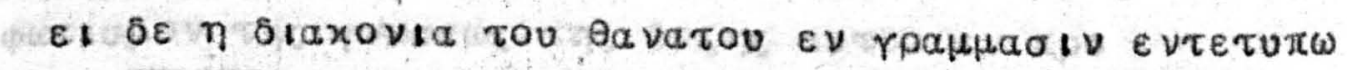

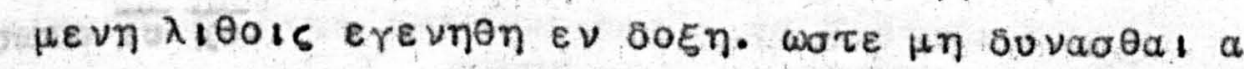

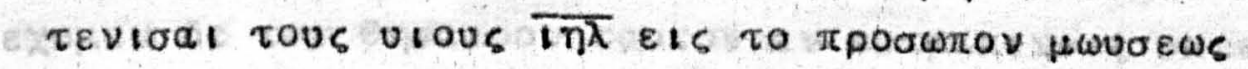

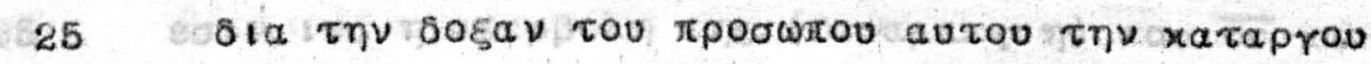

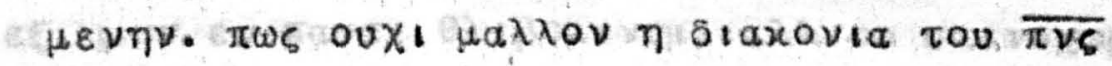

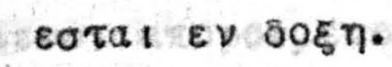

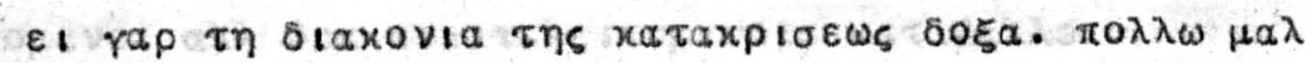

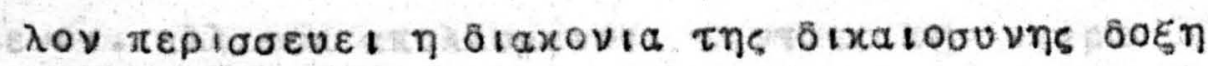

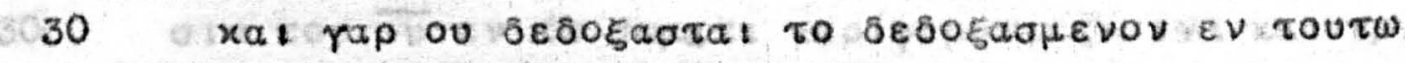

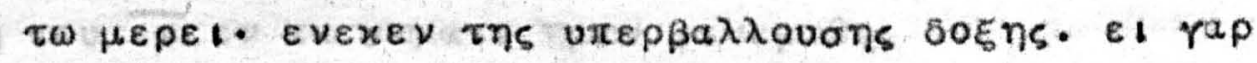

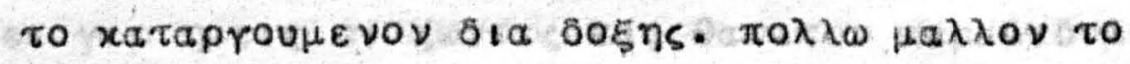

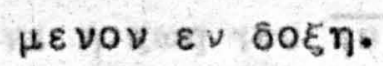

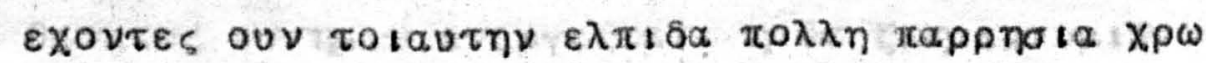

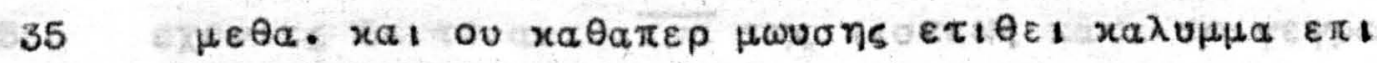

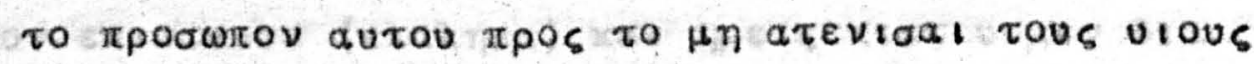

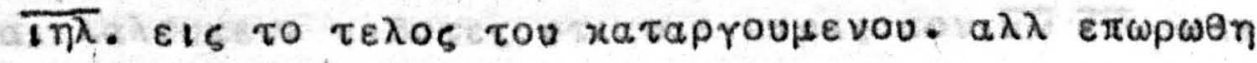

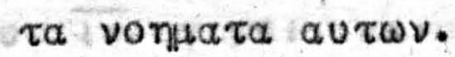

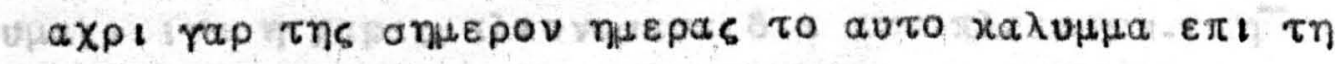

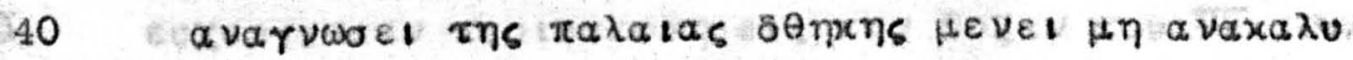

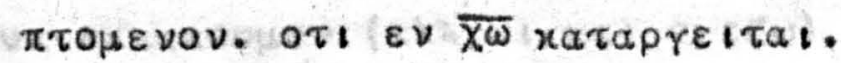




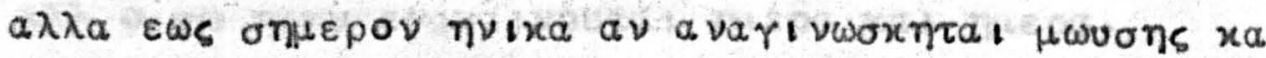

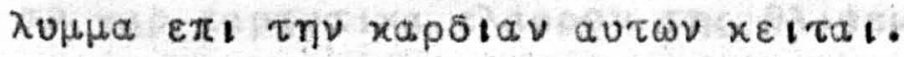

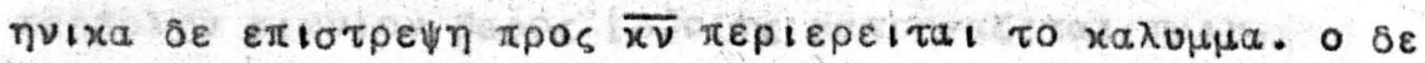

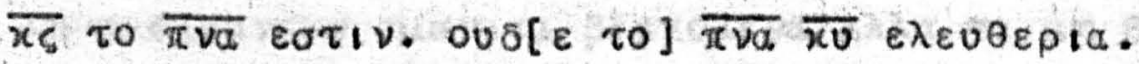

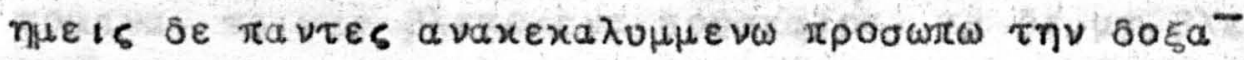

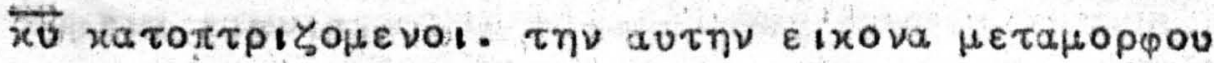

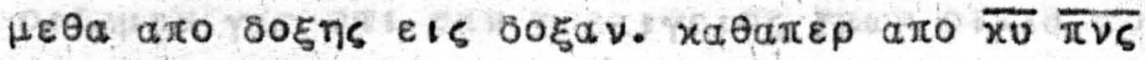

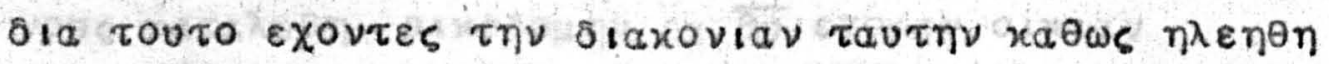

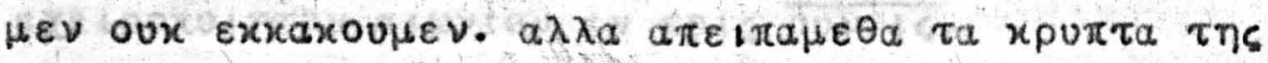

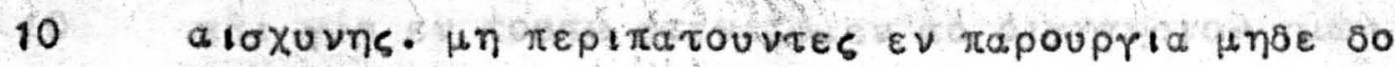

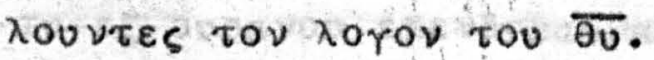

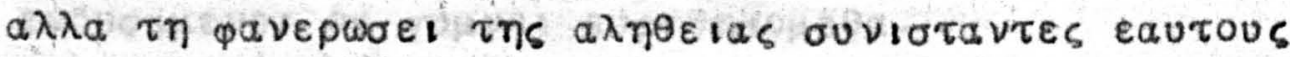

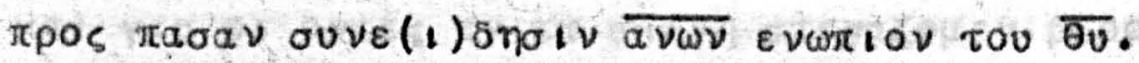

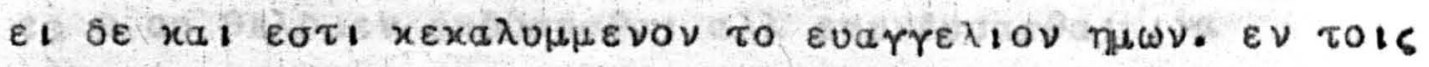

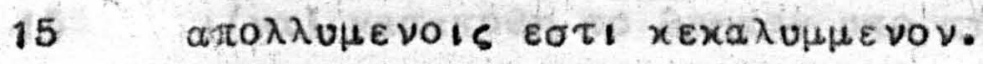

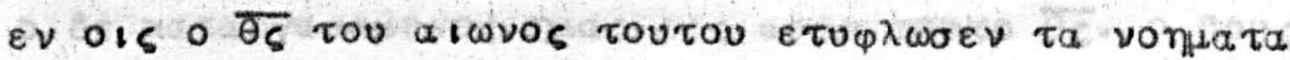

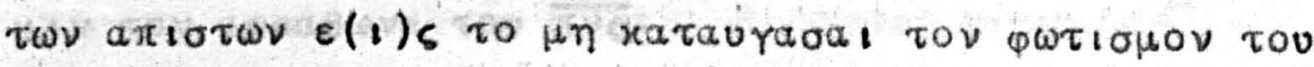

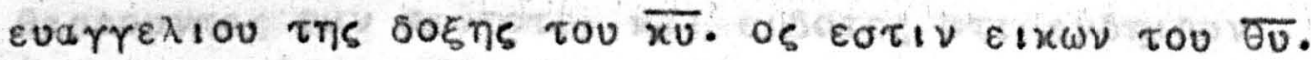

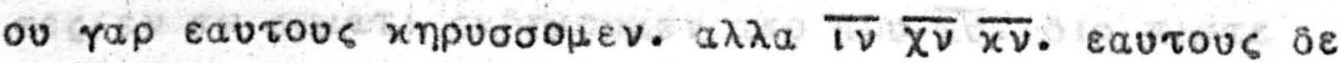

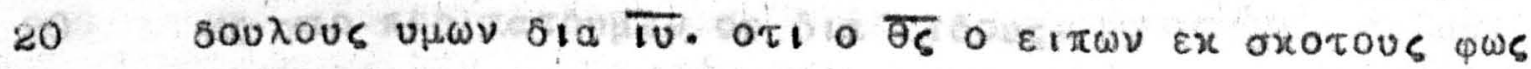

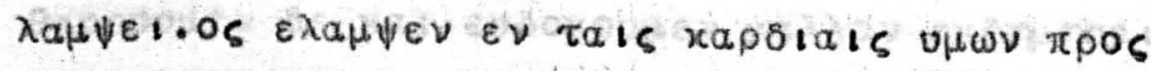

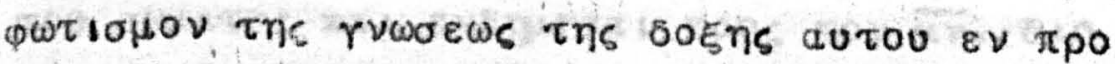
$\sigma \omega \pi \omega \overline{10} \overline{\chi v}$.

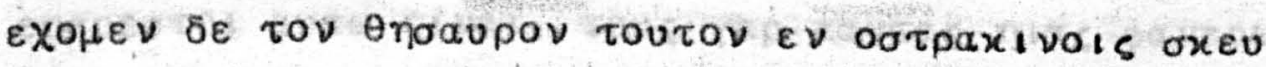
25 EoIV t va $\eta$ u

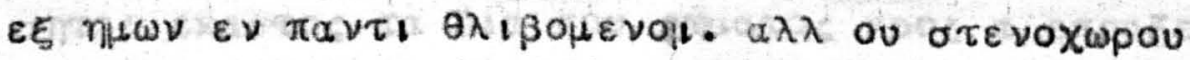

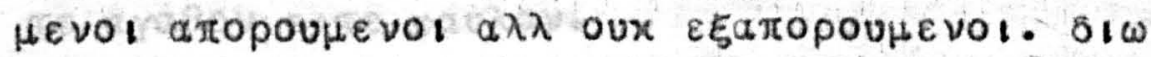

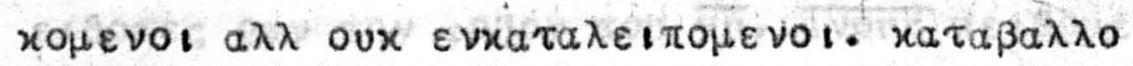

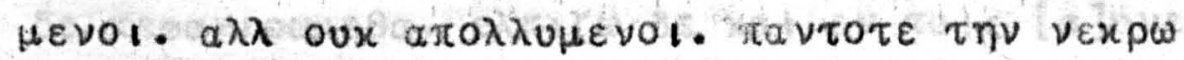

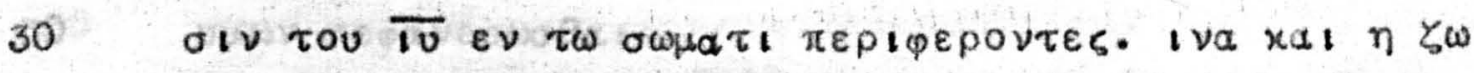

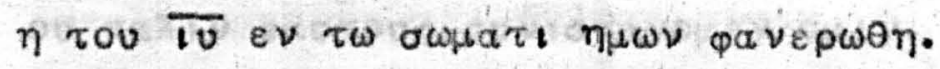

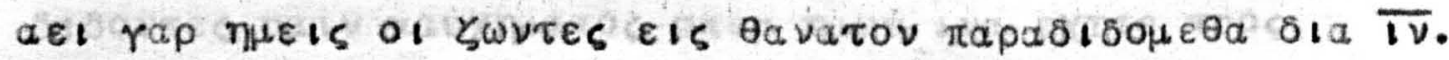

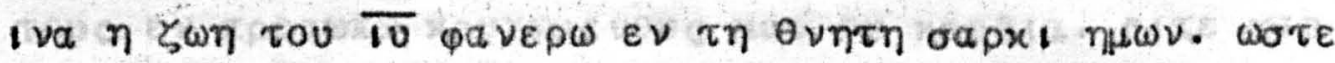

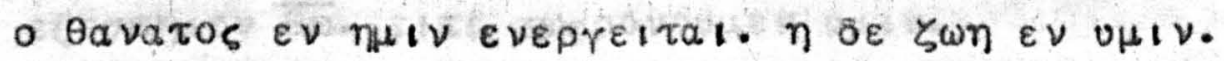

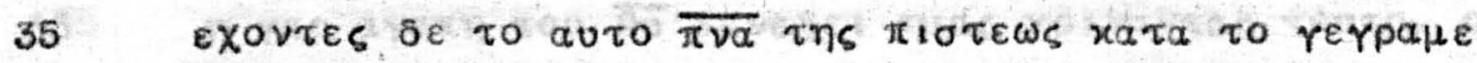

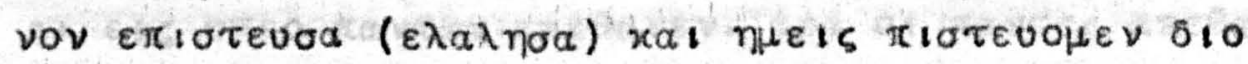

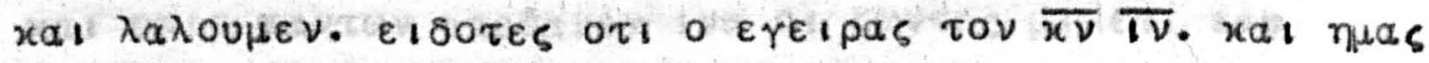

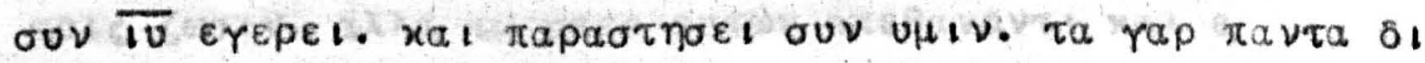
vHas. Iva $\eta$ Xapıs $\pi \lambda \varepsilon \sigma \nu a \sigma a \sigma a$ sıa $\tau \omega \nu \pi \lambda \varepsilon ı \nu \omega \nu . \tau \eta-$

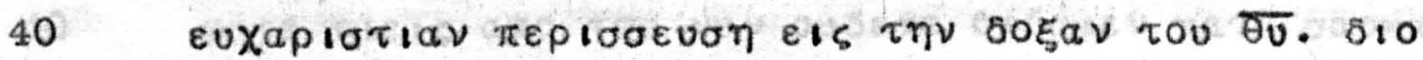

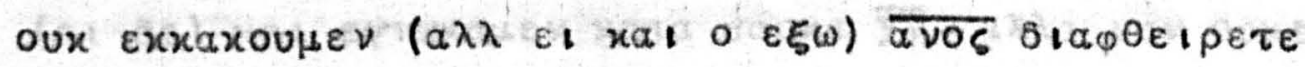




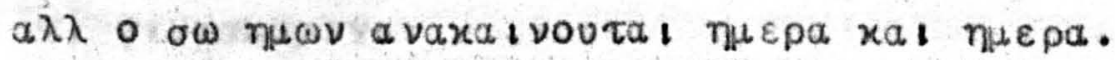

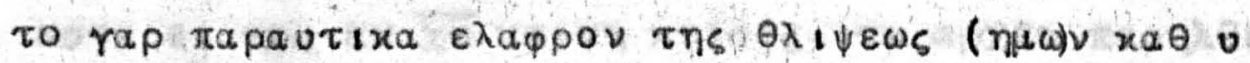

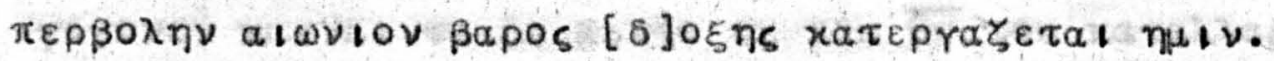

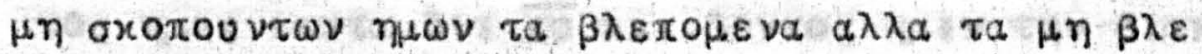

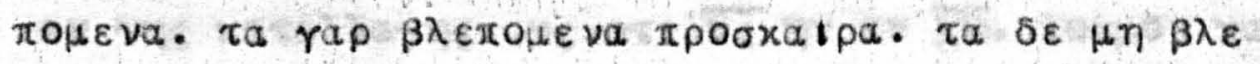

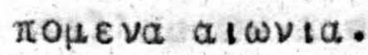

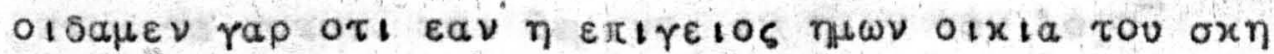

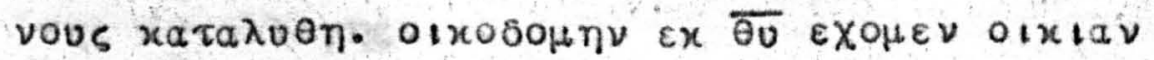
aXEipotointov alwViov \&V Tols OUVOLG.

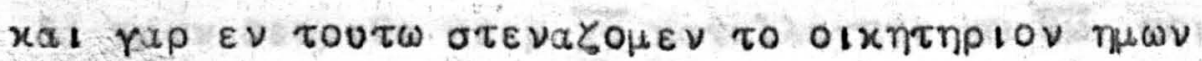

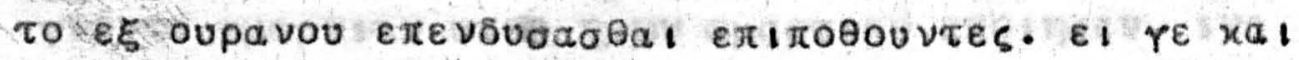

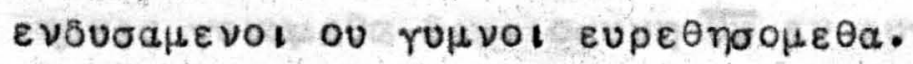

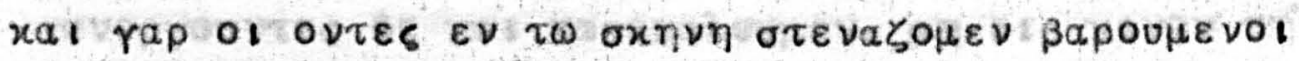

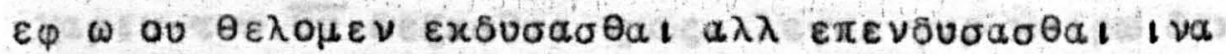

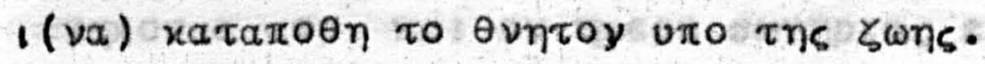

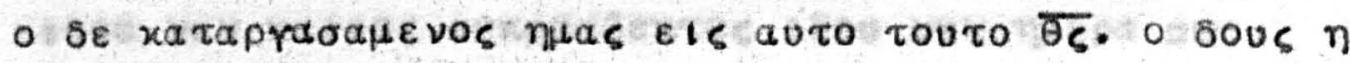

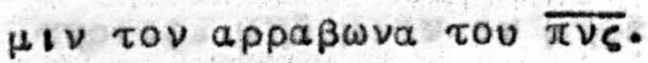

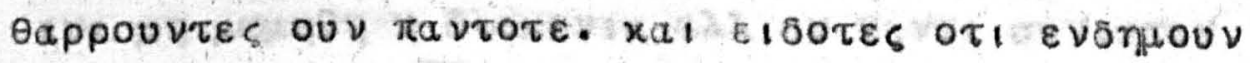

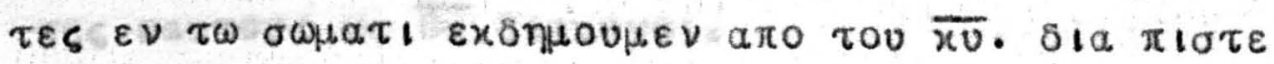

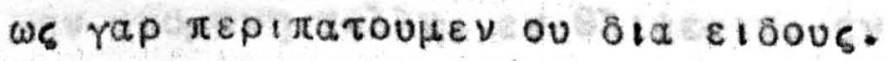

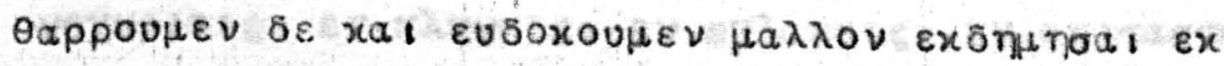

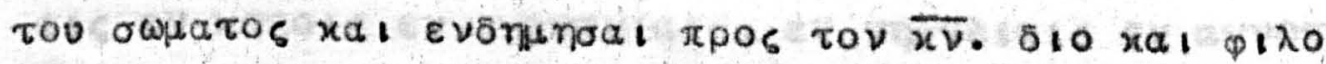

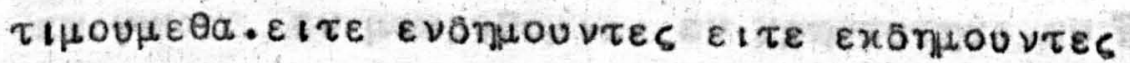

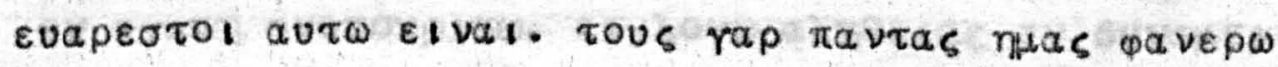

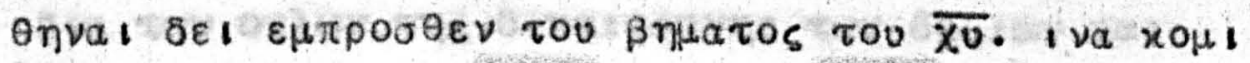

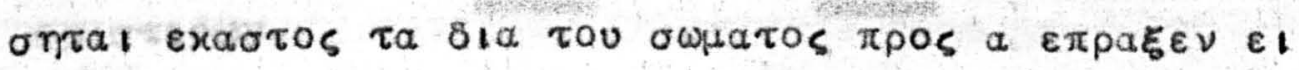

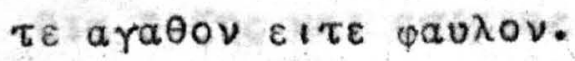

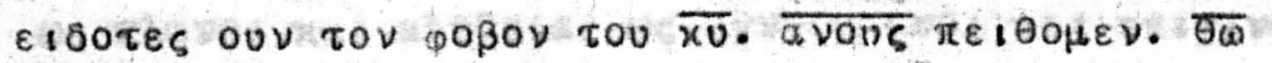

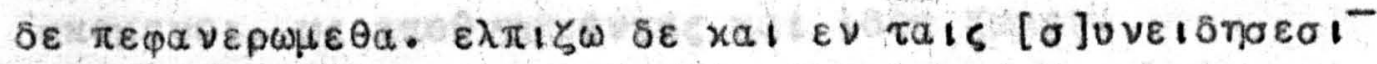

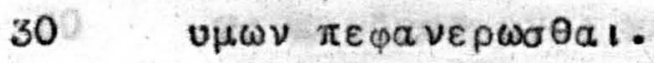

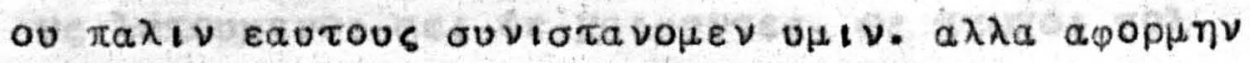

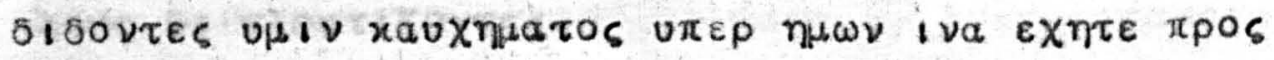

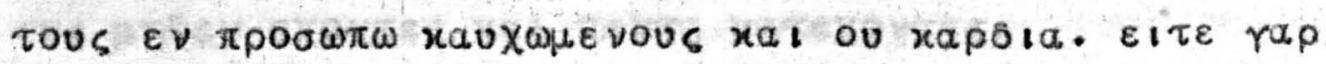

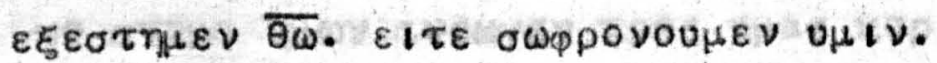

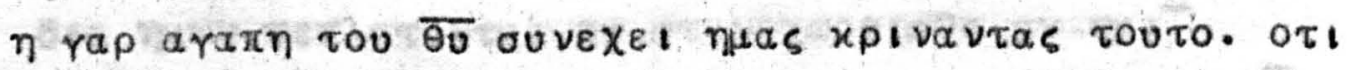

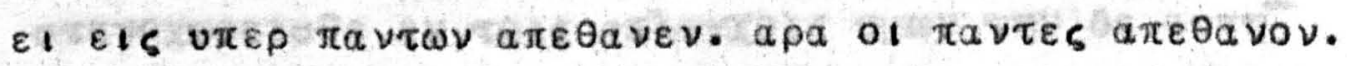

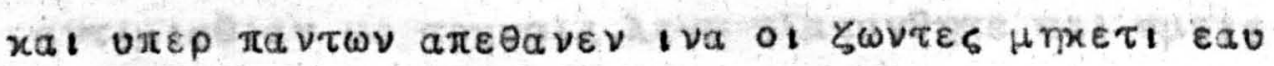

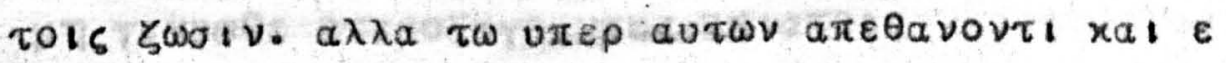

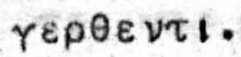

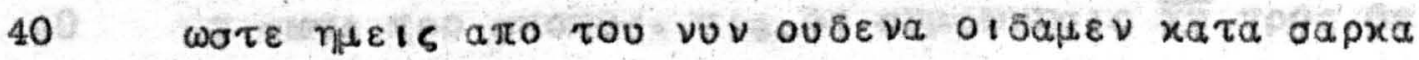

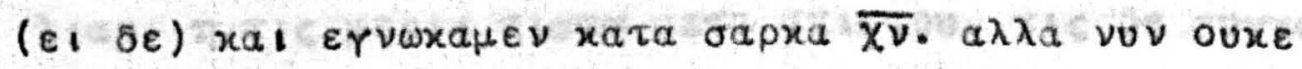


es rivarioutv.

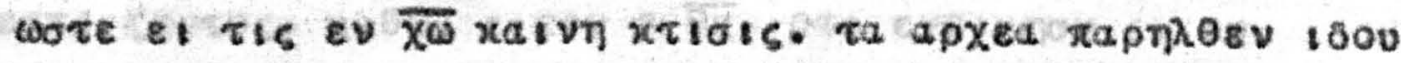

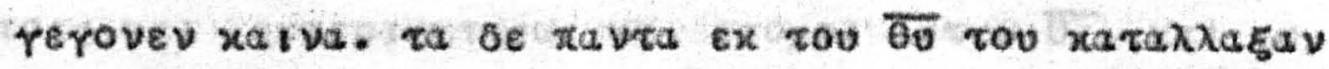

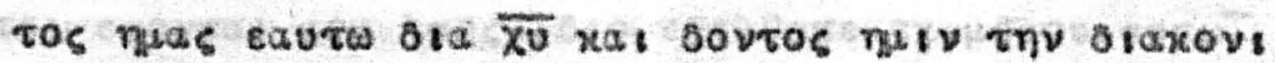

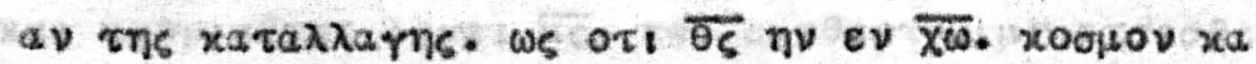

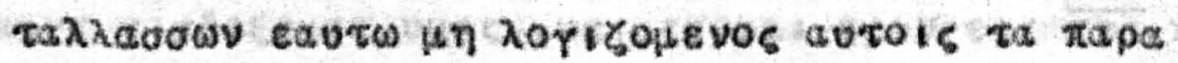

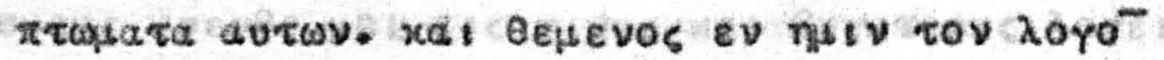

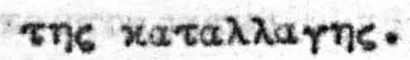

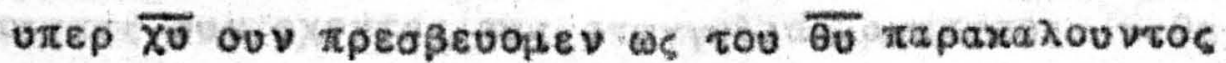

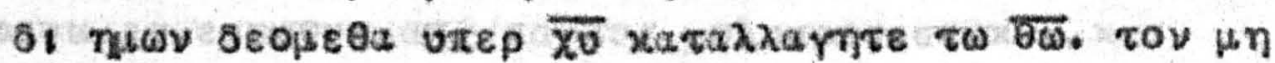

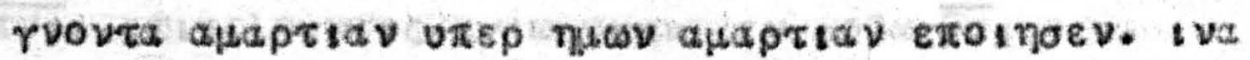
nueis revaxis $\theta$ a 1 thatoov

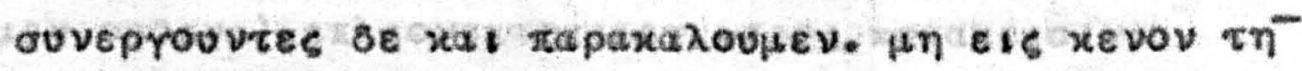

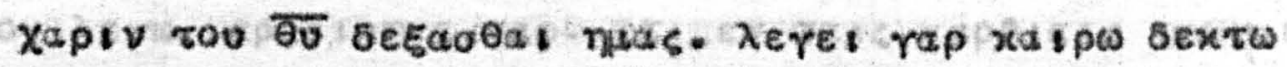

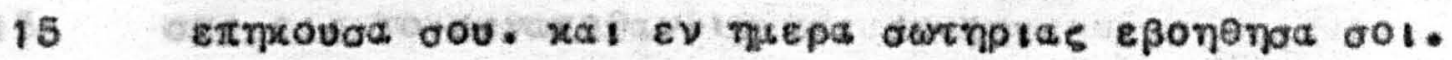
isov vov xalpos evipoosextos 1800 vov insepa ow

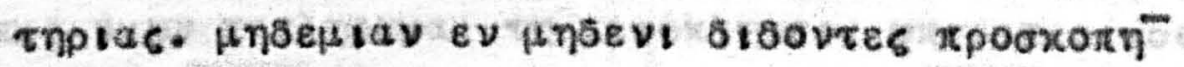

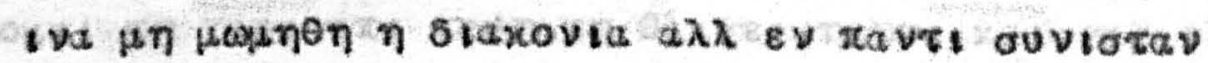
tes eavrove we $\bar{\theta}$ otaxovol.

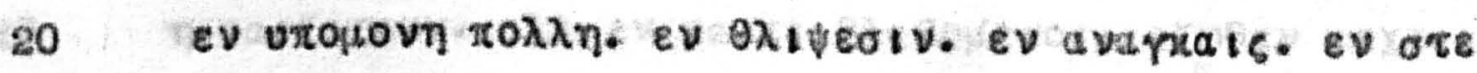

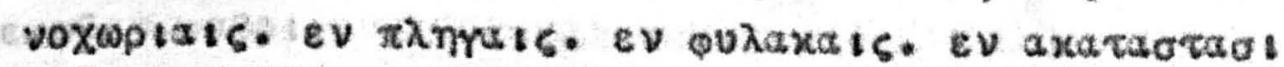

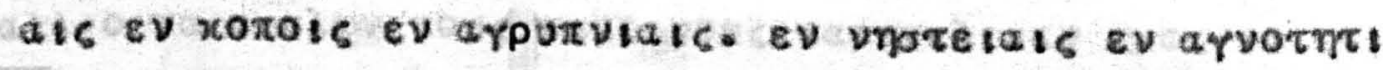

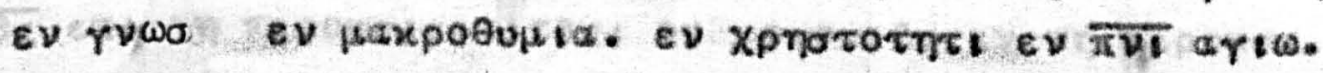

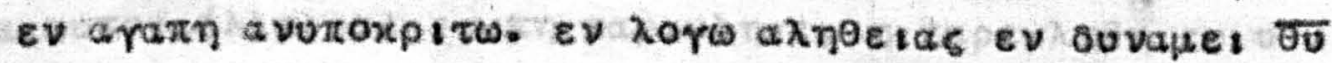

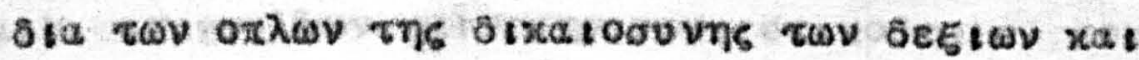
aptorepov.

ora bogns xat artutac. ota soopnulas xat evpntas

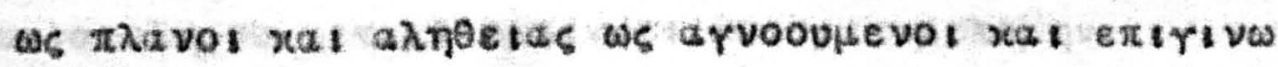

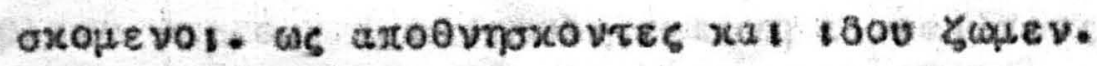

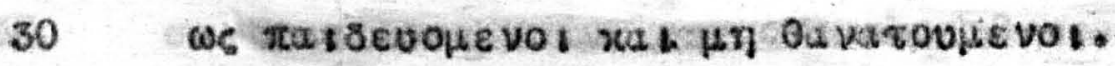

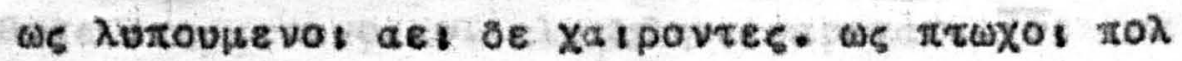

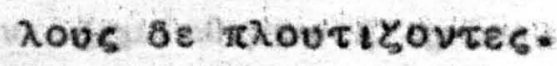

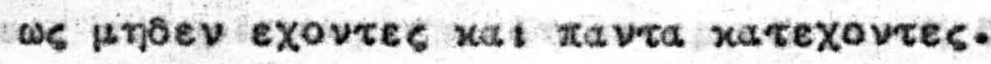

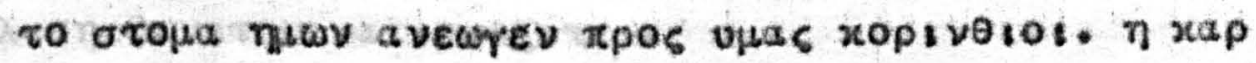

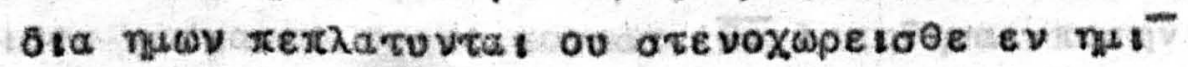

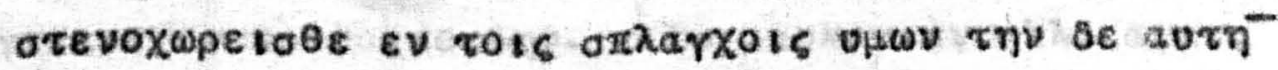
avтutatav

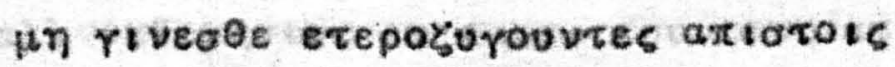

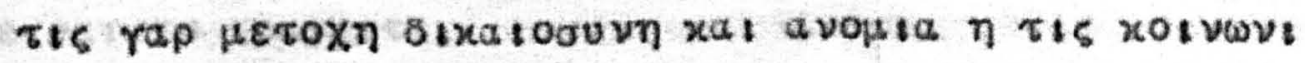

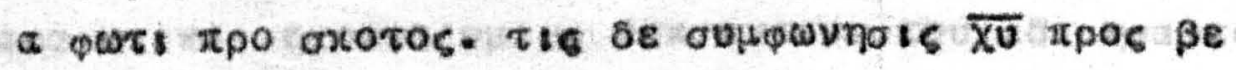

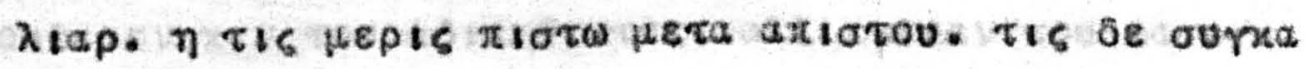




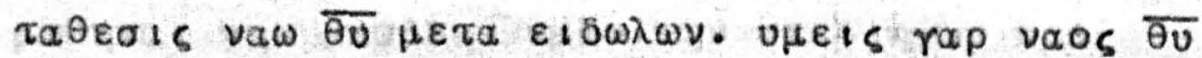

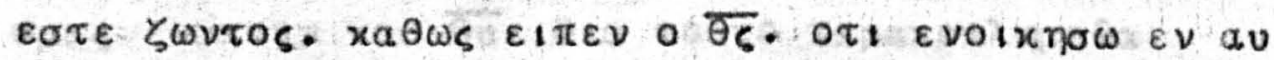

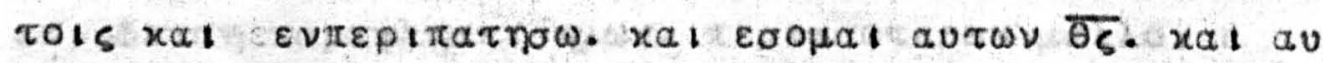

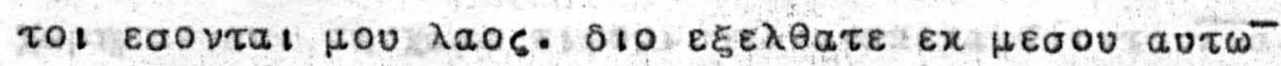

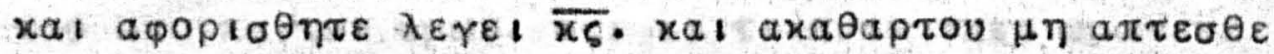

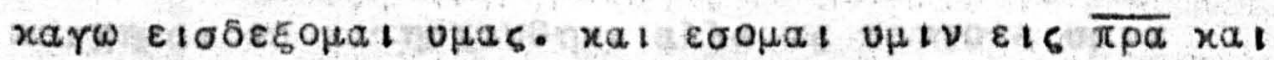

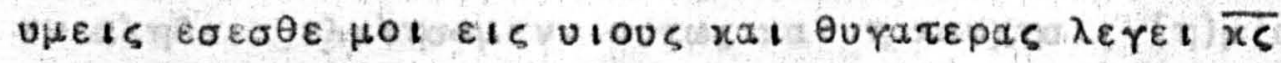

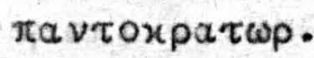

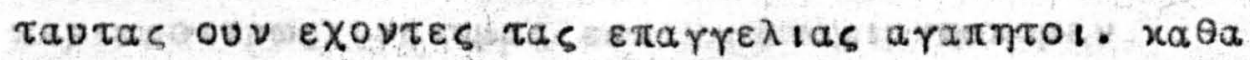

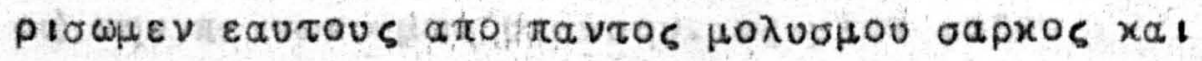

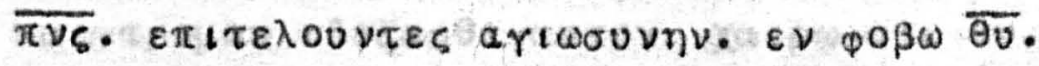

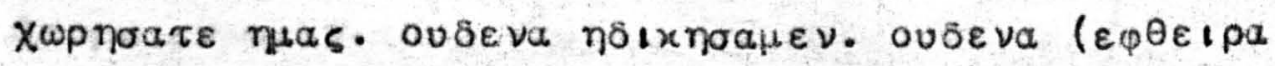

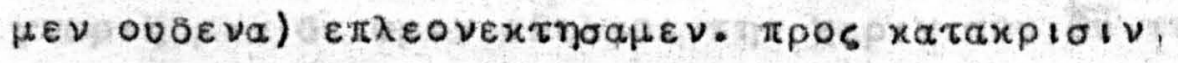

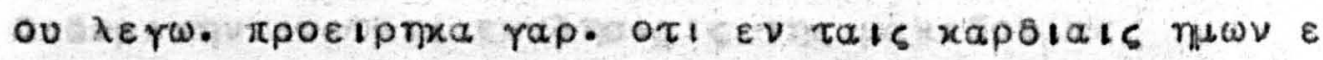

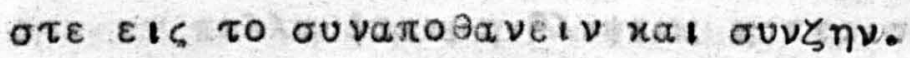

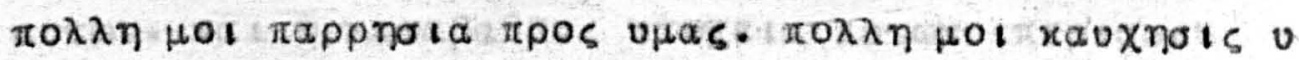

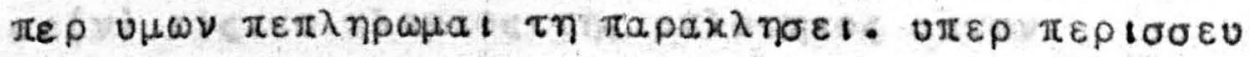

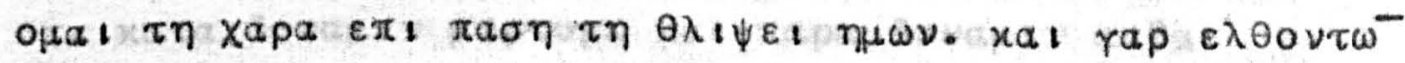

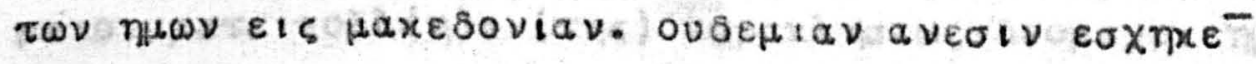

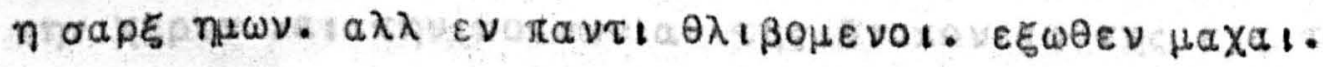
$\varepsilon \sigma \omega \theta \varepsilon \nu=\varphi O \beta O l$.

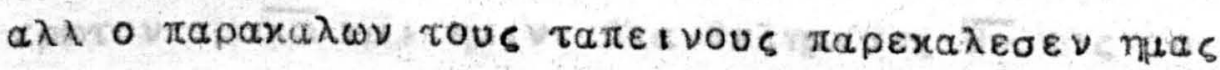

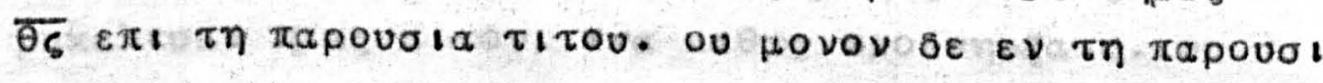

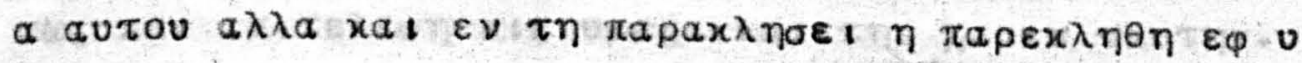

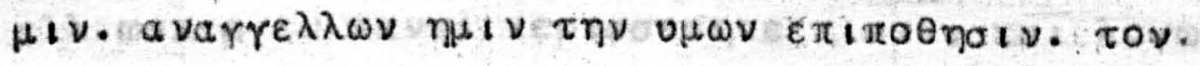

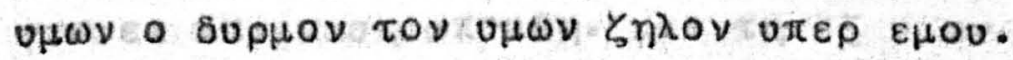

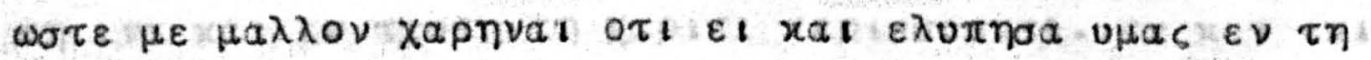
$\varepsilon \pi t \sigma \tau o \lambda \eta$ ou $\mu \varepsilon \tau a \mu \varepsilon \lambda o \mu a l$. $\varepsilon \iota$ xal $\mu \varepsilon \tau \varepsilon \mu \varepsilon \lambda \circ \mu \eta \nu$.

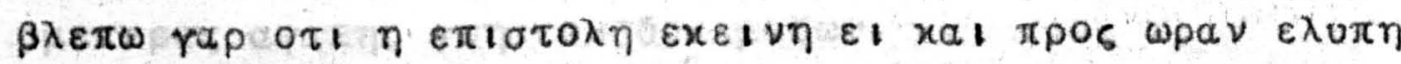

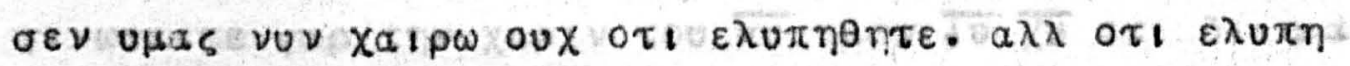

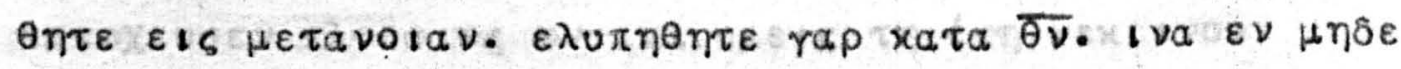

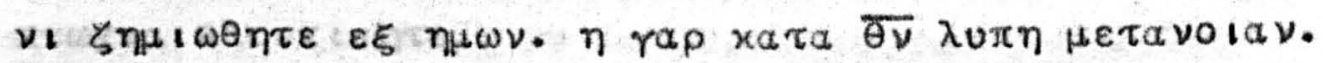

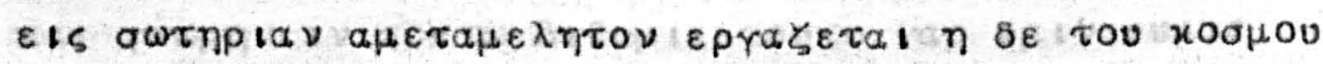

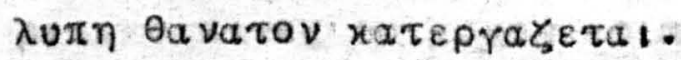

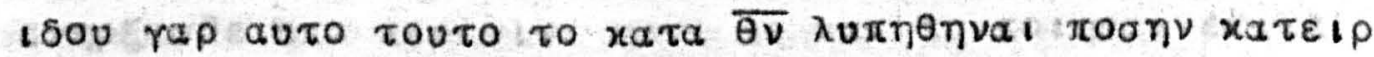

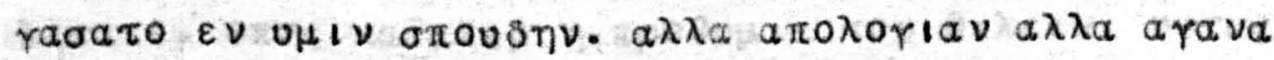

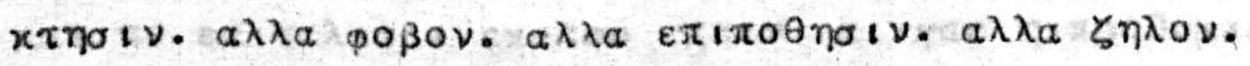

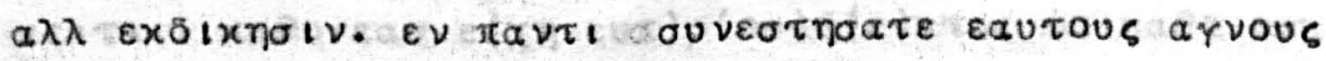

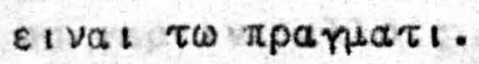

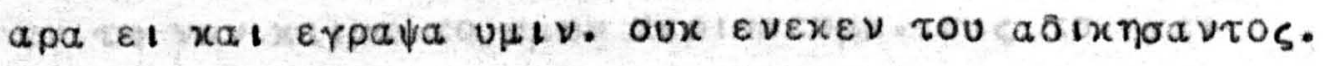

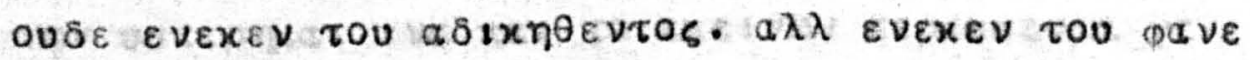




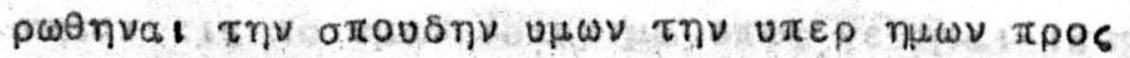

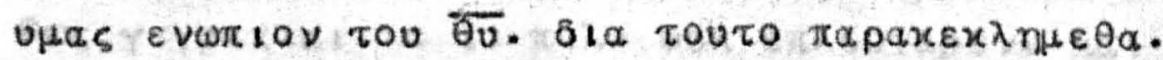

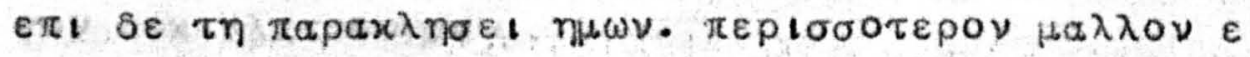

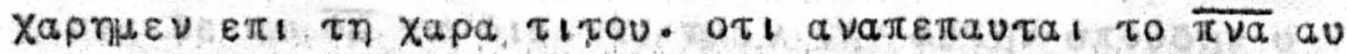

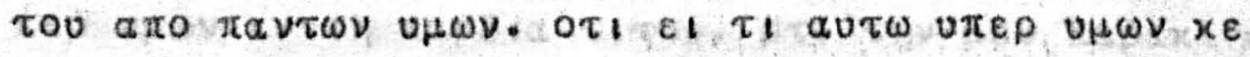

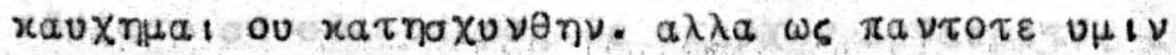

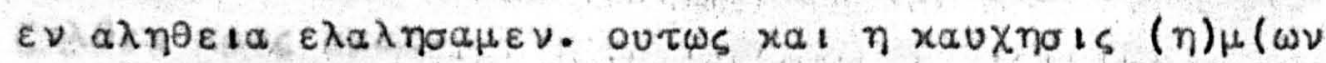

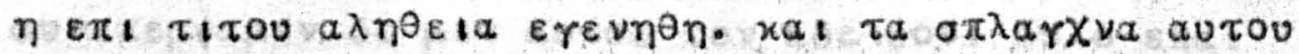

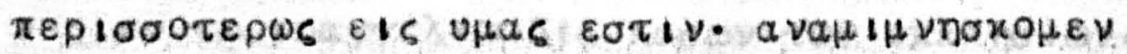

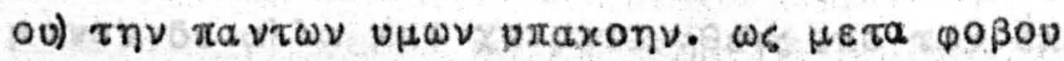

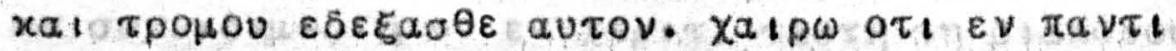
बapp $\propto$ \& $v \mu i \nu$.

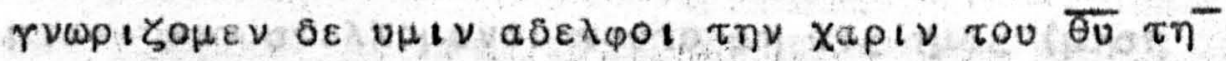

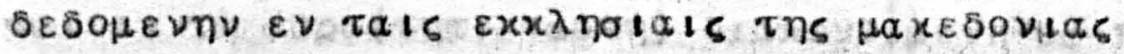

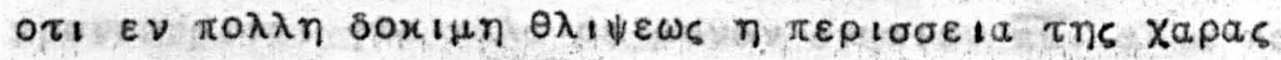

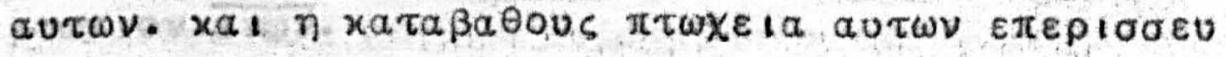

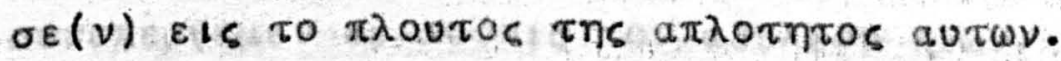

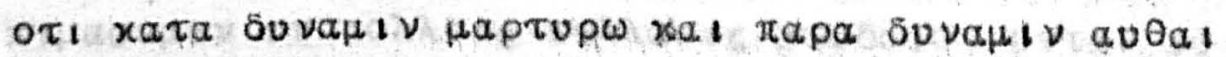

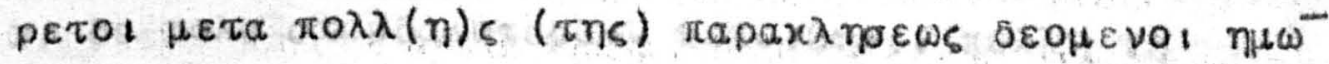

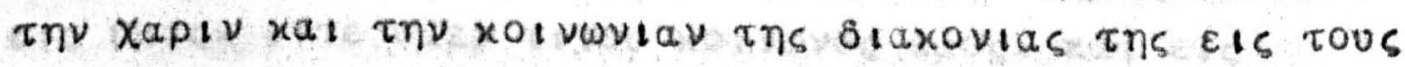

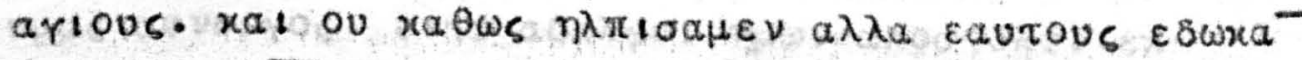
xp

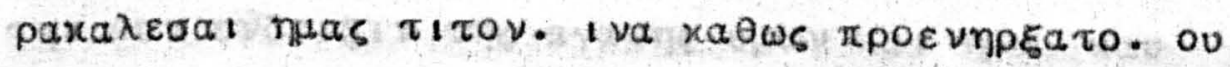

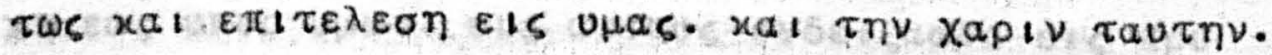

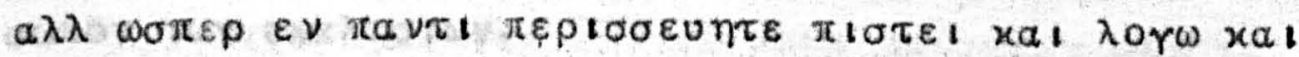

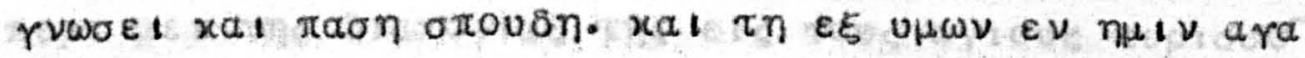

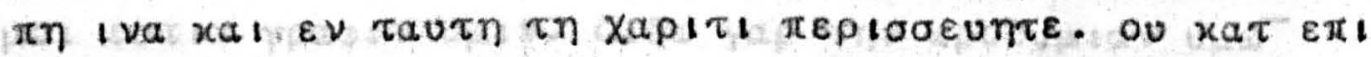

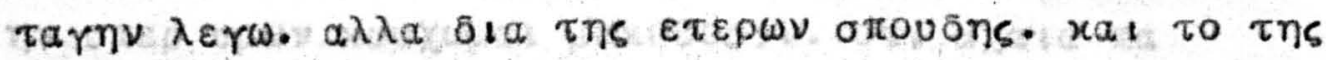

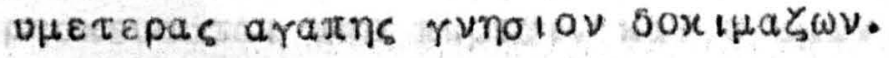

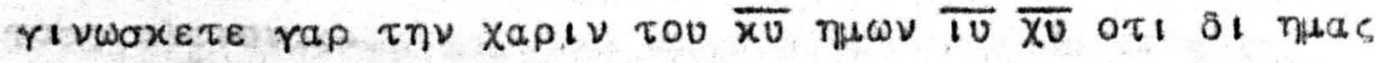

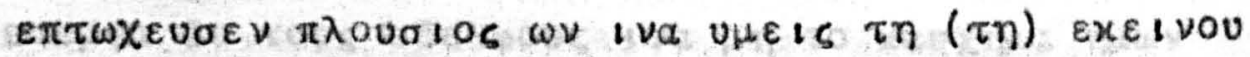

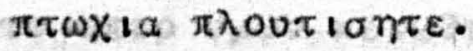

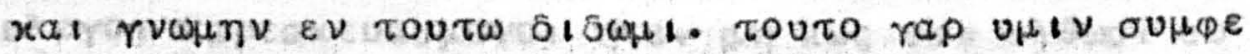

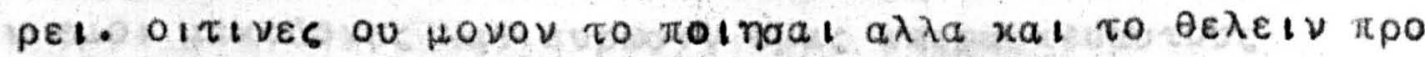

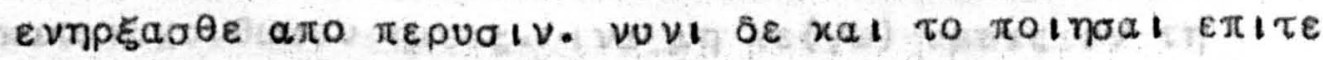
$\lambda \varepsilon \sigma a \tau \varepsilon$.

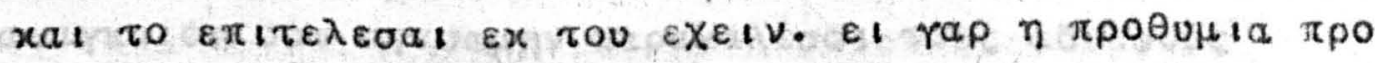

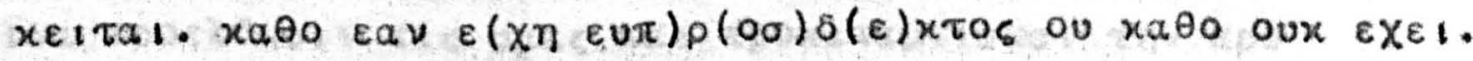

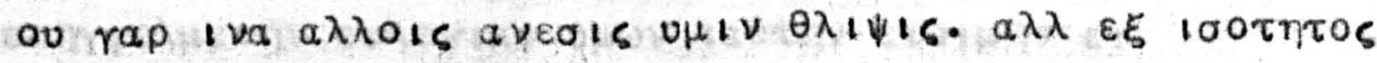

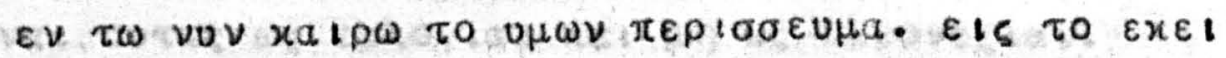

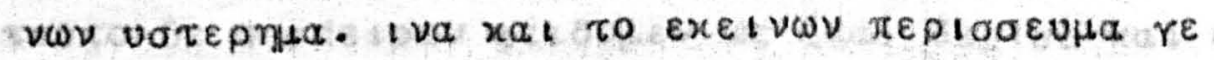




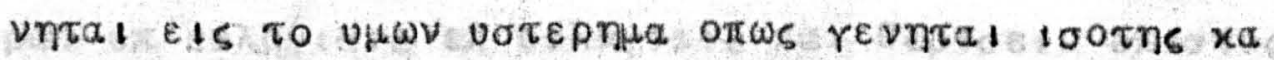

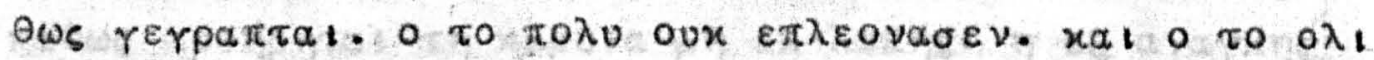

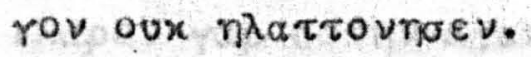

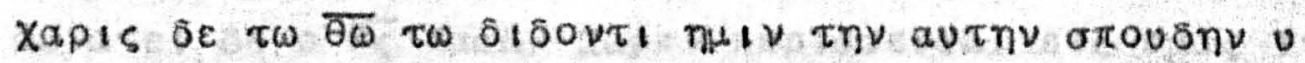

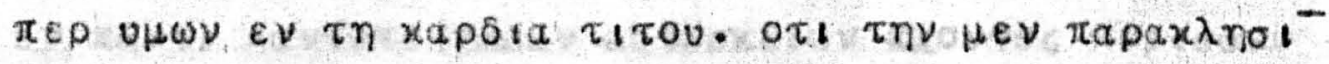

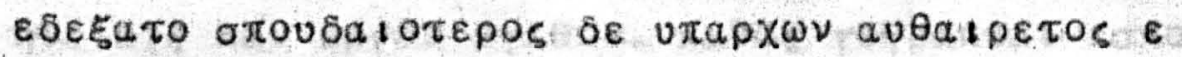
$\xi \eta \lambda \theta \varepsilon \nu \pi \rho \circ \zeta$ unas.

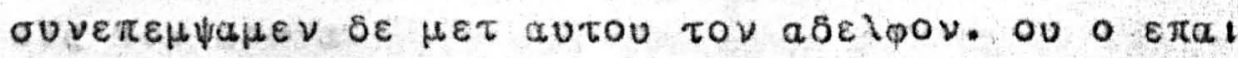

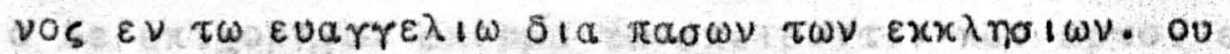

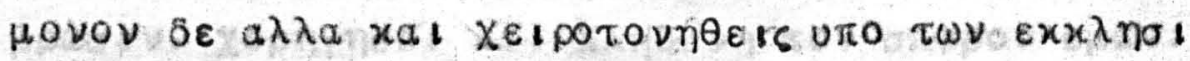

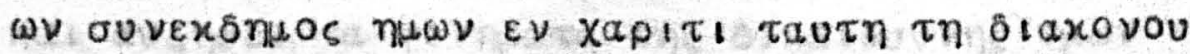

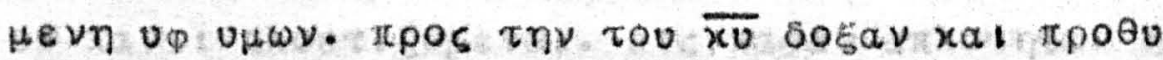

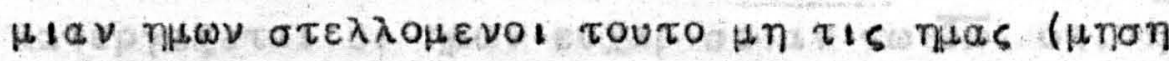

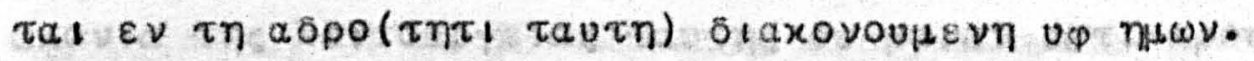

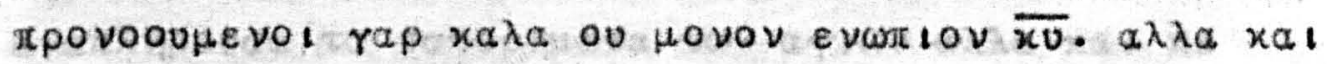
$\varepsilon \nu \omega \pi i$ ov $\bar{\alpha} \nu \omega v$.

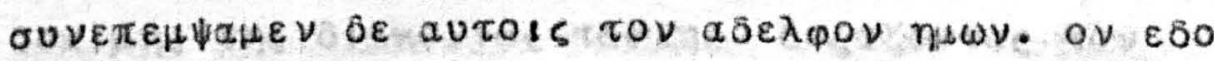

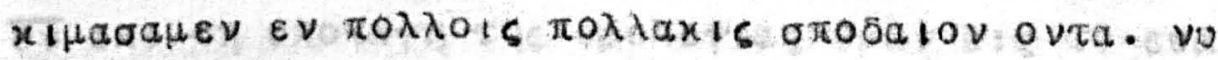

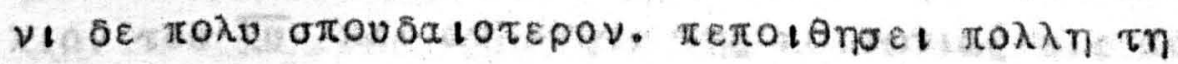

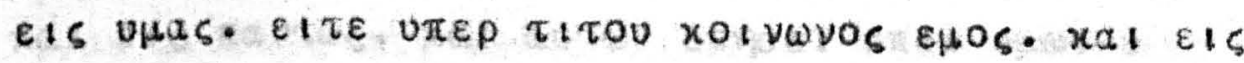

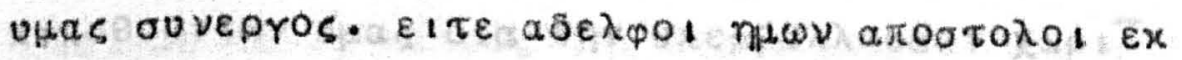

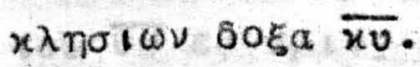

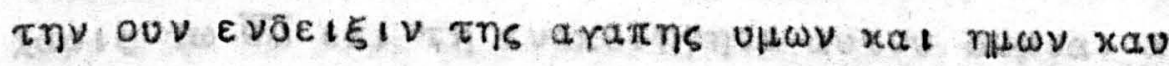

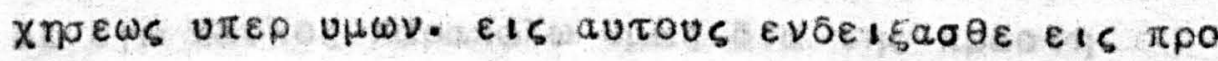

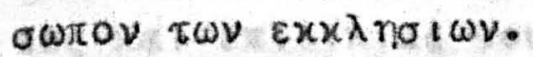

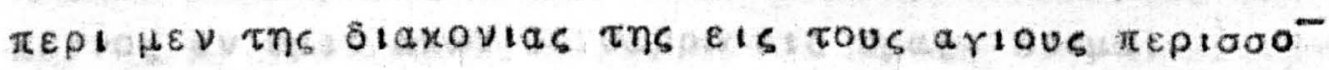

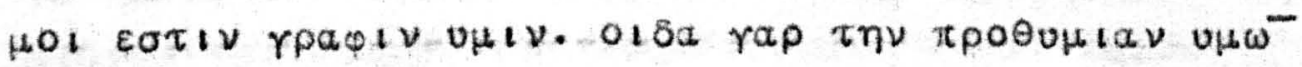

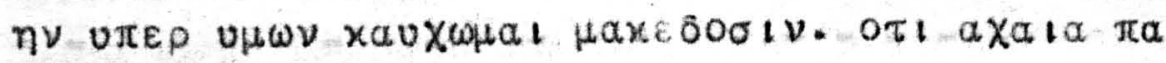

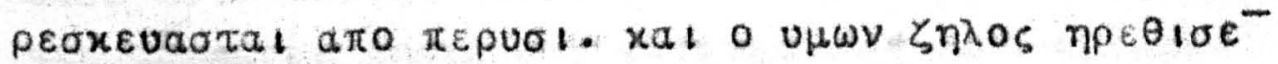
30 Tous $\pi \lambda e$ iovas.

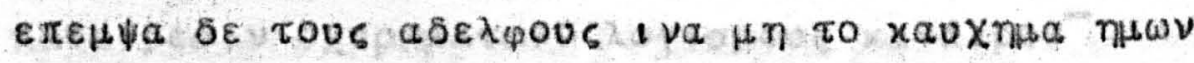

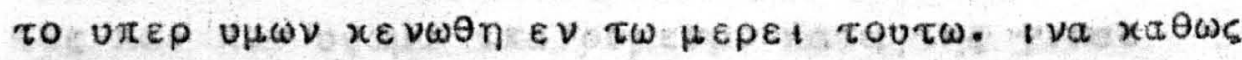

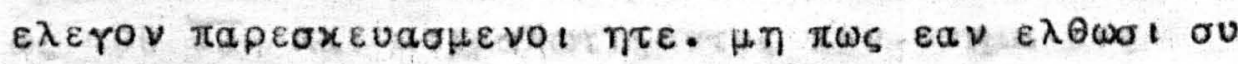

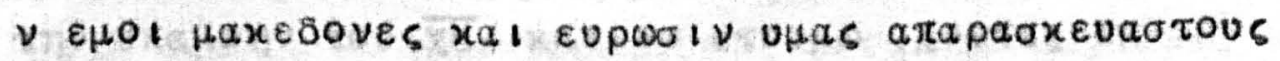

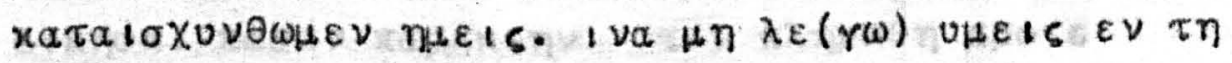

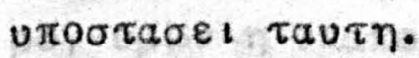

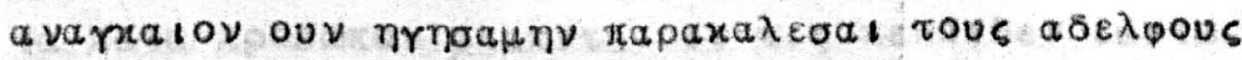

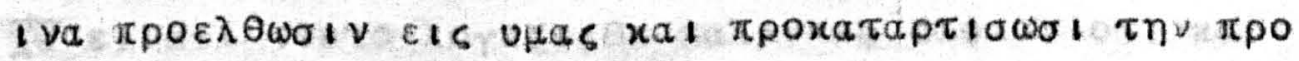

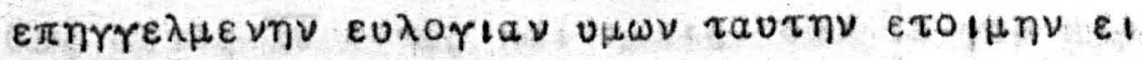

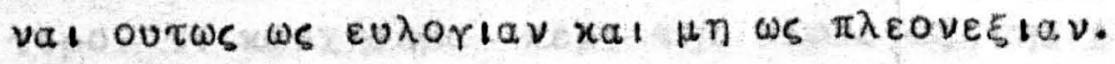

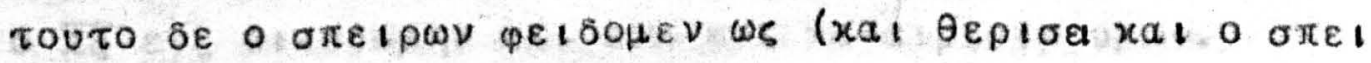




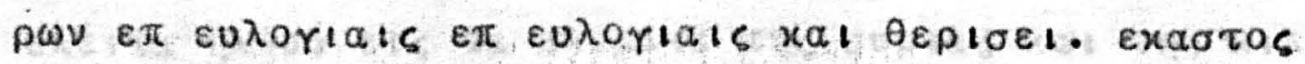

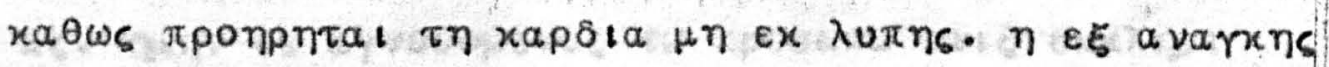
liapov rap sotnv arata o $\overline{\theta_{\zeta}}$.

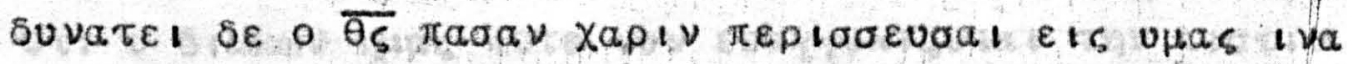

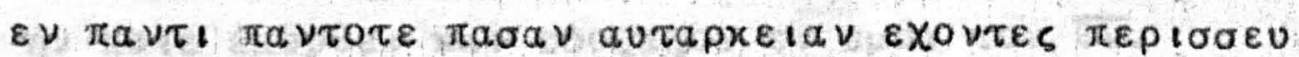

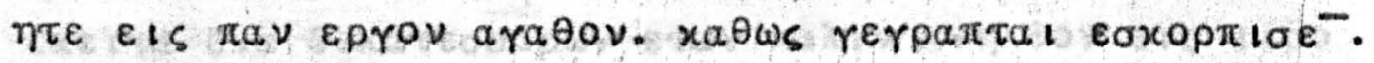

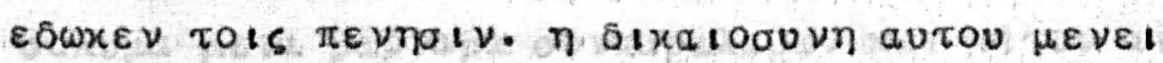
Eis rov a Iwva.

- $\delta \varepsilon \varepsilon \pi 1$ ย

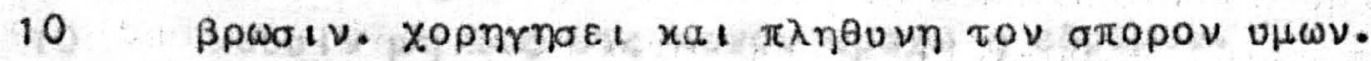

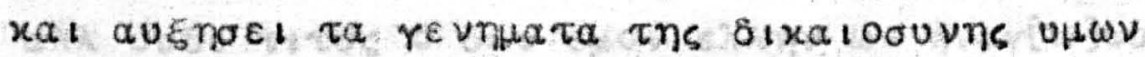

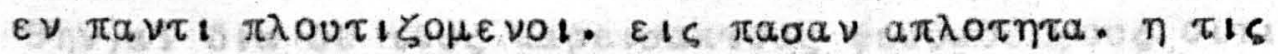

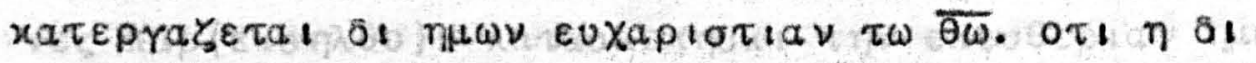

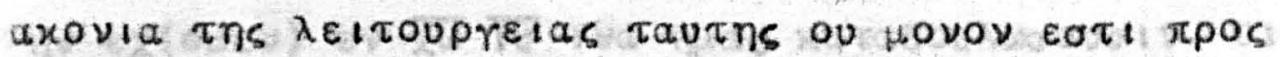

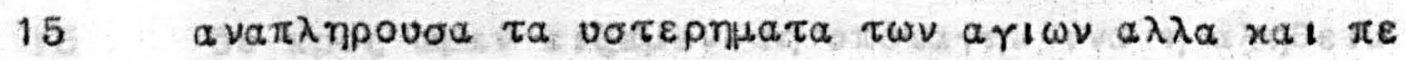

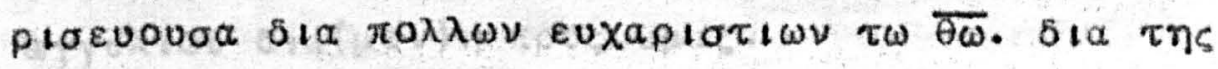

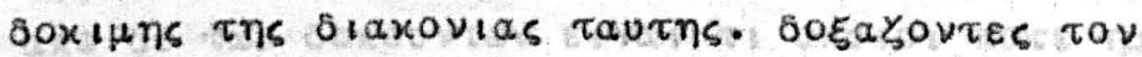

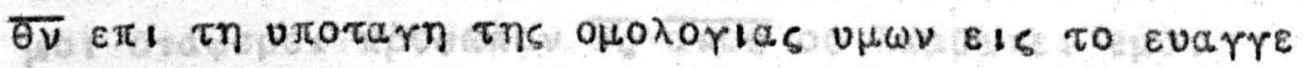

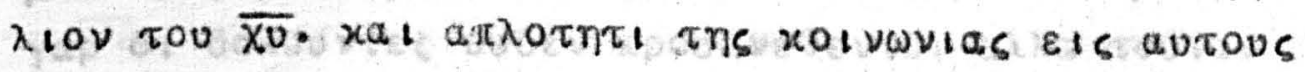

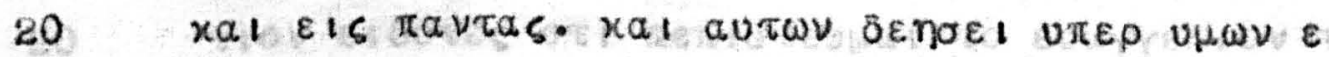

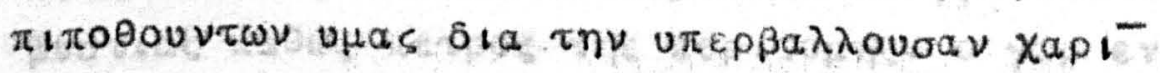

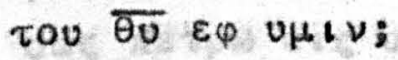

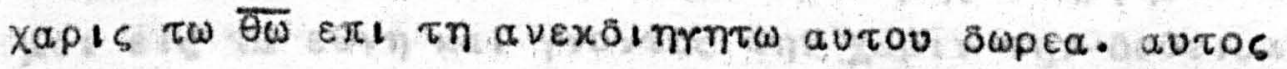

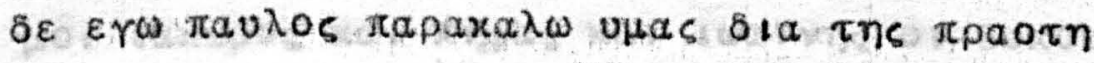

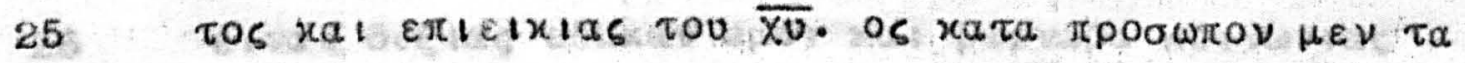

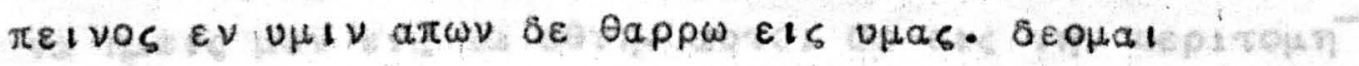

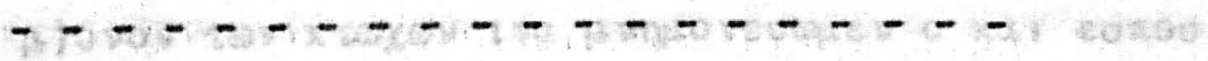

- - - - - - - - - - - - -

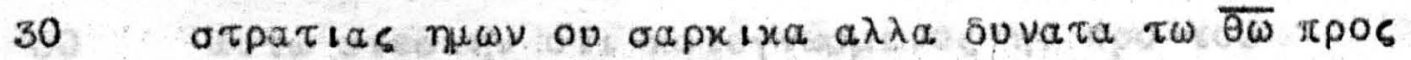

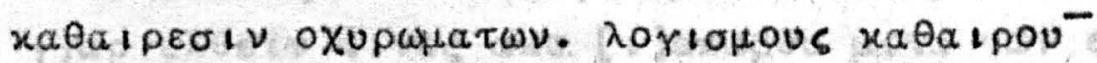

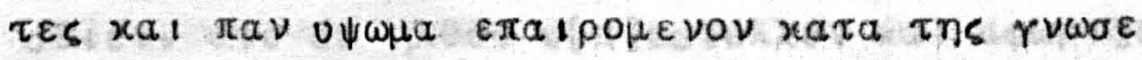

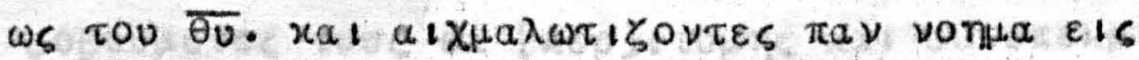

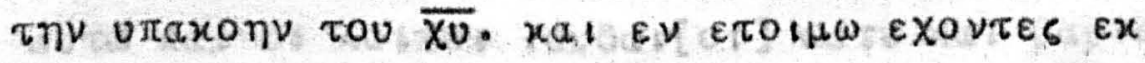

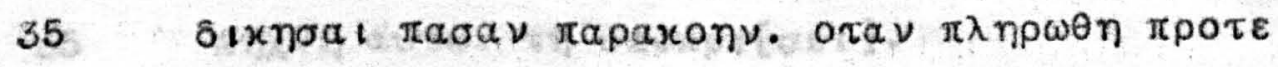
pov vHosy $\eta$ uraxon.

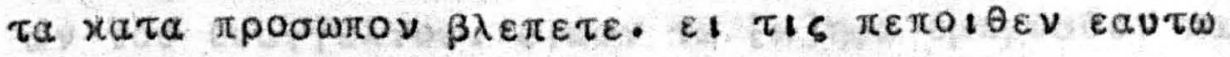

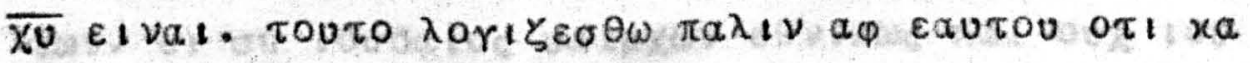

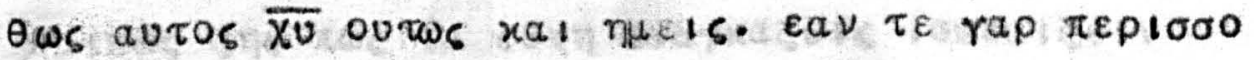

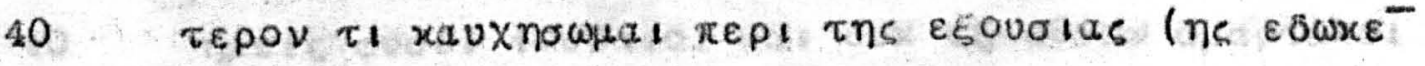

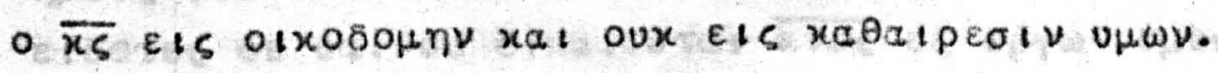




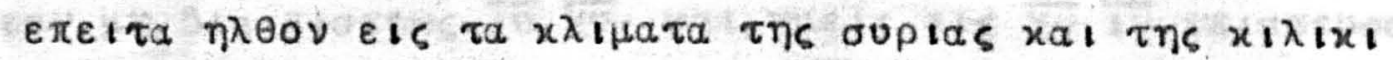

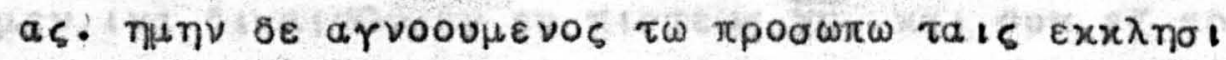

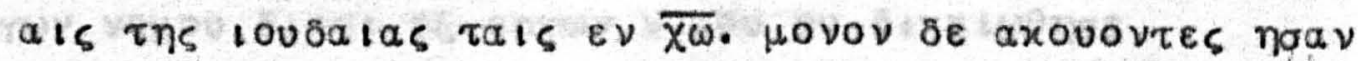

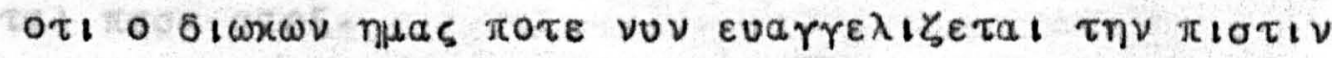

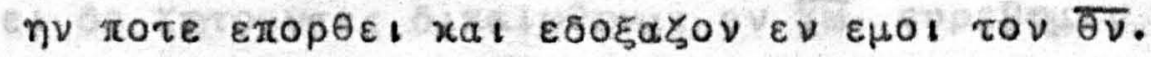
$\varepsilon \pi \varepsilon เ \tau \alpha$ oเ

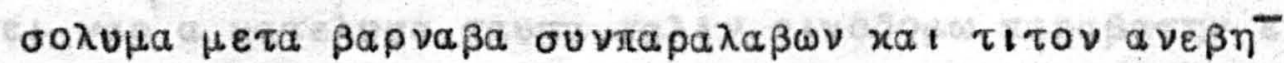

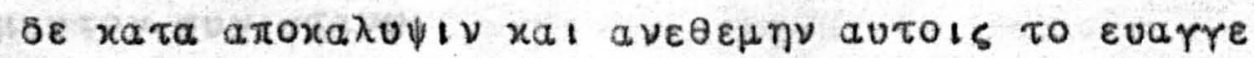

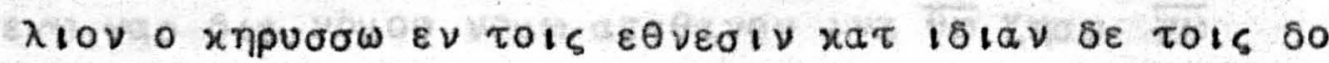

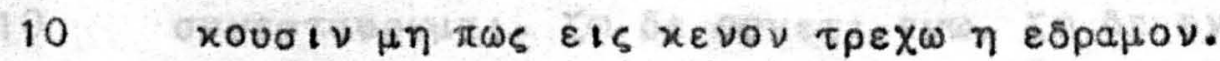

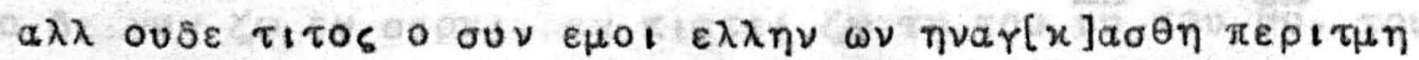

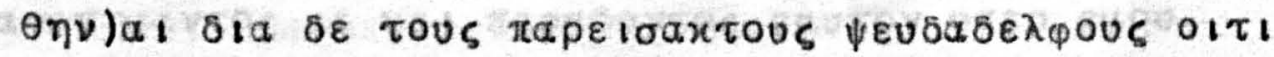

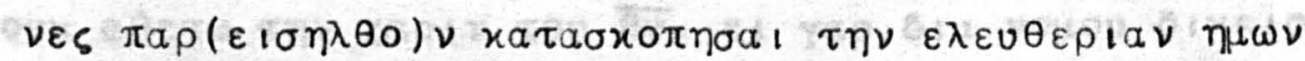
$\eta \nu \varepsilon x(0 \mu \varepsilon \nu) \varepsilon \nu \overline{\chi \omega} \overline{T v}$ iva nuas $x a \tau \alpha \delta 0 u \lambda \omega \sigma o v \sigma i v$. ois ou

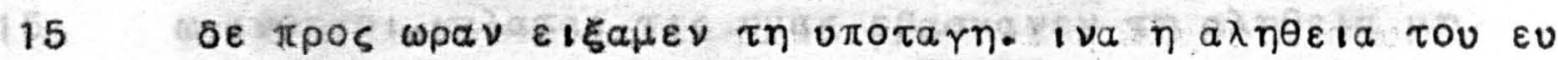

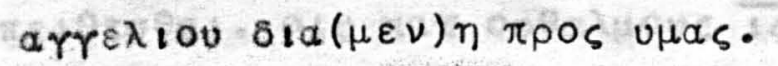

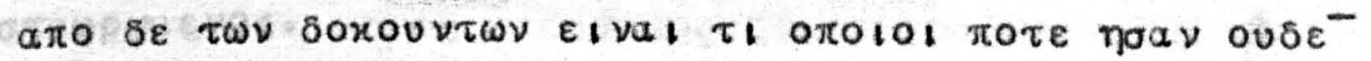

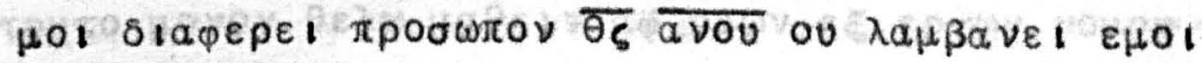

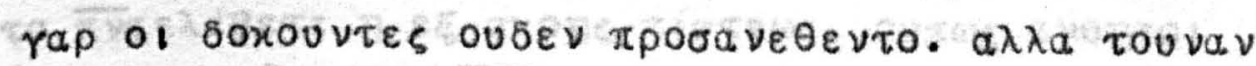

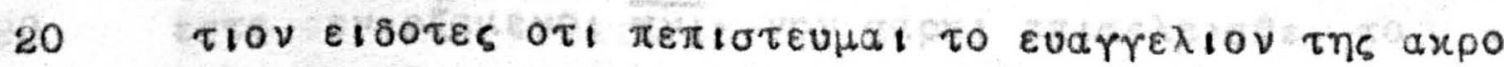

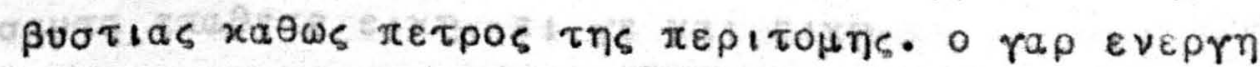

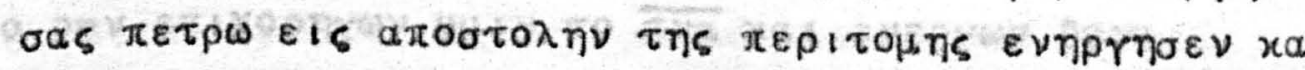

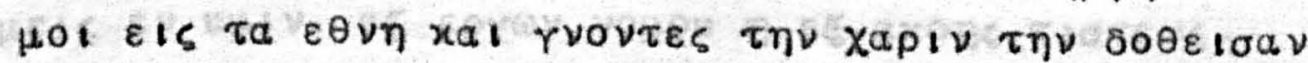

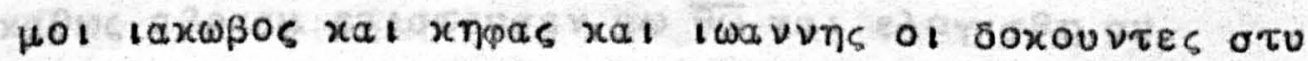

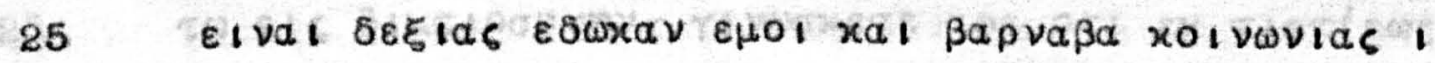

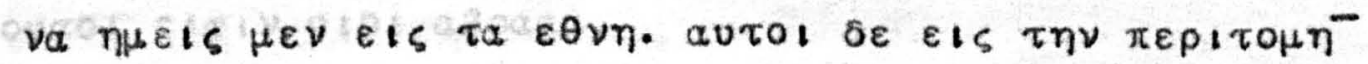

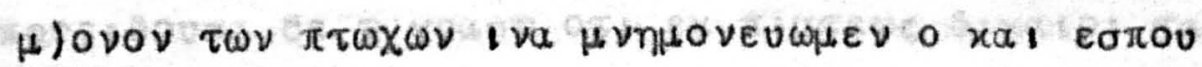

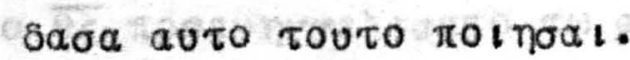

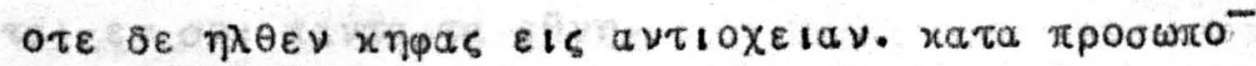

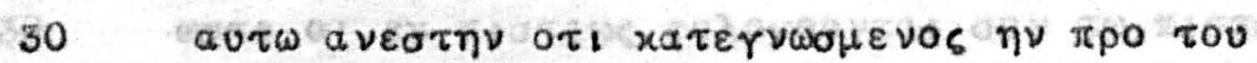

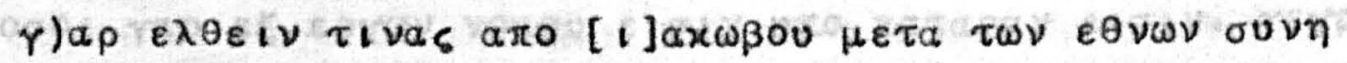

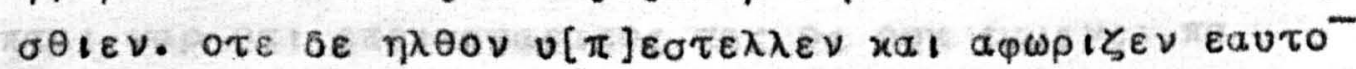

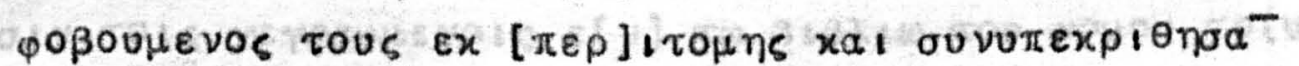

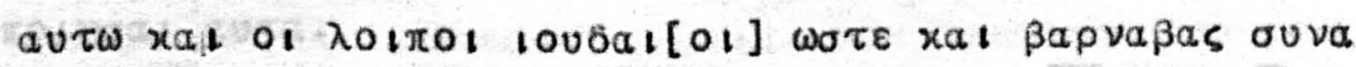

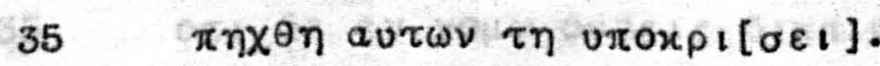

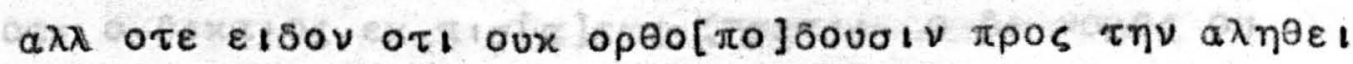

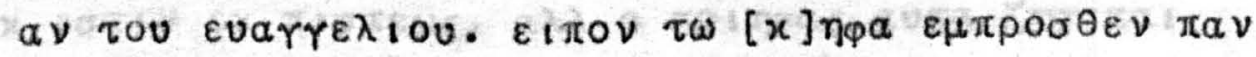

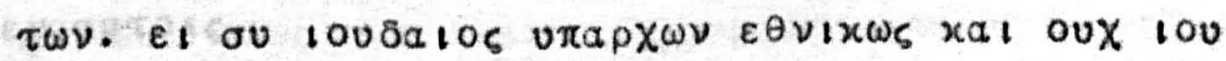

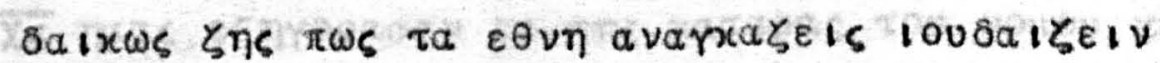

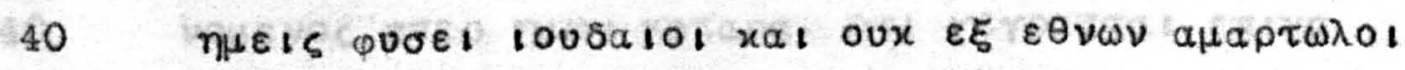

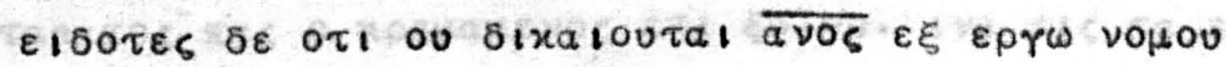




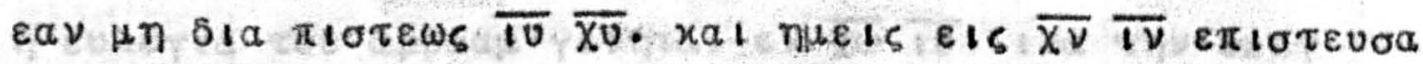

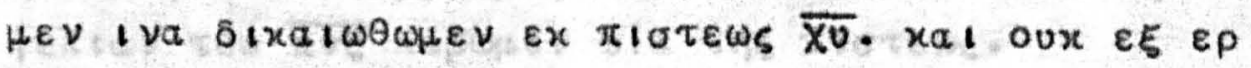

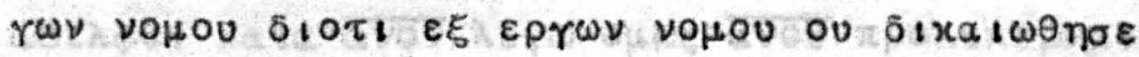

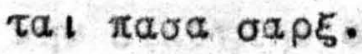

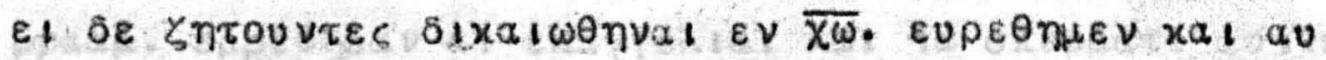

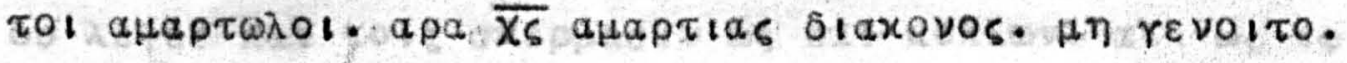

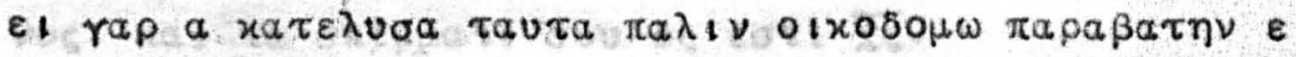

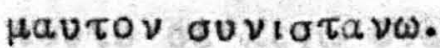

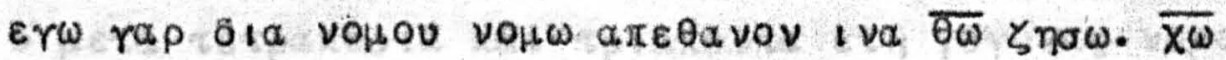

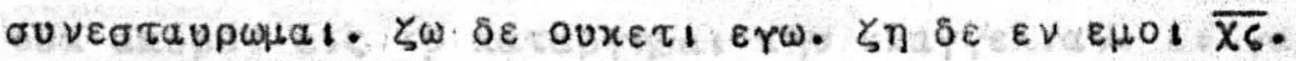

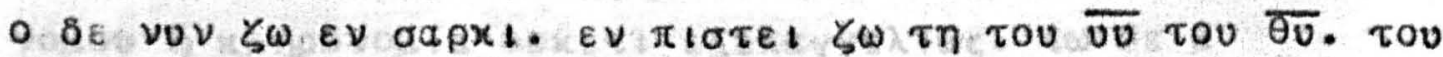

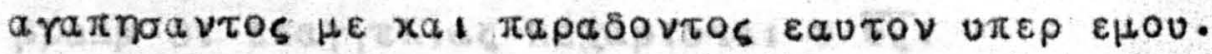

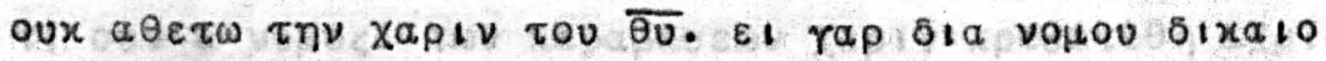

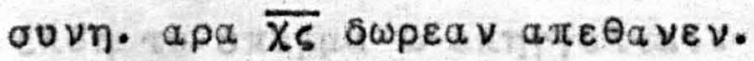

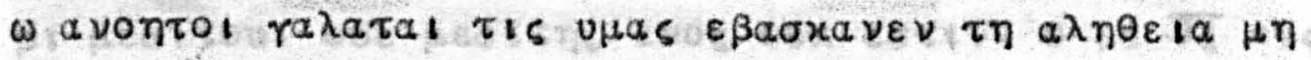

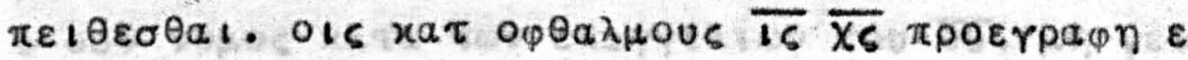
ataupur evos.

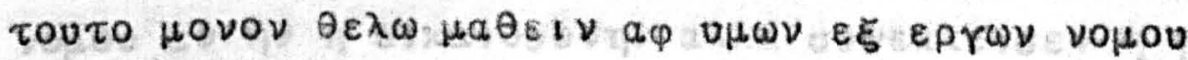

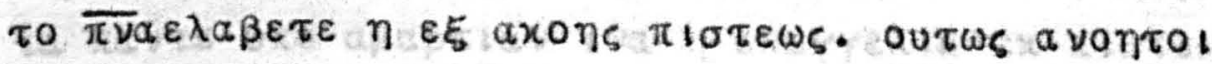

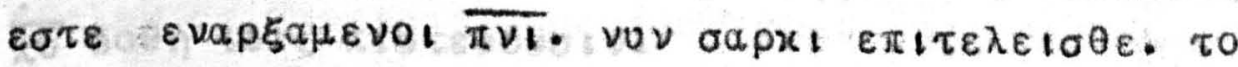

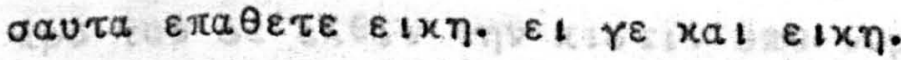

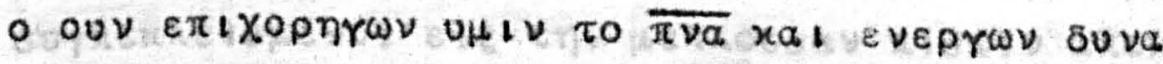

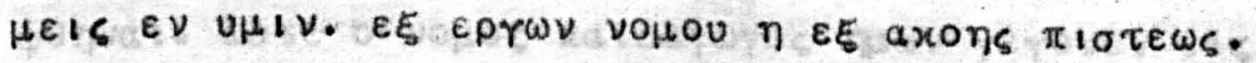

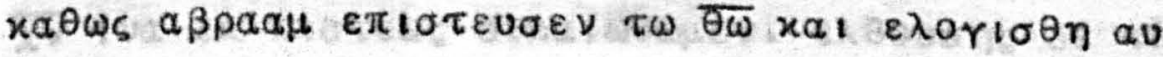

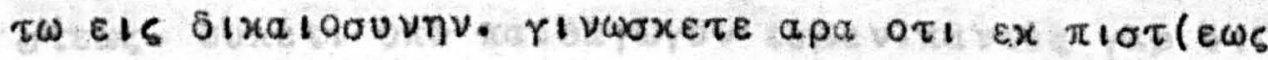

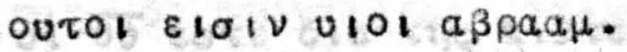

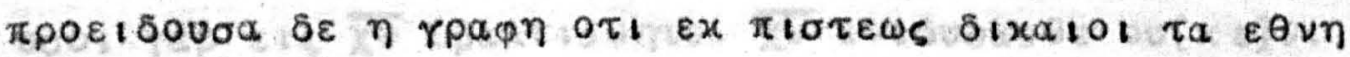

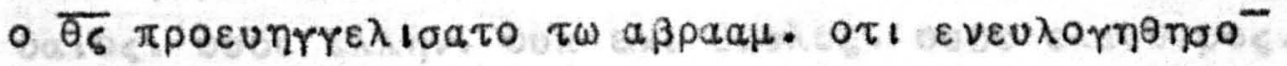
$\tau a 1 \varepsilon \nu$ o० $\pi \alpha \nu \tau a \tau a \varepsilon \theta \nu$.

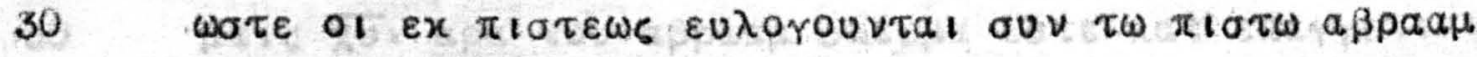

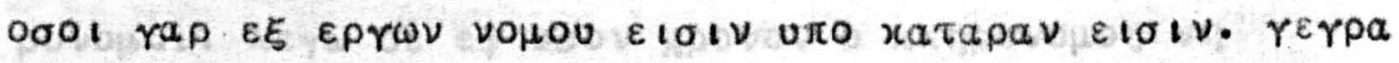

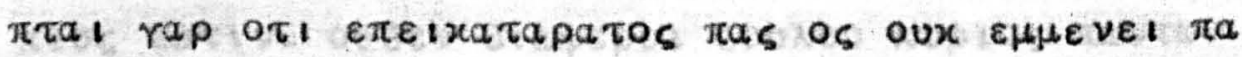

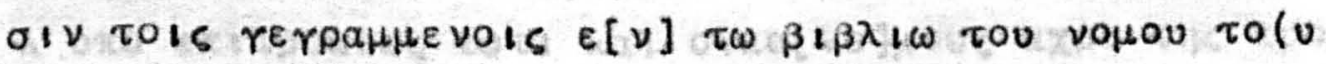
rolnoal auta.

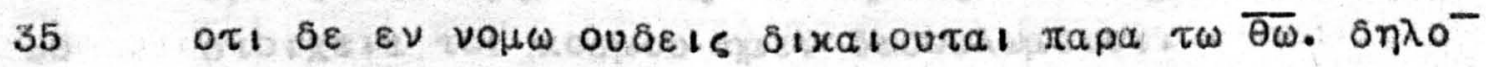

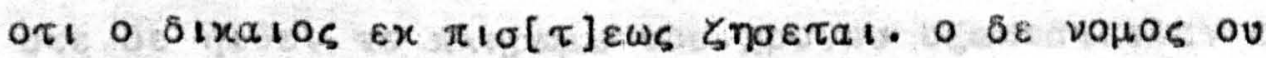

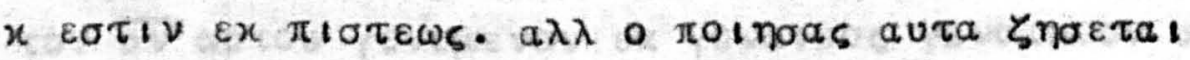
$\varepsilon \nu$ autois.

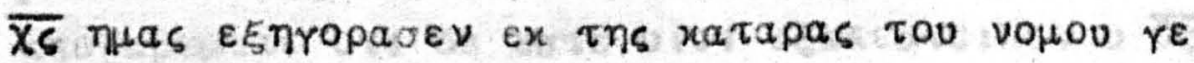

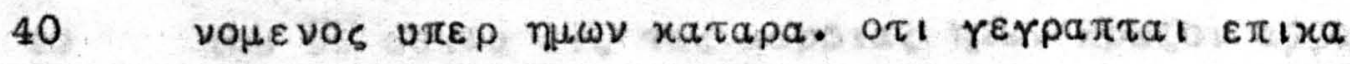

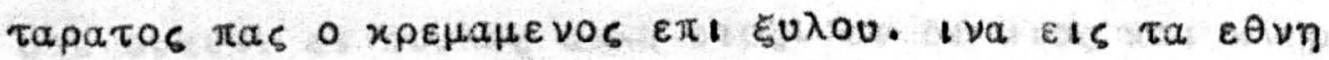




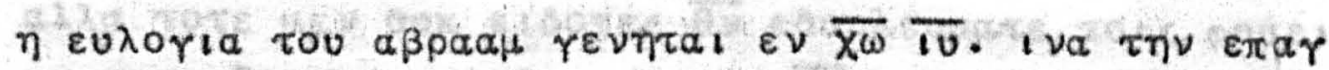

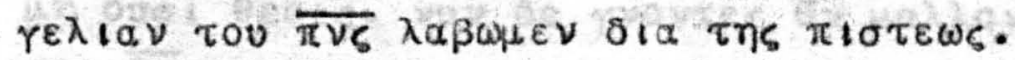

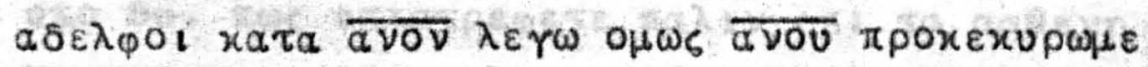

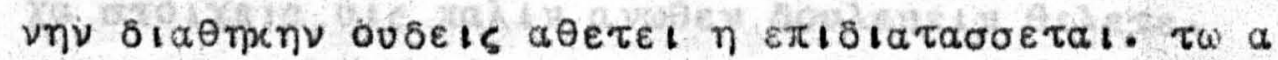

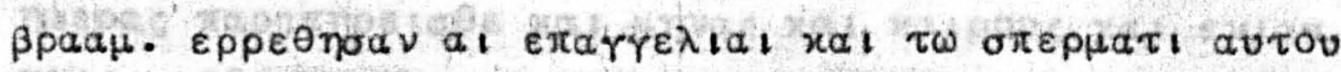

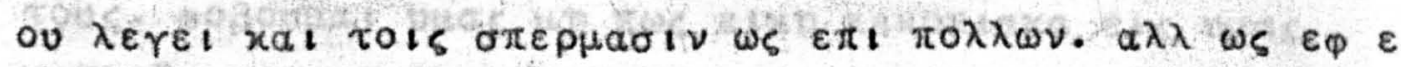

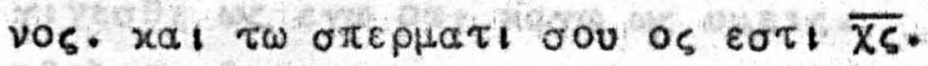

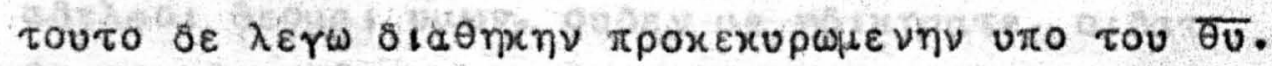

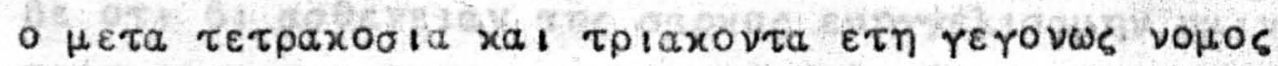

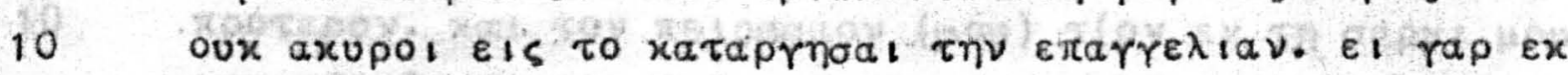

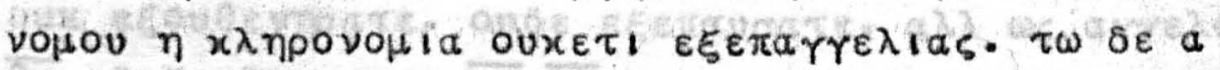

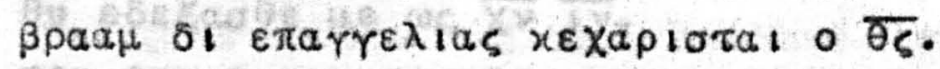

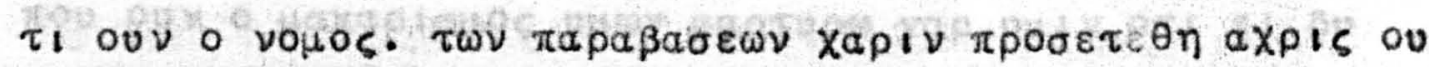

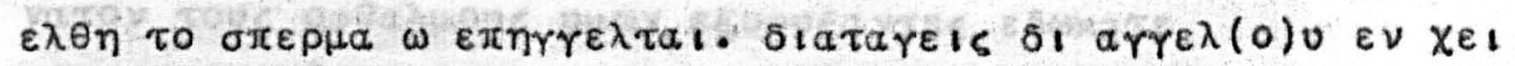

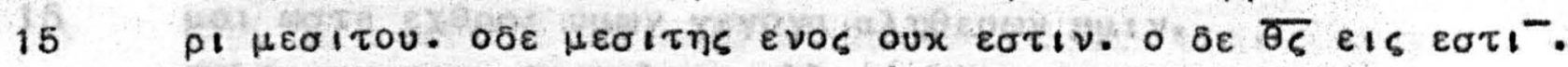

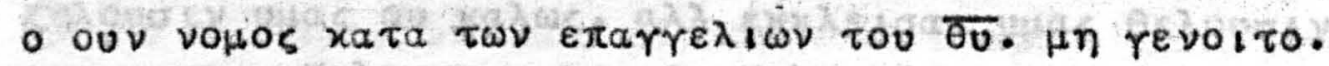

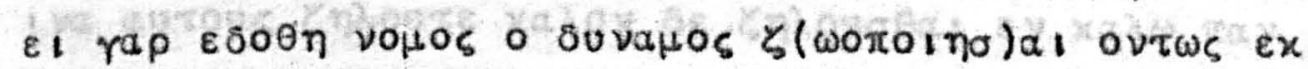

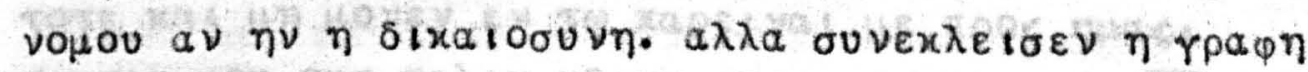

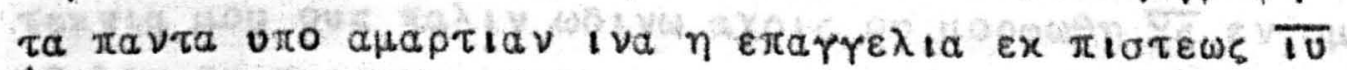

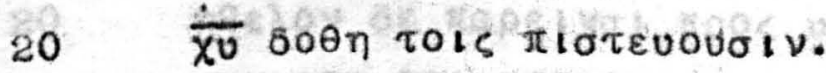

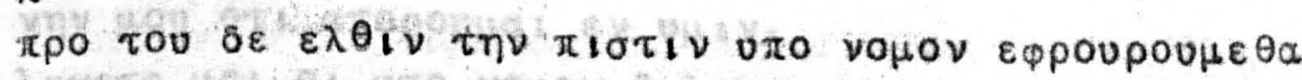

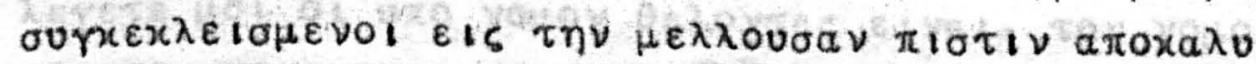

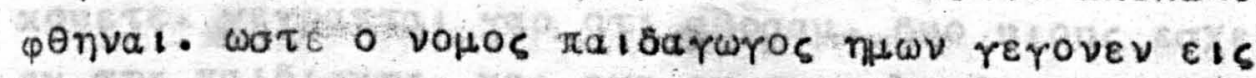

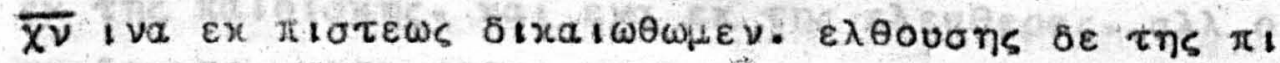

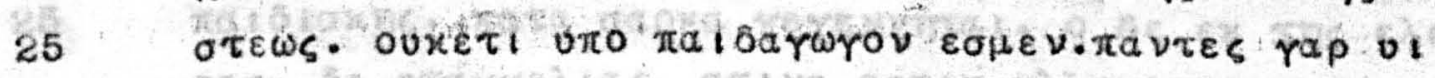

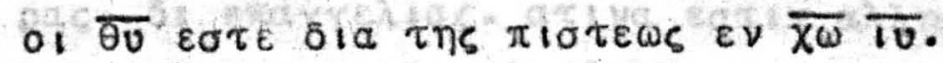

$0001 \gamma a \rho \varepsilon i \varsigma \overline{\chi \nu} \varepsilon \beta a \pi \tau 1 \sigma \theta \eta \tau \varepsilon . \overline{X V} \varepsilon \nu \varepsilon \delta \cup \sigma a \sigma \theta \varepsilon$ ou $[x \varepsilon] \nu i$ lov

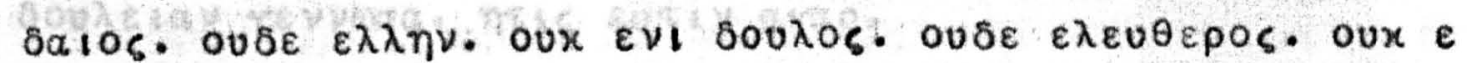

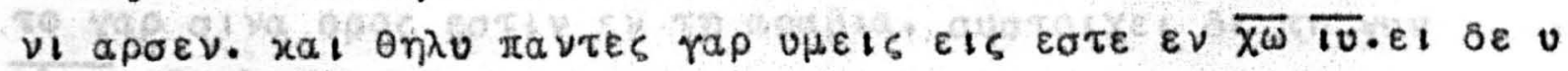

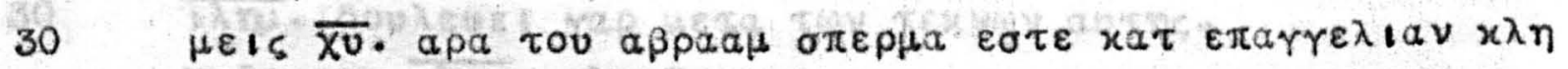

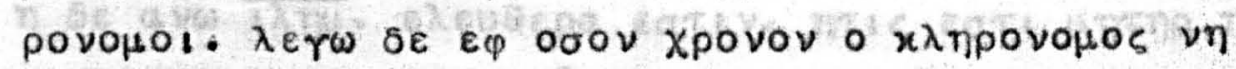

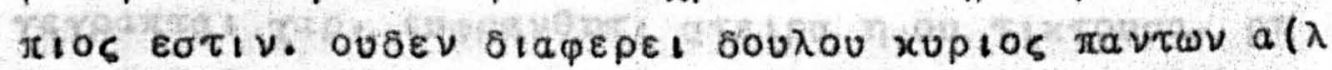

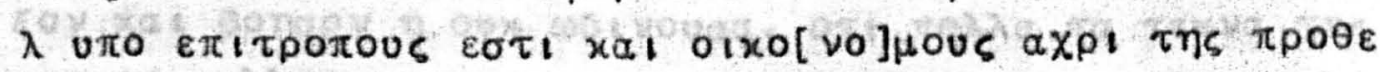

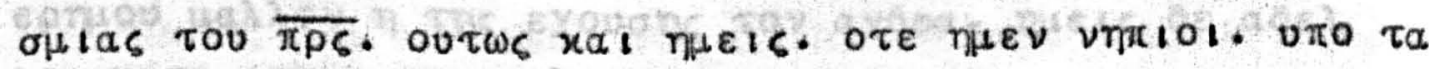

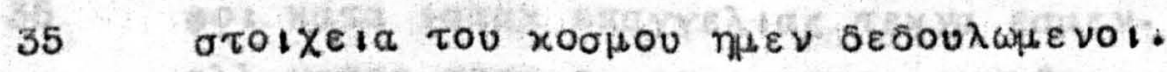

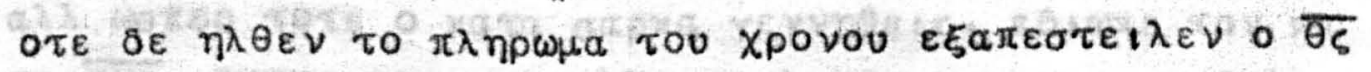

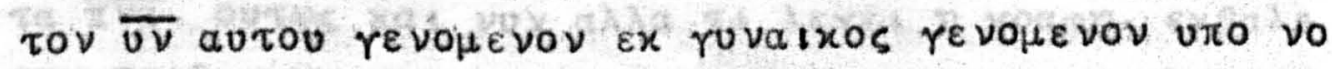

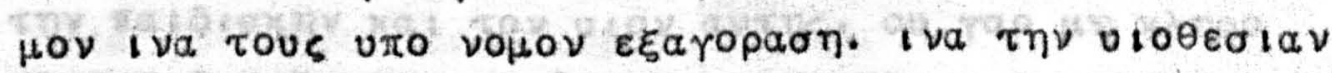

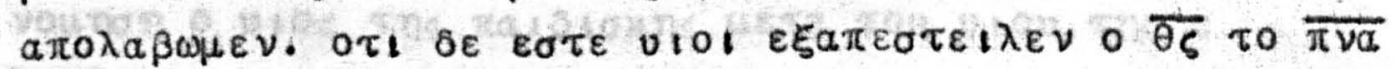

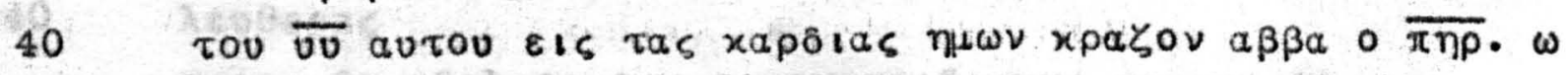

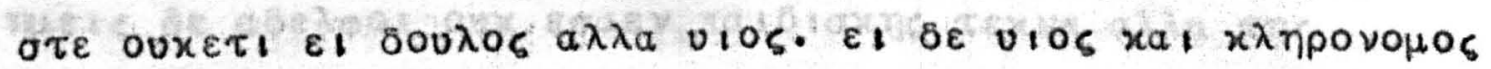
sia ब0. 


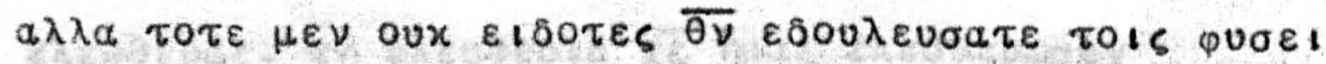

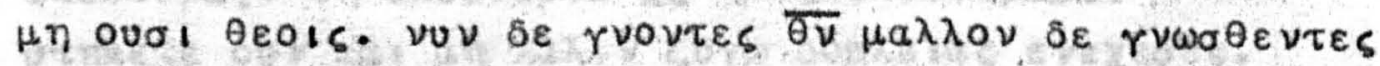

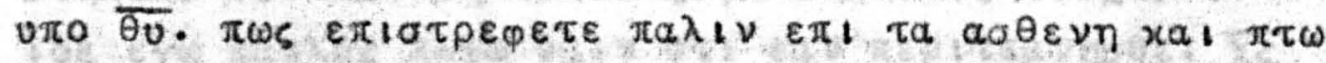

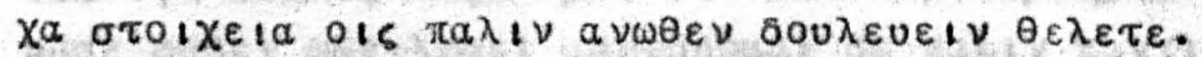

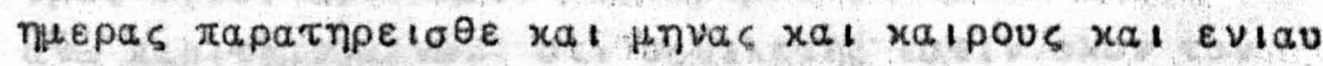

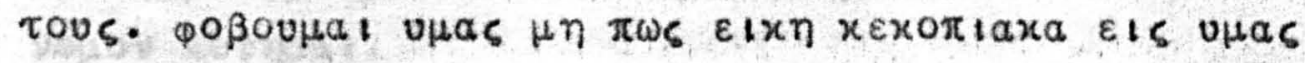

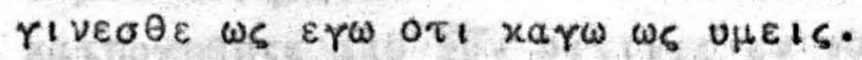

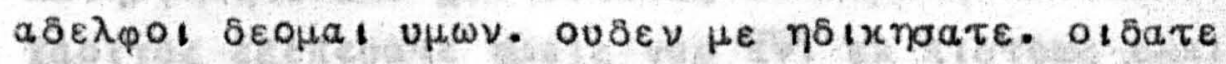

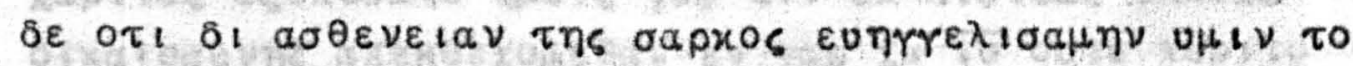

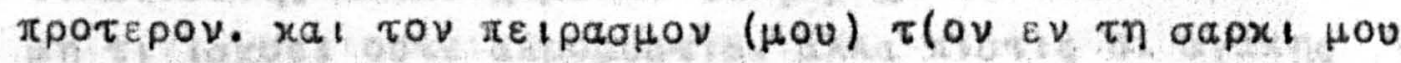

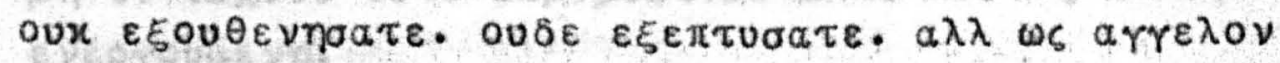
$\overline{\theta 0} \varepsilon \delta \varepsilon \xi a \sigma \theta \varepsilon \mu \varepsilon$ ws $\overline{\chi \nu} \overline{T \nu}$.

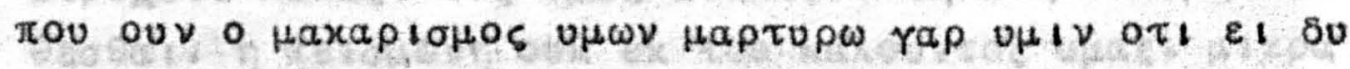

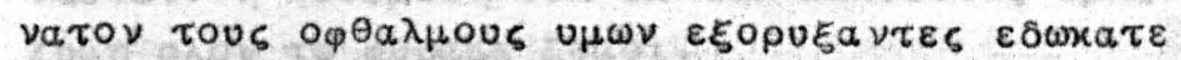

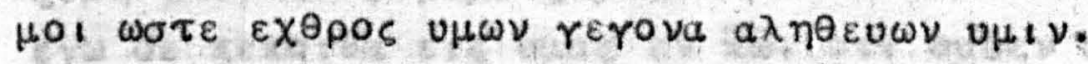

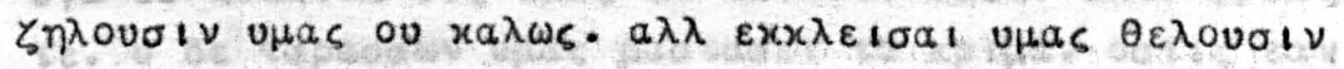

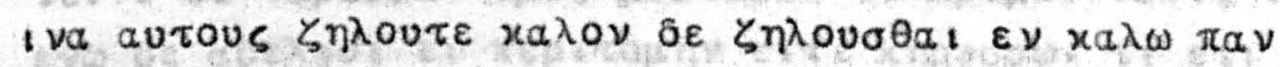

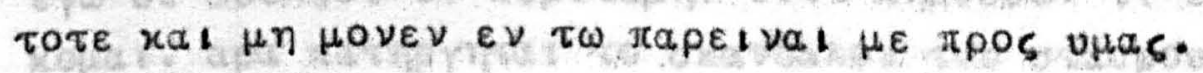

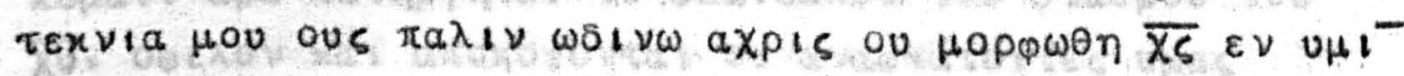

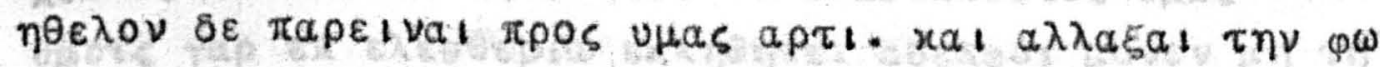

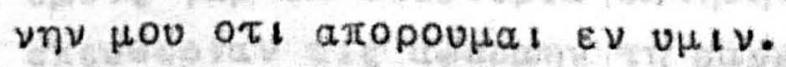

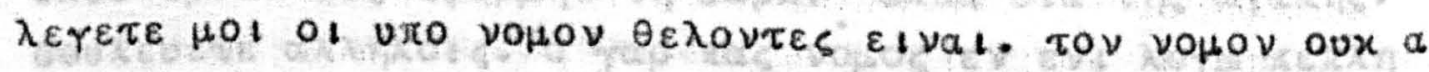

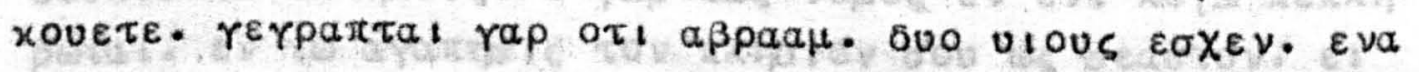

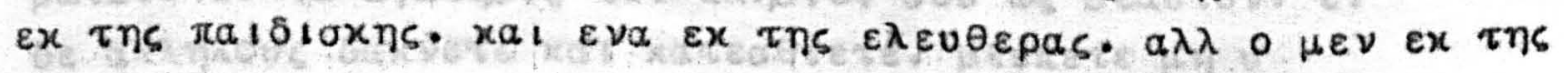

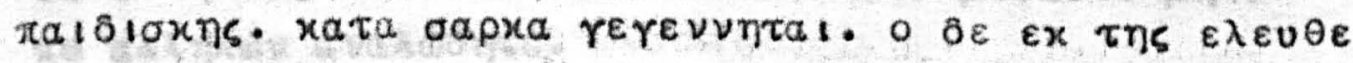

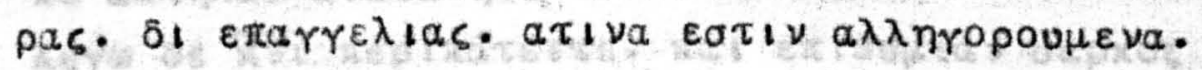

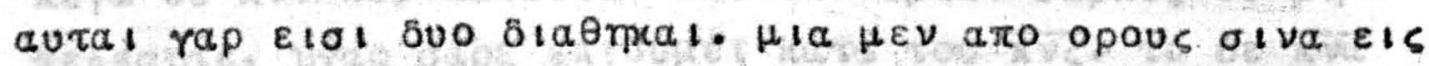

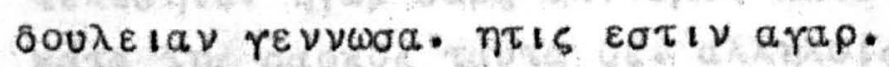

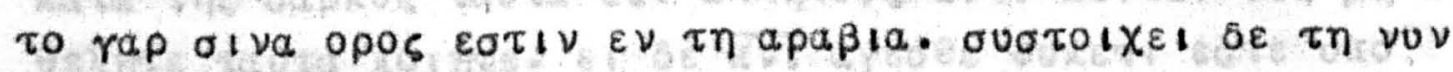

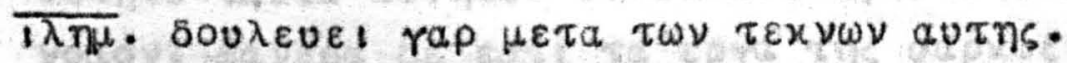

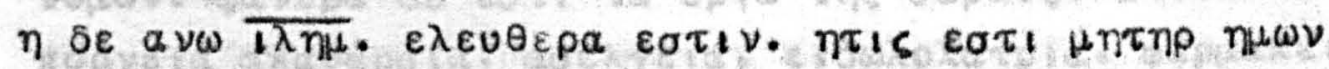

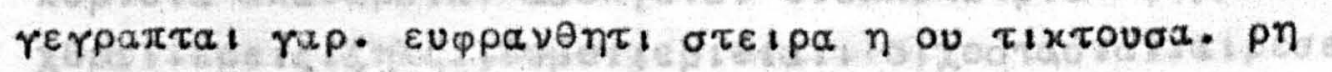

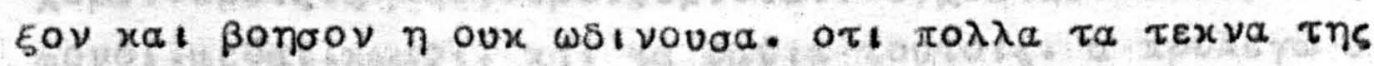

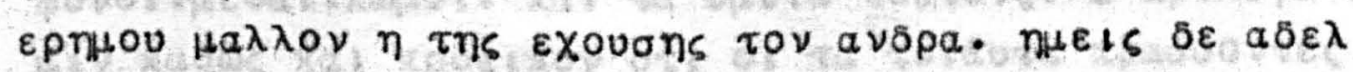

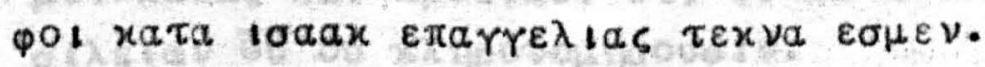

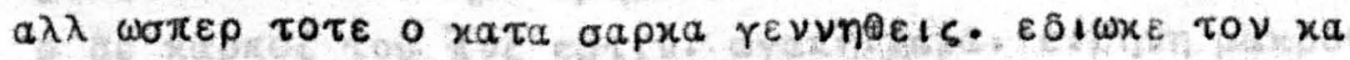

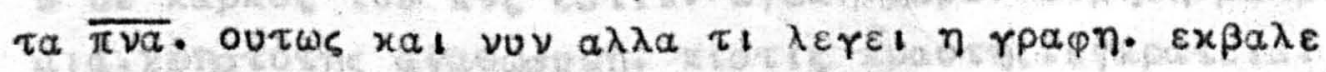

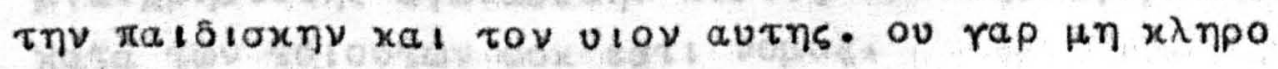

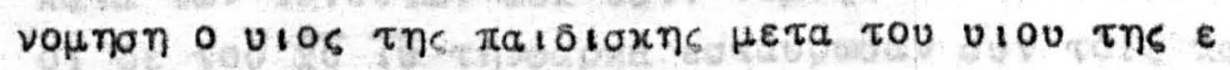




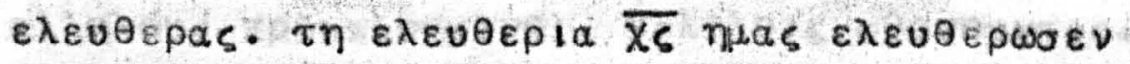

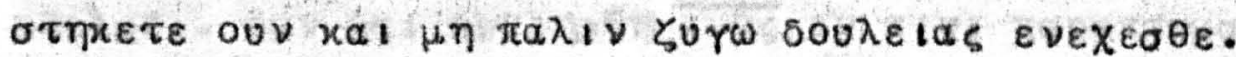

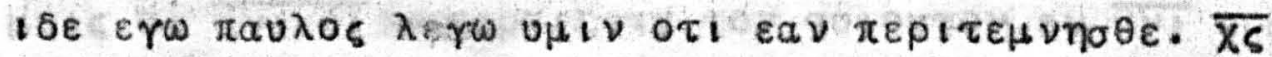

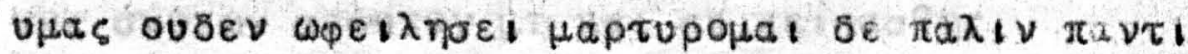

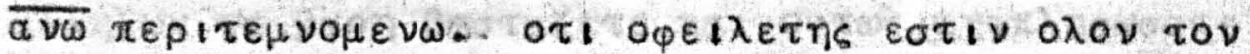
voHov roinoat.

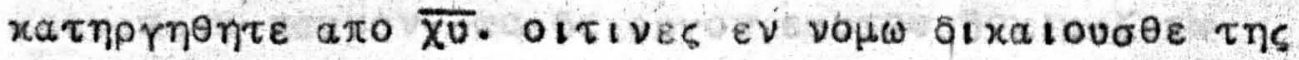

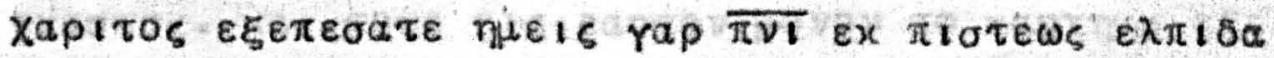

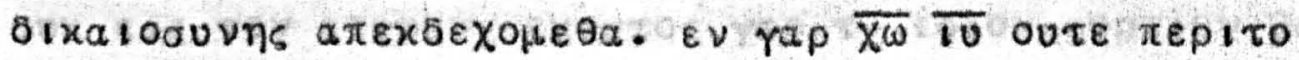

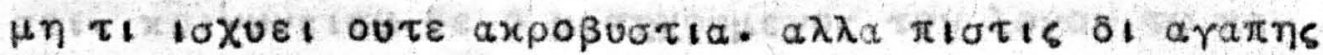
$\varepsilon \vee \varepsilon \rho$ You $น \varepsilon \eta \eta$.

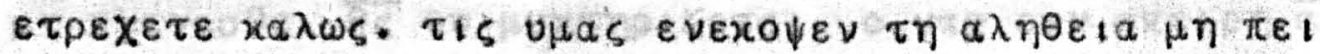

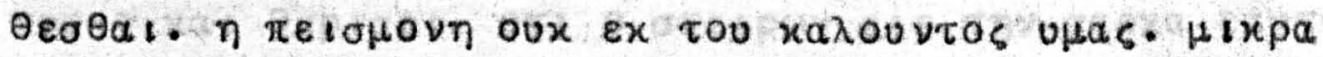

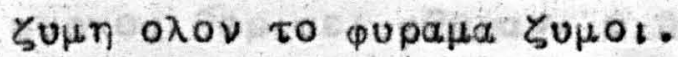

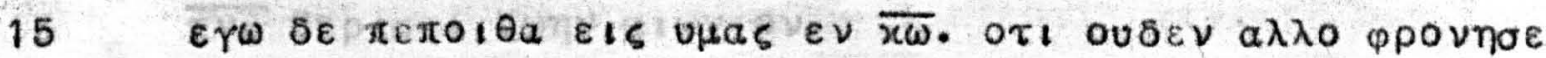

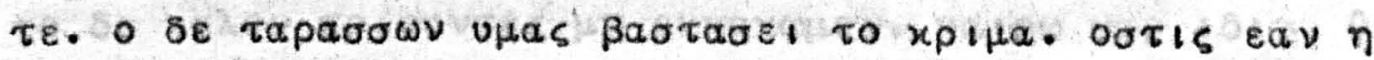

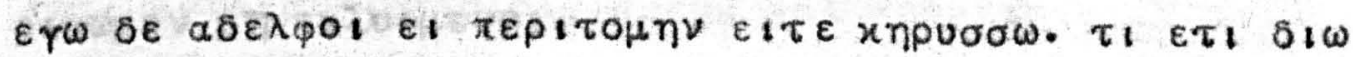

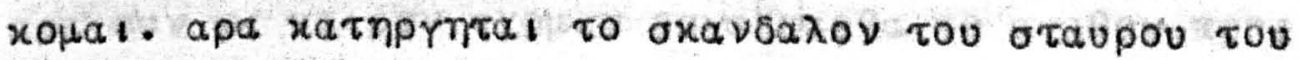

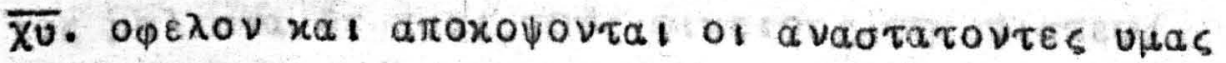

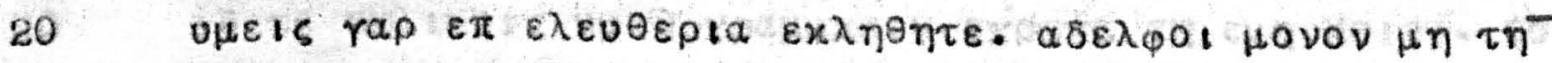

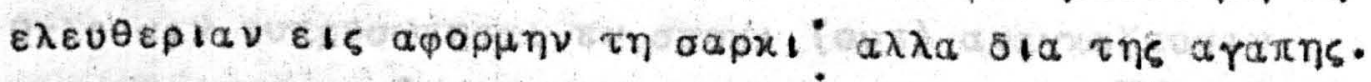

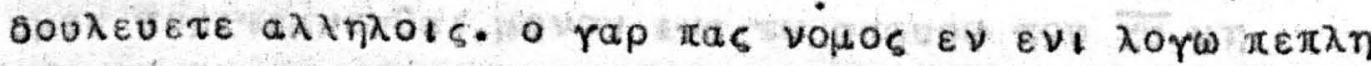

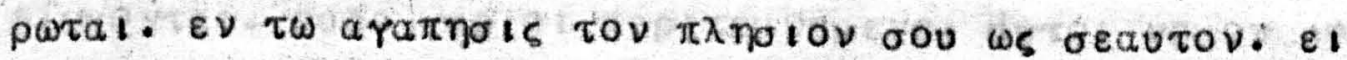

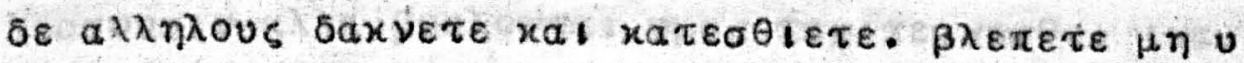

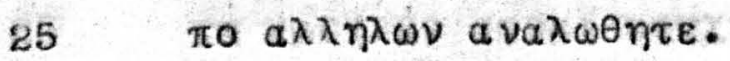

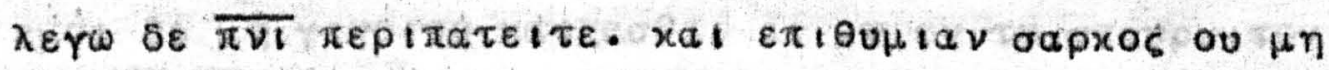

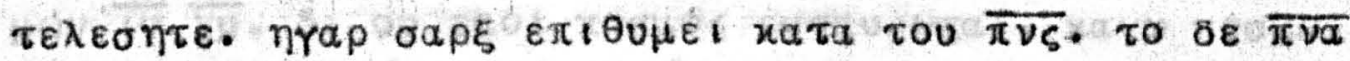

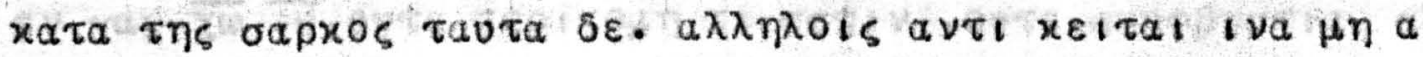

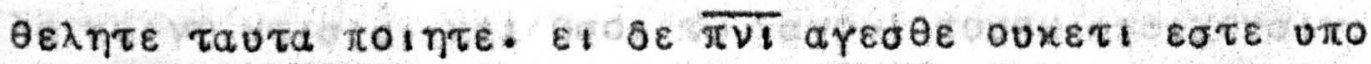

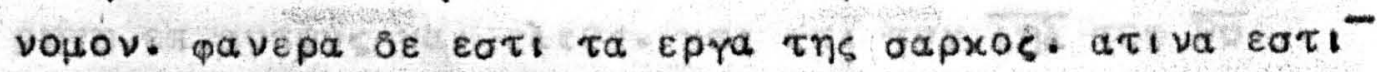

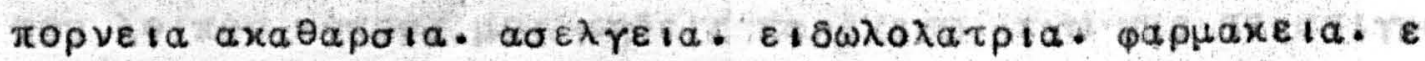

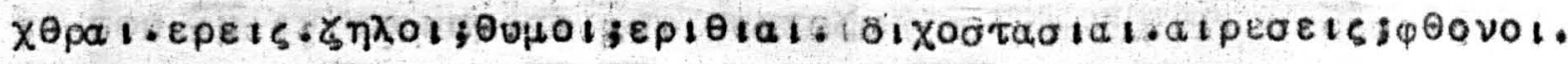

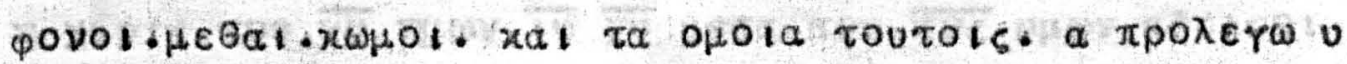

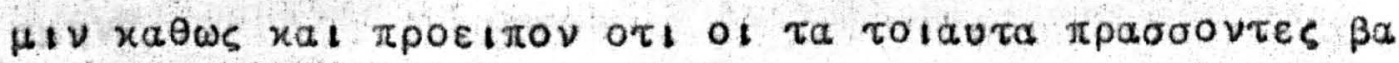

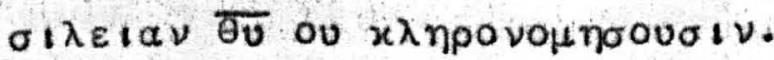

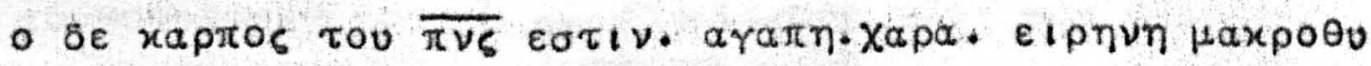

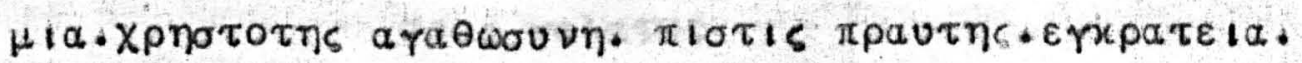

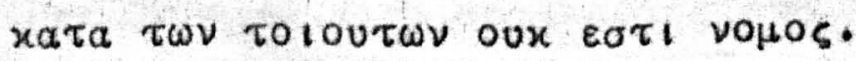

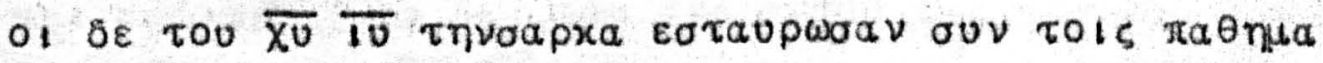

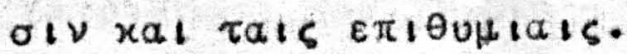

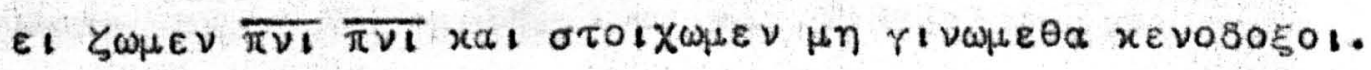




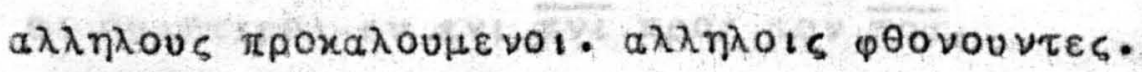

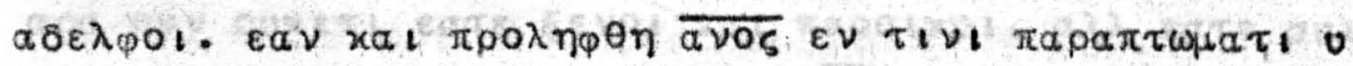

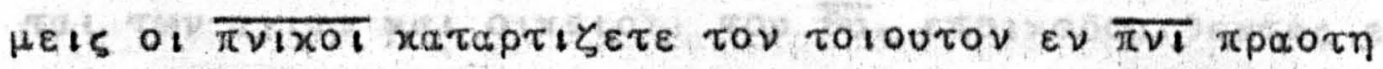

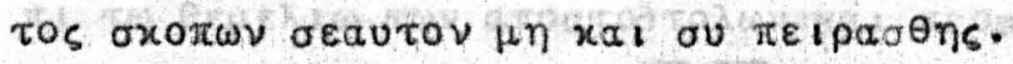

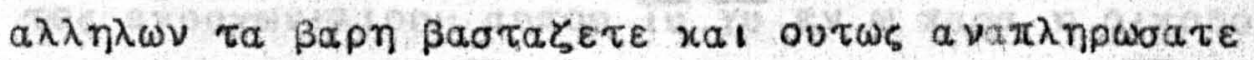

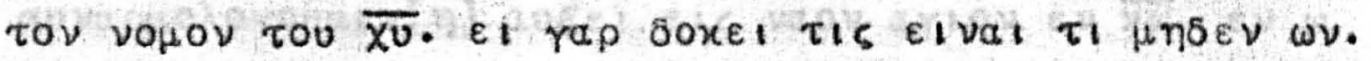

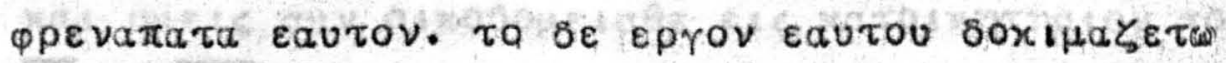

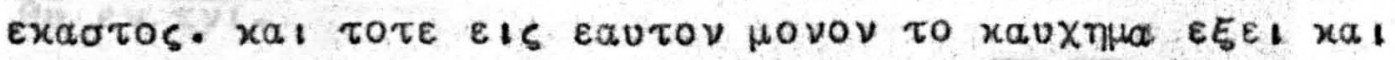

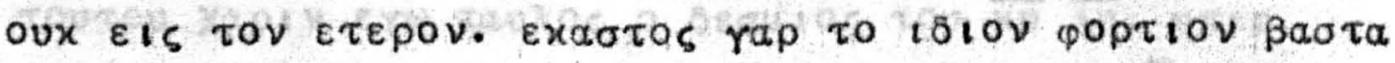

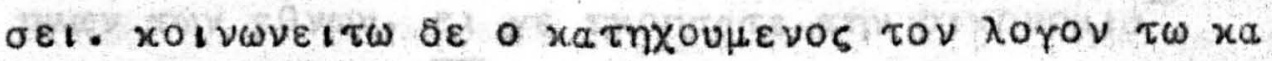

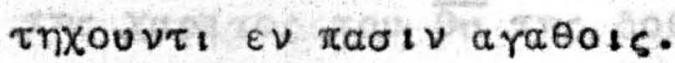

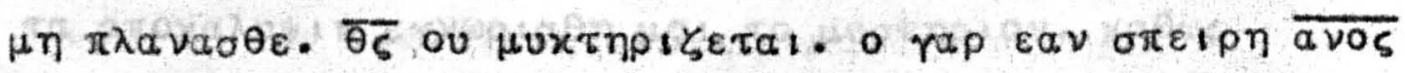

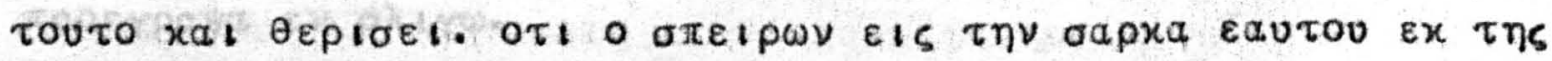

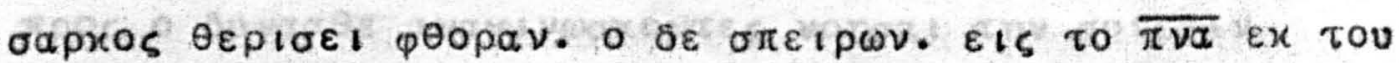

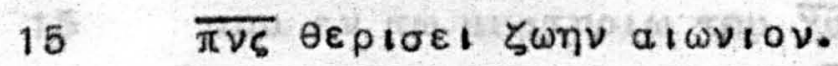

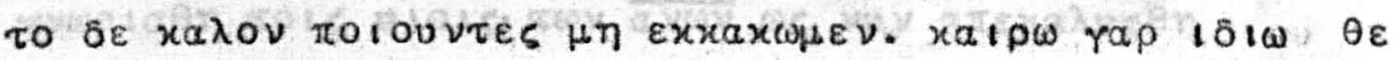

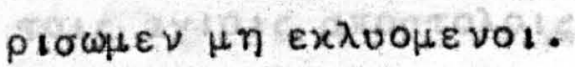

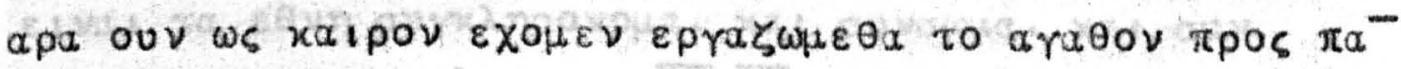

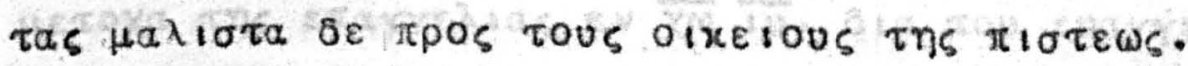

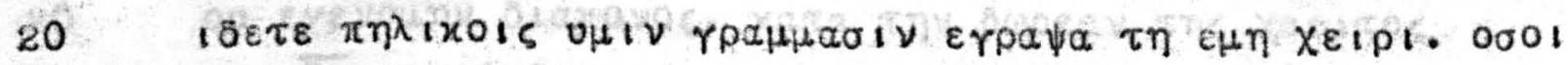

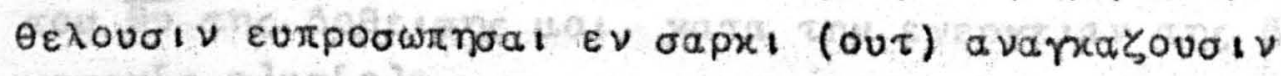

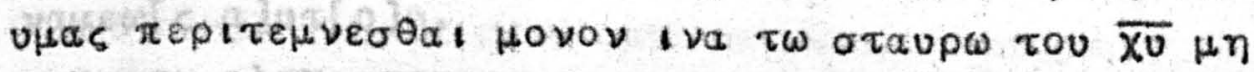

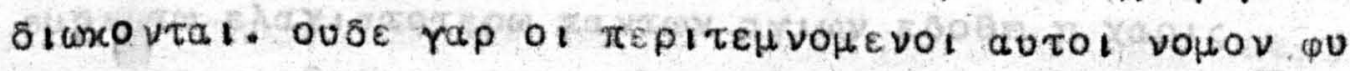

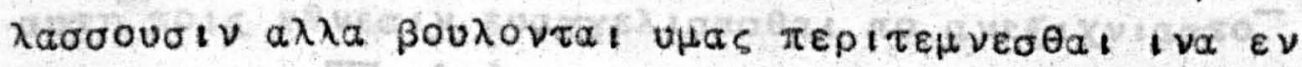

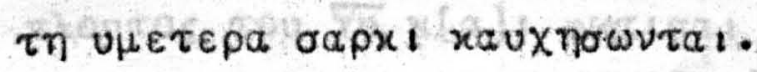

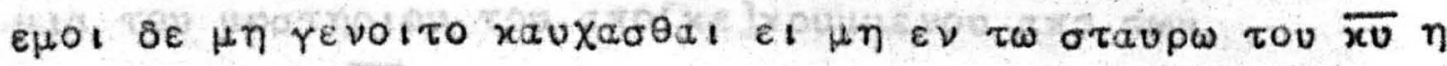

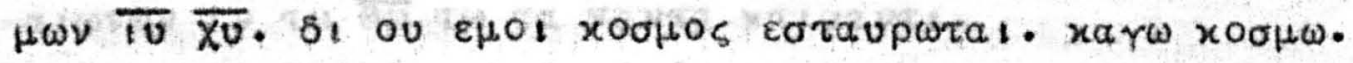

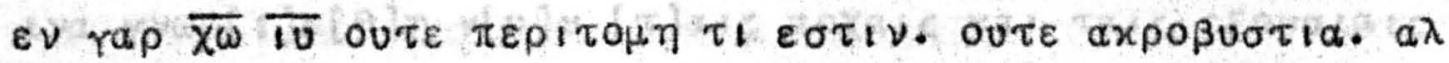

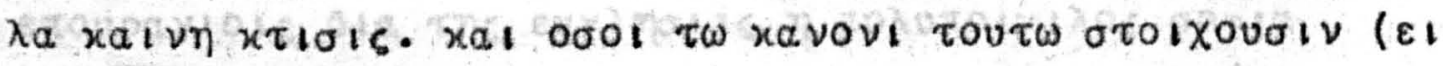

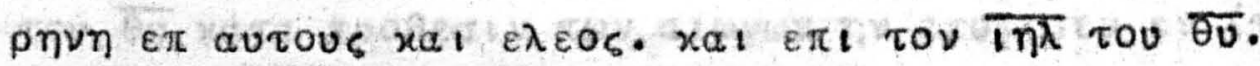

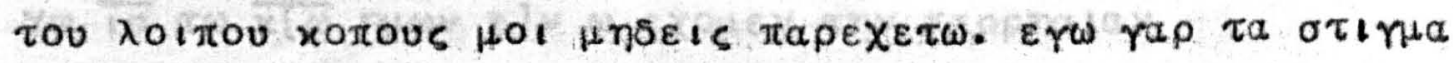

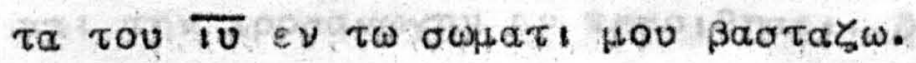

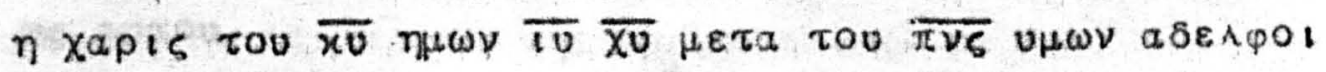
aunv.

xpos raiatas 


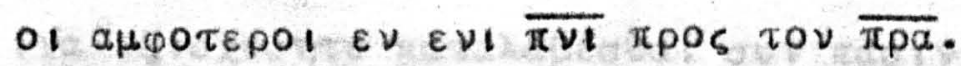

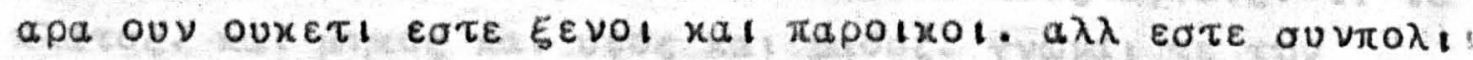

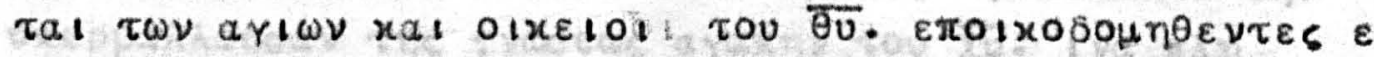

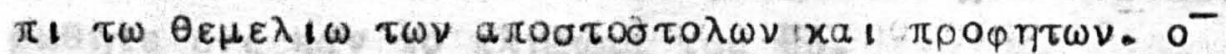

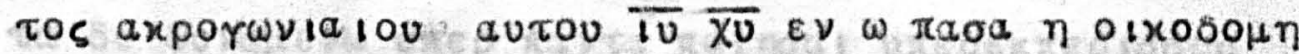

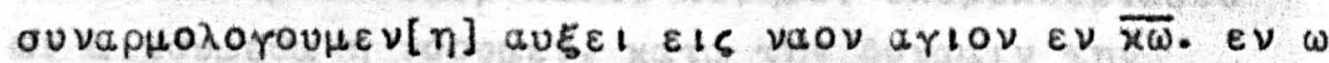

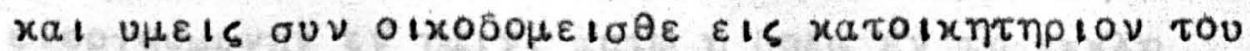

$\overline{\theta u} \varepsilon \nu \overline{\pi \nu 1}$.

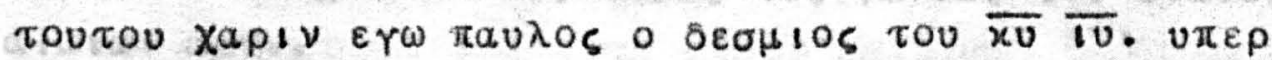

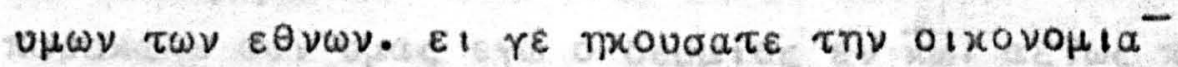

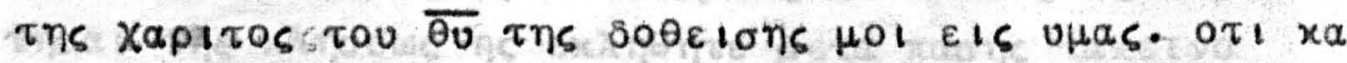

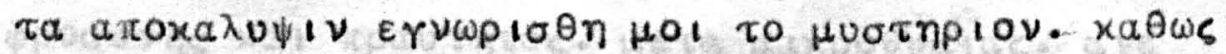

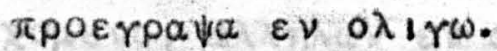

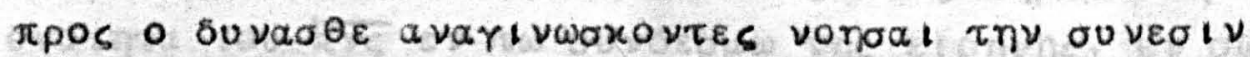

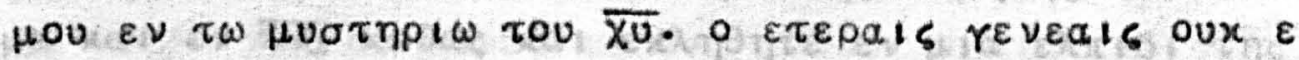

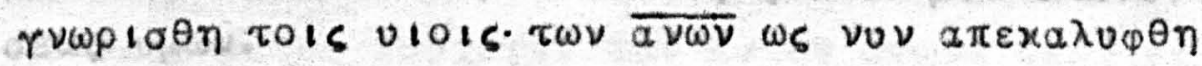

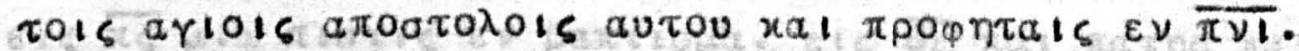

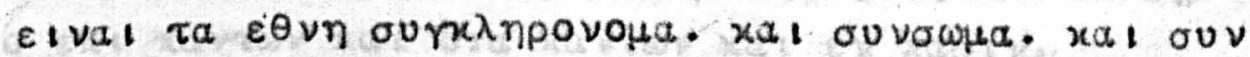

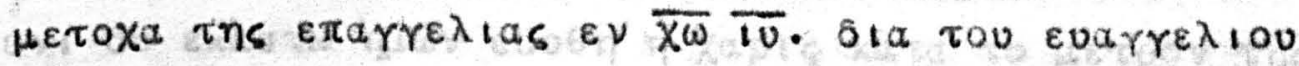

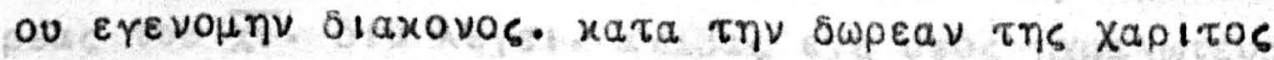

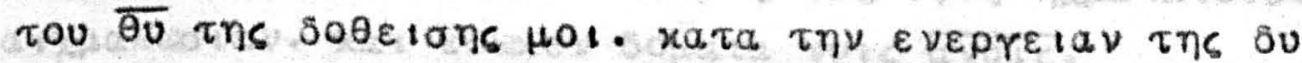

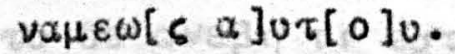

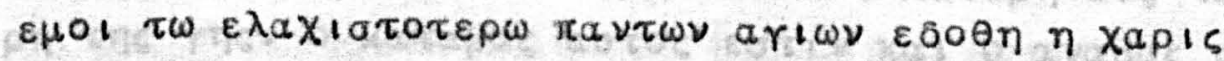

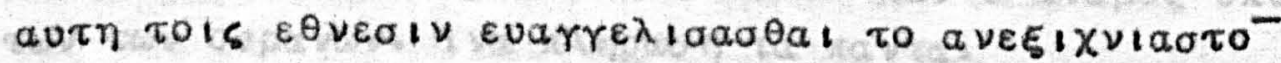

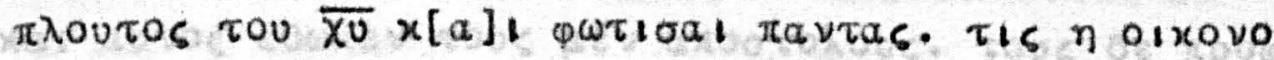

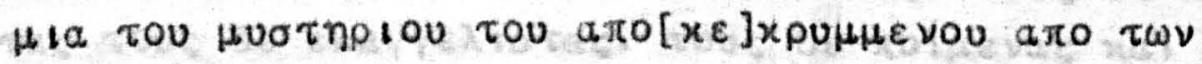

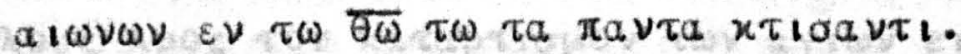

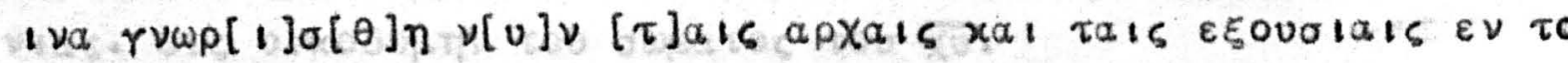

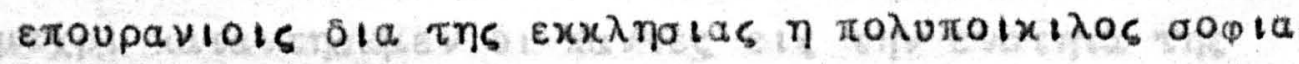

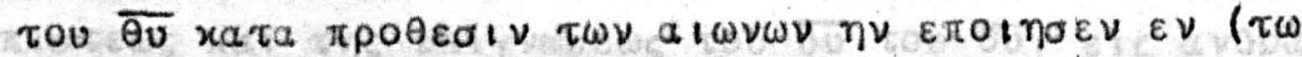

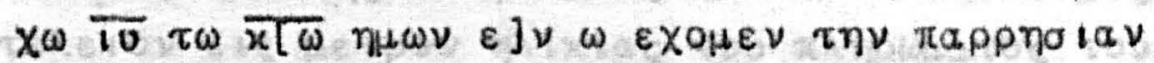

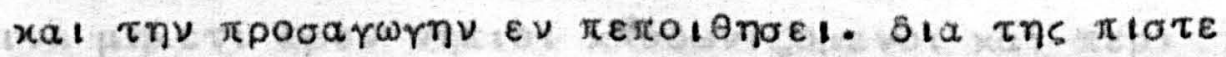
ws autou.

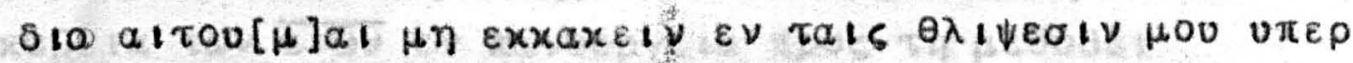

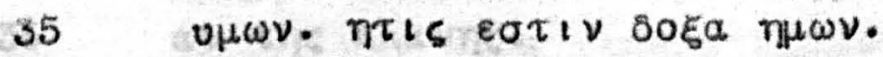

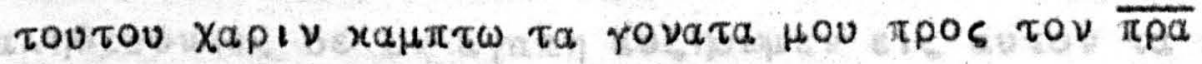

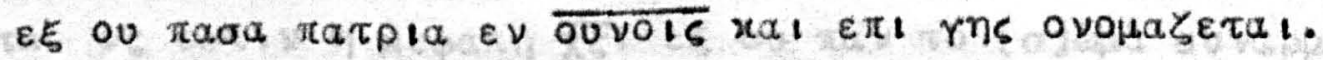

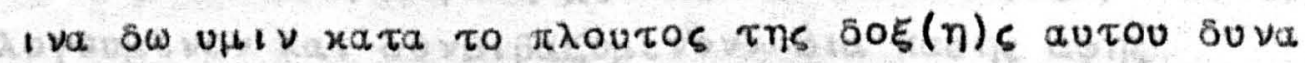

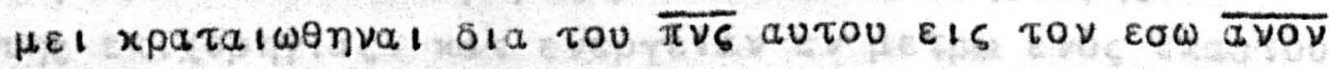

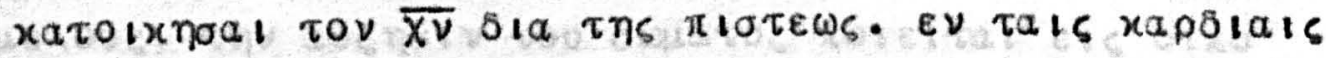

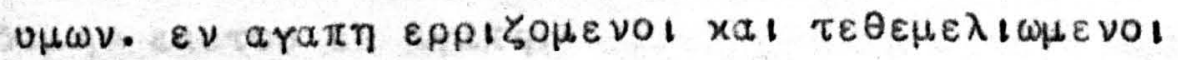




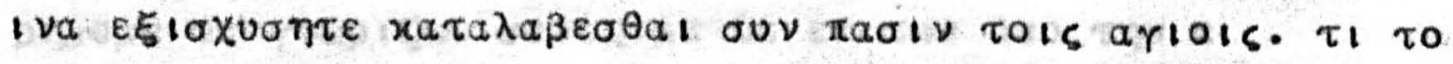

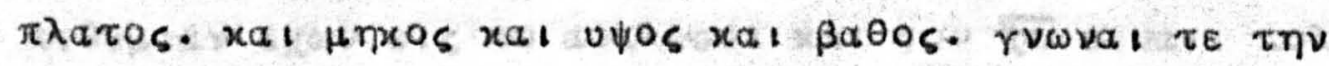

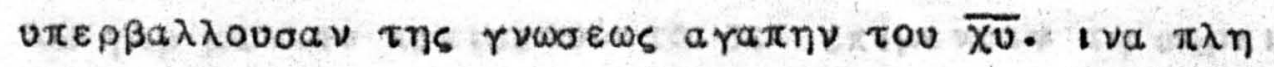

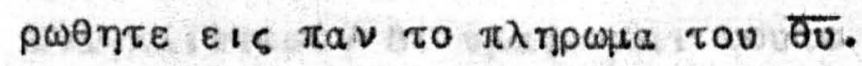

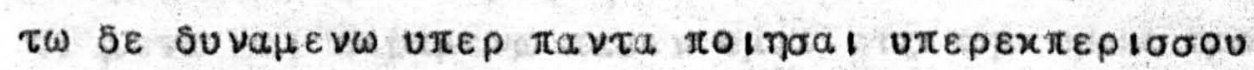

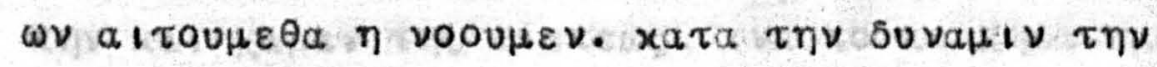

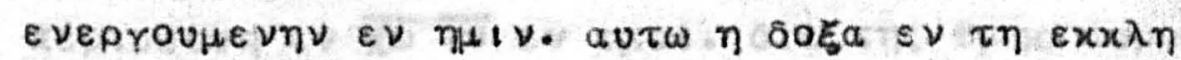

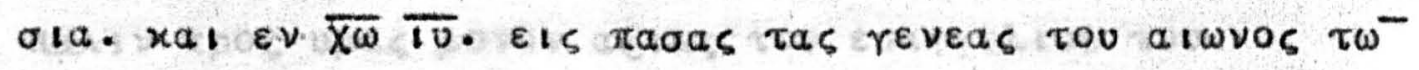

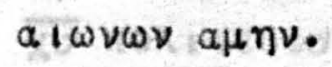

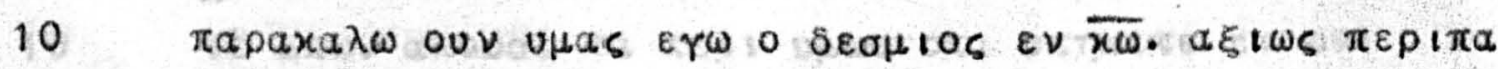

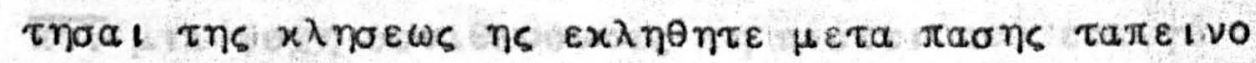

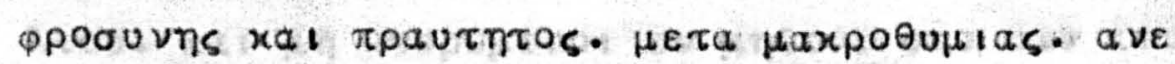

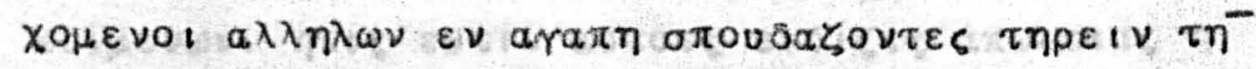

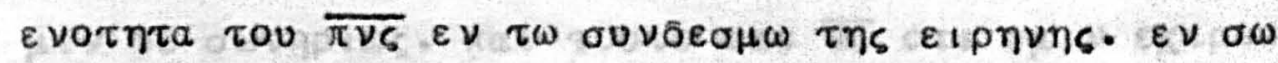

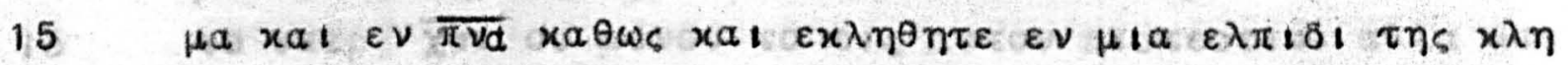

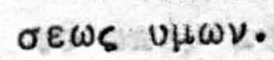

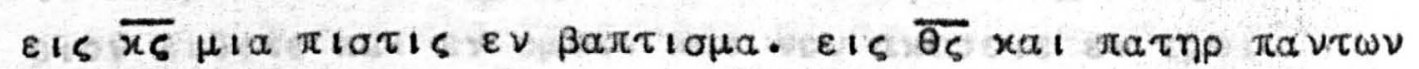

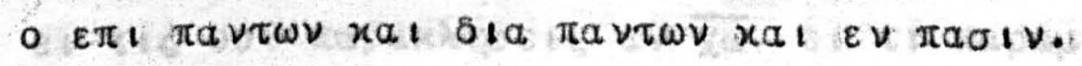

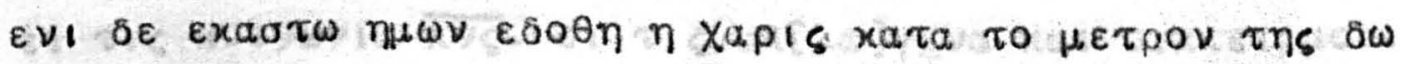

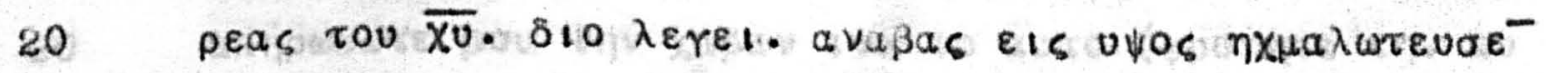

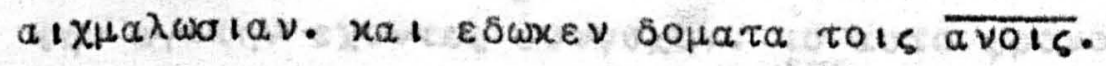

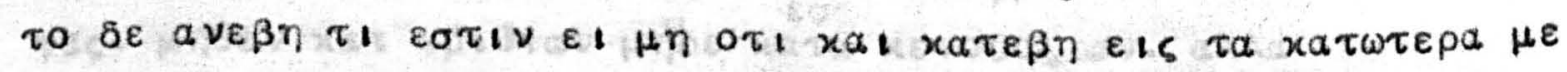

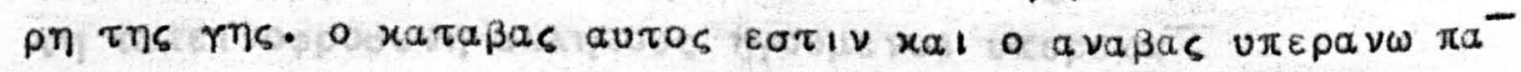

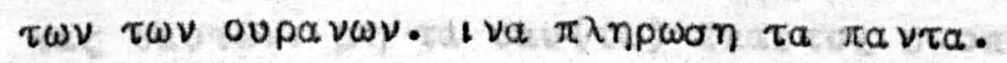

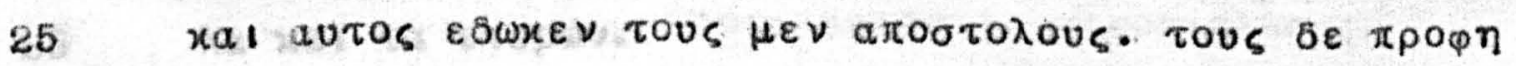

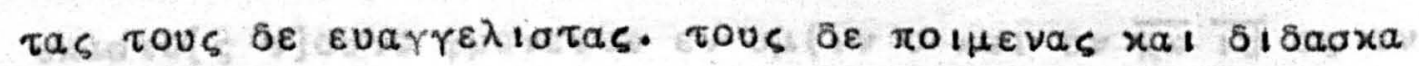

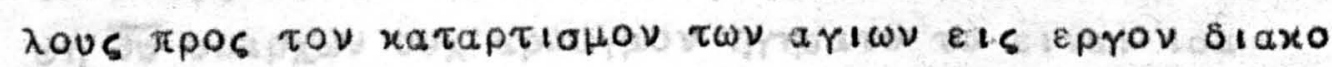
vias. Els oixoöounv tov owhatos tov $\overline{\mathrm{X}}$.

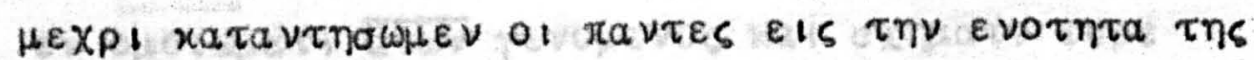

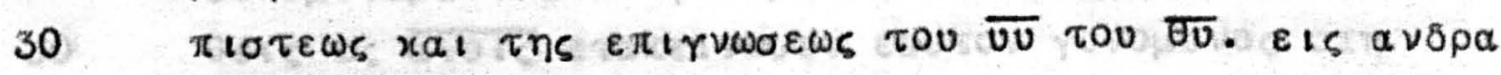

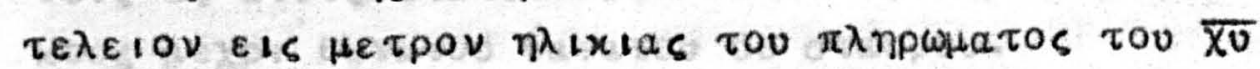

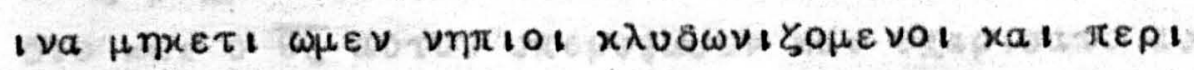

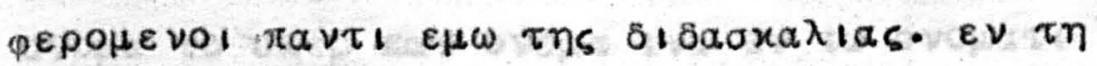

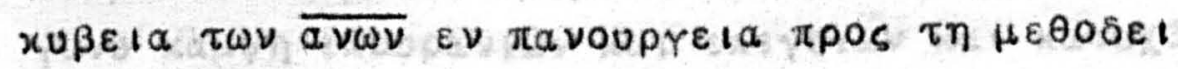

35 av $\tau$ Th $\pi \lambda a v \eta s$.

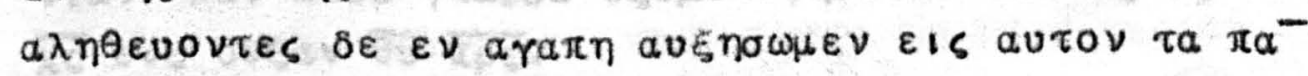

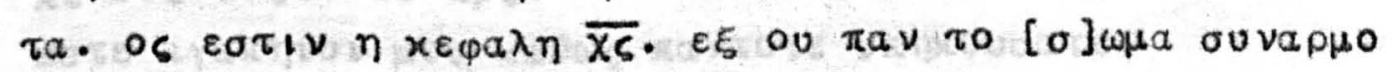

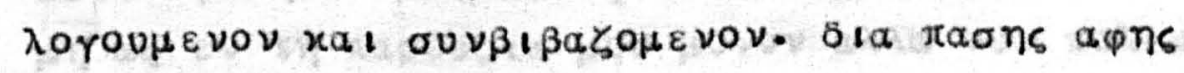

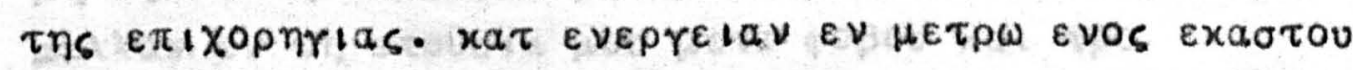

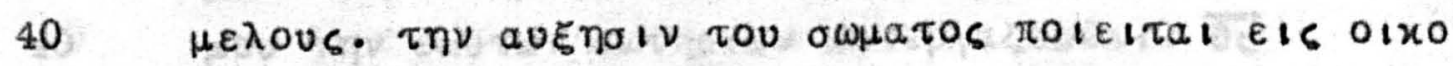

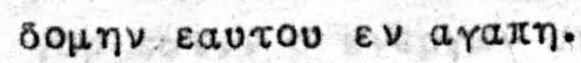




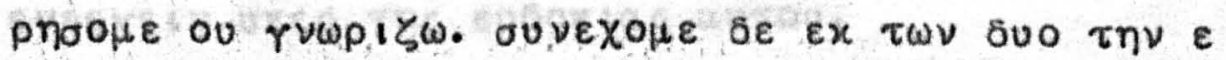

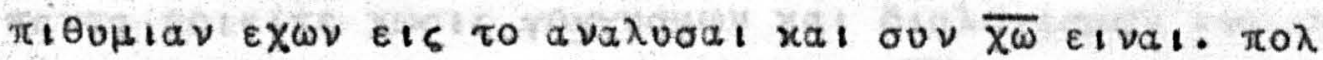

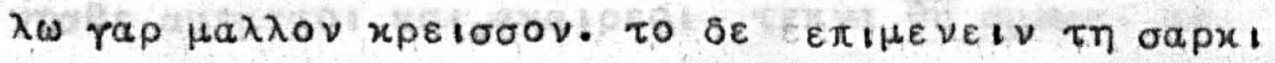

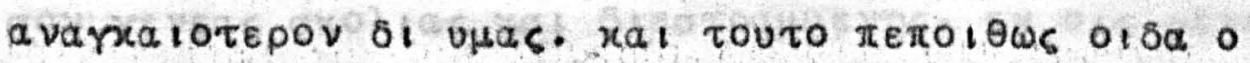

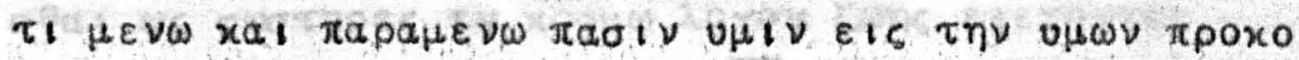

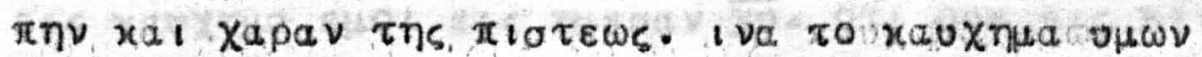

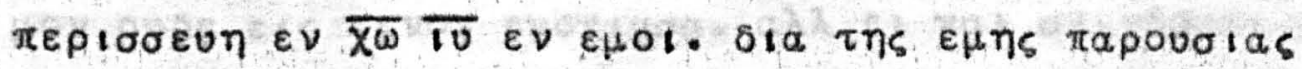

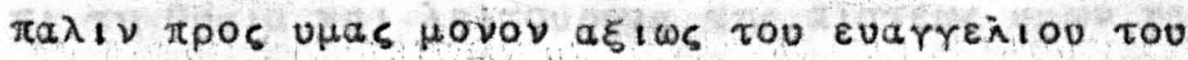

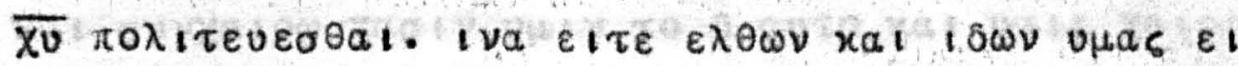

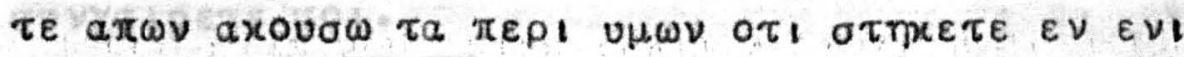

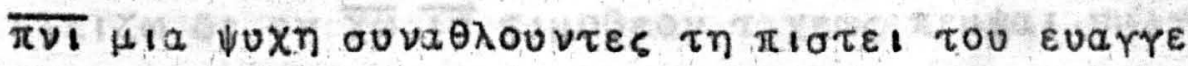

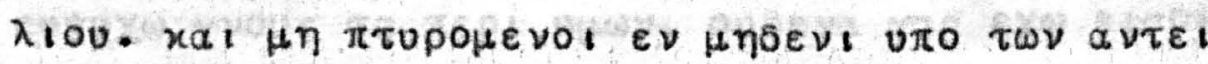

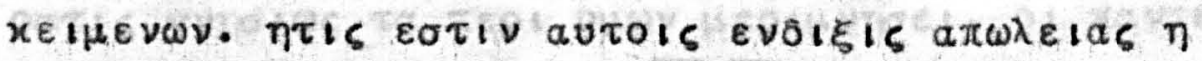

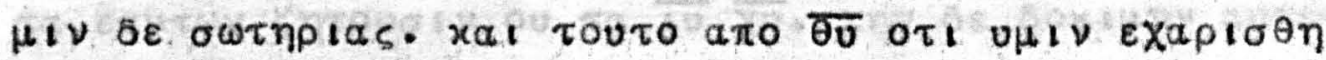

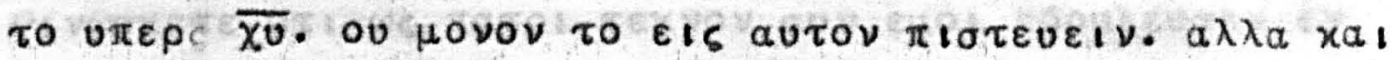

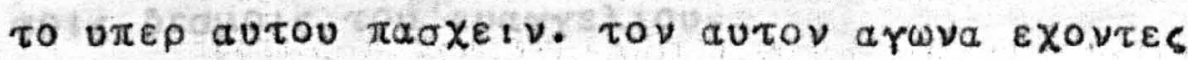

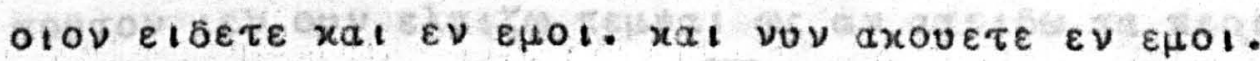

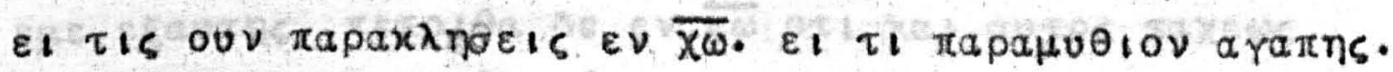

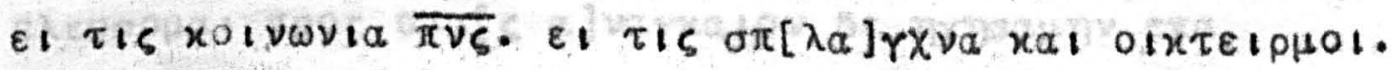

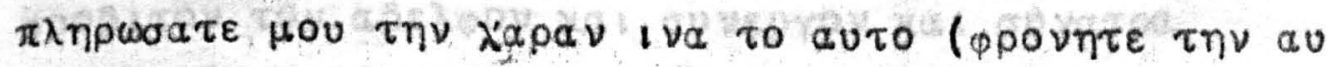

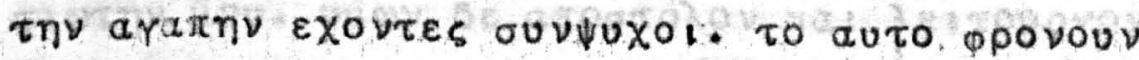

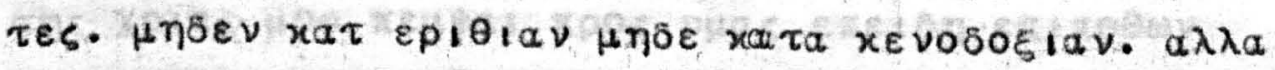

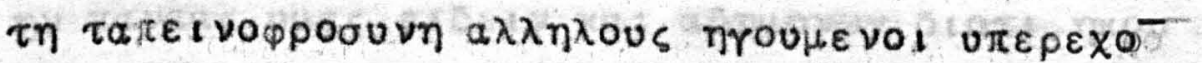

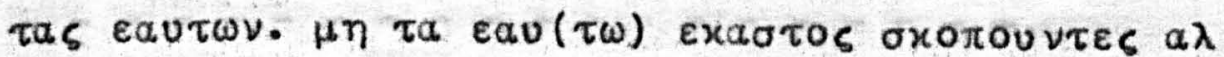

$25 \lambda \alpha$ xal $\tau \alpha \varepsilon \tau \varepsilon \rho \omega \nu$.

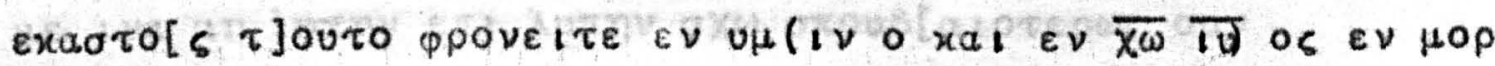

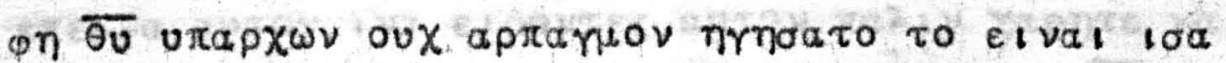

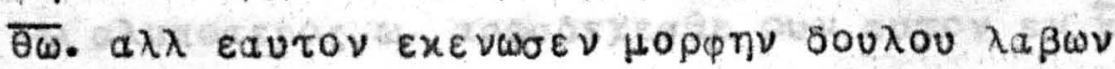

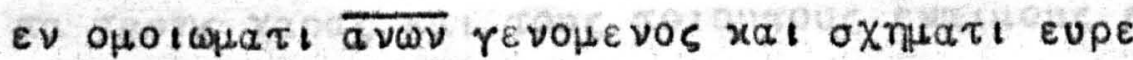

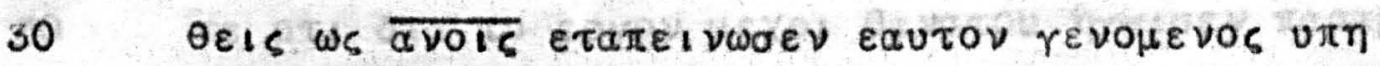

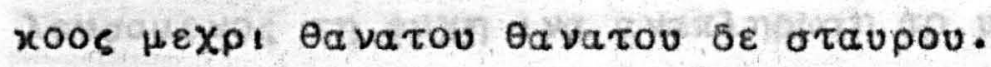

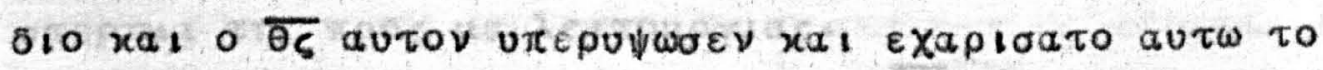

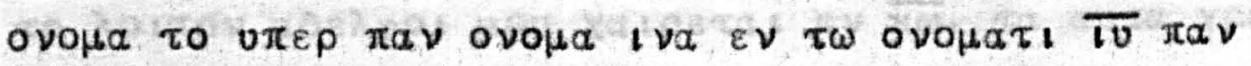

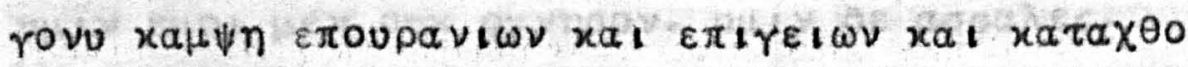

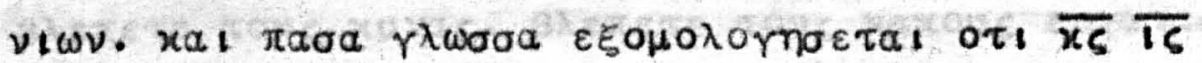

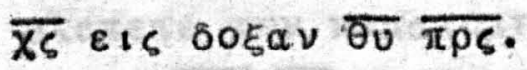

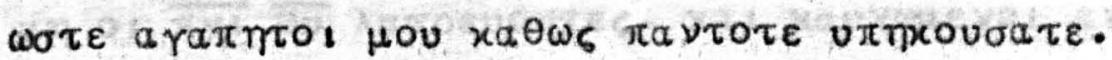

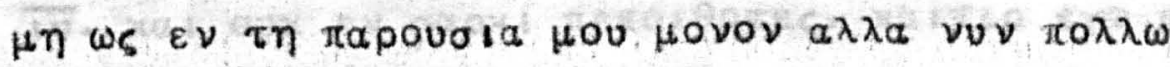

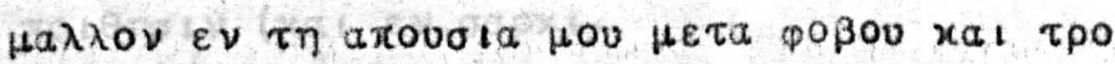

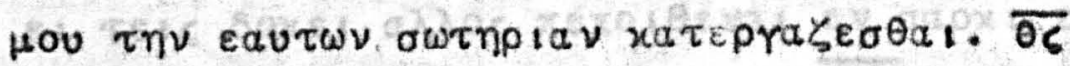

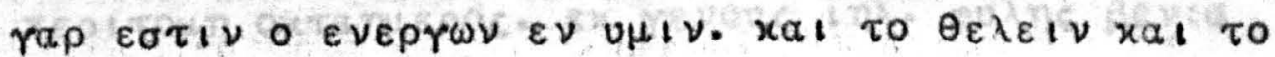




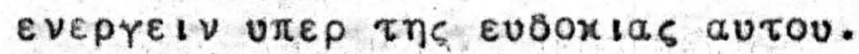

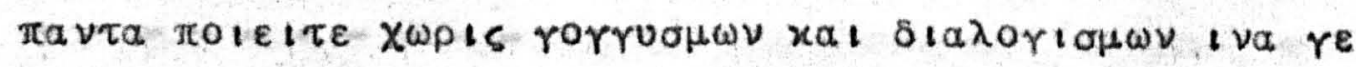

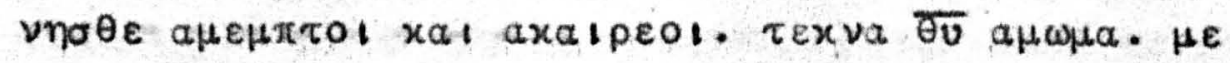

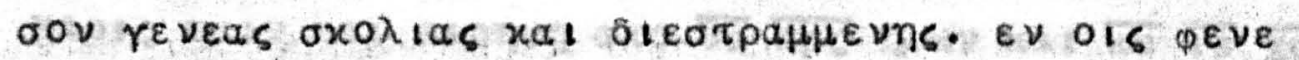

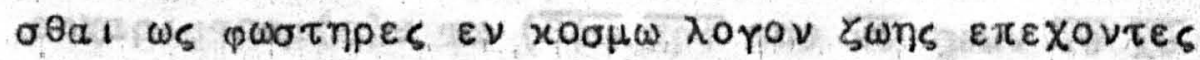

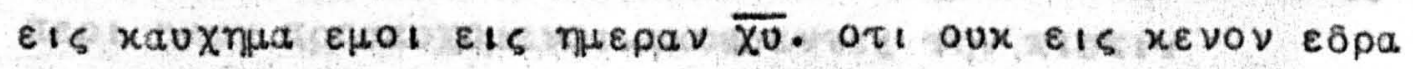

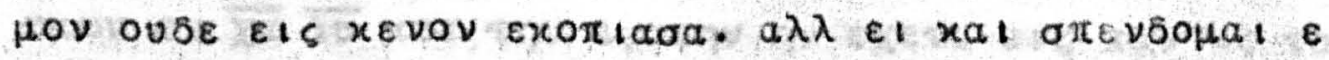

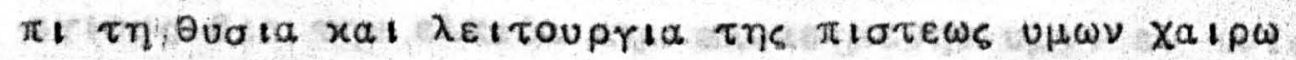

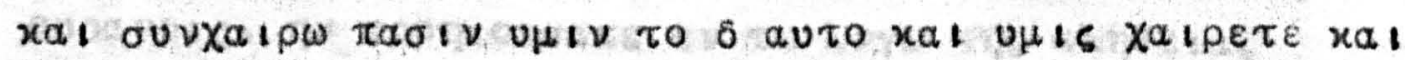

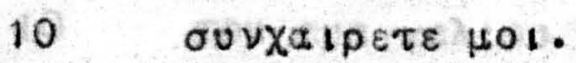

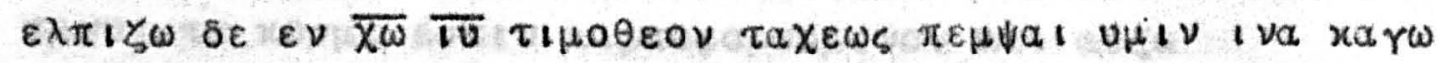

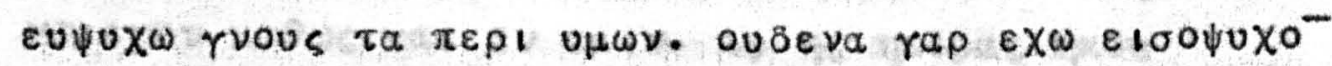

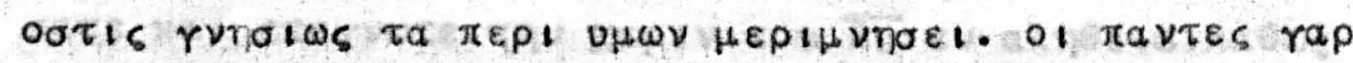

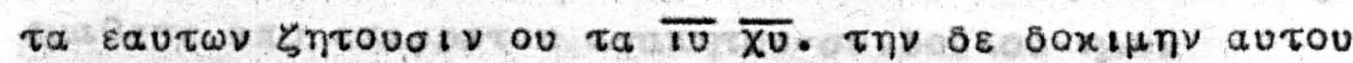

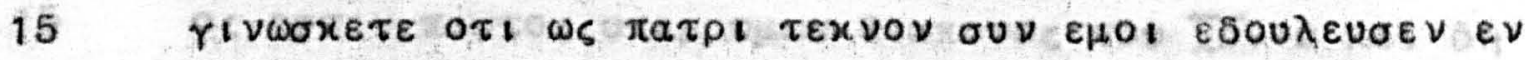

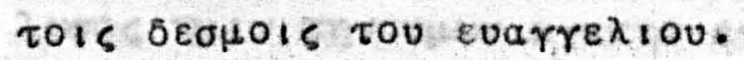

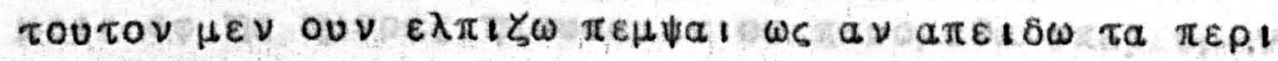

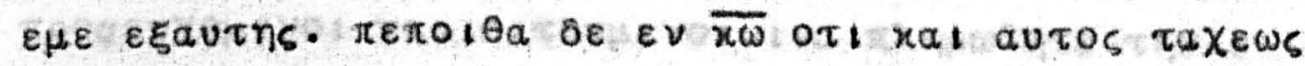

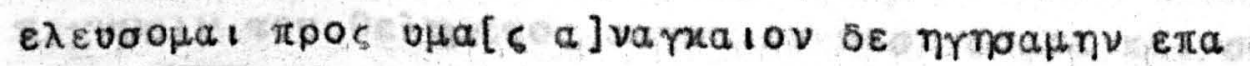

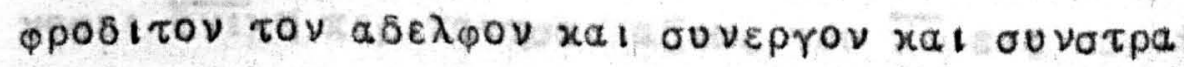

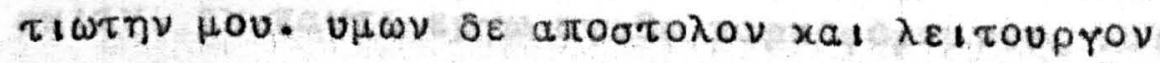

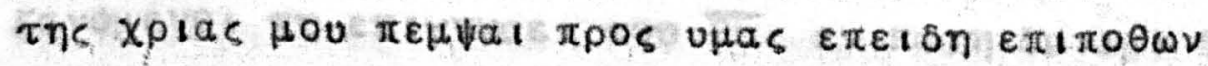

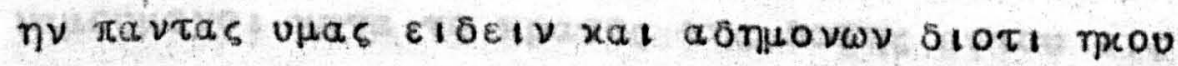
- - - - - - - - - - - - - - - -

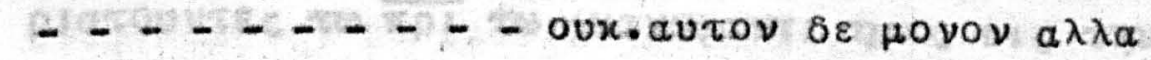

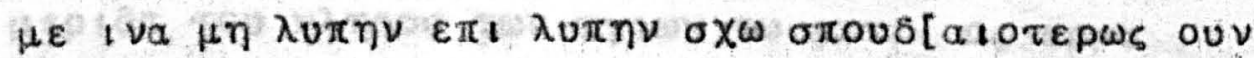

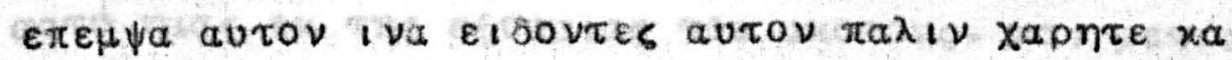

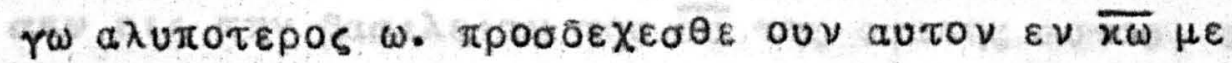

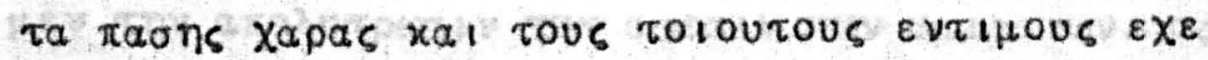

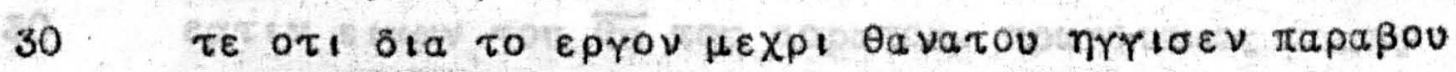

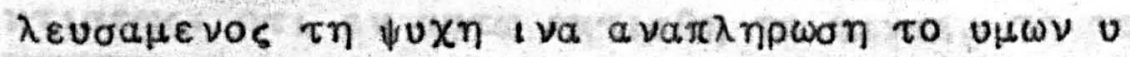

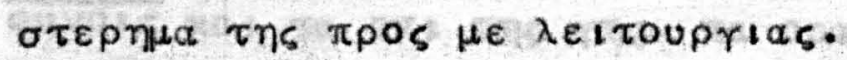

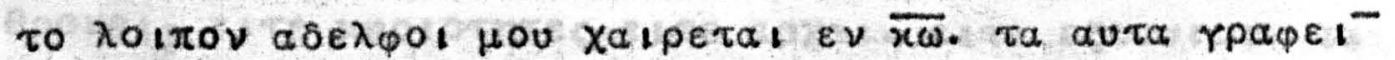

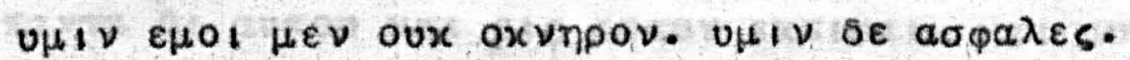

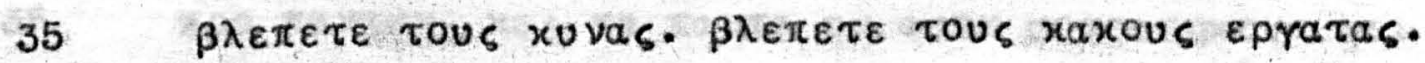

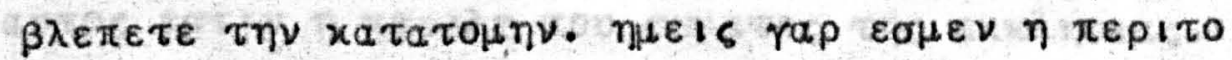

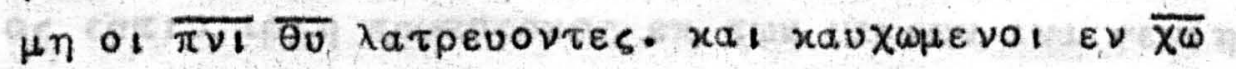

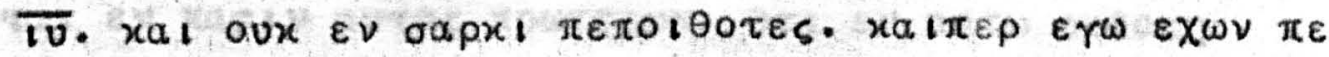
$\pi 0 \iota \theta \eta\rceil \nu(x a \downarrow \varepsilon \nu$ oapxl.

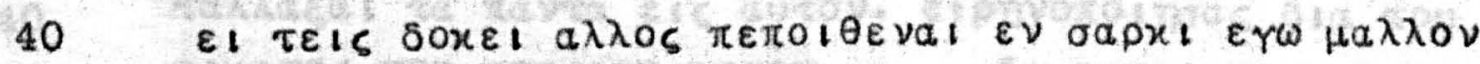

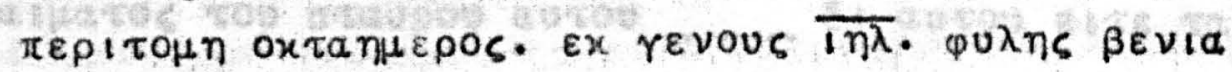




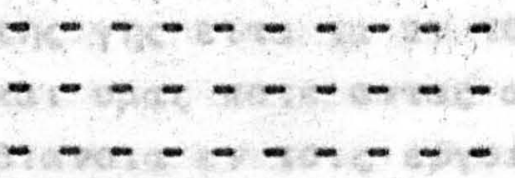

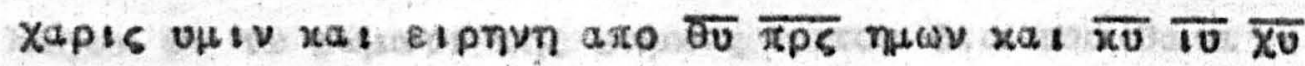

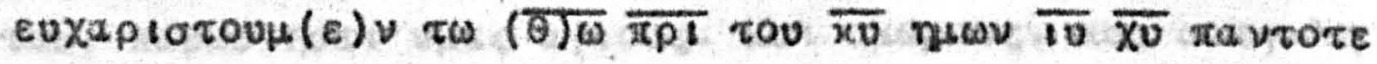

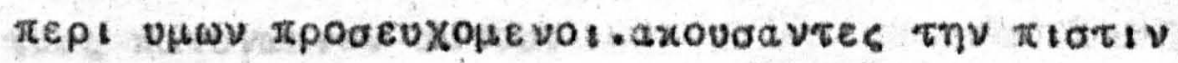

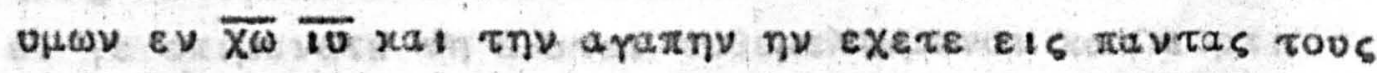

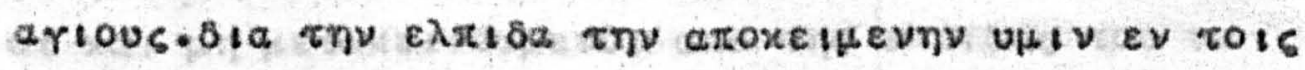

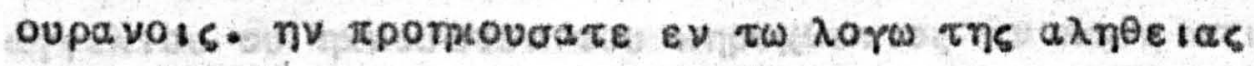

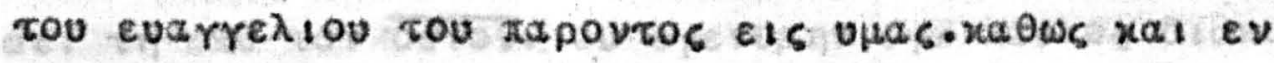

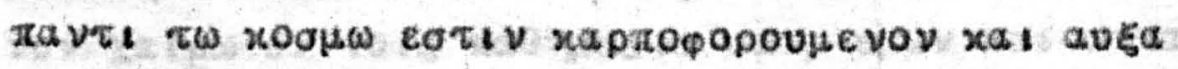

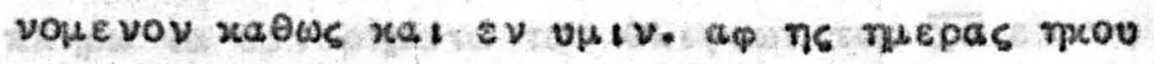

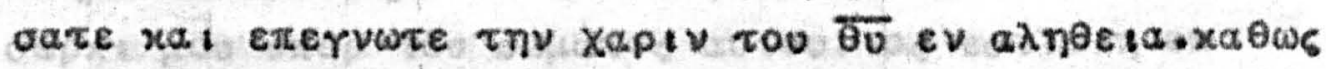

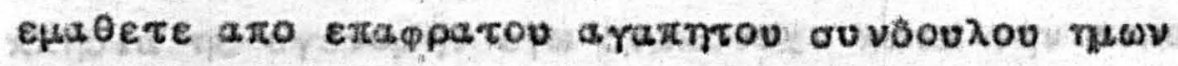

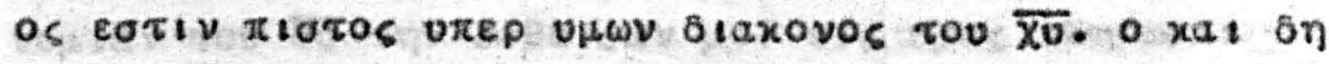

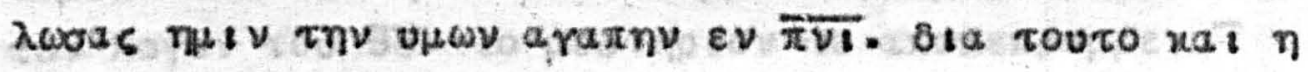

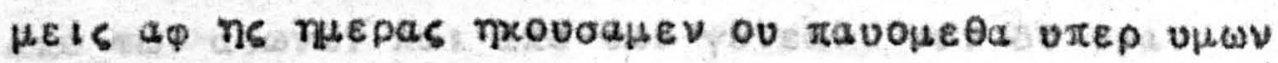

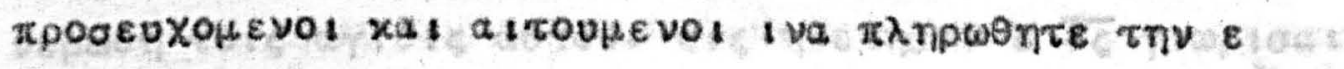

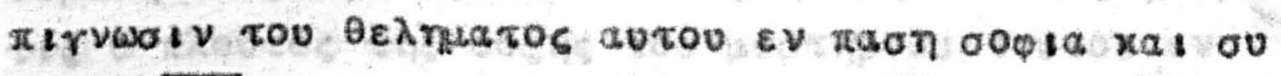

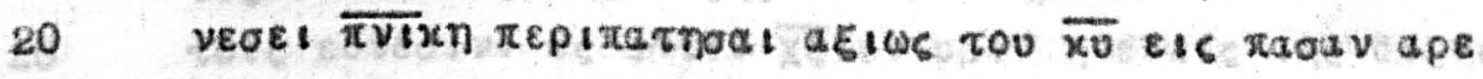

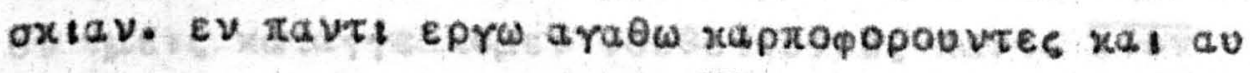

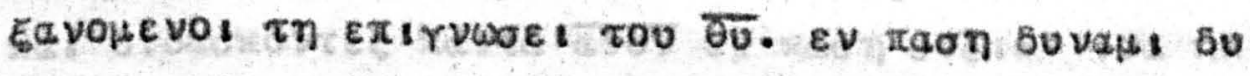

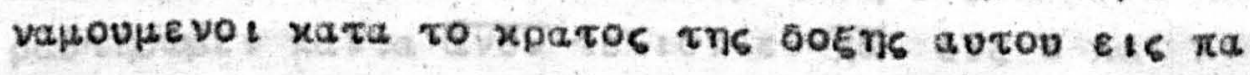

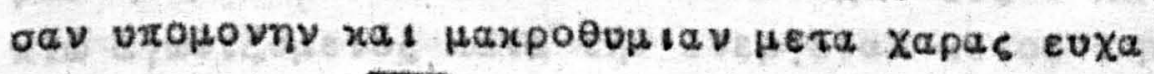

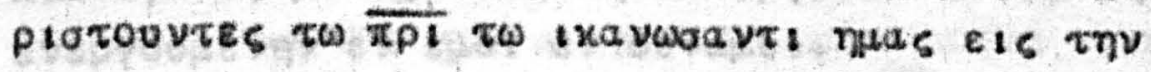

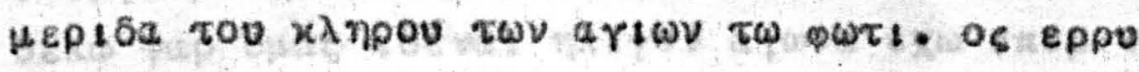

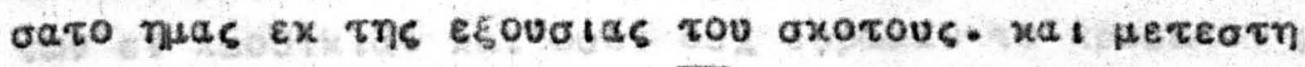

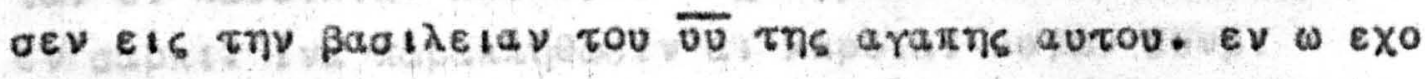

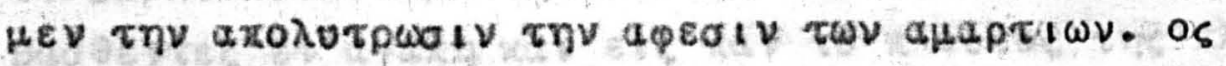

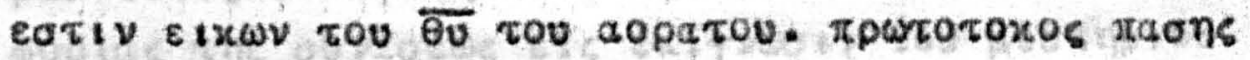

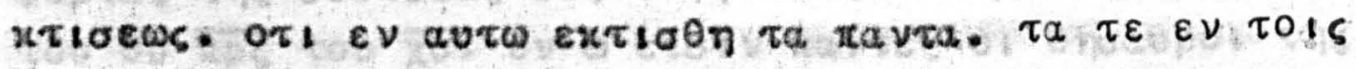

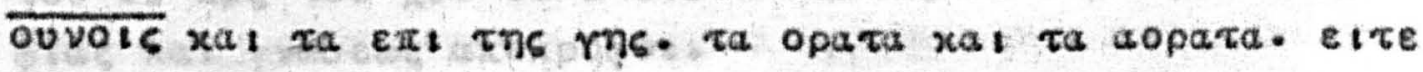

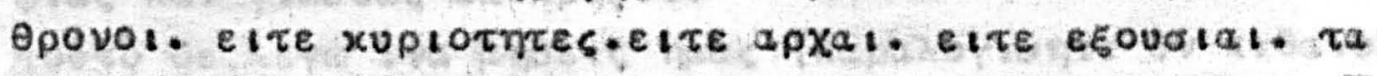

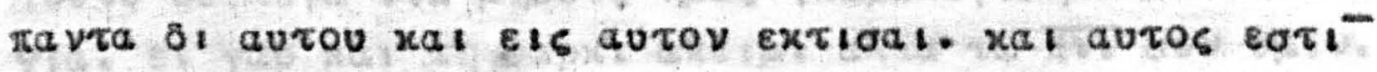

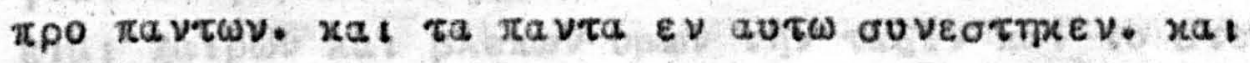

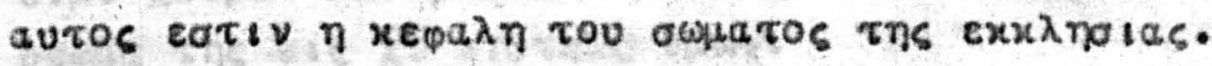

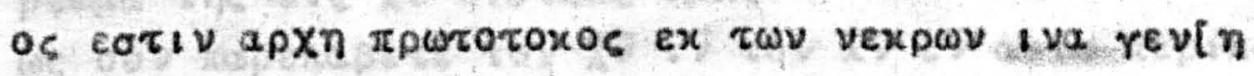

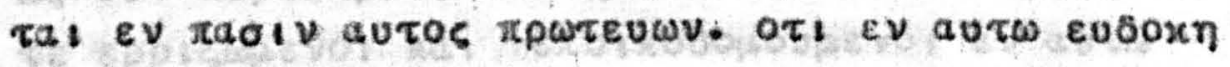

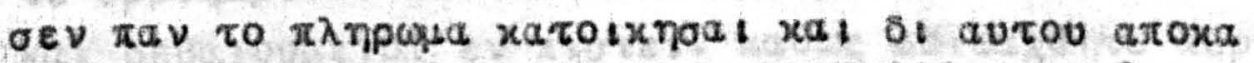

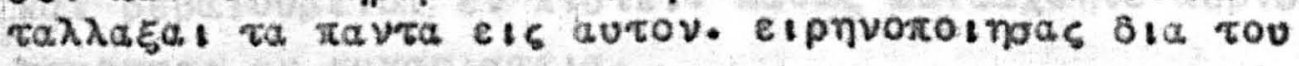
alparos rov araupov autov

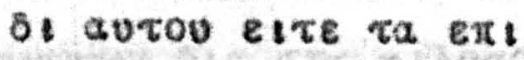




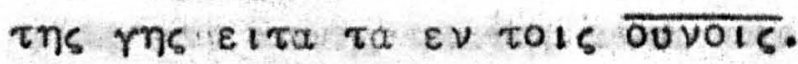

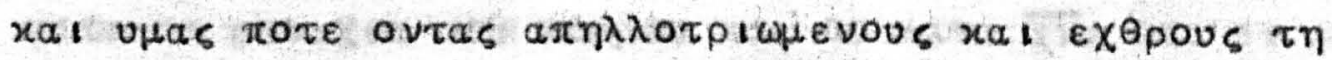

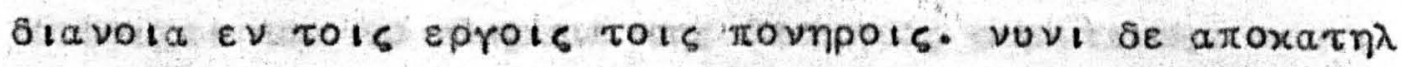

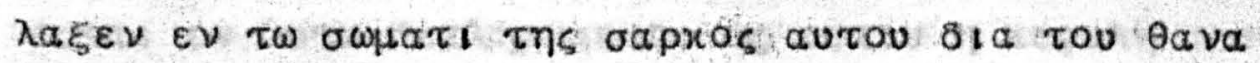

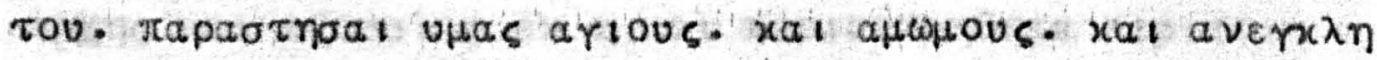

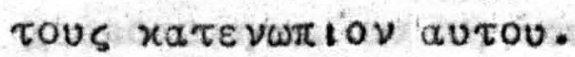

\&!

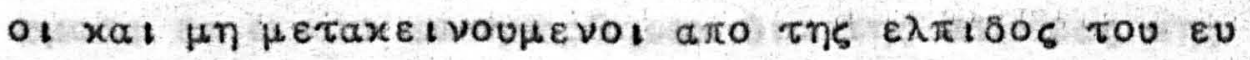

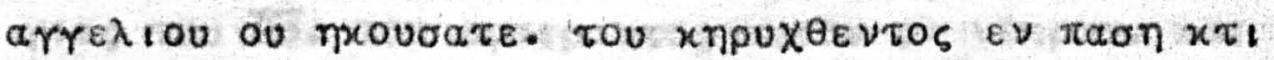

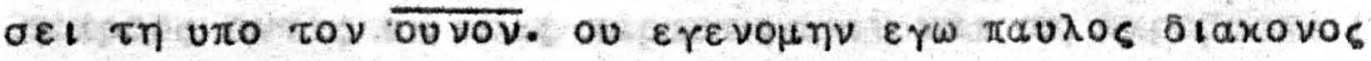

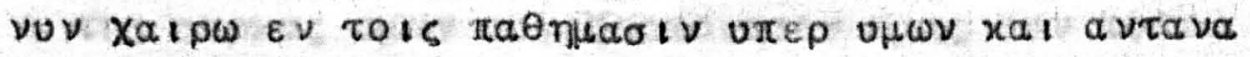

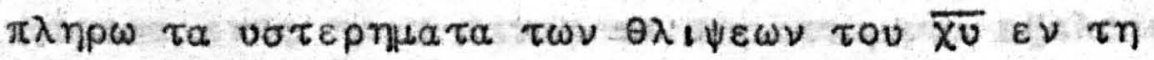

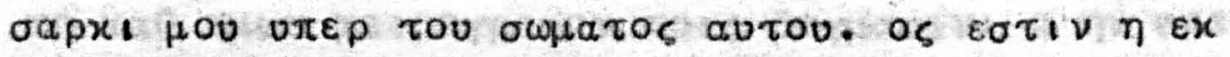

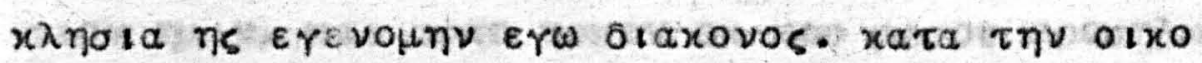

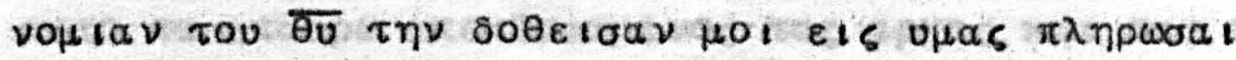

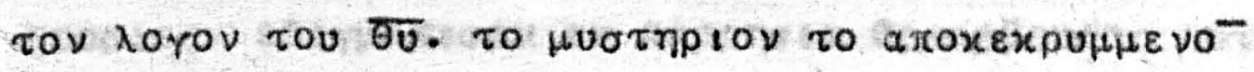

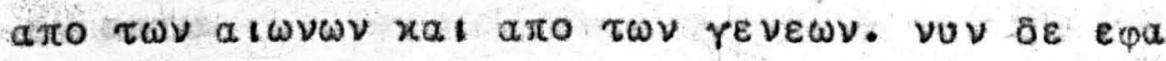

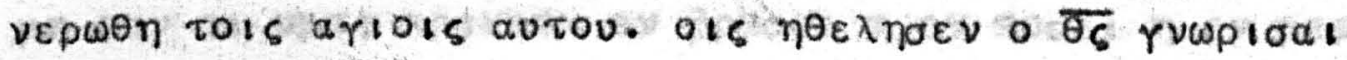

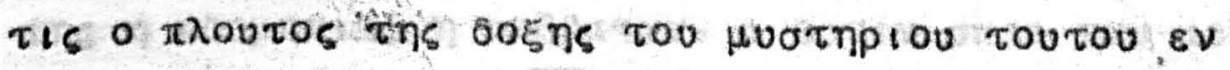

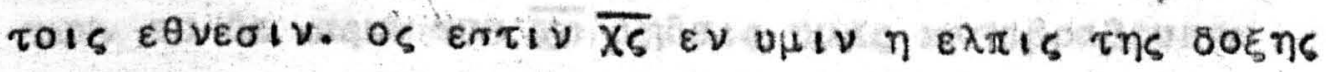

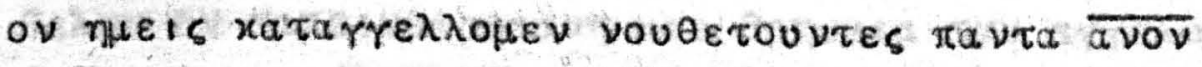

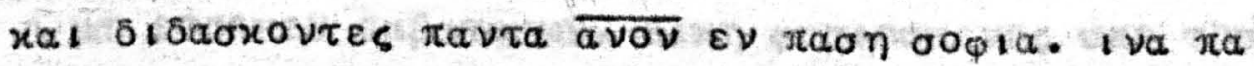

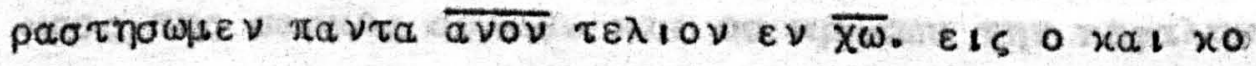

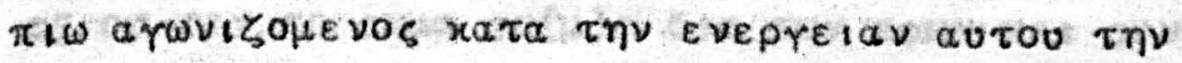
$\varepsilon v e p$ rou

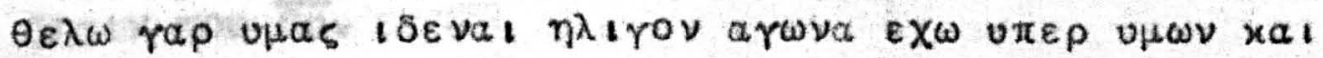

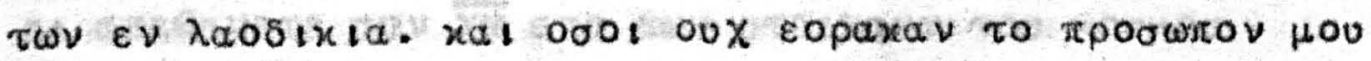

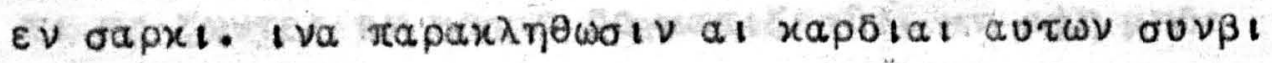

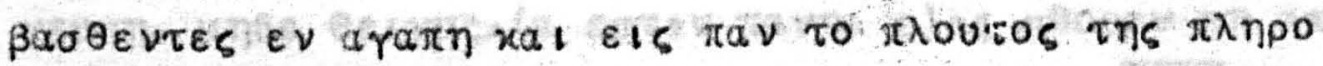

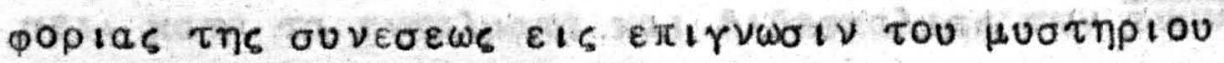

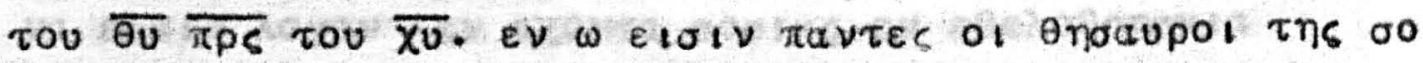
Qlas $x a$ t rvwaews aroxpupol.

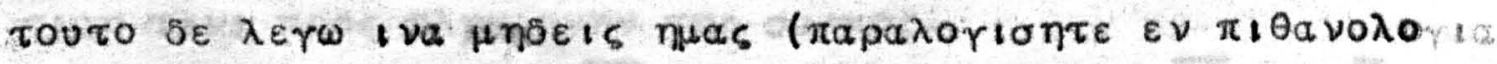

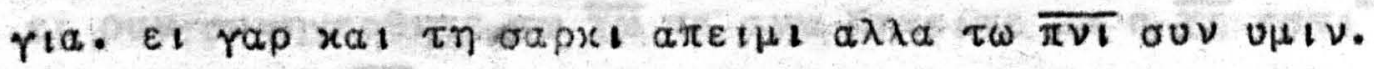

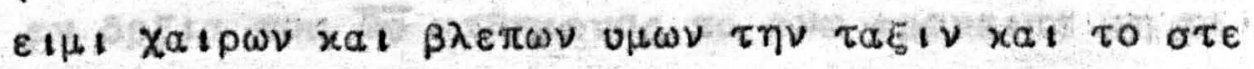

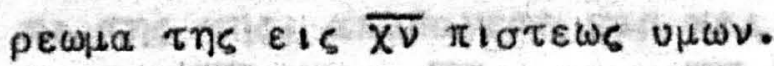

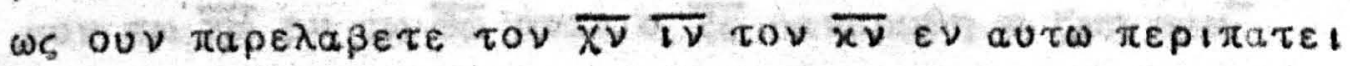

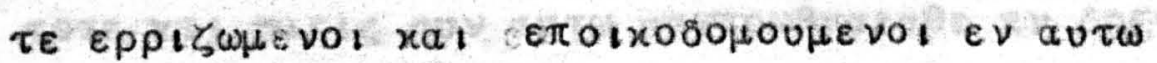

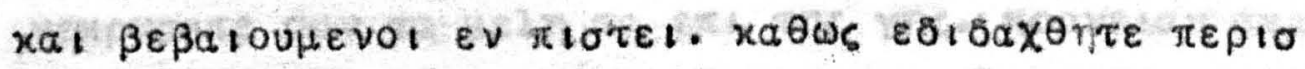

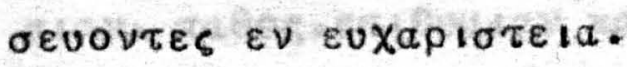

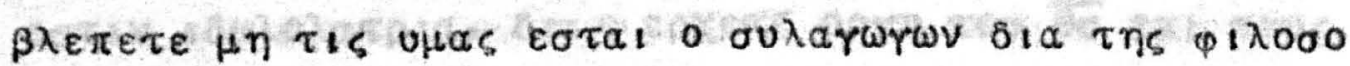




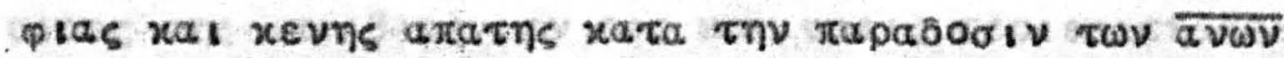

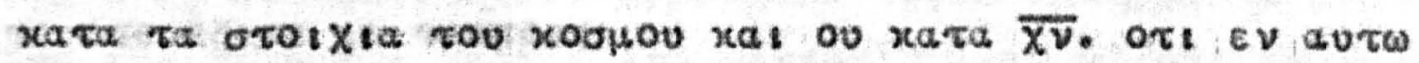

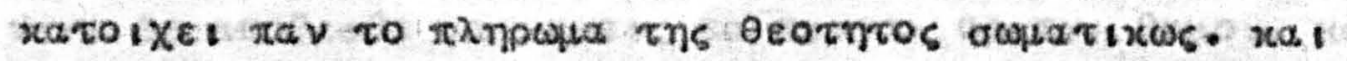

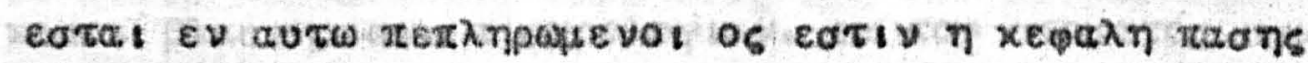

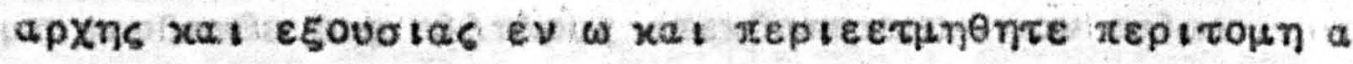

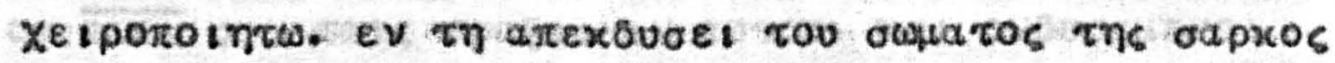

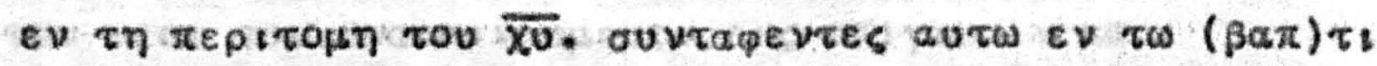

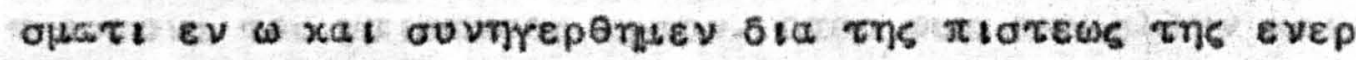

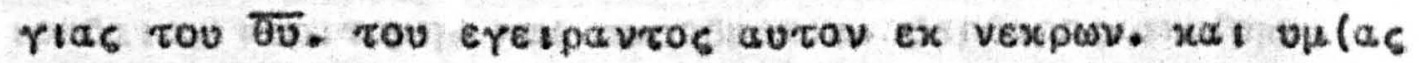

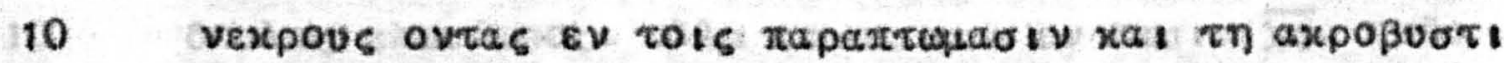

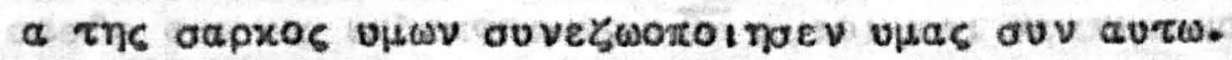

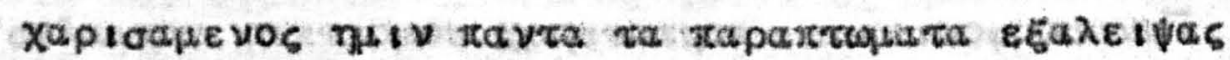

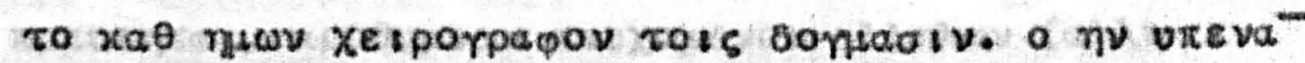

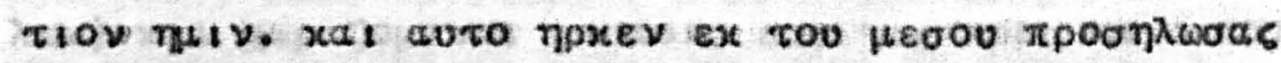

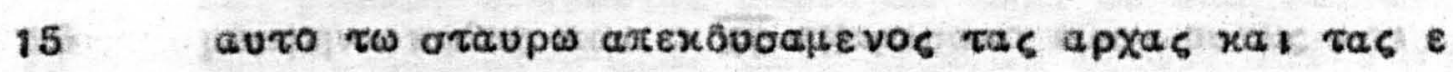

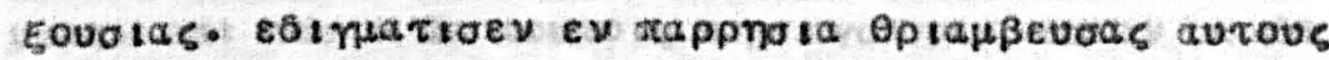
ev avrw.

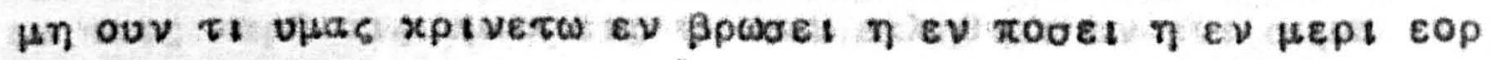

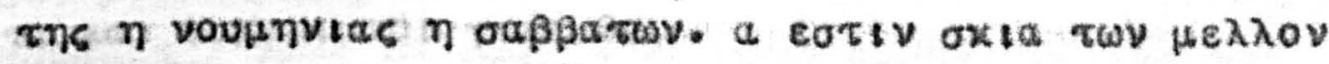

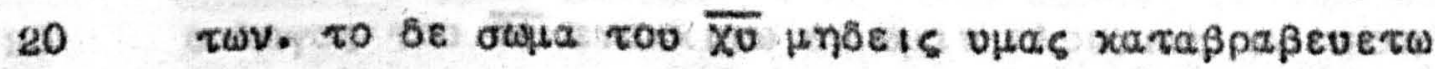

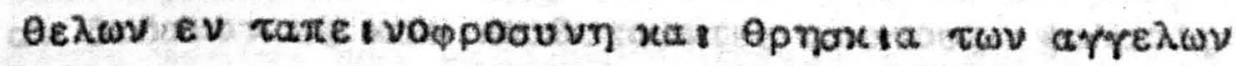

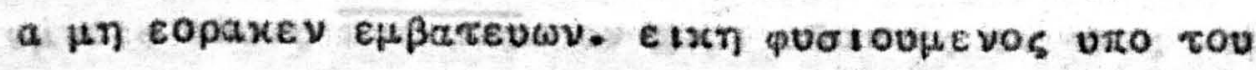

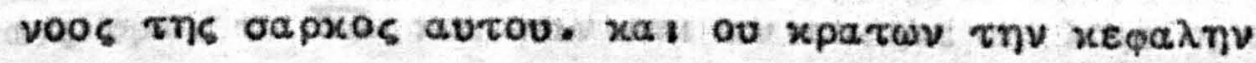

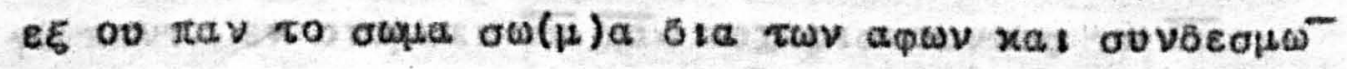

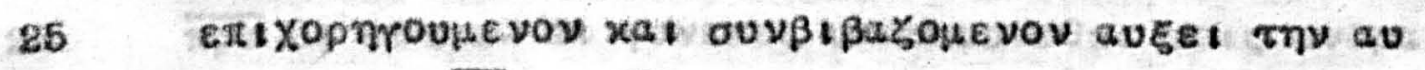
కnotv rov $\overline{\theta v}$.

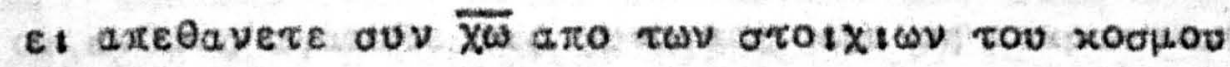

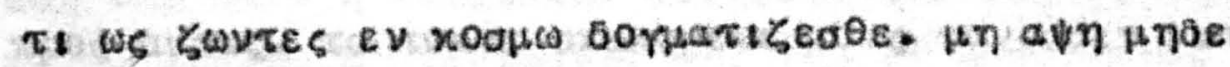

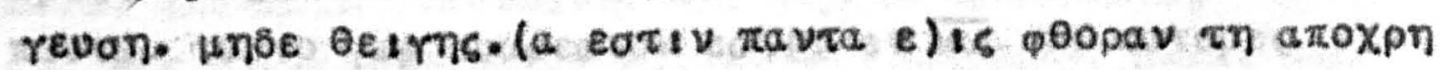

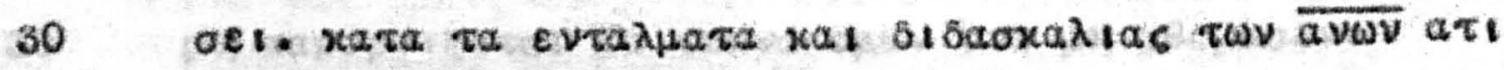

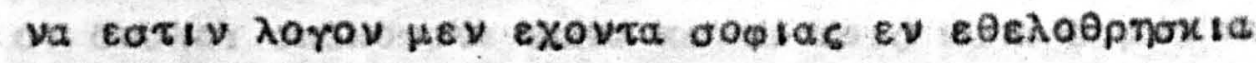

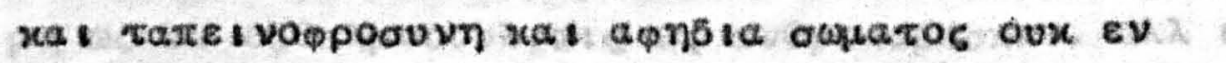

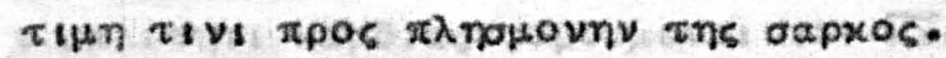

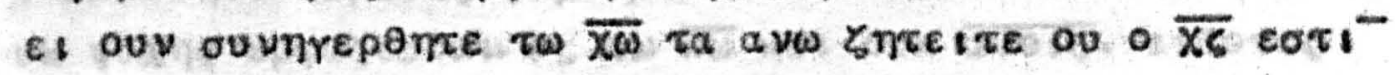

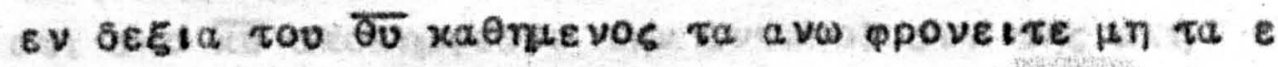

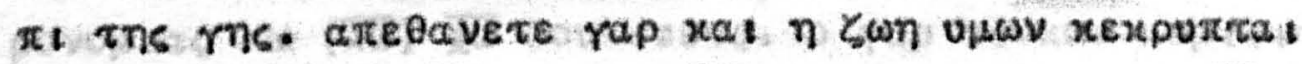

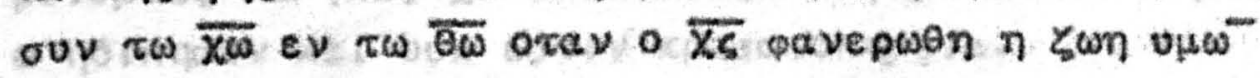

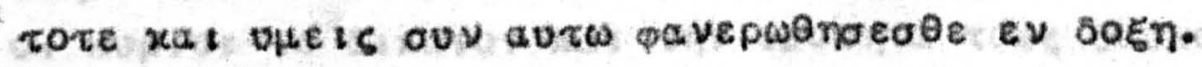

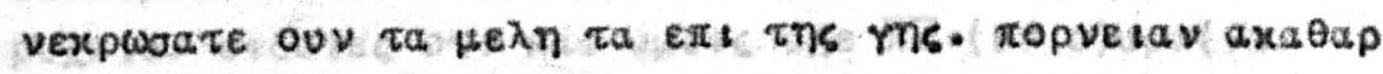

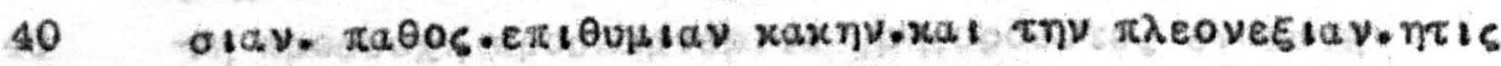

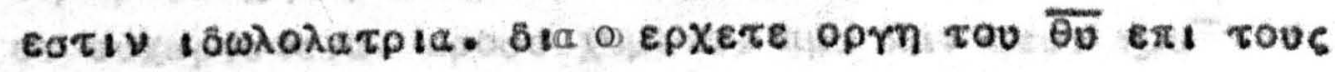




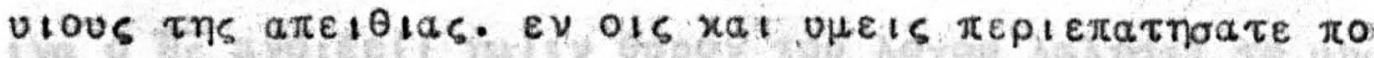

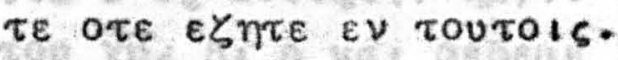

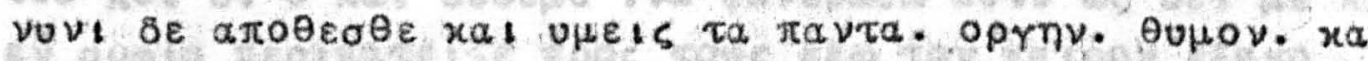

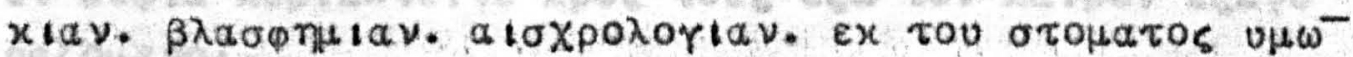

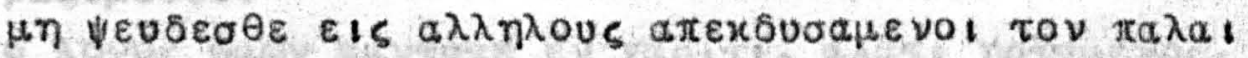

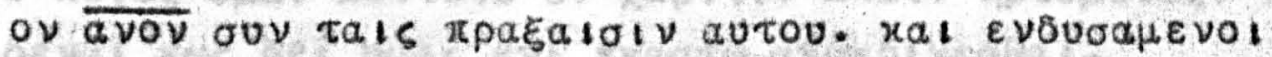

Tov veov tov a vaxa I voupevov els EIIrvwaiv $x a$

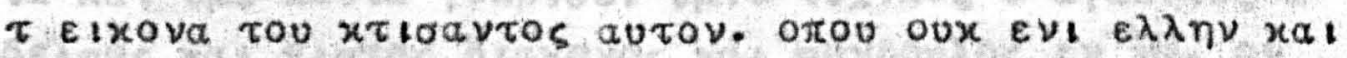

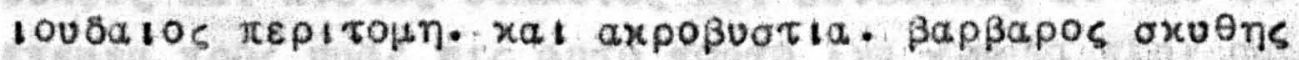

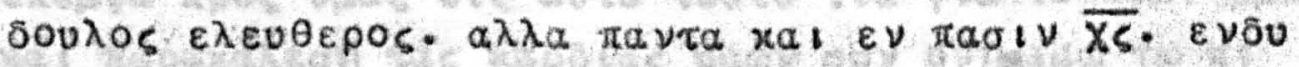

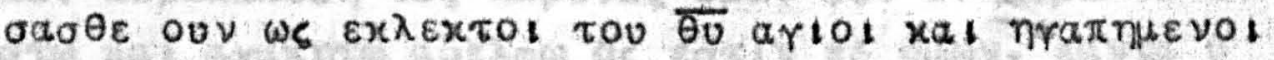

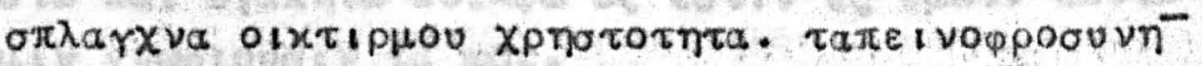

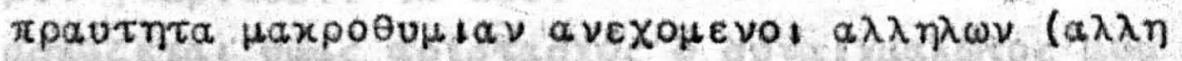

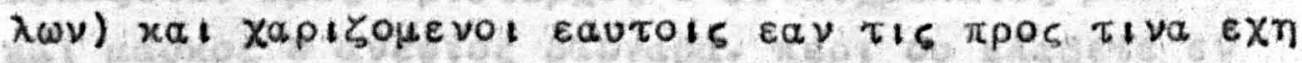

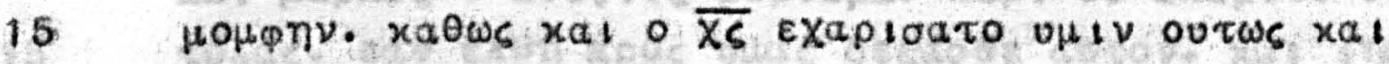

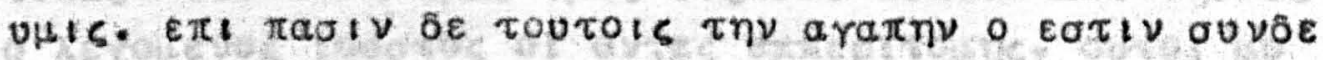

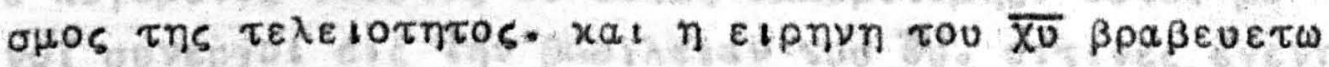

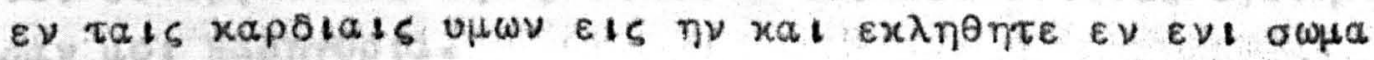

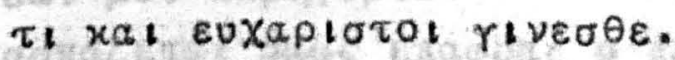

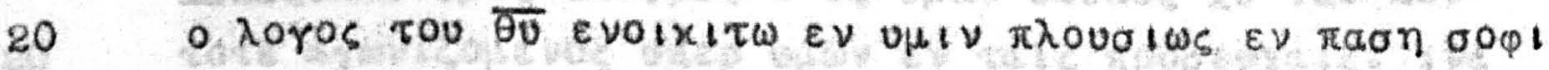

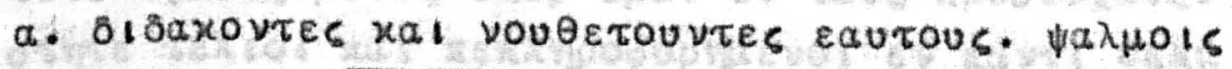

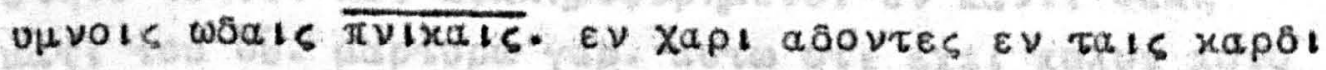

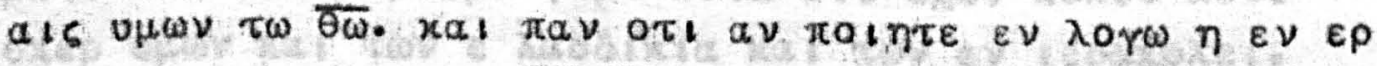

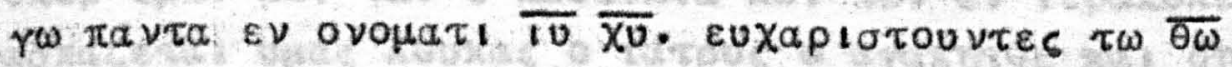

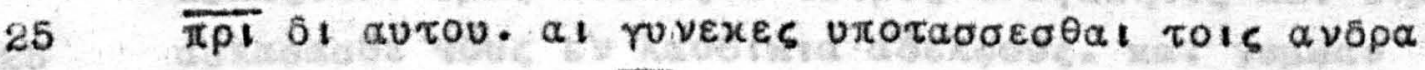

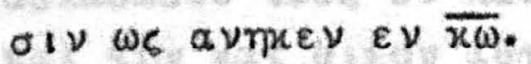

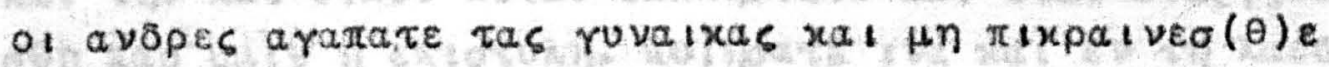

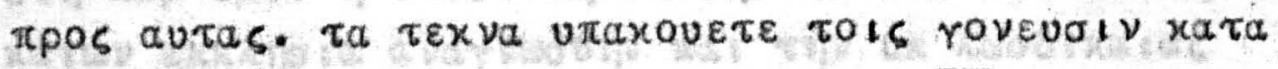

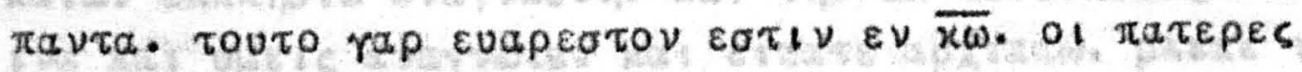

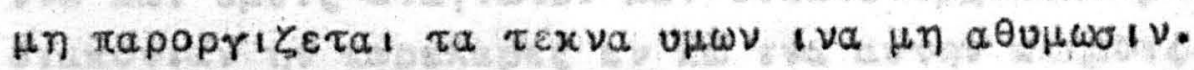

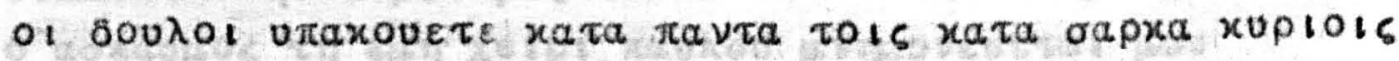

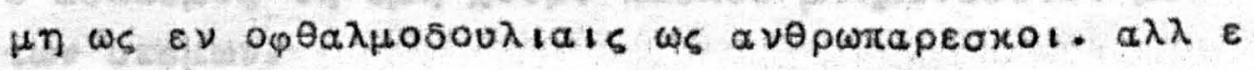

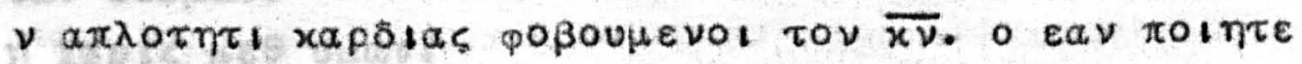

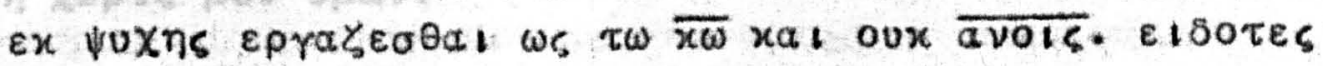

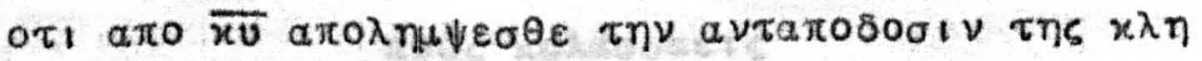

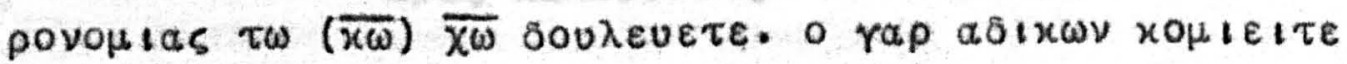

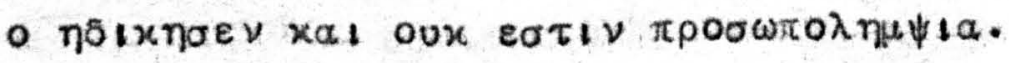

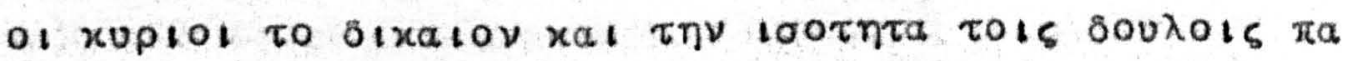

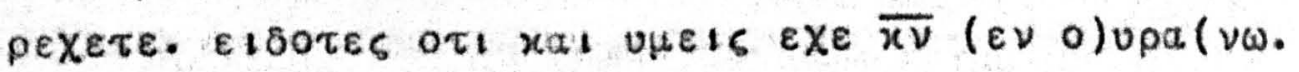

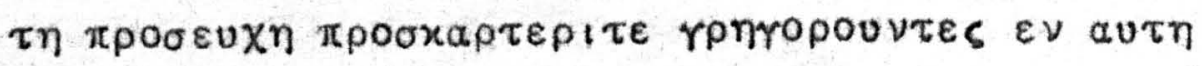

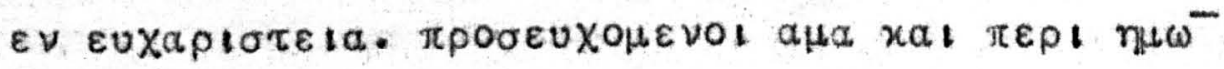




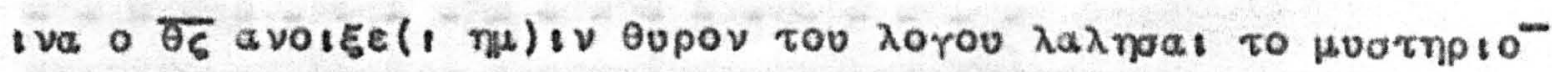

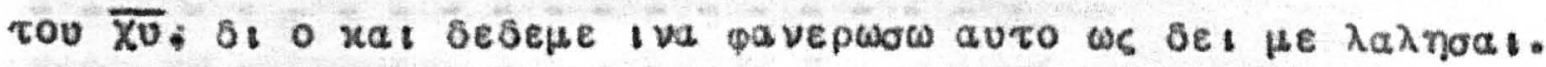

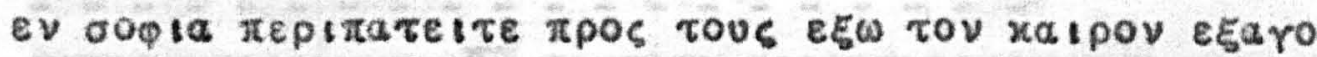
paל̧oиevol.

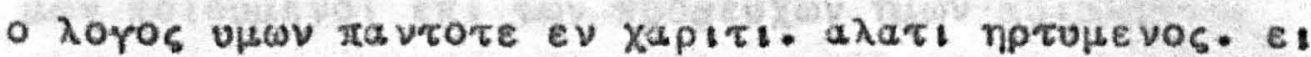

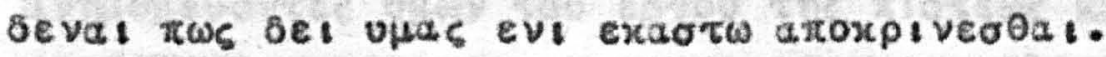

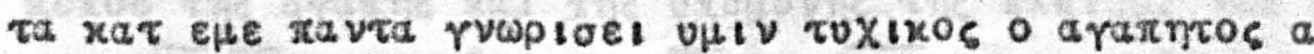

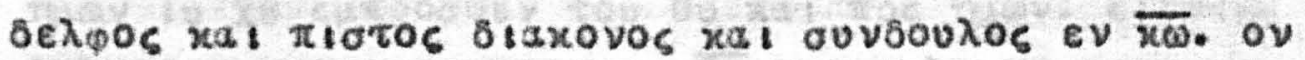

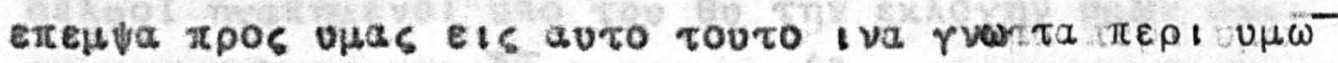

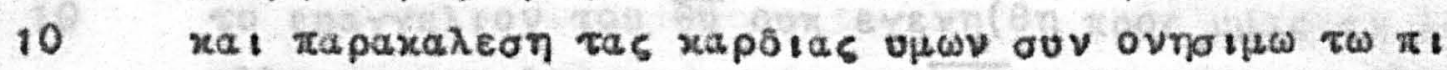

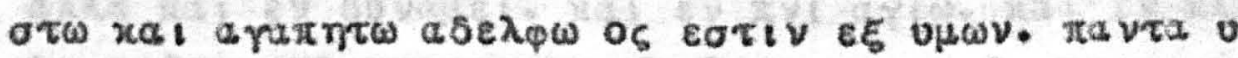

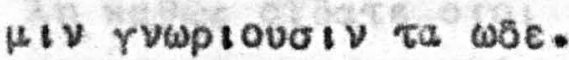

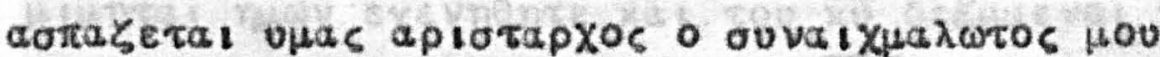

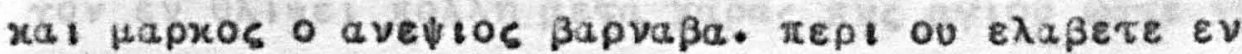

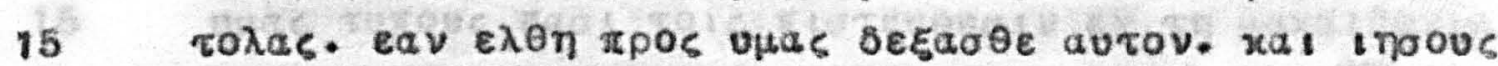

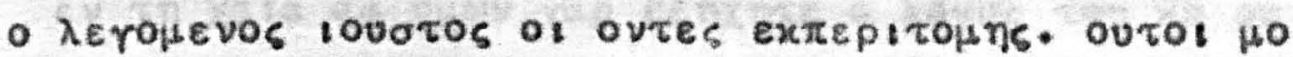

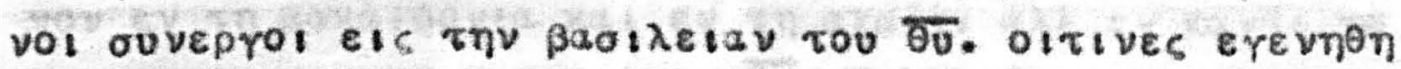
oav Hot тapทropta.

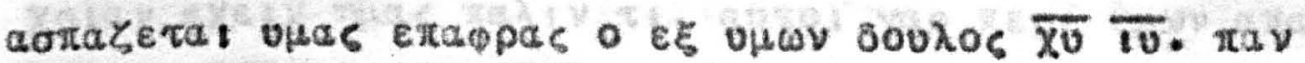

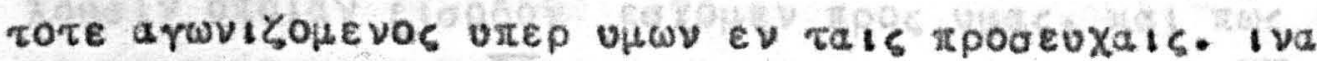

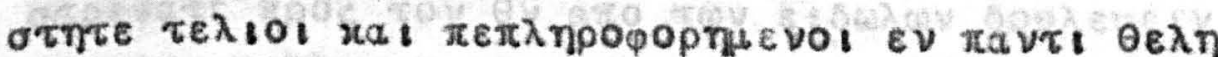

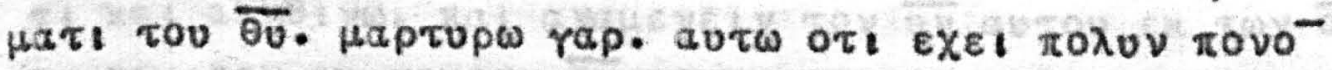

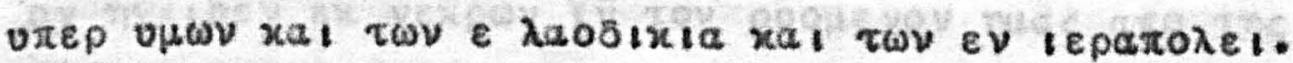

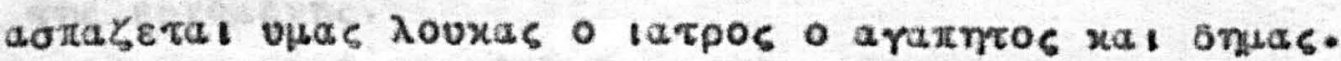

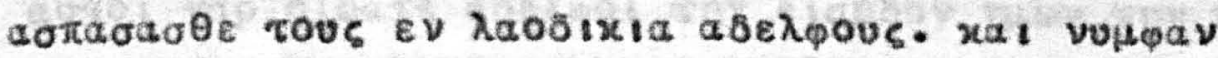

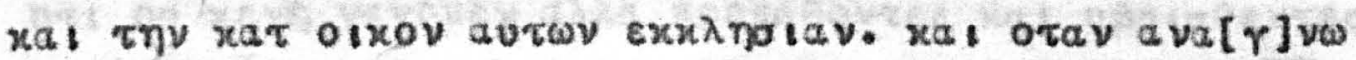

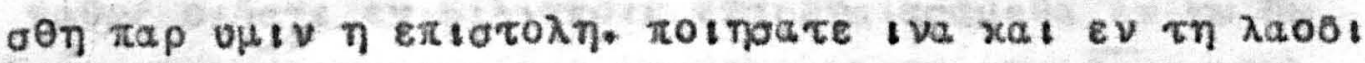

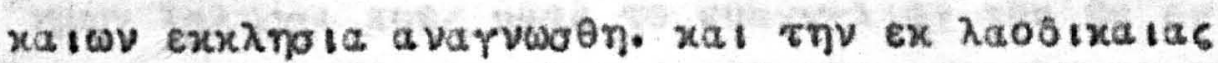

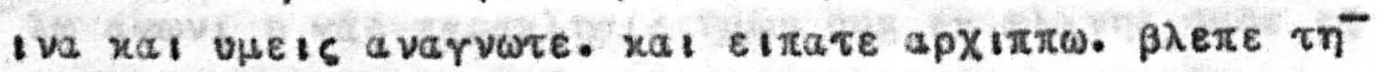

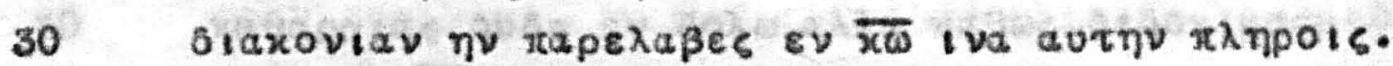

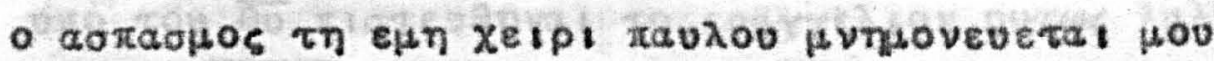

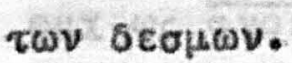
$\eta$ Xapts $\mu \varepsilon \theta$ vpwv.

$$
\text { xpos nataroaels }
$$


$\varepsilon \cup \chi a \rho$ เ

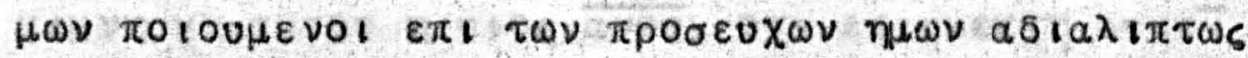

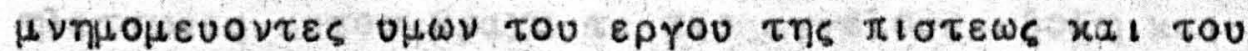

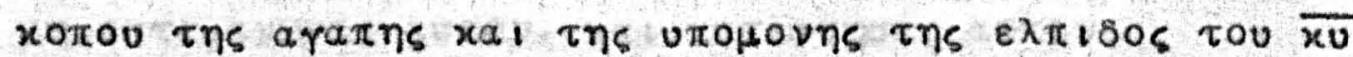

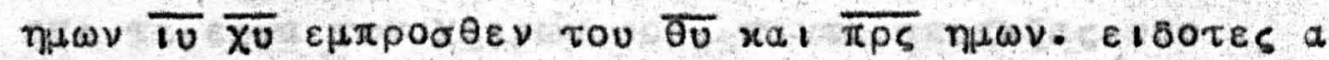

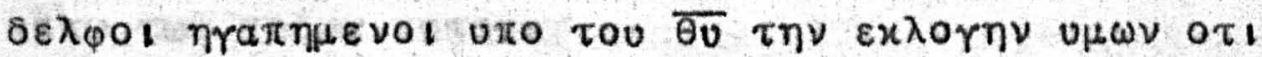

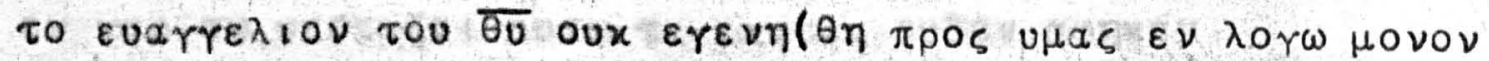

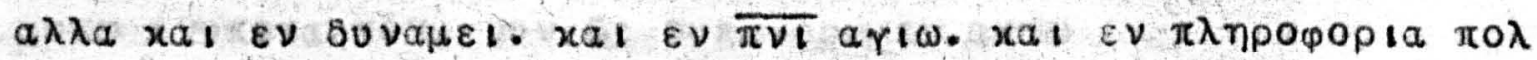

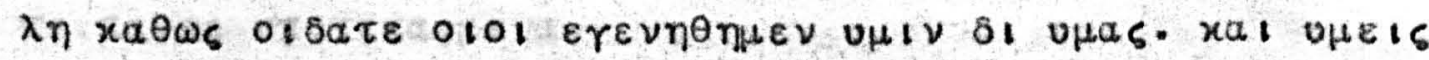

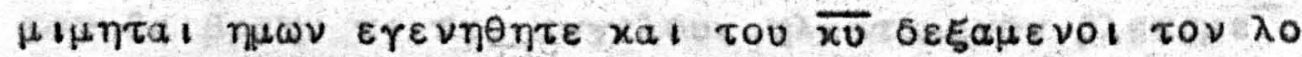

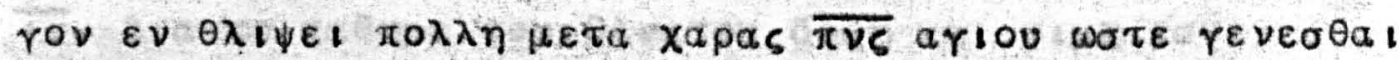

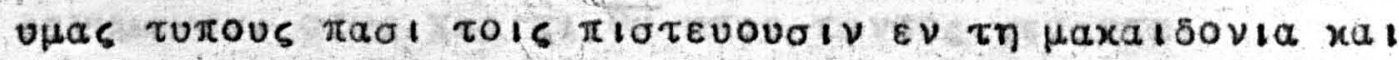

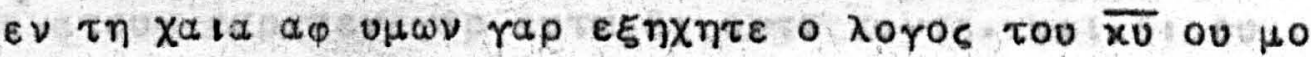

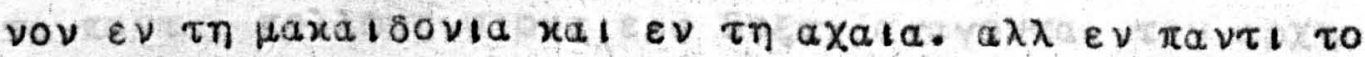

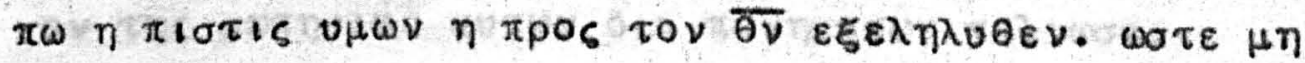

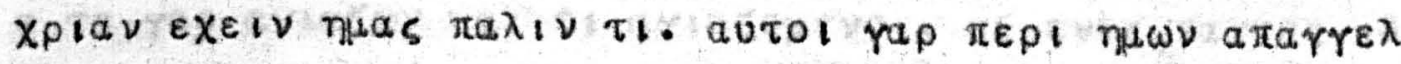

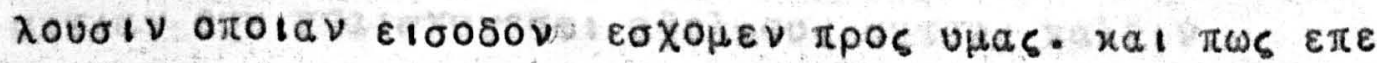

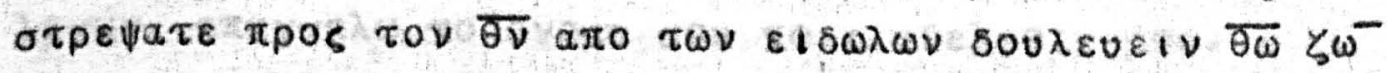

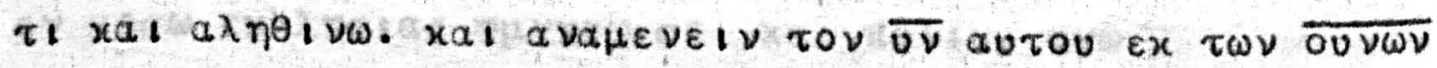

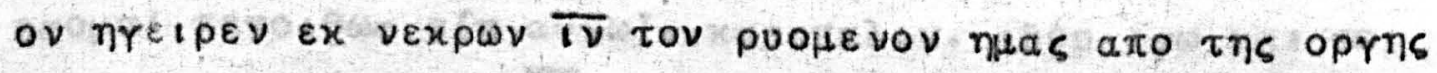

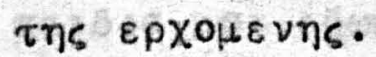

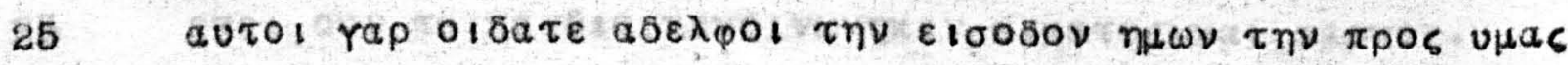

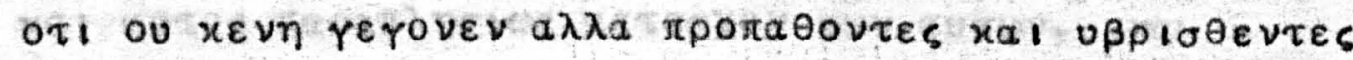

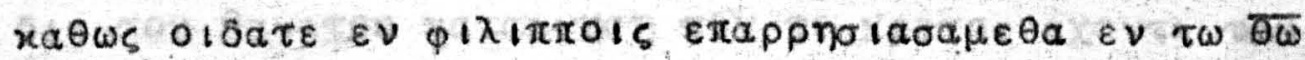

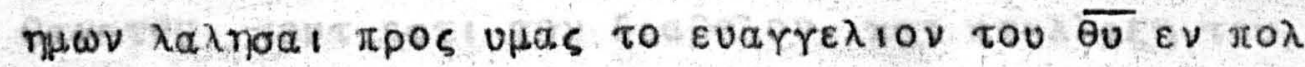

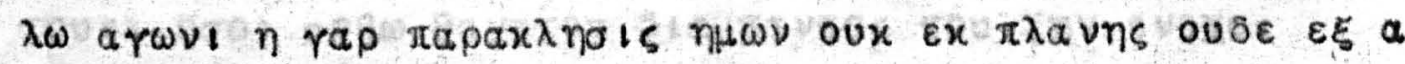

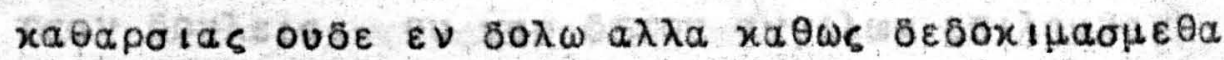

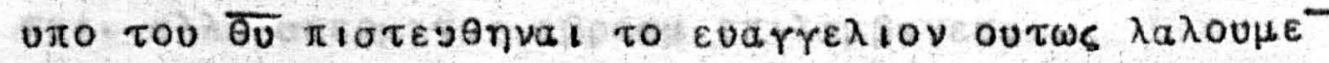

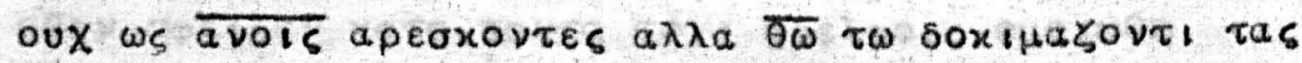

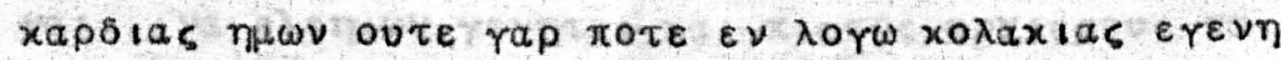

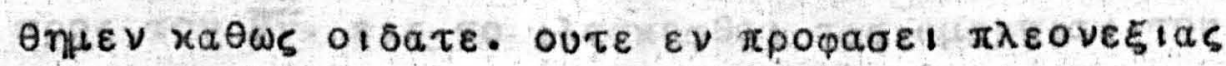

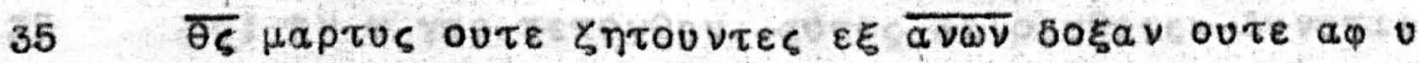

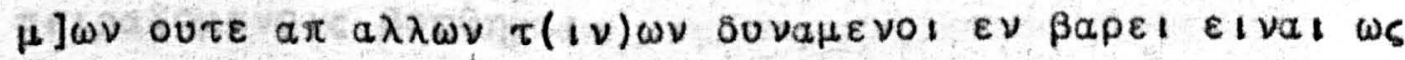
$\overline{X] 0}$ a

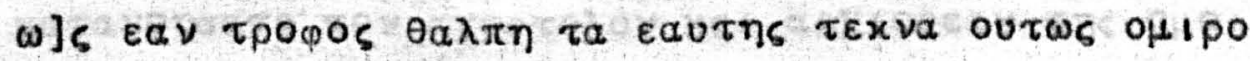

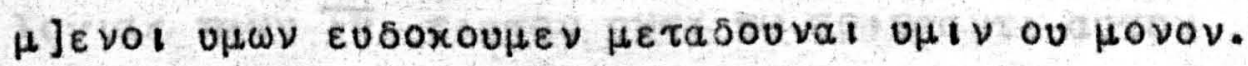

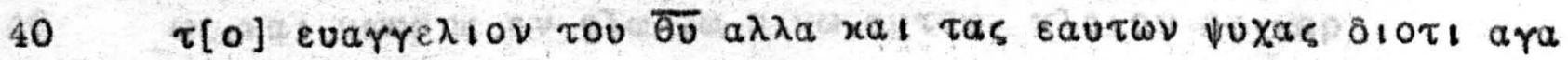

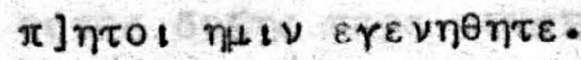




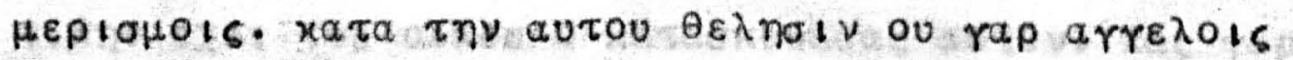

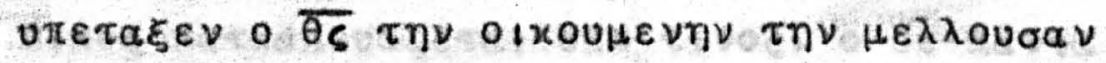

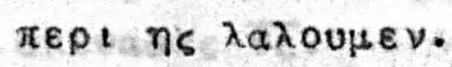

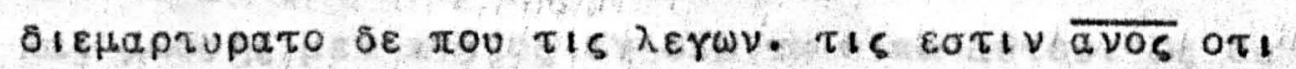

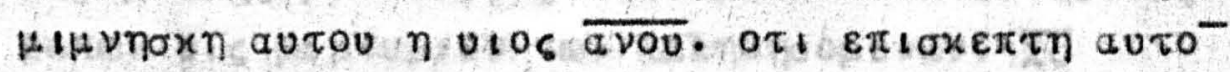

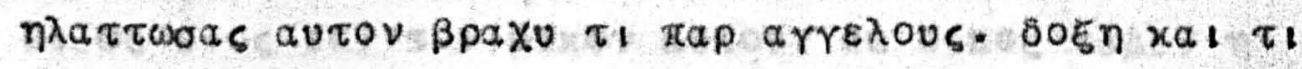

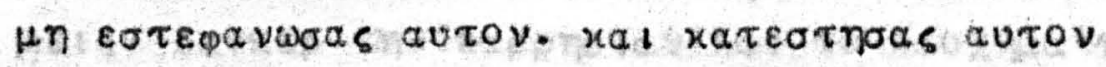

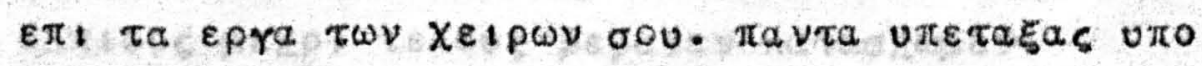

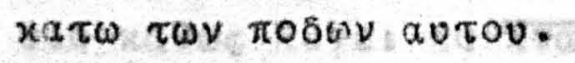

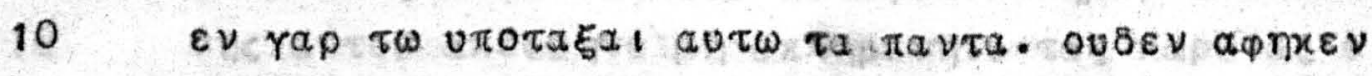

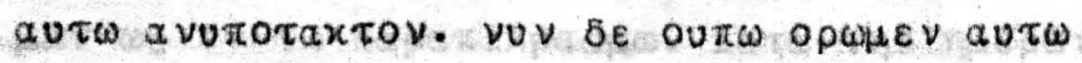

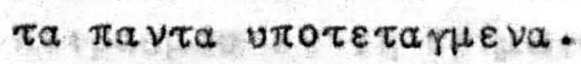

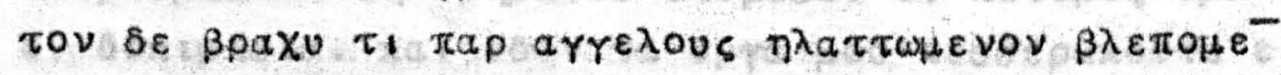

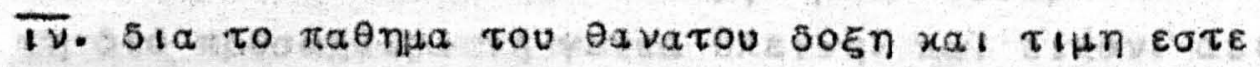

15 Q Q

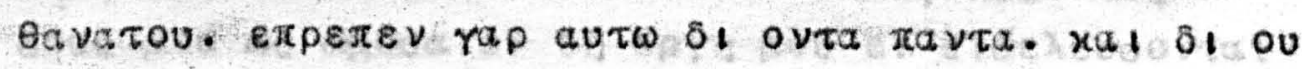

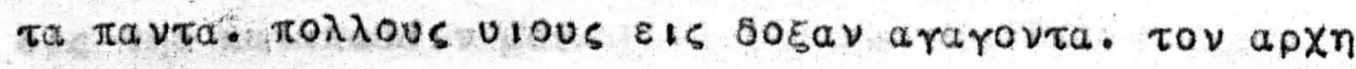

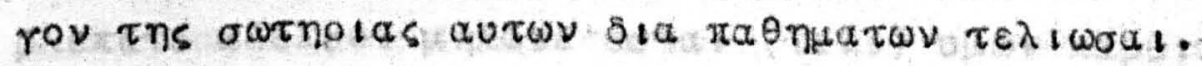

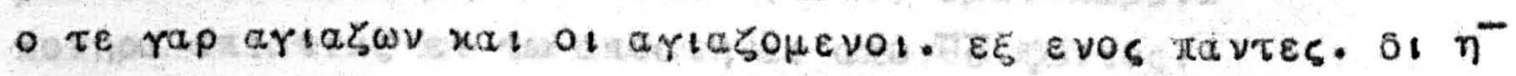

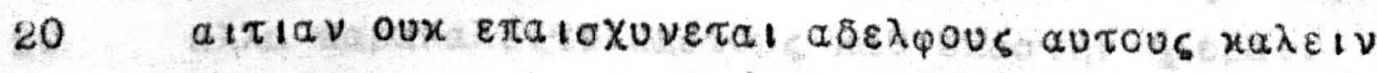

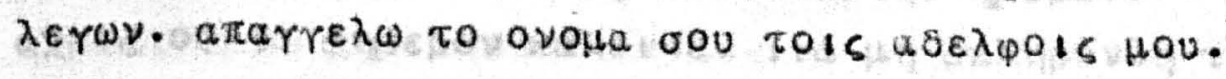

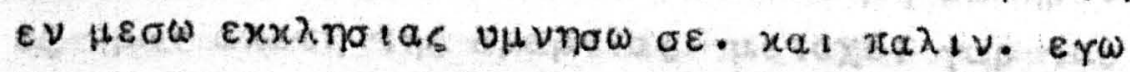

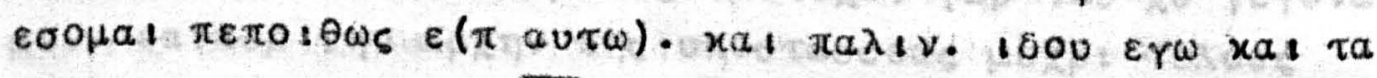
$\pi a \imath \delta 1 \alpha \mu 0 เ \varepsilon \delta \omega x \varepsilon \nu \circ \bar{\theta}$.

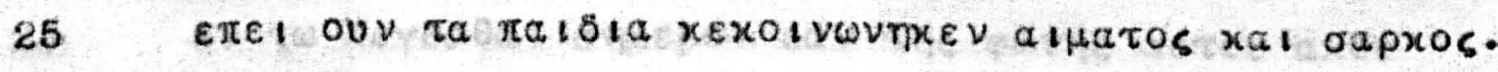

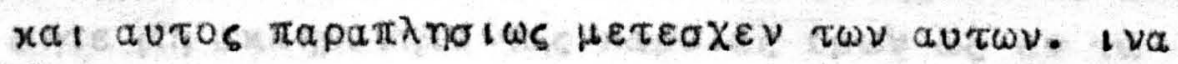

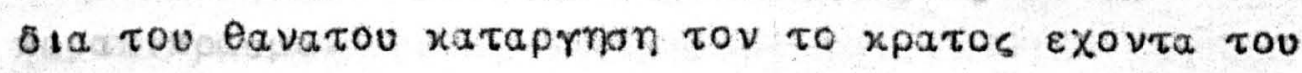

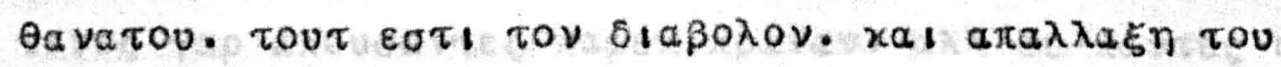

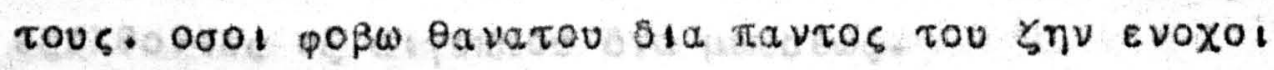

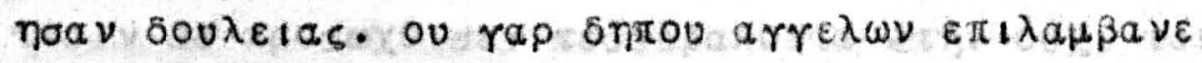

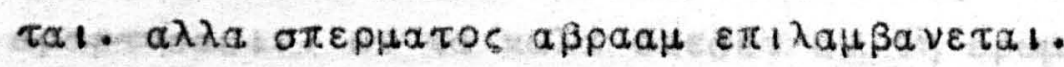

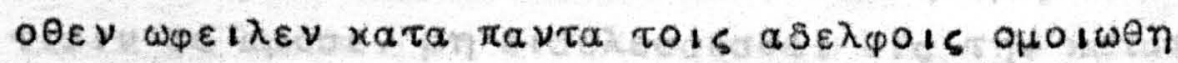

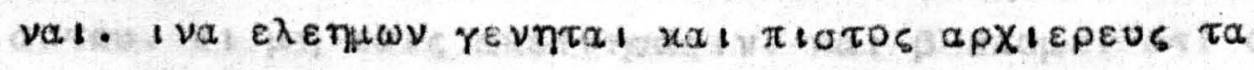

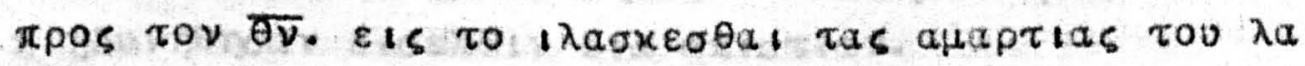

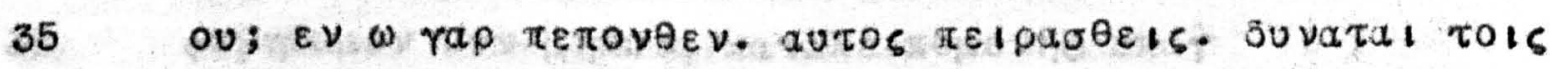
$\pi \varepsilon ı$ เ

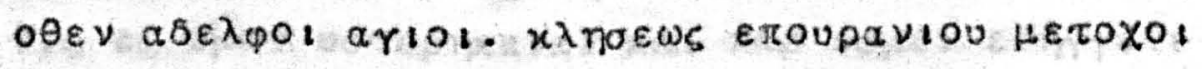

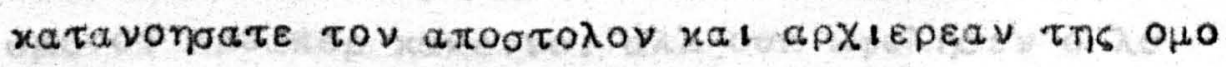

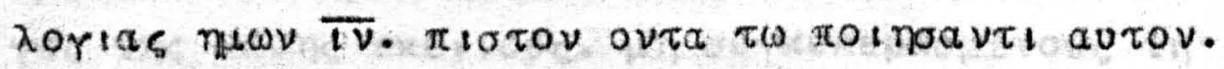

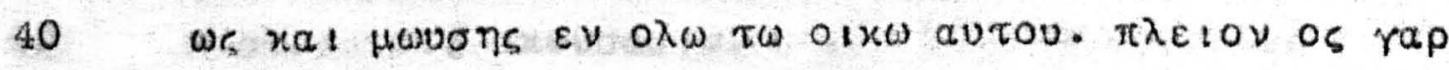

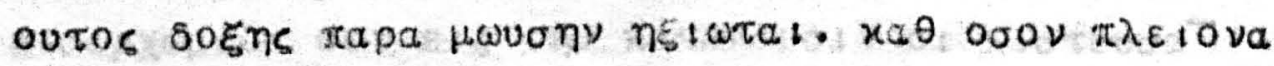




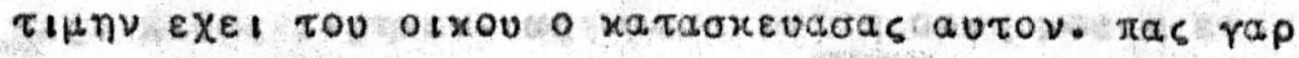

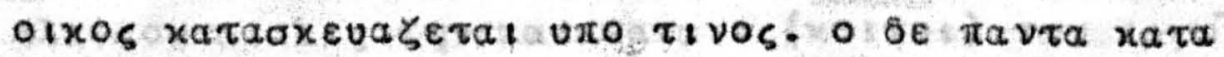
axevaras $\overline{\theta_{\zeta}}$.

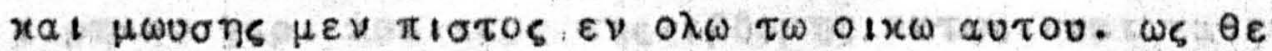

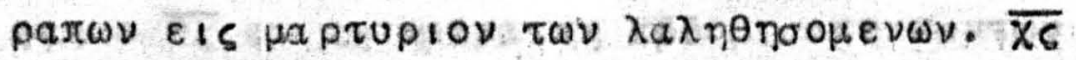

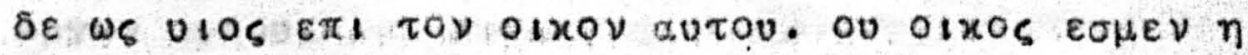

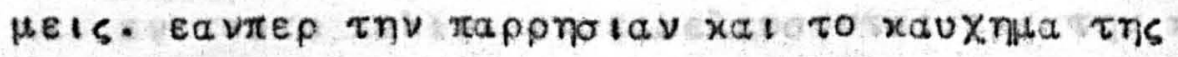

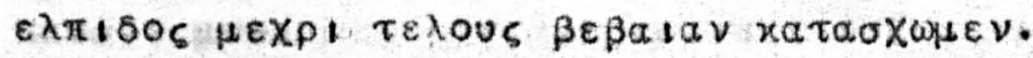

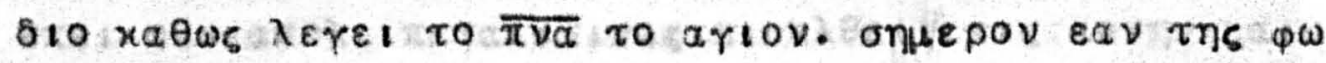

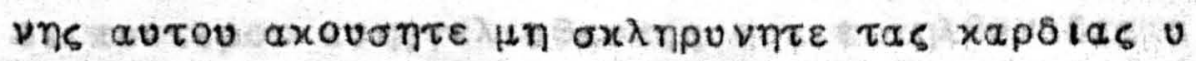

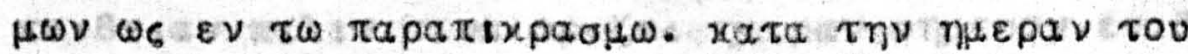

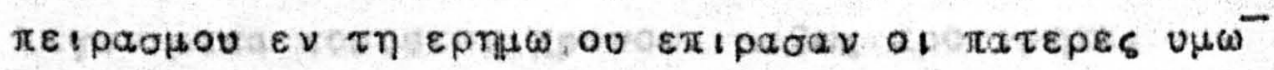

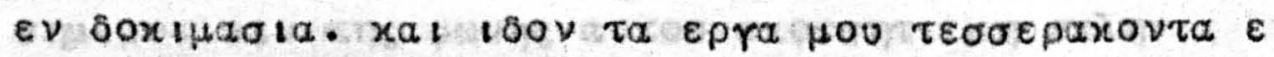

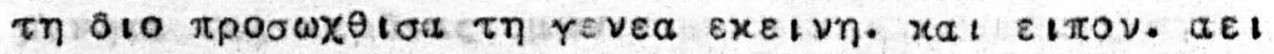

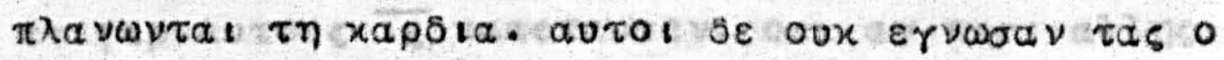

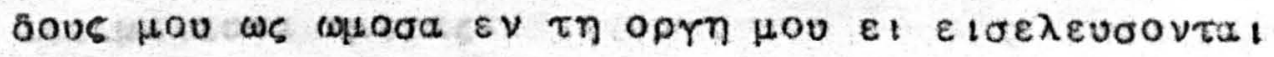

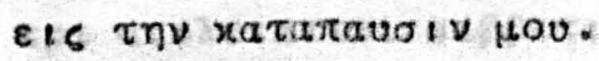

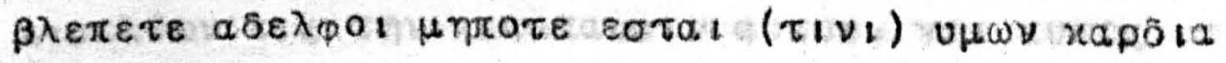

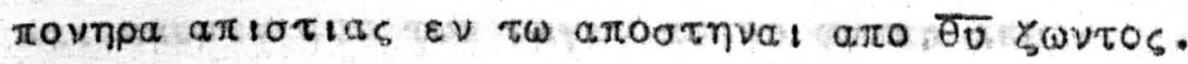

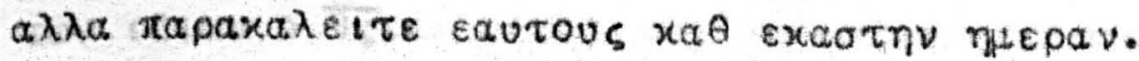

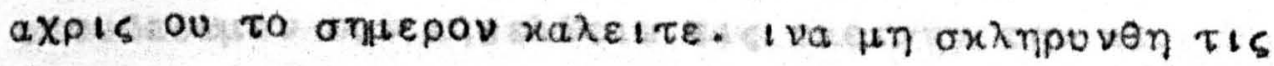

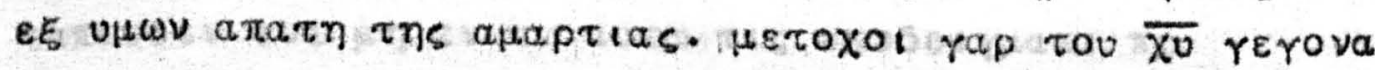

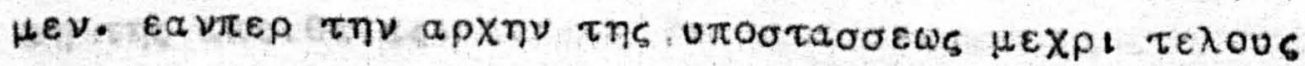

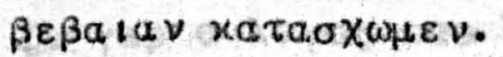

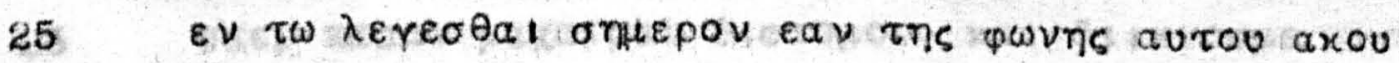

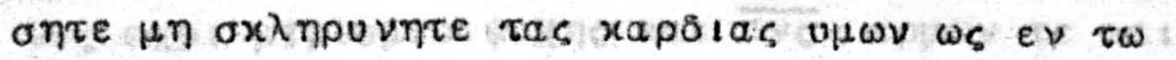

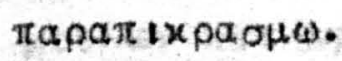

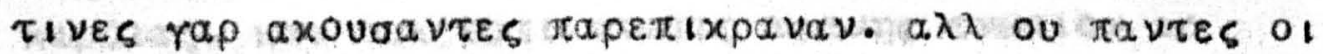

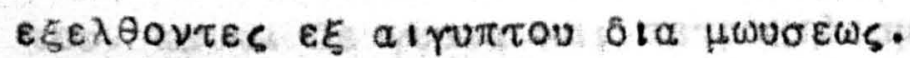

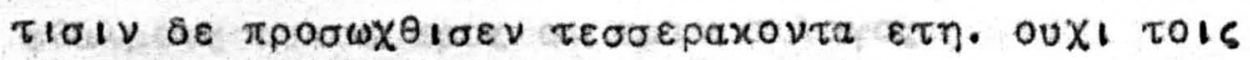

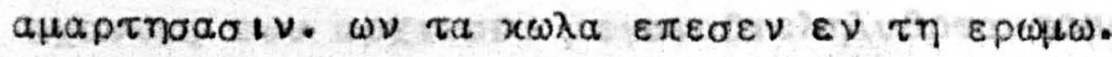

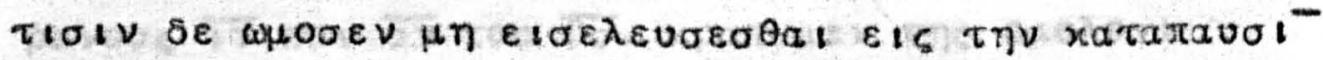

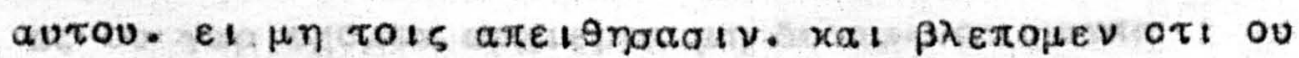

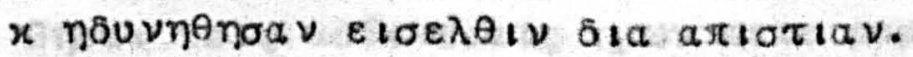

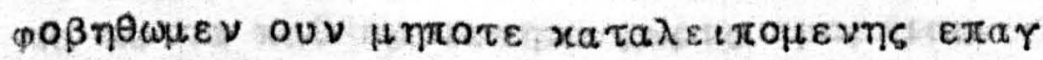

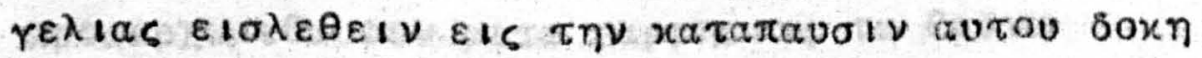

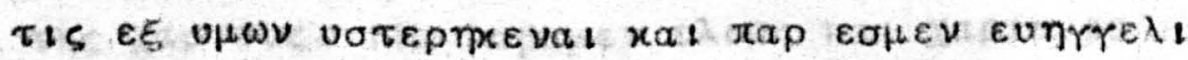

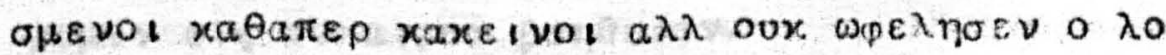

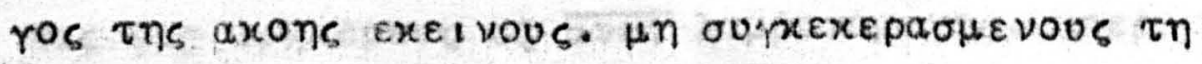

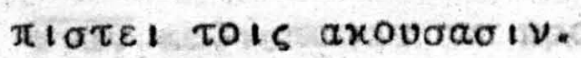

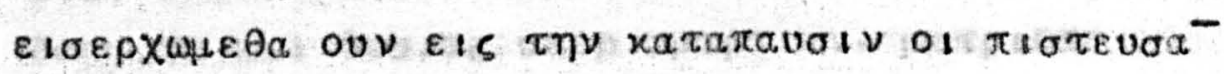




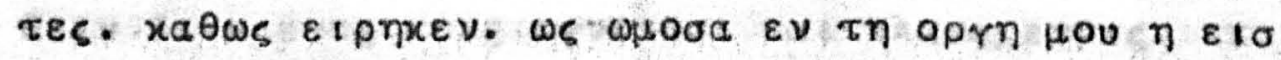

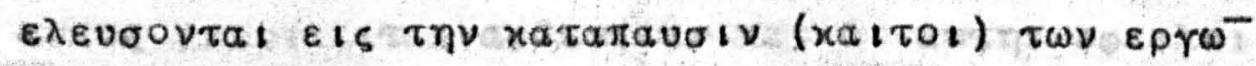

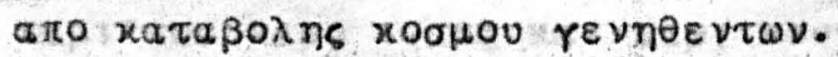

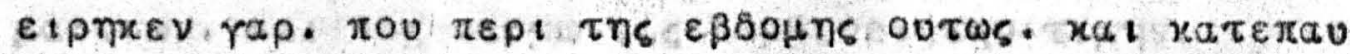

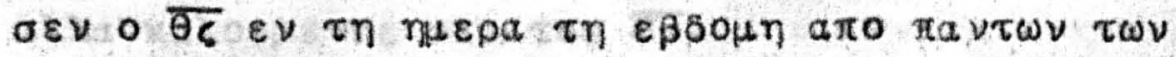
eprov avTou.

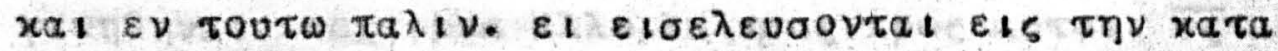

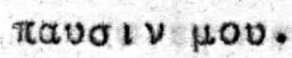

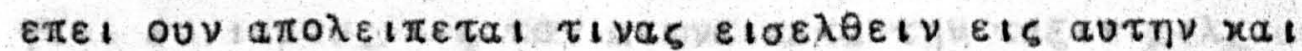

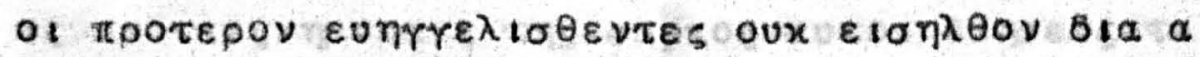

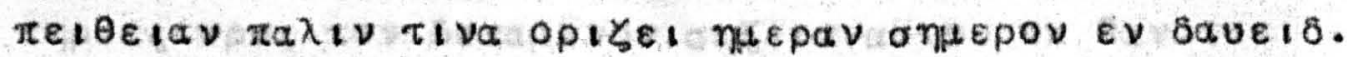

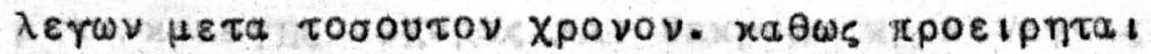

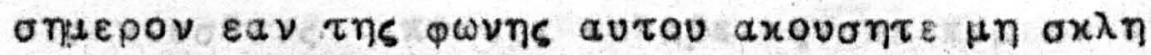

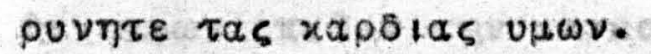

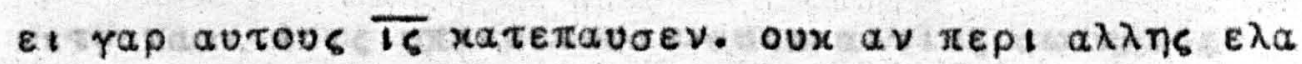

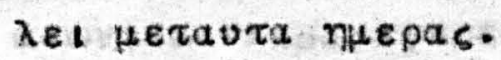

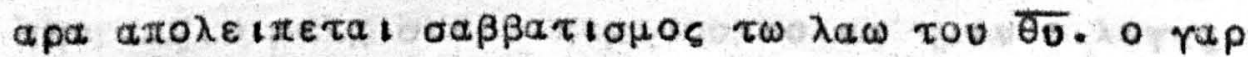

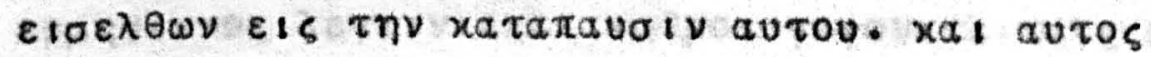

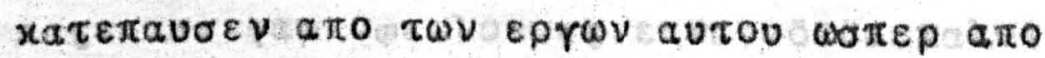

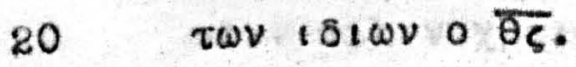

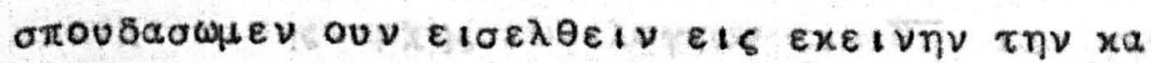

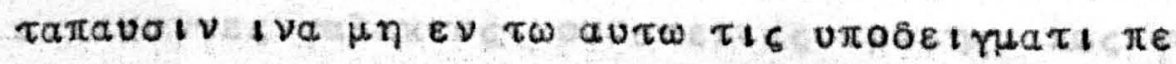
on $\tau \eta \zeta$ a a

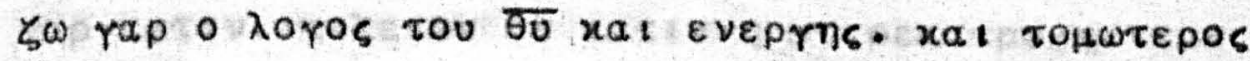

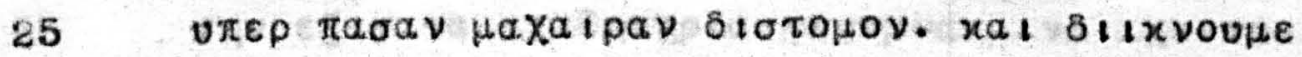

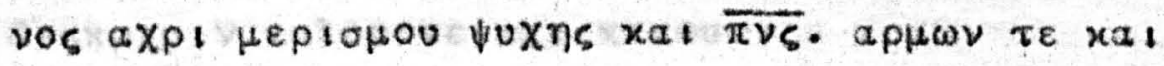

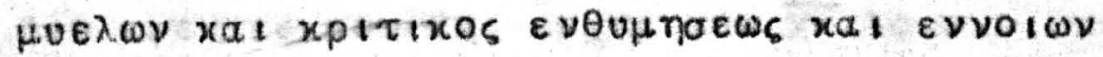

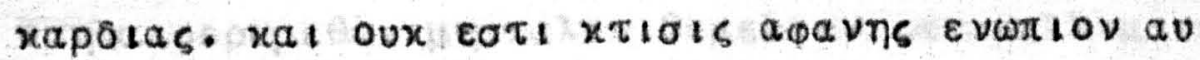

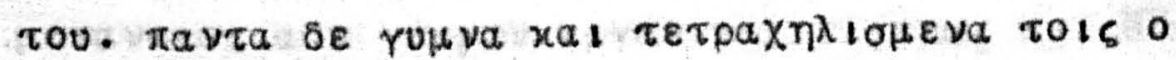

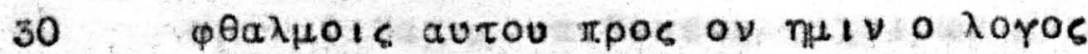

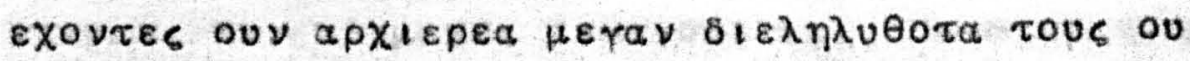

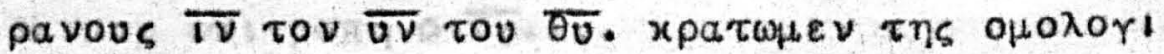

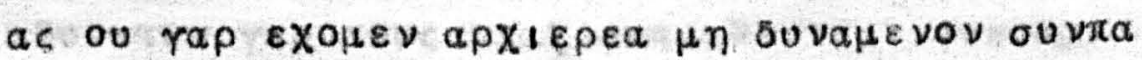

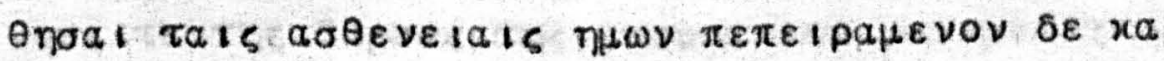

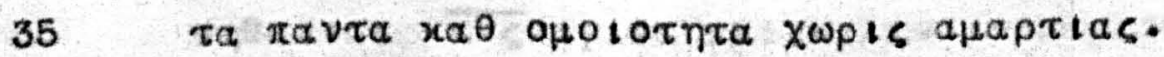

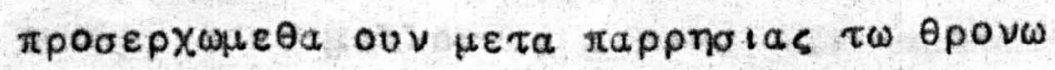

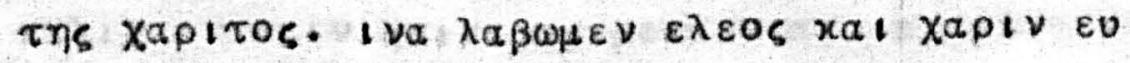

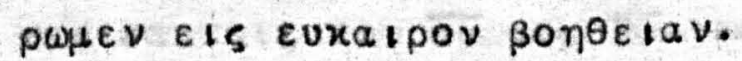

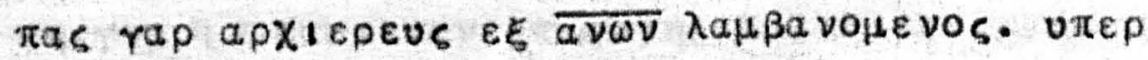

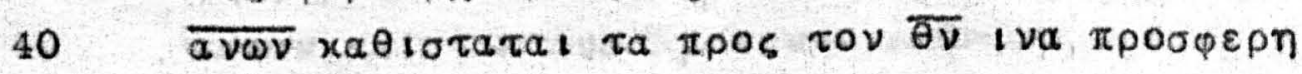

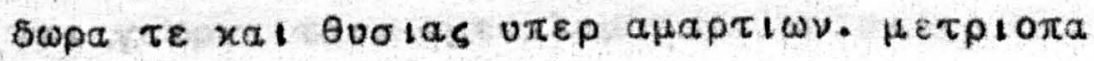




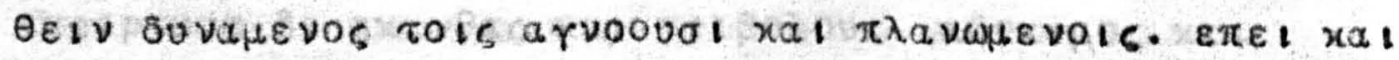

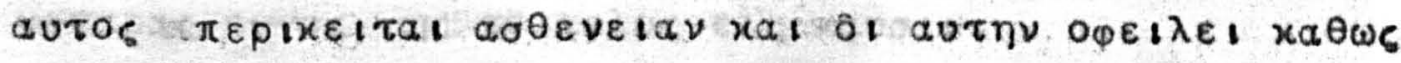

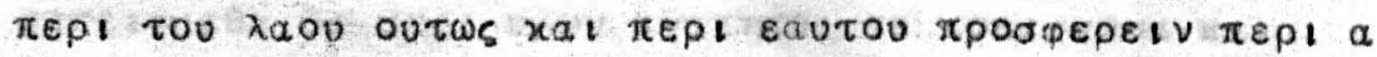

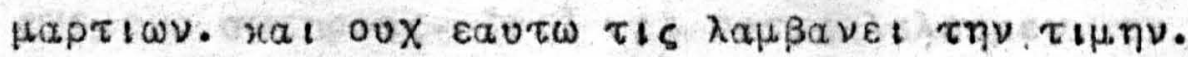

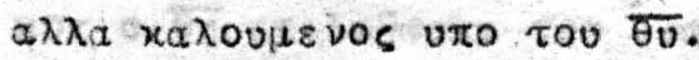

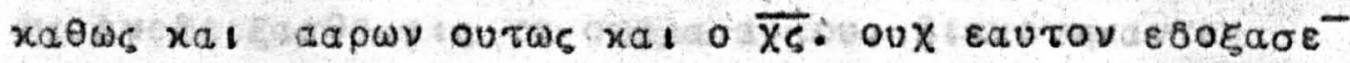

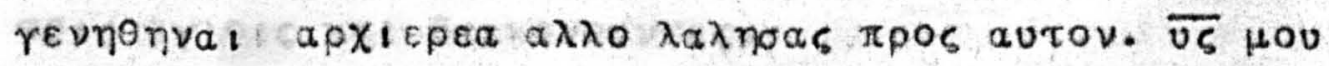

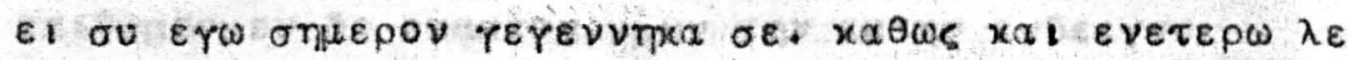

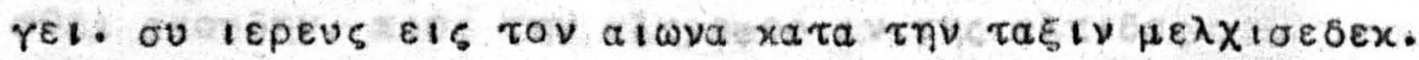

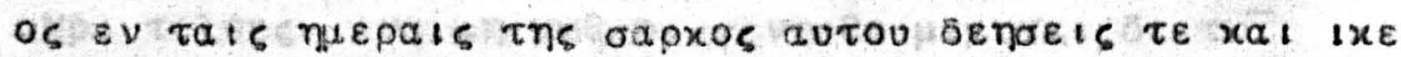

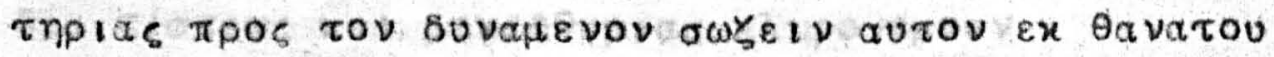

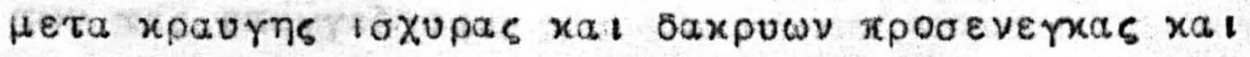

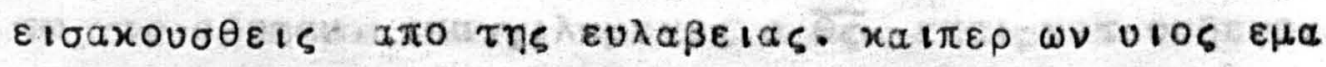

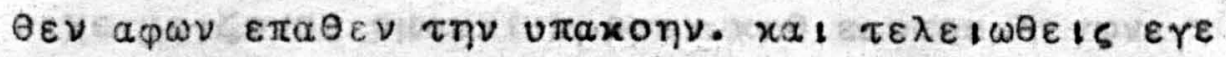

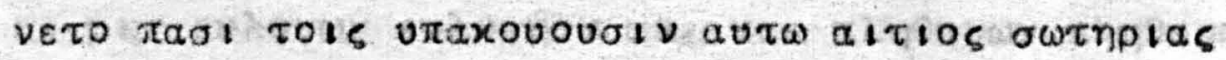

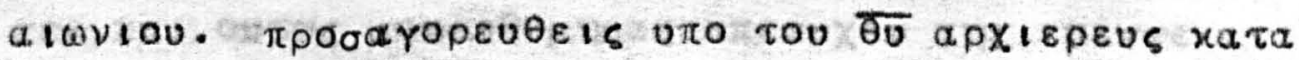

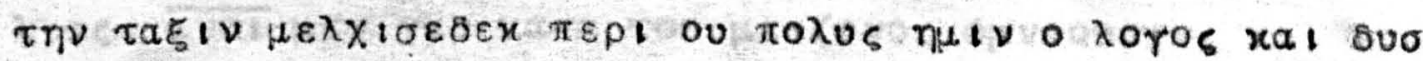

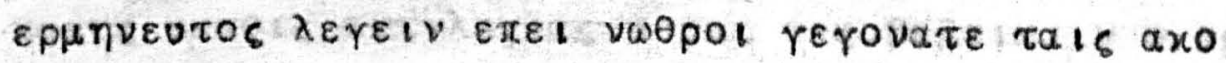

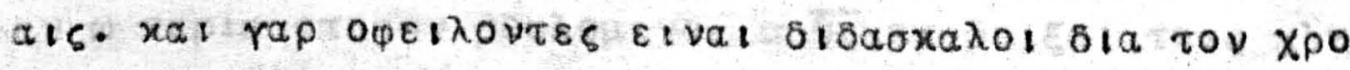

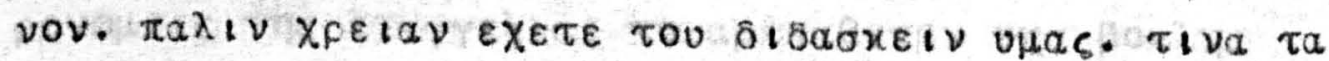

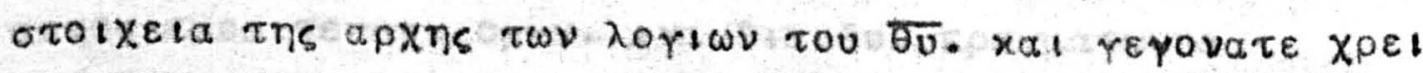

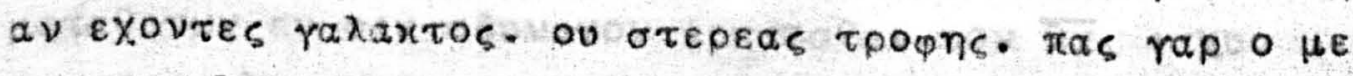

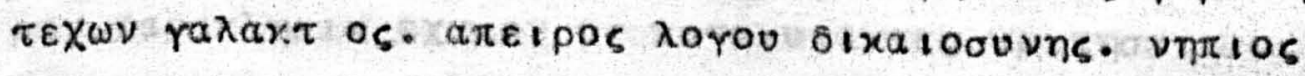

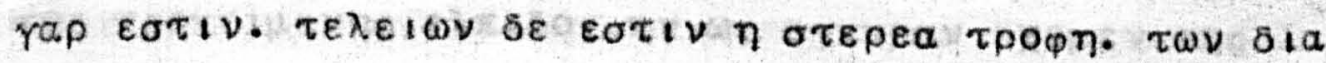

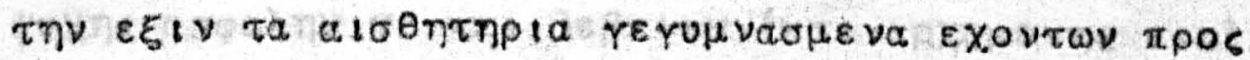

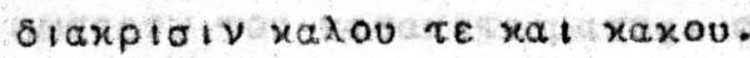

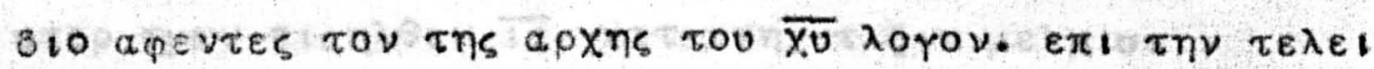

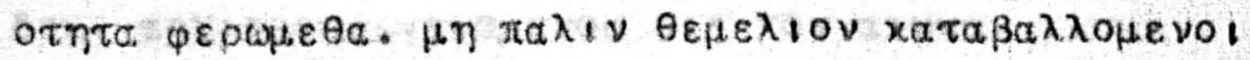

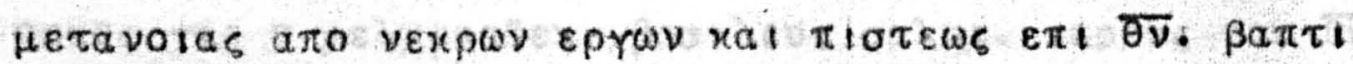

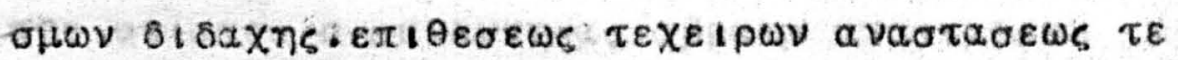

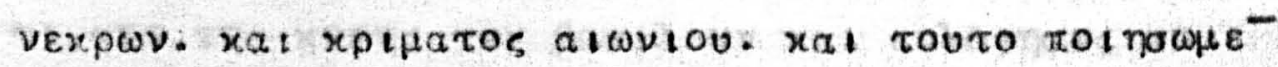
$\varepsilon a \nu \pi \varepsilon \rho \varepsilon \pi 1 \tau \rho \varepsilon \pi \eta$ ० $\overline{\theta_{c}}$.

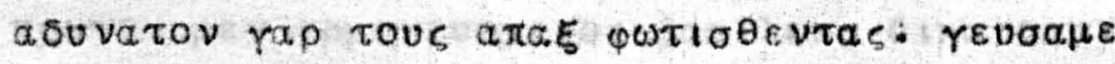

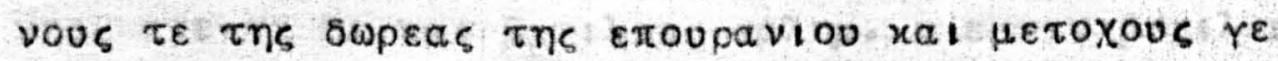

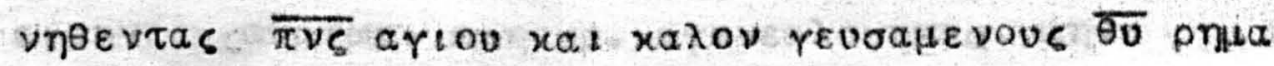

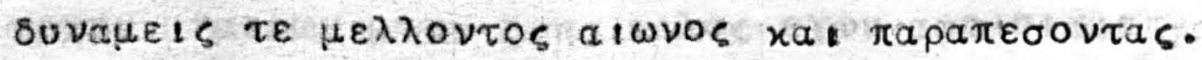

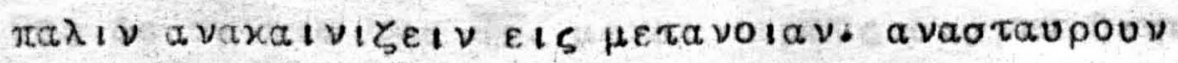

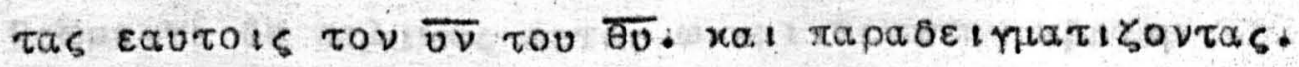

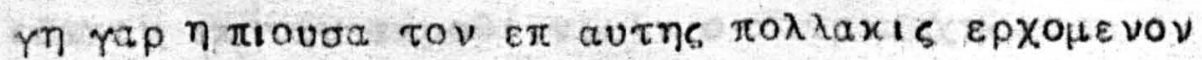

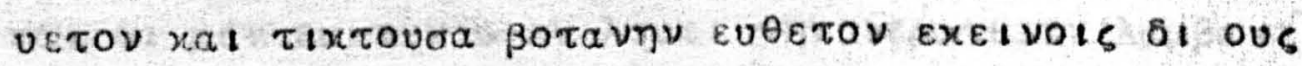

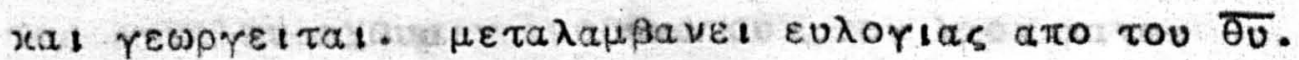




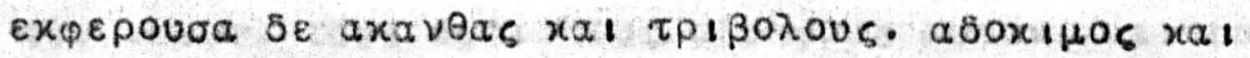

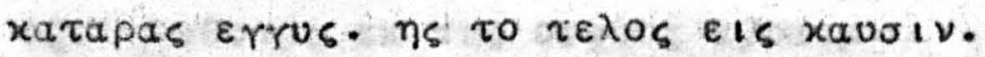

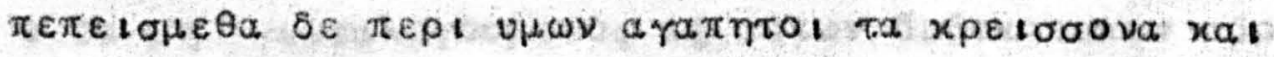

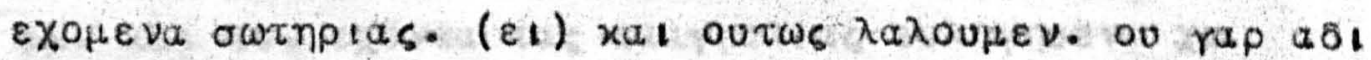

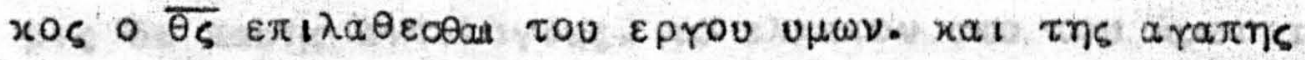

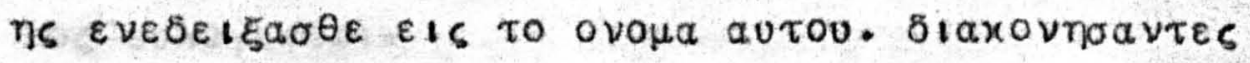
tols arlols xal staxovouves.

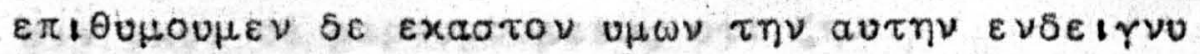

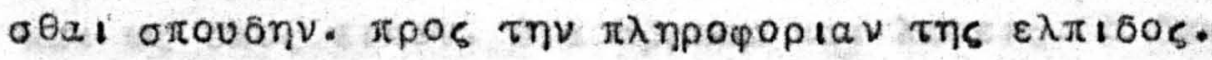

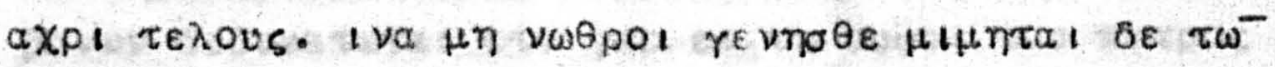

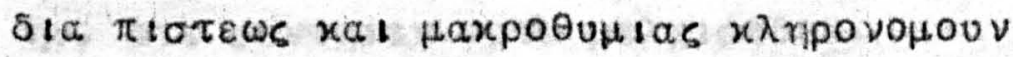

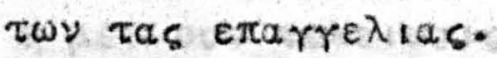

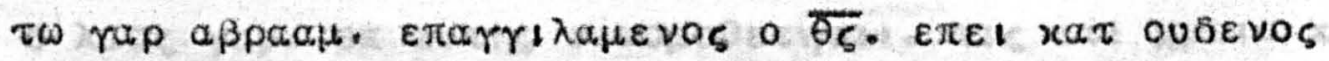

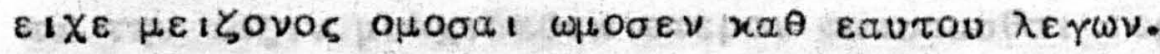

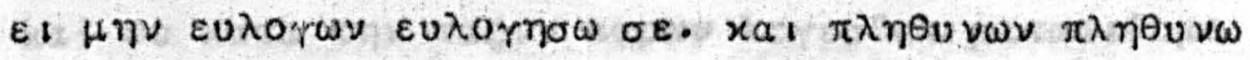

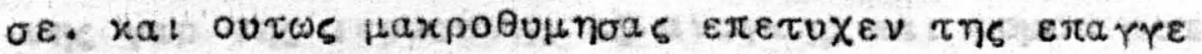

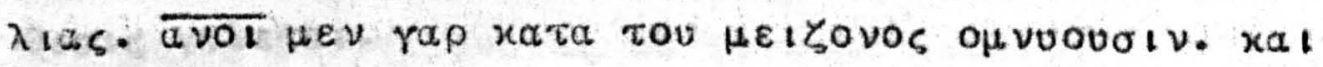

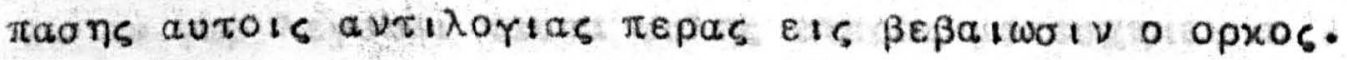

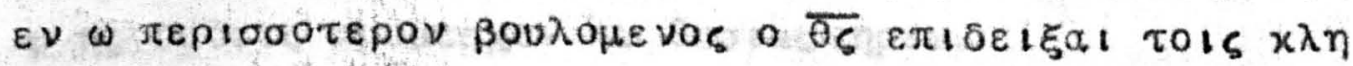

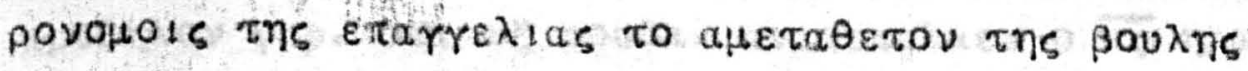

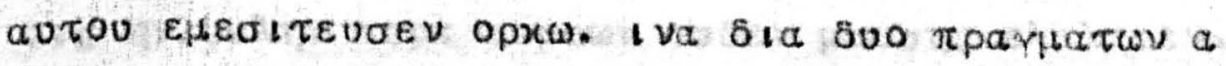

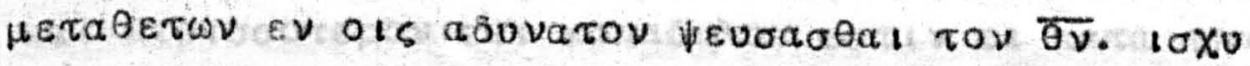

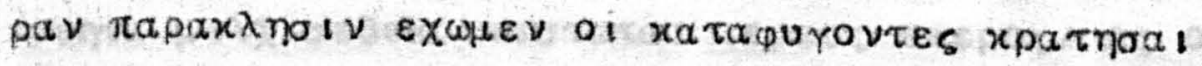

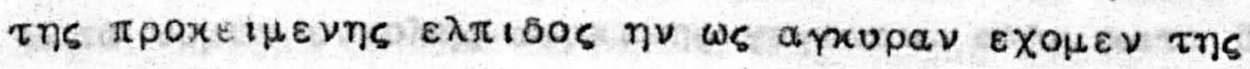

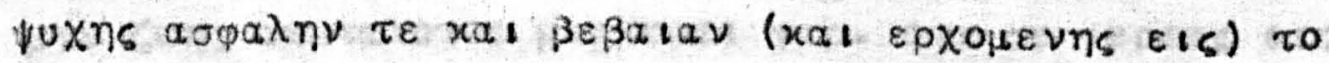

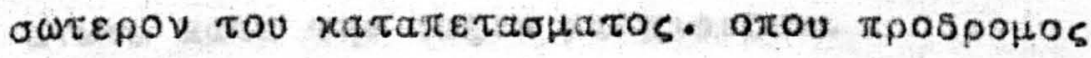

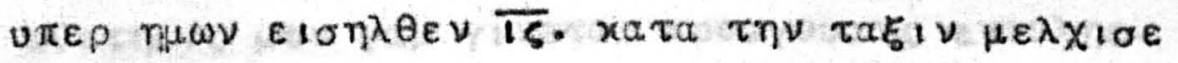

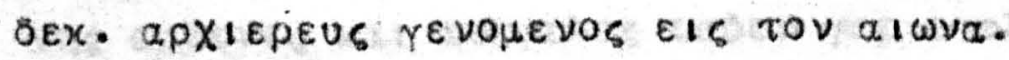

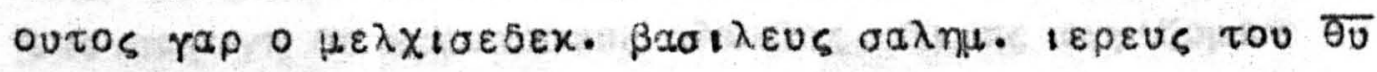

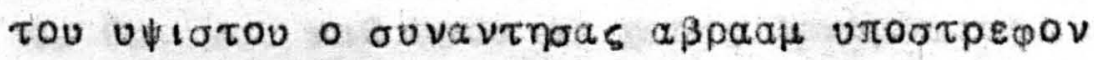

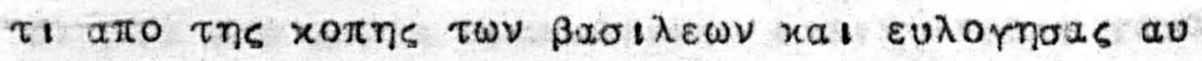

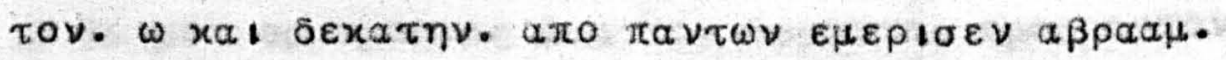

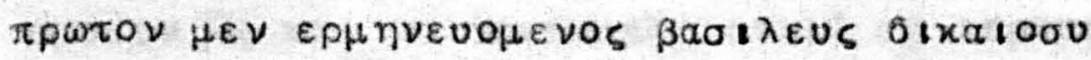

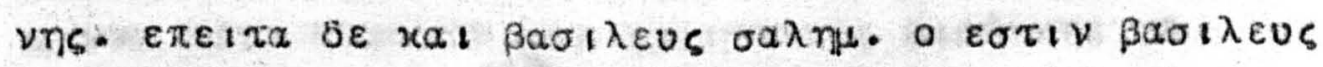

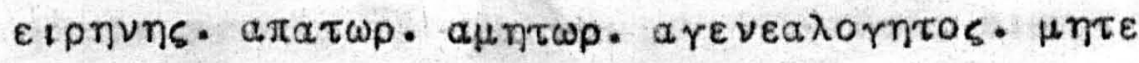

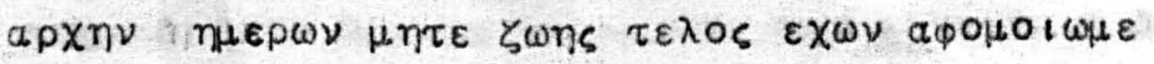

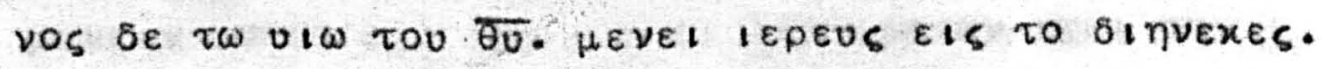

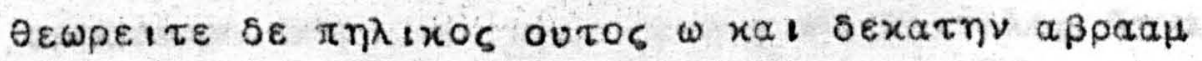

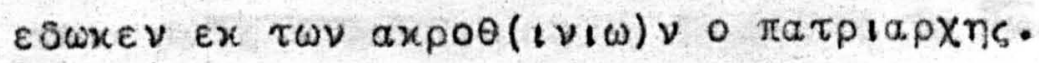

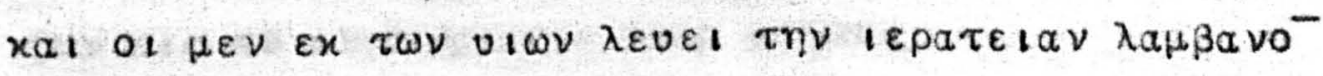

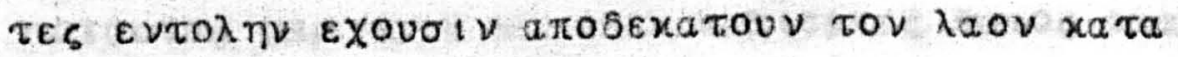




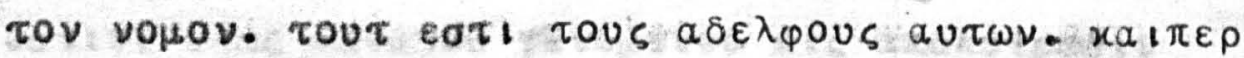

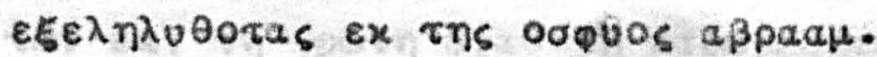

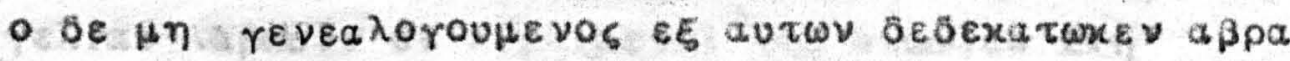

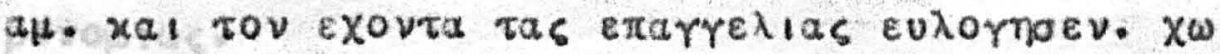

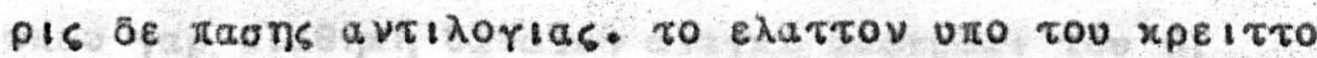
Vos evioreital.

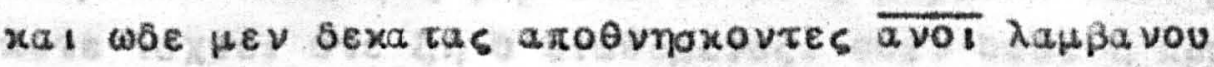

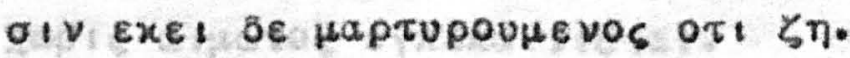

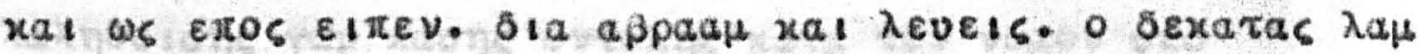

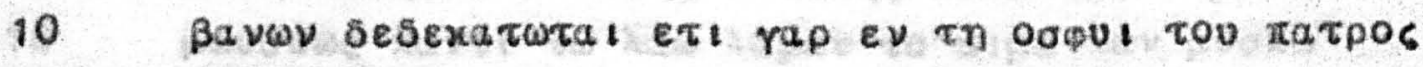

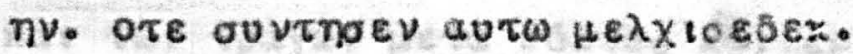

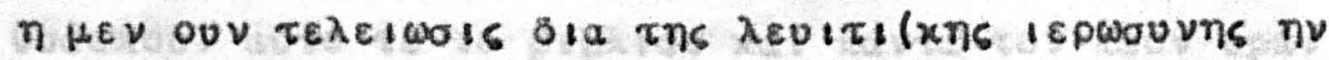

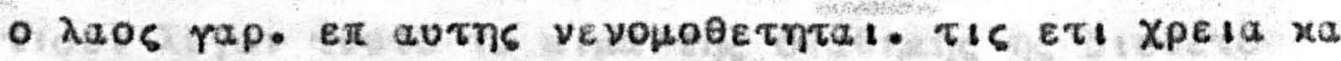

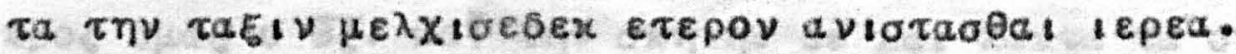

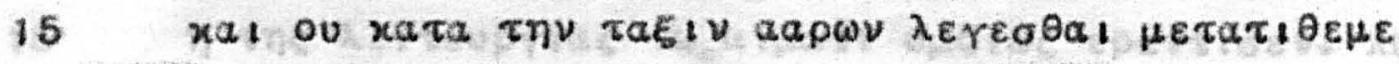

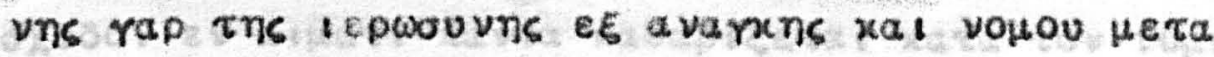
eeois rivetal.

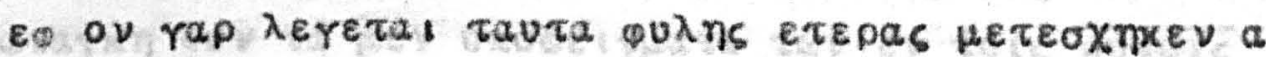

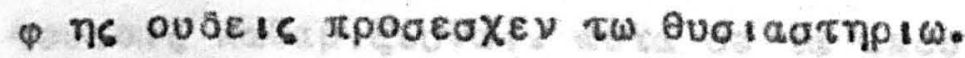

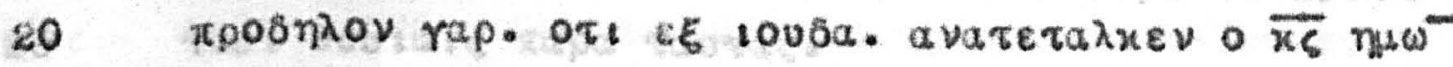

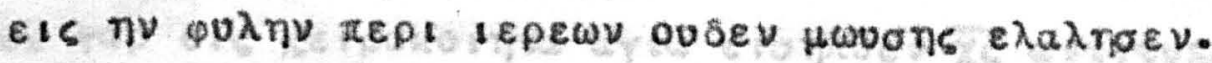

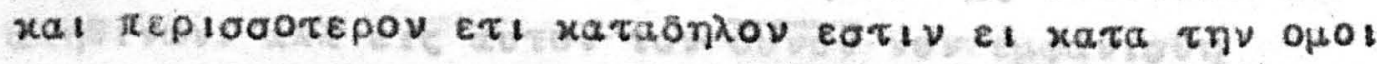

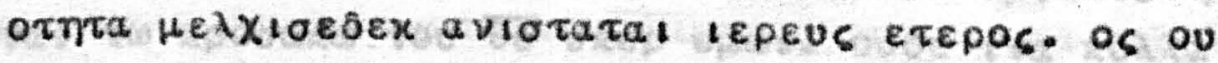

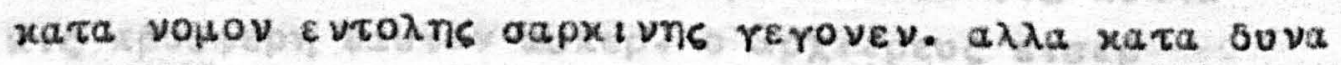

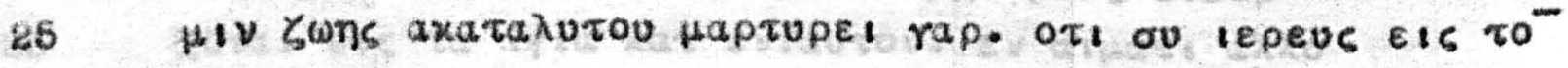

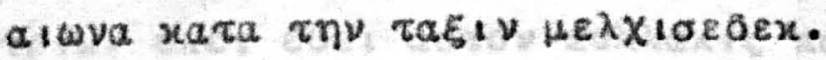

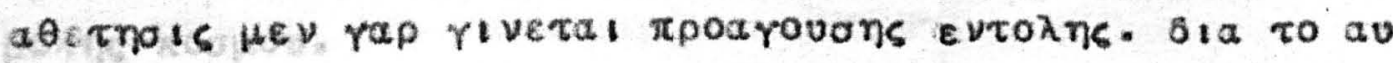

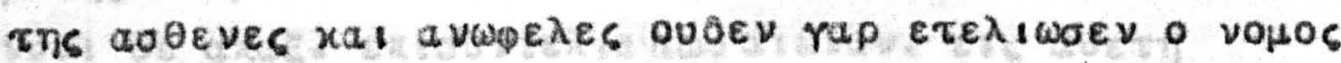

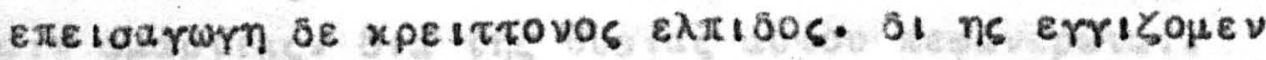

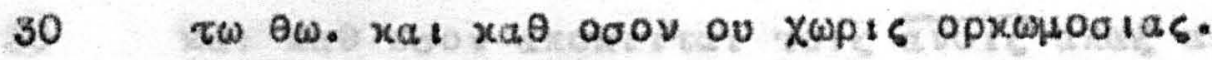
of Hev rap Xupls opxthodias. Elaiv iepels rerovotes

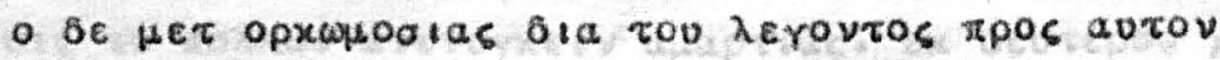

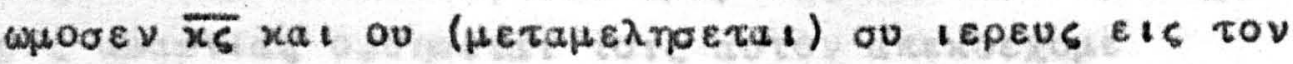

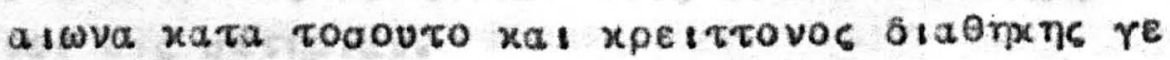

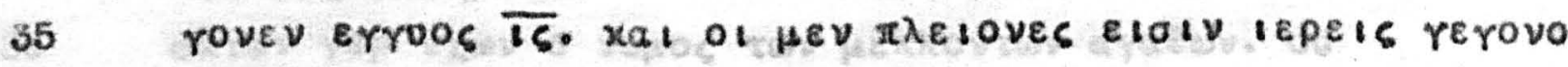

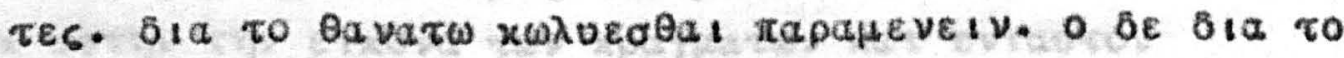

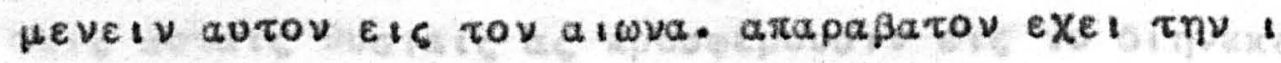

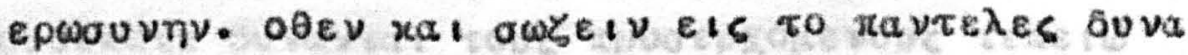

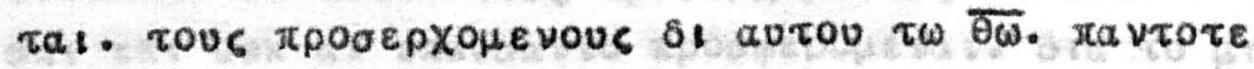

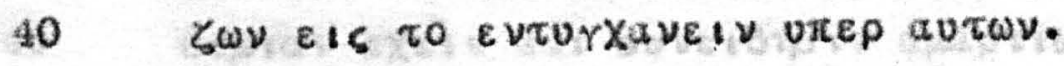

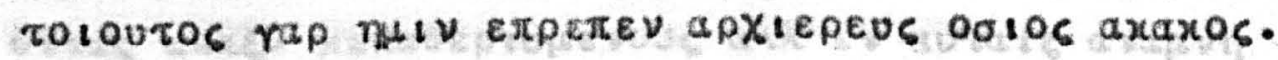




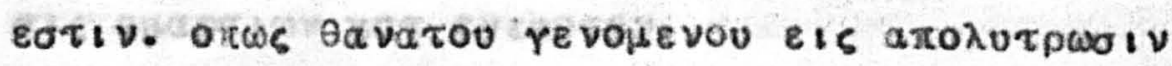

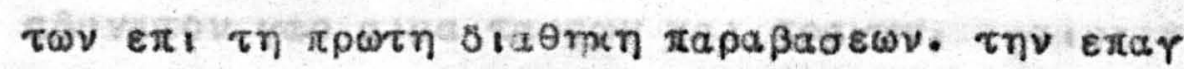

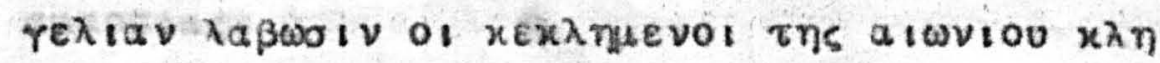
povoutas.

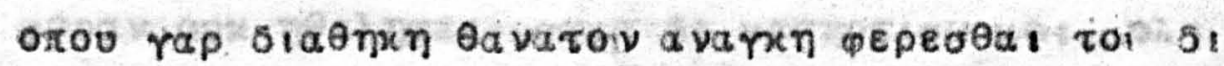

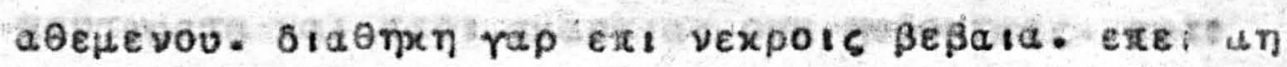

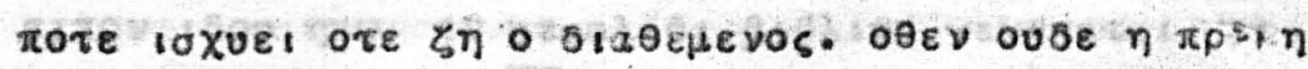
Xwpts atuaros enxexatvioral. la

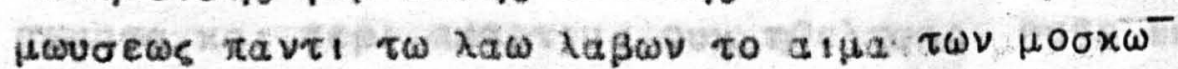

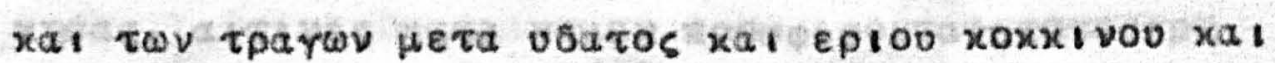

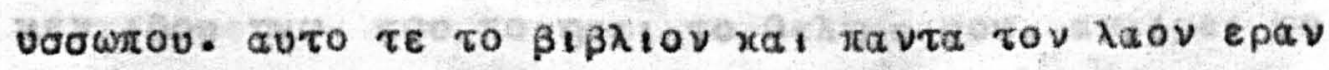

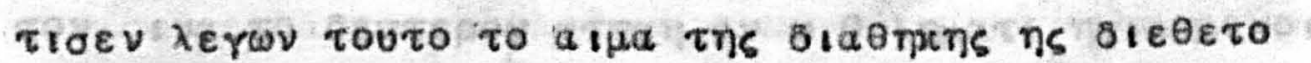

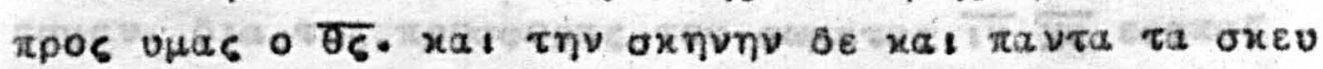

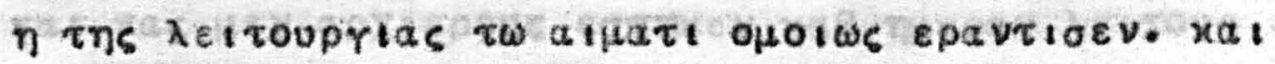

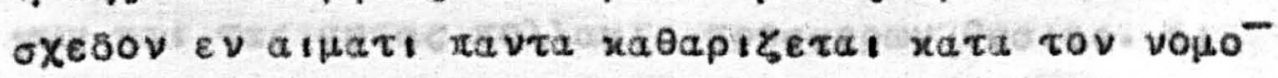
xat Xwpis atfarexXodias ou pivera! a.eots. avarin ouv ta Hev utobetruata $\tau \omega \nu$ ev tois $00 v 016$

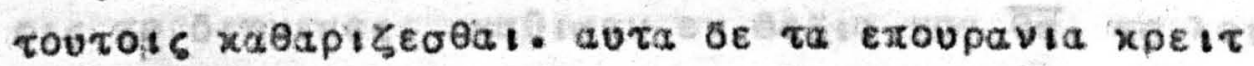

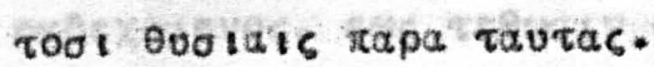
ov rap eis Xetpotointa arıa eı

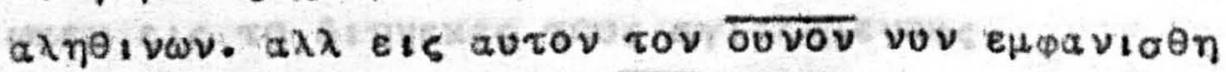

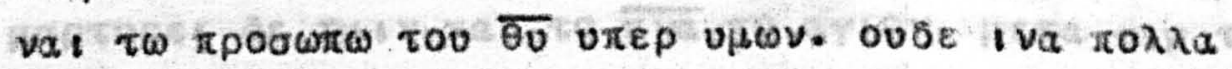

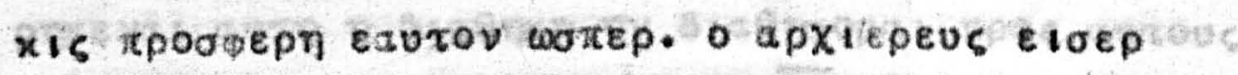

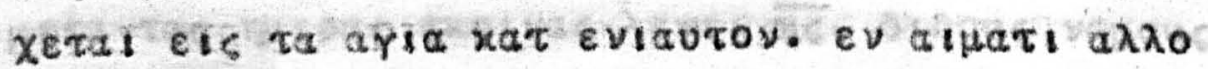

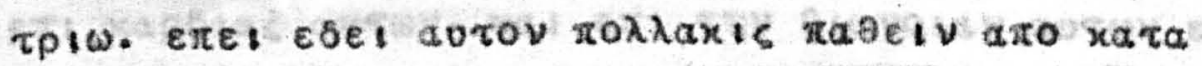
Boxns xooH

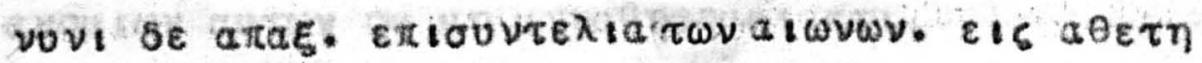

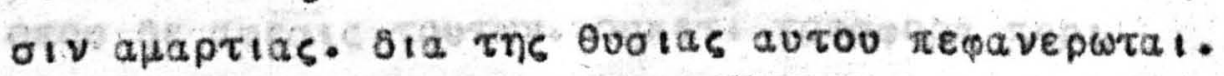

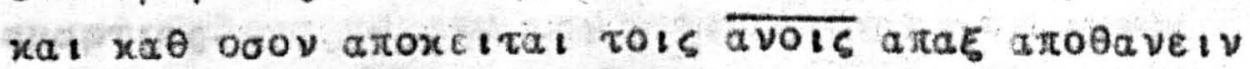

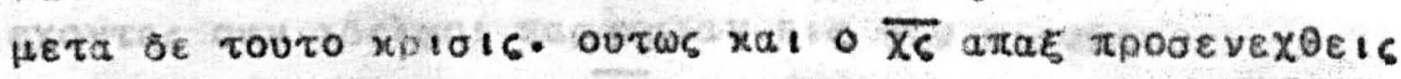

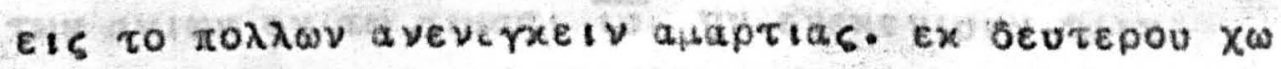

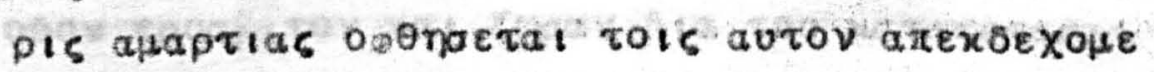
voi Ets owrnptav.

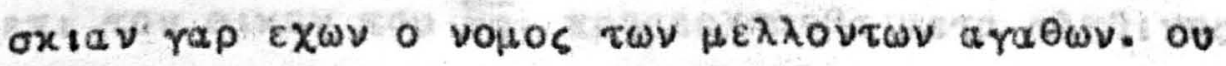

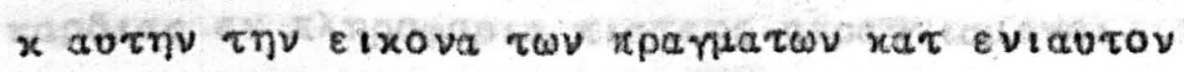

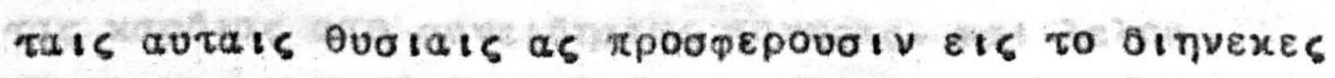

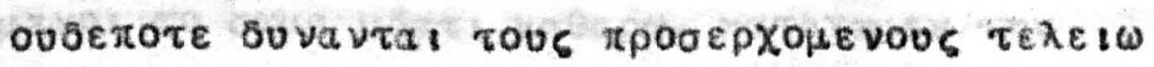

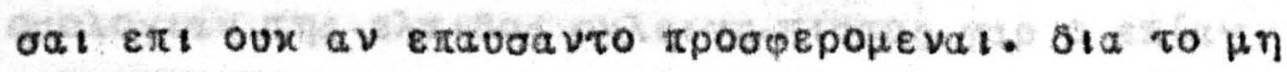

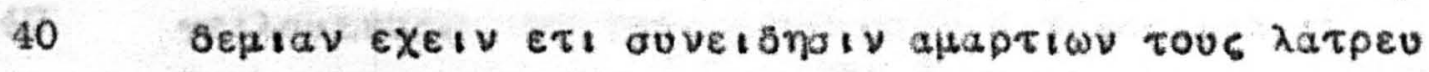

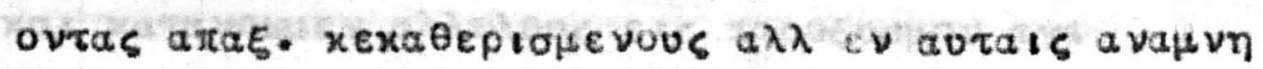




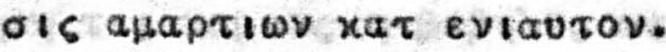

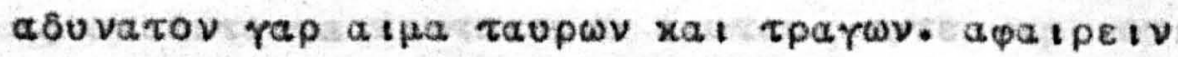

auaprtas.

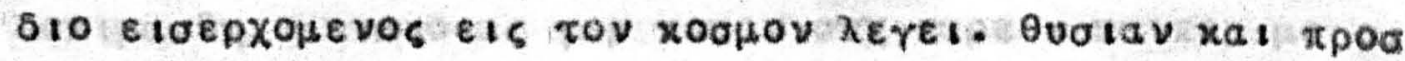

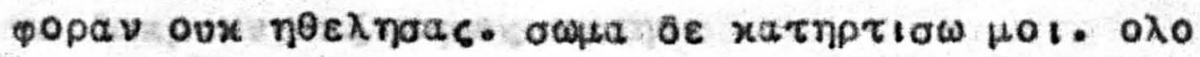

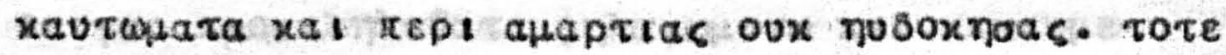

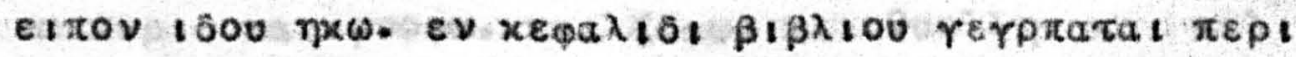

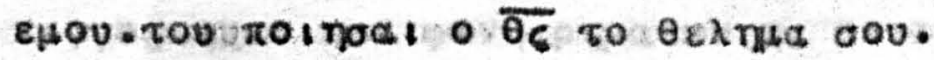

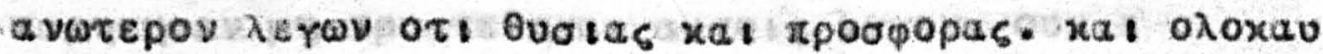

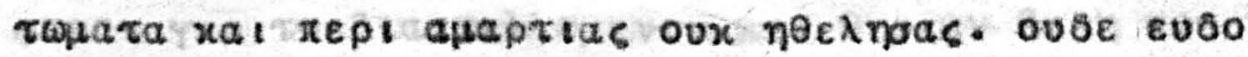

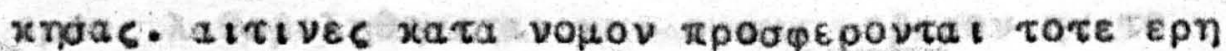

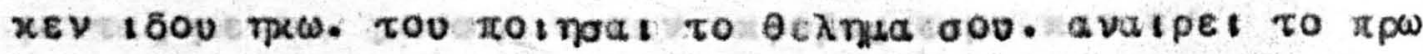

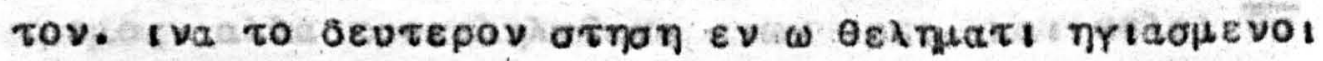

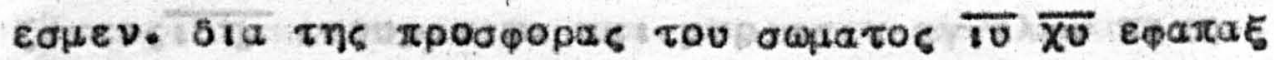

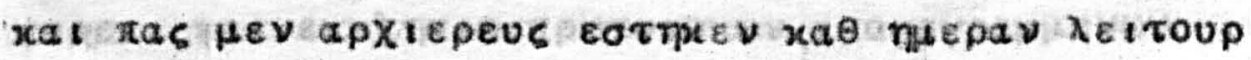

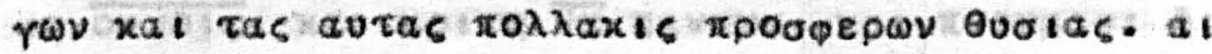

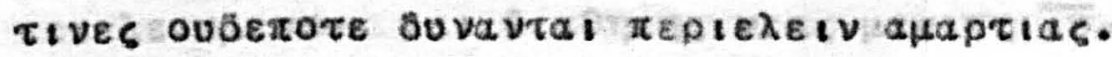

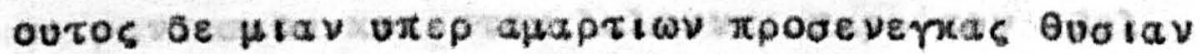

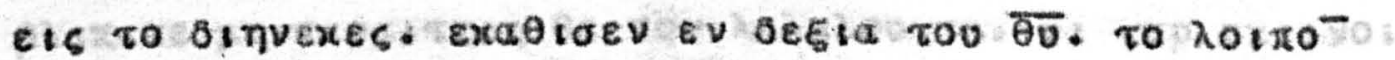

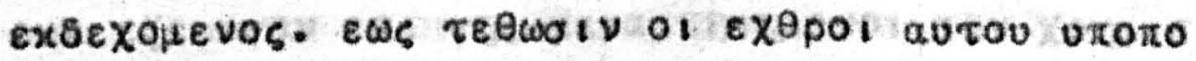
blov $\tau \omega \nu$ rosw

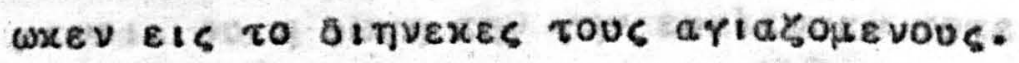

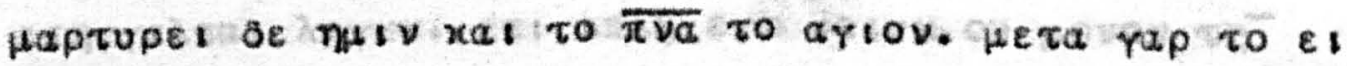

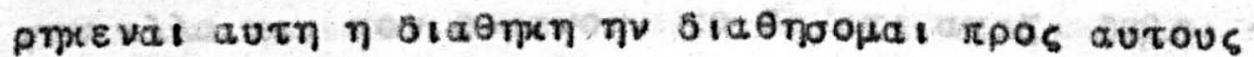

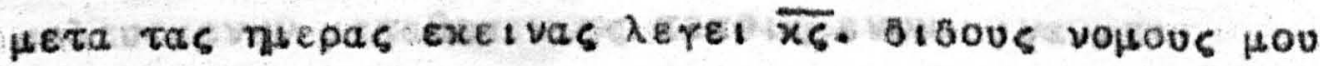

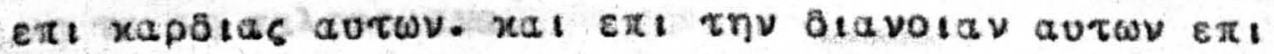

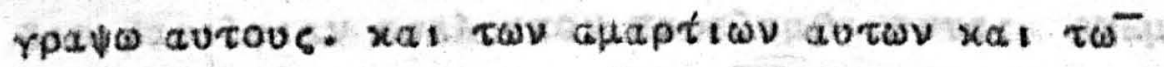

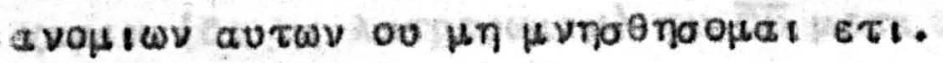

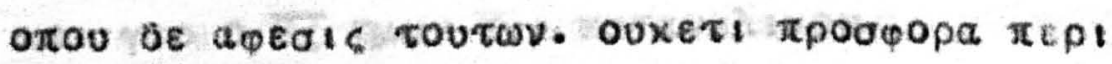
auaptias.

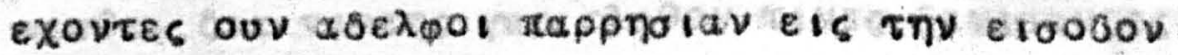

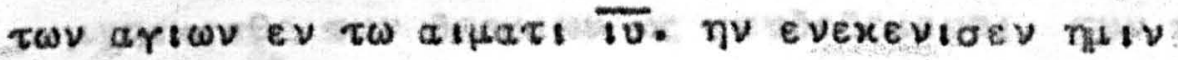

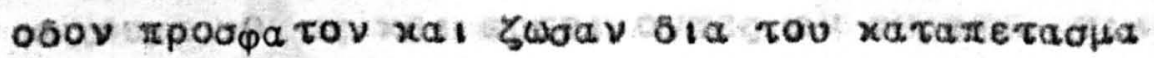

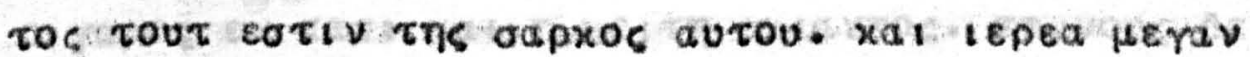

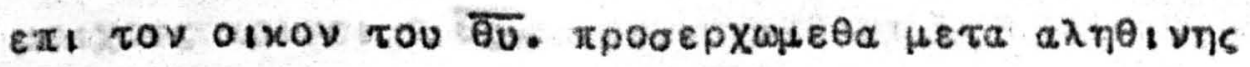

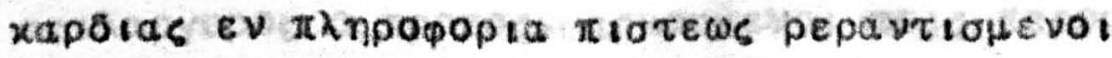

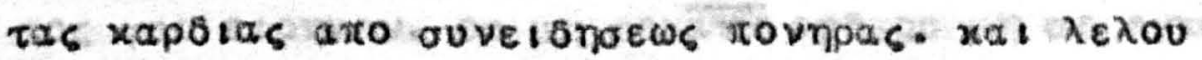

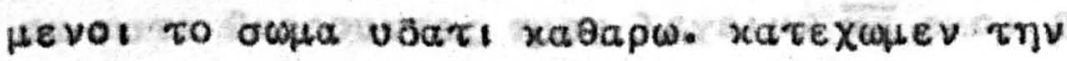

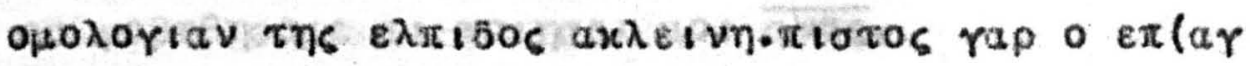
reı $\lambda$ aеvos.

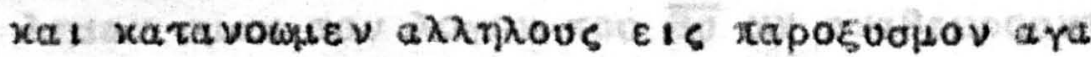




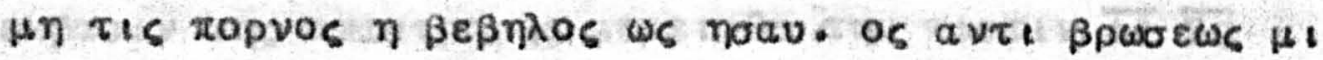

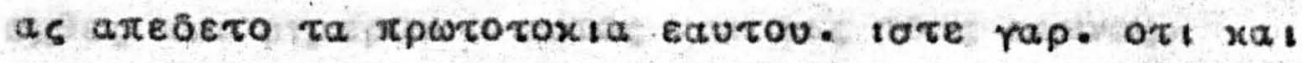

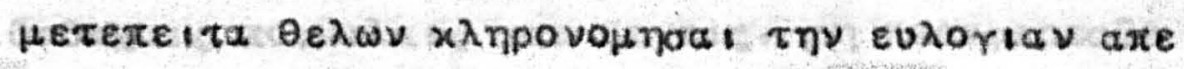

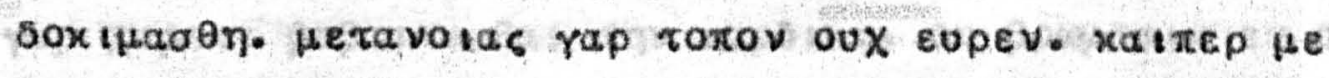

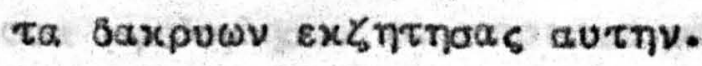

ou $\operatorname{rap} \pi \rho \circ a \lambda \eta \lambda v \theta \alpha \tau \varepsilon$ $\eta \lambda a \varphi \omega \mu \varepsilon v \omega$ xal $x \varepsilon x a v \mu e$

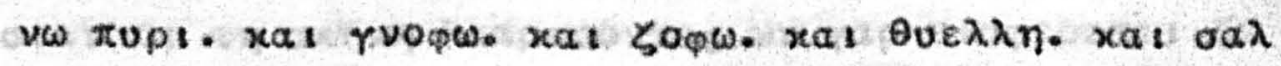

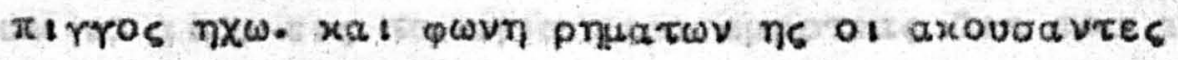

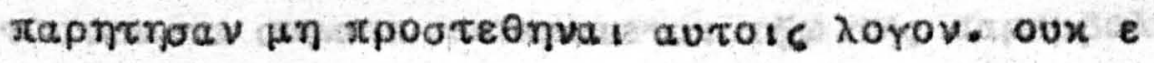

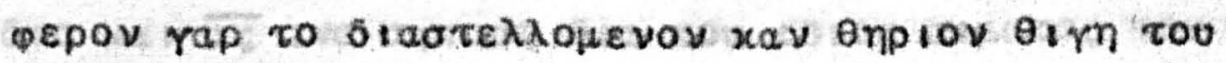

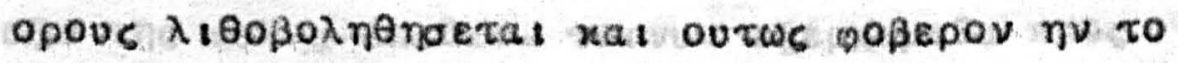

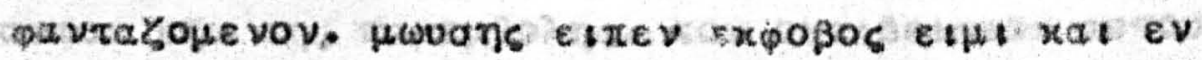

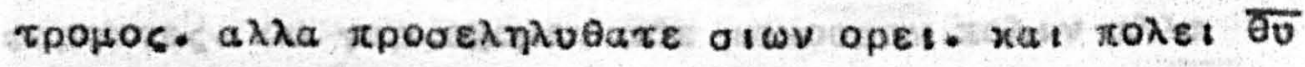

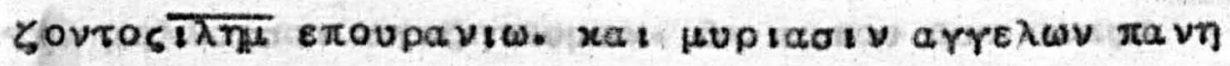

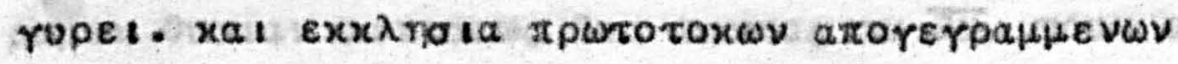

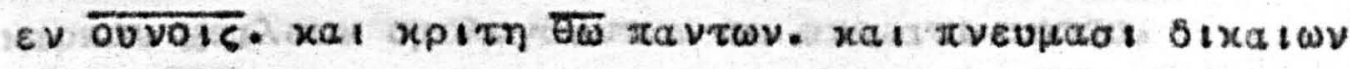

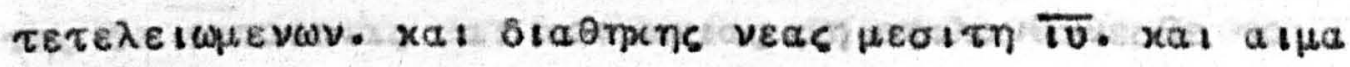

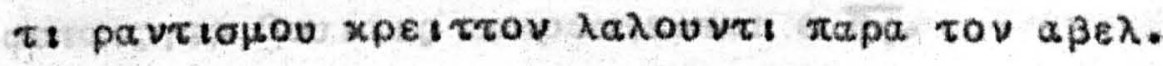

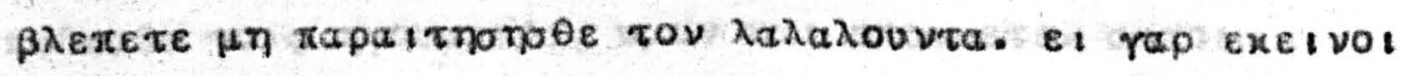

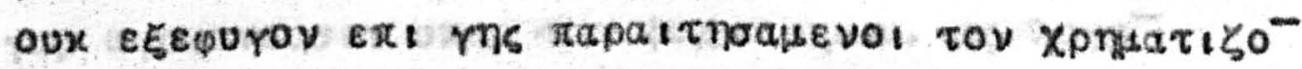

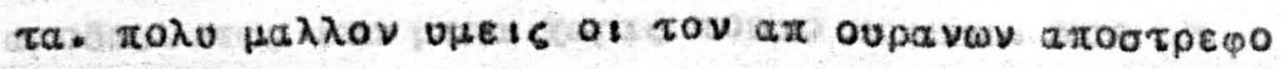

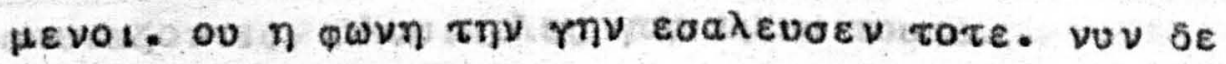

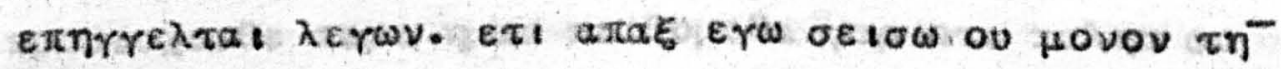

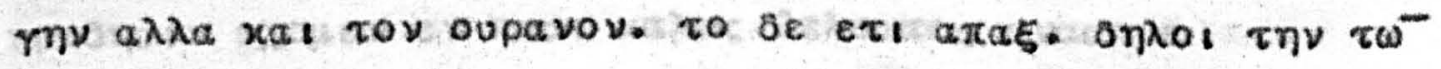

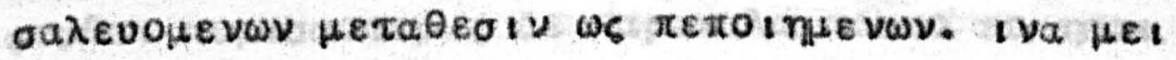

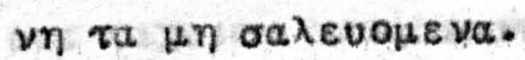

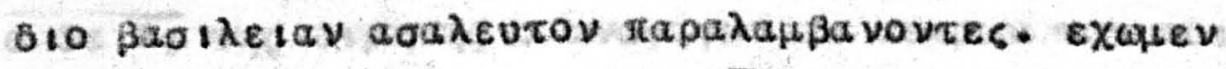

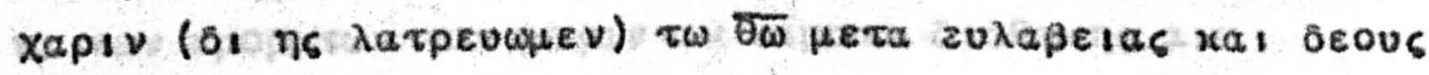

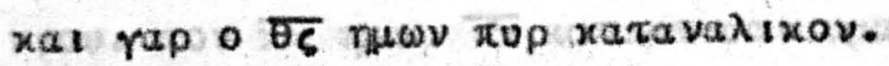

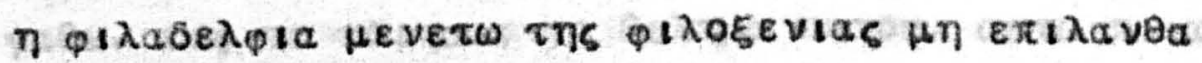

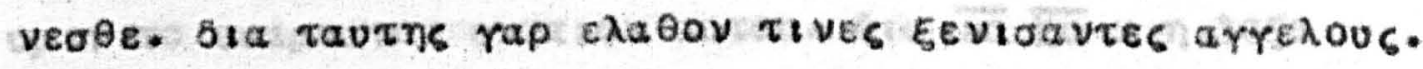

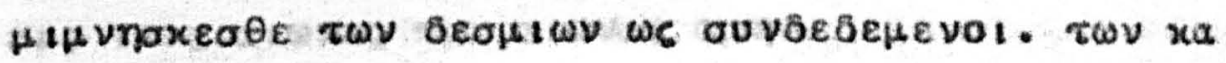

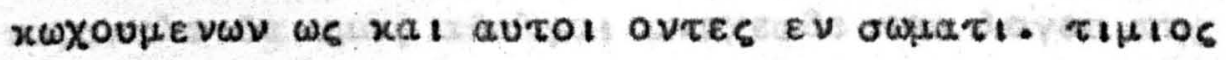

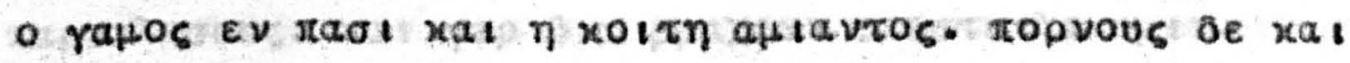
Hoixous xptvet o $\overline{\theta_{G}}$. api

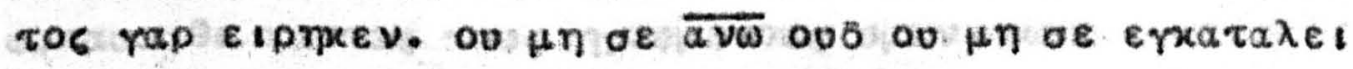

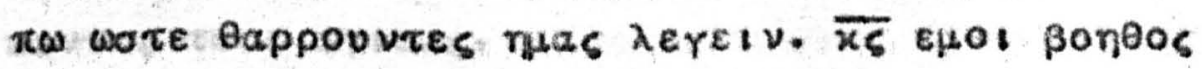

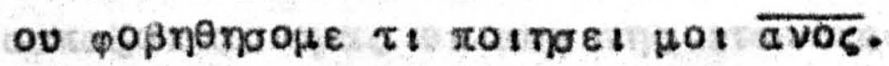

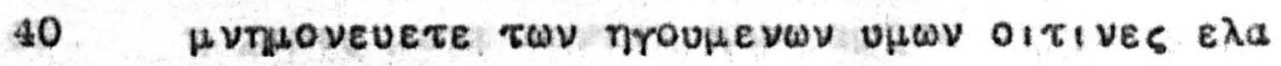

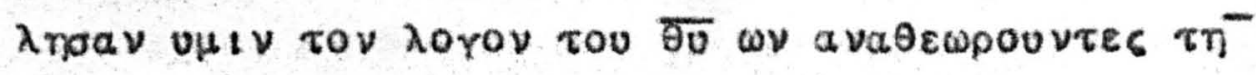




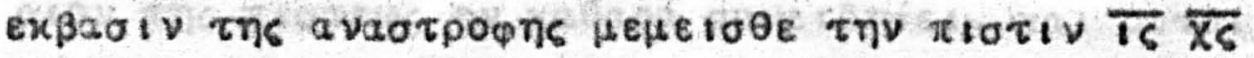
exoes xat onjepov o juros xat eis rous alwias.

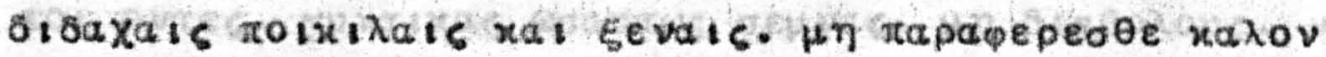

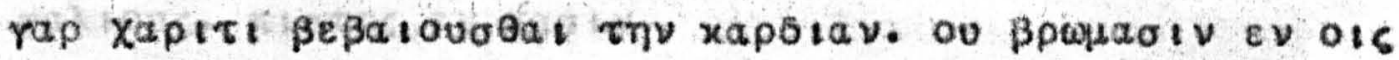
oux we

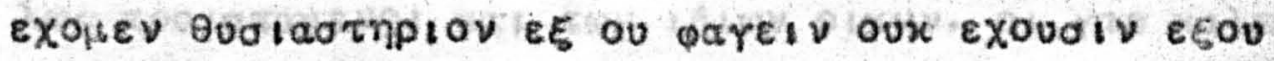

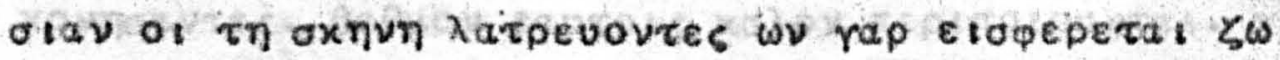

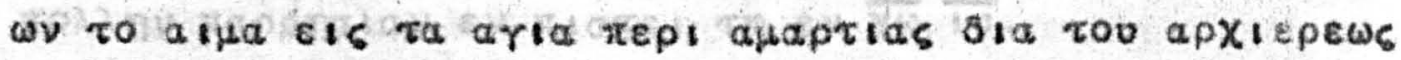

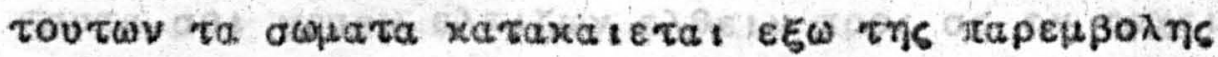
810 xal ts iva ariaon 816 tou 18100 atpatos tov Xaov

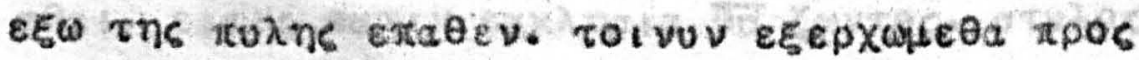

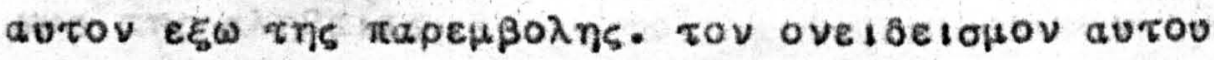

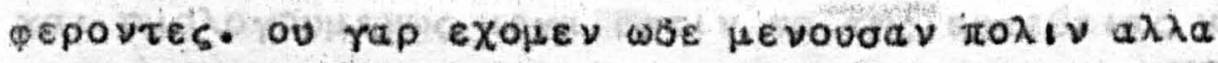

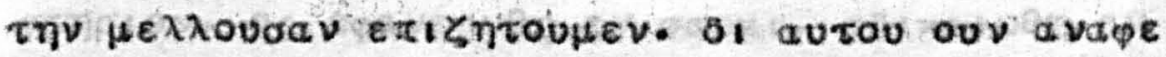

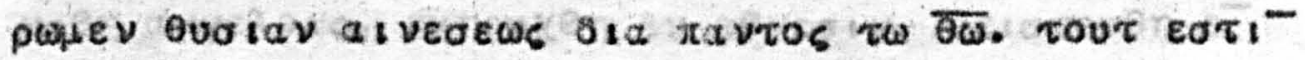

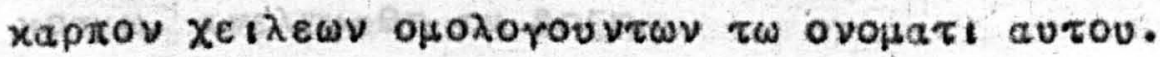

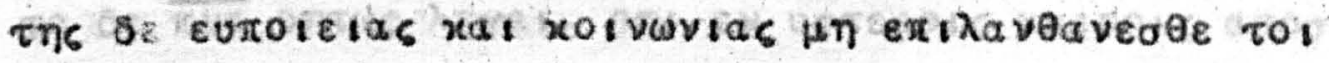

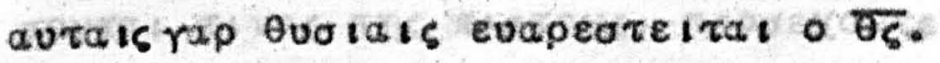

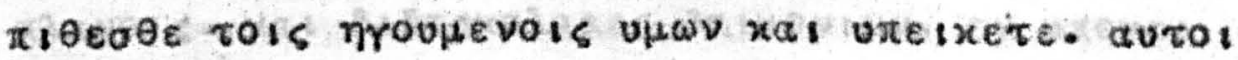

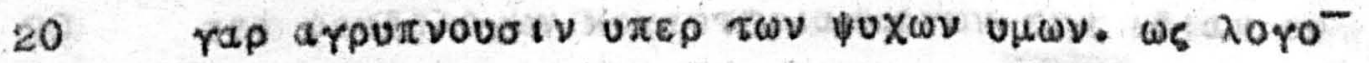

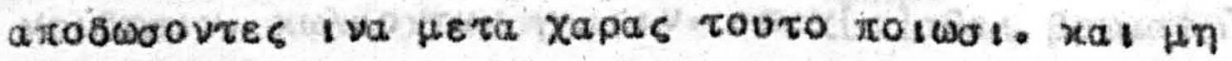

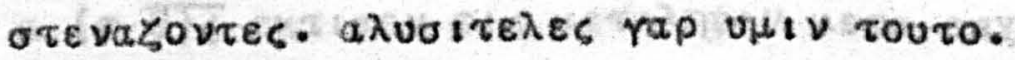

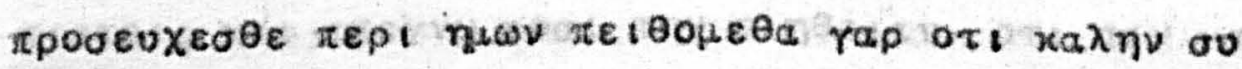

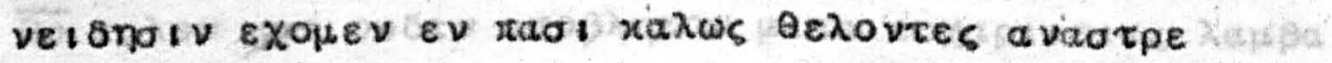

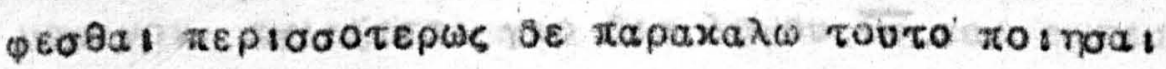
iva raxetov aroxatajta $\theta \omega$ uhiv.

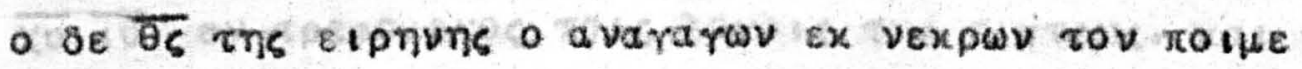

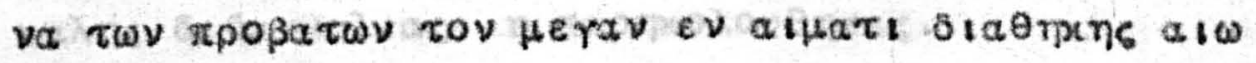

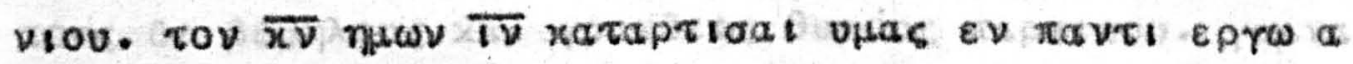

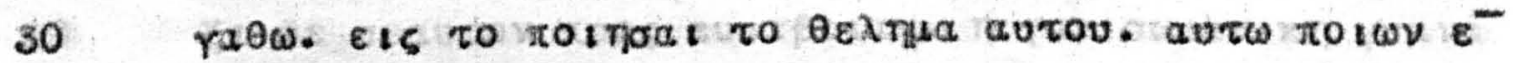

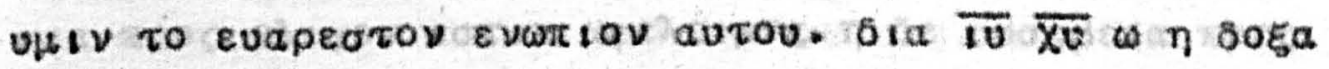
Eis rovs a lwvas rwv a I wvas ainv.

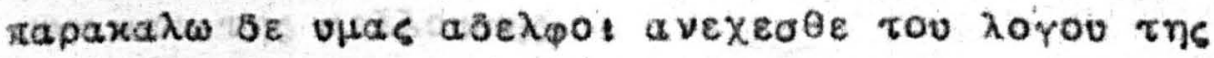

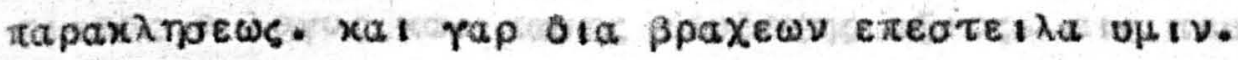

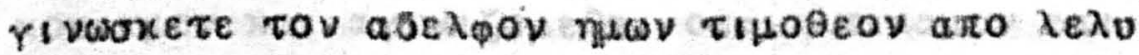

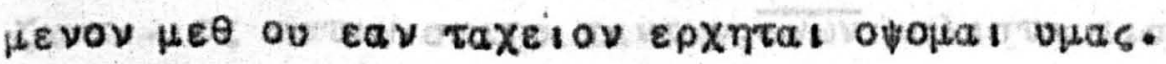

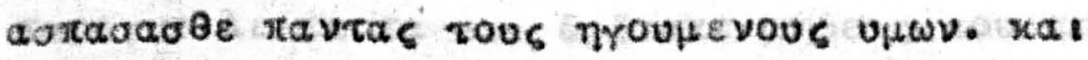
tavias rous arious.

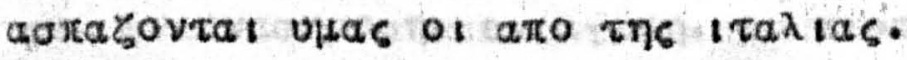

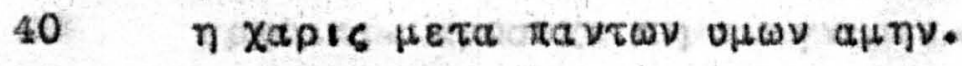

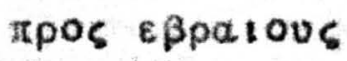




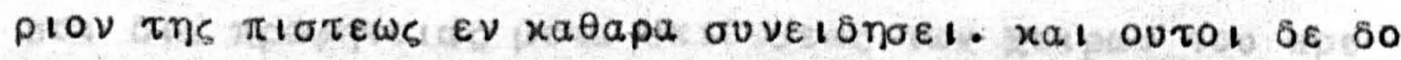

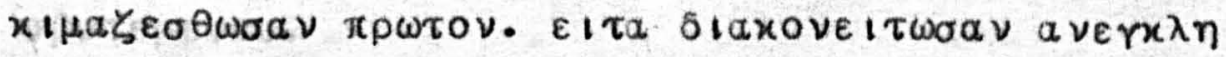

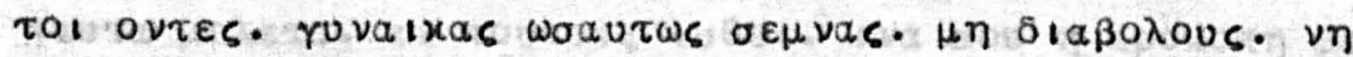

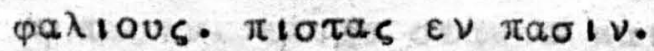

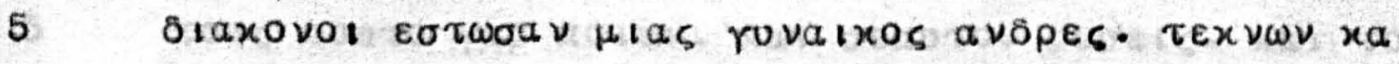

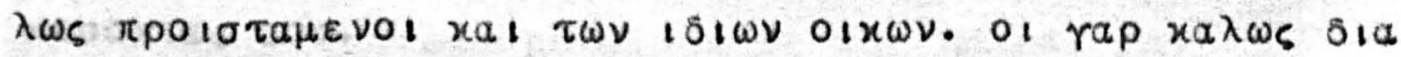

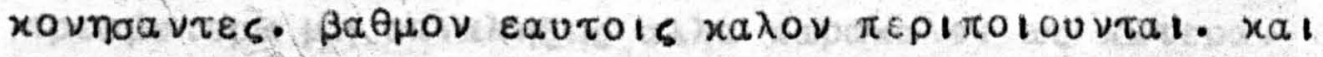

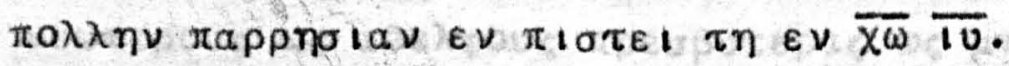

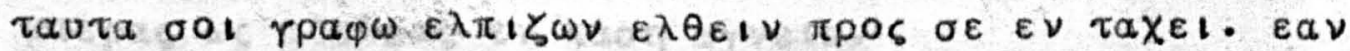

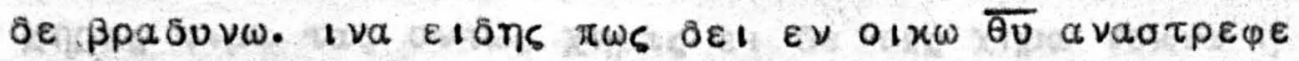

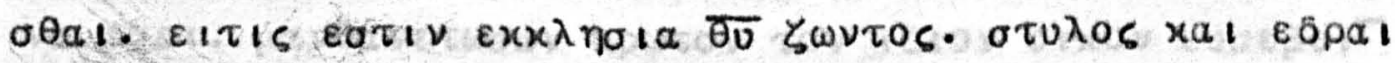

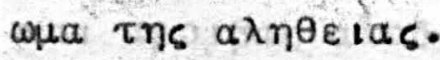

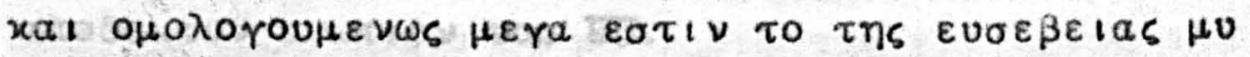

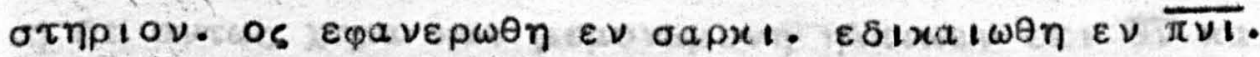

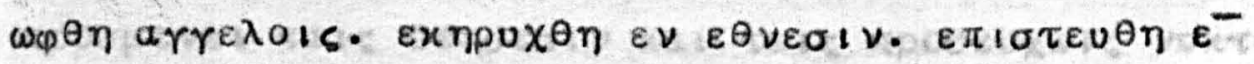
$\varkappa \circ \sigma \mu \omega . \quad \alpha \nu \varepsilon \lambda \eta \mu \varphi \theta \eta \in \nu$ $\delta \circ \eta$.

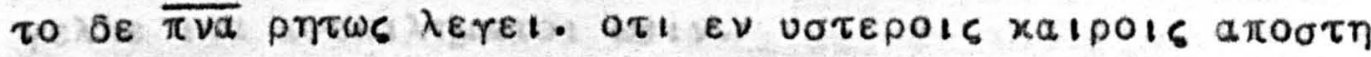

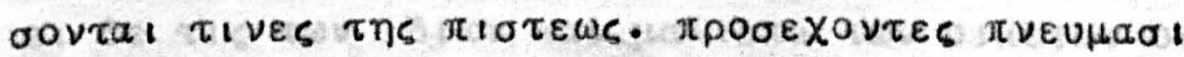

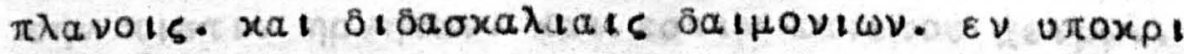

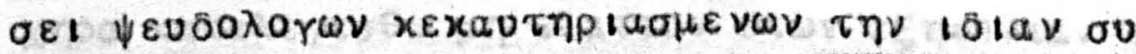

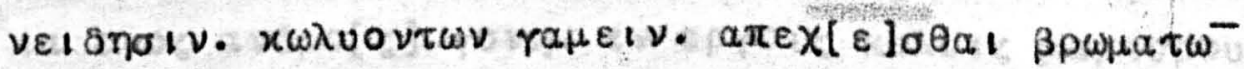

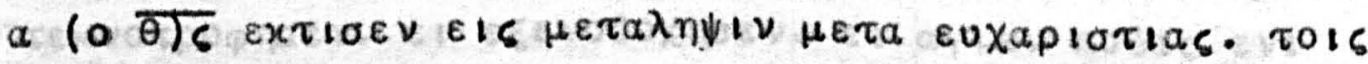

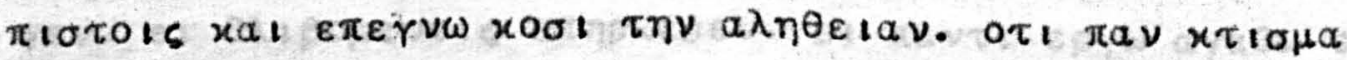

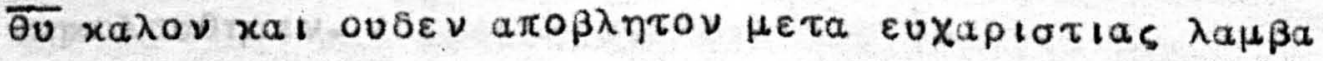

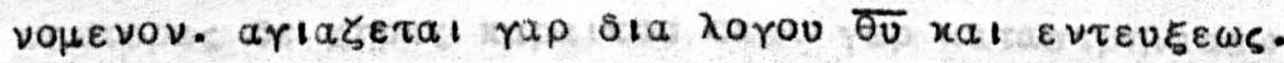

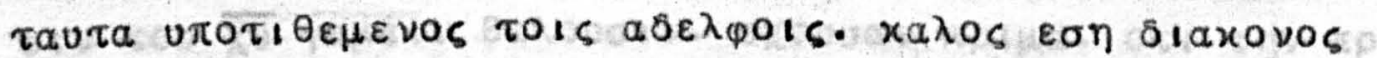

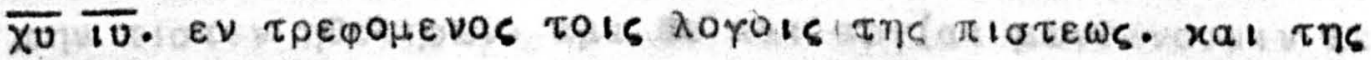

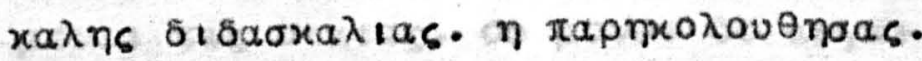

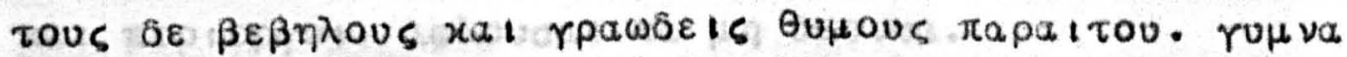

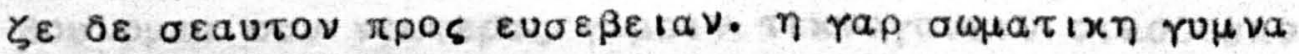

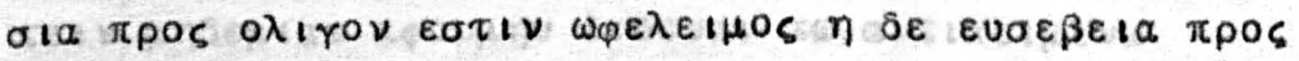

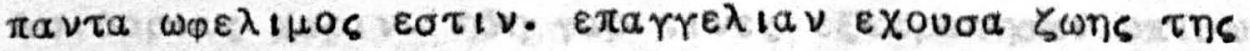
vuv xat tns $\mu \varepsilon \lambda \lambda$ ovons.

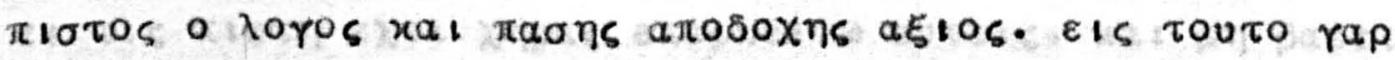

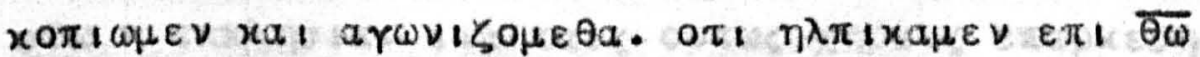

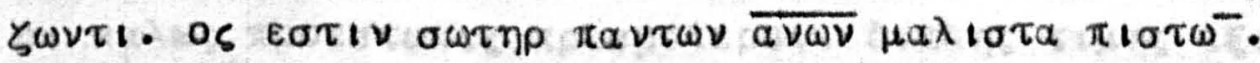

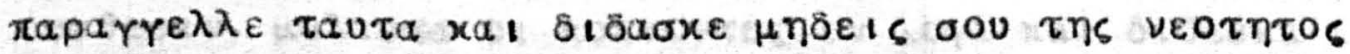

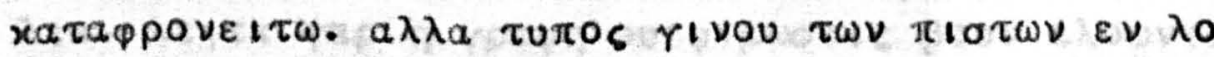

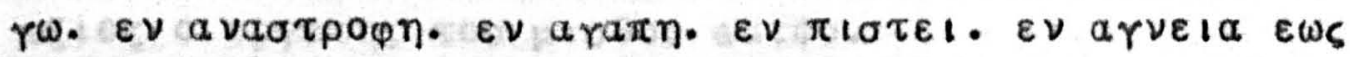

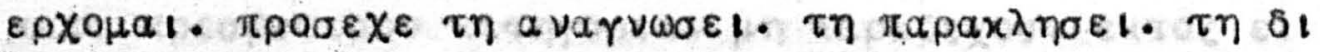

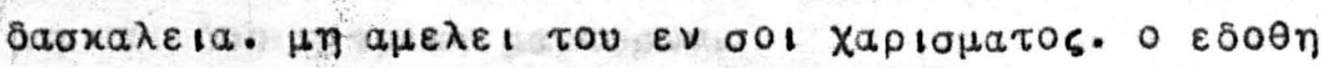




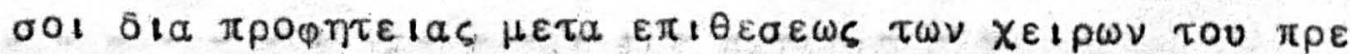

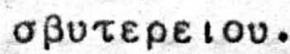

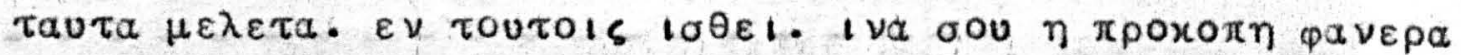
$\eta \pi a \sigma ı$. $\varepsilon \pi \varepsilon \chi \varepsilon \sigma \varepsilon a u \tau \omega$ xal $\tau \eta$

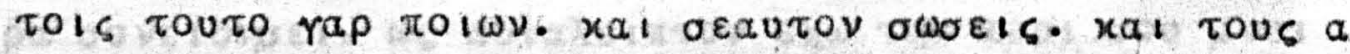
novovtas oov.

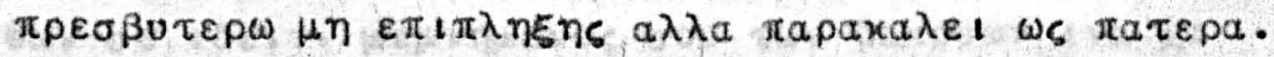

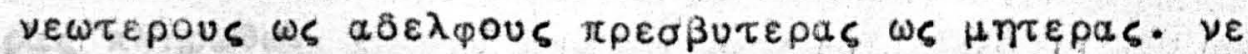

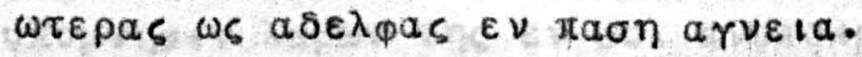

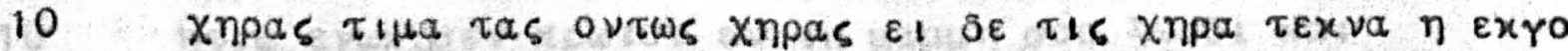

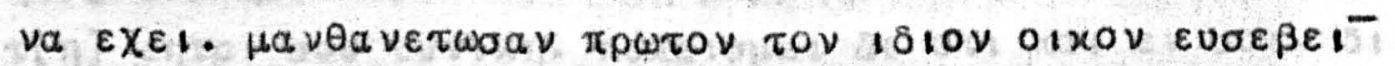

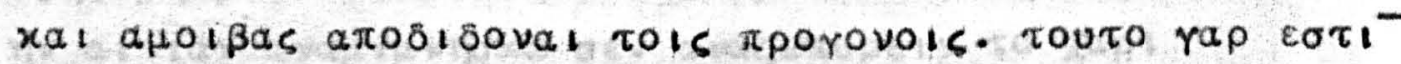

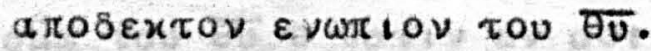

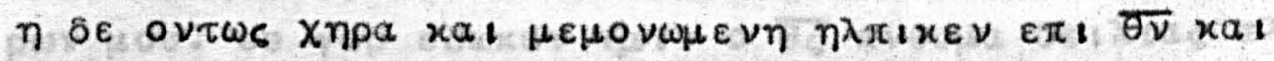

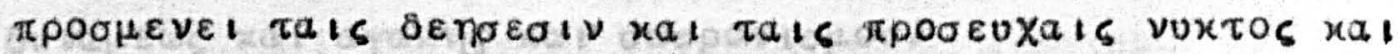

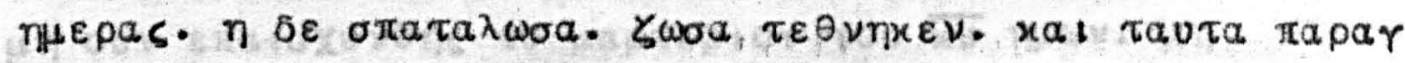

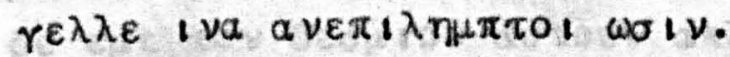

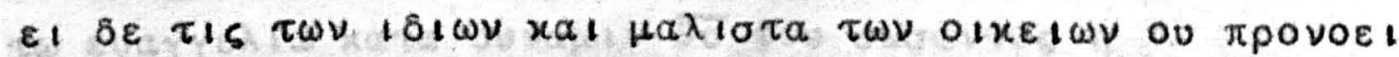

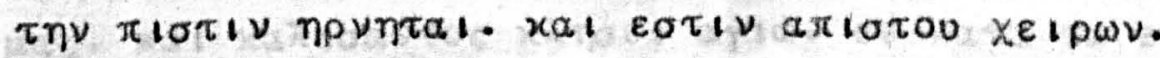

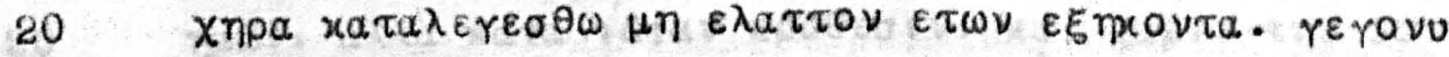

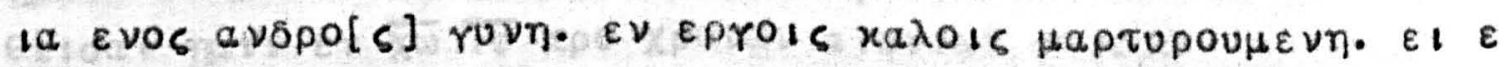

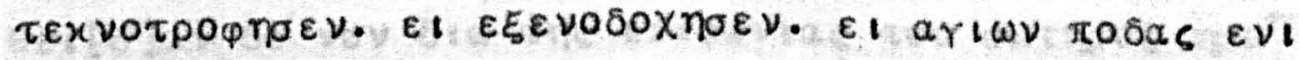

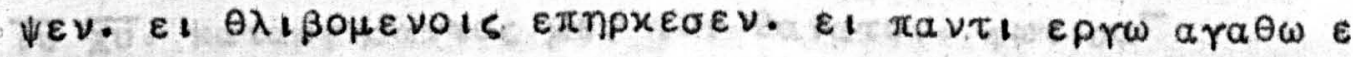

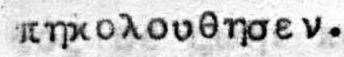

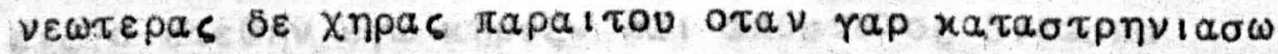

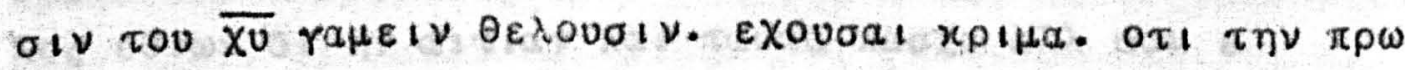

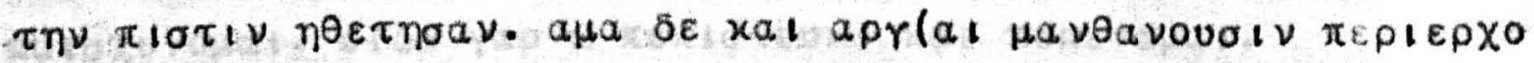

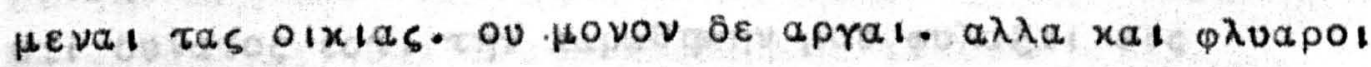

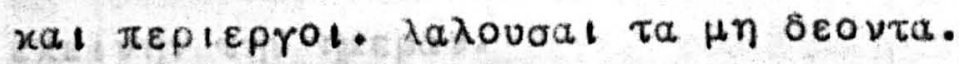

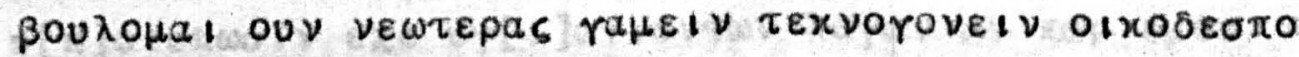

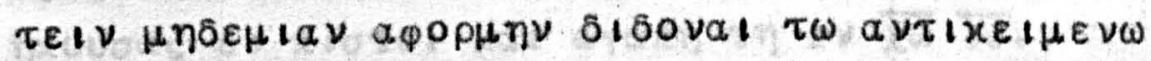

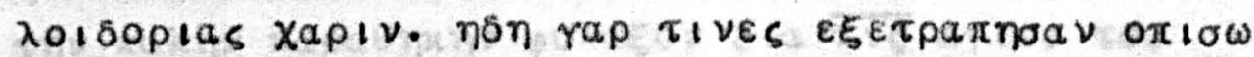

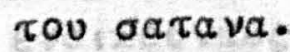

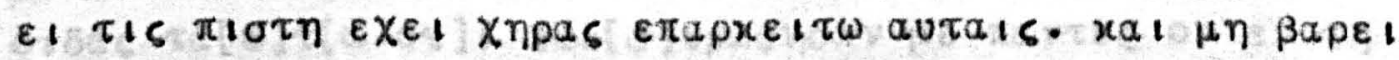

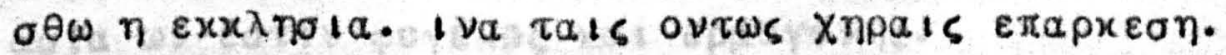

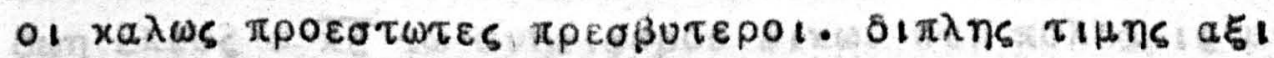

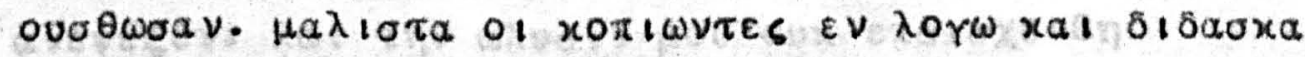

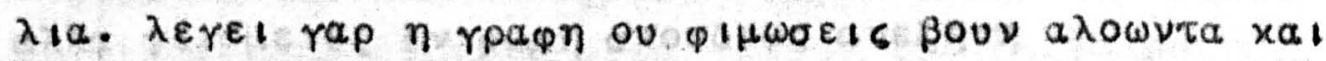
asılos o epratns tou Hiäou autou.

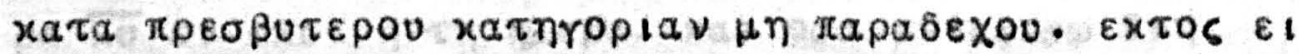

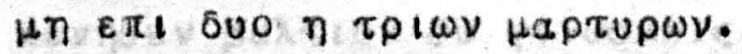




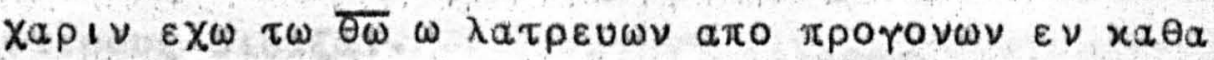

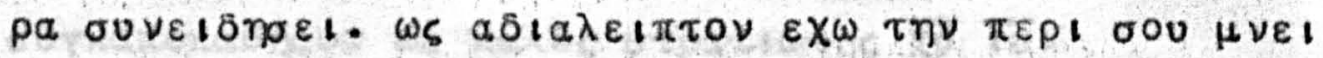

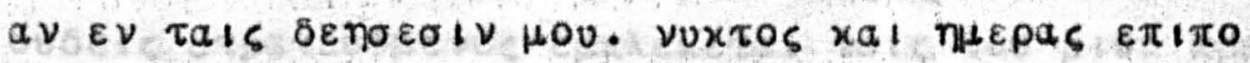

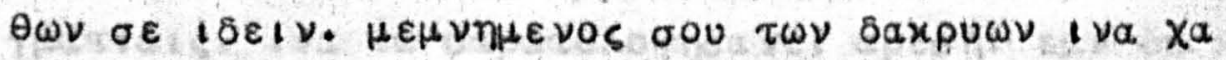
pas $\pi \lambda$ n $\rho \omega \theta \omega$.

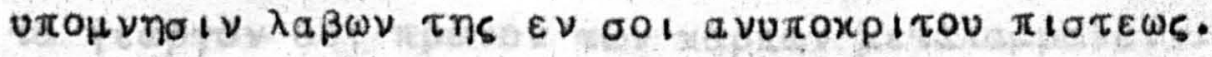

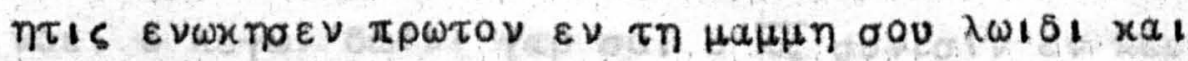

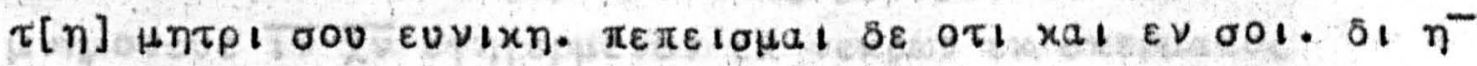

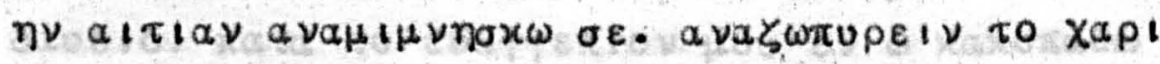

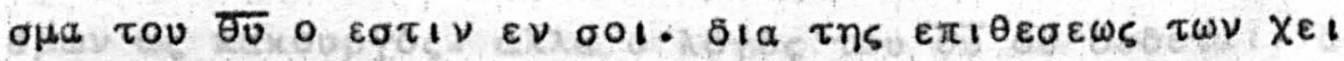

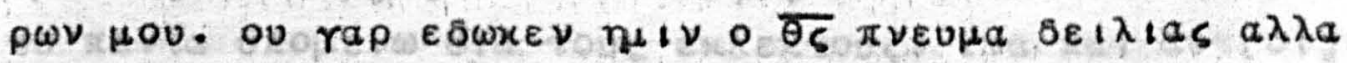

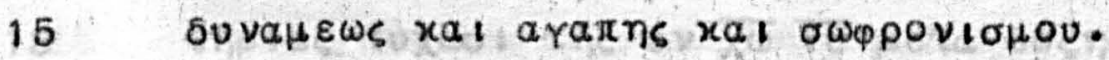

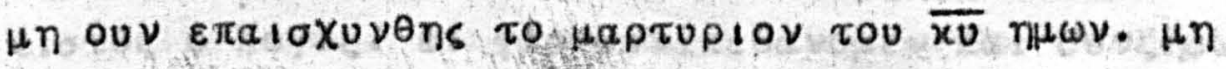

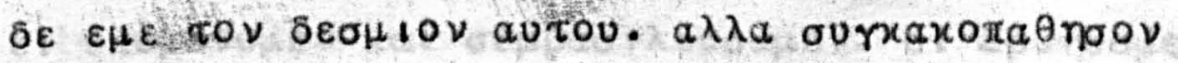

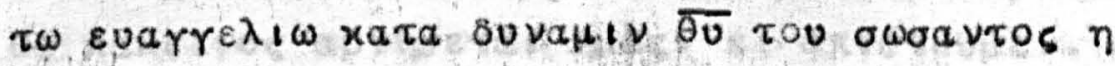

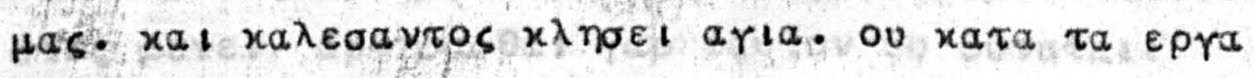

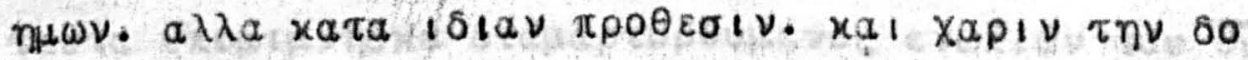

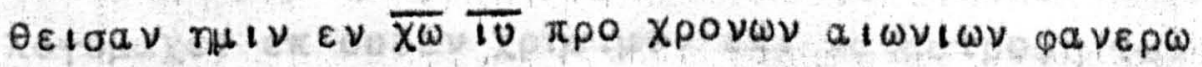

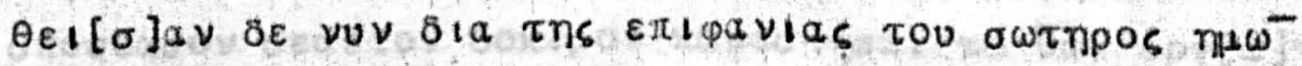

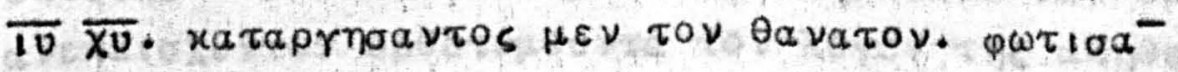

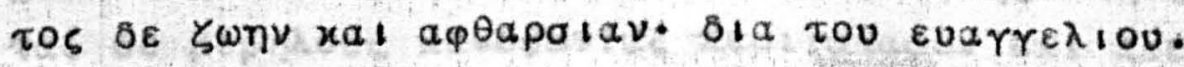

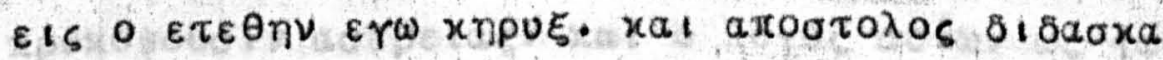

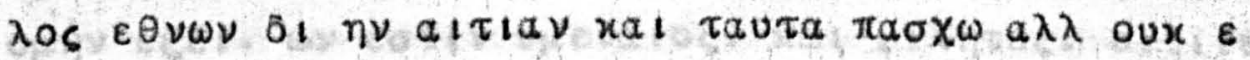

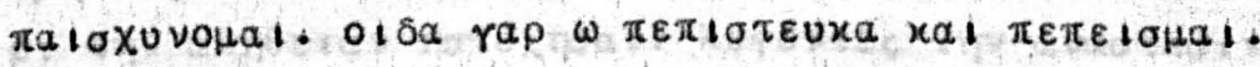

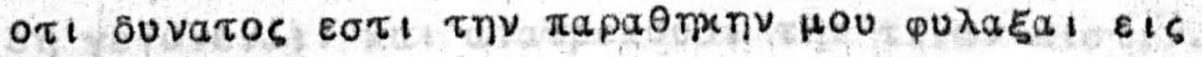

$\varepsilon x \varepsilon i \nu \eta \nu \tau \eta \nu$ ก $\varepsilon$ pav.

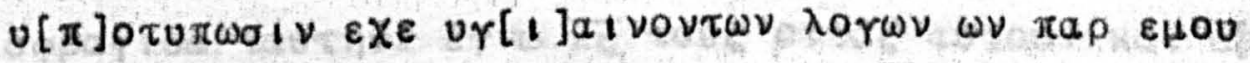

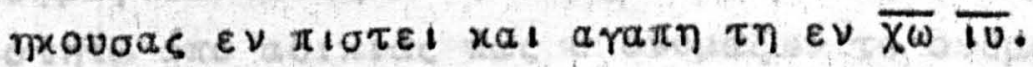

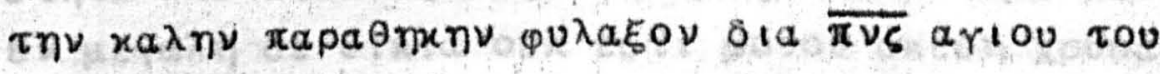

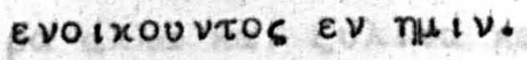

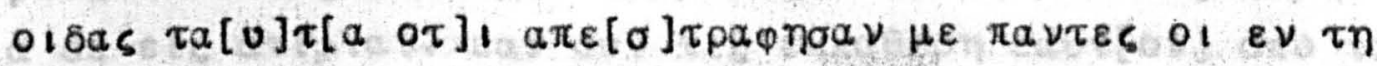

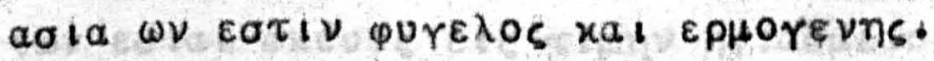

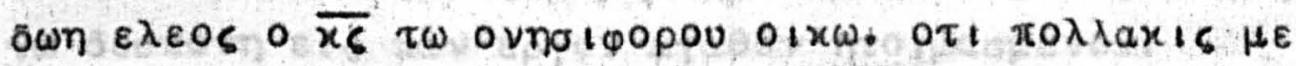

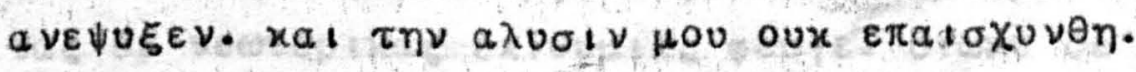

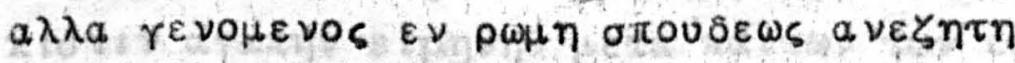

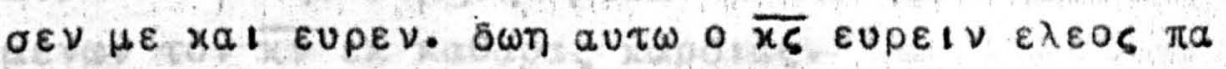

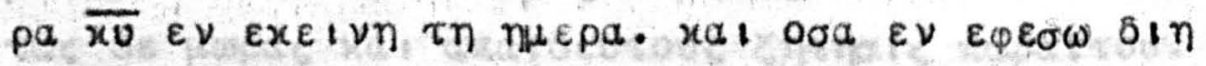

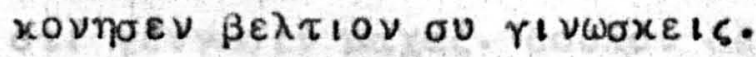




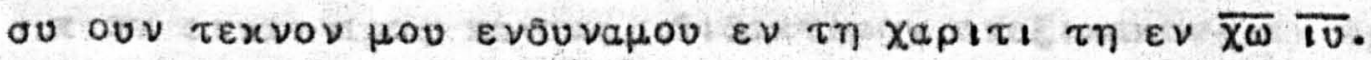

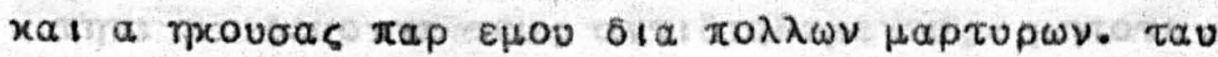

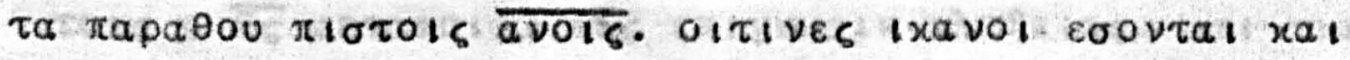

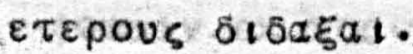

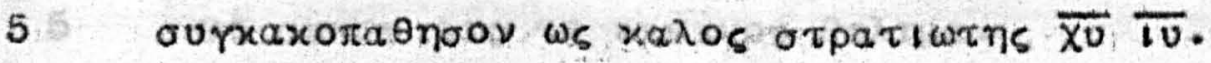

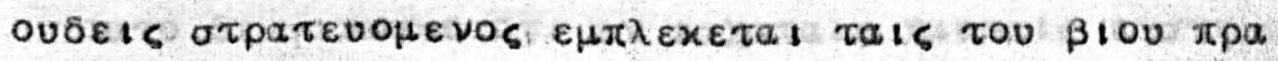

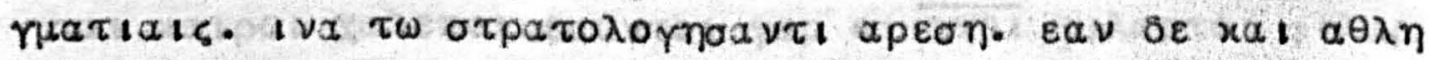

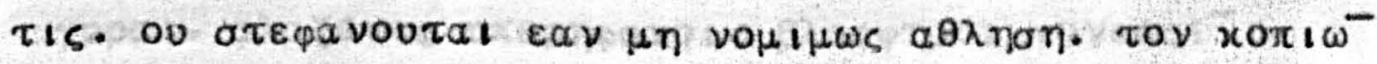

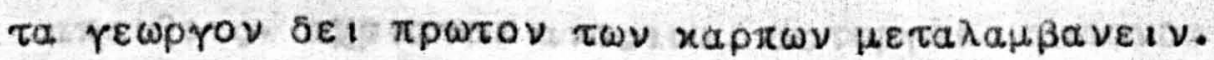

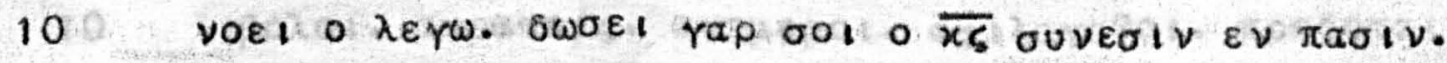

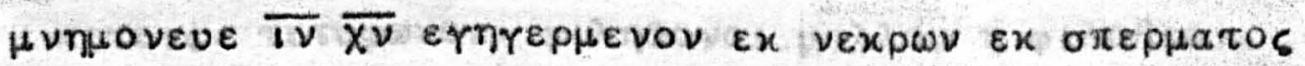

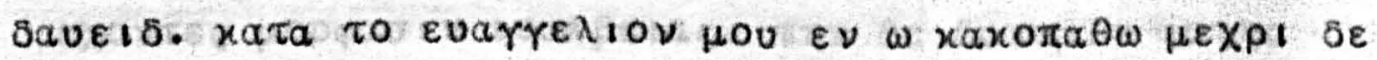

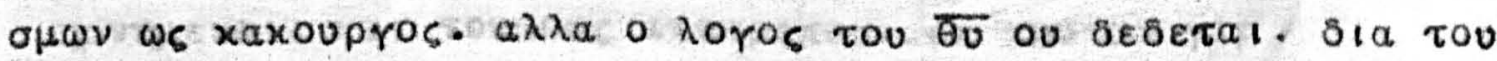

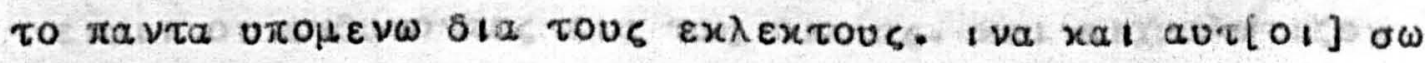

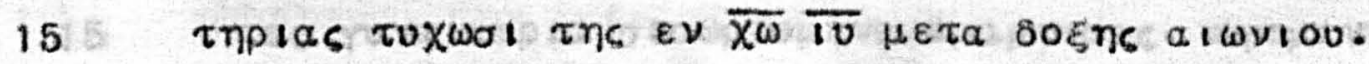

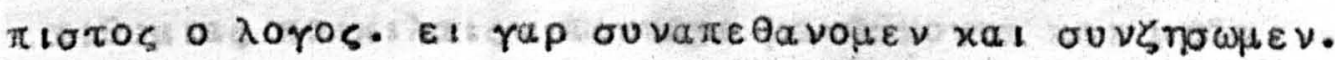
ع

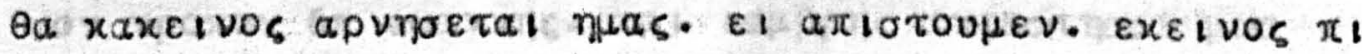

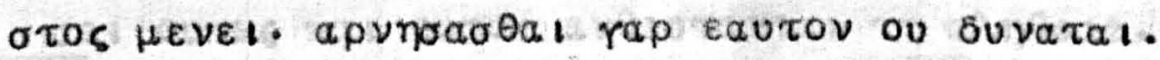

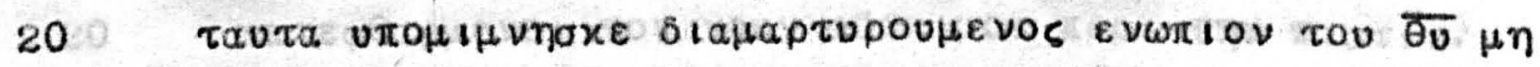

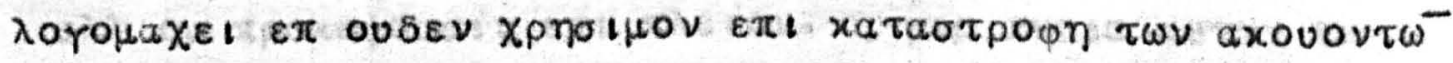

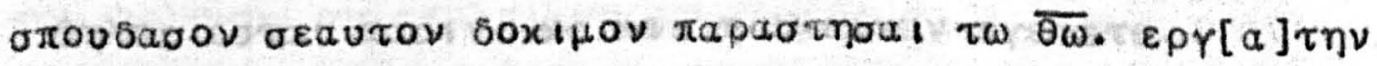

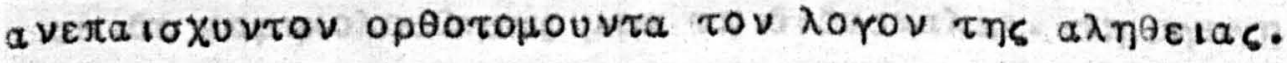

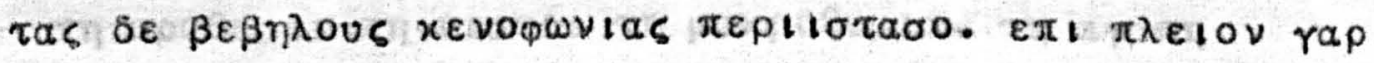

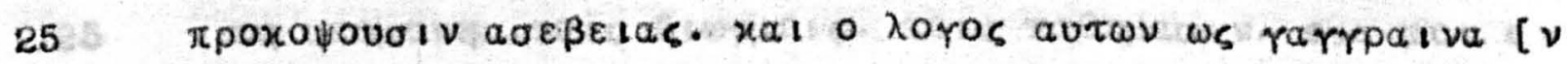

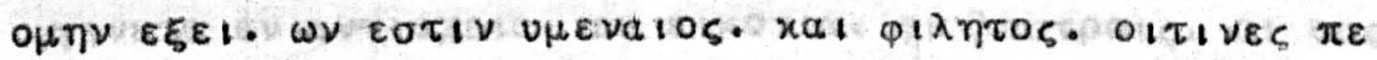

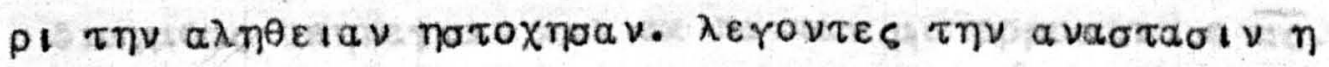

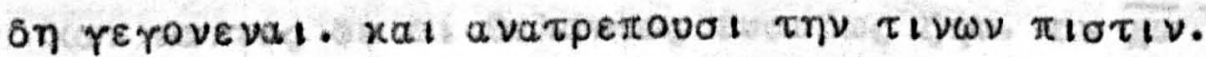

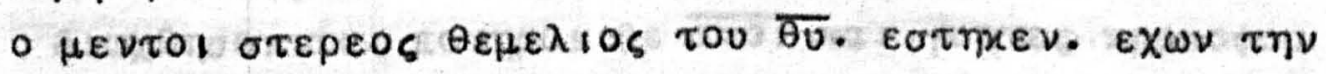

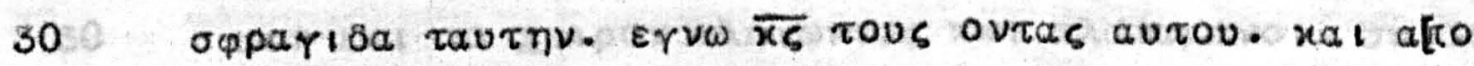

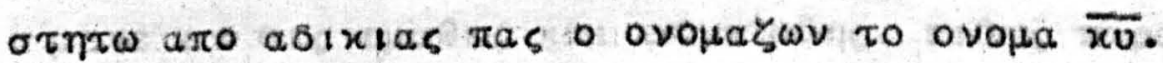

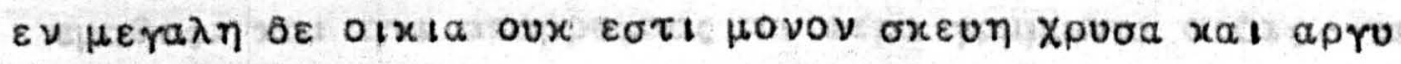

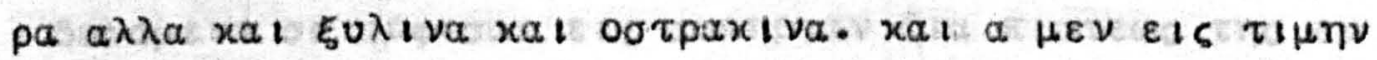

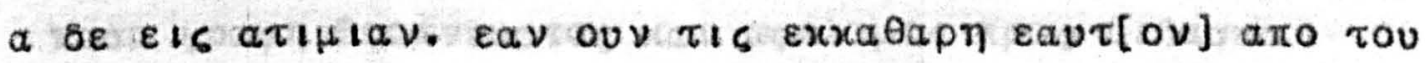

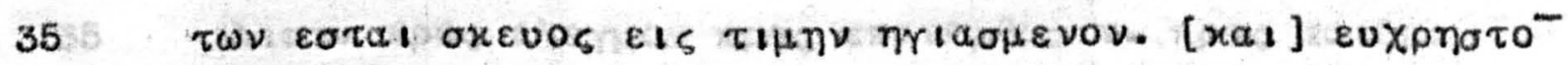

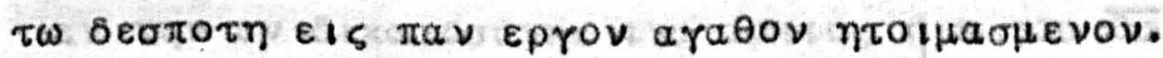

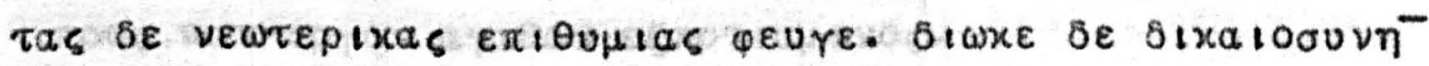

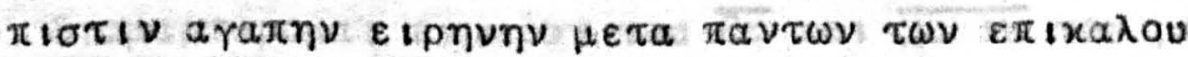

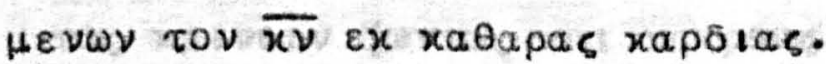

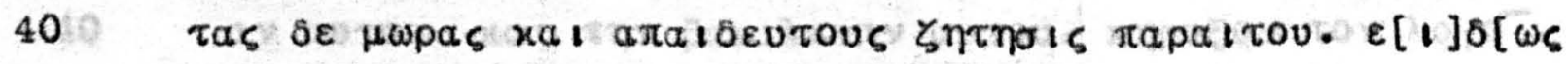

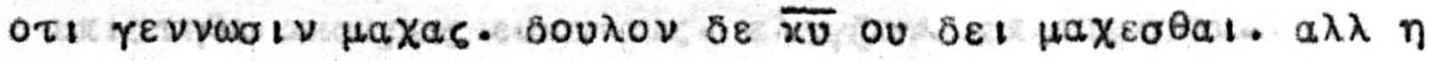




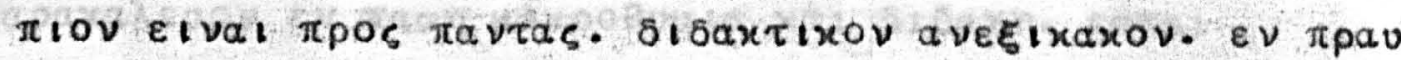

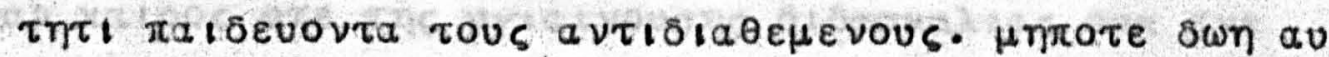

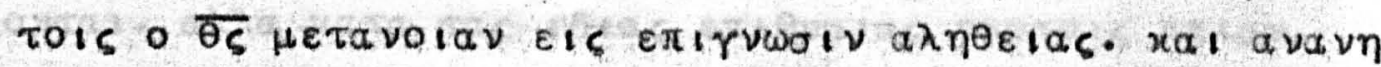

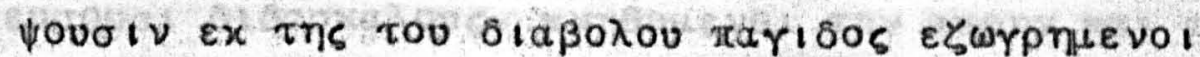
uT autou $\varepsilon l \varsigma$ to $\varepsilon x \varepsilon \downarrow$ vou $\theta \varepsilon \lambda$ nua.

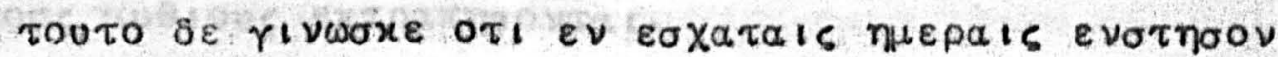

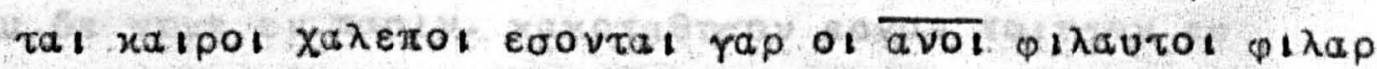

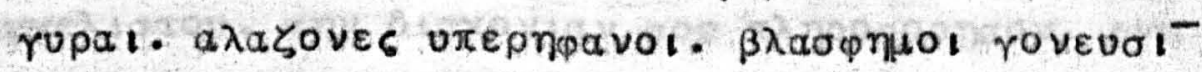

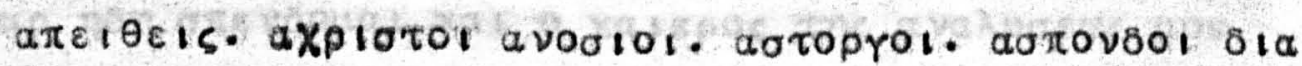

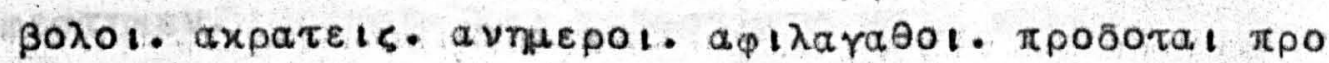

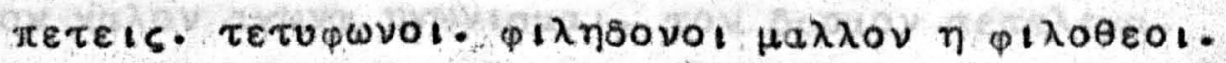

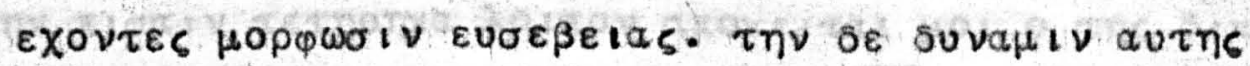

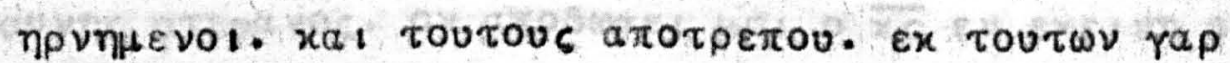

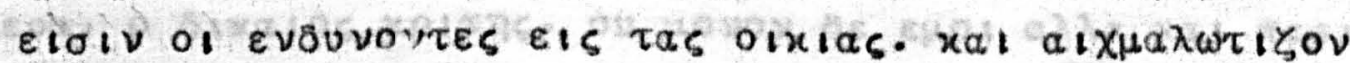

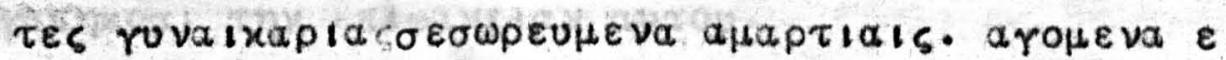

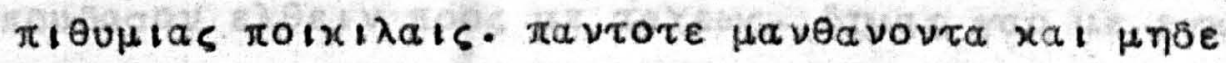

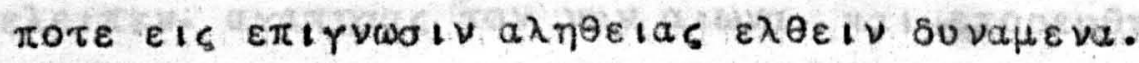

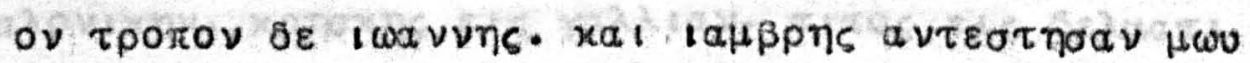

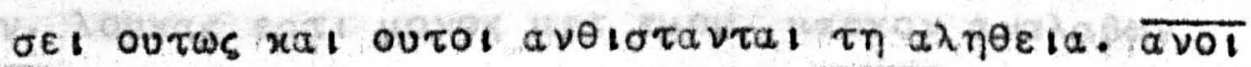

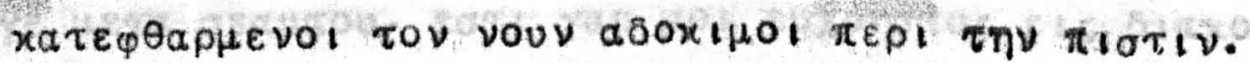

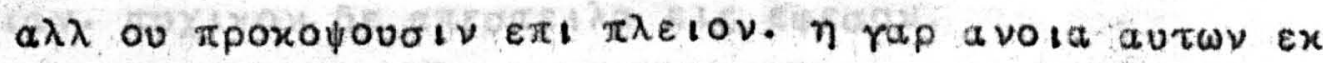

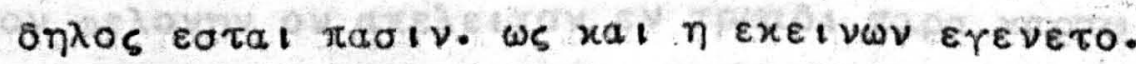

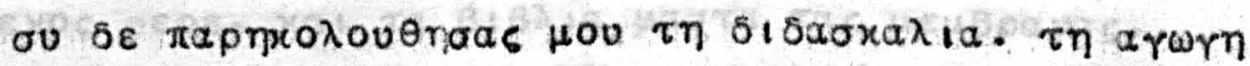

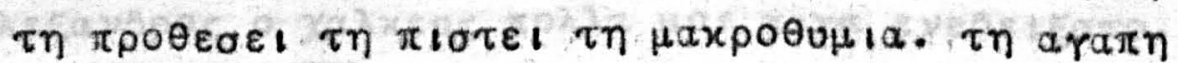

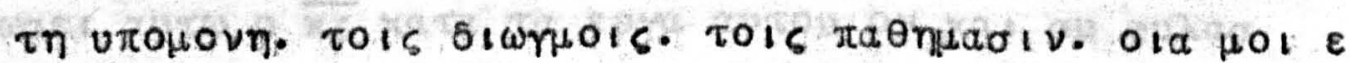

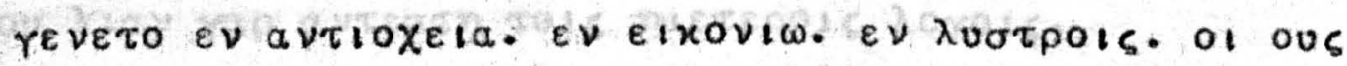

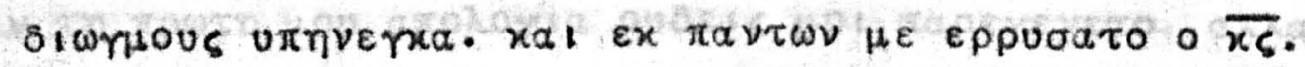

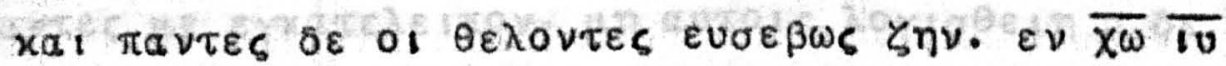

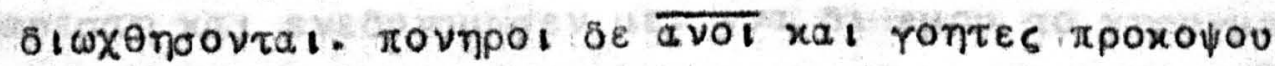

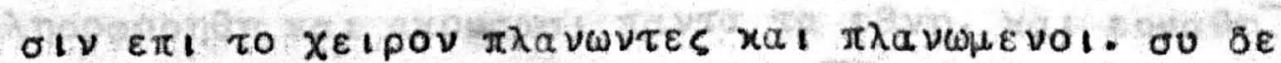

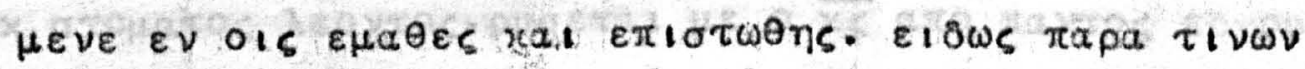

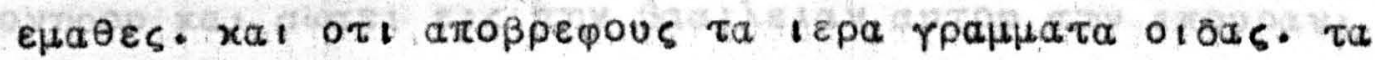

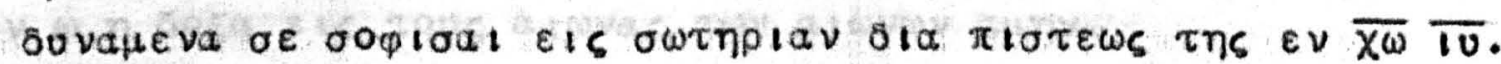

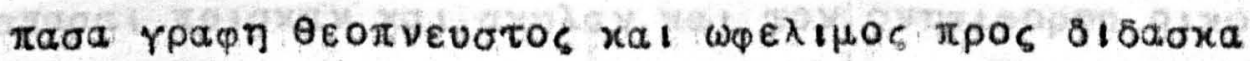

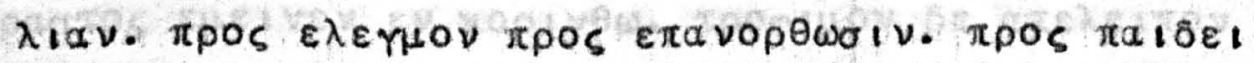

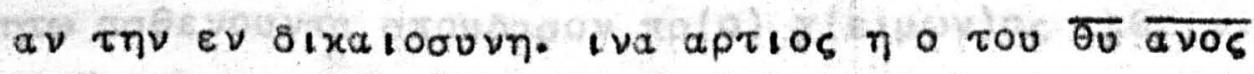

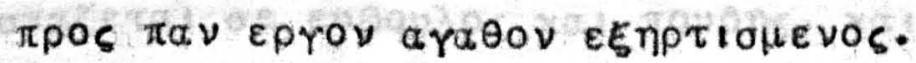

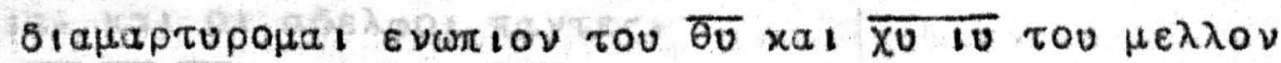

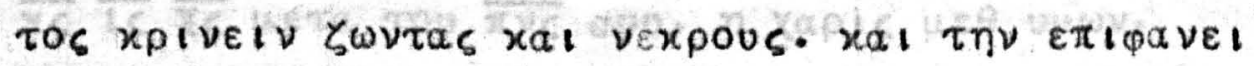

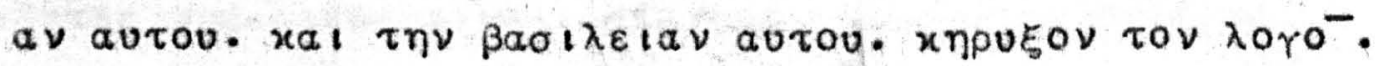

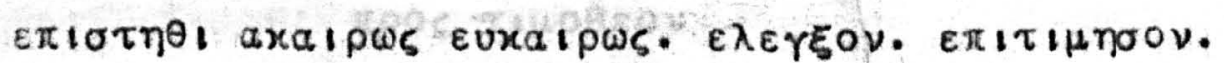




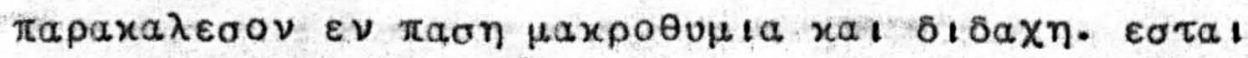
rap xalpos ote ths urialvouons $\delta 1 \delta a \sigma x a \lambda t a s$ oux $\varepsilon$ ve

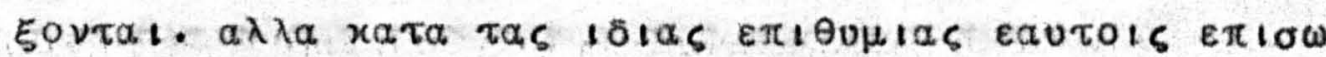

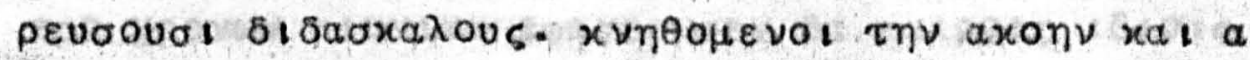

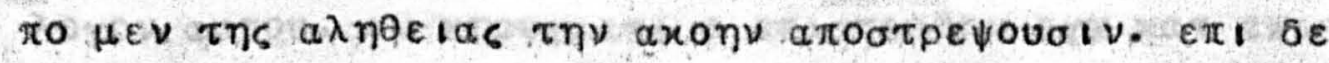

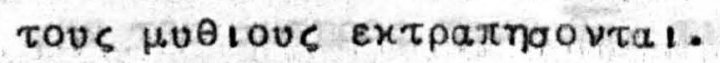

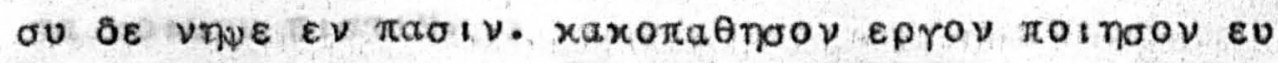

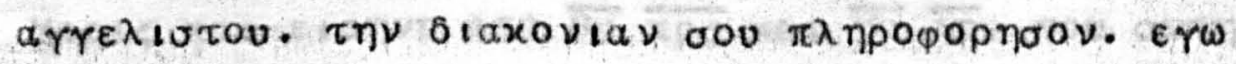

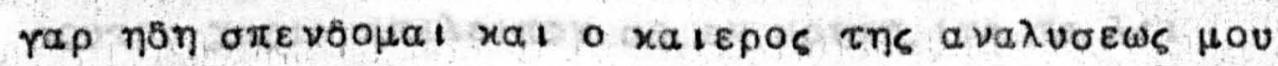
$\varepsilon \varphi \varepsilon \sigma \tau \eta x \varepsilon \nu$.

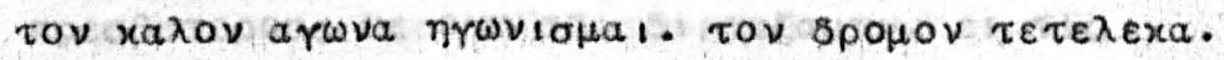

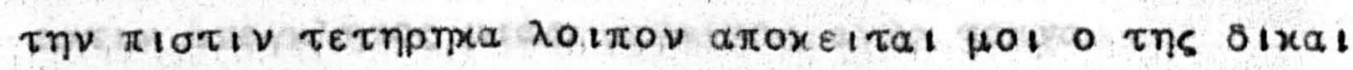

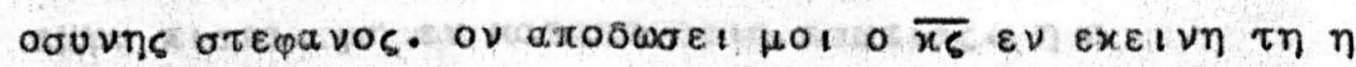

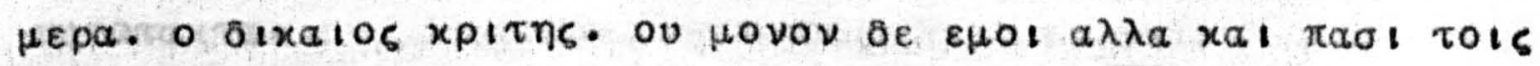

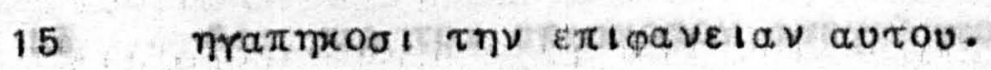

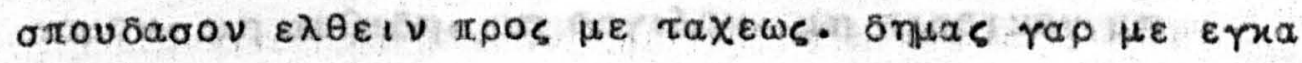

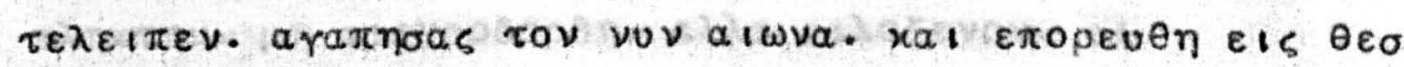

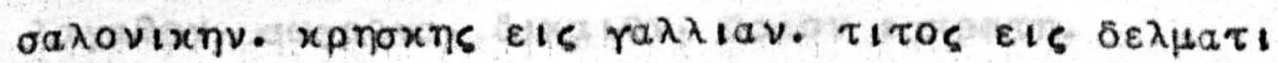

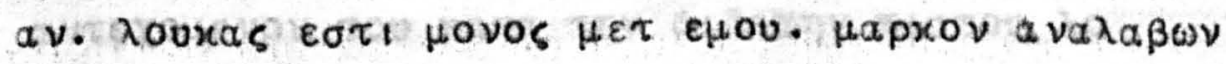

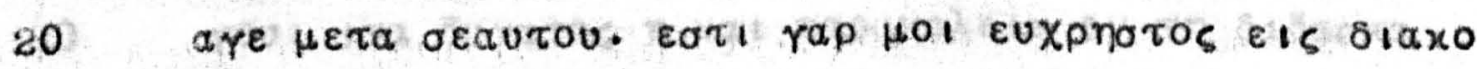

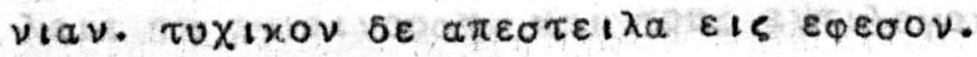

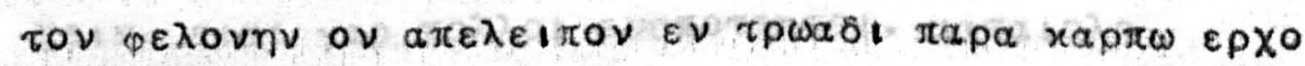

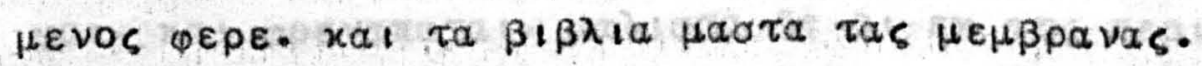

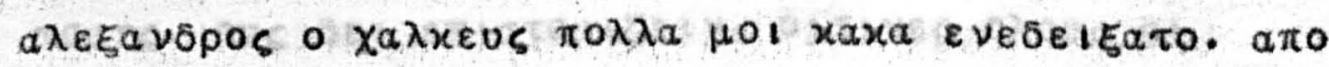

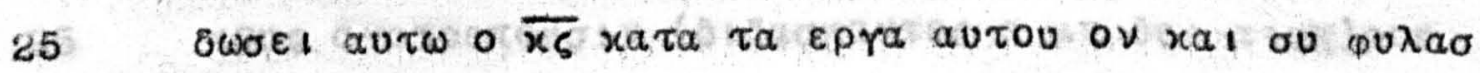

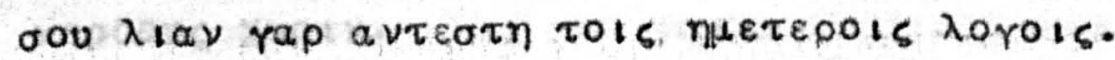

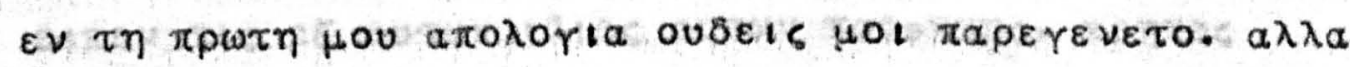

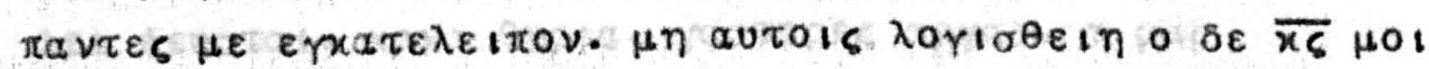

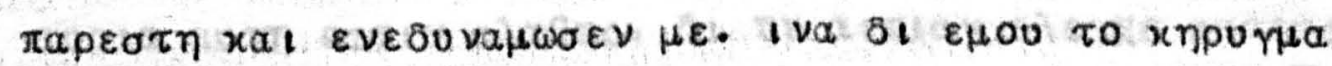

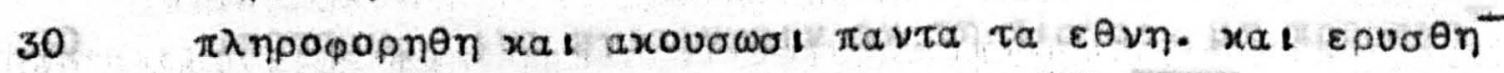

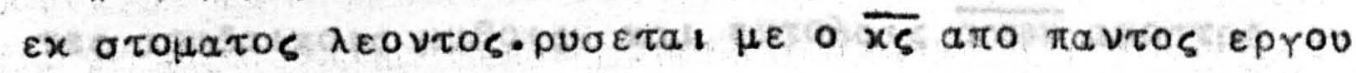

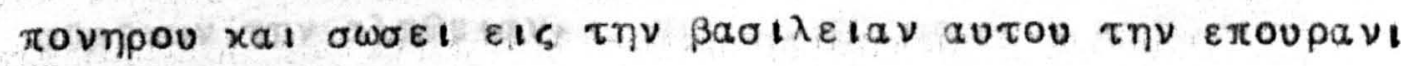

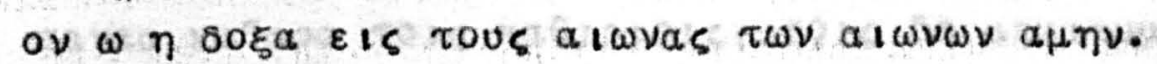

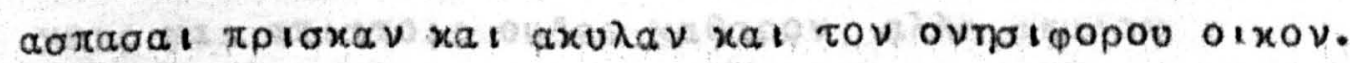

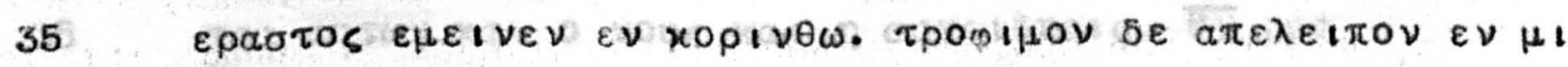

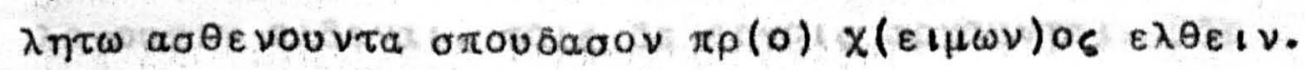

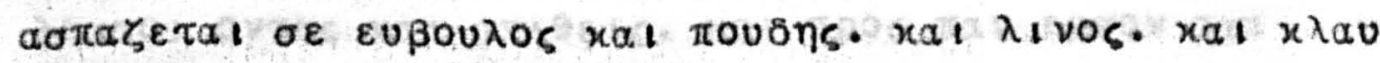

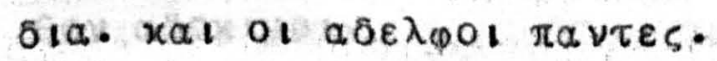

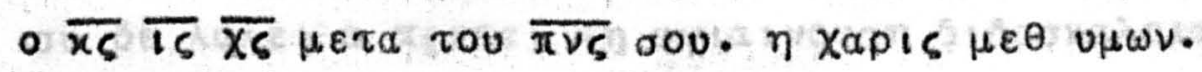




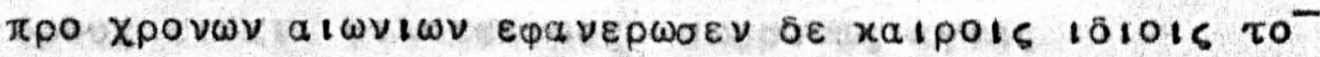

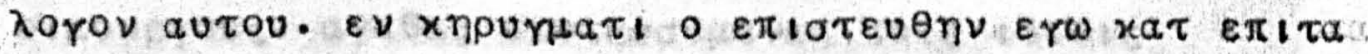

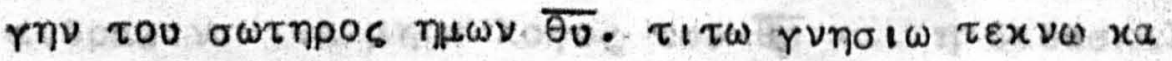

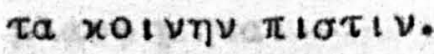

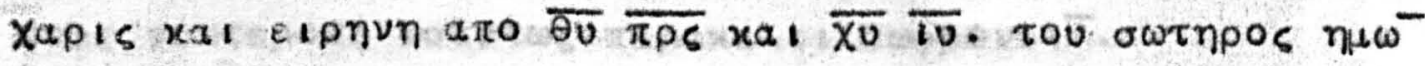

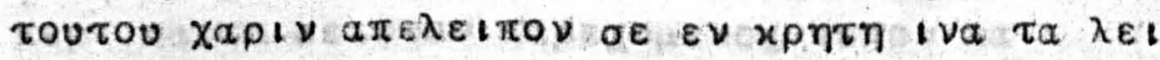

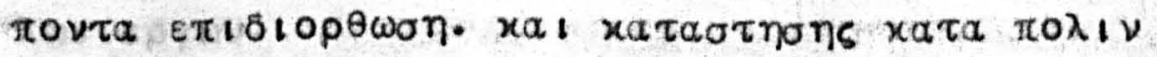

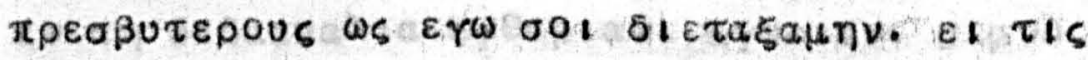

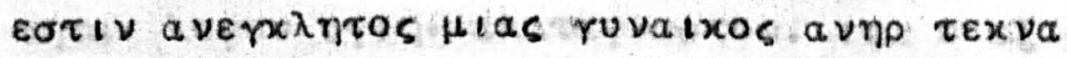

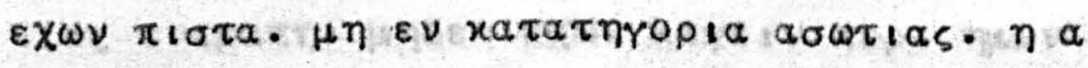

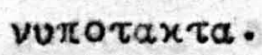

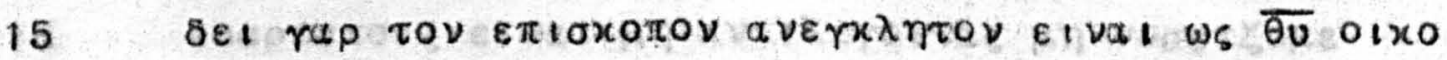

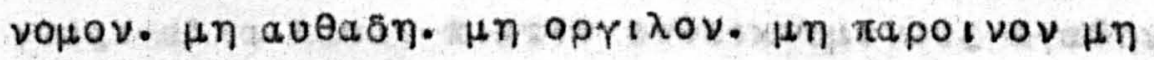

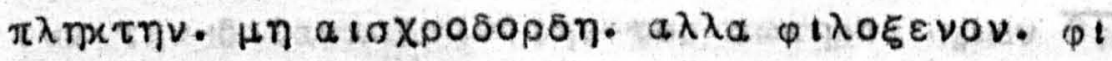

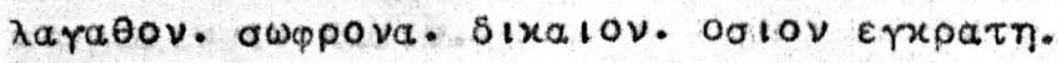

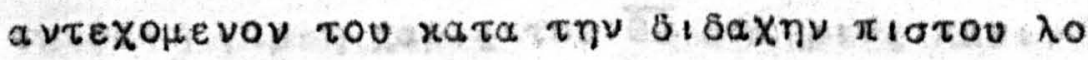

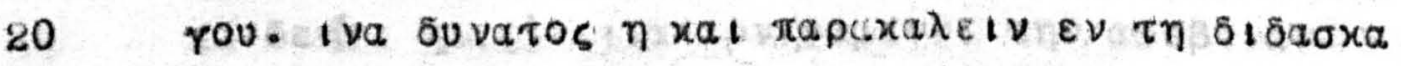

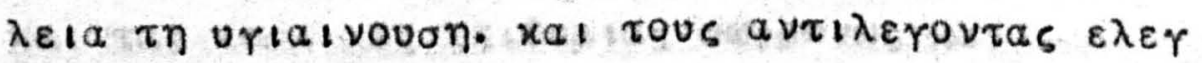

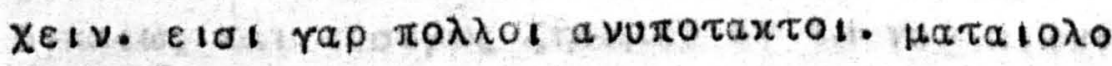

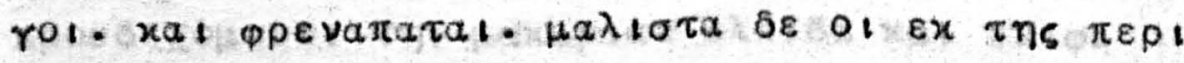

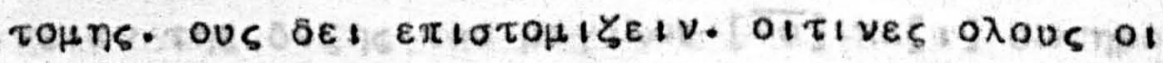

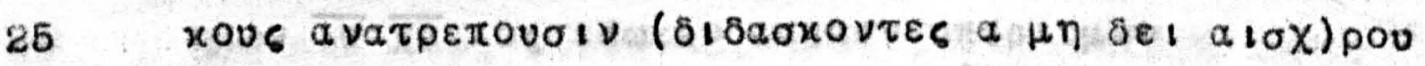
xepōous Xaptv.

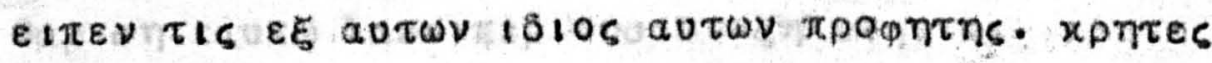

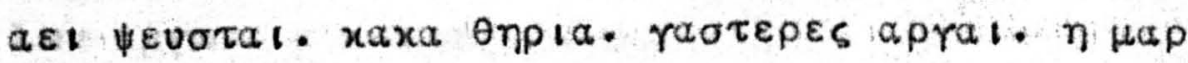

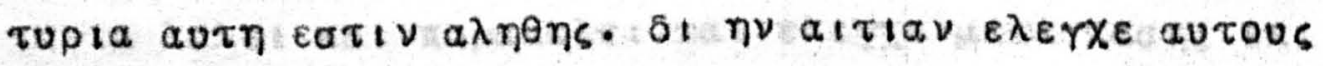

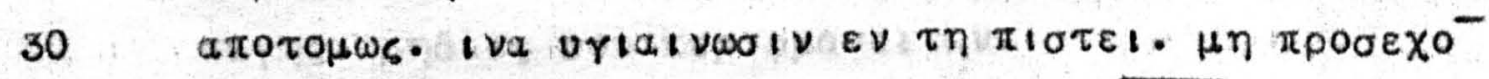

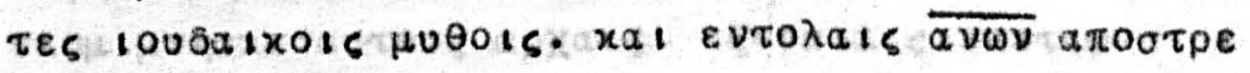

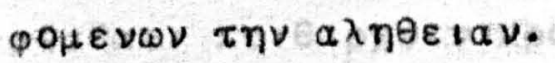

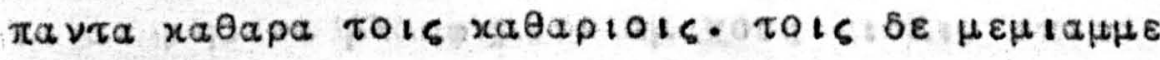

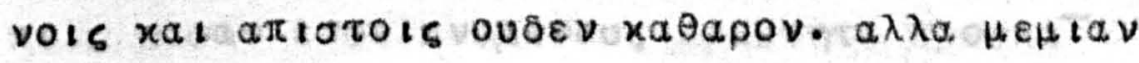

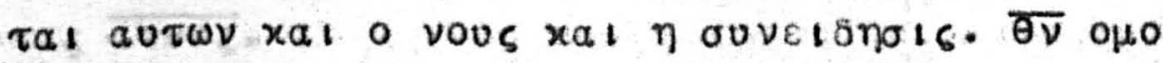

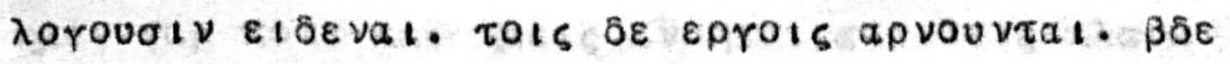

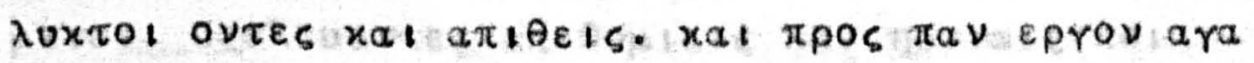

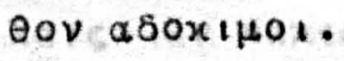

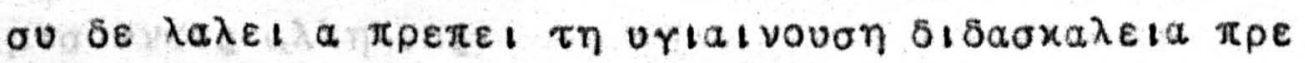

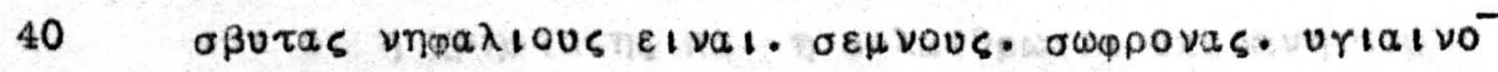

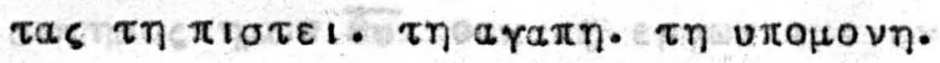




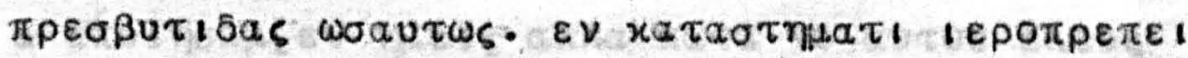

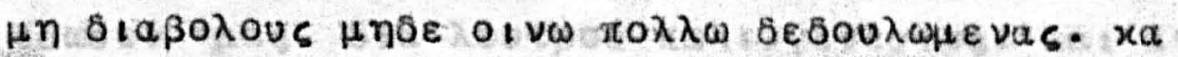

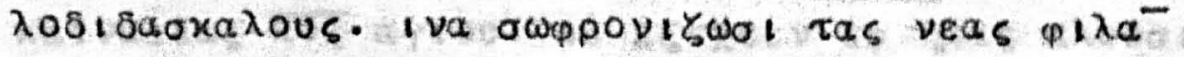
spovs eival. pitotexvous. owppovas arvas

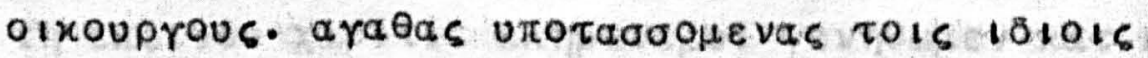

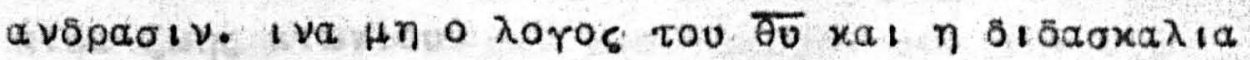
$\beta \lambda \alpha \sigma \varphi \eta \mu \eta \tau a l$.

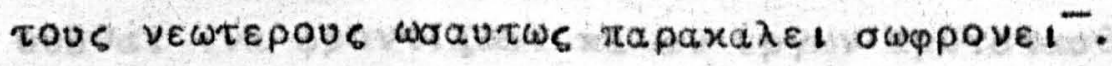

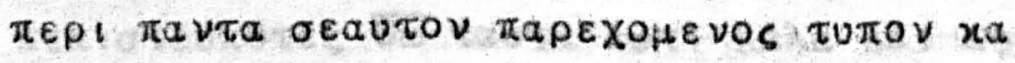

$10 \lambda \quad \lambda \omega \nu \varepsilon \rho \gamma \omega \nu$.

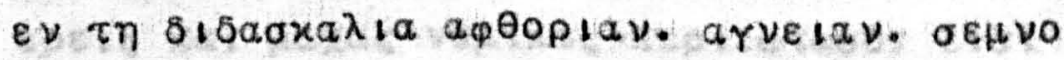

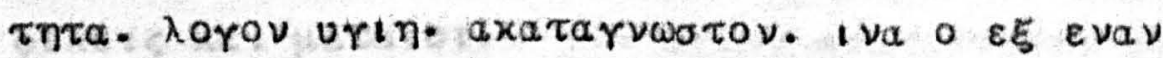

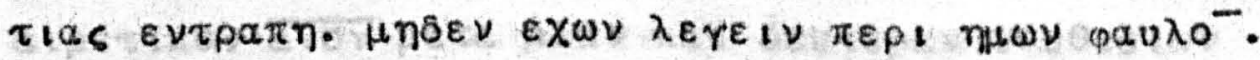

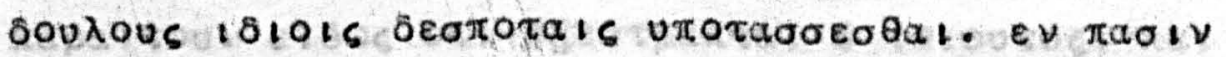

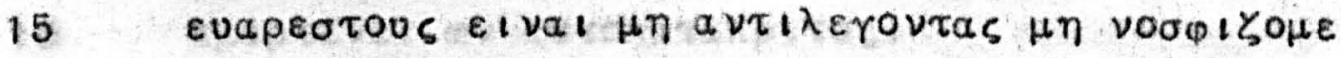

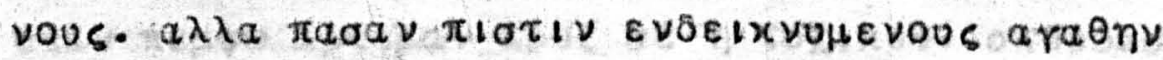

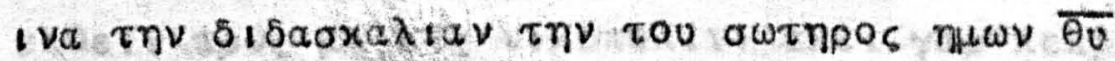

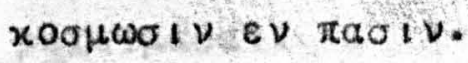

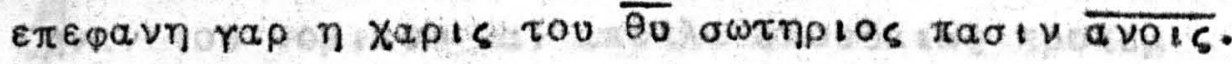

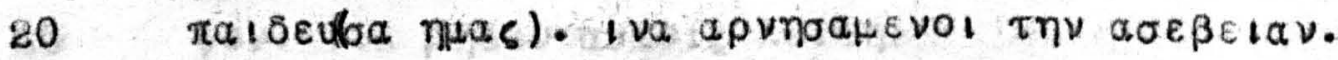

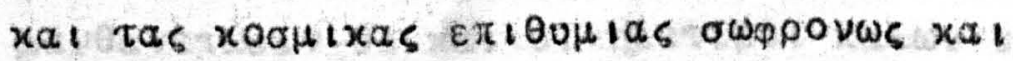

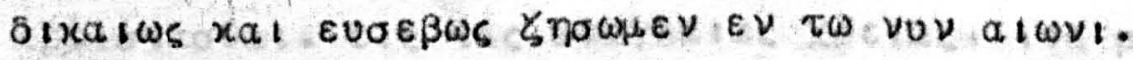

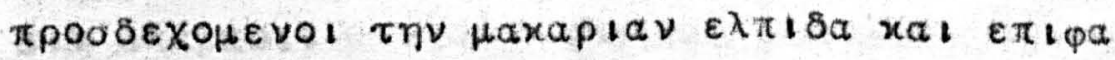

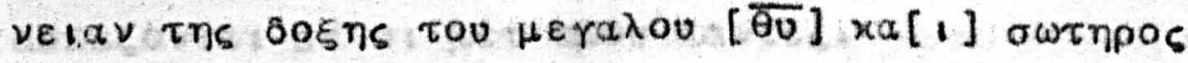

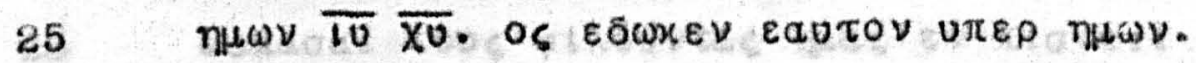

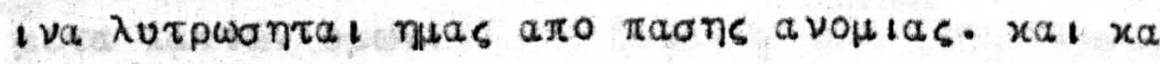

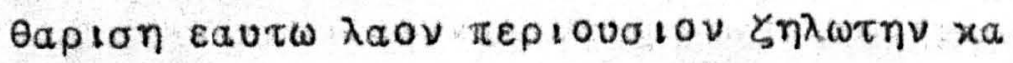

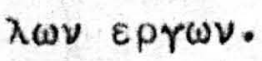

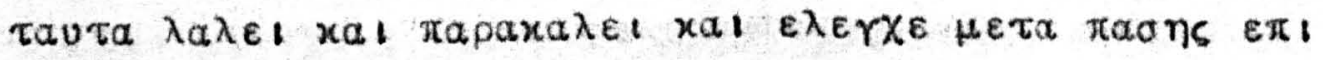

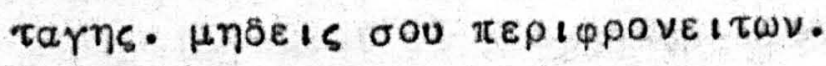

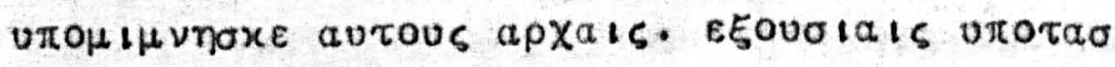

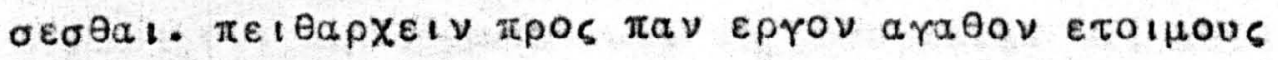

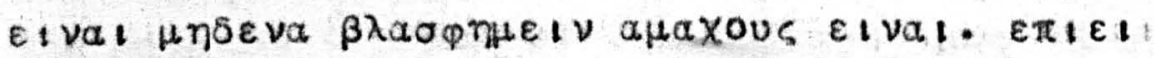

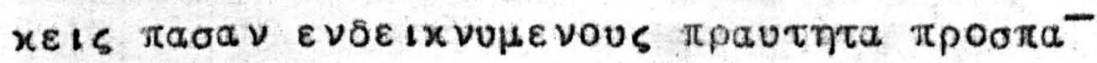
Tas avovs.

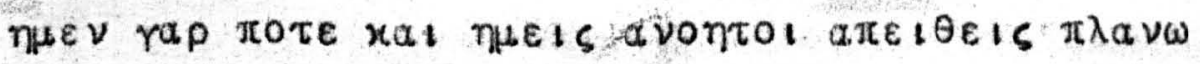

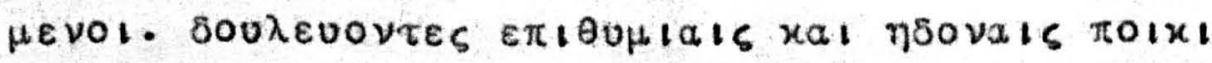

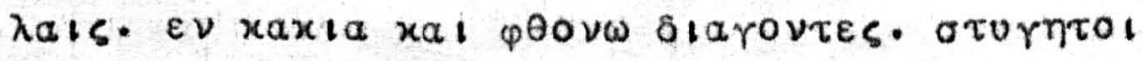

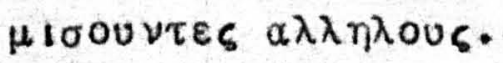

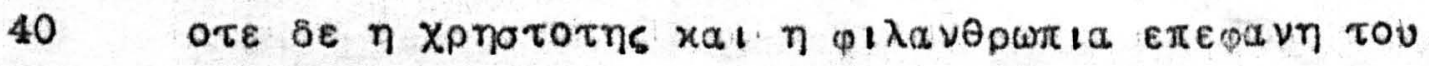

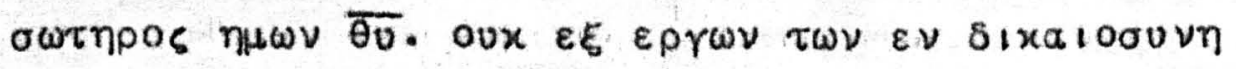




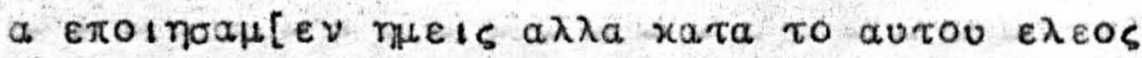

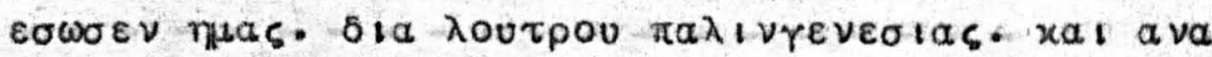

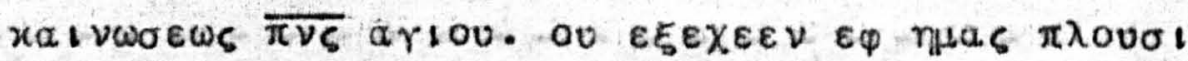

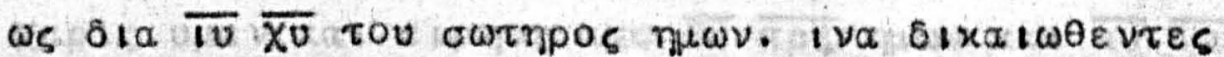

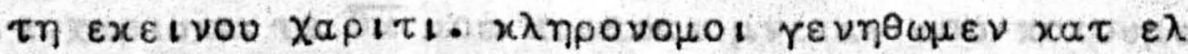

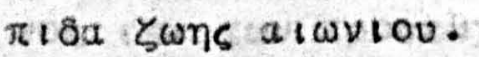

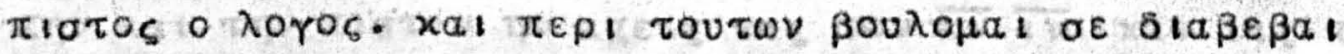

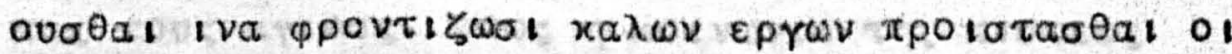

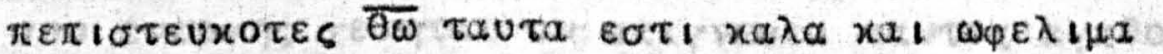

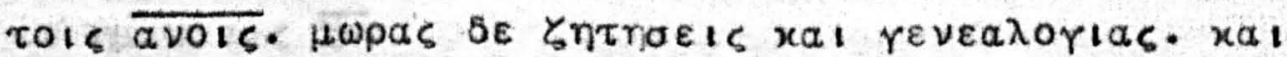

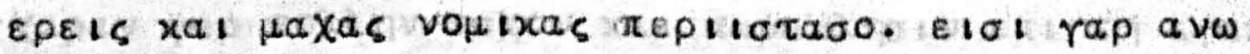

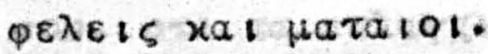

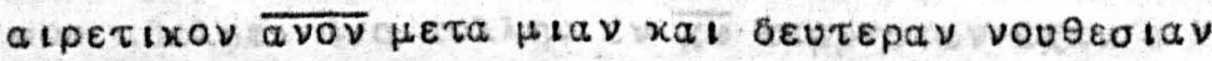

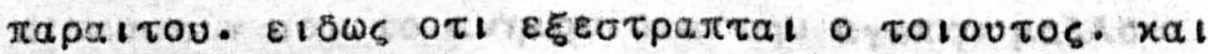

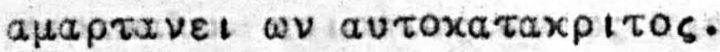

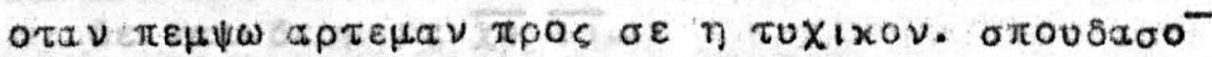

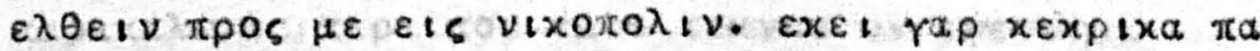

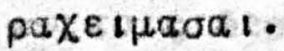

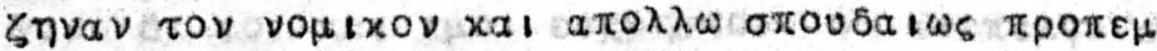

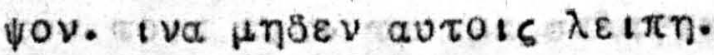

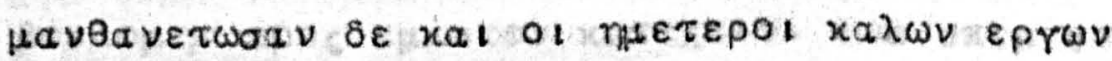

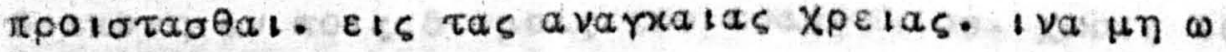
atv axaproi.

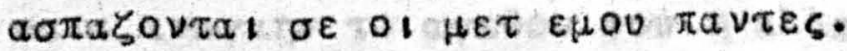

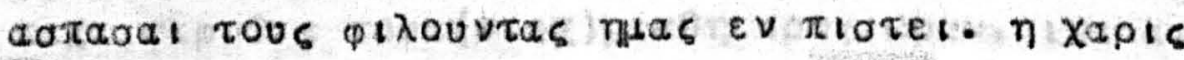

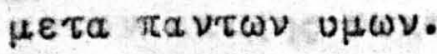

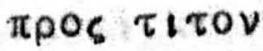

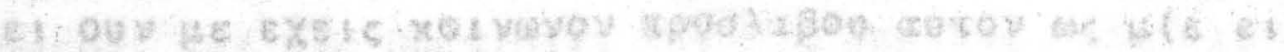

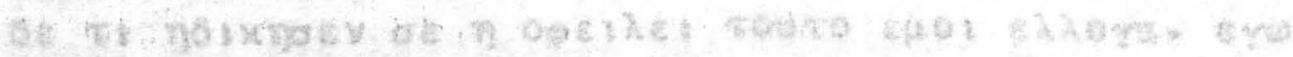

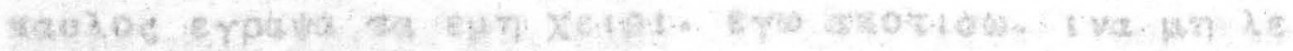

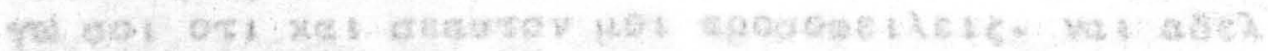

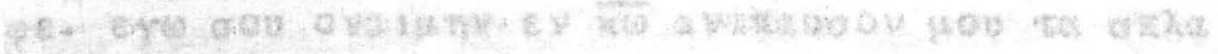

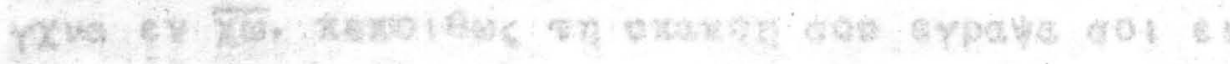

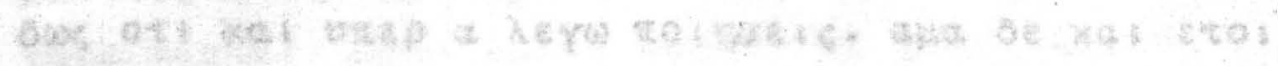

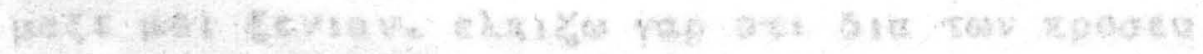

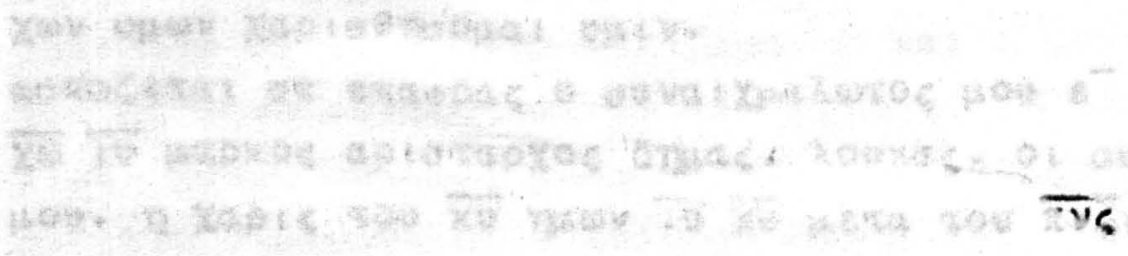




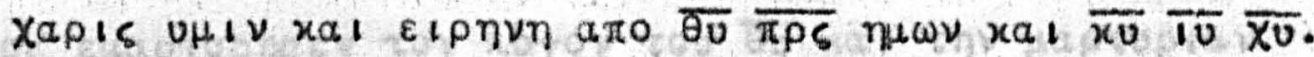

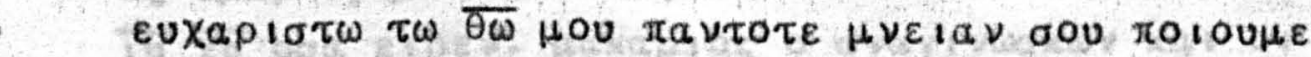

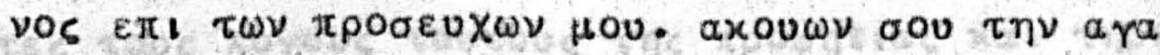

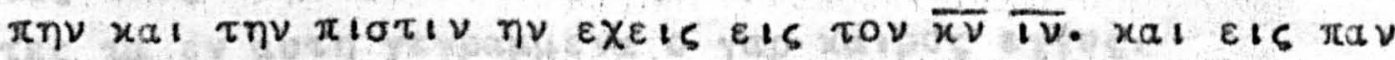

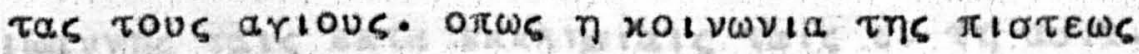

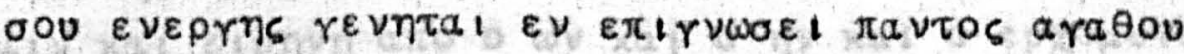
$10 \varepsilon \nu \eta \omega \mid \nu \varepsilon i s \overline{\chi \nu}$.

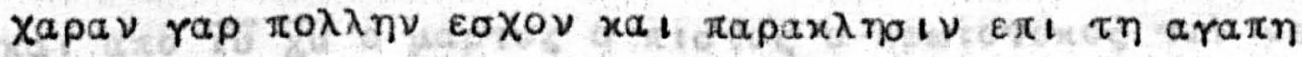

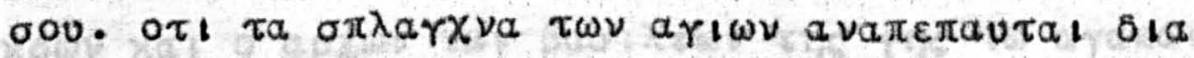
oov $a \delta \varepsilon \lambda_{\varphi} \varepsilon$. $\delta 10 \pi 0 \lambda \lambda \eta \nu \varepsilon \nu \overline{\chi \omega} \pi \alpha p p \eta \sigma \downarrow \alpha \nu \varepsilon \omega \nu \varepsilon$

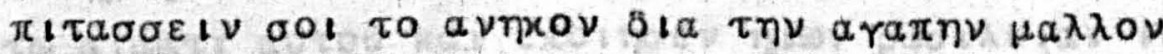

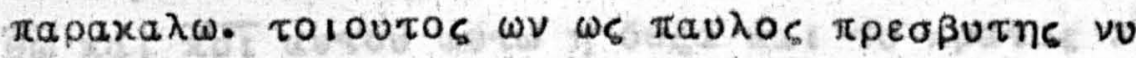
vi $\delta \varepsilon x a l \delta \varepsilon \sigma \mu l o c \overline{\chi_{0}} \overline{T 0}$.

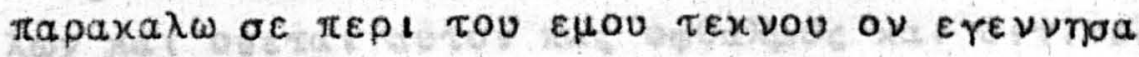

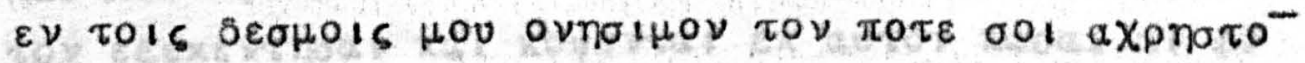

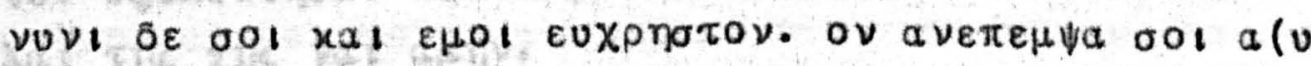

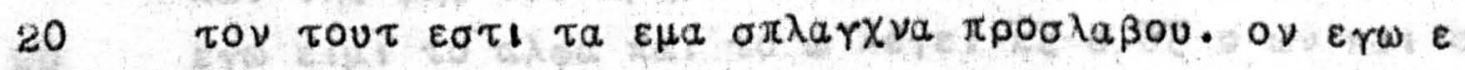

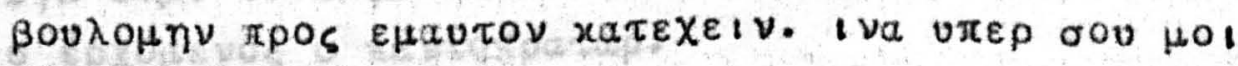

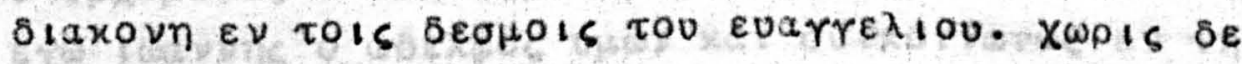

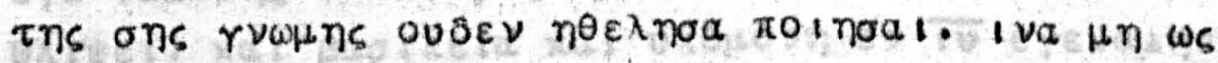

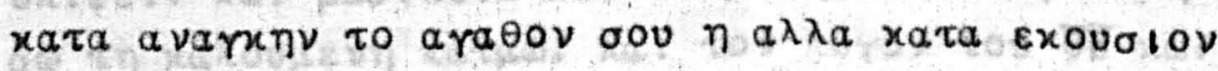

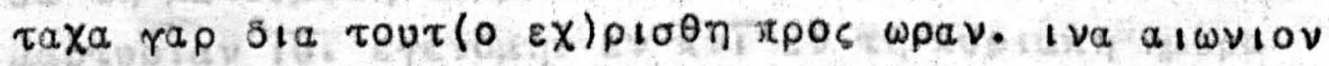

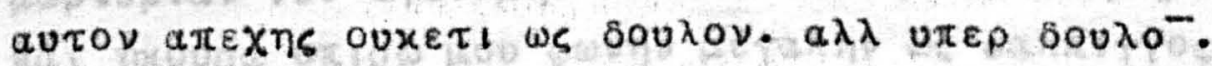

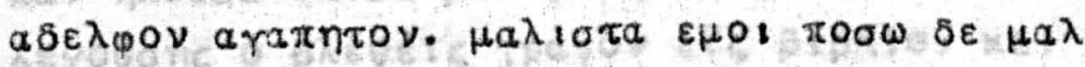
$\lambda \circ \nu$ ol $x a l \varepsilon \nu$ oapxl $x a l \varepsilon v \overline{x \omega}$.

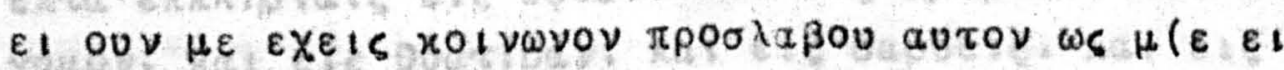

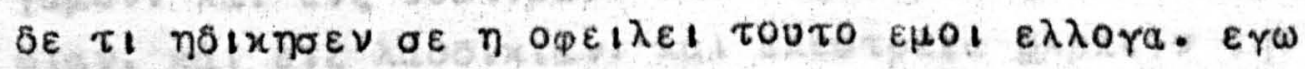

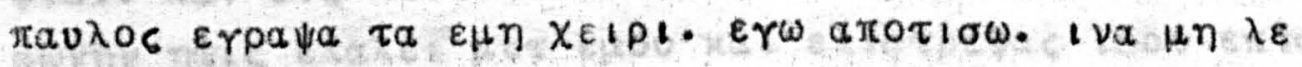

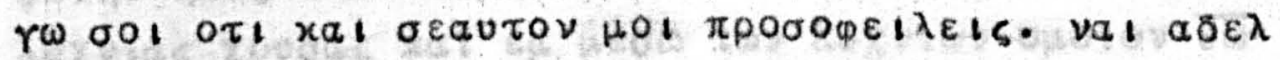

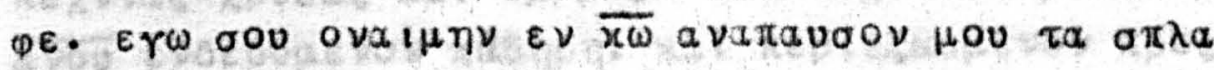

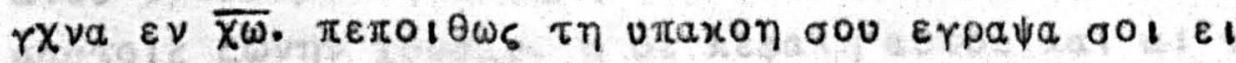

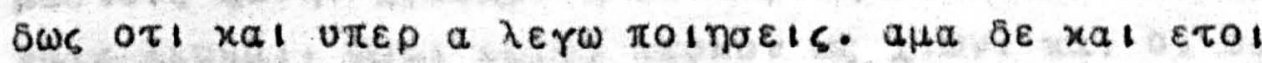

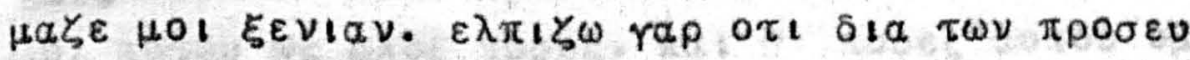

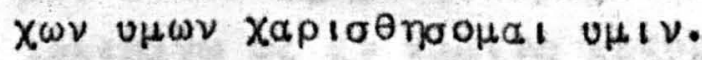

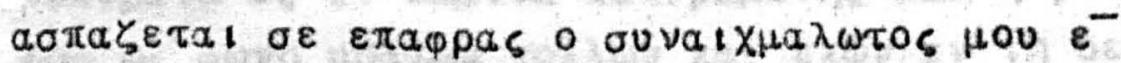

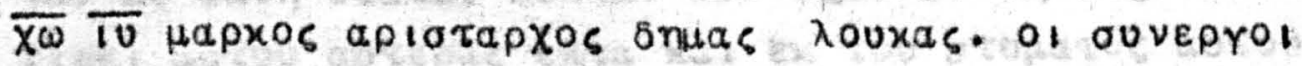

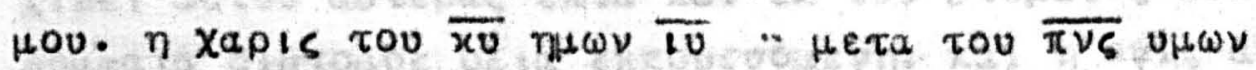

$\pi \rho \circ \varphi$ i nuova 


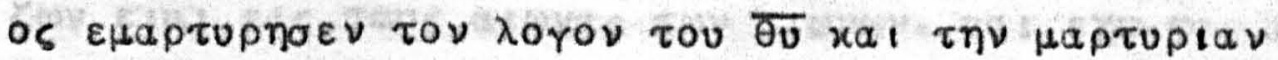

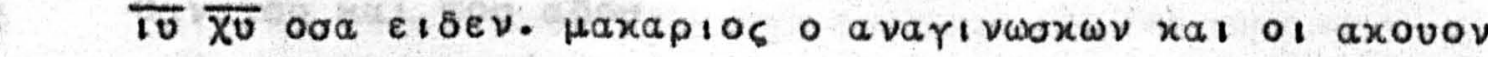

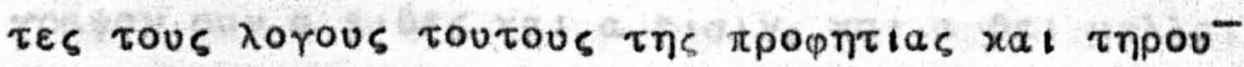

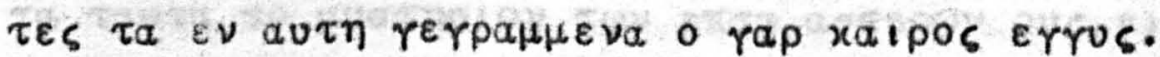

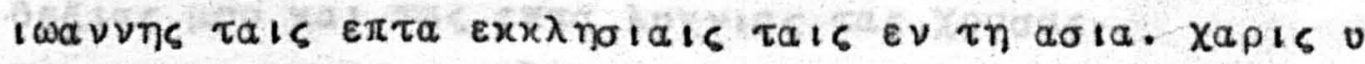

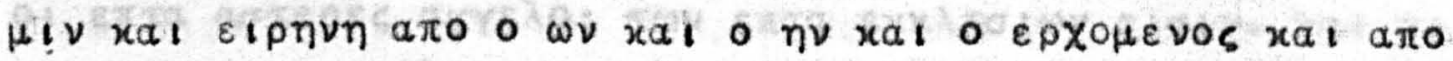

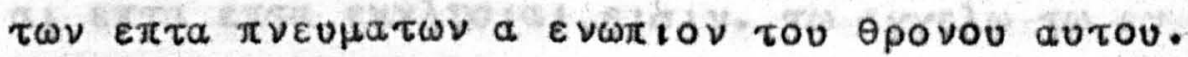

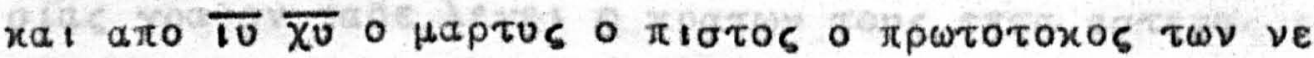

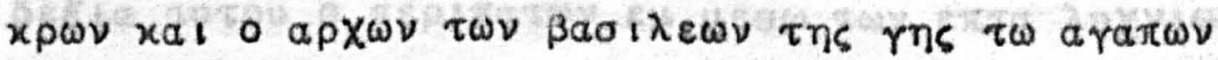

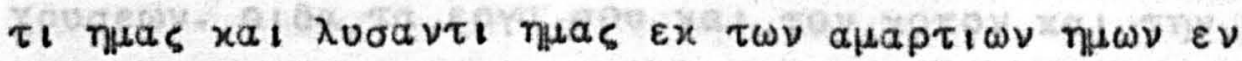

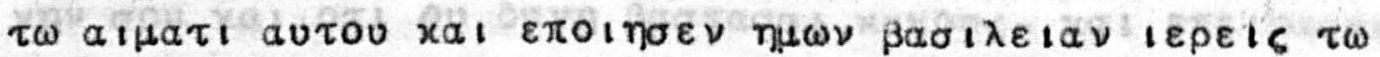

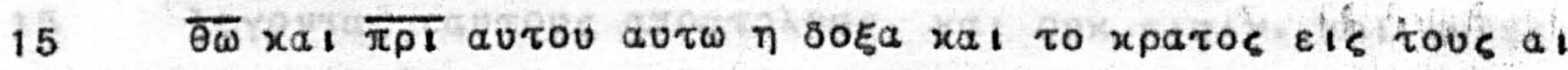

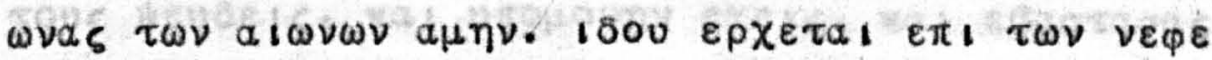

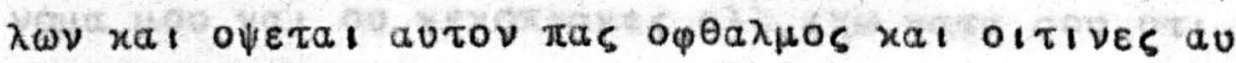

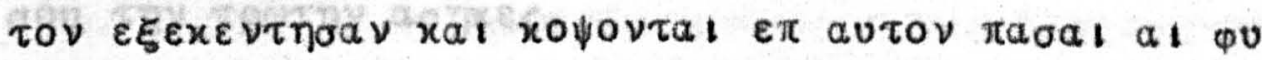

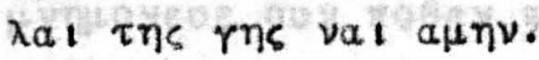

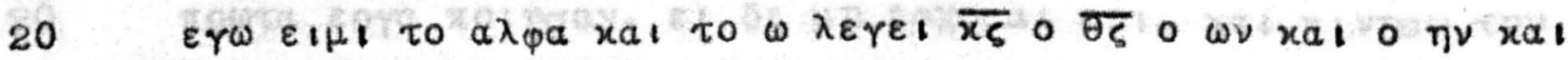
- $\varepsilon \rho \chi 0 \mu \varepsilon \nu \circ \sigma_{6}$ o $\pi \alpha \nu \tau o x \rho a \tau \omega \rho$.

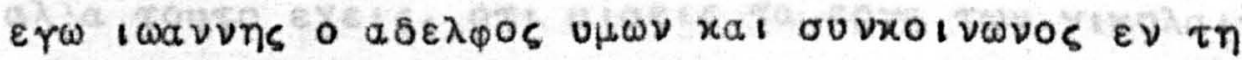

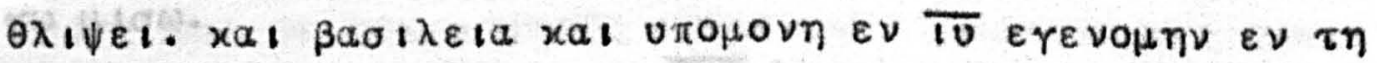

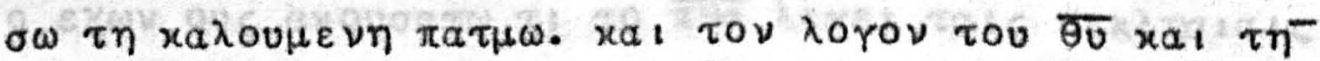

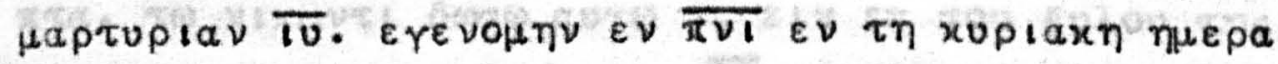

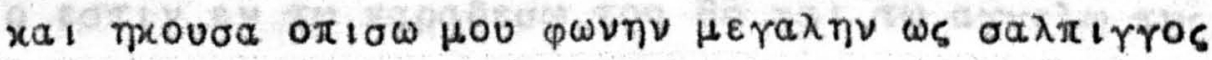

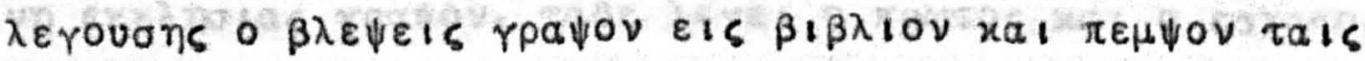

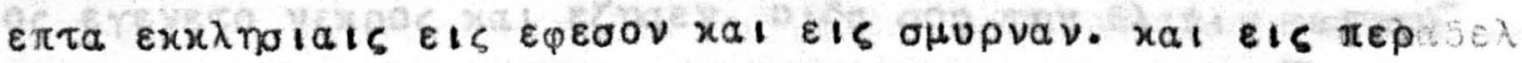

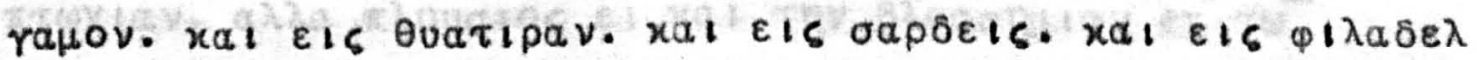

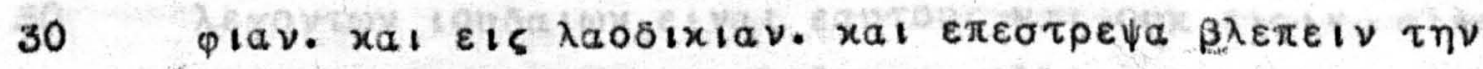

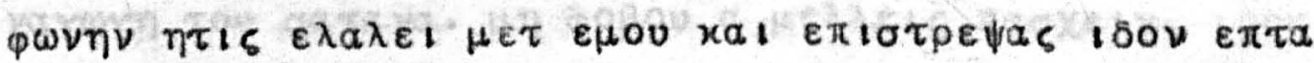

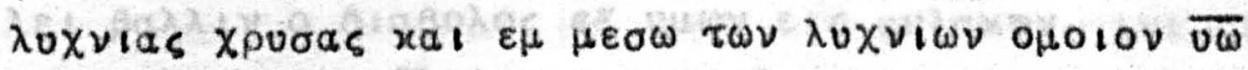

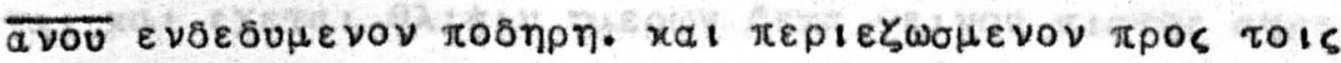

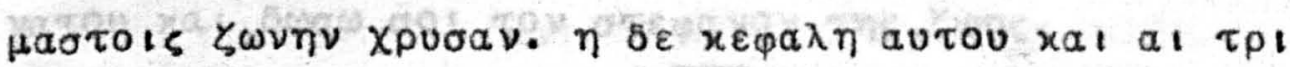

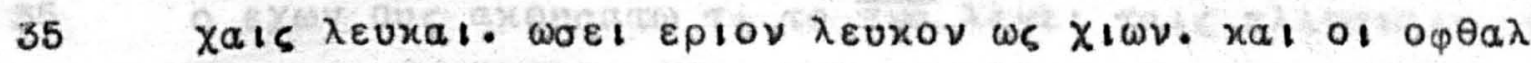

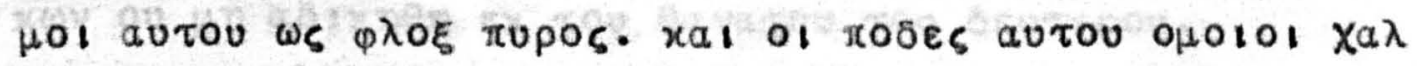

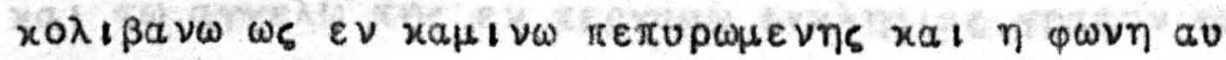

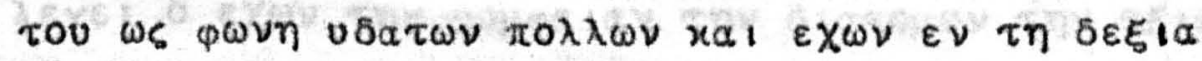

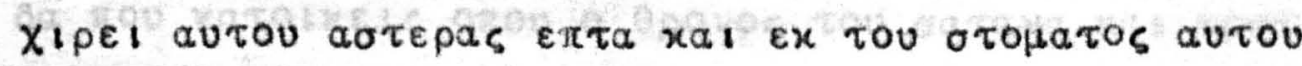

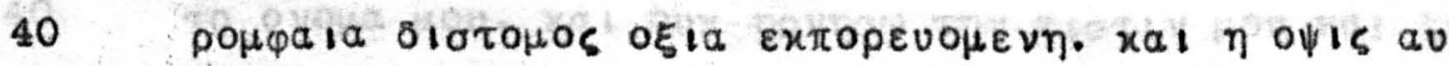

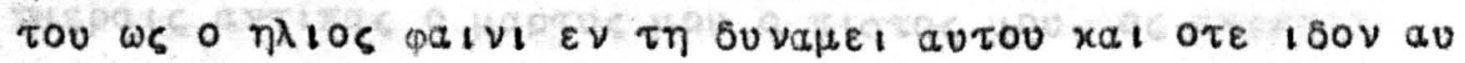




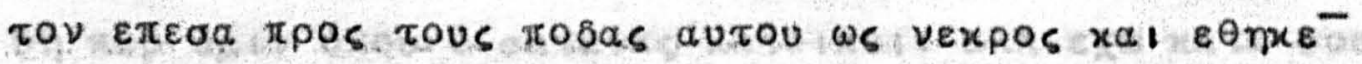

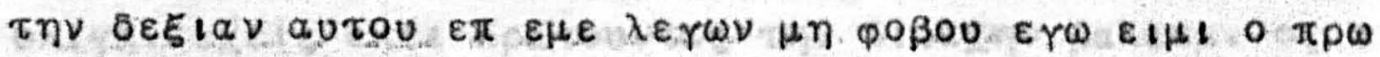

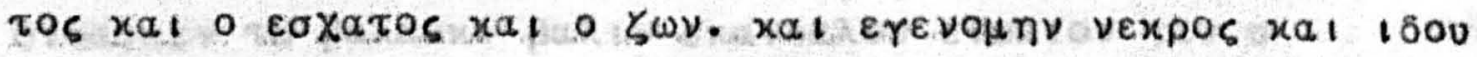

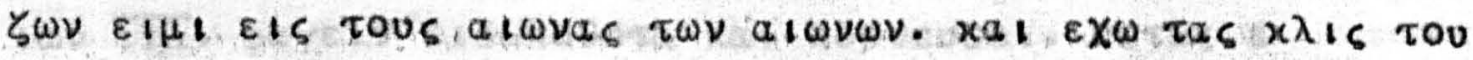

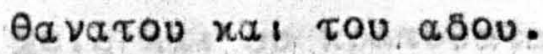

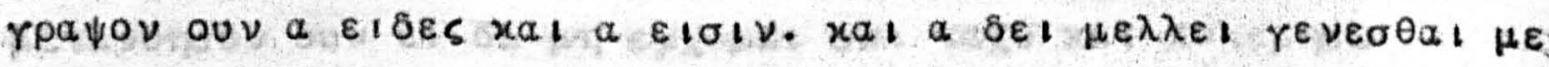

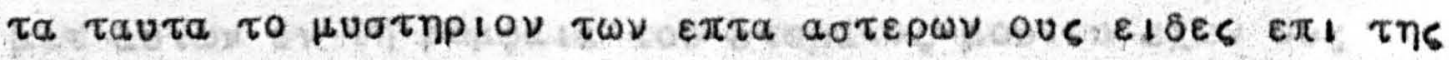

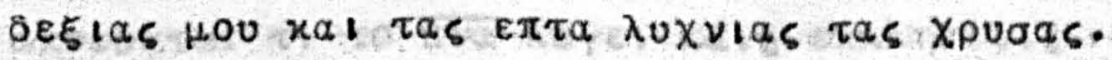

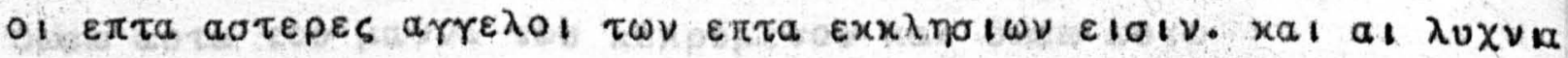

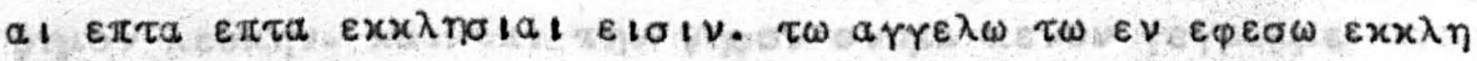

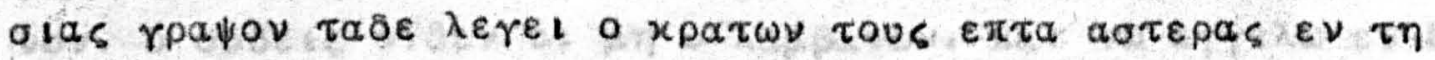

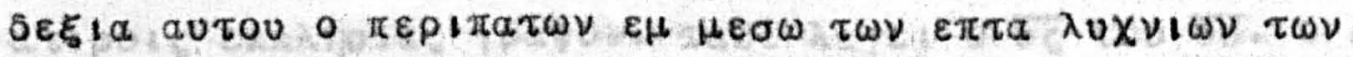

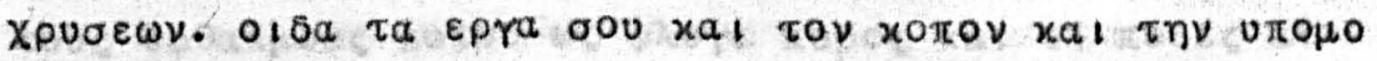

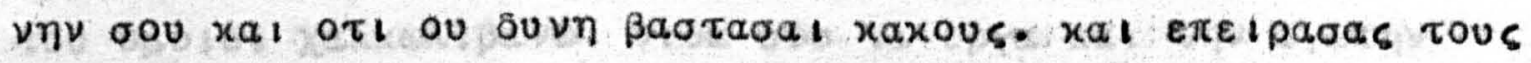

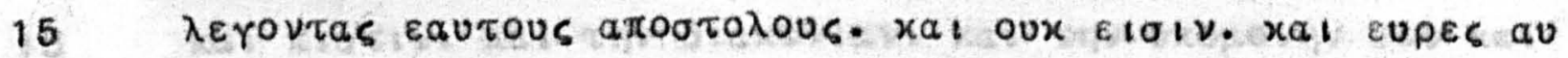

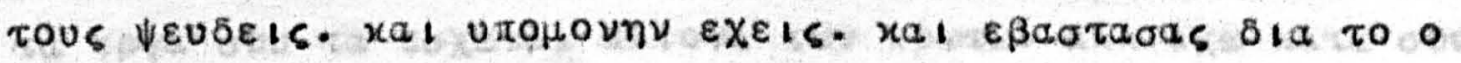

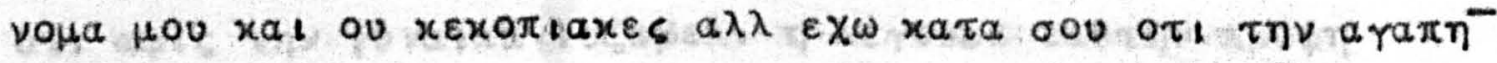

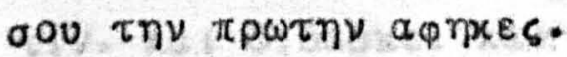

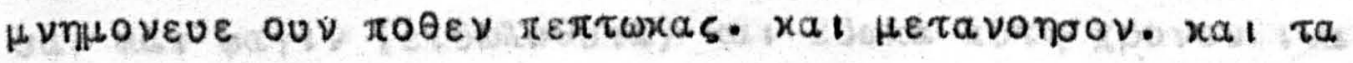

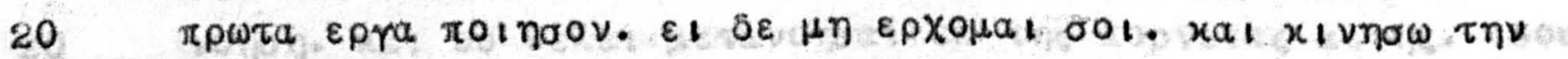

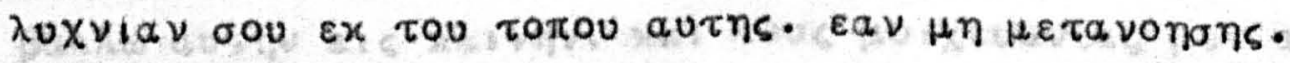

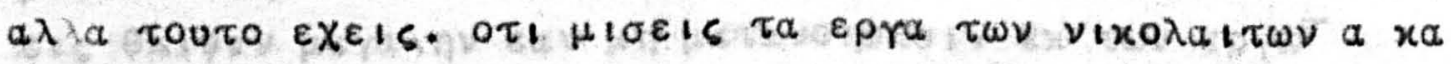
rw Hlow.

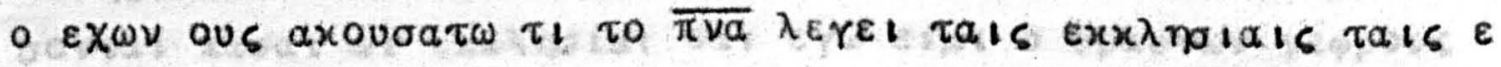

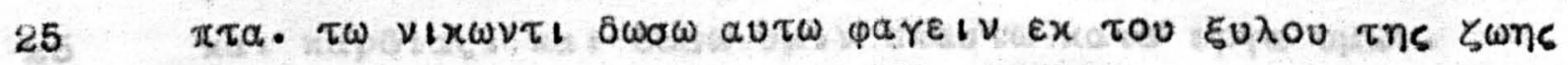

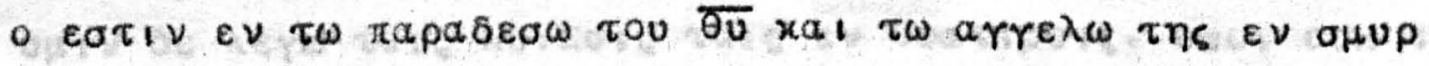

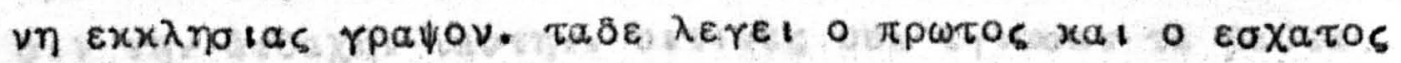

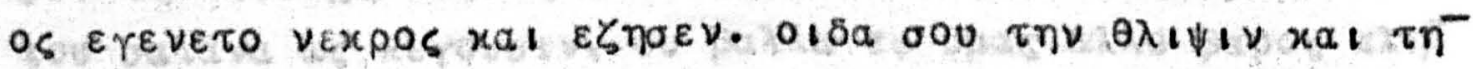

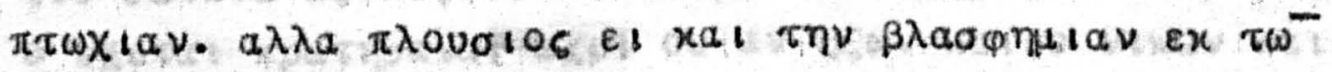

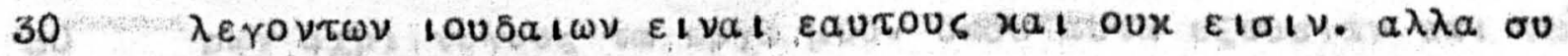

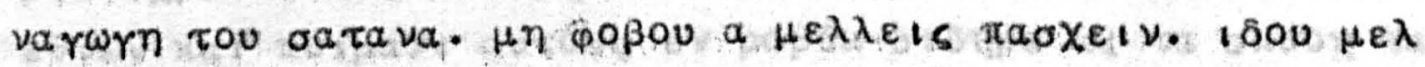

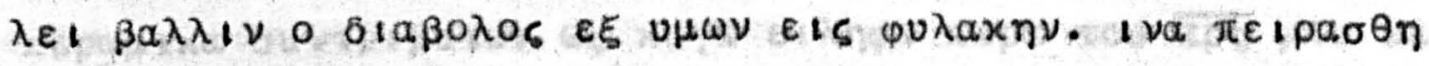

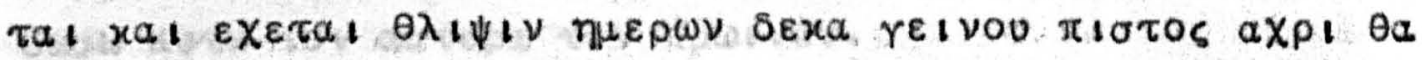

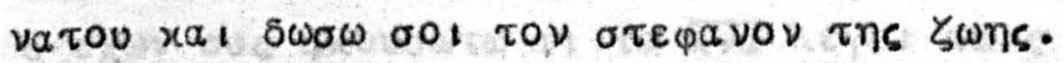

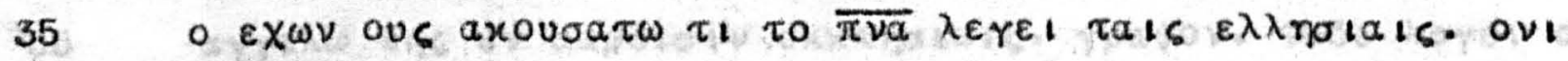

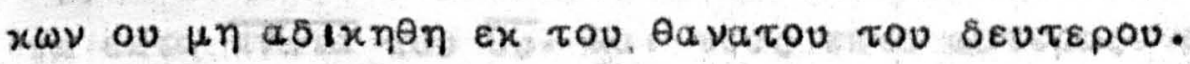

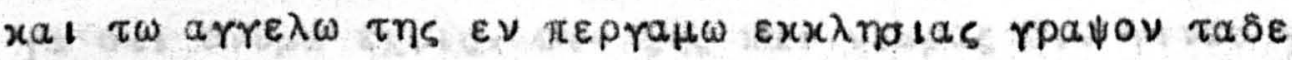

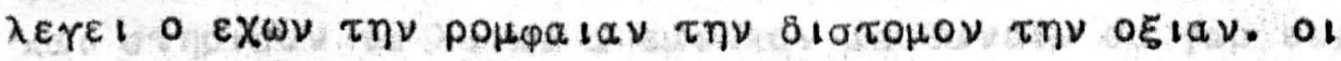

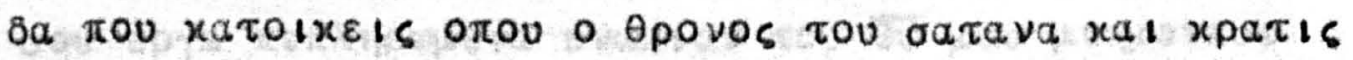

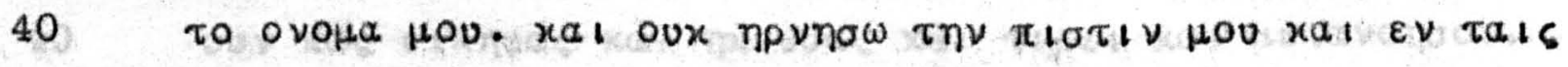

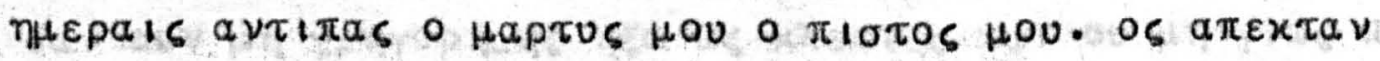




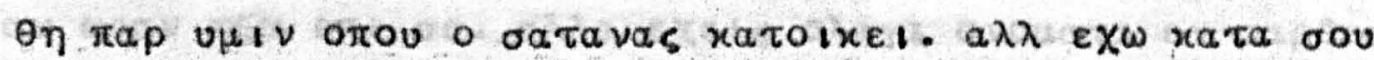

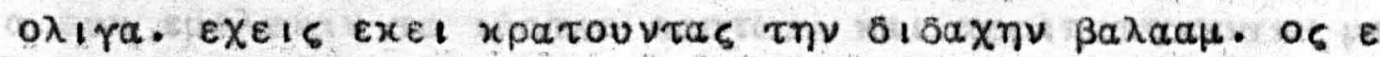

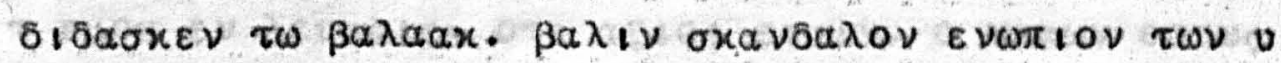

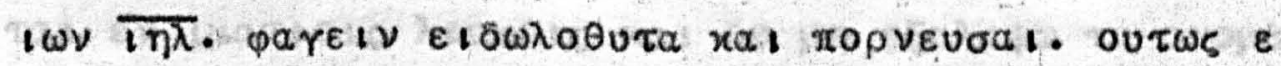

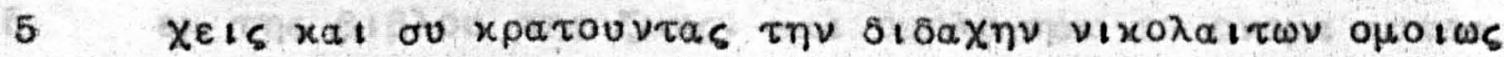

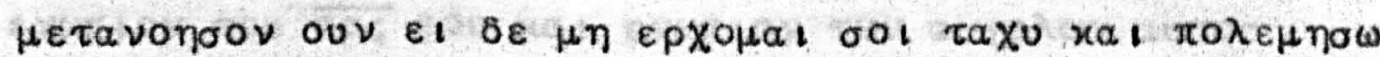

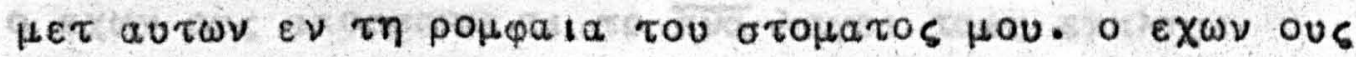

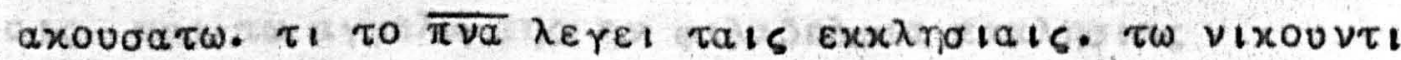

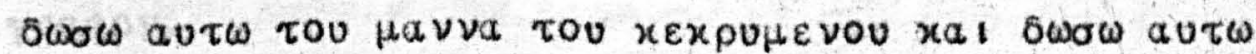

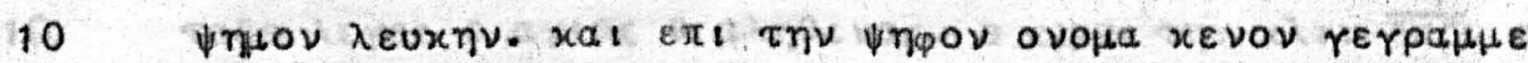

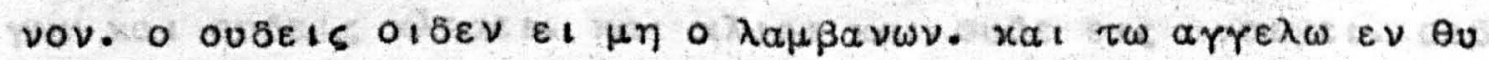

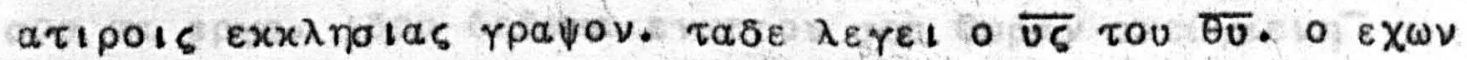

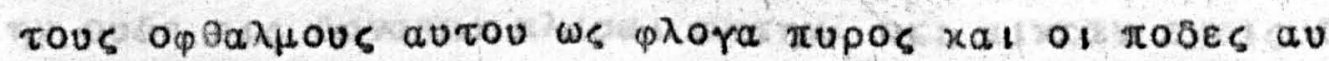

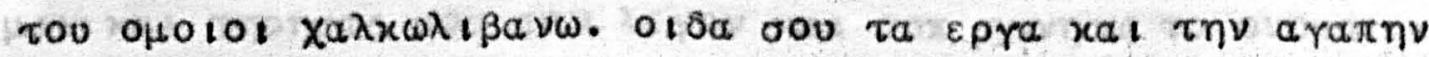

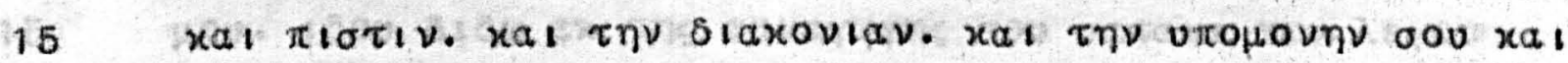

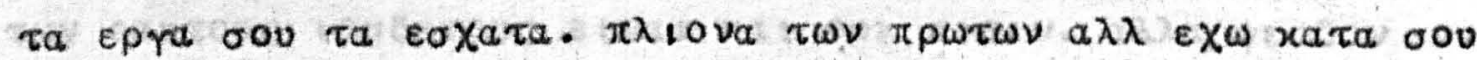

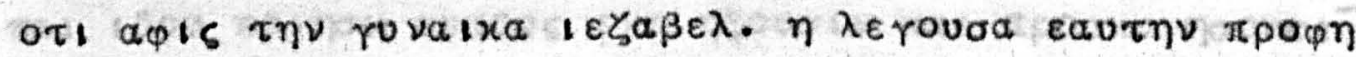

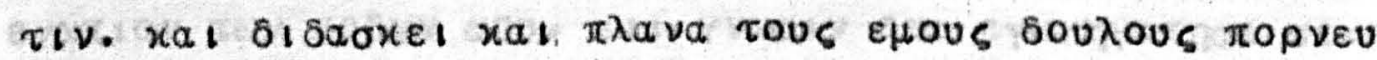

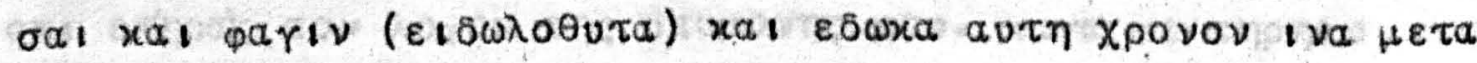

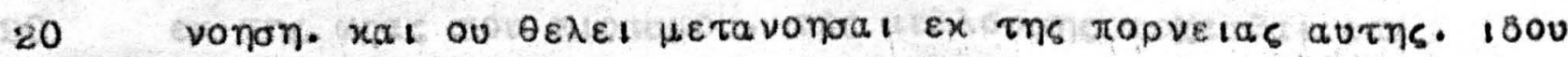

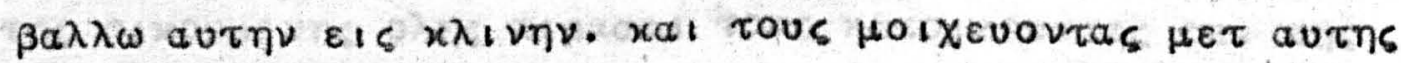

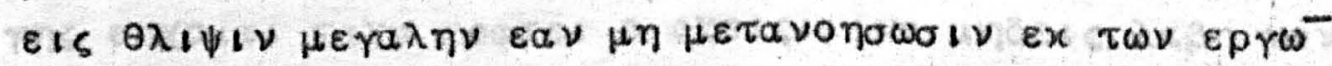

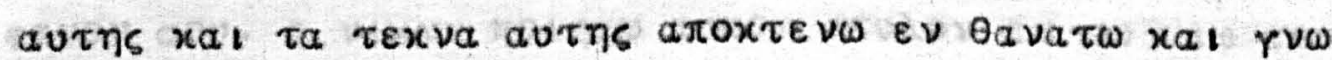

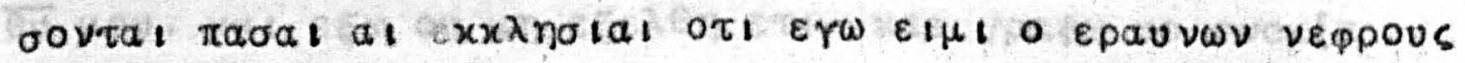

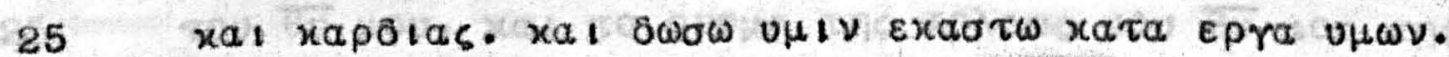

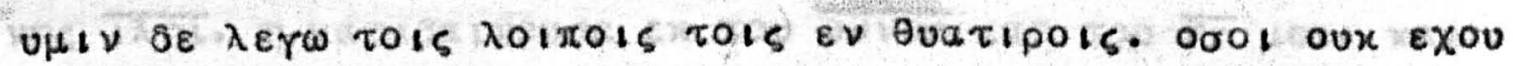

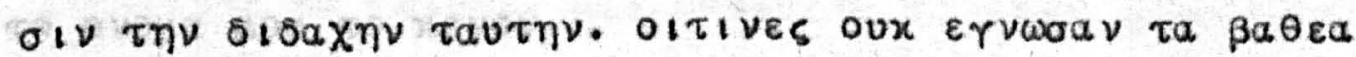

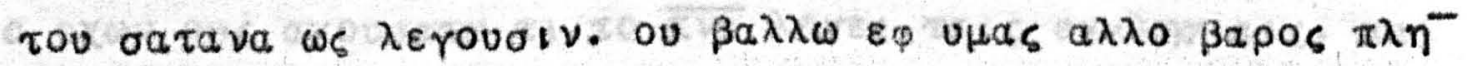

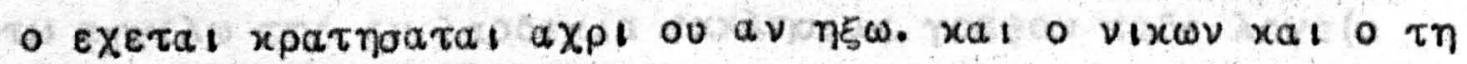

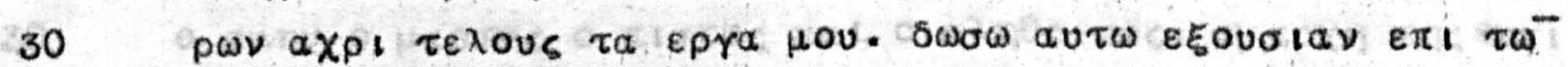

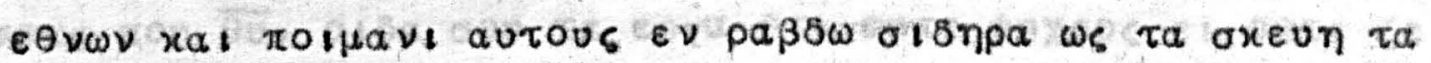

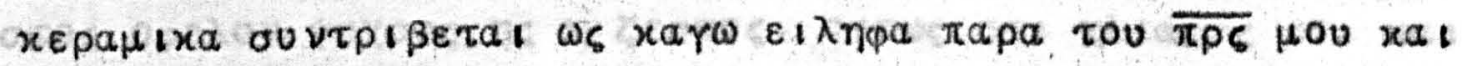

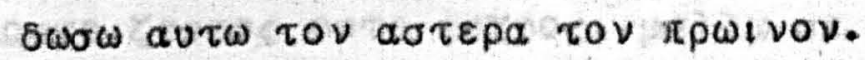

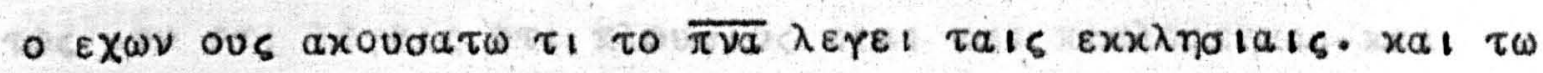

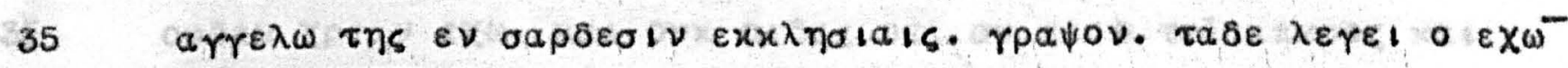

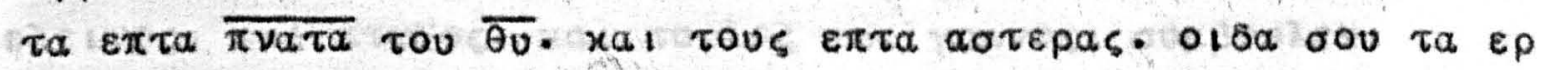

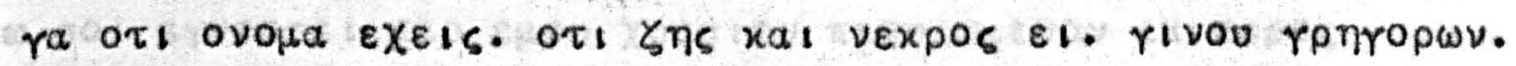

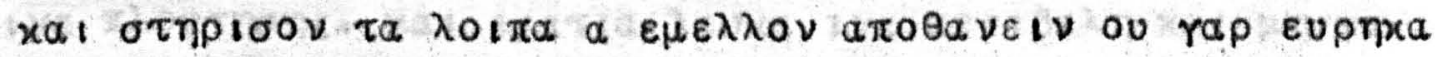

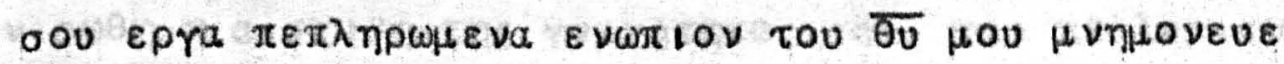

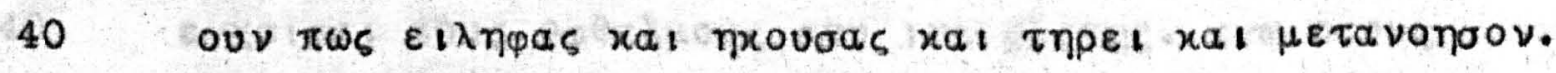

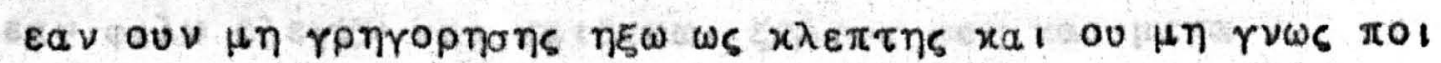




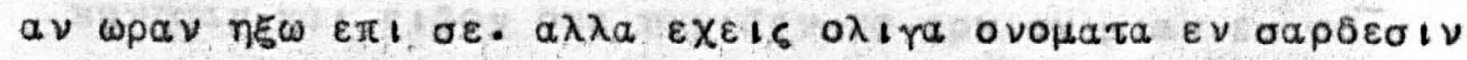

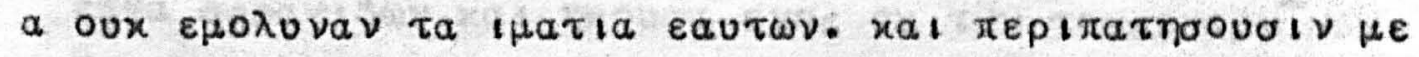

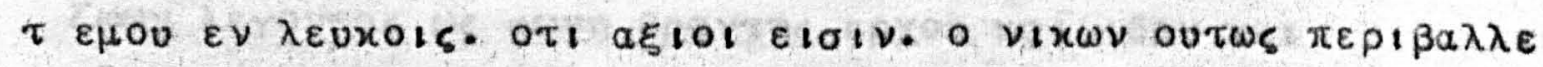

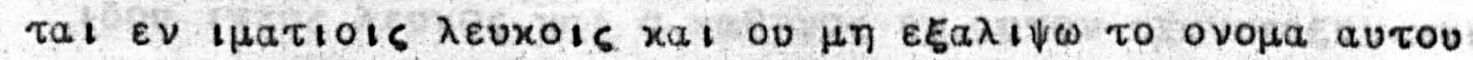

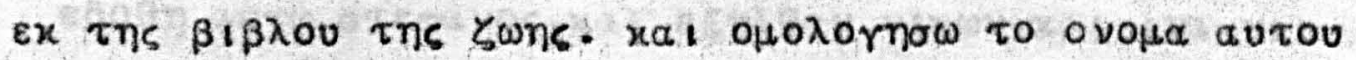

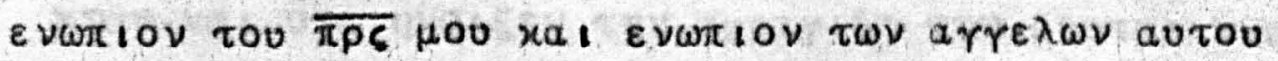

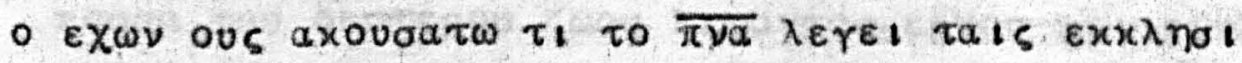

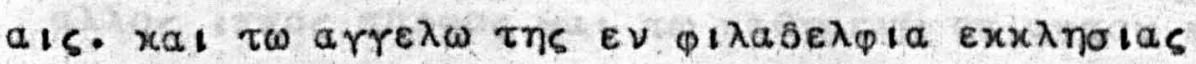
rpaঊov.

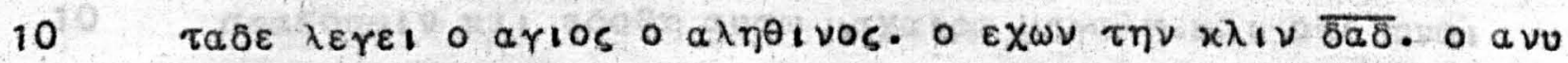

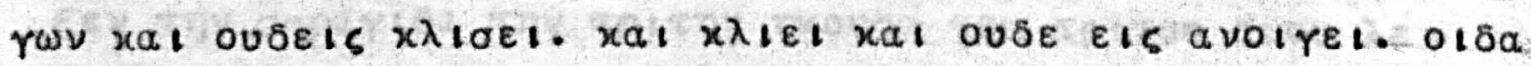

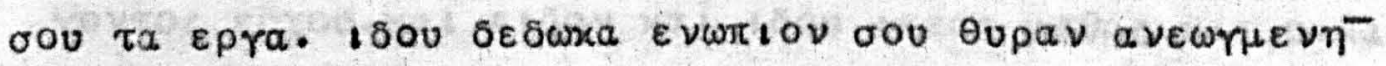

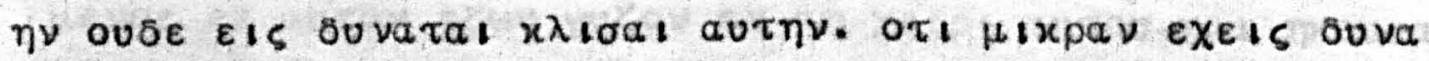

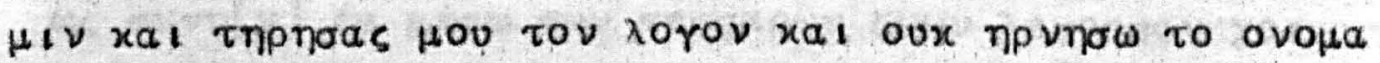

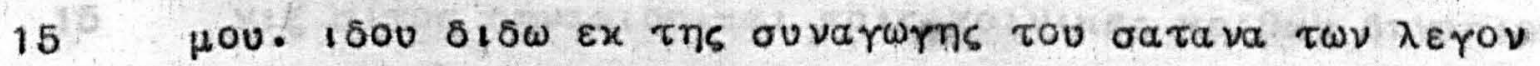

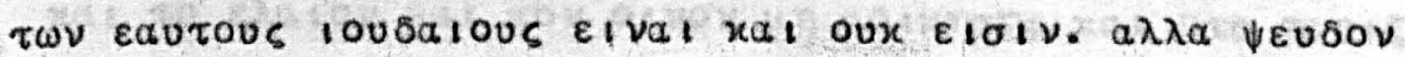

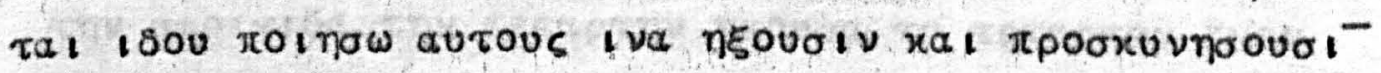

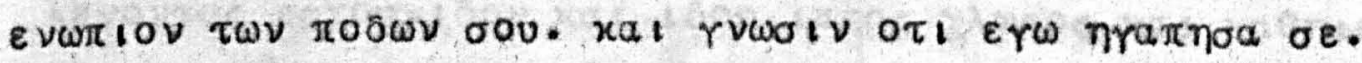

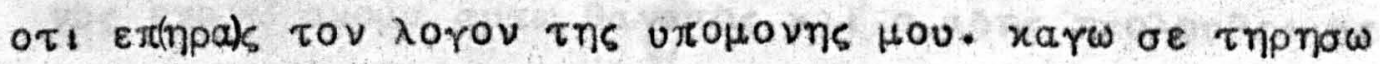

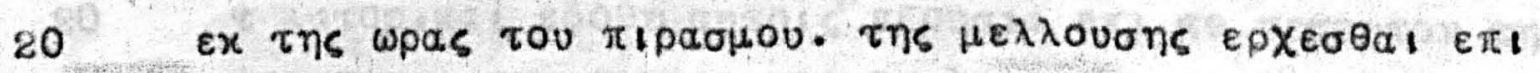

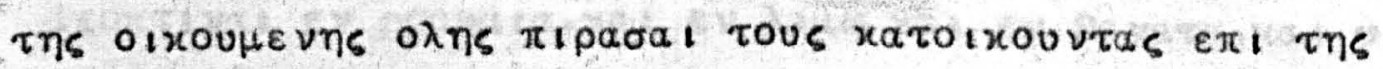

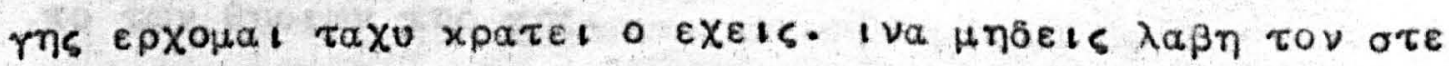

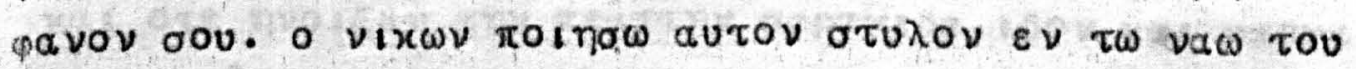

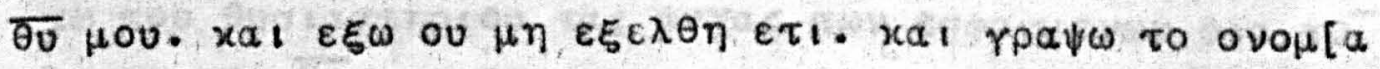

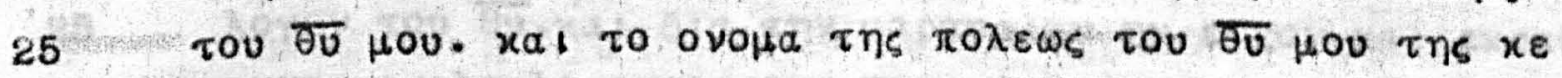

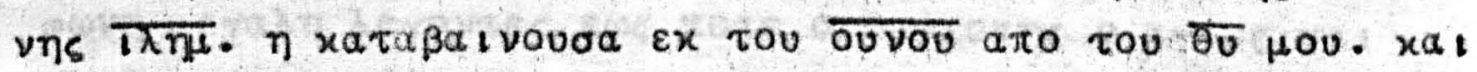
to ovoua hov to xevov.

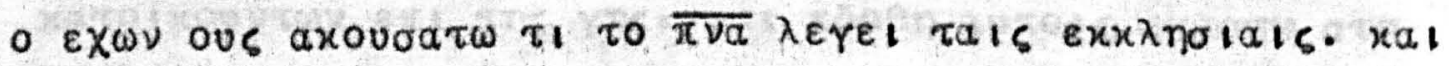

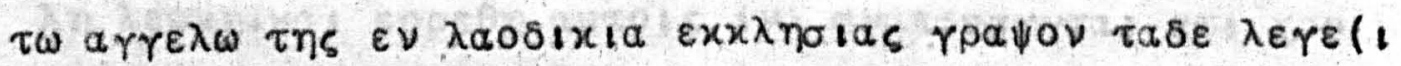

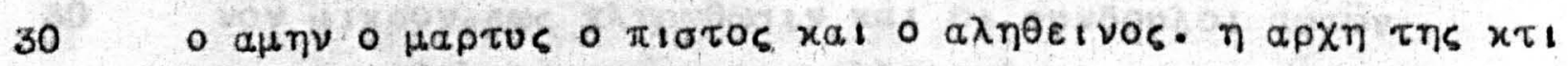

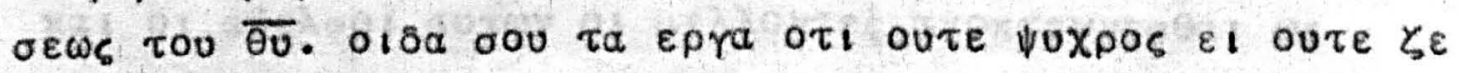

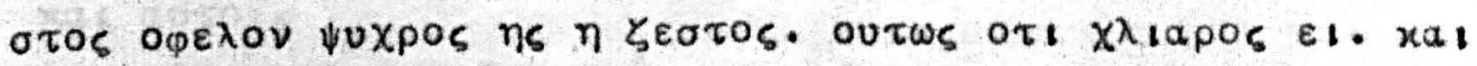

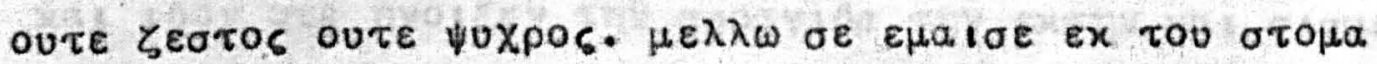

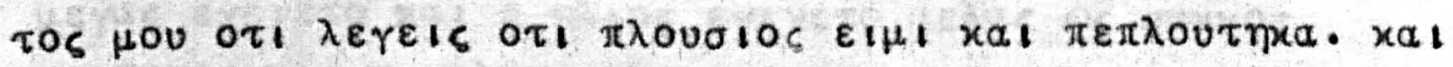

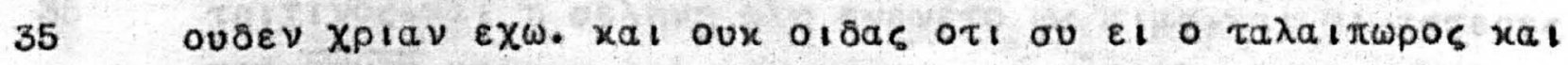

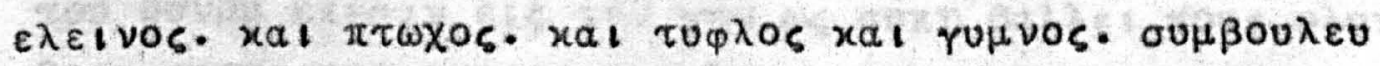

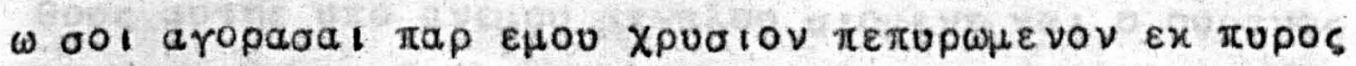

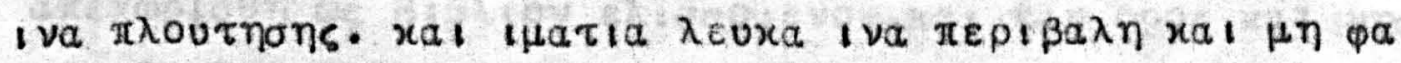

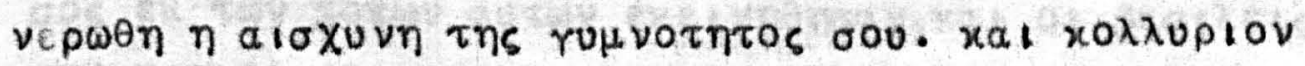

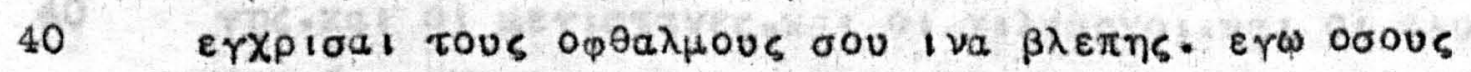

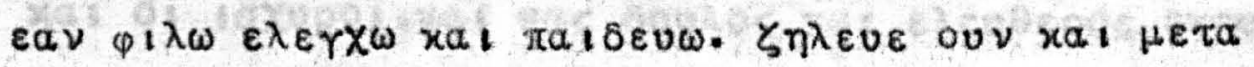




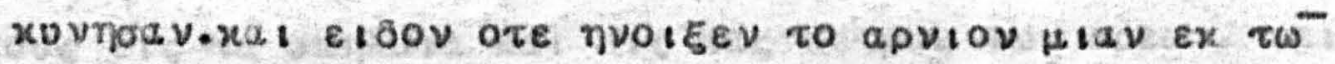

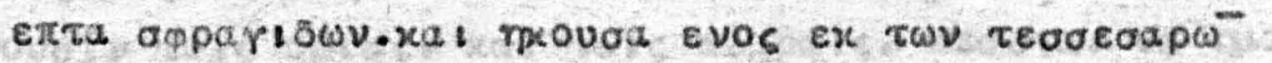

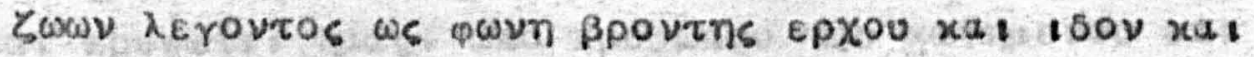

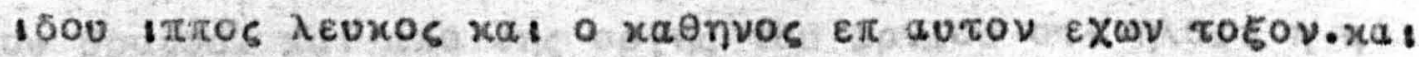

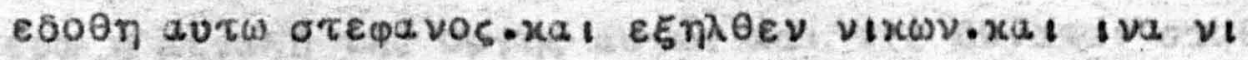

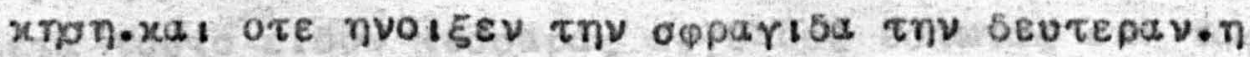

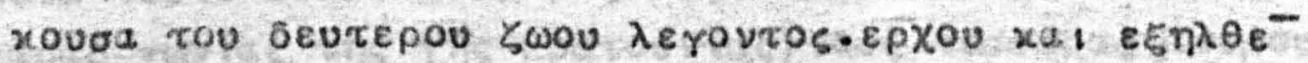

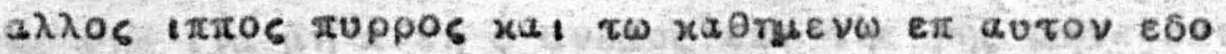

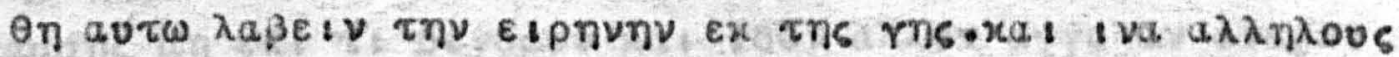

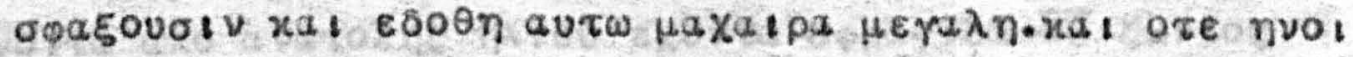

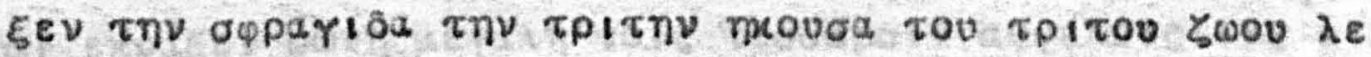

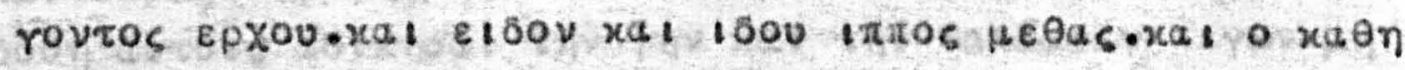

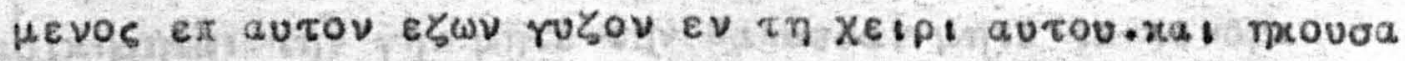

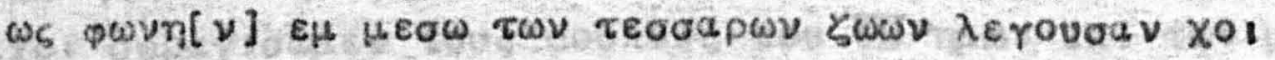

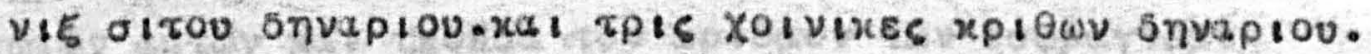

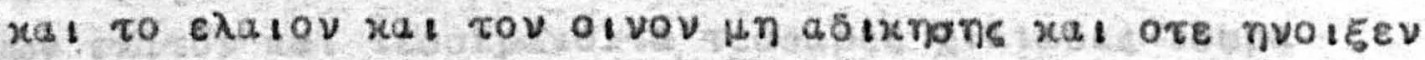

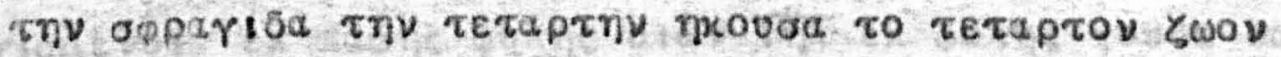

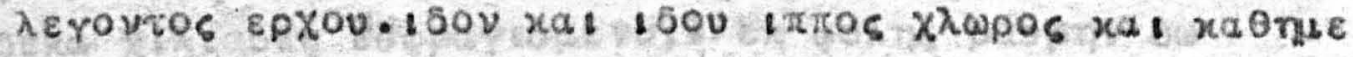

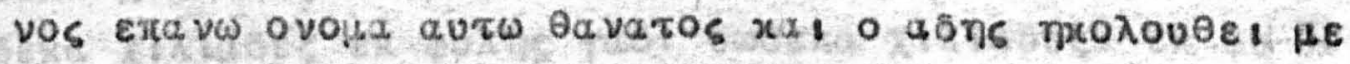

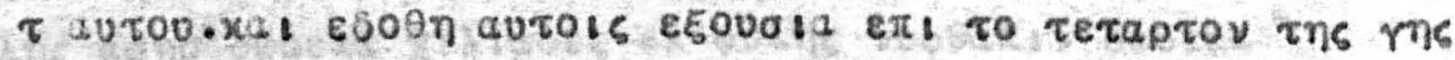
Gronetval \&v popqa

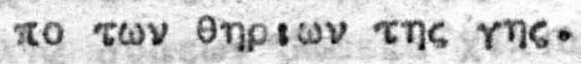

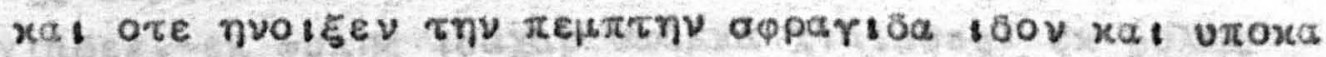

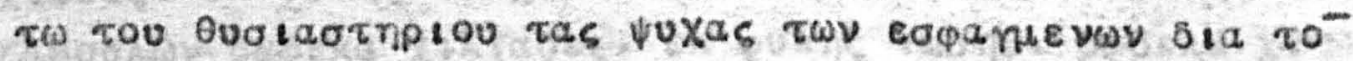

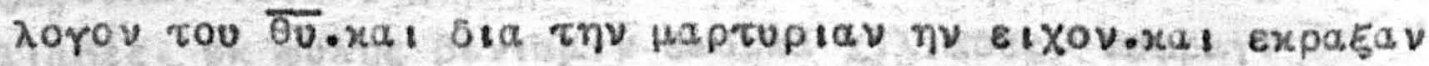

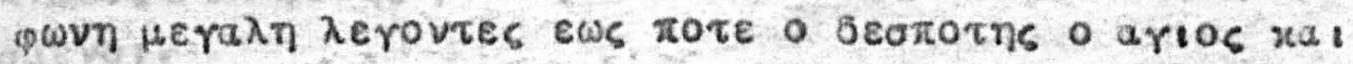

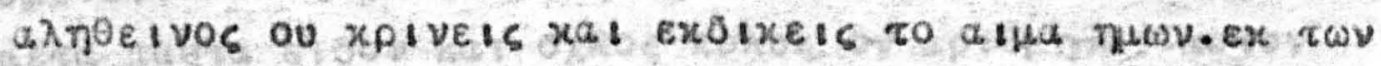

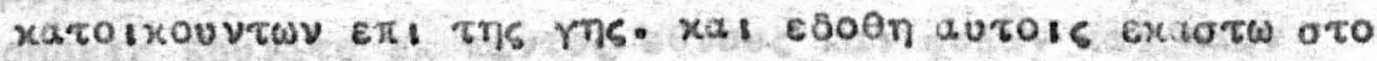

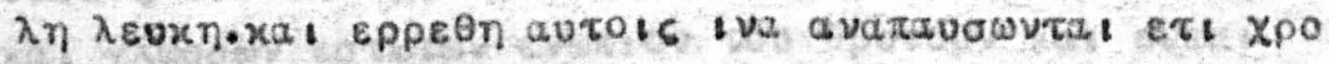

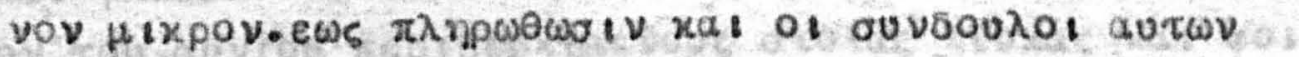

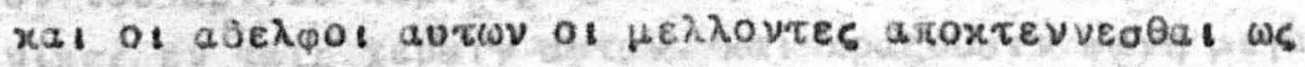
xal auroi.

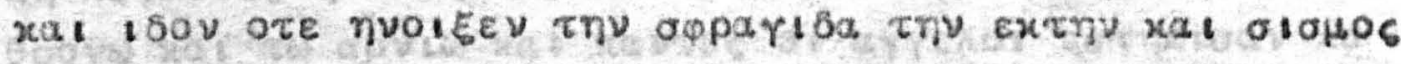

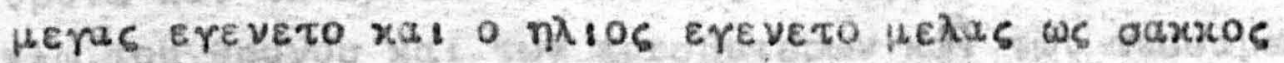

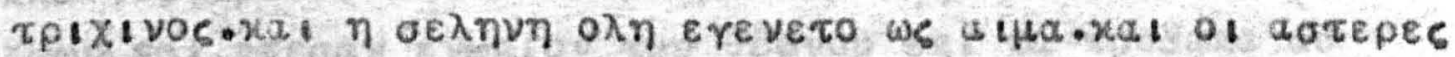

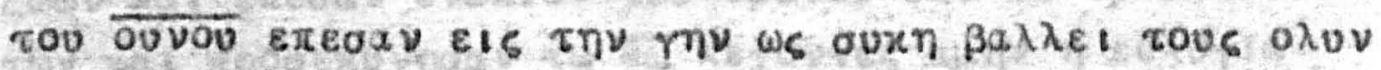

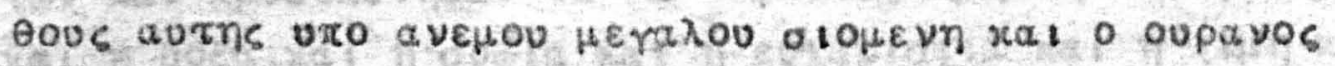

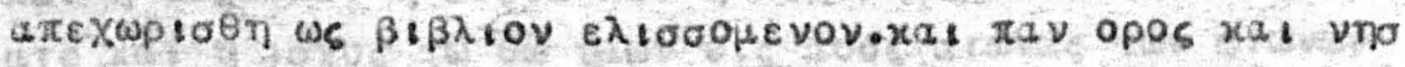

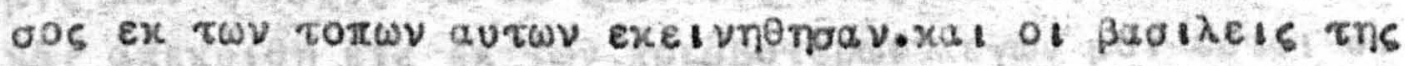

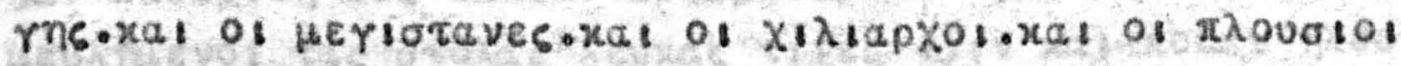

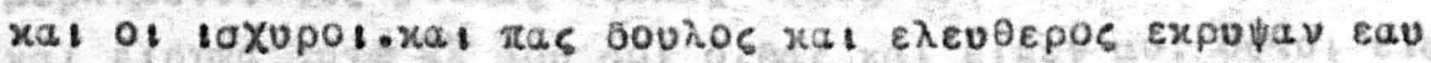




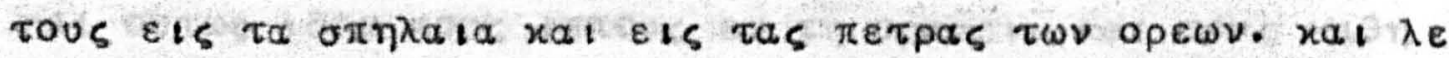

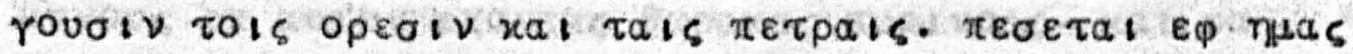

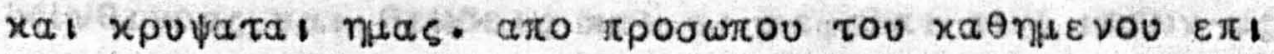

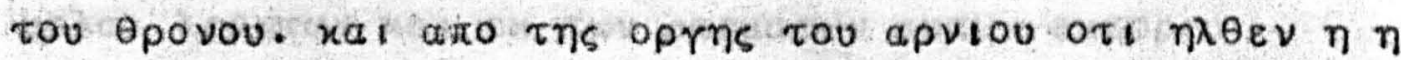

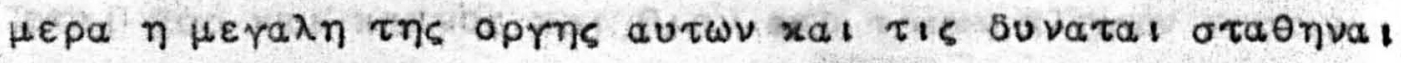

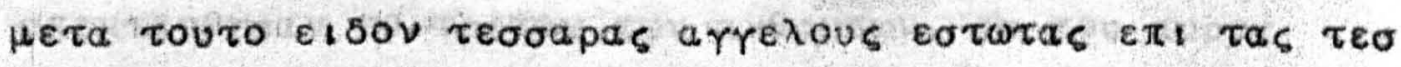

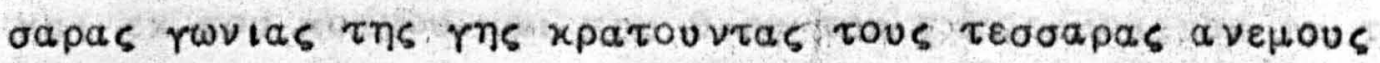

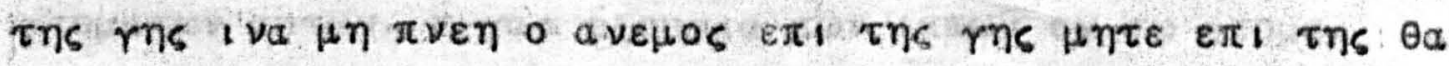

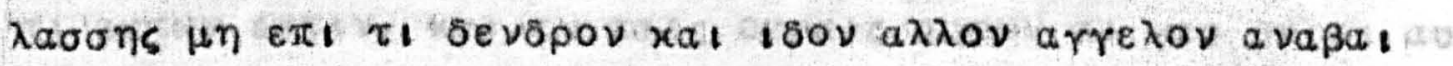

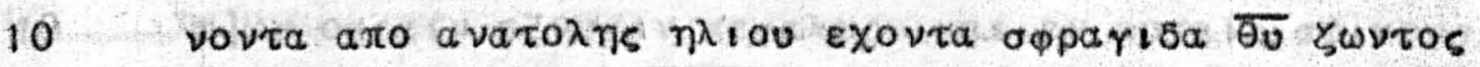
xel ExpaदEv

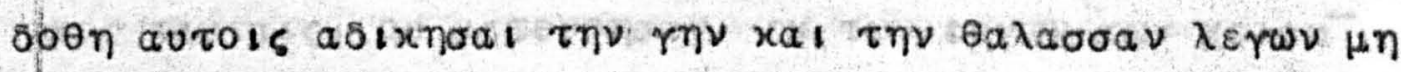

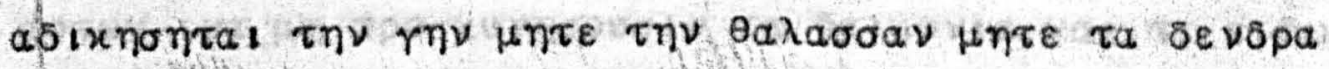

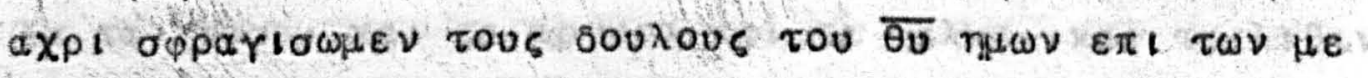

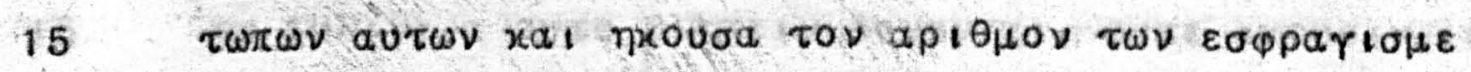

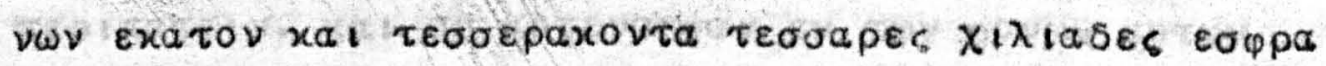

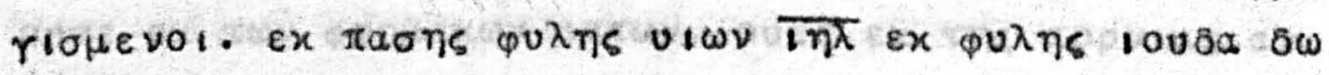

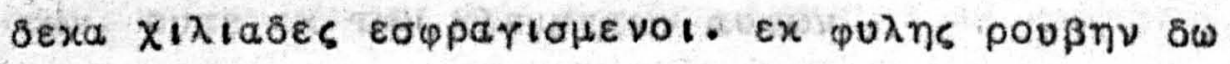

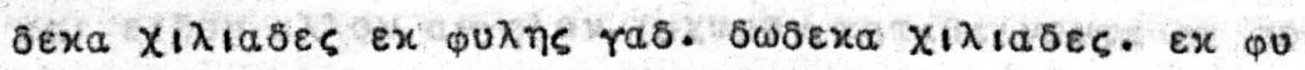

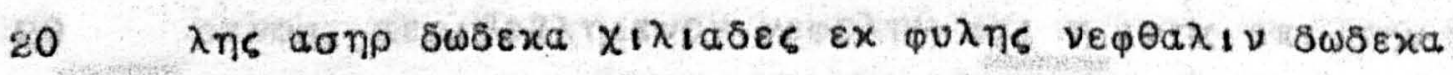

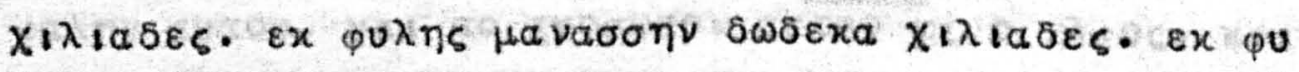

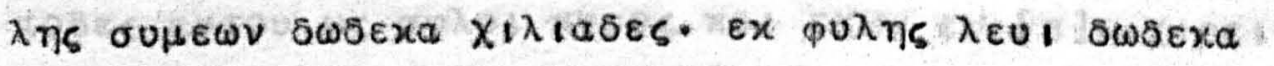

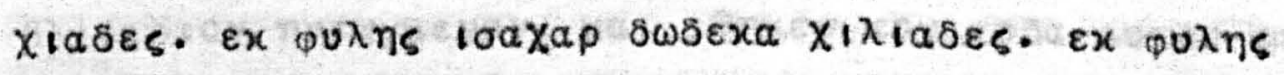

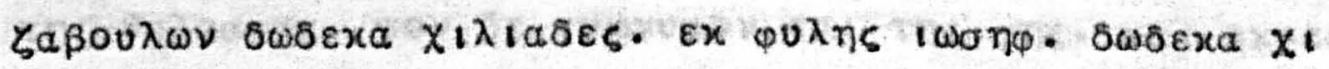

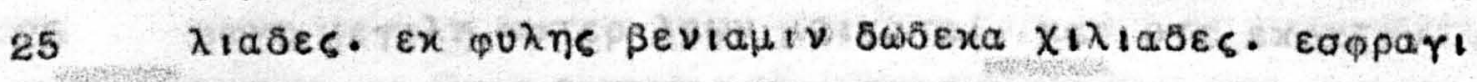

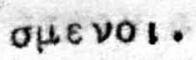

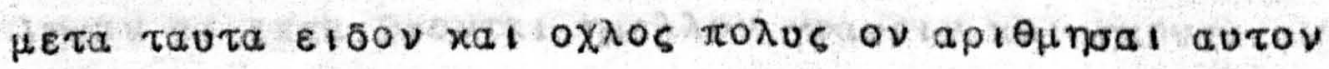

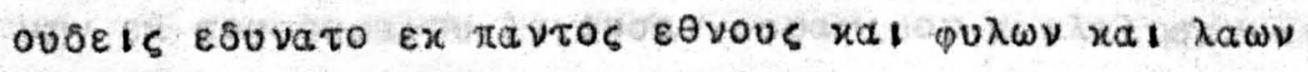

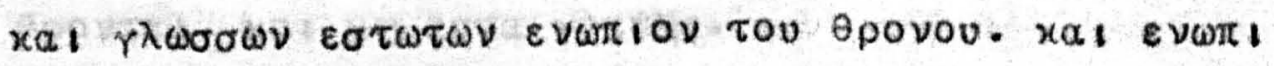

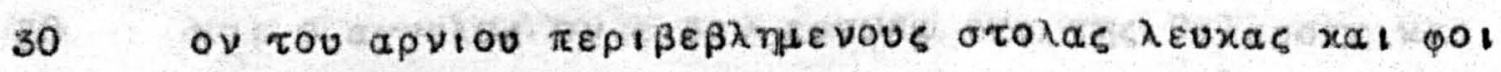

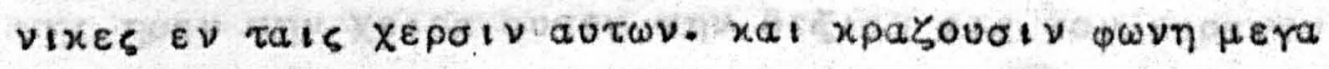

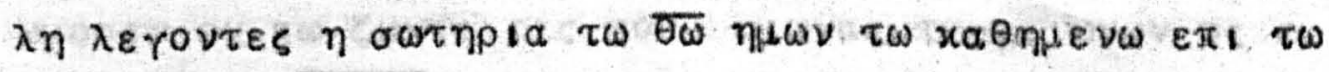

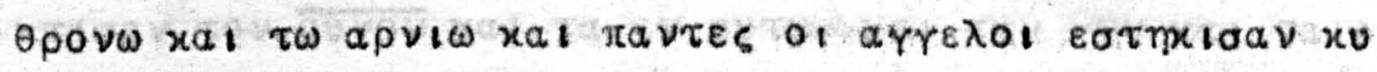

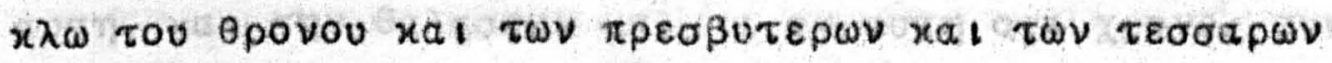

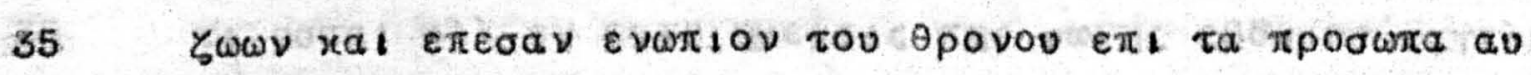

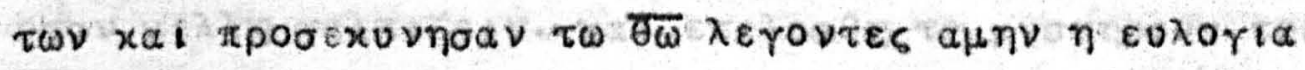

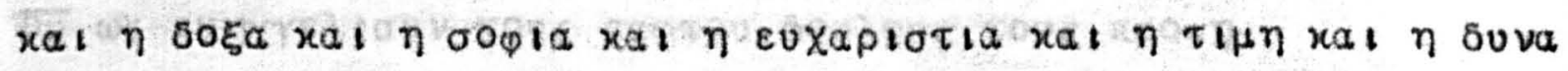

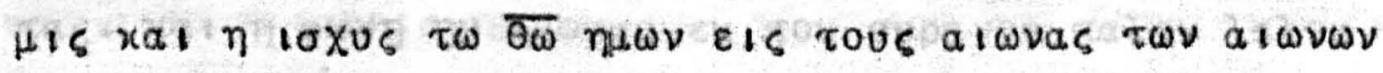

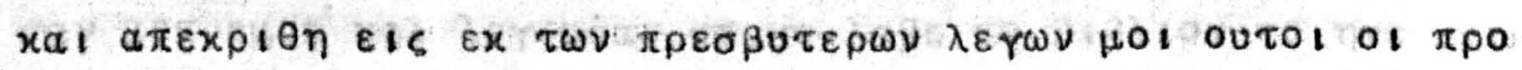

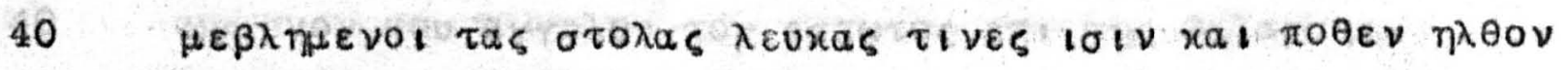

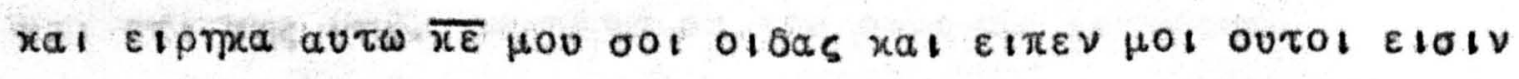




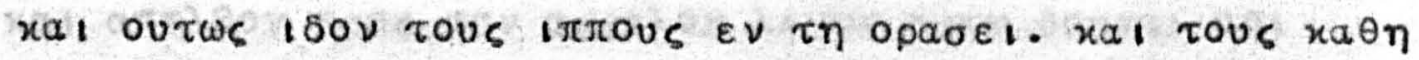

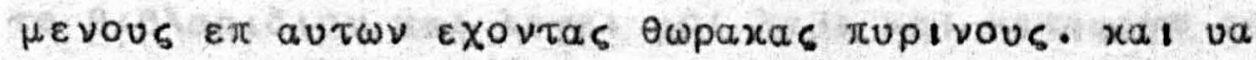

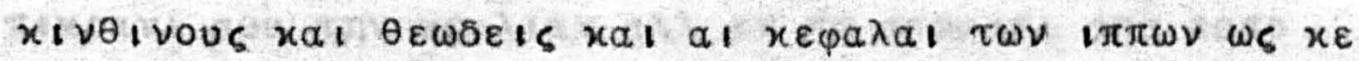

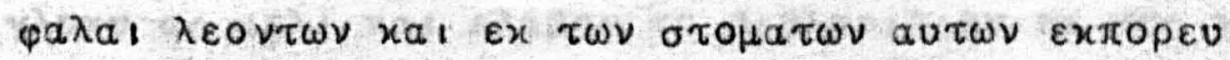

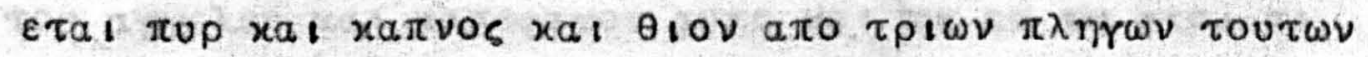

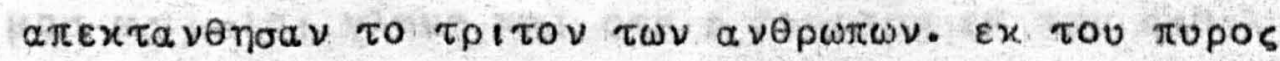

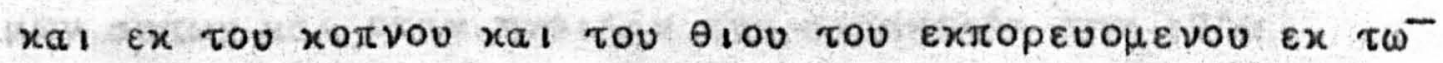

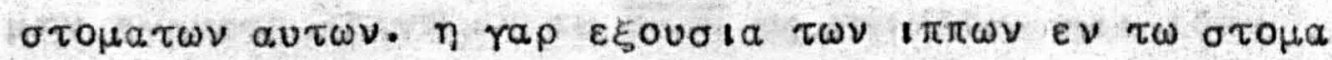

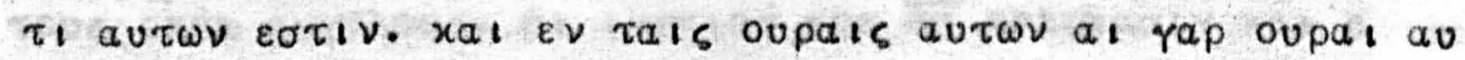

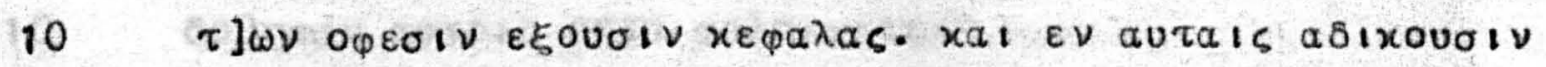

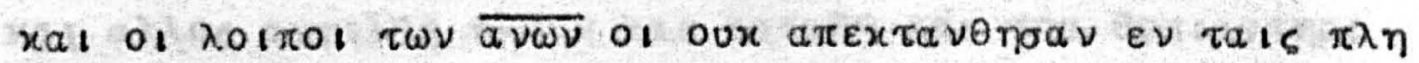

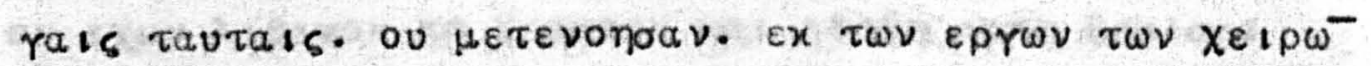

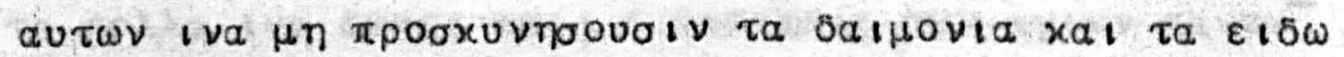

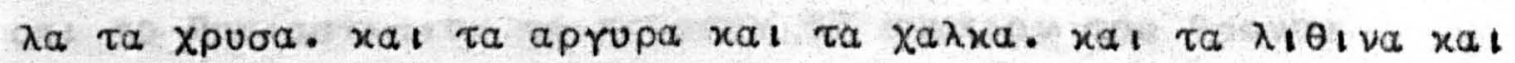

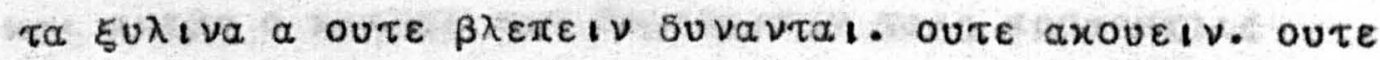

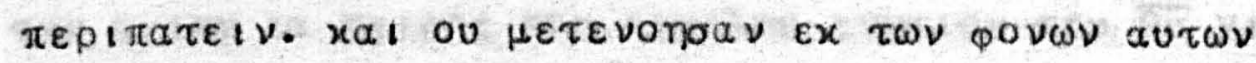

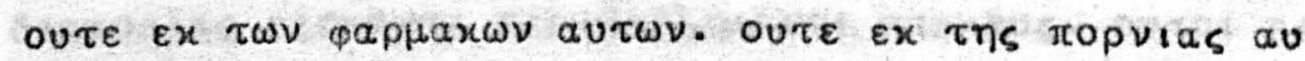

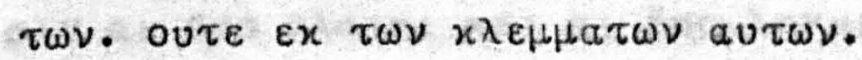

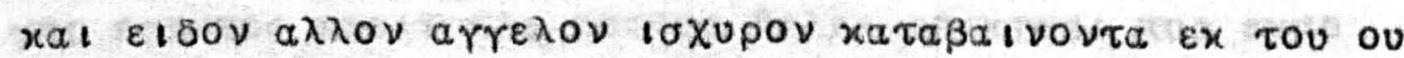

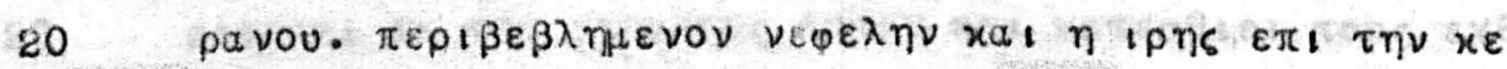

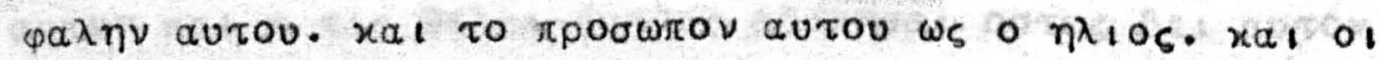

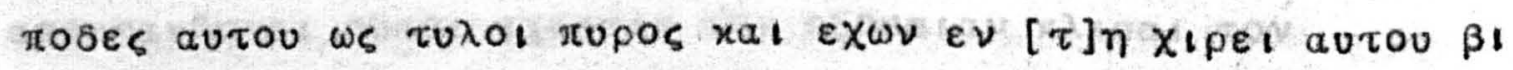

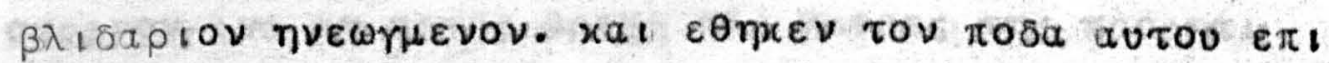

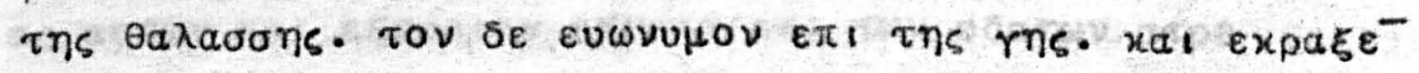

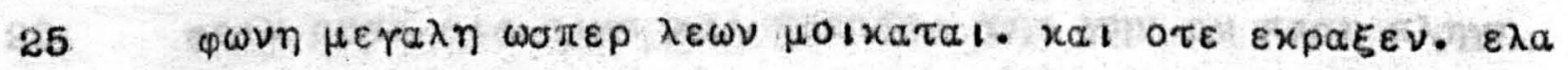

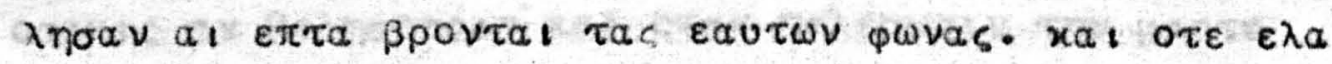

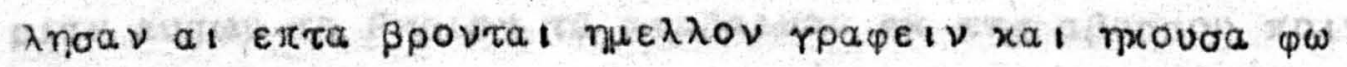

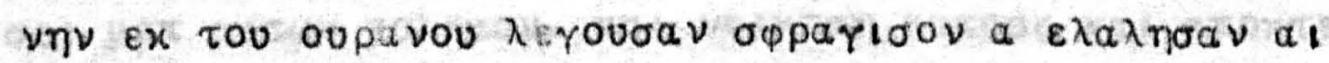

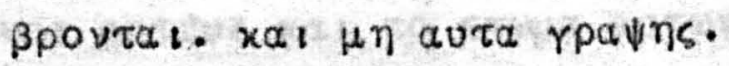

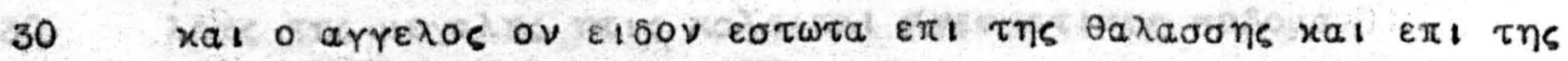

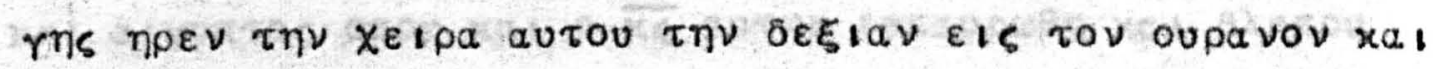

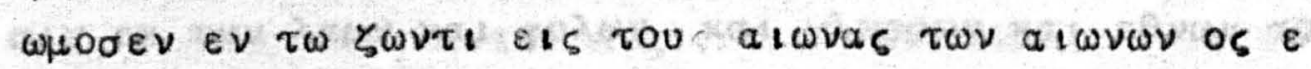

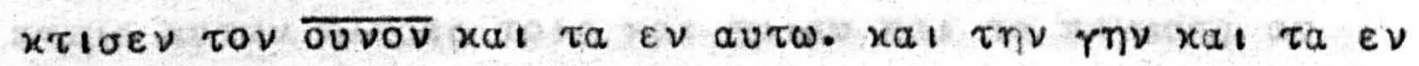

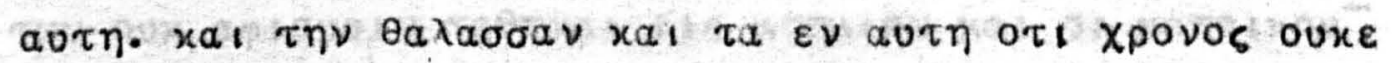

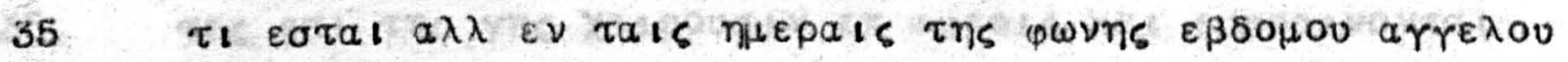

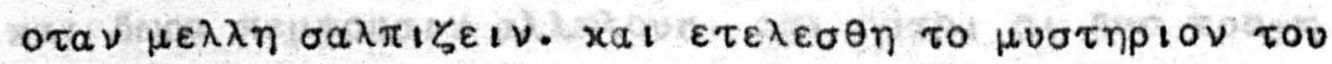

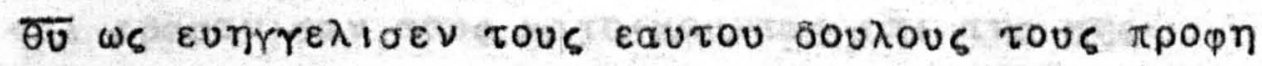

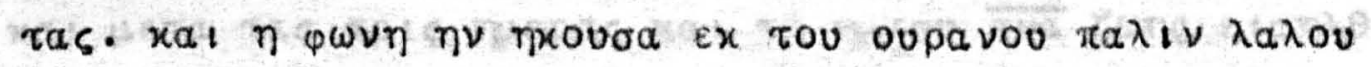

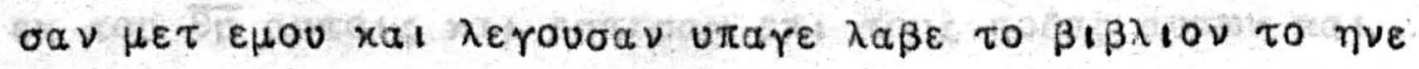

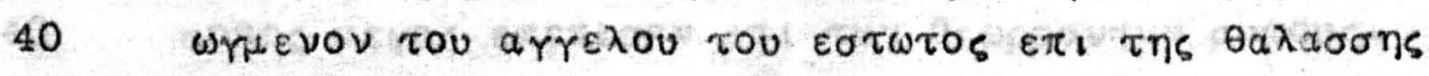
$x a, \varepsilon \pi$ tins rns. 


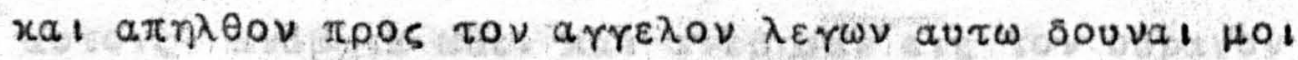

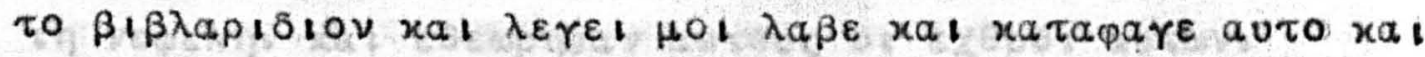

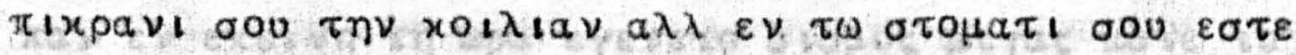

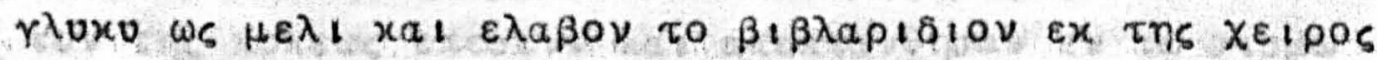

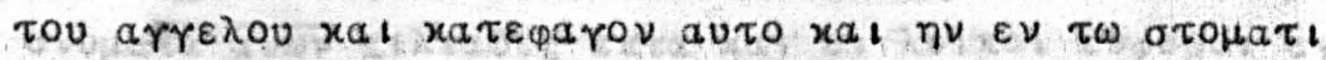

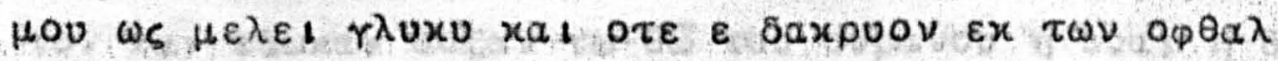

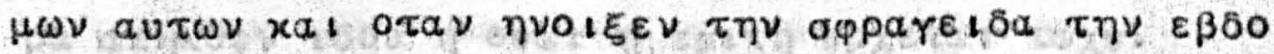

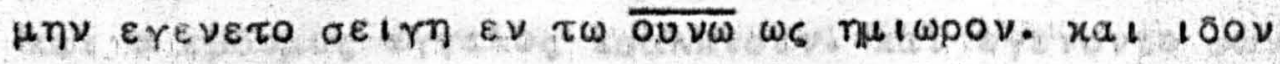

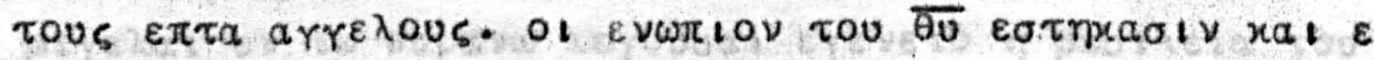

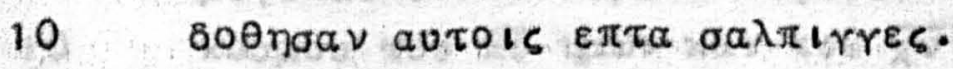

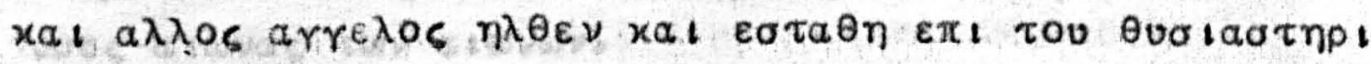

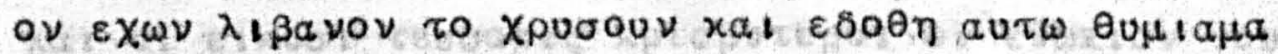

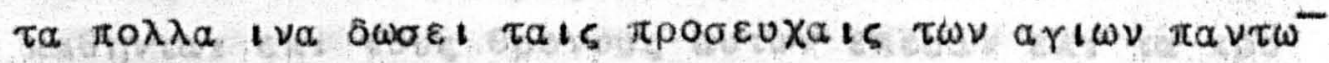

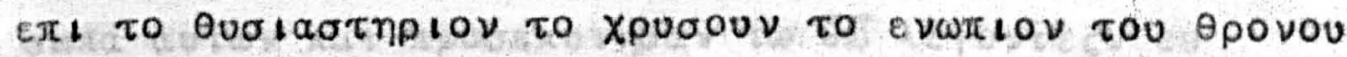

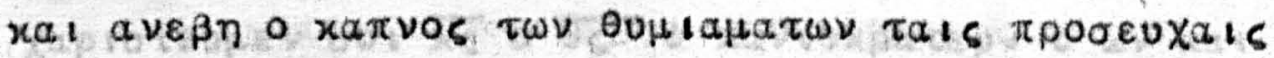

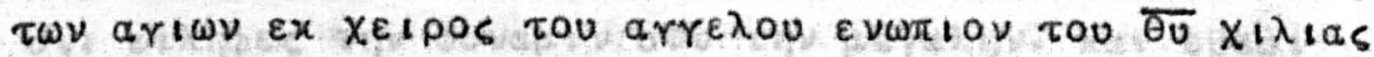

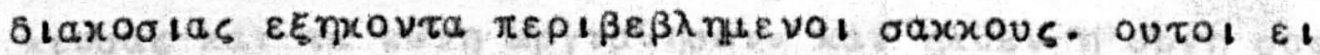

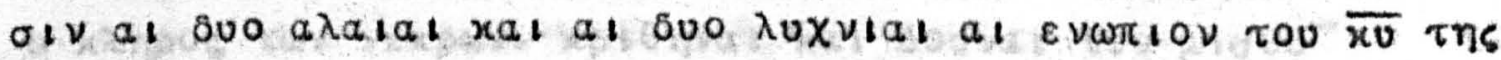

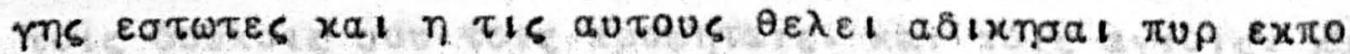

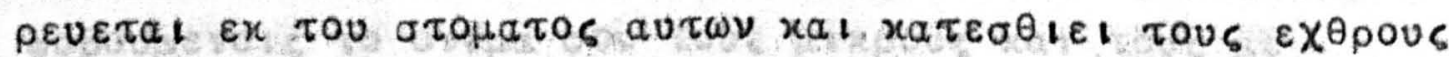

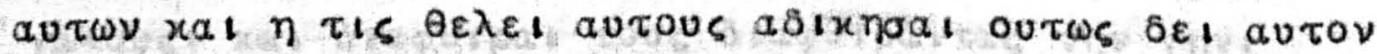

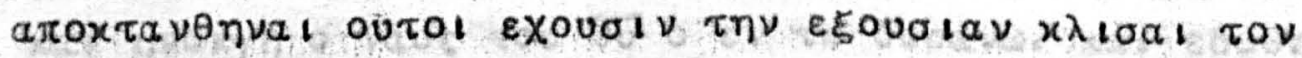

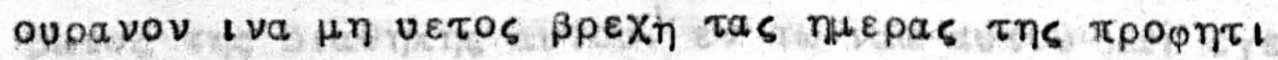

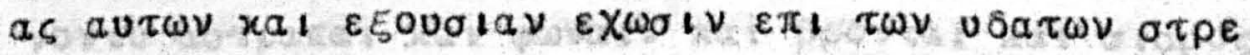

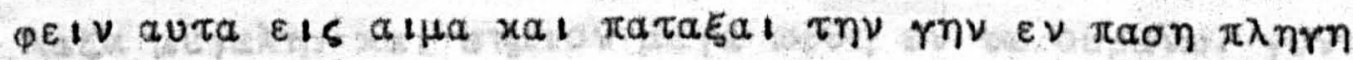

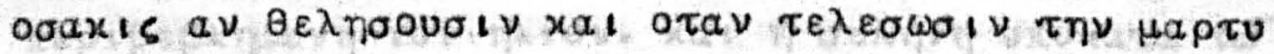

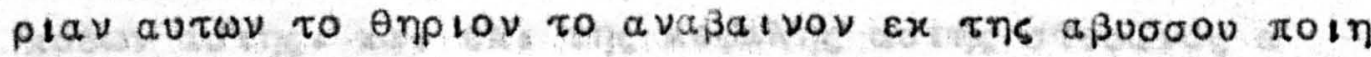

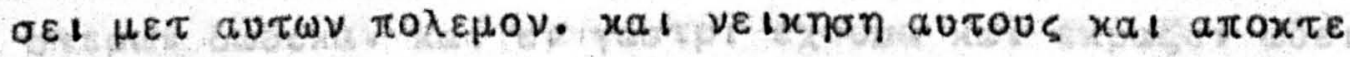

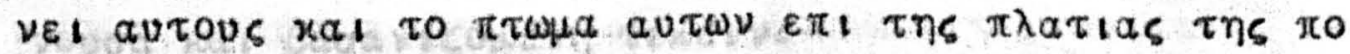

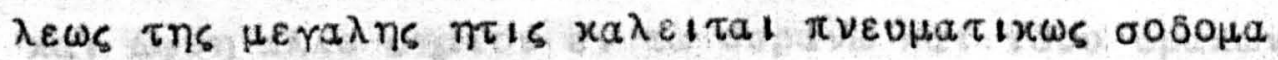

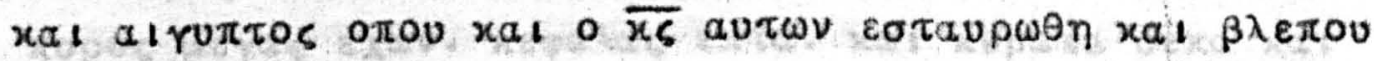

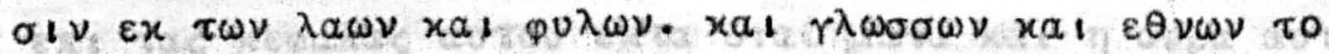

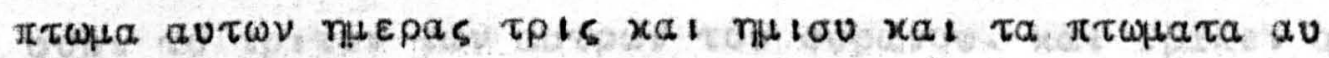

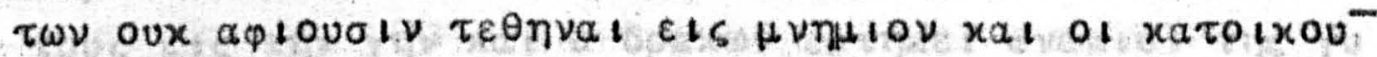

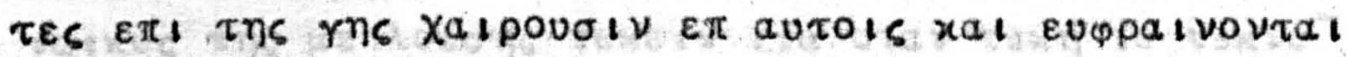

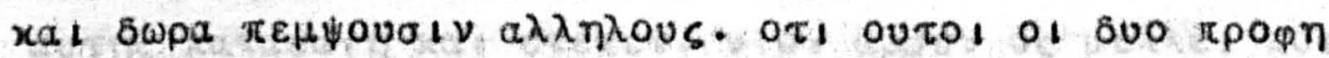

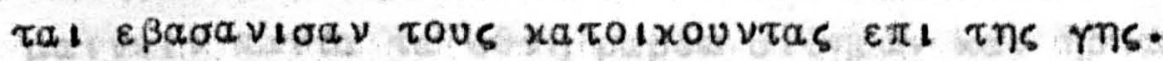

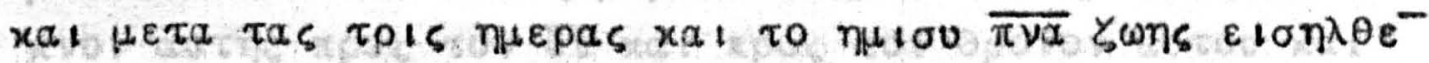

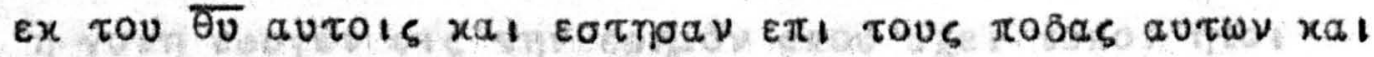

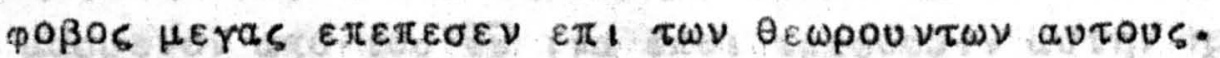

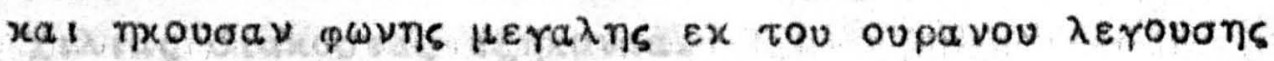




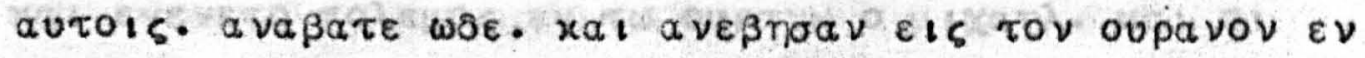

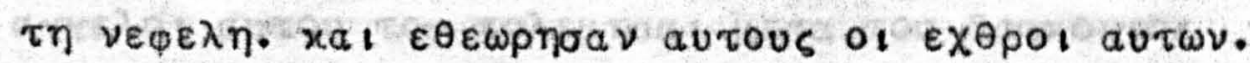

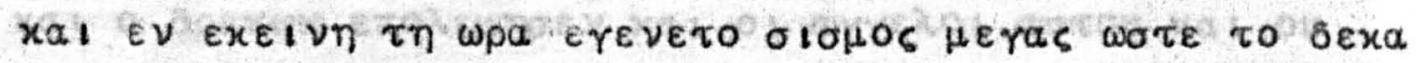

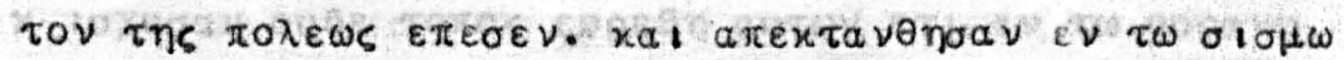

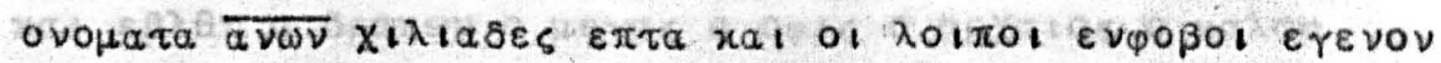

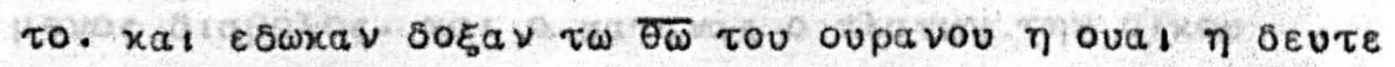

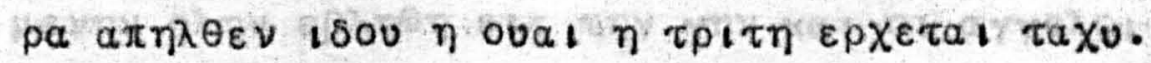

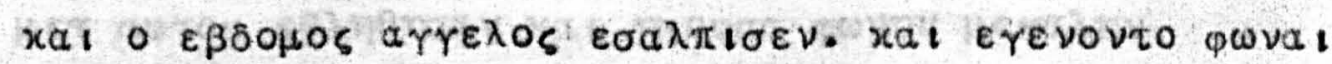

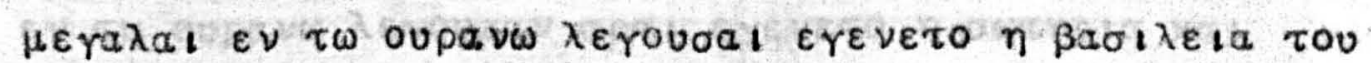

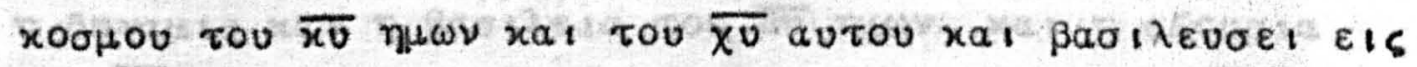

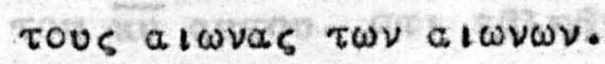

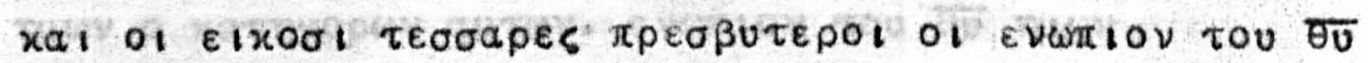

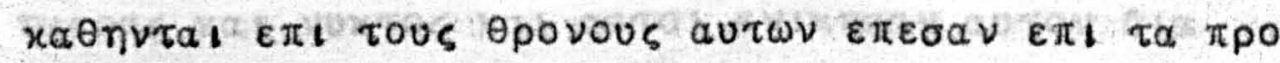

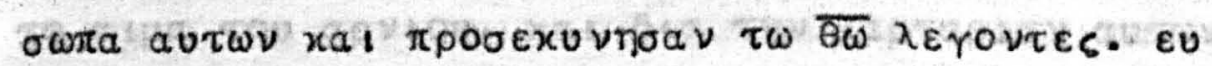

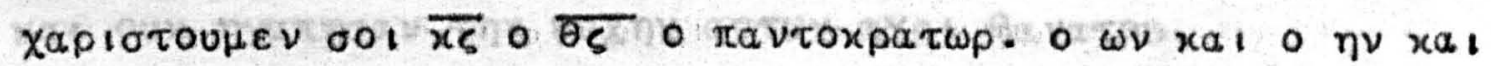

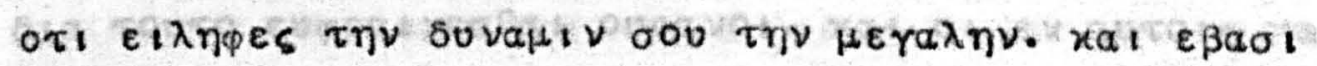

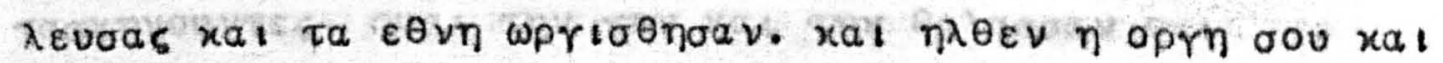

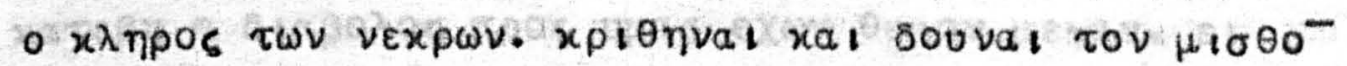

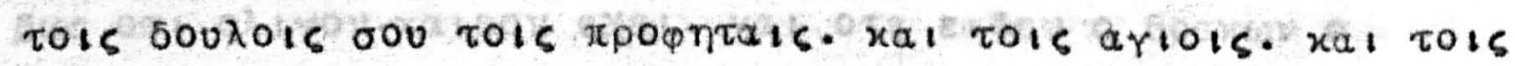

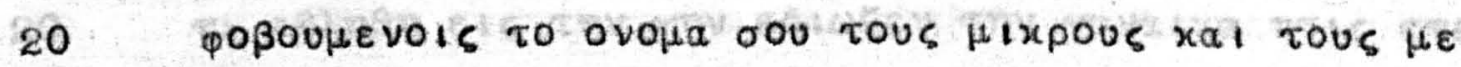

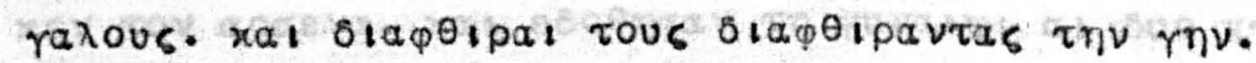

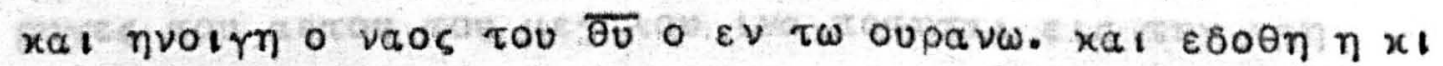

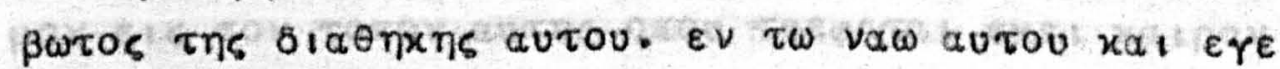

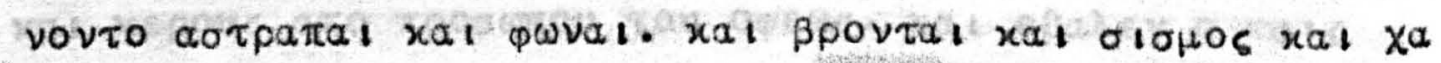

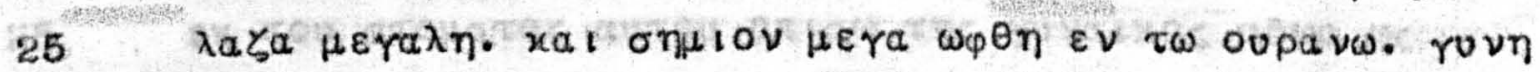

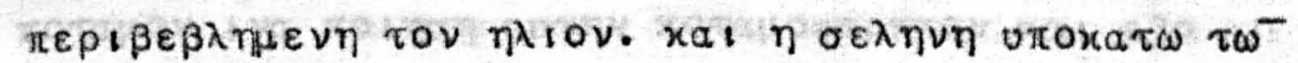

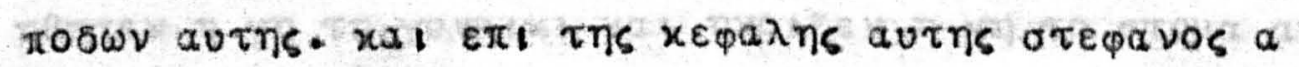

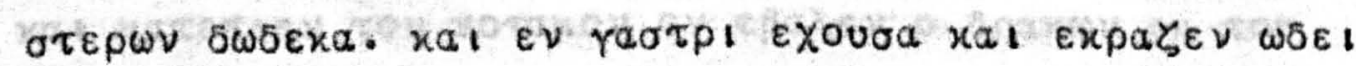
vovoa $x a l \beta a \sigma a v i \zeta o \mu \varepsilon v \eta \tau \varepsilon x \varepsilon i v$.

30 xat $\omega \varphi \theta \eta a \lambda \lambda \sigma$ onj

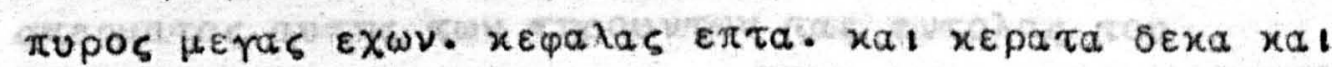

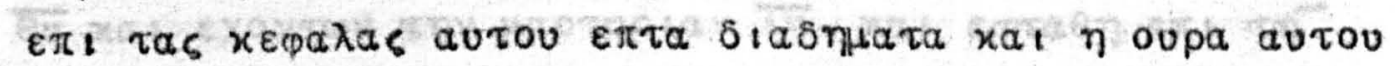

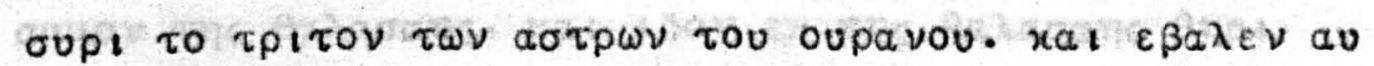

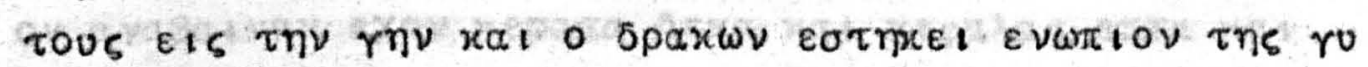

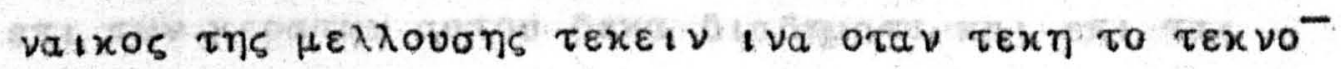

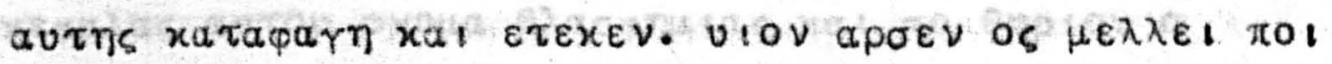

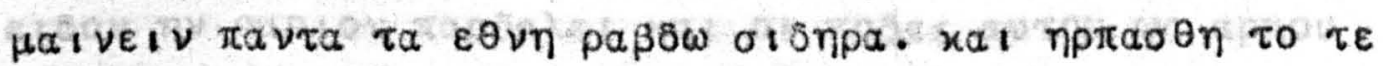

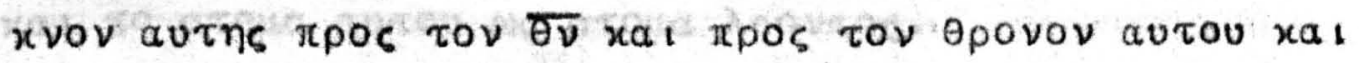

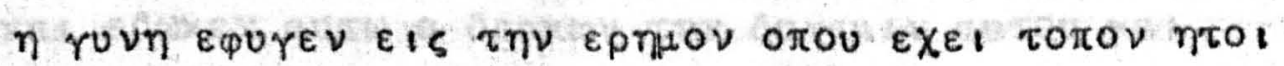

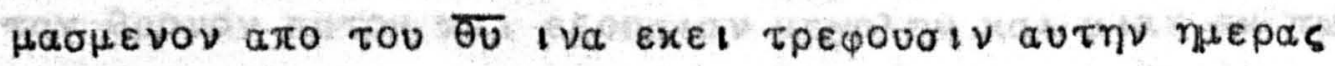

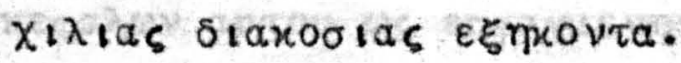




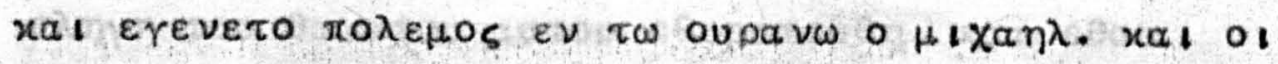

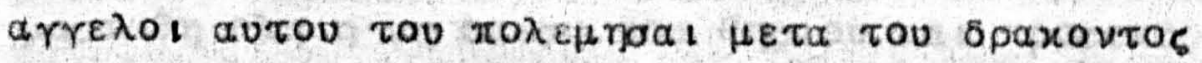

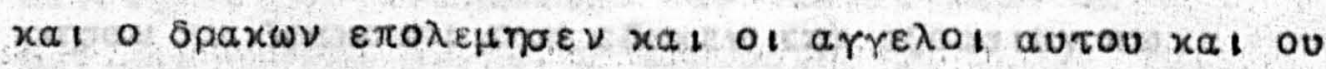

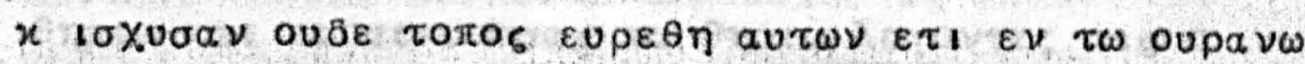

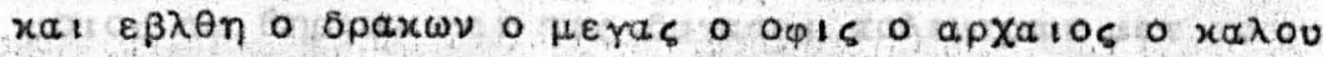

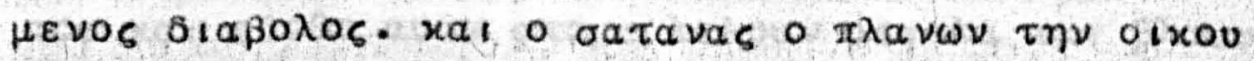

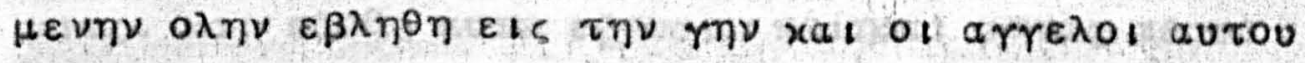

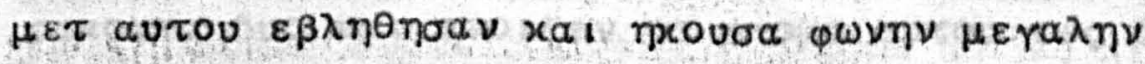

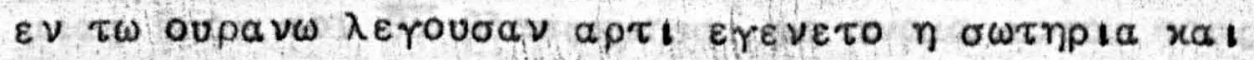

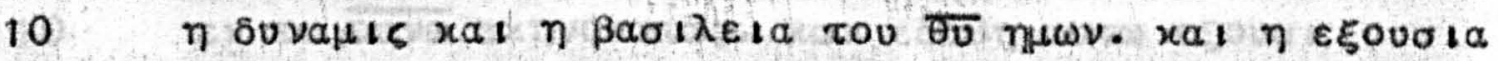

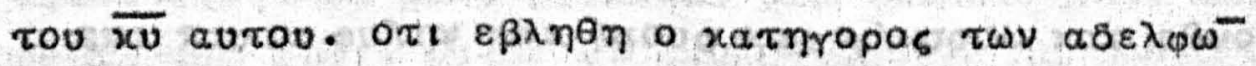

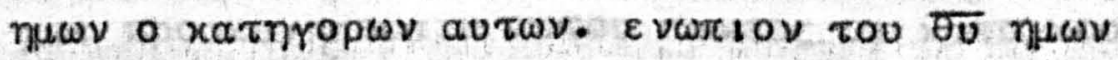

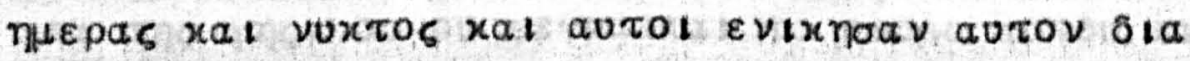

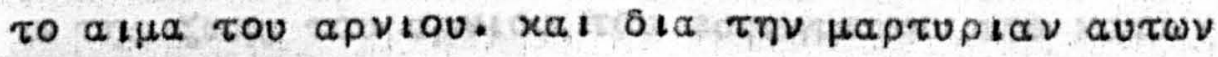

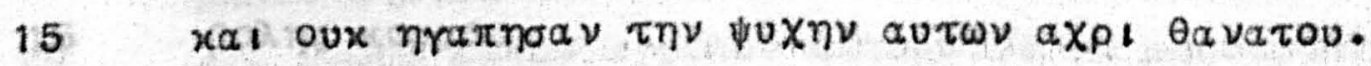

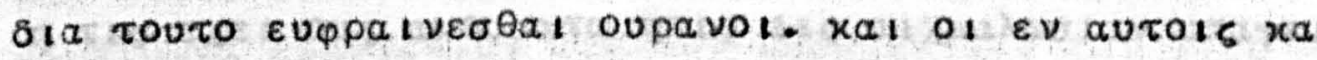

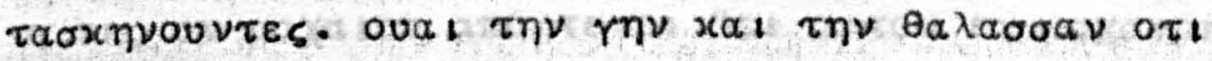

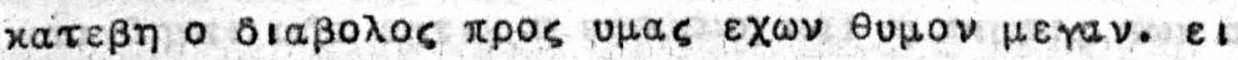

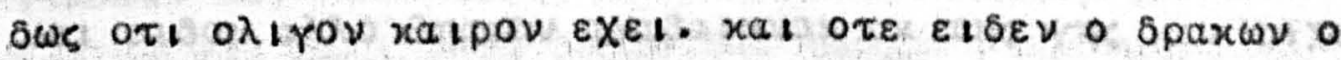

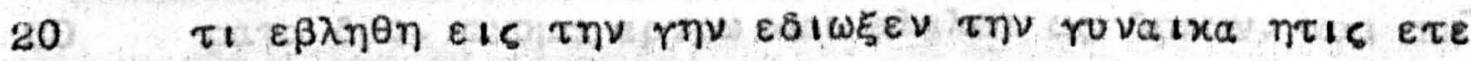

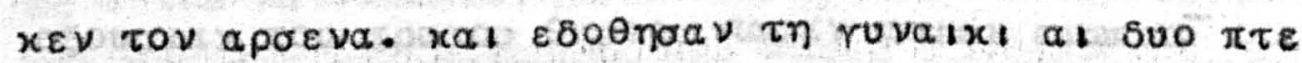

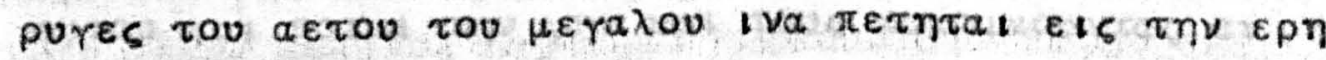
Hov Eis tov torov autns orov tpeperal exel xalpov xal xepous a

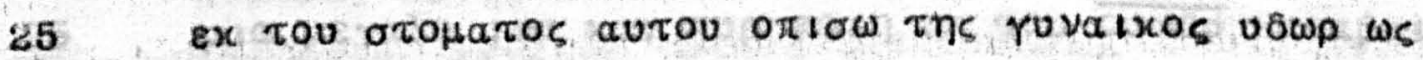

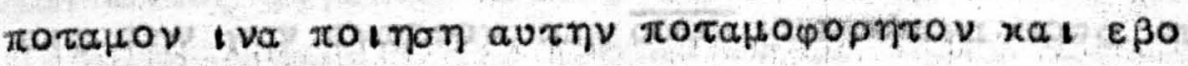

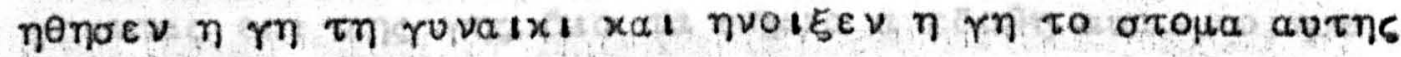

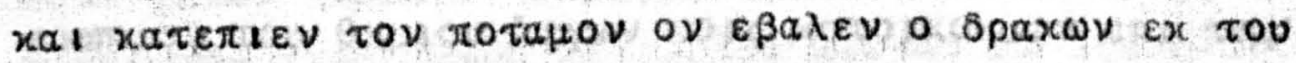

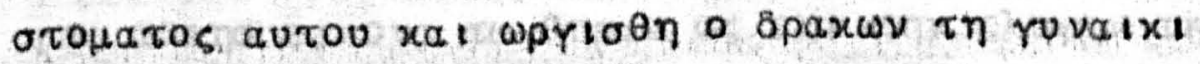

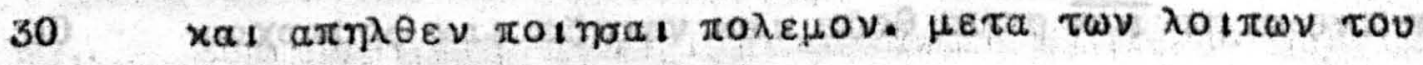

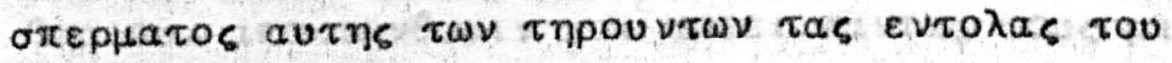

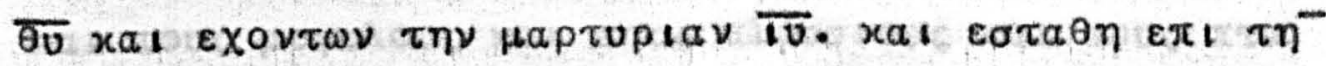

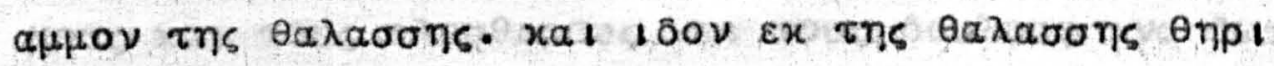

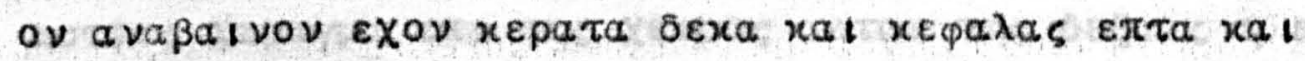

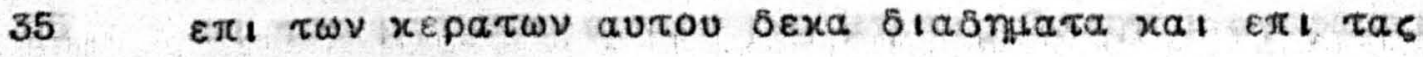
xepalas aurov ovoua $\beta$ גaopnutas xat to onpiovo

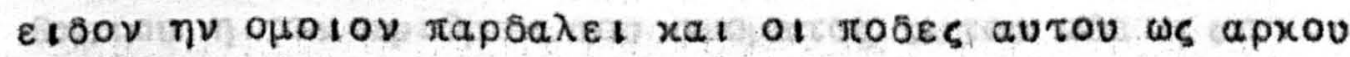

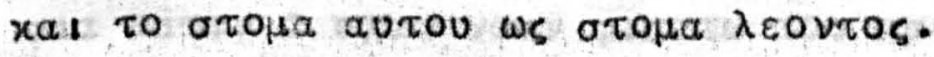
xa: $\varepsilon \delta \omega x \varepsilon v$ av

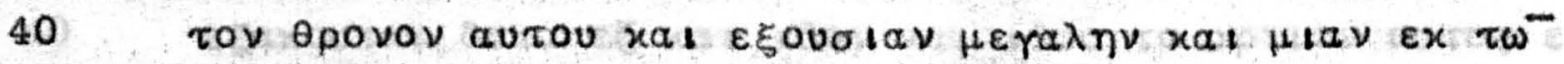

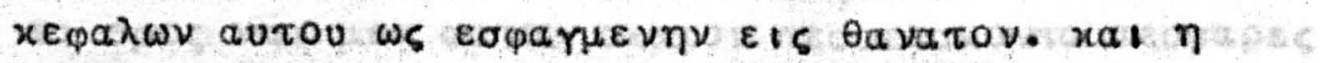




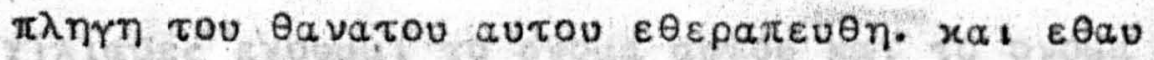

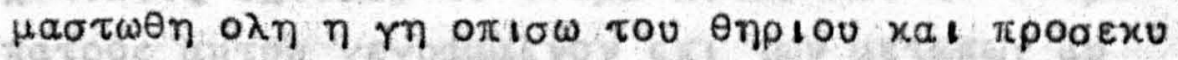

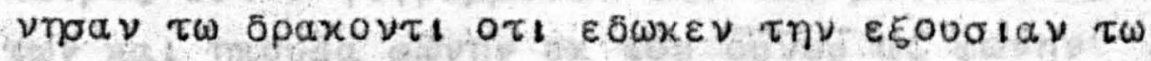

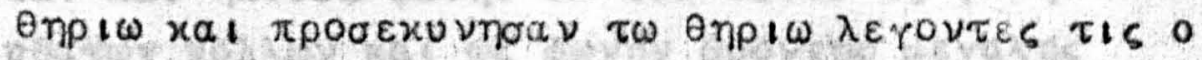

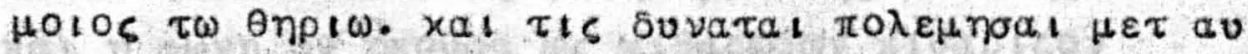

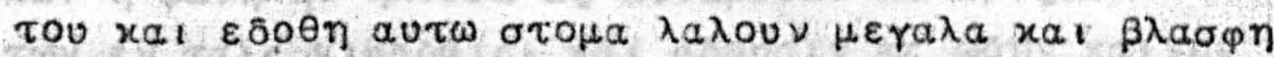

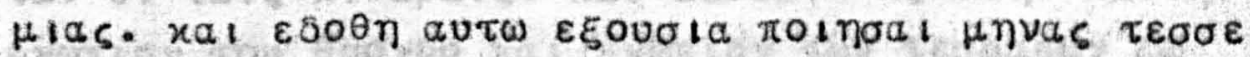

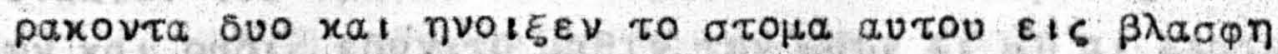

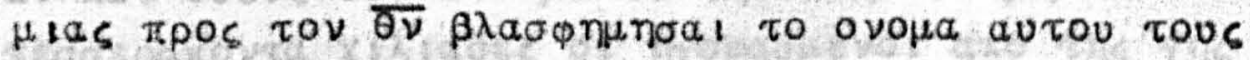

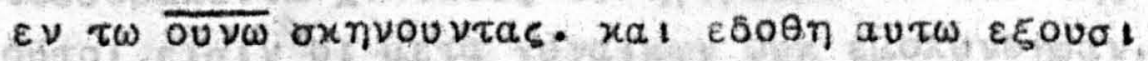

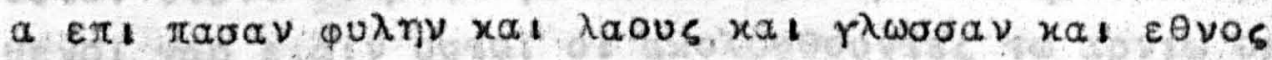

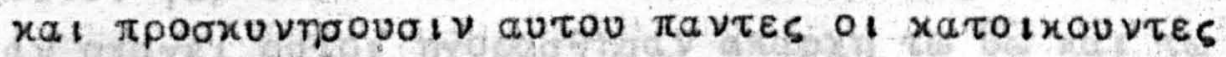

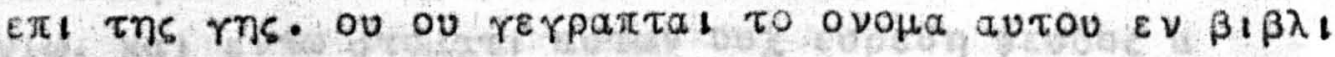

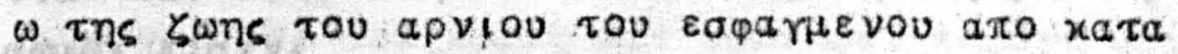

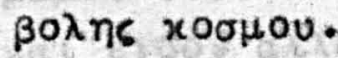

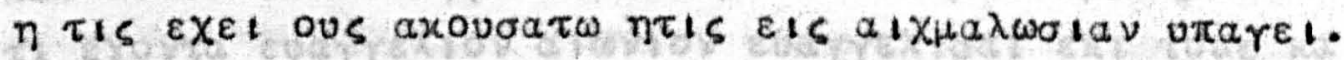

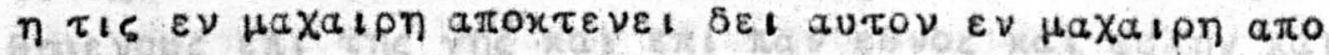

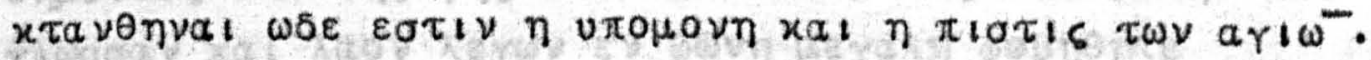

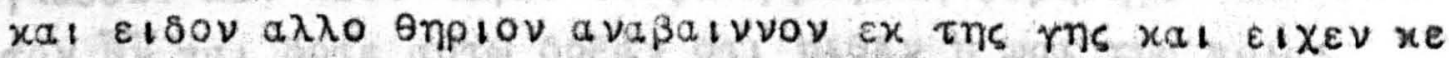

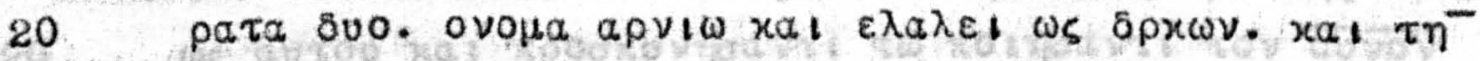

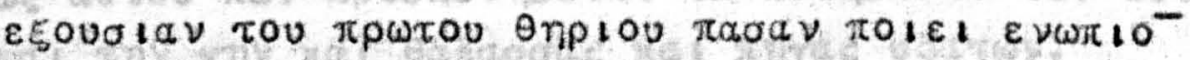

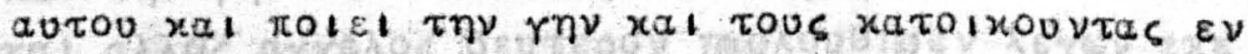

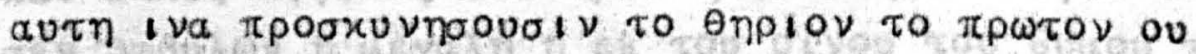

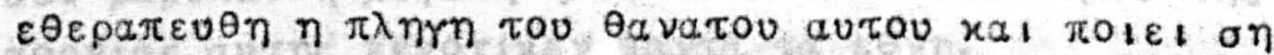

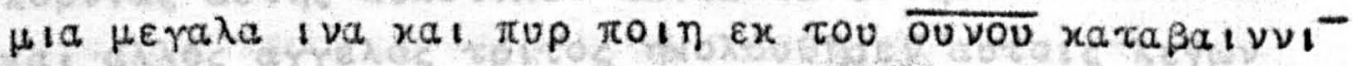

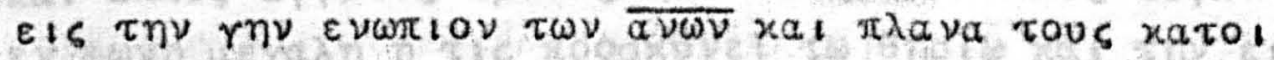

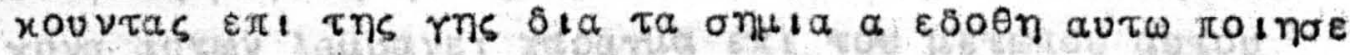

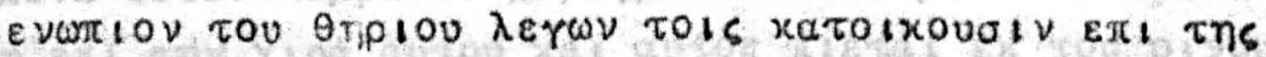

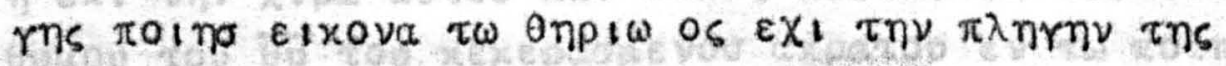

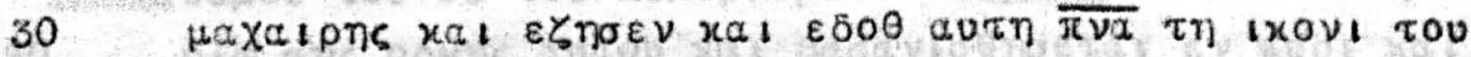

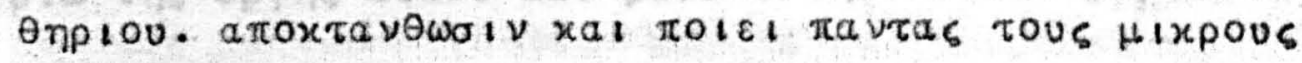

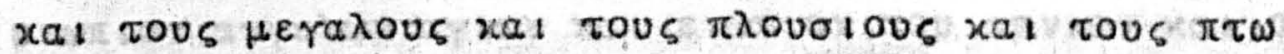

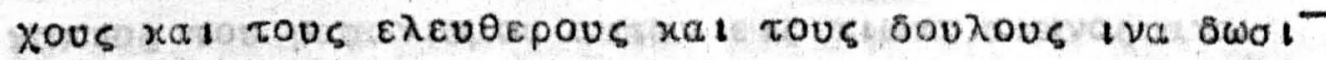

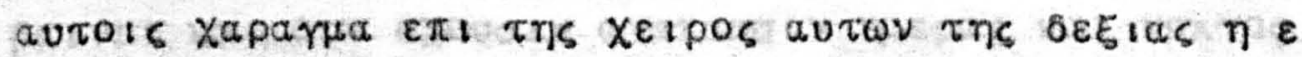

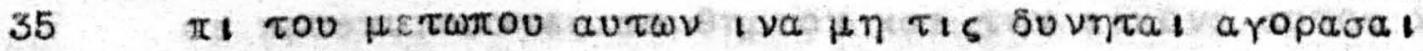

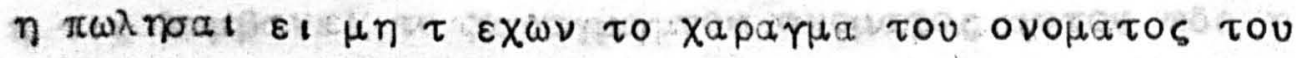

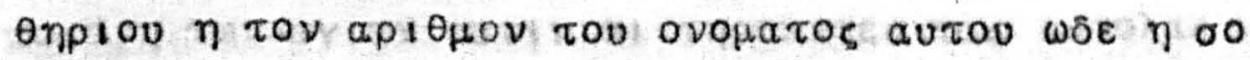

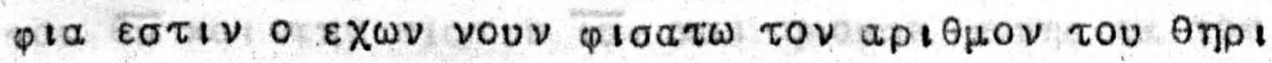

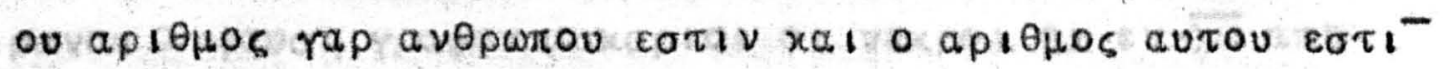

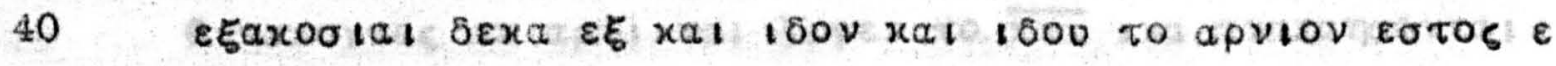

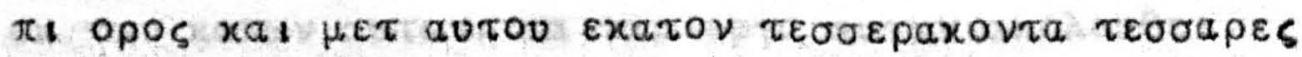




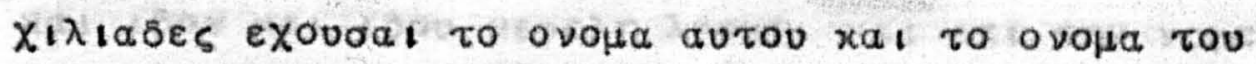

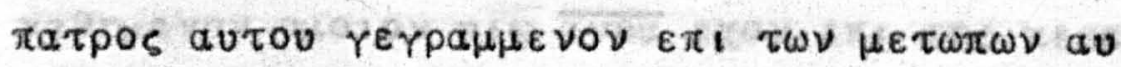

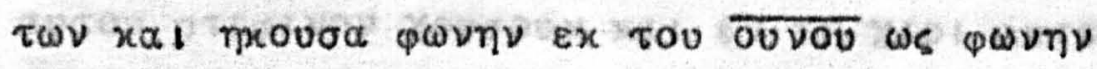

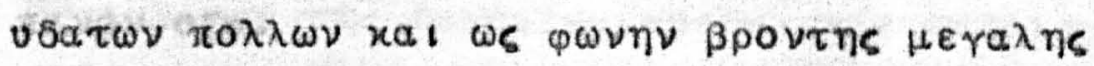

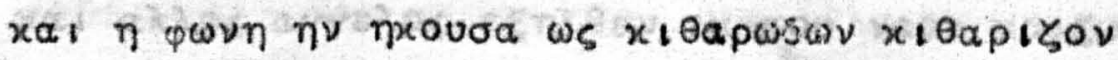

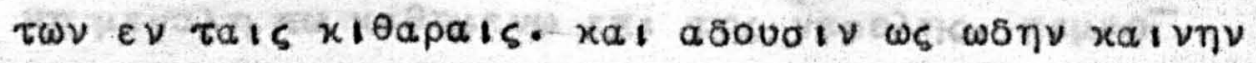

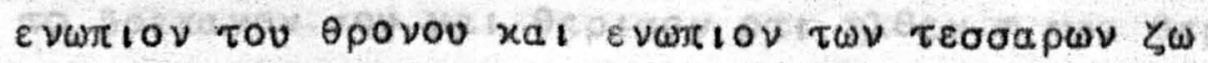

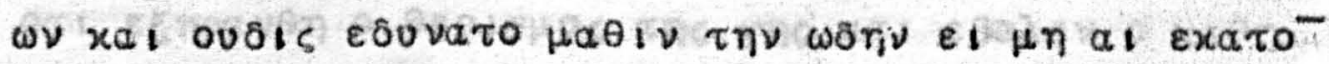
$\tau \varepsilon \sigma \sigma \varepsilon p a x \circ \nu \tau a$ Xi

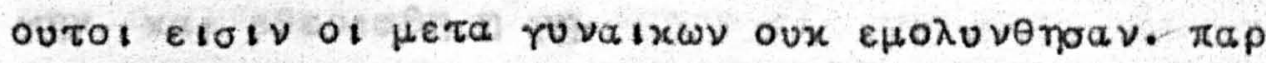

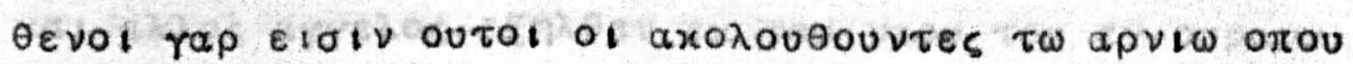

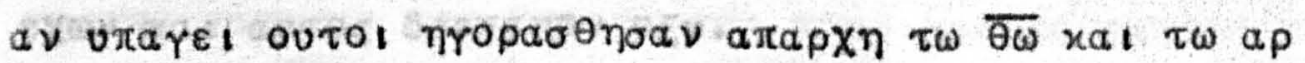

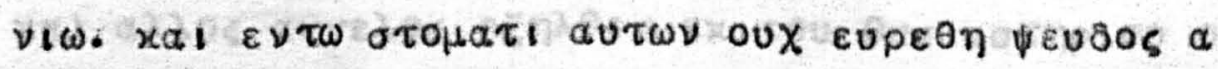
$\mu \omega \mu O t \varepsilon 101 \nu$.

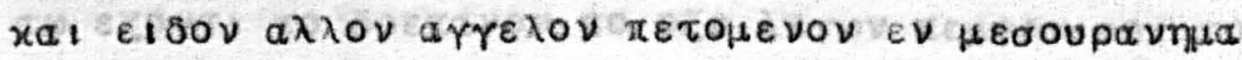

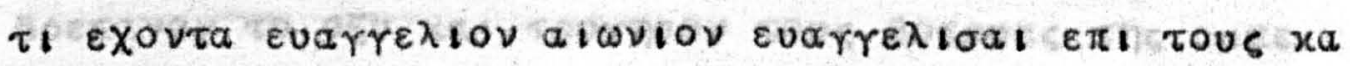

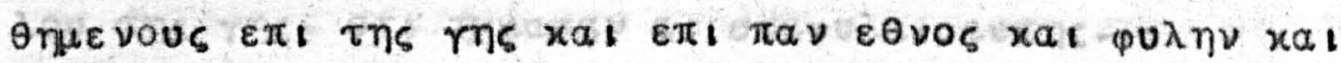

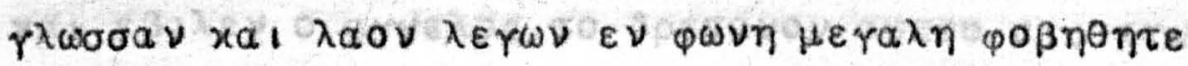

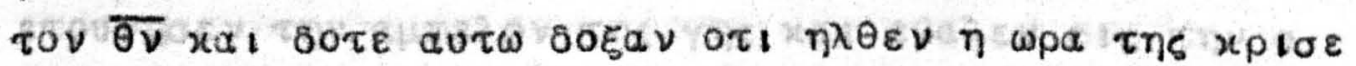

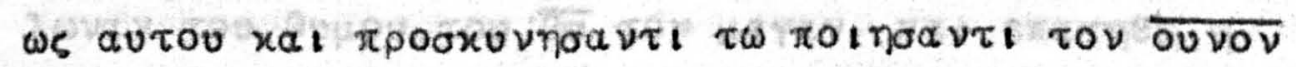

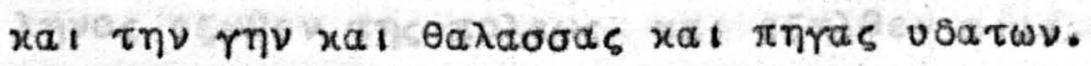

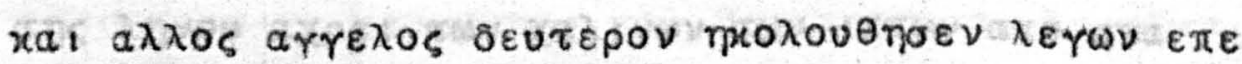

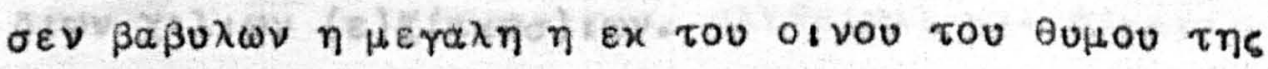

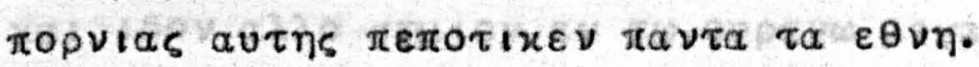

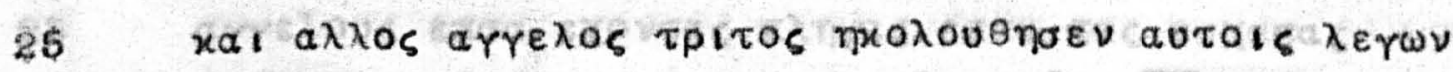

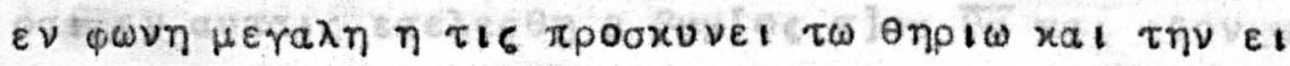

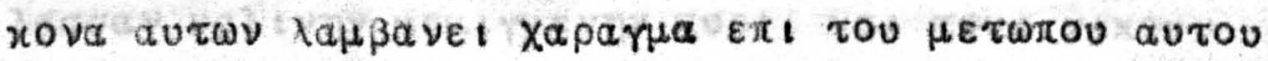

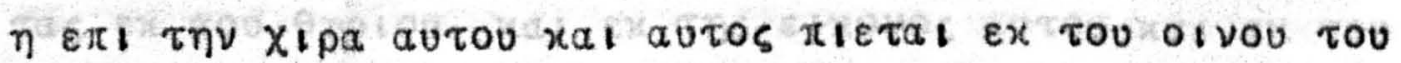

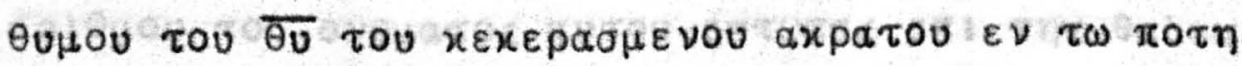

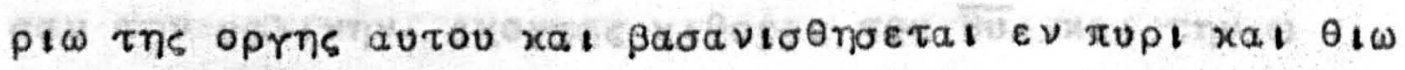

$\varepsilon \nu \omega \pi 10 \nu$ arre

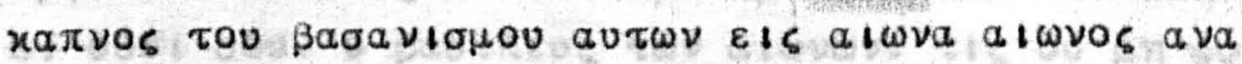

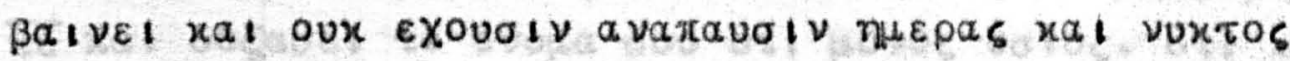

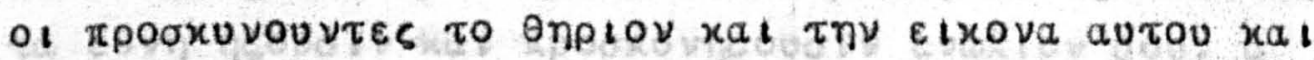

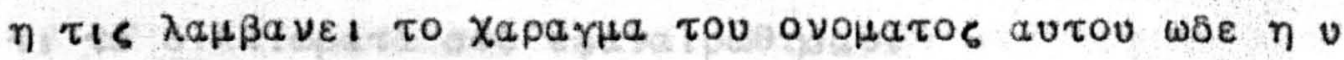

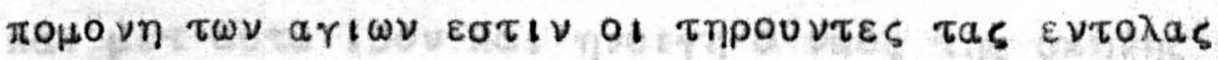

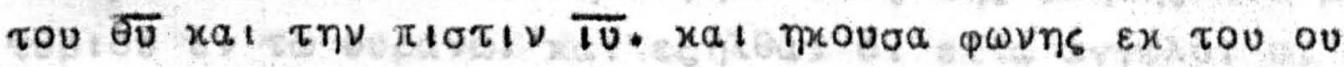

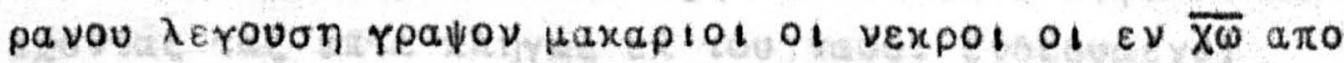

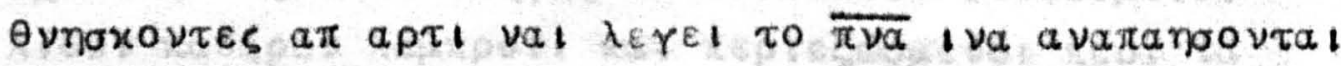

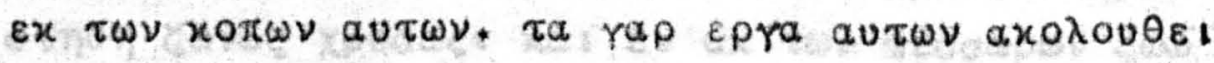

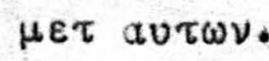




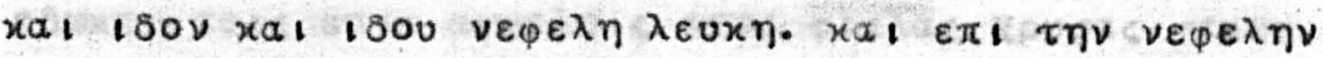

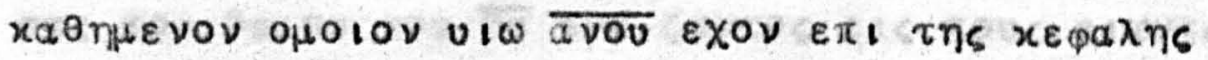

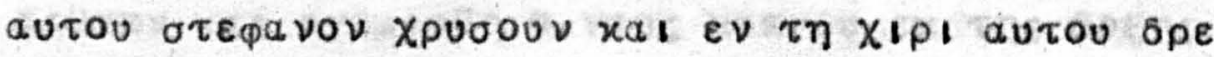
Tavov ogo.

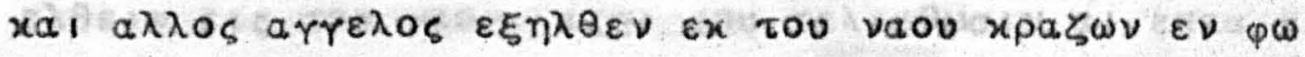

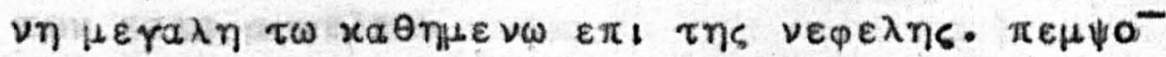

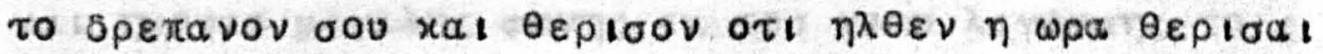

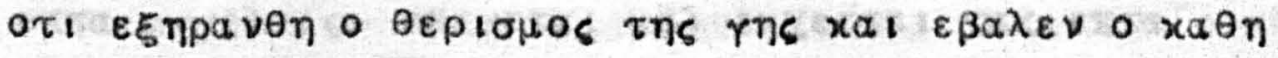

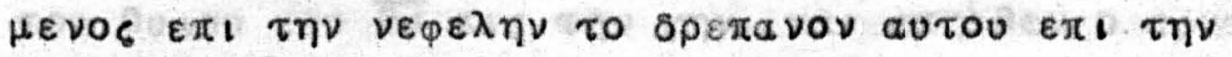

10 r $\eta v . x_{1} \varepsilon \theta \varepsilon \rho t \sigma \theta \eta \eta r$.

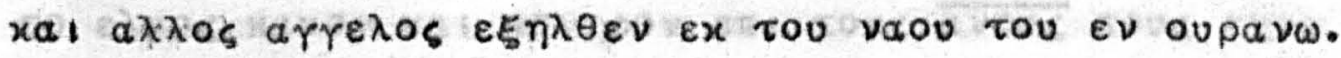

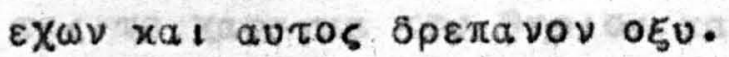

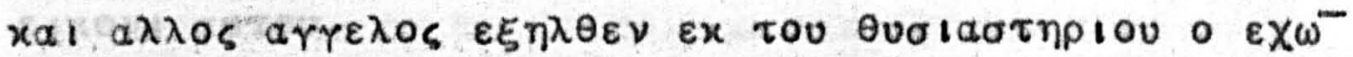

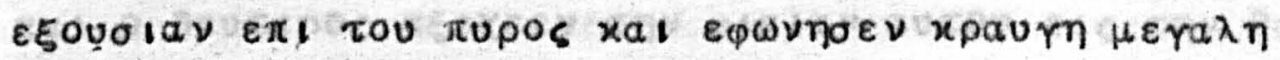

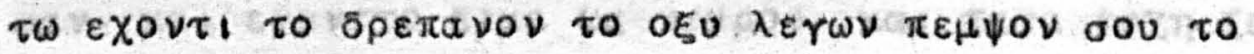

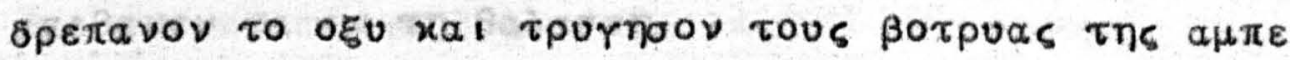

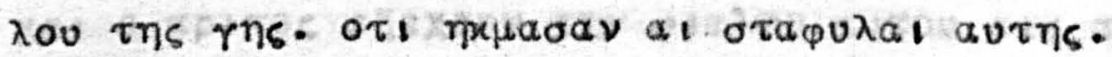

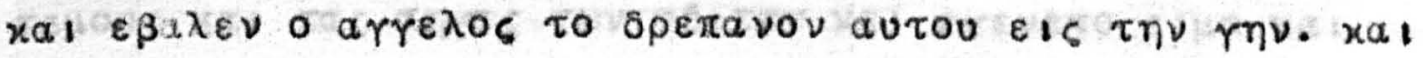

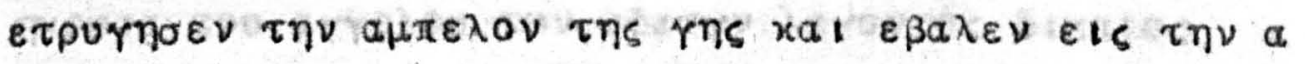

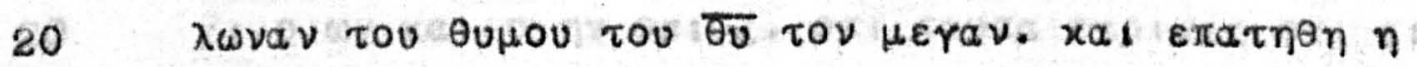

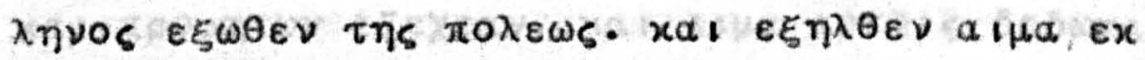

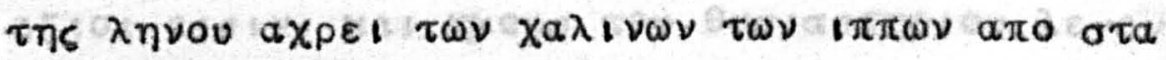
$\delta เ \omega \nu \quad X i \lambda \uparrow \omega \nu(\varepsilon) E(\alpha x \circ \sigma) i \omega \nu$.

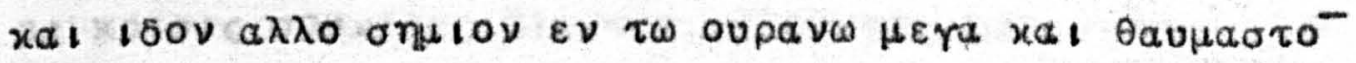

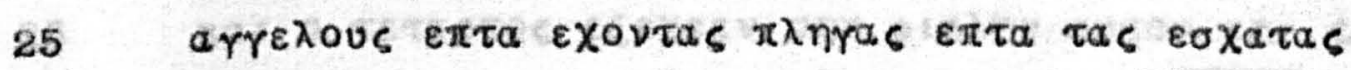

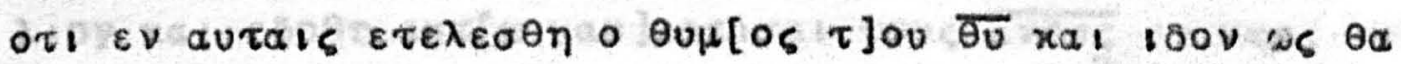

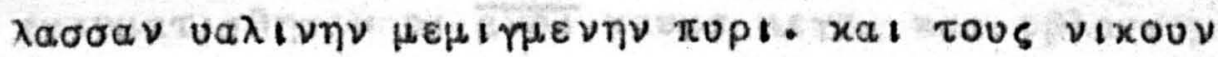

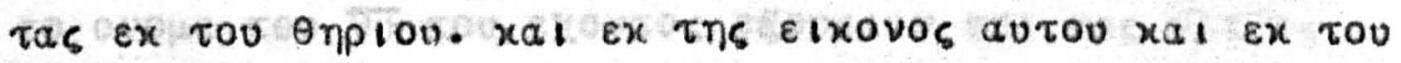

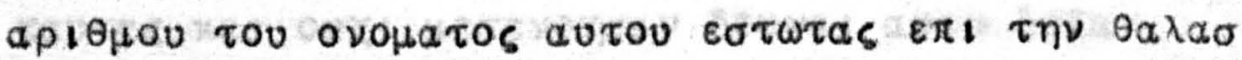

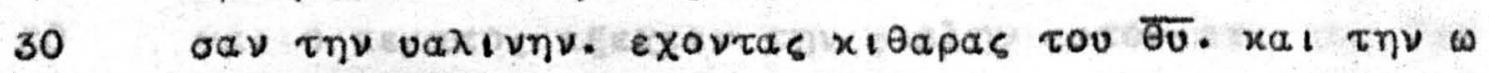

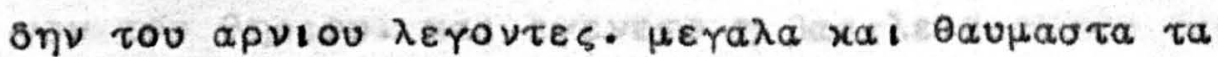

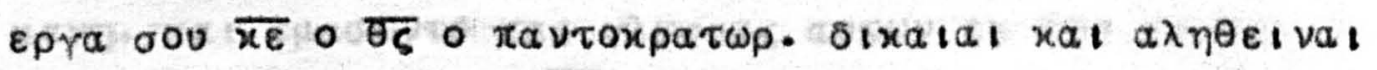

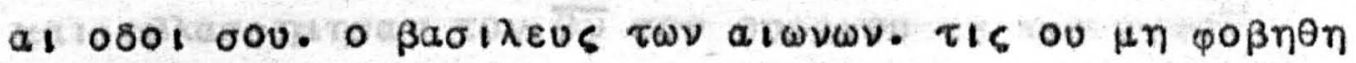

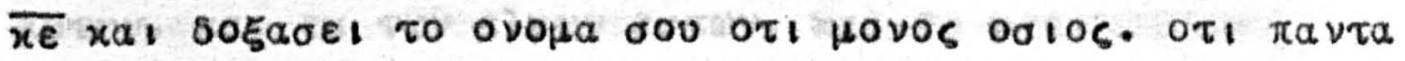

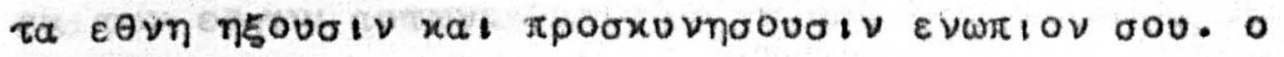

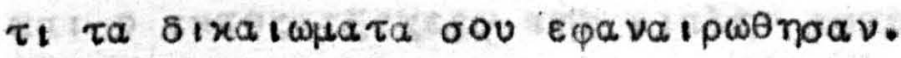

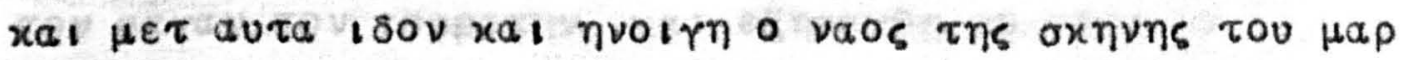

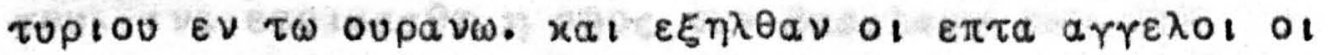

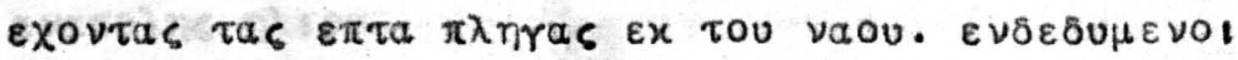

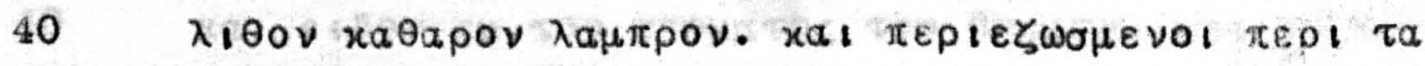

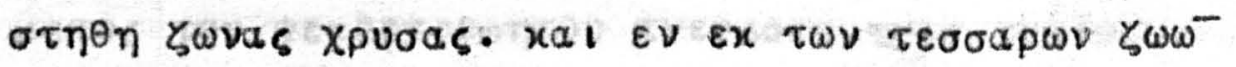




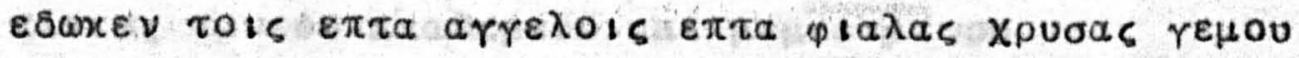

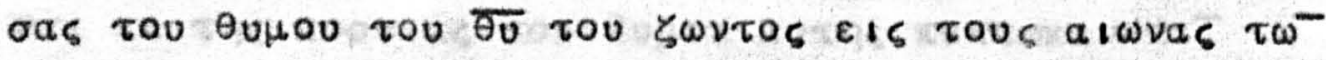
al

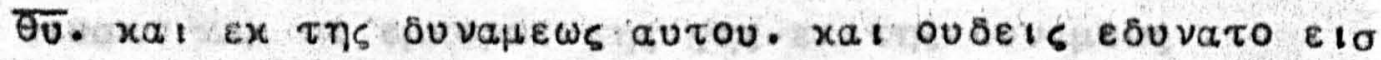

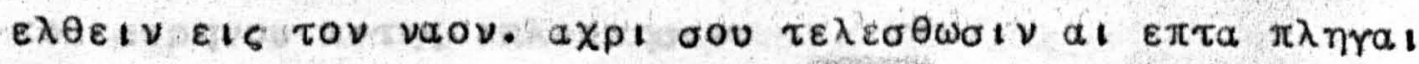

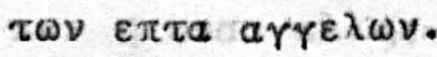

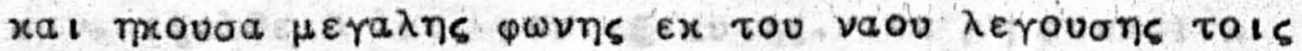

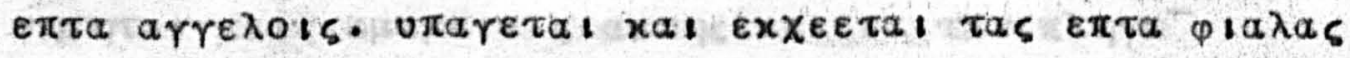

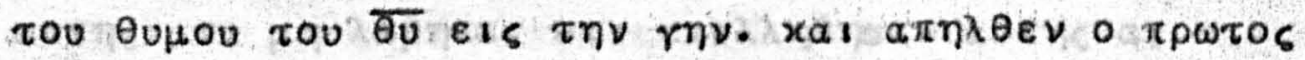

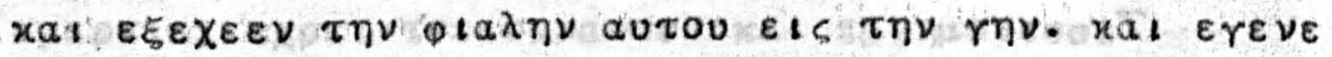

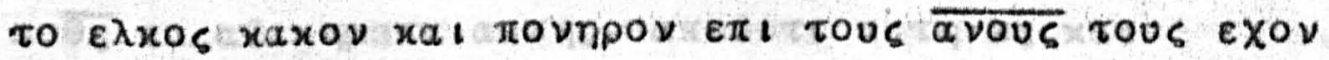

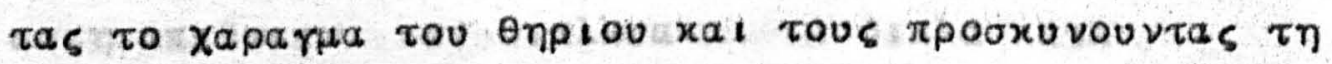
Eเxovi aurov.

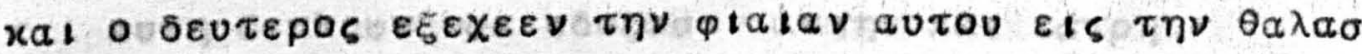

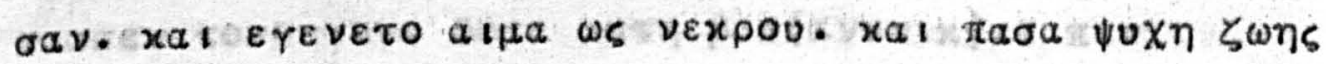
$a \pi \varepsilon \theta a \nu \varepsilon \nu \tau a \in \nu \tau \eta \theta a \lambda \alpha \sigma \sigma \eta$.

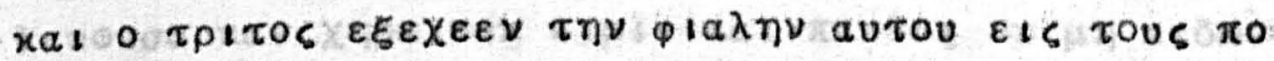

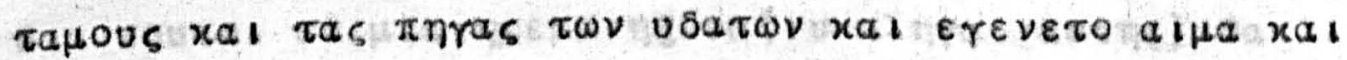

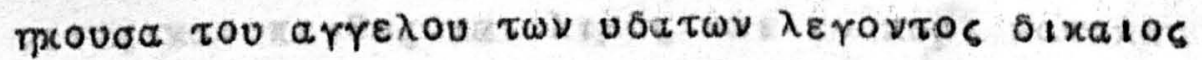

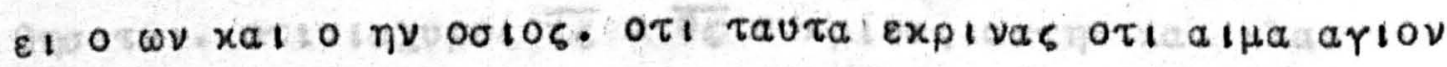

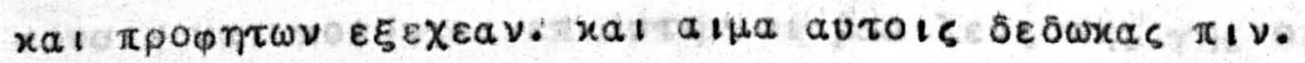

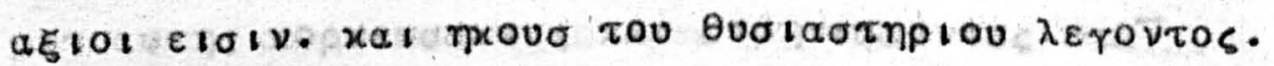

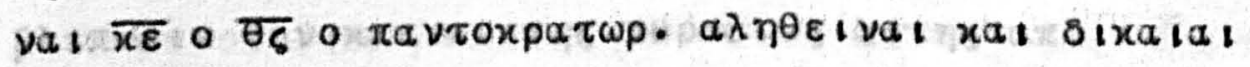
a) xplots oov.

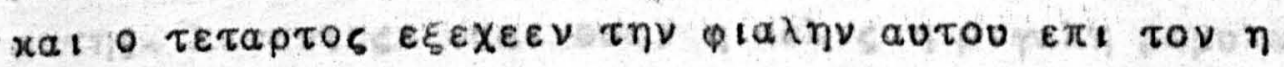

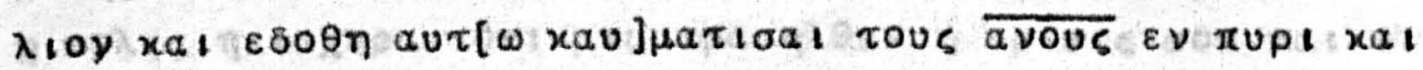

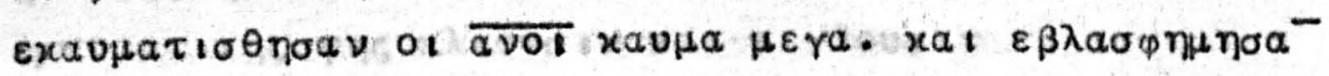

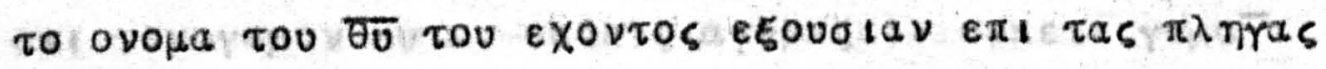

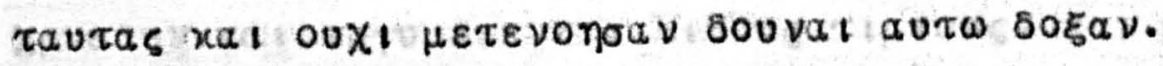

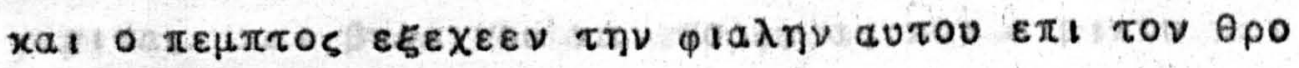

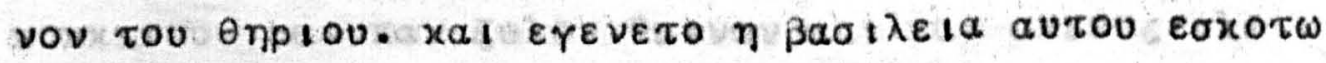

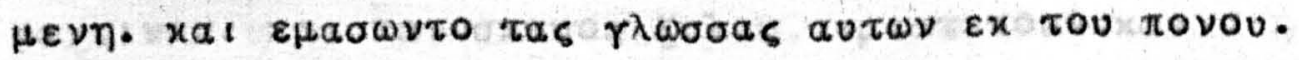

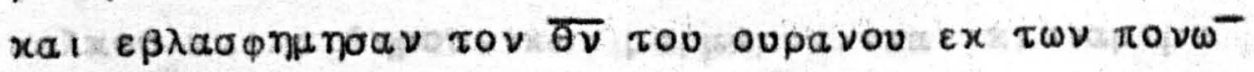

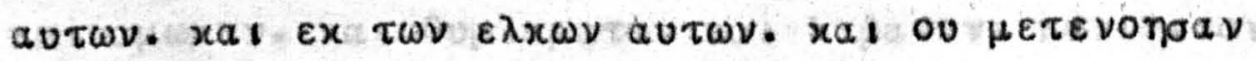
$\varepsilon x \tau \omega \nu \varepsilon \rho \gamma \omega \nu$ av $\tau \omega \nu$.

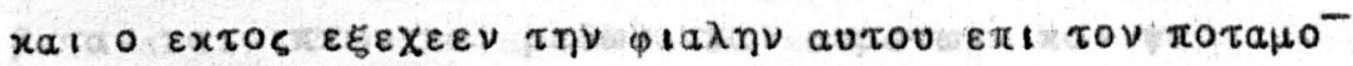

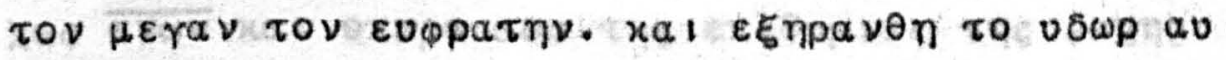

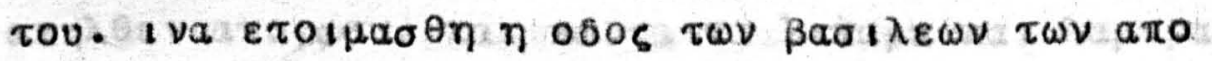

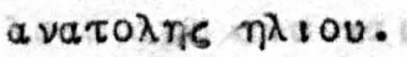

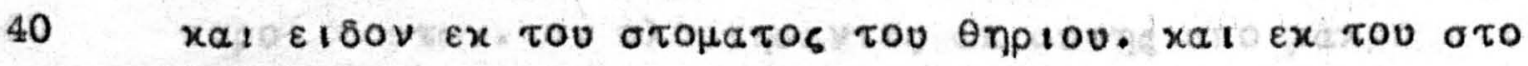

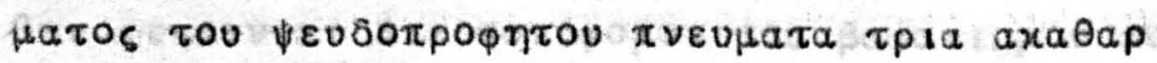




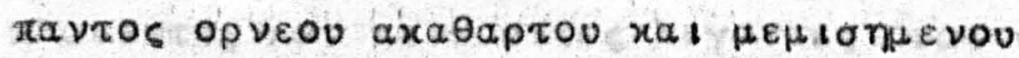

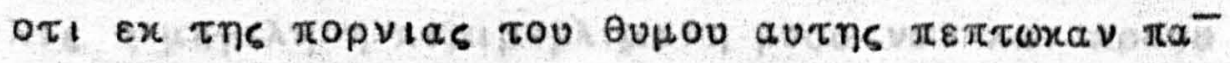

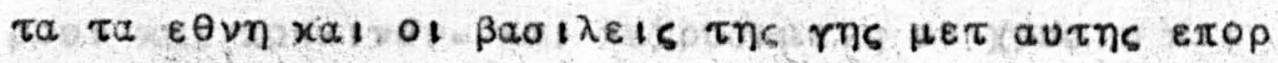

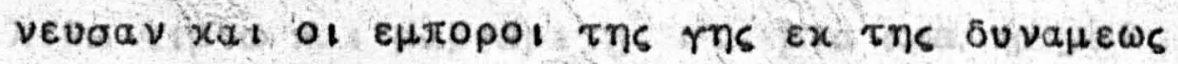

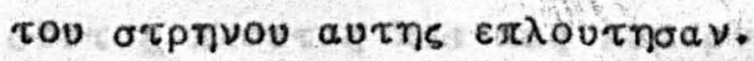

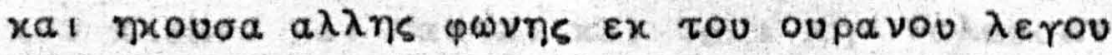

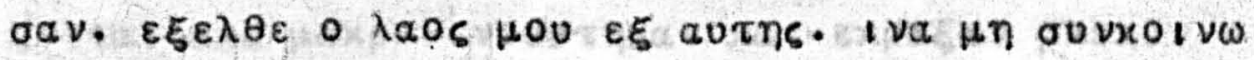

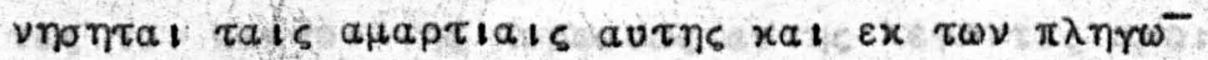

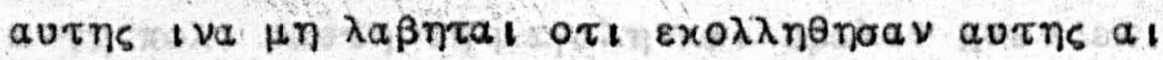

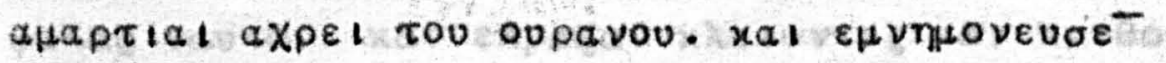

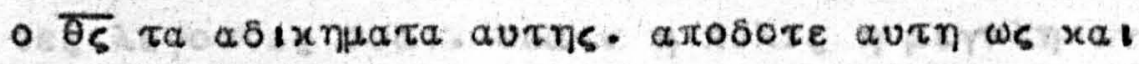

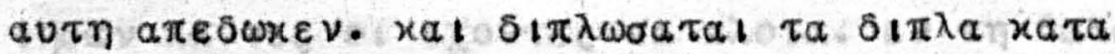

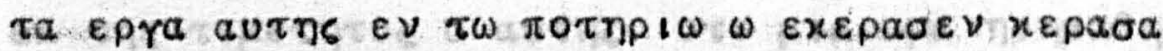

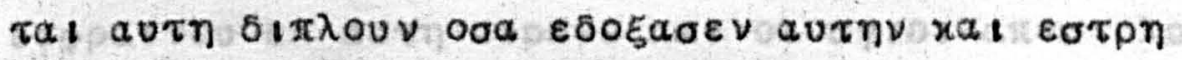

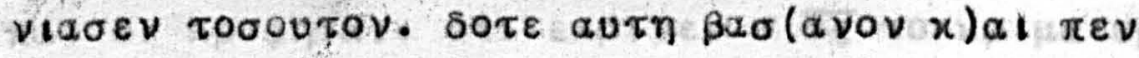

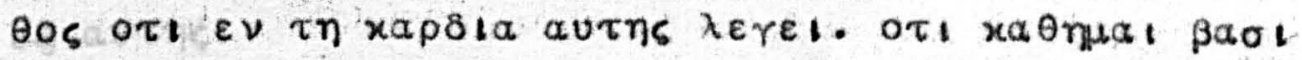

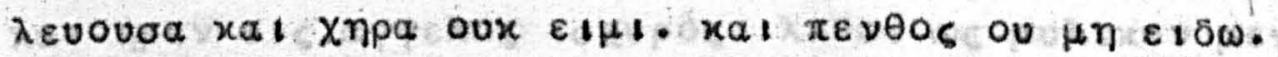

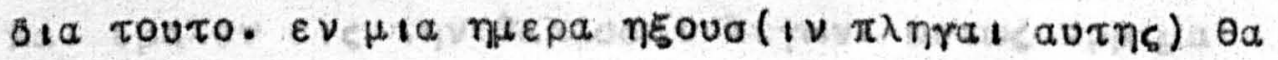

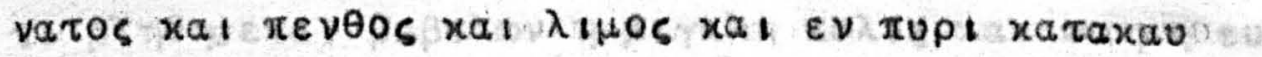

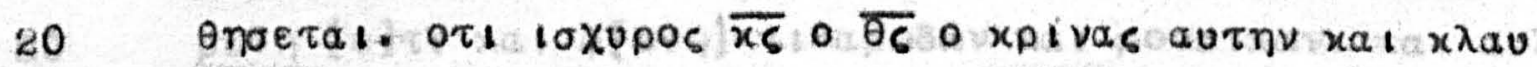

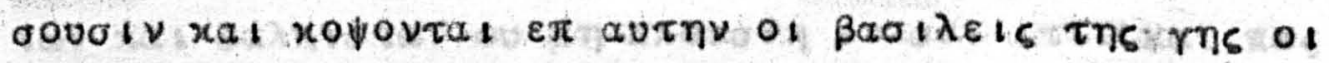

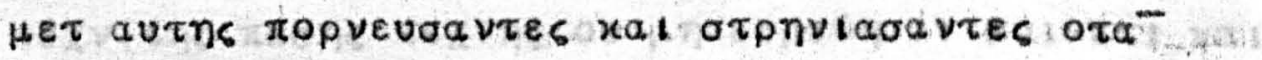

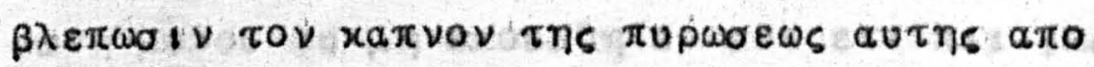

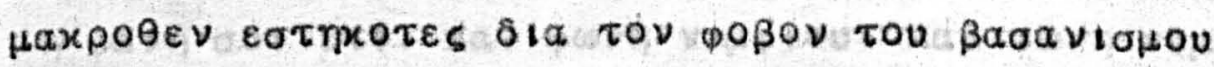

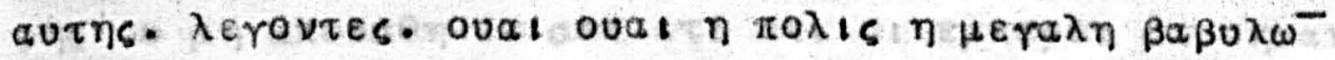

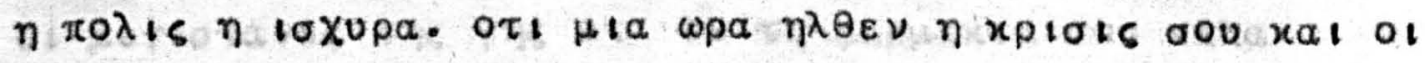

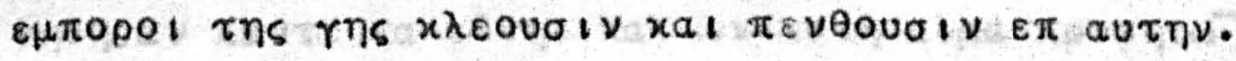

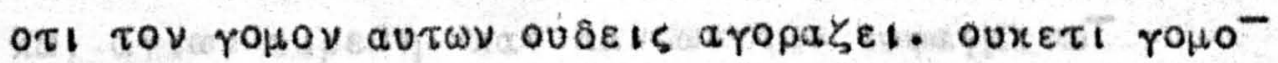

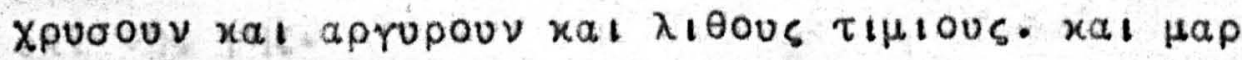

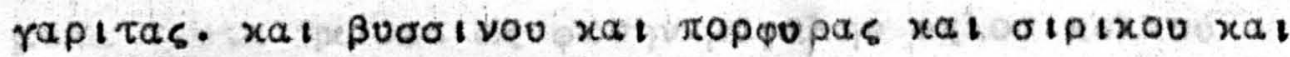

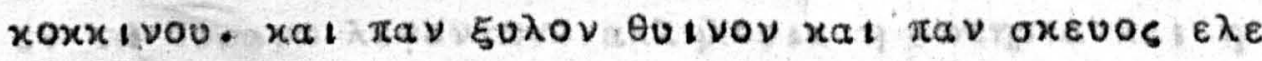

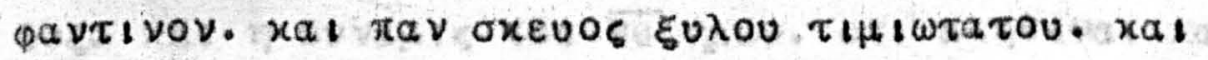

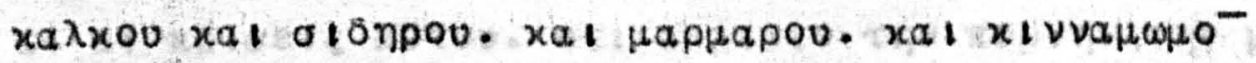

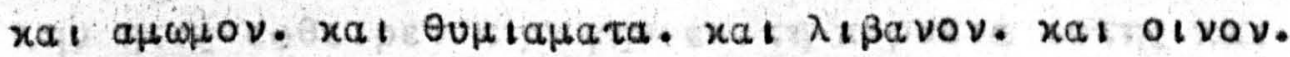

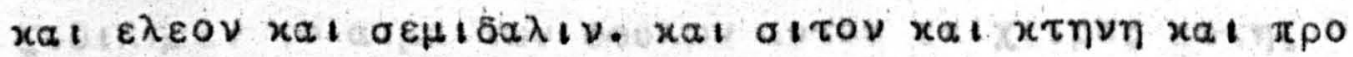

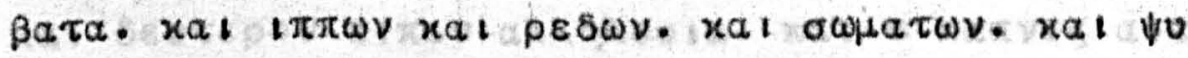

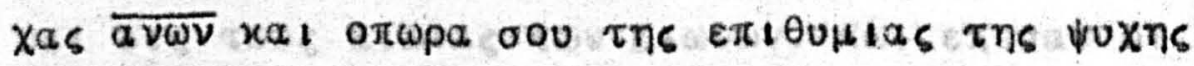

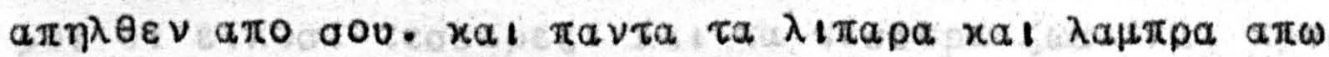

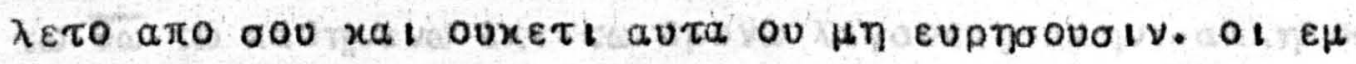

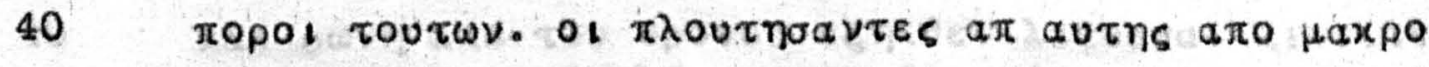

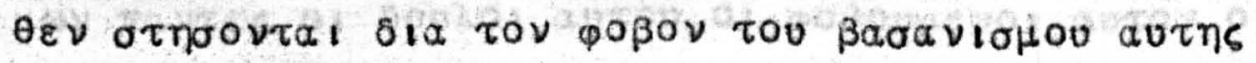




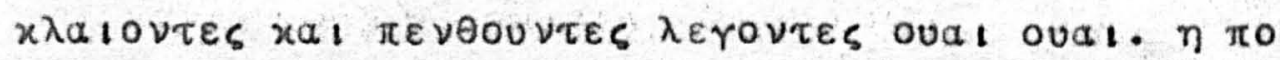

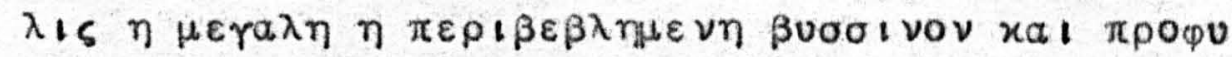

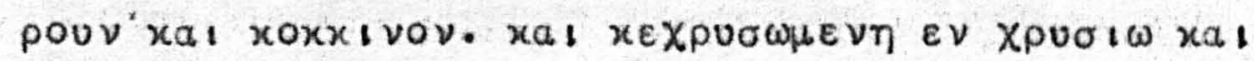

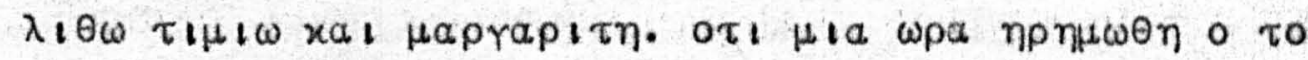

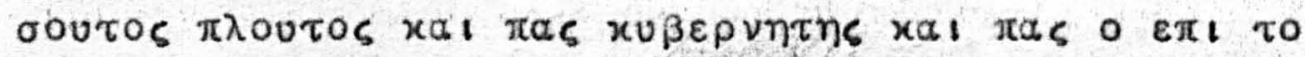

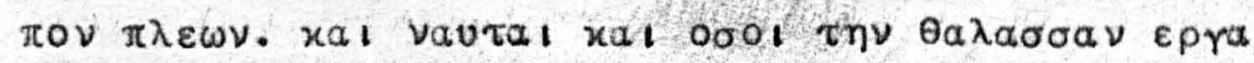

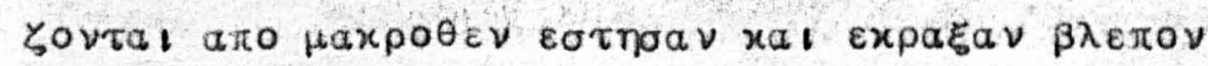

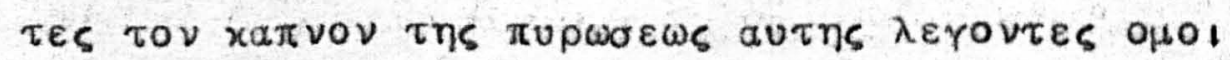

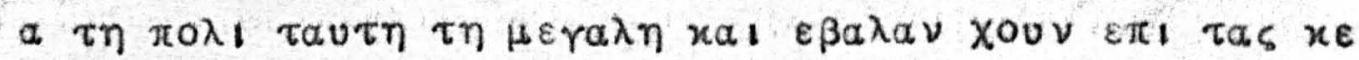

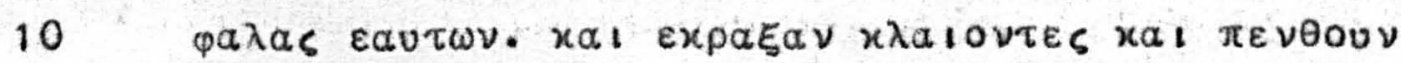

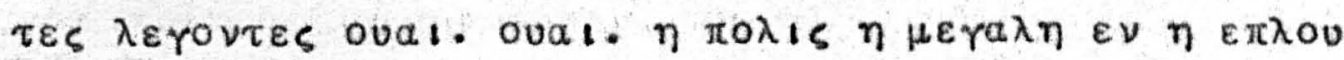

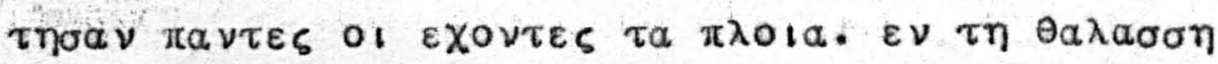

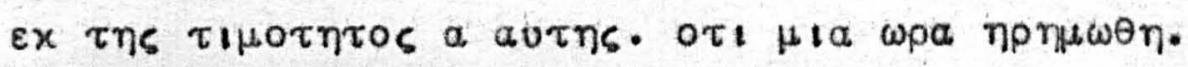

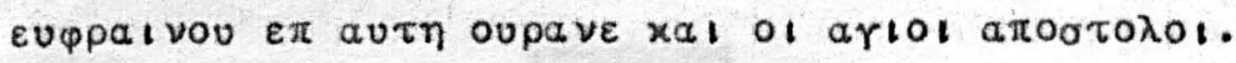

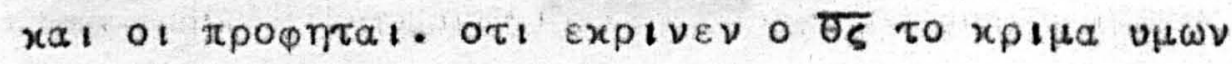
$\varepsilon \varepsilon_{3}$ autns.

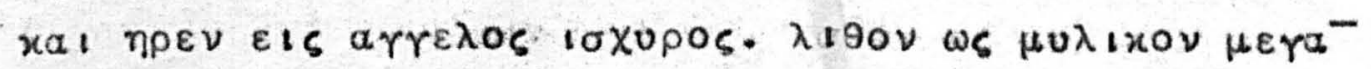

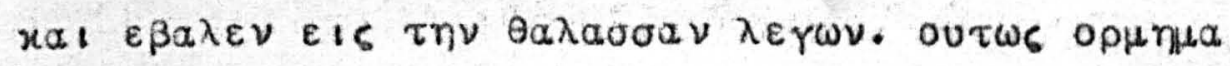

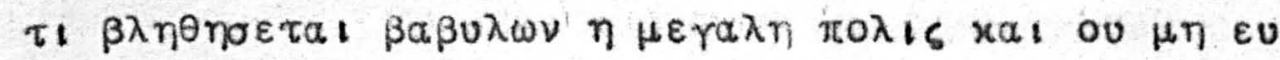

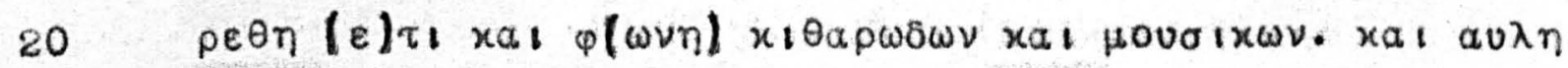

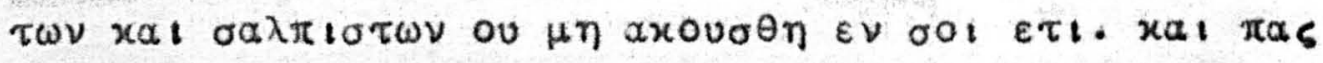

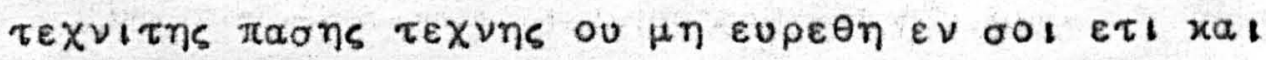

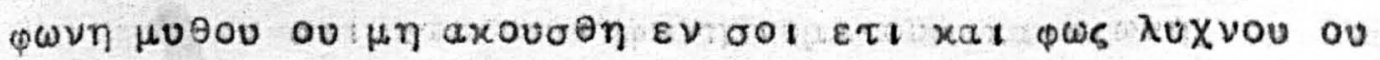

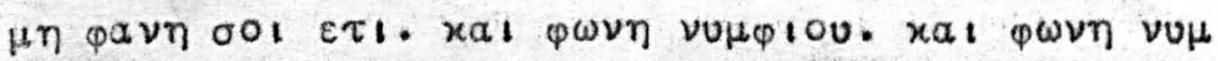

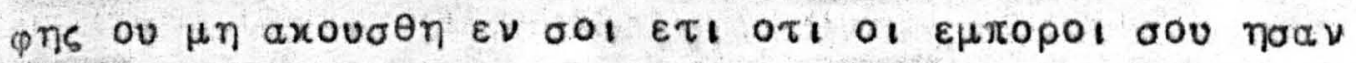

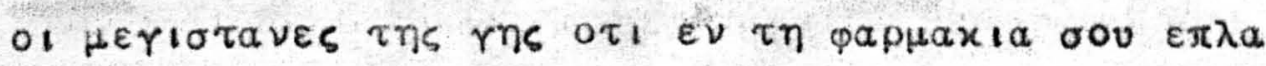

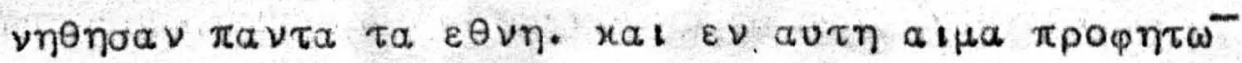

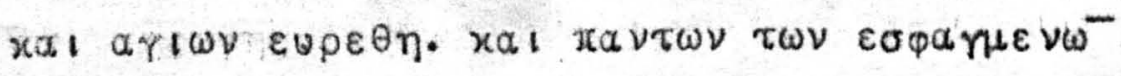
$\varepsilon \pi$ i $\tau$ ins rns.

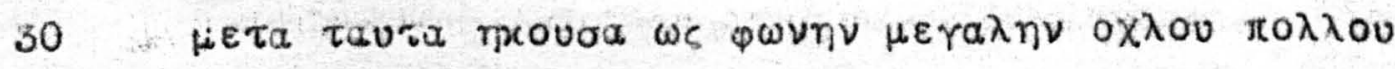

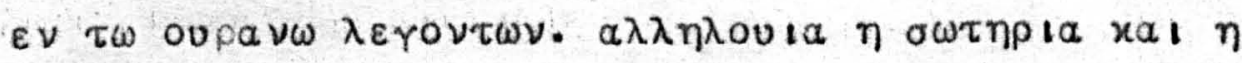

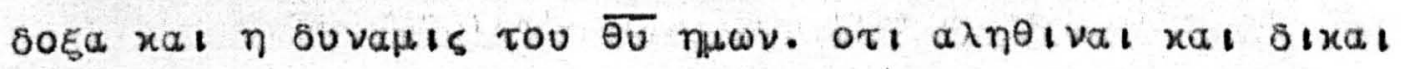

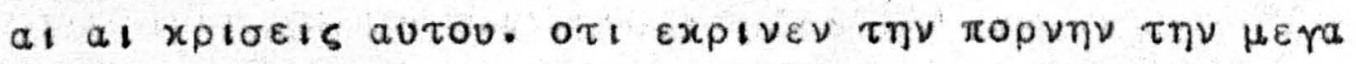

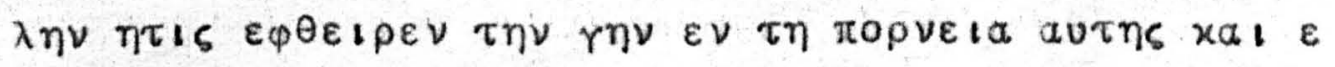

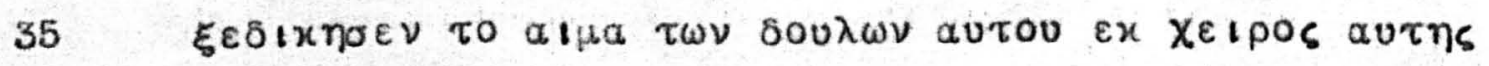

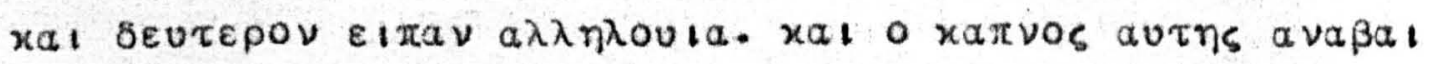

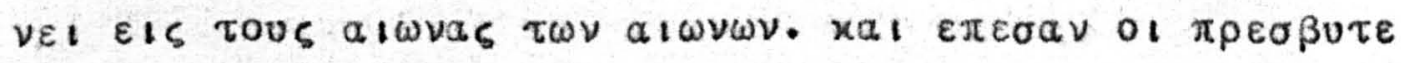

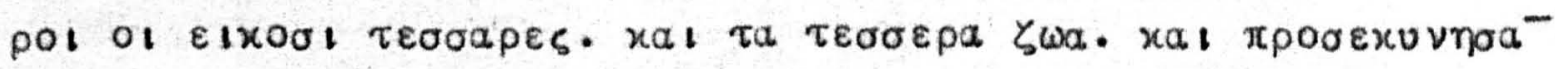

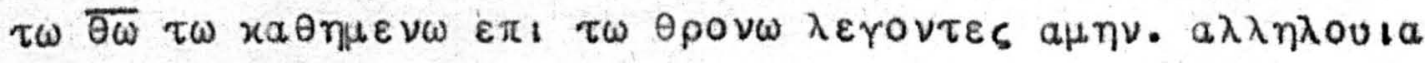

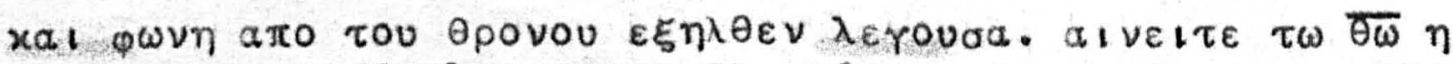

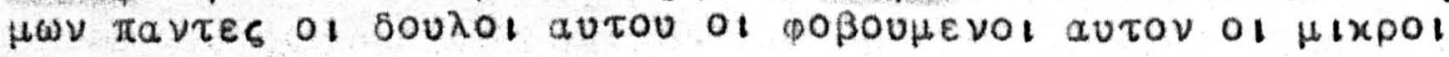


APPENDICES 
In these appendices I have boen concemed rainly with the problens of the textual critic and have formed the notes in such a way as to meet his needs. I have preferred vartous readings with different degrees of cextainty, and I hope ny attitude is revealed in the qualifying words such as "probably," "possibly," "undoubtedly," which I have used. Whenever I have corrected the work of tischendorf, I have directly stated the difference.

The following sigla are used:

A - the original scribe

$\mathrm{B}$ - the first corrector

C - the second corrector; (whenever the mamuscripts comonly designated by these letters are referred to, they are proceded by "Codex," such as Codex A, Codex B, Codex C)

I8 - Tischendorf's 8th major edition of the Greek liew Testanent, pubIished $1869-72$.

(?) - follows an omission by the first corrector to indicate that nothing is witien to prove the identity of the first corrector. Ify reasons for assigning these omissions to the first corrector are given in the Introduction; on a very fev occasions I have differed trom tischendorf. So while an elenent of doubt may remain, 1t has seomed good to indicate these erasures. This sign (?) in no way indicates the reading itself is questioned. Such questioning of a reading is always mentioned in the note. 


\section{APPENDTX A}

NOTSS ON THE TSXT OF THE ORTCTNAL SCRTBE

Matthor

9.26 - After pnun the original scribe wrote autoc, but corrected this - mistake hinself to read autnc.

26.39 - The origtnal seribe has added in the margin the text of Inke 22.43 and indicated that it is to be read after $\underline{a \lambda \lambda} \underline{\omega_{S}}$ ou.

Yarik

1.45 - The ariginal scribe wrote tov $\lambda$ o at the ond of the 1ine, but falled to wrlte yov on the next line. Neither of the correctors has supplled the needed letters.

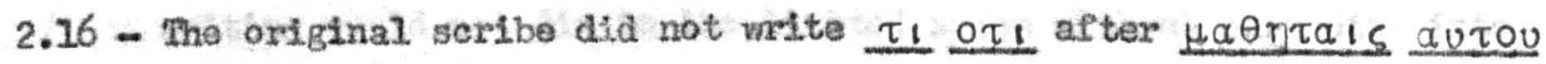
as Mschendorf has stated. A long hole in the vellum covers the beginning of this line where $\tau a$ is autou was mitten. Mischendorf's edition is as follows ...... ter is represented by one dot). I do not know how he saw the $\underline{U}$ unless he assumed some wark below the line to be vestiges of 1 t. Ne1ther do $I$ see an 1 before of 1 . ( It is, of course, possible that this hole has been enlarged during the Inst eentury.) Yet It st111 remains inpossible for $\tau_{1}$ to have been written becsuse the sosce gimply is not sufficient. In line 18 qais autou occuptes the maximun anount of space that is avallable before

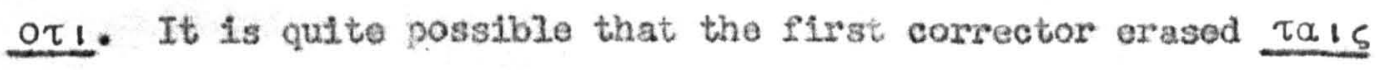
autou (hence the holes) to add $\tau_{1}$. This wovld explain IIsch-

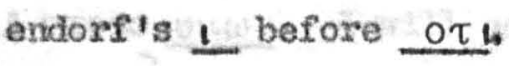

3.5 - I cannot tell whether the original scribe wrote a $a 0 x a \tau \varepsilon \sigma \tau \eta$ (with wetstein) or $a \pi \varepsilon x$. (with IIschenriorf). A small hole has removed part of the letter, and a heavy black nark covers the rest.

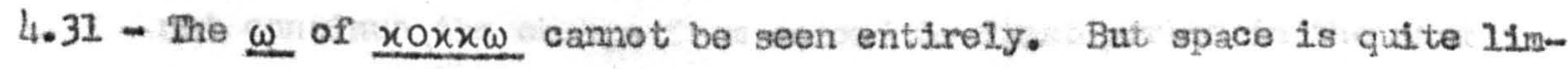
ited and not sufficient - as Tischendorf has assumed - for ov. He thought he saw 0 after $x o x x$, but this is the first part of w. There can be no doubt that he is wrong. 
Mark

9.2 - The original seribe has apparently written $\_\delta$ (or $a \lambda$ ) after quepas. What this could mean, or how it was confused wi th $\varepsilon \xi$, I cannot say. Except for the bottom of the second letter, this is very clear.

9.18 - Tisohendorf sald the orfiginal scribe wrote $x a_{1} \tau \rho \rho_{1} \varepsilon_{1}$ tovs o.ovitas after $a p \rho / \zeta \varepsilon_{1}$. This is imposstble. In the previous Iine nine letters use the same amount of space taken by TH.schendore's twenty-one letters! It is not 1ikely that A wrote ac.

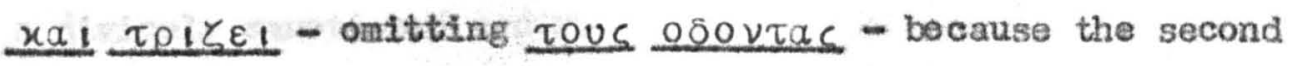
corrector has alded quTou at the end of the 1ine. A correction by the first corrector is precluded, since even his more crowded writing would not fit in the limited space without extendin into the margin. Codex $C$, therefore, containad gsolyel tous osovias (with Codex X).

John

21.13 - The original scribe mote $\varepsilon \rho x \varepsilon \tau \alpha_{1}$ 으. TC. Tischendorf rejected this reading, largely on the basis of space, and suggested either $\varepsilon \rho \times \varepsilon \tau \varepsilon$ 으 $\overline{I C}$ or $\rho \times \varepsilon \tau \alpha_{1} \overline{I C}$. I have been able to detect the article and apparently the _. Furthemore, in line 5 the $\tau_{a}$ is of $\mu a \theta \eta \tau a / s$ easily fits into the space vailable for $\tau \alpha_{1}$. Our text need not be doubted. Nestle's apparatus does not cite Codex $\mathrm{C}$ for either reading.

\section{Acts}

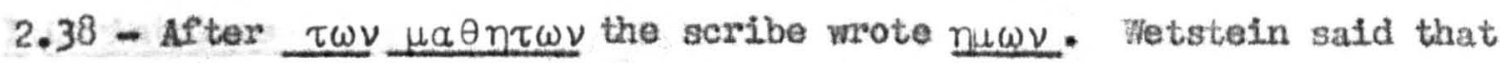
A wrote $\underline{u \mu \omega \nu}$. I will adrat that the vestiges of $\underline{U}$ appear to be seen under $\eta$, but 1 t does not resemble the $\underline{v}$ elsewhere on the same page. Furthermore the $\underline{n}$ is definitely by $A$. If it has been corrected - and I doubt it - one wonders why the corrector did not continue the change from second to first person in the next verse (with Codex D).

\section{Peter}

2.14 - Tischendorf wrote $x \tau 1 \sigma \varepsilon \downarrow$ L 
that at this point he guessed. I ar convineed the scribe wrote nuec, $a v \theta$. I ras quite certain of this when looking at the manuscript and an infra-red photograph has confirmed this. It is, as $I a r$ as I have been able to find, a reading unique to Codex $C$. If it wexe adopted ( $I$ do not advocate this) its significance might be to remove the connotation that these authorities received their powers Prom God. Cr., Arndt and Gingrich, A Greek-English Lexicon of the Now Testanent and othar early Ghristian Literature, Article xtiols, p. 456e. Peter then refers to a hwman order rather than a divinely sanctioned order.

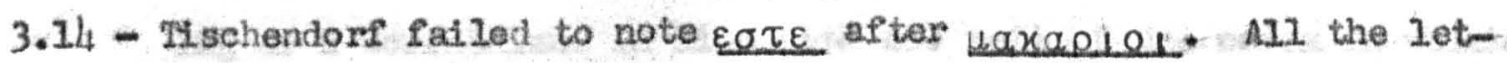
ters can be seen except the final. $\underline{\varepsilon}$.

\section{Petar}

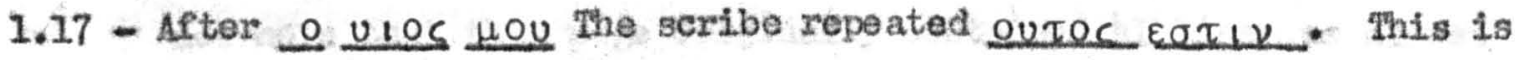
clearly seen although Tischendorf did not mention it. A noticed his nistake, erased these words, and wrote arajntoc. He apparently left the rest of the line blank and wrote - I think - عlc ov $\varepsilon \times \omega$ on the next Iine. I have no ilea why the second correct-

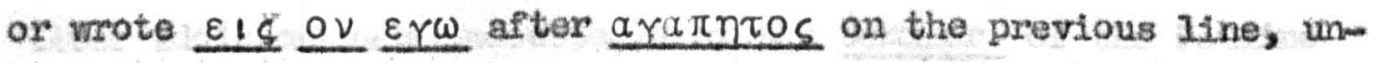
less the condition of the manuscript had deteriorated by that time. W.sohendorf's appendix mentions the various alternatives to the text at this point.

3.7 - The original scribe wrote a duplicated $\mu \varepsilon$ in $\tau \eta p o u \mu \varepsilon v$ l, $_{\text {, but }}$ erased it before golng any farther.

\section{Corinthians}

1.2 - At the top of this page Tlischendorf assuried that four lines originally written in colored ink have faded completely - apparently because the entire text of $1.2,2$ was missing* The visible writ-

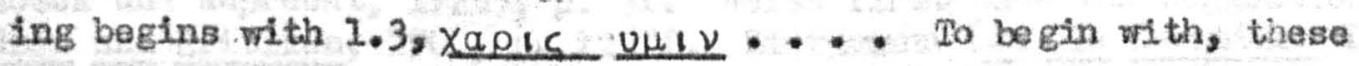
verses would probably have taken more than four lines. Nestle's text contains 187 letters (taking into consideration the nomina sacra the scribe undoubtedly used). Since the scribe averaged no more than 40 letters per line, five lines wonld have been needed. 
One may ask If it is possible that the scribe wrote these colored Iines in maller letters so that up to 50 letters could be placed on one 1ine. According to Tischendorf's edition on James 1.I the scribe did just this. But I an convinced that Tlschendorf did not see what he wrote. But even if one does accept IIschendorf's text at that place, it should be noted that only the first line has more than the normal nuber of letters. Whe three has a normal 42 letters. This line of argument, however, - Is futile because the significant fact is that only three lines have disappe ared, as a close examination of tha vellun revealst This brings the beginIng of 1 Corinthlans in line with the rest of the codex in which the beginnings of 18 of the Net Testannent books are extant: without exception they begin with three colored lines. Furthermore, we now note that this pege originally contained 41 lines as almost all the other leaves in the codex. Knowing that only three lines are risaing, is tt possible to conjecture what was omitted. In the Iight of this evidence atiention shovad be dram to the conjecture

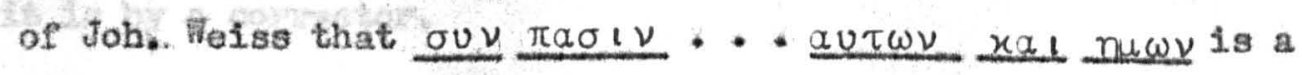
later addition made undoubtedily by the Sganter of the Paultne corpus to prepare the opistle for a wider reading. Do we now have In Codex $C$ a witness for this onission? Wh thout the second part of varse 2 the text of Hestle has 119 letters, and this cleariy is well suited for three lines. The mitor need not have filled the three lines completely, since line four could have started a new paragraph. Onty in 2 Corinthians did the original scribe fail

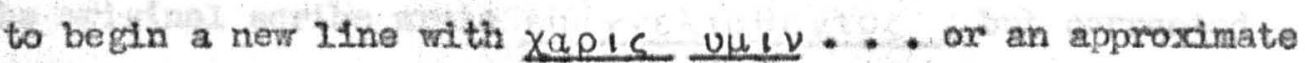

1. Joh. Weiss, Der erste Korintharbrifef, krit,-exeg. Kommentar ther das neue Testament begrundet von $\mathrm{H}_{*} \mathrm{~A}_{*}$. W. Heyer, lote Auflage (Glttingen: Vandenhoock un Ruprecht, 1925), p. 3f. Weiss first rade the suggestion in Studien und Kritiken, $1900, \mathrm{p} .125 \mathrm{f}$. Iiet zmann (An die Korinther I.II, Handbuch zum neuen lestament, 4te Auflage von $V . G$. Kurmel, p. 5 ) rejects Weiss's suggestion by saying that the phrase exhibits the Jewish $h$ abit of "katholisiorenden", and refers to such precedents in a fragment of a Palestinian text. 0 . T. Craig (The Interpretor's Bible, ad. loc.) 14kewise rejects the conjecture. He mites, "It is nore probable, however, that Paul himself used the appeal to the other cormuni ties to bring this very Independent conrregation into line," 
equivalent (in the oxtant leaves the scribe bogan a line in this way in 1 Poter, 2 Petex, I Corinthians, I Thessalonians, 2 Timothy and THtus).

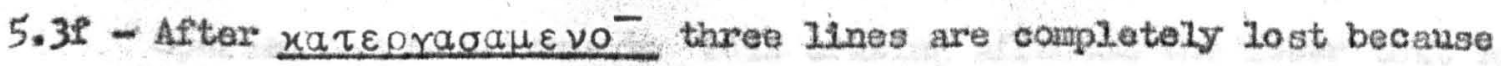
of large holes in the vellum. Nothine can be seen.

\section{Corinthians}

4.17 - Heter $\tau \eta_{S}$, $\lambda_{1} \psi \varepsilon \omega s$ nothing can be seen of the original or correeted text. But the space is quite sufficient for $n \mu \omega \nu \underline{x a}$ ט $\varepsilon \rho \beta \beta \lambda \eta \nu$. I see no reason why at cannot be assuned that $\Delta$ wrote what wave included in parentheses.

8.22 - The original scribe wrote orosalov and corrected his ow text to readojousalov

\section{Galatians}

2.9 - I an quite certain that the original scribe failed to write $\lambda \mathrm{O}_{1}$ in $\sigma \tau u \lambda 01$. The letters $\sigma \tau u$ are quite apparents $\tau U$ is much waller and has been crowded at the end of the line. Mischendorf

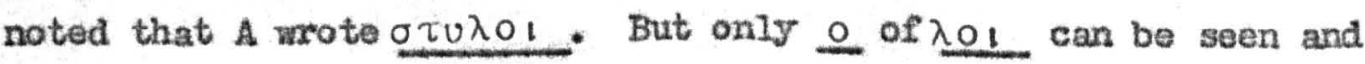
It is by a corrector.

\section{Philippians}

$2.26,27$ - After $010 \tau 1$ nxou the orfiginal text 1 s lost because of holes in the vollum. Sore of the aecepted text was antted. The corjecture of Tischendore is as good as any suggostion: A skipped fros the first no $\theta \varepsilon \nu n \sigma \varepsilon v$ to the second.

\section{Hobrews}

2.13 - See appendix B.

4.7 - The original scribe wrote $\varepsilon v a \gamma \gamma \varepsilon \lambda 1 \sigma \theta \varepsilon \nu \tau O \zeta$, but corrected his own text to read Eunry.

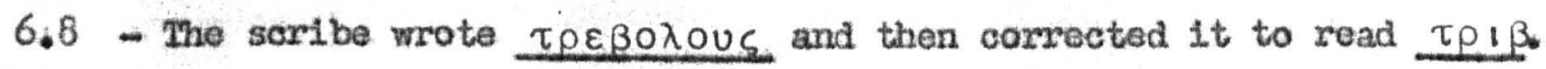
II IImothy

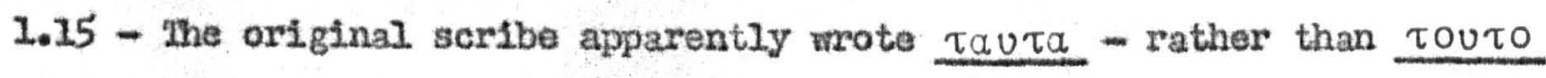
after 이의. This word is almost beyond recovery because of holes. The top of the first $\tau$ and all the second $\tau$ can be seen. of the first $\underline{a}$ only the bottom corner is available 


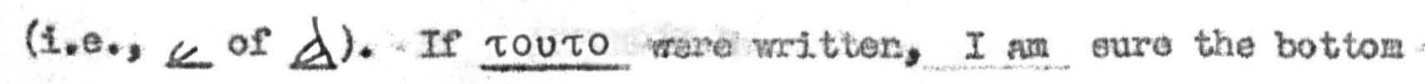
part of the on would be seen.

4.20 - Where $\mu_{1}$ (or $\mu_{\eta}$ ) of $\mu \eta \lambda \eta \tau \omega$ was written, the upper text of Ephraem has been erased; this orasting affected the lower text so that the last letter cannot be seen. Codex $\mathrm{C}$ should not be quoted for either $\mu \eta \lambda \eta \tau \omega$ or $\mu 1 \lambda \eta \tau \omega$.

\section{Titus}

$3.6-\underline{\varepsilon \varepsilon \varepsilon \varepsilon \nu}$ was originally written, but the original scribe corrected his om error by witing $\varepsilon \xi \varepsilon \times \varepsilon \varepsilon \nu$.

\section{Revelation}

13.15 - The Scribe mrote $\underline{\varepsilon 0 \theta}$ and then added a salall $\eta$ above the Iine.

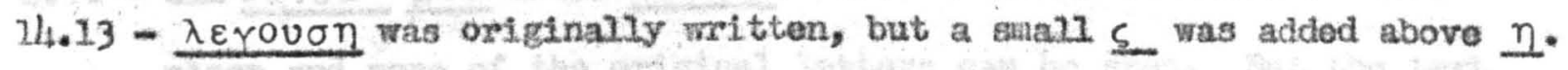
16.7 - A mrote nxovo and then added $a$ above the line. 


\section{APPBSNDIX B}

ALTBRATIONS MADE BY IHE FIRST CORRECTOR

Mat thew

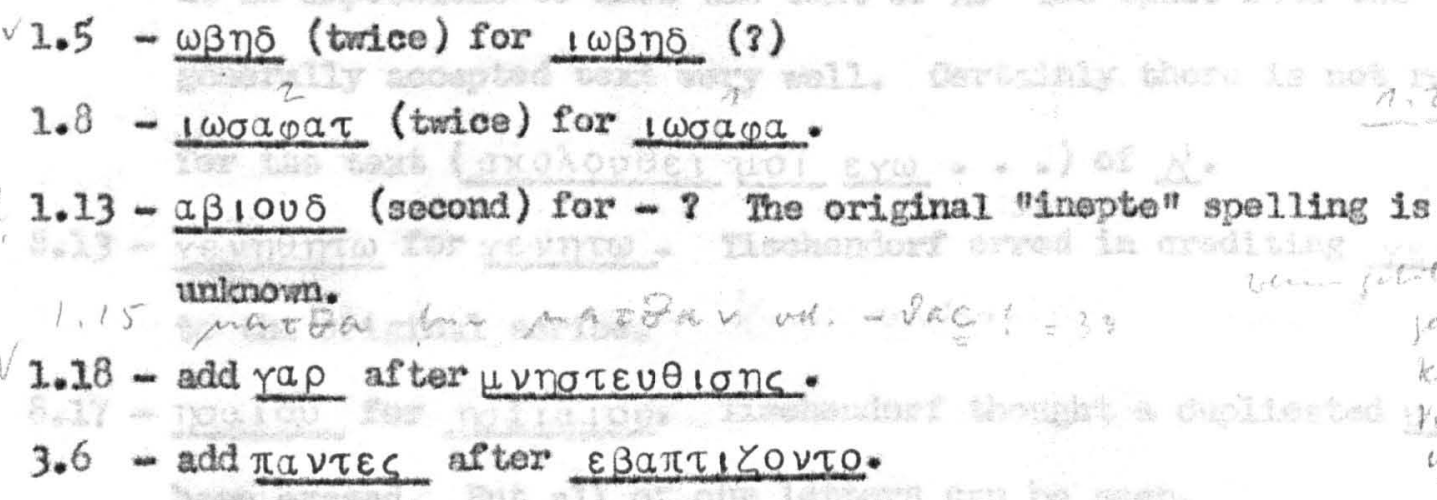


Yatther

8.4 - $\mu$ wuons for $\mu \omega \sigma \eta s$.

8.7 - B has written $\varepsilon y \omega \underline{\varepsilon \lambda \theta \omega \nu} \theta \varepsilon p \alpha$ before a hole in the vellum. It is impossible to know the text of $A$. The space fits the generally accepted text very well. Certainly thore is not room

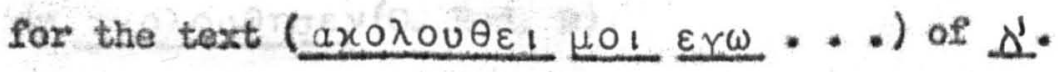

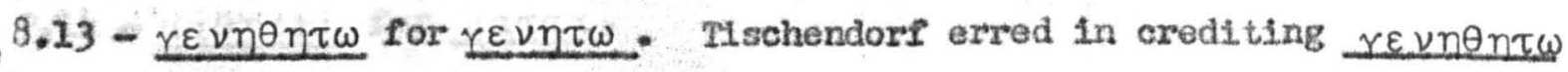
to the original scribe.

8.17 - noa 1 ou for nodialou. Tischendorf thought a duplieated $\underline{u}$ had been erased. But all of our letters can be seen.

$8.25-\pi \rho 0 \sigma \varepsilon \lambda \theta 0 \nu \tau \varepsilon \zeta$ of $\mu \alpha \theta n \tau \alpha 1$ for, partaps, $\pi \rho 0 \sigma \varepsilon \lambda . \alpha u \tau \omega$ of $\underline{\mu \alpha \theta}$ aUtou. Approxiately $1 \frac{1}{3}$ Ines have been erased and in this

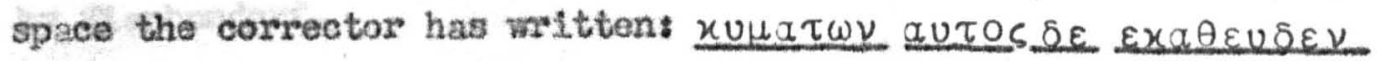
$x \alpha_{1} \pi \rho 0 \sigma \varepsilon \lambda \theta 0 \nu \tau \varepsilon \zeta$ of $\mu \alpha \theta \eta \tau \alpha_{1}$. It is cortain that the original. scribe wrote more than this. Itschendorf's suggestion that the corrector removed $\underline{\nu \tau \omega}$ after $\pi 000 \varepsilon \lambda \theta 0 \nu \tau \varepsilon s$ and $\alpha u \tau o v$ after

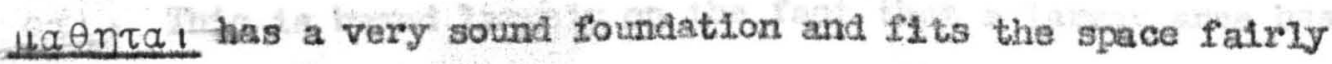
well. I have two modifleations to make, however, to his conjecture. (1) He sald Iine 33 probably ended with $01 \ldots$ and Iine 34 began with $\theta \eta \tau a l$ autou. From an infra-red photograph it is farrly clear that 1 (of 1 ) was the last lettor on the line. IAne 34

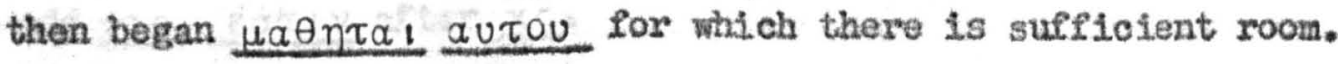
(2) Ine 33 then has several letters less than the other lines in this area. Perhaps something was duplicated this could give the corrector sufficient cause for rearranging the entire lino in-

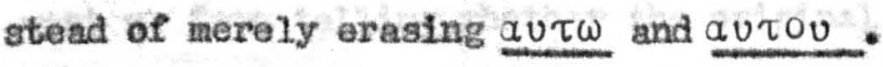

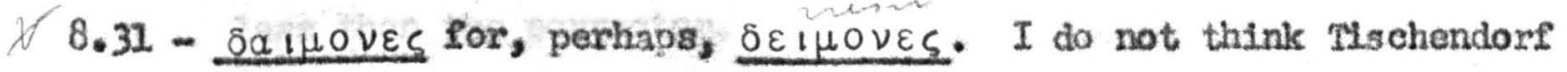
was correct in saying an original $\varepsilon$ was changed to read $a_{1}$. Neither the $\delta$ nor the $\underline{\mu}$ has been disturbed and $\underline{\varepsilon}$ does not 2111 the space well. This spelling has probably been changed more than once.

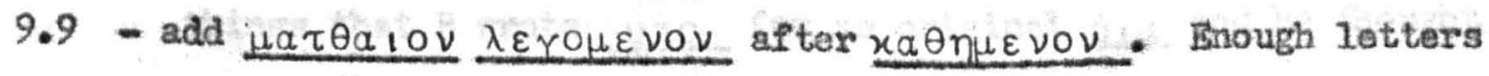
are seen to make this cortain. Tischendore did not atterapt to 
say what change had been rade. It is clear, however, that $\mathbb{A}$ wrota less than B since the end of line 37 has not been erased. This reading of codex $c^{\text {\% }}$ is a singular roading and has no other

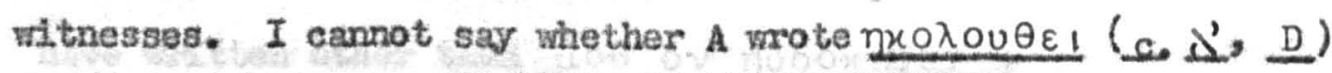

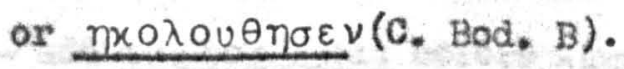

9.14 - $\mu \alpha \theta \eta \tau \alpha_{1}$ for $\mu \alpha \tau \alpha_{1}$.

9.27 - add $\underline{x \perp} \underline{\lambda \varepsilon \gamma o \nu \tau \varepsilon \zeta}$ after $x \rho a \zeta o \nu \tau \varepsilon \zeta$.

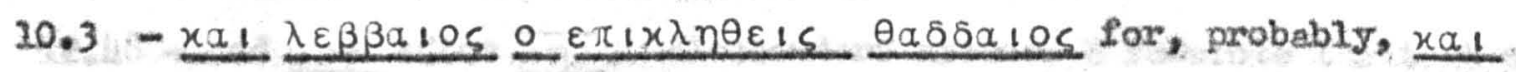
$\lambda \varepsilon \beta \beta a 105$ o $x a_{1}, \theta a \delta \delta a_{106}$. At the end of the line gatos is seen as well as $a_{1}$ (of $x a_{1}$ ?) in a position which suggests our text. This reading represents one of the possibilities put forth by Hschendore.

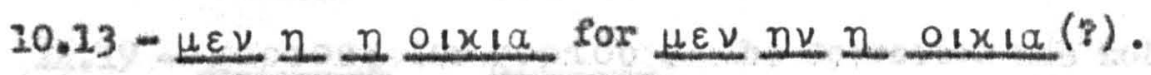

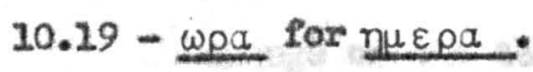

11.23 - $0 \tau_{1} \varepsilon_{1}$ for $0 \tau_{1}$. I camnot doubt that A omitted $\varepsilon_{1}$ rather than ot 1. This is based largely on the fact that a larger area has been erased than that which $\underline{\varepsilon}$ would occupy. The letters $\underline{\varepsilon}$ would not even reach the margin, whereas A apparently extended his first letter into the margin.

$12,6-\mu \varepsilon_{1} \zeta \omega \nu$ for $\mu \varepsilon_{1} \zeta{ }^{\circ} \omega \nu$ (?).

12.8 - add $\underline{x \rho} \underline{\varepsilon \sigma \tau 1 \nu}$ after $\underline{x s}$.

12.11 - add $\varepsilon \sigma \tau \alpha 1$ after $\tau 1$ s.

$12.13-\varepsilon \varepsilon \varepsilon \tau \varepsilon i \nu \varepsilon \nu, x a_{1} a \pi \varepsilon x a \tau \varepsilon \sigma \tau a \theta \eta$ vylns $\omega_{c} \eta a \lambda \lambda \eta$ for - ? Nothing can be seen of traces of the original text. Holes prevent us from telling whether the oritinal sartbe wrote more or less than the corrector.

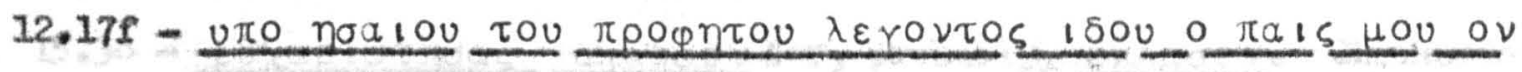

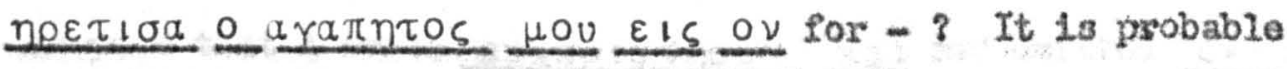
that from 6 to 10 letters have been added, but over a space this large one cannot be certain. Mischendorf suggested, anong other things that B wote $\underline{0 \pi 0}$ for an original $g$ la and he thought he saw $\varepsilon \nu \omega$ under $\varepsilon i s$ ov. To me, the latter suggestion is questionable. A curved mark ( 


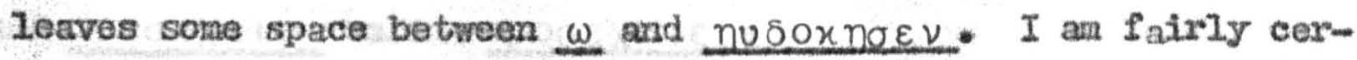
tain, however, that I have detected the $u$ of Lov on an Infra-red photograph at a point that makes it impossible for Codex $\mathrm{C}$ to have written other than ou ov nusoxngev.

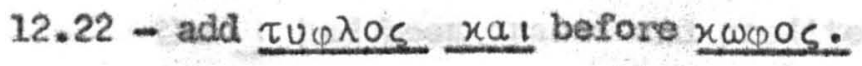

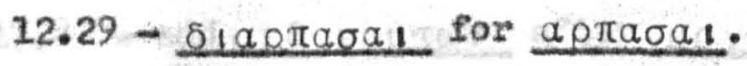

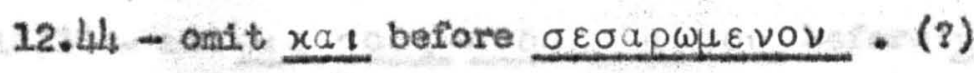

12.48 - add $\mu$ ov after $\underline{\mu \eta}$. Mschendorf assumed B remote the text of A. But A definitely wrote less than B. This place is quite difficult, but the $\rho$ is the most certain letter.

13.57 - add $\underline{\varepsilon 1}$ after $\underline{1 \tau 1 \mu O S}$. Mschendore thought this was by the original scribe.

14.19 - yoptous for yoptou. The corrector has not completed his change of the text, for tou has not been made to read tous.

14.19 - onit $x a_{1}$ before $\lambda a \beta \omega \nu$.

14.25 - $n \lambda \theta \varepsilon \nu$ for $\alpha \pi \eta \lambda \theta \varepsilon \nu$. (?)

$14.29-\underline{\varepsilon \lambda \theta \varepsilon \imath \nu}$ for, probably, $x \alpha_{1}, \eta \lambda \theta \varepsilon \nu$.

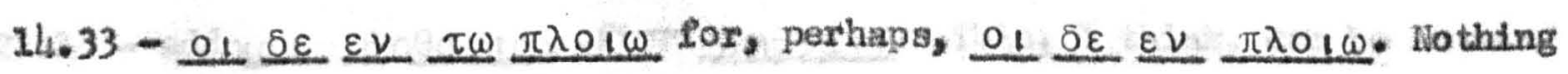
can be seen, even with the infra-red photograph, of the ariginal letters. Tho letters vandoubtedly have been added, but this is the mast we can say with any certainty.

15.4 - add gov after tov $\overline{\pi p a}$.

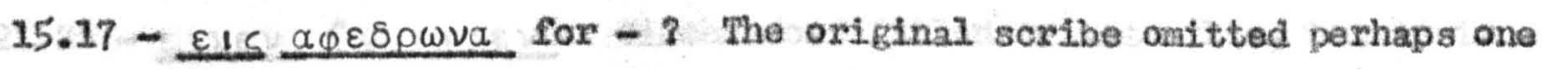
or two lotters. Certainly qov was not includod.

$15.32-\sigma \pi \lambda a \gamma \times \nu+\zeta 0 \mu \varepsilon$ for, possibly, $\sigma \pi \lambda Y \times \nu$. One of the first six letters was omitted by $A$.

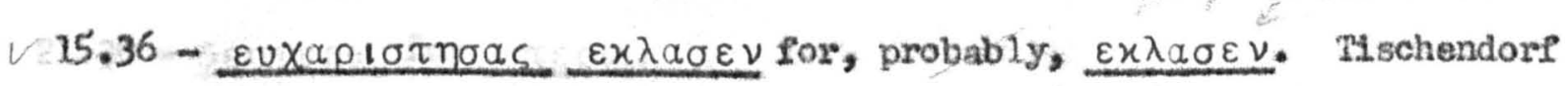

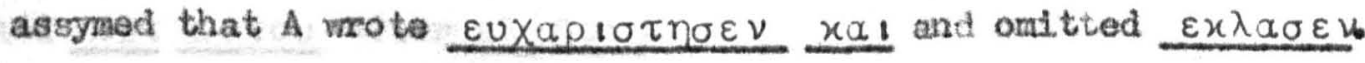
This is impossible because of the available space. The letters

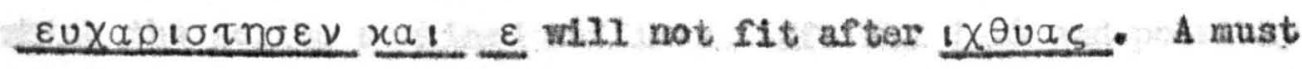
have witten $x \alpha_{1} \lambda \alpha \beta \omega \nu \ldots . \varepsilon x \lambda a \sigma \varepsilon \nu \underline{x+1} \varepsilon \delta \omega x \varepsilon \nu$.

16.3 - add $\underline{x a_{1}}$ before to $\mu \varepsilon \nu$. 


\section{Mathow}

16.11 - add $\pi \rho 0 \sigma \varepsilon \times \varepsilon 1 \nu$ before $\pi \rho 0 \sigma \varepsilon \times \varepsilon \tau \varepsilon$.

16.13 - onit $\delta \varepsilon$ bafore 0 is.

16.19 - onit $\underline{x \alpha_{1}}$ before $\underline{5 \omega \sigma \omega}$.

16.22 - $\varepsilon \pi_{1} \tau_{1 \mu \alpha \nu}$ for $\varepsilon \pi_{1} \tau_{1 \mu 1 \alpha \nu}$ (?). Mischendorf did not note that the 1 had beon orased and credited A with having $\varepsilon \pi 1 \tau \mu \mu \alpha \nu$.

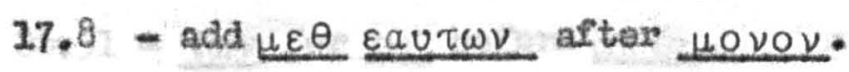

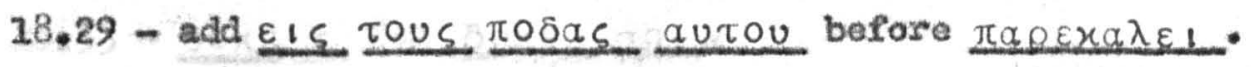

18.29 - add $\pi \alpha \nu \tau a$ before $\sigma 01$. WIschendorf erred in thinking that the corrector reversed the order of the origlnal text when he added $\pi a v \tau a$. The letters can be seen as indioated in the text.

18.35 - oupav10s for $\varepsilon \pi 0 u p$.

19.19 - add gov after $\overline{\pi \rho a_{0}}$

20.3 - onit duplicated $\tau \rho_{l}$ (2).

20.6 - omit aprovs after $\underline{\underline{\sigma} \tau \omega \tau \alpha c}$.

$20.10-\underline{\pi \varepsilon \varepsilon_{10} \nu a}$ for $\pi \lambda \varepsilon 10 \nu$.

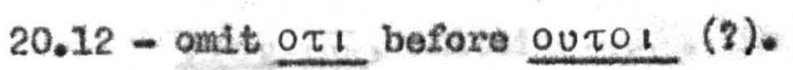

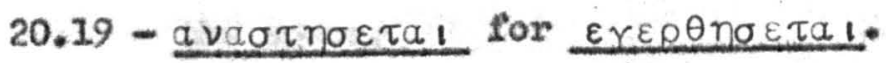

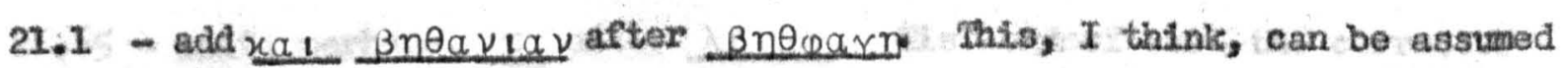
becavise of the space avatiable. A mote less than B. Other than this, It is impossiblo to know if $\mathrm{A}$ wroto $\varepsilon_{15}$ or $\pi \rho \mathrm{O}_{\mathrm{S}}$ before to opos as 98 rightly says. Nestle is questionable in quoting our MS with Codex $B$ as having E ts.

21.28 - $\varepsilon_{1} \times \varepsilon \nu$ for $\varepsilon 1 \pi \varepsilon \nu$. Tischendorf falled to note this, but it is quit te obvious. The present $\chi$ is not by A.

$21.28-\underline{\pi \rho \omega \tau \omega}$ for $\underline{\pi \rho \omega}$.

21. 28 - add $\underline{\mu O U}$ after $\underline{\alpha \mu \pi \varepsilon \lambda \omega \nu \text {. }}$

$21.30-\underline{\delta \varepsilon \cup \tau \varepsilon \rho \omega}$ for $\varepsilon \tau \varepsilon \rho \omega$.

22.15 - add $x a \tau$ autou aftor $\underline{\varepsilon \lambda \alpha \beta O \nu .}$

23.26 which the seribe combined suffixes. Cf. Mt. 7.16 and possibly Acts 4.2.

23.35 - add $\underline{a v}$ aftero $\pi \omega s$. 


\section{Hatthew}

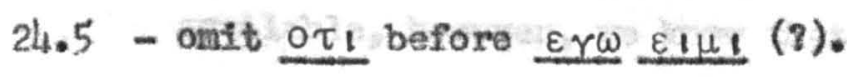

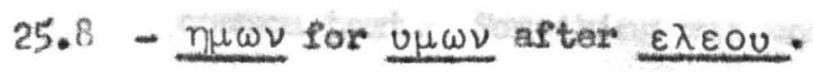

25.20 - add $\tau \alpha \lambda \alpha \nu \tau \alpha$ before $\underline{\varepsilon x \rho \rho \delta \eta \sigma \alpha}$.

26.31 - $\nu u x \tau_{1}$ for $\nu u x \varepsilon_{1}$.

$26.50-\underline{\varepsilon \tau \alpha_{1} \rho \varepsilon}$ for $\varepsilon \sigma \tau \alpha_{1} \rho \varepsilon_{\text {. }}$ (?).

26.51 - $\tau \omega \nu$ for $\tau \omega$ before $\mu \varepsilon \tau \alpha$.

26.53 - $\underline{0 x \varepsilon 15}$ for $\delta 0 x \varepsilon 1,001$.

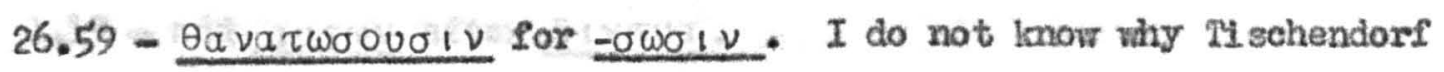
hesitated to credit gou to the corrector. By every criterion they are not the letters of the original seribe.

26.60 - add $\underline{x \alpha_{1}}$ before $\pi 0 \lambda \lambda \omega \nu$.

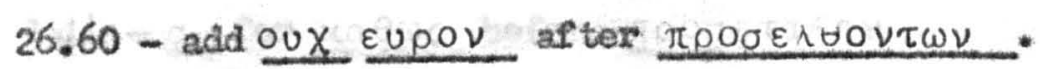

26.61 - add, perhaps, vaov, before toutov. This is based only on considerations of space. Due to homoloteleuton the scribe confused ov of $\tau 0 \nu$ for ov of vaov. The only other possibility might be vaov $\overline{\theta u}$, omitting iov. No other combination of letters fits the space.

26.65 - omt, perhaps, $\tau_{1}$ after $\lambda \varepsilon \gamma \omega \nu$. Mschendorf said the corrector erased $0 \tau l$, but without doubt the opace is not sufficient for these letters.

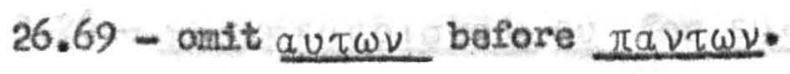

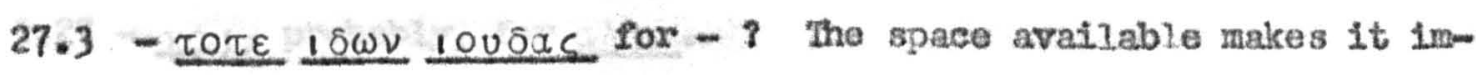
possible for more than one or two letters to have been onitted.

$27.9-1 \varepsilon \rho \varepsilon \mu 100$ for ing.

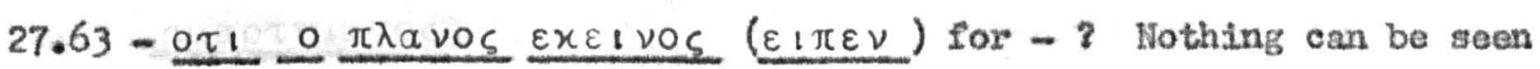
of the original letters except $\pi$ under the first $\underline{\mu}$ of $\mu \nu \eta \sigma \theta n \mu \varepsilon \nu$. After $\mu \nu \eta \sigma \theta \eta \mu \varepsilon \nu$ the vellum is so thin the uriting on the other side can be easily seen. Tischendorf said he detected ofl $\varepsilon 1 \pi \varepsilon \nu$ at the end of the line. I am certain he could not have seen anything here. I think he saw the writing on the other side of the leaf. Part of line 39 is hopelessly wrinkled and the rest has a hole so thin very little can be seen. From the space 
Matthew

avaliable, however, we lonow that $A$ wrote much more than our coramon text. Something was undoubtedly duplicated.

28.10 - Because the vellua is torn we cannot know whether A wrote $\underline{x a 1}$ $\varepsilon x \varepsilon_{1}$ or $x a x \varepsilon_{1}$. On the basis of space the formor is more preferable. But there is no indication - other than the hole itself - that a corrector changed the text to read $x a x \varepsilon_{1}$.

Maric

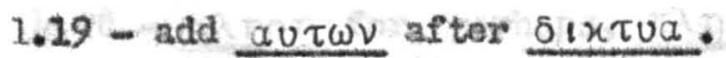

1.20 - $\zeta \varepsilon \beta \varepsilon \delta a 10 V$ for $\zeta \varepsilon \beta \varepsilon \delta a 100$. Mischendore did not note that the text had been corrected and credited $-\delta a 10 \nu$ to the original sartbe.

1.44 - $a_{\text {_ }}$ for $x a \theta \omega s$ before $\pi \rho 0 \sigma \varepsilon \tau a \xi \varepsilon \nu$.

1.45 - redundant $\nu$ between $\varepsilon \nu$ and Epnuois.

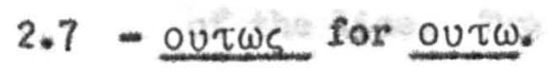

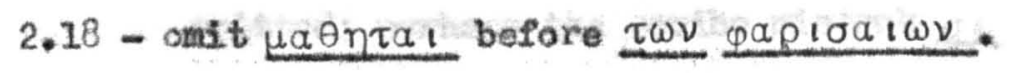

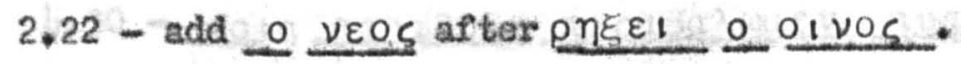

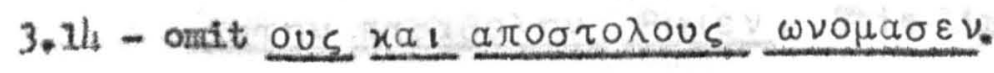

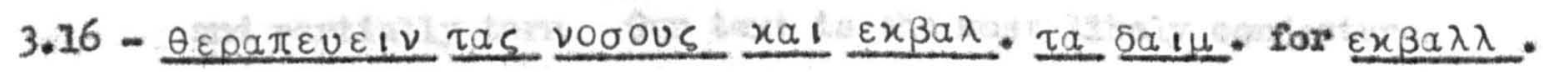

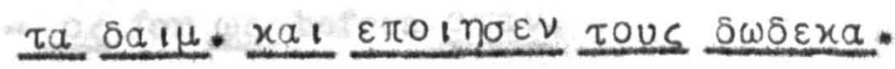

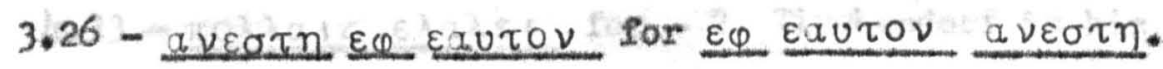

$3.26-\underline{x \alpha_{1}} \mu \varepsilon \mu \varepsilon \rho 1 \sigma \tau \alpha_{1}$ ou for $\varepsilon \mu \varepsilon \rho 1 \sigma \theta \eta \underline{n \alpha_{1}}$ ou.

$3.27-\underline{-1}$, probably, for $\underline{\alpha \lambda \lambda a}$.

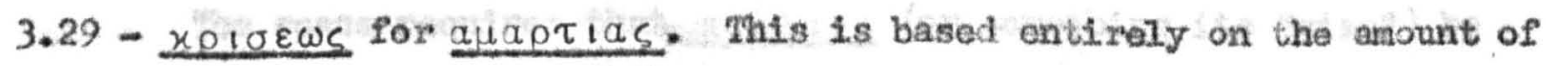
space available which precludes the possibility of an original

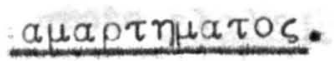

$3.31-\sigma \tau \eta x 0 \tau \varepsilon s(=\varepsilon \sigma \tau . ?)$ for $-x \circ \nu \tau \varepsilon \zeta$

4.8 - $\varepsilon \nu$ (3 times) for $\varepsilon 15$ ( 3 times). The second $\underline{\varepsilon l c}$ can be seen. The other two are assumed because no other explanation will explain the erasures that have been mado.

$4.9-0$ - $x \times \omega \nu$ for oc $\varepsilon \times \varepsilon_{1}$.

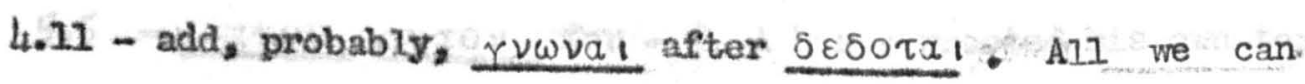
know for certain is that A wrote less than B. 
Mark

4.18 - outo1 for a

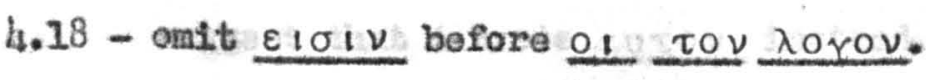

4.20 - add $\underline{\varepsilon v}$, probabiy, before $\underline{\varepsilon \eta \eta x o \nu \tau a}$. Sontething was omitted by A.

4.26 - onit o $\underline{1}$ arter $\varepsilon \lambda \varepsilon \gamma \varepsilon \nu$.

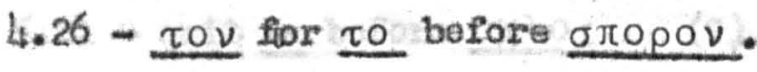

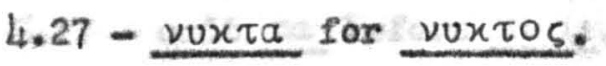

4.27 - $\beta \lambda a \sigma \tau \alpha \nu \eta$ for $\beta \lambda a \sigma \tau \alpha$.

$4.28-\pi \lambda \eta \rho \eta$ for, perhaps, $\pi \lambda \eta \rho \varepsilon \varsigma$. Tischendorf suggests, and I think It is quite 11ke1y, that $\mathbb{A}$ wrote $\rho \varepsilon \zeta$ for pDs. This helps explain why $B$ erased nore than the $S$, but doesn't explatn why the L. was also erased. Ho attempt is nade to 1 ill the space evenly.

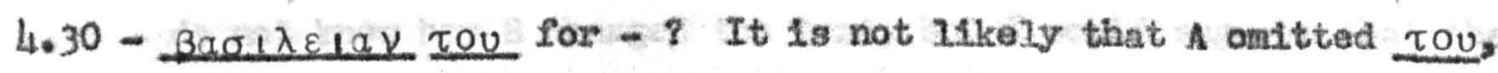
for it would merely have boen added by the corrector to the end of the 1ine. Two or three letters of $\beta \alpha \sigma\lfloor\lambda \varepsilon \downarrow \alpha \nu$ probably were aritied; perhaps the scribe nistook of for $^{*}{ }_{1}$.

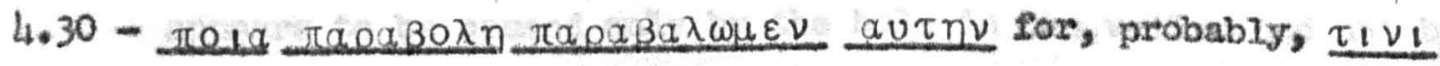

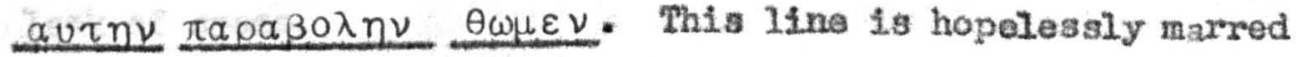

and partially torm. Our text is the most Ilkely conjecture.

4.31 - os for $\omega s$ before otav.

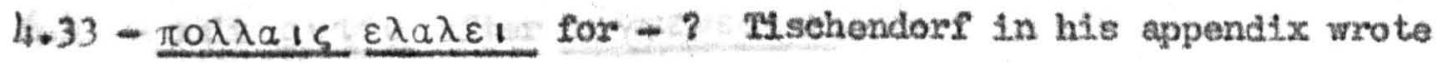
"et quiden Justo fere spatio", but in 78 he sald Codex 6 agrees with Codex I in omitting $\pi 0 \lambda \lambda \alpha / 5$. This undoubtedly is wrong. The space requires that $\pi 0 \lambda \lambda a_{1 c}$ (or something its "sizen) be included. It is posstble that $\mathrm{A}$ reversed the cormon order and

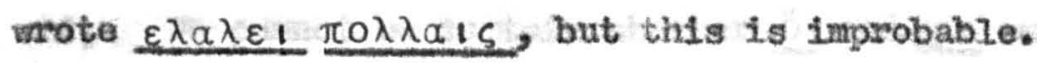

4.35 - add to before $\pi \varepsilon p a \nu$.

4.36 - add $\underline{\delta \varepsilon}$ after $\underline{\alpha \lambda \lambda \alpha}$.

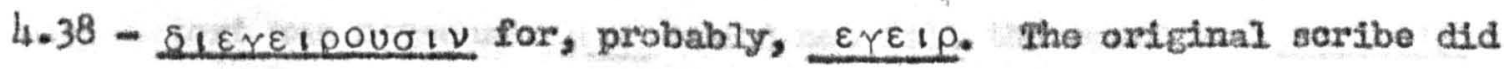
not omit $x x_{1}$ since the $x$ can be seen.

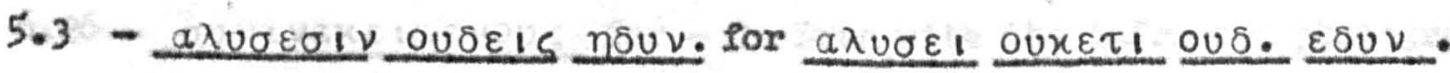

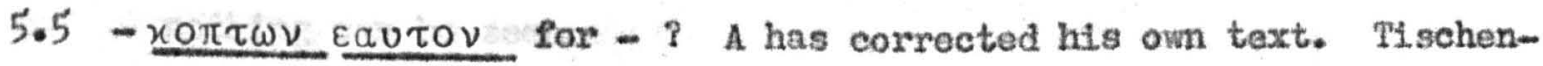

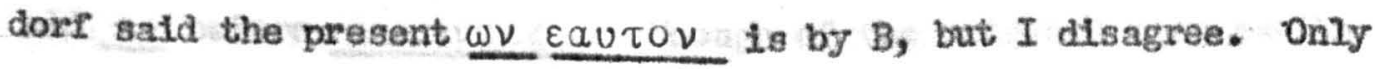


Marik

$\omega \nu \underline{\varepsilon}$ are by $B_{\xi}$ but they are not erowded in such a way as to

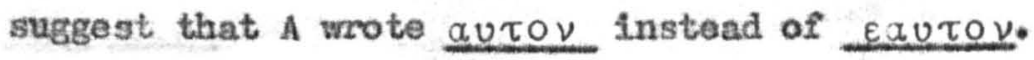

5.13 - add noav $\delta \varepsilon$ before $\omega_{S} \delta 10 x_{1} \lambda_{101}$.

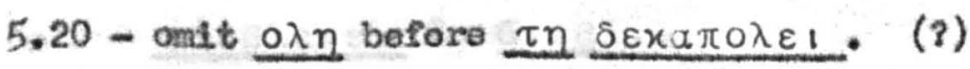

5.21 - omit o before oxios. (?)

5.27 - onft $\tau a$ before $\pi \varepsilon \rho_{1}$. (r)

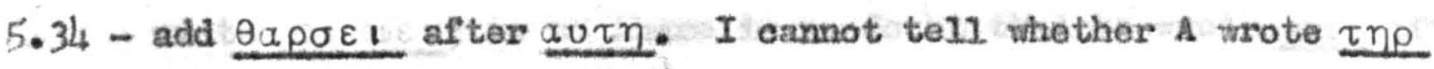
or $\underline{\varepsilon \rho}$ in $\theta$ ura $\tau \varepsilon \rho$.

$5.34-\underline{x a_{1}} 10 \theta_{1}$ for $-?$ Tischendorf satd that the original seribe probably omitted $x a l$, but there is no foundation for this assuaption. $x a_{1}, 1 \sigma \theta_{1}$ easily fits in the available space. I do not know how $B$ changed the text, but I would suggest that the

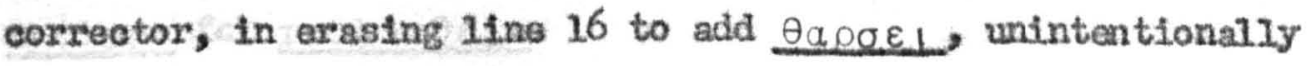
affected the writing of line 17. To support this conjecture it should be noted that the erowding of B's latters in line 17 appears to be caused only by the hole in the vellum and not by

lack of space.

6.2 - $\pi \alpha \nu \tau \alpha$ for $a \pi \alpha \nu \tau \alpha$.

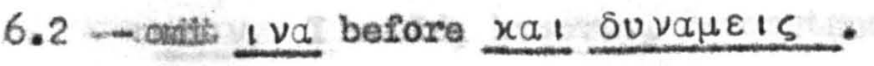

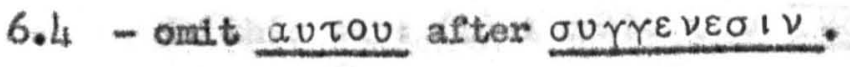

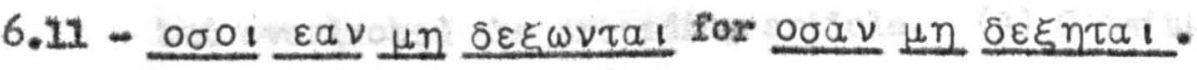

6.15 - add $\varepsilon \sigma \tau 1 \nu$ after $\pi 00 \varphi \eta \tau n s$.

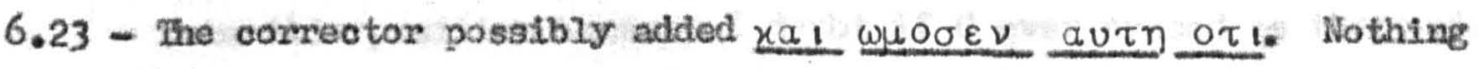
can be seen of the original text, but this fits the space admirably. We cannot be cortaln whether $\underline{z}$ and $\mu \varepsilon$ were added or omitted. On line 18 under $\mu \varepsilon$ of the corrected text two letters

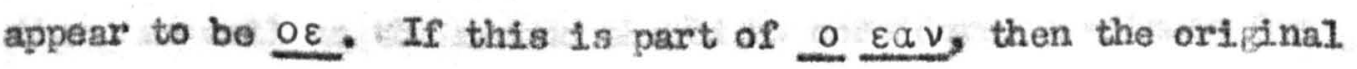
text was nonsense. $\mu \varepsilon$ would not $f 17$ the space and a ditnons is too wuch to fit.

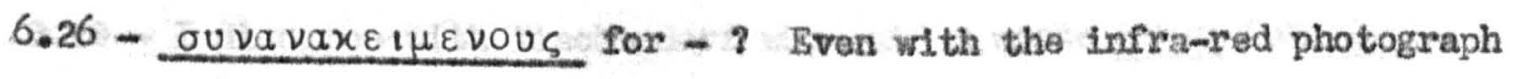
nothing can be seen.

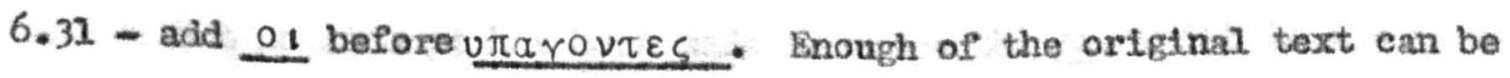
seen to make this certain. 
Lark

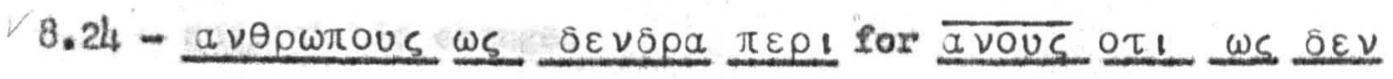
$0 \rho \omega$

$8.25-\varepsilon \pi \varepsilon \theta n x \varepsilon \nu$ for $-x \varepsilon$.

$8.25-\underline{\varepsilon \nu \varepsilon \beta \lambda \varepsilon \psi \varepsilon \nu}$ for $\underline{1 \varepsilon \beta \lambda}$.

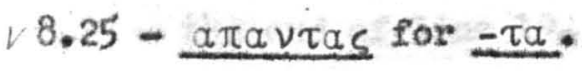

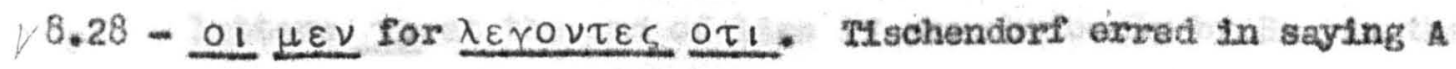
wrote $\mathrm{O}_{1} \mu \varepsilon \nu$; to me theme is no doubt that he wrote ofl after

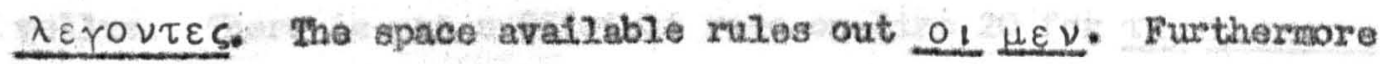
the corrector most likely would not have erased $\lambda \varepsilon y \cdot O_{1} \mu \varepsilon \nu$ and then rewritten $01 \mu \varepsilon \nu$. He has nade no attempt to Pll the spece evenly. I cannot doubt that $A$ wrote $\lambda \varepsilon y o \nu \tau \varepsilon S$ ofl.

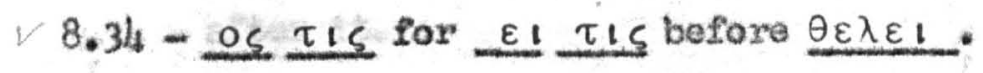

$8.34-\varepsilon \lambda \theta \varepsilon 1 \nu$ for $\alpha x 0 \lambda \sigma 0 \theta \varepsilon \mid \nu$.

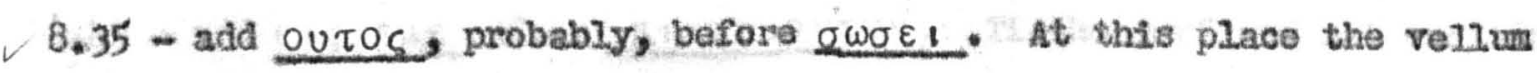
has several holes. It is certain, however, that A wrote less

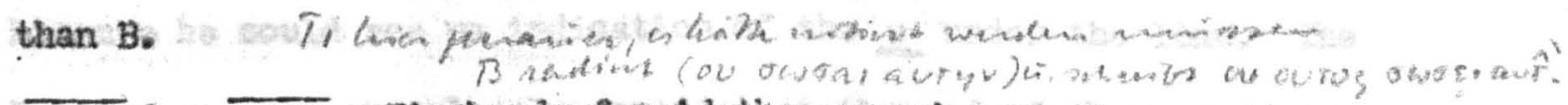

8.36 - $\overline{a V O S}$ for $\overline{\alpha \nu O \bar{V}}$. Tischendore sald the second corrector wrote 0 $\overline{a \nu O c}$ for tov $\overline{\alpha \nu O \nu}$ because $1 t$ is obvious that ho wrote 0 for Tov. I would, hovevex, reconstruct tha history of this variant

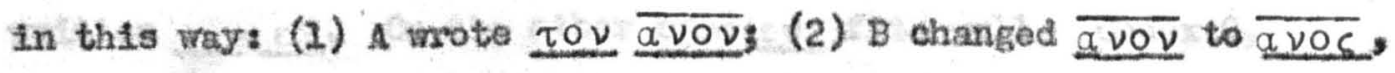
but overlooked the preceding article. (3) 0 omitted the article. Nuabers (1) and (3) are cortain. Number (2) may be questioned. I take this position because I doubt if $\mathrm{C}$ ever erased as thoroughly as the last letter of $\overline{\text { vov }}$ has been erased. 0 rojocted tov by circling 1 t with periods - as was his custora. B thoroughly erased the final $\nu$ and wrote $S$ - as was his custom. The first correotor, then, as well as the second should be constdered a witness for $\overline{\text { avoc }}$.

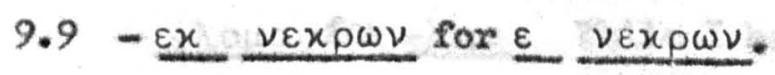

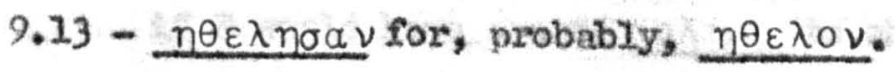

9.20 - I disagree with rischendorf who gaid B wrote $100 \mathrm{~V}$ for $10 \omega \mathrm{V}$. The word $1 \delta \omega \nu$ has not been touched. The text is not very clear, but no attempt has been made to erase the $\omega$, which is the clearest of all the letters. I cmnnot surmise what Rischendore saw to 
Marik

suggest his change.

9.23 - add $\underline{\tau \omega}$ before $\frac{\pi \sigma \tau \varepsilon v 0 \nu \tau 1}{}$. MIschendoref wrote $\pi \alpha \nu \tau \alpha$ at the end of 1 ine 19 and Suva $\tau$ a $\tau \omega$ on line 20. But this represents the corrected text of B. It is very clear that $A$ wrote $\pi \alpha^{-}$at the end of line 19. The horizontal line is quite clear. The letters $\tau_{a}$ at the end of line 19 are obviously by $B$. At the beginaing of line $20 \tau$ (by A) can be seen before a hole in the vollum. There is not sufficient room on line 20 for $\tau a$ guva $\tau a$ $\tau \omega$. That $A$ wrote has to be conjecturod: either $\delta \cup \nu a, \tau \omega$ for a similar error) or ewvaca. I prefer the latter because the I after the hole appears to be by $A$ and $\omega$ would be very crowded after it.

9.24 - add $\overline{\overline{x \varepsilon}}$ before $\beta 0 \eta \theta \varepsilon 1$.

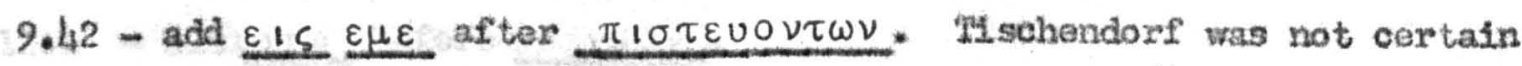

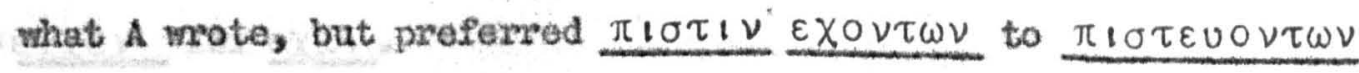
because he could see no indication of the $u$ under the line. The

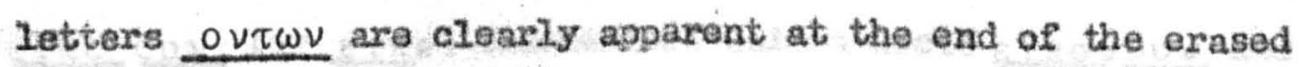
area. At the beginning of the 14ne $\tau \omega \nu, \pi 1$ can be geen as woll

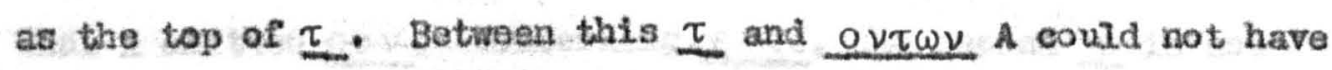
written $1 \nu \underline{\varepsilon x}$. On the other hand $\varepsilon v$ flis well. Codex $\mathrm{C}$, thorefore, agrees wth Codex 1 .

10.1 - ant $\underline{x a_{1}}$ before $\pi \varepsilon \rho a \nu$.

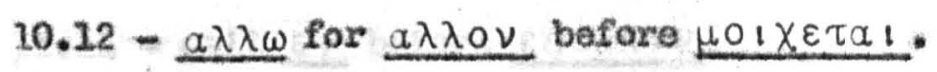

10.27 - add $\underline{\delta \varepsilon}$ after $\underline{\varepsilon \mu \beta \lambda \varepsilon \psi a s}$.

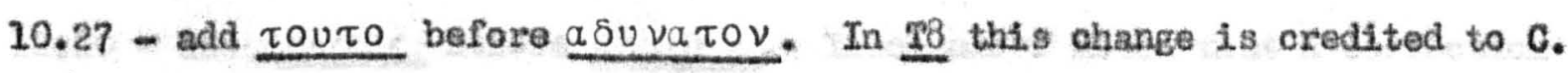

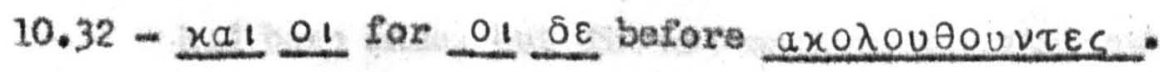

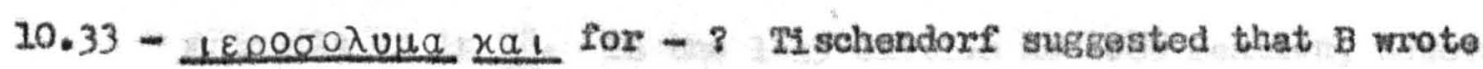
$-\lambda u \mu \alpha$ for $-\lambda \eta \mu$. While I have no better conjecture, I doubt

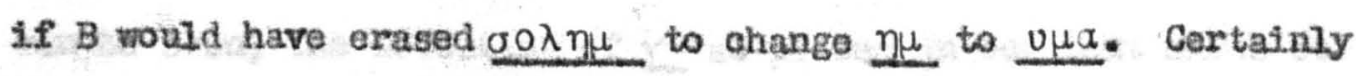
A did not omit $x a_{1}$. $1 \varepsilon \rho 0 \sigma 0 \lambda_{u} \mu a$ was probably misspelled.

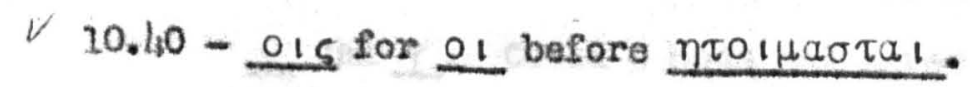

10.42 - $\mu \varepsilon \gamma a \lambda 0_{1}$ for $\beta a \sigma 1 \lambda \varepsilon i s$. 
Mark

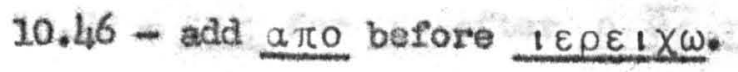

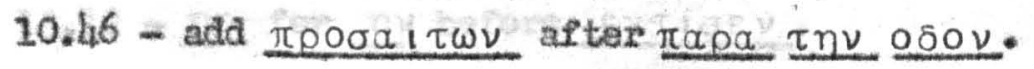

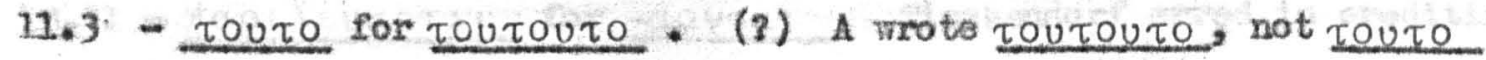
xal as both wischendorf and Wetstein conjectured. The to before $\varepsilon 1 \pi a \tau \varepsilon$ are quite clear now and evidence of the $\underline{u}$ can be seen. The first corrector, therefore, erased a redundant

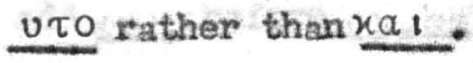

11.3 - ont $\pi a \lambda_{1} \nu$ after autov.

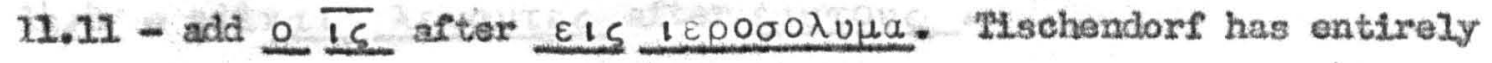
overlooked this correction.

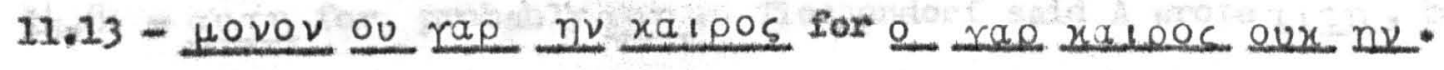

$11.25-\underline{\alpha \phi 1 \varepsilon \tau \varepsilon}$ for $\underline{\alpha \varepsilon \varepsilon \tau \text {. }}$

11.31 - add ouv before oux $\varepsilon \pi 10 \tau \varepsilon v \sigma \alpha$.

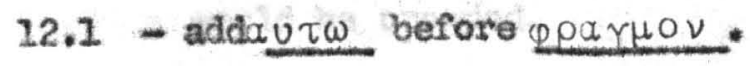

$12.6-\varepsilon 1 \chi \varepsilon \nu$ for $\underline{\varepsilon \chi \omega \nu}$.

12.9 - add toutous after vewoyous.

12.14 $-\varepsilon 1 \pi \varepsilon$ ouv for $\varepsilon 1 \pi \varepsilon$. Tischendorf said a wrote Eljoy ouv and that B simply changed $\varepsilon 1 \pi 0 \nu$ to $\varepsilon 1 \pi \varepsilon$. Thero can be no doubt that $A$ onitted ouv. Concerning $\varepsilon 1 \pi \varepsilon$, the second $\underline{\varepsilon}$ is not 1ike the usual $\underline{\varepsilon}$ of $A$, but it is strikingly simflar to the $\underline{\varepsilon}$ at the end of line 17 (in $\tau \varepsilon x \nu a$ ). I an quite aure that $A$ mote $\varepsilon 1 \pi \varepsilon$.

12.24 - omit $\varepsilon$ before $\varepsilon \xi \varepsilon \sigma \tau 1 \nu$.

12.20 - add $\underline{\underline{u \nu}}$ aftor $\underline{\varepsilon \pi \tau a}$.

12.20 - add $\pi \rho \omega \tau 0 \zeta$ before $\varepsilon \lambda a \beta \varepsilon \nu$. Hischendorf notod that a correction had been rade, but did not mention any variant elther in his appendix or in 18 . In the 1845 edition he does mention, however, that something was added by the correotor. Although very little ean be seen, the detection of $Y$ as placed in our text malces our text beyond doubt. The word $\underline{\varepsilon \alpha \beta \varepsilon \nu}$ fLts precisely between $x_{\alpha}$ $\underline{0}$ and $\mathcal{L}$. Codex $c$ does not agree with codex $\Lambda^{\prime}$ which has $\varepsilon_{i c}$. 12.23 - add ouv before a $\nu a \sigma \tau a \sigma \varepsilon 1$. 
Wark

12.29 - $\varepsilon_{1 s}$ for $\varepsilon_{1}$ after 0 도 $n \mu \omega \nu \overline{x c}$.

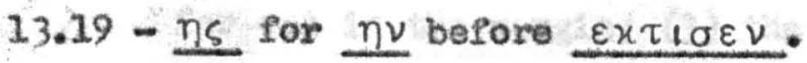

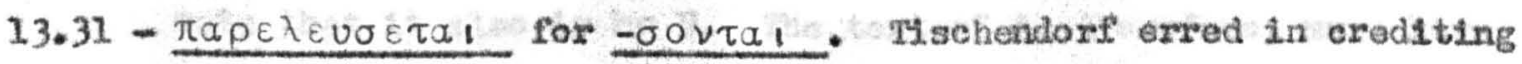
$-\sigma \varepsilon \tau a_{1}$ to the ariglnal scribe. The $\underline{\varepsilon}$ is not like the script of A. Furthermore, the area has been erased and the horizontal. stroke for the nu can be seen.

13. 34 - adis $x a_{1}$ before $\varepsilon x \omega \tau \omega$.

$14.2-\underline{\delta}$ for yaj.

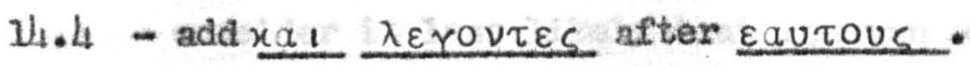

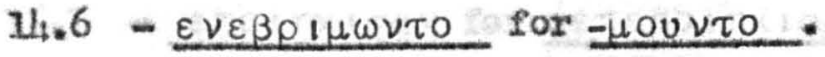

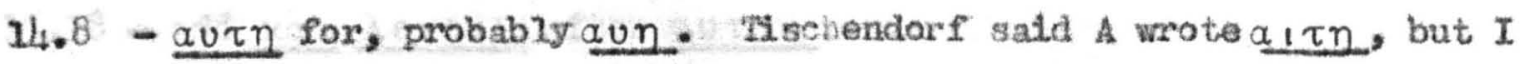
an quile certain $1 t$ was aun. The $\underline{a}$ and $\underline{\eta}$ aro clear. Remains of the $\underline{u}$ appear to survive. The letiers $1 \tau$ betreen $a$ and $\eta$ would be crowled.

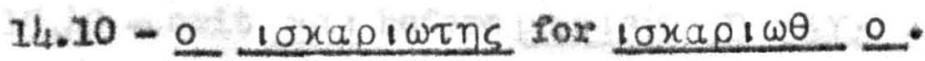

$14.13-\underline{u} a \gamma \varepsilon \tau \varepsilon$ for $u \pi a \gamma \varepsilon$. The letters aro barely visible and Tischendorf assumed they were by A. But tho present $y \varepsilon \tau \varepsilon$ is obviously quite crowded. The avallable space allows only ujaye - or some absurd spelling.

Lh. 20 - omit $\underline{\varepsilon \nu}$ before $\tau \rho \cup \beta \lambda_{1}$ ov.

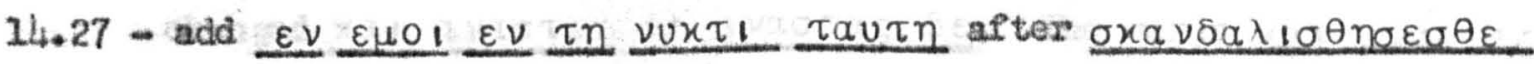

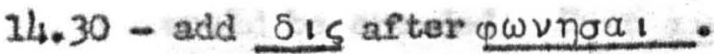

$14.45^{\circ}$ - add $\underline{x a 1 \rho \varepsilon}$ before $\underline{\rho \beta \beta} \beta \varepsilon 1$.

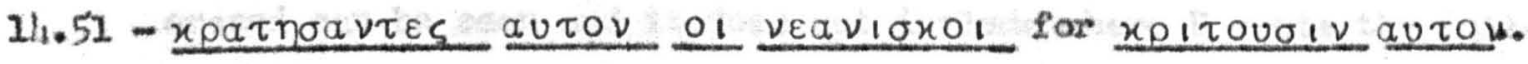

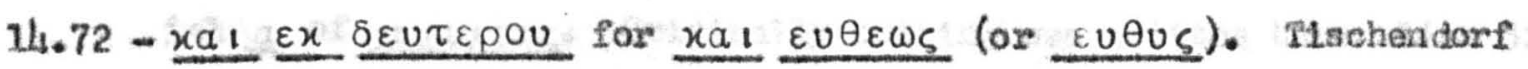

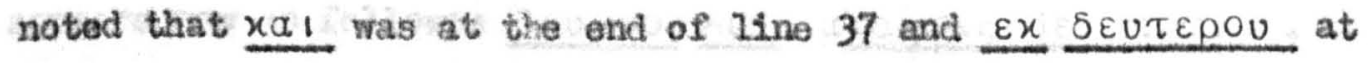
the beginning of line 38. In the margin a wodern hand (perhaps Wetstein) has verifled this by writing " $\nu a 1$ There can be no doubt, however, that this represents a correction by the first corrector. The vertical scroke of a large original $\underline{x}$ (presunably) is seen at the beginning of the iine. Otherwise none of the original viting can be seen; but the following items 
Marke

conclusively verify our text: (1) $x_{1}$ at the end of line 37 is by $B$ rather than $A$; (2) at the beginning of line $38 \underline{\varepsilon x}$ is clearly

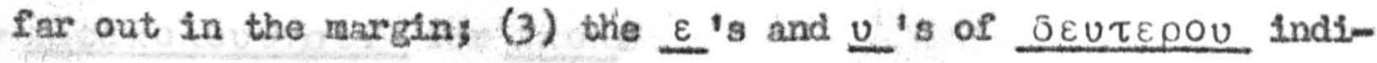
cate that it also is by $B$. The text of $A$, therefore, musi have fitted in the place occupled by $\delta \varepsilon u \tau \varepsilon \rho 0 u$ (without the marginal Ex ). Hence, $\underline{x a} \underline{\varepsilon v \theta \varepsilon \omega c}$ (or $\varepsilon v \theta u c$ ). Codex $C$, then, unites

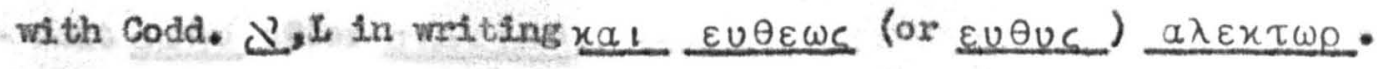

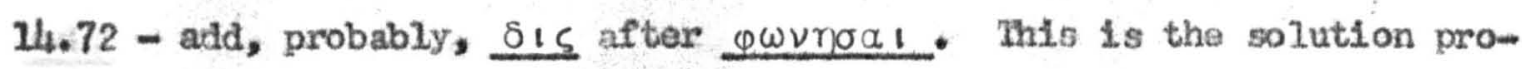
posed by Tischendorf. Something has been added to the text. I

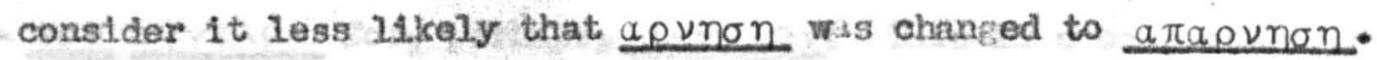

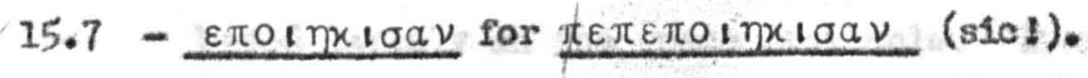

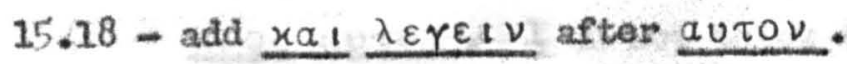

15.19 - $\varepsilon \nu \varepsilon \pi \tau u \circ \nu$ for, probabiy, $\varepsilon \nu \varepsilon \pi \tau u \sigma a \nu$.

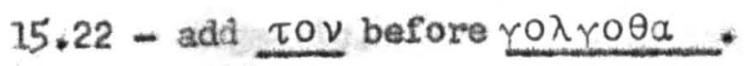

15.23 - add $\pi 1 \varepsilon 1 \nu$ after $\underline{u \tau \tau} \omega_{\text {. }}$

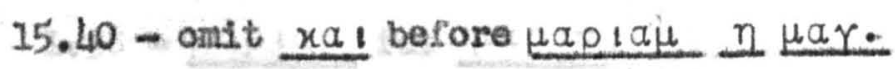

$15.46-\underline{\varepsilon \theta \eta x \varepsilon \nu}$ for $\underline{x a \tau \varepsilon \theta \eta x \varepsilon \nu .}$

$15.46-\pi \rho 0 \sigma \varepsilon x u \lambda 1 \sigma \varepsilon \nu$ for $-\sigma \varepsilon$.

16.1 - andt $\tau 00$ before $\sigma a \beta \beta a \tau o v$.

16.7 - omit, probably, $\underline{x a_{1}}$ before $\varepsilon_{1 \pi} \alpha$.

16.9 - add $\delta \varepsilon$ after $\underline{\alpha \nu \alpha \sigma \tau a \zeta}$. Tischendors erred in noting that $B$

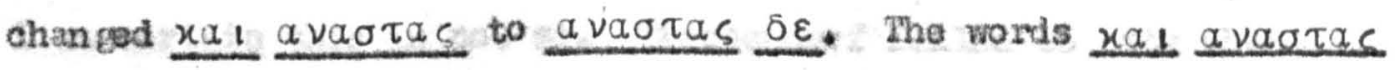
would be crowded. Bven $\underline{\text { vag } \tau a c} \delta \varepsilon$ is crowded. Our text camnot be doubted. It is bnsod on one clear fact: the area that has been erased can be seen and it does not include where B wrote the fnitial $a$ of avaotas. Originaily nothing was written here. Line 32

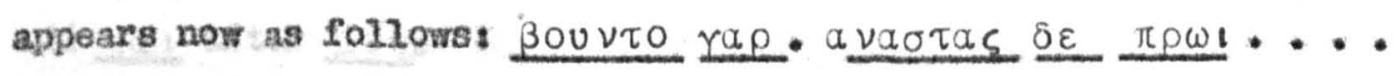
The words a vaotac $\delta \varepsilon$ are by B. But only the part occupied by vagtas $\delta \varepsilon$ has been erased. The original scribe wrote Eo0ßouvto yap and then left a short space before writing the long ending of lark. The note of many writers to the effect that Codex $C$ with many other manuscripts qives no indication that the Iong ending night be spurious must, therefore, be corrected. 
Mark

16.10 - omit $\underline{\delta \varepsilon}$ after $\underline{\varepsilon \varepsilon \varepsilon_{1} \nu \eta}$. (?)

16.17 - $\pi a \rho a x o \lambda o v \theta n \sigma \varepsilon_{1}$ for $a x a \lambda$.

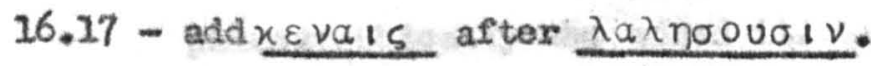

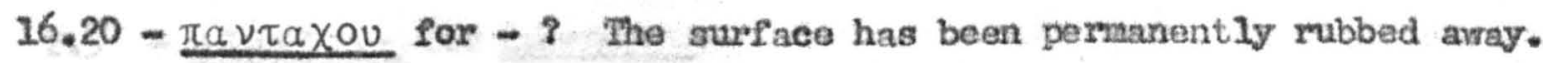
Probably A onitted one letter.

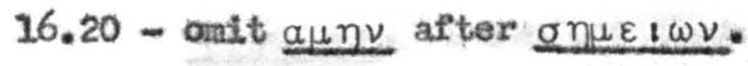

Luice

1.13 - $\underline{\delta 10 \tau 1}$ for $0 \tau_{1}$.

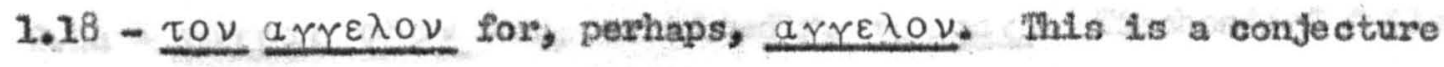
based largely on the space available. Hitschendorf said A wrote mpoc autov. I do not think this will fill all the space,

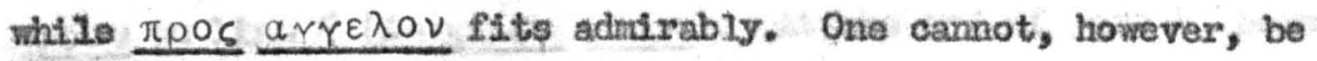
dogastic.

$1.34-\mu a \rho 1 a \mu$ for $\mu a \rho 1 \alpha$.

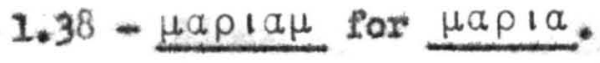

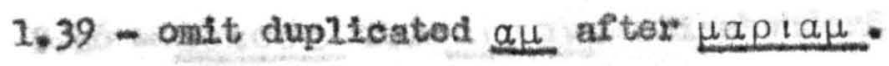

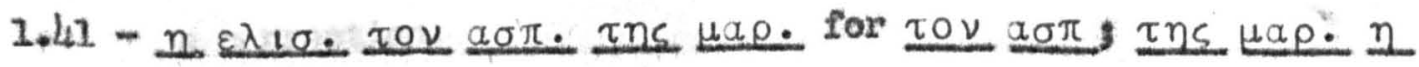
$\varepsilon \lambda 10$.

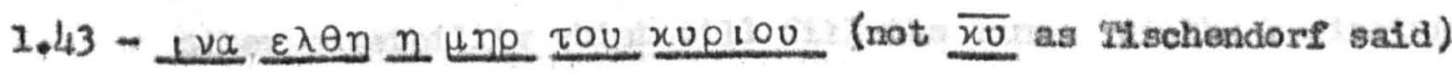
Hou for - ? Tlsohondorf sald something was previously onitied. I an certain this is not so. Flschendore failed to note that xup 100 is spelled out by B. Both lines 9 and 117 over the same area have an equal number of letters. If $\mathrm{A}$ vrote $\overline{x \mathrm{U}}$ instead of zuelov (as he has done in every other instance), then B wrote less, not more, than A.

1.45 - add $\underline{x a 1}$ after $\mu$ OU.

$1.46-\mu \alpha \rho a \mu$ for $\mu \alpha \rho 1 a$.

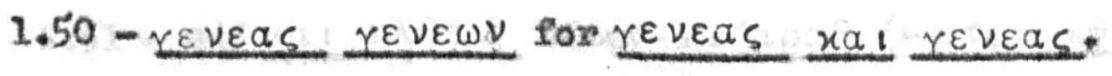

1.61 - $\varepsilon \nu$ in guvervia for $\varepsilon x$ tns ouyrevias.

1.63 - Iuvarisiov for $\pi 1 v a x i \delta a$.

$1.65-\frac{\pi \varepsilon \rho 101 x 0 u \nu \tau a c}{6}$ for $-?$ The text is permanently lost. A probably erroneously onitted two or three letters.

1.66 - omit yap before $\underline{\chi \varepsilon 1 \rho}$. 


\section{LUKE}

$1.80-a \nu a \delta \varepsilon_{1} \varepsilon \omega_{C}$ for $a \nu a \delta \varepsilon \xi \varepsilon \omega c$. This correction (an inserted L above the line) may be by elther B or C. There is no way to judge paleographically who added it.

$2.5-\underline{\mu \varepsilon \mu \nu \eta \sigma \tau . \text { for } \varepsilon \mu \nu \eta \sigma \tau .}$.

2.5 - Fischendorf said A unote $\underline{\alpha u \tau \omega}$ and $B$ corrected the text by adding ruva|x|. I confess that I can see nothing except that the second letter is $\underline{U}$. The codex is tightly bound and I camot see the nargin.

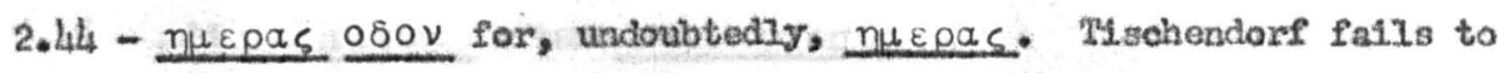
conment on this variant in his appendix, but notes that $\mu \varepsilon$ at the end of line 4 is not by A. But he notos that A wrote pas.

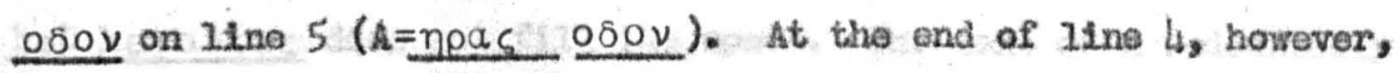

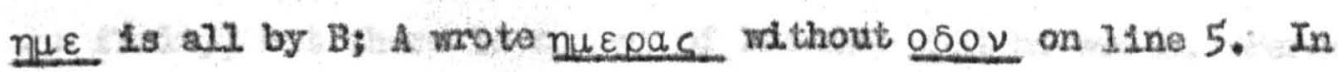
support of this the vestiges of the $\rho$ seera to appear below the line.

2.46 - autous for autov. Misehendorf did not note that autouc was a correction.

2. 48 - With Mischendorf I wrote the given text because I know of not other possibility which will sill the 2ine. At the beginning of Iine 16 oov $x a r \omega$ is certain. Our text still leaves the Iine about 4 letters shorter than any other full line on the page. $2.48-\underline{x a \gamma \omega}$ for $\underline{x a t} \underline{\varepsilon \gamma \omega}$.

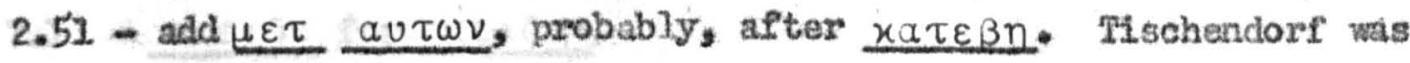

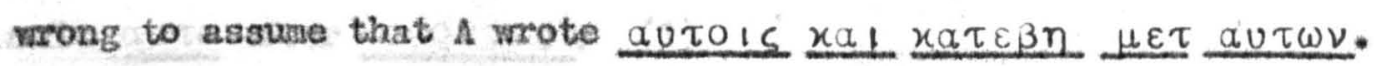
This is $60 \%$ more letters than the space can take. The onission of

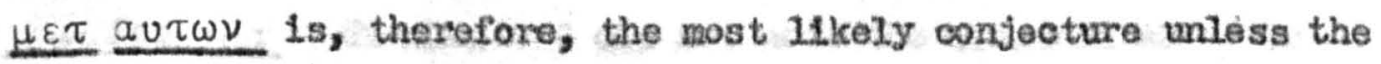
text of A was absurd.

2.51 - add $\underline{x \alpha 1} \underline{\eta \lambda \theta \varepsilon \nu}$ after $\underline{\nu \zeta \zeta a \rho \alpha \theta}(-\underline{-\varepsilon \tau},-\underline{-\varepsilon})$.

$3.11-\underline{\lambda \varepsilon \gamma \varepsilon 1}$ for $\varepsilon \lambda \varepsilon y \varepsilon \nu$.

$3.14-\varepsilon \pi \eta \rho \omega \tau \eta \sigma \alpha \nu$ for $\varepsilon \pi \eta \rho \omega \tau \omega \nu$. $\delta \varepsilon$. This is very obscure, but Iischondorf was undoubtedly wrong to have read $\varepsilon \pi n \rho \omega \tau \eta$ oav by A.. He did not oven note that the text had been correoted. 
344

Luke

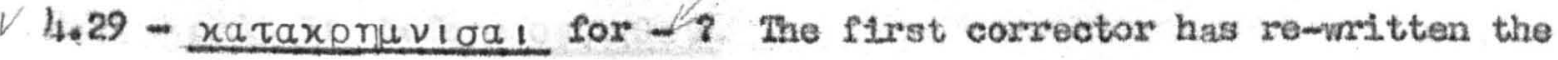
four letters $\eta_{\mu \nu !}$. Ii schendorf said A wote $1 \mu \nu \eta$, and $B$ changed

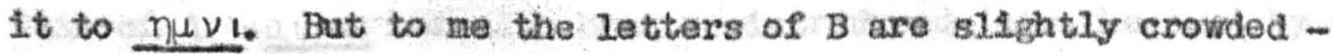
only enough to suggest that 1 was omitted by $A$. This is, of course, only a conjecture, but IIschendorf's reading must also

be so recognized. His text is less likely.

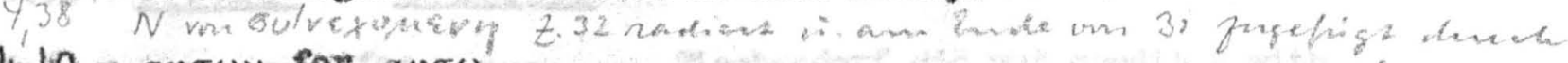

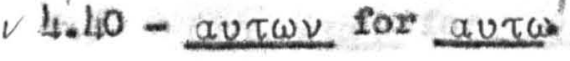

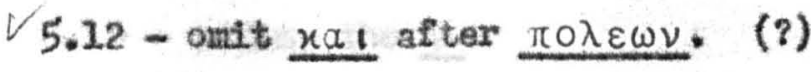
E) ounct?) vet $\operatorname{tip}, 324$.

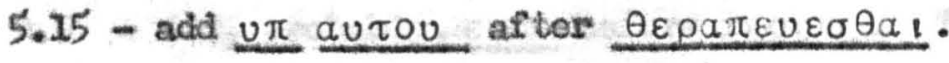

$5.28-\pi \alpha \nu \tau \alpha$ for $\pi \alpha \nu \tau a s . ~(?)$

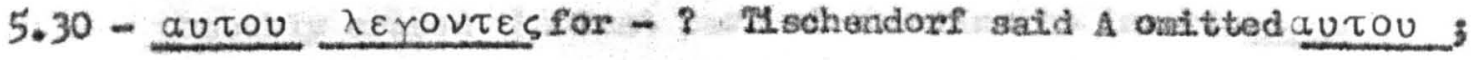
but $\lambda \varepsilon$ rovtes does not fill the space. The text of A undoubtedly was faulty, but there is no way of knowing what he wo to. Perhaps, due to haplography the scribe skipped from the first $\underline{U}$ to the second $U$ in autou. Our toxt represents an attermt to fill the apace with the number of letters it oxiginaliy contained. In any event Codex $C$ is not a witness to the onisston of autou. 5.30 - add $\underline{x \alpha}$ a $\underline{a} \rho \tau \omega \lambda \omega \nu$ after $\tau \varepsilon \lambda \omega \nu \omega \nu$. Thachendorf orrod in sayIng the present $1 \varepsilon \tau \varepsilon$ of $\varepsilon \sigma \theta i \varepsilon \tau \varepsilon$ is by A. Parts of an the letters can be seen.

5.39 - add $\varepsilon v \theta \varepsilon \omega s$ before $\theta \varepsilon \lambda \varepsilon_{1}$.

6.1 -

$7.1-\varepsilon \pi \varepsilon+\delta \varepsilon$ for $\varepsilon \pi \varepsilon ! \delta \eta$.

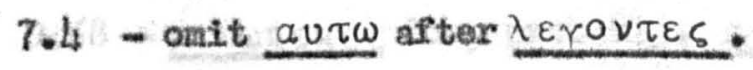

$8.29-\varepsilon \xi \varepsilon \lambda \theta \varepsilon i \nu$ for $\varepsilon \xi \eta \lambda \lambda \theta \varepsilon \nu$

8.31 - Iva for $1 \mu \alpha$ Mschendorf did not note the original error or that it had been corrected.

8.35 - add $\tau 1$ $\varepsilon \sigma \tau 1 \nu$ after $1 \delta \varepsilon 1 \nu$.

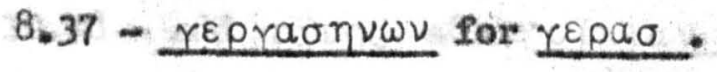

$8.38-\varepsilon \delta \varepsilon 1 \tau 0$ for $\varepsilon \delta \varepsilon \varepsilon \tau o$.

8.40 - add o before ox oxos.

8.41 - $\varepsilon 1 \sigma \varepsilon \lambda \theta \varepsilon i v$ for $1 v \alpha \varepsilon 1 \sigma \varepsilon \lambda \theta \eta$. 
Lulce

8. 44 - amit $\underline{\delta \varepsilon}$ before $0 \pi 1 \sigma \theta \varepsilon v$.

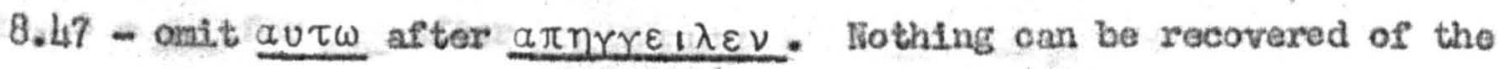

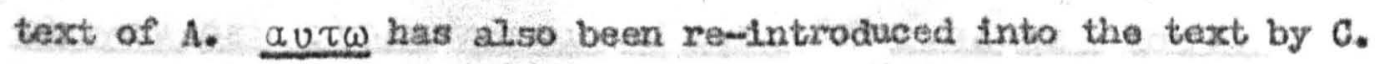
This solution as proposed by Tischendorf is most likely. Tho Pirst corrector bogen 1 ine 25 with $a \pi \eta x$.

$8.54-\underline{\varepsilon \gamma \varepsilon l \rho \varepsilon}$ for $\varepsilon \gamma \varepsilon \downarrow \rho \varepsilon \rho \varepsilon$.

$9.5-\underline{\delta \times \omega \nu \tau \alpha_{1}}$ for $\delta \varepsilon \times \omega \tau \alpha_{1}$. Tischendors did not eredit $B$ with adding the $\underline{\nu}$.

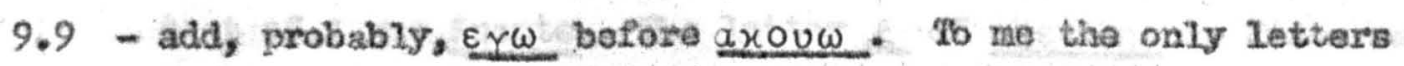
visible are $\varepsilon \gamma \omega$ and $\zeta \eta \tau \varepsilon$, , these are by B. The corrector probably erased the last part of the line to insert $\varepsilon \times \omega *$

9.12 - Tischendorf sald A apparently omitted $x a \tau a \lambda v \sigma w \sigma 1 k$. is 1 mpossible to know what $A$ wrote, but I think there is too much

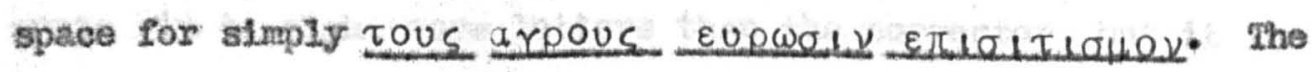
text of A was more Itkely inepte.

9.31 - omit $\delta \varepsilon$ nfter $\varepsilon \lambda \varepsilon y o \nu$.

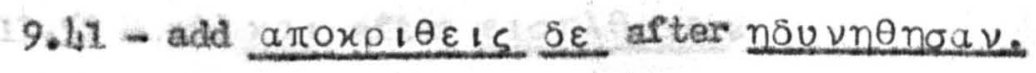

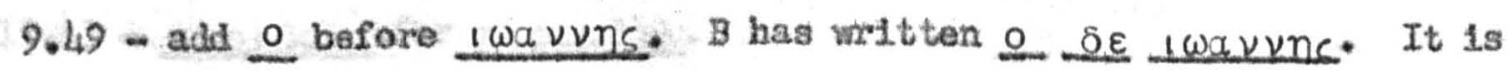
more Ilkely that $A$ onfitted 0 rather than $\delta \varepsilon$ because of the space. To onit $\delta \varepsilon$ would leave to much room.

$9.49-\varepsilon \pi 1 \sigma \tau a \tau \alpha$ for $\delta 1 \delta a \sigma x a \lambda \varepsilon$. The letters at the begining of Ine 2 are $\lambda \varepsilon$ while the last letter in line $\lambda$ was $\underline{a}$. The remaining Iotiers cannot be seen.

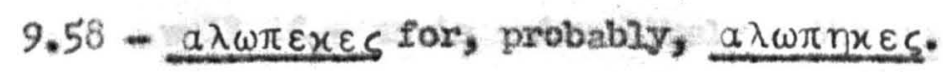

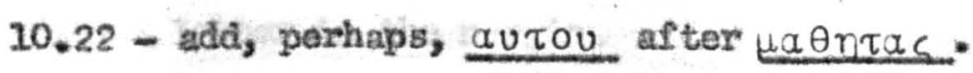

10.30 - add $\delta \varepsilon$, probably, after $0 \pi 0 \lambda \alpha \beta \omega \nu$. The limitations of apace suggest our text.

10. 35 - onit $x \alpha_{1}$ after $\varepsilon \xi \varepsilon \lambda \theta \omega \nu$.

10.38 - add autns after oix lay.

10.39- tou Tu for autou. Tisehendorf erred in stating that A wrots

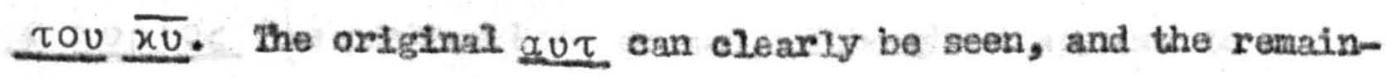
ing space is perfect for ou. Furthermore, the corrector would 
bo very unlikely to erase $\underline{\text { vo }} \overline{\overline{x U}}$ simple to change $x$ to $L$. 10.40 - $\underline{-\delta}$ for $\tau \varepsilon$ after $\varepsilon \pi i \sigma \tau \alpha \sigma a$.

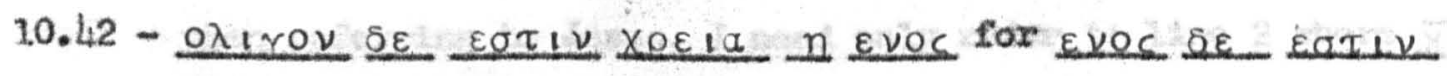

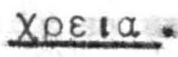

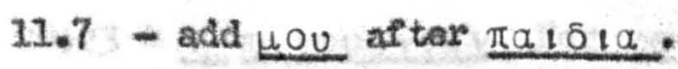

11.23 - add $\mu \varepsilon$ after $\underline{\alpha x o \rho \pi ! \zeta \varepsilon !}$. Tischendorf Palled to note this addition.

$11.24-\underline{\pi \nu \varepsilon u \mu a}$ for, probably, $\overline{\pi v a}$ rischendore said only that the

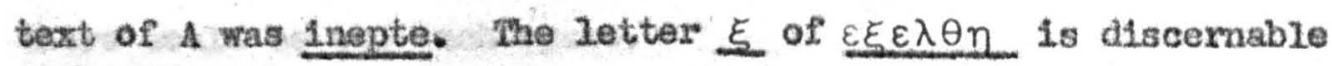
at a point wilch suggests the reading that is included in parentheses in this edition. The corrector objected to the nomina sacra for unclean spirits.

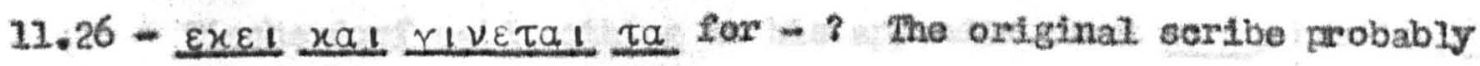
wrote six to elght nore letters than the corrector, but it is inpossible to knov what he wrote.

11. 44 - The redundant ou before oux is erased.

11.52 - add $\underline{x a l}$ after $\varepsilon 1 \sigma \eta \lambda \theta a \tau \varepsilon$.

$19.43-\pi \varepsilon \rho 1 \beta \alpha \lambda$. for $\pi \alpha \rho \varepsilon \mu \beta \alpha \lambda$.

$29.46-\underline{x \lambda n \theta n \sigma \varepsilon \tau a_{1}}$ for $\varepsilon \sigma \tau 1 \nu$

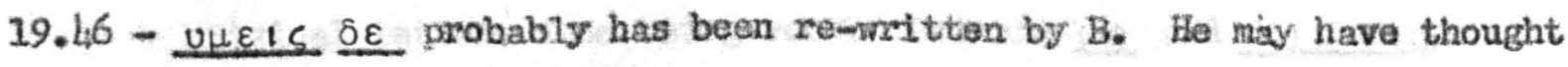

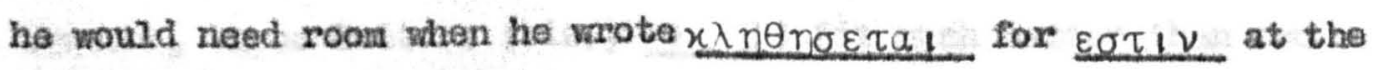
and of the preceding lines but having put all of $x \lambda r \theta n \sigma \varepsilon \tau a$ i on that line he merely remrote the text of A, Certainly this fits well if - as is quite likely - the $\underline{u}$ of $\underline{u \varepsilon}$ is was a large letter in the nargin.

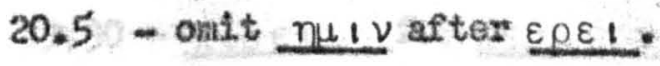

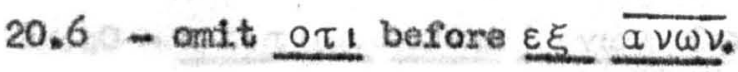

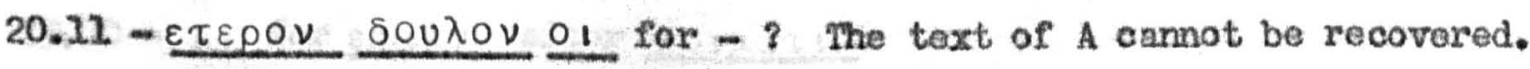
Our text, which is Tischendorf's conjecture, is most probable. It is certain that $A$ wote more than $B$.

20.16 - 을

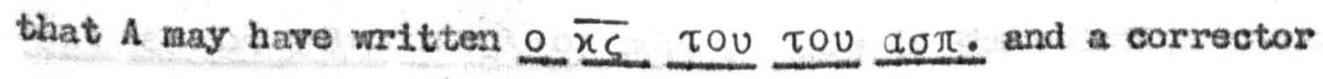


Iuke

removed the redundant $\underline{\text { Tov }}$ as well as $\mathrm{e} \overline{\mathrm{xc}}$. Though $1 \mathrm{t}$ may be objected that the noming sacra for xuploc. Is not used except when referring to Jesus, I need only refer to Iline 2 where $\overline{x 6}$ is 30 used.

20.23 - n mavoupriav for movnolav.

20.25 - ontt $\tau \omega$ before $x \alpha$ logpl. (?)

$21.34-\varepsilon \varphi \nu_{1} \delta 105$ Por, probably $\varepsilon \varphi \nu_{1} \delta 0 s$. The Jetiers $\nu_{1} \delta$ are slightzy crowded, and if any letter other than 1 were omittod, thare would be too mich space.

$21.38-1 \varepsilon \rho \omega$ for $0 \Omega \varepsilon 1$.

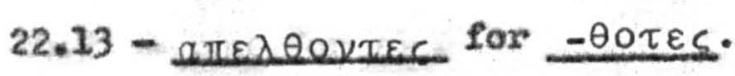

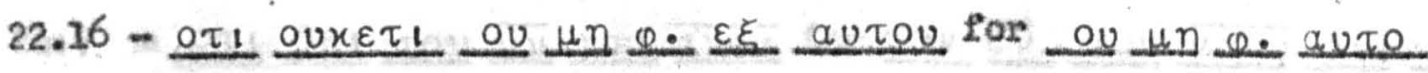

Iischendorf sald nothing in hts append $1 x$ about $0 \tau$, but in 28 renarks that the oxiginal scribe apparently omitted 1t. It is

now certain that A onitted $0 \tau_{1}$.

22.18 - add $\eta$ before $\beta a \sigma, \lambda \varepsilon, \alpha$, I an reasonably certain of this.

Mschendorf noted that A omatted ou rather than $n$. But ws at

the beginning of the Iine 18 by A. AlI the letters except $\beta$ in

Bao $\mid \lambda \varepsilon_{\perp} \alpha$ can be sees. Unfortunatsly small holes prevent the original letters frow boing seen. Batween $\omega_{S}$ and $\alpha_{\sigma} \mid \lambda \varepsilon_{1}$, however, three lotters (Viz., ou B) itt rach better than two (1.e., $\eta \underline{\eta})$. Read, therefore, $\varepsilon \omega_{S}$ ov $\beta \alpha \sigma|\lambda \varepsilon| \alpha$ Instead of $\varepsilon \omega s, \beta \alpha \sigma ı \eta_{1} \_\alpha$

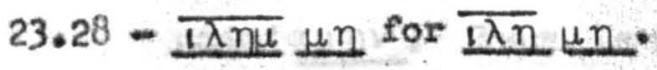

$23+29-\underline{\varepsilon \varepsilon \varepsilon \theta \rho \varepsilon \psi \alpha \nu \text { for } \varepsilon \theta \rho \varepsilon \psi \alpha \nu .}$

$23.30-\pi \varepsilon \sigma \varepsilon \tau \alpha_{1}$ for $\pi \varepsilon \alpha_{\alpha 1 \tau \alpha_{1}}$.

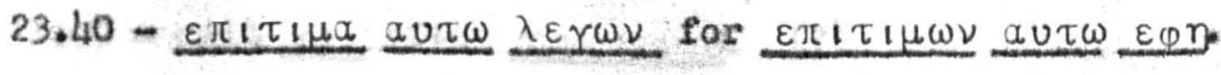

$23.40-\underline{\varepsilon l}$ for $\varepsilon \sigma \mu \varepsilon \nu$ after $x \rho\lfloor\mu \alpha \tau 1$.

$23,47-$ add $x a l$ before nuE

$23.42-\tau \omega \overline{1 v} \mu \nu . \mu 0 v \overline{x \varepsilon}$ for $\overline{t v} \mu \nu$.

23.45 - add $\tau 0 u \underline{n \lambda 100} \varepsilon x \lambda \varepsilon 1 \pi 0 \nu \tau \varepsilon S_{-}$. The second corrector then added:

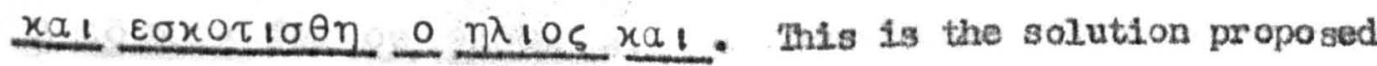


Iuke

by Tischendori, and there seens liftle reason to doubt that it is correct.

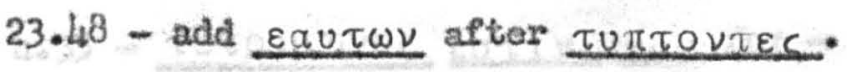

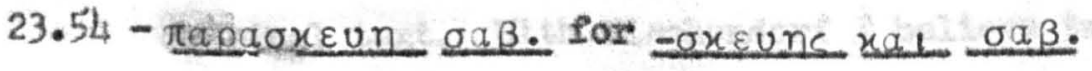

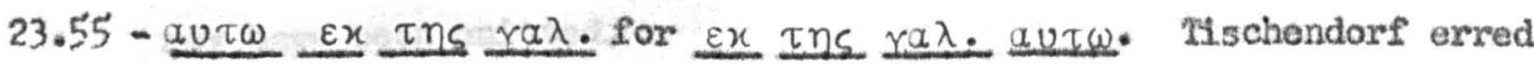
In saying the original scribe onitted $a v \tau \omega$. The letter $\underline{v}$ can be seen shortly before $\varepsilon \theta \varepsilon a \sigma$. Furthermore, all the letters easily fit in the space which would not be filled by $\varepsilon x$ Inc ra $\lambda_{1} \lambda a_{1} a_{c}$. The writing of $\mathrm{B}$ is not cramped.

23.56 - add $\underline{x \alpha_{1}}$ before $y \pi 00 \tau \rho$.

24.2 - ano for $\varepsilon x$.

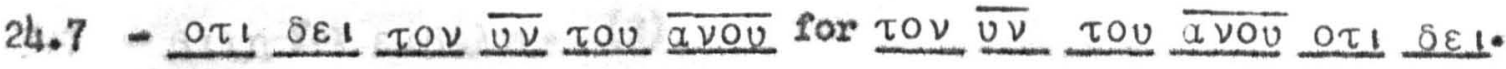
This 1 s the best conjecture since nothing can be seen of the text of A. The space is suffictent.

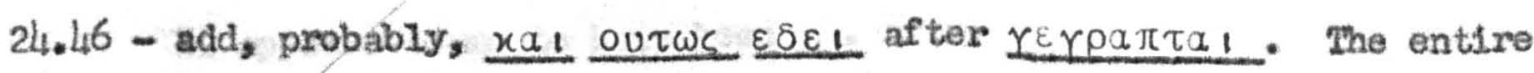
line has been erased and only the inftial $\underline{x}$ and the final $\underline{a}$ are certain. Under $\underline{\varepsilon \varepsilon 1}$ vertical strokes are apparent, but whether they are $\frac{\pi \tau}{-}$ - as Tischendorf sald - I do not know. I see no reason to doubt this text since it adequately fills the line.

24.48 - add $\underline{\delta \varepsilon}$ after $\underline{\mu \varepsilon 15 .}$

24.49 - add $\overline{1 \lambda \eta \mu}$ after $\pi 0 \lambda \varepsilon \downarrow$.

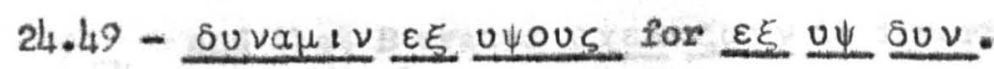

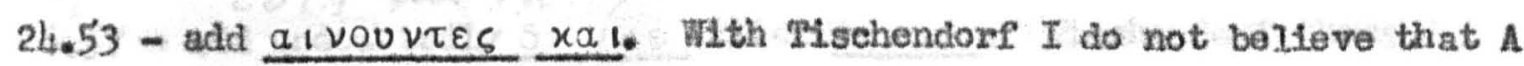
added $a \mu \eta \nu$. There is not room at the ond of the line and I see no traces of its having been arased elsewhere.

John

$$
\begin{aligned}
& 1.20 \text { - onit } \underline{x \alpha_{1}} \text { after novnoa to. } \\
& 1.26 \text { - } \mu \varepsilon \sigma o c \text { se for } \mu \varepsilon \sigma o c \text {. } \\
& 1.28 \text { - } \beta \eta \theta a \beta a \rho a \text { for } \beta n \theta \alpha \nu i \alpha .
\end{aligned}
$$

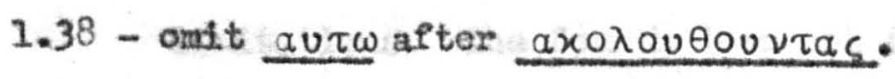

$$
\begin{aligned}
& 3.34 \text { - add o } \overline{\theta_{S}} \text { after siowolv. } \\
& 4.5 \text { - 으 } \varepsilon \delta \omega \varepsilon \nu \text { for ov } \varepsilon \delta \omega x \varepsilon \psi \text {. }
\end{aligned}
$$


John

4.16 - add $0 . \overline{15}$ after $\lambda \varepsilon v \varepsilon 1$ quin.

$4.25-\pi a \nu \tau a$ for $a \pi a \nu \tau a$.

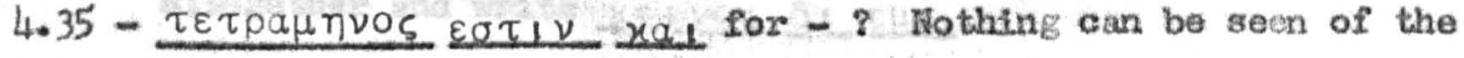
orfginal text. With Tischendorf I believe two or three letters may have been omitted.

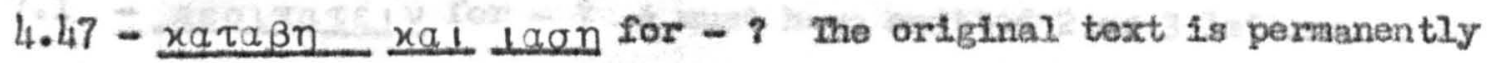
Iost. Our text is the conjecture of both IIschendorf and Wetstein, but not necessarily the only one. It is certain that A wrote less than B.

4.54 - omit $\delta \varepsilon$ before $\pi a \lambda 1 \nu$.

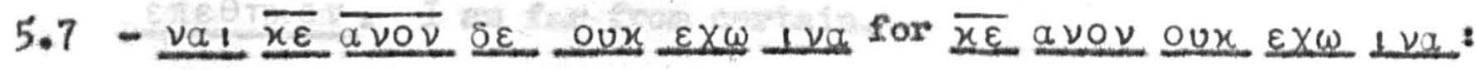

5.7 - $\beta a \lambda n$ for $\varepsilon \mu \beta a \lambda n$.

5.9 - autou for equtou.

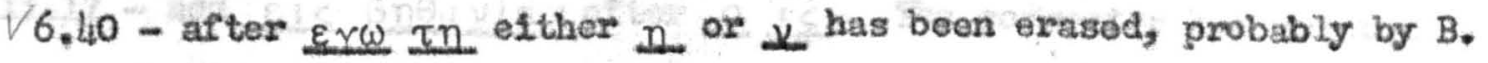
It appears to me to have been $n$.

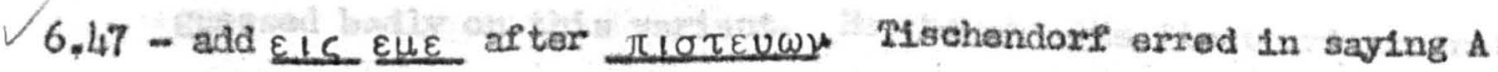
wrote $\varepsilon 15, \varepsilon \mu \varepsilon$. The page is torn at this point, but the letters, $y \omega^{-}$, that Ifschendorf noted at the end of the line are definitely by the first corrector and extand into the nargin. The original text, which has been orased, contained less than

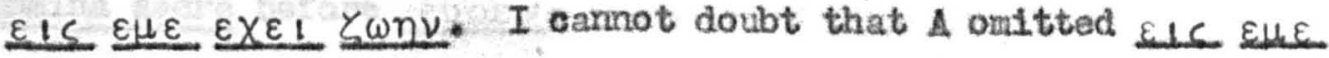

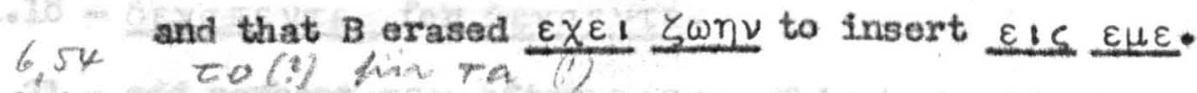

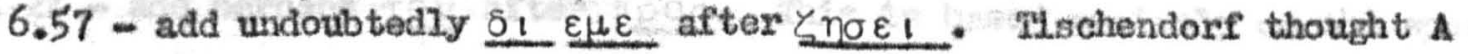
wrote $\zeta \eta$ for $\zeta \eta \sigma \varepsilon !$. He orred in saying that the letters

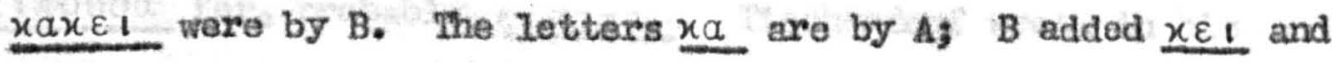
then began line 5 with vos. But since A mote $x a$ at the end of line 4 , Iine 5 must have begun in one of 4 ways: (1) $x \varepsilon$ l voc

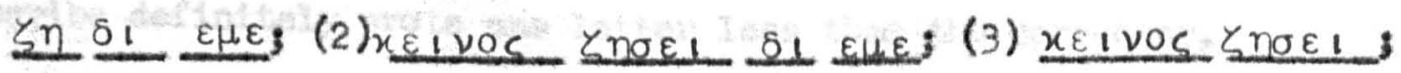
(4) $x \varepsilon_{1}$ vos $\zeta \eta$. The first two are rejected because of space limitations. The fourth does not fill the space. Iines 4 and 6 over the sane space have 9 and 10 letters respectively. I have adopted the third possibility as the most likely text of 
John

A. In adopting this text I assune - and this is not always safe to do - the text of $A$ did not mistakenly omit the verb altogether and mite $x \varepsilon i v o c$ o $\varepsilon \mu \varepsilon$.

6.72 - add $\underline{\omega \nu}$ after $\varepsilon 15$.

7.1 - omit $\underline{x \alpha_{1}}$ before $\mu \varepsilon \tau \alpha_{\text {. }}$.

7.1 - $\pi \varepsilon_{\rho} i \pi a \tau \varepsilon i \nu$ for - ? A must have omitted 2 or 3 letters.

8.45 - onit $\underline{u \mu 1 \nu}$ after $\underline{\lambda \varepsilon \gamma \omega}$.

$8.54-\delta 0 \xi a \zeta \omega$ for $\delta 0 \xi \alpha \sigma \omega$.

9.7 - add autou after $\varepsilon \pi \varepsilon \times \rho 10 \varepsilon \nu$. In his appendix Tischendorf says nothing about $\varepsilon \pi \varepsilon \times \rho 1 \sigma \varepsilon \nu$ but in TB says A apparentIy wrote $\varepsilon \pi \varepsilon \theta \eta x \varepsilon \nu$. I an Par from certain.

9.9 - add $\delta \varepsilon$ after $\varepsilon x \varepsilon \mid$ vos.

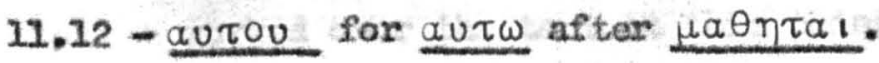

11.17 - add $\varepsilon 15$ naviav after 으 $\overline{15}$. The corrector has writton $\varepsilon \lambda \theta \omega \nu$ ouv $\overline{\text { IS }}$ at the end of the previous 1ine. Tischendorf guessed badiy on this vartant. He thought the firet witer wrote $\eta \lambda \theta \varepsilon \nu$ ouv $x a_{1} \varepsilon u p \varepsilon \nu$ but does not explain what was placed in the space occupied by these words when the corrector changed the text. Only traces of the original text can be seen, but the surest evidence for our text is the horigontal Iine for the

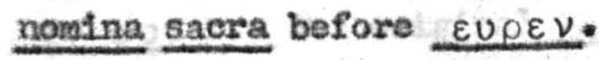

11.18 - $\underline{\delta \varepsilon x a \pi \nu \nu \varepsilon}$ for $\underline{\delta x a \varepsilon \nu \tau \varepsilon}$.

11.21 - add perhaps $\tau 0 v$ after $\pi \rho 05$. This is bused entirely on the space available.

11.28 - $\underline{\varepsilon 1 \pi 0 v \sigma a}$ for, probably, $\varepsilon 1 \pi a \sigma a$. Mschendorf wrote in his appendix "Inepte erasse videtus $\mathrm{A}_{3}$ " but in re cites Codex $\mathrm{C}$ with Codex $B$ as having originally contained $\varepsilon_{1} \pi a \sigma a$. The original saribe definitely wrote one letter less than the corrector.

11.29 - oudt $\underline{\delta \varepsilon}$ after $\underline{\varepsilon \varepsilon \varepsilon \nu \eta}$.

11.31 -

11.32 - nv IC for IC nv.

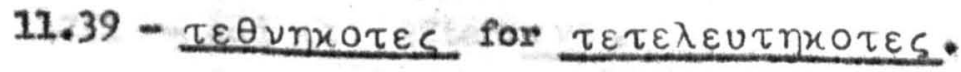




\section{John}

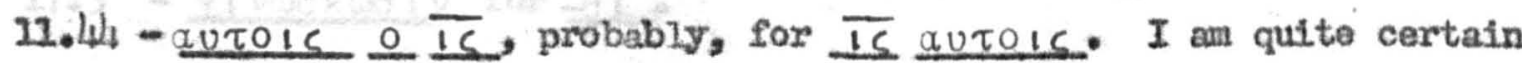
of this although I have not seen any letters woll enough to place them without brackets. To include the article would crowd the text. Also, a s, smallex than the rest of the text, indicates the text has been corrected, although Pischendorf notes nothing of the carrector's hand. Furthermore the horizontal line for the noming sacra is seen at both the beginning and end of the space. The former is almost certainly by the orifinal seribe.

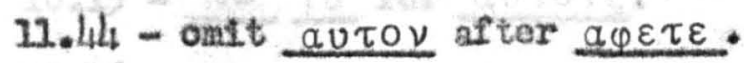

11.45 - add $0 \eta \mu \varepsilon 10 \nu$ 으 $\overline{15}$ before $\varepsilon \pi 1 \sigma \tau \varepsilon v \sigma a \nu$. THschendorf stated that $B$ also omitted $\varepsilon i c$ autov and that it was later added by c. But this Els autov is by B. The second corrector has not worked over the text at this point.

13.12 - ontt $\underline{x \alpha \text { I before } \varepsilon \lambda a \beta \varepsilon \nu}$.

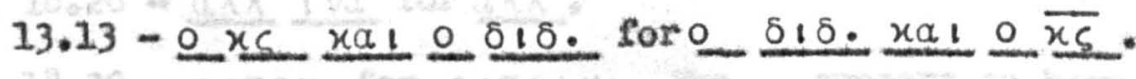

13.23 - add $\underline{\varepsilon,}$, probably, after $\underline{\eta \nu}$.

13.24 - add oUV, perhaps, after $\nu \varepsilon \cup \varepsilon$. It is more Ijkely that $\mathrm{A}$ omitted ouv than $\tau$ ou $\tau \omega$. Nothing, however, is certain.

13.31 - vu for עu.

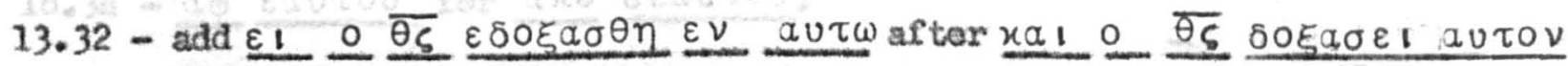
$\varepsilon v$ autw. The original $x$ at the beginning of the erased area is apparent. Though nothling olse can be seen, every conslderation supports our text. The text of $\mathrm{A}$ ended at about the middie of

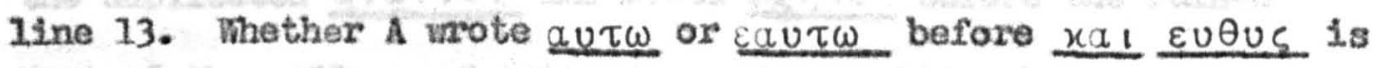
not known. It camnot be assuned that $B$, after expanding the text, re-wrote the original text.

14.2 - omit $0 \tau i$ after $\underline{u \mu l \nu}$.

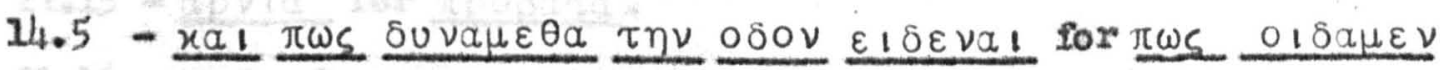
Tnv osov.

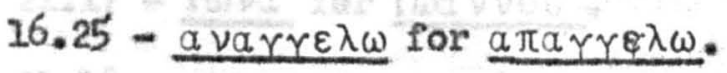

16.28 - $\pi a \rho a$ for $\varepsilon x$.

17.1 - add $\underline{x a 1}$ after $1 v a$. 
$\underline{J o h n}$

$17.3-\underline{Y 1 \nu \omega \sigma x \omega \sigma 1 \nu}$ for $=01$.

17.12 - onit $\underline{x \alpha_{1}}$ before $\underline{\varepsilon} \underline{u \lambda \alpha \xi \alpha}$.

17.23 - The redundant $\varepsilon / s$ is erased after $\tau \varepsilon \tau \varepsilon \lambda \varepsilon l \omega \mu \varepsilon \nu 0$.

18.10 - $\omega \tau$ 1 ov for $\omega \tau a \rho 10 v$. (?) Tischendorf satd this correction is by $C$. I don't know his reason for so stating. The letters $\underline{\alpha_{\rho}}$ are sirply erased as was the habit of $B$ and as Mischendorf has atso assumed throughout his edition.

$18.14-\underline{a \pi 0 \lambda \varepsilon \sigma \theta \alpha 1}$ for $a \pi 0 \theta \alpha \nu \varepsilon 1 \nu$.

$18.15-\tau \omega$ IU for auto1s.

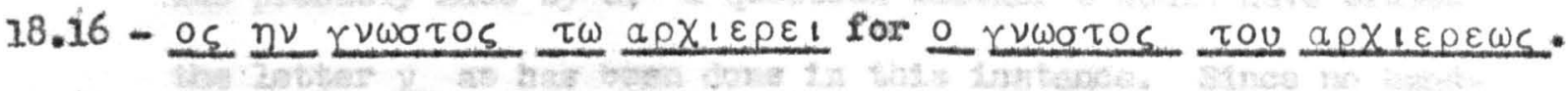
The letters $\rho \varepsilon \omega_{S}$ are clear; the rest is assumed from this and the available space.

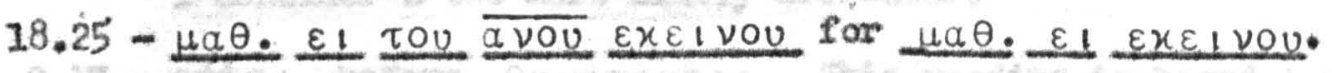

$18.27-0$ - $\pi \varepsilon \tau \rho 0 s$ for $\pi \varepsilon \tau \rho 0 s$.

$18.28-\underline{a \lambda \lambda} \underline{1 \nu a}$ for $\underline{\alpha \lambda \lambda}$.

18.32 - $\underline{\varepsilon i \pi \varepsilon \nu}$ for $\varepsilon 1 \pi \varepsilon \downarrow \nu$. The _ appears to have been partially erased. Iischendorf did not note the presence of the $\perp$ and wrote $\varepsilon 1 \pi \varepsilon \nu$ as the original text.

18.33 - onit $\pi \alpha \lambda 1 \nu$ after ouv.

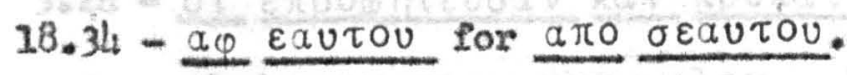

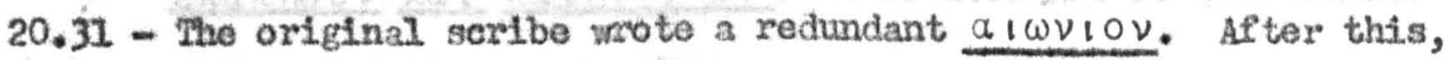
because the vellum is torn, it is impossible to know what was witten, but presumably what is included in brackets. B erased the duplicated $\alpha \_\omega \nu 1 O \nu$ and wrote $\varepsilon \times \eta \tau \varepsilon$ before the rutned part of the vellum. Conitted alwViov altogether.

21.3 -

21.15 - 1wva for 1wavvou.

21.15 - apvia for $\pi \rho 0 \beta a \tau a$.

21.16 - Iwva for 1 wa v vou.

21.17 - 1wva for 1 wa v vou.

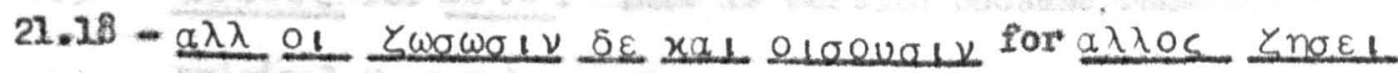
$\underline{\sigma \varepsilon} \underline{x+1} 010 \varepsilon_{1}$.

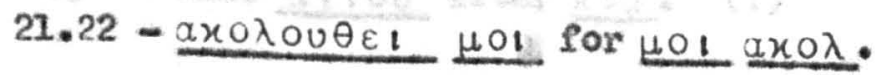


John

21.25 - o0a for $\underline{a}$.

21.25 - $\underline{x \omega \eta \eta \sigma a_{1}}$ for $\chi \omega \rho \eta \sigma \varepsilon i v_{0}$

Acts:

$1.19-\chi \omega_{0} 10 \nu$ a $1 \mu \alpha$ tos for - ? Something has been omitted by B. The text of A probably Included from 3 to 6 more lotters.

$1.22-\varepsilon \nu 0$ after $\underline{x \rho o \nu \omega}$ for $\omega$.

2.1 - Ins for $\tau$.

2.8. $-\underline{\varepsilon \varepsilon \nu \eta \theta \eta \mu \varepsilon \nu}$ for $\varepsilon \gamma \varepsilon \nu \nu \eta \theta \eta \mu \varepsilon \nu$. Tischendorf said this correction was probably aade by $\mathrm{C}$. I question whether $\mathrm{C}$ would have erased the letter $\chi$ as has been done in this instance. Since no handwriting is involved it is impossible to be certain. Nevertheloss, I consider B the more likely corrector.

2.17 - add $a_{1}$ before $\theta u$ u $\tau \varepsilon \rho \varepsilon / 5$. This reading Is based Iargely on considerations of space.

2.17 - add $\underline{\mu \omega \nu}$ after $\underline{\pi \rho \varepsilon \sigma \beta u \tau \varepsilon \rho 01}$. Again considerations of space favor this text.

3.7 - $\sigma \varphi \cup \delta \rho a$ (Not $\sigma \varphi \cup \rho \alpha$ as Tischendore said) for $\varphi \cup \delta \rho \alpha$.

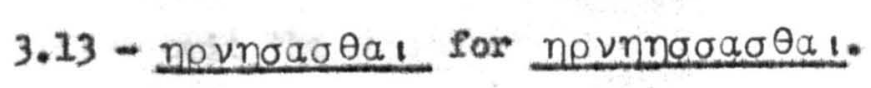

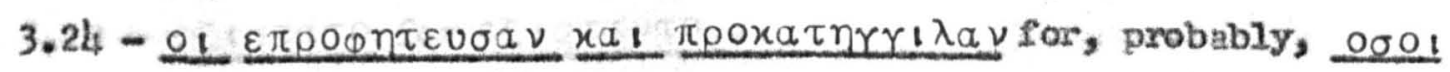

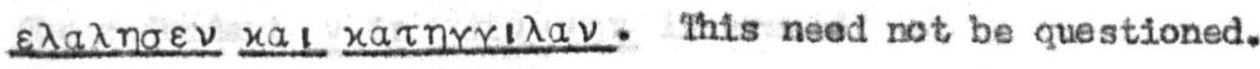

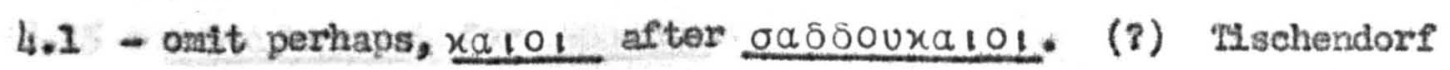
erred in saying $x \alpha_{1}$ had been erased. $x_{a}$ does not begin to fill the space. I consider a duplicated $\underline{x \mid 101}$ to be most Iikely, but it might be that A cotapounded his choices of the

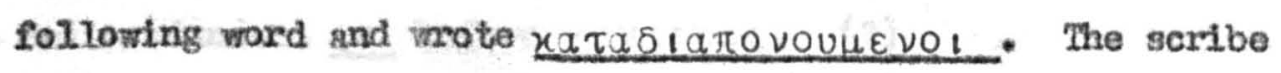
(though perhaps not the same one) has done the same elsewhere. (ce., Ht. 7.16; 23.26; Col. 3.6.)

5.36 - $\pi 000 \varepsilon x \lambda 1 \theta n$ for $\pi \rho 0 \sigma \varepsilon x \lambda \eta \theta \eta n \sigma \alpha \nu$.

5.37 - og01, probably, for 01.

5.39 - autous for auto. This is certain because remants of an original $\underline{U}$ can be seen.

6.3 - onit ayiou after $\overline{\pi \overline{v a}}$. 
Aets

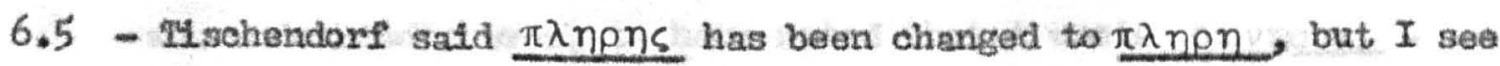
no evidence that an attempt has been made to erase the $\mathrm{c}$. I an certain Tischendore is wrong.

6.5 - $\tau_{1 \mu \rho \nu \alpha}$ for $\tau 1 \mu \omega \nu \alpha$.

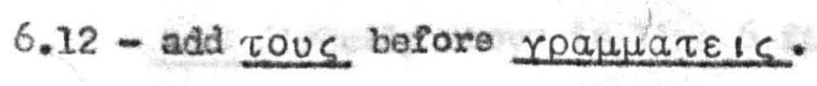

$6.12-\varepsilon \pi 10 \tau a \nu \tau \varepsilon \varepsilon$ for $-\tau \varepsilon$.

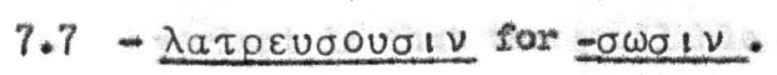

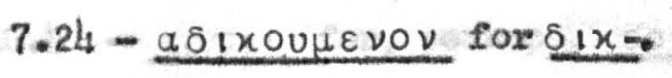

7.26 - autous for autols.

7.33 - omst gov aftor ujosnua.

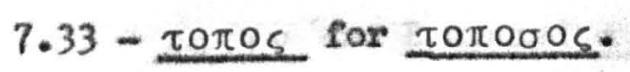

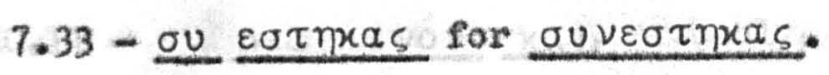

$7.34-\underline{\text { VUVI }}$ for vUV.

7.59 - onlt $\overline{x \varepsilon}$ after $\overline{x \varepsilon} \overline{10}$. Only the $\underline{\varepsilon}$ is erased. The $\underline{\chi}$ does not appear to have been touched.

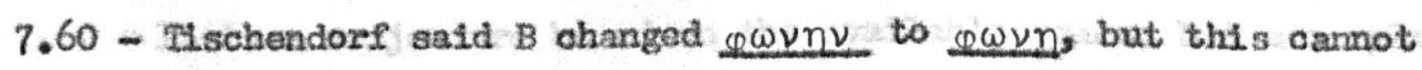
be accepted. I see no evidence that the corrector wished to omit the $\underline{\nu}$.

$8.13-\underline{\varepsilon \xi 1 \sigma \tau \alpha \tau 0}$ for $\varepsilon \xi 1 \sigma \tau \alpha \nu \tau 0$.

8.14 - The redundant $\nu$ in 1 wa $\nu \nu \nu \eta \nu$ is erased.

$8.15-x a \tau a \beta a \nu \tau \varepsilon \zeta$ for $-\beta a \tau \varepsilon s$.

8.27 - add os, probably, before $\varepsilon \lambda \eta \lambda \cup \theta \varepsilon l$. This $1 \mathrm{~s}$ most lincely since the corrector did not erase any part of autns,

$8.39-a \nu \varepsilon \beta \eta$ for $\alpha \nu \varepsilon \beta \eta \sigma \alpha \nu$. (?)

9.2 - omit redundant yu before yuva 1 xas.

9.15 - onit $\underline{\tau \omega \nu}$ before $\varepsilon \theta \nu \omega \nu$. (q)

9.19 - add $\underline{\pi a \rho \alpha x \rho \eta \mu \alpha}$ aftar $\underline{\alpha \varepsilon \beta \lambda \varepsilon \psi \varepsilon \nu} \delta \varepsilon$.

$9.19-\varepsilon v_{10 X v \sigma \varepsilon \nu}$ for $\varepsilon v_{1} \sigma \times v \theta$.

9.26 - omit, perhaps, $x \alpha_{1}$ before $\pi a \rho a y \varepsilon \nu o \mu \varepsilon \nu o s$. (3) our text is only a conjecture since nothing is visible.

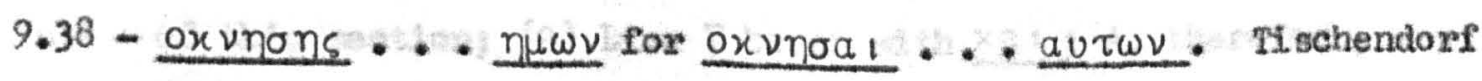


was undoubtedly urong to have noted that A wrote oxvnone... nuwV and that this apparently was changed to oxvnogl ... autwv by the second corrector. The letters gnc (for ga, ) are crowded and $\underline{\eta}$ (for $\underline{\alpha \tau}$ ) 18 unnaturally spread out. The letters aut directly above in line 6 fit perfectiy between $\underline{\varepsilon \omega s}$ and $\underline{\omega \nu}$, netther of which has been erased. The most obvious 1 tem about the

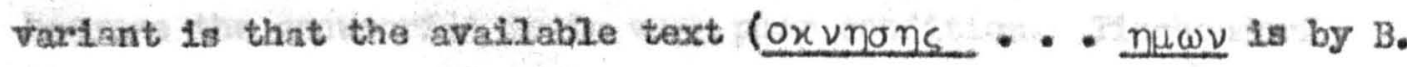
$10.4-\varepsilon \sigma \tau 1 \nu$ for, perhaps, $\varepsilon \sigma \tau 1 \varepsilon \sigma \tau 1 \nu$. (?) This is a conjecture based on the space available: A wrote $\varepsilon \sigma \tau_{l}$ and then, looking back to the previous $\tau_{L}$ vrote $\varepsilon \sigma \tau l \nu$. A thus wrote $\tau_{1} \varepsilon \sigma \tau_{l}$ $\underline{\varepsilon \sigma \tau l \nu}$ and $B$ erised $\tau_{l} \underline{\varepsilon \sigma}$.

10.11 - $\delta \varepsilon \delta \varepsilon \mu \varepsilon \nu 0 \nu$ xal, probably, before $\underline{x \theta_{1} \varepsilon \mu \varepsilon \nu o \nu}$. This is, essontially, the solution of Tischendorf at a polnt where the text of A camnot be recovered. I venture to say, also, that A (as well as the corrector B) omitted $\mu \varepsilon y a \lambda \eta \nu$, but this wust be indefinite. It is a conjecture based on the avallable space; but a complete line may vary too much in the number of letters it contains to say with any degree of assurance.

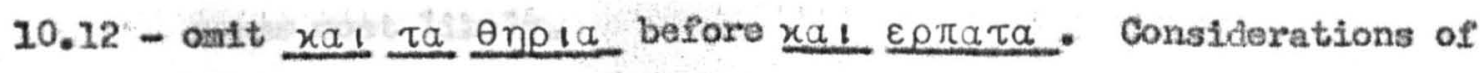
space make this virtually certain.

10.12 - ant $\tau \alpha$ before $\pi \varepsilon \tau 1 v a$. (i)

10.33. - omit redundant $\eta$ after $\varepsilon 1 \sigma \eta x 00 \sigma \theta \eta$. (?)

10.33 - ouv $\pi a \nu \tau \varepsilon s$ for either ou $\pi \alpha \nu \tau \varepsilon \zeta$ or ouv $a \nu \tau \varepsilon \zeta$. It is not possible to tell whether $\underline{\nu}$ or $\underline{\pi}$ was omitted.

10.36 - omit, perhaps, yap before oyov. Oux text of A need not be questioned. The original seribe definttely wote yap, and ample

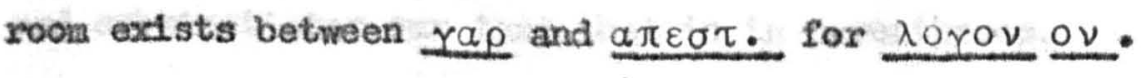

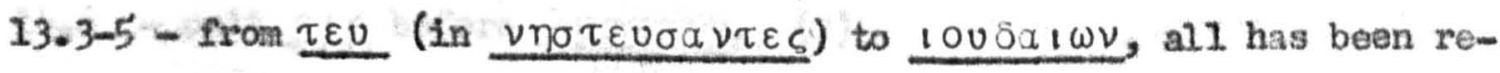
written by $B$. Several things can definitely be sald about this passage: (1) The original scribe onttted perhaps as much as $50 \%$ of this section, (2) Line 7 began with $x a$, $A$, therefore, went elther from the first $\sigma$ of $\underline{\nu \sigma \tau}$. to the first $\underline{\sigma}$ of $\pi p 0 \sigma \varepsilon v \xi$. 
Aets

or from $\underline{\varepsilon U}$ of the former to $\underline{\varepsilon U}$ of the latter; (3) line 9

ended about hatfway aeross the page.

13.6 - a redundant $\underline{x a_{1}}$ omitted. (2)

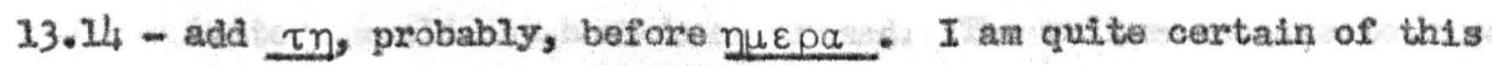
text. The corrector erased part of the text after ouvarwynv to insert sonething. It is difficult to say how much he erased because the manuscript is in very poor condstion. Tischendorf conjectured that $B$ added $\tau \omega \nu, 100 \delta a 1 \omega \nu$ but not this nany letters have been added. The letters $\tau \omega \nu, \sigma a \beta \beta \alpha$ can be seen at the end of the 1 ine and they are not crowded. The remaining space flts $\eta \underline{\varepsilon \rho \alpha}$ very well.

13.18 - ontt, porhaps, a redundant $\chi$ before $x$ bovoy. (?) II schendorf said $\mathrm{A}$ wrote $-\varepsilon \tau \eta \nu$, but $I$ an reasonably certafn this is wrong. There appears to be a crossmstroke similar to the following $\chi$. $13.18-\varepsilon \tau 00 \pi 0 \varphi$. for $\varepsilon \tau \rho 000 \varphi *$

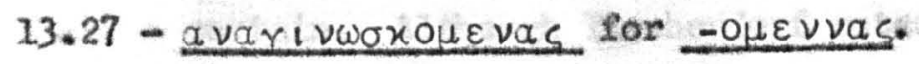

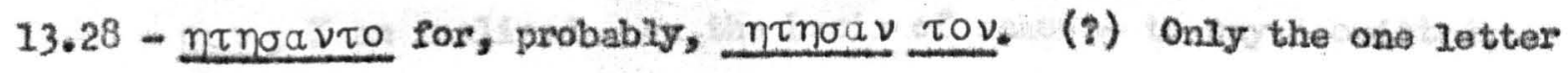
has been exased and nothing can be seen. Our text, however, geens most likely.

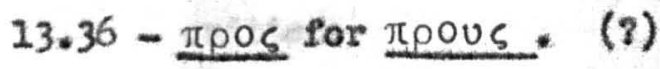

13.4h - axovoal for, probably, axovoe IV. tischendorf assumed A wrote $a x o v g a_{1}$ and did not note that this was the text of $B$. The last vertical stroke of (persumably) $\underline{\nu}$ can be seen. The Ietters geivfit perfectly.

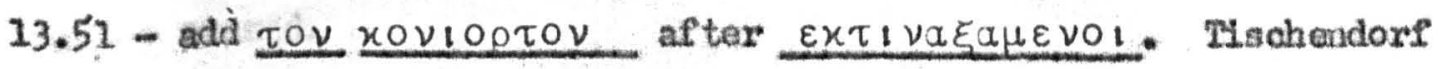
made no guess as to what was onitted. The $\xi_{-}$18 qui te apparent and substantiates our text that is in parentheses.

14.6 - omit $\varepsilon 1 \zeta$ before $\lambda v \sigma \tau p a \nu$. (?)

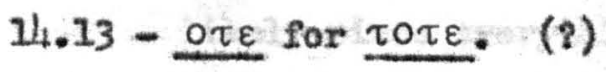

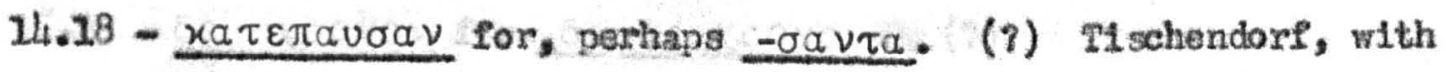
Wetstein, wrote oavio; bat the vary slight renains of the

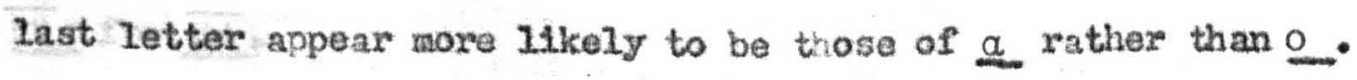


Acts

Thoir preference of course nakes more sense.

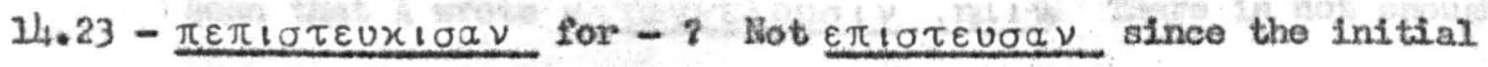
I Is preserved. Not, with Codex $D,-x a \sigma \mid \nu$ because all the letters would not have been erased. The vellum appears to have been erased to the end of the line, indicating that A wrote 5 to 10 letters more than B. The vellum is too thin now for any of the text to be recovered.

14.27 - $\pi 10 \tau \varepsilon \omega_{S}$ for $-?$ Only $\pi i$ remains of the text of $\Lambda$. Whatover It was, it extended beyond the $\pi 1 \sigma \tau \varepsilon \omega S$, written by $B$, since we can see the erased aroa. Perhaps a couple letters were re-duplicated.

15.1 - omit $\tau \omega$ before $\mu \omega \nu \sigma \varepsilon \omega s$. (?)

15.10 - add, perhaps, oUV after VUV. It appears as though the first letter had vertical strokes; it is quite certain therefore, that A wrote vUV. But whether he oralted ouv or $\tau_{1}$ is very uncertain. Although Iischendorf noted that A apparentig omitted. ouv, I am inclined - on the basis of space - to say A ontted 1. The vellum has been eraged to trangparent thinness so that It is not possible to state with any degree of certainty what was onitted.

15.16 - avo1xosounow for, probably, o1x.

15.20 - add, probably, tou before alfatos. The vellum has been very thoroughly erased. Stnce this is the end of an incoraplete line, our text is based solely on the size of the area that has been erased.

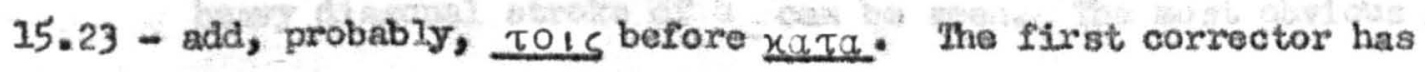
written more than $A$. The original text is impossible to recover: even the corrected text is obscure. Our text is nost

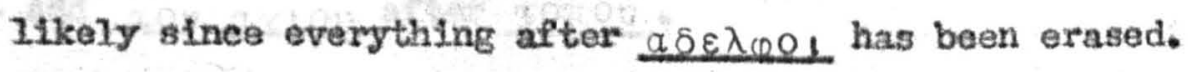

$15.33-\delta \varepsilon$ Xpovov for $\delta \varepsilon \delta \varepsilon$ Xpovov. (?)

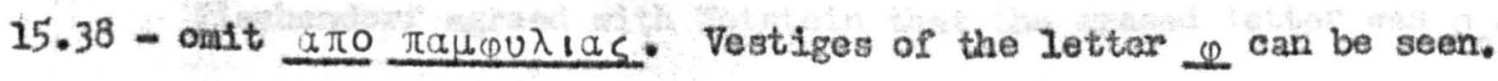
16.7 - IV for $\overline{x u}$. 
Acts

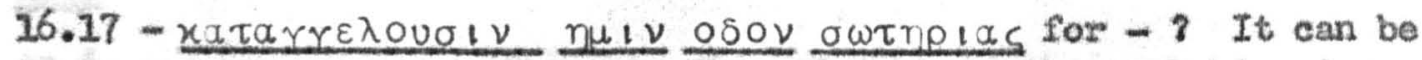
seen that A wrote $x a \tau a y r \varepsilon \lambda o v \sigma i v$ nutu there is not enough space for the accepted text. A probably inadvertently onitted 2 or 3 letters.

16.19 - add $0 \tau \downarrow$ before $\varepsilon \xi \eta \lambda \theta \varepsilon \nu_{0}$ The first corrector erased every-

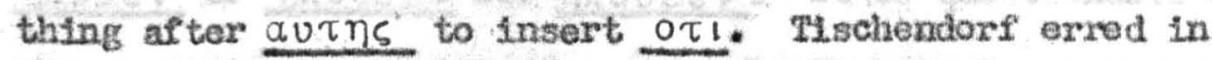
assuming the text had not been materially changed. He thought the 0 of the present $\underline{-\tau 1}$ was an original ㅇ.. The rest of

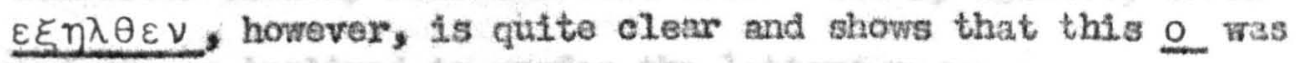
originally the inltial $\varepsilon$ of $\varepsilon \xi \eta \lambda \theta \varepsilon \nu$.

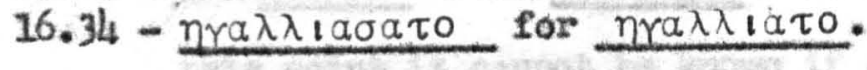

$20.16-\varepsilon \sigma \pi \varepsilon v \delta \varepsilon \nu$ for $-?$ I Imst reject forthright the suggestion by Tischendors that $\varepsilon \delta \varepsilon 1$ was origtaliy writion. It does not fill the space. Five full letters (I consider 1 a half-letter when space is concerned) were probably written. I can make no Iurther suggestion even with the ald of an infra-red photograph.

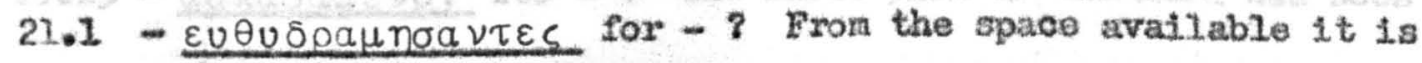
clear that A omitted 2 or 3 letters.

21.1.3 - onit probably, $\delta \varepsilon$ after $a \pi \varepsilon x \rho 1 \theta n$

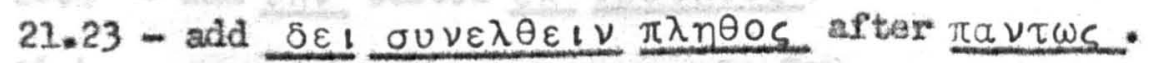

$21.25-\underline{\varepsilon \pi \varepsilon \sigma \tau \varepsilon, \lambda a \mu \varepsilon \nu}$ for $a \pi \varepsilon \sigma \tau$. TLschendorf was wrong to write $\varepsilon \pi \varepsilon \sigma \tau$ : for the first text. My original impression was that $\mathrm{A}$ wrote $a \pi$. and that $B$ changed it to read $\varepsilon \pi$. Subsequent exarination under a glass confirmed this. The $\underline{\varepsilon}$ seen by Tischendorf obviously is not by A. Furthertiore, part of the heavy diagonal stroke of $\underline{a}$ can be seen. The most obvious item at this point in the text 18 that a correction has been made: Tisohendorf did not note this.

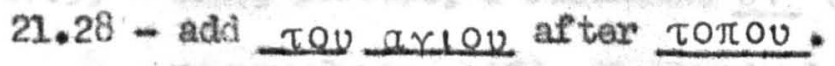

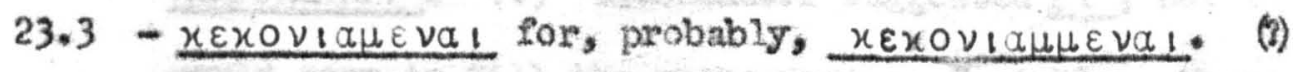

Iischendorf agreed with wotstein that the exased lettor was $\underline{\text { g. }}$ I think it was a duplicated $\mu$, since a vertical stroke can apparently be seen. 
Acts

$23.6-\varepsilon y \omega$ spelled something.

$23.7-\underline{\varepsilon \alpha \times 10 \theta \eta}$ for $\varepsilon \times 10 \theta n$.

24.24 - add 1 ola before vuva $1 x_{1}$.

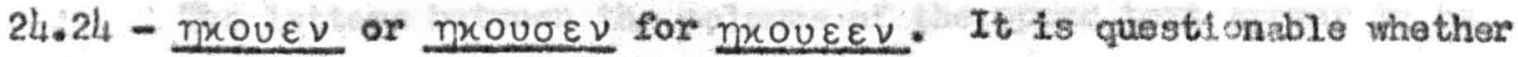
the corrector meant to erase entiroly the first $\varepsilon$, or merely to erase the middle horl gontal line and thus make o. The corrector clearly made a feeble effort and $I$, against, Tischendorf, an inclined to prefer the lattert nxovorv.

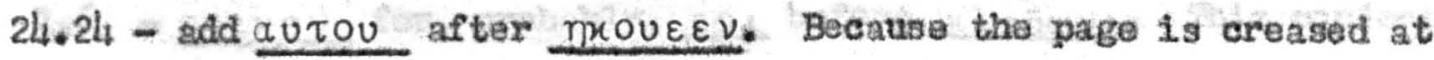
this point it cannot be known if A wrote $\overline{\chi \nu}$ or $\overline{X V} \overline{I V}$. I have no opinioh, since I cannot even see where the line originally ended.

25.13 - $\beta \varepsilon_{0 \nu 1 \times n}$ for, probably, $\beta \varepsilon \rho n \nu 1 \times n$ (or $\beta \varepsilon \rho \nu \nu 1 \times n$ ). Vertical strokes of the erased letter are seen, but nothing else.

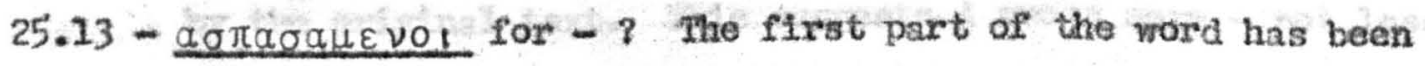
changed in some way.

25.28 - jounoa for movnoav. (?)

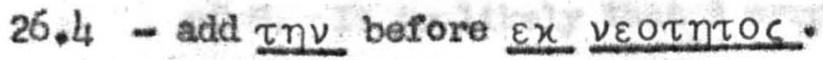

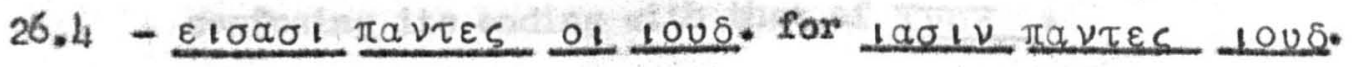

26.5 - $\pi \rho \circ \gamma i v$. for $\pi \rho \circ \varepsilon y i \nu$. See the note on this varlant in Appendix A.

26.16 - onit, probably, $\mu \varepsilon$ (not $\left.x \alpha_{1}\right)$ after $15 \varepsilon s$.

27.23 - add $\underline{\varepsilon y \omega}$ after $\underline{\varepsilon \mu \mu !}$.

27.27 - add of before vautal. Tischendorf credited $\mathrm{O}_{1}$ to the orifinal scribe.

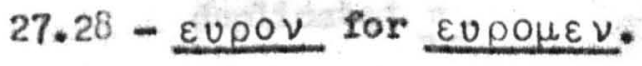

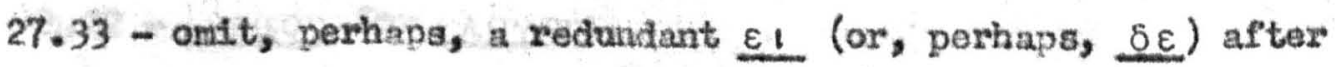

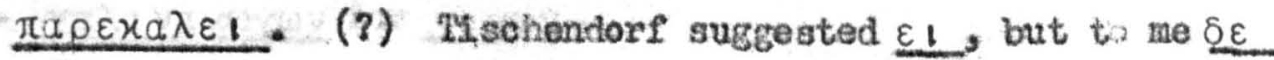
would seen to fill the space more comfortably. There is, howover, very little difference.

27.42 - add $\underline{\delta \varepsilon}$ before $\underline{\underline{\varepsilon} \rho \rho \tau 1 \omega \nu \tau \omega \nu}$. 


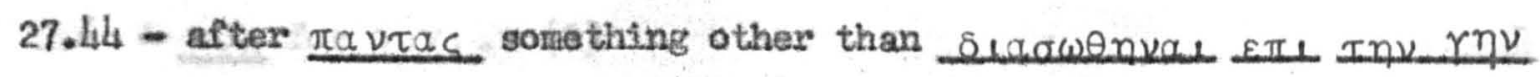
was written and appears to have beon corrected to the gtandard text which Tischendorf oredited to the original scribe. An original

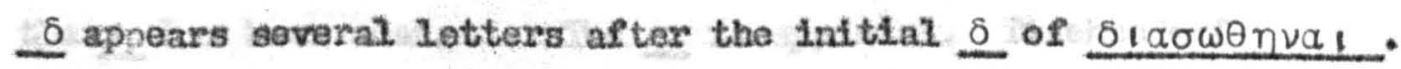
The letters between the columins of the upper text appear to be Inv. But this does not fit the pattern of the line and this area shows Indications of a correction having been made. The text was undoubtediy "inepte."

James

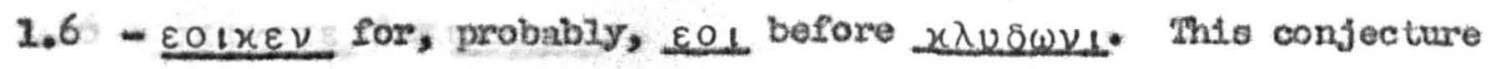
by Mschendorf fits the space well.

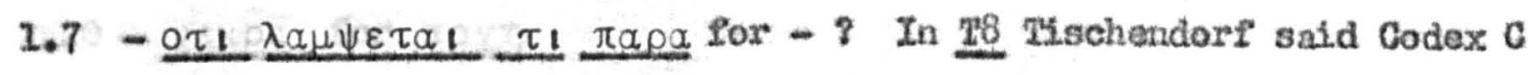
(with Codex $\perp^{\prime)}$ ) apparentiy omitted $\tau_{1}$. There is no basis for this supposition. Nothing can be read of the original text. The text of tho corrector doss not fill all the space occupled by the original text. This suggests A wote more - not jess than $B$ s this is strengthened by a comparison with line 15 where 19. Ietters occupy a space equal to that used by the 17 letters of B. It is likely that A erroneously duplicated $\varepsilon x \varepsilon$ ivos (by confusing its ending with that of avoc.

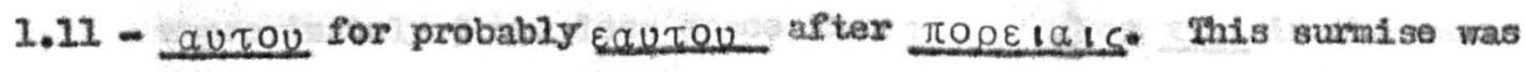
made by Tischendorf. In correcting the text $B$ wrote $\underline{a}$ and $\underline{u}$ farther apart than usual whereas the rest of the line is woll spaced.

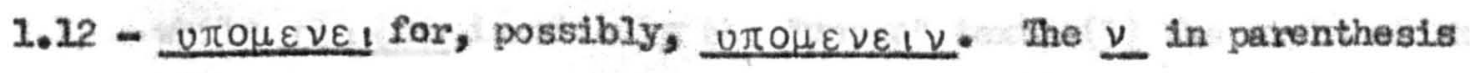
represents only a conjecture. It may equally well have been a duplicated $\pi$.

1.22 - vouou for גorou.

$1.26-\chi_{1} \lambda_{1} \nu a \gamma \omega \gamma \omega \nu$ for $-a \gamma \omega \nu$.

1.27 - omit $\tau \omega$ before $\overline{\theta \omega_{*}}$ (?)

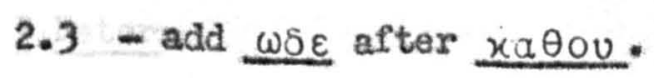

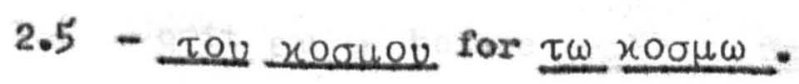


2.6 - oux for ouxt. (2)

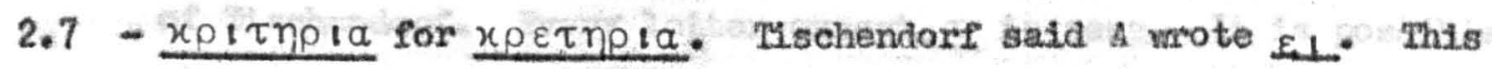
is imposstble because of the space. Nel ther the first $\_$nor the $\tau$ has been changed. The $\underline{\varepsilon}$ is apparent. Tischendorf erred in assuning this was the conmon orthographic error of $\underline{\varepsilon}$ for 1 .

$2.7-\underline{\varepsilon i x \lambda \eta \eta \theta \varepsilon \nu}$ for $\varepsilon \pi 1 x \varepsilon x \lambda \eta \theta \varepsilon \nu$.

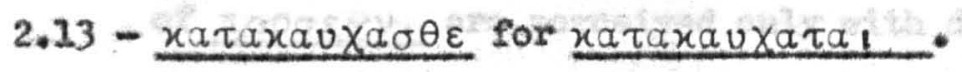

2.Il - add to before oncios.

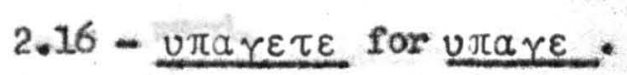

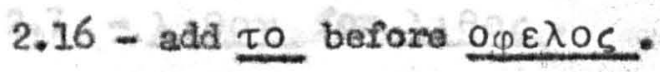

2.20 - $\nu \varepsilon x \rho a$ for $\alpha \rho \gamma \eta$.

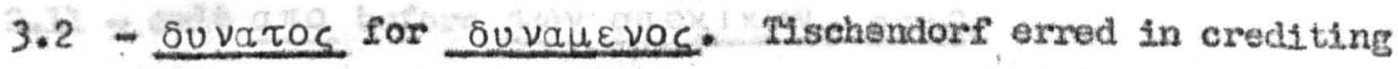
suvatos, to the orfiginal seribe.

3.5 - $\mu \varepsilon \gamma a \lambda a v \times \varepsilon_{1}$ for $\mu \varepsilon \gamma a \lambda \alpha$ aux $\varepsilon_{1}$.

3.6 - $n \lambda 1 x$ ov for o $0 \lambda_{1}$ yov. Tlschendorf agreed with wetstein that the corrector changed $\eta \lambda 1$ xov to $0 \lambda 1$ yov. Without doubt A wrote oliyov rather than $\eta \lambda i x o \nu$. I studied this place several timeg and each time became more convinced that Tlschendorf was wrong. The following items shovld be noted: (1) $\eta \lambda 1 x$ here uses approximately $3 \mathrm{~mm}$. Less space than $\eta \lambda 1 x$ after $\pi 0 p$ in the same Iine; $x$ here uses 2 moss space than the same letter in $\eta \lambda i x \eta \nu$ (2) Tischendorf and Wetstein sald $0 \lambda$ iyov is the corrected text; but this is quite obscure and partially erased while $n \lambda$ ixov is clearly the "upper" toxt; (3) the style of the writing indicates $n \lambda 1$ xov is by $B: \eta$ slants slightly to the right; the $\leq$ of $x$ is elearly not by A. Codex $C$ therefore, agrees with Codd. AKI and almost all the versions except the Latin vilgate.

3.12 - add outws after ouxa.

I Peter

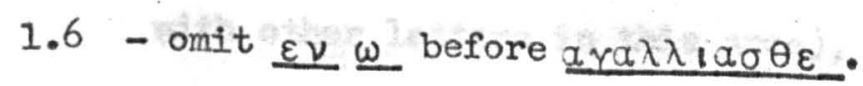




\section{Peter}

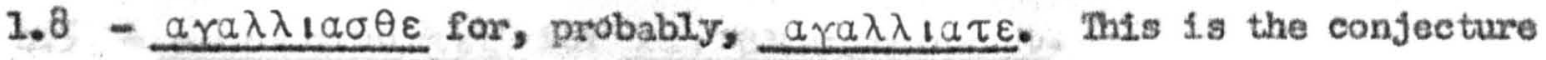
of Hischendorf. Every letter except $\tau$ is seen. It is possible however, that A wrote either $\underline{\sigma}$ or $\underline{\theta}$ and omitted the other.

1.12 - oux autots for oux EquTols.

1.20 - Tischendorf said $\pi \rho 00 \varepsilon \gamma \nu_{0}$ has been charged to read $\pi \rho 0 \varepsilon y \nu$. I do not think this is correct. The beginnings of the last few Itnes on this page are quite obscure. The first four letters of $\pi \rho 0 \sigma \varepsilon \gamma \nu$. are percelved only with difficulty, but the 0 is the clearest of them. I do not think a corrector has touched the word.

$2.7-\underline{\lambda}, \theta 0 \mathrm{v}$ for $\lambda_{1} \theta 0 \mathrm{~s}$.

2.8 - 01 for 0001 .

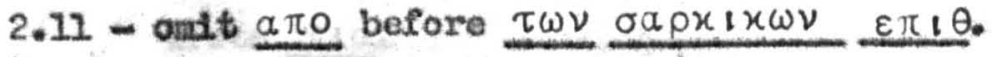

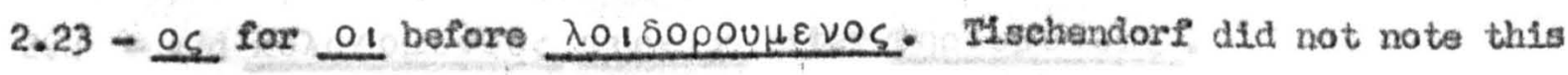
change.

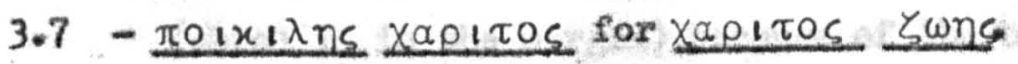

3.10 - omit $\tau \alpha$ before $x \in 1 \lambda \eta$. (3)

3.21 - omit $\delta \varepsilon$ before $\underline{a} \pi 0$ xaxou.

3.11 - add 01 before $0.0 \theta_{1} \lambda \mu O_{1}$.

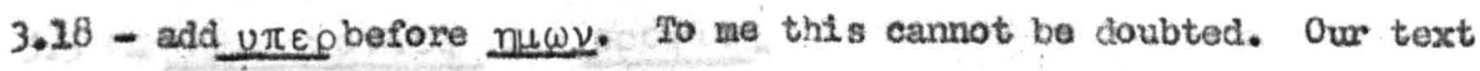
was favored by Wotstein but rejected by IIschendorf, who pre-

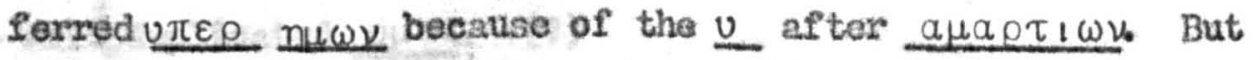
this $U$ is by B. Wo can assume, therefore, that A did not unite $u \pi \varepsilon \rho$ The space between $\alpha \mu \alpha \rho \tau l \omega \nu$ and $51 \times \alpha 10 c$ is not sufficient for $\underline{v \pi \varepsilon \rho} \eta \mu \omega \nu, a \pi \varepsilon \theta a \nu \varepsilon \nu$ (Tischendorf was apparently aware of the limited space, because he suggested a $\pi \varepsilon \theta a \nu \varepsilon$ for $-\nu \varepsilon \nu)$. But $\underline{\mu \omega \nu} a \pi \varepsilon \theta a \nu \varepsilon \nu$ fits adinirably into this space.

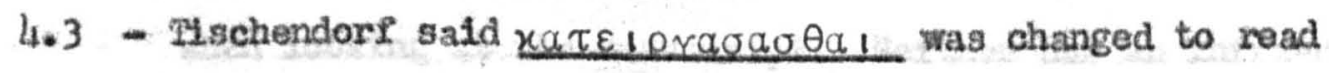

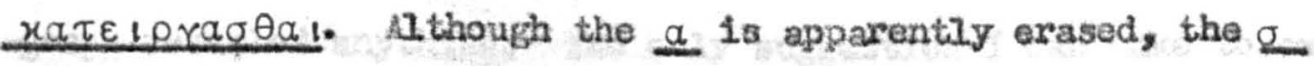
has not been touched. Whether this represents a clunsy atternt to correct the word, or whether the $a$ has merely faded (along with other letters in this area), I do not know. 


\section{Poter}

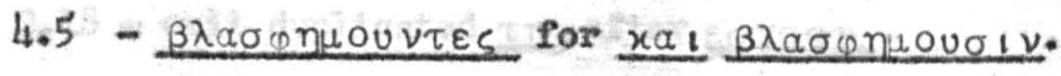

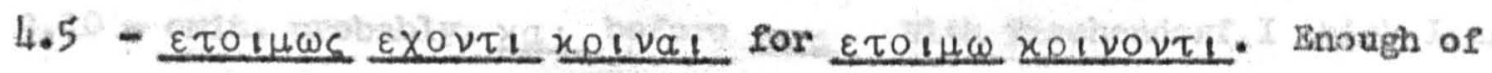 the letters are seen to make this definite. On one 1ten TIschendorf needs to be corrected. The oxiglnal scribe oraitted the $S$ at the end of $\varepsilon \tau 01 \mu \omega$.}

\section{Peter}

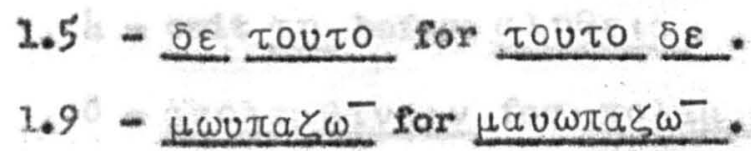

1.13 - add $\tau \omega$ after $\tau$ ou $\tau \omega$.

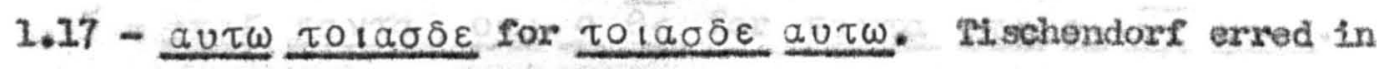
attributing this correction to the second corrector.

2.21 - $\pi 000 \eta \tau \varepsilon i a$ for $-?$ One letter was probably omitted; perhaps A wrote $\tau \perp a_{*}$

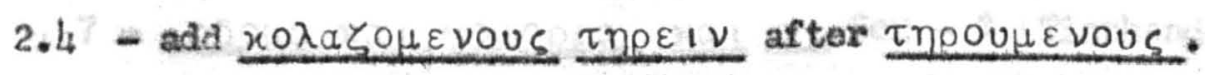

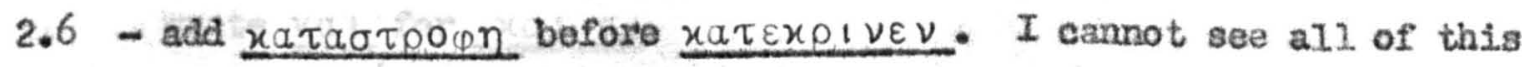
(which is in the margin) because of the binding; what $I$ see verifies the note of Tischendorf which is accepted here.

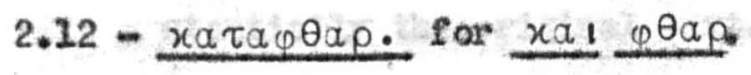

$2.22-x v \lambda 1 \sigma \mu a$ for $-\mu o v$.

$3.3-Y 1 \nu \omega \sigma x o \nu \tau \varepsilon s$ for $\pi \rho 0 y 1 \nu$.

3.7 - onit $\varepsilon \nu$ before $\pi u \rho 1 . \quad$ (3)

3.11 - onit duplicated upas. (?)

3.13 - add $\varepsilon \nu$ before o1s.

3.16 - $\sigma \tau \rho \varepsilon \beta \lambda \omega \sigma i \nu$ for $\sigma \tau \rho \varepsilon \beta \lambda \omega \sigma o v \sigma 1 \nu$.

I John

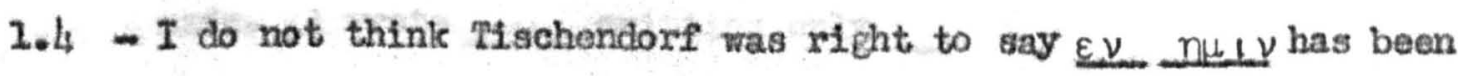

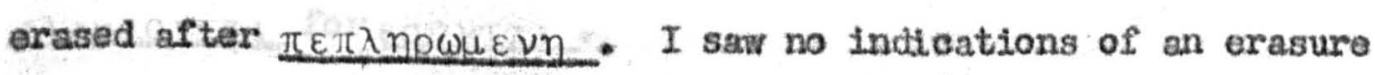
when looking at the raanuscript. Neither has an inframred photograph revealed anything. The only suggestion I can make to explain THschendorf's statement is that after $\pi \varepsilon \pi \lambda \eta \rho \omega \mu \varepsilon \nu \eta$ is a cross whose horizontal line he mistook for the middie mark of the $\varepsilon$. 1.5 - omit, parhaps, $\underline{\varepsilon \nu}$ before $\underline{u \mu \nu}$. 
I John

2.18 - omit duplicated $\underline{\tau n}$ after $\underline{\text { axa } \tau n}$.

3.10 - owt, probably, $x a_{1}$ before $\pi \alpha_{c}$. WI th Tischendorf I think I cam see vestiges of $\underline{x}$ at the begiming of the 1ine. Hetstein had suggested that either $x_{1}$ or ofl was erased.

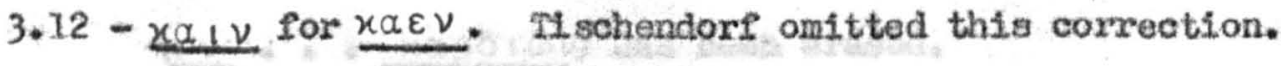

III John

4 - omit $\underline{\tau \eta}$ before $\underline{a \lambda \eta \theta \varepsilon \downarrow a}$.

8 - $a \pi 0 \lambda \alpha \mu \beta a \nu \varepsilon i \nu$ for $u \pi 0 \lambda \alpha \mu$.

Jude

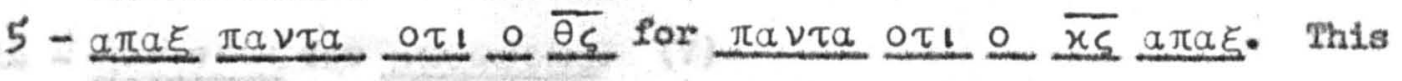
camnot be doubted. Only $\underline{x}$ cannot be seen. Tischendorf erred in

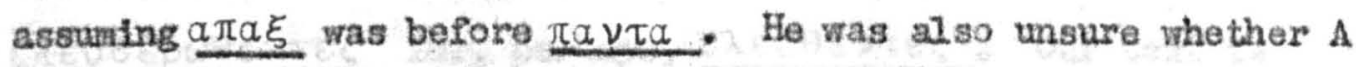
wrote $\pi \alpha \nu \tau a$ or $\tau 0 u \tau 0$.

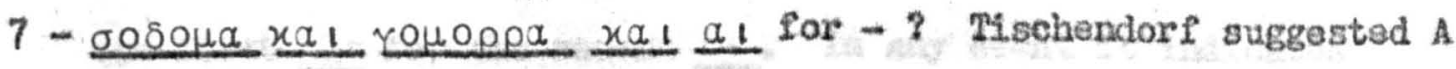
wrote $x a_{1}$ for $x a_{1} a_{1}$.

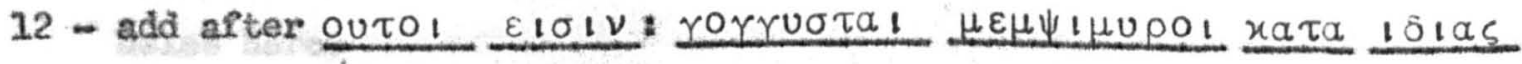

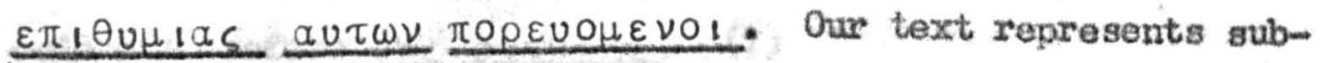
stantially the original text. It contains nothing of the addition of Codex I and others. Line 34 was not pilled the text stopped about the middle of the line. To me a large 0 appears to have been at the beginning of line 35. Because of holes it is inpossible to know if A wrote of after $\varepsilon \downarrow \sigma \downarrow \nu$. I have included it because the space appears to warrant it.

13 - Ti sehendorf said the original seribe wote $\underline{a} \alpha a$ and that $B$ corrected it to read avela. Actually no correction has been

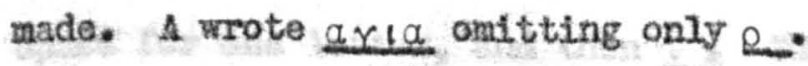

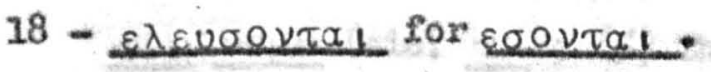

21 - arit $\overline{\theta u}$ after avann. (?)

21 - $\tau \eta p \eta \sigma \eta \tau \varepsilon$ for, probably, $\tau \eta p \eta \sigma \omega \mu \varepsilon \nu$.

22 - $\varepsilon \lambda \varepsilon \sigma \tau \varepsilon$ for $\varepsilon \lambda \varepsilon \gamma \chi \varepsilon \tau \varepsilon$. 
1.17 - omit $\mu$ ou after Sinalos. (3)

1.24 - addo $\overline{\theta_{S}}$ after autous. Ints is definite because the original Talc. Is seen. Tischendorf, however, was wrong in saying $\omega v$ xaps $1 \omega \nu$ is by A. On this line everything after autous $(\varepsilon \nu . \cdots \underline{x \rho \delta}+\omega \nu)$ has been erased.

2.1 - ondt $x \rho|\mu \alpha \tau|$ before $x \rho \mid v \varepsilon i s$.

$4.11-\pi \varepsilon \rho 1 \tau o u n s$ for $-\mu \eta v$.

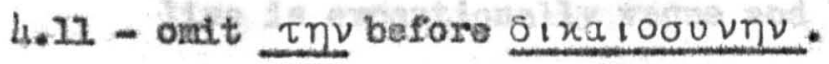

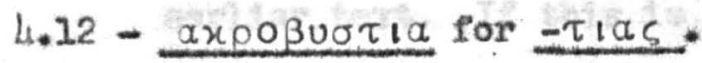

$5.14-\mu \omega v \sigma \varepsilon \omega s$ for $\mu \omega \omega \omega \sigma \varepsilon \omega s$.

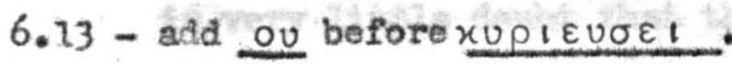

$6.18-\underline{\varepsilon \lambda \varepsilon u \theta \varepsilon \rho \omega \theta \varepsilon \nu \tau \varepsilon \zeta}$ for, perhaps, $\varepsilon \lambda \varepsilon v \theta \varepsilon \nu \tau \varepsilon \zeta$. Our text is pure conjecture based on the possibility that the scribe mistook the first $\theta \varepsilon$ for the second $\theta \varepsilon$. In any event it indicates accurately the space available. The vellum has numerous small holes here.

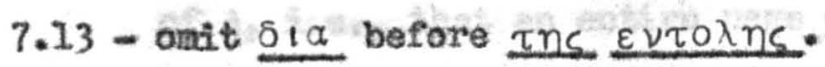

7.23 - 8.2 - Five complete 1ines $(26-30)$ as well as the end of the preceding line have been erased and re-written. The original scribe omitted almost one-half of the acceptod text in these verses. This much is knom: (1) A wrote $\varepsilon v$ at the end of line 25; (2) on line $26 \mathrm{~A}$ vrote $\tau 015 \mu \varepsilon \lambda \varepsilon \sigma i \nu \mu O U$ and left the rest of the line blank; (3) Ine 27 begins with the Initial.

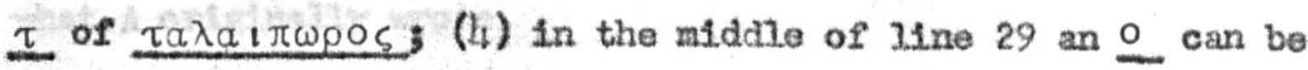
seen, followed by a space sufficient for 2 letters, then $\underline{\varepsilon v}$, space for one letter, and then $\tau$. Thts suggests $\delta] \circ[U \lambda] \varepsilon \cup[\omega]$

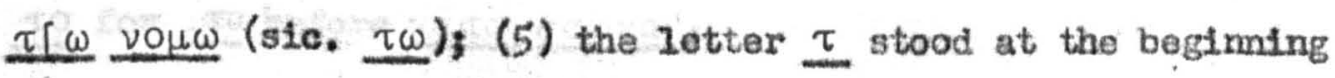
of Iine 30 and is followed by a letter vith a straight vertical. stroke. After a space sufficient for 3 letters a 2 is detected. Then about the middle of the same line a $\rho$, followed by space for 2 letters, and then $\underline{\alpha s}$ is seen. This suggests $\underline{\tau[\eta}$ 


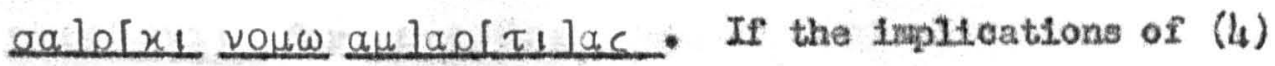
and (5) are correct - and I an certain they are - two possibilIties are clear: (a) the original scribe sisipped from auaotlac at the end of 7.25 to apaptias of 8.2 and completed the Iine

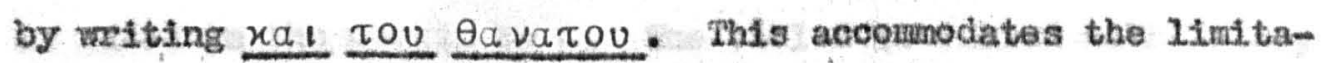
tions of space; (b) the artginal scribe ended his text in the middle of the line with guaptiac the second half of this Ine is exceptionally vague and I see no indications of an earlier text. If this is true, then A skipped 8.1, 2 entirely

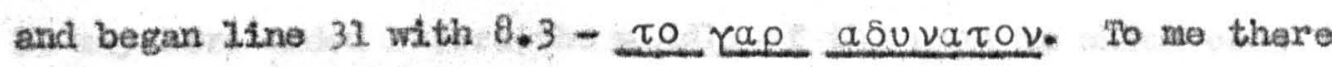
is very little doubt that this seeond altemative is correct. Nothing frota $7.2 h_{4}$ to 8.2 inclusive should be cited in a critteal apparatus (except, perhaps, $\tau \omega$ before oㅣ $\underline{\underline{\partial U}}$ ) for the original text of Codex C. It is an unjustiflable assurption to say that B re-copied the text of A. One nore Items does this onisston give us any Indication of the excomplax. of $A$, 1.e., that an entire page or column was skipoed? Against this possibility, however, we might note that it would be unusual for a leaf to begin and end at such natural places.

$8.9-\varepsilon \nu \overline{\pi \nu !}$ for $\varepsilon \overline{\pi \nu !}$.

8.9 - oux $\varepsilon \times \varepsilon 1$ for $-?$ B has rewritten $x \underline{\varepsilon \times \varepsilon}$ outos ou. The orieinal outos oux can be detected leaving room for 3 more letters than what is in parentheses. It is ixpossible to know what A originally wrote.

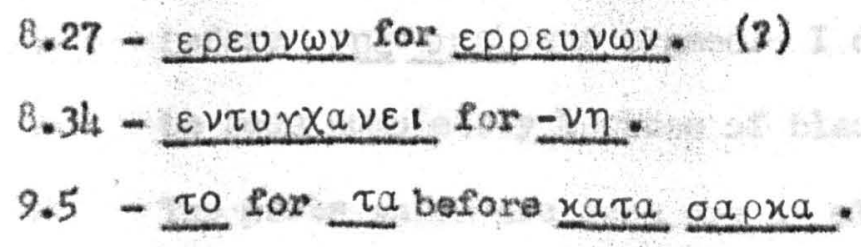

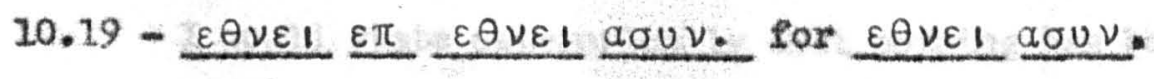

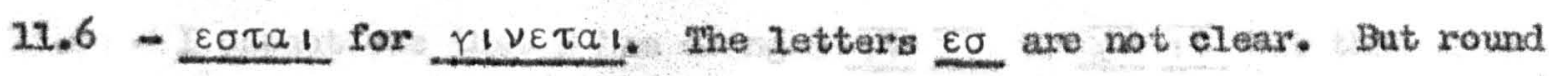
strokes appear to have been made by the first corrector.

11.16 - oait $\underline{\delta \varepsilon}$ before $\underline{\eta} \underline{a \pi \alpha \rho \times \eta}$.

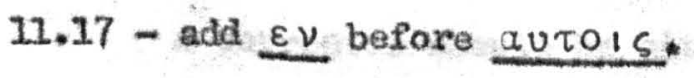


Romans

$13.11-\underline{\varepsilon \gamma \varepsilon \rho \theta \eta \nu a_{1}}$ for $\underline{\varepsilon \gamma \varepsilon \rho \theta a} \psi$

$1_{3.5,6}$ - The original seribe omitted something other than the Byzantine

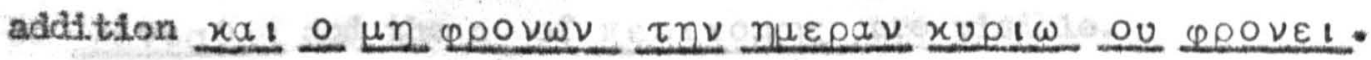
The first corrector in ro-writing the text evon oxits it, but it is added in the margin by $\mathrm{C}$. The vellum is 1 illed with holes, has been iapod, and hopelessily blackened by tincture so that very Iitcle is seen. If all the Iines were filled by $A$, I would estimate anywhere from 10 to 20 lotters of the combon text ware omitted, probably by haplography. I suspect, however, that IIne 21 way have ended before the middle of the page in which case more than 20 letters were omitted. Becanse of the frequent repetition of words it is impossible to conjecture if A stipped

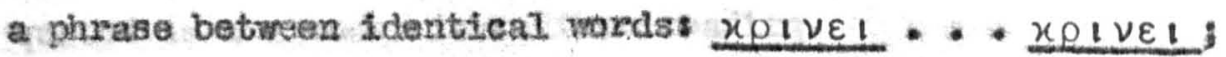

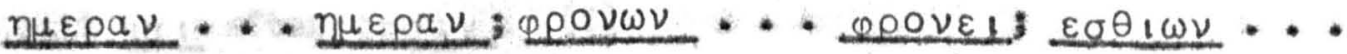

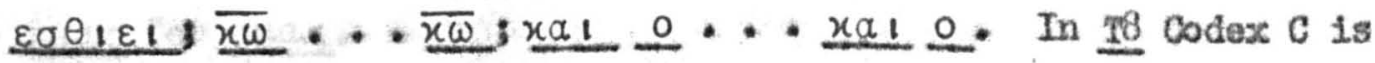
Iisted with other witnesses as having incinded yap after 으 $\mu \varepsilon \nu$. This represents the text of B. It cannot be assumed that the corrector, while adding an entire phraste, 20 mrote the origtnal texce. Codex $\mathrm{C}^{\text {th }}$ should not be eited for this variant.

$u_{4.10}-\overline{x \bar{v}}$ for $\overline{\underline{\theta u}}$

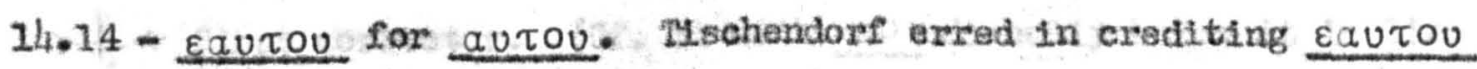
to the original scribe. The $\underline{\varepsilon}$ extends fnto the margin and is not the script of A*

15.4 - Fischendorf confectured (and statod unhesitatingly in T6) that $61 a$

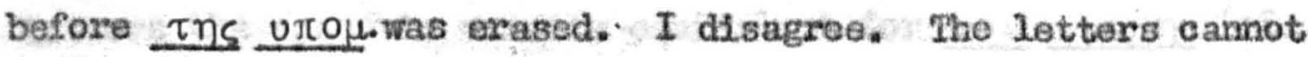
be seen completely because of black spots created by the tincture. The parts which are seen are as clear as the rest of the letters. I would state categorically that nothing has been erased.

15.6 - I cannot see that $B$ has changed $\overline{I V} \overline{X \nu}$ to $\overline{X \nu} \overline{I V}$ but this area is very indistinet. Itschendorf hinself acicnowledged in his Appendix (though not in 78 ) that he may be wrong. I don't think a correator should be cited. 
$15.8-y \varepsilon \gamma \varepsilon \nu \eta \sigma \theta \varepsilon$ for $y \varepsilon \nu \varepsilon \sigma \theta a$ l. Although the $u s$ is in poor condition at this point, our text need not be questioned. The second $\underline{\varepsilon}$ of $y \varepsilon \nu \varepsilon \sigma \theta \alpha l$ and the $p$ of $\pi \varepsilon \rho, \tau o \mu \eta s$ are visible.

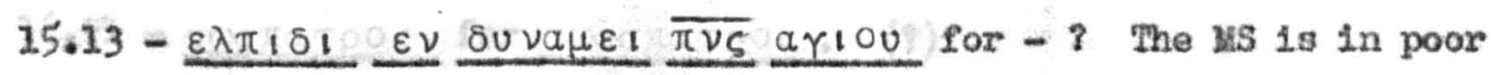
condition and nothing can be discerned about the text of $\Lambda$.

15.1. - What A wrote after $\overline{\pi V_{S}}$ eannot be known because of a hole in the vellum. Tischendorf went along with Wetstein who apparently thought he could ses the end of aylou. To whe this is very questionable. The fact that a hole is found at this point may suggest the aytou was erased in favor of the Byzantine $\bar{\theta} \bar{v}$. But this is pure conjocture, and numerous holes elsewhere on this page indicate caution is required. Codex $C$ should not be quoted as a witness for olther reading.

16.1 - omit $x a 1$ after ovoav.

16.6 - nuas for unas.

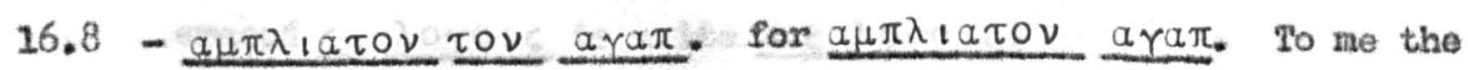
text of both $A$ and $B$ are cortain. Tischondorf apparently studied this passage several times. In doing so he gave more consideration to the avallable space and failed to note that Tov which is betwen the two columns of the upper text - is clearly by the first corrector. (cr. It with $\tau 0 \nu$ directly below it in line 31.) If schendorf's reckoning assumed $\underline{\text { Tov }}$ to be by $\mathrm{A}$ and he mentions nothing about a corrector. These letters of the original scribe

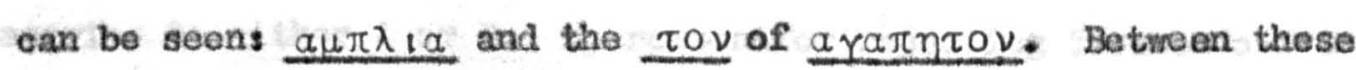
letters the space is excactly equivatent for tov ayajn (cr.,

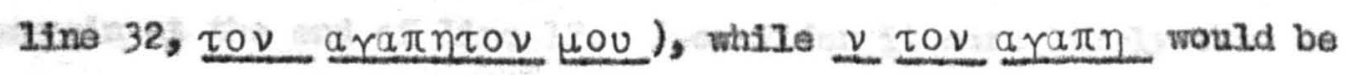
more crowded than the script of A. To be sure this represents a difference of only one letter, but I have no doubts about it. Hind Iischendorf noticed some of the corrector's text, I am certain he woutd have resched the same conclusions on the basis of space. Codex C, therefore, agrees with Codex B in writing

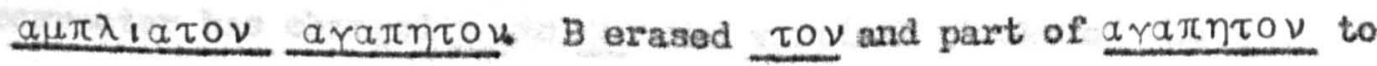


Romans

insert cov. Neither the original scribe nor the corrector mote $a \mu \pi \lambda \_a \nu$.

16.15 - Loudiav for louviav.

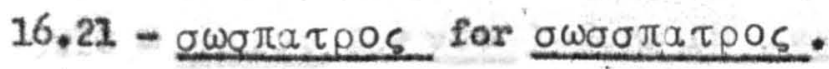

16.23 - omit redundant $\tau \eta s_{\text {before }}^{\varepsilon x \times \lambda \eta \eta \text { Ias. }}$

I Corinthians

1.11 - add $\mu 0 u$ after $\alpha \delta \varepsilon \lambda \varphi O u$.

1.24 - autols $\delta \varepsilon$ for auto $\delta \varepsilon$. The text is quite difficult to see but Tischendorf's reading erowds the text.

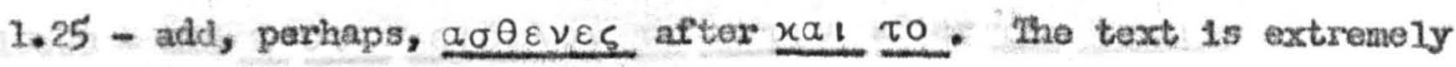
vague. Our restoration of the original toxt is based entirely on the space available.

2.4 - gosplac for gopis. The final $c$ of gop las is seen under the

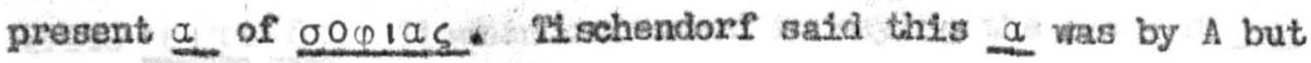
close examation reveals the 5 . The corrector, therefore,

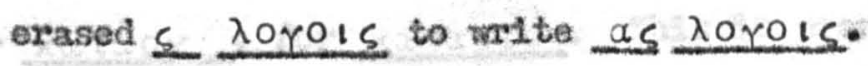

2.9 - Contrary to the opinion of rischendorf I do not think oga has been altered. The ends of the lines in this area are quite indistinct, but in this Ine the $\sigma$ of ood is the clearest of all the letters.

5.12 - unis for unas

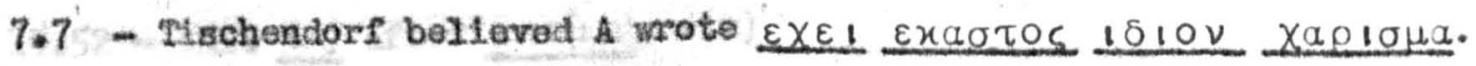
This reading is very indistinct. I am convinced a change has been made in the text, although none of the letters are clear enough to ascribe to either $A$ or B. Wy reason for rejecting Tischendorf's suggestion 1s that $\varepsilon \chi \varepsilon \mid \varepsilon$ would have extended too far into the margin at the end of Iine 17. I consider it more 1ikely that $A$

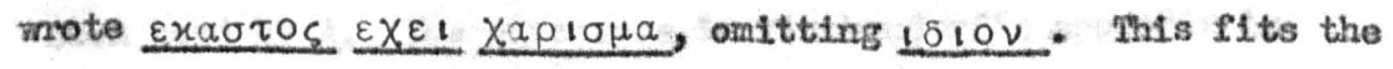
space wall.

7.9 - yaunoal for yaue $1 \nu$.

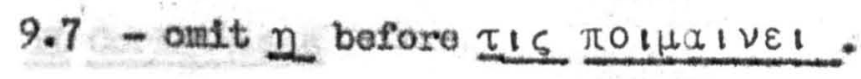

9.12 - $\overline{X U}$ for $\overline{x U}$. Mischendor onitted this correction. The first corrector has changed $x$ to $\underline{X}$; the vertical stroles of the $\underline{x}$ 


\section{Corinthians}

is cleariy visible.

9.20 - add, perhaps, ws $1000 a 105$, before 1 va. The ortinal scribe onitted something. Our text indicates the space avallable.

9.20 - youov for yov before $\mu \eta \underline{\omega \nu}$. The $\underline{\mu}$ can be seen under the second $\circ$ of vouov, thus verifying Ilschendorf's conjecture that A wrote uno vov.

$9.2 h_{4}-\lambda \alpha \beta \eta \tau \varepsilon$ for $\lambda \beta \eta \tau \varepsilon$.

10.3 - add auto after $\overline{\pi \nu i x o v .}$

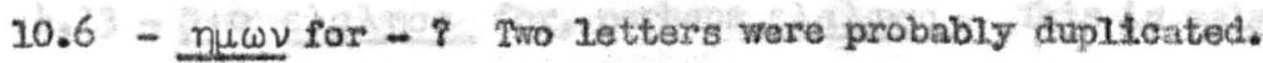

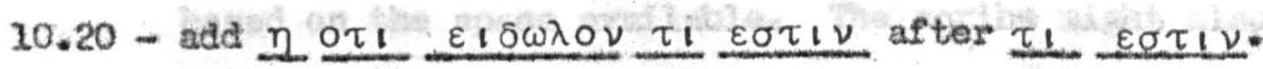

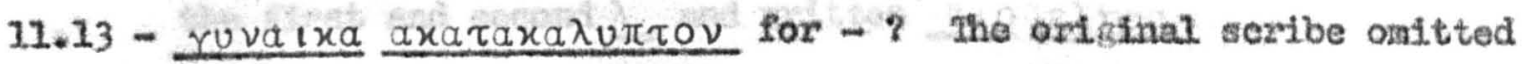
several letters possibly by passing from one $\underline{x \alpha}$ to another $\underline{x \alpha}$.

13.8 - add $\underline{\delta \varepsilon}$ before $\pi p \circ p \eta \tau \varepsilon / a$. The corrector erased $\underline{\varepsilon l \tau \varepsilon}$ to mite $\varepsilon i \tau \varepsilon \delta \varepsilon$. Tischendorf orred in saytug the present $\varepsilon_{\perp}$ was by A. B wrote the large marginal $\varepsilon$.

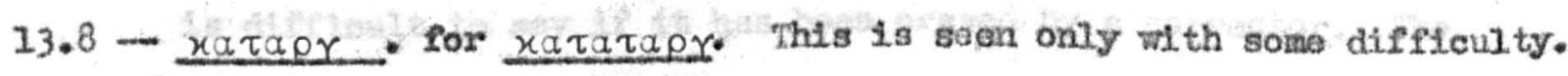
mschendorf did not note the extra $\tau a$ had been written.

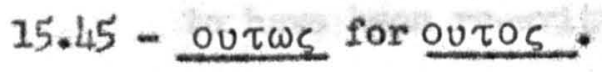

$15.50-\underline{x \lambda \eta \rho O \nu \circ \mu \varepsilon 1} \operatorname{Pos}-\mu \eta \sigma \varepsilon 1$.

15.51 - add $\mu \varepsilon \nu$ after $\pi a \nu \tau \varepsilon s$.

15.52 - omit $\omega s$ aftor $\alpha \tau o \mu \omega$. (2)

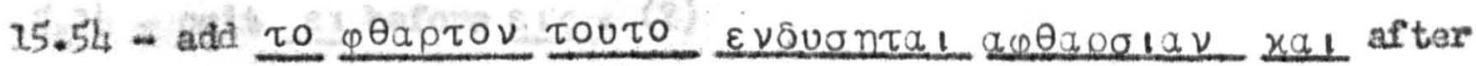

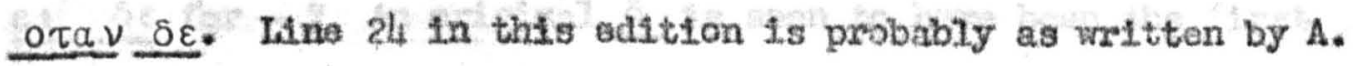
The words $a \varphi \theta \alpha \rho \sigma|\alpha \nu, x \alpha|$ can be read. In the next two lines certain letters can be detected but nothing determined from them. The letter $\varphi$ In the above phrase definitely is seen not to have been used by $A$. The discourse in Tischendorf's appendix is suffieient for the establishment of the original text.

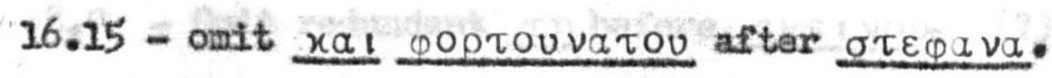

\section{Cortnthians}

$$
\begin{aligned}
& 2.10 \text { - } x a \gamma \omega \text { for } x \alpha_{1} \varepsilon \gamma \omega \text {. }
\end{aligned}
$$

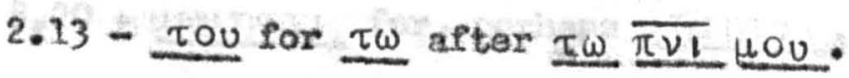




\section{Corinthians}

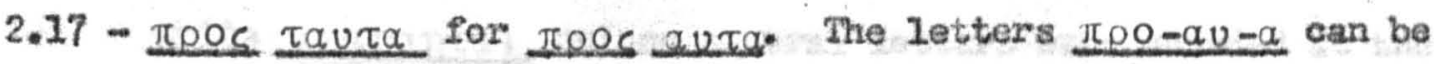
seen. It is possible that A wrote $\pi \rho 0$ qu $\tau \alpha$, but no evidence of the $\tau$ can be seen.

3.14 - $\underline{\delta a \theta \eta x \eta}$ for $\underline{\delta \eta \eta x \eta}$. The original scribe omitted $1 a$. The corrector erased $\underline{a_{1} a_{S}} \delta \theta_{\eta}$ to Ansert $1 \alpha_{\text {. }}$. The letters except $\underline{\theta} \operatorname{can}$ be seon.

4.7 - Suvauewc $\eta$ for - ? One letter was quite likely onitied in

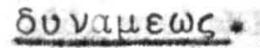

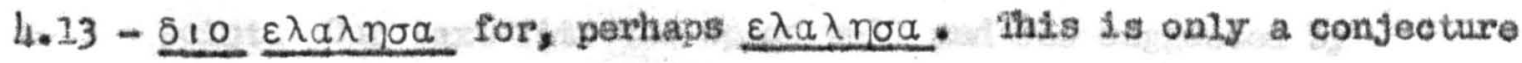
based on the apace avallable. The scribe might also have confused the first and second $\underline{\lambda}$ and written $\delta 10, \varepsilon \lambda \eta \sigma \alpha$.

4.16 - add, perisaps $\underline{n \omega \nu}$ after $\varepsilon \xi \omega_{*}$. The corrector has written 3 to 5 more letters than the original scribe. With Tischendorf I consider our text wast likely.

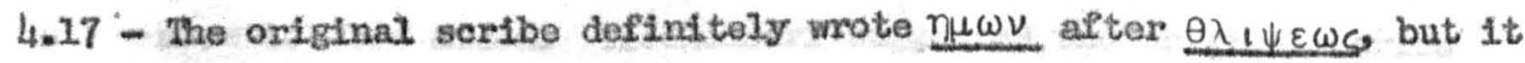
is diffleult to say if it has been arased by a corrector. The letters after nuwV havo shared the same fate, but do not appear

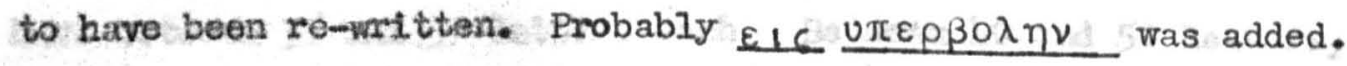

5.4 - Omit, probably, a duplieated $1 \nu \alpha$ before $x \alpha \tau \alpha$. The 1 appears to be at the beginning of the line, but it may be one of the vertical strokes of $\underline{\nu}$ or $\underline{\eta}$.

$5 . \nu_{1}$ - omit $\underline{\varepsilon_{1}}$ before $\underline{\varepsilon_{1} s_{5}}$. (?)

$5.16-\varepsilon 1$ f for $-?$ An original $\delta 13$ seen to have been the first letter on the lino. A, therefore, wrote something incorrectly. Conld it have been $\delta \varepsilon \varepsilon$ for $\varepsilon l \underline{\delta}$ ?

$7.2-\frac{\varepsilon \varphi \theta \varepsilon 1 \rho a \mu \varepsilon \nu}{\text { ou } \delta \varepsilon \nu a}$ for $-?$ Nothing can be soen of the original text. The oxiginal seribe onitted several lotters.

8.4 - Omit, perhaps, $\tau \eta s$ after $\pi 0 \lambda \lambda \eta s$.

8.9 - Oant redundant $\tau \eta$ before ExE L vou.

$8.10-\pi \varepsilon \rho v \sigma y$ for $\pi \varepsilon \rho v \sigma 1 \nu$.

8.12 - add $\tau_{15}$ aftor $\varepsilon \times \eta$

8.20 - $\mu \omega \mu \eta \tau \alpha_{1}$ for, perhaps $\mu \eta \sigma \eta \tau a_{1}$. The original scribe did not 


\section{Corinthians}

mrite $\mu \omega \mu \eta \sigma \eta \tau a_{1}$ uniess several lotters were crowded at the end of the line. Bven the letters $\mu \omega \mu \eta$ are crowded at the end of the line. Our text is the most likely conjecture. The scribe skipped from the first to the second $\underline{\mu}$.

8.20 - add, perhaps, $\tau \eta$ after $\tau a u \tau \eta$. Our text pits the space well. Tho letters were probably omitted.

$9.4-\underline{\lambda \varepsilon \gamma \omega \mu \varepsilon \nu}$ for $\underline{\lambda \varepsilon \gamma \omega}$.

9.6 - add, probably, $\unrhd \varepsilon 1 \delta 0 \mu \varepsilon \nu 0 s$. The velluz is ruined at this point. Our taxt Iits the space well and was suggested by Hschendore.

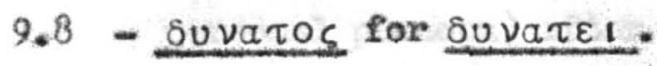

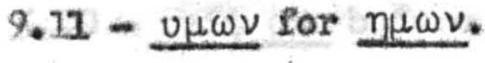

9.15 - add $\delta \varepsilon$ after $x a \rho 15$.

10.2-4 - Three full lines have been erased and approxtrately one-third more letters added. Mischendorf suggested the original scmibe stclpped frosh the first to the second $\pi \varepsilon \rho l \pi a \tau o u \nu \tau \varepsilon s$. But this still leaves too many letters. The lines of this area have between 35 and 39 letters each. Between 40 and 50 letters,

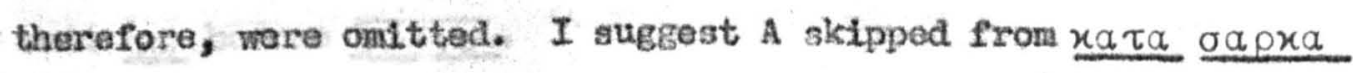
in 10.2 to $x a \tau a$ oapxa in 10.3.

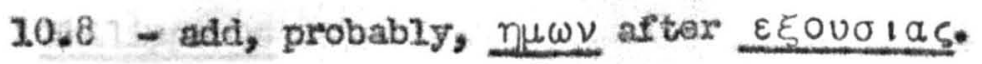

\section{Galatians}

3.7 - add oy after a $\underline{a}$ o 11.

3.7 - The end of $\pi 1 \sigma \tau \varepsilon \omega s$ has been erased, but the second witing (1e there was any) eamnot be seen.

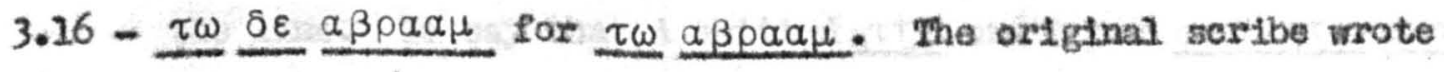
$\tau \omega \underline{a}$ at the end of Iine 4. The corrector changed it to $\tau \omega$

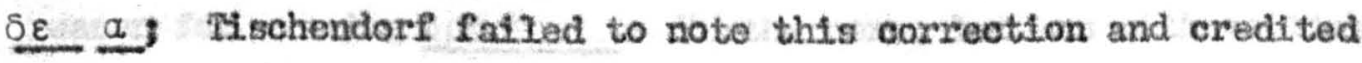
$\underline{\tau \omega} \underline{\delta \varepsilon} \underline{a}$ to the original soribe.

3.19 -

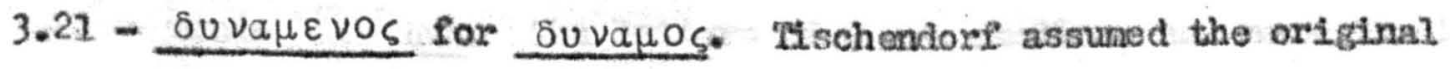

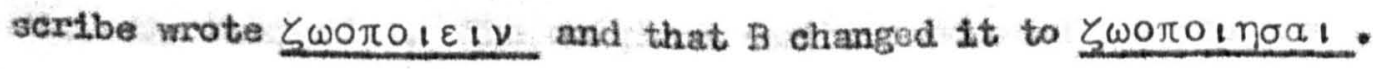


The original al, however, at the end of the erased area is quite clear. Closer observation revealed the original $\zeta$, from which it was possible to work back and note that $\mathbf{A}$ had

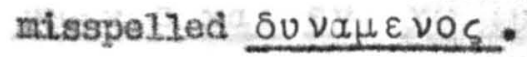

4.2 - add $\underline{\omega \nu}$ aftor $\pi \alpha \nu \tau \omega \nu$.

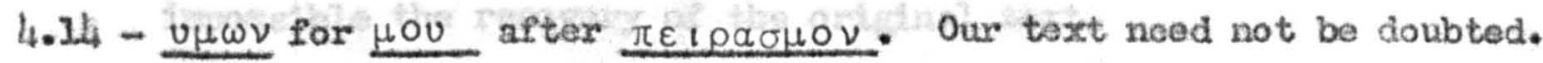
An original $\tau$ is eleariy seen under the $\underline{\nu}$ of $\underline{u \omega \nu}$. This makes room for $\mu 00$ after $\pi \varepsilon i p a \sigma \mu O \nu$ but not room for $\tau 0 \nu \underline{\varepsilon \nu}$ on the basis of the visible $\tau$ belonging to $\tau$. Furthermore, the text of $B$ is not crowded: the omission of ㅆov and uHwV (or any other personal pronoun) after $\pi \varepsilon l p a \sigma \mu o v$ would not fill the line.

5.10 - omit $\delta \varepsilon$ before $\pi \varepsilon \pi 01 \theta \alpha$. (?)

5.11 - $\varepsilon \tau 1$ for $\varepsilon 1 \tau \varepsilon$ before $\underline{x \eta p v \sigma \sigma \omega}$.

5.17 - add $\underline{a \nu}$ before $\theta \varepsilon \lambda \eta \tau \varepsilon$.

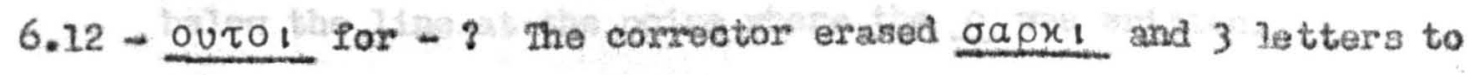
correctly spell outol. Our text indicates the space avaliable.

6.16 - $0 \tau 01 \times$ no $0001 \mathrm{v}$ for $0 \tau 01 \times 0001 \mathrm{~V}$.

Bohesians

3.11 - omit $\tau \omega$ before $\overline{\chi \omega} \overline{I v}$.

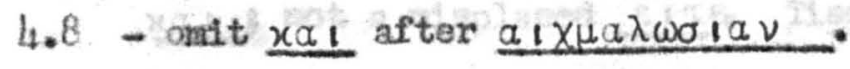

\section{Phi.ligpians}

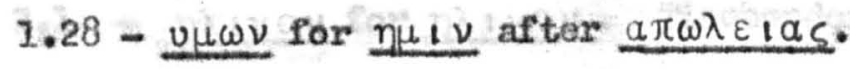

1.30 - ant $\underline{x a_{1}}$ after $\underline{\varepsilon l \delta \varepsilon \tau \varepsilon}$.

2.2 something. It was not $\underline{\alpha \tau \tau \eta \nu}$ because $\varphi \rho \circ \nu \eta \tau \varepsilon$ does not 1111

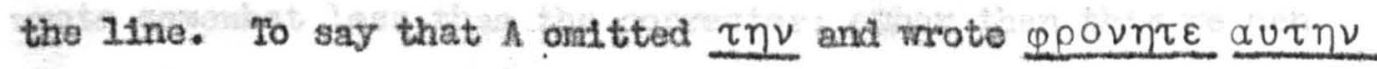
meets the requirements of space, but it leaves unexplained the reason for erasing $\varphi \rho 0 \nu \eta \tau \varepsilon$. Furthernore, letters with vertical strokes ( $\underline{\eta}, \underline{\nu})$ appear to have boen at the end of the line.

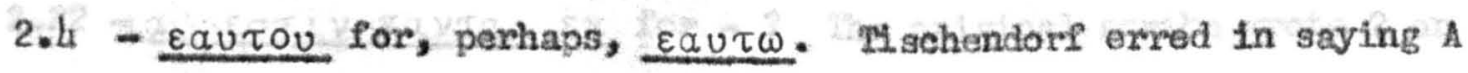
wrote $\varepsilon q u \tau \omega \nu$. The letters of $B$ are smaller, and more crowded, than the text of $A$. The original seribe undoubtedly wrote one 


\section{Philipgians}

less letter than the corrector, since only 2 letters will fit where $B$ has writion $\tau 0$. The vellum 18 creased and the toxt of A is beyond recovery.

$2.5-\underline{u \mu \nu} \circ \underline{x a_{1}} \varepsilon \nu \overline{x \omega} \overline{T U}$ for - ? The original scribe omitted 2 or 3 letters, but the vellum is sa badly damaged as to make impossible the recovery of the original text.

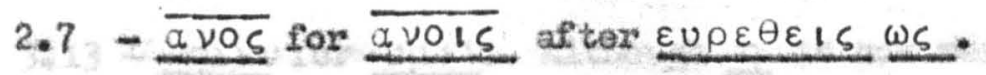

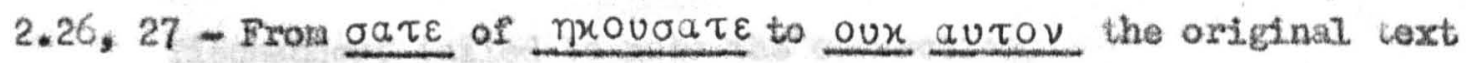

Is lost, since much of the vellum is tom away over this area. The orlginal scribe ontited something. I have no conjecture to offer other than that wade by Tischendore, nawely due to haplography the seribe sicipped from the first to the second $n g \theta \varepsilon \nu n g \varepsilon \nu$.

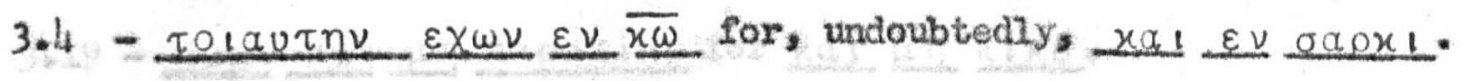
This fits the area which has been erased. The erasure extends below the line at the poine where the $\rho$ was writien.

\section{Cologsians}

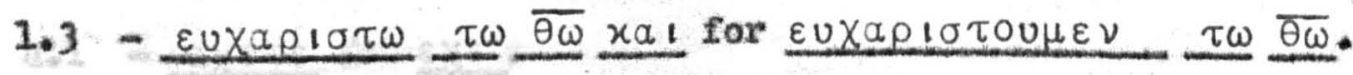

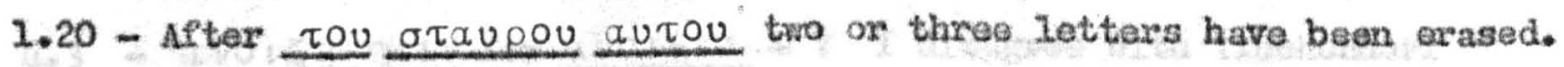
They may have beon a redundant ou. It was not Qno li nor was it nal not a misplaced $\varepsilon_{1}+\varepsilon_{*}$ Fischendore did not note this erasure.

2.1 - $n \lambda 1$ xov for $n \lambda 1$ Yov. Mischendorf eredited $n \lambda$ inov to the originat seribe. The $\underline{x}$ is crowted and the vestiges of $\underline{y}$ are seen.

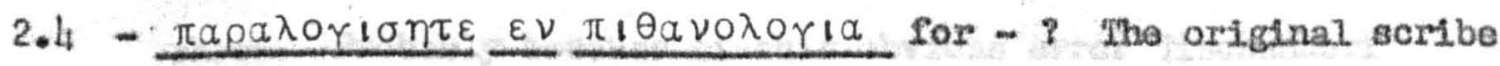
wrote somewhat less than the corrector; other than this we can lenow nothing.

$2.11-\pi \varepsilon \rho 1 \varepsilon \tau \mu \eta \theta \eta \tau \varepsilon$ for $\pi \varepsilon \rho 1 \varepsilon \varepsilon \tau$.

2.19 - omit duplicated $\sigma \omega \mu \alpha$.

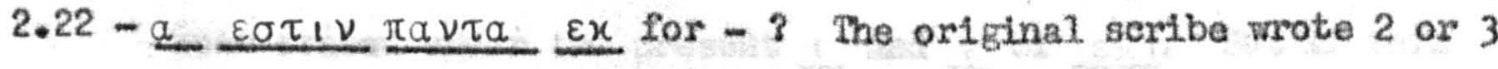
less letters. He did not oalt either $\varepsilon \sigma \tau 1 \nu$ or $\pi a \nu \tau \alpha$. 3.6 - $\underline{1}$ a for $\underline{\underline{\delta} \alpha}$ 으 (not $\underline{\delta_{1}}$ 으 as Iischendorf said). The space 


\section{Colossians}

is sufficient for aO . Even Tischendorf thought $A$ night have written an original $\omega$. One question must be raised: Did A white 51 으 or 51 and both of his choices, 1.0., 81 a. This phenomena is seen elsewhere in the lis, er., Matthew 7.16. 3.13 - The f1rst corrector has erased something after $\alpha \lambda \lambda \eta \lambda \omega \nu$. The space suggests a duplicated $\alpha \lambda \lambda \eta \lambda \omega \nu$.

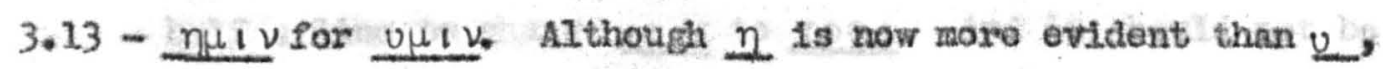
Tischendorf fasled to note the correction.

3.15 - $\underline{\overline{\theta u}}$ for $\overline{\overline{\chi U}}$.

3.16 - $\overline{\chi v}$ for $\overline{\theta u}$ (first).

3.16 - add $\underline{x \alpha}$ after $4 a \lambda_{\mu} 015$.

3.16 - $\underline{\overline{x U}}$ for $\overline{\underline{\theta U}}$ (second).

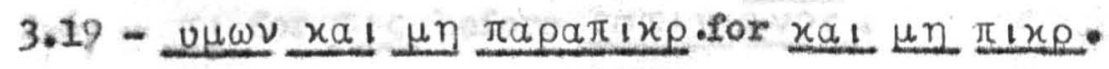

3.22 - onit $\omega_{s}$ before $\varepsilon \nu \underline{\Delta \varphi \theta a \lambda \mu}$.

$3.24-\lambda \eta \psi \psi \varepsilon \sigma \theta \varepsilon$ for $\alpha \pi 0 \lambda \eta \mu$.

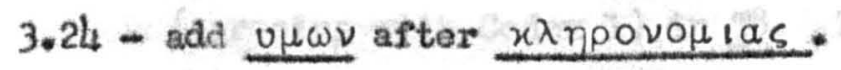

$4.1-\varepsilon X \varepsilon \tau \varepsilon$ for $\varepsilon \chi \varepsilon$.

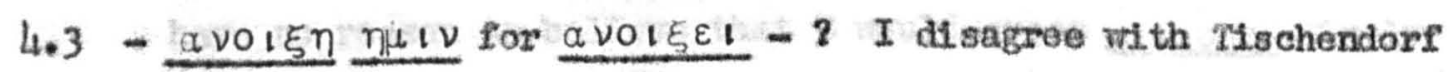
who said A corrected this hinself. Beside the strength of the stroke, the letters lean slightly to the right whereas the witing of A leans to the left. Because an $\underline{\varepsilon}$ appears after a $\nu 01 \xi$, Codex $\mathrm{C}$ appaxently agrees with Codex I in reading

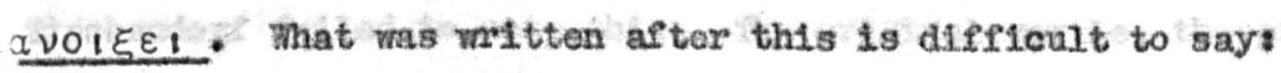
certainly nothing sensible as the $\xi$ under the present nu/v shors. Probably because of this obvious error Tischendore exedited the correction to the original soribe.

\section{Thessalonians}

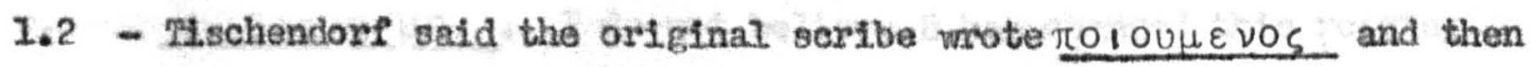
changed it to $-V_{0}$. Ihis may be so: somothing appears to have been erased under the present 1 and $\varepsilon$ of $\varepsilon \pi 1$. But if this is so, then the change was made before the scribe wrote nore, because the present spacing of letiers is even. I do not think 
I Thessalonsans

Codex $\mathrm{C}$ can be mentioned as was done in 20 as a witness for Tolounevos.

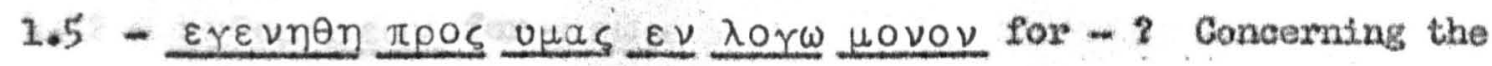
chango Tlschendorf wroto "Quid prima manus legerit, non dixerin." In 78 he quotes Codex 6 wt th the llanuscripts that road E1s rather than $\pi \rho 0 s$. It cannot be assumed that $B$ arased half a line to change $\varepsilon_{i}$ s to $\pi \rho 0 s$. And it should not bo assumed that in sileing a laxger change the corrector made this smazler change.

2.6 - onit $\underline{\tau \nu \nu \omega \nu}$ after $a \pi \underline{a \lambda \lambda \omega \nu .}$

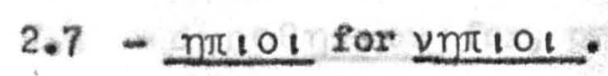

Hobrews

2.6 - $\tau_{1}$ for $\tau_{15}$ before $\varepsilon \sigma \tau 1 \nu$ avoc.

2.12 - $\varepsilon \pi \underline{a v \tau \omega}$ for - ? wischendorf said the original scribe rote

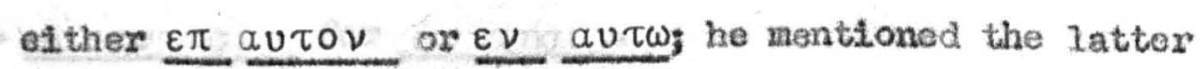

(agreeing with Codex 1) in 28. The room is not surficient for

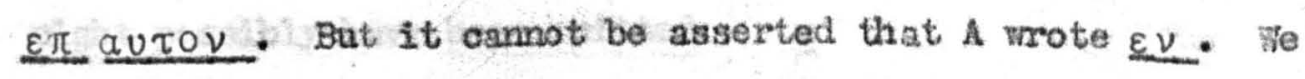

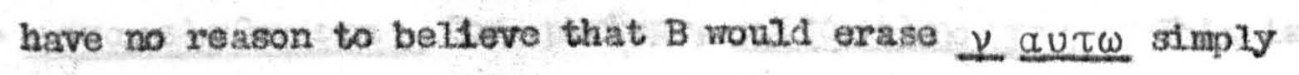
to change $\nu$ to $\pi$. I have no suggestion to offer.

2.13 - Tischendorf said the original scribe wrote $\pi a 1 \delta ! \mu{ }_{1}$ and that the corrector wrote $\pi a 1 \delta 1 a$ 으. Several letters have been rewritten by $A$. Under the prezent $\underline{01}$ the letters $\varepsilon \delta \omega$ are seen. Pischendorf failed to note this. The original scribe, therefore, omitted ㅆo1, erased $\varepsilon \delta \omega$, and then vrote $\pi \alpha 1 \delta 1 \alpha \mu 01$. None of the letters are crowded as they would be if $\alpha$ was added later. In the 1845 edtition Thischendorf changed this noto and acknowledged that A wrote $\pi a 1 \delta 1 a$ 인 but he also said B insorted a to read $\pi \alpha_{1} \delta \alpha^{\alpha} \mu_{01}$. I do not believe $B$ has changed this reading In any way. Some saall nark oan be seen.between $\pi a 1 \delta 1 \alpha$ and $\mu 0_{1}$, but if it is an $\underline{a}$, it has probably been inserted by $\mathrm{C}$.

3.12 - add $\varepsilon \nu$ before $\tau_{\mid \nu !} \underline{u \omega \nu}$.

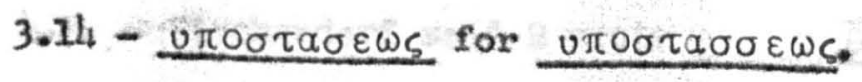


Hebrews

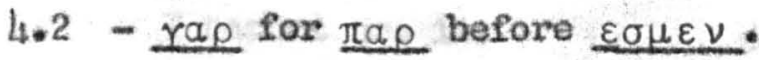

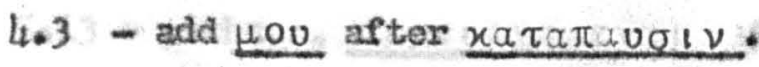

4.12 - $\varepsilon \nu \theta u \mu n \sigma \varepsilon \omega \nu$ for $-\sigma \varepsilon \omega s$.

4.16 - $\varepsilon \lambda \varepsilon o \nu$ for $\varepsilon \lambda \varepsilon O S$ after $\lambda \alpha \beta \omega \mu \varepsilon \nu$.

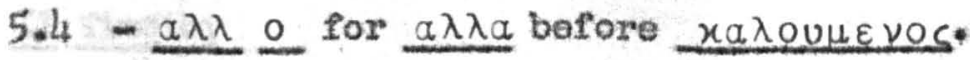

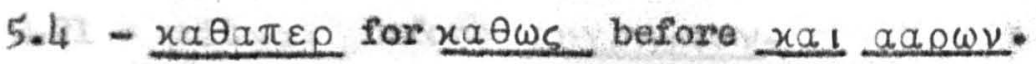

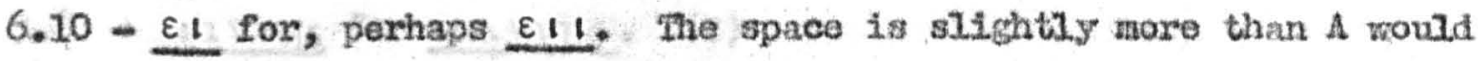
have needed of $\underline{\varepsilon l}$ and $x$ of $x \alpha_{1}$.

$6.19-\varepsilon 1 \sigma \varepsilon \rho \times 0 \mu \varepsilon \nu \eta \nu$ for, perhaps, $\varepsilon_{0 \times 0 \mu \varepsilon \nu \eta \nu}$. This fits the space we.l..

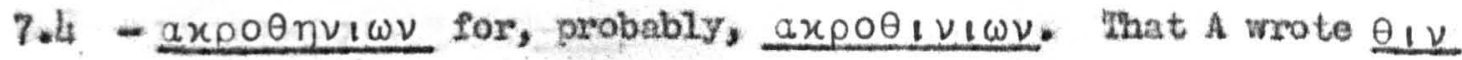
cannot be doubted. It rits the epace we11. One must not concur with 74 schendorf that the oxiginnl toxch vas "inepte".

7.9, - $\lambda \varepsilon v \varepsilon$ for $\lambda \varepsilon v \varepsilon i c$. (?)

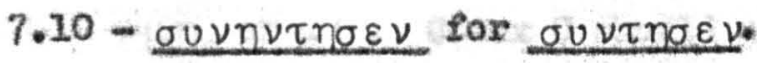

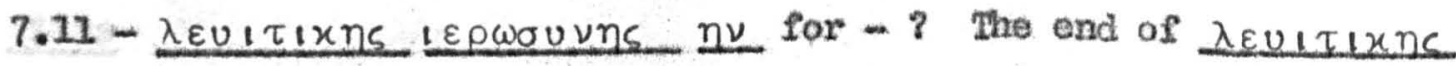
might possibly have been oralted.

7.21 - $\mu \varepsilon \tau a \mu \varepsilon \lambda \eta \theta \eta \sigma \varepsilon \tau \alpha_{1}$ for, perhaps, $\mu \varepsilon \tau \alpha_{\mu} \varepsilon \lambda \eta \sigma \varepsilon \tau \alpha_{1}$. This is the conjeoture of Itschendorfit it fits the space well.

7.22 - ondt $\underline{x a_{1}}$ after tooouto.

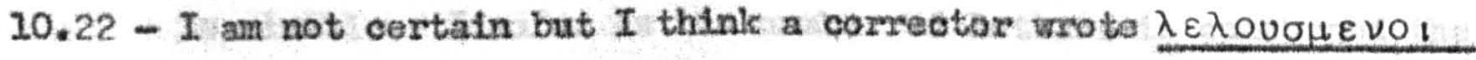

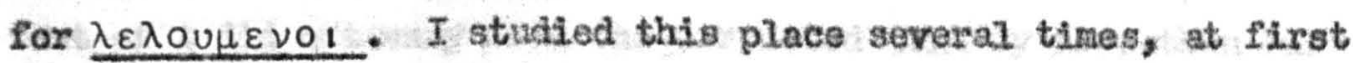
thinking A had written $\lambda \varepsilon \lambda$ ovo. I an certain he did not, but I think B did.

$12.19-\pi a \rho \eta \tau \eta \sigma a \nu \tau o$ for $-\sigma \alpha \nu$.

12.28 - add $\underline{\varepsilon v a \rho \varepsilon \sigma \tau \omega s}$ after $\lambda \alpha \tau \rho \varepsilon v \omega \mu \varepsilon \nu$.

13.6 - add $\underline{x a l}$ after $\beta 0 \eta \theta 0 c$.

13.16 - $\pi 01 \omega 01 \nu$ for $\pi 01 \omega 01$.

$13.18-\pi a \sigma 1 \nu$ for $\pi a \sigma L$.

I Tinothy

3.16 - $\underline{\bar{\theta}}$ for ocs.

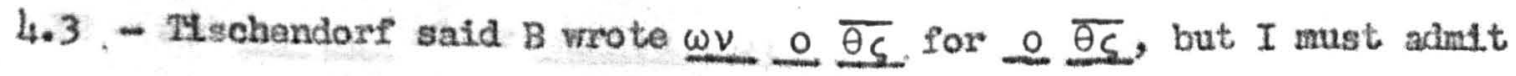




\section{Timothy}

I cannot see it.

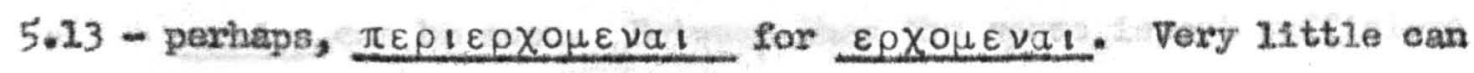
be seen of the text of either $\mathrm{A}$ or $\mathrm{B}$. The original scribe probably wrote less than the corrector.

II 2unothy

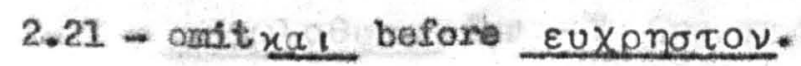

3.8 - lavvns for lwavvns. (?)

3.15 - omit $\tau a$ before $1 \varepsilon \rho a_{\text {, }}$ (?)

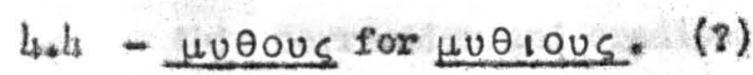

$4.6-x a 1005$ for xalepos.

$4.8-\eta$ rajnxooiv for -01 .

4.20 - concerning $\mu n \lambda n \tau \omega$ or $\mu 1 \lambda n \tau \omega$, at this point the upper text of Ephrain has been erased and the lowir text has been affected where $\mu_{n}\left(\mu_{1}\right)$ was varitten. It cannot be known whether A wrote $\underline{\mu n}$ or $\underline{1}$. Codex 0 should not be quoted el ther way.

\section{I1tus}

1.5 - Xapis $\varepsilon \lambda \varepsilon 0 s$ for Xapis xal.

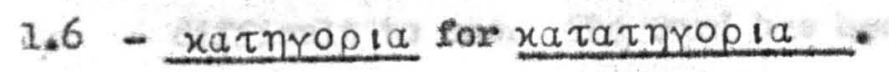

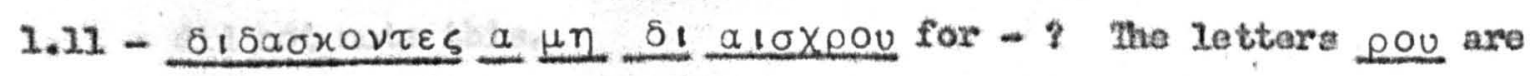
by the orifinal scribes other than this none of the original text 1s visible. Insofar as the number of letters is concerned the text of A did not vary from the corrected text by more than one or two letters.

1.15 - xaAapois for xataplois.

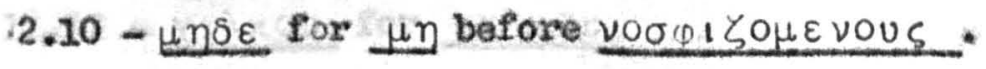

$2.12-\pi \alpha_{1} \delta \varepsilon v 0 v \sigma \alpha$ for, perhaps, $\pi \alpha_{1} \delta \varepsilon v \sigma a$. Two letters definttely were omittod by A. Our text is the conjecture of Tischendorf.

2.13 - It appears that $\underline{\overline{O U}} \underline{x} \alpha_{1}$ has been erased. The vel1um, however, is torn and I an not certain an erasure has been made.

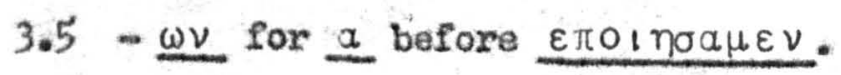

\section{Philemon}

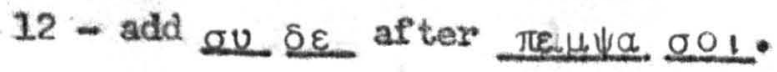




\section{Philamon}

$15-\underline{\varepsilon \chi \omega \rho} 1 \sigma \theta \eta$ for, probably, $\varepsilon \times \rho 10 \theta n$. The Lettors $\underline{\tau} 0 \cup \tau$ and o100n can be seen. Between them the space is not sufficient for 으 $\varepsilon \times \omega$.

17 - $\varepsilon \mu \varepsilon$ for $\mu \varepsilon$ efter autov $\omega s$.

\section{Revelation}

2.21 - $\varepsilon_{1} \delta \omega \lambda 0 \theta u \tau \alpha$ for - ? One or two letters ware probably onitted by the original scribes the writing of $B$ is crowded.

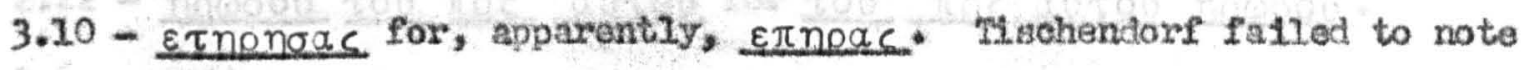

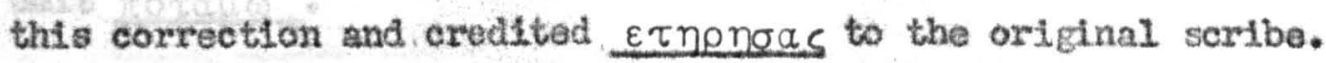

9.19 - add opola before opeo $1 \mathrm{v}$.

$10.2-\beta 1 \beta \lambda \alpha 01810 \mathrm{~V}$ for $\beta 1 \beta \lambda_{1} \alpha_{0} 10 \mathrm{~V}$. Wischendorf and Wetstein both credited this to By I do so only reluotantily. Nowhere else In this codex has a corrector so snocessfully ind tated the script of the original hand. Only the $\rho$ fails to equal the script of A*

$1 / 4.20-\underline{\varepsilon \xi a x 001 \omega \nu}$ appears to have been orased and nothing has been written in its place.

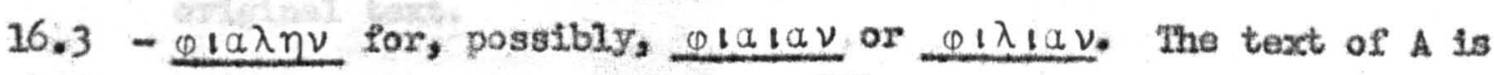
difficult to see. The word has been correctod. Ilschendore did not note this.

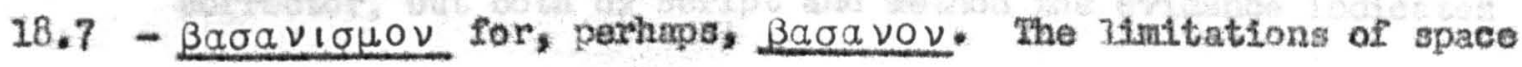
indieate 2 or 3 letters ware omitted.

18.8 - add, perhaps $a_{1}$ before $\pi \lambda \eta \gamma r a 1$. The avallable space suggests the text I have written. It is equaliy possible that the original seribe mis-spelled something.

18.12 - $\chi \alpha \lambda x 00$ for $x \alpha \lambda x 0 u$.

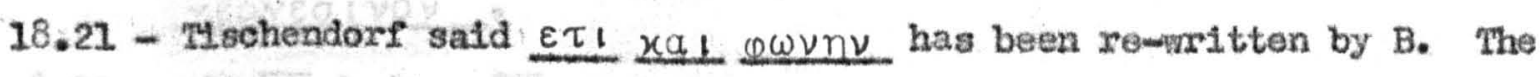
letters $\perp \underline{x \alpha_{1}} \perp$ (and posmibly $\omega_{\nu}$ ) are by the original hand. only $\underline{\varepsilon \tau}$ and $(\underline{\omega \nu}) \underline{n}$ are by B. I can make no guess, what originally was witton. 


\section{APPENDIX $\mathbf{C}$}

\section{ALTERATIONS MADE BX THE SECOND CORRECLOR}

\section{Watthew}

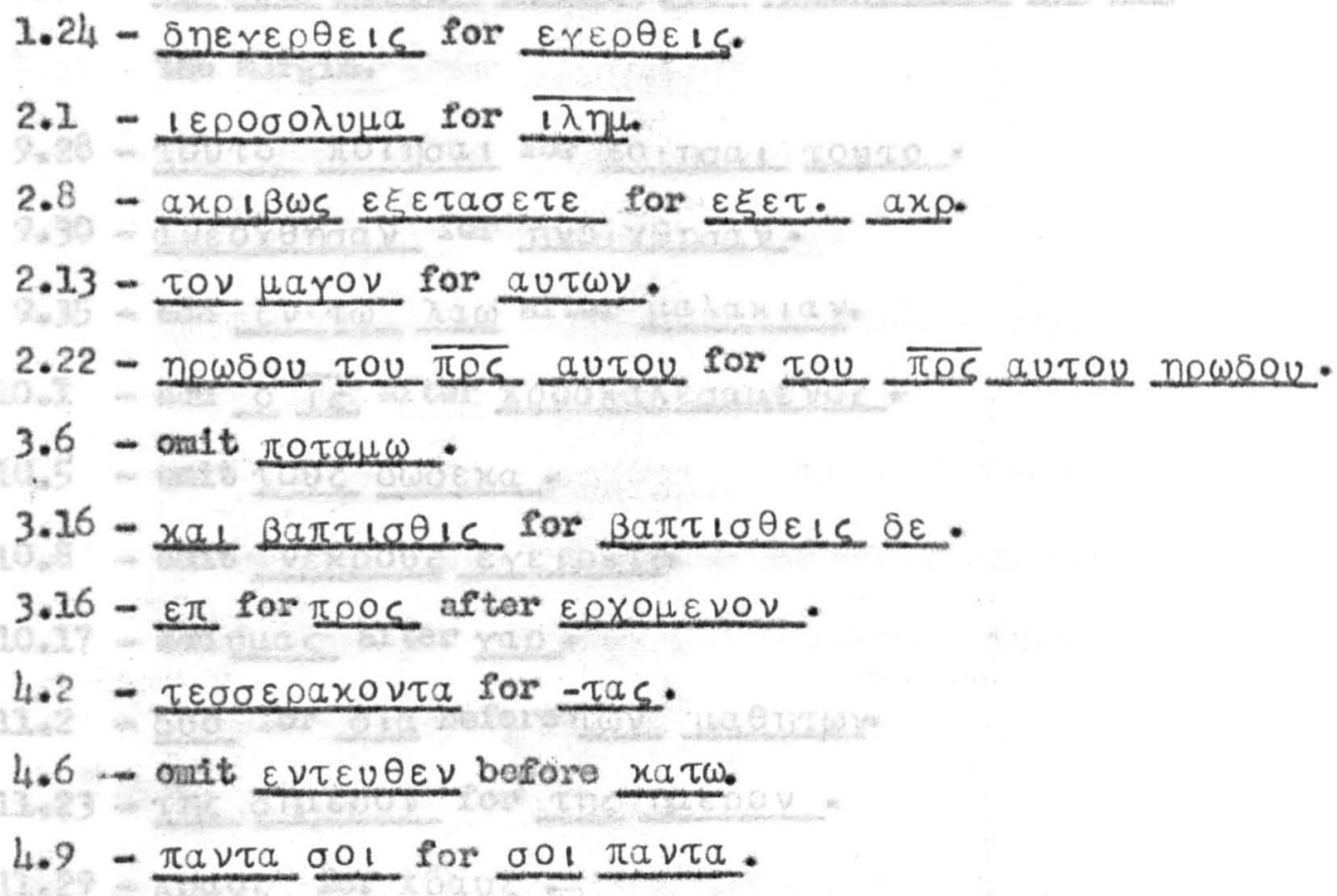
of the correction made by the first corrector and a return to the original text.

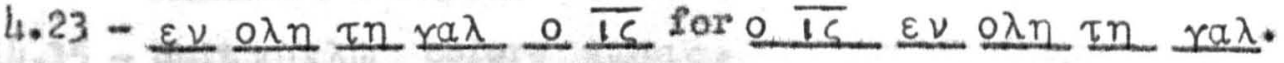

7.17 - outwe for ou $\tau \omega$. TIschendorf credited this change to the first corrector, but both by seript and method the evidence indicates the second corrector.

7.29 - onit $\underline{x \alpha 1}$ ol $\underline{\text { pap10a101. }}$

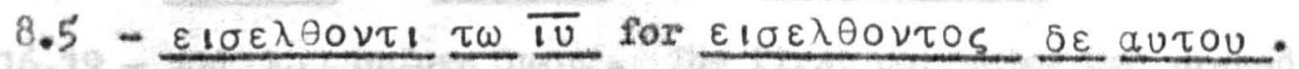

8.5 - autov for auto.

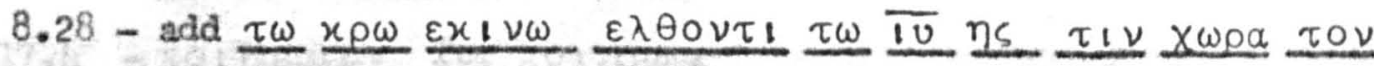
yepreoivov.

8.29 - add $\overline{T U}$ before $\overline{U \varepsilon}$.

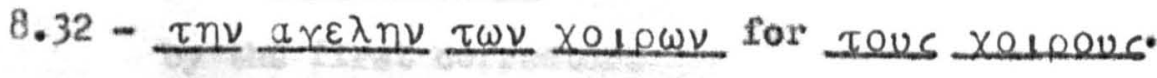

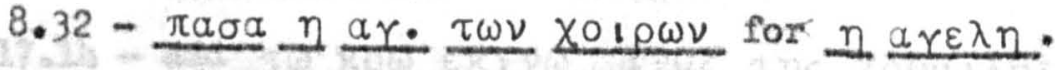

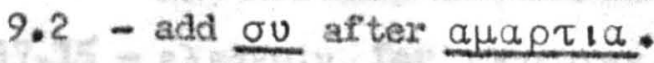

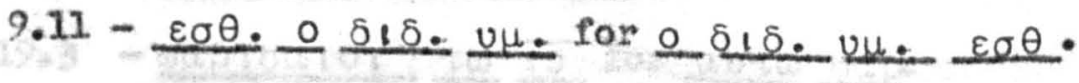

9.12 - add autus after $\underline{1 ! \pi \varepsilon \nu}$. 
Matthew

$9.13-\underline{\varepsilon \lambda \varepsilon O \nu}$ for $\varepsilon \lambda \varepsilon 0 \zeta$.

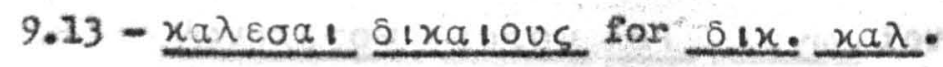

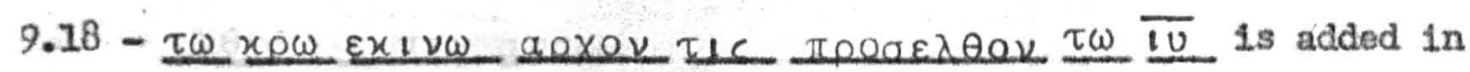
the margin.

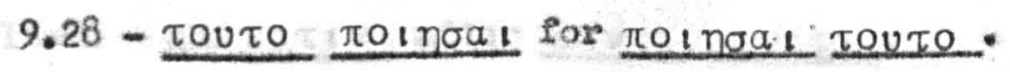

9.30 - aveoxenoav for nvoixenoqk.

$9.35-$ add $\varepsilon \nu \underline{\tau \omega} \underline{\lambda \alpha \omega}$ after $\mu \alpha \lambda \alpha x$ a $\alpha \nu$.

10.1 - add $\_\overline{i c}$ after $\pi p 0 \sigma x a \lambda \varepsilon \sigma a \mu \varepsilon v o c$.

10.5 - onit zous $\delta \omega \delta \varepsilon x a$.

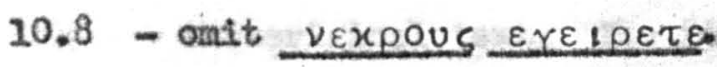

10.17 - add unac after yap.

11.2 - $\underline{\delta 0}$ for $\underline{\delta l a}$ before $\underline{\tau \nu} \underline{\mu \alpha \theta n \tau \omega \nu_{0}}$

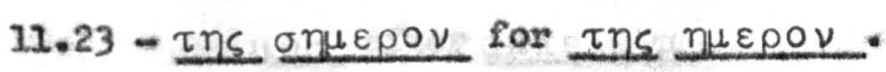

11.29 - $\pi \rho$ aOs for $\pi \rho \alpha u s$.

13.4h - $\tau \omega \nu$ for $\tau \omega$ before oupa $\nu \omega \nu$.

13.48 - aryia for $\underline{a \times r n}$.

14.4 - $\underline{\varepsilon x \varepsilon \nu \nu}$ for $\varepsilon \times \varepsilon \nu$.

14.15 - omit $x u x \lambda \omega$ before $x \omega \mu$ ac.

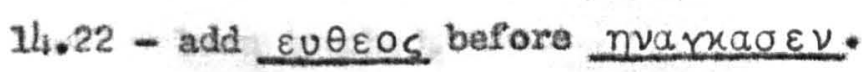

14.25 - add 015 after autous.

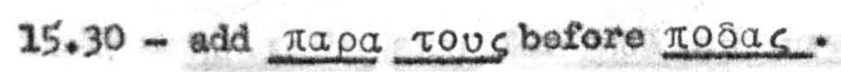

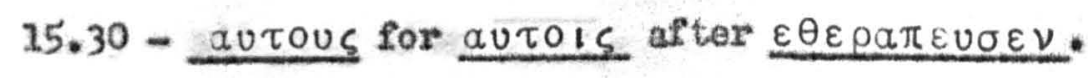

16.19 - add $\underline{x a_{1}}$ before $\underline{\delta \omega} \underline{ }$. The first corrector had erased the original $x a_{1}$. The second corrector has returned to the text of A.

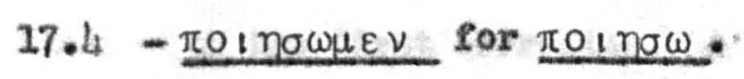

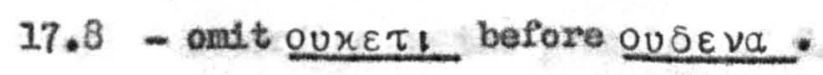

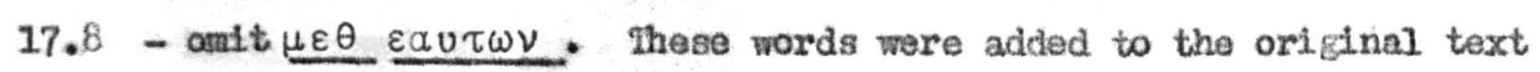
by the first corrector.

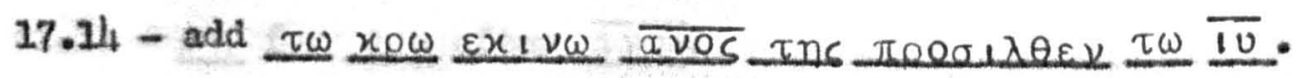

17.15 - $\underline{\varepsilon \varepsilon n \text { noov for } \varepsilon \lambda \text { noov. }}$

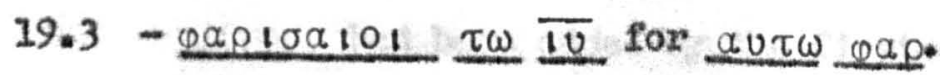

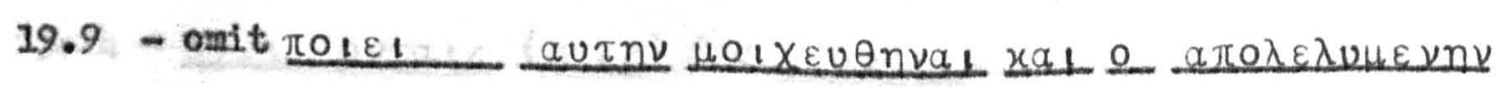

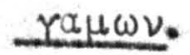


Watther

$19.16-\tau \omega \underline{x \rho \omega} \varepsilon x_{1} \nu \omega$

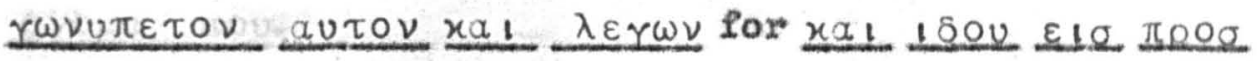

$\underline{\varepsilon \lambda \theta \omega \nu} \underline{\varepsilon i \pi \varepsilon \nu} \underline{\alpha u \tau \omega}$.

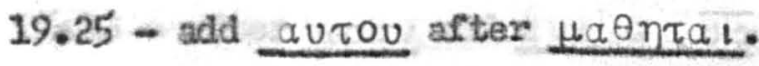

19.26 - add $\underline{\varepsilon \sigma \tau 1 \nu}$ after $\pi \alpha \nu \tau \alpha$

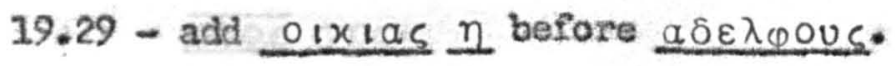

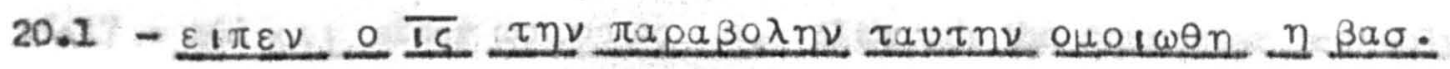

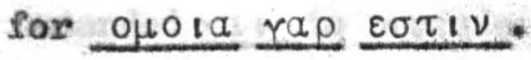

20.6 - add aprous after $\varepsilon \sigma \tau \omega \tau \alpha_{S}$. The second corrector here reasserts the original text. The first corrector had erased aprous.

IIschondori did not note that 0 had made this restoration of

the original text.

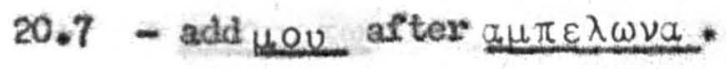

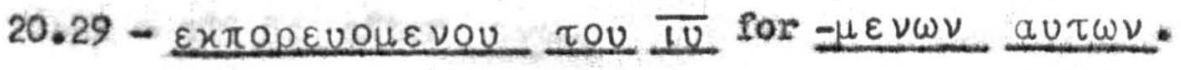

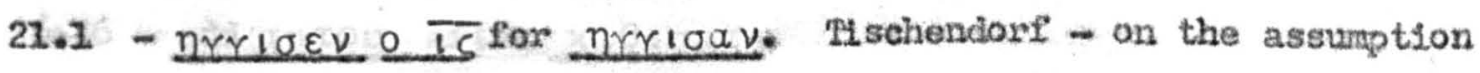
that since $C$ wrota $\eta y \gamma 1 \sigma \varepsilon v$ ho also intended to write $n \lambda \theta \varepsilon \nu$ has included in 28 the vitnese of the second corrector for $\eta \lambda \theta \varepsilon \nu$. Nestle in his apparatus has followed m8. But $n \lambda \theta 0 \nu$ has not been touched.

21.4 - add $0 \lambda \omega \nu$ before $v \varepsilon$ vovev.

21.17 - $\eta \lambda_{1} \sigma \theta \eta$, $\varepsilon x \eta$ for $\eta u \lambda_{1} \sigma \theta \eta \sigma \alpha \nu$.

21.33 - add $\tau_{15}$ after $\overline{\text { QVOS. }}$.

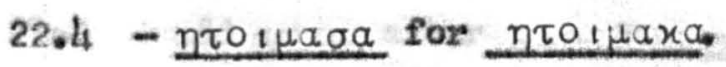

22.5 - $-\underline{0 \varepsilon}$ for $05 \underline{\delta \varepsilon}$. Tischendort erred in crediting this change to the first corrector. A line has been dram through the $S$ according to the practice of C. The first corrector probably would have erased the 5 .

22.13 - $\underline{\operatorname{vvax\varepsilon \mu \mu \varepsilon \nu \omega \nu } \text { for }-x \varepsilon \mid \nu \omega \nu .}$

$22.11-\underline{\mu n}$ for oux after $\overline{\alpha \overline{v o v}_{0}}$

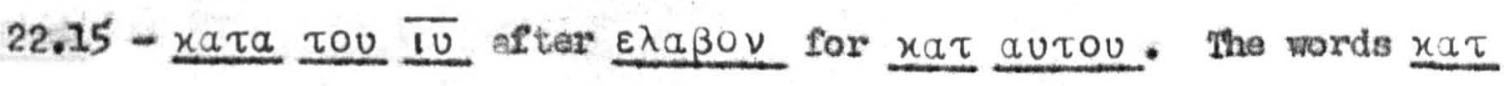
autou had been added by the first corrector.

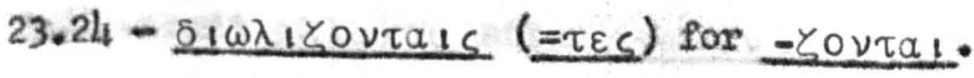


Hatther

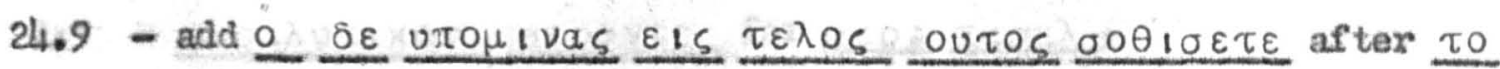
ovora Hov.

25.6 - add $\underline{\varepsilon \rho \chi \varepsilon \tau \varepsilon}$ before $\underline{\varepsilon \varepsilon \rho \rho X \varepsilon \sigma \theta \alpha+.}$

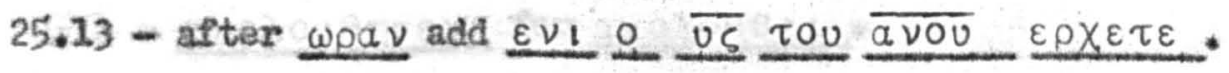

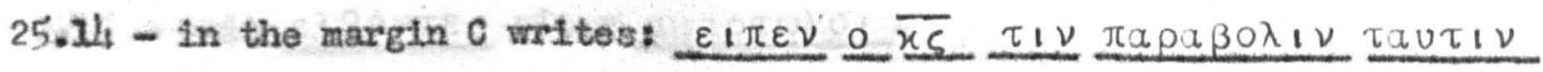
a vos Ins.

25.17 - add $\underline{x a_{1}}$ after $\underline{\omega \sigma a v \tau \omega_{5}}$-. Although the corrector probably intended $\underline{x \alpha_{1}}$ to precede 0 , the inserted letters have been placed after it.

25.17 - add $\underline{x a_{1}}$ autos after $\underline{\varepsilon \varepsilon \rho \delta n \sigma \varepsilon v}$.

25.18 - $\tau \eta \underline{x \eta}$ for $\tau \eta v$ Ynv.

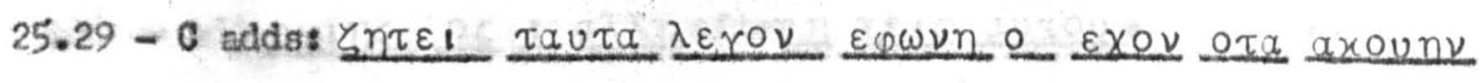
axou $\tau \omega$.

26.31 - add $\delta \varepsilon$ after $\varepsilon \gamma$ cor.

26.36 - add au

26.38 - add o $\overline{15}$ after autois.

26.39 - omit the duplicated $\varepsilon \pi \varepsilon \sigma \varepsilon \nu^{2}$. Shwen hivin

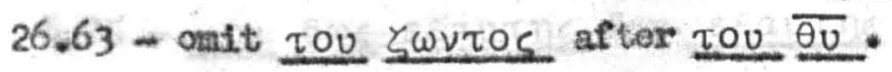

$26.67-\varepsilon x 0 \lambda a 01 \sigma a \nu$ for $-01 \lambda a g a \nu$.

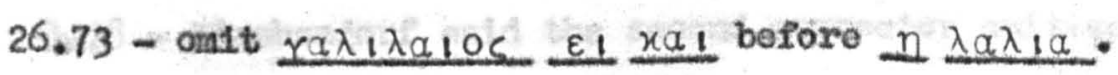

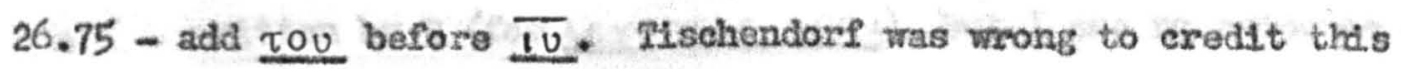

addition to $B$. The letters are added above the line.

27.2 - ada $\alpha u \tau \omega$ after $\pi \alpha \rho \varepsilon \delta \omega x \alpha \nu$.

27.51 - omit Els svo aftar $\underline{x \alpha \tau}$.

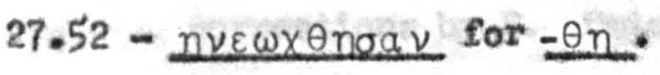

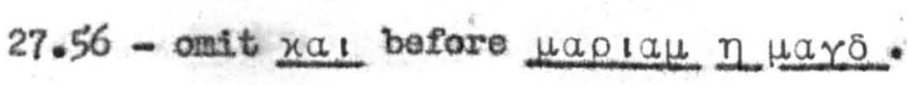

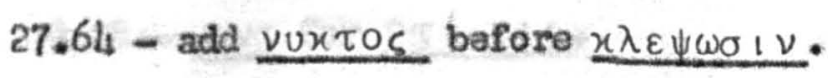

$27.64-\varepsilon \sigma x \alpha \tau \eta$ for $\sigma \chi \alpha \tau \eta$.

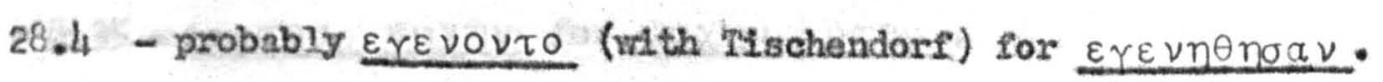

Yark

1.35 - add 0 Is after $a \pi \eta \lambda \theta \varepsilon \nu$.

1.38 - omat $\underline{a \lambda \lambda a \times 0 u}$ aftar $\underline{\alpha \gamma \omega \mu \varepsilon \nu}$. 
Jarke

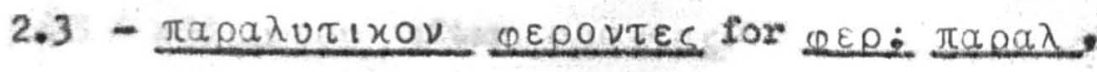

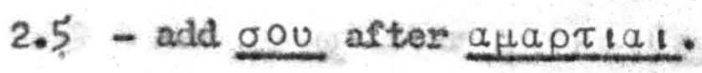

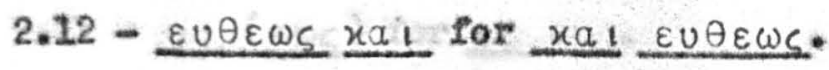

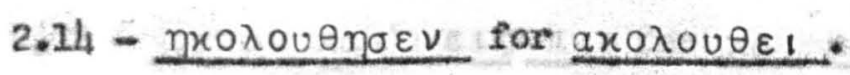

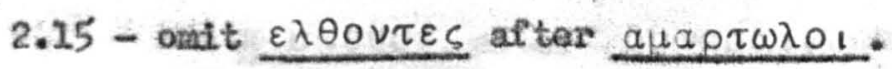

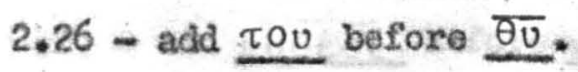

2.27 - onst $\underline{x \alpha_{1}}$ before oux.

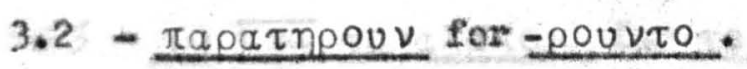

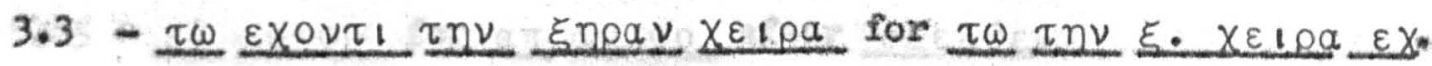
The second corrector has placed $\varepsilon$ Xov $\tau$, after $\tau \omega$, but it is linposstble to see if he has made further changas.

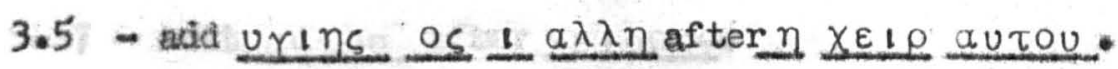

6,10 - onou for exos after gutole.

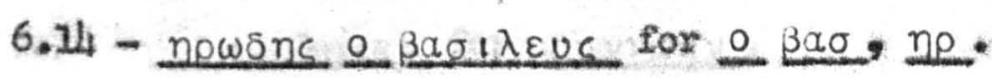

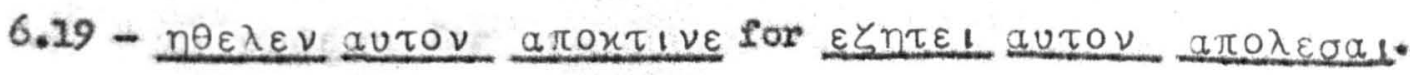

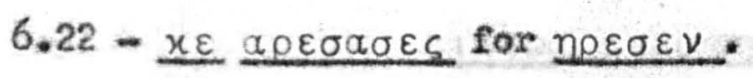

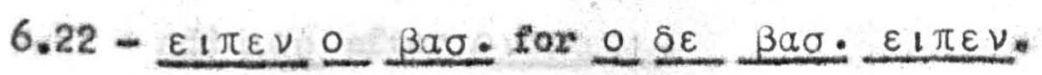

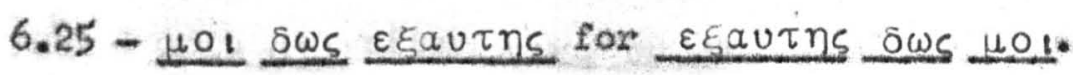

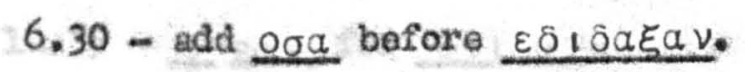

$\checkmark .28$ - Isehendorf said the second corrector omitted the 01 witten by $B$ (for $\lambda \varepsilon \gamma \circ \nu \tau \varepsilon S$. $0 \tau_{1}$ ), but I see no evidence of this. Perhaps he based this onlission on the fact that of $\mu \varepsilon \nu$ is fairly light and that it therefore had been erased by $C$. But the faintness of letters is often a charactoristif of corrections by B. Ontastons by C are generaliy noted by other narks.

$8.28-\underline{\varepsilon v a}$ for oㄴ1 $\varepsilon 15$.

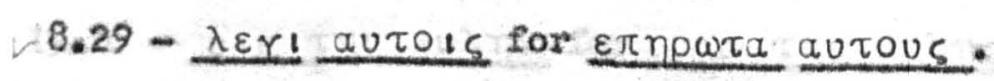

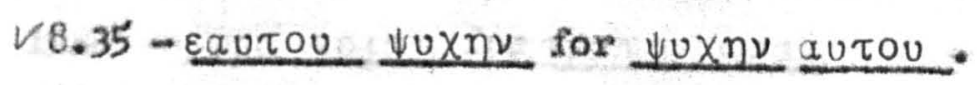

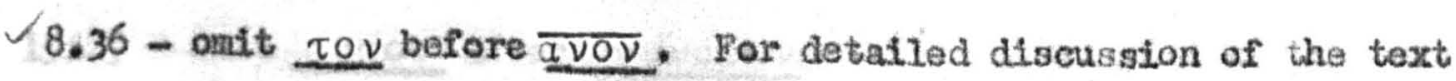
at this point see Appendiz B.

9.6 - $\lambda \alpha \lambda$ non for $a \pi$ oxp $1 \theta \eta$. 
Yark

9.18 - add aviou after osovias.

9.19 - add $\underline{\alpha u \tau \omega}$ after $a \pi 0 x \rho 1 \theta \varepsilon i c$.

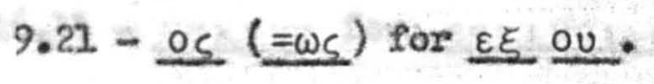

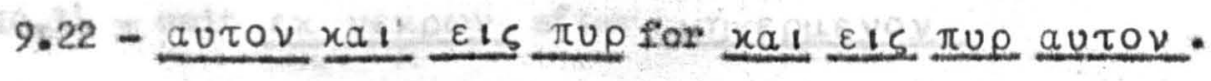

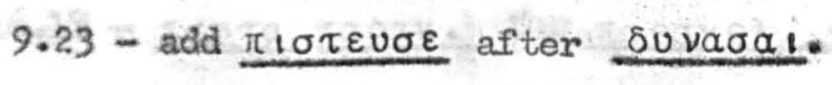

9.24 - add $\varepsilon v \theta \varepsilon 0 \mathrm{~s}$ before koakac.

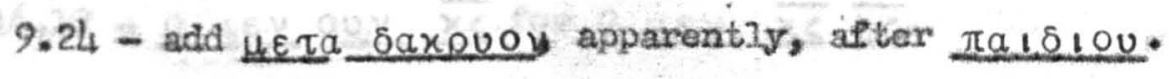

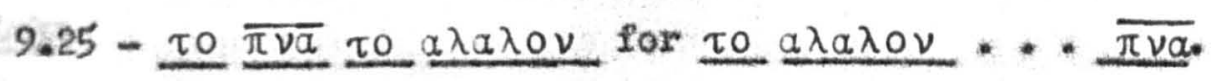

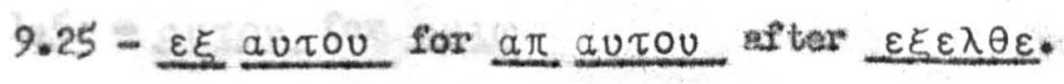

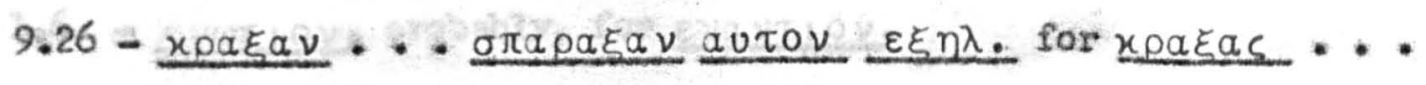

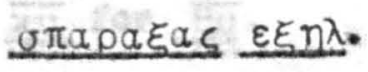

9.27 - onit auzou after $\chi$ El loos.

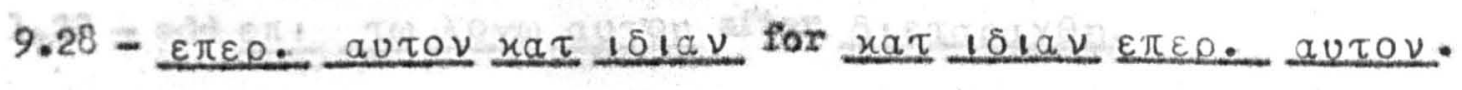

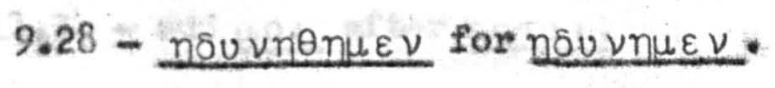

9.29 - Ev ougevn for ou.

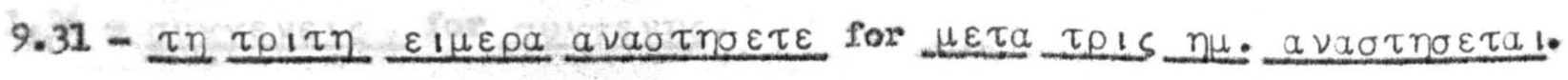

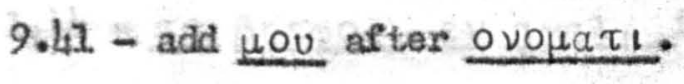

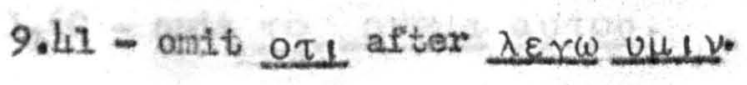

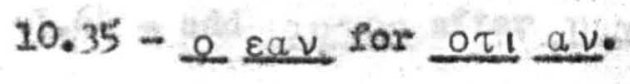

$10.37-\underline{\varepsilon i \pi 0 \nu}$ for $\underline{\varepsilon 1 \pi a \nu}$.

$10.37-\varepsilon x \underline{\delta \varepsilon \xi 1 \omega \nu}$ oov tor gov $\varepsilon x \underline{\delta \varepsilon \xi \omega \nu}$

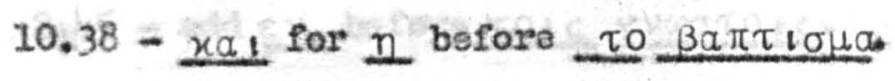

10.39 - add $\mu \varepsilon \nu$ before notnplov.

$10.43=\varepsilon \sigma \tau a_{1}$ for $\underline{\varepsilon \sigma \tau 1 \nu}$.

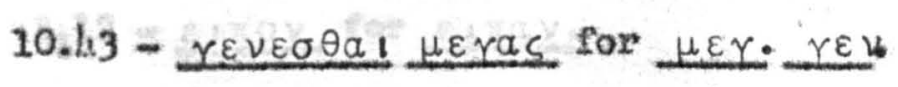

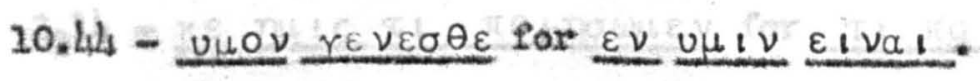

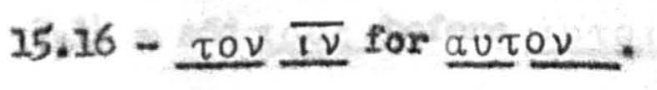

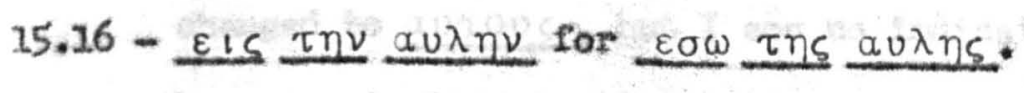

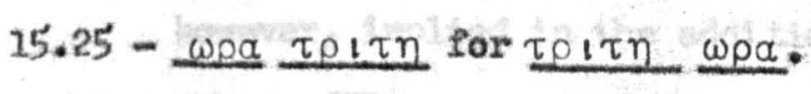

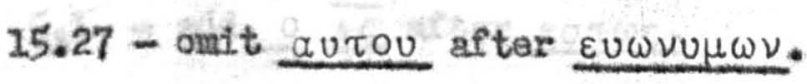

15.31 - add $\delta \varepsilon$ after ofolws.

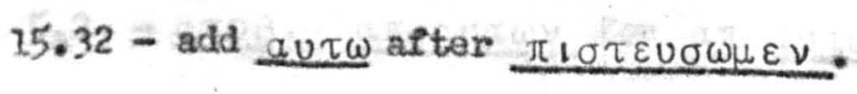




\section{Mark}

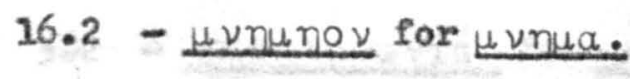

16.9 - $\underline{\alpha \rho}$ for $\pi \alpha \rho$ after $\mu \alpha \gamma \delta \alpha \lambda \eta \nu n$.

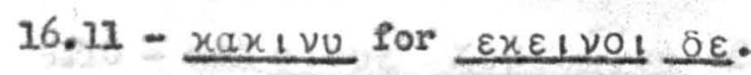

16.14 - omit $\varepsilon x \underline{\nu x \rho \omega \nu}$ after $\underline{\varepsilon \eta n y \varepsilon \rho \mu \varepsilon \nu o \nu .}$

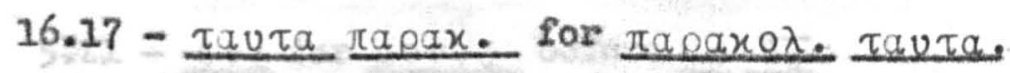

16.18 - ou $\mu \eta$ for ousev.

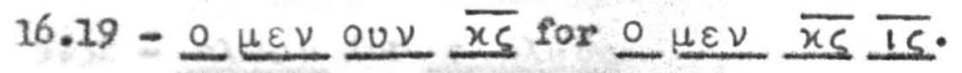

Luke

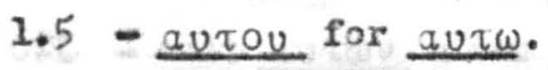

$1.6-\underline{\varepsilon v \omega \pi}$ 1ov, probabiy, for $\underline{\varepsilon v \nu \tau \tau 10 \nu}$.

1.9 - $\overline{x U}$ for $\overline{\theta u}$.

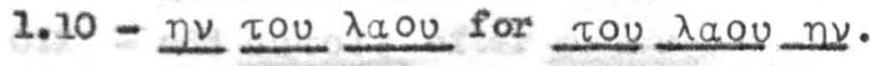

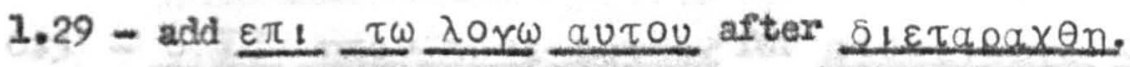

1.34 - add $\mu_{01}$ after $\underline{\varepsilon \sigma \tau_{2} l_{1}}$

1.35 - omit $\varepsilon x \underline{\sigma 0 u}$ after $r \varepsilon \nu \nu \omega \mu \varepsilon \nu 0 \nu$.

1.36 - gurrevels for gurrevns.

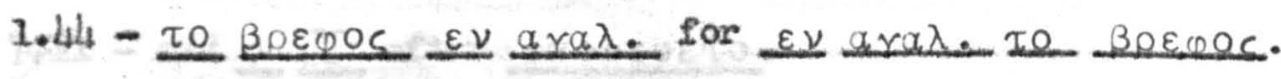

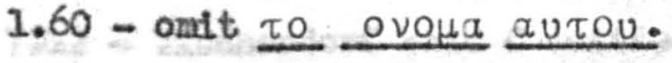

1.64 - add autou after $n \underline{y \lambda \omega \sigma o a .}$

2.1 - auruatou for aruotou.

$2.3-\pi 0 \lambda 1 \nu$ for $x \omega \rho a \nu$.

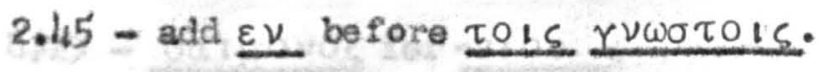

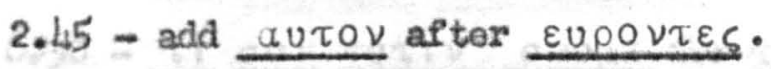

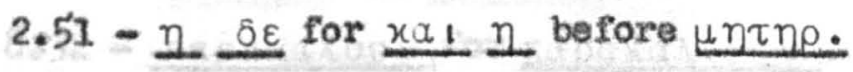

3.12 - $\varepsilon_{1 \pi 0 \nu}$ for $\varepsilon_{1} \pi a \nu$.

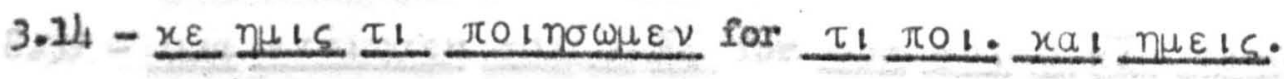

3.14 - add Looc before autols. Tischendoref said auto is was also changed to autous, but I see no Indications of this. This is, hovever, implied in the addition of $\pi \rho 05$

5.1 - add 0 Is after $\varepsilon \sigma \tau \omega s$.

$\sqrt{5.2-\pi \lambda 01 a \text { for } \pi \lambda 01 a 01 \alpha}$

$5.2-\alpha \pi 0 \beta$. 
Iuke

5.2 - $a \pi \varepsilon \pi \lambda u \nu a v t d$ for $\varepsilon \pi \lambda u \nu$.

$\checkmark \&$

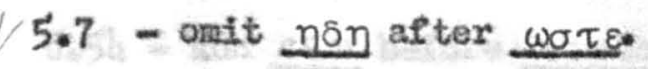

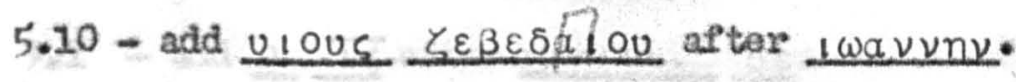

5.17 - add 으 is astor $11 \delta a 0 x \omega v$.

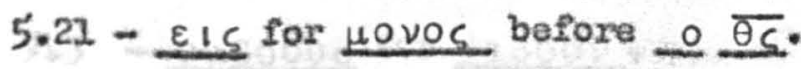

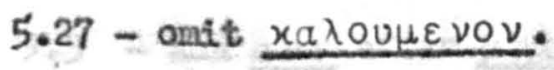

5.32 - $n \lambda \theta 0 \nu$ for $\varepsilon \lambda \eta \lambda \nu \theta \alpha$.

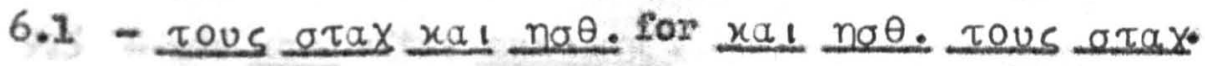

6.1 - onit $a \cup \tau \omega \nu$ after $X \varepsilon \rho \sigma i \nu$. Mischendorf omitted this correction In his edition, but in 28 he notes that the original scribe (C*)

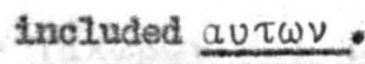

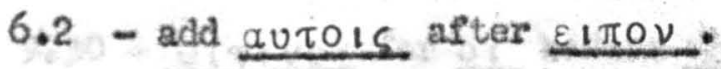

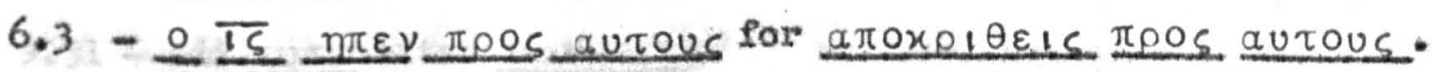

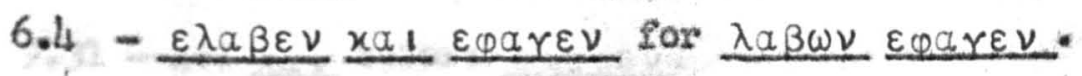

$7.1-\underline{x a \pi \varepsilon \rho v a o u \mu}$ for xasao.

7.5 - $\omega x 0 \delta 0 \mu \eta \eta E v$ for olxos.

7.11 - add 0 IS after $\varepsilon \pi 0 p \varepsilon v \varepsilon \tau 0$.

7.12 - Mschendorf said $\mathrm{C}$ omitted $\eta \nu$ before $x \eta \rho \alpha$, but I can seo no indication of such an omission. I do not knov what he thought he sar, but I am certain he 1 s mong.

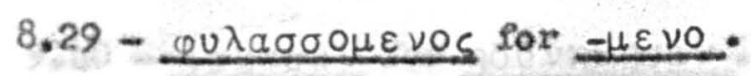

8.29 - Sa1Hovos for $-\nu 10 u$.

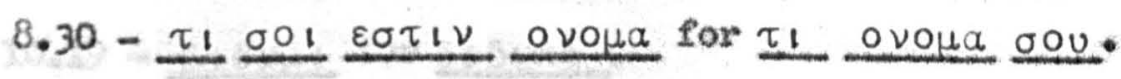

8.32 - $\pi a \rho a x a \lambda o u v$ for $\pi a \rho a x a \lambda \varepsilon \sigma a \nu$.

8.32 - onit 으 is after auto is.

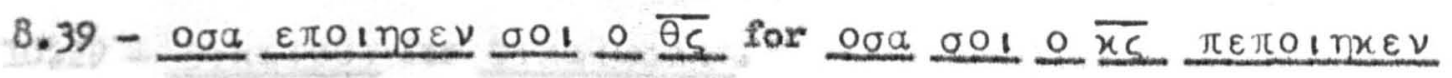
$\underline{x \alpha_{\iota}} \eta \underline{\eta \lambda \eta \eta \sigma \varepsilon \nu} \sigma$.

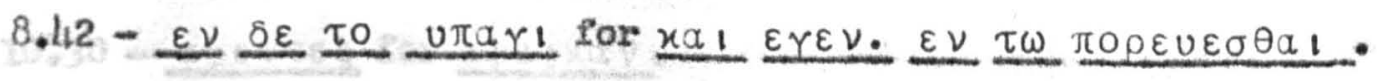

8.45 - $\tau_{15}$ for $\tau_{1}$ after $\lambda \varepsilon \gamma \varepsilon 15$.

$8.46-\underline{\varepsilon \gamma \nu \omega \nu}$ for $\varepsilon \gamma \nu \omega$.

8.47 - add $\underline{\text { a } \tau \omega}$ after $a \pi \eta \gamma \gamma \varepsilon \wedge \lambda \varepsilon \nu$. This is a restoration of the original text after the first corroctor had orased au 
Luke

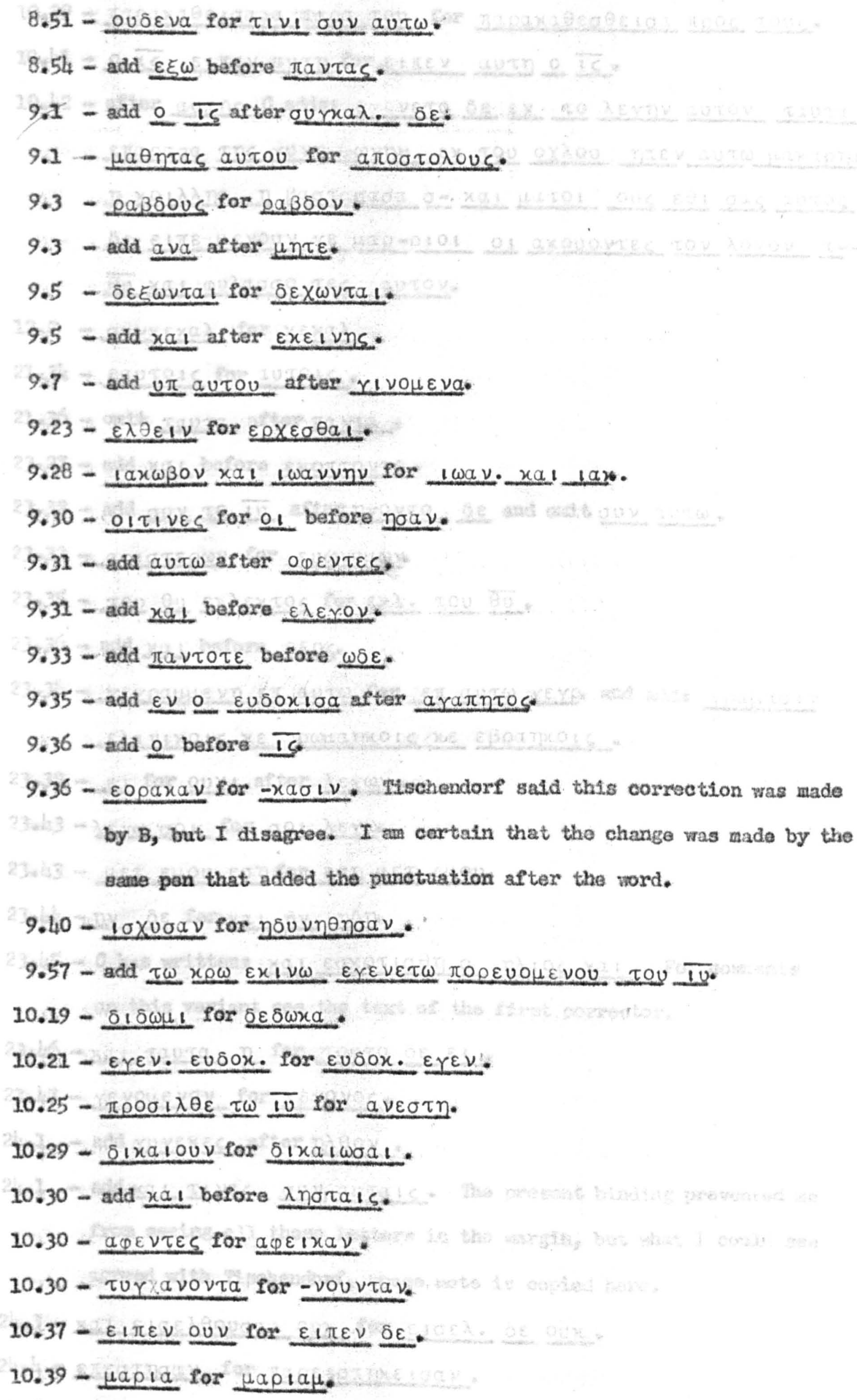


Inke

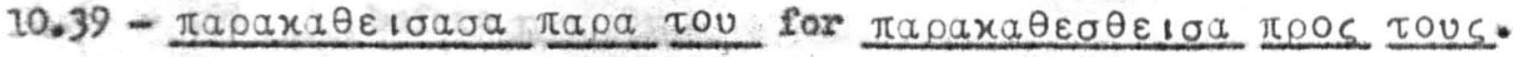

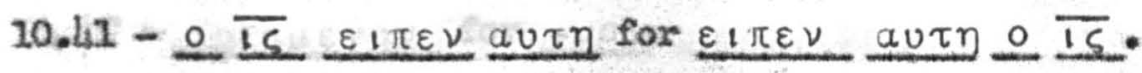

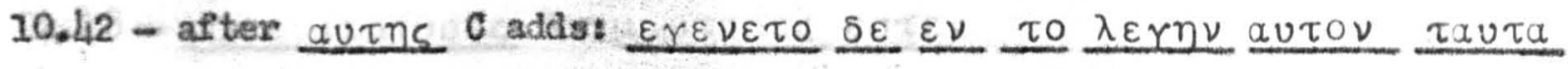

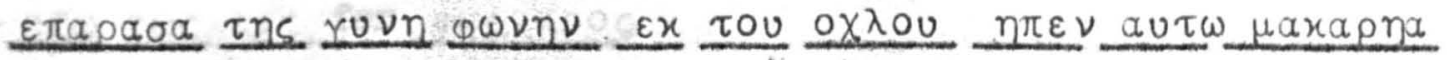

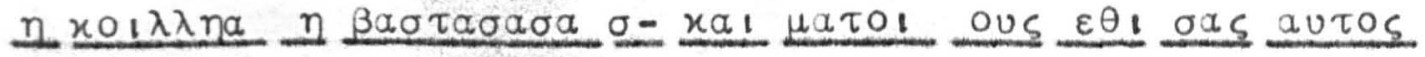

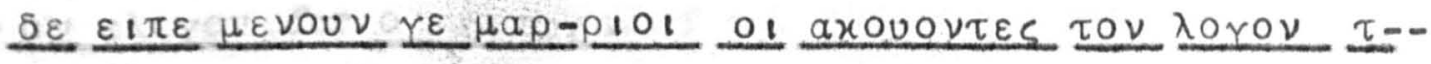

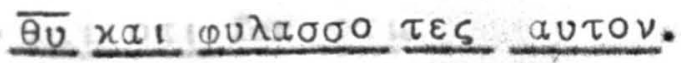

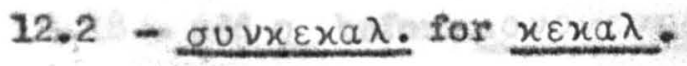

21.34 - Eautois for auto is.

21.36 - ontt $\underline{\tau \alpha u \tau \alpha}$ after $\underline{\pi \nu \nu \tau \alpha .}$

23.27 - add $x a_{t}$ before $\varepsilon x 0 \pi \tau 0 \nu \tau 0$.

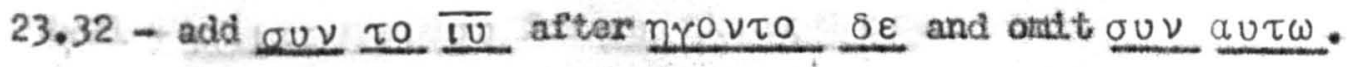

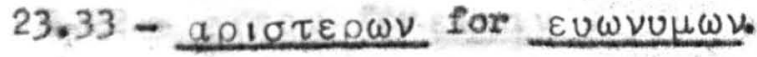

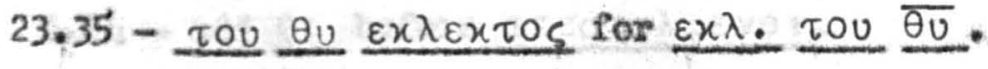

23.36 - add $\underline{x_{1}}$ bafore ogos.

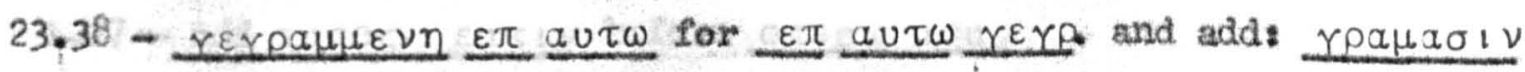

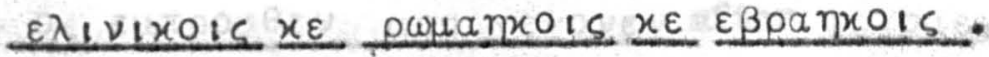

23.39 - $\varepsilon 1$ for ovXl after $\lambda \varepsilon r \omega \nu$.

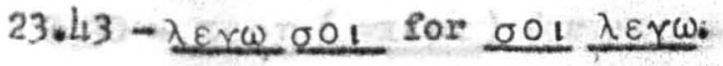

$23.43-\mu \varepsilon \tau \varepsilon \mu O U$ $\varepsilon \sigma \eta$ for $\varepsilon \sigma \eta \mu \varepsilon \tau$. $\varepsilon \mu O U$.

$23.44-n \nu$ for $x a+$ nv nôn.

23.45 - 0 has written: $x a_{1}, \varepsilon \sigma x 0 \tau_{10 \theta \eta}$ 으 $n \lambda_{1}$ os $x_{1}$. For coments on this variant see the text of the first corrector.

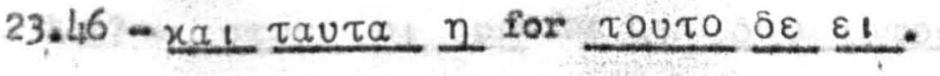

23.47 - yevouevov for yerovos.

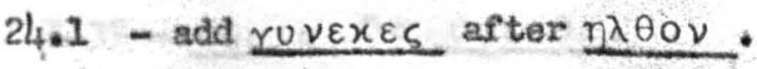

24.1 - add $\underline{x a}$ I IVES ouv auta 15 . The present binding prevented me froa seeing all these Ietters in the nargin, but what I could see agreed with Tischendorf, whose note is copied here.

24.3 - xal $\varepsilon 1 \sigma \varepsilon \lambda \theta 0 u \sigma \alpha_{1}$ oux for $\varepsilon 1 \sigma \varepsilon \lambda . \delta \varepsilon$ oux.

$24.4-\varepsilon \pi \varepsilon \sigma \tau \eta \sigma \alpha \nu$ for $\pi \alpha \rho \varepsilon 1 \sigma \tau \eta x \varepsilon 1 \sigma \alpha \nu$.

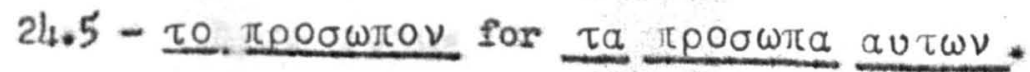




\section{Lulke}

24.6 - add $a \lambda \lambda$ before nrep $\theta n$.

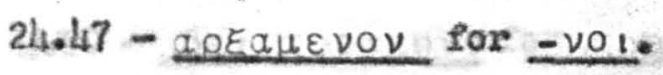

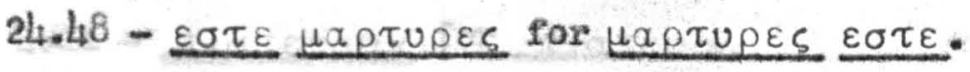

$24.50-\varepsilon \xi \omega \underline{\varepsilon \omega s} \varepsilon 1 s$ for $\varepsilon \omega_{S} \pi \rho 0 s$.

John

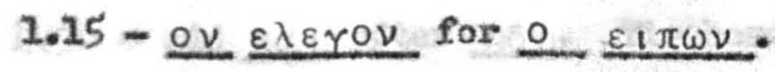

1.16 - $x a_{1}$ ofl for ofl.

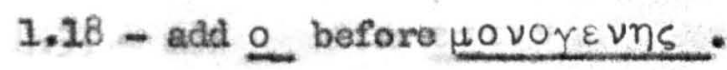

1.18 - $\overline{U S}$ for $\overline{\theta S}$.

1.19 - omit $\pi \rho 0 s$ autov.

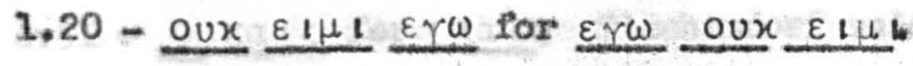

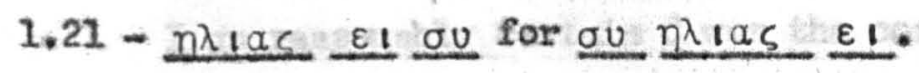

$1.22-\varepsilon 1 \pi 0 v$ for $\varepsilon 1 \pi a \nu$.

$1.24_{4}$ - add $\underline{01}$ before a $\underline{\pi \varepsilon \sigma \tau a \lambda \mu \varepsilon \nu 01 .}$

$1.24-\underline{\varepsilon} 1 \pi 0 \nu$ for $\varepsilon 1 \pi a \nu$.

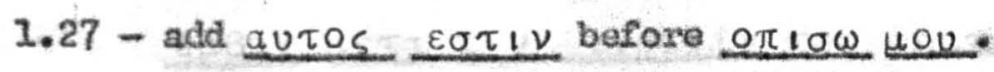

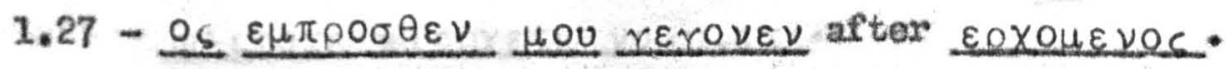

1.29 - add 0 lwavic after $\beta \lambda \varepsilon \pi \varepsilon_{1}$.

$1.30-\pi \varepsilon \rho \eta(=\pi \varepsilon \rho 1)$ for $\underline{u \varepsilon \varepsilon \rho}$.

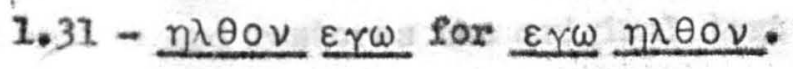

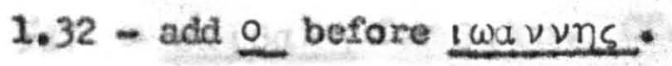

1.33 - onit $x a_{1}$ mupt.

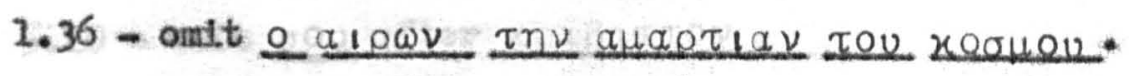

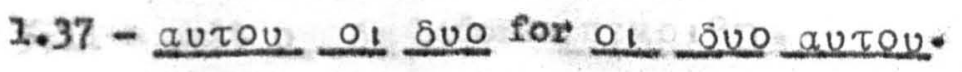

$1.38-\underline{\varepsilon} 1 \pi 0 \nu$ for $\varepsilon 1 \pi \alpha \nu$.

1.39 - $n \delta \varepsilon \tau \varepsilon$ for $0 \psi \varepsilon \sigma \sigma \theta \varepsilon$.

4.5 - 으 is 15 for ouv E1s.

$4.7-\pi \varepsilon i \eta \nu$ for $\pi \varepsilon \downarrow \nu$.

$4.9-\underline{\pi \varepsilon 1 \eta \nu}$ for $\pi \varepsilon \downarrow \nu_{0}$

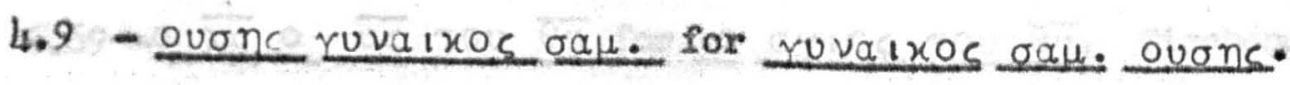

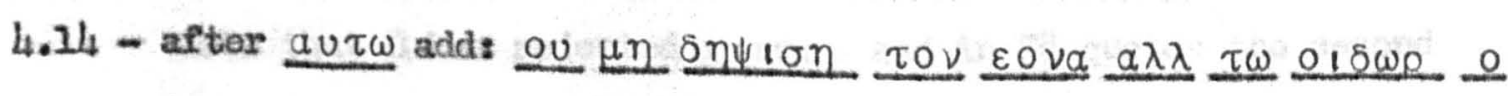
$\underline{\delta-\sigma 0} \underline{a v \tau \omega}$. 
John

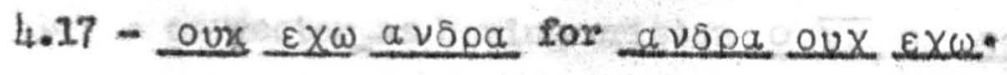

$4.20-\delta \varepsilon_{1} \pi \rho 00 x u v_{0}$ for $\pi \rho 0 \sigma x u \nu_{0} \delta \varepsilon_{1}$.

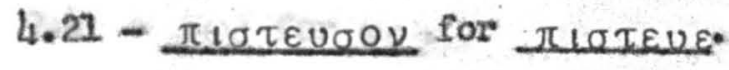

4.29 - oa for $\underline{a}$.

$4.31-\varepsilon \nu \underline{\delta} \tau \omega$ for $\underline{\varepsilon \nu} \underline{\underline{\nu}}$.

4.36 - add $x a 1$ after non. 1hschendore satd this correction was made by B.

4.37 - add 으 after $\varepsilon \sigma \tau \imath \nu$.

4.39 - oga for a.

4.42 - add 으 $\overline{x s}$ after noguou.

4.51 - 으시 for $\pi \alpha_{16}$ Ischendorf said $B$ wade this correction but I an reasonably cerbain C was the corrector.

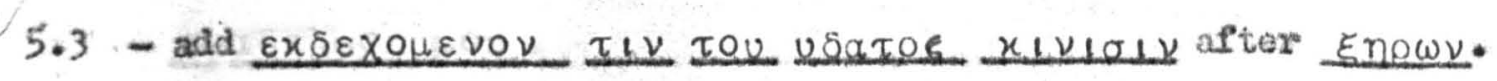

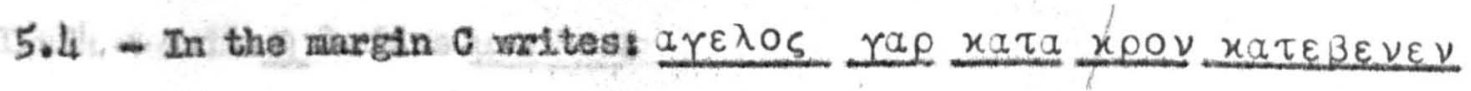

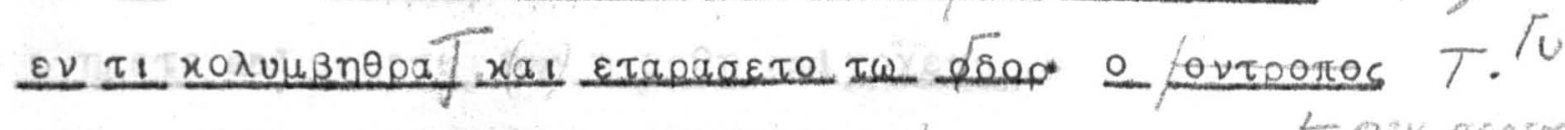

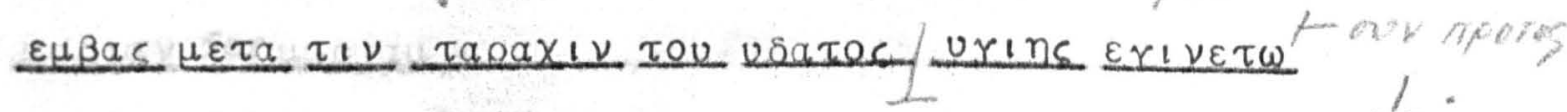

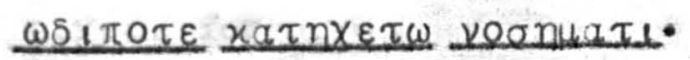

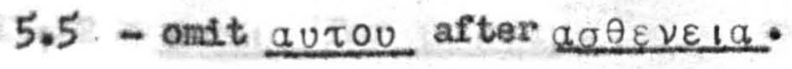

5.7 - The second corrector rejects the addstions of the first corrector

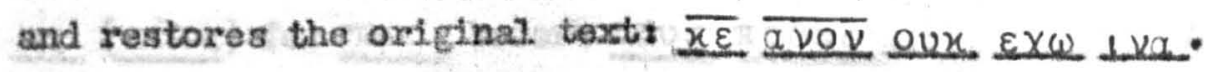

5.7. - $\varepsilon \pi 1 \beta a \lambda \eta$ for $\beta a \lambda \eta$. CP., Appendix B.

5.10 - oasit $\underline{x a l}$ after $\varepsilon \sigma \tau 1 \nu$.

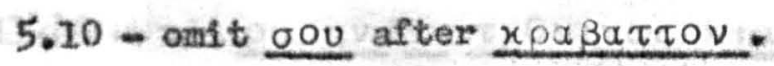

5.11 - omit 으 $\delta \varepsilon$ before $a \pi \varepsilon x \rho 1 \theta \eta$.

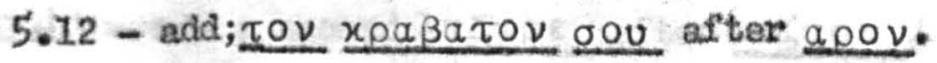

6.161 - I see no indication that $c$ changed $1 \delta \omega v$ to $15 \omega \mathrm{s}$. Tischandorf acknowledged "Sed possum fallu" in his edition but rewoves all doubt in 18.

6.65 - add $\underline{\mu \nu}$ after $\overline{\pi \rho} s$.

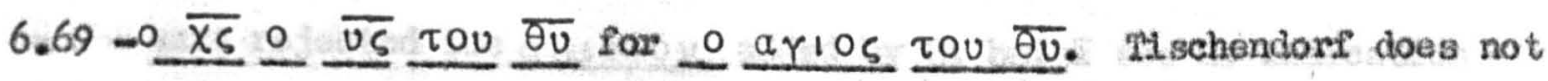
note that 6 has rejected $\mathrm{arlOS}_{\mathrm{S}}$ and $\mathrm{in} \mathrm{RB}$ quotes the second 
John

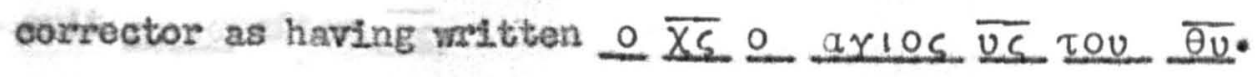

9.1 - add 0 IS aftor $\pi a \rho a r \omega v$.

$9.4-\underline{\varepsilon \omega_{c}}$ for $\omega_{c}$.

9.7 - oxit autou (which was addad by B) aftar $\varepsilon \pi \varepsilon \times \rho 10 \varepsilon \nu$.

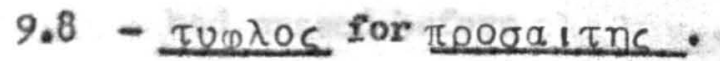

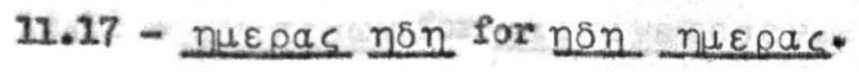

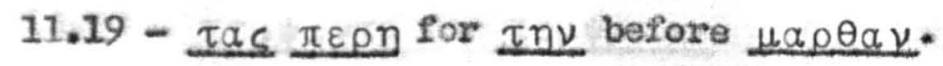

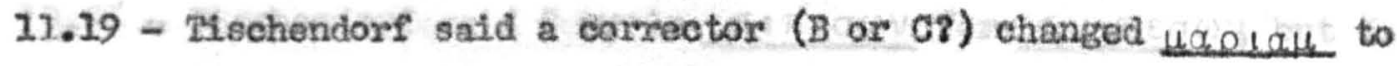
Hapiav, but I see no evidence of any change. I an cortain he is wrong. Curlously enough, Mtschendorf does not mention in 18 the corrector of codex $C$ as a witness for $\mu a p ı$ L.

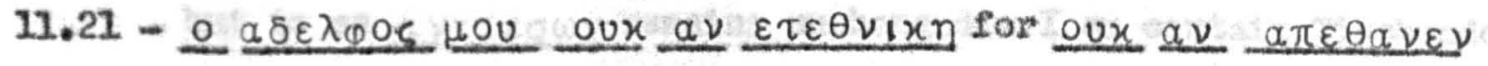
으 $\alpha_{\delta \varepsilon \lambda \varphi O S} \mu O U$.

11.2h - omit 끄 bafore $\underline{\mu \alpha \rho \theta a .}$

$11.29-\underline{\varepsilon \gamma \eta \rho \varepsilon \tau \varepsilon}$ and $\varepsilon \rho \times \varepsilon \tau \varepsilon$ for $\underline{\eta \gamma \varepsilon \rho \theta \eta}$ and $\eta \rho x \varepsilon \tau 0$.

11.31 - Haplav for uaglau.

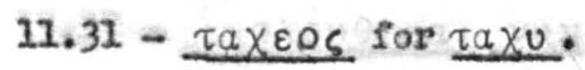

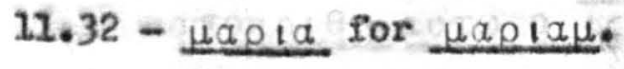

11.32 - add 으 before $\overline{I S}$.

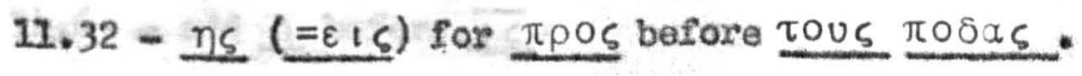

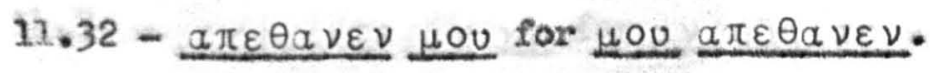

11.38 - $\varepsilon \mu \beta \rho 1 \mu \omega \mu \varepsilon \nu 0 s$ for $\varepsilon \mu \beta \rho 1 \mu n \sigma a \mu \varepsilon \nu 0 s$.

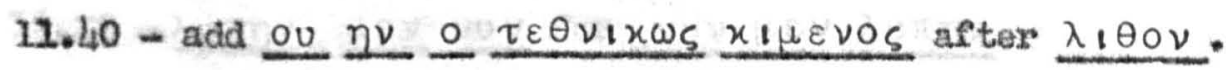

$11.43-\varepsilon x \rho a u r a \sigma \varepsilon \nu$ for $\varepsilon x \rho a \xi \varepsilon \nu$.

11.43 - add a second $\underline{\lambda \zeta \zeta a \rho \varepsilon}$ after $\underline{\lambda \zeta \zeta a \rho \varepsilon}$.

11. Wh - add $x_{1}$ before $\varepsilon \xi \eta \lambda \theta \varepsilon \nu$.

11.45 - Fiachendorf noted that $\mathrm{G}$ added $\varepsilon_{1 S}$ aujov to the text of $\mathrm{B}$ when the first corrector omitited it. But this is wrong: Els autov is by the first corrector. Iischendorf thought also that $\mathrm{C}$ may have rejected the gฤuElov added by $B$, but I see no indiation of this rejection. In 18 Mschendorf noted both $B$ and $C$ as having onue tov. 
John

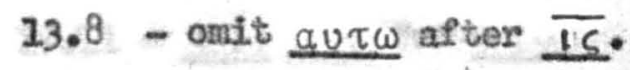

$13.10-\underline{\chi \rho \varepsilon t a \nu} \underline{\varepsilon \chi \varepsilon !}$ for $\underline{\varepsilon \varepsilon 1} \underline{\chi \rho \varepsilon t a \nu}$.

13.10 - $\underline{n}$ for $\varepsilon 1 . \mu \eta$.

13.12 - The second corrector restored the $x_{1}$ which the flrst corrector had erased before $\underline{\varepsilon \alpha \beta \varepsilon \nu}$.

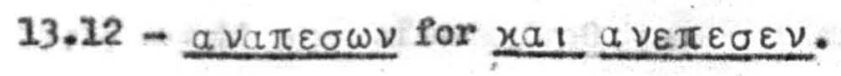

$13.20-\pi \varepsilon \mu \psi \alpha \nu \tau a$ for $\pi \varepsilon \mu \psi \alpha \tau \alpha$.

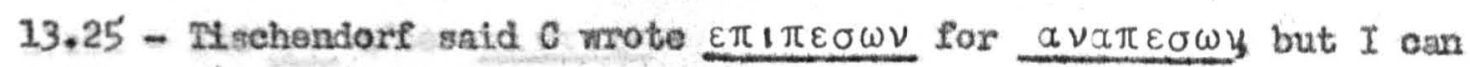
see no indications of a correction - oven with the assistance of the "pover of auggestion". It is difficult to say why Tischendorf mentioned this correction if he did not see something, but to the $\underline{\text { va } \pi \varepsilon \sigma \omega \nu}$ remains unchanged. I am cextatn Tischendore is wrong.

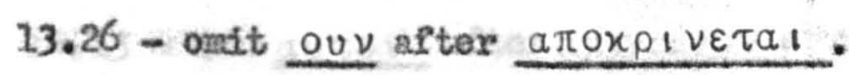

13.36 - add $\underline{a v \tau \omega}$ 으 before $\overline{15}$.

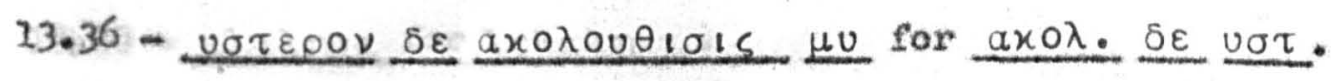

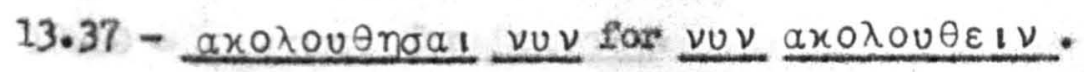

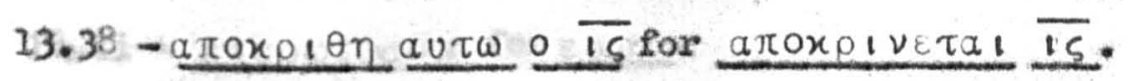

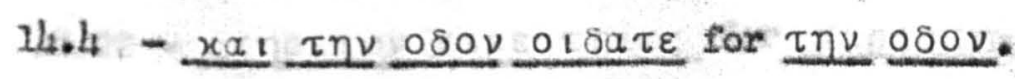

$H_{1.6}$ - add 0 before $\overline{15}$.

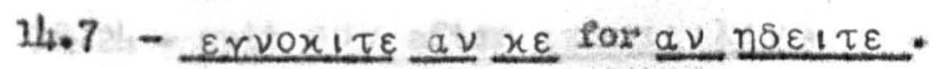

14.7 - add autov after $\varepsilon \omega p a x a \tau \varepsilon$.

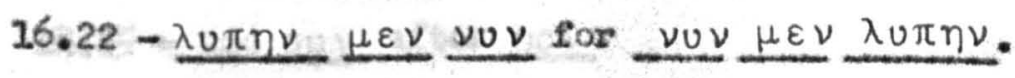

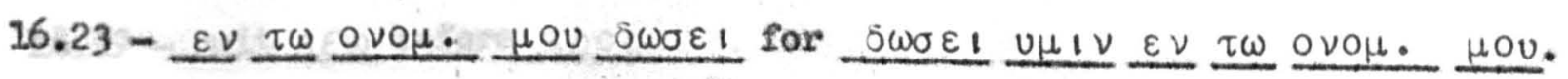
16.25 - add $\underline{a \lambda \lambda}$ before $\underline{\varepsilon \rho \varepsilon \tau \alpha_{1}}$.

$16.27-\overline{\theta v}$ for $\overline{\pi \rho s}$.

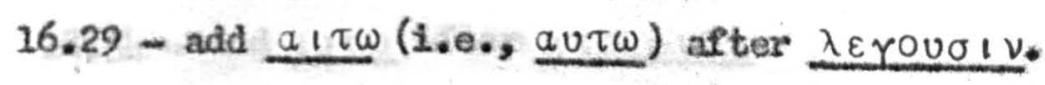

16.32 - add עuv after $x a 1$.

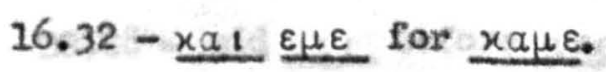

$17.1-\varepsilon \pi \eta \rho \varepsilon \nu$ for $\varepsilon \pi a \rho a s$.

17.1 - add $x \alpha_{\downarrow}$ before $\varepsilon 1 \pi \varepsilon \nu$.

17.1 - add oou after $\overline{\text { GS. }}$ 
John

$17.11-\underline{x a 1} \underline{\varepsilon \gamma \omega}$ for $\underline{x a r \omega}$.

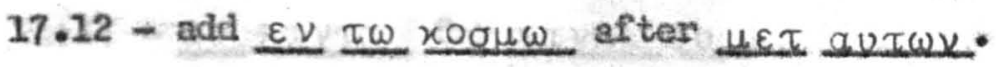

17.12 - ous for $\omega_{\text {. }}$. In his Appendix Tischendorf credited this to $\mathrm{B}_{3}$ this error has been corrected in 29 .

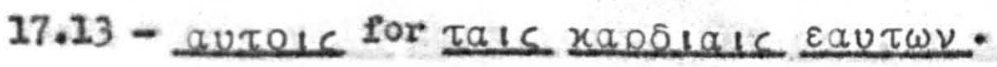

17.17 - add gov after $\underline{\alpha \lambda n \theta \varepsilon i a}$.

17.19 - $x_{1}$ avtol woiv for wolv $x a_{1}$ gever.

17.22 - add $\varepsilon \nu$ after $\pi \alpha \nu \tau \varepsilon s$.

17.21 - add $\underline{\varepsilon \nu}$ before $\omega_{01 \nu}$

17.21 - $\pi 1 \sigma \tau \varepsilon v \sigma D$ for $\pi 1 \sigma \tau \varepsilon u n$.

$17.22-\underline{x \alpha_{1}} \underline{\varepsilon \gamma \omega}$ for $\underline{x \alpha} \omega$.

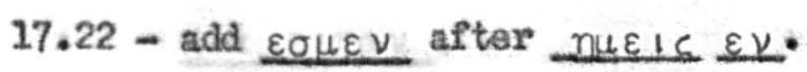

$18.4-\underline{\varepsilon \xi \varepsilon \lambda \theta 0 \nu} \varepsilon_{1} \pi \varepsilon \nu$ for $\varepsilon \xi \eta \lambda \theta \varepsilon \nu$ xal $\lambda \varepsilon v \varepsilon \perp$.

18.13 - add aucov after annvarov.

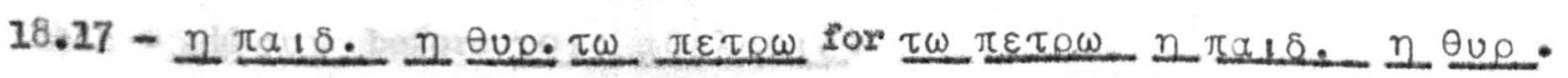

18.20 - $\varepsilon \lambda \alpha \lambda n \sigma a$ for $\lambda_{\varepsilon \lambda \alpha \lambda n x \alpha}$.

$18.20-\pi \alpha \nu \tau \omega \tau \varepsilon$ for $\pi \alpha \nu \tau \varepsilon$.

$18.21-\varepsilon \pi \varepsilon \rho \omega \tau n \sigma o v$ for $\varepsilon \rho \omega \tau$.

18.22 - $\underline{v \pi \eta \rho \varepsilon \tau \omega \nu} \pi \alpha \rho \varepsilon \sigma \tau \eta x \omega s$ for $\pi \alpha \rho \varepsilon \sigma \tau \eta x \omega \nu$ v

$18.23-0 . \overline{15}$ for $\overline{15}$.

18. 24 - ouit ouv after $a \pi \varepsilon \sigma \tau \varepsilon \mid \lambda \varepsilon \nu$.

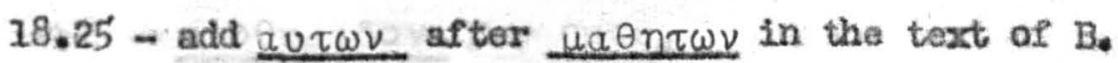

18.25 - add ouv aftar nov.

18.29 - onit $\underline{\varepsilon \xi \omega}$ before $\underline{\pi \rho 0 S}$.

18.30 - $x a x 0 \pi 01 \omega c$ (not $-0 c$ as 74 sehendorf noted) for $-\pi \omega \nu$.

18.31 - add o before $\pi \varepsilon \mid \lambda \alpha \tau O S$.

18. 34 - add $\underline{\alpha u \tau \omega}$ after $\underline{a \pi 0 x p 1 \theta n .}$

$18.34-\sigma \varepsilon \varepsilon 1 \pi 0 v$ for $\varepsilon 1 \pi 0 \nu$ o01.

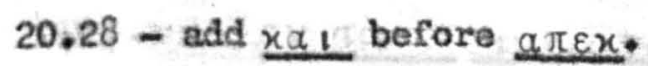

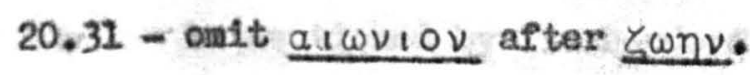

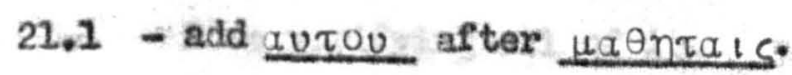

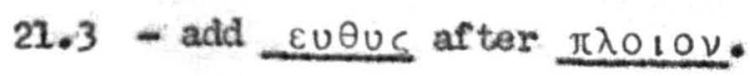


John

21.5 - omit ouv aftex $\underline{\lambda \varepsilon r \varepsilon 1 .}$.

21.17 - $\sigma u$. $\frac{\pi \nu \tau \alpha}{\text { for } \pi \alpha \nu \tau \alpha} \sigma \nu$.

21.18 - $0 \tau \varepsilon$ for $0 \tau_{1}$.

21.20 - add $\overline{\overline{x \varepsilon}}$ before $\tau i s$.

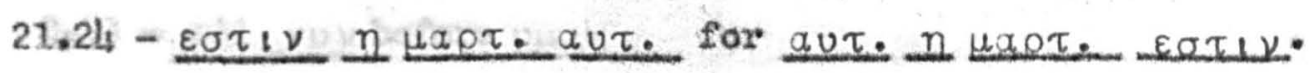

21.25 - add a $\mu \eta \nu$ (It cannot be ascertained which corrector added and then erased this word. It was not included by the original scribe.

\section{Acts}

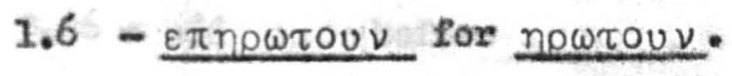

1.8 - add $\underline{\varepsilon \nu}$ before $\pi a \sigma r$.

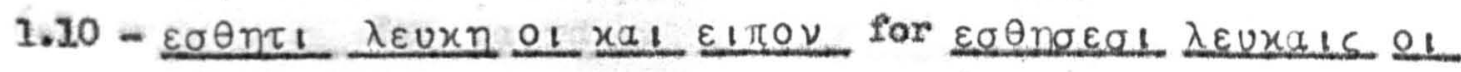
$\underline{x a} \underline{\varepsilon i \pi a \nu}$.

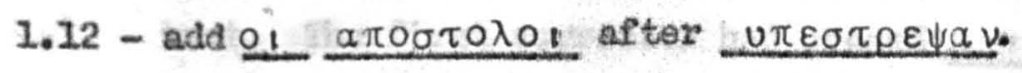

1.14 - add $x a_{1} \tau_{t} \delta \varepsilon l \sigma \varepsilon l$ after $\pi 000 \varepsilon u \times \eta$.

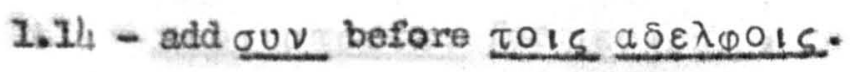

1.15 - add $\mu \alpha \theta \varepsilon \tau \omega \nu$ before $q \delta \varepsilon \lambda \omega \omega \nu$.

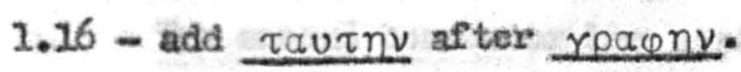

1.16 - add $\tau$ ov before $\overline{\perp \nu}$.

$1.21-\underline{\varepsilon \nu}$ for $\omega$.

$1.25-\underline{x \lambda 100 \nu}$ for zomov.

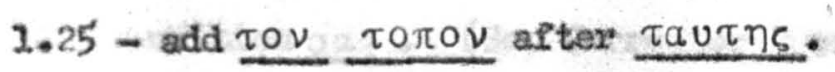

2.1 - $0 \mu 0 \theta u \mu \alpha \delta 0 \nu$ for opov.

$2.3-\underline{\tau}$ for $\underline{\delta \varepsilon}$.

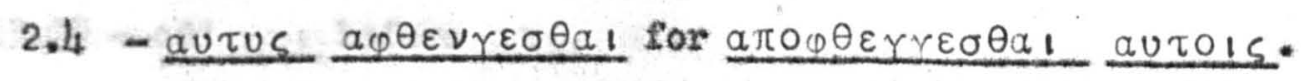

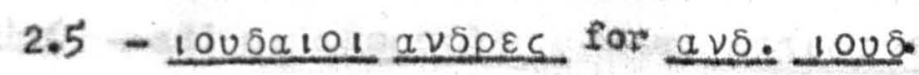

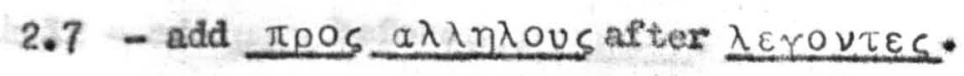

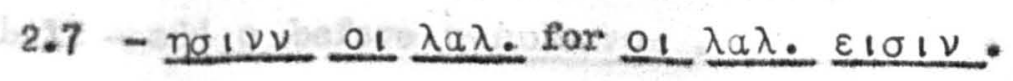

$2.9-\underline{\varepsilon \lambda \alpha \mu \eta \tau a_{1}}$ for $\underline{\varepsilon \mu \alpha \lambda \varepsilon_{1} \tau a_{1}}$.

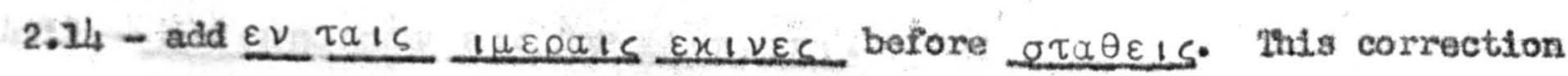
is mentioned by Tischendorf. The present binding prevents me from verifying all of it. 
Acts

2.22 - add $2 \alpha_{1}$ before autol.

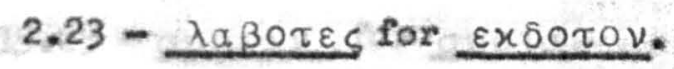

$2.23-\not \varepsilon i p o v$ for $x \varepsilon_{1}$ -

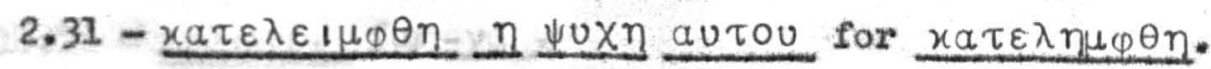

2.33 - add vuv before unts.

$2.36-\varepsilon \pi \omega_{1 n \sigma \varepsilon \nu}$ for $\varepsilon \pi 0 \eta \eta \varepsilon \nu$.

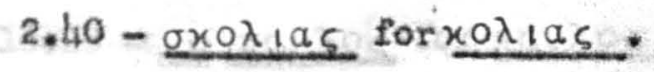

$3.19-\varepsilon \pi 1 \sigma \tau \rho \varepsilon \psi \alpha \tau \varepsilon$ for $\varepsilon \pi i \tau \rho \varepsilon \psi \alpha \tau \varepsilon$.

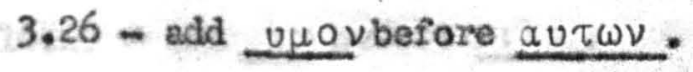

5.37 - $\pi 0 \lambda v \nu$ for $\pi 0 \lambda v$.

6.1 - Extves for tautals.

6.3 - add aviou after $\overline{\pi V S}$. This 13 a restoration of the original text. The Plrat corrector had erased aY10u.

$8.20-\pi 00 c$ for $\pi 00$.

9.3 - $\pi \varepsilon \rho 1 \varepsilon \sigma \tau \rho \varepsilon \psi \alpha \nu$ for $\pi \varepsilon \rho 1 \sigma \tau \rho$.

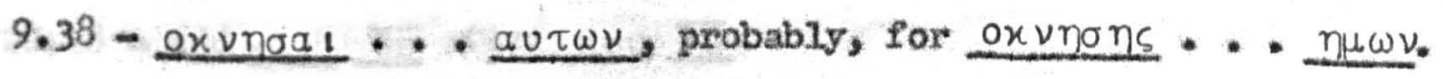
The second corrector has again reversed the judgment of the Piret corrector and restored the ortiginal text. The word $\underline{x u \omega \omega}$ is clearly witten above nuwv; the letters ons have been partially erased although $I$ ses no indieations that $C$ has written gal. (See Corrector B on this varlant.)

9.42 - add Ins after oxns.

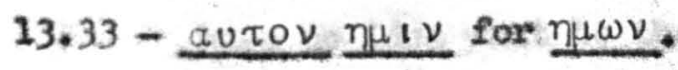

13.38 - add $\underline{x, 1}$ before a a

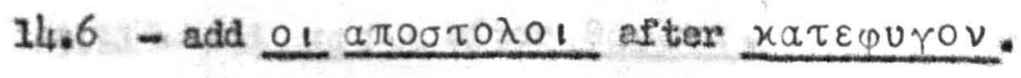

14.12 - add $\underline{\mu \varepsilon \nu}$ before $\beta a \rho \nu a \beta a \nu$.

14.12 - add o bofore nyouncvos.

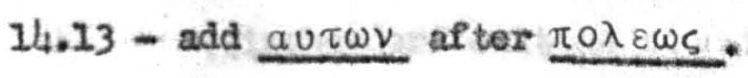

$\nu_{4.1 h}-\varepsilon t \sigma \varepsilon \pi n \delta$ ndav $\varepsilon i s$ for $\varepsilon \xi \varepsilon \pi \cdot \varepsilon \pi 1$.

$\nu_{1.17-x \alpha 1 \tau 01 v \varepsilon}$ for $x a 1 \tau 01$.

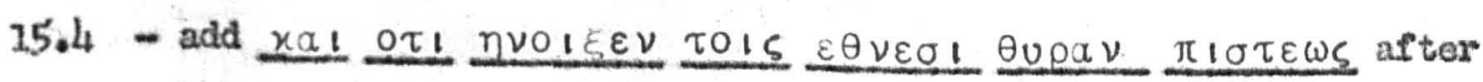

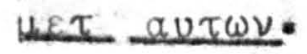




\section{Acts}

$15.23-\pi \varepsilon_{01 \varepsilon \times 0 u \sigma a v}$ for $=0$.

15.23 - ouplav for guola.

$15.2 h_{4}-\varepsilon \xi \varepsilon \lambda \theta 0 \nu \tau \varepsilon \zeta$ for $-\theta 0 \tau \varepsilon s$.

$16.16-\pi u \lambda \omega v o s$ for $-v a$.

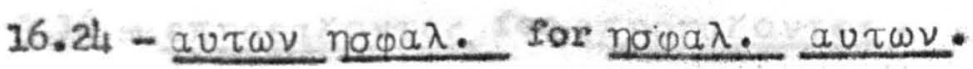

$16.27-\underline{\alpha \nu \varepsilon \beta \varepsilon t \nu}$ for $a \nu \varepsilon \lambda \varepsilon 1 \nu$.

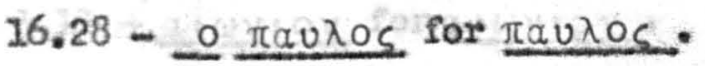

$16.29-\gamma \varepsilon \nu 0 \mu \varepsilon \nu \omega s$ for $u \pi \alpha \rho X \omega \nu$.

16.29 - add $\tau \omega$ before $\sigma 1 \lambda \alpha$.

20.10 - Probably $\lambda a \beta \omega \nu$ for $\beta a \lambda \omega \nu$. This is the conjecture of Tischendorf, it seems most Ifkely. Only $\lambda \omega \nu$ is extant at the beginning of the page, but it appears to have been rejected by $c_{*}$

20.12 - I see no indleation that $C$ rejected the $S$ of $a x p \varepsilon$ is as Tischendorf has sald.

20.16 - Expnvev for $x \varepsilon x \rho 1 x \varepsilon 1$.

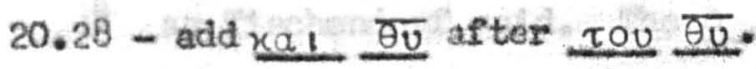

20.29 - yae o.

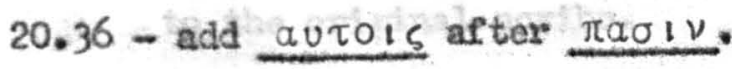

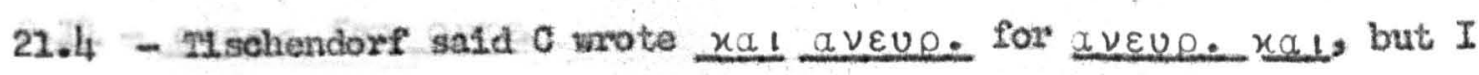
see no evidence of any change. The particle $\delta \varepsilon$ is not rejectod in the usual manner of $\mathrm{C}$, and the nargin gives no Indication that xal has been written. I an cortain Tischendorf is wrong, but I have no Idea what he thought he saw to suggest this change.

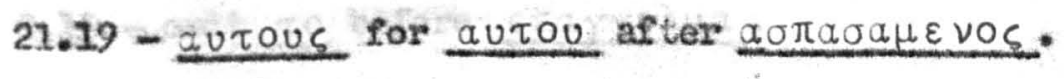

23.11 - add $\pi a v \lambda \varepsilon$ aftor $\theta a \rho \sigma \varepsilon 1$.

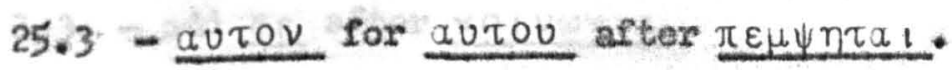

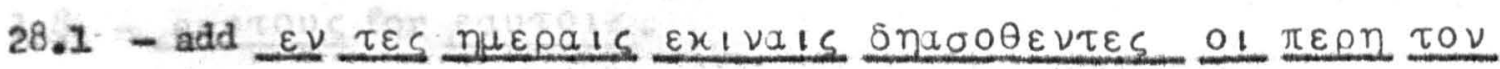

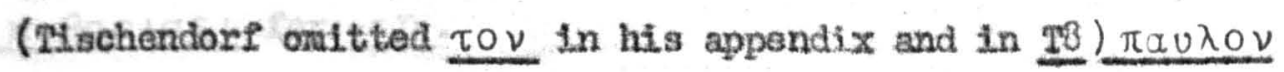

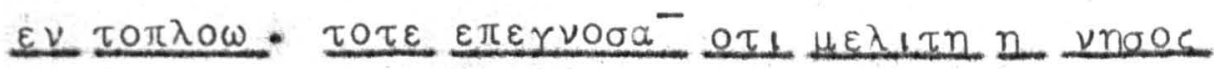
$x \alpha \lambda \cap \tau \varepsilon$. 
James

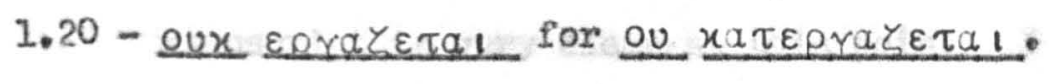

3.17 - $\varepsilon u \pi \varepsilon 1 \theta n s$ for $\varepsilon u \pi \eta \theta n s$.

3.18 - $\sigma \pi \varepsilon ! \rho \varepsilon \tau a_{1}$ for $0 \pi \varepsilon ! p \alpha_{1} \tau a_{1}$.

I Peter

3.16 - $\varepsilon \pi n \rho \varepsilon a \zeta o \nu \tau \varepsilon S$ for $\varepsilon \pi n \rho n a \zeta o \nu \tau \varepsilon \zeta$.

II Peter

1.11 - alwviov for alwviav.

1.17 - The second corrector has written $\varepsilon / 5$ 이 $\underline{\varepsilon y \omega}$ at the end of Iine 2. Whether this amounts to a change in the text I do not lnow. Hy own impression is that he wrote at the end of line 2 because they were not clearly legible at the beginning of line 3 . See Appendix A for further discussion of the text at this point.

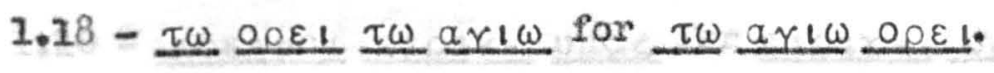

$2.1-\pi a \rho \varepsilon l \sigma a \xi o v \sigma i v$ for $\pi a \rho \varepsilon l a \xi o v \sigma i v$.

2.3 - ols for $\varepsilon 15$. I an not certain that A corrected his own text as Tischendorf said. The 0 has been written very lightly over the $\underline{\varepsilon}$. I know of no reason to credit this type of correction to the original scribe.

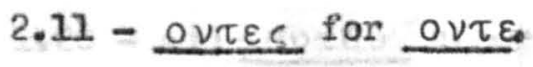

2.16 - $\overline{a \overline{V O U}}$ for, probably, $\overline{\text { avouv }}$. I do not think this erased letter whose vertical strokes can be seen - was the article $\underline{\nu}$ (for $\underline{\tau \eta}$ ) before $Q \omega \nu \eta$, but it may give a hint of the scribe's exemplar.

3.3 - $\varepsilon \sigma x a \tau \omega \nu$ for $\varepsilon \sigma \times \alpha \tau \omega$.

3.13 - omit to before $\varepsilon \pi a Y \gamma \varepsilon \lambda \mu \alpha$.

I John

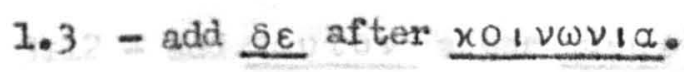

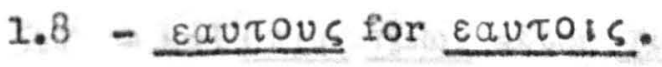

$1.8-x_{a \theta a p l \sigma \varepsilon_{l}}$ for $-\sigma \eta$.

Romans

3.25 - add $\tau \eta$ s before $\pi 10 \tau \varepsilon \omega s$.

4.1 - $\overline{\pi \rho \alpha}$ for $\pi \rho 0 \pi \alpha \tau o \rho \alpha$.

$4.18-\underline{\varepsilon \varphi}$ has not been changed to $\underline{\varepsilon \pi}$ as Tischendorf stated. The 


\section{Romans}

manuscript is very clear at this point. I have no idea what Tis chendorf had in mind.

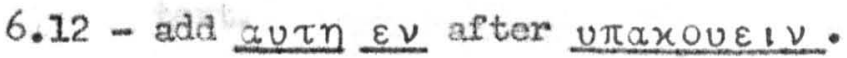

8.11 - autou for autol after \&volnouvtos.

8.25 - add o before ou $\beta \lambda \varepsilon \pi 0 \mu \varepsilon \nu$.

13.12 - $x \alpha+\varepsilon \nu \delta v \sigma$. for $\varepsilon \nu \delta v \sigma . ~ s \varepsilon$.

14.7 - add nuov after ouseis.

$y_{4.9}$ - add $x_{1}$ before $\overline{\chi_{s}}$.

16.10 - $\overline{x \omega}$ for $\overline{\chi \omega}$.

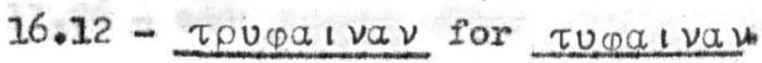

I Corinthians

1.9 - add 으 before $\overline{\theta s}$.

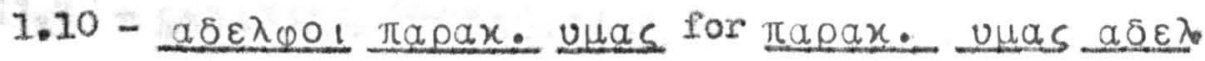

1.15 - $\varepsilon 1 \pi \eta$ for $\varepsilon 1 \mu \eta$. Tischendorf said A made this correction hinself, but I disagree. It seens to have been corrected in a very careless, unconcerned manner.

$1.15-\underline{\varepsilon \beta a \pi \tau i \sigma a}$ for $=\tau 1 \sigma \theta \eta \tau \varepsilon$.

$1.19-\underline{\sigma U \nu \varepsilon \tau \omega \nu}$ for $\underline{\underline{U} \nu \varepsilon \tau \omega}$.

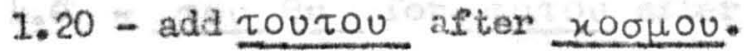

$1.23-\varepsilon \lambda \lambda$ no $1 \nu_{\text {for }} \varepsilon \theta \nu \varepsilon \sigma 1 \nu_{0}$

1.28 - add $\underline{x a}$ before $\tau a \mu \eta$ ov.

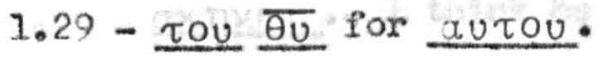

$3.1-\operatorname{san} 1 \times 015$ for - Vols.

3.10 - $\tau \varepsilon \theta 1 x \alpha$ for $\varepsilon \theta \eta x \alpha$.

3.11 - add $\overline{15}$ after $\overline{\chi_{s}}$.

3.12 - add toutov after $\theta \varepsilon \mu \varepsilon \lambda_{10}$

5.7 - add $\underline{u} \varepsilon \rho \rho \underline{\eta \omega \nu}$ after $\underline{\mu \omega \nu}$.

$5.8-a \lambda n \theta \varepsilon 1 a s$ for $-\theta 1 a s$.

6.12 - add $\mu 0_{1}$ after $\pi a v \tau a$ (third). The second corrector has added something at this point. Black spots make it impossible to decipher.

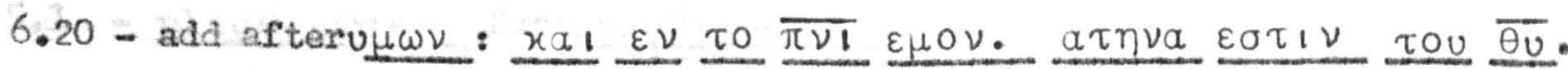


I Corinthians

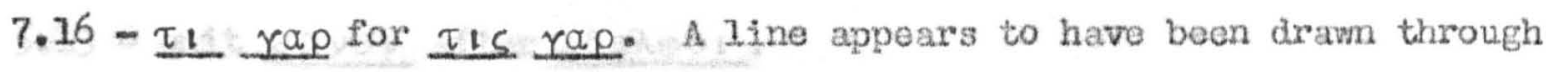
the sigma. Thischendorf erred in witing tI yap for the original text.

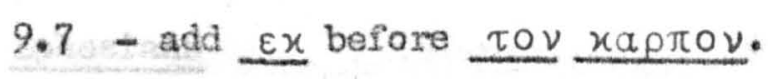

10.22 - add $\mu 01$ after $\pi \alpha \nu \tau \alpha$ (second).

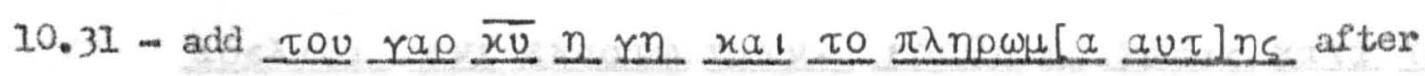
$\pi 01 \varepsilon+\tau \varepsilon$.

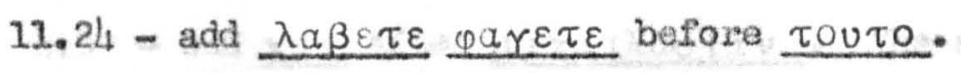

11.24 - add $x \lambda 0 \mu \varepsilon v o \nu$ after $\underline{\mu \omega \nu}$.

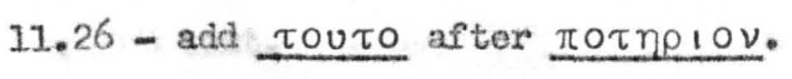

11.29 - add $\tau$ ou $\overline{x u}$ after $\sigma \omega \mu$.

$12.9-\operatorname{add} \underline{\varepsilon \nu} \tau \omega \underline{\alpha u \tau \omega} \overline{\pi \nu !}$ after $\underline{1 \alpha \mu \alpha \tau \omega \nu}$.

$13.8-\underline{\varepsilon x \pi 1 \pi \tau \varepsilon_{1}}$ for $\tilde{1}_{1 \pi \tau \varepsilon_{1}}$.

15.47 - xo1xos for $40 \times 1 \times 05$.

16.22 - add IV $\overline{X \nu}$ after $\overline{x \nu}$.

II Corinthians

1.21 - add 0 after $\overline{\theta_{c}}$.

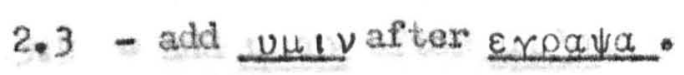

4.6 - tov $\overline{\theta u}$ for autou after sogns.

4.11 -

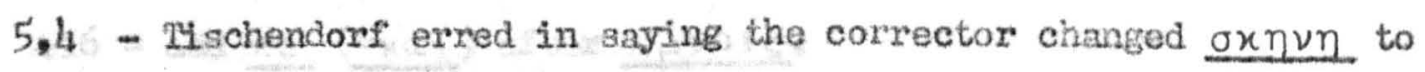
gxnve . I think he mistakenly took a mark of the upper text of Iphrain to be a correction.

$5.6-\gamma \nu \omega \sigma \varepsilon+$ for $r \nu \omega s$.

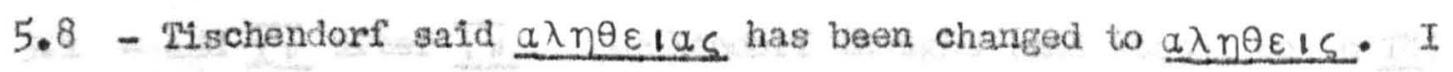
see no ovidence of any change.

$9.10-\underline{\pi \lambda \eta \theta u \nu \varepsilon_{1}}$ for $\pi \lambda \eta \theta u v \eta$.

Galatians

$4.7-\overline{\theta u} \underline{\delta \eta} \overline{\overline{x u}}$ for $\underline{\delta 1 \alpha} \overline{\underline{\partial u}}$.

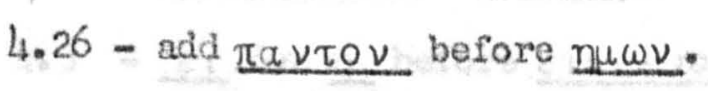

5.1 - add ouv after $\underline{\varepsilon \varepsilon v \theta \theta \varepsilon \rho ı \text {. }}$.

5.1 - $\eta \lambda \varepsilon v \theta \varepsilon \rho \omega \sigma \varepsilon \nu$ for $\varepsilon \lambda \varepsilon v \theta \varepsilon \rho \omega \sigma \varepsilon \nu$. 
Galatians

5.13 - onit $\mu 0 v 0 \nu$ after $\alpha \delta \varepsilon \lambda \varphi 01$.

6.14 - add $\tau \omega$ before $\underline{x \sigma o \mu \omega}$.

6.17 - add $\overline{x u}$ before $\overline{10}$.

Ephesians

4.7 - The following is added in the margin by the second corrector:

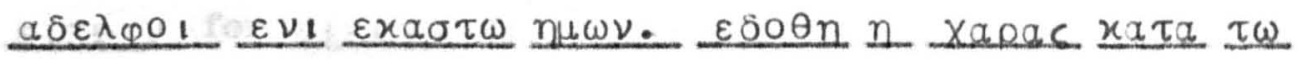

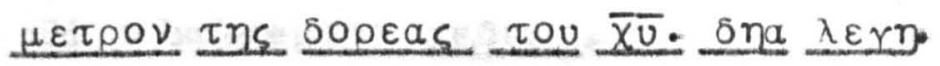

4.8 - add $\underline{x a}$ after $\underline{1} \mid x_{\mu \alpha \lambda \omega \sigma l a \nu}$. The second corrector reverses the verdict of the first corrector and restores the original text.

4.9 - add $\pi \rho \omega \tau o \nu$ after $\underline{x \alpha \varepsilon \beta \eta}$.

Philipuians

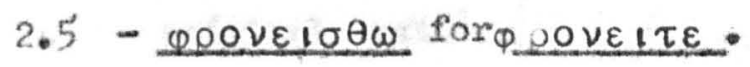

Colossians

1.12 - EuXaplotoujev (not $-\mu \varepsilon$ as Tischendorf said here and in 18 .

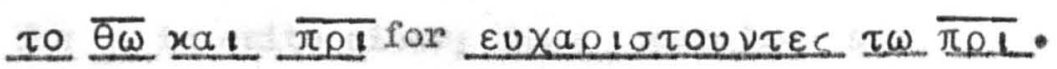

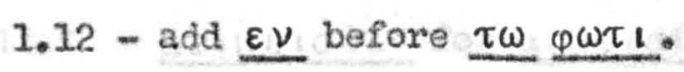

3.5 - add $\underline{u \mu \omega \nu}$ after $\mu \varepsilon \lambda \eta$.

3.6 - add $\eta$ before oprn.

3.16 - $81 \delta a \sigma x o v \tau \varepsilon s$ for $\delta 1 \delta a x o v \tau \varepsilon s$.

3.16 - add $x \varepsilon\left(=x \alpha_{1}\right)$ after upvols.

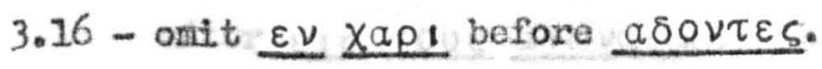

Hebrews

2.13 - See Appendix B.

3.1 - add $\overline{X \nu}$ after $\overline{I V}$. Tischendorf thought (and stated so in T8) that this addition might have been by $B$. But the miting is that of $\mathrm{C}$ and the horizontal line for the nomina sacra is his also (i.e.,, ).

3.4 - add $\tau a$ before $\pi \alpha \nu \tau \alpha$.

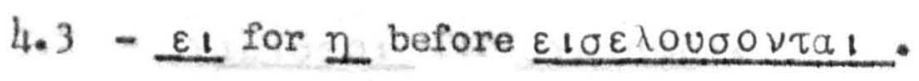

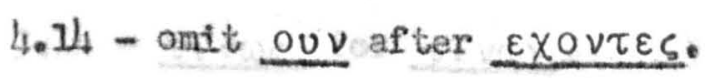


Hebrews

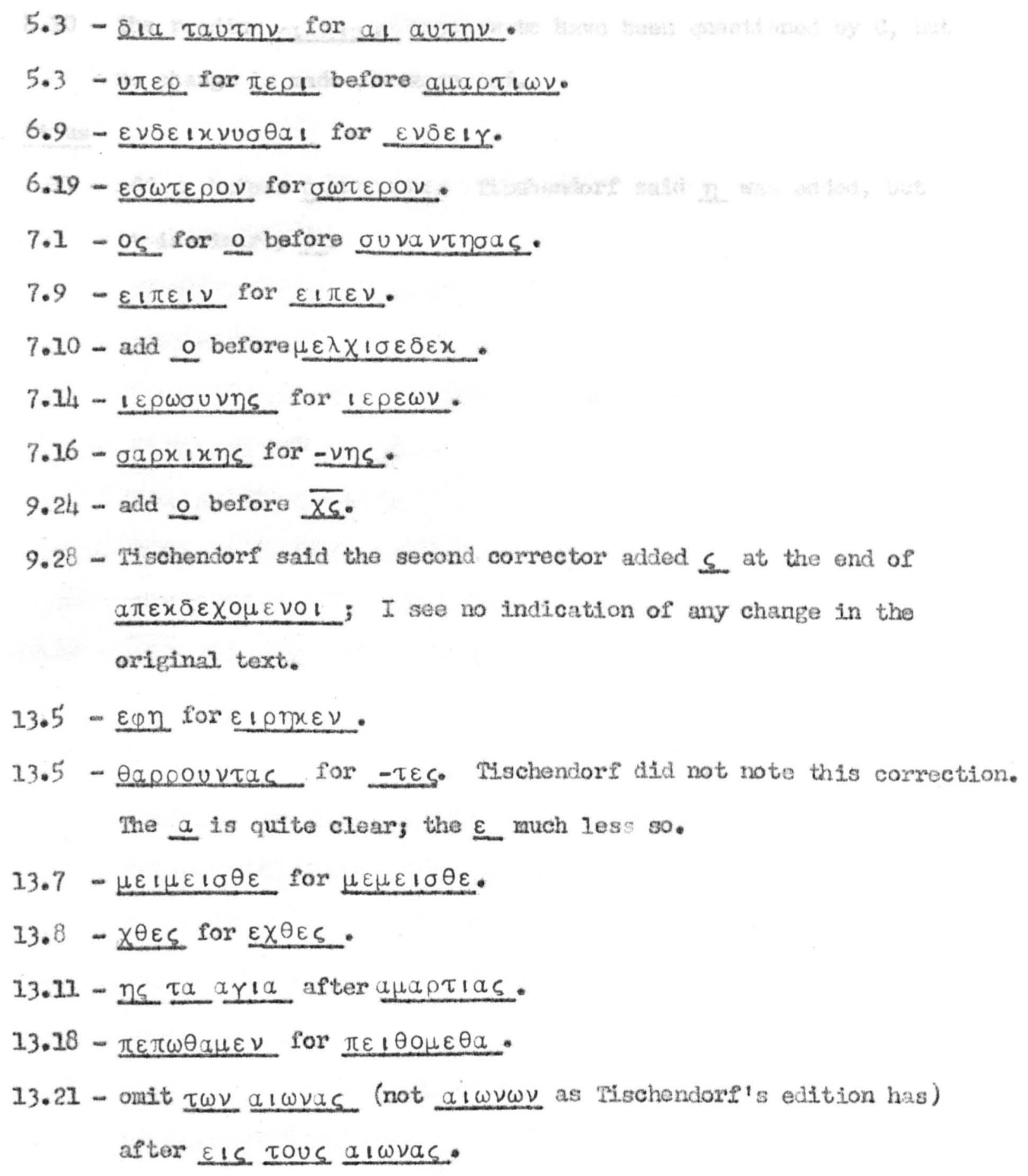
a $\pi \varepsilon \times \delta \varepsilon \times 0 \mu \varepsilon v 01 ;$ I see no indication of any change in the original text.

$13.5^{\prime}-\underline{\varepsilon \varphi \eta}$ for $\varepsilon 1 \rho \eta x \varepsilon \nu$.

13.5 - - a poouvtac for $-\tau \varepsilon s$. Tischendorf did not note this correction. The $\underline{a}$ is quite clears the $\varepsilon$ much less so.

$13.7-\mu \varepsilon \downarrow \mu \varepsilon 1 \sigma \theta \varepsilon$ for $\mu \varepsilon \mu \varepsilon \downarrow \sigma \theta \varepsilon$.

$13.8-\chi \theta \varepsilon s$ for $\varepsilon \chi \theta \varepsilon s$.

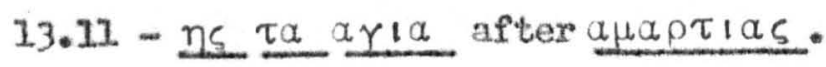

13.18 - $\pi \varepsilon \pi \omega \theta \alpha \mu \varepsilon \nu$ for $\pi \varepsilon 1 \theta 0 \mu \varepsilon \theta \alpha$.

13.21 - omit $\tau \omega \nu \underline{a l \omega \nu \alpha s}$ (not $\underline{\alpha \omega \nu} \omega \nu$ as Tischondorf's edition has) after Els tous alwvas.

I Timothy

3.12 - onit rap before $x a \lambda \omega s$.

II Minothy

$1.3-\underline{\lambda \alpha \tau \rho \varepsilon v \omega}$ for $\lambda \alpha \tau \rho \varepsilon \cup \omega \nu_{6}$

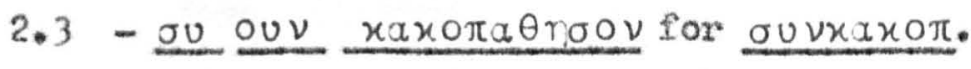

2.7 - $\delta \omega \varepsilon_{1}$ for $\delta \omega \varepsilon_{1}$.

$2 . I_{4}-\underline{\lambda o \gamma o \mu a x \varepsilon+\nu}$ for $-\chi \varepsilon_{d}$.

3.2 - $x \times a p 10 \tau 01$ for $a x p 10 \tau 01$.

$3 . \nu_{1}-\tau_{1} \nu \omega c$ for $\tau_{1} \nu \omega \nu$. 


\section{IImothy}

4.10 - The reading $y a \lambda \lambda \downarrow a \nu$ appears to have been questioned by $C$, but no change is rade or suggestad.

\section{Titcus}

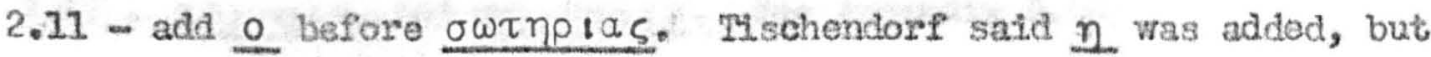
it is clearly 으. 


\section{Matthew}

$2.23-\underline{\text { sta, not } \underline{u} \pi 0 .}$.

v 7.12 - $\pi_{0} 1 \omega \sigma \downarrow \nu$, not $\pi 010001 \nu$. See Appendix B.

$v 8.6-\underline{\delta 1 \nu \omega s}$, not $\delta \varepsilon 1 \nu \omega s$.

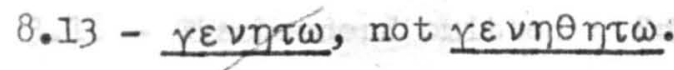

V8.17 - noata10u, not noa 1ouv.

8.31 - not $\underline{\delta \mu \mu \nu \varepsilon s, ~ b u t, ~ p r o b a b l y, ~ \delta \varepsilon 1 \mu o v \varepsilon s . ~}$

$8.34-\varepsilon \xi \eta \lambda \theta 0 \nu$, not $\varepsilon \xi \eta \lambda \theta \varepsilon \nu$.

$9.12-x \rho 1 \alpha \nu$, not $x \rho \varepsilon 1 a \nu$.

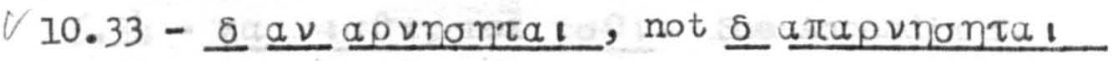

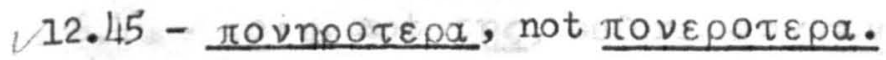

212.48 - $\underline{\mu \eta \rho}$, not $\overline{\mu \eta} \rho \underline{\mu O U}$ See Appendix B.

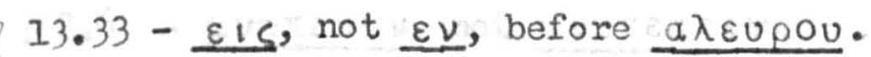

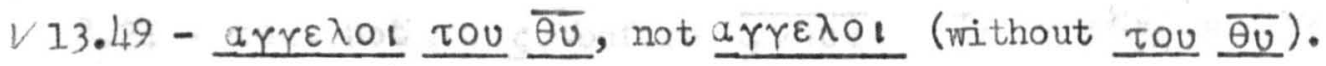

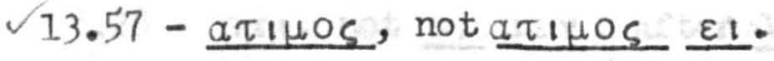

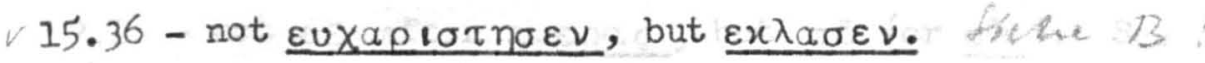

$16.22-\underline{\varepsilon \pi 1 \tau 1 \mu 1 \alpha \nu}$, not $\varepsilon \pi 1 \tau 1 \mu \alpha \nu$.

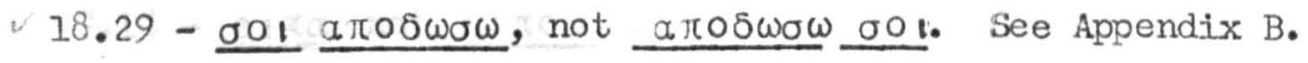

$19.16-\underline{\sigma \times \omega}$, not $\underline{\varepsilon \times \omega}$.

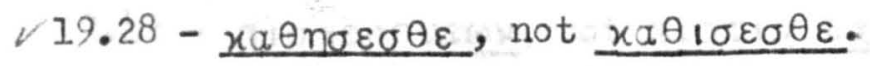

$\checkmark 20.32-\theta \varepsilon \lambda \varepsilon i s$, not $\theta \varepsilon \lambda \varepsilon \tau \varepsilon$.

$21.10-\varepsilon \sigma 1 \sigma \theta \eta$, not $\underline{\varepsilon \sigma \varepsilon 1 \sigma \theta \eta}$.

$\checkmark 21.21$ - opel, not $\omega \rho \varepsilon 1$.

$21.23-\underline{\varepsilon \lambda \theta 0 \nu \tau o \zeta}$, not $\underline{\varepsilon \lambda \theta 0 \nu \tau \varepsilon \zeta}$

$21.28-\underline{\varepsilon 1 \pi \varepsilon \nu}$, not $\underline{\varepsilon 1 \times \varepsilon \nu .}$

$21.28-\underline{\pi \rho \omega}$, not $\pi \rho \omega \tau \omega$

24.8 - $\omega \delta \varepsilon \uparrow \nu \omega \nu$, not $\omega \delta 1 \nu \omega \nu$.

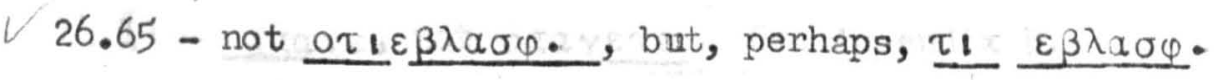

$27.5-\underline{\alpha \nu \varepsilon X \omega \rho \eta \sigma \varepsilon \varepsilon}$, not $\underline{\alpha} \pi \varepsilon \chi$.

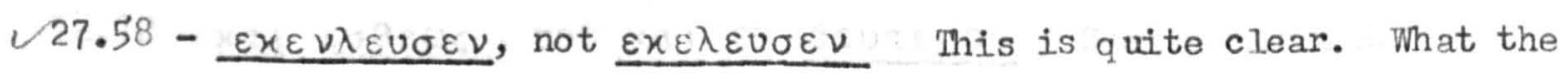
scribe thought he was writing is certainly not clear. 


\section{Matthew}

$28.5-\underline{\phi \beta \varepsilon_{1} \sigma \theta \alpha_{1}}$, not $-\theta \varepsilon$.

$28.10-\underline{\phi 0 \beta \varepsilon 1 \sigma \theta \alpha_{1}}$, not $\underline{-\theta \varepsilon}$.

Mark

1.20 -

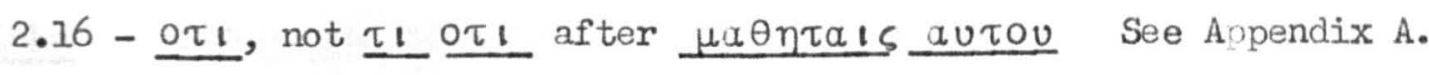

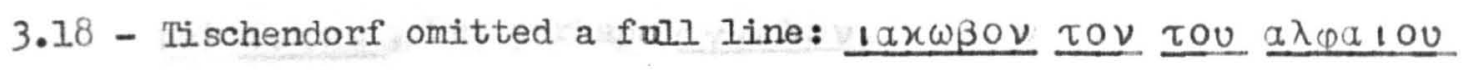

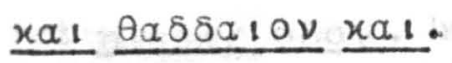

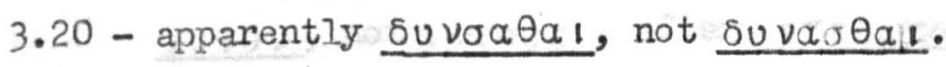

$4.31-\underline{x o x x \omega}$, not $x$ oxxov. See Appendix A.

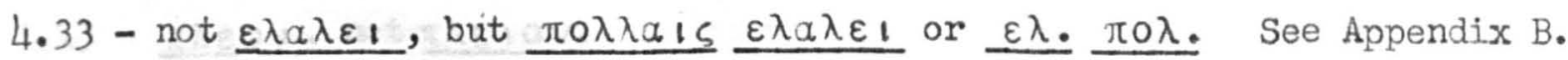

$5.34-\underline{x a l} 1 \sigma \theta_{l}$, not $\underline{1 \sigma \theta_{l}}$. See Appendix B.

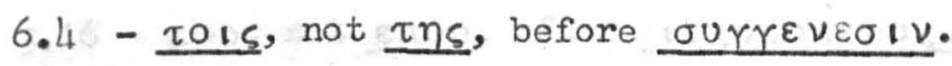

$6.13-\underline{\eta \lambda 1 \varphi O \nu}, \operatorname{not} \varepsilon \lambda_{1} \varphi O \nu$.

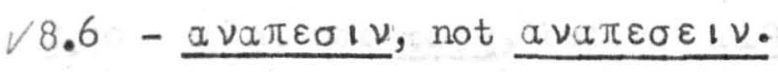

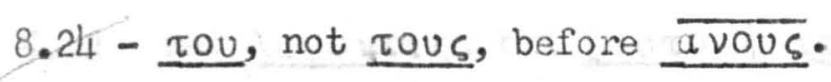

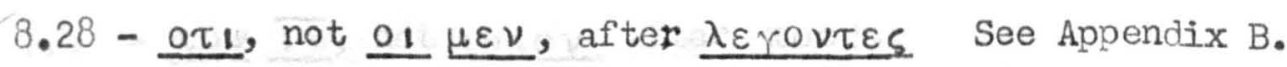

9.2 - not $\underline{\varepsilon \xi}$ after $\underline{\eta \varepsilon \varepsilon \rho \alpha_{\zeta}}$, but $\underline{\sigma \delta}$ (or $\underline{\sigma \lambda}$ ). See Appendix A.

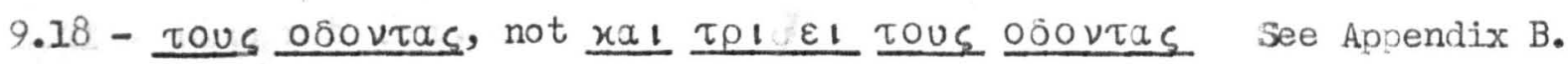

$9.18-\underline{\varepsilon_{1} \pi \alpha}$, not $\varepsilon_{1 \pi 0 \nu}$.

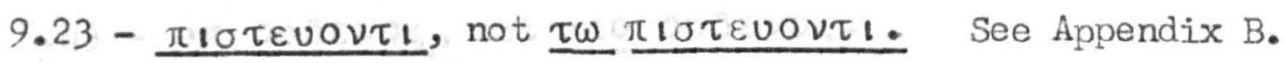

9.42 - $\pi 1 \sigma \tau \varepsilon v 0 \nu \tau \omega \nu$, not $\pi 1 \sigma \tau 1 \nu \underline{\varepsilon \chi 0 \nu \tau \omega \nu}$. See Appendix B.

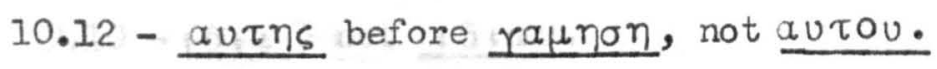

10.39 - $\beta a \pi \tau 1 \sigma \theta n \sigma \varepsilon \sigma \theta \alpha \iota$, not $-\theta \varepsilon$.

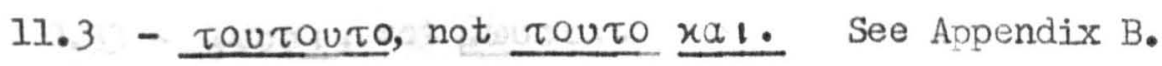

11.28 - $\tau_{15}$ o01, not $\tau_{1}$ g01.

$12.14-\varepsilon 1 \pi \varepsilon$ not $\varepsilon 1 \pi 0 \nu$ ouv.

13.31- $-\pi \alpha \rho \varepsilon \lambda \varepsilon v \sigma o \nu \tau \alpha_{1}$ not $-\sigma \varepsilon \tau \alpha_{1}$.

14.8 - not $\underline{a 1 \tau \eta}$ but $\underline{\text { aun }}$

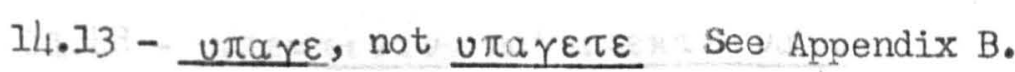

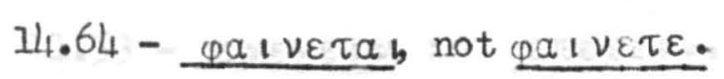

14.72 - $\underline{x a_{1}} \underline{\varepsilon v \theta \varepsilon \omega_{S}}$, not $\underline{x a_{1}} \underline{\varepsilon x} \underline{\delta \varepsilon v \tau \varepsilon p o v}$ See Appendix B. 
Mark

$15.10-\pi \alpha \rho \alpha \delta \varepsilon \delta \omega x 1 \sigma a x$, not $-x \varepsilon 10 \alpha \nu$.

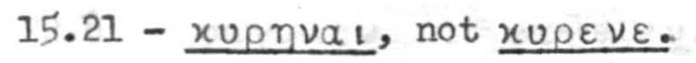

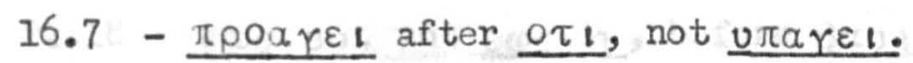

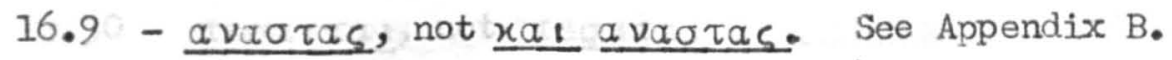

Luke

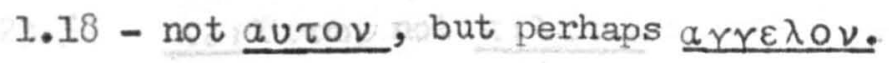

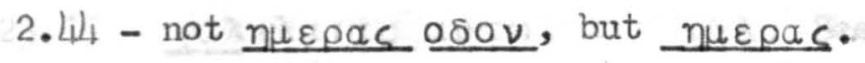

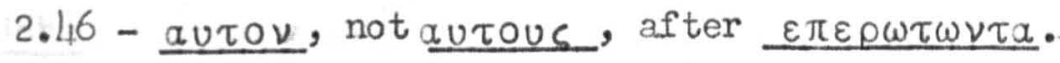

2.51 - not $\underline{x \alpha \tau \varepsilon \beta \eta} \underline{\mu \varepsilon \tau} \underline{\alpha u \tau \omega \nu}$, but $\underline{x a \tau \varepsilon \beta \eta}$.

$3.13-\underline{\pi \lambda \varepsilon o \nu}, \operatorname{not} \underline{\pi \lambda \varepsilon 10 \nu}$.

$3.14-\underline{\varepsilon \pi n \rho \omega \tau \omega \nu} \delta \varepsilon ;$ not $\varepsilon \pi \eta \eta \omega \tau \eta \eta \alpha \nu$

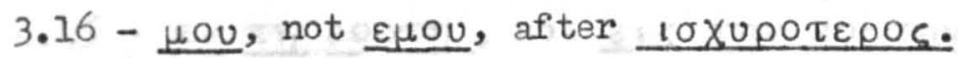

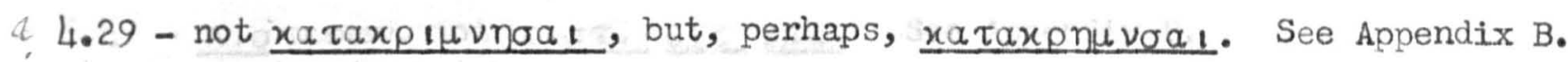

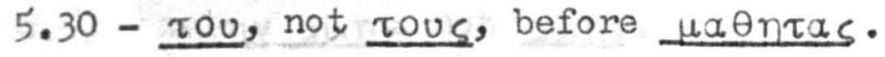

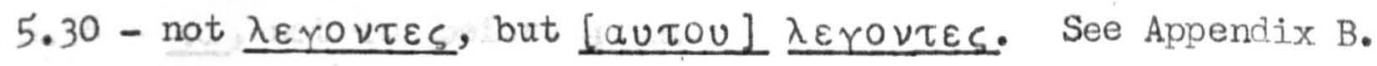

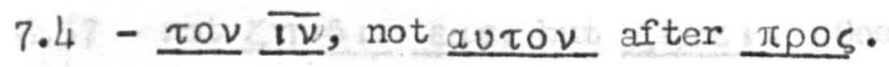

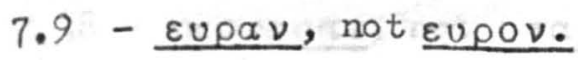

8.31 - 1ـa, not 1va.

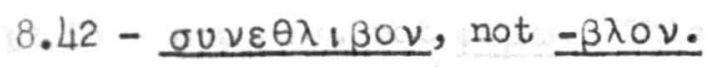

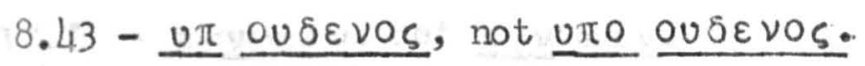

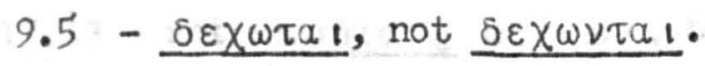

$9.10-181 \alpha$, not $181 \alpha v$.

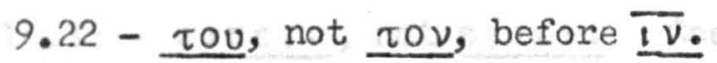

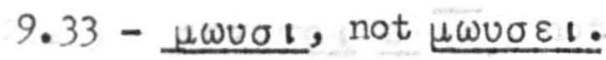

9.42 - $\pi \rho \circ \sigma \varepsilon \cup X 0 \mu \varepsilon \nu \circ u$, not $\pi \rho \circ \sigma \varepsilon \rho X$.

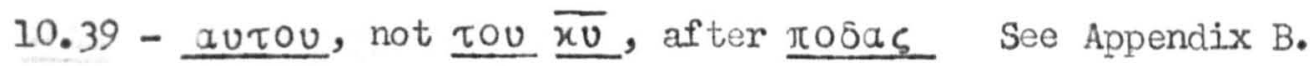

11.42 - $\alpha \lambda \lambda a$, not $\underline{\alpha \lambda \lambda}$, before oval.

$11.46-\underline{\varepsilon \nu 1}$, not $\underline{\varepsilon \pi l}$, before $\delta a x \tau u \lambda \omega \nu$.

$11.48-a \pi \varepsilon x \tau 1 v a v$, not $a \pi \varepsilon x \tau \varepsilon ! v a v$.

$11.53-\varepsilon \nu \varepsilon X \varepsilon t \nu$, not $\varepsilon \pi \varepsilon x \varepsilon 1 \nu$.

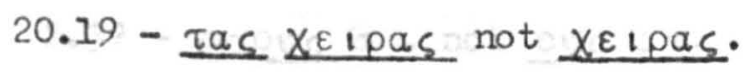


Luke

20.20 - autou, not autov before norov.

22.8 - $\varphi \omega \gamma \omega \mu \varepsilon \nu$, not $\operatorname{\omega ar\omega \mu \varepsilon \nu }$.

22.18 - $\underline{\varepsilon} \omega_{S} \underline{\text { ou}}$, not $\underline{\varepsilon \omega_{S}} \underline{\eta}$, before $\underline{\beta a \sigma i \lambda \varepsilon ! \alpha}$. See Appendix B.

$23.30-\pi \varepsilon \alpha_{1} \tau \alpha_{1}$, not $\pi \varepsilon \sigma \alpha \tau \alpha_{1}$

23.43 - $\pi \alpha \rho \alpha \delta 1 \sigma \omega$, not $\pi \alpha \rho a \delta \varepsilon 1 \sigma \omega$.

$23.52-\pi \varepsilon_{1} \lambda \alpha \tau \omega$, not $\pi 1 \lambda \alpha \tau \omega$.

$23.55-\underline{y \lambda_{1} \lambda_{\alpha 1} \alpha_{c}} \underline{\alpha u \tau \omega}$, not $\underline{\operatorname{ra} \lambda}$. See Appendix B.

John

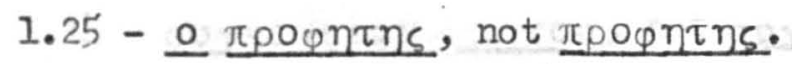

1.40 - $\tau \omega \nu \underline{a x o v \sigma \alpha \nu \tau \omega \nu}$, not axovo.

4.39 - $181 a$, not $\delta 1 \alpha$, before tov dorov.

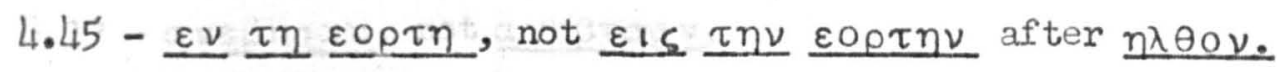

$\checkmark 6.47-\underline{\pi 1 \sigma \tau \varepsilon v \omega \nu}$, not $\pi 1 \sigma \tau$. $\varepsilon 1 s \underline{\varepsilon \varepsilon}$. See Appendix B.

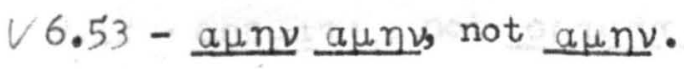

$\checkmark 6.56-\underline{\tau a}$, not $\underline{\tau 0}$ before $\underline{\alpha \mu \alpha}$

6.57 - not $\zeta \eta \underline{\delta_{1}} \underline{\varepsilon \mu \varepsilon}$, but $\underline{\zeta \eta \sigma \varepsilon_{1}}$. See Appendix B.

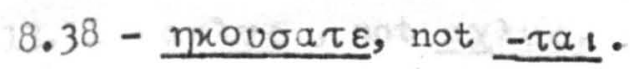

11.44 - Is autois, not auto1s 으.

13.26 - $\psi \omega \mu 10 \mathrm{~V}$, not $\psi \omega \mu \omega$. This, apparently, is corrected in T8.

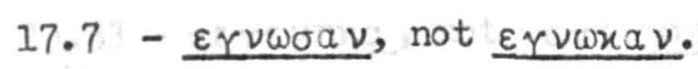

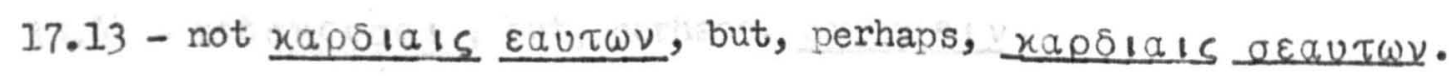

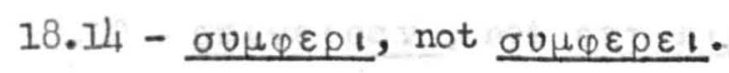

$18.32-\underline{\varepsilon i \pi \varepsilon \downarrow \nu}$, not $\underline{\varepsilon \mid \pi \varepsilon \nu .}$ See Appendix B.

$21.13-\underline{\varepsilon \rho \chi \varepsilon \tau a_{1}} \circ \overline{15}$, not $\varepsilon \rho \times \varepsilon \tau a_{1} \overline{15}$.

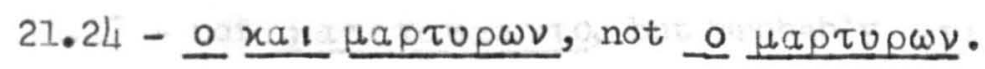

Acts

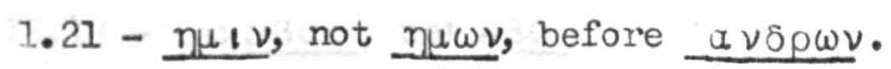

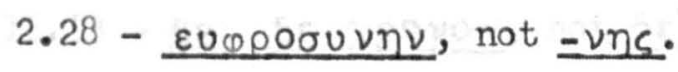

2.34 - tos, not zovs, before oupavous.

$2.38-\underline{\varepsilon \pi_{1}}$, not $\underline{\varepsilon \nu}$, before $\underline{\tau \omega}$ ovoua $\tau_{1}$.

2.39 - ogous a, not ous av. 


\section{Acts}

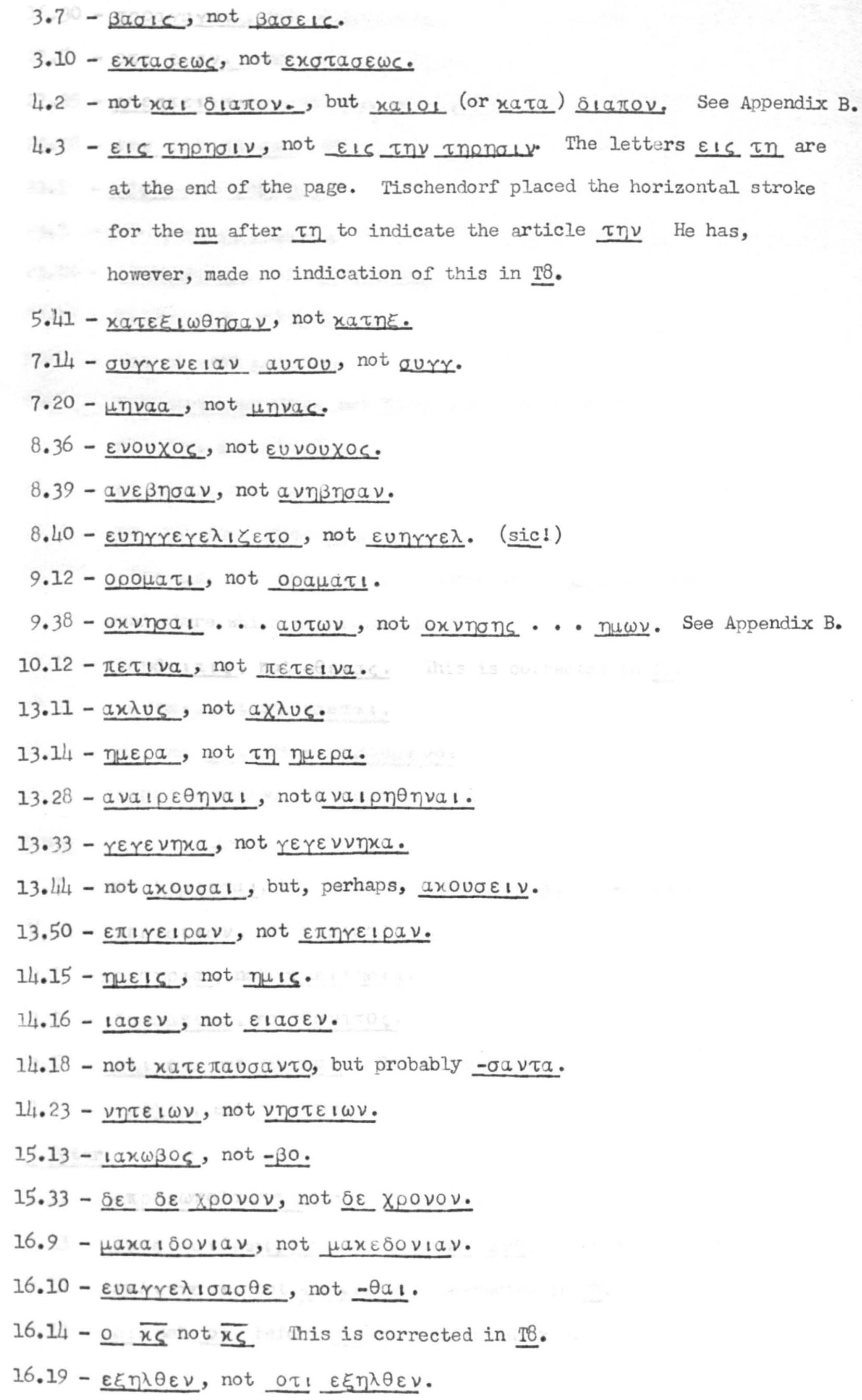


Acts

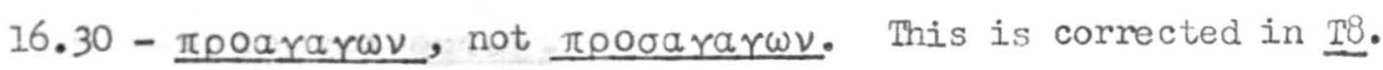

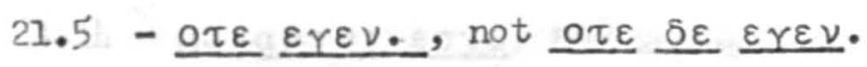

$21.25-\underline{a \pi \varepsilon \sigma \tau \varepsilon ! \lambda \alpha \mu \varepsilon \nu}$, not $\underline{\varepsilon \varepsilon \varepsilon \sigma \tau \varepsilon+\lambda a \mu \varepsilon \nu}$. See Appendix B.

22.28 - $\tau \eta \underline{\nu} \pi 0 \lambda_{1} \tau \varepsilon t a \nu$, not $\pi 0 \lambda ! \tau \varepsilon ! \alpha \nu$.

$23.1-\underline{\alpha \delta \varepsilon \lambda}$, not $\underline{a \delta \varepsilon \lambda \varphi O_{1}}$.

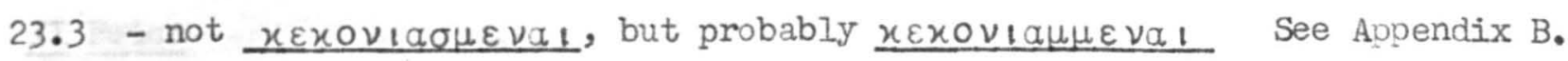

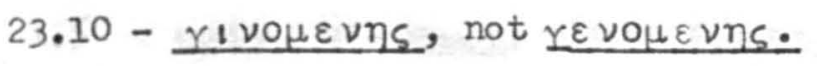

$23.15-\underline{x \varepsilon 1 \lambda t a \rho \chi \omega}$ not $x 1 \lambda$.

26.4 - lagiv, not loagiv.

26.5 - $\pi \rho 0 \varepsilon Y 1 \nu \omega \sigma x o \nu \tau \varepsilon s$, not $\underline{\pi \rho 0 \sigma \gamma 1 \nu}$. See Appendix B.

$26.7-\varepsilon \lambda_{1} \delta_{1}$, not $\varepsilon \lambda_{1} \zeta_{1}$.

$26.11-\underline{\varepsilon \delta 1 \omega x \alpha \nu}$, not $\underline{-x O \nu}$.

$26.14-\underline{\tau}$, not $\underline{\delta \varepsilon}$, after $\underline{\pi \alpha \nu \tau \omega \nu}$.

26.16 - $18 \varepsilon s \underline{\mu \varepsilon}$, not $1 \delta \varepsilon \varsigma \underline{x_{1}}$. Tischendorf's $\underline{x \alpha_{1}}$ was apparently a conjecture which he changed in T8 in the light of other NS evidence.

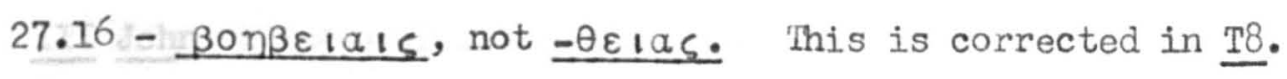

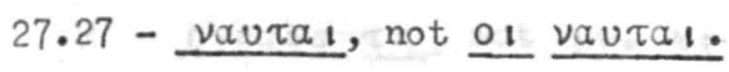

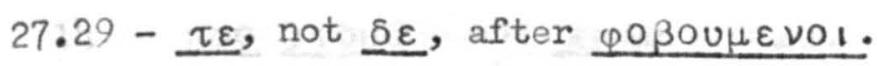

27.42 - $a \pi 0 x \tau \varepsilon+\nu \omega \sigma \mid v$, not $\underline{a} \pi \varepsilon x \tau$.

James

1.7 - not $\underline{\lambda \eta \mu \psi \varepsilon \tau \alpha_{l}}$, but probably $\underline{\lambda \eta \mu \psi \varepsilon \tau \alpha_{l}} \underline{\tau_{l}}$ See Appendix B.

1.21 - $\pi \varepsilon \rho ı \sigma \varepsilon ı \alpha \nu$, not $\pi \varepsilon \rho ı \sigma \sigma \varepsilon เ \alpha \nu$.

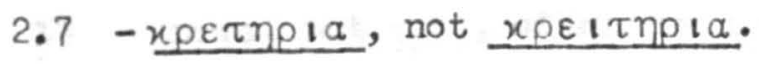

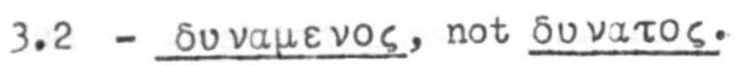

3.5 - oגirov, not $\eta \lambda 1$ xov See Appendix B.

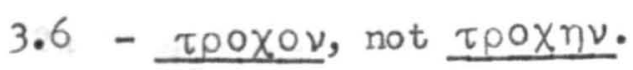

I Peter

1.17 - $a \pi \rho \circ \sigma \omega \pi 0 \lambda \eta \mu \pi \tau 0 \varsigma$, not $-\lambda \eta \mu \tau O \zeta$.

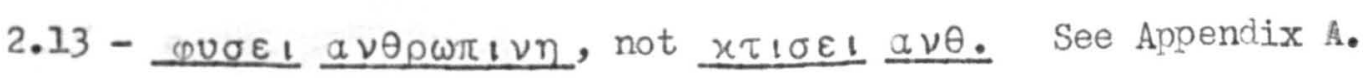

2.1 - $\Psi_{x \delta 1 x \eta \sigma i v}$ not asixnoiv. Corrected in T8.

2.23 - 이, not 오, before 스18. See Appendix B. 
I Peter

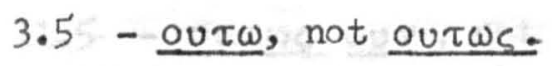

$3.14-\mu a x a \rho t 0_{1} \varepsilon \sigma \tau \varepsilon$, not $\mu a x a \rho 10_{1}$. See Appendix A.

3.18 - $\eta \omega \omega \nu$, not $\underline{u \varepsilon \varepsilon \rho} \underline{\eta \omega \nu}$. See Appendix B.

3.18 - vuas, not nuas, after iva

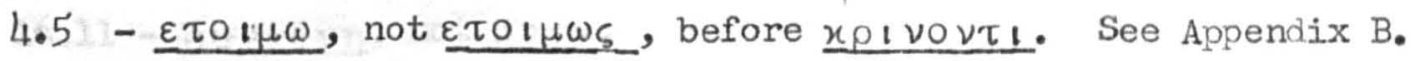
II Peter

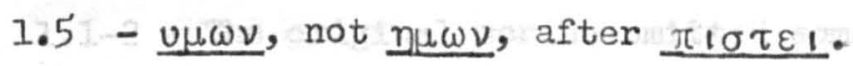

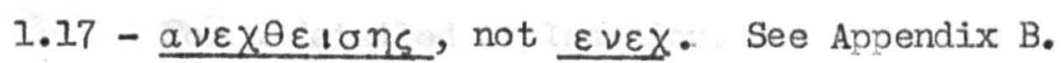

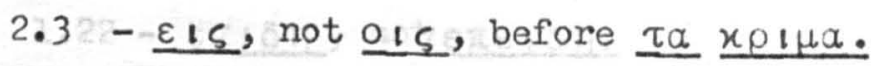

I John

$1.4-\pi \varepsilon \pi \lambda \eta \rho \omega \mu \varepsilon \nu \eta$, not $\pi \varepsilon \pi \lambda . \varepsilon \nu \underline{\eta} \underline{\eta}$. See Appendix B.

1.6 - $\frac{\pi 01 \omega \mu \varepsilon \nu}{}$, not $\underline{\pi 010 u \mu \varepsilon \nu}$. See Appendix B.

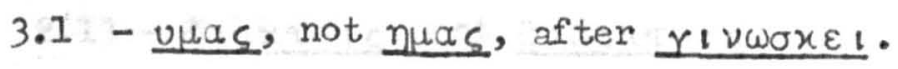

3.12 - not $\underline{x a 1 y}$ but probably $\underline{x \varepsilon \varepsilon v}$. See Appendix B.

$3.20-Y 1 \nu \omega x \varepsilon_{1}$, not $\Upsilon_{1} \nu \omega \sigma x \varepsilon_{1}$.

III John

$$
\begin{aligned}
& 9 \text { - } \underline{\operatorname{rrpa} \psi \alpha} \tau \eta \text {, not } \varepsilon \gamma_{0} \tau_{1} \tau \eta \text {. }
\end{aligned}
$$

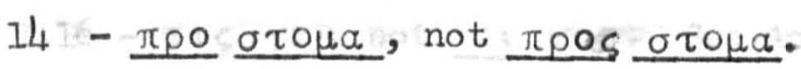

Jude

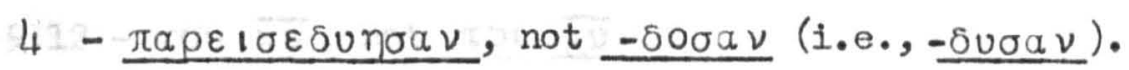

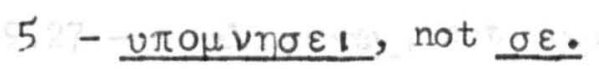

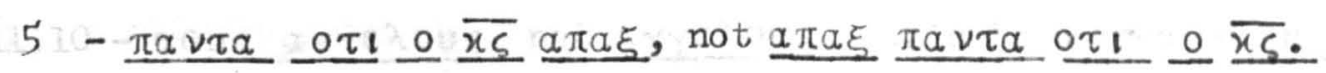

$$
\begin{aligned}
& 13 \text { - } \underline{a} 1 \alpha \text {, not } \underline{a \gamma \alpha} \text {, after } \underline{x \mu \alpha \tau a} \text {. See Appendix B. } \\
& 20-a_{Y} 10 \tau \alpha \tau \eta, \text { not } a_{Y} 1 \omega \tau \alpha \tau \eta \text {. }
\end{aligned}
$$

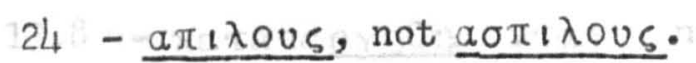

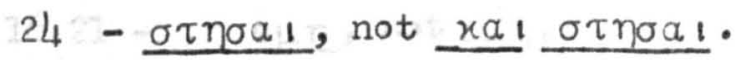

\section{Romans}

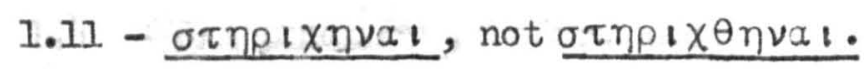

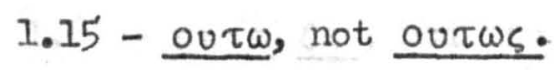

$1.18-\underline{a \pi 0 x a \lambda v \pi \tau \varepsilon \tau \alpha_{1}}$, not $\underline{a x o x}$.

$7.1-Y 1 \nu \omega \sigma x o v a 1$, not $=01 v$. 
$\underline{\text { Romans }}$

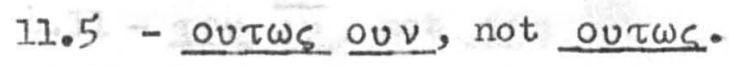

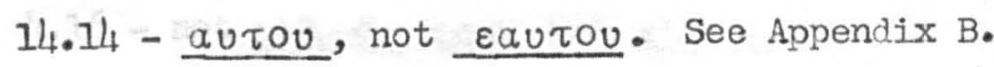

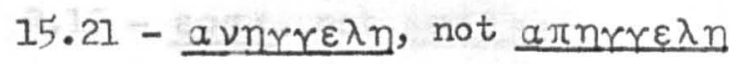

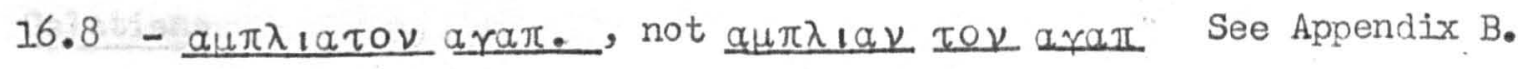
16.11 - guvrevn, not gury.

I Corinthians

1.1-2 - The original scribe omitted something from the standard text. For a detailed explanation, see Appendix A.

$1.22-\varepsilon \pi 1 \delta \eta$, not $\varepsilon \pi \varepsilon 1 \delta \eta$.

1.24 - autor $\delta \varepsilon$, not autos $\delta \varepsilon$.

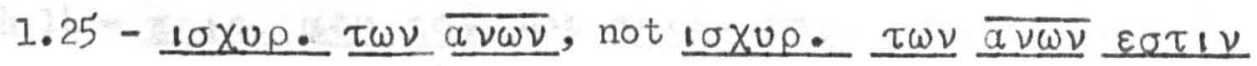

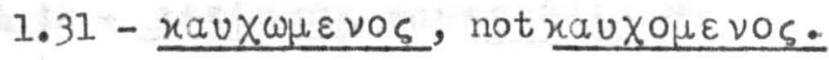

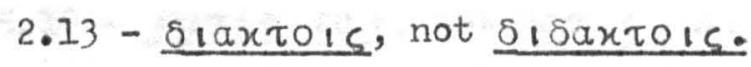

6.11 - $\underline{\alpha \lambda \lambda \alpha}$, not $\underline{\alpha \lambda \lambda}$, before $\varepsilon \delta 1 x \alpha 1 \omega \theta n \tau \varepsilon$.

$7.2-\underline{\delta 1 a} \underline{\delta \varepsilon}$, not $\underline{\delta_{1 \alpha}} \tau \varepsilon$. This is corrected in $\underline{18}$.

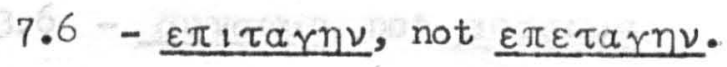

7.15 - $\underline{x \varepsilon x \lambda \eta x a \nu}$, not $\underline{x \varepsilon x \lambda \eta x \varepsilon \nu}$.

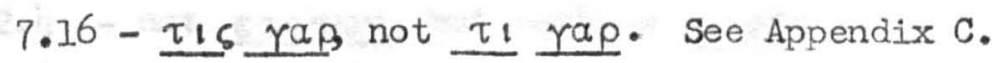

$9.10-\underline{\varepsilon \lambda \pi_{1} \delta \varepsilon_{1}}$, not $\underline{\varepsilon \lambda \pi_{1} \delta 1 .}$

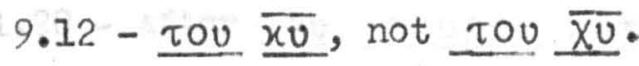

9.27 - $\underline{00 \lambda \lambda \alpha \gamma \omega r \omega}$, not $-\gamma a \gamma \omega$.

11.10 - zous aryenous, not aryenous. This is corrected in T8.

$12.25-\mu \varepsilon \rho \iota \mu \nu \omega \imath \nu$, not $-\nu \omega \sigma \downarrow \nu$.

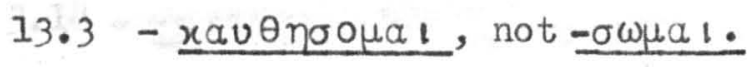

13.8 - $\underline{x \alpha \tau \tau \alpha \rho \gamma \eta \theta \eta \sigma o \nu \tau a 1}$, not $\underline{x \alpha \tau \alpha \rho \gamma}$. See Appendix B.

$16.21-\underline{\tau \eta} \underline{\varepsilon \eta \eta}$, not $\underline{\mu \eta}$.

II Corinthians

$1.4-\underline{\varepsilon \pi}$, not $\underline{\varepsilon \nu}$, before $\underline{\pi \sigma \sigma \eta}$.

$1.6-\underline{\varepsilon \downarrow \tau \varepsilon} \underline{\delta \varepsilon}$, not $\varepsilon \imath \tau \varepsilon$ before $\underline{\theta}\lrcorner \beta 0 \mu \varepsilon \theta a$.

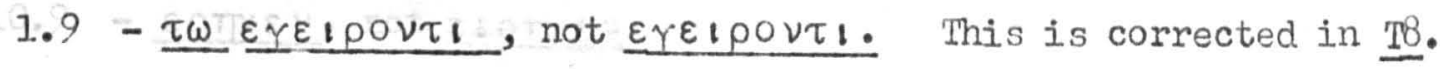

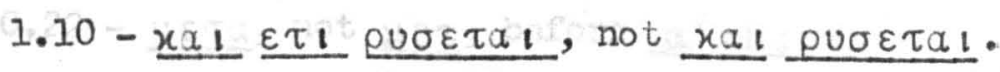




\section{Corinthians}

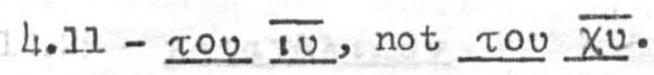

4.16 - not $\underline{a \lambda \lambda}$ 의 $\varepsilon \sigma \omega$, but $\underline{a \lambda \lambda}$ 으 $\underline{\sigma \omega}$ (or $\underline{a \lambda \lambda} \underline{\varepsilon \sigma \omega}$ ).

$9.12-\varepsilon \sigma \tau l$, not $\varepsilon \sigma \tau \downarrow \nu$.

\section{Galatians}

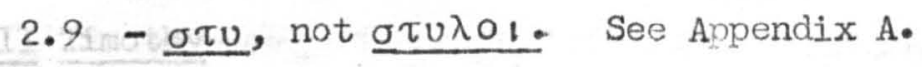

$3.10-\underline{\varepsilon \mu \mu \varepsilon \nu \varepsilon 1}$, not $\underline{\varepsilon \mu \mu \varepsilon \nu_{1}} \varepsilon \nu$.

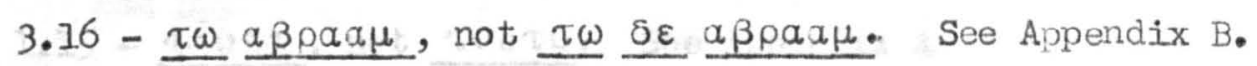

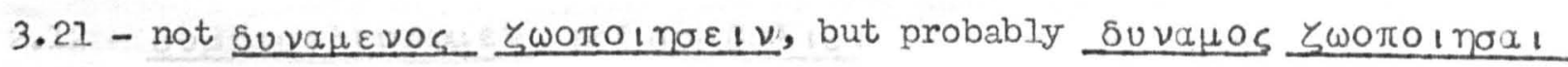

- (See Appendix B. )

4.10 - nuepas, not nu npas.

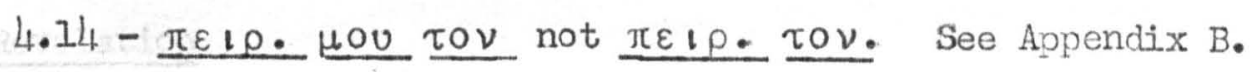

$4.14-\varepsilon \delta \varepsilon \xi a \sigma \theta \varepsilon$, not $\varepsilon \xi \varepsilon \delta \varepsilon \xi a \sigma \theta \varepsilon$.

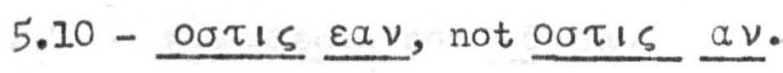

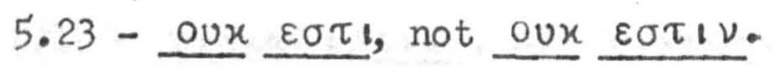

Ephesians

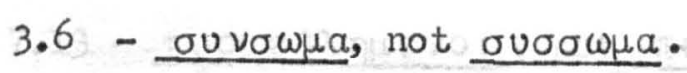

Philippians

2.4 - not $\varepsilon a u \tau \omega \nu$, but perhaps $\varepsilon a u \tau \omega$.

\section{Colossians}

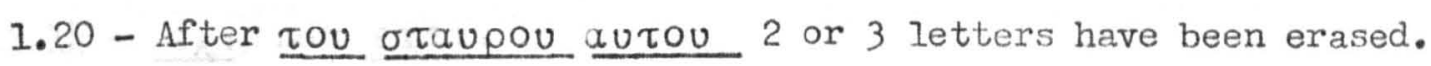
Tischendorf overlooked this erasure. See Appendix B.

2.1 - $\eta \lambda 1$ yov, not $\eta \lambda 1$ xov.

3.6 - $\delta_{1 \alpha}$, not $\underline{\delta_{1}}$ 으. See Appendix B.

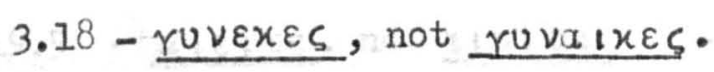

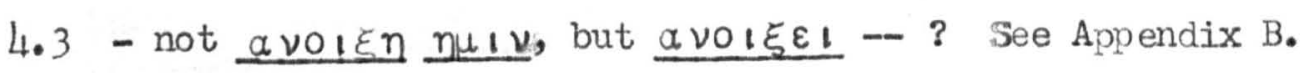
Hebrews

$2.13-\frac{\pi \alpha_{1} \delta_{1} \alpha}{\mu O_{1}}$, not $\underline{\pi \alpha_{1} \delta_{1}} \mu \mathrm{O}_{1}$. See Appendix B.

$3.1-\alpha \rho x_{1} \varepsilon \rho \varepsilon \alpha \nu$, not $\alpha \rho x_{1} \varepsilon \rho \varepsilon \alpha$.

$6.14-\underline{\varepsilon_{1}} \mu \eta \nu$, not $\underline{\varepsilon_{1}} \mu \eta$.

$10.9-\underline{\varepsilon \rho \eta x \varepsilon \nu}$, not $\underline{\varepsilon l \rho \eta x \varepsilon \nu .}$

10.22 - $\underline{\mu \tau \tau \alpha}$, not $\underline{\mu \varepsilon \tau}$, before $\alpha \lambda \eta \theta_{1}$ vn 6 .

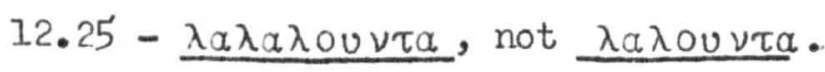


Hebrews

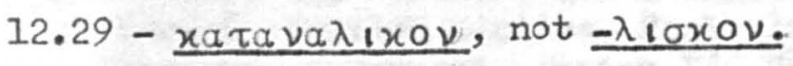

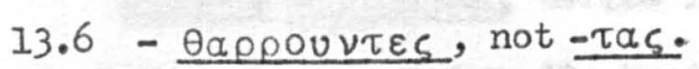

$$
\begin{aligned}
& 13.7 \text { - } \underline{\alpha \alpha \theta \varepsilon \omega \rho o u \nu \tau \varepsilon \zeta, ~ n o t ~ \rho \eta \sigma a \nu \tau \varepsilon \zeta . ~}
\end{aligned}
$$

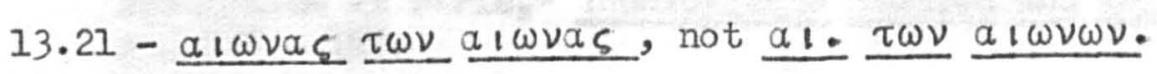

$$
\begin{aligned}
& \text { II Timothy } \\
& 1.6-\underline{\varepsilon \pi 1 \theta \varepsilon \sigma \varepsilon \omega s} \text {, not } \varepsilon \pi 1 \theta n \sigma \varepsilon \omega s .
\end{aligned}
$$

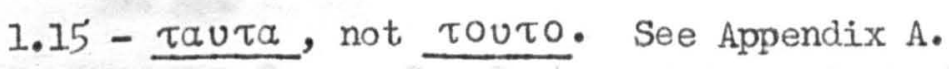

$$
\begin{aligned}
& 2.12 \text { - } \sigma u \mu \beta a \sigma i \lambda \varepsilon v \sigma \omega \mu \varepsilon \nu \text {, not } \sigma u \mu \beta a \lambda 1 \sigma . \\
& 2.20-\underline{\varepsilon \sigma \tau 1} \text {, not } \varepsilon \sigma \tau 1 \nu .
\end{aligned}
$$

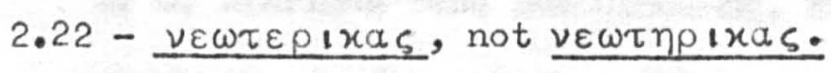

Revelation

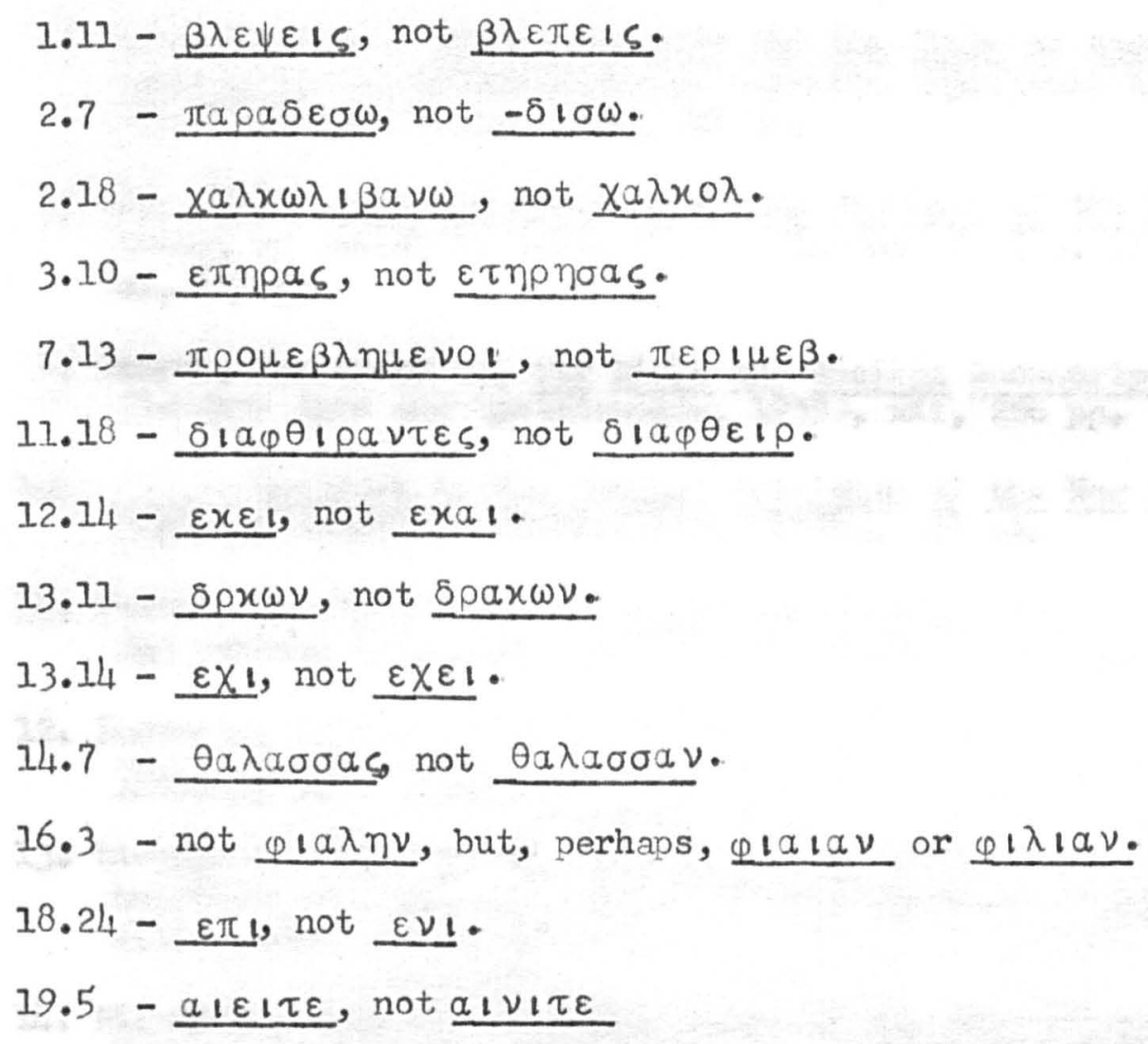


1. Ceriani, A., "Letture della Classe di Lettere e fclenze Storiche e lorali, Critica Biblica, Reale Instituto Iombardo di Scienze e Lettere Rendicont, Series IT, Vol. IXII (1800), p: $540-549$.

2. Fleck, F. F., "Ueber dite Handschrift des neuen Testamentes gew fhinIich Codes Ephraonif Syri Rescriptus genannt in dor königlichen Bibliothek zu Paris, "Theologische Studien und Kritiken, XIV (184.I), pp. $126-152$.

3. Foakes-Jackson, F. J., and Lake, Kirsopp, Bagimings of Christianity, Pari I, The Acts of the Apostles, Vol. III The Text of Mcts, by

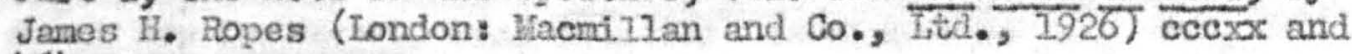
$464_{4}$ pp.

4. Gregory, Casper R., Gnon and Text of the New Testament (Edinbureh: I \& T Clark, 1907), 539 pp.

5. W., Textlaritik des neuen Testanentes, Erstor Bond (Leipzig: J. C. Hinrichs'sche Buchhand1 ung, 1900), 478 ppe

6. Oriesbach, J. J., Bymbolae Criticae, Tonus Prior (HaIee, 1785), coxxiv, $388 \mathrm{pp}$.

7. Haselden, R. B., Scientific Aids for the Study of Manuscripts, Transactions of the Bibliographical soctety, supplomont Wo. 10 (Oxford: The University Press, 1935, 108 po.

8. Hug, John I., An Introduction to the Friting og the New Testament, trans. by Daniel G. Iait, V0I. I (Iondon: C. B. Jivington, 1827) cc, $529 \mathrm{pp}$.

9. Kenyon, Frederick G., Our Btble and Ancient Manuscripts, Lth odition (Iondon: Iyre and Spottiswoode, T939), xit, $266 \mathrm{pp}$.

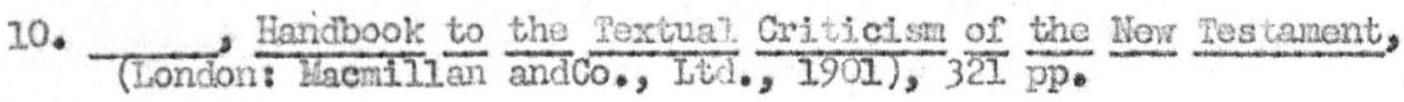

11. Kuster, Juclolphus, Novun Testanontun Graecun, atc., J. lijli's edition 2nd edition (Lipsiae: Fititi J. Fridertei Gladschti, 1723).

12. Iacham, Gar1, "Rechenschaft ther seine Ausgabe dos neven Testaments," Theologischo Studien und Mritiken, III (1830), pp. 817 - 846.

13. Lietzinann, Hans, An die Korinther I.II, Handbuch zun neven gestanent

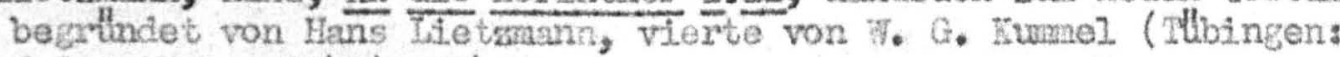
J.0.B. Yohr, 1949), 274 pp.

J. Hichaelis, John David, Introduction to the Now Testament, Vol. II, Part I, trans. from the lith edition by Herbert Tarsh (Canbridse: J. Archdeacon, 1793), $529 \mathrm{pp}$.

15. Milne, H. J. M., and Skeat, T. C., Scribes and Comectors of the Codex Sinaiticus (London: The British Huseun, 1938 ), $112 \mathrm{pp}$

16. Montfaucon, Bornardi de, Paleographia Graeca Sive de Ortu et Progressu Ifteramun Graecaman (Paris, 1708), xodx, 574 pp.

17. Nestle, Eberhard, Introduction to the Iextual Griticism of the Greek New Testanent, Prans. by William Edie (Iondon: Wilians and Norgate, 1901], xvi 351 pp.

18. 07iver, Harold H., "A Textual Transposition in Codex C," Journal of Biblical Isterature, IXXXVI (1957), pp. $233-236$. 
19. Sorivener, Frederick H. A., A Plain Introduction to the Oriticism of the Now Iestanent, 4 th edition edited by Bdward Ifinler, vol. I (Iondon: Goorge Be11 and Sons, 1894), 712 pp.

20. Soden, Hormann Fresherr van, Die Sohniften des neven Restantents, 1 . Toll (Berisint VerIag von Arthur Glate, 2902-1910), 2203 pp.

21. Mischendorf, Constantinus, Codex Bphracui Sysi Rescriptus stvo Eragmenta veteris Festanenti o Dodece Graoco Parlstenst Coreborrimo outnte ut vidotur post Christura Soculi, (Ispstao: Bemp. Tauchnitz, 1845).

22. Dragelles, Samuel Prideaux, An Account of tho Printed Toxt of the Greek How Testament, etc. (Tondon: Samuel Bagster and Dons, 1854), 274 and $945 \mathrm{pp}$.

23. Traube, Iudvig, Nomina Secre: Vorsuch giner Geschichte der christhichen Kurgung, quelion und untersuchungen zur tateintschon phIlologio des Ifittelaiters, Zweiter Band (Iinchen: C. H. Beck'sehe Verlagsbuchhandlung, 2907), $295 \mathrm{pp}$.

24. Weiss, Johames, "Der Bingang des ersten Korintherbrtefes, "Stratian und Exitiken, LXXIII (1900), pD. $125-130$.

25. … Der erste Korintherbries, Kritisch-oxsegotische Konmentar ufber das neue Testament begrtondet von F. A. W. Koyor, lote Auflage (clltingen: Vandenhoeck and Ruprecht, 1925), xlv111 and $388 \mathrm{pp}$.

26. Wetstein, J. J., Prolegonena da Hovi Pestanenti Graeci. (Austolaedami: R. \& J. Hetstens. $\overline{09}$ and D. smith, 1730), 201 pr.

27. Zuntz, Cun her, The Text of the sistzes, A Disquisition upon the Corrous Paulinum, The schweich Lectures of 19.6 (Iondon: Geoffrey trmberlege, Oxford University Press, 1953), 295 pp. 